\title{
The 21st Conference of the Asian Pacific Association for the Study of the Liver
}

\section{Oral Presentations}

\section{February, 2011 (Thursday)}

\author{
Endoscopic Course: Unique Scenarios in Pancreatic \\ obiliary Endoscopy \\ 08:15-11:45
}

Part I: Endoscopy in Cirrhosis and Portal Hypertension

\author{
EC-01 \\ Portal Biliopathy \\ D.N. Reddy, V.P. Gokak \\ Asian Institute of Gastroenterology, Hyderabad, India
}

The spectrum of abnormalities of the entire biliary tract including intrahepatic and extrahepatic bile ducts, cystic duct and gall bladder in patients with portal hypertension is called as portal biliopathy. It is also known as portal hypertensive biliopathy, pseudosclerosing cholangitis and portal cavernoma associated cholangiopathy. Most common aetiology is extrahepatic portal vein obstruction (EHPVO). Oher causes include cirrhosis of liver, NCPF and congenital fibrosis. In EHPVO, portal vein is replaced by portal cavernoma and development of choledochal and gall bladder varices. The biliary abnormalities include irregularities in CBD and hepatic ducts, localised saccular dilatations and filling defects suggestive of CBD calculi, etc. Left hepatic duct is most often affected. The incidence of portal biliopathy on ERCP is reported to be in range of $81-100 \%$, but only small percentage of these patients will be symptomatic (5-14\%). Biliary strictures develop due to ischemia and vascular bile duct injury or prolonged compression of biliary tract by portal cavernoma. Majority of the patients are asymptomatic. They can present with abdominal pain, recurrent cholangitis and obstructive jaundice. Diagnosis is established by ERCP which also deliantes biliary changes and detects if endotherapy is required. MRCP and MR Portography is as accurate as ERCP, in addition it also detects biliary changes, presence of collaterals, shuntable vein, portal cavernoma and helps to differentiate between bile duct varices and CBD stones. Endosonography is a useful addition to the battery of investigations. The natural history of biliopathy is not yet clear. At present there is no consensus on optimal treatment. Asymptomatic patients do not require any treatment. Treatment in symptomatic patients has to be individualised. Treatment options include endoscopic sphincterotomy, stone extraction, and biliary stricture dilatation with or without stenting. Care has to be taken while doing sphincterotomy due to high risk of bleeding due to ampullary and juxtra ampullary varices. Bleeding is managed like any other variceal bleeding. Endoscopic treatment is preferred for CBD stone and dominant strictures. Bilioenteric anastomosis is difficult due to surrounding collaterals. Some authors suggest shunt surgery which may itself improve biliary obstruction, if not then do biliary bypass.

\author{
EC-02 \\ Safe Sedation And Monitoring During Endoscopy In Cirrhosis \\ K.L. Goh \\ Professor of Medicine, Head of Gastroenterology and Hepatology, \\ University of Malaya Medical Centre, Kuala Lumpur, Malaysia
}

Patients with cirrhosis of the liver have a higher sedation risk during endoscopy. Alterations in liver blood flow, portosystemic shunting and drug metabolizing pathways occur which would impact adversely on the pharmacokinetics of sedative medications. Cirrhotic patients are therefore highly sensitive to drugs used for sedation. Midazolam, fentanyl and pethidine are all metabolized in the liver. As such patients with cirrhosis would have decreased metabolism of these drugs and consequent higher plasma level and longer duration of action. Furthermore, many patients with well compensated cirrhosis have subclinical encephalopathy with increased neurosensitivity to sedative drugs. The risk of sedation and analgesia is further compounded and hepatic encephalopathy may be precipitated. Renal impairment, fluid overload, and alcohol and drug abuse are additional problems to consider that may have an effect on the level of sedation, recovery time, and potential toxicity. Cirrhotic patients often undergo gastroscopy for upper gastrointestinal bleeding due to bleeding esophagogastric varices. The risk of encepaholpathy is potentiated by the bleeding and blood in the gastrointestinal tract. Owing to the risks involved, there have been recommendations for unsedated endoscopy for patients with cirrhosis. While this may be feasible for quick and uncomplicated diagnostic gastroscopy or even colonoscopy, complicated procedures involving therapy of esophagogastric varices by ligation or sclerotherapy would require some form of sedation. When sedation is administered in these patients, very close monitoring must be carried out. Half or even quarter doses of sedative and analgesic drugs should be given initially and carefully titrated. It should be remembered that the half life of such drugs in cirrhotic patients is prolonged. The principle would be to give as little sedation as required in these patients.

\section{EC-03}

Gastric Variceal Bleeding: TIPS

S. Punamiya

Department of Radiology, Tan Tock Seng Hospital, Singapore, Singapore

Amongst the treatment options described for gastric varices, TIPS and variceal obliteration using cyanoacrylate or glue are ones most commonly used. While both therapies are highly effective in controlling gastric variceal bleeding, there is sparse literature comparing 
the two. Central to the management of portal hypertension is reduction of portal venous pressure itself. Unlike glue, TIPS is known to effect direct reduction of portal venous pressure, and has, in the few published case series and studies, shown to control acute gastric variceal bleed in more than $90 \%$ patients, with a rebleed rate ranging from $11-39 \%$. The last few years have seen the emergence of glue injection for gastric varices, and it is being recommended as first line therapy for acute variceal bleed. However, there is limited data comparing it with TIPS, with insufficient evidence to support its superiority over TIPS. To date there has been only one randomized trial by Lo et al, comparing the two therapies, which clearly showed a lower rebleeding rate with TIPS (11\% v 38\%). In a retrospective comparison of both therapies in acute gastric variceal bleeding by Mahadeva et al, hemostasis was achieved with similar efficacy in both arms, but lower rebleeding rate was again noted with TIPS (15\% v $30 \%$ ). In a more recent retrospective study by Procaccini et al, no difference in rebleed and survival rates was noted with either therapy, but there was a significantly higher morbidity of TIPS due to shunt stenosis and encephalopathy. This study, although favouring glue therapy, is to be interpreted with caution, as some important aspects which could have influenced results were not considered. While it is accepted that encephalopathy is a real problem with TIPS, it can be mitigated with better patient selection. Glue is not without complications either, with various reported instances of both fatal and nonfatal embolisation causing stroke, splenic infarction, portal vein thrombosis and pulmonary embolism. Inadvertent glue embolisation into the portal vein could in fact preclude a bail out TIPS procedure, causing eventual mortality from uncontrolled bleeding.

\section{EC-04}

Glue vs. TIPS: To Debate

T. Ratanachu-ek

Department of Surgery, Rungsit University, Bangkok, Thailand

Gastric varice is less common but more severe bleeding and more difficult to manage than esophageal varices. For active bleeding, the same basic principle for managing GI bleeding such as adequate resuscitation, intensive care, correct coagulopathy, prepare blood components and so on. Both glue injection and transjugular intrahepatic portosystemic shhunt (TIPS) have effective controlled active bleeding in term of primary hemostasis, less complication and less early mortality even comparable re-bleeding rate in glue injectioj group with good protocol (good protocol means complete eradication on schedule) comparing with surgery. In general practice, most physicians use glue injection be the first line method to stop bleeding due to availibility, easy to use and more cost effective comparing with TIPS, however TIPS still has been the minimally invasive rescure procedure after failure from glue injection and may be the first way in selected center where is the experience intervention radiologist available with selected patient and, course it should be proper for chronic bleeder.

\section{Part II: Endoscopic Management of the Obstructed Biliary System}

\section{EC-05}

Diagnostic Role of Endoscopy in Autoimmune Pancreatitis

S. Pongprasobchai

Department of Internal Medicine, Siriraj Hospital, Mahidol

University, Bangkok, Thailand

Autoimmune pancreatitis (AIP) is a special form of pancreatitis presenting with pancreatic mass causing obstructive jaundice and mimics pancreatic cancer $(\mathrm{PaC})$. Due to its dramatic response to corticosteroids, diagnosis of AIP preoperatively would prevent unnecessary surgery. In addition to the imaging studies (CT scan, MRI/MRCP) and serum IgG4, endoscopy also has important role in the diagnosis of AIP. Esophagogastroduodenoscopy (EGD) may identify prominent ampulla and biopsies of ampulla, duodenum or stomach may disclose IgG4-positive plasma cells in AIP. However, due to the unpredictable diagnostic yield and the time needed for the histology, it may not be practical to do EGD, biopsy and wait another week for the histology in patient with pancreatic mass, whose treatment should not be delayed. Endoscopic retrograde cholangiopancreatography (ERCP) is very useful to diagnose AIP. The pancreatographic findings suggestive of AIP are diffuse narrowing, focal narrowing $>1 / 3$ of the length of the pancreatic duct and skipped narrowing. The cholangiographic findings suggestive of AIP are the smooth tapering of distal CBD or IgG4-associated cholangitis, which can be distinguished from primary sclerosing cholangitis by the presence of long segmental strictures of intrahepatic bile ducts in the former and the beaded or prune-tree appearance in the latter. Because pancreatography is not routinely performed in jaundiced patients, those who have any cue of AIP from cholangiography or cross-sectional imaging should have pancreatography done. Role of endoscopic ultrasonography (EUS) to diagnose AIP is less clear. EUS is inferior to ERCP in identifying narrow pancreatic duct in AIP. The parenchymal finding of tortoiseshell appearance has been suggested to indicate AIP but the accuracy is unknown. EUS-FNA is neither sensitive nor specific for AIP but useful to rule out PaC. EUS-trucut biopsy is superior to FNA and would be the best tool to diagnose AIP if the technical demand can be solved.

\section{EC-06}

Hepatolithiasis and Direct Cholangioscopic Treatment

T. Itoi, T. Tsuchiya

Department of Gastroenterology and Hepatology, Tokyo Medical University, Tokyo, Japan

Hepatolithiasis, which refers to the stone in the intrahepatic bile duct and is a common disease in East Asia, causes the recurrent cholangitis. Apart from asymptomatic hepatolithiasis, in terms of symptomatic hehatolithiasis, several therapies including operative and nonoperative procedures have been performed. Endoscopic lithotripsy is one of these nonoperative procedures. It is mainly divided into two approaches, e.g. percutaneous transhepatic and transpapillary approaches. Traditionally, percutaneous transhepatic approach by using percutanous transhepatic cholangioscopy (PTCS) have been popular for the treatment of hepatolithiasis because it is easily performed over again and again once percutaneous fistula is completed. In contrast, conventional transpapillary approach using duodenoscope and standard accessories is often ineffective because of maneuverability of such accessories or frequently presence of bile duct stricture. Therefore transpapillary lithotripsy is limited even if using peroral cholangioscopy with or without electric hydraulic lithotripsy (EHL). Nevertheless, transpapillary lithotripsy has a big advantage, namely, direct lithotripsy without making fistula. Therefore, several endoscopists have been reported the usefulness of direct peroral cholangioscopic lithotripsy using motherbaby system. Furthermore, recently, direct peroral cholagioscopic lithotripsy using ultraslim or standard upper endoscope has been tried for bile duct stones. In this lecture, I will introduce the possibility of peroral cholangioscopy for the hepatolithiasis.

\section{EC-07}

Management of Hilar Biliary Obstruction: The Endoscopist Should Do It J.H. Moon

Digestive Disease Center, Department of Internal Medicine, Soon Chun Hyang University School of Medicine, Bucheon, Republic of Korea

Hilar biliary obstruction is caused by a heterogeneous group of benign and malignant conditions. The definite diagnosis and proper management of hilar strictures remains a clinical challenge, requiring a multidisciplinary approach that includes radiologists, endoscopists, surgeons, oncologists, 
and pathologists. Endoscopic intervention provides not only initial decompression for biliary obstruction but also various diagnostic informations including tissue acquisition. Endoscopic accessment for hilar stricture can provide important clues in determining operation or other therapeutic options. For unresectable malignant hilar stricture, endoscopic biliary drainage using metallic or plastic stent is an effective palliative measure, and is less invasive. Metal stents are superior in stent patency and more cost effective in patients with anticipated survival of greater than 6 months. There have been continuous technological developments and improvements for biliary self-expandable metallic stents (SEMS). Although it seems that unilateral stenting achieved similar rates of successful drainage, bilateral stenting can be performed more easily using newly developed stents as needed. In addition to biliary drainage, endoscopic intraductal tumor ablation such as photodynamic therapy is possible and it may result in improvement in cholestasis and quality of life, and prolongation of survival. Although surgery has been the traditional treatment for benign biliary stricture (BBS), there is increasing desire for minimally invasive endoscopic management. For BBS caused by surgery such as liver transplantation, endoscopic management has become the first line approach. The preferred endoscopic approach is aggressive treatment with gradual dilation of the stricture and insertion of multiple plastic stents. The evolving role of covered SEMS placement as a more patient friendly and cost effective treatment for BBS needs further elucidation. Endoscopic procedures have been developed as an alternative to established surgical procedures, and parallel the development of endoscopy and its devices. Therefore, endoscopist will continue to occupy an important position in management of hilar biliary obstruction.

\section{EC-08}

Management of Hilar Biliary Obstruction: The Radiologist Should Do It S. Punamiya

\section{EC-09}

Management of Post Liver Transplant Strictures S. Lo

Liver Transplant Course: Liver Transplantation: Back To Basics

13:30-15:00

Session 1: Liver Transplantation-Who, When and How?

LT1-01

Indication and Timing of Liver Transplant

G.W. McCaughan

LT1-02

Measures to Increase the Application of Liver Transplantation

S. Hwang

LT1-03

Selection and Management of Deceased Donors

A. Shaked

\section{LT1-04}

Selection and Evaluation of Living Donors

C. Lo

Session 2: Optimizing the Outcome after Liver Transplantation

LT2-01

Pre- and Early Post-Transplant Recipient Management

A. Sobhonslidsuk

Department of Medicine, Ramathibodi Hospital, Mahidol University, Bangkok, Thailand
The last resort of curative treatment for decompensate cirrhosis, acute liver failure and early-stage of hepatocellular carcinoma occurring in end-stage liver diseases is liver transplantation. From a survival rate of $50 \%$ at 2 years in decompensate cirrhosis, the 1 year survival rate after liver transplantation may reach $90 \%$. A Child-Turcotte-Pugh score of 7 or higher was considered to be the minimal listing criteria eligible for liver transplantation from the policy of the United Network for Organ Sharing implemented in 1997. Since February 2002, the Model for End-Stage Liver Disease (MELD) score, a risk predictive score of death at 3 months, has been utilized as an organ allocation measure in the United States and subsequently in other countries. Pre-transplant co-morbidities such as portal hypertension, ascites, spontaneous bacterial peritonitis, renal dysfunction, malnutrition, hepatopulmonary syndrome, portopulmonary hypertension and chronic viral hepatitis have impacts on short-term and long-term outcomes of liver transplantation. Pre-liver transplant evaluation was performed thoroughly to identify significant medical diseases in potential recipients, to bar high risk patients from liver transplantation, to assure patient safety and guarantee good outcome of liver transplantation. Endoscopic variceal screening is recommended in decompensate cirrhotic patients who are on the waiting list. Arterial blood gas and doppler echocardiogram are useful tools for the evaluation of hepatopulmonary syndrome and portopulmonary hypertension. Dobutamine stress echocardiogram or cardiac catheterization is an unavoidable procedure to exclude significant coronary artery disease in some cases with underlying diseases. The medical co-morbidities should be fully managed and supported while awaiting liver transplantation. Critical care in intensive care unit for early post-operative period, appropriate immunosuppressive regimens for induction therapy and maintenance period, fluid and electrolyte administration to maintain renal function, nutritional support, the prophylaxis of opportunistic infection and the continued prophylactic treatment of chronic viral hepatitis constitute the essence of early posttransplant recipient management.

\section{LT2-02}

Surgical Complications After Living Donor Liver Transplantation C.-L. Chen Chang Gung Memorial Hospital, Kaoshiung, Republic of Korea

Complications after LDLT can be broadly classified into surgical or non-surgical causes. Surgical causes are mostly operation-related and can be further subdivided into bleeding, portal vein, hepatic artery, hepatic vein, and biliary complications. Most graft-threatening surgical complications occur within 2-4 weeks of posttransplantation. Surgical innovations have been reported for portal reconstruction in LDLT with a variety of techniques which include thrombectomy, vascular interpositions, and alternate source of portal inflow. Radiological and/or surgical intervention is the mainstay after transplantation. A noninvasive treatment for PVT after liver transplantation is systemic thrombolytic therapy using tissue plasminogen activator but its proven benefit has been reported for late-onset PVT. Early hepatic artery (HA) thombosis frequently leads to graft loss if untreated. Urgent graft rearterialization may reduce the need for retransplantation. Intraoperative and frequent early postoperative Doppler ultrasound surveillance is essential to detect HA problems. The treatment options for HA thrombosis include urgent revascularization with the native HA following thrombectomy, interposition grafting, or with gastric arteries or other alternatives. Other options include the use of intra-arterial urokinase and retransplantation. Hepatic vein obstruction in LDLT causes graft dysfunction and eventual graft loss. Free movement of the graft in the abdominal cavity during the early postoperative period may cause twisting in hepatic vessels. Tissue expander and Foley catheter have been used to reposition the graft. Refractory ascites posttransplant should alert the clinician to hepatic outflow obstruction or caval stenosis. The use or non-use of stent in biliary reconstruction is debatable. Evidence, however, shows the non-use of stent has more favorable outcome. In large series, primary radiological or endoscopic 
interventions were the treatments of choice for complications. Our current strategy in Chang Gung Memorial Hospital Kaohsiung is to do biliary reconstruction using microsurgical techniques. This has led to our overall $4 \%$ biliary anastomotic complications in the last 3 years.

\section{LT2-03}

\section{Understanding Immunosuppression After Liver Transplantation}

S.G. Lim

Chief, Dept of Gastroenterology and Hepatology, National University Health System, Singapore

One of the key issues in management of liver transplant patients is immunosuppression. The most important part of this management occurs in the first 6 months post transplant. Liver transplant immunosuppression is significantly easier to manage than other organ transplants as they do not require HLA matching, and livers are generally more resistant to rejection. Blood group matching is recommended but even then cross blood groups have been performed, albeit with less success. Rejection does occur in liver transplant recipients hence long term immunosuppression is required. The cornerstone of liver transplant immunosuppression are calcineurin inhibitors which work through inhibition of IL2 gene expression that prevents $\mathrm{T}$ cell activation. The two important drugs in this class are cyclosporine and tacrolimus, which are critical dose drugs-small differences in dose or concentration can result in therapeutic failure or adverse events. These drugs are generally combined with steroids \pm anti-metabolites such as azathioprine or mycophenolate. The general principle of immunosuppression is to start with an induction regime, usually a combination of steroids and calcineurin inhibitors \pm anti-metabolites and then slowly taper down the immunosuppresants to the minimal requirements over time. The main objective of liver transplantation is reduce acute rejection, maintain graft and patient survival, and reduce complications from disease recurrence and immunosuppression. Control of acute rejection is critical since this can lead to immediate graft loss and chronic rejection can also lead to graft loss. Management of acute rejection is usually protocolised and often successful as it usually requires induction with high dose steroids and an increase in calcineurin inhibitors or anti-metabolites. The actual induction regime for immunosuppression varies from centre to centre and there are few control studies that demonstrate significant advantage of one regime over another. Hence the selection of a particular regime is usually based on prior experience, familiarity with the drugs and its complications. However, most regimes use steroids and a calcineurin inhibitor with or without an anti-metabolite. Steroid free regimes have been successfully utilized but have not been widely used. Regimes have evolved to cater for patients particular issues such as those with renal impairment, those with autoimmune disease, hepatitis $\mathrm{C}$, and hepatocellular carcinoma but clinical trials have yet to be performed to demonstrate efficacy. Once the patient has been stabilized on an immunosuppressive regime and is discharged from hospital, a gradual reduction in immunosuppression is initiated, usually with reduction and then termination of steroids, followed by calcineurin inhibitor. During this time, acute rejection can occur, and this will require induction therapy again, followed by subsequent more careful reduction in immunosuppression. During this time, monitoring for adverse events due to immunosuppression, disease relapse and development of de novo disease is required. Eventually, patients who are stable on low dose immunosuppression can be reviewed less frequently and lead quite a normal life, the objective being to return such patients to a useful role in society.

\section{LT2-04}

Long Term Issues in Liver Transplant Recipients K.Rajender Reddy

Professor of Medicine, Director of Hepatology, Medical Director of Liver Transplantation, University of Pennsylvania

Liver transplantation is the definitive form of therapy for a subset of patients with acute liver failure and advanced chronic liver disease. Long term care of liver transplant recipients has evolved over the past 10 years. There are several causes for graft dysfunction and these include recurrence of the native disease, graft rejection, hepatic steatosis, hyperlipidemia, and diabetes, biliary and vascular complications. Recurrent hepatitis B has become a relatively minor issue because of effective post-transplant immunoprophylactic measures that include the judicious use of hepatitis B immunoglobulin and potent anti-viral therapies. Recurrent hepatitis $\mathrm{C}$ is commonly seen and is a major cause for graft dysfunction. While vascular and biliary complications occur early in the post-transplant period, a spectrum of biliary and vascular complications can be seen over the long term. Potential medical complications can emerge following LT, most of which are related to long-term use of immunosuppression. A major consequence of liver transplantation over the long term is of renal dysfunction. A number of factors that include calcineurin inhibitor use, diabetes, hypertension, and hepatitis $\mathrm{C}$ infection play a role in renal dysfunction. Additionally all transplant recipients are at higher risk than the general population of developing de novo malignancies or recurrence of previous cancers. Skin cancers, especially squamous cell carcinoma, basal cell carcinomas and melanoma can develop after LT with the peak incidence being at 3-5 years. Posttransplant lymphoproliferative disorder (PTLD) occurs in about $2 \%$ of LT recipients and most cases develop during the first year after transplantation. Epstein-Barr virus reactivation or primary infection is usually associated with PTLD. Long term care of these patients requires a fundamental recognition of drug-drug interactions. Transplanted patients should be encouraged to maintain routine dental care and discontinue tobacco. Vaccines are strongly recommended to be administered in the post transplant setting, especially inactivated vaccines.

\section{Special Lecture 1}

17:00-17:30

\section{S01-01}

Challenging in Chronic Viral Hepatitis: HBV-HCV Coinfection

C.J. Liu, P.J. Chen, D.S. Chen

Graduate Institute of Clinical Medicine, Hepatitis Research Center and Department of Internal Medicine, National Taiwan University

College of Medicine and Hospital, Taipei City, Taiwan ROC

Dual hepatitis B virus (HBV)/hepatitis C virus (HCV) infection is not uncommon in HBV or $\mathrm{HCV}$ endemic areas. In patients with dual chronic hepatitis $\mathrm{B}$ and $\mathrm{C}$, the clinical presentations and disease outcomes are usually more severe than those in patients with either single hepatotropic virus infection. However, several clinical and pathogenetic issues remain unresolved. First, clinical and in vitro studies suggest the interactions between two viruses. The dynamics of the interaction in untreated vs. treated setting, and its influence on the long-term outcomes await further studies. A key issue regarding viral interactions is whether modulation of infection occurs in the same dually infected individual hepatocyte of the liver. Clarifying this issue may help understand the reciprocal interference between HCV and $\mathrm{HBV}$, and provide clues for future immunopathogenetic studies. Second, the prevalence and clinical significance of co-existing occult HBV infection in patients with chronic HCV infection needs further investigations. Third, detailed serological and virological evaluations are required for $\mathrm{HBV} / \mathrm{HCV}$ coinfected patients before initiation of antiviral therapy. Combination therapy of peginterferon alfa-2a and ribavirin appears to be just as effective and safe for the treatment of hepatitis B surface antigen ( $\mathrm{HBsAg}$ )-positive patients chronically infected with active chronic hepatitis $\mathrm{C}$ as it is in patients with $\mathrm{HCV}$ mono-infection. Nevertheless, one third of those dually infected patients with nondetectable serum HBV DNA levels pre-treatment developed HBV reactivation post-treatment. How to prevent and treat this reactivation should be clarified. Besides, close monitoring for both viruses is recommended even for patients with HCV SVR and/or initially suppressed HBV DNA. Furthermore, about $10 \%$ of the dually 
infected patients lost HBsAg. Underlying mechanisms await further investigations. Finally, the optimal treatment strategies for dually infected patients with hepatitis B e antigen-positive chronic hepatitis $\mathrm{B}$ should be identified in future clinical trials.

\section{Special Lecture 2} 17:00-17:30

\section{S02-01}

Cholangiocarcinoma: Lessons from Thailand

B. Sripa

Tropical Disease Research Laboratory, Department of Pathology,

Faculty of Medicine and Liver Fluke and Cholangiocarcinoma

Research Center, Khon Kaen University, Khon-Khan, Thailand

Cholangiocarcinoma (CCA) is a rare primary liver cancer worldwide; however, it is prevalent in Thailand, particularly in the Northeast. Epidemiological and experimental evidence implicate the liver fluke, Opisthorchis viverrini in the aetiology. Rates of CCA in regions where the parasite is endemic are unprecedented-CCA is responsible for about $15-25 \%$ of liver cancers in the USA but represents $81.1 \%$ in the Khon Kaen Province, the highest incidence in the world. Recent national survey by Ministry of Public Health (2009) revealed a high prevalence of $O$. viverrini infection, particularly in Northeast Thailand (16.6\%) with over all national prevalence of $8.7 \%$ or approximately 6 million people are infected. This correlates with the high incidence of primary liver cancer in this region; in male, Udon Thani (ASR 115 per 100,000), Khon Kaen (ASR 87.7 per 100,000), Nakhon Phanom (ASR 78.4 per 100,000), and Ubon Ratchathani (ASR 74.9 per 100,000) compare to Songkla (ASR 10.9 per 100,000) where the liver fluke prevalence is less than $0.01 \%$. Moreover, histologically, more than $80 \%$ of the primary liver cancer in the Northeast is cholangiocarcinoma unlike those of southern Thailand that most are hepatocellular carcinoma. Indeed, $O$. viverrini and its relative Clonorchis sinensis, the Chinese liver fluke, are classified as Group 1 carcinogens-metazoan parasites that are carcinogenic to humans-by the International Agency for Research on Cancer, World Health Organization. The liver infection is associated with several hepatobiliary pathologies that predispose to CCA development. Specifically, the parasite induces severe inflammation of the bile ducts, resulting in oxidative DNA damage of the biliary epithelium. Affected cells, however, cannot undergo apoptosis and fixed genetic alterations of certain growth controlling genes occur, leading to malignancy. CCA can be classified as intrahepatic and extrahepatic or central type; the former is about $40 \%$ while the latter is $60 \%$ of the cases.

\section{February 2011 (Friday)}

\author{
Concurrent Symposium 01: From Liver Injury to Hepatic \\ Fibrosis And Portal Hypertension \\ 08:00-09:00
}

\section{C01-01}

From Chronic Hepatitis to Fibrosis and Cirrhosis: The Pathologist's View N. Theise

Department of Pathology and Medicine, Beth Israel Medical Center, Albert Einstein College of Medicine, New York, NY, USA

Pathologic assessment of liver biopsy specimens remains essential for the diagnosis and treatment of chronic hepatitis. Despite the increasing use of non-invasive methods for determination of stage of fibrosis, these techniques remain useful only for confident determination of very early or very late stage disease. The process of fibrotic progression will be considered from the point of view of mechanisms and descriptive pathology, with emphasis on the different staging schemes currently used, evaluating each of these for their respective "pros and cons." Guidance for clinicians and for pathologists in methods of reporting of staging will be discussed. Additionally, a brief review of evaluation of concomitant conditions (such as fatty liver disease, hemochromatosis) and or histologic indicators of risk for malignant progression will be offered.

\section{C01-02}

What's Next for Non-invasive Assessment of Hepatic Fibrosis?

V.W.-S. Wong

Department of Medicine and Therapeutics, The Chinese University of Hong Kong, Hong Kong, China

Hepatologists need to assess hepatic fibrosis everyday to determine the prognosis of patients, decide treatment and monitor disease progression. Traditionally, liver histology is the gold standard for assessing hepatic fibrosis. However, liver biopsy is invasive and not welcomed by many patients. Interpretation of liver specimens may also suffer from intraobserver and interobserver variability. Finally, recent studies clearly showed that sampling bias might lead to understaging of the disease, especially when the liver specimens were small. With this background, accurate non-invasive tests of hepatic fibrosis are urgently needed. Numerous serum biomarkers and formulae have been developed for the assessment of hepatic fibrosis. Some formulae, such as the aspartate aminotransferase-to-platelet ratio index (APRI), involve simple tests that are commonly performed in routine clinical practice. Other tests involve biomarkers of fibrogenesis and fibrinolysis that are not routinely measured. Overall, these serum tests have moderate accuracy in assessing different degrees of hepatic fibrosis. Liver imaging such as ultrasound scan, computed tomography and magnetic resonance imaging may detect cirrhosis when the liver is shrunken or there are signs of portal hypertension. However, they are insensitive to hepatic fibrosis and early cirrhosis. Recently, transient elastography by Fibroscan has emerged as a popular non-invasive test for hepatic fibrosis. Using Doppler technique to measure the velocity of shear wave in the liver parenchyma, liver stiffness may be measured. The test has been validated across different liver diseases such as viral hepatitis and fatty liver. One limitation of transient elastography is unsuccessful measurements in obese patients because the shear wave or ultrasound wave may fail to be transmitted to the liver parenchyma. As such, the new XL probe has been shown to increase the measurement success rate, but further calibration is needed. Magnetic resonance elastography is another novel method to assess liver stiffness and is not affected by obesity.

C01-03

From Hepatic Fibrosis to Cirrhosis and Portal Hypertension A. Alberti

Concurrent Symposium 02: Controversy and Hot Topics in Hepatitis B Immunology

08:00-09:00

\section{$\mathrm{C} 02-01$}

Overview on Innate and Adaptive Immunity Relation in Hepatitis B Infection

K. Visvanathan ${ }^{1,2}$

${ }^{1}$ Department of Medicine, Monash University, Monash Medical Centre; ${ }^{2}$ Department of Infectious Diseases, Southern Health, Clayton, VIC, Australia

This talk will examine a number of different stealth measures that the HBV virus employs to subvert the immunological response. Toll-like 
receptors (TLR's) are critical receptors that promote innate immune responses to pathogen-associated molecular patterns such as lipolysaccaride and CPG motifs. Activation of TLR's leads to production of cytokines such as TNF and IL-6. We have previously demonstrated that patients with $\mathrm{HBeAg}+\mathrm{ve} \mathrm{CHB}$ have suppressed TLR2 expression on peripheral monocytes and hepatocytes associated with decreased cytokine expression and decreased CD86 expression which plays an important role in the adaptive immune system. We will discuss this initial data as well as demonstrate the mechanism of interaction of $\mathrm{HBeAg}$ and the innate immune pathways followed by some exciting new data related to immunological response and clinical response in various HBV genotypes. In addition the biological advantages of the virus avoiding the innate immune system will be demonstrated. Data will also be presented regarding TLR mediated IFN defects in plasma dendritic cells and NK cells that have further implications in host response to HBV infections. The work provides a framework by which future treatment of CHB may be optimized by manipulation of these innate immune pathways.

\section{$\mathrm{CO} 2-02$}

\section{Immune Derangement During Hepatitis B Reactivation}

\section{A. Bertoletti}

Singapore Institute for Clinical Sciences, A*STAR, Singapore, Singapore

Chronic hepatitis B is an heterogeneous disease and patients chronically infected by HBV display strikingly different levels of HBV replication (from undetectable levels to $10^{11} \mathrm{HBV}-\mathrm{DNA}$ copies $/ \mathrm{ml}$ of serum) and often alternate periods of absence of liver inflammation and severe hepatitis. These acute exacerbations of liver damage (called hepatic flares, HF) can occur spontaneously, be induced by IFN $-\alpha$ therapy or by the withdrawal of treatment with nucleoside analogues. The mechanisms directly responsible for liver inflammation, namely the intrahepatic recruitment of inflammatory cells, orchestrated by specific chemokines, like CXCl-9 and CXCL-10 have been described, but the underlying immunological triggers are much less clear. The prevailing idea is that these episodes of acute liver inflammation result from a recovery of $\mathrm{HBV}$-specific $\mathrm{T}$ cells directly causing liver injury, but this scenario is supported by scanty experimental data, whilst recent findings raise the possibility of a contribution from innate components such as NK cells. We will also discuss the concept of immunotolerance during the natural history of HBV and whether it results from deletion or suppression of HBVspecific immunity alone or from more pervasive generalized effects on innate and adaptive immunity and which viral and host factors may be implicated in the loss of immune tolerance and on the resultant immune-mediated liver inflammation.

\section{$\mathrm{C} 02-03$}

Immunomodulatory Action of Interferons and Nucleoside/Nucleotide Analogues

H.L.A. Janssen

\section{Concurrent Symposium 03: Modern Care for Patients with Non-Alcoholic Fatty Liver Diseases 08:00-09:00}

\section{C03-01}

\section{Fitness and Fatness in Non-Alcoholic Fatty Liver Diseases}

J. George ${ }^{1,2}$, N. Johnson ${ }^{3}$

${ }^{I}$ Discipline of Exercise and Sport Science; ${ }^{2}$ Institute of Obesity, Nutrition and Exercise; ${ }^{3}$ The University of Sydney, Sydney, NSW, Australia

Non alcoholic fatty liver disease (NAFLD) and its inflammatory counterpart nonalcoholic steatohepatitis (NASH) are global health problems that are rapidly increasing in prevalence. NAFLD and NASH are a major cause of liver disease and to a lesser extent liver cancer, and an important factor contributing to cardiometabolic risk. The rising prevalence of NAFLD is largely a consequence of increases in caloric intake, altered diet composition and reduced physical activity. This has resulted in a search for effective lifestyle intervention strategies to reduce liver fat. Therapy involving diet restriction in conjunction with increased physical activity has clear hepatic benefits when weight loss is achieved. However, even with intensive programs, weight loss in excess of 3-4 kg is difficult to sustain, highlighting the need for alternative and more effective strategies. The absence of effective pharmacological approaches is a further stimulus for effective lifestyle-based therapy. Epidemiological data shows that there is an independent relationship between liver fat, physical activity and fitness and longitudinal research demonstrates that exercise participation itself reduces liver fat and liver enzymes in those with NAFLD, independent of weight loss. Mechanistic insights into the beneficial effects of exercise on liver fat are now being defined. Given the often poor practicability of weight loss strategies, and the viability of exercise therapy, clinicians should assess physical fitness and physical activity habits, educate on the benefits of fitness outside of weight loss, and focus on behavior change which promotes exercise adoption.

\section{C03-02}

Non-Alcoholic Fatty Liver Diseases: The Role of Nutritional Management J.-G. Fan, Y.-W. Chen

Department of Gastroenterology, Xinhua Hospital Affiliated to Shanghai Jiaotong University School of Medicine, Shanghai, China

Nonalcoholic fatty liver disease (NAFLD) has recently gained broader interest among physicians and scientists. Obesity, along with insulin resistance, type 2 diabetes and dyslipidemia, is a central risk factor for NAFLD. NAFLD has also been associated with total parenteral nutrition, acute starvation, intravenous glucose therapy and abdominal surgery. Iron overload is also involved in development of NAFLD. The understanding of the pathogenesis of NAFLD emphasizes a fundamental role of nutritional management in the intervention of this disorder. Specific diet and exercise guidelines for NAFLD patients have not been established yet, but several types of diets have been proposed for treatment of obesity. Increased physical activity, gradual weight reduction and in selected cases bariatric surgery remain the mainstay of NAFLD therapy. A controlled intake of carbohydrate, saturated fat and cholesterol, polyunsaturated fat and total fat is associated with remission of NAFLD. Recently, the presence of high fructose in soft drinks has received most of the attention. Higher intakes of soft drinks and a lower intake of fish rich in omega-3 are discouraged. Furthermore, methionine deficiency was associated with hepatic injury. Indeed, the quantity of ingested dietary fat does not seem to be the cause of NAFLD, although qualitative fat composition may be of concern. Although high-fat feeding can induce overnutrition and intrahepatic lipid in experimental animal and human models, it does so only with concomitant carbohydrate ingestion. A balanced nutrient composition is more important in determination of onset of NAFLD. However, Very few studies have investigated the effects of individual dietary components on NAFLD. Whether the excess of energy intake is the main factor that underlies the NAFLD epidemic, whether specific macronutrient and/or micronutrient components of the diet are involved, or whether both factors have a role still need more investigation.

\section{C03-03}

The Use of Statin in Non-Alcoholic Fatty Liver Disease: Good or Bad? A. Leerapun

Department of Internal Medicine, Chiang Mai University, Chiangmai, Thailand

Non-alcoholic fatty liver disease (NAFLD) includes various liver diseases from steatosis and steatohepatitis to cirrhosis. NAFLD frequently occurs in patients with type 2 diabetes, obesity and hyperlipidemia. NAFLD is now considered as the hepatic manifestation of metabolic syndrome. Statins are 
effective lipid-lowering agents that inhibit 3-hydroxy-3methyl-glutarylcoenzyme A reductase (HMG-CoA reductase). The benefit role of statins in primary and secondary prevention of coronary heart disease has resulted in their frequent use in clinical practice. However, concern regarding their hepatic safety profile has meant that patients with concurrent liver disease are often denied such benefits. In clinical practice, gastroenterologists and hepatologists are often asked to see patients who developed liver enzyme elevations after prescribing statins and also about the safety of prescribing statins in hyperlipidemic individuals with asymptomatic liver enzyme elevations. Several large-scale, randomized studies have shown that statininduced transaminitis is a transient phenomenon, transaminase level often return to baseline despite the treatment is continued. Furthermore, severe liver injury associated with statins use is so rare. The studies examining the safety of statins in patients with NAFLD are limited, but the existing data provide some evidence that they can use safely in patients with NAFLD who require statins for primary or secondary prevention of cardiovascular disease. It is encouraging to see that statins may not worsen nonalcoholic steatohepatitis (NASH) and can potentially improve the process. Moreover, some of clinical evidences demonstrate that patients with steatohepatitis who take statins lower their elevated liver enzymes and show evidence of improvement in fatty liver on follow-up imaging.

\section{Concurrent Symposium 04: Evidence-Base Medicine in the Practice of Hepatology} 08:00-09:00

\section{C04-01}

Factors Associated with Disease Progression in Chronic Hepatitis B: Implication for Treatment

\section{M.-F. Yuen}

Department of Medicine, The University of Hong Kong, Hong Kong, Hong Kong SAR

There are three main factors associated with disease progression, namely, the viral, host and environmental. The first two play the predominating roles. Viral replication and genomic sequences are the two key viral factors. $\mathrm{HBeAg}$ positivity is far from accurate in reflecting viral replication. HBV DNA level is the most important marker for viral replication. It has been shown in several studies that the risk of development of complications increases proportionally with the HBV DNA levels [1-3]. However, the relationship between the HBV DNA levels during immunotolerance phase and the risk of development of complications is not clear. The decision for treatment initiation in immunotolerance phase has not reached consensus. Some studies based on minimal histologic evidence suggested people not to treat patients in immunotolerance phase [4, 5]. Another viral factor is specific viral sequences. Some studies demonstrated a higher risk of disease progression in patients with genotype C compared with patients with genotype B [6, 7]. However, it has been shown that core promoter mutations that highly associated with genotype $\mathrm{C}$ virus are the independent risk factor [8]. Further studies show that mutations in enhancer II region and pre-S deletions are also associated with development of hepatocellular carcinoma $[9,10]$. Of the host factors, the more important ones include age, sex and the host responses to the viral infection. Those are reflected by aminotransferase levels and histology (fibrosis and cirrhosis). Patients with abnormal ALT levels have a higher risk of disease progression $[11,12]$. However, it is still up to debate on the level of ALT above which treatment is indicated. It is generally accepted that patients with advanced histology should be considered for treatment. Non-invasive measurements for liver stiffness e.g. transient elastography may become a useful tool to assist the decision making process for treatment initiation [13, 14].
C04-02

Does Antiviral Therapy Alter the Outcome in HBV and HCV Cirrhosis? E. Gane

Liver Unit, Auckland City Hospital, New Zealand

(i) Treatment of HBV-cirrhosis

The objective of treatment in HBV-related cirrhosis is sustained viral suppression to reduce disease progression to cirrhosis, liver failure and hepatocellular carcinoma and to prevent death, or liver transplantation. In addition, sustained viral suppression will reverse fibrosis and improve quality of life. Several recent large natural history studies have shown that high HBV DNA viral loads both at base line and throughout follow-up are associated with increased risk of developing cirrhosis, decompensated cirrhosis, HCC and liver-related mortality.In HBeAgpositive patients with $\mathrm{CHB}$, treatment with IFN-a significantly improved overall survival and complications of cirrhosis, compared with untreated patients. Lamivudine has also proved effective in advanced liver disease. The multinational study, NUCB4006, of patients with $\mathrm{CHB}$ and either cirrhosis or bridging to cirrhosis found that compared to those in the placebo group, patients who received lamivudine $100 \mathrm{mg} /$ day had reduced time to disease progression to either liver failure (defined as increase in Child-Pugh score) or HCC. Benefits were seen regardless of baseline ALT level or HBeAg status. Clinical endpoints among patients who developed YMDD mutants still occurred at a lower frequency than among patients who received placebo.Antiviral treatment in compensated HBV-cirrhosis is usually long-term. In decompensated cirrhosis, treatment with lamivudine also increases patient survival, compared with untreated controls, and improves liver synthetic function compared with pre-treatment values. Lamivudine therapy also significantly reduces hospital admissions, requirement for paracentesis and episodes of spontaneous bacterial peritonitis and encephalopathy. Adefovir dipivoxil (ADV) also has efficacy in decompensated cirrhosis as monotherapy, early rescue therapy and in lamivudine-resistant patients. Combination therapy with ADV and lamivudine reduces resistance to both drugs and should be considered for patients with cirrhosis. Today, most patients with decompensated $\mathrm{CHB}$ will be rescued by either lamivudine or adefovir. Most liver transplants in HBsAg+ candidates are now for HBV-related acute liver failure or hepatocellular carcinoma. AASLD guidelines recommend lifelong treatment in decompensated cirrhosis and the Keeffe Algorithm recommends long-term and indefinite treatment. The ACT-HBV Asia-Pacific Steering Committee suggests closely monitoring hepatic function and HBV DNA levels in patients who have a response to treatment, which may delay OLT given that patients with no response require a liver transplant.

(ii) Treatment of HCV-cirrhosis

In patients with $\mathrm{HCV}$-cirrhosis, the only factor associated with reduced rate of complications and improved survival is eradication of HCV infection through successful antiviral therapy. In a study of 96 patients with $\mathrm{HCV}$-cirrhosis treated with current standard-of-care (SOC), 48 weeks pegylated interferon plus ribavirin, $39(41 \%)$ achieved sustained virologic response (SVR). Follow-up liver biopsy (median 17 months post-treatment) demonstrated regression of cirrhosis in $44 \%$ of patients with SVR but in only $1 \%$ of those without. In those patients who achieved SVR, the rate of complications was only reduced in those patients with regression of cirrhosis. In a larger study of 307 patients with F3/F4 treated with SOC, 33\% achieved SVR which was associated with a significant reduction in risk of both hepatocellular carcinoma and of decompensation. In a meta-analysis of 8 European $\mathrm{HCV}$ treatment studies, patients with sustained biochemical response without virological response had higher rates of hepatocellular carcinoma and of decompensation to those who had sustained virologic response. Sustained virologic response was associated with a significant reduction in portal pressure $\mathrm{n}$ patients with compensated cirrhosis, whilst no decrease was observed in responder- 
relapsers. In the HALT-C study, patients with HCV cirrhosis, who failed to respond to pegylated interferon plus ribavirin, were randomised to receive long-term pegylated interferon monotherapy or no treatment. Maintenance pegylated interferon did not reduce the risk of large varices, index variceal haemorrhage, liver failure or hepatocellular carcinoma.Unfortunately the efficacy of current standard-ofcare (48 weeks duration of pegylated interferon plus ribavirin) is poor in patients with established HCV-cirrhosis, with SVR rates below $20 \%$. Preliminary results from the current Phase II studies of Telaprevir, suggest that the addition of this protease inhibitor to current SOC will significantly increase the SVR rate in patients with established cirrhosis.Unfortunately, pegylated interferon is contraindicated in decompensated $\mathrm{HCV}$-cirrhosis because of the associated risk of infection, encephalopathy.

\section{C04-03}

Transjugular Intrahepatic Portosystemic Shunt (TIPS): A Critical Review of Use and Misuse

T.D. Boyer

Department of Medicine, University of Arizona, Tucson, AZ, USA

TIPS was developed in the 1990s and subsequently numerous controlled trials have helped to define how TIPS is to be used in the management of patients with complications of portal hypertension. Accepted uses of TIPS include the control of acutely bleeding varices that are refractory to medical management, recurrent variceal bleeding in patients who have failed medical treatment and refractory cirrhotic ascites. Controlled trials or large patient series have shown that TIPS is effective in the above three situations. The limitations of TIPS has been the increased risk of hepatic encephalopathy and failure to show an improvement in survival. More recently reports have suggested that use of TIPS early in high risk patients with acute variceal bleeding may improve outcomes compared to standard medical therapy. Also, use of TIPS in patients with refractory ascites may provide a survival advantage compared to patients treated with repeated large volume paracentesis. Lastly, the development of covered stents has lead to questions as to whether TIPS should be used earlier than previously recommended in select patient populations. The misuse of TIPS is usually because of poor patient selection. TIPS is of no use in the primary prevention of variceal bleeding or in patients with veno-occlusive disease or GAVE. The risk of death in patients who have a TIPS created increases as their age and severity of liver disease increases. The difficulty with these observations is that there has been a failure to have an appropriate control group in many of these studies. When an appropriate control group has been included, for example in acute variceal bleeding, then TIPS showed a survival advantage despite the advanced nature of the patient's liver disease.

\section{Okuda Oration}

09:00-09:30

\section{OR-01}

Pathological Diagnosis of Early Stage Hepatocellular Carcinoma: Arriving at International Consensus

M. Kojiro

Department of Pathology, Kurume University, Kurume, Japan

Distinguishing small well-differentiated hepatocellular carcinoma (HCC) from high-grade dysplastic nodule (DN) is frequently difficult, and the precise pathologic diagnosis is a key issue in clinical management of the patients. However, despite extensive studies in the past decade on equivocal nodular lesions in the setting of chronic liver disease are still controversial. To improve the consistency of pathologic diagnosis of equivocal nodules in cirrhotic livers, the International Consensus Group for Hepatocellular Neoplasia (ICGHN) comprising 33 pathologists and 2 clinicians from 13 countries was convened in 2002 and the group has met several times up to 2008. Eventually the consensus document summarized the results of the meetings has been published in HEPATOLOGY in 2009. The ICGHN members discussed the diagnosis of 26 resected nodules less than $2 \mathrm{~cm}$ in diameter at the first meeting and additional set of 22 small nodules at the second meeting. The overwhelming diagnostic challenge was the differentiation of highgrade DN from well-differentiated small HCC (early HCC). In the diagnosis of equivocal nodules, it has been recognized that the presence or absence of "stromal invasion" is of key importance in deciding if a nodule is malignant or benign. After the first meeting, kappa value for HCC rose from 0.30 to 0.49. In conclusion, varying degrees of tumor cell invasion into the portal tracts within the tumor "stromal invasion" is considered the most helpful morphologic clue to distinguish early HCC from high-grade DN. In addition, immunohistochemical application of a panel of tumor markers such as GPC3, HSP70 and GS, may strengthen the diagnostic accuracy.

\section{Presidential Lecture}

10:00-10:30

\section{KL06}

Enlightening the Future: Coming Era of Individualized Treatment of Chronic Hepatitis B

\section{T. Piratvisuth}

Department of Medicine, NKC Institute of Gastroenterology and Hepatology, Songklanagarind Hospital, Prince of Songkla University, Hat Yai, Thailand

Chronic hepatitis B is the important cause of cirrhosis, hepatocellular carcinoma and liver-related death. The risk of liver-related death in patients acquiring HBV infection during early life is about $25-40 \%$. Recent studies have tried to develop risk calculator to identify patients who have high risk of disease progression requiring HBV treatment. Baseline factors such as HBV DNA level, serum ALT, HBV genotypes are used to identify patients who are likely to response to treatment. Patients with serum ALT $\geq 5$ times ULN and HBV DNA $<10 \operatorname{logs}$ copies/ml achieved HBeAg seroconversion 53\% 6 months post 48 weeks course of peginterferon treatment. Our CART analysis showed that patients with $\mathrm{HBeAg}>1.9$ PEIU, serum ALT $<130 \mathrm{IU} / \mathrm{L}$ and also HBV DNA $>9$ logs copies/ml are unlikely to response to peginterferon treatment with NPV of 93\%. Low genetic barrier anti-HBV agents such as lamivudine and telbivudine can be used in HBeAg-positive patients with baseline ALT $>2$ ULN and HBV DNA $<8$ logs IU/ml or HBeAg-negative with HBV DNA $<6$ $\operatorname{logs} \mathrm{IU} / \mathrm{ml}$. Roadmap approach according to virological response at week 24 could improve response and reduce emergence of drug resistance in long-term lamivudine or telbivudine therapy. Early serological response defined as low HBsAg level or early $\mathrm{HBsAg}$ decline at week 12 or 24 of peginterferon treatment is associated with better sustained response. Serum HBsAg decline during HBV treatment could be used as a surrogate marker to predict response and to identify poor responders who require treatment modification in order to improve treatment outcomes. HBsAg decline at the end of NA treatment may be used for predicting sustained off-treatment response. In conclusion, baseline patient characteristics and ontreatment virological and serological response can be used to guide individualized treatment for patients with $\mathrm{CHB}$ and to improve treatment outcomes. 
Plenary Session 01: Hepatitis B 11:00-12:00

\section{PS01-01}

No Resistance to Tenofovir Disoproxil Fumarate (TDF) Detected Following 192 Weeks of Treatment in Subjects with Chronic Hepatitis B Virus A. Snow-Lampart ${ }^{1}$, K. Kitrinos ${ }^{1}$, B. Chappel1 ${ }^{1}$, F. Myrick $^{1}$, J. Schawalder ${ }^{1}$, E.J. Heathoote ${ }^{2}$, P. Marcellin ${ }^{3}$, K. Borroto-Esoda ${ }^{1}$

${ }^{1}$ Gilead Sciences, Inc., Durham, NC, USA; ${ }^{2}$ University of Toronto, Toronto, ON, Canada; ${ }^{3}$ Hopital Beaujon, Clichy, France

Background/aim: To evaluate amino acid changes within HBV pol/ RT after up to 192 weeks of TDF treatment and determine their potential association with TDF resistance.

Methods: Subjects were randomized to receive TDF or adefovir dipivoxil (ADV) for 48 weeks followed by open-label TDF from studies GS-US-174-0102 and GS-US-174-0103. Subjects with confirmed HBV DNA $>400$ copies/mL at/after Week 72 could add emtricitabine (FTC) as a fixed-dose combination tablet (Truvada, TVD). HBV pol/RT population sequencing was attempted for all subjects at baseline and if viremic (HBV DNA $\geq 400$ copies $/ \mathrm{mL}$ ) at Week 192/study discontinuation or FTC addition. Virologic breakthrough (VB) was defined as confirmed HBV DNA either $>1 \log _{10}$ from nadir or HBV DNA $\geq 400$ copies $/ \mathrm{mL}$ after $<400$ copies $/ \mathrm{mL}$.

Results: 528 subjects (348 in TDF arm, 180 in ADV arm) entered Year 4 (82\% retention). Genotypic analysis was attempted on all viremic subjects randomized to TDF $(5 / 348,1.4 \%)$ at week $192 /$ last visit. Results were obtained for $3 / 5$ subjects; 1 had no change in HBV pol/RT despite VB and 2 developed individual polymorphic site changes without confirmed breakthrough. Five TDF subjects who added FTC were viremic at Week 192/last visit; 3 were unable to be genotyped and 2 had no changes in HBV pol/RT (1 with VB). Two of the $180(1.1 \%)$ subjects randomized to ADV were evaluated for genotypic changes; 1 due to addition of FTC after week 144 and 1 on TVD due to viremia at week 192. Both clinical isolates developed individual polymorphic site changes. Overall, 3/528 subjects had VB during Year 4; 2/3 were due to documented non-adherence.

Conclusions: No conserved site changes developed during Year 4 among 528 subjects treated with TDF. No 2 subjects developed the same polymorphic site change. Virologic breakthrough during year 4 was rare and attributed to non-adherence in the majority of cases.

\section{PS01-02}

Efficacy/Safety of Tenofovir Disoproxil Fumarate (TDF) in Asians with $\mathrm{HBeAg}+/ \mathrm{HBeAg}-$ Negative Chronic Hepatitis B (CHB), Four Year Preliminary Analysis

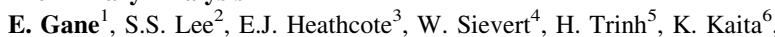
Z. Younossi ${ }^{7}$, J. George ${ }^{8}$, P. Marcellin ${ }^{9}$, D. Coombs ${ }^{10}$, J. Anderson ${ }^{10}$, E. Mondou ${ }^{10}$

${ }^{1}$ Middlemore Hospital, Auckland, New Zealand; ${ }^{2}$ University of Calgary, Calgary, AB; ${ }^{3}$ Toronto Western Hospital, University of Toronto, Toronto, ON, Canada; ${ }^{4}$ Monash University, Melbourne, VIC, Australia; ${ }^{5}$ San Jose Gastroenterology, San Jose, CA, USA; ${ }^{6}$ University of Manitoba Health Science Center, Winnipeg, MB, Canada; ${ }^{7}$ Inova Fairfax Hospital, Falls Church, VA, USA; ${ }^{8}$ Storr Liver Unit, Westmead Hospital and University of Sydney, Westmead, NSW, Australia; ${ }^{9}$ Hopital Beaujon, Clichy, France; ${ }^{10}$ Gilead Sciences, Inc., Durham, NC, USA

Background: An analysis of efficacy and safety was performed in a subset of self-reported Asian ethnicity in 2 Phase 3 pivotal trials of TDF in CHB (Studies 102/103) through week (W) 192.

Methods: Patients were randomized 2:1 to double-blind TDF $300 \mathrm{mg}$ or adefovir dipivoxil (ADV) $10 \mathrm{mg}$ daily to W48. Following liver biopsy patients were eligible for open-label TDF for 7 more years; after W72 if HBV DNA confirmed $\geq 400$ copies $(\mathrm{c}) / \mathrm{mL}(69 \mathrm{IU} / \mathrm{mL})$ emtricitabine (FTC)/TDF was an option. Elevated ALT ( $>34 / 43$ U/L women/men) and HBV DNA $>100,000 \mathrm{c} / \mathrm{mL}$ with Roche COBAS TaqMan were entry requirements. Results: Of 641 patients in both studies 189 (29\%) were Asians: 94 HBeAg-neg, $95 \mathrm{HBeAg+;} 166$ genotype B/C; 68\% men; mean age 40 years. Mean baseline HBV DNA was $7.66 \log _{10} \mathrm{c} / \mathrm{mL}$ for both Asians/non-Asians and 15 and $22 \%$, respectively, had $\geq 9 \log _{10} \mathrm{c} / \mathrm{mL}$ HBV DNA. 163 Asians started open-label TDF and 145 completed W192. HBV DNA at W192 was $<400 \mathrm{c} / \mathrm{mL}$ in $79 \%$ of all Asians (74/ $84 \% \mathrm{HBeAg}+/ \mathrm{HBeAg}-\mathrm{neg})$ and $83 \%$ of all non-Asians $(79 / 86 \%$ $\mathrm{HBeAg}+/ \mathrm{HBeAg}-\mathrm{neg}$ ) (intent-to-treat) ( $p \geq 0.2$ all comparisons). By on-treatment analysis $97 / 99 \%$ of Asians/non-Asians had HBV DNA $<400 \mathrm{c} / \mathrm{mL}$ and $86 / 79 \%$ had normal ALT $(p=0.100) .7$ Asians $(3.7 \%)$ started FTC+TDF (none in year 4), 1 discontinued, and 4/6 onstudy had HBV DNA $<400 \mathrm{c} / \mathrm{mL}$. Among those with W192 serology HBeAg loss/anti-HBe occurred in 35/26\% (Asians) and 45\%/34\% (non-Asians); $n=0$ Asians, $n=23$ non-Asians lost HBsAg. During open-label TDF serious adverse events (AEs) developed in $6 / 14 \%$ of Asians/non-Asians, Grade 3/4 AEs 10/12\%, and Grade 3/4 laboratory abnormalities $15 / 16 \%$, respectively. One Asian had phosphorus $<2$ $\mathrm{mg} / \mathrm{dL}$ (normal W192); 1 had creatinine $0.5 \mathrm{mg} / \mathrm{dL}>$ baseline and remains on study; none had creatinine clearance $<50 \mathrm{~mL} / \mathrm{min}$.

Conclusion: TDF antiviral efficacy and safety was excellent in Asians (and non-Asians) with no resistance through 4 years.

\section{PS01-03}

Week 168 Tenofovir DF (TDF) Versus Emtricitabine + TDF (FTC/TDF) in Viremic Patients Receiving Adefovir Dipivoxil for Chronic Hepatitis B (CHB)

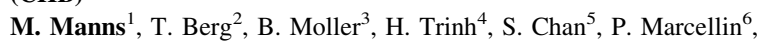
E. Suarez ${ }^{7}$, A. Snow-Lampart ${ }^{8}$, K. Peschell ${ }^{8}$, K. Borroto-Esoda ${ }^{8}$, K. Hirsch $^{8}$, D. Frederick ${ }^{8}$

${ }^{1}$ Medizinische Hochschule, Hannover, Germany; ${ }^{2}$ Universitätsklinik Leipzig, Germany; ${ }^{3}$ Private Practice, Berlin, Germany; ${ }^{4}$ San Jose Gastroenterology, San Jose, CA, USA; ${ }^{5}$ New York Hospital at Queens, Flushing, NY, USA; ${ }^{6}$ Hopital Beaujon, University of Paris University of Paris, Clichy, France; ${ }^{7}$ Hospital Universitario de Valme, Sevilla, Spain; ${ }^{8}$ Gilead Sciences, Inc., Durham, NC, USA

Background: This trial compared TDF monotherapy versus combination FTC/TDF therapy in CHB patients who had incomplete virologic response to adefovir dipivoxil (ADV).

Methods: This randomized, double-blind, multinational study enrolled patients with CHB monoinfection, randomized 1:1 to TDF or FTC/ TDF. In both treatment arms, patients were permitted to change to openlabel FTC/TDF after 24 weeks for confirmed HBV DNA > 400 copies/ $\mathrm{mL}$. Entry criteria included age 18-69 years, compensated liver disease, current treatment with ADV (lamivudine [LAM] experience allowed, TDF naïve), plasma HBV DNA ${ }^{3} 1000 \mathrm{cp} / \mathrm{mL}, \mathrm{ALT}<10$ 'the upper limit of normal. HBV DNA was measured using the Roche COBAS TaqMan HBV assay (LLOQ $=169 \mathrm{cp} / \mathrm{mL} ; 29 \mathrm{IU} / \mathrm{mL}$ ). An intentionto-treat analysis was conducted using non-completion $=$ failure.

Results: 105 patients were randomized and treated with TDF $(N=$ $53)$ or FTC/TDF $(N=52)$; 58\% had prior LAM experience. Thirteen had LAM-associated (LAM-r, rtM204V/I+/-rtL180M) and 10 had ADV-associated (ADV-r, rtN236T+/-rtA181V/T) mutations at baseline. Sixteen and 9 patients in the TDF and FTC/TDF arms, respectively, switched to combination therapy with open-label FTC/TDF after week 24. Through W168, 82\% of patients randomized to either TDF or FTC/TDF had HBV DNA $<400$ copies/mL; 13/13 with LAM-r and 9/10 with ADV-r were $<400$ copies $/ \mathrm{mL}$. ALT normalization occurred in $68 \%$ (TDF) and $67 \%$ (FTC/TDF) of patients. Three patients lost HBsAg. No resistance to tenofovir developed through week 168. No unexpected AEs and no confirmed $\geq 0.5 \mathrm{mg} / \mathrm{dL}$ increases in serum creatinine, calculated creatinine clearance $<50 \mathrm{~mL} / \mathrm{min}$ or serum phosphorus $<2.0 \mathrm{mg} / \mathrm{dL}$ were observed. Conclusions: Through 168 weeks, greater than $80 \%$ of patients on either blinded treatment or open-label FTC/TDF had HBV DNA 
$<400 \mathrm{cp} / \mathrm{mL}$. No efficacy difference was observed between initial TDF monotherapy, with the option to add FTC, or FTC/TDF combination therapy. Both TDF and FTC/TDF were well-tolerated and no resistance to tenofovir developed through week 168 .

\section{PS01-04}

Treatment Options for Lamivudine Resistant Chronic Hepatitis B Patients with Suboptimal Virological Suppression by Adefovir A. Ong ${ }^{1,2}$, V.W.S. Wong ${ }^{1}$, G.L.H. Wong ${ }^{1}$, H.L.Y. Chan ${ }^{1}$

${ }^{I}$ The Chinese University of Hong Kong, Shatin NT, Hong Kong SAR;

${ }^{2}$ University of Santo Tomas Hospital, Manila, Philippines

Background: Salvage therapy with adefovir is commonly used to treat chronic hepatitis B $(\mathrm{CHB})$ patients with lamivudine resistance but often results in incomplete virological suppression. We aimed to study the factors predicting response to adefovir in patients with lamivudine resistance and the treatment response of tenofovir and entecavir in suboptimal responders to adefovir.

Methods: Consecutive CHB patients attending the liver clinics, Prince of Wales Hospital, Hong Kong from 2008 to 2010 were screened. Patients who took adefovir for at least 6 months for lamivudine resistance were included. Early virological response (EVR) was defined as undetectable hepatitis B virus (HBV) DNA at 6 months of adefovir treatment. The primary endpoint was maintained virological response, defined as undetectable $\mathrm{HBV}$ DNA till the last follow-up visit.

Results: Among 136 patients (age $48.1 \pm 11.4$, median follow-up 39, range 5-117 months) studied, 30 (22\%) had EVR and 106 (78\%) were suboptimal adefovir responders. The 3-year cumulative probability of maintained virological response was similar between patients on adefovir monotherapy $(N=53,47.1 \%)$ and those on combination lamivudine and adefovir treatment $(N=83,37.7 \%)$. Month $6 \mathrm{HBV}$ DNA was the only independent factors associated with maintained virological response (adjusted hazard ratio 0.49, 95\% confidence interval $0.37-0.65, p<0.001) .26$ of $30(87 \%)$ early responders and 36 of $106(34 \%)$ suboptimal responders at month 6 had maintained virological response on adefovir treatment at the last follow-up $(p<0.001)$. Among 106 suboptimal responders, 18 and 11 were switched to tenofovir and entecavir, respectively. The 1-year cumulative probability of maintained virological response was higher in patients switched to tenofovir $(70.8 \%)$ than those switched to entecavir $(34.4 \% ; p=0.048)$ or continued with $\operatorname{ADV}(8.6 \% ; p<0.001)$. Conclusion: In lamivudine resistant $\mathrm{CHB}$ on adefovir salvage, HBV DNA at month 6 predicted maintained virological response at 3 years. Switching to tenofovir achieved best viral suppression among suboptimal responders to adefovir.

\section{PS01-05}

Transient Portal Hypertension in Patients of Acute-on-Chronic Liver Failure (ACLF)

H.K. Garg, A. Kumar, S.K. Sarin

Department of Hepatology, Institute for Liver and Biliary Sciences, Delhi, India

Background: Acute on chronic liver failure (ACLF) may be associated with transient but significant increase in portal pressure; however, there is scarcity of data. We studied hepatic venous pressure gradient (HVPG) during the acute phase of ACLF and subsequently after recovery.

Patient and methods: 91 consecutive patients with ACLF underwent HVPG within $48 \mathrm{~h}$ of admission. They were given standard medical therapy. None of them received beta-blockers. HVPG was repeated after 3 month in those patients who recovered from acute phase.

Results: 91 consequective patients of ACLF were recruited. Of these 34 underwent HVPG measurement during the acute phase. After a period of 3 months $61 / 91(67 \%)$ patients had died. Of the remaining 30 patients only 12 (median age 44 [range 28-67) years, males $60 \%$ ) consented for a repeat HVPG. The etiologies of cirrhosis were HBV 7 (58\%), alcohol 2 $(17 \%)$ and cryptogenic $3(25 \%)$. The acute insults were reactivation of HBV 5 (42\%), alcoholic hepatitis $1(8 \%)$, HEV 3 (25\%) and unknown 3 (25\%). The median MELD score at admission was 23.5 (19-29) which had improved to $13(7-19)$ with recovery $(p<0.05) .9 / 12(75 \%)$ patients had small esophageal varices while $3(25 \%)$ had no varices. 3 of the patients had variceal bleed. The median baseline HVPG in these 12 patients was 15.7 (range 12-21) mmHg. The median follow-up HVPG in these patients was 12 (range $2-18.5$ ) $\mathrm{mmHg}$. There was a significant reduction in HVPG with recovery $(p=0.004)$ with a median percentage reduction $21 \%$ (range -16 to $86 \%$ ).

Conclusion: ACLF in acute phase is associated with transient increase in portal pressure which may lead to variceal bleeding. The portal pressure reduces significantly after recovery from ACLF. Hence these patients merit portal pressure reducing drugs such as beta-blockers during the acute phase to prevent mortality from variceal bleeding.

\section{Plenary Session 02: Hepatitis C} 11:00-12:00

\section{PS02-01}

Hepatic Gene Expression Signature to Predict Response to Treatment in Chronic Hepatitis C

A. Ducès ${ }^{1}$, I. Bièche ${ }^{2}$, B. Jardin-Watelet ${ }^{3}$, M. Lapalus ${ }^{4}$, S. De Muynck ${ }^{4}$,

E. Dupas ${ }^{3}$, I. Molina ${ }^{3}$, N. Lambert ${ }^{5}$, E. Nicolas ${ }^{1}$, B. Sallenave ${ }^{1}$, E. Estrabaud ${ }^{4}$, N. Jullian ${ }^{6}$, M. Martinot-Peignoux ${ }^{4}, \mathrm{O}_{\text {Lada }}^{4}$, V. Paradis ${ }^{7}$, D. Valla ${ }^{4}$,

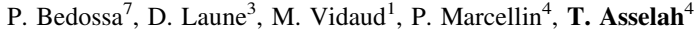

${ }^{1}$ Service de Biochimie, Clichy; ${ }^{2}$ INSERM UMR745, Paris; ${ }^{3}$ UMR3145 CNRS/Bio-Rad, Montpellier; ${ }^{4}$ Hopital Beaujon, University of Paris, Clichy; ${ }^{5}$ Biorad Laboratories, Marne-LaCoquette, ${ }^{6}$ Ariana

Pharmaceuticals, Paris; ${ }^{7}$ Department of Pathology, Clichy, France

Background and aims: The standard of care treatment of chronic hepatitis $\mathrm{C}$ (CHC) is combined pegylated interferon and ribavirin. Considering side effects and treatment cost, prediction of treatment response before therapy is important. The aim of this study was to identify an hepatic gene signature to predict sustained virological response (SVR) in patients with $\mathrm{CHC}$.

Methods: Pretreatment liver biopsies from 140 patients treated with pegylated interferon plus ribavirin were studied. Among these patients $68(49 \%)$ had SVRs, $51(36 \%)$ non response (NR) and 21 relapse (RR) (15\%). Patients were mainly infected with genotype 1, 3, 4 and 2. Real-time quantitative RT-PCR assays were used to analyse the mRNA expression of 54 genes from the literature involved in various cellular and molecular mechanisms associated with liver gene expression during hepatitis $\mathrm{C}$ infection.

Results: The expression profiles of responders differ significantly from those of NRs. The most notable changes in gene expression were mainly observed in the IFN-stimulated genes, and in growth factors/cytokines genes. Interestingly, the basal liver levels of expression of IFN-stimulated genes were higher in NRs in comparison with RR and SVRs. In NRs, the failure to respond to exogenous PEG-IFN could indicate a blunted response to IFN. This raises the possibility that, in NRs, the IFN-stimulated genes are already maximally induced.

Conclusion: Interestingly, NRs and SVRs have different liver gene expression profiles before treatment. The most notable changes occurred mainly in interferon-stimulated genes. Treatment response could be predicted with a molecular gene signature. The genes included in the signature encode molecules secreted in the serum and provide a logical functional approach for the development of serum markers to predict response to treatment. 


\section{$\mathrm{PS} 02-02$}

Boceprevir Combined with Peginterferon Alfa-2b/Ribavirin for Treatment-Experienced Patients with Hepatitis C Virus (HCV) Genotype-1: RESPOND-2 Final Results

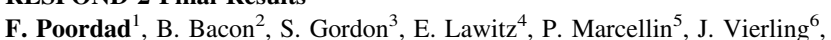
S. Zeuzem ${ }^{7}$, N. Boparai ${ }^{8}$, M. Burroughs ${ }^{8}$, C. Brass ${ }^{8}$, J. Albrecht ${ }^{8}$, R. Esteban ${ }^{9}$ ${ }^{1}$ Cedars-Sinai Medical Center, Los Angeles, CA, USA; ${ }^{2}$ Saint Louis University School of Medicine, St. Louis, MO, USA; ${ }^{3}$ Henry Ford

Hospital, Detroit, MI, USA; ${ }^{4}$ Alamo Medical Research, San Antoniz, TX, USA, ${ }^{5}$ University Clinical Centre MariborParis 7-Spital Beaujon, Clichy, France; ${ }^{6}$ Baylor College of Medicine, Houston, TX, USA;

${ }^{7}$ Universitätsklinikum des Saarlandes, Homburg/Saar, Germany;

${ }^{8}$ Merck, Whitehouse Station, NJ, USA $;{ }^{9}$ Hospital General

Universitario Vall d'Hebron, Barcelona, Spain

Background: Boceprevir is an oral inhibitor of HCV-NS3 protease. RESPOND-2 assessed the safety and efficacy of boceprevir + peginterferon/ribavirin in the re-treatment of previous non-responders and relapsers to peginterferon/ribavirin therapy.

Methods: Double-blind, placebo-controlled: randomized 403 genotype- 1 patients from the US, Canada and Europe to receive either peginterferon/ribavirin control (Arm 1), 4 weeks of peginterferon/ ribavirin (lead-in) then response guided therapy with peginterferon/ ribavirin + boceprevir (Arm 2), or peginterferon/ribavirin (lead-in) for 4 weeks followed by 44 weeks of peginterferon/ribavirin + boceprevir (Arm 3). Patients with detectable HCV-RNA at week 12 were discontinued for futility. The primary endpoint was sustained virologic response (SVR) at 24 weeks post-treatment (Roche TaqMan LLD $<9.3 \mathrm{IU} / \mathrm{mL})$.

Results: Patient distribution was $67 \%$ male, $12 \%$ black, and $12 \%$ cirrhotic. SVR was significantly higher $(p<.0001)$ in both boceprevir arms vs. control (Table). The highest SVR (80\%) was observed in patients with $\mathrm{a} \geq 1$-log decline at week 4 following the 4 week peginterferon/ribavirin lead-in who then received $800 \mathrm{mg}$ boceprevir TID for 44 weeks. Notably, SVR in Arms 2/3 was significantly higher (33-34\%) than Arm $1(0 \%)$ for patients with a $<1-\log$ decline at week 4 . The incidence of anemia was higher in boceprevir arms (43-47\%), than control (20\%) and discontinuation for anemia was infrequent $3.1 \%$ in Arm 3 only). The only adverse event that was attributed to boceprevir was dysgeusia, which was never severe or treatment-limiting.

Conclusions: Boceprevir added to peginterferon/ribavirin leads to high SVR rates in genotype 1 previous non-responders and relapsers to P/R therapy.

\begin{tabular}{|c|c|c|c|}
\hline & $\begin{array}{l}\text { Arm } 1 \\
\text { (control) } \\
\text { P/R for } 48 \\
\text { weeks* } \\
(N=80)\end{array}$ & $\begin{array}{l}\text { Arm } 2 \\
\text { P/R } 4 \text { weeks lead- } \\
\text { in BOC RGT**† } \\
(N=162)\end{array}$ & $\begin{array}{l}\text { Arm } 3 \\
\text { P/R } 4 \text { weeks lead-in then } \\
\text { P/R/BOC for } 44 \text { weeks } \\
(N=161)\end{array}$ \\
\hline $\begin{array}{l}\text { End of therapy } \\
\quad \text { response, } n(\%)\end{array}$ & $25(31)$ & $114(70)$ & $124(77)$ \\
\hline Relapse, $n / N(\%)$ & $8 / 25(32)$ & $17 / 111(15)$ & $14 / 121(12)$ \\
\hline SVR (all), $n(\%)$ & $17(21)$ & $95(59)$ & 107 (67) \\
\hline $\begin{array}{l}\text { Previous non- } \\
\text { responders, } \\
n / N(\%)\end{array}$ & $2 / 29(7)$ & $23 / 57$ (40) & $30 / 58(52)$ \\
\hline $\begin{array}{l}\text { Previous } \\
\text { relapser, } \\
n / N(\%)\end{array}$ & $15 / 51(29)$ & $72 / 105(69)$ & 77/103 (75) \\
\hline $\begin{array}{c}<1-\log _{10} \text { viral load } \\
\text { decline at week } \\
4, n / N(\%)\end{array}$ & $0 / 12(0)$ & $15 / 46(33)$ & $15 / 44(34)$ \\
\hline
\end{tabular}

Table a continued

\begin{tabular}{|c|c|c|c|}
\hline & $\begin{array}{l}\text { Arm } 1 \\
\text { (control) } \\
\text { P/R for } 48 \\
\text { weeks* } \\
(N=80)\end{array}$ & $\begin{array}{l}\text { Arm } 2 \\
\text { P/R } 4 \text { weeks lead- } \\
\text { in BOC RGT*,† } \\
(N=162)\end{array}$ & $\begin{array}{l}\text { Arm } 3 \\
\text { P/R } 4 \text { weeks lead-in then } \\
\text { P/R/BOC for } 44 \text { weeks* } \\
(N=161)\end{array}$ \\
\hline $\begin{aligned} \geq & 1-\log _{10} \text { viral } \\
& \text { load decline at } \\
& \text { week } 4, \\
& n / N(\%)\end{aligned}$ & $17 / 66$ (26) & $80 / 110$ & $90 / 114(80)$ \\
\hline
\end{tabular}

$P$ peginterferon, $R$ ribavirin, $B O C$ boceprevir

* $P$ was dosed $1.5 \mu \mathrm{g} / \mathrm{kg}$ SC weekly, $\mathrm{R}$ dose was weight-bases (600-1400 mg/day) PO divided BID, and BOC dose was $800 \mathrm{mg}$ PO TID

${ }^{\dagger}$ Response guided therapy (RGT) based on HCV negativity at week 8; (a) patient with undetectable HCV RNA received 32 weeks of triple therapy; (b) patients with detectable HCV RNA at week 8 received 32 weeks of triple therapy followed by 12 weeks of pegintereron/ribavirin. The proportion of patients achieving undetectable HCV RNA at week 8 in Arm 2 was (74/162) 46\%

\section{PS02-03}

Telaprevir in Combination with Peginterferon Alfa-2a/Ribavirin in Genotype $1 \mathrm{HCV}$ Treatment-Naïve Patients: Final Results of Phase 3 ADVANCE Study

R. Reddy ${ }^{1}$, P. Marcellin ${ }^{2}$, A.M. Di Bisceglie ${ }^{3}$, S. Zeuzem ${ }^{4}$, N.H. Bzowej, G.M. Dusheiko ${ }^{6}$, J.G. McHutchison ${ }^{7}$, A.J. Muir ${ }^{7}$, L. Bengtsson ${ }^{8}$, A.M. Dunne ${ }^{8}$, N. Adda $^{8}$, S. George ${ }^{8}$, R.S. Kauffman ${ }^{8}$, I.M. Jacobson ${ }^{9}$, ADVANCE Study Team

${ }^{1}$ University of Pennsylvania School of Medicine, Philadelphia, PA, USA; ${ }^{2}$ Hôpital Beaujon, Paris, France; ${ }^{3}$ Saint Louis University School of Medicine, Saint Louis, MO, USA; ${ }^{4}$ Johann Wolfgang Goethe University Medical Center, Frankfurt am Main, Germany; ${ }^{5}$ California Pacific Medical Center, San Francisco, CA, USA; ${ }^{6}$ Royal Free and University College, London, UK, ${ }^{7}$ Duke University Medical Center, Durham, NC, USA; ${ }^{8}$ Vertex Pharmaceuticals Incorporated, Cambridge, MA, USA; ${ }^{9}$ Weill Cornell Medical College, New York, $N Y, U S A$

Background: ADVANCE, a 3-arm double-blind, randomized, placebo-controlled Phase 3 study, compared efficacy and safety of two telaprevir $(750 \mathrm{mg} / \mathrm{q} 8 \mathrm{hr})$ response-guided regimens with peginterferon alfa-2a $180 \mu \mathrm{g} /$ week and ribavirin 1,000-1,200 mg/day (PR) in genotype $1 \mathrm{HCV}$ treatment-naïve patients.

Methods: Treatment arms: (a) 8-week T/PR, followed by PR; (b) 12week T/PR, followed by PR; (c) 48-week PR (control). Patients in T arms achieving eRVR (undetectable HCV RNA weeks 4 and 12) received 24-week therapy; those who did not received 48 weeks. Patients were randomized (1:1:1), stratified by HCV RNA $(<800,000$ $\mathrm{IU} / \mathrm{mL}, \geq 800,000 \mathrm{IU} / \mathrm{mL}$ ), and by genotype subtype. Primary endpoint was SVR.

Results: $839 / 1088$ patients $(77 \%$ ) had HCV RNA $\geq 800,000 \mathrm{IU} / \mathrm{mL}$, $631(58 \%)$ were genotype 1a, $636(58 \%)$ male, 94 (9\%) black, 117 (11\%) Latino/Hispanic, 231 (21\%) had bridging fibrosis/compensated cirrhosis. Most common $(>25 \%)$ AEs in T arms: fatigue, pruritus, nausea, headache, anemia, rash, influenza-like illness, insomnia, pyrexia, and diarrhea. Treatment discontinuations were: due to AEs 8 , 7 and $4 \%$; due to rash $0.5,1.4$ and $0.0 \%$; and due to anemia $3.3,0.8$ and $0.6 \%$ in T8PR, T12PR and PR48, respectively. 
Conclusions: A significantly greater proportion of patients achieved SVR with 12- and 8-week telaprevir-based combination regimens (75 and $69 \%$, respectively), compared with PR48 (44\%, $P<0.0001)$. Safety and tolerability of telaprevir was consistent with the previously reported profile, with improvements in treatment discontinuation rates due to adverse events, including rash and anemia.

Viral response

\begin{tabular}{|c|c|c|c|}
\hline & $\begin{array}{l}\text { T8PR } \\
(N=364)\end{array}$ & $\begin{array}{l}\text { T12PR } \\
(N=363)\end{array}$ & $\begin{array}{l}\mathrm{PR} \\
(N=361)\end{array}$ \\
\hline Patients achieving RVR, $n(\%)$ & $242(66)$ & $246(68)$ & $34(9)$ \\
\hline $\begin{array}{l}\text { Patients with HCV RNA undetectable } \\
\text { at end of treatment (EOT), } n(\%)\end{array}$ & $295(81)$ & $314(87)$ & $229(63)$ \\
\hline Patients achieving SVR, $n(\%)$ & $250(69)^{*}$ & $271(75)^{*}$ & $158(44)$ \\
\hline $\begin{array}{l}\text { Difference in SVR Rates, } \\
\text { TVR arms vs. control, } \% \text { (95\% CI) }\end{array}$ & $25(18-32)$ & $31(24-38)$ & N/A \\
\hline Patients with relapse ${ }^{\infty}, \mathrm{n}(\%)$ & $28(9)$ & $27(9)$ & $64(28)$ \\
\hline
\end{tabular}

$* P<0.0001,{ }^{\infty}$ Denominator is number of patients with HCV RNA undetectable at EOT

\section{PS02-04}

Telaprevir-Based Therapy in G1 HCV-Infected Patients with Prior Null Response, Partial Response or relapse to peginterferon/ribavirin: REALIZE trial final Results

G.R. Foster ${ }^{1}$, S. Zeuzem ${ }^{2}$, P. Andreone ${ }^{3}$, S. Pol ${ }^{4}$, E. Lawitz ${ }^{5}$, M. Diago ${ }^{6}$, S. Roberts ${ }^{7}$, R. Focaccia ${ }^{8}$, Z. Younossi ${ }^{9}$, A. Horban ${ }^{10}$, R. Van Heeswijk ${ }^{11}$, S. de Meyer ${ }^{11}$, D. Luo ${ }^{12}$, G. Picchio ${ }^{12}$, M. Beumont ${ }^{11}$

${ }^{1}$ Queen Mary University of London, Institute of Cell and Molecular Science, London, UK; ${ }^{2}$ Johann Wolfgang Goethe University Medical Center, Frankfurt am Main, Germany; ${ }^{3}$ Università di Bologna,

Bologna, Italy; ${ }^{4}$ Université Paris Descartes, INSERM Unité 567, and Assistance Publique-Hôpitaux de Paris, Cochin Hospital, Paris,

France; ${ }^{5}$ Alamo Medical Research, San Antonio, TX, USA; ${ }^{6}$ Hospital General de Valencia, Valencia, Spain; ${ }^{7}$ Department of

Gastroenterology, Alfred Hospital, Melbourne, VIC, Australia;

${ }^{8}$ Emilio Ribas Infectious Diseases Institute, São Paulo, Brazil;

${ }^{9}$ Center for Liver Disease, Inova Fairfax Hospital, Falls Church, VA, USA; ${ }^{10}$ Medical University of Warsaw, Wolska, Poland; ${ }^{11}$ Tibotec BVBA, Beerse, Belgium; ${ }^{12}$ Tibotec Inc., Titusville, NJ, USA

Background: This Phase 3 study evaluated telaprevir (T) in combination with pegylated-IFN alfa-2a (P) and ribavirin (R) in wellcharacterized G1 prior-PR treatment failure patients including prior PR non-responders (null and partial) and relapsers.

Methods: REALIZE was a randomized, international, multicentre, double-blind, placebo-controlled trial evaluating efficacy, safety and tolerability of $\mathrm{T}(750 \mathrm{mg} \mathrm{q} 8 \mathrm{~h})$ plus $\mathrm{P}(180 \mu \mathrm{g} / \mathrm{w})$ and $\mathrm{R}(1,000-1,200$ $\mathrm{mg} /$ day) compared with PR alone. The treatment arms (randomized 2:2:1, stratified by viral load and prior response) were: $12-\mathrm{T} / \mathrm{PR}$, followed by 36-week PR (T12PR48); 4-week PR followed by 12 weeks T/PR (T delayed start, DS), then 32-week PR (T12(DS)/PR48); 48-week PR (sPbo/PR48). The primary objective was efficacy of the $\mathrm{T} / \mathrm{PR}$ arms in non-responders and relapsers. Secondary objectives included evaluation of T DS and efficacy in prior-null and -partial responders. HCV RNA was quantified using COBAS TaqMan ${ }^{\circledR}$ v2.0 assay $(\mathrm{LLOQ}=25 \mathrm{IU} / \mathrm{mL})$.
Results: 833 patients were screened, and 662 treated. $70 \%$ of patients were male, $93 \%$ Caucasian, $26 \%$ had cirrhosis, and $89 \%$ had baseline $\mathrm{HCV}$ RNA $\geq 800,000 \mathrm{IU} / \mathrm{mL}$.

\begin{tabular}{|c|c|c|c|c|c|}
\hline \multirow[t]{2}{*}{$\%$} & \multirow{2}{*}{$\begin{array}{l}\text { T12/TR48 } \\
N=145\end{array}$} & \multicolumn{3}{|c|}{ T12(DS)/PR 48} & $\mathrm{Pbo} / \mathrm{PR} 48$ \\
\hline & & \multicolumn{3}{|c|}{$N=141$} & $N=68$ \\
\hline \multicolumn{6}{|l|}{ Relapsers } \\
\hline \multirow[t]{3}{*}{$\mathrm{SVR}^{* *}(P$ value*$)$} & 83 & \multicolumn{3}{|l|}{88} & \multirow[t]{2}{*}{24} \\
\hline & $<0.001$ & \multicolumn{3}{|c|}{$<0.001$} & \\
\hline & $N=121$ & \multicolumn{3}{|c|}{$N=123$} & $N=64$ \\
\hline \multicolumn{6}{|c|}{ Prior PR non-responders } \\
\hline \multirow[t]{3}{*}{$\operatorname{SVR}^{* *}(P$ value $*)$} & 41 & & \multirow[t]{2}{*}{9} \\
\hline & $<0.001$ & & & & \\
\hline & $N=49$ & & $N=$ & 48 & $N=27$ \\
\hline \multicolumn{6}{|l|}{ Partial-responders } \\
\hline \multirow[t]{3}{*}{ SVR** (P value*) } & 59 & & \multirow[t]{2}{*}{15} \\
\hline & \multirow[t]{2}{*}{$<0.001$} & \multicolumn{2}{|c|}{$<0.001$} & & \\
\hline & & $N=$ & 72 & $N=75$ & $N=37$ \\
\hline \multicolumn{6}{|c|}{$\begin{array}{l}\text { Prior Pr null- responders ( }<2 \log \text { decline in } \\
\text { HCV RNA at wk } 12 \text { of prior therapy) }\end{array}$} \\
\hline \multirow[t]{2}{*}{$\mathrm{SVR}^{* *}(P$ value $*)$} & & 29 & & 33 & 5 \\
\hline & & $<0.0$ & & $<0.001$ & \\
\hline
\end{tabular}

* In comparison to Pbo/PR48

** Assessed 24 weeks after planned treatment completion

AEs reported more frequently in $\mathrm{T}$ arms were rash, pruritus, diarrhea, anorectal disorders and anemia. 13\% of T/PR patients had premature discontinuation $(\mathrm{D} / \mathrm{C})$ of $\mathrm{T}$ due to AEs: rash $(4 \%)$ and anemia (3\%) were the most common AEs leading to T D/C.

Conclusions: T/PR SVR was significantly superior to PR in all priortreatment failure populations including null- and partial-responders. A telaprevir delayed start did not have a significant impact on SVR rates. Safety profile of T/PR was consistent with that observed in treatment naïve subjects.

\section{PS02-05}

Core Mutant Ratio is Associated with Response to PEG-IFN/RBV Treatment in HCV Genotype 1b Patients

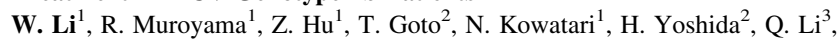
M. Omata ${ }^{2}$, K. Koike ${ }^{2}$, N. Kato ${ }^{1}$

${ }^{1}$ Unit of Disease Control Genome Medicine, Institute of Medical Science, University of Tokyo, Tokyo, Japan; ${ }^{2}$ Department of Gastroenterology, Graduate School of Medicine, University of Tokyo, Tokyo, Japan, ${ }^{3}$ Jinan Infectious Disease Hospital, Shandong University, Jinan, China

Background: Mutant type of amino acid (aa) 70 (Gln/His) in genotype $1 \mathrm{~b} \mathrm{HCV}$ core sequence was reported to be a significant and independent factor of non-virological response (NVR), however, its mechanism remains unclear. The aim of this study was to clarify how Mutant strain responds and whether Mutant ratio/quantity is related to PEG-IFN/RBV treatment response in HCV genotype $1 \mathrm{~b}$ patients.

Methods: Total 36 genotype $1 \mathrm{~b}$ hepatitis $\mathrm{C}$ patients who received 48-72 week PEG-IFN/RBV treatment were enrolled. Baseline (before treatment) Mutant ratio/quantity of each patient was examined by real-time RT-PCR with aa70 wild (W) and mutant (M) specific probes. Mutant nucleotide (nt) sequence of full-length core in each patient was determined. Moreover, treatment response of each 70W/ 
$\mathrm{M}$ and relationship between Mutant ratio/quantity and treatment response were analyzed.

Results: At baseline, 9 (25\%) patients were infected with $70 \mathrm{~W}$ only. 26 patients $(72 \%)$ were double infected with both $70 \mathrm{~W}$ and $70 \mathrm{M} .1$ patient $(3 \%)$ was infected with $70 \mathrm{M}$ only. According to mutant ratio, patients were divided into 2 groups: Major/All Mutant $(6 / 36,17 \%)$ and Minor/No Mutant (30/36, 83\%). Mutant sequence analyses showed that there were two significant nt differences between these two groups. They caused no a changes in the core protein (nt351/ aa117 and nt381/aa127) while they were possible to change F protein. During treatment, $70 \mathrm{M}$ had similar response as $70 \mathrm{~W}$. However, lower Mutant ratio patients significantly easier to achieve EVR $(P=0.003)$ and SVR $(P<0.001)$ while Mutant quantity showed no effect on antiviral responses.

Conclusions: High mutant ratio of HCV genotype $1 \mathrm{~b}$ core aa70 is associated with NVR to PEG-IFN/RBV treatment. Quantitative Mutant ratio of core aa70 is a more accurate predictor for PEG-IFN/ $\mathrm{RBV}$ treatment response than direct sequencing to determine a major type. Functional difference between $70 \mathrm{~W}$ core/F and $70 \mathrm{M}$ core/F will be further analyzed.

\section{Plenary Session 03: Molecular and Cellular Biology} 11:00-12:00

\section{PS03-01}

\section{CDKN1C/p57kip2 and its Regulator Hes1 Predict Senescence}

and Recurrence of Human Hepatocellular Carcinoma

M. Minguzzi ${ }^{1,2}$, C. Giovannini ${ }^{1,3}$, L. Gramantieri ${ }^{1,3}$, F. Fornari $^{1,3}$, P. Chieco ${ }^{1}$, L. Bolondi ${ }^{1,3}$

${ }^{1}$ Center for Applied Biomedical Research (CRBA), S.Orsola-Malpighi University Hospital; ${ }^{2}$ Department of Biology, ${ }^{3}$ Department of Clinical Medicine, University of Bologna, Bologna, Italy

Background and aims: Notch pathway is involved in proliferation, differentiation and apoptosis processes and the HES1 (Hairy/Enhancer of Split) is a major downstream target that suppresses CDKN1C/ p57kip2 expression in pancreatic cells. Since we found that Notch3 and Notch1 were aberrantly up-regulated in human hepatocellular carcinoma (HCC), we aimed at investigating the role of Hes1 in CDKN1C/p57 regulation and its function in HCC.

Methods: HepG2 and SNU398 cells were stable knocked-down for Notch3 and Notch1 and HES1 mRNA level, p57kip2, Cyclin D1 expression, cell growth and senescence analysis were assessed. Hes1 knock down (KD) cells were analysed for CDNK1C/p57 expression and senescence. CDNK1C/p57kip2 was transiently and stable overexpressed and analyses on cell proliferation, apoptosis and senescence were performed. Hes1 and p57kip2 expression was analysed in 35 surgical samples by western blot and RT- PCR respectively.

Results: Notch3 and Notch1 silenced cells reduced proliferation, upregulated p57kip2 and downregulated Cyclin D1 and Hes1. In agreement, Hes1 silencing caused p57kip2 upregulation and Cyclin D1 downregulation. P57kip2 overexpression caused G1 proliferative arrest as determined by flow cytometric analysis, but did not activate apoptosis. Analysis of p16 and SA- $\beta$ Gal expression revealed induction of senescence after ectopic p57kip2 expression. Also Notch3, Notch1 and Hes1 $\mathrm{KD}$ cells showed senescence induction as direct consequence of p57kip2 upregulation, because when it is knock down by co-infection of p57kip2 short hairpin RNA, the senescent phenotype results reverted. Negative correlation between Hes1 protein and p57kip2 mRNA levels was observed in surgical samples together with an increased time to recurrence after curative resection in patients with reduced Hes1 expression. Conclusions: We showed for the first time that Hes 1 controls senescence trough p57kip2 modulation in the liver. We hypothesize that senescence is a negative event in HCC and that Hes1 could predict HCC recurrence.

\section{PSO3-02}

Autologous $\mathrm{CD}^{+}{ }^{+}$and $\mathrm{CD} 3^{+}$Stem Cells Transplantation in Patients with End Stage Liver Disease

A.-R.N. Zekri ${ }^{1}$, H. Salama ${ }^{2}$

${ }^{1}$ National Cancer Institute, Cairo University; ${ }^{2}$ Hepatology and Tropical Medicine, National Cancer Institute, Cairo University, Cairo, Egypt

Aim: To assess the possibility of the usage of autologous $\mathrm{CD} 34^{+}$and $\mathrm{CD}_{133^{+}}$stem cells infusion as a possible therapeutic modality in patients with end-stage liver diseases.

Methods: One hundred and forty patients with end-stage liver diseases were randomized into two groups. Group 1, which included 90 patients who received granulocyte colony stimulating factor (G-CSF) for 5 days followed by autologous $\mathrm{CD} 34^{+}$and $\mathrm{CD} 133^{+}$stem cell infusion in the portal vein, and Group 2, which included 50 patients who received regular liver treatment only and served as a control group.

Results: Near normalization of liver enzymes and improvement in the synthetic function were observed in $54.5 \%$ of the studied patients, $13.6 \%$ of the patients showed stable states in the infused group; whereas none of the patients in the control group showed improvement. No adverse effects were noted.

Conclusions: Our data showed that $\mathrm{CD} 34^{+}$and $\mathrm{CD} 133^{+}$stem cells infusion can be used as supportive treatment for end-stage liver disease with satisfactory tolerability.

\section{PSO3-03}

Liver Regeneration in Heparanase Knockout Mice

A. Tryvitz, O. Ohayon, A. Pevzner, M. Paizi, G. Spira

Anatomy and Cell Biology, Faculty of Medicine, Technion, Israel Institute of Technology, Haifa, Israel

Background/aims: We have previously shown a biphasic gain in heparanase expression following $70 \%$ hepatectomy in rats. We have also demonstrated that activation of hepatic stellate cells (HSC) is associated with decreasing levels of heparanase. We now report the effect of heparanase on liver repair and HSC activation by using heparanase knockout $(\mathrm{KO})$ mice.

Methods: Seventy percent hepatectomy was performed on heparanase KO mice and control. Liver regeneration was monitored by PCNA and restituted liver mass. Heparanase uptake was performed in primary HSC isolated from heparanase KO mice. Cells were harvested and mRNA levels of HSC markers or relevant metalloproteinases monitored by RT-PCR.

Results: The capacity of heparanase KO mice to regenerate following $70 \%$ hepatectomy was similar to the control. Further, at day 5 heparanase $\mathrm{KO}$ animals replaced $60 \%$ of liver mass compared to only $30 \%$ in the control group. Cell proliferation in heparanase $\mathrm{KO}$ mice was delayed by $24 \mathrm{~h}$, peaking at $48 \mathrm{~h}$. MMP-2, MMP-3 and TIMP-1 mRNA expression significantly increased in liver cells of heparanase KO liver toward the final phase of recovery, compared to the control group. Following uptake of heparanase by primary heparanase KO HSC, the levels of MMP2, MMP3, MMP13 and TIMP1 were elevated. Interestingly, HSC activation markers ( $\alpha$ SMA, SM22, collagen) expression decreased while that of PPAR $\gamma$ gradually increased.

Conclusions: Our data suggest:

A. MMP2, MMP3 and TIMP1 are part of the liver repair compensatory mechanism in the absence of heparanase.

B. Uptake of heparanase by heparanase KO HSC is associated with inactivation of HSC. These results coincide with the recent demonstration of downregulation of PPAR $\gamma$ during HSC activation which is controlled by the MeCP2, EZH2 and miR132 pathway.

Based on the above we propose a new role for heparanase in liver cell activation. 


\section{PSO3-04}

Activation of the Inactive Hepatocyte Through Histone Acetylation: A Mechanism for Functional Compensation After Massive Loss of Hepatocyte

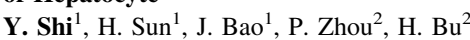

${ }^{I}$ Key Laboratory of Transplant Engineering and Immunology, Ministry of Health, West China Hospital, Sichuan University; ${ }^{2}$ Department of Pathology, West China Hospital of Sichuan University, Chengdu, China

The mechanisms by which hepatic function is maintained after extensive parenchymal loss are still unclear. By examining human and mouse liver samples, we found that under normal conditions, only a minority of hepatocytes were positively stained by acetylated histone $\mathrm{H} 2 \mathrm{~A}$ (acetyl-H2A). We also found that in human liver suffering from cirrhosis, the ratio of the positive cells was gradually increased with the developing of cirrhosis. Since acetyl-H2A is a marker of active gene transcription, we hypothesized that there might be a novel concept of "functional heterogeneity" in the hepatocytes. The DNA of the acetyl-H2A positive and negative cells were sorted and subjected to transcriptome analysis using ChIP-on-chip. The transcriptome profiles suggested that the positive hepatocytes possessed much more active transcriptional activity than that in the negative cells. Therefore, we speculated that only minority of the hepatocytes can satisfy the routine physiological requirements, and this hypothesis was confirmed in mice receiving $70 \%$ hepatectomy and their liver function remained normal even when the liver regeneration was severely inhibited by retrorsine administration. In hepatectomized liver, all of the remnant hepatocytes were marked by acetyl-H2A and the transcriptome profiles indicated that the global transcription was positively correlated with the acetyl-H2A expression. Inhibition of $\mathrm{H} 2 \mathrm{~A}$ acetylation led to transcriptional depression followed by deteriorated liver function and increased mortality. Furthermore, acetyl-H2A seemed to have the capability to choose which gene clusters should be preferentially transcribed to facilitate cells in overcoming diverse stresses. In conclusion, our data indicate a novel "functional heterogeneity" of the hepatocytes for explanation the powerful compensation capability, normally the hepatocytes are differently transcriptionally activated and a minority activated cells can satisfy the routine physiological requirements. The inactive hepatocytes, delicately regulated by acetyl- $\mathrm{H} 2 \mathrm{~A}$, act as a functional reservoir for future activation to restore the liver function after massive parenchymal loss even without liver regeneration.

\section{PS03-05}

Exogenous Recombinant Leptin Protects Neuroinflammation in a Mouse Model of Chronic Alcoholism

B. Vairappan ${ }^{1,2}$, M. Gopal $^{3}$, N. Namasivayam ${ }^{1}$

${ }^{1}$ Biochemistry, Annamalai University, Annamalainagar, India;

${ }^{2}$ Center for Hepatology, University College London, London, UK;

${ }^{3}$ Harvard Medical School, Boston, MA, USA

Background and aim: Leptin, a $16 \mathrm{kDa}$ anti-obesity hormone that can cross the blood-brain barrier (BBB) by receptor-mediated transport to exert multiple central nervous system actions. We previously demonstrated that leptin treatment reduces alcohol induced hepatic inflammation and lipid accumulation. This study aimed to determine the possible beneficial effect of leptin in an alcohol-induced neurotoxicity in mice.

Methods: CD-1 mice ( $n=15$ /group) were studied for 45 days. Four groups were studied: (1) control, (2) leptin + control $(230 \mathrm{mg} / \mathrm{kg}$ intraperitoneal every alternate day from day 15), (3) alcohol (6.32 g/ $\mathrm{kg}$ daily by gastric lavage, for 45 days) and (4) alcohol plus leptin (as prior dosing). At termination whole brain samples were processed for biochemical, cytokine, and molecular analysis.

Results: Compared to controls, ethanol supplementation significantly increased levels of brain cytokines (TNF-a, and TGF-b; $p<0.05$, respectively), which was normalized by addition of leptin $(p<0.05)$. Reactive oxygen species (ROS) and thiobarbituric acid reactive substances (TBARS) were significantly increased and the anti-oxidant capacity as determined by superoxide dismutase (SOD) and glutathione (GSH), were significantly reduced in the brain mitochondrial and cytosolic sub-fractions of mice induced ethanol $(p<0.05$, respectively). These features were also normalized by addition of leptin. Moreover, mRNA levels were increased for MMP-2 (6-fold), MMP-9 (8.4-fold) and Caspase-3 (1.2-fold) following ethanol administration as compared with control mice. Giving leptin to ethanol treated mice decreased mRNA expression of MMP-2 (2.2-fold), MMP-9 (1.5-fold) and Caspase-3 (1.3-fold) as compared with ethanol alone fed mice.

Conclusions: Leptin administration to alcohol induced neuronal subcellular organelles showed anti-inflammatory and antioxidant effects. Furthermore, leptin treatment results in a down regulation of matrix metalloproteinases and marker of apoptosis. Thus our results suggest that leptin may protect against ethanol- induced neuroinflammation warrants further mechanistic studies.

\section{Plenary Session 04: Others} 11:00-12:00

\section{PS04-01}

Replicative Study of Genome-Wide Association Studies in Primary Biliary Cirrhosis in Japanese

A. Tanaka ${ }^{1}$, P. Invernizzi ${ }^{2}$, H. Ohira ${ }^{3}$, K. Kikuchi ${ }^{4}$, S. Nezu ${ }^{5}$, A. Shibuya ${ }^{6}$ M.F. Seldin ${ }^{7}$, M.E. Gershwin ${ }^{8}$, H. Takikawa ${ }^{1}$

${ }^{1}$ Department of Medicine, Teikyo University School of Medicine, Tokyo, Japan; ${ }^{2}$ Division of Internal Medicine and Center for Autoimmune Liver Diseases, IRCCS Istituto Clinico Humanitas, Rozzano, Italy; ${ }^{3}$ Department of Gastroenterology and Rheumatology, Fukushima Medical University School of Medicine, Fukushima; ${ }^{4}$ The Fourth Department of Internal Medicine, Teikyo University Mizonokuchi Hospital, Kawasaki; ${ }^{5}$ The Third Department of Internal Medicine, Kyorin University School of Medicine, Tokyo; ${ }^{6}$ Department of Gastroenterology, Kitazato University School of Medicine, Kanagawa, Japan; ${ }^{7}$ Department of Biochemistry and Molecular Medicine; ${ }^{8}$ Division of Rheumatology, Allergy, and Clinical Immunology, University of California at Davis, Davis, CA, USA

Background and aims: Genome-wide association studies (GWAS) have been performed in patients of European ancestry to examine the genetics of susceptibility to primary biliary cirrhosis (PBC), and have demonstrated the significant associations of IL12-related signaling pathways, SPIB, IRF5-TNPO3, and 17q12-21. In the current study we tested whether these findings could be extended to a Japanese PBC cohort.

Methods: We enrolled 303 patients with PBC and 298 age- and sexmatched controls in Japanese. The diagnosis of PBC was made according to serum biochemistry, presence of serum AMA and histological studies of the biopsied liver specimens. Genomic DNA was extracted from peripheral mononuclear blood cells. TaqMan SNP assays were performed to genotype single nucleotide polymorphisms (SNPs) identified in the previous PBC GWAS.

Results: We noted significant associations at 17q12-21 loci, including IKZF3 (rs9303277; $P=0.017$ ) and ZPBP2 (rs11557467; $P=$ 0.011 ) after correction for multiple testing. SNPs at SPIB (rs3745516) and ORMDL3 (rs2305480) reached nominal significance levels but the corrected $P$ values did not reach significance. There was no genetic variance at IRF5-TNPO3 (rs10488631) in Japanese and we failed to detect significant associations with PBC for the tested $I L 12 \mathrm{~A}$ and IL12RB2 SNPs. 
Conclusions: This study replicates the association of 17q12-21 with $\mathrm{PBC}$ in a Japanese cohort. The results provide additional conformation of the importance of variation in gene(s) at this chromosomal region and extend the European results to an East Asian population group.

\section{PS04-02}

Autoantibody Profiling Using Immunoproteomic Analysis of Patient Sera With a New Type of Autoimmune-like Hepatitis Occurring After Bone Marrow Transplantation

E. Beleoken ${ }^{1,2}$, R. Sobesky ${ }^{1,2,3}$, J.-P. Le Caer $^{4}$, J.-H. Bouhris ${ }^{5}$, M. Sebagh ${ }^{1,2,6}$, B. Roche ${ }^{1,2,3}$, C. Guettier ${ }^{1,2,6}$, C. Johanet ${ }^{1,2,7}$, D. Samuel ${ }^{1,2,3}$, E. Ballot ${ }^{1,2,7}$, J.-C. Duclos-Vallée $e^{1,2,3}$

${ }^{1}$ Unité 785, Inserm; ${ }^{2}$ UMR-S 785, Univ. Paris-Sud; ${ }^{3}$ Centre HepatoBiliaire, AP-HP Hôpital Paul Brousse, Villejuif; ${ }^{4}$ Institut de Chimie des Substances Naturelles, CNRS, Gif-sur-Yvette; ${ }^{5}$ Institut Gustave Roussy; ${ }^{6}$ Service Anatomie Pathologique, AP-HP Hôpital Paul Brousse, Villejuif; ${ }^{7}$ Service Immunologie et Hématologie Biologiques, AP-HP Hôpital Saint Antoine, Paris, France

Occurrence of auto-immune-like hepatitis developed de novo is exceptionally reported after bone marrow transplantation (BMT). The purpose of this study is to characterize autoantigens recognized by sera of such patients by using serological proteome analysis.

Patients and methods: Three patients (one female, two males) received allogeneic BMT for myelodysplastic syndrome $(n=1)$ or chronic lymphocytic leukaemia $(n=2)$. In the year post-BMT, after stopping the immunosuppressive therapy, they developed an autoimmune-like hepatitis according to the hepatic histological features, different from graft-versus-host disease (GVHD), particularly without bile duct injury. Prothrombin times were between 37 and 100\%, aminotransferase levels from 13 to 72 times the upper normal limit and bilirubin from 82 to $270 \mu \mathrm{mol} / \mathrm{L}$. Serology of viral hepatitis was negative. Sera before and during the autoimmune hepatitis (AIH) occurrence were tested on immunoblots performed with two-dimensional electrophoresis resolved proteins of cytosolic, microsomal, mitochondrial and nuclear fractions obtained from rat liver homogenate. Antigenic targets were identified by mass spectrometry.

Results: Immunoreactive spots stained by sera contemporaneous with AIH were more numerous and more intensely expressed than those stained by sera taken before the hepatic disorder for all cellular fractions used as antigen. A great pattern heterogeneity depending on the patient was also noted and a total of 259 spots were stained exclusively by sera taken at the moment of hepatic disorder. 222 spots were actually identified, corresponding to 79 different proteins, some spots being isoforms of the same protein. Eleven of them were common targets of antibodies present in all patient sera and often found in auto-immune disorders.

Conclusion: This is the first immunological description of a new type of de novo-AIH occurring after BMT. The eleven common antigenic targets differ from those reported in de novo-AIH occurring after hepatic transplantation. The existence of an hepatic variant of GVHD can be put under consideration.

\section{PS04-03}

Identification of Serum Biomarkers for Transaminase-Negative Chronic Hepatitis and Nonalcoholic Fatty Liver Disease by Proteomics

K. Uchida ${ }^{1}$, H. Suzuki ${ }^{2}$, K. Meno ${ }^{1}$, T. Korenaga ${ }^{2}$, R. Nagashima ${ }^{2}$,

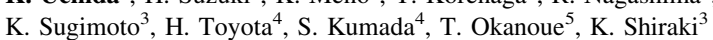

${ }^{1}$ University of Tsukuba; ${ }^{2}$ Research Division, MCBI, Inc, Tsukuba;

${ }^{3}$ School of Medicine, University of Mie, Tsu; ${ }^{4}$ Ogaki Municipal

Hospital, Ohgaki; ${ }^{5}$ Saisseikai Suita Hospital, Suita, Japan
Approximately $30 \%$ of patients with chronic hepatitis C show serum alanine aminotransferase (ALT) levels persistently within the normal range. Nonalcoholic fatty liver disease (NAFLD) is a common cause of chronic liver disease and considered the hepatic manifestation of metabolic syndrome. Based on pathologic criteria, NAFLD is categorized to simple steatosis, nonalcoholic steatohepatitis (NASH), and cirrhosis. NASH progresses to hepatocellular carcinoma (HCC) thorough cirrhosis. The entire histological spectrum of NAFLD can be seen in patients with normal ALT values, and the diagnostic ability for ALT to identify NAFLD/NASH is poor. It cannot act as a. biochemical surrogate marker to survey chronic liver disease characterized by a lowgrade inflammation. In this study, we screen for liver inflammation markers by a quantitative peptidomic methodology using twodimensional microflow HPLC (mLC)-MALDI-TOF MS/MS. Comprehensive analysis of sera from 50 patients with chronic hepatitis $(\mathrm{CH}), 108$ patients with biopsy-proven NAFLD (53 non$\mathrm{NASH}$ and $55 \mathrm{NASH}$ ) and 50 normal healthy controls has identified peptides that demonstrate remarkable diagnostic potential in diagnosis of chronic liver disease. We have identified several peptides including a glycosylated peptide showing high sensitivity and specificity in the diagnosis of chronic hepatitis and NAFLD. We verified a glycosylated peptide by an immunoprecipitation-MS assay using specific antibody and also confirmed its diagnostic value. Furthermore, differential analysis of non-NASH and NASH pooled samples performed using both non-labeling and iTRAQlabeling peptidomic methods revealed serum biomarker candidates for NASH. These results indicate that peptides circulating in blood are potentially valuable biomarkers for chronic liver disease, including NAFLD/NASH.

\section{PS04-04}

Non-Invasive Diagnosis of Steatosis Using CAP by Fibroscan®

V. de Ledinghen ${ }^{1}$, B. le Bail ${ }^{2}$, J. Foucher ${ }^{1}$, W. Merrouche ${ }^{1}$, L. Sandrin ${ }^{3}$, V. Miette ${ }^{3}$, M. Sasso ${ }^{3}$

${ }^{1}$ Hepatology, Haut Lévêque Hospital, Pessac; ${ }^{2}$ Pathology, Pellegrin Hospital, Bordeaux; ${ }^{3} R \& D$, Echosens, Paris, France

Background: Evaluation of steatosis by non-invasive means is still a challenge. Lately, the CAP (Controlled Attenuation Parameter) based on Fibroscan ${ }^{\circledR}$ principle has been devised to target specifically liver steatosis. The aim of this work is to validate CAP's performance in a cohort of patients with chronic liver disease.

Methods: All consecutive patients received in one liver unit between June 2009 and July 2010 who underwent both Liver Biopsy (LB) and Fibroscan ${ }^{\circledR}$ (M probe) were enrolled. All LB were analysed by one expert anatomopathologist. Fibrosis was assessed in Metavir or Brunt score depending on the aetiology. Steatosis was quantified as the percentage of hepatocyte with fatty accululation: S0: $\leq 10 \%, \mathrm{~S} 1$ : 11-33\%, S2: $34-66 \%$, S3: $\geq 67 \%$. Correlation was assessed using Spearman coefficient. Performance of CAP was appraised using Receiver Operating Characteristics (ROC).

Results: Characteristic of the 102 patients included were the following: 50\% male; median $\mathrm{BMI}=26 \mathrm{~kg} \mathrm{~m}^{-2}, \mathrm{HCV}=30 \%$, $\mathrm{HBV}=5 \%, \mathrm{NAFLD} / \mathrm{ALD}=33 \% ; 8 \%$ cirrhotic. Steatosis repartition was: S0: $53 \%$, S1: $20 \%, \mathrm{~S} 2: 13 \%, \mathrm{~S} 3: 15 \%$. CAP was significantly correlated to steatosis $\left(\rho=0.64, p<10^{-11}\right)$, BMI $\left(\rho=0.65, p<10^{-10}\right)$ and not significantly correlated to fibrosis and elasticity. Area Under ROC Curves (AUC) are represented in Fig. 1. Corresponding performances and thresholds are given for the maximal Youden index in Table 1. 


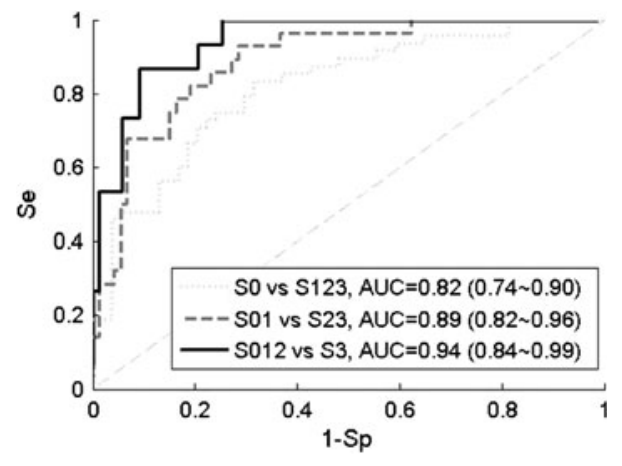

Fig. 1 AUC for steatosis grades dectection

Table 1 Performances and thresholds (max Se+Sp)

\begin{tabular}{llll}
\hline & S0 vs. S1S2S3 & S0S1 vs. S2S3 & S0S1S2 vs. S3 \\
\hline AUC & $0.82(0.74-0.90)$ & $0.89(0.82-0.96)$ & $0.92(0.84-0.99)$ \\
Optimal threshold & 215 & 237 & 297 \\
Sensitivity (Se) & $0.83(0.73-0.94)$ & $0.93(0.83-1)$ & $0.86(0.69-1)$ \\
Specificity (Sp) & $0.69(0.56-0.81)$ & $0.72(0.61-0.82)$ & $0.91(0.85-0.97)$ \\
$\begin{array}{l}\text { Positive predictive value } \\
\text { Negative predictive }\end{array}$ & $0.70(0.61-0.79)$ & $0.55(0.46-0.65)$ & $0.62(0.52-0.71)$ \\
$\quad 0.82(0.75-0.90)$ & $0.96(0.93-1)$ & $0.98(0.95-1)$ \\
\hline value & & & \\
\hline
\end{tabular}

Conclusion: It was shown that CAP is very efficient to detect even minimal steatosis $(10 \%)$. CAP being implemented on Fibroscan ${ }^{\circledR}$, both steatosis and fibrosis can be evaluated simultaneously, enlarging the spectrum of non invasive techniques for chronic liver disease management.

\section{PS04-05}

Do Beta-Blockers Reduce Energy Expenditure in Patients with Liver Cirrhosis?

W.G. Lee ${ }^{1}$, L.D. Plank ${ }^{1}$, E.J. Gane ${ }^{2}$, J.L. McCall ${ }^{2}$

${ }^{1}$ Department of Surgery, University of Auckland; ${ }^{2}$ New Zealand Liver Transplant Unit, Auckland City Hospital, Auckland, New Zealand

Background: Liver cirrhosis is associated with nutritional abnormalities including protein malnutrition and raised resting energy expenditure (REE). We previously showed that elevated REE, even within the normal range, was associated with reduced transplant-free survival in liver cirrhosis. Non-selective $\beta$-blockers reduce portal hypertension and prevent variceal bleeding but also reduce REE in acute hypermetabolic conditions such as burns and sepsis. We conducted a double-blind randomised controlled cross-over trial to investigate if non-selective $\beta$-blockers reduce REE in patients with liver cirrhosis.

Methods: Twenty-two stable cirrhotic patients (16 males, 6 females, age range $45-75$ years) not previously on $\beta$-blockers had measurements of REE and body composition before and after 3 months of treatment with nadolol or placebo, then crossed over to the alternative treatment after a 1 month washout period.

Results: Mean (SEM) REE at recruitment was 1612 (54) kcal/day. Following placebo REE decreased by $42(31) \mathrm{kcal} / \mathrm{day}(p=0.18)$ compared to a reduction of $124(30) \mathrm{kcal} / \mathrm{day}(p=0.0005)$ with nadolol ( $p=0.07$ for group comparison). Body composition changes were not significant. There was no evidence of a treatment-period interaction. There was no difference in adverse events between the groups (6 nadolol vs. 2 placebo, $p=0.29$ ).
Conclusions: Oral $\beta$-blockers reduced REE in stable cirrhotic patients and may have benefits over and above those related to phophylaxis against variceal haemorrhage.

Free Communication 01: Hepatitis B 14:00-15:00

FC01-01

1-Year Results of Telbivudine Roadmap Study Demonstrated Maximizing Antiviral Efficacy and Minimizing Resistance in HBeAg Positive Chronic Hepatitis B Patients

T. Piratvisuth ${ }^{1}$, P. Komolmit ${ }^{2}$, T. Tanwandee ${ }^{3}$, W. Sukeepaisarnjaroen ${ }^{4}$, H.L.-Y. Chan ${ }^{5}$, M. Pessoa ${ }^{6}$, E. Fassio ${ }^{7}$, S. Ono-Nita ${ }^{8}$, F. Bessone ${ }^{9}$, J. Daruich ${ }^{10}$, S. Zeuzem ${ }^{11}$, H. Cheinquer ${ }^{12}$, Y. Dong ${ }^{13}$, A. Trylesinski ${ }^{13}$

${ }^{1}$ NKC Institute of Gastroenterology and Hepatology,

Songklanagarind Hospital, Prince of Songkla University; ${ }^{2}$ Division of Gastroenterology, Department of Medicine, Chulalongkorn University; ${ }^{3}$ Siriraj Hospital, Bangkok; ${ }^{4}$ Department of Medicine, Srinagarind Hospital, Khon Kaen University, Khon Kaen, Thailand; ${ }^{5}$ Department of Medicine and Therapeutics, The Chinese University of Hong Kong, Hong Kong, Hong Kong SAR; ${ }^{6}$ Hospital das Clinicas da Faculdade de Medicina da USP, Sao Paulo, Brazil; ${ }^{7}$ Hospital Nacional Prof. Alejandro Posadas, Buenos Aires, Argentina;

${ }^{8}$ Departamento de Gastroenterologia da Faculdade de Medicina da Universidade de São Paulo, São Paulo, Brazil; ${ }^{9}$ Catedra de Clinica Medica, Universidad Nacional de Rosario, Rosario, Antigua \& Barbuda; ${ }^{10}$ Hospital de Clínicas San Martín, University of Buenos Aires, Buenos Aires, Argentina; ${ }^{11}$ Klinikum der Johann Wolfgang Goethe-Universitaet, Frankfurt am Main, Germany;

${ }^{12}$ Gastroenterology Division, Hospital de Clinicas de Porto Alegre, Universidade Federal do Rio Grande do Sul (UFRGS), Porto Alegre, Brazil; ${ }^{13}$ Novartis Pharma AG, Basel, Switzerland

Background/aims: The roadmap concept, a strategy of treatment intensification in anti-HBV therapy for patients without early virologic response has been retrospectively validated with telbivudine in the GLOBE study. We aimed to prospectively evaluate the telbivudine road-map concept with tenofovir add-on.

Patients and methods: 105 patients were recruited into this singlearm multicenter 2 year study and received telbivudine for 24 weeks (w). 103 patients were eligible for mITT analysis, at $24 \mathrm{w}$, patients with detectable ( $\geq 300$ copies/ml) HBVDNA were required to add-on tenofovir up to $104 \mathrm{w}$. 52w data (mITT, LOCF) including primary endpoint (HBV DNA at w52) are presented here.

Results: At baseline, mean HBVDNA was $9 \log _{10}$ copies $/ \mathrm{ml}$, ALT $133 \mathrm{U} / \mathrm{L}$, GFR (MDRD) $94 \mathrm{ml} / \mathrm{min}, 68 \%$ patients were Asian, 56\% genotype C. By $24 \mathrm{w}$, overall mean HBVDNA decline was $5.9 \log _{10}$ copies/mL and 55/103 (53\%) achieved undetectable HBVDNA. 57 patients stayed on telbivudine monotherapy (2 patients discontinued before $24 \mathrm{w}), 55$ of 57 with undetectable HBVDNA (2 protocol deviations); at $52 \mathrm{w}, 56$ of them (98\%) achieved undetectable HBVDNA. At 24w. 46 patients with detectable HBVDNA enrolled into tenofovir-add-on arm, with mean HBVDNA $4.1 \log _{10}$ copies $/ \mathrm{ml}$. At 52w, additional HBVDNA decline was $1.8 \log _{10}$ copies $/ \mathrm{mL}$ and $39 / 46$ (85\%) achieved undetectable HBVDNA. At 52w in the overall mITT population, 92\% had undetectable HBVDNA, 39\% $\mathrm{HBeAg}$ seroconversion, $6 \% \mathrm{HBsAg}$ clearance. 1 patient who had continued LDT-montherapy despite detectable HBVDNA at 24w experienced viral breakthrough ( $\geq 1 \log _{10}$ above nadir, protocol deviation). Overall 3/103 (3\%) patients had Serious Adverse Events (AE). 15 patients had myalgia (14\%), but none myopathy. Mean GFR increased by $6.2 \mathrm{ml} /$ min from baseline to $52 \mathrm{w}(p=0.01) .2$ patients discontinued treatment due to AE. No peripheral neuropathy, lactic acidosis or renal AEs were reported. 
Conclusion: After 1 year, telbivudine roadmap with tenofovir add-on at 24 weeks in patients with detectable HBVDNA leads to an overall higher viral suppression, 39\% $\mathrm{HBeAg}$ seroconversion and 6\% $\mathrm{HBsAg}$ clearance. The safety profile and tolerability were favorable.

\section{FC01-02}

The Cellular and Humoral Immunity Effect of Recombinant Chinese Hamster Ovary Cell Hepatitis B Vaccine in Adults

W. Zhang, L.L. Han, C.Y. Lin, X.H. Pang, J. Wu, L.Q. Li

Beijing Center for Disease Control and Prevention, Beijing, China

Objective: To evaluate the cellular and humoral immunity effect of recombinant Chinese hamster ovary $(\mathrm{CHO})$ cell hepatitis $\mathrm{B}$ vaccine in adults by randomized double-blind controlled trials

Method: A total of 642 adults aged 18-45 years old, non-vaccinated against hepatitis B, and hepatitis B five blood indicators negative were selected as the study objects from four districts in Beijing. The study objects were randomly divided into two groups, and then accept 10 and $20 \mu \mathrm{g}$ recombinant $\mathrm{CHO}$ hepatitis B vaccination respectively by $0,1,6$ month schedule. Blood sample was collected from each study object 1 month after the third dose vaccination. The anti-HBs level was detected by Abbott chemiluminescence detection method (I2000) to evaluate humoral immunity status. Of all the study objects, 153 cases were randomly selected as the cellular immunity status study objects. Their blood were collected and Lymphocyte were separated to detect the IL- 4 and IFN- $\gamma$ levels in vitro by enzymelinked immunospot (ELISPOT) method.

Results: The anti-HBs seroconversion rates in 10 and $20 \mu \mathrm{g}$ dose group were $88.8 \%$ (285/321) and $95.3 \%$ (306/321) respectively, and 95\% CI were $85.4-92.2 \%$ and $93.0-97.6 \%$ respectively. The spot forming cell (SFC) of IL-4 of the $20 \mu \mathrm{g}$-dose group (=20.31) were significantly higher than the $10 \mu \mathrm{g}$-dose group $(=8.19, t=3.27$, $P<0.01)$. With the increasing of anti-HBs titer, the SFC of IL-4 also goes up significantly. There was a positive correlation between SFC of IL-4 and anti-HBs (Spearman correlation coefficient $=0.538$, $P<0.0001$ ), There was no relationship between IFN- $\gamma$ and anti-HBs (10 $\mu$ g group: $1.49,20 \mu$ group: 0.86: $t=1.83, P>0.05)$.

Conclusion: The humoral and cellular immune effects of $20 \mu \mathrm{g}$ recombinant $\mathrm{CHO}$ hepatitis $\mathrm{B}$ vaccine are better than that of the $10 \mu \mathrm{g}$ recombinant $\mathrm{CHO}$ hepatitis $\mathrm{B}$ vaccine. $20 \mu \mathrm{g}$ recombinant $\mathrm{CHO}$ hepatitis B vaccine should be chosen as the adult's hepatitis B prevention vaccine.

\section{FC01-03}

Peginterferon Alfa-2a (180 $\mu \mathrm{g} / 48$ weeks) is Well Tolerated with High Rates of Treatment Adherence: Results of the NEPTUNE Study

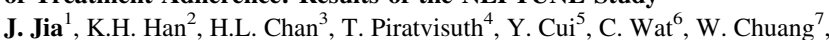
Y.-F. Liaw

${ }^{I}$ Beijing Friendship Hospital, Capital Medical University, Beijing,

China; ${ }^{2}$ Department of Internal Medicine, Yonsei University College of Medicine, Seoul, Republic of Korea; ${ }^{3}$ The Chinese University of Hong Kong, Hong Kong, China; ${ }^{4}$ NKC Institute of Gastroenterology and Hepatology, Songklangarind Hospital, Prince of Songkla University, Hat Yai, Thailand; ${ }^{5}$ Roche Product Development, Shanghai, China; ${ }^{6}$ Roche Products Ltd, Welwyn Garden City, UK; ${ }^{7}$ Kaohsiung Medical University Chung-Ho Memorial Hospital, Kaohsiung, ${ }^{8}$ Liver Research Unit, Chang Gung Memorial Hospital and Chang Gung University College of Medicine, Taipei, Taiwan $R O C$

Background/aims: The NEPTUNE study compared the efficacy and safety of peginterferon alfa-2a [40KD] (PEG-IFN $\alpha-2 a)$ for 24 or 48 weeks and at $90 \mu \mathrm{g} /$ week or $180 \mu \mathrm{g} /$ week in HBeAg-positive CHB patients $(n=524)$. It confirmed that the licensed dose $(180 \mu \mathrm{g}$ for 48 weeks) results in the highest rate of $\mathrm{HBeAg}$ seroconversion posttreatment $(36.2 \%)$.

Methods: Patients were randomized to PEG-IFN $\alpha-2 \mathrm{a}$ in a $2 \times 2$ factorial design of treatment duration ( 24 or 48 weeks) and dose (90 or $180 \mu \mathrm{g}$ ). Adverse events (AEs) were graded mild, moderate or severe. Serious AEs (SAEs) were recorded.

Results: The vast majority of patients (86-93\%) received the planned duration of PEG-IFN $\alpha$-2a, and the mean doses received were $94-98 \%$ of the total planned dose. Only $2-7 \%$ of patients discontinued treatment. Consistent with previous experience with PEG-IFN $\alpha-2 a$, the most frequent AEs were pyrexia, myalgia, alopecia, fatigue, headache, insomnia and decreased appetite. Laboratory abnormalities were consistent with previous PEG-IFN $\alpha-2 a$ experience and were clinically manageable with dose modification. The incidence of SAEs, severe AEs, AEs leading to dose modification or treatment withdrawal were low and comparable between treatment groups.

\begin{tabular}{lcccc}
\hline & $\begin{array}{l}90 \mu \mathrm{g} / \\
24 \text { week } \\
(n=144)\end{array}$ & $\begin{array}{l}180 \mu \mathrm{g} / \\
24 \text { week } \\
(n=141)\end{array}$ & $\begin{array}{l}90 \mu \mathrm{g} / \\
48 \text { week } \\
(n=132)\end{array}$ & $\begin{array}{l}180 \mu \mathrm{g} / \\
48 \text { week } \\
(n=130)\end{array}$ \\
\hline $\begin{array}{l}\text { Pts with } \geq 1 \mathrm{AE} \\
\text { Pts with } \geq 1 \text { severe AE }\end{array}$ & $\begin{array}{l}9(6 \%) \\
\text { Pts with } \geq 1 \text { SAE }\end{array}$ & $\begin{array}{c}120(85 \%) \\
7(5 \%)\end{array}$ & $\begin{array}{c}105(80 \%) \\
5(4 \%)\end{array}$ & $\begin{array}{c}117(90 \%) \\
7(5 \%)\end{array}$ \\
$\begin{array}{l}\text { Pts with dose } \\
\text { modification } \\
\text { due to AE/lab }\end{array}$ & $18(13 \%)$ & $23(16 \%)$ & $25(19 \%)$ & $31(24 \%)$ \\
$\begin{array}{c}\text { AEs leading to } \\
\text { dose } \\
\text { modification }\end{array}$ & $3(2 \%)$ & $3(2 \%)$ & $4(3 \%)$ & $1(<1 \%)$ \\
AEs leading to \\
$\quad$
\end{tabular}

PEG-IFN $\alpha$-2a was well tolerated in all groups

Conclusions: $180 \mu \mathrm{g}$ PEG-IFN $\alpha$-2a for 48 weeks was well tolerated with high rates of adherence. As neither higher dose nor longer duration affected the safety profile or adherence to therapy, the NEPTUNE study confirms that the licensed dose and duration $(180 \mu \mathrm{g}$ for 48 weeks) is the most efficacious for patients compared with lower doses and shorter durations.

\section{FC01-04}

Gene Expression Signature in PBMC that Correlated with Hepatitis B Viral Load

S. Prom-on ${ }^{1}$, P. Sodsai ${ }^{2}$, P. Tangkijvanich ${ }^{3}$, N. Hirankarn ${ }^{4}$

${ }^{1}$ Computer Engineering Department, Faculty of Engineering, King

Mongkut's University of Technology Thonburi; ${ }^{2}$ Medical

Microbiology, Interdisciplinary Program, Graduate School

Chulalongkorn University; ${ }^{3}$ Department of Biochemistry,

${ }^{4}$ Department of Microbiology, Faculty of Medicine, Chulalongkorn University, Bangkok, Thailand

In HBV infection, difference amount of viral DNA indicates different severity and may lead to different treatment outcome. Nevertheless, the mechanism of such reaction is still unknown. To study such mechanism, we have conducted a gene expression analysis experiment of patients with chronic hepatitis B that have variability in HBV viral load. 
Peripheral blood mononuclear cells were collected from 37 samples of chronic hepatitis B patients. Illumina Sentrix Humanref-8 v2 BeadChips were used for analysis of whole-genome gene expression profile. Using correlation analysis, we identified 326 genes that have their expressions significantly correlate with the viral load (absolute correlation $>0.9, p$-value $<0.05)$. Among them, 297 genes were positively correlated with the amount of HBV DNA while 29 genes were negatively correlated. The functional annotation analysis of these two opposite gene sets using DAVID and STRING shows 4 major clusters in the functional protein network that relates to mRNA processing and splicing, viral nucleoprotein, host-virus interaction and protein ubiquitination. From the significant gene list, we identified 42 interferon regulated genes using the Interferome database. These IFN regulated genes associate with regulation of protein-ubiquitin ligase acitivity and regulation of apoptosis. We further studied the association between the foldchanges of gene expression at 6 months after treatment compared to baseline and the viral load. We found 71 genes that express significantly different between the responder and nonresponder groups. With this gene expression changing signature, treatment outcome can be predicted correctly using the agglomerative hierarchical clustering. 6 genes were selected for validation by RT-PCR, including ZNF331, HIF1A, ELK4, ZNF175, IFI27, and IFNAR1, by the criterion that their expression changes differentially comparing between groups. The validation results are consistent with the result from microarray analysis. This indicates potential biomarkers for predicting the treatment outcome of interferon treatment in patients with HBV infection.

\section{FC01-05}

Microarray-based Analysis of microRNAs Expression in Severe Hepatitis B Patients

W. Chen, Y.M. Wang, B.Y. Xue, G.H. Deng

Institute of Infectious Diseases for PLA, Southwest Hospital, Third

Military Medical University, Chongqing, China

Aims: To profile the expression of microRNAs (miRNAs) and their potential target genes in the severe hepatitis B patients by monitoring miRNA and mRNA expression on a genome-wide basis.

Methods: The PBMCs of a pair of monozygotic twins with severe hepatitis B and HBV carrier separately were analyzed with Exiqon miRNA Array to detect the difference expression miRNAs. Their expressions were subsequently quantified by real-time RT-PCR. In addition, the expressions of the mRNA in monozygotic twins were detected with the Roche Nimblegen Whole Human Genome oligo microarray. The expression of the mir-16, mir-17, let-7i and let-7a were detected between 52 severe hepatitis B patients and $48 \mathrm{HBV}$ carriers. A combined approach using a computational prediction algorithm that included miRanda, PicTar, TargetScan, and the whole genome microarray experiment was performed by monitoring the mRNA:miRNA association to identify potential target genes.

Results: 45 miRNAs were found to have an increased expression and 8 miRNAs were found to have a decreased expression in the severe hepatitis B patients, which were demonstrated by Real-time RT-PCR. The expressions of mir-16 and let-7a were significantly increased in severe hepatitis B patients with 8.5-fold and 8.6-fold separately. BCL2, CARD8, EDA, IL1RAPL1, LTB and FZD10 were predicted as the target genes of mir-16. CERCAM, IGF2BP1, OPRM1 and MAP4K3 were predicted as the target genes of let-7a.

Conclusions: We firstly identified the upregulation of mir-16 and let$7 \mathrm{a}$ in the severe hepatitis $\mathrm{B}$ patients and predicted their potential target genes.
Free Communication 02: Hepatocellular Carcinoma 14:00-15:00

FC02-01

MircroRNA-148a Maintains its Own Aberrant Expression in Hepatoma through DNA Methylthansferase-1 Mediated Promoter Hypermethylation L. Yang, D. Wang, L. Chen

Union Hospital, Hust, Wuhan, China

Background and aim: MicroRNAs (miRNA) have been found to play pivotal role in malignancy including hepatocarcinogenesis. Here, role of microRNA-148a in hepatocellular carcinoma was investigated, and the mechanism of its aberrant expression was explored.

Methods: Expression of microRNA-148a and its potent target DNA methyltransferase 1(DNMT1) in normal liver (NL), cirrhotic liver (CL) and HBV-associated hepatocellular carcinoma (HCC) was evaluated with real-time RT PCR or western blot. Methylation of mir148a promoter $\mathrm{CpG}$ islands was tested with methylation-specific PCR. To evaluate imir-148a expression was inhibited by promoter CpG methylation, HepG2 was treated with 5-aza and DNMT1 in HepG2 was knockdown with RNAi respectively, mir-148a and its promoter hypermethylation was tested; Mir-148a in HepG2 was upregulated with mimic transfection, DNMT1 expression, CpG islands methylation and corresponding mir-148a m RNA expression was evaluated.

Results: MiR-148a was aberrantly lower in HCC than in NL and CL, with higher DNMT1 expression; CpG islands of mir-148a was hypermethylated in HepG2, 5-AZA and DNMT1 knockdown demethylated $\mathrm{CpG}$ islands of mir-148a, increased mir-148a expression; Upregulating 148a in HepG2 inhibited DNMT1 expression, demethylated $\mathrm{CpG}$ islands of mir-148a, and consequently increased mir-148a itself.

Conclusions: It was found that mir-148a was aberrantly inhibited in HBV-associated HCC; Mir-148a maintains its aberrant expression through a kind of self-enhancing loop: Lowly expressed mir-148a upregulated its target gene DNMT1 expression in hepatoma, which hypermethylates the promoter $\mathrm{CpG}$ islands of mir-148a, and suppressed mir-148a expression.

\section{FC02-02}

Fg12/prothrombinase Contributes to HCC Tumor Growth and Angiogenesis through the MAPK Pathway

Y. Liu, Q. Zeng, J. Wang, X. Li, X. Wang, D. Yang, X. Luo, Q. Ning

Tongji Hospital, Huazhong Science and Technology University, Wuhan, China

Fibrinogen-like protein 2(fgl2) /prothrombinase has been identified as a procoagulant protein which directly generates thrombin from prothrombin without activation of the conventional coagulation cascade. Fgl2 has been found to be overexpressed in a number of human malignant tumors including human hepatocellular carcinoma (HCC), however, its functional role in malignancy remain largely unexplored. To define the role of tumor cell-associated fgl2 in tumor growth and tumor angiogenesis, we established a fgl2 stably knock down HCCLM6 cell line, a highly aggressive HCC derived cell line in which fgl2 expression was found upregulated in response to tumor necrosis factor- $\alpha$ (TNF- $\alpha$ ) stimulation in a dose-dependent manner. Consequently, fgl 2 depletion in HCCLM6 cells led to a delayed tumor growth and vascularization of HCC xenografts in nude mice. These changes were associated with decreased phosphorylation of extra- 
cellular signal-regulated kinases (ERK) but not p38 mitogen-activated protein kinase (p38-MAPK) in vivo. In vitro, fgl2 depleted HCCLM6 cells exhibit decreased proliferation and pro-angiogenic factors expression following TNF- $\alpha$ stimulation, associated with a higher susceptibility to TNF- $\alpha$ induced apoptosis. One mechanism underlying this phenomenon that we discovered is that endogenous fgl 2 enhanced TNF- $\alpha$ induced MAPK pathway activation in HCCLM6 cells through both thrombin dependent and independent manner. However, exogenous recombinant fgl2 was found to induce phosphorylation of ERK and p38-MAPK only in thrombin dependent manner. Collectively, our data suggest that fgl2 expression in tumor is regulated by inflammatory cytokines, and that it contributes to tumor growth and tumor angiogenesis through thrombin dependent and independent activation of MAPK pathway.

\section{FC02-03}

Treatment of Tumour Recurrence after Resection of Hepatocellular Carcinoma: Does Aggressive Management Has a Role

V. Velayutham, J. Sathyanesan, S. Ramaswamy, J. Damodaran, S. Eswaran, R. Palaniappan, S. Rajagopal

Department of Surgical Gastroenterology and Liver Transplantation, Government Stanley Medical College Hospital, Chennai, India

Background and objectives: Although hepatectomy has been accepted as a therapeutic option for the primary tumor of hepatocellular carcinoma (HCC), what role the second liver resection and other modalities will play in the clinical care of patients with intrahepatic recurrence of $\mathrm{HCC}$ after the initial resection has not been well evaluated. This study aimed to assess the efficacy and safety of repeat hepatectomy and to evaluate the results of aggressive treatment of HCC recurrence for recurrent hepatocellular carcinoma (HCC).

Material and Methods: Between January 2004 and December 2009 Two hundred and twelve consecutive patients underwent hepatic resection for HCC in our hospital. Tumour recurrence was diagnosed in $98(51 \%)$ of the 193 patients with curative resection. Twenty four underwent repeat hepatic resection. Thirty patients were treated with percutaneous ablation or intra-arterial chemoembolization. Forty-four patients with a poor performance status and liver function or multiple extra hepatic recurrences did not receive any treatment. Clinical data for these patients were reviewed for operative morbidity and mortality, survival, disease-free survival, and pattern of failure.

Results: There were no operative or post-operative deaths. The cumulative overall survival after the second resection was, respectively, 90,42 and $28 \%$ at 1,3 and 5 years. There was a significant difference in survival between patients treated with repeat resection and those submitted to a non-surgical or conservative treatment $(p<0.0001)$. There were no differences in operative deaths, postoperative mortality and morbidity between the first and second hepatic resection.

Conclusion: Aggressive management with combined resection or loco regional therapy for intrahepatic recurrence may offer long-term survival in selected patients. Second liver resection for recurrence of HCC can be safely performed.

\section{FCO2-04}

Long-term Outcomes after Surgical Resection for Extrahepatic Recurrence from Hepatocellular Carcinoma

N. Takemura, K. Hasegawa, Y. Inoue, T. Ishizawa, A. Shimizu, T. Aoki, Y. Beck, Y. Sugawara, N. Kokudo

Hepato-Biliary-Pancreatic Surgery Division, Department of Surgery, Graduate School of Medicine, University of Tokyo, Tokyo, Japan
Background/aims: Extrahepatic recurrence after surgical resection of hepatocellular carcinoma (HCC) is rare, for which no effective treatment has been established. The aim of this study is to evaluate the clinical significant of resection for extrahepatic recurrence.

Methods: From 1997 to 2007, 53 patients underwent resection of extrahepatic recurrences from HCC in Tokyo University Hospital. Among them, there were 29 patients with disseminated nodules, 16 with lymph nodes metastasis, 6 with adrenal metastasis, and 2 with splenic metastasis. The long-term outcomes and prognostic factors of these patients were investigated.

Results: The 1-, 2-, and 3-year overall survival rates after resection for disseminated nodules were 64,52 , and $39 \%$, respectively. Those rates were 87,47 , and $37 \%$ for lymph nodes metastasis, 66 , 25 , and $25 \%$ for adrenal metastasis, and 50, 0, and 0 for splenic metastasis, respectively. Seven patients survived more than 5 years (5 patients with disseminated nodules, one with lymph nodes metastasis, and the other one with adrenal metastasis). The multivariate analyses show that the sites of extrahepatic recurrence did not affect the long-term outcomes. Whether the intrahepatic disease was controllable or not is the only significant prognostic factor $(P<0.001)$.

Conclusions: Surgical resection of extrahepatic recurrence of HCC may be beneficial, if the intrahepatic disease is well controlled.

\section{FC02-05}

GIDEON (Global Investigation of Therapeutic DEcisions in Hepatocellular Carcinoma and Of its Treatment with SorafeNib) Interim Results: Regional Subgroup Analysis

S.K. Yoon ${ }^{1}$, S.-L. Ye ${ }^{2}$, J. Marrero ${ }^{3}$, R. Lencioni ${ }^{4}$, A. Venook ${ }^{5}$, K. Nakajima ${ }^{6}$, M. Kudo ${ }^{7}$

${ }^{1}$ Department of Internal Medicine, The Catholic University of Korea, Seoul, Republic of Korea; ${ }^{2}$ Liver Cancer Institute, Zhongshan Hospital, Fudan University, Shanghai, China; ${ }^{3}$ Multidisciplinary Liver Tumor Clinic, University of Michigan, Ann Arbor, MI, USA; ${ }^{4}$ Division of Diagnostic Imaging and Intervention, Department of Liver Transplantation, Hepatology and Infectious Diseases, Pisa University School of Medicine, Pisa, Italy; ${ }^{5}$ University of California, San Francisco, CA, ${ }^{6}$ Global Medical Affairs, Bayer HealthCare Pharmaceuticals, Montville, NJ, USA; ${ }^{7}$ Department of Gastroenterology and Hepatology, Kinki University School of Medicine, Osaka, Japan

Background: GIDEON is an ongoing, global, prospective noninterventional study of patients with unresectable hepatocellular carcinoma (uHCC) receiving sorafenib. GIDEON provides a global database of factors that influence the management of HCC patients. The primary study objective is safety. Target accrual is 3,000 patients from $>40$ countries including 12 Asia-Pacific (AP) countries.

Methods: Demographic data and medical and treatment history are recorded at study entry. Sorafenib dosing, performance status, adverse events and liver function are recorded at follow-up. The pre-specified first interim analysis was triggered at $\geq 4$ months follow-up in 500 treated patients.

Results: Descriptive regional subgroup analyses are presented (Tables). The majority of patients in the AP region have more advanced $\mathrm{HCC}$, are younger, have $\mathrm{HBV}$ infection, and received more prior locoregional treatment. 
Conclusions: Interim analyses suggest early regional trends in patient characteristics, previous treatments for HCC and sorafenib dosing.

\begin{tabular}{|c|c|c|c|c|c|c|}
\hline $\begin{array}{l}\text { Patient } \\
\text { character- }^{\text {istics }}{ }^{\mathrm{a}}\end{array}$ & $\begin{array}{l}\text { Asia } \\
\text { Pacific, } \\
n= \\
167\end{array}$ & $\begin{array}{l}\text { Japan, } \\
n= \\
21\end{array}$ & $\begin{array}{l}\text { Europe, } \\
n= \\
143\end{array}$ & $\begin{array}{l}\text { USA, } \\
n= \\
116\end{array}$ & $\begin{array}{l}\text { Latin } \\
\text { America, } \\
n=32\end{array}$ & $\begin{array}{l}\text { Total, } \\
N= \\
479^{\mathrm{b}}\end{array}$ \\
\hline $\begin{array}{c}\text { Age, years, } \\
\text { mean } \\
\text { (range) }\end{array}$ & $55(20-86)$ & $70(57-89)$ & $67(23-88)$ & $63(31-84)$ & 65 (18-87) & $61(18-89)$ \\
\hline $\begin{array}{c}\text { ECOG PS } 1, \\
n(\%)\end{array}$ & $78(47)$ & $6(29)$ & $50(35)$ & $43(37)$ & $22(69)$ & $199(42)$ \\
\hline $\begin{array}{l}\text { CLIP score } \\
4-6, n(\%)\end{array}$ & $34(20)$ & $1(5)$ & 15 (11) & $15(13)$ & $3(9)$ & $68(14)$ \\
\hline $\begin{array}{c}\text { TNM Stage IV, } \\
n(\%)\end{array}$ & $83(50)$ & $9(43)$ & $40(28)$ & 31 (27) & $4(13)$ & 167 (35) \\
\hline $\begin{array}{c}\text { BCLC Stage C, } \\
n(\%)\end{array}$ & $123(74)$ & $5(24)$ & $73(51)$ & $43(37)$ & $9(28)$ & $253(53)$ \\
\hline $\begin{array}{l}\text { Child-Pugh } \\
\text { status } \\
\quad \mathrm{A}, n(\%)\end{array}$ & $101(61)$ & $16(76)$ & $100(70)$ & $47(41)$ & $14(44)$ & $278(58)$ \\
\hline $\begin{array}{c}\text { Hepatitis B, } \\
n(\%)\end{array}$ & $140(84)$ & $5(24)$ & 25 (18) & $23(20)$ & $1(3)$ & $194(41)$ \\
\hline $\begin{array}{c}\text { Prior TACE, } \\
n(\%)\end{array}$ & $104(62)$ & $19(91)$ & 39 (27) & 43 (37) & $7(22)$ & $212(44)$ \\
\hline
\end{tabular}

\begin{tabular}{|c|c|c|c|c|c|c|}
\hline $\begin{array}{l}\text { Sorafenib } \\
\text { safety } \\
\text { profile, } \\
n(\%)^{c}\end{array}$ & $\begin{array}{l}\text { Asia } \\
\text { Pacific, } \\
n=167\end{array}$ & $\begin{array}{l}\text { Japan, } \\
n=21\end{array}$ & $\begin{array}{l}\text { Europe, } \\
n=143\end{array}$ & $\begin{array}{l}\text { USA, } \\
n=116\end{array}$ & $\begin{array}{l}\text { Latin } \\
\text { America, } \\
n=32\end{array}$ & $\begin{array}{l}\text { Tota, } \\
N=479^{\mathrm{b}}\end{array}$ \\
\hline AEs & $139(83)$ & $21(100)$ & $123(86)$ & $111(96)$ & $21(66)$ & 415 (87) \\
\hline $\begin{array}{l}\text { Drug-related } \\
\text { AEs }\end{array}$ & $108(65)$ & $17(81)$ & $93(65)$ & $85(73)$ & $16(50)$ & 319 (67) \\
\hline SAEs & $74(44)$ & $9(43)$ & $50(35)$ & $54(47)$ & $14(44)$ & $14(44)$ \\
\hline $\begin{array}{l}\text { Drug-related } \\
\text { SAEs }\end{array}$ & $13(8)$ & $4(19)$ & 17 (12) & $13(11)$ & $4(13)$ & $51(11)$ \\
\hline $\begin{array}{l}\text { Sorafenib } \\
\quad \text { treatment } \\
\quad \text { Median } \\
\quad \text { daily } \\
\quad \text { dose, mg }\end{array}$ & 710.0 & 521.0 & 779.0 & 563.5 & 800.0 & 692.0 \\
\hline
\end{tabular}

$B C L C$ Barcelona Clinic Liver Cancer, $C L I P$ cancer of the liver Italian program, TNM tumor node metastases, $S A E$ serious adverse events

${ }^{\text {a }}$ At study entry/start of therapy

b Evaluable safety population

c Treatment emergent adverse events of all grades using NCI CTCAE, v3.0

Free Communication 03: Basic Science 14:00-15:00

\section{FC03-01}

Upregulation of PD-1 Expression on T Cells in CHB Infection is Induced by HBcAg through PI3K/AKT, ERK and JNK Activation

M. Li ${ }^{1}$, X.-H. Sun ${ }^{2}$, X.-J. Zhu' ${ }^{2}$, Y.-Q. Gao ${ }^{1}$

${ }^{1}$ Laboratory of Cellular Immunology; ${ }^{2}$ Hepatopathy, Shanghai

Shuguang Hospital Affiliated with Shanghai University of Traditional Chinese Medicine, Shanghai, China

Hyperexpression of the programmed death 1 (PD-1) molecule is a hallmark of exhausted T-cells. Previous studies showed that PD-1 expression was upregulated in chronic Hepatitis B virus (HBV) patients. How the PD-1 expression on $\mathrm{T}$ cells was upregulated in patients with chronic HBV infection remains unclear. In this study, we examined the PD-1 expression level on T cells in every phase of chronic $\mathrm{HBV}$ infection and explored the signaling pathway of PD-1 upregulation by hepatitis B core antigen ( $\mathrm{HBcAg}$ ). Seventy chronic HBV infected patients were categorized into immune tolerance group, immune clearance group and inactive virus carrier group, and 20 healthy volunteers was chosen as normal control. Peripheral blood mononuclear cells from patients and $\mathrm{T}$ lymphocytes from healthy people were separated. The mechanism of PD-1 expression induced by $\mathrm{HBcAg}$ was studied by real-time PCR, flow cytometry and westernblot. Results showed that the PD-1 expression on total CD4 ${ }^{+} \mathrm{T}$ cells in every phase of chronic HBV infection was significantly higher than that in healthy people. Such effects were not observed on total CD $8^{+} \mathrm{T}$ cells. In immune clearance phase, a positive correlation was found between serum HBVDNA level and the PD-1 expression level on total CD4 ${ }^{+} \mathrm{T}$ cells. In all phases, no correlation was shown between serum ALT level and PD- 1 expression level. The PD- 1 expression on T cells was induced by $\mathrm{HBcAg}$, which was inhibited by inhibitors of JNK, ERK and AKT, but the inhibitor of P38 didn't have that effect. The phosphorylation of JNK, ERK and AKT was induced by HBcAg. In conclusion, the PD-1 expression on total $\mathrm{CD}^{+} \mathrm{T}$ cells was upregulated in every phase of chronic HBV infection, which may be induced by $\mathrm{HBcAg}$. Moreover, JNK, ERK and AKT signaling pathways were involved in the upregulation of PD-1 induced by $\mathrm{HBcAg}$ on $\mathrm{CD} 4^{+} \mathrm{T}$ cells.

\section{FC03-02}

Analysis of Hepatitis E Virus (HEV) Binding, Entry and Replication in Hepatocytes (Huh7 Cells)

S. K. Panda, N. Kapur, S.P.K. Varma, A. Kumar, H. Durgapal Department of Pathology, All India Institute of Medical Sciences, New Delhi, India

Background: Hepatitis E virus (HEV) is a positive sense RNA virus which is the major cause of epidemic and sporadic hepatitis. The biology of HEV entry and replication are unexplored.

Methods: Virus-host interactions involving competitive binding studies were done using Ligand-linker $\left[G_{l y}{ }_{(5)}\right.$-Ala]-Reporter (EGFP/ Fluc) constructs with full-length (1-660 aa), N-terminal truncated $(\Delta$ 1-112 aa), and C-terminal truncated ( $\Delta 458-660$ aa) pORF2. Virus internalization was observed using Fluorescent virus-like particles (VLP) (in vitro assembled N-terminus 112 amino-acid deleted capsid protein (pORF2) linked to reporter (EGFP)). To understand the life cycle post entry we electroporated HEV full length replicons into Huh7 cells and quantitated virus RNA (positive- and negative-sense RNA) every 2 hours from 4 to 24 h post transfection, using strand specific quantitative real-time PCR.

Results: A single class of receptors for HEV was detected. No binding was observed when C-terminus ( $\Delta 458-660$ aa) was deleted. The fluorescent-VLPs enter the Huh7 cells 5 min post-incubation by endocytosis which was clathrin-dependent. The negative sense RNA had high copy numbers at 8 hours post transfection followed by subdued presence. The positive-sense RNA had high copy numbers at 14 hours post transfection, with higher subgenomic RNA compared to genomic RNA. There was temporal separation of negative and positive sense RNA synthesis and analysis of the subgenome copy numbers demonstrated a single subgenome. For the first time we captured replication of HEV as is happening in confocal live cell imaging using negative strand specific molecular beacons, and observed corroborative signal peaks at 8 hours post transfection.

Conclusion: Thus, we could demonstrate the entry of HEV by clathrin dependent non-caveolar endocytosis and the replication kinetics in Huh7 cells. The kinetic assay demonstrates cyclical bursts of virus replication at different time-points. These studies give significant insight into the virus biology of HEV. 


\section{FC03-03}

Packaging of Hepatitis E Virus (HEV) Genomic RNA in Trans-complemented Capsid Protein and Production of Infectious Virions

M.K. Parvez ${ }^{1},{ }^{2}$ S.U. Emerson ${ }^{1}$, R.H. Purcell ${ }^{2}$

${ }^{I}$ Molecular Hepatitis Section, Laboratory of Infectious Diseases,

NIAID, National Institutes of Health, 50 South Drive, Bethesda, MD 20892 (USA); ${ }^{2}$ Department of Pharmacognosy, King Saud University College Pharmacy, Riyadh 11451 (KSA)

Background and aim: In the absence of a robust in vitro as well as in vivo model, a recently developed HEV (sar55) genomic replicon system (pSK-GFP) has allowed to study and elucidate some important aspects of its molecular biology. However, partial swapping of capsid (ORF2)/ non-structural (ORF3) proteins by the GFP marker abolishes their expressions and hence packaging of the RNA. We therefore, intended to develop an in vitro HEV RNA packaging system and that could produce infectious virions.

Methods: A recombinant baculovirus (vBac-ORF2, titre-10E10 pfu/ $\mathrm{ml})$ ) was constructed (pSK-E2; HEVsar55-ORF3/ORF2; nt. 51307204). Expression of HEV capsid protein (pBac-ORF2) was tested in vitro (Western analysis) as well as in vBac-ORF2- transduced S10-3 cells (immune-fluorescence microscopy). In vitro transcribed (pSKGFP) RNA was transfected into S10-3 cells and viral replication was determined by GFP expression (fluorescence microscopy and FACS). Transduction was done post-transfection followed by S10-3 lysate preparation on day6. The lysates were used to infect naïve HepG2 cells and assayed for infectivity.

Results: Western analysis showed expression of expected full length ORF2 $(\sim 72 \mathrm{kDa})$ as well as its processed form $(\sim 55 \mathrm{kDa})$ in $\mathrm{S} 10-3$ cells. The FACS analysis of RNA replication showed $\sim 16 \%$ of GFP positivity in transfected S10-3 cells at day6. While HepG2 cells showed $\sim 2 \%$ positivity for GFP at day 6 post-infection, indicating production of $1 / 8$ th $(12.5 \%)$ of stable infectious particles. In accordance with our fluorescence microscope observations, naïve cells infected with lysates from moc-transfected, RNA-transfected or BacORF2-transduced cells scored negative for GFP. We however, did not detect any infectious particles in the culture media.

\section{Conclusions:}

1. Our results, for the first time, show that the HEV capsid overexpressed in mammalian system is biologically active and efficently encapsidtes HEV RNA to produce stable infectious virions.

2. This valuable system could be further used to study many aspects of HEV biology.

\section{FC03-04}

Hepatic Necrosis-induced Renal Failure: A Molecular Study

J. Schwartz ${ }^{1}$, M. Firpo ${ }^{2}$, E. Hillas ${ }^{1}$, N. Takahashi ${ }^{3}$, R. Weiss ${ }^{4}$, D. Dunn ${ }^{4}$,

J. Judkins ${ }^{1}$, X. Chen ${ }^{5}$, F. Clayton ${ }^{5}$, C. Canale ${ }^{6}$, W. Hutson ${ }^{7}$, J. Sorensen ${ }^{1}$,

A. Rohrwasser ${ }^{4}$, J.-M. Lalouel ${ }^{4}$

${ }^{1}$ General Surgery, Section of Transplantation; ${ }^{2}$ General Surgery, University of Utah School of Medicine, Salt Lake City, UT;

${ }^{3}$ Pathology and Laboratory Medicine, University of North Carolina, Chapel Hill, NC; ${ }^{4}$ Human Genetics, ${ }^{5}$ Pathology, University of Utah School of Medicine, Salt Lake City; ${ }^{6}$ Mountain West

Gastroenterology, Bountiful, ${ }^{7}$ Internal Medicine, University of Utah School of Medicine, Salt Lake City, UT, USA

Background: Renal dysfunction in the setting of acute liver failure is associated with an increased risk of death. However, studies examining the renal response to ALF are rare in the literature. As such, we present a comprehensive assessment of the differentiallyregulated renal transcriptome in the setting of massive hepatic necrosis (MHN).

Methods: C57BL6 mice received the select hepatotoxin D-(+)galactosamine $\mathrm{HCl}(\mathrm{GalN})$. Serum alanine (ALT) and aspartate (AST) levels were used to confirm hepatocellular injury. The liver and kidney were examined by light and electron microscopy. Wireless telemetry catheters were implanted intracorporeally to measure systemic and mesateric hemodynamics. Deterioration in GFR was confirmed using two independent methodologies. The renal transcriptome was analyzed using the Affymetrix ${ }^{\mathrm{M}} 4302.0$ gene chip array and perturbations measured using rank product analysis. Relative expression was confirmed using RT-qPCR and the $\Delta \Delta \mathrm{C} t$ method. Results: AST and ALT levels were markedly elevated. Histopathological study of the kidney was normal in the setting of MHN.. Serum creatinine was significantly increased. Urine output decreased by $50 \%$. Calculated and measured GFR decreased by $50 \%$ and $80 \%$, respectively. A decrease in heart rate and mean arterial pressures were noted, while portal pressures were elevated. A representative sample of differentially expressed renal transcripts are depicted below (Figure).

\begin{tabular}{|c|c|c|c|c|c|c|c|}
\hline o.1: & 60110 & crienate & Forot & UEFostay & UkFoultay & RTACR & NTACE \\
\hline Aos 14 & 50700 & $\begin{array}{l}\text { Aoyl CoA } \\
\text { synthetase } \\
\text { long-ohasin themily }\end{array}$ & $\begin{array}{l}\text { Catainio } \\
\text { aetivity } \\
\text { metabolic } \\
\text { petasess }\end{array}$ & $0.043^{\circ}$ & $0.880^{\circ}$ & 0.130 & 0.138 \\
\hline ADOal & 11800 & $\begin{array}{l}\text { member } 4 \\
\text { Appolip op rote in } \\
\text { A.1 }\end{array}$ & $\begin{array}{l}\text { prooesss } \\
\text { Cholesterol } \\
\text { transport } \\
\text { trationt }\end{array}$ & $0.700^{\circ}$ & NS & $1.274^{\circ}$ & 0.850 \\
\hline Biros & 11700 & $\begin{array}{l}\text { Baculo viral IAP. } \\
\text { repeat } \\
\text { contanining S }\end{array}$ & 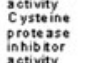 & $.0 .704^{*}$ & $1.270^{\circ}$ & $.2 .474^{*}$ & $.1 .252^{\circ}$ \\
\hline Abeal 1 & 11303 & $\begin{array}{l}\text { AT P-binf ding } \\
\text { osssetre } \\
\text { sub-tamily A }\end{array}$ & $\begin{array}{l}\text { ATP } \\
\text { binding }\end{array}$ & $0.751^{\circ}$ & 0.967. & 0.032 & $0.964^{\circ}$ \\
\hline $\operatorname{Len} 2$ & 10810 & Lipocatin 2 & $\begin{array}{l}\text { Transpenter } \\
\text { activity }\end{array}$ & $3.111^{\circ}$ & $1.307 \%$ & $2.910^{\circ}$ & 0.020 \\
\hline Apoe & 11818 & Apolipoprotein e & $\begin{array}{l}\text { Cholesterol } \\
\text { transporter } \\
\text { activing }\end{array}$ & $0.585^{\circ}$ & $1.281^{\circ}$ & 0.614 & 0.051 \\
\hline Insig 1 & 231070 & $\begin{array}{l}\text { lossuln induced } \\
\text { pene } 1\end{array}$ & $\begin{array}{l}\text { Cholesterol } \\
\text { metab olic } \\
\text { process }\end{array}$ & $.0 .032^{\circ}$ & NS & $.0 .802^{\circ}$ & $.0 .043^{*}$ \\
\hline Nphs2 & 170484 & $\begin{array}{l}\text { Nephrosis } 2 . \\
\text { hom olog. }\end{array}$ & $\begin{array}{l}\text { Prote in } \\
\text { binding }\end{array}$ & $0.050^{\circ}$ & $0.546^{\circ}$ & $.0 .578^{*}$ & $.0 .590^{\circ}$ \\
\hline Fasn & 14104 & $\begin{array}{l}\text { Fatryald } \\
\text { synthase }\end{array}$ & $\begin{array}{l}\text { F atty aoid } \\
\text { synth ase }\end{array}$ & $0.081^{*}$ & .0 .708 & .2 .34 & .0 .434 \\
\hline Hk1 & 15276 & Hexokinase 1 & $\begin{array}{l}\text { Hexokin ase } \\
\text { Heting }\end{array}$ & $0.630^{\circ}$ & NS & $0.401^{*}$ & .0 .106 \\
\hline Aqp 4 & 11829 & Aquaporin 4 & Transport & $0.783^{\circ}$ & 0.901 & $2882^{\circ}$ & .0 .218 \\
\hline Pparg & 10010 & $\begin{array}{l}\text { Peroxisome } \\
\text { proliferatoror } \\
\text { activated } \\
\text { receptor } \\
\text { gammana }\end{array}$ & $\begin{array}{l}\text { ONA } \\
\text { binding }\end{array}$ & $.0 .035^{\circ}$ & NS & 0.004 & 0.454 \\
\hline
\end{tabular}

Each value in the mieroarray experiments represent the fold ehange (in logz value) vs. the non.tieated control (" $P<0.05$ us ing ranked produot analysis) at 24 hours. F or RT.PCR's. trip licate experiments were pertormed on each or 3 separate tissue samples to obtain the mean mRNA expression level for a partioular tissue. These means were normalized a gainst the mean expression of the hous ekeeping gene GAPOH for thatparticular tissue, with results expressed as the ratio of gene:GAPOH expression

Representative mouse renal gene expression levels

Conclusions: Mice receiving GalN demonstrate simultaneous liver and kidney injury. Amongst others, energy, cell cycle regulation, and cellulare dealth pathways were altered in the kidney. These finding may provide insight into a common molecular profile central to the acute kidney injury developing in the setting of MHN.

\section{FC03-05}

Inhibition of Tissue Inhibitor of Metalloproteinase-1 by Recombinant Adeno-Associated Viruses Carrying siRNA Prevents Liver Fibrosis in Rats M. Cong, T. Liu, P. Wang, H. You, J. Jia

Beijing Friendship Hospital, Capital Medical University, Beijing, China

Background/aims: Elevated tissue inhibitor of metalloproteinase (TIMP)-1 expression contributes to excess production of extracellular matrix in liver fibrosis. The present study constructed a recombinant adeno-associated virus (AAV) carrying siRNA of TIMP-1 and investigated the effects of administration of this recombinant $\mathrm{AAV}$ in carbon tetrachloride $\left(\mathrm{CCl}_{4}\right)$-induced rat liver fibrosis model.

Methods: An siRNA which had the strongest suppression effect was cloned into the AAV vector and constructed the AAV/siRNA-TIMP1. Rats were randomly divided into olive oil control group, $\mathrm{CCl}_{4}$ treated group, AAV/EGFP administration group and recombinant AAV/siRNA-TIMP-1 administration group (10 rats/group), which were administered the recombinant AAV at the end of 1st week and 2nd week after $\mathrm{CCl}_{4}$-treatment. At the 4 th weekend, the rats were sacrificed for detection of histological changes of liver tissue, the concentration of type I and type III collagen in serum, liver tissue 
hydroxyproline content, expression of TIMP-1, MMP2, MMP13, $\alpha$ SMA and TGF- $\beta$.

Results: Accomopanied by decreased TIMP-1 expression $(55 \%$ reduction) of liver tissue in AAV/siRNA-TIMP-1 group, AAV/siRNA-TIMP-1 administration attenuated fibrosis severity, as determined by fibrosis scores $(3.8 \pm 0.8)$ compared with the $\mathrm{CCl}_{4}$-treated group $(2.5 \pm 0.7)(P=0.001)$. Compared with $\mathrm{CCl}_{4}$-treated group, the concentration of type I and type III collagen in serum, the hydroxyproline content of liver tissue in AAV/siRNA-TIMP-1 group was decreased by $35-50 \%$. Western bolt showed AAV/siRNA-TIMP-1 treatment decreased the expression of active-MMP2 and increased the expression of active-MMP13. Furthermore, administration of AAV/ siRNA-TIMP-1 resulted in a marked decrease in $\alpha$-SMA and TGF- $\beta$ staining of histological section confirmed by the detection of mRNA and protein level of these two markers in liver tissue.

Conclusions: Administration of AAV/siRNA-TIMP-1 attenuated $\mathrm{CCl}_{4}$-induced rat liver fibrosis by suppressing the expression of TIMP-1 which directly elevating the function of MMP13, and indirectly decreasing expression of Col I, MMP2 and TGF- $\beta 1$, which were potent factors in liver fibrogenesis.

\section{Free Communication 04: Miscellaneous} 14:00-15:00

\section{FC04-01}

The Safety and Efficacy of Ultrasonography-guided Percutaneous Liver Biopsy Using Coaxial Technique: Results of a Randomized, Double-blind, Controlled Trial

S.Y. Park, W.Y. Tak, Y.O. Kweon, M.K. Jung, S.W. Jeon, C.M. Cho, S.K. Kim, H.G. Park, D.W. Lee, H.S. Lee, J. Heo, K.Y. Shin

Gastroenterology and Hepatology, Kyungpook National University Hospital, Daegu, Republic of Korea

Background and aims: Main complications of liver biopsy are pain and hemorrhage which are increased in relation to the number of needle punctures. Coaxial biopsy technique has advantage of only one puncture into liver by placing a catheter into liver. We evaluated the efficacy and safety of this technique by comparing incidence and severity of adverse events.

Methods: We enrolled 212 consecutive patients (age $41.9 \pm 14.1$, male:female 133:79), undergoing liver biopsy. Patients were randomized into coaxial biopsy group or conventional biopsy group. Biopsies were performed using 16 gauge automated biopsy needle under ultrasound guidance. Pain was assessed every 30 minutes using a visual analogue scale (VAS) with vital sign monitoring. Results: One hundred seven patients were randomized to coaxial biopsy group and 105 to conventional biopsy group. There were no significant differences in baseline characteristics between two groups. The number of puncture was one in coaxial biopsy group and $4.9 \pm 0.6$ in conventional biopsy group. The most common painful or discomfort area was right upper quadrant abdomen (178 patients, $84.0 \%$ ), followed by right shoulder ( 9 patients, $4.2 \%$ ), epigastrium ( 2 patients, $0.9 \%)$. Twenty three $(10.8 \%)$ had no pain. The presence of pain is higher (86.9 vs. $89.5 \%, p=0.671)$ and requirement of analgesics lower (22.4 vs. $27.6 \%, p=0.429)$ in coaxial biopsy group. The degree of post-procedure pain were significantly different between coaxial biopsy group and conventional biopsy group; immediate after biopsy $(26.8 \pm 20.8$ vs. $38.6 \pm 22.2, p<0.001), 30$ $\min (24.8 \pm 20.0$ vs. $30.2 \pm 24.8, p=0.043), 60 \min (17.6 \pm 18.0$ vs. $19.1 \pm 16.5, p=0.542), 90 \min (14.1 \pm 15.9$ vs. $13.3 \pm 12.7$, $p=0.709)$ and $120 \min (12.5 \pm 16.2$ vs. $9.9 \pm 11.1, p=0.172)$. There were no significant differences in other adverse events including dizziness, nausea, and hypotension. There was one serious adverse events of peritoneal bleeding in each group.
Conclusion: Coaxial biopsy technique reduces the incidence and severity of pain compared with conventional multiple-puncture technique.

\section{FC04-02}

Classification of Small Atypical Nodules in Cirrhosis According to Hepatobiliary-Phase of Gd-EOB-DTPA-Enhanced-MRI: Is Hypointensity an Early Marker of Malignant Transformation?

M. Renzulli ${ }^{1}$, E. Orlando ${ }^{2}$, V. Lucidi ${ }^{1}$, B. Corcioni ${ }^{1}$, L. Grazioli ${ }^{2}$, R. Golfieri ${ }^{1}$ ${ }^{1}$ Radiology Unit, Department of Digestive Diseases and Internal Medicine, Sant' Orsola-Malpighi Hospital, University of Bologna, Bologna, Italy, Bologna; ${ }^{2}$ Radiology Unit, Spedali-Riuniti Brescia, Brescia, Italy

Background/aim: Liver nodules with atypical vascular pattern according to AASLD-criteria were classified based on their appearance in the hepatobiliary-phase (HB) of Gd-EOB-DTPA-enhanced-MRI and the results were compared to histology of surgical or biopsy specimens. Methods: We evaluated 77 consecutive MRI performed between 2007 and 2009 in cirrhotic patients bearing 111 nodules with atypical dynamic pattern (absence of arterial hypervascularity or portal-venous washout). The nodules were divided into 2 classes based on arterial vascularity: Iisovascular ( $\mathrm{n}=82$ nodules in 56 patients), II-hypervascular without portalvenous washout ( $\mathrm{n}=29$ nodules in 21 patients). The two classes were further classified into 3 subcategories, according to HB intensity: Ahypointense, B-isointense and C-hyperintense and compared to histology. Results: Histology confirmed 60 benign lesions $[n=25$ regenerative nodules (RN), $n=27$ low grade dysplastic nodules (LGDN), $n=8$ large regenerative nodules (LRN)] and 51 malignant or pre-malignant nodules [ 10 overt hepatocellular carcinomas, $n=41$ high grade dysplastic nodules (HGDN)/early hepatocellular carcinomas (HCC)].

Class IA $(n=33)$ included 31 HGDN/early HCCs $(94 \%)$ and 2 LGDN $(6 \%)$.

Class IB $(n=35)$ comprised 20 RNs (57\%), 14 LGDN (40\%) and only 1 HCC $(3 \%)$.

Class IC $(n=14)$ included only benign lesions: 11 LGDN (78.5\%) and 3 NRH $(21.5 \%)$.

Class IIA $(n=14)$ comprised 6 HGDN/early HCC and 8 overt HCCs.

Class IIB $(n=8)$ included 5 RN (62.5\%), 3 HGDN/ HCC (37.5\%).

Class IIC $(n=7)$ comprised 5 LRN $(71.5 \%)$ and 2 overt HCC $(28.5 \%)$

Conclusions: Classes A recognized the bulk (45/51: 96.7\%) of the high-risk nodules, regardless of their arterial phase behaviour. By converse, classes $\mathrm{B}$ and $\mathrm{C}$ identified the majority of benign lesions (58/ 60: 96.6\%). Hypointensity in HB of Gd-EOB-DTPA-enhanced-MRI is crucial in differentiating low-risk nodules from pre-malignant nodules, since it has $85 \%$ sensitivity for the detection of HGDN/early HCC, $94 \%$ specificity, $91 \%$ NPV, $89 \%$ PPV and diagnostic accuracy of $90 \%$.

\section{FC04-03}

Assessment Hepatic Lymph Vessels and $V_{X_{2}}$ Tumor Lymphangiogenesis with Percutaneous Transhepatic Lymphosonography with SonoVue ${ }^{\circledR}$ in vivo

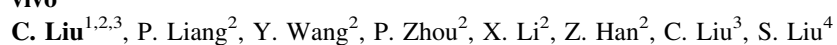
${ }^{1}$ Jinan Central Hospital, Shandong University, Jinan; ${ }^{2}$ Interventional Ultrasound, Chinese PLA General Hospital, Beijing; ${ }^{3}$ School of Control Science and Engineering, Shandong University; ${ }^{4}$ Qilu Hospital, Shandong University, Jinan, China

Purpose: To evaluate the feasibility and efficacy of percutaneous transhepatic lymphosonography (PTL) as a novel method for the detection of hepatic lymph vessels and VX2 tumor lymphangiogenesis of rabbits in vivo.

Methods and materials: Twenty health rabbits and ten rabbits injected with $\mathrm{VX}_{2}$ tumors were studied. A total of $0.5 \mathrm{~mL}$ of Sononvue was injected percutaneous transhepatic into the normal liver parenchyma for PTL. Images and/or movie clips were stored for further analysis. 
Results: The hepatic lymph vessels were visualized immediately after injection of contrast medium and continuously visualized with SonoVue ${ }^{\circledR}$ during PTL. Most of lymph vessels could be seen draining along the portal veins and bile ducts to the liver hilum and appeared around the abdominal aorta. The boundaries of VX2 tumors were hyperechoic relative to liver parenchyma and the tumors. There was a significant difference in the values of the boundaries of VX2 tumors after injection compared with the liver normal parenchyma and the tumor parenchyma during PTL.

Conclusion: PTL can be a novel method for the detection of hepatic lymph vessels and VX2 tumor lymphangiogenesis, PTL can improve the diagnostic ability of liver cancer.

\section{FC04-04}

Serum Vitamin D Concentrations and Unexplained Elevation in ALT among US Adults

S. Liangpunsakul ${ }^{1}$, N. Chalasani ${ }^{2}$

${ }^{I}$ Medicine; ${ }^{2}$ Indiana University School of Medicine, Indianapolis, IN, USA

Background: Low serum levels of vitamin D have recently been shown to be associated with cardiovascular risk and metabolic syndrome. Participants in NHANES III with unexplained elevation in ALT levels have high prevalence of metabolic syndrome. We hypothesized that the serum concentrations of vitamin D were inversely associated with unexplained elevation in ALT. The relationship between serum vitamin D concentrations and unexplained elevation in ALT was analyzed using NHANESIII dataset.

Methods: A total of 6,815 fasting subjects underwent morning physical examination. From these participants, we have constructed cases of subjects with unexplained elevation in $\operatorname{ALT}(n=308)$ and compared their serum vitamin D concentrations to matched controls without liver disease $(N=979)$. Further, we examined the prevalence of unexplained elevation in ALT level across different quartiles of vitamin D levels. The strength of association between serum vitamin D concentrations and unexplained elevation in ALT was determined using multiple regression analyses, after controlling for relevant co-variates. Results: Patients with unexplained elevation in ALT had significantly lower serum vitamin D levels compared to controls $(61.8 \pm 26.0$ vs. $66.8 \pm 27.1 \mathrm{nmol} / \mathrm{L}, p<0.01)$. The unadjusted prevalence of unexplained elevation in ALT in patients with highest to lowest quartiles of serum vitamin D levels were $21.4,21.4,25.6$, and $31.5 \%$, respectively. Compared to lowest quartile, patients with top two quartiles of serum vitamin D levels had significantly lower prevalence of unexplained elevation in ALT (OR, 95\% CI for highest quartile 0.62 [0.43-0.89] and for $3^{\text {rd }}$ quartile 0.61 [0.42-0.86]. This relationship persisted even after controlling for metabolic syndrome or insulin resistance.

Conclusions: This study suggests a significant inverse relationship between serum vitamin D levels and unexplained elevation in ALT. Further studies are needed to confirm this observation and to understand the basis for and implications of this observation.

\section{FC04-05}

Study the Associative Role of Genetic Alterations of BER Pathway Genes in Liver Disease Susceptibility in Northeast India

M. Bose, S. Bose, B. Baruah, A. Saikia, R. Deblakshmi, M. Deka Biotechnology, Gauhati University, Guwahati, India

Northeast India have high incidences of liver disease of different underlying etiology. Hepatitis infection induced oxidative stress and polymorphism in BER pathway genes may predispose patients toward liver disease susceptibility; with no data available from ethnically distinct Northeast India (NEI).

Aim: Study the associative role of polymo-rphism in BER genes hOGG1ser326cys and XRCC1codon399; and oxidative stress by virtue of $8-\mathrm{OH}-\mathrm{dG}$ status in plasma, in liver disease susceptibility.
Method: 170 liver disease patients of different viral, alcoholic and cryptogenic etiology admitted in Central Railway hospital, Guwahati; and 170 age-sex matched community controls were enrolled for the study with informed consent. hOGG1ser326cys and XRCC1codon399 gene polymorphism was studied by PCR-RFLP method after isolating DNA from whole blood. Oxidative stress was measured in plasma using 8-OH-dG ELISA kit.

Result: A statistically significant higher distribution of variant alleles was observed in patients compared to controls for both the genes \{hOGGl (patients:controls: 75.29:57.06\%, $p<0.001$ ), and XRCC1codon399 (patients:controls: 65.29:55.29\%, $p=0.06$ ) . Liver disease risk increased with the presence of mutant alleles of hOGGl $(\mathrm{OR}=2.294)$ and $\mathrm{XRCC} 1(\mathrm{OR}=1.521)$. Alarmingly higher concentration of $8-\mathrm{OH}-\mathrm{dG}$ was found in the plasma of both patients $(474.34 \pm 207.54 \mathrm{pg} / \mathrm{ml})$ and controls $(327.625 \pm 194.873 \mathrm{pg} / \mathrm{ml})$ compared to the recommended levels of $4-21 \mathrm{pg} / \mathrm{ml}$. Among the patient group 8-OH-dG concentration was highest in HAV cases and was significant for alcoholic liver disease patients $(p<0.03)$.

Conclusion: High prevalence of mutated genotype of BER pathway genes in NEI, an indicator of malfunctioning of DNA repair mechanism plays a crucial role in liver disease susceptibility. High concentration of 8-oxoG and high percentage of hOGG1 mutation are the direct indication of DNA damage and oxidative stress related liver damage in NEI population.

\section{APASL-AASLD Joint Symposium: Difference in Perspectives of Liver Diseases between Asia-Pacific and Western Region \\ 15:00-17:15}

\section{AS-01}

Differences in Perspective of Chronic Hepatitis B from Asia-Pacific Region J.-H. Kao ${ }^{1,2}$

${ }^{1}$ Graduate Institute of Clinical Medicine, National Taiwan University College of Medicine; ${ }^{2}$ Hepatitis Research Center, Natioanl Taiwan University Hospital, Taipei, Taiwan ROC

Hepatitis B virus (HBV) is a global health problem. HBV infection can cause significant morbidity and mortality, including endstage liver disease and HCC. HBV infection is endemic in Asia where the infection mostly occurs in perinatal period or early childhood. However, HBV infection is less common in Western countries where the infection is contracted after childhood through horizontal transmission. Asian HBV carriers usually run a prolonged immune tolerance phase compared to Western patients. At least $8 \mathrm{HBV}$ genotypes are identified. Genotypes A and D occur frequently in the West, while genotypes B and C are prevalent in Asia. HBV genotypes can influence the natural course of liver disease. Genotype B patients have an early and frequent $\mathrm{HBeAg}$ seroconversion than genotype $\mathrm{C}$ patients, and are associated with less progressive disease. Genotype D has a less favorable prognosis than genotype A. The difference in age of HBV infection and distribution of HBV genotypes may partly explain the different clinical course and treatment response to immunomodulatory agents between Asian and Western patients. Several regional guidelines (APASL, AASLD and EASL) for the management of chronic hepatitis B have been released. Although the indications to start anti-HBV therapy are similar, the therapeutic endpoints are somewhat different between Asian and Western guidelines, especially for $\mathrm{HBeAg}$-negative chronic hepatitis B. Regarding the prevention of $\mathrm{HBV}$, the primary goal of hepatitis $\mathrm{B}$ vaccination in Asia is to control chronic HBV infection in early childhood and prevent development of liver complications in late adulthood. In contrast, the main goal of hepatitis B vaccination in 
Western countries is to prevent acute hepatitis B in young adults. In conclusion, the implementation of universal hepatitis B immunization, interruption of possible transmission routes and wide use of antiviral treatments shall lead to the global control and even eradication of $\mathrm{HBV}$ in the foreseeable future.

\section{AS-02}

Management of Hepatitis B from a Western Perspective E.R. Schiff

Center for Liver Diseases, University of Miami, Miller School of Medicine, Miami, FL, USA

Most westerners develop chronic hepatitis B following viral exposure as an adult. The incidence is decreasing as more of the population is vaccinated. Acute icteric hepatitis B is usually not treated with antiviral therapy but there is some data suggesting the duration of this illness may be shortened by this therapy. Those with chronic hepatitis are typically immunoreactive and management is influenced by treatment guidelines. EASL guidelines are the most practical. Patients with HBV DNA $>2,000$ IU $\mathrm{ml}$ and ALT $>$ ULN whether HBeAg+ or - should be considered for therapy. Stopping rules with subsequent durability, other than HBsAg seroconversion, are debatable. The demographics of HBV patients has changed in western countries with immigration from endemic areas. All guidelines including APASL recommend not treating immunotolerant patients. Nevertheless a subset of these patients will undergo malignant transformation at a molecular level negating subsequent antiviral treatment for the prevention of hepatocellular carcinoma. Screening for HCC is variable and early detection is accomplished in less than a third of cases. How to recognize those immunotolerant patients where early treatment might prevent the evolution of HCC is an important focus of basic research. The previous concerns of resistant mutation and safety issues with long-term nucleoside/nucleotide therapy have not come to fruition. However cost and compliance are problematic. Treatment during pregnancy remains controversial. Implementation of proplylactic antiviral therapy prior to cancer chemotherapy has been more difficult because of reluctance by oncologists.

\section{AS-03}

Difference in perspective of NAFLD from Asia-Pacific region G. Farrell

\section{AS-04}

NAFLD: A Western Perspective

A.J. Sanyal ${ }^{1}$, K. Promrat ${ }^{2}$

${ }^{1}$ Department of Internal Medicine/Division of Gastroenterology, Hepatology and Nutrition, Virgin Commonwealth University Medical Center, Richmond, VA, USA; ${ }^{2}$ Gastroenterology Section, Providence VA Medical Center, Providence, RI, USA

NAFLD is a major public health problem both in the Western world and in the East. In the United States, it is estimated that about a third of the general population has NAFLD, based on population-based studies using MR spectroscopy. Autopsy studies, both in children and in adults, indicate that NAFLD is common and NASH is present in $2-5 \%$ of the population. Increasing age, obesity and the presence of multiple features of the metabolic syndrome especially diabetes are associated with a higher probability of having NASH. Conversely, NAFLD increases the risk of developing diabetes, hypertension and cardiovascular events as well as mortality. In addition, there is an increased risk of death from a variety of non-hepatocellular cancers. From a liver perspective, NAFLD is associated with a $15-20 \%$ risk of progression to cirrhosis. The disease progresses more rapidly in those with diabetes, increasing age and obesity. The PNPLA3 gene mutation at position 148 is associated with not only steatosis, but with the likelihood of having steatohepatitis and increased inflammation and fibrosis. Once cirrhosis develops, the liver disease decompensates at the rate of $3-4 \%$ per year. NASH related cirrhosis is a risk factor for hepatiocellular cancer. Recently several reports have found hepatocellular cancer with no risk factors other than fatty liver. All of these factors indicate that NAFLD is a common condition that has seignificant adverse health consequences for those who are afflicted. It is therefore a major public health hazard throughout the world.

\section{AS-05}

The Unique Feature of Autoimmune Hepatitis in Asia-Pacific M. Zeniya

Gastroenterology, Jikei University Graduate School of Medicine, Tokyo, Japan

Autoimmune Hepatitis (AIH) is an organ-specific disease characterized by chronic inflammation of liver with autoimmune phenomenon. The geographic incidence of AIH varies considerably, and in Asia involving Japan where hepatitis virus infection rate is high, AIH is rather rare. In Japan, so called juvenile cirrhosis due to AIH is rare. In Caucasoid HLA A1-B8-DR3 association was most prominent in AIH especially in young aged patients. In Japan young AIH is few and over 50 years old are most prevalent. The recent nationwide study in Japan revealed that the median age of AIH has become older. HLA DR4 was most prominent and DR3 is nearly absent in Japan. Interestingly, DR4 is a second genetic susceptible factor for AIH of older age DR3 negative Caucasoid $\mathrm{AIH}$, indicating AIH has two susceptible HLA. There are clinical differences between DR3 and DR4 positive AIH. DR3 positive AIH is younger, more therapy-resistant, high relapse rate and poor prognosis. On the other hand, DR4 positive AIH showed milder disease and respond well to corticosteroid therapy. ANA was most frequently recognized autoantibody of AIH in Japan and LKM positive type 2 AIH is rare. It might be related to the difference of genetical background. The recent nationwide study revealed that the percentages of $\mathrm{AIH}$ patients with lower titer of ANA and/or serum IgG were increased. Acute liver failure due to AIH has been reported. Acute onset or severe type of AIH showed lower titer of ANA or serum IgG. These findings suggested that early proper diagnosis and therapy of such atypical type of AIH are very important. Hepatitis C virus infected AIH has been reported. Interferon therapy originally prohibited in AIH because interferon might augment immune response resulting deterioration of AIH. However, careful interferon administration can be applied and sustained virological response is possible in Japanese patient.

\section{AS-06}

\section{Autoimmune Liver Diseases}

K. Lindor

Gastroenterology and Hepatology, Mayo Clinic, Rochester, MN, USA

Autoimmune hepatitis (AIH), primary biliary cirrhosis (PBC) and primary sclerosing cholangitis (PSC) are three distinct autoimmune liver diseases. Immunoglobulin G4 (IgG4) associated cholangitis is another immune disorder which mimics PSC and is characterized by formation of biliary strictures responsive to steroid therapy. The simultaneous or successive coexistence of PBC and AIH or PSC and AIH is called overlap syndrome. AIH is a chronic inflammatory immune-mediated liver disease characterized by elevated transaminase levels, hypergammaglobulinemia and histological features of interface hepatitis. AIH is treated with corticosteroids alone or in combination with azathioprine and both strategies are equally effective. PBC and PSC are both chronic cholestatic liver diseases with slowly progressive courses. The diagnostic criteria for PBC include serum alkaline phosphatase levels at least twice the upper limit of normal and a positive test for serum antimitochondrial antibodies. A liver biopsy specimen showing inflammatory changes involving the bile duct supports the diagnosis but is not mandatory. Ursodeoxycholic acid is the only FDA-approved therapy for PBC in a dose of $13-15 \mathrm{mg} / \mathrm{kg} / \mathrm{day}$. PSC is most commonly diagnosed with endoscopic retrograde cholangiopancreatography (ERCP), although magnetic resonance cholangiography (MRC) is rapidly emerging as the first-choice diagnostic test. There is no effective 
medical treatment for PSC and liver transplantation is the only life extending therapy for end-stage disease. IgG4 associated cholangitis is an immune disorder characterized by formation of biliary strictures and frequently involves extrahepatic bile ducts. The serum level of IgG4 is usually elevated and there is infiltration of IgG4 positive plasma cells in bile ducts. The serum level of IgG4 is usually elevated and there is infiltration of IgG4 positive plasma cells in bile ducts. Clinically patients present with abrupt onset jaundice which responds to steroid therapy. Pathogenesis and standard diagnostic criteria for the overlap syndrome have not yet been established.

\section{AS-07}

Differences in Perspective of Portal Hypertension from Asia-Pacific Region

S.K. Sarin

Department of Gastroenterology and Hepatology, Institute of Liver and Biliary Sciences, New Delhi, India

In the Asian region, the common causes of portal hypertension (PHT) include cirrhotic causes (hepatitis B and C) and non-cirrhotic portal hypertension (NCPH). While the profile of cirrhosis due to hepatits $\mathrm{B}$ and $\mathrm{C}$ in the asian region is same as that in the West, the proportion of non-cirrhotic population is high and distinct. The two common causes of NCPH include, non-cirrhotic portal fibrosis (NCPF) or Idiopathic Portal Hypertension (IPH) and extrahepatic portal vein obstruction (EHPVO). NCPF/IPH is a disease of uncertain etiology characterized by periportal fibrosis and involvement of small and medium branches of the portal vein. The liver functions and structure primarily remain normal. NCPF/IPH is an important cause of portal hypertension. About $70 \%$ present with UGI bleeding, splenomegaly and anemia. Patients have a longstanding history of mass in left hypochondrium and often hypersplenism. Signs of chronic liver disease are rare and prognosis is good. Liver functions are near normal. Gastric varices are seen in nearly $1 / 3$ cases. Hepatic venous pressure gradient (HVPG) is normal or near normal in NCPF. EHPVO is defined by obstruction of the extra-hepatic portal vein with or without involvement of the intra-hepatic portal veins. EHPVO could present as a recent attack, asymptomatic or symptomatic (severe non-colicky abdominal pain, distension, systemic inflammatory response syndrome or fever). It could also present as chronic EHPVO; with portal cavernoma and variceal bleed, splenomegaly, abnormal blood cell counts and growth retardation. Recent EHPVO rarely resolves spontaneously. Low molecular weight heparin should be started immediately followed by oral anticoagulant therapy; and given for at least 3 months. Rex bypass is useful in children with chronic EHPVO. Portal biliopathy is present in nearly all patients, and if symptomatic, is treated by sphincterotomy with stone extraction and stenting if with bile duct strictures. If possible, porto-systemic shunt surgery should be considered.

\section{AS-08}

Differences in Perspective of Portal Hypertension from Western world G. Garcia-Tsao

APASL-EASL Joint Symposium: New Era of Diagnosis and Management of Hepatocellular Carcinoma 15:00-17:15

\section{$\mathrm{AE}-01$}

New Diagnostic Tests for Hepatocellular Carcinoma Including Imaging

Modality

M. Omata

\section{$\mathrm{AE}-02$}

Prevention of Hepatocellular Carcioma

H. Wedemeyer
AE-03

Medical Ablation and Surgical Resection for Hepatocellular Carcinoma in Japan

R. Tateishi

Department of Gastroenterology, Graduate School of Medicine, The University of Tokyo, Tokyo, Japan

Japan encountered rapid increase of patients with hepatocellular carcinoma (HCC) in 1980s due to nationwide spread of chronic hepatitis C after World War II. The situation advanced surveillance, diagnostic procedures and treatment techniques for HCC including resection, ablation and transarterial embolization in Japan earlier than in other developed countries. Surveillance for HCC in chronic hepatitis is fully covered by national health insurance, which enables detection of tumor $<3 \mathrm{~cm}$ in $57 \%$ of patients in 2005 and 2006 according to national survey. Surgical resection procedures were highly established in 1990's and the mortality rate is now $<1 \%$ in high volume centers. Ethanol injection was replaced by radiofrequency ablation, which is applied to nearly $30 \%$ of naïve patients. Five-year survival rates after ablation in leading hospitals reached $70 \%$. Unlike other countries, surgical resection is indicated in cases with multiple nodules or portal hypertension in Japan. Thus there was much debate as to which treatment should be the treatment of choice for small HCC. To answer the question, nationwide randomized controlled trial was launched. In terms of long-term survival, liver transplantation is undoubtedly ideal strategy for HCC patients with chronic liver diseases. Japan is far disadvantaged in that field because of shortage of cadaveric donors. In addition to that we have serious problem of aging in hepatitis C-related patients. However our strategy including repeat resection or ablation in combination with anti-viral treatments will surely be a good reference for other counties.

\section{AE-04}

Molecular Targeted Therapy for Current and Future Approach

J.-F. Dufour

The treatment of hepatocellular carcinoma is currently after years of stagnation revolutionized. Intense research led to the development of multiple drugs blocking enzymes in signalling pathways. This new mechanism of action proved to be a formidable breakthrough in oncology. If patients affected with other tumour types profited already of these drugs, patients with hepatocellular carcinoma had to wait longer. This is due to the complexity of hepatocellular carcinoma. Hepatocellular carcinoma can be treated by other approaches, hepatocellular carcinoma arises most of the time in a cirrhotic liver and, finally, as it becomes more and more evident, that hepatocellular carcinoma is a heterogeneous cancer. Two large phase III studies with an exemplary design permitted to capture the beneficial effect of a multi-kinase inhibitor, sorafenib on the survival of patients diagnosed with hepatocellular carcinoma. Since then, numerous clinical trials have been launched testing other targeted therapies, testing combinations and testing other possible indications for patients with hepatocellular carcinoma. One of the most important challenges we are facing is to be able to match the drug to the signalling pathways at play in specific tumours.

\section{Concurrent Symposia 05: Drug Resistance In Chronic Hepatitis B - What's New?}

15:00-16:00

C05-01

Monitoring and Testing of HBV Drug Resistance

S. Locarnini

Research and Molecular Development, Victorian Infectious Disease Reference Laboratory (VIDRL), North Melbourne, VIC, Australia 
Virological breakthrough $\left(>1.0 \log _{10} \mathrm{IU} / \mathrm{ml}\right.$ from nadir) typically occurs with the emergence of antiviral drug resistant hepatitis B virus (HBV) strains (Zoulim, F \& Locarnini, S. 2009. Gastroenterology;137:1593), however selection of rtA181T/sW172* can cause flatter curves of HBV DNA (Warner $N \&$ Locarnini S. 2008. Hepatol;48:88-98). Genotypic resistance results from substitutions in the HBV genome known to confer resistance, which develop during antiviral therapy. Cross-resistance is defined as substitutions selected by one nucleos(t)ide analogue (NA) that also confer resistance to other NA. Since the case definition of antiviral drug resistance is dependent on HBV DNA measurement, most professional societies (EASL Clinical Practice Guidelines: Management of chronic hepatitis B. 2009. $J$ Hepatol;50:27) recommend HBV DNA testing 3-4 times per year to monitor efficacy and compliance. Once virological breakthrough occurs, it is recommended to perform genotypic testing for resistance. Primary drug resistance results from point mutations in the HBV DNA resulting in amino acid substitutions in the viral polymerase causing reduced susceptibility to that NA. Secondary (compensatory) mutations result in amino acid substitutions that restore the functional defects in the HBV polymerase activity (eg: replication fitness) associated with primary drug resistance. The main primary resistance substitutions for the L-nucleosides (Lamivudine/Telbivudine) are rtM204V/I (major) or rtA181T (minor); Acyclic Phosphonates (Adefovir/Tenofovir) include rtA181T/V and/or rtN236T; D-Cyclopentanes (Entecavir) include rtL180M+rtM204V/I plus one of rtT184 or rtS202 or rtM250 substitution. Several technologies are now available to detect genotypic resistance. The current methodologies includes direct PCR sequencing, INNO-LiPA, RFLP analysis and Cloning-Sequencing. The newer methodologies include Next Generation Sequencing (454 Platform, SOLID System, Genome Analyser System) and Database methodology with the overall goal of a "virtual" phenotype being generated (Yuen, $L$. et al 2007. Antiviral Research;75:64-74). These more recent developments should facilitate patient management, especially in the situation of multi-drug resistant $\mathrm{HBV}$.

\section{C05-02}

Drug resistant HBV management - oral nucleoside/nucleotide analogues modification is better than immunomodulatory drugs

\section{P. Lampertico}

\section{C05-03}

Drug resistant $\mathrm{HBV}$ management - immunomodulatory drug is better than oral nucleoside/nucleotide analogues modification

P. Marcellin

\section{Concurrent Symposium 06: Hepatocarcinogenesis - Genetic Pathway, Current and Future Therapy 15:00-16:00}

\section{C06-01}

Cancer gene discovery in hepatocellular carcinoma

R.W. Pang

\section{C06-02}

Molecular Markers in Diagnosis and Prognostic Staging of HCC M. Sakamoto

Department of Pathology, Keio University School of Medicine, Tokyo, Japan

Developmental process of human hepatocellular carcinoma (HCC) has been characterized and classified from chronic viral infection and liver cirrhosis, dysplastic nodules, early until advanced HCC clinicopathologically. Until now, many molecular mechanisms involved in multistep hepatocarcinogenesis have been reported. One of the prominent features of hepatocarcinogenesis is the gaining of hypervascularity during the process of dedifferentiation and progression, showing an importance of angiogenic switches for the progression typically from early to advanced HCC. In this symposium, I address activation of some different molecular pathways during hepatocarcinogenesis which are especially useful in the pathologic diagnosis of multistage HCC. Several genes such as HSP70, CAP2, and GPC3 have been identified as potential molecular markers for early HCC. Overexpression of stemness gene Bmi-1 was observed in early $\mathrm{HCC}$ in association with increased expression of ABCB1 (MDR1). Classical oncogene or tumor suppressor gene such as beta-catenin and p53 are mutated during the progression from early to advanced HCC. Reduced expression of TGFBR2 or increased phosphorylation of Akt has been identified as significant risk factors for early disease recurrence of HCC. Also, the presence of hepatoblastic feature like K19 in advanced HCC could be used as a predictor of aggressive HCC. Although many progresses have been made in the diagnosis of multistage hepatocarcinogenesis, we still need more useful markers to have a more precise evaluation in the each step of hepatocarcinogenesis for a better choice of treatment including current and future molecular targeted therapy.

\section{C06-03}

Molecular Targeted Therapy for Advanced Hepatocellular Carcinoma: Sorafenib and beyond

A.-L. Cheng

National Taiwan University Hospital, Taipei, Taiwan ROC

The benefit of sorafenib in treating Asian patients with advanced hepatocellular carcinoma (HCC) has been confirmed by the randomized phase III placebo-controlled "ORIENTAL" study. Compared with "SHARP" study, the Asian patients population were in general younger, HBV-related, and with more advanced stages of disease. Subgroup analyses of "SHARP" and "ORIENTAL" studies suggested that the survival benefit of sorafenib was not affected by clinicopathologic features such as age, performance status, macrovascular invasion, extrahepatic metastases, prior TACE, and etiologies (HCV-related, HBV-related, or alcohol-related). The consensus of major treatment guidelines agree upon the use of sorafenib in patients with Child-Pugh B, although with special caution for toxicities and dosages. Whether sorafenib is beneficial in earlier-stage patients undergoing TACE or curative resection/ablation therapy remains unknown. Results of the first large-scale randomized study conducted in Japan and Korea revealed no overall survival benefit for patients receiving sorafenib after TACE. The milestone "STORM" study, testing the efficacy of sorafenib in adjuvant setting (after curative local treatment) has just completed patient accrual. In addition to sorafenib, there are several multi-targeted antiangiogenic TKIs under phase II or phase III trials for HCC. Although these drugs share substantial similarity with sorafenib, there is certain dissimilarity that may worth exploring. For example, brivanib, TSU-68, BIBF-1120, and TKI258 inhibit an additional target, FGFR, which is the target of an important pro-angiogenic factor, FGF, that is often over-expressed in hosts receiving anti-angiogenic agents. Novel categories of targeted agents for HCC, such as EGFR inhibitor, m-TOR inhibitor, PI3K/Akt inhibitor, IGFR inhibitor, MEK inhibitor, HDAC inhibitor, c-Met inhibitor, Hedghog inhibitor, and proteasome inhibitor are under early-phase clinical trials. Sorafenib-based combinations may improve the therapeutic index. Pre-clinical researches have shown that MEK inhibitor, Akt inhibitor, proteasome inhibitor, and proapoptotic receptor agonist act synergistically with sorafenib. Exploratory clinical trials are now following the results of these pre-clinical researches. Combinations with cytotoxic agents have also shown promising activity in several single-arm studies. However, they have to be verified by randomized phase III trials. 
Concurrent Symposium 07: Hot Topics of HBsAg Titer in the Asia-Pacifics and Europe - 'An Old Test Revisit' 16:00-17:15

C07-01

What is the Clinical Meaning of HBV DNA versus HBsAg?

H.L.Y. Chan

Department of Medicine and Therapeutics, The Chinese University of Hong Kong, Hong Kong, Hong Kong SAR

There is always confusion on the clinical meaning of hepatitis $B$ virus (HBV) DNA and hepatitis B surface antigen (HBsAg) level. There is some correlation in the serum levels of HBV DNA and HBsAg, and the correlation is better in hepatitis $\mathrm{B}$ e antigen $(\mathrm{HBeAg})$ positive patients than in HBeAg negative patients. Virologically, HBV DNA is measuring the amount of viral particles (or Dane particles). HBsAg, on the other hand, is measuring both the Dane particles and empty subviral particles. Clinically, HBV DNA level is reflecting the replication of virus. In the natural history of chronic hepatitis B, HBV DNA fluctuates with the status of immune clearance. However, HBsAg level is reflecting the amount of virus in the liver cells or the amount of infected hepatocytes. Serum HBsAg level rarely changes rapidly, even during HBeAg seroconversion or hepatitis flare. In general, $\mathrm{HBsAg}$ levels tend to be lower in $\mathrm{HBeAg}$ negative patients than $\mathrm{HBeAg}$ positive patients. A reduction in HBsAg level usually indicates improved immune control, particularly in $\mathrm{HBeAg}$ negative patients. With the difference in the nature of these two serum viral markers, they have different clinical uses. HBV DNA is best used to monitor viral replication during therapy with nucleos(t)ide analogs. The suppression of HBV DNA during treatment can be used to guide the treatment strategy according to the roadmap concept, which may reduce the problem of drug resistance. Monitoring of HBV DNA can also pick up drug resistance for early salvage treatment. On the other hand, most data on the use of HBsAg is on the prediction of off-treatment to peginterferon therapy. It can be used to individualize peginterferon therapy. There is some early data on the use of HBsAg level to predict the off-treatment response to nucleos(t)ide analogs and it may assist to time for treatment cessation in the future.

\section{C07-02}

Role of HBsAg titer in the treatment of chronic hepatitis B M. Brunetto

\section{C07-03}

Predictive Values of Quantitative HBsAg in NUC's vs. Immune Modulatory Treatment in Chronic Hepatitis B

H. Janssen

Erasmus MC, University Medical Center Rotterdam, Rotterdam, The Netherlands

Monitoring response early during nucleos(t)ide analogs (NAs) and pegylated interferon (PEG-IFN) therapy potentially allows early differentiation between responders and non-responders, and raises the possibility of individualized, response-guided therapy in CHB.

Responders: Early favorable results demonstrating on-treatment decline in HBsAg could motivate patients to complete therapy with PEG-IFN and achieve sustained immune control. Sustained immune control is a key step towards HBsAg clearance.

Non-responders: Early indications of failing therapy could allow alterations to treatment regimen.

Individualizing therapy: PEG-IFN: In HBeAg-negative disease, an early stopping rule based on HBsAg and HBV DNA decline has been proposed in genotype D patients. In $\mathrm{HBeAg}$-positive disease, $\mathrm{HBs} \mathrm{Ag}$ decline is being investigated as a guide to management decisions.

Nucleos(t)ide analogs (NAs): An increasingly common question encountered in the clinic is 'when can therapy be stopped?' HBsAg quantification could have a role in identifying those patients who may be able to stop treatment with a low risk for relapse. Given the valuable information it provides, HBsAg monitoring is, in combination with HBVDNA measurement, likely to play a role in future studies undertaken to improve the management of $\mathrm{CHB}$ and in developing a response-guided therapy approach for individual patients.

\section{Concurrent Symposium 08: Metabolic Liver Diseases In Asia-Pacific \\ 16:00-17:15}

C08-01

Primary iron overload: A global perspective

L. Powell

\section{C08-02}

Wilson Disease

R. Mohamed

University of Malaya, Kuala Lumpur, Malaysia

Wilson disease is an autosomal recessive disorder of copper metabolism resulting in cirrhosis and neuropsychological deterioration. The genetic basis for the excessive deposition of copper in the liver and extrahepatic tissues have been identified, caused by mutations in the ATP7B gene. The classical description of cirrhosis, neurological manifestations and Kayser-Fleischer rings is present in a minority of cases. Clinical features are age-dependent: in children, liver disease predominate whereas neurologic manifestations typically present during young adulthood. The spectrum of liver disease ranges from asymptomatic elevation of aminotransferases to acute liver failure. The clinical and liver histological features of Wilson disease can be indistinguishable from autoimmune hepatitis. The diagnosis of Wilson disease relies on a combination of clinical manisfestation and biochemical parameters (low serum caeruloplasmin, high 24-hour urine copper excretion, high hepatic copper concentration). Molecular genetic testing is costly and not widely available. Treatment for Wilson disease include chelating agents such as penicillamine or trientine, zinc and dietary avoidance of high copper-containing food. Patients with fulminant presentation or decompensated cirrhosis require urgent or prompt evaluation for liver transplantation.

C08-03

Alcoholic liver diseases

M.O. Arguillas

\section{February 2011 (Saturday)}

\author{
Concurrent Symposium 09: Personalized Medicine \\ for HCV Treatment \\ 08:00-09:30
}

\section{C09-01}

Host Genetic Factors and Treatment Response of Chronic Hepatitis C Y. Tanaka

Virology and Liver Unit, Nagoya City University Graduate School of Medical Sciences, Nagoya, Japan

Chronic infection with hepatitis $\mathrm{C}$ virus $(\mathrm{HCV})$ presents a significant health problem worldwide. The spontaneous HCV clearance was observed in approximately $30 \%$ individuals of acute infection. In the therapy using a combination of pegylated interferon- $\alpha$ and ribavirin (PEG-IFN/RBV), approximately $50 \%$ of chronic hepatitis C patients 
infected with HCV genotype 1 and high viremia reached sustained viral response (SVR). These findings were strongly expected to affect variations of host genome. Recent genome-wide association studies (GWAS) on HCV infection have identified two important host genetic variants. One is the SNP in $I L 28 B$ gene, which is strongly associated with response to therapy of chronic genotype $1 \mathrm{HCV}$ infection. Four independent groups applied a GWAS to HCV infection. These groups almost simultaneously reported a strong association of IL28B polymorphisms with the response to PEG-IFN/RBV therapy as well as natural clearance of HCV. The significant SNPs could be useful for prediction prior to treatment because of the strong association with clinical outcome. In addition, the combination of $I L 28 B$ polymorphism and HCV mutations such as core 70 or ISDR had better prediction of response to PEG-IFN/ RBV. Another recent study indicated that genetic variants leading to inosine triphosphatase (ITPA) deficiency, a condition not thought to be clinically important, protect against hemolytic anemia in CHC patients receiving RBV. Our data demonstrated the association of the ITPA genotype with a higher adherence to RBV, a higher rate of SVR, and a lower rate of relapse among a subset of Japanese patients with the favorable $I L 28 B$ genotype. These findings may give a valuable pharmacogenetic diagnostic tool for the tailoring of PEG-IFN and RBV dosing to minimize drug-induced adverse events and for further optimization of the clinical anti HCV chemotherapeutics.

\section{C09-02}

\section{Individualized Treatment for Chronic Hepatitis C}

M.W. Fried

\section{C09-03}

Strategies for Managing Anemia in HCV Patients Undergoing Combination Therapy

M.L. Shiffman

Liver Institute of Virginia, Bon Secours Health System, Newport

News, VA, USA

Anemia is one of the most challenging and controversial to manage adverse events of peginterferon and ribavirin therapy. Two strategies have emerged; reducing the dose of ribavirin and/or peginterferon or adding an erythropoetic growth agent. The initial study of this topic demonstrated that reducing the dose of ribavirin resulted in a significant decline in SVR. However, reducing ribavirin exposure below $80 \%$ only occurs in patients who require dose reduction within the first 8 weeks or when several days of ribavirin dosing are missed. A double-blind, placebo-controlled trial did demonstrate that erythropoetin-alpha could correct anemia in patients receiving peginterferon and ribavirin. However, two randomized trials and a retrospective analysis of the large IDEAL study database have failed to demonstrate that the use of erythropoetic growth factors leads to an increase in SVR, except in those patients who developed significant anemia within the first 8 weeks after the onset of treatment. Retrospective analyses of other large databases have demonstrated that reducing the dose of ribavirin after a patient has become HCV RNA undetectable has minimal impact in patients after they became HCV RNA undetectable. Erythropoetic growth facts have been associated with adverse events including bone pain, red cell aplasia and venous thrombosis. Based upon these data the management of anemia in patients receiving peginterferon and ribavirin should be to reduce the dose of ribavirin by $200 \mathrm{mg}$ steps each week until the decline in hemoglobin has stabilized. Reducing the dose of peginterferon alfa-2a from 180 to $135 \mathrm{mcg} /$ week or peginterferon alfa- $2 \mathrm{~b}$ from 1.5 to $1.0 \mathrm{mcg} / \mathrm{kg} /$ week may also stabilize the decline in hemoglobin. Erythropoetic growth factors should be reserved only in those patients who have yet to become HCV RNA undetectable and have a rapid decline in hemoglobin within the first 8 weeks after treatment.
C09-04

Direct-acting Antiviral Agents for Chronic Hepatitis C and Viral Resistance: The Expected Problem of the Future

J. Pawlotsky

Concurrent Symposium 10: Autoimmune Liver Diseases: from Bench to Bed-Side

08:00-09:30

\section{C10-01}

Rationales in the treatment of autoimmune hepatitis

M.P. Manns

\section{C10-02}

What's New in Pathophysiology of Autoimmune Cholestatic Liver Disease?

V.A.C. Luketic

Section of Hepatology, Virginia Commonwealth University School of Medicine, Richmond, VA, USA

Chronic injury in cholestatic liver diseases is a two step process: first comes injury to the bile duct followed by hepatic retention of bile, most notably bile acids, that over time causes progressive fibrosis, cirrhosis, and ultimately liver failure. Primary biliary cirrhosis (PBC) and primary sclerosing cholangitis (PSC) are the most common autoimmune cholestatic diseases. Variations on the theme include AMA negative PBC, small-duct PSC, AIH-overlap syndromes, and more recently identified IgG4-associated cholangitis (IAC). Pathophysiology is best characterized in PBC. The process is initiated when a genetically susceptible individual (MHC class II) is exposed to environmental triggers (pathogens, chemicals) that lead to specific loss of tolerance to mitochondrial PDC-E2. Mediators of injury include antigen specific auto-reactive CD4+ and CD8+ T cells acting via activation of CD40, TNF, and Fas receptors. Treg defects are involved as well. Damage to biliary epithelial cells results in cholestasis than is not only due to impaired bile flow but also to defects in the secretin stimulated alkalinization and dilution of canalicular bile. Retained bile acids acting as nuclear receptor (FXR) ligands can also alter the expression - up or down - of not only bile acid responsive genes (synthesis, transport, detoxification) but also innate and adaptive immunity, further affecting cholestasis associated injury. PSC pathogenesis is less well characterized but evidence exists for involvement of genetic, autoimmune, and infectious factors. There is a strong association with specific HLA alleles with haplotypes that confer increased risk (DRB1*0301) or are protective (DRB1*0401). Susceptibility is also influenced by polymorphisms in genes encoding for TNF, MMP3, and ICAM1. While there is increased prevalence of autoimmunity and autoantibodies in PSC, evidence for direct linkage is lacking. Alternative explanations include intrinsic defects in biliary epithelial cells as well as inflammatory reaction due to bacterial antigens and toxins in the portal circulation mediated by chemokines that recruit Tregs. IAC is thought to represent the biliary manifestation of a multisystem fibro-inflammatory disorder of which autoimmune pancreatitis is the best studied manifestation. The pathogenesis is unknown. The disease is characterized by increased serum levels of IgG4 and bile duct infiltration by IgG-positive plasma cell. Its main characteristic that distinguishes IAC from PSC is its steroid responsiveness.

\section{C10-03}

\section{Overlap Syndromes \\ U. Beuers \\ Gastroenterology and Hepatology, Academic Medical Center, University of Amsterdam, Amsterdam, Netherlands Antilles}

Overlap syndromes of primary biliary cirrhosis $(\mathrm{PBC})$ and autoimmune hepatitis (AIH) have been reported in up to $10 \%$ of patients with $\mathrm{PBC}(1)$. Single cases of AIH and AMA-negative PBC overlap have also been 
reported. AIH-PBC overlap syndromes are ill-defined and still a matter of controversial discussion. Patients with overlap syndromes present with both hepatitic and cholestatic serum liver tests and have histological features of PBC and AIH. Combined treatment of these disorders with ursodeoxycholic acid and an immunosuppressive regimen is proposed in actual guidelines although controlled trials are lacking due to the low prevalence of these disorders [1,2]. Overlap syndromes of AIH with primary sclerosing cholangitis (PSC) have been reported in children, adolescents and young adults in about $8 \%$ of patients with these immunemediated disorders (1). AIH-PSC overlap syndromes are not adequately defined. Combined treatment of these disorders with ursodeoxycholic acid and an immunosuppressive regimen is proposed in actual guidelines [1]. Liver transplantation is the only curative treatment in late stage $\mathrm{AIH}-$ $\mathrm{PBC}$ and AIH-PSC overlap syndrome.

\section{C10-04}

Primary Sclerosing Cholangitis-improved Treatment Outcomes

with Medical and Surgical Intervention

P. Charatcharoenwitthaya

Department of Medicine, Siriraj Hospital, Mahidol University, Bangkoknoi, Thailand

Primary sclerosing cholangitis (PSC) is a chronic cholestatic liver disease characterized by diffuse fibrosing inflammation of the biliary tree and usually leads to biliary cirrhosis, which can be complicated by portal hypertension and hepatic failure. The etiology of PSC remains unknown, although growing evidence suggests that immunologic factors appear to play a key role in the pathogenesis of PSC. PSC is strongly associated with inflammatory bowel disease and in this setting the risk of colorectal cancer is markedly increased. The natural history of PSC is variable, but virtually always follows a progressive course; the estimated 10-year survival is approximately $65 \%$ in a population based study. During the course of PSC many patients develop dominant strictures of the bile duct and experience worsening of symptoms. Endoscopic therapy with balloon dilatation and stenting has been used to relieve biliary obstruction with a potential survival benefit in PSC patients with symptomatic dominant biliary strictures. Surgical management of dominant stricture with extrahepatic biliary resection for noncirrhotic patients is associated with low perioperative morbidity and 10-year survival more than $60 \%$. Patients with PSC are at risk for developing superimposed cholangiocarcinoma and the diagnosis of cholangiocarcinoma in the setting of PSC remains challenging. The prognosis of patients complicated with cholangiocarcinoma is dismal; however, innovative approaches using liver transplantation combined with neoadjuvant chemoradiotherapy hold some promise in highly selected patients. Effective medical management of PSC is lacking. Ursodeoxycholic acid (UDCA) has extensively investigated as a potential candidate for the treatment of PSC; however, the role of this agent in slowing the progression of PSC-related liver disease is yet unclear and indeed, high dose UDCA may be harmful. Several innovative therapies have been tried in PSC, but with scant evidence of benefit. Liver transplantation is the only successful treatment modality for advanced liver disease in this patient population.

\section{Concurrent Symposium 11: General Hepatology Session 08:00-09:30}

\section{C11-01}

Distinct Aspects of Liver Diseases in Women

V. Mahachai

Division of Gastroenterology, Chulalongkorn University, Bangkok, Thailand

Liver disorders unique to women can be considered in specific clinical settings including:
1. Liver diseases specific to pregnancy.

2. Coincidental liver diseases in pregnancy.

3. Preexisting liver diseases in pregnant women.

4. Liver diseases with female predominance.

5. Hormone-related liver diseases.

Liver diseases specific to pregnancy although uncommon can be serious and fatal include hyperemesis gravidarum, preeclampsia / eclampsia, acute fatty liver, intrahepatic cholestasis and HELLP syndrome. These conditions require prompt diagnosis for the best supportive care and timely delivery to improve maternal and perinatal outcome. Liver conditions that may be coincidental with pregnancy include acute viral hepatitis, Budd-Chiari syndrome, gallstone and drug induced liver disease. Pregnant women may have preexisting liver diseases such as chronic viral hepatitis B, C, autoimmune hepatitis, Wilson's disease and cirrhosis. Management of pregnant women with portal hypertension should be similar to that of non pregnant women although risk of variceal bleeding is increased in pregnancy. Special attention must be paid to pregnant women with chronic hepatitis $\mathrm{B}$ and $\mathrm{C}$ for the proper timing and optimal choices of antiviral therapy and the mean to prevent maternal-fetus transmission. Some liver diseases are more prevalent in women and have different impact in women. NAFLD is more prevalent in women than men especially in older women after menopause and is associated with polycystic ovary syndrome in young women. Women are more susceptible to alcoholic liver damage inspite of shorter drinking history with a more rapid progression compared to men. Both autoimmune hepatitis and primary biliary cirrhosis are strongly associated with female sex. Gallstones are twice as common in women as in men and are associated with pregnancy, obesity, $\mathrm{BCP}$ and hormone. Benign liver tumor, hepatic adenoma, has been associated with the use of oral contraceptive whereas focal nodular hyperplastic (FNH) is not affected by hormonal contraceptive use.

\section{C11-02}

Hepatopulmonary syndrome: What is the current treatment and outcome? M.B. Fallon

\section{C11-03}

Approach to Acute Kidney Injury in Cirrhotic Patients

Y.K. Chawla, S. Gupta

Department of Hepatology, Post Graduate Institute of Medical Education and Research, Chandigarh, India

Introduction: Development of renal failure in patients with cirrhosis is a poor prognostic sign associated with poor survival. Serum creatinine is one of the three variables used to calculate MELD score which is a major prognostic score in cirrhosis..

Definition and types: AKI is defined as "an abrupt (arbitrarily set at $48 \mathrm{~h}$ ) reduction in kidney function manifested by an absolute increase in serum creatinine of $0.3 \mathrm{mg} / \mathrm{dL}$ or more, equivalent to a percentage increase in serum creatinine of $50 \%$ or more (1.5-fold from baseline) or a urine output of less than $0.5 \mathrm{ml} / \mathrm{kg}$ per hour for more than $6 \mathrm{~h}$." Traditionally AKI is of three types: prerenal, renal and postrenal. Cirrhotic patients additionally may develop hepatorenal syndrome (HRS). AKI occurs in approximately $20 \%$ patients with cirrhosis and is mostly prerenal, accounting for up to $70 \%$ cases.

Diagnosis: Serum creatinine is most established, simple and easily available parameter but it has several limitations, particularly in cirrhotics. Some biomarkers which can be used as an early marker for renal dysfunction like Cystatin $\mathrm{C}$, Beta trace protein and urinary biomarkers such as interleukin-18 but use of these markers in presence of cirrhosis is still to be evaluated. HRS can be divided into type I and type II, both have different settings and outcome.

Management: Treatment depends upon the type of AKI. First step is to discontinue diuretics, lactulose, vasodilators and any potential nephrotoxic drugs. Once the diagnosis of HRS is made, specific treatment should be 
initiated as early as possible. The only definitive treatment of HRS is liver transplantation and patients must be listed once HRS has occurred.

\section{C11-04}

Management of portal hypertensive gastropathy and gastric vascular ectasia - unresolving issues

T. Kitiyakara

Concurrent Symposium 12: Expert Recommendations for NAFLD/NASH: Confronting the Eastern and Western Views

08:00-09:30

C12-01

NAFLD in the EAST and West: Same disease? Same clinical impact? V. Ratziu, E. Powell

\section{C12-02}

Confronting expert recommendations of EASL and APASL on selected challenging aspects of NAFLD management

V. Ratziu, E. Powell

\section{C12-03}

Treatment update: Are current pharmacological options for NASH of any proven benefit? - Pro and Rebuttal

V. Ratziu, E. Powell

\section{Concurrent Symposium 13: Controversy In Liver \\ Transplantation \\ 08:00-09:30}

\section{C13-01}

Minimizing Immunosuppression: Risks and Benefits

A. Shaked

University of Pennsylvania, Philadelphia, PA, USA

It is seldom that the fate of the transplanted liver is determined solely by the immune response against the allograft. Episodes of acute rejection are usually not associated with long lasting tissue damage and the incidence of graft loss due to chronic rejection is low. Instead, in many recipients, morbidity and mortality is directly related to the burden of immunosuppression as evidenced by organ system damage that is mediated by their side effects, by compromised immune surveillance resulting in the development of opportunistic infection and malignancies, and by the rapid and severe recurrence of $\mathrm{HCV}$ infection. In principle, minimization of immunosuppression is aimed at finding the most effective balance of controlling the alloimmune response while allowing the immune system to regain its surveillance function of harmful processes.

Reversal of kidney injury: Several studies have explored antibody induction with the aim to delay or reduce the exposure to CNI in the immediate post-transplant period; the initial outcomes demonstrate higher GFR early on, but the impact on long term outcomes is yet to be determined.

Immunosuppression and $\mathrm{HCV}$ recurrence: The relation between $\mathrm{CNI}$ and progression of recurrent hepatitis $\mathrm{C}$ is not well defined. Continuous suppression of the immune response is believed to contribute to the rapid progression of recurrent $\mathrm{HCV}$, leading to graft failure and decreased survival, and cirrhosis in $20 \%$ within 5 years after transplantation.

Is it safe to minimize immunosuppression in liver transplant recipients? Twenty percent or more of liver transplant recipients appear able to be withdrawn from immunosuppression without specific tolerance-induction strategies. These observations support prospective clinical trials of immunosuppression minimization in liver transplantation, and suggest that they are associated with a high level of safety for the recipient.
C13-02

Improving Outcomes of Liver Transplantation for Hepatitis C

N. Terrault

UCSF, San Francisco, CA, USA

Graft survival is reduced in HCV-infected liver transplant (LT) recipients compared with non-HCV recipients and recurrent $\mathrm{HCV}$ disease is the most common cause of graft loss. Management strategies focus on measures to prevent fibrosis progression. Careful donor selection is an important first step, as older donors ( $\geq 50$ years) significantly increase risk of cirrhosis. Prevention of acute rejection, CMV infection and diabetes are also important, as these complications are associated with accelerated fibrosis progression. Immunosuppression is believed to be an important factor but despite many studies, the "best" immunosuppressive regimen remains elusive. Current treatment strategies in $\mathrm{HCV}$ patients fall into two broad categories: pre-LT antiviral therapy with the goal of preventing recurrence, and post-LT antiviral therapy with the goal of eradicating HCV to prevent graft loss. Pre-transplant therapy antiviral is successful in preventing post-LT HCV recurrence in $\sim 15 \%$ of treated patients with genotype 1 and $30-60 \%$ for genotype 2-3. However, due to the poor tolerance of peginterferon (peg-IFN) and ribavirin (RBV) in advanced cirrhotics, pretransplant treatment is limited to those with mildly compensated cirrhosis (Childs A and MELD $<20$ ). Post-transplant antiviral therapy using peg-IFN and RBV is indicated for patients with significant inflammation (grade $\geq 3$ ) or fibrosis (stage $\geq 2$ ). Tolerability is reduced compared to non-transplant patients. Immunologic complications, including rejection and "autoimmune-like" hepatitis, occur but a low frequency. SVR rates are achieved in 30-40\% of treated patients, leaving a large pool of nonresponders. An early virologic responses is most predictive of SVR. There is a survival benefit in treated patients who achieve SVR. Clearly better-tolerated and more efficacious antiviral therapies are needed. The availability of combination therapy of peg-IFN, RBV and protease inhibitors is anticipated to improve SVR rates but tolerability and drugdrug interactions are likely to be greater than peg-IFN and RBV.

\section{C13-03}

Post-transplant HBV prophylaxis - What is best? and how can we minimize it?

G.W. McCaughan

C13-04

Transplantation for HCC: Within and Outside of Milan Criteria K.H. Kim

APASL-International Ascites Club Joint Symposium: Hyponatremia: Pathophysiology and management 08:00-09:30

Fluid-electrolyle disturbances in cirrhosis

A.J. Sanyal

Mechanisms and pathophysiology of hyponatremia in cirrhosis G. Garcia-Tsao

Relevance of hyponatremia in acute and chronic liver failure P. Angeli

Treatment of hyponatremia

S.K. Sarin 
Chainuvati Lecture 10:00-10:30

\section{KL04}

Eliminating the chance of $\mathrm{HCC}$ in CHB patients: Fact or fiction? Y.-F. Liaw

Liver Research Unit, Chang Gung Memorial Hospital, Chang Gung University College of Medicine, Taipei, Taiwan

Chronic hepatitis B virus (HBV) infection is a dynamic state of interactions between HBV, the hepatocytes and the immune system of the patient. HBV replication is the key driver of disease progression, including development of cirrhosis and hepatocellular carcinoma (HCC), during the course. Theoretically, HBV elimination or suppression would be able to reduce the risk of or slow the progression of liver diseases. Studies have shown that a finite course of conventional interferon- $\alpha$ (IFN) therapy has long term benefit in achieving cumulative response, reducing fibrosis progression and the development of cirrhosis and/or HCC. Long-term therapy with nucleos(t)ide analogs (Nuc) also resulted in fibrosis improvement or reversal of advanced fibrosis, reduction of disease progression and development of HCC. Several meta-analyses have also shown the beneficial effect of anti-HBV therapy in the reduction of $\mathrm{HCC}$ development, especially in $\mathrm{HBeAg}$ positive patients, Asian patients or in a patient population of higher $\mathrm{HCC}$ incidence. Pegylated IFN and newer Nuc may have even better long-term outcomes because of better therapeutic efficacy and/or low risk of drug resistances. However, the treatment outcomes are still far from satisfactory and the chance of HCC development is only reduced but not completely eliminated. The development of more effective and safe but affordable anti-HBV agents/strategies is needed to further improve outcomes.

\section{Keynote Lecture 1}

10:00-10:30

\section{KL01}

Stem Cells in Liver Carcinogenesis

N. Theise

Pathology and Medicine, Beth Israel Medical Center - Albert Einstein College of Medicine, New York, NY, USA

Hepatobiliary malignancy may develop in two ways. 1. They may derive from malignant transformation of facultative hepatobiliary stem/progenitor cells; or 2. they may from malignant transformation of mature hepatocytes or cholangiocytes which then adopt stem cell functions and phenotypes in the process of transformation. Postulated mechanisms for how stem cells, with protected genomes resistent to mutation, can become malignantly transformed, particularly in the setting of chronic disease, will be considered. Discussion will consider essential topics such as concepts of the stem cell niche, assymetric cell division, DNA template retention, and transit amplifying populations. These will then be explored in the specific context of the liver with identification of intraorgan hepatobiliary stem cell niches, ductular reactions in acute and chronic disease as transit amplifying populations, and the association of malignancy with such changes in liver structure and activity. Recently published findings regarding markers for stem cell functioning in human liver tumors (e.g. ckit, CD34, CD133, EpCAM, keratin 19) will also be discussed.

\section{Keynote Lecture 2}

10:00-10:30

\section{KL02}

Future perspective of liver transplantation in Asia-Pacific C.M. Lo
State-of-the-Art Lecture 1 10:00-10:30

SA01-01

Refractory Cirrhotic Ascites, Hyponatremia and Acute Kidney Injury in Cirrhosis

T.D. Boyer

Medicine, University of Arizona, Tucson, AZ, USA

The development of ascites in the patient with cirrhosis is commonly the first sign of decompensation of their liver disease. Over time the ascites becomes resistant to diuretics and the patient becomes increasingly disabled by the worsening ascites. Management of these patients is difficult and most require large volume paracentesis (LVP) to control their ascites. The other option is a TIPS. In controlled trials TIPS is more effective in controlling the ascites but at a cost of an increase in hepatic encephalopathy. It is unclear if survival is improved in patients treated with TIPS as compared to LVP. In patients with refractory ascites hyponatremia is quite common. Hyponatremia is due to the nonosmotic release of arginine vasopressin as a response to the hyperdynamic circulation that develops in patients with cirrhosis. Most patients are treated with fluid restriction. There now are available $\mathrm{V}_{2}$ receptor antagonists (vaptans) that are effective in correcting the hyponatremia. However, how they should be used in cirrhotics is unclear. As the liver disease progresses the hyperdynamic circulation worsens and patients are at risk for the development of the hepatorenal syndrome (HRS). There are many causes of acute kidney injury (AKI) in the cirrhotic with ascites including HRS, pre-renal causes, acute tubular necrosis, drug induced injury and chronic kidney disease. Most of these diseases can be differentiated by a careful urinalysis, exclusion of infection and response of renal function to volume expansion. If a diagnosis of HRS is made the patient may respond to the use of vasoconstrictors such as terlipressin plus albumin. The prognosis for the patient with HRS is however poor and the only definitive therapy is liver transplantation.

\section{Free Communication 05: Hepatitis C} 10:30-11:30

\section{FC05-01}

The IL28B Genotype is a Major Determinant in the Induction of a Virological Response by High-dose pegIFN-Ribavirin in SOC Null-Responders S. Chevaliez ${ }^{1}$, C. Hézode $^{2}$, A. Soulier ${ }^{1}$, B. Costes $^{3}$, M. Bouvier-Alias ${ }^{1}$,

S. Rouanet ${ }^{4}$, J. Foucher ${ }^{5}$, J.-P. Bronowicki ${ }^{6}$, A. Tran ${ }^{7}$, I. Rosa ${ }^{8}$, P. Mathurin ${ }^{9}$, L. Alric ${ }^{10}$, V. Leroy ${ }^{11}$, P. Couzigou ${ }^{5}$, A. Mallat ${ }^{12}$, M. Charaf-Eddine ${ }^{4}$,

G. Babany ${ }^{4}$, J.-M. Pawlotsky ${ }^{1}$

${ }^{I}$ Virology and INSERM U955; ${ }^{2}$ Hepatology and Gastro-Enterology; ${ }^{3}$ Biochemistry and INSERM U955, Faculty of Medecine UPEC, Creteil; ${ }^{4}$ Roche, Neuilly-sur-Seine; ${ }^{5}$ Hepatology and GastroEnterology, Faculty of Medecine Bordeaux 2, Bordeaux; ${ }^{6}$ Hepatology and Gastro-Enterology and INSERM U954, University Henri

Poincare, Nancy; ${ }^{7}$ Hepatology and Gastro-Enterology, University of Nice, Nice; ${ }^{8}$ Hepatology and Gastro-Enterology, Intercommunal Hospital, Creteil; ${ }^{9}$ Hepatology and Gastro-Enterology, University of Lille, Lille; ${ }^{10}$ Internal Medecine, University of Toulouse, Toulouse;

${ }^{11}$ Hepatology and Gastro-Enterology, University of Grenoble,

Grenoble; ${ }^{12}$ Hepatology and Gastro-Enterology and INSERM U955, Faculty of Medecine UPEC, Creteil, France

Polymorphisms upstream of the region encoding $I L 28 B$ have been shown to be associated with HCV treatment response. With new therapies using direct acting antivirals (DAA), a null response to IFN is associated with treatment failure. Our goal was to assess, in SOC null-responders, whether the $I L 28 B$ genotype has an influence and predictive value on the ability of retreatment with high-dose peg-IFN alpha-2a, and ribavirin to induce a virological response. 
Methods: Genotyping of IL28B SNP rs12979860 was performed in 83 genotype 1 null-responders by real-time PCR after extraction of genomic DNA from frozen sera.

Results: The IL28B genotype was determined in all patients: 3 had a CC genotype and were removed to allow comparison between CT $(n=55)$ and TT $(n=25)$ patients. The difference between reductions in HCV RNA levels between TT and CT patients was significant at week $2(<0.5$ vs. $\geq 0.5 \log , p=0.02)$, at week 4 ( $<1$ vs. $\geq 1 \mathrm{Log}, p=0.008)$, and at weeks 12 and 24 ( $<2$ vs. $\geq 2 \log , p=0.02$ ). When comparing CT and TT patients, the odds ratio were 6.46 for a more than $1 \mathrm{Log}$ drop at week $4 ; 3.30$ for a more than $2 \mathrm{Log}$ drop at week $12 ; 3.72$ for a more than $2 \mathrm{Log}$ drop at week 24; and 9.12 for an undetectable HCV RNA at week 24. Conclusions: Most patients who fail to respond to IFN-ribavirin carry either TT or CT IL28B genotypes. CT patients are significantly more likely to respond to higher doses of IFN and the difference is significant as early as at week 2 of administration. This indicates that the $I L 28 B$ genotype is a marker of host cell responsiveness to IFN. These findings will have major implications in the treatment of $\mathrm{HCV}$ infection while tailoring retreatment with higher doses of IFN-ribavirin in combination with DAAs.

\section{FC05-02}

Long-term Follow-up of Chronic Hepatitis C Patients Treated with Telaprevir in Combination with Peginterferon Alfa-2a/Ribavirin: Interim Analysis of EXTEND

A. Alberti ${ }^{1}$, K.E. Sherman ${ }^{2}$, M.S. Sulkowski ${ }^{3}$, F. Zoulim ${ }^{4}$, L.-J. Wei ${ }^{5}$, J.G. McHutchison ${ }^{6}$, B. van Baelen ${ }^{7}$, E. Martin ${ }^{8}$, J.C. Sullivan ${ }^{8}$, T.L. Kieffer ${ }^{8}$, S. De Meyer ${ }^{7}$, G. Picchio ${ }^{9}$, F. Tomaka ${ }^{9}$, C.S. Graham ${ }^{8}$, S. Zeuzem ${ }^{10}$, EXTEND Study Team

${ }^{1}$ University of Padova, Padova, Italy; ${ }^{2}$ University of Cincinnati College of Medicine, Cincinnati, OH; ${ }^{3}$ Johns Hopkins University School of Medicine, Baltimore, MD, USA; ${ }^{4}$ INSERM Unit 271, Lyon, France; ${ }^{5}$ Harvard School of Public Health, Boston, MA; ${ }^{6}$ Duke University Medical Center, Durham, NC, USA; ${ }^{7}$ Tibotec BVBA, Mechelen, Belgium; ${ }^{8}$ Vertex Pharmaceuticals Incorporated, Cambridge, MA; ${ }^{9}$ Tibotec, Inc., Titusville, NJ, USA; ${ }^{10}$ Johann Wolfgang Goethe University Medical Center, Frankfurt am Main, Germany

Background: Telaprevir (TVR), a potent, specific hepatitis $C$ virus (HCV) protease inhibitor, in combination with pegylated interferon alfa-2a and ribavirin (PR), led to higher sustained viral response (SVR) rates than $\mathrm{PR}$ alone in treatment-naïve and treatment-experienced genotype $1 \mathrm{HCV}$ patients in Phase 2 and 3 trials. EXTEND is a 3-year virology follow-up study of some of these patients. Here, we report an interim analysis of SVR durability and $\mathrm{HCV}$ variant changes in patients who had not achieved SVR. Methods: 867 patients who received both $\geq 1$ dose of TVR in PROVE1/2/3 or Study 107 and had baseline HCV sequences available were eligible; 202 enrolled. Patients who achieved $(n=123)$ or did not achieve $(n=79)$ SVR were observed for a median of 22 (range 5-35) and 25 months (range 7-36), respectively. HCV RNA levels were determined by COBAS TaqMan ${ }^{\circledR}$ HCV Test v.2.0 and the NS34a protease sequence was determined using nested RT-PCR followed by population sequencing. Amino acid positions 36, 54, 155, 156, which are associated with decreased TVR susceptibility in genotype 1 patients, were analyzed from patients who did not achieve an SVR. Results: Ninety-nine percent (122/123) of patients maintained SVR during follow-up; one PROVE2 patient experienced late relapse 44 weeks after early study dosing discontinuation. Variants were no longer detectable in $89 \%$ (50/56) of patients who did not achieve SVR. There was no evidence that suggested that time to loss of detectable variants varied by treatment arm, TVR duration, or non-response type (e.g., virologic breakthrough on-treatment versus relapse).

Conclusions: SVR after telaprevir-based therapy was durable with $122 / 123$ subjects maintaining HCV RNA undetectability at median 22 months follow-up. Variants associated with decreased sensitivity to telaprevir were no longer detectable in $89 \%$ of patients who did not achieve SVR within the study period.

\section{FC05-03}

Albinterferon-alfa-2b Every Four Weeks Plus Ribavirin: Treatment of Chronic Hepatitis C Genotype 2/3: Safety, Efficacy and Genetic Variation of IL28B

W.-L. Chuang ${ }^{1}$, S.K. Sarin ${ }^{2}$, R. Flisiak ${ }^{3}$, C.-M. Lee ${ }^{4}$, S. Shah ${ }^{5}$, P. Andreone, T. Piratvisuth ${ }^{7}$, S. Pianko ${ }^{8}$, G. Foster ${ }^{9}$, V. Bain ${ }^{10}$, M. Pang ${ }^{11}$, Y. Yin ${ }^{11}$, Y. Li ${ }^{12}$, G. Feutren ${ }^{13}$, S. Zeuzem ${ }^{14}$

${ }^{1}$ Kaohsiung Medical University, Kaohsiung, Taiwan ROC; ${ }^{2}$ G.B. Pant Hospital, Maulana Azad Memorial Medical College, New Delhi, India; ${ }^{3}$ Department of Infectious Diseases and Hepatology, Medical University of Bialystok, Bialystok, Poland; ${ }^{4}$ Chang-Gung Memorial Hospital-Kaohsiung, Kaohsiung, Taiwan ROC; ${ }^{5}$ Jaslok Hospital and Research Centre, Mumbai, India; ${ }^{6}$ Dipartimento di Medicina Clinica, Bologna, Italy; ${ }^{7}$ Songklanagarind Hospital, Hat Yai, Thailand; ${ }^{8}$ Monash Medical Center, Clayton, VIC, Australia; ${ }^{9}$ The Royal London Hospital, London, London, UK, ${ }^{10}$ University of Alberta, Edmonton, AB, Canada; ${ }^{11}$ Novartis PharmaPharmaceuticals, East Hanover, NJ; ${ }^{12}$ Novartis Institute for Biomedical Research, Cambridge, MA, USA; ${ }^{13}$ Novartis Pharma AG, Basel, Switzerland; ${ }^{14}$ J.W. Goethe University Hospital, Frankfurt, Germany

Background: Albinterferon-alfa-2b (albIFN) was investigated in this study as an every 4 weeks injection regimen $(\mathrm{Q} 4 \mathrm{w})$ in patients with chronic HCV 2-3 hepatitis to reduce injection burden and improve treatment convenience compared to standard therapy with PEG-IFN given once every week (Q1w).

Methods: Patients (391) were randomized 4:4:4:3 to one of 4 openlabel treatment groups, in combination with oral ribavirin $800 \mathrm{mg} / \mathrm{d}$ : albIFN q4wks $900 \mu \mathrm{g}, 1200 \mu \mathrm{g}$ or $1500 \mu \mathrm{g}$ or PEG-IFN $\alpha 2 \mathrm{a} 180 \mu \mathrm{g}$ q1wk for 24 weeks. HCV RNA negativity $(<20 \mathrm{IU} / \mathrm{mL}) 24$ weeks after end of treatment was the main efficacy endpoint (SVR). The IL28B SNP rs12979860 genotype was obtained in a subset of 117 patients. All analyses were performed on the intention-to-treat population.

Results: No statistically significant increase in overall or respiratory serious or severe adverse events was noted in any albIFN arm as compared to PEG-IFN $\alpha 2$ a. Rates of cough on albIFN were 21.6, 21.4 and $26.7 \%$, respectively, vs. $19.2 \%$ on PEG-IFN $\alpha 2 \mathrm{a}$ ( $p=\mathrm{NS}$ ). Alopecia rates were $35.3,37.9,47.6$ and $28.2 \%$, respectively ( $p=$ 0.05 ). There were significantly fewer hematologic reductions in albIFN arms vs. PEG-IFN $\alpha 2 \mathrm{a}\left(p=0.0279\right.$ for neutrophils $<750 \mathrm{~mm}^{-3}$ and $p=0.0175$ for hemoglobin $<10 \mathrm{~g} / \mathrm{dL}$ ), leading to fewer IFN and ribavirin dose reductions. SVR rates were 75.5, 75.7 and 78.1\%, respectively, for albIFN 900Q4w, 1200Q4w and 1500Q4w arms and $84.6 \%$ for PEG-IFN $\alpha 2$ a. The rs12979860 CC genotype was found in $61 / 117(52.1 \%)$ patients and was not significantly associated with differences in SVR in a multivariate analysis as opposed to $\mathrm{HCV}$ genotype (2 vs. 3 ) or baseline HCV viral load.

Conclusion: In this study albIFN administered Q4w resulted in numerically lower SVR rates in chronic HCV GT2/3 infections. Results were not affected by genetic variation in IL28B. Reducing the number of injections could be expected to improve quality of life but addit.

\section{FC05-04}

Ribavirin and Peginterferon Alfa-2a Retreatment of HCV Infected Thalassemia Major Patients with Previous Treatment Failure S.M. Alavian ${ }^{1}$, S.V. Tabatabaei ${ }^{1}$, B. Behnava ${ }^{1}$, M. Keshvari ${ }^{2}$, S.M. Miri ${ }^{1}$, P. Karimi Elizee ${ }^{1}$, K.B. Lankarani ${ }^{3}$

${ }^{1}$ Baqiyatallah University of Medical Sciences, Baqiyatallah Research Center for Gastroenterology and Liver Disease; ${ }^{2}$ Iranian Blood Transfusion Organization Research Centre (IBTO), Tehran; ${ }^{3}$ Shiraz University of Medical Sciences, Shiraz, Iran

Background and aims: ribavirin that is the cornerstone of anti-HCV therapy is generally contraindicated in thalassemia major patients. In this study we have evaluated safety and efficacy of ribavirin in 
combination with peginterferon alfa-2a for treatment of thalassemia patients with previous failure to interferon monotherapy.

Material and methods: 136 treatment experienced subjects were recruited for this study. Patients were treated for duration of 48 weeks with peginterferon alfa- $2 \mathrm{a} 180 \mu \mathrm{g}$ thrice weekly plus ribavirin $600-800 \mathrm{mg}$ according to weekly hemoglobin measurement $(800 \mathrm{mg}$ for $\mathrm{Hg}>10 \mathrm{~g} / \mathrm{dL}, 600 \mathrm{mg}$ for hemoglobin 8-10, and discontinuation for $\mathrm{Hg}<8$ ). Safety was ensured by weekly laboratory test and visits. Results: The mean value of age, viral load and ALT were $24 \pm 0.5$, $77,000 \pm 9,000 \mathrm{IU} / \mathrm{mL}$ and $97 \pm 8 \mathrm{U} / \mathrm{mL}$. $65 \%$ of subjects had genotype 1 infection and 19\% had compensated cirrhosis. $70(51.4 \%)$ of subjects attained SVR, 34 (25\%) relapsed and 18 (13.2\%) subjects did not respond. 3 subjects were lost to follow up, 3 patients died due to cardiac complications and $8(5.8 \%)$ patients were prematurely withdrawn due to safety reasons (severe neutropenia or intractable side effects). 119 patients experienced at least one side effect. The most prevalent were fatigue, hair loss, arthralgia, myalgia, irritability and fever. In almost all patients transfusion rate was doubled to maintain $\mathrm{Hb}$ above $10 \mathrm{~g} / \mathrm{dL}$. However, $\mathrm{Hb}<8$ was experienced by $20 \%$ of subjects.

Conclusion: our data showed that ribavirin in combination with peginterferon alfa-2a is effective and can be tolerable in treatment experienced thalassemia major patients. This regimen yields the SVR rate of $51 \%$ (95\% CI 43-60) among these patients. Daily $600-800 \mathrm{mg}$ ribavirin almost doubles transfusion need in thalassemia major patients.

\section{FC05-05}

Impact of Serum Alpha-fetoprotein Levels on the Response to Antiviral Therapy in Chronic Hepatitis C

M. Mabrouk ${ }^{1}$, W. Doss ${ }^{1,2}$, N. Zayed ${ }^{1}$, S. Afify ${ }^{2}$, G. Esmat ${ }^{1,3}$

${ }^{1}$ Endemic Medicine and Hepatology Department, Faculty of Medicine Cairo University; ${ }^{2}$ Tropical Medicine Department; ${ }^{3}$ International Research Unit, National Hepatology and Tropical Medicine Research Institute, Cairo, Egypt

Background/aim: The association between serum alpha-fetoprotein (AFP) levels during and after interferon (IFN) therapy and the response to pegylated interferon and ribavirin (Peg-IFN/RBV) combination therapy in patients with chronic hepatitis $\mathrm{C}(\mathrm{CHC})$ has been recently of great interest. The aim of this study was to investigate the potential use of serum AFP level as a predictor for treatment response in patients with $\mathrm{CHC}$ and detect its changes with therapy.

Methods: Retrospective data of 1524 CHC patients who received combined Peg-IFN/RBV therapy at the National Hepatology and Tropical Medicine Research Institute (NHTMRI), Cairo, Egypt during the year 2007 were analyzed. Detailed baseline and follow up clinical, laboratory and histological data were evaluated with special emphasis on the baseline AFP levels and their changes during therapy.

Results: Among our study population, 1113 patients completed 48 week of therapy. Early virological (EVR) and end of treatment response (ETR) were documented in 89.9 and $89.3 \%$, respectively. Baseline AFP levels were positively correlated with amino-transferase levels, stages of fibrosis and grades of inflammation. During IFN therapy, a decrease in AFP levels were observed regardless of virologic response with a significant decrease in patients with ETR. A median value for AFP; $3.5 \mathrm{ng} / \mathrm{ml}$ was considered, ETR was much higher among patients with serum AFP levels below rather than above the median value (34.3 and $30 \%$, respectively; $p$ value, 0.03 ). A positive mean of differences between baseline AFP and that measured at either 24 or 48 weeks of therapy was calculated to be 2.1 and 2.8 respectively. The former being significantly increased with ETR; $p$ value 0.034 ; in contrast to the latter; $p$ value 0.11 .

Conclusion: High baseline AFP level predicted a lower ETR rate among patients with CHC. A decrease in serial AFP levels was observed after Peg-IFN/RBV combination therapy irrespective of treatment outcome.
Free Communication 06: Portal Hypertension 10:30-11:30

FC06-01

Assessment of Adrenocortical Reserve in Stable Patients with Cirrhosis

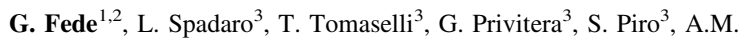
Rabuazzo $^{3}$, A. Sigalas ${ }^{2}$, E. Xirouchakis ${ }^{2}$, J. O’Beirne ${ }^{2}$, M. Garcovich ${ }^{2}$, E. Tsochatzis ${ }^{2}$, F. Purrello ${ }^{3}$, A.K. Burroughs ${ }^{2}$

${ }^{1}$ Internal Medicine, University of Catania, Catania, Italy; ${ }^{2}$ Sheila Sherlock Liver Centre, Royal Free Hospital, London, UK; ${ }^{3}$ University of Catania, Faculty of Medicine, Catania, Italy

Background/aims: Adrenal insufficiency (AI) is reported in critically ill patients with cirrhosis, and is associated with increased mortality. It is unclear if AI is an underlying condition or triggered by critical events (e.g. sepsis or shock). We investigated AI in cirrhosis without infection or hemodynamic instability.

Methods: 101 consecutive patients with cirrhosis were studied. AI was defined by a total serum cortisol (TC) $<18 \mu \mathrm{g} / \mathrm{dl}$ at 20 or 30 minutes after injection of $1 \mu \mathrm{g}$ of tetracosactrin. Transcortin, calculated free cortisol (cFC), and free cortisol index (FCI) were assessed in a subgroup of 41 patients, with FCI $>12$ representing normal adrenal function.

Results: AI was present in 38 patients (38\%). Child score (median, 10 vs. $7, \mathrm{p}<0.0001$ ), MELD score (median, 17 vs. $12, p<0.0001$ ), ascites (68 vs. $37 \%, p<0.01$ ), basal TC (median, 7.6 vs. $14.9 \mu \mathrm{g} / \mathrm{dl}, p<0.001$ ), albumin ( $28 \pm 0.8$ vs. $33 \pm 0.7 \mathrm{~g} / \mathrm{L}, p<0.0001)$, INR (median, 1.6 vs. $1.2, p<0.0001$ ), total bilirubin (median, 51 vs. $31 \mu \mathrm{mol} / \mathrm{L}, p<0.05$ ), total cholesterol (median, 120 vs. 142, $p<0.05$ ), and LDL (median, 76 vs. $81, p<0.05$ ) were significantly different between those with and without AI. ROC curves showed a basal TC $\leq 12.8 \mu \mathrm{g} / \mathrm{dl}$ to be a cut-off value closely associated with AI. cFC was significantly related to TC for baseline values $(R=0.94, p<0.0001)$, peak values $(R=0.90$, $p<0.0001)$, and delta values $(R=0.95, p<0.0001)$, in patients with and without AI. However no patient had a FCI $<12$.

Conclusions: AI defined by an abnormal response to $1 \mu \mathrm{g}$ tetracosactrin is frequent in stable patients with cirrhosis, in the absence of infections or hemodynamic instability, and is related to the severity of liver disease. However direct assays of free cortisol can help to better evaluate the true incidence of AI. Clinical consequences of AI need to be explored.

\section{FC06-02}

Hepatic Vein Transit Time Using a Microbubble Contrast Enhanced Ultrasonography Can Assess the Severity of Portal Hypertension Noninvasively in Cirrhosis

M.Y. Kim ${ }^{1}$, S.K. Baik ${ }^{1}$, H.K. Jeon ${ }^{1}$, H.J. Park ${ }^{1}$, S.Y. Park ${ }^{1}$, Y.O. Jang ${ }^{1}$, Y.J. Kim ${ }^{2}$, G.J. Cheon ${ }^{3}$, Y.D. Kim ${ }^{3}$

${ }^{1}$ Internal Medicine; ${ }^{2}$ Department of Radiology, Yonsei University Wonju College of Medicine, Wonju; ${ }^{3}$ Department of Internal Medicine, Kangneung Asan Hospital, Ulsan University College of Medicine, Kangneung, Republic of Korea

Backgrounds and aim: The hepatic venous pressure gradient (HVPG) is a gold standard for assessment of PHT. However, HVPG is limited in its clinical application because of its invasiveness. Hepatic vein transit time (HVTT) using a microbubble contrast is the time duration between bolus injection of contrast into a peripheral vein and Doppler signal enhancement of hepatic vein. HVTT is shorter in cirrhosis because of the presence of both intrahepatic and intrapulmonary shunts. We prospectively evaluated the usefulness of HVTT measurement using a microbubble contrast enhanced ultrasonography (CEUS) in non-invasive assessment of the portal hypertension in cirrhotic patients.

Methods: For ninety-four cirrhotic patients, both the measurement of HVTT and HVPG were done simultaneously and the relationship between them was analyzed. Usual clinical and biological data were 
collected. Clinical significant PHT (CSPH) and severe PHT were defined as an $\mathrm{HVPG} \geq 10 \mathrm{mmHg}$ and $\mathrm{HVPG} \geq 12 \mathrm{mmHg}$, respectively. Results: The mean age was $50.4 \pm 9.3$ years and male $(80,85.1 \%)$ was dominant. The mean HVPG and HVTT were $14.2 \pm 6.2 \mathrm{mmHg}$ and $17.4 \pm 4.0 \mathrm{~s}$, respectively. In linear regression analysis, HVPG and HVTT showed significant negative correlation $\left(P<0.001, r^{2}=0.508\right)$. In ROC curve of HVTT, the AUROC was 0.867 for the prediction of CSPH and sensitivity, specificity, positive predictive value (PPV) and negative predictive value (NPV) according to HVTT cut-off value of 18.4 s were 81.5, 83.6, 96.4 and $46.7 \%$ respectively. The AUROC for the prediction of severe portal hypertension (HVPG $\geq 12 \mathrm{mmHg}$ ) was 0.931 and sensitivity, specificity, PPV and NPV according to HVTT cutoff values of $17.0 \mathrm{~s}$ were $77.5,82.1,90.6$ and $63.2 \%$ respectively.

Conclusion: In this study, the HVTT measurement showed a strong possibility as a non-invasive alternative method in assessment of the severity of portal hypertension in cirrhotic patients and further data obtained from a larger scale of patients are needed.

\section{FC06-03}

Do Beta-blockers Worsen Insulin Resistance in Patients with Liver Cirrhosis?

W.G. Lee ${ }^{1}$, L.D. Plank ${ }^{1}$, R. Murphy ${ }^{2}$, E.J. Gane ${ }^{3}$ J.L. McCall ${ }^{3}$

${ }^{1}$ Department of Surgery; ${ }^{2}$ Department of Medicine, University of

Auckland; ${ }^{3}$ New Zealand Liver Transplant Unit, Auckland City

Hospital, Auckland, New Zealand

Background: Liver cirrhosis is frequently complicated by insulin resistance (IR), glucose intolerance and portal hypertension leading to increased mortality and hepatic decompensation. Non-selective betablockers reduce portal hypertension and prevent variceal bleeding but are known to be associated with deterioration of glucose tolerance and insulin resistance in non-cirrhotic patients with diabetes, hypertension or obesity. A double-blind randomised controlled cross-over trial was performed to investigate if a similar association was present in liver cirrhosis.

Methods: Fifteen cirrhotic patients (10 male, 5 female, age range 45-61 years) with mixed aetiology underwent a hyperinsulinaemic euglycaemic clamp for measurement of IR and a 75 g-oral glucose tolerance test for measurement of glucose tolerance as per World Health Organisation guidelines. These measurements were carried out following 3 months of treatment with nadolol or placebo and, after a 1 -month washout period, 3 months on the alternative treatment. IR was also measured in 6 healthy volunteers using the clamp procedure. Results: Prior to nadolol treatment, 6 patients had impaired glucose tolerance (IGT) and 2 had diabetes. The cirrhotic patients were more insulin resistant than healthy controls $(p=0.0002)$. Following 3 months of treatment with nadolol, IR of the patients deteriorated $(\mathrm{p}=0.001)$. Four patients developed IGT on nadolol $(p=0.08)$.

Conclusions: Three months treatment with nadolol was associated with an increase in IR and a strong trend of worsening glucose tolerance. This has implications for the prognosis and clinical management of these patients.

\section{FC06-04}

Diagnostic Accuracy of Fibrosis Biomarkers Measured in the Hepatic Vein Compared with Those Measured in the Peripheral Vein

K.T. Suk ${ }^{1}$, D.J. Kim ${ }^{1}$, S.W. Jun ${ }^{1}$, C.W. Kim ${ }^{1}$, M.S. Park ${ }^{1}$, H.S. Kim ${ }^{2}$,

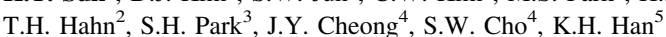

${ }^{1}$ Gastroenterology Internal Medicine, Chuncheon Sacred Hospital

Hallym University College of Medicine, Chuncheon;

${ }^{2}$ Gastroenterology Internal Medicine, Sacred Hospital Hallym University College of Medicine, Seoul; ${ }^{3}$ Department of Internal Medicine, Inje University College of Medicine, Pusan; ${ }^{4}$ Department of Internal Medicine, Aju University College of Medicine, Suwon;

${ }^{5}$ Liver Cirrhosis Clinical Research Center, Yonsei University College of Medicine, Seoul, Republic of Korea

There have been concerns about whether biomarkers which were measured in the peripheral vein $(\mathrm{PV})$ reflect the dynamic turnover of liver fibrosis. We investigated the accuracy of biomarkers checked in the hepatic vein (HV) and PV samples in predicting advanced fibrosis. A total of 101 patients with chronic viral hepatitis that underwent hepatic venous pressure gradient (HVPG), paired PV-HV samplings, and liver biopsy were prospectively enrolled. Thirty nine patients had advanced fibrosis (METAVIR F3-4). The mean level (ng/mL, [SD]) of hyaluronic acid (HA) (107.2 [144.3] vs. 43.8 [76.6]) and tissue inhibitor of metalloproteinases-1 (232.3 [82.7] vs. 194.9 [54.8]) were significantly higher in the PV than in the HV $(p<0.001)$. On the contrary, apolipoprotein A1 level (105.1 [4.6] vs. 130.4 [21.6]) was lower in the PV than in the HV $(p<0.001)$. The level of haptoglobin, matrix metalloproteinase-2 (MMP2), and procollagen III N-terminal peptide did not show difference between the PV and HV. In the multivariate analysis, haptoglobin (odds ratio [OR] 0.98, 95\% confidence interval [CI] 0.97-0.99) and MMP2 (OR 1.02, 95\% CI 1.011.03) were independent predictors of advanced fibrosis in the HV (predictive logit model: $-3.13+0.017 \times \mathrm{MMP} 2-0.019 \times$ haptoglobin). In the PV, HA (OR 1.01, 95\% CI 1.01-1.02) and haptoglobin (OR 0.98, 95\% CI 0.97-0.99) were associated with advanced fibrosis (predictive logit model: $-0.270+0.007 \times \mathrm{HA}-0.018 \times$ haptoglobin). The AUROCs (HV and PV) were 0.805 and 0.780 for predictive logit model, 0.649 and 0.653 for AST/platelet, and 0.662 and 0.665 for AST/ALT, respectively.

Conclusion: Blood sampling only in the PV may not be sufficient to evaluate liver fibrosis with a high degree of accuracy. Additional sampling in the HV during HVPG measurement may be necessary.

\section{FC06-05}

Is Third Generation Cephalosporin Still the First Choice in Spontaneous Bacterial Peritonitis?

A.B. Khalid, S. Majid, T. Hussain, H. Alishah, S. Hamid, W. Jafri Medicine, The Aga Khan University Hospital, Karachi, Pakistan

Introduction: Spontaneous bacterial peritonitis (SBP) is a serious complication in patients with decompensated liver disease. Third generation cephalosporins (TGC) have been effectively used for this infection. However resistance to TGC has been increasing for other infections. It therefore seems imperative to define the resistance of TGC in SBP.

Aims: We aim to find the TGC resistance in SBP and factors responsible for the resistance.

Material and methods: This cross sectional study was conducted on consecutive subjects who received injection Ceftriaxone 2 grams/day for SBP from November 2007 till March 2009 in our unit. SBP was diagnosed based on ascitic fluid Polymorphonuclear (PMN) count of $\geq 250$ cells $/ \mathrm{mm}^{3}$ and or positive ascitic fluid culture. All patients had a repeat diagnostic tap of ascitic fluid $48 \mathrm{~h}$ after the index tap. Failure of reduction of PMN count to less than $25 \%$ in the latter tap and/or ascitic fluid bacteria resistant to TGC were considered as nonresponders. Patients receiving any other antibiotic, prior documented resistance to TGC, hepatoma, patients receiving prophylaxis for SBP were excluded. Results: 229 patients with SBP received TGC during the study period.127 (55.5\%) were males. Hepatitis $\mathrm{C}$ was the commonest etiology in $156(68.1 \%)$ patients.185 (80.8\%) were in Child class C.178 (77\%) responded to TGC while 51(22.3\%) were nonresponders. Ascitic fluid culture was positive in $58(25.3 \%)$ patients. The predominant organism found in ascitic fluid was E. coli in $30(50 \%)$ patients.86\% of cases amongst culture negative group responded to TGC as compared to $50 \%$ of patients in culture positive group. On univariate analysis total bilirubin $>6 \mathrm{mg} / \mathrm{dl}$, prothrombin time $>22 \mathrm{~s}$ and positive Ascitic fluid culture ( $p$ value $0.04,0.02$ and $<0.001$, respectively) were associated with non-response to TGC. On multivariate analysis only ascitic fluid positivity was associated with non response to TGC.

Conclusions: We found $22 \%$ resistance to TGC in SBP. Positive ascitic fluid culture is independently associated with nonresponse to TGC. 
Free Communication 07: Metabolic and Genetic Disease 10:30-11:30H

\section{FC07-01}

Novel Role of Fibroblast Activation Protein in an Experimental Model of Obesity and Liver Steatosis

S. Chowdhury ${ }^{1}$, S. Song ${ }^{1}$, M.G. Gall ${ }^{1}$, X.M. Wang ${ }^{1}$, D.M. Yu ${ }^{1}$, K.A. Evans ${ }^{1}$, L. Lo ${ }^{2}$, G.W. McCaughan ${ }^{1,3}$, S.M. Twigg ${ }^{2,4}$, S.V. McLennan ${ }^{2,4}$, M.D. Gorrell ${ }^{1}$

${ }^{1}$ Centenary Institute; ${ }^{2}$ Bosch Institute, University of Sydney;

${ }^{3}$ AW Morrow Gastroenterology and Liver Centre; ${ }^{4}$ Endocrinology, Royal Prince Alfred Hospital, Sydney, NSW, Australia

Background: Dipeptidyl peptidase 4 (DPP4) inhibitors are a new, successful treatment for type 2 diabetes that recent preclinical studies suggest may also be a potential therapy for obesity, fatty liver and related disorders. Roles of DPP4-related proteases have not been studied in metabolism. This study aimed to investigate the role of Fibroblast Activation Protein (FAP), the closest relative of DPP4, in obesity.

Methods: Female wild type (WT) and FAP gene knockout (gko) were fed ad libitum for 20 weeks either chow or $43 \%$ total calories as fat supplemented with $5 \%$ fructose water. Glucose tolerance, insulin sensitivity, serum insulin and liver function were measured at 20 weeks. Histology and quantitative reverse-transcriptase PCR were performed on liver samples.

Results: Observations of less weight gain, adiposity, serum cholesterol, serum alanine transaminase (ALT) and hepatocellular vacuolation in FAP gko mice suggested that these mice are refractory to obesity and obesity-induced liver injury. In addition, FAP gko mice had improved glucose tolerance, insulin sensitivity and homeostasis assessment (HOMA)-insulin resistance (IR) scores compared to WT mice on the high fat diet. The FAP gko mice also had less intrahepatic mRNA expression of glucokinase, a key lipogenic gene, and CD36, a gene essential for hepatic triglyceride and fatty acid uptake.

Conclusion: These data suggest that FAP has an important role in metabolism and suggest that FAP inhibitors should be evaluated as a unique intervention for treating obesity, obesity-induced liver diseases and the metabolic syndrome.

\section{FC07-02}

Hepatic Iron Loading and Oxidative Stress in Ataxia-Telangiectasia Mice C. McDonald ${ }^{1}$, L. Ostini ${ }^{1}$, D. Wallace ${ }^{1}$, A. John ${ }^{2}$, D. Watters ${ }^{2}$,

V.N. Subramaniam ${ }^{1}$

${ }^{1}$ Membrane Transport Laboratory, Queensland Institute of Medical Research, ${ }^{2}$ Eskitis Institute for Cell and Molecular Therapies, Griffith University, Brisbane, QLD, Australia

Ataxia-Telangiectasia (A-T) is an autosomal recessive disorder resulting in a myriad of abnormalities including progressive neurodegeneration, cancer predisposition and hypersensitivity to ionising radiation. At a cellular level A-T is a disease of chronic oxidative stress (OS) resulting from reduced antioxidant defence capacity leading to the oxidative damage of proteins, lipids, and DNA. Transition metals such as $\mathrm{Fe}^{2+}$ act as pro-oxidative molecules, catalysing the conversion of weakly reactive oxygen species such as $\mathrm{H}_{2} \mathrm{O}_{2}$ to highly reactive hydroxyl radicals, causing significant oxidative damage. Cellular iron accumulation and the resultant OS has also been linked to the neurodegeneration seen in Alzheimer's and Parkinson's diseases, lymphoid tumours (which occur in approximately $30 \%$ of A-T patients), and ionising radiation hypersensitivity. To investigate iron regulation in A-T, iron indices, iron regulatory gene expression, and markers of oxidative stress were studied in livers of wild-type and Ataxia telangiectasia mutated (Atm) null mice on a control diet and when challenged with a high iron diet. At 10 weeks of age, $\mathrm{Atm}^{-/-}$mice on a control diet had increased serum iron, hepatic iron and ferritin, with significantly up-regulated hepcidin (Hamp1) expression compared to wild-type mice. When challenged with the high iron diet Bone morphogenetic protein 6, Hfe, and Ferroportin were all significantly up-regulated in $\mathrm{Atm}^{-/-}$compared to wild-type mice. $\mathrm{Atm}^{-/-}$mice also had significantly higher hepatic OS that increased disproportionately in animals fed the high iron diet.

In summary, we have demonstrated that $\mathrm{Atm}^{-1-}$ mice have a propensity to accumulate iron in spite of the appropriate regulation of genes involved in iron homeostasis. The increased hepatic iron in $\mathrm{Atm}^{-1-}$ mice is associated with a disproportionately large increase in hepatic OS. Our results suggest that increased iron status may contribute to the chronic OS seen in A-T patients.

\section{FC07-03}

Association of Low Bilirubin Levels and Promoter Variations in UGTIAI Gene with Diabetes mellitus Type 2

A. Jiraskova, J. Jovanovska, J. Skrha, L. Vítek

1st Faculty of Medicine, Charles University in Prague, Prague, Czech Republic

Introduction: Bilirubin belongs to the most potent endogenous antoxidants and we have shown that mildly elevated serum bilirubin levels protect from both coronary and carotid atherosclerosis. The objective of this study was to assess, whether serum bilirubin levels and the $(\mathrm{TA})_{\mathrm{n}}$ microsatellite variations in the promoter regions UGT1A1 gene are associated with diabetes mellitus type 2 (DM).

Methods: The study was performed on 206 patients with DM (M:F ratio $=0.96$ ) and 191 healthy controls, in whom standard biochemical tests were performed. The $(\mathrm{TA})_{\mathrm{n}}$ dinucleotide variations were determined by fragment (size-based) analysis using an automated capillary DNA sequencer (CEQ8000, Genetic Analysis System, Beckman Coulter). Logistic regression analysis was used to compare the frequency of the (TA $)_{n}$ variations in both groups.

Results: Compared to controls, both male and female DM patients had significantly lower serum bilirubin levels (9.1 vs. $10.5 \mu \mathrm{mol} / \mathrm{L}$, $p=0.003$ in males, and 9.9 vs. $13.0 \mu \mathrm{mol} / \mathrm{L}, p<0.001$ in females). Simultaneously, frequency of $\mathrm{TA}_{7}$ allele responsible for higher serum bilirubin levels was associated with significantly decreased odds for $\mathrm{DM}$ manifestation in males $(\mathrm{OR}=0.56,95 \% \mathrm{CI}=0.32-0.96, p=$ 0.037), similar, but non-significant trend was observed also in females $(\mathrm{OR}=0.86,95 \% \mathrm{CI}=0.48-1.53, p=0.60)$.

Conclusions: Results of our study suggest that (TA) $)_{n}$ repeat variations in UGT1A1 may provide protection against DM via modulations of serum bilirubin levels, especially in male population.

\section{FC07-04}

Molecular Diagnosis of Galactosemia and Characterization of Spectrum of Mutations in GALT Gene in Galactosemia Patients from North India R. Singh ${ }^{1}$, G. Kaur ${ }^{2}$, B.R. Thapa ${ }^{3}$, R. Prasad ${ }^{1}$

${ }^{1}$ Department of Biochemistry, Postgraduate Institute of Medical Education and Research; ${ }^{2}$ Department of Physiology, Government Medical College and Hospital; ${ }^{3}$ Division of Pediatric

Gastroenterology, Hepatology and Nutrition, Postgraduate Institute of Medical Education and Research, Chandigarh, India

Background: Classical Galactosemia is an autosomal recessive disorder of galactose metabolism caused by severe reduction or absence of the galatose-1-phosphate uridyl transferase (GALT) enzyme and manifests in infancy by failure to thrive, liver dysfunction, cataracts and mental retardation. No reports are available on clinical and molecular spectrum of galactosemia from Indian population. So, the study was aimed at estimation of blood GALT activity in infants with cholestasis and to establish a spectrum of mutations in GALT gene in galactosemia patients.

Methods: 450 infants ( 2 days-11 months) with cholestasis admitted in Pediatric Gastroenterology over 3.6 years were evaluated for galactosemia. Basic investigations included hemogram, liver function tests, blood culture, urine culture, urine for non-glucose 
reducing substances, eye evaluation, abdominal ultrasound, TORCH serology and mebrofenin scan. Screening for GALT deficiency was done using Perkin-Elmer neonatal GALT kit. Levels of galactose-1-phosphate were also measured. Mutation analysis for most common mutations - Q188R and N314D in GALT gene was performed by Restriction Fragment Length Polymorphism (RFLP). Single Stranded Conformational Polymorphism (SSCP) analysis and subsequently DNA sequencing were done for identification and characterization of unknown and novel mutations in GALT gene.

Results: $57(12.6 \%)$ infants were found to have reduced GALT activity with male:female: $39: 18$, jaundice in 56 (98\%), hepatomegaly in $52(91 \%)$, splenomegaly in $34(59 \%)$, coagulopathy in 24 (42\%), encephalopathy in $9(16 \%)$, septicemia in $11(19 \%)$ and cataracts in $13(23 \%)$ were observed. Increased galactose-1 phosphate levels were fraternized with reduced activity of GALT. A total of 16 mutations were detected including 9 novel mutations. N314D mutation was found in 34 patients. Q188R mutation was detected in three patients.

Conclusion: Galactosemia was detected in $12.6 \%$ of patients. Early recognition and dietary restrictions determined the better outcome.

Acknowledgments: This study was funded by Department of Biotechnology, Government of India (BT/PR6344/MED/14/783/2005).

\section{FC07-05}

Molecular Cloning of Copper Binding Protein from Indian Childhood Cirrhosis Liver Disclosed Arginine72 Variant of P53 Having Markedly Apoptotic Potential

R. Prasad ${ }^{1}$, N. Sharma ${ }^{2}$, G. Kaur $^{3}$, B.R. Thapa ${ }^{4}$

${ }^{1}$ Biochemistry, Postgraduate Institute of Medical Education and Research; ${ }^{2}$ Biochemistry, Postgraduate Institute of Medical Education and Research; ${ }^{3}$ Physiology, Government Medical College and Hospital; ${ }^{4}$ Division of Pediatric Gastroenterology, Postgraduate Institute of Medical Education \& Research, Chandigarh, India

Background: Indian Childhood Cirrhosis (ICC) is a disease of abnormal copper metabolism commonly characterized by swelling and degeneration of liver cells along with the presence of orcein staining deposits of copper. The present study was aimed at molecular cloning of reported 50KDa copper binding protein and to elucidate its role in the pathophysiology of ICC.

Methods: Liver samples of children suffering from ICC who died during their admission in the advanced stage of disease were obtained. Molecular cloning of copper binding proteins by the conventional procedure and its expression was done using Immunofloresence microscopy and RT-PCR. Apoptotic properties were assessed by expression of CD95.

Results: Molecular cloning revealed that copper binding protein belongs to Arginine72 variant of P53. Immunofloresence and Immunohistochemistry analysis documented a marked expression of p53 protein in ICC liver. p53 antibodies were also detected in the plasma of ICC patients. On functional characterization expression of p53 cDNA transfected in $\mathrm{Hep}_{2}$ cell lines was significantly increased with increasing extra cellular copper concentration. On Immunohistochemistry, p53 was localized in both cytoplasm and nucleus of transfected $\mathrm{Hep} \mathrm{G}_{2}$ cells. Marked apoptotic potential of Arg 72 p53 was demonstrated by increased expression of CD95 in ICC liver using immunoblot and RT-PCR.

Conclusion: The findings of the present study conclude that Arg form of p53 at codon 72 is found in ICC patients and it gains marked apoptotic potential when copper concentration is increased. In ICC patients' etiology of copper toxicity by consuming milk in brass, copper utensils accounts for increased tumor suppressor activity of Arg 72 form of p53 through activity of CD95.
Free Communication 08: Liver Transplantion \& Miscellaneous

10:30-11:30

\section{FC08-01}

Living Related Liver Transplant (LRLT) in HBV DNA Negative Cirrhosis without Hepatitis B Immunoglobulin (HBIG)

M. Wadhawan ${ }^{1}$, S. Gupta ${ }^{2}$, V. Vij ${ }^{2}$, N. Goyal ${ }^{2}$, P. Dargan ${ }^{2}$, M. Rastogi ${ }^{3}$, K. Vasu ${ }^{2}$, A. Kumar ${ }^{3}$

${ }^{1}$ Gastroenterology, Hepatology and Liver Transplantation, Indraprastha Apollo Hospital, Delhi; ${ }^{2}$ Liver Transplant Surgery, ${ }^{3}$ Gastroenterology and Hepatology, Indraprastha Apollo Hospital, New Delhi, India

Background: HBIG is used in the west for liver transplant in HBV CLD. With potent oral antivirals and concept of adoptive immunity, use of HBIG may not be required. We conducted prospective trial to evaluate HBV prophylaxis post liver transplant without HBIG in living related liver transplants.

Methods: 403 OLT's were performed from January 2005 to September 2010 at our centre. 56 patients had HBV related liver disease. All patients underwent LRLT. All donors were vaccinated with HBV vaccine 15 days before transplant (except ALF). All patients were given oral antivirals for HBV before transplant (except ALF) to achieve DNA negativity or HBV DNA $<2,000 \mathrm{IU} / \mathrm{ml}$. Patients with HBV DNA $<2,000 \mathrm{IU} / \mathrm{ml}$ were not given HBIG, with DNA $>2,000$ $\mathrm{IU} / \mathrm{ml}$ were given im HBIG as per Sydney protocol. Patients were monitored for HBsAg and HBV DNA at 1, 3 and 6 months and subsequently 6 monthly. Recurrence was defined as HBV DNA positive after 6 months post transplant.

Results: Fifty-six patients (age 17-63 years, M:F 49:7) having HBV related liver disease underwent LRLT. Of these 9 were HBV DNA $>2,000 \mathrm{IU} / \mathrm{ml}, 8 \mathrm{HBV}$ DNA positive but $<2,000 \mathrm{IU} / \mathrm{ml}$ and 39 were HBV DNA negative at the time of transplant. Forty-seven patients were not given HBIG, 7 received HBIG. Seventeen patients received combination of Lamivudine and adefovir, 25 baraclude, 8 tenofovir and 2 combination of baraclude and tenofovir. The median follow-up was 20 months (2-60 months). At last follow-up, all patients were HBV DNA negative. Twenty-three patients cleared HBsAg (19 in no HBIG arm, 4 in HBIG arm), 13 patients formed anti HBsAb. Rest were $\mathrm{HBsAg}$ positive with undetectable DNA.

Conclusions: HBV prophylaxis with oral antivirals without HBIG is safe and effective in LRLT setting. A proportion of patients clear HBsAg \& develop Anti HBsAb.

\section{FC08-02}

Post Liver Transplantation Metabolic Complications in Patients with Recurrent Hepatitis $\mathrm{C}$ infection

M. Gondal ${ }^{1}, \mathrm{~K}$. Mumtaz ${ }^{1}$, O. Shakil ${ }^{2}$

${ }^{1}$ Medicine, The Aga Khan University Hospital, Karachi, Pakistan,

${ }^{2}$ Medicine, University of Pittsburg, Pittsburg, PA, USA

Common post-transplantation metabolic complications (PTMC) that occur in liver transplantation (LT) recipients include diabetes mellitus $(\mathrm{DM})$, renal dysfunction (RD), arterial hypertension $(\mathrm{AH})$ and dyslipdemia (DL).

Aims:

1. To study the point prevalence and incidence of new onset PTMC, among LT recipients with $\mathrm{HCV}$ versus non HCV liver diseases.

2. To find the factors predictive of new onset PTMC in LT recipient.

Methods: This study is based on 10 year data collected from database of the University of Pittsburgh Medical Centre (UPMC). Study 
subjects were those with LT for hepatitis C related liver disease (cases) and non HCV liver disease (controls) in a ratio of 1:3.

Results: During the study period (1998 to 2008) 1280 LT were performed. $320(25 \%)$ patients underwent LT for HCV and 960(75\%) had a non HCV liver disease. The distribution of age, gender, BMI, ethnicity, race and CTP score and type of LT were similar in the two groups. The point prevalence of PTMC were equal in cases and controls except for hyperlipidemia in controls $(p$ value $=0.03$ ). Post LT-DM developed in $63 / 222(28.4 \%)$ cases as compared to $158 / 690$ $(22.9 \%)$ controls $(p$ value $=0.09) .108(40.8 \%)$ have developed post LT-RF in cases as compared to $273(34.3 \%)$ in controls ( $p$ value $=$ $0.05) .89(44.1 \%)$ developed post LT HTN as compared to 212 $(33.2 \%)$ among controls; $(p$ value $=0.005)$.

Factors predicting of post LT DM were RF (OR, 0.40; CI: 0.13-1.18; $p=0.09)$ and DL (OR, 8.18; CI: 1.62-41.21; $p=0.01)$. Factor predicting of post LT-RF were solumederol bolus (OR, 5.89; CI: 1.09-31.67; $p=0.03$ ) and graft failure (OR, 2.54; CI: 1.006-6.45; $p=0.04)$. Finally factors predicting post LT-HTN were acute rejection (OR, 11.94; CI: $2.40-59.33 ; p=0.002)$ and post LT DM (OR, 2.03; CI: 1.07-3.86; $p=0.03$ ).

Conclusions: Incidence of post LT DM, HTN and RF is high in HCV related $\mathrm{LT}$ as compared to non $\mathrm{HCV}$. Controlling factors predicting development of these may help in improving LT results.

\section{FC08-03}

Living Donor Liver Transplantation for Recurrent Hepatocellular Carcinoma after Liver Resection

T. Kaido, A. Mori, Y. Ogura, K. Hata, A. Yoshizawa, T. Iida, S. Yagi, S. Uemoto

Graduate School of Medicine, Kyoto University, Kyoto, Japan

Background/aims: Liver transplantation now plays an important role in the treatment of patients with hepatocellular carcinoma (HCC). However, there has been little information about outcomes and indications for living donor liver transplantation (LDLT) in patients with recurrent $\mathrm{HCC}$ after liver resection.

Methods: We retrospectively analyzed $176 \mathrm{HCC}$ patients who underwent LDLT at our institute between February 1999 and December 2009, focusing on patients who underwent LDLT for recurrent HCC after liver resection.

Results: One hundred and twenty-eight patients (72.7\%) received pretreatment for HCC. Among these, 19 recipients underwent liver resection before LDLT. Surgical duration in patients with pretransplant hepatectomy was significantly longer than that in patients with other pretreatments $(n=109)$ or without pretreatments $(n=48)$, while intraoperative blood loss and in-hospital mortality were not different between the 3 groups. The overall survival rate and recurrence rate were not different between the 3 groups. In patients who underwent LDLT following hepatectomy, patients meeting the Kyoto criteria ( $\leq 10$ tumors, all $\leq 5 \mathrm{~cm}$ in diameter and serum des-gamma-carboxy prothrombin levels $\leq 400 \mathrm{mAU} / \mathrm{ml})(n=15)$ showed a significantly higher survival rate than patients beyond the Kyoto criteria $(n=4)$ (5-year $93 \%$ vs. $25 \%, p=0.005)$. Similarly, patients meeting the Kyoto criteria showed a significantly lower recurrence rate than patients beyond the Kyoto criteria (5-year $10 \%$ vs. $67 \%, p=0.011$ ). In contrast, the overall survival rate as well as recurrence rate did not differ between patients who met $(n=6)$ and exceeded $(n=13)$ the Milan criteria.

Conclusions: LDLT can be safely performed in patients with HCC recurrence following liver resection. In particular, favorable longterm outcomes can be obtained in patients meeting the Kyoto criteria.

\section{FC08-04}

Intraoperative Sonazoid-enhanced Ultrasonography Changes the Surgical Management for Colorectal Liver Metastasis
K. Hakamada, D. Kudo, K. Ishido, N. Kimura, M. Nakai, N. Wajima, Y. Toyoki, S. Narumi

Gastroenterological Surgery, Hirosaki University, Hirosaki, Japan

Background and aim: Recent advances in chemotherapy and target therapy have rendered initially unresectable colorectal liver metastases (CRLM) resectable and precise visualization of smaller lesions is the key to improve treatment outcome. The aim of this study was to evaluate tumour vascularity and Kupffer cell imaging in CRLM using contrast-enhanced intraoperative ultrasonography (CE-IOUS) with Sonazoid (perfluorobutane) and to compare performance with conventional image modalities like dynamic CT, MR, and PET-CT.

Methods: We studied 59 nodules in 18 patients undergoing hepatectomy for CRLM. We observed intraoperatively tumour vascularity at the early vascular phase (10-30 s after contrast injection) and Kupffer imaging at the post-vascular phase $(15-60 \mathrm{~m})$.

Results: The detection of tumours at the post-vascular phase on CEIOUS was $92.5 \%$, while sensitivity was $75.4 \%$ with conventional extracorporeal US, $85.5 \%$ with multi-detector row CT, $92.5 \%$ with EOB-MRI, $85.7 \%$ with diffusion weighted image MRI, and $51.8 \%$ with PET-CT. Additional detection of smaller lesions by CE-IOUS had changed operative procedures in $15.6 \%$ of patients, enabling R0 resections.

Conclusion: CE-IOUS with Sonazoid is a useful tool for assessing smaller CRLMs undetected with conventional image modalities. It is expected to improve patients' outcome by introducing CE-IOUS with Sonazoid routinely for the treatment of CRLM.

\section{FC08-05}

Comparative Study between Serum Alpha-feto Protein, VEGF and SCCA in Enhancing Detection of Hepatocellular Carcinoma in Egyptian Patients T. Salman ${ }^{1}$, A.A. Raouf ${ }^{2}$, S.M. Saleh ${ }^{1}$, M. Salama ${ }^{1}$, A.A.-E. Mohammed ${ }^{1}$ ${ }^{1}$ Hepatology; ${ }^{2}$ Clinical Biochemistry, National Liver Institute, Menoufya University, Shebeen El-Kom, Egypt

Background and aims: Hepatocellular carcinoma, as a common malignant solid tumor, is characterized by fast infiltrating, early metastasis and high-grade malignancy. AFP is commonly used as a marker for HCC diagnosis. However, it is not secreted in all cases of HCC and may be normal in as many as $40 \%$ of patients with early HCC. HCC is a highly vascular tumour and consequently angiogenesis is considered to be important for its progression. The most potent and specific known angiogenic factor is vascular endothelial growth factor which is secreted by almost all solid cancers. Serpin squamous cell carcinoma antigen (SCCA) is overexpressed in liver cancer and circulating SCCA-IgM complexes have been described in patients with HCC. The present study was conducted to test the role of AFP, VEGF and SCCA in early diagnosis of HCC in Egyptian patients.

Methods: This study was performed on 90 individuals: 30 cirrhotic, 30 with HCC and 30 healthy individuals. They were subjected to assessment of LFT, AFP, AFP-L3, VEGF and SCCA. Radiological procedures was used for HCC diagnosis.

Results: AFP, AFP-L3, VEGF and SCCA were significantly higher in HCC compared to cirrhotic and control patients. The sensitivity, specificity, PPV, NPV and accuracy of AFP, at a cut off value $7.77 \mathrm{ng} /$ $\mathrm{ml}$, were $80,81.7,95.4,86.8$ and $86.3 \%$, respectively. While in VEGF, at a cut off value $437.5 \mathrm{pg} / \mathrm{ml}$, they were $73.3,61.7,48.9,82.2$ and $77.6 \%$, respectively and SCC, at a cut off value $0.53 \mu \mathrm{g} / \mathrm{L}$, they were $80,76.7,63.1,88.5$ and $84.9 \%$, respectively. The use of combination of the previously mentioned marker enhanced diagnostic possibilities. Conclusion: AFP, AFP-L3, VEGF and SCCA can help in HCC diagnosis, screening, treatment assessment and follow up. Their combination could be used in early HCC detetction especially in cases with mild elevation or normal AFP. 
Emerging Trends Symposium 13:00-15:00

Emerging Trends in the Management of Liver Diseases (Part I)

\section{ET1-01}

Emerging Trends in the Management of Hepatitis C

K.R. Reddy

ET1-02

Emerging Trends in the Management of Hepatitis B

C.-J. Liu

Graduate Institute of Clinical Medicine, Hepatitis Research Center and Department of Internal Medicine, National Taiwan University College of Medicine and Hospital, Taipei City, Taiwan ROC

Hepatitis B vaccination has been shown to preclude HBV infection effectively worldwide. Despite universal vaccination, chronic hepatitis $\mathrm{B}(\mathrm{CHB})$ continues to be a major health burden. The progress on the use of serum hepatitis B virus (HBV) DNA level and quantitative hepatitis B surface antigen ( $\mathrm{HBsAg}$ ) titer has been rapid. Serum HBV DNA levels have been shown to be important in predicting the natural history and the treatment outcomes of chronic HBV infection. Recently, baseline and on-treatment quantitative HBsAg titers have also been introduced to predict treatment response, particularly in patients receiving interferon-based therapy. Over the past decade, rapid progress has been observed with regards to the antiviral therapy for $\mathrm{CHB}$, including conventional interferon, peginterferon, and five oral agents. A common practice worldwide regarding the treatment of $\mathrm{CHB}$ is to maintain long-term viral suppression by using nucleos(t)ide analogues with potent antiviral activity and low rate of developing drug resistance (entecavir and tenofovir), or using a finite course of peginterferon. Nevertheless, one critical challenge and obstacle to the management of $\mathrm{CHB}$ in Asia is the high cost of medical care and antiviral drugs. Besides, for the general practitioners, tailoring the pharmaceutical regimen according to patient features/clinical circumstances, how to enhance the efficacy of current antiviral strategies, and how to ensure the durability of response post-treatment are still challenges. Finally, risk factors for the development of HBV-related hepatocellular carcinoma have been elucidated. Certain scoring systems or nomograms predicting the risk of developing $\mathrm{HCC}$ have just been established. Expectably, such simple-to-use risk scores combining easily measurable clinical variables for the estimation of HCC risk will be of much help in counseling patients with chronic HBV infection on their individual risk of liver disease progression.

\section{ET1-03}

\section{Practical Issues to Increase Compliance and SVR in HCV}

T. Tanwandee

Division of Gastroenterology, Department of Medicine, Siriraj

Hospital, Mahidol University, Bangkok, Thailand

Most effective of treatment for hepatitis $\mathrm{C}$ currently is the combination of peginterferon and ribavirin (PEG/RBV) which can induce the highest SVR. However, such treatment requires a moderately complex regimen of subcutaneous injections of peginterferon, twicedaily oral administration of ribavirin, frequent visits with blood tests to monitor safety, and side effects in nearly all patients. As a result, not all patients complete their course of treatment. In all, the likelihood of benefit depends on total dose exposer to both drugs and the compliance to the treatment is mandatory. Previous study has shown that exposed to more than $80 \%$ of (PEG/RBV) as well as more than $80 \%$ of treatment duration will achieve the highest SVR. In order to achieve the highest SVR, patient's motivation, adherence to treatment protocol and meticulous care to minimize side effects is crucial for the treatment. This can be done by addressing and educating all of these issues to the patients even before treatment initiation, having good support team including patient care team, help line, patient to patient support. Before treatment initiation, all of the risk factors for poor compliance such as drug and alcohol abuse, psychiatric back ground should be addressed. Risk factors for poor tolerance to such treatment should be assessed and monitored frequently including hematologic values, depression and early intervention is helpful in maintaining the treatment as planed. There were studies showing that used of growth factors such as erythropoietin and G-CSF can reduce cytopenia and maintain higher dose of ribavirin and/or peginterferon. As the result, the patient quality of life and SVR was higher. Early use of antidepressant such as citalopram and paroxetine has demonstrated better tolerance in the patients treated with PEG/RBV. In conclusion, patients education, support and early intervention of side effects crucial for improving treatment compliance and increased SVR.

\section{Emerging Trends in the Management of Liver Diseases} (Part II)

\section{ET2-01}

\section{Emerging Treatments of NASH}

A.J. Sanyal

Chairman of Division of Gastroenterology, Hepatology and Nutrition, Virginia Commonwealth University, Richmond, VA, USA

Development of rational therapy for NASH should be dictated by the pathophysiology of the condition. Studies of the pathogenesis of NASH have identified insulin resistance and intrahepatic oxidative stress as key pathophysiologic factors that play an important role in the genesis of NASH. Insulin resistance and oxidative stress are therefore attractive targets for the treatment of NASH. Several studies have evaluated the potential therapeutic value of thiazolidinediones and metformin for the treatment of NASH. A recent large scale clinical trial of metformin for the treatment of NASH in children conclusively demonstrated that it has no impact on liver histology. On the other hand several trials have shown that thiazolidinediones improve liver histology in subjects with NASH. Most trials have used pioglitazone; pioglitazone improves steatosis, inflammation and hepatocellular ballooning the key features of NASH. However, it is associated with weight gain. It is also associated with osteopenia and potentially an increased risk of congestive heart failure. Thiazolidinediones have not been shown to improve hepatic steatosis although a recent meta-analysis suggests that there may be an effect. Vitamin E (400-1200 IU/day) has also been used in trials for NASH. Vitamin E improves steatosis, inflammation and hepatocellular ballooning without affecting mean fibrosis scores. The potential risk of increased mortality with vitamin $\mathrm{E}$ is controversial with several studies showing no effect and other showing a small but significant increase in allcause mortality. Other therapeutic agents which show promise include $\mathrm{n} 3$ polyunsaturated fatty acids, pentoxyfylline, FXR agonists and incretin-mimetics. 
ET2-02

Emerging Trends in the Management of Cholestatic Liver Disease V. Luketic

ET2-03

Emerging Trends in the Management of Hepatocellular Cancer K.H. Han

Concurrent Symposium 14: HBe Seroconversion A Critical Milestone in the Clinical Course of Chronic Hepatitis B

13:00-15:00

\section{C14-01}

Immunological Changes during HBe Seroconversion

F.S. Wang

The Research Center for Biological Therapy, Beijing 302 Hospital, Beijing, China

Hepatitis B e antigen ( $\mathrm{HBe} A g$ ) seroconversion, generally defined as loss of $\mathrm{HBeAg}$ with the appearance of anti-HBe, has been recognized as an important hallmark of events during the natural course of chronic HBV infection and the drug-treatment course of CHB patients because it is found that $\mathrm{HBeAg}$ seroconversion is closely associated with favorable outcomes and is also a critical prerequisite for the clearance of HBsAg. During the course of $\mathrm{HBeAg}$ seroconversion, many important immunological changes have been observed indicating the host immunity may play important roles in the process. These immunological alterations, on one hand, reflect the restoration of the host immunity against virus contribute to $\mathrm{HBe} \mathrm{Ag}$ seroconversion. On the other hand, some early occurred immunological events in $\mathrm{HBeAg}$ seroconversion may severe as potential markers of predicting disease progression. In this report, we summarized the immunological events during HBeAg seroconversion. In particular, we discussed the roles of cytokines such as IL-10 and IL-12 and others because they can directly inhibit viral replication and indirectly determine the predominant pattern of the host immune response, and thus predicting the early, spontaneous $\mathrm{HBeAg}$ seroconversion. In addition, some immunocytes including dendritic cells, T cells and innate immune cells are also summarized in the report. More important, we also comprehensively analyzed related immunological mechanisms and factors that contribute to $\mathrm{HBeAg}$ seroconversion, such as toll-like receptors (TLRs) and programmed death-1 (PD-1) that has been demonstrated to be regulated by the presence of $\mathrm{HBeAg}$. These studies have provided some potential immunological markers to predict $\mathrm{HBeAg}$ seroconversion and further investigate the mechanisms underlying $\mathrm{HBeAg}$ seroconversion. These findings also suggest that a proper restoration of antiviral immunity would require a combination of potent suppression of HBV replication plus immunotherapy, which would amplify the host immunity against HBV to achieve sustained control of HBV replication and resolution of liver disease.

C14-02

HBV mutant breakthrough during HBe seroconversion

G.K. Lau

C14-03

HBe seroconversion - Spontaneous vs. Anti-viral therapy F. Bonino

\section{C14-04}

Should HBe seroconversion be an endpoint of treatment of chronic hepatitis $\mathbf{B}$ ?

S.G. Lim

Chief, Dept of Gastroenterology and Hepatology, National University Health System, Singapore
Background: An important endpoint of clinical treatment of chronic hepatitis $\mathrm{B}(\mathrm{CHB})$ is $\mathrm{HBe} \mathrm{Ag}$ seroconversion, however the importance of this endpoint has been questioned.

Aim: We sought to examine differences in $\mathrm{HBeAg}$ seroconversion and seroreversion rates in spontaneous compared to treatment induced seroconverters.

Method: CHB Clinic patients who were $\mathrm{HBeAg}$ positive with and without treatment with oral nucleoside analogues (lamivudine, adefovir and entecavir) were followed for $\mathrm{HBeAg}$ seroconversion and seroreversion. Durability was defined as maintained anti-HBe and $\mathrm{HBeAg}$ loss. Predictors of these $\mathrm{HBeAg}$ seroconversion were evaluated by multivariate analysis. Data were analysed by Kaplan Meier graphs, log rank test and Cox regression.

Results: There were $569 \mathrm{HBeAg}$ chronic hepatitis B patients who were followed up with $\mathrm{HBeAg}$ seroconversion developing in 246 patients. During a mean followup of 8 years, spontaneous seroconversion occurred in $59 \%$ of patients compared to $81 \%$ after oral nucleoside therapy $(p=0.0001)$. A significant difference was still maintained after Cox Regression adjustment for differences in baseline variables $(p=0.028)$. However seroreversion occurred in $22 \%$ of treated seroconverters but only $3 \%$ of spontaneous seroconverters $(p=0.0003)$. No differences in $\mathrm{HBe} A g$ seroconversion and seroreversion rates were found between patients treated with lamivudine and adefovir but there were too few entecavir treated patients who had stopped therapy to evaluate seroreversion. Predictors of $\mathrm{HBeAg}$ seroconversion were Chinese ethnicity $(\mathrm{OR}=6.9,95 \%$ CI 4.3-11.2), and a higher baseline ALT (OR $=1.1,95 \%$ CI 1.01-1.12). Out of $100 \mathrm{HBeAg}+$ treatment eligible patients, after 5 years of treatment, 37 would have sustained remission compared to only 23 who had observation only.

Conclusion: $\mathrm{HBeAg}+$ treatment eligible patients had higher sustained remission when treated versus observation, but seroreversion and $\mathrm{HBeAg}$ negative reactivation were associated with higher rates of hepatitis B flares.

Concurrent Symposium 15: Cutting-Edge Diagnostic tools in the Assessment of Hepatic Fibrosis, Cirrhosis and Portal Hypertension

13:00-15:00

\section{C15-01}

Serum fibrosis markers and transient elastography: from statistics to real-life

V. Ratziu

\section{C15-02}

MR Elastography of Liver: An Emerging Non-invasive Technique S.K. Venkatesh

Diagnostic Imaging, National University Health System, Singapore, Singapore

MR Elastography (MRE) is a new method for evaluating tissue elasticity or stiffness. MRE non-invasively measures liver stiffness by employing low frequency sound waves in combination with MRI to generate "elastograms." Elastograms are maps of tissue stiffness shown on a color scale ranging from soft to hard. Since its first application in 2006 by the Mayo Clinic Group, MRE is now routinely used in clinical practice for the evaluation of liver disease at several sites worldwide. Many studies have shown that MRE is reliable and the confidence in the information obtained with MRE grows stronger. Studies have showed that MRE is feasible and can differentiate liver fibrosis from normal liver with a high degree of accuracy. MRE can also distinguish between liver fibrosis of stage METAVIR F0-F1 versus F2-3, and F2-3 versus F4 and F0-F1 versus F4. MRE is the only technique so far to show good differentiation between different stages of liver fibrosis. MRE has good reproducibility and has been 
shown to superior to serum markers and Fibroscan. A preliminary study has also shown that MRE can be used to differentiate benign and malignant tumours of the liver with high accuracy. MRE has some technical limitations in assessment of cases with iron overload and variability due to inflammation or cholestasis. Currently efforts are being made to address these issues. The advantages of MRE includes sampling of entire liver, insensitivity to body habitus, ascites and fatty change in liver. MRE can be easily integrated into a conventional MRI protocol giving a comprehensive assessment of chronic liver diseases. MRE is an emerging technique that will play an increasing role in the assessment of chronic liver disease, can potentially replace liver biopsy for fibrosis detection and shows the most promise in reducing the need for liver biopsy for grading the severity.

\section{C15-03}

Using ${ }^{13} \mathrm{C}$-Methacetin Breath Test to Assess Advanced Fibrosis and Cirrhosis

M.L. Shiffman

Liver Institute of Virginia, Bon Secours Health System, Newport News, VA, USA

Metabolic liver function tests have long been utilized to assess hepatic fibrosis and the degree of liver dysfunction. The ${ }^{13} \mathrm{C}$-Methacetin Breath Test (MBT) utilizing the BreathID ${ }^{\circledR}$ device (Exalenz Bioscience Ltd, Israel) is an automated, non-invasive simple to perform method for assessing hepatic metabolic function (HMF). The MBT is performed by having the patient drink $150 \mathrm{~mL}$ of a tasteless solution containing $75 \mathrm{mg}$ of ${ }^{13} \mathrm{C}$-methacetin. Hepatic metabolism of the substrate produces ${ }^{13} \mathrm{CO}_{2}$ which is measured in exhaled breath through a nasal cannula attached to the Breath ID devise. The Breath ID devise calculates the percentage dose recovery (PDR) and the cumulative percentage PDR over 30 minutes (CPDR30) from the total ${ }^{13} \mathrm{C}$ dose administered. Both PDR peak and CPDR30 remain stable for patients with Ishak fibrosis scores of 0-2 (means: 33 and 11 respectively) and then decline stepwise with increasing fibrosis. Mean values for PDR and CPDR30 in patients with Ishak stage 6 were 16 and 4.7 respectively. Time to peak PDR also remained stable in patients with Ishak fibrosis scores of 0-2 (mean: 22) and then increased stepwise with increasing Ishak fibrosis score (mean for Ishak 6: $31 \mathrm{~min}$ ). $95 \%$ of patients with $\mathrm{PDR}<20, \mathrm{CPRD} 30<6.1$ and time to peak $\mathrm{PDR}>25$ had cirrhosis. In patients with cirrhosis a further decline in PDR and CPDR30 and increases in time to peak PDR were associated with increasing mortality independent of MELD. In a cohort of 395 patients monitored over 2 years patients with the lowest MBT values died despite some patients having a MELD $<15$. In contrast, all patients with high values for MBT survived despite some patients having MELD $>23$. These data strongly suggest that assessing HMF by MBT can provide information regarding fibrosis, the risk of hepatic decompensation and survival.

\section{C15-04}

Invasive or Non-invasive Methods for Assessment of Portal Hypertension S.K. Baik

Department of Internal Medicine, Yonsei University, Wonju College of Medicine, Wonju, Republic of Korea

Portal hypertension as a consequence of liver cirrhosis is responsible for serious complications such as variceal bleeding, ascites and hepatic encephalopathy. Since portal hypertension is related to the survival of cirrhotic patients as well as development of complications, measurement of portal pressure may provide prognostic information in patients with liver cirrhosis. Portal hypertension can be evaluated by measurement of hepatic venous pressure gradient (HVPG) through hepatic vein catheterization. HVPG is the difference between wedged and free hepatic venous pressure. HVPG measurement is used as diagnostic tool, i.e. portal hypertension is defined as be higher than $5 \mathrm{mmHg}$ of HVPG. The technique of HVPG measurement is safe, simple and reliable. The coefficient of variation of HVPG measurement for intraindividual assessment is 7-8\%. HVPG measurement can be useful as a prognostic marker. In addition, baseline and repeat measurement of
HVPG can provide the information whether pharmacological therapy is successful. Consequently, HVPG measurement has been accepted as the gold-standard method for the assessing the severity of portal hypertension and response to drug therapy. Doppler ultrasonography (US) allows the noninvasive investigation of hepatic and portal hemodynamics. In particular, any Doppler US parameter that could be a suitable substitute for the invasive current gold standard for assessing portal hypertension, the measurement of HVPG, would be highly desirable. Doppler's usefulness in assessment of severity of portal hypertension in terms of reproducibility, technical ease and accuracy, and response to drugs that reduce the portal pressure has been proposed. In addition, because most of the patients with cirrhosis and portal hypertension have intrahepatic shunts, they show a decrease in intrahepatic circulatory time. Doppler US using microbubble contrast agents allows measurement of intrahepatic circulatory time. Therefore, application of contrast enhanced Doppler US can be prospective for the assessment of severity of portal hypertension.

Concurrent Session 23: New guidelines in the year 2010 for portal hypertension

13:00-15:00

Primary and Secondary Prophylaxis of Variceal Bleed G.H. Lo

Cirrhotic PHT and HVOTO

S.K. Sarin

Acute Variceal Bleeding - APASL Guidelines

A. Kumar

Concurrent Symposium 16: Controversies in the Management of Portal Hypertension 15:30-17:00

C16-01

Endoscopic therapy alone or endoscopic plus pharmacologic therapy works better for secondary prevention of variceal bleeding?

F. Chan

C16-02

The Effect of Beta-Blockers in Cirrhosis beyond the Reduction of Portal Pressure

A. Kumar

Department of Hepatology, Institute of Liver and Biliary Sciences (ILBS), New Delhi, India

Beta-blockers are a class of drugs which block the action of endogenous catecholamines on beta-adrenergic receptors and are used for various indications like cardiac arrhythmias, cardioprotection after myocardial infarction, hypertension and portal hypertension. Non-selective betablocker (NSBB) significantly reduced the risk of bleeding from esophageal varices by their effect on reduction in portal pressure. More than five hundred papers have been published in the English literature on the use of NSBB in cirrhosis, both for primary and secondary prophylaxis of variceal bleeding. In fact, NSBBs have become one of the most effective preventative therapies for variceal bleeding in patients with cirrhosis. NSBBs seem to have other effects besides reduction in portal pressure. Apart from their effect on variceal bleeding prophylaxis, in many recent studies it was shown that NSBB were reported to lead to a reduction in long-term risk of developing complications of portal hypertension and improving survival. Interestingly, propranolol has been found, in several studies, to reduce the incidence of spontaneous bacterial peritonitis (SBP) 
and postsurgical infections in patients with cirrhosis. NSBBs reduce the risk for SBP and bacteremia even with a lesser reduction in hepatic venous pressure gradient than required by the definition of hemodynamic response. These appear to contribute to a reduction of bacterial translocation probably by increasing intestinal motility or decreasing bacterial overgrowth or intestinal permeability. Bacterial translocation seems to be the first step in a series of events that eventually lead to bacterial infection and several of the complications of cirrhosis and portal hypertension, such as variceal bleeding, hepatorenal syndrome, and hepatic encephalopathy. NSBBs have an important role not only in reducing the risk of variceal bleeding or rebleeding in patients with cirrhosis, but also in improving survival perhaps by reduction of bacterial translocation and subsequent complications. The advantage of using NSBBs, however, must be weighed against the risks associated with their chronic use.

\section{C16-03}

How can we do Endoscopic Screening for Esophageal Varices Costeffectively?

S. Chainuvati

\section{C16-04}

Primary and Secondary Prophylaxis of Gastric Variceal Bleeding: What Is the Difference?

S.K. Sarin

Department of Hepatology, Institute of Liver and Biliary Sciences, Delhi, India

Bleeding from Gastric Varices (GV) is generally more severe with high transfusion requirements and mortality than esophageal varices. The bleeding is more severe in patients with large GOV2 and IGV1. Prophylaxis of bleeding from varices could be primary (first bleed) or secondary (rebleed). In a recent study we treated 89 patients who had never bled from GV; by cyanoacrylate injection (Group I, $n=30$ ) or beta-blockers (BB) (Group II, $n=29$ ) or no treatment (Group III, $n=$ $30)$. The actuarial probability of bleeding from $\mathrm{GV}$ over a median followup of 26 months was $13 \%$ in cyanoacrylate compared to $28 \%$ in BB $(P=$ $0.003)$; and $55 \%$ in no treatment group $(P=0.039)$. Similarly, actuarial probability of survival was higher in the cyanoacrylate compared to BB group ( 90 vs. $72 \%, P=0.048$ ). Size of $G V>20 \mathrm{~mm}$, a MELD score $\geq 17$, presence of portal hypertensive gastropathy and treatment method predicted 'high risk' of first bleed from GV. Primary prophylaxis of GV bleeding is recommended to reduce the risk of first bleed and mortality. Cyanoacrylate injection is more effective than BB. Secondary prophylaxis of GV bleeding is needed after the control of acute GV bleeding. The GV rebleeding and complications rates are high after GV sclerotherapy and ligation. In a recent RCT we evaluated the role of cyanoacrylate injection $(n=32)$ in patients with $\mathrm{GV}$ who had bled before, and compared it with BB therapy $(n=32)$. The actuarial probability of GV bleed free rate was higher in cyanoacrylate than $\mathrm{BB}$ group ( $85 \%$ vs. $45 \%, \mathrm{P}=0.004$ ) and the mortality was lower ( 3 vs. $25 \%$, $P=0.026)$ during a median follow-up of 24 months. There were no serious adverse events with cyanoacrylate injection.

Conclusion: While secondary prophylaxis of GV bleed with cyanoacrylate injection is the standard of care, the primary prophylaxis of GV should only be done for 'high risk' GV and only by experts.

\section{Concurrent Symposium 17: Advances in HCC} Surveillance, Diagnosis, Staging and Treatment 15:30-17:00

\section{C17-01}

What's New in the Practice Guideline for HCC Surveillance

M. Sherman

Department of Medicine, University of Toronto, Toronto, ON, Canada
In 2005 the AASLD issued guidelines for the management of hepatocellular carcinoma. Guidelines for surveillance were included. These were that patients at risk for HCC should undergo surveillance at 6-12 monthly intervals with ultrasonography. Screening with alphafetoprotein (AFP) could be used if ultrasonography was not available or of insufficient quality. In 2010 new guidelines were issued that better defined the target populations for screening. The new guidelines also indicated that screening should be at six monthly intervals, not at 12 monthly intervals, and that AFP should no longer be used for screening. The basis for this change in recommendations was that:

1. Better identification of at-risk patients was possible using a new nomogram based on data from the REVEAL study, the HALT-C study and another hepatitis B study from Hong Kong.

2. Data from Korea suggested that survival was better with a 6month screening interval than a 12 -month screening interval.

3. Data from the HALT C study and another study in the USA indicated that AFP (and DCP) were insufficiently sensitive for general use as screening tests.

4. Cost efficacy analyses mostly showed that addition of AFP to regular ultrasonography increased costs, with minimal increase in benefit

\section{C17-02}

Advanced Imaging Diagnosis and Staging of HCC: How to Comply with the New Guideline?

J.M. Lee

Department of Radiology, Seoul National University Hospital, Seoul, Republic of Korea

Early detection and accurate assessment of small HCC and borderline lesions is of great importance as the therapeutic efficacy of various treatments and the survival of patients with small HCC are much more successful than those for patients with larger tumors. A variety of imaging modalities including US, CT, MRI, and PET, have used in evaluating patients with liver cirrhosis and suspected HCC. The detection of small hepatocellular nodules and precise characterization, however, remain the most challenging area in imaging the cirrhotic liver. In cirrhotic liver, for de novo $1-2 \mathrm{~cm}$ nodules ensuing in a cirrhotic context the specificity and positive predictive power of the "typical" radiological pattern of HCC, characterized by increased contrast enhancement (wash-in) on late arterial phase followed by wash-out on portal venous or delayed phases at one dynamic technique, are nearly $100 \%$, thus avoiding false positive results, although the negative predictive value was only $42-50 \%$. Therefore, any new nodule $>1 \mathrm{~cm}$ showing this vascular pattern can be confidently considered a HCC in cirrhosis. Adopting this "single technique" policy, the sensitivity for malignancy of tiny lesions improves from the $35 \%$ of the "two techniques" policy (the 2005 AASLD guidelines) to $65 \%$, thus sparing in one third of patients the use fine needle biopsy to achieve final diagnosis. A liver containing at least one HCC must be staged with an imaging technique which affords complete anatomic coverage of the liver. Dynamic CT or MRI, are the only acceptable imaging techniques for this purpose. Recent development of tissue specific MR contrast agents, have demonstrated their potential to increase the sensitivity and specificity of liver MRI in HCC. In this lecture, I will review the current guideline for diagnosis of HCC, and recent advances in imaging techniques for evaluation of hepatocellular carcinoma and borderline lesions.

\section{C17-03}

Small Liver Nodule (1-2 cm) in Cirrhosis: Biopsy or Follow up? M. Colombo

1st Division of Gastroenterology, Fondazione IRCCS Ca' Granda Ospedale Maggiore Policlinico, Università degli Studi di Milano, Milan, Italy 
The current definition of early HCC includes "very early" tumors with an indistinct nodular pattern, at pathology and no wash-in on contrast imaging, requiring therefore histological examination for diagnosis. Being dynamic contrast imaging techniques like contrast-CT, gadolinium MRI and sonovue-US (CE-US) the standard of care for a diagnosis of very early/early HCC, dual examination with CE-US and MRI demonstrating contrast wash-in + wash-out, has limited (33\%) sensitivity. Therefore, nodules with negative or discordant imaging results need to be evaluated by eco-guided liver biopsy, and, in case of failure, by enhanced follow-up (every 3 months) with imaging techniques. Ultimately, contrast imaging may be diagnostic in nodules which increase in size. The recently updated AASLD guidelines disqualify CE-US as a sole diagnostic modality for 1-2 $\mathrm{cm}$ HCC nodules, owing to the risk of false positive results in patients with an intrahepatic cholangiocarcinoma. Conversely, HCC nodules may be more efficiently diagnosed by the sequential use of one contrast imaging technique alone between CT scan and MRI, with a sensitivity of $65 \%$ and an absolute specificity (Sangiovanni 2010). Interestingly, by this approach up to $25 \%$ of liver biopsies necessary to achieve a final diagnosis of HCC in this patient population could be spared without increasing the costs. Other studies have confirmed the benefits of the sequential study with a single contrast imaging technique in 1-2 cm HCC nodules (Bolondi 2010, Sherman 2010). The fact that the accuracy of contrast imaging to diagnose early HCC in cirrhosis depends on tumor grade has prognostic implications (Iavarone 2010). Indeed, the finding of most well-differentiated tumors escaping radiological diagnosis compared to less differentiated, more aggressive HCCs, highlights the clinical importance of liver biopsy to detect early tumors with a favorable prognosis.

\section{C17-04}

Treatment of Early Hepatocellular Carcinoma: Towards Personalized Therapy

J. Bruix

Concurrent Symposium 18: Emerging Hepatitis E and Hepatitis B Virus Infection in Special Situations 15:30-17:00

\section{C18-01}

Clinical Significance of Occult Hepatitis B in Blood Donors

\section{F. Blaine Hollinger, M.D.}

Baylor College of Medicine, Houston, TX USA

Occult hepatitis $\mathrm{B}(\mathrm{OHB})$ is a covert clinical entity occurring in an HBsAg negative patient whose blood or tissue contains HBV DNA. At issue is determining the clinical relevance of this event. OHB has been implicated in the transmission of HBV through blood transfusions, in reactivation of $\mathrm{HBV}$ in immunosuppressed patients, and may impact disease and treatment outcomes in $\mathrm{HBV} / \mathrm{HCV}$ coinfected patients. These topics will be reviewed. It is well-recognized that the transfusion of blood containing HBsAg is associated with the development of posttransfusion hepatitis B. In the United States, the implementation of an all-volunteer donor population in conjunction with $\mathrm{HBsAg}$ screening in the 1970 s resulted in a dramatic reduction in the incidence of HBV infection of recipients. Subsequently, it was discovered that high-titered, anti-HBc positive blood that lacked HBsAg and anti-HBs also can transmit HBV. This prompted the addition of anti-HBc donor screening in those countries where HBV is not endemic. Later, it was discovered that units of blood from HBsAg and anti-HBc negative donor samples sometimes contained HBV DNA during the early stage of infection and that this blood is highly infectious. This risk appears to be greater during the seronegative expansion phase of HBV DNA than is seen during the recovery phase of acute disease when anti-HBs may complex with the virus leading to modulation of the disease process and a reduction in the risk of transmission. As interest intensified in evaluating the residual yield of HBV DNA positive donations in the seronegative window period, some unexpected findings emerged. For example, in 2008, the American Red Cross (ARC) tested 3.7 million units of HBsAg and anti-HBc negative blood for HBV by nucleic acid testing (NAT) and detected HBV DNA in 9 donors $(1: 410,000)$ including 6 in $\mathrm{HBV}$-vaccinated donors who developed a subclinical infection and recovered. Four of these were anti-HBs positive in the index donation (anti-HBs concentrations from 3-96 IU/L) whereas one became anti-HBs positive early in follow-up prior to the appearance of IgM anti-HBc. Viral loads were low and ranged from s11-86 IU/mL. Six of the 7 donors who were followed became $\operatorname{IgM}$ anti-HBc positive. Among the 6 vaccinated donors, 5 had non-A genotypes not typically found in the US while subgenotype A2, from which the US and European vaccines are made, was the dominant strain in the unvaccinated donors. Of interest was the fact that 5 of the donors were subsequently discovered to have sexual partners who were HBV chronic carriers with HBV DNA viral loads $>108$ copies $/ \mathrm{mL}$. The infected partners associated with 4 of the donors had the same subgenotype, and full genome sequences verified the close relationship between these isolates. Among the 5 vaccinated donors who were followed for 4-10.5 months, none developed ALT levels above $19 \mathrm{U} / \mathrm{L}$ or complained of symptoms, and all became HBV DNA negative, thereby confirming the effectiveness of vaccination in preventing clinical disease. Only 3 of the $7 \mathrm{HBV}$ DNA positive donors developed detectable $\mathrm{HBsAg}$. In contrast, both of the unvaccinated ARC donors who were followed for at least 7.5 months developed significant ALT abnormalities (119 U/L and $640 \mathrm{U} / \mathrm{L}$ ) but no chronicity. An important issue is whether antiHBs in conjunction with a low viral load in a donor are sufficient to prevent transmission of HBV to a recipient. Several studies suggest that this is the rule including Satake et al (2007) who detected no transmission in 22 recipients transfused with HBV DNA positive components that contained anti-HBs compared to 10 infections that occurred among $37 \mathrm{HBV}$ DNA positive components devoid of antiHBs $(27 \%)$. In summary, NAT is capable of detecting HBV DNA in the absence of HBsAg and anti-HBc. The issue of transmissibility from window period donations exists at some finite level in unvaccinated donors but may not be a factor in vaccinated donors. The public health benefits of HBV NAT by blood banks remain unknown. They are likely to be marginal and at considerable cost in the US and Europe, but necessary in countries where HBV is endemic.

\section{C18-02}

HBV Reactivation in Patients Receiving Chemotherapy

C. Pramoolsinsap

Department of Gastrointestinal Digestive and Liver Disease, Phaya Thai 1 Hospital, Ramathibodi Hospital, Bangkok, Thailand

Hepatitis B virus (HBV) reactivation results from increased viral replication in inactive carriers or patients with prior HBV infection, which may occur spontaneously or secondary to immunosuppressive chemotherapy. Risk of $\mathrm{HBV}$ reactivation is $50 \%$ of patients during chemotherapy, and 50-96\% in patients undergoing solid organ transplants and hematopoietic stem cell transplantation (HSCT). Patients with baseline viral load $>10^{4}$ copies $/ \mathrm{ml}$ and $\mathrm{HBeAg}$-positive, males and younger individuals are more susceptible to reactivation. HBV reactivation in HBsAg-negative/antiHBc-positive patients is lower, 6-10\% in subjects receiving chemotherapy, and over 50\% in HSCT. HBV reactivation is more common with intense chemotherapeutic regimens, i.e. corticosteroids, infliximab, and other anti-tumor necrosis factor therapies. Reactivation may occur any time during chemotherpy but is more common at therapy onset or after therapy completion because of immune reconstitution. $24-100 \%$ of patients develop acute hepatitis flares and mortality rate was $0-50 \%$. In solid organ transplantation $10-19 \%$ of HBV reactivation have serious effects, increased all-cause mortality (RR: 2.49), and increased risk for graft failure (RR 1.44). Prevention is the best managemen, hence prophylactic antiviral with nucleos(t)ide analogue (NUC) is recommended for $\mathrm{HBV}$ carriers and $\mathrm{HBsAg}$-negative/antiHBc- 
positive patients during chemotherapy and immunosuppressive therapy (regardless of HBV DNA levels) and for 6-12 months after cessation of therapy. Lamivudine or telbivudine can be used in patients with low HBV DNA levels and the anticipated duration of treatment is short $(<12$ months). The risk of lamivudine failure is associated with high baseline viral loads $>20,000 \mathrm{IU} / \mathrm{ml}$ (HR 3.91). For patients with high HBV DNA level, pre-emptive NUC with high potency and high barrier to resistance, i.e. entecavir and tenofovir should be administer initially. Recipients of anti HBc-positive liver grafts should receive NUC prophylaxis combined with HBIg. The optimal duration of combined prophylaxis is not known. IFN should be avoided due to bone marrow suppressive effect.

\section{C18-03}

\section{Epidemiology of Hepatitis E: Current Status}

\section{R. Aggarwal}

Department of Gastroenterology, Sanjay Gandhi Postgraduate Institute of Medical Sciences, Lucknow, India

Hepatitis E is caused by infection with a small RNA virus, known as hepatitis E virus (HEV). The virus has four genotypes; of these, genotypes 1 and 2 are known to infect only humans. In contrast, genotypes 3 and 4 primarily infect other mammals, particularly pigs, but occasionally cause human disease. In highly endemic areas, mostly in Asia and Africa, the disease occurs in epidemic and sporadic forms, caused mainly by infection with genotype 1 or 2 virus, acquired through fecal-oral route, usually through contaminated water supplies. Attack rates are highest in young adults. A few patients progress to acute liver failure. The disease is characterized by particularly severe course and high mortality among pregnant women. In persons with pre-existing liver cirrhosis, HEV superinfection can present as acute-on-chronic liver disease. Person-to-person spread is uncommon. Materno-fetal and transfusion-related transmission have been reported. Reservoirs of infection in hyper-endemic areas are unknown, but may include persons with subclinical HEV infection, prolonged fecal viral shedding, and animal reservoirs. In low-endemic regions (Europe, North America, Australia, Japan), occasional cases of locally-acquired HEV infection are reported; these are caused mainly by genotype 3 or $4 \mathrm{HEV}$ acquired possibly through zoonotic transmission from pigs, wild boars or deer. In these areas, chronic infection with genotype $3 \mathrm{HEV}$, which may progress to liver cirrhosis, has been reported among immunosuppressed persons. Anti-HEV antibodies have been detected in populations around the world. Seroprevalence rates are higher in highly endemic areas. However, limitations of antibody assays and waning of antibody levels with time makes interpretation of such studies difficult. Two highly effective vaccines based on recombinant truncated viral capsid proteins have been developed but are not yet commercially available. These vaccines should be of particular use in groups that are at high risk of HEV infection and/or of poor disease outcome.

\section{C18-04}

Hepatitis E in Western World: An Emerging Problem in Post-transplant Patients

M.P. Manns

\section{Concurrent Symposium 19: Hot Topics in Pediatric Liver Diseases}

15:30-17:00

\section{C19-01}

Emerging Metabolic and Genetic Diseases

S.H. Quak

Department of Paediatrics, University Children's Medical Institute, National University Hospital, National University of Singapore, Singapore, Singapore

The improvement of socio-economic status and change of life style has resulted in an outbreak of obesity in recent years. This is associated with an increasing incidence of non-alcoholic fatty liver disease. Although the impairment of liver function may be mild in the early stages, the long term outcome may be poor with development of liver cirrhosis in significant proportion of the patients and liver transplantation may be necessary. Fatty liver disease can recur in the allograft with progression to cirrhosis requiring re-transplantation. Autoimmune hepatitis is more commonly seen in recent years. It may occur as part of the more extensive autoimmune disorder such as systemic lupus erythromatosis or confined to liver alone. Early detection is essential for optimal treatment outcome. With the development of liver transplantation programme in many parts of the world, post-transplant autoimmune hepatitis is an emerging entity. It is characterized by abnormal liver function and can be mistaken for viral hepatitis or graft rejection. The main laboratory feature of this condition is the presence of the autoantibodies. The incidence is reported to be between $10-35 \%$ of patients after liver transplant. Typical histologic features include inter-face hepatitis, lobular activity, portal lymphoplasmocytic infiltration and fibrosis. Reintroduction of steroid or significant steroid increment would lead to improvement. With the advance in diagnostic technology and genetic testing, more and more inherited liver diseases are being identified. Individually genetic liver diseases are not common. Collectively, they form a significant proportion of clinical workload in major liver centres. Many metabolic liver diseases can be treated with liver transplantation and their prognosis is good.

\section{C19-02}

Chronic Viral Hepatitis in Children

M.-H. Chang

Department of Pediatrics, National Taiwan University Hospital, Taipei, Taiwan ROC

Among the hepatitis viruses, hepatitis B virus (HBV) infection and hepatitis $\mathrm{C}$ virus (HCV) infection are the two major causes of chronic viral hepatitis and related liver diseases in children, which may have long term health impact. HBV infection is an important health problem world wide, particularly in Asia where most HBV infection occurs since early childhood. Our long-term follow-up study of chronic HBV infection in children revealed that host immunity is a dominant factor in HBV evolution. While the host immunity is feeble in young children, viral nucleotide diversity is low and viral copy numbers increased. As the disease progressed toward the active or immune-clearance phase, viral diversity increased but viral copy numbers decreased. Host hormonal factor is also an important factor affecting the clinical course of HBV infection. Several new drugs are currently in clinical trials in children and hopefully more choice of therapies will be available in children soon. After primary infection of HCV in children, approximately $55-80 \%$ developed a chronic course of infection with risk for progressive liver diseases. The course of chronic HCV infection is generally benign with mild liver histologic changes in children. However, liver disease may progress with time. Evidence suggests that disease may not progress as rapidly during childhood as for those who are infected in adult life. Serial follow-up of HCV RNA levels and liver histology are helpful for the understanding of natural history and better decision of therapy in children. Combination therapy with interferon and ribavirin are the mainstream of therapy for chronic hepatitis $\mathrm{C}$ in children. High HCV RNA titers and genotype $1 \mathrm{~b}$ are risk factors for severe liver diseases and poorer response to therapy. Many children tolerate treatment better than adults. Children who are treated earlier may have better outcomes because of the shorter duration of infection.

\section{C19-03}

Occult HBV Infection during 20 Years after HB Vaccination in High Risk Neonates

Y. Poovorawan

Center of Excellence in Clinical Virology, Faculty of Medicine, Chulalongkorn University, Bangkok, Thailand

The hepatitis B (HB) vaccine became widely available in 1980 and has become more extensively used since. World Health Organization 
suggested all countries should initiate hepatitis B vaccine immunization to all newborn. In 2009, more than 160 countries worldwide implemented the vaccine in newborns and global vaccine coverage reached nearly $70 \%$. Thailand has included the hepatitis B vaccine as part of the EPI program since 1988. The vaccine showed high efficacy and continuous long term protection. The hepatitis B carrier rate of infants that were born after $\mathrm{HB}$ vaccine implementation into the Thailand EPI program is $0.7 \%$. Our studies showed that high risk infants (mother positive HBsAg) with $\mathrm{HB}$ vaccine immunization at birth, 1 and 6 months or 0,1,2 and 12 months had more than $90 \%$ effectiveness in preventing the infant from becoming an HBV carrier. The risk of infants born to mothers with positive HBsAg becoming chronically infected is associated with a delayed second dose of $\mathrm{HB}$ vaccine, even if the birth dose has been administered promptly. In addition, long term (20 years) protection was demonstrated, evidenced by the fact that there was no infant hepatitis B carrier 1-20 years following vaccination. However, high risk infants who received the vaccine showed subclinical breakthrough infection at a frequency of approximately $1 \%$ per year, as determined by anti-HBc seroconversion or transient anti-HBc positive assays with or without HBV DNA detection. Breakthrough infections may become more frequent in the second decade of life or following adolescence. Noting this risk of asymptomatic occult and breakthrough infections, a second booster of hepatitis B vaccine in adolescents needs to be evaluated.

\section{C19-04}

How to Prevent HBV Infection in Infants Born to High Viremic Carrier Mothers?

N. Leung

Consultant Physician, Alico Ho Miu Ling Nethersole Hospital, Adjunct Associate Professor, The Chinese University of Hong Kong, Taipo, Hong Kong SAR

In the Asia-Pacific region, chronic hepatitis $\mathrm{B}(\mathrm{CHB})$ disease burden mainly resulted from mother-to-child and early childhood horizontal transmission. The risk of intrauterine infection is relatively low. Universal HBV vaccination for all newborns is the key to successful control of HBV infection. Effective plasma-derived vaccines in 1980 s, followed by recombinant vaccines used in mass immunization programs recommended by the WHO have significantly reduced infection among infants and young adults. HBV vaccination with additional hepatitis B immune globulin (HBIG) for HBeAg-positive mothers in Taiwan demonstrated reduced HBV transmission and incidence of liver cancer among children. However, many endemic countries have not been able to provide good coverage due to lack of comprehensive healthcare delivery system. Children are still being infected. Vaccine failure remains a risk especially when HBIG is not available. The proposal to use oral antiviral agents in the third trimester and immediate postnatal period to reduce highly viraemic mothers from transmissing infection has been evaluated in a few small pilot studies. The infection rates in the control arm (HBV vaccination + HBIG) of two small studies were exceptionally high (28-39\%). They demonstrated some benefit of antiviral agents. Recent report in AASLD 2010 showed telbivudine given at week 20-37 of pregnancy to week 4 post partum reduced infection rate significantly from 13.0 to $2.1 \%(p=0.004)$. No safety issues were reported in infants and mothers. Successful promulgation of additional oral antiviral therapy to traditional HBV vaccination and HBIG required comprehensive public education. Mothers must be carefully assessed, counseled and monitored. The peer reviewed publication of trials is essential to properly evaluate the feasibility and benefit to the individual community.
Concurrent Symposium 20: Bridging Productive Research and a Successful Academic Career 15:30-17:00

C20-01

Critical review of the literature to formulate a research question S.G. Lim

Chief, Dept of Gastroenterology and Hepatology, National University Health System, Singapore

There are two separate but related issues. The first is the critical review of the literature and the second is formulation of the research question. The first approach is to determine if the research question has already been addressed by a previous study. If not, then what is the supporting evidence around the subject matter of interest? The researcher has to generate a focused question, or at least have already a question in mind. The best strategy to formulate a focused question comes from principles of evidence based medicine. This uses the formula: Population (P), Intervention (I), Comparator (C) and Outcome $(\mathrm{O})$. For instance, in patients with hepatitis B liver cirrhosis (P), does treatment with lamivudine (I) compared to placebo (C) reduce hepatocellular carcinoma (O). Similar types of formulas are used for diagnostic, prognostic, harm, clinical practice guidelines and other research subjects. Once the question has been formulated, then literature searching begins. Literature search strategies can only lead to selection of pertinent articles of interest and do not represent "critical reviews". Critical reviews need to be performed by critical appraisal methods, which generally are the purview of evidence based medicine and is a separate subject. Pubmed is the best and most broadly used search engine. Initially, a broad search is required so that no important articles will be missed. To do this effectively, the search should use MESH headings. This can be accessed under MESH database in pubmed. For instance under the search term "liver cirrhosis", MESH database covers all of the following terms:

Cirrhosis, Liver

- Cirrhoses, Liver

- Liver Cirrhoses

- Hepatic Cirrhosis

Cirrhoses, Hepatic

Cirrhosis, Hepatic

Hepatic Cirrhoses

Fibrosis, Liver

Fibroses, Liver

Liver Fibroses

Liver Fibrosis

Note that there are minor differences in spelling which may be critical as a single spelling difference may result in completely different search results. The MESH database allows multiple search terms to be linked in order to narrow the search subject. If one wants to search for specific types of studies, then use the "clinical queries" heading in Pubmed. This allows searches under categories of studies such as aetiology, diagnosis, therapy, prognosis, clinical prediction guide and systematic reviews. Under advanced search function"history", we can subsequently link previous searches and narrow the search subject further. Another useful tool is "related citations" which enables us to see similar studies or a closely related study. By using such tools, we are thus able to focus down on finding pertinent articles. In the absence of such articles, this is the opportunity for focused research. 
C20-02

Clinical Research: Do's and Dont's

W.R. Kim

C20-03

How to survive peer review to get papers published?

S. Locarnini

C20-04

How to lead to a successful academic career in hepatology in the Asia Pacific Region

D.S. Chen

\section{February 2011 (Sunday)}

\author{
APASL-ASIAHEP Joint Symposium: Issues \\ in the Treatment of Chronic Viral Hepatitis \\ 08:00-10:00
}

\section{AA-01}

Management of Chronic Viral Hepatitis In Pregnancy

E.J. Gane

\section{AA-02}

Chronic Viral Hepatitis in Children

M. Umar

\section{AA-03}

Persistence of HBeAg in Chronic Hepatitis B Patients with Treatment D. Amarapurkar

Department Gastroenterology and Hepatology, Bombay Hospital and Medical Reasearch Centre, Mumbai, India

$\mathrm{HBe} A g$ seroconversion is an important treatment goal in patients with $\mathrm{HBeAg}+\mathrm{ve}$ chronic hepatitis $\mathrm{B}(\mathrm{CHB})$ and is usually followed by disease remission, HBsAg loss, decreased chance of development of cirrhosis and Hepatocellular carcinoma (HCC) but continued active disease may occur in small percentage of patients. $\mathrm{HBeAg}$ seroconversion rates with different oral antiviral agents and interferons reach up to 50 to $60 \%$ at the end of 5 years of treatment. Persistence of $\mathrm{HBeAg}$ after inferferon treatment is associated with increased chance of cirrhosis, HCC and decreased survival, as contrast to that natural history in patients treated with nucleoside/ tide analogues who have persistence of $\mathrm{HBeAg}$ positivity after treatment is not clear. Patients with persistence $\mathrm{HBeAg}$ positive but very low levels of HBV DNA need to continue the treatment for a long time may be over a period of 5 to 10 years. There is no evidence to support this strategy in clinical practice. Prolonged treatment with nucleoside/tide analogues use may be associated with increased chace of adverse events as well increased risk of drug resistance. Meta analysis of treatment of HBV infection has shown interferon treatment decreases the risk of HCC especially in cirrhotic patients while oral nucleoside/tide analogues reduce the risk of HCC especially in HBe positive non cirrhotic patient. A systemic review of incidence of $\mathrm{HCC}$ in $\mathrm{CHB}$ patients receiving treatment nucleoside/tide analogues over a follow up period of 46 months (32108 months) has shown that incidence of HCC goes down in treated patients especially those with cirrhosis without resistance to the antiviral drugs and those who have persistent virological remission.
It is reasonable to continue patients with persistent $\mathrm{HBeAg}$ in $\mathrm{CHB}$ patients with treatment should be continued with the treatment achieving virological remissions and should be followed rigorously for development of antiviral resistance and side effects.

AA-04

Can Fibroscan Replace Liver Biopsy in the Asia Pacific Region?

L. Lesmana

Department of Medicine, University of Indonesia, Jakarta, Indonesia

With the introduction of Fibroscan (FS) that can measure liver stiffness, many expect that this method could replace liver biopsy (LB) which is invasive and inconvenient. Since its introduction and extensive studies by the French clinicians, now FS has shown its consistency in detecting significant liver biopsy, i.e. F2-4 by the METAVIR scoring system. Currently, normal values of FS and its cutoff levels for different fibrosis stages have gained wide acceptance among physicians. However, FS performance mostly studied in European patients with chronic hepatitis $\mathrm{C}(\mathrm{CHC})$ patients. On the other hand, in the Asia-Pacific region, chronic hepatitis $\mathrm{B}$ $(\mathrm{CHB})$ is the most prominent cause of liver fibrosis and cirrhosis. A number of studies using FS have been reported for $\mathrm{CHB}$, but data from the Asian population are scarce. Overall, FS is accepted as a promising method to detect advanced fibrosis (F3-4) in Asian CHB patients. However, in detecting F2, the performance of FS is less satisfactory. There are several issues to be addressed for Asian population, i.e. the normal range of FS in healthy population, the influence of serum alanine aminotransferase (ALT) fluctuation, metabolic syndrome, hepatic steatosis, etc. Liver stiffness reduced following resolution of acute flares in $\mathrm{CHB}$ patients, suggesting that the timing of FS assessment should be properly defined. In the management of $\mathrm{CHB}, \mathrm{LB}$ is still the gold standard to assess fibrosis regression. The use of FS in monitoring treatment response in Asian population has been studied, but more studies with adequate sample size and appropriate study design are still required. Therefore, although studies on FS have shown promising results, its application in Asian population needs to be further assessed as the current available data were not sufficient to recommend it to replace $\mathrm{LB}$.

\section{AA-05}

Managing HCV Genotype 6 Patients

A. Chutaputti

Section of Digestive and Liver Diseases, Department of Medicine, Phramongkutklao Hospital, Bangkok, Thailand

Chronic hepatitis $\mathrm{C}$ is one of the major public health problem with an estimated 180 million people infected worldwide. Most of the well known guideline minimally mention about HCV genotype 6 which commonly found in Indo-China region. The recent study from Hong Kong comparing 138 chronic hepatitis $\mathrm{C}$ genotype I and 78 chronic hepatitis $\mathrm{C}$ genotype 6 showed largely infected genotype I from blood transfusion while a major proportion of genotype 6 were infected through IVDU. Both genotype have comparable liver biochemistry, $\mathrm{HCV}$ viral load and the same rate of disease progressive and mortality.Another study from 308 in Southeast Asia patients with chronic hepatitis $\mathrm{C}$ genotype 6 who migrate to California, USA were studied. The common risk factors were history of surgery (34\%), blood transfusion $(25 \%)$ and acupuncture $(13 \%)$. There were no major differences in the clinical and virological characteristics between each genotype. 
The SVR rate from many studies showed superior result compare with genotype I with higher SVR rate with 48 weeks treatment.

Sustained virological response in hepatitis C virus-6

\begin{tabular}{|c|c|c|c|}
\hline References & $\begin{array}{l}\text { Number of } \\
\text { HCV-6 } \\
\text { patients/number } \\
\text { of HCV-1 patients }\end{array}$ & $\begin{array}{l}\text { SVR in } \\
\text { HCV-6(\%) }\end{array}$ & $\begin{array}{l}\text { SVR in } \\
\text { HCV-1 } \\
\text { as a control } \\
\text { gruoup (\%) }\end{array}$ \\
\hline Hui et al. [124] & 16 (Hong Kong)/24 & 62.5 & 29.2 \\
\hline Dev et al. [13] & 40 (Asian Australian)/21 & 82 & 61.9 \\
\hline Fung et al. [125] & 21(Hong Knog)/21 & 86 & 52 \\
\hline \multirow[t]{3}{*}{ Nguyen et al. [126] } & $\begin{array}{l}31 \text { (24 weeks IFN } \\
\text { plus ribavirin) }\end{array}$ & 51 & \\
\hline & $\begin{array}{l}23 \text { (24 weeks PEG-IFN } \\
\text { plus ribavirin) }\end{array}$ & 39 & \\
\hline & $\begin{array}{l}12 \text { (48 weeks PEG-IFN } \\
\text { plus ribavirin) }\end{array}$ & 75 & \\
\hline Li et al. [127] & 9 & 89 & \\
\hline \multirow[t]{2}{*}{ PThi [128] } & 42 naïve & 69 & \\
\hline & 33 non-responders & 60 & \\
\hline
\end{tabular}

$H C V$ hepatitis C virus, PEGIRN pegylated interferon; SVR sustained viological response

\section{AA-06}

CHC treatment in the Asia Pacific Region: Treatment response, anemic disorders and ALT flares during treatment

R. Guan

Mt Elizabeth Medical Centre, Singapore

Globally, more than 170 million people are chronically infected with Hepatitis $\mathrm{C}$ virus (HCV) and up to 4 million are newly infected yearly. HCV infection is an important cause of cirrhosis and hepatocellular cancer (HCC) worldwide, and ranks second to chronic hepatitis B infection, as a cause of the above conditions in Asia except in Japan. Cirrhosis develops in up to $15 \%$ of men and up to $5 \%$ of women twenty years after initial infection with HCV. HCC develops in up to $4 \%$ of patients with established cirrhosis. Hepatitis $\mathrm{C}$ virus infection is now the leading cause for liver transplantation worldwide. In the Asia-Pacific region, the estimated prevalence of antibodies to $\mathrm{HCV}$ (anti-HCV) range from $0.3 \%$ (New Zealand) to $5.6 \%$ (Thailand). Hyper endemic spots (anti-HCV prevalence up to 58\%) are found in Japan, Middle East, Vietnam and Taiwan. Like in the West, pegylated-interferon (IFN) plus ribavirin is the standard of care for patients in the Asia Pacific region presently. Asians have a higher likelihood of achieving a sustained virological response (SVR) than Caucasians when treated with standard of care doses and duration: $70 \%$ for $\mathrm{HCV}$ genotype 1 (HCV-1) infected patients, $90 \%$ for $\mathrm{HCV}-2 /$ 3 patients, $65 \%$ for $\mathrm{HCV}-4$ patients, and around $80 \%$ patients for HCV-6 patients. Recently a genetic polymorphism near the IL28B gene, encoding interferon-g-3 (IFN-g-3), on chromosome 19, rs12979860 has been found to be associated with treatment response. Individuals with the CC genotype have a significantly increased SVR after IFN treatment compared to individuals with the TT genotype. The CC genotype is seen more frequently in East Asians than in other regions of the world. Patients with lower baseline viral loads $(<400$ $000 \mathrm{IU} / \mathrm{mL}$ ) and rapid virological responses (RVR, undetectable HCV RNA at week 4) can be treated with shorter courses of standard of care treatment. Treatment related anaemia affects Asian patients much more than Caucasians because of pretreatment nutritional anaemia and thallasaemia. Reduction of ribavirin to the highest tolerable dose supplemented by parenteral erythropoietin if necessary helps maintain SVR in these patients. Less than $5 \%$ of patients developed ALT flares during therapy probably from ribavirin sensitivity.

Concurrent Symposium 21: Large HCC and Downstaging - A Difficult but Challenging Task

08:00-09:30

C21-01

Downstaging for Resection or Liver Transplantation

Y. Sirivatanauksorn

HPB\&Transplant Surgery Unit, Department of Surgery, Faculty of Medicine Siriraj Hospital, Mahidol University, Bangkok, Thailand

The majority of hepatocellular carcinoma (HCC) patients are diagnosed in late stages. About $10 \%$ of cases are perfectly for potentially curative treatment either hepatectomy or liver transplantation (LT). Downsizing $\mathrm{HCC}$ or the expansive term of downstaging $\mathrm{HCC}$ is the multimodality approach to advanced or unresectable HCC with the intention of cure management. The candidates for tumour downstaging therapy are primarily considered by the tumour factors, not by the conditions of patients or liver function, which are large size tumour with insufficient future hepatic remnant (FRL), extensive and multifocal bilobar tumours, and main portal vein/hepatic vein/inferior vena cava involvement. Various tumour downstaging regimens such as transarterial therapy, radiofrequency, portal vein embolisation and radiotherapy have been used to reduce tumour size, damage small tumour nodules, enlarge the FRL parenchyma, and demolish of venous thrombi. The selection of regimen depends on many factors and the patients should undergo liver resection subsequently. Though the reported outcome after salvage surgery is comparable to primary HCC resection with the 5-year survival up to $50 \%$, less than $20 \%$ of patients respond to the downstaging regimens. Moreover, with the limited publications, the factors of the predictive responders have not been established. In LT, most centres widely used 'Milan criteria' for waitlists of the candidates with HCC. In the patients who are not met the criteria, transarterial chemoembolisation (TACE) is commonly used and potentially effective downstaging modality. Nevertheless, the results of downstaging therapy to the tumour recurrence and survival after LT are arguable. Tumour progression during TACE for the LT candidates seems to be the favourable predictor and selector for the proper patients who go through LT.

\section{C21-02}

Role of Pre-operative Portal Vein Embolization

K. Hasegawa, T. Aoki, Y. Inoue, M. Ikeda, T. Ishizawa, Y. Beck,

Y. Sugawara, N. Kokudo

Hepato-Biliary-Pancreatic Surgery Division, Department of Surgery, University of Tokyo, Tokyo, Japan

Preoperative portal vein embolization (PVE) has been regarded as a useful intervention in a patient in whom major hepatic resection is needed, but the estimated future liver remnant is too small to allow its safety. Against hepatocellular carcinoma (HCC), frequently associated with liver dysfunction, major hepatic resection has high risks of postoperative liver failure. To avoid such a critical liver failure, PVE following transcatheter arterial chemoembolization (TACE) against HCC sometimes enables a surgeon to perform major hepatic resection for HCC. In addition to its anti-HCC effects, TACE is also useful to promote hypertrophy of the future liver remnant induced by PVE through occluding the arterio-portal shunts that are often accompanied with chronic liver dysfunction and HCC. In 2004, we previously reported the results of sequential preoperative TACE and PVE in 17 patients with HCC. PVE was performed a median of 9 days (range, 4-48 days) after selective TACE of the segments to be resected. In 16 patients 
among 17, we could safely perform hepatic resection a median of 21 days after PVE, which induced sufficient hypertrophy of the FLR. The 5-year overall and disease-free survival rates were 55.6 and $46.7 \%$, respectively. We have accumulated our experiences of hepatectomy for HCC after TACE + PVE, which will be shown in this presentation. In conclusion, sequential TACE and PVE is an effective preoperative procedure to enlarge surgical indication for HCC.

\section{C21-03}

Extended hepatic resection for $\mathrm{HCC}$ and pre-operative assessment R.T. Poon

\section{C21-04}

Nutritional Support in Cirrhotic Patients Undergoing Hepatic Resection D. Crawford

Department of Medicine, University of Queensland, Brisbane, QLD, Australia

Resection of hepatocellular carcinoma usually occurs in the setting of underlying cirrhosis and is associated with high rates of morbidity and mortality. Patients with cirrhosis often have underlying nutritional deficiencies characterised by reductions in muscle mass, body protein stores, functioning cell mass and body fat stores. Thus many patients who require surgical resection of hepatocellular carcinoma have significant underlying malnutrition. Malnutrition adversely impacts on post-surgical outcome, and following liver resection, patients are at risk of hepatic decompensation, postoperative sepsis, prolonged ventilation, and poor wound healing. The pathophysiological basis of malnutrition in patients with chronic liver disease is multifactorial and the nutritional deficiencies are difficult to treat. Patients have altered substrate utilisation characterised by early recruitment of protein and fat as energy substrates, as well as impaired dietary intake, malabsorption and hypermetabolism in some patients. In general, peri-operative nutritional support can reduce the rate of surgical complications and enhance surgical outcomes in malnourished patients. However, because of the unique pathophysiological basis of malnutrition in chronic liver disease and other reasons, patients with cirrhosis undergoing resection may not show the same response to perioperative nutritional support as other surgical cohorts. Fan et al. studied the impact of perioperative nutritional support in 124 patients undergoing hepatectomy for hepatocellular carcimoma. Patients who received nutritional support had reduced postoperative morbidity, fewer septic episodes, less weight loss and less deterioration in liver function. Additional studies of this nature are clearly required to further define the benefits of perioperative nutritional support in patients undergoing liver resection.

\section{Concurrent Symposium 22: Uncommon Hepatitis C Virus Genotypes}

08:00-09:30

\section{C22-01}

HCV genotype 4

G. Shiha

\section{C22-02}

\section{HCV Genotype 5}

N. Antaki

St Louis Hospital, Aleppo, Syria

$\mathrm{HCV}$ genotype 5 (HCV-5) was initially reported in South Africa where it represents $40 \%$ of all cases and it was thought that it is exclusively found there; However 4 pockets of HCV-5 have been found: in central France, in the north of Syria, in the west Flanders region of Belgium and in Spain and sporadic cases were reported elsewhere. In contrast to other $\mathrm{HCV}$ genotypes, little is known about the origin, evolution and epidemiological profile of HCV-5. Recently, a phylogenetic analysis was performed on sera from South Africa, Belgium, France and Syria and it demonstrated that the Belgian and South African strains formed two distinct clusters of similar diversity and that the strains have been spreading independently in these two areas for more than 100 years; it was speculated that the virus spread from a common pool, located outside of South Africa, in at least two directions at the same time.. For both the Spanish and Syrian populations, the skyline plots show a phase of exponential spread followed by a slowdown around 1950. This pattern is not consistent with transmission through unscreened blood products, which continued until 1990. It rather reflects other iatrogenic interventions, such as the use of unsterilized medical equipment. There are no prospective studies on the treatment of HCV-5. Only 5 non-randomized retrospective studies have been performed reporting SVR rates ranging from $48 \%$ to 83 with 48 weeks of therapy. A viral kinetic study has been conducted and suggested that a shorter duration of therapy may be warranted but this needs further evaluation and prospective studies are needed. We currently recommend treating patients with $\mathrm{HCV}-5$ for 48 weeks. If there is no EVR, we should consider discontinuation (but good data are lacking for RVR and EVR).

\section{C22-03}

Prevalence and Treatment of Hepatitis C Virus Genotype 6

P. Tangkijvanich

Faculty of Medicine, Chulalongkorn University, Bangkok, Thailand

Hepatitis C virus (HCV) genotype 6 distributed primarily in south China and Southeast Asia displays profound genetic diversity. Novel subtypes of $\mathrm{HCV}$ genotype 6 have been discovered continuously and now comprise at least 22 subtypes. The optimal duration of therapy and expected sustained virologic response (SVR) rate with a combination of pegylated interferon (PEG-IFN) and ribavirin (RBV) for genotype 6-infected patients are largely unknown. Previous retrospective studies have suggested that the SVR rate of genotype 6 is superior to that of genotype 1 and is probably similar to that of genotype 2 and 3 . However, whether 24 or 48 weeks of antiviral therapy would lead to different SVR rates in genotype 6-infected patients is not clear. Currently, early viral kinetics parameters such as rapid virologic response (RVR) and early virologic response (EVR) are regarded as important tools in the clinical management of patients with chronic hepatitis C. Preliminary data based on viral kinetics have shown that the rates of RVR, EVR and SVR in genotype 6-infected patients treated for 24 weeks are slightly lower than those in genotype 3 -infected patients treated for 24 weeks but higher than those in genotype 1-infected patients treated for 48 weeks. Thus, treatment with PEG-IFN plus RBV for 24 weeks should be sufficient for genotype 6-infected patients who achieve early viral kinetics parameters. Extending the treatment to 48 weeks may be necessary for patients who have a slow response. These data suggest that the optimal duration of antiviral therapy for genotype 6-infected patients should be individualized according to initial treatment response.

\section{Cost-Effectiveness Workshop: Consensus Parameters For Cost Effective Studies In Hepatitis B Treatment 08:00-09:30}

The Consensus Cost Effective Model - An Overview and Evaluation of Assumptions and Sensitivity Analysis

\section{Y.Y. Dan}

Department of Gastroenterology and Hepatology, Yong Yoo Lin School of Medicine National Univeristy Hospital, Singapore, Singapore

Chronic hepatitis B is endemic in Asian countries and constitutes a significant health and economic burden. While several medications have been approved for the treatment of chronic hepatitis B, there is still 
significant uncertainty with regards to treatment outcomes, cost impact, and benefits in view of the absence of long-term outcomes data and particularly in the economic setting of Asia Pacific countries. A Costeffectiveness symposium was conducted at 20th APASL in Beijing 2010 where consensus was undertaken by representatives of various countries to construct a generic cost-effectiveness model for AsiaPacific countries. This model can be customized for individual countries to take into account the unique local parameters to reflect as accurately the cost impact of Hepatitis B and treatment options. Costeffectiveness model was built using Markov model (Treeage Pro 2011). Modeling was performed for $\mathrm{HBeAg}$ and $\mathrm{HBe} \mathrm{Ag}$ negative patients who fulfill treatment criteria under the APASL guidelines. Comparison is made between no treatment, 1 year Peg-Interferon, low resistance and high resistance threshold nucleoside/nucleotide analogues and compared to the Roadmap strategy. Patients who are $\mathrm{HBeAg}$ positive receive peg-interferon for 48 weeks or continue treatment until 1 year consolidation after eAg seroconversion. Patients who are eAg negative continue oral antiviral lifelong. Rate of eAg seroconversion, HBV DNA response to treatment. reactivation and progression to cirrhosis, HCC, death or liver transplant are calculated based on projection of current best available data and validated against actual life registry and sensitivity analyses. Cost-effectiveness indices are based on USD /QALY. Modeling shows that cost-effectiveness of various drugs are dependant on local variables including cost of treatment, incidence of disease, availability of treatment options such as liver transplant as well as the willingness-to-pay threshold. The assumptions and how sensitivity analysis affect cost-effectiveness is discussed.

\author{
Application of the Consensus Cost Effective Model to Representative \\ Countries in the Asia Pacific \\ Pakistan: S. Hamid \\ Thailand: T. Piratvisuth \\ China: J. Jia \\ Taiwan: C.-J. Liu \\ Korea: K.-H. Han
}

Cost Effectiveness and Impact on Resource Utilisation by Health Care Policy Makers in Asia

K.H. Phua

\section{State-of-the-Art Lecture 2}

09:30-10:00

\section{SA02}

Summary State of the Art Lecture: Angiogenesis in Chronic Liver Disease D. Semela

Institute of Clinical Pharmacology, University of Berne, Berne, Switzerland

Angiogenesis, which is defined as formation of new vessels from preexisting vasculature, as well as vascular remodeling are tightly linked to the progression of chronic liver disease, cirrhosis and hepatocellular carcinoma (HCC). Chronic liver disease and HCC are characterized by activated angiogenic pathways, proliferating endothelial cells, increased microvessel density and an abnormal angioarchitecture. The identification of molecular signalling pathways in angiogenesis led to successful translation of antiangiogenic drugs such as the multityrosine kinase inhibitor sorafenib for treatment of patients with HCC. Multiple other angiogenesis inhibitors such as tyrosine kinase inhibitors (i.e. sunitinib, brivanib, cediranib, pazopanib, TSU-68, imatinib), monoclonal antibodies against circulating VEGF (i.e. bevacizumab), mTOR inhibitors (i.e. rapamycin, temsirolimus, everolimus) and vas- cular targeting agents (i.e. EndoTag-1) are currently in different phases of clinical testing for HCC. Combination of systemic antiangiogenic drugs with other treatment modalities for HCC such as transarterial chemoembolisation (TACE), ablative therapies such as radiofrequency ablation (RFTA) and percutaneous ethanol injection (PEI) have shown promising results in animal models and are under clinical investigation in patients with HCC. This lecture will review modes of angiogenesis (i.e. sprouting and intussusceptive angiogesis), cellular and molecular pathways regulating the formation of blood vessels in the liver. A summary on the current status and an outlook on future developments in the field of angiogenesis in chronic liver disease and $\mathrm{HCC}$ will be given. Emerging problems of angiogenesis inhibitors such as drug toxicities and resistance to antiangiogenic drugs will be also covered.

Keynote Lecture 3

09:30-10:00

KL03-01

Geographic and genetic diversity of hepatitis B virus

M.B. Mizokami

State-of-the-Art Lecture 3

10:30-11:00

SA03-01

Hepatopulmonary Syndrome and Portopulmonary Hypertension: Modern Concepts of Pathogenesis

M.B. Fallon

University of Texas Health Science Center Houston, Houston, TX, USA

Pulmonary vascular complications of liver disease comprise two distinct clinical entities, hepatopulmonary syndrome (HPS, microvascular dilatation and angiogenesis) and portopulmonary hypertension (POPH, vasoconstriction and remodeling in resistance vessels). These complications most frequently occur in patients with cirrhosis and portal hypertension and thereby share pathophysiologic environments and possibly pathogenic mechanisms. HPS is found in $15-30 \%$ of patients with cirrhosis and its presence increases mortality and the risks of liver transplantation, particularly when hypoxemia is present. No medical therapies are available, although liver transplantation is effective in reversing the syndrome. $\mathrm{POPH}$ is found in $4-8 \%$ of patients undergoing liver transplantation evaluation and the presence of moderate to severe disease significantly increases peri-operative transplant mortality. Medical therapies are increasingly effective in improving pulmonary vascular hemodynamics in POPH and may result in better peri-operative outcomes. However, whether liver transplantation itself will consistently improve or reverse underlying POPH remains undefined. HPS and POPH develop in a minority of patients with liver disease and their presence and severity are not closely correlated with the severity of liver disease or with the degree of portal hypertension. In animal models of HPS, both pulmonary microvascular dilation and angiogenesis contribute to hypoxemia and may be inhibited by blocking bacterial translocation, tumor necrosis factor activity or angiogenesis. In human studies, the efficacy of inhibition of bacterial translocation or TNF activity in HPS is less well defined and preliminary findings support that genetic variation in genes involved in angiogenesis may influence susceptibility. Human studies in POPH support that females and those with autoimmune liver disease are at increased risk and that altered estrogen signaling may contribute to susceptibility. 


\section{Special Lecture 3}

10:30-11:00

\section{S03-01}

Risk Prediction for Hepatocellular Carcinoma in Asian Chronic Hepatitis B Patients

P.J. Chen

Hepatitis Research Center, National Taiwan University and Hospital, Taipei, Taiwan ROC

Chronic hepatitis $\mathrm{B}(\mathrm{CHB})$ remains a major cause of cirrhosis and hepatocellular carcinoma (HCC), especially in Asia. The risk of CHB progression to liver cirrhosis or HCC can be significantly reduced by effective anti HBV therapies. Therefore it is important to identify high risk hepatitis $\mathrm{B}$ carriers to give timely interventions. There are many well-known risk factors for disease progress in CHB and a few risk prediction algorithms integrating these factors have been proposed. Such algorithms need to be validated before becoming in wide use, especially in different geographic areas to accommodate local factors. In addition, These algorithms identify CHB patients HCC risk in a continuous spectrum. Thus raises the question about the threshold risk to start and maintain treatments to maximize cost-effectiveness. Finally, this threshold may bridge with current well-accepted guidelines for CHB to treat more patients.

\section{Special Lecture 4} 10:30-11:00

\section{S04-01}

The Changing Trend of Global Burden of Chronic Liver Diseases W.R. Kim

\section{Special Lecture 5} 10:30-11:00

\section{S05-01}

Treatment Monitoring of Liver Cancer by Imaging Techniques

\section{B.I. Choi}

Professor of Radiology, Seoul National University Hospital, Seoul, Republic of Korea

Assessment of tumor response after loco-regional therapies is important in determining treatment success, detecting residual or recurrent tumors, identifying the complications, and guiding future therapy. Various imaging modalities such as contrast-enhanced (CE) US, CT and MRI has a fundamental role in the treatment response monitoring of HCC. Dynamic CT 1 month after ablation is currently preferred as the standard imaging modality for assessing treatment efficacy. However, CEUS is a viable alternative with several inherent advantages: no exposure to ionizing radiation, different imaging planes in addition to axial planes, real-time scanning, and, the same imaging for targeting and treatment. In addition, recent advances in the development of functional imaging techniques have provided an ability to detect microscopic changes in tumor microenvironment and microstructure, allowing assessment of tumor response after loco-regional treatment by observing alterations in tumor viability, perfusion, or vascularity. Diffusion-weighted MRI and apparent diffusion coefficient (ADC) map reflect the water molecule diffusion in tissue and can discriminate the viable tumor from the necrotic tissue. Perfusion CT or dynamic CEMR can assesses the change of the vascularity and perfusion of the tumor after loco-regional therapy. Response Evaluation Criteria in Solid Tumors (RECIST) is the worldwide standard evaluation criteria in the oncology field, thus, it should be mostly respected also in the field of interventional oncology. RECIST was revised to version 1.1 in 2009. But, the use of the RECIST guidelines for the assessment of tumor response is not considered the correct approach for HCC nowadays. The modified RECIST assessment (mRECIST) for HCC has introduced the following amendments to RECIST in the determination of tumor response for target lesions. In this session, the role of various imaging modalities including the advantages, limitations and potential in assessing treatment response of loco-regional therapies for HCC will be presented. 
The 21st Conference of the Asian Pacific Association for the Study of the Liver

\section{Poster Presentations}

\section{Poster Presentation 01: Acute Liver Failure}

\section{PP01-01}

The Study of Traditional Chinese Medicine Preparation to Promote the Differentiation of Stem Cell into Hepatocyte Vitro and in vivo Y. Tang

Experimental Class of 2006 Grade, Xiangya Medical School, Central South University, Changsha, China

Aims: To observe the promotion of traditional Chinese medicine angelica sinensis(oliv.) diels (ASD) and lycium chinense mill (LCM) preparation for the differentiation of human umbilical cord blood stem cell (HUCBSC) into hepatocyte in vitro and in vivo.

Method: Different dose ASD and LCM preparation were added into HUCBSC culture medium to screen the suitable dosage for HUCBSC growth. The rats with acute hepatic injury were randomly divided into eight groups. Some rats were treated by neonate rat blood stem cell (NRBSC) transplantation via rat caudal vein with/without ASD and LCM preparation intragastric administration and then some rats treated with cyclophosphamide intraperitoneally.

Result: Medium dosage ASD and LCM preparation was suitable for HUCBSC proliferation. The expressions of albumen mRNA and AFP mRNA in HUCBSC group added with medium dosage ASD or LCM preparation and HGF were better than those in HUCBSC group without ASD or LCM preparation. The liver function was improved after 7 days in all experimental groups $(p<0.01)$. The improvement of rat liver function in ASD or LCM alone group was better than normal saline control group. Meanwhile, ALT, AST and TBIL in group treated with both NRBSC and ASD/LCM preparation decreased even more than those in group treated with NRBSC alone $(p<0.01)$ or ASD /LCM preparation alone $(p<0.001)$. However, the difference between groups treated with/without cyclophosphamide was not obvious $(p>0.05)$. TBIL decreased more apparently in group treated with both NRBSC and ASD preparation than that in group treated with both NRBSC and LCM preparation ( $p=0.005)$. Conclusion: Suitable dose ASD or LCM preparation can promote the proliferation and differentiation of umbilical cord blood stem cell into hepatocyte in vitro and in vivo and can improve liver function in acute liver injury rats and these are beneficial for the recovery of the damaged hepatocyte.

\section{PP01-02}

A Clinical Study on Human Hepatoma Cell Line Artificial Liver in the Treatment of Liver Failure

S. Xin, H. Liu, S. You

Treatment and Research Center of Liver Failure, 302 Hospital, Beijing, China

Objective: In order to estimate the effects and safety of human hepatoma cell line bioartificial liver (BAL) in the treatment of liver failure, this paper describes the treatment using it in 14 patients with liver failure and 5 as control group.

Methods: The liver failure patients were randomly assigned to treatment and control group in the proportion of $2: 1$, the treatment group was treated with plasma exchange (PE) then treated by the BAL, and control group was only treated with PE. We observed the clinical effects, liver functional indexes and the adverse reactions on two groups.

Results: Survival rates were estimated, the 84 day's survival rate of treatment group is $71.4 \%$, control is $40.0 \%(P<0.01)$. iver function which are statistically different between treatment and control groups are: Total bilirubin $(P=0.002)$, aspartate aminotransferase $(P=$ $0.014)$, however, there are no statistical differences between two groups for alanine aminotransferase, Albumin, Acetyl cholinesterase, blood ammonia. After the bioartificial treatment and during interview, no statistical differences were found for INR $(P=0.375)$. The AFP was increasing during the bioartificial liver treatment; it reached peak value at day 10 and then gradually decreased. The AFP is statistically different between two groups $(P=0.025)$. The adverse reactions of bioartificial liver treatment mainly are: Bleeding at catheter connecting to vein $(64 \%)$, Chills (50\%), Difficult urination $(14.29 \%)$. In total, 10 patients recovered in the treatment group, one of them had liver transplantation, in the three-year interview program with those 9 treated patients and 2 control patients were examined with ultrasonic or CT, no liver cancer identified, the liver function is well.

Conclusions: The liver failure patients had better effect using hepatoma cell line derived BAL treatment, some adverse reactions appeared in treatment, and no serious advert events have been identified.

\section{PP01-03}

Chelating Agents with Antioxidants in the Diagnosis and Treatment of Putative Mercury Poisoning

D. Joshi

School of Studies in Zoology, Jiwaji University, Gwalior, India

Mercury commonly used in industrialized countries, adversely affects human and animal's physiological and biochemical functions. Mercury exposure is the second-most common cause of toxic metal poisoning. Male albino rats were administered a bolus dose of dimethylmercury $(1.0 \mathrm{mg} / \mathrm{kg}$ ) orally for 12 weeks ( 5 days/week). The chelation therapy with NAC alone and combination with antioxidants as zinc and selenium was given for (2 days/week) after toxicant administration. Animals of all groups were sacrificed after $48 \mathrm{~h}$ of last treatments and various blood \& biochemical parameters were performed. In the present study demonstrated that chronic exposure of dimethylmercury led to mark a significant rise in bilirubin, $\gamma$-GT, cholesterol, triglycerides, urea, creatinine and uric acid content with a concomitant decline in albumin concentration. Significant elevation was observed in LPO and mercury concentration in liver, kidney \& brain however a concomitant decline was observed in GSH level after toxicant administration in the same organs. A noticeable fall was observed in the brain marker enzyme acetyl cholinesterase. Combined treatment of zinc and selenium with Nacetyl cysteine to dimethylmercury-exposed rats showed a substantial reduction in the levels of DMM-induced oxidative damage and comet tail length. In conclusion, the results of this study support that the supplementation of zinc and selenium with $N$-acetyl cysteine can improve theDMMinduced blood and tissue biochemical oxidative stress and molecular alterations by recoupment in mean DNA damage.

\section{PP01-04}

Mutual Effect of $\mathrm{Tl}^{+}$and $\mathrm{Ca}^{2+}$ in Inducting of Mitochondrial Permeability Transition Pore in Isolated Rat Liver Mitochondria

S.M. Korotkov

Laboratory of Functional Biochemistry of Muscles, Sechenov Institute of Evolutionary Physiology and Biochemistry, Russian Academy of Sciences, St. Petersburg, Russia

It was found that the mitochondrial permeability transition pore (MPTP) in the inner membrane of succinate-energized mitochondria of rat liver (RLM) was stimulated in the simultaneous presence of $\mathrm{Tl}^{+}$and $\mathrm{Ca}^{2+}$ in sucrose or nitrate salt media supplemented by rotenone. Cyclosporine A (CsA) and ADP inhibited the MPTP. We studied participation of $\mathrm{Ca}^{2+}$ trigger sites both of the adenine nucleotide translocase and of respiratory complex I in opening of the $\mathrm{Tl}^{+}-\mathrm{Ca}^{2+}$-induced MPTP. State 4, state 3, and 2,4-dinitrophenol (DNP)-stimulated respiration and the membrane potential $\left(\Delta \Psi_{\text {mito }}\right)$ as well as swelling of RLM were studied in media containing mixture of $\mathrm{TINO}_{3}$ and $0-100 \mu \mathrm{M} \mathrm{Ca}^{2+}$ either with sucrose or with nitrate salts $\left(\mathrm{KNO}_{3}\right.$, or $\mathrm{NaNO}_{3}$, or $\left.\mathrm{NH}_{4} \mathrm{NO}_{3}\right)$. The $\mathrm{Tl}^{+}-\mathrm{Ca}^{2+}$. induced MPTP was shown as an increase of the swelling as well as a decrease of state 4 , or state 3 , or DNP-stimulated respiration, and as 
dissipation of $\Delta \Psi_{\text {mito. }}$ These effects of $\mathrm{Tl}^{+}$increased in the order of sucrose $<\mathrm{NH}_{4}{ }^{+} \leq \mathrm{Na}^{+}<\mathrm{K}^{+}$. They were potentiated by inorganic phosphate and decreased by MPTP inhibitors (ADP, CsA, $\mathrm{Mg}^{2+}$, rotenone, EGTA, and ruthenium red) both individually and more potent in their combinations. Maximal swelling both of nonenergized and of energized RLM in free rotenone media containing $\mathrm{Ca}^{2+}$ is accounted for a $\mathrm{Ca}^{2+}$ uptake, supported by mitochondrial endogenous substrates. Rotenone markedly decreased the swelling of the mitochondria. This indicates probable participation of $\mathrm{Ca}^{2+}$ trigger sites both the respiratory complex I and the adenine nucleotide translocase in induction of the MPTP. It is argued for that $\mathrm{Tl}^{+}$(regardless of the used respiratory substrates) can stimulate the MPTP only in the presence of $\mathrm{Ca}^{2+}$. The decrease of state 4 , state 3 , and DNP-stimulated respiration is discussed in terms of the greater increase of the swelling in the nitrate media in comparison with similar experiments free of $\mathrm{Ca}^{2+}$.

\section{PP01-05}

Efficacy of Intravenous Glycyrrhizin in the Early Stage of Acute Onset Autoimmune Hepatitis

K. Fujiwara, S. Yasui, A. Tawada, Y. Fukuda, T. Chiba, M. Arai, T. Kanda, F. Imazeki, O. Yokosuka

Graduate School of Medicine, Chiba University, Chiba, Japan

Background: There is no gold standard for making the diagnosis of acute onset autoimmune hepatitis (AIH). Therefore, losing the timing of starting immunosuppressive therapy, some of them develop into severe or fulminant form and have poor prognosis. Our aim was to elucidate the efficacy of intravenous glycyrrhizin for acute onset AIH. Methods: Thirty-four patients were defined as acute onset AIH based on our uniform criteria, and were enrolled in this study. We prospectively treated 20 patients with sufficient doses of intravenous glycyrrhizin (A glycyrrhizin-containg herbal medicine, Stronger Neo-Minophagen C, SNMC, $100 \mathrm{ml} /$ day) at an early stage, and treated 14 patients of severe disease with SNMC and corticosteroid (CS), and examined clinical, biochemical and pathological features and treatment responses.

Results: The differences in mean age, sex, mean ALT level, mean IgG level, and ANA titer were not statistically significant. Mean T-Bil level was higher and mean PT activity was lower in SNMC+CS group than in SNMC group $(p<0.001)$. The proportion of acute hepatitis was higher in SNMC+CS group than in SNMC group $(p=0.03)$. The AIH score was higher in SNMC + CS group than in SNMC group $(p=0.01)$. Recovery rate was higher in SNMC group than in SNMC + CS group $(p=0.01)$ The ALT level fell during 2-4 weeks after starting therapy in SNMC group with no significant difference compared with SNMC+CS group, and responsiveness to the therapy was determined by the disease severity at the time of starting therapy rather than the time duration from onset to therapy.

Conclusions: The early introduction of sufficient doses of intravenous glycyrrhizin might prevent disease progression of patients with acute onset AIH. Intravenous glycyrrhizin will be used safely and be of help for patients with difficult-to-diagnose acute liver disease as an 'initial' treatment tool to improve liver inflammation before starting disease-specific treatments.

\section{PP01-06}

Better Prognosis of ACLF in Patients with Compensated Cirrhosis and Acute Assault by HEV: Lesson from a Bangladeshi Prospective Study M.A. Mahtab ${ }^{1}$, F. Akbar ${ }^{2}$, S. Rahman ${ }^{1}$

${ }^{1}$ Hepatology, Bangabandhu Sheikh Mujib Medical University, Dhaka, Bangladesh; ${ }^{2}$ Medical Sciences, Toshiba General Hospital, Tokyo, Japan

Background/aims: Little is known about acute-on-chronic liver failure(ACLF) in developing countries. We conducted a prospective study in Bangladesh.
Methods: 32 patients with ACLF were enrolled. All had diagnosed or undiagnosed chronic liver disease (CLD) and developed jaundice and coagulopathy, complicated by ascites and/or encephalopathy within 4 weeks. Causes of CLD were explored in all and nature of acute assaults was assessed. Patients were treated by anti-viral drugs and broadspectrum antibiotics. Also, conservative managements with protein restriction, ensuring bowel evacuation, albumin infusion etc. were employed. None received artificial liver support or liver transplantation. Results: Serum bilirubin was $>5 \mathrm{mg} / \mathrm{dl}$ in all at admission (range $5-25.5 \mathrm{mg} / \mathrm{dl}$ ). Ascites was detected in all and 13 also developed encephalopathy. Most were suffering from compensated CLD or chronic hepatitis B. HBV was main cause of CLD (25/32; 78\%). HEV was mainly responsible for acute assault $(17 / 32 ; 53 \%) .7$ died during observation period of 90 days ( 1 within 15,1 after 45 and 5 within 60 days). Encephalopathy was detected in all dead patients. Out of 7 fatal cases, only 1 was suffering from HBV-CLD. HEV was responsible for acute assault in 1/7 fatal cases.

Conclusions: HBV is endemic in Bangladesh. HEV has replaced $\mathrm{HAV}$ as dominant agent of acute hepatitis in Bangladesh. Prognosis of ACLF was comparatively better in this cohort without any aggressive or advanced therapy. Acute assault with HEV in compensated cirrhosis due to $\mathrm{HBV}$ may result in better prognosis of ACLF. This study recommends that ACLF patients should be checked for HEV in Bangladesh and other developing countries.

\section{PP01-07}

Leptin Trend Relate to Prognosis of Acute on Chronic Liver Failure with HBV Infection

H. $\mathbf{L i}^{1}$, K. Tang ${ }^{2}$, J. Jia $^{1}$

${ }^{1}$ Liver Research Center, Beijing Friendship Hospital, Capital Medical Univeristy, China, Beijing; ${ }^{2}$ Intensive Care Unit, Tianjin Infectious Disease Hospital, Tianjin Infectious Disease Hospital, Tianjin, China

Background: Malnutrition is a common phenomenon in HBV related acute on chronic liver failure patients. But there has no consensus on objective evaluation because most nutrition indictors related to liver metabolism but most leptin is relate to fat cells. So can leptin act as an objective indicator for malnutrition in liver failure patients is still a question.

Methods: Total 31 patients with HBV related acute on chronic liver failure with same treatment strategy were enrolled. According to their prognosis, 13 patients were allocated to death group and other 18 patients were in improved group. Meanwhile, 30 inpatients with chronic hepatitis B were enrolled as control group. Leptin and subcutaneous fat was measured in all patients at day 0,14 respectively. Results: At first, leptin level was lower in improved group than death group at day $0(1,460.44 \pm 930.48$ vs. $3,046.38 \pm 1,396.91 \mathrm{pg} / \mathrm{ml}, P=$ 0.001). But triceps skin fold thickness had no statistical significance between two groups $(11.61 \pm 3.71 \mathrm{~mm}$ vs. $16.30 \pm 8.69 \mathrm{~mm}, P=$ 0.117). Compared with control group, higher leptin level in death group $(3,046.38 \pm 1,396.91$ vs. $2,219.21 \pm 997.09 \mathrm{pg} / \mathrm{ml}, P<0.05)$ and lower in improved group $(1,460.44 \pm 930.48$ vs. $2,219.21 \pm 997.09 \mathrm{pg} /$ $\mathrm{ml}, P<0.05)$ were observed. Both improved and death group had thinner triceps skin fold thickness than control group $(11.61 \pm 3.71$, $16.30 \pm 8.69,28.16 \pm 9.60 \mathrm{~mm}$, respectively). After 2 weeks treatment, leptin level decreased in improved group $(1,460.44 \pm 930.48$ vs. $552.27 \pm 375.13 \mathrm{pg} / \mathrm{ml}, P<0.05)$ while this change was not identified in death group $(3,046.38 \pm 1,396.91$ vs. $4,884.00 \pm 1,682.39$, $P>0.05)$. Meanwhile, triceps skin fold thickness of improved group became thinner $(11.61 \pm 3.71$ vs. $8.94 \pm 3.38 \mathrm{~mm}, P=0.016)$ whereas no obvious changed was observed in death and control groups $(16.30 \pm 8.69$ vs. $13.00 \pm 13.65, P=0.675,28.16 \pm 9.60 \mathrm{~mm}$ vs. $28.63 \pm 9.60 \mathrm{~mm}, P=0.978$, respectively). 
Conclusion: Altough leptin can not reflect the change of subcutaneous fat, it is a good predictor for HBV related acute on chronic liver failure patients.

\section{PP01-08}

IGF-I as a Possible Therapeutic Strategy against Increased Activity of Endonucleases and DNA Fragmentation during Fas-induced Acute Liver Failure

G. Kocic ${ }^{1}$, T. Jevtovic ${ }^{1}$, D. Sokolovic ${ }^{1}$, R. Kocic ${ }^{2}$, T. Cvetkovic ${ }^{1}$

${ }^{1}$ Biochemistry; ${ }^{2}$ Clinic for Endocrinology, Medical Faculty Nis, Nis, Serbia

Background/aim: A number of studies showed that acute liver failure (ALF), induced by toxic or viral agents is a result of apoptosis of hepatocytes, triggered mainly through the Fas-death receptors. The strategy to treat ALF would be to inhibit Fas-mediated apoptosis. Insulin-like growth factor-I (IGF-I) was found to promote proliferation, cell survival and inhibits apoptosis, while the inhibition of auto-/ paracrine action of IGF-I dramatically sensitizes cells for death ligand-induced apoptosis. Since the activation of endonucleases, which cleave genomic DNA into internucleosomal fragments, is considered to be the final hallmark of apoptosis, the aim of the present study was to evaluate the activity of alkaline-DNase I and acid DNase II in relation to DNA fragmentation.

Methods: Fas (CD95)-activating antibodies were applied i.p $(0.8 \mu \mathrm{g} /$ gBW) in BALB mice for $48 \mathrm{~h}$. To study the prevention of Fas-induced $\mathrm{ALF}$, one group of mice was injected simultaneously with IGF-I (daily dose of $6 \mathrm{ng} / \mathrm{gBW}$ ).

Results: ALT and AST were used as the markers of liver damage. DNase II was significantly increased in Fas-antibody treated mice (14.48 \pm 2.89 .vs control 8.27 $\pm 2.20 \mathrm{U} /$ gprot, $\mathrm{p}<0.001)$, more than the DNase I $(10.04 \pm 2.81$ vs control $7.79 \pm 1.85, p<0.05)$. It was followed by increased DNA-fragmentation rate $(8.99 \pm 1.12$ vs. control $5.72 \pm 1.07 \%$ /DNA). IGF-I rescued liver cells from apoptotic cell death by decreasing DNase I $(8.52 \pm 0.73, p<0.001)$ and DNase II $(9.36 \pm 2.08, p<0.001)$ as well as DNA fragmentation $(7.58 \pm 2.01$, $p<0.05$ vs. Fas-antibody treated group), while given alone did not change significantly any of investigated paramethers (DNase II $8.98 \pm 2.46$ DNase I $6.98 \pm 2.74$ and DNA fragmentation $6.17 \pm 1.24$ ). Conclusion: Our results support the role of IGF-I in the control of endonuclease activity, a final down-stream mediator of apoptosis and DNA stability. The application of IGF-I may provide an attractive therapeutic strategy for patients with ALF, one of the most important disease of humans.

\section{PP01-09}

\section{Fulminant Form of HAV Infection}

E. Vashakidze, T. Gegeshidze, T. Buachidze

Tbilisi State Medical University, Tbilisi, Georgia

Hepatitis A is an acute infectious disease, generally having a mild course. The fulminant course of the disease is described rarely $-0.01 \%$, although in the immunocompromised patients same rate is higher -0.1 to $1.0 \%$. During the last 3 years five cases of the acute hepatitis A with hepatic encephalopathy, were reported in the Tbilisi State Medical University database. Three of which had fulminant course and were lethal. Patient age was 4-11 year. 2 out of 3 patients had Wilson's disease as an underlying liver disease. Diagnosis was confirmed by decreased level of Ceruloplasmin and Copper in the blood, as well of the increased amount of copper excreted in urine during $24 \mathrm{~h}$ period. In all five case-reports the diagnosis of HAV was confirmed by the serological investigation (ELISA) with anti-HAV $\operatorname{IgM}(+)$ in the serum, although other markers of the hepatotropic viruses (B, C, D) were not detected. Liver Function Tests showed marked elevation of transaminases, bilirubin and ammonia, decrease of the Prothrombin index. Thus, clinical cases described above, proves that there is a likelihood of developing fulminant forms of the hepatitis A with the lethal outcome. All such cases require diagnostic evaluation of the other pathologic diseases, which might have negative influence on disease outcome. Therefore, making necessary to make vaccination of children against hepatitis $\mathrm{A}$.

\section{PP01-10}

Fulminant Hepatic Failure Due to Dengue Fever during Recent Epidemic in Sri Lanka -What Have We Learnt?

R.L. Satarasinghe, A.N.R. Fernandopulle, R.A.M. Rajamanthri, A.T. Jayalal, A.C.R. Navaratne

Sri Jayewardenepura General Hospital, Colombo, Sri Lanka

Background: Dengue epidemics have been affecting Sri Lanka regularly since 2002 , and dengue deaths due to fulminant hepatic failure had been extremely rare until the latest epidemic.

Aims: To identify the clinico-pathological features of dengue related deaths due to fulminant hepatic failure.

Methods: Case notes of six patients, admitted to the principal author's unit at Sri Jayewardenepura General Hospital, Kotte, Sri Lanka, who had died as a result of dengue fever related fulminant hepatic failure were retrospectively analyzed.

Results: The age range was 29-67 years with a sex distribution of male: female ratio of 1:5. All deaths have occurred between 5th and 9th day of admission. All have been clinically stable and elevation of transaminases had been less than $100 \mathrm{IU} / \mathrm{l}$ on admission. All had unpredictably developed "Dengue Shock Syndrome" and the transaminases had risen above 2,000 IU/l (maximum values AST $\approx 9,000 \mathrm{IU} / \mathrm{l}$, ALT $\approx 6,700$ IU/1). Ranges for ALP and total bilirubin had been 309-792iu/l and 2.0$4.6 \mathrm{mg} / \mathrm{dl}$ respectively. A neutrophil leucocytosis $(\mathrm{WBC}>12,000$ $\mathrm{mm}^{-3}$ ) had replaced the viral counts in all. Equal numbers had $\mathrm{O}+$ and $\mathrm{B}+$ blood group antigens. No body had drug overdoses.

Conclusions: Variations in viral virulent features could have been responsible for change in hepatic involvement in contrast to previous epidemics. Female were predominantly affected. Ischaemic liver insult could be the dominant factor for massive hepatic necrosis for which rising neutrophil leucocytosis was the only predictive factor. Further global case reports are needed to confirm our observations.

\section{PP01-11}

Changes after Extracorporeal Liver Assist Device $\left(\right.$ ELAD $\left.^{\circledR}\right)$ in Liver Failure Patients

K. Lee

Asian Centre For Liver Diseases and Transplantation, Singapore, Singapore

Introduction: Liver dialysis is still evolving. Several devices are available. It is not clear whether these devices are life-saving by themselves, even though they appear to improve liver function results. In particular, total bilirubin is reduced with MARS $^{\circledR}$ (Molecular adsorbent recirculating system). ELAD $^{\circledR}$ is a device that contains metabolically active cartridges consisting of cloned immortalized human liver cells (C3A cells). We report our initial experience.

Methods: 6 patients treated with $\operatorname{ELAD}^{\circledR}$ at our center had liver failure and were candidates for liver transplantation. They were all admitted to ICU while work-up for live donor liver transplantation was being considered.

Results: Average MELD score was 26 (range 18-39). 3 of the patients ultimately received a live donor liver transplant and survived. The other 3 died. Average age was 49 years old and the average length of ELAD ${ }^{\circledR}$ was 3.9 days (range 1.5-6 days). $50 \%$ of the liver failure was from hepatitis $B$.

All the patients experienced a significant rise in AST in the first 24 hours of treatment. Average AST was $209 \mathrm{U} / \mathrm{L}$ before treatment and was 1,380 U/L 24 hours later. This came down over the subsequent days. ALT showed no significant change. PT rose significantly during the first $24 \mathrm{~h}$ (23-43 s). Average platelet count fell from 100 ths $/ \mu \mathrm{L}$ to 74 ths $/ \mu \mathrm{L}$. There was no significant fall in total bilirubin in the first 24 hours. 
Conclusions: $\operatorname{ELAD}^{\circledR}$ can be used successfully prior to liver transplantation. Initial blood tests showed a significant rise in AST and PT with a fall in platelet. These changes probably represent cellular dysfunction of the liver cells in the cartridges, and may represent ischemic injury. These changes improve with time. It is important to look for these changes which are ELAD ${ }^{\circledR}$ induced, especially the prolonged PT.

PP01-12

EGF Can Not Reverse the Inhibiting Effects of Plasma from Patients with ACLF on Growth and Proliferation of HepG2 Cells

W. Li ${ }^{1}$, S. Lin ${ }^{1}$, Y. Takikawa ${ }^{2}$, K. Suzuki ${ }^{2}$

${ }^{1}$ Department of Infectious Disease, Zunyi Medical College, Zunyi, China; ${ }^{2}$ Division of Gastroenterology and Hepatology, Iwate Medical University, Morioka, Japan

Backgrounds and aims: The aims of this study are to explore the mechanisms by which the plasma from patients with acute on chronic liver failure (ACLF) inhibit the growth and proliferation of hepatocytes and whether Epidermal Growth Factor (EGF) can reverse this inhibition.

Methods: HepG2 cells were cultured with $50 \%$ plasma from patient with ACLF for 6, 12, 24, 48 and $72 \mathrm{~h}$ with or without 10ng/ml EGF stimulation. Cell proliferation was determined by MTT assay and intracellular cyclin D1, cyclin-dependent kinase 4 (CDK4) expressions were analyzed by western blotting. By the method of Hoechst staining we investigated the apoptosis index of HepG2 cells induced by plasma from patient with ACLF.

Results:

1. The growth and proliferation of HepG2 cells was significantly inhibited by treatment with $50 \%$ plasma from patient with ACLF from 12 to $72 \mathrm{~h}$ when compared with those cultured with $50 \% \mathrm{NC}$ plasma in a time-dependent manner.

2. 5, 10 and $20 \mathrm{ng} / \mathrm{ml}$ EGF significantly induced HepG2 proliferation in presence of NC plasma, but only $20 \mathrm{ng} / \mathrm{ml}$ EGF showed a transient promotion on growth and proliferation of HepG2 cells in presence of plasma from patient with ACLF. After stimulated with $20 \mathrm{ng} / \mathrm{ml} \mathrm{EGF,}$ the proliferation of HepG2 cells was still significantly inhibited in presence of $50 \%$ plasma from patient with ACLF when compared with that of $\mathrm{NC}$ plasma.

3. Apoptosis index of HepG2 was not significantly increased in presence of $50 \%$ plasma from patient with ACLF.

4. WB analysis showed that the intracellular expression of Cyclin D1 in HepG2 cells was obviously inhibited and the expression of CDK4 was also down-regulated after treatment with plasma from patient with ACLF from 12 to $72 \mathrm{~h}$.

Conclusion: Our results suggest that plasma from patients with ACLF inhibits the growth and proliferation of HepG2 cells in vitro and EGF can not reverse this inhibition.

\section{PP01-13}

The Expression of VEGF and TGF- $\beta 1$ in Liver Tissue of Chronic Liver Failure Case

Y.Y. Zhang, M.Y. Zou, J.G. Yao

Infectious Diseases, The 3nd People's Hospital of Nantong, Nantong, China

Objective: To evaluate the clinical significance of the expression of vascular endothelial growth factor (VEGF) and transforming growth factor- $\beta 1$ (TGF- $\beta 1$ ) in the hepatic tissue of chronic liver failure.

Methods: VEGF and TGF- $\beta 1$ antibodies in the hepatic tissues of 28 chronic liver failure (CLF) cases, 30 compensated liver cirrhosis (CLC) cases, chronic hepatitis $(\mathrm{CH})$ cases and healthy controls were detected by the method of streptavidin peroxidase (SP) immunohistochemical staining. The clinical significance of the expression of VEGF and TGF- $\beta 1$ was analyzed.

Results: There was no or very little VEGF or TGF- $\beta 1$ in liver of healthy controls. The VEGF, TGF- $\beta 1$ expression in the liver tissues of $\mathrm{CH}, \mathrm{CLC}$ or CLF cases was more than that in the liver tissues of healthy controls $(P<0.005-0.01)$. VEGF expressed more frequently in the tissues of CLC cases than in those of CH, CLF cases $(P<0.005)$, in CLF cases than in $\mathrm{CH}$ cases $(P<0.005)$; The TGF- $\beta_{1}$ expression in the liver tissues of CLC or CLF cases were more than those in CH cases. In the chronic liver failure tissues, VEGF and TGF- $\beta 1$ expressions were disproportion. The expression of them was more significant in the tissue with regeneration capacity than that of without regeneration capacity. Conclusion: The expression of VEGF and TGF- $\beta_{1}$ was closely associated with the severity of the disease and the regeneration of hepatocytes. VEGF and TGF- $\beta 1$ might act as a factor to show the regeneration capacity of hepatocytes.

\section{PP01-14}

Therapeutic Potential of Ventilago madraspatana against Carbon Tetrachloride Induced Acute Hepatotoxicity

A. Bhardwaj, M. Bhadauria, S. Shukla

SOS in Zoology, Jiwaji University, Gwalior, India

Background and aim: In realization of the interest in herbal medicines and natural products have great potential in the emerging hepatoprotective agents. In this context we have selected Ventilago maderaspatana (Rhamanaceae, commonly known as Raktavalli) against carbon tetrachloride induced acute liver failure in rats.

Methods: Acute liver failure was induced by the single administration of $\mathrm{CCl}_{4}(1.5 \mathrm{ml} / \mathrm{kg}$, ip $)$ in female rats. Treatment of Ventilago maderaspatana $(50,100,200$ and $400 \mathrm{mg} / \mathrm{kg}$, po) was given after $24 \mathrm{~h}$ of toxicant administration. Various blood and tissue biochemical and histological studies were conducted after $24 \mathrm{~h}$ of last administration. Results: Single administration of $\mathrm{CCl}_{4}(1.5 \mathrm{ml} / \mathrm{kg}$, ip) caused a sharp elevation in the activity of serum transaminases, serum alkaline phosphatase and lactate dehydrogenase after 24 hours of administration. Hepatic reduced glutathione level showed marked depletion on the contrary, hepatic lipid peroxidation was enhanced significantly. Treatment with Ventilago maderaspatana (50, 100, 200 and $400 \mathrm{mg} /$ $\mathrm{kg}$, po) after 24 hours of toxicant administration significantly recovered the alteration induced by toxicant in the markers of oxidative stress and markers of liver function test. Maximum recoupment was observed at higher doses of ethanolic extract. Bile flow and bile solids showed almost normal values indicating that Ventilago maderaspatana do not affect the functional integrity of hepatocytes. Improvement was seen in the light microscopy of liver basically supports the biochemical studies.

Conclusion: The treatment with Ventilago maderaspatana at all doses significantly prevented alterations in specific liver markers. The degree of protection was highest at the doses of 200 and $400 \mathrm{mg} / \mathrm{kg}$ after $\mathrm{CCl}_{4}$ exposure. It is suggested that Ventilago maderaspatana has hepatoprotective properties as a natural antioxidant.

\section{PP01-15}

Hepatocyte ER Stress and the Expression of Cyclin D1, MRNA of C-met in Liver of Mouse with Acute Liver Failure W. Zhong ${ }^{1}$, S. Lin ${ }^{1,2}$, C. Pan $^{1}$, Y. He ${ }^{1}$

${ }^{I}$ Department of Infectious Disease; ${ }^{2}$ The Institute of Clinical Research, Zunyi Medical College, Zunyi, China

Backgrounds: Impaired liver regeneration is related to high mortality in patients with acute liver failure, however, the mechanisms involved in the impaired liver regeneration in patients with acute liver failure are not fully understood. Recent studies demonstrated that ER stress of hepatocyte may attenuated the rate of general translation initiation by active PERK and phosphorylates eIF2a, one of three unfold protein response (UPR) subpathways. We hypothesize that sustained hepatocyte ER stress leads to impaired liver regeneration in patients with acute liver failure. In this study, we observed the hepatocyte ER stress and the expression of cyclin D1, c-met in mouse with acute liver failure. 
Methods: Acute liver failure was induced in BALB/C mouse by intraperitoneally injection with D-Galactosamine (D-Galn) and lipopolysaccharide (LPS). The expression of BIP /GRP78, cyclin D1 in liver at $0,1,3,5,7,9 \mathrm{~h}$ were determined by the method of western blotting, and the expression of mRNA of hepatocyte growth factor (HGF) receptor, c-met was investigated by the method of RT-PCR.

Results: The expression of BIP /GRP78 in liver gradually increased 3 $\mathrm{h}$ after intraperitoneally injection withD-Galn and LPS, by $9 \mathrm{~h}$, the expression of BIP /GRP78 in liver gradually decreased. the expression of cyclin D1 in liver gradually decreased from 5 to $9 \mathrm{~h}$ after intraperitoneally injection with D-Galn and LPS. The expression of cmet mRNA decreased from 7 to $9 \mathrm{~h}$ after intraperitoneally injection with D-Galn and LPS.

Conclusion: Our results suggested sustained hepatocyte ER stress exist in liver and may correlate with delayed liver regeneration in mouse with acute liver failure.

\section{PP01-16}

Acute Hepatitis A and its Outcome in a Tertiary Care Hospital Karachi O. Parkash ${ }^{1}$, S.N. Laique ${ }^{1}$, S. Bachani ${ }^{1}$, Z. Nasir ${ }^{1}$, S. Muzzamil-Uddin ${ }^{1}$, A. Memon ${ }^{2}$, S. Hamid ${ }^{1}$

${ }^{1}$ Medicine, Aga Khan University Hospital, ${ }^{2}$ Dow Medical University, Karachi, Pakistan

Introduction: HAV infection occurs worldwide but the incidence is decreasing since the introduction HAV vaccine and improving living conditions. However incidence is still hish in developing countries like in Pakistan. We aim to determine mortality and pattern of HAV infection in Pakistan

Methods: This is a descriptive retrospective cross sectional study conducted at Aga Khan university Hospital Karachi. Duration of data is from 1987 to 2009.

Results: Total of 702 patients' charts were reviewed among them $51 \%$ were male. Viral serology shows HAV IgM 100\% (702) and concomitant other viral infection like HEV IgM, 6.3\% (44), HBsAg 2.1\% (15), $1 \%$ (7) HDV Ab. In this cohort of patients $70 \%$ (488) belong to pediatric population ( $<14$ years age group) with mean age of patients was $11 \pm 8$ years. Liver function shows mean SGPT 1,912 $\pm 1,497 \mathrm{IU} /$ L, mean GGT $177 \pm 228 \mathrm{IU} / \mathrm{l}$, mean ALk.P $307 \pm 245 \mathrm{IU} / \mathrm{L}$, mean PT $17.1 \pm 8$ s. Overall mortality was $1.6 \%$ (11) and most of them $91 \%$ (10) belong to pediatric group. There is significant difference in baseline characteristics of patients who died vs. survive like respiratory rate $(31 \pm 13$ vs. $21 \pm 7 \mathrm{br} / \mathrm{min} ; P$ value 0.001$)$, serum $\mathrm{Cr}(1.75 \pm 2$ vs. $0.8 \pm 0.8 ; P$ value 0.000$)$, PT $(33 \pm 21$ vs. $16 \pm 7 \mathrm{~s} ; P$ value $0.000)$, WBC ( $25 \pm 24$ vs. $8.8 \pm 7 \mathrm{cmm} ; P$ value 0.000$)$.

Conclusion: We have seen significantly higher mortality for acute hepatitis A in this part of world and interestingly higher mortality in pediatric group as compared to adults contrary to western literature.

\section{PP01-17}

Expression of Iron Regulatory Genes in $\mathbf{C C l}_{4}$ Induced and Partially Hepatectomized Liver Injury Models

N. Sheikh ${ }^{1,2}$, I.A. Malik ${ }^{2}$, N. Naz ${ }^{2}$, G. Ramadori ${ }^{2}$

${ }^{I}$ Department of Zoology, University of the Punjab, Lahore, Pakistan;

${ }^{2}$ Department of Internal Medicine, Division of Gastroenterology and Endocrinology, Georg-August University, Goettingen, Germany

Background: A tight regulation of iron metabolism is mandatory for the living organisms as it participates in variety of metabolic processes. DMT1, DcytB, HFE, IRP1 and IRP2 genes are involved in the regulation of body iron metabolism at different levels of absorption. The current study was designed to find out the effect of direct liver injury on the iron metabolism machinery.

Methods: The animal models used in the current study to observe the hepatic changes in the expression of these genes were developed by oral administration of $\mathrm{CCl}_{4}$ or Partial hepatectomy $(\mathrm{PH})$ of the livers. Total liver RNA was isolated, reverse transcribed and studied by real time PCR.
Results: In the model of $\mathrm{CCl}_{4}$ induced acute liver injury, DMT1 gene expression was up-regulated by twofolds $(P=0.08)$ whereas DcytB and HFE gene-expression was down-regulated significantly to 0.52 fold $\left(P=0.016^{*}\right)$ and 0.27 -fold $\left(P=0.04^{*}\right)$. IRP1 and 2 gene expression was significantly affected due to damaging noxea with a decreases in IRP1 gene-expression $\left(P=0.03^{*}\right)$ and increase in IRP2 gene-expression $\left(P=0.0004^{* *}\right)$. In the model of $\mathrm{PH}$ induced direct liver injury the expression of DMT1, DcytB, HFE and IRP1 genes was down-regulated with only significant decrease of IRP1 gene expression $(P=0.014 *)$. IRP2 gene expression was significantly upregulated due to the direct liver injury $\left(P=0.0097^{* *}\right)$.

Conclusion: These findings can be summarized to conclude that acute and direct liver injury leads towards the changes in the iron metabolism genes so as to restrict the availability of iron to the microbes in order to avoid any kind of infections.

\section{PP01-18}

Clinical and Epidemiological Features of Sporadic Cases of Severe Hepatitis $\mathbf{E}$ in Nanjing Region, China

M. Wang, Y. Sui, C. He, L. Gao, C. Wang, F. Qiao, J. Xu, X. Zhang Center of Liver Diseases of PLA, the 81th Hospital of PLA, Nanjing, China

Objective: In order to investigate the clinical and epidemiological characteristics of sporadic cases of severe hepatitis E in Nanjing Region in recent years.

Methods: The clinical data of 45 patients with severe hepatitis E was collected from January, 2000 to December, 2008. The pathogenesis diagnosis was determined by EIA. The clinical diagnosis was made according to standard diagnostic criteria of viral hepatitis founded by Chinese Association of Medicine.

Results: All of 45 cases with severe hepatitis E, there are 38 male and 7 female patients respectively. The range of age is from 35 to 72 years old and the average is 58. Of all the patients, $31(68.9 \%)$ cases recovered and 14 cases died $(31.1 \%)$. There are two epidemic peaks in a year, one is from Feb to May and another one is from July to November. The duration of clinical recovery were delayed for more than 2 months for the survived patients. The survival rate was $91.3,66.7,20 \%$ for patients with severe hepatitis $\mathrm{E}$ in early stage, middle stage and terminal stage, respectively. There was $42.2 \%$ (19/45) of patients super-infected or coinfected with hepatitis A, B, C virus, and 12 of 19 (63\%) of patients super- or co-infected with other hepatitis virus accounted for hepatitis B. The survival rate was higher in the patients with elevated AFP level.

Conclusions: Hepatitis E occurred in Nanjing region for the past few years was featured with elder age, several manifestation, long recovery duration and high mortality. The factors affecting the survival rate are the age, stage of disease, super/co-infection with other hepatitis viruses. AFP level is an useful biomarker to indicate the survival rate.

\section{PP01-19}

Association of Killer Cell Immunoglobulin-like Receptors Polymorphism with Prognosis of Hepatitis B Virus-related Liver Failure

J. Qiu

The Second Hospital of Nanjing, Nanjing, China

Background: Chronic hepatitis B virus (HBV) infection remains a major global health problem, and the prognosis of patients with HBVassociated liver failure is extremely poor. Natural killer (NK) cells activation has been reported to play an important role in liver inflammation during HBV infection. However, its role in prognosis of HBV-associated liver failure is unknown. The activation of NK cells is dependent on the equilibrium between the inhibitory and activating receptors, among which immunoglobulin-like receptors (KIRs) are by far the most polymorphic.

Methods: To investigate whether KIR gene polymorphism are associated with the prognosis of the liver failure, 109 patients with liver failure were collected and divided into survival and death groups based 
on their outcomes. Case-control study was carried out in survival and death groups. 12 loci of KIR were detected by sequence specific primer polymerase chain reaction (SSP-PCR) for each patient.

Results: The frequency of KIR2DS3 was found higher in survival group than in death group $(9.7 \%$ in death group and $25.0 \%$ in survival group, $\mathrm{OR}=0.32, p=0.035$ ).

Conclusions: KIR2DS3 was associated with survival of HBV-related liver failure, suggesting that NK cells may play a role in regulating the outcomes of liver failure.

Acknowledgements: This work is founded by Jiangsu Province Department of Health Foundation (H200745).

\section{PP01-20}

Acute Flare in Chronic Hepatitis B: A Single Centre Experience

C.R.A. Lesmana ${ }^{1,2}$, L.A. Lesmana ${ }^{1,2}$, L.S. Pakasi ${ }^{2}$, B. Angga ${ }^{2}$

${ }^{1}$ Internal Medicine, Division of Hepatology, University of Indonesia,

${ }^{2}$ Internal Medicine, Digestive Disease Centre, Medistra Hospital,

Jakarta, Indonesia

Background: Patients with chronic hepatitis B (CHB) may occasionally have a sudden increase of serum alanine aminotransferase (ALT) level. Clinical studies on these patients are still limited.

Objective: To evaluate clinical characteristics and outcome of treatment in CHB patients presenting with acute flare.

Method: We reviewed medical record data of patients with $\mathrm{CHB}$ presenting with acute flare between 2008 and 2010 in Medistra Hospital, Jakarta. Patients' demographic data, initiating factors, blood chemistry test, hepatitis B serology test and outcome of treatment were recorded and analyzed descriptively.

Results: There were 27 cases of acute flare in CHB; 24 (88.9\%) were men. Median age was 46 years. Initiating events were unknown (spontaneous) in $12(44.4 \%)$ cases, viral reactivation in four $(14.8 \%)$, traditional herbal medicine in four (14.8\%), non-compliance of antiviral treatment in three $(11.1 \%)$, probably viral resistance in two (7.4\%), chemotherapy in one (3.7\%) and alcohol intake in one $(3.7 \%)$ patients. Mean serum ALT level was 1,015 $\pm 599.7 \mathrm{U} / \mathrm{L}$ and mean total bilirubin level was $15.8 \pm 13.37 \mathrm{mg} / \mathrm{dL}$. Most patients showed increased ALT levels more than 5 times upper limit of normal (ULN), but there were 3 patients with ALT levels between $2-5 \times$ ULN with very high bilirubin levels. Eighteen $(72 \%)$ patients had inverse albumin-globulin ratio. Seventeen $(63 \%)$ had negative HBeAg status. Mean log HBV-DNA level was $6.3 \pm 1.98$ copies $/ \mathrm{mL}$. During the course of flare all patients developed ascites and prolonged INR. All patients received treatment with newer nucleoside analogues, such as entecavir or entecavir plus tenofovir and correction of coagulopathy and hypoalbuminemia. Two $(7.4 \%)$ patients died due to encephalopathy, while the others showed slowly clinical improvement.

Conclusion: Acute flare in chronic hepatitis B is commonly found although the clinical course remains largely unknown in most patients. Most patients improved after treatment with newer nucleoside analogues.

\section{PP01-21}

The Study of Efficacy of Lamivudine in Patients with Fulminant Hepatitis B J.-W. Yu, L.-J. Sun

Department of Infectious Diseases, Second Affiliated Hospital, Harbin Medical University, Harbin, China

Objective: Fulminant hepatitis B is a clinical syndrome that results from massive necrosis of liver cells leading to development of hepatic encephalopathy. The aim of this study was to evaluate the efficacy of lamivudine in patients with fulminant hepatitis B and study the effects of the factors on the prognosis.

Methods: A matched retrospective cohort study using data on fulminant hepatitis B patients derived from our hospital database was conducted. 40 patients receiving lamivudine treatment were selected into the lamivudine treatment group with another 40 without lamivudine treatment studied as control. They were matched for sex, age and $\mathrm{HBeAg}$ status with lamivudine treatment group. The mortality of patients in two groups was compared. The influential factors on the mortality were studied by Cox proportional hazards model.

Results: The mortality $(63.2 \%, 24 / 38)$ of patients in the lamivudine group was significantly lower than that $(84.1 \%, 33 / 39)$ of control group $\left(\mathrm{c}^{2}=4.609, P=0.032\right)$. For patients without systemic inflammatory response syndrome (SIRS), the mortality $(52.0 \%, 13 / 25)$ of patients in the lamivudine group was significantly lower than that $(80.8 \%, 21 / 26)$ of control group ( $\left.\mathrm{c}^{2}=4.747, P=0.029\right)$. In multivariate Cox proportional hazards analyses, for patients without SIRS, age $(P=0.037)$, ratio of total to direct bilirubin $(P=0.008)$, treatment method $(P=$ $0.005)$ and the decline of HBV DNA load during therapy $(P=0.019)$ were independent predictors of prognosis.

Conclusions: Treatment with lamivudine significantly decreases the mortality of patients with fulminant hepatitis B without SIRS, and a rapid decline of HBV DNA load is one of good predictors for the treatment outcome.

\section{PP01-22}

Antioxidant and Hepatoprotective Effects of Artemisia iwayomogi Extracts J.W. Shin ${ }^{1}$, H.G. Kim ${ }^{1}$, M.K. Choi ${ }^{1}$, J.H. Wang ${ }^{2}$, C.G. Son ${ }^{1}$

${ }^{1}$ Liver and Immunology Research Center, Institute of Traditional Medicine and Bioscience of Daejeon University, ${ }^{2}$ Daejeon University, Daejeon, Republic of Korea

Oxidative stress has been interrelated increasingly to the onset or progression of number of growing human diseases such as liver disease, cancer, cardiovascular disease, inflammation, and diabetes. Food and medical herbs contains antioxidants including vitamins, polyphenolic compounds, carotenoids which are believed to prevent the oxidative stress related diseases. Artemisiea iwayomogi has been used commonly in traditional Korean medicine for liver disease patients. We therefore investigate total phenolic/flavonoid content and antioxidant activity of Artemisiea iwayomogi in vitro and its hepatoprotective effects in CCl4induced liver injury model. Total phenolic and flavonoid content of water extracts of Artemisiea iwayomogi (AIWE) were $122.4 \pm 2.3 \mathrm{mg}$ gallic acid equivalence (GAE) and $79.1 \pm 3.1$ cathecin equivalence per gram extract, respectively. Total antioxidant capacity was $405.7 \pm 10.9 \mathrm{GAE}$ per g extracts. Reducing power was $26.7 \pm 1.1 \mathrm{mg}$ GAE/g extracts. Superoxide anion scavenging activity of AIWE was observed. AIWE reduced DPPH in a dose dependent manner and IC50 was $200 \mu \mathrm{g} / \mathrm{ml}$. In NO scavenging activity, IC50 was $256 \mu \mathrm{g} / \mathrm{ml}$ whereas IC50 of gallic acid was $9.17 \mu \mathrm{g} / \mathrm{ml}$. AIWE protected $\mathrm{HepG} 2$ from $\mathrm{CCl}_{4}$ invitro. In our in vivo experiments, we treated AIWE into mice for 3 days before $\mathrm{CCl}_{4}$ injection; blood and liver were isolated at $20 \mathrm{~h}$ after $0.2 \% \mathrm{CCl}_{4}$ injection (ip). AIWE decreased AST, and ALT level significantly. Histological change by $\mathrm{CCl} 4$ injection was ameliorated by AIWE. Altogether, our findings indicate that Artemisiea iwayomogi can be used as a potential antioxidant candidate for liver disorders.

\section{PP01-23}

Validating Diagnostic Criteria in Acute Liver Failure due to Wilson's Disease

N. Patel ${ }^{1}$, D. Amarapurkar ${ }^{2}$

${ }^{1}$ Jivandeep Hospital, Anand, ${ }^{2}$ Bombay Hospital and Medical Research Centre, Mumbai, India

Introduction: Diagnosis of Wilson's disease (WD) is always been challenging, more so in setting of acute liver failure (ALF). Acute liver failure due to Wilson's disease (ALF-DW) is rapidly progressive with high mortality, unless diagnosed and treated early. For early recognition of ALF-WD, different criteria are described previously with conflicting results and are not validated in clinical practice. This study was planned to validate these criteria in patients with ALF-WD. Methods: Diagnosis of WD and ALF was based on AASLD guidelines. Previously described criteria to screen WD in setting of ALF were checked in all the patients of ALF-WD. 
Results: Of 22 patients with ALF-WD (age $=13.2 \pm 3.5[4-33]$ years, 18 males), presence of various criteria were as follows: ceruloplasmin $(<20 \mathrm{mg} / \mathrm{dl})$ in $20(90.9 \%)$; $\mathrm{KF}$ ring in $17(77.2 \%)$; combination of ceruloplasmin $\pm \mathrm{KF}$ ring in 21 (95.4\%); 24-h urinary copper $(>100 \mu \mathrm{g} /$ day $)$ in $14(63.6 \%)$; serum copper $\left(>200 \mathrm{dl}^{-1}\right)$ in 5 $(22.7 \%)$; Alkaline phosphatase to total bilirubin ratio (AP/TB) $<4$ in $10(45.4 \%)$; AST to ALT ratio (AST/ALT) $>2.2$ in $6(27.2 \%)$; combination of AP/TB \pm AST/ALT criteria in $12(54.5 \%)$; AP/TB $(<2)$ in $3(13.6 \%)$; AST/ALT $>4$ in $3(13.6 \%)$; modest aminotransferase elevation $(>2-<10$ times) in $20(90.9 \%)$; low hemoglobin $(<10 \mathrm{gm} / \mathrm{dl})$ in 17 (77.2\%); Coomb's negative hemolytic anemia in $15(68.1 \%)$.

Conclusion: Additional criteria besides standard criteria are helpful in clinical practice in very few patients of ALF-WD.

\section{PP01-24}

Living Donor Liver Transplantation for Fulminant Hepatic Failure: An Implication of Preoperative Apheresis Therapy

T. Shimamura, M. Oota, T. Suzuki, M. Taniguchi, K. Yamashita,

T. Kamiyama, S. Todo, Hokkaido Liver Transplantation Study Group

Division of Organ Transplantation, Hokkaido University, Sapporo, Japan

Background: Living donor liver transplantation (LDLTx) is a reliable therapeutic option for patients with fulminant hepatic failure (FHF). For a proper success of LDLTx, however, it would be mandatory to provide an enough time to living donor candidates for making decision and evaluation, while preventing the preoperative neurological complication in recipients. In this context, preoperative apheresis therapy is a possible modality to resolve problems. Purpose of this paper is to assess the efficacy of apheresis as a preoperative treatment, and to review our approach to the patients with FHF.

Patients and methods: From August 1997 to December 2009, 31 FHF patients were transferred to our hospital. Those were immediately treated with extra-corporeal apheresis. Apheresis therapy consisted of plasma exchange and hemofiltration.

Results: Five out of the 31 patients $(16.1 \%)$ recovered only through apheresis. Four died without transplantation. Although remaining 22 were considered to be candidates for LTx, their coma were maintained well with apheresis. Of those, 21 underwent LDLTx and one had a cadaveric LTx with a mean period of 19 (range 4-53) days after the onset of coma. One-year survival rate after LTx was $86.4 \%$ (19/ 22). Although no neurological sequelae were observed after transplantation, three died from infectious complications. Mean duration of apheresis in these 3 cases was 29 (9-45) days, and which was longer than that in recipients who had no postoperative infectious morbidity (11.5 days, range $2-53$ ).

Conclusion: Apheresis therapy is effective not only as a treatment intending spontaneous recovery, but also as a safe bridge to LTx. Although apheresis can maintain the patients well and provide an enough time for living donor candidates, attention should be paid if the duration becomes longer than approximately 2 weeks.

\section{PP01-25}

Hepatoprotective Effects of Silymarin on Liver Oxidative Stress and Inflammation Induced by Sepsis in a Rat Experimental Model K. Razavi-Azarkhiavi ${ }^{1}$, A. Allameh ${ }^{2}$, A. Mohsenifar ${ }^{1}$, M.-R. Sepand ${ }^{1}$ ${ }^{1}$ Department of Toxicology; ${ }^{2}$ Department of Clinical Biochemistry, Faculty of Medical Sciences, Tarbiat Modares University, Tehran, Iran

Background/aims: Silymarin, the major component of milk thistle has been shown to have antioxidant and anti-inflammatory properties. On the other hand, there are many documents that show oxidative stress and inflammation occurred during sepsis. In the present study, we investigated the Hepatoprotective effects of silymarin on sepsisrelated oxidative reactions leading to liver damage.
Methods: in this study, sepsis induced by Cecal Ligation and Puncture (CLP) in rats. Adult male rates were divide into 6 groups $(n=5 /$ group) as; silymarin treated (50 or $100 \mathrm{mg} / \mathrm{kg}$ b.w), sham $+\mathrm{NaOH}$, $\mathrm{CLP}+\mathrm{NaOH}, \mathrm{CLP}+$ silymarin at the doses of 50 or $100 \mathrm{mg} / \mathrm{kg} \mathrm{b} . \mathrm{w}$ groups. Silymarin were dissolved in $\mathrm{NaOH}$ and i.p injected to rats for 6 days. In two groups, after 6 days silymarin treatment, CLP operation was done. Four hours after CLP or last silymarin injection, blood was collected, liver tissues were removed and processed for biochemical and histopathological examinations.

Results: CLP operation resulted in a significant depletion in liver glutathione (GSH) when compared to that measured in sham group, whereas GSH was increased in septic rats under silymarin treatment. The level of liver thiobarbituric acid reactive substances (TBARS) was elevated in rats undergone CLP operation, however silymarin treatment caused to reduce in formation of TBARS in septic rats. Liver glutathione- $S$-transferase (GST) activity which was inhibited due to CLP, was again induced as the result of silymarin. Sepsis induction resulted in a significant elevation in Plasma tumor necrosis factor- $\alpha$ (TNF- $\alpha)$, however, it was reduced in septic rats undergone silymarin treatment. Liver histopathological examination showed inflammatory reactions in CLP rats, whereas CLP-related changes were significantly recovered as the result of silymarin.

Conclusions: Results indicate that silymarin treatment was effective against sepsis-induced oxidative damage and morphological alterations in rat, and anti-oxidative and anti-inflammatory effects of silymarin were observed in this experimental model.

\section{PP01-26}

Hyperbolic Pattern of Biochemical Parameters Evolution during Extracorporeal Elimination Treatment by Prometheus

S. Hrusovsky ${ }^{1}$, M. Zigrai ${ }^{1}$, A. Farkasova ${ }^{2}$, M. Hamilton ${ }^{2}$, M. Demes ${ }^{2}$, A. Gregusova ${ }^{2}$

${ }^{1}$ Slovak Medical University; ${ }^{2}$ University Hospital of Bratislava, Bratislava, Slovak Republic

Background: Extracorporeal elimination treatment Prometheus is used in acute and acute on chronic liver failure. We followed the evolution of blood parameters during this treatment.

Patients and methods: We used Prometheus liver support and highflux hemodialysis in 11 patients (6 men, 5 women) aged in average 39 years (from 25 to 61). The Prometheus method was used 27 times, in average 2,4 times in every patient (from 1 up to 5 times). Seven patients were treated for acute liver failure (16 procedures), three patients for acute on chronic liver failure ( 9 procedures), one patient had a chronic liver failure after OLTx (recurrence of primary sclerosing cholangitis in the graft). We made a detailed analysis of 15 procedures Prometheus, examining biochemical parameters every 3 hrs during the treatment.

Results: Serum concentration of total bilirubin fell down in average from $474 \mu \mathrm{mol} / 1$ (SD 153) to $293 \mu \mathrm{mol} / 1$ (SD 73), conjugated bilirubin from $389 \mu \mathrm{mol} / 1$ (SD 168) to $236 \mu \mathrm{mol} / \mathrm{l}$ (SD 77), ammonia from $56 \mu \mathrm{mol} / 1$ (SD 39) to $31 \mu \mathrm{mol} / \mathrm{l}$ (SD 20), creatinine from 227 $\mu \mathrm{mol} / \mathrm{l}$ (SD 204) to $117 \mu \mathrm{mol} / \mathrm{l}$ (SD 92), urea from $11 \mu \mathrm{mol} / \mathrm{l}$ (SD 8) to $4 \mu \mathrm{mol} / \mathrm{l}$ (SD 3). The most important decrease in values was observed within first hours of each procedure with hyperbolic pattern. A decrease in albumin serum levels was observed in some patients.

Conclusion: This study confirmed the effect of Prometheus procedure on bilirubin, ammonia, creatinine, and urea serum concentrations in patients with acute or acute on chronic liver failure. Hyperbolic pattern of biochemical parameters evolution was found, with a decrease (in some patients) of albumin serum levels. Further study will elucidate whether detailed evolution patterns of other factors such as regenerative intercellular mediators are similar and could be of clinical importance in these conditions. 


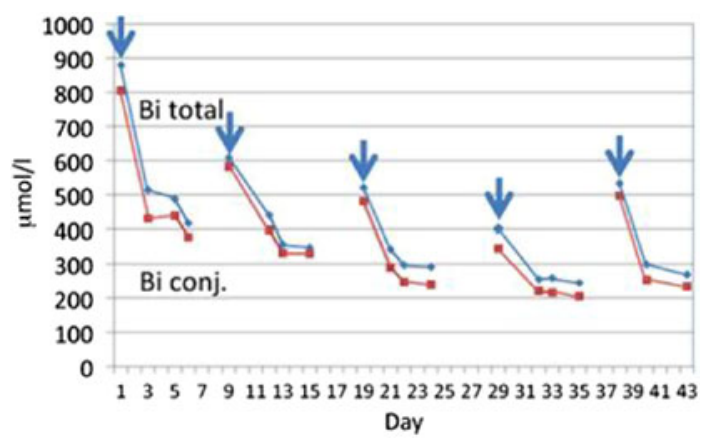

Fig. 1 Hyperbolic pattern of bilirubin serum levels during elimination procedures

\section{PP01-27}

Effect of Hepatic Stimulator Substance Encapsulated in Sterically Stable Liposomes on Fulminant Hepatic Failure in Rats Induced by Thioacetamide

F. Li, J.Y. Wang

Department of Gastroenterology, Zhongshan Hospital Affiliated to Fudan University, Shanghai, China

Background and aims: Fulminant hepatic failure (FHF) is a rare but severe complication of acute hepatitis and carries a very high mortality. There is no effect medication for FHF. Sterically stabilized liposomes (SSL) show two important characteristics of infectious target localization and prolonged blood residence time. This study was to prepare SSL encapsulating hepatic stimulator substance (HSS) (HSS-SSL) and determine its theraputic effect on FHF in rats.

Methods: HSS-SSL and SSL were prepared. FHF was induced in rats by TAA-injections $(400 \mathrm{mg} / \mathrm{kg}$, three times with a $24-\mathrm{h}$ interval). The agents (HSS-SSL, SSL alone, HSS alone and $0.9 \% \mathrm{NaCl}, n=20$ per group) were injected respectively intravenously at $2 \mathrm{~h}$ after the second and the third TAA dosage.

Results: At $24 \mathrm{~h}$ after the third TAA-injection, the survival rate of rats treated with HSS-SSL $(80 \%)$ was significantly higher than that of rats treated with $0.9 \% \mathrm{NaCl}(20 \%, P<0.001)$, SSL $(25 \%, P=0.001)$ and HSS $(50 \%, P=0.047)$. And histopathologic examination of liver specimens showed less necrosis and less inflammation in the livers of rats treated with HSS-SSL. The incidence of 3 or 4 stage hepatic encephalopathy (HE) in rats treated with HSS-SSL (25\%) was significant lower than that in rats treated with $0.9 \% \mathrm{NaCl}(100 \%, P=$ $0.003)$, SSL $(100 \%, P=0.014)$ and HSS $(70 \%, P=0.032)$. The serum biochemical parameters, serum pro-inflammatory cytokines levels and hepatic lipid peroxidation level were all significantly reduced in HSS-SSL-treated rats. In contrary, hepatocyte proliferative rate was significantly increased by HSS-SSL treatment.

Conclusion: After encapsulated into SSL, HSS liposomes (HSS-SSL) improved the prognosis of FHF in rats induced by TAA administration, which was represented as improving the survival rate and decreasing the incidence of stage 3 or $4 \mathrm{HE}$ by promoting hepatocytes proliferation, inhibiting pro-inflammatory cytokines release and ameliorating hepatic lipid peroxidation.

\section{PP01-28}

Analysis of Promoter Methylation of Oxidative-stress Related Enzyme GSTP1 and Oxidative Stress in Acute-on-Chronic Hepatitis B Liver Failure T. Li ${ }^{1}$, Q.H. Meng ${ }^{2}$, Z.Q. Zou ${ }^{2}$, Y.C. Fan ${ }^{1}$, K. Wang ${ }^{1}$

${ }^{I}$ Department of Hepatology, Qilu Hospital of Shandong University, Jinan; ${ }^{2}$ Department of Hepatology, Beijing You An Hospital, Capital Medical University, Beijing, China
Background/aims: Glutathione-S-transferases P1 (GSTP1) belongs to the family of antioxidative enzymes and is involved in oxidative stress induced liver damage in acute-on-chronic hepatitis B liver failure (ACHBLF). This study aimed to demonstrate the correlation of GSTP1 promoter methylation status and oxidative stress in ACHBLF patients. Methods: Thirty-five ACHBLF, 35 chronic hepatitis B (CHB) patients, and 10 normal controls were enrolled in this study. DNA was extracted from peripheral blood mononuclear cells (PBMCs) of the above subjects, followed by sodium-bisulfite treatment and methylation-specific PCR (MSP) analysis. Plasma Malondialdehyde (MDA) adducts level was detected by ELISA as oxidative stress marker. Model for end-stage liver disease (MELD) was employed to estimate ACHBLF severity.

Results: Eleven of $35 \mathrm{ACHBLF}$ and 3 of $35 \mathrm{CHB}$ patients displayed GSTP1 promoter methylation and the difference was significant $\left(\chi^{2}=5.71, P=0.02\right)$. No considerable difference of ALT, AST and PTA level was found between GSTP1 promoter methylated and unmethylated group of ACHBLF $(P>0.05)$, except TBIL level $(P<0.01)$. MDA adducts level was significantly higher in ACHBLF than in CHB patients $(12.44 \pm 5.38,8.42 \pm 5.49 \mathrm{pmol} / \mathrm{mg}$, $P<0.01)$ and in methylated ACHBLF group than in unmethylated group $(15.2 \pm 4.68,11.17 \pm 5.29 \mathrm{pmol} / \mathrm{mg}, P<0.01)$. Furthermore, in the ACHBLF patients, we did not find significant difference in the score of MELD between methylated and unmethylated group $(P>0.05)$. However, plasma MDA adducts level was significantly correlated with MELD in ACHBLF patients $(r=0.579, P<0.01)$. Conclusions: GSTP1 promoter methylation may facilitate oxidative stress-induced liver damage in ACHBLF and oxidative stress evaluated by MDA adducts is correlated with ACHBLF severity.

\section{PP01-29}

Dimethoate-induced Hepatotoxicity and Oxidative Damage in Male Rat: Attenuation by Vitamin C

M.M. Moawad

Biochemistry, Faculty of Science Alexandria University, Alexandria, Egypt

Background: Human exposure to organophosphorus insecticides is continuously increased leading to hepatotoxicity and excessive production of free radicals which are responsible for several cell alterations in the organism.

Aim: The present study is carried out to investigate the role of vitamin $\mathrm{C}$ in protection against oxidative damage induced by dimethoate insecticide in male rat.

Methods: Male rats were randomly divided into four groups of 7 each, group I served as controls; group II treated with vitamin C, group III received dimethoate orally and group IV treated with dimethoate plus vitamin C. Liver damage markers as amino-acid transferases (AST and ALT), alkaline phosphatase (AlP), lactate dehydrogenase (LDH) and bilirubin were evaluated. Oxidative damage biomarkers indicating extent of lipid peroxidation, antioxidant reserve indicators as reduced glutathione (GSH), antioxidant enzyme activities as glutathione peroxidase (GPx), glutathione S-transferase (GST), glutathione reductase (GR), superoxide dismutase (SOD) and catalase (CAT) were measured Results: The exposure of rats to dimethoate for 30 days promoted oxidative stress with an increase in thiobarbituric acid reactive substances (TBARS) and a decrease in glutathione (GSH) level as compared to control. Moreover, the activities of liver antioxidant enzymes like GPx, GST, GR, SOD and CAT were also diminished by oxidant damage. While, plasma transaminases, alkaline phosphatase, lactate dehydrogenase activities and bilirubin levels increased. Treatment with vitamin $\mathrm{C}$ significantly improves the liver damage from the oxidative stress caused by dimethoate and shifts the trend towards the normal status.

Conclusions: Vitamin $\mathrm{C}$ can be very effective in reducing liver injury and in overcoming oxidant damage caused by environmental stressors as pesticides. 


\section{PP01-30}

The Related Study on Plasma Exchange in Treatment of Hyperbilirubinemia Patients

G. Wang

Hepatic Cirrhosis Diseases, Dalian Sixth People Hospital, Dalian, China

Objective: To investigate the clinical value of plasma exchange in the treatment of hyperbilirubinemia patients from our hospital.

Methods: 67 cases of hyperbilirubinemia patients from our hospital were performed with plasma exchanges, and it was analyzed that the patients' clinical manifestations, liver function, plasma $\mathrm{NH}_{3}$, plasma prothrombin time before and after plasma exchanges respectively.

Results: There were significant differences to the patients' clinical manifestations, liver functions, plasma $\mathrm{NH}_{3}$, plasma prothrombin time before and after plasma exchanges.

Conclusions: Plasma exchange is one of safety and efficient measures in treating hyperbilirubinemia patients, and can win time to the patients who are waiting for liver transplantations.

\section{PP01-31}

Stability of Interferon-gamma Promoter Methylation in the Patients with Acute-on-Chronic Hepatitis B Liver Failure

K. Wang ${ }^{1,2}$, F.-C. $\mathrm{Li}^{1,2}$, X.-P. Fan ${ }^{1,2}$, B. Wang ${ }^{1,2}$, Y.-C. Fan ${ }^{1,2}$

${ }^{1}$ Department of Hepatology, Qilu Hospital of Shandong University;

${ }^{2}$ Hepatology Institute, Shandong University, Jinan, China

Background and aims: Interferon-gamma (IFN- $\gamma$ ) is demonstrated to exacerbate liver function in the progression of chronic hepatitis B. Recently, the mechanism underlining IFN- $\gamma$ mediates acute liver failure on chronic hepatitis b virus infection is still not fully understood.

Methods: We used enzyme-linked immunosorbent assay to measure the level of serum IFN- $\gamma$ in the 40 patients with acute-on-chronic hepatitis B liver failure (ACHBLF), 15 patients with chronic hepatitis $\mathrm{B}$, and 10 healthy controls; Moreover, we used the Methylation specific PCR (MSP) to identify whether the changed IFN- $\gamma$ profile was associated with its promoter methylation.

Results: The level of IFN- $\gamma$ in patients with ACHBLF was lower than that in the healthy controls, but higher than the patients with CHB. Moreover, the level of IFN- $\gamma$ had positive correlation with total bilirubin and MELD score, and a negative correlation with PTA in patients with ACHBLF. The percentage of methylation of the IFN $-\gamma$ promoter gene was higher in the ACHBLF group as compared to the control group. The IFN- $\gamma$ level was significantly decreased in methylation group as compared to unmethylaiton group in patients with ACHBLF.

Conclusions: Methylation of IFN $-\gamma$ promoter is associated with decreased level of IFN- $\gamma$ in the patients with acute-on-chronic hepatitis B liver failure.

\section{PP01-32}

Resting Energy Expenditure and Substrate Metabolism in Patients with Acute-on-Chronic Hepatitis B Liver Failure

W. Hou, H.-W. Yu, J. Li, J.-H. Wang, F.-Y. Zhang, J. Zhang, Q.-W. Yao, X. Wang, Q.-H. Meng

Serious Illness Medicine Inpatient Area, Beijing You An Hospital, Capital Medical University, Beijing, China

Goals: The present study was designed to determine the status of energy metabolism in the patients with acute-on-chronic liver failure (ACLF). Background: Protein-energy malnutrition usually occurs in the patients with chronic liver disease and is exacerbated during the progression of liver failure. However, there is limited study to fully elucidate the energy metabolism in the ACLF patients.

Study: A retrospective cohort was designed with a total of 282 patients (100 ACLF patients, 100 liver cirrhosis patients and 82 chronic hepatitis B patients). Resting energy expenditure (REE) and the oxidation rates of glucose, lipid and protein were assessed by indirect heat measurement using the CCM-D nutritive metabolic investigation system. Survival rate was estimated with the KaplanMeier method.

Results: There was no significant difference in REE among ACLF, liver cirrhosis and chronic hepatitis patients $(1,402.05 \pm 480.07 \mathrm{kcal} /$ day in ACLF patients, $1,274.27 \pm 316.36 \mathrm{kcal} /$ day in liver cirrhosis patients and $1,396.77 \pm 384.80 \mathrm{kcal} /$ day in chronic hepatitis patients). Respiratory quotient (RQ) was significantly lower in the ACLF patients than those in liver cirrhosis and chronic hepatitis B hepatitis $(P=0.000)$. In ACLF patients, RQ of the non-survival group was significantly lower than the survival group[BL1]. It is identified from ROC analysis that, a RQ cutoff value of 0.83 (area under the ROC 0.760 ) is favorable to predict good prognosis in liver failure patient, which has a sensitivity of $73.68 \%$, a specificity of $74.42 \%$, positive predicative value of $79.2 \%$ and negative predicative value of $68.1 \%$. Conclusions: In acute-on-chronic liver failure patients, respiratory quotient was significantly lower in the non-survival group than the survival group, which indicate that RQ may be used as an indicator of prognosis of liver failure.

\section{PP01-33}

Aberrant Methylation Stability of TNF- $\alpha$ Promoter in the Patients with Acute-on-Chronic Hepatitis B Liver Failure

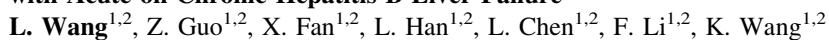

${ }^{I}$ Qilu Hospital of Shandong University; ${ }^{2}$ Hepatology Institute of

Shandong University, Jinan, China

Background and aims: Tumor necrosis factor alpha (TNF- $\alpha$ ) plays a critical role in the occurrence of liver failure. However, the exact molecular mechanism for the regulation of TNF- $\alpha$ has not been well demonstrated. The present study was designed to investigate the possible epigenetic alteration in the promoter of TNF- $\alpha$ in the patients with acute-on-chronic hepatitis B liver failure (ACHBLF).

Methods: 20 patients with ACHBLF, 12 patients with chronic hepatitis $\mathrm{B}(\mathrm{CHB})$, and 12 healthy controls were included in the present study. The methylation of $\mathrm{TNF}-\alpha$ promoter in peripheral blood mononuclear cells (PBMCs) was measured by methylation specific PCR (MSP). The level of serum TNF- $\alpha$ was determined by enzymelinked immunosorbent assay (ELISA). Model for End-stage Liver Disease (MELD) was performed for the evaluation of liver failure.

Results: The percentage of methylation of the TNF- $\alpha$ promoter was lower in the ACHBLF group (15\%) as compared to the CHB group $(50 \%)$ and the healthy control group $(66.7 \%)$. The serum level of TNF- $\alpha$ in patients with ACHBLF was higher than that in CHB and healthy controls $(P<0.05)$. Moreover, the serum TNF- $\alpha$ level was significantly decreased in methylation group as compared to unmethylaiton group in patients with $\operatorname{ACHBLF}(P<0.05)$. MELD was not significantly different between methylated and unmethylated group of ACHBLF patients $(P>0.05)$. In addition, the serum level of TNF- $\alpha$ was found to be positively correlated with serum total bilirubin $(r=$ $0.891, P<0.01)$ and MELD score $(r=0.792, P<0.01)$, but to be negatively correlated with prothrombin activity $(r=-0.511$, $P<0.05)$ in patients with ACHBLF.

Conclusion: The TNF- $\alpha$ methylation patten is stable for the liver failure, suggesting the effect of environment on methylation.

\section{PP01-34}

Aberrant Methylation Stability of IL-10 Promoter in the Patients with Acute-on-Chronic Hepatitis B Virus Liver Failure

J. Han

Department of Hepatology, Qilu Hospital of Shandong University, Jinan, China

Background and aims: The present study was aimed to determine the methylated alteration of interleukin 10 (IL-10) promoters in the progression of the hepatitis $\mathrm{B}$ virus infection. 
Methods: 25 patients with chronic hepatitis B (CHB), 25 patients with chronic acute-on-chronic hepatitis $b$ liver failure (ACHBLF), and 10 healthy controls were included in the present study. The level of serum IL-10 was determined by enzyme-linked immunosorbent assay (ELISA). The methylation of IL-10 promoter in peripheral blood mononuclear cells (PBMCs) was measured by methylation specific PCR (MSP). Model for End-stage Liver Disease (MELD) was performed for the evaluation of liver failure.

Results: Both ACHBLF and CHB patients have significant increases in serum level of IL-10 compared with healthy controls $(p<0.05)$. There were no significant difference in the serum level of IL-10 in the patients with ACHBLF and CHB $(p>0.05)$. The serum level of IL10 in ACHBLF patients has no significant correlation with level of ALT and HBV-DNA $(r=-0.022, r=0.033$, respectively; $P>0.05)$. However, serum IL-10 level was found to be positively correlated with serum total bilirubin $(r=0.566, P<0.05)$ and negatively with PTA and MELD score $(r=-0.581, r=0.443$, respectively; $P<0.05)$. Moreover, the distribution of the methylation of IL-10 promoter in ACHBLF patients is significantly different from the CHB patients, the rate of complete methylation of ACHBLF patients is obviously higher than the $\mathrm{CHB}^{\prime} \mathrm{s}$; while no significant difference between the CHB patients and the healthy controls. Furthermore, the serum level of IL-10 has a significantly negative correlation with its promoter methylation $(r=-0.878, P<0.05)$. Conclusions: The methylation of IL-10 promoter may be involved in the regulation of IL-10 in the progression of ACHBLF.

\section{PP01-35}

Change of Polymeric Immunoglobulin Receptor (pIgR) Expression in the Mucosa of the Small Intestine in Mice with Acute Liver Necrosis J.-L. Fu ${ }^{1,2}$, Y. Zhou ${ }^{2}$, B.-C. Deng ${ }^{2}$, W. Wang ${ }^{2}$, P. Liu $^{2}$

${ }^{I}$ Department of Gastroenterology, Hangzhou Normal University of Clinical Medicine, Hangzhou, ${ }^{2}$ Department of Infectious Diseases, The First Affiliated Hospital of China Medical University, Shenyang, China

Aim: The study aimed to evaluate the change to expression of polymeric immunoglobulin receptor ( $\mathrm{pIgR}$ ) in the mucosa of the small intestine in mice with acute liver necrosis.

Methods: A model of acute liver necrosis was established by intraperitoneal injection of galactosamine (GalN) and lipopolysaccharide (LPS). Sixty Balb/c mice were equally and randomly divied into one of four groups: normal saline control (NS), acute liver necrosis (LPS/ GalN), lipopolysaccharide (LPS), or galactosamine (GalN). At 9h after administration, the mice in four groups were sacrificed. The liver and the small intestine were harvested for pathology examination and microscopy. The pIgR protein expression was detected using the immunohistochemistry method and Western blotting. The mRNA expression of pIgR was assessed using real-time PCR. The content of secretory immunoglobulin A ( $\operatorname{Ig} \mathrm{A})$ in the intestinal fluid was evaluated using the radioimmunoassay.

Results: Apparent pathological lesions were presented in the liver and the mucosa of the small intestine in the LPS/GalN group, while mild lesions were observed in the LPS group and the GaIN group. Compared with the NS group, the expression of pIgR mRNA and protein showed a statistically significant reduction in the mice with acute liver necrosis $(P<0.05)$. Additionally, the sIgA content statistically significantly reduced $(P<0.05)$, and was positively correlated with the expression of $\mathrm{pIgR}$ protein in the LPS/GalN group $(P<0.01)$, as compared to the NS group.

Conclusion: The downregulation of pIgR expression in the mucosa of the small intestine with acute liver necrosis may be a functional component of the sIgA-mediated intestinal immune dysfunction.
PP01-36

Glucocorticoid Receptor Expression in Acute-on-Chronic Liver Failure S. Gao, L. Gao, B. Wang, L.Y. Chen, F.C. Li, L.H. Hu, Y.P. Yin, K. Wang Hepatology, Hepatology Institute of Shandong University, Jinan, China

Background and aims: Glucocorticoid (GC) and its ligand-glucocorticoid receptor (GR) have been widely demonstrated to be involved in inflammatory responses and may contribute to the onset of acute-onchronic liver failure (ACLF). The study was aimed to determine the expression profile of GR system in the patients of ACLF.

Methods: Twenty-four patients with ACLF (15 patients were in early stage and 9 in late stage), twenty patients with chronic hepatitis $\mathrm{B}(\mathrm{CHB})$ and sixteen health controls (HC) were enrolled in this study. Flow cytometry was used to analyze the protein expression of GR in CD3+T lymphocytes in both early and late stages of ACLF. GR $\alpha$ and GR $\beta$ mRNA expression in peripheral blood mononuclear cells (PBMCs) were determined by RT-PCR. Serum cortisol concentrations were determined using radioimmunoassay.

Results: Compared with HC, the protein levels of CD3/GR in patients with $\mathrm{CHB}$ and ACLF were gradually decreased, especially in latestage $\operatorname{ACLF}(P<0.01)$. The concentrations of serum cortisol in earlystage ACLF were significantly lower compared with $\mathrm{HC}$ and $\mathrm{CHB}$, but higher in late-stage $\operatorname{ACLF}(P<0.01,0.01)$. The expression of CD3/ GR in CD3+T cells in patients with ACLF was significantly negatively correlated with serum cortisol concentrations. Compared with $\mathrm{HC}$, the positive rates of GR $\beta$ mRNA in PBMCs in patients with $\mathrm{CHB}$ and ACLF were gradually increased, especially in ACLF group $(P<0.05)$. Conclusions: The down-regulation of GRs in CD3+T lymphocytes is associated with increased adrenal secretion of cortisol in ACLF, which suggested that glucocorticoid receptor down-regulation may be involved in altered immune status of patients with ACLF of different stages.

\section{PP01-37}

Effects of Acute Liver Damage by LPS on Sphingolipid Metabolites in Blood

Y.-M. Lee ${ }^{1}$, T.-J. Kim ${ }^{2}$

${ }^{I}$ Chungbuk National University, Chongju; ${ }^{2}$ Yonsei University, Wonju College of Medicine, Wonju, Republic of Korea

Intracellular and extracellular amount of S1P are tightly regulated by the relative enzyme activities of Sphk1 and Sphk2, S1P lyase and other lipid phosphate phosphohydrolase. Extracellular S1P exported by the aid of $\mathrm{ABC}$ transporters binds to S1P receptors to transfer its signaling. Recent studies have concluded that S1P gradient and S1P receptor signaling enable immune cells egress from lymphoid tissues. However, the study about the maintenance and regulating factors on $\mathrm{S} 1 \mathrm{P}$ level in blood is still under investigation. We investigated the effects of acute liver injury on the changes of sphingolipid metabolites in C57/BL6 mice. Anti-Fas antibody (Jo2) or combination of LPS and D-GalN treatment significantly induced liver damage and showed reduced plasma S1P level, but not plasma ceramides. The combined treatment of LPS and D-GalN greatly reduced Sphk1 activity in whole blood, while no changes were observed in plasma Sphk1 activity. Notably, the half-life of injected C17-S1P in this condition was not changed. The Sphk1 activities in red blood cells (RBCs) and cultured HUVEC cells were not significantly changed by Jo2 or LPS+D-GalN treatment. The Sphk1 activity in liver tissues was slightly increased after liver injury. However, partial hepatectomy did not contribute to reduce plasma S1P concentration. Thus, Sphk1 activity in whole blood mainly participated to maintain plasma S1P level. The future for pharmacological effects of $\mathrm{ABC}$ transporters inhibitors and Sphk2 activity will be further investigated. 


\section{PP01-38}

Hypermethylation of IL-17 Promoter in the Patients

with Acute-on-Chronic Liver Failure

Y.-C. Fan $^{1,2}$, J. Ge ${ }^{1,2}$, X.-P. Fan ${ }^{1,2}$, Z.-X. Qi ${ }^{1,2}$, F.-L. Meng ${ }^{1,2}$, L.-Y. Chen ${ }^{1,2}$, F.-C. $\mathrm{Li}^{1,2}, \mathrm{~K}$. Wang ${ }^{1,2}$

${ }^{1}$ Department of Hepatology, Qilu Hospital of Shandong University;

${ }^{2}$ Hepatology Institute of Shandong University, Jinan, China

Background/aims: We previously reported that down-regulation of interleukin 17A (IL-17A) might contribute to the acceleration of liver failure. However, the exact mechanism with regard to the epigenetic alteration of IL-17A has not been fully understood. This present study was aimed to determine the methylated alteration of IL-17A promoter in the patients with acute-on-chronic hepatitis $\mathrm{B}$ liver failure (ACHBLF).

Methods: Fifteen patients with chronic hepatitis B (CHB) and twenty ACHBLF patients were included in our present study. Methylationspecific polymerase chain reaction (MSP) was used to evaluate methylation status of IL-17A promoter. The mRNA level of IL-17A was measured with quantitative real-time polymerase chain reaction (PCR). Model for End-stage Liver Disease (MELD) was performed for the evaluation of liver failure. Ten healthy volunteers were enrolled as control.

Results: The frequency of CPG island methylation in CHB patients was significantly decreased than that in ACHBLF patients (46.7 vs. $100 \%, p<0.01)$. Moreover, we did not find any CPG island methylation in healthy controls. IL-17A mRNA expression was significantly decreased in ACHBLF patients than that in CHB patients. Furthermore, CPG island methylation of IL-17A promoter was negatively correlated with IL-17A mRNA expression $(r=-0.701, p<0.01)$. However, we did not find any significant correlations between IL-17A promoter methylation and MELD scores in ACHBLF patients.

Conclusion: The down-regulation of IL-17A due to its promoter CPG island methylation is an important event in acute-on-chronic hepatitis B liver failure.

\section{PP01-39}

Acute Liver Failure in Tertiary Care Center of Nepal

S. K.C., D. Sharma, S. Khadka

Liver Unit, National Academy of Medical Sciences, Kathmandu, Nepal

Objective: Acute liver failure (ALF) also known as fulminant hepatic failure is a multisystem illness characterized by severe and sudden liver cell dysfunction leading to hepatic encephalopathy and hepatic coagulopathy in a person without history of liver disease in past. This catastrophic illness can rapidly progress to coma and death from cerebral edema and multi organ dysfunction. The natural history of this illness is variable and survival without transplantation ranges from $10 \%$ to $90 \%$. So far, there is no published data on ALF in Nepal. This retrospective study aims to analyze the cases of acute liver failure in Nepal where conservative treatment only is available till date.

Materials and methods: Registry of the admitted patient admitted between April 2004 to March 2010 were searched and all cases of acute liver failure were included. Different factors were analyzed and significant findings were included in the result.

Results: A total of 92 (male, 72; female 20, age $38 \pm 9$ years) patients who fulfilled the criteria of ALF were analyzed. Hepatitis E was the main etiological agent. The mortality rate was high among pregnant women, specially presenting during third trimester. Injudicious use of herbal medicines was noted in $62 \%$ patients. Secondary bacterial infection, hypoglycaemia and hyponatremia were the nonhepatic factor that was associated with increased mortality. Overall survival was $59 \%$. If pregnant cases were excluded, survival rate was $75 \%$. Use of SNMC (Stronger neominophagen compound) increased the survival and lessened the hospital stay.
Conclusions: Acute liver failure is not uncommon in Nepal. It is the number one killer in pregnant women who suffered from HEV hepatitis during third trimester of pregnancy. Prevention of infection and education regarding indiscrete use of herbal medicines may decrease the mortality.

\section{PP01-40}

Etiology and Outcome of Fulminating Hepatic Failure in Rural and Urban Population of Sindh, Pakistan

S. Ali, S. Memon, M. Zaki, N. Lal, P. Kumar, P. Abbasi, F. Naz, I. Saba,

S. Gill, K. Ansari, S. Laghari

Medicine, Isra University Hospital, Hyderabad, Pakistan

Background and aim: The aim of this study was to identify the etiology for fulminating hepatic failure and to asses the outcome of fulminating hepatic failure in urban and rural population of sindh, Pakistan.

Methods: Descriptive type of study was conducted in isra university hospital over a period of 1 year belongs to all over sindh except Karachi. Patients were divided into urban and rural groups. Patients having both clinical and labortical markers suggestive of fulminating hepatic failure were included in this study and their outcome was also noted as expiry and recovery during hospitalization.

Results: Out of 65 patients, $52.3 \%$ were HBV positive. In rural 82.4 and $17.6 \%$ in urban patients with $P$ value of 0.63 . Non-B etiology including (hepatitis $\mathrm{C}, \mathrm{E}$ and toxinemia of pregnancy) was about 47.7 with $P$ value of 0.2 . Hepatitis B was significantly more prevalent in rural male (70.6\%) compared to urban male $(29.4 \%)$ then the females. Etiology was not possible in 8 patients. Mortility rate was also high about $72.5 \%$ in rural as compared to urban population $57.11 \%$. Poor outcome was associated with high level of PT $>50$, bilirubin $>3$, INR $>5$ and development of grade 4 encephalopathy.

Conclusion: Hepatitis B is more common cause of fulminating hepatic failure in rural population $(82.4 \%)$ of sindh province. Deteoriating clinical condition like hepatic encephalopathy and raised level of bilirubin, PT, INR indicates the poor prognosis and high mortility without liver transplantation.

\section{PP01-41}

Change in MELD Score Is a Useful Tool of Liver Transplantation for Patients with Fulminant Hepatic Failure under ALS

K. Inoue ${ }^{1}$, M. Yoshiba ${ }^{2}$

${ }^{I}$ Gastroenterology, Showa University Fujigaoka Hospital, Yokohama; ${ }^{2}$ Internal Medicine, Sempo Tokyo Takanawa Hospital, Tokyo, Japan

Background/aim: We have already established the effective artificial liver support system comprising of plasma exchange and hemodiafiltration using huge volume of buffer. Over $90 \%$ of patients with fulminant hepatic failure (FHF) regain consciousness under the treatment of this system. After induction of this system, we can easily discriminate the patients who recover spontaneously. Therefore, we also developed the treatment for underlying disease of FHF. The advent of emergency liver transplantation has highlighted the need for prognosis indicators. In the present study, we verify usefulness of change in MELD score as prognosis indicator under these intensive medical care.

Methods: Subject of the study were 47 patients with FHF referred to the Emergency Center at Showa University Fujigaoka Hospital. 22 patients were acute form and 25 patients were sub-acute form. These patients had presented within 8 weeks of disease onset without apparent pre-existing liver disease. These patients were placed on the artificial liver support system immediately after admission. We also did treatment corresponding to underlying disease. We calculated MELD score at admission, day 3, day 7 and day 14 and also analyzed change in MELD score and prognosis.

Results: 44 of 47 patients (93\%) regained their consciousness. 31 patients (15 of acute form and 16 of subacute form) survived and 16 
patients ( 7 of acute form and 9 of subacute form) deceased. MELD score on admission, day3, day7 and day 14 were 20.1, 10.1, 7.5 and 7 in survived patients and 22.8, 18.9, 18.1 and 18.1 in deceased patients. Conclusion: Assessment of the prognosis of FHF is essential when making decisions on the requirement for and timing of liver transplantation. Under the treatment of the artificial liver support system, we can have sufficient time to obtain a liver graft. Change in MELD is a helpful indicator for making decisions.

\section{PP01-42}

Comparison of Predictive Value of Serum Thymosin $\beta 4$ in Acute-onChronic Liver Failure Patients with the Child-pugh and MELD Scores Y. Liu, T. Han

Department of Hepatology, The Third Central Clinical College of Tianjin Medical University, Tianjin Third Central Hospital, Tianjin, China

Aim: To investigate whether decreased serum thymosin $\beta 4$ levels are associated with mortality of acute-on-chronic liver failure (ACLF) patients and decide whether the serum thymosin $\beta 4$ levels can be used in predicting the prognosis of ACLF patients by compared the predictive values with the Child-Pugh (CTP) and Model for End-Stage Liver Disease (MELD) scores.

Methods: Serum thymosin $\beta 4$ levels were measured by enzymelinked immunosorbent assay (ELISA) and CTP and MELD score were calculated for each patient on admission.

Results: Compared to survivors, higher baseline values of TBIL $(P<$ $0.001)$, INR $(P=0.014), \mathrm{Cr}(P=0.017)$, CTP scores $(P<0.001)$, MELD scores $(P<0.001)$ and lower serum thymosin $\beta 4$ concentrations $(P<0.001)$ were observed in deceased patients. Serum thymosin $\beta 4$ levels negatively correlated with TBIL, Cr, CTP scores and MELD scores $(P<0.05)$. On ROC curve analysis the area under curve (AUR) of serum thymosin $\beta 4$ values $(0.823)$ exceeded that of the CTP $(0.708)$ score $(P<0.05)$, but there was no statistically significant difference compared with the AUR of the MELD score (0.797) $(P>0.05)$. Patients with thymosin $\beta 4$ value $<0.3840 \mu \mathrm{g} / \mathrm{mL}$ had the highest relative risk of the poor prognosis. At last, the Kaplan-Meyer test showed a strong difference between survivals of patients with initial thymosin $\beta 4$ values $<0.3840 \mu \mathrm{g} / \mathrm{mL}$ (mean survived time $46.5 \pm 4.5$ days, $95 \%$ CI: $37.6-55.4)$ and $\geq 0.3840 \mu \mathrm{g} /$ $\mathrm{mL}$ (mean survived time $74.7 \pm 3.7$ days, $95 \% \mathrm{CI}: 67.4-82.1)(P<$ 0.001).

Conclusion: Serum thymosin $\beta 4$ concentration is a reliable indicator of short-term mortality in patients with ACLF.

\section{PP01-43}

The Case of the Death from Acute Hepatitis C (AHCV) of Patient after Bone Marrow Transplantation (BMT)

S. Lepkov, K.N. Melkova, G.I. Storogacov, I.N. Subortceva, A.A. Gettueva, N.V. Gorbunova, Z.T. Chernyvscay, A.N. Vorobev, S.N. Abdusalamov, A.M. Kovrigina, S.D. Kosura

The Moscow Medical University of N.N. Pirogov, Moscow, Russia

BMT is an important method in treatment hematology malignansy. Among after transplantation complications, hepatic insufficiency takes an important place. Cases of death after of AHCV described only 3 cases. Patient 48 years, in first remission of acute myeloid leucosis (AML) was made BMT from HLA-identical donor. Before BMT was examined the function of a liver, nephroses, vascular and respiratory systems it has not been revealed. Donor and recipient blood analyses on a cytomegalovirus, HCV, HBV, HGV was negative. BMT was made after standard air-conditioning. At 35 day method PSR was revealed full donor bone marrow. After 120 days of BMT growth of level of hepatic ferments has been noted. (ALT $1,100 \mathrm{U} / \mathrm{L}$, progressing level of bilirubin (to $389.3 \mathrm{mkmol} / \mathrm{l}$ ). Method PSR reveals presence RNA HCV-genotype $1 \mathrm{~b}$, level HCV $2 \times 10^{8}$ copy $/ \mathrm{ml}$. Antibodies to $\mathrm{HCV}$ is not revealed. For the purpose of reduction of hepatic and renal toxicity medicinal therapy has been reduced, made symptomatic therapy. Decrease in the immune status-CD4 absolute number $10 \mathrm{kl} / \mathrm{mkl}$. To 180 days after BMT at the patient $100 \%$ a donor hemopoiesis are conserved. Made therapy has led to moderate decrease in a cytolytic syndrome. Under vital indications it is appointed alfer-interferon in a dose of 3 million Ed 3 times a week, ribaverin on $0.4 \mathrm{~g} /$ sut taking into account kreatinine level. Has occurred an anaemia, thrombocytopenia, leucopenia, neutropenia and has demanded appointment SCF. Despite carrying out of intensive therapy, signs of hepatic insufficiency and an encephalopathy progressed, there were bradycardia episodes, appearance of spontaneous hematomas was marked. The patient has died for 225 days after carrying out BMT. At dissecting at the patient it has been revealed total destruction of hepatocytes invoked by $\mathrm{HCV}$. In bone marrow cells were absent destruction. HCV can possesses a direct cytopathic effect on hepatocytes and bone marrow cells were.

\section{PP01-44}

Etiology and Disease Characteristics of Patients with Acute on Chronic Liver Failure in Karachi, Pakistan

A.S. Butt ${ }^{1}$, S. Hamid ${ }^{2}$, W. Jafri ${ }^{2}$

${ }^{1}$ Section of Gastroenterology, Department of Medicine; ${ }^{2}$ Aga Khan University Hospital, Karachi, Pakistan

Background and aim: Acute on Chronic liver failure (ACLF) is associated with high morbidity and mortality. Hepatitis E virus (HEV) superinfection is common in patients with underlying chronic liver disease (CLD) and can lead to ACLF. Scanty data is available from Pakistan which is a hyper-endemic region for HEV virus. The aim of present study was to investigate the etiology and disease characteristics of patients presenting with Acute on Chronic Liver Failure.

Methods: Consecutive patients $\geq 18$ years of age admitted with acute liver failure in Gastroenterology wards of The Aga Khan University hospital, Karachi, Pakistan during 2008-2009 were evaluated. Those who were diagnosed to have ACLF were included. The diagnosis of ACLF was made if the patient has

1. Acute onset jaundice within last 4 weeks.

2. Serum bilirubin $\geq 2 \mathrm{mg} / \mathrm{dl}$.

3. Coagulopathy (INR $\geq 1.5$ ).

4. Clinical/histological/ radiological or serological evidence of underlying chronic liver disease.

5. Known compensated CLD and now presented with acute decompensation.

Results: Out of 200 patients 34 patients were diagnosed to have ACLF. Mean age was $40.88 \pm 12.96$ years and $25(73.5 \%)$ were males. The etiology of underlying CLD was HBV (32.4\%), HCV (17.6\%) concomitant HBV, HDV with or without HCV (23.3\%), alcohol (5.9\%), Wilson's disease (2.9\%) and cryptogenic cirrhosis (17.6\%). The etiology for acute decompensation was acute hepatitis E (44.1\%), hepatitis A (2.9\%), acute HBV (14.7\%), HDV superinfection (5.9\%), hepatotoxic drugs $(2.9 \%)$ and unknown $(29.4 \%)$. Common presentations were fever $(78 \%)$, jaundice $(100 \%)$, ascites $(58.8 \%)$ and encephalopathy $(61.8 \%)$. Mean CTP and MELD scores were $11.55 \pm 2.06$ and $28.38 \pm 9.85$, respectively. Laboratory parameters at presentation were $\mathrm{Hb} 11.9 \pm 2.3 \mathrm{mg} / \mathrm{dl}$, creatinine $1.8 \pm 1.2 \mathrm{mg} / \mathrm{dl}$, bilirubin $20.1 \pm$ $10.4 \mathrm{mg} / \mathrm{dl}$, albumin $2.2 \pm 0.6 \mathrm{mg} / \mathrm{dl}$, ALT $539.2 \pm 396 \mathrm{IU} / \mathrm{ml}$, PT $24.4 \pm 12.4$ s. Overall in hospital mortality was $55.9 \%$. On multivariate analysis, ascites, hepatic encephalopathy, renal failure, GI bleeding, total bilirubin and coagulopathy were the significant predictors of mortality.

Conclusion: HEV was the most common cause of ACLF. ACLF is associated with significant in hospital mortality. Preventive measures against HEV could prevent severe acute hepatic decompensation and mortality. 
Poster Presentation 02: Drug Induced Liver Disease (DILD)

\section{PP02-01}

Hepatotoxicity of Rifampicin in Male and Female Rats

K. Murugan, G. Vanithakumari, K. Balamurugan

Zoology, Bharathiar University, Coimbatore, India

Rifampicin is considered as the most effective drug for the treatment of tuberculosis (TB). It has also been found to be effective against several other pathogens including mycobacterium and penicillin resistant pneumococci. A variety of enzymes in the liver process either endogenous or exogenous lipophilic chemical intermediates can attack macromolecules leading to direct toxicity or hypersensitivity. Many drugs have been associated with the production of the liver toxicity. In the present study Rifampicin at $10 \mathrm{mg} / \mathrm{kg}$ given orally for 21 consecutive days to albino rats of both sexes. Drug administrated animals were supplemented with 7.5 and $500 \mathrm{mg}$ of Vitamin A and C, respectively. A withdrawal group was included. The increased level of hepatic markers such as alkaline phosphatase (ALP), bilirubin, aspartate transaminase (AST) and alanine aminotransferase (ALT) was observed in the drug administered animals, which suggested the damage to the liver. The vitamin supplementations brought about restoration of the above parameters. The regenerative capacity of the liver from the adverse effect could be seen in the withdrawal group partially. Moreover, supplementation with vitamins (A \& C) has caused a better response almost to the normal level. Further, the reduced level of protein concentration in treated rats was also revived to near normalcy by the supplementation of vitamins. The increased level of lipid profile such as total lipids, glyceride glycerol and phospholipid phosphorus, suggestive of tissue damage, was also reduced by the administration of vitamins. Liver damage was seen as hyperplasia of Kupffer cells, mild periportal infiltration and inflammation. Central vein hemorrhage, nonspecific hepatitis. Bile duct obstruction markedly visible. Further; the pathological studies also illustrated the protective effect of vitamins, in the decrease of fibroids in the portal tract caused by drug. The result suggests the combination of vitamin dosage with Rifampicin proved to be a better protective supplement.

\section{PP020-02}

Hepatoprotective Effect of Ethanolic Extract of Saffron Stigma

in Comparison with Silymarin against Rifampin Induced

Hepatotoxicity in Rats

D. Mohajeri

Department of Pathobiology, Faculty of Veterinary Medicine, Islamic Azad University, Tabriz, Branch, Tabriz, Iran

Background: Anti-tuberculosis drug rifampin is a potent hepatotoxicant. The aim of the present study was to evaluate the protective effect of ethanolic extract of Crocus sativus L. stigma (EECSL.S) in comparison with standard drug silimarin against rifampin-induced hepatotoxicity in the rats.

Methods: 40 male Wistar rats with the mean body weight of $200 \pm 20 \mathrm{~g}$ and age of 10 weeks were randomly assigned into 5 groups of 8 animals each and kept in specific cages with 12/12 h light/ dark cycle at $21 \pm 2^{\circ} \mathrm{C}$. Group I as normal control received normal saline $(10 \mathrm{ml} / \mathrm{kg})$ and group II as toxicant control received rifampin $(500 \mathrm{mg} / \mathrm{kg})$. Group III as positive control received silymarin plus rifampin $(500 \mathrm{mg} / \mathrm{kg})$ and groups IV and $\mathrm{V}(50 \mathrm{mg} / \mathrm{kg})$ received EECSL.S at $40 \mathrm{mg} / \mathrm{kg}$ and $80 \mathrm{mg} / \mathrm{kg}$ plus rifampin, respectively. All the treatments were carried out through the gavage dissolving in 10 $\mathrm{ml} / \mathrm{kg}$ normal saline daily for 1 month. At the end of experiment, levels of liver function marker enzymes (aspartate aminotransferase, alanine aminotransferase and serum alkaline phosphatase), total bilirubin, albumin and total proteins were assessed in serum of the rats.
Moreover, histopathological observation was assayed at the degree of hepatic injury.

Results: In rifampin-treated rats, silymarin and EECSL.S (40 and 80 $\mathrm{mg} / \mathrm{kg}$ ) significantly decreased the levels of serum biomarker of hepathic injury and TB and elevated the levels of Alb and TP. Histopathologically, silymarin and EECSL.S ameliorated rifampin induced hepatic injury. Histopathological changes were in agreement with biochemical findings.

Conclusion: Results indicated that EECSL.S $(80 \mathrm{mg} / \mathrm{kg})$ equals with silymarin as standard drug, point of view hepatoprotective effects against rifampin-induced hepatotoxicity.

\section{PP02-03}

Taurine in Liver Necrosis Caused by Paracetamol Overdose S. Mashayekhi, P.A. Routledge, Clinical Pharmacy, Tabriz University of Medical Sciences, Tabriz, Iran

Background: Taurine is one of the most abundant amino acids in the body. Hypertaurinemia and hypertaurineuria have already been reported after surgical trauma, X-radiation, muscle necrosis, carbon tetrachloride-induced liver damage.

Methods: Plasma taurine levels of 217 paracetamol positive and 100 paracetamol negative poisoned patients (related to non- epatotoxic agents) referred to the Toxicology lab in Cardiff Poisons unit and 90 healthy humans (age between 18 and 45) were measured by a high performance liquid chromatography method. The data were analysed using linear regression and two-tailed unpaired Student $t$ test using Prism software package.

Results: We showed that the mean plasma taurine concentration in the paracetamol-poisoned patients (mean $26.4 \pm 1.6 \mathrm{mg} / \mathrm{l}$ ) was significantly different from the control groups (mean $5.6 \pm 0.2 \mathrm{mg} / \mathrm{l})(P<0.0001)$ and the non-paracetamol group (mean $18.1 \pm 1.1 \mathrm{mg} / \mathrm{l})(P<0.01)$.

Conclusions: Taurine is produced by the liver in response to a toxic insult and subsequent leakage from damaged cells leads to increased concentrations in plasma and urine. Administration of taurine has a prophylactic as well as a therapeutic role in preventing paracetamolinduced hepatotoxicity with prevention of plasma AST, ALT, and ALP elevation. Although plasma taurine concentration may be a primary biomarker of liver damage, it may be insufficiently specific to be of value in predicting early hepatotoxicity in paracetamol poisoning.

\section{PP02-04}

Clinical Characteristics and Pathologic Findings of Patients with Toxic Hepatitis in Gyeonggi Province

J.H. Lim, J.W. Tae, Y.S. Kim, S.G. Kim, S.W. Jung, J.Y. Jang, H.S. Kim, S.H. Lee, B.S. Kim

Soonchunhyang University Medical School, Bucheon Hospital, Bucheon-Si, Republic of Korea

Objectives: Although many individual cases of toxic hepatitis have been reported, there are few comprehensive systematic studies and few reports of pathologic findings on toxic hepatitis. We studied the clinical characteristics and pathologic findings and prognosis of patients of toxic hepatitis.

Patients and methods: A retrospective analysis was performed using medical records of 184 cases of toxic hepatitis that were diagnosed from January 2004 to January 2010. Among them, 62 cases were performed liver biopsy. Patients were selected based on a RUCAM score of 4 or above.

Results: The incidence was higher in women $(n=125)$ than in men $(n=59)$. The mean age of patients was $45.9 \pm 14.4$ years. At the time of admission, the most common symptom was jaundice. The causative agents of the disease included herb medications $(43.5 \%)$, traditional therapeutic preparations $(33.7 \%)$ and the prescribed medications $(21.7 \%)$. The pathologic findings of toxic hepatitis included 
necrosis $(n=62)$, cholestasis $(n=43)$, steatosis $(n=23)$, eosinophilic infiltrations $(n=22)$, and ballooning degeneration $(n=20)$. The patients with pathologic cholestasis and necrosis went through longer hospitalization and slower recovery. The prognostic factor associated with the hospitalization and recovery periods was only serum total bilirubin at the time of admission $(p<0.001)$.

Conclusions: The common pathologic findings of toxic hepatitis were necrosis and cholestasis. But it is not specific finding of toxic hepatitis. Only the total bilirubin at the time of admission was significantly associated with hospitalization and recovery time.

\section{PP02-05}

Drug Induced Liver Injury: A Retrospective Review of 112 Patients P. Skok ${ }^{1}$, D. Ceranic ${ }^{1}$, A. Ocepek ${ }^{1}$, M. Godec ${ }^{1}$, G. Vujnovic ${ }^{1}$, R. Kavalar ${ }^{2}$ ${ }^{\text {I}}$ Gastroenterology; ${ }^{2}$ Pathology, University Clinical Centre Maribor, Maribor, Slovenia

Introduction: Early recognition of drug-induced liver injury (DILI) is a major challenge for the treating physician. This condition can be classified according to clinical presentation and laboratory features, mechanism of toxicity, and/or histological findings.

Aims: To elucidate the etiology, histological findings and outcome of patients with histologically confirmed DILI.

Patients and methods: A retrospective review of medical records of patients admitted in the period January 1998-January 2010. In the diagnostic procedure by means of laboratory investigations, imaging modalities (US, CT or MR, EUS) viral, metabolic, autoimmune and alcohol liver disease were excluded. Due to unclarified etiology liver biopsy was made in all patients.

Results: In 66 females and 56 males (av. age 59.9 years, SD 17.7) most important causes of DILI were antibiotics (leading amocillin clavulanate) in $33.9 \%$, nonsteroidal antiinflammatory drugs, NSAID's (ketoprofen, diclofenac, naproxen) in $25.9 \%$, antipsychotics (including trcyclic antidepressants) $17.9 \%$, cardiovascular drugs (statins, amiodarone, aspirin) in $14.3 \%$, and others (including acetaminophen) in $8 \%$. Histology confirmed hepatocellular (cytotoxic) patern in $48.2 \%$ of patiens, cholestatic pattern in $32.1 \%$, mixed pattern in $12.5 \%$ of patients and steatosis only in $7.2 \%$. During the follow-up period (in the range from 6 to 128 months) $8 \%$ of patients died due to factors unrelated to liver injury, among the remaining 103 patients, in $7.8 \%$ chronic liver injury developed.

Conclusion: The diagnosis of DILI remains a challenging problem. Different types of acute hepatitis, with or without cholestasis, are the most common histological pattern of liver injury. In most patients, liver injury resolves after discontinuation of the drug, however the recovery is sometimes prolonged. According to our results, in Slovenia acetaminophen is not a drug often involved in serious liver injury.

\section{PP02-06}

Study on Phenotypic and Genotypic Polymorphisms of $N$-acetyltransferase-2 Gene in Patients on Anti-Tubercular Treatment

R.P. Ola ${ }^{1}$, S.V. Rana ${ }^{1}$, S.K. Sharma ${ }^{1}$, S.K. Sinha ${ }^{1}$, S.K. Arora ${ }^{2}$, P. Pandhi ${ }^{3}$, K. Singh ${ }^{1}$

${ }^{I}$ Gastroenterology; ${ }^{2}$ Immunopathology; ${ }^{3}$ Pharmacology, PGIMER, Chandigarh, India

Introduction: $N$-Acetyltransferase-2 (NAT-2) is involved in Isoniazid (INH) metabolism. Susceptibility to anti-tubercular drug (ATD)induced hepatotoxicity may be genetically mediated with variant alleles of gene such as NAT-2 as a risk factor.

Aim: The aim of this study was to compare whether genotype pharmacogenetic test NAT2 and phenotype test of measuring INH in serum are useful to identify acetylator status of patients on anti tubercular treatment (ATT).

Materials and methods: Three hundred two patients on standard treatment for tuberculosis (TB) were enrolled in this study and ATT induced hepatotoxicity was diagnosed. NAT2 genotype was assessed by PCR with restriction fragment length polymorphism (RFLP) and serum INH levels were measured by flourometery.

Results: Three hundred two TB patients on ATT (184 males, mean age $44.7 \pm 3.9$ years) were studied. Out of 302 patients, 60 $(19.8 \%)$ developed hepatotoxicity. In hepatotoxicity group, on phenotypic estimation 21 out of $60(35 \%)$ were slow acetylators while $39(65 \%)$ fast acetylators. Genotypically, 23 out of 60 $(38.3 \%)$ were slow acetylators and $37(61.7 \%)$ fast acetylators. In the non-hepatotoxicity group, on phenotypic analysis 56 out of 242 $(23.1 \%)$ were slow acetylators \& $186(76.9 \%)$ fast acetylators and on genotypic analysis, 36 out of $242(14.9 \%)$ were slow acetylators and $206(85.1 \%)$ fast acetylators. Both the methods showed significantly higher slow acetylators in hepatotoxicity group. Slow acetylators $(25.5 \%)$ measured phenotypically were not significantly different as compared to slow acetylators (19.5\%) measured genotypically.

Conclusion: This study suggests that the acetylator status of ATT patients may be detected as efficaciously by phenotypic method as by genotypic method. Therefore, phenotypic method may be used instead of genotypic method as it is simple and inexpensive.

\section{PP02-07}

Drug-induced Liver Injury Causing Acute Liver Failure

K.B.L. Lim, Y.Y. Dan, A. Mo, S.G. Lim

Gastroenterology and Hepatology, National University Hospital, Singapore, Singapore

Background/aim: Drug-induced liver injury (DILI) is a common and potentially fatal disease, accounting for $13-30 \%$ of cases of acute liver failure (ALF). We aim to describe our experience of ALF due to DILI in a multi-ethnic tertiary referral centre.

Methods: Patients with suspected DILI had to fulfill pre-determined criteria and were classified as "definite", "probable" or "unlikely" cases. Adult patients (age $>18$ ) with ALF secondary to DILI who were referred for liver transplant evaluation from 1 Jan 1999 to 31 Dec 2008 were included and retrospectively analyzed.

Results: 25 patients (10 males, 15 females) developed ALF due to DILI. This represented $4.9 \%$ of the transplant referrals. DILI accounted for $55.6 \%$ of patients with ALF. There were 18 "definite" and 7 "probable" cases of DILI. A single agent and nonprescription medications were implicated in 84 and $68 \%$ of cases, respectively. $40 \%$ of cases were due to traditional Chinese medicines (TCM). Indications for TCM administration include hypertension, joint pain, general well-being and weight loss. Paracetamol was the causative agent in $20 \%$. Anti-tuberculosis drugs made up $66 \%$ of the implicated antimicrobial drugs. Mean peak serum biochemistries were: ALT 1998; total bilirubin 283.3; INR 4.4. There were $15(60 \%)$ hepatocellular, 5 (20\%) cholestatic, and $5(20 \%)$ mixed pattern of injury. Patients with a mixed pattern had the highest mortality. Mean MELD score was 33. Molecular adsorbent recirculating system (MARS) was used as a bridge to transplant in $50 \%$ of the patients. $16 \%$ underwent liver transplantation. Median survival $14( \pm 2)$ days and in-hospital mortality was 44\%. Transplant-free survival was $40 \%$.

Conclusion: Drug induced liver injury accounted for $55.6 \%$ of patients with acute liver failure who were referred for transplant evaluation. DILI is associated with significant mortality and a $40 \%$ transplant-free survival rate. Traditional chinese medicines were the most commonly implicated drug.

\section{PP02-08}

DRESS Syndrome Induced by Dapsone in a Patient with Vitiligo S.G. Shim, G.T. Park, G.J. Yoo, D.H. Cho, C.U. Jeong, J.E. Oh Gastroenterology and Hepatology, Samsung Changwon Hospital/ Sungkyunkwan University School of Medicine, Changwon-Si, Republic of Korea 
Dapsone (4,4'-diaminodiphenylsulfone) is used for treatment of leprosy, actinomycetoma, or malaria. It is also effective for some dermatologic and rheumatologic conditions. The common side effects are fatigue, headache, nausea, anemia, and methemoglobinemia. It can also rarely cause an idiosyncratic reaction accompanying hepatitis, infectious-mononucleosis-like with adenopathy, jauncide, a variety of dermatitis, or peripheral neuropathy. This hypersensitivity occurs frequently between 4 and 6 weeks after the initiation of the drug and has been estimated to be $0.2-0.4 \%$ of patients treated with dapsone.We recently experienced a case of severe DRESS (Drug Rash with Eeosinophilia and Systemic Symptoms) in a 39-year-old man who took $100 \mathrm{mg}$ of dapsone daily for 4 weeks for self-treatment of vilitigo. At admission, he was presented with a high fever, nausea and vomiting, throat pain with cervical lymphadenopathy, jaundice, anemia, increased LDH and transaminases, macuolopapular rash on his body and both upper and lower limbs, and eosinophilia (11.3\%). The patient completely recovered by discontinuing the drug with subsequent supportive care and steroid therapy for about 2 weeks.Dapsone should be given with great caution to patients with dermatologic disorders to avoid serious toxicities.

\section{PP02-09}

A New Diagnostic Method to Confirm the Relationship between Hepatic Sinusoidal Obstruction Syndrome and Gynura segetum

H. Gao ${ }^{1}$, J.Y. Wang ${ }^{1}$, G. Lin $^{2}$, S.C. Zhang ${ }^{1}$

${ }^{1}$ Gastroenterology, Zhongshan Hospital, Fudan University, Shanghai, China; ${ }^{2}$ School of Biomedical Sciences, Faculty of Medicine, The Chinese University of Hong Kong, Hong Kong, Hong Kong SAR

Background: Hepatic sinusoidal obstruction syndrome (HSOS) induced by Gynura segetum was reported increasingly.

Aim: To better understand the clinical characteristics of HSOS associated with Gymura segetum and investigate the possibility using serum protein-pyrrole adducts test as a confirmatory diagnostic method.

Methods: Six consecutive patients taken Gynura segetum diagnosed with HSOS admitted into Zhongshan Hospital Fudan University from Jan 2006 to Aug 2010 were included. Clinical features were collected. Protein-pyrrole adducts from pyrrolizidine alkaloids in blood sample was determined with UPLC-MS analysis.

Results: The patients (one male and five female patients) met the Seattle criteria and RUCAM. They were 41-57 years old, and ingested Gynura segetum for the periods from 3 weeks to 2 years. The discontinuation interval was between one and eight months. Ascites, jaundice and hepatomegaly were the common manifestations. Protein-pyrrole adducts were unequivocally found in all six serum samples. Adducts were determined in four patients. The imaging features were diffused patchy hepatic enhancement and periportal edema, ascites. No portal obstruction was found. Pathology ascertained that blood flow was obstructive at the site of sinusoid rather than small hepatic vein. Two patients recovered.
Two became chronic illed and two died on liver failure or gastrointestinal bleeding.

Conclusions: Blood protein-pyrrole adducts could be determined in HSOS patients caused by Gynura segetum in spite of long discontinuation. It is a useful diagnostic method for HSOS induced by Gynura segetum.

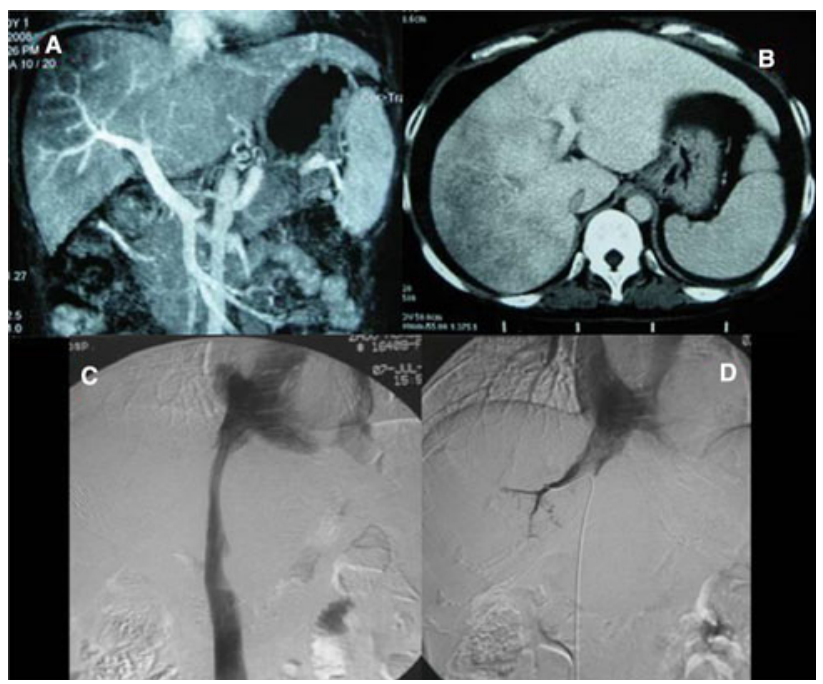

Imaging of a HSOS patient

\section{PP02-10}

Cytochrome P450 2E1-derived Reactive Oxygen Species Mediate Isoniazid Induced Hepatocytes Injury

D. Bishnu, S. Santra, S. Ghatak, A. Biswas, G.K. Dhali, A. Chowdhury, A. Santra

Center for Liver Research, Institute of Post Graduate Medical Education and Research, Kolkata, India

Background and aim: Anti-tubercular (AT) drugs are the commonest cause of serious drug induced liver disease in India. Although available data suggests involvement of oxidative stress and cytochrome P450 2E1 (CYP2E1) in isoniazid (INH) induced hepatotoxicity, evidence of a direct role for CYP2E1 in this process is still lacking. The aim of this study was to evaluate the cytotoxic effects of INH to HepG2 cells expressing CYP2E1 and compare these effects to cells which do not express CYP2E1.

Methods: This study was performed using HepG2 cells, which constitutively express CYP2E1 (E47 cells) or vector control HepG2 cells (C34 cells), which have insignificant CYP2E1 activity. Cells were cultured with or without $0.5 \mu \mathrm{M}$ INH in minimal essential medium.

Clinical feature of HSOS patients caused by Gynura

\begin{tabular}{|c|c|c|c|c|c|c|c|c|c|}
\hline No. & Gender & Age & $\begin{array}{l}\text { Intake } \\
\text { period }\end{array}$ & $\begin{array}{l}\text { Discontinuation } \\
\text { interval }\end{array}$ & Administration & $\begin{array}{l}\text { RUCAM } \\
\text { score }\end{array}$ & $\begin{array}{l}\text { Serum pyrrole- } \\
\text { protein adducts }\end{array}$ & $\begin{array}{l}\text { Adducts } \\
\text { determined }\end{array}$ & $\begin{array}{l}\text { Outcome } \\
\text { (100 days) }\end{array}$ \\
\hline 1 & $\mathrm{~F}$ & 54 & $\sim 3$ months & $\sim 8$ months & Wine infusion & 5 & + & + & Recovery \\
\hline 2 & M & 52 & $\sim 1$ month & $\sim 2$ months & Wine infusion & 6 & + & - & Death \\
\hline 3 & $\mathrm{~F}$ & 57 & $\sim 3$ weeks & $\sim 1.5$ months & Oral and decoction & 7 & + & + & Chronicity \\
\hline 4 & $\mathrm{~F}$ & 41 & $\sim 1$ month & NA & Oral & 7 & + & - & Recovery \\
\hline 5 & $\mathrm{~F}$ & 54 & $\sim 2$ years & $\sim 6$ weeks & Tea & 6 & + & + & Chronicity \\
\hline 6 & $\mathrm{~F}$ & 72 & $\sim 2$ months & $\sim 1$ month & Oral & 5 & + & + & Death \\
\hline
\end{tabular}


Intracellular reactive oxygen species (ROS), lysosomal membrane leakage (LML), mitochondrial membrane potential (MMP) and cell death were evaluated at different time points using confocal microscopy and FACS analysis. Some experiments were carried out using antioxidants and inhibitors of CYP2E1, JNK and p38.

Results: INH caused time dependent increase of ROS in E47 cells. FACS analysis revealed ROS generation in $85 \%$ E47 cells compared to $11.88 \%$ in $\mathrm{C} 34$ cells when cultured with $0.5 \mu \mathrm{M}$ INH. Enhanced LML and reduction of MMP was observed as early as $3 \mathrm{~h}$ in E47 cells with INH. INH exposure caused necrotic death of E47 cells as evidenced by confocal microscopy and FACS analysis. Antioxidants (NAC, GSH-E, Tempol) and CYP2E1 inhibitor (CMZ) pretreatment caused decreased ROS generation and cell death of E47 cells when cultured with INH.

Conclusions: These findings provide evidences to support the involvement of CYP2E1 in mediation of INH induced hepatocytes injury through oxidative damage. INH enhances LML and reduction of MMP before the onset of necrosis of the hepatocytes.

\section{PP02-11}

Involvement of Mitochondrial Membrane Potential (MMP) in Isoniazid (INH) Induced Hepatotoxicity: An in vitro Study with Mouse Hepatocytes A. Biswas, S. Ghatak, D. Bishnu, G.K. Dhali, A. Chowdhury, A. Santra Center for Liver Research, Institute of Post Graduate Medical Education and Research, Kolkata, India

Background and aims: Mitochondrial membrane potential (MMP) plays important role in maintaining physiological functions of the cells. Reduction of MMP due to various etiological conditions leads to mitochondrial injury and cell death. In isoniazid induced liver cell injury involvement of mitochondria is assumed, but detail mechanisms are still lacking. The present study is designed to explore the possible role of MMP and mitochondrial permeability transition (MPT) in INH induced hepatotoxicity.

Methods: Mouse primary cultured hepatocytes were incubated with different doses of INH to study the intracellular reactive oxygen species (ROS) generation, mode of cell death and their related signaling pathways using fluoroprobes. Involvement of mitochondria was studied by studying MMP, MPT by confocal microscopy and FACS.

Results: In hepatocytes, INH caused ROS generation in the hepatocytes in time as well as dose dependent manner. At 24-h period with $10 \mu \mathrm{M}$ dose of INH about $80-90 \%$ hepatocytes were found to generating ROS which was prevented by pretreatment with NAC. Alteration of MMP was observed (65-69 mV) was observed in INH treated hepatocytes compared to control (88-92 mV). FACS analysis also confirmed reduction of MMP in significant number of hepatocytes. The reduced MMP was associated with alteration of MPT. The reduced MMP and altered MPT were associated with apoptotic death of the hepatocytes. Cyclosporine A pretreatment prevented altered MPT and hepatocytes death due to INH. Further p38 inhibitor $(10 \mu \mathrm{M} / \mathrm{L})$ pretreatment caused significant reduction of cell death.

Conclusion: INH induced apoptotic death of the hepatocytes is mediated by the alteration of MMP and MPT.

\section{PP02-12}

Isolation, Characterization and Hepatoprotective Activity of Whole Plant of Trichosanthes lobata (Roxb) in Rats

R.A. Shastry ${ }^{1}$, K.M. Mahadevan ${ }^{2}$, P.V. Habbua ${ }^{1}$, B.S. Patil ${ }^{1}$, V.H. Kulkarni ${ }^{1}$ ${ }^{1}$ Pharmacognosy, S.E.T College of Pharmacy, Postgraduate Studies and Research Center, Dharwad; ${ }^{2}$ Chemistry, Kuvempu University, Postgraduate Studies and Research Center, Shimoga, India

Trichosanthes lobata (Roxb) (Cucurbitaceae) is commonly known as wild snake gourd. It is a Monoecious annual climber with furrowed stems, cultivated throughout India. Traditionally the plant is used in treatment of leucoderma, dyspepsia, hepatopathy and in treatment of Jaundice. In the present investigation the whole plant of trichosanthes lobata were shade dried, pulverized and successively extracted with petroleum ether, chloroform, ethylacetate and ethanol, respectively. The crude extracts were concentrated in a rotary flash evaoperator and dried in a desicator. Crude extracts were subjected for preliminary phytochemical tests and chromatographic studies which revealed the presence of steroids, tannins, proteins and aminoacids. Here an attempt has been made to isolate the tannin from ethylacetate extracts by isocratic elution using column chromatography. Isolated tannin were further characterized by I.R, N.M.R and Mass spectroscopy. Ethylacetate and ethanolic extracts were screened for hepatoprotective activity using D-galactosamine as hepatotoxic agent. The biochemical parameters such as SGOT, SGPT, SALP, Direct and total bilirubin and protein levels were determined. D-galactosamine treated group exhibited significant increase in the biochemical parameters and decreased the protein level in the blood. Ethylacetate treated group exhibited significant $(P<0.01)$ hepatoprotective activity as compared to ethanolic extracts by decreasing the SGOT, SGPT and SALP levels but increased the protein content in the blood. The results were further compared with histopathological study of the rat liver. From the results we can conclude that Crude extracts of whole plant of trichosanthes lobata can be employed in the management/ailments of hepatic disorders.

\section{PP02-13}

Pay Attention to Chinese Medicine Induced Liver Injury

Y.J. Zhang, L.R. Wei, X.M. Wang, P.P. Meng, B. Li, Y. Duan, W.N. Ou, H.C. Xing

Institute of Infectious Diseases of Capital Medical University, Beijing Ditan Hospital, Beijing, China

Background/aims: To explore the origin of 4 patients with acute liver injury and call for attention on the risk of drug-induced liver injury with Chinese herbal medicine.

Methods: Four acute liver injury patients, who had received the same Chinese herbal medicine, named YISHENWUFAKOUFUYE, shortly before, were retrospective studied, based on the history of drug-taking, clinical manifestations, liver function, serum markers of virus hepatitis, and hepatic biopsy if possible. Diagnosis of all patients was done according to the two scoring system of RUCAM and DDWJ-C, respectively.

Results: All of the patients were excluded other cause of liver injury. The score of all patients were $6-8$, which were evaluated with the two scoring systems respectively and probably drug-induced liver injury was diagnosed.

Conclusion: Some Chinese herbal medicine may be an important cause of drug induced liver injury. Monitoring the change of liver function is necessary during the treatment with Chinese herbal medicine.

\section{PP02-14}

Relative Efficacies in Mouse of Various Injection Sites for Transplantation of HMSCs as Treatment for Acetaminophen-induced Acute Hepatic Injury

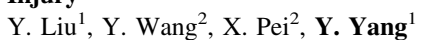

${ }^{1}$ Gastroenterology and Hepatology, Military General Hospital of China; ${ }^{2}$ Unit 9 th, Academy of Military Medical Sciences of Chinese PLA, Beijing, China

Aim: To evaluate the relative efficacies in a mouse model of human mesenchymal stem cell (hMSC) transplantation for treatment of acute acetaminophen-induced hepatic injury by injection through the portal vein, spleen or caudal vein.

Methods: An animal model of acetaminophen-induced acute liver injury was established in non-obese diabetic/severe combined 
immunodeficient (NOD/SCID) mice, and then hSMCs were transplanted by injection through the portal vein, spleen or caudal vein. Peri-transplantation liver function and histological changes were assessed.

Results: Following all three transplantation methods, hSMC could be found colonizing, differentiating and proliferating in liver. Successful transplantation was most evident in the liver following portal vein injection.

Conclusions: Different injection routes for stem cell transplantation had varying degrees of therapeutic success after acute liver injury, but of the three assessed the portal vein route of entry resulted in the most-improved liver function and best acinar structural recovery.

\section{PP02-15}

Newly Showed Ethambutol Effect on Expression of Cytochrome P-450 Isoforms in Rats Liver

G. Shayakhmetova, S. Anisimova, L. Bondarenko, A. Voronina,

V. Kovalenko

General Toxicology, Institute of Pharmacology and Toxicology, Academy of Medical Sciences of Ukraine, Kyiv, Ukraine

Background: Drug-induced liver injury is a problem of increasing significance, but has been a long-standing concern in the treatment of tuberculosis infection, especially in case of several antituberculosis medications co-administration. Nevertheless the exact mechanism and factors contributing to liver toxicity remain poorly understood.

Methods: Internal medicine literature suggests that hepatotoxic effects of ethambutol are not unusual and transient, but the exact mechanism of hepatotoxicity is unknown. In the current study, we examined effects of therapeutic dose administration of ethambutol (during 2 months) on the CYP2E1, CYP2C23 (ortholog for CYP2C19 and CYP2C9 from rat) and CYP3A2 (ortholog for CYP3A4 from rat) gene expression in male rats liver. Intensity of liver DNA fragmentation, liver microsomal $p$-nitrophenol hydroxylase activity and biochemical indices of blood serum were measured.

Results: In $24 \mathrm{~h}$ after final ethambutol administration hyperbilirubinemia and increase of alkaline phosphatase with no increase of alanine-aminotransferase activities were observed in blood serum, which indicates cholestasis. At the same times the intensity of DNA fragmentation in liver was too high. The high level of CYP2E1, CYP3A and CYP2C expression in liver has been demonstrated by reverse transcriptase-polymerase chain reaction. p-nitrophenol hydroxylase activity significantly rose as compared with control, which was additionally evidence of CYP2E1 induction.

Conclusions: The information regarding drug-drug interactions mediated by inhibition or induction of cytochrome P-450 during antituberculosis therapy is known to isoniazid and rifampicin. In addition to the reported inhibitory and inductive effects of mentioned drugs on CYP2E1, CYP3A and CYP2C activities, our results newly showed ethambutol effect on gene expression of these iso. Was significant to note that ethambutol is reported not metabolized by CYP450. Results encourage for further research toward CYP450mediated mechanisms of ethambutol hepatotoxicity and its investment to drug-drug interaction in order to develop new solutions for prediction and prevention of antituberculosis drug-induced liver injury.

\section{PP02-16}

The Effect of Sunitinib in Combination with Diclofenac: A Preliminary Study on Hepatic Histopathology and Enzymes

J.R. Tan $^{1}$, I. Segarra ${ }^{2}$, S. Chakravarthi ${ }^{3}$, J.P. Judson ${ }^{4}$, N. Haleagrahara ${ }^{4}$ ${ }^{1}$ International Medical University; ${ }^{2}$ Department of Pharmaceutical Technology; ${ }^{3}$ Department of Pathology; ${ }^{4}$ Department of Human Biology, International Medical University, Bukit Jalil, Malaysia

Background: Sunitinib is a novel multitargeted tyrosine kinase inhibitor used in the treatment of gastrointestinal stromal tumours and advanced renal cell carcinoma, which may be administered with diclofenac for pain management. Since both drugs share P450 pathway, co-administration may increase toxicity. We studied the liver sunitinib-diclofenac interaction effects evaluating liver biomarkers and histopathology.

Methods: ICR male mice $(n=6 /$ group/dose). Group A: saline; Group B: diclofenac $30 \mathrm{mg} / \mathrm{kg}$ PO; Group C: Sunitinib $(25,50,80$, $100,140 \mathrm{mg} / \mathrm{kg}$ PO); Group D: Sunitinib $(25,50,80,100,140 \mathrm{mg} / \mathrm{kg}$ $\mathrm{PO})$ and diclofenac (30 mg/kg PO). Diclofenac was administered 30 min before sunitinib and euthanized $4 \mathrm{~h}$ post sunitinib administration. Results: Serum ALT and AST levels were raised in group B $(249.60 \pm 222.72 ; 377.09 \pm 173.55 \mathrm{U} / \mathrm{L}$, respectively $)$ and slightly elevated in Group $C$ at all dose levels (range $36.75 \pm$ $12.15-75.02 \pm 20.37 \mathrm{U} / \mathrm{L}$ and $204.39 \pm 49.44-290.28 \pm 77.73 \mathrm{U} / \mathrm{L}$, respectively) and at high sunitinib doses in Group D (269.62 \pm 72.57 ; $540.30 \pm 76.09 \mathrm{U} / \mathrm{L}$, respectively). ALT and AST were lower in group D (range $\sim 182.79 \pm 64.91-221.03 \pm 112.98 \mathrm{U} / \mathrm{L}$ and $259.70 \pm 67.39-264.38 \pm 104.94 \mathrm{U} / \mathrm{L}$ ) at low sunitinib doses than Group B. Histopathological analysis showed a similar trend. Group B showed sinusoidal congestion and centrilobular necrosis and Group C had centrilobular necrosis but at a lesser extent than diclofenac at high sunitinib doses. Group D showed greater damage (vascular congestion, centrilobular necrosis) at high sunitinib doses but lesser damage than Group B at low sunitinib doses. This pattern suggests that sunitinib prevented diclofenac toxicity in lower doses, but has toxic effects on high doses. However, as sunitinib dose increased, the hepatoprotection diminished suggesting that sunitinib affects glutathione mediated detoxification.

Conclusion: Coadministration of sunitinib and diclofenac shows dose related hepatoprotective effect but may cause toxicity at high sunitinib doses.

\section{PP02-17}

The Management and Diagnosis of Antituberculosis Drugs Induced Hepatotoxicity

L. Zaidi, S. Nadir, W. Hliwa, R. Alaoui, A. Cherkaoui

Hepatology and Gastroenterology, CHU IBN ROCHD, Casablanca, Morocco

The hepatotoxicity is rather frequent in daily practice seen the endemicity of tuberculosis and the increasing number of patients under antituberculosis drugs, to determine clinicobiological forms of this complication and bring to light the difficulty of its management, we brought together during a period of 5 years, 21 patients were hospitalized in our service for hepatototoxicity of antituberculosis drugs. The average age of patients was 35 years old without sex predominance, the discovery of hepatotoxicity was made in $52 \%$ during the periodic monitoring of liver function, while in $48 \%$ of patients were symptomatic. The average time of onset of clinical and biological disturbances was 12 weeks. The clinical and biological aspects were as follows: cytolytic hepatitis in 17 cases, mixed hepatitis in 6 cases and liver failure in 5 cases; All patients received a report in search of another etiology (viral serology B and C, immunological balance such as antimitochondrial antibody..., abdominal ultrasound, liver biopsy) showing chronic hepatitis $\mathrm{C}$ in 2 patients and hepatic steatosis in 2 patients. The break of antituberculosis drugs followed by gradual reintroduction of less toxic molecules was initially recommended in 18 patients $(85 \%)$, it was continued in 2 patients in whom the cytolysis was less than 3 times the normal and 3 patients were at the end of treatment; with a good evolution of tuberculosis disease and liver disease in all patients. The hepatotoxicity of antituberculosis drugs is common in our context and can be life-threatening by the onset of fulminant hepatitis. So prevention is required by the strict rules of prescription, the search for risk factors for liver disease, early detection of signs of intolerance therapy and strict monitoring of liver function under treatment. 
PP02-18

Paracetamol Overdose: Determinants of Outcome from a Single Centre, 10 Year Experience

T.H. Lim, E. Gane, D. Orr

NZ Liver Transplant Unit, Auckland Hospital, Auckland, New Zealand

Introduction: Paracetamol overdose is a leading cause of morbidity and mortality in western countries. Prompt treatment with acetylcysteine can avert significant hepatic injury following paracetamol overdose.

Aim: To evaluate the demographic and laboratory characteristics of patients with paracetamol overdose at Auckland City Hospital and identify risk factors for severe hepatotoxicity and outcome.

Method: Patients with a diagnosis of paracetamol overdose between 1st August 2000 to 31st July 2010 at Auckland Hospital were identified. Primary outcome is significant hepatotoxicity (peak serum AST or ALT level $>1,000 \mathrm{U} / \mathrm{L}$ ). Secondary outcomes are death or referral for liver transplant. 1731 patients were identified of whom 1,419 $(82 \%)$ were intentional overdose, $312(18 \%)$ were accidental overdose. $65 / 1,419(4.6 \%)$ of the intentional group developed a severe hepatitis, compared to $31 / 312(10 \%)$ of the accidental group $(p=0.003)$. Peak INR, transaminases and inpatient stay were all significantly higher in the accidental overdose group. $3 / 266(0.3 \%)$ of those treated within $12 \mathrm{~h}$ of ingestion versus $41 / 133$ (31\%) for those treated more than $12 \mathrm{~h}$ had INR $>2 ;(p<0.0001)$. Hepatotoxicity is also more common in patients $>40$ years old: 16.5 versus $6.3 \%$ $(p=0.002)$. Only 1 patient with peak transaminases $<1,000 \mathrm{U} / \mathrm{L}$ developed an INR $>2.0$ or had evidence of liver failure.

Conclusions: Accidental overdose, a delay of $>12 \mathrm{~h}$ from paracetamol overdose and receiving $N$-acetylcysteine; and age $>40$ years are significant risk factors for severe hepatitis and progression to liver failure. All patients who present with a history of significant paracetamol overdose should be commenced on $\mathrm{N}$-acetylcysteine without delay, with close monitoring of INR and renal function. Patients with an INR $>2.0$ should be discussed with the liver transplant unit.

\section{PP02-19}

Paracetamol Overdose: Outcomes of Patients with Liver Failure and Assessment of Criteria for Liver Transplantation

T.H. Lim, E. Gane, D. Orr

NZ Liver Transplant Unit, Auckland Hospital, Auckland, New Zealand

Background: Paracetamol overdose is the leading cause of acute liver failure in the Western world. The King's College Criteria (KCC) for predicting poor prognosis in patients requiring liver transplantation have been criticised due to low sensitivity.

Aim: To evaluate the demographic and laboratory indices of patients presenting with paracetamol overdose and liver failure at Auckland City Hospital and assess the utility of Kings College Criteria.

Method: All patients diagnosed with paracetamol overdose (POD) between 1st August 2000 to 31st July 2010 at Auckland Hospital were identified. The primary outcome measure was assessment of the KCC as a predictor of poor outcome. 1731 patients with POD were identified. 45/1,731 (2.5\%) patients had an INR $>2$ (range 2-10.8; mean 4.4). The median age was 23 (range 2-62). 28 of 45 patients $(62 \%)$ were intentional overdose, $38 \%$ were accidental. Median creatinine was 130 (range 39-750); with 11 patients requiring hemodialysis. 16 patients developed hepatic encephalopathy. The median time from overdose to receiving $\mathrm{N}$-acetylcysteine was $35 \mathrm{~h}$ (range 9-168 h). 15 patients had staggered overdose. 8 of 45 patients $(17.8 \%)$ met the poor prognosis KCC. Three were transplanted, 1 was listed but died waiting, 3 patients not suitable for transplant died and 1 recovered. Sensitivity and specificity of the KCC were 100 and $97 \%$, respectively, with negative and positive predictive values of 100 and $87.5 \%$.
Conclusion: Paracetamol overdose can result in significant morbidity and mortality. Patients with a history of significant overdose should receive prompt treatment with $\mathrm{N}$-acetylcysteine. The Kings College Criteria has an excellent negative predictive value in predicting patients who will survive without transplantation, and an acceptable positive predictive value. Further study is required to assess if addition of lactate levels will improve the predictive value of the KCC.

\section{PP02-20}

An Expert System for Causality Assessment between Adverse Events and Suspected Drugs: Drug-induced Acute Liver Injuries

P. Tragulpiankit ${ }^{1}$, S. Nathaphan ${ }^{2}$, K. Lekcharoen ${ }^{2}$

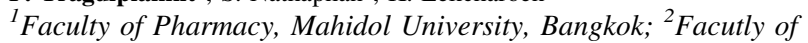
Engineering, Mahidol University, Nakhon Pathom, Thailand

Background/aims: Causality assessment is important in both patient care of the patient who has an adverse drug reaction and the determination of the relationship between an adverse event and suspected drugs of case reports. Although many causality assessment methods have been proposed to overcome this difficulty, the quality of the result still depends on the expertise of the assessor. This research uses the expert system to simulate the expertise of the human expert in solving the problem of causality assessment of drug-induced acute liver injuries by the Roussel Uclaf causality assessment method (RUCAM) as applied to acute liver injuries.

Methods: An expert system for causality assessment of adverse events: drug-induced acute liver injuries (CADIALI-expert) was developed by using a high-level programming language, Microsoft Visual Basic 6.0 enterprise edition and Microsoft Access 97 as a database management system.

Results: CADIALI-expert was validated by 35 drugs of 14 case reports of drug-induced liver injuries that were collected from medical journals. CADIALI-expert showed a correctness of $85.7 \%$. CADIALI-expert limitation was due to the inability to assess the course of the reaction of some cases that required more liver function tests data at the stop date of the drug. This expert system has 3 advantages. The first advantage is helping users to understand the causality assessment process. The second is giving users the results of causality assessment between suspected drugs and acute liver injuries. The third is decreasing the time spent in searching for previous information of suspected drugs and determining the effects of concomitant drugs.

Conclusion: Although CADIALI-expert has not been implemented in daily practice, this expert system seems to be one step of the application of causality assessment method.

\section{PP02-21}

Exploring Mesencyhmal Stem Cell Derived Exosome as a Hepatoprotective Agent in Drug-induced Liver Injury (DILI)

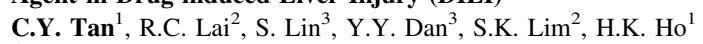

${ }^{1}$ Department of Pharmacy, National University of Singapore;

${ }^{2}$ Institute of Medical Biology, $A * S T A R ;{ }^{3}$ Department of

Gastroenterology and Hepatology, National University of Singapore, Singapore, Singapore

DILI is the most common adverse drug reaction leading to drug failure and withdrawal from the market. DILI induced by different toxicants are often idiosyncratic through mechanisms not completely understood today. Hence, management of DILI is often responsive rather than preventive. Hitherto mesenchymal stem cell-conditioned medium (MSC-CM) was shown to be effective in treating various organ failure. In recent discovery, the major component in the MSC$\mathrm{CM}$ which plays a functional role in tissue repair has been identified as exosome, a microvesicle which is secreted into the extracellular medium from the mesenchymal stem cell. This exosome has been reported to reduce myocardial ischemia/reperfusion injury. However, hepatoprotection by MSC-derived exosome has never been 
demonstrated. Thus, this study aims to explore the potential protective and treatment effect of exosome in DILI. Immortalized human hepatocytes, THLE- 2 cell was used to perform in vitro study. Three toxicants of well-defined mechanism of toxicity were treated separately to induce liver injury; chloroform, hydrogen peroxide or acetaminophen. Exosome was added to the cells before or concurrently with the introduction of toxicants. Different biochemical parameters related to the cell injury were analyzed. For in vivo experiments, three groups of mice $(n=6)$ were used. The animals of group A served as normal and were given only vehicle (olive oil). The animals of group B served as toxin control and were administered with carbon tetrachloride. The animals of group $\mathrm{C}$ received exosome $(0.1 \mu \mathrm{g} / \mathrm{ml})$ concurrently with $\mathrm{CCl}_{4}$ through intraperitoneal. Elevated levels of liver injury markers ALT/AST with $\mathrm{CCl}_{4}$ were reduced significantly with exosome treatment whereas cell viability for acetaminophen treatment increases significantly after the addition of exosome. MSC-derived exosomes is a promising hepatoprotective agent for further characterization and may find useful applications in overcoming DILI and other related liver dysfunction.

\section{PP02-22}

A Case of Toxic Hepatitis Induced by Rhamnus Cathartica

H. Diktas ${ }^{1}$, K. Arican ${ }^{2}$, A. Acar ${ }^{2}$, V. Turhan ${ }^{2}$, O. Oncul ${ }^{2}$, L. Gorenek ${ }^{2}$

${ }^{1}$ Gulhane Military Medical Academy Haydarpasa Training Hospital;

${ }^{2}$ Infectious Diseases and Clinical Microbiology, Gulhane Military

Medical Academy, Haydarpasa Training Hospital, Istanbul, Turkey

Introduction: Rhamnus Cathartica is an herbal drug used as a laxative and diuretic. In this study, a case with toxic hepatitis was presented after usage of Rhamnus Cathartica for weight loss.

Case: 44-years old woman applied to Emergency Service with abdominal pain, fatigue, nausea and vomiting symptoms. The symptoms emerged 3 days ago. No pathological sign except for conjuctival subicter was detected in physical examination of the case. The history of Rhamnus Cathartica usage during one-month period without doctor counseling was taken. In the laboratory examination, ALT: 628 (5-40 U/L), AST: 176 (5-40 U/L), ALP: 114 (35-125 U/ L), GGT: 388 (7-32 U/L), direct bilirubin: $0.5 \mathrm{mg} / \mathrm{dl}(0.01-0.3)$, indirect bilirubin: $1.8 \mathrm{mg} / \mathrm{dl}(0.18-0.7)$, PTZ: 37.4 (<14 s), INR: 3 were found. Ultrasonography showed an increase in diffuse liver parenchyma view as hepatosteatosis. Gallbladder and intrahepatic and extrahepatic bile ducts were normal. Primer and seconder viral hepatitis serology were found as negative. The patient was admitted to the hospital for one month. At the end of therapy, all the laboratory values decreased as ALT: 80 (5-40 U/l), AST: 46 (5-40 U/l), ALP: 76 (35-125 U/l), GGT: 187 (7-32 U/l), direct bilirübin: $0.38 \mathrm{mg} / \mathrm{dl}$ (0.01-0.3), indirect bilirübin: $0.92 \mathrm{mg} / \mathrm{dl}(0.18-0.7)$, PTZ: $14(<14$ sn), INR: 1.07 .

Conclusion: Unconscious use of herbal medicines can sometimes cause serious medical problems as toxic hepatitis and organ failures. It should be kept in mind that Rhamnus Cathartica use without doctor advice may develop toxic hepatitis.

\section{PP02-23}

The Protective Effects of Curcumin Extract on Liver Damage in Methotrexate Treated Rats

Y. Jafarzadeh ${ }^{1,2,3}$, A. Rezaei Moghadam ${ }^{1}$, B. Rafiei ${ }^{2}$, D. Mohajeri ${ }^{3}$

${ }^{I}$ Veterinary Medicine, Islamic Azad University, Tabriz Branch,

Tabriz; ${ }^{2}$ Science in Biotechnology, Jawaharlil Nehru Technological, Zanjan; ${ }^{3}$ Islamic Azad University, Tabriz Branch, Tabriz, Iran

Background/aims: Methotrexate (MTX) a structural analogue of folic acid, is widely used as a chemotherapeutic drug in the various malignancies and inflammatory disease. MTX treatment is often limited by severe side effects; Liver damage is one of the major toxic effects of MTX. This study was designed to investigate whether curcumin had a protective effect on methotrexate-induced liver damage.

Method: Forty-two wistar strain rats were divided into 7 groups $(n=6)$ during 8 days treatment: Group 1 control; Group 2 dimethyl sulfoxide (DMSO), vehicle drug; Group 3 MTX (20 mg kg $\left.{ }^{-1}\right)$; Group 4 DMSO with curcumin $\left(100 \mathrm{mg} \mathrm{kg}^{-1}\right)$; Group 5 DMSO with curcumin $\left(200 \mathrm{mg} \mathrm{kg}^{-1}\right)$; Group 6 low dose curcumin with MTX; Group 7 high dose curcumin with MTX. All animals were sacrificed 4 days after intraperitoneal injection of MTX then liver tissue for histopathological examination and to obtain liver tissue to study glutathione peroxidase (GPx), superoxide dismutase (SOD), malondialdehyde (MDA) levels and aminotransferase (AST) and alanine aminotransferase (ALT) were measured in serum.

Results: Administration of curcumin decreased the liver damage and MDA level and data showed that GPx and SOD levels in liver were increase by MTX treatment. The level AST and ALT were significant increase in serum level.

Conclusion: These results show that curcumin may protect the liver damage of rats from methotrexate-induced damage.

Table 1 Characteristics of patients with different patterns of liver injury in the retrospective cohort

\begin{tabular}{|c|c|c|c|c|c|}
\hline & Eatire coboet $(n=94)$ & Hepatocelluter $(n=42)$ & Cholestatile $(n=25)$ & Mixed $(n=25)$ & $p$ \\
\hline Age, mean SD & $41.02 \pm 17.51$ & $37.09 \pm 16.21$ & $41.41 \pm 21.07$ & $47.2 \pm 21.07$ & 0.1555 \\
\hline Females $n(\%)$ & $38(40.2)$ & $15(35.71)$ & $11(40.74)$ & $12(48)$ & \\
\hline \multicolumn{6}{|c|}{ Liver biochemistries at recognition } \\
\hline Total billrubin (mg/dl) & $0.99 \pm 1.85$ & $4.23 \pm 4.97$ & $6.48 \pm 7.29$ & $3.16 \pm 4.99$ & $<0.001$ \\
\hline ALT (U/L) & $44.86 \pm 49.54$ & $716.97 \pm 1316.7$ & $118.21 \pm 77.91$ & $199.4 \pm 120.54$ & $<0.001$ \\
\hline $\operatorname{ALT}(\mathrm{U} / \mathrm{L})$ & $129.37 \pm 90.03$ & $159.87 \pm 106.76$ & $519.96 \pm 623.91$ & $188.28 \pm 94.43$ & $<0.001$ \\
\hline \multicolumn{6}{|l|}{ Causality assessment $(\%)$} \\
\hline Highly probable & 1.06 & 2.3 & 0 & 0 & \\
\hline Probable & 24.46 & 28.57 & 22.22 & 20 & \\
\hline Possible & 51.96 & 54.76 & 44.44 & 52 & \\
\hline Unlliety & 23.40 & 14.28 & 33.33 & 28 & \\
\hline
\end{tabular}


Table 2 Characteristics of patients with different patterns of liver injury in the prospective cohort

\begin{tabular}{|c|c|c|c|c|c|}
\hline & Eatire coboet $(n=17)$ & Hepatocelluter $(n=9)$ & Cholestatile $(n=7)$ & Mixed $(n=1)$ & $p$ \\
\hline Age, mean SD & $46 \pm 20.51$ & $39.67 \pm 18.69$ & $52 \pm 14.17$ & 68 & 0.0491 \\
\hline Females $n(\%)$ & $6(35.29 \%)$ & $4(44.44 \%)$ & $2(28.57 \%)$ & 0 & \\
\hline \multicolumn{6}{|c|}{ Liver biochemistries at recognition } \\
\hline Total billrubin $(\mathrm{mg} / \mathrm{dl})$ & $4.39 \pm 4.93$ & $5.54 \pm 6.89$ & $3.70 \pm 2.32$ & 1.1 & 0.7962 \\
\hline ALT (U/L) & $342.06 \pm 501.42$ & $518.11 \pm 649.05$ & $131.86 \pm 92.74$ & 229 & 0.222 \\
\hline ALT (U/L) & $453.92 \pm 429 \pm 30$ & $244.8 \pm 218.2$ & $644.14 \pm 494.54$ & 168 & 0.37 \\
\hline \multicolumn{6}{|l|}{ Causality assessment $(\%)$} \\
\hline Highly probable & 17.64 & 33.33 & 0 & 0 & \\
\hline Probable & 23.52 & 22.22 & 28.57 & 0 & \\
\hline Possible & 41.18 & 33.33 & 42.85 & 100 & \\
\hline Unlliety & 17.64 & 11.11 & 28.57 & 0 & \\
\hline
\end{tabular}

\section{PP02-24}

Drug Induced Liver Injury: A Prospective and Retrospective Study of Patients from India

M.J. Choksi, N.R. Bhatt, N.D. Patel, M. Padavarat, G.P. Tahiliani,

D.N. Amarapurkar

Department of Gastroenterology, Bombay Hospital and Medical

Research Centre, Mumbai, India

Background: Drug induced liver injury (DILI), although an uncommon cause of liver injury leads to significant morbidity and mortality.

Aim: To study the incidence, etiology and outcome of patients with DILI and to compare the profile of retrospectively collected data with prospective collected data of patients with DILI.

Methods: The database of patients diagnosed with DILI from January 1999 to December 2009 was analyzed and compared with the prospectively collected database of DILI patients from January 2010 till October 14th 2010. Patients of chronic liver disease with worsening liver function tests after a newly prescribed drug intake were also included. RUCAM diagnostic scale was used for determining Drug Causality.

Results: In the retrospective cohort, 94 out of 9,845 patients $(0.95 \%)$ of liver disease were diagnosed with DILI and prospectively, 17 out of 800 patients $(2.12 \%)$ were diagnosed with DILI $(p<0.0037) .79$ out of 94 patients $(84.04 \%)$ and 6 out of 17 patients (35.29\%) had anti-tubercular drugs induced toxicity in the retrospective and prospective cohort respectively $(p<0.001)$. Albendazole, augmentin and chemotherapeutic agents were the other causes of DILI in the prospective group. There was no statistical difference in the follow up liver function tests in both groups at 1 and 6 months.

Conclusion: Prospective awareness and increased suspicion of drug as a cause of liver dysfunction significantly improves the diagnostic rate of DILI.

\section{PP02-25}

A Drug-induced Hepatitis Patient with a Manifestation of Liver Occupying Lesions on B-Ultrasound: Case Report and Literature Review D.Y. Cheng, W. Xie

Beijing DITAN Hospital, Beijing, China

Case Presentation: The patient was 31 years old. She was treated with patent herbal medicine because of pelvic inflammatory. Then she felt fatigue, poor appetite and had jaundice. So she was admitted to this hospital. The patient had abnormal liver functions along with the negative serologic markers of viral hepatitis and immunology. However a solid space-occupying lesion in the right lobe of liver was observed in abdominal ultrasound, while cirrhosis with inhomogeneous density was observed in CT. So, a liver biopsy was performed. She was finally diagnosed as drug-induced liver injury (DILI) according to the occurrence of hepatic damage directly related to drug administration, exclusion of other cause of hepatitis, and liver histology. Symptomatic and supportive treatments were given. But the result of medicaments was bad and she automatic discharge from hospital.

Discussion: The number of cases of DILI has been increasing. Traditional Chinese medicine is always considered to be safety with no side effect. Here we provide a case of hepatitis induced by herbs with a manifestation of liver occupying lesions on B-ultrasound, which was misdiagnosed as hepatocellular carcinoma (HCC). The cause of misdiagnosis as HCC on ultrasound were as follows: (1) The ultrasonography of residual liver diffuse lesions and the "tissue island in liver" was similar to that of HCC. (2) Irregular nodules of regenerating liver tissue, which influenced the reverberation. (3) The interference of flatulence and covering with the lower lobe of lung. (4) Ultrasonic examination had a relative limited scope. In most cases, liver injury would be expected to improve following discontinuation of the suspected drug. Those with severe hepatitis should be treated carefully. Corticosteroid therapy may be used in DILI cases with evident hypersensitivity, but in dose not have proven benefits. Artificial liver support system and liver transplantation are useful therapy for severe hepatic failure.

\section{PP02-26}

Carrot Seed Extract Prevents Methoteroxate-induced Liver Damage and Oxidative Stress

B. Rafiei ${ }^{1}$, A. Rezaei Moghadam ${ }^{2}$, D. Shahi ${ }^{2}$, R. Dizaji ${ }^{3}$, D. Mohajeri ${ }^{4}$

${ }^{1}$ Master of Science in Biotechnology, Jawaharlal Nehru

Technological University, Zanjan; ${ }^{2}$ Veterinary Medicine Scientific Association Member, Islamic Azad University, Tabriz Branch, Tabriz; ${ }^{3}$ Zanjan University of Medical Sciences, Zanjan; ${ }^{4}$ Associate Professor Islamic Azad University, Tabriz Branch, Tabriz, Iran

Methotrexate (MTX) was originally used as part of combination chemotherapy regimens to treat many kinds of cancers. MTX treatment is often limited by severe side effects; Hepatotoxicity, acute hepatitis, chronic fibrosis/cirrhosis is some of the major toxic effects of MTX for liver. This study was designed to investigate whether carrot seed (Daucus Carota L.) had a protective effect on methotrexate-induced liver damage. Forty-two wistar strain rats were divided into 7 groups $(n=6)$ during 8 days treatment: Group 1 control; Group 2 dimethyl sulfoxide (DMSO), vehicle drug; Group3 MTX (20 mg kg-1); Group 4 carrot seed (200 mg kg${ }^{-1}$ ); Group 5 carrot seed $\left(400 \mathrm{mg} \mathrm{kg}^{-1}\right)$; Group 6 low dose carrot seed with MTX; 
Group 7 high dose carrot seed with MTX. All animals were sacrificed 4 days after intraperitoneal injection of MTX then liver tissue for histopathological examination and to obtain liver tissue to study glutathione peroxidase (GPx), superoxide dismutase (SOD), malondialdehyde (MDA) levels and aminotransferase (AST) and alanine aminotransferase (ALT) were measured in serum. The result showed that GPx and SOD levels were increase but MDA level in liver were decrease by curcumin treatment and level AST and ALT were tend to increase in serum level but not significant Histopathological examination indicated carrot seed extract can overcome reproductive toxicity of methotrexate and decrease oxidative stress and can be protecting liver tissue. The present study indicates that curcumin may be of therapeutic benefit agains MTX-cytotoxcity.

\section{PP02-27}

Risk Factors of Anti-tuberculosis Drugs Induced Liver Injury at DOTS Clinic Hasan Sadikin Hospital Bandung, Indonesia

N. Agustanti ${ }^{1}$, A. Djumhana ${ }^{1}$, A.Y. Soeroto ${ }^{2}$, B. Bestari ${ }^{1}$, S.A. Abdurahman ${ }^{1}$ ${ }^{1}$ Internal Medicine, Division of Gastroentero-Hepatology; ${ }^{2}$ Internal Medicine, Division of Pulmonology, Padjadjaran University, Bandung, Indonesia

Background: Tuberculosis (TB) is a global pandemic disease. India, China and Indonesia contributed more than $50 \%$ of all worldwide cases, where Indonesia is in the 3rd rank. The incidence of antituberculosis drugs hepatotoxicity in developing countries is higher $(8-39 \%)$ than in developed countries (3-4\%) anti-tuberculosis drugs hepatotoxicity. The aim of this study is to determine the risk factors for anti-tuberculosis Drugs Induced Liver Injury (DILI).

Method: We retrospectively collected data from outpatients in DOTS clinic Hasan Sadikin Hospital Bandung from 2008 until 2009. Inclusion criteria were all new patients underwent anti-tuberculosis treatment and fulfilled the criteria of anti-tuberculosis DILI based on official statements of American Thoracic Society. The exclusion criteria were patients with drop out therapy ( $<2$ weeks) and had incompleted data.

Result: A total of 27 patients were diagnosed having anti-tuberculosis DILI and 54 patients with no liver injury were included in control group. In liver injury group, $11(40.7 \%)$ patients were male and 16 $(59.3 \%)$ patients were female. Mean age was 35.59 ( \pm 13.54$)$ years. Two patients were positive $\mathrm{HbsAg}, 4$ patients were positive anti-HCV and 3 patients with HIV infection. Mean BMI was $20.54( \pm 4.07)$. Mean SGOT was 256.26 U/L and SGPT was $235.22 \mathrm{U} / \mathrm{L}$. The statistic analysis using logistic regression showed the risk factors of antituberculosis DILI were age $>35$ years old $(\mathrm{OR}=4.75,95 \% \mathrm{CI}$ 1.040-21.738, $P=0.044)$ and female $(\mathrm{OR}=4.34,95 \% \quad \mathrm{CI}$ 1.033-18.270, $P=0.045)$.

Conclusion: In this study, we find that age $>35$ years old and female are the risk factors for anti-tuberculosis DILI.

\section{PP02-28}

Clinical Analysis of Drug-induced Liver Disease

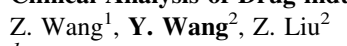

${ }^{1}$ Emergency Department of First Hospital, JiLin University;

${ }^{2}$ Gastroenterology Department, First Hospital, Jilin University, Changchun, China

Object:This paper study the common medicine and clinical feature. Method: Analysis 40 cases of drug-induced liver disease.

Result: During the medicine of drug-induced liver disease, antituberculosis drugs was 14 cases (35\%), Chinese herbal medicine was 10 cases $(25 \%)$, antibacterial agent was 6 cases $(15 \%)$ and other medicines, such as antipyretic analgesic, antipsychotic drug, antithyroid drugs which was 4 cases (10\%), and case fatality was $12.5 \%$.

Conclusion: Antituberculosis drugs and Chinese herbal medicine which made liver disfunction were most common. Preventment, diagnosing and treatment in the early time can cut down the death rate.
PP02-29

The Histophatological Effects of High Level of Testosterone Enanthate on Liver Tissue in Male Rats

A. Javadi Goavgani ${ }^{1}$, A. Davasaz Tabrizi ${ }^{2}$, G. Mousavi ${ }^{2}$, Y. Doustar ${ }^{2}$, A. Rezaei Moghadam ${ }^{3}$

${ }^{1}$ Veterinary Medicine Scientific Association Member, Islamic Azad University, Tabriz Branch, Goavgani; ${ }^{2}$ Associate Professor Islamic Azad University, Tabriz Branch, Iran; ${ }^{3}$ Veterinary Medicine Scientific Association Member, Islamic Azad University, Tabriz Branch, Tabriz, Iran

Testosterone is a steroid hormone from the androgen and it effectively, treats hypoggononadism, anemia, muscle growth, development of male reproductive tissue and atc. While high level of testosterone concentrative in blood serum have many side effects on normal activity of liver enzymes. Probably, the high level of that can be effects on alterative liver tissue. The present study was carried out to investigate the histophatological effects of high testosterone enanthate on liver in rats. Thirty-five wistar strain rats were divided into 5 groups $(n=7)$ : group1 was control; group2 was gonadectomy with take a drug for intrapretoneal, 8 weeks $(5 \mathrm{mg} / 100 \mathrm{~g}$ with $0.5 \mathrm{ml}$ vehicle drug 'alive oil'); group 3 was gonadocetomy taken equal rate of drug and vehicle drug; 4 group, taken drug whithout gonadectomy; 5 group, vehicle drug. Tissue samples of liver subjected for histology investigation stained with Haematoxylin and Eosin (H\&E) 60 days after begun experiment. Histopathological examination of liver revealed more severe and frequent abnormalities in treated group rats including testosterone enanthate compared to controls including a transformation changes with lymphocytes and kupffer cells. Moreover, sinusoidal connecting cells, necrosis and cyst formation has been shown. According to the present study, the high dose of testosterone enanthate may be of liver damage.

\section{Poster Presentation 03: Hepatitis B: Diagnosis}

\section{PP03-01}

A Preliminary Study of Expression of CD150 (SLAM) in the Non-/hypoResponders and Responders of Hepatitis B Vaccine C.S. Lin, J.G. Li, Y.F. Zhang, Y.-T. Chong, Z.L. Gao Department of Infections Diseases, The Third Affiliated Hospital of Sun Yat-sen University, Guangzhou, China

Objective: To study the association of CD150(SLAM) expression in PBMCs with the response of hepatitis $B$ vaccination.

Methods: Anticoagulant heparin blood drawn from either non-/hyporesponders or respondents of hepatitis $\mathrm{B}$ vaccine were used for the separation of peripheral blood mononuclear cells (PBMCs). PBMCs cultured and induced with rHBsAg or PHA. The expression of CD150 was subsequently analyzed using flow cytometry.

Results: The level of $\mathrm{CD} 150$ on $\mathrm{CD}^{+} \mathrm{CD}^{+}$cells in non-/hyporesponders of hepatitis $\mathrm{B}$ vaccine is significantly higher than that in responders $(t=2.297, P=0.027)$, while the levels of CD150 on both PBMC and $\mathrm{CD}^{+} \mathrm{CD}^{-}$cells in non-/hypo-responders of hepatitis $\mathrm{B}$ vaccine are not significantly different with those in responders of hepatitis $B$ vaccine. However, the levels of CD150 on both PBMCs and $\mathrm{CD}^{+} \mathrm{CD}^{+}$in non-/hypo-responders of hepatitis $\mathrm{B}$ vaccine are significantly higher than those in responders of hepatitis $\mathrm{B}$ vaccine when cells were induced with $\mathrm{rHBsAg}(t=2.086, P=0.044 ; t=2.221$, $P=0.032$ ). The same results were also can be seen when cells were induced with PHA: the levels of CD150 on both PBMCs and $\mathrm{CD}^{+} \mathrm{CD}^{+}$ in non-/hypo-responders of hepatitis $\mathrm{B}$ vaccine are significantly higher than those in responders of hepatitis B vaccine $(t=2.699, P=0.010$; $t=2.490, P=0.017)$. The levels of $\mathrm{CD} 150$ on $\mathrm{CD}^{+} \mathrm{CD}^{-}$cells in non-/hypo-responders of hepatitis $\mathrm{B}$ vaccine are higher than that in responders of hepatitis $\mathrm{B}$ vaccine, while the difference is not statistically 
different when cells were induced with either rHBsAg or PHA $(t=0.496, P=0.623 ; t=1.639, P=0.109)$.

Conclusion: CD150 could be a potential marker for the prediction of non-respond of hepatitis B vaccination, and may play a role in response of hepatitis $B$ vaccine.

\section{PP03-02}

Hepatitis B Virus Large Surface Protein in Serum as a New Candidate Test for Evaluating Hepatitis B Virus Infection

N.-Y. Wang ${ }^{1}$, D. Zhang ${ }^{2}$, W. Zhao ${ }^{1}$

${ }^{I}$ Department of Clinical Laboratory, The Second Affiliated Hospital of Southeast University, Nanjing; ${ }^{2}$ Department of Clinical Laboratory, The First Affiliated Hospital of Henan University of Traditional Chinese Medicine, ZhengZhou, China

Aim: To study the clinical application of Hepatitis B virus Large surface protein (HBV-LP) in serum for assessment of hepatitis B virus infection. Methods: HBV-LP in serum was investigated from a large-scale population with different infection phase, utilizing a new enzyme-linked immunoassay (ELISA) kit. HBVDNA, the markers of HBV (HBsAg, anti-HBsAg, $\mathrm{HBeAg}$, anti-HBeAg, anti-HBsAg, pre-s1Ag, and pre$\mathrm{s} 2 \mathrm{Ag}$ ) in serum were detected, liver function tests were performed simultaneously. The clinical value of HBV-LP was evaluated for HBVrelated hepatitis by comparing HBVDNA and other biomarkers.

Results: The ELISA system showed appropriate specificity and precision (Intra-assay and inter-assay coefficients of variation were 7.9 and $10.5 \%$, respectively). The level of HBV-LP in serum was positively correlated with HBVDNA $(r=0.743, p<0.001)$, and had significant correlation with $\mathrm{HBeAg}$ concentration $(r=0.351, p<$ $0.001)$ in HBV patients with all infection phases. There was highest accordance rate $(94.7 \%)$ between HBV-LP and HBVDNA among five HBV markers. Positive HBV-LP were found in most of patients with positive HBVDAN and negative $\mathrm{HBsAg}$ or (and) $\mathrm{HBeAg}$.

Conclusions: Serum HBV-LP was positively correlated with HBVDNA, and accurately capacity of predicting the replication levels of HBV in all infection phases. It will be useful for diagnosis, treatment and prognosis monitoring in patients with $\mathrm{HBV}$, especially, whose markers of HBV (HBeAg or HBsAg) were undetectable. The ELISA kit detecting HBV-LP is both a cost-effective and a less laborintensive alternative to PCR, enhancing its clinical utility.

\section{PP03-03}

Comparison of MELD-Na, MELDNa to Predict Short-term Prognosis of Acute-on-Chronic Hepatitis B Liver Failure

W. He, J. Hu, J. Tong, H. wang

Hospital of PLA, Beijing, China

Objective: To evaluate the short-term prognostic ability of the Model for End-stage Liver Disease-sodium (MELD-Na), End-stage Liver Disease sodium (MELDNa) and their dynamic changes in patients with acute-on-chronic hepatitis B liver failure (ACLF-HBV).

Methods: A total of 172 patients with ACLF-HBV who stayed in hospital for longer than 2 weeks were retrospectively recruited. The predictive accuracy of the MELD-Na, MELDNa and their dynamic change $(\Delta)$, were compared using the area under the receiver operating characteristic curve method.

Results: The 3-month mortality rate was $43.6 \%$. The largest concordance (c) statistic predicting 3-month mortality was MELDNa score after 2 weeks of treatment (0.79), followed by MELD-Na (0.728) scores, $\triangle$ MELDNa (0.713) and $\triangle$ MELD-Na (0.646). The average MELD-Na, MELDNa scores after 2 weeks of treatment and $\triangle$ MELD-Na, $\triangle$ MELDNa of survival group were $27.71 \pm 8.89$, $25.88 \pm 4.97,-2.12 \pm 9.97$ and $-1.89 \pm 4.20$, and those of dead group were $34.63 \pm 8.71, \quad 31.47 \pm 4.99, \quad 2.37 \pm 10.27$ and $2.37 \pm 10.27$. There was a significant difference in MELD-Na, MELDNa, $\triangle$ MELD-Na and $\triangle$ MELDNa between the survival and dead group. The 3-month mortality of the MELD-Na scores group
$<25,25-30,31-35$ and $>35$ were $18.9,37.8,57.6$ and $65.3 \%$, and the MELDNa scores group were 14.6, 33.3, 72.5 and $84.2 \%$, respectively. There was a significant difference in the 3 -month mortality between the four groups. The 3-month mortality of the $\triangle \mathrm{MELD}-\mathrm{Na}>0$ group was $58.5 \%$, and that of the $\triangle \mathrm{MELD}-\mathrm{Na} \leq 0$ group was $30 \%$; The $3-$ month mortality of the $\triangle \mathrm{MELDNa}>0$ group was $61.3 \%$, and that of the $\triangle \mathrm{MELDNa} \leq 0$ group was $29.9 \%$. there was a significant difference between these two groups.

Conclusion: MELDNa score after 2 weeks of treatment is a promising and useful predictor for 3-month mortality in patients with ACLF-HBV. The predictive value may be further improved if combining with dynamic change.

\section{PP03-04}

Comparison of SYBRGreen and Turbidimetry Methods for LAMP Product Detection in Diagnosis of Hepatitis B Virus

E. Moslemi ${ }^{1}$, M.H. Shahhosseiny ${ }^{2}$

${ }^{1}$ Biology Department, Islamic Azad University, Tehran East

(Ghiamdasht) Branch; ${ }^{2}$ Microbiology, Islamic Azad University, Shahr-E- Qods Branch, Tehran, Iran

Background/objectives: Hepatitis B virus (HBV) is one of the leading causes of hepatocellular carcinoma and liver diseases. The HBV serological and molecular detection methods have some limitations; therefore using these methods are not feasible in many diagnosis centers. In this study, SYBRGreen was compared with turbidometery assay for final detection of LAMP reaction product in diagnosis of Hepatitis B Virus.

Material and methods: Two hundred positive serum samples were obtained from 3 different groups. DNA was extracted by using DNP kit and six specific primers for LAMP technique were designed. The sensitivity and specificity tests were performed and test was optimized on samples. Loop mediated isothermal amplification (LAMP) product were evaluated by absorption and SYBR Green fluorescence studies and using electrophoresis measurements.

Result: Among 200 samples with known viral load and various titers, 172 samples (about 86\%) were LAMP positive. The analysis of the reaction products by SYBR Green fluorescence, electrophoresis and spectrophotometer showed similar results. As reaction goes to completion, Along with the DNA synthesis, $\mathrm{MgP}_{2} \mathrm{O}_{3}$ value is increased. This fact resulted in increase of turbidity in the reaction.

Conclusion: LAMP technique had more sensitivity and specificity. In addition, comparing the three final detection methods showed that the final detection of the LAMP product using SYBR Green is easier and more cost-effective and also lack of need to electrophoresis decreases carry-over of hepatitis virus.

\section{PP03-05}

Hepatitis B Virus Large Surface Protein in Serum as a Candidate Biomarker for Evaluating Hepatitis B Virus Infection

N.-Y. Wang

Department of Clinical Laboratory, The Second Affiliated Hospital of Southeast University, Nanjing, China

To study the clinical value of hepatitis B virus large surface protein (HBV-LP) for the diagnosis and monitoring of HBV infection, and develop a sandwich enzyme-linked immunosorbent assay (ELISA) for the detection of serum HBV-LP. Serum HBV-LP levels, and a panel of other HBV markers, were investigated in a large population of patients with chronic HBV. The clinical value of HBV-LP was evaluated by comparing the coincidence of detection of HBV DNA and HBV markers. An ELISA was found to be sensitive and specific for the detection of HBV-LP. The level of HBV-LP was positively correlated with HBV DNA $(r=0.743)$, and had significant correlation with $\mathrm{HBeAg}$ concentration $(r=0.542)$ in $\mathrm{HBV}$ patients. Among the five HBV markers tested, HBV-LP displayed the highest coincidence rate $(94.7 \%)$ with HBV DNA. Serum HBV-LP was strongly 
correlated with HBV DNA, and HBV-LP levels could accurately predict the levels of HBV replication. This ELISA therefore offers a promising alternative approach for the diagnosis and treatment monitoring of HBV patients.

\section{PP03-06}

Measurement of HBV DNA from Dried Blood Spots Using the Abbott m2000 System and Abbott RealTime HBV Assay

G. Cloherty ${ }^{1}$, D. Lucic ${ }^{2}$, K. Riesing ${ }^{3}$, E. Pabich ${ }^{3}$, T.P. Young ${ }^{1}$, M. Sanborn ${ }^{1}$ ${ }^{I}$ Scientific Affairs; ${ }^{2}$ Customer Support; ${ }^{3}$ Research and Development, Abbott Molecular, Des Plaines, IL, USA

Objectives: Hepatitis B virus (HBV) infection is common in developing regions lacking sufficient resources to perform standard diagnostic tests. Dried blood spots (DBS) may eliminate logistical limitations, increasing access to diagnostic tests and improving medical management. This study evaluates a HBV viral load method from DBS using the Abbott RealTime HBV (Research Use Only) assay.

Methods: A HBV dilution panel was made in citrated whole blood. $50 \mathrm{uL}$ of panel material was dispensed onto commercially available specimen collection paper (SCP) or nitrocellulose filter (NF) and air dried for $24 \mathrm{~h}$ before storage at room temperature (RT) or $2-8^{\circ} \mathrm{C}$. When tested, two whole spots were excised and placed into $1.2 \mathrm{~mL}$ of Abbott $m$ Lysis Buffer. DNA extraction and processing into RT-PCR reactions took place on the m2000sp. Amplification and viral load determination was completed by the $m 2000$ rt. Stability of HBV DNA was evaluated by testing DBS, at intervals up to 76 days.

Results: Linearity between $8.29 \log \mathrm{IU} / \mathrm{mL}$ and $3.29 \mathrm{log} \mathrm{IU} / \mathrm{mL}$, yielded an $\mathrm{r}^{2}>0.98$. A significant difference in viral load quantification was observed between NF and SCP $(\mathrm{NF}=0.61 \mathrm{log} \mathrm{IU} / \mathrm{mL}$ higher). Viral quantification correlated closely to plasma results with an estimated conversion factor of $+2.95 \mathrm{log}$ IU $/ \mathrm{mL}$ for DBS on SCP and $+2.34 \log \mathrm{IU} / \mathrm{mL}$ on NF. $100 \%$ detection at $4.29 \log \mathrm{IU} / \mathrm{mL}$ with SCP and $3.29 \log \mathrm{IU} / \mathrm{mL}$ with NF. Initial results indicate that HBV DNA is stable on SCP for at least 76 days at RT and $2-8^{\circ} \mathrm{C}$. Stability data is not available for NF beyond 21 days.

Conclusions: Excellent performance was achieved with SCP or NF with Abbott RealTime HBV (RUO) Assay. DBS on NF yielded an additional $0.61 \log \mathrm{IU} / \mathrm{mL}$ sensitivity versus SCP. Further investigation is required to establish a more exact conversion factor and limit of detection for this protocol.

\section{PP03-07}

Identification of the Risk Factor for the Development of HCC among Hepatitis B Virus Mono-infected Subjects -A Population-based Cohort Study-

A. Kusakabe ${ }^{1}$, Y. Tanaka ${ }^{2}$, M. Inoue ${ }^{3}$, F. Kurbanov ${ }^{2}$, K. Tatematsu ${ }^{2}$, S. Nojiri ${ }^{1}$, T. Joh ${ }^{1}$, S. Tsugane ${ }^{3}$, M. Mizokami ${ }^{4}$

${ }^{1}$ Department of Gastroenterology and Metabolism; ${ }^{2}$ Department of Virology and Liver Unit, Nagoya City University Graduate School of Medical Sciences, Nagoya; ${ }^{3}$ Epidemiology and Prevention Division, Research Center for Cancer Prevention and Screening, National Cancer Center, Tokyo; ${ }^{4}$ The Research Center for Hepatitis and Immunology, National Center for Global Health and Medicine, Ichikawa, Japan

Background: Hepatitis B virus (HBV) chronic infection is known as one of causes of the development of HCC. But it has been unclear that which factor is the most important for the development of HCC.

Aims: To identify the risk factor for the development of HCC, we describe results on the first large-scale population-based cohort study carried in Japan.

Methods: The Japan Public Health Center-based Prospective Study was launched in 1993-1994 among registered Japanese inhabitants (68,975 subjects) aged 40-69 years at the time of the baseline survey. We restricted subjects to those who responded to the questionnaire and for whom blood samples were available. Finally, 19,393 individuals were included. Patients with HBsAg alone were classified into the HBV mono-infected group. Enrolled subjects were followed for average 12.7 years. HBV genotype and specific mutations (nt1628-2,364) were analyzed by direct sequencing.

Results: Of 19,393 subjects, 479 were identified with HBV monoinfection $(2.5 \%)$. During the 245,923 person-years' follow-up, 13 cases of newly diagnosed HCC were documented in the HBV monoinfected group. Several factors at baseline (male, smoking, ALT, the positivity of $\mathrm{HBe}$ antigen and $\mathrm{HB}$ core-related antigen, the proportion of HBV DNA $\geq 5 \log$ copies/ml, T1753V and A1762T/G1764A) were revealed in significant association with HCC. Multivariate-adjusted Cox hazard model showed that A1762T/G1764A (hazard ratio (HR), 7.05 [95\% CI; 1.03-48.12, $P=0.046]$ ) was the only independent risk factor for the development of HCC. Kaplan-Meier method also showed that the probability of HCC occurrence-free was significantly lower in HBV mono-infected subjects with A1762T/G1764A. Compared to the no hepatitis virus-infected subjects in this cohort, the HR for HCC development in HBV mono-infected subjects with A1762T/ G1764A was almost equal to that in HCV mono-infected subjects.

Conclusion: Among HBV mono-infected subjects, those with A1762T/ G1764A could notably be a high risk group of HCC development.

\section{PP03-08}

Precore Mutation in Genotype B or Basal Core-promoter Mutation in Genotype C Correlates with Fulminant Progression in Hepatitis B

J.-H. Kang ${ }^{1}$, T. Matsui ${ }^{1}$, H. Shida ${ }^{1}$, T. Kin ${ }^{1}$, K. Tsuji ${ }^{1}$, H. Maguchi $^{1}$,

Y. Karino ${ }^{2}$, J. Akaike ${ }^{2}$, I. Ozeki ${ }^{2}$, T. Arakawa ${ }^{2}$, T. Sato ${ }^{2}$, T. Ohmura ${ }^{2}$, J. Toyoda ${ }^{2}$, Y. Tanaka ${ }^{3}$

${ }^{1}$ Center for Gastroenterology, Teine Keijinkai Hospital; ${ }^{2}$ Department of Hepatology, Sapporo Kosei General Hospital, Sapporo;

${ }^{3}$ Department of Virology and Liver Unit, Nagoya City University Graduate School of Medical Sciences, Nagoya, Japan

Background and aims: Genotype of Hepatitis B virus (HBV) influences disease progression in chronic hepatitis B. However, whether individual viral factor by different genotypes is associated with fulminant hepatitis $(\mathrm{FH})$ remained unclear. The aim of present study was to evaluate individual virus factors associated with FHB by genotypes.

Methods: During 1995 through 2010, 78 patients (55 males, median age 32 years) with acute hepatitis B (AHB) were registered in 2 hospitals located in Sapporo, Japan. Patients with acute severe hepatitis (ASH), defined to be less than $40 \%$ in prothrombin time without hepatic encephalopathy, were treated with immunosuppressive therapy to prevent fulminant progression. In this study, FH and ASH were enrolled as an object. HBV genotypes were determined using ELISA or restriction fragment length polymorphism. Mutations in precore (PC, G1896A) and basal core-promoter (BCP, A1762T or G1764A) were detected by enzyme-linked mini-sequence assay.

Results: In 78 patients with AHB, genotype A was determined in 16, $\mathrm{B}$ in $13, \mathrm{C}$ in 47 . Out of 18 patients with FH $(n=9)$ or $\mathrm{ASH}(n=9)$, genotype B was determined in 7 (53.8\%), genotype C in $11(23.4 \%)$ but genotype A in none $(p<0.01)$. In 13 genotype B patients, PC mutation was more frequent in patients with $\mathrm{FH}$ or ASH than those with self-limited hepatitis ( 85.7 vs. $16.7 \%, p<0.03$ ). As for genotype $\mathrm{C}$ infection, patients with serious hepatitis were less positive for HBeAg (36.4 vs. $75 \%, p<0.03$ ), showed more frequent $\mathrm{PC}$ or $\mathrm{BCP}$ mutation (63.6 vs. $11.1 \%, 90.9$ vs. $8.3 \%, P<0.001$ for both) in comparison with those with self-limited hepatitis. In multivariate analysis, BCP mutation was independently associated with serious outcome among genotype $\mathrm{C}$ patients $(42.3,2.10-847.2, p=0.0144)$. Conclusions: Fulminant outcome in AHB was related with PC mutation in infection of genotype $\mathrm{B}$, or associated with $\mathrm{BCP}$ mutation of genotype $\mathrm{C}$. 


\section{PP03-09}

Study on the Relationship between Alpha Fetoprotein and Liver Pathological Stages of Patients with Chronic Hepatitis B

J. Dong, B.-B. Lin, Y.-Y. Zhu, J.-J. Jiang

Center of Liver Disease, The First Affiliated Hospital of Fujian

Medical University, Fuzhou, China

Objective: To investigate the pathological meaning of AFP in patients with chronic hepatitis $\mathrm{B}$ (CHB).

Methods: Both liver biopsy samples and sera were collected in patients with CHB in our center from March 2005 to December 2009, consecutively. The pathological analysis of biopsy sample was distinguished 5 stages of inflammation $(\mathrm{G})$ and fibrosis (S), according to 2000 Chinese Guideline for Chronic Viral Hepatitis, which is similar to Metavir scoring system. Relationship was analyzed using Spearman rank correlation analysis. ROC curve was employed to evaluate the diagnostic value of AFP.

Results: Totally 621 patients with CHB were collected, male 508, female 113. There were $157(25.28 \%)$ cases with an elevated AFP (ULN is $20 \mathrm{ng} / \mathrm{ml}$ ). The mean values of AFP at different inflammation stages G0-G4 were $6.050 \pm 6.067,12.281 \pm 47.815, \quad 7.885 \pm$ $17.435,35.364 \pm 122.037,120.496 \pm 234.497 \mathrm{ng} / \mathrm{ml}( \pm \mathrm{S})$, separately. The mean values of AFP at different fibrosis stages S0-S4 were $4.806 \pm 5.010,7.302 \pm 16.854,11.226 \pm 44.708,27.551 \pm$ $80.441,99.286 \pm 219.023 \mathrm{ng} / \mathrm{ml}$, separately. ROC curve analysis showed that the level of AFP above $12.0 \mathrm{ng} / \mathrm{ml}$ revealed inflammation stages would be more than stage 3 , with area under curve (AUC) was 0.729 and specificity was $90.0 \%$. It also showed by ROC curve analysis that the level of AFP above $12.8 \mathrm{ng} / \mathrm{ml}$ indicated fibrosis stages would be more than 3 , with AUC was 0.719 and the specificity was $90.3 \%$.

Conclusions: As the stages of inflammation or fibrosis growing, the AFP levels of patients were detected also growing in a stairstepping way. Although in sub-clinical level, AFP still could be used to index pathological stage, especially when AFP is higher than $12.8 \mathrm{ng} / \mathrm{ml}$.

\section{PP03-10}

Validation of the INNO-LiPA HBV DR v2 Assay in a Treatmentexperienced Chronic Hepatitis B Patient Population

J. Doutreloigne ${ }^{1}$, R. Maertens ${ }^{1}, \mathrm{~S}$. Mirandola ${ }^{2}$, A. Alberti ${ }^{2}$

${ }^{1}$ Innogenetics NV, Gent, Belgium; ${ }^{2}$ Venetian Institute of Molecular Medicine, Padova, Italy

Background/aims: Resistance to antiviral drugs in patients with chronic hepatitis B (CHB) has become the major drawback of oral antiviral therapy which lead to viral non-responsiveness and treatment failure. Early detection of antiviral drug resistant (DR) mutations permits prompt initiation of rescue therapy. The INNO-LiPA HBV DR v2 detects mutations at codons 80, 173, 180, 181, 204 and 236 while the novel INNO-LiPA HBV DR v3 strip covers codons 184, 194, 202 and 250.

Methods: 239 clinical samples obtained from CHB patients (untreated or treated with different antivirals) covering HBV genotypes A-G were extracted with the QIAamp DNA Blood mini kit (Qiagen). Viral loads of 232 samples were known, and ranged from 173 to $2,148,813,976$ copies/mL. After INNO-LiPA HBV DR v2 amplification, 239 amplicon were tested with the INNO-LiPA HBV DR v2 strip and 103 of the 239 amplicons were also tested with the novel INNO-LiPA HBV DR v3 strip. Sequencing data was obtained for 1423 codons.

Results: After initial testing, positive test results were obtained in 231 of the 239 specimens, resulting in a sensitivity of $96.65 \%$. Repeat testing resulted in a sensitivity of $100 \%$. For the 239 clinical samples tested on INNO-LiPA HBV DR v2, 15 indeterminate results were observed on a total of 1434 codon results (1.05\%). For $1411(99.16 \%)$ of the 1423 codons for which sequencing information was available,
LiPA gave the same $(83.84 \%)$ or additional amino acid information $(15.32 \%)$ as compared to sequencing. Hundred of the 239 serum samples were tested with the novel INNO-LiPA HBV DR v3, 5 indeterminate were obtained on a total of 412 codon results $(1.21 \%)$. Conclusions: Performance testing of the INNO-LiPA HBV DR v2 showed convincing diagnostic sensitivity and accuracy. The INNOLiPA HBV DR v2 and the novel INNO-LiPA HBV DR v3 strip are useful tools for HBV therapy monitoring.

\section{PP03-11}

The Correlation of Quantitative Serum HBsAg and HBV-DNA Levels in Chronic Hepatitis B Patients in Indonesia with Regard HBeAg Status C.R.A. Lesmana ${ }^{1,2}$, L.A. Lesmana ${ }^{1,2}$, L.S. Pakasi ${ }^{2}$, R.A. Gani ${ }^{1}$, I. Hasan ${ }^{1}$, A.S. Sulaiman ${ }^{1}$, U. Budihusodo ${ }^{1}$

${ }^{1}$ Internal Medicine, Division of Hepatology, University of Indonesia; ${ }^{2}$ Internal Medicine, Digestive Disease Centre, Medistra Hospital, Jakarta, Indonesia

Introduction: Treatment response for hepatitis B virus (HBV) infection is generally measured by detecting either the presence of serum HBV-DNA. Quantitative measurement of HBV-DNA is costly and not widely available. Recently, quantitative measurement of HBV antigen (HBsAg) has been available and may be beneficial as a virological marker and this antigen has not been validated yet in Indonesia.

Objective: To find the correlation of serum HBsAg quantitative and $\mathrm{HBV}$-DNA levels in $\mathrm{HBeAg-positive} \mathrm{and} \mathrm{HBeAg}$-negative $\mathrm{CHB}$ patients.

Patients and methods: A cross-sectional study was carried out on the newly diagnosed naïve CHB patients from September 2009 to April 2010. Quantitative serum HBsAg measurement was performed using automated chemiluminescent microparticle immunoassay (Abbott Architect HBsAg QT assay). Serum HBV-DNA level was measured by COBAS TaqMan polymerase chain reaction. Correlation between serum quantitative HBsAg and HBV-DNA levels was tested using the Pearson's correlation test. All values were log-transformed before being analyzed.

Results: Seventy-two patients were enrolled in this study, 39 (54.2\%) of them were men. Patients' mean age was $40 \pm 11.84$ (range 19-70) years old. There were $33(45.8 \%)$ patients with positive HBeAg status. Log HBsAg level was significantly lower in $\mathrm{HBeAg-negative}$ compared to $\mathrm{HBeAg}$ positive patients $(2.47$ vs. $4.05 ; p<0.001)$. Quantitative serum HBsAg was well correlated with HBV-DNA levels $(r=$ of $0.58 ; p<0.001$ in HBeAg-positive and $r=0.62 ; p<$ 0.001 in $\mathrm{HBeAg}$-negative patients).

Conclusions: Serum HBsAg quantification correlates with serum HBV-DNA levels. This new virological marker is a potential surrogate marker for serum HBV-DNA levels which might be used during treatment monitoring of $\mathrm{CHB}$ patients.

\section{PP03-12}

Quantitative Measurement of Serum Hepatitis B Surface Antigen in Chronic Hepatitis B Patients Using Immunoradiometric Assay Y.J. Jung ${ }^{1}$, W. Kim ${ }^{1}$, H.-Y. Lee ${ }^{2}$

${ }^{1}$ Internal Medicine; ${ }^{2}$ Department of Nuclear Medicine, Seoul Metropolitan Government Seoul National University, Seoul, Republic of Korea

Background and aims: Quantitative measurement of serum hepatitis surface antigen (HBsAg) level is important for management of chronic hepatitis B patients in terms of monitoring the response to antiviral therapy. This study aimed to evaluate the diagnostic performance of new diagnostic kit measuring quantitative amount of serum HBsAg using immunoradiometric assay (IRMA) by comparing with the measurement using chemiluminescent microparticle immunoassay (CMIA). 
Methods: The blood samples of ninety-six patients with chronic hepatitis B were used in this study. Among them, 23 samples were also measured the copy numbers of serum hepatitis B virus (HBV) DNA. The correlation and concordance of the results from IRMA and CMIA methods were tested with Pearson correlation coefficient. $P$ values of less than 0.05 were considered as significant statistical threshold.

Results: Laboratory diagnosis based on IRMA method was in agreement with diagnosis based on CMIA method (concordance rate $100 \%$ ). Serum HBsAg level measured by IRMA method was highly correlated with result from CMIA method (correlation coefficient $R^{2}=0.838, p<0.001$ ). Serum HBsAg amount and serum HBV DNA copies show proportional relationship in some degree $\left(R^{2}=0.067, p=0.316\right.$ in IRMA method, $R^{2}=0.101, p=0.215$ in CMIA method).

Conclusions: Diagnostic performance of HBsAg measurement using IRMA method was comparable to the measurement using CMIA method in evaluating HBsAg levels.

\section{PP03-13}

The Value of HBsAg Screening in Fingertip Blood Impregnated Paper O. Karabay, A. Ogutlu, M. Karabay, B. Teker, N. Tuna, M. Yahyaoğlu Infectious Diseases, Sakarya Education and Research Hospital, Sakarya, Turkey

Aim: Hepatitis B surfage antigen (HBsAg) screening HBsAg screening is important to determine incidence of hepatitis $B$ in a community. But HBsAg screening is difficult and it is time and money consumption. In this study we aimed to investigate efficiency and usefulness at screening HBsAg with fingerprint blood absorbed by blotting paper.

Method: A drop of blood sample taken from the fingerprint of the volunteer-subjects was absorbed by blotting paper. The samples of blood have been stored at room temperature. Blood impregnated papers were solved in $\mathrm{NaCl}, 0.9 \%$ and $\mathrm{HBsAg}$ was investigated by commercial EIA method.

Results: A total $42 \mathrm{HbsAg}$ positive and $40 \mathrm{HBsAg}$ negative specimens were included in the study. A total 40 out of $42 \mathrm{HBsAg}$ positive samples $(95.2 \%)$ was found positive while 40 negative samples were all found negative $(100 \%)$.

Conclusion: We think that blood impregnated paper was more useful and more cheap method for the HBsAg screening in the community.

\section{PP03-14}

Quantitative HBsAg: New Specific Marker for the Diagnosis of HBsAg Inactive Carriage

M. Martinot-Peignoux ${ }^{1}$, M. Lapalus ${ }^{2}$, O. Lada ${ }^{2}$, A.C. Cardoso ${ }^{2}$,

M.P. Ripault ${ }^{3}$, N. Boyer ${ }^{3}$, T. Aselah ${ }^{4}$, P. Marcellin ${ }^{4}$

${ }^{1}$ INSERM U773/CRB3-Hopital Beaujon; ${ }^{2}$ INSERM U773/CRB3;

${ }^{3}$ Service D'Hépatology Hopital Beaujon; ${ }^{4}$ INSERM U773/CRB3, Service D'Hépatology Hopital Beaujon, Clichy, France

Background/aim: Accurate identification of inactive hepatitis $B$ virus (HBV) carriers (IC) from patients with HBeAg-negative chronic hepatitis $\mathrm{B}(\mathrm{CHB})$ is difficult. Identifying $\mathrm{CHB}$ is a major medical need since they could benefit from therapy with increased survival. Our aim was to evaluate the role of hepatitis B surface antigen (HBsAg) as a new marker for identification of IC.

Methods: Criteria for inclusion were: presence of HBsAg and anti$\mathrm{HBe}$ without $\mathrm{HBeAg}$. IC was defined as persistently normal ALT (during 1 year) and HBV-DNA $<2,000 \mathrm{IU} / \mathrm{ml}$. HBsAg was quantified using the Elecsys HBsAg II assay (Roche Diagnostics).

Results: 165 untreated patients were included (76 IC and $89 \mathrm{CHB}$ ). Median baseline HBsAg was 1,384 and 4,629 IU/ml in IC and CHB, respectively $(p<0.002)$; median HBV-DNA was 364 and 176,875
IU/ml in IC and CHB, respectively. Table: HBsAg/HBV-DNA baseline cut-off to identify IC.

Baseline VPP of HBSAg and HBV DNA levels

\begin{tabular}{lll}
\hline HBsAg & $<2,000 \mathrm{UI} / \mathrm{ml}$ & $<1,000 \mathrm{IU} / \mathrm{ml}$ \\
\hline HBV-DNA & & \\
$<2,000 \mathrm{IU} / \mathrm{ml}$ & $87 \%$ & $90 \%$ \\
$<1,000 \mathrm{IU} / \mathrm{ml}$ & $86 \%$ & $90 \%$ \\
$<400 \mathrm{IU} / \mathrm{ml}$ & $97 \%$ & $97 \%$ \\
\hline
\end{tabular}

Genotypes distribution was A: 40\%; B: 13\%; C: 3\%; D: 32\%; E: $17 \%$. HBsAg was lower in genotype B than in A, D, and E $(p<0.001)$. At the end of follow-up, HBsAg negativation was observed in 6 IC and 7 CHB. Fibrosis stage was $\leq 1$ in 82 vs $51 \%$ if IC and CHB, respectively $(p<0.001)$. Mean yearly decline in HBsAg was: $0.13 \pm 0.15$ vs. $0.048 \pm 0.27 \log _{10} \mathrm{IU} / \mathrm{ml}$ in patients with or without $\mathrm{HBsAg}$ negativation, respectively $(p<0.05)$.

Conclusion: Quantitative serum $\mathrm{HBsAg}$ is a specific marker for diagnosis of IC. HBsAg $\leq 1,000 \mathrm{IU} / \mathrm{mL}$ and HBV-DNA $\leq 2,000 \mathrm{IU} /$ $\mathrm{ml}$ allow identification of IC with VPP $90 \%$.

\section{PP03-15}

Application of a Newly Developed Highly Sensitivi HBsAg Chemiluminescent Enzyme Immunoassay for Hepatitis B Virus Carriers with HBsAg Seroconversion

N. Shinkai ${ }^{1,2}$, Y. Tanaka ${ }^{1}$, K. Matsuura ${ }^{1,2}$, S. Nojiri ${ }^{2}$, M. Mizokami $^{3}$ ${ }^{1}$ Department of Virology and Liver Unit, Nagoya City University Graduate School of Medical Sciences, Nogoya; ${ }^{2}$ Departments of Gastoloenterology and Metabolism, Nagoya City University Graduate School of Medical Sciences, Nagoya; ${ }^{3}$ Research Center for Hepatitis and Immunology, Kohnodai Hospital, National Center for Global Health and Medicine, Ichikawa, Japan

Background: A high sensitive CLEIA was developed for quantitative hepatitis B surface antigen ( $\mathrm{HBs} A g$ ) detection by a combination of monoclonal antibodies, each one for a specific epitope of HBsAg, and by improving the conjugation technique (Matsubara, et al. Transfusion 2009). We modified and automated this technique; the highly sensitive HBsAg CLEIA prototype (prototype) had good correlation with the quantitative HBsAg detection system (the Abbot ARCHITECT), and the sensitivity of the prototype $(5 \mathrm{mIU} / \mathrm{mL})$ was approximately tenfold higher than the Abbot ARCHITECT (50 mIU/ ml) (Rinsho Byouri, in press).

Aim: To investigate the clinical significance of prototype in patients with hepatitis B virus (HBV) infection.

Methods: We used serial serum samples of $16 \mathrm{HBV}$ carriers with HBsAg seroconversion by the Abbot ARCHITECT. Eight patients are asymptomatic carrier without treatments (ASC group). Four patients with lamivudine therapy [mean, 13 months (totally 19 months)] had HBsAg seronegative by the Abbot ARCHITECT after stopping the therapy (LVD group). Another four patients had HBsAg seronegative by the Abbot ARCHITECT during entecavir therapy (mean, 33 months) (ETV group). We compared HBsAg levels between the prototype and the Abbot ARCHITECT.

Results: There are good correlation between prototype and the Abbot ARCHITECT in 16 patients. In 14 of 16 patients prototype could detect HBsAg at the HBsAg-negative point by Abbot ARCHITECT. The differences of mean duration detectable HBsAg in ASC group, LVD group, and ETV group are 13, 3, and 4 months, respectively. In one of ASC group, even 150 months after $\mathrm{HBsAg}$ negative by Abbot ARCHITECT, the prototype could detect HBsAg. In one of ETV group, HBsAg became positive one year after HBsAg seronegative by prototype. At this point, $\mathrm{HBsAg}$ was nagative by the Abbot 
ARCHITECT and HBV DNA was undetectable by the Roche Cobas TaqMan HBV assay.

Conclusions: Prototype is convenient and precise assay for $\mathrm{HBV}$ monitoring.

\section{PP03-16}

The Clinical Significance of Liver Shear Wave Velocity on Hepatic Fibrosis Staging with Chronic HBV Carrier

D.Q. Zhou, J.S. He, M. Qiu, Y.J. Chen, G.D. Tong, P.X. Hu, Y.J. Zhen,

H.H. Tang

Hepatology, Shenzhen Hospital Affiliated to Guangzhou University of Traditional Chinese Medicine, Shenzhen, China

Aims: The aim of this study is to study the relationship between liver shear wave velocity and classification of hepatic fibrosis, and to establish a noninvasive diagnostic method on hepatic fibrosis staging with chronic HBV carrier.

Methods: All of 118 clinical diagnosised chronic HBV carriers had a biopsy and tested the liver shear wave velocity by acoustic radiation force impulse respectively. In control group, we enrolled 50 healthy people and compared the relation between hepatic fibrosis staging and liver shear wave velocity, meanwhile, we also studied some correlation factor such as age, gender, course of diseases, level of ALT, body mass index (BMI), quantities of HBV-DNA, inflammation grade and so on by Logistic regression.

Results: Liver shear wave velocity increased gradually accompany with the increasing of fibrosis staging. ROC of the diagnostic efficiency of liver shear wave velocity for S0 to S4 was $0.78,0.85,0.89,0.95,0.92$, respectively, while the liminal value cut off for liver shear wave velocity was $1.18,1.45,1.72,2.37,2.89$, respectively. Of the total, ROC $(0.95)$ for S3 was approximate to 1, and it demonstrated that the liver shear wave velocity for S3 had the maximum diagnostic sensitivity. Compared with the control group, one-way ANOVA suggested that there was a significant difference $(F=25.452, P=0.000)$ between the fibrosis staging and liver shear wave velocity, and them also had a direct correlation. The Analysis of Unconditional Logistic Regression showed that body mass index (BMI), course of diseases, and age could affect the results of liver shear wave velocity, while the level of ALT could not. Conclusions: Liver shear wave velocity could be a noninvasive reference factor for a clinical diagnosis of chronic HBV carrier to know the stage of hepatic fibrosis.

\section{PP03-17}

Elecsys HBsAg II Assay for the Quantification of Serum HBsAg and Prediction of Treatment Outcome

M. Martinot-Peignoux ${ }^{1}$, M. Lapalus ${ }^{1}$, O. Lada ${ }^{1}$, T. Aselah ${ }^{2}$, P. Marcellin ${ }^{2}$

${ }^{1}$ Hopital Beaujon, INSERM U773/CRB3; ${ }^{2}$ Service d'Hépatologie,

INSERM U773/CRB3, Hopital Beaujon, Clichy, France

Preliminary data have shown that hepatitis B surface antigen (HBsAg) is an important additive tool for patients monitoring. Most studies were performed with Architect HBsAg Assay (Architect) (Abbott, Laboratories). The aim of our study was to evaluate the Elecsys HBsAg II Assay (Elecsys) (Roche Diagnostics) for the quantification of HBsAg and prediction of patient's outcome during therapy.

Methods: 46 CHB treated patients were tested for HBsAg at baseline, weeks 12, 24, 48 (end of therapy) and weeks 72 and 96 (follow-up) with Elecsys and Architect. 32\% demonstrated a sustained undetectable serum HBV DNA (SR), 22 relapsed (RR) and 9 were nonresponders (NR).

Results: The correlation between Elecsys and Architect was $r^{2}=0.961$ $(p<0.001)$. Baseline HBsAg levels were SR: $3.53 \pm 0.83$ and $3.45 \pm 0.78$; RR: $3.50 \pm 0.63$ and $3.41 \pm 0.61$; NR: $4.261 \pm 0.432$ and $4.13 \pm 0.39 \log \mathrm{IU} / \mathrm{ml}(p=0.02)$ with Elecsys and Architect, respectively. Mean decrease in serum HBsAg levels at week 12 were $0.415 \pm 0.150$ and $0.083 \pm 0.029 ; 0.487 \pm 0,151 \mathrm{log} \mathrm{IU} / \mathrm{ml}$ and
$0.086 \pm 0.044$ in SR and RR+NR with Elecsys II and Architect, respectively $(p=0.02)$. Week 24 mean decrease in HBsAg were $0.919 \pm 0.221$ and $0.178 \pm 0.065 ; 1.075 \pm 0.240$ and $0.183 \pm 0.022$ $\log \mathrm{IU} / \mathrm{ml}$ in SR and RR+NR with Elecsys and Architect, respectively $(p=0.02)$. Table. Positive Predictive Value (PPV) and Negative Predictive Value (NPV) of a $0.5 \log _{10}$ decrease in serum HBsAg level at weeks 12 and a $1 \log _{10}$ decrease at week 24 .

Comparison Elecsys and Architect

\begin{tabular}{lllll}
\hline & $\begin{array}{l}\text { Elecsys } \\
\text { PPV (\%) }\end{array}$ & $\begin{array}{l}\text { Elecsys } \\
\text { NPV (\%) }\end{array}$ & $\begin{array}{l}\text { Architect } \\
\text { PPV (\%) }\end{array}$ & $\begin{array}{l}\text { Architect } \\
\text { NPV (\%) }\end{array}$ \\
\hline Week 12 & 83 & 75 & 70 & 84 \\
Week 24 & 70 & 77 & 75 & 82 \\
\hline
\end{tabular}

Conclusions: Elecsys and Achitect assays show excellent correlation. Elecsys provides a simple and reliable assay for the quantification of serum HBsAg.

\section{PP03-18}

Expression Analysis of miRNA in PBMC of CHB Patients from China

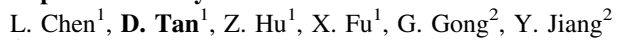

${ }^{1}$ Department of Infectious Disease, Xiangya Hospital, Central South University; ${ }^{2}$ Department of Infectious Disease, The Second Xiangya Hospital, Central South University, Changsha, China

Background: In many cases, the degree of inflammation in liver tissues is understood according to the serum level of ALT (alanine aminotransferase, ALT) in CHB (chronic hepatitis B, CHB) patients. Normal serum level of ALT is still being found in a great number of CHB patients with inflammatory activity. A new category of noncoding RNA, named miRNA, was found to be involved in human immune response. MiRNA, a new class of mediators of inflammation, plays an important role in many inflammatory diseases. The aim of this study is to investigate miRNA expression profile in PBMC (peripheral blood mononuclear cells) of CHB patients, to determine miRNA that is consistent with the pathological grade and stage of liver tissues, and to identify differentially expressed miRNA between chronic hepatitis B virus carriers and serious chronic hepatitis B patients.

Methods: The study was conducted in 8 individuals, including 4 chronic hepatitis B virus carriers and 4 serious $\mathrm{CHB}$ patients from Xiangya hospital and the Second Xiangya hospital of Central South University. PBMC recruited from $10 \mathrm{ml}$ heparin anticoagulated blood was isolated by Ficoll-Hypaque gradient centrifugation. Microarray, Agilent Feature Extraction (FE) software version 10.5 and GeneSpring GX were used to investigate miRNA expression profiles.

Results: A total of 17 miRNA expression profiles differentiated chronic hepatitis B virus carriers from serious CHB. Levels of 5 miRNAs increased in patients with severe inflammation of liver, such as miR-30a, miR-1246, miR-1305, miR-193a-3p and miR-196b. Simultaneously, levels of miR-150, miR-223, miR-574-3p, miR-197, miR-486-5p and so on 12 miRNAs reduced.

Conclusion: We propose that modulation of miRNA expression profile in PBMC is associated with the degree of inflammation in liver tissues. Perhaps, certain miRNAs can be used as marks of early serious $\mathrm{CHB}$

\section{PP03-19}

The Variations of the Cell Population Ratios of $T$ lympholeukocyte and Natural Killer Cell in Chronic Hepatitis B Patients Serum

Q. Long

The Second Hospital of Nanjing, Nanjing, China

Objective: To investigate the cell populations of natural killer cell and $\mathrm{T}$ lympholeukocyte in patients of chronic type B hepatitis serum. 
Methods: The cell population ratios (\%) in serum of 146 cases of chronic hepatitis $\mathrm{B}$ (Hepatic failure 56 cases; chronic hepatitis B with e antigen masculine 49 cases; HBV carrier with e antigen negative 41 cases) were detected by flow cytometry: the $\mathrm{CD} 3^{+} \mathrm{T}$ lympholeukocyte versus the totaled lympholeukocyte, the $\mathrm{CD} 3^{-} \mathrm{CD} 16^{+} \mathrm{CD} 56^{+}$natural killer cell versus the totaled lympholeukocyte, the $\mathrm{CD} 3^{+} \mathrm{CD} 4^{+}$and $\mathrm{CD}^{+} \mathrm{CD}^{+} \mathrm{T}$ lympholeukocyte versus respectively the $\mathrm{CD}^{+}{ }^{+} \mathrm{T}$ lympholeukocyte.

Results: The cell population ratios $(\%)$ of the $\mathrm{CD} 3^{+}$, the $\mathrm{CD} 3^{+} \mathrm{CD} 4^{+}$, the $\mathrm{CD}^{+} \mathrm{CD}^{+} \mathrm{T}$ lympholeukocyte the $\mathrm{CD}^{-} \mathrm{CD} 16^{+} \mathrm{CD} 56^{+}$natural killer cell in different groups of hepatic failure, chronic hepatitis $\mathrm{B}$ with e antigen masculine, HBV carrier with e antigen negative individually were: $38.8 \pm 17.4,54.7 \pm 11.8,45.5 \pm 14.1 ; 58.4 \pm 16.3$, $54.2 \pm 12.8,52.1 \pm 10.6 ; 33.1 \pm 13.4,33.7 \pm 11.5,38.6 \pm 8.5$ and $5.3 \pm 5.2,36.2 \pm 171.5,13.9 \pm 7.8$. It was found by tested with rank sum test that there were significant difference $(P<0.05)$ in cell population ratios $(\%)$ of the $\mathrm{CD}^{+} \mathrm{T}$ lympholeukocyte between the groups of hepatic failure and chronic hepatitis $\mathrm{B}$ with $\mathrm{e}$ antigen masculine; the $\mathrm{CD}^{-} \mathrm{CD} 16^{+} \mathrm{CD} 56^{+}$natural killer cell between the groups of HBV carrier with e antigen negative and the other two control groups.

Conclusions: The cell population ratios (\%) of $\mathrm{T}$ lympholeukocyte and $\mathrm{NK}$ in serum of hepatic failure cases were decreased while it of $\mathrm{T}$ lympholeukocyte in chronic hepatitis $\mathrm{B}$ with $\mathrm{e}$ antigen masculine cases was increased.

\section{PP03-20}

Up-regulation of CXCR3 Ligands in Hepatocytes of Chronic Hepatitis B Virus Infected Patients before Treatment

Y. Han ${ }^{1}$, X.-H. Li ${ }^{1}$, Z.-H. Yuan ${ }^{2}$, X.-X. Zhang ${ }^{1}$

${ }^{1}$ Ruijin Hospital, Shanghai Jiaotong University School of Mediciine;

${ }^{2}$ Shanghai Public Health Clinical Center Affiliated to Fudan

University, Shanghai, China

Background and aims: Chemokines regulate the infiltration of immune cells to sites of inflammation both in acute and chronic settings. Chemokine receptor CXCR3 and its ligand levels have shown a clinical utility as predictors of treatment response outcome in HCV infection. Data from CXCR3 knockout mice suggest that these pathways are important in liver injury. However, its role in HBV infection still needs to be elucidated.

Methods: We performed gene expression profiling on pre-treatment liver specimens obtained from 124 chronic HBV infected patients.
Microarray was performed using Affymetrix Human U133Plus2 arrays. Gene expression profiles were classified according to transaminase (ALT) level, Scheuer Scoring representing inflammation and fibrosis status, viral load and its serological markers. Statistical analysis was performed using oneway ANOVA Welch test with multiple correction by Benjamini-Hochberg test. Patients were followed 12 months, treatment responses were confirmed according to the KEEFFE 2008 Roadmap.

Results: We found a distinct expression pattern among different inflammatory status, both in G scoring and ALT levels. The three CXCR3 ligands (CXCL9,10,11) were significantly up-regulated (with a fold change $\geq 3$ ) in the liver tissue of patients with Scheuer score of G4 compared to G0. Similar results were obtained from different ALT groups, with the same genes significantly up-regulated in patients with 2-5 times normal ALT compared to normal. Furthermore, they were the only three up-regulated genes in ALT level higher than 5 times normal group.

Conclusions: This was an experiments performed in human hepatocytes to elucidate the different gene expression pattern in HBV patients with follow-up data, and preliminary results suggested that chemokine pathway strongly induced by interferon- $\gamma$, with activated $\mathrm{T}$ cells and NK cells effects was most involved in the high inflammatory status of hepatitis B patient and may have an underlying role in the response prediction of immune modulators based regimes.

\section{PP03-21}

High HBsAg Levels in Inactive Carriers: A Possible Predictor for HBV Reactivation

Y.J. Kim, H.C. Cho, G.-Y. Gwak, M.S. Choi, J.H. Lee, K.C. Koh, B.C. Yoo, S.W. Paik

Samsung Medical Center, Sungkyunkwan University School of Medicine, Seoul, Republic of Korea

Background/aims: There is no reliable predictor for viral replication in the inactive carriers (ICs) of hepatitis B virus (HBV). We evaluated the possibility of the serum hepatitis B surface antigen (HBsAg) level for identifying the ICs with replicative potential.

Methods: A total of 162 treatment naïve ICs of HBV were included in this retrospective study (Table). They were classified as a replicative carrier (RC)-I when their serum HBV DNA rose up between 2,000 IU/mL and 20,000 IU/mL and as a RC-II when the serum HBV DNA roes up more than $20,000 \mathrm{IU} / \mathrm{mL}$. The ICs with persistently low HBV DNA $(<2,000 \mathrm{IU} / \mathrm{mL})$ were classified as true ICs (TICs).

Table 1 Baseline characteristic of the study subjects

\begin{tabular}{llllll}
\hline & All ICs $(n=162)$ & TIC $^{*}(n=130)$ & RC-I* $(n=19)$ & RC-II $^{\S}(n=13)$ & $P$ value \\
\hline Age (mean \pm SD, year) & $55.1 \pm 9.64$ & $55.6 \pm 9.22$ & $53.2 \pm 12.21$ & $52.4 \pm 9.56$ & 0.142 \\
Male gender (\%) & $112(70)$ & $88(67.7)$ & $14(73.7)$ & $10(76.9)$ & 0.712 \\
FU period & $15.2 \pm 3.63$ & $15.4 \pm 3.74$ & $15.3 \pm 3.15$ & $13.4 \pm 2.63$
\end{tabular}

(mean $\pm \mathrm{SD}$, months)

HBsAg (median, range, IU/mL)

$\begin{array}{lllllll}\text { Baseline } & 210.3(0.08-16,247.6) & 47.0(0.0 .8-8,616.77) & 1,279.4(55.24-16,247.66) & 1,807.4(27.8-8,297.23) & <0.001 \\ \text { FU } & 54.33(0.00-7,558.14) & 5.26(0.00-7,180.02) & 1,268.40(87.58-4,479.37) & 3,523.92(823.79-7,558.14) & <0.001\end{array}$

HBV DNA (median, range, IU/mL)

\begin{tabular}{|c|c|c|c|c|c|}
\hline Baseline & $70.5(9-1,744)$ & $26.5(9-1,444)$ & $689(175-1,744)$ & $432(161-1,632)$ & $<0.001$ \\
\hline Lowest & $28.5(9-1,657)$ & $12(9-1,043)$ & $634(139-1,657)$ & $432(161-1,632)$ & $<0.01$ \\
\hline Highest & $158(9-1,955)$ & $98.5(9-1,607)$ & $1,510(257-1,955)$ & $629(161-1,632)$ & $<0.001$ \\
\hline $\mathrm{ALT}($ mean $\pm \mathrm{SD}, \mathrm{U} / \mathrm{mL})$ & $22.3 \pm 6.89$ & $21.95 \pm 6.90$ & $23.41 \pm 7.42$ & $24.23 \pm 5.73$ & 0.395 \\
\hline 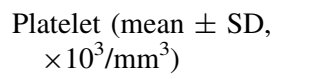 & $165.5 \pm 68.29$ & $163.3 \pm 70.87$ & $177.2 \pm 66.47$ & $170.46 \pm 40.59$ & 0.688 \\
\hline $\mathrm{PT}($ mean $\pm \mathrm{SD}, \mathrm{INR})$ & $1.09 \pm 0.13$ & $1.11 \pm 0.13$ & $1.08 \pm 0.09$ & $1.05 \pm 0.08$ & 0.256 \\
\hline
\end{tabular}


Results: With a mean follow-up period of $15.2 \pm 3.63$ months, 19 patients (11.8\%) were classified as RC-I and $13(8.0 \%)$ were classified as RC-II. One hundred thirty $(80.2 \%)$ remained as TIC. The baseline and follow-up HBsAg levels were the lowest in the TIC (47.0 and 5.26 IU/ $\mathrm{mL})$ and the highest in the RC-II $(1,807.4$ and 3,523.92 IU/mL, respectively). After follow-up, HBsAg declined in the TIC and RC-I but increased in RC-II. The cut off value of HBsAg to identify RC-II from TIC was $1,168 \mathrm{IU} / \mathrm{mL}$ with a sensitivity of $77 \%$ and a specificity of $85 \%$.

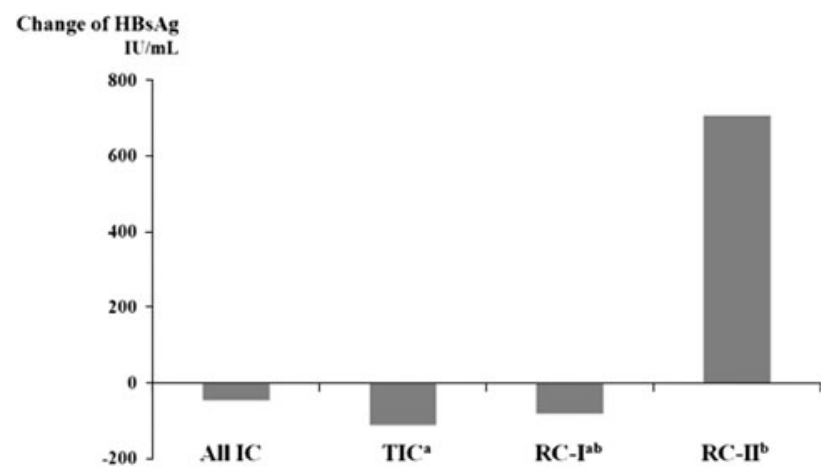

Conclusions: Serum HBsAg levels differed in the ICs of HBV and they were significantly lower in the ICs with persistent low HBV DNA levels. High HBsAg levels in ICs may be related to future HBV replication.

\section{PP03-22}

Comparison of the Performance of Direct Sequencing, DNA Microarray and Line Probe Assay in Detecting HBV Antiviral Resistance Mutations R. Yang, S. Du, X. Cong, H. Ma, L. Wei Peking University People's Hospital, Peking University Hepatology Institute, Beijing, China

Background: Sensitive and specific assays are urgently needed to monitor the HBV antiviral resistance mutations during the treatment of chronic hepatitis B (CHB) with nucleos(t)ide analogues.

Methods: The absolute sensitivity of an in-house direct sequencing (DS), a newly developed DNA microarray assay based on sequence specific primers (SSP) and a line probe assay (LiPA) v2.0. was evaluated with serially diluted HBV international standard. And 224 serum samples from $\mathrm{CHB}$ patients treated with lamivudine or adefovir were tested for the mutations of rtL180M, rtA181T/V, rtM204I/ $\mathrm{V}$ and $\mathrm{rtN2} 26 \mathrm{~T}$ by the three methods in parallel. Any inconsistent results were confirmed by clonal analysis.

Results: DS had the highest absolute sensitivity in detecting the very low serum viral load $(50 \mathrm{IU} / \mathrm{ml})$. LiPA and microarray could detect 400 $\mathrm{IU} / \mathrm{ml}$ and $1,000 \mathrm{IU} / \mathrm{ml}$, respectively. Three methods were highly consistent in detecting the common mutations at the HBV level $>10^{55}$ IU/ $\mathrm{ml}(97.1 \%)$. DS failed to detect $5 \%-15 \%$ of mixed minor mutations at HBV levels $<10 \mathrm{IU} / \mathrm{ml}$ but microarray and LiPA could detect most of these mutations. Microarray was more sensitive than LiPA in detecting 3 samples with $2-3 \%$ of minor mutations. However, 2 samples were detected by microarray as rtM204V but wild type by DS, LiPA and clonal analysis. Another 3 samples yielded indeterminate results by LiPA. Sequence analysis showed the polymorphisms in the microarray primer-binding sequence and LiPA probe hybridization sequences caused these "false positive" and indeterminate results, respectively.

Conclusions: DS is the most suitable assay when HBV DNA level was $>10^{5}$ or $<1,000 \mathrm{IU} / \mathrm{ml}$ considering its simplicity and costeffectiveness. Microarray and LiPA are especially suitable for samples with suspected minor mutations. Both assays, however, suffer from the low absolute sensitivity and polymorphisms causing the false positive or indeterminate results in about $1-2 \%$ clinical samples and should be improved in the newer version.
PP03-23

Quantitative HBsAg Levels in Long-term Lamivudine Treated Patients: The Association with the Appearance of Lamivudine Resistant Mutation Y.J. Kim, H.C. Cho, G.-Y. Gwak, M.S. Choi, J.H. Lee, K.C. Koh, B.C. Yoo, S.W. Paik

Samsung Medical Center, Sungkyunkwan University School of Medicine, Seoul, Republic of Korea

Background/aims: We surveyed quantitative HBsAg levels in longterm lamivudine treated patients and evaluated the association with the appearance of lamivudine resistance.

Methods: We included 264 chronic hepatitis B patients who received lamivudine treatment for more than 3 years. One hundred two patients were found to develop lamivudine resistance and viral breakthrough and the other 162 patients did not. We reviewed their medical record and collected their laboratory data including quantitative HBsAg level.

Table 1 Baseline clinical characteristic of the study subjects

\begin{tabular}{lllll}
\hline & All & $\begin{array}{l}\text { Lamivudine } \\
\text { resistance }(+)\end{array}$ & $\begin{array}{l}\text { Lamivudine } \\
\text { resistance }(-)\end{array}$ & $P$ value \\
\hline$N$ & 264 & 102 & 162 & \\
$\begin{array}{c}\text { Male:female } \\
(\%)\end{array}$ & $\begin{array}{c}182: 82 \\
(69: 31)\end{array}$ & $68: 34(67: 33)$ & $114: 48(70: 30)$ & 0.585 \\
$\begin{array}{l}\text { Age, year } \\
\text { HBeAg+: }-(\%)\end{array}$ & $\begin{array}{c}52.6 \pm 10.09 \\
\quad 52.9 \pm 13: 87)\end{array}$ & $23: 79(22: 78)$ & $12: 150(8: 92)$ & 0.001 \\
$\begin{array}{c}\text { Treatment } \\
\text { duration, } \\
\text { months }\end{array}$ & $62.3 \pm 24.54$ & $60.6 \pm 23.61$ & $63.29 \pm 25.31$ & 0.389 \\
$\begin{array}{l}\text { HBsAg, } \\
\text { log IU/mL }\end{array}$ & $2.58 \pm 1.07$ & $2.95 \pm 0.76$ & $2.35 \pm 1.18$ & 0.000 \\
\hline
\end{tabular}

Results: Male predominance (69\%) was observed and the mean age was $52.6 \pm 10.09$ years. The mean treatment period was $62.3 \pm 24.54$ months and $\mathrm{HBeAg}$ was positive in $13 \%$. The mean quantitative serum HBsAg was $2.58 \pm 1.07(\log \mathrm{IU} / \mathrm{mL})$ in all patients, and $2.95 \pm 0.76$ in lamivudine resistance group and $2.35 \pm 1.18$ in patients with no lamivudine resistance ( $p=0.000$ by Student's $t$ test). Multivariate analysis revealed the presence of lamivudine resistance, $\mathrm{HBeAg}$ positivity, shorter treatment duration, younger age, and female gender as predictive factors for high quantitative HBsAg level (Table 2).

Table 2 Clinical factors affecting serum quantitative HBsAg levels

\begin{tabular}{|c|c|c|c|c|}
\hline \multirow[t]{2}{*}{ Characteristics } & \multicolumn{2}{|l|}{ Univaritate } & \multicolumn{2}{|l|}{ Multivariate analysis } \\
\hline & $\operatorname{Exp}(B)(95 \% C I)$ & $P$ value & $\operatorname{Exp}(B)(95 \%$ CI $)$ & $P$ value \\
\hline $\begin{array}{l}\text { Laraiviidinc } \\
\quad \text { resistance }(+)\end{array}$ & $\begin{array}{l}0.594(0.336 \text { to } \\
0.851)\end{array}$ & 0.000 & $\begin{array}{l}0.439(0.202 \text { to } \\
0.676)\end{array}$ & 0.000 \\
\hline Age & $\begin{array}{l}-0.031(-0.044 \text { to } \\
0.019)\end{array}$ & 0.000 & $\begin{array}{l}-0.024(0.035 \text { to }- \\
0.012)\end{array}$ & 0.000 \\
\hline Female gender & $\begin{array}{l}0.264(-0.015 \text { to } \\
0.544)\end{array}$ & 0.63 & $\begin{array}{l}0.274(0.031 \text { to } \\
0.516)\end{array}$ & 0.027 \\
\hline $\begin{array}{l}\text { Treatment } \\
\text { duration }\end{array}$ & $\begin{array}{c}-0.009(0.014 \text { to } \\
-0.003)\end{array}$ & 0001 & $\begin{array}{l}-0.007(-0.012 \text { to } \\
-0.002)\end{array}$ & 0.003 \\
\hline HBeAg (+) & $\begin{array}{c}1.199(0.844 \text { to } \\
1.554)\end{array}$ & 0.000 & $\begin{array}{c}0.879(0.528 \text { to } \\
1.230)\end{array}$ & 0.000 \\
\hline
\end{tabular}

Conclusion: In long-term lamivudine treated patients, the appearance of lamivudine resistance was associated with higher HBsAg level. The possibility of high HBsAg level as a predictor of viral resistance requires further clinical studies. 


\section{PP03-24}

Multicenter Evaluation of the Elecsys HBsAg II Quant Assay

F. Bonino ${ }^{1}$, F. Moriconi ${ }^{1}$, S. Bowden ${ }^{2}$, R. Hammond ${ }^{2}$, M. Brunetto ${ }^{3}$,

S. Louisirirotchanakul ${ }^{4}$, T. Tanwandee ${ }^{4}$, P. Phisalprapa ${ }^{4}$, B. Zacher ${ }^{5}$,

K. Wursthorn ${ }^{5}$, H. Wedemeyer ${ }^{5}$

${ }^{1}$ Scientific Direction and Central Laboratory, Fondazione IRCCS Orspedale Maggiore Policlinico Mangiagalli e Regina Elena di Milano, University of Pisa, Milan, Italy; ${ }^{2}$ Melbourne Health,

Victorian Infectious Disease Reference Laboratory (VIDRL), North Melbourne, VIC, Australia; ${ }^{3}$ Laboratory of Molecular Genetics of Hepatitis Viruses, Azienda Ospedaliero Universitaria Pisana, Pisa, Italy; ${ }^{4}$ Department of Microbiology, Faculty of Medicine Siriraj Hospital, Mahidol University, Bangkok, Thailand; ${ }^{5}$ Medizinische Hochschule Hannover, Klinik für Gastroenterologie, Hepatologie und Endokrinologie, Hannover, Germany

Background/aims: Elecsys HBsAg II quant is a new quantitative electrochemiluminescence immunoassay with on-board dilution and a broad linear range optimized for clinical decision making. It reports HBsAg levels in IU/mL and is fully traceable to the WHO reference standard. This large-scale multicenter study evaluated its performance using routine serum samples from both treated and untreated patients with $\mathrm{CHB}$, in different phases of infection, and infected with all major HBV genotypes.

Methods: Samples were collected independently by five centers in Europe, Australia and Asia. HBsAg was quantified using Elecsys HBsAg II quant on a variety of analyzers. Dilution analyses (undiluted to $1: 1,000,000)$ of all sera were performed to assess the recommended mandatory dilution algorithm, determine assay range free of Hook effect, and investigate potential effect of HBV genotype. Intra- and inter-assay precision were also established.

Results: 70.0 and $85.6 \%$ of samples tested on analyzers incorporating 1:100 (Elecsys 2010 and cobas e 411) and 1:400 (MODULAR ANALYTICS E170; cobas e 601 and e 602) onboard dilution, respectively, fell within the linear range of the assay using the onboard dilution step, providing a final result on first testing. The majority of those samples above the measuring range on first analysis required only a single reanalysis using a manual predilution step of 1:10; only $\sim 2 \%$ required a second manual dilution step of $1: 100$. Using the dilution algorithm established, no high-dose Hook effect was observed up to the maximum HBsAg level tested $(870,000 \mathrm{IU} / \mathrm{mL})$. HBsAg levels were reliably determined across all genotypes and stages of disease. High precision was confirmed across all analyzers employed (CV: 1.4-9.6\%; HBsAg concentrations 0.1-37,300 IU/mL).

Conclusions: The Elecsys HBsAg II quant assay reliably quantifies HBsAg in routine clinical serum samples, including those with high HBsAg levels. Onboard dilution minimizes retesting and reduces the potential for errors.

\section{PP03-25}

Invasive Pulmonary Aspergillosis in Patients with HBV-related Liver Failure

W. Wang, C.Y. Zhao, J.Y. Zhou, Y.D. Wang, C. Shen, D.F. Zhou, H.Z. Yin Hebei Medical University, Shijiazhuang, China

Aims: Invasive pulmonary aspergillosis (IPA) has been increasingly frequent in severe liver disease. We aimed to investigate the clinical presentation, predisposing factors, and treatment of IPA in patients with liver failure caused by hepatitis B virus (HBV) infection.

Patients and methods: We retrospectively reviewed the data from 798 patients with HBV-related liver failure. A case-control study, including a total of 43 patients with probable IPA, 43 with bacterial infection and 43 without any infections, was performed to evaluate the risk factors, clinical manifestations, treatment, and subsequent outcome of IPA in patients with HBV-related liver failure.

Results: Patients with IPA had worse condition and prognosis, their MELD scores and the mortality were higher than that of the bacterial infection group and the control group $(34.84 \pm 5.47$ vs. $31.91 \pm 6.50$ vs. $28.49 \pm 6.94, P<0.01 ; 100$ vs. 65.12 vs. $41.86 \%, P<0.01)$

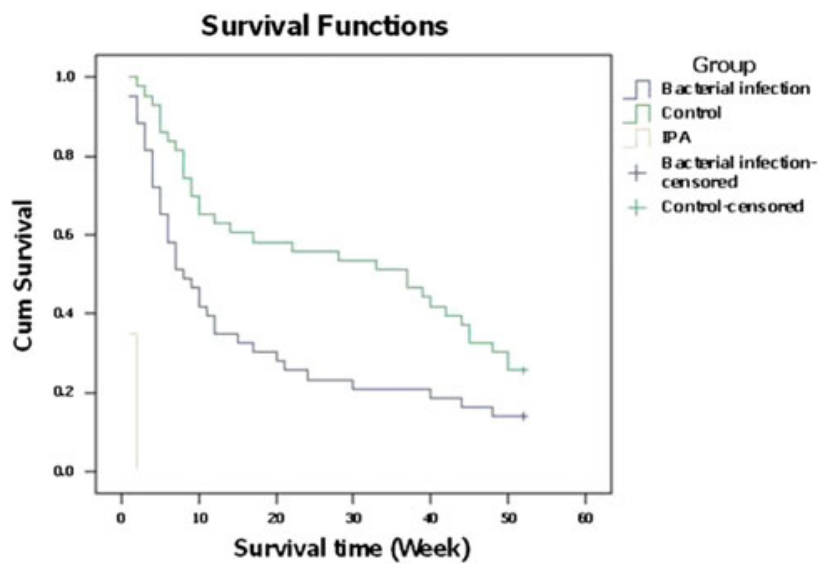

Survival curve of patients in three groups

Multiple antibiotics use and steroids exposure were significantly higher in the IPA group than the other two groups (100 vs. 62.79 vs. $20.93 \%, P<0.01 ; 83.72$ vs. 46.51 vs. $34.88 \%, P<0.01)$. On multivariate analysis, multiple antibiotics use $(\mathrm{OR}=15.59, P<0.01)$ and frequent invasive procedures $(\mathrm{OR}=0.30, P<0.01)$ predicted the occurrence of IPA in patients with HBV-related liver failure.

Conclusions: IPA is prone to be occurring in patients with HBVrelated liver failure. Once infected, the patients may have a more severe condition and poorer prognosis. We must enhance the awareness and prevent predisposing factors to reduce the risk of Aspergillus infection in patients with HBV-related liver failure.

\section{PP03-26}

Diagnostic Value of HBsAg Quantification for Predicting Clinical Course in Genotype C HBeAg Negative Carriers

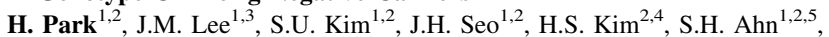
D.Y. Kim ${ }^{1,2,5}$, K.-H. Han ${ }^{1,2,5}$, C.Y. Chon ${ }^{1,2,5}$, J.Y. Park ${ }^{1,2,5}$

${ }^{1}$ Internal Medicine; ${ }^{2}$ Gastroenterology, Yonsei University College of Medicine; ${ }^{3}$ Internal Medicine, CHA University; ${ }^{4}$ Labortory Medicine, Yonsei University College of Medicine; ${ }^{5}$ Liver Cirrhosis Clinical

Research Center, Seoul, Republic of Korea

Background/aims: The quantification of HBsAg titer has been proved as a useful marker to predict the outcomes of antiviral treatment. We investigated the diagnostic value of $\mathrm{HBsAg}$ titer for predicting clinical course in genotype $\mathrm{C}$ HBeAg negative $\mathrm{HBV}$ carriers.

Methods: One-hundred and four treatment-naive $\mathrm{HBeAg}$ negative $\mathrm{HBV}$ carriers (HBV DNA $<2,000 \mathrm{IU} / \mathrm{ml}$ and ALT $<40 \mathrm{IU} / \mathrm{ml}$ ) were recruited and followed up for a median of 24 months. The study population was stratified into 2 groups: inactive carriers (IC) group with persistent $\mathrm{HBV}$ DNA $<2000 \mathrm{IU} / \mathrm{ml}$ and ALT $<40 \mathrm{IU} / \mathrm{ml}$ and $\mathrm{HBeAg}$ negative chronic hepatitis $(\mathrm{ENH})$ group with fluctuating HBV DNA or ALT during the follow-up periods. Baseline HBsAg titer was quantified using a commercial kit (ARCHITECT, Abbott Diagnostic, Germany).

Results: The mean age of study population was 25.8 years and male gender predominated $(62.5 \%)$. HBsAg titer showed a significant correlation with HBV DNA $(r=0.392 ; p<0.001)$. HBsAg titer was higher in IC group than ENH group (2.83 vs. $3.28 \log _{10} \mathrm{IU} / \mathrm{ml}, p<$ 0.001 ) and significantly predicted the future viral activation (area under the receiver operating characteristic curve (AUROC), 0.710; $p=0.001$ ). Combined use of HBV DNA and HBsAg titer slightly enhanced the performance to predict the viral activation (AUROC, 
0.750), although it was not better than the prediction accuracy of single HBV DNA (AUROC, 0.830). The optimal cutoffs were HBsAg titer $>850 \mathrm{IU} / \mathrm{ml}$ and $\mathrm{HBV}$ DNA $>850 \mathrm{IU} / \mathrm{ml}$ (sensitivity, $64.5 \%$; specificity, $93.2 \%$; positive predictive value, $80.0 \%$; and negative predictive value, $86.1 \%$ ).

Conclusions: HBsAg titer at single point is less predictable than HBV DNA in distinguishing inactive carriers from patients with the future viral activation in genotype $\mathrm{C} \mathrm{HBeAg}$ negative HBV carriers. However, combined uses of HBV DNA and HBsAg titer help predict the clinical course and decide the follow-up plan and management strategies in these patients.

\section{PP03-27}

The Development of a Biosensor-Based Microarray Assay for Necleict(s)ides Drug-Resistance Related HBV Mutations

J. Song, P. Li, L. Yao, X. Li, X. Zhou, Zhuhai Sinochips Bioscience Co., Ltd. The 5th Affiliated Hospital of Sun Yat-sen University, Zhuhai, China

Background and aims: It is very important to monitor the resistance mutation of HBV drug resistance during the HBV anti-virus treatment by nucleiti(s)des. There is less clinical assay are used for clinical practise. Here, we report a biosensor based microarray assay which aimed at developing a novel method with less instrument and its specificity, sensitivity and reproducibility were be valuated here.

Methods: Based on the biosensor based microarray technique which we reported before, we developed a microarray for hepatitis B virus drug resistance (HBV-DR) which can detected most common used nucleict(s)ides mutations of rtL180M, A181V/T/S/.P, A194T G202S/ I, M204V/I and N236T, M250V. And 40 plasmids samples containing these resistance mutations, and 150 blood samples (HBV DNA ranging from $10^{3}$ to $10^{7}$ copies $/ \mathrm{ml}$ ) from 238 patients with lamivudine or adefovir treatment were be used in our study. All the PCR amplificans were assay both by our microarray and Sanger's sequencing. The Concordance rates between our microarray and sequencing was statistically analysis by kappa test.

Results: The mutation pattern of these mutation sites were successfully detected and discriminated. And the signals of mutations were be read by naked eye or photographyed by regular digital camera as we reported before. The Concordance rates between microarray and PCR sequencing of mutated was $97 \%$ (kappa $=0.96$ ) of the plasmids. $89 \%$ (kappa $=0.795)$ of sera, and more sensitivity of the microarray for the mixture infections which approved by clone sequencing. amplicans. The sensitivity of our assay were $96 \%$ positive rate for $5 \times 10 \mathrm{E} 4$ copies/ml standard sera of China National Institute for the Control of Pharmaceutical and Biological Products of China (NICPBP).

Conclusions: Biosensor-based HBV DR chip assay was successfully developed in our study. It is a high-specificity, sensitive, reliable method for the detection of HBV drug resistance mutations. Its less instrument-dependent and easily-performed characters make it a promising method for clinical application.

\section{PP03-28}

Cellular Immunity Change and Significance Before and After Patients with Chronic Hepatitis B Developed into Severe Hepatitis G. Xibing

\section{Wuxi Hospital for Infectious Diseases, Wuxi, China}

Objective: To explore HBV specific and non-specific cellular immunity change and significance before and after patients with chronic hepatitis B (CHB) developed into severe hepatitis.

Methods: 199 cases of positive HBV DNA and HLA-A2 diagnosed as $\mathrm{CHB}$ during admission were enrolled. For 39 cases developed into severe hepatitis (group A) and 160 cases not developed into severe hepatitis (group B), dynamic changes of HBV specific CTL, nonspecific CTL and T cell subgroup were compared.
Results: 39 cases of 199 CHB patients (19.60\%) developed chronic severe hepatitis (early stage) averaging $10.05 \pm 1.72 \mathrm{~d}$ after admission. $\mathrm{CD}^{+}$level: Group A after developing into severe hepatitis $<$ Group A the next day after admission $(p<0.01)<$ Group B the next day after admission $(p<0.01)<$ normal control group $(p<$ 0.01). Group A after developing into severe hepatitis $<$ Group B $10 \mathrm{~d}$ after admission $(p<0.01) . \mathrm{CD}^{+}$and non-specific CTL level: Group A after developing into severe hepatitis $>$ Group A the next day after admission $(p<0.01)>$ Group B the next day after admission $(p<0.01)>$ normal control group $(p<0.01)$. Group A after developing into severe hepatitis $>$ Group B $10 \mathrm{~d}$ after admission $(p<0.01)$. HBV specific CTL level: There was no significant difference the next day after admission between the two groups $(p>0.05)$ and there was no significant difference in Group A after developing into severe hepatitis compared with that the next day after admission $(p>0.05)$.

Conclusion: Significant increase of peripheral blood $\mathrm{CD} 8^{+}$and nonspecific CTL in patients with chronic severe hepatitis B (early stage) suggests that immunological pathogenesis of chronic severe hepatitis $\mathrm{B}$ is related to significant increase of $\mathrm{CD}^{+}$and non-specific CTL level and if $\mathrm{CD}^{+}$and non-specific CTL of $\mathrm{CHB}$ patients increased significantly, it may develop into severe hepatitis.

\section{PP03-29}

Analyzing Holo-transcobalamin (Active Vitamin B12) in Sera of patients wIth Chronic Active Hepatitss

F. Sirmatel ${ }^{1,2}$, O. Sirmatel ${ }^{3}$, G. Bugdayci ${ }^{4}$

${ }^{1}$ Infectious Diseases, Abant Izzet Baysal University; ${ }^{2}$ Infectious Diseases Department, Medical Faculty of Abant Izzet Baysal University; ${ }^{3}$ Radiodiagnosis; ${ }^{4}$ Biochemistry, Abant Izzet Baysal University, Bolu, Turkey

Aim: In chronic hepatic failure, the increase in vitamin B12 blood levels is accepted as a result of vascular leakage. From this perspective, in chronic viral hepatitis B (HBV) and chronic viral hepatitis $\mathrm{C}(\mathrm{HCV})$ cases, the real indicator of serum active vitamin B12, namely holo-transcobalamin II (Holo TC II), is interpreted as the indicator of hepatocellular damage prior to treatment.

Material and methods: In a university hospital, Holo TC II, the best indicator of serum vitamin B12, was analysed in sera of 127 healthy blood donors and 274 chronic viral hepatitis patients (included 127 $\mathrm{HBV}$ and $17 \mathrm{HCV}$ ), which have been clinically, serologically and histologically diagnosed. Age distribution of the patients ranged between 18 and 70 . When determining the study group, patients with decompansated cirrhosis, any cardiac, metabolic, hematologic, gastrointestinal and otoimmune disorders were excluded. Analysis were performed with commercially available Holo TC II kits according to the prospectus, using macro EIA method. Results were statistically compared between the groups.

Results: In our study, no difference was detected in terms of holo TC II levels between chronic HBV (42.07 pmol/l) and HCV patients (42.8 $\mathrm{pmol} / \mathrm{l})$, while compared to the control group $(25.7 \mathrm{pmol} / 1)$, results were found significantly higher $(p<0.017)$.

Discussion and conclusion: Holo TC II can be used particularly as the earliest symptom of cellular damage in chronic viral hepatitis. Further studies on chronic hepatic failure and cellular damage may also lead to the consideration that it can be used as a diagnostic criteria.

\section{PP03-30}

Establishment of an Improved Type-Specific Nested-PCR for Four Major Genotypes (A-D) and Subgenotypes (B1, B2, C1 and C2) of HBV J.-J. Nie, K.-X. Sun, J. Wang, H. Jin, L. Wang, F.-M. Lu, T. Li, L. Yan, M.-S. Sun, J. Li, H. Zhuang

Department of Microbiology, Peking University Health Science Center, Beijing, China 
Objective: To develop a nested polymerase chain reaction (nPCR) for four major genotypes (A-D) and subgenotypes (B1, B2, C1 and C2) of hepatitis B virus (HBV).

Methods: A total of 150 entire nucleotide sequences of genotypes A$\mathrm{H}$ of $\mathrm{HBV}$ were retrieved from GenBank, and the PCR primers were designed by Primer Premier 5.0 after comparing and analyzing by DNAStar software. There were 3 multiple mixes (Mix A for genotypes B, D and subgenotypes C1, C2; Mix B for genotype A; Mix C for subgenotypes B1 and B2) in the second-round PCR after the unselected first-round amplification. The specificity and sensitivity were evaluated by plasmids and clinical samples, respectively.

Results: Of 642 serum samples genotyped by this system, 639 (99.5\%) were successfully genotyped and subgenotyped. Among the 639 samples, $117(18.3 \%)$ were selected randomly and their nPCR genotyping results were further confirmed by directed sequencing of PCR products. The genotypes of the plasmids could be identified when HBV DNA load were $\geq 10^{1.18} \mathrm{IU} / \mathrm{ml}$. Of 8 clinical samples with the viral load of $<10^{1.18} \mathrm{IU} / \mathrm{ml}, 3(37.5 \%)$ could be successfully genotyped by the nPCR method, while 15 of 38 (39.5\%) clinical samples with the viral load of $10^{1.18}-10^{2.3} \mathrm{IU} / \mathrm{ml}$ could be successfully genotyped. However, $100 \%$ of the samples could be genotyped when the viral load $\geq 10^{2.3} \mathrm{IU} / \mathrm{ml}$. When 117 serum samples were detected by the nPCR and Naito's methods, 24 were inconsistent by the two nPCR methods. The sequencing results of the 24 inconsistent samples with genotype D were agreed with the nPCR method, but not with Naito's method which was not sensitive for detection of genotype D.

Conclusions: An improved nPCR genotyping method with high sensitivity and specificity was established. It may be useful for clinical and epidemiological studies.

Acknowledgements: China National Grant 2008ZX10002-001 and 2008ZX10002-012.

\section{PP03-31}

HBsAg Level Is Useful to Separate Truly Inactive Chronic Hepatitis B Infection from those with Subsequent Reactivation

P. Kitikomonkun ${ }^{1}$, P. Charatcharoenwitthaya ${ }^{1}$, S. Chainuwatti ${ }^{1}$,

S. Nimanong ${ }^{1}$, N. Pausawasdi ${ }^{1}$, V. Prachayakul ${ }^{1}$, S. Pongprasobchai ${ }^{1}$,

S. Leelakusolvong ${ }^{1}$, S. Manatsathit ${ }^{1}$, U. Kachintorn ${ }^{1}$, S. Udompanthurak ${ }^{2}$,

T. Tanwandee ${ }^{1}$

${ }^{1}$ Division of Gastroenterology, Department of Medicine, Siriraj

Hospital, Mahidol University; ${ }^{2}$ 2. Clinical Epidemiology Unit, Office for Research and Development, Faculty of Medicine, Mahidol University, Bangkok, Thailand

Background/aims: Chronic inactive hepatitis B patients with low HBV DNA (defined as less than 2,000 IU/mL) can become active during follow up at anytime. Previous studies have demonstrated that male, younger age and higher HBV virus at entry are among predictors of subsequent virological flare. This study is aimed to evaluate whether HBsAg quantitation can better predict subsequent virological flare in inactive chronic hepatitis B infection.

Methods: This is a prospective study includes inactive chronic hepatitis B patients who were never been treated and HBV DNA $<2,000$ IU/mL $\left(\right.$ COBAS $^{\circledR}$ TaqMan $^{\circledR}$ ) from May, 2008 to December, 2009. The patients were followed every 6 months with ALT, HBV DNA and HBsAg quantitation (Abbott Architect).

Results: There were 91 patients ( 49 males, 42 females) with mean age of 50 years and mean follow up time of 11 months. Mean baseline HBV DNA, HBsAg and ALT were 364, 2,831 and $28 \mathrm{U} / \mathrm{L}$, respectively. Twenty-one patients $(23 \%)$ developed subsequent virological flare (HBV DNA $>2,000 \mathrm{IU} / \mathrm{mL}$ ) during follow-up, the only predictor of virological flare was the higher baseline HBV DNA $(p<$ 0.001). Overall, HBsAg level was not the predictor of virological flare but if the patients had baseline HBsAg level less than $250 \mathrm{IU} / \mathrm{mL}$, we found that this level can predict absent of virological flare with the sensitivity, specificity, positive predictive value (PPV) and negative predictive value (NPV) of 76.2, 55.7, 34.0, and 88.6\%, respectively [AUC of 0.66 (95\% CI, 0.54-0.77), $p=0.03$ ].

Conclusions: Low HBsAg level at baseline is useful to predict absent of subsequent virological flare in chronic inactive hepatitis B patients especially when the level of HBsAg is less than $250 \mathrm{IU} / \mathrm{mL}$ (NPV of $88.6 \%$ ) during 1 year follow up study.

\section{PP03-32}

Change of Transient Elastography in Chronic Hepatitis B Carriers Patients Compare with Healthy Volunteers

S. Likitlersuang ${ }^{1}$, T. Tanwandee ${ }^{1}$, P. Charatcharoenwitthaya ${ }^{1}$,

S. Chainuwatti ${ }^{1}$, S. Nimanong ${ }^{1}$, N. Pausawasdi ${ }^{1}$, V. Prachayakul ${ }^{1}$,

S. Pongprasobchai ${ }^{1}$, S. Leelakusolvong ${ }^{1}$, S. Manatsathit ${ }^{1}$, U. Kachintorn ${ }^{1}$,

S. Udompanthurak ${ }^{2}$

${ }^{1}$ Division of Gastroenterology, Department of Medicine, Siriraj Hospital; ${ }^{2}$ Clinical Epidemiology Unit, Office for Research and Development, Faculty of Medicine, Mahidol University, Bangkok, Thailand

Background/aims: Liver fibrosis is an important complication of chronic hepatitis B. Treatment is required if the patient has replicative virus and the risk of disease progression. It is not known whether patients with continuously low HBV DNA will be at risk of fibrosis progression. Moreover, there may be a risk of silent reactivation during 6-12 months of follow up. This study is aimed to assess liver fibrosis as determined by transient elastrography in chronic hepatitis $\mathrm{B}$ carriers compared with normal controls.

Methods: Chronic hepatitis B carrier (CHB) is defined as $\mathrm{HBeAg}$ negative, HBV DNA continuously less than 2,000 IU/mL, normal ALT and never been treated. Controls were normal subjects who were negative for hepatitis B and C viruses and had normal ALT. TE was done every 6-12 months. The patients who had HBV DNA greater than $2,000 \mathrm{IU} / \mathrm{mL}$ during follow up were excluded.

Results: There were $87 \mathrm{CHB}$ and 66 controls in the study. Mean follow-up time was 11 months. Baseline characteristics of cases and controls were comparable except the controls were 1 decade younger. The results of liver stiffness are shown in Table 1 . There was no statistical significance of liver stiffness between chronic hepatitis B carriers and controls both at baseline and last follow up. There was no relationship between liver stiffness and age, ALT, FBS, LDL, HDL, triglyceride, cholesterol and BMI in both cases and controls.

Conclusion: $\mathrm{CHB}$ with persistently low HBV DNA and normal liver stiffness by fibroscan did not have evidence of progressive liver fibrosis during 11 months of follow up. TE may provide additional benefits in ensuring the minimal risk of fibrosis progression in this group Table 1.

Table 1 Liver stiffness of chronic hepatitis B ca

\begin{tabular}{lll}
\hline & $\begin{array}{l}\mathrm{CHB}(\text { mean }+\mathrm{SD}), \\
\mathrm{kPa}\end{array}$ & $\begin{array}{l}\text { Controls } \\
(\text { mean }+\mathrm{SD}), \mathrm{kPa}\end{array}$ \\
\hline Baseline & $5.2+1.8$ & $5.0+1.3$ \\
Last follow up & $5.2+1.7$ & $5.9+0.9$ \\
\hline
\end{tabular}

\section{PP03-33}

Comparison of Two Commercial Qualitative HBsAg Assay Kits A. Lufiandati, T. Novianti

Evaluation Laboratory, PT. Prodia Widyahusada, Jakarta, Indonesia

Background: Hepatitis B Virus (HBV) infection is a global health problem. HBV surface antigen (HBsAg) is the established serological marker used routinely for the diagnosis of HBV infection. The accurate determination of HBsAg play a crucial role in the diagnosis and prevention of HBV infection, especially in endemic areas. The 
Table 1 Discrepancy results

\begin{tabular}{|c|c|c|c|c|c|c|c|c|c|c|c|c|}
\hline \multirow[t]{2}{*}{ No } & \multicolumn{2}{|c|}{$\begin{array}{l}\text { Qualitative } \\
\text { (AoSYM) }\end{array}$} & \multicolumn{2}{|c|}{$\begin{array}{l}\text { Qualitative } \\
\text { (Architect) }\end{array}$} & \multicolumn{2}{|c|}{$\begin{array}{l}\text { Quantitative } \\
\text { (Architect) }\end{array}$} & \multicolumn{3}{|c|}{$\begin{array}{l}\text { Confirmmatory } \\
\text { HBsAg (AxSYM) }\end{array}$} & \multicolumn{3}{|c|}{$\begin{array}{l}\text { Confirmatory } \\
\text { HBsAg (Architect) }\end{array}$} \\
\hline & $\mathrm{S} / \mathrm{N}$ & Interpretation & $\mathrm{S} / \mathrm{CO}$ & Interpretation & $\mathrm{IU} / \mathrm{mL}$ & Interpretation & $\mathrm{S} / \mathrm{N}$ & $\%$ Neut & Interpretation & $\mathrm{S} / \mathrm{CO}$ & $\%$ Neut & Interprretation \\
\hline Case 1 & 13.29 & $\mathrm{R}$ & 2.65 & $\mathrm{R}$ & - & - & 5.1 & N/A & $\begin{array}{c}\text { Reactive, non- } \\
\text { conformed }\end{array}$ & 3.01 & 92.63 & $\begin{array}{l}\text { Reactive, } \\
\text { confirmed }\end{array}$ \\
\hline Case 2 & 2.07 & $\mathrm{R}$ & 0.38 & NR & $<0.05$ & NR & 3.8 & N/A & $\begin{array}{c}\text { Reactive, non- } \\
\text { confirmed }\end{array}$ & 0.34 & N/A & Non Reactive \\
\hline Case 3 & 5.73 & $\mathrm{R}$ & 0.35 & NR & $<0.05$ & NR & 5.9 & N/A & $\begin{array}{l}\text { Reactive, non- } \\
\text { confirmed }\end{array}$ & 0.38 & N/A & Non Reactive \\
\hline Case 4 & 2.28 & $\mathrm{R}$ & 0.37 & NR & - & - & 2.4 & N/A & $\begin{array}{l}\text { Reactive, non- } \\
\text { confirmed }\end{array}$ & 0.40 & N/A & Non Reactive \\
\hline Case 5 & 1.43 & NR & 1.49 & $\mathrm{R}$ & 0.10 & $\mathrm{R}$ & - & - & - & 1.08 & 92.99 & $\begin{array}{l}\text { Reactive, } \\
\text { Confirmed }\end{array}$ \\
\hline Case 6 & 1.77 & NR & 5.16 & $\mathrm{R}$ & 0.11 & $\mathrm{R}$ & - & - & - & - & - & - \\
\hline Case 7 & 2.58 & $\mathrm{R}$ & 0.84 & NR & 0.05 & $\mathrm{R}$ & 2.5 & N/A & $\begin{array}{c}\text { Reactive, non- } \\
\text { confirmed }\end{array}$ & 0.79 & 91.70 & $\begin{array}{l}\text { Reactive, } \\
\text { Confirmed }\end{array}$ \\
\hline
\end{tabular}

Qualitative HBsAg AxSYM $\rightarrow \mathrm{S} / \mathrm{N}<2.0=$ non-reative; $\mathrm{S} / \mathrm{N}>2.0=$ reactive

Qualitative HBsAg Architect $\rightarrow \mathrm{S} / \mathrm{CO}<1.0=$ non reative; $\mathrm{S} / \mathrm{CO}>1.0=$ reactive

Qualitative HBsAg Architect $\rightarrow<0.05 \mathrm{IU} / \mathrm{mL}=$ non reative; $>0.05 \mathrm{IU} \mathrm{mL}=$ reactive

Confirmatory HBsAg AxSYM $\rightarrow \mathrm{S} / \mathrm{N}<1.5=$ non reative; $\geq 1.5$ and $\%$ neutralization $\geq 50 \%=$ Reactive, confirmed

$\mathrm{S} / \mathrm{N} \geq 1.5$ and $\%$ neutralization $<50 \%=$ Reactive, non-confirmed

Confirmatory HBsAg Architect $\rightarrow \mathrm{S} / \mathrm{CO}<0.7=$ non reactive; $\mathrm{S} / \mathrm{CO} \geq 0.7$ and $\%$ neutralization $\geq 50 \%=$ Reactive, confirmed

$\mathrm{S} / \mathrm{CO} \geq 0.7$ and $\%$ neutraclization $<50 \%=$ Reactive, non-confirmed

$R$ reactive, $N R$ non-reactive, \% neut \% neutralization

- Not tested with this assay because sample volume was insufficient

aim of this study was to compare the performance of 2 commercial qualitative HBsAg assay kits.

Methods: We compared the performance of AxSYM (Abbott) and Architect (Abbott) Qualitative HBsAg assay kits by evaluating 73 serum samples in parallel on both systems under routine laboratory conditions. Discordant results were tested in quantitative $\mathrm{HBsAg}$ assay (Architect). Furthermore, 19 samples also tested by AxSYM and Architect confirmatory HBsAg assay kits.

Results: The total agreement between AxSYM and Architect Qualitative HBsAg assay kits are $91.78 \%$ (67/73). In this study, we found 7 discrepancy results as mentioned in Table 1.

Conclusions: We observed good overall concordance between the 2 assays, but in differentiating reactive samples from non reactive samples, the performance of qualitative HBsAg Architect assay kit was more excellent than AxSYM, supported also by quantitative HBsAg assay.

PP03-34

Analysis of Liver Histopathology and Liver Function in Liver Cirrhosis of Patients Infected Hepatitis B with Persistently Normal ALT Levels Y.-B. Li, J. Dong, Y.-R. Liu, J.-J. Jiang

Center of Liver Disease, the First Affiliated Hospital of Fujian

Medical University, Fuzhou, China

Objective: To investigate the features of liver function, $\mathrm{HBeAg}, \mathrm{HBV}$ DNA and liver histological stage in patients diagnosed as liver cirrhosis caused by hepatitis B (HBV) with persistently normal ALT levels.

Method: Patients with liver cirrhosis infected HBV with persistently normal ALT level or abnormal ALT level were collected in this study. Alanine aminotransferase, albumin, cholinesterase, HBeAg, HBVDNA and liver histological of the patients were analyzed with rank sum test, $\chi^{2}$ test and spearman correlation analysis.

Results: Totally 58 cirrhosis patients with persistently normal ALT level and 60 with abnormal ALT level were involved in our single- center study. The liver histological changes were not significantly different between the 2 groups. HBV DNA level of the patients with persistently normal ALT levels was slightly lower than the patients with abnormal ALT level. AST/ALT, albumin, cholinesterase, $\mathrm{HBeAg}$ and liver histological were all not significantly different. In the subgroup of the patients with persistently normal ALT level, the levels of ALT about $72 \%$ patients were in the range of 30-50U/L.

Conclusion: Liver histological of the patients with persistently normal ALT level could still have obvious inflammation. HBV DNA levels of patients with persistently normal ALT level were lower than that of the patients with abnormal ALT level. It is important to emphasize surveillance on the patients group infected by HBV but with persistently normal ALT level.

\section{PP03-35}

Increased Immunosuppressive Ability of Regulatory $T$ Cells in Active Chronic Hepatitis C Patients

K.-C. Tseng ${ }^{1,2}$, Y.-C. $\mathrm{Ho}^{3}$, S.-F. $\mathrm{Wu}^{3}$

${ }^{1}$ Department of Internal Medicine, Buddhist Dalin Tzu Chi General Hospital, Chiayi; ${ }^{2}$ School of Medicine, Tzuchi University, Hualien;

${ }^{3}$ Department of Life Science, Institute of Molecular Biology, National Chung-Cheng University, Chia-Yi, Taiwan ROC

Introduction/aims: Around $55 \%$ to $85 \%$ of patients can not eliminate virus by their immune system and, thus, chronic hepatitis $\mathrm{C}(\mathrm{CHC})$ develops. Regulatory $\mathrm{T}$ (Treg) cells play a pivotal role in the persistence or clearance of $\mathrm{HCV}$ infection. Our aim was to evaluate the frequency and function of Treg cells in $\mathrm{CHC}$ patients.

Methods: Forty-four subjects were CHC patients with elevated ALT level (CHC group); thirteen subjects were $\mathrm{CHC}$ patients with persistent normal ALT level (PNALT group); eight subjects were healthy control (HS group). Treg cells were identified as CD4+, CD25+ and FoxP3+ T lymphocytes using three-color FACS. The frequency of Treg cells was 
expressed as a percentage of the total PBMC. The immunosuppressive ability of Treg cells was calculated the ratio of MFI from experimental group to positive control in CHC group and PNALT group.

Results: A higher proportion of Treg cells were found in CHC group and PNALT group, when compared with HS group (CHC vs. HS, 2.26 vs. $0.79 \%, p<0.001$; PNALT vs. HS, 2.35 vs. $0.79 \%, p<0.05$ ). The immunosuppressive ability of Treg cells was calculated based on the results from CHC to PNALT at a $2: 1 \mathrm{E} / \mathrm{T}$ ratio (45.02 vs. $13.39 \%$, $p<0.01)$, and a $5: 1 \mathrm{E} / \mathrm{T}$ ratio $(26.82$ vs. $4.68 \%, p<0.05)$ (Figure).

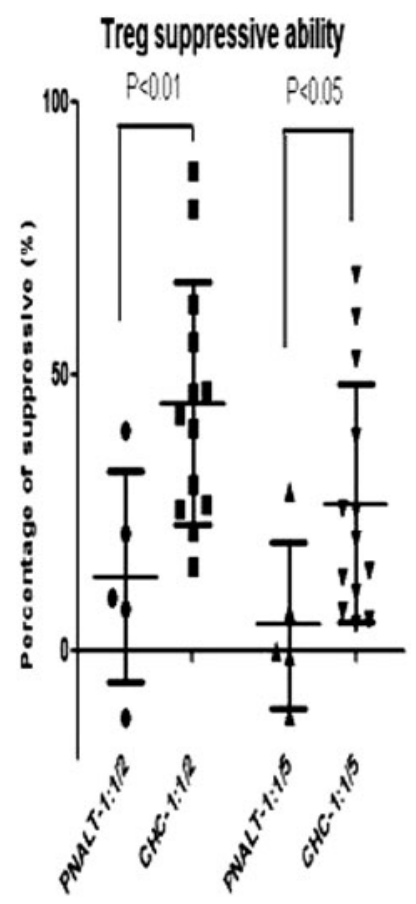

Conclusions: Our study demonstrate that the frequency of Treg cell in CHC group and PNALT group is higher than that of HS group; and the functional immunosuppression of Treg cells in CHC group is higher than that of PNALT group.

\section{PP03-36}

How Need Treatment in Chronic Hepatitis B

N. Muhammad

Medical Unit, Bilal Medical Trust Hospital Pir Baba, Buner, Pakistan

Introduction: Hepatitis B is a comman health problem, more than 2 billion peoples have been infected and about 350 million are chronic carriers. Hepatitis B virus has 4 major serotypes \{adr, adw, ayr, ayw] and eight genotypes $[\mathrm{A}-\mathrm{H}]$

Aim: The aim of the study was to look who need a treatment in chronic Hepatitis B Duration September 2009-October 2010 Source; Bilal medical trust hospital Hospital Pir Baba and Biotech lab Islamabad. Methods and materials: A total of 777 patients 558 male and 219 female aged from 15 to 70 years who were Hepatitis B surface Antigen positive both with normal and abnormal ALT were enrolled in the study. Blood sample were analysed for Hepatitis B DNA PCR Hepatitis B eAntigen and anti Be antibodies.

Results: Out of 777 cases 169 sample were positive for Hepatitis B DNA PCR [21.7\%]. 134 cases were positve for both Hepatits B DNA PCR and Hepatitis Be Antigen [17.24\%]. 523 were positive for anti Hepatitis B antibodies [67.3\% ]. 35 cases who were Hepatitis B DNA PCR positive but negative for Hepatitis B e Antigen and anti Hepatitis $\mathrm{B}$ anti bodies [4.50\%]

Conclusion: Hepatitis B is a common health problem. It is important to have a serological profile before deciding the treatment.
PP03-37

Hepatic Histological Manifestations in Nucleoside-naïve Chronic Hepatitis B Patients with Normal or Mildly Elevated ALT Levels

L. Shao, X. Du, N. Diao, L. Shen, W. Zhang

Huashan Hospital, Fudan University, Shanghai, China

Aim: To assess the epidemiologic characteristics of liver histology in search of related factors interacting with the progression of necroinflammation and fibrosis in nucleoside-naïve chronic hepatitis B (CHB) patients with normal or mildly elevated ALT levels.

Methods: Knodell HAI scoring system was used for grading and staging liver biopsy performed in 539 nucleoside-naïve patients with CHB. Three hundred and forty patients were found to be with normal or mildly elevated ALT levels, which defined as ALT normal or $<2 \times$ ULN.

Results: Age-adjusted grade was significantly increased with transaminase severity $(P<0.01)$, which was $2.10 \pm 0.07$ (95\%CI: 1.96 $2.25)$ in ALT $\leq 1 \times$ ULN group, $2.21 \pm 0.06$ (95\%CI: 2.08-2.33) in ALT $1-2 \times$ ULN group and $2.45 \pm 0.06$ (95\%CI: 2.33-2.57) in ALT $\geq 2 \times$ ULN group. While age-adjusted stage differed significantly with the mean score of $2.32 \pm 0.10$ (95\% CI: 2.13-2.51) in ALT $\leq 1 \times \mathrm{ULN}$ group, $2.05 \pm 0.08(95 \% \mathrm{CI}: 1.89-2.21)$ in ALT 1$2 \times \mathrm{ULN}$ group, and $2.00 \pm 0.08(95 \% \mathrm{CI}: 1.84-2.2)$ in ALT $\geq 2 \times$ ULN group, respectively. Furthermore, an elevation in group of age $\geq 35$ years was observed for either grade $(2.18 \pm 0.06$ in age $<35$ years vs. $2.34 \pm 0.05$ in age $\geq 35$ years; $P<0.05$ ) or stage $(1.86 \pm 0.07$ in age $<35$ years vs. $2.27 \pm 0.07$ age $\geq 35$ years; $P<$ $0.001)$.

Conclusion: Age and ALT level is closely related to necroinflammatory score. However, age, but not ALT level or HBV DNA level, is the strong predictor for fibrosis development in chronic hepatitis $\mathrm{B}$ patients. Therefore, liver biopsy is strongly recommended in $\mathrm{CHB}$ patients with age $\geq 35$ years even though in ALT normal or mildly elevated patients.

\section{PP03-38}

HBV-infection and Hepatic Steatosis

P.O. Bogomolov ${ }^{1}$, M.V. Macievich ${ }^{1}$, N.V. Voronkova ${ }^{1}$, O.S. Kuzmina ${ }^{1}$, T.V. Pavlova ${ }^{2}$

${ }^{1}$ Hepatology; ${ }^{2}$ Pathology, Moscow Regional Research Clinical Institute, Moscow, Russia

Aim: To analise the frequency of steatosis in patients with HBVinfection, its etiology and relationship with this viral infection.

Materials and methods: The retrospective analysis of 61 patients' medical histories with HBV-infection was performed. The anamnesis data, laboratory tests and liver biopsy results of these patients were analyzed. The patients were examined from 2007 till 2009. The patients with HCV, HDV, HIV-infections and alcohol abuse were excluded.

Results: We analised medical histories of 44 men and 17 women. The average age of patients was $35.5 \pm 10.6$ years. The steatosis was diagnosed in 18 persons $(29.5 \%)$. It was more common in males (72\%). The HBV-infection stage varied from inactive HBsAg carrier stage to cirrhosis. Low histological activity was the most prevalent in patients with hepatic steatosis (78\%) and without this morphological finding $(79.1 \%)$. Majority of patients were $\mathrm{HBeAg}$-negative and only $10 \%$ had $\mathrm{HBeAg}$ positive infection. The virus load fluctuated from minimum $(<500 \mathrm{cop} / \mathrm{ml})$ to high $(>10 * 8 \mathrm{cop} / \mathrm{ml})$ value. The analysis of the factors which can influence steatosis development discovered the direct correlations between being overweight and steatosis presence $(r=0.686, p<0.0001)$.

Conclusions: Preliminary retrospective analysis results of cases of steatosis indicate its high incidence among patients with $\mathrm{HBV}$ infection: approximately one third of the patients have hepatic steatosis. The mechanisms which probably influence on steatosis development are metabolic factors. 
PP03-39

Clinical Importance of Serum Hepatitis B Surface Antigen Levels in Chronic Hepatitis B

S. Togo, M. Arai, A. Tawada, T. Chiba, T. Kanda, K. Fujiwara, F. Imazeki, O. Yokosuka

Chiba University, Chiba City, Japan

Aims: In this study, based on a cohort of patients with chronic hepatitis B with long term follow-up, we investigated the HBsAg levels at various stages of chronic hepatitis $B$.

Methods: A total of 424 patients who tested positive for hepatitis B surface antigen (HBsAg) and were referred to Chiba University Hospital between February 1985 and March 2008 were included in the study and the following characteristics were analyzed: age, gender, status of hepatitis B e antigen, alanine aminotransferase level, HBV DNA level, number of platelets (PLTs) and development of hepatocellular carcinoma (HCC). Measurement of HBsAg was performed using the CLEIA method.

Results: The study group consisted of 239 men and 185 women and their average age was $40.6 \pm 14.0$ years. HBsAg showed a positive correlation with HBV DNA level (Pearson product moment correlation, $\mathrm{r}=0.586, p$ $<0.001)$ and a weak inverse correlation with age $(r=0.3325, p<$ 0.001). Univariate analysis revealed that age, number of PLTs, and HBV DNA level at baseline were predictive factors for HCC occurrence, not HBsAg titer. A control study, matched with age and sex, was performed between two groups with and without $\mathrm{HBeAg}$ seroconversion during follow-up period. Compared to the age and sex matched controls, the change of HBsAg levels per year showed a significant decrease two years before seroconversion (paired $t$-test, $p<0.05$ ). We analyzed the relationship between the level of HBsAg at 140 time points and the occurrence within two years of $\mathrm{HBeAg}$ seroconversion. In 10 of 19 patients (52.6\%), when the level of HBsAg showed more than $50 \%$ decrease compared with the previous year, $\mathrm{HBeAg}$ seroconversion occurred within 2 years, which differed significantly (Chi-square test, $p=0.003$ ).

Conclusion: The serial measurement of quantitative HBsAg level has the possibility of predicting the occurrence of HBeAg seroconversion.

\section{PP03-40}

Stimulation Index of Peripheral Blood Lymphocytes Isolated from Patients with Hepatitis B

B.A. AL-Ofairy, Y. Al-Falahy, G. Mohammed, N. Al-Khalidy Medical Microbiology, IBB University, Ibb City, Yemen

Background and aim: The cellular immune response is the most important factor that contributes to eliminate the virus from infected hepatocytes, therefore, this study had been designed to evaluate the prolferative response of T-lymphocytes in response to phytohemagglutinin (PHA) in adults Iraqi patients with Hepatitis B Virus (HBV) infection. Methods: Thirty patients with HBV [mean age: $39.2 \pm 7.4$; M:F 24: 6 ] were marked by Enzyme ELISA, duration of infection and liver function tests. These patients were attended to Al-Rashid military Hospital and Gasteroenterology Hospital, Baghdad, city and they were divided into: 8 patients (mean age: $43.17 \pm 11.41$ ) with Acute Hepatitis B (AHB), 7 patients with chronic hepatitis B-healthy carrier (CHB-HC) (mean age: $35.83 \pm 2.64$ ), and 15 patients with chronic hepatitis B (CHB),. Twenty-five volunteers had been studied as healthy groups [mean age: $36.28 \pm 8.54 ; \mathrm{M}: \mathrm{F} 16: 9]$.

Results: Our results showed that cell- mediated immunity (CMI) were decreased in patients with $\mathrm{HB}$, evidenced by a significant decreased in stimulation index (SI) values of peripheral blood lymphocytes (PBLs) after activation in vitro by PHA. SI were highly significant decreased $(P<0.01)$ in $\mathrm{AHB}(3.57 \pm 0.84), \mathrm{CHB}-\mathrm{HC}(3.93 \pm 0.76)$ and $\mathrm{CHB}$ $(3 \pm 0.25)$, when compared with healthy individuals $(5.49 \pm 1.45)$. Conclusions: The depression of T-lymphocytes proliferative response to PHA indicated that CMI in HBV patients are grossly depressed. Finally for future, this assay may be useful to assess the efficiency of an immunosuppressive therapy.
Acknowledgements: We thank Dr. Ali Ragab manager of AIDsHepatitis Viral reference laboratoty for his support, Dr. Maki hamadi fayadh, manager of Gasteroenterology Hospital also for his support and Mr. Hamdi AL-Shrgabi for secretarial assistance.

Poster Presentation 04: Hepatitis B: Epidemiology

\section{PP04-01}

Risk Factors for Intrahepatic and Extrahepatic Cholangiocarcinoma: A Case-Control Study in China

L.-Y. Tao ${ }^{1}$, X.-D. $\mathrm{He}^{2}$

${ }^{1}$ China Rehabilitation Research Center; ${ }^{2}$ Peiking Union Medical College Hospital, Beijing, China

Background/aims: The risk factors for cholangiocarcinoma are incompletely defined in China, especially for intrahepatic cholangiocarcinoma (ICC). We evaluated the risk factors for both ICC and extrahepatic cholangiocarcinoma (ECC).

Methods: A case-control study in which cases were cholangiocarcinoma patients referred to Peking Union Medical College Hospital (PUMCH) between 1998 and 2008 and controls were healthy individuals. Controls were randomly selected from an existing database of healthy individuals at the Health Screening Center of PUMCH. Data on liver disease, family history, diabetes, smoking, and drinking were collected by retrospective review of the patients' records and health examination reports or by interveiw.

Results: A total of 190 patients (61 ICC; 129 ECC) and 380 age- and sex-matched controls were enrolled. Hepatitis B surface antigen (HBsAg) $(P<0.001)$ and hepatitis B core antibody (anti-HBc) without HBsAg $(P=0.001)$ were signifcantly related to ICC. The adjusted odds ratios (OR) and $95 \%$ confidence intervals $(\mathrm{CI})$ were 18.1 (95\% CI: 7.5-44.0) and 3.6 (95\% CI 1.7-7.6), respectively. Diabetes mellitus $(P=0.007)$, cholecystolithiasis $(P=0.004)$, and previous cholecystectomy $(P<0.001)$ were signifcantly associated with ECC. Prevalence of cirrhosis was higher in ICC than ECC $(P<0.001)$. Furthermore, when excluding the ICC patients with cirrhosis, ICC patients show significant independent associations with $\mathrm{HBsAg}$ (OR: 7.3; 95\% CI: 3.1-17.2) and anti-HBc without HBsAg (OR: 2.4; 95\% CI: 1.1-5.2).

Conclusion: Liver cirrhosis and chronic hepatitis B virus infection are risk factors for ICC, while cholecystolithiasis, diabetes and previous cholecystectomy are risk factors for ECC.

\section{PP04-02}

HBV Vaccination among Health Care Workers in a Comprehensive Hospital C.-S. Lin, J.-G. Li, Y.-F. Zhang, L.-H. She, Y.-T. Chong, Z.-L. Gao Department of Infectious Diseases, The Third Affiliated Hospital of Sun Yat-sen University, Guangzhou, China

Aims: This study was to determine the vaccination status of hepatitis $B$ in health-care workers ( $\mathrm{HCW}$ ), and to determine the seroprotection in different departments of $\mathrm{HCW}$.

Methods: Retrospective study of HCW vaccinated against hepatitis B between 1999 and 2009 was carried out among HCW in a comprehensive hospital. Information of vaccination status of 801 subjects (613 of them were vaccinated, 188 of them never vaccinated) were collected through a comprehensive review of hospital HCW records. Data collected included health care workers characteristics such as date of birth, sex and department of health care work.

Results: The rate of HBsAg carriers is 5.24\% (42/801). Among 188 $(23.47 \%, 188 / 801)$ subjects who did not have vaccination, $31(16.49 \%)$ were HBsAg positive, $25(13.30 \%)$ were negative of hepatitis B marker, $102(54.26 \%)$ had anti-HBs positive only, 25 (13.30\%) had positive of anti-HBs, anti-HBe and anti-HBc, $5(2.66 \%)$ subjects had anti-HBe and anti-HBc positive. Among 613 subjects who had vaccination, $34.91 \%(214 / 613)$ had anti-HBs titer lower than $10 \mathrm{mUI} / \mathrm{mL}$. 
HCW in the physician $(59.86 \%)$ had the lowest vaccination rate. The vaccination rate of department of infection was $78.75 \%$. Status of antibody produced by natural infection and by vaccination showed that HCW in the department of infection (100.0\%) had the highest rate of antibody produced by natural infection.

Conclusion: Status of hepatitis B vaccination in HCW is low in this hospital. The phenomenon of immune memory in adults is not known yet which need further study.

\section{PP04-03}

A Study of the Carriers of Hepatitis B Surface Antigen among Patients with Schistosomal Hepatic Fibrosis

A.A.E.m. Soliman ${ }^{1}$, S.A. Zaki ${ }^{2}$, R. Zaher ${ }^{1}$, M. Abou Khatwa ${ }^{2}$

${ }^{1}$ Hepatology, Faculty of Medicine; ${ }^{2}$ Microbiology, Faculty of

Medicine, Alexandria University, Alexandria, Egypt

Introduction: Hepatitis B virus infection is recognized as a major public health problem causing acute, persistant or chronic liver infection. In Egypt, hepatitis B virus infection is considered to be endemic. The attack rate of hepatitis B was estimated to reach 8.9/ 10.000 in Alexandria. Schistosomiasis is considered the major health problem in Egypt. 12-14 million people are infected with Schistosoma mansoni when Schistosomal and non- Schistosomal patients were exposed to HBV, they were found to be equally susceptible, however follow-up studies claimed that the first group of patients tend to exhibit hepatitis B antigenaemia for longer periods than the second group and this may hugely increase the reservoir of carriers.

Aim: Determine the prevalence of HBsAg carriers, demonstration of routes of antigen excretion and its presence in liver sections\&the prevelent subtype.

Subjects and methods: Forty carriers of the hepatitis B surface antigen were selected from 539 cases of Schistosomal hepatic fibrosis. A. Detection of HBsAg in patients sera, Saliva, Urine and Stools\& Anti-HBcAg IgM.

B. Demonstration of HBsAg in infected hepatocytes.

C. Subtyping of HBsAg using the counter immuno-electrophoresis. Results:

- Hepatitis B surface antigen carriers was7.42\%, Anti-HBsAg IgM was negative.

- $5 \%$ of the salivary samples were HBsAg positive, while $50 \%$ of the Urine and Stool were HBsAg positive.

- Urine and Saliva positivity ran parallel.

- Stool samples positive for HBsAg were all positive for occult blood.

- Subtyping of the HBsAg for showed the "ay" subspecificity which is considered the constant type of North Africa.

Conclusion: Screening of Schistosomal patients for the HBs antigen is of importance as they represent a highly disseminating source of infection. (ay) is the predominant subtype of HBs Ag. Disinfection of secretions and excretions of HBsAg positive patients and their utensils is essential.

\section{PP04-04}

Molecular Characterization of HBV Strains in Morocco

W. Baha ${ }^{1,2}$, N. Dersi ${ }^{1}$, F. Lazaar ${ }^{1}$, A. El Malki ${ }^{1}$, H. Mohammed ${ }^{1}$, A. Bennani ${ }^{1}$ ${ }^{I}$ Medical Biology, Pasteur Institute of Morocco,

Casablanca, ${ }^{2}$ Faculté des Sciences et Techniques Mohammedia, University Hassan II, Mohemmadia, Morocco

Despite the availability of efficient vaccine, hepatitis B virus (HBV) infection remains one of the major causes of liver disease. Eight HBV genotypes have been identified with a distinct geographical distribution. Previous studies suggest that HBV genotype is related to severity of liver disease and treatment response. The aim of this study was to determine HBV genotypes in Moroccan patients and to characterize their precore (PC) and core promotor (CP) mutations. Serum samples from patients with chronic HBV infection were analyzed in this study.
Viral genotypes were determined using Inno-LiPA, PCR using specific primers, Multiplex-PCR and PCR-RFLP methodologies. Precore and core promoter mutation characterization was performed by line probe assay. Overall, HBV genotype D was found in $96.5 \%$ of paitents, HBV genotypes $\mathrm{A}$ was found in $2.12 \%$ and mixed genotype $\mathrm{D}$ and $\mathrm{F}$ was found in $1.4 \%$. The wild type, mixed infection, BCP mutations and precore mutant were found in $18.2,61.8,7.27$ and $12.7 \%$, respectively. The A1762T/G1764A BCP dual mutation was not found in our isolates. Four samples presented single mutation in the BCP dual mutation region, whereas six showed a novel G1764T mutation. HBV genotype D predominates in Morocco as in Mediterranean countries, genotypes $\mathrm{A}$ and $\mathrm{F}$ are quite rare; these are possibly acquired from other countries. Moroccan patients with chronic hepatitis B still represent a rather homogenous group with genotypic diversity rarely encountered. High circulation of precore and basal core promoter mutants is common in chronic hepatitis B infection in Morocco.

\section{PP04-05}

The Effect of Caffeine and Alcohol Consumption on Liver Fibrosis: A Study of 1045 Hepatitis B Patients Using Transient Elastography A. Ong ${ }^{1,2}$, V.W.S. Wong ${ }^{1}$, G.L.H. Wong ${ }^{1}$, H.L.Y. Chan ${ }^{1}$

${ }^{I}$ The Chinese University of Hong Kong, Shatin N.T., Hong Kong SAR; ${ }^{2}$ University of Santo Tomas Hospital, Manila, Philippines

Background: In patients with chronic hepatitis C, caffeine consumption is associated with reduced risk of hepatocellular carcinoma and cirrhosis. Its role in chronic hepatitis $\mathrm{B}(\mathrm{CHB})$ and the interaction with alcohol consumption are unclear.

Objective: This study aimed to investigate the relationship between caffeine and alcohol consumption and liver stiffness in CHB patients. Methods: $\mathrm{CHB}$ patients who underwent transient elastography examination in 2006-2008 were studied. Advanced fibrosis was defined as liver stiffness $>9 \mathrm{kPa}$ (normal ALT) or $>12 \mathrm{kPa}$ (elevated ALT). Caffeine and alcohol consumption was recorded using a standardized questionnaire. Excessive alcohol intake was defined as $30 \mathrm{~g}$ per day in men and $20 \mathrm{~g}$ per day in women.

Results: Among 1,045 patients who completed the questionnaire $(652$ males, age $46.5 \pm 12.2$ years), the liver stiffness was $8.3 \pm 6.2 \mathrm{kPa}$. $216(20.7 \%)$ patients had advanced fibrosis. $499(47.8 \%)$ patients drank 1 cup of coffee or more per day. $95(19.0 \%)$ patients who drank $\geq 1$ cup of coffee had advanced fibrosis, compared to $121(22.2 \%)$ patients who drank $<1$ cup $(P=0.21)$. The liver stiffness of patients who did not drink coffee and those who drank $<1,1-2$, and $\geq 2$ cups was $8.0 \pm 6.5,8.4 \pm 6.6,8.4 \pm 6.1$ and $8.2 \pm 5.5 \mathrm{kPa}$, respectively $(P=0.96)$. The amount of caffeine intake had positive correlation with the amount of alcohol intake $(R=0.167, P<0.001)$. Although $231(22.1 \%)$ patients reported alcohol consumption, only $11(1 \%)$ had excessive alcohol intake. The prevalence of advanced fibrosis among patients with mild to moderate alcohol intake $(26,18.8 \%)$ was comparable to that among non-drinkers $(190,21.0 \%)(P=0.57)$.

Conclusion: Caffeine intake does not affect liver stiffness in $\mathrm{CHB}$ patients. Patients who drink coffee regularly tend to drink alcohol. However, most CHB patients do not have excessive alcohol consumption. Mild to moderate alcohol intake does not increase the risk of advanced fibrosis.

\section{PP04-06}

Chronic Hepatitis B in Children: Retrospective Study of 46 Cases S. Berkane ${ }^{1}$, Y. Chikhi ${ }^{1}$, S. Cheraitia ${ }^{1}$, N. Ali Arous ${ }^{1}$, F. Lounes ${ }^{1}$, F. Iguer ${ }^{2}$, A. Bensalem ${ }^{2}$, S. Ait Younes ${ }^{3}$, Z.C. Amir ${ }^{3}$, F. Asselah ${ }^{3}$, H. Asselah ${ }^{1}$

${ }^{1}$ Hepatogastroenterology, Bologhine Hospital, Algiers University;

${ }^{2}$ Virology, Pasteur Institut of Algiers; ${ }^{3}$ Pathological Anatomy, Mustapha Hospital, Algiers University, Algiers, Algeria

Objectives: Algeria belongs to regions of an intermediate HBV endemicity. The prevalence of serique Hbs-Ag is $2.18 \%$ (National Survey study 1998). The systematic vaccination against hepatitis B of all new born has started only since July 2003. The main genotype is D 
in the adult population (94\%). We report clinical characteristics, therapeutic and outcomes of chronic hepatitis B in Algerian children. Method: Retrospective study of 46 children with chronic hepatitis B, included from January 1997 to December 2009.

Results: 46 children HBs-Ag positive recruited, medium age of 10.8 years [03-17], sex-ratio $1,8(30 \mathrm{H}$ and $16 \mathrm{~F})$. The Diagnosis is elaborated after family screening $(n=31)$, jaundice $(n=05)$, asthenia $(n=2)$, raised ALAT $(n=2)$, risk factors $(n=3)$, and blood donor, work check, and pre operatory check in one case, respectively. Transmission modes was: family contamination $(n=20)$, nosocomial $(n=19)$, no transmission mode identified $(n=7)$. 26 children $(56,5 \%)$ were $\mathrm{HBe}-\mathrm{Ag}$ positive, medium age 15,3 [3-17ans] and 20 (43.4\%) were $\mathrm{HBe}-\mathrm{Ag}$ negative, medium age 13.5 years [5-17]. 12 children underwent hepatic biopsy (10 Hbe Ag $+)$ which revealed stage fibrosis F1 $(n=5)$, F2 $(n=4)$ and F3 $(n=3)$. After a median follow-up of 52,6 months [1-152]; 08 children were treated, all HBe-Ag positive by lamivudine $(n=3)$, Lam $+\operatorname{ADV}(n=1)$, standard interferon $(n=3)$, Pegylated interferon $(n=1)$. Hbe-Ag seroconversion was obtained in 4 children.

Conclusion: In the Algerian children, the HVB infection spread essentially through perinatal transmission or by nosocomial way. By opposite in Asian, at diagnosis; 43\% (20 patients) have already spontaneously $\mathrm{HBe}-\mathrm{Ag}$ seroconverted with a medium age of 13 years. This study underline the interest to use appropriate surveillance, monitoring, and timely referral the children Hbs-Ag positive.

\section{PP04-07}

Association of Metabolic Profiles with Hepatitis B Virus Infection and Modifying Factors

C.-S. Hsu ${ }^{1,2,3}$, C.-H. Liu ${ }^{1}$, C.-C. Wang ${ }^{2,3}$, T.-C. Tseng ${ }^{1,2}$, C.-J. Liu ${ }^{1}$,

C.-L. Chen ${ }^{1}$, P.-J. Chen ${ }^{1}$, D.-S. Chen ${ }^{1}$, J.-H. Kao ${ }^{1}$

${ }^{1}$ National Taiwan University College of Medicine; ${ }^{2}$ Buddhist Tzu Chi General Hospital, Taipei Branch, Taipei; ${ }^{3}$ Tzu Chi University, Hualien, Taiwan ROC

Background: Metabolic syndrome may increase the risk of hepatocellular carcinoma and disease progression in chronic hepatitis $\mathrm{C}$ patients. However, the interactions between hepatitis B virus (HBV) infection and metabolic factors remain unknown. We aimed to investigate the association of HBV infection with metabolic profiles in subjects with and without chronic HBV infection, and the impacts of serum HBV DNA level on metabolic profiles from a non-obese, non-diabetes and non-cirrhotic HBV cohort.

Methods: First, a case-control analysis of HBV infected and noninfected subjects was performed. The HBV Group consisted of 365 patients with chronic HBV infection, and the Control Group consisted of 1,049 age and body mass index (BMI) matched subjects without chronic HBV infection. Fasting blood glucose, lipid profiles and adiponectin levels were compared. Second, a retrospective cohort study was performed in 122 non-obese, non-diabetes and non-cirrhotic chronic hepatitis B patients with available serum HBV DNA level and HOMA-IR index.

Results: In the case-control analysis, the HBV Group had significantly higher serum adiponectin, but lower triglyceride (TG) and HDL levels than the Control Group. These relationships already existed in subjects younger than 45 years of age and were modified by serum ALT levels. In the retrospective cohort, serum HBV DNA levels were negatively proportional to TG levels, but not to other metabolic parameters. Moreover, this relationship was more significant in subjects with higher ALT levels.

Conclusions: Patients with chronic HBV infection have higher adiponectin but lower TG and HDL levels. Hepatitis B viral load is negatively proportional to serum TG levels. All these relationships are modified by ALT levels and already exist in middle-aged HBV carriers, implying a possible interaction between chronic HBV infection and metabolic factors early in life.
PP04-08

Study on Hepatitis Viral Infection among General Population in Cambodia

M. Fujimoto ${ }^{1}$, J. Matsuo ${ }^{1}$, A. Tabuchi ${ }^{1}$, K. Katayama ${ }^{1}$, A. Nakashima ${ }^{1}$, T. Akita ${ }^{1}$, S.H. Do ${ }^{1}$, S. Svay ${ }^{2}$, J. Tanaka ${ }^{1}$

${ }^{1}$ Epidemiology, Infectious Disease Control and Prevention, Graduate School of Biomedical Sciences, Hiroshima University, Hiroshima,

Japan; ${ }^{2}$ Communicable Diseases Control Bereau, Ministry of Health, Phnom Penh, Cambodia

Aim: We aimed to recognize the prevalence of hepatitis $B$ virus (HBV) and hepatitis $\mathrm{C}$ virus (HCV) in Cambodia which might be one of Asian areas with high prevalence of $\mathrm{HBV}$. And in cooperation with Ministry of Health in Cambodia, we aimed to prevent from the infection of hepatitis viruses among general population.

Method: Sero-epidemiological survey with questionnaires about health and life were investigated in Siem Reap province, Cambodia from Feb. to Aug. 2010. All blood samples were tested for hepatitis viral markers and were determined the quantity of HBV DNA and HCV RNA with real-time PCR and their genotyping. This study is approved by the ethical committee of Hiroshima University and that of Ministry of Health in Cambodia.

Results:

1. Total subjects were 267 (120 male; 38.6 y.o. and 147 female; 40.6 y.o.), who are teachers and parents in Elementary School and people in Rohal and Chrey village.

2. 20 cases $(7.5 \%)$ were positive for both HBsAg and HBV DNA. Positive rate of HBsAg in male (10.0\%: $N=12,41.2$ y.o.) is higher than female (5.4\%: $N=8,43.4$ y.o.). There is no difference between age groups.

3. Of 20 cases with HBV DNA, 18 cases were genotype $\mathrm{C}$ and 2 cases could not be determined for genotype by EIA. According to the result of "phylogenetic tree analysis" using 4 cases of with genotype C, their type might be "China-group" and "Australiagroup". Further analysis is continued.

4. 17 cases $(6.4 \%)$ were positive for anti HCV, and 7 (2.6\%) of them were detected HCV RNA. There is no difference between sex (male $2.5 \%$, female $2.7 \%$ )

Conclusion: In this pilot study the prevalence of HBV and HCV were not so high as it is predicted. Further results will be presented such as detailed genotypes and the background of carriers and also the relation to HCC mortality in this country.

\section{PP04-09}

Patients with Negative HBeAg and Low Viraemia Can Progress to Active Disease K. Lam, A.J. Hui, R.S.Y. Chan, K.P.K. Tsang, G.S.P. Leung, W.H. Poon, N.W.Y. Leung

Medicine, Alice Ho Miu Ling Nethersole Hospital, Hong Kong S.A.R., Hong Kong SAR

Introduction: Previous reports suggested an overall $17 \%$ of $\mathrm{HBeAg}$ negative patients were viremic with evidence of chronic liver disease (e-CHB). However our previous cross sectional study showed that serum HBV DNA below 20,000 IU/mL does not exclude e-CHB.

Aim: To prospectively monitor serum HBV DNA and ALT levels in patients with negative $\mathrm{HBeAg}$ and low viramia.

Method: From July 2009, all patients with negative $\mathrm{HBeAg}$ and serum HBV DNA level below 20,000 IU/mL were monitored at 3 monthly intervals with serum ALT and HBV DNA. These patients have normal liver on ultrasonography and no co-morbidity. HBV DNA is measured by RT-PCR assay with lower limit of detectaion $<12 \mathrm{IU} / \mathrm{mL}$.

Results: - $105 \mathrm{CHB}$ patients (59 males median age 40 years, range 25-69 and 52 females median age 38 , range $23-76$ were studies.

- 11 had 3; 32 had 4, and 51 had 5 paired measurements of serum HBV DNA and ALT. 
- Serum HBV DNA rose above $20.000 \mathrm{IU} / \mathrm{mL}$.in 5 male and 4 female patients (annual incident $9.6 \%$ per patient year.)

1. M34 HBV DNA fluctuated from 36 to $380,000,000 \mathrm{IU} / \mathrm{mL}$, and ALT flared to 1,975 IU/L. He was started antiviral therapy immediately.

2. F38 HBV DNA fluctuated between 14,400 to $4,000,000 \mathrm{IU} / \mathrm{mL}$ and ALT rose to $114 \mathrm{IU} / \mathrm{L}$. She preferred traditional Chinese medicine.

3. M33 HBV DNA fluctuated from 2,800 to $640,000 \mathrm{IU} / \mathrm{mL}$ and ALT rose to $88 \mathrm{IU} / \mathrm{L}$. He was planning family and deferred therapy.

4. M36 HBV DNA fluctuated from 124 to $15,200,000 \mathrm{IU} / \mathrm{mL}$ and ALT rose to $232 \mathrm{IU} / \mathrm{L}$. He was planning family and deferred therapy. Conclusion: This prospective study showed that patients with negative $\mathrm{HBeAg}$ need careful assessment and regular monitor for $\mathrm{HBV}$ DNA and ALT to identify e CHB. Even initial low viraemic patients have risk of progressing from quiescent $\mathrm{CHB}$ to e-CHB.

\section{PP04-10}

Hepatitis B Virus Infection in Children of Chronic Hepatitis B Patients in Jakarta

M.Y. Iskandar ${ }^{1}$, H. Gunardi ${ }^{1}$, P. Gayatri ${ }^{1}$, D.H. Muljono ${ }^{2}$

${ }^{I}$ Department of Pediatrics, University of Indonesia; ${ }^{2}$ Eijkman

Institute of Molecular Biology, Jakarta, Indonesia

Background: Hepatitis B is a serious global public health problem. Chronic carrier of hepatitis B virus (HBV) is a potential source of HBV intrafamilial transmission. Nonetheless, the route of viral transmission in Indonesian family has not been reported, especially in Jakarta.

Objectives: To determine the prevalence of HBV infection, the possible routes and risk factors for HBV transmission in children of chronic hepatitis B patients in Jakarta.

Methods: An analytic cross-sectional study was conducted at Cipto Mangunkusumo Hospital from October-December 2009. Children aged 1-18 year old of chronic hepatitis B patients with hepatitis B surface antigen ( $\mathrm{HBsAg}$ ) positive (index case) were investigated for hepatitis B serological markers (HBsAg, anti-HBs, anti-HBc). A guided-questionnaire about demographic and medical information and risk factors of HBV transmission in family was applied.

Results: A total of 94 children of 64 index cases were enrolled. The prevalence of HBV infection was $20.2 \%$ (19/94) with 10 (10/19) of them were still positive for HBsAg in their blood. There was a tendency towards an increased prevalence of HBV infection in these children with advancing age. This suggested that horizontal transmission happened among them. Risk factors for HBV infection in family were having HBsAg positive sibling [OR 10.35 (IK95\% 2.62;40.84, $p=0.001$ )], both parents with HBsAg positive $(p<0.001)$, and family with $\mathrm{HBsAg}$ positive in two or more other members $(p<0.001)$.

Conclusions: High risk of HBV infection was found among children of HBsAg positive chronic hepatitis B patient. Horizontal transmission (parents-to-child, sibling-to-sibling) participated in intrafamilial transmission of hepatitis B.

\section{PP04-11}

Tumor Necrosis Factor- $\alpha-238$ A Allele Elevates the Risk of Chronic HBV Infection in European Populations

M.-H. Zheng ${ }^{1}$, D.-D. Xiao ${ }^{2}$, X.-F. Lin $^{2}$, M.-M. Peng ${ }^{2}$, K.-Q. Shi ${ }^{1}$, Y.-C. Fan ${ }^{3}$, Y.-P. Chen ${ }^{1}$

${ }^{I}$ Department of Infection and Liver Diseases, Liver Research Center, The First Affiliated Hospital of Wenzhou Medical College; ${ }^{2}$ School of the First Clinical Medical Sciences, Wenzhou Medical College,

Wenzhou; ${ }^{3}$ Department of Hepatology, Qilu Hospital of Shandong University, Jinan, China

Background and aims: Tumor necrosis factor- $\alpha$ (TNF- $\alpha$ ) plays a pivotal role in the viral clearance and host immune response to HBV, and the capacity for TNF- $\alpha$ production in individuals is influenced by

Table 1 Main results of pooled ORs in the meta-analysis when compared with spontaneously recovered population

\begin{tabular}{|c|c|c|c|c|c|c|}
\hline SNPs & Ethnicity & & Genetic model & OR $(95 \% \mathrm{CI})$ & $P$ & $P(\mathrm{Q}$-test $)$ \\
\hline TNF- $\alpha$ & Total & Co-dominant model & GA vs GG & $1.05(0.71,1.53)$ & 0.819 & 0.0001 \\
\hline \multirow[t]{11}{*}{-238} & & & AA vs GG & $1.13(0.41,3.15)$ & 0.809 & 0.971 \\
\hline & & Dominant model & $\mathrm{GA}+\mathrm{AA}$ vs GG & $1.05(0.73,1.53)$ & 0.778 & 0.0001 \\
\hline & & Recessive model & $\mathrm{AA}$ vs $\mathrm{GG}+\mathrm{GA}$ & $1.08(039,298)$ & 0.887 & 0.956 \\
\hline & Asian & Co-dominant model & GA vs GG & $0.95(0.64,1.42)$ & 0.806 & 0.001 \\
\hline & & & AA vs GG & $1.21(037,3.95)$ & 0.754 & 0.892 \\
\hline & & Dominant model & $\mathrm{GA}+\mathrm{AA}$ vs GG & $0.95(0.64,1.41)$ & 0.802 & 0.623 \\
\hline & & Recessive model & AA vs GG + GA & $1.18(036,3.86)$ & 0.785 & 0.904 \\
\hline & European & Co-dominant model & GA vs GG & $3.01(049,18.60)$ & 0.235 & 0.092 \\
\hline & & & AA vs GG & $0.93(0.12,7.19)$ & 0.946 & 0.623 \\
\hline & & Dominant model & $\mathrm{GA}+\mathrm{AA}$ vs GG & $2.22(1.07,458)$ & 0.032 & 0.183 \\
\hline & & Recessive model & AA vs GG + GA & $0.81(0.11,6.16)$ & 0.839 & 0.558 \\
\hline TNF- $\alpha$ & Total & Co-dominant model & $\mathrm{CA}$ vs $\mathrm{CC}$ & $1.05(0.79,1.39)$ & 0.741 & 0.001 \\
\hline \multirow[t]{11}{*}{-863} & & & $\mathrm{AA}$ vs $\mathrm{CC}$ & $0.96(0.76,1.22)$ & 0.748 & 0.356 \\
\hline & & Dominant model & $\mathrm{CA}+\mathrm{AA}$ vs $\mathrm{CC}$ & $1.03(0.82,1.29)$ & 0.808 & 0.007 \\
\hline & & Recessive model & $\mathrm{AA}$ vs $\mathrm{CC}+\mathrm{CA}$ & $1.05(086,128)$ & 0.655 & 0.177 \\
\hline & Asian & Co-dominant model & $\mathrm{CA}$ vs $\mathrm{CC}$ & $1.04(0$ 77, 1.43) & 0.782 & 0.0001 \\
\hline & & & $\mathrm{AA}$ vs $\mathrm{CC}$ & $0.95(0.74,1.21)$ & 0.673 & 0.300 \\
\hline & & Dominant model & $\mathrm{CA}+\mathrm{AA}$ vs $\mathrm{CC}$ & $1.02(0.79,1.31)$ & 0.882 & 0.004 \\
\hline & & Recessive model & $\mathrm{AA}$ vs $\mathrm{CC}+\mathrm{CA}$ & $1.04(0.85,1.28)$ & 0.709 & 0.131 \\
\hline & European & Co-dominant model & $\mathrm{CA}$ vs $\mathrm{CC}$ & $1.08(0.63,1.86)$ & 0.771 & - \\
\hline & & & $\mathrm{AA}$ vs $\mathrm{CC}$ & $1.63(0.32,8.28)$ & 0.559 & - \\
\hline & & Dominant model & $\mathrm{CA}+\mathrm{AA}$ vs $\mathrm{CC}$ & $1.12(0.66,1.89)$ & 0.676 & - \\
\hline & & Recessive model & $\mathrm{AA}$ vs $\mathrm{CC}+\mathrm{CA}$ & $1.58(0.31,8.00)$ & 0.578 & - \\
\hline
\end{tabular}

$\overline{S N P}$ single nucleotide polymorphism, $O R$ odds ratio, $C I$ confidence interval. $P(Q$-text $) P$ value of Q-test for hetterogeneity test, a random-effects model was used when $P$ value for heterogeneity test $<0.1$; otherwise, a fixed-effects model was used 
a major genetic component. The studies of TNF- $\alpha-238$ and 863 gene promoter polymorphism in chronic HBV infection have reported apparently conflicting results. We aimed to derive a more precise estimation of the relationship between the polymorphism of TNF- $\alpha$ 238 and 863 gene promoter and chronic HBV infection.

Methods: Nineteen studies including 5,245 chronic HBV infection cases and 3,181 controls described G238A genotypes, and 11 studies totaling 3,576 cases and 2,044 controls described C863A genotypes, were involved in the meta-analysis.

Results: The overall meta-analysis did not suggest a significant association of TNF- $\alpha-238$ and TNF- $\alpha-863$ gene promoter polymorphisms with chronic HBV infection (Table 1). However, in subgroup analysis by ethnicity, it indicated that 238A allele carriers (GA + AA) in European populations had an increased risk of developing chronic HBV infection $(\mathrm{OR}=2.22,95 \%$ CI: $1.07-4.58, \quad P=0.032$; $\mathrm{OR}=4.46,95 \%$ CI: $1.75-11.38, P=0.002$, respectively), when compared with spontaneously recovered population and healthy population, respectively. However, no significant associations were found in Asian populations in all genetic models.

Conclusion: TNF- $\alpha-238$ A allele elevates the risk of chronic HBV infection in European populations.

\section{PP04-12}

A Study on Prevalence of Antiviral Resistance Mutations in Patients with Chronic Hepatitis B in Four Northern Cities of China

C.-H. Yan, H. Ding, C.-Y. Zhao, L. Yan, B.-M. Liu, J.-X. Yang, X.-E. Liu, T. Li, H. Zhuang

Department of Microbiology, Peking University Health Science Center, Beijing, China

Introduction: Since 1999 lamivudine as the first nucleos(t)ide analogue (NA) was approved for treating chronic hepatitis B (CHB) in China, adefovir, entecavir and telbivudine have been available in clinics successively.

Aim: This study aims to analyze the prevalences of NA resistance (NAr) mutations in NA-treated and untreated CHB patients in four northern cities of China.

Methods: Six hundred and thirty-two full-length hepatitis B virus (HBV) reverse transcriptase (RT) sequences from the database of the Reference Laboratory for Viral Hepatitis of Peking University, Beijing, China were used for analyses. The RT sequences were isolated from 272 untreated and 360 NA-treated CHB patients attending five clinics in four northern cities of China (218 from Beijing, 350 from Qinhuangdao, 34 from Jining and 30 from Urumqi) between January 2007 and May 2010. Eleven classical NAr sites were analyzed and the results on rtA181T/V/S, rtM204I/V and rtN236T were reported here.
Results: The treatment regimes included NA mono-, add-on and switch-to therapies. The percentage of NA-treated patients with lamivudine, adefovir, entecavir and telbivudine exposure were $55.56 \%$ (200/360), $47.78 \%$ (172/360), 8.06\% (29/360) and 6.94\% (25/360), respectively. No NAr mutations were found in untreated group. The prevalence of NAr mutations in NA-treated group in the four northern cities of China ranged from 29.67 to $40.00 \%$, and had no statistically significant differences among cities (see Table 1).

Conclusions: RtM204I/V was predominant in accordance with high percentage of lamivudine use for NA-treated patients in four northern cities of China.

Acknowledgement: China National Grants 2008ZX10002-004 and 2009ZX10004-314.

\section{PP04-13}

Prevalence and Risk Factors of Viral B and C Hepatitis among Tunisian Inflammatory Bowel Disease: A Case Control Study

L. Kallel ${ }^{1}$, M. Fekih ${ }^{2}$, A. Ouaz ${ }^{2}$, N. Ben Mustapha ${ }^{2}$, S. Karoui ${ }^{2}$, M. Serghini², J. Boubaker ${ }^{2}$, A. Filali ${ }^{2}$

${ }^{1}$ Gastroenterology A; ${ }^{2}$ Rabta Hospital, Tunis, Tunisia

Background: Data regarding prevalence of hepatitis C (HCV) and hepatitis $\mathrm{B}(\mathrm{HBV})$ in inflammatory bowel disease (IBD) patients remains controversial.

Methods: We conducted a case control study that included 150 IBD (126 Crohn's disease [CD] and 24 ulcerative colitis [UC]) patients as well as 300 age and sex matched healthy controls recruited from blood donors.

Results: Patient's median age was 37 years (16-73 years) and median disease duration was 4.75 years ( 4 months- 28 years). Present and/or past HBV and HVC infection was found in $20 \%$ of the patients (HBsAg: $4.7 \%(n=6)$, anti-HBc: $20 \%(n=30)$, Ac anti-HBs: $8.5 \%$ $(n=13)$, Ac anti-HBe: 9.3\%, Ag HBe: $0 \%)$ and in $2 \%(n=3)$, respectively. among controls, $1.66 \%(p=0.06)$ had $\mathrm{HBsAg}$ and $0.33 \%(p=0.07)$ had anti-HVC. Among Ag HBs positive patients, HVB DNA was below $<2,000 \mathrm{IU} / \mathrm{ml}$ in 6 cases. The 7 th patient had elevated DNA level but no significant hepatic lesions (A1F0 of Metavir score). Among patients with negative $\mathrm{HBs} \mathrm{Ag}$ but positive anti-HBc, 3 had positive VHB DNA but with low load viral. Among these 10 viremic patients, 3 were receving azathioprine while no patient anti TNF therapy. No specific HVB treatment was conducted for all these patients while regular DNA HVB monitoring was instituted. Among the 3 patients with anti-HVC, 2 were viremics, of $1 \mathrm{~b}$ genotype but without significant fibrosis. When comparing patients having at least one positive viral marker with negative marker patients, we find in multivariate analysis, that age at inclusion over 35

Table 1 The prevalence offtAr mutations in TvA-treated CHB patients in four northern cities of China

\begin{tabular}{|c|c|c|c|c|c|}
\hline NAr mutation patterns & $\begin{array}{l}\text { Beijing \% } \\
(n / n)\end{array}$ & $\begin{array}{l}\text { Qihuangdao, } \\
\text { Hebei \% }(n / n)\end{array}$ & $\begin{array}{l}\text { Jining, Shandong } \\
\%(n / n)\end{array}$ & $\begin{array}{l}\text { Urumqi, Xinjiang } \\
\%(n / n)\end{array}$ & Total $\%(n / n)$ \\
\hline $\mathrm{rtM} 204 \mathrm{I} / \mathrm{V}$ & $17.58(16 / 91)^{\mathrm{a}}$ & $20.18(45 / 223)$ & $12.50(2 / 16)^{\mathrm{a}}$ & $30.00(9 / 30)^{\mathrm{a}}$ & $20.00(72 / 360)$ \\
\hline $\mathrm{rtA} 181 \mathrm{~T} / \mathrm{V} / \mathrm{S}$ & $7.69\{7 / 91)^{\mathrm{b}}$ & $5.83(13 / 223)^{\mathrm{b} *}$ & $18.75(3 / 16)^{\mathrm{b} *}$ & $3.33(1 / 30)^{\mathrm{b}}$ & $6.67(24 / 360)$ \\
\hline $\mathrm{rtN} 236 \mathrm{~T}$ & $0.00(0 / 91)^{\mathrm{c}}$ & $1.79(4 / 223)^{\mathrm{c}}$ & $0.00(0 / 16)^{\mathrm{c}}$ & $0.00(0 / 30)^{\mathrm{c}}$ & $1.11(4 / 360)$ \\
\hline $\mathrm{rtM} 204 \mathrm{I} / \mathrm{V}+\mathrm{rtA} 181 \mathrm{~T} / \mathrm{V} / \mathrm{S}$ & $2.20(2 / 91)^{\mathrm{d} *}$ & $0.00(0 / 223)^{\mathrm{d}_{* \#}}$ & $0.00(0 / 16)^{\mathrm{d}}$ & $3.33(1 / 30)^{\mathrm{d} \#}$ & $0.83(3 / 360)$ \\
\hline $\mathrm{rtN} 236 \mathrm{~T}+\mathrm{rtA} 181 \mathrm{~T} / \mathrm{V} / \mathrm{S}+$ & $1.10(1 / 91)^{\mathrm{e}}$ & $3.59(3 / 223)^{\mathrm{e}}$ & $0.00(0 / 16)^{\mathrm{e}}$ & $3.33(1 / 30)^{\mathrm{e}}$ & $2.78(10 / 360)$ \\
\hline $\mathrm{rtM} 204 \mathrm{I} / \mathrm{V}+\mathrm{rtA} 181 \mathrm{~T} / \mathrm{V} / \mathrm{S}+\mathrm{rtN} 236 \mathrm{~T}$ & $1.10(1 / 91)^{\mathrm{f}}$ & $1.35(3 / 223)^{\mathrm{f}}$ & $0.00(0 / 16)^{\mathrm{f}}$ & $0.00(0 / 30)^{\mathrm{f}}$ & $1.11(4 / 360)$ \\
\hline Total & $29.67(27 / 91)^{\mathrm{g}}$ & $32.74(73 / 223)^{\mathrm{g}}$ & $31.25(5 / 16)^{\mathrm{g}}$ & $40.00(12 / 30)^{\mathrm{g}}$ & $32.50(117 / 360)$ \\
\hline
\end{tabular}

${ }^{\mathrm{a}} \chi^{2}=2.7744, \quad P=0.4277 ;{ }^{\mathrm{b}} \chi^{2}=4.6951, \quad P=0.1955 ; \quad{ }^{\mathrm{c}} \chi^{2}=2.4850, \quad P=0.4780 ;{ }^{\mathrm{d}} \chi^{2}=\quad 6.3275, \quad P=0.0967 ;{ }^{\mathrm{e}} \chi^{2}=1.9825$, $P=0.5760 ;{ }^{\mathrm{f}} \chi^{2}=0.6283, P=0.8899 ;{ }^{\mathrm{g}} \chi^{2}=1.1184, P=0.7726$

*,\# Indicating a statistically significant difference $(P<0.05)$. rtA181T/V/S: Qinhuangdao vs. Jining, $\chi^{2}=3.9898, P=0.0458 ; \mathrm{rtM} 204 \mathrm{I} /$ $\mathrm{V}+\mathrm{rtA} 181 \mathrm{~T} / \mathrm{V} / \mathrm{S}$ : Beijing vs. Qinhuangdao, $\chi^{2}=4.9325, P=0.0264$; Qinhuangdao vs. Urumqi, $\chi^{2}=7.4628, P=0.0063$ 
years (odds ratio [OR] 3.26; 95\% confidence interval [CI] 1.25-7.29; $P=0.032$ ) and history of blood transfusion (OR 5.25; 95\% CI $1-27.57 ; P=0.05$ ) were the only hepatitis risk factors.

Conclusions: Prevalence of HBV and HCV infection in Tunisian IBD patients was rather superior to general population. No patient was had HVB vaccination.

PP04-14

Estimating the Cost of Mass Vaccination Campaign against Hepatitis B in Iranian Adolescents

B. Hajarizadeh ${ }^{1,2}$, A. Rashidian ${ }^{2}$, A.A. Haghdoost ${ }^{3}$, S.M. Alavian ${ }^{4}$

${ }^{1}$ Australian Research Centre in Sex, Health and Society, La Trobe

University, Melbourne, VIC, Australia; ${ }^{2}$ Tehran University of

Medical Sciences, Tehran; ${ }^{3}$ Kerman University of Medical Sciences,

Kerman; ${ }^{4}$ Baqiyatallah Research Center for Gastroenterology and

Liver Disease, Tehran, Iran

Introduction: In order to extend hepatitis B virus (HBV) immunization to twenty-five-year old adolescents in Iran, Ministry of Health and Medical Education (MOH\&ME) implemented HBV mass vaccination campaigns covering adolescents born in 1989-1992. The first three-round campaign of this series was implemented in 2007.

Objective: This study conducted a cost estimate and analysis of this campaign.

Methods: This cost analysis was conducted applying health system perspective. The cost variables considered in this study were recurrent costs, personnel costs, publicity costs, transportation costs and overhead costs. Data to estimate recurrent, national-level supervision and publicity costs was obtained from existing data and through interview with experts in MOH\&ME. To estimate vaccine administration, provincial supervision and outreach teams ${ }^{\prime}$ costs, data was gathered from specific provinces, and then expanded the results to the country. Transportation and overhead costs were estimated using WHO recommended proportions.

Results: Total cost for three rounds of the campaign was estimated as US\$ 8.991 million including $24 \%$ for recurrent costs, $42 \%$ for personnel costs, $31 \%$ for transportation costs, $0.1 \%$ for publicity costs and $3 \%$ for overhead costs. Total cost was estimated as US\$ 2.3 per dose administered. Sensitivity analysis indicated that increasing campaign coverage and using under 20 year-old vaccine dosage will decrease the cost to US\$ 1.9 per dose administered. On the other hand, decreasing campaign coverage and using above 20 year-old vaccine dosage will increase the cost to US\$2.5.

Conclusion: The costs in this campaign are much lower than in similar programs in developed countries and could be further decreased in some alternative scenarios. Publicity costs have been too low and needs more attention in future campaigns.

\section{PP04-15}

Comparing the Cost-effectiveness of Three-versus Two-dose Vaccination Schedule against Hepatitis B in Adolescents. Experience of a Developing Country

B. Hajarizadeh ${ }^{1,2}$, A. Rashidian ${ }^{2}$, A.A. Haghdoost ${ }^{3}$, S.M. Alavian ${ }^{4}$ ${ }^{1}$ Australian Research Centre in Sex, Health and Society, La Trobe University, Melbourne, VIC, Australia; ${ }^{2}$ Tehran University of Medical Sciences, Tehran; ${ }^{3}$ Kerman University of Medical Sciences, Kerman; ${ }^{4}$ Baqiyatallah Research Center for Gastroenterology and Liver Disease, Tehran, Iran

Introduction: A three-dose regimen is the usual protocol for hepatitis $B$ virus (HBV) vaccination. An alternate two-dose vaccination schedule was approved by FDA for adolescents affecting the compliance, effectiveness and the costs of implementing the campaign.

Objectives: This study compared the cost-effectiveness of the threeversus two-dose HBV vaccination regimen in adolescents in Iran as a model of a developing country with established primary health care network.
Methods: We attempted to estimate the costs from the perspective of the health care providers. A decision analysis model was developed to compare effectiveness between the two schedules. In terms of cost and compliance we used data from a national HBV mass vaccination campaign for adolescents implemented in 2007. The cost variables considered in this study were recurrent costs, personnel costs, publicity costs, transportation costs and overhead with administrative data to estimate vaccination coverage. Vaccine Immunogenicity rates were derived from literature. Sensitivity analysis was applied assuming different scenarios.

Results: Total cost of a three-dose campaign in the best scenario was estimated as US\$ 6.5 per person seroprotected ranging from US $\$ 5.5$ to US\$9.4 in alternative scenarios, while the cost for a two-dose campaign was estimated as US $\$ 4.5$ per person seroprotected in the best scenario ranging from US\$3.6 to US\$8.7 in alternative scenarios. To immunize one more person in a three-dose protocol compared to a two-dose protocol US $\$ 58.6$ would be spent by health system ranging from US\$9.6 to US\$155.4. In some scenarios a two-dose protocol had definite priority to the three-dose protocol given both higher effectiveness and lower cost.

Conclusion: In the context of developing countries, where vaccine costs are low, a two-dose HBV vaccination campaign in adolescents compared to a three-dose protocol is cost saving accompanied by higher compliance and without any significant reduction on vaccine efficacy.

\section{PP04-16}

A Study of Keratin Variants and Hepatitis B Virus Associated Chronic Liver Diseases

X. Gong, B. Zhong, S. Zhang, K. Chao, M. Chen

Gastroenterology, The First Affiliated Hospital, Sun Yat-sen University, Guangzhou, China

Objectives: Hepatitis B virus (HBV) infection is a common disease in China. Keratins $(\mathrm{K})$ play an important role in cytoprotective functions and keratin 8 (K8) variants have been shown to be high risk to develop several liver diseases. However no association between keratin variants with HBV associated chronic liver diseases has been reported to date.

Methods: The present study was to investigate the presence of K8 variants (Y54H/G62C) in HBV associated chronic liver diseases and its correlation to disease severity. A total of 300 Chinese patients and 300 sex- and age-matched healthy controls were involved in the present study. K8 genotypes which isolated from peripheral blood specimens were specified by polymerase chain reaction (PCR) amplification and restricted fragment length polymorphisms was used to detect the previously described variants in K8 (Y54H/G62C).

Results: Three hundred patients included HBV carriers, chronic hepatitis B, hepatitis B induced cirrhosis and primary liver carcinoma caused by HBV, which were $62,94,51,93$ cases respectively. The mean age is $48.3 \pm 12.3$ years; $79 \%$ were male. No K8 variants (Y54H/G62C) were found in any group.

Conclusion: The study didn't find $\mathrm{K} 8 \mathrm{Y} 54 \mathrm{H} / \mathrm{G} 62 \mathrm{C}$ variants in HBV associated chronic liver diseases in the Chinese population; it may due to not available of enough patients and didn't take entire DNA exon screening, so a further study is needed.

\section{PP04-17}

Association of Interleukin-17F A7488G Polymorphism with Chronic Hepatitis B Virus Infection in the Chinese Population

K. Chao, B. Zhong, S. Zhang, X. Gong, M. Chen

Division of Gastroenterology, The First Affiliated Hospital, Sun Yat-sen University, Guangzhou, China

Background: IL-17F is involved in many diseases including hepatitis $\mathrm{B}$ and functional polymorphisms of IL- $17 \mathrm{~F}$ were identified in several 
diseases, such as inflammatory bowel disease and asthma. However, its genetic association with HBV infection was rarely discussed. Our aim of this study was to investigate the association between the IL17F 7488, a A/G genetic polymorphism leading to His161Arg substitution in IL-17F protein, and HBV infection in the Chinese population.

Methods: DNA was extracted from peripheral blood cells of 222 chronic HBV infection patients (31 nonsymptom carriers, 72 chronic hepatitis B, 37 liver cirrhosis, 82 hepatocarcinoma) and 161 age- and gender- matched healthy controls. The IL-17F 7488 A $>$ G polymorphism was genotyped by polymerase chain reactionrestriction fragment length polymorphism (PCR-RFLP) and sequencing.

Results: There were significant differences in the genotype distribution of $7488 \mathrm{~A} / \mathrm{G}$ polymorphism of the IL-17F gene among cases and controls $(P=0.002) .149(67.12 \%)$ wild-type, $72(32.43 \%)$ heterozygous and $1(0.45 \%)$ homozygous mutant carriers of IL-17F 7488 $A>G$ variant were found in the case group, while in the healthy control group, there were $131(81.37 \%)$ AA wild-type carriers, $30(18.63 \%)$ AG heterozygous and zero GG homozygous carriers. Compared with AA genotype, AG+GG genotypes significantly increased the risk of chronic $\mathrm{HBV}$ infection $(\mathrm{OR}=2.139,95 \% \mathrm{CI}$ 1.316-3.477). Subgroup analysis showed that the frequencies of $\mathrm{AG}+\mathrm{GG}$ in chronic hepatitis, liver cirrhosis, hepatocarcinoma and control groups were $30.56,48.65,31.71$ and $18.63 \%$, respectively. Compared with AA, AG+GG genotypes were associated with an increased risk of chronic HBV infection in chronic hepatitis, liver cirrhosis and hepatocarcinoma groups $[\mathrm{OR} 1.921,95 \% \mathrm{CI}=$ 1.014-3.642 for chronic hepatitis, OR 4.137, 95\%CI $=1.914-8.820$ for liver cirrhosis; OR $2.027,95 \% \mathrm{CI}=1.1-3.736$ for hepatocarcinoma. However, this phenomenon was not found in the group of nonsymptom carriers, indicating a potential role of IL-17F A7488G polymorphism in liver injury.

Conclusion: The current findings indicate that the IL-17F A7488G polymorphism influence the risk of chronic HBV infection and liver injury the Chinese population.

\section{PP04-18}

Age-specific Spontaneous HBsAg Seroclearance in the Natural History of HBeAg-negative Genotype C Patients with Chronic HBV Infection

M.-S. Kwak, E.J. Cho, E.S. Jang, S.J. Yu, J.-H. Lee, Y.J. Kim, J.-H. Yoon, H.-S. Lee

Department of Internal Medicine and Liver Research Institute, Seoul National University College of Medicine, Seoul, Republic of Korea

Background and aims: In chronic hepatitis B virus (HBV) infection, $\mathrm{HBsAg}$ seroclearance, which is associated with favorable outcome, reportedly occurs at $0.1-2.1 \%$. Several factors were suggested to be associated with seroclearance including age and $\mathrm{HBeAg}$-negativity. On the other hand, HBV genotype was recently found to be a significant determinant for HBsAg kinetics during longterm telbivudine treatment. Therefore, HBV genotype may affect the HBsAg seroclearance. The present study was to evaluate the age specific HBsAg seroclearance rate in HBeAg-negative Korean patients, virtually all of whom are infected with genotype $\mathrm{C}$.

Methods: We included 852 patients who were $\mathrm{HBeAg-negative} \mathrm{and}$ HBsAg positive for at least one year from 2003 to 2005 . We reviewed baseline clinical, biochemical, and virologic factors. Then, we tested HBsAg every 1-3 years to assess HBsAg seroclearance. Multivariable cox regression was performed to find predictors.

Results: Of these 852 patients, median age was 53.0 (18-85), and follow up duration was 39.0 (2-90) months. Serum HBsAg was cleared in 82 patients $(9.6 \%)$. In multivariate analysis, the probability of HBsAg seroclearance was positively associated with age $(P=0.016)$ and with normal ALT level $(P=0.062)$. Seroclearance rate increased with age (30-49 vs. $18-29$; HR, 3.196; 95\% CI, 0.727-14.054; 50-69 vs. 18-29; HR, 5.311; 95\% CI, 1.298-21.898; 70- vs. 18-29; HR, 7.654; 95\% CI, 1.693-34.600), as presented in Fig. 1.

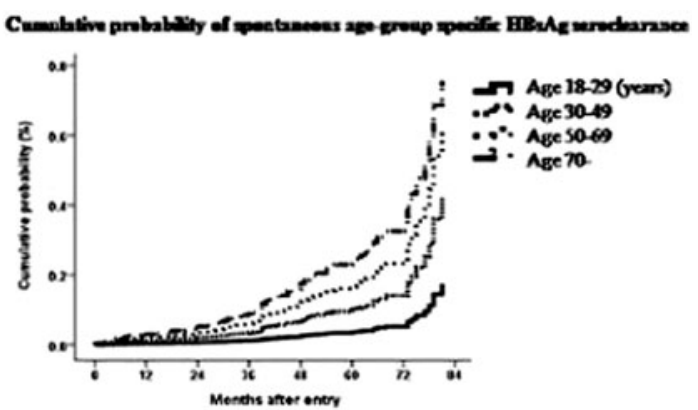

Conclusions: Serum HBsAg seroclearance rate in $\mathrm{HBeAg}$ negative patients per 1 person year was as high as $2.96 \%$ in chronic HBV genotype $\mathrm{C}$ infection and increased with age and with normal ALT levels at baseline.

\section{PP04-19}

Hepatitis B Virus Infection in Multi-ethnic Populations of Ternate S.I. Ie ${ }^{1}$, E. Sidarta ${ }^{1}$, T. Turyadi $^{1}$, M.D. Thedja ${ }^{1}$, Y.S.M. Soedarmono ${ }^{2}$, A. Sadhewa ${ }^{3}$, A.R. Harahap ${ }^{1,4}$, D.H. Muljono ${ }^{1}$

${ }^{1}$ Eijkman Institute for Molecular Biology; ${ }^{2}$ Central Blood Center, Indonesian Red Cross, Jakarta; ${ }^{3}$ School of Life Sciences and Technology, Bandung Institute of Technology, Bandung; ${ }^{4}$ Department of Clinical Pathology, Faculty of Medicine, University of Indonesia, Jakarta, Indonesia

Background: A vaccination program has been implemented nationwide in Indonesia, however, hepatitis B virus (HBV) infection is still endemic in certain areas. With multi-ethnic populations of Austronesian and non-Austronesian backgrounds, Ternate of North Moluccas offers a unique host setting for HBV infection. We assessed the prevalence of HBV infection and its public health implication in adult population (17-39 y.o) with well-defined ethnicity in Ternate. Methods: The ethnicity of the subjects was ascertained for at least three generations maternally and paternally. A total of 389 samples from 99 Austronesian (aged 17-25 y.o., mean $20.25 \pm 1.89$ years, male/female 37/62), 242 non-Austronesian $(17-39,20.06 \pm 2.95,91 /$ $151)$, and 48 mixed ethnicity $(17-34,21.27 \pm 4.29,19 / 29)$ were screened for HBsAg, anti-HBc, and anti-HBs by ELISA (Evolis ${ }^{\mathrm{TM}}$ BioRad Laboratories, Inc., Hercules, CA). Samples with HBsAg and/ or anti-HBc positive were subjected to HBV DNA detection.

Results: Among 389 samples, the prevalence of HBsAg was $16.7 \%$, anti-HBs $24.2 \%$, and anti-HBc $34.7 \%$. These markers were comparable between different ethnic populations studied. Of 135 anti-HBcpositive samples signifying past and/or ongoing HBV infection, $26.7 \%$ were HBsAg-positive and $73.3 \%$ were HBsAg-negative. Notably, of 254 samples without detectable anti-HBc, $11.4 \%$ showed $\mathrm{HBsAg}$ reactivity. HBV DNA was detected in $97.2 \%$ of anti-HBc-positive/ $\mathrm{HBsAg}$-positive samples, $42.4 \%$ of anti-HBc-positive/HBsAg-negative samples, and $93.1 \%$ of anti-HBc-negative/HBsAg-positive samples. Of the remaining 225 samples with anti-HBc-negative/ HBsAg-negative profile, $2.7 \%$ were positive for anti-HBs.

Conclusion: Hepatitis B is still endemic in Ternate with substantial percentage of occult HBV infection. Determination of HBV screening strategy is crucial since anti-HBc-negative status does not totally exclude the carriage of HBV. A review of immunization strategy including catch-up and booster vaccination could be a subject for consideration. Study is in progress on the role of host- and virusrelated factors to elucidate the discrepancy between serological and molecular assays for HBV detection. 
PP04-20

The Seroprevalence of Hepatitis B and Hepatitis C Virus at Healthy People in Izmir

S. Köse, A. Gözaydın, Y. Ulu, M. Türken, S. Topaloğlu

Infectious Diseases and Clinical Microbiology, Tepecik Educational and Research Hospital, Izmir, Turkey

Background: Viral hepatitis infections are major causes of liver disease in our country and worldwide. Hepatitis B infection is the most important problem in our country. In the worldwide there is almost 400 million people infected with hepatitis B virus (HBV) and it is nearly 4 million in our country. In recent years the role of Hepatitis $\mathrm{C}$ virus (HCV) infection at chronic viral hepatitis, has ben increasing. The present study aimed to estimate the seroprevalence of hepatitis B and hepatitis C virus among healthy people in İzmir, who has choosen randomly.

Materials and methods: The participants were chosen by İzmir local health authority, randomly. Serum samples $(n=2,942)$ obtained from healthy individuals were screened for Hepatitis B surface antigen (HBsAg), antibody to HBsAg (anti HBs), antibody to hepatitis B core antigen (anti HBc total) and antibody to HCV (anti HCV) by EIA (Liasson Diasorin, Italy).

Results: Of 2,942 participants ( $>14$ years) $2.6 \%$ were $\mathrm{HBsAg}$ positive. The rate of anti $\mathrm{HBs}$ positivity was $0.7 \%$, anti $\mathrm{HBc}$ total was $21.6 \%$, anti $\mathrm{HCV}$ was $0.1 \%$.

Conclusions: In our country most of the seroprevalence of HBV and HCV studies are among blood donors. In this study, which we used random sampling, seroprevalence of $\mathrm{HBV}$ and $\mathrm{HCV}$ is found similar with outher studies in İzmir among blood donors.

\section{PP04-21}

Isolated Antibody to Hepatitis Core Antigen Seropositivity in HIV-infected Patients

H. Kumbasar Karaosmanoglu, O. Altuntas Aydin, O. Nazlican

Infectious Diseases and Clinical Microbiology, Haseki Training and Research Hospital, Istanbul, Turkey

Aim: To determine the prevalance of isolated hepatitis B core antibody (anti-HBc) seropositivity and occult hepatitis B (HBV) infection among HIV/AIDS patients.

Method: We conducted a retrospective study on 146 HIV/AIDS patients followed by the Infectious Diseases and Clinical Microbiology Outpatient Clinic of Haseki Training and Research Hospital between January 2006 and March 2010. Age, gender, HIV transmission routes, ELISA results of $\mathrm{HBsAg}$, anti-HBc IgG, anti-HBs, anti-HCV serology, real-time PCR results of serum HBV DNA and HCV RNA levels were collected by retrospective analysis of followup records.

Results: Of 146 HIV/AIDS patients, 26 subjects (18\%) had isolated anti-HBc. Of 26 HIV/AIDS cases who had isolated anti-HBc, 20
$(77 \%)$ were men. Mean age was $38(22-79)$ years. The possible routes of HIV transmission were heterosexual contact in 17 (65\%), homosexual contact in $8(31 \%)$, intravenous drug use in $1(4 \%)$. HBV-DNA was detectable in $3(13 \%)$ of HIV/AIDS patients with isolated antiHBc. 15 of $23 \mathrm{HBV}$ DNA negative patients had been receiving HAART containing Lamivudine or Tenofovir for at least 3 months. Only one patient was hepatitis $\mathrm{C}$ co-infected.

Conclusion: The presence of isolated anti-HBc is frequent in HIVinfected patients and this is a risk factor for occult HBV infection. On this occasion, HIV/AIDS patients with isolated anti-HBc should be tested for serum HBV DNA before starting HAART.

\section{PP04-22}

Alterations in CXCR5+CD4+ TFH Cells in Different Stages of Hepatitis B Virus Infection

J.-Y. Feng, P.-W. Zhao, J. Wang, Y. Wang, Y.-F. Jiang

The First Hospital, Jilin University, Changchun, China

Background and aim: The major CD4+ T cell subset that helps B cells is the $\mathrm{T}$ follicular helper (TFH) cell, a cell deputed to help B cells in immune responses. Due to the critical role by TFH cell in the generation of antibody responses, disregulated TFH cell function has been reported in lymphoma (Angioimmunoblastic-T-cell lymphomas, Primary cutaneous CD4+ Small/Medium-sized Pleomorphic T-cell Lymphoma) and autoimmune diseases (Systemic lupus erythematosus. There is only few report about TFH cell expression in HBV infection. This study aimed to evaluate the expression and immune function of TFH cell in different stage of HBV-related liver disease. Methods: Peripheral blood was collected from chronic HBV carriers, inactive $\mathrm{HBsAg}$ carriers (post treatment), $\mathrm{HBeAg}$ positive and negative hepatitis B patients, HBV induced cirrhosis and hepatocellular carcinoma patients, and healthy individuals. PBMC was isolated from whole blood by density-gradient centrifugation. Phenotypes of TFH cells (CXCR5, ICOS, PD-1) were analyzed using flow cytometry.

Results: Changes in the level of TFH cell makers are summarized in Table 1. CD4+CXCR5+ levels were significantly altered among the different stages of HBV infection. CD4+CXCR5+ was higher in inactive HBsAg carriers (post treatment) than chronic HBV carriers $(\mathrm{p}=0.01)$, $\mathrm{HBeAg}$ positive hepatitis $\mathrm{B}(\mathrm{p}=0.046)$ and $\mathrm{HBeAg}$ negative hepatitis $\mathrm{B}(\mathrm{p}=0.036)$ patients. CD4+CXCR5+, CD4+ICOS+, CD4+PD-1+ TFH cells were all significantly higher in which group? than other HBV-related liver disease $(P<0.05)$.

Conclusion: There may be some connections between CXCR5+CD4+ TFH cells and HBV stages such as immunotolerant, immunoactive and immunotolerant after treatment. This indicates that TFH cell is a special marker in the process of liver immune. It may also indicate that TFH cell plays an immune memory function in the liver immune process.

Table 1 TFH cell makers of Healthy and HBV-related liver d

\begin{tabular}{llllrr}
\hline Group & Sample size & CD4+ & CD4+CXCR5+ & CD4+ICOS+ & CD4+PD-1+ \\
\hline Chronic HBV carriers & 14 & $29.06 \pm 11.57$ & $12.44 \pm 8.55$ & $4.06 \pm 3.50$ & $8.24 \pm 6.46$ \\
Inactive HBsAg carriers (Post treatment) & 18 & $27.81 \pm 10.38$ & $27.30 \pm 16.26$ & $5.05 \pm 8.27$ & $7.256 \pm 8.73$ \\
HBeAg positive hepatitis B & 15 & $28.19 \pm 10.01$ & $21.08 \pm 7.38$ & $3.35 \pm 2.68$ & $3.84 \pm 2.56$ \\
HBeAg negative hepatitis B & 5 & $31.02 \pm 4.62$ & $16.83 \pm 11.32$ & $2.90 \pm 2.15$ & $8.00 \pm 8.47$ \\
HBV-induced Cirrhosis & 13 & $19.90 \pm 17.17$ & $29.30 \pm 12.95$ & $5.85 \pm 3.32$ & $10.79 \pm 18.07$ \\
HBV-induced hepatocellular carcinoma & 20 & $19.26 \pm 10.33$ & $26.05 \pm 2.03$ & $4.951 \pm 2.84$ & $10.173 \pm 16.02$ \\
Healthy controls & 7 & $22.73 \pm 8.27$ & $21.45 \pm 8.52$ & $3.4243 \pm 3.14$ & $4.2671 \pm 1.87$ \\
\hline
\end{tabular}


PP04-23

Increased 2B4 and Decrease NKG2A Expression on Natural Killer Cells in Chronic Hepatitis B Patients Treated with Telbivudine

Y.-F. Jiang, J.-Y. Feng, J.-Q. Niu, J. Wang, Y. Wang

The First Hospital, Jilin University, Changchun, China

Background: Natural Killer (NK) cells, controlled by multiple activating and inhibitory receptors, are an important element of the innate immune response to viral infection including HBV. As the function of NK cells is primarily regulated by NK cell receptors (NKR), we analyzed whether decreased NK cell function in HCV infection and progression of CHB may be related to dysregulated NKR expression. Methods: A total of 53 patients of $\mathrm{CHB}$ patients were selected, treated with Telbivudine (LDT) for 48 weeks, and examined at every 12 weeks treatment for serum HBV DNA loads, ALT and AST levels. NK phenotype and function assays were analyzed by FACS, And phenotypes of peripheral blood lymphocyte subsets (T cells, NK cells, NKT cells, Treg cells) were also analysed.

Results: The results showed significantly reduced numbers of NK cells with CHB patients $(p=0.013)$, while the proportion of CD3+CD4+ T cells, Treg cells and the ratio of CD4/CD8 were significantly higher than that of healthy controls $(p=0.025, p=0.006$ and $p=0.046$ respectively). The expression of receptor 2B4+ on NK cells were lower in $\mathrm{CHB}$ patients compared to that in healthy controls $(p=0.021)$. The NK and 2B4+ expression on NK cells in posttreatment group were increased than that in pre-treatment group $(p=0.001)$. But NKG2A expression on NK cells in post-treatment group was decreased than that in pre-treatment group $(p=0.001)$. A significant inverse correlation was obtained between $2 \mathrm{~B} 4+\mathrm{CD} 56+\mathrm{NK}$ cells and HBV DNA levels $(R=0.278, p=0.035)$. The NK cell phenotype was associated with polarization of $\mathrm{NK}$ cell function towards CD107a expression as a marker of degranulation, but IFN- $\gamma$ production by NK cells was not increased.

Conclusions: These findings demonstrate that the concentration of 2B4+ and NKG2A CD56+ NK cells normalizes with LDT treatment in CHB patients, suggesting that immune response in CHB patients receiving LDT influences the target cell recognition by NK cells.

\section{PP04-24}

Toll-like Receptors and Cytokines/cytokine Receptors Polymorphisms Associated with Non-response to Hepatitis B Vaccine

X. Chen ${ }^{1}$, J. Chen ${ }^{1}$, F. Lu ${ }^{1}$, Z. Liang ${ }^{2}$, H. Zhuang ${ }^{1}$

${ }^{1}$ Department of Microbiology, Peking University Health Science Center; ${ }^{2}$ 2nd Division of Viral Vaccines, National Institute for

Control of Pharmaceutical and Biological Products, Beijing, China

Introduction: It is well documented that $5 \%-10 \%$ hepatitis B adult vaccinees are non- and hypo-responders and probably are not adequately protected against HBV infection. The genetic variations of genes involved in processes such as pathogen recognition, antigen processing and presentation, differentiation/maturation of lymphocytes may affect the duration and intensity of protective humoral response to the hepatitis $\mathrm{B}$ vaccine.

Methods: The study cohort consisted of 46 responders and 24 nonresponders was recruited from Jiangsu province, China, as part of a survey to determine risk factors of hypo-response to hepatitis $\mathrm{B}$ vaccine which had been described previously. Genomic DNA from each individual's peripheral blood leukocytes was extracted by standard protein K digestion and phenol-chloroform methods. A total of 53 known SNPs across 21 genes were chosen by SNPbrowserTM software 4.0. All candidate genes were reported with a minor allele frequency (MAF) higher than $10 \%$ in the Chinese Han population. SNP genotyping was conducted using the iPLEX MassARRAY system. Chi-square $(\chi 2)$ tests were used to examine the differences of each single nucleotide polymorphism between responders and non- responders using a software platform, SHEsis.

Results: It was found that 4 SNPs (rs2243248, rs1805015, rs1295686 and rs3804100) in IL-4, IL-4RA, IL-13 and TLR2 genes were associated significantly with vaccinees' status of serum anti-HBs response to the HBV vaccine $(P<0.05)$. Two SNPs (rs1295686 and rs1805015) also showed significant association with vaccine-induced immune response when analyzed together with risk factors such as age and gender, by multivariable logistic regression analysis $(P<0.05)$. Further haplotype analysis showed that AG haplotype constituted by rs1143633 (IL-1B; intron) and rs1143627 (IL-1B; intron) appeared more frequently in non-responders than that in responders $(P=0.035)$. Conclusion: The specific SNPs in the cytokines/cytokine receptors and TLR genes were associated with status of hepatitis B vaccineinduced protective humoral response.

\section{PP04-25}

Low Prevalence of Hepatitis C in Patients with Chronic Kidney Disease in Sri Lanka

S.N. Wai ${ }^{1}$, R.D. Lanerolle ${ }^{2}$, N.J. Beeching ${ }^{1}$, S. Mananwatte ${ }^{3}$, A.L.M. Nazar ${ }^{4}$, M.H.R. Sheriff ${ }^{5}$, M. Hopkins ${ }^{6}$, S.A.A.P. Karunanayake ${ }^{7}$

${ }^{1}$ Tropical Medicine, Liverpool School of Tropical Medicine, Liverpool, UK; ${ }^{2}$ Clinical Medicine, Dept of Clinical Medicine, Faculty of Medicine, University of Colombo; ${ }^{3}$ Virology, Central Reference Laboratory, National STD and AIDS Control Program, Colombo; ${ }^{4}$ Dialysis Unit, National hospital of Sri Lanka, Colombo; ${ }^{5}$ PGIM, Western Infirmary, Colombo; ${ }^{6}$ Virology, Clinical Virology Laboratory, Royal Liverpool University Hospital, UK, ${ }^{7}$ Clinical Medicine, National University of Colombo, Colombo, Sri Lanka

Background: Patients with chronic renal disease (CKD) are at increased risk of acquiring hepatitis $\mathrm{C}(\mathrm{HCV})$. Previous reports showed a low HCV antibody prevalence in Sri Lankan patients with CKD (Fernando et al, 2002).

Aims: To document the prevalence of $\mathrm{HCV}$ antibody positivity and associated risk factors in Sri Lankan patients with CKD.

Methods: Patients with CKD ( $\geq$ Grade 3 ) were recruited in 2 specialist clinics in Colombo in mid 2010. A questionnaire was completed and HCV antibodies determined locally using a 2nd generation ADVIA Centaur assay (Siemens Healthcare, UK), together with 18 negative samples. Generation EIA kit (Oxford Biosystems UK). 2 samples that were positive and 4 that were equivocal were retested in Liverpool by 3.

Results: 278/280 eligible patients enrolled, $186(66.9 \%)$ male and mean (SD) age 47.7 (13.5) years. HCV risk factors included haemodialysis in $253(91 \%)$, blood transfusion in $225(80.9 \%)$, and renal transplantation in $123(44.2 \%)$. All but 3 transplants had been performed in Sri Lanka. One of the 2 positives was confirmed anti-HCV positive; the other 5 reactive samples and 18 negatives were negative in the confirmatory assay, ie prevalence was $0.36 \%$ (95\% CI $0.01-1.99 \%$ ).

Discussion: Exposure of CKD patients to HCV remains very low in Sri Lanka and the initial high false positive rate is not unexpected in a low prevalence setting. The HCV prevalence resembles that a decade earlier in Colombo, when anti-HCV was found in 2/59 (1.7\%) haemodialysis patients, and in 1/94 (1.1\%) patients transplanted locally, compared to $7 / 25(28 \%)$ of Sri Lankan patients transplanted in India (Fernando et al. 2002). The low rates of HCV exposure in Sri Lankan CKD patients compared to neighbouring countries probably reflects the long-term use of volunteer rather than commercial blood and organ donors in Sri Lanka, and continued attention to good infection control practices.

\section{PP04-26}

The Study about the Compliance in Patients with Chronic Hepatitis B in Southwest of China

K. Fan, Y. Wang, Q. Mao, X. Wang Institute of Infectious Diseases for PLA, Southwest Hospital, The Third Military Medical University, Chongqing, China

Background: When patients use nucleoside analogue (NUC), the regularity of patients using drugs is a significant factor for efficacy. 
But we found that parts of patients did not complied the guidance of doctors, so this investigation is to study these patients.

Methods: The data come from 685 patients with $\mathrm{CHB}$ who are using NUC during 2002.1.1 to 2010.10.10, including male 526 and female 159. The compliance of patients was investigated. The number of patients receiving LAM, ADV, LdT, ETV, LAM+ADV, LdT + ADV, ETV + ADV were 133,174,125,99, 131,14 and 9, respectively.

Results: $31.7 \%(217 / 685)$ of the patients have a bad compliance. There are just $5(0.7 \%)$ patients who did not obtain the advice from the doctors that they should take these drugs in a long time. There is no significant difference $(p>0.05)$ between compliance of different genders and ages. The compliance of LAM, ADV, LdT, ETV, $\mathrm{LAM}+\mathrm{ADV}, \mathrm{LdT}+\mathrm{ADV}, \mathrm{ETV}+\mathrm{ADV}$ were 65.4, 63.8, 70.7, 67.2, $73.3,78.6,88.9 \%$, respectively. The rate of bad compliance patients is higher in these patients who have a longer time of treatment. The reasons of drug withdrawalling including prescribed drug could not last to the next visit $(40.78 \%)$, cost of treatment $(21.4 \%)$, patients felt better; felt symptomless; thinks treatment will not help (2.9\%), according to the advice of other people (2.9\%), busy with other things (11.7\%), other reasons $(20.4 \%)$.

Conclusion: The compliance of HBV patients with NUC in China is not satisfactory. It is necessary to enhance the guidance for patients, help the patients who stop to have drug because they cannot buy enough drug in their cities, and make the medical insurance better in the future.

\section{PP04-27}

Risk of Reactivation in Patients with Current and Past Hepatitis B Infection Treated with Rituximab-based Chemotherapy

M.M.G. Ow ${ }^{1,2}$, E. Gane $\mathrm{e}^{1,2}$

${ }^{1}$ Department of Gastroenterology, Middlemore Hospital; ${ }^{2} \mathrm{NZ}$ Liver Transplant Unit, Auckland City Hospital, Auckland, New Zealand

Introduction/aim: $\mathrm{HBV}$ reactivation may occur in both $\mathrm{HBs} A g$ positive and $\mathrm{HBsAg}$ negative/anti-HBcore positive individuals receiving chemotherapy. This retrospective analysis examined the risk of reactivation in patients with current/previous HBV treated with Rituximab-based chemotherapy.

Methods: All patients who received Rituximab-based chemotherapy for haematological malignancies at Middlemore and Auckland City Hospitals from 2005 to 2010 were analysed. Baseline HBV status was recorded as either naïve (HBsAg neg/anti-HBcore neg), current (HBsAg+), or previous HBV infection (HBsAg neg/anti-HBcore+). Serial HBsAg, HBVDNA, and ALT were recorded. Use of prophylactic Lamivudine was noted. Primary outcome was rate of $\mathrm{HBV}$ reactivation (defined as $\mathrm{HBsAg}+\mathrm{HBV}$ DNA $>5 \log _{10} \mathrm{IU} / \mathrm{mL}$ and ALT $>$ ULN).

Results: 501 patients received Rituximab, of whom 10 (2\%) had active $\mathrm{HBV}(\mathrm{HBsAg}+)$ and $72(17 \%)$ had previous HBV infection (HBsAg neg/anti-HBcore+). All $10 \mathrm{HBsAg}$ positive patients received Lamivudine pre-chemotherapy. 1 patient (10\%) reactivated after Lamivudine cessation 6 months post-chemotherapy, despite low pretreatment HBVDNA. Monitoring was performed in 45 patients with previous HBV. None of the 28 who received Lamivudine reactivated, compared to $2 / 17$ who did not receive Lamivudine $(p=0.06)$. All patients who reactivated received R-CHOP and were rescued with antiviral therapy.

Conclusion: In HBsAg positive patients who receive Rituximab, delayed reactivation may occur despite Lamivudine prophylaxis and low pre-treatment viral load. Careful monitoring post-Lamivudine cessation is imperative in this group. In patients with previous HBV, reactivation is prevented by Lamivudine prophylaxis. In patients with current or previous HBV receiving R-CHOP, Lamivudine prophylaxis should be continued at least 6 months after last dose of Rituximab and should be followed by serial monitoring of HBsAg/ALT for a further 3 months.
PP04-28

Higher Viral Load Leads to More Rapid Liver Disease Progression throughout the Natural History of Chronic Hepatitis B Virus Infection

J.-D. Chen ${ }^{1,2}$, H.-I. Yang ${ }^{3}$, U.H. Iloeje ${ }^{4}$, S.-L. You ${ }^{3}$, S.-N. Lu ${ }^{5}$, L.-Y. Wang ${ }^{6}$, J. $\mathrm{Su}^{4}$, C.-A. Sun ${ }^{7}$, Y.-F. Liaw ${ }^{8}$, C.-J. Chen ${ }^{2,3}$, for the R.E.V.E.A.L.-HBV Study Group

${ }^{1}$ Department of Internal Medicine, Bei-Hu Branch, National Taiwan University Hospital; ${ }^{2}$ Graduate Institute of Epidemiology, College of Public Health, National Taiwan University; ${ }^{3}$ Genomics Research Center, Academia Sinica, Taipei, Taiwan ROC; ${ }^{4}$ Research and Development, Bristol-Myers Squibb Company, Wallingford, CT, USA; ${ }^{5}$ Department of Gastroenterology, Kaohsiung Chang-Gung Memorial Hospital, Kaohsiung; ${ }^{6}$ Department of Medicine, Mackay Medical College; ${ }^{7}$ Department of Public Health, College of Medicine, Fu-Jen Catholic University; ${ }^{8}$ Liver Research Unit, Chang Gung Memorial Hospital and Chang Gung University, Taipei, Taiwan ROC

Background/aims: This study aimed to investigate transition rates of liver disease progression and their determinants during natural history of chronic hepatitis B.

Methods: Three subsets of the original R.E.V.E.A.L.-HBV cohort were selected according to participants' disease states:

1. persistently normal ALT (PNALT) subcohort $(n=851)$ : participants who had persistently normal serum ALT levels and no LC or HCC through 1991-1999;

2. chronic hepatitis $(\mathrm{CH})$ subcohort $(n=238)$ : participants who developed newly-diagnosed $\mathrm{CH}$ among those with normal serum ALT levels and without LC and HCC in 1991-1992;

3. LC subcohort $(n=145)$ : participants who developed newlydiagnosed LC among those with normal serum ALT levels and without LC and HCC in 1991-1992.

Outcomes of interest included $\mathrm{CH}$, LC, HCC, liver-related death (LD), and end-stage liver disease (ESLD, defined as at least one event of LC, HCC and LD). Cox regression models with or without timedependent variable were used to estimate multivariate-adjusted hazard ratios $\left(\mathrm{HR}_{\mathrm{a}}\right)$ and $95 \%$ confidence intervals (CIs).

Results: Annual disease transition rates, from PNALT to $\mathrm{CH}, \mathrm{CH}$ to LC and LC to HCC, were 6.1, 2.2, and 3.0\%, respectively, for subjects with initial-state $\mathrm{HBV}$ loads $\geq 10^{4}$ copies $/ \mathrm{mL}$; and $1.9,0.5$ and $3.4 \%$ for initial-state viral loads $<10^{4}$ copies $/ \mathrm{mL}$. A high initial-state viral load $\left(\geq 10^{4}\right.$ copies $\left./ \mathrm{mL}\right)$ was an independent risk predictor for transitions from PNALT to $\mathrm{CH}, \mathrm{CH}$ to $\mathrm{LC}$, and LC to LD with $\mathrm{HR}_{\mathrm{a}}$ (95\% CI) of $2.9(1.9-4.3), 3.9(0.9-17.2)$, and $5.6(1.6-26.1)$, respectively. The $\mathrm{CH}, \mathrm{LC}$, and $\mathrm{HCC}$ were important intermediate states for PNALT progressing to ESLD, CH to HCC/LD, and LC to $\mathrm{LD}$, with the time-dependent $\mathrm{HR}_{\mathrm{a}}(95 \% \mathrm{CI})$ of 3.1 (1.1-9.0), 7.1 (1.1-45.1), and 17.9 (6.3-50.9), respectively.

Conclusions: High viral loads lead to more rapid liver disease progression throughout the natural history of chronic hepatitis B virus infection.

\section{PP04-29}

Clinical Features of Acute Exacerbation in Chronic Hepatitis B Patients S.Y. Choi, Y.O. Kweon, S.K. Kim, W.Y. Tak, C.M. Cho, S.W. Jeon, M.K. Jung, S.Y. Park

Department of Internal Medicine, Division of Gastroenterology and Hepatology, Kyungpook National University Hospital, Daegu, Republic of Korea

Background and aims: Acute exacerbation (AE) can occur commonly in patients with chronic hepatitis $\mathrm{B}(\mathrm{CHB})$. In some cases, $\mathrm{AE}$ may be followed by $\mathrm{HBeAg}$ seroconversion and clinical remission. However, prolonged or repeated AE may also contribute to progression to liver cirrhosis or end stage liver disease such as liver failure. The purpose of this study was to identify the etiology and the clinical consequence of $\mathrm{AE}$ in Korean patients with $\mathrm{CHB}$. 
Methods: We reviewed the medical records of $\mathrm{CHB}$ patients with $\mathrm{AE}$ (defined by the increase of ALT above 400 IU/L) who were admitted to Kyungpook National University Hospital between January 2002 and December 2008.

Results: 78 (M:F $=60: 18$ ) patients were included in this study. The mean age was 41.2 years (range 18-76). Laboratory data showed the mean of the highest ALT level was 983 IU/L (range 405-3,245), total bilirubin was $8.4 \mathrm{mg} / \mathrm{dL}$ (range $0.68-41.16$ ). The most common cause of $\mathrm{AE}$ was spontaneous exacerbation $(n=45)$. The other causes are withdrawal hepatitis $(\mathrm{n}=17)$, breakthrough during antiviral treatment $(n=6)$, herbs or others with hepatotoxicity $(n=6)$ and immunosuppressive or chemotherapeutic agents $(n=4)$. There were no significant difference between patients who were $\mathrm{HBeAg}$ positive $(n=57)$ and HBeAg negative $(n=19)$ in terms of laboratory and clinical courses. $\mathrm{HBe} A g$ seroconversion at 3,12 and 24 months following $\mathrm{AE}$ was 10, 28 and $36 \%$, respectively, in $\mathrm{HBeAg}$ positive cases.

Conclusions: AE in patients with $\mathrm{CHB}$ is mainly caused by spontaneous exacerbation. Withdrawal hepatitis and breakthrough hepatitis which is associated with antiviral treatment were next common cause to spontaneous exacerbation. So, We should be considerate to stop antiviral agent as well as to start and to choose suitable antiviral agent in the treatment of $\mathrm{CHB}$ for preventing $\mathrm{AE}$ during or after antiviral treatment.

\section{PP04-30}

Prevalence of Hepatitis B among the School Going Children in Mymensingh Region

C.R. Debnath ${ }^{1}$, M.R. Debnath ${ }^{2}$, M. Shahidullah ${ }^{3}$

${ }^{1}$ Hepatology, Mymensingh Medical College Hospital; ${ }^{2}$ Blood

Transfusion Centre, Mymensingh Medical College Mymensingh;

${ }^{3}$ Department of Medicine, Mymensingh Medical College,

Mymensingh, Bangladesh

Background: Hepatitis B virus is a global health problem, especially in Asia. HBV infection is a major cause of chronic hepatitis, Cirrhosis and Hepatocellular carcinoma, that are responsible for high rate of morbidity and mortality. The aim of the study was to analyze the prevalence of HBV among the school going children of mymensingh region.

Methods: The cross sectional Data was collected from vaccination programmed among the school going children

Results: Among 10,820 school going children 388 were positive for HBs Ag (3.59\%).

Conclusion: Based on the results, HBV infection in Bangladesh has the similarity with other Asian countries. Bangladesh is one of the densely populated country of the world. The prevalence of HBV infection and its sequliae can be prevented by successful vaccination. It is noted that Bangladesh government has started incorporating the HBV programme. This will eventually reduce the prevalence of viral hepatitis and it's sequliae.

\section{PP04-31}

Low Prevalence of Hepatitis B Infection in Western India

N. Patel ${ }^{1}$, D. Amarapurkar ${ }^{2}$

${ }^{1}$ Jivandeep Hospital, Anand; ${ }^{2}$ Bombay Hospital and Medical Research Centre, Mumbai, India

Introduction: Prevalence rates of hepatitis B viral infection (HBV) in India vary from 3 to $12 \%$, which falls in intermediate prevalence zone. Prevalence of hepatitis $\mathrm{C}$ viral infection (HCV) in India vary from 0.3 to $4 \%$ among blood donor population. Previous data from western India suggests $\mathrm{HBV}$ prevalence of $1.6-5.9 \%$ and $\mathrm{HCV}$ prevalence of $0.2-15.9 \%$. We aimed to study HBV and HCV prevalence from multiple sources.

Methods: Data on prevalence of HBV and HCV was collected prospectively from multiple sources: hospital attending population (3 general hospitals), general population (Mumbai), mandatory blood bank screening ( 2 blood banks), multiple voluntary camps (4 camps for general population, 3 camps for college students).
Results: HBV prevalence was as follows: hospital attending population (1-year): 23/1486 (1.5\%), 5/350 (1.4\%), 2/468 (0.4\%); general population (1-year): 18/1168 (1.5\%); blood bank data: 154/28330 (3year) $(0.5 \%), 2046 / 126610$ (9-year) $(1.6 \%)$; voluntary camps: $2 / 860$ (general population) $(0.2 \%), 11 / 1968$ (college students) $(0.5 \%)$. HCV prevalence was as follows: blood bank data: 55/28330 (3-year) (0.19\%), 604/125600 (9-year) $(0.48 \%)$; general population (1-year): 1/1168 (0.1\%); hospital attending population (1-year): $2 / 670(0.2 \%)$; voluntary camps: 0/860 (0\%).

Conclusions: In Western India, there is very low prevalence of HBV (average 1.4\%) and HCV (average $0.4 \%$ ); making it a low prevalence area.

\section{PP04-32}

Acquirement and Disappearance of HBsAg and Anti-HCV in an Aged Cohort: A Follow-up Study in a Dual HBV/HCV Endemic Township

Y.-H. Kuo, S.-N. Lu

Division of Hepato-Gastroenterology, Department of Internal

Medicine, Chang Gung Memorial Hospital -Kaohsiung Medical Center, Kaohsiung County, Taiwan ROC

Background: HBsAg and anti-HCV are stable markers and widely used as seroprevalence of chronic hepatitis B and C infection. Incidence of seroclearance and seroreversion of these two makers are supposed to be as low as near zero.

Aims: To elucidate the incidence rates of seroclearance and seroreversion of HBsAg and anti-HCV in an dual HBV and HCV endemic area. Methods: In 2005, a total of 1002 residents were screened for hepatitis markers and liver function tests in a dual HBV and HCV endemic community. All of them, aged 57 years or more were invited to this follow-up study in 2010, and 405 (40.4\%) participated. HBsAg, anti-HCV, and ALT were checked in all participants and quantitative HBsAg, HBV DNA, anti-HBs, anti-HBc, HCV RNA, and abdominal ultrasonography were studied under different indications. Results: There were 192 male and 213 female residents with a mean age of $69.6 \pm 7.8$ years. Only one new HBsAg carrier was noted and she was 77 years old. Among those 59 old carriers, $48(81.4 \%)$ decreased concentration (IU/ml) of $\mathrm{HBsAg}$ quantification and 8 $(13.6 \%)$ cleared their HBsAg. The annual incidence of HBsAg was $2.7 \%$. (95\% confidence Interval (CI): 2.02-3.07). No cases showed anti-HCV seroconversion. Among 154 old anti-HCV subjects, 17 $(11 \%)$ showed anti-HCV seroreversion with an annual incidence of 2.2\% (95\% CI: $1.86-2.53$ ). Anti-HBc alone contributed to $32.8 \%$ (133/405), old ages ( $>65$ years) and anti-HCV-positive subjects were two significant associated factor.

Conclusions: In this aged population, only one new case of chronic hepatitis B was noted. There were no newly acquired cases of chronic hepatitis C. Estimated annual incidence rates of seroclearance, $\mathrm{HBsAg}$ and anti-HCV were 2.7 and $2.2 \%$, respectively. High prevalence of anti-HBc alone were noted among aged population and cases with chronic hepatitis $\mathrm{C}$ infection.

\section{PP04-33}

Cross-Sectional Demographical Features of HBV Infection in Young Male Patients of Turkey

L. Gorenek, V. Turhan, M. Hatipoglu, H. Diktas, A. Acar, O. Oncul Infectious Diseases and Clinical Microiology, Gulhane Military Medical Academy, Haydarpasa Training Hospital, Istanbul, Turkey

Background: This study analyzes epidemiologic, serologic, histopathologic and therapeutic data of young male patients with chronic active HBV (CAHBV) infection from five geographical regions of Turkey. Methods and results: Between October 2008 and May 2010, a total of 309 patients 18 - to 40 -years-old were included in study. This patient was divided five regions as south, east, west, north and central part of Turkey. Hepatitis B surface antigen ( $\mathrm{HBsAg}$ ), HbeAg and Anti-Hbe seropositivity, liver biopsies and therapeutic regimens which started 
before admission to hospital were analyzed. Mean age was 24,74 years. The geographical distribution of the patients with CAHBV was $6,1 \%$ (19) in north region, $12,62 \%$ (39) in central region, $11,65 \%$ (36) in south region, $30,74 \%$ (95) in east region, 38,83\% (120) in west region of Turkey. Overall, 66\% (204) of these patients are naive. The positive rates of $\mathrm{HBeAg}$ and Anti-HBe were 52\% (107) and 48\% (97) among naive cases, respectively. Of all the cases, 34\% (105) used oral antiviral or interferon therapy. Mean AST level was 43,712 \pm 4.0 (Normal 5-40 U/l) and ALT level was 77.35 \pm 4.0 U/l (Normal 5-40 U/l). Liver biopsy performed 177 of all patients. We used to Knodell classification to show liver parenchymal damage with fibrosis and histological activity index. Mean Knodell histological activity index was 4.04/18 and fibrosis degree was 1.16/6.

Conclusion: We found that, in the east region of Turkey is not different from west part about the incidence of CAHBV. Most part of the patients is naive and there are nearly same HbeAg and Anti-Hbe seropositivity. The major treatment modality is oral antiviral therapy.

\section{PP04-34}

Seroprevalence of Hepatitis C Virus and Human Immunodeficiency Virus in Blood Donors of North-Western Pakistan

S.Z. Safi, I. Qadri

NCVI, National University of Sciences \& Technology, H-12, Islamabad, Pakistan

Background: HCV and HIV infections are major health problems associated with blood transfusion practices in Pakistan. Trends and prevalence of HCV and HIV in BDs are different as reported form various cities and geographical regions. This study was conducted in a large population size to asses the epidemiology of HCV and HIV in North West Frontier Province (N.W.F.P) of Pakistan.

Methods: In this study 62,251 healthy BDs, age 17-50 were included from January 2008 to July 2009. 61,059 (98.1\%) of the study subjects were male while only $1,192(1.9 \%)$ were female. All the donors from 11 areas of N.W.F.P were screened for Hepatitis C Virus and Human Immunodeficiency Virus antibodies by ELISA (Biokit). Blood group of each individual was also determined.

Results: Frequency of HCV and HIV infection was 2.6 and $0.045 \%$ ), respectively. Prevalence of $\mathrm{HCV}$ and HIV in 2008 was $2.4 \%$ (CI $1.9-2.95$ ) and $0.049 \%$ (CI $0.035-0.063$ ), respectively. In 2009, it was $3.0 \%$ (CI 2.7-3.28,) for HCV and 0.038 (CI 0.019-0.057) for HIV. The difference in prevalence of HCV from 2008 to 2009 is highly significant $(P=0.00001)$ while for HIV it's non-significant $(P=0.56)$. Among the age groups the prevalence of HCV and HIV was not significantly different $(P=0.128)$. Only $1484(2.4 \%)$ of the donors were voluntary, the remaining were replacement and family blood donors. The difference in number of male and female donors was highly significant $(P=0.00001)$. B + blood group was the common in $30.5 \%$ BDs. Our study revealed a high prevalence of HIV than most of the previous reports.

Conclusion: Frequency of HCV infection in blood donors is higher in N.W.F.P than rest of the world and lower than other regions of the country. Transfusion of the infected blood is one of the common causes of its transmission. HIV is getting more in Pakistani population.

\section{PP04-35}

Liver Function Tests and Demography Profiles of HBV-DNA Positive Patients Referred to Naft Great Hospital in 2009-2010

M.A. Jalali Far ${ }^{1,2}$, M. Alinejadi ${ }^{3,4}$, K. Gholam Abas ${ }^{1}$, N. Saki ${ }^{1,2}$, S. Negravi ${ }^{5}$ ${ }^{1}$ Ahvaz Jondi Shapour University of Medical Sciences; ${ }^{2}$ Thallasemia and Hemoglubinopathy Research Center; ${ }^{3}$ Medical Laboratory Science, Ahvaz, Jondi Shapour University of Medical Sciences; ${ }^{4}$ Medical Laboratory, Great Naft Hospital; ${ }^{5}$ Noor and Padideh English Institute, Ahwaz, Iran

Hepatitis B virus (HBV) is a serious public health problem worldwide and important cause of chronic hepatitis, cirrhosis, and hepatocellular carinoma (HCC). Presently, more than $80 \%$ of HCC are found in $\mathrm{HBV}$ infected people. $3 \mathrm{HCC}$ is now the fifth most frequent cancer in the world, killing 300,000-500,000 people each year. Liver function tests are useful marker in determining the HBV infection status.

In this descriptive study, we studied 41 (18-70 years old) people referred to Naft great hospital. The HBs-Ag and HBV-DNA of them was positive based on enzyme immune assay and qualitative PCR. The alanine amino transferase, aspartate amino transferase and alkaline phosphatase determined for them. The data analyzed by SPSS. The 14 was female and the rest were male. In our population, 2 patients had abnormal ALK, seven patients in ALT and 7 patients in AST. The elevated ALT was two times more in male than female, but the elevated AST was more in female. According to our finding the ALT and AST was elevated in $34 \%$, but because the ALT is more sensitive in viral hepatitis, This risk factor marker for start anti viral therapy in HBV infection indicated that more than one third of HBV patients need anti viral therapy. The viral load recommended for that patients.

\section{PP04-36}

Prevalence of VHC-Ab and HBsAg among Blood Donors in Guelmim in the South of Morocco

N. Aqodad, M. Lahbabi, M. Elyousfi, I. Mellouki, D. Benajah, M. Elabkari, A. Ibrahimi

Gastroenterology Unit, Hospital University Hassan II, Fez, Morocco

Aims: To study the prevalence of VHC-Ab and HBsAg in blood donors to the province of Guelmim in the south of Morocco.

Patients and methods: It was about a retrospective study from 02/01/ 2006 to $02 / 07 / 07$, having interested the registers of the blood donors in the bank of blood in provincial hospital of Geulmim in the south of Morocco.

Results: Seven hundred and seventy seven donors were included during this period with a male prevalence (sex-ratio $=2,5$ ). $\mathrm{VHC} \mathrm{Ab}$ were positive in 6 donors $(0,8 \%)$ all male. $\mathrm{HBsAg}$ was positive in 10 cases $(1.3 \%)$ ( 8 men and 2 women, $p=0.73$ ). Co-infection $\mathrm{B}$ and $\mathrm{C}$ was noted in only one case. No co-infection with HIV has been found. Only one donor was mono-infected by the HIV.

Conclusion: Prevalence of hepatitis $\mathrm{C}$ virus among blood donors in southern Morocco was similar to that reported by other Moroccan series, while that of HBsAg was significantly lower.

\section{PP04-37}

Seroprevalence and Risk Factors for Hepatitis B Infection in JiLin, China H. Zhanghong, J.Q. Niu

Hepatology, First Hospital, Jilin University, Changchun, China

Background and aim: HBV is highly endemic in China. Accordingly, a National Expanded Program on Immunization has instituted there since 1992. The aim of the current study was to determine the seroepidemiology of HBV infection in JiLin, China to guide effective preventive measures for health policy makers.

Methods: A cross-sectional serosurvey was conducted throughout JiLin, China. A total of 3,833 people were selected, and demographic and behavioral information gathered. Serum samples were tested for HBV markers and liver enzymes.

Results: The prevalences of HBsAg, anti-HBs and anti-HBc were 4.38, 35.66 and 40.88\%, respectively. ALT and AST levels were significantly higher among $\mathrm{HBsAg}(+)$ than $\mathrm{HBsAg}(-)$ subjects and HBsAg (+) with $\mathrm{HBeAg}(+)$ than $\mathrm{HBsAg}(+)$ with $\mathrm{HBeAg}(-)$, subjects. In multivariate logistic regression analysis, smoking (odds ratio [OR] 1.61), bad sleep quality (OR 1.79), being private smallbusinessmen (OR 3.17), laborers (OR 2.37), peasants (OR 2.16), male (OR 1.79), no personal history of vaccination (OR 4.1), and age 50-69 years (OR 4.36), 30-49 years (OR 5.8), 18-29 years (OR 9) were independent predictors for chronic HBV infection. At the same time, drinking (OR 0.64), having a large family size (OR 1.35), being a private small-businessman (OR 1.39), male gender (OR 1.61), no 
personal history of vaccination (OR 25), and age 50-69 years (OR 0.5), 30-49 years (OR 0.41), 18-29 years (OR 0.33) were independent predictors for exposure to HBV (anti-HBc positive).

Conclusions: The prevalence rate of HBV infection (4.38\%) was lower than before general HBV vaccination. However, $44.59 \%$ of the population remained susceptible to HBV. Therefore, catch-up immunization for those whose HBV marker are all negative are necessary especially for the high-risk groups (having poor sleep quality, being private small-businessmen, Drinking, large family size, no personal history of vaccination, young male adults).

\section{PP04-38}

Prevalence of Hepatitis B Virus (HBV) Infection among Immigrants in Rome

A. Morrone, L. Nosotti, I. Uccella, R. Marrone, L. Pecoraro, C. Pajno,

T. D'Arca

National Institute for Health, Migration and Poverty, Rome, Italy

Background and aims: Hepatitis B virus (HBV) infection is still a highly prevalent disease worldwide, with large morbidity and mortality rate, and it has been recognized as a worldwide public health problem. In Italy, infection incidence has decreased since the introduction of universal vaccination for infants; however, there is a lack of epidemiological data regarding prevalence of HBsAg-positivity in immigrants. Aim of this study was to describe the prevalence and the patterns of HBV infection in a large cohort of immigrants in Rome. Methods: Between August 2008 and July 2009, 1,203 consecutive patients were visited at our Department (68,2\% female, 32,8\% male, mean age 30 years). 58.78\% were from Africa, $22.04 \%$ from Asia, $13.06 \%$ from Eastern-Europe and $6.12 \%$ from South-America. A screening form with demographic, clinical and laboratory data was collected for each HBsAg -positive patient, in order to identify the patterns of HBV infection and to optimize their management.

Results: The prevalence of HBsAg-positive patients was 16.5\% (199/ 1203). $71.44 \%$ of these subjects were from Africa, $16.66 \%$ from Eastern-Europe, $9.52 \%$ from Asia and $2.38 \%$ from South-America. Mean age was 27 years (61.9\% male, $38.1 \%$ female). HBeAg was positive in 373 patients (30.9\%). Respectively, 8.7 and $15 \%$ of $\mathrm{HBV}$ infected immigrants were co-infected with HCV and HDV. The diagnostic categories assigned were: inactive carrier state $58.7 \%$, chronic hepatitis B $31.6 \%$ and HBV-related cirrhosis $9.7 \%$.

Conclusions: Chronic infection with HBV is an important health issue in immigrated patients, partly as a result of absence of vaccine prophylaxis in many countries. Although the incidence of HBV in Italy has dramatically reduced, there is a risk of a rise in HBV infections, as result of the increase of imported cases through immigration from highly endemic countries. Another alarming finding of this study is the high prevalence of anti-HDV antibodies in immigrants.

\section{PP04-39}

Molecular Epidemiology of Hepatitis B Virus Transmission in Children of HBV-related Chronic Liver Disease (CLD) Patients

M.Y. Iskandar ${ }^{1}$, H. Gunardi ${ }^{1}$, T. Turyadi ${ }^{2}$, P. Gayatri ${ }^{1}$, R.A. Gani ${ }^{3}$, D.H. Muljono ${ }^{2}$

${ }^{1}$ Department of Pediatrics, University of Indonesia; ${ }^{2}$ Eijkman Institute of Molecular Biology; ${ }^{3}$ Department of Internal Medicine, University of Indonesia, Jakarta, Indonesia

Background: Hepatitis B virus (HBV) infection is a global public health problem. One HBV-infected family member is potential as a source of intrafamilial transmission. We studied HBV epidemiology and characteristics within families of chronic hepatitis B (CHB) patients at Cipto Mangunkusumo Hospital, Jakarta, Indonesia.

Methods: All family members [index cases (IC), spouses, and 1-18 y.o children] of $\mathrm{CHB}$ patients were tested for HBsAg and anti-HBc. Families with at least one anti-HBc-positive child were studied. HBV DNA isolated from all anti-HBc-positive subjects was analyzed over the surface, pre-core and basal core promoter (BCP) regions by polymerase chain reaction and sequencing. $\mathrm{HBsAg}$-positive subjects were tested for $\mathrm{HBeAg}$ and anti-HBe.

Results: Among 94 children of 46 IC, anti-HBc was detected in 19 children of 14 IC (male/female 8/6), and in 9 of $14 \mathrm{IC}^{\prime}$ s spouses. HBsAg was detected in 10 children and in 2 spouses. HBV DNA was identified in all IC, 16 children (10 with HBsAg), and 4 spouses (2 with $\mathrm{HBsAg}$ ). HBV genotype/serotype distribution in children was comparable between male and female IC. Majority of HBV DNA sequences found in children, regardless the HBsAg status, were identical to those of corresponding IC, including some mutations in the surface, pre-core and BCP regions. Mutations in HBsAg ' $\mathrm{a}$ ' determinant were detected, either singly or in combination, in 20 of 34 HBV DNA positive subjects; mutants associated with $\mathrm{HBsAg}$ detection failure-G145R, T143S/M, and Y161F- were detected in 2, 13 , and 10 subjects, respectively. Pre-core 1,896 mutants were found in 7 subjects, of whom 5 were HBeAg-negative, while BCP T1762/ A1764 mutants were identified in 11 subjects, with 6 having $\mathrm{HBeAg}$ negative phenotype.

Conclusions: Family members of HBV-related CLD patients are at risk of acquiring infection and should be monitored regardless the HBsAg status. Further study is in progress to elucidate the implications of detected mutants.

\section{PP04-40}

A Prevalence Study of Hepatitis B Vaccination among Adults in Beijing and Cost-utility Analysis of Different Vaccination Strategies

H. Wang, L. Wang, $\mathrm{H}$. Li

Department of Epidemiology Institute of Basic Medicine, Peking Union Medical College and Chinese Academy of Medical Science, Beijing, China

Hepatitis B virus (HBV) infection is one of the important global health problems, especially in China. To investigate the prevalence of $\mathrm{HB}$ vaccine among the adults in Beijing and understand its corresponding health economics will contribute to the establishment of a better hepatitis B vaccine immunization strategy for the adults' in Beijing. A populationbased multi-stage sampling as well as a hospitable based random sampling was used to collect the subjects. Questionnaire survey was conducted among 1,449 subjects with different HBV infection outcomes to estimate the costs and some of data was verified from electronic medical record from hospital. And 1,064 of them who finished EQ-5D scale was used to investigate life quality for the subjects with different health-states. A 7-health-state 1-year-cycle Markov model was developed to estimate lifetime HBV related events for a cohort of 20 -year old by TreeAge, among which annual transition probabilities between different health-states were derived from published literature. The model was calibrated using data from published literatures and national HBV serosurvey in 2006. Markov model was also used to do cost-utility analysis of different hepatitis B vaccine immunization strategy for adults in Beijing. Our study found that: Firstly, the maximum economic benefits will be gained when all the adults with age of 20 years or older are vaccinated. Both the current vaccination strategy and vaccination adults at high risk will not achieve the desired economic benefits. Secondly, the conclusion will not change with the different screening strategy. Thirdly, sensitivity analysis showed that Hepatitis B vaccination rate in adult should reach at least $60 \%$ to keep the conclusion true. So, it is recommended that hepatitis B vaccine should be inoculated among the whole vulnerable adults after serologic testing is obtained in Beijing. Also the hepatitis B vaccine rate should reach at least $60 \%$.

\section{PP04-41}

The Assessment Report for Beijing's Health Promotion in Primary Schools in Beijing, China

Y. Er ${ }^{1}$, L. Teng ${ }^{1}$, J. Duan $^{1}$, W. Cao ${ }^{2}$

${ }^{I}$ Beijing CDC; ${ }^{2}$ Haidain CDC, Beijing, China 
Objective: To assess the effects and implementation of the Beijing's health promotion program in primary school in order to provide basis for knowledge of hepatitis prevention brought into the school health education. Methods: Stratified cluster sampling from pupils and one of their parents in 12 primary schools, cast scene investigation with the self-made questionnaire in the unit of class, and interview the school doctors and head teachers, and also check the program files to understand activities. Results: The Beijing's health promotion program in primary school was well implemented according to the design covering $100 \%$ target population, and the rate of awareness in pupils was over the level of $95 \%$. The rate of hepatitis A and B vaccine has reached $94.33 \%$ and $96.47 \%$ respectively.

Conclusion: The Beijing's health promotion program in primary school get the end of promoting school heath and vaccination work, whose the intents have been achieved, discovered a new kind of model in hepatitis health promotion, and provide valuable experiences for bringing knowledge of hepatitis into school health education.

\section{PP04-42}

High Prevalence of Occult Hepatitis B virus (HBV) Infection among Blood Donors (BD) with Hypertransaminasemia in Slovakia

E. Lovrantova ${ }^{1}$, M.R. Pijak ${ }^{2}$, S. Adamcova-Selcanova ${ }^{3}$, M. Peprny ${ }^{4}$, V. Csibova ${ }^{5}$ ${ }^{I}$ Infectious Diseases, F.D.Roosevelt Faculty Hospital, Banska Bystrica; ${ }^{2}$ Slovak Medical University, Bratislava; ${ }^{3}$ Hepato-

Gastroenterology, F.D.Roosevelt Faculty Hospital; ${ }^{4}$ National Service for Blood Transfusion, Banska Bystrica; ${ }^{5}$ University Hospital, Bratislava, Slovak Republic

Background: Recently, occult HBV infection (OBI) was recognized as the absence of circulating hepatitis B surface antigen (HBsAg) in individuals positive for serum or tissue HBV DNA, irrespective of other HBV serological markers. Hence, OBI is one of the main challenges for blood transfusion services. We present our first results from a prospective pilot study on prevalence of OBI among BD in Slovakia.

Methods: From March 2008 to March 2010, 65010 consecutive BD without history of previous HBV infection or other known risk factors were screened for HBsAg and antibodies to the hepatitis B core antigen (anti-HBc). In addition, serum HBV DNA was examined in a subgroup of BD with anti-HBc positivity and elevated serum alanine aminotransferase (ALT) levels (at least 2 times the upper limit of normal). Serological markers for HBV infection were evaluated by a commercially available ELISA kits (Abbott Laboratories) and were confirmed by Western blot analysis. Serum HBV DNA levels were measured by COBAS TaqMan, Roche Molecular System with a lower limit of detection, $20 \mathrm{IU} / \mathrm{mL}$. The study was performed in four large centres from different regions of Slovakia.

Results: Anti-HBc positivity was detected in $0.77 \%(513 / 65010)$ of all examined subjects. Elevation of ALT was present in 11\% (61/442) of anti-HBc positive subjects. Among anti-HBc positive subjects with elevated ALT, low level viremia (HBV DNA below $200 \mathrm{IU} / \mathrm{ml}$ ) was found in $24.6 \%(15 / 61)$.

Conclusions: A screening of 65,010 potential BD from different regions in a low endemic country revealed a high percentage (24.6\%) of OBI among anti-HBc positive subjects with hypertransaminasemia. These results deserve special attention because in BD with positive anti$\mathrm{HBc}$ only, most reports do not mention ALT levels. Further studies are thus warranted to examine the role of virological markers in screening of $\mathrm{OBI}$ in anti-HBc positive BD with both normal and elevated ALT.

\section{PP04-43}

Occult HBV Infection among Iranian Thalassemic and Hemophilic Patients with Chronic Hepatitis C

S.M. Alavian ${ }^{1}$, N. Jonaidi ${ }^{1}$, J. Tavallai ${ }^{2}$, S.Y. Saiedi Hosseini ${ }^{1}$

${ }^{1}$ Baqiyatallah Research Center for Gastroenterology and Liver Disease (BRCGL); ${ }^{2}$ Baqiyatallah University of Medical Sciences, Tehran, Iran
Introduction: Occult infection with hepatitis B virus (HBV), defined as persistent detectable viral genome in serum or liver while HBV surface antigen ( $\mathrm{HBs} \mathrm{Ag}$ ) is undetectable. Several studies showed relation between occult $\mathrm{HBV}$ and hepatocellular carcinoma and cryptogenic cirrhosis. We studied prevalence of occult HBV infection in Thalassemic and hemophilic patients with chronic HCV.

Method and materials: We studied Thalassemic and hemophilic patients with chronic HCV in Tehran hepatitis centre (THC) between years 2009-2010. HBs Ag positive patients were excluded. We used real time PCR for detecting HBV DNA.

Results: 145 patients, $126(86.9 \%)$ male, 35(24.2\%) Thalassemic,75 (51.7\%) hemophilia A, 20 (13.8\%) hemophilia B and 15 (10.3\%) with other coagulation disorders were evaluated.HCV genotype 1a $53.8 \%$ and $3 \mathrm{a} 33.8 \%$ was the most frequent genotype. HBV DNA was negative in all patients.

Conclusion: Occult HBV infection was extremely rare in Thalassemic and hemophilic patients with chronic HCV in our study, we suggest more study about this subject with other tests.

\section{PP04-44}

A Cross Sectional Multi-center Epidemiological Study in Asian Children and Adolescents with Chronic Hepatitis B (CHB)

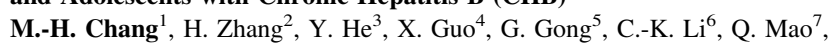
S. Dang ${ }^{8}$, P. $\operatorname{Kar}^{9}$, S. Treepongkaruna ${ }^{10}$, N. Tan ${ }^{11}$, A. Uddin ${ }^{12}$, A. Trylesinski ${ }^{13}$, C. Avila ${ }^{13}$

${ }^{1}$ Department of Pediatrics, National Taiwan University Hospital, Taipei, Taiwan ROC; ${ }^{2}$ The 302 Hospital of China Military, Beijing; ${ }^{3}$ Tongji Med. College, Union Hospital, Wuhan; ${ }^{4}$ Department of Hepatology and Endocrine, Beijing You'an Hospital, Beijing; ${ }^{5}$ The Second Xiangya Hospital, Chongsha, China; ${ }^{6}$ Prince of Wales Hospital, The Chinese University of Hong Kong, Hong Kong, Hong Kong SAR; ${ }^{7} 1$ st Affiliated Hospital of 3 rd Military Med. Univ. of PLA, Chongqing; ${ }^{8}$ The Second Affiliated Hospital of Medical School of Xian Jiaotong University, Xi'an, China; ${ }^{9}$ Maulana Azad Medical College \& Hospital, New Delhi, India; ${ }^{10}$ Ramathibodi Hospital, Mahidol university, Bangkok, Thailand; ${ }^{11} \mathrm{KK}$ Women's and Children's Hospital, Singapore, Singapore; ${ }^{12}$ Novartis Pharma, East Hanover, NJ, USA, ${ }^{13}$ Novartis Pharma AG, Basel, Switzerland

Background/aims: Nearly 400 million people worldwide are chronically infected with the Hepatitis B virus (HBV). Vertical and perinatal transmission are the predominant routes of infection in Asia. Epidemiological data in children is limited and continues to alter, especially with the introduction of hepatitis B vaccinations. We aimed to collect epidemiological data in children and adolescents with $\mathrm{CHB}$ on prevalence of $\mathrm{HBeAg}$ positive(+)/negative(-) disease, ALT levels, family history and history of HBV transmission.

Patients and methods: Multi-center, observational, cross-sectional study collecting retrospectively epidemiological data from children/ adolescents with CHB aged 2-18 years. Data was collected anonymously from 396 Asian pediatric patients from 7 Asian countries within 15 months who were either HBsAg+ or had a history of CHB. Patients were grouped in 3 age groups (2-6, 7-12, 13-18 years).

Result: Majority of patients $(n=215)$ was in age group 13-18 years $(54.3 \%)$ with mean age 12.1 years. All patients were Asian, 335 (85\%) Chinese ethnicity. 184 (46\%) patients had been immunized for HBV and 65 (15\%) had received HBIG. 199 (50\%) pediatric patients were infected through confirmed mother-to-child transmission, while route of infection was unknown for 161 patients $(41 \%)$. Biopsy was performed in $23(6 \%)$ patients. $359(91 \%)$ patients were $\mathrm{HBsAg}+$ and $251(63 \%) \mathrm{HBeAg}+(26 \% \mathrm{HBeAg}-, 11 \%$ unknown). Out of the 253 patients $(64 \%)$ with known HBV DNA levels, $162(64 \%)$ had $>10^{5} \mathrm{IU} / \mathrm{ml}$ HBVDNA, while only $60(24 \%)$ had $<10^{3} \mathrm{IU} / \mathrm{mL}$ HBVDNA. Median ALT levels were $32 \mathrm{IU} / \mathrm{ml}$ with $25 \%$ of patients with $>57 \mathrm{IU} / \mathrm{mL}$. Only a minority $(23-26 \%)$ of pediatric patients are currently being treated with no difference between age groups. During 
the last 12 months, $92-97 \%$ of patients had stable or improved disease conditions. For the majority $(53-58 \%)$ of untreated patients no treatment is currently planned.

Conclusions: In Asia the majority of pediatric CHB patients has been infected through Mother-to-child transmission and the majority of diagnosed patients do not require treatment under current therapy indication.

\section{PP04-45}

Pattern of Chronic Hepatitis B Infection in Thai Patients

T. Sriprayoon ${ }^{1}$, C. Laohaphand ${ }^{2}$, T. Tanwandee ${ }^{3}$

${ }^{1}$ Gastroenterology, Siriraj Hospital, Mahidol University; ${ }^{2}$ Medicine, Chulabhorn Cancer Center; ${ }^{3}$ Gastroenterology, Mahidol University, Bangkok, Thailand

Background: The prevalence of HBV infection varies throughout the world; it is highest in developing countries, $75 \%$ of these in Asia where hepatitis B is the leading cause of chronic hepatitis, cirrhosis and hepatocellular carcinoma (HCC). The majority of infections are contracted postnatally or perinatally and the prevalence of $\mathrm{HBeAg}$ negative chronic hepatitis B increased from recent studies.

Aim: To determine the pattern of chronic hepatitis B in Thai patients Methods: This study was a cross-sectional study. We invited all chronic hepatitis B patient aged 20-65 years both males and females to participate in this protocol. The demographic data, stage of liver disease, development of complication and survival were registered. Co-infection with HCV or HIV and decompensated cirrhosis were excluded. All patients were tested for LFT, HBeAg, HBV DNA viral load, Alpha fetoprotein, ultrasound upper abdomen.

Results: A total of 1,000 cases of chronic hepatitis B patients were enrolled (482 men, 518 female; mean age 39 year). There were 151 patients $(15.1 \%)$ age less than 30 years. Four-fifth of cases $(835 / 1,000)$ was $\mathrm{HBeAg}$ negative chronic hepatitis B. In these group, 518/835 (62\%) had HBV viral load less than 2,000 IU/ml. For HBeAg positive chronic hepatitis B, there were 165/1,000 (16.5\%) and almost all had high viral load more than 2,000 IU/ml (97\%). Liver cirrhosis was initially diagnosed by ultrasound in 119 cases $(11.9 \%)$. There were 3 cases of HCC developed ( 3 male). The mean age of HCC presentation was approximately $55 \pm 6.7$ year, range $49-63$. The incidence of cirrhosis and $\mathrm{HCC}$ appeared to be higher in $\mathrm{HBeAg}$ negative patients. Conclusions: Most chronic hepatitis B patients in Thailand are $\mathrm{HBeAg}$ negative with $38 \%$ viremic regardless of ALT. These findings suggest that HBV viral load is important to identify active viral replication.

\section{PP04-46}

Predictive Factors of Spontaneous Hepatitis B Surface Antigen Seroclearance

M. Arai, S. Togo, A. Tawada, T. Chiba, T. Kanda, K. Fujiwara, F. Imazeki, O. Yokosuka

Department of Medicine and Clinical Oncology, Graduate School of Medicine, Chiba University, Chiba, Japan

Background and aims: Chronic infection with HBV is a major cause of mortality worldwide. Loss of hepatitis B s antigen (HBsAg) usually results in normalized serum alanine aminotransferase (ALT) and decreased HBV-DNA levels, and may confer a better clinical outcome. This study aimed to examine the predictors of HBsAg seroclearance using a long-term follow-up.

Methods: Age, sex, ALT, platelet counts, status of hepatitis B e antigen, HBV-DNA levels, genotype, use of antiviral drugs, and quantitative serum HBsAg at baseline were analyzed for a total of 423 HBV carriers who were followed in Chiba University Hospital (Japan) between 1985 and 2008. The measurement of HBsAg was performed by CLEIA method using HISCL-2000i (Sysmex Corporation, Kobe, Japan). Cox proportional hazards models were used to estimate HBsAg seroclearance rate ratios associated with various determinants.
Results: HBsAg seroclearance occurred in 26 participants during 2,570 person-years of follow-up evaluation, giving a $1.01 \%$ annual seroclearance rate. Hepatocellular carcinoma was occurred in one patient after HBsAg seroclearance. 24 out of 26 patients with HBsAg seroclearance had not used the antiviral drugs. Multivariate Cox hazard regression analysis identified low levels of quantitative serum HBsAg at baseline and male as independent and predictive factor for the HBsAg seroclearance, showing an adjusted odds ratio of 0.46 (95\% confidence interval [CI], 0.31-0.68) and 3.48 (95\%CI, 1.33-9.12), respectively.

Conclusions: This study revealed that a low HBsAg level and male were important factors affecting the natural seroclearance of $\mathrm{HBsAg}$.

\section{PP04-47}

Hepatitis B Virus Genotypes in Morocco

O. Derdabi ${ }^{1}$, Y. Cherradi ${ }^{1}$, R. Afifi ${ }^{1}$, S. Benjelloun ${ }^{2}$, M. Benazzouz ${ }^{1}$, A. El Feydi Essaid ${ }^{1}$

${ }^{I}$ Department of Hepato-Gastroenterology (Medecine C), Ibn Sina Hospital, Rabat; ${ }^{2}$ Department of Virology, Pasteur Institute of Morocco, Casablanca, Morocco

Background and objective: Hepatitis B virus genotypes distribution is heterogeneous around the world. Morocco is an area of intermediate prevalence. The goal of this study is to determinate the genotype(s) present in Moroccan population, and to search an eventual correlation with various aspects of the infection

Material and methods: One hundred thirty chronically infected patients were considered prospectively. Inclusion criteria were: age $>18$ years, serum HBV DNA $>200 \mathrm{Ui} / \mathrm{ml}$ dating less than two weeks, while exclusion criteria were co-infection with HIV or hepatitis $\mathrm{C}$ or D. Participation consent was obtained from all the patients and data were collected in a pre-established form including: epidemiological aspects (comorbidities, transmission way, discovery circumstances), laboratory and abdominal ultrasonography findings, serological profile, HBV DNA level and histological lesion evaluated by liver biopsy. Sera were sent to "Pasteur institute" for genotyping. The method used was gene $\mathrm{S}$ sequencing with a phylogenetic analysis.

Results: The mean age was 42.7 years and male/female sexe ratio was 01.1. Non medical teeth care was the most frequent transmission way $(59.2 \%)$, whereas the infection was fortuitously discovered in $81.6 \%$. Thirty percent of patients had elevated transaminase level and $06.25 \%$ had cholestasis. Only two patients $(2.15 \%)$ were positive for hepatitis B e antigen. Liver histological lesions were found in $47 \%$ of the patients who benefited from a liver biopsy. Ninety three patients out of one hundred thirty were successfully genotyped (no PCR amplification, problem of sequencing...). Two genotypes were found: "D" which was predominant $(93.55 \%)$ and " $\mathrm{A}$ " found in 06 patients $(06.45 \%)$

Conclusion: Our study showed that genotype D is widely predominant $(93.55 \%)$ in our country and that it is highly associated to mutant virus $(97.85 \%)$.

\section{PP04-48}

Trends for the Chronic Hepatitis B Patients in Thailand, by Analysis of HBeAg Status: Chulabhorn Hospital Cohort Study

C. Laohapand ${ }^{1}$, T. Sriprayoon ${ }^{1}$, T. Tanwandee ${ }^{2}$, N. Kositpesat,

P. Thungsuppawattanakit, N. Triphuridet, P. Praditsuktavorn, J. Boonthai,

C. Chanachote, C. Suwanwela, P. Pattaranutaporn

${ }^{1}$ Chulabhorn Hospital; ${ }^{2}$ Division of Gastroenterology, Department of Medicine, Faculty of Medicine Siriraj Hospital, Mahidol University, Bangkok, Thailand

Background and objective: Chronic hepatitis B (CHB) infection is still one of the main health concerns. In 2010, Chulabhorn hospital launched a Cohort study to enroll Thai CHB patients for surveillance and treatment if required. This study is aimed to determine the pattern of CHB in Thailand, by analysis of Hepatitis B e-Antigen (HBeAg) Method: This is a cross-sectional study including 1,000 patients, aged 20 to 65 years. Physical examination, HBV serology, HBV DNA viral 
load and ultrasound were performed. The patients were excluded if they had co-infection with HCV, HIV, hepatic decompensation or evidence of hepatocellular carcinoma.

Result: Of these 1,000 patients, 835 cases were $\mathrm{HBeAg}$ seronegative $(83.5 \%)$ and 165 cases were $\mathrm{HBeAg}$ seropositive (16.5\%). There were no different of sex between the two groups but the HBeAg seronegative group was 10 years older than seropositive group. Moreover, HBeAg-negative patients had lower serum ALT $(p<0.001)$ and lower serum HBV DNA level $(p<0.001)$. verall, there were no difference between patients who initially found cirrhosis from ultrasonography in both groups.

Summary: These figures were different from the past understanding that Thai patients had higher rate of $\mathrm{HBeAg}$-positive $\mathrm{CHB}$ patients. The trend towards HBeAg-negative predominant of $\mathrm{CHB}$ infection has also been observed as in many countries.

Table 1 Comparison of $\mathrm{HBeAg}$-negative and $\mathrm{HBeAg}$-positive chronic hepatitis B patients

\begin{tabular}{llll}
\hline Variable & $\begin{array}{l}\text { Positive } \\
(n=165)\end{array}$ & $\begin{array}{l}\text { Negative } \\
(n=835)\end{array}$ & $P$ value \\
\hline Sex & & & NS \\
Male $(\%)$ & 47.88 & 46.26 & \\
Female (\%) & & 51.74 & \\
Age (mean+SD) (year) & $34 \pm 10.4$ & $44 \pm 20.7$ & $<.0001$ \\
Serum ALT level & & & $<.0001$ \\
$\leq 1.5 \times(\%)$ & 69.70 & 92.22 & \\
$1.5 \times-5 \times(\%)$ & 22.42 & 7.54 & \\
$>5 \times(\%)$ & 7.88 & 0.24 & \\
Viral load (log 10 IU/ml) & $7.16 \pm 1.79$ & $2.90 \pm 1.50$ & $<.0001$ \\
Ultrasound & & & 0.53 \\
Cirrhosis & 16.34 & 11.02 & \\
No Cirrhosis & 83.64 & 88.98 & \\
\hline Comparis of & & & \\
\hline
\end{tabular}

Comparison of HbeAg CHB patients

\section{PP04-49}

Working towards Development of the First Population-based Chronic Hepatitis B Registry in Thailand

W. Sukeepaisarnjaroen ${ }^{1}$, T. Tanwandee ${ }^{2}$, T. Piratvisuth ${ }^{3}$, S. Thongsawat $^{4}$, T. Sanpajit ${ }^{5}$, S. Chonprasertsuk ${ }^{6}$, W. Jeamsripong ${ }^{7}$, E. Sripariwuth ${ }^{8}$,

P. Komolmit ${ }^{9}$, T. Patcharatrakul ${ }^{10}$, R. Boonsirichan ${ }^{11}$, C. Bunchorntavakul ${ }^{12}$, S. Tuntipanichteerakul ${ }^{13}$

${ }^{1}$ Department of Medicine, Srinagarind Hospital, Khon Kaen University, Khon Kaen; ${ }^{2}$ Department of Medicine, Siriraj Hospital, Mahidol University, Bangkok; ${ }^{3}$ Department of Internal Medicine,

NKC Institute of Gastroenterology and Hepatology, Songklanagarind Hospital, Prince of Songkla University, Songkhla; ${ }^{4}$ Department of Internal Medicine, Maharaj Nakorn Chiang Mai Hospital, Chiang Mai University, Chiang Mai; ${ }^{5}$ Department of Medicine, Phramongkutklao Hospital, Bangkok; ${ }^{6}$ Department of Medicine, Thammasat Chalermprakiet Hospital, Thammasat University, Pathumthani; ${ }^{7}$ Department of Medicine, Buddhachinaraj Hospital; ${ }^{8}$ Department of Medicine, Naresuan University Hospital, Naresuan University, Phitsanulok; ${ }^{9}$ Department of Medicine, King

Chulalongkorn Memorial Hospital, Chulalongkorn University;

${ }^{10}$ Department of Medicine, Police General Hospital; ${ }^{11}$ Department of Medicine, BMA Medical College and Vajira Hospital; ${ }^{12}$ Department of Medicine, Rajavithi Hospital; ${ }^{13}$ Department of Medicine, Bhumibol Adulyadej Hospital, Bangkok, Thailand

Background: Chronic Hepatitis B (CHB) is an important health problem in Thailand which has placed significant burden to country, especially when disease progresses to cirrhosis and hepatocelluar carcinoma. Data on epidemiological and viral characteristics, treatment patterns and associated outcomes of CHB in Thailand are limited and have never been systematically collected. These data are important and can be used to provide effective management of $\mathrm{CHB}$ patients. Therefore, Liver Society (Thailand) has sponsored the study in which objectives are to establish a national registry to collect epidemiologic data of all $\mathrm{CHB}$ patients who receive treatment that reflects routine practice, to evaluate short- and long-term treatment outcomes of CHB management, and to create a model to identify predictors of treatment effectiveness.

Methods: An electronic database was developed to capture data from 13 centers throughout Thailand. Patients will be included if they are diagnosed with CHB, are to receive treatment as per institution/country standard of care, are naïve to interferon, and provide their consent.

Results: As of October 2010, data from 121 patients were captured with $80 \%$ enrolled during past 6 months. Mean age was 43.7 years and $76.9 \%$ were male. Modes of infection were unknown and from perinatal transmission in $85 \%$ and $14 \%$ of patients, respectively. Of those who were tested, 87 and $13 \%$ were infected with genotypes $\mathrm{C}$ and $\mathrm{B}$, respectively. $85 \%$ had no cirrhosis. $42 \%$ were Anti-HBe positive. Quantitative method was employed to test hepatitis B surface antigen (HBsAg) in 55 patients. Mean ALT was 8 times above upper limit of normal. Data up to week 12 of treatment period will be available during presentation.

Conclusions: This is the first population-based registry for CHB with great collaboration among centers throughout Thailand. The availability of complete data will be crucial for developing effective management of Thai CHB patients.

\section{PP04-50}

Association of TNF-related Gene Polymorphism with Liver Cancer and Cirrhosis among Chronic Hepatitis B Patients

G.H. Lee ${ }^{1}$, R. Chan ${ }^{2}$, M.O. Aung ${ }^{3}$, B. Mak ${ }^{3}$, S.G. Lim ${ }^{4}$

${ }^{1}$ Gastroenterology and Hepatology, National University Health System; ${ }^{2}$ National University Hospital; ${ }^{3}$ National University Health System; ${ }^{4}$ National University of Singapore, Singapore, Singapore

Background: The influence of host factors in the pathogenesis of chronic hepatitis B virus (HBV) infection remains unclear. Polymorphism of the tumour necrosis factor genes (TNF) was known to contribute to the pathogenesis of hepatitis $\mathrm{C}$ and HIV infections. The aim of this study was to study the relationship between single nucleotide polymorphisms in the $T N F$ related genes, and the clinical outcomes in patients with chronic hepatitis B, including liver cirrhosis and hepatocellular carcinoma (HCC).

Methods: Stored sera from phenotypically characterised chronic hepatitis B patients from an Asian tertiary centre were studied using a nested case control method. Genotyping of the $T N F-\alpha$ gene between positions 129 and 3800, TNFRSF1A gene between positions 20 to 9915, and the TNFRSF1B gene between positions 22887 and 42444 were performed, with 31 allele positions analysed. Odds ratios (OR) were analysed using SPSS software, and $\mathrm{p}$ value was derived with two-tailed Fisher Exact test.

Results: One hundred and one hepatitis B patients, with mean followup of 7.5 years, were analysed in four outcome groups: liver cirrhosis only $(n=30)$, HCC without cirrhosis $(n=15)$, both cirrhosis and HCC $(n=19)$ and control groups (HBV patients with no complication, $n=37)$. Compared with the controls, $T N F-\alpha 148 \mathrm{C}>\mathrm{M} / \mathrm{A}$ was strongly associated with liver cirrhosis with HCC (OR 11.14, 95\% CI 2.82-43.66, $p<0.001$ ), but $T N F-\alpha 1499 \mathrm{G}>\mathrm{R}$ was negative associated with cirrhosis and HCC (OR $0.039,95 \%$ CI 0.02-0.84). HCC without cirrhosis was reduced in patients with TNFRSF1A 671T $>$ G (OR 0.047, 95\% CI 0-0.89) and TNFRSF1A 9915T >C (OR 0.041, 95\% CI 0-0.79) polymorphisms. There was no significant association between TNFRSF1B polymorphisms with HBV complications in the study population. 
Conclusions: Polymorphisms in TNF-related genes are associated with increased or decreased incidence of $\mathrm{HCC}$ and cirrhosis in HBV patients, suggesting the roles of the host immunogenetic factors in disease progression.

\section{Poster Presentation 05: Hepatitis B: Treatment}

\section{PP05-01}

The Study of the Immunosuppression Effects of $\mathrm{CD}^{+} \mathrm{CD}^{+} 5^{+}$Regulatory $\mathrm{T}$ Cells on Dendritic Cells from Patients with Chronic Hepatitis B Y. Xuebing, Z. Peipei, X. Juanjuan

Department of Infectious Diseases, The First Affiliated Hospital of Xuzhou Medical College, Xuzhou, China

Objective: To investigate the rule of the regulatory $\mathrm{T}$ cells (Treg) suppressing the dendritic cells in the pathogenesis of $\mathrm{CHB}$, and to find out new therapy for CHB.

Methods: 15 cases with $\mathrm{CHB}$ and 15 health persons as normal control (NC) were enrolled in this study. PBMCs were isolated from CHB and NC with density gradient centrifugation. Dendritic cell (DC) generated from PBMC were cultured and induced by GM-CSF, IL-4, $\mathrm{TNF}-\alpha$ in vitro. MACS was applied to purify Treg and $\mathrm{CD} 4^{+} \mathrm{CD} 25^{-} \mathrm{T}$ cell from PBMCs. Mature DC were combined with $\mathrm{CD} 4^{+} \mathrm{CD} 25^{-} \mathrm{T}$ cell and cocultured with Treg which derived from different sources and with different proportionalities for three days. MTT assay was used to determine the suppressing index. Treg were added to DC at different times. Costimulatory molecules CD80, HLA-DR on DC surface were analyzed by flow cytometry.

Results:

(1) Treg from CHB and NC suppressed DC immunity. Whether DC came from $\mathrm{CHB}$ or $\mathrm{NC}$, Treg from $\mathrm{CHB}$ suppressed DC higher than from NC $(P<0.01)$. Treg from $\mathrm{CHB}$ had the highlyest suppression to DC from CHB $(P<0.01)$. The second was the treg from $\mathrm{CHB}$ to $\mathrm{DC}$ from NC. But the suppression of Treg from NC to DC which came from $\mathrm{CHB}$ or NC had no statistic significance $(P>0.05)$.

(2) DC were cultured with Treg at different proportions 1:1, 1:2, 1:10, 1:100 in three days. Treg suppressed DC immunity. Followed by the higher of the proportion, the rule was increased. The suppressing index was also increased.

(3) The expression level of CD80 and HLA-DR in DC decreased, when adding Treg to the culture system at different times the first day, the third day, the fifth day.

Conclusions: Treg from CHB can suppress the immunity of DC higher than from $\mathrm{NC}$, and it show time and dose dependent. It also can suppress the immunity of DC by decreasing the expression level of surface marker.

\section{PP05-02}

Association of Hepatitis B e-Antigen and HBV DNA Level with Humoral Immunity During Nucleoside Antiviral Treatment of Chronic Hepatitis B C. Xie, D.-Y. Xie, S.-B. Xie, L. Peng, S.-Q. Zhang, J.-Q. Xie, B.-L. Lin, Z.-L. Gao

The Third Affiliated Hospital of Sun Yat-sen University, Guangzhou, China

Objective: To evaluate the variation and relationship between humoral immunity, $\mathrm{HBeAg}$ and HBV-DNA when hepatitis B patients undergoing treatment with nucleoside / nucleotide analogues.

Methods: 157 chronic hepatitis B patients were recruited and divided into chronic hepatitis B group (CHB), liver cirrhosis group (LC) and severe hepatitis B group (SHB) according to liver function. Of which 50 patients received antiviral treatment (Lamivudine $100 \mathrm{mg} / \mathrm{d}$, or entecavir $0.5 \mathrm{mg} / \mathrm{d}$, or telbivudine $600 \mathrm{mg} / \mathrm{d}$ ). Serum levels of
Complement 3 and 4 (C3, C4), C-reaction protein (CRP), Hemolytic Complement (CH50), immunoglobulin G, M, A (IgG, IgM, IgA), hepatitis B surface antigen ( $\mathrm{HBsAg}$ ) and hepatitis B e antigen $(\mathrm{HBeAg})$ were detected by ELISA and Immunoturbidimetry. HBV DNA was quantified by real-time PCR before and after the nucleoside antiviral therapy in the $1,2,3,4$ weeks.

Results: Serum IgA, IgM and CRP levels of SHB patients were significantly higher than that of the CHB patients and LC patients. C3, $\mathrm{C} 4, \mathrm{CH} 50$ levels of $\mathrm{CHB}$ patients were significantly higher than the SHB patients and LC patients. HBeAg positive patients had a higher level of IgM, IgG and lower $\mathrm{C} 3$ level than $\mathrm{HBeAg}$ negative patients. After the treatment, all the patients of three groups had a significant decrease of immunoglobulin and HBV DNA levels. The serum complement level of the entire patient was significantly elevated since week 4. Fourty percent $\mathrm{HBeAg}$ positive patients got e antigen serum conversion. $\mathrm{C} 3$ was related to $\mathrm{HBeAg}$ and HBV DNA level $(r=0.87$, and $r=-0.78$, respectively, $P<0.05$ ).

Conclusion: The severity of hepatitis B is different at different levels of humoral immunity. CRP, C3, C4, CH50 and IgM, IgA well reflect the immune function in patients. Changes in $\mathrm{C} 3$ level can predict the efficacy of antiviral therapy. The anti-viral activity of nucleoside / nucleotide analogues may be related closely to its role of antiinflammation and immunomodulation.

\section{PP05-03}

The Profile of the Co-infected HIV and B Hepatitis Patients in the Infectious Diseases Clinic of Iasi

C. Manciuc, C. Dorobat, C. Nicolau, L.J. Prisacaru, A. Vata

HIV / AIDS Department, The Infectious Diseases Clinic of Iasi, Iasi, Romania

Aims: The aim of the study was to reveal the incidence and to determine the biological-clinical profile of co-infected HIV and hepatitis B patients.

Methods: We retrospectively analysed the clinical records of the patients monitored in the HIV AIDS Regional Centre of Iasi.

Results: In the North-East region of Romania we have monitored 1,309 patients out of whom 1,147 are active ones; $22.8 \%$ are coinfected with $\mathrm{HBV}$ and $0.9 \%$ with HVB+HVD, which represents 272 patients. The age median is 21.6 years. $83 \%$ are poliexperimented with a median of 5 therapeutic schemata. The CD4 median was $232 / \mathrm{mmc}$ with variations between 3 and 820 and the viral load from undetectable to more than 2 million copies $/ \mathrm{ml} .62 \%$ of the coinfected ones receive Lamivudine as basic therapy, $38 \%$ of them for more than five years. The new composites used in combined therapy are Tenofovir + Emtricitabine in $2.2 \%$ of cases and Raltegravir. More than $24 \%$ do not have an antiviral product B. None of the patients received Interferon. Conclusions: For the current virological resistance profile of HIV + HVB (+D) co-infected patients the therapeutic prospects recommend the new antivirals, due to the prolonged use of Lamivudine.

\section{PP05-04}

The Effectivness of Entecavir for the Treatment of Chronic Hepatitis B in Patients with Resistance to Lamivudine and Adefovir

Z. Koutoubi, M. Taha, A. Chebli

Internal Medicine, Tawam Hospital in Afiliation with Johns Hopkins Medicine International, Al-Ain, United Arab Emirates

Introduction: It is estimated that between 350 and 400 million people are chronically infected with hepatitis B virus (HBV). Chronic HBV infection leads to development of serious complications, such as cirrhosis and hepatocellular carcinoma. The ultimate goals of chronic hepatitis B therapy are to decreases the incidence of cirrhosis, endstage liver disease and hepatocellular carcinoma. Entecavir is a nucleoside analog that is active against both lamivudine and adefovir dipivoxil-resistant HBV. The aim of this study is to investigate the effectiveness of Entecavir in treating patients with chronic hepatitis B 
resistant to lamivudine and adefovir therapy in patients from United Arab Emirates.

Methods: In an observational prospective study, we followed 41 patients with chronic hepatitis B who received either lamivudine alone or in combination with adefovir. Twelve patients became resistant to lamivudine alone or in combination with adefovir therapy with significantly elevated hepatitis B viral load. Lamivudine and adefovir therapy were stopped and all 12 patients were started on entecavir $1 \mathrm{mg}$ daily, and patients were followed with viral load and liver function test.

Results: Twelve patients (29\%) with chronic hepatitis B who received either lamivudine alone or in combination with adefovir relapsed during the treatment. All 12 patients (100\%) responded to entecavir within an average of 3 months from starting the therapy. Hepatitis B e Ag was positive in 11 patients (92\%), and hepatitis B e $\mathrm{Ag}$ was negative in one patient $(8 \%)$.

Conclusions: All patients with chronic hepatitis B with resistance to lamivudine alone or in combination with adefovir responded to entecavir with significant reduction in the hepatitis B viral load. Entecavir was superior to lamivudine alone or in combination with adefovir in the treatment of chronic hepatitis B.

\section{PP05-05}

Interferon Therapy Decreased the Incidence of Hepatocellular Carcinoma in Patients with Chronic Hepatitis B: A Prospective Cohort Study J. You ${ }^{1}$, L. Zhuang ${ }^{2}$, H.Y. Chen ${ }^{1}$, X. Feng ${ }^{3}$, Y.H. Che ${ }^{4}$, Y.L. Cun ${ }^{5}$, Y.F. Zhang ${ }^{1}$, Y.S. Zhang ${ }^{6}$, B.Z. Tang ${ }^{1}$, M.L. Huang ${ }^{1}$, Y.L. Ma ${ }^{2}$, J.H. Huang ${ }^{7}$, S.M. Yan ${ }^{8}$, Q.Q. Wang ${ }^{2}$, L. Chen ${ }^{1}$, C. Chen ${ }^{1}$, J.M. $\mathrm{Li}^{1}$

${ }^{I}$ Department of Infectious Diseases, The First Affiliated Hospital of Kunming Medical University; ${ }^{2}$ Department of Hepatology, The Third People's Hospital of Kunming; ${ }^{3}$ Department of Laboratory Medicine, The First Affiliated Hospital of Kunming Medical University; ${ }^{4}$ Department of Medicine, The First People's Hospital of Kunming; ${ }^{5}$ Department of Tumor, The Third Affiliated Hospital of Kunming Medical University; ${ }^{6}$ Department of Gastroenterology, The First Affiliated Hospital of Kunming Medical University; ${ }^{7}$ Department of Infectious Diseases, The Yunnan General Hospital of The Chinese People's Armed Police Forces, Kunming; ${ }^{8}$ Department of Internal Medicine, The Third People's Hospital of Yunnan Province, Kunmking, China

Background/aims: To investigate incidence of Hepatocellular carcinoma (HCC) in patients with CHB those treated with interferon (IFN), and compare the results with that in the developing HCC from the non-IFN treated (NT) group.

Patients and methods: 262 patients with chronic HBV infection who started IFN therapy (6-48 months duration) from 1994 were enrolled in this study. The patients in the NT group were 60 patients without treatment for IFN from 1994. Presence of HCC was examined every 6-12 months by abdominal US or CT. Efficacy of IFN therapy was categorised as follows. Complete response (CR) was defined as persistent normalisation of ALT levels during IFN therapy and follow-up with a sustained serum HBV DNA-negative status. All other response patterns were regarded as non-complete response (NCR).

Results: Median follow-up periods of patients with CR (109 patients), NCR (153 patients) and NT (60 patients) were 13.6, 13.2 and 12.9 years, respectively. During follow-up, of 262 patients treated with IFN, patients developing HCC were a total of 10 patients $(3.82 \%), 4$ patients $(3.67 \%)$ in CR group, $6(3.92 \%)$ in NCR group and 12 patients $(20 \%)$ in NT group developed HCC. The 5-year rates of HCC incidence were $0 \%, 0 \%$ and $5.0 \%$ in the CR group, the NCR group and the NT group, respectively. Cumulative incidences of HCC in the patients with both CR and NCR were significantly lower than that in the patients with NT (vs. NT, OR $=5.4,95 \% \mathrm{CI}=1.5523 .98$; $\mathrm{OR}=5.06,95 \% \mathrm{CI}=1.6717 .21$, respectively).

Conclusion: The incidence of HCC in patients with $\mathrm{CHB}$ after IFN therapy was very low, and the incidences of HCC were lower in the patients with not only CR but also NCR than that in the patients with NT. The result indicates that IFN therapy decreased incidence of HCC in patients with $\mathrm{CHB}$.

\section{PP05-06}

Role of BioR in the Dynamics of Pro- and anti- Inflammatory Cytokines in Patients with Chronic Viral Hepatitis

A. Turcanu

Internal Medicine, University of Medicine and Pharmacy

'N.Testemitanu', Chisinau, Moldova

Introduction: T-cell immunoregulatory cytokines influence the persistence of hepatitis $\mathrm{C}$ and $\mathrm{B}$ virus (HCV, HBV) chronic infection and the extent of liver damage.

The aim of this study is to evaluate the biochemical, haematological parameters and profile of Th1/Th2 cytokines in HCV and HBV hepatitis before and after treatment with BioR.

Methods: Were included 42 patients with chronic viral hepatitis B and $\mathrm{C}$. The immunoenzymatic assay has been used to study the interleukins 1, 10 and TNF-alpha. Patients included in the study have been administered the injection solution of BioR, $1.0 \mathrm{ml}$ intramuscular, daily, during 10 days.

Results: The patients with HCV show a real amelioration of haemoglobin $(p<0.05)$, and of lymphocyte levels $(p<0.05)$ compared to patients with HBV. In result of the application of a therapy with BioR an evident diminution of cytolysis (ALT, AST) takes place both in patients with HBV $(p<0.05)$ and in the ones with HCV $(p<0.05)$. This indicates the fact that BioR plays the role of a hepatic protector. It has been also proven that BioR reduces gamma GTP in patients with $\mathrm{HCV}(p<0.05)$ compared to patients with $\mathrm{HBV}(p>0.05)$, indicating to the occurrence of a disintoxication. This study has shown that a treatment with BioR brings about the stimulation of production of IL 10, TNF alpha in patients with HCV and HBV. We consider this action a key mechanism of this preparate with antiviral effect, and we recommend it for treatment of persons with viral hepatitis.

Conclusions: This study indicates that the use of the BioR preparate in chronic viral $\mathrm{B}$ and $\mathrm{C}$ hepatitis is justified, given the fact that it acts as a hepatic protector, causes haematological regulation and has the disintoxication and immune-modulation effects.

\section{PP05-07}

Hepatoprotective Effects of Polygonum bistorta (Linn.) and its Active Principle against Liver Toxicity in Rats

D.K. Mittal

School of Studies in Zoology, Jiwaji University, Gwalior, India

Introduction: Many herbal preparations have been recommended in alternative system of medicine for the treatment of hepatic disorder. Drug-induced hepatotoxicity is still a significant unresolved clinical problem as liver is the most common site of damage. It is well known that overdoses of acetatminophen are hepatotoxic. The goal of the present work is to evaluate and compare the efficacy of root extract of Polygonum bistorta and tannic acid against toxicants induced damage in liver and kidney.

Methods: Male rats $(150 \pm 10 \mathrm{~g}$ b.w. $)$ were administered a single bolus dose of acetaminophen ( $2 \mathrm{~g} / \mathrm{kg}$, p.o.). Plant extract (PB) and its active principle (TA) was given at the dose of $100 \mathrm{mg} / \mathrm{kg}$ and $25 \mathrm{mg} /$ $\mathrm{kg}$, respectively (p.o.) after $24 \mathrm{~h}$ of toxicant administration.

Results: The hepatotoxicity produced by acute paracetamol administration was found to be inhibited by Polygonum bistorta and tannic acid with evidence of decreased levels of serum transaminases, alkaline phosphatase, protein, albumin, bilirubin, urea, creatinine, triglycerides and cholesterol concentration whereas increase was found in blood sugar. A significant rise was observed in lipid peroxidation level however reduced glutathione content was decreased. A concomitant fall was observed in the enzymatic activities of adenosine triphosphatase, glucose-6-phosphatase and histopathological findings. 
Discussion: Plant extract and its active principle prevent the development of acute liver damage, thus supported these biochemical and histopathological findings. The results of this study clearly indicate that PB and TA have a potent hepatoprotective action; whereas tannic acid is showed protective effect compare to Polygonum bistorta against Acetaminophen-induced toxicological profile.

\section{PP05-08}

Prediction of Response to Peginterferon Treatment by Serum HBsAg Quantification in HBeAg-positive Chronic Hepatitis B

G.L. Wong ${ }^{1}$, V.W. Wong ${ }^{1}$, A.M. Chim ${ }^{2}$, H.L. Chan ${ }^{2}$

${ }^{1}$ Medicine and Therapeutics, ${ }^{2}$ Institute of Digestive Disease, The

Chinese University of Hong Kong, Shatin, Hong Kong S.A.R.

Background and aims: On-treatment response predictors can guide individualization of therapy in chronic hepatitis B virus (HBV) infection. We aimed to investigate the use of serum HBsAg quantification to predict sustained response (SR) to peginterferon.

Methods: Ninety-two HBeAg-positive chronic hepatitis B patients received peginterferon for 32 to 48 weeks with or without lamivudine combination in previously conducted trials were studied. SR was defined as HBeAg seroconversion and HBV DNA $<10,000$ copies $/ \mathrm{ml}$ till 12 months post-treatment.

Results: Twenty-one (23\%) patients achieved SR. Patients who achieved SR had lower levels of on-treatment HBsAg than non-sustained responders. The best prediction of SR by serum HBsAg was at month 6 with an area under receiver operating characteristics curve of 0.77 (95\% CI $0.65-0.89, P<0.001)$. HBsAg cutoff of $300 \mathrm{IU} / \mathrm{ml}$ at month 6 had the maximum sum of sensitivity $(62 \%)$ and specificity (89\%) to predict SR. 13 of $21(62 \%)$ patients who had HBsAg $\leq 300$ $\mathrm{IU} / \mathrm{ml}$ versus 8 of $71(11 \%)$ patients who had $\mathrm{HBsAg}>300 \mathrm{IU} / \mathrm{ml}$ at month 6 achieved SR $(\mathrm{P}<0.001)$. Nine of $18(50 \%)$ sustained responders versus 12 of $74(16 \%)$ non-sustained responders had HBsAg reduction $>1 \log$ at month $6(\mathrm{P}=0.002)$. Combined HBsAg $\leq 300 \mathrm{IU} / \mathrm{ml}$ and $>1 \log$ reduction at month 6 had sensitivity, specificity, positive and negative predictive values of 43, 96, 75 and $85 \%$ to predict SR, respectively (Figure).

Conclusions: On-treatment serum $\mathrm{HBsAg}$ quantification can predict response to peginterferon in $\mathrm{HBe} A g-$ positive chronic hepatitis B.

Acknowledgement: This work is support by RFCID grant (08070242) to HLYC.

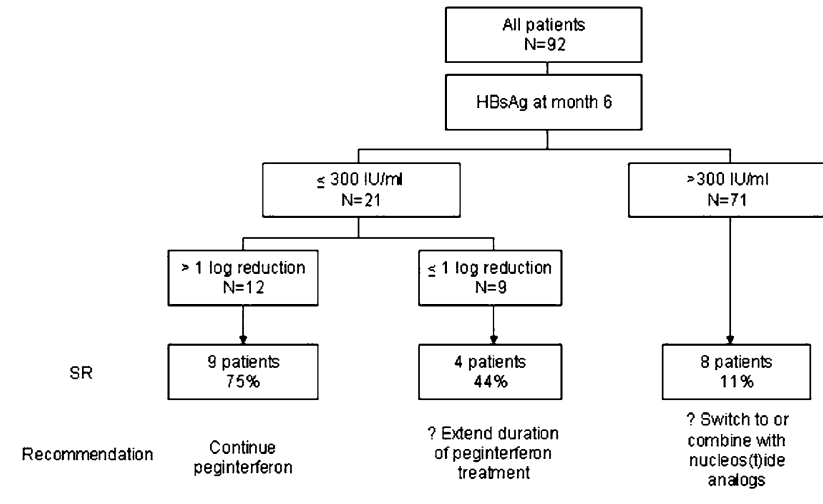

\section{PP05-09}

Role of Interferon Neutralizing Antibodies in Patients with Chronic Hepatitis B or C during Interferon Alpha Therapy

J.M. Zheng, G.F. Shi, M.Q. Chen

Department of Infectious Diseases, Huashan Hospital, Fudan University, Shanghai, China

Objective: The aims of our study were to evaluate the incidence of developing interferon neutralizing antibodies in the treatment of chronic hepatitis $\mathrm{B}$ or hepatitis $\mathrm{C}$, and to assess its relationship with response rate of antiviral treatment.

Methods: We carried out a review of the available literature to evaluate the chronic hepatitis B or hepatitis C patients treated with interferon alpha and developed interferon neutralizing antibodies (NAb).

Results: In our study, it can be seen that 147/630 (23.3\%) developed interferon neutralizing antibodies in CHB patients treated with IFN. A total of 75 out of 350 (20.9\%) CHC patients developed interferon neutralizing antibodies in the treatment. The incidences of developing interferon antibodies between $\mathrm{CHB}$ patients and $\mathrm{CHC}$ patients were not statistically significant difference, $P>0.05$. Treated with alpha 2a interferon, response rates were higher in NAb negative $\mathrm{CHC}$ patients $(94 / 151,62.3 \%)$ than those in NAb positive CHC patients $(8 /$ $43,18.6 \%)$.

Conclusions: Breakthrough occurred more in NAb positive patients than in NAb negative patients. It suggests that NAb may be a predictive factor of response during interferon treatment. Of course, more research is needed to confirm the results. We recommend that the research should also be done in those with peg-IFN treatment or who do not response to interferon well and plan to prolong interferon treatment.

\section{PP05-10}

Successful Entecavir Treatment in High Viral Load Reactivation of Occult HBV Infection Following Polichemotherapy with Rituximab for Chronic Lymphocytic Leukaemia

A. Izzi $^{1}$, B. Fimiani ${ }^{2}$, V. Iovinella ${ }^{3}$

${ }^{I}$ Emergency Infectious Diseases, 'D. Cotugno 'Hospital Naples, Naples; ${ }^{2}$ Internal Medicine Unit, 'Umberto $1^{\circ}$ Hospital,

'Nocera Inferiore; ${ }^{3}$ Internal Medicine Unit, 'San Paolo Hospital, 'Naples, Italy

Background: Reactivation of Hepatitis B Virus (HBV) occult infection (OBI) is more common when chemotherapeutic regimens including rituximab are used and fatal HBV reactivation has been reported in such patients.

Case description: In October 2008 a Caucasian patient, male, 65 years old showed a casual evidence of mediastinal lymphoadenopathy with the definitive diagnosis of B cells Chronic Lymphocytic Leukaemia CD 20+.

On January 2009 patient was HBsAg negative, Anti-HBs negative, Anti-HBe negative, Anti-HBc positive. On March 2009 patient started a six cycles of chemotherapy with cyclophosphamide + fludarabine + rituximab once monthly, that ended in August 2009. On September 2009 serum ALT levels arose 10 times x ULN and patient showed: HBsAg positive, Anti-HBs negative, HBeAg positive, Anti$\mathrm{HBc}$ IgM negative, HBV-DNA > 100 million IU/mL in RT-PCR, Anti-HIV negative, Anti-HCV negative. On October 2009 an Entecavir (ETV) treatment ad the dosage of $0.5 \mathrm{mg}$ qd orally, was started. Results: After 1 month of treatment serum ALT felt within the range of normal values and HBV-DNA levels resulted in $38000 \mathrm{IU} / \mathrm{mL}$. On December 2009 (2nd month of treatment)serum HBV-DNA was reduced over $6 \log 10$ with the value of $7,393 \mathrm{IU} / \mathrm{mL}$. On the 8 th month of treatment patient showed: HBsAg weak reactive, Anti-HBs $152 \mathrm{mIU} / \mathrm{mL}, \mathrm{HBeAg}$ negative, Anti-HBe negative, HBV-DNA 20 $\mathrm{IU} / \mathrm{mL}$, near close to undetectable $(<12 \mathrm{IU} / \mathrm{mL})$. No significant side effect reported on treatment.

Conclusions: This case report is among the first reports of HBV reactivation related to polichemotherapy with rituximab primarily treated with ETV as first-line treatment, and we strongly confirm the usefulness and safety of ETV to care this drug-induced "de novo hepatitis B “. ETV could therefore used as first choice drug in such patients, taking also in account the high mortality rate during lamivudine treatment in OBI reactivation due to rituximab therapy. 
PP05-11

Hepatitis B reactivation complicated with nephrotic syndrome in association with venom immunotherapy:need for preemptive treatment?

M.R. Pijak ${ }^{1}$, V. Csibova ${ }^{2}$

${ }^{1}$ Slovak Medical University, ${ }^{2}$ University Hospital, Bratislava, Slovak Republic

Background: Reactivation of hepatitis B virus (HBV) is a well-recognized complication in HBsAg positive patients undergoing cytotoxic or immunosuppressive therapy. However, to our knowledge, there have not been any reports describing a possible causal association between reactivation of HBV infection and non- immunosuppressive immunomodulatory interventions such as venom immunotherapy (VIT).

Case report: The patient was a 47-year-old man, professional soldier, who was known inactive $\mathrm{HBsAg}$-positive carrier for several years. $\mathrm{He}$ had recurrent generalized urticarial rash following wasp stings for the last 2 years without clear systemic symptoms. He had positive IgE antibody to bee and wasp venom but skin prick tests were positive only to wasp venom. Because of significant professional risk, VIT with wasp venom allergen was started despite the fact, that life threatening reaction was not convincingly confirmed. The VIT was discontinued after 2 years due to elevations of aminotransferases and development of nephrotic syndrome with histological findings of IgM nephropathy (variant of focal segmental glomerulosclerosis). Viral load revealed high level of HBV replication. The patient was subsequently treated with cyclosporine A and lamivudine with favourable response. He had no history of serious diseases before and results of routine laboratory test before initiation of VIT were normal. Causality assessment with the updated CIOMS (Council for International Organizations of Medical Sciences) scale showed a probable causality (+8 points) for VIT, as the primary candidate causing HBV reactivation associated with extrahepatic (renal) complication.

Conclusions: We suggest that all candidates for VIT should be screened for HBsAg and anti-HBc antibodies. HBsAg positive persons with history of life-threatening reactions should be tested for HBV DNA levels and receive pre-emptive nucleoside analogues, regardless of HBV DNA levels. The nucleoside analogues with or without immunosuppressive agent should be the first treatment option in patients with similar complications because interferon is contraindicated in autoimmune diseases.

\section{PP05-12}

Response Rates with Shorter Duration and Lower Doses of Peginterferon Alfa-2a Are Inferior to those with $180 \mu \mathrm{g}$ for 48 Weeks

Q. Xie ${ }^{1}$, J.Y. Sung ${ }^{2}$, T. Tanwandee ${ }^{3}$, K.H. $\mathrm{Han}^{4}$, C. Wat ${ }^{5}$, C.-W. Hsu ${ }^{6}$, J. Jia ${ }^{I}$ Department of Infectious Diseases, Ruijin Hospital, Shanghai, China; ${ }^{2}$ Department of Medicine and Therapeutics, Prince of Wales Hospital, Rm 114010, 9/F Clinical Sciences Building, Shatin, Hong Kong SAR; ${ }^{3}$ Department of Microbiology, Faculty of Medicine Siriraj Hospital, Mahidol University, Bangkok, Thailand; ${ }^{4}$ Department of Internal Medicine, Yonsei University College of Medicine, Seoul, Republic of Korea; ${ }^{5}$ Roche Products Ltd, Welwyn Garden City, UK; ${ }^{6}$ Liver Research Unit, Chang Gung Memorial Hospital and Chang Gung University College of Medicine, Taipei, Taiwan ROC; ${ }^{7}$ Beijing Friendship Hospital, Capital Medical University, Beijing, China

Background/aims: The non-inferiority NEPTUNE study investigated the efficacy and safety of peginterferon alfa-2a [40KD] (PEG-IFN $\alpha$ 2a) for 24 or 48 weeks and at $90 \mu \mathrm{g} /$ week or $180 \mu \mathrm{g} /$ week in HBeAgpositive $\mathrm{CHB}$ patients.

Methods: Patients were randomized to PEG-IFN $\alpha-2 a$ in a $2 \times 2$ factorial design of treatment duration ( 24 or 48 weeks) and dose (90 or $180 \mu \mathrm{g}$ ). Endpoints reported included $\mathrm{HBeAg}$ seroconversion (primary), HBV DNA $<2,000 \mathrm{IU} / \mathrm{mL}$, ALT normalization and HBeAg loss 24 weeks after the end of treatment. The null hypotheses were that 24 weeks is inferior to 48 weeks and $90 \mu \mathrm{g}$ is inferior to
$180 \mu \mathrm{g}$. The primary test statistic was odds ratio (OR) with a noninferiority margin pre-specified as 1.88 : OR $48 / 24$ or $180 / 90$ was significant if the upper $95 \%$ confidence limit (CI) was $<1.88$ at the significance level of 0.025 and the null hypothesis would be rejected. Results: Rates of response were higher with 48 weeks than with 24 weeks and with $180 \mu \mathrm{g}$ than with $90 \mu \mathrm{g}$ (Table 1). For nearly all parameters, the upper $95 \%$ CI was $>1.88$, retaining the null hypotheses. The highest response rates were achieved in the $48 @ 180$ group for the primary endpoint $(36.2 \%$; 47/130) and all secondary endpoints, including $\mathrm{HBeAg}$ seroconversion for genotype B (42.2\%) and C (38.8\%) subtypes (Table 2).

Table 1 Response 24 weeks post-treatment

\begin{tabular}{|c|c|c|c|}
\hline Response (\%) & $\begin{array}{l}90 \mu \mathrm{g}(\mathrm{n}=274) \\
180 \mu \mathrm{g}(\mathrm{n}=270)\end{array}$ & OR $(95 \% \mathrm{CI})$ & \\
\hline $\begin{array}{l}\mathrm{HBe} A g \\
\quad \text { seroconversion }\end{array}$ & $19.7 / 29.3$ & $\begin{array}{c}1.79(1.18,2.72) \\
p=0.410\end{array}$ & $\begin{array}{c}90 \mu \mathrm{g} \text { inferior } \\
\text { to } 180 \mu \mathrm{g}\end{array}$ \\
\hline $\mathrm{HBeAg}$ loss & $20.4 / 29.3$ & $\begin{array}{c}1.70(1.12,2.57) \\
p=0.0317\end{array}$ & $\begin{array}{l}90 \mu \mathrm{g} \text { inferior to } \\
180 \mu \mathrm{g}\end{array}$ \\
\hline $\begin{array}{l}\text { ALT } \\
\quad \text { normalization }\end{array}$ & $36.5 / 41.1$ & $\begin{array}{c}1.24(0.87,1.78), \\
p=0.012\end{array}$ & $\begin{array}{l}90 \mu \mathrm{g} \text { borderline } \\
\text { non } \\
\text { inferior to } 180 \\
\mu \mathrm{g}\end{array}$ \\
\hline $\begin{array}{l}\text { HBV DNA } \\
\quad<2,000 \mathrm{IU} / \mathrm{mL}\end{array}$ & $16.8 / 20.4$ & $\begin{array}{c}1.30(0,83,2.04) \\
p=0.054\end{array}$ & $\begin{array}{l}90 \mu \mathrm{g} \text { inferior to } \\
180 \mu \mathrm{g}\end{array}$ \\
\hline Response $(\%)$ & $\begin{array}{l}24 \text { weeks } \\
\quad(n=282) / \\
48 \text { weeks } \\
(n=262)\end{array}$ & OR $(95 \% \mathrm{CI})$ & \\
\hline $\begin{array}{l}\mathrm{HBe} A g \\
\quad \text { seroconversion }\end{array}$ & $18.4 / 30.9$ & $\begin{array}{c}2.17(1.43,3.31) \\
p=0.749\end{array}$ & $\begin{array}{l}24 \text { weeks inferior } \\
\text { to } 48 \text { weeks }\end{array}$ \\
\hline $\mathrm{HBeAg}$ loss & $18.8 / 31.3$ & $\begin{array}{c}2.15(1.42,3.26), \\
\quad p=0.735\end{array}$ & $\begin{array}{l}24 \text { weeks inferior } \\
\text { to } 48 \text { weeks }\end{array}$ \\
\hline $\begin{array}{l}\text { ALT } \\
\quad \text { normalization }\end{array}$ & $30.5 / 47.7$ & $\begin{array}{c}2.17(1.51,3.11), \\
p=0.779\end{array}$ & $\begin{array}{l}24 \text { weeks } \\
\text { inferior } \\
\text { to } 48 \text { weeks }\end{array}$ \\
\hline $\begin{array}{l}\text { HBV DNA } \\
<2,000 \mathrm{IU} / \mathrm{mL}\end{array}$ & $11.4 / 26.3$ & $\begin{array}{c}2.96(1.85,4.73), \\
p=0.970\end{array}$ & $\begin{array}{l}24 \text { weeks inferior } \\
\text { to } 48 \text { weeks }\end{array}$ \\
\hline
\end{tabular}

Table 2 HBeAg seroconversion 24 weeks post-treatment

\begin{tabular}{lll}
\hline \multirow{2}{*}{ Dose/duration } & \multicolumn{2}{l}{ HBeAg seroconversion, $n / N(\%)$} \\
\cline { 2 - 3 } & Genotype B & Genotype C \\
\hline $90 \mu \mathrm{g} / 24$ weeks & $12 / 49(24.5)$ & $7 / 70(10.0)$ \\
$180 \mu \mathrm{g} / 24$ weeks & $16 / 47(34.0)$ & $12 / 76(15.8)$ \\
$90 \mu \mathrm{g} / 48$ weeks & $13 / 45(28.9)$ & $16 / 68(23.5)$ \\
$180 \mu \mathrm{g} / 48$ weeks & $19 / 45(42.2)$ & $26 / 67(38.8)$ \\
\hline
\end{tabular}

Conclusions: Shorter and lower doses of PEG-IFN $\alpha-2 \mathrm{a}$ were inferior to 48 weeks and $180 \mu \mathrm{g} /$ week. The highest response rates were achieved in the $180 \mu \mathrm{g} 48$ weeks group confirming the licensed dose and duration as the most efficacious and beneficial for patients.

\section{PP05-13}

HBeAg Negative Patients Are More Common with Higher Viral Load and Fibrosis Score than HBeAg Positive Patients in Bangladesh M.B. Islam ${ }^{1}$, M.S. Alam ${ }^{2}$

${ }^{1}$ Department of Medicine, Comilla Medical College, Comilla; ${ }^{2}$ Deparment ofHepatology, Bangabandhu Sheikh Mujib Medical University, Dhaka, Bangladesh

Introduction: An estimated 350 million persons world wide are chronically infected with HBV.Around $8 \%$ of 150 million population 
of Bangladeshi are infected with HBV.15-40\% will develop serious sequelae during there lifetime. Appropriate treatment and follow up of CHB patients can prevent to develop serious sequelae.

Aim of the study: To find the different status between $\mathrm{HBeAg}$ Negative and $\mathrm{HBeAg}$ positive patient in chronic hepatitis B.

Patients and methods: This is a cross sectional study of $90 \mathrm{CHB}$ patients in Bangabandu Sheikh Mujib Medical University in the department of Hepatology during the period of January 2008 to July 2008.

Study procedure: After establishing a particular patient to be of $\mathrm{CHB}$ he/she enrolled in the study. After explanation about the necessity of the investigation blood sample was drawn and sent for AST, ALT, HBeAg, Anti $\mathrm{HBeAg}$ and HBV DNA tests. HBeAg positive/negative patients, ALT levels are between 1-2× ULN and HBV DNA > 20,000 IU/ml after taking informed written consent liver biopsy was done under admission in the hospital. Histopathology was done using Knodell's scoring system.

Results: A total of 90 cases of chronic hepatitis B were selected. Mean age of patients was 26.23 years. Among them 61(67.85\%) were $\mathrm{HBeAg}$ negative and $27(22.15 \%)$ were $\mathrm{HBeAg}$ positive. $57(63.3 \%)$ patient had DNA $>20,000 \mathrm{IU} / \mathrm{ml}$. Thirty four of patients were $\mathrm{HBeAg}$ negative. Twenty seven patients meet the criteria to treat by anti-viral drugs. Thirty three patients suggested follow up who are in inactive carrier state. Thirty patient were selected for liver biopsy. Fibrosis 1 found in patients, 2 found in 2 patients and 3 found in 7 patients. All of the patients of fibrosis score 3 were $\mathrm{HBe} \mathrm{Ag}$ negative.

Conclusions: $\mathrm{HBeAg}$ negative patients are more dangerous than $\mathrm{HBeAg}$ positive patients. I suggest mandatory liver biopsy to declare a patient inactive carrier state.

\section{PP05-14}

Combination Therapy with Entecavir and Adefovir for rtA181V/T Mutants in Previously Lamivudine-resistant Hepatitis B Virus

H.C. Cho, Y.J. Kim, G.-Y. Gwak, M.S. Choi, J.H. Lee, K.C. Koh, B.C. Yoo, S.W. Paik

Division of Gastroenterology, Samsung Medical Center, Sungkyunkwan University School of Medicine, Seoul, Republic of Korea

Background/aims: Recent studies reported the occurrence of amino acid substitutions at position 181 of the HBV polymerase, associated with a viral breakthrough under adefovir therapy following lamivudine breakthrough. The aim of this study was to characterize the virologic response to entecavir and adefovir combination therapy for $\mathrm{rtA} 181 \mathrm{~V} / \mathrm{T}$ mutants in previously lamivudine-resistant hepatitis B virus.

Methods: All hepatitis B patients who had rtA181V/T mutants after adefovir therapy following lamivudine resistance received entecavir and adefovir combination therapy for at least 1 year. Virologic responses were evaluated at 6 months and 12 months after initiation of therapy.

Results: Twenty-four patients (median age: 50 years, 22 males and 2 females) were included and all patients had had prior lamivudine resistance after lamivudine therapy. Hepatitis B rtA181V/T and/or rt236T mutants appeared after adefovir monotherapy in twenty-one patients $(87.5 \%)$ and after lamivudine and adefovir combination therapy in three patients $(12.5 \%)$. Laboratory findings of the patients were as follows; median HBV DNA: $4.8 \log _{10} \mathrm{IU} / \mathrm{ml}$ (3.0-7.1), median HBsAg quantitative: $3.4 \log _{10} \mathrm{IU} / \mathrm{ml}(2.6-4.5)$, median ALT: $35 \mathrm{IU} / \mathrm{L}$ (16-141). Twenty patients $(83.3 \%)$ were $\mathrm{HBeAg}$-positive. Mean duration of entecavir and adefovir combination was 13 months. Reduction in HBV DNA $\left(\log _{10} \mathrm{IU} / \mathrm{ml}\right)$ at 6 months were as follows; $<2$ : $16.7 \%, 2.1-3$ : $58.3 \%,>3: 25 \%$. Reduction in HBV DNA $\left(\log _{10} \mathrm{IU} / \mathrm{ml}\right)$ at 12 months were as follows; $<2: 12.5 \%, 2.1-3: 41.7 \%,>3: 45.8 \%$.

Conclusion: One-year combination of entecavir and adefovir can be beneficial for rtA181V/T mutants in previously lamivudine-resistant hepatitis B virus and long-term data is needed.
PP05-15

Antiviral Treatment in Patients with Decompensated HBV Cirrhosis R. Goyal ${ }^{1}$, N. Kalra ${ }^{1}$, A. Kumar ${ }^{1}$, D. Seth ${ }^{2}$, S.K. Acharya ${ }^{1}$

${ }^{1}$ Gastroenterology and Human Nutrition; ${ }^{2}$ Biostatistics, All India Institute of Medical Sciences, Delhi, India

Background: Patients with HBV cirrhosis have improved outcomes with antiviral treatment. The risk of potentially life threatening exacerbations following development of drug resistance in these patients merits consideration of drugs with high genetic barrier for initial therapy. Since therapy is costly and lifelong, we evaluated the efficacy and resistance profiles in decompensated HBV cirrhosis treated with adefovir alone, lamivudine/adefovir and adefovir/ Glycyrrhizin combinations.

Methods: Consecutive patients of HBV related Childs B or C cirrhosis were randomized to receive one of the three treatments. Patients were evaluated every 3 months for a period of at least 72 weeks.

Results: 63 patients enrolled from March 2007 to March 2010. Mean follow up was $46.9 \pm 34.9$ weeks, with $21(33.3 \%$ ) having 72 weeks of follow up. Mean age was $37.0 \pm 12.5$ years (male:female 58:5). At baseline, 52(82.5\%) patients were Child's B and 11(17.5\%) Child's C (mean Child-Pugh score score $8.0 \pm 1.3$ ). Improvement of $>2$ points in CTP score was seen in 61\%(17/28) patients at 48 weeks and 68\% (13/ 19) at 72 weeks. $55 \%(6 / 11)$ patients who had improved at 48 weeks maintained their response at 72 weeks. The overall mortality in the cohort was $14 \%$ (9/63). 77.8\% mortalities occurred within first 6 months of follow-up. 18 patients were $\mathrm{HBeAg}$ positive at baseline, 4 (22\%) had $\mathrm{HBeAg}$ seroconversion and one patient lost HBeAg. Mean HBV DNA levels at baseline were 45,00,000 copies $/ \mathrm{ml}$. 31(49.0\%) had HBV DNA $<1,000$ copies $/ \mathrm{ml}$ at baseline. $47 \%(9 / 19)$ of treated patients became HBV DNA negative at 48 weeks and 57\% (8/14) were HBV DNA negative at 72 weeks. 14/63 (22\%) patients had viral breakthrough.

Conclusions: Antiviral therapy leads to virological response as well as improvement in Childs score in a significant proportion of cirrhotic patients and should be considered in all patients in decompensated cirrhosis.

\section{PP05-16}

Changes to the Peripheral Blood Nature Killer Cell Subsets during Antiviral Treatment in Chronic Hepatitis B Patients

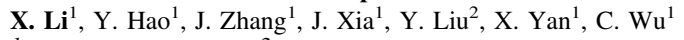

${ }^{1}$ Infectious Disease; ${ }^{2}$ Laboratory Medicine, NanJing Drum Tower

Hospital of NanJing University Medical School, Nanjing, China

Objective: To observe longitudinally the changes in peripheral blood NK cell subsets in chronic hepatitis B (CHB) patients undergoing antiviral treatment, and to explore the relationship between the percentage of NK cell subsets and serum HBV-DNA, ALT, AST levels. Methods: We studied $18 \mathrm{CHB}$ patients undergoing antiviral treatment (lamivudine, adefovir, entecavir, telbivudine) for six months. The propotion of periphral blood NK cell subsets were assessed by flow cytometry. Serum HBV-DNA load was determined by real-time fluorescent quantitative polymerase chain reaction (PCR). The investigations were focused on five time points: T0: baseline, T1: 25week; T2: 6-9 weeks; T3: 11-18 weeks; T4: 21-31 weeks.

Results: Sixteen of 18 patients had undetectable serum HBV-DNA levels by T4. At baseline, no relationship was found between NK cell subsets and HBV-DNA load or ALT, AST levels. During treatment, the proportion of CD56 $6^{\text {bright }} \mathrm{NK}$ cells showed a downward tendency, which was paralleled by the suppression of HBV replication and decreases in serum ALT, AST levels. Further correlation analysis showed CD56 $6^{\text {bright }} \mathrm{NK}$ cells were positively related with serum HBVDNA load and ALT, AST levels $(r=0.250, P=0.028 ; r=0.362$, $P=0.002, r=0.409, P=0.000)$. No such relationship was found in the percentage of CD56 ${ }^{\mathrm{dim}} \mathrm{NK}$ cells $(P>0.05)$.

Conclusions: The decrease of the proportion of periphiral blood $\mathrm{CD} 56^{\text {bright }} \mathrm{NK}$ cells may be closely correlated with virus clearance and liver inflammation lessen during antiviral treatment in CHB patients. 


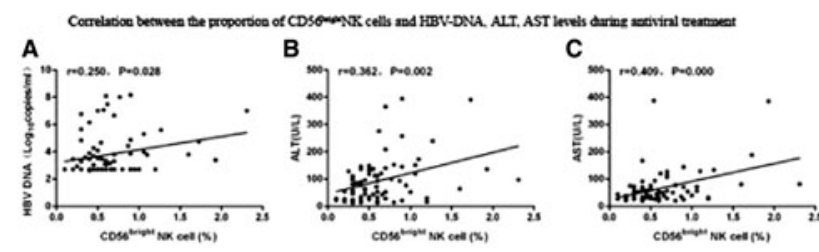

PP05-17

Does Entecavir Reduce the Glomerular Filtration Rate in Nucleos(t)ide Analogues-naive Patients with Chronic Hepatitis B?

M. Karaagac, N. Aksoy, K. Kutoglu, N. Arican, S. Kalyon, N. Yenice 3rd Internal Medicine Clinic, Okmeydanı Education and Research Hospital, Istanbul, Turkey

Objective: The aim of this retrospective study is to assess patient compliance, safety and advers effects of entecavir (ENT) treatment in nucleos(t)id analogues (NA)-naive patients with chronic hepatitis B in Turkish people.

Material and methods: The datas that present in this study have been obtained by scanning patients files retrospectively. Glomerular filtration rate (GFR) was estimated by using Cockcroft-Gault (CG) formula. Further more, informations about adverse effects on patient files at baseline and by updating at every 24 th week of treatment were determined.

Results: A total of 40 patients were involved in this study; 26 men and 14 women with a mean age of $41.12 \pm 12.56$ years and a mean duration of treatment of $96.60 \pm 32.83$ weeks. Patient compliance was detected at all of patients $(100 \%)$. Adverse effects related to treatment were mildly, generally occured and disappeared in the first two weeks and determined as fatique $(22,5 \%)$, headache $(10 \%)$, myalgia (10\%), dizziness $(7,5 \%)$, abdominal pain $(5 \%)$, rhinitis $(2,5 \%)$, cough $(2,5 \%)$, diarrhea $(2,5 \%)$ and insomnia $(2,5 \%)$. Elevation of ALT levels and reduction of platelets rates were detected 2.5 and $10 \%$, respectively. One patient had a mildly impaired renal functions at baseline because of additional diseases, but has not worsened in duration of ENT treatment. With an unexpected way, at $43.6 \%(17 / 39)$ of the patients with unimpaired renal functions, baseline GFR levels reduced mildly at the end of the treatment. But, impairment of renal functions due to treatment was detected at just $5 \%(2 / 40)$ of all patients and not required to stop treatment. Being elder was only independent parameter of reduction at GFR.

Conclusions: Although ENT is a well-tolerated drug for NA-naive patients with chronic hepatitis $\mathrm{B}$, patients should be monitored more closely, especially; in point of renal functions. Prospective, casecontrolled and well-designed more trials must be established to evaluate relation of ENT with renal functions.

\section{PP05-18}

Results of Sequential Therapy with Telbivudine with or without Add-on Tenofovir in HBeAg Negative Chronic Hepatitis B

R. Goyal ${ }^{1}$, M. Mahanta ${ }^{1}$, A. Kumar' ${ }^{1}$, S. Shalimar ${ }^{1}$, S. Duttagupta ${ }^{2}$, S.K. Acharya

${ }^{1}$ Gastroenterology and Human Nutrition, ${ }^{2}$ Pathology, All India Institute of Medical Sciences, Delhi, India

Background: We evaluated effect of telbivudine on HBeAg negative chronic hepatitis B patients.

Methods: Treatment naïve $\mathrm{HBeAg}$ negative chronic hepatitis B patients were given telbivudine $600 \mathrm{mg} / \mathrm{day}$. Treatment was initiated in patients with either raised ALT above upper limit of normal with HBV DNA $>10^{4}$ copies/ml or histological activity (HAI $>3$ and/or fibrosis stage $>1$ ). Tenofovir $300 \mathrm{mg} /$ day was added if HBV DNA was $>300$ copies $/ \mathrm{ml}$ at 24 weeks, data analyzed at 52 weeks.
Results: 31 patients (males 27, 87.1\%; mean age $29.5 \pm 7.8$ years) had taken at least 52 weeks of therapy. Mean ALT was $105.6 \pm 93.4$ (range 33-526) IU/L, with 5 patients having normal ALT. Median DNA was $7.9 \times 10^{4}$ copies/ $\mathrm{ml}$. Liver histology (26/31 patients) revealed HAI of $4.0 \pm 1.5$ and fibrosis stage of $0.8 \pm 0.9 .17$ patients had genotype D, 1 genotype A, 1 genotype C and 7 genotypes A + D. At 52 weeks, 28 patients $(90.3 \%)$ received only telbivudine. Of these, $24(85.7 \%)$ were DNA negative at 52 weeks. 22 patients $(78.6 \%)$ had normal ALT. Tenofovir was added in 3 patients $(9.7 \%)$ at 24 weeks. Two patients were HBV DNA negative at 52 weeks, one had reduction in HBV DNA levels to $<1,000$ copies/ $\mathrm{ml}$. All had normal ALT. Of all patients, $26(83.9 \%)$ were HBV DNA negative at 52 weeks. 25 patients $(80.6 \%)$ had biochemical remission.

Conclusions: Telbivudine is highly potent antiviral leading to HBV DNA undetectablity in more than $90 \%$ of treated $\mathrm{HBeAg}$ negative patients. DNA undetectablity at 24 weeks is maintained by 52 weeks in most patients. Add on tenofovir therapy might to be an efficacious approach in patients who fail to achieve DNA negativity by 24 weeks.

\section{PP05-19}

The Combination of Tenofovir + Telbivudine vs. either Monotherapy Alone over 12 Weeks

N.W.-Y. Leung ${ }^{1}$, H.L.-Y. Chan ${ }^{2}$, G.K. Lau ${ }^{3}$, T.M.-K. So ${ }^{4}$, K. Lee ${ }^{5}$, A. Trylesinski ${ }^{6}$, N.V. Naoumov ${ }^{6}$

${ }^{1}$ Department of Medicine, Alice Ho Miu Ling Nethersole Hospital; ${ }^{2}$ Department of Medicine and Therapeutics, Prince of Wales Hospital, The Chinese University of Hong Kong; ${ }^{3}$ Humanity \& Health, Unit 1309; ${ }^{4}$ Department of Medicine and Geriatrics, Princess Margaret Hospital; ${ }^{5}$ Novartis Pharmaceuticals (HK) Ltd, Hong Kong, Hong Kong SAR; ${ }^{6}$ Novartis Pharma AG, Basel, Switzerland

Background/aims: Although several monotherapy regimens are available for treatment of naive Chronic Hepatitis B (CHB) patients, there is still an unmet medical need for improved treatment regimens. Little data is available in combination therapy. This study examined the safety and efficacy of combination of telbivudine (LDT) and tenofovir disoproxil fumarate (TDF) versus LDT or TDF monotherapy in immunotolerant $\mathrm{CHB}$ patients.

Methods: In this open-label, 12-week study, 46 Chinese patients with $\mathrm{HBeAg}$ positive compensated $\mathrm{CHB}, \mathrm{HBV}$ DNA $\geq 10^{7}$ copies $/ \mathrm{mL}$ and normal ALT were randomized 1:1:1 to receive LDT $600 \mathrm{mg}$ and TDF $300 \mathrm{mg}$ in combination or LDT $600 \mathrm{mg}$ or TDF $300 \mathrm{mg}$ monotherapy. The primary objective of this study is the reduction in HBV DNA from baseline to week 12 of LDT and TDF combination therapy versus LDT or TDF monotherapy.

Results: A total of $46 \mathrm{HBeAg}$-positive patients were enrolled $(n=16$ for LDT+TDF [ 1 early termination excluded]; $n=16$ for LDT; and $n=14$ for TDF). Although all therapies had similar (median) reductions in the first 4 days, both LDT+TDF and LDT mono-therapy had greater reduction starting from Day6 (-1.91(LDT+TDF) vs. -2.00 (LDT) vs. -1.74 (TDF) $\log _{10}$ copies/ml). HBV DNA changes from baseline were slightly greater in the LDT+TDF arm at week12, while both monotherapies had similar reduction $(-4.40$ (LDT + TDF) vs. -3.92 (LDT) vs. -4.16 (TDF) $\log _{10}$ copies $\left./ \mathrm{ml}\right)(p=0.554$ for TDF vs. TDF + LDT). 1 patient achieved undetectable serum HBV DNA $(<169$ copies $/ \mathrm{mL})$ and became HBeAg negative at Week 12 in LDT + TDF arm. No serious adverse events or adverse events suspected to be related to study drug were reported during 12 weeks. No Adverse Events of renal function were reported.

Conclusion: This first randomized study showing exploratory data on LDT + TDF combination treatment vs monotherapy indicates similar potency for Tenofovir and Telbivudine monotherapy, but a potential benefit for the combination therapy. Further investigation of this combination is warranted. 


\section{PP05-20}

The Management of the Patients with Hepatitis B Virus Infection Under Immunosuppressive Therapy

A. Acar, O. Oncul, V. Turhan, Z. Karacaer, L. Gorenek, S. Cavuslu Infectious Diseases and Clinical Microbiology, Gulhane Military

Medical Academy, Haydarpasa Training Hospital, Istanbul, Turkey

Aim: In this study, we investigated the effectiveness of oral antiviral prophylactic therapy and the progress the illness in HBV seropositive patients.

Method: The patients taken immunosuppressive therapy due to several reasons between 2006 and 2010 in our hospital, were screened into three groups respectively. Group I: The patients who were initiated antiviral therapy before or simultaneously with immunosuppressive treatment and continued to after completion of antiviral therapy. Group 2: The patients who applied to our clinic with HBV reactivation during immunosuppressive therapy or after the end of this period and were started the antiviral therapy. Group 3: The patients were followed-up with no treatment because of reactivation after immunosuppressive therapy, but biochemical and virological improvement developed

Results: Five cases in Group 1, five cases in Group 2 and two cases in Group 3 were admitted to this study. Reactivation was not detected any patients initiated antiviral prophylactic therapy. Biochemical and virological response were obtained with antiviral therapy in group 2 patients in the first three months. One of the patient in Group 3 (HBsAg: Negative, anti-HBs: Positive, anti-HBc IgG: Positive) developed reactivation. Conclusion: Because immunosuppressive therapy causes HBV reactivation, prophylactic therapy should be implemented in all the patients who had anti-HBc Total positive, before undertaking immunosuppressive therapy.

\section{PP05-21}

Interferon-alpha on Expression of hTERT mRNA in Peripheral Blood Mononuclear Cells in Patients with Chronic Hepatitis B

C.-W. Zhu ${ }^{1}$, M. Chen ${ }^{2}$, X.-R. Luo ${ }^{1}$, L.-H. Wang ${ }^{2}$, H.-Y. Wang ${ }^{1}$, J.-H. Wu ${ }^{1}$, M. $\mathrm{Li}^{1}, \mathrm{~L} . \mathrm{Zhu}^{1}$

${ }^{1}$ Department of Hepatology, The Fifth People's Hospital of Suzhou, Suzhou; ${ }^{2}$ Department of Infectious Diseases, The Affiliated Hospital of Medical College of Xuzhou, Xuzhou, China

Aim: To investigate interferon alpha (IFN $\alpha$ ) therapy on human telomerase reverse transcriptase catalytic subunit (hTERT) mRNA expression in peripheral blood mononuclear cells (PBMCs) in patients with chronic hepatitis B (CHB).

Methods: PBMCs were obtained from $37 \mathrm{CHB}$ patients treated with IFN $\alpha$ (IFN $\alpha$ treated group), $36 \mathrm{CHB}$ control patients (CHB control group) and 10 healthy individuals (healthy control group). The quantitation of hTERT mRNA and $\beta$-actin mRNA in PBMCs of each group was respectively detected with real-time PCR, and the normalized hTERT mRNA (NhTERT mRNA) was calculated for each sample.

Results: NhTERT mRNA level was not significantly correlated with the subject's age, CHB patient's alanine aminotransferase (ALT) and HBV DNA load, respectively (all $P>0.05$ ), but markedly correlated with the count of $\mathrm{CD}^{+} \mathrm{T}$ lymphocytes $(P=0.026)$ and the ratio of $\mathrm{CD}^{+} / \mathrm{CD}^{+}$lymphocytes $(P=0.041)$; Mean NhTERT mRNA levels were of no significant difference among different HBV genotypes $(P=0.664)$. NhTERT mRNA mean level was $0.959 \times 10^{-3}$, $0.857 \times 10^{-3}$ and $1.042 \times 10^{-3}$ in IFN $\alpha$ treated group, CHB control group and healthy control group, respectively. The level in healthy control group was significantly higher than that in $\mathrm{CHB}$ control group $(P<0.001)$ and that in IFN $\alpha$ treated group $(P=0.041)$, whereas the level in IFN $\alpha$ treated group was significantly higher than that in CHB control group $(P=0.001)$. Subgroup analysis in IFN $\alpha$ treated group showed that mean NhTERT mRNA level was significantly higher in $\mathrm{HBeAg}$ loss subgroup as compared to $\mathrm{HBeAg}$ positive subgroup
$(P=0.015)$, while the differences of the mean level in HBV DNA less than 3log subgroup and in ALT normal subgroup did not reach statistical significance as compared to their corresponding subgroup $(P=0.059, P=0.249$, respectively).

Conclusion: The decreased hTERT mRNA is expressed in PBMCs of patients with chronic hepatitis $\mathrm{B}$, and IFN $\alpha$ therapy can upregulate the expression, especially in patients achieved $\mathrm{HBeAg}$ loss.

\section{PP05-22}

Five Years Efficacy and Safety of Telbivudine Treatment on Chinese Chronic Hepatitis B (CHB) Patients with Undetectable HBV DNA J. Jia ${ }^{1}$, D. Xu ${ }^{2}$, Q. Xie ${ }^{3}$, G. Shi ${ }^{4}$, L. Wei ${ }^{5}$, S. Wu ${ }^{6}$, M. Wan ${ }^{7}$, J. Hou ${ }^{8}$, D. Tan ${ }^{9}$, M. Wang ${ }^{10}$, Y. Chen ${ }^{11}$, C. Chen ${ }^{12}$, H. Ren ${ }^{13}$, Y. Wang ${ }^{14}$, Q. Wang ${ }^{15}$, J. Niu ${ }^{16}$ ${ }^{I}$ Beijing Friendship Hospital, Capital Medical University; ${ }^{2}$ Beijing Ditan Hospital, Beijing; ${ }^{3}$ Ruijin Hospital, Shanghai Jiao Tong University; ${ }^{4}$ Huashan Hospital, Fudan University, Shanghai;

${ }^{5}$ People's Hospital, Peking University, Beijing; ${ }^{6}$ Shanghai Infectious Diseases Hospital; ${ }^{7}$ Changhai Hospital, No.2 Military Medical University, Shanghai; ${ }^{8}$ Nanfang Hospital, Southern Medical University, Guangzhou; ${ }^{9}$ Xiangya Hospital, Central South University, Changsha; ${ }^{10}$ Nanjing Bayi Hospital, Nanjing; ${ }^{11}$ The First Affiliated Hospital of Zhejiang University, Hangzhou; ${ }^{12}$ Shanghai Liver Disease Research Center of Nanjing Military Area, Shanghai; ${ }^{13}$ The Second Affiliated Hospital of Chongqing Medical University; ${ }^{14}$ Southwest Hospital, No.3 Military Medical University, Chongqing; ${ }^{15}$ Peking University First Hospital, Beijing; ${ }^{16}$ The First Affiliated Hospital of Jilin University, Changchun, China

Background and aims: GLOBE trial has shown that telbivudine is superior to LAM in treating patients with $\mathrm{CHB}$ over a 2-year period. 4 years extension (A2303 trial) has demonstrated high rate of maintain of viral suppression and seroconversion. We report 5 years results from patients included in 2303 trial and treated for additional 52 weeks in the ACN04 study.

Methods: Chinese $\mathrm{CHB}$ patients included previously in study A2303 with undetectable HBV DNA $(<300$ copies $/ \mathrm{ml})$ for $\geq 12$ months were enrolled in ACN04 study and followed for 52 weeks. Efficacy and safety at year 5 was analyzed. Virological breakthrough was defined as an increase in serum HBV DNA by $>1 \log$ above nadir.

Results: Intension to treat population consisted of 97 patients, 67 of them were $\mathrm{HBeAg}$ positive before treatment, $30 \mathrm{HBeAg}$ negative. At 5 years telbivudine treatment, $93.65 \%$ of $\mathrm{HBeAg}$ positive patients and 96.43\% of $\mathrm{HBeAg}$ negative patients maintained undetectable $\mathrm{HBV}$ DNA. Virological breakthrough was observed in 4.84 and $3.57 \%$ of $\mathrm{HBeAg}$ positive and $\mathrm{HBeAg}$ negative patients respectively. ALT normalization was achieved in 84.6 and $79.3 \%$ of $\mathrm{HBeAg}$ positive and $\mathrm{HBeAg}$ negative patients respectively. $45.31 \%$ patients achieved $\mathrm{HBeAg}$ seroconversion at year 5 and cumulative $\mathrm{HBeAg}$ seroconversion rate over 5 years was $67.16 \%$. Six $(6 \%, 6 / 97)$ patients had HBsAg loss, 3 (3\%) of them achieved HBsAg seroconversion.

Overall safety profile was favorable. Only one patient experienced grade 3-4 CK elevation. No case of peripheral neuropathy, myopathy, rhabdomyolysis or lactic acidosis case was reported and no new safety signal was observed during year 5 .

Conclusion: Telbivudine monotherapy in the fifth year demonstrated excellent sustained virological response rate with remarkable $\mathrm{HBeAg}$ seroconversion rate. Overall safety profile is favorable.

\section{PP05-23}

Clinical Significance of the Expression of HBV Specific Granzyme and Perforin from Peripheral Blood of AHB

J. Li, Y. Han, Y. Liu, Q. Xu, F. Xie, Z. Huang Department of Infectious Diseases, The First Affiliated Hospital of Nanjing Medical University, Nanjing, China 
Objective: To dynamicly observe the relativity between the activity of granzyme, perforin and the distribution frequency of HBcAg18-27 epitope specific CTL from peripheral blood of AHB.

Methods: Construct two kinds of pentamer which recognized different HBV epitope, using multiparameter flow cytometry, associating with intracellular cytokine staining(ICS), to study the level of granzymeB, perforin secreted from HBCAg18-27 and 117-125 epitope specific CD8+CTL from peripheral blood of AHB, meanwhile to detect liver function, serum marker of HBV and serum HBV DNA.

Results: Five HLA-A0201 and six HLA-A2402 positive AHB cases, the frequency of epitope specific CD8+CTL was obviously higher in early stage than in the stage of recovery, the secretion activity of granzymeB and perforin from specific CTL was highest in the first week after hospitalization, and began to decrease after admission. With a decrease in the lever of serum ALT and HBV DNA, granzymeB, perforin gradually declined to a lower level in the fourth week after admission.

Conclusion: In the early stage of HBV infection, virus clearance is mainly depends on the granzymeB and perforin from specific CTL, the expression of granzymeB and perforin is down regulated in later period of infection, which can prevent the liver of HBV patients from further immunologic injury, thus to avoid the occurrence of severe hepatitis.

\section{PP05-24}

Hepatic Steatosis Has Negative Impact on the Outcome of Peginterferon Alfa Treatment in Chinese Patients with HBeAg-positive Chronic Hepatitis B Q. Huang, Y.-H. Xun, J.-C. Guo, J. Wu, J. Xiang, Y.-M. Jiang, G.-T. Zhu The Sixth Hospital Affiliated to Zhejiang University of Traditional Chinese Medicine, Hangzhou, China

Background and aims: Approximately $30-40 \%$ of patients with hepatitis $\mathrm{B}$ e antigen ( $\mathrm{HBeAg}$ )-positive chronic hepatitis $\mathrm{B}$ (CHB) treated with peginterferon achieve $\mathrm{HBeAg}$ seroconversion on the end of treatment. Hepatic steatosis, as a predictive factor in treatment outcome, was not investigated in CHB patients. The aim of this retrospective analysis is to determine possible relationships between hepatic steatosis and treatment response in patients with CHB.

Methods: In this study, $110 \mathrm{HBeAg}$-positive patients with biopsyproven $\mathrm{CHB}$ in 2007 received pegylated interferon $\mathrm{a}-2 \mathrm{a}$ or $2 \mathrm{~b}$ for 48 weeks and followed up for 48 weeks. 96 consecutive patients ( 74 men, age $27.96+7.98$ ) receiving PEG interferon alpha were analyzed. The virologic response rates at 48 weeks were $67.7 \%$ and $69.4 \%$ in patients with (34 cases) and without steatosis (62 cases), respectively $(P>0.05)$, while patients with steatosis were lower the sustained response rates $(30 \%)$ than without steatosis $(60 \%)$ at 96 weeks, especially in patients with severe steatosis $(9.09 \%), P<0.05$. HOMA-IR was significant decrease in patients with steatosis at 48 weeks and recover to baseline levels at 96 weeks, but patients without steatosis were not. Multivariate analysis showed that response rates at 48 weeks were independently associated with steatosis, GGT, FSG, HOMA-IR, CRP, HBV-DNA load, HBV genotype and PEG IFN. However, the sustained response rate was independently associated with steatosis, fibrosis, AST, CRP and ferritin. Hepatic steatosis was only prediction factor with the early and sustained response.

Conclusion: Hepatic steatosis has negative impact of the early and sustained response in $\mathrm{HBeAg}$-positive $\mathrm{CHB}$ patients with PEG interferon alpha therapy.

\section{PP05-25}

Preliminary Study on Specific Cellular Immune Responses Induced by HBV Envelope Proteins of Various Infected States of HBV

B. Liu, Y. Han, Q. Xu, J. Li

Department of Infectious Diseases, The First Affiliated Hospital of Nanjing Medical University, Nanjing, China

Objective: To explore the characteristic of specific cellular immune responses induced by HBV Envelope Large, Middle and Small Proteins of chronic carriers and self-limited infected individuals.
Methods:

1. We gained HBV Envelope Large, Middle and Small Proteins by transfecting 293T cells using pSW3891/LHBs, pSW3891/MHBs, pSW3891/SHBs plasmids we detected the specific expression of these plasmids through commercial diagnostic kit of HBV antigen, diagnostic kit of HBV pre-s2 envelope and Abbott, respectively.

2. Peripheral blood mononuclear cells (PBMCs) from recovered individuals and chronic carriers were separated and co-incubated with HBV Envelope Large, Middle and Small Proteins,meanwhile blank group, pSW3891 negative group and PMA + Ionomycin positive group were set as cintrols.

Cytokines INF- $\gamma$.IL-4 were detected by intracellular cytokine staining (ICS) using Flow Cytometer. Study the characteristic of specific cellular immune responses induced by HBV Envelope Large, Middle and Small Proteins.

Results:

1. We could detected Large, Middle and Small proteins from the lysate of the transfected cells but only Middle and Small proteins could be detected from the supernatant. There were no aim proteins both in the lysate and the supernatant from the cells transfected PSW3891;

2. Envelope Large, Middle and Small Proteins could induce the release of INF- $\gamma$.IL-4 by stimulating T cells of PBMC.

The response induced by Envelope Large, Middle and Small Proteins was stronger in recovered individuals than chronic carriers and the cellular response which producted INF- $\gamma$ was the major reaction.

Conclusion: HBV Envelope Large, Middle and Small Proteins could induce specific cellular immune responses; cellular immune responses induced by HBV Envelope antigen was stronger in recovered individuals than chronic carriers.

\section{PP05-26}

Preliminary Study on Specific Humoral Immune Responses Induced by HBV Envelope Large, Middle and Small Proteins

J. Li, B. Liu, Y. Han, Y. Liu, Q. Xu

Department of Infectious Diseases, The First Affiliated Hospital of Nanjing Medical University, Nanjing, China

Objective: To explore the differences of anti-HBs from individuals naturally infected and vaccinees neutralizing HBV Envelope Large, Middle and Small Proteins, then to study the characteristic of specific humoral immune responses induced by HBV Envelope Large, Middle and Small Proteins.

Methods:

1. Use ELISA made by our Lab to detect the binding reaction of anti-HBs from individuals naturally infected and vaccinees with HBV Envelope Large, Middle and Small Proteins, analyse the distinction of humoral immune responses induced by HBV Envelope Large, Middle and Small Proteins;

2. To prove the specificity of binding reaction of anti-HBS positive serums from individuals naturally infected and vaccinees with HBV Envelope Large, Middle and Small Proteins by neutralization tests, study the characteristic of specific humoral immune responses induced by HBV Envelope Large, Middle and Small Proteins.

Results:

1. Serum anti-HBS from individuals naturally infected and vaccinees could combine with HBV Envelope Large, Middle and Small Proteins. There was no difference between the binding reaction of these two groups, and there was no difference between individuals vaccinated with plasma-purified HBsAg and individuals immunized with genetic vaccine either. 
2. The result of neutralization tests indicated that anti-HBS from individuals self-limited infected and vaccinees could neutralized with HBV Envelope Large, Middle and Small Proteins, and its inhibition rate was over $50 \%$.

Conclusion: Anti-HBs producted after immunized with HBV Vaccine could neutralize HBV Envelope Large, Middle and Small Proteins, which indicated that HBV vaccine mainly containg small protein as a immunogen may provide adequate protective immune response.

\section{PP05-27}

Long-term Therapy with Adefovir Dipivoxil for Lamivudine-resistant HBeAg Positive Chronic Hepatitis B: Results from 104 Weeks Antiviral Treatment

L. Zhuang ${ }^{1}$, J. You ${ }^{2}$, S.M. Yan ${ }^{3}$, J.H. Huang ${ }^{4}$, L. Kong ${ }^{1}$, H. Lei ${ }^{1}$, Y.L. Ma ${ }^{1}$, Y.L. Li ${ }^{1}$, H.Y. Chen ${ }^{2}$, B.Z. Tang ${ }^{2}$, Q.Q. Wang ${ }^{1}$, X. Zhang ${ }^{2}$, L. Chen ${ }^{2}$, J.M. Li $^{2}$, C. $\mathrm{Chen}^{2}$

${ }^{1}$ Department of Hepatology, Third Municipal People's Hospital of Kunming; ${ }^{2}$ Department of Infectious Diseases, First Affiliated Hospital of Kunming Medical University; ${ }^{3}$ Department of Internal Medicine, Third People's Hospital of Yunnan Province; ${ }^{4}$ Department of Infectious Diseases, Yunnan General Hospital of The Chinese People's Armed Police Forces, Kunming, China

Background/aim: Adefovir dipivoxil has shown efficacy and safety in a broad range of populations with chronic hepatitis B. This study evaluates the 104-weeks efficacy of adefovir dipivoxil alone and in combination with lamivudine in treating patients with lamivudinerefractory $\mathrm{HBeAg}$-positive chronic hepatitis B.

Methods: Eighty-five $\mathrm{HBeAg-positive} \mathrm{patients} \mathrm{who} \mathrm{had} \mathrm{received}$ lamivudine treatment for various periods and had a lamivudine-resistant liver function abnormality, documented YMDD mutations and persistent viremia were randomized to adefovir dipivoxil $10 \mathrm{mg}$, lamivudine $100 \mathrm{mg}$, or addition of adefovir dipivoxil to ongoing lamivudine daily. The primary efficacy measure was virological response. The secondary efficacy measure was serological response $(\mathrm{HBeAg}$ loss rate and $\mathrm{HBeAg}$ seroconversion rate) and ALT normalization rate.

Results: After 24 weeks of therapy, mean reduction of HBV-DNA level, the percentage of patients with HBV-DNA lower than $5 \log 10$ copies/ml and the percentage of patients with HBV-DNA level decrease of more than $2 \log 10$ copies $/ \mathrm{ml}$ in patients of adefovir dipivoxil/lamivudine and adefovir dipivoxil monotherapy groups were significantly higher than those in patients of lamivudine group $(2.58,2.21 \mathrm{log}$ copies/ $\mathrm{ml}$ vs. $1.02 \log$ copies $/ \mathrm{ml}, 92.3,88.5 \%$ vs. $33.6,76.9,75.8 \%$ vs. $28.8 \%$; $P<0.001$, respectively). At the end of 52 weeks, mean reduction from baseline in serum HBV-DNA level at was $0.08,-4.25$, and $-4.12 \log 10$ copies $/ \mathrm{mL}$ in the lamivudine, adefovir dipivoxil/lamivudine, and adefovir dipivoxil groups, respectively. ALT normalization rates were significant hihger in adefovir dipivoxil/lamivudine and adefovir dipivoxil recipients than those in lamivudine recipients $(62,55$ vs. $8 \%, P<$ 0.001 , respectively). A similar pattern was observed in $\mathrm{HBeAg}$ loss among three groups. At the end of 104 weeks, mean reduction of HBVDNA level, ALT normalization and $\mathrm{HBeAg}$ loss rates were significant hihger in adefovir dipivoxil/lamivudine group than those in monotherapy group $(P<0.001$, respectively).

Conclusions: Adefovir dipivoxil is an effective treatment option for patients with lamivudine-refractory $\mathrm{HBe} A g$-positive chronic hepatitis B.

\section{PP05-28}

Efficacy and Safety of Nucleos(t)ide Analogues Agents on Chronic Hepatitis B Automated Peritoneal Dialysis Recipient Patients

H. Lu, Y. Yu

Department of Infectious Disease, Peking University First Hospital, Beijing, China

Objective: To evaluate efficacy and safety of nucleos(t)ide analogue agents on chronic hepatitis B automated peritoneal dialysis (APD) recipient patients.
Methods: Four patients were administered with $0.5 \mathrm{mg}$ per week of entecavir; one patients with adefovir dipivoxil (10 mg twice a week for four weeks, then, $10 \mathrm{mg}$ per week); one patients with lamivudine (100 mg per week). The durations of therapy were from 24 to 168 weeks. The parameters of serum HBV DNA load, live and renal function, side effects were documented.

Results: For patients administered with entecavir, tow patients were negative for HBV DNA at week 4, and two patients at week 12. HBV DNA load of the patient with adefovir dipivoxil reduced slowly (4.2 $\log 10$ copies / $\mathrm{mL}$ at week $0,3.2 \log 10$ copies $/ \mathrm{mL}$ at week 12 , negative at week 24). Serum alanine aminotransferase level of four patients reduced to normal level at 12 weeks of antiviral therapy, moreover, sustained biochemical response. One patient did not obtained normalization of serum alanine aminotransferase during the period of lamivudine antiviral therapy, and presented virological breakthrough at week 48. The serum levels of creatinine, urea nitrogen and urine protein hardly changed within the duration of antiviral treatment. No obviously side effect was seen in five patients. Conclusions: $0.5 \mathrm{mg}$ per week of entecavir or $10 \mathrm{mg}$ per week of adefovir dipivoxil could achieve effective antiviral therapy outcome, as well as with few side effects in chronic hepatitis B APD recipient patients. Lamivudine treatment could lead to viral mutation in APD recipient patients as well as in individuals with normal renal function, entecavir may be the optimal antiviral agent for APD recipient patients with chronic hepatitis B.

\section{PP05-29}

Chinese Herbal Medicine Combined with Adefovir Dipivoxil (AD) and Entecovior (EN) in Treatment of Chronic Hepatitis B with Functional Analysis

L. Min ${ }^{1}, \mathrm{Y} . \mathrm{Ye}^{2}$

${ }^{I}$ Mathematics and Physics School, University of Science and Technology Beijing, ${ }^{2}$ Traditional Chinese International Medicine Key Laboratory of China, Beijing University of Chinese Medicine, Beijing, China

Background and objectives: During 2004-2006, a 53 years old male chronic $\mathrm{HBeAg}$ positive patient received the herbs-Kuanxiongjiedu Grain (CHKG, 12 ingredients) treatment, two times daily. His baseline ALT $=106.00 \mathrm{U} / \mathrm{L}$ and $\mathrm{HBV}$ DNA $=2.09 \mathrm{e} 7 \mathrm{cp} / \mathrm{mL}$. At week 44, his HBV DNA $<1,000 \mathrm{cp} / \mathrm{mL}$ (lower detection limit), $\mathrm{ALT}=11.17 \mathrm{U} / \mathrm{L}$. During that period, his maximum $\mathrm{HBV}$ $\mathrm{DNA}=3.60 \mathrm{e} 8 \mathrm{cp} / \mathrm{mL}, \mathrm{ALT}=527.10 \mathrm{U} / \mathrm{L}$, and $\mathrm{HBeAg}$ seroconversion. After 16 week's virus suppression, his HBV DNA rebounded to $9.05 \mathrm{e} 6 \mathrm{cp} / \mathrm{mL}$. This study reports that combination scheme treating the patient can obtain good outcome.

Methods: During Feb. $2009 \sim$ now, the patient received Chinese herbal medicine CHM (15-23 ingredients, 450-600 g) treatment, two times daily (decoction). His basiline ALT $=45.7 \mathrm{U} / \mathrm{L}, \mathrm{HBV}$ $\mathrm{DNA}=2.7 \mathrm{e} 6 \mathrm{cp} / \mathrm{mL}, \mathrm{HBeAg}=450.57 \mathrm{~S} / \mathrm{CO}$, Anti-HBe $=4.02 \mathrm{~S} /$ CO. When HBeAg $<70 \mathrm{~S} / \mathrm{CO}$, switched to CHM + AD for 53 weeks. Then switched to CHM + EN. The ALT and HBV DNA tests were implemented every 4-8 weeks.

Results: At week 23, his HBeAg $=4.10 \mathrm{~S} / \mathrm{CO}$, Anti-HBe $=1.32 \mathrm{~S} / \mathrm{CO}$, $\mathrm{HBV}$ DNA $=4.1 \mathrm{e} 6 \mathrm{cp} / \mathrm{mL}$, his average ALT $=51.12 \mathrm{U} / \mathrm{L}$. After switched to CHM + AD treatment for 4 weeks, his HBV DNA $<500 \mathrm{cp} / \mathrm{mL}$ (lower detection limit). At weeks 76, switched to CHM + EN for treatment. Form the week 27 to now, his HBV DNA $<500 \mathrm{cp} / \mathrm{mL}$, average $\mathrm{ALT}=36.27 \mathrm{U} / \mathrm{L}, \mathrm{HBeAg}=1.45 \mathrm{~S} / \mathrm{CO}, \mathrm{Anti}-\mathrm{HBe}=1.01 \mathrm{~S} / \mathrm{CO}$.

Conclusion: The main function of the CHKG is to kill infected hepatocytes and the one of the CHM is to kill HBV directly. The main role of $\mathrm{AD}$ and $\mathrm{EN}$ in the scheme may be to block the replications of HBV. The CHKG may arise herbal resistance. Personality combination therapy is promising.

Acknowledgments: This work is jointly supported by the 11th 5-Year Plan Key Research Project of China (No. 2008ZX10502-006) and the NNSF of China (No. 61074192). 


\section{PP05-30}

Serum Hepatitis B Surface Antigen Quantification Cannot Predict Entecavir Treatment Response in Chronic Hepatitis B Patient

S.-H. Yoo ${ }^{1}$, H.B. Chae ${ }^{1}$, S.J. Youn ${ }^{1}$, S.-M. Park ${ }^{1}$, J.H. Han $^{1}$, S.M. Yun ${ }^{1}$, S.W. $\mathrm{Nam}^{2}$

${ }^{1}$ Internal Medicine, Chungbuk National University Hospital, Cheongju; ${ }^{2}$ Internal Medicine, National Medical Center, Seoul, Republic of Korea

We aimed to evaluate serum hepatitis B surface antigen ( $\mathrm{HBsAg}$ ) quantification as a surrogate marker for serum hepatitis B virus DNA, and as a predictor of virologic response to entecavir therapy. Twentyone chronic hepatitis B patients receiving treatment of 48-week entecavir were studied. Serum viral markers such as $\mathrm{HBsAg}, \mathrm{HBeAg}$, anti-HBe, HBV DNA were measured at the baseline, 3, 6, 9, 12 months after treatment. Virologic response was defined as HBV DNA $<100$ copies $/ \mathrm{mL}$ at the 12 months after treatment. At baseline, female, 6(28\%); mean age, 46; HBeAg positivity, 13(61\%). LogHBV DNA and HBsAg titer at the baseline vs 12 months after treatment were 7.25 vs 3.48 and 11,574 vs $4,055 \mathrm{IU} / \mathrm{mL}$. There was no association between baseline HBsAg and HBV DNA at each three months interval from baseline to 12 months after treatment. The number of responder $(\mathrm{R})$ is $12(57 \%)$. Baseline logHBV DNA and HBsAg in both R and nonresponder (NR) group were 6.86 vs $7.57(p=0.11)$ and 3,274 vs $21,315 \mathrm{IU} / \mathrm{mL}(p=0.3)$. There was no association between baseline HBsAg titer and logHBV DNA at 3, 6, 9, 12 months after treatment. Area uder ROC curve of baseline HBsAg is 0.462. Baseline HBsAg titer cannot predict the virologic response of entecavir treatment.

\section{PP05-31}

Succesfully Result in Treatment Hepatitis B Case Report T.P. Prabowo

Internal Medicine, PKT Hospital, Bontang, Indonesia

Introduction: Treatment for chronic hepatitis B was very difficult and sometime need long treatment There were many choice for treatment but unfortunately some result was not satisfied. Pegilated interferon alfa $2 b$ was one good choice for the disease. This case depicted successfully result in short term evaluation in treatment this disease.

Case description: Mrs S (37 years old) with chronic kidney disease stage $\mathrm{V}$ underwent hemodyalisis treatment twice per week for two years. In November 2009 laboratory evaluation confirm with chronic active hepatitis B and the result laboratory test were HBsAg positive, HBeAg positif, elevated ALT/AST (166/ 159) and also HBVDNA more than 40,000,000 copies $/ \mathrm{ml}(8,000,000 \mathrm{IU} / \mathrm{ml}$ Then She underwent treatment with Pegilated interferon alfa $2 \mathrm{~b} 50 \mathrm{mcg}$ per week for 6 months. Started therapy on December 2009. Seroconversion HbeAg was achieved at 24 week. Also HBVDNA reduce dramatically to 0 $\mathrm{IU} / \mathrm{ml}$. One week after the good result, surprising laboratory test result for the goal for treatment in chronic hepatitis B also found., There were HBsAg negative and Anti HBs positive $(541,9)$. Monitoring serum transaminase every month also showed reduced to normal value. Side effect treatment was not found, and continue evaluation for every next 3 month will be done for this case.

\section{PP05-32}

Clinical Study on 48-week Treatment of Entecavir versus Lamivudine in Patients with Decompensated HBV-related Cirrhrosis

\section{VIP Ward, Shanghai Public Health Clinical Center Affiliated to} Fudan University, Shanghai, China

Objective: To research clinical efficacy and safety of entecavir in treatment of decompensated cirrhrosis.

Methods: 63 patient with decompensated cirrhosis in Child-Pugh B were divided into two groups voluntarily, with 30 and 33 patients respectively. entecavir $0.5 \mathrm{mg} / \mathrm{d}$ was applied in treatment group and lamivudine $100 \mathrm{mg} / \mathrm{d}$ in control group on the basis of general treatment. Before and at 1, 12, 24 and 48 weeks after antivirus treatment, following data was obtained including Blood cell counts (WBC, RBC, PLT), liver function (ALT, AST, TBiL, ALB), PTA, level of HBV DNA, HbeAg, HbeAb, serum creatinine (Cr), ChildPugh Turcotte (CPT) as well.

Results: 24 patients among entecavir group and 30 among lamivudine group completed the therapeutic course of 48 weeks. The other 9 patients dropped out in 12 weeks because of hepatic encephalopathy and Hemorrhage upper digestive tract. The treatment has been adjusted to adefovir and lamivudine in 7 of the 30 patients of control group for lamivudine resistance. No evidence of entecavir resistanc was found. Primary hepatocarcinoma occurred, 2 from entecavir group and 3 from lamivudine group. entecavir showed similar efficacy with lamivudine in treatment of decompensated cirrhrosis $(\mathrm{P}>0.05)$. No adverse event happened to 54patients completed the therapeutic course.

Conclusion: It is effective to treat the decompensated cirrhrosis patients with entecavir for one year, and the patients presented good tolerability but no drug resistance. entecavir is an effective drug in treatment of those patients.

\section{PP05-33}

Nucleoside Analogues in Patients with Chronic Severe Hepatitis B for One Year Survival Rate

J. Chen

Liver Center, The 2nd Xiangya Hospital, Central South University, Changsha, China

Objective: To study the effects of nucleoside analogues in patients chronic severe hepatitis B for one year survival rate.

Methods: We retrospectively randomly collected the data of 301 chronic severe hepatitis B patients, 41 were treated with entecavir, 41 were treated with lamivudine, and the remaining 219 were not treated with any nucleoside analogues. One year survival rate are observed among groups.

Results: Results show no significantly difference of short-term survival rate between the group treated with entecavir or lamivudine, and that with none-nucleoside analogues $(P>0.05)$. Further more, we analyzed the MELD score and liver function in patients with different HBV-DNA levels $\left(<10^{4}\right.$ copies $/ \mathrm{ml},>10^{4}$ and $<10^{6}$ copies $/ \mathrm{ml},>10^{6}$ copies $/ \mathrm{ml}$ ), and no significant difference has been observed $(P>0.05)$. There was also no significant correlation of HBV-DNA levels and MELD scores in different periods during the therapy ( $P>0.05)$. Furthermore, The HBV-DNA level between patients with over one year and less than one year survival patients showed no significant difference either ( $\mathrm{P}>0.05)$. However, the MELD score and parameters of liver function (albumin, prothrombin time) showed significant difference $(\mathrm{P}<0.05)$ between these two groups.

Conclusions: These results suggested short-term suppression of HBV replication may not rapidly reduce the severity of liver failure and enhance the short-term prognosis in chronic severe hepatitis B patients.

\section{PP05-34}

Synthesis, Characterization and Antiviral Activity against Hepatitis B Virus of $\mathrm{Cs}_{2} \mathrm{~K}_{4} \mathrm{Na}\left[\mathrm{SiW}_{9} \mathrm{Nb}_{3} \mathrm{O}_{40}\right] \cdot \mathrm{H}_{2} \mathrm{O}$

H. Zhang ${ }^{1}$, J. Li ${ }^{2}$, Y.F. Qi ${ }^{2}$, J. Wang ${ }^{2}$, Y.F. Jiang ${ }^{1}$, J.Q. Niu ${ }^{1}$

${ }^{1}$ Hepatology, First Hospital, Jilin University; ${ }^{2}$ School of Public Health, Jilin University, Changchun, China

Objective: To explore the anti-hepatitis $\mathrm{B}$ virus (HBV) effects of $\mathrm{Cs}_{2} \mathrm{~K}_{4} \mathrm{Na}\left[\mathrm{SiW}_{9} \mathrm{Nb}_{3} \mathrm{O}_{40}\right] \cdot \mathrm{H}_{2} \mathrm{O}$ (a new Polyoxometalate) by using the HepG2 2.2.15 cell model system.

Methods: MTT assay was used to evaluate the growth inhibitory effect of $\mathrm{Cs}_{2} \mathrm{~K}_{4} \mathrm{Na}\left[\mathrm{SiW}_{9} \mathrm{Nb}_{3} \mathrm{O}_{40}\right] \cdot \mathrm{H}_{2} \mathrm{O}$ on $\mathrm{HepG}_{2} 2.2 .15$ cell lines. Extracellular HBsAg, HBeAg, and HBV DNA were measured by using ELISA or real-time PCR, respectively. Intracellular covalently closed circular DNA (cccDNA) and pregenomic RNA (pgRNA) were determined by using real-time PCR or real-time RT-PCR, 
respectively. Intracellular $\mathrm{HBsAg}$ and $\mathrm{HBcAg}$ were measured by Western blot.

Results: $\mathrm{CC}_{50}$ were 1,288 and $1,995 \mu \mathrm{mol} / \mathrm{L}$ for $\mathrm{Cs}_{2} \mathrm{~K}_{4} \mathrm{Na}[-$ $\left.\mathrm{SiW}_{9} \mathrm{Nb}_{3} \mathrm{O}_{40}\right] \cdot \mathrm{H}_{2} \mathrm{O}$ and $\mathrm{ADV}$ at cytotoxicity at day 9. In HepG2 2.2.15, $\mathrm{Cs}_{2} \mathrm{~K}_{4} \mathrm{Na}\left[\mathrm{SiW}_{9} \mathrm{Nb}_{3} \mathrm{O}_{40}\right] \cdot \mathrm{H}_{2} \mathrm{O}$ effectively suppressed the secretion of the HBV antigens and HBV DNA in a dose-dependent and time-dependent manner with $\mathrm{IC}_{50}$ values of $80 \mu \mathrm{mol} / \mathrm{L}$ for HBsAg, $75 \mu \mathrm{mol} / \mathrm{L}$ for $\mathrm{HBeAg}$ and $3.72 \mu \mathrm{mol} / \mathrm{L}$ for supernatant $\mathrm{HBV}$ DNA at day 9 . They are $1,569,1,671,173 \mu \mathrm{mol} / \mathrm{L}$, respectively, for Adefovir Dipivoxil on HepG2 2.2.15 cell. Intracellular cccDNA, pgRNA, HBsAg and $\mathrm{HBcAg}$ were also decreased in a dose-dependent manner by $\mathrm{Cs}_{2} \mathrm{~K}_{4} \mathrm{Na}\left[\mathrm{SiW}_{9} \mathrm{Nb}_{3} \mathrm{O}_{40}\right] \cdot \mathrm{H}_{2} \mathrm{O}$. At the same dose, the inhibit effect was higher than Adefovir Dipivoxil control.

Conclusion: $\mathrm{Cs}_{2} \mathrm{~K}_{4} \mathrm{Na}\left[\mathrm{SiW}_{9} \mathrm{Nb}_{3} \mathrm{O}_{40}\right] \cdot \mathrm{H}_{2} \mathrm{O}$ is a potential compound for treatment chronic HBV infection.

\section{PP05-35}

Factors of Chronic Hepatitis B Patients with Negative HBVDNA but Elevated ALT Treated with Nucleoside Analogues for 48 Weeks X. Xu, D. Zhang

The Second Affiliated Hospital of Chongqing Medical University, Chongqing, China

Aims: To study the factors of elevated alanine aminotransferase (ALT) in chronic hepatitis B (CHB) patients with negative HBVDNA treated with nucleoside analogues for 48 weeks.

Methods: A total of $130 \mathrm{CHB}$ patients whose serum HBVDNA level were negative (serum HBVDNA load $<1,000$ copies/ml) lasting for at least 6 months after treated with nucleoside analogues (Entecavir $=12$ and Adefovir dipivoxil $=118)$ for 48 weeks were enrolled, including 27 subjects with elevated ALT $(1-6 \times$ ULN $($ ULN $=40$ $\mathrm{IU} / \mathrm{ml})$, ALT positive group) and 103 subjects with normal ALT $(<40$ $\mathrm{IU} / \mathrm{ml}$, ALT negative group) at week 48 . Liver biopsy specimens were examined in 80 of them at week 0 and 37 of them both at week 0 and week 48 for pathological changes of grade and stage. A comparison of demographical, biochemical, virological, serological and histological features between the two groups were performed.

Results: there were no significant difference about the ages, ALT levels, HBVDNA loads, HBeAg statuses and histological grade and stage in the two groups at week 0 (all $p>0.05$ ). However, patients in ALT positive group had a significantly higher rate of histological fatty change than those of patients in ALT negative group both at week 0 and week $48(\mathrm{OR}=4.609,95 \% \mathrm{CI}=1.208-17.59, p=0.026$ and $\mathrm{OR}=10.5,95 \% \mathrm{CI}=1.321-83.49, p=0.025$, respectively). The histological grade was improved significantly from week 0 to week 48 in ALT negative group ( $p=0.003)$, but this could not be observed in ALT positive group. In addition, the rates of $\mathrm{HBeAg}$ seroconversion at week 48 had no significant difference in these two groups $(p>0.05)$. Conclusions: Histological fatty change was a independent factor associated with the failure of treatment with nucleotide analogues in CHB patients no matter it happened before or after the treatment. It will be necessary to perform liver biopsy in patients with negative HBVDNA but elevated ALT level after they received nucleoside analogues antiviral treatment.

\section{PP05-36}

The Research on Curative Effects of Pegasys in Treating Chronic Hepatitis B Q.H. Ling ${ }^{1}$, J.J. Chen ${ }^{1}$, W.J. Xu ${ }^{2}$

${ }^{I}$ Department of Liver Disease, ShuGuang Hospital, Shanghai University of TCM, ${ }^{2}$ Heqing Health Service Centers for Community, District of Pudong, Shanghai, China

Object: To evaluate curative effects of Pegasys (PEG-IFN- $\alpha$ ) in treating chronic hepatitis B patients.

Methods: 55 chronic hepatitis B patients were enrolled in this study and treated only with Pegasys, biochemical indicators and viral load were assessed to evaluate curative effects in four-year follow up.
Results: Of the 17 hepatitis B e antigen ( $\mathrm{HBeAg}$ ) negative patients, the viral response rate was $64.7 \%(11 / 17), 45.7 \%$ (16/35) corresponding to treated with Pegasys for 24 and 48 weeks, while the corresponding viral response rate of $37 \mathrm{HBeAg}$ positive patients was 43.24\% (16/37), 62.16\% (23/37) when treated with Pegasys for 24 and 48 weeks. The viral response rates of the 55 patients treated with Pegasys were 50.9, 56.36, 65.45, 67.27, 70.91 and $72.73 \%$ corresponding to $24,36,48,96,144,192$ weeks treatment. HBV DNA in 13 patients were once undetectable by realtime-PCR method was detectable in 4-year follow up (23.6\%). HBV DNA from the 13 patients were gene sequenced, ten cases exhibiting 1896 point mutations in pre $\mathrm{C}$ region of HBV DNA (76.9\%), no other mutations detected. The $\mathrm{HBeAg}$ negative change rate of the $37 \mathrm{HBeAg}$-positive patients treated by Pegasys in 48 weeks was $27 \%$ (10/37), but two patients were found $\mathrm{HBeAg}$ in serum 96 weeks later, even though HBV DNA detected in serum, one of which do not change on alanine aminotransferase (ALT), while another change from 54 to 33 .

Conclusions: Pegasys is effective in treating chronic hepatitis B patients for delayed and low proportion drug tolerance, leading to high viral response rate. viral response rate was higher in $\mathrm{HBeAg}$ negative patients than those with $\mathrm{HBeAg}$ positive.

\section{PP05-37}

Effect of $S$-Adenosyl-L-methionine (SAMe) in the Treatment of Chronic Hepatitis B with Hyperbilirubinemia

S. Qingshou, Y. Tu

Department of Liver and Cholic Diseases, RuiKang Hospital, Affiliated to Guangxi Traditional Chinese Medicine University, Nanning, China

Objective: To evaluate the efficiency and safety of $S$-Adenosyl-Lmethionine (SAMe) in the treatment of chronic hepatitis B with hyperbilirubinemia.

Methods: Sixty-two patients diagnosed chroic hepatitis B with TbiL $>171.1 \mu \mathrm{mol} / \mathrm{L}$ were randomized into therapy group and control group. Twenty-six patients in control group were given with routine combined therapy such as liver petecting, jaundice withdrawing, antivirus, etc for 4 weeks; 36 patients in therapy group were given with SAMe $1.0 \mathrm{~g}$, ivgtt, basis of routine combined therapy for 4 weeks. The symptoms were performed and TBiL, DBiL, ALT, $\gamma$ GT were tested for all patients at 2 weeks and 4 weeks.

Results: The total effective rate was higher in therapy group (94.4\%), than that in control group $(80.8 \%)$ at the end of the observation $(P<0.05)$. Serum TBiL, DBiL, ALT in patients in therapy group were lower than those in control group after 2 weeks and 4 weeks therapy. $\gamma \mathrm{GT}$ in therapy group was lower than that in control group after 4 weeks therapy $(P<0.01)$. No adverse reaction such as rash, fever, dizziness, headache occurred in the two groups.

Conclusion: SAMe is effective and safe in the treatment of chronic hepatitis B with hyperbilirubinemia.

\section{PP05-38}

Hyper-Cytolytic Activity of Hepatic Natural Killer Cells Correlates with Liver Injury in Chronic Hepatitis B Patients

Z. Zhang, S. Zhang, F.-S. Wang

Research Center for Biotherapy, Beijing 302 Hospital, Beijing, China

Background: Natural killer (NK) cells are abundant in the liver and serve as a major innate immune component against microbial infection. Although $\mathrm{NK}$ cells have been implicated in inducing hepatocellular damage in patients with chronic hepatitis virus infection, the roles that hepatic NK cells play in chronic hepatitis B virus (HBV) infection remain obscure.

Methods: Intrahepatic and peripheral NK cell frequency, phenotype and function were comprehensively investigated in 51 immune activation (IA) patients with chronic hepatitis $\mathrm{B}(\mathrm{CHB})$ and 27 immune tolerant (IT) carriers, as well as 26 healthy individuals. Flow 
cytometric, immunohistochemical, and immunofunctional assays were performed to characterize comprehensively the intrahepatic and peripheral NK cells and their impacts on liver pathogenesis.

Results: NK cells expressing natural killer receptors (activation receptors) preferentially accumulated in the livers of IA patients, where they were activated and skewed towards cytolytic activity but without a concomitant increase in interferon (IFN)- $\gamma$ production as compared to those of IT carriers and healthy subjects. Further analysis showed that the livers of IA patients expressed higher levels of interleukin (IL)-12, IL-15 and IL-18 in situ and lower levels of IL-10 than IT and healthy subjects, which in vitro can induce activation and degranulation of NK cells from healthy individuals. Finally, hepatic NK cells displayed more cytolytic activity than peripheral NK cells, which was found to be positively correlated with liver histological activity index and serum alanine aminotransferase levels in these IA patients.

Conclusions: Hepatic NK cells were activated and preferentially skewed towards cytolytic activity dependent on an imbalanced cytokine milieu in IA patients, and correlated with liver injury during chronic HBV infection. These findings may facilitate the rational development of immunotherapeutic strategies to enhance viral control while limiting or blocking liver injury and inflammation.

\section{PP05-39}

Curative effects of Yidu Recipe on CHB Patients with Liver-kidney Yin $\mathrm{Xu}$ and Damp-heat and Influence on T Cell Subsets

Y.-Q. Gao ${ }^{1}$, X.-J. Zhu ${ }^{1}$, X.-H. Sun ${ }^{1}$, M. Li $^{2}$

${ }^{1}$ Hepatopathy, ${ }^{2}$ Laboratory of Cellular Immunology, Shanghai

Shuguang Hospital Affiliated with Shanghai University of Traditional Chinese Medicine, Shanghai, China

To study the effect of Yidu Ricipe and the influence in T cell subsets on CHB patients. 57 cases of $\mathrm{HBeAg}$-positive $\mathrm{CHB}$ patients collected from Shuguang hospital were treated with entecavir or entecavir combine with Yidu Ricipe for 6 months. Two groups fell off a total of 7 cases. The detection indicators included ALT, AST, HBV-DNA, HBV M, the rate of normalization of liver function, HBV-DNA negative rate, $\mathrm{HBeAg}$ negative rate, Chinese medicine symptoms score. The number of NKT and the expression level of IFN- $\gamma$ and IL4 , and the number of Th1, Th2, Tc1, Tc2 cells were examined by flow cytometry. Compared with entecavir, entecavir combine with Yidu Ricipe significantly reduced the level of ALT, AST, HBV-DNA and Chinese medicine symptoms score, and improved liver function normalization rate. In Entecavir group and entecavir combined with Yidu Ricipe group, the expression level of IFN- $\gamma$ was increased and IL-4 was reduced, which led to the increase of the number of Tc1 cells and the proportion of Tc1/Tc2; in entecavir combined with Yidu Ricipe group, the number of Th1 and Tc1 cells was markedly increased and the number of Th2 and Tc 2 cells was reduced, which resulted in the increase of the ratio of Th1/Th2 and Tc1/Tc2. So the efficacy of entecavir combined with Yidu Ricipe in treating $\mathrm{HBeAg}$ positive CHB patients is superior to entecavir alone, and the Yidu Ricipe can effectively improve the liver function of $\mathrm{HBeAg}$-positive CHB patients and inhibit HBV-DNA replication and improve the clinical symptoms. The effect mechanism of Yidu Ricipe maybe play a role through increasing the number of NKT cells and the expression level of IFN- $\gamma$, and reducing the expression level of IL-4, resulting in regulation of the balance between Th1/Th2 and Tc1/Tc2

\section{PP05-40}

Outcomes of Treatment for Chronic Hepatitis B with Entecavir Therapy S.G. Lim ${ }^{1,2}$, M.O. Aung ${ }^{1}$, S. Dede ${ }^{1}$, Y.M. Lee ${ }^{1}$, G.H. Lee ${ }^{1}$, M. Fernandas ${ }^{1}$, L. Vincent ${ }^{1}$, Y.Y. $\operatorname{Dan}^{1}$

${ }^{1}$ Gastroenterology and Hepatology, National University Health

System, ${ }^{2}$ Medicine, Yong Yoo Lin School of Medicine, National

University of Singapore, Singapore, Singapore
Background: A number of clinical trials of chronic hepatitis B (CHB) patients treated with entecavir show good viral suppression and low resistance rate but few studies have reported outcomes of unselected clinic patients.

Method: CHB Clinic patients treated with entecavir were followed for viral suppression, viral breakthrough, viral resistance and clinical outcomes. Data were analysed by Kaplan Meier graphs, multiple logistic regression.

Results: There were 335 chronic hepatitis B patients but 139 were excluded due to comorbidities such as liver transplant, HCC, malignancy, renal failure or $<6$ months of entecavir therapy. Of the 196 evaluable patients, $25.7 \%$ had cirrhosis and $18.9 \%$ had prior lamivudine-resistance, $44.4 \%$ had previous exposure to lamivudine or adefovir. $\mathrm{HBeAg}$ seroconversion was $40 \%$ at 4 years, and liver disease progression was $11.36 \%$ at 5 years. $\mathrm{HBe} A g$ negative patients achieved HBV DNA negative earlier than $\mathrm{HBeAg}$ positive patients $(p<0.001)$, median duration to HBV DNA undetecability was 4.3 and 8.8 months respectively. Overall, the proportion of patients achieved undetectable HBV DNA in HBeAg negative and positive patients was 92.85 and $75.78 \%$ respectively. HCC development was higher in cirrhotic patients $12 \%$ compared to $3 \%$ at 4 years in non-cirrhotics $(p=0.045)$. Liver disease progression was related to the baseline Child-Pugh score $(p<0.001)$. In lamivudine exposed patients, those with lamivudine resistance took longer to become HBV DNA negative $(p=0.0163)$. Baseline predictors of liver disease progression include cirrhosis (OR 6.5, 95\%CI 1.3-32.3), and serum albumin (OR 1.14, 95\%CI 1.02-1.26). Only one case of entecavir resistance was found, and this was in a patient who had no prior exposure to oral antiviral agents.

Conclusion: Entecavir treated clinic patients had good outcomes despite previous nucleoside analogue therapy and a wide spectrum of disease states. HBV DNA undetectability was very high and entecavir resistance very low even in those with previous lamivudine resistance.

\section{PP05-41}

Modulation of Serum Interleukin-18 Concentrations and Hepatitis B Virus DNA Levels during Interferon Therapy in Patients with Chronic Hepatitis B S.P.E. Sylvan ${ }^{1,2}$, U.B. Hellstrom ${ }^{3,4}$

${ }^{1}$ Department of Medical Sciences, Uppsala University Hospital; ${ }^{2}$ Department of Communicable Disease Control and Prevention, Uppsala County Council, Uppsala; ${ }^{3}$ Department of Infectious Diseases, Karolinska Institutet, Karolinska University Hospital; ${ }^{4}$ Department of Communicable Disease Control and Prevention, Stockholm County Council, Stockholm, Sweden

The aims of this study: was (1) to determine plasma values of interleukin-18 (IL-18) in patients with different clinical manifestations of hepatitis B (HB) and (2) to analyze the correlation between presence of circulatory levels of IL-18 and levels of HB virus DNA during interferon-alpha induced $\mathrm{HBe}$ seroconversion in patients with chronic $\mathrm{HB}$ (CHB).

Results: The IL-18 levels in serum did not significantly differ between healthy contril subjects $(99 \pm 25 \mathrm{pg} / \mathrm{mL})$, HB-immune patients $(85 \pm 33)$, and asymptomatic carriers of HB surface antigen (144 \pm 44 $\mathrm{pg} / \mathrm{mL})$. In contrast, anti-HBe and $\mathrm{HBe}$-antigen-reactive patients with symptomatic CHB had significantly elevated levels in circulation compared with healthy control subjects. An inverse correlation was found between serum HBV DNA copies and IL-18 levels during therapy $(r=-0.54, P<0.001)$. We consistently observed an IFN-alpha induced suppression of viral replication, which was followed by the ALT flare. There was a significant increase in IL-18 production after the ALT flare, where the peak of IL-18 preceded or coincided with the time of $\mathrm{HBe}$ seroconversion in patients who cleared the virus.

Conclusions: These results suggest that IL-18 is involved in the pathogenesis of $\mathrm{CHB}$ and that IFN-alpha therapy can augment the production of IL-18 in patients with CHB. 


\section{PP05-42}

Hepatitis B Related Cirrhosis Responds Better than Chronic Hepatitis to Entecavir Therapy

A. Marengo ${ }^{1}$, D. Bitetto ${ }^{2}$, A. D'Avolio ${ }^{3}$, C. Fabris $^{2}$, G. Di Perri ${ }^{3}$, M. Rizzetto ${ }^{1}$, P. Toniutto ${ }^{2}$, A. Marzano ${ }^{1}$

${ }^{1}$ Gastro-Hepatology Unit, San Giovanni Battista Hospital, University of Turin, ${ }^{2}$ DPMSC Medical Liver Transplantation Unit, Internal Medicine, University of Udine, Italy, ${ }^{3}$ Infective Disease, University of Turin, Turin, Italy

Background: Entecavir (ETV) is a treatment option in HBV-positive patients. This retrospective study investigates efficacy of ETV monotherapy in an Italian cohort of unselected Chronic Hepatitis B $(\mathrm{CH})$ patients.

Methods: Overall, 75 patients treated with ETV were analyzed (69 compensated, $14 \mathrm{HBeAg}+/ 55$ antiHBe+ and 6 decompensated). Sixtytwo were naïve (ETV $0.5 \mathrm{mg}$ ) and 7 were Lamivudine (LAM)resistant (ETV $1 \mathrm{mg}$ ). $\mathrm{CH}$ was diagnosed in 29 patients and cirrhosis (LC) in 40. The median follow-up (FU) was 17 months (2-96) and 54 patients had a FU $\geq 12$ months. A pharmacokinetics analysis was performed in 34 patients. A cohort of 166 patients on LAM monotherapy was compared with the ETV group of compensated patients. Twelve decompensated cirrhotics (6 HBV on ETV, 6 non-HBV) were analyzed to evaluate ETV-lactic acidosis correlation.

Results: After 6-12 months 67-91\% of $\mathrm{HBeAg}$-negative patients and 46-80\% of HBeAg-positive became HBV DNA negative (Virologic Response-VR). Anti-HBe seroconversion was observed in 30\% (3/10) of patients with a $\mathrm{FU} \geq 12$ months. At univariate and multivariate analysis in the ETV group, but not in the LAM cohort, LC was found the only independent predictor of VR in patients treated for at least 12 months $(p<0.05$, OR $1.823,95 \%$ CI $1.027-3.272)$. The pharmacokinetics study did not reveal any difference in the serum ETV levels between $\mathrm{CH}$ patients and cirrhotics. Lactic acid concentrations did not show any significative difference between $\mathrm{HBV}$ and non-HBV decompensated cirrhotics.

Conclusions: Our study confirms: (1) efficacy and safety of ETV monotherapy in patients with $\mathrm{CH}$, compensated and decompensated LC, without evidence of drug-related lactic acidosis in the latter group; (2) LC was a predictor of VR in ETV but not in LAM group, not imputable to difference in serum ETV concentrations but to its strong antiviral effect in presence of a limited replicative space in patients with LC.

\section{PP05-43}

Comparison of Efficacy of LAM Combined with ADV De Novo and ETV Monotherapy in Patients with HBV-related Decompensated Cirrhosis H. Jie, L. Zheng, L. Yin, Y. Yang

Department of Infectious Diseases, 1st Affiliated Hospital, School of Medicine, Zhejiang University, Hangzhou, China

Objective: To compare the efficacy of lamivudine (LAM) combined with Adefovir Dipivoxil (ADV) de novo and Entecavir (ETV) monotherapy in treatment for patients with hepatitis B virus (HBV)related decompensated cirrhosis for 2 years.

Methods: We enrolled $60 \mathrm{HBV}$-infected patients with decompensated cirrhosis treated with LAM combined with ADV de novo and 40 patients treated with ETV. Liver and kidney function, HBVDNA, HBVM, AFP, US or CT scan of liver were tested every 1-3 month. Results: By month 12, the HBVDNA negative rates in LAM combined with ADV de novo group and ETV monotherapy group are 51.1 and $60.0 \%$ respectively. By month 24 , the HBV DNA negative rates in LAM and ADV group and monotherapy group are 86.7 and $90.0 \%$ respectively. There is no significant difference between them $(P>0.05)$. By month 24 , the HBeAg negative rates in LAM and ADV group and ETV group are 43.5 and $33.3 \%$ respectively. There is no significant difference between them $(P>0.05)$. By month 12 or 24 , ALT normalization rates in two groups are similar. Viral breakthrough happened in 2 cases $(4.4 \%)$ by month 12 and 3 cases (6.7\%) by month 24 in LAM and ADV group, and no viral resistance observed. While Viral breakthrough happened in 1 cases $(3.3 \%)$ by month 12 and 2 cases (6.7\%) by month 24 in ETV group, and viral resistance observed in one case $(3.3 \%)$ by month 24 . Improvement of liver function is obviously in both groups, for CTP and MELD scores decreased distinctly. The accumulative total mortality or liver transplantation rate are 18.3 and $17.5 \%$ respectively in LAM and ADV group and ETV group. No blood creatinine increased above the upper normal line observed in both groups.

Conclusion: Not only LAM combined with ADV de novo but also ETV monotherapy are best choice in treatment for patients with HBV-related decompensated cirrhosis.

\section{PP05-44}

The Efficacy of Peginterferon $\alpha$-2b Treatment Regimens in Chinese and South-East Asian Patients with HBeAg+ CHB Based on Genotype

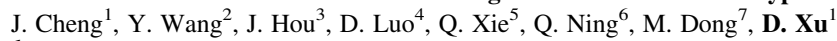
${ }^{1}$ Beijing Ditan Hospital, Capital Medical University, Beijing; ${ }^{2}$ Department of Infectious Diseases, Southwest Hospital, Third Military Medical University of PLA, Chongqing; ${ }^{3}$ Department of Infectious Diseases, Nanfang Hospital, Nanfang University, Guangzhou; ${ }^{4}$ Department of Infectious Diseases, Union Hospital, Huazhong Science and Technology University, Wuhan; ${ }^{5}$ Department of Infectious Diseases, Shanghai Ruijin Hospital, Shanghai; ${ }^{6}$ Department of Infectious Diseases, Tongji Hospital, Huazhong Science and Technology University, Wuhan; ${ }^{7}$ MSD China, Shanghai, China

Background: Genotypes B and C are the most prevalent genotypes in individuals in natives in Asian countries, including those in China, which has the largest pool of HBV-infected individuals in the world. The current study compared the effect of the three investigational treatment regimens on efficacy endpoints in subgroups of subjects based on HBV genotype.

Methods: A randomized, 3-arm, open-label, multinational interventional study in Chinese and South-East Asian selected patients with $\mathrm{HBeAg}$ positive, Anti-HBsAg negative, Anti-HBeAg negative, PCR HBV-DNA $>20,000 \mathrm{IU} / \mathrm{ml}$, HBsAg positive $\geq 6$ months, with ALT levels 2-10-fold higher than the upper limit of normal. Eligible subjects were centrally stratified by hepatitis B genotype and randomized in a 1:1:1 ratio into treatment Arm A (PEG2b $1.0 \mu \mathrm{g} / \mathrm{kg}$ QW for 24 weeks), Arm B (PEG2b $1.5 \mu \mathrm{g} / \mathrm{kg}$ QW for 24 weeks), or Arm C (PEG2b $1.5 \mu \mathrm{g} /$ $\mathrm{kg}$ QW for 48 weeks). Subjects were followed for 24 weeks after the end of treatment, and discontinued subjects were not replaced.

Results: Among 670 selected patients (including 657 from China), $304(45 \%)$ were genotype B and $357(53 \%)$ were genotype C. The PEG2b 1.5/48 weeks treatment regimen was more efficacious than the PEG2b 1.0/24 Wk regimen in treatment of HBV genotype B as well as genotype $\mathrm{C}$, as demonstrated by the statistically significant greater proportion of subjects with $\mathrm{HBeAg}$ loss, $\mathrm{HBeAg}$ seroconversion, and sustained combined response at end of follow-up. Interestingly, compared to the PEG2b 1.0/24 weeks arm, a statistically significant greater proportion of subjects with $\mathrm{HBV}$ genotype $\mathrm{B}$, but not genotype $\mathrm{C}$, in the PEG2b $1.5 / 48 \mathrm{Wk}$ arm had HBV-DNA $<20,000 \mathrm{IU} / \mathrm{mL}$ and ALT normalization at end of follow-up.

Conclusion: PEG2b $1.5 \mu \mathrm{g} / \mathrm{kg} /$ week for 48 weeks was more efficacious than PEG2b $1.0 \mu \mathrm{g} / \mathrm{kg} /$ week for 24 weeks in the treatment of Chronic Hepatitis B by HBV genotype B and genotype C.

\section{PP05-45}

Entecavir Treatment Prevents Disease Progression in HBV Related Acute-onChronic Liver Failure: Establishment of a Novel Logistical Regression Model K. Ma, W. Guo, M. Han, G. Chen, T. Chen, Z. Wu, D. Yang, J. Huang, Y. Huang, X. Zhao, J. Song, J. Qi, Q. Ning

Tongji Hospital, Huazhong Science and Technology University, Wuhan, China 
Hepatitis B related acute-on-chronic liver failure (HBV-ACLF) has a poor prognosis with high mortality. Experience in entecavir-treated patients with HBV-ACLF is limited. This study was designed to evaluate the efficacy and safety of entecavir in patients with HBVACLF and to develop a novel model.

In this retrospective study, 248 patients with HBV-ACLF were enrolled. Of these, 124 patients received standard internal medications plus treatment with entecavir; the remaining 124 patients received standard internal medications without nucleoside analogues (NA). Patient survival; biochemical markers; coagulation capacity; blood routine examination; serology markers; virological markers of HBV-DNA and complications were monitored and included in the multivariate logistical regression analysis upon which the regression model for prognostic prediction (Tongji prognostic predictory model, TPPM) was established.

The 1- and 3-month survival rates of patients in the entecavir-treated group were 72.58 and $61.29 \%$, significantly higher than that in NAfree group which were 53.23 and $45.97 \%$. The entecavir-treated group also achieved a better improvement of MELD score when compared with the controls. On multivariate logistic regression, high INR for prothrombin time, $\geq 2$ Complications and high total bilirubin, but not HBV DNA, were independent predictors of liver-related mortality. By Hosmer and Lemeshow test, TPPM for HBV-ACLF with a very good degree of fit with disease prognosis. Based on this unique group, the TPPM scoring offered a better prediction value in both specificity and sensitivity for 3-month mortality of patients with HBV-ACLF compared with MELD scoring system with statistically significant difference. In the patients with HBV-ACLF, using a cutoff of 0.22 for 3 -month predicted mortality by TPPM, the positive predictive value was $93.6 \%$, with a negative predictive value of $91.3 \%$. Entecavir treatment prevents disease progression and increased the survival of patients with HBV-ACLF. The established TPPM scoring system offers superior predictory value in both specificity and sensitivity for HBV-ACLF patients when compared with MELD.

\section{PP05-46}

The Investigation on the Compliance in Patients with Chronic Hepatitis B Treated with Nucleoside Analogue

K. Fan, Y. Wang, Q. Mao, X. Wang

Institute for Infectious Diseases of PLA, Southwest Hospital, The

Third Military Medical University, Chongqing, China

Background: When patients use nucleoside analogue (NUCs), the regularity of patients using drugs is a significant factor for efficacy. In clinical practice, we found that parts of patients did not adhere to the guidance of doctors, so this investigation is important for the treatment of CHB.

Methods: The data came from 685 patients with CHB who are using NUCs during 2002.1.1 to 2010.10.10, including 526 male and 159 female.The compliance of patients was investigated. The number of patients receiving LAM, ADV, LdT, ETV, LAM+ADV, LdT + ADV, ETV + ADV were 133, 174, 125, 99, 131, 14 and 9, respectively.

Results: $31.7 \%(217 / 685)$ of the patients have a bad compliance. There are just $5(0.7 \%)$ patients who declared did not to obtain the advice from the doctors that they should take these drugs in a long time. There is no significant difference $(P>0.05)$ between compliance of different genders and ages. The compliance of patients with LAM, ADV, LdT, $\mathrm{ETV}, \mathrm{LAM}+\mathrm{ADV}, \mathrm{LdT}+\mathrm{ADV}$ and ETV + ADV were 65.4, 63.8, 70.7, $67.2,73.3,78.6$ and $88.9 \%$, respectively. The rate of bad compliance patients is higher in these patients who have a longer time of treatment. The reasons of drug withdrawalling including that:

(1) prescribed drug could not last to the next visit (40.8\%);

(2) cost of treatment $(21.4 \%)$;

(3) the patients felt better, symptomless or thought treatment will not help $(2.9 \%)$;

(4) according to the advice of other people (2.9\%);
(5) too busy to go see doctors $(11.7 \%)$

(6) other reasons $(20.4 \%)$

Conclusion: The compliance of HBV patients with NUCs in China is not satisfactory. It is necessary to enhance the guidance for patients, help the patients who stop having drug because they cannot buy enough drug in their cities, and make the medical insurance better in the future.

\section{PP05-47}

Baseline CD4+CD25+CD127low/- Tregs and its Early Changes Predicts 24 Weeks Treatment Efficacy in E Antigen Positive Chronic Hepatitis B Patients

W. Yan, X. Wang, W. Guo, L. Xu, Y. Sun, Y. Lu, Z. Tan, T. Chen, Z. Wu, Q. Ning

Tongji Hospital, Huazhong Science and Technology University, Wuhan, China

To investigate the role of CD4+CD25+CD127low/- Tregs in telbivudine (LdT) treatment for hepatitis B e antigen ( $\mathrm{HBeAg}$ )-positive chronic hepatitis B (CHB) patients, 54 patients were enrolled in one center of an open-label, single-arm, 52-week, multicenter phase IV study in China. The median age is 27 (17-50), male $57.4 \%$, baseline median ALT of 162.5IU/L, HBV DNA of $8.2 \pm 1 \log _{10}$ copies $/ \mathrm{mL}$, $\mathrm{HBsAg}$ of $4.1 \pm 0.7 \log _{10} \mathrm{IU} / \mathrm{mL}$. Immunological analysis was performed in these 54 patients who received 24 weeks treatment of LdT. The proportional frequency of CD4+CD25+CD127low/- Tregs in $\mathrm{CD} 4+\mathrm{T}$ cells was detected by flow cytometry at baseline, weeks 12 and 24. The function of Tregs of 28 patients was assessed by examining their suppressive effect on the proliferation of core antigen-specific CD8+ T cells by CFSE staining. The percentage of CD4+CD25+CD127low/-Tregs decreased from $12.73 \pm 4.59 \%$ to 9.57 $\pm 3.27(P<0.001)$ accompanied with improved CD8+ T cells proliferation at week 24 with significant difference, particularly in patients with baseline HBV DNA $<9 \log _{10}$ copies/mL compared to patients with baseline HBV DNA $>9 \log _{10}$ copies $/ \mathrm{mL}(P=0.0007$ vs. $P=0.023$ ). Patients with baseline Tregs $<11 \%$ were more likely to achieve a significant decline of HBV DNA $>5 \log _{10}$ copies $/ \mathrm{mL}$ at week 24 compared to patients with baseline Tregs $>11 \%$ (60 vs. $23 \%$, $\left.\chi^{2}=7.282, P=0.007\right)$. Although no remarkable reduction of Tregs was found in the patients whose HBV DNA dropped $>5 \log _{10}$ copies/ $\mathrm{mL}$ or HBsAg dropped $>1 \log _{10} \mathrm{IU} / \mathrm{mL}$, the proliferation of CD8+ T cells has been improved at week 24 . Our study suggested the baseline frequency of Tregs could be used as one of the biomakers to predict LdT treatment efficacy at week 24 in HBeAg-positive patients and LdT might modulate the immune system in addition to its direct antiviral effects to achieve clinical treatment goal.

This work was supported by 2007CB512900, CLDT600ACN08T, CLD600ACN03.

\section{PP05-48}

On-treatment HBsAg and HBeAg Level Can Early Predict Sustained Virological Response in CHB, HBeAg Positive Patients Treated with Peginterferon Alpha-2a

P. Phisalprapa ${ }^{1}$, P. Charatcharoenwitthaya ${ }^{1}$, S. Chainuwatti ${ }^{1}$, S. Nimanong ${ }^{1}$, N. Pausawasdi ${ }^{1}$, V. Prachayakul ${ }^{1}$, S. Pongprasobchai ${ }^{1}$, S. Laalakusolvong ${ }^{1}$, S. Manatsathit ${ }^{1}$, U. Kachintorn ${ }^{1}$, S. Udompanthurak ${ }^{2}$, T. Tanwandee ${ }^{1}$ ${ }^{1}$ Division of gastroenterology, Department of Medicine, Siriraj Hospital, Mahidol University; ${ }^{2}$ Clinical Epidemiology Unit, Office for Research and Development, Faculty of Medicine, Mahidol University, Bangkok, Thailand

Background/aims: Treatment with peginterferon alpha-2a (PEGIFN) can achieve sustained virological response (SVR) only $32 \%$. Many predictors including baseline ALT, HBV DNA and liver histology have been proposed as predictors of response but none of these 
are strong enough to change treatment decision. This study aimed to evaluate the usefulness of these tests during treatment as early predictors of SVR.

Methods: $\mathrm{HBeAg}$ positive patients were enrolled to receive PEG-IFN $180 \mathrm{mg} /$ week for 48 weeks prospectively. Serum HBV DNA, HBsAg quantitation and $\mathrm{HBeAg}$ index were serially assessed at baseline and every 12 weeks to week 72. Treatment endpoints were $\mathrm{HBeAg}$ seroconversion and HBV DNA $<2,000 \mathrm{IU} / \mathrm{mL}$ at week 72 (SVR).

Results: There was 36 patients, mean age of 40 years and 53\% were male. Mean baseline of $\log _{10}$ HBV DNA (IU/mL), HBsAg (IU/mL), and $\mathrm{HBeAg}$ were 7.4, 4.3 and 2.7, respectively. At week 72, there were $10(28 \%) \mathrm{HBeAg}$ seroconversion and $4(11 \%) \mathrm{HBsAg}$ loss. There was no different in baseline characteristic between responders and nonresponders. Responders had more rapid decline of all 3 parameters. Using on treatment decline of these values as early predictors of SVR revealed very high negative predictive value (NPV) as shown in Table 1.

Conclusions: This study suggests that on-treatment HBsAg level and $\mathrm{HBeAg}$ index are very useful to predict SVR. With high NPV, these values may be useful for early treatment termination.

\begin{tabular}{|c|c|c|c|c|c|}
\hline Characteristics & $P$ value & Sensitivity & Specificity & PPV & NPV \\
\hline $\begin{array}{c}\text { Decrease of } \mathrm{HBsAg} \geq 0.33 \\
\log _{10} \text { at week } 12\end{array}$ & 0.006 & 100 & 67 & 46 & 100 \\
\hline $\begin{array}{c}\text { Decrease of HBsAg } \geq 1 \\
\log _{10} \text { at week } 24\end{array}$ & 0.001 & 100 & 77 & 55 & 100 \\
\hline $\begin{array}{c}\text { Decrease of } \mathrm{HBeAg} \geq 0.1 \\
\log _{10} \text { at week } 12\end{array}$ & 0.293 & 100 & 19 & 32 & 100 \\
\hline $\begin{array}{c}\text { Decrease of } \mathrm{HBeAg} \geq 1 \\
\log _{10} \text { at week } 24\end{array}$ & 0.005 & 80 & 77 & 57 & 91 \\
\hline $\begin{array}{c}\text { Decrease of HBV DNA } \geq \\
1.75 \log _{10} \text { at wk } 12\end{array}$ & 0.048 & 89 & 54 & 40 & 93 \\
\hline $\begin{array}{l}\text { Decrease of HBV DNA } \geq 3 \\
\log _{10} \text { at wk } 24\end{array}$ & 0.007 & 80 & 73 & 53 & 91 \\
\hline
\end{tabular}

\section{PP05-49}

Efficacy of the 96-week Adefovir Dipivoxil Therapy in Patients with Chronic Hepatitis B

Z. Xu, Q.-H. Xu, L.-B. Chen, H. Cao, X. Su

Infectious Disease, The Third Affiliated Hospital of Sun Yat-sen

University, Guangzhou, China

Objective: To investigate the efficacy of 96-week antiviral therapy using adefovir dipivoxil in patients with chronic hepatitis B.

Methods: 80 patients with chronic hepatitis $\mathrm{B}$ received the antiviral therapy of adefovir dipivoxil(ADV,10 mg/day). At the 12th week, 19 cases without early viral response (EVR, HBVDNA drop $<2$ $\log _{10}$ copies $/ \mathrm{ml}$ ) switched to the therapy of other nucleoside analogues. Aminotransferase (ALT) normalization, HBV DNA negative, $\mathrm{HBeAg}$ loss and $\mathrm{HBeAg}$ seroconvertion were observed at the 96th week.

Results: At week 96, rates of ALT normalization and HBV DNA negative in 61 patients with ADV therapy were $85.25 \%(52 / 61)$ and 95.08\% (58/61), while of HBeAg loss and HBeAg seroconvertion were $52.52 \%(17 / 33)$ and $42.42 \%$ (14/33) respectively. For the 19 patients switching to other nucleoside analogues, the rates of ALT normalization and HBV DNA negative came to $57.89 \%$ (11/19) and $68.42 \%$ (13/19). Both HBeAg loss and $\mathrm{HBeAg}$ seroconvertion rates in this group were $58.33 \%$ (7/12).

Conclusion: Long term ADV antiviral therapy is effective in inhibiting HBV DNA replications and benefits patients with chronic hepatits B. Switching to another nucleoside analogue is an optimal alternative for those with no EVR at week 12 in ADV therapy.
PP05-50

The Effect of Nucleoside (Acid) Analogues Therapy on Child-pugh Classification in Patients with Hepatocirrhosis

Z. Zhao ${ }^{1}$, M. Liu ${ }^{1}$, L. Yang ${ }^{2}$, Y. Zhang ${ }^{1}$, G. Zhang ${ }^{1}$, Y. Xing ${ }^{1}$

${ }^{1}$ Institute of Hepatology, Changzhi Medical College; ${ }^{2}$ Department of Gastroenterology, LuAn Group General Hospital, Changzhi, China

Aim: To investigate the effect of nucleoside (acid) analogues therapy on improvement of liver function in patients with hepatocirrhosis.

Method: A group of patients with hepatocirrhosis were evaluated by Child-Pugh classification, and received Lamivudine (100 mg per day) alone, or Lamivudine (100 mg per day) combined with Adefovir Dipivoxil (10 mg per day), or Entecavir ( $0.5 \mathrm{mg}$ per day) alone for 104 weeks. Child-Pugh grade A, B and C of 75 patients whose HBV DNA were undetectable after therapy were calculated and compared between before and after treatment. The rate of grade A in patients who got $\mathrm{HBeAg}$ seroconversion was compared to that of the nonseroconversion patients and that of naive $\mathrm{HBeAg}$-negative patients.

Results: Among 75 patients, the rate of grade A increased markedly (22.67 vs. $61.33 \%, P<0.01)$ and the grade $\mathrm{B}$ decreased obviously ( 65.33 vs. $28.00 \%, P<0.01$ ), while the grade $\mathrm{C}$ had not a significant difference $(12.00$ vs. $6.67 \%, p=0.262)$. The rate of grade $\mathrm{A}$ in patients with $\mathrm{HBeAg}$ seroconversion was higher than that of nonseroconversion (90.48 vs. $51.85 \%, P<0.01$ ) and $\mathrm{HBeAg-negative}$ ( 90.48 vs. $48.15 \%, P<0.01$ ). No patient with grade A progressed to grade $\mathrm{B}$ or $\mathrm{C}, 4$ patients with grade $\mathrm{B}$ developed to grade $\mathrm{C}, 3$ patients with grade $\mathrm{C}$ died during therapy.

Conclusion: Liver function of patients with hepatocirrhosis was improved significantly after nucleoside (acid) analogues therapy for 104 weeks. HBeAg seroconversion in $\mathrm{HBeAg}$-positive patients would be an important predictor for liver function improvement.

\section{PP05-51}

Early Decline in HBeAg Levels as Outcome Predictors during Oral Antiviral Agent Treatment for HBeAg-positive Chronic Hepatitis B

J.H. Kwon, J.W. Jang, S.H. Yoo, B.S. Oh, S.H. Bae, J.Y. Choi, S.K. Yoon, K.W. Chung

Department of Internal Medicine, College of Medicine, The Catholic University of Korea, Seoul, Republic of Korea

Aims: We investigated the quantitative change of $\mathrm{HBeAg}$ and $\mathrm{HBV}$ DNA levels during entecavir treatment for chronic hepatitis B and early decline of these titers predict the antiviral response.

Methods: Ninety-five consecutive $\mathrm{HBeAg}$ positive patients who treated more than 48 weeks with entecavir were enrolled between 2007 and 2009. The patients with decompensated cirrhosis and hepatocellular carcinoma were excluded. Serum HBeAg (CMIA, Abbott) and HBV DNA (PCR, Roche) were assessed quantitively at baseline and 4, 12 weeks and by 1-3 months interval. Virological response (Y1VR) was defined as undetectable HBV DNA $(<116$ copies/ml) at 48 weeks of therapy.

Results: Fifty-three patients $(55.8 \%)$ performed Y1VR. HBeAg and HBV DNA levels deceased during entecavir, but HBsAg decreased not significantly. At week 4 of therapy, a decrease of more than $0.5 \log _{10}$ in HBeAg levels was observed $22.9 \%$ and among them $52.6 \%$ (vs. $3.4 \%$, $p=0.000$ ) developed Y1VR. At week 4 of therapy, a decrease of more than $2 \log _{10}$ in HBV DNA levels was observed 65/88 (73.9\%) and among them, $80 \%$ (vs. $65.8 \%, p=$ non specific) developed Y1VR group. At week 12 of therapy, a decrease of $1 \log _{10}$ in $\mathrm{HBeAg}$ levels was observed $35.7 \%$ and among them $56.8 \%$ (vs. $12.1 \%, p=0.000$ ) developed Y1VR. At week 12 of therapy, a decrease of more than $4 \log _{10}$ in HBV DNA levels was observed $58.8 \%$ and among them, $70.5 \%$ (vs. $44.4 \%, p=0.019$ ) developed Y1VR. For predicting Y1VR, HBeAg levels and decrements from baseline at 4 and 12 week of therapy showed larger positive predictive value and area under ROC curve than HBV DNA levels.

Conclusions: These analyses suggest early decline of quantitative $\mathrm{HBeAg}$ levels is a useful measurement for predicting virological 
response in patients treated with entecavir when considering positive predictive value compared with HBV DNA levels.

\section{PP05-52}

Long-term Efficacy of Lamivudine/Emtricitabine and Tenofovir Combination Therapy on HBV/HIV Co-infected Patients

S. Suemura, N. Kuzushita, K. Yuguchi, H. Hasegawa, Y. Sakakibara,

T. Yoshio, S. Nakazuru, T. Toyama, E. Mita

Gastroenterology, Osaka National Hospital, Osaka, Japan

Background: The availability of highly-active antiretroviral therapy (HAART) has led to a dramatic shift in the spectrum of HIV-related morbidity and mortality. Therefore, the clinical management for hepatitis virus-related liver disease has become increasingly important. Lamivudine resistance is more frequently observed in HBV/HIV co-infected patients than in HBV mono-infected patients. In this study, we evaluated the long-term efficacy of lamivudine or emtricitabine (FTC) and tenofovir (TDF) combination administration (included in the HAART regimen) on HBV/HIV co-infected patients. Methods: From September 2006, 18 HBV/HIV co-infected patients with indications for HAART were enrolled in this study. They were treated with 3 TC $(300 \mathrm{mg})$ or FTC $(200 \mathrm{mg})$ and TDF $(300 \mathrm{mg})$ daily in the HAART regimen and followed prospectively for a mean duration of 39 months (range 11-72). Serum HBV-DNA was measured by the Amplicor HBV Monitor test, with the lower detection limit of HBV-DNA $2.6 \log _{10}$ copies/mL.

Results: Reductions of serum HBV-DNA levels and reductions of ALT levels were observed in all the patients. Suppression of HBV-DNA levels to below the limit of detection occurred in 17 of the patients (94\%) from months 1-26 (mean 11 months) except for one patient who has been followed for 21 months after HAART. The ALT levels normalized in 15 patients $(83 \%)$. Three patients had minor ALT flares, probably due to HAART toxicity. Importantly, in 7 patients (47\%), there was sero-conversion of HBe antigen and, in 5 patients $(28 \%)$, HBs antigen became negative. Additionally, in all patients, HIV viral load was below the limit of detection and CD4 counts were well controlled. Conclusions: A combination of 3TC/FTC and TDF can substantially suppress both HBV and HIV viral loads and may lead to improved outcomes of HBV related liver disease in HBV/HIV co-infected patients.

\section{PP05-53}

Baseline Peripheral NK and its Early Dynamic Changes Predicts 24 Weeks Treatment Efficacy in E Antigen Positive Hepatitis B Patients

T. Chen, L. Ding, W. Guo, G. Song, W. Yan, Z. Wu, X. Wang, Q. Ning

Tongji Hospital, Huazhong Science and Technology University, Wuhan, China

The significance of NK cells in CHB patients during antiviral treatment remains unknown. The study aim is to investigate NK cells response during telbivudine (LdT) treatment and its possible correlation with treatment efficacy. 54 hepatitis $\mathrm{B}$ e antigen (HBeAg)positive chronic hepatitis $\mathrm{B}(\mathrm{CHB})$ patients were enrolled into one center of an open-label, single-arm, 52-week, multicenter phase IV study in China. The CD3-CD56+ NK cells were sorted by flow cytometry at baseline, week 12 , and week 24 , as well as NK receptors including NKG2A, NKG2C, NKG2D, NKP30 and NKP46. The IFN$\gamma, \mathrm{TNF}-\alpha$ and CD107a were measured in standard intracellular staining procedure. Samples were also tested for HBV DNA, HBsAg, $\mathrm{HBeAg}, \mathrm{ALT}$ and AST.

The percentage of NK cells expressing activation receptors increased at Week 12 (NKG2D) and 24 (NKG2D and NKP46) $(p<0.0001$ and $p=0.003$, respectively), meanwhile the inhibitory receptor NKG2A decreased remarkably $(p=0.002)$. Despite that the expression percentage of IFN- $\gamma$ on the total CD3-CD56+ NK cells decreased slightly, the mean fluorescence index (MFI) of IFN- $\gamma$ significantly upregulated at week $12(p=0.017)$ followed by a decline at week 24 , while the MFI of CD107a increased gradually to peak at week 24 $(p<0.001)$. Stratified analysis showed that patients with NK cells $>10 \%$ at baseline achieved significantly more reduction of $\mathrm{HBV}$ DNA at week 24 than those with $\mathrm{NK}<10 \%$ at baseline $(p=0.04$, Fig. 1A). Moreover, the increase of NK cells at week 12 was positively correlated with reduction of HBV DNA at week $24(r=0.30$, $p=0.025$, Fig. 1B).

Our study suggested that LdT treatment in HBeAg-positive CHB patients led to NK cells activation in favor of viral clearance. Baseline peripheral NK and dynamic changes of its frequency and function predicts treatment efficacy of week 24 .

This study was supported by 2007CB512900, CLDT600ACN08T, CLDT600ACN03.

\section{PP05-54}

Telbivudine for Chronic Hepatitis B Bangladeshi Patients: Interim Analysis of 365 Cases

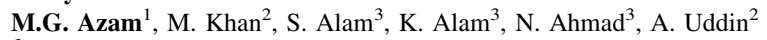

${ }^{I}$ Department of Gastroenterology and Hepatology, BIRDEM

Hospital; ${ }^{2}$ Hepatology, The Liver Centre; ${ }^{3}$ Hepatology, Bangabandhu Sheikh Mujib Medical University, Dhaka, Bangladesh

Background: Bangladesh is an intermediate prevalent zone of chronic hepatitis B (CHB) virus infection with a carrier rate of 5-7\%. Telbivudine is a new antiviral drug showing better efficacy among different clinical trials. Here, we are presenting a large cohort of treatment naive chronic hepatitis B Bangladeshi patients receiving telbivudine.

Materials and methods: We started telbivudine for chronic hepatitis B patients from February 2008 at our specialized centre in Dhaka. Upto June 2010, we have registered 365 cases. An interim analysis of biochemical and virological responses done at $6,12,18$ and 24 months. Results: At 6, 12, 18 and 24 months $135,74,18$ and 15 cases were analysed. Figure 1 showed mean S. ALT levels which is gradually declining. Figure 2 showed mean HBV DNA levels which is also gradually declining.
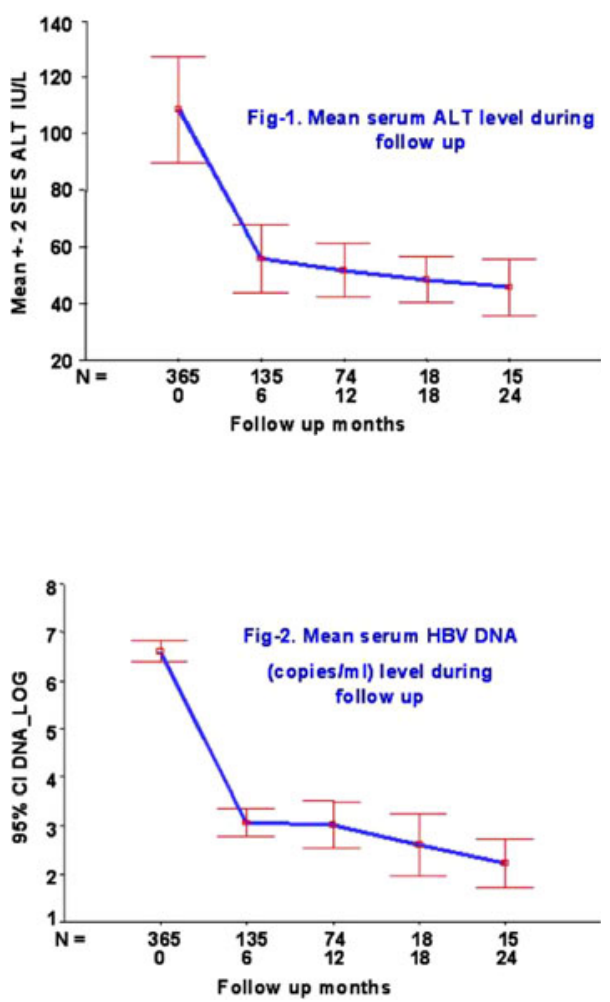

HBeAg negative cases showed significant PCR negative HBV DNA response at 12 months than that of $\mathrm{HBeAg}$ positive cases 
$(\mathrm{p}=0.000)$. Virologic breakthrough was only present among $\mathrm{HBeAg}$ positive cases at 12 months $(\mathrm{p}=0.000)$.

Conclusion: Telbivudine exerts better efficacy among HBeAg negative cases at 12 months. Virologic breakthrough is common in $\mathrm{HBeAg}$ positive cases but rare among $\mathrm{HBeAg}$ negative cases at 12 months.

\section{PP05-55}

Changes in Serum Hepatitis B Surface Antigen Level Can Be a Predictor of Antiviral Response for Treatment-naïve Patients Receiving Entecavir S.W. Lee ${ }^{1}$, H.J. Kim ${ }^{2}$, Y.K. Cho $^{3}$, B.I. Kim ${ }^{2}$

${ }^{1}$ Total Health Care Center, ${ }^{2}$ Internal Medicine, ${ }^{3}$ Sungkyunkwan University Kangbuk Samsung Hospital, Seoul, Republic of Korea

Background/aims: We investigated changes in hepatitis B surface antigen (HBsAg) level and its correlation with clinical outcomes in treatment-naïve chronic hepatitis $\mathrm{B}(\mathrm{CHB})$ patients receiving entecavir therapy.

Methods: Fifty-three consecutive patients (35 male, 18 female) with antiviral-naïve CHB who were treated with entecavir from January 2009 to July 2010 were included in our study. HBsAg levels were measured at baseline, 3, 6, and 12 months after the initiation of entecavir treatment using the Architect HBsAg QT assay (Abbott, dynamic; range $0.05-125,000 \mathrm{IU} / \mathrm{ml})$. Serum alanine aminotransferase, $\mathrm{HBeAg}$, anti-HBe, and hepatitis B virus (HBV) DNA (Cobas Taqman: low detection limit $1.84 \log _{10}$ copies/ml) were measured at baseline and every 3 months.

Results: Twenty-nine (54.7\%) patients were positive for HBeAg. The decline of $\log _{10} \mathrm{HBsAg}$ level (IU/ml) was significantly correlated with that of the $\log _{10}$ HBV-DNA level (copies/ml, Pearson correlation coefficient $0.360, p=0.023$ ) at 6 months after the initiation of entecavir treatment. However, there were no significant differences in the reduction of $\log _{10} \mathrm{HBs}$ g levels between the patients group who showed complete response (PCR-undetectability of HBV DNA at 6 Mo after treatment) and partial/non-response $(-0.23 \pm 0.83$ vs. $-0.08 \pm 0.09$ at 3 months, $-0.24 \pm 0.84$ vs. $-0.12 \pm 0.16$ at 6 months, $-0.33 \pm 0.91$ vs. $-0.11 \pm 0.16$ at 12 months). Additionally, patients group with $\mathrm{HBeAg}$ loss or $\mathrm{HBeAg}$ seroconversion showed no significant differences in the reduction of $\log _{10} \mathrm{HBsAg}$ levels compared to those of patients with $\mathrm{HBeAg}$ persistence at 3, 6, and 12 months after the entecavir treatment. Two patients $(3.8 \%)$ showed HBsAg loss during the follow-up period.

Conclusions: The changes in serum HBsAg level can be a useful parameter for assessing the response to the entecavir treatment in treatment-naïve CHB patients.

\section{PP05-56}

Creatine Kinase Elevations during 4 Years Telbivudine Anti-hepatitis B Virus (HBV) Treatment are Not Predictive of Muscle Events

M. Jung ${ }^{1}$, A. Uddin ${ }^{2}$, Y. Dong ${ }^{1}$, S. Kraev ${ }^{1}$, A. Trylesinski ${ }^{1}$

${ }^{I}$ Novartis Pharma AG, Basel, Switzerland; ${ }^{2}$ Novartis Pharma, East Hanover, NJ, USA

Background/aims: Elevated creatine kinase (CK) can occur in untreated HBV and has been reported during anti-HBV treatment. Increased rates were reported with telbivudine vs lamivudine (GLOBE study). CK increases during longterm telbivudine treatment were analyzed for a potential relationship with muscle events (ME). Methods: The overall safety population $(n=655)$ treated for 4 years with telbivudine during GLOBE+015 and 2303 studies were analyzed. CK levels/elevation-grades (G), MEs (i.e., myalgia, myopathy, (poly)myositis, muscular weakness/pain) and their potential relationship was assessed.

Results: Prior to initiating telbivudine treatment (Baseline), 81 patients $(12.4 \%)$ had elevated CK $(>1 \times \mathrm{ULN})$. During 4-year treatment, median CK increased from $101 \mathrm{IU} / \mathrm{mL}$ (Baseline) to $255 \mathrm{IU} /$ $\mathrm{mL}$ (112 weeks), decreasing thereafter to $171 \mathrm{IU} / \mathrm{ml}$ (4 years). Over 4 years, 104 patients (16\%) reported G3-4 CK elevations, 472 patients (71\%) G1-2 elevations. The majority had only $1-2$ elevation episodes $(86.6 \%)$, most were transient lasting $1-2$ visits $(\leq 3$ every months)(97.5\%). 21 patients with on treatment G3-4 elevations (26\%) had elevated CK at baseline. Increased CK had no impact on renal function (Glomerular Filtration Rate), no correlation was found with ethnicity. Muscle events are uncommon and were reported in 40 (6.1\%) patients. Baseline CKs were normal for $88 \%$ (35/40) of patients with ME. Around time of MEs, median CK, ALT and AST were not significantly increased. Only $4 \mathrm{ME}$ cases were myopathy (1) or (poly)myositis (3), 3 of which had G1-2 CK elevation at closest assessment to event. The majority (74\%) of patients with G3-4 CK elevations did not experience MEs over 4 years. $9 / 40$ patients with MEs had CK elevation within \pm 30 days of event. 12 patients had persistent $C K$ increase ( $\geq 2$ visits), only 3 of these occurred within \pm 30 days of a muscle event.

Conclusion: The majority of $\mathrm{CK}$ elevations during telbivudine treatment were asymptomatic, transient and lasted only 1-2 visits. On-treatment CK elevations were not a predictor of muscle events, which are uncommon during telbivudine treatment.

\section{PP05-57}

Clinical Improvement of Hepatitis B Virus Related Decompensated or Compensated Cirrhosis in Patients Treated with Entecavir

Y.K. Jung, J.H. Kim, I.S. Won, D.K. Kim, J.H. Kim, J.J. Lee, S.J. Lee, O.S. Kwon, D.J. Choi, Y.S. Kim

Internal Medicine, Gil Hospital, Gachon University of Medicine and Science, Incheon, Republic of Korea

Aims: The clinical effect of entecavir (ETV) treatment on hepatitis B virus related decompensated or compensated cirrhosis has not been well known yet. So we evaluated a clinical effect of ETV on naïve ETV treatment on HBV related cirrhosis patients.

Patients and methods: During study period, one hundred six patients were enrolled. All of them were HBV related cirrhosis patients and treated with ETV $0.5 \mathrm{mg}$ per day at least 6 months as first-line monotherapy. They were followed-up every 3-6 months with liver function test, prothrombin time, creatinine $\mathrm{HBeAg}$, anti-HBe, and HBV DNA levels.

Results: Age of the total patients were mean 52 years and they compromised 74 males (70\%). 53 patients $(50 \%)$ of them had decompensated cirrhosis and $40(37.7 \%)$ had $\mathrm{HBeAg}$ positivity. All patients were followed-up during median 20.5 months (range 6-39) and during follow-up periods 11 patients were died or underwent transplantation (the cumulative survival rate $89.6 \%$ ). In $\mathrm{HBeAg}$ positive patients $(n=40)$, we observed $\mathrm{HBeAg}$ seroconversion rate $35 \%$, ALT normalization rate $80 \%$, and undetectable HBV-DNA rate (PCR) $67.5 \%$ at 12 months after treatment. In HBeAg negative patients $(n=66)$, we also observed ALT normalization rate $86.4 \%$ and undetectable HBV-DNA rate $90.9 \%$. There was no difference between decompensated and compensated cirrhosis. ( $p=$ NS) In patient with ETV more than 12 months, bilirubin, prothrombin time and albumin were improved compared with baseline $(p<$ 0.001). In decompensated cirrhosis patients, Child-Turcotte-Pugh (CTP) score and model for end-stage liver disease (MELD) score were improved $(p<0.001)$. In addition, APRI score and platelet counts as indirective fibrosis markers were improved $(p<0.001)$.

Conclusion: ETV treatment as first-line monotherapy for HBV related cirrhosis was effective in both compensated or decompensated cirrhosis patients. It also improved underlying liver functions and fibrosis markers. So we expect to improve survival of HBV related cirrhotic patients treated with antiviral agents.

\section{PP05-58}

Peripheral Blood T Cell Dynamics and Natural Killer Cell Counts in Patients with Chronic Active Hepatitis B on Tenofovir Treatment Y. Ustundag ${ }^{1}$, B. Delikanli' ${ }^{2}$, M. Arasli ${ }^{3}$, F. Harmandar ${ }^{1}$, S. Aydemir ${ }^{1}$ ${ }^{1}$ Internal Medicine, Gastroenterology, ${ }^{2}$ Internal Medicine, ${ }^{3}$ Immunology, Zonguldak Karaelmas University School of Medicine, Zonguldak, Turkey 
Background/aim: We investigated serum viral kinetics and peripheral $\mathrm{T}$ cell profile, Natural Killer (NK) and NKT cell counts in patients with chronic hepatitis B viral infection on tenofovir therapy. Methods: Fifteen patients, naive to any kind of previous antiviral therapy were included. The patients received tenofovir at a daily dose of $245 \mathrm{mg}$ for 48 weeks. Immunological and virological parameters were assessed at baseline, then first, third, sixth and twelfth months. Serum HBV DNA load was measured by rt PCR, and the levels of T lymphocytes (CD3) and peripheral $\mathrm{T}$ cell subpopulations including CD4 and CD8 positive cells with flow cytometry. The decrement of HBV DNA and the changes of peripheral T-lymphocyte profile and NK-NKT cell counts were investigated.

Results: Fourteen percent of cases had serum levels of HBV DNA over $10^{7}$ copy per milliters and $12(80 \%)$ cases had undetectable HBV DNA levels after sixth months of therapy which remained negative in all these cases at the end of 1 year treatment. There was no difference in CD4/CD8 levels and NK-NKT cell counts at the beginning and throughout the study period. While HBV DNA levels were negative after 6 months of tenofovir treatment, three patients $(20 \%)$ had developed increased HBV DNA levels (range 200-180,000 IU) due to compliance problems. CD4/CD8 levels and NK-NKT cell counts in these patients did not differ significantly throughout the study period. Conclusions: Tenofovir does not change peripheral $\mathrm{T}$ cell profile and it has no effect on blood NK-NKT cell counts during its successful antiviral action period. Virologic relapse of HBV infection in our drug uncomplicant patients did not also seem to be in relation with peripheral blood $\mathrm{T}$ cell profile and NK-NKT cell counts.

\section{PP05-59}

Inhibition of Hepatitis B Virus Replication and Expression in vitro and in vivo by the Hammerhead Ribozymes Targeted Different Sites W. Dai

Institution, Shenzhen, China

Background: Hepatitis B virus (HBV) replicates asymmetrically through reverse transcription of an RNA intermediate. The $3.5-\mathrm{kb}$ pregenomic RNA serves as a template for reverse transcription and also encodes the nucleocapsid protein and the reverse transcriptase,which has the energetically flexible secondary and tertiary structure and may reduce the accessibility of ribozyme. This study was to identify accessible target sites and the effects of hammerhead ribozymes delivered by hepatitis delta virus (HDV) targeted on HBV gene expression and replication in cell culture and an animal model. Methods: To identify accessible target sites for hammerhead ribozyme, the secondary structure of $3.3 \mathrm{~kb}$ full-length HBV pregenome RNA was analyzed using a newest bioinformatic tools. Three hammerhead ribozymes for potential target sites: S, X, C genes and coexpression plasmid of replication-competent HDV-ribozyme with HBV were severally chosen to validate the inhibition of the replication and expression of HBV in transfected (or co-transfected with pTHBV) human hepatocellular carcinoma cells and hydrodynamic injection of mouse model in vivo.

Results: The encoding regions of $\mathrm{S}, \mathrm{C}, \mathrm{X}$ gene were the effective cleavage sites for hammerhead ribozyme, which inhibited the secretion of HBeAg in Huh-7 cells and in HepG2.2.15 in vitro. The ribozyme targeted $\mathrm{X}$ gene was most potential to inhibit HBV RNA accumulation. Hydrodynamic injection of the viral over length genome into mouse model in vivo leaded the expression of recombinant HDV and HBV. According to the cell culture system, the recombinant HDV-ribozyme exhibited effectively inhibition of HBV gene expression and replication in the livers and sera of the treated mice. $\mathrm{RzX}, \mathrm{RzC}$ inhibited the secretion of HBsAg by 66 and $57 \%$ respectively.

Conclusion: Encoding regions of HBV S, C, X gene were the effective cleavage sites for hammerhead ribozyme, which provides a basis for further construction of therapeutic recombinant HDV, and then the development of targeting antiviral gene therapy.
PP05-60

Blood Lactate as a Predictor of Prognosis in Acute-on-Chronic Liver Failure Caused by HBV: a Cohort Study

W. Chen, S. Lin, J. Dong, J.-J. Jiang

Center of Liver Disease, the First Affiliated Hospital of Fujian

Medical University, Fuzhou, China

Objective: To investigated the predictive value of venous blood lactate in acute-on-chronic liver failure (ACLF) caused by Hepatitis B Virus. Methods: In a single-center study, patients diagnosed as HBV-associated ACLF were collected from 2009 to 2010, consecutively. None of them underwent liver transplantation. Baseline biological parameters, such as alanine aminotransferase, aspartate aminotransferase, bilirubin, creatinine, prothrombin time and lactate were detected at admission. End-Stage Liver Disease (MELD) scores of these patients were calculated according to the data. The predictive value were analysed by receiver operating characteristics (ROC) method.

Results: A total of 48 ACLF patients were enrolled in this study, male 42 and female 6 , mean age $40.71 \pm 10.45$ years. After 3 months follow-up, all the patients were divided into 2 groups, survivors (S) group and non- survivors (NS) group. The increase of lactate occurred in $37(77.08 \%)$ patients. $22(84.81 \%)$ patients in NS group and 15 $(68.18 \%)$ in S group. Mean MELD scores of S group is 21.02, and mean MELD scores of NS group is 30.51 . The level of venous blood lactate was significantly higher in NS group than in S group (4.4 vs. $2.7 \mathrm{mmol} / \mathrm{L}, p<0.0001)$. ROC showed a threshold lactate level of $2.84 \mathrm{mmol} / \mathrm{L}$ had sensitivity of $62 \%$, and specificity of $68 \%$. Area under the curve (AUC) analysis revealed a better performance of MELD score (0.882) comparing with lactate (0.599).

Conclusion: Lactate level plays a role in ACLF, and it might be useful as a predictor of outcome in ACLF. MELD score has a better sensitivity than lactate.

\section{PP05-61}

The Clinical Observation of Telbivudine Treatment with HBeAg-positive Chronic Hepatitis B

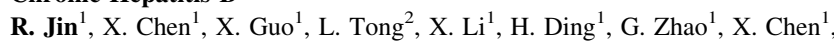
H. Zhang ${ }^{3}$

${ }^{I}$ Department of Hepatology, Beijing YouAn Hospital, Capital Medical University, Beijing; ${ }^{2}$ Centre for Hepatology, The First Hospital of Heibei Medical University, Shijiazhuang, ${ }^{3}$ Department of Pediatric Liver Diseases, 302 Military Hospital of China, Beijing, China

Background/aim: Telbivudine has shown potent HBV suppression, high $\mathrm{HBeAg}$ seroconversion and sustainable off treatment response in chronic hepatitis $\mathrm{B}(\mathrm{CHB})$ patients. This study reports the 52 weeks clinical efficacy and safety data with telbivudine treatment in nucleoside-naive $\mathrm{HBeAg}$-positive CHB.

Method: A retrospective analysis of $210 \mathrm{CHB}$ patients with $\mathrm{HBV}$ DNA load over $10^{5}$ copies $/ \mathrm{ml}$ were included. 104 of them received telbivudine $600 \mathrm{mg}$ daily and 106 received lamivudine $100 \mathrm{mg}$ daily. Treatment duration was 52 weeks. Serum HBV DNA level, anti-HBeantibodies, ALT level in serum were evaluated at baseline, week 12, 24 and 52 during therapy, and also evaluated HBV DNA levels after 12 and 24 weeks of telbivudine treatment.

Results: At week 52, the patients in telbivudine group had higher rates of undetectable serum HBV DNA level, the rates of HBeAg loss and $\mathrm{HBeAg}$ seroconversion, ALT normalization rate, viral breakthrough rate and viral rebound rate than those in lamivudine group ( 70.2 vs. $56.6 \%, 35.6$ vs. $12.3 \%, 22.1$ vs. $11.3 \%, 6.7$ vs. $16.0 \%, 2.9$ vs. $10.4 \%, p<0.05$, respectively). Patients with week 24 HBV DNA below 500 copies $/ \mathrm{ml}$ achieved the better outcomes at 52 weeks with HBV DNA negativity rate of $78.3 \%$ and viral rebound rate of $2.9 \%$ than those with HBV DNA above 500 copies $/ \mathrm{ml}$ for $\mathrm{HBeAg}$-positive patients treatment with telbivudine ( $p<0.05$, respectively). The rate of adverse incidences of telbivudine doesn't show significant difference from that of lamivudine but with more cases of elevated CK. 
Conclusion: Telbivudine is more effective in $\mathrm{HBeAg}$-positive $\mathrm{CHB}$ patients than lamivudine. It can quickly supress HBV replication and cause $\mathrm{HBeAg}$ to seroconversion. The data confirm previous findings that week 24 PCR negativity is predictive of a favorable outcome. It has relatively fewer adverse reactions and is better tolerated by patients. Thus it can be used as a long-term therapy for CHB and CK should be monitored during the treatment.

\section{PP05-62}

A Real World Study of Telbivudine Treatment for Thai Patients with Chronic Hepatitis B Infection

T. Piratvisuth ${ }^{1}$, T. Tanwandee ${ }^{2}$, W. Sukeepaisarnjaroen ${ }^{3}$, T. Sanpajit $^{4}$,

S. Thongsawat ${ }^{5}, K$ Itthikul $^{6}$, P. Promlikitchai ${ }^{7}$, C. Lertkupinit ${ }^{8}$

${ }^{1}$ Prince of Songkla University, Songkhla; ${ }^{2}$ Siriraj Hospital, Mahidol University, Bangkok; ${ }^{3}$ Srinagarind Hospital, Khon Kaen University, Khon Kaen; ${ }^{4}$ Phramongkutklao Hospital, Bangkok; ${ }^{5}$ Maharaj Nakorn Chiang Mai Hospital, Chiang Mai University, Chiang Mai;

${ }^{6}$ Anandamahidol Hospital, Lop Buri; ${ }^{7}$ Saraburi Hospital, Saraburi;

${ }^{8}$ Chonburi Hospital, Chonburi, Thailand

Background: Monitoring the drug safety and effectiveness are essential for management of chronic hepatitis B (CHB) patients. Telbivudine is a potent antiviral agent for hepatitis B virus (HBV) which available in Thailand in 2007. Its real life clinical effectiveness and resistant profile has never been evaluated.

Objectives: To evaluate the real world effectiveness and the rate of antiviral resistance among Thai patients with CHB infection who received telbivudine.

Methodology: This is a multi-center, hospital-based, retrospective chart review, observational study. Medical records of all CHB patients treated at the 8 study hospitals between 1 June 2007 and 31 May 2010 were reviewed. Patients who had been treated with telbivudine $600 \mathrm{mg}$ tablet for CHB infection for at least 24 weeks were selected.

Results: Of 307 patients, $68.7 \%$ were males, with the mean age at diagnosis of $46.2 \pm 17.6$ years. Duration under telbivudine treatment ranged from 6 to 40 months (mean $\pm \mathrm{SD}=17.6 \pm 8.3$ ). Of the 259 patients whose hepatitis $\mathrm{B}$ e antigens $(\mathrm{HBeAg})$ laboratory findings were available, 129 were positive and 130 were negative. Median HBV DNA was 1,695 and 300 copies/mL for $\mathrm{HBeAg}$-positive and $\mathrm{HBeAg}$-negative patients, respectively. Patients who had virological breakthrough were $5.8 \%$ (95\% CI 2.8-8.7). Proportion of patients with HBV DNA nondetectable by polymerase chain reaction assay $(<300$ copies $/ \mathrm{mL})$ was 78.5\% (95\% CI 73.3-83.7). Among $95 \mathrm{HBeAg}$-positive patients who had the data more than one visits, $30.5 \%$ had HBeAg loss, of whom, $62.1 \%$ had $\mathrm{HBeAg}$ seroconversion. There was $64.7 \%$ (95\% CI 58.9-70.4) of all patients with normalization of alanine aminotransferase. Serious adverse events were reported in 11 patients, $3.6 \%$ (95\% CI 1.8-6.3), grades 3/4 creatine kinase elevations were 5/206 (2.4\%). Discontinuation of the treatment was $10.8 \%$ (95\% CI 7.2-14.4).

Conclusions: In Thai patients with $\mathrm{CHB}$, telbivudine treatment provided satisfied antiviral and clinical efficacy, with a low percentage of resistance.

\section{PP05-63}

Genotype and Response to Entecavir Therapy in Nucleoside Nä̈ve HBe Negative Chronic Hepatitis B Patients in Coastal Orissa

H.S. Das, C.R. Panda

Gastroenterology, S.C.B. Medical College, Cuttack, India

Background: Hepatitis B is an important cause of CLD and contributes to one third cases of liver disease in our region. There is lack of data in chronic hepatitis B patients regarding e antigen, viral load and genotypic pattern in this region.

Objective: We prospectively evaluated nucleoside naïve chronic HBV patients who received entecavir and completed atleast 6 months of therapy. We have studied the genotype of 30 patients of chronic HBV patients undergoing entecavir therapy.
Observations: Out of $300 \mathrm{HBV}$ patients of $>18$ years age, 282 were $\mathrm{HBe}$ negative. Thirty two patients with HBe negative (nucleoside naïve) male :female 29:3 underwent therapy with $0.5 \mathrm{mg}$ entecavir daily. Patient's age ranged from 29 to 55 years, mean being 43 . Twelve out of them were Child A cirrhosis. Patients had mean ALT of 78. Baseline mean HBV DNA level was $6.2\left(\log _{10}\right.$ copies $\left./ \mathrm{ml}\right)$. Three months following therapy $86 \%$ achieved undetectable viral level and at 6 month all except one had undetectable viral load. One expired at 4th month of therapy, who developed HCC. Out of 30 patients who have undergone genotypic study, 4 had Genotype A, one genotype C, another 20 had genotype D. In five patients genotype could not be determined as the viral load was very low during testing. Out of cirrhotics 3 had genotype A and one HCC had genotype A. Other 9 with cirrhosis had genotype D. Out of 13 chronic hepatitis, 11 had genotype $\mathrm{D}$, one $\mathrm{C}$ and one $\mathrm{A}$.

Summary: More than $90 \%$ CHB beyond 18 years of age in our region is due to e negative disease and Genotype D is the commonest genotype followed by Genotype A and C. Entecavir is a potent antiviral and response does not vary in different genotypes.

\section{PP05-64}

In vivo Intracellular Injection Needle (IvIn) Technology: a New Needle for Improved Cellular Uptake of DNA in Live Animal

G. Ahlén, L. Frelin, M. Sällberg

Karolinska Institutet, Laboratory Medicine, Clinical Microbiology, Stockholm, Sweden

A major obstacle that limits the efficacy of gene transfer and genetic vaccination in large animals including humans is the poor uptake of naked nucleic acid into the cells. Several techniques such as particle bombardment and in vivo electroporation have been developed to improve on the poor uptake of DNA in humans. However, these devices require moving parts and electricity that limits the ease by which they can be used. To solve this problem we have now developed a simple and robust injection needle that takes advantage of the fact that pores opens in cellular membranes when the hydrostatic pressure in the tissue increases, termed In vivo Intracellular Injections Needle (IvIn) technology. The basic design uses 1-5 circularly oriented needles where the ends of the needles have been sealed. New openings (5-40) of various sizes have been made on the needle shaft that directs the injected liquid centrally in the circle of needles. Finally, one needle has been positioned centrally with openings in all directions.

Our data show that injection of plasmid DNA encoding hepatitis $\mathrm{C}$ virus non-structural 3/4A proteins in rabbit tibialis anterior muscles leads to a subsequent higher number of in vivo transfected muscle cells that express the transferred gene. The IvIn technology also results in an improved local inflammation at the site of injection, which help in recruiting immune cells to the injection area. The improved NS3/4ADNA uptake, antigen expression and local inflammation results in that $\mathrm{T}$ cell responses to the expressed transgene can be detected already 5 days post immunization. Importantly, the new IvIn device can be used with any commercially available syringe and does not require any advanced knowledge in injection techniques. Thus, we believe that the IvIn technology can offer a simple solution for gene transfer in vivo in larger animals and humans.

\section{PP05-65}

Long-Term Virological Suppression, Resistance and Histological Improvement in Nucleos(t)ide-naive Asian Chronic Hepatitis B Patients Treated with Entecavir in Studies ETV-022/-027/-901

T.-T. Chang ${ }^{1}$, C.-L. Lai ${ }^{2}$, S.-K. Yoon ${ }^{3}$, S.-S. Wu ${ }^{4}$, K.-H. Han ${ }^{5}$, C. Pan ${ }^{6}$, K. Kowdley ${ }^{7}$, K.-Q. Hu ${ }^{8}$, S.S. Lee ${ }^{9}$, N. Tsai ${ }^{10}$, Z.D. Goodman ${ }^{11}$, H. Zhang ${ }^{12}$, D. Cohen ${ }^{12}$, H. Tang ${ }^{13}$, M.J. Tong ${ }^{14}$

${ }^{1}$ College of Medicine, National Cheng Kung University, Tainan, Taiwan, ROC; ${ }^{2}$ Department of Medicine, Queen Mary's Hospital, University of Hong Kong, Hong Kong, China; ${ }^{3}$ Department of Internal Medicine, Kangnam St. Mary Hospital, Catholic University 
Medical School, Seoul, Republic of Korea; ${ }^{4}$ Department of Internal Medicine, Changhua Christian Hospital, Changhua, Taiwan, ROC; ${ }^{5}$ Department of Internal Medicine, Yonsei University College of Medicine, Seoul, Republic of Korea; ${ }^{6}$ Division of Gastroenterology, Mount Sinai School of Medicine, New York, NY; ${ }^{7}$ Center for Liver Disease, Virginia Mason Medical Center, Seattle, WA; ${ }^{8}$ Division of Gastroenterology, University of California, Irvine Medical Center, Orange, CA, USA; ${ }^{9}$ Foothills Provincial General Hospital, University of Calgary, Calgary, AB, Canada; ${ }^{10}$ Department of Medicine, University of Hawaii, Honolulu, HI; ${ }^{11}$ Inova Fairfax Hospital, Falls Church, VA; ${ }^{12}$ Research \& Development, Bristol-Myers Squibb Company, Wallingford, CT; ${ }^{13}$ Research \& Development, BristolMyers Squibb Company, Plainsboro, NJ; ${ }^{14}$ Pfleger Liver Institute, University of California School of Medicine, Los Angeles, CA, USA

Background: Long-term entecavir (ETV) treatment demonstrated durable virological suppression, minimal resistance, and reversal of fibrosis/cirrhosis in nucleos(t)ide-naive chronic hepatitis B (CHB) patients. We present long-term efficacy/resistance/histological results of Asian patients enrolled in ETV-022/-027 and -901 studies.

Methods: Two cohorts were analyzed. The long-term efficacy analysis cohort consisted of ETV-treated patients in ETV-022 (HBeAg+, $0.5 \mathrm{mg}$ ) who subsequently entered ETV-901 $(1 \mathrm{mg})$ with a treatment gap $\leq 35$ days. Patients with evaluable samples at week 240 were assessed for viological/biochemical/serological responses. The long-term histology analysis cohort included ETV-treated patients in ETV-022/-027/-901 with evaluable biopsies at baseline and long-term. Patients were evaluated for histologic improvement [ $\geq 2$-point decrease in Knodell necroinflammatory score (KNI) and no worsening of Knodell fibrosis score], and improvement in Ishak fibrosis score (IF) ( $\geq 1$ point decrease). Genotypic resistance was evaluated for both cohorts.

Results: Ninety-four patients were included for long-term efficacy analysis. Baseline mean HBV DNA $10.1 \log _{10}$ copies/mL; ALT 122.6 U/L. At week $240,95 \%$ had HBV DNA $<300$ and $76 \%$ had ALT $\leq 1 \times$ ULN. Cumulative rate of HBeAg loss $43 \%$. Thirty-eight patients (30 $\mathrm{HBeAg}+$ ) were included for long-term histology analysis. Baseline mean HBV DNA 9.2; ALT 124.7; KNI 7.5; and IF 2.2.

Long-term histology cohort results

\begin{tabular}{ll} 
Long-term histology Asian cohort $(N=38)$ \\
\hline Week 48 & $\begin{array}{l}\text { Long-term } \\
\text { (median 283 weeks) }\end{array}$ \\
\hline $28(74)$ & $38(100)$ \\
$11(29)$ & $33(87)$ \\
$26(68)$ & $38(100)$ \\
$23(61)$ & $30(79)$
\end{tabular}

No ETV resistance was detected through long-term treatment in these two Asian cohorts. The safety profile was consistent with previously reported experience.

Conclusions: Long-term ETV treatment resulted in powerful/durable virological/biochemical response and histological improvement with no resistance in nucleos(t)ide-naive Asian patients.

\section{PP05-66}

Outcomes of Different Adefovir Treatment Strata in Chronic Hepatitis B S.G. Lim ${ }^{1,2}$, M.M. Yee ${ }^{1}$, M.O. Aung ${ }^{1}$, M. Belinda ${ }^{1}$, G.H. Lee ${ }^{1}$, M. Fernandas ${ }^{1}$, Y.M. Lee ${ }^{1}$, L. Vincent ${ }^{1}$, S. Dede ${ }^{1}$, Y.Y. Dan ${ }^{1}$

${ }^{1}$ Gastroenterology and Hepatology, National University Health System; ${ }^{2}$ Medicine, Yong Yoo Lin School of Medicine, National University of Singapore, Singapore, Singapore

Background and aim: Treatment responses and on treatment outcomes among different strata of Adefovir treated chronic hepatitis B
(CHB) patients (treatment naïve before ADV, Lamivudine (LAM) ADV sequential and LAM ADV add on) were examined.

Patients and method: CHB Clinic patients treated with ADV were followed for achievement of HBV DNA negative, 6 months initial viral response (IVR), hepatitis B e antigen ( $\mathrm{HBeAg}$ ) seroconversion, $\mathrm{ADV}$ resistance and renal impairment. Data were analysed by Pearson Chi square, Kaplan Meier, log rank test, and Cox regression.

Results: There were 194 ADV treated patients followed for mean of 28.5 months. 81 of them were treatment naïve before ADV, where 65 were switched to ADV from LAM and 48 patients of ADV add on to LAM. Majority of patients were Chinese 181 (93.3\%), $115(59.3 \%)$ were $\mathrm{HBeAg}$ positive, $142(73.2 \%)$ were male and $65(33.5 \%)$ have cirrhosis. ADV add on patients were older than naïve \& sequential group with mean age of 52.2, 43.7 and 49.5 respectively $(P=0.001)$. Naïve ADV group achieve higher seroconversion rate and HBV DNA negative rate compare to the rest of the cohort $(P=0.017$ and $P=0.040$ ) after adjusting baseline difference with cox regression. There are no difference in 3 treatment groups in development of resistance $(P=0.483$, Cox regression), renal impairment $(P=0.172$, Cox regression), and achievement of 6 month IVR $(P=0.239$, Chisquare). Total of $4(2.06 \%)$ patients died, $7(3.60 \%)$ patients developed HCC and 3 (1.54\%) patients developed ascites. There is no difference between treatment groups by mean of death $(P=0.738)$, HCC development $(P=0.675)$ and development of ascites $(P=0.931)$.

Conclusion: Treatment naïve patients achieve higher HBeAg sero conversion and DNA negative rate compared to LAM exposed patients with ADV treatment but in term of mortality, morbidity outcomes, resistance, and renal impairments there is no significant difference between these groups.

\section{PP05-67}

Preemptive Antiviral Therapy Can Reduce Overall Treatment-related Hepatic Morbidity as well as Viral Reactivation during Transarterial Chemo-embolization

S.H. Yoo ${ }^{1}$, J.W. Jang ${ }^{1}$, B.S. Oh ${ }^{1}$, J.H. Kwon ${ }^{1}$, K.W. Chung ${ }^{1}$, H.S. Jung ${ }^{2}$

${ }^{1}$ Internal Medicine; ${ }^{2}$ Radiology, The Catholic University of Korea, Seoul, Republic of Korea

Introduction: Hepatic damage during transarterial chemo-embolization (TACE) is a critical complication in patients with hepatitis B virus (HBV)-related hepatocellular carcinoma (HCC). Apart from its role in preventing $\mathrm{HBV}$ reactivation, there are limited data on the benefit of preemptive antiviral therapy in TACE. This study aimed to evaluate the effect of HBV replication status and preemptive antiviral therapy on overall hepatic morbidity occurring during TACE.

Methods: This is a retrospective analysis of 89 prospectively enrolled patients with intermediate-stage HCC undergoing TACE. Hepatic decompensation, common toxicity criteria (CTC) $\geq$ grade 3 , and severe hepatitis ( $>5$ times ULN) of all causes during TACE were evaluated.

Results: During the follow-up, 24 (27.0\%), 24 (27.0\%), and 22 (24.7\%) patients developed decompensation, hepatic CTC $\geq$ grade 3 , and severe hepatitis, respectively. With no case of reactivation in the preemptive group, HBV reactivation occurred only in the antiviral-untreated group $(0$ vs. $33.8 \% ; P<0.05)$. Baseline viral load was independently predictive of overall decompensation during TACE $(P=0.017)$. Among antiviraluntreated patients, HBV load, albumin, prothrombin time (PT), and ECOG were significantly or marginally associated with a higher incidence of CTC $\geq$ grade 3 and severe hepatitis $(P=0.045-0.105$ ). For HBeAgpositive patients, prolonged $\mathrm{PT}$ and the absence of preemptive antiviral therapy were significantly associated with CTC $\geq$ grade 3 and severegrade hepatitis during therapy (all $P<0.05$ ), whereas no factor was statistically significant for $\mathrm{HBeAg}$-negative patients. Irrespective of $\mathrm{HBeAg}$ status, high-viremic patients tended to experience more frequent episodes of those hepatic complications during TACE $(P=0.062-0.148)$.

Conclusions: High HBV load and hepatic reserve are the important risk factors for various hepatic morbidities during TACE. In this regard, preemptive antiviral therapy is indicated during TACE, 
definitively for HBeAg-positive patients. Our findings suggest that preemptive antiviral therapy can reduce the risk of overall hepatic morbidity of all causes as well as HBV reactivation.

\section{PP05-68}

The Retrospective Cohort Study of Long-term Effects of Traditional Chinese Medicine on the Treatment of Chinese Chronic Hepatitis B Patients Q.H. Ling, C.K. Yang, J.J. Chen, Y.H. Zhuo

Department of Liver Disease, ShuGuang Hospital, Shanghai University of TCM, Shanghai, China

Objective: To evaluate the long-term effect of traditional Chinese medicine (TCM) for the treatment of Chinese chronic hepatitis B (CHB) patients. The primary endpoint was the incidence of hepatic cirrhosis after hepatitis B. Exploring the influence of TCM on the incidence of hepatic cirrhosis after hepatitis B.

Patients and methods: 1011 cases of Chinese CHB patients were enrolled. There were 220 patients (TCM group 65; control group 155) whose course of the disease were longer than 10 years. The TCM group was treated with TCM more than 5 years, and the control group was treated without TCM.

Results: Cox multivariate regression analysis suggested that the therapy of TCM was the independent prognostic factor of incidence of hepatic cirrhosis after hepatitis B. Among the TCM group of 65 patients, 4 cases $(6.2 \%)$ developed cirrhosis or liver cancer, cirrhosis, control group of 155 patients, 79 cases $(51.0 \%)$ developed liver cirrhosis or liver cancer. The outcome of the current (i.e., the incidence rates of CHB patients developed LC in the TCM group) was significantly lower than those in the control group (6.2 vs. $51.0 \%, P<0.05)$. Difference between the two groups was statistically significant survival curves. Other factors remain the same in the premise. Cox regression analysis showed that AST higher than $40 \mathrm{IU} / \mathrm{L}$ was the CHB patients with cirrhosis of the risk factors, suggesting that albumin $<43 \mathrm{~g} / \mathrm{L}$ was the CHB patients with cirrhosis of the risk factors.

Conclusions: More than 5 years of TCM treatment reduced the incidence rate of liver disease development. Chinese medicine was show to be able to reduce the incidence of liver cirrhosis, CHB extended to cirrhosis occurred during the course of time. AST levels and albumin levels were the independent factors for patients with liver cirrhosis after hepatitis B.

\section{PP05-69}

The Research on Curative Effects of Pegasys in Treating Chronic Hepatitis B Patients in a 4-year Follow up

Q.H. Ling ${ }^{1}$, J.J. Chen ${ }^{1}$, W.J. Xu ${ }^{2}$

${ }^{1}$ Department of Liver Disease, ShuGuang Hospital, Shanghai University of TCM; ${ }^{2}$ Heqing Health Service Centers for Community, District of Pudong, Shanghai, China

Objective: To evaluate curative effects of Pegasys (PEG-IFN- $\alpha$ ) in treating chronic hepatitis B patients.

Methods: 55 chronic hepatitis B patients were enrolled in this study and treated only with Pegasys. Biochemical indicators and viral load were assessed to evaluate curative effects in a 4-year follow up.

Results: The viral response rates of the 55 patients treated with Pegasys were 50.9, 56.36, 65.45, 67.27, 70.91 and $72.73 \%$ corresponding to $24,36,48,96,144,192$ weeks treatment. HBV DNA in 13 patients were once undetectable by PCR method was detectable in the 4-year follow up (23.6\%). HBV DNA from the 13 patients were gene sequenced, ten cases exhibiting 1,896 point mutations in pre $\mathrm{C}$ region of HBV DNA (76.9\%), no other mutations detected. Of the 17 $\mathrm{HBeAg}$ negative patients, the viral response rate was $64.7 \%$ (i.e., 11 out of 17), and $45.7 \%$ (i.e., 16 out of 35) corresponding to those treated with Pegasys for 24 weeks and 48 weeks, while the corresponding viral response rate of the $37 \mathrm{HBeAg}$ positive patients was $43.24 \%$ (i.e., 16 out of 37), and $62.16 \%$ (i.e., 23 out of 37) when treated with Pegasys for 24 and 48 weeks. The $\mathrm{HBeAg}$ negative change rate of the $37 \mathrm{HBeAg}$-positive patients treated by Pegasys in 48 weeks was $27 \%$ (i.e., 10 out of 37 ), but two patients were found $\mathrm{HBeAg}$ in serum 96w later, even though HBV DNA detected in serum, one of which did not change on alanine aminotransferase (ALT), while another changed from 54 to 33 .

Conclusions: Pegasys is effective in treating chronic hepatitis B patients for delayed and low proportion drug tolerance, leading to high viral response rate. Viral response rate was higher among $\mathrm{HBeAg}$ negative patients than those with $\mathrm{HBeAg}$ positive.

\section{PP05-70}

Is it Possible to Predict Lamivudine Resistance in Chronic Hepatitis B Patients Treated with Lamivudine?

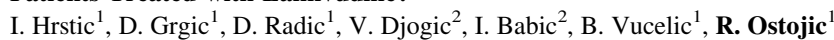
${ }^{1}$ Division of Gastroenterology, School of Medicine, University of Zagreb; ${ }^{2}$ Department of Molecular Immunogenetics, Croatian Institute of Transfusion Medicine, Zagreb, Croatia

Background: At present time treatment options for $\mathrm{CHB}$ patients in Croatia are insufficient with only available lamivudine and conventional interferon alpha. Unfortunately, prolonged lamivudine treatment is associated with increasing risk of drug resistance. Cumulative reported lamivudine resistance (LAM-R) after 3 years is around 55\%.

Aim: To detect potential baseline parameters which could serve as predictors of resistance after 3 years of LAM monotherapy.

Methods: We retrospectively analyzed patients with established eAg negative CHB. All were treated with LAM monotherapy $(100 \mathrm{mg}$ daily) for 3 years. Resistance was determined by INNO-LiPA HBV DR v2 Assay (Innogenetics N.V, Gent, Belgium). Baseline characteristics were evaluated between groups of patients with and without resistance at codons 180 or 204.

Results: Among 46 patients with all available evaluated parameters 15 were females and 29 males with age range from 22 to 69 years (mean 44, 2 years). All patients were infected with HBV genotype D. Mean serum parameters were as follows: platelets $183.50 \times 10^{9}$, bilirubin $15.85 \mathrm{mmol} / \mathrm{L}$, ALT $145.48 \mathrm{IU} / \mathrm{L}$, AST $132.83 \mathrm{IU} / \mathrm{L}$, GGT $64.07 \mathrm{IU} / \mathrm{L}$. Viral load ranged from $3.58 \times 10^{4}$ to $4.28 \times 10^{8} \mathrm{IU} / \mathrm{mL}$ (mean $\left.3.17 \times 10^{7}\right)$. Majority of patients had histologically moderate active disease (activity $1-6=7 / 46 ; 7-12=25 / 46 ; 13-18=14 / 46$ ) and moderate fibrosis $(1-2=9 / 46 ; 3-4=25 / 46 ; 5-6=12 / 46)$. Mutant viral strains were found in $36.96 \%$ of patients: isolated mutation (codon 204) in 11/46 while dual mutations (codon 204 and 180) in 6/ 11 patients. No statistical significances have been found for all parameters except liver fibrosis ( $p=0.035,95 \%$ CI $1.0595-5.1036$ ). Conclusion: Surprisingly, less resistance was found in tested Croatian CHB patients treated with LAM monotherapy. Unfortunately no baseline parameter could serve as predictor for development of mutant viral strains except baseline liver fibrosis. This significance could be consequence of longer disease duration which enables virus to escape the host immunological defense.

\section{PP05-71}

The Significant of the Serum Level of HMGB1 in the Sever Chronic Hepatitis B S. Lin

Xi'an Jiaotong University, Shanxi Xi'an, China

Aim: HMGB1 has been found that plays a main role in the pathogenesis of many diseases, extracellular HMGB1 acts as a potent proinflammatory cytokine that contributes to the pathogenesis of diverse inflammatory and infectious disorders. A growing number of studies indicate that HMGB1 is correlated with the severity and prognosis of many diseases. The aim of this study was to determine whether the serum level of HMGB1 is significance in different state of chronic hepatitis B patients,and whether it could be used as a useful prognostic biomarker in the severe chronic hepatitis B patients.

Methods: The serum level of HMGB1 was detected in 35 normal control, 79 chronic hepatitis B patients, and 70 severe chronic 
hepatitis B patients. There are 32 patients without liver cirrhosis and 47 patients with liver cirrhosis in the chronic hepatitis B patients, and the severe chronic hepatitis B patients divided into two group, 25 patients die and 45 patients survival.

Results: The serum level of HMGB1 was significant different during the normal control,the chronic hepatitis B patients and the severe chronic hepatitis B patients, it increased significantly in the severe chronic hepatitis B patients $(P<0.01)$, while there was no significant different between the patients without liver cirrhosis and with liver cirrhosis $(P>0.05)$, and otherwise in the severe chronic hepatitis B patients also there was no different between the survivors and non-survivors $(P>0.05)$.

Conclusions: Our result indicate that the serum level of HMGB1 was correlation with the state of chronic hepatitis B patients, and could represent the severity,while it could not be used as a useful prognostic biomarker in the severe chronic hepatitis B patients.

\section{PP05-72}

Treatment Response to Chronic Hepatitis C in Singapore

S.G. Lim $^{1,2}$, M.O. Aung ${ }^{1}$, M. Belinda ${ }^{1}$, G.H. Lee ${ }^{1}$, M. Fernandas ${ }^{1}$, Y.M. Lee ${ }^{1}$, L. Vincent ${ }^{1}$, S. Dede ${ }^{1}$, Y.Y. Dan ${ }^{1}$

${ }^{I}$ Gastroenterology and Hepatology, National University Health

System; ${ }^{2}$ Medicine, Yong Yoo Lin School of Medicine, National University of Singapore, Singapore, Singapore

Background/aim: There is little information on HCV genotype distribution and outcome of therapy in Singapore. Studies from blood donor populations indicate a HCV prevalence of $0.2-0.38 \%$ but little is known of the genotype distribution in Singapore.

Methods: All HCV patients seen in National University Hospital (NUH) from 2001 to 2009 were included in the study for genotype analysis and treatment response. HCV treatment response were evaluated based on genotype, and presence or absence of cirrhosis. Sustained virological response (SVR) was confirmed when HCV RNA was negative by RT PCR at least 6 months after completion of treatment. The data were presented in number (\%) and analyzed by Pearson Chi-square. Results: There were $496 \mathrm{HCV}$ patients seen in NUH from 2001 to 2009 with $42.9 \%$ being non-residents. The genotype distribution between residents and non-residents of Singapore were genotype 1: 47, 57.2\%, genotype 2: 4.9, 13.1, genotype 3: 45.9, 19.7\% and other genotypes: $2.1,4.3 \%$, respectively. A total of 198 patients were treated with interferon or pegylated interferon and ribavirin. In those who completed therapy overall SVR was $68.5 \%$. HCV genotype 2 had highest SVR, $100 \%$ compared to $62.3 \%$ in genotype 1 and only $47.1 \%$ SVR in genotype 3. Patients without cirrhosis had significantly higher SVR $(82.7 \%)$ compared to $64.0 \%$ SVR in compensated cirrhosis and $11.1 \%$ SVR in decompensated cirrhosis $(P<0.001)$. Presence of cirrhosis was significantly associated with $\mathrm{HCV}$ treatment failure, $(p=0.002$, OR 3.76 (1.5-9.1).

Conclusions: The dominant HCV genotypes in Singapore were gentoype 1 and 3 even amongst non-residents of Singapore. Sustained virologial response was seen in a high proportion of treated patients but patients with genotype 3 , cirrhosis were associated with significant treatment failure.

\section{PP05-73}

Effect of BMI on Response to Treatment with Oral Antivirals in Chronic Hepatitis B

G. Choudhuri, K. Singh, A. Verma, P. Srivastava

Gastroenterology, Sanjay Gandhi Post Graduate Institute of Medical Sciences, Lucknow, India

Background: While overweight patients of chronic hepatitis $\mathrm{C}$ are known to respond poorly to antivirals, the effect of body weight on oral antivirals in chronic hepatitis B (CHB) has not been adequately studied. We therefore evaluated response rates to oral antivirals in patients with $\mathrm{CHB}$ with normal or increased body mass index (BMI).
Methods: We retrospectively evaluated $(n=281)$ patients with compensated CHB [age 36.5 \pm 6.7 ; e+: 154 (54.8\%); males 251 $(89.3 \%)$ ] who were treated with any of the five oral drug(s) [Lamivudine, adefovir, lamivudine plus adefovir, entecavir, telbivudine, telbivudine plus tenofovir]. $189(67.2 \%)$ had normal BMI $(<23.5$ for Asians) (mean 20.8, range 17.5-23.4) and 92 (32.7\%) had elevated BMI (mean 27.3; range 25.0-37.7). Response rates in two groups were compared for each $\operatorname{drug}(\mathrm{s})$.

Results: Complete DNA suppression at 6 months in patients of normal and high BMI for each drug category were: Lamivudine 16/24 $(66.5 \%)$ vs. $5 / 8(62.5 \%)(p>0.05)$; Adefovir $9 / 29(31.0 \%)$ vs. $4 / 16$ (25.0\%) $(p>0.05)$; Lamivudine plus adefovir 12/21 (57.14\%) vs. $4 /$ $10(40 \%)(p=\mathrm{NS})$; entecavir $59 / 68(73.5 \%)$ vs. $19 / 29(65.5 \%)$ $(p=0.03)$; telbivudine $8 / 14(57.1 \%)$ vs. $8 / 9(88 \%)(p=\mathrm{NS})$; telbivudine plus tenofovir 22/34 (64.7\%) vs. $13 / 17$ (76.4\%) ( $p=$ NS). The overall complete suppression rate for all drug categories after 6 months of therapy was $66.7 \%(n=126)$ in normal BMI group and $57.6 \%(n=53)$ in high BMI group $(p=\mathrm{NS})$. However after 1 year of therapy the overall complete suppression rates were $80.2 \%$ (126 of 157 ) and $45.1 \%$ (32 of $71 ; p<0.05$ ).

Conclusion: The response to oral antiviral drugs may be less in patients of CHB with elevated BMI, and may need further studies.

\section{PP05-74}

Comparative Efficacy of Commonly Used Oral Antivirals in Naive Chronic Hepatitis B Patients in India

G. Choudhuri, P. Srivastava, K. Singh, A. Verma

Gastroenterology, Sanjay Gandhi Post Graduate Institute of Medical Sciences, Lucknow, India

Background and aims: Early good suppression of HBV-DNA may be associated with reduced risk of development of resistance when used over long periods. We analysed the efficacy of four different oral treatment regimes in naive patients with compensated CHB.

Methods: 128 patients [age $38.2 \pm 17.6$; males 117 (91.4\%), e+ 65 $(50.7 \%)]$ who had compensated liver disease due to $\mathrm{CHB}$ treated with oral antivirals at our centre were evaluated for complete DNA suppression at 6 and 12 months, and $\mathrm{HBeAg}$ seroconversion at 1 year receiving 4 commonly used treatments: lamivudine plus adefovir (LAM-ADV: 24), entecavir 0.5 mg (ETV: 71), telbivudine (LdT: 16) and telbivudine plus tenofovir (LdT-TDF: 17).

Results: Complete DNA suppression at 6 months was observed in $47.8,82,87.5,85.7 \%$ ( $p<0.05$ between LAM-ADV and others) patients respectively. At 1 year 88, 93.7, 91.8, 100\% $(p=$ NS) had undetectable DNA. $\mathrm{HBeAg}$ seroconversion rates at 1 year were 7.4, $5.9,12.5$ and $10.7 \%(p=\mathrm{NS})$.

Conclusion: In naive CHB patients undergoing treatment with oral $\operatorname{drug}(\mathrm{s}), \mathrm{HBV}$-DNA suppression was initially slow in those receiving LAM-ADV, but was above $80 \%$ and equally effective in those receiving entecavir, telbivudine or a combination of telbivudine plus tenofovir.

\section{PP05-75}

Comparison of Three Rescue Therapies for Lamivudine Resistant Chronic Hepatitis B: Entecavir 1mg, Telbivudine Plus Tenofovir, and add on Adefovir

G. Choudhuri, A. Verma, P. Srivastava, K. Singh

Gastroenterology, Sanjay Gandhi Post Graduate Institute of Medical Sciences, Lucknow, India

Background and aims: The choice of therapy in patients of CHB who have developed resistance to Lamivudine monotherapy is unclear. We compared the relative efficacies of 3 rescue therapies [add on adefovir (LAM-ADV), high dose entecavir (ETV) and telbivudine plus tenofovir (LdT-TDF) combination] for lamivudine (LAM) resistant chronic hepatitis B (CHB) in such patients who were on our follow up. Methods: 81 patients of $\mathrm{CHB}$ who developed virological breakthrough while on LAM therapy were advised one of the three regimes 
based on cost and availability considerations-Group A: ADV $10 \mathrm{mg} /$ day was added to LAM $(n=22)$; group B-LAM was switched to ETV $1 \mathrm{mg} /$ day $(n=23)$; group C-LAM was switched to LdT 600 $\mathrm{mg} /$ day + TDF $300 \mathrm{mg} /$ day $(n=36)$. Response was compared after 1 year of therapy in terms of $\mathrm{HBeAg}$ seroconversion and complete suppression of HBV DNA.

Results: All three groups were comparable in respect to mean age, $\mathrm{HBeAg}$ positivity, baseline mean ALT and mean baseline HBV DNA levels. After 6 months of therapy complete DNA suppression was better in group $\mathrm{C}(27 / 28 ; 96.4 \%)$ as compared to group A $(12 / 21$; $51.1 \%)$ and group B $(11 / 18 ; 61.1 \%)(p<0.05)$. However after 1 year of therapy suppression rate in group B $(14 / 20 ; 70 \%)$ was significantly less as compared to group A $(18 / 18 ; 100 \%)$ and group C $(20 / 20 ; 100 \%)$ on per protocol analysis $(p<0.05)$. HBeAg seroconversion after 1 year of therapy was seen in 2 (7.4\%), 1 (5.9\% and $3(10.7 \%)(p=\mathrm{NS})$. $13.1 \%(n=3)$ patients in ETV group were non-responders.

Conclusion: In LAM resistant patients nucleoside plus nucleotide combination therapy has better DNA suppression rates at 1 year as compared to high dose ETV.

\section{PP05-76}

Predictors of HBeAg Seroconversion in Chinese HBeAg-positive CHB Patients Treated with Telbivudine

J. Huang, X. Chen, X. Chen, W. Chen, R. Chen, X. Ma, X. Luo

Guangdong General Hospital, Guangzhou, China

Background/aims: To evaluate the efficacy of telbivudine in hepatitis $\mathrm{B}$ e antigen ( $\mathrm{HBeAg}$ )-positive patients with chronic hepatitis B, and determine baseline and on-treatment factors predictive of $\mathrm{HBeAg}$ seroconversion at 1 year of telbivudine treatment.

Methods: One hundred treatment-naïve $\mathrm{HBeAg}$-positive patients with hepatitis B virus (HBV) DNA levels $>10^{5}$ copies $/ \mathrm{mL}$ and alanine aminotransferase (ALT) levels $>2 \times$ the upper limit of normal received telbivudine $600 \mathrm{mg} /$ day for $\leq 1$ year. HBV serologic markers and biochemical parameters were monitored at treatment weeks 12 , 24, and 52. HBV DNA levels were quantified using Roche LightCycler ${ }^{\circledR} 480$ fluorescent quantitative polymerase chain reaction assay (lower limit of detection, $500 \mathrm{IU} / \mathrm{mL}$ ); levels of $\mathrm{HBeAg}$ and antibody to $\mathrm{HBeAg}$ were quantified by enzyme-linked immunosorbent assay (levels $<1 \mathrm{PEIU} / \mathrm{mL}$ defined HBeAg negativity).

Results: At week 52, undetectable HBV DNA ( $<500$ copies $/ \mathrm{mL}$ ), ALT normalization, HBeAg seroconversion, and viral breakthrough were achieved in $81,84,31$, and $4 \%$ of patients, respectively. The $\mathrm{HBeAg}$ seroconversion rate at week 52 was higher among patients with baseline $\mathrm{HBeAg}$ levels $\leq 500 \mathrm{PEIU} / \mathrm{mL}$ than with levels $>500$ PEIU/mL (57 vs. $20 \% ; P<0.01$ ). Declines in baseline HBeAg levels of $>1 \log _{10}$ at 12 weeks and $>2 \log _{10}$ at 24 weeks yielded $\mathrm{HBeAg}$ seroconversion rates of 100 and $95 \%$, respectively, at week 52. An $\mathrm{HBeAg}$ decline of $>2 \log _{10}$ at week 24 was the strongest predictor of HBeAg seroconversion at week 52 (odds ratio [OR] 83.928; $P<$ 0.001 ), followed by an $\mathrm{HBeAg}$ decline of $>1 \log _{10}$ at week 12 (OR 25.633; $P<0.001)$ and baseline HBeAg (OR 0.996; $P<0.01)$.

Conclusions: Telbivudine provides effective viral suppression with high rates of $\mathrm{HBe} A g$ seroconversion at 1 year. An early and profound $\left(>2 \log _{10}\right.$ from baseline) decline in $\mathrm{HBeAg}$ levels at week 24 was the strongest predictor of $\mathrm{HBeAg}$ seroconversion during telbivudine treatment.

\section{PP05-77}

REP 9AC: a Potent HBsAg Release Inhibitor that Rapidly Elicits Sustained Virologic Responses in Patients with Chronic Hepatitis B M. Al-Mahtab ${ }^{1}$, M. Bazinet ${ }^{2}$, A. Vaillant ${ }^{3}$

${ }^{I}$ Sheikh Mujib Medical University, Dhaka, Bangladesh; ${ }^{2}$ REPLICor; ${ }^{3}$ REPLICor Inc., Montreal, QC, Canada

Background: HBsAg plays a role in suppressing the immune system and may allow maintenance of chronic liver infection. REP 9AC inhibits the release of $\mathrm{HBsAg}$ from infected hepatocytes. In preclinical studies surface antigen seroclearance occurred after two weeks of treatment and four weeks of treatment resulted in 55\% of animals achieving a durable SVR. REP 9AC is currently being evaluated in patients with chronic HBV in a proof of concept clinical trial.

Methods: Patients were HBsAg+ with pre-treatment HBV DNA titers between $10^{6}$ and $10^{12}$ copies $/ \mathrm{ml}$ and had significant liver fibrosis. Safety and virologic response (HBV DNA, $\mathrm{HBsAg}$, anti-HBs) were assessed at the trial site and by confirmatory testing (HBsAg, $\mathrm{HBeAg}$, anti-HBs, anti$\mathrm{HBe}$ ) of frozen serum samples using the Architect $^{\mathrm{TM}}$ testing platform.

Results: To date, 5 of 8 patients treated have cleared serum $\mathrm{HBsAg}$ as early as 7 days and no later than 15 weeks following initiation of treatment at higher doses. Anti-HBsAg antibodies have been observed in all patients treated for at least 20 weeks. Six, patients have achieved a 3 to 7 $\log$ reduction in their HBV DNA titers from pre-treatment levels after 7-13 weeks of treatment and three of these patients have achieved complete control of their infection after 20-27 weeks of treatment (HBV $\mathrm{DNA}-, \mathrm{HBsAg}-, \mathrm{HBeAg}-$, anti-HBs+, anti-HBe+) and are being followed off treatment. All these patients are currently maintaining control over their infections 14, 27 and 52 weeks after stopping treatment.

Conclusions: REP 9AC can rapidly clear serum $\mathrm{HBsAg}$ in infected patients which appears to allow the restoration of an effective immune response, leading to the achievement of SVRs in three patients to date. These results suggest that REP 9AC may become an important new tool in the treatment of chronic hepatitis B.

\section{PP05-78}

Evaluation of Treatment Responses and Results of Lamivudine Monotheraphy in Naive Chronic Hepatitis Patients in Turkey C.P. Eyigun ${ }^{1}$, E. Gunal ${ }^{1}$, A. Ari ${ }^{2}$, I.Y. Avci ${ }^{1}$, F. Sirmatelli ${ }^{3}$, H.C. Gul ${ }^{1}$, F. Ersoz ${ }^{4}$ ${ }^{1}$ Infectious Diseases and Clinical Microbiology, Gulhane Military Medical Academy, Ankara; ${ }^{2}$ Infectious Diseases and Clinical Microbiology, State Hospital, Izmir; ${ }^{3}$ Infectious Diseases and Clinical Microbiology, Abant Izzet Baysal University, Bolu; ${ }^{4}$ Defense Science Institute, Turkish Military Academy, Ankara, Turkey

Currently, therapeutic management of chronic hepatitis is still possible with long term use of nucleoside analogs. The aim of this study is to display treatment responses and results of lamivudine monotheraphy in naive Turkish patients. Treatment naive 175 patients $[(54$ cases were women (30.9\%)] treated with lamivudine monotherapy were evaluated prospectively with participation of three therapy center in Turkey. Serum HBV DNA and ALT levels were monitored monthly until normalization then checked quarterly and biochemical and virological responses, breakthrough periods were evaluated. The mean age of the patients was $43.34 \pm 13.7$ years. 56 cases were HBeAg positive (32\%) while 119 patients were Anti-HBe positive $(68 \%)$. At the end of median 225 weeks ( $\min 27$, max 646) follow-up period, biochemical and virological responses, breakthrough periods were shown.In $\mathrm{HBeAg}$ positive patients,average ALT level was $105.33 \pm 86.39 \mathrm{IU} / \mathrm{ml}$, DNA level was $2.36 \times 10^{11} \pm 1.49 \times$ $10^{12} \mathrm{copy} / \mathrm{ml}$ at the beginning of therapy and ALT normalization period was $18.07 \pm 10.94$ weeks, DNA disappearance period was $29.4 \pm 28$ weeks, ALT breakthrough period was $113.56 \pm 74.16$ weeks, DNA breakthrough period was $92.55 \pm 65.38$ weeks.In AntiHBe positive patients,average ALT level was $108.87 \pm 106.89$ IU/ $\mathrm{ml}$, DNA level was $1.9 \times 10^{8} \pm 6 \times 10^{8} \mathrm{copy} / \mathrm{ml}$ at the beginning of therapy and ALT normalization period was $28.99 \pm 44.79$ weeks, DNA disappearance period was $19.18 \pm 36.68$ weeks, ALT breakthrough period was $120.17 \pm 98.88$ weeks, DNA breakthrough period was $141.02 \pm 91.51$ weeks. Beginning DNA levels were higher in $\mathrm{HBeAg}$ positive cases and HBV DNA breakthrough periods were longer in Anti-HBe positives significantly $(p<0.05)$. But significant corelation could not be shown between HBV DNA levels and breakthrough periods $(p>0.05)$. Although treatment responses are very good, life of lamivudine treatment is not longer than average 2-3 years in Turkish patients duo to early resistance. 


\section{PP05-79}

Long Term Resistance Rates with Lamivudine Monotheraphy in Treatment Naive Chronic Hepatitis Patients in Turkey

C.P. Eyigun ${ }^{1}$, E. Gunal ${ }^{1}$, A. Ari $^{2}$, I.Y. Avci ${ }^{1}$, F. Sirmatelli ${ }^{3}$, H.C. Gul ${ }^{1}$, F. Ersoz ${ }^{4}$

${ }^{1}$ Infectious Diseases and Clinical Microbiology, Gulhane Military

Medical Academy, Ankara; ${ }^{2}$ Infectious Diseases and Clinical

Microbiology, State Hospital, Izmir; ${ }^{3}$ Infectious Diseases and Clinical Microbiology, Abant Izzet Baysal University, Bolu; ${ }^{4}$ Defense Science Institute, Gulhane Military Medical Academy, Ankara, Turkey

As is well-known, encounter rate with antiviral resistance in lamivudine monotheraphy is very high reaching to near $76 \%$ at the end of 5 th year of therapy. The aim of this study is to display long term results of resistance to lamivudine monotheraphy in treatment naive Turkish patients. Antiviral naive 175 [54 females (30,9\%)] patients treated with lamivudine monotherapy were evaluated prospectively with participation of three therapy centers in Turkey. Serum HBV DNA and ALT levels were monitored monthly until normalization then checked quarterly.When serum HBV DNA level re-emergence up to measurable plasma levels or permanent increase was observed in normalised ALT or decreased HBV DNA levels under the treatment, lamivudine resistance was implied and investigated. The mean age of the patients was $43.34 \pm 13.7$ years. At the start of therapy, 56 cases were $\mathrm{HBeAg}$ positive (32\%) while 119 patients were Anti-HBe positive (68\%).At the end of median 225 weeks ( $\min 27$, max 646) follow-up period, distribution of lamivudine resistance by years was shown in $\mathrm{HBeAg}$ positive and Anti-HBe positive patients(Tables 1,2). Long term resistance rates in treatment naive chronic hepatitis B patients in Turkey with lamivudine monotheraphy were found very high (At the end of 3rd year resistance rate in Anti-HBe positive cases is 54.2 versus $87 \%$ in $\mathrm{HBeAg}$ positives; at the end of 5th year 77.8 versus $92,6 \%$; at the end of 10 th year, 98.7 versus $100 \%$ ). These results show that although its costeffectiveness and safety for adverse effects, long term lamivudine monotheraphy is not available in Turkey even in Anti-HBe positive cases especially after 3rd year due to resistance (Tables 1,2).

Table 1 Distribution of lamivudine resistance to

\begin{tabular}{ll}
\hline 1st year $(24-52)$ weeks & $18 / 119(15.12 \%)$ \\
2nd year (24-104) & $39 / 115(33.91 \%)$ \\
3rd year (24-156) & $58 / 107(54.2 \%)$ \\
4th year (24-208) & $67 / 96(69.79 \%)$ \\
5th year (24-260) & $70 / 90(77.78 \%)$ \\
6th year $(24-312)$ & $73 / 85(85.88 \%)$ \\
7th year $(24-364)$ & $74 / 83(89.16 \%)$ \\
8th year $(24-416)$ & $77 / 81(95.06 \%)$ \\
9th year $(24-468)$ & $77 / 79(97.46 \%)$ \\
10th year $(>468)$ & $77 / 78(98.71 \%)$ \\
\hline
\end{tabular}

Table 2 Distribution of lamivudine resistance to

\begin{tabular}{ll}
\hline 1st year (24-52) weeks & $18 / 56(32.14 \%)$ \\
2nd year (24-104) & $40 / 55(72.73 \%)$ \\
3rd year (24-156) & $47 / 54(87.04 \%)$ \\
4th year (24-208) & $48 / 54(88.89 \%)$ \\
5th year (24-260) & $50 / 54(92.59 \%)$ \\
6th year (24-312) & $52 / 54(96.3 \%)$ \\
7th year $(24-364)$ & $52 / 54(96.3 \%)$ \\
8th year $(24-416)$ & $52 / 54(96.3 \%)$ \\
9th year $(24-468)$ & $52 / 53(98.11 \%)$ \\
10th year $(>468)$ & $52 / 52(100.00 \%)$
\end{tabular}

PP05-80

Lamivudine versus Entecavir Monotherapy for Spontaneous Acute Exacerbation of Chronic Hepatitis B with 500 or more than 500 IU/L ALT T. Kande ${ }^{1}$, M. Shinozaki ${ }^{2}$, H. Kamezaki ${ }^{1}, \mathrm{~S} \mathrm{Wu}^{1}, \mathrm{~S}$. Nakamoto ${ }^{1}$, M. Arai ${ }^{1}$, K. Fujiwara ${ }^{1}$, N. Goto ${ }^{2}$, F. Imazeki ${ }^{1}$, O. Yokosuka ${ }^{1}$

${ }^{1}$ Medicine and Clinical Oncology, Chiba University, Graduate School of Medicine, Chiba; ${ }^{2}$ Gastroenterology, Numazu City Hospital, Numazu, Japan

Backgrounds and aims: Spontaneous acute exacerbation of chronic hepatitis B virus (HBV) infection occasionally occurs in its natural history and sometimes leads to fetal hepatic failure rapidly. We compared the effects of lamivudine (LAM) with those of entecavir (ETV) treatments in acute exacerbation of chronic hepatitis B with 500 or more than 500 IU/L ALT levels.

Methods: Thirty-four patients with acute exacerbation were consecutively treated with LAM or ETV. Their clinical outcomes were compared each other. Antiviral failure was evaluated based on the definition reported by A. Gallego (J Viral Hepat, 2008).

Results: None who has a reduction of $<1 \log _{10}$ IU/mL in HBV DNA after 1 or 3 months of treatment (primary antiviral treatment failure), was identified in 24 LAM-treated or $10 \mathrm{ETV}$-treated patients. Initial virological response, defined as a reduction of $4 \log 10 \mathrm{IU} / \mathrm{mL}$ in HBV DNA at month 6, of LAM and that of ETV, respectively, were $20 / 24(83.3 \%)$ and $10 / 10(100 \%)$. One patient of LAM developed hepatic encephalopathy, but all patients survived in both groups. 12 months after treatment, 10 patients of $24(41.6 \%)$ switched lamivudine to another drug or added adefovir in LAM group (55.5 \pm 25.4 months). On the other hand, patients receiving ETV did not need to change their drugs. In $\mathrm{HBe}$ antigen positive patients, however, ALT levels one month after treatment tend to be lower in LAM group than those in ETV group.

Conclusions: ETV seems to be superior to LAM in the treatment against acute exacerbation of chronic hepatitis B with 500 or more than $500 \mathrm{IU} / \mathrm{L}$ ALT levels. Further studies will be needed to reveal the rapid drop effects of LAM on ALT levels.

\section{PP05-81}

Nucleoside Analogue can Improve the Long-term Prognosis of Patients with Acute on Chronic Hepatitis B Liver Failure

H. Xiao ${ }^{1}$, Y. Zhou ${ }^{2}$, J. Wang ${ }^{3}$

${ }^{1}$ Shanghai Public Health Clinical Center Affiliated to Fudan

University; ${ }^{2}$ Shanghai Public Health Clinic Center, Fudan

University; ${ }^{3}$ Shanghai Public Health Clinical Center, Shanghai, China

Background and aims: The prognosis of patients with acute-onchronic hepatitis B liver failure (ACLF) is extremely poor. This study was designed to evaluate the effect of nucleoside analogue on survival in patients with ACLF.

Methods: We conducted a matched retrospective cohort study using data on ACLF patients derived from our hospital database from January 2006 to January 2008. A total of 126 consecutive patients were recruited. Fifty four patients receiving lamivudine, three entecavir, two adefovir dipivoxil were selected into the nucleoside analogue treatment group with another 67 without nucleoside analogue treatment studied as control. All patients were given standard medical treatment including absolute bed rest; intravenous drop albumin and plasma; maintenance water, electrolyte and acid-base equilibrium; and prevention and treatment complications, etc. They were matched for sex, age, HBV-DNA load, complications, INR, PTA and liver function in the two groups. All the patients were followed up for 6months and the survival rates were compared.

Results: The cumulative survival rates of patients in the nucleoside analogue group $(n=59)$ of 6 months were higher than those of the 
patients in the control group ( $n=67 ; 53.7$ vs. $35.6 \%$ ). The cumulative survival rates of patients in the pretreatment HBV DNA load $\geqq 10^{5}$ copies $/ \mathrm{ml}$ group $(n=71)$ were lower than those of patients in the pretreatment low HBV DNA load group $\left(<10^{5}\right.$ copies $/ \mathrm{ml}, n=55$; 35.3 vs. $67.3 \%$ ) and there was no significant difference in mortality rate between $\mathrm{HBeAg}$-positive patients $(n=36)$ and $\mathrm{HBeAg-negative}$ patients ( $n=90 ; 47.2$ vs. $52.2 \%$ ). Moreover patients with cirrhosis, age $\geqq 50$ years and MELD score $\geqq 30$ had higher mortality rate.

Conclusion: These data indicated that age, with cirrhosis foundation, high HBV-DNA load and MELD score $\geqq 30$ were the main factors that influenced prognosis. Nucleoside analogue treatment could improve the long-term prognosis of patients with $\mathrm{HBV}$-associated ACLF.

\section{PP05-82}

The Safety of Pregnant Women and Fetus during Pregnancy While Husbands with Chronic Hepatitis B Treating with Adefovir Dipivoxil W.W. Dai

The Second Hospital of Nanjing, Nanjing, China

Objective: To investigate the safety of 26 cases of pregnant women and fetus during pregnancy while women's husbands with chronic hepatitis B treating with Adefovir Dipivoxil.

Method: 26 male hepatitis B patients were treated with Adefovir Dipivoxil during the pregnancy of their wife, clinical observation of maternal complications (miscarriage, premature birth, neonatal asphyxia, fetal death and fetal malformations), abnormal development and virus carring incidence of infants were carried out.

Result: Of 26 cases of pregnant women who continued pregnancy, maternal complications occurred in 5 cases, infants infected rate was zero, no developmental abnormalities were found in infants and young children, with no difference from the control group.

Conclusion: Preliminary conclusion: while male patients with chronic hepatitis B were treating with Adefovir Dipivoxil, their wives can pregnant and it was safe both for pregnant women and fetus.

\section{PP05-83}

Long Term Outcomes of Entacavir Therapy for Chronic Hep B Patients

M. Gill, U. Adeeb, N. Mehmood, S. Naseem

Division of Digestive Diseases, Maroof International Hospital, Islamabad, Pakistan

Aims: Entacavir is considered very safe and effective treatment for chronic hep B patients. We decided to study the longterm efficacy of this compound. we were interested to determine the efficacy and the breakthrough/resistence with this compound.

Methods: we consecutively enrolled treatment naïve 90 patients who were Hep B surface antigen positive, HepB PCR $>6$ logIU/ml and ALT $>100$. Patients with established cirrhosis were not enrolled in this study. Eligible patients were given $0.5 \mathrm{mg}$ entacavir daily for total duration of 2 years. They were reviewed in out patient clinic for 3 monthly basis. LFTS and HepB PCR was done on 3 monthly basis.

Results: Seventy out of ninty patients were Hep B e antigen negative. 60 patients were male and thirty patients were female $M$ edian age was 40. Median HepBPCR was 6.4log IU/ml.Median ALT was 110.80 out of $90(88 \%)$ patients were Hep B PCR negative@ week 12. Six out $90(7 \%)$ patients were primary non responders @week 12. 84 patients continued therapy for next 36 weeks. Five patients (5\%)have break through @ week 48 overall response rate after 2 years of treatment was $82 \%$. These patients are under regular review.

Conclusion: Entacavir is very safe and effective treatment for Chronic Hep B treatment.

\section{PP05-84}

Efficacy of Tenofovir (TDF) Treatment in Chronic Hepatitis B Patients with Suboptimal Response to Adefovir (ADV)

M. Gill, U. Adeeb, N. Mehmood, S. Naseem

Division of Digestive Diseases, Maroof International Hospital, Islamabad, Pakistan

Aims: Tenofovir disproxil fumarate (TDF) has potent activity against HBV replication. It is considered very effective in chronic Hep B patients with incomplete or partial response with ADV.TDF became recently available in our region.we wanted to evaluate the efficacy and safety of TDF in ADV treated patients with suboptimal response.

Methods: We consecutively enrolled 35 patients with HBV mono infection who has HB- DNA $>6 \log I \mathrm{U} / \mathrm{ml}$ after 48 weeks of ADV 10 mg daily therapy. Patients with established diagnosis of cirrhosis were excluded from this study. The study enrollment was done between January 2010 to March 2010.All enrolled patients were given TDF $300 \mathrm{mg}$ daily before breakfast. The treatment duration for these patients is 96 weeks. These patients were seen in out patient clinic on eight weekly basis. They have baseline TSH, blood sugar, ALT, BUN creatinine and quantitative HB-DNA They had BUN/CREATININE, ALT on every visit. HB -DNA was done @ week 12 and week 24. Results: We are reporting the interim analysis of this cohort after 6 months of therapy. out of 35 patients 25 were male 10 were female. Medial ALT was 95, Medial HB-DNA was $6.8 \log \mathrm{IU} / \mathrm{ml} .25$ out of 35 (71\%) patients were HB-DNA negative @ week 12 and 24. Five (14\%) patients did not have any drop in HB- DNA. Four patients have $>2 \log$ drop in HB-DNA @ week 12 and HB-DNA negative @ week 24. One patient did not continue treatment beyond 8 weeks. Overall efficacy in this cohort @ week 24 was $(80 \%)$.we did not notice any deranged renal function in this cohort. The HB-DNA negative patients are continuing the treatment and are in close $\mathrm{F} / \mathrm{U}$.

Conclusion: Tenofovir disproxil Fumarat (TDF) is very effective and safe treatment in chronic hip B patients who have suboptimal response to Adefovir Dipivoxil (ADV).

Table 1 Correlation factors of hospital onset of mycotic infection in liver failure patients casuse by hepatitis B

\begin{tabular}{|c|c|c|c|c|c|c|c|c|c|c|}
\hline & $\begin{array}{l}\text { Broad } \\
\text { spectrum } \\
\text { antibiotic } \\
\text { using }\end{array}$ & $P$ value & Neutroperia & $P$ value & $\begin{array}{l}\text { Invasive } \\
\text { medical } \\
\text { manipulations }\end{array}$ & $P$ value & $\begin{array}{l}\text { ALSS } \\
\text { treatment }\end{array}$ & $P$ value & MELD score & $P$ value \\
\hline $\begin{array}{l}\text { Mycotic } \\
\text { infectionq }\end{array}$ & $121(87.7 \%)$ & & $87(63.04 \%)$ & 0.001 & $101(73.19 \%)$ & & $38(27.54 \%)$ & \multirow[t]{2}{*}{0.064} & $32.41 \pm 70.82$ & \multirow[t]{2}{*}{0.035} \\
\hline $\begin{array}{l}\text { No mycotic } \\
\text { infection }\end{array}$ & $590(79.94 \%)$ & 0.033 & $352(47.70 \%)$ & & $456(61.79 \%)$ & 0.011 & $151(20.46 \%)$ & & $25.54 \pm 6.45$ & \\
\hline
\end{tabular}

Correlation factors 


\section{PP05-85}

Analysis of Hospital Onset of Mycotic Infection in Liver Failure Patients Caused by Hepatitis B

L. Peng, J. Liu, J.-G. Li

Department of Infectious Diseases, The Third Affiliated Hospital of Sun Yat-Sen University, Guangzhou, China

Background: Hepatitis B is a serious global health problem. Mycotic infection is easily happened in liver failure inpatients caused by hepatitis B and will lead to worse prognosis and higher mortality. We investigated the clinical features of hospital onset of mycotic infection in liver failure inpatients caused by hepatitis B during recent 5 years. Methods: 876 hepatitis B inpatients with liver failure were recruited from June 2005 to June 2010 in our department. Hospital onset of mycotic infection was diagnosed by smearing or culturing at least twice for detection of one kind of fungus after $48 \mathrm{~h}$ at post-admission. All the clinical features of patients were analyzed retrospectively.

Results: 138 liver failure inpatients were final diagnosed for hospital onset of mycotic infection with mortality of $65.22 \%$. The most common infection strains were Candida albicans (54.35\%) and Smooth Monilia. Respiratory tract and digestive tract were most common infection positions. Susceptibility rate of all fungi to amphotericin B were $94.20 \%$, which was followed by fluconazol, Itraconazole and ketoconazole. Use of broad-spectrum antibiotic, neutropenia, invasive medical manipulations and severity of liver disease were high risk factors of fungus infection except artificial liver support system (ALSS) treatment.

Conclusions: Hospital onset of mycotic infection were common in hepatitis B inpatients with liver failure. Respiratory tract and digestive tract were the common infection position. Candida mycoderma were the main infection strains but diversification tendency had been found in the infection spectrum. Some clinical factors were high risk factors of fungus infection except ALSS treatment.

\section{PP05-86}

Study of Therapeutic Effects of Different Dosage of S-ademetionine Treating Intrahepatic Cholestasis Patients Caused by Hepatitis B with Liver Failure

L. Peng, J. Liu, J.-G. Li

Department of Infectious Diseases, The Third Affiliated Hospital of Sun Yat-Sen University, Guangzhou, China

Background and aims: Intrahepatic cholestasis is a clinical outcome of liver failure caused by hepatitis B. $S$-adenosylmethionine (SAMe) is one of most important methyl donor which takes part in polyamine biosynthesis. Our aim is to investigate the therapeutic effects of different dosage of SAMe treating intrahepatic cholestasis patients caused by hepatitis B with liver failure.

Methods: 57 intrahepatic cholestasis patients caused by hepatitis B with liver failure were recruited and received the same medical treatments. And 22 patients were in group A received 1.0 SAMe everyday, 10 patients were in group B received 2.0 SAMe everyday, other 25 patients were in group $\mathrm{C}$ without using SAMe. Improvements of ALT (alanine aminotransferase), GGT ( $\gamma$-glutamyltransferase), TBA (total bile acid), TBIL (total bilirubin) and DBIL (direct bilirubin) were compared after 4 weeks.

Results: No side effects were found. There were no significant differences in ALT and TBIL levels between 3 groups. GGT improvements in group B is significantly better than group C from 3rd week and the similar differences appeared only at 4th week between group A and C. For TBA level, significant differences only appeared at 4 th week between group A, B and C. And significant differences were found at 3rd and 4th week in DBIL levels between group A, B and C. Conclusion: SAMe have therapeutic effects on GGT, TBA and DBIL. But larger dosage using only perhaps bring better and ealier effects in GGT improvement. More samples are needed to verify the value of larger dosage using of SAMe.

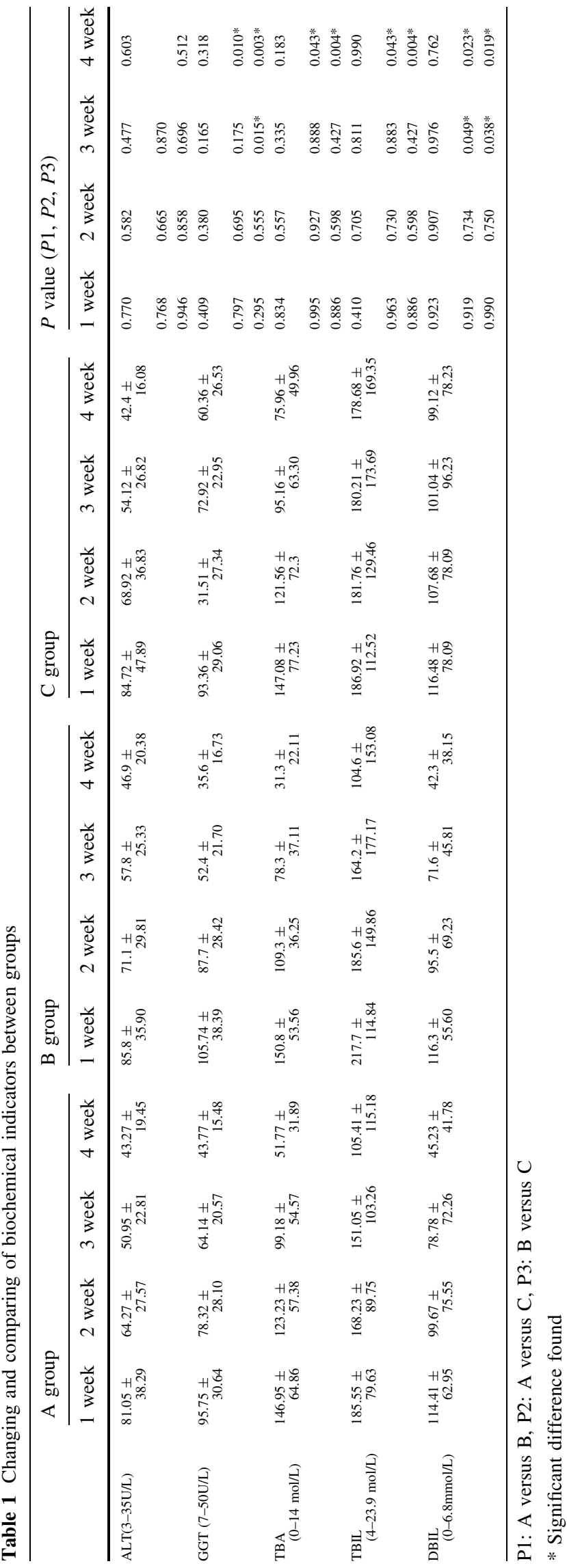




\section{PP05-87}

HBV Polymerase Substitutions Detected in Patients Failing Tenofovir Therapy: Effects on in vitro Replication and Antiviral Sensitivity

T. Sozzi, S. Bowden, S. Locarnini, T. Shaw

Hepatitis Research Unit, Victorian Infectious Diseases Reference Laboratory, North Melbourne, VIC, Australia

Although the introduction of oral nucleos $(\mathrm{t})$ ide reverse transcriptase inhibitors (NRTI) over the past two decades has dramatically improved treatment for chronic hepatitis B, their long-term effectiveness seems almost invariably to be limited by emergence of resistance. "Signature" mutations that confer primary resistance to lamivudine, adefovir (ADV), entecavir and telbivudine have been identified, but specific mutations have yet to be convincingly associated with clinical failure of tenofovir (TFV). In vitro, ADV signature mutations confer barely measurable degrees of cross-resistance to TFV, but pre-existing resistance to ADV has been associated with variable degrees of diminished responses to subsequent TFV treatment in vivo. To better characterize mechanisms of TFV resistance it is therefore crucial to have available in vitro assays that are capable of detecting small changes in viral resistance to NRTI. We have developed such assays and used them to re-examine the potential for cross-resistance between $\mathrm{ADV}$ and TFV by assessing the effects of the ADV resistance signature mutants (rtA181T/V/N236T) alone and in combination with a variety of other amino acid substitutions suspected of compromising clinical response to ADV and/or TFV. The latter included changes that affect the conserved "A" or "D" motifs, as well as several in the inter-domain regions. Corresponding panels of mutants were generated from a laboratory (HBeAg-negative genotype $\mathrm{D}$ ) $\mathrm{HBV}$ clone by site-directed mutagenesis and the in vitro drug susceptibilities and replication capacities of the parent and its mutant derivatives were compared following transient transfection of Huh-7 cells. Most substitutions resulted in measurable replication defects compared to the parent clone, but only rtD83V and rtA181T/N236T conferred statistically significant decreases in sensitivity to both ADV and TFV.

\section{PP05-88}

Treatment with Entecavir of HBV Chronic Hepatitis in Immunosuppressed Patients

G. Scotto ${ }^{1}$, G. D'Addiego ${ }^{1}$, F. Campanale ${ }^{1}$, V. Fazio $^{2}$

${ }^{1}$ Infectious Diseases; ${ }^{2}$ Laboratory, OORR Foggia, Foggia, Italy

Background/aims: In patients with chronic HBV infection, immunosuppression because of local or systemic immunosuppressive therapycan give rise to acute hepatitis and even fatal fulminant hepatitis. Several studies have shown as the antiviral therapy with a nucleosideanalogue lamivudine can avoid reactivation or worsening of hepatitis B. for other nucleoside/tide analogues there aren't many experiences in this situation. We have therefore carried out a study to assess the efficacy of entecavir in patients with HBV infection needing immunosuppressive treatment.

Patients and methods: Nine patients (two with cancer, three with haematological malignancies and four with rheumatic diseases) planned to receive immunosuppressive treatment.

All the patients presented HBV chronic active hepatitis (HBV DNA levels $>2,000 \mathrm{IU} / \mathrm{ml}$ - ALT serum levels $\times 4$ of normal value- $\mathrm{HBeAg}$ negative/HBeAb positive). Two weeks prior to onset of immunosuppressive treatment we started with antiviral therapy with Entecavir $0.5 \mathrm{mg} /$ day. All the patients are still on treatment.

Results: After 18 months of therapy, HBV DNA was negative and ALT were normal in all the patients. Entecavir was well tolerated and produced a rapid (1 month) and sustained suppression of HBV. In all cases immunosuppressive treatment was given for the planned duration of therapy.

Conclusion: This study showed good tolerance and clinical efficacy of entecavir therapy in preventing worsening of HBV chronic hepatitis in patients who received immunosuppressive treatment.
PP05-89

Efficacy of Thymosin Alfa-1 Plus Peginterferon Alfa-2a Combination Therapy Compared with Peginterferon Alfa-2a Monotherapy in HBeAg-positive Chronic Hepatitis B

B.H. Kim ${ }^{1}$, Y.-J. Lee ${ }^{2}$, W. Kim ${ }^{3}$, J.-H. Yoon ${ }^{4}$, E.U. Jung ${ }^{2}$, S.J. Park ${ }^{2}$, Y.J. Kim ${ }^{4}$, H.-S. Lee ${ }^{4}$

${ }^{1}$ Department of Internal Medicine and Hepatology Center, Bundang Jesaeng General Hospital, Seongnam; ${ }^{2}$ Department of Internal Medicine, Paik Hospital, Inje University, Busan; ${ }^{3}$ Department of Internal Medicine, Seoul Metropolitan Government Seoul National University Boramae Medical Center; ${ }^{4}$ Department of Internal Medicine and Liver Research Institute, Seoul National University College of Medicine, Seoul, Republic of Korea

Background and aims: Thymosin alfa- 1 plus interferon alfa-2a offers superior efficacy over interferon alfa-2a alone in patients with chronic hepatitis B (CHB). Thus, the aim of this study was to compare the antiviral efficacy of thymosin alfa-1 plus pegylated interferon alfa- $2 \mathrm{a}$ and pegylated interferon alfa- $2 \mathrm{a}$ alone in HBeAg-positive CHB patients.

Methods: Fifty-one treatment-naïve, $\mathrm{HBeAg}$-positive $\mathrm{CHB}$ patients were randomly assigned to either combination $(180 \mu \mathrm{g}$ of pegylated interferon alfa-2a weekly for 48 weeks and $1.6 \mathrm{mg}$ of thymosin alfa- 1 twice a week for the first 12 weeks) or monotherapy (180 $\mu \mathrm{g}$ of pegylated interferon alfa-2a weekly for 48 weeks) groups. All patients were followed for 96 weeks and analyzed based on the intention-totreat population.

Results: The rates of the combined response, defined as $\mathrm{HBeAg}$ seroconversion, HBV DNA suppression, and normalization of serum ALT were 15.4 and $12.0 \%$ for the combination group and the monotherapy group at the end of treatment $(P=0.725)$, and 23.1 and $20.0 \%$ at the end of follow-up, respectively $(P=0.789)$. Based on multiple logistic regression analysis, $>$ a $2 \log _{10} \mathrm{IU} / \mathrm{mL}$ reduction of HBV DNA at week 12 was identified as an independent predictive factor for combined response (OR 9.72; 95\% CI 1.33-71.06; $P=0.025)$ at the end of follow-up. A lower pre-treatment HBV DNA level $\left(\leq 7 \log _{10} \mathrm{IU} / \mathrm{mL}\right)$ was another predictor for combined response (OR 9.64; 95\% CI 1.23-75.32; $P=0.031$ ). Patients without any favorable predictors (pre-treatment HBV DNA level $\leq 7 \log _{10} \mathrm{IU} / \mathrm{mL}$ and $>2 \log _{10} \mathrm{IU} / \mathrm{mL}$ reduction of HBV DNA at week 12) rarely achieved a combined response at the end of follow-up (negative predictive value of $95.5 \%$ ).

Conclusions: The short-term addition of thymosin $\alpha-1$ was not superior to pegylated interferon alfa-2a alone in $\mathrm{HBeAg}$-positive CHB patients on the basis of antiviral efficacy.

\section{PP05-90}

Randomized Trial of Telbivudine or Lamivudine Treatment in Chinese Patients with Decompensated HBV Cirrhosis

F. He, T. FanXiao, H. Xiernayi

Department of Hepatology, The First Affiliated Hospital of The

XinJiang Medical University, Urumuqi, China

Background/aims: We compared the safety and efficacy of telbivudine and lamivudine in patients with chronic hepatitis $\mathrm{B}(\mathrm{CHB})$ and decompensated cirrhosis.

Methods: Ninety-four patients with $\mathrm{CHB}$ and decompensated cirrhosis were randomized to receive telbivudine $(n=42)$ or lamivudine $(n=52)$ for 48 weeks. Hepatitis B virus (HBV) DNA and alanine aminotransferase (ALT) levels and liver function were evaluated at baseline and at 12, 24, and 48 weeks of therapy.

Results: At baseline, median age was 46 years, $97 \%$ of patients were male, $41 \%$ of patients were hepatitis $\mathrm{B}$ e antigen $(\mathrm{HBeAg})$ positive, 88 and $19 \%$ of patients had Child-Pugh class B and C disease, respectively, and mean HBV DNA levels were $>4 \log _{10}$ copies/mL. At week 12, undetectable HBV DNA ( $<300$ copies $/ \mathrm{mL})$ was achieved in $100 \%$ of telbivudine- and $93 \%$ of lamivudine- 
treated patients. Polymerase chain reaction (PCR) negativity was sustained through week 48 in telbivudine-treated patients but decreased to $85 \%$ in lamivudine-treated patients. ALT normalization was reported in $86 \%$ of telbivudine-treated and $81 \%$ of lamivudinetreated patients. At week 24 , viral breakthrough occurred in $6 \%$ of lamivudine-treated patients, increasing to $15 \%$ at week 48 . None of the telbivudine-treated patients experienced viral breakthrough. At 24 weeks, a higher proportion of telbivudine- than lamivudinetreated patients experienced HBeAg loss (62 vs. $39 \% ; P<0.05)$. This increased to 75 versus $47 \%$ at 48 weeks. The HBeAg seroconversion rate was similar between groups at 24 weeks (31 vs. $30 \%$ ) and 48 weeks ( 35 vs. $31 \%$ ). At 48 weeks, a higher proportion of telbivudine- than lamivudine-treated patients (100\% vs $90 \%$ ) showed improvement in Child-Pugh score. Serious adverse events were similar between groups.

Conclusions: Telbivudine improved liver function, resulting in higher rates of PCR negativity and $\mathrm{HBeAg}$ loss and lower rates of virologic breakthrough compared with lamivudine in patients with $\mathrm{CHB}$ and decompensated cirrhosis.

\section{PP05-91}

4 Year Efficacy and Safety of Tenofovir DF Treatment in HBeAg-Negative and HBeAg-Positive Patients with Chronic Hepatitis B (CHB)

P. Marcellin ${ }^{1}$, E.J. Heathcote ${ }^{2}$, M. Buti ${ }^{3}$, E.J. Gane ${ }^{4}$, Z. Krastev ${ }^{5}$, R.A. de $\mathrm{Man}^{6}$, S. Gurel ${ }^{7}$, K. Borroto-Esoda ${ }^{8}$, D.H. Coombs ${ }^{8}$, E. Mondou ${ }^{8}$, J. Anderson ${ }^{8}$

${ }^{1}$ Hopital Beaujon, University of Paris, Clichy, France; ${ }^{2}$ University of Toronto, Toronto, ON, Canada, ${ }^{3}$ Servicio de Medicina Interna Hepatologia, Hospital General Universitari Vall d'Hebron and Ciberehd, Barcelona, Spain; ${ }^{4}$ Middlemore Hospital, Auckland, New

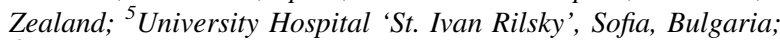

${ }^{6}$ Erasmus MC, University Medical Center Rotterdam, Rotterdam, The Netherlands; ${ }^{7}$ University of Uludag, Bursa, Turkey; ${ }^{8}$ Gilead Sciences, Inc., Durham, NC, USA

Background: Tenofovir disoproxil fumarate (TDF) is a nucleotide analog with potent antiviral activity against hepatitis B virus.

Methods: Patients with $\mathrm{HBeAg}-(N=375)$ or $\mathrm{HBeAg}+(N=266)$, CHB mono-infection were randomized 2:1 to double-blind, once daily TDF $300 \mathrm{mg}$ or adefovir dipivoxil (ADV) $10 \mathrm{mg}$. After 48 weeks, patients with a week (W) 48 biopsy were switched to openlabel TDF for up to 7 additional years with option to add emtricitabine (FTC) at/after W72 for confirmed HBV DNA $\geq 400$ copies (c)/mL (69 IU/mL). W192 (year 4) data are presented.

Results: In an Intent-to-treat analysis (ITT), $86 \%$ of $\mathrm{HBeAg}-(85 \%$ TDF-TDF; $87 \%$ ADV-TDF) and $77 \%$ of $\mathrm{HBeAg}+$ patients $(74 \%$ TDF-TDF; $84 \%$ ADV-TDF) had HBV DNA $<400 \mathrm{c} / \mathrm{mL}$ at W192. In an on-treatment analysis $99 \%(\mathrm{HBeAg}-)$ and $97 \%(\mathrm{HBeAg}+)$ had HBV DNA $<400 \mathrm{c} / \mathrm{mL} .97$ and $71 \%$ of patients $(N=129)$ with high baseline viral load (HBV DNA $\geq 9 \log _{10} \mathrm{c} / \mathrm{mL}$ ) achieved HBV DNA $<400 \mathrm{c} / \mathrm{mL}$ (on-treatment and ITT analyses, respectively). Resistance analyses showed no amino acid substitutions that could be associated with TDF resistance in patients with detectable HBV DNA at W192, at discontinuation or when FTC was added. Overall, 38 patients added FTC to TDF between W72 and W192 and of the 26 patients remaining on treatment at W192, $81 \%$ achieved HBV DNA $<400 \mathrm{c} /$ $\mathrm{mL}$. At W192 mean ALT was $33 \mathrm{U} / \mathrm{L}(\mathrm{HBeAg}-)$ and $35 \mathrm{U} / \mathrm{L}$ ( $\mathrm{HBeAg}+$ ). $\mathrm{HBeAg}$ loss/seroconversion was observed in $41 \% / 31 \%$ of $\mathrm{HBeAg}+$ patients (on-treatment analysis). Cumulatively, $10 \%$ of $\mathrm{HBeAg}+$ patients lost $\mathrm{HBsAg}$ and $7.5 \%$ seroconverted to anti-HBs (Kaplan-Meier Estimate). No $\mathrm{HBeAg}-$ patient lost HBsAg. Creatinine levels remained stable through year 4.

Conclusion: TDF was well tolerated and produced potent, continuous viral suppression through 4 years of TDF treatment and no mutations developed in association with TDF resistance in both $\mathrm{HBeAg}-$ and $\mathrm{HBeAg}+$ patients.
PP05-92

Safety and Efficacy of Telbivudine in Chinese Patients with CHB: Real-life Clinical Experience

N.J. Jiang ${ }^{1}$, G.J. Hu ${ }^{2}$, H.M. $\mathrm{Su}^{1}$, F.S. Ge ${ }^{2}$, C.Z. Jiang ${ }^{3}$, Q.M. Zhu ${ }^{4}$, H.Z. $\mathrm{Liu}^{1}$, X.Y. Liang ${ }^{1}$, W.W. Guo ${ }^{1}$

${ }^{1}$ Infectious Disease Department, The First Affiliated Hospital of Guangxi Medical University, Nanning; ${ }^{2}$ Infectious Disease

Department, Liu Zhou People's Hospital, Liu Zhou; ${ }^{3}$ Infectious

Disease Department, An Kang People's Hospital, An Kang;

${ }^{4}$ Hepatology Department, The Third Hospital of Zhen Jiang, Zhen

Jiang, China

Aim: To evaluate the efficacy and safety of 1 year of telbivudine treatment in Chinese patients with chronic hepatitis B in the clinical practice setting.

Methods: Forty-four treatment-naïve patients (31 hepatitis B e antigen $[\mathrm{HBeAg}]$ positive, $13 \mathrm{HBeAg}$ negative) with compensated liver disease, hepatitis B virus (HBV) DNA levels $>10^{4}$ copies $/ \mathrm{mL}$, and alanine aminotransferase (ALT) levels $>2 \times$ the upper limit of normal received telbivudine $600 \mathrm{mg}$ /day for up to 1 year. Virologic and biochemical parameters were monitored at baseline, 3, 6, and 12 months during treatment. HBV DNA levels were quantified using real-time fluorescence polymerase chain reaction (PCR) assay (lower limit of quantification $<10^{3}$ copies $/ \mathrm{mL}$ ). Levels of $\mathrm{HBeAg}$ and antibody to $\mathrm{HBeAg}$ were quantified using chemiluminescence immunoassay.

Results: Mean patient age was 31 years (range 18-56 years) and $77 \%$ of the patients were male. At baseline, mean HBV DNA and ALT levels were $6.61 \log _{10}$ and $86 \mathrm{U} / \mathrm{L}$ in $\mathrm{HBeAg}$-positive patients, respectively, and $5.68 \log _{10}$ copies/mL and $98 \mathrm{U} / \mathrm{L}$ in HBeAg-negative patients, respectively. The HBV DNA undetectable rate at 3,6 , and 12 months was $81.8,100$, and $100 \%$, respectively. Among $\mathrm{HBeAg}$-positive patients, the rate of $\mathrm{HBeAg}$ loss and $\mathrm{HBeAg}$ seroconversion at 3,6 , and 12 months was $35.5,45.1,54.8 \%$, respectively, and 35.5, 45.1, and 51.6\%, respectively. Initial virologic response at 3 months (IVR) correlated with $\mathrm{HBeAg}$ loss at 3, 6, and 12 months and $\mathrm{HBeAg}$ seroconversion at 3 and 6 months $(P<0.05)$. Of 23 patients with IVR to telbivudine, 14 patients $(60.8 \%)$ achieved HBeAg seroconversion at 12 months. Telbivudine was well tolerated.

Conclusions: Telbivudine induced rapid and potent HBV DNA suppression. IVR may be predictive of $\mathrm{HBeAg}$ loss and high $\mathrm{HBeAg}$ seroconversion rates after 12 months of treatment.

\section{PP05-93}

Relationship between PD-1 Expression on Peripheral Blood T Cells and HBeAg Seroconversion after Entecavir Treatment in Chronic Hepatitis B Patients

J. Xia ${ }^{1}, \mathrm{X} . \mathrm{Li}^{1}, \mathrm{Y} . \mathrm{Hao}^{1}, \mathrm{Y} . \mathrm{Liu}^{2}, \mathrm{X} . \mathrm{Yan}^{1}, \mathrm{C} . \mathrm{Wu}^{1}$

${ }^{1}$ Infectious Disease; ${ }^{2}$ Laboratory Medicine, NanJing Drum Tower Hospital of NanJing University Medical School, Nanjing, China

Objective: To observe the expression of Programmed death 1 (PD-1) on peripheral blood $\mathrm{CD} 4^{+}$and $\mathrm{CD} 8^{+} \mathrm{T}$ cells after entecavir (ETV) antiviral treatment in $\mathrm{HBeAg}$ positive chronic hepatitis $\mathrm{B}(\mathrm{CHB})$ patients, and to explore the relationship between PD-1 expression and $\mathrm{HBeAg}$ seroconversion.

Methods: We studied longitudinally $20 \mathrm{HBeAg}$-positive $\mathrm{CHB}$ patients undergoing antiviral treatment with ETV. PD-1 expression was assessed by flow cytometry, serum HBV-DNA load was determined by real-time fluorescent quantitative polymerase chain reaction. The investigations were focused on six time points: T0: baseline, T1: 2-4 weeks; T2: 5-10 weeks; T3: 11-20 weeks; T4: 21-30 weeks: T5: 31-51 weeks.

Results: 14 of the patients remained $\mathrm{HBeAg}$ positive after treatment (group 1), whereas 6 patients had $\mathrm{HBeAg}$ seroconversion (group 2). At baseline, no statistical differences were found between the two groups in PD-1 exprission, HBV-DNA load or ALT levels 
$(P>0.05)$. After treatment, the decrease rates of HBV-DNA load during period $\Delta \mathrm{T} 0-\mathrm{T} 1, \Delta \mathrm{T} 0-\mathrm{T} 2$ and $\mathrm{PD}-1$ expression on $\mathrm{CD} 8+\mathrm{T}$ cells during period $\Delta \mathrm{T} 0-\mathrm{T} 2, \Delta \mathrm{T} 0-\mathrm{T} 3$ were significantly different between the two groups $(37.3$ vs. $49.9 \%, P=0.011 ; 47.4$ vs. $56.7 \%$, $P=0.013 ;-4.2$ vs. $70.1 \%, P=0.021 ; 24.5$ vs. $66.9 \%, P=0.014)$.
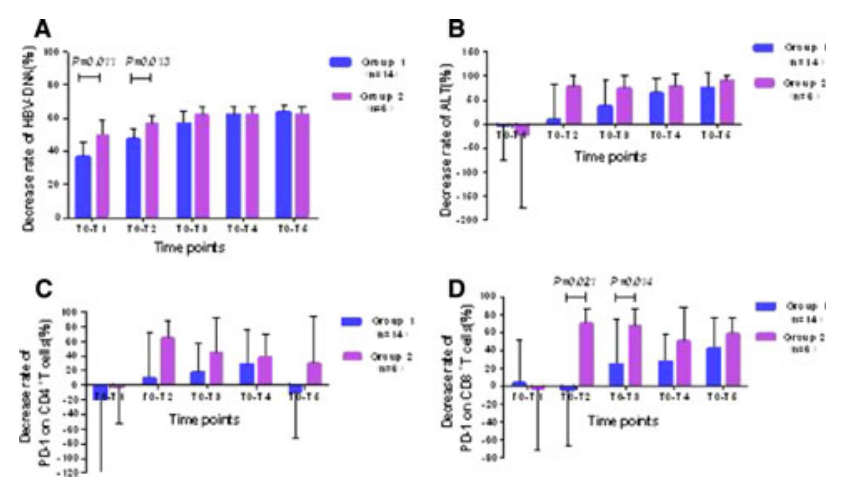

Fig. 1 The decrease rates of HBV-DNA, ALT and PD-1

Besides, the decrease in PD-1 expression on both CD4+ and CD8+ T cells was positively correlated with HBV-DNA load and ALT levels during antiviral treatment $(r=0.212, P=0.050 ; \quad r=0.377$, $P=0.000 ; r=0.279, P=0.011 ; r=0.347, P=0.001)$.

Conclusion: Treatment-induced rapid decrease of PD-1 expression on peripheral blood CD8+ T cells, which was similar to HBV-DNA load, can be one of the predict indexes of HBeAg seroconversion in $\mathrm{HBeAg}$ positive $\mathrm{CHB}$ patients.

\section{PP05-94}

Safety and Efficacy of Telbivudine in Patients with CHB-related Cirrhosis: a Single-center Experience

N. Chamroonkul, S. Attasaranya, U. Khow-Ean, B. Ovartlarnporn,

T. Piratvisuth

Department of Internal Medicine, Prince of Songkla University, Hatyai, Thailand

Background/aims: Effective treatment of patients with hepatitis B virus (HBV)-related cirrhosis is essential to prevent disease progression. We assessed the safety and efficacy of telbivudine in patients with HBV-related cirrhosis.

Methods: This retrospective observational study involved 32 patients with HBV-related cirrhosis who were treated with telbivudine $600 \mathrm{mg} /$ day from August 2008 through July 2010. Virologic and biochemical parameters were monitored at treatment weeks 12, 24, and 52.
Results: At baseline, median age was 52 years, $70 \%$ of patients were male, $56 \%$ of patients were hepatitis $\mathrm{B}$ e antigen ( $\mathrm{HBeAg}$ ) positive, and $88 \%$ of patients had Child-Pugh class A disease. Mean HBV DNA levels were $5.4 \log _{10}$ copies/mL and mean alanine aminotransferase (ALT) levels were $79.8 \pm 83.7 \mathrm{U} / \mathrm{L}$. At 24 weeks, polymerase chain reaction (PCR)-undetectable HBV DNA $(<300$ copies $/ \mathrm{mL}$ ) was achieved in $22(69 \%)$ of 32 patients $(44 \%$ of HBeAgpositive patients and $100 \%$ of $\mathrm{HBeAg}$-negative patients); $20(62 \%)$ of 32 patients had ALT levels $<1 \times$ the upper limit of normal. Of the 8 patients with detectable HBV DNA at week 24, 4 received add-on adefovir or tenofovir or switched to entecavir and 4 continued telbivudine. At 52 weeks the undetectable DNA rate increased to $89 \%$ ( 25 of 28 patients). Viral breakthrough occurred in 4 (12.5\%) of 32 patients who were followed for $>24$ weeks. Mean time of viral breakthrough was $14 \pm 3$ months. On-treatment adjustment of therapy in patients with detectable HBV DNA levels at 24 weeks was associated with a lower rate of viral breakthrough. Treatment was stopped in 1 patient who developed a progressive creatine phosphokinase elevation without clinical evidence of rhabdomyolysis.

Conclusions: Telbivudine provides effective viral suppression with ALT normalization in patients with HBV-related cirrhosis. On-treatment modification is recommended in patients who do not achieve undetectable HBV DNA at 24 weeks.

\section{PP05-95}

Efficacy and Safety of Telbivudine in Patients after OLT: a Single-center Clinical Experience

O. Gerasimova, D.A. Granov, F.K. Zherebtsov, V.V. Borovik

Russian Scientific Center of Radiology and Surgical Technologies, St. Petersburg, Russia

Background/aims: Among patients with hepatitis B virus (HBV) infection at the time of orthotopic liver transplantation (OLT), 70\% develop disease recurrence after surgery. Lamivudine combined with hepatitis B immunoglobulin is the gold standard for the treatment of these patients, as it reduces the recurrence of infection to $5 \%$ at 5 years. We sought to evaluate the efficacy and safety of telbivudine in patients undergoing OLT.

Methods: Thirteen patients ( 8 hepatitis B e antigen $[\mathrm{HBeAg}]$ positive, $5 \mathrm{HBeAg}$ negative) were treated with telbivudine for up to 24 months after OLT for prophylaxis against HBV recurrence. Efficacy parameters included levels of alanine aminotransferase (ALT), HBV DNA, hepatitis B surface antigen ( $\mathrm{HBsAg}$ ), and $\mathrm{HBeAg}$ measured every 12 weeks, and control liver biopsy after 12 months. All patients were given 3- or 2-component immunosuppressive therapy (prednisolone, calcineurin inhibitors, azathioprine) followed by monotherapy with a calcineurin inhibitor.

\begin{tabular}{|c|c|c|c|c|c|c|c|}
\hline & Group & T0 (0 week) & T1 (2-4 weeks) & T2 (5-10 weeks) & T3 (11-20 weeks) & T4 (21-30 weeks) & T5 (31-51 weeks) \\
\hline \multirow{2}{*}{$\begin{array}{l}\mathrm{DNA}\left(\log _{10}\right. \\
\text { copies } / \mathrm{ml})\end{array}$} & $1(n=14)$ & $7.54 \pm 0.67$ & $4.75 \pm 0.71$ & $3.99 \pm 0.57$ & $3.25 \pm 0.62$ & $2.85 \pm 0.32$ & $2.70 \pm 0.00$ \\
\hline & $2(n=6)$ & $7.30 \pm 0.79$ & $3.67 \pm 0.75$ & $3.04 \pm 0.41$ & $2.74 \pm 0.07$ & $2.70 \pm 0.00$ & $2.70 \pm 0.00$ \\
\hline \multirow[t]{2}{*}{ ALT(U/L) } & $1(n=14)$ & $187.26 \pm 184.15$ & $143.40 \pm 102.76$ & $110.68 \pm 95.35$ & $70.42 \pm 46.87$ & $37.53 \pm 22.65$ & $28.42 \pm 25.39$ \\
\hline & $2(n=6)$ & $272.17 \pm 215.07$ & $197.35 \pm 92.16$ & $47.90 \pm 1.56$ & $33.13 \pm 11.99$ & $26.00 \pm 15.32$ & $14.00 \pm 4.72$ \\
\hline \multirow{2}{*}{$\begin{array}{l}\text { PD-1 on CD4+ } \\
\text { T cells }(\%)\end{array}$} & $1(n=14)$ & $6.04 \pm 3.71$ & $5.36 \pm 3.62$ & $3.75 \pm 1.13$ & $3.77 \pm 1.33$ & $3.96 \pm 2.51$ & $6.54 \pm 5.26$ \\
\hline & $2(n=6)$ & $6.77 \pm 2.88$ & $5.59 \pm 3.01$ & $2.60 \pm 1.21$ & $3.07 \pm 1.62$ & $3.84 \pm 2.15$ & $2.87 \pm 1.74$ \\
\hline \multirow{2}{*}{$\begin{array}{c}\text { PD- } 1 \text { on CD8+ } \\
\text { T cells }(\%)\end{array}$} & $1(n=14)$ & $6.39 \pm 3.33$ & $5.68 \pm 2.74$ & $4.98 \pm 1.82$ & $4.21 \pm 2.69$ & $4.08 \pm 2.14$ & $4.55 \pm 4.33$ \\
\hline & $2(n=6)$ & $8.88 \pm 2.84$ & $8.29 \pm 3.86$ & $3.13 \pm 2.12$ & $3.08 \pm 2.17$ & $3.82 \pm 2.22$ & $3.03 \pm 0.67$ \\
\hline
\end{tabular}


Results: At baseline, 10 patients $(77 \%)$ had HBV DNA levels $>10^{4}$ copies/mL (median, $2 \times 10^{5}$ copies $/ \mathrm{mL}$ ) and 11 patients $(84 \%)$ had ALT levels $\geq 1.5 \times$ the upper limit of normal. Following telbivudine treatment, HBV DNA became undetectable in $6(60 \%)$ of 10 patients at 6 months and in $9(90 \%)$ of 10 patients at 12 months. Viral breakthrough was reported in $1(7.7 \%)$ of 13 patients. A significant ALT decrease $(P=0.036)$ was achieved after 3 months of treatment. At 6 months, 2 patients achieved $\mathrm{HBeAg}$ seroconversion; HBsAg clearance occurred in 3 (23\%) of 13 patients after 12 months of therapy. Treatment was well tolerated, and median serum creatinine levels remained stable $(91.3 \pm 19.4$ to $76.7 \pm 16.2$ $\mathrm{nmol} / \mathrm{mL}$ ).

Conclusions: Despite the small size of the study, the data show that telbivudine is effective in preventing recurrent $\mathrm{HBV}$ infection in patients undergoing OLT, with a favorable safety profile.

\section{PP05-96}

Early Viral Suppression at Week 8: Predicting Sustained Response at Weeks 24 and 48 in Telbivudine-treated CHB Patients

C.-T. Hu

Department of Medicine, Buddhist Tzu Chi Hospital and Tzu Chi

University, Hualien, Taiwan, ROC

Background/aims: Liver society guidelines for the management of chronic hepatitis $\mathrm{B}(\mathrm{CHB})$ recommend on-treatment adjustments in patients with suboptimal response to nucleos(t)ide analog therapy at week 24. Early viral suppression of hepatitis B virus (HBV) DNA levels at week 4 has been shown to be predictive of long-term outcomes with lamivudine. We evaluated the relationship between week 4 HBV DNA levels and HBV DNA negativity at week 24 in patients with CHB treated with telbivudine.

Methods: This retrospective observational study involved 240 treatment-naïve and lamivudine-experienced patients with CHB who received telbivudine $600 \mathrm{mg} /$ day at our center. HBV DNA levels at baseline and weeks $4,24,48$, and 72 of telbivudine treatment were quantified using Abbot real-time polymerase chain reaction (PCR) assay.

Results: A total of 110 patients (76\% male, $72 \%$ hepatitis B e antigen negative), with a median age of 52 years (range 23-82 years), baseline HBV DNA levels of $4.19 \log _{10} \mathrm{IU} / \mathrm{mL}$, and alanine aminotransferase levels of $92 \mathrm{U} / \mathrm{L}$ were included in the analysis. At 4 weeks, 53 patients $(48 \%)$ experienced a $\leq 200-\mathrm{IU} / \mathrm{mL}$ reduction from baseline in HBV DNA levels $(P<0.001)$; of these, $42(38 \%)$ were PCR undetectable $(<300$ copies $/ \mathrm{mL} ; P<0.001)$. A strong correlation was observed between HBV DNA levels at week 4 and the proportion of patients with PCR-undetectable HBV DNA at weeks 24 and 48. An HBV DNA level $\leq 200 \mathrm{IU} / \mathrm{mL}$ at week 4 was predictive of PCR undetectability at treatment weeks $24(P=0.020)$ and $48(P=0.048)$.

Conclusions: Telbivudine resulted in potent and early suppression of HBV DNA at week 4. Rapid virologic response at week 4 (HBV DNA level $\leq 200 \mathrm{IU} / \mathrm{mL}$ ) may represent an early indicator of favorable response to telbivudine in treatment-naïve and lamivudine-experienced patients with CHB.

\section{PP05-97}

HBsAg Loss and Decrease of HBsAG Levels in Patients Having Virological Response to Nucleot(S)Ide Treatment Switched to Peginterferon Alpha 2a T.B. Truong

Hepatitis, Hochiminh City University Medical Center, Hochiminh, Vietnam

Background: A lot of $\mathrm{CHB}$ patients have to continue the longterm NAs treatment. Many of them desire to stop the this longterm treatment by many reasons. We switched them to PEG-INF $\alpha 2 \mathrm{a}$.
Methods: Non-cirrhotic patients treated with NA more than 1 year, HBV DNA $<10^{2}$ copies/mL during the NA treatment period. $\mathrm{HBsAg}$ seroconversion is define as $\mathrm{HBsAg}<0.05 \mathrm{IU} / \mathrm{mL}$, antiHBs $>10 \mathrm{mIU} /$ $\mathrm{mL}$. HBsAg loss is define as HBsAg $<0.05 \mathrm{IU} / \mathrm{mL}$, antiHBs $<10$ $\mathrm{mIU} / \mathrm{mL}$. Virological relapse is define as HBV DNA $\geq 10^{5}$ copies $/ \mathrm{mL}$ in $\mathrm{CHB}$ patients with $\mathrm{HBeAg}(+)$ and $\mathrm{HBV}$ DNA $\geq 10^{4}$ copies $/ \mathrm{mL}$ in $\mathrm{CHB}$ patients with $\mathrm{HBeAg}(-)$. $\mathrm{HBsAg}, \mathrm{HBeAg}$ were quatified by Abbott Architect assay. HBV DNA was determined by real-time PCR (detectable limit $\geq 10^{2}$ copies $/ \mathrm{mL}$ ). The patients have been switched to peginterferon alpha-2a $180 \mu \mathrm{g}$ once per week.

Results: 23 patients were enrolled. 13 (56.5\%) Pts with $\mathrm{HBeAg}(+)$, $10(43.5 \%)$ Pts with $\mathrm{HBeAg}(-)$. Median time of NA treatment was 43 months (range 12-132). At the moment, there were $6(26 \%)$ Pts with HBsAg loss, 4 among them had antiHBs $>10 \mathrm{mIU} / \mathrm{mL}, 6(26 \%)$ Pts had decrease of HBsAg levels $1 \log _{10}(\mathrm{IU} / \mathrm{mL}), 8$ (34.8\%) Pts had decrease of HBsAg levels $2 \log _{10}(\mathrm{IU} / \mathrm{mL})$ and 5 among them had HBsAg loss, 2(8.7\%) Pts had decrease of HBsAg levels $3 \log _{10}$ (IU/ $\mathrm{mL}$ ) and one of them had HBsAg loss. 6(26\%) Pts had the virological relapse.

Conclusions: High rate $\mathrm{HBsAg}$ loss and decrease of HBsAg levels were observed in $\mathrm{CHB}$ patients having virological response to longterm NAs treatment then switched to PEG-IFN $\alpha$ 2a therapy. The decrease intrahepatic HBV DNA and cccDNA levels by long-term NAs treatment could explain for these very interesting results. The results need to be confirmed.

\section{PP05-98}

Initial Experience of Combination Therapy with Pegylated Interferon and Entecavir in Patients with Chronic Hepatitis B in Bangladesh

M. Mahtab ${ }^{1}$, F. Akbar ${ }^{2}$, S. Rahman ${ }^{1}$

${ }^{1}$ Hepatology, Bangabandhu Sheikh Mujib Medical University, Dhaka, Bangladesh; ${ }^{2}$ Medical Sciences, Toshiba General Hospital, Tokyo, Japan

Background/aims: Latest data shows that pegylated interferon (PegIFN) is associated with $30-40 \%$ end of treatment response (ETR) in chronic hepatitis B (CHB). The aim of this study was to assess if addition of entecavir (ETV) to Peg-IFN at baseline improves the ETR. Methods: 22 patients with $\mathrm{CHB}$ were enrolled. 16 had completed treatment to-date. In $\mathrm{HBeAg}+$ patients, $\mathrm{HBV}$ DNA was $10^{5}$ $10^{11}$ copies $/ \mathrm{ml}$ and in HBeAg- $10^{2}-10^{9}$ copies $/ \mathrm{ml}$. $12 / 16 ; 75 \%$ had $\mathrm{HBeAg}+$ and $4 / 16 ; 25 \% \mathrm{HBeAg}-\mathrm{CHB}$. Dose of Peg-IFN was 180 $\mu \mathrm{gm} /$ week in $10 / 16 ; 62.5 \%$ and $90 \mu \mathrm{gm} /$ week in $6 / 16 ; 37.5 \%$. All received ETV $0.5 \mathrm{mg} /$ day.

Results: In the $180 \mu \mathrm{gm} /$ week group, $5 / 8 \mathrm{HBeAg}+$ and $2 / 2 \mathrm{HBeAg}-$ patients had undetectable HBV DNA $(<50$ copies $/ \mathrm{ml})$ at end of treatment. $2 \mathrm{HBeAg}+$ patients had 7log and 1 had 4log HBV DNA reduction. On the other hand, in the $90 \mu \mathrm{gm} /$ week group, 2/4 HBeAg+ patients had undetectable DNA. Other 2 had $4 \log$ and $8 \log$ reduction. In both $\mathrm{HBeAg}-$ patients, HBV DNA become undetectable. Reduction in dose of Peg-IFN did not compromise the outcome.

Conclusions: The study shows that combination of Peg-IFN and ETV improves ETR in both $\mathrm{HBeAg}+$ and $\mathrm{HBeAg}-\mathrm{CHB}$ patients. However long-term follow-up with larger number of patients is required to see whether this response is sustained or not, before a 'combination' regimen with reduced dose of Peg-IFN can be recommended.

\section{PP05-99}

Tenofovir Disoproxil Fumarate (TDF) Shows Similar Virologic Suppression and Safety between Asians and Non-Asians with Chronic Hepatitis B (CHB)

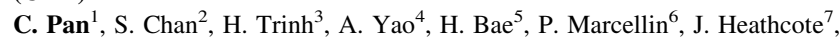
J. Anderson ${ }^{8}$, E. Mondou ${ }^{8}$, D. Coombs ${ }^{8}$, L. Lou ${ }^{8}$, B. Chiang ${ }^{8}$, E. Fagan ${ }^{8}$

${ }^{1}$ Mount Sinai Services at Elmhurst Hospital, Mount Sinai School of Medicine, New York; ${ }^{2}$ Private Office of Sing Chan MD, Flushing, NY;

${ }^{3}$ San Jose Gastroenterology, San Jose, CA; ${ }^{4} A E \& L Y$ Medical 
Associates, Flushing, NY; ${ }^{5}$ St. Vincent Medical Center, Los Angeles, CA, USA; ${ }^{6}$ Hopital Beaujon, University of Paris, Clichy, France; ${ }^{7}$ Toronto Western Hospital, University of Toronto, Toronto, ON, Canada; ${ }^{8}$ Gilead Sciences, Inc., Foster City, CA, USA

Background: Two TDF registration trials in CHB (127 Asian and 299 non-Asian patients) and an open-label trial (90 Asians) were analyzed in a cross sectional study to compare efficacy and safety of TDF in Asians versus non-Asians during the first 48 weeks (w) of treatment.

Methods: Eligibility criteria were similar between registration versus open-label trials except for HBVDNA $\geq 10^{5}$ versus $\geq 10^{4}$ copies(c)/ $\mathrm{mL}$ and ALT $>2 \mathrm{x}$ vs. $>1 \mathrm{x}$ ULN (HBeAg+ patients), respectively. All three trials were analyzed independently and compared side-by-side. Subsequently, 217 Asians were combined, and compared with 299 non-Asians stratified for $\mathrm{HBeAg}$ status.

Results: $102 \mathrm{HBeAg}$ - and $115 \mathrm{HBeAg}+$ Asians were compared with 186 and 113 non-Asians, respectively. Baseline (BL) characteristics were similar except for mean weight, height and BMI. $\mathrm{HBeAg}-$ Asians and non-Asians had mean BL-HBVDNA of 6.5 and $6.9 \log _{10} \mathrm{c} /$ $\mathrm{mL}$ which decreased by $-2.9 \log _{10}$ (both) at 4 weeks; 96 and $98 \%$ reached $<400 \mathrm{c} / \mathrm{mL}$ at 48 weeks, respectively. $\mathrm{HBeAg}+$ Asians and non-Asians had mean BL-HBVDNA of 8.4 and $8.9 \log _{10} \mathrm{c} / \mathrm{mL}$ which decreased by -3.1 and $-3.0 \log _{10}$ at 4 weeks; 83 and $79 \%$ reached $<400 \mathrm{c} / \mathrm{mL}$ at 48 weeks, respectively. Percentage of normal ALT at BL was $17 / 9$ versus $6 / 4 \%$ (HBeAg-/+) for Asians versus non-Asians, and increased to $78 / 71$ versus $81 / 74 \%$, respectively, at 48 weeks. Loss/ seroconversion for $\mathrm{HBeAg}$ and $\mathrm{HBsAg}$ at 48 weeks were lower for Asians versus non-Asians; $15 / 14$ versus $26 / 24 \%$, and $0 / 0$ vs. $4.5 / 1.8 \%$, respectively. No patient developed resistance to TDF. Mean creatinine clearances were stable and maintained within the normal range. No patient had a confirmed $\geq 0.5 \mathrm{mg} / \mathrm{dL}$ increase in serum creatinine or decrease to $<50 \mathrm{~mL} / \mathrm{min}$ in creatinine clearance from BL.

Conclusion: TDF demonstrated similar ALT normalization, rapid virologic suppression, and good safety profile in Asian and non-Asian CHB patients. No resistance to TDF developed in 48 weeks.

\section{PP05-100}

The Role Atopy and Interferon Treatment in the Development of Non-organ Specific Autoantibodies in Patients with Chronic Hepatitis B V. Csibova ${ }^{1}$, M.R. Pijak ${ }^{2}$

${ }^{1}$ University Hospital; ${ }^{2}$ Slovak Medical University, Bratislava, Slovak Republic

Background: We have previously reported that atopy can contribute to the persistence of chronic hepatitis B virus infection (CHB) and paradoxically, it may be a strong predictor of good therapeutic response in patients (pts) with a wild-type $\mathrm{CHB}(1)$. Accordingly, we sought to determine if atopy may influence the frequency of nonorgan specific autoantiboidies (NOSA) in pts with CHB treated with interferon alpha.

Methods: We retrospectively studied 38 adult pts $(32 \mathrm{M}, 6 \mathrm{~F}$, average age of 41 years) with $\mathrm{CHB}$ in a replicative phase (HBV DNA $\geq 2,000 \mathrm{IU} / \mathrm{ml}$ ). The presence of NOSA was tested by indirect immunofluorescence (IF) on rat, liver and kidney sections at 1:100 dilution. ANA positive sera were further characterized on HEp- 2 cells by IF at the same dilution. The atopic status was determined on the basis of 1 or more positive skin prick tests from a panel consisting of 7 aeroallergens.

Results: Atopy was present in $7 / 38$ (18.4\%) of all pts, in $6 / 17(35.2 \%)$ of $\mathrm{HBeAg}$ positive pts and $1 / 21(4.8 \%)$ of $\mathrm{HBeAg}$ negative pts. The frequency of ANA before treatment was $1 / 38(2.6 \%)$ and after treatment $8 / 38(21 \%)$ of all pts $(P=0.016)$. The frequency of ASMA before treatment was $4 / 38(10.5 \%)$ and after treatment 10/38 (26.3\%) of all pts $(P=0.031)$. In pts with atopy compared with pts without atopy the differences between ANA and ASMA were not significant.
No patient developed NOSA specifically associated with autoimmune liver disease such as LKM type-1, SLA and AMA.

Conclusions: These results indicate that the development of NOSA is rather due to IFN treatment than to HBV infection. Further studies on a larger group of pts are warranted to examine, if concomitant disease with predominant Th2 cytokine pattern, such as atopy, might significantly influence the formation of NOSA in patients with CHB treated with IFN alpha.

References: 1. Pijak MR et al. Hepatology Int 2007;1:O-143

\section{PP05-101}

Tenofovir Disoproxil Fumarate (TDF) Demonstrates Good Efficacy and Safety in Asians with Chronic Hepatitis B (CHB) in Real-life Settings S. Chan ${ }^{1}$, C. $\mathrm{Pan}^{2}$, H. Bae ${ }^{3}$, A. Yao ${ }^{4}$, H. Trinh ${ }^{5}$, T.-S. Leduc ${ }^{6}$, C. Hyun ${ }^{7}$, S. Wang ${ }^{8}$, J. Li ${ }^{9}$, X. Ma ${ }^{10}$, S.V. Tran ${ }^{11}$, L.-J. Mi ${ }^{12}$, M. Li ${ }^{13}$, S.S. Wu ${ }^{14},{\mathrm{M} . \mathrm{Li}^{15}}^{15}$, J. $\mathrm{Li}^{16}$, M. Kim ${ }^{17}$, D. Wu ${ }^{18}$, W. Jing ${ }^{19}$, D. Coombs ${ }^{20}$, A. Snow-Lampart ${ }^{20}$,

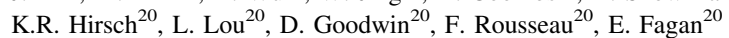

${ }^{1}$ Private Office of Sing Chan MD, Flushing; ${ }^{2}$ Mount Sinai Services at Elmhurst Hospital, Mount Sinai School of Medicine, New York, NY; ${ }^{3}$ St. Vincent Medical Center, Los Angeles, CA; ${ }^{4} A E \&$ LY Medical Associates, Flushing, NY; ${ }^{5}$ San Jose Gastroenterology, San Jose;

${ }^{6}$ Leduc Medical Group, Inc., Fountain Valley, CA; ${ }^{7}$ C.S.Hyun Clinic, Englewood, NJ; ${ }^{8}$ Charles B. Wang Community Health Center, New York; ${ }^{9}$ Maimonides Medical Center, Brooklyn, NY; ${ }^{10}$ Xiaoli Ma, MD, Philadelphia, PA; ${ }^{11}$ Sang Tran, MD, Falls Church, VA; ${ }^{12}$ New York Downtown Hospital, New York; ${ }^{13}$ Michael Li, MD, PLLC, Flushing, NY; ${ }^{14}$ Steven S.C. Wu, MD, Hacienda Heights, CA; ${ }^{15}$ Mark Li, MD, Silver Springs, MD; ${ }^{16}$ Palo Alto Medical Foundation, Mountain View, CA; ${ }^{17}$ Fairfax Family Medical, Fairfax, VA; ${ }^{18}$ Northern CA Gastroenterology Consultants, Oakland, CA; ${ }^{19}$ Wuhua Jing, MD, PhD, PC, New York, NY; ${ }^{20}$ Gilead Sciences, Inc., Foster City, CA, USA

Aim: To evaluate efficacy and safety of TDF in Asian adults with CHB.

Methods: 90 Asians were enrolled in an open-label, single-arm 48week (W48) study. Key entry criteria included HBV-DNA $\geq 10^{4}$ copies/mL; ALT $>1 \times$ and $\leq 10 \times \mathrm{ULN}$; naïve to TDF. At W48, patients could transition to commercially available CHB therapy.

Results: There were 47 males $(52 \%) .13(14 \%)$ were treatmentexperienced including lamivudine $(3 \%)$, adefovir $(7 \%)$ and interferons $(6 \%)$. Baseline mean \pm SD HBV-DNA was $7.48 \pm 1.77 \log _{10}$ copies $(\mathrm{c}) / \mathrm{mL}$. Patients were genotype B $(48 \%)$ or C $(52 \%) ; 59 \%$ $\mathrm{HBeAg}+$. At baseline, $74 \%$ had ALT $>$ ULN with mean of $103 \mathrm{U} / \mathrm{L}$. None had genotypic resistance mutations at baseline. All had HBVDNA decrease during the study. At W48, 82\% had HBV-DNA $<400$ copies $/ \mathrm{mL}(70 \% \mathrm{HBeAg}+, 100 \% \mathrm{HBeAg}) .60 \%$ with elevated baseline ALT reached normal range at W48. Six (11\%) of $53 \mathrm{HBeAg}+$ patients had HBeAg seroconversion by W48. FibroTest fibrosis scores were calculated based on age, sex and 5 laboratory parameters. Among $\mathrm{HBeAg}+$ patients, percentage of $\mathrm{F} 0$ stage increased from 60 to $67 \%$ (baseline to W48), and percentage of F3/F4 decreased from 4 to $2 \%$. Among $\mathrm{HBeAg}$ - patients, percentage of F0 decreased from 30 to $29 \%$ (BL to W48), but percentage of $\mathrm{F} 4$ decreased from 5 to $0 \%$. Genotypic analysis of HBV pol/RT was conducted on 14 patients with $\geq 400$ copies/mL at W48; no resistant mutation to TDF was identified. No subject had a marked laboratory abnormality, on-treatment hepatic flare, confirmed $\geq 0.5 \mathrm{mg} / \mathrm{dL}$ increase in serum creatinine, or confirmed creatinine clearance $<50 \mathrm{~mL} / \mathrm{min}$. 1 patient had a confirmed serum phosphorus $<2.0 \mathrm{mg} / \mathrm{dL}$ (not associated with creatinine increase).

Conclusion: This Asian study performed in HBV-infected patients confirms that, TDF is safe and potent in Asian CHB patients with $>80 \%$ achieving HBV-DNA $<400 \mathrm{c} / \mathrm{mL}$ by $\mathrm{W} 48$ and improvement of FibroTest fibrosis score. 
PP05-102

Serum HBsAg Quantification Can Predict Sustained Response to Lamivudine in Patients with Negative HBeAg: a Long-term Post-treatment Follow-up Study

H.L.-Y. Chan, G.L.-H. Wong, A.M.-L. Chim, H.-Y. Chan, S.H.-T. Chu, V.W.-S. Wong

Department of Medicine and Therapeutics, The Chinese University of Hong Kong, Hong Kong, Hong Kong S.A.R.

Background/aims: Nucleot(s)ide analogs provide potent viral suppression but the timing to stop treatment is controversial. We aimed to investigate the role of on-treatment hepatitis B virus (HBV) DNA and hepatitis B surface antigen ( $\mathrm{HBsAg}$ ) monitoring to predict the offtreatment sustained response in hepatitis $\mathrm{B}$ e antigen $(\mathrm{HBe} A g)$ negative patients.

Methods: HBeAg-negative chronic hepatitis B patients who had continuous lamivudine treatment for at least 12 months and had post-treatment follow-up for at least 12 months in previous clinical studies were studied. The primary outcome was sustained response, defined as HBV DNA $\leq 200 \mathrm{IU} / \mathrm{ml}$, at month 12 post-treatment (SR12).

Results: Fifty-three HBeAg-negative chronic hepatitis B patients received lamivudine for $34 \pm 23$ (12-76) months and had lamivudine stopped for $47 \pm 35$ (1-116) months. Nine (17\%) patients achieved SR-12. HBV DNA at baseline, month 6 and end of treatment had no association with SR-12. HBsAg level tend to reduce more dramatically during treatment among SR-12 responders. At the end of treatment, both $\mathrm{HBsAg} \leq 2 \mathrm{log} \mathrm{IU} / \mathrm{ml}$ and reduction by $>1 \log$ from baseline had sensitivity, specificity, positive and negative predictive values of $78,96,78$ and $96 \%$ for SR-12, respectively. All 5 patients with HBsAg reduction by $>1 \log$ to $\leq 2 \log \mathrm{IU} / \mathrm{ml}$ at the end of treatment achieved SR-12 and all 40 patients with $\mathrm{HBsAg}>2 \mathrm{log} \mathrm{IU} / \mathrm{ml}$ and reduction $\leq 1 \log$ did not have SR-12. The cumulative probability of sustained response and HBsAg clearance at 5 years among patients with HBsAg $\leq 2 \log$ IU/ $\mathrm{ml}$ were 88 and $72 \%$; and that among patients with $\mathrm{HBsAg}$ reduction $>1 \log$ were 74 and $61 \%$, respectively.

Conclusions: Monitoring of HBsAg level can guide the timing of stopping lamivudine in $\mathrm{HBeAg}$-negative chronic hepatitis B.

Acknowledgement: This work was supported by Research Fund for the Control of Infectious Diseases (RFCID) grant (08070242) by Food and Health Bureau, Hong Kong.

\section{PP05-103}

Very Early HBsAg Seroconversion in Chronic Hepatitis B with Peginterferon Alpha 2a Monotherapy

I. Koruk, M. Aydinli, M. Koruk

Gastroenterology, Gaziantep University Medical School, Gaziantep, Turkey

In chronic hepatitis B (CHB) aim of the therapy is to suppress the virus replication and the seroconversion of HBsAg. Nucleot(s)ide analogues or interferon(s) can be used as mono/combined therapy. We report a case of $\mathrm{HBeAg}$ negative $\mathrm{CHB}$ and his very early response to peginterferon alpha 2a monotherapy.

Case: 46-year-old male patient with a prior history of $\mathrm{HBsAg}$ positivity for 15 years is presented with elevated serum ALT levels. In his presentation the HBsAg was positive, ALT was $88 \mathrm{U} / \mathrm{ml}$ and HBV-DNA was $600,000 \mathrm{U} / \mathrm{ml}$. HBeAg was negative. The liver biopsy showed chronic changes (histologic activity was 6/18 and fibrosis score was 3/6 modified Ishak score).Pegylated interferon alpha2a $180 \mathrm{mcg} /$ week monotherapy was started. On 8th week HBV-DNA was undetectable. On the 24th week serum anti HBsAb titration was $5 \mathrm{U} / \mathrm{ml}$ and was elevated to $35 \mathrm{U} / \mathrm{ml}$ on week 28 . The changes in ALT, DNA, HBsAg and AntiHBsAb titrations were shown in Fig. 1.

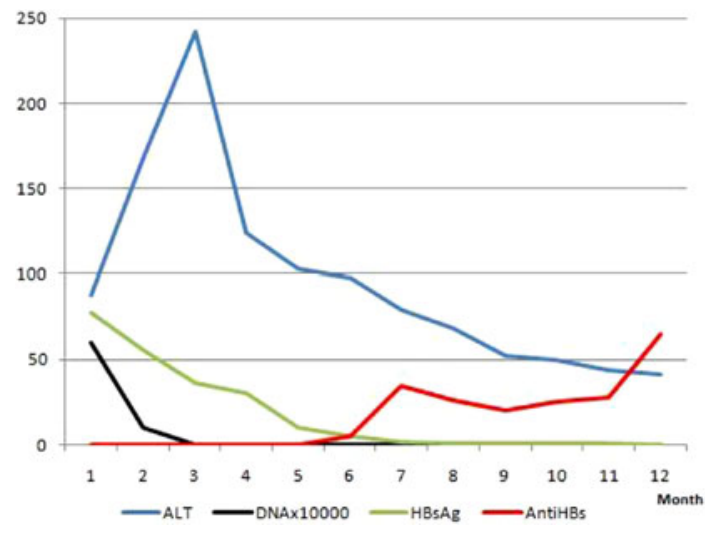

At the end of the treatment serum ALT levels returned to normal ranges, HBV-DNA and HBsAg were negative, AntiHBsAb was positive. At the end of the first year follow-up period serum ALT was normal and AntiHbsAb was positive.

Discussion: HBsAg seroconversion is defined with pegylated interferons for both during and after the treatment period. In our case we achieved the HBsAg seroconversion at a very early period of the treatment. Elevations of ALT levels during the treatment may reflect the immune-clearance of the hepatitis B virus and this may predict the good response to the treatment.

\section{PP05-104}

Evaluation of Lamivudine Effect on Prevention of Hepatocellular Carcinoma Recurrence

K.-F. Liao ${ }^{1}$, S.-W. Lai ${ }^{2}$, C.Y. Lin ${ }^{1}$, C.H. Huang ${ }^{1}$, Y.C. Chu ${ }^{1}$, Y.Y. Lin ${ }^{1}$ ${ }^{I}$ Gastroenterology and Hepatology, Buddhist Tzu Chi General Hospital, Taichung Branch; ${ }^{2}$ Family Medicine, China Medical University Hospital, Taichung, Taiwan, ROC

Background: The purpose of this study was to explore the effect of lamivudine treatment on prevention of hepatocellular carcinoma (HCC) recurrence in Taiwan.

Methods: This was a hospital-based restrospective study. We analyzed the medical records of all the subjects with HCC initially treated by hepatic resection who were hepatitis $\mathrm{B}$ surface antigen (HBsAg) positive and hepatitis $\mathrm{C}$ antibody positive at one medical center in Taiwan from 2000 to 2008. In all, 7 subjects received 100 $\mathrm{mg}$ /day lamivudine after hepatic resection (lamivudine group) and 39 subjects only receive hepatic resection (control group). The overall follow-up period was 52 weeks.

Results: The cumulative recurrence rates of $\mathrm{HCC}$ at 26 and 52 weeks in the control group were 33.3 and $53.8 \%$, respectively, while those in the lamivudine group were 28.6 and $42.9 \%$, respectively. There were no significant differences regarding the recurrence rates of HCC between two groups $(P=0.82)$.

Conclusion: Lamivudine therapy seems to be not effective on prevention of HCC recurrence after resection of primary tumor. A study with larger sample size and longer follow-up time is needed to confirm whether antiviral drugs can improve the recurrence rates.

\section{PP05-105}

Correlation of Serum HBsAg and HBeAg Levels with Outcome during 5 Years Adefovir Dipivoxil Treatment in HBeAg Positive CHB Patients Y. Mao ${ }^{1}$, M. Zeng ${ }^{1}$, H. Ren ${ }^{2}$, D. $\mathrm{Xu}^{3}$

${ }^{1}$ Shanghai Renji Hospital, Shanghai; ${ }^{2}$ Chongqing Medical University 2nd Affliated Hospital, Chongqing; ${ }^{3}$ Beijing Ditan Hospital, Capital Medical University, Beijing, China

Background: $\mathrm{HBs} A g$ clearance and $\mathrm{HBeAg}$ seroconversion are key goals of therapy in $\mathrm{CHB}$ patients. This study aimed to determine the 
correlation of $\mathrm{HBsAg}$ and $\mathrm{HBeAg}$ with long term outcome during ADV treatment.

Table 1 Correlation of the clinical characteristics with HBeAg loss and HBV DNA nudetectaable at year 5

\begin{tabular}{|c|c|c|c|c|c|c|}
\hline \multirow[t]{2}{*}{$\begin{array}{l}\text { Variable } \\
\text { studied }\end{array}$} & \multicolumn{3}{|l|}{$\begin{array}{l}\mathrm{HBeAg} \\
\text { loss }\end{array}$} & \multicolumn{3}{|c|}{$\begin{array}{l}\text { HBV } \\
\text { DNA } \\
\text { undetectable }\end{array}$} \\
\hline & $\begin{array}{l}\text { With } \\
\text { HBeAg } \\
\text { loss }\end{array}$ & $\begin{array}{l}\text { Without } \\
\text { HBeAg } \\
\text { loss }\end{array}$ & $P$ value & $\begin{array}{l}\text { HBV } \\
\text { DNA } \\
(-)\end{array}$ & $\begin{array}{l}\text { HBV } \\
\text { DNA } \\
(+)\end{array}$ & $P$ value \\
\hline $\begin{array}{l}\text { Baseline } \\
\text { HBV } \\
\text { DNA } \\
\log _{10} \\
\text { coples/ml }\end{array}$ & 8.96 & 8.80 & 0.783 & 8.96 & 8.81 & 0.871 \\
\hline $\begin{array}{l}\text { Baseline } \\
\text { HBeAg } \\
\text { PEIG/ml }\end{array}$ & 637.68 & 457.78 & 0.935 & 778.30 & 404.82 & 0.386 \\
\hline $\begin{array}{c}\text { Baseline } \\
\text { HBsAg } \\
\text { IU/ml }\end{array}$ & 14433.97 & 22339.07 & 0.249 & 22339.07 & 11404.15 & 0.371 \\
\hline $\begin{array}{l}\text { Weeks } \\
28 \mathrm{HBV} \\
\text { DNA } \log _{10} \\
\text { copies/ml }\end{array}$ & 3.96 & 5.54 & 0.051 & 3.85 & 5.54 & 0.017 \\
\hline $\begin{array}{c}\text { Weeks } 28 \\
\mathrm{HBsAg} \\
\mathrm{IU} / \mathrm{ml}\end{array}$ & 4293.05 & 7922.72 & 0.129 & 5619.57 & 6538.45 & 1.000 \\
\hline
\end{tabular}

*All data shown as median

Methods: Fifty China HBeAg positive CHB patients who participated in a 1-year $A D V$ registration trial(ADV $10 \mathrm{mg}$ daily) were offered continuation for a further 4 years. HBsAg, HBeAg and HBV DNA were analyzed at baseline, weeks 12, 28, 40, 52, 104, 156, 208 and 260 by Abbott Architect HBsAg Assay, Roche Cobas HBeAg Assay and PCR respectively.

Results: ADV treatment leads to rapid decline of both $\mathrm{HBeAg}$ (89\% decline) and HBsAg ( $74 \%$ decline) in the first 12 weeks. Both baseline HBeAg $\left(\mathrm{r}^{2}=0.419, P=0.002\right)$ and HBsAg $\left(\mathrm{r}^{2}=0.627\right.$, $P<0.001)$ were correlated with baseline HBV DNA. However, neither of them related to 5 years response. $\mathrm{HBeAg}$ loss rate presented a significant correlation with $\mathrm{HBeAg}$ levels at weeks $28(P=0.030)$, but not related to other parameters (Table 1). Significantly more patients $(78.9 \%)$ with low HBeAg levels $(<100$ PEIU/ml) than patients with intermediate (100-700 PEIU/ml, 10.5\%) and high $\mathrm{HBeAg}$ levels (>700 PEIU/ml, 10.5\%) achieved HBeAg loss at year 5. HBV DNA negativity presented a significant correlation with HBV DNA levels at weeks $28(P=0.017)$, but not related to other parameters (Table 1).

Conclusion: ADV treatment leads to rapid decline of both $\mathrm{HBeAg}$ and HBsAg levels. HBeAg loss rate and HBV DNA negativity at year 5 presented a positive correlation with $\mathrm{HBeAg}$ levels and HBV DNA at weeks 28 respectively, but there was no significant relation to HBsAg levels at baseline or weeks 28 .

\section{PP05-106}

Quantitative Serum HBsAg and HBV DNA Kinetics during Peginterferon Alfa-2a Therapy in Genotype B versus C HBeAg-positive Chronic Hepatitis B

C.-Y. Peng, H.-C. Lai, W.-P. Su, P.-H. Chuang, J.-T. Kao

Division of Hepatogastroenterology, Department of Internal Medicine, China Medical University Hospital, Taichung, Taiwan, ROC

Aim: To compare the kinetics of serum HBsAg and HBV DNA levels during peginterferon alfa-2a therapy in genotype $\mathrm{B}$ versus $\mathrm{C} \mathrm{HBeAg}-$ positive chronic hepatitis $\mathrm{B}$ patients.
Methods: One hundred and three patients were enrolled. Serum HBsAg (Abbott Architect HBsAg QT assay) and HBV DNA levels (Cobas Amplicor HBV Monitor Test) were quantified at baseline, 12, 24 , and 48 weeks during treatment and at 24 weeks after treatment. Results: The two groups (genotype $\mathrm{B}, n=64$ vs. $\mathrm{C}, n=39$ ) were similar in baseline characteristics (age $33.0 \pm 7.5$ vs. $35.5 \pm 10.6$; gender: $38 \mathrm{M} / 26 \mathrm{~F}$ vs. $28 \mathrm{M} / 11 \mathrm{~F}$; serum ALT: $122.2 \pm 79.6$ vs. $120.3 \pm 59 \mathrm{IU} / \mathrm{L} ; \log _{10}$ HBsAg: $3.83 \pm 0.53$ vs. $3.57 \pm 0.70 \mathrm{IU} / \mathrm{mL}$; $\log _{10}$ HBV DNA: $8.46 \pm 1.11$ vs. $8.22 \pm 1.14$ copies $/ \mathrm{mL}$ ). At 12,24 , and 48 weeks during treatment, and 24 weeks after treatment, serum $\log _{10}$ HBsAg and $\log _{10}$ HBV DNA declines from baseline are shown in Table 1. Therapeutic efficacy (HBeAg loss with HBV DNA $<10^{5}$ copies $/ \mathrm{mL}$ ) was similar between the two genotypes

Table 1 Serum HBsAg and HBV DNA kinetics

\begin{tabular}{llll}
\hline & $\begin{array}{l}\text { Genotype B } \\
(n=64)\end{array}$ & $\begin{array}{l}\text { Genotype C } \\
(n=39)\end{array}$ & $P$ value \\
\hline TW12 $\log$ HBsAg reduction & $0.25 \pm 0.57$ & $0.14 \pm 0.58$ & 0.33 \\
TW24 $\log$ HBsAg reduction & $0.37 \pm 0.68$ & $0.37 \pm 0.91$ & 0.99 \\
TW48 $\log$ HBsAg reduction & $0.55 \pm 1.11(28)$ & $0.50 \pm 1.18(17)$ & 0.88 \\
P-W24 $\log$ HBsAg reduction & $0.27 \pm 0.77$ & $0.13 \pm 0.72$ & 0.35 \\
TW12 $\log$ HBV DNA reduction & $2.13 \pm 1.63$ & $1.76 \pm 1.34$ & 0.24 \\
TW24 $\log$ HBV DNA reduction & $2.69 \pm 1.95$ & $2.08 \pm 1.97$ & 0.13 \\
TW48 $\log$ HBV DNA reduction & $2.84 \pm 2.05(28)$ & $3.28 \pm 2.57(17)$ & 0.52 \\
P-W24 $\log$ HBV DNA reduction & $2.18 \pm 2.10$ & $1.39 \pm 2.27$ & 0.07 \\
\hline
\end{tabular}

Conclusions: Although statistically insignificant, serum HBsAg and HBV DNA levels tended to decline more in genotype B than C early during therapy but their levels of decline were similar across genotypes later during therapy. Serum HBsAg and HBV DNA levels tended to rebound to a greater extent in genotype $C$ than $B$ after cessation of therapy.

\section{PP05-107}

An Early Decrease in Serum HBeAg Titer Is a Strong Predictor of Virological Response to Entecavir in HBeAg-positive Patients

X. Zhang, S. Lin, F. Ye, T. Chen, M. Liu, Y. Chen, S. Zheng, W. Wang, S. Zhang

Department of Infectious Diseases, The First Affiliated Hospital of Medical College of Xi'an Jiaotong University, Xi'an, China

Background and aim: Quantification of HBeAg levels has been found to be useful in monitoring and predicting the outcomes of interferon and lamivudine treatment in $\mathrm{HBeAg}$-positive patients. The aim of this study was to determine whether quantitation of $\mathrm{HBeAg}$ at baseline and on treatment could predict which patients would achieve $\mathrm{HBe} A g$ seroconversion at week 96 of entecavir therapy.

Methods: Sixty-five HBeAg-positive naïve chronic hepatitis B patients who were treated with entecavir at a dose of $0.5 \mathrm{mg}$ once daily for 96 weeks were evaluated. Serum HBV DNA levels were assessed at baseline, week 24, 48 and 96; serum $\mathrm{HBeAg}$ levels were assessed at baseline, week 12, 24, 48, 72 and 96.

Results: Serum $\mathrm{HBeAg}$ levels were associated with a higher likelihood of $\mathrm{HBeAg}$ seroconversion to entecavir at weeks 96 than serum HBV DNA levels both at baseline and on treatment (at baseline: OR 9.932, $P=0.003$ versus $\mathrm{OR} 5.045, P=0.036$; on treatment: OR $112.5, P<0.0001$ versus $\mathrm{OR} 47.782, P<0.0001)$. A maintained reduction of $\mathrm{HBeAg}>65 \%$ of pretreatment $\mathrm{HBeAg}$ values after 24 weeks treatment with entecavir was the strongest predictor for $\mathrm{HBeAg}$ seroconversion at weeks 96 (OR 70.578, $P<0.0001$ ).

Conclusions: Quantitation of $\mathrm{HBeAg}$ at the start and early during therapy showed a higher predictive value than that of HBV DNA for $\mathrm{HBeAg}$ seroconversion by entecavir. A significant decrease of serum 
$\mathrm{HBeAg}$ levels at week 24 may be an useful on-treatment measurement in the early phase for predicting $\mathrm{HBeAg}$ seroconversion and identifying patients who will most likely benefit from finite entecavir treatment.

\section{PP05-108}

A 96-weeks Study of Antiviral Therapy for Patients with HBV-related Cirrhosis

J. Yu-Sheng, X. Qi-Huan, S. Xin, C. Lu-Biao, C. Yu-Tian, X. Qi-Feng,

L. Gang

Infectious Diseases, Third Affiliated Hospital of Sun Yat-sen

University, Guangzhou, China

Introduction: Previous researches have demonstrated that the cirrhotic patients with active $\mathrm{HBV}$ replication are at high risk of developing liver failure and hepatocellular carcinoma (HCC). The study was to investigate the efficacy and safety of nucleot(s)ide analogues therapy in patients with HBV-related cirrhosis in China.

Methods: 140 patients with HBV-related cirrhosis were divided into antiviral group (76 cases, 33 entecavir, 25 adefovir dipivoxil, 15 lamivudine, 3 telbivudine) and control group (64 cases, supportive and symptomatic treatment). These two groups were matched for age, gender, and Child-Pugh score. But the baseline HBV DNA level of antiviral group was higher than that of control group $(t=2.917$, $p=0.004$ ).

Results: At the 96th week, the rate of ALT normalization in antiviral group was higher than that in control $\operatorname{group}\left(\mathrm{x}^{2}=5.260, p=0.022\right)$. The level of serum TBil was lower than that in control group $(t=$ $-2.335, p=0.021)$. HBV DNA drop $(\lg$ copies $/ \mathrm{ml})$ in antiviral group was more remarkable than that in control group $(t=-10.626$, $p=0.001)$. The rates of HBV DNA negative $(<500$ copies $/ \mathrm{ml})$ in antiviral group and control group were $77.8 \%(56 / 72)$ and $20.0 \%(11 /$ $58)$, respectively $\left(\chi^{2}=44.484, p=0.001\right) .2 .6 \%(2 / 76)$ patients in antiviral group and $9.4 \%(6 / 64)$ patients in control group developed HCC(Fisher's exact test, $p=0.142) .13 .2 \%(10 / 76)$ and $17.2 \%(11 /$ 64) underwent variceal hemorrhage, respectively $\left(\chi^{2}=0.442\right.$, $p=0.056)$. The cumulative survival rate was no significant difference between these two groups $(p=0.351)$. YMDD mutations were determined in 5 of $15(33.3 \%)$ patients with lamivudine treatment. 1 of these 5 patients died of liver failure at the 72th week, 2 received an "add-on" antiviral therapy with adefovir dipivoxil, and the other 2 were switched to entecavir therapy.
Conclusions: Nucleot(s)ide analogues are effective for HBV-related cirrhotic patients. However, prolonged observation should be done to investigate their influences on the survival and complications of cirrhosis. Viral mutation and drug resistance must be monitored to prevent the acute exacerbation of liver function during the antiviral therapy.

\section{PP05-109}

The Comparison of the Efficacy of Pegylated Interferon Alpha-2a and Alpha-2b in Chronic Hepatitis B Patients

Ü.B. Dogan, B. Kara, M.S. Akın

Gastroenterology, Adana Numune Training and Research Hospital, Adana, Turkey

Background/aim: Although 48-week therapy with pegylated-interferons has been shown to be effective for the treatment of chronic hepatitis $\mathrm{B}(\mathrm{CHB})$ virus infection, the comparison of the efficacy of pegylated interferon alpha-2a (PEG-2a) and alpha-2b (PEG-2b) in the therapy is not obvious.

Method: We reviewed 43 chronic hepatitis B patients treated with 48 weeks of PEG-2a and PEG-2b. Serum hepatitis B virus DNA (HBVDNA), HBeAg and HBsAg were assessed at baseline, during treatment (weeks 12th, 24th, and 48th), and at the 72th week to define the sustained virological response (SVR). Virological response was defined as serum HBV-DNA $<10.000$ copies $/ \mathrm{ml}$ and a normal alanine aminotransferase level.

Results: At the end-of-therapy, mean HBV-DNA level and virological response were similar between the groups. At week 72, 4 of the 16 patients $(25 \%)$ treated with PEG-2a compared with 8 of the 27 patients $(29.63 \%)$ treated with PEG-2b had sustained virological response $(p=0.75)$. HBeAg seroconversion occurred in 3 patients $(11 \%)$ in only PEG-2b group. Rate of patients having undetectable HBV-DNA ( $<400$ copies/ml) 24 weeks after a 48-week course of therapy was $18.8 \%$ for PEG-2a and $22.2 \%$ for PEG-2b and was not statistically different. Unexpectedly, HBV-DNA level before the therapy did not effect the therapy results.

Conclusions: In CHB, there was no significant difference in SVR and the rate of patients having undetectable HBV-DNA between two groups treated with PEG-2a or PEG-2b. And the level of pretreatment HBV-DNA did not effect the therapy results.

\begin{tabular}{|c|c|c|c|c|c|c|c|}
\hline \multirow[t]{2}{*}{ Groups } & \multirow{2}{*}{$\begin{array}{l}\text { Number }(\%) \\
\text { of patients }\end{array}$} & \multicolumn{3}{|c|}{ Mean HBV-DNA (IU/ml) } & \multicolumn{3}{|c|}{ Number $(\%)$ of patients with virologic response } \\
\hline & & Basement & 48 weeks & 72 weeks & $\begin{array}{l}\text { End of } \\
\text { treatment }\end{array}$ & SVR & $\begin{array}{l}\text { HBV-DNA } \\
<400 \text { copy/ml }\end{array}$ \\
\hline \multicolumn{8}{|l|}{ PEG-2a } \\
\hline HbeAg+ & $4(25)$ & 55.380 .000 & 27.627 .000 & 27.529 .000 & $2(50)$ & $1(25)$ & $1(25)$ \\
\hline HbeAg- & $12(75)$ & 28.297 .000 & 14.200 & 24.290 & $4(33.3)$ & $3(25)$ & $2(16.7)$ \\
\hline Total & $16(100)$ & 35.067 .000 & 6.917 .500 & 6.900 .500 & $6(37.5)$ & $4(25)$ & $3(18.8)$ \\
\hline \multicolumn{8}{|l|}{ PEG-2b } \\
\hline HbeAg+ & $9(33)$ & 64.995 .000 & 25.672 .000 & 12.331 .000 & $4(44.4)$ & $3(33.3)$ & $3(33.3)$ \\
\hline HbeAg- & $18(67)$ & 38.694 .000 & 117.546 & 1.744 .700 & 7 (38.9) & $5(27.8)$ & $3(16.7)$ \\
\hline Total & $27(100)$ & 47.461 .000 & 8.635 .800 & 5.273 .400 & $11(40.7)$ & 8 (29.6) & $6(22.2)$ \\
\hline
\end{tabular}

[The comparison of the results of the groups] 
PP05-110

Comparison of the Efficiency of Tenofovir and Entacavir for the Treatment of Nucleos(t)ide Naive Patients with Chronic Hepatitis B

Ü.B. Dogan ${ }^{1}$, B. Kara ${ }^{1}$, Y. Gümürdülü ${ }^{2}$, A. Soylu ${ }^{3}$, M.S. Akın ${ }^{1}$

${ }^{1}$ Gastroenterology, Adana Numune Training and Research Hospital;

${ }^{2}$ Gastroenterology, School of Medicine, Çukurova University, Adana;

${ }^{3}$ Gastroenterology, Bakırköy Training and Research Hospital,

Istanbul, Turkey

Background/aim: Chronic Hepatitis B virus (HBV) infection is an important cause of morbidity and mortality. Tenofovir Disoproxil fumarate (TDF) and entecavir (ECV) were licensed for the treatment of HBV infection. We evaluated first 12 months of chronic hepatitis B (CHB) treatments with TDF and ECV and compared their efficiencies. Methods: The study enrolled $94 \mathrm{CHB}$ patients with compensated liver disease. ECV group consisted of 29 patients who received entecavir $0.5 \mathrm{mg} /$ day; TDF group consisted of 65 patients who received tenofovir $245 \mathrm{mg} /$ day. There was no statistically significant demographic and $\mathrm{HBeAg}$ status difference between the groups. Patients returned to the clinic every 4 weeks for laboratory assessments of serum chemical and hematologic values, liver function and for documentation of any adverse events. Hepatitis B serologic markers and HBV-DNA levels were assessed every 12 weeks. The primary efficacy end point was a plasma HBV-DNA level of $<400$ copies/ ml over 48 weeks.

Results: At the end of 48 weeks, treatment with either TDF or ECV resulted in clinically important suppression of HBV-DNA as $71.3 \%$. There was no statistically difference in inducing undetectable levels of HBV-DNA in ECV (69\%) and TDF (72.3\%) groups. Also no side effect as the increasement of creatin was seen. HBeAg seroconversion was seen only one patient in ECV group, but none in TDF group.

Conclusion: In the first year of treatment for $\mathrm{CHB}$, virologic response and tolerability did not differ significantly between TDF and ECV. Both drugs are safe and efficacious for patients infected with HBV.

The comparison of the results of the groups

\begin{tabular}{|c|c|c|c|c|c|c|}
\hline \multirow[t]{2}{*}{ Groups } & \multirow{2}{*}{$\begin{array}{l}\text { Number } \\
(\%) \text { of } \\
\text { patients }\end{array}$} & \multicolumn{2}{|c|}{$\begin{array}{l}\text { Mean } \mathrm{HBV}- \\
\text { DNA (IU/ml) }\end{array}$} & \multicolumn{2}{|c|}{$\begin{array}{l}\text { Meancreatin } \\
\text { levels }(\mathrm{mg} / \mathrm{ml})\end{array}$} & \multirow{2}{*}{$\begin{array}{l}\text { Number }(\%) \\
\text { of patients } \\
\text { with } \\
\text { virologic } \\
\text { response } \\
\text { HBV-DNA } \\
<400 \\
\text { copy/ml }\end{array}$} \\
\hline & & Basement & $\begin{array}{l}48 \\
\text { weeks }\end{array}$ & Basement & $\begin{array}{l}48 \\
\text { weeks }\end{array}$ & \\
\hline \multicolumn{7}{|l|}{ Entecavir } \\
\hline HbeAg+ & $10(34)$ & 159.190 .000 & 82.482 & 0.77 & 0.78 & $7(70)$ \\
\hline HbeAg- & $19(66)$ & 26.907 .000 & 1.501 & 0.85 & 0.88 & $13(68)$ \\
\hline Total & $29(100)$ & 72.534 .000 & 29.425 & 0.82 & 0.85 & $20(69)$ \\
\hline \multicolumn{7}{|l|}{ Tenofovir } \\
\hline HbeAg+ & $29(45)$ & 43.461 .000 & 2.196 & 0.63 & 0.76 & $20(69)$ \\
\hline HbeAg- & $36(55)$ & 34.571 .000 & 2.136 & 0.76 & 0.83 & $27(75)$ \\
\hline Total & $65(100)$ & 38.537 .000 & 2.162 & 0.72 & 0.81 & $47(72.3)$ \\
\hline
\end{tabular}

PP05-111

The Correlation of HBsAg and HBV-DNA in Chronic Hepatitis B Treatment

Y. Gümürdülü ${ }^{1}$ Ü.B. Dogan ${ }^{2}$, B. Kara ${ }^{2}$, M.S. Akın², R. Kaya ${ }^{2}$, N.A. Öztürk ${ }^{2}$, B.E. Taşdoğan

${ }^{1}$ Gastroenterology, School of Medicine, Çukurova University;

${ }^{2}$ Gastroenterology, Adana Numune Training and Research Hospital, Adana, Turkey

Background/aim: Quantitative measurement of HBsAg titers may be an easy and economical reference for Hepatitis B virus (HBV) replication in Chronic Hepatitis B infection (CHB). In this study, we aimed to investigate whether quantitative $\mathrm{HBsAg}$ correlates hepatitis $\mathrm{B}$ virus (HBV) DNA levels during $\mathrm{CHB}$ treatment.

Methods: 104 CHB patients; 68 male, 36 female were recruited $(\mathrm{HBeAg}+, n=34 ; \mathrm{HBeAg}-, n=70)$. Active disease was defined as a serum alanine aminotransferase $>40 \mathrm{IU} / \mathrm{L}$ and a serum HBV-DNA $>10,000$ copies/ ml. Quantitative HBsAg was studied by automated chemiluminescent microparticle immunoassay (Architect $\mathrm{HBsAg}$, Abbott, IL). HBV-DNA was measured by TaqMan polymerase chain reaction (PCR; Erasmus MC, University Medical Center, Rotterdam, The Netherlands). Patients have been treated with entecavir or tenofovir. Serum HBV-DNA and HBsAg were assessed at baseline, during treatment (weeks 12th, 24th, and 48th), and during follow-up (weeks 60th and 72th).

Results: There was a weak correlation between quantitative $\mathrm{HBsAg}$ and HBV-DNA in the early phase of treatment. Also there was no correlation in the later phases. Correlation between HBsAg and HBVDNA concentrations was weak (correlation coefficient $=0.197, p<$ 0.05 ). There was not any effect of $\mathrm{HBeAg}$ positivity on the correlation of HBV-DNA and HBsAg.

Conclusion: There was no correlation between quantitative $\mathrm{HBsAg}$ and HBV-DNA in the CHB treatment duration.

\section{PP05-112}

Quantification of Serum HBsAg, HBeAg and HBV DNA among HBeAg Positive CHB Patients during 5 Years of Adefovir Dipivoxil (ADV)Therapy

Y. Mao ${ }^{1}$, M. Zeng ${ }^{1}$, H. Ren ${ }^{2}$, D. Xu ${ }^{3}$

${ }^{1}$ Shanghai Renji Hospital, Shanghai; ${ }^{2}$ Chongqing Medical University 2nd Affliated Hospital, Chonqing; ${ }^{3}$ Beijing Ditan Hospital, Capital Medical University, Beijing, China

Background: Serum HBsAg clearance is the ultimate goal of therapy in $\mathrm{CHB}, \mathrm{HBeAg}$ seroconversion is also a satisfactory end point for $\mathrm{HBeAg}$ positive patients. This study evaluates serum $\mathrm{HBsAg}$, HBeAg, and HBV DNA in CHB patients during ADV treatment.

Methods: Fifty China $\mathrm{HBeAg}$ positive $\mathrm{CHB}$ patients who participated in a 1-year ADV registration trial (ADV $10 \mathrm{mg}$ daily)were offered continuation for further 4 years. HBsAg, $\mathrm{HBeAg}$ and $\mathrm{HBV}$ DNA were analyzed at baseline, weeks 12, 28, 40, 52, 104, 156, 208 and 260 by Abbott Architect HBsAg Assay, Roche Cobas HBeAg Assay and PCR respectively.

Results: The baseline characteristics were: median age, 30; HBV DNA, $8.93 \log _{10}$ copies/ml; ALT, 2.7 ULN. At year 5, $52.5 \%$ 
achieved HBV DNA undetectable, 47.5\% had HBeAg loss and none had HBsAg loss. Serum HBsAg, HBeAg, HBV DNA and ALT levels decreased significantly $(P<0.001)$ in the first 12 weeks. The median HBsAg, HBeAg, and HBV DNA at baseline, weeks 12, 28, 40, 52, 104, 156, 208, 260 were 21975.74, 5628.80, 6079.01, 5850.01, 457,4.33, 5721.07, 4486.23, 4335.32, 3624.47 IU/ml and 621.01, $67.42,22.91,15.92,10.63,3.43,1.50,0.735,0.10 \mathrm{PEIU} / \mathrm{ml}$ and 8.93 , 5.70, 5.00, 4.29, 4.04, 3.75, 3.61, 2.77, $2.48 \log _{10} \mathrm{copies} / \mathrm{ml}$ respectively. Three patterns of HBsAg decline (biphasic, $57 \%$; assurgent, $11 \%$; wavy, $32 \%$ )were observed in the first year. $\mathrm{HBsAg}, \mathrm{HBeAg}$, HBV DNA and ALT levels showed the same decline pattern during the 5 years.

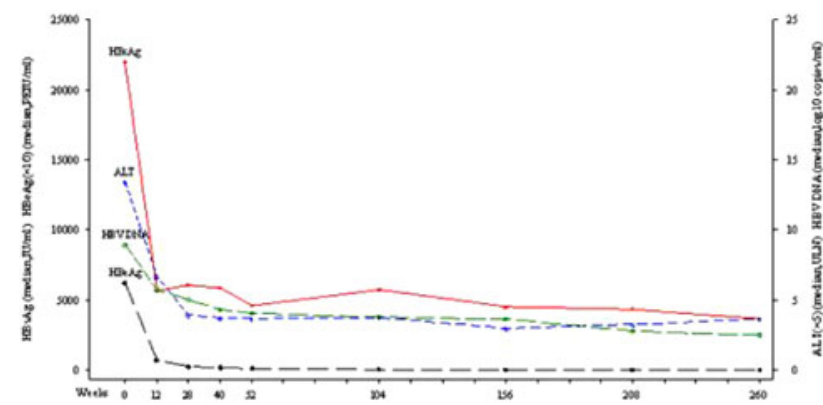

Conclusion: The median reduction of $\mathrm{HBsAg}, \mathrm{HBeAg}$, HBV DNA and ALT were significant and they showed the same decline pattern during the 5 years.

\section{PP05-113}

Lamivudine to Prevent HBV Perinatal Transmission: Viral Suppression is Often Inadequate and Induction of Lamivudine Resistance Mutations is Uncommon

M.T. Levy ${ }^{1}$, S. Manoharan ${ }^{2}$, A. Glass ${ }^{3}$, W. $\mathrm{Ng}^{2}$, G. Pavan ${ }^{4}$, M. Maley ${ }^{5}$, S.A. Locarnini ${ }^{6}$, A. Ayres ${ }^{6}$

${ }^{1}$ Gastroenterology, Liverpool Hospital, University of NSW;

${ }^{2}$ Gastroenterology, University of New South Wales;

${ }^{3}$ Gastroenterology, Liverpool Hospital; ${ }^{4}$ Gastroenterology, Royal

Prince Alfred Hospital; ${ }^{5}$ Microbiology and Infectious Diseases, South Western Area Pathology Services, Sydney, NSW; ${ }^{6}$ Research and Molecular Development, Victorian Infectious Diseases Reference Laboratory, Melbourne, VIC, Australia

Background: Perinatal transmission of HBV despite passive active immune prophylaxis still occurs. We have reported that the risk of transmission from mothers with viral loads above HBV DNA $\log 8$ copies $/ \mathrm{ml}$ approaches $10 \%$. There is some evidence that Lamivudine in the last trimester reduces this risk and is safe for baby. Is Lamivudine a good choice?

Aim: We investigated the impact of Lamivudine used in the last trimester of pregnancy on viral load and the development of genotypic nucleos(t)ide resistance.

Methods: Pregnant women with $\mathrm{HBsAg}$ and high viral $\operatorname{load}(\log 7$ $\mathrm{IU} / \mathrm{ml}$ ) were offered Lamivudine for 8 weeks (commencing at 32 weeks) until approximately 2 weeks after delivery. Viral load quantitation and HBV mutational analysis for detection of Lamivudine resistance was performed using population based sequencing and Line probe analysis on HBV DNA at the end of therapeutic period.

Results: 25 treated women who had blood tests at or within 2 weeks of stopping Lamivudine therapy, were studied. Median baseline viral load was $8.39 \log \mathrm{IU} / \mathrm{ml}$. Lamivudine was taken for a median of 50 days. Median viral load reduction was $2.82 \mathrm{log} \mathrm{IU} / \mathrm{ml}$. Viral load reduction was related to the duration of therapy $R^{2}=0.27$ but not the baseline viral load $R^{2}=0.03$. Six had a viral load at the end of treatment above $\log 7 \mathrm{IU} / \mathrm{ml}$. Two of these were treated for over 50 days. In 1 out of the 25 , a rtA181T mutation characteristic of
Lamivudine genotypic resistance was selected. The primary LMV resistance mutations $\mathrm{rtM}$ 204I/V +/- rtL180M were not detected.

Conclusions: Viral load reduction was inadequate in $24 \%$ of high viral load mothers treated with Lamivudine in the last trimester of pregnancy. Genotypic resistance was occasionally induced by this short treatment, although more sensitive assays are likely to provide additional information.

\section{PP05-114}

Durability of HBeAg Seroconversion in Treatment Induced versus Spontaneous Seroconverters

S.G. Lim ${ }^{1,2}$, M.O. Aung ${ }^{1}$, M. Belinda ${ }^{1}$, S. Dede ${ }^{1}$, Y.M. Lee ${ }^{1}$, G.H. Lee ${ }^{1}$, M. Fernandas ${ }^{1}$, L. Vincent ${ }^{1}$, Y.Y. Dan ${ }^{1}$

${ }^{1}$ Gastroenterology and Hepatology, National University Health System; ${ }^{2}$ Institute of Molecular and Cell Biology, Singapore,

Singapore

Background and aim: An important endpoint of clinical treatment of chronic hepatitis B (CHB) is $\mathrm{HBeAg}$ seroconversion, however the importance of this endpoint has been questioned. We sought to examine differences in $\mathrm{HBeAg}$ seroconversion and seroreversion rates in spontaneous compared to treatment induced seroconverters.

Patients and methods: $\mathrm{CHB}$ Clinic patients who were $\mathrm{HBeAg}$ positive with and without treatment with oral nucleoside analogues (lamivudine, adefovir and entecavir) were followed for $\mathrm{HBeAg}$ seroconversion and seroreversion. Durability was defined as maintained anti-HBe and $\mathrm{HBeAg}$ loss. Predictors of $\mathrm{HBeAg}$ seroconversion were evaluated by multivariate analysis. Data were analysed by Kaplan Meier, log rank test, Cox regression and ROC curve.

Results: There were $835 \mathrm{HBeAg}+$ chronic hepatitis B patients who were followed up with $\mathrm{HBeAg}$ seroconversion developing in 278 patients. Spontaneous seroconversoin occurred in $47 \%$ of patients compared to $61 \%$ after oral nucleoside therapy at 7 years of follow up $(P<0.001)$. A significant difference was still maintained after Cox Regression adjustment for differences in baseline variables $(p<$ 0.001 ). However seroreversion occurred in $25.8 \%$ of treated seroconverters but only $8.5 \%$ of spontaneous seroconverters $(p<0.001)$. No differences in $\mathrm{HBeAg}$ seroconversion and seroreversion rates were found between patients treated with lamivudine and adefovir but there were too few entecavir treated patients who had stopped therapy to evaluate seroreversion. Predictors of $\mathrm{HBeAg}$ seroconversion were patients with baseline ALT abnormal (OR 2.2, 95\% CI 1.6-2.8), lower baseline HBV DNA (OR 1.2, 95\% CI 1.13-1.31). Patients who maintain $\mathrm{HBeAg}$ negative status for $>15.6$ months post seroconversion (AUROC $=0.818$ ) were more likely to have durable seroconversion $(P<0.001$, OR $10.4,95 \%$ CI 4.8-22.4).

Conclusion: $\mathrm{HBeAg}$ positive $\mathrm{CHB}$ patients treated with nucleoside analogues achieved a significantly higher rate of $\mathrm{HBeAg}$ seroconversion compared to spontaneous seroconverters but this was blunted by the significantly higher rate of seroreversion. Treatment induced seroconversion was less durable than spontaneous seroconverion.

\section{PP05-115}

Re-treatment for Relapsed Chronic Hepatitis B after Lamivudine Treatment: Lamivudine Versus Entecavir C.K. Lee ${ }^{1}$, J.W. Choi ${ }^{1}$, S.M. Hong ${ }^{1}$, M.S. Kwac ${ }^{1}$, J.S. Lee $^{2}$, K.A. Kim ${ }^{2}$, W.Y. Song ${ }^{2}$

${ }^{I}$ Internal Medicine, National Health Insurance Corporation Ilsan Hospital; ${ }^{2}$ Internal Medicine, Ilsan Paik Hospital, Inje University College of Medicine, Koyang-Shi, Republic of Korea

Background/aims: Entecavir has demonstrated superior histologic, virologic and, biochemical benefit, as compared to lamivudine, in chronic hepatitis B (CHB) patients. No report has compared the efficacy of these antiviral drugs in CHB patients who replase after lamivudine treatment. We compared the clinical efficacy between 
repeat lamivudine therapy and entecavir therapy in relapsed $\mathrm{CHB}$ patients who had previous lamivudine therapy.

Methods: Between March 2000 and December 2009, 63 CHB patients relapsed following lamivudine therapy. The clinical results of two subsets of patients treated with lamivudine $100 \mathrm{mg}(n=30)$ or entecavir $0.5 \mathrm{mg}(n=33)$ were analyzed for a 2 -year period. The serum alanine transaminase (ALT) level and normalization, HBV DNA loss $(<300$ copies $/ \mathrm{mL})$, and virologic breakthrough were monitored.

Results: There were no statistically significant differences in age, sex, serum ALT, serum HBV DNA levels between the two groups. During the follow-up period, the entecavir group had a significantly higher proportion of ALT normalization at $6(70.0$ vs. $96.9 \%, p=0.047)$ and 12 (73.3 vs. $93.6 \%, p=0.028)$ months. But, at 24 months, the proportion of ALT normalization (86.9 vs. 91.6\%, $p=1.000$ ) was not different in the two groups. The serum HBV DNA levels were lower at $6\left(3.62 \pm 2.61\right.$ vs. $\left.2.12 \pm 0.49 \log _{10} \mathrm{copies} / \mathrm{ml}\right), 12(3.65 \pm 2.61$ vs. $1.96 \pm 0.29 \quad \log _{10} \quad$ copies $\left./ \mathrm{ml}\right)$, and $24 \quad(3.04 \pm 1.69$ vs. $2.15 \pm 0.78 \log _{10}$ copies $\left./ \mathrm{ml}\right)$ months and the HBV DNA loss was higher at 6 (66.7 vs. $75 \%), 12$ (63.3 vs. 88.9\%), and 24 (57.1 vs. $88.9 \%$ ) months in the entecavir group, but the difference was not significant. The incidence of virologic breakthrough was lower at 12 ( 26.7 vs. $0 \%, p=0.061)$ and $24(57.1$ vs. $11.1 \%, p=0.042)$ in the entecavir group.

Conclusions: Entecavir treatment resulted in an effective response in the serum ALT and HBV DNA levels and a low incidence of virologic breakthrough in relapsed patients following lamivudine therapy for CHB.

\section{PP05-116}

The Observation of Efficacy and Safety of Enticavir in Treatment of Acute on Chronic Hepatitis B

F. Lin, L. Feng, W. Tao, F. Ji, Q. Ping, X. Xiaozhen, C. Duyun, J. Jie Infectious Diseases, Hainan Provincial Hospital, Haikou, China

Objective: To evaluate the clinical efficacy and safety of Enticavir (ETV) in treatment of acute on chronic hepatitis B.

Methods: 37 patients with acute on chronic hepatitis B were randomly divided into two groups. 20 patients in therapy group were treated with ETV plus inter medicine; 17 patients in control group were treated only with inter medicine. Changes of serum ALT, total bilirubin (TB), albumin (ALB), prothrombin activities (PTA) and HBV DNA were detected before and after the treatment of the patientes in both groups, respectively. Improving and curing rates of the patientes in each group were monitored.

Results: Compared to the controlled group, serum ALT, TB and HBV DNA of the patients in the therapy group were significantly decreased after the treatment, and serum ALB was significantly increased after the treatment. The improving and curing rates of patientes in the therapy group and the control group were 70 and $35.9 \%$, respectively. No side effect was observed in the patients during the treatment of ETV.

Conclusions: ETV is effective and safe in treatment of patients with acute on chronic hepatitis B.

\section{PP05-117}

Telbivudine + Adefovir in HBeAg-positive Chronic Hepatitis B Patients with Established YMDD Resistance Mutation: 1 Year Results

Y. Wang ${ }^{1}$, H. Ren ${ }^{2}$, D. Tan ${ }^{3}$, Y. Chen ${ }^{4}$, M. Wan ${ }^{5}$, J. Niu ${ }^{6}$, D.-Z. Xu A. Uddin ${ }^{8}$, Y. Dong ${ }^{9}$

${ }^{1}$ Xi Nan Hospital; ${ }^{2}$ Chongqing Medical University 2nd Affiliated Hospital, Chongqing; ${ }^{3}$ XiangYu Hospital, Central-South University, Changsha $;{ }^{4}$ The First Affiliated Hospital, Zhejiang University, Hangzhou; ${ }^{5}$ Department of Infectious Disease, Shanghai Changhai Hospital, Shanghai; ${ }^{6}$ No. 1 Hospital, Jilin University, Changchun;
${ }^{7}$ Beijing Ditan Hospital, Beijing, China; ${ }^{8}$ Novartis Pharma, East Hanover, NJ, USA; ${ }^{9}$ Novartis Pharma AG, Basel, Switzerland

Background: Chronic Hepatitis B (CHB) patients with established YMDD mutation are generally difficult to treat and guidelines recommend add-on therapy. The aim of this study is to evaluate the efficacy and safety of the combination of telbivudine (LDT) + adefovir (ADV) in patients with established YMDD mutation following $2-3$ years of lamivudine (LAM) or LDT monotherapy in the GLOBE/ 015 studies.

Methods: Open label 1-year substudy of study 2303 in compensated CHB patients with consistently (median 47 weeks) detectable HBV $\mathrm{DNA}(>300$ copies $/ \mathrm{ml}) .38 \mathrm{HBeAg}+$ patients previously treated with $\operatorname{LDT}(16)$ or $\operatorname{LAM}(22)$ for a median of 165 and 104 weeks, respectively. 37/38 (97.4\%) patients had confirmed YMDD mutation (M204I, M204V or mixed). Efficacy and safety was evaluated after 52 weeks treatment with LDT + ADV. Missing visits imputed (last observation carried forward method).

Results: At baseline of substudy, median HBVDNA was $9 \log _{10}$ copies $/ \mathrm{ml}$, ALT $75 \mathrm{IU} / \mathrm{ml}$. All patients were Chinese, HBV genotype B/C. Median HBVDNA decline after 24 weeks and 1 year of LDT + ADV combination were 4.8 and $5.1 \log _{10}$ copies $/ \mathrm{ml}$, respectively. At 1 year, 53\% (20/38) of patients achieved undetectable HBV DNA ( $<300$ copies/ml), out of the remaining 18 patients, 12 had HBVDNA below $5 \log _{10}$ copies/ml. ALT normalization was achieved in 72, 24 and $16 \%$ achieved $\mathrm{HBeAg}$ loss/seroconversion. 1 patient had viral breakthrough $\left(1 \log _{10}\right.$ above nadir) but no new mutations (e.g. N236T or A181V) were found. Rate of adverse events was $21 \%$. No death occurred; 2 patients had SAEs, not drug related. 1 patient reported myalgia (resolved on treatment). 6 patients had CK elevation. No patient had myopathy or renal function impairment.

Conclusion: These data on the combination of LDT+ADV in patients with established YMDD mutation show fast and durable viral load decline with more than $50 \%$ of patients achieving undetectable $\mathrm{HBV}$ DNA after 1 year. The safety profile was favorable in these treatment experienced patients.

\section{PP05-118}

Clinical Study on the Association between Viral Replication of Chronic Hepatitis B and Occurrence of Liver Cancer

L. Li

Department of Integrated Chinese and Western Medicine, Union Hospital of Tongji Medical College, Huazhong University of Science and Technology, Wuhan, China

Aim: To investigate the association and the pathogenesis between chronic hepatitis B and liver cancer, and prevent the occurrence, development of hepatitis B in order to reduce the occurrence of liver cancer.

Methods: Analyzing the clinical data of patients with hepatocellular carcinoma from October 2008 to February 2009 of Union Hospital of Wuhan City,we researched the reasons for the current incidence of liver cancer and the relation with hepatitis $\mathrm{B}$.

Results: A total of 313 liver cancer patients were enrolled in 5 months which are suffering from hepatitis B in 212 patients, 83 without hepatitis $\mathrm{B}$, there are at least $67.7 \%$ patients with hepatitis $\mathrm{B}$, and $73.2 \%$ without liver cirrhosis direct to the liver cancer.

Conclusions: There are comparatively relevance between the occurrence of liver cancer and hepatitis B viral replication. The study highlight the early prevention and treatment of hepatitis B contract, periodic recheck HBVDNA is paramount important to control the occurrence of liver cancer, we should pay attention to the treatment strategies of anti-viral. 
PP05-119

Sustained Viral Suppression for 2 Years Following Telbivudine Switch in Lamivudine Pretreated Chronic Hepatitis B (CHB) Patients

Q. $\mathrm{Xie}^{1}$, G. $\mathrm{Shi}^{2}$, S. Thongsawat ${ }^{3}$, C.W. Hsu ${ }^{4}$, H.L.Y. $\mathrm{Chan}^{5}$, R. Safadi ${ }^{6}$, M. Wan ${ }^{7}$, S.-G. Hwang ${ }^{8}$, L. Wei ${ }^{9}$, H. Ren ${ }^{10}$, T. Tanwandee ${ }^{11}$, W. Bao ${ }^{12}$, A. Trylesinski ${ }^{13}$

${ }^{1}$ School of Medicine, Ruijin Hospital Shanghai Jiao Tong University; ${ }^{2}$ Dept. of Infectious Diseases, Huashan Hospital, Fu Dan University, Shanghai, China; ${ }^{3}$ Faculty of Medicine, Chiang Mai University, Chiang Mai, Thailand; ${ }^{4}$ Chang Gung Memorial Hospital and Chang Gung University College of Medicine, Taipei, Taiwan, ROC;

${ }^{5}$ Department of Medicine and Therapeutics, The Chinese University of Hong Kong, Hong Kong, Hong Kong S.A.R., ${ }^{6}$ Holy Family Hospital, Nazareth and Hadassah Medical Center, Jerusalem, Israel; ${ }^{7}$ Shanghai Changhai Hospital, Shanghai, China; ${ }^{8}$ Department of Internal Medicine, CHA Bundang Medical Center, CHA University, Seongnam, Republic of Korea; ${ }^{9}$ Peking University People's Hospital, Beijing; ${ }^{10}$ Chongqing Medical University 2 nd Affiliated Hospital,

Chongqing, China; ${ }^{11}$ Siriraj Hospital, Bangkok, Thailand; ${ }^{12}$ Novartis Pharma, East Hanover, NJ, USA; ${ }^{13}$ Novartis Pharma AG, Basel, Switzerland

Background/aim: Lamivudine (LAM) provides good viral suppression, but high rates of genotypic resistance to CHB patients. In the GLOBE study, Telbivudine (LDT) was superior to LAM over 2 years with significantly lower resistance rates. We aimed to evaluate a potential benefit of switching LAM patients to LDT.

Methods: ITT population was $391(237 \mathrm{HBeAg}+$ and $154 \mathrm{HBeAg}-)$ compensated CHB patients, treated for 2 years in the LAM arm of the GLOBE+015 studies, excluding patients with genotypic resistance to LAM. Patients were switched to LDT for 2 additional years. Efficacy was evaluated based on HBV DNA detectable/undetectable $(>300$ copies $/ \mathrm{mL}$ ). 2 years after switch, efficacy outcomes were evaluated in the ITT population (Last observation carried forward method). Safety was also evaluated.

Results: $81 \%$ of patients were Asian (Chinese $65 \%$ ). $57 \%$ were HBV Genotype C, 25\% genotype B. At time of switch, $171 \mathrm{HBeAg}+$ and $128 \mathrm{HBeAg}$ - patients were HBVDNA undetectable. In patients with undetectable HBV DNA at time of LDT switch, $92 \%$ of $\mathrm{HBeAg}+$ and HBeAg- patients maintained undetectable HBV DNA for 2 years, while $5 \%$ and $3 \%$ had viral failure $(\geq 1000$ copies/ml HBVDNA) respectively. $54 \%$ of $\mathrm{HBeAg}+$ patients had $\mathrm{HBeAg}$ seroconversion, 4 patients $\mathrm{HBsAg}$ clearance. $\mathrm{HBeAg}+$ and $\mathrm{HBeAg}-$ patients with detectable HBV DNA at time of switch achieved $27 \%$ and $54 \%$ of undetectable viremia, $61 \%$ and $91 \%$ of ALT normalization and $67 \%$ and $31 \%$ of VF after switching to LDT for 2 additional years, respectively. $17 \%$ of $\mathrm{HBeAg}+$ patients achieved $\mathrm{HBeAg}$ seroconversion. Safety over 2 years was similar than GLOBE study: $2.8 \%$ of serious adverse events. ALT flares $(0.8 \%)$, CK elevations $(6.6 \%)$. Muscle symptoms (2.3\%). No cases of peripheral neuropathy, lactic acidosis or rhabdomyolysis.

Conclusion: Switching well controlled lamivudine treated patients to telbivudine provides high rates of viral load suppression and $\mathrm{HBeAg}$ seroconversion combined with a favorable safety profile.

\section{PP05-120}

Efficacy of Adefovir-based Combination Therapy with Entecavir or Lamivudine for Entecavir-resistant Chronic Hepatitis B Patients S.S. Kim ${ }^{1}$, S.W. Cho ${ }^{1}$, J.Y. Cheong ${ }^{1}$, D. Lee ${ }^{1}$, S.P. Hong ${ }^{2}$, S.-O. Kim ${ }^{2}$

${ }^{1}$ Gastroenterology, Ajou University School of Medicine, Suwon,

${ }^{2}$ GeneMatrix Inc., Yongin, Republic of Korea

Background/aims: We evaluated the efficacy of adefovir (ADV) plus lamivudine (LAM) therapy or ADV add on therapy for entecavir (ETV)-resistant hepatitis B patients.

Methods: Thirty ETV-resistant and 8 patients with suboptimal response to ETV were prospectively enrolled. Twenty seven patients received ADV plus LAM therapy (ADV + LAM group) and 11 patients received ADV plus ETV therapy (ADV+ETV group) for $>24$ weeks and were monitored for virologic response. Primary nonresponse was defined as less than $1 \log _{10} \mathrm{IU} / \mathrm{ml}$ decrease in HBV DNA level from baseline at 3 months of therapy and initial virologic response was defined as a HBV DNA level less than 20,000 IU/ml after 6 months of therapy.

Results: Primary non-response was observed in $25.9 \%(7 / 27)$ in $\mathrm{ADV}+\mathrm{LAM}$ group and $27.3 \%$ (3/11) in ADV + ETV group $(P=1.00)$. Initial virologic response was observed in $29.6 \%(8 / 27)$ in $\mathrm{ADV}+\mathrm{LAM}$ group and $63.6 \%$ (7/11) in ETV + ADV group $(P=0.073)$. The mean reduction in serum HBV DNA levels at week 24 did not significantly differ between ADV+LAM group and ADV + ETV group $\left(-1.70\right.$ vs. $\left.-2.18 \log _{10} \mathrm{IU} / \mathrm{mL} ; P=0.362\right)$. Subgroup analyses of 8 patients with suboptimal response to ETV, ADV + ETV group showed significant more serum HBV DNA reduction at week 24 and 48 than $\mathrm{ADV}+\mathrm{LAM}$ group ( -0.09 vs. -2.29 and -072 vs. $-2.04 \log _{10} \mathrm{IU} / \mathrm{mL}$; $P=0.012$ and $P=0.020$, respectively). HBeAg negativity and low baseline HBV DNA were associated with an initial virologic response $(P=0.018$ and $P=0.021$, respectively). Mean duration of $\mathrm{ADV}+\mathrm{LAM}$ therapy was $14.11 \pm 5.57$ months and mean duration of ADV+ETV therapy was $12.55 \pm 7.68$ months.

Conclusions: Antiviral efficacy of ADV plus LAM therapy or ADV add on therapy for ETV-resistant hepatitis B patients is low, and not different between two groups. However, adding ADV to ETV reduces HBV DNA more effectively than ADV plus LAM in patients with suboptimal virologic response to ETV.

\section{PP05-121}

The Effect of Producing Genotypic Resistance Mutation in Different Nucleoside (Nucleotide) Analogue

P. An, L. Bian, B. Yin, D. Lv, Y.-X. Cao, X. Wu

The Sixth People's Hospital of Shenyang, Shenyang, China

Objective: To explore the influencing factors for occurring genotypic resistance for the patients in there nucleoside (nucleotide) analogue(NA) treated chronic hepatitis B (CHB).

Methods: HBV $\mathrm{P}$ gene mutation were amplified by PCR direct sequences. 320 patients of CHB treated NA were random divided into 3 groups by 1:1:1 as SPSS stochastic ways: LAM (107), ADV (105), ETV (108), the follow-up time was 192 weeks.

\section{Results:}

1. The frequency of genotypic resistance add up respectively in LAM, ADV, ETV: 1 year 20.0, 0.0, 0.0\%; 2 year 37.1, 3.8, 0.0\%; 3 years $42.8,9.5,0.9 \%$; 4 years $64.7,25.7,0.9 \%$.

2. The genotypic resistance at $\mathrm{rtL} 180 \mathrm{M}+\mathrm{rtM} 204 \mathrm{~V}$ was major in LAM, 35.2\%; but rtN236T was major in ADV, $12.3 \%$.

3. The survival analysis indicated that the incidence rate of genotypic resistance increased as the time extended in different NA,the trend increased apparently in LAM than ADV, ETV (logRank, $P<0.001$ ).

4. Cox analysis indicated that sex, age, drink, fatty liver, baseline ALT level and baseline HBV DNA level had no bearing on occurring genotypic resistance $(P>0.05)$ but it showed that family history, HBV DNA negative time and different NA treated were the independent factor on influence genotypic resistance $(P=0.003,0.040,0.000)$.

Conclusion: The accumulate incidence rate of occurring genotypic resistance in LAM was above in ADV, and was lowest in ETV for the patients with NA treated $\mathrm{CHB}$ in 4 years. The incidence rate of genotypic resistance increased as the time extended in different NA. Cox analysis indicated that HBV family history, HBV DNA negative time and different NA treated were the independent affecting factor on occurring genotypic resistance. 
PP05-122

Myalgia Misdiagnosed as an Adverse Reaction Associated with Telbivudine

Y. Zhao ${ }^{1,2}$, H.C. Wu ${ }^{3}$, X. Zhang ${ }^{3}$, S. Chen ${ }^{3}$, J. $\mathrm{Niu}^{4}$

${ }^{I}$ Department of Infectious Diseases, Shenzhen Shekou People's Hospital, Shenzhen City; ${ }^{2}$ Gastroenterology Department, The

Hospital of Jilin University, Changchun; ${ }^{3}$ Infectious Department, The Shekou People's Hospital of Shenzhen, Shenzhen; ${ }^{4}$ Hepatology

Department, The First Hospital of Jilin University, Changchun, China

Introduction: Telbivudine is a new synthetic nucleoside analogue. Because it can significantly suppresses hepatitis B virus(HBV) replication, it has been taken as a potent option for the treatment of chronic hepatitis $\mathrm{B}(\mathrm{CHB})$. However, adverse reactions (e.g., myalgia, general weakness and serum creatine kinase (CK) was elevated, etc.) associated with telbivudine have been increasing.But the rate of misdiagnosis was high too. To understand this better, we retrospectively analyzed the clinical record of one patient taking telbivudine. We hope that the result will provide clinic references for the future safe use of telbivudine.

Case description: A 54-year-old woman was diagnosed as $\mathrm{HBeAg}-$ negative CHB in 1998. In 2008, abnormal liver function and high HBV viral load were detected and she started the antiviral therapy with telbivudine $(600 \mathrm{mg} /$ day $)$. Biochemical response was achieved and HBV DNA became undetectable after 10 months of telbivudine. But she appeared the symptom of myalgia including lumbodynia, dorsalgia and difficulty of rising from bed. Laboratory tests showed that serum $\mathrm{CK}, \mathrm{CKMB}$ were not elevated. But telbivudine was withdrawed one more month later,because she can't tolerate the symptom and was suspected telbivudine-induced myopathy. However, she kept feeling backache. Biochemical and virological breakthrough (ALT 247U/L, AST $167 \mathrm{U} / \mathrm{L}$, HBV DNA $6.3 \mathrm{E}+03 \mathrm{IU} / \mathrm{ml}$ ) were identified after 5 months of telbivudine withdrawing. The tests of CK, CKMB were still normal, but rheumatoid factor and erythrocyte sedimentation rate were elevated. So she was suspected as rheumatism instead of the druginduced myopathy.And she was suggested to take follow-up in the rheumatology department.The patient determined to receive telbivudine antiviral therapy again. Meanwhile, the tests and symptoms were also followed up. Telbivudine Side effect with elevations in CK and CKMB were asymptomatic usually. Sometimes some symptoms appearing after telbivudine administration were misdiagnosised side effect. Misdiagnosis commonly took place because clinicians failed to recognize the adverse reactions of telbivudine. Future research in CHB is warranted on telbivudine, sequentially or in combination in order to maximize viral suppression. So we must recognize the side effect sufficiently.

\section{PP05-123}

The Comparison of Entecavir Monotherapy and Adefovir Add-on Therapy in Patients with Lamivudine-resistant Chronic Hepatitis B S.B. Cho, W.J. Kee, S.H. Kim, K.J. Park, H.K. Jeong, Y.E. Joo, J.S. Rew, S.K. Choi

Internal Medicine, Chonnam National University Medical School, Gwang Ju, Republic of Korea

Background/aims: Current rescue therapies for lamivudine (LAM)resistant chronic hepatitis B patients are switching to entecavir (ETV) or adding on adefovir (ADV) with continuation of LAM. But the efficacy of each rescue therapy have not been fully elucidated. We evaluated the efficacy of entecavir monotherapy and adefovir add-on therapy for at least 72 weeks in patients with LAM- resistant chronic hepatitis B

Methods: From 2006 to 2008, 92 consecutive LAM-resisitant patients were enrolled (50 LAM + ADV and $42 \mathrm{ETV} 1 \mathrm{mg}$ for at least 72 weeks).

Results: Mean age, duration of prior lamivudine treatment, baseline HBV DNA titer, AST, ALT and number of HBeAg positive patients between two groups were not different. By 72 weeks, ALT normalization (85.7 vs. $80.9 \%, p=0.563)$, loss of $\mathrm{HBeAg}(12.1$ vs. $18.6 \%$, $p=0.536), \mathrm{HBeAg}$ seroconversion ( 6.1 vs. $16.3 \%, p=0.284)$ were not significantly different between ETV group and LAM + ADV group. But the rate of undectable HBV DNA in LAM+ ADV group were higher than in ETV group (52.0 vs. $21.4 \%, p=0.003$ ). Higher ALT (OR 10.328, 95\% CI 1.058-100.796, $p=0.045$ ), and presence of primary response (OR 5.895, 95\% CI 1.239-28.047, $p=0.026$ ) were the independent predictive factor of virologic response at week 72. The occurrence of antiviral resistance to new rescue durg was lower in LAM + ADV group than ETV group but not in the statistics (4.8 vs. $2.0 \%, p=0.590$ ).

Conclusion: Adefovir add-on therapy achieved higher virologic response than entecavir-monotherapy for patients with LAM-resistant chronic hepatitis B.

\section{PP05-124}

Telbivudine versus Lamivudine for Chronic Hepatitis B Patients in Bangladesh: Results at 1 Year

M.G. Azam ${ }^{1}$, M. Khan ${ }^{2}$, S. Alam ${ }^{3}$, N. Ahmad ${ }^{3}$, K. Alam ${ }^{3}$

${ }^{I}$ Gastroenterology and Hepatology, Birdem Hospital; ${ }^{2}$ Hepatology, The Liver Centre; ${ }^{3}$ Hepatology, Bangabandhu Sheikh Mujib Medical University, Dhaka, Bangladesh

Background: Bangladesh is an intermediate prevalent zone of chronic hepatitis B (CHB) virus infection with a carrier rate of 5-7\% among general population. Globally five oral drugs are approved for suppression of viral replication and to improve liver disease. Lamivudine is available in Bangladesh for long time but telbivudine is a recent drug for $\mathrm{CHB}$ patients.

Aims: We compared the efficacy of telbivudine (group A, $n=74$ ) with lamivudine (group $\mathrm{B}, n=76$ ) among CHB Bangladeshi patients in this study. At the Liver Centre Dhaka, Bangladesh patients with hepatitis B e antigen ( $\mathrm{HBeAg}$ )-positive or $\mathrm{HBeAg}$ negative $\mathrm{CHB}$ were randomly assigned to treatment with $600 \mathrm{mg}$ of telbivudine or $100 \mathrm{mg}$ of lamivudine daily for 52 weeks. The endpoint of treatment was reduction in serum HBV DNA level, $\mathrm{HBeAg}$ negative and alanine aminotransferase (ALT) normalization at the end of treatment. Viral resistance and safety were assessed.

At week 52, mean reductions of serum HBV DNA were significantly greater in Group A than Group B [6.1 $\log (10)$ versus $5.1 \log (10), P<$ 0.001 ], and HBV DNA was polymerase chain reaction-negative in significantly more Group A than Group B (59 vs. 33\%, $P<0.001$ ), $\mathrm{HBeAg}$ loss (29 vs. $20 \%, P=0.044)$ were significantly more common in the telbivudine group. ALT normalization was also greater among telbivudine group than lamivudine group (77 vs. $63 \%$, $P=0.007)$. Viral resistance in both groups was close showing no significant difference statistically. Clinical adverse events were unremarkable in the two treatment groups.

Conclusion: In Bangladeshi patients with $\mathrm{CHB}$, telbivudine treatment for 52 weeks provided greater antiviral efficacy than lamivudine. However antiviral resistance is a great concern for both of these nucleoside analogues.

\section{PP05-125}

HBV rtN236T Mutant Subpopulations Respond Like Wild-type during Tenofovir DF (TDF) Monotherapy or Combination Therapy with Emtricitabine (FTC)

M. Curtis ${ }^{1}$, E. Svarovskaia ${ }^{2}$, Y. Zhu ${ }^{1}$, K. Peschell ${ }^{1}$, K. Kitrinos ${ }^{1}$, M. Miller ${ }^{2}$, K. Borroto-Esoda ${ }^{1}$

${ }^{I}$ Gilead Sciences, Inc., Durham, NC; ${ }^{2}$ Gilead Sciences, Inc., Foster City, CA, USA

Background/aim: The aim of this study was to evaluate the rtN236T mutation response to TDF therapy by comparing early viral load (VL) 
decay kinetics of mutant versus wild-type (WT) virus in chronic monoinfected hepatitis B patients harboring rtN236T prior to initiating TDF or TDF/FTC.

Methods: An allele-specific PCR assay was designed to detect HBV rtN236T mutant and $\mathrm{rtN} 236 \mathrm{~N}$ WT subpopulations (with $\mathrm{rtN} 236 \mathrm{~T}$ detection cut-off at $0.5 \%$ ) in serum samples with $\mathrm{VL}$ of $\geq 1,000 \mathrm{cp} / \mathrm{mL}$. Baseline (BL) samples $(n=105)$ from ADV refractory patients enrolled in Study 0106, which evaluated TDF versus FTC/TDF, were tested. Patients with BL rtN236T were tested for rtN236T levels through week 24 (W24) or until VL became $<1,000 \mathrm{cp} / \mathrm{mL}$.

Results: The rtN236T mutation was detected at BL in $13.3 \%(14 / 105)$ of ADV refractory patients (range $0.5-93.2 \%$; median $11.2 \%$ ). Patients with rtN236T responded to TDF $(n=5)$ and FTC/TDF $(n=9)$ similarly to WT $(n=90)$ with a median change in total HBV DNA from BL of $-3.9,-3.3$, and $-3.2 \log _{10} \mathrm{cp} / \mathrm{mL}$ respectively at W24; 3/5 TDF patients and 6/9 FTC/TDF patients had HBV DNA $<1000 \mathrm{cp} / \mathrm{mL}$ at $\mathrm{W} 24$. The $\mathrm{rtN} 236 \mathrm{~T}$ mutant virus showed similar rates of HBV DNA decline compared to WT; no statistical difference was observed at W4 [TDF $(p=0.375), \operatorname{FTC} / \mathrm{TDF}(p=0.109)]$. There was no significant difference in VL decline rates through W4 for the rtN236T virus when comparing TDF to FTC/TDF $(p=0.933)$. The relative proportions of $\mathrm{rtN} 236 \mathrm{~T}$ to WT $\mathrm{did}$ not increase during either treatment and the percent change from BL to W4/W12 in rtN236T was not significantly different with either therapy.

Conclusions: The rtN236T mutant virus showed similar HBV DNA decline kinetics to WT virus in ADV refractory patients who switched to TDF or TDF/FTC. Despite low levels of cross-resistance to TDF observed in vitro, TDF equally suppresses WT and rtN236T viruses in vivo.

\section{PP05-126}

DV-601, a Therapeutic Vaccine for the Treatment of Chronic Hepatitis B Infection

M. Spellman, J.T. Martin

Dynavax Technologies, Berkeley, CA, USA

Therapeutic vaccines may stimulate strong and specific cytotoxic T-lymphocyte (CTL) and B-cell antibody responses to dominant hepatitis B virus (HBV)-antigens, and thereby promote resolution of chronic hepatitis B infection (CHB). DV-601 was engineered to stimulate a broad spectrum of CTL and helper T cell epitopes, and comprises recombinant $\mathrm{HBsAg}$, subtype adw2, and $\mathrm{HBV}$ core antigen ( $\mathrm{HBcAg}$ ), with adjuvant. In this dose escalation trial, 3 cohorts of treatment-naïve or treatment-tolerant adult patients with $\mathrm{CHB}$ received 6 intramuscular doses of DV-601 $(0.1,0.25$, or $0.5 \mathrm{~mL})$ on Days 1, 15, 29, 57, 71, and 85. Additional patients, positive for HBV e antigen and treatment- naïve, were enrolled to receive the highest tolerated dose level of DV-601. Oral entecavir therapy was started prior to the first DV-601 injection, and was continued daily during the study. The primary objective was to assess the safety and tolerability of DV-601 by the evaluation of local and systemic adverse events to Day 99, including changes in laboratory analyses and in physical examinations. Secondary objectives were to evaluate virological response and immunogenicity of DV-601 based on HBV viral load, and humoral and $\mathrm{T}$ cell immunological responses. Fourteen patients were enrolled and completed all injections of DV-601, and have completed all safety evaluations. Injection site reactions were shortlived and generally resolved without treatment; frequent systemic injection reactions including fatigue, malaise, chills, and headache also were transient and rarely required intervention. Reductions in HBV DNA, and in quantitative analysis for s and e antigen are noted in all dose groups, and antibodies to $\mathrm{s}$ and e antigens have developed in the higher dose groups. DV-601 appears to be a safe and welltolerated therapeutic vaccine for the treatment of CHB, and virological response is evident.
PP05-127

Sustained Control of HBV DNA by a Novel Vaccine Containing HBcAg and HBsAg in Patients with Chronic Hepatitis B

S.M.F. Akbar ${ }^{1}$, M. Al-Mahtab ${ }^{2}$, J.C. Aguilar ${ }^{3}$, M. Onji ${ }^{4}$, S. Mishiro ${ }^{1}$

${ }^{I}$ Toshiba General Hospital, Tokyo, Japan; ${ }^{2}$ Bangabandhu Sheikh

Mujib Medical University, Dhaka, Bangladesh; ${ }^{3}$ Center for Genetic Engineering and Biotechnology, Havana, Cuba; ${ }^{4}$ Ehime University Graduate School of Medicine, Toon, Japan

Background/aims: Almost all antiviral drugs reduce or induce undetected hepatitis B virus (HBV) DNA during therapy in patients with chronic hepatitis B (CHB). However, HBV DNA negative status of CHB patients is seldom maintained after end drug therapy. Immune therapy, such hepatitis B surface antigen (HBsAg)-based vaccine therapy, has also shown similar efficacy even when this been applied with antiviral drugs. This study assessed if addition of hepatitis B core antigen ( $\mathrm{HBcAg}$ ) with $\mathrm{HBsAg}$ can afford sustained control of $\mathrm{HBV}$ replication during off treatment period in $\mathrm{CHB}$ patients.

Subjects: Eighteen treatment-naïve Bangladeshi patients with $\mathrm{CHB}$ with detectable levels of HBV DNA and elevated levels of alanine aminotransferase (ALT) were administered with a human-consumable vaccine containing both $\mathrm{HBsAg}$ and $\mathrm{HBcAg}$ (Center for Genetic Engineering and Biotechnology, Havana, Cuba) for 10 times at an interval of 2 weeks ( 5 times by nasal spray followed by 5 times by both nasal spray and subcutaneous injection). Features of safety, kinetics of HBV DNA, and levels of ALT were assessed in all patients before therapy commencement, once in every 2 weeks during therapy, and 12 months after cessation of therapy.

Results: Just after end of therapy (after 10 nasal and 5 parental vaccines), HBV DNA became undetectable in 6 patients and the levels of ALT reduced below upper limit of normal (ULN) in 16 patients with CHB. Although patients were not given any antiviral or immune modulators during follow up, 9 patients became negative for HBV DNA and the levels of ALT were within ULN in all patients 12 months after end of therapy.

Conclusions: This is the first study that showed addition of $\mathrm{HBcAg}$ in HBsAg-based vaccine induce potent antiviral and liver protection capacities in $\mathrm{CHB}$ patients during off-treatment period. A randomized-controlled trial with $\mathrm{HBs} \mathrm{Ag} / \mathrm{HBcAg}$ vaccine in $\mathrm{CHB}$ patients is warranted.

\section{PP05-128}

Roadmap-guided Lamivudine Therapy for Treatment-naive Chinese Patients with HBeAg-positive Chronic Hepatitis B

R. Jin, X. Guo, S. Zhang, Y. Zhang

Department of Hepatology and Gastroenterology, You-An Hospital, Capital Medical University, Beijing, China

Background/aim: Lamivudine is one of first-line options for chronic hepatitis B in developing countries because of its low-cost, even though its long-term efficacy may be impacted by increasing drug resistance. The "roadmap" concept using on-treatment HBV-DNA level as a predictor for drug resistance may be useful to decrease the risk of virological breakthrough. We studied the efficacy and safety of roadmap-guided lamivudine therapy for treatment-naive Chinese patients with $\mathrm{HBeAg}$-positive chronic hepatitis B.

Methods: One hundred and eighty-seven treatment-naive Chinese patients with $\mathrm{HBeAg}$-positive chronic hepatitis B and elevated ALT levels $(\geq 2 \times$ ULN) were treated with lamivudine. HBV-DNA levels were measured at week 24 to guild continuing therapy. If HBV-DNA levels turned negative, lamivudine mono-therapy was continued, otherwise, adefovir adding on and combination therapy with lamivudine was initiated. All patients were followed up for at least 12 months.

Results: At week 24, HBV-DNA levels turned negative in $74.3 \%$ $(139 / 187)$ patients and lamivudine mono-therapy was continued; and 
adefovir was added on in $25.7 \%$ (48/187) patients with measurable HBV-DNA. At week 48, HBV-DNA negative conversion and $\mathrm{HBeAg}$ conversion rates were $82.9 \%(155 / 187)$ and $16.6 \%$ (31/187), respectively. ALT levels were normalized in $76.5 \%$ (143/187) and $86.1 \%$ (161/187) patients at week 24 and 48, respectively. YMDD mutation was detected in $5.3 \%(10 / 187)$ at week 48 . There were no obvious side effects observed.

Conclusion: Roadmap-guided lamivudine therapy was safe and efficious for treatment-naive $\mathrm{HBeAg}$-positive chronic hepatitis B. The risk of drug resistance was dramatically decreased.

\section{PP05-129}

Telbivudine 2303 Studies: Sub-analysis of Taiwan Patients H.-W. Hsu ${ }^{1}$, Y.-C. Chen ${ }^{1}$, Y.-C. Chao ${ }^{2}$, C.-M. Lee ${ }^{3}$, T.-T. Chang ${ }^{4}$ ${ }^{1}$ Liver Research Unit, Chang Gung Memorial Hospital, Chang Gung University College of Medicine, Taoyuan County; ${ }^{2}$ Tri-Service Hospital, Taipei City; ${ }^{3}$ Liver Research Unit, Chang Gung Memorial Hospital, Chang Gung University College of Medicine, Kaohsiung City; ${ }^{4}$ National Cheng Kung University Hospital, Tainan, Taiwan, ROC

Background/aim: Over 4 years, continued telbivudine (LDT) treatment is an effective antiviral treatment for CHB in bothHBeAgpositive and -negative patients in GLOBE study (2303 extension study). Among $\mathrm{HBeAg}$-positivepatients, 79\% achieved undetectable HBV DNA with $51 \% \mathrm{HBeAg}$ seroconversion. For HBeAg-negativepatients $84 \%$ achieved undetectable HBV DNA and 91\% ALT normalization. We aimed to evaluate thecomparability of Taiwan subanalysis to 2303 study.

Methods: ITT population was 41 compensated CHB patients (16 $\mathrm{HBeAg}+$ and $25 \mathrm{HBeAg}$ - patients) treated for 2 years in the LDT arm of the GLOBE studies, excluding patients with genotypic resistance. Patientscontinued LDT treatment for 2 additional years. Efficacy was evaluated based on HBV DNAdetectable/undetectable ( $<300$ copies/mL), ALT normalization and $\mathrm{HBeAg}$ serconversion rate (in $\mathrm{HBeAg}+$ group).

Results: 41 (16 $\mathrm{HBeAg}+$ and $25 \mathrm{HBeAg}-)$ compensated $\mathrm{CHB}$ patients from Taiwan. $81 \%$ were HBV Genotype B, $19 \%$ genotype C. At time of entering 2303, HBV DNA levels are $4.7 \log _{10}$ copies $/ \mathrm{ml}$ for $\mathrm{HBeAg}+$ and $2.3 \log _{10}$ copies/ml for $\mathrm{HBeAg}-$ patients. After 2 additional years LDT treatment, patients who achieved PCR negative at week 24 , had $100 \%$ HBV DNA undetectable rate, $71 \%$ ALT nomalization and $57 \% \mathrm{HBeAg}$ seroconversion rate in $\mathrm{HBeAg}+$ patients. For HBeAg- patients achieved PCR negative at week 24, had $76 \%$ HBV DNA undetectable rate, $83 \%$ ALT nomalization. 4-year genotypic resistance rates are $0 \%$ and $8.7 \%$ of $\mathrm{HBeAg}+$ and $\mathrm{HBeAg}-$ patients who achieved PCR negative at week 24, respectively. Offtreatment durability of both 1 and 2 years are $100 \%$ in patients who stopped LDT treatment due to efficacy.

Conclusion: Results of Taiwan sub-analysis is comparable to GLOBE[C7] 2303 study. Both results show efficacy of LDT long term treatment with favorable HBV DNA undetectable and ALT normalization rates in both $\mathrm{HBeAg}+$ and $\mathrm{HBeAg}$ - patients, And a high[C8] $\mathrm{HBeAg}$ seronconversion rate and promising off-treatment durability in $\mathrm{HBeAg}+$ patients.

\section{PP05-130}

The Efficacy of Telbivudine in Chinese Patients with HBeAg Positive CHB S. Ji, J. Wang, Y. Xu, Y. Zhang, T. Siqing, P. Zhao, H. Guo, C. Zhou, Y. Li, J. Jiao

China-Japan Union Hospital, Jilin University, Changchun, China

Objectives: To evaluate the antiviral therapeutic efficiency of 96-weeks Telbivudine treatment for the Hepatitis B e antigen (HBeAg)-positive chronic hepatitis B (CHB) patients.
Methods: In this study, 132 treatment-naive patients with $\mathrm{HBeAg}$ positive $\mathrm{CHB}$ were randomly assigned to receive $600 \mathrm{mg}$ of Telbivudine or $0.5 \mathrm{mg}$ of Entecavir once daily. To compare the curative effect of Telbivudine and Entecavir in terms of serum HBV DNA levels,normalization of alanine aminotransferase levels (ALT), the loss and seroconversion rate of $\mathrm{HBeAg}$ and $\mathrm{HBsAg}$ in week 12, week 24, week 48 and week 96, respectively.

Results: For patients of HBeAg-positive $\mathrm{CHB}$ with Telbivudine treatment, the indices were shown in week 12 , week 24 , week 48 and week 96. The serum HBV DNA reduction was $3.95 \log _{10}$ copies $/ \mathrm{ml}, 4.99 \log _{10}$ copies $/ \mathrm{ml}, 5.16 \log _{10}$ copies $/ \mathrm{ml}$ and 5.56 $\log _{10}$ copies/ml, respectively. The proportion of patients with HBV DNA to levels undetectable by polymerase chain reaction assay was $68.6,76.9,87.5$ and $91.1 \%$, respectively. The normalization rate of ALT was 67.9, 83.9, 94.6 and $95.6 \%$, respectively. The treatment efficiency of Telbivudine was similar with that of Entecavir. For Telbivudine treatment, the serum loss and seroconversion rate of $\mathrm{HBeAg}$ were 21.4 and $12.5 \%$ in week 24 , and further increased in week $48(33.9,19.6 \%)$ and week 96 (46.4, $30.4 \%$ ); the HBsAg loss rate of Telbivudine in week 48 and week 96 were 1.6 and $4.7 \%$, and the HBsAg seroconversion rate of Telbivudine in week 96 was $1.6 \%$. The $\mathrm{HBeAg} / \mathrm{HBsAg}$ loss and seroconversion rate of Telbivudine were higher than that of Entecavir.

Conclusion: Telbivudine demonstrated powerful and rapid HBV DNA suppression after 12 weeks of treatment, and it showed higher loss and seroconversion of $\mathrm{HBeAg} / \mathrm{HBsAg}$. Telbivudine is an ideal new analogue of antiviral therapy in $\mathrm{HBe} A g$ positive chronic hepatitis B patients.

\section{PP05-131}

Virologic Response of Entecavir versus Tenofovir Therapy in Patients Following Treatment Failure with Adefovir Therapy in Chronic Hepatitis B

E. Doyuk Kartal, I. Ozgunes, N. Erben, S. Nayman Alpat, G. Usluer Eskisehir Osmangazi University, Eskisehir, Turkey

Backgrounds: The autors assessed the virologic response of Entecavir versus Tenofovir therapy in patients following treatment failure with Adefovir therapy in chronic hepatitis B.

Methods: In this prospective study a total 18 patients who received 1 mg Entecavir once daily $(n=12)$ or $245 \mathrm{mg}$ Tenofovir disoproksil once daily $(n=6)$ at least 48 weeks were included. 3 patients $(50 \%)$ in Tenofovir group and 9 patients $(75 \%)$ in Entecavir group were Lamivudine-experienced. 2 patients who have M204V mutation and 1 patient who has A 181T mutation were in Entecavir group. No patient who have mutations was in Tenofovir group.

Results: At weeks 12, 24, 48 mean reduction in serum HBV DNA levels from baseline were $-4.1,-5.2,-4.3 \log { }_{10} \mathrm{IU} / \mathrm{mL}$ in Tenofovir group and $-3.6,-3.8,-3.5 \log { }_{10} \mathrm{IU} / \mathrm{mL}$ in Entecavir group respectivelly $(p=0.515,0.346,0.540)$. The proportions of patients with undetectable serum HBV DNA levels $(<10 \mathrm{copy} / \mathrm{mL}$ by realtime PCR) at weeks $12,24,48$ were $66.7,83.3,83.3 \%$ in Tenofovir group and $25,33.3,45.5 \%$ in Entecavir group respectivelly ( $p=$ $0.141,0.131,0.336)$. The rates of HBV negativity were not significantly different between two groups during 48 weeks. The rates of ALT normalization were not significantly different between two groups at weeks 12, 24, 48 ( $p=0.627,0.615,0.515)$. HBe Ag loss or seroconversion and HBsAg loss or seroconversion were not seen both two groups.

Conclusions: Both of Tenofovir and Entecavir therapy showed a similar virologic response at week 48 in patients following treatment failure with adefovir therapy in chronic hepatitis B. 


\section{PP05-133}

The Role of Basal Core Promoter(BCP) and Precore Codon Mutations in Pegile-interferon Response

S. Köse, G. Çavdar, P. Adar, S. Yavaş

Infectious Diseases and Clinical Microbiology, Tepecik Educational and Research Hospital, Izmir, Turkey

Introduction: Pegile interferon has an important place in chronic hepatitis treatment and there are lots of factors that effect treatment. Behind these factors, studies about viral genom mutations increase recently. In this study we aimed to determine the effects of precore and basal core promoter (BCP) mutations in the response of peginterferon treatment.

Materials and methods: Chronic hepatitis $\mathrm{b}$ patient enrolled to study. Before the treatment precore and $\mathrm{BCP}$ mutations studied by Multiplex PCR and Reverse Hybridization

Methods: (Inno-Lipa HBV DR v2.Innogenetics, Belgium). Biochemical and virological response rates were calculated for patients treated with peg-interferon therapy 48 for weeks.

Results: 22 patients had pc codon 28 mutations, 10 patients had BCP (A1762/T1764) or BCP (T1762/A1764) mutations, 19 had pc codon 28 mutations with BCP mutations on the other hand 27 patients had no mutations. Virologic response rates in pc codon 28 mutation group were $68.1 \%$, in BCP mutation group $80 \%$, group with both mutations were $73.7 \%$ and group with no mutations were $70.4 \%$.

Discusion: With these results we can say that BCP mutations has an advantage in peg-interferon treatment response. However, to be an effective criteria for the response of interferon therapy there has to be new studies with much larger groups.

\section{PP05-134}

A Randomized, Controlled, Open-labeled, Multi-center Study of HBeAg-Positive Chronic Hepatitis B Patients Treated by Adefovir Dipivoxil or Combined with Bicyclol

J. Cheng ${ }^{1}$, G.-F. Shi ${ }^{2}$, H.-F. Zhang ${ }^{3}$, G.-M. Zhao ${ }^{4}$, Z.-J. Yu ${ }^{5}$, Z.-W. Lang ${ }^{6}$ ${ }^{1}$ Center for Liver Diseases, Beijing Ditan Hospital, Capital Medical University, Beijing; ${ }^{2}$ Department of Infectious Diseases, Huashan Hospital, Fudan University, Shanghai, ${ }^{3}$ Department of Pediatric Hepatology, The 302 Hospital of PLA, Beijing; ${ }^{4}$ Department of Hepatology, Tianjin Municiple Hospital of Infectious Diseases, Tianjin; ${ }^{5}$ Department of Infectious Diseases, The First Hospital, Zhengzhou University, Zhengzhou; ${ }^{6}$ Department of Pathology, Beijing Ditan Hospital, Capital Medical University, Beijing, China

Objective: To evaluate efficacy and safety of adefovir dipivoxil or in combination with bicyclol in treatment of patients with $\mathrm{HBeAg}(+)$ chronic viral hepatitis B (CHB).

Methods: Two-hundred and fifty patients with $\mathrm{HBeAg}(+) \mathrm{CHB}$ were randomized into treatment group and control group. The patients in the treatment group $(n=125)$ treated with adefovir dipivoxil $10 \mathrm{mg}$ orally qd plus bicyclol $25 \mathrm{mg}$ orally tid for 48 weeks, and those in the control group $(n=125)$ treated with adefovir dipivoxil alone for 48 weeks. The serum aminotransferase (ALT/AST), HBV DNA, $\mathrm{HBe} \mathrm{Ag} / \mathrm{HBe} \mathrm{Ab}$ and liver histology were observed before and after therapy.

Results: The serum ALT and AST levels were decreased significantly in two groups at the end of treatment comparing with baseline levels $(P<0.01)$. The serum ALT and AST levels in treatment group were lower than that of control group $(P<0.01)$. Virology responses of the treatment group were not statistically different from control group. The Knodell necroinflammatory scores were decreased significantly in two groups at the end of treatment comparing with baseline levels $(P<0.01)$. The Knodell necroinflammatory scores in treatment group were lower than that of control group $(P<0.05)$. There were no remarkable adverse events related to drug in this study.

Conclusions: Combined adefovir dipivoxil with bicyclol treatment of chronic hepatitis B is effective and safe.

\section{PP05-135}

Long-term Monitoring of HBsAg Kinetics and Prediction of HBsAg Clearance in Patients with CHB Treated with Nucleos(t)ide Analogues

S. Chevaliez ${ }^{1}$, C. Hézode $^{2}$, M. Grare ${ }^{1}$, J.-M. Pawlotsky ${ }^{1}$

${ }^{1}$ Virology and INSERM U955; ${ }^{2}$ Hepatology and Gastro-Enterology, Faculty of Medecine UPEC, Creteil, France

In patients with chronic hepatitis $B$ treated with nucleos(t)ide analogues (NUCs), the long-term kinetics of HBsAg are unknown, and the role of serial quantitative determinations of $\mathrm{HBsAg}$ levels in tailoring NUC treatment duration needs to be clarified.

Methods: HBsAg and HBV DNA levels were examined in 583 serial serum samples taken from 30 patients with chronic hepatitis B receiving various successive schedules of NUCs over several years of follow-up. The median age of the patients was 57.6 years, $80 \%$ were male and $43 \%$ of them had cirrhosis. The kinetics of HBsAg levels were plotted and the average $\mathrm{HBsAg}$ reduction per year was calculated, in order to predict the treatment duration needed in order to achieve an HBsAg clearance.

Results: The median follow-up was 93 months (range 49-123 months). For each patient, an average of $19 \pm 6$ serial samples was available (range 8-41). HBsAg levels slightly increased during therapy in 3 patients, including 2 who lost their HBV DNA and remained HBV DNA-undetectable for 28 and 71 months, respectively. One patient lost HBsAg after 72 months of undetectable HBV DNA. In the remaining 26 patients, HBsAg levels slowly and constantly decreased during therapy. Overall, 3 patients were predicted to clear HBsAg in 10 years or less, 5 within $10-20$ years, 5 within $20-30$ years and 13 in more than 30 years. The number of years needed to clear HBsAg was not related to any baseline parameter, or to the duration of undetectable HBV DNA.

Conclusions: HBsAg clearance is a rare and late event, unlikely to occur during the patient's lifetime in most cases. As a result, the goal of NUC therapy should remain to maintain HBV DNA at undetectable levels, while stopping NUC therapy is unlikely in the vast majority of patients.

\section{PP05-136}

Generation of Hepatitis B Core Antigen Exposing a Pres1-42 Sequence on the Surface

I.R. Malik ${ }^{1}$, A. Chen ${ }^{2}$, M. Sallberg ${ }^{2}$, L. Frelin ${ }^{2}$, M. Rahman ${ }^{1}$, J.A. Qureshi ${ }^{1}$ ${ }^{1}$ Health Biotechnology Division, National Institute for Biotechnology and Genetic Engineering, (NIBGE), Faisalabad, Pakistan; ${ }^{2}$ Division of Clinical Microbiology, Karolinska Institutet, at Karolinska University Hospital, Stockholm, Sweden

Background and aims: The aim of the study was to develop virus like Particles (VLPs) using the hepatitis B virus (HBV) core gene as a carrier for PreS1-42 region and to evaluate its antigenicity and immunogenicity in mice.

Methods: Viral DNA was isolated from a patient with chronic HBV. Core and Pres1-42 genes were amplified by polymerase chain reaction and cloned into the pET28a expression vector. The chimeric protein was expressed in E. coli and purified affinity chromatograph. Evaluation of antigenicity and immunogenicity of chimeric protein was carried out by ELISA, Western blot, and in C57BL/6 mice. ELISpot assay was used to determine the $\mathrm{T}$ cell response against chimeric protein.

Results: A chimeric protein was expressed and purified by affinity chromatography. Western blot revealed that $E$. coli produce chimeric protein had the expected size $(24 \mathrm{kDa})$. The chimeric protein was recognized by both classical anti-HBc antibodies and by a monoclonal antibody to pre-S1 and showed high antibody titre. ELISpot results showed that chimeric protein showed cytotoxic $\mathrm{T}$ cell specific response when they were incubated with spleen isolated from immunized mice with chimeric protein.

Conclusion: A chimeric protein combining the immunogenicity of $\mathrm{HBcAg}$ with surface exposed preS1 sequences can be a good candidate for therapeutic vaccine against hepatitis B. 
PP05-137

Decline of Intrahepatic cccDNA and Increase of Immune Cell Reactivity at Week 12 of Adefovir Dipivoxil Mono-treatment

Q. Zheng, J. Dong, Y.-Y. Zhu, J.-J. Jiang

Center of Liver Diseases, The First Affiliated Hospital of Fujian Medical University, Fuzhou, China

Background: Early viraemia clearance facilitates the recovery of antiviral $\mathrm{T}$ cell responses. The dynamic changes of intrahepatic viraemia clearance and immune cells reactivity during early phase of nucleos(t)ide therapy and the impact of these changes on $\mathrm{HBeAg}$ seroconversion are unknown.

Methods: Eight $\mathrm{HBeAg}(+)$ chronic hepatitis B patients were treated with adefovir dipivoxil for 48 weeks. Paired liver biopsies before and at week 12 of treatment were analyzed for intrahepatic HBVDNA and cccDNA by the real-time fluorescent PCR. In situ expressions of CD4, CD8 $\mathrm{T}$ cells, and $\mathrm{NK}$ cells were also analyzed by immunohistochemistry.

Results: After 48 weeks of ADV therapy, $\mathrm{HBeAg}$ loss was observed in 3 of the $8(37 \%) \mathrm{HBeAg}(+)$ patients.After 12 weeks treatment, median intrahepatic total HBV-DNA and cccDNA had decreased by $1.52 \log _{10}$ and $1.92 \log _{10}$ copies/ug, respectively. About $98 \%$ intrahepatic HBVDNA were cleared in the early phase of the anti-viral treatment. Lower baseline levels of intracellular HBVDNA and cccDNA were found in the patients with $\mathrm{HBeAg}$ loss than in those who remained $\mathrm{HBeAg+}$. Patients with $\mathrm{HBeAg}$ loss had lower cccDNA levels at week 12 than patients who remained HBeAg+. In parallel to the decline in viral load, the numbers of intrahepatic CD8+ T-lymphocytes and NK cell increased in patients with HBeAg loss compared with their baseline values. Only one patients without $\mathrm{HBeAg}$ loss has similar result.

Conclusion: The increased numbers of intrahepatic CD8+ T-lymphocytes and NK cell is associated with the lower levels of cccDNA in the early phase of anti-viral treatment, which would promote the recovery of anti-viral immunity and facilitate the HBeAg loss.

\section{PP05-138}

Enhanced Immunogenicity of Epitope Pres12-32 Inserted in the Immuno-dominant Region of Hepatitis B Core Molecule

I.R. Malik ${ }^{1}$, M. Sallberg ${ }^{2}$, L. Frelin ${ }^{2}$, A. Ghaffar ${ }^{1}$, M. Salahuddin ${ }^{1}$, N.A. Malik ${ }^{1}$, M. Rahman ${ }^{1}$, J.A. Qureshi ${ }^{1}$

${ }^{I}$ Health Biotechnology Division, National Institute for Biotechnology and Genetic Engineering, (NIBGE), Faisalabad, Pakistan; ${ }^{2}$ Division of Clinical Microbiology, Karolinska Institutet, at Karolinska

University Hospital, Stockholm, Sweden

Background and aims: The aim of the study was to develop virus like Particles (VLPs) using the hepatitis B virus (HBV) core gene as a carrier for PreS12-32 region and to evaluate its antigenicity and immunogenicity.

Methods: Viral DNA was isolated from a patient with chronic HBV. Core and Pres12-32 genes were amplified by polymerase chain reaction and cloned into the pET28a expression vector. The chimeric protein was expressed in E. coli and purified affinity chromatograph. Evaluation of antigenicity and immunogenicity of chimeric protein was carried out by ELISA.

Results: A chimeric protein was expressed and purified by affinity chromatography. SDS-PAGE results showed the exact size of protein band $(20 \mathrm{kDa})$. The chimeric protein was recognized by both classical anti-HBc antibodies and a monoclonal antibody to pre-S1.

Conclusion: A chimeric protein combining the immunogenicity of $\mathrm{HBcAg}$ with surface exposed preS1 sequences was expressed in vitro. In conclusion, the immunodominant region of $\mathrm{HBcAg}$ with the dele- tion of amino acid at position 79 may be serve as a good carrier for preS12-32 sequence of epitope and for other epitopes. This approach could generate a new type of protective vaccines against HBV.

\section{PP05-139}

A Three-year Trial with Adefovir Dipivoxil (ADV) for Patients with Chronic Hepatitis B (CHB) Related Advanced Fibrosis or Cirrhosis C.M. Lee ${ }^{1}$, S.K. Yoon ${ }^{2}$, D.D.1. Huong ${ }^{3}$, T. Tanwandee ${ }^{4}$, M. Cho ${ }^{5}$, M.Y. Lai ${ }^{6}$, B.C. Yoo $^{7}$, K.H. Han $^{8}$, S.G. Hwang ${ }^{9}$, Y.O. Kweon ${ }^{10}$, M.F. Yuen ${ }^{11}$, Y.C. Chao ${ }^{12}$, W. Choi ${ }^{13}$

${ }^{1}$ Internal Medicine, Kaohsiung Chang Gung Memorial Hospital, Kaohsiung, Taiwan, ROC; ${ }^{2}$ Department of Gastroenterology, The Catholic University of Korea, St Mary's Hospital, Seoul, Republic of Korea; ${ }^{3}$ Department of Hepatology, Medical University Hospital, Ho Chi Minh City, Vietnam; ${ }^{4}$ Department of Medicine, Division of Gastroenterology, Siriraj Hospital, Bangkok, Thailand; ${ }^{5}$ Department of Gastroenterology, Pusan National University Yangsan Hospital, Yangsan, Republic of Korea; ${ }^{6}$ Department of Internal Medicine, National Taiwan University Hospital, Taipei, Taiwan ROC;

${ }^{7}$ Department of Medicine, Sungkyunkwan University Samsung Medical Center; ${ }^{8}$ Department of Gastroenterology, Yonsei University College of Medicine, Severance Hospital, Seoul; ${ }^{9}$ Department of Gastroenterology, CHA Bundang Medical Center, CHA University, Sungnam; ${ }^{10}$ Department of Gastroenterology, Kyungpook National University Hospital, Daegu, Republic of Korea; ${ }^{11}$ Department of Medicine, Queen Mary Hospital, Hong Kong, China; ${ }^{12}$ Department of Internal Medicine, Tri Service General Hospital, Taipei, Taiwan, ROC, ${ }^{13}$ Asia Pacific Medical, GlaxoSmithKline, Seoul, Republic of Korea

Background: Data on long term antiviral therapy in CHB related compensated liver cirrhosis is limited. In this phase IV study, we evaluated the efficacy and safety of ADV in subjects with CHBrelated advanced fibrosis.

Methods: Open-label ADV $10 \mathrm{mg}$ once daily was given for 3 years to 155 subjects with CHB-related advanced fibrosis/cirrhosis (Ishak fibrosis score $\geq 4$ ) and baseline HBV DNA $\geqq 1 \times 10^{5}$ copies $/ \mathrm{mL}$.

Results: At month 36,33.5\% (52/155) of subjects had a reduction of at least 1 point in the Ishak fibrosis score and $41.3 \%(64 / 155)$ had a reduction of at least 2 points in the Knodell necroinflammation score. A HBV DNA level of $<300$ copies/mL (Amplicor Cobas ${ }^{\mathrm{TM}}$ assay) was reported in $55.0 \%(83 / 151), 66.9 \%(95 / 142)$ and $67.7 \%(90 / 133)$ of subjects at month 12, 24 and 36 respectively. Genotypic resistance with virologic breakthrough was found in $0 \%, 10.6 \%(15 / 142)$ and $10.5 \%(18 / 133)$ at months 12,24 and 36 respectively. Normalization of ALT levels were achieved in 58.4\% (87/149), 65.0\% (91/140) and $69.5 \%(91 / 131)$ of subjects at months 12,24 and 36 respectively. $\mathrm{HBeAg}$ loss/seroconversion occurred in $21.0 \%$ (17/81) $/ 12.3 \%$ (10/81), $37.0 \%(27 / 73) / 24.7 \%(18 / 73)$ and $41.2 \%(28 / 68) / 22.1 \%$ $(15 / 68)$ of subjects at months 12,24 and 36 respectively. Histological improvement was seen in similar proportions of subjects with genotype C $(33.9 \%, 42 / 124)$ and B $(30.0 \%, 9 / 30)$. Ten patients developed malignant hepatic neoplasm including one death (none considered related to ADV by the investigators). Of them, five developed the condition one year after enrolling into the study. Adverse event and laboratory profiles were consistent with advanced liver disease and associated complications, with no unexpected safety consequences. One subject had worsening diabetic nephropathy that was considered related to ADV.

Conclusions: ADV resulted in improvements in histological, virological, biochemical, and clinical parameters in CHB-related cirrhosis. 
PP05-140

Combined Responders Associated with Better On-treatment Decline of HBsAg in HBeAg-positive Chronic Hepatitis B Patients Treated with Peginterferon Alfa-2a

C.-H. Chen, S.-N. Lu, C.-M. Lee, J.-H. Wang, C.-H. Hung, T.-H. Hu

Department of Internal Medicine, Kaohsiung Chang Gung Memorial Hospital, Kaohsiung, Taiwan, ROC

Background/aims: Serum hepatitis B surface antigen (HBsAg) levels may reflect the immunomodulatory efficacy of pegylated interferon therapy. We investigated whether serum quantitative HBsAg predict treatment response to peginterferon alfa-2a in patients with hepatitis $\mathrm{B}$ e antigen (HBeAg)-positive chronic hepatitis B.

Methods: A total of $83 \mathrm{HBeAg}$-positive patients receiving peginterferon alfa-2a for 6 months and followed for at least 24 weeks were prospectively analyzed. Serum HBsAg was measured in samples taken at baseline and weeks 12, 24 of therapy and weeks 24 posttreatment.

Results: After 24 weeks of follow-up, 30.1\% (25/83) patients achieved combined response. Combined response was defined as a serum HBeAg loss and HBV DNA level below $10^{5}$ copies $/ \mathrm{mL}$ at 24 weeks posttreatment. Multivariate analysis disclosed that pretreatment $\mathrm{HBeAg}$ S/Co ratio£200 and HBV genotype B were independent factors for combined response. Combined responders experienced a significantly more pronounced decline in serum HBsAg compared to nonresponders (decline at week 12: 1.2 versus $0.6 \log \mathrm{IU} / \mathrm{mL}$, $P=0.008$, decline at week 24: 1.3 versus $0.8 \log \mathrm{IU} / \mathrm{mL}, P=0.012$, decline at week 24 posttreatment: 1.0 versus $0.3 \log \mathrm{IU} / \mathrm{mL}, \mathrm{P}<$ 0.001). Compared patients with HBV genotype $C$, patients with $\mathrm{HBV}$ genotype $\mathrm{B}$ had a significantly more pronounced decline in serum HBsAg (decline at week 12: 1.1 versus $0.4 \log \mathrm{IU} / \mathrm{mL}, P=0.001$, decline at week 24: 1.4 versus $0.5 \log \mathrm{IU} / \mathrm{mL}, P<0.001$, decline at week 24 posttreatment: 0.8 versus $0.2 \log \mathrm{IU} / \mathrm{mL}, P=0.001$ ). The only baseline factors identified by multivariate analysis that were significantly associated with decline in HBsAg at week 12 and 24 of therapy and at week 24 posttreatment were HBV genotype B and ALT $>200 \mathrm{U} / \mathrm{ml}$

Conclusions: Combined Responders had a significant decline of serum $\mathrm{HBsAg}$ in $\mathrm{HBeAg}$-positive patients treated with peginterferon alfa-2a. HBV genotype B has a strong influence on serum HBsAg decline than $\mathrm{HBV}$ genotype $\mathrm{C}$ during peginterferon treatment.

\section{PP05-141}

Efficacy and Safety of Entecavir in the Older Patient ( 250 Years of Age)

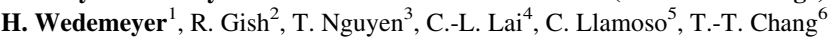
${ }^{1}$ Department of Gastroenterology, Hepatology, and Endocrinology, Hannover Medical School, Hannover, Germany; ${ }^{2}$ Division of Hepatology and Complex GI, California Pacific Medical Center, San Francisco; ${ }^{3}$ Research and Education, Inc., San Diego, CA, USA; ${ }^{4}$ Queen Mary Hospital, Hong Kong, China; ${ }^{5}$ Research and Development, Bristol-Myers Squibb Company, Wallingford, CT, USA; ${ }^{6}$ National Cheng Kung University Medical College, Tainan, Taiwan, $R O C$

Background: There are limited data on the safety and efficacy of hepatitis B virus (HBV) nucleos(t)ide analog therapy in older patients. To address this gap, we carried out a subanalysis of 48-week data from the global entecavir (ETV) registrational trials.

Methods: The subanalysis included nucleoside-naïve patients $\geq 50$ years old (at start of therapy) randomized to once-daily ETV $0.5 \mathrm{mg}$ in ETV-022 (HBeAg[+] patients) and ETV-027 (HBeAg[-] patients). Efficacy, safety, and tolerability data were assessed.

Results: 147 patients were assessed; mean age 58 years, $75 \%$ male, 69\% Caucasian; 29\% Asian; 2\% Black/African American, 69\% $\mathrm{HBeAg}(-)$. The majority $(90 \%)$ had at least one comorbidity; cardiovascular (41\%), gastrointestinal (34\%), musculoskeletal $(27 \%)$, and endocrine conditions (26\%) were the most common; 29 patients (20\%) had diabetes mellitus and 33 (22\%) had hypertension.

Summary of baseline and week 48 data

\begin{tabular}{llll}
\hline Patients aged $>50$ year, $N=147$ & $\begin{array}{l}\operatorname{HBeAg}(+), \\
n=45\end{array}$ & $\begin{array}{l}\text { HBeAg(-), } \\
n=102\end{array}$ \\
\hline Baseline & $\begin{array}{c}\text { Mean HBV DNA by PCR, } \\
\log _{10} \text { c/mL (SE) } \\
\text { Mean ALT, U/L (SE) }\end{array}$ & $9.83(0.29)$ & $7.48(0.16)$ \\
(BL) & $143.60(15.62)$ & $125.25(9.22)$ \\
\hline Week 48 & $\begin{array}{c}\text { Mean change from BL in HBV } \\
\text { DNA by PCR, } \log _{10} \mathrm{c} / \mathrm{mL}\end{array}$ & 7.08 & 5.1 \\
& $\begin{array}{l}\text { HBV DNA <300 copies/mL, } n(\%) \\
\text { ALT normalization, } \\
\quad \text { (ALT < } \times \text { ULN), n(\%) }\end{array}$ & $31(69)$ & $96(94)$ \\
& $31(69)$ & $82(80)$ \\
HBeAg loss/seroconversion. $n / N(\%)$ & $13 / 44(40)$ & - \\
Histologic improvement ${ }^{\#}, n / N(\%)$ & $35 / 42(83)$ & $70 / 97(72)$ \\
\hline
\end{tabular}

* Analysis method is non-completer $=$ failure

Missing due to no(or inadequate) Week 48 biopsy sample

\# >2-point decrease in Knodell necroinflammatory score, without worsening of Knodell fibrosis score

Serious adverse events (SAEs) were reported in 21 patients (14\%); no single SAE was reported in $>2(1 \%)$ patients. Only one patient discontinued treatment due to an adverse event. No grade $3 / 4$ renal laboratory abnormalities (creatinine) were noted.

Conclusions: In this older population, a substantial proportion of whom had comorbidities, ETV was efficacious and well tolerated through 48 weeks of treatment and consistent with results from the ETV registrational studies.

\section{PP05-142}

IIIness Perception, Quality of Life and Self Efficacy in Chronic Hepatitis B Patients

V. Sharma ${ }^{1,2}$, M.K. Sharma ${ }^{3}$, S.K. Sarin ${ }^{3,4}$

${ }^{I}$ Faculty of Health, Staffordshire University, Stoke on Trent, UK;

${ }^{2}$ Department of Health Education, Dr BL Kapur Memorial Hospital; ${ }^{3}$ Department of Hepatology, Institute of Liver \& Biliary Sciences (ILBS); ${ }^{4}$ Department of Gastroenterology, G B Pant Hospital, New Delhi, India

Introduction: Studies on illness perceptions are well-known. Less such data exists in chronic hepatitis B (CHB).

Objective: Assessment of trends in perception and other regulating factors.

Method: Chronic liver disease (CLD) outpatients were interviewed using Illness Perception Questionnaire-Revised Version (IPQ-R). IPQ-R measures nine aspects: Identity; timeline; consequence; cyclical timeline, personal controls; treatment controls; illness coherence; emotional representation and causal beliefs. Additional measures were demographic details, liver disease etiology, positive affect negative affect Scale (PANAS-X); chronic liver disease questionnaire (CLDQ); EQoL-5D and Stanford self-efficacy scale. Liver disease severity was assessed by Child's scores.

Results: 135 CLD patients, CHB $(n=67)$ and non-B CLD $(n=67)$, revealed median ages 32 versus 41 years. Lower mean ranks in illness coherence $(p<0.001)$, identity $(p=0.006)$, personal control $(p<$ 0.001) were seen in CHB when compared with non-B CLD. Factor analysis of causal beliefs confirmed highest loadings for psychological attribution $(\alpha=0.71)$.

Overall summations of CLDQ were higher in CHB (176 vs.149; $p=0.01)$ specifying higher HRQoL- confirmed by EQoL-VAS 
scores $(p=0.028)$. CLDQ measures ranked differences for abdominal symptoms $(p=0.012)$; fatigue $(p<0.001)$; systemic symptoms $(p=0.003)$ and activity $(p=0.003)$ supporting asymptomatism in CHB. However, CHB patients showed higher negative $(p=0.026)$ and lower positive $(p=0.049)$ affect from PANAS-X.

Illness coherence items confirmed lack of disease understanding in CHB by $50-60 \%(p=0.01)$. Qualitative assessment of narrative statements triangulated lack of information $(88 \%)$ and fear of deterioration (62\%) as prominent $\mathrm{CHB}$ themes.

Regression analysis showed more symptoms experienced, higher Child's scores and lower CLDQ total scores contributed to identity perceptions $\left(R^{2}=83 \% ; p=0.007\right)$ : A less relevant model for asymptomatic $\mathrm{CHB}$ chronic carriers.

Conclusions: Chronic hepatitis B patients low illness coherence from lack of information is a possibility. Other factors affecting illness perception should be explored in CHB carriers. Leventhal's model and IPQ-R may be useful in CLD-B patient management for lifestyle change.

\section{PP05-143}

Incidence of Genotypic Resistance to Lamivudine Long-term Therapy in Chronic Hepatitis B Genotype D

W. Osman ${ }^{1}$, N. Allam ${ }^{1}$, M. Hassouna ${ }^{2}$, A. Abdel-Moneim ${ }^{2}$, I. Waked ${ }^{1}$

${ }^{1}$ Hepatology, ${ }^{2}$ National Liver Institute, Menoufiya University,

Shebeen Al-koam, Egypt

Lamivudine improves patients' outcome but is reported to be associated with increasing rates of viral resistance. The long-term benefit of lamivudine therapy and rate of resistance in $\mathrm{HBeAg}$ negative genotype D patients is not fully known. The aim of the present study was to assess the incidence of genotypic resistance to lamivudine therapy in patients with chronic hepatitis B due to genotype D.

Methods: Here we analyse the data of the 85 patients with chronic hepatitis B due to genotype D who were on lamivudine $100 \mathrm{mg}$ daily for more than 1 year and showed initial response. There were 10 females, mean age was $32 \pm 8$ years. 73 (85)\% were $\mathrm{HBeAg}$ negative. Only one patient was Child C. Mean serum HBV DNA level before the start of treatment was $549,151 \pm 2,465,699$ IU/ ml. Mean follow up period was $25 \pm 10$ months (33 for 12-24 months, 27 for 24-36 months, 20 for $36-48$ and 5 for 48-60 m).

Results: ALT normalized in 35 patients and HBV-DNA decreased to $<10,000 \mathrm{c} / \mathrm{ml}$ in 25 patients $(21 \%)$, and HBV-DNA became undetectable in $42(35.7 \%)$ during the first year of treatment. The rate of relapse with either HBV-DNA reverting to positive or increasing to $>10,000 \mathrm{c} / \mathrm{ml}$ after initial response was $16 \%$ during the first year, $19 \%$ during the second year, $3.5 \%$ during the third, $1 \%$ during the fourth year of follow up. INNO-LiPA was performed for 20 patients. Wild type was found in 14 patients; mixed type in 4 and mutant (YMDD) in two patients. Mean viral load was $283,746 \mathrm{IU} / \mathrm{ml}$ compared to $5,673,000 \mathrm{IU} / \mathrm{ml}$ in the mutant.

Conclusion: Long term lamivudine therapy is associated with a high response rate with a rather low relapse rate in patients with genotype D. The incidence of YMDD mutation is low.

\section{PP05-144}

The Efficacy and the Drug-resistance of Long Term Entecavir Treatment in Nucleos(t)ide Analogues-naive Patients with Chronic Hepatitis B M. Karaagac, N. Aksoy, K. Kutoglu, N. Arican, S. Kalyon, N. Yenice 3rd Internal Medicine Clinic, Okmeydani Education and Research Hospital, Istanbul, Turkey

Objective: Many trials have been showed that entecavir (ETV) is an effective therapy with low rates of drug resistance for nucleos(t)id analogues (NA)-naive patients with chronic hepatitis B. The aim of this retrospective study is to assess these features in the patients with NA-naive chronic hepatitis B in Turkish people.

Material and methods: The datas that present in this study have been obtained by scanning the patients files retrospectively. Biochemical, serological and haematological parameters that had been achieved at the baseline and the control periods by the weeks 4, 12, 24 and the every 24th week of the treatment and the information of interferon usage before the treatment were assigned. Further more, the informations about additional diseases, consuming alcohol, smoking, weight, body mass index that had been recorded on the files at the baseline and by updating at every 24th week of the treatment were determined.

Results: A total of 40 patients were involved in this study; 30 $\mathrm{HBeAg}(-)$ and $10 \mathrm{HBe} A g(+), 26$ men and 14 women with a mean age of $41.12 \pm 12.56$ years and a mean duration of treatment of $96.60 \pm 32.83$ weeks. At the end of the treatment the proportions of undetectable HBV-DNA $(<300$ copies/ml), ALT normalization, $\mathrm{HBeAg}$ seroconversion and loss of HBsAg were 100, 95, 70\% (7/10) and $2.5 \%$ respectively. Secondary unresponsiveness to the treatment or drug-resistance was determined none of the patients. 34 of the patients achieved undetectable HBV-DNA ( $<300$ copies/ml) by the week 24. Consuming alcohol and high baseline HBV-DNA levels were the independent parameters of delayed virological response to the treatment.

Conclusions: ENT treatment can be used as an effective first line drug in the $\mathrm{HBeAg}(+)$ or (-) patients with NA-naive chronic hepatitis B in Turkish people. Although none of the patients improved drug-resistance till today, we should be on follow-up closely.

\section{PP05-145}

Serum HBV DNA and Hepatitis B Core-related Antigen in Japanese Patients with Chronic Hepatitis B during Nucleoside Analog Therapy

Y. Seo, Y. Yano, A. Miki, M. Saito, T. Azuma

Gastroenterology, Kobe University Graduate School of Medicine, Kobe, Japan

Background/aims: Serum hepatitis B core-related antigen (HBcrAg) has been reported to provide a viral marker which is independent of HBV DNA for monitoring nucleoside analogue treatment. We analyzed serum level of HBV DNA and HBcrAg during nucleoside analog therapy.

Methods: A total of 104 patients with chronic hepatitis B who were treated with nucleoside analog (4 treated with lamivudine, 23 with lamivudine + Adefovir dipivoxil, and 77 with Entecavir) for at least 6 months (median 39, range 6-108 months) in the Kobe University Hospital were enrolled in this study. The patients comprised 70 men and 34 women with a median age of 49 years (range 28-89 years). Serum concentration of $\mathrm{HBcrAg}$ was measured by commercially available CLEIA kits in 49 patients at the end of follow up. Serum level of HBV DNA was determined using a COBAS TaqMan PCR during therapy.

Results: Serum level of HBV DNA was reduced at the end of follow up (from $6.6 \pm 1.6$ to $3.8 \pm 1.7 \mathrm{log} \mathrm{copy} / \mathrm{mL}$ ). In 87 patients (83.7\%) was reduced to below $2.1 \mathrm{log}$ copy/mL (negative). In 40 of the 87 patients serum level of HBcrAg was determined. $8(20 \%)$ were $<2.9 \log \mathrm{U} / \mathrm{mL}$ (negative), and $21(52.5 \%)$ were $<4.0 \log \mathrm{U} /$ $\mathrm{mL}$ which has proposed to level may stop lamivudine administration with a low risk of reactivation. Serum HBV DNA level before therapy of 21 patients with low serum HBcrAg $(<4.0 \log \mathrm{U} / \mathrm{mL})$ during therapy were lower $(5.9 \pm 1.6$ vs. $7.2 \pm 1.4 \log$ copy $/ \mathrm{mL})$. All 21 patients with low serum $\mathrm{HBcrAg}$ developed to be $\mathrm{HBeAg}$ negative during therapy.

Conclusions: The HBcrAg assay is a sensitive and useful test for the assessment of HBV load. 


\section{PP05-146}

Efficacy and Safety of Entecavir Treatment in a Heterogenous CHB Population in a Real-life Clinical Practice Setting in China

J. Hou ${ }^{1}$, H. Ren ${ }^{2}$, Y. Wang ${ }^{3}$, Q. Xie ${ }^{4}$, D. Tan ${ }^{5}$, W. Zhao ${ }^{6}$, C. Llamoso ${ }^{7}$, D. Cohen ${ }^{7}, \mathrm{M} . \mathrm{Wu}^{8}$

${ }^{1}$ Infectious Disease, Nanfang Hospital, Southern Medical University, Guangzhou; ${ }^{2}$ Infectious Disease, Chongqing Medical University 2nd Affiliated Hospital; ${ }^{3}$ Infectious Disease, The Third Military Medical University Affiliated the First Hospital, Chongqing; ${ }^{4}$ Infectious Disease, Shanghai Jiaotong University affilated Ruijin Hospital, Shanghai; ${ }^{5}$ Infectious Disease, Central South University Xiang Ya No. 2 Hosptial, Changsha; ${ }^{6}$ Infectious Disease, Nanjing No. 2 Hospital, Nanjing, China; ${ }^{7}$ Research \& Development, Bristol-Myers Squibb, Wallingford, MA, USA; ${ }^{8}$ Research \& Development, BristolMyers Squibb, Shanghai, China

Background and aims: In patients with chronic Hepatitis B (CHB), the efficacy and safety of entecavir (ETV) was demonstrated in registrational studies. This study aimed to assess the efficacy and safety of ETV in a real-world clinical practice setting in China, to support its local manufacturing.

Methods: The study included a subset of patients enrolled in the REALM study who had received 48 weeks of treatment with ETV monotherapy $(0.5 \mathrm{mg}$ or $1.0 \mathrm{mg})$ and had baseline and on-treatment virologic and/or serologic assessments. Patients were nucleos(t)ide naïve or experienced and $\mathrm{HBe} \mathrm{Ag}(+)$ or $(-)$, and had compensated or decompensated liver disease (as assessed by the investigator).

Results: Baseline and week 48 parameters for the 2547 patients who were included and completed 48 weeks of ETV are shown in the tables.

Disease characteristics at baseline

\begin{tabular}{llll}
\hline & $\begin{array}{l}\text { Naive } \\
\text { compensated } \\
\text { liver disease }\end{array}$ & $\begin{array}{l}\text { Nucleos(t)ide } \\
\text { experienced } \\
\text { compensated } \\
\text { liver disease } \\
N=830\end{array}$ & $\begin{array}{l}\text { Decompensated } \\
\text { liver disease } \\
\text { cohort }\end{array}$ \\
& $N=1663=54$ & $N=54$ \\
\hline $\begin{array}{l}\text { Baseline } \\
\text { Mean HBV }\end{array} \quad 6.78$ & 5.97 & 4.93 \\
$\quad$ DNA $\log _{10} \mathrm{IU} / \mathrm{mL}$ & & 104 & 58 \\
Mean ALT (U/L) & 122 & 76 & 57 \\
HBeAg positive, $\%$ & 70 & &
\end{tabular}

Treatment response parameters at week 48

\begin{tabular}{llll}
\hline Week 48, n/N\# (\%) & & & \\
\hline HBV DNA* & $1135 / 1645(69)$ & $410 / 779(53)$ & $30 / 44(68)$ \\
ALT normalization & $1111 / 1284(87)$ & $421 / 508(83)$ & $15 / 21(71)$ \\
HBeAg loss & $200 / 1155(17)$ & $91 / 617(15)$ & $7 / 29(24)$ \\
HBe seroconversion & $167 / 1145(15)$ & $65 / 616(11)$ & $4 / 29(14)$ \\
\hline
\end{tabular}

*HBV DNA was analyzed using the Roche CAP/Taqman assay \#Denominator represents patients with available samples

The safety profile of ETV was consistent with previously reported experience.

Conclusions: In a real-world setting, ETV was efficacious and well tolerated through 48-weeks of treatment in a heterogenous Chinese CHB population. These data are consistent with results from the ETV registrational studies.
PP05-147

The Study about Consequence of Bad Compliance Chronic Hepatitis B Patients with Nucleoside Analogue

K. Fan, Y. Wang, Q. Mao, X. Wang Institute of Infectious Diseases for PLA, Southwest Hospital, The Third Military Medical University, Chongqing, China

Background: When patients use nucleoside analogue, the regularity of patients using drugs is a significant factor for efficacy. But we found that parts of patients did not complied the guidance of doctors and induced some bad consequences like viruses rebound and so on. So we study the causes of drug withdrawalling when patients did not to get to the best time to stop drugs.

Methods: The data come from $685 \mathrm{CHB}$ patients who are using NUC during 2002.1.1 to 2010.10.10, 218 CHB patients had a bad compliance, including male 173 and female 45 . The consequence of drug withdrawalling was researched. The number of bad compliance patients receiving LAM, ADV, LdT, ETV, LAM+ ADV, LdT + ADV, ETV + ADV were 46, 63, 41 LdT, 29, 35, 3, 1, respectively. Results: The compliance of LAM, ADV, LdT, ETV, LAM+ ADV, LdT + ADV, ETV + ADV were 65.4, 63.8, 67.2, 70.7, 73.3\% (the number of LdT + ADV and ETV + ADV were too small, so not be counted), respectively. The different between compliance of different drugs have no significant difference $(p>0.05)$. In these bad compliance patients, some ones had viruses rebound after they stopped to take medicine, the rate of viruses rebound of LAM, ADV, LdT, ETV, LAM + ADV were $72.3,59.4,60.5,50.0,70.0 \%$ respectively, and there is significant difference between LAM group and ETV group $(p<0.05)$. The average rebound quantity of viruses (lg)/average time which patients missed doses with different NUCs were 2.26 with LAM, 3.57 with ADV, 4.99 with ETV, 4.15 with LdT, 4.34 with LAM and ADV.

Conclusion: The rate of viruses rebound in LAM group is highest. The reason may be that the postmarketing time of LAM is earlier, so the time which patients used LAM is longer.

\section{PP05-148}

Transduced PEP-1-functional Domains of Hepatitis B Virus Proteins Inhibit HBV Replication in HepG2.2.15 Cells

J. Han, Y. Gao, X. Pan, X. Cong, L. Wei

Peking University People's Hospital, Peking University Hepatology Institute, Beijing, China

The functional domains of hepatitis B virus (HBV) proteins are important for HBV life cycle. Some peptides derived from HBV proteins have been showed to inhibit HBV replication. We designed and synthesized a set of fusion peptides which combined with the cellpenetrating peptide (PEP-1), linker peptide and the functional domains of HBV core protein, pre-S1 or polymerase. The fusion peptides were added to the medium of HepG2.2.15 cells. After $96 \mathrm{~h}$, the antiviral activity was assayed by intracellular and extracelluar HBV DNA, neucleocapsids, HBV pregenome RNA and viral proteins. The core protein residues 78-117 and 113-143, pre-S1 24-45, and polymerase 149-183 inhibited HBV replication at 5-50 nM, and had combined anti-HBV effects. These results suggest that peptides from HBV functional domains are potential for designing anti-HBV peptides.

\section{PP05-149}

Artificial Liver Support System Combined with Liver Transplantation in the Treatment of Patients with End-stage Severe Hepatitis: Hangzhou Experiences

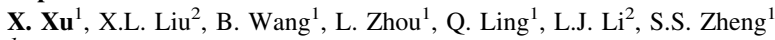
${ }^{I}$ Division of Hepatobiliary and Pancreatic Surgery, Department of Surgery, the First Affiliated Hospital, Zhejiang University School of Medicine; ${ }^{2}$ The First Affiliated Hospital, Zhejiang University School of Medicine, Hangzhou, China 
Background/aims: The search for a strategy to provide temporary liver support and salvage the patients with end-stage severe hepatitis remains an important issue. This study was designed to evaluate the experience in application of plasma perfusion-centered artificial liver support system (ALSS) combined with liver transplantation (LT) in the treatment of patients with end-stage severe hepatitis.

Methods: One-hundred and twenty-five patients with end-stage severe hepatitis who did not have a liver graft available at the time of presentation received 289 sessions of plasma exchange-centered ALSS treatment prior to LT (ALSS-LT group). The outcome of patients in ALSS-LT group was compared with those who received emergency LT (LT group), or ALSS treatment (ALSS group), or only conventional medical therapy (non-ALSS-non-LT group).

Results: ALSS treatment resulted in improvement of liver function and better tolerance to LT. The average level of serum total bilirubin before LT was significantly lower than that before the first time of ALSS treatment $(426 \pm 169 \mu \mathrm{mol} / \mathrm{L}$ vs. $557 \pm 195 \mu \mathrm{mol} / \mathrm{L}, P<0.05)$. The median waiting time for a donor liver was 12 days (2-226 days) from the first run of ALSS treatment to LT. Compared to LT group, the beneficial influences of ALSS on intraoperative blood loss and endotracheal intubation time were also observed in ALSS-LT group. The 1-year survival rates in the ALSS-LT group, LT group, ALSS group, and non-ALSS-non-LT group were 71.7, 74.6, 9.6, and 5.1\%, respectively. Conclusion: Plasma exchange-centered ALSS is beneficial in salvaging patients with end-stage severe hepatitis when a donor liver is not available and consequential LT is the fundamental treatment modality to rescue these patients and results in similar survival rate as those patients receiving emergency transplantation.

\section{PP05-150}

The Study of Adverse Drug Reaction of Tenofovir in Chronic Hepatitis B Virus Infection at Siriraj Hospital, Thailand

P. Tragulpiankit ${ }^{1}$, N. Dhana ${ }^{2}$, T. Tanwandee ${ }^{2}$, K. Kittichaisri ${ }^{1}$,

P. Sitthimongkhonkarn ${ }^{1}$

${ }^{1}$ Faculty of Pharmacy, ${ }^{2}$ Faculty of Medicine Siriraj Hospital, Mahidol University, Bangkok, Thailand

Background/aims: Tenofovir is a new nucleotide analog for chronic hepatitis B (CHB) patients. Although the safety profile has been studied in many years according to treatment of AIDS patients, tenofovir safety in CHB is relatively unknown. Therefore, the objective of this study was to determine the frequency and characteristics of tenofovir related to adverse reaction in ambulatory $\mathrm{CHB}$ patients who were prescribed tenofovir between 1st November 2006 and 30th September 2010.

Methods: Chart review and direct patient interview were performed to detect suspected adverse drug reaction (ADR). The adverse events were assessed by using Naranjo's algorithm. ADR was categorized by organ system disorder, severity and patient management associated to adverse reaction.

Results: Total 136 patients were retrospective chart reviewed, but 62 (45.6\%) patients were able to be interviewed. Forty-two (67.7\%) were male patients. Average age was $49.6 \pm 12.3$ years old. Total 53 $(85.5 \%)$ patients were prescribed tenofovir with other antiviral agents, especially lamivudine by $34(54.8 \%)$ patients. Total 24 out of 62 (38.7\%) patients suffered from $49(79.0 \%)$ events. Total 43 out of 49 (87.8\%) events were assessed by possible events. CNS, renal and GI disorders were occurred by 19 (38.8\%), $10(20.4 \%)$ and $10(20.4 \%)$ events, respectively. Severity of ADR was assessed into moderate by $3(6.5 \%)$ events and those were decreased dose adjustment.

Conclusion: Although adverse effects of tenofovir in CHB patients in the present study were not severe, ADR monitoring should be performed especially nephrotoxicity.
PP05-151

LdT + ADV Combination Therapy for Lamivudine-resistant HBV Patients

T.-H. Hu ${ }^{1}$, H.-C. $\mathrm{Yu}^{2}$, M.-C. Tsai ${ }^{1}$, M.-T. Lin $^{1}$, P.-L. Tseng ${ }^{1}$, K.-C. Chang ${ }^{1}$ ${ }^{1}$ Division of Hepato-Gastroenterology, Department of Internal Medicine, Chang Gung Memorial Hospital-Kaohsiung Medical Center, Kaohsiung County; ${ }^{2}$ Division of Gastroenterology, Department of Medicine, Kaohsiung Veterans General Hospital, Kaohsiung, Taiwan, ROC

Background/aim: In the treatment of HBV, lamivudine (LAM) has been the mainstay of treatment before. Long-term therapy may lead to emergence of drug-resistant strains, with cross resistance evident between nucleoside analogs. Adefovir (ADV) based therapy has been the mainstay of salvage treatment for lamivudine-resistant patients. However, inadequate response was occasionally observed. We aim to evaluate the efficacy of ADV + LdT combination therapy for LAMresistant patients.

Method: In this prospective studies ( $n=26)$, we evaluated second-line salvage therapy with ADV+ telbivudine (LdT) (Group 1, $n=11$ ), ADV monotherapy with inadequate response and followed by ADV+LdT (Group 2, $n=6$ ), or LAM + ADV with inadequate response followed by $\mathrm{ADV}+\mathrm{LdT}$ (Group 3, $n=9$ ) in LAM-resistant patients. Inadequate response was defined as HBV DNA $>10^{3}$ copies $/ \mathrm{ml}$ after 1 year therapy. Results: The 3 groups showed no significant differences in baseline HBV DNA levels or durations of LAM, ADV, and LDT treatments. Simple linear regression analysis showed that for each additional month of treatment, the significant viral reduction occurred in Group 1 (HBV DNA [ $\left.\log _{10} \mathrm{IU} / \mathrm{mL}\right]$ : Group 1, -0.149 ; Group 2, -0.081 ; Group 3, -0.123 ). Generalized estimating equation (GEE) analysis revealed that compared to Group 1, HBV DNA levels were 1.203 and $0.443 \log _{10}$ IU/ $\mathrm{mL}$ higher in Groups 2 and 3, respectively. Compared to ADV therapy, $\mathrm{ADV}+\mathrm{LdT}$ treatment resulted in a significant reduction in HBV DNA levels $\left(-1.593 \log _{10} \mathrm{IU} / \mathrm{mL}, p<0.001\right)$. Moreover, LdT treatment resulted in a significant viral reduction $\left(-0.05 \log _{10} \mathrm{IU} / \mathrm{mL}\right)$ for each additional month of treatment. All patients showed good antiviral response, with regressed serum ALT levels and no new mutations since the start of LdT treatment (duration 1-2.5 years).

Conclusion: It could be a consideration that LAM-resistant patients directly switched to $\mathrm{ADV}+\mathrm{LdT}$ which provides better antiviral response than either ADV alone or ADV + LAM as second-line salvage therapy.

\section{PP05-152}

Efficacy and Safety of Entecavir Treatment in Adefovir-experienced CHB Patients in a Real-life Clinical Practice Setting in China

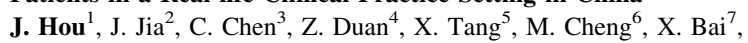
C. Llamoso ${ }^{8}$, D. Cohen ${ }^{8}$, M. Wu ${ }^{9}$

${ }^{I}$ Infectious Disease, Nanfang Hospital, Southern Medical University, Guangzhou; ${ }^{2}$ Hepatology, Capital Medical University Affiliated Friendship Hospital, Beijing, ${ }^{3}$ Hepatology, Chinese Military No.85 Hospital, Shanghai; ${ }^{4}$ Infectious Disease, Capital Medical Univeristy Affiliated Beijing You'an Hospital, Beijing; ${ }^{5}$ Infectious Disease, Guangzhou No. 8 Hospital, Guangzhou; ${ }^{6}$ Infectious Disease, Guiyang Medical College Affiliated Hospital, Guiyang, ${ }^{7}$ Infectious Disease, The Forth Military Medical University Affiliated No. 2 Hospital, Xi'an, China; ${ }^{8}$ Research \& Development, Bristol-Myers Squibb, Wallingford, MA, USA; ${ }^{9}$ Research \& Development, Bristol-Myers Squibb, Shanghai, China

Background and aims: In registrational studies, entecavir (ETV) demonstrated efficacy and safety in lamivudine (LVD)-refractory patients only. We evaluated the efficacy and safety of ETV in a cohort of patients who were pre-treated with ADV in a real-life setting in China. 
Methods: The ADV pre-treated cohort consisted of HBeAg-positive or negative patients who had received treatment with ADV monotherapy for $>12$ weeks before enrolment into the ETV arm of the REALM study and who completed 48 weeks of ETV monotherapy $(0.5 \mathrm{mg}$ or $1.0 \mathrm{mg}$ as selected by the investigator in accordance with approved prescribing information). Patients were assessed for virologic, biochemical and serologic endpoints at week 48 .

Results: Efficacy parameters for the 152 patients who were included and completed 48 weeks of ETV monotherapy are presented in the tables:

Table 1 Disease characteristics at baseline

\begin{tabular}{llll}
\hline ADV experienced compensated patients & & \\
\hline & $\begin{array}{l}\operatorname{HBeAg}(+) \\
n=121\end{array}$ & $\begin{array}{l}\mathrm{HBeAg}(-) \\
n=29\end{array}$ & $\begin{array}{l}\text { Total } \\
n=152^{*}\end{array}$ \\
\hline Baseline & & \\
Mean HBV DNA $\log _{10} \mathrm{IU} / \mathrm{mL}$ & & 5.80 \\
Mean ALT (U/L) & & 106
\end{tabular}

Table 2 Treatment response parameters at week 48

\begin{tabular}{llll}
\hline Week $48, n / N(\%)^{\dagger}$ & & & \\
\hline HBV DNA < 50 IU/mL & $65 / 115(57)$ & $20 / 21(95)$ & $85 / 138(62)$ \\
ALT & $52 / 71(73)$ & $10 / 13(77)$ & $62 / 84(74)$ \\
HBeAg loss & $19 / 120(16)$ & & $19 / 120(16)$ \\
HBe seroconversion & $14 / 120(12)$ & & $14 / 120(12)$
\end{tabular}

* For 2 patients $\mathrm{HBeAg}$ status at baseline was missing.

${ }^{\dagger}$ Denominator represents patients with available samples

The safety profile of ETV was consistent with previously reported experience.

Conclusions: Entecavir was efficacious and well tolerated through 48 weeks of treatment in Chinese CHB patients who were previously treated with ADV.

\section{PP05-153}

Budget Impact of Telbivudine versus Entecavir in the Treatment of Patients with HBeAg-positive Chronic Hepatitis B (CHB) in China W. Chen ${ }^{1}$, Q. Xie ${ }^{2}$

${ }^{1}$ Center for Pharmacoeconomics Research and Evaluation, Fudan University; ${ }^{2}$ Ruijin Hospital, Shanghai Jiao Tong University School of Medicine, Shanghai, China

Background: $\mathrm{CHB}$ infection brings heavy economic burden on China's health care system. Choosing cost effective CHB treatments is prioritized.

Objective: Assess the impact on 5-year budget of telbivudine and entecavir treatment for $\mathrm{HBeAg}$-positive $\mathrm{CHB}$, based on results of Chinese randomized clinical trials available.

Methods: From the Chinese social health insurance perspective, budget impact analysis was conducted based on a decision tree model to reflect short-term outcomes and cost of $\mathrm{CHB}$ treatment with telbuvidine and entecavir. Main outcomes including $\mathrm{HBeAg}$ seroconversion and genotypic resistance were retrieved from available literature. Treatment cost included drug cost, additional 1-year treatment cost after seroconversion and treatment cost for managing resistance with adefovir during 5-year period. Average treatment cost per patient was measured.

Results: Based on literature available, percentage of patients who have $\mathrm{HBeAg}$ seroconversion and suffer from resistance after 3-year treatment of telbivudine and entecavir was 42 vs. $27 \%$ and 39 vs. $2.6 \%$, respectively. Average treatment cost per patient with telbivudine and entecavir during 5-year period was RMB45,436 (USD\$6,781) and RMB95,097 (USD\$14,194), respectively. The treatment cost per patient with telbivudine was less than half of that with entecavir.

Conclusions: Compared to entecavir, telbivudine improves health outcomes by generating higher $\mathrm{HBeAg}$-seroconversion rate in $\mathrm{HBe} A g$-positive patients, and also leads to cost saving.

\section{PP05-154}

Switch from Adefovir and Lamivudine Therapy to Adefovir and Telbivudine Therapy for the Treatment of Lamivudine-resistant Chronic Hepatitis B Patients

Y.-J. Ahn

Gyeongsang National University School of Medicine, Jinju, Republic of Korea

Background/aims: Add on adefovir (ADV) to ongoing lamivudine (LAM) has been recommended as a standard therapy in LAM resistant chronic hepatitis B patients. Telbivudine (LdT) has demonstrated potent activity against hepatitis B with higher rate of response and superior viral suppression compared with LAM. The aims of this study were to evaluate the virologic response, biochemical and virologic breakthrough of $\mathrm{LdT}+\mathrm{ADV}$ versus LAM + ADV therapies in LAM-resistant patients.

Methods: The study population comprised 77 patients with LAMresistance, retrospectively. We divided them into 2 groups (Group 1: LAM + ADV with HBV DNA $>1000$ copies/ml after 1 year from starting treatment, $N=62$, Group 2: Switch from LAM + ADV to LdT $+\mathrm{ADV}, N=15$ ) between January 2008 and March 2010. HBV DNA levels and liver function test were assessed at baseline and 6 months during therapy.

Result: Median HBV DNA levels of group 1 and 2 were $5.61 \log _{10}$ copies $/ \mathrm{ml}$ and $5.53 \log _{10}$ copies $/ \mathrm{ml}$, respectively. In group 1 , HBV DNA changes $>1 \log _{10}$ copies/ml compared with baseline were $16.1 \%$ (10/62 patients), HBV DNA changes $<1 \log _{10}$ copies/ml compared with baseline were $17.7 \%$ (11/62), undetectable HBV DNA was $4.8 \%$ (3/62). In group 2 , HBV DNA changes $>1 \log _{10}$ copies $/ \mathrm{ml}$ compared with baseline were $0 \%(0 / 15)$, HBV DNA changes $<1 \log _{10}$ copies/ml compared with baseline were $33.3 \%$ (5/15), undetectable HBV DNA was $6.7 \%(1 / 15)$. Rate of biochemical breakthrough in group 1 was $21 \%(13 / 62)$ and group 2 was $20 \%(3 / 15)$ at 6 months of treatment. Virologic breakthrough was not detected in group 1 and group 2.

Conclusions: Our result shows switching to telbivudine and adefovir combination therapy is not inferior to lamivudine and adefovir combination therapy for the treatment of lamivudine-resistant patients. When lamivudine and adefovir therapy has suboptimal response, switching to telbivudine and adefovir therapy is one way in LAM-resistant patients. We suggest that further studies including larger number of cases are necessary to switch to telbivudine and adefovir therapy.

\section{PP05-155}

Efficacy and Safety of Entecavir in Lamivudine/Telbivudine-experienced HBeAg-negative CHB Patients in Real-life Clinical Practice Setting in China

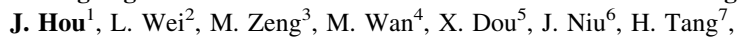
C. Llamoso ${ }^{8}$, D. Cohen ${ }^{8}, \mathrm{M} . \mathrm{Wu}^{9}$

${ }^{1}$ Infectious Disease, Nanfang Hospital, Southern Medical University, Guangzhou; ${ }^{2}$ Hepatology, Beking University People's Hospital, Beijing; ${ }^{3}$ GI, Shanghai Jiao Tong University Affiliated Renji Hospital, ${ }^{4}$ Infectious Disease, Chinese Second Military Medical University Affiliated Changhai Hospital, Shanghai, ${ }^{5}$ Infectious Disease, China Medical University Shengjing Hospital, Shenyang; ${ }^{6}$ Infectious Disease, Jilin University Affiliated No.1 Hospital, Changchun; ${ }^{7}$ Infectious Disease, Sichuan University Hua Xi Hospital, Chengdu, China; ${ }^{8}$ Research \& Development, Bristol-Myers Squibb, Wallingford, MA, USA; ${ }^{9}$ Research \& Development, Bristol-Myers Squibb, Shanghai, China 
Background and aims: In registrational studies, entecavir (ETV) demonstrated efficacy and safety in lamivudine (LVD)-refractory, $\mathrm{HBeAg}$-positive patients. However, there is limited data available on the efficacy and safety of ETV in $\mathrm{HBeAg-negative} \mathrm{patients} \mathrm{who} \mathrm{were}$ previously treated with LVD/telbivudine (LdT). We have evaluated the efficacy and safety of ETV in a cohort of LVD/LdT-experienced HBeAg-negative patients in a real-life setting in China.

Methods: The LVD/LdT pre-treated cohort consisted of $\mathrm{HBeAg}$ negative patients who had received treatment with LVD/LdT monotherapy for $>12$ weeks before enrolment into the ETV arm of the REALM study and who completed 48 weeks of ETV monotherapy $(0.5 \mathrm{mg}$ or $1.0 \mathrm{mg}$ as selected by the investigator in accordance with approved prescribing information). Patients were assessed for virologic and biochemical endpoints at week 48.

Results: Efficacy parameters for the 106 patients who were included and received 48 weeks of ETV monotherapy are presented in the tables below:

Disease Characteristics at Baseline

LVD/LdT experienced HBeAg-negative, Compensated CHB patients, $N=106$

Baseline

Mean HBV DNA $\log _{10} \mathrm{IU} / \mathrm{mL} \quad 5.16$

Mean ALT (U/L)

117

Treatment response parameter at week 48

Week $48, n / N^{\#}(\%)$

HBV DNA

$78 / 95(82)$

ALT

$54 / 63(86)$

\# Denominator represents patients with available samples

The safety profile of ETV was consistent with previously reported experience.

Conclusions: The results of this study demonstrate that ETV is efficacious and well tolerated through 48 weeks of treatment in $\mathrm{HBeAg}$-negative Chinese $\mathrm{CHB}$ patients who were pre-treated with LVD/LdT. Since HBeAg-negative disease requires long-term treatment, ETV may be a viable treatment option for switching LVD/LdT pre-treated patients.

\section{PP05-156}

Interim Analysis of Multicenter, Randomized, Controlled Trial for Lamivudine-resistant Chronic Hepatitis B: Comparing Clevudine Plus Adefovir with Lamivudine Plus Adefovir

J.S. Lee ${ }^{1}$, K.-A. Kim ${ }^{1}$, Y.J. Lee ${ }^{2}$, J.-H. Yoon ${ }^{3}$

${ }^{1}$ Internal Medicine, Inje University, Ilsanpaik Hospital, Goyang;

${ }^{2}$ Internal Medicine, Inje University, Busanpaik Hospital, Busan;

${ }^{3}$ Internal Medicine, Seoul National University College of Medicine, Seoul, Republic of Korea

Backgrounds/aims: In most of Asian-Pacific countries, limited treatment options for lamivudine (LAM)-resistant chronic hepatitis B $(\mathrm{CHB})$ are available. Clevudine has demonstrated antiviral potency in the treatment of naïve $\mathrm{CHB}$ patients but the efficacy for LAMresistant $\mathrm{CHB}$ is not reported. Furthermore, comparison between clevudine plus ADV and LAM plus ADV for LAM resistant CHB patients has not been performed in a head-to head comparison method.

Methods: The primary end point of the study is comparing HBV DNA reduction at week 48 and plan to do interim analysis when 1/3 patients of target number has been reached 48 week treatment. 23
CHB patients who developed virologi and biochemical breakthrough during LMV treatment were consecutively enrolled at 3 university hospital, received 48 week clevudine plus ADV or LAM plus ADV treatment.

Results: Baseline characteristics of the clevudine plus ADV or LAM plus ADV patients such as age, sex, HBeAg positivity, HBV DNA, AST/ALT and bilirubin were not significantly different $(p>0.05)$. In the clevudine plus ADV and LAM plus ADV groups at 48 week, degree of HBV DNA reduction $(3.86 \pm 1.39$ vs. $4.69 \pm 1.64 \log$ IU/ $\mathrm{mL}$, respectively, $p=0.64$ ), HBV DNA PCR negativity (33.3 vs. $27.3 \%$, respectively), ALT normalization (58.3 vs. $72.7 \%$, respectively, $p=0.13$ ) were not significantly different. The rate of $\mathrm{HBeAg}$ loss was greater in the LAM plus ADV than clevudine plus ADV at 48 week (40 vs. $16.7 \%$, respectively, $p=0.04$ ). In clevudine plus ADV group, one case showed viral breakthrough at 48 week. No adverse effects were reported.

Conclusions: There is no difference between clevudine plus ADV and LAM plus ADV in LAM-resistant CHB patients in terms of HBV DNA reduction, HBV DNA PCR negativity and ALT normalization at week 48. This interim analysis suggested that clevudine plus ADV did not have advantages over LAM plus ADV in LAM-resistant CHB patients. (Clinical trials.gov number, NCT00798460).

\section{PP05-157}

Pre-existing Antiviral-resistant Mutation is a Mechanism of Primary Non-response to Lamivudine among Treatment-naïve Chronic Hepatitis B Patients

S. Fung ${ }^{1}$, F. Wong ${ }^{1}$, T. Mazzulli ${ }^{1}$, V. Popovic ${ }^{2}$

${ }^{1}$ Medicine, University of Toronto, Toronto; ${ }^{2}$ Research and Development, Gilead Sciences Canada, Mississauga, ON, Canada

Background: Antiviral-resistant mutation (AVR) among treatmentnaïve chronic hepatitis $\mathrm{B}(\mathrm{CHB})$ patients has been reported previously. However, the clinical significance is unknown.

Aims: To determine the prevalence of AVR among treatment-naïve CHB patients and prevalence of rt180/204 mutations among patients treated with lamivudine (LAM) who had primary non-response $(<1 \log \mathrm{IU} / \mathrm{ml}$ decrease in HBV DNA from baseline after 3 months of therapy).

Patients and methods: Consecutive untreated adult patients with CHB attending Toronto General Hospital Liver Clinic (Canada) from 01/07 - 06/10 were tested for AVR at baseline. Those receiving antiviral therapy were monitored with serology and HBV DNA levels every 3 months. Serum samples of LAM nonresponders were analyzed separately. AVR was detected using line probe assay (InnoLiPA HBV-DR v3, InnoGenetics, Belgium).

Results: 421 treatment-naïve patients were tested for AVR: $76 \%$ men, $40 \pm 17$ years, 92\% Asian, 43\% HBeAg-positive and 13\% cirrhosis. Mean HBV DNA was $5.7 \pm 2.6 \mathrm{log} \mathrm{IU} / \mathrm{ml}$ and $\mathrm{HBV}$ genotypes A, B, C and D were found in $7 \%, 38 \%, 46 \%$ and $9 \%$ patients, respectively. The prevalence of mutations was $\mathrm{rt180/204}$, $9 \% ; \mathrm{rt} 181,1 \%, \mathrm{rtN} 236 \mathrm{~T},<1 \%$, and $\mathrm{rt} 184 / 202 / 250,2 \%$. 16/294 (5\%) patients were LAM nonresponders. Among this group, mean age $47 \pm 15$ years, ALT $129 \pm 203 \mathrm{U} / \mathrm{L}, \mathrm{HBV}$ DNA $7.4 \pm 1.5 \mathrm{log}$ IU/ $\mathrm{ml}$, and $5(31 \%)$ cirrhosis. 3/16 (19\%) nonresponders had AVR at baseline (2 rtL180M+rtM204I; 1 rtA181T). 1 nonresponder received tenofovir $300 \mathrm{mg}$ daily and another LAM+adefovir $10 \mathrm{mg}$ daily. Both patients achieved undetectable HBV DNA after 12 months $(20 \pm 8$ months mean follow-up).

Conclusions: Mutations in rt180/204 pathway were common (9\%), whereas other mutations were uncommon (1-2\%). Although primary non-response to LAM occurred infrequently, 1/5 nonresponders harbored pre-existing AVR. This may be a mechanism of primary nonresponse and should be excluded prior to the start of therapy. Salvage therapy using nucleotide agents was successful in long-term follow-up. 


\section{PP05-158}

Adefovir and Renal Impairment: 'A Bad Thing?'

J. Leo Hartono, A.M. Oo, B. Mak, G.H. Lee, M.L. Fernandes, Y.M. Lee,

V. Lai, D. Sutedja, Y.Y. Dan, S.G. Lim

Gastroenterology and Hepatology, National University Health

System, Singapore, Singapore

Background/aims: Adefovir treatment in patients with chronic hepatitis B has been reported to have a low risk of renal impairment. We studied the frequency and associated factors of renal impairment and achieving undetectable HBV_DNA during adefovir treatment.

Methods: From a clinical database of chronic hepatitis B patients, all adefovir treated patients $>6$ months, were included in the study. Exclusion criteria were malignancy, organ transplant pre-existing renal impairment. Estimated Glomerular Filtration Rate (eGFR) was calculated using the Modification of Diet in Renal Disease Study (MDRD) formula. Data were analysed using multiple logistic regression, Kaplan-Meier survival analysis, Paired Ttest, \& ROC curves with best cutoff coordinates.

Results: A total number 271 adefovir treated patients' data were analysed. During a mean(SD) followup of 28.9 (21.1) months, 33 patients developed abnormal serum creatinine. Of these, creatinine normalized in 24 patients after adefovir dose reduction $(n=13)$, adefovir discontinuation $(n=8)$, or no change in treatment $(n=3)$. Cumulative rates of abnormal creatinine were 5.3,9.4,16.3,19.5,24.9, and $33.8 \%$ at year $1,2,3,4,5$, and 6 respectively. Independent factors predicting renal impairment were Lactate Dehydrogenase (LDH) $(p=0.007)$ and eGFR $<85.5 \mathrm{mls} / \mathrm{min}(p<0.001)$ at baseline.

A higher proportion of patients who developed adefovir related renal impairment achieved undetectable HBV_DNA compared to those without renal impairment [29/32(90.6\%) vs. 142/214 (66.3\%), $p$ $=0.004]$. In patients with adefovir related renal impairment who had dose reduction to adefovir $10 \mathrm{mg}$ every other day, $80 \%$ had normalization of renal impairment. There was no change in HBV_DNA in these patients despite a reduction in adefovir dos$\operatorname{ing}(P=0.126$,paired $T$ test $)$.

Conclusion: Adefovir related renal impairment occurs in a significantly higher proportion of patients than initially reported in randomized control trials, and baseline eGFR $<85 \mathrm{mls} / \mathrm{min}$ is a predictor of future renal impairment. However, those who had adefovir dose reduction were able to normalize renal function without loss of efficacy in HBV_DNA suppression. Thus patients with adefovir renal impairment can half the dose of adefovir while maintaining viral suppression.

\section{PP05-159}

Evolution of the Hepatitis B Surface Antigen in European and Asian HBV Genotypes during the Era of Antiviral Therapy

L. Yuen, A. Ayres, M. Littlejohn, R. Edwards, S. Locarnini Researh and Molecular Development, Victorian Infectious Diseases Reference Laboratory, North Melbourne, VIC, Australia

Background/aims: Long term use of nucleos(t)ide analogues (NA) on patients with chronic hepatitis $\mathrm{B}(\mathrm{CHB})$ can select drug-resistance
(DR) associated HBV reverse transcriptase (rt) mutations, which in turn, can result in HBV surface antigen (HBsAg) changes. This can have significant clinical and public health implications. For instance, sW172stop HBV variants (rtA181T-selected) are secretion defective, lead to intracellular retention, and may be implicated in enhancing progression to hepatocellular carcinoma (HCC) (Lai et al. 2009. AVT 14:249)

Methods: A cohort of $2136 \mathrm{CHB}$ patients were selected from our SeqHepB database. The frequency of all HBsAg amino acid (aa) changes detected in the HBV isolates of their latest samples was analysed.

Results: 737 (35\%) isolates had DR-associated HBV-rt aa changes (rtI169, rtL180, rtA181, rtT184, rtS202, rtM204, rtN236 and or rtM250), and 720 (98\%) had corresponding HBsAg changes. Thirteen $\mathrm{HBsAg}$ aa positions in DR-isolates had significantly higher rates of reportable changes compared to drug-susceptible (DS) isolates; including sW172 (5\%), sL173 (9\%), sI195 (46\%), and sW196 (41\%), due to rtA181 and rtM204 changes respectively. Of these 13 a positions, $11 \mathrm{HBsAg}$ changes were detected at significantly higher rates in the DR-isolates, including the NA-associated aa substitutions sW172* (rtA181T), sL173F (rtA181V), sI195M (rtM204V), sW196L (rtM204I), sW196S (rtM204I) and sW196* (rtM204I). However, their occurrence rates varied among the HBV genotypes that are predominately identified in Europe (HBV-A, HBV-D) and Asia (HBV-B, HBV-C). In addition, HBV isolates with DR HBV-rt mutations had a higher proportion of HBsAg aa changes overall compared with those without DR HBV-rt mutations, including C-terminal truncation (after s170) mutations.

Conclusion: HBV with DR HBV-rt mutations have higher proportion of corresponding $\mathrm{HBsAg}$ changes, in particular C-terminal truncations. Since these HBsAg aa substitutions are associated with higher risk of $\mathrm{HCC}$ and their occurrence rates varied between the European and Asian HBV genotypes, they warrant further investigation.

\section{PP05-160}

Renal Impairment during Long-term Adefovir Therapy in Chronic Hepatitis B Patients

Y.J. Kim, H.C. Cho, G.-Y. Gwak, M.S. Choi, J.H. Lee, K.C. Koh, B.C. Yoo, S.W. Paik

Samsung Medical Center, Sungkyunkwan University School of Medicine, Seoul, Republic of Korea

Background/aims: Adefovir is commonly used in chronic hepatitis B patients, but renal toxicity is an important adverse effect. We evaluate the incidence of renal impairment in adefovir treated patients and the risk factors of adefovir induced renal toxicity.

Methods: We enrolled 725 adefovir (10 mg daily) treated chronic hepatitis B patients for more than 12 months. They were on adefovir mono-therapy or combination with lamivudine. Glomerular filtration rate (GFR) was estimated using Cockcroft-Gault formula. Patients with baseline GFR $<60 \mathrm{~mL} / \mathrm{min}$ and patients who were exposed to other antiviral agents were excluded. Definition or renal impairment was defined as like this: mild-GFR reduction 10-20\%, moderatedGFR reduction $20-50 \%$, severe-GFR reduction more than $50 \%$. 
Results: The baseline clinical characteristics of the patients are presented in Table 1.

Table 1 The baseline characteristics of the study subjects

\begin{tabular}{ll}
\hline Characteristics & Result \\
\hline Age, years, mean \pm SD & $48.1 \pm 10.85$ \\
Male gender, $n(\%)$ & $538(74.2)$ \\
Weight, Kg, mean \pm SD & $66.9 \pm 10.44$ \\
Baseline GFR (mL/min), mean \pm SD & $94.2 \pm 21.60$ \\
Adefovir/Adefovir+Lamivudine, $n(\%)$ & $141(19.4) / 584(80.6)$ \\
Treatment duration, month, median (range) & $27(12-83)$ \\
Liver cinhosis, $n(\%)$ & $391(53.9)$ \\
Child A/B/C, $n(\%)$ & $333(45.9) / 54(7.4) / 4(0.6)$ \\
Hepatocellular carcinoma, $n(\%)$ & $221(30.6)$ \\
CT, $n$, median (range) & $4.9(0-27)$ \\
Hypertension, $n(\%)$ & $44(6.1)$ \\
Diabetesellitus, $n(\%)$ & $74(10.2)$ \\
HBeAg positivity, $n(\%)$ & $486(67.1)$ \\
HBV DNA (log IU/mL), mean $\pm \mathrm{SD}$ & $6.1+1.5$ \\
AST (U/L), median (range) & $63(11-2051)$ \\
ALT(U/L), median (range) & $81(8-5425)$ \\
Bilirubin (mg/dL), median(range) & $1.1(0.1-36)$ \\
Albumin(g/dL), mean+SD & $3.9 \pm 0.492$ \\
Platelet(x10 $\left.{ }^{3} / \mathrm{mm}{ }^{3}\right)$, mean $\pm \mathrm{SD}$ & $144.9 \pm 72.84$ \\
Prothrombin time (INR), mean $\pm \mathrm{SD}$ & $1.15 \pm 0.17$ \\
\hline
\end{tabular}

A total of 161 patients (22.2\%) developed renal impairment during adefovir treatment. Mild renal impairment constitutes about 60\% (98 patients) and moderate impairment occurred in 63 patients. There was no case of severe renal impairment. Three patients stopped receiving adefovir and 8 patients continued adefovir treatment with increasing dose interval. The other 55 patients received adefovir $10 \mathrm{mg}$ daily with monitoring GFR. Those who continued adefovir treatment maintained stable renal function. Multivariate analysis revealed older age, longer treatment duration, and male gender as risk factors of GFR decrease more than $20 \%$ of baseline.

Conclusions: Adefovir related renal impairment is associated with longer treatment duration, old age and male gender. Careful attention and motoring of renal function is required in these patients.

\section{PP05-161}

HBsAg Kinetics in HBeAg+ CHB Patients during Long-term Telbivudine Treatment Is Dependent on HBV Genotype

K. Wursthorn ${ }^{1}$, M. Jung ${ }^{2}$, M.P. Manns ${ }^{1}$, N.V. Naoumov ${ }^{2}$, A. Uddin ${ }^{3}$, H. Wedemeyer ${ }^{1}$

${ }^{1}$ Hannover Medical School, Hannover, Germany; ${ }^{2}$ Novartis Pharma AG, Basel, Switzerland; ${ }^{3}$ Novartis Pharma, East Hanover, NJ, USA

Background/aim: HBsAg clearance is the main goal of antiviral therapy in CHB but rates are generally low and differ between patient populations and studies. We studied the influence of genotype (GT) on serum HBsAg levels during longterm telbivudine (LDT) treatment. Methods: $162 \mathrm{HBeAg}$ positive(+) patients from GLOBE/2303 studies were included. Patients completed at least 3 years (Y3) of study and had undetectable $(<300$ copies/ml) HBVDNA at year 2 and one additional visit. 32 patients were on off-treatment follow-up. Serum HBsAg levels were determined using Abbott Architect ${ }^{\circledR}$ HBsAg assay. Statistical testing was performed using ANOVA testing.
Results: HBsAg levels at BL were significantly different between genotypes A-D.

HBsAg during Telbivudine treatment by Genotype

\begin{tabular}{lllll}
\hline Genotype & $n$ & BL HBsAg & $\begin{array}{l}\text { Decline BL } \\
\text { to Y3 HBsAg }\end{array}$ & $\begin{array}{l}\text { Patients } n / N(\%) \\
\text { with HBsAg } \\
\text { clearance }\end{array}$ \\
\hline A & 6 & 4.3 & 3.5 & $3 / 16(22 \%)$ \\
B & 43 & 4.0 & 1.1 & $0 / 43(0 \%)$ \\
C & 98 & 3.6 & 0.5 & $5 / 98(5 \%)$ \\
D & 15 & 4.2 & 1.1 & $2 / 15(13 \%)$ \\
$p$ value & & $<0.0001$ & $<0.0001$ & NA \\
\hline
\end{tabular}

HBsAg kinetics over 3 years showed continuous decline (mean 0.8 $\log _{10} \mathrm{IU} / \mathrm{mL}$ ), leading to HBsAg clearance in $9 / 162(6 \%)$ of patients. HBsAg levels at $\mathrm{Y} 3$ and decline from BL to $\mathrm{Y} 3$ were significantly different between genotypes $(p<0.0001)$. GT A patients had fastest decline, decline in GT B was faster than GT C.

Conclusion: Serum HBsAg kinetics in $\mathrm{HBeAg}+\mathrm{CHB}$ patients during telbivudine treatment are significantly different between $\mathrm{HBV}$ genotypes.

\section{PP05-162}

Decreased Ratio of Treg to Th17 Cells Correlates with HBV DNA Suppression in Chronic Hepatitis B Patients Undergoing Entecavir Treatment J.-Y. Zhang, C.-H. Song, F. Shi, Z. Zhang, J.-L. Fu, F.-S. Wang Research Center for Biological Therapy, Beijing 302 Hospital, Beijing, China

Background: Treatment with nucleotide analogs is known to be effective in inhibiting HBV replication; however, patients with chronic hepatitis B (CHB) often show a wide range of clinical responses to these drugs. Therefore, the identification of an early immunologic marker associated with the clinical outcomes in such cases is critical for the improved clinical management. In our study, we aimed to investigate whether the viral load in CHB patients affected the ratio of the number of regulatory $\mathrm{T}$ cells (Tregs) to the number of interleukin-17-producing helper (Th17) cells. Further, we evaluated the clinical implications of the alterations in this ratio.

Methodology/principal findings: Nine patients seropositive for hepatitis B e antigen received entecavir monotherapy for 12 months and the percentages of Tregs and Th17 cells as well as the HBVspecific IL-17 productions in these patients were longitudinally analyzed. The entecavir-induced suppression of HBV replication was accompanied by a rapid increase in the number of Th17 cells, together with a decrease in Treg cells, which lead to a significant reduction of Treg/Th17 ratios. In addition, peripheral blood mononuclear cells (PBMCs) exhibited a decreased IL-17 production upon stimulation with the HBV core antigen in vitro.

Conclusions: The inhibition of viral replication results in an increase in Th17 cells and concomitant decrease in Treg cells. This imbalance of Treg cells to Th17 cells might have an important role in HBV persistence during entecavir treatment.

\section{PP05-163}

Hyper-activated Pro-inflammatory $\mathrm{CD}^{+}$Monocytes Correlate with the Severity of Liver Injury and Fibrosis in Patients with Chronic Hepatitis B

J.-Y. Zhang ${ }^{1}$, Z.-S. Zou ${ }^{2}$, A. Huang ${ }^{2}$, Z. Zhang ${ }^{1}$, J.-L. Fu ${ }^{1}$, X.-S. Xu ${ }^{1}$, B.-S. Li $^{2}$, F.-S. Wang ${ }^{1}$, L.-M. Chen ${ }^{1}$

${ }^{1}$ Research Center for Biological Therapy, Beijing 302 Hospital;

${ }^{2}$ Department of Infectious Diseases, Beijing 302 Hospital, Beijing, China 
Background: Extensive mononuclear cell infiltration is strongly correlated with liver damage in patients with chronic hepatitis B virus (CHB) infection. Macrophages and infiltrating monocytes also participate in the development of liver damage and fibrosis in animal models. However, little is known regarding the immunopathogenic role of intrahepatic macrophages and monocytes.

Methodology/principal findings: The frequencies, phenotypes, and functions of peripheral blood and intrahepatic monocyte subsets were analyzed in $110 \mathrm{HBeAg}$ positive $\mathrm{CHB}$ patients, including 32 immune tolerant (IT) carriers and 78 immune activated (IA) patients. Liver biopsies from 20 IA patients undergoing diagnosis were collected for immunohistochemical analysis. IA patients displayed significant increases in peripheral and intrahepatic monocytes as well as CD16 subsets, which were closely associated with the liver histological activity index (HAI) and serum alanine aminotransferase (ALT) levels. Further analysis showed that the increased $\mathrm{CD} 16^{+}$monocytes in the livers of IA patients expressed high levels of the activation marker HLA-DR and preferentially released inflammatory cytokines that not only induced liver damage and fibrosis but also that expanded Th17 cells. Finally, hepatic neutrophils positively correlated with HAI scores.

Conclusions: These distinct properties of monocyte subpopulations participate in fostering the inflammatory microenvironment and liver damage in $\mathrm{CHB}$ patients and further represent a collaborative scenario among different cell types contributing to the pathogenesis of HBVinduced liver disease.

\section{PP05-164}

Skewed Ratios between CD3+T Cells and Monocytes are Associated with Poor Prognosis in Patients with HBV-related Acute-on-Chronic Liver Failure

F. Shi ${ }^{1}$, J.-Y. Zhang ${ }^{1}$, Z. Zeng ${ }^{1}$, P. Tien ${ }^{2}$, F.-S. Wang ${ }^{1}$

${ }^{1}$ Research Center for Biological Therapy, Beijing 302 Hospital;

${ }^{2}$ Institute of Microbiology, Chinese Academy of Sciences, Beijing, China

Background: Patients with HBV-related acute-on-chronic liver failure (ACLF) share a striking similarity to the inflammatory response in septic shock where a hyperactive innate response is observed.

Aim: The present study attempted to characterize the features of CD3 + T cells and monocytes and evaluate their clinical implications in 55 patients with HBV-related ACLF, 30 patients with chronic hepatitis $\mathrm{B}(\mathrm{CHB})$ and 30 healthy controls (HC).

Results: We found that the ratio between circulating CD3+ T cells and monocytes (T/M) was decreased in ACLF patients, due to decreased CD3 counts and increased monocyte counts compared with $\mathrm{CHB}$ and $\mathrm{HC}$ subjects. We also found that the T/M ratios were decreased from the early to the intermediate stage and reached the lowest value at the late stage in ACLF patients. Analyses with clinical parameters revealed that $\mathrm{T} / \mathrm{M}$ ratios were negatively correlated with the Model for End-Stage Liver Disease Score and direct bilirubin, and positively correlated with prothrombin activity. Moreover, increased $\mathrm{T} / \mathrm{M}$ ratios were observed in patients with good prognosis, but not in patients with a poor outcome; and ACLF patients who received liver transplantation exhibited an increased T/M ratio. Importantly, we found that programmed death-1 receptor (PD-1) was drastically upregulated on both CD4+ T and CD8 $+\mathrm{T}$ cells in ACLF, which at least in part contributed to the T-cell loss in these patients. Mechanically, the in vitro coculture assay revealed that both CD4+ T and CD8+ T cells, as well as regulatory T cells, could inhibit TNF-a secretion by monocytes. In addition, the TNF-a levels in ACLF serum were negatively correlated with T/M ratios. In conclusion, our study identified the novel potential role of $\mathrm{T} / \mathrm{M}$ ratio in predicting disease progression and provided novel evidences for further studies of the immunopathogenesis in ACLF (Feng Shi and Ji-Yuan Zhang contributed equally to this work.)
Poster Presentation 06: Hepatitis B: Virology

\section{PP06-01}

Both Secretions of Interferon and Expressions of Phospho-IRF3 were Decreased in Murine Liver Dendritic Cells after Intervened by HBV J.M. Zheng ${ }^{1}$, G.F. Shi ${ }^{1}$, N. Li $^{1}$, Z.P. Qian ${ }^{2}$, M.Q. Zhu ${ }^{1}$, M.Q. Chen ${ }^{1}$, Q. Li $^{1}$, X.Y. Wang ${ }^{1}$

${ }^{I}$ Department of Infectious Diseases, Huashan Hospital, Fudan

University; ${ }^{2}$ Department of Severe Hepatitis, Shanghai Public Health Clinic Center, Fudan University, Shanghai, China

Objective: Study of the secretions of type I interferon and the expressions of phospho-IRF3 in murine liver dendritic cells after intervened by HBV.

Methods: The murine liver dendritic cells were isolated via antiCD11c microbeads, and were co-incubated with GM-CSF and IL-4 for inducing the DCs generation and proliferation. HBV virions were isolated via ultracentrifugation, and were detected by quantitative Realtime-PCR. DCs were divided into two groups at day 5. One group was cultured with HBV virons in $24 \mathrm{~h}$; the other group was cultured without HBV as a control group. Then, harvesting the cells and supernatants were detected the expressions of p-IRF3 by western blot and the concentration of IFN- $\beta$ by ELISA, after stimulated by poly $\mathrm{I}: \mathrm{C}$ in different time.

Results: IFN- $\beta$ concentration was $12.38 \pm 3.71 \mathrm{pg} / \mathrm{ml}$ at $0 \mathrm{~h}$, $88.67 \pm 9.01 \mathrm{pg} / \mathrm{ml}$ at $6 \mathrm{~h}, 69.89 \pm 5.80 \mathrm{pg} / \mathrm{ml}$ at $24 \mathrm{~h}$ in supernatants of HBV group, and was $10.83 \pm 4.11 \mathrm{pg} / \mathrm{ml}$ at $0 \mathrm{~h}$, $137.68 \pm 12.28 \mathrm{pg} / \mathrm{ml}$ at $6 \mathrm{~h}, 72.25 \pm 8.61 \mathrm{pg} / \mathrm{ml}$ at $24 \mathrm{~h}$ in supernatants of control group, respectively, in 24-well culture plates. There was no statistically significant difference at $0 \mathrm{~h}(t=0.8398$, $P>0.05)$ and at $24 \mathrm{~h}(t=0.6820, P>0.05)$ between two groups. But, there was statistically significant difference at $6 \mathrm{~h}(t=9.653$, $P<0.01)$ between two groups. The expressions of phospho-IRF3 in HBV group were lower than those in control group, when multiplicity of infection is 25 .

Conclusions: Both the secretions of type I interferon and the expressions of phospho-IRF3 were decreased in murine liver dendritic cells after intervened by HBV.

\section{PP06-02}

Interleukin-22 and Non-ELR-CXC Chemokines Reversely Correlate with Liver Inflammation and Fibrosis During Chronic Hepatitis B Virus Infection

X. Xiang ${ }^{1,2}$, H. Gui ${ }^{1}$, N.J. King ${ }^{2}$, L. Cole ${ }^{2}$, H. Wang ${ }^{1}$, Q. Xie ${ }^{1}$, S. Bao ${ }^{1,2}$

${ }^{1}$ Department of Infectious Diseases, Ruijin Hospital, Shanghai

Jiaotong University School of Medicine, Shanghai, China;

${ }^{2}$ Discipline of Pathology (D06), Bosch Institute and School of

Medical Sciences, The University of Sydney, Sydney, NSW, Australia

Aims: Hepatitis B virus (HBV) infection is still a major health issue, despite decades of research. IL-22 induces acute phase reactants and chemokines, favours anti-microbial defence, and protect tissues from damage. IL-22 is important in chronic skin inflammation, but its role in chronic hepatitis B (CHB) is unclear. This study explored the association between intra-hepatic IL-22, its relevant cytokines and the severity of liver inflammation/fibrosis in CHB patients.

Methods: IL-22, IL-17, IL-10, IL-6, non-ELR-CXC chemokines (CXCL-9, CXCL-10, CXCL-11) and FGFs and the infiltrating Kupffer cells were measured in the patients with $\mathrm{CHB}$, acute hepatitis $\mathrm{B}$ (AHB), chronic hepatitis $\mathrm{C}(\mathrm{CHC})$ and non-viral hepatitis, using immunohistochemistry.

Results: The expression of IL-22, IL-17, IL-10, IL-6 and non-ELRCXC chemokines, and Kupffer cells infiltration in liver tissues were substantially higher in AHB patients than others. The expression of IL-22, IL-6, CXCL-9, and CXCL-10 were significantly higher in 
patients with ALT levels $\leq$ twice upper limit of normal (ULN) than those with ALT levels >twice ULN, but IL-10 and IL-17 showed reverse pattern. A positive relationship was noted between IL-17 and histological activity index (HAI) score $(P<0.05)$ in CHB patients, but IL-22 was reversely correlated with HAI $(\mathrm{P}<0.01)$ (Fig. 1). A significant negative correlation between fibrosis stage and IL-22, as well as non-ELR-CXC chemokines, was observed. Immunofluorescense demonstrated that there was co-expression of IL-22, CXCL-9, CXCL-10 or CXCL-11 in the liver.
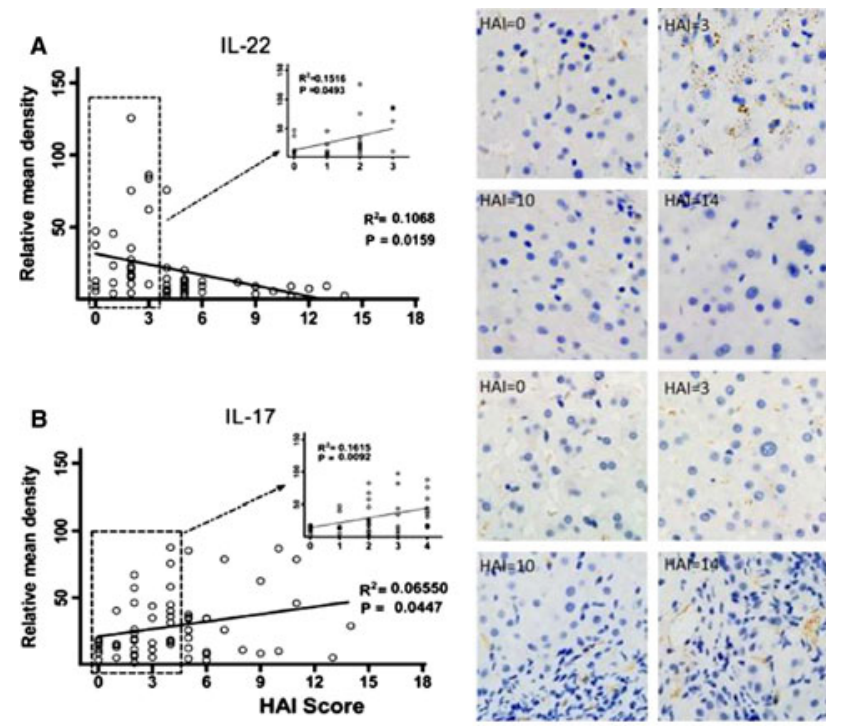

C

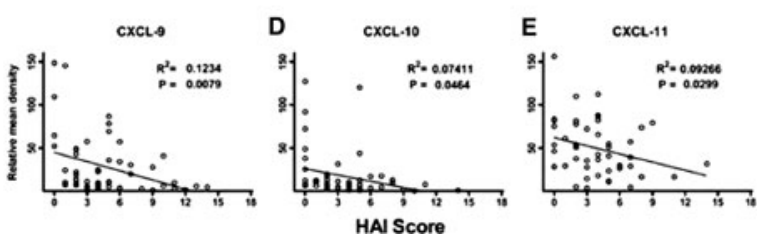

Conclusions: IL-22 and non-ELR-CXC chemokines may provide protection synergistically in liver inflammation and fibrosis during chronic HBV infection.

\section{PP06-03}

Prevalence of Infection with Delta Virus in Patients Positive for Hepatitis B Surface Antigen

S.A. Zaki, M. Abou Khatwa, H. Mikail

Microbiology, Faculty of Medicine, Alexandria University,

Alexandria, Egypt

During the past decade delta hepatitis virus has captured the attention as it causes a major health problem. Delta infection can occur in the presence of acute or chronic HBV infection.Delta superinfection can transform asymptomatic or mild chronic hepatitis B infection to severe progressive chronic active hepatitis and cirrhosis and accelerate the course of chronic active hepatitisB.

Aim: Detect the prevalence of infection with delta virus in selected groups of patients recognised to be $\mathrm{HbsAg}$ positive.

Subjectes: The study was carried out on one hundred serum samples collected from patients of different disease categories;

1-30 patients with schistosomal hepatic fibrosis.

2-22 patients on maintenance haemodialysis.

3-20 patients with acute hepatitis.

4-14 cases with hepatocellular carcinoma.

5-8 intravenous drug abusers.

6-6 chronic asymptomatic hepatitis B surface antigen carriers.
Methods: Perviously mentioned groups were tested for the presence of total antibody to hepatitis delta antigen using ELISA. Anti-delta positive samples were tested for anti-hepatitis B core antibodies of the IgM class to differentiate between superinfection and coinfection.

Results: Twenty anti-delta positive samples were detected (20\%). The highest frequency of anti-HDV positivity was found among Schistosomal hepatic fibrosis patients (46.7\%) followed by drug abusers (25\%), hepatocellular carcinoma group $(14.3 \%)$, and patients on maintenance haemodialysis $(9.09 \%)$. Non of the acute hepatitis B patients or chronic asymptomatic HBsAg carriers showed Delta antibodies. Anti-hepatitis B Core antibodies of the IgM class were absent in all the twenty samples positive for anti-delta indicating superinfection with HDV.

Conclusion: Hepatic schistosomiasis enhances HDV infection.

\section{PP06-04}

Active Recombinant Reverse Transcriptase Domain of Human Hepatitis B Virus Polymerase

D.R. Pandeya, Y. Yang, S.T. Hong

Microbiology and Genetics, Chonbuk National University, Medical School, Chonju, Republic of Korea

Hepatitis B virus polymerase plays a critical role during HBV life cycle, and polymerase/reverse transcriptase (RT) activities are critical for HBV-pol during viral replication. To investigate RT domain of human HBV polymerase, a $5^{\prime}$ end Polyhistidine tagged RT DNA (304-693 amino acids) of HBV-pol was successfully expressed in Escherichia coli. Recombinant RT was purified in native condition employing Ni-NTA affinity column. Purified RT showed a stable reverse transcriptase activity and a much stronger DNA polymerase activity, compared to RT expressed in rabbit reticulocyte lysate coupled transcriptase-translation system. The purified RT was a stable protein and showed a low selective polymerase activity. Computer modeling results also indicated that RT domain banded to nucleotide substrate in a loose mode.

\section{PP06-05}

Efficacy of Hepatitis B Vaccine in Adult HIV/AIDS Patients

O. Altuntas Aydin, H. Kumbasar Karaosmanoglu, R. Korkusuz, O. Nazlican Infectious Diseases and Clinical Microbiology, Haseki Training and Research Hospital, Istanbul, Turkey

Objectives: The aim of this study was to evaluate the efficacy of hepatitis B vaccination in HIV/AIDS patients.

Methods: Fifty-four HIV/AIDS patients, 8 women (15\%) and 46 men (85\%), 22-59 years old (37.6 \pm 9.1 ), were analyzed. Immunological status of patients at the moment of introduction of vaccination was assessed on the medical records (CD4 cell count, viral load). Efficacy of vaccination was compared with control group which consisted of 89 healthy volunteers were similar age $(35 \pm 8.9 p=0.09)$ and sex. In both groups hepatitis B infection was excluded by serologic tests. Euvax B vaccine produced by Sanofi-Pasteur, $20 \mu \mathrm{g}$ as registered for adults was injected to both groups at month 0-1-6. All post vaccine serologies completed within one year of vaccination. The patients with anti-HBs $<10 \mathrm{IU} / 1$ after finalizing the vaccination schema were accepted to be non-responder to the vaccine, those with anti-HBs $\geq 10$ IU/l were accepted to be responder.

Results: Protective level of anti-HBs was found in 76 (85.3\%) persons of the control group and $25(46 \%)$ in HIV/AIDS patients $(p<$ $0.001)$. Anti-HBs $\geq 100 \mathrm{IU} / 1$ was established in $49(55 \%)$ persons of the control group and $18(33 \%)$ of HIV/AIDS patients $(p<0.05)$. After Hepatitis B vaccination, the $25 \mathrm{HIV}$ infected patients with antiHBs $\geq 10$ IU had mean CD4 count of $381 \pm 191 / \mathrm{mm}^{3}$, whereas 29 HIV infected patients with no response to the vaccine had CD4 count of $344 \pm 237 / \mathrm{mm}^{3}$ ( $p=0.54$ ). While protective level of anti-HBs had 29\% patients with CD4 below 200 cells/pl, 54\% in CD4 above 200 cells/pl $(p=0.3)$. Differences between patients with high and low viral load were not statistically significant $(p=0.71)$. 
Conclusions: We concluded that efficacy of vaccination was statistically lower among HIV/AIDS patients in comparison with healthy volunteers. There were no statistically difference with stage of HIV infection and response to hepatitis B vaccine.

\section{PP06-06}

Induced HBs Antigenemia in Healthy Adults after Immunization with Two Different Hepatitis B Recombinant Vaccines

M. Ziaee ${ }^{1}$, M.H. Namaei ${ }^{2}$, S.A. Saadatjoo ${ }^{3}$, M. Mohamadpour ${ }^{1}$

${ }^{1}$ Internal Medicine; ${ }^{2}$ Laboratory Medicine; ${ }^{3}$ Nursing and Midwifery, Birjand University of Medical Sciences, Birjand, Iran

Background and objectives: Currently, vaccination is the most effective protective method against hepatitis B virus (HBV) infection. Some studies have shown that positive results of hepatitis B virus surface antigen ( $\mathrm{HBsAg}$ ) test could be seen after vaccination. The aim of this study was to compare the incidence of HBs antigenemia after vaccination with two different hepatitis $\mathrm{B}$ recombinant vaccines.

Methods: In this clinical trial study, 62 healthy adult volunteers were randomly allocated to vaccinate with Engerix-B or Hepavax-Gene hepatitis $\mathrm{B}$ recombinant vaccine. Blood samples were drawn 1,3 , and 5 days after vaccination and tested for HBsAg using two different commercial ELISA kits.

Results: HBsAg was detected in 5, 3, and 2 participants of Engerix-B group in the $1 \mathrm{st}$, 3rd, and 5 th day after vaccination respectively using one ELISA kit; the test was positive only in one subject in HepavaxGene group in the 5th day after vaccination. No positive result was seen in any groups when another ELISA kite was used for detection of HBsAg.

Conclusion: Our results showed that transient HBsAg antigenemia after vaccination against hepatitis $\mathrm{B}$ is possible but the time and duration of antigenemia depend on the type of vaccine and detection method.

\section{PP06-07}

Study on HBV Antigens Stimulating the Cellular Immune of HBsAg Carriers

B. Lin, D. Xie, J. Xie, X. Zhang, Z. Gao

Infectious Diseases, The Third Affiliated Hospital of Sun Yat-sen

University, Guangzhou, China

Background/aims: The exact mechanism underlying the pathogenesis of the HBsAg carrier state is not well understood, which results in an unsatisfying therapeutic approach to hepatitis B virus (HBV). The aim of the present study is to investigate the effect of HBV antigens and pathological mechanism of HBV chronic infection by analyzing the cellular immune function of peripheral blood mononuclear cells (PBMCs) from HBsAg carriers.

Methods: 60 hepatitis B virus carriers were enrolled in this study and divided into two grous: $\mathrm{HBeAg}$ positive group $(n=30)$ and $\mathrm{HBeAg}$ negative group $(n=30)$, based on their serological character, and 30 healthy people were chosen as control group. PBMCs were prepared from individuals with chronic asymptomatic HBV infection and cultured in the presence of different antigens and/ or cytokines. The level of cytokines in culture supernatants were detected by ELISA. The phenotype of the cells was detected by FACS.

Results: The level of IFN- $\gamma$ from the PBMCs of HBsAg carriers was significantly lower than the counterparts from the control group; high concentration of HBsAg cannot suppress IFN- $\gamma$ production from PBMCs of HBsAg carriers directly, PBMCs from $\mathrm{HBeAg}$ positive group produced significantly less IFN- $\gamma$ and IL-12 than that from HBeAg negative group; exogenous IL-12 in low dose combined with specific HBV antigens promoted central memory CD8+ $\mathrm{T}$ cells to produce IFN- $\gamma$.

Conclusions: $\mathrm{HBeAg}$, not $\mathrm{HBsAg}$, suppress IL-12 production from PBMCs, which may be the fundamental reason of viral persistence in HBV chronic carriers, combined IL-12 and specific HBV antigen promoted the central memory $\mathrm{CD} 8+\mathrm{T}$ cells to produce IFN $-\gamma$ and break the immune tolerance.

\section{PP06-08}

Analysis of Hepatitis B Virus Precore/Core and Polymerase Regions in the Same Viral Genome by Massive Sequencing

M. Homs ${ }^{1,2}$, M. Buti ${ }^{1,3}$, R. Jardí1, ${ }^{1,2}$, D. Tabernero ${ }^{1,2}$, M. Schaper ${ }^{1,2}$,

J. Quer ${ }^{1,3}$, I. Ortega ${ }^{4}$, A. Artacho ${ }^{4}$, P. Fernandez-Fernandez ${ }^{2}$, R. Esteban ${ }^{1,3}$, F. Rodriguez-Frias ${ }^{1,2}$

${ }^{1}$ CIBERehd. Spanish Ministry of Health and Consumer Affairs,

${ }^{2}$ Biochemistry; ${ }^{3}$ Hepatology, Hospital Vall d'Hebron;

${ }^{4}$ Bioinformatics and Statistics Unit, Institut de Recerca Vall d'Hebron, Barcelona, Spain

Aims: The main aim of the study was to analyze the possible association between Precore (PC) and Polymerase (Pol) mutations of HBV by ultra-deep-pyrosequencing (UDPS). The second aim was to confirm the thermodynamically restriction of PC mutations by the

\begin{tabular}{|c|c|c|c|c|c|c|c|c|c|c|}
\hline & & & A & B & $\mathrm{C}$ & D.1 & D. 2 & $\mathrm{E}$ & $\mathrm{F}$ & $\mathrm{G}$ \\
\hline \multicolumn{3}{|c|}{ Log HBV DNA } & $>8$ & 7.60 & $6 . S 4$ & $>8$ & 7.59 & 4.98 & 7.90 & 5.11 \\
\hline \multicolumn{3}{|c|}{ HBV genotype } & $\mathrm{D}$ & A & A & $\mathrm{D}$ & $\mathrm{D}$ & A & A & $\mathrm{D}$ \\
\hline \multicolumn{3}{|c|}{$\mathrm{HBe} A g$} & $\mathrm{~N}$ & $\mathrm{~N}$ & $\mathrm{~N}$ & $\mathrm{P}$ & $\mathrm{P}$ & $\mathrm{N}$ & $\mathrm{P}$ & $\mathrm{N}$ \\
\hline \multicolumn{3}{|c|}{ pre-Core (G1896A) } & MT & WT & MT & WT & WT & MT & WT & MT \\
\hline \multicolumn{3}{|c|}{ Polymerase (M204IV) } & M204 & M204 & M204 & M204 & M204V & M204I & M204V & M204V \\
\hline \multicolumn{3}{|c|}{ Number of sequences } & 19,421 & 29,024 & 5,585 & 34,182 & 24,645 & 20,683 & 25,175 & 31,572 \\
\hline 204 & 1 and/or 2 & 28 & \multicolumn{8}{|c|}{ Percentage of sequences } \\
\hline WT & WT & WT & 0.20 & 0.05 & 0.04 & 90.93 & 0.25 & 0.00 & 0.05 & 0.00 \\
\hline WT & MT & WT & 0.00 & 99.41 & 0.00 & 0.55 & 0.00 & 0.03 & 0.00 & 0.00 \\
\hline WT & WT & MT & 99.21 & 0.01 & 99.71 & 7.82 & 0.00 & 0.04 & 1.01 & 0.07 \\
\hline MT & WT & MT & 0.29 & 0.08 & 0.10 & 0.46 & 1.20 & 99.24 & 0.28 & 99.03 \\
\hline MT & WT & WT & 0.01 & 0.00 & 0.00 & 0.22 & 97.94 & 0.13 & 88.78 & 0.29 \\
\hline MT & MT & WT & 0.00 & 0.29 & 0.00 & 0.00 & 0.59 & 0.00 & 9.86 & 0.01 \\
\hline MT & MT & MT & 0.00 & 0.00 & 0.00 & 0.00 & 0.02 & 0.56 & 0.02 & 0.60 \\
\hline WT & MT & MT & 0.30 & 0.16 & 0.15 & 0.02 & 0.00 & 0.00 & 0.00 & 0.00 \\
\hline \multicolumn{3}{|c|}{ Core defectives (codon 1) } & 0.74 & 0.48 & 0.11 & 0.51 & 0.41 & 0.91 & 0.79 & 0.70 \\
\hline
\end{tabular}


analysis of the loop stability in the pregenomic-RNA, containing the encapsidation signal.

Patients and methods: Seven Lamivudine-treated CHB patients (A$\mathrm{G}$, Table 1) were analyzed. Pol and PC regions are separated $>1 \mathrm{~kb}$, their simultaneity analysis by UDPS is not possible. A circular construct including YMDD-Pol motif and PC/Core region was designed and UDPS analyzed by Roche-454-platform. A linkage analysis of YMDD-Pol and codon $1 / 2$ and 28 of PC was performed. The stability of pregenomic-RNA encapsidation signal was analyzed by RNAStructure-5.3 software and the correct base paring of PC codon 28 and 15 was analysed. UDPS was performed at baseline in patients A, B, C and D (D1) and at viral breakthrough in patients D (D2), E, F and G. Results: A total of 190.287 sequences were analyzed. Results are presented as percentage (Table 1).

The $99.8 \%$ (80473 cases) with the PC main mutant G1896A (Codon 28 ) were correctly paired with a T1858 (Codon 15) decreasing the stabilization energy.

Conclusions: UDPS allowed detection of minor variants $(<0.1 \%)$ in YMDD-Pol and PC regions, and confirmed the simultaneity of mutations in PC and Pol. The detection of Core defective particles evidenced the cooperation and the highly complexity of HBV quasispecies. Finally, the thermodynamically restriction of PC mutations was confirmed (Study founded by FIS-PS09/00899).

\section{PP06-09}

Correlation between Intrahepatic Hepatitis B Virus CCCDNA Levels and Other Activity Markers in Patients with HBeAG Negative Chronic Hepatitis B

R. Guner ${ }^{1}$, M. Karahocagil ${ }^{2}$, M. Büyükberber ${ }^{3}$, Ö. Kandemir ${ }^{4}$, O. Ural ${ }^{5}$, G. Usluer ${ }^{6}$, D. İnan ${ }^{7}$, I. Köksal ${ }^{8}$, N. Baykam ${ }^{9}$, K. Hizel ${ }^{10}$, T. Yamazhan ${ }^{11}$, S. Esen ${ }^{12}$, M.A. Tasyaran ${ }^{1}$

${ }^{1}$ Infectious Diseases and Clinical Microbiology, Atatürk Education and Research Hospital, Ankara; ${ }^{2}$ Infectious Diseases and Clinical Microbiology, Van 100, Yll University; ${ }^{3}$ Department of Gastroenterology, Van 100, Yil University, Van; ${ }^{4}$ Infectious Diseases and Clinical Microbiology, Mersin University, Mersin; ${ }^{5}$ Infectious Diseases and Clinical Microbiology, Selçuk University, Konya; ${ }^{6}$ Infectious Diseases and Clinical Microbiology, Osman Gazi University, Eskisehir; ${ }^{7}$ Infectious Diseases and Clinical Microbiology, Akdeniz University, Antalya; ${ }^{8}$ Infectious Diseases and Clinical Microbiology, Karadeniz Technical University, Trabzon;

${ }^{9}$ Infectious Diseases and Clinical Microbiology, Numune Education and Research Hospital; ${ }^{10}$ Infectious Diseases and Clinical Microbiology, Gazi University, Ankara; ${ }^{11}$ Infectious Diseases and Clinical Microbiology, Ege University, Izmir; ${ }^{12}$ Infectious Diseases and Clinical Microbiology, 19 Mays University, Samsun, Turkey

Purpose: We aimed to demonstrate the correlation between intrahepatic hepatitis B virus cccDNA levels and the levels of alanine aminotransferase, serum quantitative hepatitis B surface antigen levels, hepatic histopathology, staining levels of $\mathrm{HBsAg}$ and hepatitis $\mathrm{B}$ core antigen in the liver in HBeAg-negative chronic hepatitis B.

Patients and methods: Patients between 18 and 65 years old with HBsAg positivity, HBeAg negativity, serum HBV DNA levels $\geq 10^{4}$ copies/ml, and consistently or intermittently increased ALT levels for more than 6 months were included. Serum HBV DNA was measured using Cobas TaqMan. HBsAg is quantitated by chemiluminescent immunoassays. Histopathologic examinations were done according to modified Knodell. IH HBsAg and $\mathrm{HBcAg}$ were stained with immunohistochemical staining and quantified. IH DNA was isolated from needle liver biopsy using a QIAamp DNA Mini Kit. Total HBV DNA and cccDNA were measured by real-time polymerase chain reaction. Results: Fifty-nine patients were included.There was a positive correlation between the levels of IH HBV cccDNA and serum HBV DNA $(p<0.001)$. Quantitative staining groups of IH HBcAg and IH HBV cccDNA levels were significantly correlated, but there was no significant correlation between IH HBsAg staining levels $(p=0.02$ and $p=0.836$, respectively). There were positive correlations between serum HBsAg levels and serum HBV DNA and IH HBV cccDNA levels ( $\rho=0.464, p<0.001 ; \rho=0.322, p=0.017$ respectively). There was no significant correlation between serum HBsAg level and histologic activity index groups ( $p=0.691$ ), but stage 0,1 and $>2$ fibrosis groups were positively correlated $(p=0.019)$. ALT, age, fibrosis stage and serum HBsAg quantitation were the most important factors predicting IH HBV cccDNA level ( $\left.p=0.037 ; p<0.001 ; R^{2} 75.5 \%\right)$.

Conclusions: Histopathologic damage, serum HBV DNA levels and IH HBV replication markers have a more complex and dynamic process. However, a noninvasive marker, serum HBsAg quantitation, is a forefront marker in the follow-up of the disease.

\section{PP06-10}

Pre-S/S Promoter Mutants of Hepatitis B Virus Responsible for Occult Infection Are Replication Competent

G. Satpathy ${ }^{1}$, S. K. Panda ${ }^{2}$, S.K. Acharya ${ }^{3}$, S. Sengupta ${ }^{2}$, S.P. Singh ${ }^{2}$, H. Durgapal ${ }^{2}$

${ }^{1}$ Department of Occular Microbiology; ${ }^{2}$ Department of Pathology;

${ }^{3}$ Department of Gastroenterology, All India Institute of Medical Sciences, New Delhi, India

Background: Hepatitis B virus with point mutations in the pre-S1 promoter and deletions in the pre-S2/S promoter occur frequently in occult Hepatitis infection in India. The pre-S1 and pre-S2/S promoters overlap on the HBV polymerase gene. Therefore, the effect of such mutations on HBV replication was investigated.

Methods: Over length 1.86 mer wild type HBV replicon (WT-1.86) was produced in a modified promoter less vector. Pre-S1 and pre-S2/S promoters of the mutant clones 313.1 and 761.1 from patients with occult infection were used to replace the homologous region (nt 2,600-nt 3,221/1-nt 3) in the wild type replicon to produce 313.1-1.86 and 761.1-1.86 constructs. The expression patterns of viral proteins, secretion of $\mathrm{HBV}$ antigens and virus replication were investigated in transiently transfected Huh7 cells.

Results: HBV replication intermediates were observed in the mutants by southern blot which was confirmed by real time PCR. HBsAg secretion was reduced at $24 \mathrm{~h}$ in the mutant constructs compared to the wild type. Mutant 313.1-1.86 continued to have reduced secretion of $\mathrm{HBsAg}$ at all time points post-transfection, whereas mutant 761.1-1.86 secreted at levels comparable to the wild type at later time points. Diffuse cytoplasmic staining of HBsAg was observed in cells transfected with WT-1.86 compared to the peri-nuclear distribution with the mutants. Cytoplasmic and nuclear staining for Hepatitis B core antigen was observed in cells transfected with wild type and mutant constructs. WT-1.86, 313.1-1.86 and 761.1-1.86 showed consistent Hepatitis B e antigen secretion at all time points post transfection.

Conclusions: Point mutations in the pre-S1 promoter and deletions in the pre-S2/S promoter do not affect the capability of HBV to replicate.

\section{PP06-11}

A Mathematical Modeling of the Liver's Control Mechanisms in Hepatitis B A. Turgunov ${ }^{1}$, B. Aliev ${ }^{2}$, B. Hidirov ${ }^{1}$, M. Saidalieva ${ }^{1}$

${ }^{I}$ Regulatorics, Institute of Mathematics and Information Technologies of the Sciences Academy; ${ }^{2}$ Morphology, Institute of Virology, Tashkent, Uzbekistan

Background: Molecular-genetic and cellular aspects of viral hepatitis pathogenesis are definitively not established. It can be reached by 
applying modern technology for the quantitative analyzing functioning regulatory mechanisms (regulatorika) of living systems at molecular-genetic and cellular levels with using methods for mathematical modeling. Research objective is development of the quantitative methods for liver regulatorika of viral infection (viral hepatitis type B) stages using methods for mathematical modeling.

Methods: For mathematical modeling the methods of the quantitative studying regulatorika of living systems at molecular-genetic and cellular levels have been used. The equations of mathematical models for regulatorika of liver cells and hepatitis B virus (HBV) under hepatitis B based on the approaches generalization by B. Goodwin, M Eigen, V.A. Ratner and J. Smit with taking into account cooperativity, time mutual relations and presence of the combined feedback in hepatocyte regulation system are carried out.

Results: In the course of quantitative researches of viral hepatitis type $\mathrm{B}$ the following regimes of considered process have been received: clarification, symbiosis, auto-oscillations and irregular fluctuations (chaos), sharp destructive changes ("black hole" effect) which define various clinical forms of the disease (B hepatitis).

Conclusions: The developed quantitative methods based on the mathematical modeling liver regulatorika at molecular-genetic and cellular levels, allow to predict coming chaotic, repeating changes and "black hole" effect in the liver under HBV infection. This gives the chance to diagnose coming unpredictable changes phase in liver regulatorika.

[The research is supported by RUz grants (FA-F1-F011 and FA-A17F009)].

\section{PP06-12}

The Distribution of HBV cccDNA and HBV tDNA in the Liver Infected by HBV

N.J. Jiang ${ }^{1}$, W.W. Guo ${ }^{1}$, X.Y. Liang ${ }^{1}$, H.M. Su ${ }^{1}$, H.Z. Liu ${ }^{1}$, G.J. Hu ${ }^{2}$, H. Lu ${ }^{3}$, D.H. $\mathrm{Li}^{4}$, F.S. Ge ${ }^{1}$, Q.M. Zhu ${ }^{5}$

${ }^{1}$ Infectious Disease Department, The First Affiliated Hospital of Guangxi Medical University, Nanning; ${ }^{2}$ Infectious Disease Department, Liu Zhou People's Hospital, Liu Zhou; ${ }^{3}$ Infectious Disease Department, The 303 Hospital of People's Liberation Armypeople's, Nanning; ${ }^{4}$ Infectious Disease Department, The Infectious Disease Hospital of Wu Han, Wu Han; ${ }^{5}$ Hepatology Department, The Third Hospital of Zhen Jiang, Zhen Jiang, China

Aim: To investigate distribution of HBV cccDNA,HBV tDNA and HBV dynamics in the liver infected by HBV.'

Methods: 40 liver tissue samples removed from 5 liver transplant recipients with $\mathrm{HBV}$ infection were collected. Hepatocyte $\mathrm{HBV}$ cccDNA and HBV tDNA were detected by real-time quantitative polymerase chain reaction in 40 samples from 8 sections of 5 livers. Results: The level of HBV cccDNA and HBV tDNA among 8 sections for each liver were big difference. Among 5 livers the maximum intrahepatic difference of HBV tDNA was 1186.6 copy $^{\text {cell }}{ }^{-1}$, the smallest difference was 832.8 copy cell ${ }^{-1}$. The maximum intrahepatic difference of HBV cccDNA was 398.1 copy cell $^{-1}$, the smallest was 197.5 copy cell ${ }^{-1}$. The maximum intrahepatic difference of cccDNA/tDNA was 0.72 , the minimum was 0.30 . The mean of $\mathrm{HBV}$ cccDNA, HBV tDNA and cccDNA/tDNA of 8 section in 5 livers were significantly different $(p<0.05)$. The maximum mean of $\mathrm{HBV}$ tDNA was in the third section, the minimum in the sixth section, the maximum mean of $\mathrm{HBV}$ cccDNA was in the eighth section, minimum in the third section.
Table 1 The intrahepatic difference of HBV cccINA, tHBV DNA and cccDNA/tDNA

\begin{tabular}{|c|c|c|c|}
\hline $\begin{array}{l}\text { Liver } \\
\text { recipient }\end{array}$ & $\begin{array}{l}\text { t DNA difference } \\
\left(\text { copy cell }{ }^{-1}\right)\end{array}$ & $\begin{array}{l}\text { ccc DNA } \\
\text { difference } \\
(\text { copy cell } \\
-1)\end{array}$ & cccDNA/tDNA difference \\
\hline 1 & $1036.7(1162.5-125.8)$ & 119. $8(131.4-11.6)$ & $0.54(0.56-0.02)$. \\
\hline 2 & $862.6(976.1-113.5)$ & $398.1(412.5-14.4)$ & $0.39(0.42-0.03)$ \\
\hline 3 & $832.8(91.8 .3-85.5)$ & 281. 9 (291.6-9.7) & $0.30(0.37-007)$ \\
\hline 4 & $1011.2(1102.8-31.6)$ & 197. $5(256.7-59.2)$ & $0.59(0.74-0.15)$. \\
\hline 5 & $1186.6(1197.7-3.1)$ & 257. $2(259.7-2.5)$ & $0.72(0.73-001)$ \\
\hline
\end{tabular}

Table 2 The mean of HBVtDNA, HBV cccDNA and cccDNA/ tDNA of 8 sections

\begin{tabular}{|c|c|c|c|c|}
\hline $\begin{array}{l}\text { Liver } \\
\text { section }\end{array}$ & $N$ & $\begin{array}{l}\text { tDNA } \\
\left(\text { copy cell }^{-1}\right)\end{array}$ & $\begin{array}{l}\text { cccDNA } \\
\left(\text { copy cell }{ }^{-1}\right)\end{array}$ & $\begin{array}{l}\text { ccDNA/ } \\
\text { tDNA }\end{array}$ \\
\hline 1 & 5 & $934.5 \pm 792.0$ & $45.7 \pm 36.5$ & $0.16 \pm 0.11$ \\
\hline 2 & 5 & $681.0 \pm 814.9$ & $43.4 \pm 42.0$ & $0.39 \pm 022$ \\
\hline 3 & 5 & $1536.4 \pm 3002.3$ & $30.4 \pm 56.8$ & $0.14 \pm 008$ \\
\hline 4 & 5 & $9835 \pm 871.1$ & $343 \pm 33.6$ & $0.17 \pm 0.14$ \\
\hline 5 & 5 & $567.8 \pm 332.9$ & $35 \mathrm{D} \pm 27.6$ & $0.45 \pm 0.25$ \\
\hline 6 & 5 & $5549 \pm 655.5$ & $53.7 \pm 41.3$ & $0.28 \pm 0.16$ \\
\hline 1 & 5 & $6759 \pm 417.9$ & $\mathrm{~S} 92 \pm 39.3$ & $0.26 \pm 0.29$ \\
\hline 8 & 5 & $840.0 \pm 395.8$ & $124 \mathrm{D} \pm 140.6$ & $0.49 \pm 0.19$ \\
\hline
\end{tabular}

The distribution of HBV cccDNA and HBV tDNA

Conclusion: The intrahepatic distribution of HBV cccDNA and HBV tDNA were uneven in each liver. The HBV cccDNA and HBV tDNA level were not synchronized. cccDNA/tDNA may better indicate HBV dynamic in liver. There are some limitation for the evaluation of HBV dynamic in liver with traditional liver puncture biopsy in one point.

\section{PP06-13}

Clinical Significance of Core Gene Mutations during the Course of Genotype C Chronic Hepatitis B Virus Infection

G.-Y. Gwak, D.H. Lee, B.-H. Min, M.S. Choi, J.H. Lee, K.C. Koh, S.W. Paik, B.C. Yoo

Samsung Medical Center, Sungkyunkwan University School of Medicine, Seoul, Republic of Korea

Background/aims: The aim of this study was to investigate the association of HBV core gene mutations with disease severity in HBV-infected patients.

Methods: We included 249 genotype C HBV-infected patients: 39 asymptomatic carriers (AC), 68 with chronic hepatitis $(\mathrm{CH}), 75$ with liver cirrhosis (LC), and 67 with HCC. HBV DNA was extracted from patient sera and the HBV core gene was analyzed by PCR and sequencing.

Results: The overall frequency of a codon substitution, which was caused by a missense mutation in the HBV core region, was $4.5 \pm 9.0$ / patient. The codon substitutions were predominantly clustered in the mid-core regions; $22.3 \%$ of codon substitutions were found in codons $13,87,97$, and 130 . The rate of substitution for codon 13 was higher in 
$\mathrm{CH}$ and LC than in AC. For codons 87 and 130, AC had a lower substitution rate compared to the other 3 groups. The substitution rate for codon 97 was higher in $\mathrm{CH}$ and $\mathrm{HCC}$ than in AC.

Conclusions: Core gene mutations were frequently detected during the course of chronic HBV infection, and some mutational hot spots were correlated with severe forms of disease. Thus, these mutations might play a pathophysiological role in the disease progression in HBV-infected patients.

\section{PP06-14}

HBV Particles Only Elevate TGF $\beta$ Level But Not Other Proinflammatory Cytokines in Kupffer Cells in vitro

H. Li, H. Chen, Z. Xing, H. You, M. Cong, J. Jia, Dr. Tianhui Liu, Dr. Xinmin Li, Dr. Ping Wang, Dr. Xinyan Zhao

Liver Research Center, Beijing Friendship Hospital, Capital Medical Univeristy, China, Beijing, China

Background/aims: Kupffer cells play critical roles in the progress of immunotolerance of chronic $\mathrm{HBV}$ infection and subsequent liver injury by secreting many inflammatory mediators. However, it is not clear if Kupffer cells stimulated by HBV particles are responsible for this phenomenon.

Methods: HBV particles, purified from serum of chronic hepatitis B patients by gradient ultracentrifugation $(120,000 \mathrm{G})$ on the concentration of $0,4 \mathrm{log} / \mathrm{ml}, 5 \mathrm{log} / \mathrm{ml}, 6 \mathrm{log} / \mathrm{ml}$, were added into collengenase perfusion isolated Kupffer cells expressed ED1 and ED2 from wistar rats. TGF $\beta$, IL6, IL1, TNF $\alpha$ were detected by ELISA every $24 \mathrm{~h}$ in the supernatant for 7 days. Proteins and mRNA of these cytokines were also analysed by Western blot and real-time PCR at $72 \mathrm{~h}$.

Results: There is no morphological changes were observed on kupffer cells stimulation of HBV during 7 days. The results demonstrate that HBV stimulates kupffer cells over express TGF $\beta$ in a dose- and timedependent fashion. Firstly, TGF $\beta$ level was increased to $2.87 \pm 1.09$, $17.8 \pm 3.47$-folds higher after $6 \mathrm{log} / \mathrm{ml} \mathrm{HBV}$ stimulation in 3 and 7 days while only $1.22 \pm 0.57$ and $1.34 \pm 0.63$-folds $(P=0.010)$ in control. Secondly, after stimulation of $4 \mathrm{log} / \mathrm{ml}$ and $6 \mathrm{log} / \mathrm{ml} \mathrm{HBV}$ particles, TGF $\beta$ level was increased to $1.53 \pm 0.91,2.87 \pm 1.09$-folds at $72 \mathrm{~h}$ in the supernatant. Similar results were obtained in western blot analysis in Kupffer cells stimulated by HBV. Nevertheless, mRNA levels of TGF $\beta$ only slightly increased under the stimulation of different HBV titers at $72 \mathrm{~h}$ showing the mechanism of TGF $\beta$ elevated not only involve the gene transcription. Interestingly, other proinflammatory cytokines expression both in supernatant and kupffer cells, such as IL6, IL1, TNF $\alpha$, did not change after HBV treated Kupffer cells but dramatically elevated by LPS stimulation.

Conclusion: HBV can stimulate kupffer cells to secrete and express TGF $\beta$ rather than other proinflammatory cytokines, which maybe result in HBV persistent infection.

\section{PP06-15}

Prevalence of Occult Hepatitis B Infection among HIV Infected Patients in Georgia

L. Gatserelia ${ }^{1,2,3}$, L. Sharvadze $e^{1,2,3}$, M. Karchava ${ }^{1,2,3}$, N. Badridze ${ }^{1,2}$,

T. Tsertsvadze ${ }^{1,3,4}$

${ }^{1}$ Infectiouse Diseases, AIDS and Clinical Immunology Research

Center; ${ }^{2}$ Faculty of Medicine, Iv. Javakhishvili Tbilisi State

University; ${ }^{3}$ Georgian-French Joint Hepatology Clinic "HEPA",

Tbilisi; ${ }^{4}$ Faculty of Medicine, Iv. Javakhishvili Tbilisi State

University, Tbilisi, Georgia

Aims: Mortality is higher in HIV/HBV coinfected than HIV monoinfected patients. Occult HBV infection i.e. isolated positive Hepatitis B core antibody but Hepatitis B surface antigen and surface antibody negative with HBV DNA is a form of chronic HBV infection that impacts HIV management. This study estimates the prevalence and patient characteristics of occult hepatitis B infection in HIV infected patients in Georgia.

Methods: Numerator data were generated by reviewing HIV infected patients in Georgia during 2007-2009 years. Total of 342 patients with $\mathrm{HBV}$ serology showing $\mathrm{HBsAg}-, \mathrm{HBsAb}-$, anti $\mathrm{HBc}+$ were identified. 158 from these were investigated on $\mathrm{HBV}$ DNA by Real-time PCR (COBAS TaqMan, Roche) according to the manufacturer's instruction. Information on hepatitis $\mathrm{C}$ antibody, antiretroviral therapy (ARV), CD4 and HIV viral load (VL) were collected.

Results: Among the final study population, majority were males $(n=96,60.76 \%)$, mean age of $42 \pm 6$ years, on ARV $(n=110$, $69.62 \%)$, had CD4 $>200(n=118,74.68 \%), \mathrm{VL}<47 \quad(n=85$, $53.8 \%)$ and $50 \%(n=79)$ tested positive for HCV. Approximately $7 \%(n=11)$ of study population met the definition of occult HBV infection. $30 \%$ of patients not on ARV $(n=48)$ had positive HBV DNA, whereas none of patients on ARV had detectable HBV DNA. All patients on ARV $(n=110)$ were on a regimen with anti HBV activity.Of those with occult HBV infection, $54.5 \%(n=6)$ were males and mean CD4 count was $400 \pm 150$.

Conclusion: We found that prevalence of occult HBV among HIV infected patients in Georgia is about 7\%. Further, high proportion of patients with isolated anti $\mathrm{HBc}$ also tested positive for $\mathrm{HCV}$ ab (50\%). Therefore clinicians should have a high index of suspicion for occult HBV among HIV infected patients whose hepatitis panel is positive only for core antibody particularly those not on ARV and with $\mathrm{HCV}$ coinfection.

\section{PP06-16}

Plasma Myeloperoxidase Activity and Apolipoprotein A-1 Expression in Chronic Hepatitis B Patients

A. Mohamadkhani, F. Rastgar Jazii, L. Montaser-Kouhsari, G. Montazeri Digestive Disease Research Centre, Tehran University of Medical Science, Tehran, Iran

Background: Chronic hepatitis B (CHB) is a major cause of liver inflammation and the increased risk of human hepatocellular carcinoma. We investigated the level of plasma myeloperoxidase (MPO) as well as plasma proteins pattern in patients with progressive liver disease.

Methods: Thirty male subjects including nineteen CHB patients, six HBV-related cirrhotic patients and five healthy controls were included in the study. Plasma MPO was measured using enzyme-linked immunosorbent assay. Proteomic analysis of plasma proteins was performed by two-dimensional gel electrophoresis and mass spectrometry.

Results: The mean plasma MPO was significantly higher in patients with liver cirrhosis $(60 \pm 11)$, and those with CHB $(51 \pm 10)$ than those in healthy controls $(45 \pm 8)(P=0.05)$. Moreover, a positive correlation was found between plasma MPO levels,serum alanine transaminase and hepatic fibrosis stage $(r=0.4, P=0.01$, and $r=0.5$, $P=0.003$ ). Proteomic analysis showed altered plasma protein pattern in progressive hepatitis $\mathrm{B}$ as well as down-regulation of the major apolipoprotein A-1 (Apo A-1) in addition to the appearance of a variety of spots that found to be Apo A-1 isoforms with different molecular masses.

Conclusion: Progressive liver injury associated with HBV infection correlates with higher plasma MPO and altered plasma Apo A-1 pattern. 
PP06-17

Modulation of HBV Replication by Cell Cycle and Differentiation Related MicroRNAs

X. Zhang ${ }^{1}$, Z. Ma ${ }^{1}$, C. Wu ${ }^{1}$, E. Zhang ${ }^{1}$, J. Schlaak ${ }^{2}$, M. Roggendorf ${ }^{1}$, M. Lu ${ }^{1}$ ${ }^{1}$ Institute of Virology; ${ }^{2}$ Department of Gastroenterology and

Hepatology, University Hospital of Essen, Essen, Germany

Background/aims: MicroRNAs (miRNAs) are highly conserved small non-coding RNAs that participate in regulating various cellular processes. Viruses have been shown to utilize cellular miRNAs to increase their replication in host cells. Previously we have reported that miR-1 acts as a positive regulator of HBV replication and transcription through regulating the cellular gene expression (submitted, 2010). We asked whether the regulation of cell cycle and differentiation by miRNAs generally controls HBV replication.

Methods: MiRNA mimics were transfected into HBV expressing hepatoma cell lines. HBV replication and gene expression were examined. miRNA effect on cellular gene expression and hepatocytes phenotype change was checked by Microarray analysis, proliferation assay, cell cycle FACS analysis, and realtime RT-PCR.

Results: We identified another miRNA candidate, miR-449a, with a high capacity for enhancement of HBV replication. miR-449a level was lower in hepatoma cells, as compared to primary hepatocytes. Ectopic expression of miR-449a in hepatoma cells resulted in much stronger enhancement of HBV replication and transcription. Further, miR-449a antisense inhibitor had an opposite effect of decreasing viral replication and could neutralize the effect of miR-449 on HBV replication. Microarray analysis of the global cellular gene expression profile revealed that pathways related to lipid and bile acids metabolism were activated, and genes related cell cycle progression were down-regulated by miR-449a. Like miR-1, miR-449a arrested G1/S cell cycle transition and increased the expression of a number of hepatocyte-specific genes, respectively.

Conclusions: These results indicated miR-449a promotes cell differentiation and restore partially the hepatocyte phenotype, which is apparently beneficial for HBV replication. Our findings provide a new concept to understand the role of miRNAs in HBV replication and present a useful way to identify host factors involved in $\mathrm{HBV}$ infection.

\section{PP06-18}

Compare Roche COBAS AmpliPrep/COBAS TaqMan with Domestic Reagent in Hepatitis B Virus Quantitative

L. Chen, Y. Jiang, G.G. Xu, J.H. Liu, G.Y. Ren

Shanghai Public Health Clinic Center, Fudan University, Shanghai, China

Objective: To make a compare the Roche COBAS AmpliPrep/ COBAS TaqMan with domestic reagent in hepatitis B virus quantitative.

Methods: 34 serum samples of chronic hepatitis B from outpatient were tested by both Roche COBAS AmpliPrep/COBAS TaqMan and domestic reagent at same time. Detection lower limit is $6 \mathrm{IU} / \mathrm{ml}$ in Roche COBAS AmpliPrep TaqMan, and $5 \times 102$ to $5 \times 107 \mathrm{IU} / \mathrm{ml}$ in domestic reagent. Statistical analysis is by SPSS18.0.

Results:

1. Positive and negative coincidence rate between Roche COBAS AmpliPrep COBAS/TaqMan and domestic reagent is $100 \%$ in coincidencerate (34/34), respectively.

2. HBV DNA load is $6.47 \pm 1.67\left(\log _{10} \mathrm{IU} / \mathrm{ml}\right)$ in Roche COBAS AmpliPrep/COBAS TaqMan and $5.86 \pm 1.49\left(\log _{10} \mathrm{IU} / \mathrm{ml}\right)$ in domestic reagent, and the results are significant difference $(P<$ $0.0001)$.

3. 31 of $34(91.18 \%)$ samples titers of HBV DNA were higher by Roche COBAS AmpliPrep/COBAS TaqMan than that by domestic reagent $\left(0.64 \log _{10} \mathrm{IU} / \mathrm{ml}\right.$ higher on average), while only 3 of $34(8.82 \%)$ were lower than that by domestic reagent $\left(0.37 \log _{10} \mathrm{IU} / \mathrm{ml}\right.$ lower on average $)$.

4. The linear relation between two assays were good $(Y=$ $0.3087+1.0509 X, r=0.9368, P<0.0001)$ in domestic monitoring range $(5 \times 102-5 \times 107 \mathrm{IU} / \mathrm{ml})$.

\section{Conclusion:}

1. Both two methods have good linear relation, with high level of consistency, can be widely used in clinical.

2. Roche COBAS AmpliPrep/COBAS TaqMan demonstrated a better sensitivity than the domestic reagent, with a more reliable quantification result over a broad range (LOD:6IU/ml).

Therefore, It can also be used for diagnosis of occult hepatitis B, accurate assessment of antiviral therapy and prognosis.

\section{PP06-19}

Diversity Genomes in a Chronic HBV Infected Patient over 2 Years Evolution

T. Shen, M.X. Yan

Molecular Biology, Institute of Basic and Clinic Medicine, Kunming, China

To determine whether and how mutations occur within the genomes of HBV through time-course evolution, a chronic HBV infected patient was followed up for over 2 years. HBV strains were obtained from 3 time points: 1 day, half a year and 31 months.For each time point, 8-9 clones were sequenced and performed virological analysis. During at least 2 years evolution, T361A, C934A, C2351T/ A2353T, C2444T variantion and nucleotides 2846-2865 deletion of preS1 have been kept in. C339T and T770C were the new points mutation only exsited in sequenced clones of the 3rd time point, the former mutation occurred in the hydrophilic region of HBsAg and the latter caused polymerase amino acid $560 \mathrm{Val}$ to Ala. Internal deletion of coding region were obviousely appeared in the 3rd time point, the $5^{\prime}$-splice donors were at nucleotides position 2447,3018 , 3144 and 3024 , and the $3^{\prime}$-splice acceptors were at nucleotides position 489, 56, 3202 and 3193 , besides the 2447/489, all the other donors/accepters were first reported in the study. These defective genomes may produce presumptive HBV spliced proteins or truncated preS proteins. ALT, HBeAg and viral DNA load varied at the follow-up years. Combined with clinic data, the HBV variants existing in this patient may contribute to viral persistence of infection and liver pathogenesis.

\section{PP06-20}

The HBsAg Level is not Correlated with HBV DNA Level when Viral Replication is Inactive

J.J. Chen ${ }^{1}$, H.C. $\mathrm{Wu}^{2}$

${ }^{1}$ Gastroenterology; ${ }^{2}$ Chimei Medical Center, Liouying, Tainan,

Taiwan, ROC

Background and aims: There is limited information on $\mathrm{HBsAg}$ levels during the natural course of HBV-infection. HBsAg levels had been shown significant differences during the natural course of $\mathrm{HBV}$ infection. This study aimed to determine correlation between $\mathrm{HBsAg}$ levels and HBV DNA in the $\mathrm{HBeAg}$ negative phases of HBVinfection in southern Taiwan.

Methods: $40 \mathrm{HBsAg}(+)$ patients, not undergoing antiviral therapy, were analyzed in a cross-sectional study. There are 27 male and 13 female patients with age: $53.1 \pm 13.6$ years old included in this study; 9 patients co-infected with hepatitis $\mathrm{C}$ and 5 with hepatocellular carcinoma. Serum HBsAg levels were quantified using the Abbott ARCHITECT assay (Abbott Diagnostics, Abbott Park, IL, USA) and correlated with HBV-DNA. There are 10 patients who were in high replication phase (HBV DNA $>4 \log$ ), 10 patients had HBV DNA between 3 and $4 \log$ and 20 patients had HBV DNA $<3 \log$. 
Results: The HBsAg levels were higher in patients with active replication than those who HBV DNA $<4 \log (2.78 \pm 0.71$ vs. $2.03 \pm$ $\left.0.97 \log _{10} \mathrm{IU} / \mathrm{ml}, \mathrm{p}<0.05\right)$. The same trend also found when compared the HBsAg level who had HBV DNA $>3 \log$ or not $(2.64 \pm$ 0.54 vs. $\left.1.82 \pm 1.18 \log _{10} \mathrm{IU} / \mathrm{ml}\right)$. HBsAg level showed a correlation with HBV-DNA in patients with higher HBV DNA and no correlation of HBsAg and HBV-DNA were found in patients within low replication phase. $(R=0.41$ in HBV DNA $>4 \log$, and $R=0.46$ in HBV DNA $\geq 3 \log$, and $R=0.006$ in HBV DNA $<3 \log , p<0.05$ ).

Conclusions: These findings implicated that HBsAg level is correlated with HBV DNA level when viral replication is active. But poor correlated with HBV DNA when viral replication is inactive.

\section{PP06-21}

Quantitation of HBV DNA and cccDNA in Liver of Patients with HBV Related Hepatocellular Carcinoma and Chronic Hepatitis B

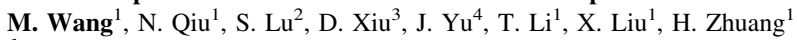

${ }^{I}$ Department of Microbiology, Peking University Health Science

Center; ${ }^{2}$ Department of Hepatobiliary Surgery and Liver

Transplantation Center, Beijing Youan Hospital, Capital Medical

University; ${ }^{3}$ Department of Surgery, Peking University Third

Hospital, Peking University, Beijing; ${ }^{4}$ Center of Liver Diseases, PLA 88 Hospital, Taian, China

Objective: The aim of this study is to quantitate total hepatitis B virus DNA (HBV tDNA) and covalently closed circular DNA (cccDNA) in liver of patients with $\mathrm{HBV}$ related hepatocellular carcinoma (HCC) and chronic hepatitis B (CHB).

Methods: The tumor and non-tumor liver samples were taken from 31 HCC patients (12 treated with antiviral drugs and 19 without treatment) during operation, and liver biopsies were collected from 13 CHB patients. HBV tDNA digested with a Plasmid-Safe ATPdependent Dnase was used for HBV cccDNA detection. The amount (copies/cell) of HBV tDNA and cccDNA were measured by a realtime PCR with use of $\beta$-globin housekeeping gene as a quantitation standard.

Results: The cccDNA level in tumor (0.069 copies/cell, $0.003-$ 27.110) was significantly lower than that in non-tumor tissues $(0.130$ copies/cell, 0.026-3.875) $(p<0.001)$, although no difference in HBV tDNA levels was found between them [2.024 copies/cell, (0.007-1255.083) vs. 1.339 copies/cell (0.084-327.720), $p=0.476]$. The ratio of cccDNA to HBV tDNA in non-tumor tissues was significantly higher than that in tumor (1:4.4 vs. 1:9.1, $p<0.05)$. The levels of HBV tDNA and cccDNA in tumor and non-tumor tissues of 12 treated HCC patients [1.945 copies/cell (0.199-14.765) and 0.031 copies/cell $(0.007-0.210)$; 0.935 copies/cell $(0.323-$ $60.857)$ and 0.068 copies/cell (0.026-1.163), respectively] were lower than that in 19 untreated [2.024 copies/cell (0.007-1255.083) and 0.080 copies/cell $(0.003-27.110) ; 2.625$ copies/cell (0.084-327.720) and 0.134 copies/cell (0.026-3.875, respectively], but there was no statistical significance $(p 0.240)$. HBV tDNA and cccDNA levels in liver biopsies of 13 CHB patients [6.199 copies/ cell (0.195-1403.120) and 0.393 copies/cell (0.031-6.482)] was significantly higher than that in tumor and non-tumor tissues of HCC patients $(p<0.05)$.

Conclusions: HBV tDNA and cccDNA levels in CHB patients were significantly higher compared with $\mathrm{HCC}$, but cccDNA levels in tumor tissues were significantly lower than that in non-tumor.

Acknowledgement: National Grants 2008ZX10002-004 and 2009 ZX10004-314.
PP06-22

HBV in Extra Hepatic Sites: Independent Evolution of HBV

in the Peripheral Blood Leukocytes

R. Chakravarty, S. Datta

ICMR Virus Unit Kolkata, Kolkata, India

Objectives: To test the hypothesis that HBV undergo independent evolution in different compartments of the body under the influence of differential immune pressure.

Methods: To examine our hypothesis, we isolated HBV DNA from lymphatic cells, the peripheral blood leukocytes (PBL), determined the HBV envelope sequences from isolated HBV DNA and performed advanced genetic, phylogenetic, and mutational analysis.

Results: Among the 84 samples with exposure to HBV which were screened for the presence of HBV DNA, 45 were detected positive for HBV DNA, in the PBL only. Although the sequence in the ' $a$ ' determinant region varied, all of them were of genotype A, subgenotype Ae/A2 along with the presence of classical immune escape mutant G145R. Notably, entirely different HBV genotypes/subgenotypes (Aa/A1, C and D) were found to predominate in the sera of the same study population. A possible molecular mechanism is proposed that explains the emergence of G145R mutation in the context of subgenotype Ae/A2 specific altered pregenomic RNA base pairing. Conclusion: So far most of the studies on vaccine efficacy or antivirals have been carried out on isolates from sera and hepatocytes. The present study documents evolution of HBV in extrahepatic sites and emergence of immune/vaccine escape variants in PBL. Thus the findings of this study have important clinical implications.

\section{PP06-23}

Hepatitis B Canr Replicate in Placental Cells Leading to Transplacental Transmission of HBV to the Babies

C. Pande ${ }^{1,2}$, P. Sakhuja ${ }^{3}$, S. Nath ${ }^{3}$, E. Gupta ${ }^{4}$, A. Kumarr ${ }^{2,5}$, S. Patra ${ }^{6}$, C.K. Mukhopadhyay ${ }^{2}$, S.K. Sarin ${ }^{1,2,5}$

${ }^{1}$ Gastroenterology, G B Pant Hospital; ${ }^{2}$ Special Centre for Molecular Medicine (SCMM), Jawaharlal Nehru University (JNU); ${ }^{3}$ Pathology, G B Pant Hospital; ${ }^{4}$ Virology; ${ }^{5}$ Hepatology, Institute of Liver \& Biliary Sciences (ILBS); ${ }^{6}$ Obstetrics \& Gynecology, Lady Hardinge Medical College, New Delhi, India

Background: During hepatitis B virus(HBV) entry into a cell, the HBV surface antigen remains outside; hence demonstration of surface antigen inside the cell by immunohistochemistry (IHC) is a reliable method of establishing HBV replication inside the cell. Whether HBV replicates in placenta and its possible pathogenetic role in transplacental transmission has not been fully explored.

Aim: To document HBV replication in placenta and to correlate this finding with $\mathrm{HBV}$ transmission in the neonates of mothers having chronic hepatitis $\mathrm{B}(\mathrm{CHB})$.

Methods: Pregnant mothers with $\mathrm{CHB}$, not on therapy,were enrolled.During delivery placenta and cord blood were collected. The babies received HBV vaccine and HBIG. Hepatitis B surface antigen was studied in the placental cells by IHC. Cord blood was studied for HBsAg and HBV-DNA.

Results: Thirty-seven mothers were studied. The median HBV-DNA level was $4 \times 10^{4} \mathrm{IU} / \mathrm{mL}$ (range $9-3 \times 10^{8} \mathrm{IU} / \mathrm{mL}$ ). Twelve $(32 \%)$ of these women were $\mathrm{HBeAg}$ positive. IHC was positive for surface antigen (Figure) in 11 of $37(30 \%)$ placenta while it was negative in $26(70 \%)$. Of the 11 mothers in whom IHC was positive $7(63 \%)$ babies were positive for HBsAg in their cord blood. Of the 26 mothers 
in whom IHC was negative, significantly less number of babies were positive for HBsAg in the cord blood (7/26 [27\%]; $p<0.05)$. The median HBV-DNA level in babies positive for HBsAg was $5 \times 10^{4}$ $\mathrm{IU} / \mathrm{mL}$ (range $81-1 \times 10^{8} \mathrm{IU} / \mathrm{mL}$ ).

Conclusion: HBV can replicate in placental cells leading to high transmission rate of $\mathrm{HBV}$ to the babies. Further studies would be needed demonstrating various replicative intermediates in placental cells to delineate the life-cycle of HBV in the placental cells.

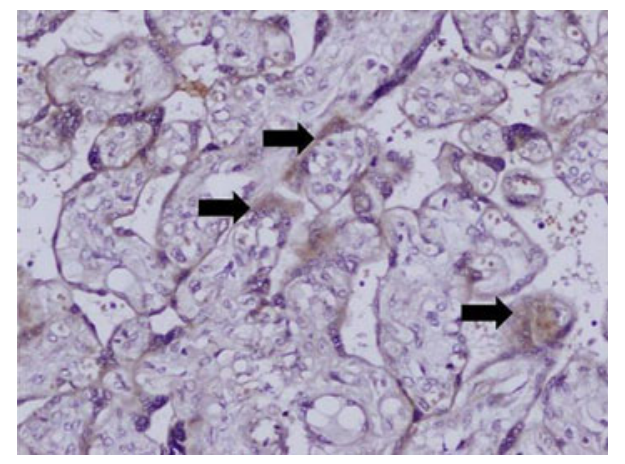

Fig. 1 Focal cytoplasmic positivity (arrows) for HBsAg

\section{PP06-24}

Occult Hepatitis C Virus Infection in Iranian Patients with Cryptogenic Liver Disease

F. Bokharaei Salim ${ }^{1}$, H. Keyvani ${ }^{1}$, S.M. Alavian ${ }^{2}$, Z. Madjd ${ }^{3}$, M. Nassiri Toosi $^{4}$, S.H.R. Monavari ${ }^{1}$, A.H.M.A. Mohammad Alizadeh ${ }^{5}$

${ }^{1}$ Department of Virology, Iran University of Medical Sciences;

${ }^{2}$ Baqiyatallah Research Center for Gastroenterology and Liver Diseases, Baqiyatallah University of Medical Sciences; ${ }^{3}$ Department of Pathology and Oncopathology Research Centre, Iran University of Medical Sciences; ${ }^{4}$ Department of Gastroenterology, Imam Khomeini Hospital, Tehran University of Medical Sciences; ${ }^{5}$ Research Center for Gastroenterology and Liver Disease, Shaheed Beheshti University of Medical Sciences, Tehran, Iran

Cryptogenic liver disease is diagnosed when an extensive evaluation has excluded recognizable etiologies of chronic liver disease. In this study, the presence of hepatitis $\mathrm{C}$ virus (HCV) RNA was tested in peripheral blood mononuclear cells (PBMCs) specimens from patients who were negative for plasma HCV RNA and antiHCV antibodies, while suffering from chronic liver disease with unknown etiology. From September 2007 up to March 2010, 69 patients with chronic liver disease with unknown etiology were enrolled in the present study From Tehran in referral centers for liver diseases. PBMCs were isolated from $10 \mathrm{ml}$ peripheral blood specimens. HCV-RNA status was tested in plasma and PBMCs samples by reverse-transcription polymerase chain reaction (RTPCR). HCV-RNA was detected in HCV-positive PBMCs specimens by RT-PCR method. HCV genotypes were subsequently analyzed in HCV-positive samples using restriction fragment length polymorphism (RFLP) assay, and then HCV genotypes were confirmed by sequencing of $5^{\prime}$ non-coding fragments after cloning PCR products into pJET1.2/blunt Cloning Vector. HCV RNA was detected in PBMCs specimens belonging to 7 (10.1\%) out of 69 patients. Genotyping of the HCV-RNA isolated from PBMCs showed that the $3(42.8 \%)$ patients with occult HCV infection had genotype $1 \mathrm{~b}, 2$ (28.6\%) patients had HCV genotype 1a, and 2 (28.6\%) patients had HCV genotype 3a. The results of this study suggest that patients with chronic liver disease with an unknown etiology may have occult $\mathrm{HCV}$ infection in the absence of anti$\mathrm{HCV}$ antibodies, and of plasma HCV-RNA. It has been suggested that in the absence of liver biopsy specimens, analysis of PBMC sample for HCV-RNA would be informative.

\section{PP06-25}

Typing HBV Accurately: Making Genotyping, Subgenotyping and Recombination Analysis Together

R. Ozaras ${ }^{1}$, F. Tabak ${ }^{1}$, M. Sayan ${ }^{2}$, B. Ceylan ${ }^{3}$, B. Mete ${ }^{1}$, M.S. Bugdaci ${ }^{4}$,

A. Cagatay ${ }^{1}$, A. Inci ${ }^{3}$, F. Kocak ${ }^{5}$, K. Gokturk ${ }^{6}$, N. Ozgunes ${ }^{7}$, L. Gorenek ${ }^{8}$,

L. Erdem ${ }^{9}$, T. Aslan ${ }^{4}$, H.K. Kumbasar ${ }^{10}$

${ }^{1}$ Istanbul University, Istanbul; ${ }^{2}$ Kocaeli University, Kocaeli;

${ }^{3}$ Istanbul Education and Training Hospital; ${ }^{4}$ Sisli Education and Training Hospital; ${ }^{5}$ Basaksehir Hospital; ${ }^{6}$ Buyukcekmece Hospital; ${ }^{7}$ Goztepe Education and Training Hospital; ${ }^{8}$ GATA Military Hospital; ${ }^{9}$ Bilim University; ${ }^{10}$ Haseki Training and Research Hospital, Istanbul, Turkey

Aim: Hepatitis B virus (HBV) has 8 genotypes (A-H) and 4 subgenotyps (D1-D4) distributed globally. Recombinations among different genotypes (A/D, A/E, C/A, C/D, G/C, E/D/A) within a given genotype (A, D, and $\mathrm{F} / \mathrm{H}$ strains) are also reported. In Turkey, the genotypes are exclusively D. Limited PCR-RFLP subgenotype studies reveal subgenotypes D1 and D2 are prevalent. However, we know little about the epidemiology and circulation of recombinant $\mathrm{HBV}$ strains.

Method: From a multicenter observational study of HBV, (The REALIST study), treatment-naive 100 patients from 11 centers were included. We aimed to obtain the accuracy in typing and we studied genotyping, subgenotyping, and recombination analyses. Following HBV DNA isolation, polymerase/reverse transcriptase gene region of the virus (between the amino acids 80 to $250,>800$ bp regions between amino-acids) it was replicated by PCR and its DNA was sequenced (ABI PRISM 3130 Genetic Analyser, Amersham-USA). The obtained DNA sequences were analyzed for genotyping, subgenotyping, and recombination by using the programs of

I. National Center for Biotechnology Information (NCBI, U.S National Library of Medicine, Bethesda-USA, http://www.ncbi. nih.gov/projects/genotyping),

II. The International Repository for Hepatitis B Virus Strain Data (HepSEQ, http://www.hpa-bioinfodatabases.org.uk),

III. Genafor/Arevir (Center of Advanced European Studies and Research, Bonn-Germany, http://coreceptor.bioinf.mpi-inf. mpg.de) and

IV. The Oxford HBV subtype tool (http://www.bioafrica.net/regagenotype/html/subtypinghbv.html)

Results: Genotype D was dominant and subgenotypes D1 was found in $88 / 100(88 \%)$, D2 in $3 / 100(3 \%), \mathrm{D} 3$ in $8 / 100(8 \%)$, and D4 in $1 / 100(1 \%)$. No any recombinant HBV strain was detected.

Conclusion: Current approach to HBV typing is based on comparing the DNA sequences to the consensus or reference sequences. However this may detect recombinant strains.

First time, we used the database containing the circulating recombinant HBV strains. HBV tying by using genotyping, subgenotyping, and recombination analyses may provide much more accurate results.

\section{PP06-26}

Primary Drug Resistance Mutations and Surface Antigen Gene Mutations in Treatment-Naïve Chronic Hepatitis B Patients

M. Sayan ${ }^{1}$, F. Tabak ${ }^{2}$, R. Ozaras ${ }^{2}$, B. Ceylan ${ }^{3}$, B. Mete $^{2}$, M.S. Bugdaci ${ }^{4}$, A. Cagatay ${ }^{2}$, A. Inci ${ }^{3}$, F. Kocak ${ }^{5}$, K. Gokturk ${ }^{6}$, N. Ozgunes ${ }^{7}$, A. Acar ${ }^{8}$,

L. Erdem ${ }^{9}$, A. Gunduz ${ }^{4}$, H.K. Kumbasar ${ }^{10}$

${ }^{1}$ Kocaeli University; ${ }^{2}$ Istanbul University; ${ }^{3}$ Istanbul Education and Training Hospital; ${ }^{4}$ Sisli Education and Training Hospital;

${ }^{5}$ Basaksehir Hospital; ${ }^{6}$ Buyukcekmece Hospital; ${ }^{7}$ Goztepe Education and Training Hospital; ${ }^{8}$ GATA Military Hospital; ${ }^{9}$ Bilim University;

${ }^{10}$ Haseki Training and Research Hospital, Istanbul, Turkey 
Aim: Hepatit B virus polymerase gene is overlapped with open reading frame of surface antigen gene. Some oral antiviral drug resistance mutations may lead to changes in surface gene amino acids. rtM204I (YIDD) mutation is emerged during lamivudin or telbivudin treatment. Recently rtM204I mutations were reported to lead to a selection in the resistance of surface gene region sW196S/L/*(stop codon). Also, some primary drug resistance mutations potentially may lead vaccine-, HBIG, and immune-escape (sG145R, sP120T) surface gene mutations.

Method: From a multicenter observational study of HBV, (The REALIST study), treatment-naive 100 patients from 11 centers were included.

Serum samples were analyzed for primary drug resistance and surface gene resistance. Following HBV DNA isolation, polymerase/reverse transcriptase gene region of the virus (between the amino acids $80-250,>800$ bp regions between amino-acids) it was replicated by PCR and its DNA was sequenced (ABI PRISM 3130 Genetic Analyser, Amersham, USA).

Results: In 5 out of 100 patients (5\%), HBV primary drug resistance and surface gene mutation were detected.

HBV genotypes/subgenotypes were as follows: Case I; D/D3, Case II; D/D4 Case III; D/D2, Case IV; D/D1, Case V; D/D1. HBV drug resistance/surface gene mutation analyses were as follows: Case I; rtM204I/sW196S + sP120S, case II; rtM204I/sW196S, case III; rtM204I + rtQ215S + rtN238T/sT126I + sG145R, case IV; rtA181V + $\mathrm{rtL180M/sP120T}$, case V; rtA181T/sW172* mutation combinations. Conclusion: Primary drug resistance mutations and surface gene mutations facilitated by these mutations can be seen even in treatment-naïve patients. This may be a potential threat to public health and should be further investigated.

\section{PP06-27}

One Silent Mutation in S2 Promoter Region Correlated with the Seroclearance of HBsAg Post-treatment

H.-R. Cheng ${ }^{1}$, J.-H. Kao ${ }^{1}$, H.-L. Wu ${ }^{2}$, P.-J. Chen ${ }^{1}$, D.-S. Chen ${ }^{1}$, C.-J. Liu ${ }^{1}$

${ }^{1}$ Graduate Institute of Clinical Medicine; ${ }^{2}$ Hepatitis Research Center, Taipei, Taiwan, ROC

Hepatitis B surface antigen (HBsAg) seroclearance usually indicates remission of hepatitis activity. However, this favorable event can be accounted for by the presence of occult hepatitis B virus (HBV) infection (OHBV). In this study, we aimed to evaluate whether the presence of $\mathrm{OHBV}$ in patients who developed HBsAg seroclearance post-treatment or spontaneously was associated with any hotspot in the S promoter region of the HBV genome. We collected 15 patients who developed HBsAg seroclearance post-treatment or spontaneously but had OHBV: 6 patients with dual hepatitis B virus (HBV)/ hepatitis $\mathrm{C}$ virus $(\mathrm{HCV})$ infection who lost $\mathrm{HBsAg}$ after peginterferon alfa-2a (PEG-IFN) therapy; $3 \mathrm{HBV}$ mono-infected patients who lost HBsAg after various antiviral therapy including lamivudine $(n=1)$ and PEG-IFN $(n=2)$; and 6 patients who lost HBsAg spontaneously. We collected another 15 control patients who were matched by age, sex, and treatment status, and remained HBsAgpositive. The subgenomic sequences within HBV S2 promoter, preS/ $\mathrm{S}$, precore and enhancer II/basal core promoter regions were amplified by nested PCR assays and then directly sequenced. We then compared the consensus sequences of these regions between the cases and the controls. We found that one silent mutation $\mathrm{C} 3050 \mathrm{~T}$ in S2 promoter region was associated with the seroclearance of $\mathrm{HBsAg}$ in the $6 \mathrm{HBV} / \mathrm{HCV}$ dually infected patients with post-treatment HBsAg seroclearance. Our data suggested that one mutation in S2 promoter region might contribute to the decreased HBsAg secretion. Further studies are needed to clarify the biologic role of this mutant.
PP06-28

HBV DNA Level is Associated with Intrahepatic HBcAg Protein Expression in Hepatocyte in Chronic B Hepatitis (CHB) Patients D. Serevina ${ }^{1}$, E. Krisnuhoni ${ }^{1}$, R.A. Gani ${ }^{2}$, L.A. Lesmana ${ }^{2}$, C. Lesmana ${ }^{2}$, I. Hasan $^{2}$, A.S. Sulaiman ${ }^{2}$, U. Budihusodo ${ }^{2}$

${ }^{1}$ Anatomy Pathology; ${ }^{2}$ Internal Medicine, Division of Hepatology, University of Indonesia, Jakarta, Indonesia

Background: Correlation between serum HBV DNA with $\mathrm{HBsAg}$ and $\mathrm{HBcAg}$ protein expression in the liver with regard to $\mathrm{HBeAg}$ status have not been well studied.

Objective: To correlate between HBV DNA levels, HBeAg serum and $\mathrm{HBsAg}$ and $\mathrm{HBcAg}$ expression in the liver.

Patient and methods: A total of 55 naive Chronic Hepatitis B (CHB) patients were enrolled. All samples were tested for $\mathrm{HBeAg}$ serum by serological test (ELISA) and HBV DNA was determined by PCR (TaqMan). HBsAg and HBcAg were evaluated immunohistochemically (Dakopatts, Copenhagen,Denmark) in the liver tissue.

Results: From 55 patients, $44(80 \%)$ were $\mathrm{HBeAg}$ positive and 11 (20\%) were $\mathrm{HBeAg}$ negative. Pathological examination of liver tissue revealed mild, moderate, and severe inflammation in 27.3, 49.1 and $23.6 \%$ of patients respectively. Furthermore, mild- moderate and severe fibrosis was showed in 38.2 and $23.6 \%$ of patients respectively. There was no correlation between serum HBV DNA and expression of HBsAg in cytoplasm $(r:-0.169 ; p 0.218)$. But there was a positive correlation between serum HBV DNA and expression of HBcAg in nuclei $(r: 0.383$; $p$ 0.004). There was no association between the expressions of $\mathrm{HBsAg}$ in cytoplasm to HBeAg serum $(p$.084) but, there was association expression of $\mathrm{HBcAg}$ in nuclei to $\mathrm{HBeAg}$ serum $(p$.017).

Conclusion: The expression of $\mathrm{HBcAg}$ in the hepatocyte nucleus can accurately reflect ongoing $\mathrm{HBV}$ replication. It can be used as a marker for quantitative estimation of HBV replication. Larger sample is needed to confirm these findings.

\section{PP06-29}

Association of Precore/Core Mutations and Genotypes in HBV Related Diseases

A. Malik, M. Asim

Medicine, Maulana Azad Medical College, Delhi, India

Background and aim: Mutant Hepatitis B with precore stop codon has been reported to be associated with severe liver damage in $\mathrm{HBeAg}$ negative patients with hepatitis B. Clinically, the biological importance of pre-core G1896A mutation is not well established. The purpose of the present study was to determine hepatitis B virus genotypes and also to elucidate the association of G1896A mutation of precore gene and the severity of liver damage.

Methods: Detection of HBV DNA sequences was carried out by polymerase chain reaction (PCR) using primers derived from the precore region of HBV genome. Ligase Chain Reaction (LCR) was performed to screen the presence or absence of G1896A mutation. Direct nucleotide sequencing was done to confirm the results of LCR. A total of 331 patients (Acute Viral Hepatitis :115), (Fulminant Hepatitis : 40), (Chronic Hepatitis:116), (Liver Cirrhosis : 30), (Hepatocellular carcinoma: 30) who were admitted in the wards of Lok Nayak Hospital, New Delhi, India.

Results: Sequence analysis of viral DNA established that precore and core mutations accounted for 24.7 and $21 \%$ in all the categories of hepatitis B related diseases. Phylogenetic analysis revealed $60 \%$ isolates belonged to genotype A, while $20 \%$ belonged to genotype D. Conclusion: The prevalence of precore mutation remains high in fulminant hepatitis cases with respect to acute viral hepatitis and chronic hepatitis. Precore mutation appears to play an important role in the development of hepatocellular carcinoma. Similar prevalence of precore stop codon G1896A (W28 stop) present in all clinical 
categories, rules out its association with any specific spectrum of liver disease. G1896A precore mutation was specifically associated with genotype D. Presence of highest rate of core gene mutations in fulminant hepatitis compared with all the other clinical categories is suggestive of acquisition of infection with already extensively mutated hepatitis B virus.

\section{PP06-30}

Identification of Hepatitis B Virus Cytotoxic T-lymphocyte (CTL) Therapeutic Vaccine Candidates

M. Gustiananda, N.K.D. Nursanty, M.D. Thedja, A. Harahap, D.H. Muljono Eijkman Institute for Molecular Biology, Jakarta, Indonesia

Introduction: Multi-epitope therapeutic vaccine capable of stimulating cellular immune responses is regarded essential for controlling and clearing hepatitis B virus (HBV) infection in chronic individuals. Such a vaccine needs to be based on epitopes derived from the prevalent viral strain and restricted by the prevalent HLA types in the related population. We aimed to identify T-cell epitopes from HBV core protein potential as vaccine candidates for Indonesian population. Methods: Twenty-eight core proteins of HBV isolates obtained from ethnically well-defined Indonesian subjects were sequenced and combined with 55 Indonesian HBV core sequences from NCBI to generate a working data set for analysis. We performed immunoinformatic analysis using NetCTLpan 1.1 Server integrating algorithm for prediction of HLA binding and cellular processing. Other prediction tools housed in the Immune Epitope Database (IEDB) Server were employed to generate a consensus result. The predicted epitopes were analyzed for conservancy among Indonesian HBV isolates using AVANA tool to identify the variants and further blasted against human proteomes to exclude those inducing autoimmune responses. Results: A set of putative T-cell epitopes (36 decamer and 31 nonamer) was identified from HBV core protein restricted by HLA allele of Indonesian population. Majority of these epitopes were promiscuous (bind to more than one HLA allele). Our analysis also revealed that certain epitopes-core 18-27-varied across HBV isolates with three variants (FLPSDFFPSI 88\%, FLPSDFFPSV 7\%, and FLPSDSFPSI $5 \%$ ). This epitope is predicted as a binder not only to HLA $A * 02$ but also to HLA A*24, HLA B*62, HLA B*07, and HLA $B * 27$, albeit with much lower affinity.

Conclusion: We discovered potential CTL epitopes by genome-tovaccine approach to develop immunotherapeutic strategies for $\mathrm{HBV}$ infection. Study is in progress to examine the ability of these peptides to elicit CTL response in chronic HBV patients.

\section{PP06-31}

Pre-core and Basal Core Promoter Mutations during the Natural History of Chronic Hepatitis B Patients in Indonesia

T. Turyadi ${ }^{1}$, M. Roni ${ }^{1}$, M.D. Thedja ${ }^{1}$, S.I. Ie $^{1}$, E. Sidarta ${ }^{1}$, A.R. Harahap ${ }^{1,2}$, D.H. Muljono ${ }^{1}$

${ }^{1}$ Eijkman Institute for Molecular Biology; ${ }^{2}$ Clinical Pathology,

Faculty of Medicine, University of Indonesia, Jakarta, Indonesia

Background: Pre-core A1896 and basal core promoter (BCP) T1762/ A1764 mutations of hepatitis B virus (HBV) genome have been correlated with severe liver diseases; however, their role in the pathogenesis of chronic hepatitis $\mathrm{B}$ (CHB) remains unclear. We assessed the prevalence and association of these mutations in different phases of CHB in Indonesian patients.

Methods: One-hundred and forty $\mathrm{CHB}$ patients, not undergoing antiviral therapy, were classified into immune-tolerance (IT), immune-clearance (IC), non/low-replicative (LR), and hepatitis B $e$ antigen ( $\mathrm{HBeAg}$ )-negative hepatitis (ENH) phases. HBV DNA was detected and quantified by polymerase chain reaction then analyzed by sequencing.

Results: ENH and LR patients were older than IC or IT patients $(p<$ 0.05). HBV DNA levels were highest in IC patients (median 5.34 $\log _{10} \mathrm{IU} / \mathrm{mL}$ ) and lowest in LR (median $1.65 \log _{10} \mathrm{IU} / \mathrm{mL} ; p<$ 0.0001). The A1896 pre-core mutants were most prevalent in LR $(53 \%, p<0.001)$ and higher in $\mathrm{ENH}(32 \%, p<0.001)$ than in IT and IC patients, while the T1762/A1764 BCP mutants were comparable between all phases. The T1762/A1764 BCP mutants were more frequently identified in genotype $\mathrm{C}$ than in genotype $\mathrm{B}(p<0.05)$, and in subtype $a d r$ than in subtypes $a d w$ and ayw $(p<0.05)$. The T1858 mutants were detected in almost all HBV isolates regardless the genotypes (B and C). No associations were observed between HBV DNA levels and pre-core as well as BCP mutations.

Conclusions: The prevalence of pre-core A1896 mutation differed in phases of $\mathrm{CHB}$ in Indonesian patients with preponderance in older ages and later stages. BCP T1762/A1764 mutations were associated with HBV genotypes and subtypes, irrespective of infection phases. These findings indicate that BCP mutations could be independent of $\mathrm{HBeAg}$ seroconversion in the natural history of chronic HBV infection.

\section{PP06-32}

Natural Occurring $\mathrm{N}$-Glycosalytion Mutations in HBsAg MHR: Prevalence and Functional Analysis

D.-M. Yu ${ }^{1}$, X.-H. Li ${ }^{1}$, X.-W. Liao ${ }^{1}$, P. Deny ${ }^{2}$, C. Pichoud ${ }^{2}$, F. Zoulim², X.-X. Zhang ${ }^{1}$

${ }^{1}$ Department of Infectious Disease, Ruijin Hospital, Shanghai Jiaotong University School of Medicine, Shanghai, China, ${ }^{2}$ INSERM U871, Lyon, France

Aims: To investigate the prevalence and function impact of natural occurring glycosalytion mutations in HBsAg Major hydrophilic region (MHR).

Methods: Hepatitis B virus (HBV) DNA from 118 patients with $\mathrm{HBsAg}$ and HBsAb coexistence were sequenced and analyzed. HBsAg gene with potential $\mathrm{N}$-glycosalytion sites were cloned into pcDNA3.1(-)/MYC-HIS C vector and then expressed in CHO cells. $\mathrm{N}$-glycosalytion was identified with Western blot and antigenicity with CMIA. Phenotypic research was performed to observe influence of insertion mutation on Rt domain after Huh7 cells transfected with replicative plasmids.

Results: $21 / 118$ patients were found to have 8 kinds of N-glycosalytion mutation in HBsAg MHR. Antigenicity was affected by additional glycosalytions. 3 amino acids insertion between $\mathrm{A}$ and $\mathrm{B}$ motif of Rt domain has no impact on viral replicative capacity.

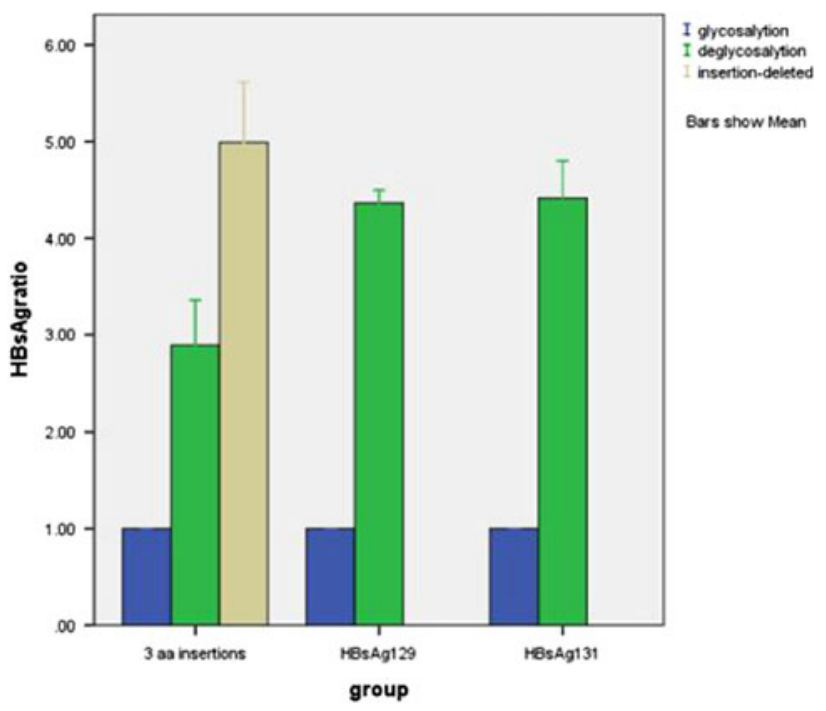

Fig. 1 Influence on antigenecities of additional gl 


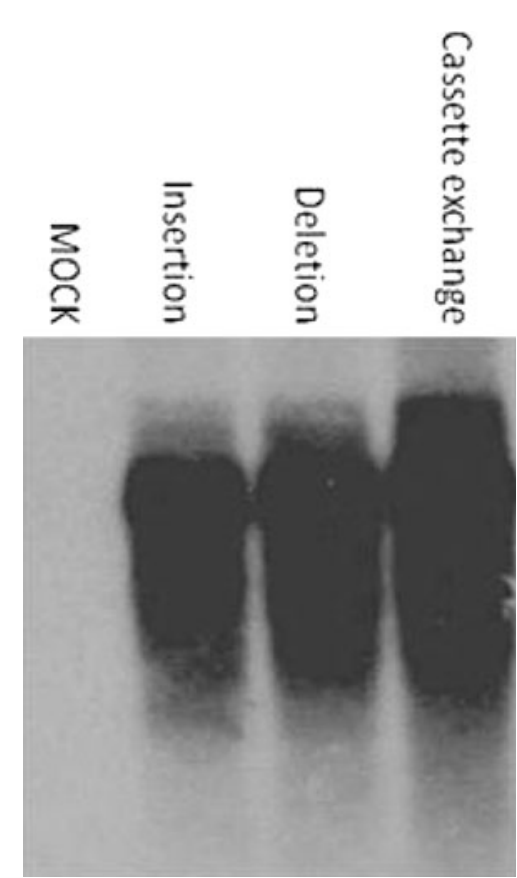

Fig. 2 Effect of 3 aminoacids insertion on replic

Conclusions: These results illustrate natural occurring glycosalytion mutations in HBsAg MHR have different impact on viral antigenicity and replicative capacity. Research on it will be helpful for disease prevention, diagnosis and vaccine designation.

\section{PP06-33}

Molecular Characterisation of Hepatitis B Virus Isolates from Chronic Hepatitis B, Cirrhotic and Hepatocellular Carcinoma Patients from Kerala, India

M. Keyter ${ }^{1}$, D. Gopalakrishnan ${ }^{1,2}$, K.T. Shenoy ${ }^{2,3}$, A. Kramvis ${ }^{1}$

${ }^{1}$ Hepatitis Virus Diversity Research Programme, University of the Witwatatersrand, Johannesburg, South Africa; ${ }^{2}$ Population Health and Research Institute; ${ }^{3}$ Gastroenterology, Sree Gokulam Medical College and Research Foundation, Kerala, India

Background: The Indian subcontinent is classified as an area of intermediate hepatitis B virus (HBV) endemicity, with predominantly two genotypes, A and D, circulating. Molecular characterisation of $\mathrm{HBV}$ in India remains limited. HBV infection is associated with the progression to advanced liver disease and the development of hepatocellular carcinoma (HCC). In a previous analysis of the $\mathrm{BCP} /$ precore region, known mutations were found predominantly in $\mathrm{HBV}$ isolates from HCC patients.

Methods: In order to determine whether there are additional mutations that may affect disease progression in these HBV isolates, the complete $\mathrm{S}$ region from $51 \mathrm{HBV}$ isolates from Kerala, India, were analysed. A $2 \mathrm{~kb}$ fragment, encompassing the complete $\mathrm{S}$ gene, was amplified by nested polymerase chain reaction (PCR) and sequenced using a series of overlapping fragments. Nine samples were selected for full genome analysis using an overlapping technique, in which a second $1.7 \mathrm{~kb}$ fragment was amplified by nested PCR and sequenced. Phylogenetic analysis of the complete $\mathrm{S}$ region was used for genotyping.

Results: Thirty three HBV isolates belonged to subgenotype A1 (65\%), 17 to genotype D (33\%) [3 D1, 13 D2, 1 D3] and one to genotype $\mathrm{C}(2 \%)$. Nine subgenotype A1 isolates, 2 from chronic hepatitis ( $\mathrm{CHB})$ and 7 from HCC patients, had pre-S2 deletions, ranging from 2 to 18 amino acid in length. One A1 isolate from a
$\mathrm{CHB}$ patient had a pre-S1 deletion. A pre-S2 deletion was also present in a one genotype D isolate. All 10 pre-S2 deletions encompassed Tand B-cell epitopes, whereas the pre-S1 deletion obliterated the preS1 start codon. Pre-S2 initiation codon mutations were detected exclusively in HCC patients.

Conclusion: The mutations and deletions detected in subgenotype A1 are similar to those previously described in other genotypes/subgenotypes and have been shown to be associated with advanced liver disease progression and HCC.

\section{PP06-34}

Genetic Variability of Hepatitis B Virus in Morocco

B. Kitab ${ }^{1}$, R. Afifi ${ }^{2}$, A. Essaid El Feydi ${ }^{2}$, M. Benazzouz ${ }^{2}$, O. Derdabi ${ }^{2}$,

Y. Cherradi ${ }^{2}$, H. Salih $\mathrm{Alj}^{3}$, K. Rebbani ${ }^{1}$, I. Brahim ${ }^{1}$, M. Hassar ${ }^{1}$, S. Ezzikouri ${ }^{1}$, S. Benjelloun ${ }^{1}$

${ }^{1}$ Laboratoire des Hépatites Virales, Institut Pasteur of Morocco, Casablanca; ${ }^{2}$ Service de Medecine C, CHU Ibn Sina, Rabat; ${ }^{3}$ Faculté des Sciences, Université Hassan II, Casablanca, Morocco

Background and aims: Morocco is a medium-level epidemic country for Hepatitis B Virus (HBV). The aim of the present study is to determine the HBV genetic variability, and its association with clinical outcome and severity of disease in Moroccan HBV carriers. Methods: The study included 250 chronic HBV infection patients at different stages of liver disease (156 male and 94 female). Serum samples were tested for serological markers and HBV DNA levels. The HBV Surface and core promoter/precore regions were amplified and directly sequenced. The clinical, virologic and phylogenetic characteristics were investigated.

Results: The mean age of patients was $44 \pm 12.2$. Most of them were HBeAg negative (90\%). Phylogenetic analysis identified $90 \%$ isolates in genotype D and $10 \%$ in genotype A. Most genotypes D isolates belonged to subgenotype D7 (80\%) followed by subgenotype D1 $(25 \%)$ and one isolate belonged to subgenotype D2. All genotype A strains belonged to subgenotype A2 and specified subtype $a d w 2$. In genotypes D strains, subtypes ayw2 (91.7\%), ayw3 (3.3\%) and ayw4 (3.3\%) were identified. A significance prevalence of mutations in the Major Hydrophilic Region (MHR) of HBsAg was found with P120T/ $\mathrm{S}$ the most frequent. In the core promoter region, the most frequent mutations are G1757A (48.9\%), T1773C (42.8\%), C1766G /T (40.8\%), T1753V (30.7\%) and A1762T/G1764A (24.4\%). In the precore region, the most common mutations are G1896A $(55.1 \%)$ and G1899A (34.6\%). Double mutation in the core promoter A1762T/ G1764A was found more frequently in HCC patients than that in non HCC patients ( 66.6 vs. $16.2 \% ; p<0.001$ ). In addition, the prevalence of $\mathrm{C} 1653 \mathrm{~T}, \mathrm{~T} 1753 \mathrm{~V}$, and G1862T mutations was significantly higher in HCC patients compared with non HCC.

Conclusion: Subgenotype D7/ayw2 is the most predominant in Morocco. The high frequency of mutations in the core promoter in patients with HCC indicates their association with severity of infection.

\section{PP06-35}

The Ratio of HbsAg Loss in Inactive Carrier Chronic Anti-HBE Positive Hepatitis B Patients During Long Term Follow Up

H. Ozkan, M. Yakut

Ankara University Medical Faculty, Ankara, Turkey

Aim: The loss of HBsAg is the golden standard indicator of virological response. In this study the ratio of HbsAg loss and related factors.

Materials and method: During 12 years from June 1998 to April $2010,862 \mathrm{HBsAg}(+)$ patients were followed up for at least 1 year. A total of $862 \mathrm{HBsAg}$ patients (557 male, 305 female; mean age $43.3 \pm 12.7$ years (range 18-77 years) were consecutively included. Of these, 679 (78.7\%) were inactive HBsAg carriers and $183(21.2 \%)$ had anti-HBe positive chronic HBV infection. 
Results: A total $862 \mathrm{HBsAg}(+)$ patients were followed up for a mean period of 51 and 6-140 months). Of these patients, 679 subjects (78.7\%)were inactive HBsAg carriers and 183 subjects (21.2\%) were anti-HBE positive CHB patients. Twenty seven subjects of 135 patients $(20 \%)$ who were receiving Lamuvudine treatment were found to have resistance. Seven of these 27 patients who developed Lamuvudine resistance had positive history for inerferon use. The history of interferon use was unrelated with lamuvudine resistance (7/ 47 vs. $20 / 88, p=0.368)$. Fifty one subjects $(5.9 \%)$ of $862 \mathrm{HBsAg}$ positive patients developed HBsAg loss during follow up period. All of these patients showed anti-HBs positiveness during follow up period. Also, 31 inactive HBVcarriers and 20 anti-HBE positive CHB patients showed HBsAg loss during that period. In that study, 183 anti-HBE positive $\mathrm{CHB}$ patients were evaluated. When baseline characteristics of anti-HBE positive CHB patients with and without HBsAg loss were compared, there was not any difference between two groups according to serum HBVDNA, ALT, Knodellscore, fibrosisscore, age,gender and lamuvudine use (6/48 vs. 14/135, $p=0.788$ ).

Conclusion: The loss of HBsAg was relatively higher than previous studies. Moreover, lamuvudine resistance was lower than anti-HBE positive patients that were followed up in previous studies.We observe that HBsAg loss was higher in anti-HBE positive CHB patients in whom SVR was obtained with interferon.It is reasonable to evaluate anti-HBE positive CHB patients annually for $\mathrm{HBsAg}$ loss.Moreover lamuvudine still reserves its importance in anti-HBE positive patients in our region because of decreased phenotypical resistance.

\section{PP06-36}

The Spontaneous Lamivudine Mutation Rate in Lamivudine-Naive Patients with Chronic Hepatitis B in China

H. Wang, Q. Xie, G. Zhao, H. Gui

Department of Infectious Diseases, Ruijin Hospital, Shanghai

Jiaotong University School of Medicine, Shanghai, China

Background/aim: Lamivudine (LAM) is a nucleoside analogue widely used to treat chronic hepatitis B $(\mathrm{CHB})$ patients. But the emergence of spontaneous LAM-resistant virus greatly limits the efficacy of therapy and induces liver injury. The aims of this study were to assess the spontaneous LAM mutation rate in LAM-naïve patients with CHB.

Methods: 102 LAM-naïve CHB patients in China were enrolled in this study. All of them had detectable viral load (Taqman Real Time PCR). Age, Weight, Height, levels of alanine aminotransferase (ALT) and HBV genotype were monitored. All the patients were randomly divided into two groups. Gene mutations were assessed by direct sequencing in group A and by INNO-LiPA in group B.

Results: Among 102 patients, 27.5\% were genotype B virus $70 \%$ were genotype C. Only $2.5 \%$ patients were genotype $(B+C)$. Mean of ALT level was $(105 \pm 149.56) \mathrm{U} / \mathrm{mL}$. Spontaneous LAM-resistant variants were detected in 10 subjects $(9.80 \%)$. The mutation of rtM204V, rtM204I and rtL180M were found. The emergence rates were $76.92,30.77$ and $7.69 \%$, respectively. The prevalence of multiple mutations $\mathrm{rtL} 180 \mathrm{M}+\mathrm{rtM} 204 \mathrm{~V}$ or rtM204I + rtM204V were observed in $3(30 \%)$ of 10 patients. Irrespective of total and subgroup, Gender, age, BMI, HBeAg status, serum viral load, ALT level and HBV genotype (B or C) were not associated with spontaneous LAMresistant mutation $(p>0.05)$. LAM mutation Rate in Group B was 13.51 versus $7.69 \%$ in Group A. There was significant difference between them $(p<0.05)$.

Conclusions: $9.80 \%$ LAM-naïve CHB Chinese patients have spontaneous LAM-resistant variants regardless of HBeAg status, serum viral load, ALT level and HBV genotype (B or C). Most of them have only one mutation. Mutation detection assessed by INNO-LipA is more sensitively than that by direct sequencing.
PP06-37

Elevation of ALT Fails to Predict Significant Histologic Abnormalities in Chronic Hepatitis B Patients

W.-K. Seto ${ }^{1}$, C.-L. Lai ${ }^{2}$, J. Fung ${ }^{1}$, J. Yuen ${ }^{1}$, D.K.-H. Wong ${ }^{1}$, M.-F. Yuen ${ }^{1}$ ${ }^{1}$ Medicine, Queen Mary Hospital, The University of Hong Kong;

${ }^{2}$ Medicine, Queen Mary Hospital, Hong Kong, Hong Kong S.A.R.

Background: We determined the association between various clinical parameters and significant liver injury in both hepatitis B e antigen (HBeAg)-positive and $\mathrm{HBeAg}$-negative patients.

Methods: From 1994 to 2008, liver biopsy was performed on 319 treatment-naïve CHB patients. Histologic assessment was based on the Knodell histologic activity index for necroinflammation and the Ishak fibrosis staging for fibrosis. Liver biochemistry, HBeAg status and HBV DNA levels were checked.

Results: $211 \mathrm{HBeAg-positive} \mathrm{and} 108 \mathrm{HBeAg}$-negative patients were recruited, with a median age of 31 and 46 years respectively. 9 out of $40(22.5 \%) \mathrm{HBeAg}$-positive patients with normal ALT had significant histologic abnormalities (necroinflammation grading $\geq 7$ or fibrosis score $\geq 3$ ). There was a significant difference in fibrosis scores among $\mathrm{HBeAg}$-positive patients with an ALT level within the Prati criteria (30 U/L for men, $19 \mathrm{U} / \mathrm{L}$ for women) and patients with a normal ALT but exceeding the Prati criteria $(p=0.024)$. Age, aspartate aminotransferase and platelet count were independent predictors of significant fibrosis in $\mathrm{HBeAg}$-positive patients with an elevated ALT by multivariate analysis $(p=0.007,0.047$ and 0.045 respectively). Serum HBV DNA and platelet count were predictors of significant fibrosis in $\mathrm{HBeAg-negative} \mathrm{disease}(p=0.020$ and 0.015 respectively). An elevated ALT was not predictive of significant fibrosis for both $\mathrm{HBeAg-positive}(p=0.345)$ and -negative $(p=0.544)$ disease. There was no significant difference in fibrosis staging among ALT 1-2 $\mathrm{x}$ upper limit of normal (ULN) and $>\chi^{2}$ ULN for both HBeAg-positive $(p=0.098)$ and -negative $(p=0.838)$ disease.

Conclusion: An elevated ALT does not accurately predict significant liver injury. Decisions on commencing antiviral therapy should not be heavily based on a particular ALT threshold. HBV DNA and platelet count are more important determinants of significance fibrosis in HBeAg-negative disease.

\section{PP06-38}

Absence of Mitochondrial Toxicity of Telbivudine in Skeletal Muscle Cells, Hepatocytes and Neuronal Cells from Rat or Human

M. Dong ${ }^{1}$, E. Persohn ${ }^{1}$, O. Grenet ${ }^{1}$, P. Francois ${ }^{1}$, A. Wolf ${ }^{1}$, S.-D. Chibout ${ }^{1}$, M. Weaver ${ }^{2}$, L. $\mathrm{Li}^{2}$

${ }^{1}$ Investigative Toxicology, Preclinical Safety, Translational Science, Novartis Institute for Biomedical Research, Basel, Switzerland;

${ }^{2}$ Preclinical Safety, Translational Science, Novartis Institute for Biomedical Research, Cambridge, MA, USA

Background/aims: Mitochondrial dysfunction is a possible toxicity of nucleoside reverse transcriptase inhibitors (NRTIs) and a black box label is included in the prescribing information for most available NRTIs. Telbivudine is a thymidine nucleoside analogue approved for the treatment of chronic HBV infection. This study compared telbivudine, lamivudine, zidovudine and zalcitabine (ddC) for their potential for mitochondrial toxicity in vitro in rat and human cells. Methods: Mitochondrial effects were studied in the following cell types: primary rat and human hepatocytes and skeletal muscle cells; rat and human neuronal cell lines (PC-12 and SH-SY5Y, respectively). Study endpoints: cellular ATP content and LDH leakages (markers of cytotoxicity), lactic acid release (mitochondrial function), mitochondrial DNA content (DNA polymerase $\gamma$ activity), and transmission electron microscopy (mitochondrial morphology ultrastructural changes), neurite outgrowth (by imaging analysis). Each cell type was treated with telbivudine or reference compounds for up to 7 days. 
Results: Telbivudine at concentrations up to $152 \mu \mathrm{M}, 10$-fold the maximal clinical exposure $\left(C_{\max } 15.2 \mu \mathrm{M}\right)$, did not cause significant changes in all endpoints in all cell systems. Similar results were obtained for lamivudine $\left(50 \mu \mathrm{M}, 10\right.$-fold $\left.C_{\max }\right)$. In contrast, Zalcitabine $(10 \mu \mathrm{M})$ caused significant reductions in ATP and mtDNA content, increased lactic acid release, along with alterations in mitochondrial morphology in proliferating hSMCs, but not in other tested cell types. Less pronounced effects were observed with zidovudine $(300 \mu \mathrm{M})$ in proliferating hSMCs. Zalcitabine $(10 \mu \mathrm{M})$ also caused a significant decrease in mtDNA in SH-SY5Y cells, without changing other parameters investigated.

Conclusion: This study demonstrated that telbivudine at concentrations that largely exceed the therapeutically relevant levels in human plasma was not associated with mitochondrial toxicity in a model of rat and human hepatocytes, skeletal muscle or neuron cells.

\section{PP06-39}

\section{Novel Combination Biomarker in Hepatitis B-Related Cirrhosis}

J.B. Chook ${ }^{1}$, R. Mohamed ${ }^{1}$, Y.F. Ngeow ${ }^{2}$, T.C. Tan ${ }^{3}$, S.F. Yap ${ }^{4}$, Hepatitis B Group

${ }^{1}$ Medicine, Faculty of Medicine, Unversity Malaya $;{ }^{2}$ Microbiology;

${ }^{3}$ Parasitology, Faculty of Medicine, University Malaya; ${ }^{4}$ Pathology, Faculty of Medicine, Unversity Malaya, Kuala Lumpur, Malaysia

Background: The mechanisms underlying hepatitis B virus (HBV) induced cirrhosis remain largely debated: HBV mutations and high iron load have been implicated.

Objective: To investigate the interplay role between iron status and HBV mutations in the development of cirrhosis.

Patients and design: Twenty patients with HBV-related cirrhosis and 20 controls were recruited. Liver profile, HBV serology, HBVDNA, serum iron and ferritin were determined in all participants. Overlapping PCR reactions were conducted to amplify the HBV genome, followed by bidirectional sequencing. Single nucleotide and codon analysis was carried out to determine candidate viral marker(s) for cirrhosis.

Results: Age, gender, ALT, HBeAg positivity, genotype, T1762/ A1764 and high viral load were not associated significantly with cirrhosis $(P>0.05)$. Six candidate nucleotides, A87, A1359, C1799, G1896, T2753 and C2964 showed the highest correlation with the presence of cirrhosis. Codon analysis showed that precore wild-type (TGG, nt 1895-1897), inclusive of the precore start codon (nt 1814-1816), gave a higher odds ratio $(35.3 ; 95 \%$ CI 3.87-321.93; $P<$ 0.001 ) than the other single codons. A combined analysis with both precore wild-type and high serum iron status increased the odds ratio further to 107.7 (95\% CI 10.21-1135.59; $P<0.001$ ).

Conclusion: These data suggested a possible complementary role between iron status and HBV precore wild-type in the development of HBV-related cirrhosis.

\section{PP06-40}

A Genetic Variant of Hepatitis B Virus Divergent from Known Human and Ape Genotypes Isolated from a Japanese Patient

K. Tatematsu ${ }^{1}$, Y. Tanaka ${ }^{1}$, F. Kurbanov ${ }^{1}$, F. Sugauchi ${ }^{1}$, T. Maeshiro ${ }^{2}$, T. Nakayoshi ${ }^{3}$, M. Wakuta ${ }^{4}$, Y. Miyakawa ${ }^{5}$, M. Mizokami ${ }^{6}$

${ }^{1}$ Department of Virology and Liver unit Nagoya City University Graduate School of Medical Sciences, Nagoya; ${ }^{2}$ Control and Prevention of Infectious Diseases Department of Medicine and Therapeutics, Faculty of Medicine, University of the Ryukyu, Okinawa, Japan; ${ }^{3}$ Heart Life Hospital Okinawa, Japan; ${ }^{4}$ Wakusan Clinic, Okinawa; ${ }^{5}$ Miyakawa Memorial Research Foundation, Tokyo; ${ }^{6}$ Research Center for Hepatitis \& Immunology, International Medical Center of Japan Kohnodai Hospital, Chiba, Japan

Back ground: Nine genotypes of HBV have been recognized, by a sequence divergence $>8 \%$ in the entire genome, and named by capital alphabet letters (A-I) in the order of discovery. An HBV isolate
(JRB34) was recovered from a Japanese patient, who was involved in military actions in Borneo inhabited by gibbons and orangutans.

Aim: To compare against human and four ape genotypes, and to investigate the infectivity of the patient serum by using urokinasetype plasminogen activator-severe combined immunodeficiency mice with the liver replaced by human hepatocytes (chimeric mice).

Methods: Full-length sequences of HBV isolates were aligned. Genetic distances were estimated and phylogenetic trees were constructed. All 1,440 complete genomes served as references for the initial alignment. Divergence between the JRB34 strain and previously reported strains was estimated. Evidence of possible recombination was investigated using reference set consisting of 228 non-recombinant $\mathrm{HBV}$ isolates. Two chimeric mice were inoculated with $10^{4} \mathrm{HBV}$ DNA copies of the patient serum. HBV-DNA in mice serum was measured by real-time PCR.

Results: Phylogenetic analyses over the full-length sequence and open reading frames indicate a close relationship of the JRB34 strain with gibbon/orangutan genotypes and human genotype C. In the fullgenome sequence, the isolated strain had $9.9-16.5 \%$ divergence. In addition, no significant evidence of recombination with known human and ape genotypes was revealed, suggesting a new genotype J (HBV/ J). Efficient infection, replication and antigen expression by $\mathrm{HBV} / \mathrm{J}$ were experimentally established in two chimeric mice with the liver repopulated for human hepatocytes. The HBV DNA sequence recovered from infected mice was identical to that in the inoculum. Conclusions: A novel HBV/J was identified. Since HBV/J is positioned in between human and ape genotypes phylogenetically, it may help to trace the origin of HBV and deserves further epidemiological surveys.

\section{PP06-41}

Hepatitis B Escape Mutants in Infants Born to Human Immunodeficiency Virus (HIV)-infected Mothers Co-Infected with Hepatitis B Virus (HBV) W. Khamduang ${ }^{1,2,3}$, C. Gaudy-Graffin ${ }^{2}$, N. Ngo-Giang-Huong ${ }^{1,3,4}$, G. Jourdain ${ }^{1,3,4}$, A. Moreau ${ }^{2}$, N. Luekamlung 5 , G. Halue ${ }^{6}$, Y.

Buranawanitchakorn $^{7}$, S. Kunkongkapan ${ }^{8}$, S. Buranabanjasatean ${ }^{9}$, C. Sureau ${ }^{10}$, M. Lallemant ${ }^{1,3,4}$, W. Sirirungsi ${ }^{1}$, A. Goudeau ${ }^{2}$, the Program for HIV Prevention and Treatment (PHPT) Group

${ }^{1}$ Faculty of Associated Medical Sciences, Chiang Mai University, Chiang Mai, Thailand; ${ }^{2}$ INSERM U966, Faculté de Médecine, Université François-Rabelais, Tours, France; ${ }^{3}$ UMI 174/Programs for HIV Prevention and Treatment (PHPT), Institut de Recherche pour le Développement (IRD), Chiang Mai, Thailand; ${ }^{4}$ Harvard School of Public Health, Immunology and Infectious Diseases, Boston, MA, USA; ${ }^{5}$ Lamphun Hospital, Lamphun; ${ }^{6}$ Phayao Hospital; ${ }^{7}$ Chiang Kham Hospital, Phayao; ${ }^{8}$ Mae Sai Hospital; ${ }^{9}$ Mae Chan Hospital, Chiangrai, Thailand; ${ }^{10}$ Institut National de la Transfusion Sanguine, Paris, France

Background/aims: Despite HBV vaccination, some perinatal HBV transmissions persist. We assessed the risk of $\mathrm{HBV}$ infection in infants born to HBV- HIV-1 co-infected mothers, and searched for HBV vaccine escape mutants in infected infants.

Methods: We used ETI-MAK-4 Diasorin kit to test HBsAg in 3,349 HIV-1 infected pregnant women participating in two perinatal HIV prevention clinical trials, during 1998-2003; and HBV DNA quantification (COBAS Amplicor HBV Monitor or Abbott real-time HBV DNA) to diagnose HBV infection in infants born to $\mathrm{HBsAg}$ positive mothers. HBV variants in infants and mothers were detected using direct sequencing and cloning of the flanking regions of the "a" determinant region of the $S$-gene.

Results: Of 237 infants born to HBsAg-positive mothers (7.1\%), 229 had samples available for the study of perinatal transmission. Eleven children $(4.8 \%$ ) were HBsAg positive within the first 6 months of life (none HIV co-infected), and longitudinal samples were available for 9 mother-child pairs. Median maternal HBV load was $7.83 \log _{10} \mathrm{IU} / \mathrm{mL}$ 
(range: undetectable to 8.24). HBsAg mutations were found in 6 infants by direct sequencing (i.e. sK122R, 2 infants; sI126T, 1 infant), including 3 born to mothers with the same mutation(s) (i.e. sI126T, sI126M + sP127S, and sT131N + sM133T + sT140I + sS204R). No HBsAg mutations were observed in the other 3 infants (one infected at birth) or in their mothers but their mothers had high viral loads $\left(>6.5 \log _{10} \mathrm{IU} / \mathrm{mL}\right)$. Cloning analysis showed that the mothers of the two infants with sK122R had minor populations of the same variant. Conclusions: This high proportion of HBV mother-to-child transmissions $(4.8 \%)$ despite vaccination may be related to higher rates of HBV replication (possibly related to HIV-1 co-infection) and/or mutation conferring the ability to escape vaccine. Further in vitro studies are needed to ascertain the capacity of these variants to escape anti-HBs antibodies neutralization.

\section{PP06-42}

Ex-vivo Phenotypig of Hepatitis B Surface Antigen

R. Szypulska, R. Tedder, S. Ijaz

Virus Reference Department, Health Protection Agency, London, UK

Background: Hepatitis B surface antigen (HBsAg) mutants have had a significant impact on hepatitis B virus (HBV) diagnostics and prophylaxis. However, there are no comprehensive data on the prevalence or type of HBsAg mutants in HBV-infected populations. Studies have shown that the effect of mutants on the HBsAg conformation is complex indicating that the virus genotype influences how individual HBsAg epitopes are displayed. Furthermore, antiviral resistance mutations occurring in the polymerase result in amino acid substitutions in the HBsAg and have been found to modulate $\mathrm{HBsAg}$ epitopes. As antivirals usage becomes more common and complex, it will be increasingly important to monitor and investigate the impact of resistance mutations on $\mathrm{HBsAg}$. Criticially, studies indicate that it is not always possible to accurately infer changes in antigenicty from direct sequencing. Therefore, phenotypic based methods are essential for investigating $\mathrm{HBsAg}$ mutants.

Methods: HBsAg epitope mapping studies have been optimised on the Luminex platform. This technology allows for individual monoclonal antibodies each recognising a discrete epitope to be investigated for their ability to bind HBsAg. The HBsAg for this assay comes directly from patients' plasma and therefore allows HBsAg to be investigated in the context of their natural genetic backbone.

Results: The HBsAg epitope portfolio has been defined for genotypes A to $\mathrm{E}$ in a panel of more than 300 sera using the methodology. The system is robust and fast, reliably detecting samples with a variety of mutations which alter the HBsAg antigenicity. G145R and other vaccine escape variants were easily detected and results from population based studies in the UK will be presented.

Conclusions: Epitope mapping using the Luminex platform provides a powerful tool for undertaking large $\mathrm{HBsAg}$ mutant prevalence studies. These data will provide a critical understanding of the impact and prevalence of HBsAg mutants in the HBV positive populations.

\section{PP06-43}

N236T-HBV Mutants Isolated from Patients with Primary Non Response to Adefovir Show a Reduction of Susceptibility to Tenofovir in vitro

O. Lada ${ }^{1}$, R. Carvalho-Filho ${ }^{1}$, F. Cluzeaud ${ }^{1}$, V. Mackiewicz ${ }^{2}$, E. Estrabaud ${ }^{1}$, M.-P. Ripault ${ }^{1}$, C. Castelnau ${ }^{1}$, M. Martinot-Peignoux ${ }^{1}$, N. Boyer ${ }^{1}$, M. Vidaud ${ }^{3}$, T. Asselah ${ }^{1}$, P. Marcellin ${ }^{1}$

${ }^{1}$ Hepatology, Inserm U773; ${ }^{2}$ Service de Virologie; ${ }^{3}$ Service de Biochimie, AP-HP Hôpital Beaujon, Clichy, France

Background/Aims: Tenofovir disoproxil fumarate (TDF) and Entecavir (ETV) have demonstrated high antiviral efficacy in treatmentnaive patients with chronic hepatitis $B$ virus (HBV) infection. However, few data are available on patients with primary nonre- sponse (PNR) (HBV-DNA viral decrease $<2 \log$ IU/mL at month 6 of therapy) to nucleotide or nucleoside analogues. Among 138 patients treated with adefovir dipivoxil (ADV) and followed from 2004 to 2008 at Beaujon hospital, we observed 10 patients with PNR to ADV. The aim of this study was to assess the in vitro drug susceptibility of HBV strains isolated from these patients.

Methods: Whole HBV genome from each patient's serum at baseline and M6 ADV therapy was PCR-amplified and cloned. HBV quasispecies populations was sequenced and analysed. The main HBV mutants were tested after transfection into HepG2 for their resistance profile to nucleoside analog. A wild type (wt) strain isolated from an untreated patient served as control.

Results: Baseline characteristics of the 10 patients were: median age 46 (range 37-63) years, 8 males, $8 \mathrm{HBeAg}$-positive, median baseline HBV DNA level $7.1 \log \mathrm{UI} / \mathrm{mL}$ (4.5-8.6). Six out 10 patients was lamivudine-experienced. Clonal analysis showed the co-localization on the same HBV genome of mixture of lamivudine (3TC) plus ADV resistance mutations. In vitro data of the main HBV mutants detected in patient's serum are summarized. Presence of rtA181V mutation may induce cross-resistance to $3 \mathrm{TC}$ and $\mathrm{ADV}$. TDF is effective on all ADV-resistant HBV strains except HBV strain carrying N236T mutation alone. ETV is effective on all ADV-resistant HBV

Conclusion: N236T-HBV mutants showed a reduction of susceptibility to tenofovir in vitro. By this clonal approach, we showed that genotypic and phenotypic analysis could be clinically relevant in the management of antiviral drug resistance.

\section{PP06-44}

The Influence of Hepatitis B e Antigen on Toll-Like Receptor 2 and 4 Expression in Peripheral Blood Mononuclear Cells

L. Jiang, J. Li

Department of Infectious Disease, the First Affiliated Hospital of Nanjing Medical University, Nanjing, China

Objective: Toll-like receptors (TLRs) play a crucial role in the innate immune response. However, the significance of TLRs expression in hepatitis B virus infection has not been clearly described. In order to investigate the relationship between the tolllike receptors and the hepatitis $\mathrm{B}$ e antigen $(\mathrm{HBeAg})$, the expression levels of TLR2/4 on peripheral blood mononuclear cells (PBMCs) of healthy and HBsAg-negative adults which stimulated by $\mathrm{HBeAg}$ were observed.

Method: Sixteen healthy and HBsAg-negative adults were enrolled, TLR2/4 and PD-1/L1 expression levels on PBMCs stimulated with $\mathrm{HBeAg}$ for $20 \mathrm{~h}$ or not stimulated were analyzed by FACS caliber and RT-PCR. Levels of cytokine productions included IL-2, IL-4, IL-6, IL-10, IL-17, TNF- $\alpha$ and IFN- $\gamma$ in supernatant were detected by CBA at the same time. Then, the human monocyte cell line (THP-1) was used to confirm the results, the expression of TLR2/4 in THP- 1 cells stimulated for $20 \mathrm{~h}$ by $\mathrm{HBeAg}$ was compared with which not stimulated.

Results: Expression of TLR2 on $\mathrm{CD} 14^{+}$PBMCs was significantly reduced when stimulated by $\mathrm{HBeAg}$ in comparison with which not stimulated, the level of TLR4 and PD-1/L1 expression did not differ significantly. These results were confirmed in THP-1 cells. Meanwhile, IL-2,IL-4 and IL-17 in supernatant were markedly reduced in the presence of the $\mathrm{HBeAg}$ compared with in the absence of $\mathrm{HBeAg}$, upregulation of IL-10 was observed following $\mathrm{HBeAg}$ stimulation,there were not significantly differences in IL-6,TNF- $\alpha$ and IFN- $\gamma$ between these groups.

Conclusion: This study demonstrates $\mathrm{HBeAg}$ down-regulates the expressions of TLR 2 on $\mathrm{CD} 14^{+}$cells from peripheral blood in healthy and HBsAg-negative adults. The proposed interaction between HBeAg and TLR2 may provide an important clue to explain the reasons of the establishment of persistent HBV infection. 


\section{PP06-45}

Current Status of HBV Genotypes and their Clinical Impact in Patients with Chronic HBV in Egypt

M.H. Zaghloul ${ }^{1}$, M.M. Elbendary ${ }^{2}$, W. El-Sherbeeny ${ }^{2}$, T.A. Besheer ${ }^{2}$, E. Tharwa ${ }^{3}$

${ }^{1}$ Clinical Pathology; ${ }^{2}$ Tropical Medicine, Mansoura University, Mansoura; ${ }^{3}$ Menofia Liver Institute, Menofia, Egypt

Introduction: Hepatitis B virus infection is a global health problem with its continuously increasing morbidity and mortality in the developing countries. Long-term infection increases the risk of developing cirrhosis and hepatocellular carcinoma. Currently hepatitis genotyping is emerging as a useful additional test that may add additional information and understanding of the natural history of hepatitis B virus.

Objectives: To know the different HBV genotypes present and their clinical impact in Egypt.

Methods: Eighty seven patients were included in the present study. They were 57 males and 30 females with ages ranging from 28.0 to 71.0 years. All patients were complaining of chronic HBV infection (cirrhotic (15) non cirrhotic (72)\}. The diagnosis of chronic hepatitis with or without cirrhosis was based on patients' history, physical examination, abdominal ultrasound and laboratory findings and confirmed by histological assessment. HBV markers, quantitative HBV DNA (Cobas amplicor) and HBV genotyping (nested PCR)were performed for all patients.

Results: HBV genotype D was the most common genotype representing $48.3 \%$ of all cases, followed by HBV genotype B representing $32.2 \%$, HBV genotype $\mathrm{C}$ representing $10.3 \%$ and HBV genotype A representing $9.2 \%$. No cases were identified in genotypes $\mathrm{E}$ or $\mathrm{F}$. Genotype $\mathrm{C}$ showed (increased serum levels of bilirubin, SGOT and SGPT and serum albumin more decreased $>$ other genotypes $(P<$ 0.05). The mean HBV DNA level is increased in genotype $B$ and $C$ compared with genotype A and D.

Conclusion: Genotype of HBV is an important entity and may have a role to play in predicting the response to various therapies and this should be taken into account as a variable before initiating any treatment.

\section{PP06-46}

Platform Comparison at Key Medical Decision Points Comparing WHO Traceability in Three HBV Viral Load DNA Tests

J. Li ${ }^{1}$, J.R. Lee ${ }^{2}$, K.-X. Sun ${ }^{1}$, Y.-H. Tian ${ }^{1}$, U. Cowen ${ }^{2}$, H. Zhuang ${ }^{1}$, B.R. Cobb ${ }^{2}$

${ }^{1}$ Department of Microbiology, Peking University Health Science Center, Beijing, China; ${ }^{2}$ Medical Affairs, Roche Molecular Systems, Pleasanton, CA, USA

Background/aims: HBV DNA (in IU/mL) is an essential marker of disease and, based on international guidelines, is critical in managing chronic hepatitis B patients. In an effort to standardize NAT testing worldwide, the WHO Expert Committee on Biological Standardization established an International Standard for HBV DNA (genotype A). This study was designed to compare two assays (the PG HBV Diagnostic Kit, v4.0, and the DaAn HBV Fluorescence Quantitative PCR Test) to the Roche COBAS ${ }^{\circledR}$ AmpliPrep/COBAS ${ }^{\circledR}$ TaqMan ${ }^{\circledR}$ (CAP/CTM) HBV Test, v2.0 to determine the traceability of each assay to the 2nd WHO standard (NIBSC Code 97/750).

Methods: A WHO standard panel comprised of 4 panel members each at clinically relevant titers $(55 ; 2,000 ; 20,000$; and 200,000 IU/ $\mathrm{mL}$ ) and near the lower limit of the assays was tested in triplicate over 3 days with each 3 assays. Average titer, minimum/maximum values, standard deviation, and observed vs. nominal titer comparison were calculated. Valid test results that were within the assay range testdefined assay limits were included for analysis. Average $\log _{10^{-}}$ transformed values (IU/mL) were compared; titers within $\pm 0.3 \log _{10}$ of the nominal titer were considered acceptable.

Results: The CAP/CTM HBV Test agreed with the WHO standard across all four titers tested. The PG assay agreed with the WHO standard at the 2,000 IU/mL titer, but failed at the other titer levels. The DaAn assay failed to agree with any WHO standard titers, which is unexpected since the WHO material should achieve traceability regardless of which test is used.

Conclusion: In comparing the 3 assays, traceability to the WHO standard at all 4 levels tested was achieved only with the CAP/CTM HBV Test. Because international treatment guidelines rely on accurate values measured in $\mathrm{IU} / \mathrm{mL}$, it may be difficult to interpret viral load results from assays that are not traceable to the WHO standard.

\section{PP06-47}

Dynamics and Clinical Significances of Serum HBV DNA Levels in Fatal Acute-on-Chronic Hepatitis B Liver Failure

J. Lai ${ }^{1}$, W.-M. Ke ${ }^{2}$, Y.-F. Zhang ${ }^{2}$, G.-L. Zhang ${ }^{2}$, W.-Q. Gan ${ }^{2}$

${ }^{I}$ Department of Infectious Diseases; ${ }^{2}$ The Third Affiliated Hospital,

Sun Yat-Sen University, Guangzhou, China

Aims: Until now, it is still not completely clear what initiates fatal acute-on-chronic liver failure (ACLF) with hepatitis B occurrence and impacts its progress. The aim of study is to investigate the dynamics and clinical significances of serum HBV DNA levels in the disease. Methods: 100 patients with fatal ACLF with hepatitis B were enrolled from January 2004 to September 2009. They had never accepted antiviral therapy or liver transplantation. At three phases including 29-90, 8-28 and 1-7 days before patients death, the HBVe system status by electrochemiluminescence immunoassay and serum HBV DNA levels by Realtime-PCR were detected.

Results: In 30 patients with $\mathrm{HBeAg}$ positive, HBV DNA levels at three phases of 29-90, 8-28 and 1-7 days before their death were $6.38 \pm 1.61 \log _{10}, 5.90 \pm 1.57$ and $5.39 \pm 2.15 \log _{10}$ copies $/ \mathrm{ml}$ in sequence. In 70 patients with anti-HBe positive, HBV DNA levels at same phases were $4.78 \pm 1.68 \log _{10}, 5.74 \pm 1.76$ and $4.70 \pm 1.68$ $\log _{10}$ copies/ml respectively. Regardless of HBVe system status, there weren't significant differences among three phases in the same group $(P>0.05)$. However, HBV DNA levels of HBeAg positive group were significantly higher than that of anti-HBe positive group at the phase of 29-90 d $(P<0.05)$. No significant difference was found at the phases of $8-28 \mathrm{~d}$ and $1-7 \mathrm{~d}(P>0.05)$.

Conclusions: To initiate ACLF occurrence, serum HBV DNA levels of $\mathrm{HBeAg}$ positive patients were higher than that of anti-HBe positive ones. Sustained high HBV DNA levels may worsen ACLF progress and be fatal to patients.

\section{PP06-48}

Indoleamine 2,3-Dioxygenase Mediates the Antiviral Effect of Gamma Interferon against Hepatitis B Virus in Human Hepatocyte-Derived Cells R. Mao

Department of Infectious Diseases, Huashan Hospital, Fudan University, Shanghai, China

Interferon-alpha (IFN-a) is an approved medication for chronic hepatitis B. Gamma Interferon (IFN-g) is a key mediator of host innate and adaptive antiviral immunity against hepatitis B virus (HBV) infection in vivo. In an effort to elucidate the antiviral mechanism of these cytokines, we tested 37 IFN-stimulated genes (ISGs), which are highly inducible in hepatocytes, for their ability to inhibit HBV replication upon overexpression in human hepatoma cells. We found that indoleamine 2,3-dioxygenase (IDO), an IFN-g-induced enzyme catalyzing tryptophan degradation, efficiently reduced the level of intracellular HBV DNA, without altering the steady state level of viral RNA. Furthermore, we showed that expression of an enzymatically inactive IDO mutant did not inhibit HBV replication, and that tryptophan supplementation in culture completely restored HBV replication in IDO-expressing cells, indicating that the antiviral effect elicited by IDO is mediated by tryptophan deprivation. Interestingly, we obtained evidence that IDO mediated tryptophan deprivation preferentially inhibited viral protein translation and genome 
replication, but did not significantly alter global cellular protein synthesis. Finally, we demonstrated that tryptophan supplementation was able to completely restore HBV replication in IFN-g, but not IFN-a, treated cells, which strongly argues that IDO is the primary mediator of IFN- $\gamma$ elicited antiviral response against $\mathrm{HBV}$ in human hepatocyte-derived cells.

\section{PP06-49}

HBs Mutations Related with YMDD Mutation Induced the Expression of hfgl2 Gene

M. Han, W. Li, Y. Li, W. Yan, X. Wang, D. Chen, X. Luo, Q. Ning

Tongji Hospital, Huazhong Science and Technology University,

Wuhan, China

Background: Mutations in the highly conserved tyrosine-methionine-aspartate-aspartate (YMDD) motif are frequently associated with resistance to antiviral treatment and often followed with hepatitis flare, representing a major concern in the treatment of hepatitis B virus (HBV) infection. Previous studies showed that highly expression of hfgl2 gene is related with necrosis of hepatocytes and development of fulminant hepatitis.

Methods: To characterize the interaction between HBs mutations resulted by YMDD mutation and the expression of hfgl2 gene, HBs mutation expression plasmids, I195M and W196S were cotransfected with a hfgl2 promoter luciferase report construct into $\mathrm{CHO}$ cells and HepG2 cells respectively.

Results: Cotransfection of I195M or W196S with hfgl2p(-1334) LUC resulted in a significant increase in relative luciferase activity with an average increase of 3.3-fold and 3.7-fold in $\mathrm{CHO}$ cells, and 3.5 -fold and 4.9-fold increase respectively in HepG2 cells when compared with pcDNA3.1 empty vector cotransfected cells. There was no change in relative luciferase activity when HBs wild plasmid was cotransfected with hfgl2p(-1334)LUC in either CHO or HepG2 cells. Conclusion: These results suggest that HBs mutations related with YMDD mutation induce hfgl2 promoter activity in both $\mathrm{CHO}$ cells and HepG2 cells. It provides new insights in the interaction between HBV mutation and host gene hfgl2 expression and the mechanism of hepatitis flare following YMDD mutation.

This work was supported by NSFC No. 30972606 and National Key Basic Research Program of China 2007CB512904.

\section{PP06-50}

A Disparate Subset of Double Negative T Cells Contributes to Outcome of Murine Fulminant Viral Hepatitis via Effector Molecule flg2

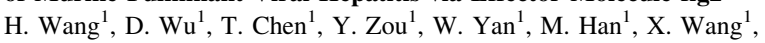

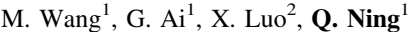

${ }^{I}$ Tongji Hospital, Huazhong Science and Technology University;

${ }^{2}$ Tongji Hospital, Wuhan, China

Fulminant viral hepatitis (FVH) is an important clinical problem worldwide. The underlying pathogenetic mechanisms have not been fully elucidated. To date, $\mathrm{CD}^{+}{ }^{+} \mathrm{CD} 4^{-} \mathrm{CD}^{-}$double negative (DN) $\mathrm{T}$ cells have been described as regulatory $\mathrm{T}$ cells (Tregs), which can suppress $\mathrm{T}$ cell responses via different mechanisms. An immune coagulant, also known as murine fibrinogen-like protein 2 (mfgl2) fibroleukin, is capable of directly cleaving prothrombin to thrombin, resulting in intra-vascular fibrin deposition within the liver and culminating in widespread hepatocyte necrosis. In this study, we present new insights into the immune mediated mechanisms involved in $\mathrm{FVH}$ caused by murine hepatitis virus strain 3 (MHV-3). After infection with MHV-3, susceptible Balb/cJ mice had increased proportions of DN T cells expressing high levels of the early activating molecule CD69, and hepatocytes were shown to recruit splenic DN T cells. Serum levels of alanine aminotransferase (ALT), aspartate aminotransferase (AST) and total bilirubin (TBil) increased, accompanied by massive hepatocyte necrosis. Distinct from previously described DN T cells, these predominantly consisted of a TCR $\alpha \beta^{+}$subset expressing high levels of CD44, but they did not secret IFN- $\gamma$, IL-10, IL-4, Fas ligand (FasL), perforin or granzyme. Following MHV-3 infection, $\mathrm{mfgl} 2 \mathrm{mRNA}$ and $\mathrm{mfgl} 2$ protein levels increased in these cells. Adoptive transfer of DN T cells from MHV-3 infected Balb/cJ mice shorten the survival time of MHV-3 infected Balb/cJ mice, mfgl2 expression, massive fibrin deposition and liver injury were found in the these adoptive mice by immunohistochemical staining of the livers. Our results suggest that DN $\mathrm{T}$ cells might migrate from spleen to liver after MHV-3 infection, and that DN T cell-derived mfgl2 may serve as an important effector molecule contributing to the pathogenesis of MHV-3-induced FVH.

\section{PP06-51}

Gibbon and Orangutan HBV Transmission Evidence by Inoculated HBV Carrier Gibbon and Orangutan Serum into uPA/SCID Mice with Human Hepatocytes

P. Sa-nguanmoo ${ }^{1}$, Y. Tanaka ${ }^{2}$, P. Ratanakorn ${ }^{3}$, K. Tatematsu ${ }^{2}$, H. Naganuma ${ }^{2}$, S. Payungporn ${ }^{4}$, A. Sommanustweechai ${ }^{5}$, M. Mizokami ${ }^{2}$, Y. Poovorawan ${ }^{1}$ ${ }^{1}$ Center of Excellence in Clinical Virology, Faculty of Medicine, Chulalongkorn University, Bangkok, Thailand; ${ }^{2}$ Department of Virology and Liver Unit, Nagoya City University Graduate School of Medical Sciences, Nagoya, Japan; ${ }^{3}$ Faculty of Veterinary Science, Mahidol University, Nakhon-Pathom; ${ }^{4}$ Department of Biochemistry, Faculty of Medicine, Chulalongkorn University; ${ }^{5}$ Dusit Zoo, Zoological Park Organization, Bangkok, Thailand

Background/aims: Replication of human HBV in non-human primates and non-human primate HBV in different non-human primates can be proof. The aim of this study is to examine whether non-human primate HBV can also be transmitted to human or not.

Methods: Three uPA/SCID mice with human hepatocytes (chimeric mice) were inoculated with $\mathrm{HBsAg}$ positive serum from gibbon or orangutan. Inoculated mice sera were subjected to detect HBV DNA by real-time PCR. Serological markers (HBsAg and HBcrAg) were quantitative by chemiluminescence enzyme immunoassay (CLEIA). Conventional PCR was used to detected cccDNA in mice liver tissues. HBV core protein and human albumin were stained by using immunofluoresence method. Phylogenetic analysis was constructed to evaluate the relationship between HBV strains before and after inoculation.

Results: HBV DNA, HBsAg, and HBcrAg were detectable 4-6 weeks after inoculation in two chimeric mice sera of each group. HBV cccDNA was detected in mice liver tissues, and core protein was stained in only human hepatocytes, not mice hepatocytes. Phylogenetic analysis revealed that HBV strains obtained from chimeric mice sera were identical to the original gibbon or orangutan $\mathrm{HBV}$ strain prior to inoculation.

Conclusions: Gibbon and orangutan HBV can be transmitted to human, suggesting that human can be also suffering from non-human primate $\mathrm{HBV}$.

Acknowledgement: The study was supported by the Thailand's Commission on Higher Education, the Chulalongkorn University Graduated Scholarship to Commemorate the 72nd Aniversary of His Majesty King Bhumibol Adulyadej, the Department of Virology and Liver Unit, Nagoya City University Graduate School of Medical Sciences, Japan and the Center of Excellence in Clinical Virology, Chulalongkorn University.

\section{PP06-52}

Evaluating the Atopy Risk in Chronic Hepatitis B Patients with High Total IgE Levels

S. Köse ${ }^{1}$, G. Çavdar ${ }^{2}$, M. Türken ${ }^{1}$, E. Ayaşlıoğlu ${ }^{3}$

${ }^{I}$ Tepecik Educational and Research Hospital, Infectious Diseases and Clinical Microbiology, Izmir; ${ }^{2}$ Tepecik Educational and Research

Hospital, Infectious Diseases and Clinical Microbiology, Ispartazmir;

${ }^{3}$ Kırlkkale University, Infectious Diseases and Clinical Microbiology, Kirikkale, Turkey 
Introduction: As for many viral infections, hepatitis viruses also increase atopy risk as a result of immune changes. In this study we attempted to assess atopy risk by determining total serum IgE levels in patients with chronic viral hepatitis B.

Methods: Among the HBs Ag positive 870 patients followed those whose total serum IgE levels were found elevated were evaluated in terms of atopy. Patients allergic and symptoms were identified and a skin prick test was performed.

Results: Among the total 870 patients total serum IgE level was found as $>100 \mathrm{IU} / \mathrm{dl}$ in 20 patients $(2.3 \%)$. Their mean age was 41.3 years (ranging 21-58 years); $12(60 \%)$ were females. Of them 12 (60\%) had chronic active hepatitis and eight (40\%) were HBV carriers. Nine patients $(45 \%)$ had symptoms of allergic rhinitis and six $(30 \%)$ had acute/chronic urticaria whereas any symptoms did not exist in another five $(25 \%)$. Consequently, skin prick test was positive in $10(50 \%)$ and negative in $10(50 \%)$. The skin prick test was negative in all the five patients who had elevated $\operatorname{IgE}$ and no allergic complaints were identified.

Discussion: In acute or chronic viral hepatitis, elevated serum IgE levels may cause atopy clinic. It was thought that allergic diseases seen in some chronic diseases and Th 1 immune response would play a role in atopy. In this study, in patients with chronic hepatitis the elevated serum $\operatorname{IgE}$ level was found consistent with atopy risk in the presence of allergic symptoms.

\section{PP06-53}

Different Pattern of Longitudinal Changes in Serum HBsAg Levels Across Different Phases During the Natural History of Chronic Hepatitis B

S.J. Yu, H.-S. Lee

Department of Internal Medicine and Liver Research Institute, Seoul National University College of Medicine, Seoul, Republic of Korea

Background/aims: Hepatitis B virus (HBV) surface antigen (HBsAg) levels vary during the natural history of chronic hepatitis B (CHB). Recently, HBsAg levels in the different phases of HBVinfection were evaluated in cross-sectional studies. However, longitudinal changing patterns of HBsAg levels have not been welldefined. This study was aimed to evaluate changing patterns of HBsAg levels during the two different phases (immune clearance and $\mathrm{HBeAg}$-negative hepatitis) of $\mathrm{CHB}$ patients with a longitudinal viewpoint.

Methods: We retrospectively analyzed the level of HBsAg and HBV DNA in the serum samples of untreated control groups which were prospectively collected during 48 weeks of two clevudine phase III clinical trials (Hepatology 2007;45:1172-1178).

Results: Serum HBsAg levels were correlated with HBV viral load weakly or positively in both $\mathrm{HBeAg}$-positive patients and $\mathrm{HBeAg}$ negative patients (98.3 vs. 100\%). Median baseline HBsAg and HBV DNA levels were higher in $\mathrm{HBeAg}$-positive patients than in $\mathrm{HBeAg}$ negative patients (HBsAg: 9,868 vs. 1,883 IU/mL, 2007;46: 1041-048). Among $84 \mathrm{CHB}$ patients who were recruited (61 HBeAgpositive patients and $23 \mathrm{HBeAg}$-negative patients), 1 of $61 \mathrm{HBeAg}$ positive patient was excluded due to the paucity of remnant serum sample to analyze; a total of 83 patients' sera were analyzed. Serum levels of HBsAg and HBV DNA were measured using ARCHITECT platform and Amplicor PCR assay, respectively. $P<0.001$; HBV DNA: 8.435 versus $6.374 \log$ copies $/ \mathrm{mL}, P<0.001$ ). Median HBsAg and HBV DNA levels decreased significantly at the end of 1year follow-up period in $\mathrm{HBeAg}$-positive patients $(P=0.007$ and $P<0.001$, respectively). In contrast, in $\mathrm{HBeAg}$-negative patients, HBsAg levels fluctuated during the 1-year follow-up period and did not decrease significantly at the end of follow-up $(P=0.455)$.

Conclusions: This study demonstrated serum HBsAg levels were naturally decreased during immune clearance phase, but not in HBeAg-negative hepatitis phase. These findings of this longitudinal study prove the results of previous cross-sectional studies.
PP06-54

Case-Control Study on Sequence Variations in the Enhancer II/Core Promoter/Precore and $\mathrm{X}$ Genes of $\mathrm{HBV}$ in Patients with Hepatocellular Carcinoma

P. Tangkijvanich ${ }^{1}$, P. Sa-nguanmoo ${ }^{2}$, V. Mahachai $^{3}$, A. Theamboonlers ${ }^{2}$, Y. Poovorawan ${ }^{2}$

${ }^{1}$ Department of Biochemistry, Faculty of Medicine, Chulalongkorn University; ${ }^{2}$ Center of Excellence in Clinical Virology, Faculty of Medicine, Chulalongkorn University; ${ }^{3}$ Department of Medicine, Faculty of Medicine, Chulalongkorn University, Bangkok, Thailand

Purpose: To evaluate the sequence variations in the enhancer II (EnhII)/ basal core promotor (BCP)/precore (PC) and X genes of hepatitis $B$ virus (HBV) in Thai patients with hepatocellular carcinoma (HCC) by conducting a cross-sectional case-control study.

Methods: Sixty patients with HCC and 60 patients without HCC, who were matched for sex, age, hepatitis B e antigen ( $\mathrm{HBeAg}$ ) status and HBV genotype were included. Viral mutations in the EnhII/BCP/ $\mathrm{PC}$ and $\mathrm{X}$ regions were characterized by direct sequencing in serum samples.

Results: The prevalence of T1753C/A, A1762T/G1764A and G1899A mutations were significantly higher in the HCC group compared to the non-HCC group (43.3 vs. $23.3 \%, P=0.02 ; 88.3 \%$ vs. $53.0 \%, P<$ 0.001 ; and 35.0 vs. $8.3 \%, P=0.001$, respectively). No significant difference between groups was found in respect to G1613A, C1653T, C1766T/T1768A, A1846T/C, T1858C and G1896A mutations. By multiple logistic regression analysis, the present of cirrhosis, A1762T/ G1764A and G1899A mutations were independently associated with the risk of HCC.

Conclusion: These data suggested that A1762T/G1764A and G1899A mutations were associated with the development of HCC in Thai patients.

\section{PP06-55}

Individualized Treatment According to Rapid Virologic Response in Hepatitis C Virus Genotype 6: Infected Patients

P. Tangkijvanich ${ }^{1}$, P. Komolmit ${ }^{2}$, V. Mahachai ${ }^{2}$, K. Poovorawan ${ }^{2}$, Y. Poovorawan ${ }^{3}$

${ }^{1}$ Department of Biochemistry; ${ }^{2}$ Department of Medicine, Faculty of Medicine, Chulalongkorn University; ${ }^{3}$ Center of Excellence in Clinical Virology, Faculty of Medicine, Chulalongkorn University, Bangkok, Thailand

Background: Chronic infection with hepatitis $\mathrm{C}$ virus (HCV) genotypes 6 is common in Southeast Asia. However, the optimal duration of therapy with a combination of pegylated interferon (PEG-IFN) and ribavirin $(\mathrm{RBV})$ in $\mathrm{HCV}$ genotype 6 is not clear. The aims of this study were to determine treatment response on the basis of rapid virologic response (RVR; defined as undetectable HCV RNA at week 4) in HCV genotype 6, and compared to those of HCV genotype 1 and 3.

Methods: Fifty-seven naïve patients were enrolled and treated with PEG-IFN alpha-2a (180 mg/week) plus RBV (800-1200 mg/day). Patients with HCV genotype 1 (group 1) and genotype 3 (group 3) were treated for a fixed duration of 48 and 24 weeks, respectively. Pateints with HCV genotype 6 who achieved RVR were treated for 24 weeks (group 6A) and the rest of the patients were treated for 48 weeks (group 6B).

Results: The mean baseline HCV RNA levels were not statistically different between groups $(6.4 \pm 0.8,5.7 \pm 1.1$ and $6.6 \pm 0.9$ $\log _{10} \mathrm{IU} / \mathrm{mL}$, in groups 1,3 and 6 , respectively). Groups 1,3 and 6 achieved an RVR in 6/12 (50.0\%), 14/16 (87.5\%) and 22/29 (75.9\%), respectively. One patient in group 1 and 3 patients in group 6 were considered non-responders (showed minimal changes in HCV RNA levels at week 12) and discontinued therapy. A sustained virologic response (SVR) was achieved in 8/12 (66.7\%) and 13/16 (81.3\%) of groups 1 and 3, respectively. Groups 6A $(n=22)$ and 6B $(n=4)$ had SVR rates of 77.3 and $75 \%$, respectively. 
Conclusions: The overall response rate of HCV genotype 6 was slightly lower than that of genotype 3 but higher than that of genotype 1. Treatment with PEG-IFN plus RBV for 24 weeks is sufficient for patients with genotype 6 and undetectable HCV RNA at week 4 .

\section{PP06-56}

The Selection of HBV PreS Mutants Is Associated with the Duration of the Immune Clearance Rather than the HBsAg Clearance

M.R. Brunetto ${ }^{1}$, P. Colombatto ${ }^{1}$, A. Stabile ${ }^{1}$, D. Cavallone ${ }^{1}$, B. Cherubini ${ }^{1}$,

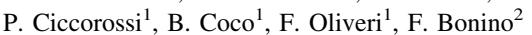

${ }^{1}$ Hepatology Unit, University Hospital of Pisa; ${ }^{2}$ Internal Medicine

Unit and Chair of Gastroenterology, University of Pisa, Pisa, Italy

Background and aims: PreS1-PreS2 variants (PreSmt) may influence HBsAg serum levels (HBsAgsl), that are relevant for monitoring therapy response and disease evolution.

Patients and methods: 32 of $450(7.1 \%)$ HBV carriers with spontaneous or therapy induced HBsAgsl decline $<100 \mathrm{IU} / \mathrm{ml}$ (12) or clearance (20) were included: 7 Inactive Carriers (IC), $5 \mathrm{HBeAg}+$ chronic hepatitis $(\mathrm{CH}), 12 \mathrm{HBeAg}-\mathrm{CH}$ and 8 cirrhosis $(\mathrm{CI})$. Quantitative HBsAg (Architect), HBV-DNA (Cobas TaqMan), PreS1 and PreS2 regions and genotype (S gene) (CEQ 2000 XLDS) were analysed at BL and EOF.

Results: At BL 20\% HBeAg+ $\mathrm{CH}$ and $55 \% \mathrm{HBeAg}-\mathrm{CH} / \mathrm{CI}$ showed PreSmt. Median HBsAgsl in HBeAg- with (11) or without (16) PreSmt were 1384 (376-4108) and $731 \quad(0.06-6,680) \quad \mathrm{IU} / \mathrm{ml}$ $(P=\mathrm{NS})$. PreS2 mutants showed deletions (del) in MHC class Irestricted T-cell epitope nt23-56(7) or mutations at PreS1(1) or PreS2(3) ATG. EOF wt-reversions (4/11) were more common than mt-selection $(1 / 20)(p=0.047)$. All untreated IC ( 2 gt A,5D; FU: 52 m, range 9-94) had wild type (wt) PreS. All HBeAg+ CH pts (3 gt A,1D,1F; FU: 38 m, range 10-60) received interferon (IFN): 4 PreSwt cleared $\mathrm{HBsAg}$, one mixed wt/PreS2 ATG mt maintained HBsAgsl $2.8 \mathrm{IU} / \mathrm{ml}$. All HBeAg- CH pts (2 gt A,10 D; FU: 52m, range 25-102) received IFN(8) or IFN+NA(4): 7 cleared HBsAg (1/5 developed wt/ PreS2 del; 1/2 PreSmt reverted to wt), 5 remained with median HBsAgsl $58 \mathrm{IU} / \mathrm{ml}$ (2/4 wt/PreS2 del reverted to wt). All HBeAg- CI pts (2 gt A,6D; FU: $94 \mathrm{~m}$, range 13-123) received $\operatorname{IFN}(2)$, IFN+NA(4) or NA(2): 4 cleared HBsAg $(1 \mathrm{wt} / \mathrm{mt}$ PreS2 ATG and 3 PreS2 del); 4 remained with median HBsAgsl 34 IU/ml (3 PreSwt, 1 wt $/ \mathrm{mt}$ PreS2 del reverted to wt).

Conclusions: Presence or emergence of PreSmt is not related to HBsAg clearance. The increasing prevalence of PreSmt from IC and $\mathrm{HBeAg}+$ pts to $\mathrm{HBeAg}-$ pts suggest that immune clearance phase duration is the major determinant for PreSmt selection.

\section{PP06-57}

Analysis of Functional Regulatory Elements within Hepatitis B Virus Post-transcriptional Regulatory Element as New Antiviral Targets N. Panjaworayan ${ }^{1}$, N. Thongtae ${ }^{1}$, C.M. Brown ${ }^{2}$

${ }^{1}$ Biochemistry, Kasetsart University, Bangkok, Thailand;

${ }^{2}$ Biochemistry, University of Otago, Dunedin, New Zealand

Background/aims: Hepatitis B virus (HBV) infection is a major cause of hepatocellular carcinoma worldwide. However, effective antiviral drugs are not available for a large number of HBV infected patients. To develop novel antiviral drugs, a better understanding of the regulation of HBV gene expression is essential. This study was conducted to identify functional elements and news siRNA target sites within post-transcriptional regulatory element (PRE) of HBV RNAs.

Methods: Comparative genomic analysis and various siRNA-predicting programmes were performed to predict conserved regulatory elements and potential siRNA target sites within PRE respectively. Deletion analysis of the PRE sequence was experimented to study effects of each identified regulatory element on luciferese activity in liver cells. Specific reporter gene plasmids were constructed to compare functional mechanism of PRE with constitutive transport element
(CTE) and Rev responsive element (RRE). To study whether polypyrimidine tract binding protein (PTB) is a trans-acting factor for PRE function, cells were transiently over-expressed with exogenous PTB as well as reduced the endogenous PTB by RNA interference (RNAi).

Results: Computational analysis revealed several conserved regulatory elements that are predicted to form local secondary structures. Potential effective siRNA target sites were identified using combined siRNA predicting programmes. Experimental deletion analysis showed that sub-elements of the PRE have different effects on the reporter activity. The specific reporter assays revealed that the PRE is involved in inhibition of splicing and it utilizes different mechanism from CTE and RRE. In addition, expression of PTB fusion protein appeared to be independent of the reporter plasmids containing HBV PRE.

Conclusions: The HBV PRE is likely to contain multiple regulatory elements and it utilizes different mechanism from CTE and RRE. In this study, PTB has an independent role for PRE function. This study has identified potential siRNA target sites that may inhibit HBV gene expression via RNAi.

\section{PP06-58}

The Related Study on Serum HBV Genotype of Hepatocellular Carcinoma Patients

G. Wang

Hepatic Cirrhosis Diseases, Dalian Sixth People Hospital, Dalian, China

Objective: To investigate the clinical value of serum hepatitis B virus genotype of hepatocellular carcinoma patients from our hospital. Methods: Serum hepatitis B virue genotype of 34 cases of hepatocellular carcinoma patients and 30 cases of asymptomic HBV carriers, 44 cases of chronic hepatitis B patients from our hospital were examed, respectively.

Results: There were 23 cases of hepatocellular carcinoma patients of serum HBV B genotype (67.7\%), 10 cases of hepatocellular carcinoma patients of serum HBV C genotype (29.4\%), 1 case of hepatocellular carcinoma patients of serum HBV non-B, C genotype (3.0\%).

Conclusions: Compared with serum HBV B genotype, serum HBV C genotype is one of the risk factors of hepatocellular carcinoma patients developing and progressing.

\section{PP06-59}

The Influence of Viral Characteristics on the Pathogenesis of Chronic Severe Hepatitis B

S.Y. Huang, D.M. Yu, F. Liu, Y. Han, X.X. Zhang

Institute of Infectious and Respiratory Diseases, Rui Jin Hospital,

Shanghai Jiao Tong University School of Medicine, Shanghai, China

Aim: To investigate the relationship between HBV (hepatitis B virus) characteristics and the onset of CSHB (chronic severe hepatitis B).

Methods: Fourteen treatment-naïve patients were included in the present study, and they were grouped into ASC group (asymptomatic carriers, 5 cases), CHB group (chronic hepatitis B, 5 cases) and CSHB group (4 cases). HBV genomes were extracted from sera samples, and cloned after PCR amplification of full-length HBV DNA. Fourteen to 16 clones per sample were sequenced. Quasispecies complexity and diversity within the full genome and each gene (S, C, X, P and RT region) were calculated and related mutations were analyzed.

Results: The quasispecies complexities in CSHB patients were significantly higher than ASC within full genome, S, P, C gene and RT region at nucleotide level, and within $\mathrm{S}, \mathrm{P}$ gene at amino acid level, while $\mathrm{X}$ gene quasispecies complexities were comparable at both levels. The quasispecies diversities within full genome, S, P, C gene and RT region were statistically different among the three groups. Nucleotides deletions and variations occurred more frequently in CSHB patients than the others. Various deletions were found in CSHB patients, such as pre-S2 deletion (nt3035 to nt17, 7/15 clones 
in 1 patient), leading to the absence of pre-S2 protein and decurtation of pre-S1 protein, and C deletion (nt2199 to nt2325, 2/14 clones in 1 patient), resulting in loss or change of several T-cell epitopes in core protein. G1896A in the precore region was found in all CSHB patients (14/14,8/15,15/15,9/16 clones), more frequent than in CHB patients $(0,2 / 15,0,15 / 15,15 / 15$ clones) and ASC patients ( $0,0,0,0,0$ clones).

Conclusions: The quasispecies complexity and diversity are higher in immune clearance stage than in immune tolerance stage. And the more frequent INDEL mutations may correlate with the development of chronic severe hepatitis B.

\section{PP06-60}

Interleukin-21 Pathway Is Associated with the Pathogenesis of Chronic Hepatitis B

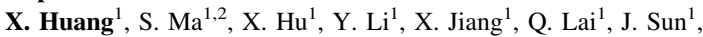
W.G.H. Abbott ${ }^{1,3}$, J. Hou ${ }^{1}$

${ }^{1}$ Hepatology Unit and Key Lab for Organ Failure Research, Nanfang Hospital, Southern Medical University, Guangzhou; ${ }^{2}$ Department of Infectious Diseases, Kunming General Hospital of PLA, Kunming, China; ${ }^{3}$ The New Zealand Liver Transplant Unit, Auckland City Hospital, Auckland, New Zealand

Background/aims: IL-21 has stimulatory effects on acquired immune responses in chronic non-hepatitis virus infections (Elsaesser et al., Science 2009a; Johnson et al., Science, 2009b). The purpose of this study was to determine whether IL-21 is associated with the evolution of the immune tolerant (IT), the inactive healthy carrier (IHC) and the active CHB phases of a chronic HBV infection.

Methods: Serum IL-21 concentration (ELISA), IL-21 and IL-21 receptor (IL-21R) mRNA in peripheral blood mononuclear cells (PBMC) (Quantitative PCR), the frequency of $\mathrm{IL}-21^{+} \mathrm{CD} 4^{+} \mathrm{T}$ cells in $\mathrm{PBMC}$, and their distribution in $5 \mathrm{~T}$ helper subsets that were either positive for IFN-g, IL-4, IL-17A, CXCR5 or negative for all 4 markers (M-ve) (Intracellular Cytokine Staining) were measured in 20 healthy control (HC), 20 IT, 58 HBeAg-positive CHB and 21 IHC subjects.

Results: IT had a non-significant increase in serum IL-21, a lower IL-21R mRNA level $(p=0.008)$, and a higher $\mathrm{IL}-21^{+} \mathrm{CD} 4^{+} \mathrm{T}$ cell frequency $(p=0.0009)$ than HC, due to higher IL-17A, CXCR5 and $\mathrm{M}$-ve subset frequencies. The breakdown of tolerance (IT vs. CHB and IHC) was associated with a further increase in serum IL-21 $(p<$ 0.008 ). HBeAg seroconversion (IHC vs. IT and CHB) was associated with a further increase in IL- $21^{+} \mathrm{CD} 4^{+} \mathrm{T}$ cells $(p<0.0001)$, due to the M-ve, IL-17A and IFN-g subsets. CHB was associated with increased IL-21 and IL21R mRNA (both $p<0.0001$ ) and decreased $\mathrm{IL}-21^{+} \mathrm{CD} 4^{+} \mathrm{T}$ cells $(p<0.0001)$ relative to IT and IHC, due to decreased CXCR5 and IL-4 subsets.

Conclusions: IL-21 may influence the evolution of chronic HBV infections. The unidentified IL-21 producing $\mathrm{CD}^{+}$subsets may be involved in viral control and its mechanism need further explored.

\section{PP06-61}

Impact of Hepatitis B Virus on the Expression of Suppressor of Cytokine Signaling -1

C. Qingxian

The Department of Infectious Diseases, The Third Affiliated Hospital of Sun Yat-sen University, Guangzhou, China

Aim: To study the correlation between the expression of suppressor of cytokine signaling (SOCS)-1 of chronic hepatitis B (CHB) and serum Hepatitis B virus (HBV)-DNA levels, and explore the impact of HBV on SOCS-1 gene expression in vitro.

Method: 50 cases of CHB were included. The expression of SOCS-1 in liver tissues was investigated by immunohistochemical staining and SOCS-1 mRNA levels in peripheral blood mononuclear cells (PBMC) were detected by real-time quantitative retroverse polymerase chain reaction (QRT-PCR). Their correlations with the serum HBV DNA levels were analyzed by SPSS statistics software.
HepG2.2.15 and HepG2 (control) cells were cultured in vitro and their SOCS-1 expressions were analyzed by QRT-PCR before and after stimulated by IL-6.

Result: Both the expression of SOCS-1 in liver tissues and the SOCS1 mRNA levels in PBMC were negatively correlated with the serum HBV DNA levels $\left(R_{1}=-0.347, P_{1}<0.05\right.$ and $R_{2}=-0.555, P_{2}<$ 0.05 , respectively). The SOCS-1 mRNA levels in hepG2.2.15 cells were significantly lower than that in hepG 2 cells after stimulated by IL-6, though no difference was found before stimulated by IL-6.

Conclusion: SOCS-1 gene expression of $\mathrm{CHB}$ was negatively correlated with HBV-DNA levels, HBV exert an inhibitory effect on the expression of SOCS-1 in vitro. This effect may modulate the intracellular signaling pathway, contributing to the pathogenesis in HBV infection.

\section{PP06-62}

Evolution of Multi-drug Resistant Hepatitis B Virus during Nucleoside Analogues Therapy

W. Zhao ${ }^{1}$, L. Chen ${ }^{2}$, Y.-L. Shao ${ }^{1}$, J.-H. Wu ${ }^{1}$, L.-L. Pan ${ }^{1}$, Y.-L. Zhu ${ }^{1}$, J.-H. Gan ${ }^{1}$

${ }^{1}$ Department of Infectious Diseases, First Affiliated Hospital of Soochow University, Suzhou; ${ }^{2}$ Infectious Diseases, Changshu No. 2 People's Hospital, Changshu, China

Aims: We investigated the evolution patterns and formation mechanisms of multi-drug resistant hepatitis B virus (HBV) during nucleoside analogues (NAs) therapy in chronic hepatitis B (CHB) patients.

Methods: $706 \mathrm{CHB}$ patients with unsatisfactory curative effect during NAs therapy were enrolled. Amino acid sequence of HBV RT domain was analysized. 15 serum samples from five representative patients were selected for clonal analysis using 'PCR-cloningsequencing' method. Additionally, dynamic detection of consecutive serum samples was carried out in four patients to determine the evolution of HBV strains.

Results:

1. The total multi-drug resistance detection rate of NAs monotherapy, sequential therapy and combination therapy was $3.5 \%$. Among which, double resistance to Lamivudine (LAM) and Adefovir (ADV) was $72 \%$; triple resistance to LAM, ADV and entecavir (ETV) was $28 \%$.

2. Multi-drug resistance can lead to viral rebound, hepatitis B recurrence or even death.

3. The evolution of quasispecies can be divided into three models. (1) From single drug resistant HBV mutations only to mixtures of clones that have multi-drug resistant mutations and single drug resistant HBV mutations.

(2) From single drug resistant HBV mutations only to mixtures of clones that have multi-drug resistant mutations.

(3) From mixtures of clones that have multi-drug resistant mutations and single drug resistant HBV mutations to the single drug resistant HBV mutations.

4. Combination therapy with ETV and ADV reduced HBV DNA to the detection limit of 500 copies $/ \mathrm{ml}$ within 12 weeks in 3 patients.

\section{Conclusion:}

1. Multi-drug resistance can be induced during NAs monotherapy, sequential therapy or combination therapy.

2. Multi-drug resistance can lead to serious clinical outcomes.

3. Mutations conferring multi-drug resistance colocate on the same viral genome.

4. Dynamic changes of HBV quasispecies had relation to the selective pressure of NAs therapy, distinct replicative advantage of the variants and replication space.

5. Combination therapy with ETV and ADV may be effective for some multi-drug resistant patients. 


\section{PP06-63}

IFIT1 and -2 are Cellular Factors that Limit Hepatitis B Virus Replication

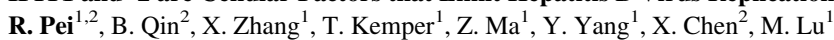
${ }^{1}$ Institute of Virology University Essen, Essen, Germany; ${ }^{2}$ Wuhan Institute of Virology, Wuhan, China

Interferon (IFN)- $\alpha$ is able to stimulate a number of cellular genes and inhibit the replication of various viruses. We attempted to identify ISGs that show antiviral activities against HBV. According to the gene array analysis and real time RT-PCR, ISGs like IFTI1 and -2 were highly expressed upon IFN-stimulation. Knock down of IFIT1 and -2 in HepG2.2.15 led to a marked increase of the amount of HBV replication intermediates. Consistently, an enhanced HBV replication occurred in transient transfection with a replication-competent clone in HepG2 and Huh7 after knock down of IFIT1 and -2. However, IFN- $\alpha$ stimulation could override the knock down by siRNAs and restore the expression of IFIT1 and -2, leading to the inhibition of HBV replication. Silencing of IFIT1 or -2 decreased the expression of both genes while other ISGs like MxA were not affected. IFIT1 and -2 was upregulated after transfection with dsDNA. Accordingly, transfection with plasmids carrying the replication-competent HBV clone enhanced IFTI1 and -2 expression, indicating that the unintended induction of IFIT1 and 2 expression in transient transfection experiments may reduce HBV replication. Further, the influence of IFTI1 and -2 on HBV gene expression was tested with reporter plasmids with HBV promoters. Cotransfection of IFIT1 and -2 expression vectors led to a decreased activity of HBVs promoters. These results were consistent with the expression of HBsAg and HBeAg data measured by ELISA assays. In summary, IFIT1 and -2 expression in hepatoma cells was significantly stimulated by IFN- $\alpha$, polyIC, dsDNA including plasmids. Silencing of these genes led to a marked increase of HBV replication in stably transfected cells and in transient transfection. IFIT1 and -2 were able to influence the HBV promoter activity and decrease HBsAg production. These results suggest that IFIT1 and -2 contribute significantly to the regulation of HBV gene expression and replication.

\section{PP06-64}

Serum Hepatitis B Surface Antigen (HBsAg) Titer and Quantitation of HBV tDNA,HBV cccDNA in Patients with HBV-Related Hepatocellular Carcinoma

Y. Li, T. Han

Tianjin Third Central Hospital, Tianjin, China

Objective: To analyze the characterization of hepatitis B surface antigen (HBsAg) titer and the quantitation of total HBV DNA, covalently closed circular DNA (cccDNA) in patients with HBVrelated hepatocellular carcinoma (HCC).

Methods: Total HBV DNA and HBV cccDNA in serum and liver samples were measured in $37 \mathrm{HCC}$ patients by a real-time PCR assay; HBsAg titer were also measured by Abbott chemiluminescence.

Results: HBsAg did not correlate with intrahepatic HBV cccDNA $(r=0.151, P=0.371)$ nor total HBV DNA $(r=0.146, P=0.388)$ in tumor tissues. Similarly HBsAg did not correlate with intrahepatic HBV cccDNA $(r=0.186, P=0.271)$ nor total $\mathrm{HBV} \mathrm{DNA}(r=0.323, P$ $=0.051)$ in non-tumor tissues. Intrahepatic total HBV DNA $(P=0.067)$, HBV cccDNA $(P=0.438)$ and the proportion of intrahepatic HBV cccDNA in total HBV DNA $(P=0.501)$ had no different between the tumor and non-tumor tissues. The intrahepatic HBV cccDNA correlated with serum HBV DNA in tumor tissues $(r=0.382$, $P=0.020)$. The intrahepatic total HBV DNA correlated with HBV cccDNA in non-tumor tissues $(r=0.327, P=0.048)$. The intrahepatic total HBV DNA of tumor tissues correlated with intrahepatic total HBV DNA of non-tumor tissues $(r=0.665, P<0.001)$.

Conclusion: HBV replication level decreased in HCC patients. The correlation was weak between HBsAg titer and intrahepatic markers of HBV replication.Compared to the non-tumor tissues, the levels of $\mathrm{HBV}$ replication in the tumor tissues had no different.
Poster Presentation 07: Non-Alcoholic Fatty Liver Disease (NAFLD): Basic

PP07-01

Adiponectin a Diffrentia L Marker between Steatosis and Steatohepatitis H.A. Abd El Moety ${ }^{1}$, D.A. Maharem ${ }^{2}$, M. El -Gandour ${ }^{1}$, A.A. Abd El Moety ${ }^{3}$ ${ }^{I}$ Chemical Pathology; ${ }^{2}$ Internal Medicine, Medical Research Institue, Alexandria University; ${ }^{3}$ Hepatology, Faculty of Medicine, Alexandria University, Alexandria, Egypt

Nonalcoholic fatty liver disease (NAFLD) becoming a world- wide public health problem.It represents a spectrum of disease ranging from simple steatosis to steatohepatitis (NASH). Adipocytokines refer to adipocyte-derived biologically active molecules TNF- $\alpha$, leptin and adiponectin, all been implicated in development of hepatic inflammation and fibrosis inNAFLD patients. This new hormone differ from its predecssors in important feature, production and concentration acutully decrease in obesity, and all adipose-derived hormone are increased.It is possible that adiponectin expression is activated during adipogenesis, a feed back inhibition on its production may occur during the development of obesity. Adiponectin may exert a hepatic protective effect.

Aim: Evaluation of adiponectin level as a differential marker between steatosis and Steatohepatitis.

Subjectes and methods: 20 NAFLD patients, 20 biopsy proved NASH and 20 control subjects, matched for age, sex and BMI. All the subjects were subjected to an abdominal ultrasonography, routine biochemical evaluation: liver function ALT and AST, lipid profile (cholesterol, triglycerides, HDL-C,LDL-C), CRP and Adipocytokines (TNF- $\alpha$, IL-6, LEPTIN, and Adiponectin).

Results:

1. Plasma adiponectin levels were significantly lower in NAFLD patients than control gp $(6.15 \pm 1.39$ vs. $12.03 \pm 3.46 \mathrm{ng} / \mathrm{ml})$.

2. Adiponctin was significantly lower in NASH than NAFLD $(1.80$ $0 \pm 0.96 \mathrm{ng} / \mathrm{ml}$ vs. $6.15 \pm 1.39 \mathrm{ng} / \mathrm{ml}$ ).

3. Leptin level was significantly higher in NAFLD than NASHgp $(69.50 \pm 18.70 \mathrm{ng} / \mathrm{ml}$ vs. $43.20 \pm 6.93 \mathrm{ng} / \mathrm{ml})$.

4. Adiponectin ROC curve showed an AUROC curve inNAFLD gp $(0.945 p=0.049)$ while inNASH was $(0.995, p=0.007)$.

5. TNF- $\alpha$ \& IL-6 was significantly higher in NASH than NAFLDgp $(79.25 \pm 13.89 \mathrm{pg} / \mathrm{ml}$ vs. $41.25 \pm 17.53 \mathrm{pg} / \mathrm{ml})$ and $(110.20 \pm 55.34$ $\mathrm{pg} / \mathrm{ml}$ vs. $43.85 \pm 16.13)$.

6. Plasma adiponectin level inNAFLD gp was inversely correlated with T. G $(r=-0.368 p=0.111)$ GOT $(r=-0.037 p=0.878)$ and GPT $(r=-0.022 p=0.926)$ while it was +ve correlated in NASH gp with Cholesterol $(r=0.317 p=0.174)$ and T.G $(r=0.042 p=0.861)$.

Conclusion: This data support a role for low circulating adiponectin in the pathogenisis of NAFLD and hypoadiponectinemia found to be a feature of NASH.ADIPONECTIN found to be a non-invasive differential marker between NAFLD \&NASH.

\section{PP07-02}

Dietary Anthocyanins-rich Purple Sweet Potato Protects Against High-glucose-Induced Lipid Accumulation by Activating AMP-Activated Protein Kinase in Human HepG2 Cells

Y.P. Hwang, J.H. Choi, H.G. Jeong

Pharmacy, Chungnam National University, Daejeon, Republic of Korea

Background/aims: Anthocyanins are a class of natural polyphenol compounds present in a wide variety of fruits, beans, cereals, and vegetables. In animal models, anthocyanins have powerful antioxidant, anti-inflammatory and anti-tumor effects. In addition, anthocyanins could prevent obesity and hyperglycemia. The present studies were performed to determine the extent to which the effect of 
anthocyanin fraction (AF) obtained from the purple sweet potato on hepatocellular lipids is mediated by AMP-activated protein kinase (AMPK) regulates lipid accumulation in insulin resistant states. The activation of AMPK protects against fatty acid synthase (FAS) induction and lipid accumulation caused by high glucose.

Methods: The expression of hepatic Nrf2 and heme oxygenase-1 (HO-1) were measured using semi-quantitative RT-PCR and Western blot analysis.

Results: AF increased Thr-172 phosphorylation of AMPKa in a doseand time-dependent manner. In parallel, phosphorylation of acetylCoA carboxylase (ACC) at Ser-79 was increased, which was consistent with decreasing ACC activity. Intracellular triacylglycerol and cholesterol contents were also decreased. An insulin resistant state was induced by exposing cells to $30 \mathrm{mM}$ glucose as indicated by decreased phosphorylation of AMPK $\alpha$ and ACC. AF substantially prevents the impairment in phosphorylation of AMPK $\alpha$ and its downstream target, ACC, elevation in expression of FAS, and lipid accumulation in human HepG2 hepatocytes exposed to high glucose. Conclusions: AF lowers hepatic lipid content and FAS expression by activating $\mathrm{AMPK} \alpha$, thereby mediating beneficial effects in hyperglycemia and insulin resistance.

\section{PP07-03}

Saponins Derived from Platycodon Grandiflorum Stimulate AMPK, Lower Lipids, and Inhibit Fatty Acid Synthesis Induction in InsulinResistant Human HepG2 Cells

Y.P. Hwang ${ }^{1}$, H.J. Park ${ }^{1}$, H.K. Kim ${ }^{1}$, E.H. Han ${ }^{1}$, J.H. Choi ${ }^{1,2}$, J.H. Yang ${ }^{1}$, J.H. $\mathrm{Im}^{1}$, J.Y. Kim ${ }^{1}$, Y.C. Chung ${ }^{3}$, H.G. Jeong ${ }^{1}$

${ }^{1}$ Pharmacy, Chungnam National University, Daejeon; ${ }^{2}$ Pharmacy, Chosun University, Gwangju; ${ }^{3}$ Food Science, Korea International University, Jinju, Republic of Korea

Background/aims: AMP-activated protein kinase (AMPK) activation by Sirtuin1 (Sirt1) protects against fatty acid synthesis (FAS) induction and lipid accumulation caused by high glucose. Sirt1 acts as the upstream of AMPK signaling and hepatocellular lipid metabolism. The present studies were performed to determine the extent to which the effect of saponins derived from roots of Platycodon grandiflorum (CKS) on hepatocellular lipids is mediated by AMP-activated Protein Kinase (AMPK) regulates lipid accumulation in insulin resistant states.

Methods: A cell model for high-glucose-induced accumulation of hepatic lipids was used by exposing HepG2 cells to a high concentration of glucose $(30 \mathrm{mM})$ for 24 . To study the molecular mechanisms underlying CKS action, we examined the function of important cellular signaling pathways using luciferase-based promoter assays and Western blot analysis following acteoside treatment.

Results: CKS increased Sirt1 and AMPK activity in human HepG2 hepatocytes exposed to high glucose. In addition, CKS increase acetyl coenzyme A carboxylase (ACC), AMPK downstream effectors, in human HepG2 hepatocytes exposed to high glucose. CKS inhibits lipid synthesis and total cholesterol synthesis in a similar manner to the AMPK activator, 5-aminoimidazole-4-carboxamide 1-b-ribofuranoside (AICAR). CKS substantially prevents the impairment in phosphorylation of AMPK and its downstream target, ACC, elevation in expression of fatty acid synthase, and lipid accumulation in human HepG2 hepatocytes exposed to high glucose. These effects of CKS are largely abolished by inhibition of Sirt1 activity, suggesting that the stimulation of AMPK. CKS lowers hepatic lipid content and inhibition of triacylglycerol synthesis by activating AMPK, thereby mediating beneficial effects in hyperglycemia and insulin resistance. Conclusions: AMPK signaling by CKS, which Sirt1 induces, may have potentail therapeutic implications for dyslipidemia and accelerated atherosclerosis in deabets and energy metabolism-related disease.
PP07-04

Saponins Derived from Roots of Platycodon Grandiflorum Attenuates Experimental Non-alcoholic Fatty Liver Disease Induced by High Fat Diet J.H. Choi ${ }^{1,2}$, K. Tilak ${ }^{1}$, B.H. Park ${ }^{1}$, Y.P. Hwang ${ }^{1}$, G.Y. Song ${ }^{1}$, Y.C. Chung ${ }^{3}$, H.G. Jeong ${ }^{1}$

${ }^{1}$ Pharmacy, Chungnam National University, Daejeon; ${ }^{2}$ Pharmacy, Chosun University, Gwangju; ${ }^{3}$ Food Science, Korea International University, Jinju, Republic of Korea

Background/aims: Non-alcoholic fatty liver disease (NAFLD) is known to be a highly relevant factor of the called metabolic syndrome, which includes obesity, diabetes, hyperlipidemia, and hypertension. Saponins derived from roots of Platycodon grandiflorum (Changkil saponins: CKS) have shown to exert anti-oxidant and anti-inflammation activity. We investigated the preventive role of CKS in an experimental NAFLD model induced by a high fat diet.

Methods: The study included 5-week-old male Sprague-Dawley rats, which were equally divided into five groups. The study was conducted for 6 weeks. Serum activity of alanine aminotransferase (ALT) and aspartate aminotransferase (AST), body weight, histopathological examinations, hepatic triglyceride (TG), total cholesterol (T-CHO), low density lipoprotein cholesterol (LDL-CHO) and high density lipoprotein cholesterol (HDL-CHO) levels were performed. Levels of expression in sterol regulatory element binding protein-1 (SREBP-1), fatty acid synthetase (FAS) and silent information regulator 1 (SIRT1) and phosphorylation of acetyl-CoA carboxylase (ACC) and AMP-activated protein kinase (AMPK) were performed. Results: Steatosis, inflammation, ballooning degeneration, and necrosis increased significantly in the HFD group, compared to the control group. These increases were inhibited by CKS treatment for last 2 weeks. CKS reduced HFD-elevated serum activity of ALT and AST, histopathological changes, hepatic levels of TG, T-CHO and LDL-CHO. Also, CKS elevated HFD-reduced hepatic level of HDLCHO. Furthermore, CKS inhibited HFD-elevated expression of SREBP-1 and induced HFD-reduced expression of FAS and SIRT1 and phosphorylation of ACC and AMPK.

Conclusions: CKS might be a critical preventive part in response to HFD-induced hepatic steatosis in an experimental NAFLD model. CKS is good candidate to act as an adjuvant agent in the development of hepatic steatosis, an important step in the development of fatty liver.

\section{PP07-05}

Histologic Evidence of NASH on Liver Biopsy Does Not Increase the Risk of Laparoscopic Bariatric Surgery

K. Herrera*1, J. Ryan*2, N. De la Cruz ${ }^{1}$

${ }^{1}$ Laparoendoscopic and Bariatric Surgery, University of Miami, Miller School of Medicine, Miami, FL; ${ }^{2}$ Hepatology and Liver

Transplantation, Cedars Sinai, Los Angeles, CA, USA

Bariatric surgery is an increasingly common procedure within the US. Patients with morbid obesity, who are candidates for the procedure, are at an increased risk of having non-alcoholic fatty liver disease (NAFLD). A sub-group of patients with NAFLD will progress to nonalcoholic steatohepatitis (NASH), which can be differentiated by the presence of inflammatory changes on liver biopsy. NASH can present with various stages of liver fibrosis and frank cirrhosis. It would be of interest to know if patients with NASH are at an increased risk of surgical complications, compared to patients who have more benign findings on liver biopsy.

Methods: We reviewed a retrospective database of $n=455$ liver biopsies from patients who had undergone laparoscopic bariatric surgery at a single center during the period of December, 2002- July, 2009. Biopsies were classified as demonstrating mild or severe histological changes. Mild histological changes were defined by the presence of minimal Steatosis and/or inflammation with no fibrosis. Severe histological changes were defined by the presence of steatosis, 
severe inflammation and/or fibrosis. The baseline characteristics and surgical complication rates of patients were determined for both categories.

Results: A total 455 liver biopsies from patients who underwent bariatric surgery were reviewed. Of these, 245 showed mild histological changes (Group A), and 210 showed advanced changes (Group B). The mean age was 39.58 in group A and 42.5 in group B. The mean BMI was similar in both groups (47.34 vs. 47.15$)$. The complication rates where $6.12 \%$ and $5.71 \%$ for groups A and B respectively ( $p=\mathrm{NS}$ ). RYGBP was the most common procedure performed in both groups $98.8 \%$ (group A) and $98.09 \%$ (group B).

Conclusions: The patients with both mild and severe histological changes on liver biopsy had similar baseline characteristics.

The surgical complication rates were similar for both groups.

\section{PP07-06}

TNF-alpha-Induced Cytotoxicity in SK-hep-1 Cells Treated with Palmitic Acid

J.M. Oh ${ }^{1}$, C.H. Kim ${ }^{1}$, S.J. Oh ${ }^{1,2}$, B.H. Kim ${ }^{1}$, S.K. Kim ${ }^{1}$

${ }^{1}$ College of Pharmacy and RCTCP, Chungnam National University, Daejeon; ${ }^{2}$ Bio-Evaluation Center, KRIBB, Chungbuk, Republic of Korea

In steatohepatitis, TNF-alpha is implicated in hepatic inflammation cell recruitment and free fatty acids may potentiate cell death signaling mediated by TNF receptor activation. To investigate effects of free fatty acids on TNF-alpha induced cytotoxicity, we were evaluated the TNF-alpha induced cell death in the presence of fatty acids including saturated fatty acids and unsaturated fatty acids. Palmitic acid (PA) but not unsaturated fatty acids such as linoleic acid and oleic acid augmented TNF-alpha-induced cytotoxicity. In addition, to elucidate mechanism(s) involved in interactions between free fatty acids and TNF-alpha, oxidative stress was evaluated using lipid peroxidation in SK-hep-1 cells treated with PA and TNF-alpha. The results showed that levels of malondialdehyde, a parameter of lipid peroxidation was more increased in cells treated with both PA and TNF-alpha, relative to cells treated with TNF-alpha only. The present study suggest that PA increased oxidative stress in cells treated with TNF-alpha, which may be responsible for augmentation of TNFalpha-induced cytotoxicity.

\section{PP07-07}

Di(2-ethylhexyl) Phthalate Induces Hepatotoxicity Via Phospholipase

D-medaited Signaling Pathways in Sprague-Dawley Rats

T.H. Kim, N. Patra, Y.J. Lee, H.J. Lim, U. De, H.S. Kim

College of Pharmacy, Pusanl National University, Busan,

Republic of Korea

Phospholipase D (PLD) is an enzyme that catalyzes the hydrolysis of phosphatidyl choline (PC) to generate phosphatidic acid (PA) and choline. PLD is believed to play an important role in cell proliferation, survival signaling, cell transformation, and tumor progression. However, it remains to be determined whether expression of PLD in the liver is sufficient to induce hepatotoxicity. The aim of this study was to investigate the possible role of PLD in hepatotoxicity induced by di(2-ethylhexyl) phthalate (DEHP) in Sprague-Dawley rats. DEHP $(500 \mathrm{mg} / \mathrm{kg} / \mathrm{day})$ was administered to prepubertal rats (4 weeks of age, weighing approximately $70-90 \mathrm{~g}$ ) by oral intubation for 1 day, 7 days, or 28 days, respectively. In this study, PLD1/2, peroxisome proliferator-activated receptor (PPAR), cytochrome P450 (CYP), and histone deacetylase (HDAC) proteins expressions were examined by Western blot analysis using specific antibodies. Liver weight was significantly increased in the DEHP treatment group. Immunohistochemistry was carried out to examine the proliferation of hepatocytes. DEHP resulted in strong staining of PCNA at 28 days of exposure. A significant increase in PLD1/2 expression was observed in the liver of DEHP-exposed rats after 7 days compared to the corresponding control groups. Moreover, PPAR, constitutive androstane receptor (CAR), and CYP2B1 protein levels were markedly increased in DEHP-treated groups. Our results suggest that PLD plays a critical role in PPAR-induced hepatotoxicity through a complex interaction with nuclear receptors, such as CAR and the HDACs.

\section{PP07-08}

The Relationship Between the Level of Adiponectin with Insulin Resistance and Severity of Liver Steatosis

V.D. Nemtsova ${ }^{1}$, E.B. Semyatichko ${ }^{1}$, O.V. Kolesnikova ${ }^{2}$

${ }^{I}$ Gastroenterology, Kharkov State Medical University; ${ }^{2}$ Hepatology, Institute of Therapy Named by ac. L.T. Maloi AMS, Kharkov, Ukraine

Background: In recent years adipose tissue hormones play a significant role in the development of nonalcoholic fatty liver disease (NAFLD) and, in particular, non-alcoholic steatosis. There are reports that the concentration of adiponectin in patients with NAFLD is reduced, however, the relationship between its content in the plasma, insulin resistance and severity of liver steatosis remains unknown.

Objective: To evaluate the relationship between the level of adiponectin, insulin resistance and severity of liver steatosis.

Methods: The study involved 72 patients with non-alcoholic steatosis and 20 healthy individuals matched by age, weight and sex. All patients underwent a study adiponectin concentration by ELISA, depending on the lipid profile of blood serum, insulin resistance index and body fat in the liver, reflecting the severity of steatosis.

Results: Cumulative assessment of the studied parameters adiponectin levels positively correlated with age over 45 years $(r=0.28$, $P<0.05)$, female sex $(r=0.36, P<0.05)$, lipoproteins of high density content $(r=0.42, P<0.05)$ and negatively- with waist circumference ( $r=-0.3, P<0.05)$, BMI $(r=-0.32, P<0.05)$, levels of triglycerides $(r=-0.48, P<0.05)$ and $\operatorname{ALT}(r=-0,46, P<0.05)$, index of HOMA-IR $(r=-0.58, P<0.05)$, severity of liver steatosis $(r=-0.62, P<0.05)$, and also predicted the presence of the metabolic syndrome. Regression analysis showed that NAFLD with a decreased level of adiponectin is associated with age, female sex, triglyceride level and severity of liver steatosis $\left(R_{2}, 0.245\right)$.

Conclusions: These data demonstrate that the reduced level of adiponectin in NAFLD is associated with insulin resistance and with the severity of liver steatosis.

\section{PP07-09}

Risk Factors for Cardiovascular Disease in Patients with Nonalcoholic Fatty Liver Disease

O.V. Kolesnikova ${ }^{1}$, I.D. Chupin ${ }^{2}$, V.D. Nemtsova ${ }^{3}$

${ }^{1}$ Hepatology, Institute of Therapy Named by ac. L.T. Maloi AMS;

${ }^{2}$ Internal Medicine, ${ }^{3}$ Gastroenterology, Kharkov State Medical University, Kharkov, Ukraine

Objective: Dyslipidemia is a common but modifiable coronary heart disease (CHD) risk factor in type 2 diabetes and the metabolic syndrome. Nonalcoholic fatty liver disease has been recognized as a novel component of the metabolic syndrome.

Aim: The aim of the present study was to examine the relationship between liver fat content and the response of postprandial triglyceride (TG)-rich lipoproteins (TRL) and adiponectin.

Methods: Sixty-eight patients with hepatic steatosis were allocated into subgroups with either low $(<5 \%)$ or high $(>5 \%)$ liver fat measured with computed tomography (CT). Subjects underwent an oral fat tolerance test with measurements of postprandial TG, cholesterol, apolipoprotein B-48 (apoB-48), and apoB-100 in TRL fractions, a euglycemic hyperinsulinemic clamp, and determination of abdominal fat volumes by CT.

Results: Patients with high liver fat displayed increased response of postprandial lipids in plasma and very-low-density lipoprotein 1 (VLDL1) fractions. Liver fat positively correlated with postprandial responses (area under the curve) of TG $(r=0,584 ; P=0.001)$, 
cholesterol $(r=0,548 ; P=0.002)$, apoB-48 $(r=0,536 ; P=$ $0.002)$, and apoB-100 $(r=0,46 ; P=0.02)$ in the VLDL1 fraction. Respective incremental areas under the curve correlated significantly with liver fat. Fasting adiponectin levels were inversely correlated with both postprandial lipids and liver fat content.

Conclusions: High liver fat content associated with postprandial lipidemia represents potential risk factors for cardiovascular disease in patients with hepatic steatosis. Low adiponectin concentration is closely linked to high liver fat content and impaired TRL metabolism.

\section{PP07-10}

Hepatic Fat Content and Serum Resistin in Patients with Nonalcoholic Fatty Liver Disease

V.D. Nemtsova ${ }^{1}$, E.B. Semyatichko ${ }^{1}$, O.V. Kolesnikova ${ }^{2}$

${ }^{1}$ Gastroenterology, Kharkov State Medical University; ${ }^{2}$ Hepatology, Institute of Therapy Named by ac. L.T. Maloi AMS, Kharkov, Ukraine

Objective: Serum resistin concentration is increased in patients with nonalcoholic fatty liver disease (NAFLD) in proportion with the histological severity of the disease, but the relevance of the contribution of fatty liver per se is undetermined. The objective of the study was to assess the relationship between serum resistin and the degree of fat accumulation in patients with NAFLD.

Methods: The hepatic fat (HF) content, measured quantitatively by means of computed tomography (CT), serum resistin, and biochemical and hormonal metabolic correlates of fatty liver and insulin resistance were assessed in 86 patients with NAFLD and 30 individuals with comparable anthropometric features served as controls. Insulin sensitivity was estimated using the homeostatic model assessment (HOMA-IR).

Results: In patients with NAFLD, the HF content $(13 \pm 8$ vs. $2 \pm 1 \%$ wet weight; $P<0.001$ ) was increased in comparison with the controls. Patients with fatty liver had lower insulin sensitivity (HOMA-IR: $5.8 \pm 2.6$ vs. $7.3 \pm 2.6 ; P<0.02$ ), serum resistin $(3.3 \pm 0.6$ vs. $4.1 \pm 1.0 \mathrm{ng} / \mathrm{ml} ; P<0.02)$, and adiponectin $(P<0.01)$ concentrations. Serum resistin was inversely correlated with the HF content $(r=-0,42 ; P<0.002)$ but not HOMA-IR.

Conclusion: This study demonstrates that excessive fat accumulation in the liver of insulin-resistant patients is associated with lower serum resistin concentration and not with hyperresistinemia.

\section{PP07-11}

Ultrasound Evaluation Results in Russian Federation National-Wide Direg Study for Non-alcoholic Fatty Liver Disease Screening

O.M. Drapkina, V.T. Ivashkin

Internal Disease, Moscow Medical University Sechenov, Moscow, Russia

Aim: To assess the liver, spleen and pancreas ultrasound picture in patients enrolled into the national population-based DIREG-L-01903 study for non-alcoholic fatty liver disease (NAFLD) screening

Methods: In total of 30787 primary care patients $(56 \%$ females, mean age $47.8 \pm 16$ years) were enrolled into open multicenter national-wide prospective study. Careful clinical examination, serum biochemistry (including ALT, AST, $\gamma$-GT, lipid spectrum and hepatitis screening) and abdominal ultrasound diagnostics with precise liver, spleen and pancreas assessment were performed in 30,754 patients.

Results: NAFLD was found in 8215 (27)\% of included patients. Within group with confirmed NAFLD liver steatosis was diagnosed in 80.3 , steatohepatitis in $16.8 \%$, and cirrhosis in $2.9 \%$ of patients. Of notice, only in $3.6 \%$ of NAFLD patients ( $1.0 \%$ in all population) the diagnosis has been established before DIREG program initiation, despite regular observations of participants in primary care centers. AST was increased $\geq 1.5 \mathrm{~N}$ in $2816(9.2 \%)$, ALT was increased $\geq 1.5$ $\mathrm{N}$ in $3144(10.2 \%)$ of patients. Results of ultrasound liver, spleen and pancreas examination : Liver enlargement 5017 (16.3\%), Liver parenchyma heterogeneity 7590 (24.7\%), Portal hypertension 150 (0.5\%), Signs of liver steatosis $7456(24.2 \%)$, Signs of liver fibrosis 692 (2.3\%), Signs of liver cirrhosis $243(0.8 \%)$, Pancreas enlargement 998 (3.2\%), Spleen enlargement 387 (1.3\%), Normal ultrasound picture, in total $16520(53.7 \%)$.

Conclusion: Taking into account high prevalence (27\%) of NAFLD in Russian Federation the attention should be given for liver ultrasound revealing high rate of liver enlargement (16.3\%) and signs of liver steatosis $(24.2 \%)$ in patients screened for NAFLD.

\section{PP07-12}

Risk Factors for Non-alcoholic Fatty Liver Disease in Russian Federation in National-wide Direg-L-01903 Studying

O.M. Drapkina, V.T. Ivashkin

Internal Disease, Moscow Medical University Sechenov, Moscow, Russia

Aim: Assess the risk factors (RF) for NAFLD in Russian Federation in the national population-based DIGER study.

Methods: Enrolled 30,787 primary care patients (56\% fem, age $47.8 \pm 16$ ). Clinical examination, serum biochemistry and abdominal ultrasound diagnostics with precise liver assessment were performed in 30,754 patients.

Results: NAFLD was found in 8215 (27\%). Within group with confirmed NAFLD liver steatosis was diagnosed in $80.3 \%$, steatohepatitis $16.8 \%$, and cirrhosis $2.9 \%$. Only in $3.6 \%$ of NAFLD patients ( $1.0 \%$ in all population) the diagnosis has been established before DIREG-L-01903 program initiation, despite regular observations of participants in primary care centers. AST increased $\geq 1.5 \mathrm{~N}$ in 2816 (9.2\%), ALT increased $\geq 1.5 \mathrm{~N}$ in $3144(10.2 \%)$ of patients. In total patients population most frequent were arterial hypertension (AH) (42\%), dyslipidemia (38\%), abdominal obesity (AO) (36\%). In total NAFLD patients population more frequent conditions were: $\mathrm{AH}$ (70\%), dyslipidemia (76\%) and hypercholesterolemia (69\%), $p<$ 0.001 compared with total population. In patients 18-29 years AO was identified as risk factor, because in was found in 45\% NAFLD patients in comparison with $14 \%$ of patients without NAFLD, $p<$ 0.001 . The significance of AO as NAFLD RF is decreased with advanced age due to relatively higher prevalence of $\mathrm{AO}$ in patients without NAFLD aged 40-80 years. NAFLD was diagnosed in $64.3 \%$ of patients with type I diabetes, $69.8 \%$ patients with type II diabetes, $45.2 \%$ of patients with $\mathrm{AH}, 61.5 \%$ of patients with $\mathrm{AO}$ and in $66.9 \%$ in those with metabolic syndrome (MS).

Conclusion: Taking into account high prevalence (27\%) of NAFLD in Russian Federation the attention should be given for NAFLD RF such as $\mathrm{AH}$, dyslipidemia and hypercholesterolemia in all age groups as well as AO in patients younger than 39 years. Metabolic factors clustering might explore an important link between MS and NAFLD.

\section{PP07-13}

The Prevalence of Non-Alcoholic Fatty Liver Disease in Russian Federation in National-Wide Direg Study

O.M. Drapkina, V.T. Ivashkin

Internal Disease, Moscow Medical University Sechenov, Moscow, Russia

Aim: To evaluate the prevalence of non-alcoholic fatty liver disease (NAFLD) in Russian Federation within the national population-based DIGER study.

Methods: In total of 30,787 primary care patients $(56 \%$ females, mean age $47.8 \pm 16$ years) were enrolled into open multicenter national-wide prospective study. Careful clinical examination, serum biochemistry (including ALT, AST, $\gamma$-GT, glucose, lipid spectrum and hepatitis screening) and abdominal ultrasound diagnostics with precise liver assessment were performed in 30,754 patients.

Results: In our study, NAFLD was found in 8,215 (27\%) of included patients. Within group with confirmed NAFLD liver steatosis was 
diagnosed in $80.3 \%$, steatohepatitis in $16.8 \%$, and cirrhosis in $2.9 \%$ of patients. The highest NAFLD prevalence $(38 \%)$ was found in patients aged between 50 and 80 years. Interestingly, only in $3.6 \%$ of NAFLD patients (1.0\% in all population) the diagnosis has been established before DIREG-L01903program initiation, despite regular observations of participants in primary care centers.

Conclusion: We have shown that NAFLD has very high prevalence $(27 \%)$ in Russian population. Direct screening explored that NAFLD prevalence is 27-folds higher comparing with anamnestic data enclosing extremely high rate of underdiagnostics. The attention should be given by primary care physicians for focus diagnosis of this potentially curable disorder.

\section{PP07-14}

Liver Disease Structure Explored in Russian Federation National-wide Direg-l-01903 Study for Non-alcoholic Fatty Liver Disease Screening

O.M. Drapkina, V.T. Ivashkin

Internal Disease, Moscow Medical University Sechenov, Moscow, Russia

Aim: To assess liver disease nosologic structure in patients enrolled into the national population-based DIREG-L-01903 study for nonalcoholic fatty liver disease (NAFLD) screening.

Methods: In total of 30,787 primary care patients (56\% females, mean age $47.8 \pm 16$ years) were enrolled into open multicenter national-wide prospective study. Careful clinical examination, serum biochemistry (including ALT, AST, $\gamma$-GT, lipid spectrum and hepatitis screening) and abdominal ultrasound diagnostics with precise liver, spleen and pancreas assessment and waist circumstence were performed in 30,754 patients.

Results: NAFLD was found in 8,215 (27)\% of included patients. Within group with confirmed NAFLD liver steatosis was diagnosed in $80.3 \%$, steatohepatitis in $16.8 \%$, and cirrhosis in $2.9 \%$ of patients. AST was increased $\geq 1.5 \mathrm{~N}$ in $2816(9.2 \%)$, ALT was increased $\geq 1.5 \mathrm{~N}$ in $3144(10.2 \%)$ of patients. In total, liver ultrasound examination revealed liver enlargement in $16.3 \%$ portal hypertension in 0.5 signs of liver steatosis in $24.2 \%$ signs of liver fibrosis in 2.3 signs of liver cirrhosis in 0.8 of total patients. Further meticulous clinical evaluation in tertiary medical centers enclosed liver diseases were: NAFLD N (\%) from a total of 30,754: 8,315 (27.0\%); $\mathrm{N}(\%)$ from pts with liver disease 8315 (71.6\%). Alcohol-induced liver disease 1608 (5.2\%); 1608 (13.9\%). Viral hepatitis (total) 1617 (5.3\%); 1617 (13.9\%). Hepatitis C 281 $(0.9 \%) ; 281$ (2.4\%). Hepatitis B $176(0.6 \%) ; 176(1.5 \%)$. Hepatocellular carcinoma $71(0.2 \%)$; $71(0.6 \%)$.Autoimmune hepatitis $89(0.3 \%)$; 89 $(0.8 \%)$. Inherent liver disease (including hemachromatosis) $111(0.4 \%)$; $111(1.0 \%)$. Toxic/drug-induced liver disease $120(0.4 \%) ; 120(1.0 \%)$. Conclusion: NAFLD seems to be most prevalent liver disease estimating $27 \%$ of screened patient, whereas viral hepatitis and alcoholinduced liver disease have prevalence of approximately $5 \%$.

\section{PP07-15}

Guggulsterone Inhibits LXR $\alpha$ Mediated SREBP-1c-Dependent Hepatic Steatosis through PKC Dependent Pathway

E.J. Cho, J.-H. Yoon, E.S. Jang, M.-S. Kwak, S.J. Yu, J.-H. Lee, H.-S. Lee Internal Medicine, Seoul National University Hospital, Seoul, Republic of Korea

Background and aims: Nonalcoholic fatty liver disease (NAFLD) is increasing in prevalence and may progress to steatohepatitis and cirrhosis. Liver X receptor $\alpha(\mathrm{LXR} \alpha)$ mediated sterol regulatory element binding protein-1c (SREBP-1c) dependent lipogenesis is an important pathway in hepatic steatosis. The plant sterol guggulsterone from Commiphora mukul has been shown to have hypolipidemic effect via inhibiting farnesoid $X$ receptor. However, the effect of guggulsterone on hepatic steatosis is unknown. Here, we investigated its effects on LXR $\alpha$ mediated SREBP-1c dependent hepatic steatosis and elucidated the underlying mechanism.
Methods: The effects of guggulsterone on SREBP-1c signaling in hepatocytes were investigated by oil red $\mathrm{O}$ stain, reporter gene assay, reverse transcriptase polymerase chain reaction (RT PCR), real time PCR, chromatin immunoprecipitation (ChIP) assay, and immunoblot analysis. Male C57BL/6 mice on high-fat-diet were treated with or without guggulsterone for 5 weeks. Hepatic steatosis and inflammatory markers were evaluated.

Results: Cellular TG content was increased when HepG2 were cultured in the presence of oleic acid, and this increase was prevented by guggulsterone treatment. Guggulsterone prevented $\operatorname{LXR} \alpha$ agonist (T0901317) induced activation of SREBP-1c, inhibited its mRNA and protein induction, thereby resulting in the down-regulation of SREBP-1c target genes. This inhibitory effect of guggulsterone was blocked by the protein kinase $\mathrm{C}$ (PKC) inhibitor. Moreover, guggulsterone enhanced LXR $\alpha$ phosphorylation at threonine residue and decreased phosphorylation at serine residue, which caused impaired DNA binding activity, leading to inhibition of $\operatorname{LXR} \alpha$ transactivation and expression. In high-fat-diet fed mice, guggulsterone treatment inhibited body weight gain, hepatic steatosis, as shown by a decrease in fat accumulation in liver. The results of blood biochemistry and histopathology showed attenuation of high-fat diet-induced liver injury by guggulsterone.

Conclusion: These results show that guggulsterone prevents LXR $\alpha$ mediated SREBP-1c dependent hepatic steatosis through PKC dependent pathway, and suggests that guggulsterone can therapeutically be used to treat NAFLD.

\section{PP07-16}

Serum Levels of Adipocytokines; Leptin and Adiponectin in Patients with Nonalcoholic Fatty Liver Disease: Potential Biomarkers M.M. El-Attar ${ }^{1}$, N.T. El-Mlegy ${ }^{2}$

${ }^{I}$ Tropical M, Assiut U Hospital; ${ }^{2}$ Biochemistry, Assiut U, Assiut, Egypt

Nonalcoholic fatty liver disease (NAFLD) occurs in 60-95\% of people with obesity. Understanding the pathogenesis of NAFLD is of great importance in ultimately finding a treatment, cure or prevention of this disease.

The aim:

1. To investigate the role of adipocytokines (leptin and adiponectin) in NAFLD and correlate them with different anthropometric and clinical variables.

2. To investigate the relationship between high-sensitivity C-reactive protein (hs-CRP) and NAFLD.

Patients and methods: Thirty patients ( 9 males and 21 females) with NAFLD (their diagnosis was based on ultrasonographic finding of bright liver). Their age (mean \pm SD) $38.00 \pm 10.42$ years. A control group included 15 healthy volunteers, who was gender, age and BMI matched. Serum levels of leptin, adiponectin, (hs-CRP), and lipid profile were assayed.

The results: NAFLD patients showed significantly higher BMI, AST/ ALT ratio, serum levels of total cholesterol, triglycerides, LDL-C, serum NEFFA levels, serum hs-CRP levels and significantly lower serum HDL-C and adiponectin levels in comparison to controls. Among NAFLD patients; these changes were significantly high in females than males patients. Serum leptin levels showed no significant difference between patients and controls. NAFLD patients with $\mathrm{BMI} \geq 30$ and those with $\mathrm{AST} / \mathrm{ALT}$ ratio $\geq 1$ showed significantly higher serum levels of NEFFA, leptin, hs-CRP and significantly lower serum levels of adiponectin in comparison to those with BMI $<30$ and AST/ALT ratio $<1$.

Conclusion: To date adiponectin and hs-CRP has yielded the most promising results. Serum leptin has contradictory results so cannot be used as a marker of NAFLD. 
PP07-17

Jiangzhi Granula Ameliorate Hepatic Steatosis Via Improving Leptin Resistace in High-fat Diet Induced NAFLD Rats

L. Zhang, P. Zheng, G. Ji

Shanghai University of TCM, Shanghai, China

Background: Nonalcoholic fatty liver disease (NAFLD) is one of the most common forms of chronic liver disease, with a prevalence ranging from 10 to $30 \%$. The current study was undertaken to determine whether Jiangzhi Granula can improve metabolic parameters in NAFLD rats and its mechamism in leptin resistance.

Methods: Six-week-old male Sprague-Dawley rats were fed either normal rat chow (normal control) or the same diet plus 10\% gard and $2 \%$ cholesterol as high fat diet (HFD). For the HFD rats, a dynamic liver histology observation was taken in every 2 weeks to evaluate the model. In the 8th week, the HFD rats were randomly divided into: model control group $(n=10)$, simvastatin group $(n=10)$ and Jiangzhi granula group $(n=10)$. After 4 weeks dug treatment, serum and liver biochemistry was analysed; liver sections were stained with hematoxylin-eosin and oil red reagent; letptin signaling factors of hypothalamus and liver were measured by RT-PCR and western bloting, while hypothalamus signal transducer and activator of transcription 3(STAT3) binding ability was tested by EMSA.

Results: Compared with HFD rats, which showed expected hepatic steatosis and leptin resistace, Jiangzhi granula treated rats exhibited reduced hepatic steatosis, serum alanine aminotransferase (ALT), serum triglyceride (TG), serum low density lipid (LDL) and liver TG content. The Jiangzhi granula produced a remarkable leptin-sensitizing effect in both the hypothalamus and liver because leptin receptor (OB-R), Janus kinase 2 (JAK2), STAT3 were up-regulated. Conclusion: These combined data indicate that NAFLD rats treated with Jiangzhi granula are protected against hepatic steatosis which via improving leptin resistance.

\section{PP07-18}

Inhibition of Lipid Synthesis Through the Activation of AMP-Activated Protein Kinase by Nectandrin B

H.-K. Choi, T.T. Hien, K.W. Kang

BK21 Project Team, College of Pharmacy, Chosun University, Gwangju, Republic of Korea

Myristica fragrans (nutmeg) has been used as a food and cosmetic as well as traditional oriental medicine against dysentery, diarrhea and analgesic. Its seed extracts possess anti-hyperlipidemic and anti-atherosclerotic activities in vivo. We isolated seven 2,5-bis-aryl-3,4dimethyltetrahydrofuran lignans from total extracts of Myristica fragrans and nectandrin B showed the strong AMP-activated protein kinase (AMPK) stimulation effect at the concentration of $5 \mu \mathrm{M}$ in differentiated $\mathrm{C} 2 \mathrm{C} 12$ cells. The present study was performed to determine whether nectandrin B inhibits lipid accumulation in hepatoma cell line. In HepG2 cells, nectandrin B increased phosphorylation intensity of AMPK and acetyl coenzyme A carboxylase (ACC). Nectandrin B substantially prevented lipid accumulation in HepG2 cells exposed to high glucose. Moreover, the protein expression and transcription of fatty acid synthase (FAS) gene was suppressed by nectandrin B in a concentration-dependent manner. These results demonstrated that nectandrin B may have potential therapeutic implications for dyslipidemia disease in liver.

PP07-19

Curcumin Ameliorates Hepatic Abnormalities Induced by Combined Consumption of High Fat Diet and Alcohol: a Molecular Mechanism

M. Bhadauria, S.K. Nirala, S. Shukla

SOS in Zoology, Jiwaji University, Gwalior, India

Background and aim: Intake of excessive energy from an energycondensed-diet with high percentage of fat is associated with increased incidence of cardiovascular disease, obesity, diabetes, insulin resistance and certain cancers. With rising obesity rates, nonalcoholic fatty liver disease (NAFLD) is predicted to become the main cause of chronic liver disease in the next decades. Similarly, alcohol dependency is one of the world's major health and socioeconomic problem and is the 3rd biggest cause of early death and illness, behind tobacco and high blood pressure. Alcohol together with high fat-containing-diet affects the extent of hepatotoxicity. Natural products or their active components may serve the best approaches to minimize these disease conditions. Therefore, curcumin (active principle of Curcuma longa) was selected to reduce the toxic effects induced by combined consumption of high-fat-diet (HFD) and alcohol.

Methods: Male rats were randomly distributed into different groups of 6 each (control, HFD, alcohol, HFD + alcohol and HFD + alcohol+ curcumin). Subchronic HFD $(30 \%)+$ Alcohol $(10 \% \mathrm{v} / \mathrm{v})$ intoxication was induced for 4 weeks. Curcumin was administered (5, 1015 and $20 \mathrm{mg} / \mathrm{kg}$, orally) daily for 4 weeks. Condition of fatty liver was measured by cholesterol, triglyceride, bilirubin, HDL and LDL content in liver/serum. Activities of transaminases, alcohol-dehydrogenase and acetaldehyde-dehydrogenase were studied. DNA damage, level of TNF- $\alpha$, Interleukin- 6 and CYP2E1 activity were measured in liver to understand molecular mechanism.

Results: All biochemical variables were significantly altered by HFD + Alcohol intoxication. DNA damage was significantly reduced and level of TNF- $\alpha$, Interleukin- 6 and CYP2E1 activity was found towards control with curcumin treatment. Activities of transaminases, alcohol-dehydrogenase, acetaldehyde-dehydrogenase and bilirubin concentration were significantly maintained towards control with curcumin at 15 and $20 \mathrm{mg} / \mathrm{kg}$ doses. Transmission electron microscopic evaluation confirmed a great extant of protection by curcumin therapy. Conclusion: Curcumin helps in mitigating hepatic abnormalities induced by combined consumption of alcohol and HFD, and may be considered seriously for clinical use.

\section{PP07-20}

The Effects of Cocoa in an Obese Animal Model of Nash M. Janevski ${ }^{1}$, K. Antonas ${ }^{2}$, R. Standish ${ }^{1}$, P. Lewandowski ${ }^{1}$ ${ }^{1}$ School of Medicine, Deakin University, Geelong; ${ }^{2}$ School of Biomedical Science, Victoria University, Melbourne, VIC, Australia

Objectives: The aim of this study was to investigate the effect of $T$. cacao (cocoa) on: biochemical parameters, glutathione (GSH), and NADPH oxidase (NOX) in obese rats on the methionine choline deficient (MCD) diet.

Methods: Obese Zucker rats were fed one of 5 diet regimes: 52 days of MCD then 28 days of MCD with cocoa (C1); 52 days of MCD then 56 days of MCD with cocoa (C2); 80 days of MCD with cocoa (C3); 108 days of MCD with cocoa (C4); or MCD diet alone for 52 days (D). Lean (L) and obese (F) Zuckers on a standard chow were controls. Liver histological examination, fasting plasma glucose, triglyceride levels, red blood cell (RBC) and liver GSH, and NOX levels (mRNA and protein) were determined.

Results: Steatosis severity increased in D rats compared to $\mathrm{F}$ rats, both showed greater steatosis than $\mathrm{L}$ rats. The $\mathrm{C} 2$ rats had lower steatosis compared to the D rats. Plasma glucose was similar between groups; triglyceride levels were higher in the $\mathrm{C} 1$ rats compared to the $\mathrm{D}, \mathrm{C} 2$ and $\mathrm{C} 4$ rats. Higher RBC GSH was observed in the D and C1$\mathrm{C} 4$ rats compared to the F rats. Lower liver GSH was observed in the $\mathrm{D}, \mathrm{C} 1$ and $\mathrm{C} 4$ rats compared to $\mathrm{L}$ rats. NOX mRNA was similar between groups. NOX protein was lower with cocoa (C1-C4) compared to the $\mathrm{L}, \mathrm{F}$ and $\mathrm{D}$ rats.

Conclusion: The MCD diet induced steatosis to a greater extent in obese Zucker rats compared to standard chow. Cocoa (C2 diet) produced less steatosis and may reduce NOX1 generated superoxide and subsequent oxidative damage. 
PP07-21

Rosiglitazone Improves Nonalcoholic Steatohepatitis Rats Via the Suppression of Expressions of Nuclear Factor-kappa B and Cyclooxygenases-2 F.S. Zhu, C.Q. Yang

Department of Gastroenterology, Institute of Digestive Disease of Tongji Hospital, Tongji University School of Medicine, Shanghai, China

Introduction: Rosiglitazone on liver nuclear factor-kappa B (NF- $\kappa \mathrm{B})$ and cyclooxygenases-2 (COX-2) has not been well illuminated.

Objective: To investigate the impact of rosiglitazone on liver NF- $\kappa \mathrm{B}$ and COX-2 expressions in nonalcoholic steatohepatitis (NASH) rats. Methods: Thirty Sprague-Dawley male rats were randomly divided into three groups: a control group, NASH group and rosiglitazone treatment group. The two latter groups were given normal diet plus $2 \%$ cholesterol and $10 \%$ lard for 12 weeks. In addition, the 8 weeks duration of rosiglitazone was intragastric intervened in the treatment group. Liver histology, antioxidant enzyme activities, tumor necrosis factor $\alpha(\mathrm{TNF}-\alpha)$, prostaglandin $\mathrm{E}_{2}\left(\mathrm{PGE}_{2}\right)$ in serum and hepatic samples were measured, respectively. The mRNA and protein expressions of peroxisome proliferator-activated receptor gamma $(\operatorname{PPAR} \gamma), \quad \mathrm{NF}-\kappa \mathrm{B}$ and $\mathrm{COX}-2$ were determined by real-time polymerase chain reaction (PCR), western blotting and immunohistochemistry, respectively.

Results: There were varying degrees of steatosis, inflammatory infiltration and fibrosis in the NASH models. The serum and hepatic levels of total anti-oxidation competence, superoxide dismutase, catalase, glutathione peroxidase and malondialdehyde in the NASH group were conspicuous disordered than those indicators in the control group. In the NASH group, the levels of serum TNF- $\alpha$ and $\mathrm{PGE}_{2}$ were significantly increased. Immunohistochemistry showed expressions of liver NF- $\kappa \mathrm{B}$ and COX-2 were significantly elevated, but $\operatorname{PPAR} \gamma$ was decreased in the NASH group. The mRNA and protein expression of COX-2 was increased in the NASH group, and after rosiglitazone intervention, all of those indicators markedly improved $(P<0.05)$.

Conclusion: Rosiglitazone plays a therapeutic role in NASH treatment, at least in part, by the suppression of expressions of NF- $\kappa \mathrm{B}$ and COX-2.

\section{PP07-22}

Functional Characterization of the Semi-Synthetic Bile Acid Derivative Int-767, a Dual FXR and TGR5 Agonist

G. Rizzo ${ }^{1}$, D. Passeri ${ }^{1}$, F. de Franco ${ }^{1}$, G. Ciaccioli $^{1}$, L. Donadio ${ }^{1}$, G. Rizzo ${ }^{1}$, S. Oralndi ${ }^{1}$, B. Sadeghpour ${ }^{1}$, X.X. Wang $2^{2}$, T. Jiang ${ }^{2}$, M. Levi ${ }^{2}$, M. Pruzanski ${ }^{3}$, L. Adorini ${ }^{1}$

${ }^{1}$ Intercept Pharmaceuticals, Perugia, Italy; ${ }^{2}$ Department of Medicine, University of Colorado, Denver, CO, ${ }^{3}$ Intercept Pharmaceuticals,

New York, NY, USA

Two receptors for bile acids have been identified, the nuclear hormone receptor farnesoid $\mathrm{X}$ receptor (FXR) and the G-proteincoupled receptor TGR5. Previous work characterized INT-747, the 6a-ethyl derivative of chenodeoxycholic acid (CDCA) and a potent and selective FXR agonist, as well as INT-777, 6a-ethyl-23(S)methyl derivative of cholic acid (CA) and a potent and selective TGR5 agonist. Here we characterize INT-767, a novel semi-synthetic 23-sulfate derivative of INT-747. INT-767 is a potent agonist for both FXR (EC $507 \mathrm{nM})$ and TGR5 $\left(\mathrm{EC}_{50} 630 \mathrm{nM}\right)$, does not show activity against 74 GPCRs (Millipore) and does inhibit 8 different ion channels (Millipore). INT-767 is the first agonist so far described to potently and selectively activate both bile acid receptors. In line with its dual activity, INT-767 induces FXR-dependent lipid uptake by adipocytes, with the beneficial effect of shuttling lipids from central to peripheral storage, and promotes TGR5dependent GLP-1 secretion by enteroendocrine cells, a validated target in the treatment of type 2 diabetes. Moreover, INT-767 improves hyperlipedimedia and hypercholesterolemia in diabetic mice. Collectively, these preclinical results indicate that INT-767 is a safe and effective modulator of FXR and TGR5-dependent pathways, suggesting potential clinical applications in the treatment of liver and metabolic diseases.

\section{PP07-23}

FXR Agonists with Potent Activity in Animal Models of Metabolic Syndrome and NASH

C. Kremoser ${ }^{1}$, E. Hambruch ${ }^{1}$, U. Deuschle ${ }^{1}$, M. Burnet ${ }^{2}$, H. Yoneyama ${ }^{3}$

${ }^{1}$ Phenex Pharmaceuticals AG, Heidelberg; ${ }^{2}$ Synovo GmbH, Tuebingen, Germany; ${ }^{3}$ Stelic Institute, Tokyo, Japan

NASH (=Non-alcoholic steatohepatitis) can develop progessively from a fatty liver and factors that predispose to this progression are hepatic insulin resistance, hyperlipidemia and obesity. Despite that fact that the incidence for NASH is dramatically rising worldwide there is no drug approved for NASH that addresses these etiologic factors in parallel.

We have developed FXR agonists $\left(\mathrm{EC}_{50}<100 \mathrm{nM}\right)$ that demonstrate potent lipid lowering, glucose lowering and insulin sensitizing effects in C57/bl6 mice prefed and maintained on a high fat diet. Moreover, when these FXR agonists were tested in a specific mouse model which closely resembles human NASH, strong anti-steatotic, anti-fibrotic and anti-inflammatory effects were observed when compared to other insulin sensitizers such as Pioglitazone. Key genes (Cyp2E1, Malic enzyme, others) which are linked to the appearance of NASH are downregulated in their expression. Thus we conclude that these FXR agonists can beneficially alter hepatic lipid and carbohydrate metabolism and exert direct hepatoprotective effects in parallel which make them ideal candidates for the treatment of human NASH.

\section{PP07-24}

Effects of Fluvastatin on CDAA Diet Induced NASH Model L.-W. Chong ${ }^{1,2}$, K.-C. Yang ${ }^{2}$, Y.-T. Huang ${ }^{3}$

${ }^{1}$ Institute of Clinical Medicine, National Yang-Ming University, Taipei, Taiwan; ${ }^{2}$ Division of Gastroenterology and Hepatology, Department of Internal Medicine, Shin Kong Wu Ho-Su Memorial Hospital; ${ }^{3}$ Institute of Traditional Medicine, National Yang-Ming University, Taipei, Taiwan, ROC

Background/aims: Oxidative stress and inflammation play important roles in the development of non-alcoholic steato-hepatitis (NASH). In addition to their cholesterol-lowering effects, statins (HMG-CoA reductase inhibitors) have been shown to exert anti-inflammatory and anti-fibrotic effects in animal models of renal fibrosis. In this study, we investigated the effects of fluvastatin on a rat model of NASH induced by choline-deficient diet.

Methods: Rat model of NASH was induced by choline deficient Lamino acid defined (CDAA) diet. Fluvastatin was administered at low ( $5 \mathrm{mg} / \mathrm{kg} /$ day $)$ and high $(10 \mathrm{mg} / \mathrm{kg} /$ day $)$ doses for 8 weeks. Assessment of therapeutic effects included:

(A) histological scoring of hepatic fibrosis,

(B) molecular markers of fibrogenesis including $\alpha$-SMA protein expression and mRNA expressions of $\mathrm{Col} 1 \mathrm{a} 2$ and TIMP-1 genes,

(C) cellular oxidative stress marker (MDA) and

(D) inflammatory cytokine (TNF- $\alpha$ ) level

Results: Fibrosis scores of livers from CDAA-treated rats receiving low dose and high dose of fluvastatin $(1.15 \pm 0.63,0.58 \pm 0.92)$ were significantly reduced in comparison with CDAA rats receiving vehicle $(1.67 \pm 0.56)$. In addition, hepatic $\alpha$-SMA protein expression and mRNA expressions of $\mathrm{Col} 1 \mathrm{a} 2$ and TIMP-1 genes in CDAA treated rats were significantly reduced by fluvastatin treatment. Elevated hepatic MDA and TNF- $\alpha$ levels in the liver tissue of CDAA rats 
were also significantly attenuated by both low-dose and high-dose fluvastatin treatments.

Conclusion: Our results showed that fluvastatin ameliorated liver fibrosis in CDAA treated rats via its anti-oxidative and anti-fibrotic effects.

\section{PP07-25}

Gender Based Changes in the Serum Biochemistry of the Weaning Rats due to Diet Composition

A.A. Syed, N. Sheikh

Department of Zoology, University of the Punjab, Lahore, Pakistan

Background/aims: Non-alcoholic fatty liver disease (NAFLD) is a syndrome of hepatic diseases ranging from simple liver inflammation to steatosis, leading to fibrosis and cirrhosis. NAFLD is considered to be related with multiple health issues including obesity. The aim of the current study was to investigate the impact of different diet compositions and some cholesterol reducing agents in weaning male and female Wister rats.

Methods: Biochemical tests for the changes in different serum parameters were performed using Kits as per manufacturer's instruction. Animals $30 \mathrm{~g}$ in weight were fed on different diet compositions. Animals were divided into 4 groups, control and three experimental groups. The experimental groups were designated as I, II and III with a prefix of M for male and F for female groups. The experimental diets were prepared in lab and designated as I-FCC, IIFCCP and III-FCCN

Composition of the experimental diets

\begin{tabular}{lll}
\hline Group & Lab codes & Composition \\
\hline Control & 0-C & Normal rat chow \\
I & I-FCC & $33 \%$ fat $+33 \%$ Carbohydrate $+34 \%$ rat chow \\
I & II-FCCP & $50 \mathrm{~g}$ Nigella sativa seeds $/ \mathrm{kg}$ of $\mathrm{I}-\mathrm{FCC}$ \\
III & III-FCCN & $50 \mathrm{~g}$ Psyllium husks $/ \mathrm{Kg}$ of $\mathrm{I}-\mathrm{FCC}$
\end{tabular}

Results: Significant changes were observed in the serum profile of the experimental groups as compared to the control $(P<0.05)$. Among females triglycerides and cholesterol were significantly increased, whereas albumin, bilirubin direct, iron and sodium were significantly decreased when compared with control using one way ANOVA. The male experimental groups showed significant changes in the serum cholesterol, iron, triglycerides with increasing pattern while albumin was decreased significantly. Serum sodium conc. was decreased in MI group whereas increased in M-II and M-III.

Conclusion: Taken together these findings we can say that the diet composition impacts variably on two genders and the blood biochemistry gets affected due to high fat contents.

\section{PP07-26}

Autophagy in Obesity Related Fatty Liver

X.Y. Duan, J.G. Fan, Q. Pan

Xinhua Hospital, Shanghai Jiao-Tong University School of Medicine, Shanghai, China

Background/aims: It is documented that autophagy plays a key role in lipid metabolism. This study aimed to explore the effects of autophagy on the development of obesity related fatty liver in rats fed with high-fat diet (HFD).

Methods: Sixty-two male SD rats were randomly divided into 2 groups: the control group $(n=24)$ and model group $(n=38)$, which were fed with a standard diet and a HFD respectively. After 5 and 9 weeks, 8 rats respectively from control group and model group weighing over mean +1.5 SD of control group were killed. Physical indexes, liver function, serum lipids levels were assayed. Hepatic histology was examined by H\&E stain. The mRNA expression of hepatic and white adipose tissue (WAT) autophagy and lipid metabolic related factors was measured by real-time PCR.

Results: Serum alkaline phosphatase, total cholesterol, low-density lipoprotein, glucose levels increased from week 5, along with observed hepatic steatosis. By week 9 , the rats developed hypertriglyceridemia and simple fatty liver. Meanwhile, in the model group, the expression of hepatic and WAT Beclin-1 and LC3-II was significantly increased at week 5 compared with the normal group, but it was contrary at week 9. In addition, hepatic fatty acid synthetase (FAS), ApoB100 and WAT perilipin increase and hepatic carnitine palmityl transferase (CPT-I), Bcl-2 and WAT hormone-sensitive lipase (HSL) decrease gradually with HFD exposure time lengthening.

Conclusions: During the development of obesity related fatty liver, autophagy was activated at initial stage, which was possibly an adaptive reflection on lipid accumulation and was associated with the down-regulation of Bcl-2 mRNA expression. However, as disease progressed, autophagy function begins decreasing, which possibly promotes fat storage via decreasing WAT hormone-sensitive lipase (HSL) and increasing perilipin mRNA expression, meanwhile causes fatty liver via increasing hepatic fatty acid synthesis and decreasing fatty acid oxidation.

\section{PP07-27}

Transition from Hepatic Steatosis to Steatohepatitis: Unique microRNA Patterns and Potential Downstream Functions and Pathways

X. Jin, Y.M. Li

Digestive Department, The First Affiliated Hospital, College of Medicine, Zhejiang University, Hangzhou, China

Aims: To explore the unique miRNA profiles of transition from hepatic steatosis to steatohepatitis in a rat model, and search for the common functions and pathways of target genes of those dys-regulated miRNAs.

Methods: Microarray and stem-loop RT-PCR were jointly utilized to detect dys-regulated miRNAs in a rat model. Significance Analysis of Microarray, Prediction Analysis of Microarray and clustering analysis were implemented to calculate significantly dys-expressed miRNAs. The targets of each dys-regulated miRNA and common targets of all down-regulated miRNAs were predicted through the web server "microrna". The functions and pathways of those predicted genes were searched against data bases of Gene Ontology and KEGG by the web server "DAVID".

Resuts: According to above mentioned bioinformatics stools, 14 upregulated and 6 down-regulated miRNAs were selected as an accurate molecular signature in distinguishing steatohepatitis from steatosis. Moreover, The Gene ontology showed 499 and 287 enriched functional categories for the target genes of up- and down-regulated miRNAs, including ion homeostasis, protein transport, monovalent inorganic cation transport and so on. Besides, KEGG pathway showed 46 and 41 enriched pathways for the target genes of up- and downregulated miRNAs, including calcium signaling pathway, apoptosis, fatty acid metabolism and so on. Finally, the analysis of common target genes of all down-regulated miRNAs showed relationship with ion transport and binding as well as the structure and component of membrane, hinting the possible change of ion channel and membrane integrity in the transition from steatosis to steatohepatitis.

Conclusion: This is by far the first report on the dys-regulated miRNAs in the transition from hepatic steatosis to steatohepatitis and showed potential clinical application in disease differentiation. This study also provides data reservoir for miRNA exploration and reveals several novel disease-specific GO functions and KEGG pathways, contributing to further researches on the pathogenesis and treatment of nonalcoholic steatohepatitis. 
PP07-28

Oleoylethanolamide Regulates Lipid Homeostasis in Steatohepatitis Cell Line

X.-Y. Lin ${ }^{1}$, Y.-Y. Zhu ${ }^{1}$, J. Dong ${ }^{1}$, J.-J. Jiang ${ }^{1}$, J. Fu ${ }^{2,3}$

${ }^{1}$ Center of Liver Disease, The First Affiliated Hospital, Fujian

Medical University, Fuzhou; ${ }^{2}$ Department of Pharmacology, Xiamen

University, Xiamen, China; ${ }^{3}$ Department of Pharmacology,

University of California, Irvine, CA, USA

Aim: Oleoylethanolamide (OEA) is a potent endogenous ligand of liver peroxisome proliferator-activated receptor-alpha (PPAR-a) that plays an important role in liver diseases. Our study is aimed at exploring the effect of OEA on steatohepatitis induced by oleic acid (OA) and lipopolysaccharide (LPS) in vitro.

Methods: We established HepG2 steatosis by 6-h stimulation with $100 \mathrm{ng} / \mathrm{ml}$ LPS following the 24-h treatment with $0.25 \mathrm{mM}$ oleic acid. Cells were treated with OEA at different concentration and the biochemical analysis was performed. Triglyceride content and lipid staining were detected by GPO/POD enzymatic reaction and Sudan oil red, respectively. The levels of gene expression were detected by real-time PCR using GAPDH as the internal standard.

Results: HepG2 cells were found with lipid accumulation and inflammation by co-treatment of LPS and OA. After $6 \mathrm{hr}$ pre-treatment with OEA, lipid accumulation in HepG2 cells was markedly reduced by triglyceride measurement and oil red lipid staining. Real-time PCR analysis showed the expression of TNF- $\alpha$ and IL- 6 were markely increased after LPS-indudcing and these two cytokines were significantly decreased with $50 \mu \mathrm{M}$ OEA's protection for $6 \mathrm{~h}(p<0.05, P<$ 0.01 respectively). SREBP-1c and SCD-1 were suppressed in OEA treatment $(P<0.001)$. In contrast, the mRNA expression of LPL was markly increased in OEA treatment $(p<0.001)$.

Conclusion: Biochemistry analysis and gene expression data further confirmed that OEA could reduced lipid accumulation in liver. In addition, the effect of OEA on reducing the expression of SCD-1 implies the possible role of OEA in preventing hepatocellular carcinoma development.

\section{PP07-29}

Jiangzhi Granula Regulats Expression of LXR $\alpha$ and SREBP-1c in NAFLD Rat Model

L. Yang ${ }^{1}$, T. Liu ${ }^{2}$, H. Song ${ }^{1}$, G. Ji ${ }^{1}$, P. Zheng ${ }^{1}$

${ }^{1}$ Institute of Digestive Diseases; ${ }^{2}$ Gastroenterology Department,

Longhua Hospital, Shanghai University of Traditional Chinese

Medicine, Shanghai, China

Background/aim: Studies have confirmed that LXR $\alpha$ and SREBP-1c play a key role in regulating the development of NAFLD. Jiangzhi Granula is a prescriptions of TCM developed by our institute. Our researches have identifided that it has a therapeutic effect for NAFLD. Our aim was to study the effect of Jiangzhi Granula on regulating LXR $\alpha$ and SREBP-1c expression in NAFLD rat model.

Methods: Forty male Wistar rats were randomly divided into control group,NAFLD group, Jiangzhi Granula group and Pioglitazone group. All rats were fed with high fat diet except control. After the NAFLD model being established (4 weeks), Jiangzhi Granula and Pioglitazone were fed to corresponding group respectively for another 4 weeks. At the end of 8 weeks, liver index was measured, liver steatosis level was observed under light microscope after HE stain; serum ALT, AST, TC, HDL, LDL and liver TG, FFA were measured, liver LXR $\alpha$ and SREBP-1c expression were determined by real-time PCR and western blot. The experimental data were analyzed by ANOVA and $t$ test.

Results: Jiangzhi Granula and pioglitazone was obviously lower liver index of NAFLD rat.Jiangzhi Gruanla improved serum ALT and AST levels which were significantly incresed in NAFLD group. Both
Jiangzhi Gruanla and Pioglitazone improved liver FFA, TG levels which were significantly increased in NAFLD group, while Pioglitazone was superior to Jiangzhi Gruanla. In NAFLD group, serum TC, LDL levels significantly increased while HDL level decreased. Jiangzhi Granula down-regulated the serum LDL. LXR $\alpha$, SREBP-1c mRNA and protein levels were notably expressed higher in NAFLD group, while the treatment with Jiangzhi Granula and Pioglitazone lowered their expression.

Conclusions: Jiangzhi Granula can improve liver pathologic changes, liver index, the levels of serum ALT, AST, LDL and liver TG, FFA.Jiangzhi Granula regulates fatty acid metabolic disorder through decreasing levels of $\operatorname{LXR} \alpha$, SREBP-1c.

PP07-30

Protective Effects of Sylimarin on Methionine-choline Deficient Diet Induced Steatohepatitis in a Rat Model

J.-W. Lee, J.I. Lee, Y.S. Kim, S.H. Ki, B.W. Bang, S. Jeong, D.H. Lee, Y.W. Shin

Internal Medicine, Inha University School of Medicine, Incheon, Republic of Korea

Background and aims: The pathogenesis of nonalcoholic steatohepatitis (NASH) is not fully understood, and the 'two-hit' hypothesis remains the prevailing theory. Sylimarin is a mixture of flavonoid anti-oxidants that has been reported to prevent liver injuries induced by various chemicals and toxins. In this study, we investigated the effects of sylimarin on methionine-choline deficient (MCD) diet induced NASH in rats with insulin resistance.

Methods: Male OLETF rats, an established animal model of obese type 2 diabetes were used. As the control animals, LETO rats were used. At 24 weeks of age, rats were divided into experimental groups and fed for 8 weeks. OLETF rats were fed on one of three different diets as follows: the standard laboratory rat chow as a control $(n=10)$, the MCD diet $(n=10)$, and the MCD-sylimarin diet (sylimarin content $0.5 \% \mathrm{wt} / \mathrm{wt}$, $n=10$ ). LETO rats were continued to be fed on the standard laboratory rat chow $(n=10)$. Histological analysis, realtime PCR and western blotting of whole liver tissue and isolated HSCs were performed.

Results: Inflammation and fibrosis was alleviated by feeding sylimarin with MCD diet. $\alpha$-SMA and $\alpha 1$-procollagen mRNA levels were significantly reduced. In addition, activation of HSCs, tested by $\alpha$ SMA mRNA level in isolated HSCs, was diminished by sylimarin. In addition, the effect of sylimarin on liver fibrosis was associated with decrease in the activation of ERK. Nuclear factor erythroid 2-related factor (Nrf)2, a key transcrioption factor that regulates the cellular antioxidant response against reactive oxygen species, was markedly increased in rats fed on MCD diet with sylimarin compared with that on MCD diet alone.

Conclusions: Sylimarin was effective in decreasing liver inflammation and fibrosis in MCD diet induced NASH. In addition, sylimarin suppressed the activation of HSCs. Our results suggested that sylimarin might be beneficial in NASH by diminishing the ERK activation and up-regulating Nrf2.

\section{PP07-31}

Vitamin D Ameliorates Stress Ligand Expression Elicited by Free Fatty Acids in Hepatic Stellate Cells

G.S. Seydel ${ }^{1,2}$, A. Beilfuss ${ }^{2}$, A. Kahraman ${ }^{2}$, K. Aksoy ${ }^{1}$, G. Gerken ${ }^{2}$, H. Akkiz ${ }^{3}$, A. Canbay ${ }^{2}$

${ }^{1}$ Biochemistry, Çukurova University, Adana, Turkey;

${ }^{2}$ Gastroenterology and Hepatology, Essen University, Essen, Germany; ${ }^{3}$ Gastroenterology and Hepatology, Cukurova University, Adana, Turkey

Background and aims: Hepatic stellate cells (HSC) play an important role as the major source of fibrillar and non-fibrillar matrix 
proteins in the process of liver fibrosis. Natural killer (NK) cells have an anti-fibrotic effect through killing activated HSC. Major histocompatibilitiy complex class I (MHC I)- related molecules, MICA and MICB, function as ligands for the NKG2D receptor and play an important role in HSC susceptibility to NK cells during hepatic inflammation. The aim of this study was therefore to investigate the effect of vitamin $\mathrm{D}_{2}$ and free fatty acids (FFA) on stress ligands and pro-fibrotic activity in LX-2 cells and human primary HSC.

Methods: LX-2 cells and primary human HSC were treated with vitamin $\mathrm{D}_{2}\left(10^{-6} \mathrm{M}\right)$ and FFA at different concentrations $(0.25,0.5$, and $1 \mathrm{mM}$ ) for $24 \mathrm{~h}$ and expression of the stress ligands MICA/B as well as TGF- $\beta, \alpha$-SMA, COL $1 \alpha$ were assessed by quantitative real time-PCR.

Results: Treatment of cells with $0.5 \mathrm{mM}$ and $1 \mathrm{mM}$ FFA induced aSMA and TGF-b expression in LX-2 cells. Moreover, 1mM FFA resulted in increased expression of MICA. Surprisingly, COL1a expression was reduced after addition of FFA. MICA/B expression in primary HSC was not affected by FFA treatment. Vitamin $\mathrm{D}_{2}$ treatment significantly downregulated the FFA-induced expression of TGF- $\beta$ and $\alpha$-SMA in LX- 2 cells. Also, in HSC a significant decrease of MICA/B mRNA by $\mathrm{VD}_{2}$, independent of FFA treatment, was detectable.

Conclusion: These results indicate that vitamin $\mathrm{D}_{2}$ may reduce inflammatory and pro-fibrogenic activity of stellate cells in vitro.

\section{PP07-32}

Proinflammatory Cytokines and Visfatin in Non Alcoholic Fatty Liver Disease of Asian Indians

M.A. Sadik ${ }^{1}$, G. Radhika ${ }^{1}$, M. Sasikala ${ }^{1}$, R.K. Mukarjee ${ }^{1}$, P.N. Rao ${ }^{2}$, D.N. Reddy ${ }^{3}$

${ }^{1}$ Institute of Basic Science and Translational Research; ${ }^{2}$ Hepatology; ${ }^{3}$ Gastroenterology, Asian Institute of Gastroenterology, Hyderabad, India

Background/aim: Non- alcoholic fatty liver disease (NAFLD) is the hepatic manifestation of obesity which is now considered to be due to constant low grade inflammation. Several adipocytokines have been implicated in the pathogenesis of NAFLD and visceral adiposity is known to be a major risk factor for NAFLD in Asian Indians. Visceral adipose tissue is demonstrated to preferentially secrete a $56 \mathrm{kDa}$ protein, Visfatin, previously known as NAMPT. The aim of the present study is to understand comprehensive role of proinflammatory cytokines and adipokine visfatin in NAFLD.

Methods: Patients clinically diagnosed to have NAFLD $(n=69)$ by standard biochemical and imaging methods formed the study group and subjects $(n=38)$ with no evidence of liver disease were control group. Plasma visfatin levels were measured using ELISA kit. Tumor necrosis factor $\alpha$, Interleukin- 6 and Interleukin- 8 were measured by Flowcytometer (BD FACS). Fasting Blood glucose, Lipid profile and Liver enzymes were estimated using standard autoanalyser (Randox) procedures, the data was analyzed by SPSS 13.0 version and a $P$ value $<0.05$ was considered significant.

Results: Compared to controls the levels of proinflammatory cytokines TNF $\alpha$, IL- 8 , and IL- 6 have been found to be higher by 25,21 and $21 \%$ respectively. On the other hand the Visfatin levels have been found to be lower by $20 \%$ in NAFLD subjects.

A significant positive correlation between serum IL-6 and IL-8 $(p<$ $0.0001)$ and a negative correlation between visfatin and High Density Lipoprotein (HDL) $(r=.341, p=0.006)$ were observed.

Conclusion: The results of this study indicate that increased levels of $\mathrm{TNF} \alpha$ and decreased levels of Visfatin are independently associated with NAFLD. The protective/fecilitatory role of visfatin in NAFLD needs to be further explored.
PP07-33

Level of Adiponectin and soluble Tumor Necrosis Factor Alfa Receptor II Effect on Insulin Resistance Subject Obese with Fatty Liver

A. Sulaeman ${ }^{1}$, S. Wahid ${ }^{2}$, A. Sulaiman ${ }^{3}$, G.S. Lawrence ${ }^{4}$

${ }^{1}$ Doctoral Program, Faculty of Medicine; ${ }^{2}$ Pathology Anatomy,

Hasanuddin University, Makassar; ${ }^{3}$ Hepatology Unit, University of

Indonesia, Jakarta; ${ }^{4}$ Vascular Research Unit, Department of

Pathology, Hasanuddin University, Makassar, Indonesia

Background: Reactive oxygen species due to obesity which affecting the lipid peroxidation and trigger inflammation to occur as can be seen in increase of cytokines such as sTNF $\alpha$ RII and TGF-b1 and the reduction of. The level of sTNF $\alpha$ RII as inflammation and adiponectin as an antiinflammmation cytokine effect on insulin resistance which is thought to make some of the feature of obesity to obesity with fatty liver.

Aim: to investigate effect on resistance insulin from level of sTNF $\alpha$ RII and adiponectin in obese patients with and without fatty liver.

Method: This study is an observational research with case control design. Ninety four subjects of this study are obese patients with criterias is waist ccircumference for Asian $>90 \mathrm{~cm}$ (IDF criteria) and fatty liver feature which are detected by ultrasonography. The subjects are divided into two groups, i.e. obesity with fatty liver as the case group (50 subjects), and obesity patients without fatty liver as the control group (44 subjects). Blood sample were then further analysed for adiponectin, sTNF $\alpha$ RII and insulin level using ELISA method, insulin resistance measure with HOMA index. Blood examination was conducted in Prodia Clinical Laboratory.

Result: Level of adiponectin and sTNF $\alpha$ RII are different between obese with and without fatty liver Adiponectin: $3.54 \pm 1.06$ (no FL) $3.08 \pm 1.12$ (FL) sTNF $\alpha$ RII: $20.57 \pm 5.32$ (no FL) $23.79 \pm 5.99$ (FL). Combination between Low level of adiponectin $(<3.43 \mu \mathrm{g} / \mathrm{l})$ with high level of sTNF $\alpha$ RII $(>22.10 \mathrm{ng} / \mathrm{dl})$ effect on insulin resistance and occur fatty liver highest than others.

Conclusion: Low level of adiponectin and high level of sTNF $\alpha$ RII effect on insulin resistance and occur fatty liver in obese patient

\section{PP07-34}

Steatosis and Hyperglycaemia Are Associated with Altered StoreOperated $\mathrm{Ca}^{2+}$ Entry in Hepatocytes

C.H. Wilson ${ }^{1}$, J. Castro ${ }^{1}$, G.Y. Rychkov ${ }^{2}$, G. Barritt ${ }^{1}$

${ }^{1}$ Medical Biochemistry, Flinders University; ${ }^{2}$ Physiology, University of Adelaide, Adelaide, SA, Australia

Background/aims: In hepatocytes, intracellular $\mathrm{Ca}^{2+}$ regulates glucose and lipid metabolism and many other pathways. Store-operated $\mathrm{Ca}^{2+}$ entry (SOCE) plays an essential role in maintaining intracellular $\mathrm{Ca}^{2+}$. The STIM 1 and Orai 1 proteins are the molecular components of SOCE in primary hepatocytes as well as in other cell types.. The isoforms STIM2, Orai2 and Orai3 may also be involved in hepatocyte SOCE. Intracellular $\mathrm{Ca}^{2+}$ homeostasis is altered in hepatocyes and in other cell types in diabetes. The aim was to determine whether SOCE is altered in hepatocyes isolated from livers exhibiting steatosis and insulin resistance.

Methods: Hepatocytes were isolated from obese ( $f a / f a)$ and lean $(\mathrm{Fa} /$ fa) Zucker rats. SOCE was measured by patch clamp recording, and using fura-2 and fluorescence microscopy. Expression of STIM and Orai mRNA was assessed by quantitive PCR. Plasma concentrations of liver marker enzymes, glucose and lipids were measured.

Results: Hepatocytes isolated from obese rats $(24 \pm 2 \mathrm{mM}$ blood glucose) exhibited a significant decrease in the amplitude of $\mathrm{I}_{\mathrm{SOC}}$ (SOCE current) activated by $25 \mu \mathrm{M}$ ATP compared to the $\mathrm{I}_{\mathrm{SOC}}$ in control hepatocytes. No difference was observed in expression of STIM1, STIM2, Orai1 and Orai3 mRNA in hepatocytes from obese compared with lean rats. Orai2 was expressed at low levels compared 
with Orai 1 and an approximately 2-fold increase in Orai2 expression in hepatocytes from obese compared with lean rats was observed. Conclusions: Steatosis is associated with an impairment of SOCE in hepatocytes. This may be due to changes in the distribution, rather than expression, of STIM1, and to changes in the expression of Orai2.

\section{PP07-35}

Remission Rate and Influence Factors of Fatty Liver in a Cohort Study Population of Shanghai China

Y.-W. Chen, Q. Zhou, J.-G. Fan

Department of Gastroenterology, Xinhua Hospital Affiliated to

Shanghai Jiaotong University School of Medicine, Shanghai, China

Background and aims: Nonalcoholic fatty liver disease (NAFLD) is a common condition and may be the most prevalent liver disorder. However, researches about the remission rate of NAFLD were less and it needs further investigation. The aim of the current study was to investigate the remission rate of fatty liver in Shanghai Bao-Steel Company-based population of China.

Methods: 5860 employees underwent health examination in 1999 and 2001 in Shanghai Bao-Steel Company were reviewed. Fatty liver was diagnosed with B-type ultrasonography. The remission rate of fatty liver in the different sex and age during the study period were determined, and the relationships between the remission rate of fatty liver and baseline of body mass index (BMI), triglyceride, total cholesterol and the change of them were studied.

Results: 426 men and 32 women diagnosed with NAFLD in 1999 were studied. Fatty liver was relieved in 63 of these patients after 2 years. By the analysis of age and sex, it was found that the remission rate of fatty liver increased among women younger than 50 years old $(P<0.01)$. Logistic regression analysis demonstrated that the remission rate of fatty liver was positively correlated to baseline of BMI and triglyceride, the change of body mass index. By the analysis of baseline of BMI, it was found that remission rate of fatty liver among employees with normal body weight, overweight, obesity and severe obesity were 18.2, 17.1, 13.4 and $1.7 \%$. By the analysis of baseline of triglyceride, it was found that triglyceride increased significantly in fatty liver onset patients without hypertriglyceridemia $(P<0.01)$

Conclusion: The remission rate of fatty liver in two years was $13.8 \%$. It was low among women older than 50-year-old, decreased with BMI baseline, positively correlated to baseline of BMI, triglyceride and the change of BMI and triglyceride.

\section{PP07-36}

Non-alcoholic Fatty Liver Disease in Chronic HBV Transfected Mice

Z. Zhang, J. Fan, Q. Pan

Gastroenterology, Xinhua Hospital, School of Medicine, Shanghai

Jiao Tong University, Shanghai, China

Background: Chronic viral hepatitis B (CHB) and nonalcoholic fatty liver disease (NAFLD) have long been presented to be major public health concern in China. Patients suffered from both diseases increase greatly for decades. The mechanisms underlying CHB accompanying NAFLD, however, are rarely understood, which may attribute to the lack of suitable experimental model to a large extent.

Methods and results: Transgenic mice with sustaining HBV production were established by microinjection of ocyte. These mice were randomly assigned into four groups and treatment with High fat diet (HFD) and common forage respectively. Following HFD, the body and liver weight, liver index in $\mathrm{HBV}$ transgenic mice were significantly increased regardless the gender. And the levels of serum cholesterol, glucose, AST, ALT were higher in HFD groups compared with control mice. Lipid droplets could be observed in most lobules of HFD groups after 8-week administration, which was consistent with features of simple steatosis. Fatty degeneration of hepatocytes, patch necrosis, mild to moderate chronic inflammatory infiltration were observed after
16 weeks of HFD feeding, reflecting the emerge of steatohepatitis. At the time point of 24-week, liver steatosis, cytologic ballooning, as well as perisinusoidal fibrosis and local fibrosis, occurred in HFD groups. Immunohistochemical staining and real-time PCR analysis of the liver tissues showed positive signal of HBsAg in all groups of mice, though no significant difference was documented.

Conclusion: Our study suggests that animal model of NAFLD can be established in HBV transgenic mice and provides a nice animal model for further studies on Non-alcoholic Fatty Liver Disease Accompanying Chronic HBV Transfection. Their histopathological disorders undergo obviously progresses throughout the period of HFD administration and seem no significant alternation by HBV production.

\section{Poster Presentation 08: Non-Alcoholic Fatty Liver Disease (NAFLD): Clinical}

\section{PP08-01}

The Usefulness of Body Mass Index and Liver Function Test in the Diagnosis of Non-alcoholic Fatty Liver Disease

Z. Koutoubi, M. Taha, A. Chebli

Internal Medicine, Tawam Hospital in Afiliation with Johns Hopkins Medicine International, Al-Ain, United Arab Emirates

Background/aims: NAFLD refers to a condition where there is accumulation of excess fat in the liver. Most patients are suspected to have NAFLD because of characteristic appearance on hepatic ultrasonography (US) and/or elevation of liver enzymes. The aim of this study is to investigate the usefulness of BMI and LFT in diagnosing NAFLD suspected by US in patients living in the United Arab Emirates and its association with diabetes and dyslipidemia.

Methods: In an observational prospective study, we evaluated the LFT and the BMI levels of 118 patients (55 men and 63 women) living in the United Arab Emirates (91 nationals, 27 non-national), who had fatty liver diagnosed by liver ultrasound. We investigated the levels of body mass index (BMI), aspartate aminotransferase (AST), alanine aminotransferase (ALT), gamma-glutamyl transferase (GGT), and alkaline phosphatase (ALP). We calculated also the number and the percentage of diabetics and dyslipidimic patients.

Results: The BMI was elevated in 111 patients $(96 \%)$, the ALT level was elevated in 23 patients $(19 \%)$, AST level was elevated in 22 patients (19\%), the ALP level was elevated in 3 patients $(3 \%)$ and the GGT level was elevated in 52 patients $(45 \%)$. Diabetes was present in 35 patients $(30 \%)$ and dyslipidemia was present in 35 patients $(30 \%)$

Conclusions: Only BMI was helpful in the diagnosis of NAFLD. The ALT, AST, ALP and GGT levels appear to be not very helpful in the diagnosis of NAFLD in our group of patients. There is a small association between NAFLD, diabetes and dyslipidemia

\section{PP08-02}

Thiazolidinediones in Non-alcoholic Steatohepatitis: a Meta-Analysis E.V.B. Razon-Gonzalez, J. Ong, M.A. De Lusong, M.E. Velasquez, E.S. Tripon, A.D. Salvana, E. Dela Cruz, R. Forroza Section of Gastroenterology, Philippine General Hospital, Manila, Philippines

Introduction/objectives: Thiazolidinediones (TZDs) improve insulin resistance in non-alcoholic steatohepatitis (NASH). This study aims to compare the efficacy of TZDs compared to placebo in improving hepatic steatosis and fibrosis in NASH.

Methods: PUBMED and Cochrane database were searched for trials involving patients with NASH given TZDs or placebo. A metaanalysis of all randomized, double-blind, placebo-controlled trials was performed. Primary outcome measures were improvement in steatosis and fibrosis. 
Results: Four randomized controlled trials (RCTs) including 334 participants were assessed. Three RCTs evaluated pioglitazone while one assessed rosiglitazone. TZDs led to significant improvement in steatosis with $64 \%$ of subjects on TZD showing improvement in steatosis versus $29 \%$ for placebo. (RR 2.29, 95\% CI 1.77-2.96, $p<$ $0.00001, \mathrm{i}^{2} 44 \%$ ). There was a trend towards improvement of fibrosis with TZDs with $36 \%$ of subjects on TZD showing improvement in fibrosis versus $28 \%$ for placebo. (RR 1.31 , 95\% CI $0.97-2.96$, $\left.p=0.08, \mathrm{i}^{2} 29 \%\right)$. Restricting the analysis to non-diabetic subjects did not change the results. TZDs led to significant improvement in steatosis (RR 2.04, CI 1.57-2.6, $p<0.00001$ ) but improvement in fibrosis was likewise not significant (RR 1.28, 95\% CI 0.93-1.76, $p$ $0.13)$. Sensitivity analyses were performed. Exclusion of one study which had a shorter duration of treatment (6 months) compared to the other studies $\left({ }^{3} 12\right.$ months) and exclusion of the study that used rosiglitazone (other 3 studies used pioglitazone) did not alter the results. Conclusions: Compared to placebo, TZDs cause significant improvement in steatosis. There is a trend towards improvement in fibrosis.

\section{PP08-03}

Study of the Relationship between Chronic Hepatitis C Virus,

Non Alcoholic Fatty Liver Disease and Insulin Resistance A.A. Abd Elmoety ${ }^{1}$, N. Mashall ${ }^{2}$, E. Shaat ${ }^{3}$, R. El Sheikh ${ }^{2}$

${ }^{1}$ Hepatology, Faculty of Medicine; ${ }^{2}$ Internal Medicine;

${ }^{3}$ Biochemistry, Alexandria University, Alexandria, Egypt

Background: Hepatitis $\mathrm{C}$ infection is considered the leading cause of chronic liver disease in Egypt. Hepatic steatosis (NAFLD) has been reported in $30-70 \%$ of patients with chronic hepatitis $\mathrm{C}$ (CHC).

Aim of the work: Assess the relationship between chronic hepatitis $\mathrm{C}$ infection, NAFLD and insulin resistance in Egyptian patients.

Material: 20 Patients with chronic HCV infection and hepatic steatosis were proven by liver biopsy were included in the study. Other 20 healthy adults were enrolled as a control group.

Methods: Liver function tests, HCV antibodies by third generation ELISA, HCV-RNA by PCR, fasting serum insulin and serum ferritin. Insulin resistance was evaluated using the homeostasis model assessment (HOMA-IR) method. Also, ultrasound examination of the abdomen.

Results: insulin resistance (IR) by (HOMA) index was significantly higher in patients than in control group (mean \pm SD) $8.36 \pm 6.61$ and $2.17 \pm 0.08)$, respectively). Fasting insulin level was significantly higher in chronic HCV patients than in control group $(P=0.000)$. Serum ferritin level was significantly higher in patients $(124.04 \pm$ $123.18 \mathrm{ng} / \mathrm{ml})$ than in control group $(65.22 \pm 46.27 \mathrm{ng} / \mathrm{ml})$. HOMAIR positively correlated with staging $(P=0.034, r=0.477)$ and with grading $(P=0.022, r=0.509)$ in HCV infected patients.

Conclusion: $\mathrm{HCV}$ infected patients have high degree of IR. IR facilitates fibrogenesis in chronic HCV patients. High serum ferritin may be implicated in (IR).

\section{PP08-04}

Waist-to-Hip Ratio is a Superior Predictor for Nonalcoholic Fatty Liver Disease

R. Zheng ${ }^{1}$, L. Jiang ${ }^{1}$, X. Chen ${ }^{1}$, J. Meng ${ }^{1}$, L. Lu ${ }^{2}$

${ }^{I}$ Research and Therapy Center for Liver Diseases, Dongnan Affiliated Hospital of Xiamen University, Zhangzhou; ${ }^{2}$ Departent of Gastroenterology, First Affiliated People's Hospital of Shanghai Jiaotong University, Shanghai, China

Objective: To investigate the most effective anthropometric index as a predictor for nonalcoholic fatty liver disease (NAFLD).

Method: This retrospective case-control study was comprised of 127 patients from Centers for Liver Disease in Dongnan affiliated hospital of Xiamen University. Liver ultrasound B scans and Liver biopsy were analyzed for each patient. 77 patients were diagnosed as
NAFLD, and 50 patients were diagnosed as non- NAFLD. Data of height, weight, waist circumference, hip circumference, waist-to-hip ratio (WHR), waist-to-height ratio (WHtR) were collected from each patient. Risk Factors were determined by Mantel-Haenszel Chisquare test and analyzed using logistic regression models and empirical receiver-operating characteristic curves.

Results: Compared with non-NAFLD group, NAFLD group has significantly higher $\operatorname{BMI}(t=8.541, P<0.001), \operatorname{WHR}\left(t^{\prime}=10.783\right.$, $P<0.001)$ and $\operatorname{WHtR}\left(t^{\prime}=9.113, \quad P<0.001\right)$. Moreover, $\operatorname{BMI}\left(\chi^{2}=25.017, P<0.001\right)$,WHR $\left(\chi_{M H}^{2}=56.793 ; P<0.001\right)$ and $\mathrm{WHtR}\left(\chi_{M H}^{2}=42.695 ; P<0.001\right)$ are also risk factors of NAFLD examined by $M-H$ chi-square test. A Linear forward binary logistic regression was performed to evaluate the relationship between NAFLD and its related factors. BMI $\left({ }_{j}=11.757\right)$ and $\operatorname{WHR}\left({ }_{j}=3.094\right)$ were chosen as predictors for NAFLD, reaching an overall predicting percentage as $85 \%$. Receiver-operating characteristic curves showed that BMI, WHR, WHtR and WC had area under curves as 0.854 , $0.916,0.878$ and 0.876 , respectively. Therefore, WHR values most for diagnosing NAFLD. Especially when choosing 0.891 as the cutoff point, the sum of sensitivity and specificity maximizes.

Conclusion: WHR serves as the most effective anthropometric index for predicting NAFLD.

\section{PP08-05}

Elevated Persistent GGT and TGP in Corelation to CT Scan Density Measurement of Severe Steatosis and Evaluation of Fibrosis L.M. Mos ${ }^{1}$, C. Zorila ${ }^{1}$, G. Pribac ${ }^{2}$, A. Frentiu ${ }^{3}$, A. Ardelean ${ }^{2}$

${ }^{1}$ Internal Medicine; ${ }^{2}$ Cell Biology, West University Vasile Goldis Arad; ${ }^{3}$ Internal Medicine, Clinical Municipal Hospital, Arad, Romania

Background and aim: Our aim was to evaluate the causes of isolated elevation of gamma glutamil transpeptidase (GGT).

Material and method: We studied 83 patients diagnosed with NASH 45 with elevated GGT, and TGP and 39 with normal values of GGT and TGP, by biochemical, imagistic (ECHO, CT) and functional biochemical liver tests, folowed over a period of 12 month. We applied index GUCI index (Göteborg University Cirrhosis Index) ratio of the multiplication of AST with prothrombin international normalized ratio (INR) to the count of platelet calculated according to the formula $\mathrm{GUCI}=\mathrm{ASAT} \times \mathrm{PT}-\mathrm{INR} \times 100 /$ thrombocyte count $\left(\times 10^{9} / \mathrm{L}\right)$. Patients with hipersplenism were not included in the study. Results: All had liver steatosis, 43 have elevated TGO. Elevation of TGO was present in associated contributing factors: disglygemic status, methabolic syndrome. We excluded patients with hepatotoxic drugs intake, Cytomegalovirus chronic infections and alcohol. The presence of hepatic steatosis was significantly correlated with total abdominal fat area and visceral fat area. There was a significant difference in the craniocaudal liver span between patients with NASH (mean $18 \mathrm{~cm})$ and patients with steatosis (mean $14 \mathrm{~cm})(P<0.01)$. Conclusions: Liver-to-spleen $\mathrm{CT}$ attenuation ratio of $<1$, indicating hepatic steatosis. Elevated persistent GGT, in corelation with elevated of TGP and GUCI index values can indicate severe steatosis and severe fibrosis.

\section{PP08-06}

Association between 25-OH Vitamin D Concentrations and Risk of Coronary Artery Disease in Patients with Non Alcoholic Fatty Liver Disease

W. Nseir ${ }^{1}$, N. Assy ${ }^{2}$

${ }^{1}$ Internal Medicine, Holly Family Hospital, Nazareth; ${ }^{2}$ Liver Unit, Ziv Medical Center, Safed, Israel

Background: Vitamin D deficiency may be involved in the development of atherosclerosis and coronary artery diseases. Vitamin D deficiency is very common in patients with chronic liver disease. The 
contribution of Vitamin D concentration to the risk of CAD in obese patients with NAFLD is unknown.

Aim: To evaluate the association between vitamin D concentration, fatty liver, and coronary artery disease (CAD).

Methods: 60 patients with fatty liver (NAFLD, aged 50土9) were compared to 30 sex, age matched healthy individuals. Coronary artery disease (CAD) was defined as a stenosis of $>50 \%$ in at least one major coronary artery by cardiac CT. Metabolic syndrome according to ATPIII Guidelines, Diffuse fatty liver was defined by liver minus spleen density $\geq-10 \mathrm{HU}$ (CT), Biomarkers of insulin resistance, inflammation, oxidant-antioxidants, vitamin D levels, were measured. Results: Patients with NAFLD showed higher prevalence of coronary soft plaques (55 vs. $28 \%, P<0.001$ ), higher prevalence of coronary stenosis ( 35 vs. $15 \%, P<0.001)$, higher HOMA ( $4.5 \pm 3.7$ vs. $1.5 .0 \pm 2.2, P<0.001)$ and higher triglyceride levels $(280 \pm 60$ vs. $170 \pm 40, P<0.005)$, and lower vitamin $\mathrm{D}$ concentration $(13 \pm 8$ $\mathrm{ng} / \mathrm{ml}$ vs. $31 \pm 4, P<0.001)$ than healthy controls. Multiple logistic regression showed that diffuse fatty liver (L-S density $>-10 \mathrm{HU}, \mathrm{OR}$ $3.7,95 \%$ CI $2.3-5.9, P<0.001)$ and severe vitamin D deficiency $(<12 \mathrm{ng} / \mathrm{ml}$, OR $2.5,95 \%$ CI $1.5-4.6, P<0.01)$ predicts coronary artery disease independently by metabolic syndrome.

Conclusion: Severe vitamin D deficiency and diffuse fatty liver are associated with higher risk for CAD. This finding may help to optimize the cardiovascular risk stratification.

\section{PP08-07}

Does the Chronic Intermittant Hypoxia Link between Obstructive Sleep Apnea and Fatty Liver Disease?

C. Türkay, B. Kasapoglu, D. Ozol, I. Kirbas

Fatih University Hospital, Ankara, Turkey

Introduction: Currently the common pathogenetic mechanisms in Nonalcoholic fatty liver disease (NAFLD) and Obstructive sleep apnoea (OSA) are targeted growing attention. The aim of this study is to find out the influence of $\mathrm{CIH}$ and OSA related parameters to severity of NAFLD with liver functions tests.

Methods: We examined the liver functions tests and ultrasonographic data of liver as well as markers of OSA severity (AHI, oxygen desaturation index, nadir oxygen saturation, percentage of time spent with $\mathrm{SpO} 2<90 \%$ [\% $\%<90]$ ) of 106 subjects.

Results: Fatty liver disease was diagnosed in 71 subjects (group-1) and remaining 35 subjects were taken as controls (group-2). As NAFLD severity increased from mild to severe form, mean AHİ and ODI values also increased significantly. Our multivariate analysis showed that AHI, ODI, lowest desaturation values and percentage of sleep duration with $\mathrm{SpO} 2<90$ were independent predictors of NAFLD after adjustment for BMI, weight and insulin resistance. Furthermore, the most correlated parameter for the severity of NAFLD was found as the duration of hypoxia.

Conclusion: We postulate that sleep fragmentation, or disruption because of frequent hypopnoeic and apnoeic episodes in sleep apnoea patients, may result in elevated levels of pro-inflammatory cytokines that may promote oxidative stress.

\section{PP08-08}

The Treatment of Nonalcoholic Fatty Liver Disease in Patients with Type 2 Diabetes Mellitus

O. Lavrynenko, L. Zhuravlyova

Kharkiv National Medical University, Kharkiv, Ukraine

Objectives: Nonalcoholic fatty liver disease (NAFLD) develops in $75-90 \%$ in patients with type 2 diabetes mellitus (DM2) and obesity.
NAFLD has been recognized as a major cause of liver fibrosis. There is no proven medical therapy for NAFLD.

Aim: This study looked at treatment in patients with NAFLD and DM2.

Materials and methods: We examined 40 patients with confirmied NAFLD and DM2 with dynamic monitoring before and after complex corrective therapy with biguanide (metformin $1,000 \mathrm{mg} /$ day), ursodeoxycholic acid (UDCA $10 \mathrm{mg} / \mathrm{kg} /$ day) and lactulosae $(25 \mathrm{mg} /$ day). Serum levels of distant markers of fibrosis and nonspecific inflammation: matrix metalloproteinase type 1 (MMP-1), tissue inhibitor of metalloproteinase type 1 (TIMP-1), tumor necrosis factor- $\alpha$ (TNF- $\alpha$ ), interleukin-1 $\beta$ (IL-1 $\beta$ ), fibronectin (FN) were determined by immunoassay ELISA method.

Results: After treatment we had significant changes in carbohydrate and lipid metabolism: significant decrease the level of glycemia $(27.6 \%)$ and the level of HBA1C (33.9\%), tendency to reduce total cholesterol (29.9\%), increase high-density lipoprotein $(52.9 \%)$, decrease in low-density lipoprotein $(38 \%)$ and significant decrease of triglyceride levels $(57.6 \%)$. Study of liver function tests revealed a significant reduction in ALAT activity (35.5\%) and ASAT (28\%), alkaline phosphatase (46.9\%), reducing total and conjugated bilirubin (from 19.7 to $38.7 \%$ ). Analyzing the dynamics of distant fibrosis markers and nonspecific inflammation, we can observe lower levels of MMP-1 (38.9\%), TIMP-1 (12.9\%), FN (14.1\%), TNF- $\alpha$ (39.7\%) and a decrease in the level of IL-1 $\beta(35.2 \%)$.

Conclusions: For NAFLD patients with DM2, we recommend combining diet and exercise with this therapeutic medical treatment. It has a clearly designed corrective effect, good tolerance and safety.

\section{PP08-09}

The Prevalence and Significance of Autoantibody in Non-alcoholic Fatty Liver Diseases

A. Sobhonslidsuk ${ }^{1}$, N. Wisedopas ${ }^{2}$, P. Sornmayura ${ }^{3}$, C. Setthaudom ${ }^{3}$ A. Pornvoranunt ${ }^{3}$

${ }^{1}$ Medicine, Ramathibodi Hospital, Mahidol University; ${ }^{2}$ Pathology, Chulalongkorn University; ${ }^{3}$ Pathology, Ramathibodi Hospital, Mahidol University, Bangkok, Thailand

Background: Non-alcoholic fatty liver disease (NAFLD) is usually presented in non-alcoholics with abnormal liver enzymes and negative viral hepatitis markers. Positive autoantibody is a criteria for diagnosis of autoimmune hepatitis (AIH) but autoantibody can be found in other conditions. We aimed to identify prevalence of autoantibody in NAFLD and clinical application.

Method: A retrospective study of patients with NAFLD confirmed with liver biopsy was done. Brunt criteria was used for grading and staging. Clinical data and results of tests performed as parts of investigation were collected. Antinuclear antibody (ANA) was detected by indirect immunofluorescene (IIF) on HEp-2 cell lines. Anti-smooth muscle antibody (ASMA) was measured by IIF on murine stomach and kidney. Titers of 1:40 and above were considered positive. Immunoglobulin (Ig) $\mathrm{G}$ level was determined by immunonephelometry. Simplified diagnostic criteria for $\mathrm{AIH}$ was applied. Statistical analysis was done with t-test and Fisher exact.

Result: Fifty-eight with NAFLD were enrolled. Six were excluded due to incomplete data. Mean age was $56.6(10.6)$ years. The number of male: female was 35 (67.3\%): 17 (32.7\%). Non-alcoholic steatohepatitis (NASH) was found in $39(75 \%)$. Twenty $(40 \%)$ had positive for ANA and/or ASMA. Nineteen (36.5\%) had positive ANA, and 6 $(11.8 \%)$ had positive ASMA. Six (11.5\%) had ANA titer over 1:320, 
and $2(3.9 \%)$ had ASMA titer over 1:320. The most common ANA pattern was homogeneous $(47.4 \%)$. Positive autoantibody was not associated with age, sex, ALT, ferritin, IgG, grading and staging, and NAFLD type. None had IgG level over 1.5 times of normal. Only 2 cases had simple criteria score for AIH of 6 ('probable AIH').

Conclusion: The prevalence of autoantibody in NAFLD is higher than normal but its clinical significance cannot be demonstrated. IgG and simplified diagnostic criteria help exclude AIH in NAFLD with positive autoantibody and reduce the need of liver biopsy.

\section{PP08-10}

Short- and Long-Term Effects of a Dietary Intervention on Abdominal Obesity in Men and Women with Non-Alcoholic Fatty Liver Disease

O.N. Korneeva, O.M. Drapkina, V.T. Ivashkin

Internal Disease, Moscow Medical University Sechenov, Moscow, Russia

Introduction: Weight loss is key element of NAFLD treatment Aim: Assess prospectively the short and longterm effects of caloriereduced diet on anthropometric measures like body-mass index, waist circumference, waist-hip ratio and compliance to the diet in NAFLD patients Methods: 60 obese NAFLD patients (36 men), age $48 \pm 13$ years. BMI at baseline $=33.4 \pm 4.9 \mathrm{~kg} / \mathrm{m}^{2}$ both sexes; $\mathrm{WC}=115.3 \pm$ $11.3 \mathrm{~cm}$ men, $=108.8 \pm 9.6 \mathrm{~cm}$ women; $\mathrm{WHR}=1.02 \pm 0.06 \mathrm{men},=$ $1.00 \pm 0.07$ women. We compared obesity anthropometric changes in Supervised 3 weeks short-term hypocaloric program $1800 \mathrm{kcal}$ and Home-based 6 months diet

Results: At 3 weeks Supervised group significant reducted in all obesity measures in both sexes $(p<0.0003)$; men achieved larger reduction in WC and WHR; $\Delta \mathrm{WC}$ men $=4.6 \pm 1.7 \mathrm{~cm}$ versus $\Delta \mathrm{WC}$ women $=3.7 \pm 2.1 \mathrm{~cm}$; $\Delta \mathrm{WHR}$ men $=0.03 \pm 0.01$ versus $\Delta \mathrm{WHR}$ women $=0.027 \pm 0.01 .6$ month Home-based diet group had less WC and WHR reductions-WC men 112.1 $\pm 10.7 \mathrm{~cm}$ versus $110.6 \pm 10.4 \mathrm{~cm}$; WC women $103.0 \pm 6.9 \mathrm{~cm}$ versus $104.7 \pm 9.3 \mathrm{~cm}$; WHR men $=1.0 \pm 0.05$ versus $0.99 \pm 0.05$; WHR women $=0.96 \pm 0.05$ versus $0.98 \pm 0.06$. Compliance of Home-based diet related to sex (women better than men) and associated with greater reduction in WC and WHR in women. At 6 months home-based diet men had a significant increase in abdom.obesity $(\triangle \mathrm{WC}=-1.44 \pm 2.9 \mathrm{~cm}$ men, $p=0.02 ; \Delta \mathrm{WHR}=-0.014 \pm 0.02, p=0.003$ ).

Conclusion: Supervised short-term hypocaloric program is more effective than long Home-based diet. Predictive value of gender in outcomes: men greater reductions in WC and WHR in short-term supervised program, due to the less men compliance to the long-term home-based diet, women had greater reduction in WC and WHR after 6 months.

\section{PP08-11}

Hepatic Resistin Overexpression is Related to Histological Severity of Liver Disease in Patients with Non-Alcoholic Steatohepatitis

C. Shen, C.-Y. Zhao, L.-B. Kong, Y.-D. Wang, W. Wang, H.-H. Che, B. Yang Department of Infectious Disease, Third Affiliated Hospital of Hebei Medical University, Shijiazhuang, China

Background and aims: Resistin has been shown to induce insulin resistance and inflammation. We aimed to investigate whether hepatic expression of resistin might contribute to the development of nonalcoholic steatohepatitis (NASH).
Methods: Thirty NASH patients, 28 simple steatosis (SS) patients, and 43 controls were recruited. Blood resistin, biochemical parameters, and insulin were determined. Hepatic resistin mRNA expression was examined by reverse transcription-polymerase chain reaction. The localization of resistin was analyzed by immunohistochemistry.

Results: Mean level of serum resistin in NASH was 2-fold higher than controls $(6.3 \pm 1.5$ vs. $3.1 \pm 1.2 \mathrm{ng} / \mathrm{ml}, P<0.05)$, but was similar to SS. Hepatic resistin mRNA expression was dramatically increased in NASH compared with SS $(P<0.05)$ and controls $(P<$ $0.05)$.

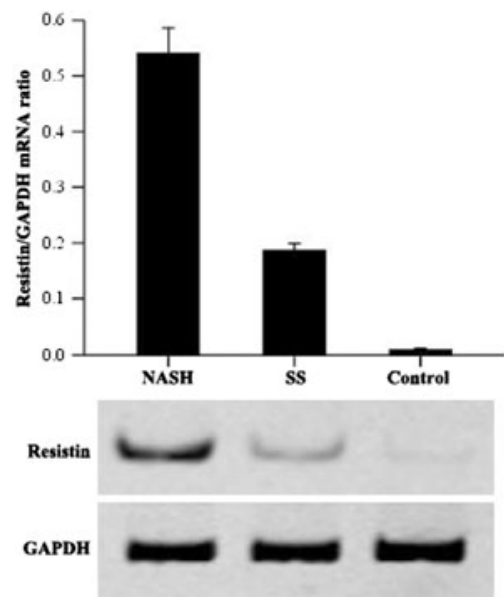

Fig. 1 Resistin mRNA expression

Resistin staining was predominantly found in perisinusoidal cells, which was similar to pattern of alpha-smooth muscle actin.

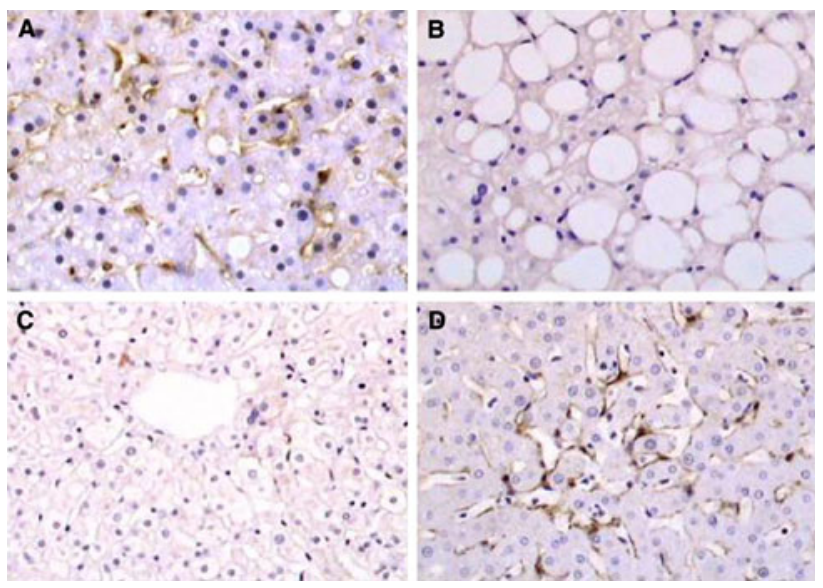

Fig. 1 Immunohistochemistry analyses 
On multivariate analysis, hyperresistinemia (OR 3.4, $P=0.04)$ was associated with steatosis. Increased hepatic resistin mRNA expression predicted the severity of necroinflammation (OR 4.4, $P=0.02$ ) and fibrosis (OR 3.8, $P=0.02$ ). Neither serum resistin nor hepatic resistin gene expression correlates with insulin resistance.

Conclusions: Hepatic resistin overexpression in NASH is associated with more severe histological changes. Liver produces resistin that may be involved in the pathogenesis of human NASH.

\section{PP08-12}

Diagnostic Value of Hepatorenal Index in the Patients with Non-Alcoholic Fatty Liver Disease

O.V. Kolesnikova ${ }^{1}$, I.D. Chupin ${ }^{2}$, V.D. Nemtsova ${ }^{3}$

${ }^{1}$ Hepatology, Institute of Therapy Named by ac. L.T. Maloi AMS,

${ }^{2}$ Internal Medicine; ${ }^{3}$ Gastroenterology, Kharkov State Medical

University, Kharkov, Ukraine

Background: Not so long ago, hepatorenal index (HRI) started to used as a criterion for diagnosis of liver steatosis. A distinctive feature of the HRI is an opportunity to determine small grades (from $5 \%$ ) liver steatosis, whereas conventional ultrasound scanning of hepatic parenchyma verifies steatosis, ranging from $25-30 \%$ of liver injury.

Objective: To determine the diagnostic value of (HRI) on the basis of the relationship between its value, biochemical and anthropometric parameters in patients with nonalcoholic fatty liver disease (NAFLD). Methods: The study involved 102 patients with NAFLD in age from 35 to 65 years; $53.5 \%$ males and $46,5 \%$ females. All patients underwent determination of anthropometric parameters [waist circumference, hips circumference, body mass index (BMI)], liver function tests (ALT, AST), carbohydrate (fasting glucose serum, glycosylated hemoglobin) and lipid metabolism (total cholesterol, triglycerides, lipoproteins of high density), as well as levels of serum adipocytokines.

Results: Analysis of the received data showed that the HRI value associated with body mass index $(r=0.52, P<0.001)$, waist circumference $(r=0.48, P<0.001)$, as well as the level of liver enzymes (ALT) $(r=0.46, P<0.001)$, triglycerides $(r=044, P<$ $0.001)$, insulin $(r=0.32, P<0.001)$, resistin $(r=0.38, P<0.001)$. The negative correlation was observed between HRI and the level of adiponectin $(r=-0.26, P<0.001)$. BMI $\left(26,29,32 \mathrm{~kg} / \mathrm{m}^{2}\right)$, waist circumference $(89,100,106 \mathrm{~cm})$ and the rate of ALT $(0.62 \pm 0.2$, $0.96 \pm 0.24,1.56 \pm 0.32 \mathrm{mmol} / \mathrm{l})$ significantly increased with the increasing severity of liver steatosis, defined by HRI $(\leq 5 \%, 5 \%-$ $25 \%, \geq 25 \%)(P<0.001)$.

Conclusions: Hepatorenal index has significant relationship with indicators that are known to have associated with the development of liver steatosis.

\section{PP08-13}

The Role of Products of Colon Microflora in Pathogenesis of Nonalcoholic Fatty Liver Disease

L.A. Zvenigorodskaja, E.A. Cherkashova, N.G. Samsonova, T.V. Nilova, S.J. Seliverstova, S.G. Kchomerici

Central Scientific Research Institute of Gastroenterology, Moscow, Russia

Introduction: Nonalcoholic fatty liver disease (NAFLD) is one of the important factors of cardiovascular risk. However the diagnosis of NAFLD is frequently late.

Aim: To devise noninvasive method to diagnose NAFLD.

Methods: 74 patients were examined in the age of 37-65 years. All patients before and after treatment were examined by several points: biochemical blood analysis (lipids, parameters of liver function), endotoxin and nitric oxide in blood, analysis of shortchain fat acids
(SFA) in faeces. We also made biopsy of liver. Patients have been divided into 2 groups depending on activity of hepatitis: (1) steatosis; (2) steatohepatitis and into 3 groups depending on therapy: (1) statin $10 \mathrm{mg}$; (2) probiotic (Bifiform complex 3 caps); (3) probiotic in combination with statin.

Results: We did not revealed direct correlation between biochemical parameters of liver function and heaviness of morphological changes in liver (appearance of morphological attributes of steatohepatitis precedes development of cytolytic and cholestatic syndrome). However patients with steatohepatitis had increased level of endotoxin $(0.37 \pm 0.01)$ and nitric oxide $(72.25 \pm 2.22)$ in blood. Total quantity of SFA in faeces was also decreased in those patients. There was more effective decrease of lipids, endotoxin and nitric oxide in those patients, who received probiotic in combination with statin. If patient received only probiotic there was not any changes of lipids, but the level of endotoxin and nitric oxide became lower. If NAFLD progressed in steatohepatitis and there was high level of $\gamma$-GTP and transaminases we didn'n notice such effective decrease of cholesterol.

\section{Conclusion:}

1. The increase level of endotoxin and nitric oxide in blood is the marker of является steatohepatitis.

2. Application of statin in combination with probiotic is more effective in achievement of target levels of lipids and decreasing endotoxin and nitric oxide.

3. If patient has steatohepatitis, effectiveness in the hypolipidemic therapy decreases (in comparison with steatosis), because the metabolism of drugs in liver decrease.

\section{PP08-14}

Clinical Correlation of Nonalcoholic Fatty Liver Disease in a Chinese Taxi Drivers Population in Taiwan: Experience at a Teaching Hospital T.-H. Tung ${ }^{1,2}$, T.-H. Lin ${ }^{2}$, W.-H. Chiu ${ }^{3}$, H.-C. Shih ${ }^{4}$, M.-H. Chang ${ }^{1}$, J.-H. Liu ${ }^{1}$ ${ }^{1}$ Cheng-Hsin General Hospital; ${ }^{2}$ Fu-Jen Catholic University;

${ }^{3}$ National Yang-Ming University, Taipei; ${ }^{4}$ Kaohsiung Armed Forces General Hospital, Kaohsiung, Taiwan, ROC

Purpose: To explore any gender-related differences in prevalence of and condition-associated factors related to non-alcoholic fatty liver disease (NAFLD) amongst Taiwanese taxi drivers in Taipei, Taiwan. Methods: We studied a total of 1,635 healthy taxi drivers $(1,541$ males and 94 females) voluntarily admitted to physical check-up between January 2006 and December 2006. Blood samples and ultrasound-proved fatty liver sonography results were collected.

Results: The prevalence of NAFLD for this sub-population was found to be $66.4 \%$, the prevalence revealing no statistically significant decrease with increasing population age $(p=0.58)$. Males exhibited a greater prevalence of NAFLD than did females $(67.5$ vs $47.9 \%, p<$ 0.0001). Using multiple logistic regression analysis, higher BMI, higher AST, higher ALT, higher fasting plasma glucose, presence of hypertension, hyperuricemia, and hypertriglyceridemia were the significant factors associated with NAFLD. Gender-related differences as regards associated factors were also revealed. For males, hypertension (OR 1.37, 95\% CI 1.04-1.93), hyperuricemia (OR 1.73, 95\% CI 1.07-2.89), higher AST (OR 2.70, 95\% CI 1.43-5.00), higher ALT (OR 2.83, 95\% CI 1.86-4.30), hypertriglyceridemia (OR 2.22, 95\% CI 1.54-3.20), and higher fasting plasma glucose (OR 2.23, 95\% CI 1.31-3.79) were significantly related to NAFLD but these were not so for females.

Conclusion: Several gender-related differences were noted pertaining to the prevalence of and relationship between hypertension, hyperuricemia, higher AST, higher ALT, hypertriglyceridemia, and higher fasting plasma glucose and NAFLD among taxi drivers population. 
PP08-15

The Associations between Anthropometric Measures, Liver Enzymes and Cardiovascular Risk Factors in Koreans

J.I. Yang ${ }^{1}$, J.M. Kang ${ }^{1}$, J.-H. Lee ${ }^{2}$, H.J. Byun ${ }^{3}$, W. Kim ${ }^{2}$, D. Kim ${ }^{1}$, Y.J. Kim², J.-H. Yoon ${ }^{2}$, C.S. Shin ${ }^{1}$, S.-H. Cho ${ }^{1}$, H.-S. Lee ${ }^{2}$

${ }^{I}$ Department of Internal Medicine, Seoul National University Hospital Gangnam Healthcare Center; ${ }^{2}$ Department of Internal Medicine and Liver Research Institute; ${ }^{3}$ Department of Dermatology, Seoul National University College of Medicine, Seoul, Republic of Korea

Background and aims: As data about the associations between anthropometric measures, liver enzymes and cardiovascular risk factors in Asians are limited, we explored the associations between them using large scale data from Korean subjects.

Methods: Data were gathered from 64,383 subjects who had been enrolled voluntarily in general health examination at Seoul National University Hospital Gangnam Healthcare Center between 2003 and 2009 but not having any serologic evidence of hepatitis B or hepatitis C. Gathered and analyzed data were as follows: body weight, height, waist circumference, AST, ALT, gamma gultamyltransferase (GGT), systolic and diastolic blood pressure, $\mathrm{Hb} \mathrm{Alc}$, fasting blood glucose and lipid profiles. Linear regression analysis was used to examine the associations between anthropometric measures, liver enzymes and cardiovascular risk factors.

Results: Both the liver enzyme concentrations and cardiovascular risk factors were associated with BMI or waist circumference (all $p<$ 0.001). After adjusting age, sex, and BMI or waist circumference, liver enzyme concentrations were also associated with cardiovascular risk factors (all $p<0.001$ ). The slopes of cardiovascular risk factors against AST, ALT and GGT in women were different from those in men: they were all similar in women; however, the slope against AST was highest in men.

Conclusions: There were significant associations between anthropometric measures, liver enzymes and cardiovascular risk factors in Asians. The associations between liver enzyme concentrations and cardiovascular risk factors may be different according to gender.

\section{PP08-16}

Non-alcoholic Fatty Liver Disease Patients and the Metabolic Syndrome in a Malaysian General Hospital

R.M. Koo ${ }^{1}$, S.T. $\mathrm{Kew}^{2}$, G.N. Somaweera ${ }^{3}$, S. Venugopal ${ }^{4}$

${ }^{1}$ Research and Postgraduate Department; ${ }^{2}$ Department of Internal

Medicine, International Medical University, Kuala Lumpur;

${ }^{3}$ International Medical University; ${ }^{4}$ Diagnostic Imaging Department, Tuanku Ja'afar Hospital, Seremban, Malaysia

Introduction/aims: Non-alcoholic fatty liver disease (NAFLD) refers to a clinicopathological spectrum of liver diseases ranging from frank steatosis to cirrhosis. NAFLD is closely associated with metabolic abnormalities such as obesity, type 2 diabetes mellitus (DM) and dyslipidemia-which form the components of the metabolic syndrome (MetS). We sought to determine the proportion of patients diagnosed with NAFLD who have the MetS in the setting of a general hospital in Malaysia.

Methods: This study examined 84 patients diagnosed with fatty liver on ultrasonography. All subjects had their alcohol consumption habits and medication history determined. Subjects referred for an ultrasonographic examination to specifically determine if they have NAFLD were grouped as 'NAFLD found intentionally'. Subjects found to have fatty liver incidentally and later deemed to have NAFLD were grouped as 'NAFLD found incidentally'. Subjects with NAFLD were screened for the MetS according to the modified ATP III and IDF definitions of the MetS by conducting a standard interview and appropriate anthropometric and biochemical studies.

Results: 9 subjects had alcoholic liver disease and 5 subjects consumed steatotic drugs, leaving 70 individuals with NAFLD. Median age of the cohort was 49 years (range 12-74 years) and 60\% were males. Using the modified ATP III and IDF definitions, the overall prevalence of MetS in all NAFLD subjects was $88.6 \%$ and $87.1 \%$ respectively (Kappa value $0.799 \pm 0.22, p<0.001$ ). The prevalence of the MetS was more common in subjects with NAFLD found intentionally (modified ATP III: 100 vs. $78.9 \%(p<0.01)$, IDF: 93.8 vs. $81.6 \%)$. The prevalence of DM (37.5\%), Dyslipidemia $(68.8 \%)$ and Hypertension $(71.9 \%)$ were all greater in individuals with NAFLD found intentionally.

Conclusion: NAFLD is strongly associated with the MS, especially in patients who were referred for ultrasonographic examinations specifically to determine if they have NAFLD.

\section{PP08-17}

Frequency of Non Alcoholic Fatty Liver Disease (NAFLD) Among Visitors of a Hepatitis Awareness Program

A. Saeed ${ }^{1}$, Z. Abbas ${ }^{1}$, N.H. Luck ${ }^{1}$, M.A. Khan ${ }^{2}$, S.M. Hassan ${ }^{1}$, M.N. Zafar ${ }^{3}$, S.A.A. Naqvi ${ }^{4}$, S.A.-U.-H. Rizvi ${ }^{4}$

${ }^{1}$ Hepatogastroenterology; ${ }^{2}$ Hepatobiliary Surgery; ${ }^{3}$ Biochemistry Lab; ${ }^{4}$ Urology, Sindh Institute of Urology and Transplantation, Karachi, Pakistan

Background: The prevalence of non-alcoholic fatty liver disease (NAFLD) in local population is unknown. The aim of this study was to evaluate the occurrence of NAFLD in visitors of a screening camp on World Hepatitis Day.

Methods: Attendees of a hepatitis awareness program was offered screening to rule out any liver ailments. The participants were evaluated on history, BMI measurement, blood tests for HBsAg, anti$\mathrm{HCV}$, alanine aminotransferase (ALT) and abdominal ultrasound.

Results: Among 392 participants without any history of liver disease, $251(64.0 \%)$ were males and $141(36 \%)$ females, with a mean age of $36.7 \pm 14.6$ years (range 7-80 years). Eleven (2.8\%) subjects were found out to be HBsAg positive, 30 (7.7\%) were Anti HCV positive. Nineteen $(4.8 \%)$ serology negative subjects had history of regular alcohol intake leaving behind 332 subjects. Among these fatty liver was present in 70 subjects ( $17.9 \%$ of total visitors) on ultrasonography. Comparing them with rest of the serology negative alcohol negative subjects, there were more house wives $(p=0.008)$ and more diabetics $(p=0.012)$ with fatty liver. They were older with mean age $42.4 \pm 10.8$ compared to $34.6 \pm 15.9(p=0.000)$ and had higher BMI $28.6 \pm 5.2$ versus 21.8 versus $4.8(p=0.000)$. Regression analysis suggested BMI as the only significant independent risk factor $(P=0.000)$. However, Seventeen subjects with fatty liver had BMI $<25$ and only one of them was diabetic. Eleven patients (15.7\%) with fatty liver had raised ALT level and possible NASH which accounts for $2.8 \%$ (11/392) of the total visitors.

Conclusion: This analysis revealed that our general population is at risk of having NAFLD and NASH and it should be looked into even in non-obese persons.

\section{PP08-18}

A Study of the Relationship of between Cardiac Diastolic Function and Live Function in Patients with Nonalcoholic Steatosis Hepatitis J. Huang

Gastroenterology, Shenzhen Futian People’s Hospital, Shenzhen, China

Aim: To explore changes in cardiac diastolic function in patients with nonalcoholic steatosis hepatitis, NASH) by color Doppler Echocardiography and analyze the relationship between liver function and cardiac diastolic function in these patients.

Methods: 64 patients with Simple fatty liver (SFL) and 56 patients with NASH, treated at our hospital from July 2005 to June 2008 and equal number of matched healthy controls were included in this study. All participants were subjected to routine liver function tests. Meanwhile, B-mode ultrasound diagnosis of fatty liver 
and color Doppler echocardiographic evaluation of cardiac diastolic function were carried out. Diastolic cardiac dysfunction (DCD) was defined when the patient had an E/A ratio $<1$.

Results: The incidence of diastolic cardiac dysfunction in patients with NASH was significantly higher than that in normal controls (44.64 vs. 10.94 vs. $5.00 \%, P<0.01$ ). As the fatty liver disease progressed, the E/A ratio decreased gradually $(P<0.01)$. Significant differences were noted in ALT, AST, GGT and IBIL levels between normal controls and patients with SFL or NASH (both $P<0.01$ ). The patients with NASH had a significantly higher level of TBIL,IBIL and DBIL compared with SFL group $(P<0.01, P<0.05)$.In NASH patients with DCD, the levels of GGT was significantly higher than those in patients without DCD $(P<0.05)$.

Conclusion: DCD is commonly seen in patients with NASH. The levels of GGT significantly increase in NASH patients with DCD. Elevated GGT may be a novel risk factor for DCD or coronary artery disease.

\section{PP08-19}

High Prevalence of Advanced Colorectal Neoplasms in Patients with Nonalcoholic Steatohepatitis: a Prospective Study V.W.-S. Wong ${ }^{1}$, G.L.-H. Wong ${ }^{1}$, S.W.-C. Tsang ${ }^{2}$, T. Fan ${ }^{2}$, J.J.-Y. Sung ${ }^{1}$, H.L.-Y. Chan

${ }^{I}$ Department of Medicine and Therapeutics, The Chinese University of Hong Kong; ${ }^{2}$ Department of Medicine, Tseung Kwan O Hospital, Hong Kong, China

Background/aims: Nonalcoholic fatty liver disease (NAFLD) is the most common chronic liver disease worldwide and is the hepatic manifestation of metabolic syndrome. Because of shared risk factors, we aimed to study the prevalence of advanced colorectal neoplasms in NAFLD patients.

Methods: Subjects aged 40-70 years were prospectively recruited for full colonoscopic screening from 2 study cohorts:

1. community subjects who underwent metabolic and liver screening and

2. consecutive patients with biopsy-proven NAFLD.

In the former group, fatty liver was defined as hepatic triglyceride content exceeding $5 \%$ as measured by proton-magnetic resonance spectroscopy. Advanced colorectal neoplasm was defined as adenomas with villous architecture, high-grade dysplasia or size bigger than $1 \mathrm{~cm}$.

Results: The prevalence of colorectal adenomas in NAFLD subjects $(N=199)$ and controls $(N=181)$ was 34.7 and $21.5 \%$ $(P=0.005)$, prevalence of advanced neoplasms was 19.1 and $7.2 \%$ $(P=0.001)$, and prevalence of cancer was 1.0 and $0.6 \%$ $(P=1.0)$, respectively. After adjustment for age and gender, NAFLD remained independently associated with adenomas (odds ratio $1.63,95 \%$ confidence interval $1.02-2.63, P=0.043$ ) and advanced neoplasms (odds ratio $2.66,95 \%$ confidence interval 1.34-5.25, $P=0.005)$. 38 of $70(54 \%)$ advanced neoplasms were found in the right-sided colon of NAFLD subjects, compared to 5 of $17(29 \%)$ in controls $(P<0.001)$. Among patients with biopsyproven NAFLD, the prevalence of adenomas in patients with simple steatosis $(N=83)$ and nonalcoholic steatohepatitis (NASH; $N=54)$ was 20.5 and $55.6 \%(P<0.001)$, prevalence of advanced neoplasms was 13.3 and $33.3 \%(P=0.005)$, and prevalence of cancer was 1.2 and $1.9 \%(P=1.0)$, respectively.

Conclusions: NAFLD is associated with high prevalence of colorectal adenomas, which commonly involve the right-sided colon. One-third of NASH patients have advanced neoplasms. Colorectal cancer screening is strongly indicated in this high-risk group. (This study was supported by the Health and Health Services Research Fund [Reference 07080081].)
PP08-20

Clinical, Etiological and Biochemical Evaluation of Newly Detected Diabetics and IGT Patients with Elevated Serum Alanine

Aminotransferase Level

M.G. Azam, M. Rahman, A. Rahman, M. Kabir, T. Bhuiyan, N. Hoque, I.K. Datta, A.A. Mamoon

Department of Gastroenterology and Hepatology, Birdem Hospital, Dhaka, Bangladesh

Background: Among diabetics, raised alanine aminotransferase (ALT) level is frequently observed. It might be an indicator of significant liver disease.

Aims and objectives: This study was aimed to explore clinical, etiological and biochemical correlation among newly detected diabetics and IGT patients with elevated serum ALT level in Bangladesh.

Materials and methods: A total of 3,862 newly detected diabetics (93.3\%) and IGT (6.7\%) patients (male 61.7\%; age $43.1 \pm 11.1$ years) were registered at the outpatient department of BIRDEM hospital, Dhaka, Bangladesh from 1st August 2009 to 31st October 2009. Of them, $120(3.1 \%)$ subjects with raised serum ALT level $\left({ }^{3} 1.5 \times\right.$ ULN $)$ were included for this study after meeting the exclusion and inclusion criteria. Results: Mean body mass index of the study subjects was $25.5 \pm 4.0$ (mean $\pm \mathrm{SD}$ ) $\mathrm{Kg} / \mathrm{m}^{2}$. Hepatomegaly was found in $5.8 \%$ of the study subjects. Raised serum ALT level was more prevalent in male $(61.7 \%)$ than female $(38.3 \%)$ subjects $(p=0.025)$. Dyslipidaemia was found in $98.3 \%$ cases. Majority $(76.67 \%)$ of the study subjects had nonalcoholic fatty liver disease (NAFLD), 8.33\% had HBsAg, 4.17\% had anti-HCV, and 3.33\% had combined NAFLD and HBsAg and $7.5 \%$ of unknown etiology. Raised serum ALT level had a significant correlation with the age of the study subjects $(p=0.008)$. Similarly, a significant correlation was found between raised serum ALT level with metabolic syndrome $(p=0.016)$. No significant correlation was found between raised serum ALT level with nonalcoholic fatty liver disease (NAFLD) $(p=0.681)$, BMI $(p=0.891)$, waist circumference $(p=0.166)$ and waist hip ratio $(p=0.284)$.

Conclusion: Mild to moderate elevation of serum ALT level is a common scenario in type II DM. Most of the patients remain asymptomatic or had non-specific symptoms. Raised serum ALT level has significant correlation with age and metabolic syndrome. Nonalcoholic fatty liver disease (NAFLD) is the most common etiological factor.

\section{PP08-21}

Serum Osteocalcin Levels in Patients with Nonalcoholic Fatty Liver Disease: Association with Ballooning Degeneration

Y. Yilmaz ${ }^{1}$, O. Yonal ${ }^{1}$, R. Kurt ${ }^{1}$, F. Eren ${ }^{2}$, Y.O. Alahdab ${ }^{1}$

${ }^{1}$ Department of Gastroenterology, Marmara University School of

Medicine; ${ }^{2}$ Institute of Gastroenterology, Marmara University,

Istanbul, Turkey

Introduction: Osteocalcin ( $\mathrm{OCN})$, the most abundant non-collagenous protein found in bone, may display several metabolic actions.

Aim: Our aim was to examine relations of serum osteocalcin $(\mathrm{OCN})$ levels to clinical, biochemical, and histological characteristics of patients with biopsy-proven nonalcoholic fatty liver disease (NAFLD). Methods: We carried out a case-control study including 99 patients with biopsy-proven NAFLD and 75 age- and sex-matched controls. Concentrations of OCN were measured in aprotinin-treated serum samples using a solid-phase enzyme amplified sensitivity immunoassay. Results: Serum OCN levels were significantly lower in patients with NAFLD than in healthy controls. In patients with NAFLD, serum OCN levels were inversely associated with ALT $(r=-0.36, P<$ $0.001)$, AST $(r=-0.39, P<0.001)$, HOMA-IR $(r=-0.30, P<0.01)$ and the degree of hepatocyte ballooning $(r=-0.20, P<0.05)$. Serum OCN was the only independent predictor of the degree of hepatocyte ballooning in NAFLD patients $(\beta=-0.24 ; t=-2.146, P<0.05)$. 
Conclusions: Compared with controls, NAFLD patients have a decrease in serum OCN concentrations, which is closely associated with serum transaminases and the extent of hepatocyte ballooning.

\section{PP08-22}

The Presence of Liver Steatosis in Chronic Hepatitis B and its Association with Disease Severity

L.A. Lesmana ${ }^{1,2}$, C.R.A. Lesmana ${ }^{1,2}$, L.S. Pakasi ${ }^{2}$, E. Krisnuhoni ${ }^{3}$

${ }^{1}$ Internal Medicine, Division of Hepatology, University of Indonesia;

${ }^{2}$ Internal Medicine, Digestive Disease Centre, Medistra Hospital;

${ }^{3}$ Anatomy Pathology, University of Indonesia, Jakarta, Indonesia

Background: Liver steatosis is a common finding in patients with chronic hepatitis $\mathrm{B}(\mathrm{CHB})$. However, its impact on the natural course of $\mathrm{CHB}$ is less recognized compared to those known in chronic hepatitis $\mathrm{C}$ patients.

Objectives: To know the prevalence of liver steatosis in CHB patients and to evaluate whether its presence was associated with a more progressive disease.

Patients and methods: A cross-sectional study was carried out in Medistra Hospital, Jakarta on CHB patients between 2007 and 2009. Data on patients' demography, anthropometry, liver function test and hepatitis B serology were carefully collected. All patients underwent a liver biopsy using a Menghini needle. The association between liver steatosis and disease severity (serum alanine aminotransferase [ALT], serum HBV-DNA, hepatitis B e antigen (HbeAg) status, grading of necroinflammation and staging of liver fibrosis according to Metavir score) was analyzed.

Results: There were 155 patients included in this study; $89(57.4 \%)$ among them were men. Patients' mean age was $39.9 \pm 10.69$ years, ranging from 16 to 70 years. The prevalence of liver steatosis was $26.5 \%$. Patients with liver steatosis had significantly higher body weight $(68.1 \mathrm{vs}$. $60.1 \mathrm{~kg} ; p<0,001)$, body mass index $\left(24.5 \pm 3.4 \mathrm{vs} .22 .7 \pm 3.22 \mathrm{~kg} / \mathrm{m}^{2}\right.$, $p=0.003$ ), and waist circumference ( 85.9 vs. $79.3 \mathrm{~cm} ; p=0.005)$. No association between liver steatosis and HBeAg status $(p=0.702)$. The mean serum ALT $(52.1 \pm 26.97$ vs. $70.3 \pm 103.59 \mathrm{U} / \mathrm{L} ; p=0.275)$ and $\log$ HBV-DNA (6.15 vs. $6.18 ; p=0.932$ ) levels were not different between patients with and without liver steatosis. The presence of moderate-to-severe liver fibrosis (68.3 vs. $66.7 \% ; p=0.849)$ and necroinflammation ( 68.3 vs. $64 \% ; p=0.624)$ were not different between patients with and without liver steatosis.

Conclusions: Liver steatosis was found in $26.5 \%$ of chronic hepatitis $\mathrm{B}$ patients. Its presence was mostly associated with central obesity. Steatosis in chronic hepatitis B did not related to a more progressive disease.

\section{PP08-23}

Clinical Study on Old Patients with Non-Alcoholic Fatty Liver Disease L. Jian

Department of Gastroenterology, The Second Affiliated Hospital, Zhengzhou University, Henan Province, Zhengzhou, China

Objective: To investigate the etiology and risk factors of non-alcoholic fatty liver disease (NAFLD) in older patient.

Methods: The biochemical marker of FPG (fasting plasma glucose), TG (triglycerides), TC (total cholesterol), alanine aminotransferase (ALT), aspartate aminotransferase (AST) and the clinical risk factors of Body Mass Index (BMI), obesity, hypertension disease, coronary heart disease and diabetes was detected in 65 cases of NAFLD group and 58 case of healthy control group with old patient.

Results: Comparisons of the control group, the patients with NAFLD group had significantly more risk factors (BMI, hypertension, obesity, diabetes, coronary heart disease), $P<0.05$, and the level of FPG, TG, TC, ALT and AST also in NAFLD group was significantly more higher, $P<0.05$.

Conclusion: The obesity, hypertension disease, coronary heart disease and diabetes and the higher level of hyperglycemias, hyperglycemias are the major risk factors in old patient of NAFLD and have closed relationship.

\section{PP08-24}

Relationship between Mitochondrial DNA Copy Number, Metabolic Abnormalities and Hepatic Steatosis

D.W. Jun ${ }^{1}$, T.Y. Kim ${ }^{1}$, J.H. Sohn ${ }^{1}$, W.K. $\mathrm{Cho}^{2}$, H.J. Kwon ${ }^{2}$, H.J. Jeon ${ }^{1}$

${ }^{1}$ Hanyang University; ${ }^{2}$ Celltrion Pharma, Seoul, Republic of Korea

Background: Insulin resistance plays a central role in fatty liver, a part of the metabolic syndrome. This study examined the relationship between fatty liver, metabolic abnormalites and mitochondrial DNA (mtDNA) copy number in peripheral blood that is correlated with diabetes or metabolic markers.

Method: Fatty liver was assessed by questionnaire on alcohol consumption and abdominal ultrasonography. MtDNA copy number in peripheral leukocytes was measured by a real-time quantitative polymerase chain reaction [PCR].

Results: Among 445 subjects, 148 subjects had hepatic steatosis and 297 were shown normal liver echogenecity. mtDNA copy number was significantly lower in fatty liver group in comparison with that of normal finding group (6.08 vs. 8.30, $p<0.001)$. MtDNA copy number of alcoholic fatty liver patients were lower than in the case of non-alcoholic fatty liver disease $(5.28$ vs. $6.63, p<0.05)$. In case of increased liver chemistry ( $>40 \mathrm{IU} / \mathrm{L})$ the mtDNA copy number in peripheral blood was lower than normal liver chemistry group. MtDNA copy number was inversely correlated with alanine aminotransferase $(r=-0.168, p<0.001)$, aspartate aminotransferase $(r=-0.114, p<0.001)$, gamma-glutamyltransferase $(r=0.11, p<$ $0.001)$, body mass index $(r=-0.127, p=0.007)$, waist circumference $(r=-0.19, p=0.021)$, diastolic blood pressure $(r=-0.17$, $p=0.024)$, and free fatty acid $(r=-0.114, p=0.021)$.

Conclusion: MtDNA copy number in peripheral leukocytes was associated with fatty liver and insulin resistance related factors.

\section{PP08-25}

Lifestyle Modification: the Vital Approach of Steatohepatitis (Case Report)

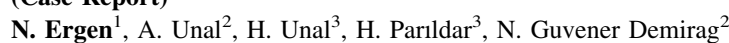

${ }^{1}$ Exercise Physiology; ${ }^{2}$ Endocrinology; ${ }^{3}$ Baskent University, Istanbul, Turkey

A 28-year-old overweight man referred to our hospital for routine medical examination and elevated liver transaminases and hypertriglyceridemia are found in his laboratory tests. After excluding the viral toxic and autoimmune causes that may underly elevated liver enzymes, grade 2 hepatosteatosis was diagnosed, confirmed by the abdominal ultrasonography. When the 75-g oral glucose tolerance test was performed to reveal insulin resistance (Plasma levels of glucose at $0,30,60$ and $120 \mathrm{~min}$ were: $80,159,176$ and $141 \mathrm{mg} / \mathrm{dl}$. Plasma levels of insülin at $0,30,60$ and 120 min were: 11.68, 98.1, 201 and $111.3 \mu \mathrm{I} / \mathrm{ml}$, respectively) impaired glucose tolerance (IGT) was diagnosed. The patient denied the recommended medical therapy and was referred to the exercise physiology department for lifestyle modification. After anthropometric analysis was performed, his resting metabolism rate (RMR) was measured. As RMR was found within the normal range $(1,894 \mathrm{kcal} /$ day $)$, he was treated with medical diet plus exercise treatment as lifestyle modification between March 2010 and August 2010. İn this 6 months' period, he lost $12.2 \mathrm{~kg}$ of body weight and $8 \mathrm{~kg}$ of his body fat mass. During to follow up; ALT level was decreased remarkably (from 278 to $46 \mathrm{U} / \mathrm{L}$ ). The laboratory results of the initial and the final biochemical and anthropometric values are shown in Tables 1 and 2 .

Conclusion: Lifestyle modification with the medical diet and exercise program is an indispensable method of steatohepatitis. Strictly, adherence to this treatment may not require any pharmacological treatment as in our patient. 


\section{PP08-26}

A Pilot Study on the Effect of Resistance Exercise Training in Nonalcoholic Steatohepatitis (NASH) Patients

C.W. Chan ${ }^{1}$, N.C. Yeung ${ }^{2}$, S.P. Cheung ${ }^{2}$, W.K. Wong ${ }^{3}$, C.W.K. Wong ${ }^{4}$, K.L. $\mathrm{Ng}^{2}$, K.H. Chan ${ }^{1}$, Y.M. Choi ${ }^{3}$, W.H. Chow ${ }^{1}$, W.K. Kwan ${ }^{1}$

${ }^{I}$ Department of Medicine, Yan Chai Hospital, Hong Kong;

${ }^{2}$ Department of Physiotherapy; ${ }^{3}$ Department of Dietetics;

${ }^{4}$ Department of Radiology, Yan Chai Hospital, China, Hong Kong S.A.R.

Introduction: There exists scanty data on the effect of resistance exercise on NASH patients. We performed a pilot study to evaluate the effect of supervised resistance exercise in Hong Kong asian patients.

Methods: NASH patients with alanine amino-transferase (ALT) $\geq 1.5$ times upper limit of normal were invited to participate a 12-week lifestyle program jointly designed by dietitians, physiotherapists and hepatologists in Yan Chai Hospital. All participants were advised to follow low sugar and weight loss diet (total daily energy intake $\sim 25 \mathrm{kcal} / \mathrm{kg} / \mathrm{day}$, low fat $<30 \%$ ). Resistance exercise group received additional supervised resistance exercise training (70-80\% 1RM maximum for 3 sets/exercise, $8-10$ exercises/day, 2-3 nonconsecutive days/week). Compliance was monitored with diet diary, physiotherapy record and international physical activity questionnaires.

Results: From September 2009 to February 2010, eleven NASH patients (10 male, 1 female) were recruited, mean age 42 (SD 8.76). Six participants received diet plus resistance exercise (RE) program, five were on diet alone. At the end of treatment program, significant improvements in health parameters were observed in both groups (Table). Diet plus resistance exercise program produces greater reduction in body weight ( $\mathrm{RE}-3.2 \mathrm{~kg}$ vs. diet $-2.7 \mathrm{~kg}$ ), percentage body fat (RE $-3.85 \%$ vs. diet $-1.23 \%$ ), abdominal circumference (RE $-4.63 \mathrm{~cm}$ vs. diet $-2.17 \mathrm{~cm}$ ), ALT (RE $-51 \mathrm{IU} / \mathrm{L}$ vs. diet $31 \mathrm{IU} / \mathrm{L})$ than diet alone.

Conclusion: In this pilot study, 12 weeks diet plus resistance exercise produces greater improvement in liver function and health parameters in NASH patients than diet alone. Further studies are needed to evaluate the treatment efficacy of resistance exercise in NASH patients.

\begin{tabular}{|c|c|c|c|c|}
\hline Parameters & Baseline & $\begin{array}{l}\text { 2nd of } \\
\text { Program } \\
\text { [mean (SD)] }\end{array}$ & $\begin{array}{l}\text { Mean } \\
\text { change } \\
(\mathrm{SBM})\end{array}$ & $P$ value \\
\hline Body weight (kg) & $80.3(10.1)$ & $76.3(10.9)$ & $-295(0.54)$ & $<0.001$ \\
\hline Body mass index & $28.4(34)$ & $26.8(3.4)$ & $-1.03(0.18)$ & $<0.001$ \\
\hline $\begin{array}{l}\text { Abdominal } \\
\quad \text { circumference }(\mathrm{cm})\end{array}$ & $98.8(9.9)$ & $94.0(9.8)$ & $-.36(0.52)$ & $<0.001$ \\
\hline Percentage fat $(\%)$ & $32.4(5.5)$ & $29.8(5.4)$ & $-2.2(0.87)$ & 0.035 \\
\hline ALT (IU/L) & 90 (27) & $50(28)$ & $-43(5)$ & $<0.001$ \\
\hline AST (IU/L) & $47(15)$ & $30(9.9)$ & $-19(11)$ & $<0.001$ \\
\hline GGT (IU/L) & $67(52)$ & $46.4(30.7)$ & $-23(9)$ & 0.037 \\
\hline $\begin{array}{l}\text { Total cholesterol } \\
\quad(\mathrm{mmol} / \mathrm{L})\end{array}$ & $5.2(1.2)$ & $4.6(0.3)$ & $-0.4(0.5)$ & 0.446 \\
\hline $\begin{array}{l}\text { HDL-cholesteroll } \\
\quad(\mathrm{mmol} / \mathrm{L})\end{array}$ & $0.89(0.14)$ & $0.91(0.11)$ & $-0.08(0.06)$ & 0.192 \\
\hline $\begin{array}{l}\text { IDL-cholesterol } \\
(\mathrm{mmol} / \mathrm{L})\end{array}$ & $3.60(1.04)$ & $3.10(0.89)$ & $-0.39(0.42)$ & 0.398 \\
\hline fasting sugar $(\mathrm{g} / \mathrm{L})$ & $5.95(0) 95)$ & $5.92(1.53)$ & $-0.10(0.39)$ & 0.808 \\
\hline Insulin $(\mathrm{uU} / \mathrm{ml})$ & $14(7.0)$ & $11.0(7.9)$ & $-0.85(1.63)$ & 0.639 \\
\hline HOMA & $3.90(1.99)$ & $3.04(1.08)$ & $-0.36(0.34)$ & 0.379 \\
\hline
\end{tabular}

PP08-27

Non-alcoholic Fatty Liver Disease is Closely Associated with Sub-clinical Inflammation: a Case-Control Study on Asian Indians in North India

P. Nigam ${ }^{1,2}$, S.P. Bhatt ${ }^{3}$, M. Vaidya ${ }^{2}$, J. Dasgupta ${ }^{1}$, D.S. Chadha ${ }^{4}$, A. Misra ${ }^{1,5}$ ${ }^{I}$ Center for Diabetes, Obesity, and Cholesterol Disorders $(C-D O C)$, National Diabetes Obesity and Cholesterol Foundation (N-DOC), New Delhi; ${ }^{2}$ Food and Nutrition, Govt $M$ H College of Science and Home Science and Science for Women, Jabalpur; ${ }^{3}$ Medicine, All India Institute of Medical Sciences, New Delhi; ${ }^{4}$ Cardiology, Command Hopita, Banglore; ${ }^{5}$ Diabetes and Metabolic Diseases, Fortis Hospital, Vasant Kunj, New Delhi, India

Objectives: Association between sub-clinical inflammation and nonalcoholic fatty liver disease (NAFLD) has not been studied in Asian Indians. In this case-control study, we aimed to analyse association of NAFLD with the metabolic syndrome, sub-clinical inflammation and metabolic profile in Asian Indians in north India.

Methods: One hundred and twenty cases of NAFLD were compared to 152 healthy controls without NAFLD. Anthropometric profile, high-sensitivity C-reactive protein (hs-CRP), metabolic profile [fasting blood glucose (FBG), lipid profile] and hepatic function tests [alanine aminotransferase and aspartate aminotransferase] were recorded.

Results: Metabolic parameters (FBG, total cholesterol, serum triglycerides), hs-CRP and prevalence of the metabolic syndrome were higher in cases as compared to controls ( $p$ value $<0.05$ for all). The median (range) of hs-CRP (mg/L) for cases [2.6(0.2-13.4)] were significantly higher than in controls [1.4(0.03-11.4)]. Similarly, higher values of hs-CRP were obtained when subgroups of cases with obesity, abdominal obesity and the metabolic syndrome were compared to controls $[2.75(0.03-14.3)$ versus $1.52 \quad(0.04-14.3)$, $p=0.0010 ; 2.8(0.03-14.3)$ vs. $1.5(0.06-14.3), p=0.0014$ and 2.7 (0.5-14.3) vs. $1.6(0.06-8.5), p=0.0013$, respectively. On multivariate logistic regression analysis BMI $(p=0.00)$, WC $(p=0.00)$, HC $(p=0.00)$, FBG $(p=0.00)$, TC $(p=0.00)$, TG $(p=0.00)$, LDL-c $(p=0.00)$, AST $(p=0.01)$, ALT $(p=0.00)$ and hs-CRP $(p=0.00)$ were significantly and independently associated with NAFLD. After adjustment with aforementioned variables, the association between high hs-CRP and NAFLD remained large and statistically significant [adjusted OR 1.17, 95\% confidence interval (CI) 1.05-1.29].

Conclusions: In this cohort of Asian Indians in North India, presence of NAFLD showed independent relationships with sub-clinical inflammation.

\section{PP08-28}

PPAR- $\gamma$ C161T Polymorphism Affected Prevalence but Not Incidence of NAFLD

Y.-Y. Li, Y.-J. Zhou, Y.-Q. Nie, C.-Y. Cao

Department of Gastroenterology, Guangzhou First Municipal

People's Hospital, Guangzhou Medical College, Guangzhou, China

Objectives: We investigated the effect of PPAR- $\gamma$ C161T single nucleotide polymorphism (SNP) on the prevalence and incidence of nonalcoholic fatty liver diseases (NAFLD) in Chinese people to determine the genetic influence on NAFLD phenotype.

Methods: Population based surveys were performed in 2005 and 2009 in same group of subjects from 6 areas of Guangdong Province, southern China (median follow-up 4 years). Each subject received questionnaires, anthropometric measurements, biochemical tests and abdominal ultrasonography at baseline and endpoint. PCR-RFLP was applied to detect PPAR $\gamma$ C161T single nucleotide polymorphisms (SNP) from peripheral blood leukocytes. 
Results: In 2005 study of 3543 subjects, 530 (15.0\%) were diagnosed as having NAFLD, among which 96 cases of NAFLD (age 18-70) and 96 matched cases of non-NAFLD were randomly selected for SNP study. The genotype distributions of CC, CT and TT 55.2, 39.6 and $5.2 \%$ in NAFLD group were significantly different from those of $74.0,25.0$ and $1.0 \%$ in controls $(p=0.015)$. Allele's frequencies of C and $\mathrm{T} 75.0$ and $25.0 \%$ in NAFLD group also differed significantly from those of 86.5 and $13.5 \%$ in controls $(p=0.004)$. We followedup 233 subjects (age 22-74) who had no NAFLD in 2005, but developed NAFLD in 2009 and found the genotype of CC, CT and TT were $52.4,40.3,7.3 \%$ in NAFLD group, compared to $61.2,26.5$ and $12.3 \%$ in controls of 297 subjects, who remained non-NAFLD from baseline to endpoint. The difference was not significant $(p>0.05)$. Allele's frequencies of $\mathrm{C}$ and $\mathrm{T} 72.5$ and $27.5 \%$ also did not differ from those 74.5 and $25.5 \%$ in controls.

Conclusion: The SNP of PPAR- $\gamma$ C161T significantly affected the prevalence but not the incidence of NAFLD in Chinese people, suggesting that the gene influence on NAFLD susceptibility might occur in childhood.

\section{PP08-29}

Adiponectin-45 Polymorphism Affected Prevalence but Not Incidence of NAFLD

Y.-Y. Li, Y.-J. Zhou, Y.-Q. Nie, C.-M. Huang

Department of Gastroenterology, Guangzhou First Municipal

People's Hospital, Guangzhou Medical College, Guangzhou, China

Objectives: We investigated the effect of adiponectin-45 single nucleotide polymorphism (SNP) on the prevalence and incidence of nonalcoholic fatty liver diseases (NAFLD) in Chinese people to determine the genetic influence on NAFLD phenotype.

Methods: Population based surveys were performed in 2005 and 2009 in same group of subjects from 6 areas of Guangdong Province, southern China (median follow-up 4 years). Each subject received questionnaires, anthropometric measurements, biochemical tests and abdominal ultrasonography at baseline and endpoint. PCR-RFLP was applied to detect adiponectin SNP from peripheral blood leukocytes. Results: In 2005 study of 3543 subjects, 530 (15.0\%) were diagnosed as having NAFLD, among which 106 cases of NAFLD (age 18-70) and 106 matched cases of non-NAFLD were randomly selected for SNP study. The genotype distributions of TT, TG and GG 64.2, 23.6 and $12.2 \%$ in NAFLD group were significantly different from those of $54.7,38.7$ and $6.6 \%$ in controls $(p=0.04)$. We followed-up 185 subjects (age 22-74) who had no NAFLD in 2005, but developed NAFLD in 2009 and found the genotype of TT, TG and GG were 47.6, 45.4, 7.0\% in NAFLD development group, compared to 50.9, 42.3 and $6.8 \%$ in controls of 322 subjects, who remained non-NAFLD from baseline to endpoint. The difference was not significant $(p>0.05)$.

Conclusion: The SNP of adiponectin-45 significantly affected the prevalence but not the incidence of NAFLD in Chinese people, suggesting that the gene influence in NAFLD might occur in childhood.

\section{PP08-30}

Homocysteine Levels in Patients with Liver Cirrhosis, Non-alcoholic Steatohepatitis (Nash) and Hepatosteatosis

A. Varma ${ }^{1}$, K.J.B.S. Gaur ${ }^{2}$, S.S. Kansal ${ }^{3}$

${ }^{1}$ Internal Medicine, SGR Institute of Medical and Health Sciences, Dehradun; ${ }^{2}$ Medicine, Himalayan Institute of Medical Sciences, Jolly Grant Dehradun; ${ }^{3}$ Medicine, Himalayan Institute of Medical

Sciences, Dehradun, India

Objectives: Homocysteine is an atherogenic risk factor which has been proposed to be involved in hepatic fibrogenesis. The study was designed with the aim to evaluate the effectiveness of serum homocysteine levels as a marker of liver cirrhosis, non-alcoholic steatohepatitis and hepatic steatosis
Methods: The study involved patients of liver cirrhosis, non-alcoholic steatohepatitis and hepatosteatosis which were diagnosed using ultrasonography. We evaluated the serum homocysteine levels and attempted to establish the possible correlation with these forms of liver injury.

Results: The mean serum homoscysteine levels in patients with liver cirrhosis were $23.36 \pm 6.6$; steatohepatitis $11.21 \pm 4.39$ and hepatosteatosis $6.86 \pm 2.02$. Control $=6.71 \pm 1.66$.

Conclusion: Homocystein levels were found to be significantly higher in cirrhosis and non alcoholic steatohepatitis. It, along with ultrasonography may be a good non-invasive fibromarker which can stage the degree of liver fibrosis, monitor the impact of therapy, is readily available, reproducible and a useful tool for early diagnosis of cirrhosis and steatohepatitis.

\section{PP08-31}

Liver Stiffness Measurement and the Pediatric NAFLD Fibrosis Index in Thai Obese Children

R. Lorchatchawankul, V. Chongsrisawat, K. Setrkraising, P. Sintusek, A. Wongteerasut, Y. Poovorawan Pediatrics, Chulalongkorn University, Bangkok, Thailand

Background: With the recent epidemic of childhood obesity, nonalcoholic fatty liver disease (NAFLD) has become an emerging problem and a common cause of chronic liver disease in children. NAFLD encompasses a spectrum of disease from simple steatosis, non-alcoholic steatohepatitis (NASH), fibrosis and ultimately to cirrhosis. This study aimed to correlate liver stiffness measurement (LSM) and the pediatric NAFLD fibrosis index (PNFI) with serum alanine transaminase (ALT) level and body mass index (BMI) in Thai obese children.

Methods: Between September 2009 and October 2010, 45 obese children $\left(33 \mathrm{M}\right.$, aged $11.8 \pm 3.3$ years, BMI $\left.32.2 \pm 6.6 \mathrm{~kg} / \mathrm{m}^{2}\right)$ and 45 normal controls $(18 \mathrm{M}$, aged $10.8 \pm 3.4$ years, BMI $19.2 \pm 4.0$ $\mathrm{kg} / \mathrm{m}^{2}$ ) were enrolled into the study. All obese children had fatty liver diagnosed by ultrasonography. The PNFI was calculated based on age, waist circumference, and level of triglyceride. All subjects were evaluated for LSM by transient elastography (FibroScan). The relationship of LSM and PNFI with serum ALT level and BMI was analyzed with Spearman correlation coefficients.

Results: Obese children had significantly higher LSM score than normal controls $(7.43 \pm 2.89$ vs. $4.71 \pm 1.08 \mathrm{kPa}, p<0.001)$. The mean \pm SD of PNFI in obese children was $8.81 \pm 1.89$. The PNFI was correlated with BMI $(r=0.46, p=0.001)$. Abnormal ALT level $($ ALT $>40 \mathrm{U} / \mathrm{L})$ was detected in 26 of 45 obese children. LSM was correlated with serum ALT level $(r=0.58, p<0.001)$. Whereas the PNFI was not correlated with serum ALT level $(r=0.23, p=0.14)$. No correlation was found between LSM and PNFI in obese children. Conclusions: Liver stiffness measurement is a simple noninvasive tool for monitoring of chronic liver disease. It is feasible in obese children and is related to hepatic inflammation. This study shows that PNFI, a good predictor of liver fibrosis in children, is not related to hepatic inflammation.

\section{PP08-32}

Natural History of NAFLD in Population: a Prospective Cohort Study Y.-J. Zhou, Y.-Y. Li, C.-M. Huang, Y.-Q. Nie

Department of Gastroenterology, Guangzhou First Municipal People's Hospital, Guangzhou Medical College, Guangzhou, China

Objective: The aim of the present study was to investigate the incidence and natural course of nonalcoholic fatty liver disease (NAFLD) in population in southern China.

Methods: On the base of our previously epidemiological survey in 2005, we followed-up 3,545 cases for median 4 years. Among them, 624 were willing to participate in re-survey group. Interview, physical examinations, biochemical tests and ultrasonography were performed 
again for each subject. NAFLD diagnosis was based on ultrasonographic findings with combination to medical history and laboratory. Survey for survival and causes of death was applied to all 3,543 cases, among which 219 (6.2\%) dropped out, and the remaining 3,324 were recruited into mortality data group. The results were obtained with analyzing the data from the two groups.

Results: In re-survey group of 624 cases, there were 117 NAFLD at baseline, among which 51 cases $(43.6 \%)$ remained unchanged; 26 (22.2\%) became worse and 40 (34.2\%) improved. Among 507 cases of non-NAFLD at baseline, 185 (36.5\%) developed NAFLD at endpoint. The standard incidence was $4.9 \%$ per year. (male $5.1 \%$ vs. female $4.7 \%, P<0.05$; urban $43.1 \%$ vs. rural areas $27.4 \%, P<0.05)$. In mortality data group, 4 in 467 NAFLD cases $(8.6 \%$ ) died of cardiocerebral vascular diseases compared with 22 in 2857 non-NAFLD cases $(7.7 \%$ ) died of cardio-cerebral vascular diseases and malignancy of other organs. There was no significant difference between these two subgroups. $(P>0.05)$. Age and several variables related with metabolic syndrome (MS) were risk factors for NAFLD progression.

Conclusion: The incidence of NAFLD in southern China was high. NAFLD in the population had a comparatively benign prognosis. Some variables related with metabolic syndrome (MS) were risk factors for NAFLD progression.

\section{PP08-33}

Survey on Relation of NAFLD to Metabolic Syndrome in Elderly

Y.-J. Zhou, Y.-Y. Li, C.-Y. Cao, Y.-Q. Nie

Department of Gastroenterology, Guangzhou First Municipal

People's Hospital,Guangzhou Medical College, Guangzhou, China

Objective: To investigate the relation of nonalcoholic fatty liver disease (NAFLD) to metabolic syndrome (MS) in Chinese elderly.

Methods: We followed-up 1,149 Chinese elderly (69.5 \pm 12.8 years) undergoing routine annually physical check-up at this hospital from 2005 to 2008 (median follow-up 4 years). Interview, physical examinations, biochemical tests and ultrasonography were performed in each subject for every year. NAFLD diagnosis was based on ultrasonographic findings with combination with medical history and laboratory tests.

Results: The standard prevalence of NAFLD at endpoint (22.7\%) was significantly higher than that $(16.6 \%)$ at baseline $(P<0.01)$. However, the prevalence of MS at endpoint (17.2\%) was significantly lower than that $23.5 \%$ at baseline $(P<0.01)$. The Non-MS subjects who had NAFLD at baseline were more likely to develop MS at endpoint compared to those without NAFLD (19.0 vs. $4.4 \%, P<$ 0.01 ), and the Non- NAFLD subjects who had MS at baseline were also more likely to develop NAFLD at endpoint compared to those without MS (25.6 vs. $8.1 \%$ ) with a RR value of 3.592 .

Conclusion: The natural course of NAFLD and MS might not be necessarily parallel in Chinese elderly, although NAFLD and MS were closely related to each other.

\section{PP08-34}

Influence of PPAR- $\gamma$ Genetic Polymorphisms Three Positions on NAFLD Susceptibility

Y.-Y. Li, Y.-J. Zhou, Y.-Q. Nie, C.-Y. Cao

Department of Gastroenterology, Guangzhou First Municipal

People's Hospital, Guangzhou Medical College, Guangzhou, China

Objective: To investigate the influence of three positions of genetic polymorphisms of peroxisome proliferator-activated receptors- $\gamma$ (PPAR- $\gamma$ ) on non-alcoholic fatty liver disease (NAFLD) susceptibility in Chinese people.

Methods: Population based survey was performed in 6 areas of Guangdong Province, southern China. Each subject received questionnaires, anthropometric measurements, biochemical tests and abdominal ultrasonography. NAFLD subjects and matched non-NAFLD people were randomly selected for single nucleotide polymorphisms (SNP) study. PCR-RFLP was applied to detect three positions of PPAR- $\gamma$ SNP from peripheral blood leukocytes.

Result: The proportions of subjects who had different genotypes in three SNP positions of PPAR- $\gamma$ gene in NAFLD group and controls were showed in Table 1.

Table 1 Genotypes and allelic frequencies of three positions SNPs of PPAR- $\gamma$ gene

\begin{tabular}{lllllllll}
\hline & $\begin{array}{l}\text { Genotype } \\
(\%)\end{array}$ & & & & $\begin{array}{l}\text { Allele's } \\
\text { frequency(\%) }\end{array}$ & P \\
\hline C161T & $\mathrm{n}$ & CC & CT & TT & C & T & $<0.05$ \\
NAFLD & 96 & $55.2 \%$ & $39.6 \%$ & $5.2 \%$ & $75.01 \%$ & $25.0 \%$ & \\
Controls & 96 & $74.0 \%$ & $25.0 \%$ & $1.0 \%$ & $86.5 \%$ & $13.5 \%$ & \\
Pro12Ala & $\mathrm{n}$ & CC & CG & GG & C & G & $>0.05$ \\
NAFLD & 317 & $95.0 \%$ & $5.0 \%$ & 0 & $97.5 \%$ & $2.5 \%$ & \\
Controls & 379 & $93.7 \%$ & $6.3 \%$ & 0 & $96.5 \%$ & $3.2 \%$ & \\
C681G & $\mathrm{n}$ & CC & CG & GG & C & G & $>0.05$ \\
NAFLD & 317 & $37.9 \%$ & $45.4 \%$ & $16.7 \%$ & $60.6 \%$ & $39.4 \%$ & \\
Controls & 379 & $44.3 \%$ & $44.3 \%$ & $11.3 \%$ & $66.5 \%$ & $33.5 \%$ & \\
\hline
\end{tabular}

Conclusion: SNP in position of C161G of PPAR- $\gamma$, but not positions of Pro12Ala and C681G was associated with NAFLD susceptibility.

\section{PP08-35}

Analysis of Clinical Features and Prognostic Factors in Patients with Nonalcoholic Fatty Liver Disease

Y.T. Yao, W.H. Sha

Guangdong General Hospital, Guangzhou, China

Objective: The aims of this study were to evaluate the clinical features and prognostic factors of inpatients with nonalcoholic fatty liver disease (NAFLD).

Methods: Clinical datas of 4,437 inpatients with NAFLD in Guangdong Provincial hospital were collected. The distributions of concomitant diseases, causes of admission and risk factors of NAFLD related liver injury and death were analyzed.

Results: Major admission causes of NAFLD were non-diabetic related angiopathy $(34.1 \%)$, diabetes-related complications $(24.0 \%)$, liver injury (7.8\%), biliary-pancreatic diseases (4.8\%). Common concomitant diseases of NAFLD were hypertension (42.0\%), diabetes mellitus $(32.4 \%)$, cerebrovacular disease $(26.3 \%)$, coronary heart disease $(16.6 \%)$, cholelithiasis $(11.2 \%)$ and acute pancreatitis $(6.3 \%)$. The distributions of NAFLD concomitant diseases were different in different age groups. The peak age of diabetes mellitus, hypertension and coronary heart disease in patients with NAFLD were from 60 to 70 years old. Whereas the cerebrovascular disease, cholelithiasis and pancreatitis were from 70 to 80 years old and $<40$ years old, repectively. The risk factors of liver damage were pancreatitis (OR 3.0), biliary infection (OR 2.6), diabetic peripheral vascular complication (OR 1.7) and age (OR 0.8), all $p<0.01$. The risk factors of death were diabetic peripheral vascular complication (OR 29.5), cardiovascular events (OR 24.6), tumor (OR 9.8), diabetes-related infection (OR 4.7) and age (OR 1.7), all $p<0.01$.

Conclusion: The clinical manifestations and prognosis of NAFLD are closely related to age and its concomitant diseases. Age should be paid more attention when interventions are made. 


\section{PP08-36}

Body Mass Composition in Kazakh Patients with Non-alcoholic Fatty Liver Disease

K.P. Oshakbayev ${ }^{1}$, E.A. Izatullayev ${ }^{1}$, A.V. Nersesov ${ }^{1}$, D.A. Kaibullayeva ${ }^{1}$, B.S. Ilyassova ${ }^{1}$, G.B. Isakova ${ }^{2}$, M.G. Kurbanova ${ }^{2}$, Hepatology

${ }^{1}$ Gastroenterology and Hepatology; ${ }^{2}$ Therapy Department, Scientific and Research Institute of Cardiology and Internal Diseases, Almaty, Kazakhstan

Background: Non-alcoholic fatty liver disease (NAFLD) is associated with overweight.

Aim: To study the body mass composition in patients with NAFLD. Methods: Study design was randomized, clinical, comparative, controlled. 32 adults people ( 17 females) were included. The sample was divided on two groups adjusted by age, sex: 32 patients with NAFLD (patients group), and 20 healthy (controls). Body composition was evaluated by professional analyzer «Tanita SC-330» (Japan).

Results: In patients group: BMI was $30.0 \pm 0.8 \mathrm{~kg} / \mathrm{m}^{2}$ (compared $24.2 \pm 1.3$ in controls; $ð=0.0003)$, fat mass was $34.1 \pm 1.1$ $(20.3 \pm 2.6$ in controls; $ð<0.0001)$, muscle mass was $62.6 \pm 1.1$ (in controls $75.7 \pm 2.5$; $ð<0.0001$ ), TBW was $46.3 \pm 0.7$ (in controls was $56.2 \pm 1.9$; $ð<0.0001$ ), bone mass was $3.3 \pm 0.1$ (in controls $4.1 \pm 0.1 ; \partial<0.0001$ ), metabolic age was $52.0 \pm 1.2$ (in controls $42.4 \pm 1.6$; $\partial<0.0001$ ), BMR was $1628.0 \pm 56.0$ (in controls $1419.9 \pm 49.3 ; ð=0.004)$. Raised total weight result more in increasing of fat mass $\left(r_{1}=0.69 ; \partial<0.01\right)$, rather than muscle mass $\left(r_{2}=0.57 ;\right.$ б $\left.<0.01\right)$ and TBW $\left(r_{3}=0.31 ; ð<0.01\right)$.

There was revealed the inverse regression dependence between fat mass and muscle mass $(y=99.5-1.05 \times x, r=-0.99 ; p<0.0001)$, and TBW $(y=95.3-1.31 \times x, r=-0.98 ; p<0.0001)$. The more fat mass the less both muscle mass and TBW.

Raised fat mass result in direct increase of BMR $(r=0.42$; $p=0.005)$, of metabolic age $(r=0.67 ; ð<0.0001)$, and of bioimpedance $(r=0.14 ; p=0.3)$. BMR raised when fat mass increased up to $>20 \%$ of it. When fat mass increased up to 35-40\% BMR started slow down. In case of fat mass $>40 \%$ the BMR started go down.

Conclusion: In patients with NAFLD an accumulation of fat and increasing of fat mass percentage result in significantly decrease muscle, bone, and water percentages $(\partial<0.0001)$. It can be scientific base for the «fatty replacement» concept. Raised fat mass changes a body composition in patients with NAFLD.

\section{PP08-37}

Overweight Loss Therapy in Patients with Non-alcoholic Fatty Liver Disease

K.P. Oshakbayev ${ }^{1}$, E.A. Izatullayev ${ }^{1}$, A.V. Nersesov ${ }^{1}$, M.N. Nugmanova ${ }^{2}$

${ }^{1}$ Gastroenterology and Hepatology; ${ }^{2}$ Imaging Department, Scientific and Research Institute of Cardiology and Internal Diseases, Almaty, Kazakhstan

Background: Main cause of Non-alcohol Fatty liver disease (NAFLD) is considered metabolic syndrome (MS), but care of MS is differing.

Aim: To study the efficiency of overweight loss therapy in patients with NAFLD under concomitant MS.

Methods: 37 patients were randomized in research with NAFLD under MS, who receiving a symptomatic hormonal and nonhormonal sugar-lower therapy, antihypertensive therapy. Before study 10 patients were received insulin dozed from 4 to 68 unit per day, 27 patients received nonhormonal sugar-lower therapy. In 37 patients was arterial hypertension (AH). Fatty liver disease was evaluated by ultrasonography method of M. Hamaguchi et al. (2007). As overweight loss therapy used the analimentary detoxication technique is based on starvation diet with a control of endogen metabolic intoxication (Invitation Patent of the Republic of Kazakhstan N13868 dated 16.07.2007). The weight loss therapy last for 13-26 days. SPSS 17.0 for Windows were used.
Results: Overweight loss has been achieved in all 37 patients from 8 to $17 \mathrm{~kg}$ due to abdominal component of fatty deposits [body analyzer "Tanita SC-330" (Japan)]. Liver function tests, blood glucose level, and blood pressure became as normal in all patients. On 30 day after the weight loss therapy shown absence of fatty liver ultrasonography. Dozes of hormonal and non-hormonal sugar-lower, and anti-hypertensive preparations were reduced under control of blood glucose and blood pressure levels. Improvements of a common health of patients were marked.

Conclusion: The weight loss therapy based on the analimentary detoxication method reduce the criterions of fatty liver ultrasonography, liver function tests, blood glucose level, and blood pressure in patients with NAFLD under concomitant MS.

\section{PP08-38}

Steatohepatitis in Symptomatic Gallstone with and Without Metabolic Syndrome

N.Y. Bayramov ${ }^{1}$, A. Novruzov ${ }^{2}$

${ }^{1}$ Surgery, Azerbaijan Medical University, Baku; ${ }^{2}$ Surgery, Agsu City Hospital, Agsu, Azerbaijan

The aim of this study was to investigate the incidence of steatohepatitis in gallstone disease.

Material and methods: A total of 85 patients with symptomatic gallstone were enrolled for this study. All patients underwent laparoscopic cholecystectomy. During operation the liver biopsy was taken, which patient's preoperative permission. Patients with acute complications of gallstone (acute cholecystitis, cholangitis, pancreatitis), viral infection and cirrhosis were excluded. Patients were divided into two groups: with and without metabolic syndrome.

Results: A total of 85 patients $58(68 \%)$ had metabolic syndrome. Of these patients with metabolic syndrome and symptomatic gallstone 29 (50\%) had steatosis, 22 (38\%) had steatohepatitis, 7 (12\%) had fibrosis. Of the 27 patients with symptomatic gallstone and without metabolic syndrome 12 (44\%) had normal liver, 8 (29\%) had steatosis, $6(22 \%)$ had steatohepatitis and $1(3 \%)$ had fibrosis.

Conclusion: Gallstone and metabolic syndrome are high risk factors for fatty liver disease, especially for steatohepatitis.

\section{PP08-39}

Nonalcoholic Fatty Liver Disease in Metabolic Syndrome Among Patients Attending in a Tertiary Care Center in Bangladesh

M.M.R. Khan ${ }^{1}$, M.A.J. Chowdhury ${ }^{2}$, M.H. Rashid ${ }^{3}$, M.K. Rahman ${ }^{1}$, M.M.H. Chowdhury ${ }^{1}$, M.A. Alim ${ }^{4}$, Q.T. Islam ${ }^{5}$

${ }^{1}$ Internal Medicine, Rajshahi Medical College, Lxsmipur; ${ }^{2}$ Internal Medicine, Bangabandhu Sheikh Mujib Medical University, Dhaka;

${ }^{3}$ Hepatology, Rajshahi Medical College, Rajshahi;

${ }^{4}$ Gasteroenterology, Rajshahi Medical College, Laxsmipur;

${ }^{5}$ Medicine, Dhaka Medical College, Bangladesh, Dhaka, Bangladesh

Background: Non-alcoholic fatty liver disease (NAFLD) affects a substantial proportion of the general population and is frequently associated with many features of the metabolic syndrome. NAFLD is now considered the hepatic manifestation of metabolic syndrome and is increasingly recognized as a major contributor to the burden of hepatic morbidity world-wide. The aim was to assess the potential association between metabolic syndrome and NAFLD.

Methods: This hospital based study done on 334 nonalcoholic subjects of both sex and age of ${ }^{3} 20$ years. All subjects were prospectively assessed. History taking, clinical examination and necessary laboratory investigations were performed. Prevalence of the metabolic syndrome was defined by using modified (waist circumference: men $90 \mathrm{~cm}$ and women $80 \mathrm{~cm}$ ) National Cholesterol Education ProgramAdult Treatment Panel III criteria. Fatty liver diagnosis was based on the ultrasound examination of liver.

Results: Among 334 studied subjects 27.2\% ( $n$ 91) had the metabolic syndrome and was more commonly seen in women than in men (32.4 
vs. $21.7 \%, P<0.05)$. Majority of the patients $(59.3 \%)$ were in the age group 40-60 years. Overall frequencies of components of metabolic syndrome were in the order of abdominal obesity $(97.8 \%)>$ low high density lipoprotein $(83.5 \%)>$ hypertension $(71.4 \%)>$ triglycerides $(67 \%)>$ fasting plasma glucose $(44 \%)$. Abdominal obesity appeared to be the highest frequent components of the metabolic syndrome in both sexes (men 100\% and women 96.4\%). Most of those with metabolic syndrome had three components $(48.3 \%), 38.4 \%$ had four, and $13 \%$ had five components. Prevalence of NAFLD among studied subjects (n 334) was 44\% ( $n$ 148). Metabolic syndrome was found in $61.5 \%$ of cases with NAFLD and was more commonly seen in men than in women ( 54 vs. $46 \%$ ).

Conclusion: NAFLD is common among population and significantly increased in subjects with the metabolic syndrome.

\section{PP08-40}

Efficacy of Low-Calorie Diet Modified on Carbohydrate and Fat Components on Clinical and Blood Chemistry in NAFLD Patients N. Topilskaya, S. Morozov, V. Isakov, B. Kaganov

Research Institute of Nutrition, Moscow, Russia

Introduction: Development of NAFLD is closely connected to obesity and insulin resistance. Decrease of body weight is a nonpharmacologic way of correction of insulin resistance and one of most effective methods of NAFLD treatment.The aim of this study was to evaluate the efficacy of low-caloric diet in patients with NAFLD.

Methods: 128 NAFLD patients (BMI $36 \pm 4.31 \mathrm{~kg} / \mathrm{m}^{2}$ ) were enrolled to the study. According to clinical, laboratory and liver biopsy findings they were divided into 2 groups: the NASH group (77 patients) and 51 controls (simple steatosis group). A low-calorie diet modified on carbohydrate and fat components was prescribed to the patients of the main group (caloric content of 2,000-2,200 kcal/day). The control group received isocalorie diet (daily caloric content of $2,642 \pm 246.62 \mathrm{kcal})$. The BMI, ALT, AST and HOMA-IR were evaluated before and 6 months after the beginning of dietary treatment.

Results: In the NASH group significant reduction of initially elevated laboratory tests were achieved: $119 \pm 10$ vs. $56 \pm 3.6, p=0.03$ for ALT, $119 \pm 10$ vs. $56 \pm 3.6, p=0.03$ for AST and $119 \pm 10$ vs. $56 \pm 3.6, p<0.01$ for HOMA-IR. The significant decrease of BMI was found after treatment in both the NASH $\left(32.24 \pm 2.15 \mathrm{~kg} / \mathrm{m}^{2}\right.$ initially vs. $28.37 \pm 3.23 \mathrm{~kg} / \mathrm{m}^{2}$ after treatment, $\left.p<0.01\right)$ and the control ( $34.25 \pm 3.21$ vs. $30.21 \pm 3.5 \mathrm{~kg} / \mathrm{m}^{2}$ accordingly, $\left.p<0.01\right)$ groups. The mean proportion of BMI reduction was higher in the NASH group compared to controls: $(\mathrm{M} \pm \mathrm{m}) 13.2 \pm 2.3 \%$ in NASH versus $11.1 \pm 1.9 \%$ in controls, $p<0.05$. The proportion of its decrease from the basic values did not differ significantly between studied groups: $10.6 \pm 1.2 \%$ in NASH and $10.4 \pm 1.4$ in controls, $p=0.54$.

Conclusions: Low-calorie diet leads to significant improvement of clinical and laboratory (ALT, AST, HOMA-IR) results in patients with NAFLD and NASH.

\section{PP08-41}

NAFLD as a Risk Factor for Ischemic Heart Disease, Cerebrovascular Disease and Cancer

N. Patel ${ }^{1}$, D. Amarapurkar ${ }^{2}$, M. Padaravat ${ }^{3}$, S. Joshi ${ }^{3}$

${ }^{1}$ Jivandeep Hospital, Anand; ${ }^{2}$ Gastroenterology; ${ }^{3}$ Bombay Hospital and Medical Research Centre, Mumbai, India

Introduction: Non-alcoholic fatty liver disease (NAFLD) is associated with increased cardiovascular and cancer-mortality. This study aimed to evaluate risk factors in NAFLD with occurrence of these events.

Methods: All the consecutive patients of NAFLD seen during 2007-2010 were included. Prevalence of Ischemic heart disease (IHD), cerebrovascular disease (CVD) and cancers in NAFLD was compared with age-sex-matched irritable bowel syndrome (IBS) and hepatitis B (HBV). Risk factors like diabetes, obesity, dyslipidemia, hypertension, insulin resistance (IR), tobacco and family history of $\mathrm{IHD} / \mathrm{CVD} /$ cancer $(\mathrm{FH})$ were evaluated.

Results: A total of 286 NAFLD (age $47.2 \pm 13.4$ (16-82) years; 69 females; 46 cirrhosis) were included. There were $9(3.1 \%)$ CVD, 38 (13.2\%) IHD and $6(2 \%$; pancreas, 3 HCC, 1 breast, 1 acute lymphocytic leukemia) cancer. Among risk factors, diabetes was present in $96(33.5 \%)$, hypertension $75(26.2 \%)$, dyslipidemia $87(30.4 \%)$, tobacco $11(3.8 \%)$, obesity $155(54.1 \%)$, FH in $44(15.3 \%)$ and IR (non-diabetic) $75(26.2 \%)$. In IBS $(n=286$, age $=46.2 \pm 12.4$ (26-78) years; 72 females), CVD was present in 1 (0.34\%); IHD 22 $(7.6 \%)$ and cancer $1(0.34 \%$; breast $)$; risk factors were: diabetes 80 (27.9\%), hypertension $45(15.7 \%)$, dyslipidemia $66(23 \%)$, tobacco 50 (17.4\%), obesity $130(45.4 \%)$, FH 5 (1.7\%) and IR (non-diabetic) 50 (17.4\%). In HBV $(n=286$, age $=45.8 \pm 14.3(24-65)$ years; 71 females; 50 cirrhosis), CVD was present in 2 (0.68\%); IHD $18(6.2 \%)$ and cancer 6 ( $2 \%$; 4 HCC, 1 breast, 1 gastric); risk factors: diabetes 66 (23\%), hypertension $70(24.4 \%)$, dyslipidemia 56 (19.5\%), tobacco 40 (13\%), obesity $100(34 \%)$, FH $10(3.4 \%)$ and IR (non-diabetic) $20(6.8 \%)$. On correlating risk factors with IHD/CVD/cancer events, diabetes was significantly more present in NAFLD versus IBS versus HBV group with complications (31.2 vs. 13.7 vs. 10.6\%); hypertension (33.3 vs. 15.5 vs. $7.1 \%$ ); dyslipidemia (17.2 vs. 9 vs. $14.2 \%)$, tobacco ( 9 vs. 28 vs. $20 \%$ ), obesity (16.7 vs. 10 vs. $11 \%)$, FH (27.2 vs. 80 vs. 30$)$ and IR(non-diabetic) (2.6 vs. 6 vs. $5 \%$ ).

Conclusions: NAFLD had high rate of IHD/CVD/cancer events (18.5 vs. 8.3 vs. 9\%). Except diabetes and hypertension, other risk factors were lower or non-significant in NAFLD.

\section{PP08-42}

The Sexual Dimorphism of Adipocytokine Alterations and Alanine Aminotransaminase Activity in Nonalcoholic Fatty Liver Disease

M.-L. Chang, C.-M. Sung, C.-T. Chiu, C.-T. Yeh

Liver Research Unit, Department of Hepatogastroenterology, Chang Gung Memorial Hospital, Taoyuan, Taiwan, ROC

How gender affects adipocytokines and ALT activity in nonalcoholic fatty liver disease (NAFLD) is crucial to disease outcome but is often confounded by body mass index (BMI) or age. We aimed to elucidate these gender differences in NAFLD. A case-control study of 155 patients with NAFLD and 264 sex- and age-matched controls from a medical center in Taiwan was conducted to analyze the data after distributing the subjects into various groups by gender, BMI, age or ALT activity. Both leptin and adiponectin, but not plasminogen activator inhibitor-1 (PAI-1), correlated with gender (male: negatively, $p<0.001)$, BMI $(p<0.001)$ and ALT activity $(p<0.001)$ in NAFLD patients. Among all of the adipocytokines, PAI-1 had the highest area under the receiver operating characteristics curve $(0.880)$ for diagnosing NAFLD, with a cutoff point of $10.7 \mathrm{ng} / \mathrm{ml}$. No difference in ALT level was noted between the male and female patients. Among the males, regardless of the group, those with NAFLD had higher ALT, leptin and PAI-1 but lower adiponectin levels than the controls $(p<0.001)$. Similar differences were seen among the female patients $\geq 45$ years. Among the female patients $<45$ years, no difference in any adipocytokine was noted. Among the female patients with $\mathrm{BMI} \geq 24(p=0.027)$, BMI $<24(p<0.001)$, or normal ALT activity $(p<0.001)$, higher PAI-1 was the only difference between patients with NAFLD and controls.

Conclusion: Sexual dimorphism causes various alterations in adipocytokines but not ALT activity in NAFLD patients. After grouping the patients, hyperleptinemia, hypoadiponectinemia and high PAI-1 indicated the presence of NAFLD in males and older females but not females $<45$ years. Beyond the influence of gender, BMI and ALT activity, PAI-1 is a reliable, independent predictor of NAFLD, but caution must be taken against potential bias in females $<45$ years. 


\section{PP08-43}

Prevalence and Etiology of Abnormal Liver Tests: a Population-based Survey in JiLin, China

H. Zhang, J.Q. Niu

Hepatology, First Hospital, Jilin University, Changchun, China

Background: Serum biochemical liver tests (LTs) (alanine aminotransferase, aspartate aminotransferase, and gamma glutamyltransferase) and platelet counts are often used to screen for chronic liver disease. We determined the prevalence and etiologies of abnormal LTs in an adult population in Jilin, China.

Methods: A total of 3,791 individuals between the ages of 18 and 79 years were interviewed and then underwent ultrasonography and blood tests.

Results: The prevalence of abnormal LTs was $14.77 \%$ (560 out of 3791 subjects). The most noticeable risk factor for abnormal LTs was non-alcoholic fatty liver disease (NAFLD), which accounted for $11.61 \%$, metabolic syndrome for $25 \%$, or both for $22.14 \%$. Abnormal LTs were more common in male than in female subjects. The development of abnormal LTs was correlated with older age males, increased daily alcohol intake, fasting plasma glucose, body mass index, triglyceridemia, and low-density lipoprotein. Abnormal LTs were more common in the HCV (42\%) than in the HBV (32\%), metabolic syndrome $(32 \%)$, NAFLD $(31 \%)$, and alcohol alone subgroups $(30 \%)$. Abnormal LTs in patients with metabolic syndrome, NAFLD were associated with high fasting plasma glucose, high triglycerides, high body mass index, and low-density lipoprotein. However, abnormal LTs in patients with hepatitis B virus were associated with gender and increased age.

Conclusions: The results from the current study demonstrated that the prevalence of abnormal LTs is high in the population $(14.77 \%)$. Metabolic syndrome, NAFLD, and alcohol intake appear to be potentially important causes of the observed abnormal LTs.

\section{PP08-44}

Etiology and Prevalence of Abnormal Serum Alanine Aminotransferase Levels in an Adult Population in Jilin, China

H. Zhang, J.Q. Niu

Hepatology, First Hospital, Jilin University, Changchun, China

Background: Chronic liver diseases are a big burden in China. Alanine aminotransferase (ALT) is a useful indicator of hepatocyte damage.

Aim: We determined the prevalence and etiologies of elevated ALT in an adult population in Jilin, China.

Methods: A total of 4,072 individuals aged between 18 and 79 years were first interviewed, and then underwent ultrasonography and blood tests.

Results: The prevalence of elevated ALT was $9.79 \%$. The most noticeable risk factor for ALT elevation was non-alcoholic fatty liver disease (accounting for 11.28\%), metabolic syndrome $(17.29 \%)$, or both $(22.81 \%)$. The development of non-alcoholic fatty liver disease was related to females of increased age, fasting plasma glucose, body mass index, triglyceridemia, and low-density lipoprotein; and decreased high-density lipoprotein. The elevated ALT was related to young males with increased low-density lipoprotein who had NAFLD and/or metabolic syndrome. However, elevated ALT with infection of hepatitis B or C virus was not associated with metabolic disorders, but rather with gender and increased age.

Conclusions: The results from the current study demonstrated that elevated ALT is fairly high in the population $(9.7 \%)$ and that the cause of its elevation is mostly due to NAFLD and metabolic syndrome.
PP08-45

Relationship between Serum Aminotransferase Levels and Metabolic Disorders in Jilin Province, China

H. Zhang, J.Q. Niu

Hepatology, First Hospital, Jilin University, Changchun, China

Background: Increasing evidence suggests an association between elevated serum aminotransferase levels and metabolic disorders (metabolic syndrome, hyperlipemia and diabetes mellitus). However, the significance of relatively low levels of aminotransferases in relation to metabolic disorders has not been fully investigated in the general population. We investigated the association between serum amiontransferase levels and metabolic disorders using data from a survey in Jilin province, China.

Methods: In 2007, a cross-sectional survey was conducted throughout Jilin, China, covering both urban and rural areas. A total of 4,072 people, 18-79 years old, were undergoing real-time ultrasonography, $\mathrm{CT}$, blood tests, and interviews with a structured questionnaire.

Results: Serum aminotransferase levels within the normal range were associated with metabolic syndrome independent of age, body mass index, waist circumference, smoking, and alcohol intake. Compared with the lowest level $(<20 \mathrm{IU} / \mathrm{L})$, the adjusted odds ratios for ALT levels of $20-29,30-39,40-49$ and $>50$ IU/L were $1.83,1.88,1.97$, and 3.34 in men, and $1.49,1.46,3.13$, and 2.46 in women, respectively. Near-normal serum aminotransferase levels asso-ciated with hyperlipemia, NAFLD, DM were also found in the study.

Conclusions: Normal to near-normal serum aminotransferase levels are associated with metabolic disorders.

\section{PP08-46}

Insulin Resistance and Non-alcoholic Fatty Liver Disease in Egyptian Women with Polycystic Ovary Syndrome: a Silent Tragedy

M.S. Hassan, N. Mousa, O. Warda, M. Elshamy, N. El-Lakkany

Obestetric and Gynecology, Mansoura University, Mansoura, Egypt

Although non-alcoholic fatty liver disease (NAFLD) and polycystic ovary syndrome (PCOS) have not been extensively linked in previous studies, the two conditions do share a common attribute. Both are associated with a substantial occurrence of insulin resistance. Thus, women with PCOS may have an increased prevalence of nonalcoholic fatty liver disease, including nonalcoholic steato-hepatitis (NASH).

Objective: to determine the frequency and the characteristics of NAFLD in Egyptian women with PCOS and evidence for etiological association.

Patients and methods: One hundred and thirty non pregnant patients with diagnosis of PCOS according to Rotterdam criteria (97 overweight and obese women with mean age, $27.43 \pm 3.53$, BMI $29.08 \pm 1.58$ and 33 lean women with mean age, $26.31 \pm 3.14$, BMI $21.90 \pm 1.42$ ) attending Gynecology department, Mansoura university hospital participated in the study. NAFLD was diagnosed by abdominal ultrasound following exclusion of other causes of chronic liver disease. All cases were examined thoroughly anthropometric measurement, BMI, waist hip ratio, qualitative assessment of fatty liver by ultrasound, laboratory investigations for, fasting blood glucose, serum total cholesterol, triglycerides, LDL, HDL, ALT, HOMA index was determined.

Results: 58 of 97 overweight and obese patients with PCOS $(60 \%)$ had insulin resistance and $51(52.6 \%)$ had NAFLD. 6 of 33 lean patients with PCOS (18.2\%) had insulin resistance and NAFLD. The presence of steatosis was associated with a greater body mass index (BMI) $(P<0.001)$ and HOMA-IR $(P<0.001)$, a lower fasting highdensity lipoprotein $(P<0.001)$, a higher ALT, fasting total cholesterol, triglycerides and LDL cholesterol $(P<0.001)$ and a greater prevalence of impaired fasting glucose $(P<0.001)$. 
Conclusion: NAFLD is frequent in patients with PCOS especially the obese suggesting an association between these two conditions and the necessity of hepatic screening among PCOS patients for potential NAFLD.

\section{PP08-47}

Blood Urea Nitrogen Is Elevated in Patients with Nonalcoholic Fatty Liver Disease

X. Liu, H. Zhang, Y. Jiang, Y. Tu, J. Liang

Department of Liver Disease, Ruikang Hospital of Guangxi

Traditional Chinese Medicine University, Nanning, China

Background: Nonalcoholic fatty liver disease (NAFLD) is strongly associated with many predictors of cardiovascular disease such as hypercholesterolemia, hypertriglyceridemia, insulin resistance, central obesity, and the metabolic syndrome. Activation of reninangiotensin-aldosterone system (RAAS) has been proved in patients with NAFLD. Blood Urea Nitrogen elevation is high risk factors and biomarker of RAAS activation of heart failure and coronary heart disease. The aim of the current study was to investigate the BUN in patients with NAFLD.

Methods: BUN and creatinine $(\mathrm{Cr})$ values of 85 patients with NAFLD and of 30 age- and sex-matched healthy individuals were compared prospectively. Liver/spleen ratio of computed tomography (CT) values were used to determine fatty liver and to evaluate fatty deposition. Alanine aminotransferase (ALT) and aspartate aminotransferase (AST) were employed to estimate inflammation. We analyzed the correlation between BUN and fatty deposition or inflammation.

Results: NAFLD patients had significantly higher BUN compared to the control cases $(5.0353 \pm 1.1271$ vs. $4.1263 \pm 1.0095 \mathrm{mmol} / \mathrm{L}$; $t=3.898, p<0.001)$. However, there is no difference of $\mathrm{Cr}$ $(84.0941 \pm 14.4062$ vs. $79.4667 \pm 16.7120 \mu \mathrm{mol} / \mathrm{L} ; t=1.45, p$ $=0.15)$. BUN was negatively correlated with Liver/spleen ratio of CT values $(r=0.009, p=0.1974)$, ALT $(r=-0.012, p=0.941)$ and AST $(r=-0.009, p=0.592)$

Conclusion: We have shown for the first time in the literature that, patients with NAFLD have higher BUN. It may have prognostic value in NAFLD patients indicating a possible cardiovascular disease (CVD) risk increase.

\section{PP08-48}

Circulating Levels of Fetuin in Patients with Biopsy-Proven Nonalcoholic Fatty Liver Disease: Relation with Liver Fibrosis

Y. Yilmaz ${ }^{1}$, E. Ulukaya ${ }^{2}$

${ }^{1}$ Department of Gastroenterology, Marmara University, School of Medicine, Istanbul; ${ }^{2}$ Department of Biochemistry, Uludag University Medical School, Bursa, Turkey

Background: Serum concentrations of fetuin A/ $\alpha 2 \mathrm{HS}$-glycoprotein (AHSG) have been linked to human metabolic alterations and can serve as an indicator of liver cell function. We assayed serum levels of AHSG in patients with nonalcoholic fatty liver disease (NAFLD), a hepatic manifestation of the metabolic syndrome, and examined their association with clinical, biochemical, and histological phenotypes.

Methods: Serum AHSG levels were determined by ELISA in 99 patients with biopsy-proven NAFLD and 75 age- and gender-matched controls. Correlations among the study variables were tested by the Spearman's correlation coefficient. Linear regression was used to identify independent predictors of histological parameters after adjustment for potential confounders

Results: Serum AHSG levels were significantly higher in patients with NAFLD $(940 \pm 120 \mu \mathrm{g} / \mathrm{mL})$ compared with healthy controls $(800 \pm 130 \mu \mathrm{g} / \mathrm{mL}$, Student's $t$ test, $P<0.001)$. Bivariate analyses (Spearman's rank correlation) in patients with NAFLD showed a statistically significant association between AHSG levels and insulin resistance as assessed by the HOMA index $(r=0.31, P<0.01)$ and the liver fibrosis score index $(r=0.36, P<0.001)$. The association between AHSG and fibrosis remained statistically significant even after adjustment for potential confounders, including the HOMA index ([beta] $=1.65, t=2.38, P<0.05$ ).

Conclusion: The main finding of this study is that serum AHSG levels are significantly higher in patients with biopsy-proven NAFLD and may serve as a biochemical marker of fibrosis in this patients group. The association between serum AHSG levels and fibrosis was independent of potential confounders, including HOMA. Our data suggest that high serum AHSG levels in patients with NAFLD do not merely reflect the effects of insulin resistance, but also a more extensive distorsion of liver architecture. Measurements of this biomarker might potentially help identify those patients with the highest risk of liver fibrosis for liver biopsy.

\section{PP08-49}

The Efficacy of $\omega-3$ Unsaturated Fatty Acid on NASH Rats D. Wenjin

Department of Gastroenterology, Xinhua Hospital, Affiliated to Shanghai Jiaotong University, Shanghai, China

Objective: To observe the efficacy of $\omega-3$ unsaturated fatty acid on lipid and glycometabolism, as well as adipocytokines of NASH rats. Methods: Divided rats into 3 groups (control/NASH/therapy group). Control group was fed with normal diet,the other two with high-fat diet. Add $\omega-3$ unsaturated fatty acid to therapy group from 8 to 12 weeks. Weight, FBS, ALT, AST,TC, TG and adipocytokines were measured.

Results: NASH model was established successfully. Weight, TC, TG, ALT etc. decreased in therapy group, as compared with those in NASH one. The reduction of AST and TC had significant difference. Therapy group showed lower leptin, resistin and higher adiponectin levels than NASH group.

Conclusion: Unsaturated fatty acid may improve lipid and glycometabolism on NASH rats, as well as regulate the expression of adipocytokines.

\section{PP08-50}

Pattern of Liver Function Tests in Morbidly Obese Saudi Patients Undergoing Bariatric Surgery

A.M. Al Akwaa

Medicine, King Abdulaziz Hospital, Al Ahsa, Saudi Arabia

Background: Morbidly obese patients have a high prevalence of fatty liver disease and its serious complications, and high prevalence of abnormal liver function tests (LFT). The LFT can give a clue to the liver damage and correlate with activity. We aim to look at pattern of LFT in morbidly obese Saudi patients undergoing bariatric surgery in Eastern region Saudi Arabia.

Methods: Medical records of patients undergoing bariatric surgery were reviewed. Demographic data, co morbid conditions, medications taken were recorded. Intraoperative liver appearance was noted. Patients with alcohol intake or without LFT excluded.

Results: Out of 113 charts, 15 patients excluded. Remaining 98 patients were analyzed. (58.2\%) were females. Mean age $33.1 \pm 8.87$ years. Mean BMI was $53.7 \pm 1.27 \mathrm{~kg} / \mathrm{m}^{2}$. Abnormal LFT (ALT, AST, ALK and GTT) were observed in (17.3\%), with 1.5-2 times the upper limit of normal. ALT was the most elevated in (12.2\%). Abdominal ultrasonography was done in 67 (68.4\%) patients of whom $51(76 \%)$ had fatty liver.All patients had normal coagulation profiles, $\mathrm{Hb}$ and platelets.

Co morbid conditions including diabetes mellitus, hypertension, hyperlipidemia, bronchial asthma, obstructive sleep apnea were observed in $51(51.5 \%)$ patients, 8 of them $(16.3 \%)$ had abnormal LFT.

No intraoperative changes of cirrhosis was observed

Conclusion: The prevalence of abnormal liver function tests is low in our morbidly obese patients undergoing bariatric surgery. A 
prospective study, with a larger sample and liver biopsy is needed to clarify the findings. This is the first report from our area.

\section{PP08-51}

Nonalcoholic Fatty Liver Disease in Menopausal Women: Influence Hormone Replacement Therapy

G. Florentino ${ }^{1,2}$, H. Cotrim ${ }^{3}$, C. Padilha ${ }^{3,4}$, A. Florentino ${ }^{5}$, C. Gadelha ${ }^{5}$, A. Marinho ${ }^{2}$, P.A. Paschwingel ${ }^{6}$

${ }^{1}$ Universidade Fereral da Bahia, Salvador; ${ }^{2}$ Universide Federal de Campina Grande-Paraiba, Campina Grande; ${ }^{3}$ Universidade Federal da Bahia, Salvador; ${ }^{4}$ Universidade Federal de Campina Grande, Campina Grande-Paraiba; ${ }^{5}$ Universidade Federal de Campina Grande, Campina Grande; ${ }^{6}$ Universide Federal da Bahia, Salvador, Brazil

Background: Alterations in body composition, fat distribution and/or hormonal or metabolic changes occur following menopause and obesity, dyslipidemia, diabetes and metabolic syndrome (MS) are common. However, the characteristics of nonalcoholic fatty liver disease (NAFLD) in menopause need to be better understood.

Aim: To evaluate the frequency of NAFLD in postmenopausal women; the correlation of menopause with features of MS; the association of hormone replacement therapy (HRT) with NAFLD.

Methodology: A series-case study evaluated menopausal women from April/2009 to April/2010. All the participants had clinical and laboratorial evaluations (HBsAg, anti-HCV, ALT, AST, GGT, CPK, lipids, glucose, insulin, auto antibodies, ferritin, transferring saturation), abdominal ultrasound (AUS). HOMA-IR $\geq 3$ was considered insulin resistance (IR). NAFLD criteria: negative or occasional history of ethanol intake ( $\leq 20 \mathrm{~g} /$ day); exclusion of others liver diseases; hepatic steatosis on AUS.

Results: The study included 179 menopausal women. NAFLD criteria were observed in 60 (33. 5\%) and they presented: the mean age of $57.0 \pm 6.7$ years and the means of BMI and waist circumference of $30.6 \pm 4.4$ and $99.0 \pm 9.3$ respectively. Hepatic steatosis was observed in $100 \%$ of these cases on AUS. Metabolic syndrome features observed were: central obesity in $30(51.8 \%)$, hypertension in $22(36.7 \%)$, elevated triglycerides in $35(60.3 \%)$ and low HDL-C in $37(66.1 \%)$ respectively. History of HRT used was referred by $12.8 \%$ $(23 / 179)$ of the menopausal women and $8.7 \%(2 / 23)$ of them had NAFLD. The presence of MS features was similar in women with and without history of HTR.

Conclusions: The frequency of NAFLD in menopausal women (MMP) was high; it was associated with features of metabolic syndrome; the correlation of NAFLD with hormone replacement therapy (TRH) was not observed. The results suggest that the frequency of NAFLD in menopausal women was not influenced by the TRH.

\section{PP08-52}

LDL-cholesterol Serum Levels Inversely Correlate with Oxysterols Plasma Levels in HCV Infected Patients

M. Arciello ${ }^{1,2}$, S. Petta ${ }^{3}$, B. Conti ${ }^{1}$, V. Leoni ${ }^{4}$, G. Iannucci ${ }^{5}$, G. Labbadia ${ }^{5}$, S. Sofia ${ }^{2}$, C. Cammà ${ }^{3}$, A. Craxì ${ }^{3}$, C. Balsano ${ }^{1,2}$

${ }^{1}$ Andrea Cesalpino Foundation, Rome; ${ }^{2}$ Department of Internal Medicine and Public Health, University of L'Aquila, L'Aquila; ${ }^{3}$ Cattedra \& Unità Operativa di Gastroenterologia, Di.Bi.M.I.S., University of Palermo, Palermo; ${ }^{4}$ Genetic Unit of Neurodegenerative and Metabolic Diseases, IRCCS National Institute of Neurology "C.Besta", Milan; ${ }^{5}$ Department of Internal Medicine, University of Rome 'La Sapienza', Roma, Italy

Background/aims: Hepatitis C virus (HCV) infection is characterized by increased oxidative stress (OS), which is well evaluable testing oxysterols plasma concentration. These molecules, interfering with different cellular functions, may affect lipid metabolism through Liver X Receptors (LXRs) modulation. HCV exploits host lipid metabolism to facilitate its replication and its diffusion; however, their plasma levels have not yet been studied in chronically $\mathrm{HCV}$ infected patients (CHC). Thus, we aimed to evaluate and highlight the potential pathogenetic role of oxysterols, 7-ketocholesterol (7KC) and 7-beta-hydroxycholesterol (7betaOH), in HCV-related lipid dysmetabolism.

Methods: The study was carried out on 80 consecutive patients, 42 Chronic Hepatitis C infected (93\% of which by HCV genotype 1b) and 38 Non Alcoholic Fatty Liver Disease (NAFLD). Plasma oxysterols, $7 \mathrm{KC}$ and 7 betaOH, levels were determined by isotope dilution gas chromatography/ mass spectrometry (GC-MS).

Results: GC-MS revealed higher $7 \mathrm{KC}(71.2 \pm 77.3$ vs. 30.4 $\pm 14.5, p<0.005)$ and 7 betaOH $(23.7 \pm 20.6$ vs. $11.5 \pm 4.9, p<$ 0.001) plasma levels in HCV respect to NAFLD patients. Furthermore, multivariate regression analysis highlighted an inverse independent correlation between high oxysterols and low LDL-cholesterol ( $p=0.01$ for 7 betaOH; $p=0.02$ for $7 \mathrm{KC}$ ) plasma levels in HCV group; in contrast, NAFLD group showed a direct correlation between oxysterols levels and LDL-cholesterol ( $p<0.001$ for 7 be$\mathrm{taOH} ; p=0.002$ for $7 \mathrm{KC}$ ).

Conclusions: Our work for the first time correlates oxysterols plasma levels, markers of oxidative stress, to lipid dysmetabolism, in HCV infected patients. In addition, our data further support a different regulation of lipid metabolism in HCV infected and NAFLD patients, suggesting that the non alcoholic liver steatosis (NAFLD) and steatosis due to HCV-related lipid dysmetabolism, have different features, probably due to oxysterols, and thus cannot be considered equally.

\section{PP08-53}

Liver Histopathology versus Biochemical Markers in Hepatic Steatosis S.Z. Mohamed ${ }^{1}$, I.I. Ramzy ${ }^{1}$, M.E.S. El Raziky ${ }^{1}$, O.G. Shaker ${ }^{2}$, M.M.F. Nosseir ${ }^{3}$, Y.S. Ibraheim ${ }^{1}$

${ }^{1}$ Tropical Medicine; ${ }^{2}$ Medical Biochemistry, Faculty of Medicine Cairo University, Cairo; ${ }^{3}$ Pathology, Theodor Bilharz Research Institute, Giza, Egypt

Background: Non-alcoholic fatty liver disease (NAFLD) is recognized as one of the most frequent causes of chronic liver disease and abnormal liver function tests.

Aim: To study the pattern of serum leptin, adiponectin, apolipoprotein AI, TNF alpha and hepatic tissue TNF alpha in patients with NAFLD and to assess the validity of these Biomarkers versus histopathology in diagnosis of different types of hepatic steatosis with special emphasis on the correlation with the severity of illness.

Patients and methods: A prospective study of 70 patients with NAFLD and 15 normal individuals as controls was carried out. Complete history taking, clinical examination, laboratory parameters, abdominal ultrasonography, needle liver biopsy and histopathologic examination were done for all cases. Scores of fibrosis (BAAT and modified APRI) were also done.

Results: Hepatic steatosis is a disease of both sexes with female predominance, mostly in the fourth decade. The main risk factors for NAFLD are obesity, hypertriglyceridemia and diabetes mellitus. Serum leptin, apolipoprotein AI and TNF alpha are capable to differentiate between NAFLD patients and normal individuals with specific cutoff. BMI $>25.6$ indicate hepatic steatosis with high sensitivity and specificity.

Conclusions: Elevated transaminases in NAFLD especially AST could indicate the development of NASH with no correlation with severity of lesion. Despite the significant correlation between high serum level of leptin, apolipoprotein AI and TNF alpha and low serum level of adiponectin in NAFLD patients; no single marker succeeded to predict the development of non-alcoholic steatohepatitis (NASH). BAAT and modified APRI scores are good diagnostic tools for diagnosis of hepatic fibrosis in NAFLD patients. 
Combination of serum markers of hepatic steatosis raises the issue of noninvasive method for prediction of NASH or fibrosis. Moreover, grading of liver brightness by abdominal ultrasonography can be validated as a simple and sensitive method for diagnosis of NAFLD, $\mathrm{NASH}$ and fibrosis.

\section{PP08-58}

Role of Fat CT on ALT Prediction on NAFLD Patients

S.B. Kim, H.Y. Kang, M.S. Kang, S.H. Moon, H.D. Shin, J.E. Shin, H.J. Kim, I.H. Song

Dankook University, Cheonan, Republic of Korea

Background: Nonalcoholic fatty liver disease (NAFLD) has become the common disease with the increasing prevalence of obesity in South Korea. Recent studies have been showing that visceral fat is more important risk factor than subcutaneous fat on NAFLD. We investigated the role of fat CT on NAFLD patients.

Patients and methods: We analyzed the 360 subjects who have taken fat CT in Dankook University Hospital health promotion center. Fat CT measured visceral fat amount at three levels (supraumbilicus, umbilicus, infraumbilicus). We divided fatty liver on abdominal sonography into normal, mild fatty, moderate fatty, severe fatty liver. Alcohol history and blood tests such as cholesterol, fasting blood glucose, liver function test were evaluated.

Results: We analyzed 296 subjects after excluding significant alcohol intake (42) (more than $140 \mathrm{~g} /$ week in men or $70 \mathrm{~g} /$ week in women), positive HBsAg (11), positive anti-HCV (1), NIDDM patients (6), inadequate data (4). Fat CT on supraumbilicus level was strongly correlated with severity of fatty liver and ALT level. The amount of visceral fat according to severity of fatty liver on abdominal sonography showed increasing tendency (normal liver $104.13 \mathrm{~cm}^{2}$, mild fatty liver $162.91 \mathrm{~cm}^{2}$, moderate fatty liver 152.38 $\mathrm{cm}^{2}$, severe fatty liver $209.35 \mathrm{~cm}^{2}$ ). Correlation coefficient with ALT level was most high in visceral fat amount (0.458) compared to other factors such as obesity (0.339), waist circumference (0.407), BMI (0.352), cholesterol (0.250), triglyceride (0.319). When we divided the visceral fat amount into low group $\left(<100 \mathrm{~cm}^{2}\right)$ and high group $\left(\geq 100 \mathrm{~cm}^{2}\right)$, the proportion of abnormal ALT level( $\left.>40 \mathrm{IU} / \mathrm{L}\right)$ was higher in low group $(3.7 \%, 4 / 108)$ than high group $(20.1 \%, 37 /$ 184)

Conclusions: Visceral fat is the most powerful predictor for ALT level elevation in NAFLD. Fat CT can be the useful indicator for controlling the NAFLD.

\section{PP08-59}

Predictors of Liver Fibrosis among Filipino Patients with NAFLD

J. Tupas-Edano ${ }^{1}$, I.H. $\mathrm{Cua}^{1}$, M. Dofiles ${ }^{1}$, J. Bocobo ${ }^{1}$, J. Gopez-Cervantes ${ }^{2}$, St. Lukes Liver Study Group

${ }^{1}$ Institute of Digestive and Liver Diseases; ${ }^{2}$ Institute of Digestive and Liver Diseases/Center for Liver Diseases, St. Luke's Medical Center, Quezon, Philippines

Background and aims: With increasing prevalence of obesity and diabetes mellitus worldwide, non-alcoholic fatty liver disease (NAFLD) has become a common cause of chronic liver disease. Liver biopsy remains the gold standard in diagnosing NASH. Therefore, the aim of this study was to identify the independent predictors of fibrosis among Filipino patients with NAFLD.

Methods: Sixty one patients diagnosed with NAFLD who underwent liver biopsy were included. Clinical and biochemical parameters were collected. Patients with significant alcoholic intake and other liver diseases were excluded.

Results: Majority of the study population were females (54\%) with mean age of $46 \pm 11$. Only $34 \%$ were obese (BMI $\geq 30$ ), $19.7 \%$ had hypertension, $16.4 \%$ with diabetes and $29.5 \%$ had dyslipidemia. Forty seven patients $(77 \%)$ had elevated ALT levels. Fibrosis is seen in $72 \%$. Age (OR 5.0, $p=0.08$ ), female sex (OR 4.8, $p=0.04$ ), BMI (OR 8.9, $p=0.03$ ) and HDL (OR 0.2, $p=0.09$ ) were found to be significant predictors of fibrosis.

Conclusion: Age, female sex, BMI and HDL are significant predictors of fibrosis in Filipino patients with NAFLD.

\section{PP08-60}

FIB-4 Is More Accurate in Predicting Advanced Fibrosis Among Filipino Patients with NAFLD

D.C. Uy, I.H. Cua, J. Edano, J. Bocobo, J. Cervantes

St. Luke's Medical Center, Quezon City, Philippines

Background: Accurate evaluation of liver fibrosis in patients with non-alcoholic fatty liver disease (NAFLD) is important in order to identify those who will develop complications. The aim of this study is to compare the diagnostic performances of simple non-invasive scoring systems in identifying advanced fibrosis among Filipino patients with biopsy-proven NAFLD.

Methods: Patients with biopsy-proven NAFLD enrolled in the St. Luke's Medical Center Liver Data Bank from 2007 to 2010 were included in this study. The FIB-4, BARD, AST/ALT ratio, and the AST to platelet ratio index were calculated for each patient.

Results: A total of 61 patients were included. Majority are females (54\%) with mean age of 46 years \pm 11 . The mean body mass index was $29.1 \mathrm{~kg} / \mathrm{m}^{2} \pm 4.3$. Ten subjects $(16.4 \%)$ had diabetes. All subjects had biopsy-proven non-alcoholic steatohepatitis. Six (9.8\%) had advanced fibrosis (stage 3-4). In this study, the FIB-4 had the highest and only significant AUC $(0.76,95 \%$ CI $0.62-0.89)$ compared to APRI $\quad(\mathrm{AUC}=0.74, \quad 95 \% \quad \mathrm{CI} \quad 0.62-0.86) . \quad$ ALT/AST ratio $(\mathrm{AUC}=0.57,95 \%$ CI $0.41-0.74)$ BARD $(\mathrm{AUC}=0.4495 \% \mathrm{CI}$ $0.27-0.62$ ).

Conclusions: The FIB-4 is more accurate in identifying NAFLD patients with advanced fibrosis compared with other scoring systems. Therefore, allowing liver biopsy to be used more in an appropriate manner.

\section{PP08-61}

Histopathological Profile of NAFLD Patients in Coastal Eastern India S.P. Singh ${ }^{1,2}$

${ }^{I}$ Department of Gastroenterology, Digestive Diseases Centre;

${ }^{2}$ Department of Gastroenterology, S.C.B. Medical College, Cuttack, Orissa, Cuttack, India

Introduction: NAFLD has emerged as the commonest liver disorder in both the developed and developing nations. The histopathological staging/classification of NAFLD is important since this correlates with progression/outcome of the disease. The present study was conducted to analyze the histological profile of the NAFLD patients in coastal eastern India.

Materials: The liver biopsy slides of 46 consecutive NAFLD subjects attending the Gastroenterology outpatient department constituted the study material. The histopathological findings in these liver biopsy slides of NAFLD were analyzed.

Results: Of the 46 NAFLD patients, only 6 were females with a male female sex ratio of $7: 1$. The mean age was 42.3 years. 25 of 46 [54.3\%] had mild steatosis [ $<33 \%], 41.3 \%$ had moderate steatosis and only $4.4 \%$ had severe steatosis [ $>66 \%$ ]. Matteoni's classification of the histological findings showed that while 22.2 and $13.3 \%$ had Class 1 and Class 2 disease respectively, Class 3 disease was present in $55.6 \%$ NAFLD patients and $8.9 \%$ had class 4 disease. Fibrosis seen in only 4 subjects was mild [stage 1].

Conclusions: The study showed that half of the NAFLD patients in coastal eastern India which is a developing region had only mild 
steatosis. However two thirds of NAFLD patients had Class 3 or 4 disease which is believed to be progressive. Fibrosis was quite uncommon, and even when seen was only mild.

\section{PP08-62}

Non Alcoholic Fatty Liver Disease and Framingham Cardiovascular Risk Factors

C. Türkay, B. Kasapoglu, K.S. Yalçın

Fatih University Hospital, Ankara, Turkey

Non alcholic fatty liver disease (NAFLD) is regarded as the hepatic manifestation of metabolic syndrome. The 2 exactly defined risk factors for coronary artery disease, obesity and Type 2 diabetes are also defined as risk factors for NAFLD. The aim of this study is the evaluation of presence of NAFLD and its association with inflammatory markers and Framingham cardiovascular risk score among non-obese and non-diabetic patients.

Framingham cardiovascular risk scoring was performed to all patients according to the age, gender, systolic blood pressure and antihypertensive medication history. From the blood samples glucose, uric acid, AST, ALT, GGT, crp and lipid profile were studied. Insulin resistance was calculated with the Homeostasis Model Assesment (HOMA) method.

Two thousand and fifty-eight cases were included in the study. Their ages were ranging in between 21 and 70 years (mean age $49.7 \pm 13.2$ years). Among those 1,563 were women while 495 were men. According to the ultrasonographic findings, patients were grouped as; without any fatty infiltration of the liver (control group) $(n=982)$, mild steatosis $(n=473)$, moderate steatosis $(n=363)$ and severe steatosis $(n=240)$ groups. There was no statistically significant difference in regards to the age between groups $(p>0.05)$. However, in gender evaluation, in severe steatosis group male/ female ratio was statistically significantly higher than other groups. With the increase in steatosis stage, ALT, AST, GGT, CRP, ferritin and uric acid levels also significantly increased. In severe steatosis group, the mean Framingham cardiovascular risk score was significantly higher than that of other groups. In correlation analysis, there was a positive correlation between GGT, CRP, uric acid and ferritin levels with Framingham cardiovascular score.

In our study we determined that in also non-diabetic or non-obese NAFLD patients cardiovascular disease risk increases and in these patients subclinical inflammatory markers are also higher.

\section{PP08-63}

Evaluation of Serum Adiponectin, Leptin, Resistine, and Retinol Binding Protein-4 Levels in Non-diabetic, Non-obese Patients with Non-alcoholic Fatty Liver Disease

B. Kasapoglu, C. Türkay, K.S. Yalçın

Fatih University Hospital, Ankara, Turkey

Insulin resistance, oxidative stress and inflammatory processes thought playing a key role in pathogenesis and progression of Non alcoholic fatty liver disease (NAFLD). In this aspect serum adiponectin, leptin, resistin and retinol binding protein (RBP)4 levels gain importance in both pathophysiology and targeted therapy regimens. We aimed to evaluate the levels of these adipokines among patients with fatty liver disease and control subjects top evaluate their role in lipotoxicity. This study was carried out among eighty non-diabetic and non-obese (Body mass index $<30$ ) outpatients. Thirty nine patients were diagnosed by liver biopsy, 18 patients diagnosed by ultrasound included in the study and 23 volunteers enrolled as a control group. The results are shown in Tables 1 and 2 .
Table 1 Leptin, adiponectin, resistin and RBP4 levels of participants \begin{tabular}{l} 
Özellik \\
\hline CONTROL $(n=23)$ \\
NAFLD $(n=57)$ \\
$P$ \\
Adiponectin \\
$\mathbf{1 9 . 1} \pm \mathbf{1 0 . 1}$ \\
$\mathbf{1 7 . 1} \pm \mathbf{9 . 0}$ \\
$\mathbf{0 . 4}$ \\
Leptin \\
$13.6 \pm 11.2$ \\
$16.8 \pm 11.4$ \\
0.4 \\
Resistin \\
$22.3 \pm 12.3$ \\
$22.6 \pm 12.4$ \\
0.9 \\
RBP 4 \\
$83.2 \pm 28.2$ \\
$80.9 \pm 30.9$ \\
0.75 \\
\hline
\end{tabular}

Table 2 The association of leptin, adiponectin, resistin and RBP4 levels with fibrosis among patients diagnosed with biopsy

\begin{tabular}{l}
\hline Ozzellik \\
\hline Fibrozis Evre $\mathbf{1}(\mathbf{n}=\mathbf{4})$ \\
Fibrozis Evre $\mathbf{2}$ \\
$(\mathbf{n}=\mathbf{8})$ \\
Fibrozis Evre $\mathbf{3}(\mathbf{n}=\mathbf{6})$ \\
Adiponektin \\
$19.9 \pm 4.5$ \\
$16.8 \pm 3.5$ \\
$13.2 \pm 4.2$ \\
Leptin \\
$15.2 \pm 7.1$ \\
$17.4 \pm 9.3$ \\
$25.2 \pm 11.1$ \\
Rezistin \\
$15.4 \pm 4.2$ \\
$17.9 \pm 4.6$ \\
$19.2 \pm 7.1$ \\
RBP 4 \\
$66.1 \pm 12.4$ \\
$69.2 \pm 10.2$ \\
$62.4 \pm 12.4$ \\
HOMA-IR \\
$1.8 \pm 0.9^{\mathrm{a}, \mathrm{b}}$ \\
$2.6 \pm 0.8^{\mathrm{c}}$ \\
$3.5 \pm 0.6$ \\
\hline
\end{tabular}


When diabetes and obesity are excluded leptin, adiponectin, resistin and RBP4 levels were not higher among patients with NAFLD than the control group and moreover there was no association of with these parametes and the levels of fibrosis among patients diagnosed with biopsy.

\section{Poster Presentation 09: Other Viral Hepatitis}

\section{PP09-01}

Molecular Epidemiology of Emerging Hepatitis Viruses: Their Association with Aplastic Anemia

M. Idrees ${ }^{1}$, S.A. Sha ${ }^{2}$

${ }^{1}$ Punjab University; ${ }^{2}$ Pathology, Sheikh Zayed Medical University, Lahore, Pakistan

Background: Aplastic anemia (AA) is a serious and rare disorder characterized by a hypocellular bone marrow. Hepatitis associated aplastic anemia (HAAA) is a variant of aplastic anemia in which aplastic anemia follows an acute attack of hepatitis.

Aim: To investigate the role of hepatitis viruses in the development of AA and characterize the illness.

Method: Total 44 patients with AA were enrolled between 2006 and 2009 and studied who were referred to the Sheikh Zayed Medical Complex, Lahore. Hematology and biochemical analysis were done on all samples. Serum samples were assayed for antibodies, and/or antigens related to hepatitis $\mathrm{A}, \mathrm{B}, \mathrm{C}, \mathrm{E}$, viruses and for the nucleic acid detection of hepatitis A, B, C, Delta, E, GB virus C, TTV and $\mathrm{B} 19$ viruses by the polymerase chain reaction. The quantification for positive samples were done using Real-time PCR. Genotyping of $\mathrm{HCV}$ was carried out using chain termination sequencing method (ABI Genetic Analyzer).

Results: Out of total 44 cases that had the typical features of this syndrome of HAA, 10 (22.7\%) were hepatitis associated. Four (9.1\%) each cases were due to HCV and HEV and 1 (2.3\%) each with HAV and HCV + HEV co-infection.

Conclusion: HAAA is an uncommon but severe condition which may occur following idiopathic cases of acute hepatitis. In patients presenting with pancytopenia after an episode of acute hepatitis, the diagnosis should be considered and confirmed by PCR, RT-PCR and if possible by bone marrow biopsy.

PP09-02

Detection of Transfusion Transmitted Virus (Tt Virus) in Body Fluids and Secretions of Egyptian Volunteer Blood Donors

F. El Ghamry ${ }^{1}$, M. Alboraie ${ }^{1}$, M. Goda ${ }^{2}$

${ }^{1}$ Department of Internal Medicine, Al Azhar Faculty of Medicine, Cairo; ${ }^{2}$ Department of Clinical Pathology, Faculty of Medicine, Benha University, Benha, Egypt

Background: Transfusion transmitted virus (TT virus) was identified in patients with post transfusion hepatitis in Japan. It shares several characteristics with parvovirus. Several genotypes and subtypes of TT virus have been isolated, also it replicates in a manner that generates extensive sequence heterogenicity so it is a chronic virus that can persist for years and can mutate inside the body. TT viraemia is frequent in blood donor populations and high prevalence of TT virus has been found in patients exposed to blood.

Objective: Detection of TT virus in serum, some body fluids and secretions of Egyptian volunteer blood donors.

Patients and methods: Serum samples were collected from 65 apparently healthy blood donors without clinical or biochemical (normal transaminases) evidence of hepatitis (45 males and 20 females with mean age $32 \pm$ SD 10 years). Serum samples from all subjects were tested by PCR (polymerase chain reaction) to detect TT virus pure gene DNA. Positive subjects were further studied for detecting TT virus DNA in urine, saliva, breast milk (from lactating mothers) and semen (from adult males).

Results: The results showed that 12 out of $65(18.4 \%)$ serum samples were positive for TT virus ( 8 males $12.3 \%$ and 4 females $6.1 \%$ ). Saliva and urine (from 12 positive patients) were positive for TT virus DNA in 50 and $58.3 \%$ respectively. All tested breast milk and semen samples were negative for TT virus.

Conclusion: TT virus was common in a group of Egyptian volunteer blood donors and TT virus might be transmitted by urine and saliva.

\section{PP09-03}

Hepatitis E Antibodies in Adolescent Females

S.A. Zaki ${ }^{1}$, A. Amer ${ }^{1}$, A. Nagaty ${ }^{2}$, M. Darwish ${ }^{3}$

${ }^{1}$ Microbiology, Faculty of Medicine; ${ }^{2}$ Obstetrics and Gynecology, Alexandria University; ${ }^{3}$ Microbiology, Ain Shams University, Alexandria, Egypt

Introduction: Hepatitis E virus (HEV) found to be a frequent cause of acute sporadic hepatitis in the pediatric population. HEV caused epidemic outbreaks that can generally be traced to faecal contamination of drinking water.

In Egypt,infection with hepatitis E virus closely resembles that with hepatitis A virus, both in pattern of transmission and in causing an acute self-limiting illness with no chronic sequelae. Hepatitis E affects mainly young adults (15-40 years of age) and seems to be responsible for high mortality in pregnant women, especially when it occurs in the third trimester.

Aim: To evaluate the seroprevalence of HEV in Egyptian adolescent females.

\section{Material and methods:}

- 95 healthy adolescent females

- A standardized questionnaire was administered to each

- venous blood sample obtained from each. All samples were tested by an enzyme immunoassay (EIA) for specific anti-HEV antibody. Two recombinant antigens (SG-3 and 8-S) derived from open reading frame 2 (ORF2)or ORF3 of the Burmese strain of HEV and expressed in E. coli used as solid phase antigens.

Results: The mean age of the study subjects was $21.81 \pm 2.5$ (SD) range 16-25 years. Eighty $(84.2 \%)$ subjects resided in Alexandria, while $15(15.8 \%)$ came from semiurban villages.

Antibodies to HEV were found in 37 (38.9\%) of the 95 study participants. $10(55.6 \%)$ pregnant females, as well as $48(62.3 \%)$ non pregnant females of the child bearing age lacked evidence of HEV infection and may be susceptible.

Conclusion: The endemicity of HEV infection in Egypt warrants the search into the epidemiologic risk factors associated with anti-HEV positivity through large scale population based studies. Puplic health measures, are needed to control HEV and other enteric infections.

\section{PP09-04}

The First Case of Genotype 4 Hepatitis E Related to Wild Boar in South Korea

Y.M. Kim ${ }^{1}$, S.-H. Jeong ${ }^{1}$, J.Y. Kim ${ }^{1}$, J.C. Song ${ }^{1}$, J.H. Lee ${ }^{1}$, J.-W. Kim ${ }^{1}$, H. Yun ${ }^{2}$, J.S. Kim ${ }^{2}$

${ }^{1}$ Seoul National University Bundang Hospital, Seongnam-Si; ${ }^{2}$ Korea National Institute of Health, Seoul, Republic of Korea

Introduction: Several cases of serologically diagnosed hepatitis E have been reported in South Korea. However, there have been no documented cases of zoonotic transmission with virologic characterization of hepatitis $\mathrm{E}$ virus (HEV). We report on a sporadic case of autochthonous acute hepatitis $\mathrm{E}$ after ingestion of raw bile juice obtained from a wild boar living on a domestic mountain in a southern part of Korea. 
Case report: A 54-year-old male was transferred to our hospital due to severe hepatitis. Before Six days ago, the patient had presented with myalgia, anorexia, and dark colored urine. He denied taking any medications or herbs. The patient was a heavy alcohol drinker. He had not traveled outside Korea in the preceding year. One month before symptom onset, he had ingested raw bile juice obtained from a wild boar captured on Giri-mountain in the southern part of South Korea. Laboratory data showed total bilirubin $4.7 \mathrm{mg} / \mathrm{dL}$, AST/ALT 512/ $1,328 \mathrm{IU} / \mathrm{L}, \gamma$-GT $441 \mathrm{IU} / \mathrm{L}$, PT $118 \%$. Viral marker showed IgM/IgG anti-HAV $(-/+), \operatorname{HBsAg}(-)$, anti-HBs(+), anti-HCV(-), HCV RNA $(-), \operatorname{IgM} / \operatorname{IgG}$ anti-HEV $(+/+)$. Abdominal CT was unremarkable, except for wall thickening of the gallbladder, which suggested secondary change of hepatitis. With supportive care, the patient recovered rapidly, and discharged after 5 days. After one month, laboratory results was normalized. HEV RNA was detected in patient's serum and stool, and viral sequences analysis is identified Korean HEV strain, named 2010-BSNU-AYC (HM769726) belongs to genotype 4. Conclusions: It is the first case of hepatitis $\mathrm{E}$ acquired through zoonotic transmission from the raw bile juice of a wild boar living on a domestic mountain, which was verified as genotype $4 \mathrm{HEV}$ in South Korea. HEV should be a differential diagnosis of acute viral hepatitis in South Korea.

\section{PP09-05}

Bilirubin is Related to Severity of Acute Kidney Injury in Patients with Viral Hepatitis A

M.-J. Kim

Department of Internal Medicine, Inha University Hospital, Incheon City, Republic of Korea

Background: Flu-like outbreaks of viral hepatitis A(VHA) were occured in South Korea for recent years. The existence of VHArelated renal failure as a distinct disease entity is controversial. The purpose of this study was to analyze the clinical features of acute renal injury(AKI) in adults with hepatitis A.

Methods: Clinical and laboratory data of patients with AHA from January 2005 to December 2009 were analysed in our hospital. The diagnosis of AKI was based on appropriate laboratory investigations and RIFLE criteria.

Results: Total 1231 patients with VHA were admitted during 5 years and the incidence of AHA was explosively increased since 2008. AKI was noted in 40 cases in those hepatitis A patients (3.24\%). Mean ages of AKI patients were in the middle of 4th decade, male was 33 (82.5\%). In AKI group serum creatinine levels were peak $(6.3 \pm 5.7$ $\mathrm{mg} / \mathrm{dl}$ ) at 3rd hospital day. Serum AST, ALT and total bilirubin were $2353 \pm 2547 \mathrm{IU} / \mathrm{l}, 2887 \pm 2052 \mathrm{IU} / \mathrm{I}$, and $9.3 \pm 5.4 \mathrm{mg} / \mathrm{dl} \mathrm{respec}-$ tively at that time. The serum creatinine $(11.4 \pm 5.5$ vs. $3.1 \pm 1.8$ and total bilirubin $(8.7 \pm 4.6$ vs. $4.5 \pm 2.1 \mathrm{mg} / \mathrm{dl})$ were significantly higher in patients of AKI group than non-AKI group. Oliguria was seen in $85 \%$ of AKI group with three biopsy showing interstitial nephritis. Mean $4.3 \pm 2.3$ sessions of HD were performed in oliguric AKI with high level of bilirubin and renal function was completely recovered early than liver function.

Conclusion: AKI is not rare complication of VHA. The renal function and morbidity were determined by the liver dysfunction in hepatitis A, and level of total bilirubin seems to be related to severity of AKI.

\section{PP09-06}

Humanized uPA/SCID Mice as a New Animal Model for the Study of Hepatitis Delta Infection

M. Lütgheetmann ${ }^{1}$, L.V. Mancke ${ }^{1}$, T. Volz ${ }^{1}$, T. Bornscheuer ${ }^{1}$, L. Allweiss ${ }^{1}$, A.W. Lohse ${ }^{1}$, J. Petersen ${ }^{2}$, M. Dandri ${ }^{1}$

${ }^{I}$ I Department of Internal Medicine, Medical Centre Hamburg

Eppendorf; ${ }^{2}$ Institut for Interdisciplinary Medicine, Asclepios Clinic St. Georg, Hamburg, Germany
Background: Hepatitis delta virus (HDV) is a subviral agent which can cause severe liver disease in association with chronic hepatitis B virus (HBV) infection. Although HDV requires HBV envelope proteins for assembly and infectivity, little information is available about the pathophysiology of HDV infection and whether HDV can directly inhibit HBV replication in vivo.

Aim of this study was to establish a small model of HDV infection using uPA/SCID mice repopulated with primary human hepatocytes. Methods: We performed HDV infection experiments using either naïve $(N=13)$ or HBV-chronically infected $(N=5)$ human liver chimeric mice. In both experimental settings, animals received HDV/ HBV human infectious serum containing $10^{6} \mathrm{HDV}$ (genotype1) and $10^{\wedge} 8 \mathrm{HBV}$ (genotype D) genome equivalents. Serological and intrahepatic HBV (rcDNA, cccDNA) and HDV-RNA viral levels were measured over time by quantitative RT-PCR. Presence of HBsAg, $\mathrm{HBcAg}$ and HDAg was ascertained by immunohistochemistry.

Results: De novo synthesis of genomic HDV-RNA was determined both in serum and liver of 5/5 HDV super-infected mice. HDV-RNA viremia rose up to $1 \times 10^{\%}$ copies $/ \mathrm{ml}$ within 8 weeks after HDV inoculation. Notably, a median $0.5 \log$ reduction of HBV levels was determined in HDV super-infected animals as HDV replication increased, suggesting that HDV may hinder HBV replication in vivo. Establishment of HDV infection in co-infected mice was rapid and efficient (13/13 mice), with HDAg-positive staining already detectable after 3 weeks of infection. Remarkably, 6 weeks post infection mice developed high viral titres (both $10^{8}$ copies $/ \mathrm{ml}$ ) with the majority of human hepatocytes staining HDAg-positive, although HBV infection was still detectable in a minority of human hepatocytes $(0.2$ cccDNA/cell), confirming that HDV can replicate in human hepatocytes in the absence of HBV.

Conclusions: We established a new and efficient model of chronic $\mathrm{HDV} / \mathrm{HBV}$ infection for studying HDV replication mechanisms, as well as for in vivo preclinical antiviral drug evaluations.

\section{PP09-07}

Low Levels of HDV-RNA Replication and High Levels of HBsAg Expression are Associated to Liver Decompensation in Chronic Hepatitis D R. Romeo ${ }^{1}$, B. Foglieni ${ }^{2}$, L. Raffaele ${ }^{2}$, M. Spreafico ${ }^{2}$, D. Prati $^{2}$, M. Colombo ${ }^{1}$ ${ }^{1}$ Gastroenterology Unit, IRCCS Fondazione Cà Granda Ospedale Maggiore Policlinico, Milan, ${ }^{2}$ Department of Transfusion Medicine and Hematology, Ospedale A. Manzoni, Lecco, Italy

Background and aim: Hepatitis D is considered the most severe form of viral hepatitis in humans. Recent findings indicate that the progression of chronic hepatitis D can be related to hepatitis B virus (HBV) replication.. Aim of the study was to explore the possible associations of hepatitis B virus surface antigen ( $\mathrm{HBsAg}$ ) and hepatitis D virus RNA (HDV-RNA) levels with the outcome of chronically infected HDV patients.

Patients and methods: HDV-RNA and HBsAg were measured in base-line serum samples of 195 consecutive anti-HDV positive patients (149 males, mean age 54 years, 138 anti-HBe positive) followed for a mean of 256 months. HDV-RNA was quantified by quantitative real-time PCR (Roche Diagnostics), using a plasmid containing 596-bp fragment of the 5'UTR HDV-RNA as quantification standard. HBsAg was quantified by a commercially available chemoluminescent immuno assay (CMIA, Abbott Diagnostics).

Results: Liver cirrhosis was present in 122 patients (62\%). The mean HDV-RNA and HBsAg levels in cirrhotic patients were $1.4 \times 10^{6} \mathrm{cp} /$ $\mathrm{mL}$ and 5,736 IU/mL, respectively, as compared to $1.1 \times 10^{6} \mathrm{cp} / \mathrm{mL}$ and 7,502 IU $/ \mathrm{mL}$, respectively in non cirrhotics $(p=\mathrm{ns})$. During follow-up, 58 cirrhotic patients $(47 \%)$ experienced a liver related complication (29 HCC, 29 liver decompensation). The mean HDV RNA and HBsAg level in 64 cirrhotics without complication were 1.2 $\times 10^{6} \mathrm{cp} / \mathrm{mL}$ and $4,525 \mathrm{IU} / \mathrm{mL}$, respectively, as compared to cirrhotics who developed HCC $2.0 \times 10^{6} \mathrm{cp} / \mathrm{mL}$ and $4,937 \mathrm{IU} / \mathrm{mL}$ 
( $p=\mathrm{ns}$ ), and cirrhotics who developed liver decompensation $6.2 \times$ $10^{5} \mathrm{cp} / \mathrm{mL}$ and $8847 \mathrm{IU} / \mathrm{mL}$, respectively ( $p<0.001$ for both comparisons). 28 (14\%) patients died of liver related causes (14 HCC, 14 liver decompensation).

Conclusions: Low levels of HDV-RNA are associated to less progressive, event-free chronic liver disease. Liver decompensation is associated to significantly lower levels of HDV-RNA replication but significantly higher HBsAg expression.

\section{PP09-08}

Prevalence of Hepatitis E in Immigrants: a Seroepidemiological Survey in the Area of Foggia (Southern Italy)

G. Scotto ${ }^{1}$, V. Fazio $^{2}$, G. Daddiego ${ }^{1}$

${ }^{1}$ Infectious Diseases, ${ }^{2}$ Laboratory, OO RR Foggia, Foggia, Italy

Background: Hepatitis E virus (HEV) is the etiologic agent of an enterically transmitted viral hepatitis associated with inadequate hygienic conditions. HEV is endemic in developing countries where it occurs both in sporadic and endemic form. The aim of the present study is to investigate the prevalence of anti-HEV among immigrants from developing countries in the province of Foggia.

Methods: The seroprevalence of HEV was determined in a cohort of 412 asymptomatic immigrants (mostly from countries in Sub-Saharan Africa) who had recently arrived in Foggia. Serum samples were tested for anti-HEV by a commercial enzyme immunoassay (EIA) based on recombinant proteins; repeatedly positive obtained results were confirmed with a Western blot assay.

Results: A total of 90 (21.8\%) of the 412 serum samples taken from immigrants were reactive to anti-HEV IgG. Eighty-three of these (prevalence $20.1 \%$ ) were confirmed by Western blot. Anti-HEV IgM was found in 11/90 (12.0\%) of the anti-HEV Ig-positive serum samples. All anti-HEV positive immigrants were asymptomatic, and alanine transferase values were elevated only in $7 / 11$ patients with anti-HEV IgM-positive.

Conclusions: The results of this seroepidemiological study indicate a high circulation of HEV in immigrant population. The elevated prevalence of acute hepatitis interested mainly subjects arrived in Italy in the same period and coming from the same country (Eritrea).

\section{PP09-09}

RNA Silencing Suppressors (RSS) are Absent in the Genome of Hepatitis E Virus (HEV)

S.K. Panda, A. Kumar, H. Durgapal

Pathology, All India Institute of Medical Sciences, New Delhi, India

Background/aims: HEV is the major cause of sporadic and epidemic hepatitis which has a single stranded positive sense RNA genome that replicates via negative sense intermediate. The dsRNA intermediates can elicit innate RNAi response against viral genome. Thus, potential of HEV protein/s as RNA silencing suppressors cannot be ruled out. Here we report the absence of RSS activity in the HEV proteome.

Methods: SiRNAs against Firefly luciferase (Fluc) gene were designed, synthesized, converted into shRNA cassette and cloned. HEV genes encoding Methyltransferase, Helicase, Replicase, ORF2 and ORF3 proteins were cloned individually in eukaryotic expression vector. Fluc shRNAs were validated by co-transfection with target (pcDNA3-Fluc) in Huh7 cells. Inhibition was determined by dual luciferase assay at $48 \mathrm{~h}$ post transfection.

Suppressor assay was performed by co-transfecting Fluc shRNA along with target and individual viral protein expressing constructs into Huh7 cells. shRNA against Enterovirus 70 was used as unrelated control. Empty vector transfected along with target was used as reference control. Flock house Virus (FHV) protein B2 was used as a positive control for silencing suppression.

HEV protein which shows any RSS activity was further evaluated as enhancer of HEV replication in the presence of HEV specific shRNA. HEV RNA was co-transfected with individual HEV protein expressing vector and pre-validated shRNA designed against 3' NCR of the virus. Post $48 \mathrm{~h}$, RSS activity of viral protein was determined by Real Time PCR.

Results: Methyltransferase, Helicase, Replicase and ORF2 proteins did not show any RSS activity in dual luciferase RSS assay. pORF3 gave a strong RSS activity comparable with that of FHV B2 protein. But when HEV genome was targeted with pre-tested shRNA against HEV along with pSG1-ORF3, no enhancement effect was observed as demonstrated by Real Time PCR.

Conclusion: Therefore, we concluded that the HEV genome does not encode any RNA silencing suppressors.

\section{PP09-10}

The Stability and Inactivation of Hepatitis $\mathbf{E}$ Virus Grown in Cell Culture T. $\mathbf{L i}^{1}$, S. Song ${ }^{2}$, Q. Yang ${ }^{2}$, K. Ishii ${ }^{1}$, N. Takeda ${ }^{3}$, T. Wakita ${ }^{1}$

${ }^{I}$ National Institute of Infectious Disease, Tokyo, Japan; ${ }^{2}$ Hangzhou City Yuhang District Center for Disease Control and Prevention, Hangzhou, China; ${ }^{3}$ RCC-ERI, Bangkok, Thailand

Purpose: Hepatitis E virus (HEV), a small round-structured virus consists of a 7.2-kb positive-sense RNA genome packaged within a non-enveloped capsid, is a causative agent of hepatitis E. The human HEV strains appear to fall into at least four major genetic groups. Although the cell culture system and experimental animal models are indispensable techniques for the clarification of the mechanisms of viral replication and infection, these mechanisms of HEV are not elucidated because of the lack of these techniques. For these purposes we developed an efficient culture system of HEV and examined the stability of the virus by using this system.

Materials and methods: Virus: Genotype $3 \mathrm{HEV}$ was propagated in PLC/PRF/5 cells. HEV was efficiently secreted in the culture supernatant.

To examine the stability, HEV was inoculated onto PLC/PRF/5 cells after heat treatment or treatments with UV, NaCIO, Ethanol or chloroform, respectively. If HEV was inactivated by these treatments, viral antigen could not be detected from culture supernatant by ELISA system. The test period is 2 months.

Results: Heat treatment at $65^{\circ} \mathrm{C}$ for 5 min resulted in a complete inactivation of HEV. HEV is also inactivated by the treatment with $\mathrm{NaCIO}$ with concentration above $125 \mathrm{ppm}$ or $50 \mathrm{~mW}$ UV-irradiation for $30 \mathrm{~min}$. HEV showed resistance in alcohol and chloroform.

Discussion: Because of the lack of efficient cell culture system of $\mathrm{HEV}$, the stability of HEV virions had not been well examined. Information of HEV stability obtained by this study is useful for the prevention of $\mathrm{HEV}$ infection and $\mathrm{HEV}$-associated food poisoning.

\section{PP09-11}

Nitric Oxide Synthase and Hepatitis E Infection

R.K. Hazam, M.P. Sarma, P. Deka, S. Medhi, P. Kar

Maulana Azad Medical College, New Delhi, India

Background and aim: Nitric oxide (NO) is one of the versatile mediators in the control of viral infections, acting as a pro-apoptotic inducer in some cell types or as an antiapoptotic modulator in other cell types including hepatocytes makes it reasonable to consider this molecule as a potential player in Hepatitis E virus (HEV) infection. Correlation between iNOS, eNOS polymorphism, expression and severity of disease in hepatitis B related hepatitis has been reported earlier. The study was aimed to determine the role of iNOS and eNOS gene polymorphism and their expression in HEV related liver disease.

Methods: 197 AVH, 37 FHF and age and sex matched 256 healthy controls were included. PCR amplification for iNOS and eNOS genes was performed using specific primers. RFLP analysis was carried out by restriction digestion using enzymes TSP509I for iNOS and Ban II for eNOS to detect the polymorphism. Expression study was done using commercially available ELISA kits and by rtPCR. 
Results: The frequency of iNOS $(\mathrm{CT}+\mathrm{TT})$ and eNOS $(\mathrm{GT}+\mathrm{TT})$ genotypes was non significantly higher in FHF compared to AVH and healthy controls. iNOS and eNOS expression in FHF (56.5 $\pm 6.2 \mathrm{IU} /$ $\mathrm{ml}, 61.1 \pm 1.7)$ cases were significantly increased as compared to AVH $(17.2 \pm 8 \mathrm{IU} / \mathrm{ml}, 23.3 \pm 4.2)$ and controls $(7.7 \pm 3.2 \mathrm{IU} / \mathrm{ml}, 16$ $\pm 3.6)$ both by ELISA $(p<0.005)$ and semi quantitative rt-PCR. Conclusion: iNOS and eNOS may be a potential determinant of susceptibility to fulminant hepatitis, but larger population based studies are required to confirm the observations.

\section{PP09-13}

Seroepidemiology and Genetic Characterization of Hepatitis E Virus among Different Animals in Beijing, China

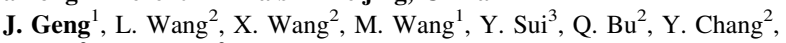

Y. Zhu ${ }^{2}$, H. Zhuang ${ }^{2}$

${ }^{1}$ Institute of Hepatology, The 81 st Hospital of PLA, Jiangsu Province, Nanjing, ${ }^{2}$ Peking University Health Science Center, Beijing;

${ }^{3}$ Institute of Hepatology, The 81st Hospital of PLA, Nanjing, China

Background/aims: Anti-HEV has been detected in numerous animal species, and accumulating evidence support that HEV genotype 4 is the dominant genotype in China since year 2000. The aim of this study is to investigate the prevalence of HEV genotypes among different species of animals, general population and workers in pig farms and slaughter-houses in Beijing.

Method: Anti-HEV in sera was detectedby enzyme immunoassays. All fecal samples and some blood samples were detected for HEV RNA by a nested RT-PCR assay. PCR products were cloned and sequenced, and analyzed genetically.

Results: The prevalences of anti-HEV in serum of swine, rabbits, cattle, milk cow, horse, sheep, donkey, dog, duck, chicken, pig farm workers, slaughterhouse workers and general population were $81.17 \%$ (802/988), 54.62\% (65/119), 25.29\% (66/261), $14.87 \%$ (40/269), $14.29 \%$ (40/280), 9.3\% (53/514), 0 (0/25), 0 (0/20), $2.53 \%(8 / 316)$, $3.03 \%$ (7/231), 67.31\% (35/52), 35.9\% (70/195) and 20.29\% (522/ 2572), respectively. The anti-HEV prevalence of adult ( $\geq 6$ months) and younger swine ( $\leq 3$ months) were $91.49 \%$ (591/646) and $61.7 \%$ (211/342) respectively. The positive rates of HEV RNA in young swine was $47.94 \%(93 / 194)$. Ninety three isolates shared $87.8-100 \%$ nucleotide homology with each other and had identities of 75.6-78.9, $73.9-76.1,76.4-80.6$ and $83.1 \%-95.0 \%$ with the corresponding regions of HEV genotypes 1, 2, 3, 4, respectively. Swine HEV isolates showed the highest similarity with the human isolate T1 $(95.0 \%)$ from a patient in same area. Phylogeneticaly, all HEV isolates belong to subgenotype 4 days.

Conclusion: HEV genotype 4 infection is widely spread among many species of animals and human beings in Beijing, China. Swine is the principle animal reservoir of HEV may play an important role in HEV transmission from swine to humans.

\section{PP09-14}

Do Patients with Chronic Hepatitis B Infection Are Aware of Delta Hepatitis?

Y. Gumurdulu ${ }^{1}$, U.B. Dogan ${ }^{2}$, B.E. Tasdogan ${ }^{1}$, B. Kara ${ }^{2}$

${ }^{1}$ Cukurova University Faculty of Medicine Department of

Gasroenterology; ${ }^{2}$ Adana Numune Training and Research Hospital

Department of Gastroenterology, Adana, Turkey

Introduction: Delta hepatitis which is seen as coinfection or superinfection with chronic hepatitis B have a similar clinical course like the other hepatitis, but it progresses more severely and have a higher risk of fulminant course. With delta superinfection it takes at least 10 years lesser to develop liver cirrhosis than with only chronic hepatitis B. Delta virus is responsible for $30 \%$ of fulminant hepatitis cases seen in patients with HBV. For these reasons, we think that $\mathrm{HbsAg(+)}$ patients must be aware of the delta virus and protect themselves from the contamination ways.
Patients and method: We prepared a questionnaire for the patients diagnosed with chronic hepatitis B who admitted to our out-patient clinic to find out whether they are informed about the delta virus. In this survey the patients' age, gender, the duration of chronic hepatitis $\mathrm{B}$ diagnosis and whether information about delta virus/hepatitis was given or not were asked. Also the sources of the information were asked to the patients who were informed before.

Results: Totally 255 patients participated to our questionnaire. The mean duration of hepatitis B diagnosis was $81.7 \pm 69$ (1-360) months. While $96.1 \%(n=245)$ of the patients did not have any information about the hepatitis D virus and/or delta hepatitis and have not heard about it, 3.9\% $(n=10)$ had information about this topic. None of the patients were given information about the delta virus during their follow-up.

Discussion: It is known that the prognosis of the patients with chronic hepatitis B worsens when infection with HDV adds on. These patients mostly are not informed about the delta virus. For these reasons, we think that more information about delta hepatitis and virus must be given to the patients with hepatitis B infection by their clinicians and they must be warrented about the protection from the contamination sites.

\section{PP09-15}

Prevalence of Hepatitis A in Iranian Patients with Chronic Hepatitis B or Hepatitis C Infection

L. Zeidabadi Nejad ${ }^{1}$, P. Shoaei ${ }^{1}$, A. Babak ${ }^{1}$, M. Yaran ${ }^{1}$, B. Ataei ${ }^{1}$, M. Forughifar ${ }^{1}$, N. Kassaian ${ }^{2}$

${ }^{I}$ Infectious Diseases Research Center, Isfahan University of Medical Sciences; ${ }^{2}$ Infectiouse Diseases, AIDS and Clinical Immunology Research Center, Isfahan, Iran

Background: Acute hepatitis A is a critical disease in patients with chronic hepatitis B or hepatitis $\mathrm{C}$ infection which may lead to more severe complications. Therefore, routine vaccination against HAV infection is recommended in these patients in many countries. Nevertheless, studies of HAV seroprevalence and exposure predictors in populations with chronic hepatitis $\mathrm{B}$ or hepatitis $\mathrm{C}$ infection are scanty in our region.

Patients and methods: We studied on 135 patients with chronic hepatitis B and 59 patients with hepatitis $C$ infection who referred in Isfahan Infectious Diseases and Tropical Medicine Research Center during spring 2007-spring 2008. HAV-Ab was measured using ELISA method and the seroprevalence was obtained based on demographic characteristics. Logistic regression analysis was used to identify independent risk factors in patients with HAV infection in our cases.

Results: HAV seropositivity was detected in 188 (97\%) ones (95\% in Hepatitis B and $100 \%$ in hepatitis C patients). There was no independent risk factor for HAV infection neither in hepatitis $\mathrm{B}$ nor in hepatitis $\mathrm{C}$ patients.

Conclusion: According to the findings, hepatitis A is common in patients with chronic hepatitis $B$ and $C$. So, vaccination against HAV infection is recommended in these patients.

\section{PP09-16}

The Seroprevalence and Clinical Significance of Hepatitis D Virus Infection in Korean Chronic Hepatitis B Patients

H.S. Kim, S.J. Kim, H.W. Park, W.G. Shin, K.H. Kim, J.H. Lee, H.Y. Kim, M.K. Jang

Department of Internal Medicine, Kangdong Sacred Heart Hospital of Hallym University Medical Center, Seoul, Republic of Korea

Background/aims: Hepatitis D virus (HDV) infection can cause more severe liver disease in chronic hepatitis B (CHB) patients. Korea is an endemic area of hepatitis B virus infection. However, little has been known about the HDV prevalence and clinical features of HDV infection in Korean CHB patients. This study was aimed to identify the seroprevalence and clinical features of HDV Infection in Korean CHB Patients. 
Methods: A total of $940 \mathrm{CHB}$ patients were consecutively enrolled from January 2008 to August 2010. All patients were hepatitis B surface antigen (HBsAg) positive for at least six months and tested for anti-HDV. A portion of the HDV delta antigen was amplified, sequenced and subjected to molecular and phylogenetic analysis using serum from the patients with positivity to anti-HDV. Clinical features and virologic markers were evaluated.

Results: The median age was 47 (15-94) years and $64.4 \%$ of the patients were male. $\mathrm{HBeAg}$ was positive in 347 patients (36.9\%). Inactive $\mathrm{HBsAg}$ carrier, CHB, cirrhosis and hepatocellular carcinoma accounted for 29, 45.6, 17.8 and $7.6 \%$, respectively. Anti-HDV was positive in three patients among the 940 subjects, giving a $0.32 \%$ positive rate. All patients with positivity to anti-HDV were inactive HBsAg carrier. HDV RNA could be detected in two patients. Phylogenetic analysis revealed that two patients were infected by genotype 1 of HDV.

Conclusions: HDV seroprevalence is very low $(0.32 \%)$ in Korea and all patients were infected by genotype 1 of HDV. HDV infection does not impact clinical course in Korean CHB patients.

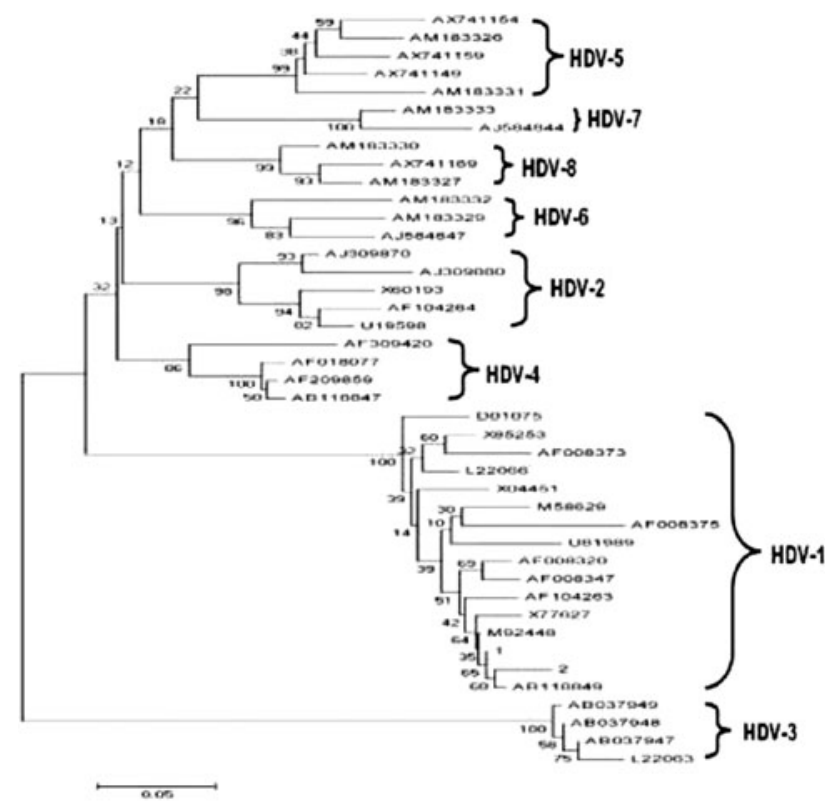

Fig. 1 Phylogenetic analysis od=f a 356-bp fragment from two patients using the $\mathrm{NJ}$ method

\section{PP09-17}

Comparison of Clinical Features of Hepatitis A between HBsAg-Positive and HBsAg-Negative Patients

K.M. Kim, S.J. Eo, G.-Y. Gwak, M.S. Choi, J.H. Lee, K.C. Koh, B.C. Yoo, S.W. Paik

Division of Gastroenterology, Samsung Medical Center, Sungkyunkwan University School of Medicine, Seoul, Republic of Korea

Background/aims: The notion that acute hepatitis A superimposed on chronic hepatitis B infection leads to a worse outcome than acute hepatitis A alone remains controversial. The aim of this study was to determine the influence of the presence of hepatitis B surface antigen (HBsAg) on the severity of acute hepatitis A.

Methods: We analyzed the medical records of 449 patients hospitalized for acute hepatitis A from January 2000 to February 2010 and compared clinical outcomes after dividing these patients into two groups according to the presence of $\mathrm{HBsAg}$.

Results: Of the 449 patients, there were 30 patients in the HBsAgpositive group and 419 in the $\mathrm{HBsAg}$-negative group. The HBsAg- positive group was older than the HBsAg-negative group $(36.1 \pm 8.3$ vs. $31.8 \pm 8.5$ years, $P=0.004$ ), but other baseline characteristics were similar between the two groups. Mean platelet count at the time of admission was significantly lower, and mean peak values of protrombin time and serum total bilirubin during admission were significantly higher, in the HBsAg-positive group. When comparing clinical outcomes between the two groups, gastrointestinal bleeding, acute renal failure, and acute liver failure were more frequently observed in the HBsAg-positive group. In particular, the incidence of acute liver failure was approximately ninefold higher in the HBsAgpositive group than in the HBsAg-negative group ( 7 vs. 14 , OR 8.80 , $P=0.000)$. Multivariate analysis showed that the presence of $\mathrm{HBsAg}$ (OR 7.43, 95\% CI 2.56-21.57) and age (OR 1.07, 95\% CI 1.02-1.13) were independent risk factors for occurrence of acute liver failure. Conclusions: In patients with chronic hepatitis B infection, acute hepatitis A is associated with more severe clinical outcomes, including acute liver failure, compared with patients with acute hepatitis A alone. Consequently, hepatitis A vaccination is strongly recommended for individuals with chronic hepatitis B infection, especially older adults.

\section{PP09-18}

Raising Monoclonal Antibodies against Hepatitis E Virus (HEV) Proteins and Their Uses in Immunohistochemistry

S.K. Panda, P. Gupta, H. Durgapal

Department of Pathology, All India Institute of Medical Sciences, New Delhi, India

Background and objectives: HEV is one of the major causes of acute viral hepatitis worldwide. Monoclonal antibodies (MAbs) can prove very effective and useful for detection of HEV in historical pathologic material.

Method: MAbs against HEV pORF2 (aa 1-660) and pORF3 (aa 1-123) were generated by conventional hybridoma technique. Briefly, 6-8 weeks old female BALB/c mice were immunized using DNA prime protein boost regime. Splenocytes harvested from the immunized animals were fused with SP2/O-Ag14 mouse myeloma cells in the presence of polyethylene glycol 1500 (Sigma). The culture supernatants were then screened by ELISA. Reactive clones were then subcloned three times by limiting dilution and expanded. The MAbs were produced by growing cells in serum free media (Sigma) followed by ammonium sulfate precipitation. The lmmunoglobulin isotypes of the MAbs were determined using mouse monoclonal antibody isotyping reagents (Sigma) as per the manufacturer's instructions. The MAbs generated against pORF2/pORF3 were used to detect these antigens in liver biopsy of HEV infected patients by Immunohistochemistry using standard protocol.

Results: HEV anti-ORF2 and anti-ORF3 MAbs from different clones were produced and characterized by ELISA, Immunofluorescence, Western Blotting and Isotyping. The majority of antibodies tested were of IgG1 and IgG2a class. The anti-ORF2/ORF3 MAbs could detect pORF2/pORF3 respectively in liver biopsy of HEV infected patients. This study is first to demonstrate HEV antigens directly in liver biopsies.

Conclusion: The MAbs generated against HEV pORF2/pORF3 may be used for direct detection of HEV in liver biopsies of chronic HEV and HEV superinfected patients.

\section{PP09-19}

Epidemiological and Genetic Analysis of a Diffuse Outbreak of Hepatitis A in Japan, 2010

K. Ishii

Department of Virology II, National Institute of Infectious Diseases, Tokyo, Japan

Background/aims: Hepatitis A virus (HAV) is still one of the common causative agents for acute hepatitis in Japan. Most of the 
infections occurred in Japan were sporadic with exceptional occurrence of small scale outbreaks. In 2007, 2008 and 2009, relatively low number of cases (approximately 100-150) of acute hepatitis A was reported. In 2010, however, 308 cases have been reported as of week 38 . For the purpose of epidemiological investigation of $2010 \mathrm{HAV}$ resurgence, we genotyped HAV specimens obtained from 60 cases. Methods: Viral RNA from a total of 98 serum specimens of 60 patients from whom serum and stool samples had been obtained was extracted and a 230-bp fragment of VP1/2A region was amplified by RT-PCR. Nucleotide sequences of the amplified products were aligned with those of HAV genotypes available from GenBank by using ClustalW2 software. Phylogenetic trees were constructed with the MEGA software by the Neighbor-Joining method.

Results: Analysis of the VP1/2A region sequence revealed that 60 HAV strains were divided into 3 genotypes: 1A (43 cases), 1B (1 case) and $3 \mathrm{~A}$ (16 cases). The genotype $1 \mathrm{~A}$ consisted of 2 genomic clusters.

Conclusions: The sequences of one of the two 1A clusters (31 cases) were very similar and 26 of them had $100 \%$ identity. These sequences were similar to strains isolated from the river and sewage of Philippines. The other $1 \mathrm{~A}$ cluster consisted of the endemic strains in Japan. The sequences of $3 \mathrm{~A}$ strains in Japan were similar to the strains that cased recent large epidemic in the Republic of Korea. The resurgence of HAV in 2010 can be attributed to importation of the HAV of the newly emerged two genotypes.

\section{PP09-20}

Putative Host Range and Disease Severity Determinants in Hepatitis E Virus

Y. Zhu, H. Fu, L. Wang, J. Geng, L. Li, X. Wang, H. Zhuang

Department of Microbiology, Peking University Health Science

Center, Beijing, China

Hepatitis $\mathrm{E}$ is a worldwide public health problem, particular in areas where hygiene conditions are poor. Hepatitis E virus (HEV) has at least four genotypes: genotypes 1 and 2 exclusively infect human; while genotypes 3 and 4 , is considered to be a zoonotic agent, infecting both humans and animals. This study was aimed at determining why genotype 3 and $4 \mathrm{HEV}$ strains isolated from swine are able to cross species borders, whereas genotype 1 and 2 strains isolated from humans are not. The full length genome of the swine HEV isolate CHN-XJ-SW13 was amplified as overlapping fragments using reversetranscription-nested polymerase chain reaction (RT-nPCR) and rapid amplification of cDNA ends (RACE). The sequence of CHN-XJ-SW13 was compared with those of 86 HEV strains covering genotype 1-4 retrieved from GenBank. Possible regions of the viral genome, specifying the host range of HEV or associated with the severity of hepatitis E disease, were then screened for with the aid of the ALIGNX sequences alignment software package. The CHN-XJSW13 swine HEV isolate was determined to be a novel subtype of genotype 4 , whose sequence provided several valuable clues for tracing the sources of human HEV infection. 25 specific nucleotide positions were identified to possibly being involved specifying the host range of $\mathrm{HEV}$ or determining the severity of hepatitis E disease.

\section{PP09-21}

Hepatitis A Seropositivity among Fresh Man Medical Students, Isfahan (Iran), 2007

P. Shoaei ${ }^{1}$, H. Rahimi ${ }^{1}$, M. Yaran ${ }^{1}$, A. Babak ${ }^{1}$, M. Ataie ${ }^{2}$, M. Foroughifar ${ }^{1}$

${ }^{1}$ Infectious Diseases and Tropical Medicine Research Center, Isfahan University of Medical Sciences; ${ }^{2}$ Najaf Abad Islamic Azad University, Isfahan, Iran

Background and aims: Health science students are commonly exposed to some infectious agents, including hepatitis A virus (HAV) which may cause substantial morbidity and even death. This serological survey was performed among medical students of Isfahan (the second big city in Iran) to determine the prevalence of $\operatorname{IgG}$ antibody against $\mathrm{HAV}$, and as a secondary objective to determine risk factors for acquisition of this infection.

Patients and methods: All participants completed a structured questionnaire that assessed demographic and socio-economic characteristics. Anti-HAV IgG was tested using DIA-PRO Elisa Kit.

Results: A total of 403 students, $252(62.5 \%)$ male and 151 (37.5\%) female, were included in the study. The prevalence of Anti-HAV IgG was detected as $67.5 \% .89 .3 \%$ of them used healthy water, $7.4 \%$ had a history on icter, $0.5 \%$ hepatitis A history, and $0.5 \%$ had over injected antiHAV immunoglobulin. Correlation of hepatitis A infection with residency and drinking water had significant factors $(P$ value 0.01 and 0.018 respectively).

Conclusion: It was suggested that the routine HAV-vaccination for medical students is not necessary. Health's care instructions and safe water limits the infection.

\section{PP09-22}

The Dynamic Changes of Humoral Immunity in Patients with Hepatitis E Superinfection in Chronic Hepatitis B

Z. Xiaohong, W. ZheBin, Z. Ying, C. Hong, G. ZhiLiang

The Third Affiliated Hospital of Sun Yat-Sen University, Guangzhou, China

Aims: The present study was carried out to evaluate dynamic changes of the serum immunoglobulins level (IgG, IgM, IgA) and complement components (C3, C4,CH50), and cellular components (CD8+, CD4+, $\mathrm{CD} 4+/ \mathrm{CD} 8+$ ) in hepatitis $\mathrm{E}$ superinfection in chronic hepatitis $\mathrm{B}$ patients in comparison with acute exacerbation of chronic hepatitis $\mathrm{B}$ patients, and acute hepatitis E patients.

Patients: 22 cases of hepatitis E superinfection with prior chronic hepatitis B virus infection were enrolled in the study group, 19 cases of acute exacerbation of chronic hepatitis B infection, and 17 cases of acute hepatitis $\mathrm{E}$ virus infection were enrolled as compared groups.

Results: In the group of patients with hepatitis E superimposed on chronic hepatitis B infection, the serum IgG and $\operatorname{IgA}$ level is higher than normal. According to the duration of the disease, the serum IgG and IgA level is higher and higher, there was a significant difference between the level of $\operatorname{IgG}$ and $\operatorname{IgA}$ at the beginning and on the 4 weeks after onset $(P<0.05)$. There were no significant differences of the serum IgM, C3, C4, CH50 level, and the count of CD8+, CD4+ T lymphocytes in the peripheral blood from the beginning to the 4 weeks after onset.Compared with acute hepatitis E,the patients with hepatitis $\mathrm{E}$ superimposed on chronic hepatitis B infection is associated with higher level of serum $\operatorname{IgG}, \operatorname{IgA}(P<0.05)$.Compared with acute exacerbation of chronic hepatitis $\mathrm{B}$,the patients with hepatitis $\mathrm{E}$ superimposed on chronic hepatitis B infection is only associated with higher serum $\operatorname{IgG}$, IgA level $(P<0.05)$ on the 4 weeks after onset. Conclusions: In the cases of hepatitis $\mathrm{E}$ superimposed on chronic hepatitis B infection, the serum IgG and IgA may play an important role in the damage of hepatocytes.

\section{PP09-23}

Clinical and Virological Characteristics of Hepatitis B\&D in Chronically Infected Patients with Respect to Level of Education in Pakistan

N. Lal, M.S. Memon, M. Zaki, K. Ansari, P. Abbasi, B. Tufail, S. Tarar, S. Gill, S. Solangi, S. Laghari

Section of Gastroenterology and Hepatology, Isra University Hospital, Hyderabad, Pakistan

Background and aim: Hepatitis B virus (HBV) infection and hepatitis delta virus (HDV) present worldwide and affects all age groups. It is more prevalent in rural population. Little is known about the level of education and clinical and virological characteristics of $\mathrm{HBV}$ and HDV.

Method: We prospectively investigated above characteristics in 426 HBSAg positive patients at Hepatology clinic from 2006 to 2009. 
The participants were divided into two groups; group $\mathrm{A}<10$ years and group B more than 10 years of education. Both groups were matched from mean age, ethnicity, mean weight, BMI at the first visit.

Results: Out of 426 patients, 193 (45.3\%) were coinfectioned with delta virus. The sero prevalence of delta antibody and HCV antibody were significantly higher in group " $\mathrm{A}$ " with $p$ value of 0.002 and 0.04 respectively. There was no difference in mean level of HBV DNA, presence of HBeAg and mean ALT levels in both groups. There was significantly increased morbidity of liver disease (presence of cirrhosis and advanced Child's Class) associated with group "A", independent of co-infection with HDV or HCV.

Conclusion: Lower the level of education is associated with significantly higher prevalence of delta antibody, HCV antibody and advanced liver disease in HBSAg positive patients.

\section{PP09-24}

Virological and Clinical Characteristics of Delta Virus Infection in Rural and Urban Population of Sindh, Pakistan

S. Ali, M.S. Memon, K. Ansari, M. Zaki, P. Abbasi, T. Bhatti, S. Gill, S. Solangi, S. Laghari

Section of Gastroenterology andHepatology, Isra University Hospital, Hyderabad, Pakistan

Background and aim: Hepatitis "D" virus infection or delta hepatitis has been studied in various regions of Pakistan, but prospective data comparing rural and urban population with respect to clinical and virological characteristics is sparse.

Method: We prospectively investigated 426 the HBSAg positive patients at our Hepatology clinic belongs to all over Sindh except Karachi. We divided patients in urban and rural groups. All the participants under went testing for HBeAg, HBV DNA both qualitative and quantitative assays, delta antibody testing and delta RNA where appropriate. Both groups were matched in age, weight and BMI.

Results: Out of 426 patients, 193(45.3\%) were reactive for delta antibody. In rural it was 37 and $8.2 \%$ in urban with $p$ value of 0.003 . HDV RNA was tested in 140 anti HDV reactive patients. It was detected in 48/114 (42\%) rural and 12/26 (46\%) urban patients with $p$ value of 0.2 . Hepatitis delta was significantly more prevalent in rural male compared to urban male with $p$ value of 0.01 . No difference in female sex seen. More advance disease is seen in rural patients \{Cirrhosis with anti HDV positive. Rural 72/158 (45.6\%), urban $11 / 35(31.4 \%) P$ value $<0.001\}$. There was no difference in urban and rural groups with respect to presence of HBV DNA, HBeAg, anti $\mathrm{HCV}$ antibody and ALT levels in females.

Conclusion: Significantly higher anti HDV is prevalent $(37 \%)$ in rural patients of Sindh province. HDV co infection is associated with increased morbidity of liver disease in both rural and urban patients.

\section{PP09-25}

An Experience of Pegylated Interferon in Patients with Chronic

Hepatitis D

Z. Abbas ${ }^{1,2}$, H. Mithani ${ }^{3}$, S. Raza ${ }^{4}$

${ }^{1}$ Hepatogastroenterology, Sindh Institute of Urology

and Transplantation; ${ }^{2}$ Orthopaedic and Medical Institute;

${ }^{3}$ Civil Hospital; ${ }^{4}$ Dow Medical University, Karachi, Pakistan

Background and aims: Hepatitis D is a difficult to treat infection. Standard interferon-alpha is not an ideal treatment. Recent few trials with pegylated interferon have shown better response. The aim of this study was to evaluate the local experience of the efficacy of pegylated interferon for the treatment of chronic hepatitis D.

Methods: An observational study. Data of the HDV RNA positive patients treated with pegylated interferon in the last 2 years was analyzed. Patients were treated for 48 weeks. Virological and biochemical responses were noted.
Results: Total number of patients treated so far is 65 . Three patients decompensated during treatment. Thirty three patients have completed the 48 weeks treatment. Thirty-one are males. Mean age 28 years (range 11-55). Eighteen patients received pegylated interferon alpha-2a and rest alpha-2b. Clinical cirrhosis was present in 14 (42\%). End of treatment viral clearance was seen 16/33 (48\%) cases. ALT normalization was seen in $17(51 \%)$. Nine patients have completed 6 months post-treatment. Three had sustained response. The treatment was well tolerated by most of these patients.

Conclusion: Patients of hepatitis D should be considered for treatment with Pegylated interferon as a significant proportion of patients do respond to treatment.

\section{PP09-27}

First Report of HDV Genotype II in Iran

P. Meysami ${ }^{1}$, H. Keyvani ${ }^{1}$, S.H. Monavari ${ }^{1}$, S.M. Alavian ${ }^{2}$

${ }^{1}$ Department of Virology, Iran University of Medical Sciences, Tehran, Iran; ${ }^{2}$ Baqiyatallah Research Center for Gastroenterology and Liver Disease, Tehran, Iran

Background: Hepatitis D virus is a defective satellite virus that uses the surface antigen of hepatitis B virus for transmission and propagation. Epidemiological studies have shown that prevalence of HDV infection among HBs antigen carriers is approximately $5 \%$ all over the world. Recently, Sequence analysis of numerous isolates has led to classification of HDV into eight major clades.

Methods: In this study, 150 patients who were tested for HBs Ag and HDV antibody were analyzed by nested polymerase chain reaction (n PCR). Genotypes of HDV were determined in PCR positive patients by restriction fragment length polymorphism (RFLP) method.

Results: HDV RNA was detected in serum samples of $45(30 \%)$ out of 150 patients when tested by nested PCR. Genotyping of HDV RNA isolated from positive PCR patients showed that $40(88.9 \%)$ patients had genotype I, 3(6.7\%) patients had genotype II while $2(4.4 \%)$ patients' genotypes remained undetermined by RFLP method. The latter will be subject for sequencing in further studies.

Conclusion: Our data indicate that the majority of hepatitis delta virus isolates in HBs Ag carrier are genotype I and II. Since 6\% of virus genotype still remains undetermined, further studies using more precise methods, such as sequencing, is nessacery. This data indicate that the distribution of genotype II is not restricted to East of Asia, but is spread over the different parts of Asia.

\section{PP09-28}

Clinical Characteristics of Acute Hepatitis A in Patients with Chronic Hepatitis B

M.S. Lee, S.R. Shin, K.T. Suk, H.S. Kim, M.K. Jang, S.H. Park, D.J. Kim, C.K. Park Hallym University Medical Center, Seoul, Republic of Korea

Background/aims: To date, it has been controversy over the impact of chronic hepatitis $\mathrm{B}(\mathrm{CH}-\mathrm{B})$ on clinical outcome of acute hepatitis $\mathrm{A}$ (AH-A). This study was aimed to evaluate clinical characteristics in patients with $\mathrm{AH}-\mathrm{A}$ on underlying $\mathrm{CH}-\mathrm{B}$ compared to patients with AH-A alone.

Methods: Data of 719 patients diagnosed with AH-A from January 2007 to September 2010 in Hallym University Medical Center were reviewed retrospectively.

Results: Twenty four patients (3.3\%) had history of CH-B and showed HBsAg positive. Among them, 8 were HBeAg-positive and 16 were $\mathrm{HBeAg}$-negative including 4 non-replicative HBsAg carriers. Demographic features were not significantly different between patients with $\mathrm{AH}-\mathrm{A}$ superimposed on $\mathrm{CH}-\mathrm{B}$ and $\mathrm{AH}-\mathrm{A}$ alone. Duration of illness before hospital visit and clinical symptoms such as fever, jaundice, skin rash, anorexia, nausea, vomiting, epigastric pain, diarrhea, and myalgia were similar in two groups. Laboratory findings including $\mathrm{CBC}$, prothrombin time (PT), aminotransferases, bilirubin, 
and albumin level were not different between both groups, but serum creatinine level was higher in patients with AH-A on CH-B (1.12 vs. $0.93 \mathrm{mg} / \mathrm{dL}, p=0.018$ ). Acute kidney injury defined as an increase in serum creatinine to more than 2 folds from baseline was more frequent in patients with $\mathrm{AH}-\mathrm{A}$ on $\mathrm{CH}-\mathrm{B}$ (8.3 vs. $1.3 \%, p=0.049$ ). Frequencies of acute liver failure, prolonged cholestasis, and relapsing hepatitis were not significantly different between both groups. Hospital stay and time to PT normalization were similar in two groups. There were no significant differences in clinical and laboratory findings according to $\mathrm{HBeAg}$ status or HBV DNA level.

Conclusions: Our data suggest that clinical course and outcome of AHA superimposed on $\mathrm{CH}-\mathrm{B}$ were not significantly different from $\mathrm{AH}-\mathrm{A}$ alone, but renal impairment occurred more frequently in patients with underlying $\mathrm{CH}-\mathrm{B}$. In addition, $\mathrm{HBeAg}$ status or level of HBV replication did not affect clinical outcome of $\mathrm{AH}-\mathrm{A}$ in $\mathrm{CH}-\mathrm{B}$ patients.

\section{PP09-29}

Hepatitis E Virus in Cryptogenic Liver Cirrhosis

F. Akyuz ${ }^{1}$, B. Pinarbasi ${ }^{2}$, M. Bozaci ${ }^{3}$, B. Baran ${ }^{4}$, U. Akyuz ${ }^{5}$, A. Uyanikoglu ${ }^{4}$, K. Demir ${ }^{4}$, F. Besisik ${ }^{4}$, S. Ozdil ${ }^{4}$, G. Boztas ${ }^{4}$, Z. Mungan ${ }^{4}$, S. Badur ${ }^{4}$,

S. Yenen ${ }^{4}$, S. Kaymakoglu ${ }^{4}$

${ }^{1}$ Istanbul Faculty of Medicine; ${ }^{2}$ Gastroenterohepatology;

${ }^{3}$ Microbiology; ${ }^{4}$ Istanbul University, Istanbul Faculty of Medicine;

${ }^{5}$ Gastroenterology, Yeditepe University, Istanbul, Turkey

Background and aim: Hepatitis E virus(HEV) is one of the well known causes for acute hepatitis that does not progress to chronic hepatitis and cirrhosis. Few immunosupressed patients in whom chronic hepatitis developed were reported in recent years. In this study we aimed to investigate presence of $\mathrm{HEV}$ infection in cryptogenic cirrhosis.

Material-methods: Twenty-one healthy donors (12 male) and 35 cryptogenic cirrhotic patients (19 male) were studied. All patients were negative for any known etiology of chronic liver diseases. Stored serum samples of patients at $-85^{\circ} \mathrm{C}$ were analyzed. A commercially available diagnostic anti-HEV ELISA kit(Dia Pro, Milan, Italy) was used to detect anti-HEV antibodies. HEV RNA isolated by QIAMP Viral RNA mini kit (QIAGEN, Hilden, Germany). HEV RNA quantification was performed by Geno Sen's HEV(Rotor Gene) Quantitative Real Time PCR Kit (Genome Diagnostics Pvt. Ltd, Netherlands) using Rotor Gene 3000 real time PCR system.

Results: Mean age of patients and controls were comparable, $46 \pm 12.1$ years, $37.5 \pm 9.7$ years, respectively. Mean ALT, AST, ALP and GGT levels were $36 \pm 15,50 \pm 21,220 \pm 90,67 \pm 85 \mathrm{IU} / \mathrm{L}$ respectively in patients. Anti HEV positivity rate was 9.5 and $25.7 \%$ in control and patients, respectively $(p>0.05)$. Although, we did not find any HEV RNA positivity in control group, HEV RNA was positive in 3 patients $(8.6 \%)(p>0.05)$. HEV RNA titers and AST levels at that time of sera collection were 326.461 copies/ml, $91 \mathrm{IU} / \mathrm{L}, 480$ copies $/ \mathrm{ml}, 68$ IU/L and 72 copies/ml, $42 \mathrm{IU} / \mathrm{L}$, for each HEV RNA positive patient respectively. There was a positive correlation between HEV RNA titers and AST levels ( $p=0.04, r=0.642)$. However, we do not know yet whether these HEV infections were chronic.

Conclusion: This study showed that anti-HEV and HEV RNA positivity seems to be high in cryptogenic cirrhosis. HEV RNA might be responsible for inflammatory-biochemical activity in cryptogenic cirrhosis.

\section{PP09-30}

\section{Retrospect Case Analysis of Hepatitis E in Elderly Chinese}

Y. $\mathbf{L i}^{1}$, J. Yu ${ }^{2}$, T. Tang ${ }^{1}$

${ }^{1}$ Department of Gastrointestinal Diseases; ${ }^{2}$ Department of General Surgery, Hospital No. 1 of Jilin University, Changchun, China

Aim: To analyze the characters of hepatitis E in elderly Chinese compare to young patients.

Methods: Retrospect analysis of hepatitis E cases in elderly and young patients treated between 2007 and 2010. Elderly group contains 53 cases between age of 61 and 82 (average 66.5), of which 42 are male, 11 are female. Young group contains 64 cases between age of 17 and 56 (average 36.3), of which 41 are male, 23 are female. We compared symptoms, liver function, hospitalization period and prognosis between two age groups.

Results: Compare to young group, elderly hepatitis E patients have milder fever and vomiting. However, elderly patients showed more severe symptoms in other aspects. The percentage of patients showed fatigue and itchy skin is higher in the elderly group (90.6\%) than in the young patients $(84.9 \%)$. Serum bilirubin level in elderly patients $(308.4 \pm 311.3 \mu \mathrm{mol} / \mathrm{L})$ is higher than in young patients $(168.3 \pm 81.4 \mu \mathrm{mol} / \mathrm{L})$. Elderly patients were hospitalized longer (45 \pm 15 days) than young patients $(25 \pm 10$ days). The percentage of patients with jaundice and severe hepatitis is higher in elderly (92.4 and $9.4 \%$, respectively) than in young patients (76.6 and $0 \%$, respectively) $(P<0.05) .58 \%$ of elderly patients also have other diseases, mostly are hypotension, coronary heart disease, diabetes, gallbladder stones, and fatty livers. $34.0 \%$ have complications, such as cardiac muscle damages, hepatic coma, lung infection, gastric haemorrhage and hepatic-renal symptoms. In young patients, $15.6 \%$ also have other diseases, mostly are duodenal ulcers, diabetes, gallbladder stones, and fatty livers. $7.81 \%$ of young patients have complications, mostly are gastric haemorrhage. The difference between elderly and young patients are significant $(P<0.01)$.

Conclusions: Cases of elderly hepatitis $\mathrm{E}$ patients are increasing. Compare to young patients, elderly patients have more severe symptoms, more complications and higher percentage of elderly patients develop severe hepatitis and jaundice, and the hospitalized period is longer.

\section{PP09-31}

Study on Prevalence of Superinfection/Co-infection in Patients with Acute Viral Hepatitis

X. Wang, L. Wang, Q. Bu, P. Liu, J. Geng, H. Fu, Y. Zhu, H. Zhuang Microbiology, Peking University Health Science Center, Beijing, China

Aim: To investigate the seroprevalence of hepatitis virus infection and superinfection/coinfection in patients with acute hepatitis or chronic active hepatitis caused by hepatitis viruses in China.

Method: 433 blood samples, collected from 5 areas of China (Jilin110, Hebei-112, Shandong-54, Jiangsu-100, Xinjiang-57) during the time of Jan 2007- July 2009, were detected for anti-HAV IgM, $\mathrm{HBsAg}$, anti-HBs, $\mathrm{HBeAg}$, anti-HBe, anti-HBc, anti-HCV, $\mathrm{HDAg}$ and anti-HEV IgM/IgG by EIA.

Results: Seroprevalence of HAV, HBV, HCV, HEV infection and Superinfection/Coinfeciton were 3.93\% (17/433), 35.80\% (155/433), $16.40 \%$ (71/433), $56.35 \%$ (244/433), and 27.94\% (121/433), respectively. In group of superinfection/coinfection, the prevalence of dual infection and triplet infection of hepatitis virus were $86.78 \%(105 / 121)$ and $13.22 \%(16 / 121)$. The incidence of superinfection with HBV/HEV and $\mathrm{HCV} / \mathrm{HEV}$ was $52.07 \%$ (63/121) and $29.75 \%$ (36/121).

Conclusion: Acute hepatitis caused by HEV was predominant among 5 areas in China. And the most common superinfection was HBV/ $\mathrm{HEV}$, followed by HCV/HEV mode.

\section{PP09-32}

Virological and Clinical Characteristics of Hepatitis Delta Virus in South Asia

A. Khawaja ${ }^{1}$, K. Mumtaz ${ }^{1}$, U.S. Ahmad ${ }^{1}$, S. Memon ${ }^{2}$, M.T. Usmani ${ }^{1}$, S. Hamid ${ }^{1}$, W. Jafri ${ }^{1}$

${ }^{1}$ Medicine, The Aga Khan University Hospital, Karachi; ${ }^{2}$ Medicine, Isra University Hospital, Hyderabad, Pakistan

Background and aims: Data is scarce on impact of hepatitis D virus (HDV) in patients with hepatitis B virus (HBV) infection from South Asia. We studied the impact of HDV co-infection on virological and clinical characteristics.

Methods: We collected data of 480 patients with HBsAg positive and HBV DNA PCR detectable who presented to Aga Khan University 
and Isra University, Pakistan in last 5 years. HDV co-infection was diagnosed on the basis of anti-HDV; ALT, HBeAg, HBeAb and HBV DNA PCR quantitative levels were checked in all patients. We divided all patients into two groups-based on anti-HDV and compared their biochemical, serological and virological labs and clinical spectrum. Clinical spectrum of disease included asymptomatic carrier (AC), chronic active hepatitis (CAH), immuno-tolerant phase (IP), and compensated cirrhosis (CC).

Results: HDV co-infection was found in 169 (35.2\%). There were $164(34.6 \%) \mathrm{HBeAg}$ positive and 316 (65.4\%) HBeAg negative patients. Mean ALT level was $66 \pm 73$ IU; $233(48.5 \%)$ had raised ALT. HBV DNA level was $\geq 10 \mathrm{e} 5$ in $103(21.5 \%)$ patients. Overall, among HBV/HDV co-infection, 146/169 (86.4\%) had suppressed HBV DNA PCR as compared to $231 / 311$ (74.3\%) patients in HBV mono-infection; $p$ value $=0.002$. Among $\mathrm{HBeAg}$ negative patients 71/128 (55.5\%) had raised ALT levels among HBV/HDV co-infection as compared to $71 / 188(37.8 \%)$ in $\mathrm{HBV}$ mono-infection ( $p$ value $=0.002$ ); levels of HBV DNA were equal in two groups; there were $27 / 128(21 \%)$ patients with CC among HBV/HDV coinfection as compared to $23(12 \%)$ in HBV mono-infection $(p$ value $=0.009)$; there were less AC $(p$ value $=0.009)$ and more CAH $(p$ value $=0.009)$ among HBV/HDV co-infection patients. Among $\mathrm{HBeAg}$ positive patients, serum ALT, HBV DNA levels and the spectrum of HBV was similar in the two groups.

Conclusions: HBV/HDV co-infection results in suppression of HBV DNA. A fair proportion of HBV/HDV co-infected patients with $\mathrm{HBeAg}$ negative have active hepatitis B infection and even cirrhosis as compared to those with mono-infection.

\section{PP09-33}

Clinical Features of Cholestatic Hepatitis with Acute Viral Hepatitis A in Chunbuk Province of Korea: Multicenter Experience G.M. Jung ${ }^{1}$, Y.K. Cho ${ }^{1}$, I.H. Kim², C.S. Choi ${ }^{3}$, E.Y. Cho ${ }^{4}$ ${ }^{1}$ Presbyterian Medical Center, Jeon-ju; ${ }^{2}$ Department of Internal Medicine Chonbuk National University and Hospital, Jeonju; ${ }^{3}$ Digestive Disease Research Institute, Department of Internal Medicine, Wonkwang University College of Medicine, Iksan; ${ }^{4}$ Department of Internal Medicine, Wonkwang University School of Medicine, Kunsan, Kunsan, Republic of Korea

One of important atypical clinical manifestations of acute hepatitis A is prolonged cholestasis. The clinical course of cholestatic hepatitis is usually characterized by severe jaundiced, pruritus, and fever, Biochemical abnormality show marked elevation of the serum bilirubin $(>10 \mathrm{mg} / \mathrm{dL})$ and ALP, minimal elevation of serum aminotransferase. The jaundice may last for 10 weeks. However, diagnostic clinical criteria was some different in each authors. There was no study of large number patients with this disease and no multi-center study. So, the aim of this study was to evaluate the clinical feature and incidence in one local province area.

Methods: We reviewed the medical records of 421 patients who were diagnosed as acute hepatitis A between January 2007 and January 2010 at the 4 hospital in Chunbuk province. 30 patients were diagnosed as acute cholestatic hepatitis A. This was based on the following criteria: clinically jaundice for at least 10 weeks, with peak serum bilirubin $>10 \mathrm{mg} / \mathrm{dL}$; positive for HAV IgM; no evidence of previous liver disease, no exposure to hepatotoxins; and normal finding of the biliary tree.

Result: The Incidence of cholestatic hepatitis A was $7.1 \%$. The median age of them was 32.3 years. The frequent symptoms were myalgia and nausea. Mean peak total bilirubin level was $17.2 \mathrm{mg} / \mathrm{dL}$. Mean duration of hospitalization was 31.6days, mean duration of recovery to normal bilirubin was 93 days. In all of cases ursodeoxycholic acid was used, and steroid was used two cases. There was no case of death. They were all recovered completely.
Conclusions: This study showed that incidence of cholestatic hepatitis A was not rare, and It is clinical course need long duration of hospitalization and recovery time. Because of social and financial loss by this disease, this result also support the necessity of vaccination in Korean society even though this disease progression was not fatal.

\section{PP09-34}

Clinical Manifestations and Outcomes of Acute Hepatitis A in Korea: a Multi-Center Study, 2007-2009

S.Y. Kwon ${ }^{1}$, S.H. Park ${ }^{2}$, J.E. Yeon ${ }^{3}$, S.H. Jeong ${ }^{4}$, O.S. Kwon ${ }^{5}$, J.W. Lee ${ }^{6}$, H.S. $\mathrm{Kim}^{7}$, Y.S. $\mathrm{Seo}^{3}$, Y.S. Kim ${ }^{8}$, W.H. Choe ${ }^{9}$, K.S. Byun ${ }^{10}$, C.H. Lee ${ }^{9}$

${ }^{1}$ Internal Medicine, Konkuk University; ${ }^{2}$ Hallym Univesity; ${ }^{3}$ Korean University, Seoul; ${ }^{4}$ Seoul National University Bundang Hospital, Bundang; ${ }^{5}$ Gachon University; ${ }^{6}$ Inha University, Incheon;

${ }^{7}$ Soonchunhyang University, Cheonan; ${ }^{8}$ Soonchunhyang University, Bucheon; ${ }^{9}$ Konkuk University; ${ }^{10}$ Korea University, Seoul, Republic of Korea

Background/aims: The seroprevalence of anti-HAV has been changing rapidly in the recent 30 years in Korea. Symptomatic hepatitis $\mathrm{A}$ in adulthood is increasing recently. The aim of this study was to investigate the clinical characteristics and outcomes of acute hepatitis A in Korea from 2007 to 2009.

Methods: The data of patients diagnosed with acute hepatitis A from 2007 to 2009 were collected retrospectively from 21 tertiary hospitals. The demographic data, clinical findings, serological results, and outcomes were analyzed.

Results: A total of 4,215 patients with hepatitis A were included. Mean age of the patients was 32.3 years, and 2,630 (62.4\%) of the patients were male. The average admission duration in admitted cases was 9 days $( \pm 5.1)$. The most frequent symptoms were nausea, fatigue, fever. Laboratory data showed the mean of the highest ALT level was $2,829 \mathrm{IU} / \mathrm{L}$, total bilirubin was $7.3 \mathrm{mg} / \mathrm{dL}$, albumin was $3.8 \mathrm{~g} / \mathrm{dL}, \mathrm{PT}$ INR was 1.3 , creatinine was $1.2 \mathrm{~g} / \mathrm{dL}$. HBsAg was positive in $3.7 \%$ of the patients, and anti-HCV positive in $0.73 \%$. Renal insufficiency occurred in 108 cases $(2.74 \%)$, and hepatic failure occurred in 31 cases $(0.79 \%)$. Relapsing hepatitis and cholestatic hepatitis were observed in $5.16 \%(199 / 3854)$ and $0.65 \%$ (25/3956), respectively. Nineteen patients $(0.47 \%, 19 / 4043)$ died (12 cases) or were transplanted (7 cases). A total of 993 patients were surveyed with questionnaire for risk factors of HAV infection. A history of contact with hepatitis A patients was observed in $9.9 \%$ of the patients.

Conclusions: The age of patients with acute hepatitis A has been increased in the recent years. Most patients recover uneventfully, however, the morbidity and mortality rates are increasing. These results provide that active immunization should be adapted immediately. Catch-up vaccination of HAV should be considered in young adults to reduce morbidity or to prevent community-wide outbreaks.

\section{PP09-35}

Tumor Necrosis Factor Alpha Level in Hepatitis E Infected Pregnant Women

G.D. Salam ${ }^{1}$, A. Kumar ${ }^{2}$, P. $\mathrm{Kar}^{3}$

${ }^{1}$ Maulana Azad Medical College,University of Delhi; ${ }^{2}$ Obs and Gynecology; ${ }^{3}$ Maulana Azad Medical College, University of Delhi, New Delhi, India

Background: The host response in HEV related liver disease of pregnant women is unclear.

Aims: This prospective study was carried out to evaluate the role of tumor necrosis factor alpha (TNF- $\alpha$ ) in HEV related acute viral hepatitis (AVH) and fulminant hepatic failure (FHF) in pregnant women. Methods: The study included HEV related pregnancy cases: $24 \mathrm{AVH}$ and 10 FHF; Non-HEV related pregnancy cases: 48 AVH and 3 FHF; HEV related non pregnant cases: $16 \mathrm{AVH}$ and $4 \mathrm{FHF}$; Non-HEV related non pregnant cases: $44 \mathrm{AVH}$ and $5 \mathrm{FHF}$; 60 healthy pregnant women and 50 healthy non pregnant women. Serum TNF- $\alpha$ level was 
assayed by commercially available ELISA kits according to manufacture's protocol.

Results: Pregnant cases had significantly higher HEV viral load in both $\mathrm{AVH}$ and FHF cases than non pregnant cases $(p=0.0001$ and 0.044 respectively). Increase level of TNF- $\alpha$ was observed in AVH and FHF of HEV infected pregnant women than Non-HEV pregnant women ( $p=0.001$ and 0.106 respectively). TNF- $\alpha$ level was significantly higher in AVH and FHF of HEV infected pregnant women compared with AVH and FHF of HEV infected non pregnant women $(p=0.006$ and 0.001 respectively) and healthy pregnant women $(p=0.0004$ and 0.0001 respectively). Increase TNF- $\alpha$ level was observed in HEV infected women having preterm delivery and low birth weight newborn compared with Non-HEV and healthy pregnant women.

Conclusion: Increase serum concentration of TNF- $\alpha$ observed in HEV infected FHF and AVH pregnant cases shows that pregnancy with HEV infection increases TNF- $\alpha$ secretion. TNF- $\alpha$ might be an important factor in the different outcomes of HEV infection during pregnancy.

\section{PP09-36}

Prevalence and Genotype of TTV in North India

S. Singh, M.A. Ansari, A. Singh, P. Nag, M. Irshad

Laboratory Medicine, All India Institute of Medical Sciences, Delhi, India

Background/aim: Torque Teno Virus (TTV), a ssDNA virus, is reported to be prevalent all over the world. Its forty genotypes have already been identified. Present study is aimed to investigate the prevalence and genotypes of TTV in different liver diseases in North India. Methods: A total number of 90 sera with different liver diseases and 50 healthy controls were included in this study. PCR was done targeting N22 region of TTV. Fourteen TTV-DNA positive samples were subjected to genotyping by Restriction Fragment Length Polymorphism (RFLP). PCR products were cloned in TA-sequencing vector and confirmed by colony PCR and sequencing. Hepatitis viral markers (HBV and HCV) were tested by EIA.

Results: TTV-DNA was detected in 51 of 90 (56.6\%) liver disease cases and 13 of $50(26 \%)$ healthy control. The sequence analysis of the PCR product failed to show a significant sequence divergence when compared with known isolates of TTV genotype1 (G1). The results of genotyping in showed the presence of G1 in 11 of 14 $(78.5 \%)$ and genotype $2(\mathrm{G} 2)$ in 3 of 14 cases $(21.4 \%)$. Other genotypes were not identified in these patients subgroup. HBsAg, IgM anti-HBc and IgG anti-HCV was found to be comparable with each other among the studied disease groups. Whereas, presence of HCVcore antigen was found to be slightly raised in FHF and CIR cases as compare to $\mathrm{AVH}$ and $\mathrm{CVH}$ cases.

Conclusion: The results indicate that TTV is moderately present among Liver disease and healthy individuals with G1 to be the major genotype in North India. This study could not show any association of TTV-DNA with HBV and HCV infection or severity of disease caused by these viruses. TTV appears to be a silent pathogen with no sign of liver damage.

Acknowledgement: We thank ICMR for the financial grant to complete the study.

\section{PP09-37}

To Study The Etiology and Clinical Profile of Acute Viral Hepatitis among Children of Indian State of Punjab

D. Bhat, H.S. Bains, G.S. Dhooria

Dayanand Medical College, Ludhiana, India

Background: Viral hepatitis is an important public health problem in our country. In children also it is an important cause of morbidity. Viral hepatitis is endemic in Indian state of Punjabcausing high morbidity.

Objectives: To study the etiology and clinical outcome of acute viral hepatitis among children in Punjab, India.
Methods and results: One hundred and nine consecutive children who presented with featuresof acute hepatitis during the period March 2005-March 2007 at dayanand medical college andhospital were studied.Out of these 109 cases,specific etiological agents were isolated in $99(90.8 \%)$ cases. Of these $67(67.6 \%)$ were due to single, whereas $32(32.3 \%)$ were due to morethan one virus.Hepatitis A was the infecting agent in majority of the cases i.e.76 (69.7\%) cases,followed by Hepatitis E in $13(11.9 \%)$ cases. Mixed infections were due to HAV and HAEin $10(9.1 \%)$ cases, HAV and HAC in $4(3.6 \%)$ cases and $\mathrm{HAV}+\mathrm{HCV}+\mathrm{HEV}$ in $0.9 \%$ cases. In $8(7.3 \%)$ cases typhoid fever was also present. In $10(9.1 \%)$ cases none of the markers could beidentified.Acute Hepatitis can be due to single viral agent or at times more than one virus caninfect the same person. In some cases typhoid fever can exist as coinfection.

Conclusion: Acute viral hepatitis is an important health issue in children of Punjab. Active immunization and maintenance of personal hygiene can go a long way in reducing the burden ofdisease.

\section{PP09-38}

Seroprevalence of Subclinical HEV Infection in Pregnant Women with and Without Chronic HCV Infection in Dakahlya Governorate, Egypt N.H. Mousa ${ }^{1}$, M.S. Hassan ${ }^{2}$, Y.Z. $\mathrm{Gad}^{3}$, A. Elewa ${ }^{4}$

${ }^{I}$ Tropical, Mansoura University, Mansoura, Ecuador; ${ }^{2}$ Obestetric and Gynecology; ${ }^{3}$ Internal Medicine; ${ }^{4}$ Clinical Pathology, Mansoura University, Mansoura, Egypt

Background: Hepatitis E virus (HEV) is a major public health problem in the developing countries. HEV infection in pregnant women is more common and fatal in the third trimester. The present study was designed to determine the seroprevalence of subclinical HEV infection in pregnant women with and without chronic HCV infection.

Methods: A total of 116 asymptomatic pregnant women divided into group 1 included 56 pregnant women with chronic HCV infection and group 2 included 60 pregnant women with negative HCV serology were included in this study. Prevalence of anti-HEV antibodies and anti-HCV were determined by an enzyme linked immunosorbent assay (ELISA) kit.

Results: The overall prevalence of anti-HEV IgG was high significant among pregnant women with chronic HCV infection 40/56 (71.42\%) than pregnant women free from chronic HCV infection 28/60 (46.7\%) $(p=0.006)$. Chronic HCV infection of pregnant women appeared to be a risk factor associated with $\mathrm{HEV} \mathrm{IgG}$ antibody (OR 2.86, CI 1.24-6.6). The seropositivity of HEV IgG was significantly high in rural areas than urban areas (62.5 versus $37.5 \%)$ in group 1 and $(78.58$ versus $21.42 \%)$ in group $2(p=0.15)$ and OR 2.2 , CI $0.65-7.7)$. A significant decrease in albumin $(p=0.047)$ and increase in bilirubin $(p=0.025), \operatorname{ALT}(p=0.032)$, and AST $(p=0.044)$ in pregnant women with positive $\mathrm{HCV}$ and $\mathrm{IgG}$ anti HEV than pregnant women with negative $\mathrm{HCV}$ with positive IgG anti HEV.

Conclusions: The seroprevalence of anti-HEV $\mathrm{IgG}$ in pregnant women is high in Egypt especially in rural areas. Chronic HCV infection is associated with more increase in anti-HEV IgG serological marker. With chronic HCV co-infection marked worsening of biochemical liver indices was detected than pregnant women with IgG anti-HEV alone.

\section{PP09-39}

Shock in the Liver Herpes Encephalitis

G. Totolyan ${ }^{1}$, G.I. Storozhakov ${ }^{1}$, S. Kosyura ${ }^{1}$, I. Fedorov ${ }^{1}$, L. Ilchenko ${ }^{2}$, N. Malishev ${ }^{3}$, E. Dekonenko ${ }^{3}$

${ }^{1}$ Russian State Medical Univesity, Moscow; ${ }^{2}$ Russian Academy of Medical Sciences, Chumakov Institute Poliomyelitis and Viral Encephalitides, Moscow Region; ${ }^{3}$ Infection Hospital, Moscow, Russia

Defeat in the CNS HSV infection most often occurs in the form of encephalitis or meningoencephalitis. The share of herpetic encephalitis (HE) accounts for $10-20 \%$ of the total number of viral 
encephalitis. The disease may be associated with skin lesions, mucous membranes and internal organs (lungs, liver, etc.).

Objective: To determine the frequency and characteristics of liver damage in the HE.

Materials and methods: Retrospectively analyzed the clinical and laboratory data of 112 patients with $\mathrm{HE}$ who were treated at the department neuroinfection Infection Hospital No. 1. Blood serum (BS) and cerebrospinal fluid (CSF) in addition the determination of antibodies $(\mathrm{Ab}) \operatorname{IgM}$ and $\mathrm{IgG}$ antibodies of herpes simplex virus 1,2 (HSV 1,2) and HSV DNA 1,2 (PCR).

Results: In 28/112 (25\%) patients with HE showed liver damage. Concomitant chronic hepatitis $(\mathrm{CH})$ in $6 / 28(21.4 \%)$ from them (in $3-\mathrm{CH}$ alcoholic etiology $(\mathrm{ACH})$, in $2-\mathrm{CH} \mathrm{B}+\mathrm{C}$, and 1 with $\mathrm{CH}$ $\mathrm{B}+\mathrm{C}-\mathrm{HDV}$ superinfection). Herpetic hepatitis $(\mathrm{HH})$ are without icterus, hepatomegaly $(4 / 28 ; 14 \%)$, increase in aminotransferase (from 2 to 9 standards) in 27/28 (96.4\%), when combined with HDV superinfection - up to 26 rules. In $9(32 \%)$ cases in the BS and in 11 (39\%) in the CSF IgM ab detected HSV in $11(39 \%)$ and $12(43 \%)$ HSV IgG ab in BS and CSF, respectively. HSV DNA was detected in $3 / 28(11 \%)$ patients. In 11/28 (39\%) patients developed a coma with and a fatal outcome in 8 patients (29\%) patients, 2 of whom had ACH. Conclusions: In the presence of liver disease in patients with HE mortality is higher than in patients with HE without liver disease (29\% (8/28) against $17 \%$ (14/84), respectively).

\section{PP09-40}

Role of PIBF and Altered Cytokine Profile in HEV Related Pregnancy Outcome

P.D. Bose ${ }^{1,2}$, B.C. Das ${ }^{3}$, A. $\mathrm{Kumar}^{4}$, P. $\mathrm{Kar}^{1,2}$

${ }^{1}$ Dept of Medicine, LNJP Hospital; ${ }^{2}$ Faculty of Medical Sciences;

${ }^{3}$ ACBR, University of Delhi; ${ }^{4}$ Department of Gynecology, LNJP

Hospital, New Delhi, India

HEV infection is detrimental in pregnancy, particularly in 3rd trimester when hormonal and cytokine levels are altered. Successful allopregnancy is a Th2 phenomenon. PIBF an anti-abortive $34 \mathrm{kDa}$ protein induces a $\mathrm{TH} 2$ dominant cytokine production and in a cytokine-mediated way blocks NK activity.

Aim: Study the role of altered PIBF expression and subsequent immune deregulation in determining pregnancy outcome in $\mathrm{HEV}$ infected cases.

Methods: Clinically proven cases of $\mathrm{HEV}$ related $\operatorname{AVH}(n=100)$ and $\operatorname{FHF}(n=43)$ who were in pregnancy were enrolled in the study, and were followed till delivery or till mortality, with details; along with healthy pregnancy $\operatorname{cases}(n=100)$ and healthy, age matched non-pregnant volunteer's $(n=30)$. Viral load was estimated by Realtime PCR. PIBF differential mRNA expression by semiquantitative rt-PCR. IL12 (Th1 response) and IL10 (Th2 response) expression analysis was studied ELISA.

Results: HEV viral load was significantly higher in FHF (139994.0 \pm 103104.17 copies/ml) compared to AVH (768.92 \pm 1105.40 copies/ $\mathrm{ml}),(p=0.046)$. Compared to AVH cases, maternal mortality $(58.14 \%, p<0.001)$ and fetal mortality $(86.67 \%$ delivered cases) was significantly higher $(p<0.001)$ in FHF cases, which also showed the presence of high serum viral load $(p=0.046)$. Patients of FHF mostly had preterm delivery (22/30 delivered cases, $73.33 \%$ ), compared to $\operatorname{AVH}(42 / 100,42 \%) ; p<0.05$. Viral load is significantly higher in cases with fetal mortality in both AVH $(p=0.018)$ and $\operatorname{FHF}(p=0.001)$. PIBF mRNA expression was significantly downregulated in $\mathrm{FHF}$ cases $(p=0.006)$ and $\operatorname{AVH}(p=0.022)$ compared to controls. Downregulated PIBF expression status correlated with Th1 biased status and HEV related fetal outcome of pregnancy especially in FHF. Higher IL-12/IL-10 ratio was observed in HEV infected cases compared to controls (FHF $>$ AVH $>$ non-pregnant controls $>$ healthy pregnant cases). In FHF group, higher IL12/IL10 (Th1/Th2) ratio is associated with maternal mortality, $p=0.264$.
Conclusion: Lower expression of PIBF, high viral load influences HEV related disease severity and outcome in pregnancy. The Th1 biased immune status may be responsible for reduced fetal protection, probably due to higher activity of NK cells, eventually leading to fetal death.

\section{PP09-41}

Antigen Presenting Cells in the Circulation of Chronic HBV Patients Do Not Constitutively Cross-Present HBV Antigens

A.J. Gehring ${ }^{1}$, M. Haniffa ${ }^{2}$, S. Konduru ${ }^{1}$, F. Ginhoux ${ }^{2}$, A. Bertoletti ${ }^{1}$

${ }^{1}$ Infection and Immunity, Singapore Institute for Clinical Sciences, Agency for Science Technology and Research; ${ }^{2}$ Singapore Immunology Network, Agency for Science Technology and Research, Singapore, Singapore

Background and aims: Chronic exposure to large quantities of circulating antigen is thought to play a primary role in the dysfunction and depletion of virus-specific $\mathrm{T}$ cells in chronic HBV patients. However, where $\mathrm{T}$ cells encounter antigen and whether repeated activation can occur in the periphery are unknown. The goal of our study is to determine whether antigen presenting cells (APCs) in the circulation of chronic HBV patients constitutively cross-present HBV antigen to virus-specific CD8 $\mathrm{T}$ cells.

Methods: We isolated CD11c+ dendritic cells, CD14+ or CD16+ monocytes and CD20+ B cells from HLA-A2+ healthy donors or chronic $\mathrm{HBeAg}+\mathrm{HBV}$ patients with $10^{8} \mathrm{IU} \mathrm{HBV} \mathrm{DNA} / \mathrm{ml}$ serum and ALT $>100$. APCs were co-incubated overnight with TCR-redirected $\mathrm{T}$ cells specific for HLA-A2 restricted HBc18-27 and HBs183-91 epitopes. We monitored $\mathrm{T}$ cell activation by intracellular cytokine staining for IFN- $\gamma$. Peptide loaded APCs served as positive control for $\mathrm{T}$ cell activation.

Results: We were able to sort, with greater than $98 \%$ purity, each population of APC from frozen PBMC isolated from healthy and chronic HBV patients. We did not observe any HBc18-27- or HBs183-91-specific T cell activation following incubation with APCs from healthy donors. Similar to healthy donors, we did not see T cell activation after co-culture with any of the APCs isolated from chronic HBV patient PBMCs. We did observe T cell IFN- $\gamma$ production with peptide loaded APCs, indicating APCs were functional and able to stimulate TCR redirected $\mathrm{T}$ cell cytokine production.

Conclusion: Our data suggests that APCs in the peripheral blood of chronic HBV patients do not constitutively cross-present antigen to virus-specific CD8 $\mathrm{T}$ cells and therefore may not contribute to the chronic $\mathrm{T}$ cell activation thought to be responsible for $\mathrm{T}$ cell dysfunction.

\section{Poster Presentation 10: Alcoholic Liver Disease}

\section{PP10-01}

Cobalamin (Vitamin B12) and Holotranscobalamin Changes in Plasma and Liver Tissue in Alcoholics with Liver Disease

\section{Z. Adwan}

Gastroenterology, Sms Hosp, Allepo, Syria

Objective: To know if alterations in plasma cobalamin $\left(B_{12}\right)$ concentration and $\mathrm{B}_{12}$ carriers, e.g., holotranscobalamins (holo $\mathrm{TC}$ ), occur in blood and liver tissue from patients with severe alcoholic liver disease. purpose was to test the hypothesis that liver disease may disrupt $\mathrm{B}_{12}$ distribution.

Method: Total $\mathrm{B}_{12}$, as well as $\mathrm{B}_{12}$ bound to transcobalamin I, II, III (holo TC), were measured to determine their concentration in plasma and in liver tissue; Poteriochromonas malhamensis-a protozoan reagent served to measure only metabolically active (true) $B_{12}$. Total $\mathrm{B}_{12}$ as distributed in holo $\mathrm{TC}$ in plasma and liver tissue of healthy subjects (controls) were compared to patients with severe alcoholic liver disease. 
Results: Severe liver disease initiates highly elevated $B_{12}$ levels in plasma and a lowered liver tissue total $B_{12}$ concentration. The $B_{12}$ distributed to holo TC II is significantly depleted during liver disease. In contrast, holo TC I and III are elevated in plasma during liver disease and contain more $\mathrm{B}_{12}$ than controls. Total $\mathrm{B}_{12}$ and $\mathrm{B}_{12}$ distributed to $\mathrm{TC}$ are lower in diseased liver tissue.

Conclusion: Severe alcoholic liver disease involves leakage of total $\mathrm{B}_{12}$ from liver tissue into the plasma. Holo TC I and III concentration increases in plasma; this preserves the high plasma $\mathrm{B}_{12}$ from being excreted. However, plasma holo TC II $\mathrm{B}_{12}$ distribution is decreased, indicating that there is a depression of exogenous $B_{12}$ entering the plasma and tissues. In severe liver disease, liver tissue $B_{12}$ binding and storage by TC is disrupted and causes $B_{12}$ to leak out of the liver into the circulation. Eventually liver disease could produce enough severe tissue $B_{12}$ deficits to cause metabolic dysfunction despite elevated plasma total $\mathrm{B}_{12}$. Elevation of plasma $\mathrm{B}_{12}$, accompanied by a lowering of holo TC II distribution, seemed to be a useful index of liver disease severity suggesting preventative treatment.

\section{PP10-02}

Alcohol-metabolizing Enzyme Gene Polymorphisms in Alcohol Dependence and Alcoholic Liver Cirrhosis Among Polish Individuals H. Cichoż-Lach, E. Lis, M. Słomka

Department of Gastroenterology, Medical University of Lublin, Lublin, Poland

Background: Alcoholic liver disease is estimated to occur in 20-30\% of individuals who abuse alcohol consumption. Genetic polymorphism of enzymes involved in alcohol metabolism plays a relevant role in etiopathogenesis of alcoholism and alcoholic liver disease.

Aim: The aim of the study was to find in the Polish population the alcohol dehydrogenase 1B (ADH1B), ADH1C and aldehyde dehydrogenase 2 (ALDH2) genotypes, which are likely to be responsible for higher susceptibility to alcohol dependence and alcoholic liver cirrhosis.

Methods: The ADH1B, ADH1C and ALDH2 genotype and alleles frequencies were examined in 202 patients: 141 alcohol dependants (77 with alcoholic liver cirrhosis, 64 alcoholics without damage to gastrointestinal organs) and 61 nondrinkers-as a control group. Genotyping of the ADH, ALDH2 was performed using polymerase chain reaction-restriction fragment length polymorphism method on white cell DNA.

Results: The genotype $\mathrm{ADH} 1 \mathrm{C}^{*} 1 /{ }^{*} 1$ and allele $\mathrm{ADH} 1 \mathrm{C}^{*} 1$, ADH1B*1 were found to be significantly more frequent in alcohol dependants compared to nondrinkers. Frequency of ADH1C*1 allele in alcoholic liver cirrhosis group was $62.8 \%$, and $\mathrm{ADH} 1 \mathrm{C}^{*} 1 / * 1$ genotype was observed in $45,4 \%$ and was significantly higher than in the control group. The differences between alcohol dependants groups were not statistically significant. In the group of nondrinkers $\mathrm{ADH} 1 \mathrm{~B} * 2$ and $\mathrm{ADH} 1 \mathrm{C} * 2$ alleles were more frequent in comparison to the alcoholic liver cirrhosis patients and alcohol dependants. All examined patients were ALDH $2 * 1 / * 1$ homozygotic.

Conclusion: Our studies suggest that in the Polish population examined $\mathrm{ADH} 1 \mathrm{C}^{*} 1, \mathrm{ADH} 1 \mathrm{~B} * 1$ allele and $\mathrm{ADH} 1 \mathrm{C}^{*} 1 / * 1$ genotype favor developing alcohol dependence and alcoholic liver cirrhosis. However $\mathrm{ADH} 1 \mathrm{~B} * 2$ and $\mathrm{ADH} 1 \mathrm{C} * 2$ allele are likely to protect against them. Genetic polymorphism of ALDH2 shows no correlation with alcohol dependence or alcoholic cirrhosis, Polish is monomorphic $\mathrm{ALDH} 2 * 1$.
PP10-03

Genetic Polymorphism of CYP2E1 and Alcoholic Liver Cirrhosis

H. Cichoż-Lach, E. Lis, M. Słomka

Department of Gastroenterology, Medical University of Lublin, Lublin, Poland

Background and aims: Genetic polymorphism of enzymes involved in alcohol metabolism plays a relevant role in etiopathogenesis of alcoholic liver disease. The aim of this study was to find in the Polish population the CYP2E1 alleles and genotypes, which are likely to be responsible for higher susceptibility to alcohol dependence and alcoholic liver cirrhosis and to examine if this genotype differentiates age, at which the patients have become alcohol dependant.

Methods: The CYP2E1 alleles and genotypes frequency were examined in 176 alcohol dependants: 94 with alcohol liver cirrhosis, 82 without damage to gastrointestinal organs, and 95 healthy volunteers who do not drink alcohol - as a control group. Genotyping of the CYP2E1 was performed using PCR-RELP methods on white cell DNA. Results: In the examined population the frequency of CYP2E1*c2 allele was $2.45 \%$ and CYP2E $1 * \mathrm{c} 1-97.55 \%$. CYP2E $1 * \mathrm{c} 1 / *_{\mathrm{c} 1}$ genotype occurred in $95.10 \%$, CYP2E $1 * \mathrm{c} 1 / *_{\mathrm{c} 2}$ in $4.90 \%$ of examined subjects. Nobody had CYP2E $1 * \mathrm{c} 2 / * \mathrm{c} 2$ genotype. CYP2E $1 * \mathrm{c} 1$ allele and $\mathrm{CYP} 2 \mathrm{E} 1 * \mathrm{c} 1 / *_{\mathrm{c} 2}$ genotype were statistically more frequent among the alcohol dependants (with alcoholic liver cirrhosisand without damage to gastrointestinal organs) than in the controls. Differences in the CYP2E1 allele and genotype distribution between alcohol dependants groups were not significant. Average age of the patients with CYP2E1*c1/*c2 genotype who became alcohol dependant was 26.19 years and in patients with CYP2E $1 *^{*} 1 / *^{*} 1$ genotype was 37.60 years $(p<0.001)$.

Conclusion: Our studies suggest that the presence of $c 2$ allele promotes alcohol dependence and alcohol liver cirrhosis amongst Poles. The persons with $\mathrm{CYP} 2 \mathrm{E} 1 *^{\mathrm{c}} 1 / *_{\mathrm{c} 2}$ genotypes became alcohol dependant at a considerably younger age than the subjects with CYP2E1*c1/*c1 genotypes.

\section{PP10-04}

Prevalence of Helicobacter Pylori and its Possible Implications in the Prognosis of Fibrosis in Patients with Alcoholic Liver Disease L. Mos, C. Zorila, C. Cotoraci, G. Pribac, C. Pop, A. Ardelean West University Vasile Goldis Arad, Arad, Romania

Premises: Dyspeptic syndrome is a major cause of hospitalization and discomfort in patients with alcoholic liver diseases (ALD).

Objective: Due to increased incidence of infection with H. Pylori in dyspeptic syndrome we followed Ac-specific incidence in patients with dyspeptic syndrome and ALD admitted to our medical department in 2009-2010.

Methods: We compared the incidence of $\mathrm{H}$. pylori infection in 58 patients diagnosed with ALD with 56 patients hospitalized for acute dyspeptic syndrome, in which were originally collected Ac. anti $\mathrm{H}$. Pylori and in which ALD was excluded. We applied fibrotest

Results: H. Pylori Ac was present in $71 \%$ of patients with ALD and $52 \%$ in the control group. GUCI index values was over 0.26 $(p<0.010)$ in the presence of HP in patients with ALD compared with patients without HP infection. Immunological status showed lower numbers of circulating CD4+ and CD8+ T cells $(p<0.005)$. None of the patient with ALD and HP positive infection have had peptic ulcers $(p<0.010)$. Fibrotest results were over 0.58 , indicates moderate fibrosis. 
Conclusions: H. pylori prevalence is higher in patients with ALD, greater than in the general population, associated with low numbers of circulating $\mathrm{CD} 4+$ and $\mathrm{CD} 8+\mathrm{T}$ cells. The presence of HP infection was not statistic related with the presence of peptic ulcer or severe gastritis in ALD. HP can influence and determine the prognosis of liver disease by influence the progression of fibrosis in infected patients. It is important to determine and cure HP infection in patients with chronic liver diseases with ALD, when the diagnosis is made.

\section{PP10-05}

The Modulation of Garlic Oil on Fatty Acid Metabolizing Factors in the Liver of Acute Ethanol-Exposed Mice

T. Zeng, C.-L. Zhang, X.-L. Zhao, K.-Q. Xie

Shandong University, Jinan, China

Background: The hypolipidemic effects of garlic has been demonstrated in numerous studies, and garlic oil (GO) has been shown to partially attenuate ethanol-induced fatty liver. The current study was designed to investigate the effects of GO on the hepatic fatty acidmetabolizing factors in acute ethanol-exposed mice.

Methods: Male Kun Ming mice were pretreated with different doses of $\mathrm{GO}(50,100,200 \mathrm{mg} / \mathrm{kg} \mathrm{bw})$ at $2 \mathrm{~h}$ before exposure to a single dose of ethanol $(4.8 \mathrm{~g} / \mathrm{kg} \mathrm{bw})$. The animals were sacrificed at 4,8 , and 16 hrs after ethanol treatment, respectively. The hepatic free fatty acid (FFA) levels were detected by using commercial kits. The protein levels of sterol regulatory element binding protein-1 (SREBP-1), peroxisome proliferators-activated receptor- $\alpha$ (PPAR- $\alpha$ ), phosphorylated AMP-activated protein kinase (p-AMPK), phosphorylated acetyl-CoA carboxylase (p-ACC), and fatty acid synthase (FAS) were determined by western blotting.

Results: Ethanol significantly increased the hepatic FFA levels, which was blocked by GO $(100,200 \mathrm{mg} / \mathrm{kg} \mathrm{bw})$ pretreatment. The hepatic FAS protein levels were dramatically and dose-dependently reduced in GO-pretreated mice. In addition, GO significantly increased the p-AMPK protein levels and inhibited ethanol-induced decrease of the p-ACC protein levels at $4 \mathrm{~h}$ time point. Furthermore, GO significantly reduced the mSREBP-1 protein levels at $8,16 \mathrm{~h}$ time points, but increased the PPAR- $\alpha$ protein levels at $4,8 \mathrm{~h}$ time points. Compared with those of the ethanol group, mSREBP-1 protein levels were decreased by $34.28,45.41$ and $53.53 \%(P<0.01)$ at $8 \mathrm{hrs}$ time points in three GO groups, respectively, while the PPAR- $\alpha$ protein levels were increased by 69.52, 62.66 and $70.22 \%(P<0.01)$, respectively.

Conclusion: These data suggested that GO could inhibit the rising of hepatic FFA levels induced by ethanol, which might be associated with the activation of AMPK and PPAR- $\alpha$, and the inhibition of SREBP-1 and FAS.

\section{PP10-06}

Antioxidant and Hepatoprotective Activity of the Phytoconstituents of Flaveria Trinervia Against Alcoholic Liver Damage

V. Krishna, P. Suresh Babu, H. Joy Hoskeri

Department of Biotechnology and Bioinformatics, Kuvempu

University, Shimoga, India

Alcoholic liver disease is the major cause of morbidity and mortality in the world. Flaveria trinervia (Asteraceae) is a potent hepatoprotective herb used to cure liver diseases by the traditional medicinal practitioners of the central Western Ghats (Karnataka, State) of India. The sequential soxhlet extraction and column chromatographic separation of petroleum ether and chloroform extracts of $F$. trinervia resulted two fatty acids namely, tridecan-1-ol and tricosan-1-ol and from the methanol extract a triterpenoid betulinic acid and two flavonoids namely, quercetin and rutin were isolated. These compounds were characterized based on UV, IR, ${ }^{1} \mathrm{H}-\mathrm{NMR},{ }^{13} \mathrm{C}-\mathrm{NMR}$ and Mass spectral studies.

Phytoconstituents of $F$. trinervia were screened for free radical scavenging activity and in vivo antioxidant and hepatoprotective property against alcoholic liver damage using Wistar albino rats. $\mathrm{LD}_{50}$ for the extracts and the constituents was determined to be 500 and $200 \mathrm{mg} / \mathrm{kg}$ body weight, one tenth of these doses were considered as the safer dose for administration. Extracts and the constituents were administered orally once daily with an interval of $3 \mathrm{~h}$ after the administration of $30 \%$ ethanol at dose of $10 \mathrm{~g} / \mathrm{kg}$ for a period of 45 days. Silymarin was used as standard reference.

The results of pharmacological studies revealed significant protection of liver in the animals treated with quercetin. Total bilirubin, total protein, AST, ALT and ALP levels were restored as $0.43 \pm 0.05, \quad 7.06 \pm 0.38, \quad 222.13 \pm 29.96, \quad 104.02 \pm 8.70$ and $134.12 \pm 19.14$ respectively, as similar to the control and standard groups. Restoration of oxidative stress markers levels also evidenced the in vivo antioxidant properties of the constituents. Moderate hepatoprotective activity observed in the animals treated with the methanolic extract and the constituent's rutin and tridecan-1-ol. Histology of liver sections evidenced the presence of normal hepatic cords, absence of fatty infiltration, and cirrhosis in the animals treated with methanolic extract and quercetin.

\section{PP10-07}

Exploration of a New Role of Syringic Acid on Liver Injury and Cytokine Response Induced by the Hepatotoxin-Ethanol

N. Saravanan ${ }^{1}$, N. Menaka ${ }^{2}$, N. Nalini ${ }^{2}$

${ }^{I}$ Division of Biochemistry, Rani Meiyammai College of Nursing, Annamalai University, Chidambaram; ${ }^{2}$ Department of Biochemistry and Biotechnology, Annamalai University, Annamalai Nagar, India

Background and aim: Alcohol consumption has been steadily increasing in developing countries. Alcohol is responsible for a liver disorder that affects around one in four people in developed countries. Bioflavonoids are used as herbal medicines, and exhibit various biological activities. Syringic acid, a bioflavonoid, is known to have antioxidant and hepatoprotective activities but the mechanism of action in the molecular level is not well known. The aim of this study was to evaluate the effect of syringic acid on the cytokine response in the liver of ethanol fed rats.

Methods: Male albino Wistar rats were gavaged with $20 \%$ ethanol ( $5 \mathrm{gm} / \mathrm{kg}$ body weight) for 8 weeks and/or naïve rats fed with glucose only. Four weeks later, a group of alcoholic rats were coadministered with $10 \mathrm{mg} / \mathrm{kg}$ syringic acid (orally) for the next 4 weeks or syringic acid alone (naïve group). On the $8^{\text {th }}$ weeks all the rats in different groups were sacrificed, and plasma and liver tissue collected for analysis.

Results: The concentration of liver marker enzymes, lipid peroxidation marker, expressions of COX-2, PGE2 and of TGF- $\beta$ levels as well as the alterations in the proinflammatory cytokines such as TNF$\alpha$ and IL- 6 were studied. Liver marker enzymes such as AST, ALT, ALP, GGT and lipid peroxidation marker TBARS, LOOH and CD were significantly increased $(p<0.05)$. The expressions of COX-2, PGE2 and of TGF- $\beta$ levels as well as the expressions of TNF- $\alpha$ and IL-6 were significantly increased $(p<0.05)$ in ethanol treated animals when compared to control. These values were significantly reverted by the coadministration of syringic acid with ethanol when compared to naive rats fed glucose.

Conclusion: These results suggest that syringic acid treatment to ethanol fed rats enhances the liver function and down regulates the oxidative stress and inflammations, which are participating in the pathogenesis of ethanol-induced liver injury. 


\section{PP10-08}

Therapeutic Effect of Integrative Medicine Therapy on Alcoholic Cirrhosis Complicated with Hepatic Encephalopathy M. Shi ${ }^{1}$, Y. Liu ${ }^{1}$, F. Qian ${ }^{2}$

${ }^{I}$ Changning Hospital; ${ }^{2}$ Infectious Diseases, Changning Hospital, Shanghai, China

Objective: To investigate the clinical effect of omithine-aspartate and naloxone combined with Xingnaojing injection on hepatic encephalopathy in patients with alcoholic cirrhosis.

Methods: 47 patients with alcoholic cirrhosis complicated with hepatic encephalopathy were randomly divided into control $\operatorname{group}(n=22)$ and treatment group $(n=25)$. The control group was treated with arginine and glutamate in addition to conventional treatment. The treatment group was treated with omithine-aspartate and naloxone combined with Xingnaojing injection in addition to conventional treatment. By observing serum ammonia and liver function of two groups, the clinical effect can be evaluated.

Results: The serum ammonia in the treatment group was also significantly decreased than that in the control group $(P<0.05)$. In the treatment group, total efficacy rate was $88.00 \%$, and in the control group, it was $63.64 \%$. There was a significant difference in total efficacy rate between the two groups $(P<0.05)$. Detected liver function of two groups of patients before and after treatment, AST, ALT, GGT and TBIL Vary degrees of recovery, but in the same group before and after treatment, and between the two groups after treatment, there was no significant difference in liver function $(P>0.05)$.

Conclusion: On treating hepatic encephalopathy in patients with alcoholic cirrhosis, omithine-aspartate and naloxone combined with Xingnaojing injection are effective in down-regulating serum ammonia and can increase therapeutic effect, which is worth popularizing in clinical practice.

\section{PP10-09}

Corticosteroids Plus Pentoxifylline Versus Corticosteroids Alone in Severe Alcoholic Hepatitis: a Prospective Randomised Trial with Long-term Follow up

S.S. Sidhu ${ }^{1}$, O. Goyal ${ }^{1}$, P. Singla ${ }^{2}$, D. Gupta ${ }^{2}$, R.S. Chhina ${ }^{1}$, A. Sood ${ }^{1}$

${ }^{I}$ Gastroenterology; ${ }^{2}$ Medicine, DMC and Hospital, Ludhiana, India

Aims: Corticosteroids (CS) and pentoxifylline (PTX) decrease shortterm mortality in severe alcoholic hepatitis (SAH), but not to the desired extent. Combining both drugs may lead to better survival, but has not been studied so far. Aim of this study was to compare the efficacy of combination of CS and PTX, versus CS alone in decreasing short-term and long-term mortality in SAH.

Methods: Seventy patients with SAH \{Maddrey's Discriminant Function $(\mathrm{DF}) \geq 32\}$ were enrolled over 3 years. Exclusion criteria were- renal failure; positive viral serology (HAV, HBV, HCV or $\mathrm{HEV}$ ); concomitant infections; active gastrointestinal haemorrhage; and decline in DF with symptomatic treatment for 5-7 days. Patients were randomised into group A $\{n=36$; prednisolone $40 \mathrm{mg} /$ day orally plus PTX $400 \mathrm{mg}$ thrice/day orally $\}$, and group B $\{n=34$; prednisolone $40 \mathrm{mg} /$ day orally $\}$ for 4 weeks. Data is expressed as median (range).

Results: Baseline characteristics of two groups were similar (age-40 and 42 years respectively; all males). At 4 weeks, mortality rate in group A was 25\% (9/36), while in group B was 26.5\% (9/34) $(p=1.00)$. Both groups showed significant reduction in DF group A-78.5 (36.8-140.9) to $32.7(3.5-108.5)$; group B-74.9 (45.6-140.2) to $40.6(9.4-110.6)\}$. Treatment was stopped in 2 patients in group A (acute pancreatitis and abdominal pain), and one patient in group B (SBP). On long-term follow up (9- 44 months), mortality in group A $(66.67 \% ; 24 / 36)$ was not significantly different from group B $(73.5 \% ; 25 / 34)(p=0.607)$. Using multivariate analysis, age and creatinine correlated with mortality in group B.
Conclusions: In patients with SAH, CS alone or in combination with PTX lead to a decrease in short-term mortality and a significant reduction in DF. However, PTX does not offer any advantage over CS alone in decreasing short-term mortality. The long-term mortality in SAH remains high despite treatment.

\section{PP10-10}

Prevalence of Bleeding Oesophageal Varices in Alcoholic Upper Gastrointestinal Bleeders in Adult Sri Lankans Admitted to a Tertiary Referral Centre

R.L. Satarasinghe, A.C.R. Navaratne, A.T. Jayalal, C. Subasinghe, K. Dias Sri Jayewardenepura General Hospital, Colombo, Sri Lanka

Aims: To elucidate the prevalence of bleeding oesophageal varices in adult Sri Lankan, alcoholic upper gastrointestinal bleeders.

Methods: Case notes of adult Sri Lankan alcoholics who underwent consented endoscopy for upper gastrointestinal bleeding in principal author's unit at Sri Jayewardenepura General Hospital, Kotte, Sri Lanka from 01.01.2007 to 30.09.2010 were retrospectively analyzed. Results: Total population comprised 118 patients with an age range of 28-78 years. $93.2 \%$ of the population showed endoscopic evidence of a recent variceal bleeding. $7 \%$ had bled from peptic ulcers, while $3.4 \%$ from erosions and $0.8 \%$ from gastric varices. The following table depicts the important laboratory parameters of the patient population.

\begin{tabular}{lc}
\hline & Mean $\pm \mathrm{SD}$ \\
\hline Age (years) & $55.3 \pm 11.6$ \\
Blood urea $(\mathrm{mg} / \mathrm{dl})$ & $47.3 \pm 40.1$ \\
Creatinine $(\mu \mathrm{mol} / \mathrm{L})$ & $125.3 \pm 99.4$ \\
SGOT $(\mathrm{U} / \mathrm{L})$ & $96.3 \pm 99.1$ \\
SGPT $(\mathrm{U} / \mathrm{L})$ & $55.1 \pm 59.0$ \\
Albumin $(\mathrm{g} / \mathrm{dl})$ & $2.8 \pm 0.6$ \\
INR & $2.0 \pm 0.6$ \\
APTT (seconds) & $49.1 \pm 13.1$ \\
Platelet $\left(10^{9} / \mathrm{L}\right)$ & $108.8 \pm 55.3$ \\
\hline
\end{tabular}

Conclusions: Oesophageal varices have been the dominant source of bleeding in alcoholics. Thrombocytopenia, hypoalbuminemia, deranged clotting have been important associations while transaminases were unreliable predictors. Nevertheless all the parameters mentioned reflect presence of advanced liver disease and loss of liver reserve.

\section{PP10-11}

Infection in Patients with Severe Alcoholic Hepatitis I.M. Dumitru ${ }^{1}$, S. Rugina ${ }^{1}$, R.C. Cernat ${ }^{2}$, E. Dumitru ${ }^{3}$, C.N. Rugina ${ }^{2}$ ${ }^{1}$ Clinical Infectious Diseases Hospital, Ovidius University; ${ }^{2}$ Clinical Infectious Diseases Hospital; ${ }^{3}$ Ovidius University, Constanta, Romania

Background/aim: Despite the widespread use of broad spectrum antibiotics and antifungal, infection is responsible for up to a quarter of the deaths of patients with liver disease and the most susceptible seems to be those with alcoholic cirrhosis. This study's aims were to evaluate the incidence of infections in patients with severe alcoholic hepatitis and to focus on predictive factors of development of infections.

Methods: At admission, systematic screening of infection consisted of chest X-ray and blood, ascites, urinary cultures and in several cases CSF analysis.

Results: One hundred twenty patients with severe alcoholic hepatitis were included. Infections at admission were as follows: 78 infections $(65 \%)$ were diagnosed: $24(30.7 \%)$ spontaneous bacterial 
peritonitis or bacteremia, $26(33.3 \%)$ pulmonary infections (14 patients with acute community-acquired pneumonia, 8 with hospitalacquired pneumonia, and 4 patients with pulmonary tuberculosis), $18(23.1 \%)$ urinary tract infections, $2(2.6 \%)$ acute meningitis and 8 $(10.3 \%)$ skin and soft parts infections (erysipelas, cellulites). The following germs were isolated: Escherichia coli 12 cases, Klebsiella pneumoniae 8 cases, Enterococcus spp. 6, Candida spp. 6, Acinetobacter spp. 4, Streptococcus pneumoniae in 2 cases with severe meningitis. The mortality was significantly higher in patients with bacterial infection than in non-infected patients (32.4 vs. $13.2 \%$; $p=0.02$ ). The independent factors associated with mortality were bacterial infections, the score of Child-Pugh and creatininemia $>1.5$ $\mathrm{mg} / \mathrm{dl}$.

Conclusions: Severe alcoholic hepatitis is associated with high risk of infection. In these cases there is a high mortality rate. Infections, liver failure and renal failure are independent risk factors of mortality.

\section{PP10-12}

Prior Knowledge Fitting of 2D L-COSY in Hepatic Encephalopathy: Differentiation of Glutamine from Glutamate

M.K. Sarma ${ }^{1}$, R. Nagarajan ${ }^{1}$, A. Huda ${ }^{2}$, N. Wilson ${ }^{3}$, A. Singhal ${ }^{4}$, N. Binesh ${ }^{5}$, R.K. Gupta ${ }^{6}$, S.-H. Han $^{7}$, S.A. Castellon ${ }^{8}$, A.D. Thames ${ }^{8}$, C.H. Hinkin ${ }^{8}$, M.A. Thomas ${ }^{1}$

${ }^{1}$ Radiological Sciences, UCLA School of Medicine, Los Angeles; ${ }^{2}$ Physics, California State University, Fresno; ${ }^{3}$ Biomedical Physics, UCLA School of Medicine, Los Angeles, CA; ${ }^{4}$ Radiology, St. Vincent's Medical Center, Bridgeport, CT; ${ }^{5}$ Imaging, Cedars-Sinai Medical Center, Los Angeles, CA, USA; ${ }^{6}$ Radiodiagnosis, SGPGIMS, Lucknow, India; ${ }^{7}$ Medicine; ${ }^{8}$ Psychiatry, UCLA School of Medicine, Los Angeles, CA, USA

Aims: Hepatic encephalopathy (HE), a complex neuropsychiatric disorder, is normally diagnosed by neuropsychological (NP) tests. The goals of this study were to quantify cerebral metabolites and to separate glutamate from glutamine in HE patients and healthy subjects using the prior-knowledge fitting of 2D localized correlated spectroscopic (L-COSY) data acquired on $1.5 \mathrm{~T}$ MRI scanners manufactured by two different vendors, and to correlate the metabolite changes with NP tests.

Methods: We studied $18 \mathrm{HE}$ patients (age $=53.3 \pm 10.6$ years) and 21 healthy controls (age $=52.7 \pm 12.6$ years) using a GE $1.5 \mathrm{~T}$ Signa MRI scanner. Another group of $14 \mathrm{HE}$ patients (age $=49.6 \pm 14.7$ years) and 13 healthy controls (age $=50.1 \pm 10.8$ ) were studied using a Siemens $1.5 \mathrm{~T}$ Avanto MRI scanner. The following parameters were used for 2D L-COSY: TR/TE $=2 \mathrm{~s} / 30 \mathrm{~ms}, 3 \times 3 \times 3 \mathrm{~cm}^{3}$ voxel size, $96 \Delta \mathrm{t} 1$ increments with 8 averages per $\Delta \mathrm{t} 1$. A priorknowledge fitting (ProFit) algorithm has been developed for the quantitation of 2D L-COSY.

Results: After quantifying the brain metabolites using the ProFit algorithm we were able to differentiate glutamine(Gln) from glutamate(Glu) on the GE $1.5 \mathrm{~T}$ acquired 2D L-COSY data in the medial frontal gray matter. The ratios of myo-inositol(mI), Glu, total choline, scyllo-inositol(sI), phosphoethanolamine(PE) and total NAA showed statistically significant decline in HE patients compared to healthy controls, while the ratio of Gln was significantly increased. Similar trend was seen in the ProFit quantified Siemens 1.5 T data in the frontal and occipito-parietal white regions. Significant differences were found between the NP domain scores of patients and healthy volunteers for motor functioning, cognitive speed, executive functions and global neuropsychological score. Significant correlations between various NP domains and metabolite ratios were also observed.

Conclusion: ProFit processed 2D L-COSY data demonstrated unambiguous quantitation of glutamine from glutamate, mI, NAA, Cho groups, sI and PE, and their respective changes in HE patients.
PP10-13

Genetic Polymorphism of Alcohol Metabolizing Enzymes in North Indians A.K. Dutta ${ }^{1}$, Y. Chawla ${ }^{2}$, P. Kulhara ${ }^{3}$, N. Marwaha ${ }^{4}$, K.K. Kohli ${ }^{1}$

${ }^{I}$ Biochemistry; ${ }^{2}$ Department of Hepatology; ${ }^{3}$ Psychiatry;

${ }^{4}$ Transfusion Medicine, Postgraduate Institute of Medical Education and Research, Chandigarh, India

The association between six functionally relevant genetic polymorphisms namely $\mathrm{ADH} 2 * 2$ (rs2066702), $\mathrm{ADH} 2 * 3$ (rs1229984), ADH3*2 (rs698), ALDH2*2 (rs671), CYP2E1*5c (rs2031920) and CYP2E1*1d and alcohol dependence was studied in 100 healthy controls and 100 alcohol dependents (50 non-cirrhotics, 50 cirrhotics) from North India. DNA was isolated from blood and various genetic polymorphisms were diagnosed by PCR-RFLP. Genotype frequencies of normal homozygotes (NH), heterozygotes (HE) and mutant homozygotes $(\mathrm{MH})$ of various polymorphisms were $0.93,0.07$ and 0 $(\mathrm{ADH} 2 * 2), 1,0$, and $0(\mathrm{ADH} 2 * 3), 0.45,0.44,0.11,(\mathrm{ADH} 3 * 2), 1,0,0$ $(\mathrm{ALDH} 2 * 2), 0.997,0.003,0(\mathrm{CYP} 2 \mathrm{E} 1 * 5)$ and $0.74,0.21,0.05$ (CYP2E1*1d), respectively among controls. The allele frequencies of various alleles were $0.96(\mathrm{ADH} 2 * 1), 0.04(\mathrm{ADH} 2 * 2) ; 1(\mathrm{ADH} 2 * 3)$; $0.67 \quad(\mathrm{ADH} \quad 3 * 1), \quad 0.33 \quad(\mathrm{ADH} \quad 3 * 2) ; 1 \quad(\mathrm{ALDH} 2 * 2) ; 0.995$ (CYP2E1*5b), $0.005 \quad$ (CYP2E1*5c); 0.85 (CYP2E1*1c), 0.15 (CYP2E1*1d) respectively. $\mathrm{ADH} 2 * 2, \mathrm{ADH} 3 * 2$ and $\mathrm{CYP} 2 \mathrm{E} 1 * 1 \mathrm{~d}$ demonstrated genetic polymorphism in North Indians. ADH $2 * 2$ genetic polymorphism was associated with alcohol dependence $(p=0.02$ and OR 0.08). None of the alcoholics were carrying $\mathrm{ADH} 2 * 2$ allele. As both $\mathrm{ADH} 2 * 2$ and $\mathrm{ALDH} 2 * 2$ alleles are known to be protective against alcohol dependence, low frequency (0.04) of $\mathrm{ADH} 2 * 2$ in healthy controls and absence of ALDH $2 * 2$ in all the groups could explain the high prevalence of alcoholism in North Indians. Among all the polymorphisms studied only the CYP2E1*1d allele was associated with alcoholic cirrhosis $(p=0.01$, OR 2.3).

\section{PP10-14}

Assessment of Severity of Alcoholic Steatohepatitis

I. Copaci, L. Micu, M. Voiculescu

Center of Internal Medicine, Fundeni Clinical Institute, Bucharest, Romania

Introduction: Excepting histologically proven alcoholic steatohepatitis (ASH), indication for corticotherapy is given by a Maddrey index $\geq 32$ or on the presence of hepatic encephalopathy. However this score neglects the $15 \%$ mortality rate when it is under 32 .

Patients and methods: We performed a retrospective study over a period of 3.5 years, performed on 224 patients with histologically proven ASH (mean age 53 years $\{29-70\}$; M/F = 152/72; acute alcoholic hepatitis 93 pts of which 43 with severe form, remaining patients with cirrhosis). All patients who had a Maddrey index $\geq 32$ or who presented hepatic encephalopathy received steroids in the form of metilprednisolon $32 \mathrm{mg}$ over a period of 28 days.

Results: $72 \%$ of patients received steroids. Global survival at 3 months was $74 \%$. 60 patients died after a mean period of 26 days (1-88) due to liver failure $(N=29)$, infection $(N=14)$, bleeding $(N=6)$ and other causes $(N=11)$. In univariate analysis, Maddrey $\geq 32(p<0.001)$, Child-Pugh $>10(p<0.001)$, MELD $>19(p<$ $0.001)$, hepatic encephalopathy $(p<0.001)$, digestive bleeding $(p<$ $0.001)$, age $>50(p<0.05)$, C reactive protein $>30(p<0.02)$, creatinine $>1.5 \mathrm{mg} / \mathrm{dL}(p<0.001)$, factor $\mathrm{V}(p<0.04)$ were associated with mortality at 3 months. In multivariate analysis, age $>50(0.003$; $4\{1.45-6.25\})$, presence of hepatic encephalopathy (0.007; $2.3\{1.25-4.25\})$, creatinine $>1.5 \mathrm{mg} / \mathrm{dL}(0.001 ; 4.6\{2.5-8.7\})$, MELD score $>19(0.001 ; 4\{1.8-9.25\})$ are independent predictive factors of mortality. In ROC curve analysis, the AUC of MELD was 0.846 (SE 0.027). A cut-off of 19 for the MELD score predicts mortality at 3 months, with a sensitivity of $86 \%$ and a specificity of $59 \%$. 


\section{Conclusions:}

1. Performance of the MELD score is superior to the Maddrey index for predicting death at 3 months, with a good sensitivity and specificity.

2. Decrease of serum bilirubin levels after 7 days of corticotherapy is a predictive factor of survival at day 30 , with negative predictive value of $88.2 \%$.

\section{PP10-15}

Dynamic Changes of uPA, PAI-1, MMPs and TIMP-1 Expression and Role of PAI-1 in Development of Alcoholic Liver Fibrosis

H. Song, J. Chen, L. Wang, G. Ji

Institute of Digestive Disease, Longhua Hospital, Shanghai

University of Traditional Chinese Medicine, Shanghai, China

Background/aims: Imbalance of extracellular matrix synthesis and degradation is the major cause for liver fibrosis. Among this regulating system, urokinase-type plasminogen activator (uPA), type-1 plasminogen activator inhibitor (PAI-1), matrix metalloproteinase (MMP) and tissue inhibitor of metal proteinase-1 (TIMP-1) were reported important factors. This study is to clarify whether and how these molecules participate in alcoholic liver fibrosis (ALF) development.

Methods: SD rats were randomized into control, $\mathrm{CCl}_{4}$ and model group. Model rats were intragastric administrated with a mixture (alcohol,corn oil and pyrazole) once a day and peritoneal injection with low dose $\mathrm{CCl}_{4}$ twice a week. $\mathrm{CCl}_{4}$ group was only injected with $\mathrm{CCl}_{4}$. At 4, 8, 10 and 12 weeks, changes in Serum ALT and AST, liver histopathology were observed; uPA, PAI-1, MMP-2, MMP-9, TIMP-1 were measured. For in vitro experiment, we silenced PAI-1 expression in HSC-T6 cells through plasmid pSilencer-PAI-1. Cell viability and cycle were detected by MTT and flowcytometry, uPA, PAI-1, MMP-2, TGF- $\beta 1$ levels were measured in control and PAI-1RNAi cells after Acetaldehyde activation.

Results: Little difference was observed in liver fibrosis degree between $\mathrm{CCl}_{4}$ and control group. While in model group compared with control, fibrosis was much more severed; serum ALT, AST increased significantly; uPA and PAI-1 levels rose significantly and PAI-1increasing rate was higher than uPA, especially at 10 and 12 weeks; MMP-2, MMP-9 and TIMP-1 rose, TIMP-1 increased more than MMP-2 and MMP-9, especially at 10 and 12 weeks. In vitro, compared with control, PAI-1RNAi cells had lower viability, significant increase in G1 phase while decrease in G2 and S phase. PAI-1 and TGF- $\beta 1$ expression were downregulated after Acetaldehyde stimulation in PAI-1-RNAi cells, while uPA and MMP-2 expression changed little.

Conclusion: Increasing expression imbalance of more PAI-1 than uPA, TIMP-1 than MMP-2 and MMP-9, plays an important role in the ALF development. PAI-1 may contribute to ALF through promoting HSC cell viability, cell proliferation and TGF- $\beta 1$ expression.

\section{PP10-16}

Alcoholic Cirrhosis and Chronic Heart Failure

I. Komissarenko, L. Lazebnik, E. Golovanova, E. Hanina, M. Gusejnzade

Central Research Institute of Gastroenterology, Moscow, Russia

NT-proBNP Is a reliable noninvasive marker of early dysfunction of a myocardium against a volume overload of chambers of heart at progressing of a portal hypertension at sick of a cirrhosis.

Research objective: To reveal signs of early heart failure at patients with an alcoholic cirrhosis (AC).

Material and methods: 25 patients (from them women of $28 \%$, men of $72 \%$ ) with an alcoholic cirrhosis are surveyed. Middle age of patients $54.9 \pm 8.9$ years. All patient conducts research of level NTproBNP on an empty stomach, the ECHO-CG with definition systolic and diastolic myocardium dysfunctions. Patients are divided into 3 groups: I group patients from the AC at class A on Child-Pugh (16\%). II group patients from the AC on Child-Pugh at class B (48\%). III group of the AC Child-Pugh at class $\mathrm{C}(36 \%)$ cases.

Results: Average maintenance NT-proBNP in I group average index LVDS $73 \pm 38 \mathrm{ml}$, LVDD $122 \pm 54 \mathrm{ml}$, LVEF $69 \pm 36 \%$ has made $84 \pm 45.2 \mathrm{fmol} / \mathrm{l}$, by data the ECHO-CG, at all dysfunction is marked diastolic. In II group NT-proBNP $88 \pm 53 \mathrm{fmol} / 1$, by data the ECHO-CG average index LVDS $62 \pm 26 \mathrm{ml}$, LVDD $127 \pm 40 \mathrm{ml}$, LVEF $66 \pm 25 \%$, at all are marked diastolic dysfunction. In III group NT-proBNP $97 \pm 29 \mathrm{fmol} / \mathrm{l}$, by data the ECHO-CG average index LVDS $68 \pm 30 \mathrm{ml}$, LVDD $151 \pm 30 \mathrm{ml}$, LVEF $67 \pm 27 \%$, at all are marked diastolic dysfunction.

Conclusion: The present research confirms presence of dysfunction of a myocardium at patients with an alcoholic cirrhosis. Decrease systolic functions LV at patients with cirrhosis Ch-P A,B,C is marked at $100 \%$ of patients infringement diastolic relaxations LV (diastolic dysfunction), increase of level NT-proBNP is revealed. The increase in level NT-proBNP can be the high-sensitivity indicator preclinic myocardium dysfunctions at a cirrhosis.

\section{PP10-17}

Fibroscan in Comparison with Other Noninvasive Tests for Evaluation of Alcoholic Liver Disease

E. Dumitru, L. Alexandrescu, I.M. Dumitru, A.I. Suceveanu, A.P. Suceveanu Ovidius University, Constanta, Romania

Introduction: Because chronic, excessive alcohol consumption is a major public health problem, screening for liver fibrosis in chronic alcohol-drinking patients is mandatory. The transient elastography (Fibroscan) allows evaluation of liver fibrosis by measuring liver stiffness. The aim of this study was to assess Fibroscan for noninvasive diagnosis of liver fibrosis in alcohol abuse patients and to compare performance of Fibroscan with other laboratory tests.

Methods: Ninety five alcoholic patients were studied (patients aged more than 18 years old, who admitted to chronic consumption of more than $50 \mathrm{~g} /$ day of alcohol for more than 5 years). Fibroscan and aspartate aminotransferase (ASAT) to platelet ratio index (APRI) tests were performed. Spearman's correlation coefficient, univariate analysis and multiple regression analysis were used to study the relationship between liver stiffness and laboratory parameters.

Results: The recorded liver stiffness values were between 3.9 and 68 $\mathrm{kPa}$, with a mean of $15.4 \pm 19.1 \mathrm{kPa}$. The mean measurement success rate was $85.1 \pm 28.4 \%$, with an average of $11.4 \pm 2$ valid measurements. In univariate analysis, 9 parameters were significantly correlated with liver stiffness. In a multiple regression analysis only prothrombin time $(p<0.01)$ and alkaline phosphatase $(p<0.01)$ were associated with liver stiffness. The correlations between Fibroscan and APRI tests was $0.74(0.51-0.94)$ for F1, $0.51(0.38-0.57)$ for F2, $0.41(0.31-0.49)$ for F3, and $0.52(0.35-0.69)$ for F4 $(p<0.01$ for F2, F3 and F4).

Discussion/conclusion: The diagnostic performance of Fibroscan was better than the performance of APRI. The various combinations of the Fibroscan with non-invasive laboratory tests did not improve diagnostic performance relative to Fibroscan alone. Ultrasound-based transient elastography can be used on its own for the non-invasive diagnosis of alcoholic fibrosis.

\section{PP10-18}

Liver Stiffness Changes in Recovering Acute Alcoholic Hepatitis E. Dumitru ${ }^{1}$, L. Alexandrescu ${ }^{2}$, I.M. Dumitru ${ }^{2}$, A.I. Suceveanu ${ }^{2}$, A.P. Suceveanu ${ }^{2}$

${ }^{1}$ Faculty of Medicine; ${ }^{2}$ Ovidius University, Constanta, Romania

Introduction/aim: Liver tissue alterations other than fibrosis (such as hepatic necroinflammation) may have an impact on liver stiffness measurement. The aim of this study was therefore to evaluate the 
dynamic changes in liver stiffness measurement (LSM) during the course of acute alcoholic hepatitis (AAH).

Methods: We prospectively recruited consecutive patients with acute alcoholic hepatitis. In each patient, aminotransferase, bilirubin (BIL), the international normalized ratio (INR) and LSM were performed on the same study day, at admission and then every 2 days, until the peak levels of all parameters were identified. Subsequently, these parameters were measured every 1 week until they had normalized.

Results: In all patients, the degree of liver stiffness at the time of the peak increase in aminotransferases exceeded the cutoff values proposed for the prediction of significant fibrosis or cirrhosis. A progressive significant reduction in liver stiffness values was observed $(p<0.01)$ in the follow-up period in parallel with the reduction of aminotransferase levels $(p<0.01)$. Moreover, a statistically significant, positive correlation between aminotransferases and LSM was found $(r=0.63, p<0.05$ and $r=0.61, p<0.05$ for alanine aminotransferase and aspartate aminotransferase, respectively). INR and BIL were significantly associated with peak LSMs $(p<0.05)$.

Conclusion: LSMs changed dynamically during the course of AAH. LSM should be assessed after normalization of ALT levels in order to accurately assess the degree of fibrosis.

\section{PP10-19}

Disease Dependent QUALITATIVE and QUANTITATIVE Differences in the Inflammatory Response to Ascites

D.H. Van Thiel ${ }^{1}$, M. George ${ }^{1}$, N.I. Nedelcu ${ }^{2}$, G. Ramadori ${ }^{3}$, B.M. Attar ${ }^{4}$, S. Dhillon ${ }^{1}$, E. Letinas ${ }^{5}$, J. Fareed ${ }^{5}$

${ }^{1}$ Medicine/Hepatology/Liver Transplant, Rush University Med Center, Chicago, IL, USA; ${ }^{2}$ Medicine/Infection Diseases/ Epidiamology, Victor Babes infection Clinic, Bucharest, Romania; ${ }^{3}$ Hepatology/Transplant/GI, August Georg University, Gottingen, Germany; ${ }^{4}$ Hepatology/GI, Cook County Hospital, Chicago;

${ }^{5}$ Pharmacology/Pathology, Loyola University Medical Center, Maywood, IL, USA

Ascites is associated with an inflammatory response manifest in ascitic fluid.

Aims: (1) Determine if qualitative differences in the biomarker measures of inflammation due to ascites differ between diseases. (2) Quantify the differences in the biomarkers measure in ascites as a function of the specific liver disease.

Methods: 41 cirrhotics with differing etiologies, with massive ascites requiring a large volume paracentesis. Ascitic fluid was assayed for biomarkers utilizing the BioMerieux assay for Procalcitonin (PCT) and Randox biochips to identify and quantify 12 different cytokines, interleukins and growth factors. The data were compared to normal human plasma (NHP).

Results: The 41 ascitic patients consisted of 21 with alcoholic cirrhosis, 8 with extra hepatic malignancy, 4 with cardiac cirrhosis and 8 with HCV. The PCT plasma level in all groups studied was increased 7-209X that in NHP. The PCT plasma level was consistently greater than in ascitic fluid 4-65X more than NHP. The only cytokine greater in plasma of ascitic cirrhotics other than PCT was IFN- $\gamma(p<0.01)$. The alcoholic had increased of IL-4,IL-6, IL-8,VEGF,TNF- $\alpha, \mathrm{MCP}-1$, and EGF; the metastatic cancer had increased of IL-4,IL- 6 and TNF- $\alpha$; the HCV had increased levels of IL-6, MCP-1, and EGF; the congestive heart failure only had an increased level of IL-6. The greatest level for IL-2 was found in the malignancy group. The alcoholic group had the greatest values for IL-4, IL-1a, IL-1b.The HCV group had the greatest level for IL-6, IL-8, VEGF, MCP-1; and the cardiac cirrhotic group had the greatest level of EGF.

Conclusions: (1) Significant qualitative and quantitative differences in ascitic fluid cytokine exist between cirrhotics with primary hepatic diseases, (2) Alcoholic cirrhotics have the greatest cytokine response to ascites, (3) Cardiac cirrhotics have the least evidence for an ascitic inflammatory response, (4) Ascites associated with HCV and hepatic metastatic disease manifest intermediate inflammatory responses.

\section{PP10-20}

Ethanol Administration Intensifies the Sepsis-Related Changes in Liver Oxidative and Inflammatory Reactions in an Experimental Model K. Razavi-Azarkhiavi ${ }^{1}$, A. Allameh ${ }^{2}$, A. Mohsenifar ${ }^{3}$, M. Jamali-Zavarei ${ }^{4}$ ${ }^{1}$ Department of Toxicology, Faculty of Medical Sciences, Tarbiat Modares Faculty of Medical Sciences, Tarbiat Modares, ${ }^{2}$ Department of Clinical Biochemistry; ${ }^{3}$ Department of Toxicology, Faculty of Medical Sciences, Tarbiat Modares University; ${ }^{4}$ Imam Khomeini Hospital, Tehran University of Medical Sciences, Tehran, Iran

Background: Ethanol as a hepatotoxic agent can increase the risk of bacterial infection. On the other hand sepsis is associated with different oxidative and inflammatory reactions in liver.

Aims: In the present study, we investigated the interaction of ethanol toxicity with polymicrobial infection on oxidative stress factors in liver.

Methods: Polymicrobial sepsis was induced by Cecal Ligation and Puncture (CLP) in rats. Experimental groups were divided as follows: control (saline-injected); sham operated; CLP; ethanol treated (1.0 and $2.0 \mathrm{~g} / \mathrm{kg} \mathrm{b.w}$ ) and CLP + ethanol ( 1.0 and $2.0 \mathrm{~g} / \mathrm{kg} \mathrm{b} . \mathrm{w})$ groups. A single dose of ethanol was injected i.p, to animals immediately after sepsis onset. Blood and livers were removed after $4 \mathrm{~h}$ and processed for biochemical and histopathological examinations.

Results: Administration of ethanol alone caused a significant increase in liver thiobarbituric acid reactive substances (TBARS). However liver glutathione (GSH) and glutathione-S-transferase (GST) were reduced due to ethanol. Plasma tumor necrosis factor- $\alpha$ $(\mathrm{TNF}-\alpha)$ was also decreased due to higher dose of ethanol. The microsomal cytochrome P4501A1 (CYP1A1) activity was unaffected in ethanol-treated rats. Interestingly, there was a dramatic elevation in TNF- $\alpha(\sim 84 \%)$ in CLP group. In addition to TNF- $\alpha$, liver TBARS was also increased significantly as the result of CLP. However, GSH, GST and CYP1A1 were decreased in CLP group. The interactions of ethanol with sepsis-related changes were studies by using septic rats treated with ethanol. In this case, TBARS were further increased in CLP rats treated with ethanol, whereas, GSH and GST were further reduced. Changes in TNF- $\alpha$ and CYP1A1 in this group can be attributed to CLP operation. Histopathological observations carried out on liver biopsies $4 \mathrm{~h}$ after CLP attest to the biochemical changes in this group.

Conclusion: It is concluded that the experimental sepsis which is associated with inflammatory and oxidative reactions in liver, is potentiated with ethanol treatments.

\section{PP10-21}

Zieve Syndrome Is a Rare Form of Alcoholic Liver Disease

G.I. Storozhakov, M. Chichkina, S. Kosyura, I. Fedorov, L. Ilchenko Russian State Medical Univesity, Moscow, Moscow, Russia

Aims: To establish the frequency and describe the syndrome Zieve have examined patients with alcoholic liver disease (ALD).

Material and methods: The study included 111 patients with ALD at the age of 20-81 (average age $50.2 \pm 8.6$ years). In 78 (70\%) patients diagnosed with liver cirrhosis (LC), alcoholic etiology, 33 (30\%) acute alcoholic hepatitis (AAS). Was studied iron metabolism, the content of reticulocytes, Coombs' test was performed sternal puncture.

Results: The frequency of anemia was $28.8 \%(n=32)$. In three (2.7\%) of them (1 man and 2 women) conducted a differential diagnosis of obstructive jaundice due to severity, presence of abdominal pain, severe jaundice and subfebrile. Zieve syndrome suggested on the basis of the availability of the following symptoms: long-term chronic alcohol intoxication admissions to hospital after 
another alcoholic excess; subfebrile; moderate anemia (hemoglobin 7.9-10.3 g/l) increase in the content of reticulocytes (25-53\%) increase in serum bilirubin for by the predominance of the indirect fraction (140-204 $\mu \mathrm{mol} / \mathrm{l})$; triglitseridemii (2.4-3.2 mmol/l), hypercholesterolemia (7.0-7.2 mmol/l), negative Coombs test. These patients were also observed elevated levels of iron in serum (28-35 $\mu \mathrm{mol} / \mathrm{l})$. In this case, hemolysis was not accompanied by an increase in the spleen.

Conclusions: Frequency syndrome Zieve have surveyed our patients was $2.7 \%$. The emergence of this symptom in patients with chronic alcohol intoxication impairs the course and prognosis LC that determines whether the inclusion Zieve syndrome in the diagnostic search for ALD. Complete abstinence and the use of antioxidants (e.g. tocopherol) can achieve resolution of clinical manifestations of this syndrome.

\section{PP10-22}

Structure of Morbidity and Mortality in Patients with Liver Cirrhosis G.I. Storozakov ${ }^{1}$, S. Kosyura ${ }^{2}$, N. Merzlikina ${ }^{2}$, I. Fedorov ${ }^{2}$, G. Totolyan ${ }^{1}$, L.Y. Ilcenko ${ }^{3}$, M. Chicykina ${ }^{2}$

${ }^{1}$ Russian State Medical Univesity, Moscow; ${ }^{2}$ Russian State Medical University, City Clinical Hospital 12; ${ }^{3}$ Russian State Medical Univesity, Moscow, Institute Poliomyelitis and Viral Encephalitis of M.P. Chumakov, Moscow, Russia

Objective: To assess the structure of complication and cause of death in patients with liver cirrhosis (LC).

Materials and methods: The study included 246 patients with liver cirrhosis by Child-Pugh (men 163, women 83) at the age of 37-70 years. All patients had integrated clinical, laboratory and instrumental examinations in City Clinical Hospital No. 12, Moscow. Observation period is 24 months.

Results: Mortality among this group of patients with LC with class of Child-Pugh (12-15 points), $7.1 \%(n=21)$. From: alcoholic etiology LC (including surrogates alcohol) installed-16 (76.2\%) patients alkogol-viral-5 (23.8\%). Hospitalization half of the patients $(n=10$; $50 \%$ ) was due to the clinical with manifestation of esophageal variceal bleeding and/or development of ascites. The cause of hospitalization in other cases was: acute alcoholic hepatitis, infectious processes, edema-ascites syndrome. $11(57.1 \%)$ patients over the LC was development of infection, $6(28.5 \%)$-marked leukemoid reaction (leukocytosis $35 \times 10^{9}-70.4 \times 10^{9} / 1$ ). Index Maddrey of 6 patients (37.5\%) monitoring over 32 (33-87.8). Causes of death were: hepatic coma $(n=6)$, esophageal variceal bleeding $(n=3)$, hepatorenal syndrome $(n=6)$ pancreonecrosis $(n=1)$.

Conclusions: Decompensation LC of the patients has been associated with delayed treatment care, low social status, presence of chronic alcoholism and manifestation of encephalopathy. Progressive deterioration of the patient in hospital due to the development of complications: esophageal variceal bleeding, infections, and worsening hepatic encephalopathy with systemic inflammatory response syndrome.

\section{PP10-23}

Influence of Stem Cell Autotransplantation on Expression of Anti-apoptotic Protein Bcl-2 in Patients with Alcoholic Liver Cirrhosis

G. Burganova $^{1,2}$, S. Abdulkhakov ${ }^{1,3}$, A. Gumerova ${ }^{1}$, I. Gazizov ${ }^{1}$, T. Yilmaz ${ }^{1}$, M. Titova ${ }^{1}$, A. Odintcova ${ }^{4}$, A. Kiassov ${ }^{1}$

${ }^{1}$ Department of Normal Human Anatomy, Kazan State Medical University; ${ }^{2}$ Surgery Department, Kazan Medical Academy for Postdegree Education; ${ }^{3}$ Central Scientific Laboratory, Kazan State Medical University; ${ }^{4}$ Department of Gastroenterology, Republican Clinical Hospital, Kazan, Russia

Background: Expression of anti-apoptotic protein Bcl-2 is considered as one of the signs of malignant transformation of cells. In many cases of chronic liver disease apoptosis is a significant mechanism of cell death, and very often the outcome of such diseases is primary liver cancer. Furthermore the possibility of tumor development as a result of stem cell (SC) transplantation is widely discussed recently. Aim: In this regard the aim of our study was to investigate the effect of autologous hematopoietic SC transplantation on the expression of Bcl-2 in patients with alcoholic liver cirrhosis (ALC).

Material and methods: The study was performed on liver biopsies of 10 patients with ALC. Biopsies were taken before the injection of autologous peripheral blood SCs mobilized by granulocyte colonystimulating factor (G-CSF) into celiac trunk and 3 months after the procedure. Paraffin-embedded liver biopsy specimens were stained immunohistochemically with antibodies against Bcl-2.

Results: A significant number of Bcl-2-positive cells were found in liver biopsies before transplantation; they were localized in liver parenchyma and in inflammatory infiltrates around the portal tracts. Positive cells could be divided into 3 types of cells: inflammatory cells with round or oval nucleus, cells with processes (sinusoidal cells) and single hepatocytes. Cells with processes were predominantly located in the parenchyma and in small infiltrates. We also observed weak expression of Bcl-2 in cholangiocytes. Three months after transplantation along with the improvement of liver structure expression of Bcl-2 diminished: in large infiltrates the number of Bcl-2-positive cells decreased, and in small infiltrates they disappeared. Conclusions: These results might indicate a reduction of liver carcinogenesis risk as a result of SC therapy.

Acknowledgements: Our research was performed as a part of the Program «Development of Cell Medicine in Tatarstan» on the base of Republican Clinical Hospital.

\section{PP10-24}

Expression of CD 34 and a-SMA in Patients with Alcoholic Liver Cirrhosis before and after Autologous Stem Cell Transplantation

G. Burganova ${ }^{1,2}$, S. Abdulkhakov ${ }^{1,3}$, A. Gumerova ${ }^{1}$, I. Gazizov ${ }^{1}$, T. Yilmaz ${ }^{1}$, M. Titova ${ }^{1}$, A. Odintcova ${ }^{4}$, A. Kiassov ${ }^{1}$

${ }^{I}$ Department of Normal Human Anatomy, Kazan State Medical University; ${ }^{2}$ Surgery Department, Kazan Medical Academy for Postdegree Education; ${ }^{3}$ Central Scientific Laboratory, Kazan State Medical University; ${ }^{4}$ Department of Gastroenterology, Republican Clinical Hospital, Kazan, Russia

Introduction: Accumulation of extracellular matrix observed in liver fibrosis and cirrhosis is due to the activation of hepatic stellate cells and their transdifferentiation to myofibroblasts. Alpha-smooth-muscle actin (a-SMA) is a marker of myofibroblasts. Capilliarization of sinusoids is also one of significant steps in liver fibrosis which leads to portal hypertension development. CD34 - a marker of endothelial and hematopoietic stem cells (SC) - is absent in normal sinusoidal endothelium and appears only in case of their capilliarization.

Aim: The purpose of the study was evaluation of the effect of hematopoietic SC autotransplantation on liver fibrosis in patients with alcoholic cirrhosis before and after 3 months of SC transplantation. Material and methods: The study was performed on liver biopsies of 10 patients with alcoholic liver cirrhosis (Child-Pugh class A). Biopsies were taken before the injection of autologous peripheral blood SC mobilized by granulocyte colony-stimulating factor (G$\mathrm{CSF}$ ) into celiac trunk, and 3 months after the procedure.

Liver biopsy specimens were stained immunohistochemically with antibodies against CD 34 and a-SMA.

Results: Before transplantation we observed a great number of $\mathrm{CD}$ 34-positive cells in liver biopsies especially in portal tracts and in the infiltrates around the portal tracts. We also observed CD 34-positive cells in porto-portal and porto-central septa and some isolated cells in liver parenchyma. Three months after transplantation the number of CD 34 positive cells markedly decreased but they were also located mainly around the portal tracts. 
Before transplantation a-SMA-positive cells were mainly localized in periportal zones and portal tract infiltration areas. Three months after transplantation the number of myofibroblasts in liver parenchyma reduced.

Conclusion: Our data suggest that autologous intra-celiac transplantation of hematopoietic SC in patients with alcohol liver cirrhosis is an effective procedure as it can reduce liver fibrosis development and restore endothelial sinusoids.

\section{PP10-25}

Influence of Drinking Pattern on Morbidity and Mortality in Rats

N. Abramishvili, I. Rtskhiladze, D. Metreveli

Laboratory, Medical Centre Mrcheveli, Tbilisi, Georgia

Objective and aims: We studied the influence of drinking pattern on morbidity and mortality in rats.

Methods: 75 wistar rats were randomly allocated in 5 groups after 14 days run in ("quarantine") period. 20 rats allocated in group "A" consumed $7.5 \mathrm{ml} 40 \%$ alcohol (vodka) $=3 \mathrm{~g}$ ethanol once per week. 20 rats allocated in group "B" consumed $0.55 \mathrm{ml} 40 \%$ alcohol $($ vodka $)=0.22$ g ethanol once per day. 4 rats allocated in group " $\mathrm{C} 1$ " (control group) consumed $7.5 \mathrm{ml}$ water once per week. 11 rats allocated in group " $\mathrm{C} 2$ " (control group) consumed $3.75 \mathrm{ml}$ water once per week. 20 rats allocate in group " $D$ " consumed $3.75 \mathrm{ml} 40 \%$ alcohol $($ vodka $)=1.5 \mathrm{~g}$ ethanol once per week. Liquid was introduced via gastric catheter. Duration of study was 30 days.

Statistical analysis: Kaplan-Meier survival analysis.

Results: On 30th day of study in group "A" survived 1 (from 20), in group "B"-3 (from 20), in group " $\mathrm{C} 1$ " -3 (from 4), in group "C2"-10 (from 11) and in group "D"-16 (from 20) respectively, s. Kaplan-Meier Plots.

Conclusions: Survival rates in wistar rats differ significantly in relation to drinking pattern. Consuming ethanol (1.5 g) once per week resulted in significantly less mortality rate than consuming of $0.22 \mathrm{~g}$ ethanol [the same total amount of ethanol $(0.22 \mathrm{~g} \times 7=1.5 \mathrm{~g})]$ once per day.

\section{Poster Presentation 11: Hepatitis C: Diagnosis}

\section{PP11-01}

Fibrosure, APRI and Forns Score versus Liver Biopsy in Chronic HCV Infection in Egypt

H.A. Abd El Moety ${ }^{1}$, S.A. Zaki ${ }^{2}$, A.A. Abd El Moety ${ }^{3}$, S. Baioumy ${ }^{4}$

${ }^{1}$ Chemical Pathology; ${ }^{2}$ Microbiology; ${ }^{3}$ Hepatology, Faculty of Medicine, Alexandria University, Alexandria; ${ }^{4}$ Pathology, Tanta University, Tanta, Egypt

Introduction: Chronic infection with $\mathrm{HCV}$ a major health problem. Clinical management of chronic HCV dependent on extent liver fibrosis. Liver biopsy gold stander an invasive procedure responsible for severe complications and sample variability interpretation. Serum biomarkers for inflammation/fibrosis investigated to wave liver biopsy.

Aim: Diagnostic accuracy panel of Non-invasive serum biomarkers for hepatic fibrosis

[Fibrosure, APRI score, Forn's score] versus liver biopsy.

Method: $20 \mathrm{HCV}$ patients subjected for: APRI, Forn's, Fibrosure scores

PCR quantitative HCV-RNA

Liver functions

Lipid Profile

CBC

Ultrasound guided liver biopsy.

\section{Results:}

- FORNS score; AUROC (0.917) with 95\% CI (0.791-1.042) for $\left(f_{0} f_{1}\right)$ versus $\left(\mathrm{f}_{2} \mathrm{f}_{3} \mathrm{f}_{4}\right)$ while $(0.688)$ with $95 \%$ CI $(0.464-0.91)$ for $\left(f_{o} f_{1} f_{2}\right)$ versus $\left(f_{3} f_{4}\right)$. Cutoff $(>6.9)$ sensitivity for significant fibrosis $\left(\mathrm{f}_{2} \mathrm{f}_{3} \mathrm{f}_{4}\right)$ and extensive fibrosis ( $\left.\mathrm{f}_{3} \mathrm{f}_{4}\right)$ were $(100 \%)$ specificity $(0 \%)$ with accuracy $(40 \%)$ and $(20 \%)$, respectively.

- APRI score; AUROC (0.792) with 95\% CI (0.568-1.015) comparing $\left(\mathrm{f}_{0} \mathrm{f}_{1}\right)$ versus $\left(\mathrm{f}_{2} \mathrm{f}_{3} \mathrm{f}_{4}\right)$ while was $(0.875)$ with $95 \% \mathrm{CI}$ $(0.703-1.047)$ for $\left(\mathrm{f}_{0} \mathrm{f}_{1} \mathrm{f}_{2}\right)$ versus $\left(\mathrm{f}_{3} \mathrm{f}_{4}\right)$. Cutoff $(<0.5)$ sensitivity $(0 \%)$ and specificity $(100 \%)$ with accuracy $(60 \%)$ for significant fibrosis and $(80 \%)$ for extensive fibrosis.

- Fibrosure (fibro-acti test); showed best AUROC (1.00) in different fibrotic stages with 95\% CI (1.00-1.00). Cutoff ( $>0.59)$ sensitivity $(50 \%)$ for significant fibrosis and $(100 \%)$ for extensive fibrosis while specificity $(100 \%)$ in all fibrotic stages. The PPV $(100 \%)$ for significant and extensive fibrosis. NPV and accuracy (75, 80\%),respectively for significant fibroses, while NPV and accuracy $(100 \%)$ for extensive fibrosis.

Significant correlation between liver biopsy and Fibro-test $(P$ 0.002) and Acti-test ( $P$ 0.000).

Significant correlation between liver biopsy hepatitis activity score and APRI ( $P$ 0.047) and FORNS score $(P$ 0.000).

Conclusion: FORNS score was not considered since does not discriminate between significant and extensive fibrosis. Low sensitivity of APRI prohibtes detection of minmal fibrosis and allow undetermined results.

Fibrosure classified all cases of chronic HCV sufficient to wave liver biopsy.

\section{PP11-02}

Evaluation of Serum Angiopoietin- II in HCV Related Glomerulonephrities H.A. Abd El Moety ${ }^{1}$, G.H. Magour ${ }^{1}$, D.A. Maharem ${ }^{2}$, A.H. Mohamed ${ }^{2}$

${ }^{I}$ Chemical Pathology; ${ }^{2}$ Internal Medicine, Medical Research Institue, Alexandria University, Alexandria, Egypt

Although cryoglobulins and HCV antigen-antibody complexes are typically blamed in the pathogenesis of HCV nephropathy, they cannot explain all the reported lesions. Angiogenesis has been reported to play a significant pathogenic role to liver damage during CHC. CHC is associated with elevated serum levels of angiogenesis markers namely vascular endothelial growth factor (VEGF), angiopoietin-2 (Ang-2). Abnormal angiogenesis also occurs in diabetic nephropathy, and is associated with elevated level of both VEGF and Ang-2.The aim of this work was to investigate the role of angiogenesis and the angiogenic markers (VEGF and Ang-2) in the pathogenesis of HCV related nephropathy.

Methods: Serum levels of vascular endothelial growth factor (VEGF) and angiopoetin 2 were measured by ELISA. Rheumatoid factor, cryoglobulins and urinary albumin creatinine ratio were estimated in 24 patients with chronic hepatitis $C$ virus without nephropathy and 16 patients with chronic hepatitis $\mathrm{C}$ virus with nephropathy.

Results: Serum levels of both VEGF and Ang-2 was significantly higher in patients with nephropathy (1060.00-9000.00) and (3705.00-150000.00 pg/ml) respectively than their levels in non nephropathic group (1000.00-6800.00) and (500.00-8805.00 pg/ml), respectively. There was a significant correlation between serum level of VEGF and UACR $\left(r=0.552^{*}, p=0.027\right)$. Also a significant correlation was found between serum levels of Ang-2and UACR $(r=0.829 *, p=<0.001)$.

Conclusions: Circulating levels of angiogenic factors, VEGF and Ang-2, are significantly higher in patient with HCV nephropathy than those without nephropathy. The correlation between circulating levels of VEGF, Ang-2 and UACR a marker of renal injury points to the potential role of these angiogenic factors in the pathogenesis of $\mathrm{HCV}$ nephropathy. 


\section{PP11-03}

Study of the Cellular Immunity in Patients with Hepatic Schistosomiasis with and without Hepatitis C Viral Infection

S.A. Zaki ${ }^{1}$, A.A.E.M. Soliman ${ }^{2}$, M.S. el Rafie ${ }^{3}$, M.I. Abd El Hamied ${ }^{3}$, M.Z. Houssein ${ }^{3}$

${ }^{1}$ Microbiology, Faculty of Medicine; ${ }^{2}$ Hepatology, Faculty of Medicine, Alexandria University, Alexandria; ${ }^{3}$ Microbiology, Faculty of Medicine, Tanta University, Tanta, Egypt

Introduction: The host granulomatous response to Schistosoma mansoni eggs in the liver is an immunological reaction of the cellmediated type. In Egypt HCV detected in all stages of the life cycle of Schistosoma mansoni.

Aim: To measure the cell mediated immune competence of patients with hepatic Schistosomiasis with and without HCV infection.

Subjects and methods:

Group A: 20 patients with hepatic Schistosomiasis.

Group B: 20 patients with mixed hepatic Schistosomiasis and $\mathrm{HCV}$ infection.

Group C: 20 patients with chronic hepatitis C.

Group D: 10 controls. Patients subjected to: A-clinical and ultrasonic examination, stool analysis, Liver function tests, Liver biopsy, $\mathrm{HBs} \mathrm{Ag}$ and anti $\mathrm{HBc}$ and $\mathrm{HCV}$ antibodies.

B-Immunological studies a-Quantification of total T-Iymphocyte and its subpopulations. b-Lymphocyte blastogenesis to: *Nonspecific (mitogens). *Specific: SEA, SWAP, $\mathrm{HCVc}_{22}$ and $\mathrm{HCVc}_{33}$.

Results: (1) $\mathrm{CD}^{+}$: significantly decreased. (2) $\mathrm{CD}^{+}$: significantly decreased and a difference between $\mathrm{A}$ and $\mathrm{B}(3) \mathrm{CD} 8^{+}$: No significant difference. (4) $\mathrm{CD} 4^{+} / \mathrm{CD}^{+}$: significantly decreased and a differenc between except A and C. (5) PHA, Con - A and PW mitogens significantly decreased. (a) PHA: significant differences except between $\mathrm{A}$ and C. (b) Con -A and PWM: significant difference between $\mathrm{A}$ and B. (6) $\mathrm{HCVc}_{22}$ and $\mathrm{HCVc}_{33}$ antigens: significant increase $\mathrm{B}$ and $\mathrm{C}$ and significant difference between groups except A and D. (3) SEA and SWAP. Mean levels of SI significantly increased in A and B and a significant difference between groups except $\mathrm{C}$ and $\mathrm{D}$.

Conclusion: $\mathrm{T}$ cell subsets and Iymphoproliferative assays to mitogens and specific antigens could be a valuable tool for measuring the cell mediated immune competence of hepatic Schistosomiasis with and without $\mathrm{HCV}$ infection.

\section{PP11-04}

Diagnostic Value of Saliva for Detection of Hepatitis C Virus Infection among Thalassemic Patients

S.M. Miri ${ }^{1}$, M. Firuzmandi ${ }^{2}$, P. Karimi Elizee ${ }^{1}$, B. Hooshmand ${ }^{2}$, H. Keyvani ${ }^{3}$, A. Azar-Keyvan ${ }^{4}$, M. Keshvari ${ }^{5}$, B. Hajibeigi ${ }^{5}$, S.M. Miri', S.M. Alavian ${ }^{1}$

${ }^{1}$ Baqiyatallah University of Medical Sciences, Research Center for Gastroenterology and Liver Disease, Tehran, Iran; ${ }^{2}$ DDS, Dentistry Faculty, Shahid Beheshti Medical University, ${ }^{3}$ Iran University of

Medical Sciences, Tehran, Iran, ${ }^{4}$ Iranian Blood Transfusion

Organization, ${ }^{5}$ Tehran Blood Transfusion Research Centre,

${ }^{6}$ Dentistry Faculty, Shahid Beheshti Medical University, Tehran, Iran

Background and aims: Thalassemic patients due to necessity for multiply transfusion are highly exposed to Hepatitis C virus (HCV) infection than normal population. The role for $\mathrm{HCV}$ transmission through saliva had been discussed in different studies but assessing its danger as a hazardous risk for oral health workers can be discussed further.

Patients and methods: To further evaluation HCV salivary shedding, saliva and blood samples were collected from 44 thalassemic patients with $\mathrm{HCV}$ infection compared with 18 non-HCV thalassemia registered in a national clinical trial and referred to hepatitis clinic in Iranian blood transfusion center during March and April 2008. Stimulated saliva and fresh plasma were centrifuged and stored at $-70^{\circ} \mathrm{C}$, after the addition of guanidine isothiocyanate RNA extraction buffer. HCV-RNA was detected by RT-nested-PCR. HCV genotyping was carried out by RFLP.

Results: The dominant genotype in our patients was 1a in both male and female (42 and $41 \%$ respectively) $(p>0.05)$ as well as in major thalassemia $(44 \%, p<0.05)$ and in patients who had positive results in PCR of blood $(60 \%)$ or saliva $(30 \%)(p>0.05)$. Positive PCR results for saliva had been shown in ten patients $(16.1 \%)$ while others $(n=52,83.9 \%)$ were negative and there were not any relationship with previous treatment for $\mathrm{HCV}$ and type of treatment regimen $(p>0.05)$. Among patients with positive blood PCR results, 36 patients $(82 \%)$ had negative saliva PCR results and $8(18 \%)$ were positive. Conversely, for those with negative blood PCR results $(n=18), 16$ patients $(89 \%)$ had negative saliva PCR results while 2 patients $(11 \%)$ were negative $(p>0.05, R=0.087)$

Discussion: Saliva may serve as a possible, but low, nonparenteral transmission route of $\mathrm{HCV}$. These results provide a biological basis for saliva as a possible source of HCV infection, but do not necessarily imply transmission by this route.

\section{PP11-05}

Search for HCV RNA Positive, Anti HCV Negative Donor among Ahwaz Blood Donors

H. Bahrami

Hematology, Amir-Allmomnin Hospital, Ahwaz, Iran

Background and objective: In Diagnosis of hepatitis $\mathrm{C}$ virus infection usually begins with the detection of anti-HCV antibodies and only seropositive blood donors are usually confirmed by RIBA. Because of the period of over 2 months between infection and seroconversion, serological assays are not very efficient for diagnosis of the early stages of HCV infection. Since HCV RNA appears in blood as early as 2 weeks after infection, the use of NAT can substantially shorten the diagnostic window. In order to identify acutely infected blood donors before seroconversion and to reduce the potential risk of transfusion-associated hepatitis $\mathrm{C}$, the detection of HCV RNA by NAT has therefore been introduced recently in several developed countries. In this study, a molecular screening of HCV RNA among anti HCV negative blood donors were carried out in Iranian Blood Transfusion Organization, Research center, Tehran.

Materials and methods: 20,000 blood donors samples which were negative for anti HCV (EIA, third generation) screened for $\mathrm{HCV}$ RNA in 25 mini-pools during June 2008-March 2009. A total of 800 mini-pools were tested using manual RT-PCR method for qualitative detection of HCV RNA, with a lower limit of detection of $200 \mathrm{IU} / \mathrm{ml}$. Results: All of samples were negative for HCV RNA by RT-PCR method. On initial testing, two false positive results were defined as positive but on repeat single testing were negative.

Conclusion: HCV RNA detection by PCR can be carried out routinely in blood donor screening without significant delay of release of the components. The residual risk of transmission can be reduced by identification of early infection, which can lead to an improved safety of blood components. It was shown that combined screening using anti- HCV and 25 mini-pools HCV RNA testing can also be both useful and cost effective.

\section{PP11-06}

A Suggested Algorithm for Using Serum Biomarkers for the Diagnosis of Liver Fibrosis in Chronic Hepatitis C Infection

M.M. El-Attar ${ }^{1}$, E.M. Sweefy ${ }^{2}$, H.G. Rashed ${ }^{3}$, H.E. Hasan ${ }^{4}$

${ }^{I}$ Tropical M; ${ }^{2}$ Internal $M ;{ }^{3}$ Clinical Pathology; ${ }^{4}$ Pathology, Assiut $U$ Hospital, Assiut, Egypt

Hepatitis $\mathrm{C}$ infection is pandemic in Egypt. Liver biopsy is the gold standard for diagnosis and staging of fibrosis in chronic hepatitis $\mathrm{C}$ patients. However, liver biopsy is invasive, associated with sampling 
error, and potential complications. Finding non invasive alternative is really needed.

This study aims to assess the accuracy of biochemical markers and ultrasonography in predicting the stage of fibrosis in chronic hepatitis C patients.

Patients and methods: 65 Patients with chronic HCV were enrolled in this study. Ultrasonographic examination, blood count and liver function tests were done. Serum level of Hyaluronic acid (HA), IL-12 and YKL-40 were also assayed. Liver biopsy was taken. The correlation of the stage of fibrosis (according to METAVIR score) with biochemical markers and ultrasonographic findings was tested. ROC and accuracy tests of these parameters were examined.

Results: Histolopathological examination showed that 39 patients (60\%) had F1, 9 (14\%) had F2, 17 (26\%) had F3 and none had F0 or F4. A value of ALT index $<0.38$ or HA $<9.7 \mathrm{ng} / \mathrm{ml}$ or portal vein cross sectional area $<25.8 \mathrm{~mm}^{2}$ exclude significant fibroses $(\geq \mathrm{F} 2)$. AST+ALT $<39.5$ or APRI $<0.235$ or HA $\times 100 /$ Platelet $<9.534$ exclude the presence of advanced fibrosis with $100 \%$ NPV. Using these values advanced fibrosis could be excluded in $72 \%$ of our patients. APRI value of $(\geq 1.1)$ can diagnose advanced fibrosis with $100 \%$ PPV in $10 \%$ of our patients. So only $18 \%$ of our patients were not classified by these parameters for whom liver biopsy was recommended. Neither IL-12 nor YKL-40 helped in diagnosis of advanced fibrosis.

Conclusion: Applying a simple algorithm based on ALT, AST, platelet count, PV cross sectional area, in addition to Hyaluronic acid level may eliminate the need for liver biopsies in more than $80 \%$ of chronic HCV patients.

\section{PP11-07}

Hepatic Expression of Nitric Oxide Isoforms and Serum Nitrites/Nitrates in Chronic Hepatitis $\mathbf{C}$ and Schistosomal Liver Disease

A.A. Badawy ${ }^{1}$, O.A. Hammam ${ }^{1}$, R. Atta ${ }^{2}$, N. Elesily ${ }^{3}$

${ }^{1}$ Pathology; ${ }^{2}$ Tropical Medicine; ${ }^{3}$ Clinical Chemistry, Theodor Bilharz Research Institute, Cairo, Egypt

Elevated Nitric Oxide (NO) production is assumed to be responsible for pathological changes in many inflammatory conditions. Serum nitrites/nitrates representative to NO release as well as hepatic tissue immunohistochemical expression of $\mathrm{NO}$ isoforms: inducible $\mathrm{NO}$ synthase (iNOS) and endothelial NO synthase (eNOS); which have important function in inflammation and vasoregulation; were assessed in 72 patients with hepatitis $\mathrm{C}$ infection (out of them 24 cases were co-infected with Schistosomiasis). Intact hepatic tissue from outer most part of segmentectomy specimens performed for focal hepatic malignancy, and serum from healthy individuals served as control. Serum viral load was quantitated by PCR, and hepatic necroinflammatory activity and fibrosis were scored by Metavir Score. Control hepatic tissue expressed eNOS in hepatocytes, and in sinusoidal and vascular endothelium, and expressed iNOS as faint cytoplasmic staining in periportal hepatocytes. In diseased liver: staining intensity of both iNOS and eNOS increased. Extent of iNOS expression expanded to involve areas in zone 2 and 3 of hepatic acinus, inflammatory cells, and endothelial lining of bile ducts and sinusoids. Serum nitrites/nitrates raised variably in diseased cases without definite correlation with grades of hepatitis activity, stages of fibrosis, hepatic tissue expression of iNOS or eNOS, or blood viraemia level. Evidenced by Spearman correlation test extent of iNOS expression correlated with increasing grade of hepatitis activity, upstaging of fibrosis, and steatosis. Associated schistosomal affection correlated with presence of splenomegally, and more eNOS expression. PCR positivity and viraemia level had no definite correlation with studied parameters.

In conclusion: Schistosomiasis is a risk factor for haemodynamic disturbance in chronic viral hepatitis. Blood NO level do not reflect necro-inflammatory activity in chronic liver diseases and histologic study of liver biopsy is a must to monitor therapy.
PP11-08

Hepatitis C Virus Core Antigen Was Correlated with RNA Load and had Similar Dynamics during Treatment for Chronic Hepatitis C

R. Yang, H. Rao, L. Wei

Peking University People's Hospital, Peking University Hepatology

Institute, Beijing, China

Background: Compared with hepatitis C virus (HCV) RNA assay, $\mathrm{HCV}$ core antigen assay is simple, rapid and reproducible. But the low sensitivity and narrow dynamic range often limited its use. Here a new sensitive and automated chemiluminescent microparticle immunoassay (CMIA) was introduced to observe the dynamics of $\mathrm{HCV}$ core antigen in chronic hepatitis $\mathrm{C}(\mathrm{CHC})$ patients during antiviral treatment.

Methods: Replicates of WHO standard dilution series and serum panel comprising all HCV genotypes were tested by Abbott HCV core antigen CMIA. Then serial serum samples from 61 consecutive genotype $1 \mathrm{~b}$ CHC patients treated with PEG-IFN $\alpha$-2a and ribavirin were tested by antigen CMIA and by Roche Cobas Taqman HCV RNA in parallel at baseline, week 2,4 and 12 of treatment, respectively.

Results: The functional sensitivity of $\mathrm{HCV}$ core antigen assay was $1.64 \mathrm{fmol} / \mathrm{l}$ (equivalent to $400 \mathrm{IU} / \mathrm{ml}$ of RNA) and genotype independent. HCV core antigen was closely correlated to RNA load with a correlation coefficient $(r)$ of $0.94(P<0.001)$ and had similar dynamics as that of RNA during the first 12 weeks of treatment in all patients with or without early virological responses (EVR). In 26 patients with rapid virological responses (RVR), all had undetectable core antigen; in the other 35 patients without RVR, 29 had positive core antigen and 6 had undetectable core antigen at week 4 . In 47 patients with EVR, all had undetectable core antigen; in the other 14 patients without EVR, thirteen had positive core antigen and the only one patient with undetectable antigen had a very low level of HCV RNA (63 IU/ml). Conclusions: HCV core antigen can be used as a marker of viral replication and long-term follow up will be needed to clarify its dynamics during and after antiviral treatment and to define its usefulness in predicting the sustained virological response.

\section{PP11-09}

Diagnostic Accuracy of Serum Alt for Grades of Liver Inflammation and Liver Fibrosis in Naive Chronic Hepatitis C Patients

S. Mirza ${ }^{1}$, A.R. Siddiqui ${ }^{1,2}$, M. $\mathrm{Umar}^{3}$, S. Hamid $^{4}$

${ }^{1}$ Community Health Sciences, Aga Khan University, Karachi,

Pakistan; ${ }^{2}$ Department of Family and Community Medicine, College of Medicine, King Saud University Riyadh, Riyadh, Saudi Arabia;

${ }^{3}$ Department of Medicine, Holy Family Hospital, Rawalpindi;

${ }^{4}$ Department of Medicine, Aga Khan University, Karachi, Pakistan

Background: Liver biopsy is an invasive procedure related to risks and discomfort to the Chronic Hepatitis $\mathrm{C}(\mathrm{CHC})$ patients. Its role prior to antiviral therapy is now debatable. Serum ALT is a less invasive biochemical marker which may indicate not only grade of liver inflammation but also fibrosis on liver biopsy.

Objectives: To determine the diagnostic accuracy of serum ALT for grades of liver inflammation and liver fibrosis on pretreatment liver biopsy in naïve $\mathrm{CHC}$ patients.

Methods: A cross section of 595 naive $\mathrm{CHC}$ patients who completed Interferon $\alpha$ and Ribavirin therapy for 24 weeks at the liver clinic in Holy Family hospital, Rawalpindi and outpatient department of Aga Khan University hospital, Karachi was studied. Grades of liver inflammation and stage of fibrosis was reported by modified Histological activity index (HAI) and Batts-Ludwig scoring on pretreatment liver biopsy. One-way ANOVA was conducted to look for the difference of mean serum ALT for grades of liver inflammation (minimal, mild, moderate and severe) and liver fibrosis (none, portal fibrosis, bridging fibrosis and cirrhosis). ROC curve analysis was done and Area Under Curve (AUC) calculated for assessing diagnostic accuracy.

Results: As the grade of liver inflammation increased, mean serum ALT also increased ( $p$ value 0.002). Same was observed for liver 
fibrosis ( $p$ value $<0.001$ ). ROC curve analysis showed that serum ALT was a less accurate test for mild and moderate liver inflammation as well as for portal fibrosis and bridging fibrosis. It was found be moderately accurate for severe liver inflammation and cirrhosis (AUC 0.77 for each).

Conclusion: Serum ALT can be used to indicate severe liver inflammation and cirrhosis in naive $\mathrm{CHC}$ patients.

\section{PP11-10}

A Novel Rapid Non-invasive Screening Device (C-FAST) for Hepatitis C Virus with High Sensitivity and Specificity

G. Shiha ${ }^{1}$, A. Amien ${ }^{2}$, W. Samir ${ }^{3}$, H. Bader ${ }^{2}$

${ }^{1}$ Faculty of Medicine Mansoura University, Mansoura; ${ }^{2}$ Engineering Authority-Ministry of Defence, Cairo; ${ }^{3}$ Specialized Medical Hospital, Mansoura University, Mansoura, Egypt

Introduction: Chronic hepatitis $\mathrm{C}$ virus (HCV) infection is a major health problem with around 200 million individuals affected worldwide. The screening test for detection of $\mathrm{HCV}$ was the determination of the anti-HCV antibodies by ELISA method. However, RT-PCR was still the accurate and standard technique for detection of HCV. All substances consist of molecules, each molecule has a specific frequency (Molecular Signature). We developed a novel, rapid, noninvasive screening device C-FAST (trade mark), which is patented Bio-sensor slash spectroscopy based technology for the detection of hepatitis $\mathrm{C}$ virus. When the signal is picked up a pivoting antenna of the C-FAST is drawn like a compass needle, to indicate alignment between the patient and the frequency card in the C-FAST.

Aim: Evaluation of the sensitivity and specificity of HCV detection using C-FAST device as compared to RT-PCR.

Methods: The study included 879 cases seen at out-patients hepatology clinic at Mansoura, Egypt. HCV RNA was detected for all cases using RT-PCR. C-FAST device was applied for all cases to detect the infected patients with HCV. Sensitivity, specificity, positive and negative predictive values were determined for comparison between the two methods.

Results: 879 cases ( 868 male and 11 female) with age ranged from 18-46 years were included in the study. 159 cases were positive for HCV-RNA and 720 cases were negative for HCV-RNA using RT-PCR. The results of C-FAST showed that 178 cases were infected with HCV while 701 cases were not infected with HCV. The efficacy of C-FAST was $97.8 \%$, sensitivity $100 \%$, specificity $97.3 \%$, PPV $89.3 \%$ and NPV $100 \%$.

Conclusion: C-FAST is rapid, non-invasive, accurate, simple, highly sensitive and specific tool for screening of hepatitis $\mathrm{C}$ virus and have the potential for early detection of viral load which will enhance the therapeutic outcome.

\section{PP11-11}

Extrahepatic Manifestations in Chronic Hepatitis C

\section{Coldea}

Internal Medicine, Railway Hospital, Sibiu, Romania

The purpose: The chronic hepatitis $\mathrm{C}$ is a frequently problem in worldwide, the number of infected individuals is high and go on, getting a public health problem. In Romania there are nearly 1 million persons infected with hepatitis $C$ virus. Without hepatic manifestations in chronic hepatitis $\mathrm{C}$ appears other extrahepatic manifestations such as cryoglobulinemia mixed is the most frequently.

The aim of study: I analysed the distribution of chronic hepatitis $\mathrm{C}$ in Clinical Hospital Sibiu, between 2001-2004, the aim of study is to establish the characteristic and frequency of extrahepatic manifestations. At the same time I followed to establish the correlations between age, sex, residence, the possible infection moment and extrahepatic manifestations.
Material and methods: The study is retrospective based on 162 cases of chronic hepatitis with antibodies HCV positive hospitalized in Departments of Clinical Hospital Sibiu.

Results and discussion: The group of 162 cases with chronic hepatitis with antibodies $\mathrm{HCV}$ positive are composed by 102 females $(63 \%)$ and 60 males $(37 \%)$. In my study I observed that it was a preponderant affection of age $60-69$ years $(27.16 \%)$. The average age was $54.46 \pm 13.15$ years. In my group. In my group, 53 patients $(33 \%)$ present without hepatic manifestation, extrahepatic manifestations, and 109 patients present only hepatic manifestations. The distribution of cases with extrahepatic manifestations is: 16 patients with endocrine manifestations $(30.18 \%), p$ value $=0.008,22$ patients with cryoglobulinemia $(41.53 \%), p$ value $=0.085 ; 5$ patients with skin manifestations $(9.43 \%), p$ value $=0.0000044,8$ patients with hematological manifestations $(15.09 \%), p$ value $=0.00006$; and 2 patients other manifestations (3.77\%).

Conclusions: The number of extrahepatic manifestations in chronic hepatitis $\mathrm{C}$ is in continues growth and became a open filed of research.

\section{PP11-12}

Correlation of Iron Loading and Severity of Liver Damage in Thalassemic with $\mathrm{HCV}$ by Using MRI

S.M. Alavian ${ }^{1,2}$, B. Behnava ${ }^{1}$, M. Keshvari ${ }^{3}$, B. Hajibeigi ${ }^{3}$, L. Mehrnoosh ${ }^{2}$, P. Karimi Elizee ${ }^{1}$, S.M. Miri ${ }^{4}$, K.B. Lankarani ${ }^{5}$, S.-Y. Saeedi ${ }^{4}$

${ }^{1}$ Baqiyatallah University of Medical Sciences, Baqiyatallah Research Center for Gastroenterology and Liver Disease (BRCGL), Tehran, Iran; ${ }^{2}$ Tehran Hepatitis Center; ${ }^{3}$ Iranian Blood Transfusion Organization Research Centre (IBTO), Tehran, Iran; ${ }^{4}$ Baqiyatallah Research Center for Gastroenterology and Liver Disease; ${ }^{5}$ Shiraz. University of Medical Sciences, Tehran, Iran

Background and aims: MRI T2* is an non-invasive assay which can estimate the iron loading of liver in thalassemic patients. The aim of this study, was to evaluate the relationship between the iron loading by MRI T2* assay and the degree of liver damage in thalassemic patients with $\mathrm{HCV}$ infection.

Methods: Thirty thalassemic patients with chronic hepatitis c were enrolled. The iron loading was evaluated by MRI T2*assay. Also, liver biopsy was done in all of them and histopathologic findings were gradedaccording to the Knodell's criterial.

Results: The iron loading of liver was normal in 6 (20\%), mild in 13 (43.3\%), moderate in $10(33.3 \%)$ and seveer in $1(3.3 \%)$ of the patients. There was a significant correlation between the iron loading and the grade of liver inflammation $(p$ value $=0.0$ ). Also, a significant correlation was seen between the hepatic iron loading and the stage of liver fibrosis 9 ( $p$ value $=$ ) too.

Conclusion: Previous studies have indicated that in thalassemic patients with chronic hepatitis $\mathrm{c}$, the degree of iron deposition in liver which has been estimated by atomic absorption assay, correlates with the severity of liver damage. This study indicated that,the iron loading of liver by MRI T2* is also directly related to the degree of hepatic necroinflammation and the extent of liver fibrosis.

\section{PP11-13}

Expression Profiles of Plasma microRNAs in Patients with CHC Genotype $1 \mathrm{~b}$ before and after Treatment with Peg-IFN $\alpha$-2a Plus Ribavirin

L.-L. Gu, G.-M. Gong, Y. Lin, X.-W. Liao, X.-H. Li, Y. Han, X.-X. Zhang Department of Infectious Disease, Rui Jin Hospital, Shanghai Jiao Tong University, School of Medicine, Shanghai, China

Backgroud and aims: Circulating miRNAs are promising biomarkers for diseases evolution, and affect the function of recipient cells. Interaction between viral and host factors causes the persistent $\mathrm{HCV}$ 
infection in human. To providing evidence for application of circulating miRNAs in explaining pathogenesis, diagnosis and therapy of $\mathrm{HCV}$ infection, we characterized the expression profiles of plasma miRNAs in CHC patients by using microarray analysis.

Patients and methods: We enrolled five CHC patients (genotype 1b) treated with peg-IFN $\alpha$-2a plus ribavirin and responded completely, and five healthy donors. Patients' peripheral blood was drawn in citric acid tubes before and 12 weeks after treatment. Isolated plasma samples were divided into three groups: healthy, untreated and treated group. Then we pooled plasma together in each group for further analysis. Agilent human microRNA microarrays of plasma were performed for comparison of expression levels of miRNAs among distinct groups.

Results: Over 100 miRNAs emerged in plasma of the healthy group, while approximately 200 miRNAs arose in plasma of the untreated and treated group, respectively. Lots of miRNAs changed (fold change $\geq 2$ ) when compared their levels among different groups including 89 plasma miRNAs were up-regulated and 48 were downregulated in the untreated group compared with the healthy group. These altered miRNAs mostly likely related to immunity, inflammation and metabolism. Notably, several increasing miRNAs in the untreated group decreased after antiviral therapy.

Conclusions: The diverse expression profiles of plasma miRNAs in CHC patients before and after treatment may be potential biomarkers for CHC. Their involvement in some major mechanisms contributing to $\mathrm{HCV}$ infection may shed new light on the causes of chronic HCV infection and offer novel therapeutic targets.

\section{PP11-14}

ANA as a Marker for the Induced HCV Chronicity in HCV and S. mansoni Co-infected Patients

E. El-Ahwany ${ }^{1}$, H. Mari ${ }^{2}$, R. Atta ${ }^{3}$, S. Zada ${ }^{2}$

${ }^{1}$ Immunology Department, Theodor Bilharz Research Institute, Giza;

${ }^{2}$ Biology Department, The American University in Cairo, New Cairo;

${ }^{3}$ Gastrentrology Department, Theodor Bilharz Research Institute,

Giza, Egypt

Background/aim: Egypt has the highest hepatitis C (HCV) prevalence rate worldwide. This virus, which is known to target the liver cells possibly leading to liver failure, has long been associated with schistosomiasis (schisto). Schistosomiasis has been observed to induce the chronicity of $\mathrm{HCV}$ in co-infected patients. On the other hand, high anti-nuclear antibodies (ANA) serum level has been reported in patients with chronic HCV. Merging both findings, the aim of this study was to determine if ANA can be used as a marker for the schisto-induced HCV chronicity and if its intensity can give a clue to the stage of infection in co-infected patients with $\mathrm{HCV}$ and S. mansoni.

Method: This was done by semi-quantifying the ANA serum levels following the guidelines established by the CDC, Atlanta, USA. The study included 200 subjects, which were divided into four groups (50 subjects in each group): Group 1 as a control (-ve $\mathrm{HCV},-\mathrm{ve}$ Schisto.); Group 2 (+ve HCV, -ve Schisto.); Group 3 (-ve HCV, +ve Schisto.); Group 4 (+ve HCV, +ve Schisto.). The serum level of ANA was determined using an indirect fluorescent antibody test on HEp-2 cells. All co-infected subjects (100\%) were positive for ANA. In addition a higher ANA fluorescence intensity was observed in co-infected patients compared to the other three groups.

Conclusions: These findings support the documented effects of schistosomiasis on the response of the immune system against HCV infection in co-infected patients. It also suggests that ANA can be used as a marker to detect the induced chronicity of HCV in coinfected patients and that the fluorescence intensity can be of clinical significance in determining the stage of infection.
Poster Presentation 12: Hepatitis C: Epidemiology

PP12-01

Demographic, Virological and Histological Profile of Patients with Chronic Viral Hepatitis of the Complex University Hospital Professor Edgard Santos

T.D. Alves ${ }^{1}$, E.A. Bomfim ${ }^{1}$, D.L. Andrade ${ }^{1}$, T.P. Souza ${ }^{1}$, J.S. Pompa ${ }^{1}$, C.A. Silva ${ }^{1}$, R. Paraná ${ }^{2}$, M.I. Schinoni ${ }^{2}$

${ }^{1}$ Faculty of Medicine, Federal University of Bahia; ${ }^{2}$ Complex University Professor Edgard Santos, Hepatology Unit, Federal University of Bahia, Salvador, Brazil

Background: The Complex University Hospital Professor Edgard Santos (C-HUPES) is state reference center for treatment of viral hepatitis,so,it's necessary to report the prevalence and characteristics of this population.

Aims: To study the prevalence of types of chronic viral hepatitis(B$\mathrm{HBV}$ and $\mathrm{C}-\mathrm{HCV}$ ) and analyze the virological and histological profile of the patients of C-HUPES.

Methods: A retrospective descriptive study based cases diagnosed as having $\mathrm{HBV}(\mathrm{HBs} A \mathrm{~g}$ positive and detection of HBV-DNA) and HCV(Anti-HCV positive and detection of HCV-RNA), with report of liver biopsy.Sample consisted of 456 patients (HBV $=156 / \mathrm{HCV}=$ 300) was categorized: 1-patients with mild fibrosis (META$\mathrm{VIR}=\mathrm{F} 0-\mathrm{F} 1-\mathrm{F} 2) \times$ advanced $($ METAVIR $=\mathrm{F} 3-\mathrm{F} 4)$, 2-mild inflammation $\quad($ METAVIR $=\mathrm{A} 0-\mathrm{A} 1) \quad \times$ advanced $($ METAVIR $=$ A2 - A3), 3-presence $\times$ abcense of iron and 4presence $\times$ absence of steatosis. The chi-square test was used for categorical variables (significance level $=5 \%$ ).

Results: The demographic,histological and laboratory findings are presented in Table 1.There was statistic inference between steatosis and the type of hepatitis $(p=0.000)$; steatosis and antiHBe $(p=0.000)$ and between HBeAg and inflammation $(p=0.015)$.

\begin{tabular}{|c|c|c|}
\hline & $\begin{array}{l}\text { HBV } \\
(n=156 / 34.2 \% \\
\text { of the sample })\end{array}$ & $\begin{array}{l}\mathrm{HCV} \\
(n=300 / 65.8 \% \\
\text { of the sample })\end{array}$ \\
\hline Sex (male/female) & $65.4 \% / 34.6 \%$ & $55.15 \% / 44.85$ \\
\hline $\begin{array}{l}\text { Average age in years } \\
\text { (male/female) }\end{array}$ & $37.68 / 36.06$ & $49.47 / 50.46$ \\
\hline $\begin{array}{l}\text { Degree of fibrosis } \\
\text { (METAVIR score: } \\
\text { F0-1-2/F3-4) }\end{array}$ & $76.2 \% / 23.8 \%$ & $69.3 \% / 30.7 \%$ \\
\hline $\begin{array}{l}\text { Degree of inflammation } \\
\text { (METAVIR score: } \\
\text { A0-1/A2-3) }\end{array}$ & $68 \% / 32 \%$ & $73.7 \% / 26.3 \%$ \\
\hline $\begin{array}{l}\text { Iron overload } \\
\quad \text { (present/absent) }\end{array}$ & $21.6 \% / 78.4 \%$ & $17.7 \% / 82.3 \%$ \\
\hline Steatosis (present/absent) & $32.7 \% / 67.3 \%$ & $63.2 \% / 36.8 \%$ \\
\hline $\begin{array}{l}\text { Average Transaminases } \\
\text { in U/L (AST/ALT) }\end{array}$ & $66.08 / 97.06$ & $79.83 / 100.88$ \\
\hline $\begin{array}{l}\text { AntiHBe positive }(n=54) / \\
\text { AgHBe positive }(n=52)\end{array}$ & $77.78 \% / 46.20 \%$ & - \\
\hline Genotype $(1 / 2+3+4+5)$ & - & $74.4 \% / 25.6 \%$ \\
\hline
\end{tabular}

Conclusions: Our sample had a higher prevalence of HCV than HBV, which can be explained by greater effort to detect hepatitis $\mathrm{C}$ in our midst.Among patients with $\mathrm{HCV}$, there was a higher frequency of genotype 1; higher than that demonstrated in other centers in the country. Found a higher proportion of steatosis in patients with $\mathrm{HCV}$ than HBV. In both groups, the mild fibrosis was more prevalent, which could be explained by the efforts being employed in the early diagnosis of chronic viral hepatitis. 


\section{PP12-02}

Discovery of the Missed Link between Schistosomiasis and HCV Infection A.A.E.M. Soliman ${ }^{1}$, S.A. $\mathrm{Zaki}^{2}$, A.A. Abd El Moety ${ }^{1}$, H.A. Abd El Moety ${ }^{3}$ ${ }^{1}$ Internal Medicine; ${ }^{2}$ Microbiology, Faculty of Medicine, Alexandria University; ${ }^{3}$ Chemical Pathology, Medical Research Institue, Alexandria University, Alexandria, Egypt

Schistosomiasis is a term covering infestation of man by blood fluke trematode parasite.

Egypt S. mansoni occurs intensively in the Nile Delta, while $S$. hematobium is endemic in Nile Valley.

Studies in Egypt found the highest risk of HCV infection in whom infested with schistosomiasis. HCV-Ab prevalence reported $70 \%$ in adults suffering from Schistosomiasis and without history of blood transfusion.

Aim: Determine the link between schistosomal infestation and HCV, whether the parasite could be vector of transmission of the virus to human.

Methods: Different stages of life cycle of $S$. mansoni provided by Theodore Bilharzias Institute.

Specimens of living S. mansoni worms and Biomphalaria alexandria snails were grounded separately in sterile mortar after adding $5 \mathrm{ml}$. sterile saline. After centrifugation sterile supernatant tested for

Detection of HCV-RNA by RT-PCR

HCV-RNA quantitation.

Results: HCV-Antigen: Worms, Miracidia, Snails and Cercariae of S. mansoni were Positive for HCV-Ag. The Snails gave strong positive result. Eggs gave negative result.

HCV-RNA by RT-PCR: Worms, Miracidia, Snails and Cercariae of S. mansoni tested for HCV-RNA By Qualitative RT-PCR were Positive. The eggs gave negative result.

HCV-RNA Quantitation: Miracidia were positive (800 Copies/ml) and Snails were positive $(1,100$ Copies $/ \mathrm{ml})$ other specimens gave negative results.

Conclusion: Existence of virus and its replication in parasite.

S. mansoni parasite carries $\mathrm{HCV}$ and considered as a non-human vector for transmission of $\mathrm{HCV}$ infection

Parasitic and viral co-infection change pathology of hepatic schistosomiasis from periportal fibrosis to cirrhosis and the development of $\mathrm{HCC}$

\section{PP12-03}

The Association between Genetic Polymorphisms of IFNAR2, IL10RB and $I L$-28RA Gene and Outcomes of HCV Infection in High-risk Chinese Population

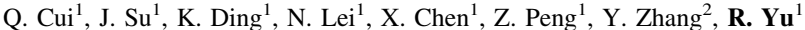

${ }^{I}$ Department of Epidemiology and Biostatistics, Nanjing Medical University; ${ }^{2}$ Center for Disease Control and Prevention, Nanjing Military Region, Nanjing, China

Background: The outcomes of chronic HCV infection vary, depending on viral and host factors. Those mechanisms involved in the control of the innate and adaptive response could have an influence on outcome of infection. Single-nucleotide polymorphisms (SNPs) located on the IFNAR2, IL1ORB and $I L-28 R A$ gene have been associated with autoimmune diseases and HBV infection. The aim of the study was to investigate a possible association of these genes with susceptibility of hepatitis $\mathrm{C}$, and to assess whether they could influence the outcomes of HCV infection in a high-risk Chinese population.

Methods: Genomic DNAs of 552 patients with anti-HCV seropositive and 421 uninfected controls were extracted from the peripheral blood leukocytes. Genotyping of IFNAR2, IL1ORB and $I L-28 R A$ gene polymorphisms were performed using TaqMan ${ }^{\circledR}$ assays method.

Results: The distribution of IFNAR2 and ILIORB genotypes among the control, persistent infection, and spontaneous clearance groups did not differ. However, $I L-28 R A$-rs 10903035 A allele was over-represent in persistent infection as compared with uninfected controls and spontaneous clearance, respectively $(\mathrm{OR}=1.54,95 \% \mathrm{CI}=1.23$ $1.92, P=0.000 ; \mathrm{OR}=1.42,95 \% \mathrm{CI}=1.12-1.81, P=0.002)$, and negative effect of the AA genotype were significantly different in different strata except for the females $(P<0.05)$. IL-28RArs11249006 GG genotype seemed to protect from susceptibility of persistent $\mathrm{HCV}$ infection $(\mathrm{OR}=0.53,95 \% \mathrm{CI}=0.31-0.91$, $P=0.022$ ), while AG genotype enhances persistent of HCV infection $(\mathrm{OR}=1.54,95 \% \mathrm{CI}=1.07-2.24, \quad P=0.022)$, and the protective effect of GG genotype was significantly different among subgroups stratified by age and source of subjects $(P<0.05)$. In addition, stratified analysis also showed that IL1ORB-2834167 AG/GG genotype remained significant different between spontaneous clearance and persistent infection patients in the terms of females and patients infected with non-1 viral genotype $(P<0.05)$.

Conclusions: Two SNPs in IL28RA appeared to be associated with susceptibility of $\mathrm{HCV}$ infection and natural viral clearance respectively, which implicate a primary role for IL28RA in the outcomes of $\mathrm{HCV}$ infection.

\section{PP12-04}

Hepatitis C Virus Antibody Positive Cases in Multitransfused Thalassaemic Patients in South of Iran

A. Akbari, M. Imanieh, M. Karimi, H. Tabatabaee

Shiraz University of Medical Sciences, Shiraz, Iran

Backgroud: Hepatitis $\mathrm{C}$ virus (HCV) infection is the common cause of liver disease among thalassaemic patients who receive recurrent blood transfusion. Recent studies in Iran has shown a high prevalance of HCV among Iranian thalassaemic patients, inspite of low HCV seroprevalence in general population. In this study we investigated the prevalence of new cases of hepatitis $\mathrm{C}$ and its risk factor in the group of thalassaemia major patients.

Method: This study was carried out in thalassaemic Ward of Shiraz University of Medical Sciences southern Iran. Cases were interviewed using a standard questionaire including demographic, clinical history and $\mathrm{HCV}$ related risk behaviors. Then three blood samples were investigated by serum marker of anti-HCV antiboby (anti- $\mathrm{HCV} \mathrm{Ab)} \mathrm{via}$ Eliza-3th method in times zero, 45 days and after 6 months follow up. Results: A total number of 200 thalassaemic patients randomly participants in our study. The mean age of patients were $15.2 \pm 6.3$ (1-36 years old) and the proportion of male to female 1 to 1 . Splenectomy was done in $\% 35$ of cases. The mean serum ferritin level was 2,288 \pm 932 nonogram in mililitre. The rate of HCV antibody positivity in the first sample was $25 \%$. All of second and third blood samples were negative. There was significant correlation between age and being positive for anti- $\mathrm{HCV} \mathrm{Ab}(P<0.00)$ and also between being positive anti-HCV Ab and the time they began to receive blood transfusion. The patients who started blood transfusion before 1996 (The year we started screening program for blood donors in Blood Bank in Iran) had 12.5 times more risk for being positive for anti$\mathrm{HCV}$ ab (odds ratio $=12.5$ ).

Conclusion: Our results approved the effect of screening program for blood donors in blood transfusion centers.

\section{PP12-05}

Seroprevalence Study of HCV Genotypes in Two Tertiary Hospitals and its Association with Risk Factors

M.H. Hasmoni ${ }^{1,2}$, H.A. Hamzah ${ }^{3}$, R. Salleh ${ }^{2,4}$

${ }^{1}$ Department of Internal Medicine, Kulliyyah of Medicine, International Islamic University Malaysia, Kuantan; ${ }^{2}$ Department of Medicine, Hospital Raja Perempuan Zainab II, Kota Bharu; ${ }^{3}$ Department of Basic Medical Sciences, Kulliyyah of Medicine, International Islamic University Malaysia, Kuantan; ${ }^{4}$ Department of Medicine, Hospital Sultanah Nur Zahirah, Kuala Terengganu, Malaysia 
Background: This study aim to identify HCV genotypes and associated risk factors in a group of $\mathrm{HCV}$ infected patients from two tertiary hospitals in Malaysia.

Methods: This is a seroprevalence study of HCV genotypes amongst infected patients in Hospital Tengku Ampuan Afzan, Kuantan, Pahang and Hospital Sultanah Nur Zahirah, Kuala Terengganu, Terengganu. Patients were screened using ELISA by detecting the anti$\mathrm{HCV}$ in the sera. All negative first-round PCR products were re-tested by nested PCR. The base sequence of the PCR products was determined using the same primers as for the RT-PCR. By comparing the obtained nucleotide sequence data with sequences of known genotypes from the NCBI homepage, we deduced that our local isolates could be assigned to genotypes $1,3,4$ and 6 . We correlated the mode of transmission, basic demographic characteristics and their risk factors with the predominance of different genotypes.

Results: Out of 171 patients diagnosed positive for anti-HCV by ELISA, 116 patients agreed for HCV genotypes evaluation. $31.9 \%$ $(n=37)$ were of genotype $1,58.6 \%(n=68)$ genotype $3,6.0 \%$ $(n=7)$ genotype 4 and $3.5 \%(n=4)$ genotype 6 . There was no statistically significant difference between the risk factors analyzed and the acquisition of HCV infection. We also found out that only $53.4 \%(n=62)$ of these patients were treated and $27.6 \%(n=32)$ had liver biopsy. Interestingly we noted that patients with genotype 4, one of them was an Egyptian and another four had exposure to risk factors on their travel to the Middle East countries.

Conclusion: Genotype 3 and 1 were the most prevalent genotypes (58.6 and $31.9 \%$, respectively) followed by genotype 4 and 6 . Our study results are consistent with an unpublished data from the Malaysian Society of Gastroenterology and Hepatology [Genotype 3 (56\%), genotype 1(39\%)].

\section{PP12-06}

Spontaneous Viral Clearance of Chronic HCV Infection in Upper Egypt: Community-based Study, Ten Years Follow-up

M.M. El-Attar ${ }^{1}$, M.H. Shehata ${ }^{1}$, M.A. Mohammed ${ }^{1}$, M. Abdel-Hammed ${ }^{2}$, A.M. Nasr ${ }^{1}$

${ }^{1}$ Tropical M, Assiut U Hospital; Assiut, ${ }^{2}$ Elmenia U, Elmenia, Egypt

Background: Hepatitis C virus (HCV) infection is a global blood borne disease with the highest estimated prevalence in Egypt (11-14\% of the population chronically infected). The natural course of $\mathrm{HCV}$ infection is highly variable. The reason why the infection persists in some patients and resolves spontaneously in others is not known.

Aim: To detect the frequency of spontaneous clearance of chronic $\mathrm{HCV}$ infection in Upper Egypt and to determine the predictors of persistence of $\mathrm{HCV}$ infection.

Patients and methods: In 1997, a community-based study was performed in Upper Egyptian Sallam village. Out of the 329 patients who had positive HCV RNA, only 200 patients were included in this study, after excluding cirrhotic patients at the start of the study and who received specific treatment for $\mathrm{HCV}$ infection. We followed up them every 6 months "between 2004 to 2008 " clinically, ultrasonographically, laboratory (liver functions, $\mathrm{CBC}$, prothrombin time and concentration) and HCV-RNA.

Results: After 10-year follow-up, spontaneous clearance of chronic $\mathrm{HCV}$ infection was detected in $35(17.5 \%)$ out of 200 patients. Eighteen $(51.4 \%)$ cases with HCV-RNA clearance were symptomatic, while $104(63 \%)$ cases with HCV-RNA persistence were symptomatic. Most of the physical and laboratory findings were detected more in patients with HCV-RNA persistence than who had cleared HCVRNA. By ultrasonographic examination, normal liver echo pattern was found in $30(85.7 \%)$ cases with HCV-RNA clearance and in 75 $(45.5 \%)$ patients who had HCV-RNA persistence. Evidence of liver cirrhosis was found only in cases with HCV-RNA persistence [9 $(5.5 \%)$ persons]. Old age $>60$ was the strongest predictor of persistence of $\mathrm{HCV}$ infection followed by obesity and blood transfusion.
Conclusions: Spontaneous clearance of chronic HCV infection could happen without any specific antiviral therapy in $17.5 \%$ of patients. Older age was the strongest predictor of persistence of $\mathrm{HCV}$ infection followed by obesity and blood transfusion.

\section{PP12-07}

Transmission of Hepatitis $\mathrm{C}$ between Spouses an Epidemiological Study at National Liver Institute Hospital

W.S. Morad

Comunity Medicine, National Cancer Institute, Menoufiya University, Shebin El Kom, Egypt

Background/aims: In Egypt, relatively higher rates of sexual transmission have been reported and reflect the higher background prevalence in this country. In rural Egypt, sexual transmission between monogamous spouses ranged between 3 and 34\%. This is a cross sectional hospital based study evaluated the non-sexual and sexual risk factors of $\mathrm{HCV}$ transmission from index male and female cases to their spouses, to assess proportionate morbidity rate of $\mathrm{HCV}$ disease and to assess the relationship between the degree of $\mathrm{HCV}$ viremia in index cases and the transmission of HCV infection.

Methods: One hundred index males and 100 index females with HCV and their spouses provided informed consent to participate. Participants were interviewed to gather risk factor information using multiple question models previously prepared questionnaire and serological tests (anti HCV Abs, Hbs Ag and HCV-RNA PCR).

Results: Proportionate morbidity rate of HCV disease in NLI was $80 \%$ and HCV transmission from wife to husband including sexual relationship occurred in $46 \%$ of cases while from husband to wife was 25 and $35.5 \%$ overall $\mathrm{HCV}$ transmission between spouses.

Conclusion: This study documented previously uncertain life-style risk factors for $\mathrm{HCV}$ transmission between spouses, confirmed the high prevalence of $\mathrm{HCV}$ infection (17-26\%) in Egypt which is higher than other parts of the world, and provided evidence of sexual transmission of HCV among spouses but we can not ignore the significant role of non sexual risk factors of $\mathrm{HCV}$ among couples such as sharing clippers or nail cutters and others.

\section{PP12-08}

Prevalence of Blood Transfusion Induced Hepatitis among Patients with Clotting Factor Deficiencies in Tabriz

S.O. Mashayekhi ${ }^{1}$, D. Sheykhi ${ }^{2}$, A. Nikanfar ${ }^{3}$, A. Hosseyn Pour Feizi ${ }^{3}$, M. Nazari ${ }^{4}$, R. Dolatkhah ${ }^{3}$, M. Ghandforoush-Sattari ${ }^{1}$

${ }^{1}$ Hematology and Oncology Research Center, Faculty of Pharmacy, Tabriz University of Medical Sciences, Iran; ${ }^{2}$ Faculty of Pharmacy, Tabriz University of Medical Sciences, Iran; ${ }^{3}$ Hematology and Oncology Research Center, Faculty of Medicine, Tabriz University of Medical Sciences, Iran; ${ }^{4}$ Pediatric Hospital, Tabriz University of Medical Sciences, Iran, Tabriz, Iran

Background/aims: Hemophilia is an inherited coagulation disorder. About 6,560 hemophilia patients are registered in Iranian Hemophilia Organization (prevalence of 6-7 in 100,000). Due to the chronicity of the disease, it demands life-long treatment including numerous drugs and blood products (clotting factors). The present study examines prevalence of treatment-induced hepatitis in hemophilic patients and patients with other clotting factor disorders.

Methods: Patients registered in two hematology clinics in Tabriz were invited to the study. Those who volunteered were asked to complete a simple questionnaire. The questionnaire was consisted of demographic information, the treatments and patients' hepatitis/HIV status. The questionnaire was completed by reviewing patients records and direct questions from the patients. The data were analyzed by using SPSS (ver. 13) software.

Results: Data collection was conducted between November 2008 and July 2009. Eighty patients attending two hematology centers in Tabriz were enrolled to the study. The median age of subjects was 
$19.03 \pm 11.88$, and $29 / 6 \%$ were female. Fifty four patients had hemophilia type A and 6 were affected by hemophilia type B and 10 were affected by Von wilbrand and 11 were affected by other clotting factor deficiencies. Eighty four percent had received hepatitis vaccination previously. No patients were HIV positive while $5 \%$ had been diagnosed with hepatitis B and $25 \%$ were affected by hepatitis C.

Conclusions: Our study reveals the prevalence of hepatitis caused by blood transfusion in patients with clotting factors deficiencies. All patients treated by clotting factors prior to 1985, were positive for hepatitis $\mathrm{C}$, of which 4 had hepatitis $\mathrm{B}$. These patients received contaminated-imported blood products. After initial spread of hepatitis, protocols for examining blood products were designed and employed, which reduced number of new cases to zero. Hepatitis causes increase in the number of medication and their cost and possibility of side effects.

\section{PP12-09}

Prevalence of Blood Transfusion Induced Hepatitis among Patients with Thalassemia in Tabriz

S.O. Mashayekhi ${ }^{1}$, D. Sheykhi ${ }^{2}$, A. Nikanfar ${ }^{3}$, A. Hosseyn Pour Feizi ${ }^{3}$ M. Nazari ${ }^{4}$, R. Dolatkhah ${ }^{3}$, M. Ghandforoush-Sattari ${ }^{1}$

${ }^{1}$ Hematology and Oncology Research Center, Faculty of Pharmacy, Tabriz University of Medical Sciences, Iran; ${ }^{2}$ Faculty of Pharmacy, Tabriz University of Medical Sciences, Iran; ${ }^{3}$ Hematology and Oncology Research Center, Faculty of Medicine, Tabriz. University of Medical Sciences, Iran; ${ }^{4}$ Pediatric Hospital, Tabriz University of Medical Sciences, Iran, Tabriz, Iran

Background/aims: Beta thalassemia is an autosomal recessive disease, which is inherited with Mendelian rules. It is characterized by absent or braid in both constructive beta chain genes. Besides severe anemia; which is the most important symptom of this disease, different signs like facial changes, growth and developmental disturbance are also seen in the patients. Iran is placed on the universal thalassemia belt and has 15,000 beta thalassemia major patients. The main thalassemia treatment is regular blood transfusion. The aim of the study was to examine prevalence of hepatitis B and $\mathrm{C}$ caused by blood transfusions.

Methods: Patients registered in two thalassemic clinics in Tabriz were invited to the study. Those who volunteered were asked to complete a simple questionnaire. The questions were about demographic information and blood transfusion-induced hepatitis. The questionnaires were completed by reviewing patients' records and direct questions from the patients. The data were analyzed by using SPSS (ver. 13) software.

Results: Data from 100 patients were collected between August 2008 and July 2009. The median age of subjects was $14.0 \pm 11.32$ and $43 \%$ were female. 81 patients visited the clinics once a month while the number of visits was more for the rest. A few numbers of respondents were infected with hepatitis B virus $(2 \%)$ or hepatitis $\mathrm{C}$ virus $(3 \%)$ but no one was HIV infected. $80 \%$ of patients had vaccinated against hepatitis B.

Conclusions: Fortunately our study revealed a low prevalence of hepatitis $\mathrm{B}$ or $\mathrm{C}$ among thalassemic patients. These were patients who received contaminated imported blood transfusion prior to 1985 . No new cases were diagnosed after introducing preventative methods for examining blood products. This shows the necessity of testing blood products before administrating it to a patient in need.
PP12-10

Burden of Viral Hepatitis C in Japan: A Propensity Analysis of Patient Outcomes

G.G. Liu ${ }^{1}$, M.D. Di Bonaventura ${ }^{2}$, Y. Yuan ${ }^{3}$, J.-S. Wagner ${ }^{2}$, G.J. L'italien ${ }^{3,4}$, P. Langley ${ }^{5}$, I. Kamae ${ }^{6}$

${ }^{1}$ Guanghua School of Management, Peking University, Beijing, China; ${ }^{2}$ Health Sciences Practice, Kantar Health, New York, NY;

${ }^{3}$ Global Health Economics and Outcomes Research, Bristol-Myers Squibb Company, Princeton, $N J ;{ }^{4}$ School of Medicine, Yale University, New Haven, CT; ${ }^{5}$ College of Pharmacy, University of Minnesota, Minneapolis, MN, USA; ${ }^{6}$ Graduate School of Health Management, Keio University, Fujisawa, Japan

Background: Viral hepatitis C (HCV) affects 170 million patients worldwide and 2 million patients in Japan. However, many patients do not receive adequate diagnosis and treatment. The objective of the current study is to examine the burden of HCV in Japan from a patient perspective.

Methods: Using data from the 2008 and 2009 Japan National Health and Wellness Surveys, patients who reported a HCV diagnosis $(n=306)$ were compared to a propensity-score-matched non-HCV control group ( $n=306$ ) on measures of quality of life (using the SF$12 \mathrm{v} 2$ ), work productivity (WPAI questionnaire), and healthcare resource use in the past 6 months. All analyses applied sampling weights to project to the whole population in Japan.

Results: The propensity-matching process eliminated differences between the two comparison groups on gender, age, ethnicity, income, employment status, insurance, smoking behavior, alcohol use, exercise behavior, anxiety and depression. Relative to non-HCV controls, HCV patients reported significantly lower levels of quality of life (SF-12v2) as measured by the bodily pain (72.07 vs. 76.28$)$, general health (44.64 vs. 48.61$)$, and mental health ( 66.50 vs. 70.32$)$ subscales $(p<.05$ for all). Workplace absenteeism ( 8.59 vs. $4.12 \%$ ), overall work impairment $(26.08$ vs. $17.32 \%)$, and the frequency of healthcare provider visits in the past six months (14.8 vs. 9.74) were significantly higher among HCV patients relative to matched non-HCV controls $(p<.05$ for all). Conclusions: The results of this study suggest that HCV can be a substantial burden on patients in terms of quality of life at both physical and mental health measures. Furthermore, the results find that $\mathrm{HCV}$ can be a significant economic cost driver in terms of healthcare use and lost productivity.

\section{PP12-11}

Anti-HCV Positivity in Cases with History of Intravenous Drug Abuse Via Community Announcement: A Useful Experience

R. Fadaei Nobari ${ }^{1}$, P. Adibi ${ }^{2}$, B. Ataei ${ }^{3}$, N. Kassaian ${ }^{3}$, M. Meshkati ${ }^{1}$,

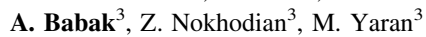

${ }^{1}$ Health Center, Isfahan University of Medical Sciences; ${ }^{2}$ Research Chancelor, Isfahan University of Medical Sciences; ${ }^{3}$ Infectious Diseases Research Center, Isfahan University of Medical Sciences, Isfahan, Iran

Background: Injection drug use plays the most important role in transmission of hepatitis C. In Iran, surveys have been conducted on various high risk groups but this is the first announcement based study for anti-HCV prevalence among cases with history of intravenous drug using (IVDU) in the country.

Methods: The announcement-based detection and follow up of patients with anti-HCV positive project in volunteers with history of IVDU was conducted during Nov 2008-March 2009 in Isfahan province. At the 
first step, six focus groups were conducted to answer the main question: "How can we discover IVDUs in community and detect HCV in them?" 2 pilot studies were carried out in two cities and the results were used for the main study. Comprehensive community announcement was done in all of public placed and for physicians. The volunteers were invited to Isfahan reference laboratories and the serum samples were sent to Infectious Diseases Research Center Laboratory in standard conditions and HCV-Ab was tested by ELISA method. Data was analyzed using descriptive methods and content analysis.

Results: In this study, 1,747 ones that are estimated $50 \%$ of all expected IVDUs in the community were presented themselves. The most reasons of coming volunteers in this study were the prefect propaganda, appropriate encounter of lab staffs with them, continuous evaluation and good cooperation in Isfahan province administrations. $\mathrm{HCV}-\mathrm{Ab}$ was detected in $34 \%$ of them and the HCV-Ab positives were sent for further follow-up procedures including confirmatory test, education and treatment.

Conclusion: In spite of some limitations to select real cases, this study considered as a successful experience. Compared to the surveys in Iran on HCV prevalence in IVDUs, the results of this study which was based on volunteers by announcement, seems to be admirable.

\section{PP12-12}

Hepatitis C in Patients with Multi Blood Transfusion in Isfahan, Iran N. Kassaian ${ }^{1}$, B. Ataei ${ }^{1}$, A. Babak ${ }^{1}$, Z. Nokhodian ${ }^{1}$, P. Adibi ${ }^{2}$, S. Mobasherizadeh ${ }^{3}$

${ }^{I}$ Infectious Diseases Research Center, Isfahan University of Medical Sciences; ${ }^{2}$ Research Chancelor, Isfahan University of medical Sciences; ${ }^{3}$ Isfahan University of Medical Sciences, Isfahan, Iran

Frequent transfusion of blood and blood products to patients with thalassemia major, hemophilia and hemodialysis patients may cause infections such as hepatitis infection in them.

Hepatitis $\mathrm{C}$ was the most common blood transfusion induced hepatitis until blood screening began in 1992.

Although the infection rates are diminished, but still different prevalence rates are reported from various countries.

This study aimed to determine the prevalence of hepatitis $\mathrm{C}$ and associated risk factors in these patients in Isfahan, the second big province in Iran.

Materials and methods: In a descriptive study all patients with hemophilia, thalassemia and hemodialysis in Isfahan province, winter 2009 were enrolled.

A questionnaire, including demographic and risk factors of hepatitis $\mathrm{C}$ was completed through a structured interview with closed questions by a trained interviewer for each patient and $\mathrm{HCV}-\mathrm{Ab}$ test results were extracted from patient records. Data were analyzed by SPSS v. 15 software using descriptive statistics and multivariate logistic regression analysis.

Results: In this study, 60 of 570 patients with thalassemia major (10.5\%), 232 out of 350 patients with hemophilia A and B (66\%) and 17 of the 800 hemodialysis patients $(2.1 \%)$ were positive for hepatitis C. Based on Multivariate Logistic Regression model, no independent risk factor was found.

Conclusion: Prevalence of hepatitis $\mathrm{C}$ in patients with recurrent blood transfusion in Isfahan is high. Since no independent risk factor for hepatitis $\mathrm{C}$ disease was found in these three groups, it is concluded that multitransfusion is the only predictor for hepatitis $\mathrm{C}$.

\section{PP12-13}

Hepatitis $C$ in IVDUs in Isfahan, Iran, 2008

B. Ataei ${ }^{1}$, Z. Nokhodian ${ }^{1}$, N. Kassaian ${ }^{1}$, A. Babak ${ }^{1}$, P. Shoaei ${ }^{1}$, M. Meshkati ${ }^{2}$, M. Ataie ${ }^{3}$, P. Adibi $^{4}$

${ }^{1}$ Infectious Diseases Research Center, Isfahan University of Medical Sciences; ${ }^{2}$ Province Health Center, Isfahan University of Medical Sciences; ${ }^{3}$ Najafabad Islamic Azad University; ${ }^{4}$ Research Chancelor, Isfahan University of Medical Sciences, Isfahan, Iran
Background: In the last decade the incidence and pattern of drug abuse in Iran has changed into IDU especially in young population. Detection of HCV infection and its main risk factors among IVDUs will be useful to create harm reduction strategies and there are limited studies about it in the country. Hence, this was conducted to assessment HCV seropositivity and related risk factors in IVDUs in Isfahan, Iran.

Methods: This cross-sectional study was carried out between November 2008 and March 2009. Voluntary IDUs referred to drug treatment and harm reduction centers in Isfahan province were enrolled into study. After obtaining written informed consent, a validated questionnaire was completed by interview to assess the probable risk factors involved in $\mathrm{HCV}$ infection. $5 \mathrm{ml}$ blood was drawn from each subject, the sera were separated and HCV Ab was detected using ELISA.. Chi-square, Mann-Whitney and Logistic Regression were used for data analysis and $p$ value $\leq 0.05$ was considered significant. Results: Five-hundred and thirty nine subjects including 511 male and 28 female with mean of age $32 \pm 8.5$ years were enrolled. Overall $47.1 \%$ were positive for HCV-Ab. HCV seropositivity was higher among IVDUs with history of tattooing, surgery, tooth filling, illegal sex and injecting drug in prison compared to ones without these. Also, it was higher in non occupied and alcoholic persons. The mean of age, duration of intravenous drug abuse, duration and time of incarceration were higher in HCV-positives compared to HCV-negatives. Multivariate Logistic Regression analysis showed significant statistical associations of $\mathrm{HCV}$ seropositivity with cupping $(\mathrm{OR}=3.2,95 \% \mathrm{CIs}=1.3-8.1)$.

Conclusion: The high prevalence of $\mathrm{HCV}$ infection found in the IVDUs in our study, calls for preventive measures to be taken by the Iranian Ministry of Health in drug treatment and harm reduction centers routinely.

\section{PP12-14}

Hepatitis C Virus Infection and Type 2 Diabetes Mellitus: A Systematic Review

W. Zhang, B. Feng, H.-Y. Rao, F. Liu, L. Wei Peking University People's Hospital, Peking University Hepatology Institute, Beijing, China

Background: Type 2 diabetes mellitus (T2DM) is a frequent complication of hepatitis $\mathrm{C}$ virus (HCV) infection. However, some studies found no significant associations between them. We performed this systematic review to discuss whether hepatitis $\mathrm{C}$ infection increases risk of T2DM and whether current interferon-based treatment for adults with $\mathrm{HCV}$ infection induces the onset or development of T2DM. Methods: We identified eligible studies through keyword searches in relevant databases including PubMed and Embase from January 1990 to March 2010. Selection of papers was carried out using the system (Evidence-based Medicine Levels of Evidence) and the inclusion criteria are established before the articles were identified.

Results: We identified 53 eligible studies. This systematic review found that significant T2DM risk in HCV-infected adults in comparison to non-infected controls from both 11 prospective and 17 retrospective studies. The prevalence of T2DM in HCV-infected adults was $4.5-32.4 \%\left(\mathrm{HR}_{\text {adjusted }} 1.7-5.6\right)$ in prospective cohort investigations, and that was $14.7-54 \%\left(\mathrm{OR}_{\text {adjusted }} 2.2-3.27\right)$ in retrospective cohort investigations. It also observed that factors, such as age ( $\geq 50$ years, HR 1.0 ), BMI ( $\geq 24$ and $<27 \mathrm{~kg} / \mathrm{m}^{2}, \mathrm{HR} 1.9 ; \geq 27 \mathrm{~kg} /$ $\mathrm{m}^{2}$ HR 1.7), family history of T2DM (HR 3.20), fibrosis (HR 7.32-12.5), hepatic steatosis (OR 2.3-2.684), genotype (1b/2b, OR 0.9 ; 3a, OR 1.0; 4a, OR 0.8), viral load (OR 0.62), and gender (HR 0.9-2.61), have increased the risk for T2DM in HCV-infected adults. In 4 prospective cohort investigations, interferon therapy was indicated to be a risk factor for T2DM in $\mathrm{HCV}$-infected adults $\left(\mathrm{HR}_{\text {adjusted }}\right.$ 0.44-2.78).

Conclusions: Hepatitis $\mathrm{C}$ infection is an independent risk factor for T2DM. It suggests that the interferon therapy induce the onset or 
development of T2DM in adults with HCV infection. Larger and more prospective investigations are needed to confirm these findings. More investigations are needed to perform in Asian populations.

\section{PP12-15}

Analysis of the High Risk Groups of HCV Infection in Poland M. Pawlowska ${ }^{1}$, W. Halota ${ }^{1}$, R. Flisiak ${ }^{2}$, A. Horban ${ }^{3}$, J. Juszczyk ${ }^{4}$, K. Simon ${ }^{5}$ ${ }^{I}$ Department of Infectious Diseases and Hepatology, Collegium Medicum N.Copernicus University, Bydgoszcz; ${ }^{2}$ Department of Infectious Diseases and Hepatology, Medical University of Bialystok, Bialystok; ${ }^{3}$ Hospital of Infectious Diseases, Medical University in Warsaw, Warszawa; ${ }^{4}$ Department of Infectious Diseases, University of Medical Sciences in Poznan, Poznan; ${ }^{5}$ Department of Infectious Diseases, Hepatology and Aquired Immunodeficiency, Wroclaw University of Medicine, Wroclaw, Poland

$\mathrm{HCV}$ infection is one of the most epidemiological and clinical healthcare problems, affecting an estimated 170 million people worldwide.

The aim of this study was to assess the prevalence of HCV infection in a part of Polish population and to define high risk group of HCV infection.

Methods: In sera samples from 26057 adult people the anti-HCV antibodies presence was examined with Elecsys Anti-HCV test (Roche Diagnostics $\mathrm{GmbH}$ )

In patients with anti-HCV antibodies presence assessing the serum $\mathrm{HCV}$ RNA with PCR method with COBAS AMPLICOR Hepatitis C Virus (HCV) Test, version 2.0 (Roche Molecular Systems, Inc.) was performed. Accordingly, examined patients filled special questionnaires.

Multivariate logistic regression model was used to investigate the association between independent covariates and positive antyHCV. $5 \%$ level of significance was used.

Results: The presence of anti-HCV antibodies was detected in sera samples of 496/26,057 (1.9\%) patients. HCV RNA presence was confirmed in $110 / 354(31.07 \%)$ anti-HCV positive patients.

The main risk factors of anti-HCV antibodies presence were: the male sex 2.01 [1.43, 2.83], hospitalizations more then 3 times in life 1.71 $[1.28,2.28]$, blood transfusion before 1992 years 3.12 [2.24, 4.35] and intravenous drug using 6.78 [4.1, 11.2] OR [95\% CI]. The main risk factor for HCV RNA serum presence was intravenous drug use 9.2 $[2.9,29.2]$ OR $[95 \% \mathrm{CI}]$

Conclusions: The real prevalence of HCV infection in Poland seems to be about $1.9 \%$.

HCV RNA was detected in $31 \%$ patients with anti-HCV serum presence.

Patients often hospitalized and intravenous drug users are in the high risk of $\mathrm{HCV}$ infections.

\section{PP12-16}

Seroprevalence of Hepatitis C (HCV) Infection among Street Children in Isfahan, Iran

B. Ataei ${ }^{1}$, Z. Nokhodian ${ }^{1}$, A. Babak ${ }^{1}$, P. Shoaei ${ }^{1}$, M. Mohhammadzadeh ${ }^{2}$, S. Sadeghi ${ }^{2}$, M. Ataie ${ }^{3}$

${ }^{1}$ Infectious Diseases and Tropical Medicine Research Center, Isfahan University of Medical Sciences; ${ }^{2}$ Isfahan Welfare Organization;

${ }^{3}$ Najaf Abad Islamic Azad University, Isfahan, Iran

Background and aims: There are millions of children around the world living on the street. They are at higher risk of physical, sexual and drug abuse, and have no access to health care facilities. Therefore they are at risk of viral infections such as HCV. The aim of this study was determining the prevalence of $\mathrm{HCV}$ infection in Isfahan (the second big city in Iran) street children (2005-2007).

Methods: The cross-sectional study was taken place on 386 street children through a nonprobable-convenience sampling method. They were requested to answer a questionnaire (demographic and behavioral data), and then they were tested for anti HCV antibodies.
Results: Among 386 street children, 270 (70\%) were boys and the mean age was $12.62 \pm 3.23$ years. The majority of them, 267 cases $(69 \%)$, were on the street for financial reasons. 353 (91.7\%), 366 (94.8\%) and 375 (97.2\%) of them had no history of smoking, using alcohol or substance addiction, respectively. $40(34.5 \%)$ of girls and $12(4.4 \%)$ of boys $(p<0.0001)$ were engaged in sex and $79(68 \%)$ of girls and $46(17 \%)$ of boys $(p<0.0001)$ were involved in physical fighting. Four of them $(1 \%)$ were positive for $\mathrm{HCV} \mathrm{Ab}$.

Conclusion: The knowledge of street children about high risk behaviors and the infectious diseases should be improved through educational programs. They need also legal, social and health support.

\section{PP12-17}

A Novel Hepatitis C Virus Genotyping Method Using RT-PCR Reverse Dot Hybridization Technique and its Application

Y. Zhou ${ }^{1}$, J. Wei ${ }^{1}$, T. Zhang ${ }^{2}$, W. Li ${ }^{1}$, H. Huang ${ }^{1}$, Y. Zeng ${ }^{1}$, F. Zhang ${ }^{2}$, J. Wang ${ }^{1}$, B. Zhou ${ }^{1}$, Y. Wu ${ }^{3}$, S. Liu ${ }^{4}$, J. Hou ${ }^{1}$

${ }^{I}$ Department of Infectious Diseases, Nanfang Hospital, Southern Medical University; ${ }^{2}$ Da-An Diagnostic Center, Sun Yat-Sen University; ${ }^{3}$ School of Biotechnology, ${ }^{4}$ School of Pharmaceutical Sciences, Southern Medical University, Guangzhou, China

Background/aims: To develop a rapid and specific hepatitis $\mathrm{C}$ virus (HCV) genotyping method with the reverse dot hybridization technique and investigate the distribution of $\mathrm{HCV}$ genotypes and subtypes in Guangdong area, China.

Methods: The primers and the probes targeting the 5'untranslated region (5'UTR) and Core region of HCV genotypes $1 \mathrm{~b}, 2 \mathrm{a}, 3 \mathrm{a}, 3 \mathrm{~b}$ and $6 \mathrm{a}$ were specially designed. And then the RT-PCR Reverse Dot Hybridization (PCR-RDH) method for HCV genotyping was established. A total of 115 patients with hepatitis C from Nanfang Hospital, Southern Medical University, were genotyped by the method, and 38 of them were also genotyped by sequencing and phylogenetic analysis to evaluate the accuracy and specificity of PCR-RDH method.

Results: Of 115 patients, 111 were successfully genotyped to be $1 \mathrm{~b}, 2 \mathrm{a}$, $3 \mathrm{a}, 3 \mathrm{~b}, 6 \mathrm{a}$ and mix-infection of $1 \mathrm{~b} / 2 \mathrm{a}$ at frequencies of $56.8,8.1,3.6,5.4$, 25.2 and $0.9 \%$, respectively. 15 healthy control samples were all negative. The accuracy and reliability of genotyping method of PCR-RDH was confirmed by amplification of HCV Core and NS5B regions from 38 cases followed by DNA sequencing and phylogenetic analysis.

Conclusions: The PCR-RDH method for HCV genotyping, with high reliability and specificity, is suitable for clinical and epidemiological investigations in developing countries. The prevalence of $\mathrm{HCV}$ genotypes $1 \mathrm{~b}$ and $2 \mathrm{a}$ decreases while $1 \mathrm{~b}$ remains the dominant genotype in Guangdong, where the prevalence of 6 a significantly increases as compared with that 10 years ago.

Acknowledgements: This work was supported by grant from the National Natural Science Foundation of China (30771899).

\section{PP12-18}

Screening for Hepatitis C Results in Detection of Early "Stage" of the Disease and Better Response to Peg-interferon and Ribavarin M. Sharma ${ }^{1}$, S.A. Kaabi ${ }^{1}$, N.A. Dweik ${ }^{1}$, A. John ${ }^{1}$, K. Matar ${ }^{1}$,

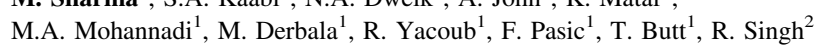
${ }^{1}$ Division of Gastroenterology,Department of Internal Medicine; ${ }^{2}$ Department of Medical Research, Hamad Medical Corporation, Doha, Qatar

Background: Screening for Hepatitis $\mathrm{C}$ has not been found to be beneficial in healthy individuals.

Aim: To compare the disease pattern and response to treatment among two groups of patients with Chronic Hepatitis C.

Group 1(Screening Group) comprised of patients detected from the screening survey and Group 2 (Referral Group) were those referred for treatment in the same time period.

Secondary aim was to compare the above variables in the average and high risk population (Group 1). 
Methodology: A survey was done to screen for Hepatitis $\mathrm{C}$ antibodies in the average and high risk population over a 16 months period in the State of Qatar. Screening was done using a rapid immunochromatographic assay. All patients detected to have at least grade 1, stage 1 disease in the liver biopsy, (as per Scheuer classification) were offered treatment.

Results: 13,704 people were screened and 272 people were detected to have positive antibodies and 237 patients were referred. Both the groups were similar in baseline characteristics. ALT and AST were significantly lower in the Group 1 than the Group $2(p=0.01)$. Liver histology revealed milder grade of necroinflammation and significantly lesser fibrosis in the screening group. The response to treatment was significantly better in the Screening Group than in the referral Group (72\% versus $54.1 \%, p=0.03$ ) (Table 1). Among the Group 1, only ALT was found to be significantly higher $(p=0.04)$ in the high risk group.

Table 1

\begin{tabular}{llll}
\hline & $\begin{array}{l}\text { Group 1 } \\
\text { (screening } \\
\text { group) }\end{array}$ & $\begin{array}{l}\text { Group 2 } \\
\text { (referral } \\
\text { group) }\end{array}$ & $P$ value \\
\hline Total number of patients & 272 & 237 & \\
Patients participating & $148(62 \%)$ & $234(98 \%)$ & \\
Consent for treatment & $69(46.6 \%)$ & $126(53.8 \%)$ & $\mathrm{ns}$ \\
Mean viral load (million) & 3.2 & 3.4 & \\
Genotype 1, 4 & 7,132 & 23,160 & 0.01 \\
ALT & $63.4+49.5$ & $78.6 \pm 60.98$ & 0.11 \\
AST & $44.5 \pm 35.12$ & $44.5 \pm 35.12$ & \\
Liver histology & $78.3 \%(n=98)$ & $72.0 \%(\mathrm{n}=203)$ & 0.00 \\
$\quad$ necroinflammation & & & 0.03 \\
$\quad$ grade 0-2) & $78.5 \%$ & $72.9 \%$ & \\
$\begin{array}{l}\text { Fibrosis score (stage 0-2) } \\
\text { Rapid /early virological }\end{array} \quad 37 / 51(72.4 \%)$ & $65 / 120(54.2 \%$ & \\
$\quad$ response & & &
\end{tabular}

Conclusion: Screening results in detection of Hepatitis $\mathrm{C}$ at an early stage and results in better response to the treatment.

\section{PP12-19}

Prevalence of Hepatitis B and C Virus in Barbers, Isfahan (Iran), 2007 B. Ataei ${ }^{1}$, P. Shoaei ${ }^{1}$, Z. Nokhodian ${ }^{1}$, A. Babak ${ }^{1}$, M. Yaran $^{1}$, N. Kassaian ${ }^{1}$, M. Ataie ${ }^{2}$, M. Foroughifar ${ }^{1}$

${ }^{1}$ Infectious Diseases and Tropical Medicine Research Center, Isfahan University of Medical Sciences; ${ }^{2}$ Najaf Abad Islamic Azad University, Isfahan, Iran

Background and aims: Hepatitis B virus (HBV) and hepatitis C virus (HCV) infections are among the most threatening health problems in the world including Iran. The transmission route of HBV and $\mathrm{HCV}$ is mainly parenteral. Small numbers of epidemiological studies demonstrate that prenatal, sexual, household and occupational transmission occurs. Contact of patient's blood or bodily fluids with non-intact skin is another mode of HBV and HCV transmission. Barbers in Iran may often be exposed accidentally to the blood and bodily fluids of their customers.

The aim of this study was to determine the prevalence of HBV and $\mathrm{HCV}$ in barbers.

Patients and methods: The study targeted the barber's population of Isfahan (the second big city in Iran). They administered a structered questionnaire and a blood sample was collected for anti HCV, HBs $\mathrm{Ab}, \mathrm{HBc} \mathrm{Ab}$ and HBs Ag.

Results: 479 barbers ( 246 women and 233 men) were screened for $\mathrm{HBs} \mathrm{Ag}, \mathrm{HBs} \mathrm{Ab}, \mathrm{HBc} \mathrm{Ab}$ and $\mathrm{HCV} \mathrm{Ab}$. Their mean age was
$39 \pm 11.4$ years. Four $(0.8 \%)$ were positive for $\mathrm{HBs} \mathrm{Ag}, 6(1.3 \%)$ were positive for $\mathrm{HBc} \mathrm{Ab}$ and 276 (57.7\%) were positive for $\mathrm{HBs} \mathrm{Ab}$. No significant relationship was found between these seropositivities and factors such as sex, vaccination against HBV, health training classes, and special services (such as tattooing, ear puncture manicuring and so on).

Conclusion: Both HBV and HCV infections may constitute occupational hazards for barbers. The infection sources could be not only personal risk factors such as sharp injuries and scissor cuts but may also include other unknown factors but our data didn't show significant values about it and need more studies.

\section{PP12-20}

Chinese HCV Genotype and Subtype Distribution Study: Interim Analysis W. Ju, S. Yang, Q. Wang, X. Wang, H. Xing, W. Xie, Y. Xie, J. Cheng Beijing Ditan Hospital, Capital Medical University, Beijing, China

Aim: To determine HCV genotype and subtype distribution in China mainland.

Methods: Clinical data and samples of $\sim 3,000$ chronic hepatitis $\mathrm{C}$ patients are to be collected from 100 hospitals in China. Nucleotide sequence analysis of the NS5B and/or CORE-E1 regions of the HCV genome was performed on serum samples, and phylogenetic analysis is used for analysis of HCV genotype and subtype.

Results: As an interim analysis, a total of $641 \mathrm{HCV}$ RNA positive serum samples were collected from chronic $\mathrm{HCV}$ infected patients from 12 geographic areas in China during 2009.10-2010.10. HCV genotype was determined in 616 samples [96.1\% (616/641)]. Genotype 1, 2, 3 and 6 were detected, at frequencies of 75.2, 18.2, 4.2 and $2.4 \%$, respectively. No genotype 4 and 5 strains were found. We detected subtypes 1b, 2a, 3a, 6a, 3b, 6n, and 1a at frequencies of 74.8, $18.2,2.8,1.9,1.5,0.5$, and $0.3 \%$, respectively. In general, subtype $1 \mathrm{~b}$ was the predominant [74.8\% (461/616)] followed by $2 \mathrm{a}[18.2 \%$ (112/ 616)].Detailed subtype distribution is showed in graph 1.

Conclusions: Interim analysis of this study demonstrated a genetic heterogeneity of $\mathrm{HCV}$ infection in China, with at least four HCV genotypes and seven subtypes. Clinical trials of direct anti-HCV agents (DAA) should consider this genetic heterogeneity.

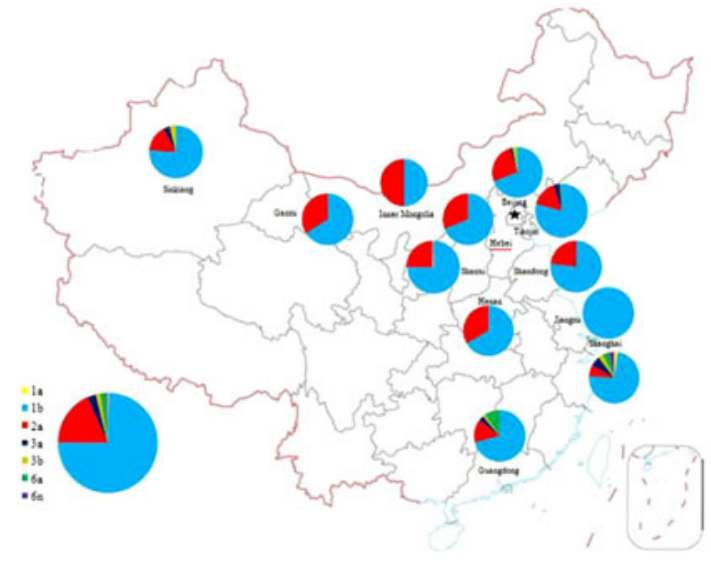

Graph 1

PP12-21

Altered Surface Expression and Cytolytic Function of Natural Killer Cell Receptors in Hepatitis C Virus Infection

Y.-F. Jiang, J.-Y. Feng, Y. Pan, M.-L. Wang, T. Jiang, J.-Q. Niu, X.-D. Shi The First Hospital, Jilin University, Changchun, China

Background: Natural killer (NK) cells are essential early after infection, not only for viral containment but also for timely and efficient induction of adaptive responses. NK cells have been 
proposed as a contributing mechanism to viral persistence in $\mathrm{HCV}$ infection. As the function of NK cells is primarily regulated by NK cell receptors (NKR), we analyzed whether decreased NK cell function in infection and progression of chronic HCV may be related to dysregulated NKR expression. Therefore, the aim of this study was to characterize the function of NK cells during the chronic phase of $\mathrm{HCV}$ infection and compare with self-limited outcomes.

Methods: HCV patient were divided into four groups according to their viral load and ALT level:

Group 1. HCV-RNA $>10^{5}$ Copies $/ \mathrm{ml}$, ALT $>80 \mu / \mathrm{L}(n=22)$;

Group 2. HCV-RNA $>10^{5}$ Copies/ml, ALT $<40 \mu / \mathrm{L}(n=12)$;

Group 3. HCV-RNA $<10^{2}$ Copies/ml, ALT $<40 \mu / \mathrm{L}(n=5)$;

Group 4. Self-limited infection $(n=12)$, and 10 healthy donors were included as controls.

NK phenotype and function assays were analyzed by FACS.

Results: There was a significant reduction in total NK cell frequency and, proportion of NKp30 and NKp46 expressing NK cells in Group 1 patients compared to healthy controls and other groups. There was low expression of natural cytotoxicity receptor (NCR) in all HCV patients compared to healthy controls. The NK cell phenotype was associated with polarization of $\mathrm{NK}$ cell function towards CD107a expression as a marker of degranulation, but IFN$\gamma$ production by CD56dim NK cells was not increased. Furthermore, the proportion of NK cells expressing CD94/NKG2A was higher in hepatitis $C$ patients with viral load than the self-limited patients.

Conclusion: Defective expression of NKR represents a novel mechanism that contributes to the impaired function of NK cells during chronic hepatitis $\mathrm{C}$ infection. CD94/NKG2A is related to the viral clearance and may play an important role in the innate immune response against $\mathrm{HCV}$ infection.

\section{PP12-22}

Prevalence of Hepatitis C Virus Infection in Lymphoproliferative Disorders Patients

M. Farahani ${ }^{1}$, H. Keyvani ${ }^{2}$, M. Ghane ${ }^{3}$, A. Baci ${ }^{4}$

${ }^{1}$ Department of Microbiology, Tonekabon Azad University;

${ }^{2}$ Virology, Iran University of Medical Sciences, Tehran; ${ }^{3}$ Department of Microbiology, Tonekabon Azad University, Tonekabon;

${ }^{4}$ Department of Oncology, Iran University of Medical Sciences,

Tehran, Iran

Background and aims: Hepatitis $\mathrm{C}$ virus (HCV) infection is a global health problem, being the second most common chronic viral infection in the world with a global prevalence of about 3\% (about 180 million people). $\mathrm{HCV}$ is both an hepatotropic and a lymphotropic virus; and chronic infection could cause, on one hand, chronic hepatitis, cirrhosis and hepatocellular carcinoma and on the other hand several extrahepatic diseases including, first, mixed cryoglobulinemia and lymphoma.

Materials and methods: A cross-sectional analysis was performed on 100 lymphoproliferative disorder patients serum samples was collected from 12 ALL (acute lymphoblastic leukaemia), 20 CLL (chronic lymphocytic leukaemia),42 NHL (Non-Hodgkin's lymphoma), 14 Hd (Hodgkin's disease),12 MM (multiple myeloma). Blood samples screened for anti-HCVantibody by using the enzymelinked immunosorbent assay (ELISA) method.

Results: HCV antibody was found in serum samples of $3(2.5 \%)$ out of 100 patients when tested by ELISA method.Two positive HCV-Ab patients had NHL (Non-Hodgkin's lymphoma) and one patient had ALL (Acute lymphoblastic leukaemia).

Conclusions: Based on our data the prevalence of hepatitis $\mathrm{C}$ virus $(\mathrm{HCV})$ is higher in lymphoproliferative disorders compared the other groups.
PP12-23

Genetic Variation and Serum Level of IL28B in Chinese with Hepatitis C Virus Infection

X. Shi, D. Wang, P. Yu, T. Jiang, P. Zhang, M. Wang, Q. Jin, J. Sun,

J. Jiang, J. Niu

First Hospital, Ji Lin University, Changchun, China

Background and aims: Some single nucleotide polymorphisms near the IL28B gene (rs12979860, rs 8099917and rs12980275) were significantly associated with spontaneous clearance of HCV. This study amied to detect these variants in $\mathrm{HCV}$ infected patients, and present its association with serum IL28B,transaminase level and liver fibrosis. Methods: IL28B gene variants were detected by pyrosequencing and anti-HCV was tested. Viral RNA load and serum IL28B were measured.Clinical transaminase and Liver fibrosis were performed. The subset analysis by subdividing the subjects into groups according to $\mathrm{HCV}$ infection and transaminase were performed. Differences of IL28B in genotypes were evaluated by ANOVA.

Results: SNPs of IL28B were in complete linkage disequilibrium $\left(D^{\prime}=1.0\right)$ in 464 patients, the frequency of major alleles(rs12979860C allele, rs 8099917T allele, rs12980275A allele) was $96.85 \%$, the one of minor alleles (T, G, G alleles) was 3.45\%. There was no difference for frequency of $\mathrm{C} / \mathrm{C}$ genotype between spontaneous clearance and persistent infection $(P=0.5)$. Serum IL28B in individuals with $\mathrm{C} / \mathrm{C}$ genotype was higher than T/C genotype. FibroScan was performed in 107 patients with C/C, high serum IL28B was found in patients with stage $\mathrm{F} 1-2(n=84)$ [87.82 $(66.35-106.51) \mathrm{pg} / \mathrm{ml}]$ than F3-4 $(n=23)$ [65.0 (55.66-98.66) pg/ml] $(P=0.02)$.

Conclusions: Genetic variations in IL28B may affect the immune response and contribute towards virus clearance or development of liver disease.

IL28B serum level among different HCV patients

\begin{tabular}{llrll}
\hline Genotype & $\begin{array}{l}\text { Conditions of } \\
\text { HCV infection } \\
\text { and transaminase }\end{array}$ & $N$ & $\begin{array}{l}\text { Serum level } \\
\text { (pg/ml) }\end{array}$ & $P$ value \\
\hline $\mathrm{C} / \mathrm{C}$ & Total & 198 & $77.45(57.81-100.73)$ & $0.004^{\&}$ \\
$\mathrm{C} / \mathrm{C}$ & HCV persistence & 162 & $79.41(57.39-102.74)$ & $0.011^{*}$ \\
$\mathrm{C} / \mathrm{C}$ & HCV clearance & 36 & $72.25(65.70-94.71)$ & $0.048^{*}$ \\
$\mathrm{C} / \mathrm{C}$ & Normal transaminase & 107 & $73.11(56.17-98.11)$ & $0.01^{\#}$ \\
$\mathrm{C} / \mathrm{C}$ & Abnormal transaminase & 91 & $83.93(60.18-104.81)$ & $0.004^{\#}$ \\
T/C & Total (a) & 16 & $50.63(37.56-93.13)$ & \\
T/C & HCV persistence (b) & 14 & $50.63(36.21-96.19)$ & \\
T/C & Normal transaminase (c) & 10 & $54.47(32.01-101.56)$ & \\
\hline$\&$
\end{tabular}

\& Compared with a, * compared with $\mathrm{b},{ }^{\#}$ compared with $\mathrm{c}$

\section{PP12-24}

Peculiarities of the HIV/HCV and/or HIV/HBV Co-infection in Patients Living with HIV/AIDS

I.G. Baltadzhiev ${ }^{1}$, A. Kevorkian ${ }^{2}$

${ }^{1}$ Infectious Diseases; ${ }^{2}$ Department of Epidemiology, Hygiene and Social Medicine, Medical University, Plovdiv, Bulgaria

Due to overlapping transmission routes, VHC-related liver disease has become a leading cause of morbidity in HIV/AIDS patients. The aim of the study was to point on some peculiarities of HIV/HCV and HIV/HBV co-infected patients.

A cohort of 145 HIV/AIDS patients (124/85.51\% men and 21/14.48\% women) were tested for HCV (HCV-AB and/or HCVRNA) and HBV/HDV (HBsAg, HBcIgM, HBcIgG, HDV-AB). Epidemiological and clinical studies and abdominal ultrasonography were done. 
Thirty one (21.37\%) HIV-patients, mainly heterosexual young people were $\mathrm{HCV}$ and HBV negative; 108 (74.48\%) were HCV positive IDUs; $2(1.37 \%)$ was HBsAg positive, 3 (2.06\%) MSM IDUs were $\mathrm{HCV} / \mathrm{HBV}$ co-infected and one MSM who undergone a severe icteric form of viral hepatitis and ended to cirrhosis was $\mathrm{HCV} / \mathrm{HBV} / \mathrm{HDV}$ positive. The HIV/HCV chronically infected cohort consisted almost entirely of IDU young men from 18 to 33 years old. Most of these individuals had been or were imprisoned at the time of the study and most of them were from the Gipsy-ethnic group, consisting of 50,000 peoples in a city of 350,000 inhabitants. The HIV/HCV positive patients were anicteric and only 15 $(13.88 \%)$ of them had ALT/AST twice to three times the normal levels. GGT (44\%), AP (23\%), and HE (9\%) showed pathological values. Hepato-splenomegaly were usual for most cases in this group. They received HAART, accordingly their clinical and monitored medical records, starting from $\leq 500 \mathrm{CD} 4+$ cells $/ \mu$ l. However the adherence was not sure in most cases we did not observed more treatment bound side effects in the HIV/HCV than in the just HIV infected patients. Not worsening of the hepatic functional tests was observed.

We focused on the need of screening, management and counseling for $\mathrm{HCV}$ and/or HBV infected HIV-patients, immunization of $\mathrm{HBsAg} / \mathrm{HBcIgG}$ negative one, and adequate treatment of $\mathrm{HIV} / \mathrm{HCV}$ and/or HIV/HCV/HBV chronically infected patients. An occult VHB needs to be excluded among none immunized HIV-living people.

\section{PP12-25}

Significance of Metabolic Risk Factors for Hepatic Steatosis Varies Independently in Asians Compared to Non-Asians with HCV Genotype 2/3 Infection

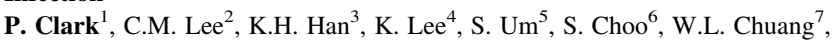
S.R. Shah $^{8}$, C.Y. Peng ${ }^{9}$, S. Thongsawat ${ }^{10}$, T. Tanwandee ${ }^{11}$, V. Mahachai ${ }^{12}$, C. Pramoolsinsap ${ }^{13}$, M. Cho ${ }^{14}$, G.R. Foster ${ }^{15}$, M. Torbenson ${ }^{16}$, E. Pulkstenis ${ }^{17}$, G.M. Subramanian ${ }^{17}$, J.G. Mc Hutchison ${ }^{1}$, K. Patel ${ }^{1}$, for the ACHIEVE 2/3 Investigators

${ }^{I}$ GI/Hepatology, Duke Clinical Research Institute, Durham, NC, USA; ${ }^{2}$ Chang-Gung Memorial Hospital-Kaohshiung, Kaoshiung Hsien, Taiwan R.O.C.; ${ }^{3}$ Severance Hospital, Yonsei University;

${ }^{4}$ Yongdong Severance Hospital, ${ }^{5}$ Anam Hospital, Korea International University, Seoul; ${ }^{6}$ Ajou University Hospital, Suwon, Republic of Korea; ${ }^{7}$ Kaohsiung Medical University, Kaohsiung, Taiwan R.O.C.; ${ }^{8}$ Jaslok Hospital Research Center, Mumbai, India; ${ }^{9}$ China Medical University Hospital, Seoul, Taiwan R.O.C.; ${ }^{10}$ Chiang Mai Hospital, Chiang Mai, ${ }^{11}$ Siriraj Hospital, Mahidol University; ${ }^{12}$ Chulalongkorn Hospital; ${ }^{13}$ Ramathibodi Hospital, Mahidol University, Bangkok, Thailand; ${ }^{14}$ Pusan National University College of Medicine, Busan, Republic of Korea; ${ }^{15}$ Queen Mary's University of London, London, UK; ${ }^{16}$ Johns Hopkins University School of Medicine, Baltimore;

${ }^{17}$ Human Genome Sciences, Rockville, MD, USA

Background: Steatosis in HCV-G3 is predominantly mediated by viral factors and by metabolic risk factors in HCV non-G3. We hypothesized metabolic factors may contribute less to steatosis for Asian patients than non-Asians.

Methods: 932 treatment-naïve chronic HCV-G2/3 patients enrolled in a phase 3 study of albinterferon/RBV. Diabetics and outliers were excluded. 696 patients had data for primary analysis. 37\% (260/696) were Asian. Baseline data included fasting triglycerides, BMI, homeostasis model assessment-insulin resistance (HOMA-IR). A single expert pathologist scored baseline liver biopsy steatosis (grade $0<5 \%$ steatosis, $1=6-30 \% ; 2=31-60 \% ; 3>61 \%$ ) and METAVIR fibrosis. Bivariate/multivariable (MVA) models analyzed steatosis (binary variable $</>$ grade $2 / 3$ ), comparing Asians with nonAsians.
Results: Despite non-Asians having higher mean BMI (27 vs. $24 \mathrm{~kg} / \mathrm{m}^{2}$, $p=0.0001)$, higher proportion of HOMA-IR $>4$ (20 vs. $11 \%$, $p=0.001)$ and being more likely to have HCV-G3 than Asian patients (64 vs. $35 \%, p<0.0001$ ), steatosis prevalence was similar to Asians (11 vs. $10 \%, p=0.712$ ). Non-Asians with HOMA-IR $>4$ had higher steatosis prevalence than those with HOMA-IR $<4$ (18 vs. $10 \%, p=0.040$ ) but a similar relationship was not evident amongst Asians (10 vs. $11 \%, p=0.579)$. Similarly, in non-Asians steatosis rates increased with higher BMI $(</>25,8$ vs. $14 \% p=0.042)$ and higher viral load $(</>600,000 \mathrm{IU}, 7$ vs. $14 \%, p=0.015)$, but these were not significant for Asians. On MVA for non-Asians: HCV-G3, BMI $>25$ and viral load were significant independently associated variables but only HCV-G3 for Asians.

Logistic MVA (steatosisGr2/3)

\begin{tabular}{lcc}
\hline & Non-asian & Asian \\
\hline BMI $>25$ & $2.171(1.099,4.287)$ & $0.666(0.257,1.726)$ \\
& $p=0.0256^{*}$ & $p=0.4026$ \\
HOMA $>4$ & $1.869(0.918,3.806)$ & $0.919(0.236,3.577)$ \\
& $p=0.0847$ & $p=0.9036$ \\
HCV RNA & $2.574(1.221,5.426)$ & $1.717(0.665,4.433)$ \\
$>600,000$ IU & $p=0.013^{*}$ & $p=0.2638$ \\
HCV genotype & $3.323(1.531,7.214)$ & $6.605(2.545,17.143)$ \\
(G3 vs. G2) & $p=0.0024^{*}$ & $p=0.0001^{*}$ \\
\hline
\end{tabular}

* Significant at $95 \% \mathrm{CI}$, ** TG/HOMA $>4 />\mathrm{F} 2$ were not significant

Conclusions: Metabolic risk factors are independently associated with steatosis in HCV-G2/3 for non-Asians but not Asians, perhaps reflecting ethnic differences in HCV-G3 prevalence or host-viral interaction.

\section{PP12-26}

Hepatitis C Virus (HCV) and Human T-cell Lymphotropic Virus Type 1 (HTLV-1) Coinfection in Thalassemic Patients in Iran

S.M. Alavian ${ }^{1}$, B. Behnava ${ }^{2}$, S.-Y. Saeedi ${ }^{2}$

${ }^{I}$ Research Center for Gastroenterology and Liver Disease, Baqiyatallah University of Medical Sciences; ${ }^{2}$ Baqiyatallah Research Center for Gastroenterology and Liver Disease, Tehran, Iran

Background and aims: Both HCV and HTLV1 infections can transmit through blood transfusion. However, the interactions between HCV and HTLV1 infections have been unclear yet. Our aim was to evaluate the seroepidemiology of these two infections in Iranian thalassemic patients and to assess the effect of $\mathrm{HCV}$ infection on the natural history of HTLV1.

Methods: In a cross- sectional study, 257 transfusion dependent thalassemic patients were tested for anti HCV(ELISA third generation) and anti HTLV1(ELISA and confirmatory Blot). The seropositive cases for HTLV1 were tested for HTLV1 RNA (RTPCR). The correlations between anti HTLV1 and also HTLV1 RNA positivity and $\mathrm{HCV}$ infection were evaluated.

Results: The seroprevalence of anti HTLV1 in thalassemic patients was 11.3 and $21.4 \%$ of them were HTLV1 RNA positive. The seroprevalence of anti $\mathrm{HCV}$ in thalassemic patients was $40.1 \% .12 .6 \%$ of anti HCV seropositive patients were coinfected with HTLV1. The prevalence of anti HTLV1 and HTLV1 RNA did not differed in HCV positive or HCV negative patients.

Conclusion: In spite of, similar routes of viral transmission, it seems that HCV infection does not correlate with the seroprevalence of HTLV1 in thalassemic patients. Also, HCV infection could not change the natural history of HTLV1 infection. 
PP12-27

Previous Hepatitis B Virus Infection Is Strongly Associated with the Severity of Inflammatory Activity and Fibrosis in Chronic Hepatitis C G. Teuber ${ }^{1}$, A. Schäfer ${ }^{2}$, S. Kriener ${ }^{3}$, K. Sprinzl ${ }^{4}$, A. Berger ${ }^{5}$, D. Gangwar ${ }^{4}$, S. Zeuzem ${ }^{4}$, M. Scheuerlen ${ }^{2}$, M.R. Kraus ${ }^{6}$

${ }^{1}$ IFS, Frankfurt/Main, ${ }^{2}$ Medizinische Klinik und Poliklinik II, Universität Würzburg, Würzburg; ${ }^{3}$ Senckenbergisches Institut für Pathologie; ${ }^{4}$ Medizinische Klinik I; ${ }^{5}$ Institut für Medizinische Virologie, Klinikum der Johann Wolfgang Goethe-Universität, Frankfurt/Main; ${ }^{6}$ Medizinische Klinik II, Kliniken AltöttingBurghausen, Burghausen, Germany

Background and aims: Chronic hepatitis $\mathrm{C}$ is affecting worldwide more than 170 Mio. patients. Due to similar routes of transmission, previous hepatitis B virus (HBV) infection is frequently observed in these patients. The impact of previous HBV infection on the natural course of chronic hepatitis $\mathrm{C}$ is controversially discussed. Therefore, we investigated the influence of recent $\mathrm{HBV}$-infection on the severity of inflammatory activity and the extent of fibrosis.

Patients and methods: 686 patients (mean age $45.3+12.7$ years, 418 males [60.9\%] and 268 females [39.1\%) with chronic hepatitis $\mathrm{C}$ were included. In all patients, anti-HBc, and-if positive- $\mathrm{HBsAg}$ and anti-HBs were determined. HBsAg positive patients were excluded from further analysis. Liver biopsy was available in all enrolled patients and assessed according to the Ishak Score.

Results: Anti-HBc was detectable in 273 [37.9\%] of our patients and 134 [48.4\%] of these patients were anti-HBs positive. In patients with chronic hepatitis $\mathrm{C}$ and previous HBV infection, the inflammatory activity (HAI) was significantly higher compared to those without evidence for previous HBV infection $(p=0.012)$. Thus, of the anti-HBc positive patients $17.3 \%$ showed a minimal inflammatory activity compared to $20.5 \%$ of the anti-HBc negative patients, 67.8 versus $72.5 \%$ had a mild inflammatory activity, 14.1 versus $7.0 \%$ had a moderate inflammatory activity and 0.8 versus 0 had a severe inflammatory activity. Similarly, the degree of fibrosis as well as the prevalence of cirrhosis were significantly higher in patients with previous HBV infection than in anti-HBc negative patients $(p=0.049 ; p=0.03)$. HCV-RNA levels and ALT-levels were similar in both patient groups. Conclusions: Previous HBV infection appears to be a strong risk factor for a higher histologic inflammatory activity and a higher degree of fibrosis in patients with chronic hepatitis $\mathrm{C}$ emphasizing the need for $\mathrm{HBV}$-vaccination in anti-HBc negative patients with chronic hepatitis $\mathrm{C}$.

\section{PP12-28}

Hepatic Iron Overload and Chronic Hepatitis C

E.A. Bomfim ${ }^{1}$, A.C.P. Oliveira ${ }^{1}$, C.A.C. Silva ${ }^{1}$, D.L. Andrade ${ }^{1}$, J.F.B. Santos ${ }^{1}$, T.F.D. Alves ${ }^{1}$, T.F.P. Souza ${ }^{1}$, R. Paraná ${ }^{2}$, M.I. Schinoni ${ }^{3}$

${ }^{I}$ Faculty of Medicine, Federal University of Bahia; ${ }^{2}$ Internal Medicine Department, Faculty of Medicine, Federal University of Bahia; ${ }^{3}$ Biofunction Department, Health Sciences Institute, Federal University of Bahia, Salvador, Brazil

Background: Hepatitis $\mathrm{C}$ is a worldwide liver chronic disease and different factors are associated to the increased progression to severe liver fibrosis, such as alcohol intake $>30 \mathrm{~g} /$ day, older age at infection, co-infection; nevertheless, different research centers have found conflicting data concerning the liver iron overload fibrogenic role. Iron ions act as reactive oxygen species, leading to lipid peroxidation, activating Ito cells, which produce collagen matrix.

Aim: To assess the association between hepatic iron overload and fibrosis stage grades in Hepatitis C Virus (HCV) carriers.

Methods: Descriptive study; positive anti-HVC and qualitative $\mathrm{HCV}$ RNA treatment naive chronic Hepatitis C outpatients $(n=290)$ registered from 2007 to 2009 at the Federal University of Bahia Teaching Hospital. The variables studied were: liver biopsy results with fibrosis grading according to METAVIR score, presence or absence of iron stores and steatosis, aspartate transaminase (AST), alanine transaminase (ALT) and gamma-glutamyl transpeptidase (GGT) levels, alcohol intake assessment. Fibrosis grades were divided into two categories: mild/moderate (F0-F2), severe (F3-F4). Exclusion criteria: VHB/HIV coinfection, primary/secondary hemochromatosis. Statistical analysis: Chi-square and Student's t-test; significance: $p<0.05$; software: SPSS 17.

Results: Mean age: $49.58+/-10.09$ years; males: $55.7 \%$; AST: 76.82 +/-71.87; ALT: 92.66 +/-68.17; GGT: $114.30+/-132.03$. These enzymes' levels and the mean age were statistically related to severe fibrosis, as well as iron overload stores $(p=0.003)$ and steatosis $(p=0.01)$. HCV genotype 1 was related to mild/moderate fibrosis $(p=0.025)$. Alcohol intake data were lost in $51.7 \%$ of the subjects, which may have affected the negative result $(p=0.17)$ for this variable.

Conclusions: In this study hepatic iron overload and hepatic steatosis were associated with severe hepatic fibrosis (METAVIR F3-F4).

\section{PP12-29}

Hepatitis $B$ and $C$ among Visitors of a Hepatitis Awareness Program A. Saeed ${ }^{1}$, Z. Abbas ${ }^{1}$, S.A.A. Naqvi ${ }^{2}$, S.A.-U.-H. Rizvi ${ }^{2}$

${ }^{1}$ Hepatogastroenterology; ${ }^{2}$ Urology, Sindh Institute of Urology and Transplantation, Karachi, Pakistan

Background: The aim of this study was to evaluate the occurrence of hepatitis B and C in visitors of a screening camp on World Hepatitis Day.

Methods: Attendees of a hepatitis awareness program was offered screening for hepatitis B and C. The participants were evaluated on history, physical examination, blood tests for $\mathrm{HBsAg}$, anti-HCV, alanine aminotransferase (ALT) and abdominal ultrasound.

Results: Among 392 participants without any history of liver disease, $251(64.0 \%)$ were males and $141(36 \%)$ females, with a mean age of $36.7 \pm 14.6$ years (range 7-80 years). Eleven (2.8\%) subjects were found out to be HBsAg positive, $30(7.7 \%)$ were anti-HCV positive. Comparing them with rest of the serology negative subjects, history of blood transfusion was the only statistically significant risk factor $(p=0.022)$. ALT levels were elevated in $3 / 11 \mathrm{HBsAg}$ positive and $14 / 30$ anti-HCV positive subjects. Ultrasound examination showed increased echogenicity in $2 / 11$ of $\mathrm{HBsAg}$ positive and $4 / 30$ of anti$\mathrm{HCV}$ positive subjects. One $\mathrm{HBsAg}$ positive subject was having irregular liver margins and splenomegaly.

Conclusion: This analysis reveals that our general population is still having a significant number of asymptomatic apparently healthy hepatitis $\mathrm{B}$ and $\mathrm{C}$ patients. There is a need to adopt measures to identify such patients to offer early treatment and prevent further spread of these infections.

\section{PP12-30}

Proteinuri and Glomeruler Filtration Rate in Patients with Non-diabetic Chronic Hepatitis C

E.S. Horasan, G. Ersoz, A. Kıykım, A. Kaya

Mersin University, Mersin, Turkey

Background: Previous studies supports an association between hepatitis $\mathrm{C}$ virus (HCV) infection and glomerulonephritis. However, there are limited and different studies about the association between Chronic Hepatitis $\mathrm{C}$ (CHC) and chronic kidney disease (CKD). In the current study, we investigated the possible association between HCV infection, kidney function tests, estimated glomerular filtration rate (eGFR) and micro-albuminuria by analyzing data from individuals healthy control.

Methods: We conducted a prospective case-control study to examine the relationship between nondiabetic subjects with hepatitis $\mathrm{C}$ infection and kidney function tests. Patients with exclusions criteria's were Hepatitis B surface antigen ( $\mathrm{HBs} A g$ ), Human immunodeficiency virus (HIV) and cryoglobuline positive in the CHC group. Data were 
evaluated with SPSS (ver.16.0). Statistical analyses were made with $T$ test, Chi-squared test and Mann-Whitney $U$ when appropriate. $P<0.05$ was considered significant.

Results: A total of 300 patients were analyzed; $150 \mathrm{CHC}$ and 150 healthy control; $66 \%$ of patients were male, the median age was $50.6 \pm 11$ years. There was no difference in the prevalence of the macroalbuminuri, age, gender, blood urea nitrogen (BUN) between two groups $(p>0.005)$.In comparison with healthy individuals, the prevalence of low eGFR, serum creatinin level and microalbuminuria was significantly greater in individuals with $\mathrm{HCV}$ infection. Prevalence of microalbuminuria in patients with hepatitis $\mathrm{C}$ infection was $2.6 \%$ and it was significantly higher than control group $(0 \%)(P=0.018)$. Mean eGFR was $129.7 \pm 46.3$. It is significantly low than control group (174.44 $\pm 146.87, p=0.001)$. Serum creatinin level was significantly higher than control $(0.62 \pm 0.16$ vs. $0.56 \pm 0.18, p=0.004)$.

Conclusion: Our data suggest that $\mathrm{HCV}$ infection are associated with microalbuminuri, increased serum creatinin levels, decresed eGFR. Other factors may have different impacts on chronic kidney disease. More research is needed to explore the implications of these observations.

\section{PP12-31}

Drug Resistance and HCV Co-infection in Former Blood Donors Infected with HIV-1

J. Liu ${ }^{1}$, X. Chen ${ }^{1}$, Q. Xie ${ }^{1}$, W. Zhang ${ }^{2}$, L. Wei ${ }^{3}$, T. Shen ${ }^{1}$, Q. Xu ${ }^{1}$, H. Zhuang ${ }^{1}$, F. Gao ${ }^{1,4}, \mathrm{~F} \cdot \mathrm{Lu}^{1}$

${ }^{1}$ Department of Microbiology, Peking University Health Science Center, Beijing; ${ }^{2}$ Department of Epidemiology, College of Public Health, Zhengzhou University, Zhengzhou; ${ }^{3}$ Hepatology Institute, Peking University People's Hospital, Beijing, China; ${ }^{4}$ Human Vaccine Institute, Duke University Medical Center, Durham, NC, USA

Background: Unhygienic blood collection in the late 1980s and early 1990s caused an outbreak of HIV-1 and HCV infections among blood donors in rural areas in Henan province, China.

Methods: Partial HIV-1 pol and HCV NS5b gene sequences, as well as $\mathrm{CD}^{+}$cell counts, HIV-1 and HCV viral loads were obtained from 97 persons infected with HIV-1 to determine the efficacy of treatment, the prevalence of drug-resistant mutations, and the impact of $\mathrm{HCV}$ infection on disease progression of infection with HIV-1.

Results: After treatment with AZT, DDI and NVP for 23 months, plasma HIV-1 RNA levels were reduced to an undetectable level $(<1,000$ copies $/ \mathrm{ml})$ in $61.9 \%$ of patients $(60 / 97)$, and $\mathrm{CD}^{+}$cell counts in these patients were significantly higher $(P<0.01)$ than those with viral loads $\geq 1000$ copies $/ \mathrm{ml}$. The K103N mutation was detected most frequently $(72.7 \%)$. Nearly two-thirds of treatment failure patients were found without detectable drug-resistant mutations, suggesting that poor adherence also played a role in treatment failure in this cohort. The majority of patients infected with HIV-1 were also infected with HCV (91.8\%) and about half of the patients were infected with either HCV subtype $1 \mathrm{~b}$ or $2 \mathrm{a}$ ( 47.5 and $52.5 \%$, respectively). There was no significant difference in HIV-1 viral loads and $\mathrm{CD}^{+}$cell counts between patients co-infected with either HCV subtype $1 \mathrm{~b}$ or $2 \mathrm{a}$. However, HCV viral loads were significantly higher in patients infected with subtype $2 \mathrm{a}$ than in patients infected with HCV subtype $1 \mathrm{~b}$.

Conclusions: These results suggest that a full understanding of the prevalence of drug-resistant mutations in treated patients and an improvement in adherence to treatment regimens will be critical to effectively treat patients in resource-limited areas who are infected with HIV-1 or co-infected with HIV-1 and HCV.

Acknowledgment: China National Grants 2005CB523103 and 2008ZX10002-013
PP12-32

HCV Profile in HIV Coinfection in Manipur, India (Based on RIMS Study)

N. Thongam ${ }^{1}$, B. Singh ${ }^{2}$, K. Singh ${ }^{2}$, G. Singh ${ }^{2}$

${ }^{1}$ Biochemistry, Jawaharlal Nehru Institute of Medical Sciences;

${ }^{2}$ Medicine, Regional Institute of Medical Sciences, Imphal, India

Aims and background: Manipur is one of the six high HIV prevalent states of India neighbouring Myanmar, Thailand and Laos (Golden triangle).HIV-HCV coinfection is very high in Manipur (18-35\%) as intravenous drug users (IDUs) are very common.

Materials and methods: After getting Institutional Ethical Committee clearance, RIMS and informed consent of patients the present study was done between May 2006 to September 2009.

Results and discussions: Out of 2720 HIV, 435 (16\%) were HIV$\mathrm{HCV}$ with $52 \%$ chronic alcoholism, $83 \%$ of HIV-HCV had CD4 count $<200$ cells/cumm showing more advance AIDS cases.24 (6\%) of 435 HIV-HCV had CD4 $<100 / \mathrm{cmm}$ even after HAART. 32 (7\%) had thrombocytopenia $<1$ lac, $57 \%$ of the HIV-HCV presented with HIV specific OI manifestations like Toxoplasmosis, CMV, TBM which were diagnosed from CSF analysis.51 (11.7\%) had pulmonary TB,7 of 51 HIV-TB developed gynaecomastia,23 (5.2\%) had cryptominingitis, 1 had Kaposi Sarcoma, 2 had P. Marneffi, 3 had HCC, 1 had pellagra like features, 1 had PML. AST > ALT were seen in $63 \%$. The number of DM was $23 \%$ in HIV-HCV coinfection.BMI of diabetic cases was found to be higher than BMI of non-diabetic cases. 52 died in spite of HAART due to liver complication and ART failure. HCV genotyping study were done in 69 cases, genotype- 3a (59 cases), genotype- 1(5 cases), genotype- 6 ( 2 cases), genotype- 3 and 4 ( 3 cases).

Conclusions: HCV should be screened in cases of Hepatomegaly with AST $>$ ALT or thrombocytopenia with history of risk behavior. HCV-HIV coinfection increases failure to ART. Detection of AIDS specific OIs leads to early diagnosis of HIV. Liver complications of HCV including HCC and metabolic complication DM increases mortality. HCV genotype $3 \mathrm{a}$ is commonest in Manipur, a northeast state of India. Facilities to provide HCV management should be provided in ART centers.

\section{PP12-33}

Status of HCV-types/Subtypes in Patients with Liver and Renal Disease M.A. Ansari, S. Singh, A. Singh, P. Nag, M. Irshad

Laboratory Medicine, All India Institute of Medical Sciences, Delhi, India

Background/aim: Hepatitis C Virus (HCV), a single stranded positive sense, enveloped RNA virus with $9.6 \mathrm{~kb}$ genome, is prevalent in $\sim 3 \%$ population worldwide and emerging cause of liver/renal diseases. The present study was planned to investigate the status of genotypes in liver and renal diseases patients in North India.

Methods: In this study, 50 sera with liver and renal diseases were screened for HCV-RNA using core region specific primers. Genotyping was done by Restriction Fragment Length Polymorphism (RFLP) in 35 $\mathrm{HCV}$ positive patients. Restriction enzymes MboI, AccI and BstNI were used for digestion of amplicon (399 bp). Core amplicons were sequenced and aligned with known HCV sequences. Phylogenetic analysis was done by neighbor joining method. Presence of anti-HCV antibodies and HCV core protein in sera was estimated by EIA.

Results: Of $35 \mathrm{HCV}$ positive cases, 23 (65.7\%) belonged to genotype 3, $10(28.6 \%)$ to genotype $1,1(2.9 \%)$ each to genotype 2 and genotype 4 . None of them was found to be genotype 5 or genotype 6 . Sequence alignment studies also confirm the genotypes observed by RFLP. Similarly, phylogenetic analysis supports the finding of RFLP analysis. Conclusion: HCV genotype 3 was found to be the dominant genotype prevalent in this part of India. Sequence alignment and phylogenetic 
analysis also confirm the result of RFLP. There was no significant corelation between $\mathrm{HCV}$ genotypes and $\mathrm{HCV}$ core protein in serum.

\section{PP12-34}

Il28b Polymorphisms Are Associated with Acute Presentation and Spontaneous Clearance in Acute Hepatitis C Virus Infection S. Beinhardt ${ }^{1}$, P. Steindl-Munda ${ }^{1}$, M. Strasser ${ }^{2}$, M. Gschwantler ${ }^{3}$, A. Maieron ${ }^{4}$, A. Kreil ${ }^{5}$, K. Rutter ${ }^{1}$, A.F. Staettermayer ${ }^{1}$, T.M. Scherzer ${ }^{1}$, R. Strassi ${ }^{6}$, H. Holzmann ${ }^{6}$, P. Ferenci ${ }^{1}$, H. Hofer ${ }^{1}$

${ }^{1}$ Department of Gastroenterology and Hepatology, Medical University of Vienna, Vienna; ${ }^{2}$ Paracelsus Medical University, Salzburg; ${ }^{3}$ Wilheminenspital, Vienna; ${ }^{4}$ Hospital Elisabethinen, Linz; ${ }^{5}$ Otto Wagner Spital; ${ }^{6}$ Department of Virology, Medical University of Vienna, Vienna, Austria

Background: Single nucleotide polymorphisms (SNP) in the IL28B gene region influence outcome of hepatitis $\mathrm{C}$ virus infection. This study evaluated the prevalence of rs12979860 and rs8099917 genotypes in Austrian patients with pedigreed symptomatic acute (AHC) or chronic hepatitis $\mathrm{C}(\mathrm{CHC})$ and healthy controls ( $\mathrm{HC})$.

Methods: Clinical data and DNA was obtained from 785 subjects: 96 HC, 103 AHC, 586 CHC, HCV-genotype 1. The rs12979860 and rs8099917-region of the IL28B-gene was investigated by StepOnePlus Real time PCR System.

Results: 49 (48\%) of 103 patients with AHC cleared HCV spontaneously (SC). The genotype distribution for rs12979860 in AHC versus CHC was: C/C 62 vs. $37 \%$, C/T 35 vs. $50 \%$, T/T 3 vs. $13 \%$. For rs 8099917 it was T/T 82 vs. $60 \%, \mathrm{G} / \mathrm{T} 17$ vs. $36 \%, \mathrm{G} / \mathrm{G} 1$ vs. $4 \%$. The highest frequency of C/C genotype for rs12979860 and T/T genotype for rs8099917 were observed in patients with AHC and spontaneous clearance (71 and 90\%). The C/C genotype in patients with SC was nearly twice the frequency of CHC patients ( 71 vs. $37 \%, p<0.001)$ and more frequent than in AHC patients without SC $(51 \%, p<0.01)$. The C-allele frequency of rs 12979860 was higher in patients with AHC compared to CHC patients ( 80 vs. $62 \%, p<0.001$ ). Similarly, the Tallele of rs8099917 was more prevalent in patients with AHC compared to $\mathrm{CHC}$ ( 90 vs. $78 \%, p<0.001$ ). Jaundice in AHC was more common in patients with genotype $\mathrm{C} / \mathrm{C}$ of rs12979860 compared to T-allele carrier $(p=0.038)$. Viral load or transaminase levels were not different. Conclusion: In symptomatic AHC rs12979860 C/C and rs8099917 T/ $\mathrm{T}$ carriers are more frequent than in $\mathrm{CHC}$, whether they clear the virus spontaneously or not. Nevertheless, together with virological data determinations of rs12979860 and rs8099917 may help to predict spontaneous clearance of $\mathrm{HCV}$.

\section{PP12-35}

Hepatitis C Virus (HCV) Risk Factors: Lack of Knowledge q Main Reason for Epidemics in Pakistan

J.A. Saadat ${ }^{1,2}$, H. Bashir ${ }^{3}$, I.A. Khawar ${ }^{4}$, Z. Khalii ${ }^{5}$, N.U. Ain ${ }^{1}$, M. Sajjad ${ }^{5}$, U.B. Aamir ${ }^{5}$, S.Z. Safi ${ }^{5}$, Q. Bashir ${ }^{5}$

${ }^{I}$ National University of Sciences and Technology; ${ }^{2}$ Margalla Institute of Health, Islamabad; ${ }^{3} \mathrm{MDU}$, Khuzdar; ${ }^{4}$ University of the Punjab, Lahore; ${ }^{5}$ National University of Sciences and Technology, H-12, Islamabad, Pakistan

Background/Aims: Hepatitis $\mathrm{C}$ is one of the major health care problems being faced by the world today. Unfortunately most of the world's population living in the third world countries below the poverty line is a major victim of this disease. Lack of awareness and insufficient knowledge are important contributing factors for such a rapid spread of virus.

Methods: In order to determine the extent of the awareness about $\mathrm{HCV}$ risk factors among $\mathrm{HCV}$ infected and uninfected population, an awareness survey was conducted in the Rawalpindi/Islamabad zone.
Results: According to survey conducted among uninfected population, regarding risk factors, about $\sim 70 \%$ of the population was aware of the risk factors for HCV like blood transfusion, needle sharing, sexual contact etc. While in patients, less than $50 \%$ of the infected people were aware of the $\mathrm{HCV}$ risk factors. It was also observed that people both infected and uninfected have more awareness for $\mathrm{HCV}$ transmission through blood but high degree of variability was observed in the rest of risk factors.

Conclusions: The studies concluded that the awareness level regarding risk factors and $\mathrm{HCV}$ transmission routes are important factors affecting the mounting HCV infected toll.

\section{PP12-36}

The Prevalence of Anti-HCV among Family Members of Patients Infected with HCV

N. Aqodad, M. Lahbabi, M. Elyousfi, I. Mellouki, D. Benajah, M. Elabkari, A. Ibrahimi

Gastroenterology Unit, Hospital University Hassan II, Fez, Morocco

Introduction and aim of study: If transmission of hepatitis $\mathrm{C}$ virus (HCV) infection through parenteral exposure is well documented, intra-familial transmission of $\mathrm{HCV}$ is still debatted. Our aim is to determine whether $\mathrm{HCV}$ infection of index cases increases intrafamilial transmission (sexual and nonsexual) of $\mathrm{HCV}$.

Methods: A cross-sectional study was conducted with $18 \mathrm{HCV}$ positive patient (index cases) and 54 familial contact, from October 2002 to August 2003, in the gastroentrology unit of the Fes University Hospital.

Results: Seropositivity was found in $7.4 \%$ of the contacts. No difference was found betwen index cases and their contacts for anterior risk factors of $\mathrm{HCV}$ infection (dental care, parenteral injection and surgery). HCV seropositivity was greater in spouses $(21.4 \%)$ than in familly member without sexual contact $(2.5 \%)$, but this difference was not significative (OR 10.6 CI 95\% [0.83-295]).

Conclusion: $\mathrm{HCV}$ prevalence is relatively elevated in familly memebre to pateient infected with $\mathrm{HCV}$, especially in spouses, but without formal arguemnts for intrafamilial transmission of $\mathrm{HCV}$.

\section{PP12-37}

HCV Re-infection after Successful Therapy among Asian and Caucasian Patients in Vancouver, Canada

J.D. Farley, T. Maung Maung, A. Truong, E. Paripovic, S. Mayronne, T. Nguyen, W. Shum

Dr. John Farley Inc, Vancouver, BC, Canada

Background: Hepatitis C Virus infection (HCV), an important public health problem in Canada, can be effectively treated with a combination regimen of Pegylated interferon and Ribavirin. Re-infection, however, can occur after successful therapy. Asians account for $40.7 \%$ of the increasing immigrant population in Vancouver, BC. Our study focuses on the re-infection rate of this group.

Methods: We conducted a retrospective chart review of all patients who successfully completed standard HCV therapy at our clinics (achieved SVR). Participants were categorized as Asian or Caucasian based on self-reported ethnicity status. Data analysis was performed with PASW (SPSS Version 18).

Results: 354 patients were included in the analysis (cases). $11.3 \%$ were Asians and $88.7 \%$, Caucasians. $19.5 \%$ were females and $80.5 \%$, males. Average age was $47.54(\mathrm{SD} \pm 10.013)$ years. $70.1 \%$ of cases had a history of intravenous drug use (IDU). $12.1 \%$ of cases became re-infected. On univeriate analysis, we did not find any significant differences in gender, age, HCV genotypes, or HIV co-infection on comparing the two ethnicities. However, a history of IDU was significantly lower in Asians (OR $0.206,95 \%$ CI $0.1035,0.4102$ ). The 
HCV re-infection rate was also lower in Asians (OR 0.001, 95\% CI $0.0001,0.014)$ than in Caucasians.

Genotype distribution and re-infection after SVR

\begin{tabular}{lcc}
\hline Variable & Asian & Caucasian \\
\hline G1 & 22 & 151 \\
G2 & 4 & 48 \\
G3 & 14 & 110 \\
Re-infected & 1 & 42 \\
\hline
\end{tabular}

Conclusion: In our study population, $\mathrm{HCV}$ genotype distribution in both Asians and Caucasians was similar; so was response to $\mathrm{HCV}$ therapy by risk category. The durability of SVR was better in Asians after successful treatment; this is likely because of a siglificantly lower re-infection rate, attributed to less IDU.

\section{PP12-38}

Prevalence of HCV Infection and Liver Dysfunction in a Cohort of Non-Hodgkin's Lymphoma (NHL) Patients Treated with Chemotherapy A. Morrone ${ }^{1}$, L. Nosotti ${ }^{1}$, M. D'Andrea ${ }^{2}$, M.C. Petti ${ }^{2}$

${ }^{1}$ National Institute for Health, Migration and Poverty; ${ }^{2}$ Regina Elena Institute, Roma, Italy

Background: Several studies have reported a higher prevalence of $\mathrm{HCV}$ infection in patients with B-cell non-Hodgkin's lymphoma (NHL) than in the general population. Treatment for NHL include the use of chemotherapeutic agents such as cytotoxic drugs, corticosteroids and rituximab, that can be hepatotoxic and immunosuppressive. Although reactivation of $\mathrm{HBV}$ undergoing immunosuppressive therapy for hematological malignancies is a well-documented complication, data on $\mathrm{HCV}$ reactivation or liver function impairment after chemotherapy for NHL are controversial.

Methods: From January 2006 to December 2009 we observed 207 consecutive patients with NHL treated with chemotherapy; all patients were screened for $\mathrm{HCV}$ infection and baseline liver function tests. HCV-positive individuals were closely monitored for serum transaminase, bilirubin and HCV-RNA levels during treatment and for 6 months after anticancer therapy.

Results: The prevalence of HCV infection was 9.17\% (19 out of 207 patients). Among the HCV-infected subjects, the prevalence of hepatotoxicity was $31.5 \%$ (6 out of 19) versus $1.53 \%$ (3 out of 188) among the HCV-uninfected individuals. Most of the patients showed a low-grade liver dysfunction, but a patient developed a fulminant hepatitis.

Conclusions: Although less frequent and less severe than in HBVinfected subjects, liver dysfunction can occur as a consequence of chemotherapy in $\mathrm{HCV}$-infected patients with NHL, particularly with rituximab. The frequency and the severity of this complication varies among the different reports, and in many cases it is difficult to determine whether liver injury is caused by $\mathrm{HCV}$ reactivation or chemotherapy toxicity. In our study we describe a high prevalence of hepatotoxicity in $\mathrm{HCV}$-infected subjects; in the majority of them it doesn't represented a significant clinical problem, but in one case it caused a fulminant hepatitis.Therefore we recommend to screen all patients with NHL for HCV infection before starting chemotherapy and to monitor liver function test and HCV-RNA serum levels during treatment.
PP12-39

The Elevated Liver Function Tests Prevalence among HCV-RNA Patients in Great Naft Hospital

K. Gholam Abas ${ }^{1}$, M.A. Jalali $\mathrm{Far}^{2,3}$, M. Alinejadi ${ }^{2,4}$, N. Saki ${ }^{1,3}$

${ }^{I}$ Ahvaz Jondi Shapour University of Medical Sciences; ${ }^{2}$ Medical

Laboratory Science, Ahvaz Jondi Shapour University of Medical

Sciences; ${ }^{3}$ Thallasemia and Hemoglubinopathy Research Center;

${ }^{4}$ Medical Laboratory, Great Naft Hospital, Ahwaz, Iran

The worldwide prevalence of $\mathrm{HCV}$ infection is $3.0 \%$, corresponding to 170 million persons. Currently, HCV infection causes an estimated $8,000-10,000$ deaths annually in the United States, and that number is predicted to triple in the next 10-20 years. Current estimates suggest $2-5 \%$ of patients with liver cirrhosis due to HCV develop HCC annually. An elevated liver function tests is one factor Potential factors influencing progression of fibrosis and help us to estimate the percent of patient who susceptible.

Our descriptive study included 24 patients admitted to Great Naft Hospital during April 2009 till Jan 2010. The HCV-Ab and then qualitative HCV-RNA of them was positive. The liver function tests were done and data analyzed by SPSS.

Our patients were in 20-57 years old. The $29.2 \%$ (7/24) was female and $69.8 \%(17 / 24)$ male. The $29.2,4.2$ and $37.5 \%$ of our population had abnormal levels in ALT, AST and ALK respectively. The ALT elevated was seen in male. The $1 \mathrm{a}$ and $3 \mathrm{a}$ were the frequent $\mathrm{HCV}$ genotypes in our population.

According to our finding, similar to other region of country, the 1a and 3a was prevalent genotypes. Although AST abnormality was more frequent in our population, because the ALT is most sensitive indicator for viral infection, the $1 / 3$ of our patients needs to examine for liver damage. Viral load and other diagnostics method recommended.

\section{PP12-40}

No Reappearance of HCV RNA in Patients with Sustained Virological Response by Long-Term Follow-Up Study

D. Maruoka, F. Imazeki, K. Fujiwara, M. Arai, T. Kanda, O. Yokosuka Department of Medicine and Clinical Oncology, Graduate School of Medicine, Chiba University, Chiba, Japan

Background/Aims: Patients with chronic hepatitis $\mathrm{C}$ have high risk of HCC, but sustained virological response (SVR) after interferon (IFN) treatment induced not only durable normal liver function but also improvement of liver fibrosis and further reduced the rate of HCC development significantly. We investigated the sequel of laboratory data in patients with chronic hepatitis $\mathrm{C}$ who achieved SVR with IFN treatment by long-term retrospective cohort study.

Methods: Five hundred eighty-one consecutive patients with $\mathrm{C}$-viral chronic liver disease (CLD) who underwent liver biopsy at our department between January 1986 and December 2005 were enrolled in this study. Four hundred sixty-seven of these patients were treated with IFN and 207 of them achieved SVR with follow-up periods of $8.36 \pm 5.13$ years, and the other 114 were IFN-untreated. ALT level, albumin level, platelet count and APRI value were serially examined during follow-up period. Results: None of 207 patients with SVR became HCV RNA positive during follow-up period. The duration between the date of confirmation of SVR and the date of last examination of HCV RNA was $7.5 \pm 5.3$ years ( $0-21.3$ years). HCV RNA was examined by TaqMan method in 119 of these patients and the duration was $8.2 \pm 5.5$ years (0.5-21.3 years). But 14 of these patients showed transient elevation of ALT levels. Platelet count and albumin level increased only in those who eradicated HCV. APRI value decreased in 
patients with SVR more than in those with non-SVR. Patients who achieved SVR and had fibrosis stage $2-4$ at enrolment showed that platelet count continued to increase by $5.49 \pm 4.53 \times 10^{3} / \mu 1$ and APRI value decreased by $2.50 \pm 2.26$ for 10 years.

Conclusions: HCV-RNA remained negative in all patients with SVR and laboratory data including APRI also improved during a long term follow-up period.

\section{PP12-41}

Evolution Epidemiology of Hepatitis C Virus 3a Infection in Thailand S. Akkarathamrongsin ${ }^{1}$, P. Hacharoen ${ }^{1}$, K.P. Praianantathavorn ${ }^{1}$, P. Tangkijvanich ${ }^{2}$, A. Theamboonlers ${ }^{1}$, Y. Tanaka ${ }^{3}$, M. Mizokami ${ }^{4}$, Y. Poovorawan ${ }^{1}$

${ }^{1}$ Center of Excellence in Clinical Virology; ${ }^{2}$ Department of Biochemistry, Faculty of Medicine, Chulalongkorn University, Bangkok, Thailand; ${ }^{3}$ Department of Virology and Liver Unit, Nagoya City University Graduate School of Medical Science, Nagoya; ${ }^{4}$ Research Center for Hepatitis and Immunology, National Center for Global Health and Medicine, Ichikawa, Japan

Approximately $2.2 \%$ of the entire Thai population has been infected with $\mathrm{HCV}, 50 \%$ with the predominant HCV-3a. Dissemination of HCV-3a, the most frequent subtype among IVDU, in the population may be associated with a similar mode of transmission. This study has investigated the molecular epidemiology and epidemic history of HCV-3a in Thailand. Altogether 356 serum samples were collected from HCV RNA positive patients, with the majority residing in the central part of the country. HCV genotypes were determined by phylogenetic analysis based on the core and NS5B sequences. Subtype 3a $(38.5 \%)$ was the most predominant, followed by $1 \mathrm{a}(21 \%), 1 \mathrm{~b}$ (13.8\%), genotype 6 (20.1\%) which comprises subtypes 6e (0.3\%), $6 \mathrm{f}$ (11\%), 6i (1.9\%), 6j (1.9\%) and 6n (4.8\%), and 3b (5.6\%). The most risk factor of HCV-infection was a history of blood transfusion (33.1\%), followed by tattooing (27.7\%), IVDU (26.9\%), unsafe medical injection (23.8\%), shaving performed by a barber $(23.1 \%)$ and exposure to other risk factors. Phylogenetic analyses indicated the existence of a specific cluster of HCV-3a strains in the Thai population which may be consistent with a particular route of HCV transmission in Thailand. Molecular evolutionary analyses estimated the date of tMRCA to the year 1914 (1874-1950), indicating that HCV-3a has existed in this area for approximately 100 years. The Bayesian skyline plot showed that this particular strain spread during the 1960s-1970s, which coincides with the period of the Vietnam War (1955-1975) and the widespread use of injection stimulants introduced by the US army. These results suggest that the future burden of HCV-related disease will increase over the next few decades.

\section{PP12-42}

Genotype of Hepatitis C Virus among Blood Donor in Udon Thani, the Northeast Thailand

S. Akkarathamrongsin ${ }^{1}$, P. Hacharoen ${ }^{1}$, K. Praianantathavorn ${ }^{1}$, A. Theamboonlers ${ }^{1}$, K. Chaiear ${ }^{2}$, P. Tangkijvanich ${ }^{3}$, Y. Poovorawan ${ }^{1}$

${ }^{1}$ Center of Excellence in Clinical Virology, Chulalongkorn University, Bangkok; ${ }^{2}$ Udon Thani Hospital, Udon Thani;

${ }^{3}$ Department of Biochemistry, Faculty of Medicine, Chulalongkorn University, Bangkok, Thailand

Background/aim: Data on $\mathrm{HCV}$ epidemiology and prevailing genotype in rural areas of Thailand are scarce. Udon Thani is a rural province in the north-east of Thailand, bordering directly on Laos. Both countries share a history of cultural connection and transmigration. Thus, this study was aimed at determining HCV genotype distribution among Thai blood donors in Udon Thani.

Methods: One hundred and ninety six anti-HCV positive serum samples of Thai blood donors were obtained from Udon Thani Hospital. RNA extraction and RT-PCR were performed in order to amplify the HCV $5^{\prime}$ UTR. HCV RNA positive samples were further subjected to amplification of the NS5B region, direct sequencing and genotype determination by phylogenetic analysis.

Results: Of the anti-HCV positive sera, 125 were positive for $\mathrm{HCV}$ RNA. Phylogenetic analysis based on the NS5B sequence revealed HCV genotypes 1a (22.1\%), 1b (10.6\%), 3a (37.5\%), 3b (3.9\%) and 6 (25.9\%); subtype 6i (10.6\%), 6f (9.6\%), 6n (4.8\%) and 6v (0.9\%).

Conclusion: HCV-3a was highly prevalent in this region. Genotype 6 showed rather profound diversity and high frequency. In addition, one sample of subtype $6 \mathrm{v}$ was found in this study suggesting that this genotype may have been accumulating in this area and subsequently undergone variations. This study may be useful for surveillance and monitoring of the incidence of hepatitis and related diseases in this region of Thailand.

Acknowledgements: This research was supported by the Chulalongkorn University Research Fund, the National Research Fund, the RGJ program of the Thailand Research Fund and the Commission on Higher Education.

\section{PP12-43}

Distribution of HCV Genotypes in Patients Infected by Different Sources N. Aqodad, M. Lahbabi, I. Mellouki, M. Elyousfi, D. Benajah, M. Elabkari, A. Ibrahimi

Gastroenterology Unit, Hospital University Hassan II, Fez, Morocco

Introduction: Several studies reported that there is a relationship between the hepatitis $\mathrm{C}$ virus (HCV) genotype and the source of infection. The aim of our study was to determine the distribution of HCV genotypes in our population.

Patients and methods: Retrospective study of all cases of chronic hepatitis C followed in our department between 05 January 2001 and 08 January 2010. All clinical and biological data were collected and analyzed from the files and registry of patients treated and followed for $\mathrm{HCV}$.

Results: 234 Patients with HCV infection were seen in our department. The average age was 45.4 years [17-80]. Sex ratio 0.8 . HCV genotypes were found: genotype $1[43 \%(N=101)]$, genotype $2[27 \%$ $(N=63)]$, genotype $3[0.4 \%(N=1)]$, genotype $4[0.4 \%(N=1)]$ and mixed genotype in $0.8 \%$ of patients. The mode of contamination could be determined in $51.7 \%(N=121)$ patients. The genotype of $\mathrm{HCV}$ most predominant in patients already transfused was genotype I: $66.6 \%(N=18)[51.8 \% \mathrm{Ib}(N=14)]$. For patients already operated the most predominant genotype of HCV was genotype I: $50 \%(N=33)$ [37.8\% Ib $(N=25)]$. Among 113 patients in whom no risk factor for $\mathrm{HCV}$ infection was found $40.7 \%(N=46)$ were infected with genotype I [76\% Ib $(N=35)]$.

Conclusion: The genotype $\mathrm{lb}$ is predominant after blood transfusion and surgery. However, among 113 patients without obvious risk factor $76 \%(N=35)$ are infected with genotype lb. The genotype 3 usually secondary to intravenous drug use (IVD) was observed in only one patient already operated, but not users IVD. The low frequency of genotype 3 in our population is related to the low frequency of this route of infection in our country.

\section{PP12-44}

Evaluation Of HCV Infection Rate in Hemodialysis Patients of South Khorasan in Comparison with Other Blood Born Viruses M.H. Namaei, M. Ziaee, R. Azizee, H. Eshraghi Birjand University of Medical Sciences, Birjand, Iran

Background and aims: Hemodialysis patients are at risk of several blood born diseases such as viral hepatitis (A, B, C) and HIV infection. This study was performed to evaluate the prevalence of $\mathrm{HCV}$ infection in hemodialysis patients in our region.

Material and methods: A cross sectional study was performed on 41 hemodialysis patients recruited to vali Asr hospital hemodialysis site. Previous history of infection to HBV, HDV, HIV, and HTLV1and2 was evaluated in all patients using ELISA method. The 3rd generation 
of ELISA kit was used for evaluating anti $\mathrm{HCV}$ in serum. $\mathrm{HCV}$ Viremia was checked in all patients using RT-PCR method.

Results: Mean age of our patients was 64.93 years (68.3 in males and 31.7 in females). Four out of 41 patients were $\mathrm{HBsAg}$ positive, and one was Anti-HTLV1 positive; none of them were HDV or HIV positive. HCV infection was not detected in none of patients by ELISA method, but RT-PCR demonstrated the viremia in one patient. This patient was HBsAg positive and had previous history for surgery.

Conclusion: Although HCV infection is less common than HBV infection in our patients; as ELISA method can not demonstrate all hemodialysis patients with HCV infection it is needed to evaluate this group of patients for HCV infection using RT-PCR.

\section{PP12-45}

Assessment of Awareness and Knowledge Regarding Hepatitis C among Pakistani Men

S. Hamid ${ }^{1}$, J.A. Saadat ${ }^{1,2}$, H. Bashir ${ }^{3}$, I.A. Khawar ${ }^{4}$, U.B. Aamir ${ }^{1,5}$, Q. Bashir ${ }^{1}$ ${ }^{1}$ NUST, $H-12 ;{ }^{2}$ Margalla Institute of Health, Islamabad; ${ }^{3} \mathrm{MDU}$

Khuzdar, Khuzdar; ${ }^{4} I B B$, University of the Punjab Lahore, Lahore;

${ }^{5}$ Department of Virology, NIH, Islamabad, Pakistan

Background: Hepatitis C is infecting 3\% of world's population. Pakistan, a third world country, with total population of over 169 million has 10 million people infected with HCV and the number is still mounting. Lack of awareness regarding HCV might be a leading cause of virus spread.

Methods: In order to evaluate awareness level about HCV and its role in infection spread, an awareness survey was conducted in Rawalpindi/Islamabad zone targeting men. Men being supportive as well as the most dominant personality in a house constituting about $48 \%$ of Pakistani population with about $63 \%$ literacy rate can proved to be helpful for combating the disease. A survey was designed with basic information included regarding $\mathrm{HCV}$.

Results: Just $20 \%$ of the people were aware of the fact that HCV is caused by virus. Illiteracy $(72 \%)$ and age $(17 \%)$ were blamed to be the cause of HCV. Along with blood transfusion, blades, and syringes, other risk factors have been identified like utensils, coughing, casual contact, saliva, mother to baby and sexual contact as the main transmission factors for HCV. 58\% population was aware that there is no vaccination available for $\mathrm{HCV}$ while $30 \%$ have misperception that $\mathrm{HBV}$ vaccine can also protect against $\mathrm{HCV}$. $84 \%$ believe that $\mathrm{HCV}$ is treatable while $77 \%$ says that one can have more than one type of hepatitis at one time. Just $50 \%$ of men undergo HCV testing. Surveys were given weight age by $91 \%$ of men to bring about awareness about $\mathrm{HCV}$. Liver, Stomach, heart and skin were the main targets of HCV as marked in survey.

Conclusion: The low level of awareness of HCV in the dominant factor i.e., man in Pakistani society is a major risk of HCV epidemic.

\section{PP12-46}

Comparison of Risk Factors for the Spread of Chronic HBV and Chronic HCV Infection (a Local Experience: Southern Punjab, Pakistan)

M.A. Qazi ${ }^{1}$, H. Raza ${ }^{1}$, A. Imran ${ }^{1}$, S. Anjum ${ }^{1}$, G.M.-U.-d. Chaudhary ${ }^{2}$

${ }^{1}$ Medicine, Quaid-e-Azam Medical College/Bahawal Victoria

Hospital, Bahawalpur; ${ }^{2}$ Medicine, Nishtar Medical College, Multan, Pakistan

Background: Chronic Hepatitis C virus (HCV) infection and hepatitis B virus (HBV) infection are common causes of liver cirrhosis in Pakistan. Aim of the study was to compare the risk factors for the spread of these viral infections.

Methods: A descriptive analytical study conducted in Bahawal Victoria Hospital, Bahawalpur (1June 2005 to 30 July 2006). Twenty two hundred patients having dyspepsia were screened for HCV and HBV infection with Enzyme linked immunosorbant assay. Demo- graphics and the following risk factors were noted: history of $(\mathrm{H} / \mathrm{O})$ injection, $\mathrm{H} / \mathrm{O}$ blood transfusion, $\mathrm{H} / \mathrm{O}$ operation, family history of $\mathrm{HCV} / \mathrm{HBV}$ infection, $\mathrm{H} / \mathrm{O}$ sexual contacts, $\mathrm{H} / \mathrm{O}$ shaving by the barbers (in males) and $\mathrm{H} / \mathrm{O}$ dental procedure. The patients were divided into three groups: Group-A anti-HCV +ve, Group-B HBsAg +ve, Group-C who were both anti-HCV +ve and HBsAg +ve. Risk factors in Group-A and Group-B were compared and chi-square test was applied using SPSS version 17.

Results: Age range was $15-85$ years. Over all sero-prevalence was $20.1 \%$, Group-A, Group-B, Group-C 78.1, 16.3, 5.6\% respectively. In Group-A, $62.3 \%<40$ years of age and Group-B $57.5 \%(p=0.448)$, $58.9 \%$ males in Group-A and Group-B $64.4 \%(p=0.381), 62.9 \%$ from rural area in Group-A and Group-B 64.4\% $(p=0.806), \mathrm{H} / \mathrm{O}$ injection $88.6 \%$ in Group-A and Group-B $94.5 \%(p=0.130), \mathrm{H} / \mathrm{O}$ blood transfusion $13.4 \%$ in Group-A and Group-B $8.2 \%(p=0.221)$, $\mathrm{H} / \mathrm{O}$ surgical procedure $25.4 \%$ in Group-A and Group-B $17.8 \%$ $(p=0.166)$, family history $16.9 \%$ in Group-A and Group-B $27.4 \%$ (0.036), $\mathrm{H} / \mathrm{O}$ drug abuse $12.3 \%$ in Group-A and Group-B $8.2 \%$ $(p=0.323), \mathrm{H} / \mathrm{O}$ sexual contact $17.1 \%$ in Group-A and Group-B $9.6 \%(p=0.108), \mathrm{H} / \mathrm{O}$ shaving $85 \%$ in Group-A and Group-B 76.6\% $(p=0.165)$, and $\mathrm{H} / \mathrm{O}$ dental procedures $70.9 \%$ in Group-A and Group-B 35.6\% $(p<0.001)$.

Conclusion: Among the risk factors, family history is significant in $\mathrm{HBV}$ spread while history of dental procedures is significant in $\mathrm{HCV}$ spread.

\section{PP12-47}

The New Epidemiological Features of HCV Infection in Southern China

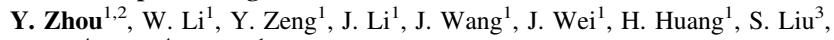
Y. $\mathrm{Wu}^{4}, \mathrm{C} \cdot \mathrm{Li}^{4}, \mathrm{~J} . \mathrm{Hou}^{1}$

${ }^{1}$ Infectious Diseases, Nanfang Hospital; ${ }^{2}$ Hepatology Unit; ${ }^{3}$ School of Pharmaceutical Sciences; ${ }^{4}$ School of Biotechnology, Southern Medical University, Guangzhou, China

Background: In recent years, the changes in the distribution of $\mathrm{HCV}$ genotypes were reported to have important impacts on therapy and prognosis in some countries.

Objective: To investigate the current distribution and epidemiological features of HCV genotypes in Southern China.

Methods: A total of 247 chronic hepatitis $\mathrm{C}(\mathrm{CHC})$ patients, from three provinces in Southern China, were enrolled in the study. HCV genotypes/subtypes were determined by nucleotide sequencing of the $\mathrm{HCV} 5^{\prime} \mathrm{UTR}$ and NS5b regions followed by phylogenic analysis. The logistic regression model was used to analyze relationship between HCV genotype distribution and patients' age, gender and modes of risk exposure.

Results: Seven subtypes were detected in Southern China. Subtype $1 \mathrm{~b}$ $(59.1 \%)$ and $6 \mathrm{a}(21.9 \%)$ were the most common subtypes, followed by subtype $2 \mathrm{a}(8.5 \%), 3 \mathrm{a}(4.5 \%), 3 \mathrm{~b}(4.0 \%), 1 \mathrm{a}(1.6 \%)$ and $2 \mathrm{~b}(0.4 \%)$. $\mathrm{HCV}$ genotype distribution varied with the year of infection. The prevalence of subtype $1 \mathrm{~b}$ decreased from $82.4 \%$ before 1993 to $62.1 \%$ after $1993(P=0.006)$, whilst the frequency of subtype 6a increased from $10.3 \%$ before 1993 to $25.4 \%$ after $1993(P=0.022)$. The multivariate logistic regression showed that subtype $1 \mathrm{~b}$ was independently associated with blood transfusion (OR 2.962, $P=0.012$ ) and appeared more frequently in patients aged over 40 years (OR $2.063, P=0.036$ ). Subtype 6 a was significantly less frequent in patients infected through blood transfusion (OR 0.174, $P=0.001$ ).

Conclusion: The prevalence of subtype $1 \mathrm{~b}$ decreased significantly and appeared more frequently in older chronic hepatitis $\mathrm{C}$ patients whose risk exposure mainly associated with blood transfusion. And the frequency of subtype 6a increased due to the rise of sources of IDU among younger patients. The new features in distribution were associated with changes in the modes of HCV acquisition in Southern China. 
Acknowledgements: This work was supported by grant from the National Natural Science Foundation of China (30771899).

\section{PP12-48}

Risk Factor for HCV Infection Among HIV Infected Patients in Sanglah General Hospital Bali, Indonesia

K. Mariadi, D.N. Wibawa, T. Parwati, A. Somia

Internal Medicine, Udayana University Faculty of Medicine,

Denpasar, Indonesia

Background: The estimated worldwide prevalence of Hepatitis C viral (HCV) infection is $2.2 \%$. Risk factors associated with acquiring $\mathrm{HCV}$ infection include transfusion of blood and blood products and transplantation of solid organs from infected donors, illegal injection drug use, unsafe therapeutic injections, occupational exposure to blood (primarily contaminated needle sticks), birth to an infected mother, sex with an infected partner, and sex with multiple partners. However, there are temporal and geographic differences in the extent to which these risk factors have contributed to HCV transmission. Among the estimated 40 million persons infected with Human immunodeficiency virus (HIV) worldwide, an estimated 4-5 million are chronically infected with HCV. This study aim to know the risk factor for HCV infection in HIV infected patients in Sanglah General Hospital Bali.

Methods: We performed a case-control study in HIV infected patients. We use Chi square test to know the significant risk factor for $\mathrm{HCV}$ infection.

Results: We involved 62 HIV patients in Sanglah General Hospital in this study. The sample was collected consecutively. Fifty five $(88.7 \%)$ out of 62 was male and the rest was female. The prevalence of HCV infection among HIV patients was $45.2 \%$ (28 out of 62 ). We found intravenous drugs user (IDU) and sexual transmission as a risk factors for HIV-HCV infection in $33(53.2 \%)$ and 29 (46.8\%) of patients,consecutively. The frequency of IDU and sexual transmission in HCV infected patients was 96.4 and 3.6\%, consecutively. IDU has a significant association with $\mathrm{HCV}$ infection $\left(\chi^{2} 38.27 ; p<0.001\right)$ with OR 125 (95\% CI 14.3-1,000) if compared with sexual transmission. Conclusion: IDU was significant risk factor for $\mathrm{HCV}$ infection among HIV infected patients.

\section{Poster Presentation 13: Hepatitis C: Treatment}

PP13-01

A Study on the Effect of China Healthcare Insurance Policy on the Quality of Life of Chronic Hepatitis C Patients

J. Liu, C.-S. Lin, L. Mai, L. Peng, Z.-L. Gao

Department of Infectious Diseases, The Third Affiliated Hospital of Sun Yat-sen University, Guangzhou, China

Objective: To evaluate the effect of PEG-Interferon $\alpha$-2a therapy on the quality of life(QOL) of patients with chronic hepatitis $\mathrm{C}(\mathrm{CHC})$. And to study the influence on the QOL of patients after PEG-Interferon $\alpha$-2a including in the China healthcare insurance.

Methods: A study based on $72 \mathrm{CHC}$ patients with PEG-Interferon $\alpha$ 2a plus Ribavirin therapy for one year and $30 \mathrm{CHC}$ patients (group C) without any antivirus treatment was carried out using the general quality of life inventory (GQOLI-74) questionaire. Theses 72 cases include group A $(n=42)$ whose medical expenses were in charge of China healthcare insurance and group $\mathrm{B}(n=30)$ at those own expenses. QOL of patients in these three groups were investigated twice using GQOLI-74 questionaire before (T0) and after PEGInterferon $\alpha$-2a plus Ribavirin therapy (T1). QOL of these patients were compared on $\mathrm{T} 0$ and $\mathrm{T} 1$, respectively.

Results: On T0, there were no statistic differences on all scales and total scores of the GQOLI-74 questionaire among these three groups
( $Z=-0.377$ to $1.177, P>0.05)$. On T1, group A had higher QOL on all scales and total scores than group $\mathrm{C}(P=0.000-0.006)$; group B had higher QOL $(P=0.004-0.024)$ on other scales and total scores except for material life $(P=0.305)$; group A had higher QOL on psychological function, material life and total scores than group B $(P=0.004-0.039)$. Group A on T1 had higher QOL on all scales and total scores than that on T0 $(P=0.002-0.031)$. Group B on T1 had higher QOL on Physical function, social function and total scores than that on $\mathrm{T} 1(P=0.025-0.043)$.

Comparison of QOL among three groups before PEG-In

\begin{tabular}{llllll}
\hline Groups & $\begin{array}{l}\text { Physical } \\
\text { function }\end{array}$ & $\begin{array}{l}\text { Psychological } \\
\text { function }\end{array}$ & $\begin{array}{l}\text { Social } \\
\text { function }\end{array}$ & $\begin{array}{l}\text { Material } \\
\text { life }\end{array}$ & $\begin{array}{l}\text { Total } \\
\text { scores }\end{array}$ \\
\hline $\begin{array}{c}\text { Group A } \\
(n=42)\end{array}$ & 55.33 & 58.75 & 61.88 & 60.63 & 58.45 \\
$\quad$ median & & & & & \\
$\begin{array}{c}\text { (interquartile } \\
\text { distance })\end{array}$ & $(50.2,64.9)$ & $(50.8,66.7)$ & $(55.6$, & $(52.0$, & $(52.6$, \\
$\begin{array}{c}\text { Group B } \\
\quad(n=30)\end{array}$ & 57.50 & 60.44 & 61.06 & 55.22 & 58.27 \\
$\quad$ median & & & & & \\
$\begin{array}{c}\text { (interquartile } \\
\text { distance })\end{array}$ & $(53.0,65.6)$ & $(52.5,68.5)$ & $(55.6$, & $(49.8$, & $(52.0$, \\
$\begin{array}{c}\text { GroupC } \\
\quad(n=30)\end{array}$ & 58.63 & 60.25 & $58.1)$ & $65.9)$ & $68.0)$ \\
$\quad$ median & & & & 54.82 & 56.38 \\
(interquartile & $(46.9,62.7)$ & $(54.8,66.9)$ & $(49.3$, & $(40.9$, & $(46.7$, \\
distance) & & & $63.3)$ & $61.7)$ & $64.6)$ \\
$Z$ value & -1.177 & -0.846 & -1.062 & -0.377 & -1.085 \\
$P$ value & 0.239 & 0.398 & 0.288 & 0.706 & 0.278 \\
\hline
\end{tabular}

Comparison of QOL among three groups after PEG-Int

\begin{tabular}{|c|c|c|c|c|c|}
\hline Groups & $\begin{array}{l}\text { Physical } \\
\text { function }\end{array}$ & $\begin{array}{l}\text { Psychological } \\
\text { function }\end{array}$ & $\begin{array}{l}\text { Social } \\
\text { function }\end{array}$ & $\begin{array}{l}\text { Material } \\
\text { life }\end{array}$ & $\begin{array}{l}\text { Total } \\
\text { scores }\end{array}$ \\
\hline $\begin{array}{l}\text { Group A } \\
\quad(n=42) \\
\text { median }\end{array}$ & 67.13 & 76.38 & 68.13 & 70.09 & 72.63 \\
\hline $\begin{array}{c}\text { (interquartile } \\
\text { distance) }\end{array}$ & $\begin{array}{c}(54.5 \\
75.3)\end{array}$ & $(58.2,79.3)$ & $\begin{array}{c}(61.6, \\
73.5)\end{array}$ & $\begin{array}{c}(52.4 \\
78.1)\end{array}$ & $(57.6,79.6)$ \\
\hline $\begin{array}{l}\text { Group B } \\
\qquad(n=30) \\
\text { median }\end{array}$ & 65.06 & 65.00 & 69.63 & 56.30 & 64.30 \\
\hline $\begin{array}{c}\text { (interquartile } \\
\text { distance) }\end{array}$ & $\begin{array}{l}(55.7 \\
68.1)\end{array}$ & $(58.6,68.9)$ & $\begin{array}{r}(57.8 \\
73.9)\end{array}$ & $\begin{array}{r}(51.0 \\
61.4)\end{array}$ & $\begin{array}{r}(49.2, \\
71.8)\end{array}$ \\
\hline $\begin{array}{l}\text { Group C } \\
\qquad(n=30) \\
\text { median }\end{array}$ & 54.56 & 54.00 & 53.31 & 57.53 & 54.64 \\
\hline $\begin{array}{c}\text { (interquartile } \\
\text { distance) }\end{array}$ & $\begin{array}{r}(46.3 \\
58.1)\end{array}$ & $(50.8,62.5)$ & $\begin{array}{r}(45.8 \\
58.1)\end{array}$ & $\begin{array}{r}(48.0, \\
67.6)\end{array}$ & $\begin{array}{r}(43.4 \\
59.8)\end{array}$ \\
\hline$Z$ value & -2.274 & -3.211 & -2.725 & -2.795 & -5.222 \\
\hline$P$ value & $0.008 ※$ & $0.003 ※$ & $0.006 \times$ & $0.005 ※$ & $0.000 \times$ \\
\hline
\end{tabular}

Conclusions: After PEG-Interferon $\alpha$-2a therapy, QOL of CHC patients is higher than before. Patients whose medical expenses were in charge of China healthcare insurance have higher QOL than patients at those own expenses.

PP13-02

Recombinant Erythropoietin in the Treatment of Ribavirin Induced Hemolytic Anemia in Chronic HCV Patients

A.F. Elhassi, B. Elhadad

Faculty of Medicine, Benghazi-Libya, Benghazi, Libya 
Aim: Hemolytic anemia is well known complication of treatment of chronic HCV by combination therapy with interferon including ribavirin. Dose reduction was first implemented to prevent this complication on the expense of viral response. Accumulating evidence now supports the use of recombinant human erythropoietin to manage this type of anemia in those patients. Our aim in this to determined the response of this type of anemia by treatment with erythropoietin.

Method: 146 patient of chronic HCV were eligible for treatment by combination therapy with interferon Alfa and ribavirin. All patients included were assessed for their base line hemoglobin level. Post 8 weeks anti HCV treatment hemoglobin and after four weeks from initial recombinant human erythropoietin therapy in the dose between 4,000-8,000 IU per week were measured.

Result: Hemoglobin level dropped by more than $3 \mathrm{~g} / \mathrm{dl}$ in initial 8 weeks of treatment in 75 patients $(51.3 \%)$. Their average base line $\mathrm{Hb}$ level was $12.8 \mathrm{~g} / \mathrm{dl}(13.8 \mathrm{vs}$. $12.7 \mathrm{~g} / \mathrm{dl})$ for male and female respectively in comparison to average post treatment $\mathrm{Hb}$ which was $10.0 \mathrm{~g} /$ $\mathrm{dl}(p<0.05)$. The drop was more in males than females $(3.5 \mathrm{vs}$. $2.8 \mathrm{~g})$.Post erythropoietin $\mathrm{Hb}$ level was $10.7 \mathrm{~g} / \mathrm{dl}$, the raise was by $0.4 \mathrm{~g} / \mathrm{dl}$ for males and $0.8 \mathrm{~g} / \mathrm{dl}$ for females $(P=0.0418) .11$ patient did not shows any response to erythropoietin therapy and stop anti HCV treatment.

Conclusion: Ribavirin induced anemia is a common finding in our $\mathrm{HCV}$ patients treated by combination This high rate of anemia probably due to large induction dose of ribavirin.The drop of hemoglobin was seen in the first 8 weeks of treatment and it was more in males than females. Drop out rate was clearly reduced when recombinant erythropoietin administered early in the course of treatment which preserve $\mathrm{Hb}$ level of at $10 \mathrm{~g} / \mathrm{dl}$ and prevent ribavirin dose reduction.

\section{PP13-03}

Investigation of Quality of Life in Patients with Chronic Hepatitis C after PEG-interferon $\alpha$-2a Therapy

C.S. Lin, J. Liu, L.H. She, Z.X. Zhao, Z.L. Gao

Department of Infectious Diseases, The Third Affiliated Hospital of Sun Yat-sen University, Guangzhou, China

Objective: To explore the quality of life (QOL) for the patients with chronic hepatitis $\mathrm{C}$ (CHC) and evaluate the intervention effect of PEG-Interferon $\alpha$-2a therapy on QOL in CHC patients.

Methods: A study based on 102 CHC patients (group A, before PEGInterferon $\alpha$-2a therapy, T0) and 44 healthy persons (group B) was carried out using the general quality of life inventory (GQOLI-74) questionnaire. QOL of questionnaire were compared between these two groups. Patients with group A were divided into group A1 (72 patients) with therapy by PEG-Interferon $\alpha$-2a plus Ribavirin for one year and group A2 (30 patients) without antivirus therapy. QOL of patients in these two groups were investigated using GQOLI-74 questionnaire at the end of PEG-Interferon $\alpha$-2a plus Ribavirin therapy (T1) and half year after the end of therapy (T2). QOL of CHC patients (group A1 and group A2) were compared at T0, T1 and T2 respectively.

Results: Compared with healthy controls, CHC patients had lower QOL $(Z=-2.069$ to $5.492, P=0.000-0.039)$ on other scales and total scores of theGQOLI-74 questionnaire except for psychological function $(Z=-1.508, P=0.132)$. Both T1 and T2, patients of group A1 had higher QOL on physical function, psychological function, social function and total scores than patients of group A2 at the same time $(Z=-2.274$ to $5.325, P=0.000-0.038)$. Patients of group A1 at $\mathrm{T} 1$ had higher QOL on physical function, psychological function, social function and total scores than those at T0 $(Z=-2.011$ to $-2.239, P=0.020-0.042)$. Patients of group A1 at T2 had higher QOL on social function than those at T1 $(Z=-2.645, P=0.018)$.
Conclusions: QOL of $\mathrm{CHC}$ patients is more impaired than healthy persons. PEG-Interferon $\alpha$-2a therapy will improve the QOL of the patients.

\section{PP13-04}

Spontaneous Weight Changes during HCV Treatment: Effects on Virological Response Rates

H.R. Alwakeel ${ }^{1}$, H.E. Zaghla ${ }^{1}$, N.A. $\mathrm{Omar}^{1}$, H.A. Alshinnawy ${ }^{1}$,

E.A. Rewisha ${ }^{1}$, L.E. Matarese $^{2}$, A.A. Taha ${ }^{3}$, H.M. Kandil ${ }^{4}$

${ }^{1}$ Hepatology, Nationtional Liver Institute, Shibin Alkom, Egypt;

${ }^{2}$ Surgery, University of Pittburgh Medical Center, Pittsburgh, PA, USA; ${ }^{3}$ Public Health, Medical College, Shibin Alkom, Egypt;

${ }^{4}$ Medicine, University of Pittsburgh Medical Center, Pittsburgh, PA, USA

Aim: We examined whether treatment associated weight loss during chronic hepatitis $\mathrm{C}$ therapy is associated with improved response.

Methods: Virological response rates were compared among patients with or without weight loss during $\mathrm{CHC}$ therapy.

Results: Among 438 who provided consent, 184 cases started CHC therapy and were included. Median pretreatment body mass index (BMI) was 27.7 (18.4-51.3) with $38 \%$ overweight and $31 \%$ obese (BMI 25-30 and $\geq 30$, respectively). Among patients with liver biopsy $(n=90)$, steatosis was present in $37(31.6 \%)$ and fibrosis grade was $1-2 / 6$ in $46 \%, 3-4$ in $37.3 \%$ and 5-6 in $14.7 \%$. After 1 and 3 months of treatment, 38 and $54.3 \%$ had BMI decrement of $\geq 0$ [ns1] $.5 \mathrm{~kg} /{ }^{2}$, with mean loss of $1.2 \pm 3.7$ and $2.6 \pm 5.2 \mathrm{~kg} /{ }^{2}$, respectively. For genotype 1, weight loss at 1 and 3 months was associated with higher EVR ( 90.9 vs. $71.0 \%$ at 1 month and 85.9 vs. $70.8 \%$ at 3 months, respectively, $p=0.01$ and 0.05 , respectively). RVR and SVR among those with or without weight loss at 1 month were 42.9 vs. $26.0 \%$ and 55.2 vs. $34.8 \%$, respectively, $p=0.08$ ). Independent predictors of response were: pre-treatment ALT and non-genotype 1 for RVR, weight loss at 1 month, non-genotype 1 and longer treatment for EVR and non-genotype 1, younger age, longer treatment, and lower pre-treatment RNA load for SVR.

Conclusion: Spontaneous weight loss during the first 3 months of IFN therapy was associated with improved EVR and ETR in patients with CHC genotype 1 . We postulate that weight loss programs at the beginning of CHC therapy may improve treatment response

\section{PP13-05}

Inhibitory Effect of Recombinant Full Length Camel Lactoferrin and its N-lobe on Hepatitis C Virus Infection of Hepatoma

E.M. Redwan ${ }^{1}$, Y. Liao ${ }^{2}$, E. El-Fakkarany ${ }^{1}$, B. Lönnerdal ${ }^{3}$

${ }^{1}$ Protein Research, Mubarak City for Scientific Research and

Technology Applications, Alexandria, Egypt; ${ }^{2}$ Nutrition; ${ }^{3}$ Department of Nutrition, University of California, Davis, CA, USA

Objectives: Lactoferrin has been shown to have anti-viral activity against hepatitis $\mathrm{C}$ virus (HCV). The objective of this study was to examine and compare the effects of recombinant and native camel lactoferrin as well as their $\mathrm{N}$ - and $\mathrm{C}$-terminal fragments on continuous steps of HCV infection in HepG2 cells.

Methods: Native camel lactoferrin was purified by heparin-sepharose beads. Proteolytically generated $\mathrm{N}$ - and C-lobes from native fulllength camel lactoferrin were purified by heparin-sepharose beads and Sephadex G-50 chromatography. Recombinant camel lactoferrin and its fragments were synthesized by a Bac-to-Bac baculovirus expression system. The inhibitory effects on HCV entry into HepG2 cells were evaluated by incubation of $\mathrm{HCV}$ with lactoferrin prior to infection or pretreatment of the cells with lactoferrin prior to infection. Inhibitory effect on HCV amplification in HepG2 cells was determined by lactoferrin treatment of HCV infected cells. RT-nested PCR was performed to amplify intracellular HCV $5^{\prime}$ non-coding RNA sequences. 
Results: Native and recombinant camel lactoferrins and their fragments were purified, but the C-lobe of recombinant camel lactoferrin was insoluble. Recombinant and native camel lactoferrin and their fragments could prevent HCV entry into HepG2 cells by direct interaction with the virus instead of causing significant changes in the target cells. They were also able to inhibit virus amplification in HepG2 cells infected with HCV.

Conclusions: Camel lactoferrin is an effective novel anti-HCV molecule. The $\mathrm{N}$ - and $\mathrm{C}$-lobes of native camel lactoferrin are both sufficient to elicit anti-HCV effects in HepG2 cells. Recombinant camel lactoferrin and its N-lobe displayed similar HCV inhibitory effects as their native counterpart, and may constitute an efficient and cost-effective approach for potential clinical appliations.

\section{PP13-06}

Caucasians versus Egyptians HCV-4 Patients with Elevated Baseline HCVRNA Treated with PEG-IFN $\alpha 2 a$ and Ribavirin. Role of RVR and EVR

D. Dimitroulopoulos ${ }^{1}$, I. Elefsiniotis ${ }^{2}$, D. Xinopoulos ${ }^{1}$, K. Tsamakidis ${ }^{1}$,

S. Patsavela ${ }^{1}$, D. Kypreos ${ }^{1}$, A. Ferderigou ${ }^{3}$, D. Korkolis ${ }^{4}$, S. Koutsounas ${ }^{5}$,

E. Paraskevas ${ }^{1}$

${ }^{1}$ Gastroenterology, Agios Savvas Hospital; ${ }^{2}$ Internal Medicine,

"Helena Venizelou" Hospital - University of Athens; ${ }^{3}$ Biochemistry;

${ }^{4}$ First Surgical Department, Agios Savvas Hospital; ${ }^{5}$ Reference

Center for Viral Hepatitis, IKA, Athens, Greece

Background: Despite the recent spread of hepatitis $\mathrm{C}$ virus genotype 4 (HCV-4) into European countries, very little is known about the influence of ethnicity on treatment outcomes in patients with HCV-4. Aim: The aim of the study was to compare the virologic response rates: rapid (RVR), early (EVR), at 24 weeks of treatment, at the end of treatment and sustained (SVR), between two groups, Caucasians and Egyptians, of HCV-4 patients.

Patients and methods: Sixty (30 Caucasians - Group A and 30 Egyptians - Group B) chronic HCV-4, subtype a, adult patients with elevated baseline viral load $(>800,000 \mathrm{IU} / \mathrm{m} \mathrm{L})$ were treated for a fixed period of 48 weeks with PEG-IFN $\alpha$-2a and ribavirin. During the study HCV-RNA levels were measured at weeks 4, 12, 24, 48 and 72. Results: Baseline characteristics, including liver histology, were similar between the two groups. SVR rates were $36.7 \%$ (11/30) for Group A and $30.0 \%(9 / 30)$ for Group B $(p=0.784)$. No statistically significant differences were observed comparing RVR, EVR and HCV-RNA negativity at weeks 24 and 48 between Group A and Group B (26.7 vs. $30.0 \% p=1.000,50.0$ vs. $46.7 \% p=1.000,53.3$ vs. $43.3 \% p=0.605$ and 56.7 vs. $36.7 \% p=0.196$, respectively). For group B, RVR was weakest indicator for SVR as compared with RVR of group A.

Conclusion: Ethnic origin non influence the treatment outcomes nor the intermediate response rates of chronic $\mathrm{HCV}-4 \mathrm{a}$ infected patients with elevated baseline HCV-RNA.

\section{PP13-07}

Transfer Factor Activation of the Natural Killer Cell in Patients with Chronic Hepatitis C Infection

A.A. Abd Elmoety ${ }^{1}$, S.A. Zaki ${ }^{2}$, A.A.E.m. Soliman ${ }^{1}$, Z. Mourad ${ }^{3}$, N. Farahat ${ }^{3}$ ${ }^{1}$ Hepatology, Faculty of Medicine; ${ }^{2}$ Microbiology, Faculty of Medicine; ${ }^{3}$ Clinical Pathology, Faculty of Medicine, Alexandria University, Alexandria, Egypt

Background: Infection with hepatitis $\mathrm{C}$ virus (HCV) is a leading cause of chronic liver disease, little is known about how this virus is able to persist or whether this persistance might be because of its ability to alter the early innate immune response. The major HCV envelope protein $E_{2}$ has been shown to bind to CD81, this leads to restricted cytotoxicity mediated by NK cells. Transfer factor advanced formula plus is formed of bovine and egg colostrum and found to increase NK cell activity by $437 \%$ above the base line. It is produced by 4 life research company/USA. Also, it contains growth factors as insulin growth factors (IGF I), (IGF II), Transforming growth factor beta (TGF-B) and epidermal growth factor (EGF).

Objectives: Assessment of Natural Killer (NK) cell activation by transfer factor in patients with chronic $\mathrm{HCV}$ infection whom not candidate for standard therapy.

Material: 30 patients with chronic $\mathrm{HCV}$ infection whom are not candidate for standard therapy.

Methods: History and clinical examination. Liver function tests. Viral markers (HBS Ag, HCV Ab). (4) HCV RNA by PCR in the serum. (5) Flow cytometric analysis of NK cells in blood sample. (6) Patients were given transfer factor plus capsules twice daily before meals for 3 months then re-evaluation was done.

Significant reduction of Mean alanine aminotransferase (ALT) from $79.27 \pm 71.47$ to $30.53 \pm 6.21$, aspartate aminotransferase (AST) from $80.73 \pm 46.88$ to $36.40 \pm 3.23$ and serum Bilirubin from $1.58 \pm$ 0.72 to $0.86 \pm 0.33$. Significant elevation of serum albumin from 3.19 \pm 0.70 to $3.59 \pm 0.54$ and No significant change in serum HCV RNA by PCR nor NK cell count by flow cytometry.

Conclusion: Transfer factor advanced formula plus is an effective new therapeutic option for patients with chronic HCV infection who are not candidate for standard therapy.

\section{PP13-08}

The Desease and Management of Acute Hepatitis C

H.L. Ghazinyan, H. Ghazinyan, A. Asoyan, T. Asatryan,

G. Melik-Andreasyan

Hepatology Department, Nork Clinical Hospital of Infectious

Deseases, Yerevan, Armenia

Background: Symptomatic acute hepatitis C occurs in only $15 \%$ of patients who are infected with hepatitis $\mathrm{C}$ virus. The symptoms are often mild and not specific just acute hepatitis $\mathrm{C}$ making the diagnosis less possible.

The aim of this study was to present description and management of acute hepatitis $\mathrm{C}$.

Methods: From 2007-2009 294 patients with hepatitis C, acute hepatitis $\mathrm{C}$ were diagnosed in $25(8.5 \%)$ of them. The mean age of them was 45 years (18-72). 21 (84\%) were men. The average time from exposure to the first signs of hepatitis was 67 days (14-120). The observation period for the possibility of spontaneous viral clearance was chosen 12 weeks. 11 of 25 patients were treated with pegylated interferons for 12 weeks.

Results: All 25 patients were symptomatic with jaundice and elevated serum bilirubin levels (median 168 mcmoll, 36-360). Most common symptoms included weakness, abdominal discomfort, and flu-like symptoms. The median ALT level was 1,915 IUl (500-3,330). The predominany risk factor for acute hepatitis C was Iv drug use $48 \%$. The other common risk factors were medical procedures $28 \%$, sexual exposure $12 \%$, unknown $12 \%$. HCV genotypes $1(44 \%)$ and $3(36 \%)$ were predominant. Baseline viral load was $>800,000 \mathrm{IUml}$ in 6 patients and $<800,000 \mathrm{IUml}$ in 5 patients. $9(36 \%)$ out of 25 patients had spontaneous resolution during the observation period. $9(82 \%)$ out of 11 patients, who had received antiviral treatment, achieved rapid viral response and $10(91 \%)$ sustained viral response, without regarding baseline viral load and viral genotype.

Conclusions: Symptomatic acute hepatitis $\mathrm{C}$ was mostly associated with I/v drug use and had high rates of spontaneous resolution. Early treatment with PEGIFN-alpha 12 week's duration had $91 \%$ sustained viral response. No correlation was found between treatment response and baseline viral load and viral genotype. 


\section{PP13-09}

Comparative Efficacy and Overall Safety of Different Doses of Consensus Interferon for Treatment of Chronic HCV Infection: Meta-analysis

S.-M. Alavian, B. Behnava, S.V. Tabatabaei

Baqiyatallah University of Medical Sciences, Baqiyatallah Research Center for Gastroenterology and Liver Disease, Tehran, Iran

Background: About half of genotype 1 and one third of genotype 2/3 infections have treatment failure with peginterferon alpha and ribavirin. Consensus Interferon (CIFN) is an option for retreatment of these patients.

Methods: We did a search Medline, Scopus, ISI and Cochran central registry of clinical trials for randomized clinical trials for different regimens of CIFN and we included the HIV and HBV seronegative patients with positive HCV-RNA during 6 months before starting the study. Studies were appraised based on methods of random sequence generation, allocation concealment and blinding. Random effects model of Dersimonian and Laired method was employed to run metaanalysis. The end point was sustained virological response (SVR).

Results: Data of 10 RCTs containing 1600 subjects were extracted. High daily induction dose regimen of CIFN did not yield higher rate of SVR than Low daily induction dose treatment regimen, $\mathrm{RR}=0.83$ (95\% CI 0.58-1.17). $9 \mu \mathrm{g}$ thrice weekly (tiw) was associated with significantly higher rate of SVR compared with $3 \mu \mathrm{g}, \mathrm{RR}=3.14(95 \%$ CI 1.68-5.58). Withdrawal rate was similar $\mathrm{RR}=1.28$ (95\% CI $0.65-$ 2.50) but dose modification was higher in $9 \mu \mathrm{g} \mathrm{RR}=3.22(95 \% \mathrm{CI}$ $1.08-9.60) .18 / 15 \mu \mathrm{g}$ tiw was not more effective than $9 \mu \mathrm{g}$ with similar treatment duration RR $=1.02(95 \%$ CI $0.87-1.19)$ inadequate reporting of methodological information and side effects, lack of publication bias assessment and sensitivity analysis due to small number of studies in each analysis were our limitation in this study.

Conclusions: High dose daily induction therapy with CIFN is not superior to low dose therapy in terms of SVR. It seems that $9 \mu \mathrm{g}$ t.i.w is optimal treatment dose of CIFN for treatment of HCV infection. Optimal duration and safety profile of CIFN therapy are not elucidated yet.

\section{PP13-10}

Outcomes of CINF/Riba for Chronic Hep C G 3 Patients Who Relapsed with Previous Peg INF/Riba Therapy

U. Adeeb, M.L. Gill

Division of Digestive Diseses, Maroof International Hospital, Islamabad, Pakistan

Aims and objectives: There are not many studies about the efficacy of C IFN and Riba combination in chronic hep C(G3) relapsers wiith Peg INF Riba combination therapy. To evaluate the efficacy of cons INF and Riba in patients previously treated with Peg interferon and Riba combination therapy. We consecutively enrolled 70 patients to study the efficacy of Con INF and Riba.

Methods: The study was conducted between Feb 2009 to July 2010. Inclusion criteria: Chronic hep C G 3 relapsers with Peg INF and Riba therapy for at least 6 months.

Exclusion criteria: Active psychiatric history, platelet count $<75,000$, INR $>1.5$, S. Albumin $<2.5$, portal vein diameter $>13 \mathrm{~mm}$ on ultrasound.

All enrolled patients have CBC, ALT, HCV PCR, HCV genotype, thyroid function tests and blood sugar at baseline. Patients were given $15 \mathrm{ug}$ thrice weekly CINF and Riba $400 \mathrm{mg}$ bid.The treatment duration was for 9 months. Patients were reviewed 4 weekly basis for Clinical exam, CBC and TSH level, HCV PCR quantitative @week 12. The patients who had HCV PCR negative or $50 \%$ reduction in HCV PCR at week 12 were given further 6 months of therapy.

Results: Among 70 patients 45 were male and 25 were female. Median age was 45 .

60 out of $70(85 \%)$ patients were HCVPCR negative @ week 12 of therapy. These 60 patients completed further 24 weeks of therapy and remained HCVPCR negative @ end of total 24 weeks of therapy. Five patients stopped treatment because of side effects and financial constrains.

Conclusion: C INF (infergen) Riba combination is very optimistic option for HEP C G 3 relapsers with Peg INF Riba combination. We did give 9 months of therapy because of financial consideration. Perhaps one year therapy will be most optimal duration.

PP13-11

Optimizing Outcomes with Epoetin Beta during Treatment with Pegylated Interferon and Ribavirin in Chronic Hepatitis C Patients: Experience from Thailand

T. Tanwandee ${ }^{1}$, P. Charatcharoenwitthaya ${ }^{1}$, S. Chainuvati ${ }^{1}$, W. Chotiyaputta ${ }^{1}$, S. Nimanong ${ }^{1}$, P. Pamornpiboon ${ }^{2}$, I. Sukarom ${ }^{2}$

${ }^{1}$ Division of Gastroenterology, Department of Medicine, Faculty of Medicine, Siriraj Hospital, Mahidol University; ${ }^{2}$ Medical

Department, Roche Thailand Ltd., Bangkok, Thailand

Background/aims: Successful treatment of chronic hepatitis $\mathrm{C}$ (CHC) with pegylated interferon plus ribavirin (Peg-IFN + RBV) is often limited by anemia which can lead to dose reduction or discontinuation of treatment. The aim of this study was to determine the impact of epoetin beta (EPO-beta) on treatment outcomes in $\mathrm{CHC}$ patients receiving Peg-IFN + RBV in routine practice.

Methods: At Siriraj Hospital, medical records of $\mathrm{CHC}$ patients receiving Peg-IFN + RBV during January 2005-February 2009 were retrospectively reviewed. Patients were included if they were treatment naïve, completed standard course of Peg-IFN + RBV, and had available 24-week follow-up data. Data on baseline characteristics, treatments and outcomes were captured.

Results: 149 patients were included. 85 patients did not receive EPO-beta (non-EPO-beta group) while 64 patients received EPO-beta (EPO-beta group). 94\% in EPO-beta group received EPO-beta after the start of Peg-IFN+RBV with a median time to start of 2 months. Patients in EPO-beta group were significantly older, were likely to be female, and had lower weight than those in non-EPO-beta group. Hemoglobin levels $(\mathrm{Hb})$ at baseline and end of follow-up were significantly lower in EPO-beta group (13 vs. $14.4 \mathrm{~g} / \mathrm{dL}$ and 12 vs. 13.8 $\mathrm{g} / \mathrm{dL}$ ) which resulted in significantly higher change in mean $\mathrm{Hb}$ in this group. Change in mean RBV dose from start to end of treatment was significantly higher in EPO-beta group. Proportion of patients who could maintain RBV dose was significantly higher in non-EPO-beta group (75.3 vs. $42.2 \%)$. Proportions of patients in EPO-beta and nonEPO-beta groups who achieved sustained virological response (SVR 74.6 vs. $78 \%$ ), early virological response (EVR 92.9 vs. $93.8 \%$ ), and end of treatment response (EOTR 91.9 vs. 94.4\%) were not significantly different.

Conclusions: Patients with poorer prognosis were likely to receive EPO-beta to maintain $\mathrm{Hb}$ throughout the course with Peg-IFN+RBV. Treatment outcomes, which were SVR, EVR, EOTR, were likely to be maintained by using EPO-beta.

\section{PP13-12}

Risk Factors For Non Response to Standard Interferon and Rivavirin Therapy in Chronic HCV Gynotype 3 in Bahawalpur (Punjab Pakistan) M.A. Qazi ${ }^{1}$, H. Raza ${ }^{1}$, A. $\operatorname{Imran}^{1}$, S. Anjum ${ }^{1}$, G.M.-U.-d. Chaudhary ${ }^{2}$

${ }^{I}$ Medicine, Quaid-e-Azam Medical College/Bahawal Victoria Hospital, Bahawalpur; ${ }^{2}$ Medicine, Nishtar Medical College, Multan, Pakistan

Aim: To find out the risk factors associated with non-response to combination therapy in patients with chronic $\mathrm{HCV}$ infection genotype 3.

Methods: An experimental descriptive study, conducted in Bahawal Victoria Hosptial/Quaid-e-Azam Medical College, Bahawalpur, Pakistan (1 July 2007-15 August 2009). A total of 455 patients who were PCR qualitative positive for HCV RNA with genotype 3 . Complete history, physical examination, laboratory tests and 
ultrasonography was done in all the patients. Treatment with standard interferon 3MIU thrice a week and ribavirin 1,000-1,200 mg daily was started. By the end of 3 months, HCV RNA PCR (qualitative) was done for early virological response (EVR) and the patients were labeled as non-responders who were positive for HCV RNA PCR. The following variables were analyzed: age, sex, body mass index (BMI), area of residence, marital status, diabetes mellitus (DM) and platelet count. Two Groups were made: Group-A, HCV RNA PCR (qualitative) negative and Group-B, HCV RNA PCR (qualitative) positive (non-responders). Both groups were compared. Statistical analysis was done using SPSS version 17 and Chi square test was applied for statistical significance.

Results: The age range was $18-65$ years $($ mean $=36.29)$. Majority of the patients were $<40$ years $(60.9 \%)$, males $(65.3 \%)$, BMI $<25$ $(58.9 \%)$, married $(80 \%)$, from rural area $(69.9 \%)$ and without DM $(87.5 \%)$. EVR was noted in $85.5 \%$. When both groups were compared, EVR was reduced in Group-B with BMI $\geq 25(p<0.001)$, belonging to rural area $(p=0.010), \mathrm{DM}(p=0.033)$ and platelet count $<150(p=0.004)$ which was statistically significant whereas all other variables were insignificant.

Conclusion: Treatment of chronic HCV infection genotype 3 with combination therapy is successful but EVR is reduced in patient with $\mathrm{DM}$, BMI $\geq 25$, decreased platelet count and patients from rural area.

\section{PP13-13}

IL28B Genotype Distribution and Treatment Outcome among Patients with Chronic Hepatitis C (CHC): Impact Evaluation in a Mediterranean Population

S. Madonia ${ }^{1}$, M.C. Renda ${ }^{2}$, R. Ruggeri ${ }^{1}$, G. Malizia ${ }^{3}$, G. Perricone ${ }^{1}$, E. Sinagra ${ }^{1}$, S. Plano ${ }^{1}$, G. Giannuoli ${ }^{3}$, F. Tine, ${ }^{3}$, M. Olivo ${ }^{1}$, A. Maggio ${ }^{2}$, M. Cottone ${ }^{1}$

${ }^{1}$ UOC Medicina II, Ospedali Riuniti 'Villa Sofia-Cervello'; ${ }^{2}$ UOC Ematologia II, Ospedali Riuniti "Villa Sofia-Cervello"; ${ }^{3}$ UOC Gastroenterologia, Ospedali Riuniti Villa Sofia-Cervello, Palermo, Italy

Background and aims: Different studies described the role of IL28B-polymorphism in predicting treatment outcome among patient with CHC. Alleles distribution varies in different ethnic groups contributing to differences in sustained response (SVR) observed in different populations.

The aim of the study is to analyze IL28B genotypes in our population and impact on SVR.

Patients and methods: All CHC patients consecutively observed at our department between May 1st and July 31st 2010, who had completed treatment with PEG-interferon/ribavirin according to the assigned schedule and dosage and had a post treatment follow up of at least 24 weeks were included.

Genomic DNAs were extracted from peripheral blood. IL28B -3kbC $>\mathrm{T}$ and $\mathrm{K} 70 \mathrm{R}(\mathrm{A}>\mathrm{G})$ variants were investigated by direct genomic sequencing, endonuclease digestion and reverse dot-blot hybridization. PCR primers were home-made on the basis of IL28B map (GENATLAS) and FASTA-SNP sequences.

Results: 108 patients were included: 70 males; $84 \mathrm{G} 1 ; 38$ cirrhotics. IL28B $-3 \mathrm{kbC}>\mathrm{T}$ and K70R $(\mathrm{A}>\mathrm{G})$ variants resulted CC/AA in 32 (29.6\%), CT/AG in $63(58.3 \%)$ and TT/GG in $13(12 \%)$.

Overall, 58 had a SVR (53.7\%), 21 relapsed (19.4\%), 24 were nonresponders $(22.2 \%)$ and 5 had a breaktrough $(4.6 \%)$.

Thirthy-one/32 with a CC/AA genotype obtained a SVR $(96.8 \%)$ compared with $21 / 63$ with CT/AG $(33.3 \%)$ and 6/13 with TT/GG (46\%). Frequencies of CC/AA genotype resulted $53.4 \%(31 / 58)$ among SVR and $20 \%$ (1/5) among patients with breaktrough. No CC/
AA patients were observed among 21 relapsers and 24 nonresponders.

Among 81 G1 patients, 23/35 with SVR were CC/AA (65.7\%), compared with 0/46 without SVR.

Twenty-nine patients had been previously treated without SVR, 4 CC/AA (13.7\%) and 25 CT/AG or TT/GG (89.2\%).

Conclusions: In our population, one-third of $\mathrm{CHC}$ patients have a favorable IL28B genotype. The role of IL28B genotype in predicting treatment outcome in CHC patients is confirmed, especially in G1 patients.

\section{PP13-14}

Treatment Outcomes for Chronic HCV Therapy among HCV Monoinfected and HIV/HCV Co-infected Patients in a Community-based Clinic, Vancouver, Canada

J. Farley, T. Maung Maung, A. Truong, E. Paripovic, S. Mayronne,

T. Nguyen, W. Shum

Dr. John Farley Inc, Vancouver, BC, Canada

Introduction: Because of similar risk settings, Hepatitis C Virus (HCV) and Human Immuno-deficiency Virus (HIV) co-infection is relatively common. $\mathrm{HCV}$ treatment is effective; those with coinfection respond less well however. The aim of this study was to evaluate outcomes of HCV therapy in HCV mono-infected and HCV/ HIV co-infected individuals treated at our clinic.

Methods: Retrospectivechart reviews of 526 patients who completed HCV therapy (cases). Data analysis was performed using PASW. Univariate analysis was done comparing HCV treatment outcomes between the two groups. For significance tests, chi-square test with cut-off $p$ value $<0.05$ was used.

Results: $87.6 \%$ were HCV mono-infected and $12.4 \% \mathrm{HIV} / \mathrm{HCV}$ coinfected. $81 \%$ were males and $19 \%$ females. $58 \%$ were Genotype 1; $12.7 \%$ Genotype 2; $27.9 \%$ Genotype 3 and $1.4 \%$ others. Mean age was 43.24 years. Mean HCV viral load was 1826091.96 . There were no significant differences in age, HCV viral load, gender or genotype distribution between two groups. The discontinuation of treatment, lost to follow up, and relapse rates were similar. HIV co-infected cases was less likely to attain SVR (OR 0.329, $P$ value 0.000 ), more likely to be non-responders (OR $0.951, P$ value 0.019 ), and, to be reinfected after SVR (OR 2.430, $P$ value 0.009).

Treatment outcome for HCV therapy

\begin{tabular}{lllll}
\hline & $\begin{array}{l}\text { HCV/HIV } \\
\text { co-infected } \\
\text { patients } \\
(N=65)\end{array}$ & $\begin{array}{l}\text { HCV } \\
\text { mono- } \\
\text { infected } \\
\text { patients } \\
(N=461)\end{array}$ & $\begin{array}{l}\text { Odds } \\
\text { ratio }\end{array}$ & $\begin{array}{l}P \text { value } \\
\text { (Chi square) }\end{array}$ \\
\hline $\begin{array}{l}\text { Non-responder } \\
\begin{array}{l}\text { Sustain virological } \\
\text { response (SVR) }\end{array}\end{array}$ & $10(15.4 \%)$ & $74(16.0 \%)$ & 0.951 & 0.019 \\
$\begin{array}{l}\text { Re-infection after SVR } \\
\text { (2) }\end{array}$ & $13(20 \%)$ & $273(59.2 \%)$ & 0.329 & 0.000 \\
\hline
\end{tabular}

Discussion: Overall SVR rates in theHIV/HCV co-infected cases (32.2\%) were lower in the HCV mono-infected. Our results compare favourably with regstration trials, and encouraging from public health and clinical perspectives. Unfortunately, HCV re-infections in coinfected individuals are not insignificant and are likely associated with IDU. For more effective HCV treatment programs for HIV/HCV co-infected individuals, harm-reduction intervention strategies must be emphasized. 


\section{PP13-15}

Efficacy and Tolerability of Pegylated Interferon Plus Ribavirin Therapy in Thai Patients with Chronic Hepatitis C: Real World Practice

T. Jongboonyanuparp ${ }^{1}, \mathrm{~T}$. Witeerungrot ${ }^{2}, \mathrm{~N}$. Chamroonkul ${ }^{1}$,

N. Netinatsunton ${ }^{2}$, J. Sottisuporn ${ }^{2}$, T. Piratvisuth ${ }^{2}$

${ }^{1}$ Gastroenterology Unit, Department of Internal Medicine; ${ }^{2} \mathrm{NKC}$ Institute of Gastroenterology and Hepatology, Prince of Songkla University, Songkla, Thailand

Background: Chronic hepatitis $\mathrm{C}$ infection is one of the leading causes of liver-related morbidity and mortality in Thai patients. Most of the studies of chronic hepatitis $\mathrm{C}$ have been reported from Western countries. There are many different factors between Caucasian and Thai patients which can affect the treatment response such as genotype, BMI and genetic.

Aim: To evaluate the efficacy and tolerability of pegylated interferon and ribavirin therapy in Thai patients.

Methods: We retrospectively evaluated 150 chronic hepatitis C patients who had completed essential data and treated with pegylated interferon alfa $2 \mathrm{a}$ or $2 \mathrm{~b}$ plus ribavarin at Songklanagarind hospital from 2004-2010.

Results: Among 150 patients, 52\% were male, mean age of 45.6 years, $41.3 \%$ had cirrhosis and $58.7 \%$ had no cirrhosis. HCV genotype 3 is the most common genotype seen in $67.3 \%$ of our patients. The rest were infected with genotype 1 and other genotypes in 30.7 and $2 \%$, respectively. The treatment duration was usually 24 weeks in genotype 3 and 48 weeks in genotype non 2 and 3. Response guided therapy has been applied to our patients since 2007. SVR was achieved in $68.7 \%$ of all patients. SVR was $71.3 \%$ in genotype 3 and $60.9 \%$ in genotype 1 . SVR was higher in patients without cirrhosis (78.4\%) than in those with cirrhosis $(58 \%)$. In patients without cirrhosis SVR was achieved $79.6 \%$ in genotype 3 and $74.2 \%$ in genotype 1 . There were 2 patients $(1.3 \%)$ who could not tolerate the side effects resulted in treatment discontinuation.

Conclusions: Pegylated interferon plus ribavirin therapy is effective in Thai patients with chronic hepatitis C with overall SVR of $68.7 \%$. Genotype 3 has better SVR than genotype 1, 71.3 versus $60.9 \%$. Patients without cirrhosis have a higher SVR than those with cirrhosis. Despite having a lot of side effects, Peginterferon plus ribavirin is well tolerated.

\section{PP13-16}

Predictive Factors of Sustained Virological Response in Treated-HCV Carriers in Moroccan Patients

Y. Cherradi ${ }^{1}$, A. Rajaa ${ }^{1}$, H.O. El Malki ${ }^{2}$, H. Benbrahim ${ }^{1}$, M. Benazzouz ${ }^{1}$, A. Essaid ${ }^{1}$

${ }^{1}$ Department of Hepatogastroenterology-Médecine"C", Ibn Sina

Hospital; ${ }^{2}$ Laboratory of Clinical Research, Mohammed V University, Rabat, Morocco

Introduction: Hepatitis "C" (HCV) is a real public health problem. Antiviral treatment involved its management and improved patients' prognosis. Sustained virological respone (SVR) is the major treatment endpoint. The aim of this study is to define predictive factors of SVR in Moroccan population.

Patients and methods: It's a monocentric study. We included 266 consecutives HCV-carriers treated in our department by pegylated INF and ribavirine with at least 72 weeks-follow up. We considered age of HCV diagnosis, gender, body masse index values (BMI), initial serum ALT, baseline viral loads (BVL), genotype and cumulated doses of peg INF and ribavirine. Data were then analyzed statistically.

Results: On our patients, $52 \%$ patients were female and $48 \%$ male. Mean age is 55 years. Genotypes 1 and 2 are the more prevalent (respectively 52 and $45.5 \%$ ), although genotypes 3 and 4 have been found. $44.3 \%$ of patients presented severe fibrosis (F3-F4) at diagnosis and 55\% had high baseline viral loads. SVR was achieved in $70 \%$ of our patients. Age $<50$ years and male gender were very significant factors of SVR (respectively $p=0.004$ and $p=0.005$ ). They are independently predictors of SVR. Risk of non-SVR is respectively 2 and 2.3 times more important in patients aged over 50 years and female gender $(\mathrm{OR}=2.3$, IC $1.3-4, \quad \mathrm{OR}=2,3$, $\mathrm{IC}=1-3.5)$. BMI $>25 \mathrm{~kg} / \mathrm{m}^{2}$, high BVL and initial cytolysis were not significant predictive factors of SVR (respectively $p=0.49$, $p=0.35$ and $p=0.8$ ). Genotype 1 is significantly more associated to SVR than 2. Cumulated doses of peg INF and ribavirine- which is an objective indicator of treatment adherence- and cEVR were significant factor of SVR (with respectively $p=0.001$ and $p=0.002$ ).

Conclusion: In our series, genotypes 1 and 2 are more prevalent. Male gender, age $<50$ years at diagnosis, genotype 1 , treatment adherence and cEVR are significant predictive factors of SVR. They must be particularly considered in our practice.

\section{PP13-17}

Interferon and Ribavirin Therapy for Chronic Hepatitis

C (HCV) in Patients with Sickle Cell Anemia (SCA): Safety, Tolerability and Efficacy

H. Issa

Internal Medicine/Gastroenterology Section, King Fahad Specialist Hospital-Dammam, Dammam, Saudi Arabia

Aim: Sickle cell anemia is one of the common hemoglobinopathies in the world and one of the common complications of SCA is chronic hepatitis C. Ribavirin however is considered to be contraindicated in patients with hemolytic anemia. This study evaluates the safety, tolerability and efficacy of pegylated interferon and full dose of ribavirin for the treatment of $\mathrm{HCV}$ in patients with SCA.

Patients and methods: Fifty-nine consecutive SCA patients with $\mathrm{HCV}$ were evaluated. All were treated with pegylated interferon and standard full dose of ribavirin.

Results: Fifty-nine (35 F:24 M) SCA patients were treated. Their mean age was 29 years (15-54 years). All tolerated the treatment well and none dropped their $\mathrm{Hb}$, required blood transfusion pre, during or after therapy. There were no hematological side effects attributable to ribavirin at the usual recommended full dose. Their mean $\mathrm{Hb}$ at the start of treatment was $10.1 \mathrm{~g} / \mathrm{dl}(7.5-11.5 \mathrm{~g} / \mathrm{dl})$, at 3 months was 10.2 $\mathrm{g} / \mathrm{dl}(8.9-11.2 \mathrm{~g} / \mathrm{dl})$, at 6 months was $10.4 \mathrm{~g} / \mathrm{dl}(8.5-11.9 \mathrm{~g} / \mathrm{dl})$ and at the end of treatment was $10.3 \mathrm{~g} / \mathrm{dl}(8.2-11.7 \mathrm{~g} / \mathrm{dl})$. None of the patients experienced major voaso-occlusive crisis during therapy.Forty-three (72.9\%) achieved SVR. The remaining 16 were nonresponders. They had a relatively significant HCV-RNA viral load and eleven of them had genotype 1, four had genotype 4, and one genotype 5 .

Conclusions: Patients with SCA and HCV can be treated with pegylated interferon and full dose of ribavirin. The treatment is safe with excellent hematological tolerance and the response rate is comparable to those without SCA. This study is the largest to be reported to date with excellent hematological tolerance to full dose of ribavarin among SCA patients.

\section{PP13-18}

24-week Therapy in Rapid Virologic Responders for Chronic Hepatitis C Genotype 1 Patients

M.N. Kim, J.Y. Park, D.Y. Kim, S.H. Ahn, C.Y. Chon, K.-H. Han Yonsei University College of Medicine, Seoul City, Republic of Korea

Background and aims: A rapid virologic response(RVR) at 4 weeks has been the single best predictor of a sustained virologic response(SVR) and several studies have demonstrated that shortening the length of therapy to 24 weeks in patients with RVR at week 4 has been effective as the standard 48 -week regimen in hepatitis $\mathrm{C}$ virus genotype $1(\mathrm{HCV}-1)$ patients. We assessed whether 24-week regimen 
is as effective as standard treatment in HCV-1 patients with RVR in Korea.

Methods: A total 45 patients were treated with peginterferon alpha2a or alpha-2b and ribavirin between May 2005 and June 2009. RVR was defined as PCR-negative serum HCV RNA at 4 weeks of therapy. Patients with RVR were treated for either 24 or 48 weeks by open randomization and patients without RVR were treated for 48 weeks, with a 24-week follow-up period.

Results: Among 45 patients, 27 patients (60\%) had RVR and 29 patients (64.4\%) had SVR. Among 27 patients with RVR, 22 patients $(81.5 \%)$ had a SVR and among 18 patients without RVR, 7 patients $(38.9 \%)$ had a SVR. The SVR rate was significantly higher in patients with RVR ( $p=0.005)$ Among 27 patients with RVR, 18 patients were treated for 24 weeks and 9 patients were treated for 48 weeks. The SVR rate of patients treated for 24 weeks was $83.3 \%(15 / 18)$ and the SVR rate of patients treated for 48 weeks was $77.8 \%$ (7/9). The SVR rate did not differ significantly $(p=1.000)$. Multivariate analysis in all patients showed that RVR and low viral load were independent factors associated with SVR.

Conclusion: RVR is the most useful predictor of SVR and HCV-1 patients with RVR can be treated with 24-week therapy without compromising the chances for SVR. Checking RVR at 4 weeks will be needed for tailoring the length of treatment.

\section{PP13-19}

Long Term Follow-up for the Patients Treated with Pegylated İnterferon Plus Ribavirin for Chronic Hepatitis C

M. Yakut ${ }^{1,2}$, N. Örmeci ${ }^{1}$, H. Ozkan ${ }^{1}$, A. Dökmeci ${ }^{1}$

${ }^{1}$ Ankara University Medical Faculty; ${ }^{2}$ Gastroenterology, Ankara University Medical Faculty, Ankara, Turkey

Aim: The purpose of this study is to present long term results of pegylated interferon alpha plus ribavirin treatment in CHCI.

Patients and methods: In this study, we evaluated a total of 65patients with chronic hepatitis C patients who obtained a SVR and 77 patients patients without SVR with PEG-IFN plus ribavirin combination antiviral therapy among the 411 patients chronically infected with $\mathrm{HCV}$.

Results: Of 411 patients with HCV,161 (39\%) patients were treated with Peg-IFN plus ribavirin therapy.Nineteen of 161 patients were treated with Peg- IFN-plus ribavirin therapy due to not participate in routine follow up were excluded. This resulted in a 142 (63 men and 79 women) patients who were available for the SVR analysis. In the evaluation of 72 weeks a SVR was achieved by 65 of $142(46 \%)$ patients.Among CHCpatients 47 patients had minimal fibrosis (F0F1), and 19 patients were F3-F4 Advanced fibrosis (F3 and F4) were only factor that were significantly associated with non-SVR (5 vs.14, $p=0.038$ ). A total of 54 CHCpatients who achieved a SVR with Peg-IFN plus ribavirin therapy were eveluated.They had a median age of $53.6 \pm 10$ years and included 15 male. At baseline no patients had clinical liver cirrhosis.None of the patients with longterm SVR developed liver decompensation and HCC during followup period. SerumHCV-RNA was negative at all the follow-up evaluations in 53 patients who achieved SVR (Persistent SVR), and persistently positive in 1 patient after SVR (late virological relapse). 55 patients who no obtained a SVR to PEG-IFN and ribavirin therapy and who had a follow-up of more than 48 weeks were evaluated for developed liver cirrhosis and hcc. The mean follow-up duration of non-SVR patients was $49.9 \pm 10.5$. 9 of the 55 patients with nonSVR developed liver decompensation during follow-up, and 2 patients developed hec in this period.One patient who developed cirrhosis underwent liver transplantation.

Conclusion: Pegylated interferon alpha plus ribavirin treatment in $\mathrm{CHCI}$ is effective. Late virological relpse was seen in only 1 patient after SVR.
PP13-20

Evaluation of Rapid Virological Response to Pegylated Interferon and Ribavirin in HCV Infected Transfusion Dependent Thalassemic Patients

M. Keshvari ${ }^{1}$, F. Zamani ${ }^{2}$, S. Salimi ${ }^{2}$, B. Behnava ${ }^{3}$, S. Saiedi ${ }^{3}$, S. Alavian ${ }^{3}$ ${ }^{1}$ Research Center of Iranian Blood Transfusion Organization;

${ }^{2}$ Iran Research Center of Gastroenterology and Liver Disease;

${ }^{3}$ Baqiyatallah Research Center for Gastroenterology and Liver

Disease, Tehran, Iran

Background/aim: Hepatitis C is the leading cause of mortality and morbidity in transfusion dependent thalassemic patients (TDT). Epidemiologic studies have shown that around 20 to $40 \%$ of Iranian TDT patients are infected with $\mathrm{HCV}$ virus. In recent years, rapid virologic response (RVR) is regarding as an indicator of sustain virologic response. There is limited data about RVR in thalassemic patients. The aim of this study was assessment of factors which influence RVR in TDT patients.

Methods: This is a non randomized clinical study started from February 2010 until now conducted at two centers in Tehran. The patients were investigated for liver function tests, HCV viral load by amplicor and HCV genotyping by RFLP methods. Liver biopsy was done for all patients and scored according to Knodell scoring system. All patients were treated by Peg - INF alpha 2a and Ribavirin (Roche) and drug dosage was adjusted regarding to patients weight and $\mathrm{HCV}$ genotype. HCV RNA by PCR method (sensitivity less than $50 \mathrm{IU} / \mathrm{ml}$ ) was done one month after beginning of treatment to detect RVR.

Results: During the study period, 148 TDT patients were enrolled at the two centers. 92 patients were treated and evaluated to detect $\mathrm{HCV}$ RNA after treatment. 62 persons $(67.4 \%)$ and 30 patients $(32.6 \%)$ were infected by genotype $1 \mathrm{a}$ and genotype $3 \mathrm{a}$. There was no relation between patients gender, HCV RNA load, liver histology and biochemical parameters before treatment to RVR (HCV RNA was undetectable at 1 month after treatment). There was a statistic relationship between getting RVR and HCV genotype. 31 persons (50\%) and 23 patients $(76.7 \%)$ sequentially in genotype 1 and genotype 3 groups got RVR after treatment $(p<0.05)$

Conclusion: In TDT patients, among several host and viral factors, RVR had just a relation to HCV genotype.

\section{PP13-21}

Extent of Liver Inflammation as Predictor of Response to Interferon A and Ribavirin in Chronic Hepatitis C Patients in Pakistan

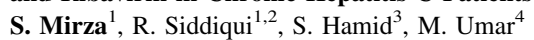

${ }^{I}$ Department of Community Health Sciences, Aga Khan University, Karachi, Pakistan; ${ }^{2}$ Department of Family and Community Medicine, King Saud University, Riyadh, Saudi Arabia; ${ }^{3}$ Department of Medicine, Aga Khan University, Karachi; ${ }^{4}$ Department of Medicine, Holy Family Hospital, Rawalpindi, Pakistan

Background: The relationship between pretreatment grades of liver inflammation and End of Treatment Response (ETR) to Interferon $\alpha$ and Ribavirin has not been studied well for Chronic Hepatitis C (CHC) infection, particularly in genotype 3 which is prevalent in Pakistan. Serum ALT is a less invasive biomarker for liver inflammation than liver biopsy.

Aims: To compare ETR in CHC patients with high grades to those with low grades of pretreatment liver inflammation and to determine the diagnostic accuracy of pretreatment ALT for grades of liver inflammation. Methods: A retrospective treatment cohort of naïve CHC patients, who received Interferon $\alpha$ and Ribavirin for 24 weeks, was studied. Pretreatment grade of inflammation on liver biopsy was classified as high if there was moderate or severe inflammation and low grade if there was minimal or mild inflammation. Multivariable logistic regression modeling was performed. Diagnostic accuracy of 
pretreatment ALT for liver inflammation grades was determined by computing Area Under the Receiver Operator Curve (AUROC).

Results: Of 672 patients, 103 had high and 569 had low grade of liver inflammation. Mean age was 36.9 (SD 9.1) years, with patients with high grade being older than those with low grade inflammation $(p=0.03)$. High grades of liver inflammation were associated with ETR (RR 1.17, 95\% CI 1.12-1.18) adjusting for age, TLC and pretreatment ALT. AUROC of pretreatment ALT for males and females for mild, moderate and severe liver inflammation were 0.629 , $0.69,0.87$ and $0.638,0.68$ and 0.74 respectively.

Conclusion: ETR in patients with high grades of pretreatment liver inflammation was $17 \%$ higher than those with low grades. Pretreatment ALT was moderately accurate for predicting only severe inflammation on liver biopsy, and did not correlate well with mild or moderate inflammation.

\section{PP13-22}

Efficacy of Pegylated Interferon and Ribavirin Combination Treatment in Elderly Patients with HCV Genotype 1b and High Viral Load

A. Miyasaka ${ }^{1}$, I. Kumagai ${ }^{1}$, R. Endo ${ }^{1}$, K. Abe ${ }^{1}$, Y. Takikawa ${ }^{1}$, K. Suzuki ${ }^{1}$, S. Sainokami ${ }^{2}$

${ }^{1}$ Department of Gastroenterology and Hepatology, Iwate Medical University, School of Medicine, Morioka; ${ }^{2}$ Department of Internal Medicine, Fussa Hospital, Tokyo, Japan

Background: In Japan, patients infected with the hepatitis $C$ virus (HCV) tend to be older than those in American and European countries. The combination treatment of pegylated interferon (PEGIFN) and ribavirin leads to a high sustained virological response (SVR) rate in patients with genotype $1 \mathrm{~b}$ and high viral load. However, adverse events associated with this combination treatment have been observed frequently in elderly patients.

Objective: In this study, we evaluated the efficacy of PEG-IFN and ribavirin combination treatment in elderly patients with HCV genotype $1 \mathrm{~b}$ and high viral load.

Methods: Forty-two patients with HCV genotype $1 \mathrm{~b}$ and high viral load were treated with PEG-IFN alpha-2b/ribavirin for 48 weeks. The patients were divided into two groups, those more than 60 years old (group A, $n=12$ ) and those under 60 years old (group B, $n=30$ ).

Results: There were no significant differences in sex, red blood cells, hemoglobin, platelet counts, albumin, ALT and HCV-RNA levels between the groups before treatment. The complication rate was significantly higher in group A than in group B $(p=0.031)$ at baseline. The rate of treatment discontinuation and PEG-IFN alpha-2b and/or ribavirin dose reduction because of adverse events were also higher in group A than in group B. On intention-to-treatment analysis, the SVR rate was slightly but not significantly lower in group A than in group B (33\% (4/12) vs. 50\% (15/30), respectively, $P=0.404)$.

Conclusion: In this study, the SVR rate of PEG-IFN and ribavirin combination treatment is lower in elderly patients than in young patients with $\mathrm{HCV}$ genotype $1 \mathrm{~b}$ and high viral load. However, if measures for controlling complications and avoiding treatment discontinuation and dose reduction are taken, this combination treatment might improve the SVR rate of elderly patients with HCV genotype $1 \mathrm{~b}$ and high viral load.

PP13-23

Predictors of Hematological Complications in Chronic Hepatitis C Egyptian Patients Treated with Interferon and Ribavirin

G. El Azab ${ }^{1}$, M. Assem ${ }^{1}$, M. Abdel Fattah ${ }^{2}$

${ }^{1}$ Nationa Liver Institute, Menoufia; ${ }^{2}$ Central Lab., Tanta, Egypt

Background: Hematological complications including anemia, neutropenia, and thrombocytopenia are commonly seen in patients with chronic hepatitis $\mathrm{C}$ treated with pegylated interferon and ribavirin. This may necessitate dose reduction or discontinuation of therapy in substantial number of patients with subsequent reduction of viral response. The aim of this study was to identify factors predicting the development of hematological complication in Egyptian patients with chronic hepatitis $\mathrm{C}$ treated with pegylated interferon and ribavirin.

Methods: One seventy and eight-naive CHC Egyptian patients eligible to start antiviral combination therapy were included. Patients were treated with either Peg-IFN $\alpha$-2a 180 mcg weekly or Peg-IFN $\alpha$ $2 \mathrm{~b} 1.5 \mathrm{mcg} / \mathrm{kg} /$ week with ribavirin based on patient body weight. The duration of treatment was 48 weeks. All subjects were monitored during the combination therapy and for a further period of 24 weeks after the treatment ended.

Results: 114 (64\%) patients were males. Mean age of the patients was 37.8 years Seventy-six (42.7\%) patients developed one or more of hematological abnormality during the HCV treatment. Anemia, neutropenia, and thrombocytopenia were reported in 46 (25.8\%), 35 (19.7\%), and $22(12.4 \%)$ patients, respectively. Using multivariate analysis; low baseline hemoglobin, low baseline neutrophils, lower creatinine clearance, body weight less than $60 \mathrm{~kg}$ in Peg-INF $\alpha$-2a treated patients, and presence of schistosomal antibodies were significantly associated with the development of hematological abnormalities.

Conclusion: The study identified CHC patients having high risk of hematological abnormalities development during treatment. Those patients may benefit from aggressive monitoring for early detection and proper management of these complications.

\section{PP13-24}

Options for Treatment Difficult to Treat Patients with Chronic Hepatitis C in Countries with Limited Access to Pegylated Interferon I. Hrstic ${ }^{1}$, D. Radic ${ }^{1}$, M. Morovic ${ }^{2}$, M. Coric ${ }^{3}$, B. Vucelic ${ }^{1}$, R. Ostojic ${ }^{1}$ Division of Gastroenterology, School of Medicine, University of Zagreb, Zagreb; ${ }^{2}$ Infectious Disease Ward, General Hospital Zadar, Zadar; ${ }^{3}$ Department of Pathology, School of Medicine, University of Zagreb, Zagreb, Croatia

Background: The "difficult-to-treat" patients with chronic hepatitis $\mathrm{C}$ (CHC) are those in whom no sustained virologic response (SVR) is achieved with the standard of treatment. In Croatia there is substantial number of CHC patients with no-SVR after combination of standard interferon (stdIFN) and ribavirin (RBV). According to Croatian National Health Insurance Company those patients cannot be retreated with pegylated IFN (pegIFN).

Aim: To evaluate and compare the efficacy of re-therapy in previous NR CHC patients with 2 regimens.

Patients and methods: 51 pts with biopsy proven $\mathrm{CHC}$ and genotype 1 infection where included. Group A consisted of 25 pts (7F/18M; age $45.24 \pm 6.04$; mean fibrosis 5 of 6 according to Ishak score) treated with triple induction therapy (stdIFN alfa-2a, RBV and amantadine (AMT) for 48 weeks. Group B consisted of 26 pts $(8 \mathrm{~F} / 18 \mathrm{M}$; age $41.69 \pm 10.44$; mean fibrosis 3 of 6 ) treated with pegIFN and RBV for 48 weeks. Serum HCV RNA clearance was considered as virological response.

Results: SVR was achieved in $32 \%$ of patients in group A and in 50\% of patients in group B. No statistical difference in SVR for both groups was found for gender, age, basal ALT and AST. Only fibrosis score differed in both groups: pts with fibrosis level $\leq 3$ more often achieved SVR (group A $p=0.001$; group B $p=0.047$ ). Surprisingly no statistical difference has been found for basal viral load.

Conclusion: Although new therapeutic regimen with pegylated interferon is available for difficult to treat patient, in Croatia is not every day practice. Obtained SVR with re-therapy of NR CHC pts is excellent and even higher than those reported in the literature. Higher SVR can be effect of small numbers and probably too large difference between 2 regimens can be due to lower fibrosis score in group of pts treated with pegIFN and RBV. 


\section{PP13-25}

Does the Viral Load at the Twelfth Week Predicts Sustained Virologic Response in Patients with Chronic Viral Hepatitis C?

N. Aqodad ${ }^{1}$, H. Irhoudane ${ }^{1}$, M. Lahbabi ${ }^{1}$, M. El Yousfi ${ }^{1}$, I. Mellouki ${ }^{1}$, D.A. Benajah ${ }^{1}$, M. El Abkari ${ }^{1}$, A. Ibrahimi ${ }^{1}$, A. Najdi ${ }^{2}$, C. Nejjari ${ }^{2}$

${ }^{1}$ Hepatology; ${ }^{2}$ Epidemiology, Hassan II University Hospital, Fez, Morocco

The determination of viral load (VL) at the twelfth week (W12) from the beginning of the treatment is recommended in patients with chronic viral hepatitis $\mathrm{C}(\mathrm{HCV})$ genotype 1 receiving pegylated interferon plus ribavirin. It is used to predict the sustained virologic response (SVR) and adjust the duration of treatment. The aim of this work is to study the influence of the VL at W12 on the SVR in our patients with chronic viral hepatitis $\mathrm{C}$ genotype I.

Materials and methods: This is a retrospective, descriptive and analytical study of patients having a viral hepatitis $\mathrm{C}$ genotype $\mathrm{I}$. We determined two groups of patients: group A: Had a decrease of VL more than $2 \log$ and groupe B had no decrease of VL more than $2 \mathrm{log}$ at W12, and we compared the SVR in the two groups.

Results: Forty seven patients were included with a mean age of 57.39 +/-9.81 [17-77], of which 52.3\% were female. The determination of quantitative RNA at W12 was performed in 24 patients, a decrease of more than $2 \log$ VL has been found in 21 patients (group A) of which 10 had an SVR. An undetectable serum HCV-RNA at W12 was noted in 10 patients, 5 of which had a SVR. Three patients did not decrease by more than $2 \log$ of VL at W12 (group B), 2 of them had a SVR. The difference between the two groups was not significant concerning the SVR (Fisher's exact test $p<0.515$ ).

Conclusion: SVR was similar among patients who have reduced the VL of more than two log and those who have undetectable serum HCV-RNA at W12. However, it is important to note that among three patients in whom VL did not decrease of more than $2 \log$ at W12, tow patients had a SVR.

\section{PP13-26}

Analysis of Current Status of Chronic Hepatitis C in Guangxi

J. Yu, Q. Shi, Y. Chen, F. Zeng, A. Yang, D. Mao

Hepatology, Guangxi Chinese Medical University, Nanning, China

Background and aims: Chronic hepatitis $\mathrm{C}(\mathrm{CHC})$ is one of the major cause of chronic liver disease and liver cancer. The standard therapy for $\mathrm{CHC}$ is the combination of pegylated interferon- $\alpha$ and ribavirin. The present study is aimed to assess the current status, underlying risk factors and virological response to the standard $\mathrm{CHC}$ treatment in Guangxi province.

Methods: 100 patients from our university hospital (56 males, mean age 44 years) were selected from May 2009 to Aug. 2010. Patients' biochemical, virological profile and HCV genotypes were analyzed. 23 patients were treated with peginterferon alfa-2a $180 \mu \mathrm{g}$ per week plus ribavirin $1,000 \mathrm{mg} /$ day for 48 weeks.

Results: $60 \%$ patients were older than 41 years. The clinical diagnoses were chronic hepatitis with normal ALT in $14 \%$, with persistently elevated ALT level in $72 \%$; cirrhosis in 11 and $3 \%$ associated with primary liver cancer. The blood transfusion was found as a leading risk factor in 54\% of cases and history of injection drug use in $12 \%$. Risk factor unidentifiable cases accounted for $26 \%$. In patients 31 to 40 -year-old,drug user accounted for $38 \%$. Distribution of genotypes: genotype-1a $10 \%$, genotype- $1 \mathrm{~b} 58 \%$, genotype-2a $11 \%$, genotype- $3 \mathrm{~b} 16 \%$, genotype- $6 \mathrm{a} \%$. The mean viral load is $2.8 \mathrm{E}+07$ $\pm 3.8 \mathrm{E}+07 \mathrm{copies} / \mathrm{ml}$. The sustained virological response (SVR) rate was $85 \%$ in genotype- $1 \mathrm{~b}, 100 \%$ in genotype-1a, genotype- $3 \mathrm{~b}$ and genotype-6a. The patients failed to achieve SVR were those with high viral load (HCV RNA > 7.16E+07), old age ( $>54$ years), and genotype-1b.

Conclusion: Blood donors screen since 1990s for anti-HCV reduce the risk of $\mathrm{CHC}$, but injetion drug use become one of important risk in the young. The screening of population with the predictive factors including adults with history of blood transfusion (age $>41$ years), drug use can detect $\mathrm{HCV}$ infection and treatment with standard therapy early is benefit patients in Guangxi.

\section{PP13-27}

The Effect of IL28B Genotype and Core Region Mutations of Hepatitis C Virus Genotype 1b on Response to Anti-viral Therapy

S. Nakamoto, F. Imazeki, T. Kanda, S. Wu, A. Tawada, M. Arai, K. Fujiwara, K. Saito, Q.H. Yuan, T. Muto, N. Yamahata, H. Shirasawa, O. Yokosuka Graduate School of Medicine, Chiba University, Chiba, Japan

Aims: In chronic hepatitis $\mathrm{C}$ patients, several viral and host factors have been associated with response to combination therapy of peginterferon and ribavirin. These factors include age, gender, ethnicity, viral genotypes, metabolic factors, genetic variation of the host gene, and viral sequence variations. In this study, we assessed the effect of steatosis, IL28B genotype, and hepatitis C virus (HCV) core sequence on treatment response in Japanese patients infected with HCV genotype $1 b$.

Methods: We analyzed 57 patients (28 males) with high viral load $(>5 \log \mathrm{IU} / \mathrm{ml})$ treated with peginterferon and ribavirin combination therapy. The data of glucose intolerance, serum lipid, and liver function was obtained. Liver biopsy specimens at baseline were evaluated for fibrosis, activity, and steatosis. The patterns of IL28B-related single nucleotide polymorphism, rs8099917, and amino acid patterns at positions 70 and 91 in the HCV core region were also determined. Partial EVR (pEVR) was defined as more than $2 \log$ decrease of HCV-RNA during the first 12 weeks of therapy.

Results: At baseline, higher platelet count $(p=0.006)$, higher white blood cell count $(p=0.03)$, absence of glucose intolerance ( $p=0.01)$, hepatic steatosis $(<5 \%, p=0.01)$, double-wild-type ( $70 \mathrm{R}$ and $91 \mathrm{~L})$ in the $\mathrm{HCV}$ core region $(p=0.01)$, and TT genotypes at rs8099917 (major-type, $p<0.0001$ ) were associated with pEVR. In multivariate analysis, higher platelet count (odds ratio 1.21 [1.03-1.43]), and IL28B major-type (OR 41.2 [6.55-259]) were independently associated with pEVR. When IL28B-rlated factor was excluded, higher platelet count (OR 1.17 [1.05-1.31]) and DW-type in the core region (15.4 [1.70-140]) were significant factors to predict pEVR.

Conclusions: In Japanese patients with genotype $1 \mathrm{~b} \mathrm{HCV}$, hematologic factors and metabolic factors were associated with response to peginterferon and ribavirin combination therapy. Furthermore, it may be useful to evaluate HCV core amino acid pattern and IL28B-related genotypes to predict treatment outcome more accurately.

\section{PP13-28}

The Treatment Responses According to ALT Variability with Peg-interferon and Ribavirin Combination Therapy for Chronic Hepatitis C Patients

C.W. Park, Y.J. Kim, Y.J. Kim, K.I. Lee, B.K. Jang, W.J. Chung, J.S. Hwang Internal Medicine, Keimyung University Dongsan Hospital, Daegu, Republic of Korea

Background: The purpose of successful treatment of $\mathrm{HCV}$ is to prevent progression of chronic hepatitis to liver cirrhosis and hepatocellular carcinoma. Responsive factors about treatment such as genotype, age, sex, viral titer are already known, but, relationship between ALT variability during treatment and treatment response is indefinite, So, we evaluated the interactive relationship and clinical importance.

Methods: Between May 2005 and Feb 2009, we reviewed 344 patients data who confirmed as chronic hepatitis $\mathrm{C}$ and received pegIFN and ribavirin combination treatment. Among these patients, 145 patients who followed up to more than 6 months after finishing treatment were enrolled. Patients were divided as group 1 (initial high 
ALT, abnormal ALT during treatment), 2 (initial high ALT, normalized ALT during treatment), 3 (initial normal ALT, abnormal ALT during treatment), 4 (initial normal ALT, normal ALT during treatment). We compared the result of early virological response (EVR), end of treatment response (ETR), sustained virological response (SVR) between each groups.

\section{Results:}

1. Among 145 patients, mean age was 54.8 years old. Male:Female ratio was 75:70. Genotype 1 patients were 68 , and non-genotype 1 were 77.

2. The results of EVR were 32, 47, 10,30\% and ETR were 38, 52, $12,28 \%$, and SVR were 25, 49, 7, 21\% in group 1,2,3, and 4 . SVR rates were significantly different between group 1 and $2(54$ vs. $90 \%, p=0.001$ ), and insignificant between group 3 and 4 .

3. Group 1 and 3 which got abnormal ALT levels during treatment had significantly low $\operatorname{EVR}(p=0.01)$ and $\operatorname{SVR} \operatorname{rates}(p=0.01)$ compared than group 2 and 4.

Conclusion: Normalized ALT levels during treatment had significant relationship between treatment response of chronic hepatitis $\mathrm{C}$ patients who received peg-IFN and ribavirin combination therapy, and can be used as important predisposing factor for treatment response.

\section{PP13-29}

Chronic Hepatitis $C$ Infection in Injection Drug Users in Singapore S.G. Lim ${ }^{1,2}$, W. Eric ${ }^{1}$, M.O. Aung ${ }^{1}$, M. Winslow Rasiah ${ }^{3}$, S. Arthur Lee Kiat ${ }^{3}$ ${ }^{1}$ Gastroenterology and Hepatology, National University Health System; ${ }^{2}$ Medicine, Yong Yoo Lin School of Medicine, National University of Singapore; ${ }^{3}$ Institute of Mental Health and Wood Bridge Hospital, Singapore, Singapore

Background/aim: Hepatitis $\mathrm{C}$ virus(HCV) infection is a potential problem within the injection drug abuse community. We evaluated the biochemical, virological and risk factor profile of injection users with HCV infections in Singapore.

Methods: Injection drug users from a rehabilitation programme were voluntarily screened for human immunodeficiency virus, hepatitis B and hepatits $\mathrm{C}$ after signing informed consent. Their liver function and biochemical profiles were analyzed.

Results: Of 623 injection drug users, $71.1 \%$ were positive for hepatitis $\mathrm{C}, 1.1 \%$ for hepatitis $\mathrm{B}, 2.5 \%$ for hepatitis B and C co-infection, and $0.4 \%$ for HIV, hepatitis B and C infections.

Of the 433 anti-HCV positive subjects, $34.6 \%$ attended our hepatitis C clinic. $(92.7 \%$ males, $7.3 \%$ females; mean age 43 years; $47.3 \%$ Chinese, $39.3 \%$ Malays, $6.7 \%$ Indians).

Risk factors: needle use (100\%), spouses with hepatitis C (2.7\%), sexual promiscuity $(58.7 \%)$, tattoos $(63.3 \%)$ and prior blood transfusion $(2.7 \%) .55 \%$ shared needles and $79.8 \%$ abused multiple drugs.

Virological analysis on 66 subjects showed, $28.7 \%$ had undetectable HCV RNA(median RNA 396,000 IU/ml). 20.9\% had genotype 1 ( 2 subtype $1 \mathrm{a} ; 2$ subtype $1 \mathrm{~b} ; 2$ subtypes $1 \mathrm{a} / 1 \mathrm{~b}$ ), 2.3\% had genotype 2 (subtypes $2 \mathrm{a} / 2 \mathrm{c}$ ), $74.4 \%$ genotype 3 (96\% subtype $3 \mathrm{a}$ ), and $2.3 \%$ genotype 4 (subtype $4 \mathrm{c} / 4 \mathrm{~d}$ ).

Biochemical analysis was normal (median total bilirubin $10 \mu \mathrm{mol} /$ L, albumin 44g/L, AST $42 \mathrm{U} / \mathrm{L}$, ALT $65 \mathrm{U} / \mathrm{L}$, ALP $77 \mathrm{U} / \mathrm{L}$, prothrombin time $13.9 \mathrm{~s}$, platelet count $266 \times 10^{9} / \mathrm{L}$, AFP $\left.3.8 \mu \mathrm{g} / \mathrm{L}\right) .54$ subjects underwent liver ultrasound (39 normal; 10 fatty livers; 4 hepatic cysts; 1 coarse echotexture).

Conclusion: There is a high prevalence of HCV infection in Injection drug users in Singapore with $71.1 \%$ anti-HCV positive but in those tested, active HCV infection was present in $71.3 \%$. Genotype $3 \mathrm{a}$ is the commonest, suggesting transmission of a single strain. Liver function tests are frequently normal and cannot be used to screen injection drug users for active $\mathrm{HCV}$ infection.
PP13-30

Should Patients with Liver Cirrhosis due to HCV Genotype 3 in Developing Countries Be Offered Antiviral Therapy?

G. Choudhuri, A. Verma, T.S. Negi

Gastroenterology, Sanjay Gandhi Post Graduate Institute of Medical Sciences, Lucknow, India

Background and aims: While HCV related liver cirrhosis (HCVLC) in west is usually due to genotype 1 , are difficult to treat with antivirals and hence offered liver transplantation early, the infection in resource scarce countries like India, often due to favorable genotypes, may respond better often obviating the need for liver transplanatation. We evaluated tolerability and outcome of interferon (conventional or pegylated) and ribavarin in patients with HCV-LC genotype 3.

Methods: From 1998 to 2010 in a single unit experience of 228 patients, who were offered combined antiviral treatment with interferon and ribavarin with response guided individualized treatment for at least 24 weeks, 99 had cirrhosis and 129 were without cirrhosis (CHC). Additional supportive co-therapy with erythropoietin, blood transfusions or colony stimulating factors was given whenever required.

Results: Of $99 \mathrm{HCV}-\mathrm{LC}$ patients $74(74.7 \%)$ were due to genotype 3 [median age 47.5, range 26-71 years, 33 (45.5\%) males; 43, 27 and 2 (60, 37.5 and 2.7\%] patients were in Child A, B and C groups respectively. $16(28.5 \%)$ patients dropped out due to adverse effects. Sustained virological response (SVR) was achieved in $46(64.8 \%)$ in those who completed atleast 24 weeks of therapy. 27 (35\%) patients required support with co-factors to complete treatment.

Conclusion: Patients with HCV related Child A and B cirrhosis due to genotype 3 have relatively good response rates. In developing countries where OLT is expensive and not easily available, liver cirrhosis patients with favorable genotypes may benefit from a trial of antiviral therapy.

\section{PP13-31}

Response to Interferon Plus Ribavarin Combotherapy in Chronic

Hepatitis $C$ in Indian Patients: Genotype 1 versus 3

G. Choudhuri, T.S. Negi, A. Verma

Gastroenterology, Sanjay Gandhi Post Graduate Institute of Medical Sciences, Lucknow, India

Background: Genotype is the most important determinant of treatment outcome in chronic hepatitis $\mathrm{C}(\mathrm{CHC})$. As compared to western population very little data exists regarding treatment outcomes in various $\mathrm{HCV}$ genotypes in Indian patients. We compared response to standard therapy in patients with chronic hepatitis $\mathrm{C}(\mathrm{CHC})$ genotype 3 with genotype 1 .

Methods: Data was retrieved from the prospective followed up case files of patients treated for $\mathrm{CHC}$ at our centre. After exclusion of patients with cirrhosis, response rates were analysed in patients with CHC with genotype 3 or genotype 1 .

Results: Of 131 patients $96(73 \%)$ had geno 3 and $20(15.4 \%)$ had geno1 [mean age 39.1 vs. 37.7 years $(p=\mathrm{NS})$; males 52.2 vs. $55 \%$ $(p=\mathrm{NS})$; mean body mass index (24.1 vs. $\left.24.5 \mathrm{~kg} / \mathrm{m}^{2}\right)$, alcohol intake 10.2 vs. $10.0 \%(p=\mathrm{NS})$, smokers 10 vs. $12.4 \%(p=\mathrm{NS})$ and mean baseline HCV RNA level $3.6 \times 10^{6}$ vs. $\left.7.4 \times 10^{6}(p=N S)\right]$. $14,18,36$ and $34(13.7,17.6,35.2,31.3 \%)$ patients received plain interferon alpha2b and $2 \mathrm{a}$ and pegylated interferon alpha $2 \mathrm{~b}$ and $2 \mathrm{a}$ respectively along with ribavarin $(800 \mathrm{mg} / \mathrm{d}$ for $<50 \mathrm{~kg}, 1000 \mathrm{mg} /$ days for $50-70 \mathrm{~kg}$ and $1,200 \mathrm{mg}$ OD for $>70 \mathrm{~kg}$ ). Duration of therapy was 24 weeks for geno 3 and 48 weeks for geno1. Dropout rates were more in patients with geno 1 (7.2 vs. $35.0 \%$; $p<0.05)$. Rapid virological response (RVR) was comparable in 2 groups $(75 / 89 ; 84.2 \%$ vs. $10 / 13 ; 76.9 \%$ ) and sustained virological response (SVR) rates were $97.1 \%(82 / 85)$ and $84.2 \%(11 / 13)$ were similar in the 2 groups 
$(p=\mathrm{NS})$ on per protocol analysis and $82 / 96(85.4 \%)$ and $11 / 20$ $(55.0 \%)$ on intention to treat analysis $(p=0.004)$.

Conclusions: Although more patients with genotype 1 dropped out due to adverse effects caused by longer duration of treatment, the response rates of Indian patients with genotypes 1 and 3 did not differ much.

\section{PP13-32}

Predictability of Therapeutic Outcome in Patients with Hepatitis C Virus Infection Using Interferon-beta Administration Prior to the Standard Combination Therapy

T. Nakatani, E. Kikuchi, K. Seki, Y. Sawada, Y. Sato, K. Hashimoto, Y. Maekawa, H. Matsuo

Gastroenterology, Nara Prefectural Nara Hospital, Nara, Japan

Aims: Longer treatment using pegylated interferon-alpha $2 \mathrm{~b}$ (PEG) plus ribavirin (RBV) for patients with hepatitis $\mathrm{C}$ virus $(\mathrm{HCV})$ has enabled higher SVR (HCV-RNA negative 24 weeks after termination of the therapy). On the other hand, early prediction of therapeutic outcome is desirable to avoid expense and inconvenience of unnecessary therapy. In this study, it is evaluated whether treatment efficacy is predictable early in treatment among patients using IFN-beta administration prior to the combination therapy.

Methods: Twenty-nine patients with high load $1 \mathrm{~b}$ HCV undergoing IFN-beta induction (3MU IFN-beta twice-daily for 2 weeks) prior to the combination therapy were divided into 2 groups

(A: complete achievement of IFN-beta induction,

B: incomplete achievement of induction therapy such as dose reduction etc.). Predictability was evaluated in terms of the level of HCV-RNA reduction during IFN-beta induction and the rebound of HCV-RNA after initiation of combination therapy.

Results: In all patients, SVR was $55.2 \%$ (16/29). In the monovariate analysis, the factors significantly associated with SVR were 'reduction in HCV-RNA by at least $2 \log$ during induction period', 'no rebound of HCV-RNA after initiation of combination therapy' and 'EVR (HCV-RNA negative at weeks 12) achievement'. In each group, EVR (A/B; $61.9 \%(13 / 21) / 50.0 \%$ (4/8)), SVR (A/B; $66.7 \%$ $(14 / 21) / 25.0 \%(2 / 8): P<0.05)$ and drug adherence $(\mathrm{A} / \mathrm{B} ; 93.3 \%$ / $77.3 \%$ in PEG-IFN: $P<0.05,93.3 \% / 63.6 \%$ in RBV) were higher in group A. Among group A, when comparing between group A-1 (achieving more than $2 \log$ decrease in HCV-RNA during induction period) and group A-2 (not achieving $2 \log$ decrease), SVR (A-1/A-2; 81.3\%(13/16)/20.0\%(1/5): $P<0.05)$ was significantly higher in group A-1. Finally, in group A-1, patients without any rebound of HCV-RNA after initiation of combination therapy achieved $100 \%$ $(10 / 10)$ of SVR.

Conclusion: Therapeutic outcome could be predicted depending on the response against IFN-beta induction prior to the combination therapy for chronic hepatitis $\mathrm{C}$.

\section{PP13-33}

Is Rapid Virological Response a Good Predictor of Sustained Virological Response in HCV Genotype 3 Related Cirrhosis?

G. Choudhuri, T.S. Negi, A. Verma

Gastroenterology, Sanjay Gandhi Post Graduate Institute of Medical Sciences, Lucknow, India

Background and aims: Rapid virological response (RVR) has been extensively validated as one of the best predictors of sustained virologic response (SVR) to treatment in chronic hepatitis $\mathrm{C}(\mathrm{CHC})$ especially in patients with genotype 1 . Its usefulness in patients with $\mathrm{HCV}$ related liver cirrhosis (HCV-LC) undergoing therapy is unclear. We evaluated efficacy of RVR in predicting response to treatment in patients with $\mathrm{CHC}$ with and without cirrhosis.
Methods: Data of all the patients of CHC genotype 3 who completed 24 weeks of response guided individualized treatment with interferon (conventional or pegylated) plus ribavarin at our centre was divided in two groups and compared. Group A comprised patients without cirrhosis (based on clinical or radiological or histological features) while Group B comprised those with liver cirrhosis (HCV-LC).

Results: Of 168 patients $96(57.1 \%)$ were in group A and $72(42.8 \%)$ in group B [mean age in years 39.1 and $46.9(p<0.05)$; mean BMI 24.0 and $24.1(p=\mathrm{NS})$; males 52.4 and $48.6 \%(p=\mathrm{NS})$ alcohol intake 14.2 and $13.8 \%(p=\mathrm{NS})$; smoking 14.2 and $13.8 \%(p=\mathrm{NS})$; pre-treatment mean HCV RNA levels $3.6 \times 10^{6}$ and $1.7 \times 10^{6}$ $\mathrm{IU} / \mathrm{mL}(p=\mathrm{NS})] .7(7.3 \%)$ and $16(28.5 \%)$ patients respectively dropped out due to adverse effects $(p=0.01)$. RVR rates in two groups were $84.2 \%(75 / 89)$ and $82.4 \%(10 / 46)(p=0.9)$ respectively. The SVR rates were $92.4 \%(82 / 89)$ in those without cirrhosis and $62.7 \%(34 / 56)$ in those who had liver cirrhosis $(p<0.01)$. The Positive Predictive Value of RVR was 0.96 and 0.7 respectively in the 2 groups.

Conclusions: In HCV genotype 3 related chronic liver disease undergoing antiviral treatment, RVR is not a good predictor of SVR in those who have developed liver cirrhosis.

\section{PP13-34}

Retreatment of Chronic Hepatitis C, Genotype 3 Patients with Pegylatedinterferon and Ribavirin

A.S. Butt, K. Mumtaz, S. Hamid, H.A. Shah, W. Jafri

Section of Gastroenterology, Department of Medicine, Aga Khan University Hospital, Karachi, Pakistan

Background: Re-treatment of patients with chronic hepatitis $\mathrm{C}$ virus $(\mathrm{HCV})$, non-responders or relapser to interferon alfa and ribavirin is difficult.

Aim: To evaluate the sustained virological response (SVR) after retreatment with pegylated interferon and ribavirin in chronic $\mathrm{HCV}$, non-responder (NR) or relaper patients with genotype 3 infection.

Methods: Data of patients visiting our Gastroenterology clinics from 2006 to 2008 was retrieved. All patients who were non-responders or relapsers to initial treatment with standard IFN and ribavirin were studied. All patients received pegylated interferon alfa 2 a $(180 \mathrm{mcg} /$ $\mathrm{kg} /$ week) with ribavirin (400mg TID). Diagnosis of cirrhosis was made by liver biopsy and/or on biochemical and ultrasonological findings.Patients were given treatment for 6 or 12 months based on rapid virological response(RVR). Primary end point was SVR. Predictors of SVR were also analyzed and adverse events were recorded. Results: A total of 63 patients were re-treated.There were $54.1 \%$ males and $53(84 \%)$ were $>35$ years of age. 35 patients had child's Acirrhosis.There were $25(39.67 \%) \mathrm{NR}$ and $38(60.3 \%)$ relapsers. Duration of treatment was 6 months in 43 patients and 12 months in 18. Two patients were unable to complete treatment due to adverse events. Following re-treatment, the SVR was achieved by $38(60.3 \%)$ patients. RVR and ETR was achieved in $44(72.1 \%)$ and $40(65.6 \%)$ cases respectively. Moreover, SVR was achieved in $31 / 43(72 \%)$ and 9/18 (50\%) patients who received 6 and 12 months treatment respectively.

RVR ( $p$ 0.006), non-cirrhotic status ( $p$ 0.007), relapse with initial treatment $(p<0.05)$, re-treatment within 1 year $(p<0.05)$ were predictors of SVR. The most frequent adverse events were fever $(55 \%)$, fatigue $(40 \%)$, and anorexia (35\%).Six patients were supported with GCSF and 10 with erythropoietin for leucopenia and anemia respectively with modification in dosage of peg-IFN and ribavirin.

Conclusion: Re-treatment with pegylated interferon and ribavirin in non-responder HCV genotype 3 patients is effective. Rapid virological response, non-cirrhotic status, relapse with initial treatment, retreatment within 1 year were predictors of SVR. 


\section{PP13-35}

11 Years of Sustained Virological Responses in Turkish Patients Treated with IFN Alpha Plus Ribavirine Combination

C.P. Eyigun ${ }^{1}$, E. Gunal ${ }^{1}$, I.Y. Avci ${ }^{1}$, H.C. Gul ${ }^{1}$, F. Ersoz ${ }^{2}$, G. Mert ${ }^{1}$, O. Coskun ${ }^{1}$, A.B. Besirbellioglu ${ }^{1}$

${ }^{I}$ Infectious Diseases and Clinical Microbiology, Gulhane Military Medical Academy; ${ }^{2}$ Defense Science Institute, Turkish Military Academy Defense Science Institute, Ankara, Turkey

Aim of this study is to put forward the sustained virological responses of long term theraphy in genotype 1 chronical hepatitis $\mathrm{C}$ patients.63 $(88.7 \%)$ naive $8(11.3 \%)$ nonresponder or relapser, total 71 chronic hepatitis patients were treated with IFN alpha $(5 \mathrm{MU} /$ day or $3 \times 3 \mathrm{MU} /$ week) + ribavirin (800-1,200 mg/day) or PEG IFN alpha $(1.5 \mu \mathrm{g} / \mathrm{kg} /$ day $)+$ ribavirin $(10.6 \mathrm{mg} / \mathrm{kg})$ combinations between 2000-2006 years during 52 weeks. Patients were checked monthly during treatment and semiannual after treatment for routin biochemical examinations and quantitative serum HCV RNA. 29 $(40.8 \%)$ of patients were women while $42(59.2 \%)$ of them were men. Mean age of patients was $48.25 \pm 10.63$ years, mean HCV RNA level at the beginning was $4 \times 10^{8} \pm 9 \times 10^{8} \mathrm{copy} / \mathrm{ml}$, mean ALT level was $79.63 \pm 50.54 \mathrm{IU} / \mathrm{ml}$. At the end of therapy, HCV RNA disappeared in 63 patients $(88.7 \%)$ but $4(5.63 \%)$ patients were relapser within 6 month after treatment while $4(5.63 \%)$ patients were nonresponder. $6(8.5 \%)$ patients were followed up 11 years, $7(9.6 \%)$ patients 10 years, $7(9.6 \%)$ patients 9 years, $8(11.3 \%)$ patients 7 years, $14(19.7 \%)$ patients 6 years, $9(12.7 \%)$ patients 5 years, $8(11.3 \%)$ patients atleast 4 years after treatment. Sustained virological response rate was $87.3 \%(55 / 63)$ in naive patients, $50 \%$ (4/8) was in nonresponder plus relapser patients and $83.1 \%(59 / 71)$ was in whole of patients. Our sustained response rates are very high against literature. Younger infection age in patients, abcense of complicated cases, good patient compliance and giving almost all of target drug dosage can be reasons of this. It wasn't observed virological breakthrough in any of patients who had sustained virological response 6 months later from endtreatment. Absence of HCV RNA at end of 6thmonth after treatment goes on to be most important sign of cure chance for chronical hepatitis $\mathrm{C}$.

\section{PP13-36}

Peginterferon Alfa-2b/Ribavirin Treatment for 16 Weeks Is Inferior to 24 Weeks in Japanese Patients Infected with HCV Genotype 2

A. Tawada ${ }^{1}$, T. Kanda ${ }^{1}$, F. Imazeki ${ }^{1}$, R. Azemoto ${ }^{2}$, S. Mikami ${ }^{3}$, K. Kita ${ }^{4}$, M. Takashi ${ }^{5}$, M. Sunaga ${ }^{6}$, S. Nakamoto ${ }^{1}$, S. Wu ${ }^{1}$, M. Arai ${ }^{1}$, K. Fujiwara ${ }^{1}$, O. Yokosuka ${ }^{1}$

${ }^{I}$ Medicine and Clinical Oncology, Chiba University, Graduate School of Medicine, Chiba; ${ }^{2}$ Gastroenterology, Kimitsu Chuo Hospital, Kisarazu; ${ }^{3}$ Gastroenterology, Kikkohman General Hospital, Noda; ${ }^{4}$ Gastroenterology, Chiba Kaihin Hospital, Chiba;

${ }^{5}$ Gastroenterology, Saiseikai Narashino Hospital, Narashino;

${ }^{6}$ Gastroenterology, Chiba Chuo Medical Center, Chiba, Japan

Backgrounds and aims: Current standard of care for patients infected with $\mathrm{HCV}$ of genotype 2 is the combination of peginterferon (PEG-IFN) plus ribavirin (RBV) for 24 weeks. We assessed the sustained virological response (SVR) rates in a predominantly hepatitis $\mathrm{C}$ virus (HCV) genotype 2 infected Japanese population.

Methods: Between 2006 and 2009, 93 patients who were treated for chronic hepatitis $\mathrm{C}$, genotype 2 infection at Chiba Prefecture area, were included. Epidemiological data and treatment outcomes were evaluated in all treated patients. 71 patients were treated with PEGIFN alpha-2b plus RBV for 24 weeks. Another 22 patients, who obtained HCV RNA negativity at week 4, were randomized to 16 or 24 weeks of PEG- IFN alpha-2b plus RBV.

Results: 58 of 71 patients $(81.6 \%)$ completed the treatment with an overall SVR rate of $81.0 \%$. The SVR rate was $88.5 \%$ in male, and $80.0 \%$ in female patients. Patients younger than 60 years had higher
SVR rates than older patients (84.7 vs. $66.6 \%)$. SVR was also statistically significantly lower when adherence of PEG-IFN and ribavirin are lower than 80 and $80 \%$ (54.5 vs. $87.2 \%, P=0.0261)$. In RCT, SVR rates of 16 weeks and those of 24 weeks were 6 of $9(67 \%)$ and 13 of $13(100 \%)$, respectively.

Conclusion: The SVR rates of PEG-IFN plus RBV in our study are similar to those in previous studies. PEG-IFN plus RBV treatment for 16 weeks in Japanese patients infected HCV genotype 2 is overall inferior to 24 weeks of treatment but might be useful in some patients. Further studies will be needed.

\section{PP13-37}

Outcomes of CINF and Riba Therapy for Hep C G3 Patients who Relapsed with Previous Peg INF and Riba Therapy

M. Gill, S. Naseem, U. Adeeb

Division of Digestive Diseases, Maroof International Hospital, Islamabad, Pakistan

Aims and objectives: There are not many studies about the efficacy of CINF and Ribavirin combination in chronic HepC G3 relapsers with Peg interferon and Ribavirin combination therapy. We wanted to evaluate the efficacy of CIFN and riba in these patients.

Methods: The study was conducted between Feb 2009 to July 2010. We consecutively enrolled 60 patients who were chronic hepatitis C relapsers with Peg interferon and Ribavirin therapy. Patients with active psychiatric history, platelet count $<75,000$, INR $>1.5$, S. Albumin $<2.5$, portal vein diameter $>13 \mathrm{~mm}$ on ultrasound were excluded from the study. All enrolled patients have CBC, ALT, HCV PCR quantitative, HCV genotype, thyroid function tests and blood sugar at baseline. Patients were given $15 \mu \mathrm{g}$ thrice weekly CINF and Ribavirin $400 \mathrm{mg}$ bid for 9 months. Patients were reviewed on 4 weekly basis for clinical examination and $\mathrm{CBC}$, TSH level and blood sugar. The patients who had HCV PCR negative or $50 \%$ reduction in HCV PCR at week 12 were given further 6 months of therapy.

Results: Among 60 patients 45 were male and 15 were female. Median age was 45.50 out of $60(80 \%)$ patients were HCV PCR negative @ week 12 of therapy. These 50 patients completed further 24 weeks of therapy and remained HCV PCR negative @ end of total 36 weeks of therapy. 45 out of $60(75 \%)$ patients were HCVPCR negative 6 months (SVR) after completing the treatment.

Conclusion: CINF (Infergen) Riba combination is very optimistic option for Hep CG3 relapsers with peg INF and riba combination.we did give 9 months of therapy because of financial consideration perhaps 1 year therapy will be most optimal duration.

\section{PP13-38}

The Nutrition Assessment of Interferon Treatment in Patients with Chronic Hepatitis

H. Tanaka, S. Takamatsu, H. Koga

Gastroenterology, Kishiwada Tokusyuukai Hospital, Kishiwada, Japan

Introduction: The treatment of hepatitis C with Peg Interferon (Peg IFN) and ribavirin is highly effective. However, IFN treatment has various side effects and encounter the difficulty in completion of the therapy. Fever and loss of appetite frequently are those side effects, which may affect the nutritional influence and disturbance of the metabolic effects on the patients during IFN treatment. With the indirect calorimetry, we evaluated the nutritional and metabolic effects before and after IFN treatment on the patients with chronic hepatitis. Subjects and methods: The subjects were eight patients with chronic hepatitis. 7 patients with chronic hepatitis $\mathrm{C}$ were treated with pegIFN- $\alpha 2 b$ and ribavirin, 1 patient were done with IFN- $\alpha 2 b$. All patients were admitted and collected $24 \mathrm{~h}$ urine to measure the nitrogen balance. During the test period, the amount of the nutritional intake was carefully monitored. By indirect calorimetry after overnight bed rest and fasting, resting energy expenditure(REE) was measured and non- 
protein respiratory quotient (npRQ) was calculated to all patients before treatment and after 1 weeks of treatment.

Results: Though no significant differences showed in several serum parameter (Aibumin, T-Bililubin, protrombin time) between pretreatment and after 1 week of treatment, AST and ALT were significantly lower, compared with the pretreatment level. Body weight and REE after 1 week of treatment were significantly lower than those of pretreatment. However, REE/Body weight after 1 week of treatment showed the higher tendency than that of pretreatment $(p=0.0578)$. The other nutrition parameter (npRQ, \%Carbon, \%Fat, and \%Protein) had no significant changes.

Conclusion: By using indirect calorimetry, it was suggested that patients with chronic hepatitis showed relatively loss of energy during interferon treatment.

\section{PP13-39}

Cerebral Glucose Metabolism and Neuropsychiatric Symptoms in Chronic Hepatitis C Patients under Antiviral Therapy

K. Sawara, Y. Yoshida, T. Oikawa, A. Miyasaka, R. Endo, Y. Takikawa,

A. Kato, K. Suzuki

Gastroenterology and Hepatology, Iwate Medical University, Morioka, Japan

Recently,antiviral therapy such as Interferon and Ribavirin combination therapy is one of the most important treatment of chronic hepatitis $\mathrm{C}$ patients and widely used all over the world. However, adverse effects of this therapy that Depression or neuropsychiatric symptoms might make it difficult to complete.

The aim of this study is to evaluate neuropsychiatric symptoms associated with antiviral therapy and its correlation of effects on cerebral glucose metabolism(CMRglu) in chronic hepatitis $\mathrm{C}$ patients. Seven patients with HCV infection including one cirrhotic patient and six chronic hepatitis patients undergoing antiviral therapy (interferon $\alpha$ or interferon $\alpha$-2b or Peg-interferon $\alpha$-2b with Ribavirin)were prospectively evaluated neuropsychiatric symptoms by neuropsychiatric test and Self-rating Depression Scale(SDS) and assessed cerebral glucose metabolism(CMRglu)using [18F]deoxyglucose positron emission tomography (FDG-PET) before and at the 8th week of treatment.

Compare to before and at 8th week of treatment, SDS points of all patients were worsened. Viral load of $\mathrm{HCV}$ of six patients were decreased. Viral load of HCV of one patient was unchanged. Digit symbol test(DST) of two patients were improved. DST of two patients were unchanged. DST of one patient was worsened. Block design test(BST) of one patient of was improved. BST of four patients of were worsened. CMRglu of six patients were $1-24 \%$ decreased in whole of the brain region. CMRglu of one patient was increased in the most of all brain regions. These results suggest that antiviral therapy affects on cerebral glucose metabolism and Depression or neuropsychiatric symptoms and its abnormalities in chronic hepatitis $\mathrm{C}$ patients. This study is undergoing and needed further study to evaluate these effects before and after antiviral study.

\section{PP13-40}

Antiviral Therapy in HCV Cirrhotic Patients: Pre-treatment and Early On-treatment Haematological Parameters Predict Therapy Response

I. Carey, A. Mendes, D. Joshi, S. Knighton, A. Gera, M. Al-Freah, A. Suddle, K. Agarwal

Institute of Liver Studies and Transplantation, King's College London School of Medicine at King's College Hospital, London, UK

Lower treatment response rates to pegylated interferon-alpha (PegIFN) + ribavirin are noted in patients with chronic hepatitis $\mathrm{C}(\mathrm{CH}-\mathrm{C})$ with compensated cirrhosis, but only limited information is available on treatment outcome predictors.
Aims: To assess impact of pre-treatment, on-treatment haematological, biochemical and clinical characteristics of patients with $\mathrm{CH}-\mathrm{C}$ cirrhosis without portal hypertension on therapy response.

Patients: Sixty-six patients with $\mathrm{CH}-\mathrm{C}$ cirrhosis without portal hypertension (96\% Child-Pugh A, median MELD 13 and UKELD 44), median age 51 years, 50 males; treated with Peg-IFN-2a and weight based Ribavirin( $13 \mathrm{mg} / \mathrm{kg} /$ day) according to their genotype (24-72 weeks) between July 2006-December 2009 were divided into 3 groups by response: sustained responders (SVR) $n=20$ (30\%), relapsers (Rel) $n=24$ (37\%) and non-responders (NR) $n=22$ (33\%).

Methods: HCV-RNA viral load, HCV genotype (G), sodium, bilirubin, creatinine and albumin levels, prothrombin time, haemoglobin (Hb) levels and neutrophil (ANC), platelets (PLT) counts, ChildPugh, MELD and UKELD scores were assessed at baseline and at different time-points: treatment week 4 (TW4), TW8, TW12, TW24 (G1and4) and at the end of therapy (EOT) and compared with therapy outcome.

Results: Baseline HCV-RNA viral load was similar in SVR, Rel and NR. SVR was significantly lower (13 vs. .54\%, $p<0.01$ ) in G1 and 4 patients. No differences (all median, range) in Child-Pugh $(5,5-12)$, $\operatorname{MELD}(13,10-16)$ and $\operatorname{UKELD}(44,39-50)$ scores at any point were detected. Baseline median ANC and PLT [both $\times 10^{9} / \mathrm{ml}$ ] were lower in NR than SVR and Rel (ANC 2.43 vs. 3.67 and 3.21, $p=0.04$ and PLT 122 vs.142 and 156,p=0.05). Baseline $\mathrm{Hb}$ levels [g/dl] were similar in all patients, but decreased significantly during therapy at TW4, TW8 and TW12 in SVR than in NR and Rel (TW4 1.9 vs. 1 vs. $1.2, p=0.03$; TW8 2.6 vs. 1.9 vs. $2.2, p=0.03$ and TW12 3.5 vs. 2.8 vs. $3, p=0.04$ ). There was no difference in Peg-IFN/Ribavirin dose reductions and use of haematological growth factors between groups.

Conclusions: HCV genotype, early Hb decrease by TW12 (perhaps reflecting inter-individual ribavirin metabolism); and higher baseline ANC and PLT counts were predictive of response.

\section{PP13-41}

Liver Histology of Chronic Hepatitis C Patients who Relapsed or Not Responded to Conventional Interferon and Ribavirin Therapy N. Butt ${ }^{1,2}$, A. Abbasi ${ }^{2}$, A.-R. Bhutto ${ }^{2}$, S.M. Munir ${ }^{1,2}$

${ }^{I}$ Gastroenterology, Aga Khan University Hospital; ${ }^{2}$ Hepatology Section, Medical Unit III, Jinnah Postgraduate Medical Centre, Karachi, Pakistan

Background: Liver biopsy is an important tool to determine the response of treatment in chronic hepatitis $\mathrm{C}$ nonresponders and relapsers of conventional interferon and ribavirin therapy.

Objective: To evaluate liver histology of chronic hepatitis $\mathrm{C}$ patients, nonresponders and relapsers to previous conventional interferon and ribavirin therapy.

Design: Descriptive case series

Patients and methods: This study was conducted in Hepatology section of Medical Unit-III, Jinnah Post Graduate Medical Centre Karachi, Pakistan from January 2008 to December 2008. We recruited 109 hepatitis $\mathrm{C}$ patients who had relapsed or not responded to conventional interferon and ribavirin for at least 24 weeks. Patients had previous course of conventional interferon monotherapy, age below 17 years, concomitant evidence of other viral infection, disease or alcohol abuse and decompensated cirrhosis were excluded. All the patients were subjected to liver biopsy. The inflammatory activity and fibrosis of liver biopsies were assessed according to Batts-Ludwig classification. Computer program SPSS version 15 was used to manage and analyze data.

Results: A majority of the 109 patients were female with HCV genotype 3. Among these $100(91.7 \%)$ patients were nonresponders and $9(8.3 \%)$ were relapsers. Mean age of nonresponders and relapsers were $38.9 \pm 8.8$ years. Nonresponders had elevated levels 
of serum aminotransferases. According to Batts-Ludwig classification Grade 0 inflammation was not present in nonresponders and relapsers, grade 1 in $51(46.8 \%)$, grade 2 in $47(43.1 \%)$, grade 3 in $10(9.2 \%)$ and grade 4 in $1(0.9 \%)$ of nonresponders and relapsers. Stage 0 fibrosis was present in $10(9.2 \%)$, stage 1 in 34 (31.2\%), stage 2 in 36 $(33.0 \%)$, stage 3 in $13(11.9 \%)$ and stage 4 in $16(14.7 \%)$ of nonresponders and relapsers.

Conclusion: Our data suggest that even not eradicating HCV, we are able to halt the progress of necroinflammation and fibrosis with combination of conventional interferon and ribavirin therapy.

\section{PP13-42}

Evidence-based Antiviral Therapies of a Patient with Hepatitis C after Kidney Transplantation

F. Ao, Q. He, W. Tang

Shenzhen Third People's Hospital, Shenzhen, China

Backgroud: To formulate an evidence-based treatment plan for a patient with hepatitis $\mathrm{C}$ after kidney transplantation with combined interferon- $\alpha$ and ribavirin;

Methods: Based on an adequate assessment of the patient's condition and using the principle of PICO, we searched The Cochrane library (Issue 1, 2009), PubMed (1995 to March 2009) and CHKD (1995 to end of 2008);

Results: 5 case reports, 11 cohort studies, 1 meta-analysis report, and total 17 papers in English; 1 study report in Chinese. To summarize the above case series (or case) reports, one meta analysis and one article in Chinese, the treatments using IFN $\alpha$ or combined with Ribavirin, sustained virological response rate was approximately $28.1 \%$, the occurrence rate of transplanted renal function impairment was approximately $23.3 \%(\mathrm{NNH}$, transplanted kidney function impairment or loss occurred in roughly one out of four cases). And among post-kidney transplantation patients, the risk of death in anti-HCV positive patients was 1.41 times of that in anti-HCV negative patients. Therefore, with treatments, the relative risk reduction $(\mathrm{RR})$ was $1.41 \times 28.1 \%=39.6 \%$; the number of patients needed to treat (NNT) in order to have one effective case was $1 / \mathrm{RR} \approx 2.5$; the likelihood of being helped versus harmed $(\mathrm{LHH})$ was $1 / \mathrm{NNT}: 1 / \mathrm{NNH}=1 / 2.5: 1 / 4=1.6$, indicating that patients could obtain more benefits than harms with treatments. According to the current evidence, PEG-IFN $\alpha-2 \mathrm{~b} 50 \mu \mathrm{g} /$ week plus Ribavirin $600 \mathrm{mg} /$ day was given to the patient for 12 months. No adverse effect occurred. Beginning at the completion of 2 months of treatment, HCV RNA turned to negative, liver and kidney function were maintained normal. Follow-up for 40 week after end of treatment, liver and kidney function were normal, and HCV RNA is negative too.

Conclusion: Peginterferon alfa plus Ribavirin for the treatment is good for hepatitis $\mathrm{C}$ after kidney transplantation.

\section{PP13-43}

A Case Report - Increased Serum Alpha-fetoproten (AFP) Level Depending on the Pegylated Interferon (PEG-INF) Plus Ribavirin H. Maeno

Surgery, Tokunoshima Tokushukai Hospital, Ooshima-Gun, Japan

Combination pegylated interferon-2a (PEG-INF) plus ribavirin are standard therapy for chronic hepatitis C. Most references were reported combination therapy improved serum aminotransferase level and lower serum alpha-fetoprotein (AFP) level. Especially, serum AFP level is good indicator for biological responder or hepatocarcinogenesis. We report an experienced case extraordinary serum AFP level patient for PEG-INF plus ribavirin combination therapy. A 63year-old man who had received S6 hepatocellular carcinoma (HCC) resection since 6 months, he has chronic hepatitis $C$ (genotype 1b) without HCC. The serum AFP level was slightly higher than normal range (AFP $20.9 \mathrm{ng} / \mathrm{ml}$ : cut off $10 \mathrm{ng} / \mathrm{ml}$ ) befor treatment PEG-INF plus ribavirin. Two months later, serum AFP level was increased about 20 times level (AFP $201.5 \mathrm{ng} / \mathrm{ml}$ ) compared with normal level. We had suspected a HCC recurrence. The patients were performed several examination, but there was no recurrence HCC intrahepatic, extrahepatic and the hole body. Finally, AFP level was increased 100 times level (AFP 1079.2ng/ml), we interrupted PEG-INF and ribavirin combination therapy. Because, HCC recurrence was not complete contradicted. However, after three months later interrupted combination therapy, AFP level was reverted normal level (AFP $3.9 \mathrm{ng} / \mathrm{ml}$ ). There was no report further increased AFP level by PEGINF and ribavirin combination therapy, we report on this case with some bibioraphical comments.

\section{PP13-44}

Re-treatment, with Peginterferon-alpha 2a, of Patients with Chronic Hepatitis C Non Responders to Previous Treatment with Interferon-alpha 2b

G. Scotto ${ }^{1}$, V. Fazio $^{2}$

${ }^{1}$ Infectious Diseases, OO RR Foggia; ${ }^{2}$ Laboratory, OORR Foggia, Foggia, Italy

Background: HCV-related chronic infection refractory to previous therapy is common. The data about retreatment of non responders are, at moment, inadequate. Aim of this study was to determine the efficacy and tolerability of pegylated interferon a-2a plus ribavirin in patients who have failed to respond to the combination pegylated interferon $a-2 b$ plus ribavirin

Patients and methods: 24 patients non-responders, all with genotype $1,14 / 24(58.3 \%)$ with high viral load (>800,000 IU/ml), 6/24 (25\%) with cirrhosis were enrolled. The patients received one vial of PegIFN alpha-2a weekly $(180 \mu \mathrm{g})$ subcutaneously. Interferon was combined with ribavirin (1,000 or 1,200 mg/day) and all patients were treated for 48 weeks, with a 24-week follow-up.

Results: By intent-to-treat analysis, 3/24 (12.5\%) patients discontinued treatment due to severe side-effects. At the end of therapy, HCV-RNA was undetectable in 4/24 (16.7\%) patients, three with low viral load and one with high viral load. Among the patients with $\mathrm{HCV}$ RNA $<800,000 \mathrm{IU} / \mathrm{ml}$, two presented a rapid virological response (RVR) and one early virological response (EVR), while the other patient (HCV RNA $>800,000 \mathrm{IU} / \mathrm{ml}$ ) achieved a late virological response (after week 24). The patients with viral load $<800,000 \mathrm{UI} / \mathrm{ml}$ presented lower baseline fibrosis scores (F0-F3) and in the same group of patients a $>2-\log _{10}$ decrease in HCV RNA load with previous treatment was achieved. When terminating follow-up, a sustained virological response (SVR), defined as undetectable $(<34 \mathrm{IU} / \mathrm{ml}) \mathrm{HCV}$ RNA levels, was observed in all responder patients.

Conclusions: Retreament of pegylated interferon $a-2 b$ and ribavirin non responders with pegylated interferon a-2a and ribavirin is safe and efficacious and can be considered a very promising retreatment strategy for subjects failing previous therapy, especially in patients with lower base-line fibrosis

\section{PP13-45}

Categorical Week 4 Response Predicts SVR Rates in HIV-HCV Patients Receiving Peginterferon Alfa-2a Plus Ribavirin in the PARADIGM Study M.S. Sulkowski ${ }^{1}$, J. Slim ${ }^{2}$, R.K. Sterling ${ }^{3}$, T. Hassanein ${ }^{4}$, R. Solà ${ }^{5}$, R. Serrão ${ }^{6}$, C. Martorell ${ }^{7}$, A. Bertasso ${ }^{8}$, S. Stancic ${ }^{8}$, M. Rodriguez-Torres ${ }^{9}$, On Behalf of the PARADIGM Study Investigators

${ }^{1}$ Johns Hopkins University School of Medicine, Baltimore, MD;

${ }^{2}$ St. Michael's Medical Center, Newark, NJ; ${ }^{3}$ Virginia Commonwealth University, Richmond, VA; ${ }^{4}$ University of California San Diego, San Diego, CA, USA; ${ }^{5}$ Hospital del Mar, Barcelona, Spain; ${ }^{6}$ Hospital São João, Porto, Portugal; ${ }^{7}$ The Research Institute, Springfield, MA; ${ }^{8}$ Roche, Nutley, $N J ;{ }^{9}$ Fundación de Investigación De Diego, Santurce, PR, USA 
Background: In HIV-HCV co-infected patients, a rapid virologic response (RVR) with peginterferon plus ribavirin is highly predictive of achieving a sustained virologic response (SVR). We examine whether, in co-infected non-RVR patients, the probability of achieving an SVR is influenced by the magnitude of decline in HCV RNA at week 4.

Methods: The PARADIGM study randomised 410 co-infected genotype 1 patients to 48 weeks of $180 \mu \mathrm{g} /$ week peginterferon alfa-2a (40KD) plus ribavirin ( 800 or $1,000 / 1,200 \mathrm{mg} /$ day based on weight). In this analysis, patients were grouped by RVR status at week 4 . NonRVR patients were subdivided into mutually exclusive categories based on the decrease in HCV RNA at week 4, and within each category the proportion of patients with complete early virological response (cEVR) and SVR was calculated. In PARADIGM, RVR, cEVR and SVR rates were similar for both ribavirin doses, thus data were combined.

Results: Co-infected patients with an RVR had higher SVR rates versus non-RVR patients (71 vs. 17\%, respectively). In non-RVR patients, the probability of an SVR and a cEVR was graded according to the magnitude of the decrease in HCV RNA at week 4, with the highest being in those with at least a $2-\log _{10}$ decrease. Among nonRVR patients that achieved a cEVR, 64\% achieved an SVR (Table 1). Conclusion: Non-RVR co-infected patients who achieve at least a 2$\log _{10}$ decrease in HCV RNA by week 4 of treatment, or a cEVR, have good rates of SVR when treated with peginterferon alfa-2a (40KD) plus ribavirin.

Acknowledgement: Funded by Roche, Basel, Switzerland.

Table 1 Data from 22 patients were missing values lor HCV RMA after week 4

\begin{tabular}{lccc}
\hline WSC4HCVRNA & $\begin{array}{l}n(\%) \\
n=410\end{array}$ & $\begin{array}{l}\text { eEVR/SVR according } \\
\text { to response at week 4 } \\
\text { and 12, respectively }\end{array}$ \\
\cline { 2 - 4 } & & cEVR & SVR \\
\hline $\begin{array}{l}\text { RVR (HCV RNA } \\
\quad<2 \text { IU/mL) }\end{array}$ & $31(8)$ & $26 / 31(84)$ & $20 / 26(77)$ \\
No RVR & $357(87)$ & $74 / 357(21)$ & $47 / 74(64)$ \\
$\geq 3$-log drop & $32(8)$ & $29>32(91)$ & $18 / 29(62)$ \\
$\geq 2$ to $<3-\log$ drop & $60(15)$ & $27 / 60(45)$ & $19 / 27(70)$ \\
$\geq 1$ to $<2-\log$ drop & $115(26)$ & $16 / 115(14)$ & $8 / 16(50)$ \\
$<1-\log$ drop & $150(37)$ & $2 / 150(1)$ & $2 / 2(100)$ \\
\hline
\end{tabular}

\section{PP13-46}

Genotype-specific Predictors of Early Viral Clearance among Chronic Hepatitis C Patients Treated with Peginterferon Alfa-2a (40KD) Plus Ribavirin

M.L. Shiffman ${ }^{1}$, D. Nelson ${ }^{2}$, P. Ferenci ${ }^{3}$, M. Bourliere ${ }^{4}$, M. Curescu ${ }^{5}$,

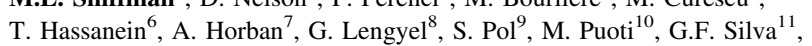
G.M. Dusheiko ${ }^{12}$, M. Schmitz ${ }^{13}$, F. Tatsch ${ }^{14}$, A. Mangia ${ }^{15}$

${ }^{1}$ Liver Institute of Virginia, Bon Secours Health System, Newpot News, VA; ${ }^{2}$ University of Florida, Gainsville, FL, USA; ${ }^{3}$ Medical University of Vienna, Vienna, Austria; ${ }^{4}$ Hospital Saint Joseph, Marseille, France; ${ }^{5}$ University of Medicine and Pharmacy Timisoara, Timisoara, Romania; ${ }^{6}$ University of California, San Diego, CA, USA; ${ }^{7}$ Medical University Clinic of Infectious Diseases, Warsaw, Poland; ${ }^{8}$ Semmelweis University, 2nd Dept.of Medicine, Budapest, Hungary; ${ }^{9}$ Hopital Necker-Enfants Malades, Paris, France; ${ }^{10}$ University of Brescia, Brescia, Italy; ${ }^{11}$ Botucatu School of Medicine, Botucatu,
Brazil; ${ }^{12}$ Royal Free and University College School of Medicine, London, UK, ${ }^{13}$ IST GmbH, Mannheim, Germany; ${ }^{14}$ Roche, Basel, Switzerland; ${ }^{15}$ Hospital Casa Sollievo Della Sofferenza, Rotondo, Italy

Background: The PROPHESYS trial is a large, multinational study of patients treated for chronic hepatitis C. According to countryspecific requirements, PROPHESYS 1 follows patients prescribed peginterferon alfa-2a (40KD) $($ PegIFN $\alpha-2 a)+$ ribavirin. PROPHESYS 2 and 3 include combination therapy with $\operatorname{PegIFN} \alpha-2 \mathrm{a} /$ ribavirin or PegIFN $\alpha$-2b/ribavirin. Here, we present the results of an interim analysis investigating predictors of viral clearance during the first 12 weeks of treatment with PegIFN $\alpha$-2a/ribavirin.

Methods: HCV mono-infected treatment-naive patients with hepatitis C virus (HCV) genotype (G) 1, 2 or 3, initiating treatment on/ before 31 Oct 2009 with available baseline HCV RNA data were included in this analysis. Rapid and complete early virological response (RVR and cEVR; mutually exclusive groups) were defined as undetectable $\mathrm{HCV}$ RNA $(<15 \mathrm{IU} / \mathrm{mL})$ at weeks 4 and 12 , respectively. Generalised additive model and multiple logistic regression analyses (stepwise selection) explored baseline predictors associated with RVR.

Results: This interim analysis included 5,730 patients. RVR was achieved by 21,75 and $65 \%$ of G1, G2 and G3 patients, respectively, and cEVR by 32,11 and $18 \%$ of G1, G2, G3 patients, respectively. Baseline factors significantly associated with RVR are presented in the table.

Conclusion: Baseline factors associated with RVR varied according to genotype. When making treatment decisions for patients infected with $\mathrm{HCV}$, clinicians should consider different factors depending on the patient's genotype.

Acknowledgement: Funded by Roche, Basel, Switzerland.

Baseline predictors of RVR Odds ratios $\{95 \% \mathrm{Cl})$ significantly different in 1 in trie final models $(p<0.05)$

\begin{tabular}{llll}
\hline & $\begin{array}{l}\mathrm{G} 1 \\
(N=3,867)\end{array}$ & $\begin{array}{l}\mathrm{G} 2 \\
(N=825)\end{array}$ & $\begin{array}{l}\mathrm{G} 3 \\
(N=1,038)\end{array}$ \\
\hline Age (per 10-year $\downarrow)$ & $1.20(1.11-1.29)$ & - & $1.36(1.18-1.55)$ \\
HCV RNA (per 1-log $\downarrow)$ & $3.13(2.82-3.50)$ & - & $1.38(1.18-1.62)$ \\
Weight (per 10-unit $\downarrow)$ & $1.11(1.04-1.18)$ & - & - \\
Platelets $(\geq 140$ vs. & $2.22(1.61-3.05)$ & $1.98(1.28-3.09)$ & \\
$\left.\quad<140 \times 10^{9} / \mathrm{L}\right\}$ & & & - \\
ALT ratio $(>3$ vs. $\leq 3)$ & $192(1.57-2.36)$ & - & - \\
Male vs. female & $157(1.29-1.91)$ & - & - \\
Asian vs. white & $2.40(1.20-4.82)$ & - & - \\
Black vs. white & $0.49(0.23-0.84)$ & - & $1.78(1.19-2.67)$ \\
No cirrhosis vs. cirrhosis & $1.89(1.48-2.42)$ & - & \\
Histology not assessed & $2.58(197-3.38)$ & - & - \\
$\quad$ vs. cirrhosis & & & \\
\hline
\end{tabular}

$\downarrow$ Decrement

PP13-47

Durability of Sustained Virological Response (SVR) and Incidence of Clinical Events Following Peginterferon Alfa-2a \pm Ribavirin: PEGASYS Long-term Follow-up Study

M.G. Swain ${ }^{1}$, M.Y. Lai ${ }^{2}$, M.L. Shiffman ${ }^{3}$, W.G.E. Cooksley ${ }^{4}$, S. Zeuzem ${ }^{5}$,

D.T. Dieterich ${ }^{6}$, A. Abergel ${ }^{7}$, M.G. Pessoa ${ }^{8}$, A. Lin $^{9}$, F. Tatsch ${ }^{10}$, E. Connell ${ }^{9}$, M. Diago ${ }^{11}$

${ }^{1}$ University of Calgary, Calgary, AB, Canada; ${ }^{2}$ National Taiwan University College of Medicine and National Taiwan University Hospital, Taipei, Taiwan R.O.C.; ${ }^{3}$ Liver Institute of Virginia, Bon Secours Health System, Newport News, VA, USA; ${ }^{4}$ Royal Brisbane 
Hospital, University of Queensland, Brisbane, QLD, Australia;

${ }^{5}$ J. W. Goethe-University Hospital, Frankfurt, Germany; ${ }^{6}$ Mount Sinai School of Medicine, New York, NY, USA; ${ }^{7}$ Hôpital Hotel Dieu, Clermont-Ferrand, France; ${ }^{8}$ University of São Paulo School of Medicine and Instituto de Infectologia Emilio Ribas, São Paulo, Brazil; ${ }^{9}$ Roche, Nutley, NJ, USA; ${ }^{10}$ Roche, Basel, Switzerland;

${ }^{11}$ Hospital General de Valencia, Valencia, Spain

Background: SVR in hepatitis C virus (HCV)-infected patients is associated with improvements in liver histology and reduced risk of hepatocellular carcinoma and liver-related mortality. Data regarding long-term durability of SVR and incidence of liver-related morbidity/ mortality following peginterferon-based treatment are limited. This prospective long-term follow-up study of a large diverse cohort of patients assessed the durability of SVR and the incidence of significant clinical events in patients achieving an SVR following interferon-based treatment.

Methods: HCV-infected patients recruited from 10 trials of interferon-based therapies \pm ribavirin were assessed for significant liverrelated clinical events. In HCV RNA serum-negative patients $(<50 \mathrm{IU} / \mathrm{mL}$ at the final week 24 follow-up assessment), the durability of undetectable serum HCV RNA was assessed. The efficacy population consisted of (a) all patients previously treated with conventional/pegylated interferon \pm ribavirin and (b) the subset of naive patients previously treated with peginterferon alfa-2a $(40 \mathrm{kD})$ \pm ribavirin, all with undetectable HCV RNA at the last visit of the original study.

Results: Among 1,724 patients evaluable for safety, 25 significant clinical events were detected in $21(1.2 \%)$ patients: ascites $(n=5)$, encephalopathy $(n=4)$, hepatic malignancy $(n=1)$ and death ( $n=15 ; 3$ hepatic related). Of the 21 patients, 8 had significant hepatic-related clinical events, of whom 4 had baseline cirrhosis. No liver transplantations were reported. In the efficacy population, 99\% $(1331 / 1343)$ of patients previously treated with peginterferon alfa-2a \pm ribavirin continued to have undetectable HCV RNA after mean follow-up 3.9 years. Less than $1 \%(12 / 1343)$ of patients in the peginterferon alfa-2a \pm ribavirin group became HCV RNA detectable after a mean of 1.8 years.

Conclusion: SVR achieved with peginterferon alfa- $2 \mathrm{a} \pm$ ribavirin should be considered a cure for HCV and is associated with a low incidence of significant clinical events.

Acknowledgement: Funded by Roche, Basel, Switzerland.

\section{PP13-48}

The Frequency and Grade of Leukopenia and Neutropenia in Patients with Chronic Hepatitis C during Different Combinations of Antiviral Therapy

I. Bakulin, A. Sharabanov

Gastroenterology, State Institute of Physicians' Post-Graduate

Education, Moscow, Russia

Background: Antiviral therapy in patients with chronic hepatitis $\mathrm{C}$ (CHC) has been associated with a risk of leukopenia (LP) and neutropenia (NP). IFN-dose reduction or discontinuation is the way for management of such adverse events (AE). These measures reduces the probability to achieve a sustained virological response. This study was aimed to estimate the frequency of different LP/NP grades during antiviral therapy.

Methods: 78 patients with $\mathrm{CHC}$ (61.5\% men, 48.7\% 1 genotype), median age $33.5 \pm 7.6$, treated with PEG-IFN $\alpha 2 \mathrm{a}$ /ribavirin $(74.3 \%)$ or IFN $\alpha 2 \mathrm{a} /$ ribavirin were included in a retrospective study. LP was estimated for 4 grades $\left(\times 10^{9} / 1\right)$ : mild $(2.0-2.5)$, moderate $(1.5-1.99)$, severe $(1.0-1.49)$ and potentially life threatening $(<1.0)$; the same was for NP: mild (1.0-1.3), moderate $(0.75-0.99)$, severe $(0.5-0.74)$ and potentially life threatening $(<0.5)$. Latent grade was considered to be $2.51-4.1$ for LP and 1.31-2.03 for NP.

Results: $42.3 \%$ patients $(n=33)$ meet LP,the frequency of NP was $62.8 \%(n=49)$. Both LP and NP were diagnosed most frequent on 12 week of antiviral therapy-32.9 and $42.1 \%$ of patients respectively. Minimal count of WBC was $1.3 \times 10^{9} / 1$, neutrophils$0.21 \times 10^{9} / 1$. The frequency and severity of LP/NP were higher in patients treated with PEG-IFN $\alpha 2 \mathrm{a} /$ ribavirin

LP/NP grades and frequency (\%)

\begin{tabular}{llll}
\hline & $\begin{array}{l}\text { All patients } \\
(n=78) \text { LP/NP }\end{array}$ & $\begin{array}{l}\text { PEG-IFN-a2a } \\
(n=57) \text { LP/NP }\end{array}$ & $\begin{array}{l}\text { IFN-a2a } \\
(n=21) \\
\text { LP/NP }\end{array}$ \\
\hline Mild & $39.7 / 55.1$ & $54.4 / 66.7$ & $0 / 8.8$ \\
Moderate & $19.2 / 37.2$ & $26.3 / 47.4$ & $0 / 3.5$ \\
$\begin{array}{l}\text { Severe } \\
\text { Potentially life } \\
\quad \text { threatening }\end{array}$ & $0 / 5.1$ & $5.3 / 19.3$ & $0 / 0$ \\
Total & $42.3 / 62.8$ & $0 / 7.0$ & $0 / 0$ \\
\hline
\end{tabular}

There were no cases of infectious complications associated with LP/NP.

Conclusion:

1. $\mathrm{LP} / \mathrm{NP}$ are one of the most frequent hematological $\mathrm{AE}$ of antiviral therapy

2. The most severe frequency of LP/NP was fixed on 12 week of therapy

3. Severe and life threatening grades are more typical for NP.

\section{PP13-49}

Peginterferon Alpha-2a in Chronic Hepatitis C Bangladeshi Patients Treated for 24 Weeks Irrespective of Genotypes: Results of 112 Cases

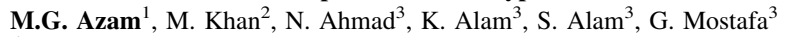
${ }^{1}$ Department of Gastroenterology and Hepatology, BIRDEM

Hospital; ${ }^{2}$ Hepatology, The Liver Centre; ${ }^{3}$ Hepatology, Bangabandhu Sheikh Mujib Medical University, Dhaka, Bangladesh

Background/aims: In Bangladesh, about $1 \%$ of apparently healthy populations are positive for antibody against hepatitis $\mathrm{C}$ virus (antiHCV). Its long-term consequences lead to chronic hepatitis, cirrhosis of liver and hepatocellular carcinoma. This study was carried out to compare efficacy and safety of peginterferon $\alpha$-2a alone or in combination with ribavirin treated for 24 weeks irrespective of genotypes.

Methods: A total of 112 patients were included in this study after meeting the following criteria: age 18-60 years, anti-HCV+ve for $>6$ months, ALT $>2$ XULN, detectable HCV-RNA. Genotype 3 was seen in $83(74.1 \%)$ patients, genotype 1 was found in $24(21.4 \%)$ patients, $5(4.4 \%)$ patients were infected with genotype 4 . The patients were divided into two groups. Group A comprised of 55 patients who received peginterferon alpha-2a 180 mgm subcutaneously weekly 
while group B comprised of 57 patients who received peginterferon alpha-2a in same dose with oral ribavirin $800-1,000 \mathrm{mg}$ daily for 24 weeks. The patients were followed up monthly up to 6 months after completion of therapy.

Results: Of the total 112 patients, $10(9.1 \%)$ patients were withdrawn prematurely from the study for adverse events. In both groups with similar pattern, $85 \%$ (86/102) patients were negative for HCV RNA at end of treatment (ETR). So far 74 patients completed 48 weeks follow up (group A 38 and group B 36 patients). Among group A, four (4/ $38 ; 10.5 \%)$ patients relapsed while all patients of group B maintained sustained viral response (SVR) $(p<0.01)$.

Conclusions: Peginterferon alpha-2a is a well-tolerated drug with promising ETR and encouraging SVR. Among chronic hepatitis C patients in Bangladesh, combination therapy with ribavarin is more effective than peginterferon monotherapy. However, cost of treatment is high for a developing country like us.

\section{PP13-50}

Intensified Peginterferon Alfa-2a (40KD) Dosing Increases Sustained Virological Response Rates in Genotype 1 Hepatitis C Patients with Elevated Low-density Lipoprotein

S.A. Harrison ${ }^{1}$, D. Abdurakhmanov ${ }^{2}$, M.L. Shiffman ${ }^{3}$, I. Bakulin ${ }^{4}$, W. Mazur ${ }^{5}$, M. Rodriguez-Torres ${ }^{6}$, G.F. Silva ${ }^{7}$, H. Cheinquer $^{8}$, M. Hohmann ${ }^{9}$, F. Tatsch ${ }^{10}$, K.R. Reddy ${ }^{11}$, OnBehalf of the PROGRESS Investigators

${ }^{1}$ Brooke Army Medical Center, Fort Sam Houston, TX, USA;

${ }^{2}$ Moscow Medical Academy, Moscow, Russia; ${ }^{3}$ Liver Institute of Virginia, Bon Secours Health System, Newport News, VA, USA;

${ }^{4}$ State Postgraduate Medical Institute, Ministry of Defence of the Russian Federation, Moscow, Russia; ${ }^{5}$ Medical University of Silesia, Chorzów, Poland; ${ }^{6}$ Fundacion de Investigacion De Diego Santurce, Santurce, PR, USA; ${ }^{7}$ Botucatu School of Medicine, Botucatu; ${ }^{8}$ Hospital de Clinicas de Porto Alegre, Porte Alegre, Brazil; ${ }^{9}$ IST

\section{GmbH, Mannheim, Germany; ${ }^{10}$ Roche, Basel, Switzerland;}

${ }^{11}$ University of Pennsylvania, Philadelphia, PA, USA

Background: Patients infected with hepatitis C virus (HCV) who have elevated low-density lipoprotein (LDL) levels achieve higher sustained virological response (SVR) rates following peginterferon/ ribavirin treatment versus patients with lower LDL. Whether SVR rates in patients with low/elevated LDL can be improved by dose intensification is unclear.

Methods: In PROGRESS, genotype 1 patients were randomised to 48 weeks of $180 \mu \mathrm{g} /$ week peginterferon alfa-2a $(40 \mathrm{kD})$ plus ribavirin (Arm A: 1,200 mg/day; Arm B: 1,400/1,600mg/day) or 12 weeks of $360 \mu \mathrm{g} /$ week peginterferon alfa-2a (40 kD) followed by 36 weeks of $180 \mu \mathrm{g} /$ week plus ribavirin (Arm C: 1,200mg/day; Arm D: 1,400/ $1,600 \mathrm{mg} /$ day). This retrospective analysis of PROGRESS assessed SVR rates among patients with low $(<100 \mathrm{mg} / \mathrm{dL})$ or elevated $(\geq 100 \mathrm{mg} / \mathrm{dL}$ ) fasting LDL.

Results: Patients with high LDL $(n=266)$ had higher baseline HCV RNA $\left(6.0 \times 10^{6} \mathrm{IU} / \mathrm{mL}\right)$ versus patients with low LDL $(n=271$; $\left.4.2 \times 10^{6} \mathrm{IU} / \mathrm{mL}\right)$. Apart from Arm A, SVR rates were higher in patients with elevated LDL versus low LDL (A 35 vs. 38\%, B 53 vs. $42 \%$, C 59 vs. $42 \%$, D 60 vs. $27 \%$ ). Multiple logistic regression analysis detected significant interaction $(p=0.011)$ between intensified peginterferon alfa-2a dose and LDL on SVR. Further analysis identified younger age, genotype $1 \mathrm{~b}$ (vs. genotype 1a), low steatosis score and low viral load to be significantly associated with SVR $(p<0.05)$; a significant relationship between treatment and SVR was identified in patients with high LDL.

Conclusion: This retrospective analysis demonstrates that intensified dosing of peginterferon alfa-2a (40 kD) increases SVR rates among genotype 1 patients with elevated LDL. Further confirmatory studies are recommended.
Acknowledgement: Funded by Roche, Basel, Switzerland.

Patients with high LDL ( $\geq 100 \mathrm{mg} / \mathrm{dL})$

\begin{tabular}{|c|c|c|c|c|}
\hline Parameter & $\begin{array}{l}\text { PegIFN } \\
\text { alfa-2a } \\
180+\text { RBV } \\
1,200 \\
(n=46)\end{array}$ & $\begin{array}{l}\text { PegIFN } \\
\text { alfa-2a } \\
180+\mathrm{RBV} \\
1,400 / \\
1,600 \\
(n=45)\end{array}$ & $\begin{array}{l}\text { PegIFN } \\
\text { alfa-2a } \\
360 / \\
180+\mathrm{RBV} \\
1,200 \\
(n=91)\end{array}$ & $\begin{array}{l}\text { PegIFN } \\
\text { alfa-2a } \\
360 / \\
180+\mathrm{RBV} \\
1,400 / 1,600 \\
(n=64)\end{array}$ \\
\hline \multicolumn{5}{|l|}{ Baseline (median) } \\
\hline Male, $n(\%)$ & $38(83)$ & $39(87)$ & $73(80)$ & $70(83)$ \\
\hline $\begin{array}{c}\text { Mean age, } \\
\text { years }\end{array}$ & 45.8 & 43.8 & 45.5 & 44.2 \\
\hline $\begin{array}{c}\text { Caucasian, } \\
n(\%)\end{array}$ & $41(89)$ & $33(84)$ & $79(87)$ & $80(95)$ \\
\hline Weight, kg & 938 & 98.4 & 93.3 & 94.0 \\
\hline $\begin{array}{c}\text { HCV RNA, } \\
\text { lU/mL }\end{array}$ & $5,134,313$ & $5,678,644$ & $5,518,347$ & $7,283,369$ \\
\hline \multicolumn{5}{|l|}{ Response, $n$} \\
\hline RVR (week 4) & $8(17)$ & $8(18)$ & $31(34)$ & $25(30)$ \\
\hline EVR (week 12) & $27(59)$ & $31(69)$ & $64(70)$ & $66(79)$ \\
\hline SVR & $16(35)$ & $24(53)$ & $54(59)$ & $50(60)$ \\
\hline Relapse & $10(40)$ & $8(25)$ & $13(19)$ & $10(17)$ \\
\hline
\end{tabular}

\section{PP13-51}

Analysis for the Predictors of Rapid Virological Response in Therapy of Patients with Chronic Hepatitis C of High Viral Loads

Z. Xu, X.-H. Zhang

The Third Affiliated Hospital of Sun Yat-sen University, Guangzhou, China

Objective: To survey the predictors of rapid virological response(RVR) during antiviral therapy of patients with chronic hepatitis $\mathrm{C}$ of high viral loads.

Method: 55 patients with chronic hepatitis $\mathrm{C}$ of high viral loads were treated with peg-interferon $\alpha$-2a and ribavirin. All patients received a combination of peg-interferon $\alpha$-2a $(180 / 135 \mu \mathrm{g} /$ week,im) and ribavirin (900-1,200 mg/day) for therapy. Liver functions and the level of HCV-RNA in baseline and the 4th week were detected during the therapy. Logistic regression analysis then was taken and comparison between groups of different viral loads was processed.

Result: Negative correlation between the baseline level of HCV RNA and EVR was shown. Patients with baseline HCV RNA $<1 \times$ $10^{7}$ copies/mL got better RVR rate $(86.49 \%, 32 / 37)$, while the RVR rate of patients with baseline HCV RNA $\geq 1 \times 10^{7}$ copies $/ \mathrm{mL}$ was $61.11 \%(11 / 18)$. Stasistical analysis showed significant difference $(P<0.05)$.

Conclusion: The baseline HCV RNA load can be the predictor of RVR in antiviral therapy of patients with chronic hepatitis $\mathrm{C}$ of high viral loads.Patients with baseline HCV RNA level $<1 \times 10^{7}$ copies/ $\mathrm{mL}$ will reach better efficacy.

\section{PP13-52}

Efficacy of Conventional Interferon and Ribavirin Therapy in Chronic Hepatitis C Patients

A. Abbasi ${ }^{1}$, A.-R. Bhutto ${ }^{1}$, N. Butt ${ }^{1,2}$, S.M. Munir ${ }^{1,2}$

${ }^{1}$ Hepatology Section, Medical Unit III, Jinnah Postgraduate Medical Centre; ${ }^{2}$ Gastroenterology, Aga Khan University Hospital, Karachi, Pakistan 
Introduction: Hepatitis $\mathrm{C}$ virus is a major cause of chronic liver disease worldwide. Chronic hepatitis $\mathrm{C}$ is a tremendous health problem in Pakistan.

Objective: To find out efficacy of interferon-ribavirin therapy for chronic Hepatitis C patients in Pakistan.

Design: A Quasi experimental.

Patients and methods: This study was conducted in Hepatology section of Medical Unit-III, Jinnah Postgraduate Medical Centre, Karachi, Pakistan from August 2006 to December 2007. Eligible participants were patients with a positive serum antibody to HCV and had HCV-RNA detectable in serum by reverse transcription polymerase-chain-reaction (PCR). Patients were excluded from the study if there was an evidence of decompensated cirrhosis, serum $\alpha$-fetoprotein concentration of more than $50 \mu \mathrm{g} / \mathrm{L}$, HIV, co infection with hepatitis B, previous organ transplantation, psychiatric disease or poorly-controlled coexisting medical conditions. Patients were given Interferon-alfa-2b 3 million international units three times a week subcutaneously and oral ribavirin at $1,000-1,200 \mathrm{mg}$ in two to three divided doses a day for a 24 weeks. Hepatitis C virus, PCR has been performed pre treatment and at the end of the treatment. At the end of treatment over all efficacy of therapy and pretreatment predictors of therapy like age, gender, and serum Alanine aminotransferase (ALT) were assessed.

Results: Total of 404 patients was offered combination therapy that satisfied the inclusion criteria. Among these females were 243 $(61.1 \%)$ and males were 161 (39.9\%) had age range 13-60 years with mean of $36.03 \pm 9.30$ years. Out of 404,336 (83.2\%) showed response to combined Interferon and Ribavirin therapy depicted by HCV RNA by PCR at the end of 24 weeks treatment.

Conclusion: Combination therapy of interferon and ribavirin in chronic hepatitis $\mathrm{C}$ patients has still better response rate in Pakistani population. Younger age and female gender are favorable predictors while a base line ALT level is not reliable predictor of response to combination therapy

\section{PP13-53}

HCV RNA Decline within First Weeks of HCV Treatment is a Strong Predictor for SVR among Genotype 1 Patients

T. Tsertsvadze $e^{1,2,3}$, L. Sharvadze $e^{1,2,3}$, M. Karchava ${ }^{1,2,3}$, E. Dolmazashvili ${ }^{1,2,3}$, M. Zhamutashvili ${ }^{1,2}$, A. Abutidze ${ }^{1,2,3}$, L. Gatserelia ${ }^{1,2,3}$, N. Chkhartishvili ${ }^{1,2,3}$ ${ }^{I}$ Georgian-French Joint Hepatology Clinic "HEPA"; ${ }^{2}$ Department of Virology, Infectiouse Diseases, AIDS and Clinical Immunology

Research Center; ${ }^{3}$ Faculty of Medicine, Iv. Javakhishvili Tbilisi State University, Tbilisi, Georgia, Tbilisi, Georgia

Background: We evaluated the predictive value of early phase HCV RNA decline of HCV treatment for SVR among G1 patients with difference baseline viral load and moderate liver fibrosis.

Methods: 39 chronic hepatitis $\mathrm{C}$ patients infected with genotype 1 and with moderate liver fibrosis were enrolled. Patients were given peginterferon alfa and weight based ribavirin for 48 weeks. HCV RNA was measured by RT PCR with the detection limit of $25 \mathrm{Iu} / \mathrm{ml}$. Liver fibrosis was evaluated by transient elastography. HCV viral load was measured at baseline, 3, 24 and $72 \mathrm{~h}$ after initial interferon injection and at 1, 2, 4, and 12 weeks accordingly. Sensitivity, specificity, positive and negative predictive value with corresponding 95\% confidence intervals were calculated.

Results: According to the results, SVR was obtained among 16 (41\%) patients out of total 39. Of 39 patients enrolled, significant HCV RNA decline $\left(>2 \log _{10} \mathrm{Iu} / \mathrm{ml}\right)$ was observed in $7,13,16$ and 21 patients at weeks $1,2,4$ and 12 accordingly.

Table 1 Prognostic values ofviral responses at week 1, 2, 4 and 12

\begin{tabular}{lcccc}
\hline Week & $\begin{array}{l}\text { Sensitivity, } \\
\%(95 \% \text { CI })\end{array}$ & $\begin{array}{l}\text { Specificity, } \\
\%(95 \% \text { CI })\end{array}$ & $\begin{array}{l}\text { PFV, \% } \\
(95 \% \text { CI })\end{array}$ & $\begin{array}{l}\text { KPV, \% } \\
(95 \% \text { CI })\end{array}$ \\
\hline 1 & $44(19,70)$ & $100(85,100)$ & $100(56,100)$ & $72(53,86)$ \\
2 & $75(47,92)$ & $96(76,99)$ & $92(62,99)$ & $85(64,95)$ \\
4 & $81(54,95)$ & $87(65,96)$ & SI $(54,95)$ & $87(65,96)$ \\
12 & $100(76,100)$ & $78(56,92)$ & $76(52,91)$ & $100(78,100)$ \\
\hline
\end{tabular}

Conclusion: Our study showed that one week response had greater positive prognostic value compared to 2, 4 and 12 week response for SVR. However, two week response had high positive prognostic value and high sensitivity. Dramatic decline of HCV viral load at week one and two after initial interferon injection is a strong predictor for SVR among G1 patients. Prediction of SVR at initial phases of interferon treatment may be useful for better patient management. Findings of our study need to be further justified in larger cohort.

\section{PP13-54}

Chronic Hepatitis C: Detection, Prevalence of the Genotype and Treatment in Azerbaijan (Based on the Data of Baku' Private Clinics)

\section{G.S. Aghayeva}

Internal Diseases, Azerbaijan Medical University, Baku, Azerbaijan

Aims: There is not enough information about chronic hepatitis $\mathrm{C}$ (CHC) in the former USSR countries. The aim of present retrospective research is to study dynamics of revealed patients with $\mathrm{CHC}$, prevalence of genotypes, results of treatment based on data from private clinics during the period of 2005-first half of 2010 .

Methods: 1,446 patients (55\% men) with $\mathrm{CHC}$ were examined. All patients had positive PCR, genotyping was done on Roche Genotyping Kit. For all patients was calculated BMI, fasting glucose level, HOMA-IR.

Results: 1st genotype, 74\%; 2nd, 8\%; 3rd, 18\%. SVR was achieved in $74.7 \%$ of patients (genotype 1, 68\%; genotypes 2 and 3, 88\%). High percentage of diabetes mellitus $(7.5 \%)$, infringement of carbohydrates metabolism such as developed insulin resistance $(71 \%)$, increase of fasting glucose $(46 \%)$. Influence of initial fasting glucose on results of antiviral therapy was studied in the process of this research. Thus, CHC patients with initial fasting glucose $<5.5 \mathrm{mmol} / \mathrm{l}$ achieved SVR in $86 \%$ cases. Patients with fasting glucose $5.6-6.0 \mathrm{mmol} / \mathrm{l}$ achieved SVR in $68.8 \%$ cases, and only $33.3 \%$ of patients with fasting glucose $>6.6 \mathrm{mmol} / \mathrm{l}$ have achieved SVR.For the purpose of glucose metabolism correction in the group of patients with genotype 1 metformin was added to standard antiviral treatment scheme. In comparison with the group of patients received standard treatment, higher SVR (80\%) was achieved in the group with added metformin (vs. $60.9 \%$ in group with standard treatment), $p<0.05$.

Conclusion: $\mathrm{CHC}$ is mostly determined at age of $25-40(42 \%)$, prevalent genotype in Azerbaijan is genotype 1 (74\%). 68\% of patients with the genotype 1 and $88 \%$ of patients with the genotypes 2 and 3 achieved SVR. Infringements of glucose metabolism influence SVR achievement. Adding metformin to standard antiviral treatment scheme can improve results of therapy. 


\section{PP13-55}

Influence of Interferon- $\alpha$ Antibodies and 2', 5'-Oligoadenylate Synthetase 1 (OAS1) Gene Expression on liver Injury in Hepatitis C Infected Patients P. Kar ${ }^{1}$, P.K. Gumma ${ }^{1}$, P. Bharti ${ }^{1}$, B.C. Das ${ }^{2}$

${ }^{1}$ Medicine, Maulana Azad Medical College; ${ }^{2}$ Ambedkar Center for Biomedical Research, University of Delhi, New Delhi, India

Aim: To study the influence of interferon- $\alpha$ antibodies concentrations on expression levels of OAS1 mRNA in liver tissue of Chronic HCV patients and to correlate with disease severity.

Method: A total of HCV infected $(N=32)$ and healthy individuals $(N=32)$ were included in this study to quantify interferon antibodies (IFN $\alpha-\mathrm{Ab})$ using anti-IFN- $\alpha$ quantitative ELISA kit. Expression analysis of OAS1 was done by relative quantification method $\left(2^{-\Delta \Delta \mathrm{Ct}}\right)$. Severity of liver disease was assessed according to Knodell score for histologic activity index (HAI) and Ishak's fibrosis staging using sections from formalin-fixed and paraffin-embedded liver tissue samples by hematoxylin-eosin staining.

Results: The IFN $\alpha-\mathrm{Ab}$ were present in $14(43.75 \%)$ chronic hepatitis $\mathrm{C}$ patients and $3(9.38 \%)$ healthy individuals respectively $(p$ value $=$ $0.002)$. The antibody levels were higher $(76.80 \pm 117.01 \mathrm{ng} / \mathrm{ml})$ in the treated patients than naive $(6.8 \pm 10.01 \mathrm{ng} / \mathrm{ml})$ patients. IFN therapy induced high OAS1 expression ( $76 \pm 70.15$ fold) compared to naïve $(19.40 \pm 28.45$ fold). In naïve, when antibodies were high $(5.10 \pm 9.37 \mathrm{ng} / \mathrm{ml})$ OAS1 expression levels $(2.29 \pm 0.99$ fold $)$ decreased ( $p$ value 0.03 ). Though the values are not statistically significant in treated patients, the IFN $\alpha-\mathrm{Ab}$ and OAS1 expressions are showing same trend of antagonism. We observed higher OAS1 (25 fold rise) expressions in 22 cases when HAI (1-8) was minimal to moderate. As the inflammation increases to moderate to remarkable inflammation (HAI 9-18) there is a decrease in trend of OAS1 (5 fold rise) expression.

Conclusion: Preexisting IFN $\alpha$-Abs does occur and elevated during the IFN therapy. IFN $\alpha$-Abs down regulates the OAS1 expression levels in HCV patients by blocking the interferon. This could be a matter of concern particularly for the patients with progressing liver disease.

\section{PP13-56}

Retrospective Pooled Safety Analysis of Standard- versus High-dose Peginterferon Alfa-2a (40KD) in Chronic Hepatitis C Genotype 1 or 4 Patients

P. Marcellin ${ }^{1}$, S.K. Roberts ${ }^{2}$, K.R. Reddy ${ }^{3}$, S.A. Harrison ${ }^{4}$, D.M. Jensen ${ }^{5}$, S.J. Hadziyannis $^{6}$, G.J. Dore ${ }^{7}$, M. Diago ${ }^{8}$, M. Weltman ${ }^{9}$, D. Messinger ${ }^{10}$, F. Tatsch $^{11}$, M. Rizzetto ${ }^{12}$

${ }^{1}$ Hôpital Beaujon, Clichy, France; ${ }^{2}$ The Alfred Hospital, Prahan,

VIC, Australia; ${ }^{3}$ University of Pennsylvania, Philadelphia, PA;

${ }^{4}$ Brooke Army Medical Center, Fort Sam Houston, TX; ${ }^{5}$ Center for

Liver Diseases, Chicago, IL, USA; ${ }^{6}$ Henry Dunant Hospital, Athens,

Greece; ${ }^{7}$ National Centre in HIV Epidemiology and Clinical

Research, Sydney, NSW, Australia; ${ }^{8}$ Hospital General de Valencia,

Valencia, Spain; ${ }^{9}$ Nepean Hospital, Sydney, NSW, Australia; ${ }^{10}$ IST

GmbH, Mannheim, Germany; ${ }^{11}$ Roche, Basel, Switzerland;

${ }^{12}$ University of Torino, Torino, Italy

Background: The safety profile of standard-dose peginterferon alfa2a $(40 \mathrm{kD})(180 \mu \mathrm{g} / \mathrm{week})$ is well established. Recent trials have explored a 12-week induction regimen of $360 \mu \mathrm{g} / \mathrm{week}$ peginterferon alfa-2a $(40 \mathrm{kD})$ in difficult-to-cure patients infected with hepatitis $\mathrm{C}$ virus (HCV). This retrospective analysis examined the safety profile of standard- versus high-dose peginterferon alfa-2a (40 kD).

Methods: Data were pooled from five randomised multicentre trials in which treatment-naive and treatment-experienced patients infected with $\mathrm{HCV}$ genotype 1 or 4 received peginterferon alfa- $2 \mathrm{a}(40 \mathrm{kD})$ $180 \mu \mathrm{g} /$ week (standard-dose group; $n=1,672$, including 94 [5.6\%] oriental patients) or $360 \mu \mathrm{g} /$ week (high-dose group; $n=1,268$, including 73 [5.8\%] oriental patients) plus ribavirin 1,000/1,200 mg/ day for 12 weeks; all received standard dose after 12 weeks. This safety analysis is mainly limited to the first 12 weeks of treatment.

Results: Demographics in both groups were comparable. The frequency of adverse events (AEs), serious AEs and discontinuations due to safety were similar in the first 12 weeks (Table). Both groups had comparable incidence of cough, dyspnoea and psychiatric disorders and low incidence of pneumonia, but in the high-dose group more patients reported weight decrease versus standard dose $(p<0.0001)$ and laboratory abnormalities (thrombocytopenia and neutropenia) were more common (Table 1$)$. Significant $(p<0.05)$ baseline predictors for discontinuation due to safety within the first 12 weeks were male gender, older age and lower albumin and neutrophil count, but not starting dose of peginterferon alfa-2a $(40 \mathrm{kD})$.

Conclusion: Peginterferon alfa-2a (40 kD) has a wide safety margin, even when administered at twice the recommended dose during the first 12 weeks of treatment.

Acknowledgement: Funded by Roche, Basel, Switzerland

Table 1 AEs and discontinuations during the first 12 wetks of treatment

\begin{tabular}{|c|c|c|c|}
\hline & $\begin{array}{l}\text { Standard- } \\
\text { dose group } \\
(n=1,672)\end{array}$ & $\begin{array}{l}\text { High-dose } \\
\text { group } \\
(n=1,268)\end{array}$ & $\begin{array}{l}\text { Hazard } \\
\text { ratio } \\
(95 \% \mathrm{Cl})\end{array}$ \\
\hline Any $\mathrm{AE}, n(\%)$ & $1,609(96.2)$ & $1,233(97.2)$ & $1.09(1.01-1.17)$ \\
\hline Number of AEs/patient & 6.6 & 6.9 & \\
\hline Seriqus AEs, $n(\%)$ & $53(3.2)$ & $53(4.2)$ & $1.33(0.91-1.95)$ \\
\hline $\begin{array}{c}\text { Number of serious } \\
\text { AEs/patient }\end{array}$ & 0.038 & 0.050 & \\
\hline $\begin{array}{l}\text { Number of severe/life } \\
\text { threatening } \\
\text { AEs/patient }\end{array}$ & 0.298 & 0.284 & \\
\hline \multicolumn{4}{|l|}{ AEs, $n(\%)$} \\
\hline Cough & $210(12.6\}$ & $173(13.8)$ & $1.11(0.91-1.35)$ \\
\hline Dyspnoea & $207(12.4)$ & $160(12.6)$ & $1.03(0.84-1.26)$ \\
\hline Pneumonia & $12(0.7)$ & $9(07)$ & $1.00(0.42-2.37)$ \\
\hline Psychiatric disorder & 487 (29.1) & $357(28.1)$ & $0.96(0.84-1.10)$ \\
\hline $\begin{array}{l}\text { Infection and } \\
\text { infestation }\end{array}$ & $312(18.9)$ & $262(20.7)$ & $1.13(0.96-1,34)$ \\
\hline Weight decrease & $55(3.3)$ & $96(7.7)$ & $2.42[1.74-3.36)$ \\
\hline \multicolumn{4}{|l|}{$\begin{array}{l}\text { Lab abnormalities, } \\
n(\%)\end{array}$} \\
\hline Neutropenia & $36(2.2)$ & $72(5.7)$ & $2.71(1.81-4.04)$ \\
\hline Thrombocytopenia & $42(2.5)$ & $59(4.7)$ & $1.90(1.28-2.82)$ \\
\hline $\begin{array}{l}\text { Treatment distontinuation } \\
\quad \text { for safety, } n(\%)\end{array}$ & $47(2.8)$ & $37(2.9)$ & $1.05(0.68-1.61)$ \\
\hline
\end{tabular}

\section{PP13-57}

Associations between Two SNPS (rs12979860, rs12980275) and Complete Early Virological Response for PegIFNa-2a (40KD) Based Treatment in Naive and Non-responders

T. Asselah ${ }^{1}$, P. Marcellin ${ }^{1}$, M.W. Fried ${ }^{2}$, D.M. Jensen ${ }^{3}$, S. Germer ${ }^{4}$, R.

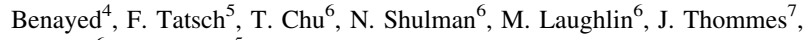
D. Chin ${ }^{6}$, L. Essioux ${ }^{5}$

${ }^{1}$ Hopital Beaujon, Clichy, France; ${ }^{2}$ University of North Carolina at Chapel Hill, Chapel Hill, NC; ${ }^{3}$ Center for Liver Diseases, Chicago, IL; ${ }^{4}$ Roche, Nutley, NJ, USA; ${ }^{5}$ Roche, Basel, Switzerland; ${ }^{6}$ Roche, Palo Alto; ${ }^{7}$ Genentech, San Francisco, CA, USA

Background: Two SNPs (rs12979860, rs12980275) in the IL28B promoter (chromosome 19) were strongly associated with virological response in treatment-naive and previous non-responders (NR) to 
PegIFN $\alpha$-2b/ribavirin (RBV) in two multinational phase III studies of PegIFN $\alpha-2 \mathrm{a} / \mathrm{RBV}$. Here we further examine associations between these SNPs and cEVR in genotype 1 patients.

Methods: DNA samples from 406 treatment-naive patients and 426 prior NR to PegIFN $\alpha-2 b / R B V$ enrolled in the two trials were genotyped with Illumina 1M Omni chips (containing 1,016,423 SNPs). The genome-wide association scan (GWAS) endpoint of interest was cEVR (undetectable HCV RNA at week 12 of interferon-based treatment).

Results: Overall cEVR rate was $49.6 \%$ (126/254) in treatment-naive patients and $13.1 \%(51 / 388)$ in prior NR. In treatment-naive patients, cEVR rates were 65.5 and $19.2 \%$, respectively, in those with $\mathrm{C} / \mathrm{C}$ and $\mathrm{T} / \mathrm{T}$ genotypes for rs 12979860 , and 59.4 and $28.2 \%$, respectively, in those with $\mathrm{T} / \mathrm{T}$ and $\mathrm{G} / \mathrm{G}$ genotypes for rs8099917. In previous NR, cEVR rates were 34.6 and $8.3 \%$, respectively, in those with $\mathrm{C} / \mathrm{C}$ and $\mathrm{T} / \mathrm{T}$ genotypes for rs12979860, and 20.9 and $5.6 \%$, respectively, in those with $\mathrm{T} / \mathrm{T}$ and $\mathrm{G} / \mathrm{G}$ genotypes for rs8099917. The association between both SNPs and cEVR was stronger among treatment-naive patients $\left(\right.$ rs12979860 C > T, $p=5 \times 10^{-8} ;$ rs8099917 $\mathrm{T}>\mathrm{G}$, $\left.p=2 \times 10^{-6}\right)$ than previous $\mathrm{NR}(\mathrm{rs} 12979860 \mathrm{C}>\mathrm{T}, p=0.004$; rs8099917 $\mathrm{T}>\mathrm{G}, p=10^{-4}$ ). The two alleles are in tight linkage disequilibrium: all treatment-naive individuals with $\mathrm{C} / \mathrm{C}$ at rs 12979860) were also $\mathrm{T} / \mathrm{T}$ at $\mathrm{rs} 8099917$, and all who were $\mathrm{T} / \mathrm{T}$ at rs12979860 were G/G at rs8099917.

Conclusion: Two closely associated alleles (rs12979860, rs12980275) in the $I L 28 B$ region of chromosome 19 are important determinants of cEVR in treatment-naive patients and previous NR. These two alleles are in tight linkage disequilibrium. The associations were strongest in treatment-naive patients, suggesting that other factors may mitigate the effect of $I L 28 B$ polymorphism on outcomes in patients who fail standard therapy.

Acknowledgement: Funded by Roche, Basel, Switzerland

\section{PP13-58}

Safety and Efficacy of Interferon and Ribavirin for Chronic Hepatitis C (HCV): Comparison between Normal and Sickle Cell Anemia (SCA)

\section{H. Issa}

Internal Medicine/Gastroenterology Section, King Fahad Specialist Hospital-Dammam, Dammam, Saudi Arabia

Aim: To evaluate the safety and efficacy of pegylated interferon and ribavirin for the treatment of $\mathrm{HCV}$ in normal patients and patients with SCA.

Patients and methods: Fifty-five SCA patients with HCV were compared to 67 normal patients with $\mathrm{HCV}$ in term of response to treatment and side effects. All were treated with pegylated interferon and standard dose of ribavirin.

Results: Fifty-five (33 F:22 M) SCA patients were treated. Their mean age was 29.5 years (15-54 years). Only eight were receiving hydroxyurea. All tolerated the treatment well and none dropped their $\mathrm{Hb}$, required blood transfusion pre, during or after therapy. Their mean $\mathrm{Hb}$ at the start was $10.2 \mathrm{~g} / \mathrm{dl}(7.5-11.5 \mathrm{~g} / \mathrm{dl})$, at 3 months was $10.4 \mathrm{~g} / \mathrm{dl}(8.9-11.2 \mathrm{~g} / \mathrm{dl})$, at 6 months was $10.35 \mathrm{~g} / \mathrm{dl}(8.5-11.9 \mathrm{~g} / \mathrm{dl})$ and at the end of treatment was $10.3 \mathrm{~g} / \mathrm{dl}(8.2-11.7 \mathrm{~g} / \mathrm{dl})$. Forty $(72.7 \%)$ achieved SVR. The remaining 15 were non-responders. They had a relatively significant HCV-RNA viral load and ten of them had genotype 1 , four had genotype 4 , and one genotype 5 . Sixty-seven ( 35 F:32 M) normal patients with $\mathrm{HCV}$ were compared to those with SCA. Their mean age was 47 years (22-67 years). All tolerated the treatment but $7(10.44 \%)$ developed anemia, 4 (5.9\%) neutropenia, 1 (1.4\%) arthropathy and $1(1.4 \%)$ thrombocytopenia. Forty-eight $(71.64 \%)$ achieved SVR. The remaining 19 were non-responders. They had a relatively significant HCV-RNA viral load and twelve of them had genotype 1, five had genotype 4, and two had genotype 3. Conclusions: Patients with SCA and $\mathrm{HCV}$ can be treated with pegylated interferon and ribavirin at the usual recommended dose.
This is even so in those who are not receiving hydroxyurea. The treatment is safe, the side effects are less and the response rate is comparable to those without SCA.

\section{PP13-59}

TMC435 in Combination with Peginterferon and Ribavirin in Treatmentnaïve Genotype-1 HCV Patients (PILLAR study): 24-week Interim Safety and Efficacy Results

M.W. Fried ${ }^{1}$, M. Buti ${ }^{2}$, G.J. Dore ${ }^{3}$, P. Ferenci ${ }^{4}$, I. Jacobson ${ }^{5}$, P. Marcellin ${ }^{6}$, S. Zeuzem ${ }^{7}$, O. Lenz ${ }^{8}$, M. Peeters ${ }^{8}$, V. Sekar' ${ }^{9}$, G. De Smedt ${ }^{8}$

${ }^{1}$ University of North Carolina at Chapel Hill, Chapel Hill, NC, USA; ${ }^{2}$ Hospital Vall d'Hebron and Ciberehd, Barcelona, Spain;

${ }^{3}$ St Vincent's Hospital, Sydney, Australia and National Centre in HIV Epidemiology and Clinical Research, University of New South Wales, Sydney, NSW, Australia; ${ }^{4}$ Allgemeines Krankenhaus der Stadt Wien, Vienna, Austria; ${ }^{5}$ Weill Cornell Medical College, New York, NY, USA; ${ }^{6}$ Hopital Beaujon, Clichy, Paris, France; ${ }^{7}$ Klinikum der Johann-Wolfgang-Goethe-Universität-Med. Klinik I, Frankfurt, Germany; ${ }^{8}$ Tibotec BVBA, Beerse, Belgium; ${ }^{9}$ Tibotec Inc., Titusville, NJ, USA

Background/aims: PILLAR (NCT00882908) is an ongoing, international, Phase IIb, randomized, double-blind study in treatmentnaïve $\mathrm{HCV}$ genotype-1 patients to assess the safety and efficacy of TMC435 (an oral investigational HCV NS3/4A protease inhibitor) in combination with peginterferon $\alpha-2 \mathrm{a}(\mathrm{PegIFN})$ and ribavirin (RBV). A planned interim 24-week safety and efficacy analysis was performed. Methods: Patients $(n=386)$ were randomized to one of 5 arms: TMC435 (75 or $150 \mathrm{mg}$ qd)+PegIFN $(180 \mathrm{mg} /$ week $) / \mathrm{RBV}(1,000$ $1,200 \mathrm{mg} /$ day) for 12 weeks, then PegIFN/RBV for 12 weeks; TMC435 (75 or $150 \mathrm{mg} \mathrm{qd)} \mathrm{+} \mathrm{PegIFN/RBV} \mathrm{for} 24$ weeks; or placebo + PegIFN/RBV for 24 weeks then PegIFN/RBV for 24 weeks. Total treatment duration was 24 or 48 weeks in TMC435 arms (response-guided; treatment ended if HCV RNA $<25 \mathrm{IU} / \mathrm{mL}$ detectable/undetectable at Week 4 and $<25 \mathrm{IU} / \mathrm{mL}$ undetectable at Weeks 12,16 , and 20) and 48 weeks in control arm.

Results: At week 4, HCV RNA $<25 \mathrm{IU} / \mathrm{mL}$ (undetectable) was achieved by $68-79 \%$ of patients in TMC435 groups compared to $5 \%$ with placebo. Between $79-86 \%$ of patients in TMC435 groups were able to stop therapy after 24 weeks. In TMC435 groups, $88-97 \%$ of patients who discontinued therapy at or before week 24 and reached week 12 after planned end of therapy achieved SVR12. Higher responses were apparent with TMC435 irrespective of patient demographics, geographic region, or disease characteristics (HCV RNA level, genotype-1 subtype, IL28B genotype, metavir score). There were no differences between TMC435 and placebo groups in incidence of rash, anemia and gastrointestinal events, or adverse events leading to treatment discontinuation. Mild, reversible increases in bilirubin (direct and indirect) were noted in TMC435 $150 \mathrm{mg}$ group. Serum ALT concentration decreased over time.

Conclusions: In this interim analysis, TMC435 combined with PegIFN/RBV achieved undetectable HCV RNA rapidly in the majority of patients. TMC435 was well tolerated and associated with a low treatment discontinuation rate, comparable to placebo.

\section{PP13-60}

Safety and Efficacy of Locally Manufactured Pegylated Interferon in Hepatitis C Patients

M. Nassiri Toosi, H. Jabbari, S. Merat, A. Bayatian

Gastroenterology and Hepatology Ward, Tehran University, Tehran, Iran

Background: To evaluate the safety and effectiveness of locally produced pegylated interferon-2a in treatment-naïve patients with chronic hepatitis C.

Methods: All treatment-naïve patients diagnosed with chronic hepatitis $\mathrm{C}$ who referred to two university based outpatient clinics in 
Tehran from December 2007 to May 2008 were enrolled. Exclusion criteria included the presence of a debilitat- ing disease, decompensated cirrhosis, or refusal to participate in the study. Patients were treated with $180 \mathrm{~g}$ pegylated interferon-2a (Pegaferon) weekly and $800-1,200 \mathrm{mg}$ ribavirin daily for 24 or 48 weeks depending on genotype and weight. Viral and biochemical response and adverse drug reactions were recorded.

Results: A total of 108 patients were enrolled; 63 with genotype 1 and 45 with genotypes 2 and 3 . The mean age of the patients was 39 years (range 19-65). Ninety-seven patients completed the study and 76 achieved sustained viral response. The sustained viral response among patients completing the study was $67 \%$ for genotype 1 and $95 \%$ for geno-types 2 and 3. Adverse events were well tolerated and none led to discontinuation of treatment, however dose adjustment was necessitated in 16 patients. The most common adverse events were fatigue $(73.5 \%)$, poor appetite $(66.2 \%)$, and feverishness $(57.4 \%)$. The mean hemoglobin drop was $2.9 \mathrm{~g} / \mathrm{dL}$.

Conclusions: Locally produced PEG-IFN in Iran is safe and effective in treatment-naïve chronic hepatitis $\mathrm{C}$.

\section{PP13-61}

Impact of the IL28 Polymorphism on Response Guided Therapy in Chronic Hepatitis C

P. Ferenci ${ }^{1}$, T. Scherzer ${ }^{1}$, R. Stauber ${ }^{2}$, H. Laferl ${ }^{3}$, K. Rutter ${ }^{1}$, A. Maieron ${ }^{4}$, A.F. Staettermayer ${ }^{1}$, S. Beinhardt ${ }^{1}$, H. Hofer ${ }^{1}$, M. Gschwantler ${ }^{5}$, C. Datz ${ }^{6}$, Austrian Hepatitis Study Group

${ }^{1}$ Internal Medicine 3, Medical University of Vienna, Wien; ${ }^{2}$ Internal Medicine, Medical University of Graz, Graz; ${ }^{3}$ Internal Medicine 1, Kaiser Franz Josef Hospital, Wien; ${ }^{4}$ Internal Medicine 4, Elisabetinen Hospital, Linz, Austria; ${ }^{5}$ Internal Medicine 4, Wilhelminen Hospital, Wien, Azerbaijan; ${ }^{6}$ Internal Medicine, Krankenhaus Oberndorf, Oberndorf, Austria

Response guided therapy (RGT) allows individualisation of treatment duration according to the virological response (VR) at weeks 4 and 12. A single nucleotide polymorphisms (SNP) in the IL28 B region (rs 12979860) predicts the early VR and the outcome of antiviral combination therapy in chronic hepatitis $\mathrm{C}$. The aim of this study was to evaluate the impact of rs 12979860 genotype for RGT in patients who participated in randomized controlled multicenter trial (Ferenci et al. Gastroenterology 2010) in HCV genotype 1/4 patients.

Methods: According to their VR patients were assigned to various length of treatment prospectively. All patients were treated with 180 mcg peginterferon alpha-2a/week and weight based ribavirin. Patients with a rapid VR (RVR, HCV-RNA undetectable at week 4) were treated for 24 weeks (group D), patients with early VR (EVR) for 48 (group A) or 72 weeks (group B). Treatment was discontinued in nonresponders (NR, group C) at week 24. The rs12979860 SNP was analyzed in 334 of the 551 study participants by the StepOnePlus Real time PCR System (Applied Biosystems, Foster City, USA).

Results: The frequency of the genotype differed among the study groups (RVR: C/C: $59.7 \%$; T/C or T/T: $40.3 \%$; EVR: C/C: $30.4 \%$; T/C or T/T: $69.6 \%$; NR: C/C: $2.5 \%$; T/C or T/T: $97.5 \%)$. Overall, relapse rates were lower in patients with $\mathrm{C} / \mathrm{C}$ than in T-carriers (RVR: 9.2 vs. $15.2 \%$; EVR: 19.5 vs. $43.1 \% ; p<0.01)$. Treatment extension decreased relapse rates in $\mathrm{T}$ carriers (72 weeks: 17.0\%; 48 weeks: $41.8 \% ; p<0.005)$. In $\mathrm{C} / \mathrm{C}$ patients' treatment extension relapse rates were similar ( 20 vs. $26.9 \%$ )

Conclusions: The IL28 genotype identifies patients who benefit from treatment extension to 72 weeks. Furthermore, patients with RVR and the C/C genotype need only 24 weeks of treatment with SVR rates similar to those obtained by triple therapy with protease inhibitors.

\section{PP13-62}

The Comparison of the Efficacy of Pegylated Interferon Alpha-2a and Alpha-2b in Chronic Hepatitis C Genotype 1

Ü.B. Dogan, B. Kara, M.S. Akın

Gastroenterology, Adana Numune Training and Research Hospital, Adana, Turkey

Background/aim: The purpose of this study was to assess the efficacy of treatment with pegylated interferon alpha-2a (PEG-2a) versus pegylated interferon alpha-2b (PEG-2b), plus ribavirin, in inducing rapid, early and sustained virological response (RVR, EVR and SVR) in chronic hepatitis $\mathrm{C}$.

Methods: Patients with the following characteristics were included: Anti-HCV(+); normal and/or elevated serum transaminase levels; positive HCV-RNA by quantative PCR and at least stage 1 fibrosis according to Ishak Scoring System on liver biopsy. Patients were assigned to PEG-2a (180 $\mu \mathrm{g} / \mathrm{week} ; n=17)$ or PEG-2b $(1.5 \mu \mathrm{g} / \mathrm{kg} /$ week; $n=27)$, plus RBV $(1,000-1,200 \mathrm{mg} /$ day $)$ for 48 weeks. All patients were genotype 1 . At week 24 , the treatment was discounted in patients positive for HCV-RNA by PCR, while patients negative for HCV-RNA continued treatment up to 48 weeks. The end of the treatment and sustained virological responses of the patients were ascertained by assessing HCV-RNA levels at the end of the treatment and after 24 weeks follow up after the cessation of treatment.

Results: The RVR (29.4 vs. $29.6 \%$ ) and EVR (76.5 vs. $77.8 \%$ ) rates were nearly identical for PEG-2a and PEG-2b groups. At the end of week 48 , HCV-RNA was undetectable in 10 of 17 patients $(59 \%)$ receiving PEG-2a and in 15 of 27 patients $(56 \%)$ receiving PEG-2b. The group sustained virologic response rates were 47.1 and $48.2 \%$ for PEG-2a and PEG-2b groups, respectively. No significant differences were noted between the two groups. The incidence and severity of adverse events were similar in two groups. Multivariate analysis revealed that $\mathrm{HCV}$ viral load was significantly associated with RVR, EVR and SVR inversely.

Conclusion: In patients infected with HCV genotype 1, the rates of sustained virologic response and tolerability did not differ significantly between the two available peginterferon-ribavirin regimens and HCV viral load was important in RVR, EVR and SVR.

\section{PP13-63}

Viral Kinetics Modeling of Short-term Monotherapy Data of ACH-1625, an HCV Protease Inhibitor

A. Agarwal, B. Zhang, M. Huang, M. Deshpande

Achillion Pharmaceuticals, Inc., New Haven, CT, USA

Background: ACH-1625 is a potent inhibitor of HCV NS3 protease. Administration of ACH-1625 in HCV patients at doses of 200, 500, and $600 \mathrm{mg}$ BID and $600 \mathrm{mg}$ QD for 5 days resulted in 3.86, 4.25, 3.94, and $3.81 \log _{10}$ mean maximum drop of plasma HCV RNA, respectively. The objective of this work was to model HCV kinetics and estimate viral kinetics parameters. A second objective was to use these estimated parameters and predict RVR with ACH-1625 in combination with pegasys.

Methods: A single population viral dynamics model was applied to the HCV RNA data obtained from treatment with ACH-1625 and viral kinetics parameters were estimated. Subsequently, viral kinetics parameter estimates of ACH-1625 and pegasys were 
analyzed to determine their probability distributions in the drugtreated patient population. These parameters were then assigned at random in a set of 1,000 patients in silico within probability distributions identified. Simulations were run to estimate viral load in the in silico patients at day 28 of combination treatment using Matlab 7.9.0.

Results: All dose cohorts of ACH-1625 demonstrated a sharp first phase decline which is reflected in the high drug efficiency observed: 0.9991, 0.9985 and 0.9987 with 500, 200 and $600 \mathrm{mg} / \mathrm{QD}$ dosing, respectively. The virion clearance rate, time delay and infected cell death rate were estimated to be $8.3 \pm 2.1$ day $^{-1}, 3.1 \pm 3.1 \mathrm{~h}$, and 0.27 \pm 0.14 day $^{-1}$, respectively. When combined with pegasys, ACH1625 at $200 \mathrm{mg}$ BID or $600 \mathrm{mg}$ QD is predicted to increase RVR $>2$ fold as compared to SOC.

Conclusions: (1) Viral kinetics parameter estimates obtained from modeling of ACH-1625 montherapy data are consistent with literature values, validating the high drug efficiency estimates obtained with ACH-1625 (2) Antiviral therapy with ACH-1625 in combination with SOC is predicted to enhance achievement of RVR in $\mathrm{HCV}$ patients.

\section{PP13-64}

Phase 2 Study of Pegylated Interferon Lambda for HCV: Safety, Viral Response, and Impact of Host Genotype through Week 12

M. Rodriguez-Torres ${ }^{1}$, E. Lawitz ${ }^{2}$, R. Ghalib ${ }^{3}$, N. Sussman ${ }^{4}$, F. Anderson ${ }^{5}$, G. Everson ${ }^{6}$, I. Jacobson ${ }^{7}$, J.C. Lopez-Talavera ${ }^{8}$, M.A. Horga ${ }^{9}$, J. Hillson ${ }^{10}$, T. Gray ${ }^{10}$, D. Fontana ${ }^{11}$, E. Ramos ${ }^{10}$, A.J. Muir ${ }^{12}$

${ }^{I}$ Fundacion de Investigacion De Diego Santurce, Santurce, Puerto Rico; ${ }^{2}$ Alamo Medical Research, San Antonio; ${ }^{3}$ North Texas Research Institute, Dallas; ${ }^{4}$ Baylor College of Medicine, Houston, TX, USA;

${ }^{5}$ Liver and Intestinal Research Centre, Vancouver, BC, Canada;

${ }^{6}$ University of Colorado Hospital, Aurora, CO; ${ }^{7}$ Weill Cornell Medical College, New York, NY; ${ }^{8}$ Global Development/Medical Affairs; ${ }^{9}$ Virology, Bristol-Myers Squibb Company, Wallingford, CT; ${ }^{10}$ Clinical Development; ${ }^{11}$ Porfolio and Program Management, ZymoGenetics, Inc., Seattle, WA; ${ }^{12}$ Duke University Medical Center, Durham, NC, USA

Background: PEG-IFN- $\lambda$ exerts antiviral effects through a unique receptor with limited distribution and is expected to have an improved safety profile vs. PEG-IFN-alfa2a.

Methods: 55 patients received a single dose of PEG-IFN- $\lambda$ 80, 120 , 180 , or $240 \mathrm{mg}(n=45)$ or PEG-IFN-alfa2a $180 \mathrm{mg}(n=10)$ for PK, followed 2 weeks later by weekly doses plus RBV for 24 (G2/3) or 48 (G1/4) weeks. Safety data were analyzed through 12 weeks. Host IL-28b genotype was determined for 48 pts (CC $[n=15]$ or CT/TT $[n=33]$ at rs12897986).

Results: Viral response (VR; < LLQ of 25IU/ml) varied with HCV genotype, dose, race and host genotype. For HCV G1/4, combining the 3 highest doses of PEG-IFN- $\lambda$, the proportion with VR at weeks $2 / 4 / 12$ was $71 / 71 / 86 \%$ with CC host genotype, compared to $8 / 25 / 50 \%$ with CT/TT.

No deaths or dose-limiting toxicities occurred. Three PEG-IFN- $\lambda$ patients (7\%) required dose reduction ( $2 \mathrm{on} 240 \mathrm{mg}, 1 \mathrm{on} 180 \mathrm{mg}$ ), all due to ALT/AST elevations which resolved within 1 week. One (10\%)
PEG-IFN-alfa2a patient required dose reduction for depression. Adverse events were similar among dose/treatment groups. PEG-IFN- $\lambda$ caused little change in platelet or WBC counts; observed decreases in hemoglobin were less severe compared to PEG-IFN-alfa2a.

Conclusions: PEG-IFN- $\lambda$ was associated with rapid viral decline and was well-tolerated at doses up to $240 \mathrm{mg}$, with less hematologic toxicity compared to PEG-IFN-alfa2a. PEG-IFN- $\lambda$ shows promise across a broad range of doses and viral genotypes, and in difficult to treat host genotypes.

\begin{tabular}{lllllllll}
\hline Dose & HCV & VR & VR & VR & HCV & VR & VR & VR \\
& Type & week & week & $\begin{array}{l}\text { week } \\
\text { Type }\end{array}$ & week & week & week \\
& G1, 4 & $2(\%)$ & $\begin{array}{l}4 \\
(\%)\end{array}$ & $\begin{array}{l}12 \\
(\%)\end{array}$ & G2,3 & $2(\%)$ & $4(\%)$ & 12 \\
& & & $(\%)$ & & & \\
\hline IFN- $\lambda$ 80 $80 \mathrm{~g}$ & $n=7$ & 0 & 0 & 14 & $n=5$ & 40 & 60 & 80 \\
IFN- $\lambda 120 \mu \mathrm{g}$ & $n=7$ & 29 & 43 & 71 & $n=4$ & 75 & 100 & 100 \\
IFN- $\lambda 180 \mu \mathrm{g}$ & $n=6$ & 33 & 33 & 67 & $n=5$ & 60 & 80 & 80 \\
IFN- $\lambda 240 \mu \mathrm{g}$ & $n=7$ & 29 & 43 & 43 & $n=4$ & 75 & 100 & 100 \\
IFN- $\alpha 180 \mu \mathrm{g}$ & $n=5$ & 20 & 40 & 40 & $n=5$ & 80 & 100 & 100 \\
\hline
\end{tabular}

VR by genotype

\section{PP13-65}

Effect of Food on the Pharmacokinetics of Boceprevir in Japanese and Caucasian Healthy Subjects

C. Kasserra ${ }^{1}$, M. Treitel ${ }^{1}$, E. Hughes ${ }^{2}$, S. Gupta ${ }^{1}$, E. O'Mara ${ }^{1}$

${ }^{I}$ Merck Research Laboratories; ${ }^{2}$ Former Employee of Merck

Research Laboratories, Kenilworth, NJ, USA

Aim: To determine the effects of ethnicity (Caucasian vs. Japanese) and food (meal fat content and timing) on the pharmacokinetics of boceprevir, a novel HCV protease inhibitor.

Methods: In this open-label, 3-period study, matched healthy subjects were randomized to receive boceprevir 200,400 , or $800 \mathrm{mg}$ as a single dose following an overnight fast (period 1), single dose with a high-fat breakfast (period 2), single dose with a Japanese meal (lowfat, first day of period 3), followed by TID dose with a Japanese meal for 7 days (single dose on last day of period 3).

Results: 38 healthy adult males were randomized (Caucasian $n=18$; Japanese $n=20$ ). Overall, pharmacokinetics of boceprevir were similar in Caucasian and Japanese subjects (Table). Steadystate AUC and $C_{\max }$ increased in a less than dose-proportional manner in both Caucasian and Japanese subjects. Mean AUC and $C_{\max }$ values increased similarly with either a high-fat breakfast or a Japanese meal compared with fasted conditions in both ethnic groups, with the increased exposure more apparent at higher dose levels. In addition, another open-label single-dose study in healthy subjects $(n=12)$ showed that timing of a meal relative to dosing (5 min before, during, or immediately after) did not impact boceprevir pharmacokinetics. 
Conclusions: Boceprevir should be administered with food. No dose adjustment of boceprevir is warranted for Japanese patients.

Effect of food on single dose PK of boceprevir

\begin{tabular}{|c|c|c|c|c|c|c|}
\hline \multirow[t]{2}{*}{ Race } & \multirow[t]{2}{*}{ Dose (mg) } & \multirow[t]{2}{*}{ Parameter } & \multirow[t]{2}{*}{$n$} & \multicolumn{2}{|c|}{ LS means } & \multirow{2}{*}{$\begin{array}{l}\text { Fed vs. fasted } \\
\text { Ratio }(90 \% \mathrm{CI})\end{array}$} \\
\hline & & & & Fasted & Fed & \\
\hline \multirow[t]{9}{*}{ Caucasian } & \multirow[t]{3}{*}{200} & AUC (I) & 6 & 842 & 979 & $116(99-136)$ \\
\hline & & AUC (tf) & 6 & 834 & 971 & $116(99-137)$ \\
\hline & & $\mathrm{C}_{\max }$ & 6 & 330 & 324 & $98(69-139)$ \\
\hline & \multirow[t]{3}{*}{400} & AUC (I) & 5 & 1,336 & 1,979 & $148(123-178)$ \\
\hline & & AUC (tf) & 5 & 1,315 & 1,966 & $150(125-179)$ \\
\hline & & $\mathrm{C}_{\max }$ & 5 & 438 & 645 & $147(119-183)$ \\
\hline & \multirow[t]{3}{*}{800} & AUC (I) & 6 & 3,959 & 6,315 & $159(130-196)$ \\
\hline & & AUC (tf) & 6 & 3,914 & 6,301 & $161(131-198)$ \\
\hline & & $\mathrm{C}_{\max }$ & 6 & 1,134 & 1,684 & $148(102-216)$ \\
\hline \multirow[t]{9}{*}{ Japanese } & \multirow[t]{3}{*}{200} & AUC (I) & 6 & 878 & 1,080 & $123(106-143)$ \\
\hline & & AUC (tf) & 6 & 865 & 1,074 & $124(107-144)$ \\
\hline & & $\mathrm{C}_{\max }$ & 6 & 386 & 457 & $119(81-173)$ \\
\hline & \multirow[t]{3}{*}{400} & AUC (I) & 5 & 1,327 & 2,265 & $171(144-202)$ \\
\hline & & AUC (tf) & 5 & 1,307 & 2,255 & $173(146-205)$ \\
\hline & & $\mathrm{C}_{\max }$ & 5 & 432 & 741 & $172(126-234)$ \\
\hline & \multirow[t]{3}{*}{800} & AUC (I) & 6 & 3,251 & 6,277 & $193(162-229)$ \\
\hline & & AUC (tf) & 6 & 3,190 & 6,263 & $196(165-233)$ \\
\hline & & $\mathrm{C}_{\max }$ & 6 & 1,002 & 2,001 & $200(156-255)$ \\
\hline
\end{tabular}

\section{PP13-66}

Antiviral Activity of Boceprevir Monotherapy in Treatment-naive Subjects with Chronic Hepatitis C Genotype 2/3 M. Silva ${ }^{1}$, C. Kasserra ${ }^{2}$, S. Gupta ${ }^{2}$, M. Treitel ${ }^{2}$, E. Hughes ${ }^{3}$, E. O'Mara ${ }^{2}$ ${ }^{1}$ Hospital Universitario Austral, Pilar, Argentina; ${ }^{2}$ Merck Research Laboratories; ${ }^{3}$ Former Employee of Merck Research Laboratories, Kenilworth, NJ, USA

Aim: To examine the effect of boceprevir, a novel hepatitis $\mathrm{C}$ virus (HCV) protease inhibitor, on HCV-RNA levels in subjects with $\mathrm{HCV}$ genotype (G) 2/3 infection.

Methods: This was a randomized, placebo-controlled study evaluating the antiviral activity of boceprevir monotherapy $200 \mathrm{mg}$ BID $(n=11), 400 \mathrm{mg}$ BID $(n=12)$, and $400 \mathrm{mg}$ TID $(n=6)$, or placebo $(n=10)$ for 14 days in treatment-naive subjects.

Results: The maximum mean decrease in HCV-RNA was $-0.37 \mathrm{log}$, $-0.24 \mathrm{log},-1.60 \mathrm{log}$, and $-0.21 \mathrm{log}$ in the $200 \mathrm{mg}$ BID, $400 \mathrm{mg}$ BID, $400 \mathrm{mg}$ TID, and placebo groups, respectively (Fig. 1). Decreases in HCV-RNA in subjects receiving $200 \mathrm{mg}$ BID and 400 mg BID were similar to placebo; however, the decrease in HCV-RNA in subjects receiving $400 \mathrm{mg}$ TID was markedly different from placebo. At end of treatment, 4 of 6 subjects receiving $400 \mathrm{mg}$ TID had a $>1 \log$ drop in HCV-RNA. Although the sample size was small, responses appeared similar in $\mathrm{G} 2(n=9)$ and $\mathrm{G} 3(n=30)$ subjects. Pharmacokinetic analysis revealed a less than dose-proportional increase in bioavailability. Overall, boceprevir was well tolerated. Conclusion: Boceprevir (400 mg TID) is associated with a marked decrease in HCV-RNA in subjects with HCV G2/3 infection, comparable to the viral load drop seen in G1 subjects receiving the same monotherapy dose in a previous study. Evaluation of the clinical dose of boceprevir ( $800 \mathrm{mg}$ TID) as a component of triple therapy for G2/3 slow responders (detectable HCV-RNA at week 4) is of potential clinical interest.

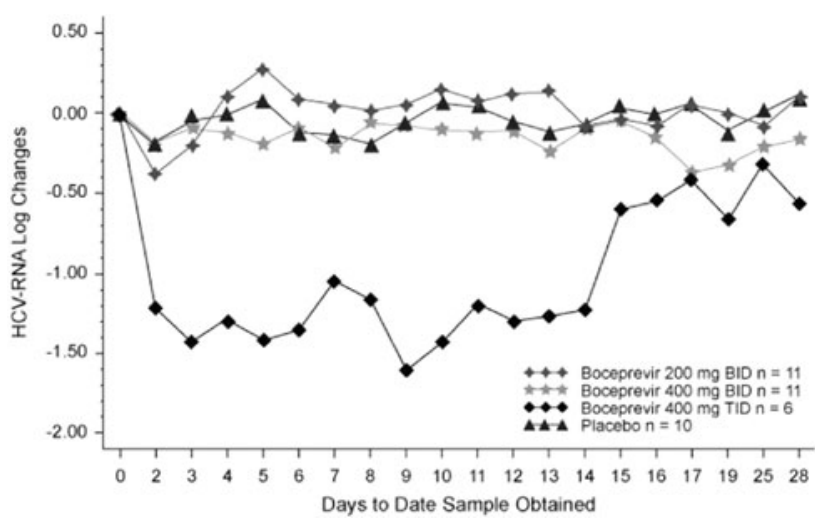

Fig. 1 HCV-RNA profiles in treatment-naive G2/3 patients

PP13-67

Hepatitis B Virus Coinfected Chronic Hepatitis C Patients: Clinical Features and Treatment Efficacy of Peginterferon Alfa Plus Ribavirin S.H. Park ${ }^{1}$, Y.J. Kim ${ }^{2}$, Y.S. Kim ${ }^{3}$, H.J. Yim ${ }^{4}$, Y.M. Park ${ }^{5}$, Y.S. Kim ${ }^{6}$, S.H. Jeong ${ }^{7}$, J.W. Lee ${ }^{8}$, J.W. Choi ${ }^{9}$, M.S. Lee ${ }^{1}$, C.K. Park ${ }^{10}$, KIPECT ${ }^{1}$ Internal Medicine, Hallym University Kangnam Sacred Heart Hospital; ${ }^{2}$ Department of Medicine, Samsung Medical Center, Sungkyunkwan University; ${ }^{3}$ Department of Medicine, Gil Medical Center, Gacheon University; ${ }^{4}$ Department of Medicine, Ansan Medical Center, Korea University, Seoul; ${ }^{5}$ Department of Medicine, Bundang Jesaeng Genenal Hospital, Seongnam-Si; ${ }^{6}$ Department of Medicine, Bucheon Medical Center, Soon Chun Hyang University, Bucheon-Si; ${ }^{7}$ Department of Medicine, Seoul National University Bundang Hospital, Seongnam-Si; ${ }^{8}$ Department of Medicine, Inha University Medical Center, Incheon; ${ }^{9}$ Department of Medicine, National Health Insurance Medical Center, Goyang-Si; ${ }^{10}$ Internal Medicine, Hallym University Sacred Heart Hospital, Anyang-Si, Republic of Korea

Background/aims: Cross-sectional studies have documented than $2-10 \%$ of chronic hepatitis $\mathrm{C}(\mathrm{CHC})$ patients are also hepatitis B surface antigen (HBsAg) positive. But in Korea, there was no sufficient data about coinfection of these two hepatotrophic viruses. This study aimed to evaluate the clinical characteristics, the treatment efficacy of peginterferon alfa plus ribavirin, and the change of hepatitis $\mathrm{B}$ virus (HBV) infection after treatment in $\mathrm{HBV}$ coinfected $\mathrm{CHC}$ patients.

Methods: Eighteen $\mathrm{HBsAg}$ positive $\mathrm{CHC}$ patients were selected from the KIPECT study which evaluated the treatment efficacy and safety of peginterferon alfa plus ribavirin in $\mathrm{CHC}$ patients. Additional information about HBV infection was provided by attending physicians from eight university hospitals.

Results: The prevalence of HBV coinfection in $\mathrm{CHC}$ patient was $2.4 \%$. HCV genotype Ib was the most common (44\%). Two of 18 patients were positive for $\mathrm{HBeAg}$ and most of the subjects $(83.3 \%)$ had baseline HBV DNA less than 2,000 IU/mL. The overall sustained virologic response rate was $72.2 \%$ in all patients, and 60 and $87.5 \%$ in genotype 1 and genotype 2, respectively. Two of the three who had baseline HBV DNA more than 2,000 IU/mL showed no detectable DNA after treatment. Two of the 13 whose baseline HBV DNA was less than $2,000 \mathrm{IU} / \mathrm{mL}$ developed hepatitis B viral replication.

Conclusion: The prevalence of HBV coinfection in $\mathrm{CHC}$ patients was $2.4 \%$ and most of the patients were inactive carrier of HBsAg. Almost 
of them remained as an inactive carrier, but some showed rise of serum HBV DNA. The treatment efficacy profile was similar with that of HCV mono-infection.

\section{PP13-68}

To Compare the Virological Responses in Naïve Hepatitis C Patients Treated with Conventional Interferon/Ribavirin and Pegylated Interferon/ Ribavirin

H. Sharif ${ }^{1}$, A. Naeem ${ }^{1}$, M. Umar ${ }^{1}$, M. Ahmed ${ }^{1}$, S. Ambreen ${ }^{1}$, M. Arif ${ }^{2}$ ${ }^{1}$ Gastroenterology and Hepatology Division; ${ }^{2}$ Gastroenterology \& Hepatology Division, Holy Family Hospital, Rawalpindi, Pakistan

Objective: To conduct a retrospective review of virological response rates (RVR, EVR, ETR and SVR) in chronic hepatitis C patients treated with combination of standard interferon with ribavirin and compare their responses to patients treated with combination of pegylated interferon and ribavirin.

Study design: Comparative retrospective observational study

Place of study: Gastroenterology and Hepatology Division, Department of Medicine, Holy Family Hospital, Rawalpindi, Pakistan.

Methods: Patients of chronic hepatitis C treated at Gastroenterology and Hepatology Unit of Holy Family Hospital, Rawalpindi between 2006-2009 were identified through Liver Clinic database. Baseline demographic characteristics, biochemical and virological responses data and details of treatment were collected. 431 who fulfilled the inclusion criteria were included in the study. They were divided in two groups depending on the treatment received. 206 patients received standard interferon and ribavirin for 24 weeks while 225 patients were treated with pegylated interferon and ribavirin for six months PCR (Qualitative) for HCV RNA was done at 4 weeks, 12 weeks, 24 weeks and 48 weeks after the initiation of therapy to asses the treatment. Virological responses in the two groups were then compared in the cohort.

Results: Virological Responses Standard IFN/Ribavirin Peg IFN plus Ribavirin

RVR $68.9 \% 68.0 \%$

EVR $77.6 \% 75.8 \%$

ETR $70.9 \% 76.6 \%$

SVR $63.2 \% 60.5 \%$

Conclusion: Pegylated interferon and ribavirin is not superior to standard interferon and ribavirin in treating Chronic Hepatitis $\mathrm{C}$ in Pakistani cohort.

\section{PP13-69}

HCV Genotypes/ Subtypes in Oman and Treatment Responses

E.F. Serebour ${ }^{1}$, A. Saeed ${ }^{2}$, I.S. Al-Bulushi ${ }^{1}$, S.A. Al-Harthy ${ }^{1}$, A. Dovlo ${ }^{3}$, Z. Muharrmi ${ }^{4}$

${ }^{1}$ Microbiology Department, Sultan Qaboos University Hospital;

${ }^{2}$ Medicine; ${ }^{3}$ Mathematics, Sultan Qaboos University; ${ }^{4}$ Microbiology, Sultan Qaboos University Hospital, Muscat, Oman

Background: HCV genotype 4 predominance ranging from $70 \%$ in Saudi Arabia to $90 \%$ among Egyptian HCV patients are reported. Other Middle East countries have reported similar genotype 4 predominance. Very little is known about HCV genotypes and their response to PEG-2a and Ribavirin among Omani patients.

Aims: To determine HCV genotypes/subtypes and predictive factors including efficacy and safety of treatment among Omani patients.

Methods: Demographic data from $247 \mathrm{HCV}$ patients (M:F; 1.6:1) age range, 6-80 years, consecutively recruited over a 5-year period (Jan 2005-2010) from Gastroenterology referral clinics were tested. Viral factors, serum ALT at weeks 0, 12, 48 and 72 weeks for monitoring treatment and host factors were included. The Innolipa HCV Genotype Assay was used from Jan 2005 to Dec 2007. The Abbott RealTime HCV Genotype II, an in-vitro RT-PCR Assay using genotype specific fluorescent labeled oligonucleotide probes used from Jan 2008 to Jan 2010.
Results: HCV genotype $1(46.2 \%)$ is the predominant genotype among chronic HCV patients in Oman. Other genotypes 2 (11.3\%), 3 (20.2\%), 4 (20.2), $5(0.4 \%)$ and $6(0.8 \%)$ were detected. HCV subtype 1 a was found to be $46 \%$ among genotype 1 cases. In Oman, there was no association between gender and genotype ( $p$ value $=0.690$ ). However there is an association between genotype and age ( $p$ value $=0.022$ ). Patients (under 36 years) seem to have more of genotype 1 than the older patients ( 56.8 to $38.3 \%$ ). $58 \%$ of treated HCV patients had sustained response to standard treatment. Treatment was discontinued in four patients. Two developed decompensated cirrhosis.One patients had severe skin rash after 24 weeks. One patient had generalized lymphadenopathy.

Conclusion: HCV Genotype 1 instead of 4 is predominant in Oman. Genotype 1 is more common in young patients. About $50 \%$ of patients respond to treatment

\section{PP13-70}

To Determine the Prevalence and Impact of Previous HBV Infection in Chronic Hepatitis C Patients Treated with Conventional Interferon/ Ribavirin

A. Naeem, M. Umar, Z. Butt, I. Akbar

Gastroenterology \& Hepatology Division, Holy Family Hospital, Rawalpindi, Pakistan

Objective:To assess the prevalence and impact of previous HBV infection on clinical, biochemical, virological and histological features in patients with chronic hepatitis $\mathrm{C}$ being treated with combination therapy of conventional interferon and

Study design: Retrospective observational study

Duration \& place of study: 12 months (01 August 2008-31 July 2009) at Gastroenterology and Hepatology Division, Holy Family Hospital, Rawalpindi

Methodology: 665 chronic hepatitis $\mathrm{C}$ patients were included in the study. The patients were divided into two groups based on anti $\mathrm{HBc}$ status. Serum alamime transaminase and liver biopsy prior to treatment was carried out. Patients were treated with conventional interferon/ribavirn therapy for 6 months. PCR for HCV RNA after six months of completion of therapy was done to know SVR.

Results: Out of $665 \mathrm{HCV}$ infected patients, $46 \%(n=305)$ patients were positive for anti HBcIgG. Gender ration with $46.2 \%$ male and 45.6\% female were positive for anti $\mathrm{HBcIgG}$.

Patients with positive anti-HBcIgG status, $82.4 \%$ had either minimal or mild inflammation on liver biopsy while with anti-HBcIgG negative patients, $43.6 \%$ had minimal and $56.3 \%$ had mild inflammation on liver biopsy. Average ALT level in anti-HBcIgG cases was $73.82 \mathrm{IU} / \mathrm{ml}$ while it was $73.89 \mathrm{IU} / \mathrm{ml}$ in anti-HBcIgG negative patients. Considering the SVR status in anti-HBcIgG positive cases was $65.5 \%$ in comparison with $67.7 \%$ in anti-HBcIgG negative patients.

Conclusion: Anti-HBcIgG has no significant impact on liver histology, ALT levels and treatment response in chronic hepatitis C patients

\section{PP13-71}

Thyroid Dysfunction Assessment in Hepatitis C Virus Infected Patients Treated with Combination of Interferon- $\alpha_{2} b$ and Ribavirin, Isfahan, Iran L. Zeidabadi Nejad ${ }^{1}$, P. Shoaei ${ }^{1}$, B. Ataei ${ }^{1}$, A. Babak ${ }^{1}$, M. Ataie ${ }^{2}$, M. Foroughifar $^{1}$

${ }^{I}$ Infectious Diseases and Tropical Medicine Research Center, Isfahan University of Medical Sciences; ${ }^{2}$ Najaf Abad Islamic Azad University, Isfahan, Iran

Objective: The objective of this study was to assess the patterns of thyroid response to combination interferon and ribavirin therapy in chronic hepatitis $\mathrm{C}$ (CHC) patients. Thyroid dysfunction (TD) includes hyperthyroidism and hypothyroidism and their possible sign and symptoms. 
Patients and methods: This study was conducted at research center of tropical and infectious diseases in Isfahan city from September 2006 to 2008. In a retrospective study, 130 cases of CHC proven by anti HCV Ab and positive HCV RNA, were examined with TSH, Anti-TPO Abs and T4 levels at baseline, 12 weeks and 24 weeks during therapy, and till 12 months after treatment.

Results: Among the 130 patients, overt thyroid disease had developed in $3(2.3 \%)$ which one and two ones had hyper- and hypothyroidism, respectively. The hyperthyroid patient had elevated Anti-TPO $(90 \mathrm{u} / \mathrm{ml})$. $97.7 \%$ of HCV patients had intact thyroid functions at the end of treatment. They had not hyper/hypothyroidism goiter or thyrotoxicosis (TTX). Conclusion: Thyroid dysfunction in combination therapy with Interferon- $\alpha 2 \mathrm{~b}$ and Ribavirin in patients with chronic hepatitis $\mathrm{C}$ infection is rare, thus treatment with this drugs can be safely continued in them, because thyroid disease, if manifested, responds well to suitable therapies. People with pre-existing thyroid auto antibodies should be closely monitored for thyroid disorders.

\section{PP13-72}

Predictive Factors for Sustained Response to Peginterferon $\alpha$-2a and Ribavirin Treatment of Patients with Genotype 2/3 Chronic Hepatitis C H.K. Jeong, Y.J. Kim, Y.A. Song, S.B. Cho, C.H. Park, H.S. Kim, J.S. Rew, S.K. Choi

Internal Medicine, Chonnam National University Medical School, Gwang Ju, Republic of Korea

Background/aims: Peginterferon plus ribavirin is the standard treatment for chronic hepatitis $C$. The sustained virological response (SVR) is the most important factor of decision to initiate and continue therapy for chronic hepatitis $\mathrm{C}$ infection. This study identified predictive factors for SVR and efficacy with peginterferon $\alpha-2 \mathrm{a}$ and ribavirin in patients with patients with genotype $2 / 3$ chronic hepatitis $C$.

Methods: A total of 125 treatment-naive, HCV RNA-positive patients with genotype $2 / 3$ chronic hepatitis $\mathrm{C}$ were treated with peginterferon $\alpha$-2a $180 \mathrm{mcg} /$ week and $800 \mathrm{mg} /$ day for 24 weeks. The end of treatment virologic response (ETVR), the sustained virologic response (SVR), the end of treatment of biochemical response (ETBR), the sustained biochemical response (SBR) and the predictive factors for SVR were analyzed.

Results : The ETVR and SVR were 97.1 and $90.6 \%$. The ETBR was $81.0 \%$ and the SBR was $95.8 \%$. Multivariate analysis showed that body mass index of 25 and over was the only independent factor that affected sustained virologic response $(\mathrm{OR}=0.03,95 \%$ CI: $0.003-0.345, p=0.005)$. Sixteen patients $(12.8 \%)$ dropped out at the end of treatment, and $8(6.4 \%)$ stopped treatment due to treatmentrelated adverse events.

Conclusions: The peginterferon and ribavirin as an initial treatment for genotype $2 / 3$ chronic hepatitis $C$ is extremely effective and safe. The body mass index may be regarded as one of the significant predictive factors influencing the SVR.

\section{PP13-73}

Significance of ANA,SMA,LKM1 and M2 Detection in Chronic Hepatitis C Patients Treated with PEG-IFN $\alpha$-2a

J. Jiao, J. Wang, P. Zhao, H. Bo

China-Japan Union Hospital,Jilin University, Changchun, China

This study analyzed the variation of ANA,SMA,LKM1 and M2 antibodies in chronic hepatitis c patients who treated with PEG-IFN $\alpha$ $2 \mathrm{a}$, discussed the association between PEG-IFN $\alpha-2 \mathrm{a}$ response and ANA,SMA,LKM1 and M2 occurrence.

One hundred and fifty six chronic hepatitis $\mathrm{C}$ patients (84 patients were infected with genotype $1 \mathrm{HCV}, 72$ patients were infected with genotype non-1 HCV) were treated with PEG-IFN $\alpha$-2a and ribavirin for 48 weeks and followed up to 24 weeks. Fluorescence quantity analysis, immunofluorescence technique, and ELISA were used to detect serum HCV RNA and ANA,SMA,LKM1 and M2 antibodies of chronic hepatitis $\mathrm{C}$ patients who treated with PEG-IFN $\alpha-2 \mathrm{a}$ and ribavirin .At least 4 time points after PEG-IFN $\alpha$-2a application ( 0 weeks, 12 weeks, 48 weeks, end of 24 weeks follow up) were analyzed.

Results showed that ANA,SMA,LKM1 and M2 antibody positive rate (any one of them positive was regard as "antibody positive") in chronic hepatits C patients was $32.69 \%$, which ANA was $17.95 \%$, SMA was $14.10 \%$, LKM1 was $3.85 \%$, M2 was $2.56 \%$ respectively. No significant difference could be found in the distribution of these antibodies between type 1 and non-1 HCV infection. EVR, ETVR and SVR in those ANA/SMA/LKM1 positive (before treatment) patients were higher than those antibody negative patients. Occurrence of ANA,SMA,LKM1 at the end of treatment (48 weeks) were increased compared with those before PEG-IFN $\alpha$ - $2 \mathrm{a}$ treatment, and then decreased in 24 weeks of follow-up. Occurrence of M2 continues to rise with PEG-IFN $\alpha-2$ a application, no restore even if the withdrawal, some patients presents symptoms of primary biliary cirrhosis(PBC). In those obtained SVR patients,positive rate of ANA/SMA/LKM1 at the end of treatment was lower than that of patients who didn't obtain SVR.

\section{PP13-74}

Predictive Factors for Response to Antiviral Therapy of Chronic Hepatitis C L.S. Orlova, I.V. Malov

Department of Infectious Diseases, Irkutsk State Medical University, Irkutsk, Russia

Background/aims: Sustained virological response (SVR) on treatment of pegylated interferon and ribavirin is observed at $50-80 \%$ of patients and depends on different factors. Genotype of HCV is the most significant predictor of SVR. Influences of host's predictors are contradictory and demand future research. The aim of our study is to evaluate the association between host's predictive factors and SVR. Methods: 150 patients were treated by the interferon and ribavirin, $46 \%$ of them was Mongoloid. SVR is loss RNA HCV at 24 weeks of follow up. The role of some factors was researched using methods of statistics.

Results: In total SVR was $67.3 \%$. Among the patients with $\mathrm{HCV}$ genotype non- $1 \mathrm{~b} 84.2 \%$, and among the patients with genotype $1 \mathrm{~b}$ was $54.2 \%$. Patients with low viral load $\left(<3.3 \times 10^{6}\right.$ copies $\left./ \mathrm{ml}\right)$ had response in $74.2 \%$. Patients under 50 years old more often had SVR-73\% $(p<0.01)$. In our research the duration of disease had important significance. Patients with duration of disease under 10 years had SVR in $81.5 \%$ cases, and in patients with a longer history in only $46.2 \%$ of cases. Patients with a weight under $70 \mathrm{~kg}$, more frequently responded on treatment $(80.4 \%, p<0.05)$. People with second blood group achieved SVR in $100 \%$ of cases compared with first, third and fourth groups 61.1, 40.0 and $50.0 \%$ accordingly $(p<0.05)$. From all patients, four had $\mathrm{Rh}$ negative and all these patients achieved SVR, but it wasn't statistically (small sample). All the rest factors have not been associated with the achieving of a sustained virological response $(p>0.05)$.

Conclusions: Nowadays in the world role of HLA in SVR is studying very actively. One of simple and affordable methods for analysis genetic system is systems of $\mathrm{AB} 0$ and $\mathrm{Rh}$. In this connection data about role these factors are very interesting and demand future research.

\section{PP13-75}

Frequencies of Resistance-associated Amino Acid Variants (RAV) Following Treatment with Boceprevir (BOC) Plus PegInterferon Alfa-2b and Ribavirin (P/R)

J. Albrecht ${ }^{1}$, J. Vierling ${ }^{2}$, P. Kwo ${ }^{3}$, E. Lawitz ${ }^{4}$, J. McCone ${ }^{5}$, E. Schiff ${ }^{6}$, D. Pound ${ }^{7}$, M. Davis ${ }^{8}$, J. Galati ${ }^{9}$, S. Gordon ${ }^{10}$, N. Ravendhran ${ }^{11}$, L. Rossaro ${ }^{12}$, F. Anderson ${ }^{13}$, I. Jacobson ${ }^{14}$, R. Rubin ${ }^{15}$, L. Pedicone ${ }^{1}$, E. Chaudhri ${ }^{1}$, X. Tong ${ }^{1}$, P. Qiu ${ }^{1}$, R. Barnard ${ }^{1}$, C. Brass ${ }^{1}$, P. Mendez ${ }^{1}$, R. Ralston ${ }^{1}$ ${ }^{1}$ Merck, Whitehouse Station, NJ, ${ }^{2}$ Baylor College of Medicine, Houston, TX, ${ }^{3}$ Indiana University School of Medicine, Indianapolis, IN, ${ }^{4}$ Alamo Medical Research, San Antonio, TX, ${ }^{5}$ Mt. Vernon Endoscopy Center, Alexandria, VA, ${ }^{6}$ University of Miami, Miami, FL, ${ }^{7}$ Indianapolis Gastroenterology Research Foundation, Inidianapolis, 
IN, ${ }^{8}$ DigestiveCARE-South Florida Center of Gastroenterology, Wellington, FL, ${ }^{9}$ Liver Specialists of Texas, Houston, TX, ${ }^{10}$ Henry Ford Hospital, Detriot, MI, ${ }^{11}$ University of Maryland Medical System and Digestive Disease Associates, Baltimore, MD, ${ }^{12}$ University of California-Davis Medical Center, Sacramento, CA, USA, ${ }^{13}$ Liver and Intestinal Research Centre, Vancouver, BC, Canada, ${ }^{14}$ Weill Cornell Medical College, New York, NY, ${ }^{15}$ Liver Center of Atlanta, Atlanta, GA, USA

Background: BOC, an NS3 protease-inhibitor, added to P/R leads to high rates of sustained-virologic-response (SVR) in chronic hepatitis C G1 patients. We evaluated treatment-naïve G1 patients who received BOC/P/R in a Phase 2 trial (SPRINT-1) to determine factors associated with a differential frequency of RAVs.

Methods: 595 patients were randomized to control [48 weeks of $\mathrm{P}$ $(1.5 \mu \mathrm{g} / \mathrm{kg})+\mathrm{R}(800-1,400 \mathrm{mg} / \mathrm{day})]$ or one of 5 BOC treatment arms, including $\mathrm{P} / \mathrm{R}$ for 4 weeks (lead-in) followed by BOC $800 \mathrm{mg}$ TID plus $\mathrm{P} / \mathrm{R}$ for 44 weeks and $\mathrm{P}+$ low-dose $\mathrm{R}$ (400-1,000mg/day) followed by BOC. HCV-RNA was detected using Roche Taqman $(\mathrm{LLD}<15 \mathrm{IU} / \mathrm{mL})$. Amino acid variants at BOC resistance loci in the NS3/4A protease were detected using population sequencing.

Results: SVR was significantly higher in all BOC arms (54-75\%) compared to control (38\%). The 109 patients who developed RAVs on-study (those without RAVs at baseline) were G1a ( $n=78)$, G1b $(n=30)$ and 1 not subtyped. The most frequently detected on-study RAVs in G1a patients were R155K (77\%), V36M (68\%), and T54S $(37 \%)$. The most frequently detected in G1b patients were T54S (57\%), T54A (37\%), A156S (43\%), and I170A (43\%). Of these 109 patients, 2 achieved SVR (both from BOC arms) and 107 were nonresponders, relapsers or breakthroughs. Patients randomized to lowdose $\mathrm{R}$ had the highest frequency of on-study RAVs (41\%) whereas those assigned 4 week P/R lead-in followed by 44 weeks BOC/P/R had the lowest frequency $(11 \%)$. Notably, $4 \%$ of patients had known RAVs at baseline, and the majority (71\%) achieved SVR (all from BOC arms). Among the 3 most frequent on-study RAVs, T54S was less frequent in patients receiving lead-in therapy.

Conclusions: The profiles of on-study RAVs differed between G1a/ G1b patients. Lead-in therapy may reduce on-study mutations, such as T54S. Despite having RAVs at baseline, the majority of such patients achieved SVR.

\section{PP13-76}

Combination BMS-790052, BMS-650032 and PegIFN/RBV Provides Undetectable HCV RNA through 12 Weeks of Therapy in HCV Genotype 1 Null Responders

D.F. Gardiner ${ }^{1}$, A. Lok ${ }^{2}$, E. Lawitz ${ }^{3}$, C. Martorell ${ }^{4}$, G. Everson ${ }^{5}$, R. Ghalib ${ }^{6}$, R. Reindollar ${ }^{7}$, V. Rustgi ${ }^{8}$, P. Wendelburg ${ }^{1}, K . Z^{2} u^{1}$, V. Shah ${ }^{1}$, D. Sherman ${ }^{1}$,

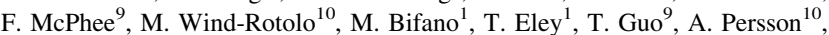
R. Hindes ${ }^{9}$, D. Grasela ${ }^{1}$, C. Pasquinelli ${ }^{1}$

${ }^{I}$ Research and Development, Bristol-Myers Squibb, Hopewell, NJ;

${ }^{2}$ University of Michigan, Ann Arbor, MI; ${ }^{3}$ Alamo Medical Research, San Antonio, TX; ${ }^{4}$ The Research Institute, Springfield, MA;

${ }^{5}$ University of Colorado-Denver, Aurora, CO; ${ }^{6}$ North Texas Research Institute, Arlington, TX; ${ }^{7}$ Carolinas Center for Liver Disease,

Statesville, NC; ${ }^{8}$ Metropolitan Research, Fairfax, VA; ${ }^{9}$ Research and Development, Bristol-Myers Squibb, Wallingford, CT; ${ }^{10}$ Research and Development, Bristol-Myers Squibb, Princeton, NJ, USA

Background/aims: BMS-790052 is a potent NS5A inhibitor with broad genotypic coverage while BMS-650032 is a potent HCV NS3 protease inhibitor with coverage of HCV genotypes (GT) $1 \mathrm{a}$ and $1 \mathrm{~b}$. Studies combining these compounds are underway to determine their safety and efficacy.

Methods: The Phase 2a study AI447011 compares BMS-790052 (60 $\mathrm{mg}$ QD) and BMS-650032 (600 mg BID) alone (Group A) or with pegIFN/RBV (Group B) for 24 weeks in HCV GT 1 null responders. The primary aim was determining the proportion of subjects achieving undetectable HCV RNA $(<10 \mathrm{IU} / \mathrm{mL})$ at Weeks 2 and 4 of therapy and 24 weeks post-treatment. A Week 12 interim analysis was performed.

Results: 21 subjects (11 Group A, 10 Group B) were randomized; virologic responses are presented in Table 1. 6 (54.5\%) Group A subjects experienced viral breakthrough while Group B subjects maintained viral suppression. Viral breakthrough occurred only with GT 1a and these subjects had pegIFN/RBV added to their regimen. HCV RNA fell to UD in two subjects, to $<25 \mathrm{IU} / \mathrm{mL}$ in another 2 subjects, and by $\geq 1.5 \mathrm{log}$ in anther 2 subjects. Diarrhea was the most common $\mathrm{AE}$ and was mainly mild to moderate in severity.

\begin{tabular}{lll}
\hline & $\begin{array}{l}\text { Group A } \\
\text { BMS-650032 } \\
\text { and BMS-790052 } \\
n=11\end{array}$ & $\begin{array}{l}\text { Group B } \\
\text { BMS-650032, } \\
\text { BMS-790052, } \\
\text { pegIFN/RBV } n=10\end{array}$ \\
\hline $\begin{array}{l}\text { Median HCV } \\
\text { RNA decline }\end{array}$ & $-5.1 \log 10$ & $-5.3 \log 10$ \\
Week 2 IU/mL & & \\
RVR $n(\%)$ & $7(63.6 \%)$ & $6(60 \%)$ \\
eRVR $n(\%)$ & $4(36.4 \%)$ & $6(60 \%)$ \\
$\operatorname{cEVR} n(\%)$ & $5(45.5 \%)$ & $9(90 \%)$ \\
\hline
\end{tabular}

Conclusions: Treatment with BMS-790052 and BMS-650032 with or without PegIFN/RBV demonstrated similar RVR rates in HCV infected GT 1 null responders, however, a four-drug combination maintained viral suppression in all subjects through week 12. Should this activity predict SVR, these results will have significant implications for future combination HCV antiviral therapy.

\section{PP13-77}

Pegylated Interferon, Ribavirin Combination Is Associated with Serious Psychiatric Manifestation in HCV Patient with Wilson disease, a Case Report

A. Elyamany, S. Lashin

Faculty of Medicine, Alexandria University, Alexandria, Egypt

Chronic infection with the hepatitis $\mathrm{C}$ virus ( $\mathrm{HCV})$ is a common and growing problem. Neuropsychiatric symptoms are commonly associated with chronic HCV infection, its sequelae, and its treatment. In particular, interferon, a primary component of treatment for chronic hepatitis $\mathrm{C}$ and well known to be associated with significant adverse effects as depressive symptoms.

Case report: We report a 24-year-old female who developed psychiatric symptoms after the second pegylated interferon dose and ribavirin for treatment of chronic hepatitis $\mathrm{C}$ infection, genotype 4 . Therapy was promptly discontinued. Correction of possible etiological factors did not improve the condition. Wilson disease was found to be the undiagnosed etiological factor for these psychiatric manifestations, and was presented for the first time. D- Penicillamine treatment started with marvelous response.

Recommendation: Consideration for a possible etiology for psychiatric manifestations during Interferon therapy is advisable.

\section{PP13-78}

Genetic Polymorphism in $I L 28 B$ Gene Associated with Clinical Course of Hepatitis C Virus Infection in Japanese Population

K. Matsuura ${ }^{1,2}$, Y. Tanaka ${ }^{1}$, N. Nishida ${ }^{3}$, M. Sugiyama ${ }^{1}$, M. Kurosaki ${ }^{4}$,

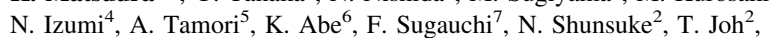

H. Tsubouchi ${ }^{8}$, K. Tokunaga ${ }^{3}$, M. Mizokami ${ }^{9}$

${ }^{1}$ Department of Virology \& Liver Unit; ${ }^{2}$ Department of

Gastroenterology and Metabolism, Nagoya City University Graduate School of Medical Sciences, Nagoya; ${ }^{3}$ Department of Human 
Genetics, Graduate School of Medicine, the University of Tokyo, Tokyo; ${ }^{4}$ Division of Gastroenterology and Hepatology, Musashino Red Cross Hospital, Musashino, ${ }^{5}$ Department of Hepatology, Osaka City University Graduate School of Medicine, Osaka; ${ }^{6}$ Department of Gastroenterology and Hepatology, Iwate Medical University,

Morioka; ${ }^{7}$ Department of Gastroenterology, Nagoya Koseiin Medical Welfare Center, Nagoya; ${ }^{8}$ Kagoshima University Graduate School of Medical and Dental Sciences, Kagoshima; ${ }^{9}$ Research Center for Hepatitis and Immunology, Kohnodai Hospital International Medical Center of Japan, Ichikawa, Japan

Background/aim: We reported that single nucleotide polymorphisms (SNPs) around IL28B gene are strongly associated with response to pegylated interferon (PEG-IFN) plus ribavirin (RBV) therapy for hepatitis $\mathrm{C}$ virus (HCV)-genotype 1 patients. Afterward, it is reported that the genetic variants in $I L 28 B$ gene are associated with spontaneous clearance of HCV. On the other hand, it hasn't known yet whether the genetic variants in $I L 28 B$ gene are associated with HCV-related hepatocellular carcinoma (HCC). In this study, we examined whether the genetic variants in $I L 28 B$ gene are associated with clinical course including spontaneous clearance of $\mathrm{HCV}$, response to IFN treatment and HCC.

Methods: We enrolled 490 individuals treated at 17 hospitals throughout Japan, including 107 spontaneous clearance (SC), 186 virological response (VR) and 86 null virological response (NVR) to PEG-IFN/RBV treatment who were infected with genotype 1 , and 111 HCV-related HCC. The representative 16 SNPs around IL28B gene were genotyped by DigiTag2 assay. A case-control study was conducted among these groups.

Results: Compared SC to the other groups of persistent infection (PI), including VR, NVR and HCC group, rs8099917 showed the strongest association in the minor allele dominant model $\left(p=6.91 \times 10^{-9}\right.$; $\mathrm{OR}=7.47)$; the proportion of minor allele $(\mathrm{T} / \mathrm{G}$ or $\mathrm{G} / \mathrm{G})$ in $\mathrm{SC}$ and PI group were 6 and $31 \%$, respectively. On the other hand, compared among VR, NVR and HCC by genotyping of rs8099917, the proportion of minor allele were 11,77 and $29 \%$, respectively. There were significant differences in the minor allele dominant model between VR vs. NVR $\left(p<2.2 \times 10^{-16} ; \mathrm{OR}=26.9\right)$. There was no difference of the allele frequency between HCC and PI group.

Conclusions: Our data indicated that genetic variants around the $I L 28 B$ gene were associated with spontaneous clearance of $\mathrm{HCV}$ as well as response to $\mathrm{HCV}$ treatment in Japanese population. However, it is considered that these were not associated with HCC.

\section{PP13-79}

Interferon Alfa Antibody a Useful Predictive Marker of Sustained Viral Response (SVR) for Therapy in Chronic Hepatitis C

P.P. Basu ${ }^{1,2}$, N. Krishnaswamy ${ }^{2}$, N.J. Shah ${ }^{2}$, T. Pacana ${ }^{2}$, S. Foulstin ${ }^{2}$, C. Tang ${ }^{2}$, R.J. Brown ${ }^{1}$

${ }^{I}$ Columbia NY Presbyterian College of Physicians and Surgeons, NY, Manhattan; ${ }^{2}$ North Shore Forest Hills Hospital New York, Forest Hills, NY, USA

Purpose: Interferon alfa is the mainstay of therapy for hepatitis $\mathrm{C}$ infection. Sustained viral response (SVR) remains $46 \%$ in all genotypes. On treatment, rapid viral response (RVR) and early viral response (EVR) are predictive markers of SVR and treatment modification. We propose a novel marker, IFN alfa $\mathrm{Ab}$, in predicting response of interferon alfa therapy on chronic hepatitis $\mathrm{C}$ naïve genotype 1 patients.

Methods: Forty $(n=40)$ patients (age: $45.2 \pm 7.2$ years, mean \pm SD; range: $26-56$ years) were recruited. Serum levels of IFN alfa $\mathrm{Ab}$ were obtained initially at 0 week, then $2 \mathrm{nd}$, 4th, 12th, 24th, 48th, and 72nd week, respectively.

Exclusion: Co-infection, hemolytic syndromes, severe cardiac disease, severe depression, uncontrolled diabetes mellitus, decompensated cirrhosis, and patients actively using illicit drugs and those with alcohol consumption $>50$ g per day.
Results: 25/40 (62.5\%) achieved SVR. 31/40 (77.5\%) developed IFN alfa Ab during the treatment (14 at 12th week, 16 at 24th week, and 1 at 48th week);15/31(48.4\%) had higher titers ( $\geq 1: 150)$. 13/14 (92.9\%) with $\mathrm{Ab}$ at 12 th week had higher titers. The presence of high-titer $\mathrm{Ab}$ at 12th week predicted failure of SVR with $86.7 \%$ sensitivity, $100 \%$ specificity, $100 \%$ positive predictive value, and $92.6 \%$ negative predictive value. 11 Afro-Americans developed high-titer $\mathrm{Ab}$ at 12 th week and failed SVR. 22/40 (55\%) with high viral load $>400 \mathrm{k}$ failed SVR.

Conclusion: Majority of patients during interferon therapy developed IFN alfa $\mathrm{Ab}$ at some period. This study postulates the early presence of higher titer $\mathrm{Ab}$ at week 12 that predicts the failure of SVR with high sensitivity and specificity. Afro-Americans developed high-titer $\mathrm{Ab}$ early and failed SVR. Ab titers do not correlate with BMI, age, sex, and fibrotic score but predict failure of SVR. This study may modify the recent treatment paradigm with cost benefits. A larger prospective trial is needed.

\section{PP13-80}

Individualized Antiviral Efficacy of Pegylated Interferon $\alpha$-2a in Chinese Patients with Chronic Hepatitis C

Y. Xu, J.-B. Wang, T.-N.-L. Siqing, J. Jiao, Y.-G. Zhang, P. Zhao, H.-H. Guo, Y. Li, C.-Y. Zhou, S.-W. Ji, Y.-X. Lang

Gastroenterology, China-Japan Union Hospital,Jilin University, Changchun, China

Background: Interferon plus ribavirin (RBV) is the standard treatment for hepatitis $\mathrm{C}$ virus (HCV) infection. While the severity of chronic hepatitis $\mathrm{C}(\mathrm{CHC})$ is closely associated with viral genotype and genetic susceptibility, antiviral efficacy differs between individuals. This study evaluated the efficacy of individualizing therapy with pegylated interferon $\alpha$-2a (Peg-IFN $\alpha-2 a) / R B V$ in 121 Chinese patients with CHC.

Methods: Patients initially received conventional Peg-IFN $\alpha$-2a $180 \mu \mathrm{g}$ (sc) once weekly together with $800-1,200 \mathrm{mg} / \mathrm{d}$ RBV for 12 weeks. Treatment response [complete early virologic response (cEVR); partial early response (pEVR); rapid virologic response (RVR); end-of-treatment virologic response (ETVR)] was assessed at Weeks 4 and 12, and sustained virologic response (SVR) was assessed at 24-week follow up. Individualized treatment approaches (increasing treatment duration, increasing RBV dose, or combination therapy with thymosin- $\alpha$ ) were established in patients who did not achieve cEVR at Week 12.

Results: 13 patients (10.7\%) received RVR and $100 \%$ received SVR; 55 patients $(45.5 \%)$ received cEVR and $78.6 \%$ received SVR; Sixty six patients $(54.5 \%)$ did not achieve cEVR at Week 12; $40(60.6 \%)$ were infected with $\mathrm{HCV}$ genotype 1 . Of all patients without a 12 -week cEVR: 18 (27.2\%) continued treatment with standard dose Peg-IFN $\alpha$ 2a/RBV to Week 48; 19 (28.8\%) continued treatment with standard dose Peg-IFN $\alpha-2 \mathrm{a} / \mathrm{RBV}$ to Week $72 ; 16$ (24.3\%) received thymosin- $\alpha$ and Peg-IFN $\alpha-2 \mathrm{a} / \mathrm{RBV}$ to Week 24; and $13(19.7 \%)$ received PegIFN $\alpha-2 \mathrm{a} / \mathrm{RBV} 1,600 \mathrm{mg}$ for 48 weeks. Increasing standard treatment duration to 72 weeks produced a SVR of $73.7 \%$ at follow up compared with $33.3 \%$ in patients receiving conventional treatment to 48 weeks $(p=0.01)$. Combination therapy with thymosin- $\alpha$ resulted in a SVR of $50.0 \%$, while increasing RBV dose produced a SVR of $53.8 \%$.

Conclusions: Individualizing therapy by extending the treatment duration to 72 weeks in $\mathrm{CHC}$ patients who did not demonstrate a cEVR with Peg-IFN $\alpha-2 \mathrm{a} / \mathrm{RBV}$ at 12 weeks was effective in improving SVR rates.

\section{PP13-81}

IFN Therapy Complications in Patients with Chronic Hepatitis C: Prevalence and Correlation

I. Ivanikov ${ }^{1}$, A. Ilyankova ${ }^{1}$, M. Shapina ${ }^{2}$

${ }^{1}$ Central Clinical Hospital of the Presidential Administration of the Russian Federation; ${ }^{2}$ Moscow State University, Moscow, Russia

Objectives and goals: To estimate the prevalence and clinical significance of pegylated interferon (PegIFN-alpha) with ribavirin 
treatment complications in patients with chronic hepatitis C. To reveal the relation between changes in blood tests and sex, age, degree of activity and duration of the disease, genotype of a virus, HCVRNA concentration.

Material and methods: 50 patients with genotype 1 or 3 were taken as a research group. They were treated by PegIFN-alpha associated with ribavirin during the period 2004-2009. Blood values (leukocytes, red blood cells, hemoglobin, platelets, neutrophils, eosinophils, lymphocytes, monocytes, ESR) were analyzed three times: prior to the beginning of treatment, on the sixth week of treatment and in one month after the treatment. Stats processing with SPSS18.

Results: Among all patients leucopenia was diagnosed in $85 \%$ of cases, neutropenia in $75 \%$, thrombocytopenia in $55 \%$, erythropenia in $40 \%$. During the research statistically significant $(p<0.05)$ difference between all estimated blood values taken on the sixth week of treatment, the same blood tests taken prior to the beginning of treatment, and the results received after the termination of treatment $(p<0.05)$ has been shown. Correlation between HCVRNA concentration and decrease of leukocytes $(p=0.012)$, red blood cells $(p=0.047)$, platelets $(p=0.007)$, neutrophils $(p=0.007)$, increase of lymphocytes $(p=0.015)$ has been discovered. Statistically significant correlation between duration of disease prior to the beginning of treatment and decrease of leukocytes $(p=0.0003)$, red blood cells $(p=0.0009)$, platelets $(p=0.0006)$, neutrophils $(p=0.00001)$, increase of lymphocytes $(p=0.003)$ has also been shown. Correlation between age and decrease of leukocytes $(p=0.0001)$, platelets $(p=0.01)$, neutrophils $(p=0.009)$, increase of monocytes $(p=0.001)$ and between a virus genotype and decrease of leukocytes $(p=0.02)$, neutrophils $(p=0.007)$ was also statistically significant. We found statistically significant dependence of platelets decrease from sex $(p=0.045)$. No correlation between changes of other laboratory tests (hemoglobin, eosinophils, ESR) and investigated parameters was found.

\section{PP13-82}

Methods of Correction of by-effects Combined Antiviral Therapy(AVT) O. Golovchenko

Medical Clinical Research Center 'Health Clinic', Vinnytsya, Ukraine

Background: Using In modern therapy of a chronic virus Hepatitis $\mathrm{C}$ combination Pegylated Interferon- $\alpha(\mathrm{PIFN}-\alpha)$ with Ribavirin is peculiar numerous and dose-depends collateral reactions because of which $25 \%$ of patients don't finish it. One of the least shined still have a question on the various autoimmune phenomena is most frequance thyroid gland defeat.

Methods: 50 patients were examined under the preparation program to AVT, restrictions and contrindications were not revealed. AVT was performed out-patient under the standard scheme: PIFN- $\alpha$ once a week and Ribavirin daily (800-1,200 mg/day).

Results: At the first injections development of flu-similar syndrome at $90 \%$ of patients was noted. It was adequately corrected by reception liquid (to $3 \mathrm{~L}$ ), interferon introduction in second half of day, and Paracetamol reception. Further expressiveness of the given symptoms has decreased. Similar symptoms have ceased to disturb after $6-7$ th injections. From 15 to 18 th weeks of therapy at $70 \%$ gradual decrease haemoglobin level to $90-100 \mathrm{~g} / \mathrm{L}$,in this connection it was appointed $\mathrm{r}$-HuEPO in a dose of $100 \mathrm{IU} / \mathrm{kg} 3$ times a week hypodermically till the stable normal level of haemoglobin is noted, that has allowed to avoid a dose reduction Ribavirin at $80 \%$ of patients. Increase of level of Anti-TPO (from 5 to 20 norms) has been registered at $40 \%$ of patients. Thus: level T3-within norm, T4-below, TSH-Above. Symptoms of expressed hypothyreosis were absent. AVT was continued with additional appointment L-thyroxin in a dose $100 \mathrm{mcg}$ a day daily till 1 month after termination AVT, was cancelled after normalisation of laboratory indicators of a condition of a thyroid gland. Summary. Correction of a syndrome and haematology infringements does not make problems, but compensation variety autoimmune disorders demands the further studying.

\section{PP13-83}

Pan-Asian Variability of Interleukin 28B (IL28B) Gene Polymorphism and Relationship to Sustained Viral Response (SVR) in Chronic

Hepatitis C

P.P. Basu ${ }^{1,2}$, N.J. Shah ${ }^{2}$, N. Krishnaswamy ${ }^{2}$, S. Foustin ${ }^{2}$, C. Tang ${ }^{2}$, T. Pacana ${ }^{2}$, R.J. Brown ${ }^{1}$

${ }^{1}$ Columbia NY Presbyterian College of Physicians and Surgeons, NY, Manhattan; ${ }^{2}$ North Shore Forest Hills Hospital New York, Forest Hills, NY, USA

Background and objectives: The overall SVR achieved with treatment of chronic hepatitis $\mathrm{C}(\mathrm{CHC})$ genotype 1 with peginterferon plus ribavirin is $42-46 \%$ in pivotal trials. The IL28B single nucleotide polymorphism (SNP) was recently discovered, and the likelihood of achieving an SVR was doubled in patients with the IL28B C/C variant versus the $\mathrm{C} / \mathrm{T}$ or $\mathrm{T} / \mathrm{T}$ variants. The prevalence of the $\mathrm{C} / \mathrm{C}$ allele predicts the SVR rate in different ethnic groups, and whites have a higher frequency than African Americans with Hispanics an intermediate frequency. Asians have the highest prevalence rate of the favorable $\mathrm{C} / \mathrm{C}$ allele, but there is little data on the distribution of different alleles among a pan-Asian population. This retrospective study investigated the pan-Asian diversity of IL28B gene and SVR rates in a population of Asians with CHC G1 infection.

Methods: Thirty three patients, with a mean age 46 years, from a large pan-Asian distribution were retrospectively analyzed from the ERAIS C trial with IL28B testing. All patients had CHC genotype 1 infection. The ethnic diversity of the Asians were classified into Middle Asians (MA) (11/33, 33.3\%); central Asian (CA) (7/33, $21.2 \%)$; and East Asian (EA) $(15 / 33,45.5 \%)$ groups. The overall SVR rate was 22 of $33(66.67 \%)$. The allele frequency was $\mathrm{C} / \mathrm{C}$ in $24 / 33$ (72.7\%), $\mathrm{C} / \mathrm{T}$ in $7 / 33(21.2 \%)$, and $\mathrm{T} / \mathrm{T}$ in $2 / 33(6 \%)$.

Results: Subanalysis of the allele frequency in three groups (see table).

Conclusion: From this retrospective study, a Pan-Asian population has a higher $\mathrm{C} / \mathrm{C}$ allele predominance with a higher SVR in genotype 1 CHC. A larger population based analysis is needed to confirm the hypothesis.

Subanalysis of the allele frequency in three group

\begin{tabular}{|c|c|c|c|c|c|c|}
\hline & $\begin{array}{l}\mathrm{CC} \\
(n)\end{array}$ & $\begin{array}{l}\text { SVR } \\
(\%)\end{array}$ & $\begin{array}{l}\mathrm{CT} \\
(n)\end{array}$ & $\begin{array}{l}\text { SVR } \\
(\%)\end{array}$ & $\begin{array}{l}\mathrm{TT} \\
(n)\end{array}$ & $\begin{array}{l}\text { SVR } \\
(\%)\end{array}$ \\
\hline $\begin{array}{l}\text { Middle Asian (Turkey, } \\
\text { Turkmenistan, } \\
\text { Iran, Iraq), } \\
n=11\end{array}$ & 6 & $3(50)$ & 5 & $2(40)$ & 0 & - \\
\hline $\begin{array}{l}\text { Central Asian } \\
\text { (Uzbekistan, } \\
\text { Tajikistan, } \\
\text { Kazakhstan, } \\
\text { Afghanistan), } \\
n=7\end{array}$ & 5 & $4(80)$ & 0 & - & 2 & $0(0)$ \\
\hline $\begin{array}{l}\text { East Asian } \\
\text { (China, Tibet, } \\
\text { Pakistan, } \\
\text { India, Bangladesh, } \\
\text { Philippines), } \\
n=15\end{array}$ & 13 & 11 (84.6) & 2 & $2(100)$ & 0 & - \\
\hline $\begin{array}{l}\text { Percentage of } \\
\text { SVR attained }\end{array}$ & & $\begin{array}{c}\text { 18/24 (75\% } \\
\text { SVR) }\end{array}$ & & $\begin{array}{c}4 / 7(57.1 \% \\
\text { SVR) }\end{array}$ & & $\begin{array}{r}0 / 2(0 \% \\
\text { SVR) }\end{array}$ \\
\hline
\end{tabular}




\section{PP13-84}

Association between IL28B-genotype and Insulin Resistance in Treatmentnaïve Patients with Chronic Hepatitis C

P. Steindl-Munda ${ }^{1}$, A.F. Staettermayer ${ }^{1}$, K. Rutter ${ }^{1}$, S. Beinhardt ${ }^{1}$, T.M. Scherzer ${ }^{1}$, K. Zinober ${ }^{1}$, H. Hofer ${ }^{1}$, F. Wrba ${ }^{2}$, P. Ferenci ${ }^{1}$

${ }^{1}$ Dep. of Gastroenterology and Hepatology; ${ }^{2}$ Institute of Clinical Pathology, Medical University of Vienna, Vienna, Austria

Background: A SNP (rs12979860), 3kb upstream of the IL28B-gene has a significant influence on treatment-outcome of patients with chronic hepatitis $\mathrm{C}$. Insulin resistance is also known to be a significant predictor of outcome of interferon/ribavirin combination therapy. The aim of our study was to evaluate the role of the IL28B-genotype on insulin resistance in treatment-naïve Austrian HCV patients.

Methods: At time of writing this abstract, data of $142 \mathrm{HCV}$ (GT1:105, GT2:2, GT3:9, GT4:26; $m=93, f=49$ ) and of $30 \mathrm{HBV}$ infected patients $(m=11, f=7)$ were available. Patients on medication for diabetes were excluded. Blood samples for fasting insulin and glucose were taken shortly before liver biopsy was performed, HOMA-IR was calculated. All biopsies were read by a single pathologist unaware of the clinical data.

The rs12979860 region of the IL28B-gene was investigated by StepOnePlus Real time PCR System (Applied Biosystems, Foster City, USA) after written informed consent of the patients.

Results: Insulin resistance (HOMA-IR $\geq 2.0$ ) was associated with the rs12979860 genotype, the presence of advanced fibrosis and steatosis. In patients with the C/C-genotype the HOMA-IR was $2.14 \pm 1.70$ (mean $\pm \mathrm{SD}$ ), in patients carrying the T-allele it was $3.41 \pm 3.37$ $(p=0.0083)$. Patients carrying the T-allele had more pronounced steatosis $(22.3 \pm 20.4 \%$ of hepatocytes $)$ than those with $\mathrm{C} / \mathrm{C}$ $(10.9 \pm 12.4 \% ; p=0.0018)$. Advanced fibrosis (F3-4) was associated with higher HOMA-IR $(4.21 \pm 3.17)$ than mild fibrosis $(\mathrm{F} 0-2)$ $(2.32 \pm 2.37, p=0.001)$.

Patients with HCV genotype $1(n=105)$ tended to have a lower HOMA-IR $(2.56 \pm 2.50)$ than those with genotype $4(n=26)$ $(3.74 \pm 3.51, p=0.112)$.

No difference in HOMA-IR was seen between patients with HCV and HBV $(2.84 \pm 2.75$ vs. $2.84 \pm 3.66)$.

There were no significant differences in body mass index between the studied subgroups.

Discussion: The IL28B-genotype has an influence on insulin resistance in patients with chronic HCV. However, both the pathogenetic causality and the clinical relevance need further investigation.

\section{PP13-85}

Improved Fibrosis Staging by Liver Stiffness Evaluation after Treatment with Natural Interferon (Alpha-N3) + Ribavirin in Patients with Chronic Hepatitis-C

A.M. Popescu, M. Voiculescu

Depart. of Internal Medicine, Fundeni Clinical Institute, Bucharest, Romania

Background: Liver stiffness (LS) measurement by transient elastography is a well-validated method for assessment of fibrosis in patients with chronic hepatitis $\mathrm{C}(\mathrm{CHC})$. Studies have shown that prolongation of treatment with interferon (IFN) plus ribavirin (RVB) over 48 weeks may prevent relapse in HCV genotype-1 patients, who did not achieve RVS, also improving fibrosis staging.

Aim: To evaluate the safety and efficacy of natural IFN alpha from normal human leucocytes (IFN alpha-N3) plus RBV, to treat CHC in marginal categories of patients with intolerance to pegylated-IFN, thrombocytopenia, heart or dermatological diseases, depressive syndrome, thyroiditis, age over 65 years.

Methods: A prospective case-control study was conducted, enrolling 22 CHC patients with co-morbidities or old age, currently considered ineligible for Peg -IFN+RBV, all genotype 1, who received IFN alpha-N3, 3 MU. X 3 /week +RBV 800-1,200 mg/day. 22 untreated matched patients served as controls. LS, transaminases, blood cells count, heart and thyroid functional tests, HCV-RNA levels, were monitored at baseline and at weeks: 4, 12, 24, 48, 72, and 6- 12 months after therapy.

Results: 22 patients (M/F 15/7, mean age 62 years) treated with IFN alpha-N3 plus RBV, responded as follows: RVR in 7 patients, SVR in 10 patients, relapse in 7, non-responders 5 . In 10 slow responding patients, treatment was prolonged to 72 weeks, with an improvement in SVR of $13.6 \%$. LS did not differ between the patients with SVR and non-SVR (10.8 vs. $11.1 \mathrm{KPa})$. On treatment, LS dynamics was independent to the RVR and SVR. In slow responders, prolongation of treatment over 48 weeks significantly decreased LS from a mean of $13-8.9 \mathrm{KPa}, p<0.001$ (improved fibrosis by 1 stage), continuing 6-12 months post-treatment.

Conclusion: Personalized treatment in $\mathrm{CHC}$ difficult-to-treat patients using natural IFN alpha+ RBV is improving liver fibrosis, independently of the virological response type.

\section{PP13-86}

Cost-benefit Analysis of Response Guided Therapy: Dynamic Disease Markov Modeling for Patients with Chronic Hepatitis C Virus by Fibrosis Stages

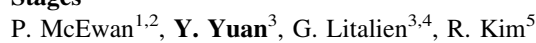

${ }^{1}$ Cardiff Research Consortium, Cardiff; ${ }^{2}$ School of Human \& Health Sciences, Swansea University, Wales, Swansea, UK; ${ }^{3}$ Global Health Outcomes and Outcomes Research, Bristol-Myers Squibb, Princeton, NJ; ${ }^{4}$ Yale University School of Medicine, New Haven, CT; ${ }^{5}$ Mayo Clinic College of Medicine, Rochester, MN, USA

Background/aims: Measuring viral kinetics to determine optimal duration of therapy in patients with rapid virological response (RVR) or early virological response (EVR) has emerged; however, comprehensive economic evaluation of response guided therapy (RGT) is lacking. This study aims to quantify costs and benefits of RGT compared to standard duration of therapy (SDT) in patients with HCV genotype-1. Methods: The study utilises a Markov lifetime simulation; patients enter the model at Metavir disease stages F0 through F4 and are partitioned: RVR; complete Early Virological Response (cEVR); partial Early Virological Response (pEVR), and non-response (NR). Progression though fibrosis stages is controlled via dynamic age dependent transition probabilities. Sustained virological response (SVR) by fibrosis stage with RGT was contrasted with SDT in treatment naive $\mathrm{HCV}$ genotype-1 patients. Average age of patients in stages F0-F2 was assumed 35 years and 45 for F3-F4, in the reference case. UK 2010 costs were used; costs and benefits discounted at $3.5 \%$. Results: RGT was associated with cost saving and modest improvement in QALYs; Table 1 details results for predicted lifetime per patient costs and benefits for RGT versus SDT, stratified by fibrosis stages at treatment.

Results for RGT versus standard duration therapy

\begin{tabular}{|c|c|c|c|c|}
\hline & \multicolumn{2}{|l|}{ SDT } & \multicolumn{2}{|l|}{ RGT } \\
\hline & F0-F2 & F3-F4 & F0-F2 & F3-F4 \\
\hline Total cost & $£ 21,524$ & $£ 32,362$ & $£ 19,246$ & $£ 31,531$ \\
\hline $\begin{array}{l}\text { Cost difference } \\
\text { (RGT-SDT) }\end{array}$ & & & $-£ 2,278$ & $-£ 830$ \\
\hline Total QALYs & 16.56 & 11.41 & 16.61 & 11.42 \\
\hline QALY difference (RGT-SDT) & & & 0.048 & 0.008 \\
\hline
\end{tabular}

Conclusion: RGT compared to SDT offers the potential for substantial cost savings with patients treated earlier in their disease offering the greatest potential saving; however, initiating therapy 
sooner risks treating patients destined to have a benign or slow progression. Cost savings in treating advanced disease are limited due to reduced SVR rates.

\section{PP13-87}

Effects of Interferon Alpha on Parathyroid Gland in Chronic Hepatitis B and C: An Observational Study

P.P. Basu ${ }^{1,2}$, N.J. Shah ${ }^{2}$, N. Krishnaswamy ${ }^{2}$, S. Foustin ${ }^{2}$, C. Tang ${ }^{2}$, T. Pacana ${ }^{2}$, R.J. Brown ${ }^{1}$

${ }^{1}$ Columbia NY Presbyterian College of Physicians and Surgeons, NY, Manhattan; ${ }^{2}$ North Shore Forest Hills Hospital New York, Forest Hills, NY, USA

Objectives: Interferon alpha (IFN $\alpha$ ) has been the mainstay of therapy for chronic hepatitis C. Side events of IFN-based therapy includes autoimmune thyroiditis $(10.7 \%)$. The effects of IFN gamma on calcium homeostasis have been well documented. This study evaluated IFN alpha induced autoimmune hyperparathyroidism, with or without eucalcemic state.

Methods: Ninety patients were evaluated, 45 patients with chronic hepatitis C (group A), 36 patients with chronic hepatitis B (group B), and nine patients with multiple sclerosis (MS) (group C) who were undergoing pegylated IFN alfa-2a for chronic hepatitis B or C or IFN beta therapy for MS were recruited. Baseline and 24-week ionized calcium, serum parathyroid hormone intact (PTH intact), 24-h urine calcium, bone densitometry, and parathyroid antibody (PTH Ab) were measured on all subjects. Exclusion criteria: Parathyroid dysfunction, multiple endocrine neoplasia, hypercalcemic syndromes, hypervitaminosis D, renal failure, osteoporosis and sarcoidosis

Results: Twenty-three of 90 patients $(25.5 \%)$ had high normal serum calcium $(>9.6 \mathrm{mg} / \mathrm{dL})$. The $24-\mathrm{h}$ urine and total ionized calcium levels were elevated in 2/36 (5.6\%) patients in Group B. Baseline serum PTH-intact and PTH Ab were normal in all groups. Nine of 45 patients (20\%) in group A and six of 36 patients (16.7\%) in group B receiving peginterferon IFN alfa-2a developed elevated PTH levels versus none in group C. PTH scan (radionucleotide scan) identified 1/ $36(2.8 \%)$ patients with an adenoma in group B. PTH Ab was found 9/ $45(20 \%)$ in group A, 5/36 (13.9\%) in group B, and none in Group C. Conclusion: Interferon alpha has subclinical autoimmune effects on parathyroid gland expressing elevated PTH level with PTH Ab without clinical manifestation of hyperparathyroidism. A larger sample size is required to confirm the potential effect of IFN alpha on calcium homeostasis, which might potentially explain the symptoms of fatigue in chronic hepatitis $\mathrm{C}$ associated with IFN therapy.

\section{PP13-88}

Intravenous Silibinin as "Rescue Treatment" for on Treatment Nonresponders to Peginterferon/Ribavirin Combination Therapy K. Rutter ${ }^{1}$, S. Beinhardt ${ }^{1}$, T.M. Scherzer ${ }^{1}$, A.F. Staettermayer ${ }^{1}$, H. Hofer ${ }^{1}$, T. Popow-Kraupp ${ }^{2}$, P. Steindl-Munda ${ }^{1}$, P. Ferenci ${ }^{1}$

${ }^{1}$ Internal Medicine $3 ;{ }^{2}$ Clinical Virology, Medical University of Vienna, Wien, Austria

Background: Intravenous Silibinin (SIL) is a potent antiviral agent against the hepatitis $\mathrm{C}$ virus (HCV). In this study we tested the efficacy of iv. SIL in on-treatment nonresponders to SOC.

Methods: 27 on treatment nonresponders to $180 \mu \mathrm{g}$ peginterferonalfa2a+ 1,000-1,200 mg ribavirin/d (SOC) (mean age: $52.6 \pm 6.9$ years; male/female:19/8; genotype1:19, 3a:4, 4:4; fibrosis F4:14, F3:5, F2:2) received $20 \mathrm{mg} / \mathrm{kg} / \mathrm{d}$ SIL (Legalon-SIL ${ }^{\circledR}$, RottapharmMadaus, Monza, Italy) for $15(n=11)$ or 21 days $(n=16)$. Nonresponse was defined either by a $<2 \log$ drop in viral load after 12 weeks or HCV-RNA positivity after 24 weeks. Fifteen patients were treatment naïve, 12 were nonresponders. SOC was continued in all patients responding to SIL. HCV-RNA was quantified (detection limit: $15 \mathrm{IU} / \mathrm{mL}$ ) by TaqMan $^{\circledR}$ (Roche Diagnostics, USA).
Results: At the end of SIL treatment $23 / 27$ patients $(85.1 \%)$ had undetectable HCV-RNA (17 after 7days, 6 after 15 days). After SIL administration patients were followed for at least 12 weeks. HCV RNA became detectable in 5 patients 3 weeks after the end of SIL. Two patients received a second course of intravenous SIL and became HCV-RNA became negative again, one relapsed thereafter. One of the $4 \mathrm{HCV}$-RNA pos. patients became HCV-RNA neg. 8 weeks after SIL on SOC. Sixteen patients reached one treatment endpoint (end of treatment HCV-RNA undetectable $n=8$, nonresponse $n=8$ ), 11 patients have not completed SOC yet (all of them are HCV-RNA negative). Nine patients with end of treatment response completed 24 weeks of treatment free follow up, five patients remained HCV-RNA negative (all with detectable, but unquantifiable HCV-RNA before SIL), four relapsed. Thus by ITT analysis $5 / 15$ patients $(31.3 \%)$ had a SVR. Except or a slight increase in bilirubin $(0.98 \pm 0.35$ (SD) to $2.12 \pm 0.99 \mathrm{mg} / \mathrm{dl}$ ) treatment was well tolerated.

Conclusion: Intravenous silibinin is an effective "rescue treatment" for on treatment nonresponders to full dose of SOC.

\section{PP13-89}

Telaprevir in Combination with Peginterferon Alfa-2a/Ribavirin in Treatment-naïve Genotype 1 HCV Patients: Final Results of Phase 3 ILLUMINATE Study

N.H. Afdhal ${ }^{1}$, D.R. Nelson ${ }^{2}$, G.T. Everson ${ }^{3}$, M.S. Sulkowski ${ }^{4}$, M.W. Fried ${ }^{5}$, S.L. Flamm ${ }^{6}$, F. Poordad ${ }^{7}$, K. Kleber ${ }^{8}$, M. Martin ${ }^{8}$, A.J. Sankoh ${ }^{8}$, R.S. $\mathrm{Kauffman}^{8}$, S. George ${ }^{8}$, C. Wright ${ }^{8}$, K.E. Sherman ${ }^{9}$, ILLUMINATE Study Team

${ }^{I}$ Beth Israel Deaconess Medical Center, Boston, MA; ${ }^{2}$ University of Florida, Gainesville, FL; ${ }^{3}$ University of Colorado Denver, Aurora, CO; ${ }^{4}$ Johns Hopkins University School of Medicine, Baltimore, MD; ${ }^{5}$ University of North Carolina at Chapel Hill, Chapel Hill, NC; ${ }^{6}$ Northwestern University, Chicago, IL; ${ }^{7}$ Cedars-Sinai Medical Center, Los Angeles, CA; ${ }^{8}$ Vertex Pharmaceuticals Incorporated, Cambridge, MA; ${ }^{9}$ University of Cincinnati College of Medicine, Cincinnati, OH, USA

Background: The Phase 3 open-label study, ILLUMINATE, evaluated 24- versus 48 -week treatment in patients achieving extended rapid viral response (eRVR, undetectable HCV RNA at weeks 4 and 12).

Methods: HCV genotype 1 treatment-naïve patients received telaprevir (12 weeks, $750 \mathrm{mg} \mathrm{q} 8 \mathrm{~h}$ ), peginterferon alfa-2a (P, $180 \mu \mathrm{g} /$ week) and ribavirin ( $R, 1,000-1,200 \mathrm{mg} /$ day). eRVR+ patients were $1: 1$ randomized (week-20) to receive a total of 24 or 48 weeks PR. eRVR- patients received a total of 48 weeks PR. Patients with $<2$ $\log _{10}$ HCV RNA decline at week 12 or detectable HCV RNA at 24 weeks discontinued treatment. The primary study endpoint was the proportion of randomized patients achieving SVR; the study was powered to rule out non-inferiority ( $10.5 \%$ interval) of 24 -week to 48 week treatment.

Results: Among 540 patients enrolled (60.2\% male, $79.1 \%$ Caucasian, $13.5 \%$ black, $11.3 \%$ with cirrhosis, median HCV RNA of 6.5 $\left.\log _{10} \mathrm{IU} / \mathrm{mL}\right), 72 \%(n=389)$ and $65.2 \%(n=352)$ achieved RVR and eRVR, respectively. Among 322 (59.6\%) randomized eRVR+ patients, 92\% (24 weeks, $n=162$ ) and $87.5 \%$ (48 weeks, $n=160$, $\Delta 4.5 \%, 2$-sided $95 \% \mathrm{CI}=-2.1 \%$ to $+11.1 \%$ ) achieved SVR. Overall, SVR was $71.9 \%$ (ITT analysis). 36 patients (6.7\%) and 94 patients (17.4\%) discontinued treatment due to virologic failure and AEs, respectively. Most common AEs leading to discontinuation were fatigue $(n=22)$ and anemia $(n=12)$. After randomization, $1(0.6 \%)$ and $20(12.5 \%)$ patients in the 24-week and 48-week arms discontinued due to AEs. During telaprevir treatment phase, $3(0.6 \%)$ and 6 (1.1\%) patients discontinued treatment due to anemia and rash, respectively.

Conclusions: Among eRVR patients, a 24-week telaprevir-based regimen was non-inferior to a 48-week telaprevir-based regimen 
(SVR 92 and 87.5\%). More AEs and AE-related treatment discontinuations were observed in the latter arm. The overall study achieved a $71.9 \%$ SVR rate. Permanent discontinuation of all study drugs due to AEs occurred in $17.4 \%$ of patients.

\section{PP13-90}

Boceprevir Combined with Peginterferon alfa-2b/Ribavirin for Treatment-naïve Patients with Hepatitis C Virus (HCV) Genotype-1: SPRINT-2 Final Results

F. Poordad ${ }^{1}$, J. Mccone ${ }^{2}$, B. Bacon ${ }^{3}$, S. Bruno ${ }^{4}$, M. Manns ${ }^{5}$, M. Sulkowski ${ }^{6}$, I. Jacobson ${ }^{7}$, R. Reddy ${ }^{8}$, N. Boparai ${ }^{9}$, V. Sniukiene ${ }^{9}$, C. Brass ${ }^{9}$, J. Albrecht ${ }^{9}$, J.-P. Bronowicki ${ }^{10}$

${ }^{1}$ Cedars-Sinai Medical Center, Los Angeles, CA; ${ }^{2}$ Gastroenterology/ Hepatology/Certified Endoscopy Centers, Alexandria, VA; ${ }^{3}$ Saint Louis University School of Medicine, St. Louis, MO, USA,

${ }^{4}$ A.O. Fatebenefratelli e Oftalmico, Milan, Italy; ${ }^{5}$ Medical School of Hannover, Hannover, Germany; ${ }^{6}$ Johns Hopkins University School of Medicine, Baltimore, MD; ${ }^{7}$ Center to the Study of Hepatitis C Joan and Sanford I. Weill Medical College of Cornell University, New York, NY; ${ }^{8}$ University of Pennsylvania, Philadelphia, PA; ${ }^{9}$ Merck, Whitehouse Station, NJ, USA; ${ }^{10}$ Centre Hospitalier Universitaire de Nancy, Université Henri Poincaré-Nancy-1, Vandoeuvre-lès-Nancy, France

Background: Boceprevir is a HCV-NS3 protease inhibitor. SPRINT2 assessed the safety and efficacy of boceprevir in treatment-naïve gentoype-1 patients.

Methods: This double-blind placebo-controlled trial randomized 938 non-black and 159 black patients to receive either peginterferon/ ribavirin control (Arm 1), 4 weeks of peginterferon/ribavirin (lead-in) then response guided therapy (RGT) with peginterferon/ribavirin plus boceprevir (Arm 2), or 4 weeks peginterferon/ribavirin lead-in and 44 weeks peginterferon/ribavirin plus boceprevir (Arm 3). Patients with detectable HCV RNA at week 24 were discontinued for futility. The primary endpoint was sustained virologic response (SVR) 24 weeks post-therapy in all patients receiving $\geq 1$ dose of any study medication (Roche TaqMan LLD $<9.3 \mathrm{IU} / \mathrm{mL}$ ). Non-black and black patients were enrolled and analyzed separately per-protocol.

Results: SVR in non-black and black patients was significantly higher in both boceprevir arms vs. control (Table). For non-black patients completing the lead-in and receiving $\geq 1$ dose of boceprevir or placebo, respective SVRs were 42,70 and $71 \%$. At the end of lead-in period, $\sim 25 \%$ of patients had $<1-\log$ decline in baseline HCV RNA. Regardless of week 4 decline, SVR were consistently higher in the boceprevir arms than controls. Discontinuation for adverse events occurred in 16, 12 and $16 \%$ in the 3 arms, respectively. Anemia was reported in $29 \%$ of controls versus $49 \%$ in the boceprevir arms.

Conclusions: Boceprevir with peginterferon/ribavirin significantly increased SVR in both the RGT \& 48-week treatment arms over standard of care by $\sim 70 \%$. Anemia rarely led to treatment discontinuation. RGT produced comparable SVR to 44 weeks of triple therapy.

\section{PP13-91}

Hepatic Toxicity in Patients with Diffuse Large B-cell Lymphoma (DLBCL) with Hepatitis C Virus RNA Undergoing Chemotherapy with Rituximab

S. Lepkov, G.I. Storogakov, A.M. Kovrigina, O.A. Kolomiycev, S.D. Kosura, G.S. Tumyan, I.N. Subortseva, P.A. Zeynalova, T.T. Kondratieva,

A.A. Gettueva

The Moscow Medical University of N.N. Pirogov, Moscow, Russia

Persistent human hepatitis virus HCV play an important role in the tumorigenesis of DLBCL. In this research we studied liver dysfunction during chemotherapy R-CHOP with rituximab and level RNA of a hepatitis $\mathrm{C}$.

18 patients (pts) with DLBCL have been included In this studies. Median age was 48 years. HCV-RNA was present in all 18 pts. Serum level of HCV-RNA before of the beginning of treatment was from $1 \times 10^{3}$ to $8.2 \times 10^{5} \mathrm{copy} / \mathrm{ml}$. The median level HCV-RNA was $1.2 \times 10^{4} \mathrm{copy} / \mathrm{ml}$. At diagnosis, ALT value was above UNL in 6 patients. Seventeen patients had advanced stage III/IV of disease, and 1pts had I-E stage. All pts undergoing chemotherapy by R-CHOP (with rituximab). Complete remission has been reached 4 pts DLBCL. Median remission was 7 months. Partial remission was achieved 9 pts. Increased level of HCV-RNA during chemotherapy were observed in all $18 \mathrm{pts}$ and was from $5.3 \times 10^{5}$ to $5.7 \times 10^{7}$ copy $/ \mathrm{ml}$, median level HCV-RNA was $4.8 \times 10^{6} \mathrm{copy} / \mathrm{ml}$. Increased level of transaminases (ALT, AST) was simultaneously with increased level of HCV-RNA in 17 pts . The level of ALT after 4-6 chemotherapy was from 120 to 3,000 ED-median 16 norm. 6 pts cannot got all chemotherapy as a result of liver toxicity. In 17 patients with lymphoma and hepatitis $\mathrm{C}$ after standard courses of chemotherapy (R-CHOP) was developed III-IV degree of citopeniya. This was the reason for an increase in the intervals between the courses.

A significant proportion of patients with $\mathrm{HCV}+\mathrm{NHL}$ develop liver toxicity leading to interruption of chemotherapy. Lymphomas + HCV represent a distinct clinical subset of NHL that deserves specific clinical approach to limit liver toxicity and ameliorate survival.

\begin{tabular}{|c|c|c|c|c|c|c|}
\hline & \multicolumn{3}{|l|}{ Non-black patients } & \multicolumn{3}{|l|}{ Black patients } \\
\hline & $\begin{array}{l}\text { Arm } 1 \text { (Control): } \\
\text { P/R for } \\
48 \text { weeks } * \\
N=311\end{array}$ & $\begin{array}{l}\text { Arm } 2 \mathrm{P} / \mathrm{R} \\
4 \text { week lead-in } \\
\text { then BOC RGT* } \\
N=316\end{array}$ & $\begin{array}{l}\text { Arm } 3 \text { (control): } \\
\text { P/R for } \\
48 \text { weeks* } \\
N=311\end{array}$ & $\begin{array}{l}\text { Arm } 1 \text { (Control): } \\
\text { P/R for } \\
48 \text { weeks* } \\
N=52\end{array}$ & $\begin{array}{l}\text { Arm } 2 \text { P/R } 4 \\
\text { week lead-in } \\
\text { then BOC RGT* } \\
N=52\end{array}$ & $\begin{array}{l}\text { Arm } 3 \text { (control): } \\
\text { P/R for } \\
44 \text { weeks* } \\
N=55\end{array}$ \\
\hline SVR, $n(\%)$ & $125(40)$ & 211(67) $P<0.001$ & $213(68) P<0.0001$ & $12(23)$ & $22(42) P=0.04$ & $29(53) P=0.004$ \\
\hline $\begin{array}{l}\text { End of Therapy } \\
\text { Response, } n(\%)\end{array}$ & $176(57)$ & $235(74)$ & 241(77) & $15(29)$ & $26(50)$ & $36(65)$ \\
\hline Relapse, $n / N(\%)$ & $37 / 162(23)$ & $21 / 232(9)$ & $18 / 230(8)$ & 2/14 (14) & $3 / 25(12)$ & $6 / 35(17)$ \\
\hline \multicolumn{7}{|c|}{ SVR by decline in HCV RNA after 4-week lead-in $n / N(\%)$} \\
\hline$<1 \log$ declline & $3 / 62(5)$ & $21 / 73(29)$ & $31 / 79(39)$ & $0 / 21(0)$ & $6 / 24(25)$ & $5 / 16(31)$ \\
\hline$>1 \log$ decline & $121 / 234(52)$ & $187 / 228(82)$ & $178 / 218(82)$ & $12 / 26(46)$ & $16 / 24(67)$ & $22 / 36(61)$ \\
\hline
\end{tabular}

$P$ peginterferon, $R$ ribavirin, $B O C$ boceprevir 


\section{PP13-92}

Very Early Viral HCV Kinetics - Response Predictor of SVR

P. Migdalski, P. Mozer-Lisewska

Department of Infectious Diseases, University of Medical Sciences in Poznan, Poznan, Poland

Aim of the study: SVR predicting after first dose of PegIFN based on very early viral HCV kinetics.

Material and methods: Two groups of patients with chronic hepatitis C (CHC), genotype $1 \mathrm{~b}$ were compared. 22 patients with SVR $(M=16, F=6$, mean age $42 \pm 6)$ and 20 individuals $(M=12$, $F=8$, mean age $44 \pm 8$ ) without SVR. In both groups Peg IFN alfa 2 with ribavirin were administrated for 48 weeks. Viremia HCV was evaluated before treatment and after 2 days and 6 months after therapy (Cobas Amplicor HCV Monitor test v2, Roche). In analyzing results we used statistical normalization method. The value of $\mathrm{HCV}$ viremia in time 0 was taken as $100 \%$, the remaining results; 2 days and 6 months after treatment were the percent of the initial value. TStudent test for independent variables was used $(p>0.05)$

Results: In patients with SVR mean value of viremia after 2 days of therapy was $33.1 \pm 6.1 \%$ and in group without SVR $47.7 \pm 6.8 \%$ of the initial pretreatment value. There was statistically significant difference between compared groups $(\mathrm{p}=0.0374)$.

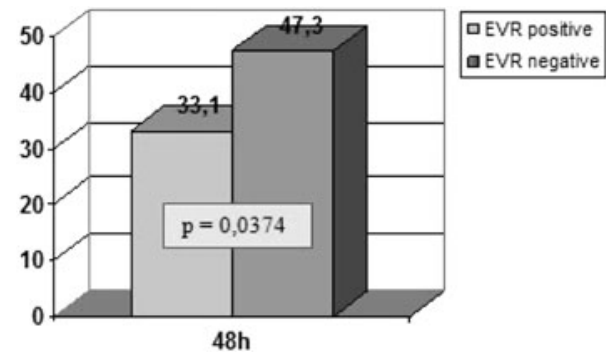

\section{Conclusions:}

1. Reduction of HCV viremia two days after beginning of treatment is good response predictor. In patients with SVR the value is statistically significant lower than in group without SVR.

2. Evaluation of early $\mathrm{HCV}$ kinetics is good response (SVR) prognostic factor

\section{PP13-93}

Peginterferon Plus Ribavirin for 48 or 72 Weeks in Asian Hepatitis C Virus Genotype 1 Patients without Rapid Virologic Response

C.-H. Liu ${ }^{1,2,3}$, C.-C. Liang ${ }^{4}$, C.-J. Liu ${ }^{1,2,3}$, C.-L. Lin' ${ }^{5}$, S.-S. Yang ${ }^{6}$, S.-J. Hsu', T.-C. Tseng ${ }^{8}$, T.-H. Su ${ }^{1,2,3}$, J.-H. Chen ${ }^{9}$, P.-J. Chen ${ }^{1,2,3}$, D.-S. Chen ${ }^{1,3}$, J.-H. $\mathrm{Kao}^{1,2,3}$

${ }^{1}$ Internal Medicine, National Taiwan University Hospital, ${ }^{2}$ Graduate Institue of Clinical Medicine, National Taiwan University College of Medicine, ${ }^{3}$ Hepatitis Research Center, National Taiwan University

Hospital, ${ }^{4}$ Internal Medicine, Far Eastern Memorial Hospital,

${ }^{5}$ Internal Medicine, Taipei Municipal Hospital, Ren-Ai Branch, Taipei, " Internal Medicine, Taichung Veterans General Hospital, Taichung, ${ }^{7}$ Internal Medicine, National Taiwan University Hospital, Yun-Lin Branch, Douliou, ${ }^{8}$ Internal Medicine, Buddhist Tzu Chi General Hospital, Taipei Branch, Taipei, ${ }^{9}$ Pathology, National Taiwan University Hospital, Yun-Lin Branch, Douliou, Taiwan R.O.C.

Background/aims: Although extending the treatment duration to 72 weeks may improve sustained virologic response (SVR) in Caucasian hepatitis $\mathrm{C}$ virus (HCV) genotype 1 slow responders with peginterferon plus ribavirin, the efficacy of Asian HCV genotype 1 slow responders with extended therapy is not clear.

Methods: In this multicenter trial, 271 treatment-naïve HCV genotype 1 Asian patients who failed to achieve rapid virologic response (RVR), defined as undetectable HCV RNA at week 4 of therapy, were randomized to receive either 48 or 72 weeks of peginterferon alfa-2a ( $180 \mu \mathrm{g} /$ week) plus ribavirin $(1,000-1,200 \mathrm{mg} /$ day $)$. Serial HCV RNA levels were evaluated at weeks $8,12,24$, at end-of-treatment and at week 24 off therapy. The primary endpoint was SVR, defined as undetectable serum HCV RNA at week 24 off therapy, by intentionto-treat (ITT) analysis. Furthermore, the optimized treatment duration was determined in patients with different viral kinetic by per-protocol (PP) analysis.

Results: The SVR rate of 72-week treatment was significantly higher than those of 48 -week treatment ( 67 vs. $52 \%, p=0.02$ ). In patients who had undetectable HCV RNA at week 8 , the SVR rates were similar in 72 -week and 48 -week treatment ( 85 vs. $82 \%, p=0.64$ ). However, the SVR rate of 72-week treatment was significantly higher than that of 48week treatment in those who had detectable HCV RNA at week 8 but undetectable HCV RNA at week 12 (86 vs. 33\%, $p<0.001$ ).

Conclusions: In Asian HCV genotype 1 patients who fail to achieve RVR after peginterferon alfa-2a plus ribavirin, 72-week therapy has a superior SVR rate to 48 -week therapy. Checking week 8 HCV RNA level can help clinicians decide extending treatment duration to 72 weeks or not.

\section{PP13-94}

Treatment of Chronic Hepatitis C with Pegylated Interferon Alfa-2a and Ribavirin: The Efficacy and Predictors of Response to Therapy M. Bozic, K. Bojovic, I. Milosevic Institute for Infectious and Tropical Diseases, Clinical Center of Serbia, Belgrade, Serbia

Introduction: The current standard for therapy of chronic hepatitis $\mathrm{C}$ $(\mathrm{CHC})$ is combination of pegylated interferon alpha $2 \mathrm{a}$ or $2 \mathrm{~b}$ (PEGIFN- $\alpha 2 \mathrm{a}$ or PEG-IFN- $\alpha 2 \mathrm{~b})$ and ribavirin (RBV). The Sustained Virologic Response (SVR) means successful treatment. Certain factors are good predictors of treatment outcome and they provide optimal, individual therapeutic approach.

Objectives: Evaluation of efficacy of $\mathrm{CHC}$ treatment and predictors of treatment response.

Methods: 194 patients with CHC were treated with PEG-IFN- $\alpha 2 \mathrm{a}+$ RBV in Institute for Infectious and Tropical Diseases, Belgrade. Virological examinations: anti-HCV antibodies (ELISA), serum HCV RNA levels and HCV genotypes (PCR). Liver biopsy specimens were staged in accordance with the METAVIR score. Predictors with influence on treatment outcome were estimated by multivariable logistic regression analysis.

Results: There were more patients of male gender (59.3\%), older than 40 years $(58.2 \%)$, with high basal HCV RNA level (63\%), with G1 $\mathrm{HCV}(67 \%)$ in treated group of patients with CHC. Bridging fibrosis and cirrhosis were found in $54(27.84 \%)$ of patients. SVR was achieved in $69.2 \%$, relapse was found in $11.9 \%$ of patients. $19.8 \%$ of patients had no response on therapy. Predictors for achievement of SVR were: non-1 HCV genotype, achievement of early virological response (EVR), and patients younger than 40 years. In oposite to these facts, treatment failure in form of relapse was influenced by high basal viral load, G1 HCV, patients age ( $>40$ years), presence of cirrhosis and interrupted continuity of treatment.

Conclusion: The therapy was efficient in $69.2 \%$ of our patients. The most important predictors for successful treatment were: non-1 HCV genotype, achievement of EVR and patients age. Predictors for failure of treatment were: G1 HCV, high basal viral load, older age $(>40$ years), presence of cirrhosis and discontinuity of treatment. 


\section{PP13-95}

8-week Efficacy and Safety of ABT-333 or ABT-072 with Standard-ofCare, Following 3-day Monotherapy in Genotype $1 \mathrm{HCV}$-infected Treatment-naïve Subjects

I.A. Gaultier, D.E. Cohen, E.O. Dumas, L.M. Larsen, T.J. Podsadecki, B. Bernstein

Global Research and Development, Antivirals, Abbott Laboratories, Abbott Park, IL, USA

Background: ABT-333 and ABT-072 are NS5B non-nucleoside polymerase inhibitors being developed for the treatment of $\mathrm{HCV}$ genotype 1 infection.

Methods: This ongoing, randomized, blinded, placebo-controlled, dose-ranging study is assessing safety, tolerability, pharmacokinetics, and antiviral activity of ABT-333 (400 or $800 \mathrm{mg}$ twice-daily) or ABT-072 (100, 300 or $600 \mathrm{mg}$ once-daily) or placebo monotherapy for 3 days, followed by ABT-333, ABT-072 or placebo + pegylated interferon alpha-2a $180 \mu \mathrm{g} /$ week + weight-based ribavirin $1,000-1,200 \mathrm{mg} /$ day (SOC) for 12 weeks. After week 12, subjects receive SOC alone for 36 weeks.

Results: 50 subjects were randomized to ABT-333, ABT-072 or placebo. $8(16 \%)$ subjects were female, $6(12 \%)$ were black, and 2 were Asian (4\%). Mean baseline characteristics: age 50 years; BMI $28.3 \mathrm{~kg} / \mathrm{m}^{2}$; HCV RNA $6.7 \log _{10} \mathrm{IU} / \mathrm{mL}$ (82\% with HCV RNA > $800,000 \mathrm{IU} / \mathrm{mL}) .36(72 \%)$ subjects were infected with genotype 1a. All subjects have passed Week 8. Safety profile of ABT-072 or ABT$333+$ SOC was generally comparable to that of SOC alone, with headache, fatigue, neutropenia and depression being the most frequent adverse events. No subject on ABT-333 or ABT-072 discontinued due to an adverse event. One subject on ABT-072 300 $\mathrm{mg}$ discontinued due to virologic rebound at Week 8. Efficacy results are shown below. Table 1

Conclusions: Both ABT-333 and ABT-072 were well tolerated in combination with SOC, and had antiviral activity significantly greater than SOC alone. Response in both ABT-333 dose groups was similar. These agents are candidates for development in combination with other antiviral agents.
PP13-96

Analysis of the Efficacy of Treatment with Peginterferon Alfa-2a and Ribavirin in Elderly Patients with Chronic Hepatitis C

J.-W. Yu, L.-J. Sun

Department of Infectious Diseases, Second Affiliated Hospital, Harbin Medical University, Harbin, China

Objective: The aim of this study was to evaluate the efficacy and safety of treatment with peginterferon alfa-2a and ribavirin in elderly patients with chronic hepatitis $\mathrm{C}$ (CHC).

Methods: The medical records of $119 \mathrm{CHC}$ patients treated with peginterferon and ribavirin were retrospectively analyzed. These patients were divided into two groups according to age: patients aged $\geq 65$ years $(n=40)$ and patients aged $<65$ years $(n=79)$. The incidence of side events, the rate of reduction or discontinuation of ribavirin and virilogical response rate of two groups were compared.

Results: Compensated liver cirrhosis was more frequent in patients aged $\geq 65$ years than patients aged $<65$ years $(12.5 \%, 5 / 40$ vs. $\left.2.5 \%, 2 / 79 ; X^{2}=4.766, P=0.029\right)$. Patients aged $\geq 65$ years had significantly higher incidence of side events than patients aged $<65$ years $\left(72.5 \%, 29 / 40\right.$ vs. $\left.48.1 \%, 38 / 79 ; \mathrm{X}^{2}=6.425, P=0.011\right)$. Reduction or discontinuation of ribavirin was more frequent in patients aged $\geq 65$ years than patients aged $<65$ years $(37.5 \%, 15 / 40$ vs. $\left.20.3 \%, 16 / 79 ; X^{2}=4.100, P=0.043\right)$. Patients aged $\geq 65$ years with genotype 1 had significantly higher relapse rate than patients aged $<65$ years $\left(54.2 \%, 13 / 24\right.$ vs. $29.4 \%, 15 / 51 ; c^{2}=4.275, P=$ 0.039 ). Patients aged $\geq 65$ years with genotype 1 had significantly lower SVR rate than patients aged $<65$ years $(36.7 \%, 11 / 30$ vs. $\left.60.0 \%, 36 / 60 ; c^{2}=4.364, P=0.037\right)$. There were no significant differences in virilogical response rates between two groups for patients with genotype 2 .

Conclusions: Compared with patients aged $<65$ years, the incidence of side events of patients aged $\geq 65$ years was high. The rate of reduction or discontinuation of ribavirin and relapse rate of patients aged $\geq 65$ years with genotype 1 were high, and the SVR rate was low. Age had no impact on virological responses rates for genotype 2 .

Table 1 Virologic Response through week 8

\begin{tabular}{|c|c|c|c|c|c|c|c|c|}
\hline \multirow[t]{2}{*}{ Compound/dose } & \multicolumn{4}{|l|}{ ABT-072 } & \multicolumn{3}{|l|}{ ABT-333 } & \multirow[t]{2}{*}{ PBO } \\
\hline & $100 \mathrm{mg}$ QD & 300 mg QD & $600 \mathrm{mg}$ QD & Total & 400mg QD & $800 \mathrm{mg}$ QD & Total & \\
\hline Monotherapy, N & 8 & 8 & 7 & 23 & 8 & 8 & 16 & 11 \\
\hline $\begin{array}{l}\text { Mean maximum HCV RNA } \\
\text { change from } \mathrm{BL}(\mathrm{SD}), \log _{10} \\
\mathrm{IU} / \mathrm{mL}\end{array}$ & $\begin{array}{r}-1.14^{*} \\
(0.99)\end{array}$ & $\begin{array}{r}-1.07 * \\
(0.41)\end{array}$ & $\begin{array}{r}-1.57^{*} \\
(0.94)\end{array}$ & $\begin{array}{r}-1.25^{*} \\
(0.81)\end{array}$ & $\begin{array}{r}-1.08 * \\
(0.68)\end{array}$ & $\begin{array}{l}-0.95 \\
\quad(0.68)\end{array}$ & $\begin{array}{l}-1.02 \\
\quad(0.66)\end{array}$ & $\begin{array}{l}-0.36 \\
\quad(0.13)\end{array}$ \\
\hline Week $4, N$ with data & 8 & 8 & 7 & 23 & 8 & 8 & 16 & 9 \\
\hline $\begin{array}{l}\text { Subjects with HCV RNA <25 IU/ } \\
\text { mL } \\
(\%)[\mathrm{RVR}]\end{array}$ & $3 / 8(37.5)$ & $1 / 8(12.5)$ & $3 / 7(42.9)$ & $7 / 23(30.4)$ & $4 / 8(50)$ & $4 / 8(50)$ & $8 / 16(50)$ & $1 / 9(11.1)$ \\
\hline Week $8, \mathrm{~N}$ with data & 8 & 7 & 7 & 22 & 8 & 8 & 16 & 8 \\
\hline $\begin{array}{l}\text { Subjects with HCV RNA }<25 \text { IU/ } \\
\text { mL } \\
(\%)[\text { RVR }]\end{array}$ & $3 / 8(37.5)$ & $3 / 7(42.9)$ & $6 / 7(85.7)$ & $12 / 22(54.5)$ & $6 / 8(75)$ & $5 / 8(62.5)$ & $\begin{array}{l}11 / 16 \\
\quad(68.8)\end{array}$ & $\begin{array}{l}3 / 8 \\
\quad(37.5 .1)\end{array}$ \\
\hline
\end{tabular}

* Statistically significantly different from placebo $(P<0.05)$ 


\section{PP13-97}

Impact of Ribavirin Cumulative Dose on Virological Responses in Hepatitis C Virus Patients with Peginterferon Alfa-2a and Ribavirin Treatment

L.-J. Sun, L.-J. Yu

Department of Infectious Diseases, The Second Affiliated Hospital, Harbin Medical University, Harbin, China

Objective: To study the impact of ribavirin cumulative dose on virological responses rates in hepatitis $\mathrm{C}$ virus genotype 1 patients with peginterferon alfa- $2 \mathrm{a}$ and ribavirin treatment.

Methods: The medical records of 225 chronic hepatitis $\mathrm{C}$ genotype 1 patients treated with peginterferon and ribavirin were retrospectively analyzed. These patients were divided into four groups according to ribavirin cumulative dose: $>97 \% ; 97-80 \% ; 79-60 \% ;<60 \%$. The relationship between ribavirin cumulative dose and virological responses rates was studied.

Results: More patients had reduction of ribavirin than peginterferon alfa-2a $\left(43.1 \%, 97 / 225\right.$ vs. $\left.27.1 \%, 61 / 225 ; X^{2}=12.641, P=0.001\right)$. The SVR rates of ribavirin cumulative dose $>97 \%$ group, $97-80 \%$ group, $79-60 \%$ group and $<60 \%$ group were $65.6 \%$ (84/128), $60.5 \%$ (26/43), $58.3 \%(21 / 36)$ and $27.8 \%(5 / 18)$, respectively, and the SVR rates of patients with less than $60 \%$ ribavirin cumulative dose were significantly lower than those of other groups $\left(c^{2}=9.538, P=0.023\right)$. The relapse rates of ribavirin cumulative dose $>97 \%$ group, $97-80 \%$ group, $79-60 \%$ group and $<60 \%$ group were $20.0 \%$ (21/105), $23.5 \%$ $(8 / 34), 27.6 \%(8 / 29)$ and $61.5 \%(8 / 13)$, respectively, and the relapse rates of patients with less than $60 \%$ ribavirin cumulative dose were significantly higher than those of other groups $\left(\mathrm{c}^{2}=10.837, P=\right.$ 0.013). The SVR rates of ribavirin cumulative dose $>97 \%$ group, 97-80\% group, $79-60 \%$ group and $<60 \%$ group for patients with rapid virologic response were $92.0 \%$ (23/25), 88.9\% (8/9), 85.7\% (6/ 7) and $75.0 \%(3 / 4)$, respectively, showing no significant differences between different groups $\left(c^{2}=1.098, P=0.778\right)$.

Conclusions: Minor ribavirin dose reductions do not affect SVR adversely. The relapse rate of patients with less than $60 \%$ ribavirin cumulative dose was high, and the SVR rate was low.

\section{PP13-98}

Hepatocellular Carcinoma Risk in Chronic Hepatitis C Patients with Sustained Viral Response: A Long-term Prospective Study

C.-H. Wang, L.-R. Mo, K.-K. Chang, R.-C. Lin, J.-J. Kuo

Hepatogastroenterology, Tainan Municipal Hospital, Tainan, Taiwan R.O.C.

Background: Eradication of hepatitis $\mathrm{C}$ virus (HCV) with antiviral therapy significantly inhibits development of hepatocellular carcinoma (HCC) in patients with chronic HCV infection. However, HCC sporadically developed even after achievement of sustained viral response (SVR). The aim of the study was to examine which factors would contribute to the occurrence of $\mathrm{HCC}$ in $\mathrm{HCV}$ patients with SVR.

Methods: From 2003 to 2005, a total 166 patients (male: 94; female: 72 ; mean age: $58.8 \pm 9.8$ years old) with chronic $\mathrm{HCV}$ infection were enrolled into the study. Liver biopsy was performed in each patient. All patients were treated with pegylated interferon (peg-IFN) plus ribavirin (RBV). Diagnosis of HCC was made using liver biopsy, $\alpha$-fetoprotein level or combined two dynamic liver imaging studies. We selected several pre-treatment valuables of patients to assess risk factors for development of HCC, including age, sex, AST, ALT, BMI, WBC, hemoglobin, platelet, prothrombin time (PT), HCV RNA level, genotype, diabetes mellitus, liver histological features (bridging necrosis, lobular inflammation, piecemeal necrosis, fatty change, fibrosis, and portal tract inflammation).

Results: 129 patients $(77.7 \%)$ achieved SVR after peg-IFN/RBV therapy. Among patients with SVR, seven patients (5.4\%) developed HCC during seven years' follow-up period. Based on multivariate logistic analysis (Table 1), age $(p=0.003)$, lobular inflammation activity $(p=0.04)$, platelet $(p=0.008)$, and PT $(p=0.001)$ are predictors for HCC occurrence.

Conclusion: The results in the current study suggest that patients with chronic HCV infection after eradication who have one of factors of older age, liver histological hallmark of lobular inflammation, thrombocytopenia, and prolonged PT would be still risky for developing HCC.

\section{PP13-99}

Effect of PSE on the HCV Cirrhosis and Discussion PSE as Pretreatment Prior to Peg-IFN $\alpha$-2a

W. Qi, J. Wang

Digestive Department, China-Japan Union Hospital,Jilin Province, Changchun City, China

Objective: To analyze the efficacy of partial splenic artery embolization on hypersplenism within hepatitis $\mathrm{C}$ virus cirrhotic patients, and evaluate antiviral treatment on the hepatitis $\mathrm{C}$ virus cirrhotic patients after PSE.

Methods: From January 2006 to January 2010, 76 hepatitis C virus cirrhotic patients with severe hypersplenism (neutrophils $<1.0 \times 109$ $\mathrm{L}^{-1}$ and / or PLT $<50 \times 109 \mathrm{~L}^{-1}$ ) admitted to Department of Gastroenterology, China-Japan Union Hospital. In 23 cases out of the total 76 cases,PSE was operated .PSE can release the hypersplenism. If neutrophils of the cirrhotic patients $>1.0 \times 109 \mathrm{~L}^{-1}$ and PLT $>50 \times$ $109 \mathrm{~L}^{-1}$ after PSE, we would use antiviral treatment.The patients were divided into three groups.In group one, the full dose of Peg-IFN $\alpha-2 a$ $(180 \mu \mathrm{g})$ plus ribavirin $(800-1,200 \mathrm{mg})$ was used within 48 weeks, and patients was followed up for 24 weeks after the end of antiviral treatment. In group two: The low dose of Peg-IFN $\alpha-2 \mathrm{a}(90 \mu \mathrm{g})$ plus ribavirin had been used for 72 weeks, when significant bone marrow suppression showed. Group three concluded the rest 53 patients who disagreed PSE as control.

Results: After partial splenic artery embolization 1 week, white blood cell and platelet count were significantly increased, $P<0.05 .21$ out of 23 cases after PSE could use antivriral treatment RVR, EVR, SVR of Non-genotype 1 patients was $16.67,75$ and $75 \%$ which were higher than genotype 1 patients (RVR $11.11 \%$, EVR 66.67\%, SVR $55.56 \%$ ). The patients in group one obtained a $66.67 \%$ rate of sustained viral clearance(SVR) versus patients in group two $50 \%, P>$ 0.05. All patients with antiviral treatment had better liver function.PSE was safe in with few sever complications.

Conclusion: PSE was safe.And hepatitis $\mathrm{C}$ viral cirrhotic patients with hypersplenism who were difficult in using antiviral treatment can use Peg-IFN $\alpha$-2a plus ribavir.Full dose of Peg-IFN $\alpha$-2a and low dose obtained similar SVR.

\section{PP13-100}

Sustained SVR Is Maintained during Long Term Follow-up Regardless of Genotype after Chronic Hepatitis C Treatment

J.E. Oh, S.G. Shim, D.H. Cho, C.U. Jeong, G.J. Yu, G.T. Park

Sungkyunkwan University Samsung Changwon Hospital, Chang Won, Republic of Korea

Background: The standard of care for chronic hepatitis $\mathrm{C}$ therapy is based on Pegylated interferon alpha and ribavirin .This combination therapy has led to a sustained virological response rate (SVR) of 50 to $80 \%$ depending on genotype.

Method: Seventy six patients with chronic hepatitis C had treated PEG-IFN and ribavirin therapy. 65 patients with sustained virologic response (SVR) after treatment were enrolled in a long-term clinical follow-up study. We determined long term clinical, virologic and biochemical outcomes this patients with SVR. According to genotype(genotype 1 and genotype 2,3), we assessed the durability of SVR during long- term follow-up.

Result: Four patients had not obtained a RVR and only 1 patient had not obtained a EVR in 65 patients who had obtained SVR. There's 
genotype was all genotype 1.SVR was maintained in 63 patients while HCV- RNA became positive in 2 patients after SVR follow-up period. One became within 1 year after SVR, the other became within 3 months after SVR and the patients had obtained EVR and RVR. These patients had all genotype 1 .

Conclusion: SVR to PEG-IFN and ribavirin is maintained in $99 \%$ of patients during long term follow up regardless of genotype.

\section{PP13-101}

Anti-HCV Treatment of Thalassemia Major Adolescents with Peginterferon Alfa-2a and Ribavirin

S.V. Tabatabaei ${ }^{1}$, S.M. Alavian ${ }^{1}$, B. Behnava ${ }^{1}$, M. Keshvari ${ }^{2}$, S.M. Miri ${ }^{1}$, P. Karimi Elizee ${ }^{1}$, K.B. Lankarani ${ }^{3}$

${ }^{1}$ Baqiyatallah University of Medical Sciences, Baqiyatallah Research Center for Gastroenterology and Liver Disease, Tehran, Iran;

${ }^{2}$ Iranian Blood Transfusion Organization Research Centre (IBTO), Tehran, Iran; ${ }^{3}$ Shiraz University of Medical Sciences, Shiraz, Iran,

Tehran, Iran

Background and aims: There is little information about outcome of anti-HCV treatment in thalassemia major children with chronic HCV infection. In addition, ribavirin, the cornerstone of anti-HCV therapy is contraindicated in thalassemia patients.here we aimed to assess safety and efficacy of ribavirin in combination therapy with peginterferon alfa-2a for treatment of thalassemia major children.

Material and methods: 30 thalassemia major patients aged between 11 and 18 years were recruited to this study. Subjects received $180 \mu \mathrm{g}$ peginterferon alfa subcutaneously thrice weekly with or without $600-800 \mathrm{mg}$ of ribavirin according to serum hemoglobin $(800 \mathrm{mg}$ for $\mathrm{Hg}>10 \mathrm{~g} / \mathrm{dL}, 600 \mathrm{mg}$ for hemoglobin 8-10, discontinuation for $\mathrm{Hg}<$ $8)$. Safety was ensured by weekly laboratory test and visits.

Results: Twenty two patients received oral ribavirin. Mean ALT, serum HCV-RNA and serum ferritin were $100 \pm 10 \mathrm{IU} / \mathrm{L}, 650,000 \pm$ $120,000 \mathrm{IU} / \mathrm{mL}$, and $2,383 \pm 330 \mathrm{ng} / \mathrm{mL}$, respectively. Furthermore, half of children had genotype 1 infection in both groups and their ALT, HCV-RNA and serum ferritin levels were also similar. Regarding virological response, 14 out of $22(64 \%)$ children that received oral ribavirin attained SVR, while SVR was observed in 6 out of $8(75 \%)$ children who received monotherapy. Withdrawal causes were as follows: four children in ribavirin group were prematurely withdrawn due to non-response, two because of laboratory abnormalities, and one because of severe depression. Among subjects in peginterferon monotherapy, one subject due to severe depression and one for familial reasons were withdrawn from the treatment. Twenty one $(70 \%)$ children experienced at least one clinical adverse event, while the most prevalent adverse events were flue like syndrome $(70 \%)$, arthralgia (17\%), and myalgia (20\%). Transfusion was doubled among those who received ribavirin.

Conclusion: Ribavirin and peginterferon for treatment of thalassemia major children are safe and effective but their combination therapy does not seem to be significantly better than monotherapy.

\section{PP13-102}

Outcome of Follow up of Sustained Virologic Response in Chronic Hepatitis C Patients Treated with Pegylated Interferon and Ribavirin S.B. Choi ${ }^{1}$, Y.J. Lee ${ }^{1}$, S.J. Park ${ }^{1}$, E.U. Jung ${ }^{1}$, J.I. Lee ${ }^{1}$, Y.J. Song ${ }^{1}$, J.H. Kim ${ }^{1}$, B.J. Choi ${ }^{1}$, S.H. Lee ${ }^{2}$, J.H. Kim ${ }^{1}$, J.S. Choi ${ }^{1}$, S.R. Jee ${ }^{1}$, S.Y. Seol ${ }^{1}$

${ }^{I}$ Department of Gastroenterology and Hepatology, Pusan Paik Hospital, College of Medicine, Inje University; ${ }^{2}$ Lee, Busan, Republic of Korea

Background: Standard therapy of patients with chronic hepatitis $\mathrm{C}$ virus (HCV) infection is the combination of pegylated interferon alfa and ribavirin. Several studies have shown that the reappearance rate of HCV RNA in serum after the achievement of sustained virological response (SVR) is $1-2 \%$. But recently, the Korean study reported that the reappearance rate of HCV RNA after SVR is over $10 \%$. Therefore, we evaluated the reappearance rate of $\mathrm{HCV}$ RNA after SVR in chronic hepatitis $\mathrm{C}$ patients treated with pegylated interferon alfa and ribavirin at our hospital.

Methods: Three hundred and forty three patients who were treated with pegylated interferon alfa and ribavirin at Pusan Paik Hospital between April in 2004 and December in 2008 were retrospectively analyzed.

Results: Among the 343 patients, 338 patients (98.5\%) achieved the end of treatment response(ETR) and 292 patients [85.1\%, genotype-1: 75.5\% (114/151), genotype non-1: 92.7\% (178/192)] had SVR. Patients who have been followed for more than 6 months after SVR were 184 (genotype-1: 72, genotype non-1: 112) of the 292 patients and 82 patients (genotype-1: 33, genotype non-1: 49) could follow up with serum HCV RNA and liver function test for more than 2 years after SVR. The mean age of these 184 patients was $48.41 \pm 11.85$ years, and 105 patients were male. The mean ALT was $104.96 \pm$ $120.00 \mathrm{IU} / \mathrm{L}$, body mass index was $23.38 \pm 2.63 \mathrm{~kg} / \mathrm{m}^{2}$ and baseline serum HCV RNA titer was $3.16 \pm 7.45 \times 10^{6}$ copies $/ \mathrm{ml}$. These 184 patients have been followed for an average of $19.70 \pm 12.67$ months. The reappearance rate of HCV RNA in these 184 patients was $0 \%$. Conclusions: Sustained virological response was maintained for an average of $19.70 \pm 12.67$ months in chronic hepatitis $C$ patients who achieved SVR after treatment with pagylated interferon alfa and ribavirin. To evaluate the durability of SVR, future long-term prospective studies are needed.

\section{PP13-103}

25[OH]D Deficiency Is Common in Patients with Genotype 1 Chronic Hepatitis C, but Is Not Associated with a Lower SVR

S. Davison ${ }^{1}, \mathrm{R} . \mathrm{Nguyen}^{1}, \mathrm{~S}$. Connor ${ }^{1}, \mathrm{~W} . \mathrm{Ng}^{1}, \mathrm{~T}$. Dobbins ${ }^{2}, \mathrm{M} . \mathrm{Maley}^{3}$, S. Monaharan ${ }^{2}$, J.W. Quin ${ }^{4}$, M.T. Levy ${ }^{1}$

${ }^{1}$ Gastroenterology, Liverpool Hospital, Liverpool; ${ }^{2}$ University of New South Wales, Sydney; ${ }^{3}$ Microbiology, Liverpool Hospital; ${ }^{4}$ Bigge Park Centre, Liverpool, NSW, Australia

Introduction: Abu-Mouch et al. reported that 25[OH]D supplementation with interferon/ribaviron treatment for chronic hepatitis $\mathrm{C}$ (CHC) was associated with an increase in sustained virological response (SVR). This study aims to determine if higher a pre-treatment serum 25[OH]D level is associated with an increased likelihood of SVR with interferon/ribaviron for CHC.

Method: We retrospectively analysed patients who received treatment for genotype 1 CHC between January 2007 and December 2009 at the Gastroenterology Department, Liverpool Hospital, NSW and Bigge Parke Centre, Liverpool, NSW. 72 patients treated with standard interferon/ribaviron regimens for at least 12 weeks were included. Stored initial serology specimens had 25[OH]D levels measured. Patients were divided into two groups, above and below the median $25[\mathrm{OH}] \mathrm{D}$ level of $59 \mathrm{nmol} / \mathrm{L}$.

Results: Two patients with incomplete HCV follow up (1 death, 1 withdrew from care) were excluded from analysis. 37 patients $(53 \%)$ were of Asian background. 46 subjects (66\%) achieved SVR. $25[\mathrm{OH}] \mathrm{D}$ deficiency was common, mild $(25-50 \mathrm{nmol} / \mathrm{L})$ in $22 \mathrm{sub}-$ jects $(31 \%)$ and moderate $(<25 \mathrm{nmol} / \mathrm{L})$ in 3 subjects $(4 \%)$ (MJA position statement 2005). SVR was achieved in 23/34 (68\%) patients with higher $25[\mathrm{OH}] \mathrm{D}$ compared with $23 / 36$ patients $(64 \%)$ with lower $25[\mathrm{OH}] \mathrm{D}(p=0.7)$. No association between 25[OH]D group and SVR was found after adjusting for Asian background $(p=0.6)$. Of 29 patients investigated for liver fibrosis with biopsy or multiphasic CT, 8 had advanced fibrosis (F3 or higher, cirrhosis on CT). 4 of these patients $(50 \%)$ had Vitamin D deficiency at baseline.

Conclusion: $25[\mathrm{OH}] \mathrm{D}$ deficiency was common in genotype $1 \mathrm{CHC}$ patients. There was no evidence of a difference in SVR according to baseline 25[OH]D levels. Vitamin D deficiency was very common in advanced liver fibrosis or cirrhosis. Further studies of the treatment of CHC with concomitant $25[\mathrm{OH}] \mathrm{D}$ supplementation are needed to determine the safety and efficacy of this novel approach. 


\section{PP13-104}

Efficacy and Safety of Entecavir versus Adefovir in Chronic Hepatitis B Patients with Evidence of Hepatic Decompensation: Week 96 Results

H. Cheinquer ${ }^{1}$, M. Raptopoulou-Gigi ${ }^{2}$, S.K. Sarin ${ }^{3}$, T. Tanwandee ${ }^{4}$, N. Leung ${ }^{5}$, C.-Y. Peng ${ }^{6}$, R.P. Myers ${ }^{7}$, R.S. Brown Jr ${ }^{8}$, L. Jeffers ${ }^{9}$, N. Tsai ${ }^{10}$,

J. Bialkowska ${ }^{11}$, S. Tang ${ }^{12}$, Y.-C. Chen ${ }^{13}$, S. Beebe ${ }^{12}$, E. Cooney ${ }^{12}$,

Y.-F. Liaw ${ }^{13}$

${ }^{1}$ Universidade Federal Do Rio Grande Do Sul, Porto Alegre, Brazil;

${ }^{2}$ Department of Internal Medicine, Aristotle University of

Thessaloniki, Thessaloniki, Greece, ${ }^{3}$ Department of

Gastroenterology, G. B. Pant Hospital, New Delhi, India;

${ }^{4}$ Department of Medicine, Siriraj Hospital, Mahidol University,

Bangkok, Thailand; ${ }^{5}$ Alice Ho Miu Ling Nethersole Hospital, Hong

Kong, China; ${ }^{6}$ China Medical University Hospital, Taichung, Taiwan

R.O.C.; ${ }^{7}$ Liver Unit, University of Calgary, Calgary, AB, Canada;

${ }^{8}$ Center for Liver Disease and Transplantation, Columbia University Medical Center, New York, NY; ${ }^{9}$ Miami VA Medical Center, Miami, FL; ${ }^{10}$ John A. Burns School of Medicine, University of Hawaii, Honolulu, HI, USA; ${ }^{11}$ Department of Infectious Diseases, Medical University, Lodz, Poland; ${ }^{12}$ Research and Development, BristolMyers Squibb Company, Wallingford, CT, USA; ${ }^{13}$ Chang Gung Memorial Hospital, Chang Gung University College of Medicine, Taipei, Taiwan R.O.C.

Background: We present the Week 96 results of a randomized, openlabel, comparative study of ETV versus ADV in patients with chronic hepatitis $\mathrm{B}$ infection $(\mathrm{CHB})$ and hepatic decompensation.

Methods: Adult subjects with $\mathrm{CHB}(\mathrm{HBeAg}[+]$ or $\mathrm{HBeAg}[-])$ and a Child-Pugh score $\geq 7$ were randomized to ETV $1.0 \mathrm{mg}$ or ADV $10 \mathrm{mg}$ daily and treated for up to 96 weeks. No prior anti-HBV therapy was allowed, except LVD and interferon-alpha.

Results: Among 191 randomized and treated subjects 101 completed 96 weeks of treatment (ETV $n=60$, ADV $n=41$ ). The study population was: $74 \%$ male; mean age 52 years; $54 \%$ Asian, $33 \%$ Caucasian; 54\% $\mathrm{HBeAg}(+)$; and 34\% LVD-resistant. Table 1

\begin{tabular}{|c|c|c|c|}
\hline & & $\begin{array}{l}\text { ETV }(1.0 \mathrm{mg} \\
\text { QD) } N=100\end{array}$ & $\begin{array}{l}\text { ADV (10 mg } \\
\text { lePara> }\end{array}$ \\
\hline \multirow[t]{3}{*}{$\mathrm{BL}$} & $\begin{array}{l}\text { Mean HBN DNA by PCR, } \\
\log _{10} \text { copies/mL(SE) }\end{array}$ & $7.53(0.18)$ & $8.16(0.23)$ \\
\hline & Mean ALT, U/L(SE) & $99.2(11.1)$ & $100.0(8.6)$ \\
\hline & Mean CTP score (SE) & $8.8(0.21)$ & $8.3(0.19)$ \\
\hline \multirow[t]{7}{*}{$\begin{array}{c}\text { Week } \\
96\end{array}$} & $\begin{array}{l}\text { Mean change from BL } \\
\text { in HBV DNA by PCR, } \\
\log _{10} \text { copies/mL (SE)* }\end{array}$ & $\begin{array}{l}-4.79 \\
\quad(0.241)\end{array}$ & $\begin{aligned}-4.51 \\
\quad(0.449)\end{aligned}$ \\
\hline & $\begin{array}{c}\text { HBV DNA }<300 \text { copies } / \mathrm{mL} \\
n / N(\%) \mathrm{NC}=\text { Failure* }\end{array}$ & $46 / 100(49)$ & $23 / 91(25)$ \\
\hline & $\mathrm{NC}=$ Missing $*$ & $49 / 60(82)$ & $23 / 41(56)$ \\
\hline & $\begin{array}{c}\text { Normal ALT }(<1 \times \text { ULN }) \\
n / N(\%) \mathrm{NC}=\text { Failure* }\end{array}$ & $46 / 78(59)$ & $22 / 71(31)$ \\
\hline & $\mathrm{NC}=$ Missing $*$ & $46 / 49(94)$ & $22 / 71(31)$ \\
\hline & $\begin{array}{c}\text { CTP score }>2 \text { point reduction, } \\
n / N(\%) \mathrm{NC}=\text { Failure } *\end{array}$ & $37 / 100(37)$ & $17 / 91(19)$ \\
\hline & $\mathrm{NC}=$ Missing $*$ & $37 / 59(63)$ & $17 / 41(41)$ \\
\hline
\end{tabular}

CTP Child Turcotte Pugh, $N C$ non-completer

Treatment difference: ${ }^{*} p=0.0007 ;{ }^{\dagger} p=0.0008 ;{ }^{\S} p=0.0028$

$\dagger$ Subjects with abnormal ALT at baseline

Rates of AEs, SAEs, and discontinuations due to AEs were comparable between the treatment groups. Cumulative rates for death and HCC were ETV 25\%; ADV 34\% and ETV 14\%; ADV 20\%, respectively.

Conclusions: Through 96 weeks of treatment ETV demonstrated superior antiviral activity to ADV in subjects with $\mathrm{CHB}$ and hepatic decompensation. ETV was well tolerated and safety results were comparable between treatment groups with death rates consistent with expectations for this population.

\section{PP13-105}

Evaluation of Long Term Renal Impacts of Adefovir Dipivoxil Plus Lamivudine in Chronic Hepatitis B Patients

C.P. Eyigün ${ }^{1}$, E. Günal ${ }^{1}$, H.C. Gül ${ }^{1}$, I.Y. Avc1 ${ }^{1}$, F. Ersöz ${ }^{2}$, G. Mert ${ }^{1}$, Ö. Coşkun $^{1}$, A.B. Beşirbellioğlu ${ }^{1}$

${ }^{1}$ Infectious Disease and Clinical Microbiology, Gulhane Military Medical Academy, ${ }^{2}$ Operations Researches Department, Turkish Military Academy Defense Science Institute, Ankara, Turkey

The aim of this study is to analyze renal impacts and safety of long term adefovir dipivoxil plus lamivudine use in patients who have no renal insufficiency preceding treatment. This retrospective study was carried on 76 patients [ 17 females $(24 \%), 59$ males $(76 \%)]$.The mean age of the patients was $43.41 \pm 13.27$ years. After the detection of YMDD mutation, all patients were added adefovir dipivoxil $10 \mathrm{mg} /$ day on lamivudine given $100 \mathrm{mg}$ daily. All the patients were followed up for median 235 weeks ( $\min 71$, max 358).Serum creatinine levels were checked at the adding of adefovir dipivoxil therapy with threemonthly intervals and GFR levels were calculated at the pretreatment and posttreatment with Modified Modification of Diet in Renal Disease (MDRD) formula. The mean GFR level at the start of therapy was $88.39 \pm 15.66 \mathrm{mg} / \mathrm{dL}$ and the final mean level was $80.34 \pm 14.85$ $\mathrm{mg} / \mathrm{dL}$. The average decrease in GFR level was calculated as $8.05 \pm 11.04 \mathrm{mg} / \mathrm{dL}$. There was significant difference between the pretreatment and posttreatment GFR levels in patients receiving adefovir dipivoxil $(p<0.05)$ but this difference wasn't significant in patients who had advanced age or longer theraphy period $(p>0.05)$. The periods of previous studies evaluating the renal toxicity of adefovir dipivoxil were generally limited and it was reported that renal impairment due to this drug is dose dependent.The results of this study disclosed that long term combined use of adefovir dipivoxil and lamivudine affects GFR levels significantly even as independent from advanced age or elongation of theraphy more. We need to use this combination with monitoring patients carefully.

\section{PP13-106}

Should Indian Patients with Liver Cirrhosis due to HCV Genotype 3 Be Treated?

A. Verma, T.S. Negi, G. Choudhuri

SGPGIMS, Lucknow, India

Background and aims: While HCV related liver cirrhosis (HCV-LC) in west is usually due to genotype 1 , are difficult to treat with antivirals and hence offered liver transplantation early, the infection in resource scarce country like India is usually due to genotype 3 which may respond better. We evaluated tolerability and outcome of pegylated interferon (Peg IFN) and ribavarin in patients with HCVLC genotype 3 and compared it with chronic $\mathrm{HCV}$ without cirrhosis of same genotype.

Methods: From 1998 to 2010 in a single unit experience of 228 patients who were offered combined antiviral treatment with Peg IFN and ribavarin with response guided individualized treatment for at least 24 weeks, 99 had cirrhosis and 129 were without cirrhosis (CHC). Wherever required, supportive treatment with erythropoietin, blood transfusion and colony stimulating factors was given.

Results: Of 99 patients 72 had genotype 3 related LC. Median age was 47.5 years (range 26-71); 33 (45.5\%) were males; 43, 27 and 2 (60, 37.5 and $2.7 \%$ ) patients were in Child A, B and C groups respectively. The dropout rates were 22.2 and $8.4 \%$ whereas sustained 
virological response (SVR) rates were 64.2 and $92.0 \%$ in LC and without cirrhosis, respectively. 35.7 and $14.8 \%$ required support with stimulating factors to complete treatment in $\mathrm{LC}$ and $\mathrm{CHC}$ groups respectively.

Conclusion: Patients with HCV related Child A and B cirrhosis due to genotype 3 have relatively good response rates. So we suggest that Indian patients in this category should be offered antiviral therapy.

\section{PP13-107}

Accordion Index: A Useful Way for the Assessment of Combinated Therapy in Chronic Hepatitis C

A. Martie ${ }^{1}$, C. Serban ${ }^{2}$, A. Popescu ${ }^{1}$, M. Ardelean ${ }^{1}$

${ }^{I}$ Gastroenterology and Hepatology; ${ }^{2}$ Pathophysiology, University

of Medicine and Pharmacy, Timisoara, Romania

Introduction: The etiological treatment in chronic hepatitis $\mathrm{C}$ is the main objective to eliminate hepatitis $\mathrm{C}$ virus.

Aim: The assessment of the efficacy of combinated therapy in chronic hepatitis C.

Material and method: 57 naive patients with chronic hepatitis $\mathrm{C}$ were treated for 48 weeks with combinated therapy, in Gastroenterology Department from Timisoara, beetwen 2004-2007. We performed viral load at the beginning of treatment, at 12 weeks or 24 weeks, at the end of treatment and also, we performed sustained virological response (SVR). From 57 patients, 32 received therapy with PegInterferon alpha 2A and Ribavirin, while 25 patients received PegInterferon alpha 2B and Ribavirin. We performed the Accordion Index at all patients, as a predicted tool for the efficacy of PegInterferon and Ribavirin therapy. So we used: the IFN-AC ratio= (total IFN dose given during the entire treatment period)/(total IFN dose required to achieve hepatitis $\mathrm{C}$ virus HCV-RNA negativity) and the $\mathrm{RBV}-\mathrm{AC}$ ratio= (total RBV dose given during the entire treatment period)/(total RBV dose required to achieve HCV-RNA negativity). Results: From the 57 patients, we obtained SVR at 38 patients (66.6). In 50 patients, the Accordion Index was 4, while 7 patients had the Accordion Index less than 4. 36 patients with 4 value of Accordion Index achieved SVR and only 2 patients with Accordion Index $<4$ achieved SVR. At the Accordion Index cut-off value of 4 , the quality of SVR prediction was as following: the sensibility was $79 \%$, the specificity was $92,1 \%$, the positive predictive value was $83.3 \%$, the negative predictive value was $89.7 \%$ and accuracy was $87.7 \%$; $p<$ 0.001 .

Conclusions: The Accordion Index can be a useful way for the assessment of SVR at patients with chronic hepatitis C .

\section{PP13-108}

Tolerance of Hepatitis C Treatment at the Hassan II Hospital University, Fez, Morocco

N. Aqodad, M. Lahbabi, R. Zamharir, M. Elyousfi, I. Mellouki, D. Benajah, M. Elabkari, A. Ibrahimi

Gastroenterology Unit, Hospital University Hassan II, Fez, Morocco

Introduction: The treatment of viral chronic hepatitis $\mathrm{C}$ is currently based on the combination therapy pegylated interferon alpha-2a or alpha- $2 b+$ ribavirin which allows sustained virological response (SVR) rate ranging from 54 to $61 \%$ depending on tolerance and aderance to therapy. The objective of this study is to assess therapeutic tolerance in chronic viral hepatitis $\mathrm{C}$.

Patients and methods: Retrospective study of cases of chronic hepatitis $\mathrm{C}$ treated with combination therapy in our department. Data were collected and analyzed from the registry of patients treated and monitored for chronic hepatitis C.

Results: 234 patients were collected in our department, of which 101 were treated. The average age was 57.4 years $(17-80)$. We have selected for the evaluation of virologic response 88 patients who completed treatment and follow-up. Mortality secondary to treatment was $0.8 \%$. The sustained viral response was $38 \%$ in case of occurrence of side effects leading to reduction or discontinuation of anti viral treatment versus $51.6 \%$ in their absence. Discontinuation of antiviral treatment for side effects is observed in 7\% of cases $(N=7)$ : [ $2 \%$ when the treatment duration is 24 weeks $(N=2)$ and $5 \%$ when the duration treatment is 48 weeks $(N=5)]$. In addition, there was a decrease in dose of pegylated interferon and or ribavirin in $21.7 \%$ of cases $(N=22)$ : $(10 \%(N=10)$ when the duration is 24 weeks and in $11.8 \%(N=12)$ of cases when the duration is 48 weeks). The decrease of treatment interested more pegylated interferon that ribavirin at 24 weeks ( 31.8 vs. $27.2 \%$ ) but the decrease at 48 weeks interested more ribavirin that pegylated interferon (45.4 vs. $22.7 \%)$.

Conclusion: The tolerance of pegylated interferon + ribavirin combination therapy can get the best chance of successful treatment (51.6 vs. $38 \%)$.

\section{PP13-109}

Treatment of Chronic Viral Hepatitis C at the Hassan II Hospital University, Fez, Morocco

N. Aqodad, M. Lahbabi, M. Elyousfi, I. Mellouki, D. Benajah, M. Elabkari, A. Ibrahimi

Gastroenterology Unit, Hospital University Hassan II, Fez, Morocco

Introduction and aim of study: During the last decade, significant progress has been made in the treatment of chronic viral hepatitis $\mathrm{C}$. Currently the standard treatment is combination of pegylated interferon (peg-IFN) alpha $2 \mathrm{a}$ or $2 \mathrm{~b}$ with ribavirin. This treatment gives $50 \%$ of sustained viral eradication. The objective of this study is to report the experience of the Hassan II university hospital of Fez in the treatment of chronic viral hepatitis $\mathrm{C}$.

Methods: This is a retrospective study of cases of chronic hepatitis $\mathrm{C}$ treated in our department. All clinical, laboratory and histology data were collected and analyzed from the files and registry of patients treated and followed for chronic viral hepatitis $\mathrm{C}$ infection.

Results: 234 patients with chronic hepatitis $\mathrm{C}$ virus were seen in our department, and 101 were treated. Eighty-eight patients completed their treatment and their follow-up 6 months after the end of treatment, their average age was 57,3 years (17-77), with female predominance (sex ratio 0.9), genotype 1: $53 \%$ (47/88), genotype 2: $42 \%$ (37/88). Eight percent of patients (7/88) were cirrhotic. The viral load at the end of treatment was negative in $72.7 \%(64 / 88)$ of patients for all genotypes. The sustained viral response was $47.7 \%$ of patients for all genotypes. (34\% (16/47) for genotype 1 and 62\% (23/37) for genotype 2). In univariate analysis predictors factors of SVR was genotype 2 and pegylated combination therapy but they are not independent factors of SVR in multivariate analysis.

Conclusion: The average age of patients was 57.3 years, fibrosis was severe in $27 \%$ of cases. There was a predominance of genotype 1 (53\%) and genotype $2(42 \%)$. The SVR was $47.7 \%$. In univariate analysis predictors factors of SVR were genotype 2 and pegylated combination therapy but they were not independent factors of SVR in multivariate analysis.

\section{PP13-110}

Indicators Energy Carriers and Lipoproteins for Chronic Hepatitis C S. Gramatyuk

Kharkov National Medical University, Kharkov, Ukraine

Objective: ATP energy carriers, are necessary as cofactors to drive numerous enzymatic reactions necessary for life. ATP synthesized within the mitochondria is exchanged by an ADP-ATP antiporter for ADP accumulating in the cytoplasm, where most of these reactions occur. Although this process maintains a fairly high intracellular ATP/ADP ratio at any given time, the actual cytoplasmic ATP is not especially high. Consequently, many critical or energy-intensive reactions rely on other energetic cofactors, typically generated in a separate ATP-dependent reaction, which can accumulate at a higher concentration to store energy for that specific reaction. Changes in the 
abundance and properties of blood lipoproteins are generally considered major causes for varied pathological conditions and diseases. Aim: Demonstrate differences in membrane binding between lipoproteins LDL, and HDL and differences ATP/ADP from hepatitis C patients versus healthy controls.

Material and methods: The study included 140 patients with HCV chronic hepatitis. The chromatic assays, complemented by biophysical techniques and electron microscopy.

Results: Point to significant reduction of surface membrane binding of the lipoproteins as a consequence of genotype viruses hepatitis $\mathrm{C}$. Abbreviations ATP $(p=0.006)$ and high density lipoprotein $(p=$ $0.005)$; increase depending on the genotype of the virus ADP $(p=$ $0.07)$ and LDL $(p=0.005)$. Imbalance in ATP / ADP rapidly converts pyruvate to acetyl $\mathrm{CoA}$, releasing $\mathrm{CO} 2$ as a by-product. This acetyl CoA joins the acetyl CoA produced from fatty acids to fuel the citric acid cycle increasing oxidative stress.

Conclusion: Overall, our results indicate that the substantial modulation of membrane interactions revealed by the chromatic assays may be used as a new and potentially powerful marker for screening and prediction of diseases associated with $\mathrm{HCV}$ chronic hepatitis.

\section{PP13-111}

Peginterferon Alfa-2a and Ribavirin for 24 Weeks in Hepatitis C Type 1 and 4 Patients with Rapid Virological Response

A.I. Shaban ${ }^{1}$, M.H. Gabr ${ }^{1}$, A. Burwaiss ${ }^{2}$, A. Bani ${ }^{2}$, S. Sannuk ${ }^{2}$, M. Al-Naagi ${ }^{2}$, E. Shaban ${ }^{2}$, N. Hmeda ${ }^{2}$, M. Fathi ${ }^{2}$, A. Bisheya ${ }^{2}$

${ }^{1}$ Hepatology and Gastroentrology; ${ }^{2}$ Tripoli Medical Center, Tripoli, Libya

Background and aims: This analysis reports the rate of sustained virological response (SVR) in patients infected with hepatitis $C$ virus (HCV) genotype 1 or 4 who were assigned to 24 weeks of treatment with pegylated interferon(peginterferon alfa-2a 180 ugweek plus ribavirin 400-1,200 mg/day after achieving a rapid virological respose (RVR; HCV RNA level $<15 \mathrm{IU} / \mathrm{mL}$ ) at week 4 in a prospective trial investigating response guided therapy in a Libyan population.

Method: Non-RVR patients with an early virological response (EVR) were given 72 weeks of therapy (this is still ongoing trial).

Results: A total of 63 of 162 patients (38.8\%) had an RVR; all of them completed 24 weeks of treatment. The SVR rate was $88 \%$. The SVR rate was $92.3 \%$ in patients infected with genotype 4 and $38.3 \%$ in those infected with genotype 1 . Treatment was well tolerated.

Conclusion: This prospective study confirms that a 24 -week regimen of peginterferon alfa-2a plus ribavirin $400-1,200 \mathrm{mg}$ per day is apropriate in genotype 1 and 4 patients who achievea an RVR by week 4 of therapy.

Abreviations used in this paper: EVR early virological response, PCR, polymerase chain rection, peginterferon, pegylated interferon, RVR, rapid virological response

\section{PP13-112}

Efficacy and Safety of Peginterferon and Rbavirin in Patients Aged 70 Years or Older with Chronic Hepatitis C

S.J. Park, Y.Y. Koh, B.H. Kim, Y.M. Park

Gastroenterology, Bundang Jesaeng General Hospital, Sungnam-si, Republic of Korea

Background \& aim: Recently, age of sixties is often regarded as relatively young age because of the increased life expectancy. Although a few studies showed the reduced efficacy of peginterferon and ribavirin combination treatment in chronic hepatitis $\mathrm{C}$ patients over 60 years old, we planned to analyze the efficacy and safety of the combination treatment in far more elderly patients more than 70 years old.

Materials and methods: Eighty eight patients with chronic hepatitis $\mathrm{C}$ who had been treated with peg-IFN and RBV were retrospectively analyzed and divided into two groups according to age: $<60$ years old (Young, $n=64$ ) and $\geq 70$ years old (Elderly, $n=24$ ).
Results: Baseline hemoglobin, platelet counts and albumin levels were significantly lower and baseline prothrombin time was more prolonged in the elderly group compared with the young group $(p<$ 0.05), but serum AST, ALT, total bilirubin levels and the rate of treatment discontinuation didn't significantly differ between two groups. Dose reduction (less than $75 \%$ of the standard dosage) was more frequent in the elderly group (Young vs. elderly : 32.8 vs. $75.0 \%, p<0.01)$. Although sustained virologic response (SVR) rate in the elderly group showed a decreased tendency of efficacy but there was no statistical difference. (Young vs. elderly: 45.5 vs. $39.3 \%, p=$ 0.768 in all genotypes; 34.1 vs. $29.4 \%$ in genotype $1, p=0.374 ; 56.5$ vs. $50.2 \%$ in genotype non-1, $p=0.611$ ). Adverse events such as fatigue, anemia and neutropenia were more often in the elderly (Young vs elderly: 40.6 vs. $62.5 \%, p=0.067$ ).

Conclusion: Dose reduction caused by adverse events was more common in the elderly. However, although the sample size of the elderly was small and the efficacy showed a decreased tendency, this study showed the efficacy of combination treatment in the far more elderly $\mathrm{HCV}$ patients over 70 years old was comparable to the younger patients.

\section{PP13-113}

Importance of Medication Adherence to Peginterferon-Ribavirin Combination Therapy in Patients with Chronic Hepatitis $\mathbf{C}$ Y.J. Kim, B.S. Lee, S.H. Kim, H.Y. Lee, E.S. Lee, E.S. Kim, S.Y. Lee Chung-Nam National University, Daejeon, Republic of Korea

Background/aims: Reduction of medication adherence in combination therapy of peginterferon and ribavirin frequently occur. The aims of this study were to assess influences of reduction of medication adherence in the combination therapy of chronic hepatitis $\mathrm{C}$ patients. Methods: We retrospectively analyzed 82 patients with chronic hepatitis $\mathrm{C}$ who received peginterferon and ribavirin combination therapy by reviewing their medical records. They were categorized into 3 subgroups on the basis of medication adherence. Group 1 comprised patients that received $\geq 80 \%$ of the recommended dosage of both peginterferon and ribavirin. Group 2 comprised patients that received $\geq 80 \%$ of the recommended dosage of only $1 \mathrm{drug}$; the other drug was administered at $<80 \%$ of the recommended dosage. Group 3 comprised patients that received $<80 \%$ of the recommended dosage of both peginterferon and ribavirin.

Results: SVRs of all patients were $85.4 \%$ (41/48), 85.7\% (18/21), and $38.5 \%(5 / 13)(p=0.002)$. SVRs of genotype 1 patients were $84.2 \%$ $(16 / 19), 75 \%(9 / 12)$, and $14.3 \%(1 / 7)$ in groups 1,2 , and 3 , respectively $(p=0.003)$. SVRs of genotype non-1 patients were $86.2 \%$ (25/ $29), 100 \%(9 / 9)$, and $66.7 \%(4 / 6)$ in groups 1,2 , and 3 , respectively $(p=0.196)$. Further, SVR differed significantly with the degree of medication adherence to either peginterferon or ribavirin $(p=0.003$ and 0.021 ,respectively). In multivariate analysis, the peginterferon dose was significant independent factors associated with SVR.

Conclusions: Medication adherence to peginterferon and ribavirin combination therapy is a very important factor in achieving SVR. In particular, we think that genotype 1 patients should maintain a higher adherence than genotype non-1 patients.

\section{PP13-114}

Heterologous T Cells Can Help Restore Function in Dysfunctional Hepatitis C Virus Non-structural (NS) 3/4A-specific T-cells during Therapeutic Vaccination

L. Frelin ${ }^{1}$, A. Chen ${ }^{1}$, G. Ahlén ${ }^{1}$, E.D. Brenndörfer ${ }^{1}$, A. Braß ${ }^{1}$, F. Holmström ${ }^{1}$, M. Chen ${ }^{2}$, J. Söderholm ${ }^{3,4}$, D.R. Milich ${ }^{5}$, M. Sällberg ${ }^{1}$

${ }^{1}$ Laboratory Medicine; ${ }^{2}$ Dental Medicine, Karolinska Institutet, Stockholm; ${ }^{3}$ Microbiology, University of Gothenburg, Sahlgrenska Academy, Gothenburg, Sweden; ${ }^{4}$ Inovio Biomedical; ${ }^{5}$ Vaccine Research Institute of San Diego, San Diego, CA, USA

It is well known that hepatitis $\mathrm{C}$ virus (HCV)-specific $\mathrm{T}$ cell responses in patients with chronic $\mathrm{HCV}$ are dysfunctional. In this 
study we aimed at restoring the immunological function through therapeutic vaccination in a transgenic mouse model with impaired $\mathrm{HCV}$-specific $\mathrm{T}$ cell responses due to a chronic presence of hepatic HCV nonstructural (NS) 3/4A antigens. The HCV-specific T cells have an actively maintained dysfunction reflected in a reduced frequency, impaired cytokine production, and impaired effector function in vivo, which can be partially restored by blocking regulatory $\mathrm{T}$ cells (Tregs) or programmed cell death ligand 1 (PD-L1). Our hypothesis was that the impairment could be overcome by adding gene sequences that created a normal priming environment by recruiting "healthy" heterologous $\mathrm{T}$ cells and by activating innate signaling. Our previous results shows that endogenously expressed hepatitis B core antigen $(\mathrm{HBcAg})$ can recruit heterologous $\mathrm{T}$ cells and activate Toll-like receptor (TLR7) signaling. Therefore, we decided to combine $\mathrm{HCV}$ NS3/4A with different forms of $\mathrm{HBcAg}$. Our results show that heterologous sequences somewhat improved activation and expansion of NS3/4A-specific T cells in a wild type host. Importantly, the signals provided by $\mathrm{HBcAg}$ effectively restored the activation of $\mathrm{HCV}$-specific $\mathrm{T}$ cells in an immunological tolerant NS3/4A-transgenic mouse model. The adjuvant effect detected in the NS3/4A-transgenic mice could be transferred to the priming of dysfunctional human leukocyte antigen (HLA)-A2-restricted NS3-specific T cells in vivo. In conclusion, the recruitment of "healthy" heterologous $\mathrm{T}$ cells to the site of $\mathrm{T}$ cell activation may also help to restore $\mathrm{HCV}$-specific responses present in chronically infected patients.

\section{PP13-115}

The Safety and Efficacy of Pegylated Interferon Treatment for Elderly Patients with Chronic Hepatitis C

H. Yoshida ${ }^{1}$, H. Taniguchi ${ }^{1}$, M. Omata ${ }^{2}$, R. Nakata ${ }^{1}$

${ }^{I}$ Gastroenterology and Hepatology, Japanese Red Cross Medical Center, Tokyo; ${ }^{2}$ Yamanashi Prefectural Hospital Organization, Kofu, Japan

Background/aim: Elderly patients with $\mathrm{HCV}$ infection is increasing in Japan. The aim is to clarify the safety and efficacy of pegylatedinterferon treatment for elderly patients.

Methods: Patients with chronic hepatitis $\mathrm{C}$ treated with pegylatedinterferon at our center between 2005 and 2009 were enrolled. Peginterferon alpha 2a (180 mcg weekly, 24 or 48 weeks), peg-interferon alpha 2a plus ribavirin ( $180 \mathrm{mcg}$ weekly, $600-1,200 \mathrm{mg}$ daily 24 or 48 weeks), or peg-interferon alpha $2 \mathrm{~b}$ plus ribavirin $(1.5 \mathrm{mcg} / \mathrm{kg}$ weekly, $600-1,200 \mathrm{mg}$ daily 24 or 48 weeks) was administered. The rate of dose reduction and termination of interferon between elderly patients (over 65 yo) and younger patients (under 65 yo) were compared. Sustained virological response (SVR) rate was compared between two groups.

Results: One hundred seventy-eight patients with chronic hepatitis $\mathrm{C}$ were enrolled between 2005 and 2009. Twenty-six patients before the determination of SVR were excluded. Thirteen patients were excluded for loss of follow up. In total 139 patients were analyzed. Of 139 patients, 37 were elder than 65 yo. One hundred two patients were 65 yo or younger. Twenty-one patients $(57 \%)$ needed dose reduction of IFN and 13 patients (35\%) needed termination of treatment in elderly group. Forty-one patients (40\%) needed dose reduction and 19 patients $(19 \%)$ needed termination in younger group. Sixteen patients $(43 \%)$ in elderly group and 65 patients $(64 \%)$ in younger group obtained more than $80 \%$ of scheduled IFN dose $(p=0.0487)$. There was no patient suffered from adverse effect after the end of treatment in both groups. SVR was achieved in twenty patients (54\%) in elderly group and in 56 patients $(55 \%)$ in younger group. SVR rate among genotype 1 patients was $44 \%$ in elderly group, $37 \%$ in younger group. Conclusion: Treatment with pegylated-interferon for elderly chronic hepatitis $\mathrm{C}$ patients was safe and effective if it is carried out under close follow up.
PP13-116

Biphasic Thyroid Dysfunction in Patient with Chronic Hepatitis C Treated with Interferon-Alpha and Ribavirin

N. Sargsyants, V. Sargsyan, A. Pepanyan

Infectious Diseases, Armenicum Clinical Center, Yerevan, Armenia

Background: Different type of thyroid dysfunction may be a side effect of treatment of chronic HCV-infection with interferon (IFN) and ribavirin $(\mathrm{RBV})$. We describe an interesting case of biphasic thyrotoxicosis associated with Peg-IFN a-2a and RBV.

Methods: Patient V.S., female, 37 years old with chronic hepatitis C was treated with Peg-IFN a-2a plus RBV. Routine evaluations, include thyroid gland function (TSH, FT4, TPO-Ab - were normal) were done initially. ALT и AST normalized already on 4th week of the treatment. From 9th week antiviral therapy patient mentioned severe fatigue, malaise, irritation, persistent subfebrile fever, generalized tremor, palpitation, difficulty on swallowing. Blood analysis revealed decreasing of TSH (0.07, normal range: 0.34-5.60), increasing of FT4 (3,33, normal range: $0.58-1,64)$, FT3 (3,04, normal range: $0.87-1.78)$, Ab-TPO (693,8, normal level < 35.0). Level of $\mathrm{TgAb}$ was normal. Ultrasonography had shown signs of destructive thyroiditis. IFN-therapy was stopped because of severe hyperthiroiditis. Endocrinologist prescribes thiamazole. Interestingly, that Abbot Real-Time PCR HCV-RNA was less than $12 \mathrm{IU} / \mathrm{ml}$ on 12th week (EVR). After 2 months treatment with thyrostatic hyperfunction was following by hypofunction (TSH increased up to 16,9 and FT4 decreased to 0.3 ) and treatment was changed on L-thyroxine.

Results: Early IFN-induced thyroid dysfunction with biphasic autoimmune thyroiditis was diagnosed. Typical course is a primary phase of destructive thyrotoxicosis following with phase of transitory hypothyreosis and subsequent recovery of thyroid gland function in majority of patients.

Conclusion: Screening for autoantibodies and serum thyroid-stimulating hormone is recommended before, during and after interferonalpha treatment. Patients should be informed about risk of thyroid dysfunction before starting interferonotherapy.

\section{PP13-117}

Clinical Study of Renal Transplantation in Patients with Hepatitis C X.-M. Ding ${ }^{1}$, W.-J. Xue ${ }^{2}$, P.-X. Tian' ${ }^{2}$, X.-M. Pan ${ }^{2}$, H. Yan², J. Hou², X.-S. Feng ${ }^{2}$, H.-L. Xiang ${ }^{2}$, X.-H. Tian' ${ }^{2}, \mathrm{Y} \mathrm{Li}^{2}$

${ }^{1}$ Renal Transplant; ${ }^{2}$ The First Affiliated Hospital, Medical College, $X i$ 'an Jiaotong University, Xi'an, China

Objective: A considerable portion of patients with end-stage renal disease accompanied by hepatitis $\mathrm{C}$ needs kidney transplantation. So we want to explore clinical strategies improving renal transplantation effect in patients with hepatitis $\mathrm{C}$ infection.

Methods: We retrospectively analyzed the clinical data of 106 patients with hepatitis $\mathrm{C}$ who received renal transplantation in this center. The patients with HCV-RNA copy number greater than 1000 $\mathrm{IU} / \mathrm{ml}$ preoperatively received interferon- $\alpha(\mathrm{INF}-\alpha)$ and oral ribovirin (RIBA) antiviral therapy before kidney transplant. The patients and renal survival rates, liver function and acute rejection rate were monitored after renal transplantation.

Results: Among 106 patients with hepatitis C, the survival rates of 1 , 3 and 5 years were 93.3, 82.1 and $79.2 \%$, respectively; the renal survival rates of 1,3 and 5 years were $92.4,80.2$ and $78.3 \%$. Based on the preoperative treatment of viral hepatitis, strengthening the postoperative hepatotheragy treatment and application of low-dose CNI or CNI-free immunosuppressive programs, the liver function and quantitative HCV-RNA in the majority of hepatitis $\mathrm{C}$ patients receiving renal transplantation remained stable and were close to the preoperative level. Only nine patients had an acute rejection confirmed by transplant renal biopsy pathological examination with an occurrence rate of $8.5 \%$. Of the 106 patients 23 died. Among these 
patients, 15 received CSA-AZA-Pred triple immunosuppressive program treatment, accounting for $65.2 \%$.

Conclusions: Rational use of immunosuppressive agents, especially regimen with a relatively low liver toxicity, is the key for successful kidney transplantation in patients with hepatitis $\mathrm{C}$ and their health and survival.

\section{PP13-118}

Successful Antiviral Treatment with Combination of PEG-IFN Alfa-2b and Ribavirin in a 3 Year Old Child with Chronic Hepatitis C

H.L. Ghazinyan, T. Asatryan, A. Asoyan, T. Galstyan

Hepatology Department, "Nork" Clinical Hospital of Infectious

Diseases, Yerevan, Armenia

Introduction: Progression of liver disease in children with chronic hepatitis $\mathrm{C}$ is slow, severe fibrosis and cirrhosis may develop in approximatelly $5 \%$ of infected children by the time they reach adulthood. Therapy with combination of PEG-IFN alfa- $2 b$ plus ribavirin in children with chronic hepatitis $\mathrm{C}$ offers favorable efficacy and acceptable safety profile.

Case description: A 3 year old woman was admitted due to tiredness, weakness and discomfort of epigastrium. The baseline results of laborator investigations were ALT $468 \mathrm{iu} / 1$, AST $385 \mathrm{iu} / 1$, total bilirubin $12,9 \mathrm{mg} / \mathrm{dl}, \mathrm{HBsAg}$, anti-HAV $\mathrm{IgM}$ and $\mathrm{IgG}$, anti-HBeAg, antiHBcAg negative, anti-HCV positive, $\mathrm{HCV}$ genotype $1 \mathrm{~b}, \mathrm{HCV}$ viral load $113 \mathrm{kiu} / \mathrm{ml}$.

The results of instrumental investigations :ultrasound of liver and $\mathrm{x}$ ray of chest without pathology. The patient was diagnosed with moderate chronic hepatitis $\mathrm{C}$ as determined by liver biopsy,recovery state of tuberculosis in lungs and antiviral treatment was started with weight -based PEG-IFN alfa-2b in combination with ribavirin. Due to 24 weeks antiviral treatment viral load was decreased $3,4 \mathrm{kiu} / \mathrm{ml}$. Instant of interrupting treatment the dose of PEG-IFN alfa-2b was increased $2 \mathrm{mcg} / \mathrm{kg}$ weekly, after wich 2 months later there was confirmed undetectable level of virus. The antiviral treatment with high dose of PEG-IFN alfa- $2 b$ with combination of ribavirin had been keeping on for 16 weeks.overall the duration of the antiviral treatment was 48 weeks. The results of treatment was sustained viral response.

\section{PP13-119}

Treatment Results of Hepatitis C with Peg-interferon Alpha and Ribavirin in Turkey

S. Köse, G. Çavdar, A. Gözaydın

Tepecik Educational and Research Hospital, Infectious Diseases and Clinical Microbiology, Izmir, Turkey

Introduction: $\mathrm{HCV}$ infectioncan be chronic in $85 \%$. Due to difficulty of eradication $\mathrm{HCV}$ infection, the purpose of antiviral treatment is supression of replication and prevention of late complications at cirossis and hepatocellular carsinoma. In this study we aimed to present the treatment results of chronic hepatitis $\mathrm{C}$ patients with peg-interferon and ribavirin.

Methods: We planned a combination therapy of peg-interferon and ribavirin treatment for 48 weeks to 61 patients who had Anti-HCV and HCV-RNA of positivity. We test the HCV-RNA of patients at 12 th week (early virological response), at 48th week (end of treatment virological response) and 6th month after the treatment (sustained virological response).

Results: 61 patients enrolled the study and $26(42.6 \%)$ of them were women. The average age was 50.8. The early virological response was $73.8 \%$, virological treatment at the and of treatment was $57.4 \%$ and sustained virological response was calculated as $54.1 \%$.

Discussion: In recent years peg-interferon and ribavirin combinaiton is the gold treatment of hepatitis C. We think that our results can provide contributions to the literature.
PP13-120

Role of Hepatic HCV-RNA on Severity of Chronic Hepatitis C and Response to Antiviral Therapy

S. Maylin ${ }^{1}$, M. Martinot-Peignoux ${ }^{2}$, C. Laouénan ${ }^{3}$, M. Lapalus ${ }^{1}$, T. Asselah ${ }^{4}$, P. Marcellin ${ }^{4}$

${ }^{I}$ INSERM U773/CRB3; ${ }^{2}$ INSERM U773/CRB3-Hopital Beuajon, Clichy; ${ }^{3}$ Hopital Bichat, INSERM U738 Faculté de Médecine D. Diderot, Paris; ${ }^{4}$ Service d'Hépatologie, INSERM U773/CRB3Hopital Beaujon, Clichy, France

Background and aims: Few data are available on the correlation between hepatic HCV RNA and serum HCV RNA, severity of the liver disease and response to therapy. The aim of the study was to assess the influence of hepatic HCV RNA level on the severity of liver disease and the response to antiviral therapy in a large cohort of chronic hepatitis $\mathrm{C}(\mathrm{CHC})$ patients.

Methods-patients: HCV RNA was measured in frozen liver biopsies and serum samples from 130 patients the day of liver biopsy prior to antiviral treatment. Liver fibrosis was assessed by Ishaq scoring. A Sustained virological Virological response (SVR) was observed in $48 \%$ of the patients, non-response (NR) in 34\%. Genotype 1 was found in $42 \%$ of the patients.

Results: Mean hepatic HCV RNA level was $7.69 \pm 0.67 \log _{10}$ copies/ $\mathrm{mg}$ of liver. Mean serum HCV RNA level was $6.21 \pm 0.72 \log _{10}$ copies $/ \mathrm{ml}$. There was a strong correlation between hepatic and serum HCV RNA $(r=0.40, p<0.0001)$ and hepatic HCV RNA and age $(p<$ 0.01). Hepatic HCV RNA was $8.04 \pm 0.68 ; 7.44 \pm 0.47 ; 7.43 \pm 0.49$ and $7.44 \pm 0.71 \log _{10}$ copies/mg of liver in genotypes $1,2,3$ and 4 , respectively, $(p=0.0001)$, higher in women than in men $(p=0.04)$. Mean hepatic HCV RNA was $7.58 \pm 0.63,7.61 \pm 0.55$ and $7.89 \pm$ $0.76 \log _{10}$ copies/mg in SVR, relapsers and NR, respectively $(p=$ 0.12). Mean hepatic HCV RNA was $7.70 \pm 0.69$ versus $7.67 \pm 0.68$ $\log _{10}$ copies/mg of liver, in patients with Ishaq $>3$ versus those with Ishaq $\leq 3$, respectively, $(p=0.72)$. The results of the multivariate analysis showed that genotype 1 was independently associated with non-response to therapy and high level of hepatic HCV RNA.

Conclusions: Hepatic HCV RNA level was not associated with severity of liver disease. High level of hepatic HCV RNA was strongly associated with HCV genotype 1 independently from response to therapy.

\section{PP13-121}

Early Virological Effects and Safety of Combined DFPP and IFN-beta Therapy Followed by PEG-IFN and Ribavirin for Chronic Hepatitis C

K. Nakamura ${ }^{1}$, K. Nagata ${ }^{2}$, M. Tsuchimochi ${ }^{1}$, H. Iwakiri ${ }^{1}$, K. Kusumoto ${ }^{1}$,

S. Hasuike ${ }^{1}$, B. Sakota ${ }^{3}$, N. Komada ${ }^{3}$, K. Shimoda ${ }^{1}$

${ }^{1}$ Gastroenterology and Hematology; ${ }^{2}$ Liver Disease Center, University of Miyazaki, Miyazaki; ${ }^{3}$ Gastoroenterology,

Fujimotohayasuzu Hospital, MIyakonojo, Japan

Background and aims: Double-filtration plasmapheresis (DFPP) is a newly developed technique that was approved in Japan in 2008. The aim of this study is to determine the efficacy and safety of induction therapy in combination with DFPP and interferon (IFN)-beta followed by pegylated IFN-alpha and ribavirin in patients with chronic hepatitis C.

Methods: We evaluated 55 patients who were chronically infected with hepatitis $\mathrm{C}$ virus (HCV) genotype 1 and had a high viral load. For 38 patients, DFPP was performed five times on days 1, 2, 4, 7 and 8 , and PEG-IFN and ribavirin therapy was initiated immediately after DFPP on day 1 (group A). The remaining 17 patients were treated with DFPP plus 3 MU of IFN-beta twice daily and ribavirin for 14 days (group B). Thereafter, both groups received weekly PEG-IFN injections and daily intake of ribavirin and statin. We compared the early virological response (EVR) and adverse events between groups $\mathrm{A}$ and $\mathrm{B}$. 
Results: The complete EVR (cEVR) in groups A and B was 52.6\% (20/ 38 ) and $82.4 \%$ (14/17), respectively. Most of the adverse events were caused by the interferon and ribavirin combination therapy. There were no differences in the cEVR between males and females in both groups. Conclusion: IFN-beta induction therapy resulted in acceptable SVR rates despite the short therapy duration. DFPP can be safely performed concomitantly with interferon and ribavirin combination therapy in chronic hepatitis $\mathrm{C}$ patients. This treatment combination may contribute to an early virological response. The effects of DFPP on the SVR and its significance remain to be clarified. DFPP plus consecutive intravenous IFN-beta for 2 weeks is a promising treatment for refractory patients.

\section{PP13-122}

The Most Accurate and Convenient Genotyping Methods, and Most Appropriate SNP Associated with IL28B for Japanese Chronic Hepatitis C Patients

K. Ito ${ }^{1}$, K. Higami ${ }^{1}$, N. Masaki ${ }^{1}$, M. Sugiyama ${ }^{1}$, M. Mukaide ${ }^{1}$, H. Saito ${ }^{1}$, Y. Aoki ${ }^{1}$, Y. Sato ${ }^{1}$, M. Imamura ${ }^{1}$, K. Murata ${ }^{1}$, Y. Tanaka ${ }^{2}$, M. Mizokami $^{1}$

${ }^{I}$ The Research Center for Hepatitis and Immunology, National Center for Global Health and Medicine, Ichikawa; ${ }^{2}$ Nagoya City University Graduate School of Medical Science, Nagoya, Japan

Background and aims: Recently, we reported from genome-wide association studies (GWAS) that several highly correlated common single nucleotide polymorphisms (SNPs), located in the vicinity of the IFN-lambda 3 (IL28B) gene on chromosome 19, are implicated in NVR (non-virological response) to PEG-IFN/RBV among patients with HCV genotype 1 . In this study, we focused on determining the most accurate and convenient genotyping methods and most appropriate SNP among four such polymorphisms associated with IL28B in order to design tailor-made therapy for Japanese chronic hepatitis $\mathrm{C}$ patients.

Methods: Firstly, five different methods (direct sequencing, high-resolution melting analysis (HRM), Hybridization probe (HP), InvaderPlus assay (Invader), and TaqMan SNP genotyping assay (TaqMan)) were developed for genotyping four SNPs (rs11881222, rs8103142, rs8099917 and rs12979860) associated with IL28B and their accuracy was compared in 292 Japanese patients. Next, the four SNPs associated with IL28B were genotyped by Invader in 416 additional Japanese patients and the response to PEG-IFN/RBV treatment was evaluated when the four SNPs were not in linkage disequilibrium (LD).

Results: HRM failed to genotype one of the four SNPs in five patients. In two of 287 patients, the results of genotyping rs 8099917 by direct sequencing differed from the results of the other three methods. The methods of HP, TaqMan and Invader were accurate for determining the SNPs associated with IL28B. In ten of the $708(1.4 \%)$ patients, the four SNPs were not in LD. Eight of nine (88.9\%) patients whose rs 8099917 was homozygous for the major allele were virological responders, even though one or more of the other SNPs were heterozygous.

Conclusions: The methods of HP, TaqMan and Invader were suitable to determine the SNPs associated with IL28B. The rs8099917 polymorphism should be the best predictor for the response to the PEGIFN/RBV treatment among Japanese chronic hepatitis $\mathrm{C}$ patients.

\section{PP13-123}

Clinical Factors Associated with the IL28B Polymorphism Prediction Failure to PEG-IFN/RBV Treatment in Chronic Hepatitis C Japanese Patients

H. Saito, K. Ito, Y. Aoki, K. Higami, M. Sugiyama, Y. Sato, M. Mukaide, K. Murata, M. Imamura, N. Masaki, M. Mizokami

The Research Center for Hepatitis and Immunology, National Center for Global Health and Medicine, Chiba, Japan

Background and aims: We identified several single nucleotide polymorphisms (SNPs) associated with interleukin (IL)-28B as predicting factors for the non-responders (NR) to PEG-IFN- $\alpha / \mathrm{RBV}$ therapy by genome-wide association study in Japanese CHC patients.
The IL28B polymorphism could predict the response to PEG-IFN- $\alpha$ / RBV therapy in about $80 \%$ Japanese CHC patients. However, the IL28B polymorphism fails to predict the response to PEG-IFN- $\alpha$ / RBV therapy in about $20 \%$ patients. Therefore, we investigated the other factors that may cause the prediction failure by the IL28B polymorphism.

Patients and methods: A total of 219 chronic hepatitis C patients with genotype $1 \mathrm{~b}$ and high viral load were enrolled. Several clinical factors were analyzed. The factors associated with discrepancy between the prediction by IL28B polymorphism and virological response to PEG-IFN- $\alpha /$ RBV therapy.

Results: Age $(p<0.001), \mathrm{Hb}(p=0.0043), \operatorname{PLT}(p=0.010), \gamma$-GTP $(p=0.029)$, T-Chol. (0.046), LDL-Chol $(p<0.001)$, FBS $(p=0.038)$, s-aa $70(p=0.002)$, and rs8099917 polymorphism $(p<0.0001)$ were significantly different between VR and NR. Multiple logistic regression analysis showed that rs8099917 polymorphism (TG or GG genotype) and age were significantly associated with NR. High LDLChol. level $(p=0.003), \gamma$-GTP $(p=0.025)$ and wild type of aa 70 $(p<0.0001)$ were significantly associated with the rs8099917 polymorphism. Age $(p=0.002)$, gender $(p=0.001), \mathrm{Hb}(p=0.006)$, PLT $(p=0.010)$ and fibrosis score $(p=0.003)$ were significantly different between NR and VR with TT allele genotype of rs8099917. Age ( $p=$ $0.007)$ and $\gamma$-GTP $(p=0.013)$ were significantly different between NR and VR with TG or GG allele genotype of rs8099917.

Conclusion: The TT genotype of rs8099917 polymorphism near IL28B gene was associated with high LDL cholesterol level, wild type of core aa 70 and high $\gamma$-GTP levels. Elder age and lower LDLChol. levels were associated with the NR who have TT genotype. Lower age and lower HCV-RNA levels were associated with the VR who have TG or GG genotype.

\section{Poster Presentation 14: Hepatitis C: Virology}

\section{PP14-01}

Identification of Amino Acid Residues Involved Hepatitis C Virus NS5A and Human TRAF2 Protein-protein Interaction

M. Faheem, S. Zaidi, U. Niazi, I. Qadri

NUST Center of Virology \& Immunolgy, National University of Sciences \& Technology, Islamabad, Pakistan

Hepatitis $\mathrm{C}$ virus (HCV) is a major cause of non-A and non-B hepatitis across the world. $\mathrm{HCV}$ is a member of family Flaviviridae which have positive sense RNA genome (Choo et al., 1989; Kuo et al., 1989; Houghton., 1996). RNA genome encodes three structural and seven nonstructural proteins. HCV non-structural gene NS5A is suggested to be involved in HCV replication and RNA-activated protein kinase repressor (PKR) (Kato et al., 1997; Tanimoto et al., 1997; Gale et al., 1997. TRAF2 is a ubiquitously expressed 56KD adaptor protein that is involved in the Tumor Necrosis Factor Receptor superfamily associated pathways. It leads to activation of both NF-kB and JNK pathways. HCV NS5A has been shown to interact with TRAF domain of TRAF2 and thus inhibit TRAF2 mediated NF- $\kappa \mathrm{B}$ activation and synergistically activate c-Jun N-terminal kinase (JNK) induced through TNF $\alpha$ (Park et al., 2002; Park et al., 2003). Since NS5A modulates TRAF2 mediated pathways the present study focuses on computational identification of residues within NS5A and TRAF2 that are involved in their interaction. NS5A 3D structure was modeled using modeler while TRAF domain structure was downloaded from PDB. Docking of both proteins was performed and interacting residues in the docked complex were identified. We found that 13 residues of NS5A and 13 residues of TRAF domain are involved in interaction making 16 bonds, out of which 6 are hydrogen bonds, 7 hydrophobic and 3 are ionic bonds. These residues provide insight and can be targeted for therapy through the use of different inhibitors in future. 


\section{PP14-02}

Genomic Heterogeneity of Hepatitis C Virus Infection in Myanmar N.K.T. Hlaing ${ }^{1}$, K.M. Win ${ }^{2}$

${ }^{1}$ Hepatology, University of Medicine (I); ${ }^{2}$ Hepatology, Yangon GI and Liver Centre, Yangon, Myanmar

Background and aims: Hepatitis $\mathrm{C}$ virus (HCV) has marked genetic diversity and high mutation rate, existing as quasispecies in the infected host. To date, HCV is classified into six major genotypes with several subtypes on the basis of nucleotide sequence similarity. HCV genotype not only determines the antiviral treatment duration but also predicts the response to antiviral therapy. This study is to evaluate the actual prevalence of different genotypes in $\mathrm{HCV}$ infected Myanmar patients.

Methods: HCV genotype data of 1,124 patients who attended the Yangon GI and Liver Center, Myanmar, from February 2006 to July 2010 were studied. HCV genotypes were determined by the commercially available line probe hybridization assay, VERSANT ${ }^{\mathrm{TM}}$ HCV Genotype Assay (LiPA) version 1.0 which is directed at $5^{\prime}$ untranslated region ( $\left.5^{\prime} \mathrm{UTR}\right)$ of HCV RNA genome, and version 2.0 which is focused at both $5^{\prime} \mathrm{UTR}$ and core region.

Results: Genotyping with LiPA 1.0 detected genotype 1 in $42.5 \%$, genotype 3 in $40.9 \%$, genotype 6 in $0.3 \%$ and indeterminate result in $14.6 \%$ of 650 patients. Genotyping with LiPA 2.0 detected genotype 1 in $20.5 \%$, genotype 3 in $40 \%$, genotype 6 in $27.9 \%$ and indeterminate in $10.6 \%$ of the other 474 patients.

Conclusion: Our study showed the better differentiation of genotype 6 from 1 by LiPA 2.0 with the dominant genotype in Myanmar 3/3b, followed by genotype 6 and 1 . Indeterminate genotypes are still noted in our study by both LiPA versions, indicating the necessity of further molecular studies on HCV genetic diversity in Myanmar.

\section{PP14-03}

Frequency of IL-10 Promoter Polymorphisms among Chronic Hepatitis C Virus Patients with and without Type 2 Diabetes Mellitus M.M. Elbendary ${ }^{1}$, S.M. Abdelaal ${ }^{2}$, T.A. Besheer ${ }^{1}$, A.A. El-Gendy ${ }^{3}$

${ }^{1}$ Tropical Medicine Department; ${ }^{2}$ Medical Microbiology \& Immunology; ${ }^{3}$ Department of Medical Physiology, Mansoura University, Mansoura, Egypt

Background /aims: HCV infection primarily causes liver disease, but also has been linked to other conditions including type 2 diabetes mellitus $\left(\mathrm{T}_{2} \mathrm{DM}\right)$. The association of interleukin (IL)-10 production capacity with $\mathrm{T}_{2} \mathrm{DM}$ was established. In this study, we determined the genotypic and allelic frequencies of the IL-10 $-1082_{\mathrm{G} / \mathrm{A}}, \mathrm{IL}-10-819_{\mathrm{C} / \mathrm{T}}$ and IL-10-592 $\mathrm{A} / \mathrm{C}$ polymorphisms and their association with IL-10 serum levels and the risk to develop $\mathrm{T}_{2} \mathrm{DM}$ among $\mathrm{HCV}$ patients groups.

Methods: Two groups of chronic hepatitis $\mathrm{C}$ infection positive patients ( 29 non diabetic and 42 with $\mathrm{T}_{2} \mathrm{DM}$ ) were included in this study in addition to 19 healthy controls. IL-10 serum levels were assayed using ELISA technique for all the studied groups. IL-10 promoter polymorphisms at positions $-1082,-819$ and -592 were examined using PCR-RFLP.

Results: The mean serum levels of IL-10 were significantly higher in $\mathrm{HCV}$ patients than that of controls and in HCV diabetic patients than $\mathrm{HCV}$ non-diabetic patients. Analysis of genotypes and alleles frequency revealed involvement of three nucleotides positions -1082 , -819 and 592 with low and high levels of IL-10 production. Moreover, genotypes $-1082_{\mathrm{GG}},-819_{\mathrm{CC}}$ and $-592_{\mathrm{CC}}$, were significantly higher in diabetic $\mathrm{HCV}$ infection patients than that of controls. Additionally only IL-10 - 1082 GG genotype was significantly higher in non diabetic $\mathrm{HCV}$ infection versus controls. No significant differences of these genotypes frequencies were observed between diabetic hepatitis $\mathrm{C}$ infection and non diabetic HCV infection patients.

Conclusion: Mutation at positions $-1082,-819$ and -592 of IL-10 promoter gene region may be one of the factors participating to $\mathrm{HCV}$ pathogenicity. Nevertheless, no association was observed between these polymorphisms and $\mathrm{T}_{2} \mathrm{DM}$.

\section{PP14-04}

Differential in vitro Effects of Intravenous Versus Oral Formulations of Silibinin on the HCV Life Cycle and Inflammation

J. Wagoner ${ }^{1}$, C. Morishima ${ }^{1}$, T.N. Graf ${ }^{2}$, N.H. Oberlies ${ }^{2}$, E. Tessier ${ }^{3}$, E.-I. Pécheur $^{3}$, J.E. Tavis ${ }^{4}$, S.J. Polyak ${ }^{1}$

${ }^{1}$ University of Washington, Seattle, WA; ${ }^{2}$ University of North Carolina at Greensboro, Greensboro, NC, USA; ${ }^{3}$ Université Lyon 1, Lyon, France; ${ }^{4}$ Saint Louis University, St. Louis, MO, USA

Introduction: Silymarin prevents liver disease in many experimental models, and is the most popular botanical medicine consumed by patients with hepatitis C. Silibinin is a major component of silymarin, consisting of the flavonolignans silybin A and silybin B, which are insoluble in aqueous solution. A chemically modified and soluble version of silibinin, SIL, has been shown to potently reduce HCV RNA levels in vivo when administered intravenously. We have previously shown that silymarin and silibinin inhibit $\mathrm{HCV}$ infection in cell culture by targeting multiple steps in the virus lifecycle.

Aim: In this study, we compared the hepatoprotective profiles of SIL and silibinin.

Methods: We used multiple assays that measure antiviral and antiinflammatory functions.

Results: Both mixtures inhibited fusion of HCV pseudoparticles with fluorescent liposomes in a dose-dependent fashion. SIL inhibited 5 clinical genotype 1b isolates of NS5B RNA dependent RNA polymerase (RdRp) activity better than silibinin, with IC50 values of $40-85 \mu \mathrm{M}$. The enhanced activity of SIL may have been in part due to inhibition of NS5B binding to RNA templates. However, inhibition of the RdRps by both mixtures plateaued at $43-73 \%$, suggesting that the products are poor overall inhibitors of RdRp. Silibinin did not inhibit HCV replication in subgenomic genotype $1 \mathrm{~b}$ or $2 \mathrm{a}$ replicon cell lines, but it did inhibit JFH-1 infection. In contrast, SIL inhibited $1 \mathrm{~b}$ but not 2a subgenomic replicons and also inhibited JFH-1 infection at high doses. Silibinin but not SIL inhibited NF-kB- and IFN-Bdependent transcription in Huh7 cells. However, both mixtures inhibited $\mathrm{T}$ cell proliferation to similar degrees.

Conclusion: These data underscore the differences and similarities between the intravenous and oral formulations of silibinin, which could influence the clinical effects of this mixture on patients with chronic liver diseases.

\section{PP14-05}

Prospective Monitoring of Hepatitis C Viral Load among Anti-viral Treatment Naive Living Donor Renal Transplant Recipients

S. Justa ${ }^{1}$, R.W. Minz ${ }^{1}$, M. Minz ${ }^{2}$, A. Sharma ${ }^{2}$, S. Anand ${ }^{1}$, A. Das ${ }^{3}$, Y. Chawla ${ }^{4}$ ${ }^{1}$ Immunopathology; ${ }^{2}$ Renal Transplant Surgery; ${ }^{3}$ Histopathology;

${ }^{4}$ Hepatology, PGIMER, Chandigarh, India

Background: There is a high prevalence of chronic hepatitis $\mathrm{C}$ virus (HCV) infection among various groups of immunosuppressed patients. Renal transplant (RT) recipients represent an important group with high prevalence of $\mathrm{HCV}$ infection rates ranging from 10 to $50 \%$. To determine future treatment strategies in Hepatitis $\mathrm{C}$ positive $\mathrm{RT}$ recipients, we document for the first time serum viral load upto 6 months post-transplantation among these patients group.

Methods: Serum viral load was serially monitored by quantitative real time PCR among $25 \mathrm{HCV}$-positive living donor renal transplant recipients (antiviral treatment naive) at three time points i.e. pretransplantation as well as day 10 and 6th month post transplant. A liver biopsy obtained, under vision at the time of transplantation, was also analyzed for viral load and underwent histological grading and staging by modified Knodell system of Ishak et al. We also analyzed the HCV genotypes among our patient cohort by direct sequencing of the 5 'non coding region of $\mathrm{HCV}$ islolates. 
Results: There is an increase in viremia immediately post-transplant (day 10) followed by a significant ( $2 \operatorname{logs}$ ) reduction at 6 months posttransplant. A significant positive correlation exists between pretransplant serum viral load and intra-hepatic viral load $(r=0.727$, $p=0.001)$. The latter also shows a positive correlation with liver fibrosis score $(r=0.423, p=0.05)$. Genotype 1 was found to be the predominant genotype among our patient cohort. Serum viral load, intra hepatic vial load and liver histology failed to show a significant correlation with any of the biochemical parameters at the time of transplantation.

Conclusion: At 6-month post transplant, a probable synergy exists between immunomodulatory effect of immunosuppressive drugs and host immune response leading to the reduction in viral load among $\mathrm{HCV}$-positive living donor renal transplant recipients. However, larger prospective studies are needed to verify this hypothesis

\section{PP14-06}

The Outcome of Hepatitis C Virus Infection in Chinese Paid Plasma Donors: A 12-19-Year Cohort Study

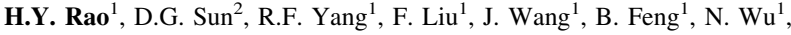
J.L. Fang ${ }^{1}$, G.J. Song ${ }^{1}$, H. Ma ${ }^{1}$, F. Guo ${ }^{1}$, J.H. Wang ${ }^{1}$, X.B. Li ${ }^{1}$, Q. Jin ${ }^{1}$, H. Qin ${ }^{1}$, H. Zhuang ${ }^{3}$, L. Wei

${ }^{1}$ Peking University People's Hospital, Peking University Hepatology Institute, Beijing; ${ }^{2}$ Guan County Center for Disease Control and Prevention, Hebei; ${ }^{3}$ Department of Microbiology, Peking University Health Science Center, Beijing, China

Background and aims: Commercial plasma donation was introduced in China in the 1970s. Cases of non-A, non-B hepatitis (hepatitis C) continued to occur and multiple outbreaks among plasma donors in Guan Country, Hebei Province between 1972 and 1990. The outcomes of hepatitis $\mathrm{C}$ virus (HCV) infection in these paid plasma donors from six villages of Guan Country were followed up for 12-19 years.

Methods: A total of 402 plasma donors with $\mathrm{HCV}$ infection were enrolled since screening by anti-HCV-positive in 1991 or 1998. Follow-up was maintained until death, or the end of the observation period (May 2010). No antiviral treatment was applied to them during the period of infection.

Results: Follow up was lost in 23 cases. After 12-19-year follow-up, 31 died, with death directly related to liver disease in 15 cases, with an overall mortality of $8.18 \%$. The incidence of liver cirrhosis was $10.03 \%$ and hepatocellular carcinoma (HCC) was 2.90\%. In May, 2010 , detections were performed in 348 cases of this cohort. The rate of viral spontaneous clearing was $21.29 \%$ (76/348), $13.84 \%$ in male and $27.27 \%$ in female. Abnormity of liver function was related to $\mathrm{HCV}$ viraemia. Gender and alcohol intakes impacted the outcome of $\mathrm{HCV}$ infection. There was no correlation between the viral spontaneous clearance with age of infection and genotype.

Conclusions: This area has a high rate of chronicity in HCV infection due to plasma donation. Twenty-five years after virus infection, liver cirrhosis or HCC has developed in a tenth of patients, with an overall mortality of $8.18 \%$.

This work was supported by National S\&T Major Project for Infectious Diseases Control (2008ZX10002-013).

\section{PP14-07}

IL28B Genetic Variants Are Associated with Spontaneous Clearance of Hepatitis C Virus Infection in Chinese Paid Plasma Donors

H.Y. Rao ${ }^{1}$, D.G. Sun ${ }^{2}$, D. Jiang ${ }^{1}$, R.F. Yang ${ }^{1}$, F. Guo ${ }^{1}$, J.H. Wang ${ }^{1}$, F. Liu ${ }^{1}$, H.Y. Zhang ${ }^{1}$, H.H. Zhang ${ }^{1}$, S.C. Du ${ }^{1}$, Q. Jin ${ }^{1}$, H. Qin ${ }^{1}$, L. Wei ${ }^{1}$

${ }^{1}$ Peking University People's Hospital, Peking University Hepatology Institute, Beijing; ${ }^{2}$ Guan County Center for Disease Control and Prevention, Heibei, China

Background and aims: Single nucleotide-polymorphisms (SNPs) near the IL28B gene have been associated with response to treatment of chronic hepatitis $\mathrm{C}$ and also with spontaneous clearance of acute hepatitis C. We analyzed the association between IL28B genetic variants and spontaneous clearance of hepatitis $\mathrm{C}$ virus (HCV) infection in 376 Chinese paid plasma donors.

Methods: Genotyping was performed by the iPLEX system (Sequenom, MassARRAY ${ }^{\circledR}$ SNP Genotyping) in 376 donors, and sequencing assays were used to validate the genotyping in 240 donors-all 80 donors who cleared HCV and 160 of 296 donors who did not cleared HCV.

Results: Eighty (21.2\%) patients spontaneously cleared HCV. Spontaneous HCV clearance was more common in patients with the rs8099917 TT, rs8105790 TT, rs12980275 AA, and rs10853728 CC genotypes compared to rs8099917 GT, rs8105790 CT, rs12980275 $\mathrm{AG}$, and $\mathrm{rs} 10853728 \mathrm{CG}$ or $\mathrm{GG}(\mathrm{OR}=15.27,14.88,7.92,2.32$, respectively, $P<0.05)$. For the same IL28B SNPs, such as rs8099917 TT, rs8105790 TT, and rs12980275 AA, spontaneous viral clearance was more likely to occur in women than in men $(\mathrm{OR}=2.06,2.05$, 2.21 , respectively, $P<0.05$ ). No association between rs12979860 CC genotype with spontaneous HCV clearance was found. There was no association between IL28B SNPs and HCV genotype.

Conclusion: Our results suggest that IL28B rs8099917, rs8105790, rs12980275, and rs10853728 polymorphism but not rs12979860 polymorphism is associated with spontaneous HCV clearance in Chinese subjects. Ongoing studies will determine whether these same genetic variants are associated with response to interferon treatment for chronic hepatitis $\mathrm{C}$.

This work was supported by National S\&T Major Project for Infectious Diseases Control (2008ZX10002-013).

\section{PP14-08}

Methylation Profile of HCC-candidate Genes in Huh-7 Cells Expressing the Hepatitis C Virus Core Protein

M. Ripoli ${ }^{1}$, R. Barbano ${ }^{2}$, P. Parrella ${ }^{2}$, V. Brunetti ${ }^{3}$, G. Mazzoccoli ${ }^{4}$, V. Fazio $^{2}$, A. Andriulli ${ }^{1}$, M. Vinciguerra ${ }^{5}$, V. Pazienza ${ }^{1}$

${ }^{1}$ Gastroenterology Unit; ${ }^{2}$ Oncology Research Laboratory, Casa Sollievo della Sofferenza Hospital, San Giovanni Rotondo; ${ }^{3}$ Italian Institute of Technology, Center for Bio-Molecular Nanotechnology, Lecce; ${ }^{4}$ Internal Medicine, Casa Sollievo della Sofferenza Hospital, San Giovanni Rotondo; ${ }^{5}$ Mouse Biology Unit, European Molecular Biology Laboratory (EMBL), Monterodondo (Rome), Italy

Background/aim: The mechanisms of hepatocarcinogenesis induced by Hepatitis $\mathrm{C}$ virus remain unclear. Our aim was to investigate the effect of the $\mathrm{HCV}$ core protein on the methylation status of selected genes potentially involved in the Hepatocellular carcinoma (HCC).

Materials/methods: We evaluated the methylation levels of the ECadherin (E-CDH1), the glutathione S-transferase p1 gene (GSTP1), adenomatosis polyposis coli (APC), tissue inhibitor of metalloproteinase 3 (TIMP3), catenin (cadherin-associated protein) beta 1 (CNNTB1) genes by a quantitative methylation-specific polymerase chain reaction (QMSP) in an in vitro model of Huh-7 cells expressing the HCV core protein of genotype $1 b$.

Results: We found that E-CDH1 promoter was hypermethylated in genotype $1 \mathrm{~b} \mathrm{HCV}$ core protein-positive cells as compared to control cells expressing the GFP protein alone (HCV core $1 \mathrm{~b}$ vs. GFP $p=0.00$, $\mathrm{HCV}$ core $1 \mathrm{~b}$ vs. Huh- $7 p=0.03$ ). This resulted in reduced levels of $\mathrm{E}-$ $\mathrm{CDH} 1$ protein as evaluated by immunoblot and by Immunofluorescence. On the other hand no significant changes were observed for the other genes investigated. Furthermore we present evidence that genotype $1 \mathrm{~b} \mathrm{HCV}$ core protein expression induces SIRT1 overexpression and that treatment with SIRT1 inhibitor decreases the methylation levels of E-CDH1 $(1 \mathrm{~b}+$ Sirtinol vs. $1 \mathrm{~b} \quad p=0.05 ; 1 \mathrm{~b}+$ Sirtinol vs. GFP + Sirtinol $p=$ NS) resulting in 1.7 fold increased E-CDH1 mRNA expression $(1 \mathrm{~b}+$ Sirtinol vs. $1 \mathrm{~b} p=0.05)$.

Conclusions: Our findings suggest that $\mathrm{HCV}$ core protein could play a role in $\mathrm{HCC}$ at least in part by altering the methylation status of 
CDH1 protein. These findings could also suggest a novel therapeutic approach for HCC.

\section{PP14-09}

To Study the Endoscopic Pattern of Portal Hypertensive Changes and their Correlation with Liver Histology in Chronic Hepatitis C Patients A. Naeem, M. Umar, M. Ahmed, Z. Minhas

Gastroenterology and Hepatology Division, Holy Family Hospital, Rawalpindi, Pakistan

Aims and objectives: To determine the frequency and pattern of portal hypertensive changes and its correlation with to degree of inflammation and fibrosis on liver biopsy in chronic hepatitis $\mathrm{C}$ patients.

Material and methods: 210 cases of chronic hepatic $\mathrm{C}$ were included as per protocol. All patients had liver biopsy and endoscopy of Gastroenterology and Hepatology division, Holy Family Hospital, Rawalpindi. Study duration was May 2008 to Feb 2010. Data was recorded on designed perform. SPSS 16 was used to analyze the data. Results: Mean age was $35.72+8.405$ years. $43.33 \%$ were male and $56.67 \%$ female. $42.38 \%(n=89), 47.14 \%(n=99)$ and $8.57 \%$ $(n=18)$ patients were having minimal, mild, and moderate disease activity respectively on liver biopsy. According to the Knodell system, inflammation with grades less than $0-4,5-7,8-10,11-13$ were $46.2 \%(n=97), 33.8 \%(n=71), 17.6 \%(n=37)$ and $14 \%(n=3)$ respectively. Staging showed $68.1 \%(n=143)$ having no fibrosis, $20 \%(n=42)$ having portal fibrosis, $4.3 \%(n=9)$ having periportal fibrosis and $4.8 \%(n=10)$ patients were having bridging fibrosis and only $2.9 \%(n=6)$ had cirrhosis. Minimal to mild disease activity was present in $89.52 \%(n=188)$ of the cases on liver biopsy [42.38\% $(n=89)$ minimal and $47.14 \%(n=99)$ mild disease activity]

Considering disease activity in patients with portal hypertension $41.2 \%(n=7)$ and $58.8 \%(n=10)$ cases were having minimal and mild disease activity respectively while correlation of disease activity with endoscopic portal hypertensive changes was not statistical significant $(P=0.442)$.

Endoscopy data revealed that $91.83 \%(n=193)$ cases were having no signs of portal hypertension and $8.17 \%(n=17)$ patients were having portal hypertension. F1, F2 and portal hypertensive gastropathy was present in $4.3 \%(n=9), 1.9 \%(n=4)$ and $1.9 \%(n=4)$ cases, respectively. The correlation between the grade and stage of liver biopsy with the endoscopic findings at $95 \%$ Confidence Interval was $P=0.879$ and $P=0.12$ respectively that is beyond the significance level

Conclusion: This study concluded that majority of chronic hepatitis $\mathrm{C}$ patients don't have portal hypertensive changes and there is poor correlation between the liver biopsy and endoscopic findings in fully compensated HCV infection.

\section{PP14-10}

Hepatitis C Virus Nonstructural Protein 2 Protein Activates NS2 Trans-regulated Protein

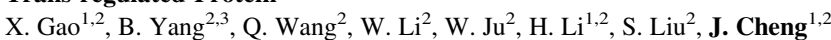

${ }^{1}$ Beijing Ditan Hospital, Peking University Health Science Center;

${ }^{2}$ Beijing Ditan Hospital, Capital Medical University, Beijing;

${ }^{3}$ Department of Microbiology, Dalian Medical University, Dalin, China

Background and aim: The hepatitis $\mathrm{C}$ virus (HCV) nonstructural protein 2 (NS2) is a multifunctional protein with an essential but poorly understood role in human hepatocytes. The aim of this study is to evaluate the biological function of NS2 in HCV pathogenesis.

Methods: Suppression subtractive hybridization ( $\mathrm{SSH}$ ) was used for screening differently expressed genes in HepG2 cells cDNA library. The expression plasmid of NS2 was then transient transfected into HepG2 cells. NS2 trans-regulated protein (NS2TP) mRNA and protein expression were detected by real-time PCR and western blot, respectively. The promoter of NS2TP was cloned into the luciferase reporter plasmid pGL4.10. The effect of NS2 on NS2TP transcription was tested by luciferase assay. The gel electrophoresis mobility shift assay (EMSA) and chromatin immunoprecipitation assay (CHIP) were used to identify the transcriptional factor that specifically interact with the NS2TP promoter.

Results: NS2TP was identified in SSH. NS2TP was named alternatively coiled-coil-helix-coiled- coil-helix domain containing 2 (CHCHD2). We confirmed that NS2 can up-regulate NS2TP expression by real-time PCR and western blot. Further studies showed that NS2 increased the transcription of NS2TP as measured by promoter activities. A minimal promoter contained within $350 \mathrm{bp}$ (nucleotides $-257 \mathrm{bp}$ to $+93 \mathrm{bp}$ ) was determined. Mutagenesis assays and overexpression revealed that CREB positively regulated the NS2TP promoter.

Conclusion: These results indicate that HCV NS2 can up-regulate NS2TP expression by increasing transcription. CREB played an important role in NS2TP transcription activation by HCV NS2.

\section{PP14-11}

Screening and Cloning of the Target Genes Transactivated by HCVFTP1 Protein Using Suppression Subtractive Hybridization Technique J.-L. Chen, J. Guo, J. Cheng Beijing Ditan Hospital, Capital Medical University, Beijing, China

Background: To clone and identify human genes transactivated by homo sapiens HCVFTP1 by constructing a cDNA subtractive library with suppression subtractive hybridization technique.

Methods: Suppression subtractive hybridization ( $\mathrm{SSH}$ ) and bioinformatics techniques were used for screening and cloning of the target genes transactivated by HCVFTP1. The mRNA was isolated from HepG2 cells transfected pcDNA3.1(-)-HCVFTP1 and pcDNA3.1(-) empty vector respectively, and SSH method was employed to analyze the differentially expressed DNA sequence between the two groups. After restriction enzyme Rsa I digestion, small sizes cDNAs were obtained. Then tester cDNA was divided into two groups and ligated to the specific adaptor 1 and adaptor 2, respectively. After tester cDNA was hybridized with driver cDNA twice and underwent two times of nested PCR and then was subcloned into T/A plasmid vectors to set up the subtractive library. Amplification of the library was carried out with $E$. coli strain DH5 $\alpha$. The cDNA was sequenced and analyzed in GenBank with Blast search after PCR.

Results: The subtractive library of genes transactivated by HCVFTP1 was constructed successfully. The amplified library contains 71 positive clones. Colony PCR shows that 56 clones contain $200-1,000$ bp inserts. Sequence analysis was performed in 24 clones randomly,and the full length sequences were obtained with bioinformatics method. Altogether 20 coding sequences were gotten,which consisted of 19 known and 1 unknown.

Conclusions: The obtained sequences may be target genes transactivated by HCVFTP1, and some genes coding proteins involved in cell cycle regulation, metabolism, and cell apoptosis.

\section{PP14-12}

Screening and Identification of Genes Trans-regulated by HCV F Protein with Microarray Assay

J. Guo, J. Cheng

Beijing Ditan Hospital, Capital Medical University, Beijing, China

Background: To investigate the biological functions of hepatitis $\mathrm{C}$ virus $\mathrm{F}$ and to use cDNA microarray technique to screen genes regulated by $\mathrm{F}$.

Methods: The HCV F coding DNA fragment was amplified with polymerase chain reaction (PCR) technique by using PBRTM plasmid containing the full length of $\mathrm{HCV}$ genome as the template. The expressive vector of pcDNA3.1-F was constructed by routine 
molecular biological methods. The HepG2 cells were transfected by pcDNA3.1(-) and pcDNA3.1-F, respectively using FuGENE6 transfection Reagent. The total RNA was isolated and reverse transcribed. The cDNAs were subjected for microarray screening with 1,152 cDNA probes.

Results: The expressive vector has been constructed and confirmed by restriction enzyme digestion and DNA sequencing analysis. High quality mRNA and cDNA had been prepared and successful microarray screening had been conducted. From the scanning results, it was found 1 genes were up-regulated and 22 genes were down-regulated by $\mathrm{F}$ protein of $\mathrm{HCV}$.

Conclusions: HCV F protein is a trans-regulator. The expression of $\mathrm{F}$ protein affected the expression spectrum of $\mathrm{HCV}$ infected hepatocyte.

\section{PP14-13}

Molecular Epidemiological Study of Hepatitis B Virus in Iran, the Unique Pattern of Genotypic Homogeneity; Results of a Nationwide Study H. Karimzadeh ${ }^{1}$, M. Norouzi ${ }^{2}$, S. Alavian ${ }^{3}$, R. Malekzadeh ${ }^{4}$, G. Montazeri ${ }^{4}$, A. Nejatizadeh ${ }^{5}$, M. Ziai ${ }^{5}$, F. Abedi ${ }^{5}$, B. Ataei ${ }^{5}$, B. Sayyad ${ }^{5}$, M. Somi ${ }^{5}$, G. Sarizade ${ }^{5}$, E. Sanei ${ }^{5}$, F. Mansoir-Ghanai ${ }^{6}$, F. Rafatpanah ${ }^{5}$, M. Judaki ${ }^{5}$, S. Ghamari ${ }^{5}$, A. Khedive ${ }^{5}$, E. Domingo ${ }^{7}$, S. Jazayeri ${ }^{2}$

${ }^{1}$ Baqiyatallah University of Medical Sciences, Baqiyatallah Research Centre for; ${ }^{2}$ Hepatitis B Molecular Laboratory-Department of Virology, School of Public Health, Tehran University of Medical Sciences; ${ }^{3}$ Baqiyatallah Research Center for Gastroenterology and Liver Disease; ${ }^{4}$ Digestive Disease Research Center, Shariati Hospital; ${ }^{5}$ Iran Hepatitis Network, Tehran; ${ }^{6}$ Iran Hepatitis Network, Giulan, Iran; ${ }^{7}$ Centro de Biología Molecular-Severo Ochoa-(CSICUAM), Universidad Autónoma de Madrid, Cantoblanco, Madrid, Spain

Aims: The aim of this study was to characterize the hepatitis B virus surface protein genotypes and sequence variations among $\mathrm{HBsAg}$ positive chronic patients in different ethnic groups in Iran.

Methods: 330 patients enrolled in this study. The surface gene was amplified and directly sequenced. Genotypes and nucleotide/amino acid substitutions were identified compared to the sequences obtained from the database.

Results: All strains belonged to genotype D, subtype ayw2. Subgenotypes D1, D2 and D3 accounted for $98.2,1.2$ and $0.6 \%$, respectively. Of a total, 856 "mutations" occurred at 382 nucleotide positions, 464 (54\%) were silent (no amino acid altering) and 392 (46\%) were missense (amino acid changing). At the amino acid level, 385 mutations were found, 302 (78.4\%) occurred within known immune epitopes of HBsAg. The average mutation frequency (the ratio between silent/ missense) was 1.18 , indicating a negative selection.

Conclusion: The similarity in amino acid distribution showed that considerable constraints must exist against $\mathrm{HBV}$ variability in a particular genotype infecting a person of a particular background and the lack of a positive selection advantage or a strong bias towards maintaining that amino acid. The uniqueness pattern of HBV genetics hemogeniety together with the low mutational frequency indicated that HBV was introduced into Iran recently and isolation of people in the absence of intermixing with other genotypes let to a homologous pattern.

\section{PP14-14}

Enhanced Expression of Divalent Metal Transporter 1 (DMT1) in the Duodenum of Hepatitis C Virus (HCV) Infected Patients

C.M. El Younis, Y. Zhang

Medicine - Gastroenterology, Brookdale University Hospital, Brooklyn, NY, USA

Introduction: Studies documented increased hepatic iron deposition in some HCV infected patients, and some implicated this histologic changes in the progression of liver disease or nonresponse to therapy. The mechanism remains elusive. DMT1 and FP1 in duodenal mucosa may play an important role in the pathogenesis of $\mathrm{HCV}$-associated iron overload.

Aim: Assess the duodenal expression of DMT1 and FP1in patients with hepatitis $\mathrm{C}$ infection.

Methods: 36 speciemens (24 HCV infected patients and 18 Non$\mathrm{HCV}$ infected). All underwent gastroscopy for other gastrointestinal pathology and had biopsies taken from the second part of duodenum. Hematologic parameters and serum iron indices measured. Biopsies were snap frozen in liquid nitrogen immediately and stored at $-70^{\circ} \mathrm{C}$ for total RNA extraction at a later date. Total RNA were extracted from two pooled duodenal biopsy specimens from each subject using TRIzol reagent. Real Time PCR were performed on total RNA extracted from specimens. For quantification of DMT1 complementary DNA (cDNA), the following primers were used: $5^{\prime}$ GTG GTC AGC GTG GCT TAT CTG-3', 5'-GAT GCT TAC CGT ATG CCC ACA GT-3'; the TaqMan probe $5^{\prime}$ TGT TCT ACT TGG GTT GGC AAT GTT TGA TTG C- $3^{\prime}$ were labeled with the reporter fluorescent dye 6-carboxyfluorescein (FAM) at the $5^{\prime \prime}$ end and with the quencher 6-carboxytetramethyl-rhodamine (TAMRA) at the $3^{\prime}$ end. For quantification of FP1, the primers 5'TGA CCA GGG CGG GAG A-3'. 5' GAG GTC AGG TAG TCG GCC AA- $3^{\prime}$ and the FAM/TAMRAlabeled TaqMan probe $5^{\prime}$-CAC AAC CGC CAG AGA GGA TGC TGTG- $3^{\prime}$ were used. GAPDH-sense: $5^{\prime}$-CAA GGC GCC CAA TAC G-3'; GAPDH antisense: 5'-CCA GGC GCC CAA TAC G-3'; GAPDH-probe (FAM) 5'-AAG GTG AAG GTC GGA GTC AAC GGA TTT G-3' (TAMRA) were used internal control.

Results: HCV patients have a significantly higher DMT1 Gene expression than the Non-HCV patients 1.075 versus 0.507 fold, $\mathrm{SD} \pm 0.488,(p<0.0001)$

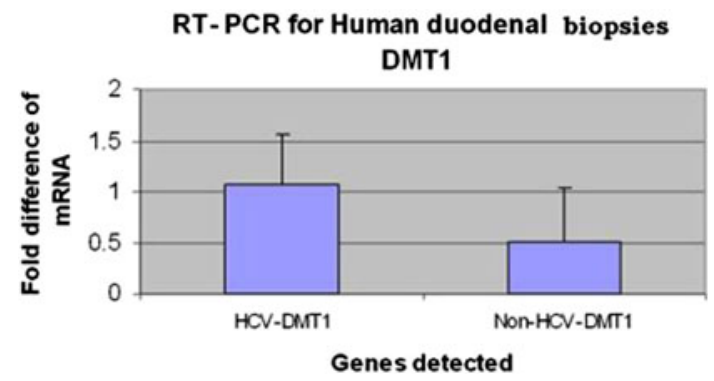

PP14-15

Serum Alpha-fetoprotein Levels in Egyptian Patients with Chronic Hepatitis C with or without Steatosis

N.H. Mousa ${ }^{1}$, Y.Z. $\mathrm{Gad}^{2}$, A.A. Soliman ${ }^{3}$, E.A. Abdelal ${ }^{4}$, M.A. Hamed ${ }^{2}$

${ }^{I}$ Tropical Medicine; ${ }^{2}$ Internal Medicine; ${ }^{3}$ Pathology;

${ }^{4}$ Clinicalpathology, Mansoura - Egypt, Mansoura, Egypt

Background/aims: Hepatic steatosis is a frequent histological finding in chronic hepatitis $\mathrm{C}(\mathrm{CHC})$ infection. Both host and viral factors contribute to steatogenesis. The prognostic importance of $\alpha$-fetoprotein (AFP) level elevation in patients with chronic hepatitis $\mathrm{C}$ (CHC), and its clinical significance in steatosis associated with $\mathrm{HCV}$ infection remain to be determined. The present study assessed the risk factors for, and the clinical significance of elevated AFP in patients with $\mathrm{CHC}$ with and without steatosis.

Methods: This study includes one hundred patients with CHC. Liver biopsy was done to all patients and they were divided into 50 patients with $\mathrm{CHC}$ and steatosis and 50 patients with $\mathrm{CHC}$ and no steatosis based on liver biopsy.

Results: AFP was significantly increased in $\mathrm{CHC}$ with steatosis than patients without steatosis $(p<0.0001)$. Highly significant positive correlation was found between serum AFP and necroinflammation as well as the severity of fibrosis/cirrhosis and negative significant correlation with albumin level in chronic HCV with steatosis $(p<0.0001)$, but negative non significant correlation with ALT and 
AST level ( $p \leq 0.778$ and 0.398 ), respectively. Highly significant increase were found in chronic hepatitis patients with steatosis than $\mathrm{CHC}$ without steatosis regarding necroinflammation as well as the severity of fibrosis/cirrhosis and AFP $(p<0.0001)$, glucose $(p<0.044)$, AST $(p<0.0001)$, ALT $(p<0.002)$, total bilirubin $(p<0.023)$, Prothrombin time $(p<0.003)$. No significant difference as regarding triglyceride $(p=0.323)$, albumin $(p=0.115)$, BMI $(p \leq 695)$ and HCV quantitative PCR $(p=302)$ were found between $\mathrm{CHC}$ with steatosis and without steatosis.

\section{PP14-16}

Hepatic miRNA Signature Predicting Sustained Virological Response in Chronic Hepatitis C

E. Estrabaud ${ }^{1,2}$, I. Bièche ${ }^{3}$, M. Lapalus ${ }^{2,4}$, S. De Muynck ${ }^{2,4}$, O. Lada ${ }^{2,4}$, M. Martinot-Peignoux ${ }^{4}$, A. Duces ${ }^{3}$, D. Valla ${ }^{4}$, P. Bedossa ${ }^{5}$, P. Marcellin ${ }^{2,4}$, M. Vidaud ${ }^{3}$, T. Asselah ${ }^{2,4}$

${ }^{1}$ Service d'Hépatologie, Pôle des Maladies de l'Appareil Digestif, Beaujon Hospital, Clichy; ${ }^{2}$ CRB3/INSERM U773 Bichat Hospital, Paris; ${ }^{3}$ Service de Biochimie et Génétique Moléculaire; ${ }^{4}$ Service d'Hépatologie, Pôle des Maladies de l'Appareil Digestif; ${ }^{5}$ Service d'Anatomie Pathologique, Beaujon Hospital, Clichy, France

Introduction: The standard of care for the treatment of chronic hepatitis $\mathrm{C}$ consists in the combination of pegylated-interferon and ribavirin. Considering side effects and treatment cost, prediction of treatment response before therapy is important. Since the identification of RNA silencing, micro RNAs (miRNAs) have been implicated in the regulation of many cellular pathways. Viral infections have been correlated to a modification of cellular miRNAs expression. Moreover, among the different cellular miRNAs, HCV specifically recruits miR-122 to increase its replication, in vitro. The aim of this study was to identify a liver miRNA signature associated to sustained virological response (SVR) in patients with CHC.

Methods: Pre-treatment liver biopsies from 111 patients treated with pegylated interferon plus ribavirin were studied. Among these patients 57 had SVRs, 36 non response (NR) and 18 relapse (RR). Patients were mainly infected with genotype 1-4 and (68). Metavir Fibrosis score is known for all the patients (34 were F1, 40 F2, 18 F3 and 17 F4). Real-time quantitative RT-PCR assays were performed to analyse the expression of 851 human miRNAs.

Results: The cellular miRNAs expression profiles of SVR differ significantly from those of NRs. In particular, the miRNAs mir-99a, mir-181a-2*, mir-23a et mir-217 were significantly deregulated, in two independent groups of patients. Interestingly, expression of mir224 appears to be associated to fibrosis progression. The levels of expression of miRNAs involved in tumoral processes were higher in the liver of NRs in comparison with SVRs and RRs. Moreover several genes targeted by those miRNAs or involved in the same pathway, present different liver expression in NRs and SVRs.

Conclusion: NRs and SVRs present different miRNAs hepatic expression, before treatment. In particular, the miRNAs mir-99a, mir181a-2*, mir-23a et mir-217 were significantly deregulated in NRs compared to SVRs. Thus, treatment response could be predicted with a molecular miRNAs signature.

\section{PP14-17}

Detection of Acute HCV Infection among Different Risk Groups L. Gatserelia ${ }^{1,2,3}$, L. Sharvadze $e^{1,2,3}$, M. Karchava ${ }^{1,2,3}$, N. Badridze ${ }^{1,2}$, T. Tsertsvadze ${ }^{1,2,3}$

${ }^{1}$ Infectiouse Diseases, AIDS and Clinical Immunology Research Center; ${ }^{2}$ Faculty of Medicine, Iv. Javakhishvili Tbilisi State University; ${ }^{3}$ Georgian-French Joint Hepatology Clinic "HEPA", Tbilisi, Georgia

Aims: To investigate acute HCV infection from the very first days of the infection among blood donors, injecting drug users (IDUs), health care workers and persons who had first life time sexual intercourse with the chronic HCV infected persons in orders to asses clinical laboratory variants of infection, viral replication kinetic, disease outcome.

Methods: Study was performed among ELISA negative persons in three groups: 7,500 blood donors, 3,200 IDUs and 70 exposed health care workers. All groups were investigated on HCV RNA by Real Time PCR using pool method and genotyped by line probe assay according to the manufacturer's instructions. The results were analyzed by age, sex, duration of infection, modes of transmission and other variables.

Results: Total of 17 patients with acute HCV infection were identified: 7 from blood donors, 9 from IDUs and 1 from health care workers and. All HCV RNA positive persons revealed within the study were followed on viral load dynamic at detection moment and at 2, 4 and 8 weeks and after 3 and 6 months from their possible exposure. Among them: 5 were symptomatic and 12 asymptomatic. Among 17 subjects: 8 had genotype $-1 b ; 4,2 a / 2 c$ and 5 , 3a. Out of 17 patients 5 cleared the virus ( 4 were symptomatic: 1 , genotype $1 \mathrm{~b}$; 1 , genotype $2 \mathrm{a} / 2 \mathrm{c} ; 2$, genotype $3 \mathrm{a}$; and 1 asymptomatic: 1 , genotype $1 \mathrm{~b} ; 1$, genotype $3 \mathrm{a}$. While 12 developed chronic infection. In all patients viremia increased rapidly and reached a peak by week 4 . After week 10 the viremia rapidly decreased. It became either undetectable by weeks $16-18$, or virus was not eliminated.

Conclusions: We found that HCV viral clearance was correlated with high viral titers and symptomatic form of disease. Study revealed no correlations between viral genotype and viral clearance rates.

\section{PP14-18}

Impact Of HCV - Core Expression on Pathogenesis of HCV Infection

P. Nag, S. Singh, M.A. Ansari, A. Singh, M. Irshad

Laboratory Medicine, All India Institute of Medical Sciences, New Delhi, India

Background/aims: Present investigation describes the role of hepatitis $\mathrm{C}$ virus $(\mathrm{HCV})$ core protein expression, as indicated by the presence of $\mathrm{HCV}$ core protein in serum, in the diagnosis and pathogenesis of HCV infection.

Methods: A total number of 432 patients with various liver diseases including acute and chronic/end stage liver diseases were analysed for HCV-related markers lipid profile and oxidants/anti oxidants levels.

Results: Of them, HCV-core protein was found to be a better serum marker compared to anti- HCV and HCV-RNA for the diagnosis of $\mathrm{HCV}$-infection in chronic liver diseases. HCV-core expression shows a low prevalence in patients with acute infection. To explore the possibility of core-expression in liver pathology via its impact on oxidative stress and steatotic changes, we studied the sera level of lipids, lipoproteins, apoproteins and anti-oxidant levels in relation to $\mathrm{HCV}$-core. The analysis of data could not demonstrate any significant change in the level of these metabolites with presence or absence of $\mathrm{HCV}$-core protein. This was explained as HCV-core expression having no insignificant role in inducement of oxidative stress and development of steatosis to cause liver damage. This study also describes the varied prevalence of different $\mathrm{HCV}$-genotypes in relation to $\mathrm{HCV}$-core protein in these patients, thus, explaining that $\mathrm{HCV}$ genotypes do not have any relation or role in expression of HCV-core. Conclusion: We could not observe a significant relation between $\mathrm{HCV}$-core expression and liver steatosis, oxidative stress status or $\mathrm{HCV}$-genotypes in different types of liver diseases.

Acknowledgement: We thank ICMR for the financial grant to complete the study.

\section{PP14-19}

The Prevalence of Autoantibodies in Chronic Hepatitis C

C.A.C. Silva, V.S. Nunes, A.C.P. Oliveira, M. Rios, M.I. Schinoni, R. Paraná Federal University of Bahia, Salvador, Brazil

Introduction: The presence of auto-antibodies in the hepatitis $\mathrm{C}$ virus chronic infection (VHC) is frequent, but it is not associated with 
autoimmune disease. Most of the studies did not demonstrate the relation with viral genotype or severity of the hepatic. Patients who are positive for anti smooth muscle and ANA have more active hepatic disease and a bigger rate of anti-viral treatment fail. Moreover, hepato-cellular injury by itself exposes host epitopes probably implicated in the emergence of auto-antibodies in infected patients. Methods: Cross sectional study of patients with VHC in the Hepatology Unit in the University Hospital Professor Edgar Santos. Included patients $>18$ years, anti-HCV positive by ELISA, detection of serum HCV-RNA by qualitative and quantitative PCR and without coinfection or manifested autoimmune diseases. Chi-square test.

Results: 103 patients: 49 (47.6\%) females and 54 (52.4\%) males. 47.9 and $41.5 \%$ of the patients presented elevation of AST and ALT, respectively, higher than $1.5 \times$ the reference value $(n=94)$. From the 60 patients who had realized hepatic biopse, $41(68.3 \%)$ presented mild fibrosis (F0 to F2, according to METAVIR) and 19 (31.7\%), advanced fibrosis (F3/F4). Anti-TPO was positive in only one of 82 patients and Anti-Thireoglobulin was positive in $3(5 \%)$ of 60 . Anti$\beta 2$ Glicoprothein was studied in 60 patients, wich $10 \%$ were positive for IgA fration and 2 (3.3\%) for IgG. $2.98 \%$ of the patients had AntiCardiolipin IgA positive, but all were negative for IgM and IgG $(n=67)$. Anti-SSA and Anti-SSB $(n=28)$ and Anti-Mitochondria $(n=25)$ were negative in all patients. ANCA was positive in 2 $(6.7 \%)$ of 30 patients. Presence of autoantibodies was not associated with the degree of fibrosis or elevation of liver transaminasis.

Conclusion: Preliminar results revealed high prevalence of autoantibodies in patients with VHC. Further analysis are necessary to evaluate the impact of the autoantibodies in VHC.

\section{PP14-20}

Possible Pathogenesis of Autoimmune and Cryoglobuline-related Disease in HCV Infection

Y. Kondo, Y. Ueno, O. Kimura, K. Kobayashi, E. Kakazu, K. Fukushima, J. Inoue, Y. Wakui, T. Shimosegawa

Tohoku University Hospital, Sendai, Japan

Background: Several kinds of autoimmune diseases and immunoglobuline-related diseases are associated with $\mathrm{HCV}$ infection. Replication of $\mathrm{HCV}$ in B cells induced the immunoglobuline hypermutation and IL6 secretion (JV2008). Moreover, HCV-replication in $\mathrm{T}$ cells affected Th commitment and T cell development (JG2010, JID2009, Virology2007).

Aim: The aim of this study is to analyze the possible pathogenesis of autoimmune diseases by using a lymphotropic HCV strain and patients' samples.

Methods: Serum from 40 selected $\mathrm{CHC}$ patients and other liver disease (CHB and NASH and Healthy) were used for IL6, TGF- $\beta$, IL21 and IL17 quantification by ELISA. The SB strain was used for infection of several kinds of primary lymphocyte subsets. The analysis of Th1, Th2 and Th17 commitment was carried out by detecting cytokines-secreting cells in several cytokine conditions. Transfection of HCV E1, E2, Core, NS3, NS4B, NS5A, NS5B expression plasmids was carried out to analyze the proteins that could affect Th development by nucleorfector. Moreover, HCV-core expressive Lenti-virus was infected into naive $\mathrm{T}$ cells to analyze the expression levels of T-bet, GATA-3, ROR $\gamma$ t mRNA after long-term culture.

Results: CHC patients with higher amounts TGF- $\beta$ and IL6 had higher rates of ANA and/or ASMA antibody-positive and higher amounts of IL17 and IL21 in comparison to those with lower amount of TGF- $\beta$ and IL6. Among CD4+ cells, naive CD4+ cells were most susceptible to replication of the SB strain. HCV-core and NS5A were responsible proteins in suppressing IFN $-\gamma$ signaling. On the other hand, IL-17 secreting CD4+ cells were significantly increased. HCVcore was the protein responsible for the enhanced Th17 commitment. Conclusion: Lymphotropic HCV replication affected the replication and development from naive $\mathrm{T}$ cells. $\mathrm{HCV}$ infection in naive $\mathrm{T}$ cells could enhance Th17 commitment. These results suggest that lymphotropic $\mathrm{HCV}$ strains might contribute to the induction of autoimmune diseases.

\section{PP14-21}

Marked Impact of IL28B Genotype in the Natural Clearance of HCV-RNA Clerance in Patients with Hemoglobinopathies M.C. renda ${ }^{1}$, S. Madonia ${ }^{2}$, R.F. Ruggeri ${ }^{2}$, G. Perricone ${ }^{2}$, A. Piazza ${ }^{1}$, D. Renda ${ }^{1}$, E. Sinagra ${ }^{2}$, G. Restivo ${ }^{1}$, S. Plano ${ }^{2}$, M. Cottone ${ }^{2}$, A. Maggio ${ }^{1}$ ${ }^{1}$ UOC Ematologia II, Ospedali Riuniti “Villa Sofia-Cervello"; ${ }^{2}$ UOC Medicina II, Ospedali Riuniti "Villa Sofia-Cervello", Palermo, Italy

Aim: Patients with transfusion-dependent hemoglobinopathies, have an high risk of hepatitis $\mathrm{C}$ virus (HCV) infection, being chronic hepatitis $\mathrm{C}$ the major cause of liver disease. Genome-wide association studies (GWAS) identified a single nucleotide polymorphism (SNP) on chromosome 19q13, 3 kilobases $(\mathrm{kb})$ upstream IL28B gene $(-3 \mathrm{kbC}>\mathrm{T})$. This SNP is associated to HCV-RNA spontaneous clearance and it is in linkage disequilibrium with a variant in the IL28B exon2 (K70RA > G). The 2 DNA variants were analyzed in a cohort of thalassemia-major and sieckle/ $\beta$-thalassemia patients to evaluate allele frequencies and correlation with HCV clearance.

Patients and methods: Forty-two treatment-naive patients, were included in this study. Genomic DNAs were extracted from peripheral blood. IL28B $-3 \mathrm{kbC}>\mathrm{T}$ and $\mathrm{K} 70 \mathrm{R}$ variants were investigated by direct genomic sequencing, endonuclease digestion and reverse dotblot hybridization. PCR primers were home-made on the basis of IL28B map (GENATLAS) and FASTA-SNP sequences.

Results: Allele frequencies of $-3 \mathrm{kbC}>\mathrm{T}$ polymorphism were $63.1 \%$ for the favourable $\mathrm{C}$ allele and $36.9 \%$ for the $\mathrm{T}$ allele. Allele frequencies for the K70R variant were $63.1 \%$ for the A allele and $36.9 \%$ for the $\mathrm{G}$ allele. Twenty of 42 patients $(47.6 \%)$ showed a spontaneous HCV-RNA clearance: 15/20 (75\%) had an IL28B genotype $-3 \mathrm{kbCC} / \mathrm{K} 70 \mathrm{RAA}, 5 / 20(25 \%)$ had an IL28B genotype $-3 \mathrm{kbCT} / \mathrm{K} 70 \mathrm{RAG}$. Twenty-two of 42 (52.4\%) showed an HCV-RNA persistence: 13/22 (59.1\%) showed an IL28B genotype -3kbCT/ K70RAG; $4 / 22(18.2 \%)$ showed an IL28B genotype $-3 \mathrm{kbCC} /$ K70RAA; 4/22 (18.2\%) showed an IL28B genotype -3kbTT/ K70RGG and 1 was homozygous for $-3 \mathrm{kbC}$ allele but was heterozygous for the K70R mutation.

Conclusions: It seems that L28B genotype has a marked impact on natural HCV-RNA clearance: $15 / 20(75 \%)$ patients with spontaneous HCV-RNA clearance had an IL28B genotype promoting the viral defence (IL28B-3kbCC/K70RAA). These data are in agreement with Thomas et al (Nature 461,2009) that showed a -3kbCC genotype in the $60 \%$ of patients who clear HCV.

\section{PP14-22}

Association of Human Leukocyte Antigen Class I (HLA-A, and HLA-B) with Chronic Hepatitis C Virus Infection in Egyptian Patients Y.M. Mosaad ${ }^{1}$, R.E.-S. Farag ${ }^{2}$, M. Arafa ${ }^{3}$, S. Eletreby ${ }^{4}$, H. Elalfy ${ }^{3}$, B.S. El-Deek ${ }^{5}$, Z. Tawhid ${ }^{6}$

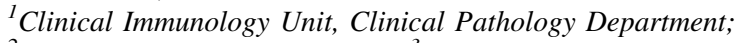

${ }^{2}$ Tropical Medicine Department; ${ }^{3}$ Tropical Medicine;

${ }^{4}$ Gastroentrology Unit, Internal Medicine Department; ${ }^{5}$ Community Medicine and Statistics Department; ${ }^{6}$ Clinical Immunology Unit,

Clinical, Mansoura University, Mansoura, Egypt

Objective: To investigate the association between the frequencies of Human leukocyte antigen (HLA)-Class I (HLA-A, and HLA-B) and chronic hepatitis $C$ virus infection in Egyptian patients and to find out whether there is a relation between certain HLA-Class I antigens and HCV viral load, Degree of fibrosis, activity and ALT level.

Subject and Methods: A case control study was conducted on 100 chronic HCV patients and 150 healthy controls. HLA-A, and HLA-B typing by complement-dependent micro-lympho-cytotoxicity assay was done for both groups. 
Result: HLA-A11 antigen was significantly increased in chronic $\mathrm{HCV}$ patients versus controls (OR 3.98; 95\% CI $=1.85-8.89$; $p=0.001 ;$ and $\mathrm{pc}=0.021)$. HLA-B12, HLA-B13, HLA-B17, and HLA-B40 were higher in patients and HLA-A32, HLA-B14 were higher in controls. However, the significance was lost after correction for multiple testing and no other HLA-Class I antigens were associated with chronic HCV infection. HLA-A9 was significantly associated with low viral load $(p=0.008, \mathrm{pc}=0.048)$.

Conclusion: The results of the present work implicate that HLA-A11 antigen may influence chronic HCV infection and may play a role in viral persistence. Different HLA-Class I antigens are not associated with degree of liver fibrosis, grades of activity, level of ALT, or HCV viral load. However, HLA-A9 is associated with low HCV viral load in chronic HCV Egyptian patients.

\section{PP14-23}

Interferon Alpha Stimulate Gene Tetherin/BST2 Inhibits Hepatitis C Virus Release

X.-B. Pan ${ }^{1,2}$, L. Wei ${ }^{1}$, J.-T. Guo ${ }^{2}$, T.M. Block ${ }^{2}$

${ }^{1}$ Peking University People's Hospital, Peking University Hepatology Institute, Beijing, China; ${ }^{2}$ Drexel Institute for Biotechnology and

Virology Research, Doylestown, PA, USA

HCV chronically infects more than 170 million people worldwide. IFN $-\alpha$ and ribavirin combination therapy is the current standard care for chronic hepatitis C. It is well known that IFN- $\alpha$ induces more than 900 IFN-stimulated genes (ISGs) in the cells. However, the ISGs that mediate the antiviral effect of the cytokine against HCV remain to be identified. In this report, we found that individual expression of an IFN-induced cell membrane protein BST2 (also called tetherin) greatly inhibits $\mathrm{HCV}$ infection in a human hepatoma-derived cell line Huh7.5 at a MOI of $0.05 \mathrm{HCV}$ infection. While these BST2 expressing Huh7.5 cells were infected with HCV at a MOI of 3 , all the cells could be infected with $\mathrm{HCV}$, the intracellular levels of HCV NS3 protein, HCV RNA and virus titer were similar as that of the parent Huh7.5 cells, whereas the supernatant $\mathrm{HCV}$ titer decreased by about 15 folds. While the cells were seeded at high cell density and cultured with $0.5 \%$ agars DMEM containing of CD81 antibody, which block free virus infection but not cell to cell transmission. The foci size of HCV infected cells were also greatly inhibited by about 10 folds in BST2 expressing Huh7.5 cells. SMARTpool siRNA targeting the mRNA of BST2 efficiently attenuated the antiviral activity of IFN- $\alpha$ against $\mathrm{HCV}$. In conclusion, our study showed that BST2 inhibits HCV release in Huh7.5 cells and also functions to limit HCV cell to cell transmission. BST2 plays an important role in interferon mediated anti HCV activity.

\section{PP14-24}

Genotype Distribution of Hepatitis C in the Kocaeli City, Turkey M. Sayan ${ }^{1}$, S. Akhan ${ }^{2}$, O. Senturk ${ }^{3}$

${ }^{1}$ PCR Unit; ${ }^{2}$ Infectious Diseases; ${ }^{3}$ Gastroenterology, Kocaeli University, Kocaeli, Turkey

Background: Hepatitis $\mathrm{C}$ virus (HCV) genotype has an important prognostic value that guides the duration and success of treatment.

Aims: To analyze the distribution of genotypes in HCV-positive patients and linking them with the viral load before and after treatment, evaluating sustained viral response.

Patients and methods: We retrospectively analyzed the results of genotyping and HCV viral load of 352 patients during the period 2004 to 2010. Serum HCV RNA levels were quantified by using the COBAS AMPLICOR HCV Monitor 2.0 and the AmpliPrep/COBAS TaqMan (Roche Diagnostics GmbH, Mannheim, Germany). The HCV strain was determined by RT-PCR amplification of the genome followed by sequencing for genotyping of the strain. Genotyping was carried out by using freshly isolated RNA. The RNA was reverse transcribed with random primers and genotyping was done by using type-specific primers based on core and NS5 regions.

Results: The median age of the patients was $50 \%$ of patients were women. The frequency of genotypes were: $12 \%$ type $1 \mathrm{a}, 76 \%$ type $1 \mathrm{~b}$, $4 \%$ type $1 \mathrm{c}, 1 \% 1 \mathrm{a} / 1 \mathrm{~b}, 1 \%$ type $1 \mathrm{~b} / 1 \mathrm{c}, 3 \%$ type $2 \mathrm{a}, 1 \%$ type $2 \mathrm{~b}, 1 \%$ type $3 a, 2 \%$ type $4 a$ and $0,2 \%$ type $5 a$. The patients with genotype 1 had a higher number of geometric mean titer (GMT) than those of other genotypes.

Table 1 The geometric mean titer of HCV RNA according to the $\mathrm{HCV}$ genotype

\begin{tabular}{ll}
\hline Genotype & Mean HCV RNA \\
\hline $1 \mathrm{a}$ & $5719134 \mathrm{IU} / \mathrm{ml}$ \\
$\mathrm{lb}$ & $5205875 \mathrm{IU} / \mathrm{ml}$ \\
$1 \mathrm{c}$ & $2087022 \mathrm{IU} / \mathrm{ml}$ \\
$1 \mathrm{a} / \mathrm{lb}$ & $1550966 \mathrm{IU} / \mathrm{ml}$ \\
$\mathrm{lb} / 1 \mathrm{c}$ & $180350 \mathrm{IU} / \mathrm{ml}$ \\
$3 \mathrm{a}$ & $2671750 \mathrm{IU} / \mathrm{ml}$ \\
$4 \mathrm{a}$ & $1347350 \mathrm{IU} / \mathrm{ml}$ \\
\hline
\end{tabular}

Conclusions: The genotype $1 \mathrm{~b}$ was the most prevalent type in our population. Genotype 1 is the most difficult treatable one.

\section{PP14-25}

NS3 Sequences of HCV Subtype 1a from Brazilian Patients Form A Distinct Group within Clade 1

E. Lampe, A.J. De-Almeida, A. Peres-da-Silva

Oswaldo Cruz Institute, Oswaldo Cruz Foundation, Rio de Janeiro, Brazil

The NS3 region has been one of the molecular targets to new directly acting antivirals (DAAs), which block specific steps in viral replication. However, the emergence of drug-resistant viruses may limit the efficacy of these antiviral agents. Based on phylogenetic analysis of subtype 1a full-length genome sequences, HCV isolates can be separated into two distinct clades. Several informative sites for this distinction are located proximal or within codons associated with resistance to NS3 inhibitors. The aim of this study was to analyze the genomic variability of Brasilian HCV NS3 sequences.

Methods: 48 samples from therapy-naïve Brazilian patients chronically infected with HCV subtype 1a were studied. The region encompassing the NS3 serine protease domain was sequenced and aligned with 240 sequences from other geographical regions. Phylogenetic analysis was performed by using the Neighbor-Joining algorithm of Mega 4.0 program.

Results: The phylogenetic tree reconstructions revealed that Brazilian sequences formed a distinct group clustering within the clade 1 . In Brazilian sequences, the majority of amino acid located at the position 40 was $\mathrm{T}$, differing substantially from those found in clade 1 which possesses the $\mathrm{A}$ as the majority amino acid. In addition, clade 1 presented at the position 80 the TMC-435 inhibitor drug-resistant amino acid $\mathrm{L}$ in $60 \%$ of its sequences in contrast to 93.8 and $97.5 \%$ of amino acid $\mathrm{G}$ found in Brazilian sequences and in clade 2, respectively. Mutations T54S associated with low to medium levels of resistance to the inhibitor telaprevir were identified in $4.1 \%$ of Brazilian isolates.

Conclusions: The Brazilian HCV-1a sequences formed a distinct group inside the clade 1 with potentially different phenotypic characteristics that may reflect differences in treatment response. These results demonstrate the importance of considering the genome 
heterogeneity of HCV subtype 1a in the context of antiviral agents directed to the NS3 serine protease domain.

\section{PP14-26}

Predictors of Treatment Failure in the Patients with Chronic Hepatitis C Treated with Gold Standard Therapy

I. Hrstic ${ }^{1}$, B. Grahovac ${ }^{2}$, B. Mohar ${ }^{2}$, B. Kolaric ${ }^{3}$, R. Ostojic ${ }^{1}$, B. Vucelic ${ }^{1}$

${ }^{1}$ Division of Gastroenterology, School of Medicine, University of

Zagreb, Zagreb; ${ }^{2}$ Department of Pathology, School of Medicine,

University of Rijeka, Rijeka; ${ }^{3}$ Social Medicine and Gerontology

Service, Zagreb County Institute of Public Health, Zagreb, Croatia

Background: HCV genotype is so far assigned as the most important predictor of a successful treatment response.

Aims:

1. verify the quasispecies in each patient; and

2. create the predictive model for treatment failure.

Methods: Scanning for HCV quasispecies variants was done with DNA melting analysis. Melting curve profile was defined for each sample with following parameters: melting temperature $(\mathrm{Tm})$, height $(\mathrm{H})$, width $(\mathrm{W})$ and peak area (A) of derivative melting curves. All parameters were analyzed against patient's baseline characteristics. Patients were treated with pegylated interferon and ribavirin according to genotype.

Results: Mean age of 109 enrolled patients was $39.6 \pm 13.3$ years; $58.7 \%$ were males, majority $(93.58 \%)$ had abnormal ALT; predominance of G1 infection (59.6\%) and high viral load (55.96\%) with mean fibrosis score (Ishak) of $4 \pm 1$. Obtained quasispecies parameters were: $\mathrm{Tm}=86.8 \pm 2.10 ; H=1.64 \pm 0.74 ; W=3.38 \pm 0.14$ and $A=4.42$ (range 5.99). Statistically significant correlation was found only for Tm and genotype $(p=0.05)$. Patients with $\mathrm{G} 1$ had the highest Tm. Predictors of non-SVR were: A $(p=0.033)$, age $(p=0.002)$, viral genotype $(p=0.002)$, viral load $(p=0.012)$ and fibrosis $(p=0.007)$. The logistic model for non-SVR is shown in Table. Only A, out of all parameters, was significant in the model. Age and gender were included in the model as biological confounders.

Logistic model for non-SVR

\begin{tabular}{lllrlll}
\hline & $\begin{array}{l}\text { Odds } \\
\text { ratio }\end{array}$ & $\begin{array}{l}\text { Standard } \\
\text { error }\end{array}$ & $Z$ test & $P$ value & $\begin{array}{l}95 \% \text { CI } \\
\text { for OR }\end{array}$ \\
\hline & 1.433 & 0.254 & 2.03 & 0.042 & 1.013 & 2.029 \\
Female & 0.491 & 0.21 & -1.66 & 0.097 & 0.213 & 1.137 \\
Age & 1.047 & 0.171 & 2.84 & 0.005 & 1.014 & 1.081 \\
\hline
\end{tabular}

Conclusions: Baseline DNA melting parameter, peak area (A), can serve as predictor for treatment failure. The formula calculates the probability of tretament failure with given area, age and gender. Chance to have treatment failure rices 1.43 times for increase of Area for 1 unit and 1.05 times for increase of age for 1 year.

\section{PP14-27}

SEN Virus Infection in Egyptian Patients with Chronic Hepatitis C and Patients Undergoing Hemodialysis

M.A. Saber ${ }^{1}$, M. Omar ${ }^{2}$, M. Hassan ${ }^{3}$, M. Akl ${ }^{4}$

${ }^{1}$ Biochemistry; ${ }^{2}$ Microbiology; ${ }^{3}$ Tropical Medicine Department;

${ }^{4}$ Pathology Department, Theodor Bilharz Research Institute, Giza, Egypt

The SEN virus has been tentatively linked to transfusion-associated non-A to E hepatitis. The aim of the present study was to

(1) determine the prevalence of SEN virus among Egyptian patients with hepatitis $\mathrm{C}$ virus (HCV)-related chronic liver disease and patients undergoing hemodialysis and
(2) demonstrate the clinical effect of SEN virus infection on coexistent hepatitis $\mathrm{C}$ in terms of severity and probability of developing hepatocellular carcinoma.

Polymerase chain reaction was used to detect SEN virus-D and SEN virus-H DNA in serum samples of 74 patients with HCV-related chronic liver disease, 45 uremic patients undergoing maintenance hemodialysis, and 28 healthy controls. SEN virus-D/H DNA was detected in $13.5 \%$ of patients with chronic liver disease, $11.1 \%$ of patients undergoing hemodialysis, and $7.1 \%$ of healthy controls, with no significant differences between patients and the control group. Clinical and biochemical measures did not significantly differ between SEN virus-infected and noninfected patients in the chronic liver disease group or the hemodialysis group. The rate of SEN virus infection was significantly higher in patients with chronic liver disease and hepatocellular carcinoma $(33.3 \%)$ than in those with chronic liver disease only $(8.5 \%)(P<.05)$. In conclusion, SEN virus does not seem to be a common infection in Egyptian patients. It has no apparent influence on the severity of coexistent HCV-related chronic liver disease but could be a risk factor for hepatocellular carcinoma in such patients. Further studies are needed to define the etiopathogenic role of SEN virus infection in the development of hepatocellular carcinoma.

\section{Poster Presentation 15: Liver Fibrosis: Basic}

\section{PP15-01}

Prevalence of HCV among OST Clients in Kathmandu Nepal

A. Karki, Hepa Foundation

Hepa Foundation, Kathmandu, Nepal

Background: Hepatitis C is one of the 'hidden' public health problems in Nepal and IDUs just like in other parts of the world is the leading risk behavior for transmission of $\mathrm{HCV}$ through sharing of contaminated injecting equipments among people who inject drugs. Methodology: This study was conducted among 200 OST clients as well as Current drug users in Kathmandu. The study was conducted in compliance with both ethical and human rights standards which included participant's anonymity and pre and post test counseling.

Results: Reactive HCV noted in $80.5 \%$, Analysis of predictors of HCV positively indicated that IDUs. Significant number of IDUs are unemployed, nearly 58. $2 \%$ are jobless. Knowledge on HCV was uniformly low, majority of IDUs around $81.3 \%$ have never heard about HCV. Nearly $48.9 \%$ reported using alcohol and drugs while they were in OST treatment. And, $97 \%$ reported alcohol consumption within one month time interval. Majority of IDUs, $67.2 \%$ have been arrested by police and $23.6 \%$ have been put in jails while using illicit drugs. $50.9 \%$ attended different treatment programs before joining OST.

Conclusions: IDUs may bridge HCV infection within and outside OST networks as they frequently inject drug and share injecting equipments. Therefore, there is a growing need to establish Hepatitis $\mathrm{C}$ as a public health issue in HIV/AIDS policies and programs and to develop and strengthen its link with Drug and HIV policies, programs and practices. Reduction of transmission of HCV through education, improved awareness of risks and access to harm reduction programs is recommended.

\section{PP15-02}

Relationship between Hepatic Iron and Fibrosis Level in Patients with Chronic Hepatitis B

T.D. Alves ${ }^{1}$, T.P. Souza ${ }^{1}$, J.S. Pompa ${ }^{1}$, A.C.P. Oliveira ${ }^{1}$, V.S. Nunes ${ }^{1}$, L.R. Freitas ${ }^{2}$, R. Parana ${ }^{3}$, M.I. Schinoni ${ }^{3}$, Hepatology Unit, Federal University of Bahia

${ }^{1}$ Faculty of Medicine, Federal University of Bahia; ${ }^{2}$ Oswaldo Cruz Foundation, Federal University of Bahia; ${ }^{3}$ Complex University Professor Edgard Santos, Hepatology Unit, Federal University of Bahia, Salvador, Brazil 
Background: Iron overload in liver tissue has been suggested as an important predictor of progression to liver fibrosis in various diseases, but studies on the subject are still inconclusive.

Aims: To study the association of hepatic iron overload, in patients with chronic Hepatitis B virus(HBV), with: stage of fibrosis and inflammation-measured by the METAVIR score,levels of transaminases and steatosis. To study the association of HBeAg and antiHBe with: iron overload, stage of fibrosis and inflammation, and steatosis. Methods: Study based on a series of cases collected retrospectively diagnosed as having HBV (serology HBsAg positive and detection of HBV-DNA), with report of liver biopsy. Sample consisted of 156 patients with biopsy showing the presence or absence of iron overload. It was categorized in groups: patients with mild fibrosis $($ METAVIR $=$ F0-F1-F2) and patients with advanced fibrosis (METAVIR $=$ F3-F4). It wasn't possible to analyze the quantitative HBV-DNA,because at the time that the biopsies were performed this test wasn't performed routinely. The chi-square test was used for categorical variables (significance level of 5\%).

Results: $65.4 \%$ were male(mean age $=37.68 \pm 11.45$ years). $34.6 \%$ were female (mean age $=36.06 \pm 11.63$ years). Of the total, $23.8 \%$ had advanced fibrosis, $32 \%$ severe inflammation (A2-A3), $21.6 \%$ iron overload and $32.7 \%$ steatosis. Mean levels of transaminases found, in $\mathrm{U} / \mathrm{L}$, were: $\mathrm{AST}=66.08 \pm 64.635$ and $\mathrm{ALT}=97.06 \pm 106.542$. $\mathrm{HBeAg}$ were investigated in 52 patients, of these $46.2 \%$ were positive. AntiHBe were investigated in 54 patients, $77.78 \%$ were positive. There wasn't statistically significant between iron overload with: stage of fibrosis $(p=0.709)$, degree of inflammation $(p=0.156)$ and levels of transaminases (ALT, $p=0.388$ /AST, $p=0.871$ ). However, there was statistically significant between iron overload and presence of steatosis $(p=0.046)$. Values were significant in the relationship between $\mathrm{HBeAg}$ and inflammation $(p=0.015)$ and between antiHBe and steatosis $(p=0.000)$.

Conclusions: It wasn't found association between iron overload and liver fibrosis in patients with HBV.Data in the literature corroborate our findings. The different findings obtained were the association of steatosis with: iron overload, presence of antiHBe; and the association between $\mathrm{HBeAg}$ and liver inflammation.These results may help understand the evolution of hepatic fibrogenesis caused by HBV.

\section{PP15-03}

Retinoic Acid Induction of BAMBI Expression Suppresses TGF- $\beta$ Mediated Activation of Hepatic Stellate Cells

M.S. Kwak, J.-H. Yoon, J.-H. Lee, E.J. Cho, E.S. Jang, S.J. Yu, Y.J. Kim, H.-S. Lee

Department of Internal Medicine and Liver Research Institute, Seoul National University College of Medicine, Seoul, Republic of Korea

Background and aims: Hepatic fibrosis is associated with deposition of extracellular matrix, and hepatic stellate cell (HSC) is the primary source of these matrix proteins. TGF- $\beta$ signaling plays a key role in the activation of HSCs, and bone morphogenetic proteins and activin membrane-bound inhibitor (BAMBI) is known to antagonize TGF- $\beta$ singnaling. Retinoic acid (RA) is known to have anti-fibrotic effect in hepatic fibrogenesis, while the mechanistic link between retinoic acids and HSC activation has not been fully elucidated. A recent report showed that RA can induce BAMBI expression in avian embryos. Therefore, we hypothesized in this study that RA may modulate HSC activation by inducing BAMBI expression, thereby leading to TGF- $\beta$ signaling suppression.

Methods: LX-2 cells, an immortalized HSC cell line, and all-trans retinoic acid (RA) were used in this study. BAMBI expression was measured by RT-PCR, and its expression was suppressed by siRNA transfection. Phospho-Smad, which is a downstream molecule of TGF- $\beta$ signaling, was examined with immunoblot analysis and collagen- $\alpha$ expression was evaluated by real time RT-PCR and ELISA. Cell proliferation was measured by MTS assay.

Results: RA treatment suppressed TGF- $\beta$-induced Smad phosphoryation and collagen- $\alpha$ synthesis in LX- 2 cells. As anticipated, RA treatment increased BAMBI expression in LX-2 cells, while BAMBIspecific siRNA transfection restored TGF- $\beta$-induced Smad phosphoryation and collagen production in these cells in the presence of RA as compared to control siRNA-transfected cells. However, RA had no effect on the viability of HSCs $(P=0.600)$.

Conclusions: These results demonstrate that RA suppresses TGF- $\beta$ induced HSC activation by inducing BAMBI expression, thereby attenuating extracellular matrix deposition. Therefore, the induction of BAMBI expression in HSCs is expected to be utilized as an effective anti-fibrotic strategy in preventing liver cirrhosis.

\section{PP15-04}

Anthocyanin Isolated from the Purple-fleshed Sweet Potato Attenuates Hepatic Fibrosis and Hepatic Stellate Cells Proliferation K.Y. Lee ${ }^{1}$, J.H. Choi ${ }^{2,3}$, Y.P. Hwang ${ }^{3}$, Y.C. Chung ${ }^{4}$, H.G. Jeong ${ }^{3}$ ${ }^{1}$ Pharmacy, Chonnam National University; ${ }^{2}$ Pharmacy, Chosun University, Gwangju; ${ }^{3}$ Pharmacy, Chungnam National University, Daejeon; ${ }^{4}$ Food Science, Korea International University, Jinju, Republic of Korea

Background/aims: Hepatic fibrosis is an outcome of many liver diseases, characterized by excessive deposition of extracellular matrix proteins. The proliferation of HSCs is a critical step in hepatic fibrogenesis. A PDGF is the most potent mitogen for HSCs. We investigated the inhibitory effect of anthocyanin fraction (AF) isolated from the purple-fleshed sweet potato in dimethylnitrosamine (DMN)-induced hepatic fibrosis and PDGF-BB-induced HSCs proliferation.

Methods: The study included 5-week-old male Sprague-Dawley rats. To induce hepatic fibrosis, we administered DMN as an intraperitoneal injection three times per week for 4 weeks. Rats were intragastrically administered 50,100 , and $200 \mathrm{mg} / \mathrm{kg}$ of AF per day six times per week for 4 weeks. The animals were sacrificed on day 29. Serum activity of ALT and AST, body and liver weights, histopathological examinations and collagen content were performed. $\alpha$ SMA, collagen type I and III, PDGFR- $\beta$, TNF- $\alpha$, and TGF- $\beta 1$ expression levels were performed. PDGF-BB induced HSCs proliferation was evaluated by cell proliferation. Expression of $\alpha$-SMA and phosphorylation of MAPK and PDGF receptor-beta (PDGFR-b) was determined by western blot analysis.

Results: The anti-fibrosis of AF on rats was associated with:

(1) inhibition PDGFR- $\beta$ activation;

(2) inhibition of inflammatory cells recruitment; and

(3) reduction of DMN-induced $\alpha$-SMA and collagen type I and III expression and collagen accumulation.

The anti-proliferation of AF on HSCs was associated with:

(1) blocking PDGFR- $\beta$ signal transduction;

(2) inhibition of phosphorylation of AKT and ERK1/2 pathways; and

(3) reduction of PDGF-BB-induced $\alpha$-SMA expression and HSCs proliferation.

Conclusions: AF reduces PDGF-BB-induced $\alpha$-SMA expression and HSCs proliferation by inhibiting the PDGFR- $\beta$ phosphorylation and subsequent AKT and ERK1/2 activation. AF inhibits DMN-induced hepatic fibrosis in an animal model. AF is an excellent candidate for preventing the development of hepatic fibrosis and hepatic stellate cells proliferation, an important step in chronic liver disease. 


\section{PP15-05}

Bone Morphogenetic Protein-7 Suppresses the Development of Hepatic Fibrosis in Rats

S.-L. Wang, Division of Gastroenterology and Institute of Digestive Diseases Tong ji University School of Medicine, Shanghai, China

Aim: Liver cirrhosis caused by the accumulation of extracellular matrix materials, is a serious clinical problem that can progress to hepatic failure. Transforming growth factor-beta (TGF- $\beta 1$ ) plays a pivotal role in extracellular matrix production. Bone morphogenetic protein-7 (BMP-7) is a member of the TGF- $b_{1}$ superfamily. Recent studies indicate that BMP-7 could reverse established renal fibrosis in mice. In this study, we investigate the possible effect of bone morphogenetic protein-7 on rat hepatic fibrosis.

Methods: Sprague-Dawley (SD) rat liver fibrosis model was induced by intraperitoneal injection of pig serum, the rats received injection of pig serum $0.5 \mathrm{ml}$ twice a week; in early treatment group, the rats received injection of BMP-7 $100 \mu \mathrm{g} / \mathrm{kg}$, three times a week from the second week; in later treatment group, the rats received injection of BMP-7 $100 \mu \mathrm{g} / \mathrm{kg}$, three times a week from the fourth week; in prevention group, the rats received injection of BMP-7 $100 \mu \mathrm{g} / \mathrm{kg}$ every other day at the beginning of the experiment; in control group, the rats received injection of equal volume saline every other day; 8 weeks, rat liver fibrosis was evaluated in vivo, and the expression of TGF- $b_{1}$ in the liver tissues of rats and control groups was detected by immunohistochemistry and Western blot.

Result: The grade of hepatic fibrosis in treatment group was attenuated by BMP-7 compared with the control group $(P<0.05)$; the expression of TGF- $b_{1}$ was significantly decreased in prevention and treatment groups.

Conclusions: BMP-7 can prevent and attenuate hepatic fibrosis in the experimental rats, the mechanism is associated with that BMP-7 could inhibit the expression of TGF- $b_{1}$ in the fibrotic liver tissues.

\section{PP15-06}

Mallory Denk Bodies in Chronic Hepatitis and its Importance

M. Basaranoglu, N. Turhan

Ankaraa YIH, Ankara, Turkey

Background and aim: Mallory Denk Bodies (MDB) are important since some investigators suggested $\mathrm{MDB}$ as a sign of histologic severity of the disease in various type of chronic hepatitis, such as hep $\mathrm{C}$ and nonalcoholic fatty liver disease (NAFLD). In an earlier classification system, Matteoni et al. scored MDB in patients with NAFLD as none, rare and many and reported that MDB plays a prominent role in this classification scheme. However, frequency of MDB in patients with NAFLD is reported from 0 to $90 \%$.

Material and methods: In this study, we evaluated 258 patients with chronic hepatitis due to metabolics, autoimmune and viral etiologies which included such as NAFLD, Wilson disease, primary biliary cirrhosis, hepatitis B and C, and hepatocellular carcinoma. Liver biopsy samples were evaluated with Hematoxylin and Eosin, periodic acid-Schiff-diastase, Gordon and Sweet's reticulin, Masson's trichrome, and iron stains. Both staging and grading were performed for each condition. Differences on MDB was further evaluated and discussed for each disease.

Results: We examined patients with NASH (50), alcoholic hepatitis (10), primary biliary cirrhosis (PBC; 50), Wilson disease (WD; 20), hepatitis B (50) and hepatitis C (50), and hepatocellular carcinoma (HCC; 30).

Conclusion: We found that there is no relationship between MDB and any chronic hepatitis form regarding histologic severity.

1. NASH: 10 pts. Mild in $60 \%$ and Moderate in $40 \%$. MDB observed in every stage.

2. PBC 11 pts. Mild in $10 \%$,Moderate in $70 \%$, and cirrhosis in $20 \%$. Frequently seen in later stages with zone 1 .
3. WD 16 pts. Moderate and severe in $60 \%$ and cirrhosis in $40 \%$. Frequently seen in later stages with zone 1.

4. Hep B 3 pts. Mild in $66 \%$ and severe in $34 \%$ Mild case with steatosis.

5. Hep C 7 pts. Mild in $40 \%$ and Moderate in $60 \%$. MDB observed in every stage.

\section{PP15-07}

Distribution of Hepatic Myofibroblasts and of Type I and III Collagens in Cirrhotic Rat Induced by Thioacetamide

R. Poonkhum ${ }^{1}$, W. Padidarcheep ${ }^{1}$, S. Nilbu-Nga ${ }^{1}$, S. Chaunchaiyakul ${ }^{1}$, O. Ohtani ${ }^{2}$

${ }^{I}$ Department of Anatomy, Faculty of Medicine, Srinakharinwirot

University, Bangkok, Thailand; ${ }^{2}$ Department of Anatomy, Faculty of Medicine and Postgraduate School of Medicine and Pharmaceutical Sciences, University of Toyama, Toyama, Japan

Background: The thioacetamide (TAA), a hepatotoxin, could induce various types of cirrhosis in rat including bridging fibrosis, biliary fibrosis, perisinusoidal/pericellular fibrosis and centrilobular fibrosis which are involvement of different populations of hepatic myofibroblasts (MFs). The hepatic MFs could be classified into 3 groups including (a) portal/septal MFs, (b) activated hepatic stellate cell myofibroblasts (HSC/MFs), and (c) interface myofibroblasts (IF/ MFs).

Aims: To study the morphology and localization of hepatic MFs in relation to the distribution of type I and type III collagen in cirrhotic rat livers.

Methods: Immunohistochemistry with $\alpha$-smooth actin ( $\alpha$-SMA) was employed to demonstrate the morphology and localization of the three subpopulations of hepatic MFs. In addition, the distribution of type I, III collagens was investigated by using specific corresponding antibodies.

Results: It was found that the portal and septal MFs had windmill in shape contributed type I, III collagens surrounding both the tributaries of portal and hepatic veins. HSC/MFs with arachnoid in shape produced type I collagen into spaces of Disse and spaces between neighboring hepatocytes. The IF/MFs presented as arachnoid cells distributing along the margin of fibrous septa and synthesized type I collagen. Apart from the three subpopulations of MFs mentioned above, we also found other hepatic MFs which had polygonal in shape found around wall of hepatic sinusoids and margin of fibrous septa and surrounding portal tract. They were probably the cells in the transforming stage into one of the three mature MFs.

Conclusions: Our data suggest that the morphology and localization of hepatic MFs are related to fibrogenesis and possibly neoangiogenesis.

\section{PP15-08}

Effects of Ursolic Acid on TIMP-1 and MMP-1mRNA Expression and JAK2-STAT3 Signal Transduction in Hepatic Stellate Cells

G.Y. Liu ${ }^{1}$, X. Zhu' ${ }^{2}$, K.H. Zhang ${ }^{1}$, B.M. Li ${ }^{1}$, Z.J. Liu ${ }^{1}$, J. Cheng ${ }^{1}$, W.H. He ${ }^{1}$ ${ }^{1}$ The First Affiliated Hospital of Nanchang University; ${ }^{2}$ Department of Gastroenterology, The First Affiliated Hospital of Nanchang University, Nanchang, China

Background and aim: Ursolic acid (UA) is a natural pentacyclic triterpenoid carboxylic acid and possess a antioxidative, anti-inflammation, and anticancer activities, our previously studies initially indicated its antifibrotic actity but mechanisms are not entirely understood. To investigate the potential offect of Ursolic acid on leptin-induced JAK-STAT signal transduction pathway and mRNA expression of MMP-1 and TIMP-1 in Hepatic stellate cells (HSCs). Materials and methods: After HCS-T6 cells(rat HSCs line)were preincubated for 30 minutes with buffer, UA(50uM), DPI $(20 \mu \mathrm{M})$, $\mathrm{AG} 490(50 \mu \mathrm{M})$ or $\mathrm{NAC}(10 \mathrm{mM})$ and then stimulated with leptin $(100$ 
$\mathrm{ng} / \mathrm{ml}$ ) for different times, TIMP-1, MMP-1mRNA expression;intracellular ROS, p-JAK2 and p-STAT3 protein expression was analyzed in HCS-T6 cells.

Results: Leptin stimulated ROS production, increased of p-JAK1 and p-STAT3 protein expression, increased the TIMP-1 mRNA expression but decreased the MMP-1 mRNA expression in HCS-T6 cells. Pretreated with U A suppressed leptin-induced ROS formation, blocked JAK2 and STAT3 phosphorylation,down-regulated the TIMP-1 mRNA expression and up-regulated the MMP-1 mRNA expression. NADPH oxidase inhibitor diphenylene-iodonium (DPI) completely inhibited ROS production, but slightly decreased p-JAK1 and p-STAT3 protein expression. JAK inhibitor AG 490, significantly attenuated leptin-stimulated ROS production, blocked JAK2 phosphorylation with concomitant inhibition of STAT3 activation, inhibited the TIMP-1 mRNA expression and increased the MMP-1 mRNA expression in HCS-T6 cells. Pretreated with NAC decrease the ROS level in HCS-T6 cells, but had no significant effect on JAK2 and STAT3 phosphorylation as well as TIMP-1 mRNA and MMP-1 mRNA expression.

Conclusions: UA can inhibit leptin induced ROS generation, JAK2 STAT3 activation inhibit the expressions of TIMP-1 mRNA, and increase the expression of MMP-1 mRNA. inhibit leptin induced. Our results demonstrate its potential role as a pharmacological antifibrotic drug.

\section{PP15-09}

The Role and Mechanism of NADPH Oxidase Participate in Leptininduced Reactive Oxygen Species Production in Hepatic Stellate Cells H.W. He, X. Zhu, B. Li, K.H. Zhang, B.M. Li, G.Y. Liu, J. Wang The First Affiliated Hospital of Nanchang University, Nanchang, China

Objectives: To investigate the NADPH oxidase (NOX) whether or not participate in leptin-induced reactive oxygen species (ROS) production in hepatic stellate cells (HSC), and explore the mechanism by survey the NOX activity and the mRNA expression of NOX subunits Racland $\mathrm{p} 22^{\text {Phox }}$.

Methods: HSC-T6 cells (rat hepatic stellate cells line) were divided into nine groups: leptin group; leptin together with inhibitors that block different ROS-producing systems. Intracellular ROS were measured with dichlorodihydrofluorescein diacetate (DCFH-DA) dye assay. NOX activity was analyzed by using spectrophotometer.mRNA levels of Rac1 and p22 $2^{\text {phox }}$ were evaluated by RT-PCR.

\section{Results:}

1. Leptin increased ROS production significantly in HSC-T6 cells. Both the NADPH oxidase inhibitor DPI $(20 \mu \mathrm{mol} / \mathrm{L})$ and AG490 $(50 \mu \mathrm{mol} / \mathrm{L})$ blocked the ROS production, inhibitors of other ROS producing systems had no significant effect on ROS production.

2. The NOX activity of HSC-T6 cells increased significantly after stimulate with leptin for $1 \mathrm{~h}$, furthermore, the NOX activity were higher in 12 and $24 \mathrm{~h}$ than $1 \mathrm{~h}$; leptin-induced NOX activity upregulation were inhibited by pretreat with DPI or AG490.

3. The RT-PCR results indicated that mRNA expression of Rac1 and $\mathrm{p} 22^{\text {Phox }}$ in HSC-T6 cells with leptin stimulate for 12 hour were increased significantly compared with normal control group.DPI and AG490 had no effect on mRNA expression of Rac1 and $22^{\text {Phox }}$.

Conclusion: The NOX is the main cellular source of the reactive oxygen species (ROS) generated by HSC in response to leptin. The mechanism is probably that leptin can directly activate NOX through JAK signal transduction, and induce the expression of NOX subunit to promote the activity of NOX, which generates considerable ROS in HSC.
PP15-10

Effects of Ursolic Acid on the ROS Production in HSC and the Relationship to Apoptosis

B. $\mathrm{Li}^{1}$, X. Zhu ${ }^{2}$, W.H. $\mathrm{He}^{2}$, K.H. Zhang ${ }^{1}$, B.M. Li $^{1}$, Z.J. Liu ${ }^{1}$, J. Cheng ${ }^{1}$, G.Y. $\mathrm{Liu}^{1}$

${ }^{I}$ The First Affiliated Hospital of Nanchang University; ${ }^{2}$ Department of Gastroenterology, The First Affiliated Hospital of Nanchang University, Nanchang, China

Objective: To observe the effect of Ursolic acid (UA) on the reactive oxygen species (ROS) production in hepatic stellate cells (HSC), nuclear translocation of NF- $\kappa \mathrm{B}, \mathrm{mRNA}$ expression of X-linked inhibitor of apoptosis protein (XIAP), to explore the possible mechanisms of HSC apoptosis of induced by UA.

Methods: Randomization of exponential phase of growth hepatic stellate cells(HSC-T6) were: leptin $(100 \mathrm{ng} / \mathrm{ml})$, leptin together with UA $(50 \mu \mathrm{M})$, leptin together with NAC $(10 \mathrm{mM})$, blank group. When HSC-T6 cells were treated for different times, the intracellular ROS and rate of HSC-T6 apoptosis were measured by flow cytometry, the nuclear translocation of NF- $\kappa \mathrm{B}$ subunit P65 was evaluated by immunocytochemical staining assay; the expressions of XIAP mRNA were evaluated by RT-PCR.

Results:

1. Leptin induced ROS production significantly, pretreated with UA time-dependly decreased the ROS levels in HSC-T6 cells.NAC also attenuated leptin-stimulated ROS production in short-term.

2. After treating HSC-T6 cells with leptin for $48 \mathrm{~h}$, the apoptosis rate decreased significantly;pretreated with UA, the apoptosis rate increased and higher than pretreated with NAC.

3. The analysis of immunocytochemistry techniques indicated that NF- $\kappa \mathrm{B}$ in HSC-T6 were activated by treated with leptin for $2 \mathrm{~h}$. The nuclear translocation of NF- $\kappa \mathrm{B}$ were lower in pretreated with UA and NAC.

4. When HSC-T6 was treated by leptin, the expressions of XIAP mRNA were significantly higher than blank group in $24 \mathrm{~h}$ $(P<0.01)$ the expressions of XIAP mRNA decreased both by pretreated with UA or NAC.

Conclusions: UA can inhibit ROS production induced by leptin in HSC, and can induce HSC apoptosis. The mechanism of HSC apoptosis effected by Ursolic acid is probably related to decreasing production of ROS, inhibiting activation of NF- $\kappa \mathrm{B}$, and down-regulating the expression of XIAP mRNA.

\section{PP15-11}

Effects of Ursolic Acid on Subunits Gene Expression and Enzymatic Activity of NADPH Oxidase in Hepatic Stellate Cells

W.H. He ${ }^{1}$, X. Zhu' ${ }^{2}$, B. Li $^{1}$, K.H. Zhang ${ }^{1}$, B.M. Li ${ }^{1}$, Z.J. Liu ${ }^{1}$, G.Y. Liu ${ }^{1}$ ${ }^{1}$ The First Affiliated Hospital of Nanchang University; ${ }^{2}$ Department of Gastroenterology, The First Affiliated Hospital of Nanchang

University, Nanchang, China

Background and aims: Recent studies have shown that NADPH oxidase plays a central role in liver fibrogenesis, and represents a target for antifibrotic therapies. To define antifibrotic mechanism of Ursolic acid (UA),we investigate the effect of UA on NADPH oxidase activity, ROS generation and NADPH oxidase subunit expression in Hepatic stellate cells (HSCs).

Methods: Hepatic stellate cells (HSC-T6) were pretreated with $\mathrm{UA}(50 \mathrm{uM})$, NAC(10mM), DPI(20uM), AG490(50uM) or culture medium, then exposed to leptin for different time. Intracellular ROS, NADPH oxidase activity and the expression of NADPH oxidase subunit Rac1mRNA and P2 ${ }^{2 \text { Phox }}$ mRNA were evaluated in HSCs;

Results: Leptin-induced NADPH oxidase activation and ROS production was blocked in HSCs by the NADPH oxidase inhibitor diphenylene-iodonium (DPI), Janus kinase (JAK) inhibitor, AG490 and UA as well; $N$-acetyl-l-cysteine (NAC) decrease intracellar ROS, 
but have no significant effect on NADPH oxidase activatity. UA down-regulate the expression of Rac1 mRNA and p22Phox mRNA, which Regulating the NADPH oxidase activatity and ROS generation. DPI,AG490 and NAC had no significant effect on Rac1 mRNA expression, but DPI can suppress p22 ${ }^{\text {Phox }}$ mRNA expression.

Conclusions: UA can inhibit NADPH oxidase activity and ROS production induced by leptin in HSCs, the mechanism is probably that UA downregulation the expression of NADPH oxidase subunits gene expression.Our results, in conjunction with previous studies of UA On proliferation and apoptosis of HSCs in vitro, demonstrate UA as a antifibrotic triterpenoid.

\section{PP15-12}

Modification of Sinusoids Area as Target in the Progression of Liver Disease. Preliminary Study

F. Marinozzi ${ }^{1}$, S. Novelli ${ }^{1}$, V. Morabito ${ }^{2}$, M. Rossi ${ }^{2}$, G. Novelli ${ }^{2}$

${ }^{1}$ Bioclinical Engineering, Sapienza University of Rome, Roma;

${ }^{2}$ General Surgery and Organs Transplant, Sapienza University of

Rome, Rome, Italy

Introduction: Liver biopsy is the only method that allows an assessment of fibrosis and necrotic and inflammatory activity of the disease, giving an important indication of status at a given time and therefore at this particular time is important to obtain as much information as possible. The study of modification of the sinusoids could contribute to the impaired exchange of substrates between hepatocytes and blood perfusing the liver with increase of hepatic venous pressure gradient (HVPG). The objective of this study is to highlight a correlation between the severity of liver disease and image analysis of histological findings.

Materials and methods: For this study were enrolled 90 patients including 30 patients with normal histological examination performed for suspected disease, 30 patients with positive histology for steatosis and 30 patients with histological positive cirrhosis. Eight areas of sinusoids were evaluated for each image. We conducted an analysis of the geometrical images of individual histological changes of sinusoids.

Results: In the images of patients free of disease, we have highlighted a sinusoidal mean area of $0.0044 \pm 0.024 \mathrm{~mm}^{2}$. HPVG evaluation confirmed the finding of a mean value in the normal range of $1.83 \pm 0.42 \mathrm{mmHg}$. In subjects with steatosis, results showed a significant difference. In fact there was evidence of a reduction of the sinusoidal areas, with a mean value of $0.0013 \pm 0.1678 \mathrm{~mm}^{2}$. The interesting thing is the evaluation of HPVG (mean $2.6 \pm 0.59 \mathrm{mmHg}$ ) was still within normal range. In images of patients with cirrhosis has highlighted a further reduction of the sinusoids (mean $0.00029 \mathrm{~mm}^{2}$ ) in relation to a high average value dell'HPVG $(10.4 \mathrm{mmHg})$ sign of portal hypertension, confirming the presence of a pathological condition in an advanced state.

Conclusions: The results seem possible diagnosis of disease progression by determining the small changes in sinusoidal area.

\section{PP15-13}

Effect of Salidroside on the Expression of Na+-Ca2+ Exchanger in Rats of Carbon Tetrachloride-induced Hepatic Fibrosis

X.L. Wu ${ }^{1}$, W.Z. Zeng ${ }^{1}$, G.S. Xu ${ }^{2}$, J.P. Qin ${ }^{1}$, H. Xu ${ }^{1}$, Z. Wang ${ }^{1}$, M.D. Jiang ${ }^{1}$ ${ }^{I}$ Department of Digestion; ${ }^{2}$ Emergency Ward, Chengdu Military General Hospital, Chengdu 610083, Sichuan Province, China, Chengdu, China

Methods: 120 rats were divided into three groups: control group $(\mathrm{C}, n$ $=40)$, Salidroside treated group $(\mathrm{S}, n=40)$ and hepatic fibrosis model group (M, $n=40$ ). Hepatic fibrosis was induced by 8 -week subcutaneous injection of CCl4. The rats in group S received Salidroside via celiac injection at a dosage of $10 \mathrm{mg} / \mathrm{kg}$. The deposition of collagen fiber was detected with VG collagen staining. The content of serum TGF $\beta 1$ was assayed with ELISA. The expression of
$\mathrm{Na}+\mathrm{Ca} 2+$ exchanger(NCE) and $\alpha 1(\mathrm{I})$ mRNA were detected with in situ RT-PCR.

Results: The area of collagen in group $\mathrm{C}$ was only $23.31 \pm 6.87 \mu \mathrm{m}^{2}$, it was increased to $190.86 \pm 29.17 \mu^{2}$ in group M. It was reduced to $74.82 \pm 11.54 \mu \mathrm{m}^{2}$ in $\mathrm{S}$ group. The serum content of TGF $\beta 1$ was only $0.34 \pm 0.15 \mu \mathrm{g} / \mathrm{L}$ in group C. In M group, TGF $\beta 1$ was elevated to $3.59 \pm 0.83 \mu \mathrm{g} / \mathrm{L}$, it was significantly decreased to $1.32 \pm 0.51 \mu \mathrm{g} /$ $\mathrm{L}$ in group $\mathrm{S}$. The expression of NCE and $\alpha 1(\mathrm{I})$ mRNA in group $\mathrm{C}$ was feeble. In group M, the O.D value of NCE and $\alpha 1$ (I) mRNA were increased to $1.95 \pm 0.31$ and $2.31 \pm 0.48$, respectively. In group $\mathrm{S}$, the $\mathrm{OD}$ value were decreased to $0.97 \pm 0.21$ and $1.26 \pm 0.17$ respectively.

Conclusions: Salidroside could reduce the production and deposition of collagen fiber in the liver. The serum content of TGF $\beta 1$ was decreased, the expression of NCE and $\alpha 1$ (I) mRNA were inhibited. Since NCE was important in controlling the activation of hepatic stellate cells(HSC), the probable anti-fibrosis mechanisms of Salidroside would contributed to the inhibiting of HSC activation and reducing the collagen production or deposition.

\section{PP15-14}

Parthenolide Modulates Hepatic CD38 Expression and Ameliorates Fibrosis in an in vivo Rat Model

D.-G. Kim ${ }^{1}$, I.H. Kim ${ }^{1}$, G.-R. Yu ${ }^{1}$, K.-H. Park ${ }^{2}$, U.-H. Kim ${ }^{2}$

${ }^{1}$ Chonbuk National University Medical School and Hospital;

${ }^{2}$ Biochemistry, Chonbuk National University Medical School, Jeonju, Republic of Korea

Background/aims: Parthenolide (PT), a sesquiterpene lactone derived from the plant feverfew, has pro-apoptotic activity in a number of cell types. We assessed whether PT modulates CD38 expression and induces the apoptosis of rat hepatic stellate cells (HCSs) and examined its effects on hepatic fibrosis in an in vivo model.

Methods: The effects of PT on rat HSCs (RI-T cell line) were investigated in relation to cell growth inhibition, apoptosis, nuclear factor kappa $\mathrm{B}(\mathrm{NF}-\kappa \mathrm{B})$ binding activity, intracellular reactive oxygen species (ROS) generation, and glutathione (GSH) levels. In addition, the anti-fibrotic effects of PT were investigated in a thioacetamidetreated rat model.

Results: PT induced growth inhibition and apoptosis in RI-T cells, as evidenced by MTT assays, flow cytometric detection of sub- $\mathrm{G}_{1}$ DNA content, and annexin V staining assays. PT increased the expressions of Bax proteins during apoptosis, but decreased the expressions of Bcl-2 and Bcl- $\mathrm{X}_{\mathrm{L}}$ proteins. PT also decreased mitochondrial membrane potential and enhanced poly (ADP-ribose) polymerase cleavage, and caspase- 3 activation. The pro-apoptotic activity of PT in RI-T cells was associated with increased intracellular oxidative stress as evidenced by increased intracellular ROS levels and depleted intracellular GSH levels. PT also inhibited TNF- $\alpha$-stimulated NF- $\kappa$ B binding activity in RI-T cells. Furthermore, PT inhibited hepatic CD38 expression and ameliorated hepatic fibrosis significantly in a thioacetamide-treated rat model.

Conclusions: PT exhibited pro-apoptotic effects in rat HSCs, inhibited hepatic CD38 expression, and ameliorated hepatic fibrosis in a thioacetamide rat model.

\section{PP15-15}

The Effect of Interferon- $\gamma$ Encapsulated in the Targeted Liposomes through Platelet Derived Growth Factor Receptor $\beta$ on Hepatic Fibrosis

Q.-H. Li, J.-Y. Wang, F. Li

Gastroenterology and Hepatology, Zhongshan Hospital,Fudan

University, Shanghai, China

Backgroud and aims: The role of the activeted hepatic stellate cells(HSCs) is the key in both hepatic fibrogenesis and expression of platelet derived growth factor receptor $\beta(\operatorname{PDGFR}-\beta)$. The aim of this 
study was to explore a new approach to selectively deliver drug to HSCs and investigate the more available effect to treat hepatic fibrosis.

Methods: We constructed a cyclic peptide(pPB) with affinity for the PDGFR- $\beta$-modified sterically stable liposomes (SSLs) to deliver interferon (IFN)- $\gamma$ to HSCs. The binding of pPB to receptor and cellular internalization were confirmed by flow cytometry and immunofluorescent images with a confocal laser microscope in primary cultured activated HSCs (pHSCs). The tissue biodistribution and pharmacokinetics of pPB-SSL-IFN- $\gamma$ were detected by ELISA.The relative fibrotic markers were analyzed by western blot,immunohistochemistry in the pHSCs and rat fibrotic livers induced by thioacetamide (TAA).

Results: The binding of pPB to HSC was in a dose-dependent manner and counteracted competitively by free pPB. In pHSCs treated with pPB-SSL-FITC, green fluorescence appeared in the cytoplasm. IN normal rats pPB-SSL-IFN- $\gamma$ mostly accumulated in the liver in contrast to other organs $24 \mathrm{~h}$ after injection,meanwhile more than the accumulation of IFN- $\gamma$. In vivo the histological ishak stage,quantity of collagen I-positive area in fibrotic liver of pPB-SSL-IFN- $\gamma$-treating group were less than those of SSL-IFN- $\gamma$, IFN- $\gamma$ and saline group. In vitro pPB-SSL-IFN- $\gamma$ was also more effective in suppressing activated HSC proliferation and the expression of $\alpha$-smooth muscle actin protein.

Conclusions: The data suggests that pPB-SSL may be applied as a new choice of drug-carrier which could selectively deliver antifibrotic agents to HSC to improve drug therapy. The approach of targeting drug-delivered might increase the efficacy of drug therapy for human hepatic fibrosis.

\section{PP15-16}

Alpha-Mangostin Preserves Expression of Ammonia-Metabolizing Enzymes in Thioacetamide-induced Fibrotic and Cirrhotic Rats

M. Khunvirojpanich ${ }^{1}$, W. Pradidarcheep ${ }^{2}$, S. Nilbu-Nga ${ }^{2}$,

U. Showpittapornchai ${ }^{2}, \mathrm{~S}$. Wattanasirichaigoon ${ }^{3}$

${ }^{1}$ Biomedical Sciences, Mahidol University, Salaya; ${ }^{2}$ Department of Anatomy; ${ }^{3}$ Department of Surgery, Srinakharinwirot University, Bangkok, Thailand

Background/aim: Ammonia metabolizing enzymes, carbamyol phosphate synthetase 1(CPS1) and glutamine synthetase (GS), are expressed in a fashion which follows hepatic zonation. CPS1 resides in the periportal region and metabolizes ammonia into urea in a high capacity, low affinity manner. Remaining ammonia is scavenged by GS expressed in pericentral hepatocytes, converting it into glutamine in a low capacity, high affinity manner. CPS1 and GS function complementary to one another to ensure complete ammonia metabolization into nontoxic forms. Immunohistochemical analysis confirmed the decline of both CPS1 and GS in cirrhotic rat liver induced by thioacetamide (TA). Alpha-mangostin, a major derivative of xanthone from mangosteen, has been reported to possess a wide range of pharmacological properties. The present study aimed to test the effects of alpha-mangostin on CPS1 and GS expression in fibrotic and cirrhotic rats induced by TA over the course of 4 months.

Method: Antibodies against CPS1 and GS were used for immunohistochemical study.

Results: Immunohistochemical staining for CPS1 and GS showed an increasing decline from month 2-4 under pure-TA treatment. Fibrous bridgings, nodule formations, and regenerative nodules were detected at months 2, 3, and 4, respectively. Pure-alpha-mangostin treatment yielded strongly stained CPS1 and GS hepatocytes in a fashion similar to the control throughout experimental period. Testing for protective effects, TA and alpha-mangostin were given concurrently to mimic the natural damage of human liver from external toxins or viral infections. Results showed a decreasing decline of CPS1 and GS density level from month two to four as compared to pure-TA treatment. Fewer fibrous portal-caval bridgings were observed in month 4 and a continuous ring of CPS1-positive hepatocytes were not absent as observed in pure-TA treatment.

Conclusion: Alpha-mangostin could partially preserve the normal expression of ammonia-metabolizing enzymes under TA-induced fibrotic and cirrhotic conditions.

\section{PP15-17}

Antifibrogenic Property of Alpha-mangostin in Thioacetamide-treated Rat K. Wannason ${ }^{1}$, S. Asuvapongpatana ${ }^{1}$, U. Showpittapornchai ${ }^{2}$,

S. Pongmayteegul ${ }^{2}$, W. Pradidarcheep ${ }^{2}$

${ }^{1}$ Department of Anatomy, Mahidol University; ${ }^{2}$ Department of Anatomy, Srinakharinwirot University, Bangkok, Thailand

Background/aims: Reiteration of liver injury triggers hepatic parenchymal and nonparenchymal cells to secrete pro-inflammatory cytokines resulting in inflammation and progressive fibrogenesis. Alpha-mangostin, major constituents from the xanthone fraction of mangosteen fruit, has been reported to possess various pharmacological properties including antioxidant and anti-inflammation. The present study aimed to elucidate the antifibrogenic effect of alphamangostin on thioacetamide-induced hepatic injury.

Methods: Thioacetamide $(200 \mathrm{mg} / \mathrm{kg}$ ) was first administered intraperitoneally to male Wistar rats over the time course of three months. Alpha-mangostin was then applied intraperitoneally to each group of the animals for another 1 month, respectively. Collagen deposition in the liver was evaluated by Sirius red staining and hepatic stellate cells activation was determined by $\alpha$-smooth muscle actin ( $\alpha$-SMA) expression by immunohistochemistry.

Results and conclusions: The results show that the thioacetamide progressively induced extensive hepatic damage, enhanced $\alpha$-SMA expression and fibrogenesis in liver at months 2-3. The alpha mangostin could lower the oxidative stress-related hepatic damage and collagen accumulation associated with thioacetamide toxicity. The present study showed that alpha-mangostin possesses antifibrogenic property following thioacetamide-induced hepatic injury in rats.

\section{PP15-18}

Bone Marrow Mesenchymal Stem Cells Regulate Hepatic Stellate Cells Activation Through LPS-TLR4 Pathway

C. Xie, L. Peng, G.-L. Zhang, Z.-L. Gao

The Third Affiliated Hospital of Sun Yat-sen University, Guangzhou, China

Objective: Experimental and clinical evidence proved that LPS elevated in patients with liver fibrosis and LPS can be directly activated HSC in vivo. The main LPS receptor is TLR4 which is an important member Toll-like receptor family. TLR4 single nucleotide polymorphism T399I site changes can significantly reduce the risk of hepatic fibrosis. So LPS-TLR4 pathway plays an important role in liver fibrosis. The aim of the study is to investigate whether bone marrow mesenchymal stem cells (MSC) affect the hepatic stellate cells (HSC) through intervention LPS-TLR4 pathway, and to clarify the mechanism of LPS caused liver fibrosis through HSC activation. Methods: Human MSC, HSC were isolated and cultured in vitro alone or co-cultured in Transwell under different treatment in four groups, which was $2 \times 105 \mathrm{MSC}$ and $2 \times 105 \mathrm{HSC}$ (with $50 \mu \mathrm{g} / \mathrm{ml}$ LPS stimulation) co-culture group, $2 \times 105 \mathrm{MSC}$ and $2 \times 105 \mathrm{HSC}$ (no LPS stimulation) co-culture group, $2 \times 105 \mathrm{HSC}$ (with $50 \mu \mathrm{g} / \mathrm{ml}$ LPS stimulation) group, $2 \times 105 \mathrm{MSC}$ (with $50 \mu \mathrm{g} / \mathrm{ml}$ LPS stimulation) group. Collection of supernatant at $6,12,36$ and $48 \mathrm{~h}$ was detected for IL- 8 and TGF- $\beta 1$ expression by ELISA. The TLR-4, Myd88 and NF-KB gene expression of HSC were detected by RTPCR and western blotting. The $\alpha$-SMA, TGF- $\beta 1$ and collagen I expression were detected by western blotting.

Results: LPS can stimulate HSC activation. Activated HSC secreted more LI-8 $(0.88 \pm 0.09 \mathrm{ng} / \mathrm{ml})$ and TGF- $\beta(0.74 \pm 0.05 \mathrm{ng} / \mathrm{ml})$ than 
static HSC $(0.31 \pm 0.04$ and $0.41 \pm 0.03 \mathrm{ng} / \mathrm{ml}$ respectivly, $P<0.05)$. The gene expression of $\alpha$-SMA, TGF- $\beta 1$ and collagen I were increased also. After co-culture with MSC, LPS caused HSC activation was inhibited significantly as the $\alpha$-SMA, TGF- $\beta 1$ and collagen I were reduced. The protein expression of TLR-4, Myd88 and NF-KB of HSC decreased.

Conclusion: MSC can interfere with HSC activation through inhibited LPS-TLR4 pathway.

\section{PP15-19}

Salidroside Inhibits PDGF Signaling to Reduce Migration of LX-2 L. $\mathbf{X u}^{1,2}$, Z. Zhang ${ }^{2}$

${ }^{1}$ Hepatopathy, Shuguang Hospital Affiliated to Shanghai University of Traditional Chinese Medicine; ${ }^{2}$ Institute of Liver Diseases, Shanghai University of Traditional Chinese Medicine, Shanghai, China

Aims: To investigate the potential mechanism underlying the inhibitory activity of Salidroside, a compound of Chinese herb medicine which could anti-fibrosis, to reduce migration of hepatic stellate cell line LX-2 induced by PDGF.

Methods: LX-2 was incubated with $10^{-5}$ M Salidroside for $12 \mathrm{~h}$. Then

1. transfer the cells to a Transwell Chamber system which membrane was coated with Collagen type I. $10 \mathrm{ng} / \mathrm{ml}$ PDGF$\mathrm{BB}$ was added to the low chamber. LX-2 was cultured with Salidroside for $6 \mathrm{~h}$ and inhibitors were added into media last $1 \mathrm{~h}$. The migrated cells were counted;

2. the cells were cultured in plastic with above culture condition.

The kinases were detected using Western blotting analysis.

Results: Salidroside significantly inhibited migration of LX-2 induced by PDGF-BB but had no remarkable effect on the expression of PDGF receptor. Ras/MEPK signaling pathway of PDGF-BB is not involved in the regulation of LX-2 migration. FAK-PI3K signaling pathway regulates the migration of LX-2. FAK inhibitor PF573228 could inhibited the migration of LX-2 $(P<0.05)$, but Salidroside had no effect on the expression of FAK and p-FAK. Rac and PI3K both is downstream signaling pathway of FAK. The migration of LX-2 can be inhibited by Rac1 inhibitor $(P<0.05)$ and PI3K inhibitor LY294002 $(P<0.05)$. Although Salidroside were not regulated the expression of PI3K and p-PI3K but could attenuate the expression of p-Rac1 $(P<0.05)$ and p-AKT $(P<0.01)$, both are downstream kinas of PI3K. Furthermore, MLC signaling pathway, another subpathway of PI3K, could mediate migration of LX-2 but CDC42 inhibitor, Toxin $\mathrm{B}$, could significantly reduce the migration of LX-2 $(P<0.05)$. Salvianolic had no effect on the expression of CDC42, PKA1 and MLC, but it remarked inhibited the expression of p-MLC, p-PAK1 $(P<0.05)$ and the expression of p-CDC42 $(P<0.01)$.

Conclusion: Salidroside significantly reduced migration of LX-2 through inhibiting PDGF signaling pathway.

\section{PP15-20}

The Influence of Activated Rat Hepatic Stellate Cells on the Profile of CD4 T Lymphocyte Subsets

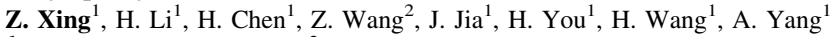

${ }^{1}$ Liver Research Center; ${ }^{2}$ Department of Nephropathy, Beijing

Friendship Hospital, Capital Medical University, Beijing, China

Background and aims: Liver fibrosis usually results from chronic inflammation induced by all kinds of injury factors. Each CD4 T lymphocyte subset participates in the process of chronic inflammation and fibrosis, and the effects on liver fibrosis are different among all the subsets. The profile of CD4 T lymphocyte subsets affects the development and outcome of inflammation. Hepatic stellate cells (HSCs) play a central role in development of liver fibrosis. And it was found that there was a direct interaction between activated HSCs or liver myofibroblasts and lymphocytes, which prevent the resolution of inflammation. The aim of the study was to detect the effect of activated rat HSCs on the profile of rat CD4 T lymphocyte subsets.

Methods: Rat CD4 $\mathrm{T}$ lymphocytes were cocultured with activated HSCs for $48 \mathrm{~h}$, with the CD4 T lymphocytes cultured alone as controls. The proportions of CD4 T lymphocyte subsets were detected through flow cytometry analysis. The galectin- 9 expression of activated HSCs was determined by cellular immunofluorescence, enzyme linked immunosorbent assay (ELISA), Western blot analysis and realtime PCR.

Results: The proportion of type 1 helper T lymphocytes (Th1 cells) decresaed at the end of coculture $(-1.73 \%, p=0.026)$ compared with the controls, while the proportions of $\mathrm{CD} 4^{+} \mathrm{CD} 25^{+} \mathrm{T}$ lymphocytes (natural regulatory $\mathrm{T}$ cells, nTregs) $(-0.73 \%, p=0.60)$ and type 2 helper T lymphocytes (Th2 cells) $(-0.47 \%, p=0.37)$ were similar to those of the controls. Activated rat HSCs expressed and secreted galectin- 9 , and the expression of galectin- 9 decreased when cocultured with CD4 T cells.

Conclusions: Activated rat HSCs cause change of CD4 T lymphocyte subsets profile and relatively suppress Th1 cells-induced response, the mechanism involved is still unclear. Activated rat HSCs express galectin-9 that can induce apoptosis of Th1 cells, and CD4 T lymphocytes downregulate galectin-9 expression in activated rat HSCs.

\section{PP15-21}

Macrophage-secreted Products Induce a Transient Phenotypic Change in Hepatocytes: Implications for Fibrogenesis

M. Melino ${ }^{1}$, V. Gadd ${ }^{1}$, R. Skoien ${ }^{1,2}$, G. Walker ${ }^{1}$, A. Clouston ${ }^{1}$, E. Powell ${ }^{1,2}$, J. Jonsson ${ }^{1}$

${ }^{1}$ Centre for Liver Disease Research, The University of Queensland;

${ }^{2}$ Gastroenterology and Hepatology, Princess Alexandra Hospital,

Brisbane, QLD, Australia

Background: Liver macrophages (Kupffer cells) play a key role in the progression of liver injury however their influence on hepatocyte function and viability remains unknown. The aim of this study was to investigate whether macrophage-secreted products alter the phenotype of these epithelial cells.

Methods: Macrophages were differentiated from THP-1 cells (monocyte cell line) via phorbol myristate acetate stimulation. The resulting macrophage-conditioned media $(\mathrm{M} \phi \mathrm{CM})$ was added to two different hepatocyte cell lines, HepG2 and Huh7. Expression of the EMT markers E-cadherin, vimentin, S100A4, collagen, transforming growth factor- $\beta$ (TGF- $\beta$ ) and $\alpha$-smooth muscle actin $(\alpha \mathrm{SMA})$ were measured using real-time PCR and immunofluorescence. Comparisons between untreated and M $\phi C M$-treated cells were analysed using a one-way ANOVA and Tukey's multiple comparison test. A $p$ value of $<0.05$ was considered statistically significant.

Results: Hepatocytes treated with $\mathrm{M} \phi \mathrm{CM}$ for $24 \mathrm{~h}$ acquired mesenchymal-like properties, with reduced E-cadherin $(p<0.05)$ and enhanced TGF- $\beta(p<0.001)$ mRNA expression. Huh7 and HepG2 cells demonstrated partial EMT due to the significant increase in mesenchymal markers, vimentin $(p<0.05)$ and S100A4 $(p<0.001)$ respectively. However $\alpha$ SMA and collagen, two markers associated with complete EMT, showed no significant change. Interestingly, hepatocyte phenotypic changes were transient as reversal of the mesenchymal-like properties was seen following a further 7 days in culture in normal media. No change in gene expression was observed in hepatocytes treated with conditioned media isolated from undifferentiated THP- 1 cells.

Conclusions: This in vitro study provides evidence that macrophagesecreted products induce transient phenotypic changes in hepatocytes that may contribute to fibrogenesis. Importantly, the data raise the possibility that hepatocytes may contribute to TGF- $\beta$ production during inflammatory liver injury with recruitment and activation of liver macrophage populations. 


\section{PP15-22}

Protective Effect of Low Molecular Weight Heparin-pluronic Nanogels on DMN-induced Hepatic Fibrosis Va TGF- $\beta$ /Smad Pathway

J.-H. Lee, H. Lee, K.H. Jung, S.-W. Hong, M.-K. Son, G.-Y. Lee, S.-S. Hong School of Medicine, Inha University, Incheon, Republic of Korea

Low molecular weight heparin $(\mathrm{LH})$ has been reported to have antifibrotic and anticancer effects. Herein, to develop a novel agent with enhanced efficacy and minimized adverse effects, we synthesized a novel low molecular weight heparin-Pluronic nanogel (LHP) by conjugating carboxylated pluronic F127 to LH. The formation and characterization of these nanogels were confirmed by ${ }^{1} \mathrm{H}-\mathrm{NMR}$, dynamic light scattering (DLS) and zeta potential measurements. LHP showed reduced anti-coagulant activity of about $33 \%$ of the innate activity. The efficacy of the LHP was evaluated in rats with liver fibrosis, in comparison with LH. Liver fibrosis was induced in rats by injection of $1 \% \mathrm{DMN}(1 \mathrm{ml} / \mathrm{kg} / \mathrm{i} . \mathrm{p}$.) for three consecutive days per week and LH or LHP (1,000 IU/kg body weight) was treated once a day for 4 weeks. LHP administration prevented DMN-mediated liver weight loss and decreased the values of aspartate transaminase, alanine transaminase, total bilirubin, and direct bilirubin and increased albumin. LHP markedly reduced the fibrotic area compared to LH. Also, LHP potently inhibited mRNA or protein expression of alpha-smooth muscle actin, collagen type I, matrix metalloproteinase-2, and tissue inhibitor of metalloproteinase-1 compared to $\mathrm{LH}$ in DMN-induced liver fibrosis. In addition, LHP decreased the expression of transforming growth factor- $\beta_{1}$ (TGF- $\beta_{1}$ ), p-Smad 2, and p-Smad 3, which are all important molecules of the TGF- $\beta /$ Smad signaling pathway. Taken together, these results are the first reports that LHP shows an anti-fibrotic effect in liver via inhibition of the TGF- $\beta /$ Smad pathway as well as elimination of extracellular matrix.

\section{PP15-23}

Aqueous Extract of Amomum xanthoides Ameliorate Dimethylnitrosamine Induced Chronic Liver Injury in Rats

J.H. Wang, J.W. Shin, H.B. Raghavendran, N.H. Lee, H.G. Kim, H.J. Park, M.K. Choi, C.G. Son

Liver and Immunology Research Center, Institute of Traditional Medicine and Bioscience of Daejeon University, Daejeon, Republic of Korea

Aim: Amomum xanthoides belong to tropical edible and medicinal plant that frequently used for various digestive disorders in Asia. In this present study, we investigate the protective effects of Amomum xanthoides and their action mechanism in DMN induced liver fibrosis in rats.

Materials and methods: Forty-eight Sprague-Dawley rats were divided into six groups of eight rats each (Normal, DMN, WAX50, WAX100, WAX200, DDB) and induced liver fibrosis by dimethylnitrosamine (DMN, $10 \mathrm{mg} / \mathrm{kg}$, ip) on three times per week for 3 weeks. Water extract of Amomum xanthoides (WAX 50, 100 or 200 $\mathrm{mg} / \mathrm{kg}$, po) was administered once a day for 3 weeks, and DDB (Dimethyl Dimethoxy Biphenyl Dicarboxylate, $2.5 \mathrm{mg} / \mathrm{kg}$, po) was used as a positive control.

Results: In vitro experiment, WAX showed low cytotoxicity and high capability of free radical scavenging compared to the positive control (silymarin and DDB). In vivo experiment, WAX significantly attenuated the DMN-induced excessive release of ALT, AST, ALP and total bilirubin $(p<0.01)$ in serum, and hydroxyproline and malondialdehyde contents in liver tissue $(p<0.01)$. WAX restored the content of total protein and albumin. Histophathological results also revealed that WAX markedly ameliorated the inflammation, necrosis and collagen accumulation.

Conclusion: Altogether, our results for the first time indicate that WAX exerts the hepatoprotective and anti-fibrotic properties against DMN-induced liver fibrosis in animal model.
PP15-24

The Regulation of Cyclooxygenase-2 Expression in Hepatic Stellate Cells and its Functional Role in the Cell Biology

M. Wang, J. Guo, C. Tu, J.M. Luk, S. Chen, J. Wang, S.L. Friedman Division of Digestive Diseases, Zhong Shan Hospital, Shanghai Medical College, Fu Dan University, Shanghai, China

Background and aims: Cyclooxygenase-2 (COX-2) is a rate-limiting enzyme of prostaglandin synthesis that implicated in inflammatory signaling, cell survival and ECM remodeling. The present study investigated the regulation of COX-2 expression in hepatic stellate cells (HSC) in vitro by lipopolysaccharide (LPS), the nature ligand of Toll like receptor 4 (TLR4) signaling, and vascular endothelial growth factor (VEGF), an important fibrogenic cytokine, and the functional impact of COX-2 iinhibition on the cell biology.

Methods and results: LX-2 cells (a human HSC line) were administrated with $100 \mathrm{ng} / \mathrm{ml} \mathrm{LPS}$, or $10 \mathrm{ng} / \mathrm{ml}$ VEGF for 12 and $24 \mathrm{~h}$, they were found to up-regulate the mRNA and protein expression of COX2 in the cells, as determined with real time quantitative PCR and Western Blot, respectively. Pretreated the cells with siRNA (100 mM) to TLR4 abrogated the upregulation of COX-2 by LPS stimulation. Similarly, the enhanced expression of COX-2 in the VEGF treated cells was inhibited by co-administration of LY294002 $(20 \mathrm{mM})$, a phosphatidylinositol 3-kinase (PI3K) inhibitor, or pre-transfection with dominant-negative AKT expression plasmids. Ectoricoxib, a highly selective inhibitor of COX-2, in doses of 1 or $10 \mathrm{mM}$ inhibited the induction of inflammatory and pro-fibrogenic cytokines expression in LX-2 cells exposed to LPS, including MCP-1, IL-1b and VEGF. It also inhibited HSC proliferation in a dose- and timedependent manner as determined by ${ }^{3} \mathrm{H}$ thymidine incorporation, and induced significant HSC apoptosis in LPS primed cells, as assayed by FACS and Annexin-V/PI staining, which was accompanied by decreased induction of anti-apoptotic molecules (phospho-ERK, phospho-AKT and Bcl-2).

Conclusion: COX-2 is inducible in activated HSC exposed to LPS, which is dependent on TLR4 signaling. It also interacts with VEGF signaling in the cells, with the involvement of PI3K and AKT. The induction of COX-2 triggered an LPS-mediated inflammatory cascade response in HSC and promoted cell survival.

\section{PP15-25}

Primary Isolated Kupfer Cells Could Maintain their Characters and Purify in Two Weeks Culture

H. Li, Z. Xing, H. Chen, J. Jia

Liver Research Center, Beijing Friendship Hospital, Capital Medical Univeristy, China, Beijing, China

Background: Kupffer cells are one of the most important mesenchymal cells in liver, which play a crucial role in liver fibrosis in recent reports. We report in detail a reliable and reproducible methodology for the isolation of $\mathrm{KC}$, which maintain highly purify and function in cell cultures.

Materials and methods: Wistar rats, male, about $300 \mathrm{~g}$ were selected as objects. Firstly, a 20G catheter was inserted into the portal vein of rat and secured with silk stitch, and then the liver was perfused and digested with DHANKS and HANKS containing 0.05\% collagenase IV respectively; secondly, the Kupffer cells isolated by 30 and $60 \%$ percoll gradient; and last step, isolated Kupffer cells were purified by selective adherence after $2 \mathrm{~h}$ of cultivation in high glucose DMEM containing $20 \%$ FBS. The kupffer cell identified by monoclonal antibody ED-2 and ED1. At same time, GFAP as a control antibody for telling apart HSC and Kupffer cells in 2 weeks culture. Kupffer cells function was evaluated by India ink and ED1 stain.

Results: This method resulted in a satisfactorily high yield of $6-18 \times 10^{6} \mathrm{KCs}$ per rat liver, over $95 \%$ positive for ED2 and ED1, while negative for GFAP which is positive in HSC. And kupffer cells keep highy purity (over 95\%) and function (over 95\%) in two weeks, 
providing ideal observe window for further research. After then $\mathrm{Ku}$ pffer cells isolated from rats became high rate of apoptosis and some myofibroblasts-like cells became growing. Besides kupffer cells have a character of resistance to $0.2 \%$ trypsin digestion.

Conclusion: The method for isolating and culturing kupffer cells in this study is effective and stable, and the biological characters are preserved in 2 weeks for further research.

\section{PP15-26}

Propolis Prevents Hepatic Fibrosis and Oxidative Damage Induced by Carbon Tetrachloride

S.K. Nirala, M. Bhadauria, R. Mathur

SOS in Zoology, Jiwaji University, Gwalior, India

Background and aim: Carbon tetrachloride $\left(\mathrm{CCl}_{4}\right)$ is a well known hepatotoxicant and exposure to this chemical is known to induce oxidative stress and liver fibrosis via formation of free radicals. The present study had been conducted to confirm the protective role of propolis extract on $\mathrm{CCl}_{4}$ induced hepatorenal oxidative stress and resultant liver fibrosis.

Methods: Animals were exposed to carbon tetrachloride $(0.15 \mathrm{ml} / \mathrm{kg}$, i.p.) for 5 months ( 5 days/week) followed by treatment with propolis extract $(200 \mathrm{mg} / \mathrm{kg}$, p.o.) for consecutive 1 month (5 days/week). Various blood and tissue biochemical and histological studies were conducted after $48 \mathrm{~h}$ of last administration.

Results: $\mathrm{CCl}_{4}$ exposure caused significant rise in level of transaminases, alkaline phosphatase, lactate dehydrogenase protein, urea, albumin, bilirubin, creatinine, triglycerides, cholesterol in serum and reduced blood sugar level. Lipid peroxidation and GSSG were also enhanced significantly, whereas GSH was decreased in the liver of carbon tetrachloride intoxicated group. Ethanolic extract of propolis successfully prevented these alterations in the experimental animals. Activities of catalase, superoxide dismutase, adenosine triphosphatase, glucose-6-phosphatase, acid and alkaline phosphatase were maintained towards normal with propolis therapy. Hydroxyproline and glycogen contents were altered due to $\mathrm{CCl}_{4}$ intoxication and propolis treatment recovered them towards control in a significant manner. Ultra and light microscopical studies showed considerable protection in liver with propolis treatment thus, substantiated the biochemical observations.

Conclusion: Our study demonstrated that propolis extract could protect the liver tissues against oxidative stress and hepatic fibrosis probably by regulating antioxidative defense activities and other metabolic processes.

\section{PP15-27}

Identification of a Novel Molecular Candidates-glia Maturation Factorbeta for Inhibiting the Activation of Hepatic Stellate Cells

H.Y. Rao, J.H. Wang, F. Liu, R. Fei, Z.D. Liu, L. Wei

Peking University People's Hospital, Peking University Hepatology Institute, Beijing, China

Background and aims: To further explore the mechanism of the inhibitory effect of interferon-beta-1a (IFN- $\beta$-1a) on the activation of human hepatic stellate cells (HSCs) LX-2, we investigated changes in the proteome associated with IFN- $\beta$-1a treatment of LX-2 cells.

Methods: Two-dimensional gel electrophoresis (2-DE) was performed to compare protein patterns of control IFN- $\beta$ - 1 a-untreated and treated LX-2 cells. Western blotting, immunohistochemical staining, and RT-PCR were used to validate the expression of the changed proteins.

Results: The results showed that 31 proteins spots displayed quantitative changes in expression after IFN- $\beta$-1a treatment. For the 31 spots, 20 proteins were identified, of which, glia maturation factorbeta (GMF- $\beta$ ) was existed in IFN- $\beta$-1a-treated LX- 2 cells only, but not in controls. Western blot analysis confirmed the results that GMF$\beta$ protein levels increased significantly in IFN- $\beta$-1a-treated LX-2 cells. The expression of GMF- $\beta$ mRNA and protein were more in normal liver tissue than the cirrhotic liver tissue. The expression of GMF- $\beta$ mRNA and protein were strengthen in rat brain, spleen, liver, and kidney tissues. GMF- $\beta$ transfection inhibited the expressions of alpha-smooth muscle actin and collagen type I (COLI) on LX-2 cells. Conclusion: The present study shows that GMF- $\beta$ can inhibit the activation of HSCs and perhaps play a role in protecting from liver fibrosis.

This work was supported by National S\&T Major Project for Infectious Diseases Control (2008ZX10002-013).

\section{PP15-28}

Anti-fibrotic Effect of L-2-Oxothiazolidine-4-Carboxylic Acid in a Dimethylnitrosamine Rat Model

I.H. Kim ${ }^{1}$, B.J. Jeon ${ }^{2}$, H.I. Kim ${ }^{1}$, S.H. Kim ${ }^{1}$, S.W. Kim ${ }^{1}$, S.O. Lee ${ }^{1}$, S.T. Lee ${ }^{1}$, D.G. $\mathrm{Kim}^{1}$

${ }^{1}$ Internal Medicine; ${ }^{2}$ Chonbuk National University Medical School and Hospital, Jeonju, Republic of Korea

Backgroud/aims: L-2-Oxothiazolidine-4-carboxylic acid (OTC) is a cysteine prodrug that maintains glutathione (GSH) in tissues. The present study was designed to investigate whether the OTC was active against dimethylnitrosamine (DMN) induced liver fibrogenesis.

Methods: Male Sprague-Dawley rats were divided into 4 groups including control, DMN, OTC 250 and OTC 500 groups. DMN was intraperitoneally injected as dissolved in sterile saline at a dose of 10 $\mathrm{mg} / \mathrm{kg}$ of body weight per day three times per week for 8 weeks. OTC 250 or OTC 500 group was treated with injections of DMN and daily with OTC at a dose 250 or $500 \mathrm{mg} / \mathrm{kg}$ by oral gavage for the length of the study, respectively. Anti-fibrotic effect of OTC was investigated in relation to blood chemistry, histopathology, immunohistochemistry, and western blot analysis.

Results: Treatment with OTC (250 or $500 \mathrm{mg} / \mathrm{kg}$ ) was found to reduce total bilirubin levels but to increase albumin levels. Treatment with OTC ( 250 or $500 \mathrm{mg} / \mathrm{kg}$ ) significantly ameliorated liver fibrosis as compared to the DMN group. Rats in the DMN group showed a typical histological picture of bridging fibrosis or cirrhosis. However, OTC-treated rats did not develop cirrhosis and suffered only from periportal fibrosis. When we assessed the stage of fibrosis and grade of lobular activity using Knodell pathological scores, the OTC-treated rats showed significantly less fibrosis and inflammatory activities than DMN-treated rats. Immunohistochemical staining for $\alpha$-SMA showed that its expression in the DMN group was significantly greater than in the control group. However, OTC-treated groups showed significantly lower $\alpha$-SMA expression than the DMN group. Furthermore, the OTC treatment groups showed markedly lower levels of $\alpha$-SMA and TGF$\beta 1$ expression than the DMN-treated model group on western blot analysis.

Conclusions: OTC significantly ameliorated liver fibrosis in a DMN rat model.

\section{PP15-29}

Differential Expression of NADH Oxidase in the Progression of Liver Fibrosis due to $\mathrm{CCl}_{4}$ Intoxication

B.C. Chakraborty, S. Ghatak, A. Biswas, K. Basu, A. Chowdhury, A. Santra Center for Liver Research, Institute of Post Graduate Medical Education and Research, Kolkata, India

Background and aim: Phagocytic NADPH oxidase plays an important in the progression of liver fibrosis, however little information is available the involvement of different isoforms of NADPH oxidase in the activation and perpetuation of hepatic stellate cells leading to the development of hepatic fibrosis. The present study was aimed to determine the involvement of different isoforms of NADPH oxidase in hepatic fibrogenesis.

Methods: Male C57BL/6 mice were injected with $\mathrm{CCl} 4$ intraperitoneally $(0.3 \mathrm{ml} / \mathrm{kg})$ thrice in a week for 9 weeks. Batches of mice were 
sacrificed sequentially after $1 \mathrm{st}, 3 \mathrm{rd}, 6$ th and 9 th week of exposure. To investigate the role of different isoforms of NADPH oxidase in hepatic fibrogenesis, we performed microscopical examination, immunohistochemistry, immunoblotting, RT-PCR, hepatic hydroxyproline and NADPH oxidase assay. In some experiments Gadollium chloride $\left(\mathrm{GdCl}_{3}\right)$, lipopolysaccharides (LPS) and poly I:C were treated before $\mathrm{CCl}_{4}$ administration.

Results: $\mathrm{CCl}_{4}$ treatment caused profound elevation of NADPH oxidase in liver associated with $\mathrm{p} 22^{\text {phox }}$ mRNA expression, activation of HSCs and senescence of some activated HSCs after 1st week of treatment. Continuation of $\mathrm{CCl}_{4}$ treatment gradually decreased NADPH oxidase activity with decrease of $\mathrm{p} 22^{\text {phox }}$ mRNA expression, but increased gp91 ${ }^{\text {phox }}$ mRNA expression with time. Liver histology showed evidence of fibrosis after 6 weeks of $\mathrm{CCl}_{4}$ treatment. Pretreatment with $\mathrm{GdCl}_{3}$ abrogated expression of p22 ${ }^{\text {phox }}$, senescence of HSCs and improvement of liver histology, while LPS pretreatment accelerates the fibrogenic process.

Conclusions: Thus it can be concluded that the increased expression of phagocytic NADPH oxidase p22 $2^{\text {phox }}$ leads to increase accumulation of senescent HSCs within the injured liver. These senescent HSCs causes increased expression of non-phagocytic form NADPH oxidase gp91 ${ }^{\text {phox }}$ that ultimately results in liver fibrosis if the exposure is continued.

\section{PP15-30}

Senescence of Activated Hepatic Stellate Cells Activates and Proliferates Hepatic Myofibroblast at an Early Phase of Liver Injury

S. Ghatak, B.C. Chakraborty, A. Biswas, K. Basu, G.K. Dhali, A. Chowdhury, A. Santra

Center for Liver Research, Institute of Post Graduate Medical Education and Research, Kolkata, India

Background and aim: Senescent Hepatic Stellate Cells (HSCs) accumulate in the fibrotic liver and induce secretion of the inflammatory mediators. At an early phase of liver injury, the HSCs undergo stress induced senescence. The aim of this study was to evaluate the functional contribution of senescent HSCs in liver pathology.

Methods: Male C57BL6/J mice were injected with $\mathrm{CCl}_{4}$ intraperitoneally $(0.3 \mathrm{ml} / \mathrm{kg})$ thrice in a week. The mice were sacrificed 48 hours after the last dose of $\mathrm{CCl}_{4}$. Liver histology by $\mathrm{H} \& \mathrm{E}$ staining, immunohistochemistry for CCL5, SMA, p53 and p21 were performed. RT-PCR for proinflammatory cytokines and iNOS were also carried out. Cellular senescence was evaluated by SA b-gal staining. Some experiments were also carried out with aminoguanidine pretreatment, an inhibitor of $\mathrm{NO}$ before $\mathrm{CCl}_{4}$ exposure.

Results: In C57BL/6 mice liver histology showed eosinophilic areas around the portal zones. These areas showed green birefringence with congo red staining when observed under polarized microscope and were suggested to be amyloid. Possibly the amyloid deposition was due to development of hepatic nitrostative stress, as evidenced by increased mRNA expression of iNOS. Aminoguanidine pretreatment significantly reduced $\mathrm{CCl}_{4}$ induced liver injury and amyloid deposition. SA b-gal staining confirmed senescence of both hepatocytes and HSCs. HSCs senescence was confirmed by co localization of SMA, p53 and p21 proteins. Further $\mathrm{CCl} 5$ was also localized in proximity of activated and senescent HSCs indicating the fact that they might play an important role in HSCs activation. The mRNA expressions of proinflammatory cytokines like IFN- $\gamma$, IL-12, IL- 6 and TNF- $\alpha$ were highly expressed.

Conclusion: Thus it can be concluded that $\mathrm{CCl}_{4}$ treatment in $\mathrm{C} 57 \mathrm{BL} /$ 6 mice caused hepatic nitrostative stress, amyloid deposition and senescence of liver cells including HSCs at an early phase. The senescent cells may facilitate further activation and proliferation of HSCs within the liver.
PP15-31

Sox9 Down-regulates Wnt/ $\beta$-catenin Signaling to Attenuate Liver Fibrogenesis in vitro and in vivo

W. Li, Y. Li, R. Gao

Infectious Diseases, Anhui Provincial Hospital, Hefei, China

Background and aim: $\mathrm{Wnt} / \beta$-catenin signaling was reported to play an important role in hepatic fibrosis, Prior studies showed that Sox 9 acted as a strong negative regulator of $\mathrm{Wnt} / \beta$-catenin signaling, Whether Sox 9 can inhibit hepatic fibrosis remains unknown. To address this issue, we determined the effect of Sox 9 on hepatic fibrosis induced by $\mathrm{CCl}_{4}$ in rats.

Methods: Recombinant plasmid pIRES-hrGFP-1-Sox9 was transfected into liver in vivo and HSC-T6 cells in vitro. Changes in liver pathology were examined by Hematoxylin and eosin (H\&E staining) and Masson's trichrome staining. Meanwhile, The levels of ALT, AST, LDH, HN and LA in serum and Hyp in liver tissue were measured, We also measured the expressions of $\alpha$-SMA, TIMP- 1 and $\beta$-catenin in HSCs and liver by real-time PCR and western-blotting respectively.

Results: Sox 9 obviously attenuated the histological changes, decreased the content of hydroxyproline (Hyp) in liver. Sox9 also markedly reduced the mRNA and protein expressions of $\alpha$-SMA, $\beta$-catenin in liver and HSC-T6 cells. More importantly, Sox9 significantly reduced the viability of HSC-T6 cells. These result suggested that Sox9 attenuates $\mathrm{CCl}-4$ induced liver fibrogenesis through down-regulating the canonical Wnt signaling pathway.

Conclusions: Our results revealed that Sox 9 attenuated the process of hepatic fibrosis by down-regulating the canonical Wnt signaling pathway. It provides us a new liver anti-fibrosis drug at a genetic level.
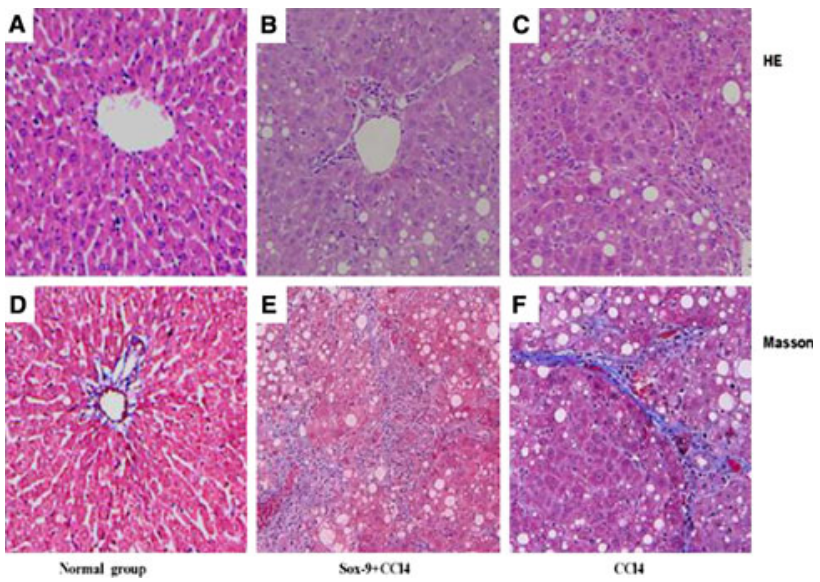

Fig. 1 Sox 9 attenuated liver pathology induced by $\mathrm{CCl}_{4}$

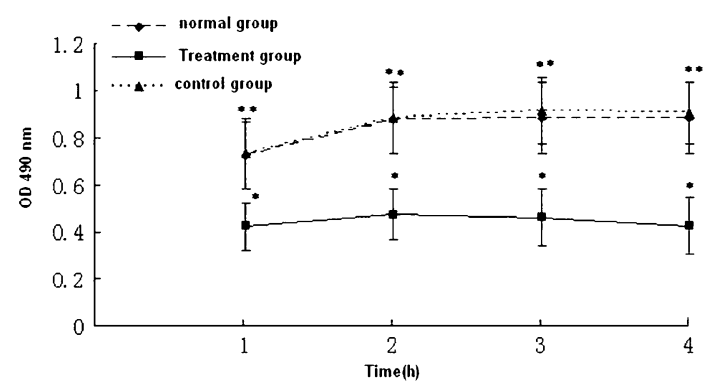

Fig. 2 Sox-9 reduced the viability of HSC-T6 cells 


\section{PP15-32}

Serum Retinol Binding Protein 4 as a Unique Surrogate Marker of End Stage Liver Disease with Complications. A Pilot Trial

P.P. Basu ${ }^{1,2}$, N. Krishnaswamy ${ }^{2}$, N.J. Shah ${ }^{2}$, T. Pacana ${ }^{2}$, C. Tang $^{2}$, S. Foulstin ${ }^{2}$, R.J. Brown ${ }^{1}$

${ }^{1}$ Columbia NY Presbyterian College of Physicians and Surgeons, NY, Manhattan; ${ }^{2}$ North Shore Forest Hills Hospital New York, Forest Hills, NY, USA

Background: Retinol binding protein 4 (RBP-4),a 21 kilodalton plasma protein, is secreted from the liver and adipose tissue, and is known to transport retinol in the blood. RBP-4 levels have an inverse relationship with disease progression and decompensation. This study evaluates the role of RBP-4 on assessing the severity of decompensated liver disease

Methods: 85 patients $(n=85)$ (age $=49.6 \pm 11.4$, mean \pm SD) with moderate to -severe liver disease were recruited into three groups: Group A, Chronic liver disease with cirrhosis $(n=30): 20 / 30$ HCV $(67 \%)$ 8/30 HBV (27\%) 2/30 Misc (6.6\%); Group B, non-cirrhotic $(n=30)$ 13/20 HCV (43\%), 4/20 HBV (13.3\%), 5/30 Alcoholics (17\%), and 5/30 misc (17\%). Group C, controls $(n=25)$. Liver biopsy, insulin resistance (HOMA-score), MELD score, adiponectin, Leptin and serum RBP-4 (ELISA), APRI, Hyaluronic acid, and Hepascore for liver fibrosis were analyzed

Results: There was a significant difference in RBP-4 across the cirrhotic, non-cirrhotic, and control groups $[F(2,82)=47.9, p<0.001$; one-way ANOVA]. Posthoc Bonferroni tests over controls $(n=25$, $4.21 \pm 1.60$; mean $\pm \mathrm{SD}$ ). RBP4 is significantly decreased in Cirrhotics $(p<0.001 ; n=30,1.46 \pm 0.51)$ over the non-Cirrhotics $(p=0.015 ; n=30,3.37 \pm 0.97)$ and controls. Cirrhotics also had decreases in HOMA $(1.09 \pm 0.09$ vs. $1.51 \pm 0.60 ; p=0.001$, Students $t$ test),adiponectin $(0.93 \pm 0.40$ vs. $1.26 \pm 0.53 ; p=0.01)$, but with an increase in TNF-alpha (11.23 \pm 3.81 vs. $3.72 \pm 3.37$; $p<0.001)$ compared to the non-cirrhotic patients. Leptin $(8.20 \pm 3.23$ vs. $10.93 \pm 9.44 ; p=0.14)$ and hepatic steatometry $(0.73 \pm 0.83$ vs. $1.10 \pm 1.21 ; p=0.19$ showed no difference. RBP 4 has no relevant correlations with TNF-alpha $(r=-0.62, p<$ $0.001)$, leptin $(r=0.12, \quad p=0.38)$ and hepatic steatometry $(r=0.12, p=0.41)$ RBP-4 correlated negatively with MELD score $(r=-0.57, p=0.001)$

Conclusions: This pilot study proposed that RBP-4 has a role as a biomarker for endstage liver disease, with an independent, inverse relationship with severity of fibrosis, hepatic decompensation, MELD score, and complications of liver failure. There is a positive correlation with insulin resistance, metabolic syndrome, and Adipokines, but not with intra-hepatic steatometry

\section{PP15-33}

LPS, Immune Activation and Liver Disease in HIV-HBV Co-infection and the Effects of HBV-active HAART

M. Crane ${ }^{1,2}$, R. Rajasuriar ${ }^{1,2,3}$, A. Avahingsanon ${ }^{4}$, G. Matthews ${ }^{5}$, N. Skinner ${ }^{1}$, P. Tangkitvanich ${ }^{4}$, G.J. Dore ${ }^{5}$, K. Visvanathan ${ }^{1}$, K. Ruxrungtham ${ }^{4}$, S. Lewin ${ }^{1,2,6}$

${ }^{1}$ Monash University; ${ }^{2}$ Burnet Institute, Melbourne, VIC, Australia; ${ }^{3}$ University of Malaya, Kaula Lumpur, Malaysia; ${ }^{4}$ Chulalongkorn University, Bangkok, Thailand; ${ }^{5}$ University of New South Wales, Sydney, NSW; ${ }^{6}$ Alfred Hospital, Melbounre, VIC, Australia

Background: Patients with HIV-HBV co-infection have accelerated progression of HBV-related liver disease. HIV infection leads to increased microbial translocation (circulating LPS). LPS can directly activate fibrogenic liver cells and has been associated with liver disease in HIV-HCV co-infection. We aimed to determine if LPS was elevated in HIV-HBV co-infection, whether this was associated with liver disease severity and if the effecof HBV-active HAART.
Materials and methods: Patients were recruited as part of two prospective, randomized controlled trials of $\mathrm{HBV}$-active HAART in Bangkok, Thailand $(n=55)$. After 48 weeks all eligible patients were placed on a tenofovir (TDF) based regimen $(n=47$, median follow up 4 years). Controls included Thai treatment naive HBV mono-infected patients $(n=20)$ and Thai uninfected controls $(n=20)$. We measured the amount of LPS and immune activation markers (sCD14, IL-10, -6, -17, IFN-g, TNF-a, CXCL-10 and CCL-2) in treatment naïve patients and controls and in co-infected patients on therapy. Liver biopsies were scored using the Metavir scale in a subset of co-infected patients $(n=26)$.

Results: LPS, sCD14, CXCL-10 and CCL-2 were significantly elevated in treatment naïve HIV-HBV co-infection compared with uninfected controls $(p<0.001)$. Levels of LPS, CXCL-10 and sCD14 remained significantly elevated after 1 year of HAART $(p<0.001$ for all) but after 4 years there was no significant difference compared to uninfected controls. In contrast, CCL-2 remained significantly elevated even after 4 years on HAART ( $p=0.001)$. Levels of IL-10, IL-17, TNF-a, IL-6 and IFN-g were not significantly different from controls. We found no significant correlation between LPS levels and liver disease severity, based on biopsy $(r=0.11 ; p=0.42)$.

Conclusion: LPS, sCD14 and CXCL10 are elevated in HIV-HBV coinfection. Levels return to normal following HBV-active HAART. There was no significant association between LPS and liver disease severity. Persistent elevation of CCL-2 on HAART may potentially play a role in ongoing disease progression.

\section{PP15-34}

The Role of IAH on the Expression of Aquaporin 1 and Aquaporin 5 in the Lung of Cirrhotic Mice

C.-Q. Yang, Z.J. Zhang

Division of Gastroenterology and Institute of Digestive Diseases, Tongji Hospital, Tongji University School of Medicine, Shanghai, China

Objective: To investigate the role of intra-abdominal hypertension caused by ascites on expression of AQP1 and AQP5 in lung of cirrhotic mice.

Methods: 50 male ICR mice, 10 mice were for the control group (IAP $=0 \mathrm{~cm} \mathrm{H}_{2} \mathrm{O}$ ), The model of cirrhosis were prepared by subcutaneous injection Carbon tetrachloride for 40 mice and then randomly divided into 4 groups: cirrhosis (IAP $=0 \mathrm{~cm} \mathrm{H}_{2} \mathrm{O}$ ) group, cirrhosis (IAP $=5 \mathrm{~cm} \mathrm{H}_{2} \mathrm{O}$ ) group, liver cirrhosis (IAP $=10 \mathrm{~cm}$ $\mathrm{H}_{2} \mathrm{O}$ ) group, liver cirrhosis (IAP $=20 \mathrm{~cm} \mathrm{H} \mathrm{H}_{2} \mathrm{O}$ ) group, different volume of albumin in saline were injected into the peritoneal of each mouse in order to form different intra-abdominal pressure. All mice were sacrificed after $24 \mathrm{~h}$, then the blood gas analysis, lung pathology, lung wet/dry ratio were determined; the expression of AQP1 and AQP5 at the protein and mRNA levels were analyzed by IHC and qRT-PCR.

\section{Results:}

1. Compared with the normal mice, the expression of AQP1 and AQP5 in lungs of cirrhotic mice were significantly decreased;

2. As the intra-abdominal pressure increased, lung W/D ratio were increased, and the expression AQP1 and AQP5 were correspondly increased.

\section{Conclusion:}

1. Cirrhosis can affect the expression of AQP1, AQP5 in lungs;

2. in mice with abdominal pressure increases, the expression of AQP5, AQP1 in lungs of cirrhotic mice were increased, and correlated closely with the severity of pulmonary edema. 


\section{PP15-35}

Effects of Magnesium Isoglycyrrhizinate on TGF- $\beta /$ Smad Signal Expression in Hepatic Stellate Cells in Rats

Z.-T. Peng ${ }^{1}$, S.-J. Long ${ }^{1}$, Y.-X. Liu ${ }^{2}$, J. Li ${ }^{1}$, P. Wang ${ }^{1}$

${ }^{1}$ Infectious Diseases, The First Affiliated Hospital of Nanhua

University, Hengyang; ${ }^{2}$ Infectious Diseases, The Third People's

Hospital of Shenzhen, Shenzhen, China

Objective: To investigation the effects of Magnesium isoglycyrrhizinate (MI) on TGF- $\beta /$ Smad signal expression in cultured HSC stimulated by TGF- $\beta_{1}$ and then to reveal the possible molecular mechanism of MIG on anti-fibrosis.

Methods: The rat HSC-T6 were cultured with or without MIG $(0 \sim 10 \mathrm{mg} / \mathrm{ml})$ in vitro after TGF- $\beta_{1}$ stimulation. The inhibitory rate of proliferation was measured by MTT method, the viability of cells was tested by evaluation of supernant LDH. The mRNA level of TGF- $\beta_{1}$, Smad3, Smad 7 were assessed by semi-quantitative RT-PCR method.

Results: MTT method showed an obvious inhibition of HSC proliferation after MIG $(0-10 \mathrm{mg} / \mathrm{ml})$ treatment. Different concentrations of MIG did not affect the vitality on HSC by LDH measurement. Different concentrations of MIG increased the mRNA level of TGF$\beta_{1}$, Smad 3, Smad7 in HSC-T6, and showed a certain trend of doseeffect relationship, and the mRNA of TGF- $\beta 1$ and Smad3, Smad7 expression were consistent changes.

Conclusions: MIG has significant impact on rat hepatic fibrosis liver TGF- $\beta /$ Smad signaling expression Inhibited rat hepatic stellate cell growth and proliferation, and its mechanism may be reduce the expression of TGF- $\beta 1$, Smad3, Smad7 mRNA.

\section{PP15-36}

Long-term Transforming Growth Factor- $\beta 1$ Exposure Induced a Sustained Epithelial-mesenchymal Transition Process in Hepatic Oval Cells with an Increased $\alpha$-fetoprotein Expression

P. Wang, T. Liu, M. Cong, H. Ma, B. Wang, J. Jia, H. You

Liver Research Center, Beijing Friedship Hospital, Beijing, China

Background: Transforming growth factor- $\beta 1$ (TGF- $\beta 1$ ) is the key regulator in the initiation and progression of liver fibrosis, and we have learned TGF- $\beta 1$ could induce an increased expression of extracellular matrix $(\mathrm{ECM})$ in hepatic progenitors within 2 days through epithelial-mesenchymal transition (EMT). The aim of the present study is to analyze the long-term effects of TGF- $\beta 1$ exposure on hepatic progenitors in vitro.

Methods: Hepatic oval cells were isolated from rats fed a cholinedeficient diet supplemented with ethionine and characterized by flow cytometry. For TGF- $\beta 1$ treatment, the cells were cultured in $10 \%$ FBS-DMEM/F12 medium containing $1 \mathrm{ng} / \mathrm{ml}$ TGF- $\beta 1$ for 16 days with the medium replaced every 2 days.

Results: Hepatic oval cells were positive for the progenitor-specific markers OV-6, $\alpha$-fetoprotein (AFP) and Dlk, as well as hepatocyte marker albumin and cholangiocyte marker cytokeratin 19. In the first four days of TGF- $\beta 1$ exposure, the cell number gradually reduced, the cell size continuously became larger and more and more cell filaments appeared in the cytoplasm, while the cell number and cell morphology sustained in the following days. Real-time PCR results showed TGF- $\beta 1$ upregulated snail expression and kept downregulation of e-cadherin, with 3 times more snail and $20 \%$ e-cadherin at 16th day, indicating the sustained EMT effects of TGF- $\beta 1$. Furthermore, TGF$\beta 1$ increased the ECM expression by collagen I, collagen III, connective tissue growth factor, and tissue inhibitor of metalloproteinase, in a time-dependent manner, which confirmed the sustained EMT process. Interestingly, the expression of AFP and cytokeratin 19 increased time-dependently to 10 folds and nearly 30 folds, respectively, at 16 th day after TGF- $\beta 1$ exposure.

Conclusion: Long-term TGF- $\beta 1$ exposure induces a sustained EMT process in hepatic oval cells with increased AFP expression, which might partially interpret the increased serum AFP level in cirrhosis patients.

\section{PP15-37}

Erythropoietin Decreases Carbon Tetrachloride-induced Hepatic Fibrosis by Inhibiting Transforming Growth Factor-beta

S.Y. Park, W.Y. Tak, Y.O. Kweon, M.K. Jung, S.W. Jeon, C.M. Cho,

S.K. Kim, J. Heo, D.W. Lee, H.S. Lee, H.G. Park, K.Y. Shin

Gastroenterology and Hepatology, Kyungpook National University Hospital, Daegu, Republic of Korea

Background and aim: In addition to hematopoietic effect, the erythropoietin is known as a multifunctional cytokine with antifibrosis and organ-protective activities. The purpose of this study is to evaluate the effect of recombinant human erythropoietin (rhEPO) on hepatic fibrosis and hepatic stellate cells (HSCs).

Methods: Carbon Tetrachloride $\left(\mathrm{CCl}_{4}\right)$ induced hepatic fibrosis mice models were used for in vivo study and hepatic stellate cells (HSCs) line for in vitro study. $\mathrm{CCl}_{4}$ and $\operatorname{rhEPO}(0,200$ or $1,000 \mathrm{U} / \mathrm{kg})$ was injected intraperitoneally in $\mathrm{BALB} / \mathrm{c}$ mice three times a week for 4 weeks. Immunohistochemistry and immunoblotting were performed to evaluate expressions of transforming growth factor- $\beta 1$ (TGF- $\beta 1$ ), $\alpha$-smooth muscle actin ( $\alpha$-SMA), and fibronectin in explanted liver. Immunoblotting of $\alpha$-SMA, phophorylated Smad- 2 and Smad-2/3 was performed in HSCs treated with TGF- $\beta 1$ and/or rhEPO.

Results: Expressions of TGF- $\beta 1, \alpha$-SMA, and fibronectin were increased in $\mathrm{CCl}_{4}$ injected mice livers, but significantly attenuated by co-treatment with $\mathrm{CCl}_{4}$ and rhEPO. Co-treatment of rhEPO markedly suppressed fibrosis in Masson's trichrome compared to treatment of only $\mathrm{CCl}_{4}$. By identifying the expression of rhEPO receptors in HSCs by RT-PCR, we confirmed the involvement of rhEPO receptor dependent anti-fibrosis effect in experiment animal models. TGF- $\beta 1$ increased phosphorylated $\alpha$-SMA, Smad- 2 expressions in HSCs, which were decreased by rhEPO co-treatment.

Conclusions: Treatment of rhEPO effectively suppressed fibrosis in $\mathrm{CCl}_{4}$-induced liver fibrosis mice models. Anti-fibrosis effect of rhEPO could be related to inhibition of TGF- $\beta 1$ induced activation of HSCs.

\section{PP15-38}

Human MMP-1 Transgene Protects Experimentally Induced Hepatic Fibrosis in Mice

J. George ${ }^{1,2}$, J. D'Armiento ${ }^{2}$, M. Tsutsumi ${ }^{1}$

${ }^{1}$ Department of Gastroenterology and Hepatology, Kanazawa Medical University, Uchinada, Ishikawa, Japan; ${ }^{2}$ Division of Molecular Medicine, Department of Medicine, Columbia University, New York, NY, USA

Hepatic fibrosis is characterized by excessive synthesis and deposition of fibrillar collagens in the liver with impaired turnover. Since the major collagenolytic enzyme, matrix metalloproteinase-1 (MMP-1) is absent in mice, MMP-13 alone is responsible for collagen remodeling during fibrogenesis. In the present study, we examined whether transgenic expression of human MMP-1 could protect the N-nitrosodimethylamine (NDMA) induced hepatic fibrosis in mice. Hepatic fibrosis was induced in wild-type and MMP-1 transgenic mice through intraperitoneal injections of NDMA in doses of $1 \mathrm{mg} / 100 \mathrm{~g}$ body weight on 3 consecutive days of every week over a period of 4 weeks. NDMA administrations resulted in marked elevation of serum AST, ALT, hyaluronic acid (HA), transforming growth factor-b1 (TGF-b1) and procollagen-III peptide in wild-type mice. There was marked activation of hepatic stellate cells, deposition of collagen-1 and HA in the liver. However, these processes were markedly decreased in NDMA administered MMP-1 transgenic mice. qRT-PCR and Western blotting for collagen I, a-smooth muscle actin (a-SMA), and TGF-b1 demonstrated marked upregulation of mRNA and protein levels respectively, in NDMA treated wild-type mice but not in 
similarly treated transgenic mice. Our study demonstrated that transgenic expression of MMP-1 protects the liver from NDMA induced hepatic fibrosis in mice by preventing excessive deposition of collagens in the liver. Our results further indicate that methods to upregulate the activity of MMP-1 could provide an option for therapeutic intervention of human hepatic fibrosis.

\section{PP15-39}

Nitrix Oxide Plays a Role in Mediating the Effects of Adiponectin on Hepatic Stellate Cell Proliferation, Migration and Apoptosis

L. Su, J. George, Y. Liu, J. Wang, Storr Liver Unit, Westmead Millennium Institute, University of Sydney

Westmead Millennium Institute, Westmead, NSW, Australia

Introduction: Adiponectim ameliorates liver fibrosis in rodent models, but the mechanisms have not been completely elucidated. Nitric oxide (NO) has anti-fibrogenic properties and adiponectin is known to stimulate endothelial Nitric Oxide Synthase (eNOS) and $\mathrm{NO}$ production in non-liver cells. We hypothesized that adiponectin may promote NOS expression and NO production in hepatic nonparechymal cells and thereby modulate the anti-fibrogenic effects of adiponectin.

Methods: Rat HSCs, KCs and SECs were isolated and cultured. Recombinant adiponectin $(5 \mu \mathrm{g} / \mathrm{mL})$ was added to HSCs, KCs and SECs for $24 \mathrm{~h}$. For inhibition experiments, adiponectin $(5 \mu \mathrm{g} / \mathrm{mL})$ or adiponectin and the NO inhibitor (L-NAME $1 \mathrm{mM}$ ) were added to HSCs for $24 \mathrm{~h}$. iNOS gene and protein expression in HSCs and KCs as well as eNOS phosphoprotein expression in SECs were analyzed by qPCR, immunblot and flow cytometry. NO metabolite concentrations $\left(\mathrm{NO}_{2} / \mathrm{NO}_{3}\right)$ in culture medium were measured using the $\mathrm{NO}$ Assay Kit. HSC proliferation was determined by BrdU incorporation. HSC migration was examined by wound healing assay. HSC apoptosis was assessed by Annexin V flowcytometry and DAPI staining. Results: Adiponectin enhanced HSC and KC iNOS gene (1,000 and $8,000$ fold respectively, $p<0.0001)$ and protein expression $(7.2$ and 7.7 fold respectively, $p<0.001)$. Adiponectin increased SEC eNOS protein $(1.7$ fold, $p<0.05)$. As expected, adiponectin increased NO $\left(\mathrm{NO}_{2} / \mathrm{NO}_{3}\right)$ concentration in $\mathrm{HSC}, \mathrm{KC}$ and SEC medium. Adiponectin attenuated HSC proliferation and migration $(p<0.01)$ as well as augmented HSC apoptosis. These effects were partially reversed after L-NAME treatment $(p<0.01,0.05$ and 0.01$)$.

Conclusions: Adiponectin up-regulates iNOS/eNOS/NO expression in hepatic nonparechymal cells. Adiponectin inhibits HSC proliferation and migration as well as enhances HSC apoptosis. The latter effects are at least, in part, mediated through adiponectin-induced NO production. Further experiments to determine the signaling pathways which modulate adiponectin-mediated iNOS/eNOS/NO expression are under investigation.

\section{PP15-40}

CYP2E1 Inhibitor (DDC) Significantly Upregulates MMP-1 Expression through ERK1/2, p38MAPK and AKT Signaling Pathways

T. Liu, M. Cong, P. Wang, J. Jia, H. You

Liver Research Center, Beijing Friendship Hospital, Capital Medical University, Beijing, China

Background/aims: Cytochrome P450 2E1 (CYP2E1)-derived reactive oxygen species from hepatocyte mediate paracrine stimulation of collagen I protein synthesis by hepatic stellate cells, and CYP2E1 inhibitors block this effect, but the mechanism has not been fully clarified.

Methods: A model was developed using co-cultures of C3A cells, which do express CYP2E1 (C3A-2E1) or do not express CYP2E1 (C3A) with human hepatic stellate cells (LX-2). The co-culture systems were treated by diethyldithiocarbamate (DDC, CYP2E1 inhibitor), and the effects of DDC on collagen I, MMP-1 and activation of MAPK pathway in LX-2 were evaluated.

Results: Compared with LX-2 co-cultured with $\mathrm{C} 3 \mathrm{~A}$, both the intracellular $\mathrm{H}_{2} \mathrm{O}_{2}$ level and collagen I expression increased in LX-2 co-cultured with $\mathrm{C} 3 \mathrm{~A}-2 \mathrm{E} 1$, and DDC inhibited the upregulation of $\mathrm{H}_{2} \mathrm{O}_{2}$ and collagen I. During this process, the transcription of MMP-1 was pronounced at $24 \mathrm{~h}$ (12-fold) and $48 \mathrm{~h}$ (10-fold) after exposed to DDC in LX-2 co-cultured with C3A-2E1, while the collagen I transcription kept stable, suggesting a post-transcription regulation of collagen I. Accordingly, DDC induced a time-dependent upregulation of MMP-1 both in the supernatant and in LX-2 cells co-cultured with C3A-2E1, while the secretion of collagen I were significantly reduced, suggesting the downregulation of collagen I due to the upregulation of MMP-1. Furthermore, the results of Phospho-MAPK Array Proteome showed that when exposed to DDC the AKT signaling pathway was activated and the ERK1/2 and p38 signaling pathways were inhibited in LX-2 co-cultured with C3A-2E1, and western blot results further confirmed these changes on the signal pathways.

Conclusions: These results suggested that increased expression and secretion of MMP-1 by DDC was responsible for the decrease in collagen I protein produced by the $\mathrm{C} 3 \mathrm{~A}-2 \mathrm{E} 1$ co-culture, and the upregulation of MMP-1 by DDC may be associated with the modulation of ERK1/2, p38 and AKT signaling pathways.

\section{PP15-41}

Regulation of Inflammation-Driven ECM Remodelling by CD147 and its Interaction with Extracellular Cyclophilins

S. Calabro ${ }^{1,2}$, A. Potter ${ }^{1,2}$, A. Lee ${ }^{1,2}$, A. Morgan ${ }^{1}$, V. Wen ${ }^{1}$, F.J. Warner ${ }^{1,2}$, S. McLennan ${ }^{2}$, N.A. Shackel ${ }^{1,3}$

${ }^{1}$ Centenary Institute; ${ }^{2}$ The University of Sydney, Camperdown;

${ }^{3}$ AW Morrow Gastroenterology, Royal Prince Alfred Hospital, Sydney, NSW, Australia

Introduction: CD147 is a multifunctional glycoprotein and is involved in inflammatory cell recruitment as the only known receptor for pro-inflammatory extracellular cyclophilins A and B (CyPA). Blocking this interaction has previously been reported to reduce tissue pathology in murine models of asthma, lung inflammation and rheumatoid arthritis. The current study addressed the role of CD147 in the inflammatory response to chronic liver injury and down-stream effects on ECM remodelling in mice of two genetic backgrounds (C57bl/6 and balb/c) treated with $\mathrm{CCl}_{4}$ for 4 weeks.

Methods: 8 week old $\mathrm{C} 57 \mathrm{bl} / 6$ or balb/c mice were treated with $\mathrm{CCl}_{4}$ (12\% in paraffin oil) twice weekly as well as either an anti-CD147

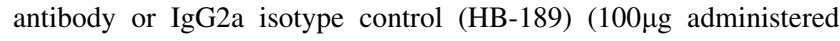
twice weekly). Injury was assessed by LFTs and histology. Inflammation was assessed by IHC staining of CD45 and, ECM remodelling was assessed by analysis of tissue Hydroxyproline content and net tissue MMP activity. qPCR was used to analyse expression of TNF- $\alpha$, IFN- $\gamma$, TGF- $\beta, \alpha$-SMA, Col1a, Col4a, MMP-2, MMP-9 and MMP-13. Results: After 4 weeks of $\mathrm{CCl}_{4}$ administration, anti-CD147 intervention in $\mathrm{C} 57 \mathrm{bl} / 6$ mice led to a $37 \%$ reduction in inflammatory cell aggregates in liver tissue, associated with a decrease in serum ALT and a reduction in Hydroxyproline. In balb/c mice, a 50\% reduction in inflammatory cell clusters was also observed as well as reduced serum AST, however this was associated with increased tissue Hydroxyproline and decreased gene expression of TGF- $\beta, \alpha$-SMA and MMP13 and increased IFN- $\gamma$.

Conclusion: These studies demonstrate the importance of the novel interaction between CD147 and Cyclophilins in the formation of inflammatory cell clusters, associated with progressive injury. Further, the downstream effects of blocking this interaction are dependent on the genetic background of mice. 


\section{PP15-42}

Hepatocyte Derived MMPs in Chronic Liver Injury and Regulation by CD147

S. Calabro ${ }^{1,2}$, A. Potter ${ }^{1,2}$, A. Lee ${ }^{1,2}$, A. Morgan ${ }^{1}$, V. Wen ${ }^{1}$, F.J. Warner ${ }^{1,2}$, S. McLennan ${ }^{2}$, N.A. Shackel ${ }^{1,3}$

${ }^{1}$ Centenary Institute; ${ }^{2}$ The University of Sydney, Camperdown; ${ }^{3} \mathrm{AW}$ Morrow Gastroenterology, Royal Prince Alfred Hospital, Sydney, NSW, Australia

Introduction: The ECM remodelling response to chronic liver injury is characterised by both ECM deposition and degradation, mediated by MMP activity. CD147 is a highly abundant glycoprotein involved in regulation of MMPs which we have previously reported as being predominantly localised to the hepatocytes. The current study examined hepatocyte expression of CD147 and MMPs in the chronic liver injury and the hepatocyte and studied their regulation in hepatocytes in vitro.

Methods: Tissue localisation of net MMP activity was assessed in both human cirrhotic liver explants using in situ zymography with DQ-labelled gelatin, co-localised with CK18 for hepatocytes and $\alpha$-SMA for Hepatic Stellate cells. MMPs-2, -9, 13 and -14 were assessed by qPCR in the TAA murine model of liver injury $(300 \mathrm{mg} /$ $\mathrm{L}$ in drinking water for 4, 8 and 20-week time points) in whole liver and isolated hepatocytes. In-vitro studies on H2.35 murine hepatocytes using TNF- $\alpha$ stimulation $(100 \mathrm{ng} / \mathrm{ml})$ and over-expression of CD147 to assess regulation of MMP expression.

Results: In situ zymography assessment of MMP activity in human donor and end-stage cirrhosis demonstrated significant activity across the lobule which co-localised with CK18 in both donor and cirrhotic tissue, and $\alpha$-SMA in cirrhotic tissue only. qPCR analysis of gene expression of MMPs in the TAA model of injury demonstrated increased expression of MMPs-2, -9, -13 and -14 in whole liver, and MMPs-9 and -14 in primary hepatocytes. In vitro, H2.35 cells were found to increase expression of MMP-9 in response to TNF- $\alpha$ stimulation. CD147 over-expression resulted in increased MMP-14, but no induction of MMP-2 or -9 .

Conclusion: These studies demonstrate increasing MMP activity associated with progressive liver injury, with increase hepatocytederived MMP-9 and -14 expression at end-stage (20 weeks TAA). Further, regulation of MMP expression was shown to be differentially regulated, with only MMP-14 inducible by increased CD147.

\section{PP15-43}

$2^{\prime}, 4^{\prime}, 6^{\prime}$-Tris (Methoxymethoxy) Chalcone Increases Fas-ligand through Inhibiting Histone Deacetylase and Induces Apoptosis of Activated Hepatic Stellate Cells

S.H. Lee, D.H. Sohn, X.-H. Che, Y.-Z. Zhao, B.-H. Jeon, W.-Y. Jiang College of Pharmacy, Wonkwang University, Iksan, Republic of Korea

Background/aims: Activated HSCs are proliferative and fibrogenic, with accumulation of extracellular matrix. Therefore, suppression of HSC activation and proliferation as well as induction of apoptosis in activated HSCs has been proposed as therapeutic strategies for the treatment and prevention of hepatic fibrosis. We have previously shown that $2^{\prime}, 4^{\prime}, 6^{\prime}$-tris(methoxymethoxy) chalcone (TMMC), a synthetic chalcone derivative, inhibits the proliferation of rat HSCs. Thus, the aims of this study were to examine the effect of TMMC on apoptosis in activated HSCs.

Methods: Rat HSC and hepatocyte were used in the in vitro experiments. An ELISA and immunohistochemistry were used to detect the expressions of FasL and a-smooth muscle actin, respectively. Expressions of apoptosis-related genes by real-time PCR.
Results: We showed that TMMC induces apoptosis in activated HSCs at higher concentrations $(30-50 \mathrm{mM})$, but is not cytotoxic to primary hepatocytes. Moreover, TMMC induces hyperacetylation of histone by inhibiting histone deacetylase (HDAC) in activated HSCs. Interestingly, TMMC treatment remarkably increased Fas-ligand (FasL) mRNA expression in a dose-dependent manner. Cycloheximide treatment reversed the induction of TMMC on apoptosis, indicating that de novo protein synthesis was required for TMMC-induced apoptosis in activated HSCs. In addition, FasL synthesis by TMMC is closely associated with maximal procaspase-3 proteolytic processing. In vivo, TMMC reduced activated $\mathrm{HSCs}$ in $\mathrm{CCl}_{4}$-intoxicated rats during liver injury recovery, as demonstrated by a-smooth muscle actin expression in rat liver. TMMC treatment also resulted in apoptosis, as demonstrated by cleavage of poly(ADP-ribose) polymerase in rat liver.

Conclusion: In conclusion, we found that TMMC enhances FasL expression through HDAC inhibition, resulting in caspase-3 activation and subsequent induction of apoptosis in activated HSCs in vitro. Our results also show that TMMC reduces hepatic fibrosis in the $\mathrm{CCl}_{4}$ acute rat in vivo. Thus, TMMC may have therapeutic potential by inducing HSC apoptosis and inhibiting liver fibrogenesis.

\section{PP15-44}

Evaluation of IL10 Gene Polymorphisms in Chronic Hepatitis B Infection in the Kazakh Population

B.S. Ilyassova ${ }^{1}$, L.Y. Yermekova ${ }^{2}$, K.I. Yesmembetov ${ }^{3}$

${ }^{I}$ Department of Gastroenterology and Hepatology, Institute of Cardiology and Internal Diseases; ${ }^{2}$ Department of Immunology, Kazakh-Russian Medical University, Almaty, Kazakhstan;

${ }^{3}$ Hepatology department, the E.M.Tareyev Nephrology, Internal and Occupational Diseases Clinic, I.M.Sechenov FMSMU, Moscow, Russia

The chronic hepatitis B (CHB) is current problem in the case of spreading, asymptomatically on the early stage and the heavy clinical picture and prognosis on late stage. In Kazakhstan the epidemiology date of CHB is 3.9 per 100,000 of population (2009). The speed of progression of $\mathrm{CHB}$ to the cirrhosis depends on the multitude factors. One of them is the activity of the regulatory interleukine-10 (IL-10). In this study, we evaluated the effects of functionally relevant cytokine polymorphisms in the promoters of the IL-10 (-1082G/A, -819C/ $\mathrm{T}$, and $-592 \mathrm{C} / \mathrm{A})$ genes on the course of HBV infection in the Kazakh population.

Material and methods: investigated 180 samples of the patients in $\mathrm{CHB}$ and 50 healthy volunteers. Of 170 patients, 56 were considered have asymptomatic clinic, normal level of ALT, negative of HBV DNA and positive of anti-HBe-antibody results and 64 were found to have $\mathrm{CHB}$ and 50 have HBV- cirrhosis. Polymorphisms in the IL-10 gene promoter were analyzed by sequencing in both directions using BigDye Terminator v3.1 Cycle Kit (Applied Biosystems).

Results: The frequency of C/C genotype at position -592 was not significant in the DNA-negative patients than in the persistence groups and health group. In CHB frequency of homozygotes $\mathrm{G} / \mathrm{G}$ was significant higher than in the liver cirrhosis $(15.1$ vs. $8.5 \%, p=0.034)$. In the study of the promoter -819 the T/T genotype significantly predominated in the cirrhosis that the another groups $(p<0.001)$. The G/G genotype at position -1082 has not associated with the persistent and progression of the $\mathrm{HBV}$ infection. The frequency of $\mathrm{A} / \mathrm{A}$ at the 1082 gene was higher in CHB and cirrhosis that in clearance of HBVinfection and control group $(p<0.01)$.

Conclusion: Inheritance of the IL-10 gene promoter (-1082A/A, $-819 \mathrm{~T} / \mathrm{T}$ ) polymorphisms in Kazakh population is genetic factor to progression in chronic HBV infection. 


\section{PP15-45}

Impact of Pro-inflammatory Interleukins on Blood-Brain-Barrier Permeability and the Adenoviral Cerebral Transduction in a Damage-Associated Neurotoxic Experimental Cirrhosis Model

J.F. Floresvillar-Mosqueda ${ }^{1,2}$, A.S. Sandoval-Rodriguez ${ }^{1}$, M.G. MenaEnriquez $^{1}$, J. Gonzalez-Cuevas ${ }^{1}$, J.J. Garcia-Bañuelos ${ }^{1}$, J. ArmendarizBorunda ${ }^{1,2}$

${ }^{1}$ Molecular Biology, Institute of Molecular Biology in Medicine and Gene Therapy/Universidad de Guadalajara; ${ }^{2}$ O.P.D. Hospital Civil de Guadalajara 'Juan I. Menchaca', Guadalajara, Mexico

Background: Gene therapy for neuronal diseases deal with a main limitation for vector transduction, which is passing through the blood-brain barrier (BBB). Use of adenovectors seems promising since inflammation and neurotoxicity appears to increase BBB-permeability and consequently adenovectors access to the brain.

Objective: Assess in an experimental model of cirrhosis with neurotoxic damage the brain-levels of IL- $1 \beta$, IL-6 and TNF- $\alpha$ and its effects in BBB permeability and therefore on adenoviral transduction. Methods: Wistar male rats were subjected to cirrhosis with neurotoxic damage model (bile duct ligation + manganese intoxication in drinking water). Cirrhotic animals were divided in bile duct ligation (BDL), BDL + Ad (BAd) and BDL + Ad + Mn (BAM), with their respective sham control groups. 4 weeks after BDL animals were injected systemically with $6 \times 10^{11} \mathrm{pv} / \mathrm{kg}$ of AdhuPA and sacrificed 3 and 6 days after treatment. BBB permeability (Evans Blue assay), transgene expression (IHQ) and cytokine brain-levels (ELISA) were analyzed, besides liver fibrosis (Morphometric analysis).

Results: Neurotoxic damage increase $75 \%$ BBB-permeability. BAd and BAM groups showed a $24-26 \%$ reduction in hepatic fibrosis compared to untreated controls. uPA transgene was detected in brain at day 3 and 6 in cirrhotic and in cirrhotic + neurotoxic damage groups. IL-1 $\beta$ protein levels showed an increase of 100-200\% in cirrhotic groups, while mRNA shows a 15 and 20 fold increase in cirrhotic and neurotoxic damaged animals treated with AduPA. IL-6 protein diminished significantly at day 6 in cirrhotic neurotoxicdamaged animals compared to other groups. TNF- $\alpha$ gene expression remained with no changes in all groups, while protein increased in cirrhotic groups treated with uPA with or without neurotoxic injure. Conclusions: Gene therapy using AduPA reverted hepatic fibrosis. A positive correlation between increments of interleukin levels and BBB-permeability was observed in cirrhotic groups. Adenovirus crossed the BBB in cirrhotic and cirrhotic with neurotoxic-damage animals and is capable of transgene expression.

\section{PP15-46}

Application UPLC/Q-TOF MS-based Metabonomics in the Study of Fecal Metabolic Profiling of Human Hepatocellular Carcinoma and Liver Cirrhosis

H. Cao, H. Huang, W. Xu, J. Yu, D. Chen, L. Li

State Key Laboratory for Diagnosis and Treatment of Infectious

Disease, The First Affiliated Hospital, College of Medicine, Zhejiang University, Hangzhou, China

In this study, feces metabolites from hepatocellular carcinoma (HCC) and liver cirrhosis patients and healthy humans were studied by a metabonomic method based on ultra performance liquid chromatography coupled with Q-TOF mass spectrometry (UPLC/Q-TOF MS). Partial least squares to latent structure-discriminant analysis (PLSDA) models were built to separate the healthy humans from HCC and liver cirrhosis patients and to find potential biomarkers that are expressed in significantly different amounts among the three populations. Scores plot of pattern recognition analysis were capable of distinguishing liver cirrhosis and HCC patients from healthy humans. Six metabolite ions were considered as potential biomarkers according to the variable importance in the project (VIP) value and S-plot: a strong increase in lysophosphatidylcholines and a dramatic decrease in bile acids and bile pigments in patients with Hepatocellular carcinoma and liver cirrhosis. These results indicate that fecal UPLC/QTOF MS spectra combined with pattern recognition analysis techniques offer an efficient, convenient way of depicting tumor biochemistry, which may be of great benefit to early diagnosis of human Hepatocellular carcinoma using single feces samples.

\section{PP15-47}

Establishment and Stability of Hepatic Cirrhosis Rat Models

Z.H. Wang ${ }^{1}$, Y.K. Wang ${ }^{2}$, Z.Z. Liu ${ }^{2}$

${ }^{1}$ Emergency Department of First Hospital JiLin University, Changchun, China; ${ }^{2}$ Gastroenterology Department of First Hospital, JiLin University, Changchun, China

Objective: To study the establishment and stability of hepatic cirrhosis animal model (CM).

Methods: The methods of compound factor (A group), carbon tetrachloride $\left(\mathrm{CCl}_{4}\right)$ plus alcohol (B group), corn flour and cholesterol plus alcohol ( $\mathrm{C}$ group) were used to construct the $\mathrm{CM}$. A group: $50 \mathrm{Wistar}$ rats whose fodder feed was $80 \%$ corn flour, $20 \%$ animal oil, and $15 \%$ cholesterol, $5 \%$ alcohol's beverage. The rats were administered with $40 \% \mathrm{CCl}_{4}$-olive oil mixture by subcutaneous injection $\left(5 \mathrm{~mL} \mathrm{~kg}^{-1}\right)$, then hypodermically injected with $40 \% \mathrm{CCl}_{4}$-olive oil mixture (3 $\mathrm{mL} \mathrm{kg}^{-1}$ ) every 3 days for 6 weeks. At the end of the 6 th week, ten rats were executed randomly and made into hepatic smears ( 2 escaped, 6 died). The rest were randomly divided into two groups. 16 rats were treated with normal fodder feeding in each group. Meanwhile one group were hypodermically injected with $40 \% \mathrm{CCl}_{4}$-olive oil mixture( 3 $\mathrm{mL} \mathrm{kg}^{-1}$ ) every 7 days. The pathological changes at the end of 7 th and 8th week were observed. B group: 25 rats were fed with normal fodder food. Remaining conditions were the same as those which used in the first 6 weeks of A group.C group: 25 Wistar rats weren't hypodermically injected with $\mathrm{CCl}_{4}$ and remaining conditions were same as B group.

Results: The rat of A group were established CM completely at the end of 6th week. Then fedded with normal fodder food, pseudolobuli were all disappeared after 2 weeks. And continute hypodermically injected with $\mathrm{CCl}_{4}$ for 2 weeks after establishment of CM, pseudolobuli were mainly existed. Typical CM were not constructed with other two methods.

Conclusion: The forming rate of hepatic cirrhosis model constructed with compound factor is high and $\mathrm{CCl}_{4}$ can keep the relative stability of the models.

\section{PP15-48}

Quercetin Inhibits Activation of Hepatic Stellate Cells in Experimental Hepatic Cirrhosis

A.M. Salazar Montes, L.D. Hernández Ortega, L.A. Ruiz Corro,

A.A. Sobrevilla Navarro, J. Armendariz Borunda

Molecular Biology and Genomics, Universidad de Guadalajara,

Guadalajara, Mexico

Liver cirrhosis is a distortion of normal tissue architecture which develops when the liver is chronically damaged. The inflammatory process in the liver caused by oxidative stress starts on Kupffer cells and continues with the activation of hepatic stellate cells (HSC), highly producers of collagen rendering in the development of fibrosis. On the other hand, we have showed that quercetin, an natural flavonoid and potent antioxidant prevents liver damage by decreasing oxidative stress and inflammation. However, quercetin effect on HSC activation has not been elucidated

Aim: To dilucidate the effect of quercetin on HSC activation in an experimental model of cirrhosis.

Methodology: Wistar male rats were chronically intoxicated with $\mathrm{CCl}_{4}$ for eight weeks and concomitantly treated with quercetin $(150 \mathrm{mg} / \mathrm{kg} / \mathrm{day})$ for the same period of time. Animals were sacrificed, liver samples were taken for histology (Masson stain, sirious 
red and immunohistochemistry), gene expression of profibrogenic genes (Col-1 TGF-b and CTGF) and anti-fibrogenic gene TGF-b3.

Results: Expression of profibrogenic genes Col-1 TGF-b and CTGF greatly increased when the animals were chronically intoxicated with $\mathrm{CCl}_{4}$ compared to healthy rats. Animals concomitantly treated with quercetin presented significantly less expression of all these genes. Cirrhotic animals presented a fibrosis index of $22.5 \%$ while rats treated with quercetin showed a fibrosis index of $10.76 \%$. Activation of HSC determinated by positive cells to a-sma by immunohistochemistry showed $40 \%$ less activated HSC in animals treated with quercetin respect to control animals without this treatment.

Conclusions: Treatment with quercetin prevents liver injury in an animal model of cirrhosis and inhibits HSC activation with the consequent decrease in fibrosis index and expression of pro-fibrogenic genes like Col-1 TGF-b and CTGF.

\section{PP15-49}

Caffeine Is a Preventive Therapy in Experimental Liver Cirrosis D. Gordillo-Bastidas ${ }^{1}$, E. Oceguera-Contreras ${ }^{1}$, A. Salazar-Montes ${ }^{1}$, J. Armendáriz-Borunda ${ }^{1,2}$

${ }^{I}$ Molecular Biology, Institute of Molecular Biology in Medicine and Gene Therapy/Universidad de Guadalajara.; ${ }^{2}$ O.P.D., Hospital Civil de Guadalajara 'Juan I. Menchaca', Guadalajara, Mexico

Background: Liver fibrosis is determined by activation of hepatic stellate cells (HSC); wich is perpetuated by growth factors and proinflammatory molecules. Caffeine (CFA) has been shown to be capable of modifying this signaling pathways in vitro.

Aim: To determine whether CFA prevents experimentally induced hepatic cirrhosis.

Methods: Liver cirrhosis was induced in Wistar rats by intraperitoneal application of thioacetamide (TAA) during 7 weeks. Animals were treated with CFA by orogastric route. Rats were sacrificed, liver biopsies were taken and blood was obtained. The degree of fibrosis was evaluated by morphometric analysis in histological sections stained with Masson trichrome. Inflammatory cell infiltrate was estimated in histological sections stained with hematoxylin and eosin. Gene expression was analyzed by real time Q-PCR of Col-1, CTGF, TGF $\beta 1$, TNF- $\alpha$, IL- $1 \beta$, IL-6, SOD and CAT.

Results: The groups that received CFA showed decreased inflammatory infiltrate. Fibrotic index was significantly decreased by $80 \%$ $(p<0.0001)$ in the TAA+CFA group compared with cirrhotic control group. CTGF, Col-1 and TGF $\beta 1$ gene expression were lowered by $3.5(p<0.01), 3.5(p<0.05)$ and $3(p<0.01)$ fold respectively in the TAA+CFA group compared to TAA. Expression of proinflammatory genes TNF- $\alpha$, IL- 6 and IL- $1 \beta$ was diminshed by $1.6(p<0.06), 6.0$ $(p<0.05)$ and $9(p<0.01)$ fold, respectively, in TAA+CFA group compared with control group. Regarding the antioxidant enzymes it was found that the expression of SOD and CAT was greater in 1.5 $(p<0.05)$ and $9(p<0.05)$ fold respectively in the TAA+CFA as compared with cirrhotic group.

Conclusions: Our results suggest a potent effect of CFA to limit profibrogenic and proinflammatory response despite the presence of an inducer of liver cirrhosis. The mechanism by which CFA is acting, involves down-expression of growth factors, proinflammatory cytokines and increased antioxidant enzyme systems.

\section{PP15-50}

Study on the Mechanism of Apoptosis of Hepatic Stellate Cells Induced by Nerve Growth Factor

J. Shu, X. Lv, H. Zhu

Guangzhou Red Cross Hospital, Guangzhou, China

Objective: To investigate the effect of NGF on apoptosis of HSC in vitro and explore the possible mechanism.

Methods: HSC was incubated with different concentrations of NGF. HSC apoptosis was identified by FCM. Expressions of NGF and
p75NTR were detected by immunofluorescence. The expressions of apoptosis-regulating proteins Caspase-3, P53 and Bcl-2 of HSC after apoptosis induced by NGF were examined by immunohistochemical staining.

Results: Apoptosis index of HSC was higher than that of control group $[22.36 \pm 9.51$ vs. $5.88 \pm 1.36 \%]$ afer theated with NGF $(100 \mathrm{ng} / \mathrm{mL})$ detected by flow cytometry $(P<0.05)$. When HSC was stimulated with $100 \mathrm{ng} / \mathrm{mL}$ NGF for $24 \mathrm{~h}$, immunofluorescence assay showed that the average optical density of NGF increased significantly compared with the control group $(6.53 \pm 1.40$ vs. $1.77 \pm 0.17)$ $(P<0.05)$, while the expression of p75NTR was not significantly changed $(3.52 \pm 0.36$ vs. $4.24 \pm 0.38)(P>0.05)$. After incubating with $100 \mathrm{ng} / \mathrm{mL}$ NGF for $24 \mathrm{~h}$, the positive expression rates of P53 and Caspase- 3 of HSC increased significantly compared with those of control group: $(78.41 \pm 4.00)$ vs. $(34.96 \pm 3.84) \%$, $(39.26 \pm 1.57)$ vs. $(9.27 \pm 1.01) \%$, respectively $(P<0.05)$. The positive expression rate of Bcl-2 protein of HSC decreased significantly compared with that of control group $[18.12 \pm 1.38$ vs. $91.53 \pm 2.98 \%](P<0.05)$. Conclusions: NGF could be used as an initiating factor and effect factor to increase the expression of NGF on HSC, but no significant effect on p75NTR expression.The mechanism of NGF to induce HSC apoptosis may be associted with the up-regulating expression of Caspase-3, P53 and down-regulating expression of Bcl-2 on HSC.

\section{PP15-51}

Effects of Nerve Growth Factor on Proliferation of Hepatic Stellate Cells J. Shu, X. Lv, H. Zhu Guangzhou Red Cross Hospital, Guangzhou, China

Objective: To investigate the effect of NGF on proliferation of HSC in vitro.

Methods: HSC was incubated with different concentrations of NGF. The effect of NGF on cell proliferation was observed by XTT colormetric assay. HSC cell cycle was analyzed by flow cytometry (FCM). The concentration of lactate dehydrogenase (LDH) in the supernate of medium were detected by automatic biochemistry analyzer.

Results: 100, 200, $400 \mathrm{ng} / \mathrm{mL}$ NGF incubated with HSC for $24 \mathrm{~h}$ could inhibit proliferation of HSC $(P<0.05)$ without concentrationdependent $(P>0.05)$ : XTT colormetric assay showed that optical density of NGF groups were $0.66 \pm 0.03,0.69 \pm 0.03$ and $0.66 \pm 0.03$, respectively, but in control group it was $0.73 \pm 0.01$. After treating HSC with NGF at the concentration of 100, 200, $400 \mathrm{ng} / \mathrm{mL}$, the number of $\mathrm{HSC}$ in $\mathrm{G}_{2}$ phase were $14.83 \pm 5.41$, $14.73 \pm 2.50$ and $14.87 \pm 2.06 \%$, respectively. They were significantly higher than control group in which the number of HSC in $\mathrm{G}_{2}$ phase was $7.47 \pm 4.39 \%(P<0.05)$. There was no significant difference of the level of LDH in the supernate of medium between control group and NGF groups $(P>0.05)$.

Conclusions: NGF could make HSC cell cycle arrest in $\mathrm{G}_{2}$ phase, it maybe the mechanism of NGF inhibited the proliferation of HSC.

\section{PP15-52}

Effects of NGF on the Morphology of Hepatic Stallete Cells

J. Shu, X. Lv, H. Zhu

Guangzhou Red Cross Hospital, Guangzhou, China

Objective: To investigate the effects of curcumin on the morphology of hepatic stallete cells.

Methods: The morphological changes of human hepatic stallete cells treated by different concentrations of NGF for $24 \mathrm{~h}$ were observed by inverted microscopy, acridine orange staining, and transmission electron microscopy (TEM).

Results: After HSC was stimulated with $100 \mathrm{ng} / \mathrm{mL}$ NGF for $24 \mathrm{~h}$ :

1. The inverted microscopy revealed that the population of HSC lessened, cells grew down, and morphous of cells became round or oval gradually. 
2. Acridine orange staining showed fluorescence enhancement, dense concentration of dark green or yellowish green fluorescence, the shape of HSC became shrinkage and irregular in NGF group.

3. Cell shrinkage, chromatin condensation and (or) ranked along inside of nuclear membrane and apoptosis body could be found by transmission electron microscope.

Conclusion: After treated with NGF, typic morphologic changes of proliferative inhibition and apoptosis of HSC could be identified.

\section{PP15-53}

Dipeptidyl Peptidase IV and Fibroblast Activation Protein Involvement in NAFLD and Fibrosis

X.M. Wang ${ }^{1}$, S. Song ${ }^{1}$, S. Chowdhury ${ }^{1}$, D.M. Yu ${ }^{1}$, M.G. Gall ${ }^{1}$, F.M. Keane ${ }^{1}$, S. Cordoba ${ }^{1}$, L. Lo ${ }^{2}$, N.A. Nadvi ${ }^{1,3}$, T.-W. Yao ${ }^{1}$, K.A. Evans ${ }^{1}$, S.V. McLennan $^{2,4}$, S.M. Twigg ${ }^{2,4}$, G.W. McCaughan ${ }^{1,5}$, M.D. Gorrell ${ }^{1}$

${ }^{1}$ Centenary Institute; ${ }^{2}$ Bosch Institute; ${ }^{3}$ Faculty of Pharmacy, University of Sydney; ${ }^{4}$ Endocrinology; ${ }^{5} \mathrm{AW}$ Morrow

Gastroenterology and Liver Centre, Royal Prince Alfred Hospital, Sydney, NSW, Australia

Fibroblast activation protein (FAP) and dipeptidyl peptidase IV (DPP4) are type II cell surface proteins of the prolyl oligopeptidase gene family. FAP has both DPP and collagen type I (CN-I) specific gelatinase activities. DPP4 has many known substrates and binds to fibronectin. Both proteins associate with beta 1 integrin and influence cell adhesion and migration on extracellular matrix in vitro. FAP is selectively expressed by activated, but not resting, hepatic stellate cells and myofibroblasts in tumors, arthritis and lung and liver fibrosis. The role of DPP4 in metabolic regulation has been exploited by a new type 2 diabetes therapy that selectively inhibits the peptidase activity of DPP4.

To further investigate the roles of FAP and DPP4 in liver fibrosis, chronic liver injury was induced in wild type, FAP gene knockout (gko) and DPP4 gko mice by $\mathrm{CCl}_{4}$ injection or by high fat diet $(45 \%$ total calories as fat for 20 weeks). Both FAP and DPP4 gko mice developed less obesity, fatty liver, liver fibrosis and inflammation compared to wild type C57B1/6 mice. DNA microarray detected 22 genes up-regulated and 172 genes down-regulated in FAP gko fibrotic mice, and 12 genes up-regulated and 37 genes down-regulated in DPP4 gko fibrotic mice, compared to fibrotic wild type mice. Reduced fibrosis severity was associated with reduced intrahepatic B cell densities. Reduced fatty liver was associated with less glucokinase, less CD36 and improved glucose tolerance. FAP and DPP4 substrates identified by MALDI-TOF and differential gene expression measured by qPCR suggest differing metabolic mechanisms in FAP gko compared to DPP4 gko livers.

The data suggest pro-fibrotic roles for FAP and DPP4 in experimental chronic liver injury, and potential for targeting either DPP4 or FAP as obesity, fatty liver and anti-fibrotic therapies.

\section{PP15-54}

Curcumine Suppresses Experimental Hepatic Fibrosis by Suppresses NF- $\kappa$ B and Caspases Signaling Pathway

L. Kong ${ }^{1}$, Y. Sun ${ }^{2}$, X. Zhang ${ }^{3}$, X. Sang ${ }^{1}$, M. Meng ${ }^{1}$, Y. Zheng ${ }^{1}$

${ }^{1}$ Department of Traditional and Western Medical Hepatology, Third Hospital of Hebei Medical University; ${ }^{2}$ Department of Traditional Chinese Medicine, Hebei Geriatric Hospital, Shijiazhuang;

${ }^{3}$ Department of Hepatogy, Cangzhou Infectious Disease Hospital, Cangzhou, China

Aims: To elucidate the effect and the mechanism of curcumine on hapatic stellate cells activation and liver tissue NF- $\kappa$ B pathway and apoptosis-related genes in experimental mice hepatic fibrosis.

Methods: Mice hepatic fibrosis model induced by carbon tetrachloride $\left(\mathrm{CCL}_{4}\right)$ was established. Blood and liver tissue was harvested from the mice administered $\mathrm{CCL}_{4}$ with or without curcumine treatment and the animals of control group. The expression of $\alpha$-SMA, $\mathrm{NF}-\kappa \mathrm{B} / \mathrm{I} \kappa \mathrm{Bp} 65$, ICAM, VCAM, and apoptosis-related genes Bcl-2/ Bax in liver tissue was measured by immunohistochemistry and RT-PCR assay. Serum ALT and HA were measured.

Results: $\mathrm{CCL}_{4}$ induced hepatic fibrosis models showed increased serum ALT and HA levels, and progressive hepatic injury including inflammatory infiltration and fibrosis, which associated with enhanced $\alpha$-SMA, NF- $\kappa$ B, I $\kappa$ BP65 protein expression, and up-regulated ICAM, VCAM protein and gene expression. Apoptosis-related gene bax expression was increased, bcl-2 expression was decreased, caspases 3 protein and gene expression were up-regulated. Administration of Curcumine significantly decreased serum ALT and HA levels and ameliorated hepatic necroinflammation and fibrosis. These effects were associated with repressed hepatic HSC activation, lowered NF$\kappa \mathrm{B}$ activity through reducing hepatic ICAM-1 and VCAM- 1 protein and gene expression. In addition, curcumine also up-regulated antiapoptotic gene $\mathrm{Bcl}-2$, and down-regulated pro-apoptotic gene Bax, down-regulated caspases 3 protein and mRNA expression.

Conclusions:The present study provide morphological and molecular biological evidence for the protective role of the Curcumine in hepatic necroinflammation, hepatic apoptosis, and hepatic fibrosis in experimental hepatic fibrosis. It could be a hopeful medicine for improving hepatic fibrosis.

\section{PP15-55}

Increased Expression of Claudin-1 and Claudin-7 in Liver Cirrhosis and Hepatocellular Carcinoma

A. Kiss ${ }^{1}$, A. Holczbauer ${ }^{1}$, G. Lotz ${ }^{1}$, A. Szijarto ${ }^{2}$, P. Tatrai ${ }^{1}$, P. Kupcsulik ${ }^{2}$, I. Kovalszky ${ }^{3}$, Z. Schaff ${ }^{1}$

${ }^{1}$ 2nd Department of Pathology; ${ }^{2} 1$ st Department of Surgery; ${ }^{3} 1$ st Department of Pathology, Semmelweis University Budapest, Budapest, Hungary

Background: Claudins have been reported to be differentially regulated in malignancies and implicated in the process of carcinogenesis and tumor progression. Claudin-1 has been recently described as a cofactor in the entry of hepatitis $\mathrm{C}$ virus (HCV) into hepatocytes.

Aims: The objective of the current study was to characterize claudin mRNA and protein expression in hepatocellular carcinoma (HCC) as well as in HCC-surrounding and normal liver samples with respect to cirrhosis and $\mathrm{HCV}$ infection.

Methods: Expression of claudin-1, -2, -3, -4-, and -7 was measured by real-time RT-PCR, immunohistochemistry, and Western blotting in thirty HCCs (both HCV negative and HCV positive cases) with corresponding non-tumorous tissues and 6 normal livers. Result of IHC was evaluated by morphometrical analysis. Beta-actin was used as reference gene for relative quantification of mRNA expression.

Results: Claudin-1 and -7 mRNA and protein expression was found significantly elevated in cirrhosis regardless of etiology compared with normal liver and non-cirrhotic surrounding liver. HCC developed in cirrhotic livers showed even higher expression of claudin-1 contrary to decreased CLDN-7 expression in HCC when compared to cirrhosis. HCCs developed in cirrhotic livers revealed significantly higher expression of both claudin-1 and -7. HCVnegative cirrhotic and HCC samples did not significantly differ from HCV-infected specimens. Western blot analysis confirmed results of IHC.

Conclusion: Cirrhosis increases the expression of claudin- 1 and -7 in the surrounding liver and developing HCCs. Elevated claudin-1 and 7 expressions are thought to result indirectly from $\mathrm{HCV}$ infection via induction of cirrhosis. This would be a novel mechanism whereby $\mathrm{HCV}$ infection and consequently developing cirrhosis could enhance the effectivity of viral entry and therefore boost the chronicity of the infection. 


\section{Poster Presentation 16: Liver Fibrosis: Clinical}

\section{PP16-01}

PF-03491390 Pan-caspase Inhibitor Decreases Liver Injury and Fibrosis in a Murine Model of Non-alcoholic Steatohepatitis (NASH) F. Barreyro

Laboratorio de Metabolismo del Oxigeno, Hospital de Clinicas, Capital Federal, Argentina

Hepatocyte apoptosis contributes to liver injury and fibrosis. Although, both apoptotic pathways are involved in the pathogenesis of NASH, the final common step is executed by a family of cysteineproteases termed caspases. Thus, our aim was to ascertain if administration of PF-03491390-pan-caspase inhibitor, ameliorates liver injury and fibrosis in a murine model of NASH.

Methods: C57/BL6J mice were fed-regular chow or high fat diet (HFD) for 20-weeks. All mice were treated with vehicle or PF-03491390. Apoptosis was assessed by TUNEL-assay and biochemical measurement of caspase-3 activity. Liver injury was evaluated by histopathology and serum-transaminases. Steatosis was measured by liver-triglyceride content. Quantitative-PCR (qPCR) was used to measure markers of hepatic inflammation (IL 1-b), HSC activation (alpha-smooth muscle actin, aSMA), and fibrosis (Coll-1). Immunofluorescence (IF) for aSMA was performed to identify HSC activation. Collagen was quantitated by Sirius red staining.

Results: HFD-fed-mice demonstrate an 5-fold increase in hepatocyteapoptosis by the TUNEL-assay and an 1.6-fold increase in caspase-3activity; this increase in apoptosis was attenuated in HFD-mice treated with PF-03491390 (HFD-PF) $(p<0.05)$. Likewise, HFD-mice had elevations of both serum-ALT $(70 \pm 10)$ and AST $(144 \pm 9)$ levels which were normalized in the PF-03491390-treated HFD-animals $(p<0.05)$. Liver injury was reduced in HFD-PF-mice as compare to HFD by measuring histologic inflammation score and IL1-b qPCR $(p<0.05)$. These differences could not be attributed to differences in hepatic steatosis as liver-triglycerides content were similar in both HFD-groups. aSMA a marker for HSC activation was decreased by IF and qPCR $(p<0.05)$ in HFD-PF compared to mice receiving a HFD. Hepatic fibrosis was reduced in PF-03491390 versus vehicle-treated HFD-animals by decreasing Sirius red staining and Coll-1 qPCR $(p<0.05)$.

In conclusion, these data demonstrate that in a murine model of NASH, liver injury and fibrosis are suppressed by inhibiting hepatocytes-apoptosis and suggests that PF-03491390 may be an attractive anti-fibrotic therapy in NASH.

\section{PP16-02}

A Polymorphism of Antizyme Inhibitor 1 Gene Is Associated with Liver Cirrhosis Risk in Chinese Hepatitis B Patients

L. Peng, J. Guo, Z. Zhang, L. Liu, Y. Cao, H. Shi, J. Wang, J. Wang Division of Digestive Diseases, Department of Internal Medicine, Zhongshan Hospital, Shanghai Medical College, Fu Dan University, Shanghai, China

Aims: Genetic association studies have linked a number of single nucleotide polymorphisms (SNPs) with outcomes of liver diseases. The present study was designed to validate the association of emerging SNPs with development of liver cirrhosis in a Chinese population infected with hepatitis B virus (HBV).

Methods: A total of 563 Chinese subjects with persistent HBV infection (349 with evident liver cirrhosis and 214 without cirrhosis clinically or pathologically) were studied. Fourteen SNPs in ten candidate genes were detected with matrix-assisted laser desorption/ ionization time-of-flight mass spectrometry (MALDI-TOF MS) method. The distribution of genotypes and alleles for each polymorphic site was compared between the age-matched cirrhosis and noncirrhosis subjects.
Results: The polymorphism of rs2679757 in antizyme inhibitor 1 (AZIN1) gene was found to be associated with the risk of liver cirrhosis (odds ratio for GG+AG versus AA 1.63, 95\% confidence interval 1.13-2.35, $P=0.008$ ) and $\mathrm{G}$ was the risk allele. Rs886277 in transient receptor potential cation channel subfamily $\mathrm{M}$, member 5 (TRPM5) gene had a tendency of association with liver cirrhosis $(P=0.06)$. Genotypic or allelic frequencies of other SNPs were not different in the comparison (see the graph). A documented signature termed cirrhosis risk score (CRS) was not proved valuable for identifying liver cirrhosis among these patients by comparing the median CRS values $(P=0.295)$.

Conclusions: AZIN1 gene and its polymorphism may be associated with HBV-related liver cirrhosis in Chinese. The emerging SNPs and associated genes warrant further mechanism studies and clinical validation in hepatitis B patients to reveal their roles in fibrosis progression.

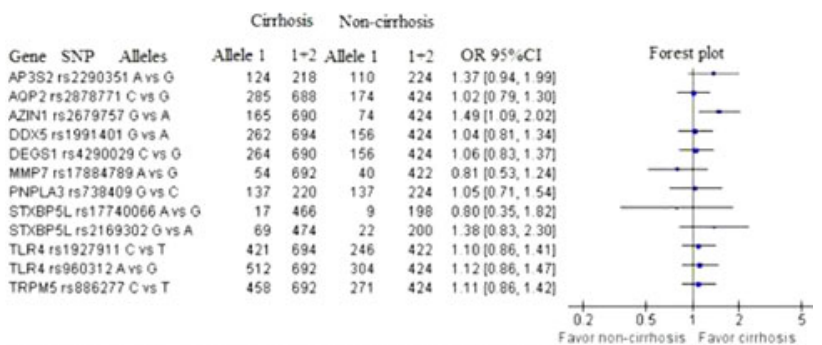

Allebic frequency in the cirrbosis and non-cirmosis group and per-allele OR for each SNP with genotype diversiny. SNP, single nucleotide polymorphism; OR odds ratio, CI, confidence interval; AP3S2, adaptor-telated protein complex 3, sigma 2 subunit, AQP2, aquaporin 2; AZIN1, antizyme inhabitor 1; DDXS, DEAD box polypepoide 5; DEGS1, degenerative spermatocyte homolog 1, lipid desaturase, MMIP7, matrix metalloproteinase 7; PNPLA3. patatin- Hke phospholipase domain containing 3; STXBP5L, syntaxin binding protein 5-Lke; TLR4, toll-Lke receptor 4. TRPMS, transient receptor potential cation charnel subfamily M, member 5

Allele 1 denotes the frst allele of the two compared alleles for each SNP and 1-2 denotes the total number of the two alleles:

\section{PP16-03}

Comparison of Clinical Characteristics between Patients with Viral Cirrhosis and Alcoholic Cirrhosis: A Retrospective Analysis Y.-L. Zhou ${ }^{1}$, Z.-J. Duan ${ }^{2}$

${ }^{1}$ Gastroenterology; ${ }^{2}$ Dalian Medical University, Dalian, China

Objective: Compared and analyzed the variances of clinical features, complications and prognosis between patients with viral cirrhosis (VC) and those with alcoholic cirrhosis (AC). It was aimed to explore the different tendency of treatment for the two diseases.

Methods: 952 admission data of patients with cirrhosis in the First Affiliated Hospital of Dalian Medical University from April 2004 to May 2009 were collected. Sex, age, history, manifestations, liver function, complications, and the death cause were compared.

Results: Compared to VC, the AC had younger age and was commonly seen in male patients. Facial telangiectasia and spider nevus presented obviously in group $\mathrm{AC}$, and the incidences of splenomegaly and dull complexion enhanced in $\mathrm{VC}$ group compared to that in $\mathrm{AC}$ group $(P<0.05)$. In $\mathrm{VC}$ and $\mathrm{AC}+\mathrm{VC}$ group, the top three incidences of complications respectively were upper gastrointestinal bleeding, hepatocellular carcinoma and hepatic encephalopathy. Upper-gastrointestinal bleeding, hepatic encephalopathy and hepatocellular carcinoma were the most common complications in AC group. The prevalence of hepatocellular carcinoma in VC group were higher than AC group $(P<0.01)$, moreover, the combined etiologies could lead to much higher prevalence. The occurrence of spontaneous bacterial peritonitis in AC group was more than in VC group $(P<0.01)$. The hepatic encephalopathy was the common death cause in both VC and $\mathrm{AC}$ group. In $\mathrm{AC}+\mathrm{VC}$ group, hepatocellular carcinoma was the main death cause. 


\section{Conclusions:}

1. The most common complication was upper gastrointestinal bleeding and the most common death cause was hepatic encephalopathy in either viral cirrhosis or alcoholic cirrhosis. Hepatocellular carcinoma was the most common death cause in combined etiologies group.

2. Compared to viral cirrhosis, the occurrence of spontaneous bacterial peritonitis was much more than that in alcoholic cirrhosis.

3. Complications were the main important variance among the three groups, which guided us to give different tendency of prophylaxis and treatment so as to reduce the morbidity.

\section{PP16-04}

The Levels of Serum TIMP-1 Are Significantly Correlated with the Degrees of Liver Fibrosis in Patients with Chronic Hepatitis B C. $\mathbf{Z h u}^{1}, \mathrm{Y} . \mathrm{Li}^{2}, \mathrm{~W} . \mathrm{Li}^{1}, \mathrm{Y} . \mathrm{Li}^{1}, \mathrm{R} . \mathrm{Gao}^{1}$

${ }^{1}$ Department of Infectious Disease; ${ }^{2}$ Department of Pediatrics, Affiliated Provincial Hospital, Anhui Medical University, Hefei, China

Background: Chronic hepatitis B (CHB) is a severe liver disease and can progressively develop liver fibrosis. Notably, high levels of tissue inhibitor of metalloproteinases-1 (TIMP-1) expression are observed in patients with liver fibrosis. However, the potential relationship between the levels of serum TIMP-1, hepatic TIMP-1 and the degrees of hepatic fibrosis has not yet been illustrated.

Methods: A total of $159 \mathrm{CHB}$ with varying degrees of liver fibrosis were recruited and subjected to liver biopsy for the analysis of their liver fibrotic stages and inflammatory activities. The levels of TIMP-1 expression in the liver tissues and serum TIMP-1 in those patients were determined by immunohistochemistry and enzyme-linked immunoabsorbent assay (ELISA), respectively.

Results: The concentrations of serum TIMP were positively correlated with the levels of TIMP-1 expression in the liver tissues $(R=0.9521)$ and the degrees of liver fibrosis (0.704) in CHB patients with inflammation at grade 2. More importantly, the concentrations of serum TIMP-1 were independent of the degrees of inflammation in those patients.

Conclusions: TIMP-1 may be a valuable biomarker and detection of serum TIMP-1 concentrations may be a safe and cheaper measure for the evaluation of liver fibrosis at least in CHB patients.

Acknowledgement: This study was supported by the Science and Technology Foundation of Anhui Province (No.07010302195), and the Science and Technological Fund of Anhui Province for Outstanding Youth (No.10040606Y14).

\section{PP16-05}

Diagnostic Potential of Serum Direct Markers and Noninvasive Fibrosis Models in Patients with Chronic Hepatitis B

Ö. Gümüşay ${ }^{1}$, S. Özenirler ${ }^{2}$, A. Atak ${ }^{3}$, C. Sönmez ${ }^{3}$, S. Özkan ${ }^{4}$, A.F. Tuncel ${ }^{5}$, G. Yilmaz ${ }^{6}$, G. $\mathrm{Akyol}^{6}$

${ }^{1}$ Internal Medicine; ${ }^{2}$ Gastroenterology; ${ }^{3}$ Immunology; ${ }^{4}$ Public

Health; ${ }^{5}$ Biochemistry; ${ }^{6}$ Pathology, Gazi University Faculty of

Medicine, Ankara, Turkey

Background: Liver biopsy is recommended in the majority of patients with chronic viral hepatitis for fibrosis evaluation. Because of the disadvantages and invasiveness of liver biopsy, many studies related to noninvasive biomarkers and scores have been performed. In this study, we aimed to assess the diagnostic value of hyaluronic acid (HA), tissue inhibitor of matrix metalloproteinase 1 (TIMP-1), aminoterminal peptide of pro-collagen III (PIIINP) and noninvasive fibrosis models to predict liver fibrosis in the treatment-naive chronic hepatitis B (CHB) patients and to compare their diagnostic performance.

Methods: This study included 58 patients with a diagnosis of $\mathrm{CHB}$ infection and 30 healthy controls. HA, TIMP-1 and PIIINP were mea- sured by ELISA; and the OELF, ELF panel, PP score, APRI, FIB-4 indexes were calculated using the formulas taken from previous publications. Fibrosis stage was determined using Ishak's scoring system. Results: Among all the markers analyzed the mean values of APRI, FIB-4, OELF, ELF were significantly higher in chronic hepatitis B patients than in healthy controls. $(p<0.05)$ The diagnostic value of all the noninvasive indeces was low to detect mild fibrosis. The diagnostic accuracy of predicting $\geq \mathrm{F} 3$ fibrosis by using HA, OELF, ELF, PP score, APRI, and PIIINP were very good with an AUROC of $0.932,0.833,0.830,0.831,0.823,0.807$, respectively $(p<0.05) \mathrm{We}$ demonstrated that hyaluronic acid has highest AUROC for predicting $\geq$ F3 fibrosis, and PP score, OELF and ELF were superior to other fibrosis prediction models. In our study the results from a combination of tests showed that ELF \&APRI had the highest diagnostic value for detection of moderate to severe fibrosis.

Baseline characteristics of patients

\begin{tabular}{llll}
\hline Characteristics & $\begin{array}{l}\text { Patient group } \\
(n=58)\end{array}$ & $\begin{array}{l}\text { Healthy controls } \\
(n=30)\end{array}$ & $P$ \\
\hline Gender (male:female) & $33 / 25$ & $15 / 15$ & 0.652 \\
BMI $\left(\mathrm{kg} / \mathrm{m}^{2}\right)$ & $25.9 \pm 5.1$ & $25.3 \pm 4.2$ & 0.721 \\
Platelet count $\left(\times 10^{3} / \mu \mathrm{L}\right)$ & $208.7 \pm 70.5$ & $235.1 \pm 52$ & 0.031 \\
Protrombin time $(\%)$ & $96.8 \pm 6.3$ & $97.5 \pm 7.6$ & 0.681 \\
AST (IU/L) & $40.5 \pm 27$ & $20.6 \pm 5.9$ & 0.0001 \\
ALT (IU/L) & $63.2 \pm 52.4$ & $21.6 \pm 9.4$ & 0.0001 \\
GGT (IU/L) & $32.9 \pm 32.5$ & $24.2 \pm 12.2$ & 0.145 \\
Albumin (g/dl) & $4.5 \pm 0.5$ & $4.5 \pm 0.4$ & 0.537 \\
HBeAg (+) & $11(19 \%)$ & & \\
\hline
\end{tabular}

Diagnostic performance of noninvasive indexes

\begin{tabular}{|c|c|c|c|c|c|c|}
\hline & \multicolumn{2}{|c|}{ F0 vs. F1-6 } & \multicolumn{2}{|c|}{ F0 vs. F1-2 } & \multicolumn{2}{|c|}{ F0-2 vs. F3-6 } \\
\hline & AUROC & $P$ & AUROC & $P$ & AUROC & $P$ \\
\hline $\mathrm{HA}(\mathrm{ng} / \mathrm{dl})$ & 0.668 & 0.074 & 0.593 & 0.341 & 0.902 & 0.0001 \\
\hline TIMP-1 (ng/dl) & 0.487 & 0.893 & 0.472 & 0.775 & 0.548 & 0.636 \\
\hline PIIINP (ng/dl) & 0.739 & 0.011 & 0.690 & 0.051 & 0.807 & 0.002 \\
\hline APRI & 0.620 & 0.319 & 0.618 & 0.225 & 0.823 & 0.001 \\
\hline FIB-4 & 0.771 & 0.007 & 0.606 & 0.273 & 0.771 & 0.007 \\
\hline OELF & 0.651 & 0.211 & 0.571 & 0.567 & 0.833 & 0.002 \\
\hline ELF & 0.663 & 0.177 & 0.588 & 0.481 & 0.830 & 0.002 \\
\hline PP score & 0.746 & 0.009 & 0.697 & 0.043 & 0.831 & 0.001 \\
\hline
\end{tabular}

Diagnostic values of combination tests

\begin{tabular}{llccccc}
\hline & $\begin{array}{l}\text { Sensitivity } \\
(\%)\end{array}$ & $\begin{array}{l}\text { Specificity } \\
(\%)\end{array}$ & PPV & NPV & +LR & -LR \\
\hline HA and PIIINP & 85.7 & 97.2 & 85.7 & 97.2 & 30.6 & 0.15 \\
HA and FIB-4 & 83.3 & 100 & 100 & 97.4 & NA & 0.17 \\
PIIINP and FIB-4 & 71.4 & 93.9 & 71.4 & 93.9 & 11.7 & 0.30 \\
HA and APRI & 85.7 & 100 & 100 & 97.5 & NA & 0.14 \\
PIIINP and APRI & 83.3 & 92 & 62.5 & 97.1 & 10.4 & 0.18 \\
ELF and FIB-4 & 80 & 100 & 100 & 92.6 & NA & 0.20 \\
ELF and APRI & 80 & 100 & 100 & 96.4 & NA & 0.10 \\
PP and HA & 85.7 & 97.2 & 85.7 & 97.2 & 30.6 & 0.15 \\
\hline
\end{tabular}


Conclusion: In chronic hepatitis B patients, combination of ELF\&APRI has a better diagnostic value in predicting moderate to severe fibrosis. Further studies with a greater number of patients are needed to validate the usefulness of these markers and their combinations.
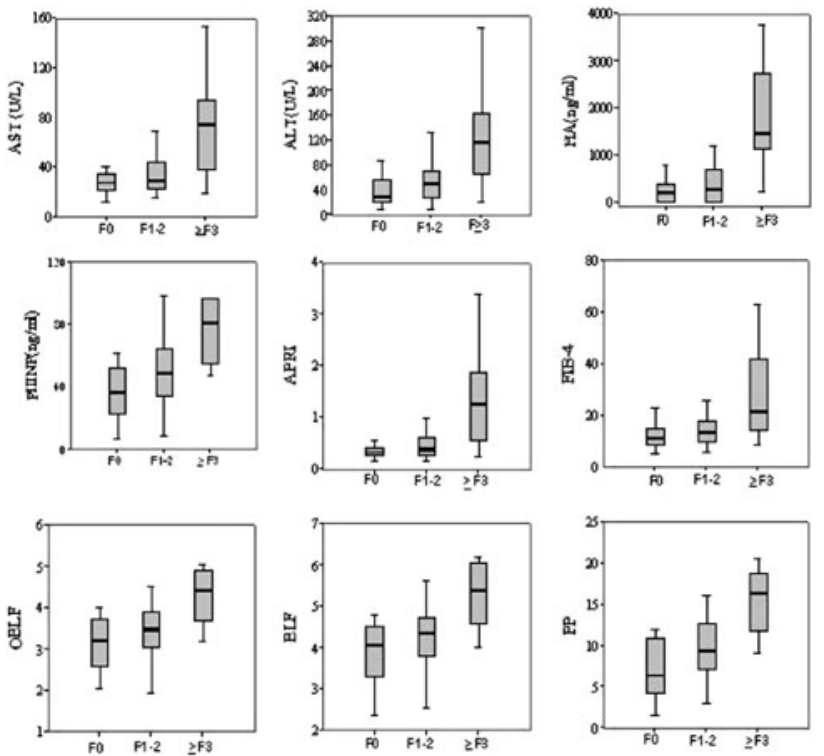

Fig 1 Box plots of each marker of fibrosis

\section{PP16-06}

Detection of Hepatic and Serum TGF- $\beta 1$ Levels in Patients with HBV-induced Chronic Liver Diseases and its Clinical Significances F. He, R. Gao, R. Zhang, X. Zhang, J. Zhu

Department of Hepatology, First Hospital, Jilin University, Changchun, China

Objective: To investigate the relationship between the severity of liver histology and serum TGF- $\beta 1$ level in patients with $\mathrm{HBV}$-induced chronic liver diseases (CLD-B).

Methods: ELISA was used to measure TGF- $\beta 1$ in sera from 89 patients with chronic hepatitis $\mathrm{B}(\mathrm{CHB})$ and 66 patients with hepatitis $\mathrm{B}$-induced liver cirrhosis and 30 healthy individuals. Liver samples from 26 patients with $\mathrm{CHB}$ and 5 patients with hepatitis Binduced liver cirrhosis were examined for TGF- $\beta 1$ mRNA levels by in situ hybridization, and computer imaging analysis was performed to measure integrated optimal density (IOD) of TGF- $\beta 1$ mRNApositive cells in liver tissues. HE staining and Van Gieson's method were used to evaluate histological inflammation grading and fibrosis staging.

Results: The levels of TGF- $\beta 1$ in sera from patients with CHB and active liver cirrhosis were significantly higher than those in healthy controls $(P<0.01)$. There was good consistency between the levels of TGF- $\beta 1$ in sera and TGF- $\beta 1$ mRNA expression in liver tissues. The levels of TGF- $\beta 1$ in sera were positively correlated with the degree of inflammation and the degree of earlier fibrosis stages in patients with CLD-B $(r=0.721, r=0.683$, both $P<0.05)$.

Conclusion: Detection of serum TGF- $\beta 1$ in patients with CLD-B may have clinical significance for the diagnosis of hepatic inflammation and early stage fibrosis.
PP16-07

Improving Performance of Liver Stiffness Measurement Detecting Liver Cirrhosis in Chinese Patient with Compensated Chronic Hepatitis B

Y. Chen ${ }^{1}$, X. Liang ${ }^{1}$, L. Dai ${ }^{1}$, Q. Zhang ${ }^{1}$, J. Peng ${ }^{1}$, Y. Zhu ${ }^{1}$, H.L.-Y. Chan ${ }^{2}$, J. Hou ${ }^{1}$

${ }^{1}$ Department of Infectious Disease, Nanfang Hospital, Southern Medical University, Guangzhou, China; ${ }^{2}$ Department of Medicine and Therapeutics and Institute of Digestive Disease, The Chinese University of Hong Kong, Hong Kong, Hong Kong S.A.R.

Background and aim: Liver stiffness measurement (LSM) by FibroScan ${ }^{\circledR}$ is a well-established non-invasive method to detect liver cirrhosis. We aimed to developed algorithm using serum indexes to improve the performance of LSM in detecting liver cirrhosis in patients with compensated chronic hepatitis B.

Methods: A total of 389 patients (322 men and mean age 31.3 years) between July 2007 and March 2010 were included. All patients had had liver biopsy, LSM, ultrasonography and blood tests for analysis. LSM with positive likelihood ratio $>9.0$ and LSM with negative likelihood ratio $<0.1$ were considered as cirrhosis determining and excluding cutoffs, respectively.

Results: Seventy-four patients were excluded from analysis due to inadequate biopsy sample size or hepatic decompensation. LSM was correlated with liver fibrosis stage with Kendall's tau_b 0.597 $(P=0.000)$. The area under the receiver operating characteristic curves (AUROC) of LSM for predicting liver fibrosis $\geq \mathrm{F} 2, \geq \mathrm{F} 3$ and F4 were 0.87 [95\% confidence interval (CI) $0.83-0.91$ ], 0.89 (95\% CI $0.86-0.93)$ and 0.89 (95\% CI $0.85-0.93)$, respectively. The best LSM cutoffs to exclude and confirm F4 fibrosis were 10.4 and $17.0 \mathrm{kPa}$ in patients with alanine aminotransferase (ALT) $<5 \times$ ULN, and $13.7,24.7 \mathrm{kPa}$ in patients with ALT $\geq 5 \times \mathrm{ULN}$, respectively. LSM based cirrhosis index (LSM-CI) combining LSM, ultrasonography and platelet could predict cirrhosis with AUROC 0.92 , which was significantly higher than LSM alone $(Z=2.14$, $P=0.0314$ ), and discriminated $74.6 \%$ of patients with or without liver cirrhosis. Sequentially combined with age platelet index (API) and LSM would provide best discrimination to exclude and confirm cirrhosis; $78.7 \%$ of patients could avoid liver liver as compared to $73.3 \%$ with LSM ALT stratified cutoff.

Conclusion: Sequentially combined with API can significantly improve the performance of ALT-based LSM algorithm to predict cirrhosis in compensated hepatitis B.

\section{PP16-08}

Carboxyhemoglobin May Be Used as an Index to Estimate Therapeutic Effects on Cirrhostic Patients with HE

X. Sun, Z. Duan

Gastroenterology, First Affiliated Hospital of Dalian Medical University, Dalian, China

Objective: To detect the carboxyhemoglobin $(\mathrm{COHb})$ level and its significance in the patient of hepatitis B related cirrhosis(HBC) accompanying hepatic encephalopathy (HE).

Methods: 63 inpatients with HBC occurring HE in our department from January 2002 to April 2010 were enrolled as group H, and they were included in group P-H after treatment. 20 healthy volunteers were in group $\mathrm{N}$. The information of ill history, the grade of HE, and the data of $\mathrm{COHb}$ in each patient were collected. The data of total bilirubin, creatinine and international normalized ratio were gathered to count MELD scores. Compared the horizontal differentiation of $\mathrm{COHb}$ in $\mathrm{HE}$ patients between pre and post treatments, analyzed the relationships between $\mathrm{COHb}$ level and grade of HE,/MELD scores respectively. SPSS software was used for statistic analysis. 
Results:

1. The level of $\mathrm{COHb}$ in Group $\mathrm{H}$ and $\mathrm{P}-\mathrm{H}$ was higher than in Group $\mathrm{N}(P<0.01)$, with statistical difference between Group $\mathrm{H}$ and $\mathrm{P}-$ $\mathrm{H}(P=0.01)$.

2. The $\mathrm{COHb}$ level had positive correlation with Grade of $\mathrm{HE}(P=$ $0.004, r=0.357)$.

3. There was no correlation between the $\mathrm{COHb}$ level and MELD scores.

Conclusions: $\mathrm{COHb}$ could be regarded as one of indexes to estimate therapeutic effects on HE in liver cirrhostic patients. It related to the grade of HE, but had no relationship with the severity of cirrhosis.

\section{PP16-09}

Liver Fibrosis Associated with Hepatogenic Ulcers and Erosions O.M. Horlenko ${ }^{1}$, E.I. Arshiy ${ }^{2}$, O.M. Moskal ${ }^{2}$, V.I. Rusyn ${ }^{3}$, F.V. Horlenko ${ }^{3}$ ${ }^{1}$ Pediatrics with Infectious Diseases; ${ }^{2}$ Propedeutic Internal Diseases; ${ }^{3}$ Surgical Diseases, Uzhgorod National University, Medical Faculty, Uzhgorod, Ukraine

Our investigation included the study the complication of patients with the Liver fibrosis, (LF) middle age $47.9 \pm 1.1$ years. Were identificated hepatogenic ulcers in 33 patients with LF, in other contingents were erosive process. Ulcers and erosions were localized in the antrum area of stomach. The group of patients used basic treatment of LF which included hepatoprotective and immunomodular medicines- "Ursofalk" and "Dufalac" during a month. Immunocorrective effect of medicines which contain bifidobacterium and lactobacterium was correct of disorder of T- and B-cellular of immune through the action of system of interleucines. Identificated the increase of level of proinflammatory cytocines (IL- $1 \beta$, IL-2, IL-8, and FNP- $\alpha$ ) and antiinflammatory cytocines IL-4 in the blood serum in the patients with $\mathrm{LF}$ associated with hepatogenic ulcers and erosions. Concentration of $\mathrm{IL}-1 \beta$ is elevated in the LF patients comparatively with a control group on the amount in 10 times and was $40.76 \pm 4.41 \mathrm{mmol} / \mathrm{l}$ $(p<0,001)$, IL-2 - in 12 times $(120.5 \pm 40.54 \mathrm{mmol} / \mathrm{l}, p<0.05)$, IL4 - in 4 times $(21.41 \pm 3.0 \mathrm{mmol} / \mathrm{l}, p<0.001)$, IL-8 in 4 $(103.83 \pm 18.9 \mathrm{mmol} / \mathrm{l}, p<0.01), \mathrm{FNP}-\alpha-$ in 8 times $(9.36 \pm 1.37$ $\mathrm{mmol} / \mathrm{l}, p<0.001)$. As a result the concentration of IL- $1 \beta$ decreased in 7 times comparatively with an initial level $(p<0.001)$, IL-2 in 1.9 times $(p>0.05)$, IL-4 in 1.8 times $(p<0.01)$, IL-8 in 2.2 times $(p<0.05)$, FNP- $\alpha$ in 9 times $(p<0.001)$. Under act of treatment with including only "Ursofalk" concentration of IL-1 $\beta$ did decreased in 2 times comparatively with an initial level $(p<0.01)$, concentration of IL-2 in 1.7 times $(p<0.05)$, concentration of IL-4 in 1.2 times $(p>0.05)$, concentration of IL-8 in 1.4 times $(p>0.05)$, concentration of FNP- $\alpha$ in 1.3 times $(p>0.05)$. A suggested treatment approach is illustrated decrease levels of IL- $1 \beta$, IL- 4 and FNP- $\alpha$ to the reference ranges.

\section{PP16-10}

Metabolic Investigation in Patients with Hepatatis B with or without Diabetes Mellitus to Determine the Impact of Anti-oxidant Activity B.R. Devrajani ${ }^{1,2}$

${ }^{1}$ Medicine, LUMHS; ${ }^{2}$ Liaquat University of Medical and Health sci, Hyd, Pakistan

Objective: To determine the impact of antioxidant activity by metabolic investigations in patients with hepatitis B with or without diabetes mellitus.

Patients and methods: Fifty patients each were included in this study with chronic hepatitis $\mathrm{B}$, and $50 \mathrm{HBV}$ patients with diabetic mellitus. Eligibility criteria included: $\mathrm{HBsAg}, \mathrm{HBeAg}$, and HBV DNA positivity in blood for 6 months or more; elevation of serum aspartate aminotransferase or alanine aminotransferase levels for 6 months or more and exclusion of co-infection with hepatitis delta or acquired immunodeficiency virus, metabolic or autoimmune disorders. All subjects underwent blood testing after a 10-h overnight fast to measure serum levels of fasting plasma glucose, total cholesterol, triglyceride, and alanine aminotransferase. The subjects were also assessed for urea, creatinin, iron, copper, zinc and magnesium and total anti-oxidative activity was measured by reducing $\mathrm{Fe}+++$ to $\mathrm{Fe}++$ using vitamin $\mathrm{c}$ as reducing agent at $\mathrm{pH} 4$ to 5 .

Results: The urea, uric acid and creatinine levels are significantly increased in HBV, cholesterol (total, HDL and LDL) all decreased in HBV patient where as triglyceride levels significantly increased in HBV patients with DM. The AST increases significantly in both groups of HBV patients. Magnesium is significantly decreased in diabetic HBV patients and unchanged in HBV patients. Iron is increased and zinc is decreased in both groups of HBV patients. Copper remained unchanged in both groups and total antioxidant activity is decreased in both groups of HBV patients.

Conclusion: Additional factors in elevated level of iron resulting into low antioxidative activity. Urea, uric acid and creatinine levels are significantly increased that shows not only heart and pancreas but kidney can be affected in HBV patients.

\section{PP16-11}

Nitric Oxide Production Levels in Patients with Different Stages of Hepatosplenic Scistosomiasis

T. Besheer ${ }^{1}$, M.M. Elbendary ${ }^{1}$, O.S. Salama ${ }^{2}$

${ }^{1}$ Tropical Medicine Department; ${ }^{2}$ Clinical Pathology Department, Mansoura University, Mansoura, Egypt

Background/aims: Nitric oxide (NO) implicated in many physiological processes and has dual roles in infections in host defense and pathogenesis. Schistosomiasis is a parasitic infection that affects 200 million people and is directly responsible for an annual death of 20,000 patients. We aimed to determine nitric oxide levels in response to different stages of Hepato-Splenic Schistosoma (HSS) infection to predict its severity.

Methods: The study was carried out on 72 Egyptian cases and subclassified into: clinically (HSS with and without ascites), ultrasonic degrees of hepatic fibrosis (Degree I, II and III), intensity of schistosomal infection by Kato thick smear (Grade I, II, III) and Control Group (36 subjects). NO was determined by a standard Griess reaction.

Results: NO levels are significantly increased in HSS with ascites than HSS without ascites group $(P<0.001)$. NO levels show significant increase in degree III fibrosis (88. $27 \pm 8.42)$ than both degree II fibrosis $(64.77 \pm 11.05)$ and degree I fibrosis $(53.77 \pm 9.72)$ $(P<0.001)$. Also there are significant increased between degrees of schistosomal hepatic fibrosis (SHF) (I, II, III) and control group $(P<$ $0.001)$. NO levels in G III schistosomal intensity of infection (63.11 \pm 16.37) is less than G II $(67.36 \pm 18.13)$ and G I (64.62 \pm 18.30$)$ and there are no significant correlation between different grades $(P=0.817)$. ROC curves show the diagnostic performance of NO level in degree III SHF studied cases to differentiate degree III from other degrees. The best cut-off value of NO level was $78.85 \mu \mathrm{mol} /$ L.with sensitivity $88.9 \%$ and specificity $96.3 \%$. Regression analysis coefficient was done to predict degree of SHF according to NO and albumin levels by equation $[Y=2.914-0.633$ albumin + $0.017(\mathrm{NO})]$.

Conclusion: There was a significant increase in NO level with progression of hepatic fibrosis.

\section{PP16-12}

Transient Elastography: Noninvasive Screening Test for Discrimination of HBsAg Positive HBeAg-negative Patients

I. Pirvulescu, L. Gheorghe, S. Suciu, C. Gheorghe, M. Diculescu Gastroenterology and Hepatology, Fundeni Clinical Institute of Digestive Diseases and Liver Transplantation, Bucharest, Romania

Background: Transient elastography (TE) is a new non invasive tool for measuring liver stiffness (LS), which is correlated to the histologic 
stage of liver fibrosis TE can be used in screening patients to identify those with significant fibrosis or cirrhosis and may be useful in discriminating HBV inactive carriers from chronic hepatitis B patients (CHB).

Aims: To asses the utility of TE for differentiate HBV inactive carriers from $\mathrm{CHB}$.

Methods: $116 \mathrm{HBsAg}$ positive/ $\mathrm{HBeAg}$ negative patients were analyzed. The patients were divided in two groups:

A. 54 patients $\mathrm{HBV}$ inactive carriers (normal aminotransferases and/or DNA-HVB $<2,000 \mathrm{UI} / \mathrm{ml}$ );

B. 62 patients with persistently or intermittent elevation of aminotransferases and/or DNA-HVB >2,000 UI/ml).

We measure LS in two different intercostal spaces for each patients. Results: Regarding mean stiffness, aminotransferases and DNAVHB, statistically significant differences was found between two groups $(4.66 \pm 0.99 \mathrm{kPa}$, respectively $923.23 \pm 1131.9 \mathrm{UI} / \mathrm{ml}$ in group A vs. $7.36 \pm 3.89 \mathrm{kPa}$, respectively $1,289,449 \pm 3.2 \mathrm{milUI} / \mathrm{ml}$ in group B) $(p=0.001$, respectively $p=0.000)$. No correlation between the mean variation of LS measured in two different intercostal spaces $(0.715 \pm 0.87$ in group $\mathrm{A}$ and $1.28 \pm 0.80$ in group B) and among mean age and BMI was found between the two groups $(36.74 \pm 12.66$ vs. $43.38 \pm 13.84$ years, respectively $24.09 \pm 3.46$ vs. $27.14 \pm 4.39$ ).

Conclusions: This study shows that mean hepatic stiffness by elastography is significantly lower in patients with inactive hepatitis B compared to those with $\mathrm{HBeAg-negative} \mathrm{disease.TE} \mathrm{can} \mathrm{differ-}$ entiate the inactive carrier from chronic hepatitis B and it could be used together with ALT measurement as a quick screening test for a large proportion of the patients with $\mathrm{HBsAg}$ positive/ $\mathrm{HBeAg}$ negative.

\section{PP16-13}

XL versus M FibroScan Probe: Feasibility and Results for Liver Stiffness Measurement. A Prospective Study

V. de Ledinghen ${ }^{1}$, J. Vergniol ${ }^{1}$, P.-H. Bernard ${ }^{2}$, M. Patanchon ${ }^{1}$,

W. Merrouche ${ }^{1}$, J. Foucher ${ }^{1}$

${ }^{1}$ CHU Bordeaux, Pessac; ${ }^{2}$ CHU Bordeaux, Bordeaux, France

Background: Liver stiffness measurements (LSM) are uninterpretable in nearly one in five cases. The principal reason is increased waist circumference. A new probe, named the XL probe, was designed specifically for obese patients. The aim of this prospective study was to assess the feasibility and comparison of LSM using M and XL probe of FibroScan in a large cohort of patients with chronic liver disease and BMI $>25 \mathrm{~kg} / \mathrm{m}^{2}$.

Methods: 353 consecutive patients with BMI $>25 \mathrm{~kg} / \mathrm{m}^{2}$ (199 males, mean age 60 years, mean BMI $31.7 \mathrm{~kg} / \mathrm{m}^{2}$ ) with chronic liver disease underwent LSM using M Probe. Two groups were defined: group A $(n=137)$, patients with correct measurement, and group B $(n=216)$ patients with failure of measurement (no valid shot, $n=100$ ) or uninterpretable result. Thereafter, all patients had LSM using XL probe.

Results: In group A (mean BMI $30 \mathrm{~kg} / \mathrm{m}^{2}$ ), a strong correlation was found between LSM using M and XL probe. Median LSM was 10.1 $\mathrm{kPa}$ with $\mathrm{M}$ probe and $7.7 \mathrm{kPa}$ with XL probe $(p<0.0001)$. In 123 cases, LSM using XL probe was lower than LSM using M probe. LSM using M probe was strongly correlated with Fibrotest, APRI and FIB-4. In group B (mean BMI $32.6 \mathrm{~kg} / \mathrm{m}^{2}$ ), with XL probe, 6 patients had failure of measurement $(2.7 \%)$ and 48 patients had uninterpretable result $(22.2 \%)$. No factor was associated with uninterpretable result using XL probe. In this group, median LSM was $7.6 \mathrm{kPa}$. In this group, LSM using XL probe was strongly correlated with Fibrotest, APRI and FIB-4.

Conclusion: In patients with BMI $>25 \mathrm{~kg} / \mathrm{m}^{2}$, when LSM using M probe is uninterpretable, LSM using XL probe is possible in $75 \%$ of cases. Since XL probe values are significantly lower than $M$ values, new cut-offs for XL probe should be defined for the estimation of liver fibrosis.

\section{PP16-14}

Prospective Evaluation of FibroScan for the Diagnosis of Hepatic Fibrosis in Patients with Chronic Hepatitis B Virus Infection

X. Zhu, L.-C. Wang, E.-Q. Chen, X.-B. Chen, L.-Y. Chen, L. Liu, X.-Z. Lei, C. Liu, H. Tang

Center of Infectious Diseases, West China Hospital of Sichuan University, Chengdu, China

Background and aims: The Fibroscan (FS), the aspartate aminotransferase-to-platelet ratio index (APRI), and the FIB-4 index are simple and inexpensive methods to detect liver fibrosis. The primary objective was to evaluate the performance of FS for the noninvasive diagnosis of hepatic fibrosis in Western Chinese patients with chronic hepatitis B virus (HBV) infection compared with APRI and FIB-4; the secondary objective was to determine liver stiffness measurement (LSM) cutoff values for the noninvasive diagnosis of significant fibrosis and liver cirrhosis.

Methods: Overall, 178 consecutive patients with chronic HBV infection, successful liver biopsy, and alanine aminotransferase levels $\leq 2 \times$ ULN were prospectively studied. Liver fibrosis was graded by an independent pathologist using the METAVIR (F0-F4) classification. APRI and FIB-4 were calculated from laboratory data.

Results: The area under receiver operating characteristics curves (AUROC) for LSM for significant fibrosis (METAVIR F2-3) and cirrhosis (F4) was 0.95 (95\% confidence interval 0.91-0.98) and 0.98 (0.96-0.99), respectively $(n=175)$. For the detection significant fibrosis and cirrhosis, the AUROC of APRI were $0.81(0.74-0.87)$ and $0.83(0.77-0.90)$; the AUROC of FIB-4 were $0.86(0.80-0.91)$ and $0.77(0.68-0.85)$. Thus, FS was more accurate than APRI and FIB-4 for the diagnosis of significant liver fibrosis and cirrhosis. FS optimal cutoff values for the identification of significant fibrosis and cirrhosis were 7.9 and $13.8 \mathrm{kPa}$, respectively.

Conclusions: FS is a reliable predictor of significant fibrosis and cirrhosis in Western Chinese patients with chronic HBV infection, superior to APRI and FIB-4.

Acknowledgement: This work was supported by the National Key Technologies Research and Development Program of China during the 11th Five-Year Plan Period (No.2008ZX10002-006), National S\&T Major Project for Infections Diseases Control (No.2009ZX10004-905), and the National Basic Research Program of China (No.2007 CB512902)

\section{PP16-15}

XL Probe, a New Probe for Liver Stiffness Measurement. Comparison with M Probe, Liver Biopsy, Fibrotest and APRI

V. de Ledinghen ${ }^{1}$, J. Vergniol ${ }^{1}$, B. Le Bail ${ }^{2}$, N. Branca ${ }^{1}$, W. Merrouche ${ }^{1}$, J. Foucher ${ }^{1}$

${ }^{I}$ CHU Bordeaux, Pessac; ${ }^{2}$ CHU Bordeaux, Bordeaux, France

Background: Liver stiffness measurements (LSM) are uninterpretable in nearly one in five cases. The principal reason is increased waist circumference. A new probe, XL probe, was designed specifically for obese patients. The aim of this prospective study was to assess the performance in measuring liver fibrosis using $\mathrm{M}$ and XL probe of FibroScan, Fibrotest and APRI score performed the same day as liver biopsy in patients with chronic liver disease.

Methods: 79 patients (39 males, mean age 55 years, mean BMI 27 $\mathrm{kg} / \mathrm{m}^{2}, \mathrm{HCV} n=27$, NAFLD $n=19$ ) had LSM using XL probe and liver biopsy were included. Fibrosis was scored according to METAVIR. Diagnostic performances were assessed using areas under the receiving operating characteristic curve (AUROC).

Results: The fibrosis score according to METAVIR split was: F0-F1 $n=33$, F2 $n=22$, F3 $n=13$, F4 $n=11$. Mean LSM using M probe and XL probe were 14.3 and $12.3 \mathrm{kPa}(p<0.01)$, respectively. 
The AUROCs (95\% CI) are shown in the table. With a sensitivity of $80 \%$, cut-offs for M versus XL probes were 6.9 versus $6 \mathrm{kPa}$ for F2F3F4 fibrosis, 9.4 versus $7.6 \mathrm{kPa}$ for F3F4 fibrosis and 16.5 versus $14.5 \mathrm{kPa}$ for cirrhosis. For XL probe, with these cut-offs, PPV and NPV were 73 and $70 \%$ for F2F3F4 fibrosis, 59 and $89 \%$ for $\mathrm{F} 3 \mathrm{~F} 4$ fibrosis and 62 and $98 \%$ for cirrhosis, respectively.

\section{AUROCs}

\begin{tabular}{lllll}
\hline & XL probe & M probe & Fibro test & APRI \\
\hline$\geq$ F2 & $0.78(0.68-0.89)$ & $0.75(0.64-0.87)$ & $0.80(0.71-0.91)$ & $0.73(0.62-0.84)$ \\
$\geq$ F3 & $0.88(0.78-0.97)$ & $0.85(0.75-0.96)$ & $0.74(0.61-0.86)$ & $0.76(0.62-0.90)$ \\
Cirrhosis & $0.96(0.90-1.00)$ & $0.96(0.91-1.0)$ & $0.72(0.57-0.88)$ & $0.84(0.69-0.98)$ \\
\hline
\end{tabular}

Conclusion: XL probe of FibroScan seems a reliable method for the assessment of liver fibrosis. Its performance is of the same order as that of M probe. LSM with XL probe are lower than values with M probe. Therefore, new cut-offs for the diagnosis of liver fibrosis with $\mathrm{XL}$ probe are needed in large studies.

\section{PP16-16}

Impact of Helicobacter pylori Infection on Liver Pathology in Egyptian Patients with Chronic Hepatitis C

M.M. Ragheb ${ }^{1}$, M.M. Awad ${ }^{2}$, L.A. Tag Eldin ${ }^{3}$, T.M. Dosoki ${ }^{2}$

${ }^{1}$ Internal Medicine, Unit of Endemic and Infectious Diseases, Faculty of Medicine, Suez Canal University, Cairo; ${ }^{2}$ Internal Medicine;

${ }^{3}$ Biochemistry, Faculty of Medicine, Suez Canal University, Ismailia, Egypt

Background/aims: Chronic liver disease due to $\mathrm{HCV}$ is a common health problem in Egypt where Helicobacter pylori (HP) infections is prevailing. This study aimed to determine the prevalence of HP infection in patients with chronic hepatitis $\mathrm{C}(\mathrm{CHC})$ and its impact of on liver pathology.

Patients and methods: A descriptive; cross sectional study was carried out in Suez Canal University Hospital during 2009 and included 60 patients with CHC. All were interviewed for sociodemographics and risk factors and tested for liver functions, alpha fetoprotein (AFP), serology for schistosomiasis and HP. Liver biopsy was histologically examined and tested for HP DNA by PCR.

Results: Patients ${ }^{\prime}$ age had a median of 44.5 years (range $=26-58$ ), $75 \%$ were males, and $80 \%$ were rural. The study revealed elevated ALT and/or AST in $66.7 \%$, thrombocytopenia in $26.7 \%$, anti-schistosomal antibody in $45 \%$ and anti HP antibody in $61.7 \%$. According to Ishak scoring criteria, $\mathrm{CHC}$ had a mean fibrosis stage of $2.8 \pm 1.36$ and a mean grade of activity of $6.07 \pm 2.6$. Sequencing proven Helicobacter pylori DNA in liver tissue was found in $10 \%$ of patients, ( 3 of 9 with cirrhosis and 3 of 51 without cirrhosis, $p=0.038$ ). The median viral load was 337,000 and $165,000 \mathrm{IU} / \mathrm{ml}$ in patients positive and negative for Helicobacter DNA in liver $(p=0.349)$. While, the frequency of advanced fibrosis (F4,5 and 6) showed no significant association with seroreactivity to HP $(p=0.496)$, advanced fibrosis was significantly more frequent in patients positive for HP DNA in liver compared to negative patients ( 83.3 vs. $20.4 \%, p=0.004)$. By multivariate logistic regression, blood transfusion, cirrhosis and HCV viral load were independent predictors for presence of HP DNA in liver of patients with chronic hepatitis $\mathrm{C}$.

Conclusion: Dual infection of the liver with HCV and HP is associated with more severe pathology compared to infection with $\mathrm{HCV}$ alone.
PP16-17

Can Serum Fibrosis Markers Predict Large Oesophageal Varices in Patients with Liver Cirrhosis?

G.M.K. Galal ${ }^{1}$, N.F. Amin ${ }^{2}$, H.A. Abdel-Hafeez ${ }^{3}$, M.A.H. El-Baz ${ }^{4}$

${ }^{1}$ Tropical Medicine and Gastroenterology, Sohag Faculty of Medicine, Sohag University, Sohag, ${ }^{2}$ Internal Medicine; ${ }^{3}$ Clinical Pathology; ${ }^{4}$ Biochemistry, Assiut Faculty of Medicine, Assiut University, Assiut, Egypt

Background and aim: Non invasive predictors of large oesephageal varices (LOV) could reduce the number of screening endoscopies. As portal hypertension is a consequence to liver fibrosis. Therefore, serum fibrosis markers would be evaluated together with clinical, laboratory and sonographic variables as possible non invasive predictors of large oesophageal varices.

Patients and methods: One hundred and fifty-four cirrhotic patients with splenomegaly and 30 healthy controls were recruited in a prospective study in 2 Gastroenterology centers in Upper Egypt. Clinical parameters assessed included Child-Pugh class, liver size, ascites. Laboratory parameters included complete blood count, liver function tests, AST/platelet ratio. Transforming growth factor- $\beta 1$ (TGF- $\beta 1$ ), alpha2 macroglobulin (A2M) and hyaluronic acid (HA) were assayed. Ultrasonographic examination was done for assessment of liver span, portal vein diameter, detection of minimal ascites.

Results: Fifty-four patients (35\%) had no or small varices, 100 (65\%) patients had medium to large varices by endoscopy. On multivariate analysis, the independent predictors of LOV were the presence of ascites $(\beta=0.258, \quad P=0.047)$ and serum HA $(\beta=0.449$, $P=0.009)$. The receiver operating characteristic curve for HA showed 0.916 area under curve. The sensitivity, specificity, positive and negative predictive values and diagnostic accuracy of HA at a cut off value $207 \mu \mathrm{g} / \mathrm{l}$ were $94,77.8,88.7,87.5$ and $88.3 \%$, respectively. Conclusions: The presence of ascites and serum HA level higher than $207 \mu \mathrm{g} / \mathrm{l}$ can predict the presence of LOV in cirrhotic patients. This would help physicians to identify patients who would most likely benefit from screening endoscopy and thus, reducing costs and discomfort for patients with low probability for LOV.

\section{PP16-18}

Validation of $\mathrm{P} 2 / \mathrm{MS}$ for Predicting Liver Fibrosis in Hepatocellular Carcinoma Patients

G.E. Chung ${ }^{1}$, J.-H. Lee ${ }^{2}$, E.J. Cho² ${ }^{2}$ E.S. Jang ${ }^{2}$, M.S. $\mathrm{Kwak}^{2}$, J.-H. Yoon ${ }^{2}$, Y.J. Kim ${ }^{2}$, H.-S. Lee ${ }^{2}$

${ }^{I}$ Department of Internal Medicine and Healthcare Research Institute, Seoul National University Hospital Healthcare System Gangnam Center; ${ }^{2}$ Department of Internal Medicine and Liver Research Institute, Seoul National University College of Medicine, Seoul, Republic of Korea

Background/aims: P2/MS was reported to be a simple, accurate and inexpensive method for the assessment of liver fibrosis in the chronic hepatitis B patients. However, the clinical significance in patients with hepatocellular carinoma (HCC) was limited due to the lack of validation. In this study, we aimed to validate the P2/MS index in patients with HCC.

Methods: HCC patients who underwent surgical resection between June 2007 and March 2009 at Seoul National University Hospital, Seoul, Korea were enrolled in this study. Fibrosis stage was assessed according to the METAVIR scoring system by a single pathologist who was unaware of the patients' information.

Results: The P2/MS were inversely correlated with the stages of fibrosis according to the METAVIR fibrosis score (adjusted $r=0.448$, $P<0.001)$. The area under the receiver-operating characteristic curve 
(AUROC) of P2/MS was 0.804 (95\% confidence interval [CI] $0.681-0.927)$ for detecting significant fibrosis $(\geq \mathrm{F} 2), 0.749$ (95\% CI $0.669-0.829)$ for detecting severe fibrosis $(\geq \mathrm{F} 3)$, and $0.769(95 \% \mathrm{CI}$ $0.698-0.839$ ) for detecting historical cirrhosis (F4). At a value $<62$, P2/MS detected significant fibrosis with a specificity of $85.7 \%(95 \%$ CI 42.0-99.2) and a positive likelihood ratio (PLR) of 100 (95\% CI 14.22-703.10) and at a value $>115, \mathrm{P} 2 / \mathrm{MS}$ ruled out significant fibrosis with a sensitivity of $90.2 \%$ (95\% CI 91.4-98.2) and a negative likelihood ratio (NLR) of 8 (95\% CI 2.84-22.50). At a value $<45$, P2/MS detected histological cirrhosis with a specificity of $81.0 \%$ (95\% CI 70.3-88.6) and a PLR of 3.6 (95\% CI 2.26-5.73) and at a value $>85, \mathrm{P} 2 / \mathrm{MS}$ ruled out cirrhosis with a sensitivity of $89.1 \%$ (95\% CI 80.4-94.38) and a NLR of 0.30 (95\% CI 0.17-0.53). Conclusion: P2/MS is an accurate and simple index with comparable outcome to other non-invasive method in patients with HCC.

\section{PP16-19}

Ligase Chain Reaction as a Modality for the Detection of Point Mutation in Precore Region of HBV Related HCC Cases

A. Malik ${ }^{1}$, M. Asim ${ }^{1,2}$

${ }^{I}$ Medicine, Maulana Azad Medical College; ${ }^{2}$ Delhi University, New Delhi, India

Background and aim: Mutant Hepatitis B with precore stop codon has been reported to be associated with severe liver damage in $\mathrm{HBeAg}$ negative patients with hepatocellular carcinoma. Clinically, the biological importance of pre-core G1896A mutation is not well established. The purpose of the present study was to determine hepatitis B virus genotypes and also to elucidate the association of G1896A mutation of precore gene and the severity of liver damage in HBV related HCC cases.

Methods: Detection of HBV DNA sequences was carried out by polymerase chain reaction (PCR) using primers derived from the precore region of HBV genome. Ligase Chain Reaction (LCR) assay was performed to screen the presence or absence of G1896A mutation. Direct nucleotide sequencing was done to confirm the results of LCR. A total of $116 \mathrm{HBV}$ related cases who attended the medical Out Patients Department and wards of Lok Nayak Hospital, New Delhi, India were screened over the period of 3 years.Patients having superinfection with $\mathrm{HDV} / \mathrm{HCV} / \mathrm{HIV}$ and past history of interferon therapy were excluded.

Results: Sequence analysis of viral DNA established that the G1896A mutation was observed in 32 cases in HCC cases. Phylogenetic analysis revealed $60 \%$ isolates belonged to genotype A, while $20 \%$ belonged to genotype $\mathrm{D}$ and $20 \%$ belonged to genotype $\mathrm{E}$.

Conclusion: The present data suggests that precore G1896A mutations is responsible for $27.2 \%$ of the patients of Asian Indian origin suffering from HBV related HCC cases and these cases are more symptomatic and aggressive in patients with the mutant form of the virus as compared with the wild form.

PP16-20

Spontaneous Fungal Peritonitis: A Fatal Complication in Patients with Advanced Liver Cirrhosis

J.-H. Kwon ${ }^{1}$, J.-H. Lee ${ }^{1}$, S.Y. Hwang ${ }^{1}$, S.J. Yu ${ }^{1}$, J.S. Kim ${ }^{1}$, E.J. Cho ${ }^{1}$, M.-S. Kwak ${ }^{1}$, E.S. Jang ${ }^{1}$, Y.J. Kim ${ }^{1}$, J.-H. Yoon ${ }^{1}$, E.-C. Kim ${ }^{2}$, H.-S. Lee ${ }^{1}$

${ }^{1}$ Internal Medicine and Liver Research Institute; ${ }^{2}$ Laboratory Medicine, Seoul National University College of Medicine, Seoul, Republic of Korea

Purpose: Antibiotic treatment for cirrhotic patients with spontaneous peritonitis sometimes fails: Fungal infection may be one of the causes. In this study, we aimed to evaluate the clinical significance and characteristics of the spontaneous fungal peritonitis (SFP).

Methods: Consecutive cirrhotic patients with spontaneous peritonitis treated between 2000 and 2005 at a tertiary care center in Seoul, Republic of Korea, were included. We analyzed the clinical characteristics and the prognosis of SFP patients and compared them to spontaneous bacterial peritonitis (SBP) patients.

Results: During the study period, 416 patients developed spontaneous peritonitis and $15(3.6 \%)$ revealed SFP. As compared to SBP, nosocomial peritonitis (peritonitis developed after hospitalization for $>48$ h) were more common and Child-Pugh score was higher in SFP patients (both $P<0.01$ ). Ten patients were infected by Candida spp. (C. albicans, $8 ;$ C. tropicalis, $1 ;$ C. glabrata, 1 ), and 5 by Cryptococcus neoformans. Eleven patients were co-infected with bacteria (e.g., Klebsiella oxytoca, Eneterococcus faecium and Escherichia coli), which were sensitive to administered antibiotics. However, only 4 patients were treated with adequate antifungal agent. Consequently, 1-month mortality rate of SFP patients was $73.3 \%$ [11 of 15 ; median time-to-death, 2 days (range 0-22)], which was significantly higher than patients with SBP alone $(28.7 \%, P=0.0007)$.

Conclusions: These results indicate that SFP is a fatal complication in patients with advanced liver cirrhosis. Longer admission duration and higher Child-Pugh score may be a risk factor of SFP. Immediate and adequate antifungal treatment is warranted in patients with spontaneous peritonitis, once fungus is found in ascitic fluid.

\section{PP16-21}

Pentoxifylline Action in Liver Fibrosis

S. Chooklin

Medical University, Lviv, Ukraine

Background: Fibrosis is a dynamic process from chronic liver damage to cirrhosis. Pentoxifylline, a methyl xanthine phosphodiesterase inhibitor, is one of several agents, which have been tested as a potential antifibrogenic treatment.

Methods: Pentoxifylline was used for treatment of 9 patients with liver fibrosis. Seven patients received placebo. Liver sections were stained with hematoxylin-eosin or azan stain for histological analysis of the liver fibrosis. The extent of cytolysis was assessed by alanine aminotransgerase (ALT) analysis in serum. TNF- $\alpha$ were measured by ELISA. Immunohistochemical studies were performed by the indirect immunoperoxidase method using monoclonal antibodies specific for $\alpha$-smooth muscle actin ( $\alpha$-SMA), desmin and polyclonal antibody against TGF- $\beta 1$.

Results: In patients that had received placebo smooth muscle $\alpha$-actin positive cells and TGF- $\beta 1$-positive cells were detected in a linear pattern along the fibers, and also scattered along the sinusoidal walls. In contrast, the liver fibrosis treated with pentoxifylline showed markedly fewer smooth muscle $\alpha$-actin positive cells and TGF- $\beta 1$ positive cells. Pentoxifylline markedly reduced the expression of desmin in HSCs. Patients treated with pentoxifylline had lower levels of TNF- $\alpha$ and ALT. Pentoxifylline increase TNF- $\alpha$ production through inhibition of nuclear factor $\kappa \mathrm{B}(\mathrm{NF} \kappa \mathrm{B})$ activation.

Conclusion: Pentoxifylline was able reduce cytokine activation, fibroproliferation and myofibroblastic differentiation and to reduce hepatocellular damage and the inflammatory response.

\section{PP16-22}

A Comparative Analysis of Liver Fibrosis in HIV-mono-Infected and HIV/Hepatitis C Co-infected Patients

I.M. Dumitru ${ }^{1}$, E. Dumitru ${ }^{2}$, S. Rugina ${ }^{1}$

${ }^{1}$ Clinical Infectious Diseases Hospital, Ovidius University; ${ }^{2}$ Ovidius University, Constanta, Romania

Background/aim: Since the advent of highly active antiretroviral therapy (HAART) there have been dramatic reductions of morbidity and mortality from all causes among HIV-infected persons. One of the exceptions to this trend is liver related deaths which now represent one of the leading causes of death among HIV-infected individuals with undetectable HIV viremia. Much of this excess mortality has been driven by the Hepatitis $\mathrm{C}$ (HCV) co-infection. HCV progresses more rapidly in patients co-infected with HIV. 
Objective: To evaluate the stage of liver disease in HIV-infected patients with and without HCV using Fibroscan.

Material and method: This is a prospective study of 33 HIV monoinfected and $13 \mathrm{HIV} / \mathrm{HCV}$ co-infected patients; we investigated hepatic fibrosis in the two groups of patients by measuring liver stiffness using Fibroscan. The results were integrated in the clinical context of HAART therapy.

Results: All the patients have CD4 $>200 \mathrm{cell} / \mathrm{mm}^{3}$, and undetectable HIV-RNA. Aminotransferases where more frequently increased in coinfected patients compared with HIV mono-infected patients (53 vs. $9 \%, p<0.05)$. We noticed a significantly increased liver stiffness according to Fibroscan in patients with co-infection compared with HIV mono-infected patients $(8.3 \pm 2.6$ vs. $5.9 \pm 1.8 \mathrm{kPa})$.

Conclusions: According to our results, treatment for HCV infection may be required to slow the growing epidemic of end-stage liver disease in this population.

\section{PP16-23}

Non-invasive Index for Predicting Significant Liver Fibrosis: Comparison of Diagnostic Performances in Patients with Chronic Hepatitis B and C

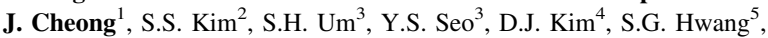
Y.J. Lee ${ }^{6}$, M. Cho ${ }^{7}$, J.M. Yang ${ }^{8}$, S.W. $\mathrm{Cho}^{2}$

${ }^{1}$ Gastroenterology, Ajou University; ${ }^{2}$ Ajou University School of Medicine, Suwon; ${ }^{3}$ Korea University College of Medicine, Seoul; ${ }^{4}$ Hallym University College of Medicine; Chuncheon; ${ }^{5}$ Pochon CHA University, Sungnam; ${ }^{6}$ Inje University College of Medicine; ${ }^{7}$ Pusan National University College of Medicine, Pusan; ${ }^{8}$ Catholic University College of Medicine, Suwon, Republic of Korea

Background/aims: The majority of non-invasive markers of liver fibrosis have been developed in patients with chronic hepatitis $\mathrm{C}$. We aimed to develop a formula for predicting significant fibrosis in patients with chronic viral hepatitis and to compare the usefulness of published models derived from the data obtained from patients with chronic hepatitis $\mathrm{C}$.

Methods: Serum markers and the METAVIR stage of fibrosis in liver biopsy specimens were compared prospectively in patients with chronic hepatitis B and C (estimation set 367, validation set 159).

Results: In the estimation set, multiple forward stepwise logistic regression analysis identified $\gamma$-glutamyl transpeptidase, haptoglobin, a2-macroglobulin, matrix metalloproteinase-2, and tissue inhibitor of metalloproteinase- 1 as independent predictors of significant fibrosis. A formula termed the SF index was constructed with the aforementioned five variables. The area under the receiver operating characteristic curves of the SF index for predicting significant fibrosis were 0.828 , 0.776 , and 0.827 in the estimation, validation, and total sets, respectively. Using cut-off scores of 2.2 and 3.3, significant fibrosis was predicted with considerable accuracy. The diagnostic performances of the SF index and the Zeng index derived from chronic hepatitis B patients were much better than indices derived from chronic hepatitis $\mathrm{C}$ patients, such as the APRI, Forns index, and FIB-4.

Conclusions: We developed a novel formula for predicting significant fibrosis in patients with chronic viral hepatitis. Serum indices from studies in patients with chronic hepatitis $C$ were less accurate in patients with chronic hepatitis B for predicting significant fibrosis.

\section{PP16-24}

Factors Affecting the Rate of Fibrosis and Their Significance in HCV Infection

A. Naeem, M. Umar, M. Ahmed, S. Ambreen, H. Sharif, U. Ismail Gastroenterology and Hepatology Division, Holy Family Hospital, Rawalpindi, Pakistan

Objective: Correlation of age and ALT with the histological changes in HCV patients (fully compensated) in a Pakistani cohort.

Methodology: 850 patients of chronic hepatitis $\mathrm{C}$ were that were treated at Division of Gastroenterology and Hepatology, Holy Family
Hospital, Rawalpindi, Pakistan between January 2006 to August 2009. After the consent, patients underwent workup including CBC, ALT levels, and Liver biopsy. Data was analyzed with SPSS 16. $T$ test was used for significance. Regression equations estimated the progression of disease on yearly basis.

Results: 69 patients dropped of 850 patients leaving 781cases. $45.09 \%$ were male and $54.01 \%$ female with $87.2 \%$ married and $12.8 \%$ single. Mean age was 35.7 years. Blood counts before treatment showed mean hemoglobin, TLC and platelets of $13.68+$ $5.0 \mathrm{mg} / \mathrm{dl}, 7.46+2.12$ and $225+65.39$ respectively. Alanine aminotransferase levels were high in $76.70 \%$ while $23.30 \%$ had levels less than $40 \mathrm{IU}$. Liver biopsy was analyzed according to modified histological activity index. Grade 3, 5, 4 were present with $18.3,17.9$ and $13.8 \%$ respectively. Maximum cases $88.47 \%$ had minimal or mild disease activity. Maximum patients had stage1 disease i.e. $61.58 \%$ cases with stage1 fibrosis, stage 2 and 3 in 22.02 and $8.57 \%$ respectively.

Multiple linear regression model analysis showed that one year increase in age will increase the liver biopsy grade by 0.05 and rise in 100 units ALT will worsen the biopsy grade by 0.6 . (95\% CI, $P<0.000$ ). In general,to increase the biopsy grade by 1 , it will take 20 years and 166.67 units of ALT independently.

Similarly stage of fibrosis will increase at rate of 0.028 per year that will take 25 years of age and 500 IU increase in ALT levels to increase stage by one degree (95\% CI, $P<0.000)$. Other variable like gender, baseline blood counts did not had significant impact on histology $(P 0.011,0.109,0.461,0.65)$

Conclusion: Age and ALT levels are the independent factors that are significantly correlated with disease progression.

\section{PP16-25}

Prediction of Liver Fibrosis in Chronic Hepatitis B Using Transient Elastography with Multiple Cut Off Value

I. Hasan, S. Salim, A.S. Sulaiman

Division of Hepatology, Department of Internal Medicine, University of Indonesia, Jakarta, Indonesia

Background and aims: In contrast to chronic hepatitis $\mathrm{C}$, few studies had been conducted in assessing transient elastography (TE) for predicting significant fibrosis or cirrhosis in chronic hepatitis B (CHB).The aims of this study were to evaluate TE for diagnosing significant fibrosis/cirrhosis in CHB patients, and to find stiffness level for every stage of fibrosis using multiple cut off approach.

Methods: Liver biopsies and TE were performed on naive $\mathrm{CHB}$ patients, which evaluated histologically by a pathologist using Metavir score. Area under receiver operating characteristics curve (AUROC) was assessed and the optimal cut off was derived to discriminate $\mathrm{F} 2-4$ to $\mathrm{F} 0-1$ and $\mathrm{F} 4$ to non F4. Clinical application of TE using multiple cut off was constructed to identify F2 or F4.

Results: $75 \mathrm{CHB}$ patients were recruited. The AUC of TE to dicriminate $\mathrm{F} 2-4$ to $\mathrm{F} 0-1$ was $0.729 \pm 0.116$ with optimal cut off $6.25 \mathrm{kPa}$ (Sensitivity $62.8 \%$; Specificity $86.7 \%$ ). Using multiple cut off approach, TE $<2.85 \mathrm{kPa}$ could be categorized as non F2-4, and $\mathrm{TE}>15.7 \mathrm{kPa}$ as $\mathrm{F} 2-4$.

The AUC of TE to discriminate F4 to non F4 was $0.99 \pm 0.023$ with optimal cut off was $18.95 \mathrm{kPa}$ (Sensitivity 100\%; Specificity $94.1 \%$ ). Using multiple cut off, TE $<18.95 \mathrm{kPa}$ is categorized as non $\mathrm{F} 4$, and those with TE $>25.5 \mathrm{kPa}$ as F4.

Conclusion: TE is an adequate tool to replace liver biopsy to diagnose significant fibrosis $( \pm \mathrm{F} 2)$ in $\mathrm{CHB}$ patient and it showed excellent performance to detect F4. Using TE with multiple cut off value would significantly reduce the need of liver biopsy. 


\section{PP16-26}

Serum Levels of Leptin and TGF- $\beta 1$ in Patients with Chronic Hepatitis B and their Correlations with Hepatic Fibrosis

J. Long ${ }^{1}$, S. Lin ${ }^{2,3}$, F. Yang ${ }^{1}$

${ }^{1}$ Department of Infectious Disease; ${ }^{2}$ Zunyi Medical College;

${ }^{3}$ The Institute of Clinical Research, Zunyi Medical College, Zunyi, China

Backgrounds: Leptin and TGF- $\beta$ 1have gained attention in the pathogenesis of fibrogenesis in various chronic liver diseases. The purposes of this study are to evaluate possible associations of serum levels of leptin with progression of hepatic fibrosis in patients with chronic HBV infection.

Subjects and methods: Serum levels of leptin and TGF- $\beta 1$ were measured by an enzyme-linked immunosorbent assay. Subjects included in this study were 35 patients with chronic hepatitis B (CHB) and 24 normal controls (NC). Knodell fibrosis were scored in patients with $\mathrm{CHB}$, patients with and under F2 stage were included as mild fibrosis and patients above F2 stage were included as advanced fibrosis.

\section{Results:}

1. Serum levels of TGF- $\beta 1$ in patients with mild fibrosis $(6,538.03 \pm 4,100.45 \mathrm{pg} / \mathrm{ml})$, advanced fibrosis $(36,347.81 \pm$ $16,155.80 \mathrm{pg} / \mathrm{ml}$ ) were significantly higher than those in $\mathrm{NC}$ $(2,456.97 \pm 1,373.12 \mathrm{pg} / \mathrm{ml})$.

2. Serum levels of leptin in patients with mild inflammation $(10,227.78 \pm 8,807.92 \mathrm{pg} / \mathrm{ml})$ were higher than those of patients with advanced inflammation $(7,465.83 \pm 7,103.44 \mathrm{pg} / \mathrm{ml})$ and $\mathrm{NC}(8,829.03 \pm 7,982.95 \mathrm{pg} / \mathrm{ml})$. but the differences were not significant among those three groups.

Conclusion: Our results indicated that serum levels of TGF- $\beta 1$ but not leptin correlate more closely stages of hepatic fibrosis in patients with chronic HBV infection.

\section{PP16-27}

Normal Values of Transient Elastography (Fibroscan) in Children without Evidence of Liver Disease: Comparison of Adult versus Pediatric Probe

R. Francavilla ${ }^{1}$, V. Cecinati ${ }^{1}$, S. Castellaneta ${ }^{1}$, L. Brescia ${ }^{1}$, M. Barone ${ }^{1}$, F. Indrio ${ }^{1}$, L. Cavallo ${ }^{1}, \mathrm{~S}$. Dembinski ${ }^{2}$

${ }^{1}$ Biomedicina Età Evolutiva, University of Bari, Bari; ${ }^{2} A X A N$, Milano, Italy

Transient elastography (Fibroscan) is a novel non-invasive method for the assessment of hepatic fibrosis in patients with chronic liver diseases by measuring liver stiffness (LS); however, no data are available in children although a pediatric probe is available. Aim of our study was to assess the normal value for this technique in children without liver disease and assess the performances of the two probes. Methods: 175 children [mean age 8 years \pm 3.6 (CI $95 \%$ 7.5-8.6 years)] with:

a. no history of liver disease,

b. normal liver function test in the last 12 months,

c. liver ultrasound (USS) negative for liver disease and

d. BMI normal for the age underwent a Fibroscan determination using two different probes [adult $(\mathrm{M})$ and pediatric $(\mathrm{P})$ ].

All measurements were preceded by a liver USS to select the site of the test.

Results: Determination was successful in all with a success rate of $85 \pm 17 \%$ (CI 95\% 82-87\%) for the M versus $80 \pm 18 \%$ [(CI 95\% $78-83 \%$ ); IQR $0.9 \pm 0.8$ ] for the P probe $(p<0.02)$. LS (Kpa) was $4.5 \pm 1.4$ (CI 95\% 4.3-4.7) for the $\mathrm{M}$ versus $4.8 \pm 1.9$ [(CI95\% 4.6-5.3); IQR $1 \pm 1.1]$ for the P probe $(p<0.01)$. The distribution of 3rd, 10th, 25th, 50th, 75th, 90th, and 97th centile of LS were: 2.8, 3.3,

3.7, 4.3, 4.9, 5.9 and 7.0 for the $M$ and 3.0, 3.3, 3.9, 4.5, 5.6, 6.5 and
8.0 for the $\mathrm{S}$ probe. We found a significant difference in the LS measurement with the two probes ranging from -20 to $28 \%$ $[$ Mean \pm SD $5.2 \pm 5.9 \%$ (CI 95\% 4.3-6\%)].

Conclusion: Our data show that Fibroscan is feasible in children and LF in children without liver disease may vary from 3,0 to 7,0. The LS measured with the adult probe may be significantly differ to that measured with the pediatric probe.

\section{PP16-28}

Can a Valid Liver Stiffness Value Be Obtained with 5 Compared to the Standard 10 Measurements?

D. Tan, J. Chang

Gastroenterology and Hepatology, Singapore General Hospital,

Singapore, Singapore

Introduction: The manufacturer's recommendation for liver stiffness measurement(LSM) using transient elastography (Fibroscan ${ }^{\circledR}$ ) is to take the median value of at least 10 measurements (shots). There is limited data on whether a valid LSM result can be obtained with fewer than 10 shots.

Aim: To evaluate if a valid LSM can be obtained with 5 shots compared to 10 shots for diagnosis of significant fibrosis.

Methods: The median LSM of the first 5 shots (LSM5) was compared with the median LSM of 10 shots (LSM10) in a cohort of 209 patients who underwent paired liver biopsy and Fibroscan for evaluation of liver fibrosis.

Results: Mean age was $48.8 \pm 12.6,52.2 \%$ were males and $87 \%$ Chinese. Primary etiologies were hepatitis B (42\%), non-alcoholic steatohepatitis $(16 \%)$ and chronic hepatitis C (10\%), among others. $50.2 \%$ had significant fibrosis(METAVIR $\geq$ F2) on liver biopsy. Correlation between LSM10 and LSM5 was high $(r=0.992)$, with no significant difference between LSM10 and LSM5 $(p=0.515)$. Mean difference between LSM10 and LSM5 was small $(0.016 \pm 1.68 \mathrm{kPa})$. A significant difference (defined as $\geq 10 \%$ difference between LSM10 and LSM5) was seen in 41 patients $(19.6 \%)$. Correlation between the inter-quartile range-to-median LSM ratio(IQR/M) using 5 versus 10 shots was poor $(r=0.665)$ with a significant difference between the IQR/M $(p=0.004)$. AUROC for significant fibrosis $(\mathrm{F} \geq 2)$ was $0.833(95 \%$ CI $0.777-0.889)$ for LSM5 and $0.842(95 \%$ CI 0.787-0.896) for LSM10. An optimal cut-off of $8.8 \mathrm{kPa}$ was identified based on LSM10 (sensitivity $81.4 \%$, specificity $76.7 \%$, PPV $77.1 \%$, NPV 79.0\%). Using this cut-off, LSM5 and LSM10 agreed in $95.2 \%$ of cases.

Conclusion: The use of 5 shots to obtain a median LSM value appears to be a feasible alternative to the recommended 10 shots. This would reduce the time taken for each Fibroscan examination and facilitate its use in mass community screening. However, the accuracy using 5 shots is less robust compared to the recommended 10 shots, particularly in patients with a high IQR and wide variation in LSM values.

\section{PP16-29}

Non-invasive Fibrosis Assessment in Chronic Hepatitis C: FibroScan Is Better than APRI Score and Hyaluronic Acid for Identifying Liver Fibrosis

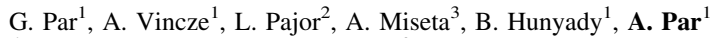

${ }^{1}$ First Department of Medicine; ${ }^{2}$ Department of Pathology;

${ }^{3}$ Department of Laboratory Medicine; University of Pecs, Pecs, Hungary

Background: Although liver biopsy is the gold standard for the diagnosis of liver disease, recently non-invasive tests may also play a role in the evaluation of liver fibrosis. We have studied aspartateaminotransferase/platelet ratio index (APRI), serum hyaluronic acid (HA) and liver stiffness (LS) measurement to assess fibrosis in chronic hepatitis $\mathrm{C}$ virus (HCV) infection. 
Patients and methods: Out of $119 \mathrm{HCV}$-infected patients 75 had biopsy-proven chronic hepatitis C, 24 had HCV-cirrhosis, 20 individuals were symptom-free $\mathrm{HCV}$-carriers with persistently normal alanine-aminotransferase and 30 healthy blood donors served as controls. Serum HA level was measured by ELISA, Wai's APRI score was calculated from aspartate-aminotransferase (AST) and platelet number, for LS measurement transient elastography (FibroScan) was applied. METAVIR fibrosis score were determined by liver biopsy.

Results: In HCV patients all fibrosis markers were significantly elevated compared with normal controls and were highest in HCVcirrhosis. Results of symptom-free HCV-carriers corresponded to those of healthy controls. APRI, HA and LS results correlated with the METAVIR score. LS identified significant $\left(\mathrm{F}^{3} 2\right)$ fibrosis better than APRI and HA. (LS $>7.48 \mathrm{kPa}$ : sensitivity $90.56 \%$, specificity 74.2\%, PPV 85.7, NPV 82.1, accuracy $84.5 \%$; APRI $<0.5$ :sensitivity 74.4\% specificity 70\%, PPV 79.5, NPV 63.6, accuracy 72.7\%; HA: sensitivity $52.9 \%$, specificity $100 \%$, PPV 100, NPV 54.28, accuracy $69.8 \%$ ). Using a novel sequential algorithm that comprises APRI and LS for assessment of fibrosis, $47.8 \%$ of HCV patients did not need biopsy for diagnosing significant $\left(\mathrm{F}^{3} 2\right)$ fibrosis.

Conclusion: The three fibrosis markers particularly in combination, may represent a useful option in the non-invasive assessment of fibrosis in HCV infection, but alone LS is better than APRI or HA.

\section{PP16-30}

A New Score for Fibrosis Prediction in Chronic HCV Hepatitis

S. Bota ${ }^{1}$, R. Sirli ${ }^{1}$, I. Sporea ${ }^{1}$, M. Focsa ${ }^{2}$, A. Popescu ${ }^{1}$, M. Danila ${ }^{1}$, M. Strain ${ }^{1}$, M. Sendroiu ${ }^{1}$, A. Deleanu ${ }^{1}$, A. Tudora ${ }^{1}$, I. Dan ${ }^{1}$

${ }^{1}$ Gastroenterology and Hepatology; ${ }^{2}$ Biophysics and Medical Informatics, University of Medicine and Pharmacy, Timisoara, Romania

Introduction: Liver biopsy (LB) is still considered the "gold standard" for assessment of liver fibrosis.

Aim: To evaluate the effectiveness of various non-invasive methods for predicting liver fibrosis (Transient Elastography-TE, APRI score, Lok score, Forns score, FIB-4 score, FI - Fibrosis Index score, King score) (using as gold-standard the LB) and to create a new score for fibrosis prediction.

Methods: The study included 212 patients, mean age $49.9 \pm 9.9$ years. In each patient LB, TE and biological tests were performed in the same session. The biological tests were used to calculate the scores above according to the known formulas.

Results: All the tests for predicting liver fibrosis were directly, statistically significant correlated with histological fibrosis. TE $(r=0.62, p<0.0001)$, King score $(r=0.57, p<0.0001)$ and APRI score $(r=0.56, p<0.0001)$ correlated best with the severity of fibrosis. From our data were obtained the following formula: $0.956+0.084 \times \mathrm{TE}-0.004 \times$ King score $+0.124 \times$ Forns score $+0.202 \times$ APRI score. A direct, linear, strong correlation $(r=0.68)$ was found between the new score and liver fibrosis $(p<0.0001)$. The cut-off values of the new score for various stages of liver fibrosis were $F \geq 1: 1.77$ (AUROC $=0.76$ ), $F \geq 2: 2.18$ (AUROC $=0.78), F \geq 3$ : 2.47 (AUROC $=0.86$ ) and $F=4: 2.98$ (AUROC $=0.97$ ). The new score had a better predictive value than TE for significant fibrosis $(F \geq 2$ Metavir): AUROC $=0.78$ vs. $0.74, p=0.02$; also for severe fibrosis $(F \geq 3$ Metavir): AUROC $=0.86$ versus $0.81, p=0.003$; while for cirrhosis the predictive values were similar AUROC $=0.97$ versus $0.97, p=0.28$. The new score was also superior to APRI, King and Forns score for the prediction of significant and severe fibrosis.

Conclusion: The new score for predicting fibrosis was better correlated with liver fibrosis than the other tests alone. For predicting significant and severe fibrosis, the new score is better than TE and for predicting liver cirrhosis they have similar value.
PP16-31

The Value of Acoustic Radiation Force Impulse Elastography (ARFI) in our Daily Practice

I. Sporea, S. Bota, R. Sirli, A. Popescu, M. Danila, M. Sendroiu Gastroenterology and Hepatology, University of Medicine and Pharmacy, Timisoara, Romania

Introduction: ARFI elastography is a new method used mainly for the assessment of liver fibrosis.

Aim: To assess the value of liver stiffness (LS) measurement by means of ARFI as a predictive factor for the severity of fibrosis, for the presence of cirrhosis complications and for the etiology of the ascites.

Methods: We performed ARFI measurements in 460 subjects, mean age $52.2 \pm 15.3$ year: 10 ARFI measurements in each patient and a median value was calculated, expressed in meters/second $(\mathrm{m} / \mathrm{s})$. We considered as reliable only measurements with IQR $<30 \%$ and SR $\geq 60 \%$.

Results: In 13 patients (2.9\%), we did not obtain valid measurements. We included 76 healthy volunteers, 77 patients with HCV and 37 with HBV chronic hepatitis (with liver biopsy), 246 patients with liver cirrhosis and 24 patients with non-cirrhotic ascites. The mean LS value determined by ARFI in healthy volunteers was $1.15 \pm 0.21 \mathrm{~m} / \mathrm{s}$. In $\mathrm{HCV}$ chronic hepatitis, the cut-off value for $\mathrm{F} 2$ was $1.33 \mathrm{~m} / \mathrm{s}$ $($ AUROC $=0.74)$, for $\mathrm{F} 3-1.64 \mathrm{~m} / \mathrm{s} \quad($ AUROC $=0.74)$ and for $\mathrm{F} 4-1.79 \mathrm{~m} / \mathrm{s}($ AUROC $=0.90)$. In cirrhotic patients there were no statistically significant differences between the mean values of ARFI measurements in patients with grade 2 and 3 esophageal varices versus those with no or grade 1 esophageal varices $(p=0.07)$, between those with or without hepatocellular carcinoma $(p=0.15)$, but we found a statistically significant different values in patients with ascites vs. those without ascites $(3.09 \pm 0.69$ vs. $2.68 \pm 0.67 \mathrm{~m} / \mathrm{s})$ $(p<0.001)$. The best cut-off value for predicting ascites in cirrhotic patients was $2.52 \mathrm{~m} / \mathrm{s}$ (AUROC 0.64). ARFI had $94.2 \%$ accuracy for predicting the cirrhotic or non-cirrhotic etiology of ascites.

Conclusion: ARFI is a feasible method in most of the patients $(97.1 \%)$. It has a very good predictive value for the presence of cirrhosis, for the cirrhotic etiology of ascites and in cirrhotic patients it can be a predictor of vascular decompensation.

\section{PP16-32}

Real-time versus Transient Elastography for Assessment of Liver Fibrosis in Chronic Hepatitis C Patients

C. Mihai, G. Stefanescu, M. Dranga, B. Mihai, C. Lacatusu, C. Cijevschi Prelipcean

University of Medicine and Pharmacy 'Gr. T. Popa', Iasi, Romania

In the last years liver histology-considered in the past "gold standard" in the evaluation of liver fibrosis (LF) is going to be replaced by non-invasive methods like liver elastography.

Aim: To compare real-time elastography (RTE) — Hitachi with transient elastography-Fibroscan (FS) in evaluation of LF in patients with chronic hepatitis $C$.

Material: We studied 40 patients with chronic hepatitis $C$ which were evaluated for antiviral treatment in Institute of Gastroenterology and Hepatology, Iasi, Romania. All patients received RTE, FS and liver biopsy. The scorification for fibrosis was:

Liver biopsy F0-F4 according to Metavir score; FS: F0-F1 $<7.1 \mathrm{kPa}$, F2 $7.1-9.5 \mathrm{kPa}$, F3 9.5-13 kPa, F4 > $13 \mathrm{kPa}$;

$R T E$ Median elastic ratio in each stage were: F0: $1.20, \mathrm{~F} 1: 1.11, \mathrm{~F} 2$ : 1.07, F3: 0.8 F4: 0.41.

Results: According to histology 3 patients had F0, 8 F1, 15 F2, 7 F3 and $7 \mathrm{~F} 4$. According to FS 5 patients $(12.5 \%)$ had a wrong staging of fibrosis: 1 with F0, F1 and respectively F3 and 3 with F2. According to RTE 12 patients (30\%) had a wrong staging: 1 with F0, 3 with F1 and F3 and 5 with F2. The predictor factors for wrong classification of $\mathrm{LF}$ were mild fibrosis and $\mathrm{BMI}>30 \mathrm{~kg} / \mathrm{m}^{2}(p<0.05)$. 
Conclusions: Hepatic elastography is a useful tool for detecting mild or severe fibrosis in chronic hepatitis C. FS is superior to RTE in staging hepatic fibrosis. Obese patients are difficult to assess by realtime or transient elastography.

\section{PP16-33}

Insulin Resistance Correlates with the Severity of Fibrosis in Chronic Hepatitis B

J.H. Sohn ${ }^{1,2}$, T.Y. Kim ${ }^{1}$, J.Y. Jeong ${ }^{1}$, D.W. Jun ${ }^{2}$, C.S. Eun ${ }^{1,2}$, Y.C. Jeon ${ }^{1,2}$, D.S. $\operatorname{Han}^{1,2}$

${ }^{1}$ Gastroenterology, Hanyang University Guri Hospital, Guri;

${ }^{2}$ Internal Medicine (Gastroenterology), Hanyang University College of Medicine, Seoul, Republic of Korea

Background/aim: This study was aimed to investigate the correlation of insulin resistance and liver fibrosis in patients with chronic hepatitis B (CHB).

Methods: We reviewed the data of 71 non-diabetic patients with CHB (male 52, female 19, mean age $39.6 \pm 11.8$ ) and evaluated the relationship between histologic findings, non-invasive markers and insulin resistance determined by the homeostasis model assessment (HOMA-IR). Liver fibrosis was staged according to METAVIR scoring system. Various non-invasive serum markers were evaluated, including AST/platelet ratio (APRI), hyaluronic acid (HA), type IV collagen. Diagnostic accuracy of HOMA-IR for predicting hepatic fibrosis was evaluated using area under ROC curves (AUC) analysis. Results: The numbers of CHB patients with F0-F1 were $6(8.5 \%), \mathrm{F} 2$ $16(22.5 \%)$, F3 27 (38.0\%), F4 22 (31.0\%), respectively. HA, type IV collagen, and HOMA-IR were significantly correlated with the progression of fibrosis stage $(r=0.37,0.38,0.33, p<0.01)$ but APRI was not $(r=0.22, p=0.06)$. The AUC values of four parameters for predicting significant (F2-4) and advanced (F3-4) fibrosis were shown in Table 1. The 1.72 cutoff value of HOMA-IR had $42.9 \%$ sensitivity, $90.9 \%$ specificity, 4.71 positive likelihood ratio, and 0.63 negative likelihood ratio for the advanced fibrosis. Especially, all patients (14/49) with HOMA-IR $>1.72$ and HA $>50 \mathrm{ng} / \mathrm{ml}$ had advanced fibrosis $(p=0.01)$.

Conclusion: Insulin resistance (HOMA-IR) correlates with the severity of fibrosis in $\mathrm{CHB}$, indicating a simple and useful predictor of fibrosis progression. Also, combination of HOMA-IR and HA reduces the need for liver biopsy in about $30 \%$ of patients with advanced fibrosis.

AUC values of HOMA-IR and serum markers

\begin{tabular}{lcc}
\hline & $\begin{array}{l}\text { Significant fibrosis } \\
(\mathrm{F} 2-4)\end{array}$ & $\begin{array}{l}\text { Advanced fibrosis } \\
(\mathrm{F} 3-4)\end{array}$ \\
\hline HOMA-IR & $0.76(0.60-0.93$, & $0.68(0.55-0.81$, \\
& $p=0.04)$ & $p=0.02)$ \\
HA & $0.64(0.35-0.94$, & $0.68(0.54-0.82$, \\
& $p=0.28)$ & $p=0.01)$ \\
Type IV & $0.77(0.61-0.92$, & $0.67(0.57-0.80$, \\
collagen & $p=0.03)$ & $p=0.02)$ \\
APRI & $0.77(0.60-0.95$, & $0.62(0.46-0.77$, \\
& $p=0.03)$ & $p=0.11)$ \\
\hline
\end{tabular}

PP16-34

Liver Stiffness in Patients with Extra Hepatic Portal Vein Obstruction P. Sharma ${ }^{1}$, S.R. Mishra ${ }^{2}$, M. Kumar ${ }^{1}$, H. Garg ${ }^{1}$, B.C. Sharma ${ }^{3}$, S.K. Sarin ${ }^{1}$ ${ }^{1}$ Institute of Liver and Biliary Sciences (ILBS), Delhi; ${ }^{2} G$ B Pant Hospital, Delhi; ${ }^{3}$ G B Pant Hospital, Delhi, India

Introduction and aims: Liver stiffness (LS) measurement by transient elastography (TE) is fairly accurate in predicting fibrosis in patients with hepatitis B and C. There is no data in patients with extrahepatic portal vein obstruction (EHPVO). We assessed LS and spleen stiffness (SS) in patients with EHPVO.

Patients and method: LS and SS measured in consecutive EHPVO patients. Patients underwent endoscopy, liver biopsy, liver function tests, ultrasound abdomen, detailed history and examination. Fifty age matched healthy controls also taken for LS measurement.

Results: 65 patients (bleeder, $n=45$, nonbleeder, $n=20$ ) of EHPVO were enrolled (age $25.4 \pm 10.7, \mathrm{M}: \mathrm{F}$ 29:36). Twenty two (34\%) had hypersplenism. LS in EHPVO patients as compared to controls was $(6.7 \pm 2.3$ vs. $4.6 \pm 0.7 \mathrm{kPa}, p=0.01)$. Patients who had bled $(n=45)$ had higher LS $(7.2 \pm 2.7$ vs. $5.5 \pm 1.5 \mathrm{kPa}$, $p=0.01)$ and SS $(60.4 \pm 5.4$ vs. $30.3 \pm 14.2 \mathrm{kPa} p=0.01)$ than non bleeder. There was no significant difference in age $(26.7 \pm 10.4$ vs. $22.5 \pm 9.8$ years, $p=0.8$ ) and median duration of disease in bleeder versus non bleeder[( 4.5 year, range $1-26$ year) vs. (6.0 year, range 1-22 year), $p=0.23$ ]. Taking a cut off $6.05 \mathrm{kPa}$ for LS, sensitivity and specificity for bleed in EHPVO patients was 62 and $81 \%$ respectively [AUC $0.701(0.557-0.845)$ ]. Similarly for SS of $42.8 \mathrm{kPa}$ the sensitivity and specificity for bleed was 87 and $94 \%$ respectively [AUC 0.888 (0.779-0.997)]

Conclusion: Liver stiffness in patients with EHPVO is higher than in healthy controls.

\section{PP16-35}

Steatohepatitis and Fibrosis in Chronic Hepatitis C Patients: Is there any Association?

M.H. Hasmoni ${ }^{1,2}$, R. Salleh ${ }^{2}$, K.A. Jaafar ${ }^{1,2}$, M.H. Jaafar ${ }^{2}$, A. Abas ${ }^{3}$

${ }^{I}$ Department of Internal Medicine, Kulliyyah of Medicine, International Islamic University Malaysia, Kuantan;

${ }^{2}$ Gastroenterology Unit, Department of Medicine; ${ }^{3}$ Department of Radiology, Hospital Raja Perempuan Zainab II, Kota Bharu, Malaysia

Background: Hepatic steatosis and disturbances in lipid metabolism has been identified as one of the metabolic disorders in hepatitis $\mathrm{C}$ (HCV) infection. This study was to examine the possible association of steatohepatitis with the degree of fibrosis in chronic HCV patients. Methods: Retrospective analysis of data from 72 patients with chronic HCV who underwent liver biopsy from January 2008 to March 2010 in two tertiary hospitals in Malaysia was performed. Patients' demographic data and their body mass index (BMI), liver function tests and lipid profiles, histological findings of steatosis, staging and grading of biopsy specimen were made. Staging and grading of liver disease was done using modified histological activity index staging (score 0-6) and grading (score 0-18) respectively. Univariate analysis was done to assess the association between the degree of fibrosis and steatohepatitis.

Results: $79.2 \%$ were males and the mean age for the study population was $40.7 \pm 12.0$ years. There were 13.9 and $9.7 \%$ cases of coinfection with HIV and hepatitis B respectively, while $76.4 \%$ patients 
were infected with HCV alone. The table showed the superimposed steatohepatitis that was present with severe fibrosis and mild or no fibrosis in the two different groups. However, the univariate analysis assessing the association between the degree of fibrosis and the presence of steatohepatitis in both the two groups could not find any significant result.

Conclusion: There was no significant association between steatohepatitis and the degree of fibrosis in patients with chronic HCV infection, either co-infection with other viruses or $\mathrm{HCV}$ infection alone.

Steatohepatitis and liver fibrosis

\begin{tabular}{lll}
\hline & $\begin{array}{l}\text { Severe fibrosis } \\
\text { with steatohepatitis, } \\
n(\%)\end{array}$ & $\begin{array}{l}\text { Mild or no } \\
\text { fibrosis with } \\
\text { steatohepatitis, } n(\%)\end{array}$ \\
\hline $\begin{array}{l}\text { HCV with co-infection } \\
\text { HCV infection alone }\end{array}$ & $8(47)$ & $2(11)$ \\
\hline
\end{tabular}

\section{PP16-36}

Transient Elastographic Evaluation in Adult Subjects without Overt Liver Disease: Influence of Alanine Aminotransferase Levels

R. Khanna, M. Kumar, V. Tomar, P. Sharma, H. Garg, R. Kumar, V. Bhatia, S.K. Sarin

Hepatology, Institute of Liver and Biliary Sciences, New Delhi, India

Background and aims: Studies on normal values of liver stiffness (LS) in subjects at 'low risk' for liver disease are scant. The aim of the present study was to assess liver stiffness values in the subjects without overt liver disease with normal alanine aminotransferases (ALT) and to determine potential factors, which may influence these values with special reference to newly suggested updated upper limits of normal for ALT.

Methods: LS measurements were performed in 445 subjects without overt liver disease [mean age $41.1 \pm 13.6$, male $73.5 \%$ ] and normal liver enzymes.

Results: Mean LS value was $5.10 \pm 1.19 \mathrm{kPa}$. LS values were higher in men than in women $(5.18 \pm 1.67$ vs. $4.86 \pm 1.24 \mathrm{kPa}$, respectively, $p=0.008$ ); in subjects with higher body mass index (BMI) category [Normal, overweight and obese subjects; $4.10 \pm 0.75$, $5.08 \pm 0.66$, and $6.05 \pm 1.28 \mathrm{kPa}$ respectively; $p<0.001]$; in subjects with metabolic syndrome than in those without $(5.63 \pm 1.37$ vs. $5.01 \pm 1.14 \mathrm{kPa}, p=0.001)$; and in subjects with ALT levels more than updated limits of normal compared to subjects with ALT levels less than updated limits of normal $[5.68 \pm 1.21$ vs. $4.77 \pm 1.05 \mathrm{kPa}$, $p<0.001]$. On multiple linear regression, BMI and ALT was found to be significant predictor of liver stiffness.

Conclusions: Liver stiffness values in subjects without overt liver disease with normal ALT are influenced by BMI and ALT levels. Subjects with ALT levels less than updated limits of normal have lower LS values as compared to those with higher levels.

\section{PP16-37}

HBeAg Negative Chronic Hepatitis: Determinants of Fibrosis

R. Goyal ${ }^{1}$, A. Kumar ${ }^{1}$, S. Shalimar ${ }^{1}$, M. Mahanta ${ }^{1}$, D. Seth ${ }^{2}$, S. Duttagupta ${ }^{3}$, S.K. Acharya ${ }^{1}$

${ }^{1}$ Gastroenterology and Human Nutrition; ${ }^{2}$ Biostatistics; ${ }^{3}$ Pathology, All India Institute of Medical Sciences, Delhi, India

Introduction: $\mathrm{HBeAg}$ negative chronic hepatitis has been assuming increasing importence among HBV related liver diseases. Data from
India is based on small series with limited patients. Predictors of fibrosis in $\mathrm{HBeAg}$ negative patients are not described from India.

Methods: We have been prospectively following patients with chronic hepatitis B since January 2007. They are subjected to complete workup including liver function tests, HBeAg, HBV DNA Quantitation, Genotyping and baseline Liver biopsies. Predictors of fibrosis were assessed in consecutive $\mathrm{HBeAg}$ negative patients using one way ANOVA and chi square tests, as appropriate.

Results: Complete evaluation including liver biopsy was done in 146 patients (mean age $29.5+8.28$ years, $14.4 \%$ females). Mean ALT at baseline was $73.5+85.67$ IU/L. Median HBV DNA at baseline was 5,365 copies/ml and 65 (44.5\%) had HBV DNA > 104 copies/ml. 19 patients were genotype A, 39 genotype D, 26 genotype $\mathrm{A}+\mathrm{D}$ and 32 insufficient serum DNA for genotyping. $24.0 \%$ had significant fibrosis on biopsy (HAI $>3$ and/or fibrosis stage $>1$ ).

The univariate analysis showed that the important predictors of fibrosis in chronic hepatitis were age ( $p$ 0.004), serum ALT levels ( $p$ 0.010), serum AST levels $(p<0.001)$ and serum HBV DNA levels $(p$ 0.003). Patient's BMI ( $p 0.95)$, sex ( $p 0.52)$, platelet count $(p 0.98)$, bilirubin ( $p 0.51)$, serum albumin ( $p 0.29)$, serum AFP levels $(p 0.27)$ and genotype $(p 0.11)$ did not correlate with the histological fibrosis. Conclusions: Advanced age, HBV DNA load and transaminase levels may predict advanced fibrosis in patients with $\mathrm{HBeAg}$ negative chronic hepatitis $\mathrm{B}$, while BMI, platelet count, serum albumin or HBV genotypes did nor correlate with fibrosis severity.

\section{PP16-38}

Liver Stiffness Measurements by Means of Transient Elastography in Clinical Practice-A Monocentre Experience on 5678 Cases

I. Sporea, R. Sirli, I. Ratiu, M. Sendroiu, D. Gerhardt, A. Popescu Gastroenterology, University of Medicine and Pharmacy Timisoara, Timisoara, Romania

The aim of this paper was to evaluate the indications and results of transient elastographic evaluation of the liver stiffness in patients from our Department.

Material and method: we retrospectively assessed all the liver stiffness measurements (LSM) (FibroScan) made from 2007 to 2010. Results: From the 5,678 LSM, in 385 cases we could not obtain 10 valid measures, our failure rate being $6.8 \%$. In 633 cases $(11.1 \%)$ the SR was $<60 \%$ and/or the IQR was $>30 \%$, so only in approximately $82 \%$ of the cases TE is reliable.

360 patients with chronic HCV hepatitis and 157 patients with HBV hepatitis were evaluated by means of TE and LB. For the same severity of fibrosis, the mean values of LS in HBV patients were similar to those in $\mathrm{HCV}$ patients: F1 $6.5 \pm 1.9$ versus $5.8 \pm 2.0 \mathrm{kPa}$ $(P=0.05), \quad \mathrm{F} 2 \quad 7.2 \pm 2.8 \quad$ versus $\quad 6.8 \pm 2.5 \mathrm{kPa} \quad(P=0.151), \quad \mathrm{F} 3$ $9.1 \pm 3.5$ versus $10 \pm 5.1 \mathrm{kPa}(P=0.762), \mathrm{F} 415.7 \pm 85.9$ versus $16.1 \pm 6.4 \mathrm{kPa}(P=0.765)$.

A significant direct correlation of LS measurements with fibrosis was found in HCV patients (Spearman's $r=0.570, P<0.0001$ ), stronger than in HBV patients $(r=0.407, P<0.0001)$.

The mean LS in 140 inactive $\mathrm{HBsAg}$ carriers was $5.6 \pm 2.1 \mathrm{kPa}$, statistically significant higher than in normal subjects (140 patients: $4.8 \pm 1.2 \mathrm{kPa}, p=0.0002)$.

In 1,000 cirrhotic patients with recent gastroscopies, for a cut-off value of $28.4 \mathrm{kPa}, \mathrm{LSM}$ had $60 \% \mathrm{PPV}$ and $85 \%$ NPV for the presence of at least grade 2 esophageal varices (AUROC 0.781), and for a cutoff value of $46.4 \mathrm{kPa}$, it had $69 \% \mathrm{PPV}$ and $68 \% \mathrm{NPV}$ (AUROC $=0.692$ ) for variceal bleeding.

Conclusion: LSM is a useful method for assessing chronic hepatitis. It is feasible in almost $93 \%$ of the cases, but in approximately $11 \%$ of the cases the results are not reliable (SR $<60 \%$, IQR $>30 \%$ ). In patients with cirrhosis it can be an indicator of significant portal hypertension. 


\section{PP16-39}

How Good Can a Liver Fragment Be Depending on the Size of Needle? I. Sporea ${ }^{1}$, D. Gherhardt ${ }^{1}$, A. Popescu ${ }^{1}$, R. Sirli ${ }^{1}$, M. Danila ${ }^{2}$, M. Cornianu ${ }^{3}$, C. Vernic ${ }^{4}$

${ }^{1}$ Gastroenterology and Hepatology; ${ }^{2}$ Department of

Gastroenterology and Hepatology; ${ }^{3}$ University of Medicine and

Pharmacy "Victor Babes"; ${ }^{4}$ Department of Medical Informatics and Biostatistics, University of Medicine and Pharmacy "Victor Babes", Timisoara, Romania

Background and aim: The aims of our paper were to establish if size of needle does influence the number of portal tracts obtained through liver biopsy.

Material and method: We conducted a retrospective study on 671 echoassisted percutaneous liver biopsies performed in the Department of Gastroenterology and Hepatology, Timisoara during January 2006-October 2010. The liver fragments were analyzed by a senior morpho-pathologist; Knodell score was used to describe fibrosis.

Results: The mean length of the fragment obtained was $3.3 \pm 0.9 \mathrm{~cm}$ (it was specified for 584 out of 671 biopsies). There were no significant statistical differences between the lengths of the fragment obtained with needle size 1.4 versus $1.6(3.3 \pm 0.8$ vs. $3.3 \pm 0.9$, $p=0.81355$ ). Liver fragments obtained with Menghini needle type 1.6 had a higher number of portal tracts compared to those obtained with needle type 1.4 from a statistical significant point of view $(24.1 \pm 10.6$ vs. $21.5 \pm 9, p=0.0323)$. Both types of needles were used in case of cirrhosis, but the most frequent one was type1.6 as it can be seen in Tables 1 and 2 .

Table 1 Stage of fibrosis on liver biopsy fragmen

\begin{tabular}{lcccccc}
\hline $\begin{array}{l}\text { Type of needle } \\
\text { (diameter) }\end{array}$ & $\begin{array}{l}\text { Total number } \\
\text { of biopsies }\end{array}$ & F0 & F1 & F2 & F3 & F4 \\
\hline 1.4 & 84 & 12 & 47 & 4 & 20 & 1 \\
1.6 & 587 & 26 & 357 & 8 & 187 & 9 \\
\hline
\end{tabular}

Table 2 Mean number of portal tracts according $\mathrm{t}$

\begin{tabular}{|c|c|c|c|c|c|c|}
\hline $\begin{array}{l}\text { Type of } \\
\text { needle }\end{array}$ & & F0 & $\mathrm{F} 1$ & $\mathrm{~F} 2$ & F3 & $\mathrm{F} 4$ \\
\hline 1.4 & $\begin{array}{l}\text { Mean } \\
\text { number } \\
\text { of portal } \\
\text { tracts }\end{array}$ & $15.5 \pm 5.9$ & $20.8 \pm 9.6$ & $35.2 \pm 5.3$ & $23.6 \pm 5.9$ & $\begin{array}{c}\text { Sample too } \\
\text { small }\end{array}$ \\
\hline 1.6 & $\begin{array}{l}\text { Mean } \\
\text { number } \\
\text { of portal } \\
\text { tracts }\end{array}$ & $16.7 \pm 9.6$ & $23.3 \pm 10.3$ & $24.3 \pm 8.5$ & $26.4 \pm 10.6$ & $30.4 \pm 11.8$ \\
\hline$p$ & & 0.715 (NS) & 0.1219 (NS) & $0.0440(\mathrm{~S})$ & 0.250 (NS) & \\
\hline
\end{tabular}

Conclusion: Good liver fragments, in terms of length, were obtained with both Menghini needle sizes 1.4 as well as 1.6. Whenever Menghini needle 1.6 was used for liver biopsy, the number of portal tracts was higher compared to Menghini needle 1.4. Maybe it would be a good idea to use 1.6 needle only in case of suspicion of advanced fibrosis based on non-invasive techniques, while 1.4 could be helpful for the majority of cases.
PP16-40

Serum Soluble E-selectin Correlates to Hyaluronic Acid and Laminin Levels in Patients with Chronic Hepatitis B

M.-X. Yan, W.-Q. Wang, S.-L. Li, Y.-G. Wang

Department of Gastroenterology, Shandong Qianfoshan Hospital, Shandong University, Jinan, China

Introduction: Permanent inflammation lead to hepatic fibrosis and cirrhosis in patients with chronic HBV infection, but the underlying pathogenesis remains unclear. The purpose of our study was to investigate the relationship between serum concentration of soluble E-selectin (sE-selectin) and serum markers of hepatic fibrosis, hyaluronic acid (HA) and laminin (LN), in patients with chronic hepatitis $\mathrm{B}(\mathrm{CHB})$, and discuss their significances.

Methods: Fourty-eight patients with chronic hepatitis B and fourteen controls were studied. Serum soluble E-selectin (sE-selectin), hyaluronic acid (HA) and laminin (LN) were detected by enzyme-linked immunosorbent assay (ELISA) and radio-immunity assay, respectively. Results: sE-selectin level in patients with $\mathrm{CHB}$ increased significantly than that in controls $(p<0.01)$. sE-selectin level significantly related to levels of serum alanine aminotransferase (sALT) and aspartate aminotransferase (sAST) $(p<0.01$, respectively), but had no relationship with HBV level. Serum levels of HA and LN increased significantly in CHB patients than in controls $(p<0.05$, respectively). Both $\mathrm{HA}$ and $\mathrm{LN}$ had strong relations with serum $\mathrm{sE}$ selectin concentration in CHB patients.

Discussion/conclusion: sE-selectin correlates to hepatic inflammation and fibrosis serum markers HA and LN. E-selectin is involved in development of inflammation and fibrogenesis in patients with chronic HBV infection.

\section{PP16-41}

Role of Liver Fibrosis in Virologic Response to Combination Pegylated Therapy in the Treatment of Genotype 1 Chronic Hepatitis C

N. Aqodad, M. Lahbabi, I. Mellouki, M. Elyousfi, D. Benajah, M. Elabkari, A. Ibrahimi

Gastroenterology Unit, Hospital University Hassan II, Fez, Morocco

Introduction and aim of study: Several factors affect the viral response to treatment with pegylated combination therapy in chronic liver disease $\mathrm{C}$, such as viral genotype and liver fibrosis. The aim of this study was to determine the role of liver fibrosis in response to combination pegylated therapy in the treatment of genotype 1 chronic hepatitis C.

Patients and methods: Retrospective study of all cases of chronic hepatitis $\mathrm{C}$ virus genotype I treated with pegylated combination therapy in our department. Hepatic fibrosis was classified using the METAVIR score based on histological liver biopsies. Patients were divided into two groups according to fibrosis stage: group F1-F2 and F3-F4 group.

Results: Forty patients were collected, we have selected for this study 26 patients whom liver biopsy had performed and who completed their treatment and their follow-up 6 month after the end of treatment: group1: F1-F2 $(N=10)$ and Group 2: F3-F4 $(N=16)$. Apart from sex, and HCV viral load, both groups were comparable regarding age, smoking, alcohol consumption, clinical symptoms, positive anti-HBc, the presence of fatty liver in abdominal ultrasonography, the appearance of treatment side effects leading to stop therapy. There was a female predominance in group $\mathrm{F} 1-\mathrm{F} 2$ (60 vs. 55\%). The average viral load in group F1-F2 was higher than that of group F3F4 (2,186,610 vs. 1,190,040 IU/ml). Virologic response in group F1$\mathrm{F} 2$ and F3-F4 was 70 and $75 \%$ respectively but the difference was not 
statistically significant $(p=0.5)$. The sustained virological response in group F1-F2 and F3-F4 were similar (50\%).

Conclusion: Although advanced fibrosis is a predictor of poor response to antiviral therapy, in our study patients with severe fibrosis (F3-F4) showed a sustained virological response comparable to that of patients with minimum or moderate fibrosis (F1F2) $(50 \%)$.

\section{PP16-42}

Biopsy or Biomarkers: Which Is the Sample of Choice in Assessment of Liver Fibrosis?

S. Atef

Ain Shams University, Cairo, Egypt

Aim: To assess the diagnostic value of fibrotest and hyaluronic acid in discriminate between insignificant and significant fibrosis. Also, to find out if these parameters could replace liver biopsy which is currently used for selection of chronic hepatitis $\mathrm{C}$ patients eligible for antiviral therapy.

Study design: This study was conducted on 52 patients with HCV RNA detected by polymerase chain reaction (PCR) who had undergone liver biopsy and attending the internal medicine clinic at Ain Shams University Hospital. Liver fibrosis was evaluated according to the METAVIR scoring system on a scale of F0 to F4. Biochemical markers assessed were: alpha-2 macroglobulin $(\alpha 2-\mathrm{MG})$, apolipoprotein A1 (Apo-A1), haptoglobin, gamma-glutamyl transferase (GGT), total bilirubin (TB) and hyaluronic acid (HA). The fibrotest score was computed after adjusting for age and gender. Predictive values and ROC curves were used to assess the accuracy of fibrotest and HA results.

Results: For fibrotest, the observed area under curve for the discrimination between minimal or no fibrosis (F0-F1) and significant fibrosis (F2-F4) was 0.6736 for cutoff value 0.19 with sensitivity of $84.2 \%$ and specificity of $85.7 \%$. For HA, the sensitivity was $89.5 \%$ and specificity was $85.7 \%$ and area under curve was 0.540 at the best cutoff value $71 \mathrm{mg} / \mathrm{dL}$. Multi-use of both parameters, HA at $71 \mathrm{mg} / \mathrm{dL}$ with fibrotest score at 0.22 give a sensitivity $89.5 \%$, specificity 100 and efficacy $92.3 \%$ (AUC 0.895).

Conclusion: The use of both fibrotest score and HA could be as alternative to biopsy in most patients with chronic hepaitis $\mathrm{C}$ putting in consideration some limitations of the proposed markers in evaluating liver fibrosis.

\section{PP16-43}

Patients Older than 45 Years with over Ten-year CHB History Were High Risk for Developing Liver Cirrhosis

Q.H. Ling, J.J. Chen, J. Guo, X.D. Zhang, Y.H. Zhuo

Department of Liver Disease, ShuGuang Hospital, Shanghai

University of TCM, Shanghai, China

Background: Chronic HBV infection is the leading cause of liver cirrhosis. The paper aimed to evaluate the effect of risk factors on the development of cirrhosis in patients with over 10-year chronic hepatitis B histories.

Material and methods: We retrospectively assessed 390 patients which chronic HBV hepatitis histories were over 10 years, including 195 CHB patients with liver cirrhosis and 195 without liver cirrhosis. Results: The average age of LC group was significantly older than that of non-LC group $(p<0.001)$. Despite of the same CHB history, the number of young patients (18-35 years) developed in LC was less than that of old patients (45-65 years) $(p=0.041)$. In LC group, the average age of patients after hepatic decompensation was older than that of patients in hepatic compensation (54.35 vs.51.02, $p=0.032$ ). Conclusion: Old age played a crucial role in the development of cirrhosis in patients with chronic hepatitis B. Patients older than 45 years with over 10-year CHB history were high risk for developing liver cirrhosis.
PP16-44

Noninvasive Measurement of Liver Fibrosis by Transient Elastography and Influence Factors in Patients with Chronic Hepatitis B

H. Ding, T. Wu, K. Ma, Z. Wu, J. Song, J. Qi, Q. Ning

Tongji Hospital, Wuhan, China

Objective: To evaluate the noninvasive measurement of liver stiffness (LS) by transient elastography and analysis the possible influence factors from the patients clinical situation including age and gender, liver inflammation including ALT level, total billirubin level (TBil), HBV replication (HBV DNA level), portal vein pressure (portal vessel diameter, PVD), splenic thickness (SPT), body mass index (BMI) in patients with chronic hepatitis B (CHB).

Methods: A total of $466 \mathrm{CHB}$ patients including 31 patients with Acute on chronic liver failure and 82 patients with liver cirrhosis (LC) were enrolled at Tongji hospital from April 2009 to December 2010. A measurement of liver stiffness was performed by a FibroScan device (EchoSens, France). Simultaneously patients' serum biochemistry, HBV DNA level, PVD, and SPT, BMI were also tested. A group of 41 healthy volunteers were enrolled as control.

Results: The liver stiffness value in CHB patients correlated positively with their age, with a significantly higher value in male than that in female. Patients with BMI $>28 \mathrm{~kg} / \mathrm{m}^{2}$ (obesity) and abnormal level of ALT and TBil had a significantly increased liver stiffness value when compared with those of normal range of ALT and TBil, with the patients of ACLF the highest value. Furthermore, the liver stiffness of the patients with LC is significantly higher than that in patients without LC as evidenced by PVD and SPT and patients with ACLF. The differences are statistically significant.

Conclusion: Noninvasive Measurement of liver fibrosis by Transient Elastography provides an autonative way for patients with CHB. In order to properly evaluate the stage of liver fibrosis, the results of stiffness should be analyzed together with patients clinical situation including liver inflammation (level of ALT, TBil), gender, BMI.

\section{PP16-45}

APRI in Predicting Severe Fibrosis in Chronic Hepatitis B versus Chronic Hepatitis C

Y. Thitichettrakul ${ }^{1}$, A. Sobhonslidsuk ${ }^{1}$, A. Thakkinstian ${ }^{2}$, N. Wisedopas ${ }^{3}$, P. Sornmayura ${ }^{4}$

${ }^{1}$ Medicine; ${ }^{2}$ Section for Clinical Epidemiology and Biostatistics, Ramathibodi Hospital, Mahidol University; ${ }^{3}$ Pathology, Chulalongkorn University; ${ }^{4}$ Pathology, Ramathibodi Hospital, Mahidol University, Bangkok, Thailand

Background: Liver biopsy, the gold standard for assessing staging of fibrosis, can result in severe complications. AST-Platelets ratio index (APRI), a non-invasive marker requiring simple blood tests, may substitute liver biopsy. The comparative study of APRI in chronic hepatitis $\mathrm{B}(\mathrm{CHB})$ and chronic hepatitis $\mathrm{C}(\mathrm{CHC})$ was never done. Objectives: To identify the cutoff of APRI in predicting severe fibrosis in $\mathrm{CHB}$ and $\mathrm{CHC}$ and to compare the predicting values of APRI in $\mathrm{CHB}$ and $\mathrm{CHC}$ staging.

Methods: Medical records of CHB and CHC patients who underwent liver biopsy between 1 Jan 2004-31 Jan 2010 were reviewed. Histological staging was done with Knodell scoring system. APRI was calculated from the formula of AST/upper normal limit of AST $\times 100 /$ platelet count $\left(10^{9} / \mathrm{L}\right)$. ROC curve analysis was used to identify the cutoff of APRI. Likelihood ratio and an area under ROC (AUC) were reported.

Results: Two hundred and thirteen patients were enrolled. Mean age was 45.6 (11.1). CHB and CHC were found in 124 (58.2\%) and 89 $(41.8 \%)$ patients. Severe fibrosis (=Stages 3 and 4 ) were seen in 62 (29.1\%). The APRI was higher in severe than non-severe fibrosis with the medians of $1.17(0.44-5.52)$ and $0.58(0-5.38)$. ROC analysis revealed that the APRI fairly classified severe from non-severe fibrosis with AUC of 0.762 (95\% CI 0.698, 0.828). The suggested 
cutoffs of $\geq 0.84$ and $\geq 1.74$ yielded likelihood ratio of 2.02 (95\% CI $1.54,2.65)$ and 4.06 (95\% CI 2.12, 7.79). Sensitivity and specificity for the corresponding cutoffs were 70.97 and $64.90 \% ; 32.26$ and $92.05 \%$. The cutoffs tended to be better in discriminating severe fibrosis in $\mathrm{CHC}$ (AUC $=0.76 ; 95 \%$ CI 0.66, 0.86) than in $\mathrm{CHB}$ $(\mathrm{AUC}=0.63 ; 95 \%$ CI $0.69,0.83)(p=0.087)$.

Conclusions: APRI of $\geq 0.84$ and $\geq 1.74$ are good predictive values for severe hepatic fibrosis. APRI seems to be more accurate in CHC than $\mathrm{CHB}$

\section{PP16-46}

Discordance between Liver Stiffness Measurement Using FibroScan ${ }^{\circledR}$ and Liver Biopsy in Assessing Liver Fibrosis in Patients with Chronic Hepatitis B

S.U. Kim ${ }^{1}$, Y.N. Park ${ }^{2}$, J.Y. Park ${ }^{1}$, D.Y. Kim ${ }^{1}$, S.H. Ahn ${ }^{1}$, K.-H. Han ${ }^{1}$, C.Y. Chon ${ }^{1}$

${ }^{1}$ Department of Internal Medicine, Yonsei University College of Medicine; ${ }^{2}$ Department of Pathology, Yonsei University College of Medicine, Seoul, Republic of Korea

Background/aims: Few studies investigated the predictors of discordance between liver biopsy (LB) and liver stiffness measurement (LSM) using FibroScan ${ }^{\circledR}$. Thus, we determined the predictors of discordance between LB and LSM in patients with chronic hepatitis B (CHB).

Methods: One hundred and fifty patients were enrolled. Only LSM with $\geq 10$ valid measurements was considered reliable. Liver fibrosis was evaluated using Batts and Ludwig system. LB specimens $<15$ $\mathrm{mm}$ were considered ineligible. Reference cutoff LSM values to determine discordance were calculated from our cohort $(6.0 \mathrm{kPa}$ for $\geq \mathrm{F} 2,7.5 \mathrm{kPa}$ for $\geq \mathrm{F} 3$, and $9.4 \mathrm{kPa}$ for $\mathrm{F} 4$ ).

Results: The mean age (107 men and 43 women) was 40.9 years. A discordance (defined as discordance of at least 2 stages between LB and LSM) was identified in $40(26.7 \%)$ patients. Success rate and interquartile range $(\mathrm{IQR}) /$ median value $(\mathrm{IQR} / \mathrm{M})$ were not selected as significant factors associated with discordance $(P=0.236$ and $P=0.150$, respectively). In multivariate analyses, fibrosis stage (F0-2) and activity grade (A3-4) were identified as independent predictors of discordance $(P<0.001$; hazard ratio [HR], 0.051; 95\% confidence interval [CI], 0.012-0.226 for F3-4 and $P=0.042$; HR, 2.250; 95\% CI $1.028-5.457$ for A3-4). The estimated odds ratios of discordance were 0.044 in patients with F3-4 $(P<0.001 ; 95 \%$ CI $0.010-0.191)$ and 3.346 in those with A3-4 ( $P=0.003$; 95\% CI 1.492-7.504).

Conclusions: Fibrosis stage (F0-2) and activity grade (A3-4) were the independent risk factors to predict discordance between $\mathrm{LB}$ and LSM in patients with CHB.

\section{PP16-47}

Normal Liver Elasticity Values with ARFI Imaging: A Pilot Study in Healthy Living Liver and Kidney Donors

S.U. Kim ${ }^{1}$, C.Y. Son ${ }^{1}$, H. Park ${ }^{1}$, W.K. Han ${ }^{2}$, G.H. Choi ${ }^{3}$, S.C. Yang ${ }^{2}$, J.S. Choi ${ }^{3}$, J.Y. Park ${ }^{1}$, D.Y. Kim ${ }^{1}$, S.H. Ahn ${ }^{1}$, K.-H. Han ${ }^{1}$, C.Y. Chon ${ }^{1}$ ${ }^{1}$ Department of Internal Medicine; ${ }^{2}$ Department of Urology, Yonsei University College of Medicine; ${ }^{3}$ Department of Surgery, Yonsei University College of Medicine, Seoul, Republic of Korea

Background/aims: Acoustic radiation force impulse (ARFI) elastography is a novel non-invasive method for providing a liver elasticity measurement. However, no studies on the normal values of liver elasticity in healthy subjects are reported. The aim of this study is to identify the normal range of ARFI velocity by recruiting healthy living liver and kidney donors in South Korea.

Methods: Liver elasticity measurements were performed in 39 consecutive healthy living liver and kidney donors who were admitted for transplantation. None of subjects had history of diabetes mellitus, hypertension, hepatitis $\mathrm{B}$ or $\mathrm{C}$ virus infection, liver dysfunction, heart dysfunction and metabolic syndrome.
Results: The mean age of our study population was 35.5 years (22 men and 17 women), with a mean ARFI velocity of $1.09 \pm 0.13 \mathrm{~m} / \mathrm{s}$ (range $0.80-1.33 \mathrm{~m} / \mathrm{s}$ ). ARFI velocity were not significantly different between subjects with body mass index (BMI) $<23.5 \mathrm{~kg} / \mathrm{m}^{2}(1.08 \pm$ $0.12 \mathrm{~m} / \mathrm{s})$ and $\mathrm{BMI} \geq 23.5 \mathrm{~kg} / \mathrm{m}^{2}(1.12 \pm 0.14 \mathrm{~m} / \mathrm{s}, P=0.366)$ nor were they significantly different according to age $(P=0.683)$ and gender $(1.12 \pm 0.10 \mathrm{~m} / \mathrm{s}$ in men vs. $1.07 \pm 0.16 \mathrm{~m} / \mathrm{s}$ in women; $P=$ 0.153 ). Using the 5 th and 95 th percentiles, we determined the normal range of ARFI velocity to be $0.88-1.26$ (mean $1.09 \pm 0.11 \mathrm{~m} / \mathrm{s}$ ).

Conclusions: We identified the normal range of ARFI velocity and found that ARFI velocity was not significantly influenced by BMI, age and gender.

\section{PP16-48}

Nilotinib $20 \mathrm{mg} / \mathrm{Kg}$ Is a Safe and Potent Antifibrotic Therapy in Tetrachloride $\left(\mathrm{CCl}_{4}\right)$ Rate Model

G. Shiha ${ }^{1}$, M. Shaker ${ }^{2}$, K. Zalata ${ }^{3}$, T. Ibrahim ${ }^{2}$

${ }^{1}$ Internal Medicine, Faculty of Medicine Mansoura University;

${ }^{2}$ Pharmacology and Toxicology, Faculty of Pharmacy, Mansoura University; ${ }^{3}$ Pathology Department, Faculty of Medicine, Mansoura University, Mansoura, Egypt

Introduction: Liver fibrosis is a major worldwide medical problem with significant morbidity and mortality. Tyrosine kinase inhibitors are relatively new class of therapeutic agents used for chronic myeloid leukemia. We have confirmed a promising anti-fibrotic activity for nilotinib and imatinib in thioacetamide-induced hepatic fibrosis. Aim: Evaluation and comparison the effects of imatinib and nilotinib to that of silymarin (reference standard) on established hepatic oxidative stress, injury and fibrosis in the $\mathrm{CCl}_{4}$ rat model.

Method: Male Wistar rats received intraperitoneal injections of $\mathrm{CCl}_{4}$ twice weekly for 8 weeks, as well as daily intraperitoneal treatments during the last 4 weeks of $\mathrm{CCl}_{4}$-intoxication.Hepatic damage was evaluated by analysis of liver function tests and hepatic oxidative stress parameters. Hepatic fibrosis was evaluated by histopathology and morphometry, as well as collagen and 4-hydroxyproline contents. Results: Nilotinib $(20 \mathrm{mg} / \mathrm{kg})$ was the most effective treatment to counteract $\mathrm{CCl}_{4}$-induced hepatic injury and oxidative stress. Moreover, nilotinib $(10 \mathrm{mg} / \mathrm{kg})$, nilotinib $(20 \mathrm{mg} / \mathrm{kg})$ and silymarin $(100$ $\mathrm{mg} / \mathrm{kg}$ ) treatments reduced in $\mathrm{CCl}_{4}$-treated rats the mean score of hepatic fibrosis by 31,68 and $47 \%$, respectively, and the hepatic collagen content by 47,49 and $18 \%$, respectively. Hepatic morphometric evaluation and 4-hydroxyproline content revealed that $\mathrm{CCl}_{4}$-induced fibrosis was ameliorated pronouncedly by nilotinib (20 $\mathrm{mg} / \mathrm{kg}$ ) and imatinib $(20 \mathrm{mg} / \mathrm{kg})$. Unlike nilotinib, imatinib $(20 \mathrm{mg} /$ $\mathrm{kg}$ ) showed some sort of hepatic injury evidenced by characteristic anisonucleosis visualized.

Conclusion: Nilotinib $20 \mathrm{mg} / \mathrm{kg}$ has a potent and safe anti-fibrotic effect in $\mathrm{CCl}_{4}$ rat model which supports our earlier conclusion that nilotinib exerts strong antifibrotic activity in thioacetamide-induced hepatic fibrosis.

\section{PP16-49}

The Usefulness of Multiple Biomarkers for the Prediction of Significant Fibrosis in Chronic Hepatitis B

S.H. Park ${ }^{1}$, T.G. Kim ${ }^{1}$, E.H. Seo ${ }^{1}$, D.J. Kim ${ }^{2}$, J.Y. Cheong ${ }^{3}$, S.W. Cho ${ }^{3}$, S.G. Hwang ${ }^{4}$, Y.J. Lee ${ }^{5}$, M. Cho ${ }^{6}$, J.M. Yang ${ }^{7}$

${ }^{1}$ Inje University Haeundae Paik-Hospital, Busan; ${ }^{2}$ Chuncheon Sacred Heart Hospital, Chuncheon; ${ }^{3}$ Ajou University School of Medicine, Suwon; ${ }^{4}$ Pochon CHA University College of Medicine, Sungnam; ${ }^{5}$ Inje University College of Medicine, ${ }^{6}$ Pusan National University College of Medicine, Busan; ${ }^{7}$ Catholic University College of Medicine, Suwon, Republic of Korea

Background/aims: It has not been explicitly addressed whether new biomarkers, in addition to readily available clinical data, make a contribution to the prediction of liver fibrosis. 
Methods: A total of 209 patients with chronic hepatitis B who underwent liver biopsy were recruited. The Clinical Score (CS) model using only routine clinical data and the Biomarker Score (BS) model using 7 putative biomarkers were derived from the derivation set $(n=105)$, and these models were applied to a separate patients group ( $n=104)$ to investigate whether the addition of BS improved the diagnostic accuracy in predicting significant fibrosis beyond an assessment based solely on CS.

Results: The most informative biomarkers for predicting significant fibrosis were hyaluronic acid and matrix metalloproteinase-2. The BS was an independent predictive factor for significant fibrosis even after accounting for CS in both sets. Among the derivation set, the incorporation of the BS into the CS model did not significantly increase the ROC area, with only a small improvement of about $2 \%(p=0.11)$. Similarly, in the validation set, a combined model with CS and BS showed no superior diagnostic accuracy over the CS model alone, with an improvement of about $2 \%[0.83(0.75-0.92)$ vs. 0.81 $(0.70-0.91) ; p=0.16]$.

Conclusions: The simultaneous addition of several biomarkers adds only modestly to clinical predictive factors for risk assessment of individual patients. These results highlight the need for the models to be validated in another cohort with a broader distribution of fibrosis severity.

\section{PP16-50}

Prediction of Liver-related Event Using Liver Stiffness Measurement $\left(\right.$ FibroScan ${ }^{\circledR}$ ) in Patients with Advanced Liver Fibrosis or Cirrhosis J.H. Lee, S.U. Kim, J.Y. Park, D.Y. Kim, S.H. Ahn, K.-H. Han, C.Y. Chon Department of Internal Medicine, Yonsei University College of Medicine, Seoul, Republic of Korea

Background/aims: Advanced liver fibrosis and cirrhosis are closely related to the development of liver-related death, decompensation, and hepatocellular carcinoma (HCC). Liver stiffness measurement (LSM) using FibroScan ${ }^{\circledR}$ has been known as a noninvasive tool to assess liver fibrosis in patients with chronic liver disease (CLD). Thus, this study investigated the usefulness of LSM in predicting the development of liver-related event (LRE) in patients with advanced liver fibrosis and cirrhosis.

Subjects and methods: A total of 113 patients with CLD who underwent liver biopsy (LB) and LSM between March 2006 and April 2010 and showed advanced liver fibrosis or cirrhosis on LB (F3-4, Batts and Ludwig system), were consecutively enrolled. LB specimen $\geq 15 \mathrm{~mm}$ and LSM with $\geq 60 \%$ of success rate, $\geq 10$ valid measurements, and interquartile range/median $<0.3$ were selected for analysis. After LB and LSM were performed, patients attended surveillance program for the detection of HCC [3-6 months interval with ultrasound and alpha-fetoprotein (AFP)]. During the study period, LRE development (defined as liver-related death, decompensation, or HCC) was checked.

Results: The mean age of the patients ( 60 men, 53 women) was 52.4 years and the median LSM value was $14.0 \mathrm{kPa}$. During the follow-up period [median 21.1 months (range 6.4-55.5), LRE developed in 16 $(14.2 \%)$ patients [liver-related mortality in none, liver-related decompensations in five (two hepatic encephalopathy, two ascitic decompensation, and one variceal bleeding], and HCC in 11). On multivariate analysis, together with AFP, platelet count, Child-Pugh score, a higher LSM value ( $>16 \mathrm{kPa}$ ) were identified as independent predictors of LRE development, with a hazard ratio of 3.508 $[P=0.048 ; 95 \%$ confidence interval 1.009-12.191], as compared to LSM value $\leq 16 \mathrm{kPa}$.

Conclusions: Our data suggest that LSM can be a useful predictor of LRE development in patients with advanced liver fibrosis or cirrhosis.
PP16-51

Pirfenidone Decreases TGF- $\beta$, IL-6, TNF- $\alpha$ and Increases CB2 Receptor Gene Expression in Patients with Chronic Hepatitis C

L. Flores-Contreras ${ }^{1}$, A.S. Sandoval- Rodriguez ${ }^{1}$, S. Lucano-Landeros ${ }^{1}$, I. Arellano-Olivera ${ }^{1}$, J. Armendáriz-Borunda ${ }^{1,2}$

${ }^{1}$ Molecular Biology, Institute of Molecular Biology in Medicine and Gene Therapy/Universidad de Guadalajara; ${ }^{2}$ O.P.D. Hospital Civil de Guadalajara 'Juan I. Menchaca', Guadalajara, Mexico

Background: Hepatitis $\mathrm{C}$ virus (HCV) chronic infection increases serum levels of TGF- $\beta$, IL- 6 and TNF- $\alpha$, stimulating liver inflammation and fibrogenesis, declining quality of life of patients. Furthermore, cannabinoids receptors (CB1 and $\mathrm{CB} 2$ ) may regulate fibrosis progression in patients with chronic hepatitis $\mathrm{C}$ (CHC). Pirfenidone (PFD) is an anti-fibrogenic and anti-inflammatory drug. Objetive: To assess serum levels of TGF- $\beta$, IL- 6 and TNF- $\alpha$, liver gene expression of CB1 and CB2, necro-inflammation score, fibrosis index and quality of life in patients with $\mathrm{CHC}$ before and after 24 months of treatment with PFD.

Methods: 28 CHC patients received Pirfenidone (1200 mg/day) for 24 months. Liver biopsies and serum samples were obtained at the beginning and at the end of the treatment. In liver, CB1 and CB2 gene expression were evaluated by Real Time-PCR, along with necroinflammation and fibrosis using Modified histological activity index (HAI) of Knodell and Ishak. TGF- $\beta$, IL- 6 and TNF- $\alpha$ serum levels were measured using ELISA. HCV genotype was established and viral load was measured every 6 months, quality of life was assessed by SF36 questionnaires.

Results: At the end of 24 months of treatment, TGF- $\beta$ and IL- 6 serum levels decreased significantly in 73 and $91 \%$ of patients $(p<0.05)$, respectively. TNF- $\alpha$ showed tendency to diminish in $79 \%(p>0.05)$. Necroinflamation score was reduced an average of 3 points in $70 \%$ of patients $(p<0.05)$ and fibrosis index decreased 2 points average in $55 \%$ of patients $(p<0.05)$. ALT levels showed a tendency to normalize in $81 \%$ of patients and quality of life was improved in $100 \%$. In 15 representative patients, CB2 mRNA increased (85.71\%), while CB1 diminished in $28.57 \%$.

Conclusions: TGF- $\beta$, IL-6, TNF- $\alpha$ diminution and mRNA CB2 increase due to PDF treatment improves liver function in patients with $\mathrm{CHC}$ and reduces inflammation and fibrosis improving patients quality of life.

\section{PP16-52}

Validation of Laminin-based Score and Fibroscan in Prediction of Liver Fibrosis in Egyptian Patients with Chronic Hepatitis C

T. Salman ${ }^{1}$, N. Allam ${ }^{1}$, G. Youssef ${ }^{2}$, B. Abdel-Naby ${ }^{1}$, M. Salama ${ }^{1}$

${ }^{1}$ Hepatology; ${ }^{2}$ Clinical Pathology, National Liver Institute, Menoufiya University, Shebeen El-Kom, Egypt

Background and aims: The constraints of liver biopsy and the limitations of single parameters in assessing liver fibrosis have boosted the development of scores combining the results of markers. The laminin-based score devised by our group in 2006 to assess liver fibrosis in 60 patients. The equation $=[13.0331 \times S$. laminin $(\mu \mathrm{g} /$ $\mathrm{ml})]-[0.5681 \times S$. albumin $(\mathrm{g} / \mathrm{dl})]+[0.2001 \times \mathrm{PV}$ diameter $(\mathrm{mm})]+[0.0079 \times$ AST $(\mathrm{IU} / \mathrm{l})]-3.8876$. This study was conducted to validate the diagnostic accuracy of this score and transient elastography in prediction of liver fibrosis in another group of ninety patients with chronic hepatitis $\mathrm{C}$.

Methods: Ninety patients were biopsied and subjected to assessment of S.albumin, AST, prothrombin concentration, laminin, PV diameter and estimation of liver stiffness using Fibroscan.

Results: Patients were classified according to stage of fibrosis using modified Knodell score into 7 groups (stage 0-6). A significant 
incremental relation between the stage of fibrosis and portal vein diameter, AST, laminin was detected and a significant decremental relation with albumin. A significant relation between stage of fibrosis and LBS was found $(p<0.01)$. AUC for detection of significant fibrosis (F3-F6) was 0.929 . At a cut off value of 0.545 , sensitivity was 86 and specificity $87 \%$. For the detection of cirrhosis, area under the curve was 0.985 and at a cut off value of 1.24 , sensitivity was $100 \%$ and specificity $98.7 \%$. There was a highly statistically significant incremental relation between liver stiffness and staging of fibrosis $(p<0.01)$ and a highly significant correlation between liver stiffness and LBS $(r=0.43, p<0.01)$.

Conclusion: LBS and TE proved their value in assessing fibrosis and may reduce needs for repeated liver biopsies. Because of their correlation with each other, they complement each other and the presence of the limitations of one will allow the use of the other.

\section{PP16-53}

Low Liver Stiffness in Cirrhotic Patients with Persistent Normal Alanine Aminotransferase and Effective Antiviral Therapy

J.-H. Wang, H.-W. Xu, S.-N. Lu, C.-H. Hung, K.-C. Chang, T.-H. Hu

Division of Hepato-Gastroenterology, Chang Gung Memorial

Hospital -Kaohsiung Medical Center, Kaohsiung, Taiwan R.O.C.

Background: Liver stiffness measurement (LSM) is a non-invasive method for diagnosis of hepatic cirrhosis. The aims of this study were to evaluate the influences of hepatitis activity and antiviral therapy on LSM in cirrhotic patients.

Methods: Consecutive patients with compensated hepatic cirrhosis were enrolled for LSM, using FibroScan system (Echosens, Paris, France). The medical records of hepatitis activity and antiviral therapy in the two-year period before enrollment were reviewed. Patients were stratified into inactive, fluctuated and active groups by hepatitis activity. For chronic hepatitis $\mathrm{C}$ virus (HCV), patients was stratified into sustained virological response (SVR) and non-sustained virological response (NSVR) by results of antiviral treatment. The LSM results were compared among different groups.

Results: A total of 163 patients (mean age 57.2, male/female 90/73) was enrolled. The mean LSM value was $10.0,13.8$ and $18.8 \mathrm{kPa}$ in inactive, fluctuated and active groups, respectively. Patients in active group had significantly higher LSM value than those in inactive or fluctuated groups $(p=0.002$ and $p=0.013)$. For chronic HCV, mean LSM value was $21.9,11.8$, and $23.0 \mathrm{kPa}$ in patients without antiviral therapy, in SVR and in NSVR groups, respectively. Patients in NSVR had significantly higher LSM value than those in SVR $(p=0.030)$. For chronic hepatitis B, mean LSM value was $6.4,17.4$ and $14.6 \mathrm{kPa}$ in patients without antiviral therapy, with therapy $<12$ and $\geq 12$ months, respectively. There is significantly higher LSM value in patients with therapy less than 12 months $(p=0.020)$.

Conclusions: There were low LSM values in cirrhotic patients without hepatitis activity, with SVR and long-term antiviral therapy for chronic viral hepatitis

\section{PP16-54}

The Frequency and Determinants of Liver Stiffness Measurement Failure D. Ji, P. Han, Q. Shao, J. Zhang, F. Li, B. Li, G.-F. Chen

Liver Fibrosis Noninvasive Diagnosis and Treatment Center, Beijing 302 Hospital, Beijing, China

Objective: To investigate the frequency and determinants of liver stiffness measurement (LSM) with transient elastography (FibroScan) and unreliable results.

Methods: A total of 4,000 patients, including asymptomatic carrier, chronic hepatitis $\mathrm{B}(\mathrm{CHB})$, chronic hepatitis $\mathrm{C}(\mathrm{CHC})$, alcoholic liver disease (ALD), liver cirrhosis, autoimmune liver disease (AILD), hepatocellular carcinoma (HCC) and liver transplant recipients, was enrolled. Liver fibrosis was evaluated by FibroScan detection. LSM failure was defined as zero valid shots, an interquartile range (IQR) greater than or equal to one third of the median value, or a success rate (SR) less than $60 \%$.

Results: LSM failure occurred in $6.5 \%$ of all examinations (260 patients out of 4000). LSM was unable to achieve the valid values in 91 cases $(2.3 \%)$, SR was less than $60 \%$ in 108 cases $(2.7 \%)$, and IQR was larger than $30 \%$ of median value in 61 cases $(1.5 \%)$. LSM failure was independently associated with body mass index (BMI) greater than $28 \mathrm{~kg} / \mathrm{m}^{2}$, female sex, age greater than 50 years, intercostal spaces less than $9 \mathrm{~mm}$, and HCC patients. There were no significant differences among other diseases. By changing another skilled operator, success was achieved on 64 cases out of 260 , which reduced the failure rate to $4.9 \%$.

Conclusions: The principal reasons of LSM failure are obesity and narrow of intercostal space. The failure rates of HCC, elder or female patients are higher. These results emphasize the need for adequate operator training and for technological improvements in specific patient subpopulations.

\section{PP16-55}

Chinese Patients Older than 45 Years with Over Ten-year CHB History Have High Risk for Developing Liver Cirrhosis

Q.H. Ling, J.J. Chen, J. Guo, X.D. Zhang

Department of Liver Disease, ShuGuang Hospital, Shanghai

University of TCM, Shanghai, China

Background: Chronic HBV infection is the leading cause of liver cirrhosis. This paper aimed to evaluate the effect of risk factors on the development of cirrhosis among Chinese patients with over ten-year chronic hepatitis B histories.

Material and methods: We retrospectively assessed 390 patients whose chronic HBV hepatitis histories were over 10 years, including 195 CHB patients with liver cirrhosis and 195 without liver cirrhosis. Results: The average age of LC group was significantly older than that of non-LC group $(p<0.001)$. Despite of the same CHB history, the number of young patients (18-35 years) developed in LC was less than that of old patients (45-65 years) $(p=0.041)$. In LC group, the average age of patients after hepatic decompensation was older than that of patients in hepatic compensation (54.35 vs. 51.02, $p=0.032$ ). Conclusion: Old age played a crucial role in the development of cirrhosis among patients with chronic hepatitis B. Patients older than 45 years with over 10-year CHB history had higher risk for developing liver cirrhosis.

\section{PP16-56}

Evaluation of Virtual Touch Tissue Quantification for Noninvasive Staging of Liver Fibrosis in Chronic Liver Disease

H. Kuroda, K. Sawara, Y. Miyamoto, K. Oikawa, A. Miyasaka, Y. Takikawa, K. Suzuki

Iwate Medical University, School of Medicine, Morioka, Japan

Background/aims: Virtual Touch Tissue Quantification (VTTQ) is a new technology for performing liver elastography. However, use of this technique to estimate the degree of fibrosis in chronic liver disease (CLD) related to hepatitis $\mathrm{C}$ virus (HCV) infection has not yet been established. We evaluated the validity, accuracy and flexibility of the VTTQ method in CLD.

Methodology: Subjects comprised 30 patients with chronic hepatitis $(\mathrm{CH}), 30$ patients with liver cirrhosis (LC) and 10 healthy subjects (controls). All patients had HCV infection. Elastography of the liver was performed at different sites using the VTTQ method. Relationships between shear wave velocity (SWV) obtained by VTTQ, clinical diagnosis, serum parameters of liver function and fibrosis in the liver were evaluated. Moreover, cut-off values for differential diagnosis between $\mathrm{CH}$ and $\mathrm{LC}$ were estimated using area under the receiver operating characteristics (AUROC).

Results: Mean SWV ( \pm standard deviation) was $2.67 \pm 1.18$, $1.33 \pm 0.54$ and $0.99 \pm 0.21 \mathrm{~m} / \mathrm{s}$ in the $\mathrm{LC}, \mathrm{CH}$ and control groups, 
respectively. SWV was significantly higher in the LC group than in the $\mathrm{CH}$ and control groups $(p<0.0001)$, and in the $\mathrm{CH}$ group than in the control group $(p=0.0023)$. SWV in each stage of fibrosis was $1.09 \pm 0.22 \mathrm{~m} / \mathrm{s}$ in F0-1, $1.24 \pm 0.52 \mathrm{~m} / \mathrm{s}$ in F2, $1.61 \pm 0.79 \mathrm{~m} / \mathrm{s}$ in $\mathrm{F} 3$, and $2.35 \pm 1.11 \mathrm{~m} / \mathrm{s}$ in F4. SWV correlated significantly with serum total bilirubin, alanine aminotransferase, cholesterol, albumin, platelet counts, prothrombin time, fibrosis markers, hyaluronic acid and type IV collagen. A steady stepwise increase in elasticity correlated significantly with severity of hepatic fibrosis $(\rho=0.9772$; $p=0.002$ ). Diagnostic ability for LC was superior in VTTQ (AUROC, 0.930 in VTTQ; 0.846 in aspartate aminotransferase to platelet ratio index; 0.829 in Forns' index; and 0.785 in platelet count). Conclusions: VTTQ is extremely useful for distinguishing between $\mathrm{CH}$ and LC, and SWV might correlate significantly with degree of progression in CLD with $\mathrm{HCV}$ infection.

\section{PP16-57}

Change of Transient Elastography Value after Antiviral Treatment in Patients with Chronic Hepatitis B: Two Years Data

J.H. Kim, Y.K. Jung, I.S. Won, J.H. Kim, J.J. Lee, Y.S. Kim, O.S. Kwon Gastroenterology, Gil Hospital/Gachon University, Incheon, Republic of Korea

Background/aims: Transient elastography (TE, Fibroscan) is a useful method to evaluate liver fibrosis. Antiviral treatments with neucleot(s)ide analogues improve liver fibrosis in hepatitis B virus (HBV) related chronic liver diseases. This study aimed to evaluate the improvement of TE values after antiviral treatment.

Methods: Patients with HBV related chronic liver diseases, who had TE values for 2 years were retrospectively enrolled. Among them, patients who had been treated with neucleot(s)ide analogues were selected. TEs were done as baseline and then repeated after 2 years. During this period, the presence of antiviral resistance and the maintenance of HBV DNA $<2,000$ copies $/ \mathrm{mL}$ were investigated.

Results: A total of 56 patients with HBV related chronic liver disease was enrolled. Forty five of them had been treated with neucleot(s)ide analogues. The median TE value was $9.1(3.3-75)$ at baseline and decreased to $6.9(3.4-61.5) \mathrm{kPa}$ at 2 years $(p=0.004)$. The median TE value in patients with antiviral resistance $(n=11)$ was 7.1 $(3.3-42.8)$ at baseline and $5.3(3.4-21.8) \mathrm{kPa}$ at 2 years. The median TE value in patients without antiviral resistance $(n=34)$ was 10.7 $(4.1-75)$ and $7.5(3.5-61.5) \mathrm{kPa}$ at 2 years. The improvement of TE value was higher in patients without antiviral resistance $(p=0.052)$. The median TE value in patients with maintenance of HBV DNA $<2,000$ copies $/ \mathrm{mL}(n=17)$ was $14.4(4.1-75)$ at baseline and 6.9 (3.5-61.5) $\mathrm{kPa}$ at 2 years. The median TE value in patients without maintenance of HBV DNA $<2,000$ copies $/ \mathrm{mL}(n=28)$ was 7.5 $(3.3-42.8)$ at baseline and $6.9(3.4-27) \mathrm{kPa}$ at 2 years. The improvement of TE value was higher in patients with maintenance of HBV DNA $<2,000$ copies/mL $(p=0.02)$.

Conclusion: Although there is a limitation of TE in evaluating liver fibrosis, patients without antiviral resistance and with maintenance of HBV DNA $<2,000$ copies/mL will show improvement of liver fibrosis by antiviral treatment.

\section{PP16-58}

How Many Valid Measurements Are Necessary to Assess Liver Fibrosis Using FibroScan ${ }^{\circledR}$ in Patients with Chronic Viral Hepatitis?

H.S. Shin ${ }^{1}$, H.W. Jang ${ }^{1}$, S.U. Kim ${ }^{1}$, J.Y. Park ${ }^{1}$, S.H. Ahn ${ }^{1}$, K.-H. Han ${ }^{1}$,

C.Y. Chon ${ }^{1}$, Y.N. Park ${ }^{2}$, E.H. Choi ${ }^{3}$, D.Y. Kim ${ }^{1}$

${ }^{1}$ Department of Internal Medicine; ${ }^{2}$ Department of Pathology;

${ }^{3}$ Department of Biostatistics, Yonsei University College of Medicine, Seoul, Republic of Korea

Background/aims: More than 10 valid measurements (VMs) is one of the prerequisites for reliable liver stiffness measurement (LSM) using FibroScan ${ }^{\circledR}$. However, this prerequisite is only manufacturer's recommendations, not based on the scientific evidences. We investigated how many VMs are required to assess liver fibrosis without significant loss of performance in patients with chronic hepatitis $\mathrm{B}$ (CHB) and $\mathrm{C}$ (CHC).

Methods: From January 2005 to December 2009, a total of 179 patients with $\mathrm{CHB}$ or 66 with $\mathrm{CHC}$ who underwent liver biopsy (LB) and LSM before starting antiviral treatment were enrolled. More than $10 \mathrm{VMs}$, more than $60 \%$ of success rate, and interquartile range/ median values $<0.3$ were considered reliable. Batts and Ludwig scoring system was used for histologic assessment.

Results: The mean age and body mass index was 46.0 years and $23.4 \mathrm{~kg} / \mathrm{m}^{2}$ in patients with $\mathrm{CHB}$ and 49.7 years and $23.1 \mathrm{~kg} / \mathrm{m}^{2}$ in those with $\mathrm{CHC}$. The median LSM value was $5.4 \mathrm{kPa}$ for F0F1, $6.8 \mathrm{kPa}$ for $\mathrm{F} 2,8.1 \mathrm{kPa}$ for $\mathrm{F} 3$, and $14.3 \mathrm{kPa}$ for $\mathrm{F} 4$ in $\mathrm{CHB}$ where it was $4.5 \mathrm{kPa}$ for F0F1, $6.3 \mathrm{kPa}$ for F2, $10.4 \mathrm{kPa}$ for $\mathrm{F} 3$, and $26.6 \mathrm{kPa}$ for $\mathrm{F} 4$ in $\mathrm{CHC}$. The median value of elasticity score from the first 3, 5, and all VMs significantly predicted $\geq \mathrm{F} 2$ and F4 fibrosis stage (all $P<$ 0.05 ) without significant differences (all $P>0.05$ by DeLong's method). Alanine aminotransferase (ALT) was the only predictor of the discordance in fibrosis stage estimated by the median value of elasticity score from the first $3 \mathrm{VMs}$ and LB in CHB, whereas no significant predictor was identified in CHC.

Conclusions: Three VMs may be enough to assess liver fibrosis using LSM without significant loss of the performance in patients with $\mathrm{CHB}$ and CHC. However, ALT should be considered in the interpretation of LSM results.

\section{Poster Presentation 17: Metabolic And Genetic Disease}

\section{PP17-01}

Comparative Evaluation of Iron Overload in Patients with Chronic Liver Disease

M. Radicheva

Medical Faculty, Trakia University, Stara Zagora, Bulgaria

Objective: To assess and compare the presence and significance of iron overload in patients with chronic liver disease.

Methods: The study of 220 patients with chronic liver diseases, which were examined for frequency and correlation between elevated liver enzymes, ultrasound diagnosed steatosis, liver biopsy, features of metabolic syndrome and indices of iron metabolism.

Results: The value of serum iron $(22.3 \pm 8.3$ vs.17.6 \pm 3.5$)$, serum ferritin ( $225.4 \pm 213.8$ vs. $132.6 \pm 65.2)$ and transferrin saturation $(32.4 \pm 9.2$ vs. $27.0 \pm 3.8)$ were significantly higher in patients with chronic liver diseases compared with controls. Serum iron and ferritin were significantly higher in non-alcoholic fatty disease compared with controls $(p=0.039)$ and chronic hepatitis B (HHV) $(p=0.001)$, alcoholic fatty disease (AFLD) compared with controls $(p=0.0001)$ and chronic hepatitis B (HHB) $(p=0.0001)$, chronic hepatitis C (HHC) compared with controls $(p=0.05)$.

Conclusion: Iron overload was highest in patients with alcoholic etiology, followed by non-alcoholic fatty liver disease and chronic hepatitis C. In chronic hepatitis B, as well as autoimmune diseases, abnormalities were detected in individual patients.

\section{PP17-02}

Assessment of Metabolic Inborn Diseases

A. Bajraktarevic ${ }^{1}$, V. Pavlovic ${ }^{1}$, S. Putica ${ }^{1}$, M. Miokovic ${ }^{1}$, A. Mahinic ${ }^{1}$,

S. Maglajlija ${ }^{1}$, S. Majstorovic ${ }^{2}$, S. Kurtagic ${ }^{2}$, A.J. Causevic ${ }^{3}$, Z. Zvizdic ${ }^{4}$,

G. Sulejmanpasic ${ }^{5}$

${ }^{1}$ Pediatrics Department, Public Health Institution of Canton Sarajevo; ${ }^{2}$ Gastrohepatology Department, Pediatrics Clinic

Sarajevo; ${ }^{3}$ Department for Biochemistry, Medical Faculty Sarajevo;

${ }^{4}$ Surgery for Children Sarajevo, Clinical Medical Center; ${ }^{5}$ Institute 
for Radiology, Clinical Medical Center Sarajevo, Sarajevo, BosniaHerzegovina

Introduction: Hepatic transplantation for metabolic or genetic diseases of the liver produces a definite cure of the liver disease. Up to $70 \%$ have abnormal biochemical tests of liver function in early infancy. The mean age at recognition of icteric hepatitis is between 2 and 3 weeks.

Aims: It is not clear whether growth failure can be reversed by liver transplantation.

Methods: Laboratory-based and translational research has greatly increased our understanding of inherited metabolic liver disease. Specific diagnosis will usually depend on selecting laboratory tests in a logical manner, having defined the phenotype and considered the potential metabolic causes.

Results: The benefits of this treatment are not only the prolongation of and improvement in the quality of life for the patient, it has also given insight into the basic metabolic defects of many disorders. Liver biopsies for demonstrate inflammation, giant cell transformation, and lobular and portal fibrosis.

Discussion: The rapid expansion of molecular genetics and the development of effective gene therapy may well displace liver transplantation as appropriate treatment of these disorders in the future.

Conclusion: Liver transplantation for inborn errors of metabolism not only replaces the diseased organ, but also leads to complete reversal of the metabolic defect. Until the promise of specific gene or enzyme replacement therapy is realized, liver and hepatocyte transplantation offers the best chance of achieving metabolic control in these challenging patients.

\section{PP17-03}

Relationship between Hemochromatosis Gene Mutation and Degree of Fibrosis in Liver Disease Associated with Chronic Hepatitis B and C G.S. Bagir ${ }^{1}$, M. Oksuz ${ }^{2}$, Ü.B. Dogan ${ }^{2}$, T. Egesel ${ }^{2}$, A. Canataroglu ${ }^{1}$

${ }^{1}$ Internal Medicine; ${ }^{2}$ Gastroenterology, Adana Numune Training and Research Hospital, Adana, Turkey

There are various factors that affect the liver fibrosis degree in chronic hepatitis $\mathrm{B}(\mathrm{CHB})$ and chronic hepatitis $\mathrm{C}(\mathrm{CHC})$. One of these is the deposition of iron in the liver. Although hemochromatosis gene (HFE) mutation is determined as heterozygote, it is thought that it causes the deposition of iron in the liver. However, the effect of the deposition of iron as a result of HFE gene mutation that leads to the progression of fibrosis in patients with chronic viral hepatitis is still uncertain. The purpose of this study is to evaluate the association of HFE gen mutation with fibrosis in patients with $\mathrm{CHB}$ and $\mathrm{CHC}$. We evaluated 144 patients ( 53 with $\mathrm{CHB}, 39$ with $\mathrm{CHC}, 52$ with cirrhosis) and 50 controls. ALT, AST, serum iron, ferritin and transferrin saturation tests were measured and the presence of HFE mutations were investigated for all individuals. Liver biopsy was performed to all patients. Histopathological findings in the liver were scored as necroinflammatory activity and fibrosis according to the Knodell scoring scale. The C282Y mutation was detected as homozigot wild type in all individuals. H63D heterozygous and homozygote mutation were detected in $27(18.8 \%)$ and $2(1.4 \%)$ patients, in $12(24 \%)$ and $2(4 \%)$ controls, respectively $(p>0.05)$. Presence of HFE gen mutation was not associated with serum iron measures and advanced hepatic fibrosis $(p>0.05)$.

Consequently, presence of H63D mutation does not seem to play a major role in the progression of fibrosis in the patients with $\mathrm{CHB}$ and CHC.

\section{PP17-04}

The Effect of Treadmill Exercise on Experimental Diabetic Hepatopathy in Rats

Y. Doustar

Faculty of Veterinary Medicine, Islamic azad University, Tabriz Branch, Scientific Association Faculty of veterinary Medicine, Tabriz, Iran

Regular exercise by increasing insulin sensitivity and improving the glucose uptake and lowering body adiposity is a powerful nonpharmacological tool for prevention and treatment of diabetes mellitus and therefore is highly recommended in the type I and type $\Pi$ diabetics. Diabetic hepathopathy is the main cause of liver failure. The purpose of this study was to evaluate the effect of regular exercise on diabetic hepathopathy. In this study 56 wistar rats with approximate age of 12 weeks and 200-300g weight were allocated into two equal groups (treatment and control). For induction of diabetes, these two groups were injected by streptozotocin $(50 \mathrm{mg} /$ $\mathrm{kg}$ ) intraperitoneally. The treatment group were kept in normal conditions of management with regular exercise (treadmill) for 12 weeks; 5 days in a week, an hour every day. In control group we have normal conditions (food and place) without any physical activity/regular exercise. After 12 weeks liver tissues were sampled in both groups and 5-6 micron tissue sections were prepared through H\&E staining method. Histopathological study of representative sections in control group showed hepathosclerosis, central vein perivascular cuffing of mononuclear inflammatory cells, hepathocellular degeneration, sclerosing hyaline necrosis, apoptosis, dense perisinusoidal, and periportal and perivenular fibrosis. Mild pathological changes in treatment group were observed. Differences of histopathological changes between the experimental groups were significant. Treadmill exercise is capable in reduction in pathological changes and improvement of diabetic hepathopathy to near normal

\section{PP17-05}

Gender Difference of the Prevalence and Associated Risk Factors of Metabolic Syndrome among Hospital Staff

C.-T. Hsu ${ }^{1}$, Y.-J. Chao ${ }^{2}$, J.-Y. Chen ${ }^{2}$, Y.-S. Wu ${ }^{3}$, K.-C. Chen ${ }^{1}$, C.-J. Wei ${ }^{3}$, F.-L. Chen ${ }^{3}$, T.-H. Tung ${ }^{1}$

${ }^{1}$ Cheng-Hsin General Hospital; ${ }^{2}$ Chang-Gang Memorial Hospital;

${ }^{3}$ Fu-Jen Catholic University, Taipei, Taiwan R.O.C.

Objectives: To explore the gender difference of the prevalence and associated risk factors of metabolic syndrome among hospital staff based on the health examination.

Methods: To understand the morbidity of metabolic syndrome, we analyzed the database of the health examination of the workers in a medical center on the northern Taiwan. The definition of metabolic

The association of H63D gen mutation with fibrosis

\begin{tabular}{|c|c|c|c|c|c|c|c|}
\hline H63D & Stage 0 & Stage 1 & Stage $2(1-3)$ & Stage 3 & Stage 4 (Cirrhosis) & Total patients & Controls \\
\hline $\mathrm{Wt} / \mathrm{H} 63 \mathrm{D}$ & - & $4(15.4 \%)$ & $4(14.8 \%)$ & $10(26.3 \%)$ & $9(17.3 \%)$ & $27(18.8 \%)$ & $12(24.0 \%)$ \\
\hline H63D/H63D & - & - & $1(3.7 \%)$ & - & $1(1.9 \%)$ & $2(1.4 \%)$ & $2(4.0 \%)$ \\
\hline $\mathrm{Wt} / \mathrm{Wt}$ & $1(100 \%)$ & $22(84.6 \%)$ & $22(81.5 \%)$ & $28(73.7 \%)$ & $42(80.8 \%)$ & $115(79.9 \%)$ & $36(72.0 \%)$ \\
\hline Total numbers & $1(100 \%)$ & $26(100 \%)$ & $27(100 \%)$ & $38(100 \%)$ & $52(100 \%)$ & $144(100 \%)$ & $50(100 \%)$ \\
\hline
\end{tabular}


syndrome is according to the criteria proposed by Department of Health in 2007, Taiwan. Due to the limitation of the examination, the total cholesterol is used to replace the HDL-C value.

Result: There are 1,203 subjects [male $424(35.2 \%)$ and female 779 $(64.8 \%)$ ] included in this study. The mean age is $40.20 \pm 10.59$ years. The prevalence of metabolic syndrome is $16.3 \%$ and male $(24.8 \%)$ is significant higher than female $(11.7 \%)(p<0.01)$. Based on the components of the abnormal criteria of metabolic syndrome, the results show that the highest proportion is higher blood pressure, higher triglyceride, and higher total cholesterol (3.5\%) in male and higher waist circumference, higher blood pressure, and higher total cholesterol (2.2\%) in female. From the multiple logistic regression, gender difference is found in associated factors related to metabolic syndrome after adjustment for confounding factors. The abnormal waist circumference is the most significant risk factor related to metabolic syndrome both in the male $(\mathrm{OR}=1.16,95 \%$ CI $1.09-1.24)$ and in the female (OR $=1.12,95 \%$ CI 1.04-1.21).

Conclusion: The prevalence of metabolic syndrome showed the gender difference in this study. Higher waist circumference is the most significant risk factor related to metabolic syndrome. In order to prevent the incident metabolic syndrome, to encourage hospital staff with the exercise habit, dietary improvement, and controlled central obesity is important.

\section{PP17-06}

Roles of Acid Sphingomyelinase on Glucose and Lipid Metabolism in Hepatocytes

Y. Osawa ${ }^{1,2}$, M. Nagaki ${ }^{2}$, Y. Yasuda ${ }^{2}$, H. Moriwaki ${ }^{2}$, M. Seishima ${ }^{1}$

${ }^{1}$ Informative Clinical Medicine; ${ }^{2}$ Gastroenterology, Gifu University Graduate School of Medicine, Gifu, Japan

Background and aims: Acid sphingomyelinase (ASM) regulates homeostasis of sphingolipids including ceramides and sphingosine-1phosphate. Although the sphingolipids regulate AKT activation, the role of ASM on hepatic glucose and lipid metabolism is unclear. To explore this issue, we examined the roles of ASM on glucose and lipid metabolism in hepatocytes.

Methods: ASM was overexpressed by adenovirus vector (Ad5ASM) in mouse liver and in primary cultured hepatocytes. Glucose tolerance test was performed on the Ad5ASM infected mice. The effects of Ad5ASM on glycogen deposition and lipid accumulation in the liver and primary cultured hepatocytes were examined. To investigate the mechanisms of the anabolic effects on glucose metabolism by ASM, the effects of ASM overexpression on AKT and AMP-activated protein kinase (AMPK) in the liver and primary cultured hepatocytes were examined.

Results: Overexpression of ASM by adenovirus vector in hepatocytes improved glucose tolerance and increased glycogen and lipid accumulation in the livers of mice. ASM activated AKT, and inhibition of AKT decreased glycogen deposition, and lipid accumulation by Ad5ASM in primary cultured hepatocytes. In addition, ASM decreased AMPK phosphorylation, resulting in the up-regulation of glucose transporter 2, and constitutively active AMPK expression decreased the glycogen deposition, and lipid accumulation by Ad5ASM.

Conclusion: ASM modulates AKT activation and AMPK inactivation, thereby regulating glucose and lipid metabolism in the liver.

\section{PP17-07}

Evaluation of Liver Dysfunction in Type2 Diabetic Patients in Tertiary Level Hospital in Bangladesh

M.H.-O.- Rashid ${ }^{1}$, M.A. Islam ${ }^{2}$, H. Tarik², M.A. Haque ${ }^{2}$, M.Z. Haque ${ }^{2}$, M.R. Khan ${ }^{2}$, S. Ekram ${ }^{2}$

${ }^{1}$ Hepatology Department; ${ }^{2}$ Medicine Department, Rajshahi Medical College, Rajshahi, Bangladesh

Background/aim: Diabetic patients often have altered liver function.This study was designed to find out the liver dysfunction as evidenced by liver function tests and to observe the extent and severity of abnormalities of LFTs.

Methods: This was a descriptive cross-sectional comparative study carried out in Department of Medicine, Rajshahi Medical College Hospital and Rajshahi Diabetic Association Hospital from July 2008 to June 2010. One hundred (100) diagnosed type 2 diabetic patients and 30 apparently healthy people were included. All of those study population were free from taking any hepatotoxic drugs and free from any preexisting liver disease.

Results: The prevalence of abnormal serum bilirubin, ALT, AST, Alkaline phosphatase, prothrombin time and S. albumin were 6, 30, 7, 6,54 and $12 \%$ respectively in type 2 diabetic patients and $0,3.3,0$, $6.7,10$ and $3.3 \%$ respectively in normal people and the differences were statistically significant.

All the LFTs of type 2 diabetic patients were mildly abnormal except 2 patients $(2 \%)$ had moderate elevation of ALT, 7 patient $(7 \%)$ had markedly prolonged PT, and 1 patient $(1 \%)$ had moderately decreased s. albumin. In normal people all LFTs abnormalities were mild.

Conclusion: A high proportion of patients with type 2 diabetes mellitus in our country have abnormal liver function. Such patients would thus warrant more intensive glycaemic control to prevent progression of significant liver diseases.

\section{PP17-08}

A Meta-analysis of Randomized Clinical Trials on Chinese Herbal Medicines for Treatment of Hypercholesterolemia

Z.L. Liu ${ }^{1}$, J.P. Liu ${ }^{1,2}$, A.L. Zhang ${ }^{3}$, Q. Wu ${ }^{4}$, Y. Ruan ${ }^{5}$

${ }^{1}$ Centre for Evidence-Based Chinese Medicine, Beijing University of Chinese Medicine, Beijing, China; ${ }^{2}$ NAFKAM, University of Tromso, Troms $\varnothing$, Norway; ${ }^{3}$ Discipline of Chinese Medicine, School of Health Sciences World Health Organization, Collaborating Centre for Traditional Medicine, Bundoora, VIC, Australia; ${ }^{4}$ School of Humanities, Beijing University of Chinese Medicine; ${ }^{5}$ Beijing University of Chinese Medicine, Beijing, China

Background: Hypercholesterolemia is a common heart disease risk factor and is often accompanied with other heart disease risk factors including age, blood pressure, family history, and diabetes. Chinese herbal medicines have been used for long time in lipid-lowering purpose.

Objectives: To assess the effects of Chinese herbal medicines for hypercholesterolemia.

Search methods: We searched the following databases until July, 2010: The Cochrane Library, MEDLINE, EMBASE, Chinese BioMedical Database, Traditional Chinese Medical Literature Analysis and Retrieval System, China National Knowledge Infrastructure, Chinese VIP, Chinese Academic Conference Papers and Chinese Dissertation Database, Allied and Complementary Medicine.

Selection criteria: Randomised clinical trials comparing Chinese herbal medicines with placebo, no treatment or pharmacological in participants with hypercholesterolemia were included. Data collection and analysis: Two authors extracted data and assessed study quality independently and any disagreement was resolved through discussion. Trials were assessed for risk of bias against key criteria: random sequence generation, allocation concealment, blinding of participants, incomplete outcome data, selective outcome reporting and other sources of bias.

Results: 22 randomised trials (2,130 participants) were included. The mean treatment duration were $2.3 \pm 1.3$ months (ranging from 1 to 6 months). Twenty trials were conducted in China and 18 trials were published in Chinese, and the methodological quality was assessed as generally low. Five different herbal medicines were tested in the included trials, which compared herbs with western medicine in six comparisons (20 trials), or placebos (2 trials). No outcomes of incidence of complications, quality of life and no serious adverse events were reported. One trial reported economic costs. Significant effect of 
Xuezhikang was shown on total cholesterol comparing with inositol nicotinate and marine triglycerides respectively. Xuezhikang had higher cost-effectiveness than pravastatin in lowering lipid profiles. Compared with pravastatin, Daming showed significant effect on lowing lipid.

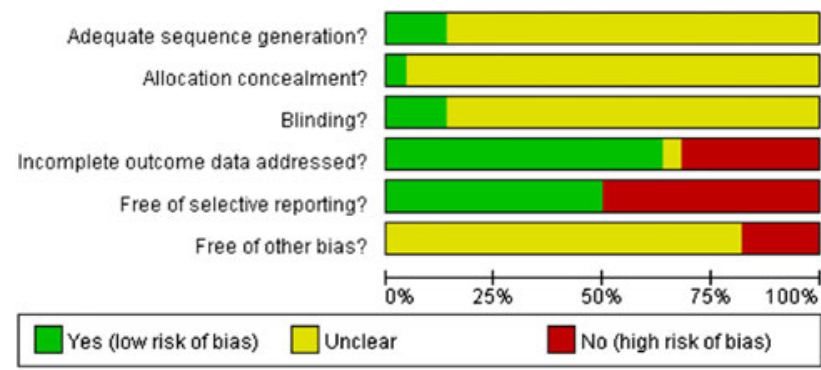

Methodological quality graph:authors' judgement

Conclusions: Some herbal medicines may have positive effect on cholesterol-lowering.

\section{PP17-09}

Wilson's Disease with Coexisting Autoimmune Hepatitis. A Case Report E. Vashakidze, T. Gegeshidze, T. Buachidze Tbilisi State Medical University, Tbilisi, Georgia

Wilson's disease [WD - an inherited disorder of copper metabolism in individuals with mutant ATP7B genes, occurs in every ethnic and geographic population, with worldwide prevalence of about 30 in million. WD can present clinically as liver disease, as a progressive neurological disorder or as psychiatric illness. The spectrum of liver disease encountered in patients with WD can be highly variable, ranging from asymptomatic with only biochemical abnormalities to acute liver failure. Because at present de novo genetic diagnosis is expensive and not universally available,a combination of clinical findings and biochemical testing is usually necessary to establish the diagnosis if WD. We describe 19 year old female, who presented with classical features WD and several features of autoimmune hepatitis $(\mathrm{AIH})$. The patient was initially diagnosed as having $\mathrm{AIH}$ and treated with standard corticosteroid therapy [prednisolone $60 \mathrm{mg}$ daily] with caused, initial clinical improvement, but subsequent deterioration. The diagnosis of WD was made 4 moons after initial diagnosis of AIH. Subsequent diagnosis of WD and introduction of penicillamine [1 g daily] gave excellent improvement and normalization of liver function tests. We suppose that in this case concurrent WD and AIH cannot be excluded. Thus, in patients with $\mathrm{AIH}$, a thorough screening for WD is necessary, particularly when the response to steroid therapy is poor. Conversely, in patients suffering from WD with superimposed features of $\mathrm{AIH}$, a combination of steroids and penicillamine may be of benefit

\section{PP17-10}

ABCB4 Gene Mutations in Chinese Children with Chronic Intrahepatic Cholestasis and High Gamma Glutamyltransferase

L. Fang, X. Wang, Q. Zhu, J. Wang

The Center for Pediatric Liver Diseases, Children's Hospital of Fudan University, Shanghai, China

Aims: To elucidate the existence and characterize ABCB4 gene mutations in mainland Chinese children with chronic intrahepatic cholestasis and summarize the clinical features and response to therapy.
Methods: Thirteen children presenting with chronic intrahepatic cholestasis and persistently elevated gamma glutamyltransferase (GGT) were referred to the Fudan University Children's Hospital from March 2004 to October 2009. All encoding exons and flanking areas of the ABCB4 gene were sequenced. Their clinical features, biochemical parameters, hepatic histopathology and response to therapy were analyzed.

Results: Six mutations of ABCB4 were detected in three out of thirteen patients. Apart from R47X, the remainder were novel, including IVS5+2_+3insT, D459G, R582Q, P693HfsX698, M1276WfsX1308. All three patients had compound heterozygous mutations. Liver function tests (mean \pm SEM) showed that GGT was lower $(162.0 \pm 44.8$ vs. $439.5 \pm 355.9 \mathrm{U} / \mathrm{L}, P=0.037)$ and total bile acid was higher $(352.5 \pm 97.0$ vs. $55.9 \pm 50.4 \mu \mathrm{mol} / \mathrm{L}, P=0.000)$ in patients with $\mathrm{ABCB} 4$ mutations than those in patients without mutations. The ratios of serum GGT and TBA were less than 1 in all patients with $\mathrm{ABCB} 4$ mutations (range 0.25-0.72); whereas in those patients without $\mathrm{ABCB} 4$ mutations the ratios were greater than 2 (range 2.14-17.73). No correlation between mutations and histological changes was noted. After ursodeoxycholic acid (UDCA) treatment in all patients with $\mathrm{ABCB} 4$ mutations, pruritus significantly diminished or disappeared and serum aminotransferases levels declined.

Conclusions: Some cases of chronic intrahepatic cholestasis with high GGT could be attributed to ABCB4 mutations in China. The ratio of serum GGT and TBA seems to be a promising parameter in differentiating progressive familial intrahepatic cholestasis type 3 (PFIC3) from other causes. UDCA therapy could improve clinical symptoms and liver function.

\section{PP17-11}

Diagnosis and Analysis of Hepatolenticular Degeneration in 37 Cases J. Zhu, Gastroenterological Study Group Digestive Department, Guiyang Medical College, Guiyang, China

Object: To explore the clinical feature, values of various accessory examination in the diagnosis of patients with hepatolenticular degeneration (HLD) in order to improve diagnosis of the disease.

Methods: The clinical features and various accessory examination of 37 cases with the confirmed diagnosis of HLD according to Sternlieb standard who were hospitalized in our hospital during December 1998 to October 2007 were analyzed retrospectively.

\section{Result:}

(1) In 37 cases, the age was from 7 to 39 ,including 27 males and 10 females. The average age is $16 \pm 6.8$ years old.

(2) The most clinical features were liver dysfunction in 19 cases,central nervous system symptoms in 11 cases, hematuria in 3 cases, anemia in 2 cases and joint pain in 2 cases.

(3) The 3 cases had the positive family history. The ceruloplasmin (CP) in 31 patients was below $200 \mathrm{mg} / \mathrm{L}$. The positive rate of cornea Kayser-Fleischer ring was $89.19 \%$. The urinary copper excretions in all cases was higher than $100 \mu \mathrm{g} / 24 \mathrm{~h}$.

(4) The 8 cases of 12 patients examined by cranial CT showed abnormal low density lesion in basal ganglia,lentiform nucleus, and so on.

The 5 cases of 8 patients examined by cranial MRI showed abnormal signs in basal ganglia, lentiform nucleus, and so on.

Conclusion: Early diagnosis and early treatment is key to the prognosis. In order to avoid misdiagnosis, with the patients who fail to get a clear diagnosis and are liver dysfunction; manifestation of central nervous system; hematuria; anemia, and so on, it is essential to checking cornea Kayser-Fleischer ring, copper metabolism and 
requiring carefully the family history. Cranial CT, MRI should be adopted when necessary.

\section{PP17-12}

Clinical Study of Hereditary Tyrosinemia Type 1 from a Single Center in Egypt

H. El Karaksy ${ }^{1}$, M. El-Raziky ${ }^{1}$, N. El-Koofy ${ }^{1}$, R. El-Sayed ${ }^{1}$, M. Rashed ${ }^{2}$, H. El-Kiki ${ }^{3}$, A. El-Hennawy ${ }^{4}$, N. Mohsen ${ }^{1}$

${ }^{1}$ Pediatrics, Cairo University, Cairo, Egypt; ${ }^{2}$ Genetics, King Faisal Specialist Hospital and Research Center, Riyadh, Saudi Arabia;

${ }^{3}$ Radio-diagnosis; ${ }^{4}$ Pathology, Cairo University, Cairo, Egypt

Background: Hereditary tyrosinemia type 1 (HT1) is an increasingly recognized inborn error of metabolism among Egyptian children.

Aim: To define the presenting clinical, biochemical and imaging features and outcome of NTBC therapy and liver transplantation in a cohort of Egyptian children diagnosed with HT1.

Methods: The study was carried out at the Pediatric Hepatology Unit at Cairo University Children's Hospital. HT1 was diagnosed by quantification of succinylacetone (SA) in dry blood spots.

Results: Twenty-two cases were diagnosed with HT1 over a period of 3 years from August 2006 to July 2009. Infants presenting with focal hepatic lesions and hepatomegaly $(n=13)$ were significantly younger at diagnosis than those presenting with rickets $(n=5)$ (median age 3.25 vs. 10 months; $p=0.05$ ). Alpha fetoprotein was highly elevated in all cases. Seven children died within few weeks of diagnosis before therapy was initiated. Ten patients were treated with NTBC. The response to NTBC treatment was apparent by a steep drop in serum AFP and undetectable SA in urine within 2 months. Three children underwent living donor liver transplantation after treatment with NTBC for 10, 18 and 22 months respectively, despite adequate response to therapy because of financial issues. The explanted livers were all cirrhotic with no dysplasia or malignant transformation.

In conclusion: Focal hepatic lesions are the commonest presentation of HT1 patients and they present at an earlier age than rickets. NTBC is effective but very expensive. Liver transplantation is still considered in HT1 patients.

\section{PP17-13}

The Association of Metabolic Syndrome, Insulin Resistance and Nonalcoholic Fatty Liver Disease in Overweight/Obese Children

N. El-Koofy ${ }^{1}$, G. Anwar ${ }^{1}$, M. El-Raziky ${ }^{1}$, A. El-Hennawy ${ }^{2}$, F. El-Mougy ${ }^{3}$, H. El-Karaksy ${ }^{1}$

${ }^{1}$ Pediatrics; ${ }^{2}$ Pathology; ${ }^{3}$ Chemical Pathology, Cairo University, Cairo, Egypt

Objective: To study the prevalence of metabolic syndrome (MS), insulin resistance (IR) and non-alcoholic fatty liver disease (NAFLD) in overweight/obese children with clinical hepatomegaly and/or raised alanine aminotransferase (ALT).

Patients and methods: Thirty-three overweight and obese children, aged 2-13 years, presenting with hepatomegaly and/or raised ALT, were studied for the prevalence of MS, IR and NAFLD. Laboratory analysis included fasting blood glucose, serum insulin, serum triglycerides, total cholesterol, high density lipoprotein cholesterol (HDL-c), low density lipoprotein cholesterol (LDL-c) and liver biochemical profile, in addition to liver ultrasound and liver biopsy.

Results: Twenty patients $(60.6 \%)$ were labeled with MS. IR was present in $16(48.4 \%)$. Fifteen $(44 \%)$ patients had biopsy proven NAFLD. Patients with MS were more likely to have NAFLD by biopsy $(p=0.001)$. Children with NAFLD had significantly higher body mass index, waist circumference, ALT, total cholesterol, LDL-c, triglycerides, fasting insulin, and lower HDL-c compared to patients with normal liver histology $(p<0.05)$ and fitted more with the criteria of MS ( 80 vs. $44 \%$ ). IR was significantly commoner among NAFLD patients (73 vs. $28 \%$ ).
Conclusion: There is a close association between obesity, MS, IR and NAFLD. Obese children with clinical or biochemical hepatic abnormalities are prone to suffer from MS, IR and NAFLD.

\section{PP17-14}

Insulin Resistance among Chronic HCV Infected Hemophilia Patients M. Keshvari ${ }^{1}$, S.V. Tabatabaei ${ }^{2}$, B. Behnava ${ }^{2}$, S.M. Alavian ${ }^{2}$ ${ }^{1}$ Iranian Blood Transfusion Organization Research Centre (IBTO), Tehran, Iran; ${ }^{2}$ Baqiyatallah University of Medical Sciences, Baqiyatallah Research Center for Gastroenterology and Liver Disease, Tehran, Iran

Background and aims: It is described that chronic HCV infection might be a risk factor for development of insulin resistance in chronic infected patients. In addition diabetic mellitus and insulin resistance can reduce virological response to anti-HCV therapy via impairment of host immune system. In current study we aimed to determine prevalence of insulin resistance among chronic $\mathrm{HCV}$ infected hemophilia patients and its association with viral markers.

Material and methods: 53 hemophilia patients were recruited. Fasting blood glucose and insulin, and liver enzymes were measured by automated analyzers. Patients with previous diagnosis of DM or those taking anti-hyperglycemia medications were excluded. Significant insulin resistance defined as HOMA-IR $\geq 2$.

Results: The mean age, viral load, insulin, FBS and ALT were respectively $30 \pm 1$ years, $1,330,000 \pm 124,000 \mathrm{IU} / \mathrm{mL}, 10 \pm 1.4$ microU/mL, $94 \pm 2 \mathrm{mg} / \mathrm{mL}$ and $53 \pm 6 \mathrm{IU} / \mathrm{mL}$. 23 subjects $(43 \%)$ had significant insulin resistance. By applying student $t$ test, Pearson correlation and Chi-squared test, there were no significant association between HCV genotype, viral load, ALT, FBS and fasting insulin level or HOMA-IR score.

Conclusion: $43 \%$ (95\% CI 31-57) of chronic HCV infected hemophilia patients have significant insulin resistance. Among these patients, viral markers do not seem to be associated with this insulin resistance.

\section{PP17-15}

Liver Histology in Children with Wilson's Disease. A Study of 64 Cases T. Chetkina, A. Potapov, E. Tumanova, V. Senyakovitch Gastroenterology, Scientific Centre of Children's Health Russian Academy of Medical Science, Moscow, Russia

Background: Wilson's disease (WD)—rare hereditary disorder, which characterize by copper accumulation in liver, brain and in other organs and tissues.

Aim: To estimate morphological liver changes at children with WD. Patients and methods: 64 children with WD (36 boys, 28 girls) at the age of 5-17 years (average age $12.5 \pm 3.18$ years). All children underwent liver puncture biopsy with deducing parameters of histologic activity index by Knodell and histologic fibrosis index by Desmet.

Results: In all observations there were degenerative changes in hepatocytes: muddy cytoplasmic swelling-at 41 (64\%), small and/ or large vacuoles-35 (54.7\%), balloon dystrophy-20 (31.4\%), adipose degeneration-16 (25.0\%), double nuclei hepatocytes-33 $(51.6 \%)$, anisocariosis-20 (31.3\%), glycogenic nuclei degeneration-12 (18.8\%), hepatocytes nuclei vacuolization-15 (23.4\%) children. Swelling of Kupffer cells was marked at 17 (26.6\%), their proliferation $-9(14.1 \%)$, proliferation of bile ducts in portal tracts and septa-20 (31.3\%) patients. Morphological liver fibrosis was revealed at 58 patients: minimal-in $13(22.4 \%)$, moderate-19 (32.8\%), severe-15 (27.8\%) specimens. Cirrhosis indications were found at $11(20.9 \%)$ children. Rubean acid (RA) stain biopsy specimens was positive in $6(25 \%)$ from 24 cases. Quantitative copper determining in liver tissues was conducted in 25 patients, in 17 cases $(68 \%)$ it was increased (medium $361.66 \pm 145.97 \mathrm{mkg} / \mathrm{g}$ ). Chronic hepatitis indications of minimal activity were determined 
at $27(42.2 \%)$, of low activity-24 (37.5\%), moderate and high activity figures were equal-7 (10.9\%) patients. Inflammatory changes were absent at six $(9.4 \%)$ children.

Conclusion: Morphological features of chronic hepatitis at children with WD were predominance of minimal and low activity degrees of the process $(79.7 \%)$ combined with a high expression fibrosis changes (48.7\%). Morphological detection WD by rubean acid stain established diagnose only in $25 \%$ cases.

\section{PP17-16}

Effect of Chronic Active Herpesvirus Infections on the Expression of Cytolysis Syndrome in Children with Liver Disease Forms of Accumulation

G. Volynec, T. Chetkina

Gastroenterology, Scientific Centre of Children's Health Russian

Academy of Medical Science, Moscow, Russia

The study of the impact of chronic active herpesvirus infections on the cytolytic activity in 25 children with liver disease forms of accumulation ( 15 children with Wilson's disease and 10 children with glycogen disease). Conducted a serological examination of blood by ELISA for the presence of markers of EBV, CMV, HSV1-2, VZV. PCR was carried out identification of DNA HSV1-2, HHV6, CMV and EBV in blood cells and liver biopsy. Severity of cytolytic activity was assessed by increasing the activity of transaminases - alanine aminotransferase (ALT) and aspartate aminotransferase (AST) serum at the onset of the disease. Analysis of expression of cytolytic activity in children with liver disease a form of savings, depending on the presence of active herpesvirus infections showed that in chronic active EBV infection, HSV1-2 infection and HHV6-infection cytolytic activity was significantly higher than that in the absence of these infections (Table 1). Chronic active CMV infection is cytolytic activity in children with liver disease a form of accumulation does not increase.

Table 1 Indicators cytolytic activity

\begin{tabular}{lll}
\hline Infection kind & $\begin{array}{l}\text { Illnesses of } \\
\text { accumulation } \\
\text { ALT Ed/ml }\end{array}$ & $\begin{array}{l}(\mathrm{n}=25) \text { AST } \\
\text { Ed/ml }\end{array}$ \\
\hline $\begin{array}{l}\text { Chronic active EBV an } \\
\text { infection }\end{array}$ & $249.4 \pm 28.8^{* * * *}$ & $180.0 \pm 18.8$ \\
$\begin{array}{l}\text { Chronic active EBV infections } \\
\text { are not present }\end{array}$ & $141.5 \pm 15.2$ & $179.8 \pm 16.2$ \\
$\begin{array}{l}\text { Chronic active CMV an } \\
\quad \text { infection }\end{array}$ & $118.9 \pm 10.8^{* * *}$ & $121.6 \pm 11.4^{* * *}$ \\
$\begin{array}{l}\text { Chronic active CMV infections } \\
\text { are not present }\end{array}$ & $213.6 \pm 16.3$ & $209.0 \pm 18.8$ \\
$\begin{array}{l}\text { Chronic active infection } \\
\quad \text { HSV1-2 }\end{array}$ & $284.5 \pm 25.6^{* * * *}$ & $203.2 \pm 28.8$ \\
$\begin{array}{l}\text { Chronic active infection HSV1- } \\
\quad \text { is not present }\end{array}$ & $147.8 \pm 14.2$ & $172.1 \pm 28.8$ \\
$\begin{array}{l}\text { Active infection HHV6 } \\
\text { Active infection HHV6 is not } \\
\text { present }\end{array}$ & $155.7 \pm 12.4$ & $117.3 \pm 10.9$ \\
\hline
\end{tabular}

$* p<0.05, * * p<0.01, * * * p<0.001$ at comparison with indicators at children who do not have chronic active herpesvirus infections

Studies have shown that the active herpesvirus infection negatively affect the cytolytic activity in the hepatic forms of Wilson's disease and glycogen disease.
PP17-17

The Epidemiologic Study of the Prevalence and Associated Factors of Metabolic Syndrome among Hospital Staff

K.-C. Chen ${ }^{1}$, Y.-J. Chao ${ }^{2}$, J.-Y. Chen ${ }^{2}$, C.-J. Wei ${ }^{3}$, F.-L. Chen ${ }^{3}$

${ }^{1}$ Cheng-Hsin General Hospital; ${ }^{2}$ Chang-Gang Memorial Hospital;

${ }^{3} \mathrm{Fu}$-Jen Catholic University, Taipei, Taiwan ROC

Objectives: Metabolic syndrome is a cluster of cardiovascular risk factors. From the viewpoint of preventive medicine, it is essential for early diagnosis and appropriate treatment to subjects with metabolic syndrome. The objective in this study is to explore the prevalence and associated risk factors of metabolic syndrome among hospital staff based on the health examination.

Methods: Based on the cross-sectional study design, the database of the health examination of the workers in a medical center on the northern Taiwan is analyzed. The definition of metabolic syndrome is according to the criteria proposed by Department of Health in 2007, Taiwan. Due to the limitation of the examination, the total cholesterol is used to replace the HDL-C value.

Results: The prevalence of metabolic syndrome is $16.3 \%(24.8 \%$ in male and $11.7 \%$ in female) among 1,203 screened subjects. The highest proportion of abnormal index of metabolic syndrome is central obesity combined with higher blood pressure and total cholesterol. Based on the multinomial logistic regression, the significant factors related to 1-2 abnormal index of metabolic syndrome include gender (male vs. female, $\mathrm{OR}=1.85,95 \% \mathrm{CI}$ : 1.31-1.47), age $(\mathrm{OR}=1.04,95 \% \mathrm{CI}: 1.02-1.06)$ and obesity $(\mathrm{OR}=1.39,95 \% \mathrm{CI}$ : 1.31-1.47). In addition, the significant factors related to $3-5$ abnormal index of metabolic syndrome also include gender (male vs. female, $\mathrm{OR}=2.08,95 \% \mathrm{CI}: 1.28-3.45)$, age $(\mathrm{OR}=1.12,95 \% \mathrm{CI}: 1.09-1.14)$ and obesity $(\mathrm{OR}=1.73,95 \% \mathrm{CI}: 1.60-1.87)$.

Conclusion: To promote the health promoting hospital, the priority of metabolic syndrome prevention is controlled waist circumference and body weight.

PP17-18

Influence of Inherited Liver Disorders on Viral Hepatitis C Clinical Outcome

L. Piekuse $^{1}$, V. Sondore ${ }^{2}$, J. Keiss ${ }^{2}$, M. Kreile ${ }^{1}$, A. Zarina ${ }^{1}$, B. Lace ${ }^{3}$, A. Krumina ${ }^{1}$

${ }^{1}$ Scientific Laboratory of Molecular Genetics, Riga Stradins University; ${ }^{2}$ Infectology Center of Latvia; ${ }^{3}$ Latvian Biomedical Research Center, Riga, Latvia

Chronic hepatitis $\mathrm{C}$ is worldwide distributed disease, that has antiviral treatment, but in many cases it is ineffective and doesn't cause viral clearance, and even in some cases cause severe adverse effects. Most common inherited liver disorders - Gilbert syndrome, Wilson disease, Alpha-1-antitrypsin deficiency and Hereditary haemochromatosis influence development of chronic hepatitis, and also could be involved in cirrhosis development in chronic hepatitis patients.

Materials and methods: DNA from 240 chronic hepatitis C (VHC) patients and 240 healthy individuals were analyzed for main function decreasing mutations in genes UGT1A1, HFE, ATP7B, SERPINA1, that were detected using PCR, RFLP, Bi-pasa, direct sequencing methods. Results: Gilbert syndrome in VHC patient group wasn't significantly different from control group. ATP7B and SERPINA 1 gene mutations were more frequent in VHC group, and correlated with changed liver lipid metabolism and cirrhosis development. Mutations in HFE gene correlated with iron and ferritin concentration in VHC patients. There was correlation between inherited liver disorders and viral clearance after antiviral therapy.

Conclusions: Inherited liver disorders (Wilson disease, Gilbert syndrome, Alpha-1-antitrypsin deficiency and Hereditary haemochromatosis) has impact on VHC progression and antiviral therapy efficiency. 


\section{PP17-19}

Hepatoerythropoietic Porphyria (HEP): Case Report

K.R. do $\mathbf{O}^{1}$, F.R. d'Albuquerque e Castro ${ }^{2}$, L.H. Spielmann ${ }^{3}$, L.A.R. Freitas ${ }^{4}$ ${ }^{1}$ Liver Unit, Hospital Alcides Carneiro; ${ }^{2}$ Medicina, Faculdade de Medicina de Petrópolis; ${ }^{3}$ Radiology, Hospital Alcides Carneiro, Rio de Janeiro; ${ }^{4}$ Laboratório de Patologia e Biologia Celular, Fundação Oswaldo Cruz, Bahia, Brazil

Porphyrias are a group of infrequent metabolic diseases caused by partial deficiencies of the activity of seven sequentially acting enzymes in the biosynthesis pathway of haem. They are inherited diseases with the exception of Sporadic Porphyria Cutanea Tarda (PCT) Type I the most frequent form of porphyria. PCT type is the result of inactivation of hepatic Uroporphyrinogen (URO-D) enzyme. Porphyria appears in individuals in all human race. The prevalence has been evaluated in a variable percentage of $0.5-10$ per 100,000 in different populations.

Purpose: We report on a 48-year-old men coming from Petrópolis, Rio de Janeiro,Brazil with a history of 8 years of duration, characterized by pruritus and burning on sun-exposed areas. His personal and family history were inconspicuous. Examination disclosed a slightly pale men in otherwise general good health with stirking and thick dark brown crusts in and ulcers with prominent scarring on dorsa of the fingers on both hands. Besides, he had shallow ovoid and lineal smallpox like scars on her cheeks. Biochemical investigation included the study of porphyrin (uroporphyrin, coproporphyrin and protoporphyrin) in blood plasma. Microscopic examination - histopathological examination diagnosis - intense colestasis; liver changes consistent with erytropoietic.

\section{Poster Presentation 18: Molecular and Cellular Biology: Biliary Tract Pathophysiology}

\section{PP18-01}

CD147 enhances cell proliferation of liver fluke-induced cholangiocarcinoma cell line

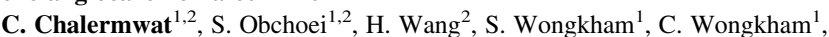
Q. $\mathrm{Yao}^{2}$, C. Chen ${ }^{2}$

${ }^{I}$ Department of Biochemistry, Liver Fluke and Cholangiocarcinoma Research Center, Faculty of Medicine, Khon Kaen University, Khon Kaen, Thailand; ${ }^{2}$ Molecular Surgeon Research Center, Division of Vascular Surgery and Endovascular Therapy, Michael E. DeBakey Department of Surgery, Baylor College of Medicine, Houston, TX, USA

Background: Liver fluke-induced Cholangiocarcinoma (CCA) is a common cancer in the northeast of Thailand. It has no specific treatments. Outcome of current treatment is unfavorable. CD147 is a transmembrane glycoprotein which plays an important role especially in growth process of tumor cells. However, the role of CD147 on CCA cell proliferation has not yet been reported.

Methods: Firstly, CD147 expression on CCA tissues was demonstrated by immunohistochemistry staining. CD147 mRNA and protein expression in five CCA cell lines and an immortalized bile duct cell line were further evaluated by qRT-PCR and western blot, respectively. To evaluate CD147 and proliferative function, the stable clones of KKU-M213-CCA cell line were established. CD147 and scrambled-control shRNA were transected to KKU-M213-CCA cell line and then continued clonal selection with puromycin antibiotic. Then, CD147 expression was determined, using qRT-PCR and western blot, in the CD147-knockout and scrambled-control clone. Both of selected clones were used for MTT-proliferative study and wound healing assay.

Results: Over-expressions of CD147 were observed in cancerous bile duct when compared to adjacent normal bile duct. However, high
CD147 mRNA and protein expression was found in certain CCA cell lines. After CD147 silencing, decreased cell proliferation and delayed wound healing were observed in CD147 knockout clone of KKU-M213-CCA cells.

Conclusion: Our results have demonstrated that CD147 is expressed in liver fluke-induced CCA tissues. However, CD147 is highly expressed in certain liver fluke-induced CCA cells and is required for tumor cell proliferation. The mechanism in which CD147 controlled CCA cell proliferation is under investigated.

\section{PP18-02}

Effects of Relief of Obstructive Jaundice by Internal and External Biliary Drainage on theExpression of CD14 by Kupffer Cells

X. Huang ${ }^{1}$, J. Xiao ${ }^{2}$, W. $\mathrm{Li}^{2}$

${ }^{I}$ The Chinese People Liberation Army Navy General Hospital,

${ }^{2}$ The Chinese People Liberation Army General Hospital, Beijing, China

Aim: We studied the effects of internal and external biliary drainage on the expression of CD14 by Kupffer cells in rats with obstructive jaundice $(\mathrm{OJ})$, in order to explore the mechanism of internal biliary drainage (ID) being superior to external drainage (ED).

Methods: In the first part, sixty male adult Sprague-Dawley rats were randomly assigned to four groups: OJ $(n=15)$, ID $(n=15)$, ED $(n=15)$ and sham operation (SH $n=15)$. The expression of CD14 by Kupffer cells was measured as the semiquantitative values (the average integral optical density, IOD/area) by an image analysis system with an immunohistochemistry method. In the second part, forty rats were randomly assigned to four groups(OJ $n=10$,ID $n=10$,ED $n=10$,SH $n=10$ ). Kupffer cells were isolated by a method of in situ hepatic perfusion and digestion with collagen IV and were cultured with endotoxin in vitro for $15 \mathrm{~h}$. The expression of Kupffer cells CD14 mRNA was detected by reverse transcription polymerase chain reaction.

Results: The expression of CD14 by Kupffer cells was significantly stronger in $\mathrm{OJ} \operatorname{rats}(0.015557 \pm 0.002135)$ than that in $\mathrm{SH}$ rats $(0.00135 \pm 0.000822)(p<0.01)$. The expression of CD14 by Kupffer cells was significantly reduced in ID rats $(0.001548 \pm$ $0.001024)$ compared with OJ rats $(p<0.01)$, but not reduced in ED $\operatorname{rats}(0.008570 \pm 0.001893)$ (ED vs. OJ $p=0.5907)$. Under the stimulation of endotoxin, the expression of Kupffer cells CD14 mRNA was significantly increased in ED rats $(6.104 \pm 2.171)$ compared with $\mathrm{SH}$ rats $(1.388 \pm 0.683)$, OJ rats $(1.998 \pm 0.740)$ and ID rats(1.018 \pm 0.489$)$ respectively, $(p<0.01)$. The difference of the expression of CD14 mRNA by Kupffer cells between ID and SH, OJ group was not statistically significant(ID vs. SH $p=0.944$, ID vs. OJ $p=0.513$ ). These results indicated that external biliary drainage enhanced the sensibility of CD14 genes to endotoxin, while internal biliary drainage didn't.

Conclusions: The mechanism of internal biliary drainage being superior to external drainage seems to be related to the transcription process of CD14 genes.

Poster Presentation 19: Molecular and Cellular Biology: Cell Cycle and Apoptosis

\section{PP19-01}

PI3K and mTOR Inhibitors Alone or in Combination with Chemotherapy Effectively Inhibits Hepatocellular Carcinoma Growth

D. Pothiraju, M.P. Manns, A. Vogel

Hannover Medical School, Hannover, Germany

Background: Hepatocellular carcinoma (HCC) is the fifth most frequent cancer and third most lethal cancer worldwide. Activated PI3K/ AKT/ mTOR pathway has recently emerged as a pivotal contributor 
to HCC development. Aberrant mTOR signaling has been identified in up to 50-60\% of HCC cases, associated with insulin-like growth factor pathway activation and PTEN dysregulation. The aim of this study was to delineate the role of PI3K and mTOR inhibitor in HCC. Methods: HuH7 cells were treated with PI3K (NVP BKM120-AA), mTOR (RAD001), PI3K and mTOR (NVP BEZ235-AN) inhibitors and cytotoxic drugs which are Doxorubicin, Cisplatin, 5Fluoro Uracil $(5 \mathrm{FU})$ and Irinotecan alone or in combination. Cell proliferation was measured using MTT assay. Cell cycle (PI staining) and apoptosis (AnnexinV vs. PI) analysis were carried out by FACS. Signaling mechanism of these drugs was confirmed by western blots. In in vivo studies, nude mice were transplanted with Huh7 cells, tumor size was measured, tissue pathological analysis was done by HE staining, proliferation was done by IHC using Ki67 antigen and pHis3 anitibody and apoptosis was measured by Tunnel staining.

Results: All inhibitors had an effect on cell proliferation inhibition. Combination with cisplatin significantly decreased the viability of hepatoma cells compared to Doxorubicin, Irinotecan and 5FU. Interestingly, RAD001 and BEZ treatment led to an increased phosphorylation of AKT where as in BKM, it was decreased. Dephosphorylation of 4E-BP1 and p70S6R were observed in RAD001, BKM and BEZ treatment. Higher concentration of BEZ led to decreased pAKT expression. Tumor size and cell proliferation were significantly decreased and apoptosis was increased in combination treated mice compared to single treated mice.

Conclusion: Our results illustrate that, PI3K/ AKT/ mTOR inhibitors effectively induce loss of cell viability. Additionally, combination with cisplatin showed synergistic effect on cell proliferation inhibition. Xenograft models confirmed reduced tumor growth upon treatment.

\section{PP19-02}

Lipopolysaccharide Triggered Tumor Necrosis Factor - Alpha Induced Liver Cell Injury in Acute Arsenic Toxicity in a Murine Model

S. Ghosh ${ }^{1}$, S. Ghatak ${ }^{1}$, A. Biswas ${ }^{1}$, K. Basu ${ }^{2}$, A. Chowdhury ${ }^{1}$, A. Santra ${ }^{1}$

${ }^{I}$ Center for Liver Research; ${ }^{2}$ GI Pathology, Institute of Post Graduate Medical Education and Research, Kolkata, India

Background and aim: Co-exposure to arsenic and alcohol increases liver injury. Alcohol affects arsenic kinetics and retention in the liver. Further, elevated lipopolysaccharide (LPS) is a major independent risk factor in alcoholic liver injury. However its role in arsenic intoxication is not clear. We investigated possible synergistic effects of LPS in arsenic induced liver injury.

Methods: Rapid arsenic induced liver injury was developed in mice by three doses of arsenic ( $2 \mu \mathrm{g} / \mathrm{g}$ followed by two $1 \mu \mathrm{g} / \mathrm{g}$ body weight) through i.p route at an interval of $3 \mathrm{~h}$. Some mice were pretreated with LPS ( $25 \mu \mathrm{g} / \mathrm{g}$ b.w) $1 \mathrm{~h}$ before first dose of arsenic to evaluate its synergistic effects. In some experiments pretreatment with inhibitor of macrophage function such as gadolinium chloride $\left(\mathrm{GdCl}_{3}\right)$ were done. Evaluation of hepatic oxidative stress, pathological lesions, mode of cell death and signaling pathways were done. In vitro experiments on primary mouse hepatocytes were carried out to validate the in vivo experiments.

Results: Pathological evaluation showed severe steatosis only in liver sections from mice in the LPS plus arsenic group; blood transaminase levels, 4-HNE adducts, activities of caspase-3 and -9 and TUNEL positive cells were significantly elevated in the combination group. Hepatic TNF- $\alpha$ and TNF-R1 levels, nuclear translocation of p50 subunit of $\mathrm{NF} \kappa \beta$ and mRNA expression of inducible nitric oxide synthase (iNOS) were significantly elevated in the combined group but not in LPS and arsenic group. $\mathrm{GdCl}_{3}$ pretreatment caused significantly less liver injury in the combined group. In vivo findings were recapitulated in in-vitro experiments.

Conclusions: The enhanced hepatic steatosis as well as injury appears to involve an increase in oxidative and nitrosative stress generated by the combination of LPS and arsenic.
PP19-03

Evolutionary Model for Study of Human Liver Diseases

S. Konovalova, M. Savina

Laboratory of Comparative Biochemistry of Inorganic Ions, I.M. Sechenov Institute of Evolutionary Physiology and Biochemistry of Russian Academy of Sciences, Saint Petersburg, Russia

Adult lampreys, in contrast to the larval forms and all others vertebrates, have neither a gall-bladder nor a bile duct system that causes accumulation of the bile acids and bile pigments in the hepatocytes. In addition during the period of pre-spawning migration when lampreys switch off exogenous feeding hepatocytes filled with lipid droplets that are expended in spring before spawning. Thus the liver of the adult lamprey represents a unique natural model imitating the pathological states of the human liver diseases (billiary atresia, steatosis and cholestasis). The aim of our work was to investigate bioenergetics and biochemical characteristics of lamprey liver during pre-spawning migration. In winter period of pre-spawning migration ATP concentration in lamprey liver tissue (measured with High-performance Liquid Chromatography and Luciferin-luciferase method) and mitochondrial membrane potential in lamprey hepatocytes (investigated by a confocal microscopy and flow cytometry using potentialdependent fluorescent probes -TMRM, DiOC6, and MitoTracker Green) decreased and the Atkinson energy charge reaches extremely low level 0.2-0.3. In this period the concentration of cytosolic $\mathrm{Ca}^{2+}$ (detected by Fura 2-AM) was very high $(160 \mathrm{nM})$ as compared with spring period $(60 \mathrm{nM})$. Moreover, in spring before the spawning and the death (lampreys are the monocyclic animals) we observed a sharp increase of apoptotic hepatocytes (by caspases 3, 7, 9 activities) and elevation of serum aminotransferase activity (estimated according to the method of Ritman and Frankel). So in the period of pre-spawning migration the lamprey liver enables one to study all bioenergetic changes in cells that are characteristic of pathological conditions of the human liver.

The work is supported by Russian Foundation for Basic Research (project No. 08-04-00564).

\section{PP19-04}

Effects of the Inhibition of Hypoxia Dependent Factors on Cell Growth and Apoptosis in Hepatocellular Carcinoma Cell Lines

S.H. Choi ${ }^{1}$, Y.B. Won ${ }^{2}$, S.H. Kim ${ }^{2}$, J.Y. Park ${ }^{2,3}$, D.Y. Kim ${ }^{2,3}$, S.H. Ahn ${ }^{2,3}$, W.S. Ro ${ }^{2,4}$, C.O. Yun ${ }^{5}$, K.-H. $\operatorname{Han}^{2,3,4}$

${ }^{I}$ Brain Korea 21 project for Medical Science; ${ }^{2}$ Department of Internal Medicine, College of Medicine, Yonsei University; ${ }^{3}$ Yonsei Liver Cancer Special Clinic, Yonsei University Health System; ${ }^{4}$ Brain Korea 21 Project for Medical Science, College of Medicine, Yonsei University; ${ }^{5}$ Brain Korea 21 Project for Medical Science, Institute of Cancer Research, Yonsei Cancer Center, Seoul, Republic of Korea

Objectives: Hypoxia-inducible factor- $1 \alpha(\mathrm{HIF}-1 \alpha)$ is a central transcription factor involved in various aspects of cancer biology, including proliferation, survival, angiogenesis, and extracellular matrix metabolism under hypoxia. Interleukin 8 (IL-8) induced survival of endotherial cells on hypoxia. This study evaluated whether adenovirus-mediated small hairpin HIF- $1 \alpha$ (shHIF- $1 \alpha)$ and IL- 8 (shIL-8) inhibited cell growth and apoptosis in the hepatocellular carcinoma (HCC) cell lines.

Method: HCC cell lines were infected with adenovirus expressing shRNA and cultured under hypoxic conditions $\left(1 \% \mathrm{O}_{2}, 24 \mathrm{~h}\right)$. Following infection, the expression levels of HIF-1 $\alpha$, apoptotic factors, and growth factors were examined through real-time quantitative PCR and immunoblot, while tumor growth was measured by cell proliferation assay (MTT assay) and apoptosis was measured by TUNEL assay and concentration of cytochrome C.

Results: The inhibition of HIF- $1 \alpha$ by adenovirus-mediated shRNA led to the repression of tumor growth, while the inhibition of IL-8 did not. It also up regulated the expression of apoptotic factors and down- 
regulated growth factors. Inhibition of HIF-1a and IL-8 increased concentration of cytochrome $\mathrm{C}$ and DNA fragmentation in HCC cell lines.

Conclusions: These data suggest that adenovirus-mediated shHIF- $1 \alpha$ and shIL-8 inhibit anti-apoptosis and cell proliferation in HCC cell lines.

\section{PP19-05}

The Expression of Survivin Protein in Normal and Pathologic Liver Tissues

S. Guo, Q. Xie, X. Zhou, S. Jiang, H. Zhou

Infectious Diseases Department, Shanghai Jiaotong University,

School of Medicine, Shanghai, China

Introduction: Survivin is a recent described anti-apoptosis protein which is overexpressed in most of the human cancers and some developmental cells.

Aims: To investigate the expression of survivin protein in normal and various pathologic liver tissues, included human chronic hepatitis (HBV), cirrhosis, HCC tissues, as well as in mice damaged liver tissues in different phases.

Methods: Liver specimens are from 20 patients ( 1 healthy, 6 hepatitis, 5 cirrhosis, 8 carcinoma) while mice liver injury models was induced by the combination of D-galactosamine (D-Gal) and lipopolysaccharide (LPS). Immunohistochemistry staining was performed to evaluate the expression and distribution of survivin protein in liver tissues.

Results: Immunohistochemistry staining showed positive in $8 / 8$ HCC, 4/5 in cirrhosis while 3 of 6 hepatitis tissues showed little detectable staining, and there was no staining (1 of 1$)$ in normal human liver tissue. Furthermore, at the cellular level, the expression of survivin was observed primarily in the cytoplasm. In contrast, in mice hepatocellular injury tissues, the expression of survivin showed positive as well, with an increase in the intensity of staining by time. The expression of survivin in normal mouse liver tissue was undetectable.

Conclusions: The survivin proteins is undetectable in normal human tissues, whereas its expression increased from hepatitis tissues to HCC tissues, which suggest the transformation of liver tissue from inflammation to HCC. Furthermore, survivin can not only suppress the cell apoptosis in human liver tissues, have an effect of carcinogenesis, but also highly correlated with the cell regeneration in liver injury tissues of mouse as well.

\section{PP19-06}

Free fatty Acids Induce Lipoapoptosis in HepG2 Cells

X.-H. Che, S.H. Lee, Y.-Z. Zhao, B.-H. Jeon, W.-Y. Jiang, D.H. Sohn

Pharmacy, Wongkwang University, Iksan, Republic of Korea

Background and aims: Nonalcoholic fatty liver disease (NAFLD) is the most common liver disease in Western and non-Western countries, but its exact molecular/cellular mechanisms remain obscure and effective therapeutic strategies are still limited. It is well-accepted that free fatty acid (FFA)-induced lipotoxicity plays a pivotal role in the pathogenesis of NAFLD. However, the mechanism by which FFAs mediate lipoapoptosis is unclear. Oleic $(\mathrm{C} 18: 1)$ and palmitic $(\mathrm{C} 16: 0)$ acids are the most abundant fatty acids in liver triglycerides in both normal subjects and patients with NAFLD. The aim of this study is to elucidate the possible mechanisms of FFAs (oleic or palmitic acids) induced liver apoptosis in human hepatocellular in vitro model.

Methods: HepG2 cells were treated with oleic or palmitic acids. We developed a convenient assay by using an Oil-Red-O-based colorimetric measurement to qualify the degree of oleic or palmitic acidsinduced steatosis in HepG2 cells. The effects of oleic or palmitic acids on HepG2 cells apoptosis, cell cycle and other related mechanisms were systematically examined. Poly (ADP-ribose) polymerase (PARP) and caspase-3 cleavage studies were executed to the presence of apoptosis, and flow cytometric quantification for cell population.
Results: We demonstrated that oleic or palmitic acids induce the lipid accumulation in HepG2 cells in a dose-dependent manner at $24 \mathrm{~h}$ that can be assessed by Oil-Red-O-based colorimetric measurement. Moreover, $2 \mathrm{mM}$ oleic or $0.5 \mathrm{mM}$ palmitic acids induce apoptosis for $24 \mathrm{~h}$ in HepG2 cells. The induced apoptosis of HepG2 cells was associated with phosphatidylserine externalization, poly (ADP-ribose) polymerase (PARP) and caspase-3 cleavage, increase of Bax and decrease of Bcl-2. Furthermore, oleic or palmitic acids induced cell cycle arrest at G1 and S phases in HepG2 cells.

Conclusions: Our results demonstrated that FFAs (oleic or palmitic acids) induced lipoapoptosis by different mechanisms in human hepatocellular in vitro steatosis model.

\section{PP19-07}

P53 Is Regulated by Notch3 Signalling via MDM2 in Hepatocellular Carcinoma

M. Minguzzi ${ }^{1,2}$, C. Giovannini ${ }^{1,3}$, L. Gramantieri ${ }^{1,3}$, P. Chieco ${ }^{1}$, C. Mastroleo ${ }^{1}$, K.B. Marcu ${ }^{1,3,4,5}$, L. Bolondi ${ }^{1,3}$

${ }^{1}$ Center for Applied Biomedical Research (CRBA), S.Orsola-Malpighi University Hospital; ${ }^{2}$ Department of Biology; ${ }^{3}$ Department of Clinical Medicine, University of Bologna, Bologna, Italy;

${ }^{4}$ Biochemistry and Cell Biology Department, Stony Brook University, Stony Brook, NY, USA; ${ }^{5}$ Laboratory of Immunorheumatology and Tissue Regeneration, Rizzoli Orthopaedic Institutes, Bologna, Italy

Background and aims: Notch genes encode single-pass transmembrane receptors involved in proliferation, differentiation and apoptosis. We previously showed that Notch 3 and Notch 1 were aberrantly expressed in human hepatocellular carcinoma (HCC) and in the present study we aimed at investigating their function by analyzing which pathways are activated and deregulated by Notch in HCC.

Methods: HCC cell lines were stably silenced for Notch3 and Notch1 expression. Proliferation was analysed by FACS and p53 mRNA and protein expression evaluated by real time PCR and western blot respectively. Mdm2 and CyclinG1 mRNA expression were analyzed by semi-quantitative RT-PCR and CyclinG1, MDM2, phosphoMDM2 and phospho-AKT protein expression were detected by western blot. P53 was silenced by siRNA transfection in HepG2 and SNU398 cell lines.

Results: Notch3 and Notch1 silenced cells showed reduced proliferation, which was associated with p53 up-regulation only in Notch3 knock-down (KD) cells. Notch3 silencing resulted in p53 up-regulation at the transcriptional level but mainly by post-transcriptional mechanisms. Total levels of $\mathrm{Mdm} 2$ protein and phosphorylated MDM2 were reduced in Notch3 silenced cells and MDM2 phoshorylation status was AKT independent. Mdm2 transcription was not affected by Notch3 status and Cyclin G1 protein was down-regulated in Notch3 silenced cells. Importantly, p53 silencing showed that Mdm2 and CyclinG1 transcription were both independent of p53 and these observations were confirmed in Hep3B HCC cells lacking the p53 gene.

Conclusion: Our results in HCC cell lines shows that Notch3 controls p53 at the transcriptional level but mainly at post-transcriptional level by acting on CyclinG1 and MDM2 pathways. Thus, our data support the hypothesis that Notch3 has a crucial role in HCC cancer development and progression through $\mathrm{p} 53$ regulation.

\section{PP19-08}

In vitro and In vivo Evaluation of Hepatoprotective Activity from Cordyceps militaris

B.-S. Wang, C.P. Lee, P.-D. Duh

Department of Food Science and Technology, Chia Nan University of Pharmacy and Science, Tainan, Taiwan ROC

The protective effects of Cordyceps militaris on tert-butyl hydroperoxide (t-BHP) induced oxidative damage in hepatocytes of HepG2 cells and in rat liver induced by carbon tetrachloride $\left(\mathrm{CCl}_{4}\right)$ were 
investigated. The results in vitro showed that pre-treatment with water extracts of Cordyceps militaris (CME) protected the hepatocytes against t-BHP induced oxidative injury and the results were comparable to the treatment with water extracts of Cordyceps sinensis (CSE). The mechanism of hepatoprotection against cells death was achieved mainly by decreasing ROS generation, TBARS formation, restoring GSH levels, increasing Bcl-2/Bax ratio, modulating mitochondria membrane potential as well as reducing caspase-3 activation. In addition, administration of dried powder of Cordyceps militaris (DPCM; 0.1, 0.3 and $0.5 \mathrm{~g} / \mathrm{kg}$ of bw) to rats for consecutive 28 days before a single dose of $50 \% \mathrm{CCl}_{4}(2 \mathrm{~mL} / \mathrm{kg}$ of bw, ip $)$ significantly reduced the liver injury by lowering serum levels of serum glutamate oxaloacetate transaminase (GOT) and glutamate pyruvate transaminase (GPT). The restoration of GSH levels in rat liver observed may in part explain DPCM reduced the liver injury in rat liver induced by $\mathrm{CCl}_{4}$. Taken together, these results suggest that the hepatoprotective actions of Cordyceps militaris on hepatotoxicity in vitro and in vivo may be related to their maintenance of the normal redox status of cells and stabilize antioxidant systems in the cell.

\section{PP19-09}

HCVNS5A Protein Down-regulates Hepcidin Gene Expression

G. Gong, Y.-Z. Liu, X.-Q. Xiao, D. Cheng, Y.-F. Jiang

Infectious Diseases, The Second Xiangya Hospital, Changsha, China

Objective: To investigate whether the nonstructural protein 5A(NS5A)encoded by the hepatitis C virus RNA genome affects the expression of hepcidin gene which is a major regulator of iron metabolism.

Methods: HCV NS5A expression plasmid (pCN5A) and the blank pRc/CMV plasmid were individually transfected into QSG7701 cells, RT-PCR was employed to detect the HCV NS5A and hepcidin mRNAs. Western blot was used for detection of HCVNS5A and hepcidin proteins.

Results: HCV NS5A plasmid was successfully transfected into QSG7701 cells, which was evidenced by finding HCVNS5A mRNA and protein from the transfected cells. The transcription of hepcidin mRNA decreased remarkably in the cells transfected with pCN5A plasmid comparing to the untransfected cells and pRc/CMV transfected cells; The level of hepcidin protein expression was also found lower in the pCN5A plasmid transfected cells in a dose dependent way, comparing to the untransfected cells and pRc/CMV transfected cells.

Conclusions: HCV NS5A inhibits the transcription of hepcidin mRNA and expression of hepcidin protein., and may be a reason of high iron load in HCV infected patients.

\section{PP19-10}

A Novel Action of Dipeptidyl Peptidase 9 in Akt Dependent Epidermal Growth Factor Signalling and Apoptosis

T.-W. Yao ${ }^{1}$, D.M. Yu ${ }^{1}$, W.-S. Kim ${ }^{2}$, M.G. Gall ${ }^{1}$, G. Sharbeen ${ }^{1}$,

G.W. McCaughan ${ }^{1,3}$, K.-Y. Choi $^{2}$, P. Xia ${ }^{1}$, M.D. Gorrell ${ }^{1}$

${ }^{1}$ Centenary Institute, University of Sydney, Sydney, NSW, Australia;

${ }^{2}$ Biotechnology, Yonsei University, Seoul, Republic of Korea; ${ }^{3} A W$

Morrow Gastroenterology and Liver Centre, Royal Prince Alfred

Hospital, Sydney, NSW, Australia

Dipeptidyl peptidase IV (DPP4), DPP8, DPP9 and FAP (fibroblast activation protein), are serine proteases of the DPP4 gene family that exhibit unique peptidase and extra-enzymatic activities. These enzymes have been implicated in various diseases including cancer and chronic liver injury. We report here a novel role of DPP9 in regulating cell survival and proliferation through modulating a molecular signalling pathway. Akt (protein kinase B) activation was significantly inhibited by human DPP9 overexpression in human hepatoma cells and human embryonic kidney cells, whereas extracellular signal-regulated kinase (ERK1/2) activity was unaffected, suggesting a pathway-specific effect. Interestingly, the inhibitory effect of DPP9 on PI3K/Akt pathway activation was growth factor dependent. DPP9 overexpression caused significantly less epidermal growth factor (EGF)-induced Akt activation, and a marked reduction in EGF-mediated cell survival and cell proliferation. However, such inhibitory effect was not observed in cells stimulated with CTGF, HGF, insulin or PDGF-BB. The effect of DPP9 on Akt activation did not occur when DPP9 enzyme activity was ablated by either mutagenesis or inhibition. In addition, we showed that DPP9 associated with H-Ras, a key signal molecule of the EGF receptor signalling pathway. These findings suggest an important signalling role of DPP9 in the regulation of EGF-dependent survival and proliferation.

\section{PP19-11}

Hepatocyte Apoptosis and Oxidative Stress in Patients with Chronic Hepatitis B

\section{J. Zhao}

Department of Hepatology, Qilu Hospital of Shandong University, Jinan City, China

Backgound and aims: Hepatocyte apoptosis is involved in the pathogenesis of liver diseases, while at the same time oxidative stress plays an important role in the liver cell damage. This present study was aimed to evaluate the possible relationship between hepatocyte apoptosis and oxidative stress in patients with chronic hepatitis B (CHB).

Methods: CHB patients were placed into group A (ALT $>40 \mathrm{IU} / \mathrm{L}$ ) and $\mathrm{B}(\mathrm{ALT} \leq 40 \mathrm{IU} / \mathrm{L})$. Healthy controls was considerated as group C (all ALT $\leq 40 \mathrm{IU} / \mathrm{L}$ ). Serum concentrations of $\alpha$-tocopherol, superoxide dismutase (SOD), glutathione peroxidase (GSH-Px) and malondialdehyde (MDA) were determined while liver cell apoptosis was evaluate by using terminal deoxynucleotydil transferase-mediated d-UTP biotin nick-end labeling (TUNEL).

Results: SOD, GSH-Px, and MDA were not different between groups. $\alpha$-Tocopherol was significantly decreased in groups A $(P<0.01)$ and $\mathrm{B}(P<0.05)$ when compared with group $\mathrm{C}$ and was found to be negatively correlated with the apoptosis index $(r=$ $-0.575, p<0.01)$.

Conclusions: The decreased plasma concetration of $\alpha$-tocopherol was negatively correlated with liver apoptosis index in patients with normal alanine aminotransferase levels, suggesting that $\alpha$-tocopherol may be a possible markers to reflect liver cell damage especially in the absence of serum aminotransferase elevation.

\section{PP19-12}

Studies of Catechin Induced Apoptosis on Human Liver Cancer Cell Lines HepG2

P. Vasanth Raj ${ }^{1}$, K. Nitesh ${ }^{2}$, S. Sagar Gang ${ }^{1}$, J. Hitesh ${ }^{1}$, J. Venkata Rao ${ }^{1}$, C. Mallikarjuna Rao ${ }^{2}$, N. Udupa ${ }^{3}$

${ }^{1}$ Department of Pharmaceutical Biotechnology; ${ }^{2}$ Department of Pharmacology; ${ }^{3}$ Manipal College of Pharmaceutical Sciences, Manipal, India

Background and aims: Hepatocellular carcinoma (HCC) ranks the fifth most common and the third most deadly cancer worldwide. Catechin, a well known flavonoid has been used as natural remedy for liver diseases. We examined the effect of catechin on human liver derived cancerous cell line HepG2.

Methods: HepG2 cells were routinely grown and subcultured as monolayers in MEM supplemented with $10 \%$ newborn calf serum. Concentrations of catechin greater than $210 \mu \mathrm{g} / \mathrm{ml}$ were found to the toxic to the cells; hence two concentrations 200 and $100 \mu \mathrm{g} / \mathrm{ml}$ were used. HepG 2 cells were grown on $25 \mathrm{~cm}^{2}$ bottles at $1 \times 10^{6} \mathrm{cells} / \mathrm{ml}$ concentrations for $24 \mathrm{~h}$ at $37^{\circ} \mathrm{C}$ in a humidified atmosphere of $5 \%$ $\mathrm{CO}_{2}$. The cells were then exposed to catechin 200 and $100 \mu \mathrm{g} / \mathrm{ml}$. After $24 \mathrm{~h}$ of drug treatment total RNA was extracted from these cells and expression of Bax, Bcl-2 and P53 levels were studied. Further flow cytometric studies in catechin treated cells were carried out using propidium iodide. 
Results: Reverse transcriptase PCR was used to analyze the levels of Bax, Bcl-2 and p53 mRNA levels in HepG2 cells treated with catechin $(200$ and $100 \mu \mathrm{g} / \mathrm{ml})$. Amplification of Bax, Bcl-2 and p53 was done with help of specific primers using cDNA as template strand prepared from HepG2 cells. Catechin down regulated Bcl-2, and up regulated Bax mRNA levels in HepG2, thus inducing apoptosis. No significant change was observed in p53 expression. Further flow cytometric analysis confirmed the significant increase in apoptotic cells before and after catechin treatment.

Conclusion: Together, these results identify the ability of catechin to induce apoptosis against HepG2 cells, which may involve a p53 independent pathway to induce apoptosis. Hence, catechin merits further investigation to study its anti cancer mechanism.

\section{PP19-13}

In vitro Anti Cancer Activity of Silymarin Against Human Liver Cancer Cell Lines HepG2

K. Nitesh ${ }^{1}$, P. Vasanth Raj ${ }^{2}$, J. Hitesh ${ }^{2}$, S. Sagar Gang ${ }^{2}$, C. Mallikarjuna Rao ${ }^{1}$, J. Venkata Rao ${ }^{2}$, N. Udupa ${ }^{3}$

${ }^{I}$ Department of Pharmacology; ${ }^{2}$ Department of Pharmaceutical Biotechnology; ${ }^{3}$ Manipal College of Pharmaceutical Sciences, Manipal, India

Background and aims: Hepatocellular carcinoma (HCC) ranks the fifth most common and the third most deadly cancer worldwide. Silymarin, a well known flavonoid has been used as natural remedy for liver diseases. We examined the effect of Silymarin on human liver derived cancerous cell line HepG2.

Methods: HepG2 cells were routinely grown and subcultured as monolayers in MEM supplemented with $10 \%$ newborn calf serum. Concentrations of silymarin greater than $120 \mu \mathrm{g} / \mathrm{ml}$ were found to the toxic to the cells; hence two concentrations 100 and $50 \mu \mathrm{g} / \mathrm{ml}$ were used. HepG 2 cells were grown on $25 \mathrm{~cm}^{2}$ bottles at $1 \times 10^{6}$ cells $/ \mathrm{ml}$ concentrations for $24 \mathrm{~h}$ at $37^{\circ} \mathrm{C}$ in a humidified atmosphere of $5 \%$ $\mathrm{CO}_{2}$. The cells were then exposed to silymarin 100 and $50 \mu \mathrm{g} / \mathrm{ml}$. After $24 \mathrm{~h}$ of drug treatment total RNA was extracted from these cells and expression of Bax, Bcl-2 and P53 levels were studied. Further flow cytometric studies in silymarin treated cells were carried out using propidium iodide.

Results: Reverse transcriptase PCR was used to analyze the levels of Bax, Bcl-2 and p53 mRNA levels in HepG2 cells treated with silymarin $(100$ and $50 \mu \mathrm{g} / \mathrm{ml})$. Amplification of Bax, Bcl-2 and $\mathrm{p} 53$ was done with help of specific primers using cDNA as template strand prepared from HepG2 cells. Silymarin down regulated Bcl-2, and up regulated Bax mRNA levels in HepG2, thus inducing apoptosis. No significant change was observed in p53 expression. Further flow cytometric analysis confirmed the significant increase in apoptotic cells after silymarin treatment.

Conclusion: Together, these results identify the ability of silymarin to induce apoptosis against HepG2 cells, which may involve a p53 independent pathway to induce apoptosis. Hence, silymarin merits further investigation to study its anti cancer mechanism.

\section{PP19-14}

The Suppression of gen5 may be a Potential Mechanism for Retrorsine Induced Hepatocytes Cell Cycle Block

H. Sun ${ }^{1}$, Y. Shi ${ }^{1}$, J. Bao ${ }^{1}, \mathrm{H} . \mathrm{Bu}^{2}$

${ }^{1}$ Key Laboratory of Transplant Engineering and Immunology, Ministry of Health, West China Hospital, Sichuan University;

${ }^{2}$ Department of Pathology, West China Hospital, Sichuan University, Chengdu, China

It has been demonstrated that low and long term administration of retrorsine (RS), a member of pyrrolizidine alkaloid family, will block proliferation of hepatocyte even when encounter a strong proliferative stimulus for several months. But the mechanism is still in debate. In our study, we found that the cell cycle progression of mice hepatocytes, which were treated with RS according to the protocol, was arrested in late G1 phase or at G1/S transition after a standard 2/3 partial hepatectomy $(\mathrm{PH})$. In addition, the expression of a significant histone acetyltransferase (HAT)- gen5, which acts as a promotor in the cell cycle progression, was markedly decreased in RS-treated mice compared with the controls. Furthermore, Lysine 14 in histone H3 (H3K14), a gen5 preferential substrate, was hypoacetylated in contrast with the controls during regeneration. In conclusion, the suppressed expression of gcn 5 and a series of consequences may be a potential mechanism for RS induced cell cycle block.

Poster Presentation 20: Molecular and Cellular Biology: Gene Transfer/Therapy

\section{PP20-01}

The Effect of Over Expression of CCAAT/enhancer-Binding Protein on the Metabolism of Human Hepatoma Cell Line HepG2 Cells

Y. Chen, W. Li, H. Zhang, M. Ding, Z. Duan

Artificial Liver Center, Beijing You'an Hospital,Capital Medical

University, Beijing, China

Objective: The CCAAT/enhancer-binding protein (C/EBP) $\alpha$ gene was transferred into human hepatoma cell line HepG2 cells by plasmid vector, and the cells with high expression of $\mathrm{C} / \mathrm{EBP} \alpha$ was selected. In order to explore the different functions of the HepG2-C/ $\mathrm{EBP} \alpha$ cells, the cellular morphology, glyconeogenesis, urea production as well as secretion and intracellular levels of albumin were detected.

Methods: The plasmid of pLHCX-CEBPa was transfected into the HepG2 cells by Liposomes. High expression of $\mathrm{C} / \mathrm{EBP} \alpha$ cells was selected with medium contained of hygromycin. The morphological changes of HepG2 cells were observed by use of phase-contrast microscope; Western blot was used to evaluated the expression of $\mathrm{C} / \mathrm{EBP} \alpha$ protein as well as intracellular albumin protein; The intracellular glycogen was revealed by periodic acid Schiff staining (PAS); The metabolism function of urea synthesis was detected by automatic biochemical detector; The albumin secretion was measured by an enzyme-linked immunosorbent assay (ELISA).

Results: After transfected pLHCX-CEBPa and got positive cells, the western blot result revealed that the $\mathrm{C} / \mathrm{EBP} \alpha$ protein levels were at least twofold higher in HepG2-C/EBP $\alpha$ cells than in HepG2 cells in culture, which suggested that we had successful got C/EBP $\alpha$-positive HepG2 cells. The cultured $\mathrm{C} / \mathrm{EBP} \alpha-\mathrm{HepG} 2$ cells still remained a polygon shape with the cell size smaller than that of the HepG2 cells. Compared with the control cells, the HepG2-C/EBP $\alpha$ cells accumulated more intracellular polysaccharides such as glycogen as revealed by periodic acid Schiff staining (PAS). The urea production were sustained at higher levels in HepG2-C/EBP $\alpha$ cells. However, the albumin production and albumin secretion still remained the same levels in HepG2-C/EBP $\alpha$ cells.

Conclusions: The study succeeded in establishing HepG2 cells with high expression of the C/EBP $\alpha$. The HepG2- C/EBP $\alpha$ cells had an influence on glycogen synthesis as well as urea synthesis. This results provided useful information for artificial liver with HepG2 cells.

\section{PP20-02}

Hepatitis B Virus HBx Protein Deregulates the Expression of MiR-15a/16 in Hepatocellular Carcinoma Cells via HBx-c-Myc- MiR-15a/16 Pathway J. Wang, G. Wu

Department of Hepatobiliary Surgery, Sun Yat-Sen Memorial Hospital of Sun Yat-Sen University, Guangzhou, China

Purpose: Chronic hepatitis B virus (HBV) infection is the main cause of human hepatocellular carcinoma (HCC) in China. HBV encodes 
four virus proteins of which HBx plays a key role in HBV related hepatocarcinogenesis. We postulated that HBx might transform liver cells by altering the expression mode of miRNAs in host cell.

Methods: HCC cell line HepG2 was stably transfected with the pcdna3.1-HBx plasmid which contains the $\mathrm{HBx}$ open reading frame (ORF) (HepG2-hbx) or pcdna3.1 plasmid (HepG2-vc). We used microarray to profile the expression differences $(\uparrow / \downarrow>$ twofold) of miRNAs between HepG2-hbx and HepG2-vc, while the expression of selected miRNAs was detected by qRT-PCR.

Results: $\mathrm{HBx}$ induced widespread down-regulation of miRNAs in HepG2-hbx including miR-15a/16 compared with HepG2-vc in microarray assay, whereas few miRNAs (e.g. miR-21) were up-regulated. Of these, the down-regulation of miR-15a/16 was validated by qRT-PCR in pcdna3.1-HBx plasmid stably/transiently transfected HCC cells (HepG2/Huh7). Silencing of c-Myc by siRNA retrieved the expression of miR-15a/16 in HepG2-hbx, while HBx could significantly up-regulate the expression of c-Myc at both mRNA and protein level in HCC cells and tissues. Ectopic expression of miR-15a/16 mimics suppressed the growth and clonogenicity of HepG2-hbx in vitro.

Conclusion: $\mathrm{HBx}$ can interfere with the expression of miRNAs in HCC cells. The activation of c-Myc by HBx is responsible for the down-regulation of miR-15a/16. Restoring the expression of miRNA$15 \mathrm{a} / 16$ has a potential to be a prospective treatment of HBV infected HCC.

\section{PP20-03}

Construction and Identification for the Recombinant Plasmids to inhibit the Expression of Pokemon Gene

Y. Wu ${ }^{1}$, S.J. Zhang ${ }^{2}$

${ }^{1}$ Hepatobiliary Surgery, ${ }^{2}$ The First Affiliated Hospital of Zhengzhou University, Zhengzhou, China

Background: Proto-oncogen Pokemon is one of the transcriptional inhibition family members of POK. It is not only participate in regulating the cell-cycle and cell apoptosis but also be found the first specific transcript inhibitor of alternative reading frame (ARF) in suppressor gene which excessively express in multiple tumors. As proto-oncogene, the most specific aspect of Pokemon is the function to promote other oncogene. Because of the function in Pokemon during the course of tumorigenesis, it can be regarded to be the new effectively target gene in tumor therapy. In this topic, we successfully constructed the plasmids which can effectively inhibit the expression of Pokemon gene by using the technique of siRNA.

Methods: By using the internet design soft of Ambion company to design two strips siRNA sequences aiming at Pokemon gene, and synthesis two pairs complementory DNA fragments of siRNA. Directional cloning into the sites of BamHIand Hind III of the pSilencer 3.1-H1 neo vector by using the T-4 DNA ligase and directional cloning into the sites of BamHIand EcoRIof the RNAi-Ready pSIREN-RetroQ vector for constructing the recombinant plasmids, separately named as pSliencer- Pok-1, pSliencer-Pok-2, pSIREN-Pok1 and pSIREN-Pok-2. Then transformed into competent cells of escherichia coli DH5 $\alpha$, separately select by using resistance to benzyl chloride and puromycin aminonucleoside, select positive clones to amplify, and these amplified vectors were confirmed by sequencing. Result:

1. Successfully constructed the plasmid pSilencer-Pok-1and pSliencer-Pok-2 which could inhibit the expression of Pokemon protein by using of pSilencer3.1-H1 neo vector.

2. Successfully constructed the plasmid pSIREN-Pok-1 and pSIREN-Pok-2 which could inhibit the expression of Pokemon protein by using of RNAi-Ready pSIREN-RetroQ vector.

3. The positive clones which be selected out are correct through sequencing.
Conclusion: Successfully constructed the plasmid pSIREN-Pok-1 and pSIREN-Pok-2 which could inhibit the expression of Pokemon protein by using of different plasmid vector.

\section{PP20-04}

Induction of Specific Cytotoxic T Lymphocyte Responses against Hepatitis B Virus by Hepatitis B Virus Core Antigen Gene-modified Dendritic Cells C. Huang ${ }^{1}$, L. Zhang ${ }^{1}$, L. Ou-Yang ${ }^{2}$

${ }^{1}$ Infectious Disease; ${ }^{2}$ Experimental Medicine, 1st Affiliated Hospital, Nanchang University, Nanchang, China

Aim: To explore whether hepatitis B virus (HBV) core antigen genemodified dendritic cells (DCs) are able to induce a specific cytotoxic $\mathrm{T}$ lymphocyte (CTL) response.

Methods: The recombinant adenoviruses, $\mathrm{Ad}-\mathrm{HBc}$ (carrying $\mathrm{HBcAg}$ genes), were prepared and transfected into DCs generated from cord blood. The efficacy of transfection was observed through the expression of enhanced green fluorescent protein (EGFP) in DCs and the expression of HBV core antigen were detected by Immunohistochemistry and RT PCR. HBV core antigen gene-modified DCs were co-cultured with $\mathrm{T}$ lymphocytes from cord blood and the proliferation of $\mathrm{T}$ cells were detected using mixed lymphocyte reaction (MLR). Lactate dehydrogenase (LDH) release assay was carried out to assess the killing ability of CTL cells against $\mathrm{HepG}_{2} 22.1 .5$ cells.

Results: HBV core antigen genes were expressed in DCs with a high efficacy by recombinant adenoviral vector. EGFP were observed in $90 \%$ transfected DCs and DCs kept their typical forms after transfection. Above $80 \%$ transfected DCs were expressed for HBcAg. The result of MLR showed that HBV core gene-modified DCs effectively stimulated naive T lymphocytes to proliferate. The specific CTL cells generated by HBV core antigen gene-modified DCs had obvious cytotoxity against $\mathrm{HepG}_{2} 22.1 .5$ cells, and the cytotoxity in Ad-HBc transfection group was significantly higher than that in control group $(P<0.001)$.

Conclusion: HBV core gene-modified DCs are able to effectively stimulate naive $\mathrm{T}$ lymphocytes to proliferate and enhance the specific CTL response at the same time, showing its promising future for developing anti-viral vaccine.

\section{PP20-05}

Effect of siRNA Targeting EphA7 Gene on the Biological Behavior of Hepatocellular Carcinoma

S. Zhang, G. Zhang

The First Affiliated Hospital of Zhengzhou University, Zhengzhou, China

Background: As a key member of the Eph receptor family, EphA7 gene was involved in the early vertebrate embryo development, axon guidance and many important physiological processes. Many recent studies indicate that abnormal expression of EphA7 was found in lung, colon, gastric, prostatic cancers. The purpose of this study was to investigate the changes of proliferation activity, cell cycle and invasive ability of SMMC-7721 hepatoma cell line after transfected with recombinant vector pRNA-siEphA7.

Methods: By using the SMMC-7721 cells transfected with empty vector as control, the pRNA-siEphA7 vector was constructed and transfected into SMMC-7721 hepatoma cells by using liposome. G418 screening method was used to obtain the stable transfection SMMC7721/siEphA7 hepatoma cells. Real-time PCR and Western blot were used to detect the changes of EphA7 gene and protein in the stable transfection SMMC-7721 cells. MTT assay was used to detect the proliferative transfection was detected by flow cytometry assay. The changes of invasion and migration abilities after transfection were measured by Transwell chamber invasion assay.

Results: The expression of EphA7 gene and protein in SMMC-7721 was significantly inhibited after transfected with interference vector pRNA-siEphA7. MTT assay showed that the speed of cell 
proliferation was lower in the transfection group than in the nontransfection and mock-vehicle groups $(P<0.05)$. Flow cytometry results showed that the distribution of the proportion of cells in G1, G2 and S phase are basically similar. Experiments on the in vitro adherency and invasiveness showed the cell adhesion and invasion ability was lower in the transfection group compared to that in the non-transfection and mock-vehicle group $(P<0.05)$.

Conclusion: EphA7 may play an important role in tumor cell proliferation, invasion and metastasis, it may be used in gene therapy for HCC.

\section{PP20-06}

Prolonged Antitumor Effects of Repeated Gene Therapy by Intra-muscular Electroporation in a Woodchuck Model with Hepadnavirus-induced Hepatocelluar Carcinoma

K.-W. Huang

Department of Surgery and Hepatitis Research Center, National

Taiwan University Hospital, Taipei, Taiwan ROC

Purpose: Antiangiogenic gene therapies have proven effective in many implanted hepatocellular carcinoma (HCC) models with small tumor burdens. These models, however, may not realistically reflect human HCCs, which are typically large, multifocal, and associated with viral hepatitis. This study utilized woodchucks which bore spontaneously-occurring HCCs induced by woodchuck hepatitis virus infection to investigate the efficacy of a repeated gene therapy strategy.

Experimental design: Adenoviruses encoding antiangiogenic factors (pigment epithelium-derived factor and endostatin) were delivered into woodchuck livers bearing HCCs via the intrahepatic artery and then the followed gene delivery via intramuscular electroporation every weeks for 10 weeks.

Results: Monotherapy with antiangiogenic or cytokine genes exhibited therapeutic effects on woodchuck HCCs. Yet, the repeated gene therapy strategy which contained antiangiogenic gene therapy via adenoviral vector and via repeated intra-muscular electroporation displayed significantly prolonged antitumor effect than either monotherapy. Mechanistic analysis revealed that the combined therapies induced more significant reduction of tumor vasculature than monotherapy by adenoviral vector. The antiangiogenic effect will be kept during the course of repeated gene therapy by electroporation. The hepatitis status of woodchucks during treatment did not show any flare up, the tolerance to repeated gene therapy is fair.

Conclusions: Combination of two gene therapies by viral delivery and followed electroporation could elicited prolonged antitumor effects than monotherapy via viral delivery alone.

\section{PP20-07}

Truncated Receptor Type II TGF- $\beta$ Decreases Hepatic Fibrosis and Steatosis Modulating Gene Expression of TGF- $\beta$, Col-1, PAI-1 and Cannabinoid Receptors

M.G. Mena-Enriquez ${ }^{1}$, A.S. Sandoval-Rodriguez ${ }^{1}$, J. Santillan-Montes ${ }^{1}$, J. Armendariz-Borunda ${ }^{1,2}$

${ }^{I}$ Institute of Molecular Biology in Medicine and Gene Therapy/ Universidad de Guadalajara; ${ }^{2}$ O.P.D. Hospital Civil de Guadalajara 'Juan I. Menchaca', Guadalajara, Mexico

Introduction: Transforming growth factor $\beta$ (TGF- $\beta$ ) is the main profibrogenic cytokine; consequently strategies to block its signal pathway have been developed. Gene therapy using a truncated type II receptor for TGF- $\beta$ (T $\beta$ RII $\Delta$ cyt) had showed to reduce hepatic fibrosis; but its effect on the expression of cannabinoid receptors (CB1 and CB2) which participate in the progression of liver cirrhosis and steatosis has not been elucidated.

Objective: Evaluate Ad-T $\beta$ RII $\Delta$ cyt treatment on fibrosis, steatosis and gene expression of cannabinoid receptors and profibrogenic molecules in the liver.
Material and Methods: Cirrhosis was induced administrating $\mathrm{CCl}_{4}$ for 8 weeks. Cirrhotic rats were divided into groups $(n=6)$, control, therapeutic gene (Ad-T $\beta$ RII $\Delta$ cyt) and irrelevant gene (Ad-GFP). At fourth week of $\mathrm{CCl}_{4}$ intoxication, $2 \times 10^{10} \mathrm{pi} / \mathrm{kg}$ of Ad-T $\beta \mathrm{RII} \Delta$ cyt or Ad-GFP was administrated via iliac vein. Animals were sacrificed at day 2, 3 and 28 after treatment. Fibrosis index, steatosis, hydroxiproline content, gene expression of Col-1, TGF- $\beta$, PAI-1, CB1 and CB2 was analyzed, as well as, ALT and AST serum levels.

Results: Treatment with Ad-T $\beta$ RII $\Delta$ cyt decreased at day $28 ; 35 \%$ of fibrotic tissue and $42 \%$ of liver steatosis $(p<0.001)$; also suppressed the expression of profibrogenic molecules: TGF- $\beta$ (11-fold), Col-1 (5fold) and PAI-1 (19-fold) at day 3 compared to controls $(p<0.05)$. At day 2, mRNA levels of CB1 (profibrogenic) decreased, just as, CB2 mRNA increased (anti-fibrogenic); although, CBs changes are not statistically significant. Hydroxiproline content decreased threefold $(p<0.01)$; AST $22 \%$ and ALT 37\% $(p<0.05)$ at day 28 in the Ad-T $\beta$ RII $\Delta$ cyt group compared to control group.

Conclusion: Expression of TGF- $\beta$-truncated receptor II prevents fibrosis progression and improves liver function by lowering gene expression of profibrogenic molecules (TGF- $\beta$, Col-1, PAI and CB1). Nevertheless, cannabinoid receptors expression is not significantly affected; a reduction in steatosis is also observed when Ad-T $\beta$ RII $\Delta$ cyt therapy was employed in this experimental model of cirrhosis.

\section{PP20-08}

Silencing of Gli2 Sensitizes the Human Hepatocellular Carcinoma Cells to TRAIL-induced Apoptosis

T. Chen, J. Liu, Y. Wang, J. Chen

Hepatobiliary Surgery, Sun Yat-Sen Memorial Hospital, Sun Yat-Sen University, Guangzhou, China

Background: Recent reports have demonstrated that many tumor cells, including human hepatocellular carcinoma (HCC) cell, acquire resistance to the apoptotic effects of TRAIL chemotherapy and that this resistance appears to be closely related to the deregulated expression of anti-apoptotic molecules.

Aims: To investigate the chemosensitivity of Gli2 knockdowned HCC cell and the possible mechanism.

Materials and methods: The components of the Hh pathway were investigated by RT-PCR and western blot. Vectors expressing shRNA targeting Gli2 mRNA were constructed by gene recombination, and transfected into HCC. Cell proliferation in TRAIL was determined by MTT and colony formation assays. Apoptosis was measured by flow cytometry and analyzed by caspase enzymatic assays.

Results: Ptc1, Smo, Gli1 and Gli2 expression levels in HCC were much higher than in the normal human liver cell. HCC cells transfected with Gli2 shRNA had significantly lower rates of cell proliferation and colony formation activity compared to controls. The transfected cells were susceptible to TRAIL with decreased $50 \%$ inhibition concentration. Bcl-2 and c-FLIP levels, and the activities of caspase-3, -8, and -9 were all decreased.

Conclusions: Knockdown of Gli2 sensitizes HCC cell to chemotherapy. Silencing of Gli2 combined with TRAIL chemotherapy enhanced caspase-mediated apoptosis.

\section{PP20-09}

The Novel Gene KCTD9 Contributes to Immunologically Mediated Liver Injury through NK Cell Activation in Virus Induced Liver Failure

T. Chen, Y. Zhou, L. Zhu, B. Pi, X. Liu, G. Deng, R. Zhang, Y. Wang, Z. Wu, M. Han, X. Luo, Q. Ning

Tongji Hospital, Huazhong Science and Technology University, Wuhan, China

Acute liver failure (ALF) or acute on chronic liver failure (ACLF), mainly caused by hepatitis virus (especially HBV) infection in China, exhibits rapid progression, poor clinical prognosis and high mortality, while immune response mediated injury plays a pivotal role during 
the process. Gene differential expression analysis based on microarray technology showed that KCTD9 gene had a notably higher expression in PBMCs from HBV-ACLF patients in comparison with asymptomatic HBV infection. The up-regulated KCTD9 gene expression was elucidated to be in both liver tissues and PBMCs in amplified cases with HBV-ACLF at both mRNA and protein levels. The extraordinary elevated expression of KCTD9 was found in peripheral and hepatic NK cells of patients with HBV-ACLF, and the percentage of peripheral NK cells expressing KCTD9 correlated with the severity of liver injury in the patients as displayed by their positive correlation with ALT and AST levels. NK92 cells with overexpressed KCTD9 exhibited marked increase in CD69 expression and cytotoxicity, furthermore a significantly decrease of NKG2A receptor, suggesting the involvement of KCTD9 in NK cell activation in vitro. An abundant expression of KCTD9 in hepatic NK cells was also observed in Balb/cJ mice with MHV-3 induced fulminant viral hepatitis (FVH). A shRNA specifically targeting KCTD9 significantly inhibited KCTD9 expression in vitro and in vivo, and ameliorates MHV-3 induced FVH in Balb/cJ mice with an increased survival rate from 0.0 to $22.2 \%$, improvement of liver function and histopathology. In contrast, delivery of KCTD9 expression plasmid to MHV-3 infected mice leads to rapid progression of liver disease as displayed by shortening of survival time, acceleration of liver function and histopathology. In conclusion, our study firstly described the novel KCTD9 gene contributes to immune mediated liver injury through NK cell activation in human and mice hepatitis virus induced liver failure.

\section{PP20-10}

Induction of WHs/cAg Specific $T$ cell Responses by DNA Vaccine in the Newly Constructed WHV Transgenic Mouse Model

Z. Meng ${ }^{1,2}$, E. Zhang ${ }^{1}$, X. Zhang ${ }^{1}$, A. Kosinska ${ }^{1}$, Y. Xu ${ }^{3}$, D. Yang ${ }^{4}$, T. Zhou ${ }^{5}$, W. Mason ${ }^{5}$, M. Roggendorf ${ }^{1}$, M. $\mathrm{Lu}^{1}$

${ }^{1}$ Institute of Virology, University Hospital of Essen, University

Duisburg-Essen, Essen, Germany; ${ }^{2}$ Department of Infectious

Diseases, Taihe Hospital, Hubei Medical University, Shiyan;

${ }^{3}$ Department of Microbiology, Tongji Medical College, Huazhong

University of Science and Technology; ${ }^{4}$ Division of Clinical

Immunology, Tongji Hospital, Tongji Medical College, Huazhong

University of Science and Technology, Wuhan, China; ${ }^{5}$ Fox Chase

Cancer Center, Philadelphia, PA, USA

The woodchuck model is informative for studies of hepadnaviral infection and pathogenesis, antiviral drugs, and hepatocellular carcinoma. However, immunological studies are challenging due to a lack of reagents to identify components of the woodchuck immune response. In this present work, we attempted to establish a complement to the woodchuck model: two lineages of WHV transgenic ( $\mathrm{tg}$ ) C57BL/6 mice, 1217 (with a WHV wild type transgene) and 1281 (with a WHsAg minus WHV transgene). These transgenes transcribed WHV RNAs (3.7 and $2.1 \mathrm{~kb}$ ) and produced WHV replicative intermediates in the liver. Lineage 1217 mice, as expected, secreted progeny virus into the serum. In both WHV-tg lineages, male mice harbored higher levels of WHV replication intermediates than females, consistent with gender-dependent hepadnaviral replication. Mice of lineage 1281 developed significant levels of anti-WHc of subtype $\operatorname{IgG} 2 \mathrm{a}$, the frequency increasing with age. In addition, WHcAg-specific CTLs were detected in 6 of 35 mice of this lineage. DNA-based vaccination with WHsAg and WHcAg were tested in both lineages. Anti-WHs was induced by vaccination with pWHsIm only in lineage 1281 but not in lineage 1217, suggesting that mice of wtWHV lineage 1217 have B cell tolerance to WHsAg. Surprisingly, WHsAg-specific CTLs were induced in the 1217 mice. In addition, immunization with pWHcIm induced high level of anti-WHc as well as WHcAg-specific CTLs in 1217 mice. Thus, a DNA vaccine was able to break significant components of the putative WHV-specific immune tolerance in these mice. Vaccine-induced immune responses did not lead to a reduction of WHV replication; analyses of immunopathogenesis are ongoing. Taken together, our results in the newly established WHV tg mice show a promising effect of DNA vaccine, inspiring further analysis of therapeutic vaccination against hepadnaviral infection. The spontaneous development of autoimmunity to WHcAg in the 1281 line of $\mathrm{tg}$ mice may also warrant further investigation.

\section{PP20-11}

Matrine Enhances Protective Effect on Hepatocellular Oxidative Stress via Upregulation of Liver Fatty Acid Binding Protein (L-FABP)

Z.-L. Ai ${ }^{1,2}$, S.-K. Yao ${ }^{1}$

${ }^{1}$ Department of Gastroenterology, China-Japan Friendship Hospital, Ministry of Health; ${ }^{2}$ Graduate School, Peking Union Medical College and Chinese Academy of Medical Sciences, Beijing, China

Introduction: L-FABP has an important function as an effective endogenous cytoprotectant against oxidative stress. Moreover, clofibrate treatment can improve the expression of L-FABP to protect hepatocytes from oxidative stress. However, whether other drugs also possess such a characteristic is not known yet. We have always been focused on matrine, which has a wide range of pharmacological actions.

Aim: To investigate effects of matrine on L-FABP's antioxidative property.

Methods:

(1) After HepG2 cell line was treated with matrine for 24, 48 and $72 \mathrm{~h}$, we employed MTT assay to detect cell viability.

(2) Based on MTT assay, cells were treated with three different doses of matrine $(0.4,0.8$ and $1.6 \mathrm{mg} / \mathrm{ml})$ for 24,48 and $72 \mathrm{~h}$ before exposed to $400 \mu \mathrm{M} \mathrm{H}_{2} \mathrm{O}_{2}$ for $20 \mathrm{~min}$. Then, reactive oxygen species (ROS) assay was evaluated the optimal dose of matrine.

(3) We applied RT-PCR and western bloting to explore the influence of matrine on the expression level of L-FABP.

\section{Result:}

(1) MTT assay showed matrine treatment under $1.6 \mathrm{mg} / \mathrm{ml}$ for 24 , 48 and $72 \mathrm{~h}$ was feasible $(p<0.05)$.

(2) ROS assay showed dichlorofluorescin in all matrine-treated cells after stress was significantly reduced $(p<0.05)$. Moreover, $0.8 \mathrm{mg} / \mathrm{ml}$ matrine treatment for $48 \mathrm{~h}$ was the optimal condition against ROS generation $(p<0.05)$.

(3) RT-PCR and western bloting both indicated $0.8 \mathrm{mg} / \mathrm{ml}$ matrine treatment could increase the level of L-FABP expression under the oxidative stress condition $(p<0.05)$.

Conclusion: Matrine is able to enhance L-FABP expression, and therefore, protects hepatocytes from oxidative stress.

\section{PP20-12}

Effects on the Tumor Cell Growth in Hepatocellular Carcinoma Cell Lines after Inhibition of HIF Dimerization

S.H. Choi ${ }^{1}$, M.S. Lee ${ }^{2}$, J.Y. Park ${ }^{2,3}$, D.Y. Kim ${ }^{2,3}$, S.H. Ahn ${ }^{2,3}$, W.S. Ro ${ }^{1,2}$, K.-H. Han ${ }^{1,2,3}$

${ }^{1}$ Brain Korea 21 Project for Medical Science; ${ }^{2}$ Department of Internal Medicine; College of Medicine, Yonsei University, ${ }^{3}$ Yonsei Liver Cancer Special Clinic, Yonsei University Health System, Seoul, Republic of Korea

Objectives: HIF-1 $\beta$ (ARNT) formed dimerization of HIF- $1 \alpha$, the various aspects of cancer biology, including proliferation, survival, angiogenesis, and extracellular matrix metabolism, under hypoxia. This study evaluated whether small interference HIF-1 $\beta$ (siHIF-1 $\beta$ ) inhibited dimerization with HIF- $1 \alpha$ and repressed tumor cell growth in the hepatocellular carcinoma (HCC) cell lines. 
Method: HCC cell lines (HepG2 and Hep3B) were treated with HIF$1 \beta$ coding siRNA and cultured under hypoxic conditions $\left(1 \% \mathrm{O}_{2}\right.$, $24 \mathrm{~h}$ ). Following treatment, the expression levels of HIF- $1 \alpha$, HIF- $1 \beta$ and growth related factors were examined through real-time quantitative PCR and immunoblot assay, while tumor growth was measured by cell proliferation assay (MTT assay).

Results: The inhibition of HIF- $1 \beta$ by small interference RNA (siHIF$1 \beta$ ) led to the day dependent repression of tumor cell growth through inhibition of HIF dimerization under hypoxia incubation. Inhibition of HIF dimerization through treatment of siHIF- $1 \beta$ also regulated the expression of tumor growth related factors (VEGF, EGF and HGF). Tumor cell motlility was inhibited by treatment of siHIF- $1 \beta$ under hypoxia.

Conclusions: These data suggest that siHIF- $1 \beta$ inhibit dimerization of HIF under hypoxia, and Inhibition of HIF- $1 \beta$ decreased survival ratio and tumor cell growth in HCC cell lines under hypoxia.

\section{Poster Presentation 21: Molecular and Cellular Biology: Liver Regeneration}

\section{PP21-01}

Liver Injury at Crush Syndrome and the Role of Proline Rich Peptide as a Protector

G.R. Karapetyan, N.S. Kukurtchyan, G.A. Kevorkian

Department of Pathological Biochemistry and Radioisotopes,

H. Buniatian Institute of Biochemistry Natl. Acad. of Sci of RA,

Yerevan, Armenia

At Crush Syndrome (CS) develop the immobilization stress which is activated by toxic peptides formed in ischemic muscle. Toxemia is one of the factors lead to hepatocytes injury and possible early using of antioxidants decrease disease progression at CS. Proline Rich Peptide (PRP-1) hinder proapoptotic capasas 3 and 9, stimulate the immunocompetent cells and held a neuroprotective activity against many toxins formed in organism. The aim of this work was to study CS earlier period influence on liver tissue and PRP-1 administration effect as a protector.

As a material for this study was used liver of 160-200 $\mathrm{g}$ white rats of "Wistar" line. Crush syndrome was produced by compression of femoral soft tissues using a special press with a compression force $100 \mathrm{~kg} / \mathrm{kg}$ during $2 \mathrm{~h}$. Animals were divided into three groups:

I: $2 \mathrm{~h}$ compression,

II: $4 \mathrm{~h}$ decompression and

III: injection of $10 \mathrm{mkg}$ PRP-1 before decompression.

At the end of experiment small pieces of liver were put in cold solution of parapharmaldehid and then in an osmium tetraoxide. Further treatment of material was performed by traditional method used in transmission electron microscopy.

At $2 \mathrm{~h}$ compression no ultrastructural changes of organelles were observed however catecholamine level increasing in blood leads to its physiological statement changes as an adaptation to stressors influence. At $4 \mathrm{~h}$ decompression under the influence of toxins take place some destructive changes of organelles which signalize about apoptosis develop processes. At PRP-1 administration membrane structures of cell do not lose their safeness and are close to intact rat.

PRP-1 administration before decompression lead to neutralization of toxic peptides formed by ischemic muscle and decreases the catecholamine influence on liver ultrastructure.
PP21-02

Analysis of Gene Transcription Regulation in Mouse Liver Regeneration by Partial Hepatectomy

Z.-H. Tang ${ }^{1}$, Z.-W. Quan ${ }^{1}$, Y.-B. Liu ${ }^{1}$, W.-P. Zhang ${ }^{2}$

${ }^{1}$ General Surgery, Xinhua Hospital, School of Medicine, Shanghai

Jiaotong University; ${ }^{2}$ Pathophysiology, Second Military Medical

University, Shanghai, China

Background and aims: Transcription factor play a important role in liver regeneration but its mechanism is not very clear. Liver regeneration can be induced by surgical resection, liver transplantation and chemical medicine. Partial hepatectomy (PHx) in rodents is the main paradigm of studying liver regeneration, but there are fewer literatures about mouse liver anatomy and resection technique in this field. The aim of this study is to explore the traits of mouse anatomy, establish the mouse liver regeneration model by $\mathrm{PHx}$ and analyze the transcription factor mRNA expression changes during liver regeneration. Methods: Adult C57BL/6 mice were utilized by hepatic perfusion technique to study the liver anatomy. Three anterior liver lobes equivalent to $68 \%$ of the mice liver weight were resected to establish liver regeneration model. The remains of liver after $\mathrm{PHx}$ were removed at $0,0.5,1,2,4,24,48,72 \mathrm{~h}$ time points . Total RNA of liver tissue was extracted and Real-time PCR was performed to analyze the gene expression changes of c-myc,AFP,GPC3,ZHX2,DPZF during liver regeneration.

Results: The liver regeneration model in mice with PHx was established successfully. C-myc mRNA started to increase at $0.5 \mathrm{~h}$ time point after PHx, peaked at $4 \mathrm{~h}$ and recovered to normal expression level at $24 \mathrm{~h}$. AFP mRNA increased gradually at $24 \mathrm{~h}$ and GPC3 mRNA increased at $48 \mathrm{~h}$. ZHX2 and DPZF mRNA declined at $24 \mathrm{~h}$ and recovered to nomal expression level at $72 \mathrm{~h}$.

Conclusions: As a immediate-early transcription factor, $\mathrm{C}$-myc gene participates in the initial phase of liver regeneration. AFP and GPC3 gene may have the effect in the DNA synthesis phase and make positively regulation in cell proliferation. Transcription repressor gene ZHX2 and DPZF have the inhibitory action on the mRNA expression of AFP,GPC3 during liver regeneration after PHx.

\section{PP21-03}

Liver Regeneration: Potential Roles of Mesenchymal Stem Cells and Toll Like Receptors

H. Kocak, I.F. Tas, Z. Tokcaer-Keskin, I. Gursel, K.C. Akcali

Molecular Biology and Genetics, Bilkent University, Ankara, Turkey

Background and aim: Liver cell transplantation is a powerful alternative to orthotopic cell transplantation in the treatment of liver failures. Recently considerable effort is being channeled to understand the nature, kinetics and directing stem cells to effectively accumulate at the regenerating liver site. Mesenchymal stem cells are one of the promising cell sources modulating liver regeneration process. Present work was designed to study how mesenchymal stem cells might modulate liver immune behaviors by changing TLR expression and increase regenerative potential during liver regeneration in rats.

Methods: Normal and partially hepatectomized rats were treated with mesenchymal stem cells isolated and expanded from rat bone marrows. Accumulation of mesenchymal stem cells was confirmed by RT-PCR, FACS, and IF.

Results: Our data showed that mesenchymal stem cells expressed several TLRs, and their accumulation during regeneration was depended on the timing of injury. Mesenchymal stem cells isolated from bone marrow of normal rats were observed at the injured liver 
3 days after the injection. There were no labeled mesenchymal stem cells in the liver sections of uninjured animals. Mesenchymal stem cells administration significantly altered the expression of TLR2, 3 and 9 while retaining their migration potential to regenerating liver. Conclusion: Our findings implicated that mesenchymal stem cells administration during liver regeneration modulate the immune response through changing the expression of the TLRs in remaining livers into which they are recruited or infused. This alteration may contribute to the regeneration process following partial hepatectomy.

\section{PP21-04}

Immunophenotype of Ductular Reaction in Human Livers with HCV E. Prakoso ${ }^{1,2}$, D. Bowen ${ }^{1,2}$, J. Kench ${ }^{3}$, A. Clouston ${ }^{4}$, A. Lee ${ }^{1}$, G. McCaughan ${ }^{1,2}$, N. Shackel ${ }^{1,2}$

${ }^{1}$ Liver Immunobiology Laboratory, Centenary Institute; ${ }^{2} A W$ Morrow Gastroenterology \& Liver Centre; ${ }^{3}$ Department of Anatomical Pathology, Royal Prince Alfred Hospital, Sydney, NSW; ${ }^{4}$ Envoi Pathology, Brisbane, QLD, Australia

Background/aim: The phenotype of the ductular reaction (DR) in human is still controversial. We aimed to characterize DR immunophenotype in human livers with HCV.

Methods: Four-marker immunolabelling, confocal microscopy and 3-dimension image analysis were utilized to assess DR immunophenotype in human livers with HCV and donor livers.

Results: The DR was not present in the donor livers $(n=3)$. In HCV livers $(n=3)$, DR was seen radiating from the portal tract extending into the proximate third of the hepatic lobule consistent with the location of Canal of Hering $(\mathrm{CoH})$. The dominant DR were irregular strings of $\mathrm{ck} 7+/ \mathrm{ck} 19+/ \mathrm{ck} 18+/ \mathrm{EpCAM}+/ \mathrm{NCAM}+$ cholangiocyte-like cells with poorly defined lumens, which either communicated with bile ductules/canaliculi (ck7+/ck19+/ck18+/EpCAM+/NCAM-), or in continuation with hepatocyte plates in the periportal area. With the latter, NCAM disappeared first followed by ck19 and as the cells acquired the morphology of mature hepatocytes, ck7 became weaker then disappeared and EpCAM switched from cytoplasmic to membranous pattern. These clusters of membranous EpCAM+ cells with or without weak $\mathrm{ck} 7+$ in the periportal area were Albumin+ and defined intermediate hepatocytes. A gradient of intermediate hepatocytes periportally and more mature hepatocytes pericentrally was observed consistent with the 'streaming liver' hypothesis. Additionally, small triangular single cells with ck7+/ck19-/ck18-/EpCAM-/ NCAM- were observed and these probably represented the true hepatic progenitor cells (HPCs). However, ck19+/ck7-/ck18-/EpCAM- cells were not seen. AFP expression was consistently negative. Finally, the DR did not co-localize with $\alpha$-SMA or Thy- 1 although a very close spatial relationships was demonstrated with such cells.

Summary/conclusions: In HCV livers, DR consists of proliferating HPCs, which reside in the $\mathrm{CoH}$ and is not present in the normal livers. DR does not co-localize with fibrosis. Further studies are warranted to investigate the role of the DR in pathogenesis of HCV-related liver injury and regenerative responses.

\section{PP21-05}

Altered Microcirculatory Blood Flow is Associated with Delayed Liver Regeneration in TLR4-mutant Mice

M. Marlini ${ }^{1,2}$, A. Mabuchi ${ }^{1}$, B. Mallard ${ }^{1}$, M.N. Hairulhisyam ${ }^{2}$, A. Wheatley ${ }^{3}$ ${ }^{I}$ Department of Physiology, University of Otago, Dunedin, New Zealand; ${ }^{2}$ Department of Medical Sciences, Faculty of Medicine and Health Sciences, Islamic Science University of Malaysia, Kuala Lumpur, Malaysia; ${ }^{3}$ Department of Physiology, School of Medicine, National University of Ireland, Galway, Ireland

Background and aims: Haemodynamic events and the changes to hepatic blood flow are the least studied and least understood aspects in liver regeneration. It has been previously reported that liver regeneration is delayed in $\mathrm{C} 3 \mathrm{H} / \mathrm{HeJ}$ mice, a mouse that is hypo- responsive to lipopolysaccharide (LPS), due to a point mutation in trl4 gene. Using IVFM, we aimed to determine whether delayed liver regeneration in these TLR4-mutant mice was associated with altered hepatic microcirculation during liver regeneration.

Methods: The hepatic microcirculation of the regenerating liver of $\mathrm{C} 3 \mathrm{H} / \mathrm{HeN}$ or $\mathrm{C} 3 \mathrm{H} / \mathrm{HeJ}$ mice was studied using intravital fluorescence microscopy (IVFM), at days 3 and 7 after hepatectomy. All data were analysed using one way analysis of variance (ANOVA) with Dunnett's and/or Bonferroni's post-hoc tests. $P$ value $<0.05$ was considered statistically significant.

Results: Liver mass restoration was significantly delayed in $\mathrm{C} 3 \mathrm{H} / \mathrm{HeJ}$ mice at all time points after $\mathrm{PH}$ compared to $\mathrm{C} 3 \mathrm{H} / \mathrm{HeN}$ mice. At day 3 after $\mathrm{PH}$, sinusoidal red blood cell (RBC) velocity increased by $100 \%$ in $\mathrm{C} 3 \mathrm{H} / \mathrm{HeN}$ but only by $40 \%$ in $\mathrm{C} 3 \mathrm{H} / \mathrm{HeJ}$ mice $(p<0.001)$. Estimated sinusoidal blood flow increased by 50 and $70 \%$ at days 3 and 7 after $\mathrm{PH}$ respectively in $\mathrm{C} 3 \mathrm{H} / \mathrm{HeN}$ mice. In contrast, estimated sinusoidal blood flow was similar to the control group in $\mathrm{C} 3 \mathrm{H} / \mathrm{HeJ}$ mice at day 3 and was lower by $20 \%$ at day 7 after PH. Sinusoidal diameter and functional sinusoidal density were reduced by $20 \%$ at day 3 post-hepatectomy in both strains.

Conclusions: The present study indicates that hepatic blood flow might play a pivotal role in liver regeneration. Altered sinusoidal blood flow and velocity in TLR4-mutant mice may contribute to the delay in regenerative response in these mice.

Acknowledgement Study supported by a grant from the Royal Society of New Zealand Marsden Fund

\section{PP21-06}

Epigenetic Control of Hepatic Gene Expression Induced by Oxidative Stress

E.R. Gracía-Trevijano ${ }^{1}$, E. Fernández ${ }^{2}$, J.R. Viña ${ }^{1}$, L. Torres $^{3}$

${ }^{1}$ Biochemistry and Molecular Biology, Universidad de Valencia;

${ }^{2}$ INCLIVA, Valencia; ${ }^{3}$ Biochemistry and Molecular Biology,

Universidad de Valencia, Burjassot, Spain

The study of the molecular mechanisms involved in the regulation of c-myc expression has been of special relevance. We show in experimental models of liver regeneration and cirrhosis the important role played by Id 2 as part of a repressor complex on c-myc promoter that affects chromatin structure. On quiescent hepatocytes the complex Id2/Sin3A (HDAC)/ E2F4/p130 remains bound to the c-myc promoter repressing its expression. Upon stimulation, Id 2 and Sin $3 \mathrm{~A}$ are released from the promoter, inducing c-myc expression. Moreover, since Id 2 expression is induced by c-Myc, we suggest a possible regulatory loop between both c-myc and Id2 Fluctuations in GSH content have been demonstrated to be involved in many of the processes where c-myc is over-expressed. We show the effect of in vivo GSH depletion by BSO on the activity of general components of chromatin remodeling complexes such as $\mathrm{Sin} 3 \mathrm{~A}$ or $\mathrm{CBP} / \mathrm{p} 300$ affecting $\mathrm{c}-\mathrm{Myc}$ and Id2 expression. We explore further the molecular mechanisms for the effect of GSH on Id2 gene expression in a pathological model of acetaminophen-induced toxicity. Id 2 was repressed in the liver of rats after acetaminophen overdose. We propose a novel mechanism for Id2 repression mediated by the proteasome pathway and protection by $N$-acetylcysteine. Since both Id 2 and c-Myc take part of the transcriptional regulatory pathway of multiple genes, the significance of the mechanism proposed here, might be extended to the modulation of other genes. We performed genome-wide ChIP on-chip experiments with antibodies specific for mouse Id2 and E2F4 in liver samples under basal conditions. Analysis of preliminary data for each factor and a comparison of Id2 and E2F4 target genes indicated a limited overlap of 274 target genes for both factors. These data confirm the existence of other Id2/E2F4-target genes that might be modulated by the same mechanism as c-myc.

Acknowledgments: FIS PS09/0236, GVPROMETEO2010-075 


\section{PP21-07}

Role of Hepatocyte Growth Factor Activator in Liver Biopsies of Egyptians with Hepatitis $\mathrm{C}$ and Non Hepatitis C-related Chronic Hepatitis

D.M. Taie ${ }^{1}$, N. Ehsan ${ }^{2}$, D. Maher ${ }^{2}$, R. Samaka ${ }^{1}$, H. Aiad ${ }^{1}$, M. Kandil ${ }^{1}$

${ }^{1}$ Department of Pathology, National Liver Institute and Faculty of

Medicine, Menoufiya University; ${ }^{2}$ Department of Pathology, National

Liver Institute, Menoufiya University, Ashmoon, Egypt

Background: Egypt has the highest prevalence of Hepatitis C Virus (HCV) infection in the world, with the eventual development of chronic hepatitis, cirrhosis and hepatocellular carcinoma (HCC). Hepatic repair after injury requires fragmentation of scar, resolving vascular derangements and parenchymal regeneration. Hepatocyte growth factor activator (HGFA) plays a potent role in hepatic repair after injury.

Objectives: To investigate tissue expression of HGFA from patients with HCV and HBV-related chronic hepatitis and to correlate its expression with the grade of activity, stage of fibrosis and tissue transforming growth factor beta1 (TGF- $\beta 1$ ).

Methods: Paraffin embedded sections prepared from 31 liver biopsies of patients with HCV ( $41 \pm 8$ years), 23 liver core biopsies obtained from patients with chronic hepatitis negative for $\mathrm{HCV}$ infection $(17.5 \pm 15)$ and 20 normal subjects, donors for liver transplantation ( $29 \pm 7.5$ years) were evaluated for grading and staging of chronic hepatitis and applied for immunostaining with HGFA and TGF- $\beta 1$ antibodies. Semiquantitative analysis of immunoreactive cells ${ }^{\prime}$ intensity and percentage for HGFA and TGF $\beta 1$ were interpreted to calculate the $\mathrm{H}$-score.

Results: Liver tissues from patients with $\mathrm{HCV}$ and non-HCV infection revealed HGFA around sites of injury and regeneration. HGFA $\mathrm{H}$-score correlated significantly with the grade of activity, stage of fibrosis ( $p<0.01$ each) and demonstrated a highly statistical significa.

\section{PP21-08}

TEC Protein Tyrosine Kinase is Involved in the Erk Signaling Pathway Induced by HGF

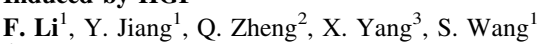

${ }^{1}$ Pathophysiology, Anhui Medical University, Hefei, China;

${ }^{2}$ Rush University Medical Center, Chicago, IL, USA; ${ }^{3}$ Academy of Military Medical Sciences, Beijing, China

Background/Aims: TEC, a member of the TEC family of nonreceptor type protein tyrosine kinases, has recently been suggested to play a role in hepatocyte proliferation and liver regeneration. This study aims to investigate the putative mechanisms of TEC kinase regulation of hepatocyte differentiation, i.e. to explore which signaling pathway TEC is involved in, and how TEC is activated in hepatocyte after hepatectomy and hepatocyte growth factor (HGF) stimulation.

Methods: We performed immunoprecipitation (IP) and immunoblotting (IB) to examine TEC tyrosine phosphorylation after partial hepatectomy in mice and HGF stimulation in WB F-344 hepatic cells. The TEC kinase activity was determined by in vitro kinase assay. Reporter gene assay, antisense oligo nucleotide, TEC dominant negative mutant $\left(\mathrm{TEC}^{\mathrm{KM}}\right)$, and specific kinase inhibitors were used to examine the possible signaling pathways in which TEC is involved. The cell proliferation rate was evaluated by ${ }^{3} \mathrm{H}-\mathrm{TdR}$ incorporation.

Results: TEC phosphorylation and kinase activity were increased in $1 \mathrm{~h}$ after hepatectomy or HGF treatment. TEC enhanced the activity of Elk and serum response element (SRE). Inhibition of MEK1 suppressed TEC phosphorylation. Blocking TEC activity dramatically decreased the activation of Erk. Reduced TEC kinase activity also suppressed the proliferation of WB F-344 cells. These results suggest that TEC is involved in the Ras-MAPK pathway and acts between MEK1 and Erk.

Conclusions: TEC promotes hepatocyte proliferation and regeneration and is involved in HGF-induced Erk signaling pathway.
PP21-09

Predictors of Hepatocyte Proliferative Activity in Chronic Hepatitis B \& C Versus Steatohepatitis as Assessed by the Monoclonal Antibody MIB1-KI67

M. Elbendary ${ }^{1}$, H. Elalfy ${ }^{1}$, K. Zalata $^{2}$

${ }^{1}$ Endemic Medicine; ${ }^{2}$ Pathology, Mansoura University, Mansoura, Egypt

Background: Chronic hepatitis is characterized by increased regenerative cell proliferation, a process that makes cells more susceptible to gene mutations and development of HCC. Evaluation of the proliferative index could be a useful tool for identifying patients at risk for $\mathrm{HCC}$.

Aim of the work: The current study was planned to evaluate the hepatocyte proliferation in predominant causes of chronic liver disease in attempt for investigating predictors of proliferation.

Subject and methods: This study included 84 patients with chronic liver diseases, they were classified into three groups; chronic hepatitis C (50 patients), NASH (20 patients) and chronic hepatitis B (14 patients). All cases were investigated by liver function tests PCR $\mathrm{HCV}$ and HBV, routine abdominal ultrasound and liver biopsy with detection of the proliferative index using the monoclonal antibody MIBI-Ki67.

Results: The proliferative index was significantly higher in chronic hepatitis $\mathrm{C}$ group than chronic hepatitis $\mathrm{B}(P$ value $=0.007)$. There were significant correlations of the Ki-67 index in both zone 1 \& zones (2 and 3) with ALT, AST, and HAI score. Using the multiple regression analysis on the variables affecting proliferation, it was found that predictors of zone 1 proliferation were the following variables, ALT, age, AST and etiological factor in that order.

Conclusion: HCV etiology had significantly higher proliferation index while NASH had the least index. Increased HAI score is associated with higher proliferative index in either zone1 or zones 2 and 3. Predictors of proliferation index in zone 1 were ALT, age, AST and etiological factor.

PP21-10

SOCS3 Gene Is Not Regulated by its Promoter Methylation during Liver Regeneration after Partial Hepatectomy

J. Xia ${ }^{1}$, H. Sun ${ }^{1}$, Y. Shi ${ }^{1}$, H. Bu ${ }^{2}$

${ }^{1}$ Key Laboratory of Transplant Engineering and Immunology, Ministry of Health, West China Hospital, Sichuan University;

${ }^{2}$ Department of Pathology, West China Hospital of Sichuan

University, Chengdu, China

Suppressor of cytokine signaling 3 (SOCS-3) is an inhibitor of a number of growth factor signaling pathway. The promoter region of socs3 gene is rich of $\mathrm{CpG}$ islands which are targets of DNA methyltransferase. The methylation of $\mathrm{CpG}$ leads to SOCS-3 transcriptional blockage, which in turn fails to inhibit the cell signaling pathway and is closely correlated with the development of a variety of tumors. SOCS-3 also plays a crucial role as an inhibitor in the negative feedback loop to terminate liver regeneration. It is not clear whether the expression of SOCS-3 during liver regeneration is also regulated through $\mathrm{CpG}$ methylation. We aimed to investigate the dynamic changes of SOCS-3 expression and its CpG methylation during liver regeneration after partial hepatectomy $(\mathrm{PH})$. The remnant liver tissues of $2 / 3$ hepatectomized mice were harvested at different time point. SOCS3 mRNA was examined by qPCR. The sodium bisulfate converted DNA was amplified by PCR using specific primers and was cloned into $\mathrm{T}$ vector, and then was transformed into E. coli for DNA sequencing and identification of each $\mathrm{CpG}$ methylation. Our results showed that SOCS-3 mRNA was rapidly induced after $\mathrm{PH}$, and the peak of SOCS-3 mRNA emerged between 2-8 h after PH. A second mRNA peak emerged at day 5 after operation. Fifty-eight $\mathrm{CpG}$ islands were identified in the promoter region. There was no regular change of the general methylation level of SOCS-3 
gene at each time point. Additionally, none of the individual $\mathrm{CpG}$ island was found to be regularly changed during the liver regeneration.

Conclusion: The expression of SOCS-3 is closely correlated with the process of liver regeneration after $\mathrm{PH}$, however, the alteration of SOCS-3 gene does not seem to be regulated by its $\mathrm{CpG}$ methylation.

PP21-11

HDAC1/2 Redundantly Regulate Liver Regeneration

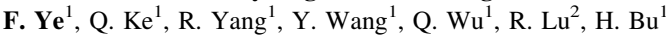

${ }^{1}$ Key Laboratory of Transplant Engineering and Immunology,

Sichuan University Hua Xi Hospital, Chengdu, China;

${ }^{2}$ Developmental Biology, UT Southwestern Medical Center, Dallas,

TX, USA

Background: Epigenetic chromatin remodeling events such as histone modification are essential for many biological processes. HDACs (Histone Deacetylases) are properly the most understood enzymes that remove the acetyl group from the histone of target genes. Here we are trying to answer two questions: whether HDAC1/2 is indispensible in the liver development and how they play roles in the process of liver regeneration.

Method: We generate liver specific deletion of HDAC $1 / 2$ mice by crossing $\mathrm{HDAC} 1^{\text {flox/flox flox/flox }}$ with Alb-Cre transgenic mice. $2 / 3$ or HDAC2partial hepatectomy $(\mathrm{PH})$ surgery was conduct on these mice (WT; HDAC1 KO; HDAC2 KO; HDAC1/2dKO). Brdu was injected via the intraperitoneally $1 \mathrm{~h}$ before harvesting the tissue. Real-time PCR and western blot were used to analyze the expressed genes.

Results: All genotypes (WT; HDAC1 KO; HDAC2 KO; dKO) of liver specific knock out mice were born in normal Mendelian ratios. All of them can alive, and grow up to be fertile adults. We sectioned the liver and stained with H.E., TUNEL, caspase3, Brdu and PCNA. No obvious difference was observed between the four groups. Liver function test, e.g. direct bilirubin, total bilirubin, total protein, Globulin, albumin, AST, ALT and ALP shows no difference between these four groups. We conduct $\mathrm{PH}$ on these four groups. Liver/body weight ratio shows HDAC1 $\mathrm{KO}$ and HDAC2 $\mathrm{KO}$ are as the same as WT during liver recovery process, but $\mathrm{dKO}$ mice liver regeneration was delayed. qPCR data shows the Cylin A, Cyclin D1 and Cyclin E expression were delayed. P21, P27 and P53 which were the inhibitors for cell cycle are not changed. We also confirmed these results by western blot.

Conclusion: Liver specific deletion of HDAC $1 / 2$ genes does not affect liver development and liver function. HDAC1/2 can redundantly regulate liver regeneration through regulating Cyclin A, Cyclin D1 and Cyclin E expression.

PP21-12

Sustained Repression and Translocation of Ntcp and Expression of Mrp4 for Cholestasis after Rat $90 \%$ Partial Hepatectomy

T. Miura ${ }^{1}$, N. Kimura ${ }^{1}$, T. Yamada ${ }^{2}$, T. Shimizu ${ }^{2}$, N. Nanashima ${ }^{2}$,

D. Yamana ${ }^{1}$, K. Hakamada ${ }^{1}$, S. Tsuchida ${ }^{2}$

${ }^{1}$ Department of Gastroenterological Surgery; ${ }^{2}$ Biochemistry and Genome Biology, Hirosaki University Graduate School of Medicine, Hirosaki, Japan

Background and aims: To clarify the mechanism of persistent cholestasis after massive hepatectomy, the relationship between such cholestasis and the expression and localization of organic anion transporters for bile acids was examined in a rat model.

Methods: Male Sprague-Dawley rats were subjected to $90 \%$ hepatectomy, and tissues were harvested on $0,1,3$, and 7 days for microarray analysis, the quantitative real-time polymerase chain reaction (RT-PCR), Western blotting and immunohistochemistry to examine the expression of multidrug resistance protein 4 (Mrp4), bile salt export pump (Bsep) and sodium-dependent taurocholate cotransporting polypeptide (Ntcp).
Results: Persistently elevated serum bile acids were observed on days 3 and 7. RT-PCR and Western blotting indicated that the expression of Mrp4, a bile acid export pump located in the basolateral membrane, was increased on day 3. Ntcp, a transporter used to uptake bile acids from the sinusoids, was significantly decreased throughout the period. Bsep, an export pump localized to the canalicular membrane, was unchanged. Immunohistochemistry revealed the localization of Mrp4 and Bsep in the basolateral and canalicular membranes, respectively. On the other hand, Ntcp was localized in the cytoplasm on days 3 and 7 and was hardly detected in the basolateral membrane.

Conclusions: These results suggested that the sustained repression and translocation of Ntcp and the expression of Mrp4 at the basolateral membrane seemed to be responsible for the high blood bile acids levels after massive hepatectomy.

\section{Poster Presentation 22: Molecular and Cellular Biology:} Stem Cell Research

\section{PP22-01}

Decellularized Liver Matrix Provides a Natural Platform for the Survival and Differentiation of Transplanted Hepatic Progenitor Cells

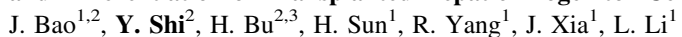
${ }^{1}$ Department of Surgery, Division of Experiment Surgery, Mayo Clinic, Rochester, MN, USA $;{ }^{2}$ Key Lab of Transplant Engineering and Immunology; ${ }^{3}$ Department of Pathology, West China Hospital, Sichuan University, Chengdu, China

Orthotopic liver transplantation is the only available treatment for severe end-stage liver disease, but organ shortage is the main limit. Recent evidences indicated decellularized liver matrix (DLM) is a novel approach to generate transplantable liver graft as a potential treament for liver disease. We investigated the effect of DLM on the differentiation of rat fetal liver cells, which contain stem/progenitor cells. The perfusion decelluarlization process preserves the structural and functional characteristics of native microvascular network and intact three-dimensional chamber geometry of Lewis rat liver matrix. Layer-by-layer heparin deposition was carried out to make the surface of the matrix thromboresistant. The isolated Lewis rat fetal liver cells cultured on DLM adhered along the extracellular matrix (ECM) and increased liver-specific gene expressions compared with cells cultured on collagen. The hepatic progenitor cells that were transplanted into Lewis rat portal system with DLM could survive, express hepatocyte marker alpha-fetoprotein and synthesis of albumin and glycogen after 3 days. These findings demonstrate that DLM supports cytodifferentiation from immature progenitor cells to hepatocytes and maintain its differentiated phenotypes in vitro and in vivo. DLM and fetal liver cells could be used to generate a noval transplantable liver graft as a potential treatment for liver disease and also could be used for the preliminary evaluation of the toxicity of drugs and chemicals.

\section{PP22-02}

Xenogeneic Decellularized Liver Matrix Supports Perfused Cultured HepG2 Cells Adhesion, Proliferation and Hepatic Specific Functions in vitro

J. Bao ${ }^{1,2}$, Y. Shi ${ }^{1}$, H. Bu ${ }^{3}$

${ }^{1}$ Key Lab of Transplant Engineering and Immunology, West China Hospital, Sichuan University, Chengdu, China; ${ }^{2}$ Department of Surgery, Division of Experiment Surgery, Mayo Clinic, Rochester, MN, USA; ${ }^{3}$ Department of Pathology, West China Hospital, Sichuan University, Chengdu, China

Hepatic tissue engineering encompasses several approaches to develop adjunct internal liver support methods, such as hepatocyte transplantation and implantable hepatocyte-based devices, as well as temporary 
extracorporeal liver support techniques, such as bioartificial liver (BAL). Recent evidences indicated decellularized liver matrix is a novel approach to generate transplantable liver graft as a potential treatment for liver disease. Here we investigated the availability of human hepatoblastoma cell line (HepG2) co-cultured with a xenogeneic decellularized liver matrix from rat. The perfusion decellularization process preserves the structural and functional characteristics of native microvascular network and intact three-dimensional chamber geometry, allowing efficient recellularization of the liver matrix with HepG2 cells and subsequent serum-free medium perfusion for in vitro culture. Most of HepG2 cells adhered on the matrix and proliferated to a high cell density at 5 days of culture. The xenogeneic liver matrix supports HepG2 cells liver-specific function including albumin secretion, urea synthesis and cytochrome P450 (CytP450) expression at higher level than the control monolayer and also. Interesting, HepG2 cells co-cultured with xenogeneic liver matrix recovered the ammonia removal activity that was lost in monolayer culture. HepG2 cells co-cultured with xenogeneic liver matrix also metabolized lidocaine and diazepam. These results suggest that xenogeneic liver matrix can support the human hepatocytes as a potential approach to generate noval tissue engineered liver or BAL for liver disease treatment.

\section{PP22-03}

Cytochrome P450 Epoxygenase 2C9 Inhibitor Sensitizes Cancer Stem Cell Apoptosis under Hypoxia

S.J. Myung ${ }^{1}$, J.-H. Yoon ${ }^{1}$, J.-H. Lee ${ }^{1}$, S.-J. Yu ${ }^{1}$, Y.-J. Kim ${ }^{1}$, H.-S. Lee ${ }^{1}$, K.A. $\mathrm{Kim}^{2}$

${ }^{1}$ Internal Medicine, Seoul National University College of Medicine, Seoul; ${ }^{2}$ Internal Medicine, Ilsanpaik Hospital, Inje University, Goyang, Republic of Korea

Background and aims: Cancer stem cells (CSCs) are believed to be the key drivers in tumor growth and therapy resistance. Although hypoxia has been shown to induce tumor progression through activating multiple signals such as modifying angiogenesis and invasion and help maintain multiple normal stem cell population, its roles in CSCs are largely unknown. In this study, we tried to evaluate the role of hypoxia in the proliferation and therapy resistance of CSCs.

Methods: Side population (SP) cell analysis and sorting were used to detect subpopulations that function as CSCs. To find out specific signals activated in CSCs under hypoxic condition, we compared gene expression of hypoxic CSCs with that of normoxic CSCs using microarray. Huh-7 cell, a human hepatocelluar carcinoma cell line, doxorubicin, cytochrome $\mathrm{P} 450$ epoxygenase 2C9 (CYP 2C9) inhibitor sulfaphenazole were used in this study. Cell growth and apoptosis were assessed using MTS assay, and apoptotic and kinase signaling pathways were explored by immunoblot analysis.

Results: Hypoxia induced CSC proliferation and microarray showed that the expression of CYP 2C9, which was known to be implicated in hypoxia-induced endothelial cell migration and angiogenesis, was significantly increased in hypoxic CSCs compared to normoxic CSCs. Treatment with doxorubicin induced CSC apoptosis via the activation of mitochondrial apoptotic signals, including caspase-9 activation, whereas hypoxic CSCs were less sensitive to doxorubicin-induced apoptosis. The CYP 2C9 inhibitor sulfaphenazole pre-treatment sensitized hypoxic CSCs to doxorubicin cytotoxicity, and this was attributed to more profound augmentation of JNK and caspase-9 activation.
Conclusions: These results indicate that hypoxia promotes CSC proliferation and hypoxia-inducible CYP 2C9 expression is responsible for doxorubicin resistance in CSCs under hypoxia. Thus, the selective interruption of CYP 2C9 may therapeutically be implicated in sensitizing CSCs to anti-cancer treatment, particularly in the advanced case exposed to hypoxic environment.

PP22-04

Acute Liver Failure-derived Bone Marrow Mesenchymal Stem Cells Exhibit Liverst Stem Cell Transcriptional Profiling

J. Li, L. Zhang, J. Xin, L. Jiang, H. Cao, X. Hong, L. Li

State Key Laboratory for Diagnosis and Treatment of Infectious

Diseases, The First Affiliated Hospital, School of Medicine, Zhejiang University, Hangzhou, China

Introduction: Hepatic progenitor cell (HPC) transplantation is a promising alternative to liver transplantation for patients with endstage liver disease. However, the precise origin of HPCs is unclear. Aims: This study aimed to determine whether bone marrow mesenchymal stem cells (BMSCs) isolated from rats in acute hepatic failure (AHF) possess hepatic potential and/or characteristics.

Material and methods: BMSCs were isolated from normal rats as well as rats in which AHF was induced by D-galactosamine. HPCs and primary hepatocytes were isolated from normal rats for comparison. The Affymetrix GeneChip Rat Genome 2302.0 Array was used to perform transcriptome profiling of the AHF-derived BMSCs and HPCs.

Results: The results showed that AHF-derived BMSCs had a gene expression profile significantly different from that of control BMSCs. More than $87.7 \%$ of the genes/probe sets that were upregulated more than twofold in AHF-derived BMSCs were expressed by HPCs, including 12 genes involved in liver development, early hepatocyte differentiation and hepatocyte metabolism. Confirmatory quantitative RT-PCR analysis yielded similar results. In addition, 940 probe sets/genes were expressed in both AHFderived BMSCs and HPCs but were absent in control cells. Compared to the control cells, AHF-derived BMSCs shared more commonly expressed genes with HPCs.

Conclusions: AHF-derived BMSCs have a hepatic transcriptional profile and express many of the same genes expressed by HPCs, strongly suggesting that BMSCs may be a resource for hepatocyte regeneration, and further confirming their potential as a strong source of hepatocyte regeneration during severe hepatic damage.

\section{PP22-05}

Experimental Study on Bone Marrow Derived Mesenchymal Stem Cells Repairing Injured Liver and Tracing in vivo

B. Lin, Y. Huang, D. Xie, J. Xie, H. Cao, Z. Gao

Infectious Diseases, The Third Affiliated Hospital of Sun Yat-sen University, Guangzhou, China

Background and aims: There is no effective therapy for liver failure but liver transplantation, Some previous studies showed bone marrow-derived mesenchymal stem cells (BMSCs) may play a role in the liver regeneration. The aim of this reseach is to study the effectiveness of BMSCs in repairing injured liver in rats and to trace BMSCs by in vivo bioluminescence technology (BLI). 


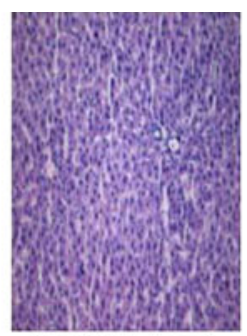

Liver histology of normal

SD (HE stain 200x)

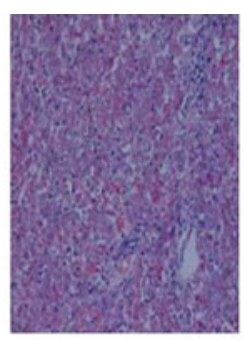

Liver histology of D.GalN+LPS SD model (HE stain 200x)

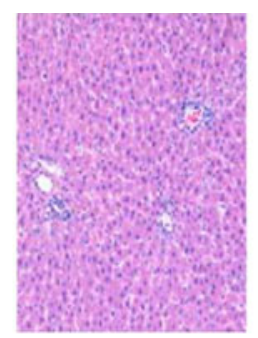

Liver histology of SD atter BMSCS transplantation (HE stain 200x)
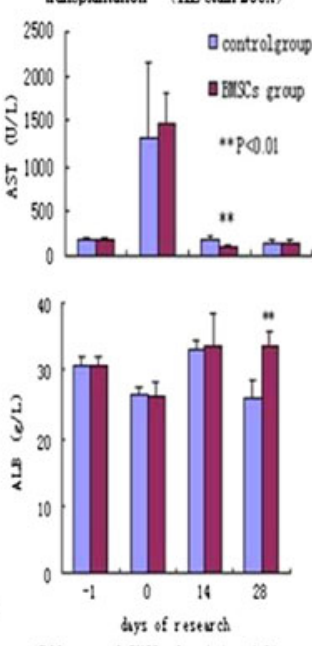

Effecacy of alls for injured live: MUSCs conize vein injection, they are still traced in liver at day 21 .

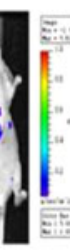

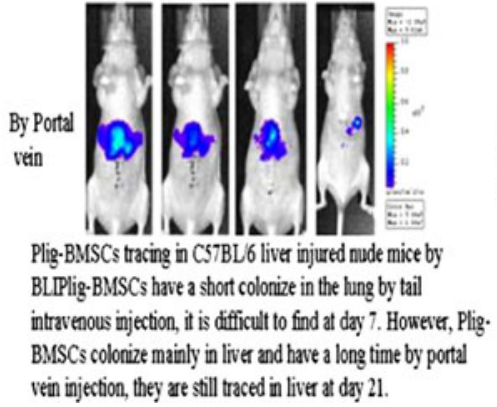

Experimental study on bone marrow-derived mesenchy

Methods: Thirty inbred rats with liver failure were divided into two groups of 15 rats: BMSCs group and control group, based on BMSCs transplantation or not. Liver functions were tested at 4 time point of the research: the day before injection of D-GalN+LPS, baseline, day 14 , and day 28. and the BMSCs were traced by in vivo bioluminescence technology at $1 \mathrm{~d}, 3 \mathrm{~d}, 7 \mathrm{~d}$ and $21 \mathrm{~d}$.

Results: Serum AST levels in BMSCs transplantation group were significantly lower than that in control group at $14 \mathrm{~d}(p<0.01)$, and serum albumin levels were significantly higher than that in control group at $28 \mathrm{~d}(p<0.01)$. Liver inflammation in transplantation group were alleviated more than that in control group at $28 \mathrm{~d}$ and liver HAI index of liver histology is close to normal. BLI tracing showed BMSCs with portal vein injection could colonize in the liver, but luminous intensity decayed over time. However, BMSCs with tail intravenous injection had no bioluminescence in liver but in lung.

Conclusions: BMSCs transplantation can repair liver damage and improve liver funtion and liver histology, and BMSCs injection through portal vein can colonize in the injured liver.

\section{PP22-06}

Connective Tissue Growth Factor Induced WB-F344 Cells Differentiation Towards Hepatocytes

A. Yang, P. Wang, T. Liu, M. Cong, H. You

Liver Research Center, Beijing Friendship Hospital, Capital Medical University, Beijing, China

Background: Connective tissue growth factor (CTGF) plays an important role in hepatic progenitor cells proliferation during liver regeneration, but less attention has been focused on CTGF influence on WB-F344 cells differentiation in vitro.
Methods: Recombination human CTGF stimulated WB-F344 cells in serum-free medium for $24 \mathrm{~h}$ and observed CTGF influence on cell viability and differentiation. CTGF plasmid stable transfected with WB-F344 cells and investigated endogenous CTGF influence on differentiation. Markers of differentiation including $\alpha$ - fetoprotein (AFP), albumin and cytokeratin-19 (CK-19) were detected. Two inhibitors of CTGF, Iloprost or/and WP-631, was/were administered to WB-F344 cells to investigate the effects of inhibitors on WB-F344 cells differentiation.

Results: Recombination human CTGF could inhibit the viability of WB-F344 cells mildly. High dose $(10 \mathrm{ng} / \mathrm{ml})$ of CTGF could change the phenotype markers of WB-F344 cells. Recombination human CTGF could downregulate the expression of fetal hepatocyte marker (AFP), while upregulate the expressions of mature hepatocyte marker (albumin) and cholangiocyte marker (CK-19). The effects of endogenous CTGF on WB-F344 cells differentiation were consistent with CTGF directly stimulated WB-F344 cells, including decreased of AFP and increased of albumin, but the degree of differentiation was less obvious than recombination human CTGF. Furthermore, suppression of CTGF induction by inhibitors was associated with a significant inhibition WB-F344 cells differentiation towards hepatocytes.

Conclusions: Both recombination human CTGF and endogenous CTGF could be able to induce WB-F344 cells differentiation towards hepatocytes, while suppression of CTGF could inhibit WB-F344 cells differentiation.

\section{PP22-07}

Hepatogenic Differentiation of Adult Mesenchymal Stem Cells Is Associated with Increased Expression of Major Xenobiotic Metabolizing Factors A. Allameh, S. Ismaeili, M. Ghaderi Clinical Biochemistry, Tarbiat Modares University, Tehran, Iran

Background: Stem cells are probably able to regenerate the damaged liver tissues, but due to their proliferating nature, they are predisposed to toxic and carcinogenic agents. The susceptibility of cells to toxic agents before and after differentiation can be assigned to the developmental aspects of the drug metabolizing factors.

Aim: To study the developmental changes in xenobiotic metabolizing factors during the hepatogenic differentiation of adult stem cells.

Methods: Hepatogenic induction of human bone marrow mesenchymal stem cells (MSCs) and human Umbilical Cord Blood (UCB) were performed to obtain hepatocyte-like cells. After characterization, the cells were processed for measurement of major xenobiotic metabolizing enzymes during 2-week differentiation.

Results: Expression of CYP3A4 specific mRNA (RT-PCR) was significantly higher in hepatocyte-like cells obtained on day 14 of differentiation as compared to the respective progenitor MSCs. The glutathione S-transferase (GST) activity was surged in the cells undergone differentiation. Changes in GST were associated with alterations in expression of major GST isoforms (GSTA1-1 and GSTP1-1) as shown by western blotting. RT-PCR data showed that both the GSTs are expressed in differentiated hepatocytes, whereas, GSTP1-1 expression was confined to the MSCs. This data suggest that unlike GSTP1-1, the expression of GST A1-1 commence after differentiation of MSCs to hepatocyte lineage. Differences in CYP3A4 in cells before and after differentiation were corroborated with the extent of cytotoxicity and DNA damage (Comet assay) in cells exposed to aflatoxin B1.

Conclusion: The hepatocyte-like cells derived from adult stem cells are potentially active in phase-I and II drug metabolizing factors and the cells exhibit different sensitivities to DNA damaging agents before and after differentiation. 
PP22-08

Hepatic Progenitor Cells, Bone Morphogenic Protein and Gremlin in the Pathogenesis of Hepatocellular Carcinoma on Top of Chronic HCV N. Baddour ${ }^{1}$, M. Gumei ${ }^{1}$, Y. Taher ${ }^{2}$, L. Abdou ${ }^{1}$

${ }^{1}$ Pathology; ${ }^{2}$ Hepatologty, Internal Medicine, Alexandria Faculty of Medicine, Alexandria, Egypt

Background: The role of hepatic progenitor cells (HPCs) HCC on top of chronic $\mathrm{HCV}$ is questionable. Our working hypothesis was that Gremlin which inhibits Bone Morphogenic protein-7 (BMP-7), is secreted by fibroblasts, will block BMP-7 differentiating effect on stem cells, arrest their maturation and promote proliferation in an immature state.

Patients and methods: Immunohistochemical expression of CK19, and FGF 2, and mRNA expression of BMP-7 and Gremlin were studied in chronic $\mathrm{HCV}$, cirrhosis and $\mathrm{HCC}$ patients.

Results: CK 19 positivity was higher in cirrhotics (0.009) and correlated with grade $r=0.64, p=0.009$ in group $\mathrm{I}, r=0.75$, $p=0.001$ in group II, and stage $(r=0.71, p=0.001)$. No CK19 positivity was observed in HCC patients . Ductular reaction was higher in cirrhotics $(p=0.001)$. It correlated with CK 19 positivity $(r=0.42, p=0.012)$, grade $(r=0.56, p=0.024)$ and stage $(0.66$, $p=0.006)$. FGF 2 expression increased in HCCs . FGF-2 correlated with grade $(r=0.6, p=0.013)$, stage $(r=0.72, p=0.002)$, CK 19 $(r=0.71, p=0.002)$ and ductular reaction $(r=0.68, p=0.004)$. BMP7 was expressed in hepatitic, cirrhotic and cancerous tissues. BMP-7 was higher in cirrhotics . It correlated with ductular reaction $(r=0.576, \quad p=0.031)$ and negatively with CK19 $(r=0.6$, $p=0.023)$. A $(p=0.009)$ higher number of cirrhotics and HCC cases showed positive Gremlin expression, which correlated with the stage $(r=0.7, p=0.002)$. A positive correlation was noted between BMP-7 and gremlin mRNA expression $(r=0.77, p=0.045)$. Thus, a negative feedback loop exists which controls BMP 7 expression. Gremlin expression correlated with CK19, FGF2 positivity and steatosis in hepatitics $(r=0.75, p=0.001)$.

Conclusion: CK19 is not a valid marker of stem cell activation in $\mathrm{HCC}$ on top of chronic HCV. Cirrhosis promotes carcinogenesis by fibroblast secreted gremlin blocking BMP function and promoting stem cell activation and proliferation which may contribute to carcinogenesis in this setting.

\section{PP22-09}

Safety and Efficacy of the Hepatic Stem Cell Transplantation as Supportive Modality in the Management of End-stage Decompensated Liver Cirrhosis A.A. Khan, N. Parveen, A. Rajendraprasad, M. Vali, M. Aejaz Habeeb CLRD, Hyderabad, India

Background: Globally, 1.4 million deaths occur annually as a result of chronic liver diseases, Liver transplantation is the only existing modality for treating decompensated liver cirrhosis. The major limitation is being shortage of donors, operative risks and post transplant rejection. Cell therapy using human fetal liver derived stem cells can provide great potential to conservatively manage end stage liver diseases. These cells possess high proliferation, less immunogenic, more resistant to cryopreservation and ischemic injury, properties that could enhance their engraftment within the recipient liver. Therefore the present aimed to prove the safety and efficacy of transplantation of liver stem cells in cases of end-stage liver cirrhosis and were followed more than 2 years.

Method: This current study was approved by Institutional Ethics Committee. Informed consent was obtained from each patient to carry out the study. 60 patients with liver cirrhosis of different etiologies having average MELD score of 25 were infused with human fetal liver derived stem cells (EpCAM+ve) were infused through the hepatic artery to the liver using femoral artery.

Results: There was no hypersensitive or febrile reaction, nausea and vomiting were seen following cell infusion. No complications related to hepatic encephalopathy recurred in any of the subjects up to 2 years follow up. There was marked clinical improvement observed in terms of all clinical and bio-chemical parameters. Majority of the cases showed reduction in the grading of varices from 3 to 1 after cell infusion.

Conclusion: Human fetal liver stem cell therapy offers a potentially supportive modality to organ transplantation in the management of liver diseases. The data generated of 2 years follow-up shows the safety and efficacy of this cell based therapy.

\section{PP22-10}

Cord Blood: A Potential Source of Stem Cells for the Treatment of Liver Disease

N. Parveen, A.A. Khan, A. Rajendraprasad, M.V. Shaik, M.A. Habeeb Centre for Liver Research and Diagnostics, Hyderabad, India

Introduction: Liver transplantation is the choiceable and potent modality for the treatment of acute and chronic liver failure. The limitations are multidisciplinary major surgical procedure, shortage of donor organs, immunosuppressive drugs and the cost. Hepatocyte transplantation bridges the gap between the trauma and survival. However the scarcity of donors, low proliferation, big size and the poor viability of cells after cryopreservation and the requirement of large number of cells in the management of hepatic failure are the major limiting factors. Stem cells offer a new approach for the treatment of acute and chronic liver failure. Stem cells can be isolated from various sources. Cord blood is one of the potential sources of stem cells.

Methodology: Human umbilical cord blood samples were collected in sterile tubes. MNCs were isolated from umbilical cord blood using the density gradient method and the cells were labeled with human anti CD 34. The cells positive for CD 34 were sorted using MACS. The viability of the sorted cells was checked by Trypan Blue dye exclusion method and MTT assay. The sorted cells were cultured on matrigel and the cells were induced to differentiate into hepatocyte like cells. Hepatogenesis was assessed at time points as indicated by RT-PCR, flow cytometry, immunofluorescence techniques.

Results: RT-PCR results indicate that the cord blood derived CD34+ cultured cells expressed hepatic stem cells markers such as albumin, AFP and CK18 at different days.

Conclusions: Umbilical cord blood is a precious source for isolating stem cells, and transplantation of CD34 +ve cells will significantly improve the liver disease condition.

\section{PP22-11}

The Effects of Bone Marrow Mesenchymal Stem Cells on Liver Regeneration and Repair after 80\% Hepatectomy in Rat

S. Hong-Li, S. Zhongyang, G. Huibin, Z. Weiping, D. Chong, S. Xiaojing, Z. Jing

Department of Organ Transplantation, Tianjin First Center Clinic Hospital, TianJin, China

Objective: To investigate the effects of rat bone marrow mesenchymal stem cells on liver regeneration and repair after $80 \%$ heatectomy.

Methods: BMSCs were isolated and purified by density gradient centrifugation combined adherent method. To established $80 \%$ hepatectomy model with rat aged 6-8 weeks and weight of 200-220 g. Experimental group contains 16 rats with BMSC injection via their portal veins and control group contains 20 rats with saline by the same way. Liver function test by biochemical methods, and the flow cytometry and immunohistochemistry to detect hepatocytes cycle and proliferation cell nuclear antigens.

Results: BMSCs were isolated, cultured and proliferated successfully in vitro; Survival rate after operation: survival rate was $93.7 \%$ in the experimental group and $75 \%$ in control group; Liver tissue display that in the experimental group after $1^{\text {st }} \mathrm{d}, 3^{\text {th }} \mathrm{d}, 7^{\text {th }} \mathrm{d}$, hepatic sinusoid mild expansion,less inflammatory cell infiltration, necrosis was lighter than 
the control group,especially between $3^{\text {th }} \mathrm{d} ; 1^{\text {st }} \mathrm{d}, 3^{\text {th }} \mathrm{d}, 7^{\text {th }} \mathrm{d}$ after operation, the levels of ALT and AST were decreased more significantly in experimental group than in the control group $(P<0.05)$; The $S$ phase of hepatocytes cycle was significantly higher in experimental group than in the control group $(P<0.05)$; Remnant liver immunohistochemistry showed that PCNA expression was higher in the experimental group than in the control group significantly $(P<0.05)$. Conclusion: BMSCs can improve rat liver regeneration and repairment significantly after $80 \%$ hepatectomy.

\section{PP22-12}

Clinical Study of Autologous Bone Marrow Mesenchymal Stem Cell Transplantation in Patients with Decompensated Liver Cirrhosis C.-H. Park ${ }^{1}$, S.H. Bae ${ }^{1,2}$, H.Y. Kim ${ }^{1}$, M.J. Song ${ }^{1}$, S.H. Jo ${ }^{1}$, J.Y. Han ${ }^{1}$, J.M. Yang ${ }^{1}$, J.Y. Choi ${ }^{1}$, S.K. Yoon ${ }^{1}$

${ }^{1}$ Division of Hepatology, Department of Internal Medicine; ${ }^{2}$ Catholic High Performance Cell Therapy Center, The Catholic Univ. of Korea, Seoul, Republic of Korea

Liver transplantation(LT) is the only curative treatment for end-stage liver disease, but unavailability of the donor liver is its major limitation. Here, we report the results of the autologous transplantation of BM derived MSCs to improve liver functions in five patients with decompensated liver cirrhosis(LC), who were not able to undergo LT. The causes of LC were hepatitis B (HBV) in two patients, and hepatitis $\mathrm{C}(\mathrm{HCV})$ with autoimmune hepatitis $(\mathrm{AIH})$, toxic hepatitis and Wilson's disease in one patient each. As soon as the MSCs were obtained by BM aspiration and FACS analysis, the hepatic artery infusion was done under fluoroscopic guidance. Serum albumin levels before the treatment were 2.8 and $2.4 \mathrm{~g} / \mathrm{dL}$ in each hepatitis $\mathrm{B}$ patients, and 2.7 and $1.9 \mathrm{~g} / \mathrm{dL}$ in toxic hepatitis and Wilson's disease, and after 1 week, these levels improved to $3.3,3.1,2.8$ and $2.6 \mathrm{~g} / \mathrm{dL}$, respectively. Fibroscan stiffness improved from 65,33 , and $34.8 \mathrm{kPa}$ to $46.4,19.8$ and $29.1 \mathrm{kPa}$ on the 4th week day in the patients with Wilson's disease, HCV with $\mathrm{AIH}$, and in one of the HBV patients, respectively. The stiffness was unable to achieve from the other HBV patient owing to the severe shrinkage of the liver, but 4 weeks later, fibroscan was performed with ease resulting in $67.8 \mathrm{kPa}$. The patient with toxic hepatitis underwent cadaveric LT at another hospital before the first follow-up. Subjective healthiness and quality of life were assessed by EORTC QLQ-C30 questionnaire which improved in all patients. One of the HBV patients underwent LT due to hepatic failure resulted from severe infection four months after the infusion, and the expression of hepatic progenitor markers (CK 7,8,9,18,19, Ckit, EpCAM) were observed by immunohistochemical staining in the explanted liver. These findings may lay out a promising future in MSC transplantation as a bridge for LT.

\section{PP22-13}

Effects of Let-7 microRNA on Chemosensitivity in Human Hepatocellular Cancer Stem Cells

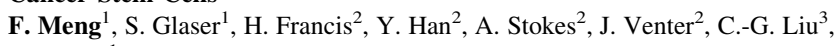
G. Alpini ${ }^{1}$

${ }^{1}$ Department of Medicine and Scott \& White Digestive Disease Research Center, Texas A\&M Health Science Center College of Medicine and Scott \& White Hospital, ${ }^{2}$ Digestive Disease Research Center, Scott \& White Hospital, Texas A\&M Health Science Center College of Medicine, Temple, ${ }^{3}$ Department of Experimental Therapeutics, Division of Cancer Medicine, University of Texas M. D. Anderson Cancer Center, Houston, TX, USA

MicroRNAs are endogenous small noncoding RNAs that regulate gene expression and cancer development. A rare population of hepatocellular cancer stem cells (HSCs) holds the extensive proliferative and self-renewal potential necessary to form a liver tumor. We postulated that specific transcriptional factors might regulate the expression of microRNAs and subsequently modulate the target genes involved in phenotypic characteristics of HSCs. The expression of microRNAs was evaluated in human HSCs by microArray profiling, and the target genes and functional effects of the group of up-regulated microRNAs by IL- 6 were further defined. We demonstrated that conserved let-7 family members were up-regulated in HSCs by global microarraybased microRNA profiling followed by validation with real-time PCR. Importantly, inhibition of let-7 increases the chemosensitivity of HSCs to sorafenib and doxorubicin. Knocking down IL-6 in HSCs significantly reduced let-7 expression and subsequently inhibited chemoresistance. We have also demonstrated that let-7 directly targets SOCS-1 and caspase-3 through dual-Luciferase miRNA reporter assay. Therefore, the modulation of aberrantly expressed miRNA in HSCs may be a useful strategy to limit cancer stem cell growth or improve responses to cytotoxic therapies. Furthermore, the identification of specific miRNA gene targets that are involved in tumor cell stemness, such as SOCS1 and Caspase-3; elucidation of the mechanisms by which they are regulated; and their functional effects will provide potential targets to reduce chemoresistance. Knowledge of specific processes that are regulated in a miRNA manner and identification of critical targets for individual miRNA in HCC stem cells will yield useful information and novel insights into the mechanisms of tumorigenesis and chemoresistance in HCC and other cancers.

\section{PP22-14}

Hepatogenic Differentiation and Genetical Stability of Human Amniotic Fluid Derived Mesenchymal Stem Cells Transduced with Lentivirus Vectors

Y.B. Zheng, D.Y. Xie, B.L. Lin, S.B. Xie, L. Peng, C.S. Lin, Y.F. Zhang, X.H. Zhang, Z.X. Zhao, Y.T. Chong, Z.L. Gao

Department of Infectious Diseases, The Third Affiliated Hospital of Sun Yat-Sen University, Guangzhou, China

Background/aims: Recent observations indicate that stem cells derived from human second-trimester amniocentesis (AF-hMSCs) are pluripotent stem cells capable of differentiating into multiple lineages, including representatives of all three embryonic germ layers. In recent years, lentiviral vectors ( $L V s$ ) have emerged as a robust and practical experimental platform for gene delivery and rational genetic reprogramming of stem cells. However, hepatogenic differentiation and genetical stability of AF-hMSCs with lentivirus-mediated expression of green fluorescent protein (GFP) has not yet been clearly elucidated. The aim of the present study was to investigate the in vitro biological characterization and hepatic differentiation potential of AF-hMSCs transduced with LVs containing the GFP gene (GFP/AFhMSCs), in order to assess the safety and capabilities of LVs.

Methods: AF-hMSCs were isolated from the second-trimester amniocentesis, and AF-hMSCs were enriched by transducing with LVs containing the GFP gene. Morphology, Reverse transcriptionpolymerase chain reaction (RT-PCR), Immunofluorescence (IF) and Cytogenetic studies were used to investigate comparatively the invitro biological characterization and hepatic differentiation potential of AF-hMSCs and GFP/AF- hMSCs.

Results: AF-hMSC demonstrate extensive expansion and differentiation capacities and are hence being studied for use in stem cell therapeutics, including gene delivery. GFP/AF-hMSCs possess the same proliferation and self-renewal capacity as AF-hMSCs. After incubation with definite hepatogenic agents, GFP/AF-hMSCs exhibited stable hepatic differentiation potential same as AF-hMSCs. That is to say, the differentiated GFP/AF-hMSCs were same in expressions of specific markers of hepatocyte differentiation in genes and protein levels as the differentiated AF-hMSCs. Cytogenetic analyses indicates that GFP/AF-hMSCs are as genetically stable as AF-hMSCs.

Conclusions: AF-hMSCs still maintained stable hepatic differentiation and cytogenetics after transduced with LVs containing the GFP gene. These results demonstrate the ability of LVs to efficiently transduce AF-hMSCs. Modifications to the LVs design were 
performed to optimize the vector as a tool with superior biosafety for the treatment of diverse terminal liver diseases.

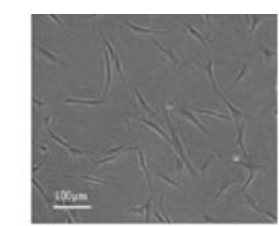

AF-hMSCs Passage3

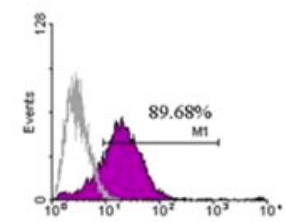

Flow cytometry using GFP marker

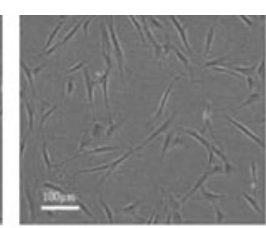

GFP/AF-hMSCs Passage 1

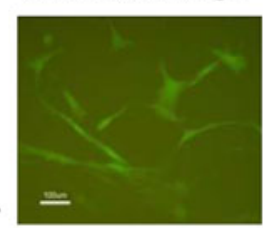

GFP/MSC MOI(20)96h

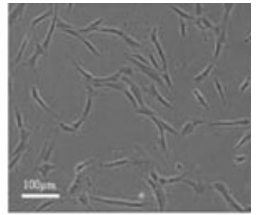

GFP/AF-hMSCs Passage 3

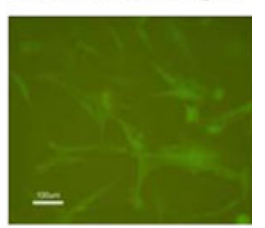

GFP/MSC MOI(20) Passage 3

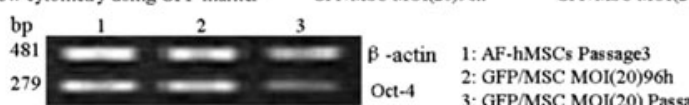

Yubao Zheng Figure 1 for APASL 2011

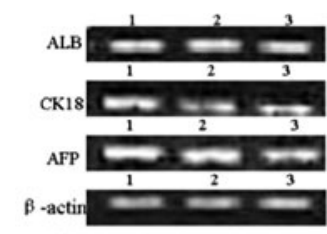

1: Differentiated AF-hMSCs(day14)

2: Differentiated GFP/AF-hMSCs(day14)

3: Differentiated GFP/AF-hMSCs(day21)

$$
\begin{array}{ll}
\text { AF-hMSCs } & \text { GFPIAF-hMSC MOI } \\
\text { passage3 } & \text { (20) passage } 3
\end{array}
$$

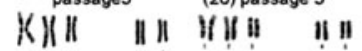

|| || ||$x \|||||$ |i $\mid$

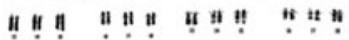

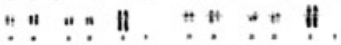

Cytogenetic analyses: normal

karyotype. in Both MSCs
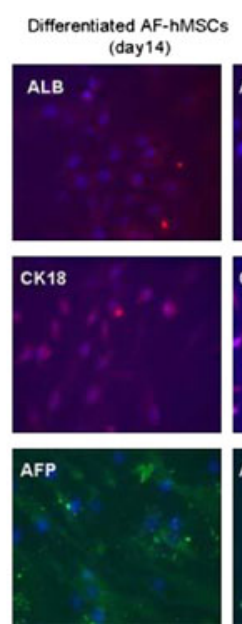

Differentiated GFP/AFhMSCs (day 14)
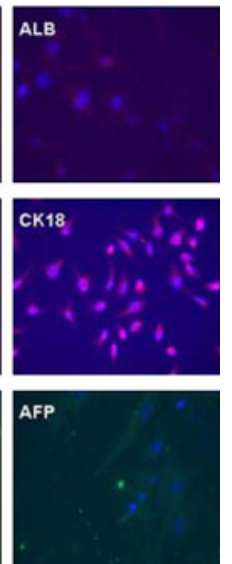

Yubao Zheng Figure 2 for APASL 2011

PP22-15

Comparison of Bone Marrow Derived Mesenchymal Stem Cell and Mononuclear Cells on the Treatment of CCl4 Induced Hepatic Fibrosis Rats F. Liu, N. Wu, W. Zhang, H.-Y. Rao, X. Cong, R. Fei, H.-S. Chen, L. Wei Peking University People's Hospital, Peking University Hepatology Institute, Beijing, China

Background: To compare the effects of transplantations of bone marrow mesenchymal stem cells (MSC) and mononuclear cells (MNC) on hepatic fibrosis rats.

Methods: Hepatic fibrosis rats were induced in rats by $\mathrm{CCl}_{4}$. In vitro cultured MSCs and MNCs were transplanted by intraportal vein into rats 8 weeks after initiation of carbon tetrachloride treatment. 4 weeks after transplanted, all of rats were killed. The clinical blood biochemical parameters were examined. The degree of liver injury and regeneration was evaluated by detecting blood biochemical parameters from serum and the expression of $\alpha$-SMA, PCNA. Sry (sexdetermining region for $\mathrm{Y}$ chromosome) gene was detected by in-situ hybridization of liver tissue to define the origin of proliferating cells.
Results: Compared with 8 week hepatic fibrosis group, in MSCs and MNCs transplanted group, the levels of ALT, AST and TBIL in serum were lower; the expression of $\alpha$-SMA and Collagen III were higher $(P<0.05)$; the expression of PCNA was higher $(P<0.05)$. Expression levels of MMP-2, MMP-9 and MMP-13 were also higher in MSCs and MNCs group. Sry+ cells were detected in liver tissue of MSCs and MNCs groups. Compared with MNCs group, histological activity index (HAI) was slightly improved in MSCs groups.

Conclusion: Transplantation of BMNCs or MSCs alone could populate in the injured liver, both showed similar population ability and improving fibrosis in the $\mathrm{CCl} 4$ induced hepatic fibrosis model rats. Transplantation of MSCs could improve the histological scores of chronic liver injury. These results indicate that bone marrow-derived MSCs will provide information on an appropriate cell source for transplantation therapy for hepatic fibrosis.

\section{PP22-16}

Genetic Regulation of Progenitor Cells in Fetal Liver Y.Y. Dan ${ }^{1}$, A. Sagayaraj ${ }^{2,3}$, S.G. Lim ${ }^{1}$

${ }^{1}$ Gastroenterology and Hepatology, National University Health System; ${ }^{2}$ Yong Loo Lin School of Medicine; ${ }^{3}$ NUHS, Singapore, Singapore

Human Liver progenitor cells may be used in a wide variety of clinical applications such as cellular transplantation. We aimed to determine the key message signals that drive progenitor cell proliferation and differentiation in developing fetal liver and determine their implication in regenerative medicine. Human fetal liver from 11 to 24 weeks gestation were obtained with full consent and subjected to immunohistochemistry and qRT-PCR (SABiosciences) for growth factor, extracellular matrix and liver specification factors. Comparison was made with adult end stage cirrhotic livers where progenitor cell proliferation was seen as contrasted with adult normal livers. These progenitor cells were transplanted into Rag -/- animals treated with CCL4 and the degree of repopulation was analysed and correlated with growth factors. EPCAM/CD44+ progenitor cells appear in small clusters at 10 weeks and undergo surge in proliferation at 18-21 weeks of gestation before dwindling in frequency. This was followed by increase in albumin, alphafetoprotein and CYP450 enzymes. The key transcription factors that show correlating upregulation at 18-21 weeks were Sonic Hegehog members as well as EGF, FGF1 and BMP3. Venn diagrams with cirrhotic model showed consistent increase in FGF and EGF. FGF and EGF supplemented in the cultures of fetal liver progenitor cells increase cell proliferation while maintaining immunophenotype. In vivo experiment with transplantation of progenitor cells showed positive correlation of percentage of human cells engraftment and FGF expression and not HGF over 9 months follow up. FGF and EGF appear to play a key role in the proliferation of liver progenitor cells in developing fetal liver, Similar trend is seen in cirrhotic livers where progenitor cells proliferation is seen. Corroboration is noted with both in vivo and ex vivo models. These growth factors may be potential targets of intervention to expand progenitor cells for regenerative purposes.

\section{Poster Presentation 23: Portal Hypertension} and Cirrhosis: Clinical

\section{PP23-01}

Comparative Analysis of Clinical Symptoms and Laboratory Results Affecting Life Prognosis of Patients with Resistant Ascitis

V. Ivashkin, M. Maevskaya, E. Fedosina

Hepatology, 1 I.M. Sechenov Moscow Medical University, Moscow, Russia 
Aim: To identify independent risk factors influencing death rate of patients with resistant ascitis (RA).

Materials and Methods: 66 RA patients were examined in the clinic. The diagnosis was made in compliance with the criteria of the International Ascites Club. The average age of the patients was $48.0 \pm 14.23$ (from 22 to 78). Male to female ratio was 1.4:1.0. Based on the results of the examination the patients were grouped in Class B Child-Pugh (22 people) and Class $C$ ( 44 people). On the basis of the analysis of indicators included in MELD system the patients were divided into two groups - 40 patients (10-20 points) and 26 (20-30 points).

Results: Of the 66 liver cirrhosis patients complicated by RA 35 people survived, 31 died by the end of the study ( 2 years). The main clinical symptoms and the rate of life-threatening complications of liver cirrhosis were not significantly different. Several parameters were studied using the method of binary logistic regression. The relative risk of death of patients with RA was determined to be significantly higher in patients with reduced level of serum $\mathrm{Na}$ $(p=0.01,95 \%$ CI $0.9-1.1)$, albumin $(p=0.01,95 \%$ CI $0.4-0.9)$, increased creatinine $(p=0.03,95 \%$ CI $1.4-4.5)$, bilirubin $(p=0.04$, 95\% CI 3.1-3.9), INR ( $p=0.01,95 \%$ CI $0.42-0.89$ ).

Conclusion: Regression analysis shows that in addition to wellknown indicators included in prognostic systems, the level of serum $\mathrm{Na}$ definitely influences the life-span of patients with RA which makes it important to take into account the results of this analysis when patients with RA are put on a waiting-list for liver transplantation.

\section{PP23-02}

Etiology and Long-term Outcome of Extrahepatic Portal Vein Obstruction in Children

B. Weiss ${ }^{1}$, E. Shteyer ${ }^{2}$, A. Vivante ${ }^{1}$, D. Berkowitz ${ }^{3}$, S. Reif ${ }^{4}$, Z. Weizman ${ }^{5}$, Y. Bujanover ${ }^{1}$, R. Shapiro ${ }^{6}$

${ }^{1}$ Division of Pediatric Gastroenterology Hepatology and Nutrition, Edmond and Lily Safra Children's Hospital and Sackler Faculty of Medicine, Tel-Aviv University, Tel Aviv; ${ }^{2}$ Division of Pediatric Gastroenterology Hepatology and Nutrition Jerusalem, Hadassah Medical Center, The Hebrew University School of Medicine, Jerusalem; ${ }^{3}$ Division of Pediatric Gastroenterology Hepatology and Nutrition, Meyer Children's Hospital, Rambam Medical Center, and Rappaport School of Medicine, Technion, Israel Institute of Technology, Haifa; ${ }^{4}$ Division of Pediatric Gastroenterology Hepatology and Nutrition, Dana Children's Hospital, Souraski Medical Center, and Sackler Faculty of Medicine, Tel-Aviv University, Tel-Aviv; ${ }^{5}$ Division of Pediatric Gastroenterology Hepatology and Nutrition, Soroka Medical Center, Ben Gurion University School of Medicine, Beer Sheva, ${ }^{6}$ Department of Pediatric Gastroenterology and Nutrition, Division of Pediatric

Transplantation, Schneider Children's Hospital, and Sackler Faculty of Medicine, Tel-Aviv University, Petach-Tikva, Israel

Aim: To study the experience of management and the outcome of children with extrahepatic portal vein obstruction (EHPVO) in a whole country population.

Methods: A nationwide multicenter retrospective case series of children with EHPVO. Data on demographics, radiographic studies, laboratory workup, endoscopic and surgical procedures and growth, were extracted from charts. Characteristics of clinical presentation, etiology, management and outcome were analyzed.

Results: Thirty patients (13 males), 19 (63.3\%) Israeli and 11 (36.7\%) Palestinians, were included in the analysis. Age of presentation was $4.8 \pm 4.6$ years. Mean follow-up was $4.9 \pm 4.3$ years. The EHPVO incidence for Israeli children ages $0-14$ years was $0.72 /$ million. Risk factors were detected in $13(43.3 \%)$ patients, including 9 with perinatal risk factors, and 4 with prothrombotic states: two low levels of protein $\mathrm{S}$ and $\mathrm{C}$, one lupus anticoagulant, and one homozygous for MTHFR mutations. In $56.6 \%$ of patients no predisposing factor for was found. The most common presenting symptoms were an incidental finding splenomegaly (43.3\%), and UGI bleeding (40\%). No difference was found between Israeli and Palestinian children in regard to age at presentation, etiology and clinical symptoms. Bleeding occurred in 18 patients $(60 \%)$, at a median age of 3 years. Sclerotherapy or esophageal banding was performed in 20 patients. No complications of sclerotherapy were reported. Portsystemic shunts were performed in 11 patients $(36.6 \%)$, at a median age of 11 (range 3-17) years: splenorenal in 9, mesocaval in 1, and meso-Rex shunt in 1 patient. One patient underwent splenectomy. Patients were followed up for a median of 3 (range 0.5-15) years. One patient died at age 3 years because of mucopolysaccharidase deficiency type III. None died because of gastrointestinal bleeding.

Conclusion: EHPVO is a rare disorder. The etiological factors are still mostly unknown, and the endoscopic and surgical treatment options ensure a good long term prognosis.

\section{PP23-03}

Study of Portal and Systemic Levels of Nitric Oxide, Endothelin-1 and Procollagen III Peptide in Chronic Liver Disease in Egypt

H.A. Abd El Moety ${ }^{1}$, M.H. Kandil ${ }^{1}$, G.H. Magour ${ }^{1}$, G. Fadaly ${ }^{2}$, M. Elgendy ${ }^{3}$, M. Abd El Baky ${ }^{3}$

${ }^{1}$ Chemical Pathology; ${ }^{2}$ Pathology; ${ }^{3}$ Surgery, Medical Research

Institute, Alexandria University, Alexandria, Egypt

Egypt has one of the highest incidence of liver diseases in the world with prevalence of schistosomiasis. NO diffuses into cytosol of adjacent vascular smooth muscle cells play a role in the pathogenesis of vasodilation. Endothelial cells also produce the most potent vasoconstrictor agent endothelin (ET-1).

Aim: Evaluation of nitric oxide and endothelin-1 and procollagen III peptide in patients with chronic liver disease and portal hypertension in both systemic and portal blood samples together with the histopathological scoring of liver biopsies.

Subjects: The control group 15 subjects free from any liver disease. The patient group 30 patients with chronic liver disease and schistosomal portal hypertension.

Methods: Clinical examination, abdominal ultrasonography, measurement of portal venous pressure and histopathological examination of liver biopsy. Laboratory investigations included evaluation of total nitric oxide (NO), endothelin-1 (Et-1) and type III procollagen (PIIINP) in both portal and systemic blood. In addition prothrombin, serum alanine, aspartate aminotransferase (AST, ALT), g glutamyl aminotransferase (gGT) activities, serum bilirubin, albumin, serodetection of hepatitis B surface antigen ( $\mathrm{HBsAg}$ ) and hepatitis B core antibody and (anti-HCVAb).

\section{Conclusion:}

-NO and ET-1 levels in both systemic and portal blood of SHF patients were significantly higher than in the control group.

$\mathrm{NO}$ is a potent vasodilator.

ET-1 increase may be a compensatory mechanism to antagonize the vasodilatory effect of NO.

Child class B subgroup had higher NO and ET-1 than class A.

NO and ET-1 levels did not differ between anti HCV positive and negative SHF patients.

\section{PP23-04}

Platelet Count and Spleen Size: Avoidance of Screening Endoscopy for Varices in Patients with Hepatitis C Cirrhosis

J. Gerada, E. Gerada, S. Sen, W.J. Griffiths

Department of Hepatology, Addenbrooke's Hospital, Cambridge, UK

Background: Many patients with liver cirrhosis, screened for oesophageal varices $(\mathrm{OV})$ are found to have either no or insignificant varices, resulting in unnecessary procedures and financial burden. We had previously shown that the non-invasive parameters of platelet count 
and spleen size, measured by transabdominal ultrasound, were highly sensitive for the prediction of $\mathrm{OV}$ in patients with hepatitis $\mathrm{C}$ cirrhosis. Aim: To determine in a subsequent cohort of patients with hepatitis $\mathrm{C}$ cirrhosis whether such a clinical tool was accurate in determining which patients require endoscopic screening.

Method: 246 outpatients with biopsy-proven hepatitis C cirrhosis over a 3-year period were studied retrospectively. Endoscopy should have been performed, according to our protocol, if any of the following parameters were met: platelet count $<100 \times 10^{9} / 1$, spleen size ${ }^{3} 14 \mathrm{~cm}$ or specific ultrasound findings indicative of portal hypertension (recanalised paraumbilical vein, ascites, splenic hilar varices).

Results: Of 246 patients, 98 patients underwent upper GI endoscopy, 76 of whom met criteria (78\%). Of 148 patients who did not undergo endoscopy, 63 met criteria (43\%). Endoscopic findings are summarized in the table below.

Conclusion: Using simple non-invasive criteria, screening endoscopy could be avoided in around $44 \%$ of patients with hepatitis C cirrhosis. Treatable varices would not be missed in this group.

\begin{tabular}{lllll}
\hline & OGD & & Predictive \\
\cline { 3 - 4 } & & $\begin{array}{l}\text { Significant } \\
\text { varices } \\
\text { Value }\end{array}$ & $\begin{array}{l}\text { Insignificant } \\
\text { varices } \\
\text { (grade 1 or nil) }\end{array}$ & \\
\hline $\begin{array}{c}\text { Pr more) } \\
\begin{array}{c}\text { Platelet count/ } \\
\text { Us flen size/ }\end{array}\end{array}$ & Criteria met & 14 & 62 & $18 \% \mathrm{PPV}$ \\
& $\begin{array}{c}\text { Criteria } \\
\text { not met }\end{array}$ & 0 & 22 & $100 \% \mathrm{NPV}$ \\
\hline
\end{tabular}

Endoscopic findings for patients who had an OGD

\section{PP23-05}

Chemical Pleurodesis for the Management of Symptomatic Hepatic Hydrothorax

H.J. Kim, Y.K. Cho, B.I. Kim

Internal Medicine, Sungkyunkwan University Kangbuk Samsung

Hospital, Seoul, Republic of Korea

Background/aims: Hepatic hydrothorax in the setting of decompensated liver cirrhosis is a challenging problem. We report the outcome of eight patients with refractory hepatic hydrothorax who underwent chemical pleurodesis with or without video-assisted thoracoscopic surgery (VATS).

Methods: Data for eight consecutive patients presenting to our institution between July 2007 and November 2009 with refractory hepatic hydrothorax who underwent chemical pleurodesis with or without VATS were retrospectively reviewed.

Results: All patients have right sided pleural effusion and ascites refractory to medical therapy. The mean (standard deviation) sessions of chemical pleurodesis was 4.8 (3.4). Six patients underwent only chemical pleurodesis via chest tube and two patients underwent chemical pleurodesis with VATS. Success defined as control of symptomactic hydrothorax in the first 30 days after the procedure was achieved in six of eight patients $(75.0 \%)$. Three $(37.5 \%)$ of these patients remained asymptomatic and hydrothorax free at a median follow-up of 104 (13-577) days after the procedure. However, three patients experienced symptomatic recurrence of hydrothorax 116, 212, and 227 days after the initial procedure. Complications occurred were low grade fever/leukocytosis $(n=8,100.0 \%)$, pneumonia
( $n=1,12.5 \%)$, pneumothorax $(n=3,37.5 \%)$, azotemia/acute renal failure $(n=5,62.5 \%)$ and hepatic encephalopathy $(n=3,37.5 \%)$. Morbidity including PCD site pain and chest pain during chemical pelurodesis occurred in all eight patients. Three patients were suspected to have procedure-related mortality $(37.5 \%)$ due to the occurrence of acute renal failure.

Conclusions: Refractory hepatic hydrothorax can be controlled with multiple sessions of chemical pleurodesis via chest tube with or without VATS in as many as $75 \%$ of patients. However, a proportion of these patients experienced significant procedure related morbidity and mortality. The procedure may be considered as a palliative alternative in a limited proportion of patients needing frequent thoracentesis.

\section{PP23-06}

The Association of Helicobacter Pylori with Gastritis in Patients with Portal Hypertention due to Hepatic Shistosomiasis

S.A. Zaki ${ }^{1}$, A.A.E.m. Soliman ${ }^{2}$, S. Baioumy ${ }^{3}$, S. Farid ${ }^{1}$, I. El Ghazzawy ${ }^{1}$, A. Ghanem ${ }^{2}$, M. Rashed ${ }^{4}$

${ }^{1}$ Microbiology, Faculty of Medicine; ${ }^{2}$ Hepatology, Faculty of Medicine, Alexandria University; ${ }^{3}$ Pathology, Tanta University;

${ }^{4}$ Medicine, Alexandria University, Alexandria, Egypt

Introduction: Gastritis is common in portal hypertension of all aetiologies.

In recent years a newly recognized pathogen, Helicobacter pylori was found to be associated with gastritis, duodenitis and duodenal ulceration.

Aim: To investigate the probable role of $H$. pylori in gastritis in patients with portal hypertension.

Subjects and methods: 53 patients with portal hypertension due to hepatic Schistosomiasis. All patients were examined clinically and liver biopsy was done to exclude non schistosomal causes of portal hypertension .

Endoscopy: From each patient four mucosal antral biopsies were taken,two for histopathology and two for culture.

Histopathology: The organisms identified in Gram stain sections by their spiral shape, their location beneath the mucous layer.

Microbiology: The samples were ground\& inoculated on Christensen $\mathrm{s}$ urea medium, blood agar with $7 \%$ citrated human blood and Skirrow's medium.

Results: All the specimens from the antral mucosa of patients with portal hypertension due to hepatic Schistosomiasis showed histological evidence of gastritis, mild in $24.53 \%$, moderate in $64.15 \%$, severe in $11.32 \%$ of cases .

Helicobacter pylori was recognized in $35(66.04 \%)$ cases, in their specimens either by culture, Gram staining of histopathological sections or both. The organism was isolated from $32(60.38 \%)$ of 53 patients, 30 were also histopathologically positive for organism on Gram staining. The organism was detected on Gram staining of histopathological sections in 33 (62.26) patients, 30 of whom were culture positive.

\section{Conclusion:}

I: Helicobacter pylori is detected more by histopathology than by cultures

II: Helicobacter pylori appears to be an important aetiological or probably a precipitating factor in the pathogenesis of gastritis associated with portal hypertension due to hepatic Schistosomiasis.

\section{PP23-07}

A Study on the Natural History of Portal Biliopathy

V. Ramachandran ${ }^{1,2}$, T.K. Chattopadhyay ${ }^{3}$, P. Sahni ${ }^{3}$, S. Pal ${ }^{3}$, S.K. Acharya ${ }^{4}$, R. Sharma ${ }^{5}$

${ }^{1}$ Surgical Gastroenterology, KMC Hospital, Mangalore; ${ }^{2}$ Ex Senior Resident, Department of Gastrointestinal Surgery and Liver Transplantation; ${ }^{3}$ Department of Gastrointestinal Surgery and Liver 
Transplantation; ${ }^{4}$ Department of Gastroenterology, All India Institute of Medical Sciences; ${ }^{5}$ Department of Radiology, All India Institute of Medical Sciences, Delhi, India

Introduction: Portal biliopathy refers to the biliary abnormalities in patients with portal hypertension. Little is known about its natural history as well as the effects of portosystemic decompression and other interventions.

Objectives:

1. To study the frequency of portal biliopathy in patients with Extrahepatic Portal Venous Obstruction (EHO)

2. The Clinical, Biochemical and Morphological abnormalities in Portal biliopathy

3. Natural course of symptomatic and asymptomatic Portal biliopathy and effects of interventions

Methods: Between March 2006 and December 2007, consecutive patients with EHO were evaluated for Portal biliopathy.

Inclusion criteria: All patients who had evidence of intrahepatic biliary radicle dilatation (IHBRD) on Ultrasound abdomen or had an elevated Alkaline Phosphatase level were subjected to MRCP. Patients were included in study if MRCP revealed features of Portal biliopathy.

Exclusion criteria: Patients with secondary portal vein thrombosis, primary bile duct calculi and surgical obstructive jaundice.

Investigations: Haematological and Biochemical tests, Viral marker studies for Hepatitis B and C, Upper G.I.endoscopy, Ultrasound Doppler study and MRCP (at recruitment and then annually if portal biliopathy existed) was done for all patients.

Interventions: ERC and Stenting was done for symptomatic portal biliopathy with cholangitis. Lienorenal Shunt was offered to patients with EHO who presented with UGI bleeding refractory to endoscopic treatment, hypersplenism or had high grade varices. Three monthly follow up was done and alterations in abnormalities (Biochemical and Radiological) was noted.

Statistical analysis: Chi-square test, Fisher's exact test and Mann Whitney tests were performed and a $p$ value of $<0.05$ was considered statistically significant. SPSS $12, ., 0$ was used for calculations

Conclusions: Proportion of EHO patients with Portal biliopathy was $32.8 \%$. With the above screening, a third of patients had MRCP evidence of Portal biliopathy. Of these, a third manifested with symptomatic biliary obstruction. IHBRD and Extrinsic impressions on Common bile duct were the common abnormalities noted on MRCP.

\section{PP23-08}

Prediction of Large Esophageal Varices in Cirrhotic Patients Using Classification and Regression Tree Analysis

W.-D. Hong, Q.-H. Zhu

Department of Gastroenterology and Hepatology, The First Affiliated Hospital of Wenzhou Medical College, Wenzhou, China

Objectives: The predictive accuracy of available noninvasive markers of large esophageal varices (LEV) is still considered to be unsatisfactory. This study aimed to develop a decision model based on classification and regression tree analysis for the prediction of LEV in cirrhotic patients without a history of variceal bleeding.

Methods: 309 cirrhotic patients were enrolled. Study participants were randomly assigned to the training sample and test sample in a 3:2 ratio. Within the training sample, the classification and regression tree (CART) analysis was used to identify predictors and prediction model of LEV. The prediction model was then applied to the test sample.

Results: The prevalence of LEV in cirrhotic patients was $50.8 \%$. A tree model consisted of just three variables (spleen width, portal vein diameter, prothrombin time) was developed by CART analysis provided a simple algorithm for prediction of LEV with a diagnostic accuracy of $84 \%$. The cut-off values were $44.5 \mathrm{~mm}, 11.75 \mathrm{~mm}$,
$17.05 \mathrm{~s}$ for spleen width, portal vein diameter, prothrombin time in the model, respectively. The tree model was validated in the test sample and it achieved a diagnostic accuracy of $84.4 \%$.

Conclusions: A decision tree model that consists of spleen width, portal vein diameter, and prothrombin time may be useful for prediction of LEV in cirrhotic patients.

PP23-09

Mesenteric Venous Thrombosis: Presentation \& Outcomes in a Tertiary Referral Center in South India

V. Ramachandran, B.V. Tantry, S. Shenoy

Gastroenterology and Surgical Gastroenterology, KMC Hospital, Mangalore, India

Introduction: Mesenteric Ischaemia due to Mesenteric Venous Thrombosis (MVT) is associated with a mortality of $15 \%$. However, early intervention can reduce mortality due to this disease. We present a series of three cases of Mesenteric Venous Thrombosis which were successfully managed in our Department between January 2008 and December 2009.

Case description:

Case 1: A 41-year-old female presented with abdominal pain of 2 days duration and had peritonism on examination. Diagnosis of MVT was made on Ultrasound Doppler study and confirmed on Contrast Enhanced CT Scan (CECT) of abdomen. Upper GI Endoscopy did not reveal varices. Thrombophilia evaluation was negative. Patient responded to anticoagulant therapy. However, she presented with Recurrent episodes of Pulmonary Embolism due to Femoral venous thrombosis necessitating placement of IVC Filter.

Case 2: A 26-year-old male presented with abdominal pain and vomiting of one week duration and had distended, tender abdomen on examination. He gave a past history of Tanner's procedure for Oesophageal varices 10 years back. Upper G.I.Endoscopy revealed Grade 3 Oesophageal varices and Thrombophilia evaluation revealed Protein S deficiency. He was diagnosed to have MVT on Ultrasound Doppler Study and was anticoagulated with Heparin. However, his peritonism worsened necessitating laparotomy and resection of $1 \mathrm{~m}$ of gangrenous jejunum.

Case 3: A 26-year-old male presented with symptoms of intestinal angina of 1 month duration. Upper G.I.Endoscopy revealed Grade 3 Oesophageal varices and Thrombophilia evaluation revealed polycythaemia. PCR Study revealed JAK 2 mutation. Diagnosis of MVT was made on Ultrasound Doppler Study and confirmed by CECT Scan of abdomen. He responded to anticoagulation and phlebotomy and later started on Hydroxyurea and Aspirin.

All three patients are doing well on follow up and are on oral anticoagulants. This series is being presented to highlight the favourable outcome in all three cases.

\section{PP23-10}

Liver Stiffness Measurement and Serum Fibrosis Marker for Predicting Esophageal/Gastric Varices in Children with Biliary Atresia

V. Chongsrisawat ${ }^{1}$, P. Vejapipat ${ }^{2}, K$. Setrkraising ${ }^{1}$, R. Lorchatchawankul ${ }^{1}$, P. Sintusek ${ }^{1}$, A. Wongteerasut ${ }^{1}$, Y. Poovorawan ${ }^{1}$

${ }^{1}$ Pediatrics; ${ }^{2}$ Surgery, Chulalongkorn University, Bangkok, Thailand

Background/aims: Biliary atresia (BA) is an idiopathic, necroinflammatory process resulting in obliteration of the extrahepatic biliary tree. Even with established bile drainage after portoenterostomy, patients may develop liver fibrosis and portal hypertension (PH). The potential fatal complication of $\mathrm{PH}$ is ruptured esophageal/gastric varices $(\mathrm{EV} / \mathrm{GV})$. The standard diagnostic tool for EV/GV is endoscopy which is an invasive procedure in children. Transient elastography (FibroScan) is a noninvasive technique to assess liver fibrosis by measuring liver stiffness in patients with chronic liver diseases. The aim of this study was to explore the accuracy of liver stiffness measurement 
(LSM) and clinical parameters in predicting the presence of EV/GV in children with BA following portoenterostomy.

Methods: Post portoenterostomy BA patients and normal children were recruited. Splenomegaly and presence of EV/GV were determined by physical examination and endoscopy, respectively. Aspartate transaminase to platelet ratio index (APRI) was used as a serum fibrosis marker. LSM was performed using FibroScan. Data was expressed as mean $\pm \mathrm{SD}$.

Results: Forty-six BA patients (age $10.46 \pm 5.19$ years) and 47 controls (age $11.03 \pm 3.47$ years) were enrolled. Liver stiffness score of the BA patients was significantly higher than the controls $(32.78$ \pm 22.87 and $4.7 \pm 1.15 \mathrm{kPa} ; p<0.001)$. Patients with EV/GV had significantly higher liver stiffness score and APRI than those without EV/GV. The sensitivity (and specificity) of FibroScan (using cutoff value of $7.4 \mathrm{kPa}$ ) and APRI (using cutoff value of 1.2) in predicting of $\mathrm{EV} / \mathrm{GV}$ were 97 (25\%) and 84.8\% (75\%), respectively. Whereas the sensitivity (and specificity) of splenomegaly in predicting of EV/GV were 90.9 and $83.3 \%$.

Conclusions: LSM is a useful tool for predicting the presence of EV/GV. Nonetheless, basic physical examination, routine biochemical and hematological tests are still worthwhile; they are well correlated with the presence of $\mathrm{EV} / \mathrm{GV}$ in patients with post portoenterostomy BA.

\section{PP23-11}

Early Transjugular Intrahepatic Portosystemic Shunt (TIPS) in Acute Variceal Bleeding Among Cirrhotics: A Meta-analysis

E.V.B. Razon-Gonzalez, M.A. De Lusong, V. Banez, M.E. Velasquez, A. Salvana, E. Santiago-Tripon, R. Forroza, E.A. Dela Cruz Section of Gastroenterology, Philippine General Hospital, Manila, Philippines

Background and aims: Transjugular intrahepatic portosystemic shunt (TIPS) has been proven to lower portal pressure, to effectively treat and to prevent recurrence of variceal hemorrhage among cirrhotic patients. A meta-analysis of 12 randomized controlled studies (RCTS) of TIPS versus endoscopic therapy in 2008 shows that TIPS is associated with a significantly decreased incidence of variceal bleeding and mortality. The significance of the timing of TIPS, whether elective or emergency (within 24-72 h of onset of variceal bleed), however, has not been evaluated. This study aims to compare early TIPS versus vasoactive drugs with endoscopic treatment with or without elective TIPS in terms of treatment failure and mortality rates among cirrhotic patients with acute variceal bleed.

Methods: We searched PUBMED RCTS involving early TIPS versus standard of care from inception among cirrhotic patients with acute variceal bleed. Primary outcome measures were treatment failure rate in terms of rebleeding and mortality arte. Seven studies were identified but only three were retrieved after excluding the rest. One study was excluded because it was not an RCT. Studies were reviewed by two independent reviewers after assessing eligibility. Dispute was settled by a third reviewer. Methodologic quality of each study was high. Data extraction and analysis were done using Review Manager version 5 .

Results: Only two studies involving 115 patients were included. Early use of TIPS, compared to standard of care, was associated with decreased treatment failure (OR $0.08 ; 95 \%$ CI $0.03-0.26$; $P<0.0001)$ and lower mortality rate (OR $0.22 ; 95 \%$ CI $0.08-0.57$; $P=0.002$ ).

Conclusion: In cirrhotic patients with acute variceal bleeding, early use of TIPS is associated with reduction in treatment failure and mortality.
PP23-12

Frequency of Poor Health Related Quality of Life (HRQOL) and Factors of HRQOL in Patients with Cirrhosis

O. Parkash ${ }^{1}$, R. Iqbal ${ }^{2}$, I. Azam ${ }^{3}$, W. Jafri ${ }^{1}$

${ }^{1}$ Medicine; ${ }^{2}$ Medicine and Community Health Sciences; ${ }^{3}$ Community Health Sciences, Aga Khan University, Karachi, Pakistan

Introduction: Chronic Liver Disease /cirrhosis (CLD) in individuals produces variety of symptoms which in turn lead to a negative impact on Health Related Quality of Life (HRQOL). However no such work has been carried out in Pakistan. The general aim of this study was to evaluate the magnitude of poor HRQOL and to assess factors related with HRQOL in patients with CLD.

Methods: This was cross sectional study conducted at Gastroenterology outpatient clinics of Aga Khan University Hospital, Karachi. All adult cirrhotic patients based on clinical and radiological criteria were recruited. Chronic Liver Disease Questionnaire (CLDQ) was used to assess HRQOL of these patients. Patients were categorized into two groups based on CLDQ score; $<5$ as poor and $\geq 5$ as good score for determination of frequency of poor HRQOL. CLDQ score was used as an outcome measure to determine factors related with HRQOL.

Results: Total of 273 participants were recruited in the study; among them $155(57 \%)$ were males. Mean age of participants was 49 years $(\mathrm{SD} \pm 11)$. In this study the most common cause for cirrhosis was viral infection 247 (91.5\%). Mean CTP score was $8 \pm 1.85$ and mean MELD score was $12.6 \pm 6.8$. Two thirds of patients $209(76.6 \%)$ had advanced cirrhosis in CTP B or C stage. Poor HRQOL was seen in $187(69 \%)$ of the participants. Mean CLDQ score was 4.36 (SD \pm 1.1$)$. Among the domains, fatigue domain had significantly lower CLDQ score. On multivariable analysis Hemoglobin $(\beta=0.09$ [SE $=$ 0.04]), Albumin $(\beta=0.32$ [SE $=0.09])$, Diastolic Blood Pressure (DBP) $(\beta=0.01[0.005])$ prior history of decompensation $(\beta=0.98$ $[\mathrm{SE}=0.39])$ were significant factors associated with HRQOL in patients with liver cirrhosis.

Conclusion: Frequency of poor health related quality of life determined by CLDQ score is high in patients with liver cirrhosis. Hemoglobin, serum albumen, prior history of decompensation (like encephalopathy and upper gastro intestinal bleed), are associated with HRQOL.

\section{PP23-13}

Branched Chain Amino Acids (BCAAS) and Zinc in the Treatment of Ascites in Liver Cirrhosis

K. Gruengreiff

Private Practice of Hepatology, Magdeburg, Germany

Introduction: Liver cirrhosis is frequently accompanied by malnutrition and zinc deficiency. Poor nutrition and protein deficiency are cloosely associated with the development of ascites.

Aim: With reference to this facts I would like to report my own experience with optimised nutrition treatment of patients with liver cirrhosis in a hepatological practice.

Patients: 25 patients with liver cirrhosis, 18 male, and 7 male, have been included in the study. The most frequent cause of the cirrhosis was alcohol $(n=18)$ followed by chronic hepatitis $\mathrm{C}$ $(n=3)$. Eleven of the 25 patients had ascites for the first time and 14 were experiencing a repeat occurrence. All main existing forms of treatment were used. Depending of their serum albumin level, the patients received BCAAs $(n=18)$ if they could afford them. Five patients used probiotic yoghurt (actimel) and two patients took neither of these. All patients exhibited zinc deficiency and zinc was therefore substituted in all cases (zinc histidine or zinc aspartate). 
Results: After 12-16 weeks, 13 patients no longer had ascites (mean albumin $36.27 \mathrm{~g} / \mathrm{l}$ ). Five patients had minor ascites (albumin $32.32 \mathrm{~g} / \mathrm{l}$ ) and seven patients had initial of ascites or more (albumin $27.38 \mathrm{~g} / \mathrm{l}$ ). The serum zinc levels at this time was still low in 18 and normal in 7 patients. After 6-9 months, it was possible to evaluate data for 17 patients. Ten patients had no ascites (albumin $38.5 \mathrm{~g} / \mathrm{l}$; zinc $11.7 \mu \mathrm{mol} /$ 1. Four patients exhibited minor acites (albumin $32.2 \mathrm{~g} / \mathrm{l}$, zinc $9.8 \mu \mathrm{\sigma}$ $\mathrm{mol} / \mathrm{l})$. In three patients, the acites quantity had increased further.

Conclusions: These findings underlie the importance of the nutrional state-indicate here by albumin — and of zinc levels for the treatment of ascites.

PP23-14

Clinical Study of Miniature Ultrasonic Probes in Diagnostic and Follow-up with Gastric Varices

H. Xu, Z. Zhao, J. Zhang, S. Wu

Norman Bethune First Hospital of Jilin University, Changchun, China

Objective: To evaluate the efficacy and safety of miniature ultrasonic probes (MUPs) in gastric varices" diagnosis and follow-up.

Method: Endoscopic ultrasonic procedures were performed in 84 suspicious gastric varices patients by method of miniature ultrasonic probes of $20 \mathrm{MHz}$ together with U-type reverse gastroscopy. And endoscopic therapy was performed according to the Endoscopic ultrasonography diagnosis.

Result: The diagnostic accuracy of gastric varices by miniature ultrasonic probes together with U-type reverse gastroscopy and conventional endoscopy finding were $82(69 / 84)$ and $100 \%(84 / 84)$, respectively.After the treatment of patients in the follow-up endoscopy ultrasonography may diagnose residual or recurrence of varices and continue treatment.

Conclusion: Miniature ultrasonic probes $(20 \mathrm{MHz})$ performance endoscopic ultrasonic procedures together with U-type reverse gastroscopy is demonstrated to be significantly superior to conventional endoscopic diagnosis in both accurate diagnosis ability of gastric varices and evaluation of efficacy of endoscopic therapy in the follow-up patients. It is very useful and more efficacy and safety.

\section{PP23-15}

Hyponatraemia, Hepatitis C, and Diabetes Are Risk Factors for Hepatic Encephalopathy in Cirrhosis: Prospective Study in a Large Patient Series H.R. Watson ${ }^{1}$, H. Vilstrup ${ }^{2}$, F. Wong ${ }^{3}$, P. Gines ${ }^{4}$

${ }^{I}$ Asia-Pacific R\&D, Sanofi-Aventis, Chilly-Mazarin, France;

${ }^{2}$ Department of Hepatology and Gastroenterology, Aarhus University Hospital, Aarhus, Denmark; ${ }^{3}$ Department of Medicine, Toronto

General Hospital, Toronto, ON, Canada, ${ }^{4}$ Liver Unit, Hospital Clinic, Barcelona, Spain

Introduction: Hepatic encephalopathy (HE) is one of the most common manifestations of decompensated cirrhosis, but there are no large studies assessing frequency, characteristics, and predictive factors.

Methods: Ambulant patients included in controlled trials of ascites management were followed for up to 1 year with visits every 4 weeks. Patients with HE >grade 1 at screening or a TIPS were excluded. Complications of cirrhosis were documented at every visit. Kaplan-Meier analysis was used for estimating 1-year event rates and a multivariate Cox model for relative risk (RR).

Results: 1,198 patients were included: $69.6 \%$ male and $30.4 \%$ female, with a mean age 57.2 years (SD 9.8), mean MELD score 13.3 (SD 4.1) and 26\% in Child-Pugh class C. Most frequent aetiologies were alcohol $(68.4 \%)$, HCV (21.1\%), HBV (8.4\%). 139 patients $(11.6 \%)$ were hyponatraemic $(\mathrm{Na} \leq 130 \mathrm{mmol} / \mathrm{l})$ on entry and 306 patients $(25.6 \%)$ had a history of HE. Median duration of observation was 240 days. In total 395 episodes of HE were recorded. 264 patients $(22.0 \%)$ experienced at least one episode (1-year event rate $27.1 \%$
(95\%CI 24.2, 29.9)) and 169 patients (14.1\%) had at least one episode leading to hospitalisation or death. The highest grade of $\mathrm{HE}$ was grade 4 in 28 patients $(2.3 \%)$, grade 3 in 44 patients $(3.7 \%)$ and grade 2 in 94 patients $(7.9 \%)$. Precipitating factors reported $(n=239)$ included infections $(24.7 \%)$, renal insufficiency $(9.2 \%)$, dehydration $(8.4 \%)$ and variceal bleeding $(7.5 \%)$. Episodes of HE were associated with previous history of HE (RR 2.22), hyponatraemia (RR 2.01), serum bilirubin $>51 \mu \mathrm{mol} / 1$ (RR 2.11), diabetes (RR 1.40) and hepatitis C aetiology of cirrhosis (RR 1.65).

Conclusion: This large study demonstrated that infections are the most common precipitating factors of HE. In addition to previous history of HE and poor liver function, this study identified hyponatraemia, hepatitis $\mathrm{C}$ infection, and diabetes as risk factors for $\mathrm{HE}$.

\section{PP23-16}

Oral Supplementation of Symbiotic to Decrease Ammonium Levels in Cirrhotic Patients with or without Minimal Hepatic Encephalopaty L.E. Moreno-Luna ${ }^{1}$, M. Fafutis-Morris ${ }^{2}$, V. Delgado Rizo ${ }^{3}$, V. Zuñiga ${ }^{4}$, J.E. Segura-Ortega ${ }^{5,6}$, Intestine and Cirrhosis

${ }^{1}$ Clinicas Médicas; ${ }^{2}$ Departamento de Inmunología; ${ }^{3}$ Departamento de Fisiología; ${ }^{4}$ Centro Universitario de Ciencias Exactas e

Ingeniería; ${ }^{5}$ Clínicas Quírurgicas, Universidad de Guadalajara;

${ }^{6}$ Departamento de Gastroenterología, Hospital Civil de Guadalajara Fray Antonio Alcalde, Guadalajara, Mexico

High ammonium is associated with minimal hepatic encephalopathy (MHE) and hepatic encephalopathy (HE). The inulin-type fructane prebiotic stimulates colonic bifidobacteria and inhibits proliferation of other bacteria. Probiotics (lactobacillus and bifidobacteria) increase the fecal content of bacilli and other beneficial bacteria. Symbiotic includes prebiotics and probiotics.

Materials and methods: The study was accepted by the institution's IRB. Patients gave informed consent; were $\geq 18$ years, without HE, no HE treatment, no severe complications in the last 3 months. Liver function tests, psychometric tests and arterial ammonium levels were performed at the beginning and after 90 days. From the liver clinic patients were randomly selected to received the symbiotic (inulintype fructan, lactobacillus rhamnosus plus bifidobacterium lactis) as an oral supplementation during 90 days. The AIM of this study was to determine the effects of the symbiotic in patients with cirrhosis with high ammonium with or without MHE.

Results: Thirty-two cirrhotic patients were included, $75 \%$ women, mean age 54 years (range 28-82), 23 (72\%) patients with HCV positive, $2(6 \%)$ autoimmune hepatitis, and $7(22 \%)$ alcohol and others. Five (16\%) patients had MHE with high ammonium levels and 27 (84\%) had high ammonium levels without MHE at the beginning of the study. Patients classified as Child-Pugh A were: 22 (69\%), B: 7 (22\%), C: $3(9 \%)$. Arterial ammonium levels before treatment were 24.48 to 272.88 mean 83.60 and after the symbiotic $15-196$ mean 40 $(P=<0.05)$. In $25(78 \%)$ patients ammonium decreased, in $5(16 \%)$ ammonium remained unchanged, and in $2(6 \%)$ patients ammonium increased. $(P=<0.05)$.

Conclusions: Patients receiving the symbiotic had a significant decrease in ammonium levels. No patient developed HE, 4 (80\%) patients with MHE improved the psychometric tests after receiving the symbiotic. Longer follow up should be done to evaluate if the incidence of $\mathrm{HE}$ events decreases.

\section{PP23-17}

Long-term Anthropometry of Cirrhosis Patients Administered Food Plus Aminoleban during Treatment Esophageal Varices

N. Nagata, S. Hirose, K. Shiraishi, T. Kagawa, T. Mine

Hepatology and Gastroenterology, Tokai University School of Medicine, Isehara, Japan

The aim of this study was to investigate the effect of branched chain amino acid (BCAA) on the nutritional improvement in malnutrition 
cirrhosis patients after the treatment procedures for esophageal varices, we performed the study where a regular diet and two packages of Aminoleban (BCAA containing products) were administered to the patients, and nutritional assessments were performed 2 weeks and 3 months after the treatment procedures to esophageal varices. Ten patients who were admitted to the Tokai University Hospital. In our previous study of diet only, alb was significantly reduced, but alb did not decreased this study two weeks after the treatment. Alb was increased significantly 3 month after the treatment $(p=0.0342)$. BCAA/Tyr ratio (BTR) did not elevate despite the administration of Aminoleban, and there was no difference in its value before the treatment and 3 months after the treatment. Body weight (BW) and Body mass index (BMI) were significantly lower 2 weeks after the treatment, as compared before the treatment. In our previous study of diet only, BW, BMI, \% TSF and \%AMC were significantly reduced, but $\%$ TSF and \%AMC did not decreased in this study two weeks after the treatment.

Nutritional treatment with diet plus Aminoleban improved malnutrition for cirrhosis patients after the treatment esophagus varices.

\section{PP23-18}

Transjugular Intrahepatic Portosystemic Shunts (TIPSS)-Auckland Hospital Experience

J. Hsiang ${ }^{1}$, A. Holden ${ }^{2}$, E. Gane ${ }^{3}$

${ }^{I}$ NZ Liver Transplant Unit; ${ }^{2}$ Radiology Department, Auckland City

Hospital; ${ }^{3}$ NZ LIver Transplant Unit, Auckland, New Zealand

A retrospective analysis was performed on efficacy and clinical outcome of all Transjugular intrahepatic portosystemic shunts (TIPSS) procedures performed at Auckland Hospital. The clinical records on all patients who underwent TIPSS at Auckland Hospital between 1996 and 2010 were reviewed. Clinical indices included in this analysis were aetiology and severity of liver disease, TIPSS indication, pre- and post-procedure serum creatinine, recurrence of gastrointestinal (GI) bleeding, ascites, stent patency rates, procedural complication and survival rates.

88 TIPSS procedures were attempted in 81 patients. Indication for TIPSS was refractory ascites in $36(41.9 \%)$, variceal bleeding in 24 $(27.9 \%)$, hydrothorax in $8(8.1 \%)$, hepatopulmonary syndrome in 2 $(2.3 \%)$, acute Budd Chiari syndrome in $1(1.2 \%)$ and hypersplenism in 4. $39.5 \%$ of the cases subsequently underwent liver transplantation. Perioperative blood transfusion requirements were not reduced in patients with pretransplant TIPSS. Procedure failure rate was $8.3 \%$. Primary patency was 63,56 , and $25 \%$ at 3,6 and 12 months respectively. Secondary assisted patency was $100 \%$ at 3, 6 and 12 months. Mean serum creatinine improved following TIPSS $(p=0.001)$. No procedure-related mortality occurred. Cumulative mortality was $20 \%$ at 1 year and $8 \%$ at 5 years. Eleven patients $(12.5 \%)$ developed hepatic encephalopathy, one died from coma, seven treated and responded to bowel cares. All TIPSS patients awaiting transplantation survived to transplant. Mean time to transplantation after TIPSS was 168 days. Expanded polytetrafluoroethylene covered stents were used in 45 patients, 33 of these placed after 2006. Successful TIPSS improves all complications of portal hypertension and may facilitate management of patients on the waiting list for transplantation. Hepatic encephalopathy is uncommon and long-term patency satisfactory with current surveillance protocols.

\section{PP23-19}

Serum Hepcidin in Patients with Advanced Liver Cirrhosis: Relationships with Iron Markers and Disease Severity

T.C.H. Tan ${ }^{1,2,3}$, M. Franklin ${ }^{4}$, P. Taylor ${ }^{4}$, L. Fletcher ${ }^{1}$, D. Crawford ${ }^{2,3}$

${ }^{I}$ Gastroenterology and Hepatology, Princess Alexandra Hospital, Woolloongabba; ${ }^{2}$ School of Medicine, University of Queensland, Brisbane; ${ }^{3}$ Gallipoli Medical Research Foundation, Greenslopes Private Hospital, Greenslopes; ${ }^{4}$ Clinical Pharmacology, Princess Alexandra Hospital, Woolloongabba, QLD, Australia
Background: Altered iron metabolism and elevated serum ferritin (SF) are common in advanced cirrhosis and may adversely influence survival. Hepcidin is the central iron regulatory hormone. The relationship between hepcidin homeostasis and iron status in advanced cirrhosis remains relatively unclear.

Aims:

(1) To prospectively evaluate serum hepcidin ( $\mathrm{SH})$ concentration in patients with advanced cirrhosis

(2) To determine the relationships between $\mathrm{SH}$, SF and hepatic iron concentrations (HIC) in patients with end-stage cirrhosis.

Methods: Thirty-eight cirrhotic patients were recruited. Severity of liver disease was assessed using the MELD score. Fasting serum biochemistry, iron indices and hepcidin were measured. SH concentration was quantified by a high performance liquid chromatographytandem mass spectrometry using an appropriate radiolabelled internal standard. HIC was measured using an MRI technology $\left(\right.$ Ferriscan $\left.^{\mathrm{R}}\right)$. Results: Male:female ratio was 35:3. Mean age was $52 \pm 7.5$ years. Cirrhosis aetiologies were alcohol $(n=7)$, hepatitis $\mathrm{C}$ virus (HCV) $(n=9), \mathrm{HCV} / \mathrm{alcohol}(n=12)$, non-alcoholic fatty liver disease $(n=4)$, chronic hepatitis B $(n=3)$ and cholestatic liver diseases $(n=3)$. Median MELD score was 13 . Median HIC was $13 \mathrm{mmol} / \mathrm{kg}$ dry tissue (range 5-113). HIC was $<20 \mathrm{mmol} / \mathrm{kg}$ in 29 patients (76\%), 20-40 mmol/kg in five patients $(13 \%)$ and $>40 \mathrm{mmol} / \mathrm{kg}$ in one patient (3\%). Mean SH was $15 \pm 1.9$ (range $<5-56.3) \mathrm{ng} / \mathrm{ml}$ and was below the lower limit of detection $(<5 \mathrm{ng} / \mathrm{ml})$ in $18(47 \%)$ patients, consistent with reduced hepatic synthesis, but did not correlate with the underlying MELD score $(r=-008, p=0.63)$ or HIC $(r=0.05, P=0.75)$. SH correlated significantly with SF (Spearman $r=0.74, P=<0.001$ ), Patients with SF above $400 \mu \mathrm{g} / 1$ had significantly higher hepcidin compared to those with SF lower than $200 \mu \mathrm{g} /$ 1 ( 27 vs. $8 \mathrm{ng} / \mathrm{ml}, P<0.0001)$. Patients with higher SF $(>400 \mu \mathrm{g} / \mathrm{l})$ had higher HIC ( $r=0.68, P=0.06)$.

Conclusion: Decreased hepcidin appears common in advanced cirrhosis, correlates well with SF and may be an important pathogenic mechanism underlying cirrhosis-associated iron loading.

\section{PP23-20}

The Design and Application of Circumferential Ligation below the Cardia on Gastroesophageal Varices (GOV1)

H. Xu, G. Chen, Y. Sun, Y.T. Tang, K.Y. Wang

Department of Gastroenterology, The First Hospital of Jilin

University, Chang Chun, China

Objective: To evaluate the effects of circumferential ligation below the cardia on gastroesophageal varices (GOV1).

Methods: A total of 245 patients with gastroesophageal varices (GOV1) were enrolled in the study from october 2006 to october 2010, they randomly allotted into two groups, 165 in the A group with endoscopic variceal ligation (EVL) in routine method,and 180 in the B group with circumferential ligation below the cardia by " $U$ " retroflexion (EVLR),to assess efficacies of EVL and EVLR for gastroesophageal varices.

Results: During the period of following-up (eighteen months), Markedly effective rate had significant difference between two groups $(P<0.05)$, B was better to A (64.4 vs. $48.5 \%)$. Averaging therapy times were $2.31 \pm 1.20$ in group A, $1.69 \pm 0.98$ in group B, respectively, B can significantly decrease the average therapy times $(P<0.05)$. and hemostasis rate of emergency in B was $100 \%$ better than A $(91.67 \%)$. However,relapsed bleeding rate, varices recurred rate,complications rate and mortality had not significant difference in both groups $(P>0.05)$.

Conclusion: Circumferential ligation below the cardia is more effective and safer than ligation in routine method for GOV1,it can improve the severity of gastroesophageal varices, raise the rate of emergency hemostasis, and decrease the averaging therapy times. 
PP23-21

The Survival of Escherichia coli Nissle 1917 in the Gastrointestinal Tract after the Twelve Days Treatment

J. Lata ${ }^{1}$, J. Jurankova ${ }^{2}$, V. Pribramska ${ }^{3}$, H. Skacelova ${ }^{2}$, L. Gerzova ${ }^{2}$,

T. Stosova ${ }^{4}$

${ }^{1}$ Department of Internal Medicine, Faculty of Medicine, Ostrava University, Ostrava, ${ }^{2}$ Department of Clinical Microbiology,

${ }^{3}$ Department of Internal Medicine, University Hospital, Brno,

${ }^{4}$ Department of Microbiology, University Hospital, Olomouc, Czech Republic

The aim of study: To establish survival of EcN after 12 days therapy in healthy volunteers.

Material and methods: $\mathrm{EcN}$ in the dose $2.5-25 \times 10^{9}$ of bacteria in one capsule (Mutaflor, Ardeypharma, Germany) was given to 18 healthy volunteers (6 males, 12 females, average age 25.8 /24-28/ years). One capsule was taken for four days and than two capsules before the breakfast for the 8 days. Microbiological examination of the stool and genotyping of EcN by Pulsed Field Gel Electrophoresis (PFGE) were done before and at 3rd, 6th, 9th, 12th, 15th, 18th, 22nd and 24th day after the initiating of the treatment.

Results: Microbiological examination was negative for $\mathrm{EcN}$ in all subjects before the treatment. Positive findings of E. coli (and PFGE proved $\mathrm{EcN}$ ) in the day 3rd was find in six subject (33\%) and in all subject except one in the day 12 th. In the day 15 , in 4 subject $(22 \%)$ was EcN already absent in the stool and in the day 24, positive findings was found just in five subjects $(28 \%)$.

Conclusion: After the 12 days therapy, EcN survive in the stool in $78 \%$ of subjects 4 days after the stopping of treatment and just in $28 \%$ of subjects after the 12 days of completion the therapy. If the treatment has to be effective, $\mathrm{EcN}$ has to be dosed on a long-term basis.

The research was supported by the Internal Grant Agency of the Ministry of Health of the Czech Republic (NS 9868-3).

\section{PP23-22}

The Prediction Values of Transient Elastography for Clinically Significant Esophageal Varices in Patients with Cirrhosis

T. Chaojin, Teerha Pirathvisuth

Medicine, Prince of Songkla University, Hat Yai, Thailand

Background: Variceal bleeding is a common complication and leading cause of death in cirrhotic patients. Endoscopic variceal surveillance is recommended in patients with cirrhosis. However the procedure requires experience endoscopist and is quite invasive procedure. Recent study had shown that liver stiffness measurement (LSM) was correlated with cirrhosis and portal hypertension. The aim of this study was to evaluate the role of Transient elastography in predicting presence of esophageal varices that require primary prophylaxis

Methods: All patients with cirrhosis who never has history of upper gastrointestinal bleeding underwent LSM with Transient elastography and followed by upper gastrointestinal endoscopy on same days by endoscopist who did not know the result of LSM. The clinically significant esophageal varices defined as esophageal varices $\geq$ grade II and/or presence of red wale sign. The relationships between LSM and clinically significant esophageal varices were analyzed in all patients Results: There were 52 patients with median age of 58 years, 35 patients were male and 47 patients had CTS class A. Clinically significant esophageal varices were seen in 14 patients. Means of LSM were 30.2 and $15.5 \mathrm{kPa}$ in patients with and without clinically significant esophageal varices, respectively. Patients with clinically significant esophageal varices had significant high liver stiffness scores than those without $(P<0.001)$. LSM value $>16.2 \mathrm{kPa}$ high predict the presence clinically siginicant esophageal varices (Se: $85 \%$, PPV: $41 \%$, NPV: $91 \%$ ).

AUROC values of LSM were 0.81 (95\% CI: 0.69-0.94) for clinically significant esophageal varices.
Conclusions: LSM is highly correlated with the clinically significant esophageal varices in patients with cirrhosis. LSM less than $16.2 \mathrm{kPa}$ highly predict absence of clinically significant esophageal varices with negative predictive value of $91 \%$.It, therefore, may be the potential tool for selecting patients who do not require endoscopic procedure.

\section{PP23-23}

25 Grams Versus 50 Grams of 25\% Albumin in Preventing Renal Impairment after Large Volume Paracentesis in Cirrhosis

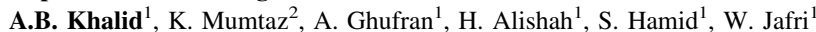

${ }^{1}$ Medicine, Aga Khan University Hospital; ${ }^{2}$ Medicine, Aga Khan University, Karachi, Pakistan

Background: Large volume paracentesis (LVP) in patients with cirrhosis may lead to significant hemodynamic changes termed as paracentesis induced circulatory dysfunction (PICD). PICD is marked by increased renin, impaired renal function (IRF), and decreased serum sodium. Intravenous albumin is effectively used to prevent PICD and IRF. The exact dose of intravenous albumin however, is not yet established.

Aims and objectives: To compare 25 and $50 \mathrm{~g}$ of $25 \%$ intravenous (IV) albumin to prevent IRF in patients with cirrhosis undergoing LVP. Methods: This was a cross sectional study of patients with cirrhosis who underwent LVP from January 2007 to July 2009 in our Gastroenterology unit. Patients with spontaneous bacterial peritonitis, creatinine $>1.5 \mathrm{mg} / \mathrm{dl}$, hepatoma, coexisting malignancy or paracentesis of $<51$ were excluded. Demographics, etiology, and laboratory parameters along with amount of LVP and dose of albumin received were noted. Moreover, serum creatinine and serum sodium at baseline and one week post paracentesis were recorded.

Results: 141 patients met the inclusion criteria and were included for analysis. 110 patients received $25 \mathrm{~g}$ of IV albumin while 31 received $50 \mathrm{~g}$ of IV albumin after LVP. Mean age,CTP scores and other biochemical parameters were comparable between the two groups Mean ascitic fluid removed was 6.71 in $25 \mathrm{~g}$ albumin group while 8.51 in $50 \mathrm{~g}$ albumin group. Serum creatinine at baseline and 1 week post LVP was $1.04 \pm 0.02$ and $1.07 \pm 0.03$ in the $25 \mathrm{~g}$ albumin group while $1.12 \pm 0.04$ and $1.4 \pm 0.17$ in $50 \mathrm{~g}$ albumin respectively, ( $p$ value 0.35 ). Similarly serum sodium at baseline and 1 week post LVP was $130.44 \pm 3.7$ and $129.62 \pm 4.4$ in the low dose albumin group while $129.62 \pm 5.6$ and $128.7 \pm 6.0$ in $50 \mathrm{~g}$ albumin respectively, ( $p$ value 0.14$)$.

Conclusion: We conclude that $25 \mathrm{~g}$ of $25 \% \mathrm{IV}$ albumin may be as effective as $50 \mathrm{~g}$ in preventing renal impairment after LVP in cirrhosis.

\begin{tabular}{|c|c|c|c|c|c|}
\hline & \multicolumn{2}{|c|}{$\begin{array}{l}\text { Albumin } 25 \mathrm{~g} \\
(n=110)\end{array}$} & \multicolumn{2}{|c|}{$\begin{array}{l}\text { Albumin } 50 \text { grams } \\
(n=31)\end{array}$} & \multirow[b]{2}{*}{$p$ value } \\
\hline & Pre LVP & Post LVP & Pre LVP & Post LVP & \\
\hline $\begin{array}{l}\text { Serum } \mathrm{Cr} \\
(\mathrm{mg} / \mathrm{dl})\end{array}$ & $1.04 \pm 0.02$ & $1.07 \pm 0.03$ & $1.12 \pm 0.04$ & $1.41 \pm 0.17$ & 0.35 \\
\hline $\begin{array}{l}\text { Serum Sodium } \\
\text { (meq/lit) }\end{array}$ & $130.44 \pm 3.5$ & $129.6 \pm 4.1$ & $129.62 \pm 5.6$ & $128.7 \pm 6.0$ & 0.14 \\
\hline
\end{tabular}

Serum creatinine and sodium before and after LVP

\section{PP23-24}

Phosphodiesterase-5 Inhibitor in Liver Cirrhosis with Portal Hypertension S. Chooklin

Medical University, Lviv, Ukraine

Introduction: As there is no satisfactory medical therapy for portal hypertension, there is a need for new pharmacological strategies.

Methods: We investigated the effects of phosphodiesterase-5 (PDE-5) inhibitor - sildenafil on hepatic and systemic hemodynamics. To 10 healthy subjects and 12 patients with Child-Pugh A liver cirrhosis 
$10 \mathrm{mg}$ sildenafil were administered orally. Measurements of hepatic and splanchnic blood flow by Doppler ultrasonography. The following haemodynamic parameters were measured: diameter of the portal vein, flow volume in the portal and splenic veins; resistivity indices in the common hepatic artery, splenic artery, and superior mesenteric artery. Results: Cardiac output and heart rate remained constant. After a short dip, mean arterial blood pressure again increased. Systemic vascular resistance transiently decreased slightly. Sildenafil significantly increased portal blood flow in healthy and in liver cirrhosis patients. The proportion of antegrade flow to retrograde flow increased. Inhibition of PDE-5 lowers intrahepatic portal resistance. In liver cirrhosis the resistivity indices of the common hepatic artery, of the splenic artery increased significantly.

Conclusion: Sildenafil increases portal flow and lowers portal pressure in patients with Child-Pugh A liver cirrhosis without relevant systemic side effects. Inhibition of PDE-5 may represent a novel principle for the treatment of portal hypertension.

\section{PP23-25}

Modified Percutaneous Transhepatic Variceal Embolization with 2-octylCyanoacrylate for Secondary Prophylaxis of Gastric Variceal Bleeding: Compared with Endoscopic Cyanoacrylate Injection

C. Zhang, X. Tian, Q. Wang, F. Liu, Y. Cui, F. Liu, J. Liu

Department of Gastroenterology, Provincial Hospital Affiliated to

Shandong University, Jinan, China

Aims: This retrospective study was to compare the efficacy of a modified percutaneous transhepatic variceal embolization (PTVE) with 2octyl-cyanoacrylate (2-OCA) and endoscopic injection sclerotherapy (EIS) with 2-OCA in prophylaxis of gastric variceal rebleeding.

Methods: Patients with history of gastric variceal bleeding who underwent either EIS or PTVE in our hospital from January 2006 to December 2008 were retrospectively reviewed. The rebleeding rate, survival rate and complications were compared between the two groups.

Results: Seventy-seven cases were included in this study. EIS was performed in 45 patients; PTVE was performed in 32 patients. During the follow-up $(19.78 \pm 7.70$ months in EIS group, $21.53 \pm 8.56$ months in PTVE group), rebleeding occurred in 17 patients (37.78\%) in EIS group, and in 4 patients $(12.5 \%)$ in PTVE group $(P=0.014)$. The cumulative rebleeding free rate was $74.9,59.3$, and 49.4 in 1,2 , and 3 years respectively for EIS; 93.4, 83.9, 83.9\% for PTVE $(P=0.011)$. Followup computed tomography $(\mathrm{CT})$ revealed that the PTVE with 2-OCA could achieve a long-lasting obliteration in the entire varices as well as all the feeding veins. The survival rate was not different in the two groups. No severe complications occurred in both groups.

Conclusions: With extensive and permanent obliteration of both gastric varices and its feeding veins, the modified PTVE with 2-OCA is considered to be an effective and safe method for gastric varices. It is superior to endoscopic 2-OCA injection in terms of preventing gastric variceal rebleeding.

\section{PP23-26}

Portal Hypertensive Polyps a New Entity in Portal Hypertension M.J. Choksi ${ }^{1}$, N.R. Bhatt ${ }^{1}$, V.S. Gudsoorkar ${ }^{1}$, D.N. Amarapurkar ${ }^{1}$, A.D. Amarapurkar ${ }^{2}$

${ }^{I}$ Department of Gastroenterology, Bombay Hospital and Medical Research Centre; ${ }^{2}$ Department of Pathology, BYL Nair Hospital, Mumbai, India

Background: Portal hypertensive polyps (PHPs) with charactersitic congested and proliferating capillaries in the lamina propria has been rarely described. This is the first largest study on PHPs.

Aim: To compare the prevalence and etiology of polyps seen on UGI scopy in patients with portal hypertension $(\mathrm{PH})$ with the polyps seen on UGI scopy done for all causes.
Methods: A retrospective analysis of all patients undergoing UGI scopy was done to compare the etiology of the polyps in the portal hypertensive group versus those without $\mathrm{PH}$.

Results: In patients with PH $(n=631)$, polyps were noted in 16 patients (PHPs $n=9$, hyperplastic polyp $n=6$ and fundic gland polyp $n=1)$. In the patients without $\mathrm{PH}(n=3,180)$ polyps of various etiologies were noted in 105 patients (hyperplastic $n=42$, leiomyomatous $n=7$, adenocarcinoma $n=7$, fundic gland polyp $n=7$ and others $n=42$ ). The prevalence of polyps of all causes was similar both groups ( 2.5 vs. $3.3 \%, p=0.3957$ ). The prevalence of hyperplastic polyps was similar in the portal hypertensive $(0.95 \%)$ and non portal hypertensive group (1.3\%). All PHPs were located in the UGI tract and were seen in patients cirrhotic PH. Fifty-five percent $(n=5)$ of patients with PHPs had history of prior endotherapy for variceal bleed.

Conclusion: Portal hypertensive polyps are a definite identifiable lesion in patients of cirrhosis with portal hypertension. Hypergastrinemia and angiogenesis may be a mechanism for development.

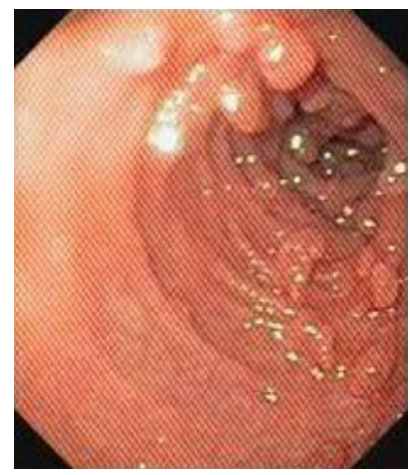

Multiple portal hypertensive polyps in duodenum

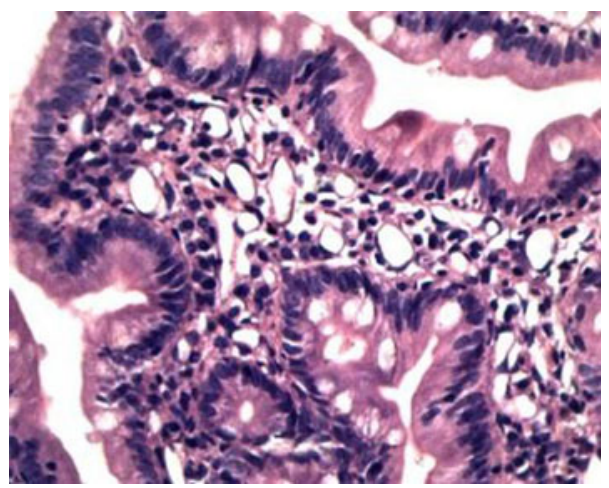

Histopathology image of portal hypertensive polyp

PP23-27

Novel Strategy for Management of Incarcerated Umbilical Hernia in Cirrhotic Patients

H.M. Moustafa ${ }^{1}$, M.A.-A. Nafady ${ }^{2}$, M.H. Othman ${ }^{3}$, A.F. Murad ${ }^{4}$

${ }^{1}$ Gastroenterology and Hepatology; ${ }^{2}$ Radiology, Al Azhar University;

${ }^{3}$ Radiology, Asyut University; ${ }^{4}$ Radiology, South Egypt Cancer

Institute, Assiut, Egypt

Objectives: The optimal management of umbilical hernia in patients with liver cirrhosis with or without ascites remains a matter of debate and poses unique and specific management problems due to the patho physiology of cirrhosis. 
The purpose of this study is to demonstrate the efficacy of non surgical reduction of incarcerated umbilical hernia in high risk cirrhotic patients by aspiration of fluid in the hernia sac and deflation of intestinal loop within the sac under sonographic guidance.

Patients and methods: We recruited 21 cirrhotic patients with incarcerated umbilical hernia referred to our radiology center from different surgeons within the period from January 2008 to January 2010. Color Doppler Sonography of the herniated bowel loops, aspiration of the fluid within the sac and deflation of the loops under sonographic guidance were done followed by of reduction of the hernia contents through the abdominal wall defect.

Results: The present study included 21 cirrhotic patients $[18$ males $(85.7 \%)$ and 3 females $(14.3 \%)]$. Three $(14.3 \%)$ cases proved inappropriate vasculature of the intestine by Color Doppler Sonography, so those were referred back to their surgeon for immediate operation. In the remaining $18(85.7 \%)$ patients, the Color Doppler proved appropriate vasculature, Complete aspiration of the fluid was done, followed by deflation of the distended loops inside the sac using 18 gauge syringe under Sonographic guidance. The hernias could be reduced completely in 15 out of the 18 patients $(83.3 \%)$. In the remaining three $(16.7 \%)$ cases, the hernia could not be reduced so the patients were referred back to their surgeons.

Conclusion: Cirrhotic patients with incarcerated umbilical hernia could be managed safely with that procedure as an alternative to surgery or at least to be a bridge for later safe elective surgery. The possibility of invalidity and failure of the procedure should be considered.

\section{PP23-28}

Follow-up of Esophageal Varices, Which Were Treated with Endoscopic Variceal Ligation Using Conventional Endoscopy and High-frequency Intraluminal Ultrasound

W.H. Choe ${ }^{1}$, S.Y. Kwon ${ }^{1}$, S.Y. Ko ${ }^{1}$, J.D. Lee ${ }^{2}$, C.H. Lee ${ }^{1}$

${ }^{1}$ Department of Internal Medicine, Konkuk University Hospital, Seoul; ${ }^{2}$ Department of Internal Medicine, Konkuk University Hospital, Choong-Joo, Republic of Korea

Background: The role of endoscopic-ultrasonography (EUS) for follow-up of esophageal varices treated with endoscopic variceal ligation (EVL) is not established.

Methods: Endoscopic follow-up with synchronous esophagogastroduodenoscopy (EGD) and high-frequency intra-luminal ultrasound (HFIUS) was performed in 38 patients underwent EVL for hemorrhage from esophageal varices (EV) We examined postEVL varices by EGD regarding variceal grades and red color (RC) signs, and by HFIUS regarding the largest variceal cross-sectional surface area (CSA). In 30 patients, esophageal varices disappeared or were reduced to grade 1 by EVL on EGD findings, and they were prospectively followed for 1 year from when synchronous endoscopic follow-up was performed and we evaluated the predictive risk factors of future their re-bleedings, based on EGD and HFIUS findings.

Results: EGD grading of post-EVL EV had good correlation with their HFIUS measurements $(0.77 ; p<0.01)$. However, there was no significant difference in the largest CSA of post-EVL EV between patients who would experience future re-bleeding and those would not $\left(7.2 \pm 3.9,5.3 \pm 4.2 \mathrm{~mm}^{2}, p=0.31\right)$. EGD findings of EV were more predictive than their HFIUS measurements (Rebleeding rate: $57 \%$ in Grade 1 with $\mathrm{RCS}(+)$ vs. $10 \%$ in Grade 0 or Grade1 without $\mathrm{RCS} ; p=0.03)$.

Conclusions: Conventional EGD was sufficient modality to follow up of EV treated with EVL. Therefore, it might not be necessary that EUS were performed to evaluate post-EVL varices.
PP23-29

A Study on Prevalence of Hepatopulmonary Syndrome among Patients of Portal Hypertensive Gastropathy

M. Murmu, E. Chandrasekaran, A.K. Malhotra, H. Wardhan, P. Jain, P. Tyagi, A.K. Agarwal, N.K. Chaturvedi

P.G.I.M.E.R. and Dr. Ram Manohar Lohia Hospital, Delhi, India

Background and aim: To study the prevalence of Hepatopulmonary Syndrome (HPS) among a cohort of patients with Portal Hypertensive Gastropathy (PHG) diagnosed at endoscopy in a tertiary-care hospital in central Delhi, India. The two complications of portal hypertension, i.e., PHG and HPS have common patho-physiological pathway and biological factors responsible for their development.

Method: Forty patients, both outpatients and in-patients, found to have PHG, with the background of chronic liver disease and portal hypertension were selected for the study. Detailed history, physical examination and relevant laboratory investigations were done. All patients underwent pulmonary function tests, measurement of oxygen saturation through pulse oximetry, measurement of partial pressure of oxygen and alveolar arterial oxygen gradient through arterial blood gas analysis and 2-dimensional contrast echocardiography was done to visualize the intrapulmonary vascular dilatations.

Results: Out of forty patients, $37(92.5 \%)$ were males with alcohol being the main aetiology of chronic liver disease $(85 \%)$. Twelve patients fulfilled the definition of HPS, out of whom ten (83.33\%) were males and two $(16.66 \%)$ were females. Maximum patients with PHG presented in the third and fourth decade of life and also HPS is more prevalent in this age group only. Prevalence of HPS in our patients with PHG is $30 \%$. Dyspnea and clubbing were the most significant findings in patients with HPS ( $p=0.000$ and 0.007 , respectively). Hypoxemia and orthodeoxia $\left[\mathrm{P}(\mathrm{A}-\mathrm{a}) \mathrm{O}_{2}>15 \mathrm{mmHg}\right]$ was present in $30 \%$ of the patients ( $p=0.000$ and 0.031 , respectively). Patients with HPS had statistically significant Child-Turcotte-Pugh and Model for End-stage Liver Disease (MELD) Score $(p=0.003$ and 0.028 , respectively). Patients with mild PHG were 65\%, of whom 27\% had HPS, whereas in $35 \%$ patients of severe PHG, $35.7 \%$ had HPS $(p=0.059)$.

Conclusion: HPS is more common in patients with severe PHG.

\section{PP23-30}

Efficacy of the 1st vs.2nd HVPG Measurements and Two MELDs for Predicting the Survival in Patients with Decompensated Liver Cirrhosis K.T. Suk ${ }^{1}$, D.J. Kim ${ }^{1}$, S.H. Jun ${ }^{1}$, C.W. Kim ${ }^{1}$, O.K. Kwon ${ }^{1}$, H.S. Kim ${ }^{2}$, S.R. Shin $^{2}$, S.H. Park ${ }^{2}$, M.S. Lee ${ }^{2}$, C.K. Park ${ }^{2}$, S.H. Park ${ }^{3}$

${ }^{1}$ Gastroenterology Internal Medicine, Chuncheon Sacred Hospital Hallym University College of Medicine, Chuncheon;

${ }^{2}$ Gastroenterology Internal Medicine, Sacred Hospital Hallym University College of Medicine, Seoul; ${ }^{3}$ Department of Internal Medicine, Inje University College of Medicine, Pusan, Republic of Korea

The prognostic value of the HVPG for survival is still a controversial issue and few data are available for the efficacy of the 2nd HVPG for predicting patient survival. We aimed to evaluate the efficacy of a 2 nd HVPG measurement compared with the 1st HVPG measurement and two MELD (MELD and MELD-Na) for predicting the survival of decompensated liver cirrhosis. Between January 2006 and January 2010, 57 patients with decompensated liver cirrhosis, all of whom underwent more than two HVPG measurements (mean interval: $9.6 \pm 10.4$ months), were enrolled. The prognostic accuracy of the 1 st and 2nd HVPGs, MELD, and MELD-Na scores were analyzed by means of the AUROC. Ten patients (17.5\%) died from cirrhosis. Reasons of the 2nd HVPG were varix rebleeding $(n=17)$ and confirmation of the adequacy of beta-blocker dosing $(n=40)$. Differences between the 1 st and 2nd examinations were not related 
with death. AUROCs (1st and 2nd) were 0.843 and 0.863 in HVPG, 0.663 and 0.587 in MELD, and 0.715 and 0.678 in MELD-Na, respectively. Of the six measurements, only the 1st and 2nd HVPGs ( $p=0.001,95 \%$ CI $0.72-0.97 ; p=0.000,95 \%$ CI $0.75-0.98)$ and the 1st MELD-Na ( $p=0.03,95 \%$ CI $0.49-0.86)$ had prognostic power for the death of patients with decompensated liver cirrhosis. Survival using each cut-off was significantly different in the 1 st and 2nd HVPG and the 1st MELD-Na $(p<0.05)$. In multivariate analysis, gender (Female; OR 4.75, 95\% CI 1.23-18.33) and the 2nd HVPG (OR 1.11, 95\% CI 1.05-1.17) were positively related with death.

Conclusions: Although HVPG had a limited role in the prediction of death in decompensated cirrhosis, a high level of the 2nd HVPG and the female gender were risk factors of death. Therefore, regular follow-up HVPG, especially in females, may be needed for the evaluation of prognosis in patients with decompensated liver cirrhosis.

\section{PP23-31}

Treatment Outcomes of Gastric (Fundus) Variceal Bleed by Endoscopic Sclerotherapy with N-Butyl-2 -cyanoacrylate

M. Gill, U. Adeeb, S. Naseem, N. Mehmood

Division of Digestive Diseases, Maroof International Hospital, Islamabad, Pakistan

Aims: Gastric variceal bleed is a very challenging clinical emergency. Routine endoscopic band ligation is not feasible and has questionable efficacy. We wanted to study the efficacy of endoscopic injection therapy with $N$-Butyl-2-cyanoacrylate (EBC) in gastric variceal hemorrhage $(\mathrm{GVH})$.

Methods: We consecutively enrolled 20 patients who presented with hemetemesis or melena and on endoscopy had large fundal gastric varices with, Spurting/oozing from the varices or with cherry red spot or adherent clot. The study period was from july2009 to August 2010.we injected 3-5 cc of $N$-Butyl-2-Cyanoacrylate mixed with equal amount of lipoidal into fundal $\mathrm{v}$ arices until all the lesions became hardened and bleeding stopped. These patients were kept under regular review for 12 months.

Results: Fifteen out of 20 patients were male, median age was 44 . All were child C cirrhotics because of Hep C. 12 out of $20(60 \%)$ had GOV 2 and $8(40 \%)$ had GOV 1 and GOV 2.Initial homeostasis was achieved in 16 out of $20(80 \%)$ patients. Four out of 20 (20\%) patients did not respond to this therapy, had massive bleed and did not survive. Six out of 20 patients rebled after median F/U of 10 months. Four were successfully treated with second session of EBC, two patients have massive bleed and EBC did not help. After one year 14 out of $20(70 \%)$ patients did not rebleed and they are under continuous F/U. Active bleeding on initial EBC was significant predictor of recurrent bleed. Conclusion: EBC is an effective modality of treatment in GVH to achieve homeostasis. However in some patients other options like urgent TIPS is required who do not respond to this modality.

\section{PP23-32}

Long-term Prognosis of Patients with Gastric Fundal Varices Receiving Balloon-occluded Retrograde Transvenous Obliteration

M. Nakazawa, K. Hamaoka, Y. Hujii, Y. Uchida, T. Handa, K. Hirahara, H. Uchiya, M. Yamaoka, T. Chikayama, K. Watanabe, S. Ando, Y. Mizuno, K. Yoshino, K. Sugawara, D. Motoya, K. Hujimori, M. Inao, N. Nakayama, Y. Imai, S. Nagoshi, S. Mochida

Gastroenterology and Hepatology, Faculty of Medicine, Saitama Medical University, Iruma-gun, Japan

Background and aims: Balloon-occluded retrograde transvenous obliteration (B-RTO) is a standardized procedure for patients with gastric fundal varices, but the effect of major shunt obliteration on clinical course of the patients remains uncertain. Thus, the long-term prognosis of the patients receiving B-RTO therapy was evaluated. Patients and methods: Objects were 101 liver cirrhosis patients that had undergone B-RTO for gastric fundal varices between 1999 and 2010. Forty-three patients received B-RTO therapy following rupture of the varices (hemorrhage cases) and 58 patients for the prophylaxis of hemorrhage (prophylaxis cases). According to the Child-Pugh classification, 37, 56 and 8 patients were diagnosed as grade A, B, and $\mathrm{C}$, respectively. Hepatocellular carcinoma (HCC) was found in 26 patients. The prognosis of the patients was assessed in relation to the demographic and clinical features of the patients.

Results: B-RTO was successfully performed in 97 patients $(96 \%)$. Recurrence of gastric fundal varices was not found in these patients. Overall cumulative survival rates were 71,65 and $59 \%$ at 3,5 and 7 years, respectively, following B-RTO. The rates did not differ between hemorrhage cases and prophylaxis cases. Multivariate analysis revealed that the presence of HCC and Child-Pugh classification (grade A vs. B and C) were the significant factors to affect the survival rates $(p<0.05)$. Esophageal varices were aggravated in 20 patients (21\%), and the rupture of esophageal varices found in 5 patients $(5 \%)$. Liver function tests, such as serum albumin and bilirubin concentrations and prothrombin time, were not deranged and intractable ascites did not develop following B-RTO in any of the patients.

Conclusions: B-RTO is a useful therapeutic procedure for gastric fundal varices both in hemorrhage and prophylaxis cases, which may produce excellent prognosis except for patients with $\mathrm{HCC}$ and those diagnosed as grade $\mathrm{B}$ and $\mathrm{C}$ according to the Child-Pugh classification.

\section{PP23-34}

A New Approach to Spontaneous Bacterial Peritonitis Diagnostics in Patients with Liver Cirrhosis

V. Ivashkin, M. Maevskaya, E. Fedosina

Hepatology, 1 I.M. Sechenov Moscow Medical University, Moscow, Russia

Introduction: Spontaneous bacterial peritonitis (SBP) is one of the most frequent bacterial infections in patients with decompensated cirrhosis and ascites and is associated with high (20-40\%) mortality. Aim: To determine clinical importance of investigation of blood serum cytokines and ascitic fluid in patients suffering from SPB.

Materials and methods: 38 patients with liver cirrhosis and ascites were examined in the clinic. The diagnosis was made in compliance with the criteria of the IAC. The average age of the patients was $49.1 \pm 13.2$ (from 23 to 74 ). Male to female ratio was $1.5: 1.0$. The patients were grouped in Class B (12 people) and Class C (26 people) of Child-Pugh system. In MELD system the patients were divided into two groups-14 patients (10-20 points) and 24 patients (20-30 points). In addition to routine methods of diagnosing patients with clinical SPB manifestations had the levels of cytokines (IL-1, IL-6, $\mathrm{TNF}-\alpha$ ) in blood serum and ascitic fluid measured ('Protein Contour') before the administration of antibiotics treatment.

Results: Terminal patients had higher levels of cytokines (TNF- $\alpha$ and IL-6) compared to patients of Class B irrespective of etiology of the disease. In the group of patients where the survival rate was not more than 6 months, the average levels of TNF- $\alpha$ and IL- 6 accounted for $922.3 \pm 190.6$ and $123.41 \pm 9.1 \mathrm{pg} / \mathrm{ml}$ and exceeded the relative figures in patients with liver cirrhosis of Class B (303.4 \pm 47.1 and $55.3 \pm 8.1 \mathrm{pg} / \mathrm{ml}$, respectively).

Conclusion: The SPB development was accompanied by higher levels of TNF- $\alpha$ and IL- 6 in blood serum and ascitic fluid in patients with liver cirrhosis of Class $\mathrm{C}$ than in patients with Class B $(p<0.001)$. The measurement of TNF- $\alpha$ and IL-6 levels gives 
additional clinical information which contributes to making a proper diagnosis and more effective treatment of SPB patients, thus, giving the possibility to prolong the life of patients.

\section{PP23-35}

Prediction of Compensated Liver Cirrhosis by Ultrasonography and Routine Blood Tests in Chronic Viral Hepatitis

S.W. Cho, H.S. Lee, J.Y. Cheong, S.S. Kim

Gastroenterology, Ajou University School of Medicine, Suwon, Republic of Korea

Background/aims: It has been demonstrated that liver biopsy is a standard method for the diagnosis of liver cirrhosis in chronic hepatitis. Because the liver biopsy is an invasive method, non-invasive methods have been used to diagnose compensated liver cirrhosis in chronic hepatitis. The current study was designed to evaluate ultrasonography and routine blood tests for diagnosing compensated liver cirrhosis in chronic viral hepatitis.

Materials and methods: A total of 203 patients with chronic viral hepatitis who underwent liver biopsy were included in this study and ultrasonography and routine blood tests were analyzed retrospectively. Ultrasonography findings such as surface nodularity, parenchyma echogenecity and spleen size were evaluated. The diagnostic accuracy of ultrasonography and routine blood tests were examined.

Results: Discriminant analysis with forward stepwise selection of the variables showed that liver surface nodularity and platelet count, albumin level were independently associated with compensated liver cirrhosis $(p<0.05)$. Cross tabulation displays that all four variables of platelet counts $<100,000 / \mathrm{uL}$, albumin levels $<3.5 \mathrm{~g} / \mathrm{dL}$, INR $>1.3$, and surface nodularity had $>95 \%$ specificity. If at least one of the four variables is found in patients, we can predict liver cirrhosis with $90 \%$ specificity and $61 \%$ sensitivity.

Conclusions: These results suggest that four variables including platelet counts $<100,000 / \mathrm{uL}$, albumin levels $<3.5 \mathrm{~g} / \mathrm{dL}$, INR $>1.3$, surface nodularity can be used to identify liver cirrhosis in chronic viral hepatitis with high specificity.

\section{PP23-36}

Effectiveness of Adefovir Dipivoxil-based Rescue Therapy in Lamivudineresistant Hepatitis B Virus-related Liver Disease

H.J. Lee, H.C. Lee, J.R. Eun

Internal Medicine, Yeungnam University College of Medicine, Daegu, Republic of Korea

Background/aims: This study was conducted to evaluate the risk of liver disease-related events according to the baseline liver status and on-treatment viral response during adefovir dipivoxil (ADV)-based rescue therapy in patients with lamivudine-resistant hepatitis $B$ virus (HBV)-related liver disease.

Materials/methods: Between March 2004 and April 2009, a total of 475 patients were treated with ADV for more than 1 year. A total of 408 patients with lamivudine resistance prior to 2005 were enrolled as historical controls because ADV was unavailable at that time.

Results: The median follow-up was 36 and 23 months in the ADV group and the control, respectively. For patients with Child-Pugh class A cirrhosis, one $(0.9 \%)$ died from hepatic encephalopathy in the non-sustained viral suppression (persistently PCR negative, SVS) group, whereas five $(3.4 \%)$ in control group. The incidence of hepatocellular carcinoma (HCC) was 5.9 (1/51), 7.9 (9/114), and $10.2 \%(15 / 147)$ in patients with SVS, non-SVS, and control group, respectively $(p=\mathrm{NS})$. For patients with Child-Pugh class B or C, the incidence of death was $8.0(2 / 25), 16.1(9 / 56)$ and $30 \%(30 / 100)$ in patients with SVS, non-SVS and control group, respectively $(p<0.001)$. The incidence of hepatic encephalopathy was $8.7(2 / 23)$, $13.5(7 / 52)$ and $25.5 \%(24 / 94)$ in each group $(p=0.006)$. The incidence of HCC was $13.6(3 / 22), 24.0(12 / 50)$ and 26.0\%(25/96) in each group (SVS vs. control, $p=0.040$ ).
Conclusion: ADV-based rescue therapy reduced the risk of liver diseaserelated events such as death, $\mathrm{HCC}$, and hepatic encephalopathy in patients with lamivudine-resistant hepatitis B virus-related decompensated cirrhosis. Sustained viral suppression with PCR negative might be more beneficial. Therefore, strict and sustained viral suppression is important in cirrhotic patients, in particular, when they are decompensated.

\section{PP23-37}

A Case of Acute Portal Vein Thrombosis in Patient with Protein C, S Deficiency Syndrome Totally Recanalized by Anticoagulant Therapy B.K. Choi, S.H. Yang, J.H. Kim, J.W. Seo, E.M. Kang, S.H. Kim, J.S. Jang Seoul Veterans Hospital, Seoul, Republic of Korea

Portal vein thrombosis is a form of venous thrombosis affecting the hepatic portal vein, which can lead to portal hypertension. Portal vein obstruction is a rare condition with an overall incidence of $0.05-0.5 \%$ in autopsy studies. Treatment of portal vein thrombosis includes anticoagulants, thrombolysis, insertion of shunts, bypass surgery to the portal vein and even transplantation. Especially, anticoagulant therapy is not curable but associated with reduction in new thrombotic episodes. We experienced a 68-year-old man of acutely proceeded total occlusion of intrahepatic and main portal vein thrombosis on abdominal CT and doppler sono, with multiple microabscesses. The presenting symptoms were general myalgia, fever, nausea, vomiting and poor oral intake. The laboratory test showed mild leukocytosis and elevated liver enzyme than three times of normal limit. The coagulation test was compatible with the protein $\mathrm{C}, \mathrm{S}$ deficiency syndrome (factor $\mathrm{C} 41 \%$, protein $\mathrm{S}$ $14 \%$ ). We diagnosed the portal vein thrombosis with protein $\mathrm{C}, \mathrm{S}$ deficiency syndrome. We started the anticoagulant therapy with warfarin 5mg and low molecular weight heparin (enoxaparin: $1 \mathrm{mg} / \mathrm{kg}$ $\mathrm{q} 12 \mathrm{~h}$ ). After 2 weeks, we had switched enoxaparin into warfarin $2.5 \mathrm{mg}$, and maintained antibiotics for 4 weeks. The symptoms were improved and liver abscesses were disappeared on next CT. Since then, we had followed the clinical progress with INR, doppler sono, abdominal $\mathrm{CT}$ and confirmed the total recanalization after 6 months. As ever, we have done continuously follow up of progress. So we report the case because we have experienced total resolution of portal vein thrombosis by only anticoagulant therapy.

\section{PP23-38}

Histological Sub-classification of Cirrhosis Correlates with Clinical Stage and Grade of Portal Hypertension in Patients with Cirrhosis

M.Y. Kim ${ }^{1}$, S.K. Baik ${ }^{1}$, H.K. Jeon ${ }^{1}$, H.J. Park ${ }^{1}$, S.Y. Park ${ }^{1}$, Y.O. Jang ${ }^{1}$, Y.J. Kim ${ }^{2}$, G.J. Cheon ${ }^{3}$, Y.D. Kim ${ }^{3}$

${ }^{1}$ Internal Medicine; ${ }^{2}$ Department of Radiology, Yonsei University Wonju College of Medicine, Wonju; ${ }^{3}$ Department of Internal Medicine, Kangneung Asan Hospital, Ulsan University College of Medicine, Kangneung, Republic of Korea

Backgrounds/aim: It has been suggested that histologic further subclassification of cirrhosis is required because of heterogenecity of severity within cirrhosis. We aimed to determine the relationship between histological subclassification and clinical stage of liver cirrhosis as well as grade of portal hypertension.

Methods: One hundred-twenty three patients who had biopsy-proven cirrhosis and were able to estimate clinical stage of cirrhosis and hepatic venous pressure gradient (HVPG) were included. Histologic subclassification of cirrhosis using Laennec fibrosis scoring system was scored semiquantitatively without knowledge of clinical stage of cirrhosis and HVPG results were evaluated. Laennec fibrosis scoring system subclassified cirrhosis as mild; thin septa, moderate; at least two broad septa, and severe; at least one very broad septum or many minute nodules. The clinical stages of cirrhosis were defined as stage 1: no varix and ascites, stage 2: varix without ascites, stage 3: ascites without variceal bleeding and stage 4 : variceal bleeding irrespective of ascites. Biologic and laboratory data were also collected from all included patients. 
Results: Histologic subclassification of cirrhosis using Laennec fibrosis scoring system significantly correlated with both the clinical stage of cirrhosis $(P<0.001)$ and HVPG (mild: $8.1 \pm 2.6 \mathrm{mmHg}$, moderate: $12.4 \pm 3.3 \mathrm{mmHg}$, severe: $16.3 \pm 4.0 \mathrm{mmHg}, \quad P<0.001)$. With higher grade of histologic subclassification of cirrhosis, increased frequency in both severe portal hypertension (HVPG $>12 \mathrm{mmHg}$ ) and an episode of variceal hemorrhage was observed $(P<0.001)$.

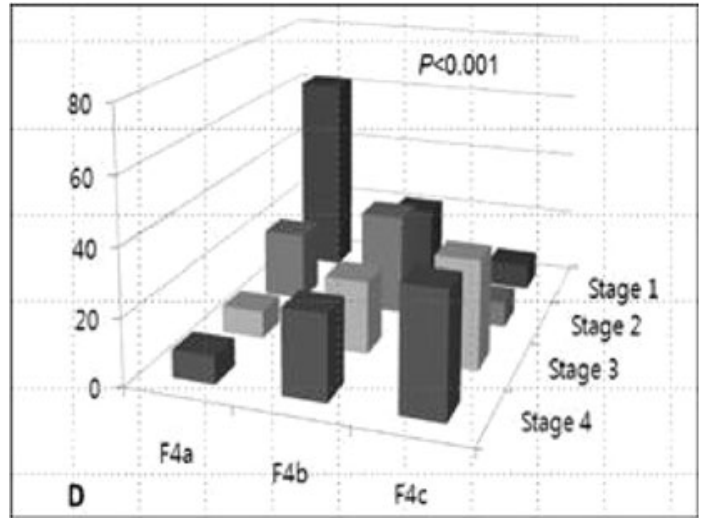

Laennec stage and clinical stage

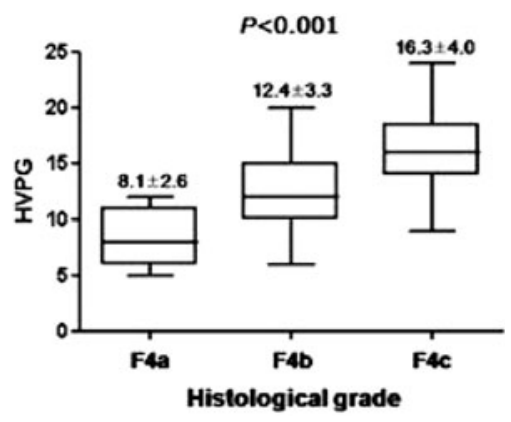

Laennec stage and HVPG

Conclusions: Histologic subclassification of cirrhosis using Laennec fibrosis scoring system is well correlated with both the clinical stage of cirrhosis and grade of portal hypertension. It suggests that cirrhosis should be subclassified into different stage according to its histologic severity.

\section{PP23-39}

Evaluation of New Methods for Assessing Recurrent Ascites in Liver Cirrhosis in Therapeutic Studies

H.R. Watson ${ }^{1}$, P. Minini ${ }^{2}$, G. Lecorps ${ }^{2}$, F. Wong ${ }^{3}$, P. Gines ${ }^{4}$

${ }^{1}$ Asia-Pacific R\&D; ${ }^{2}$ Biostatistics, Sanofi-Aventis, Chilly-Mazarin,

France; ${ }^{3}$ Department of Medicine, Toronto General Hospital,

Toronto, ON, Canada $;{ }^{4}$ Liver Unit, Hospital Clinic, Barcelona, Spain

Background and aims: Patients with liver cirrhosis often require long-term treatment for the control of ascites. Quantification of the recurrence of ascites over the long-term is therefore important for the evaluation of new treatments, but methods reported are more appropriate for short-term effects. The aim was to compare standard time to first event methods of analysis with alternatives for the long-term assessment of recurrent ascites.

Methods: 887 patients with liver cirrhosis and recurrent ascites were studied in three placebo-controlled clinical trials of a treatment for ascites. Durations of treatment ranged from 12 to 52 weeks. Patients underwent a therapeutic paracentesis at baseline. Paracenteses and changes in body weight were recorded throughout the studies. The following variables were calculated: time to first paracentesis, the total volume of ascites accumulated and number of paracenteses. Statistical methods used were Kaplan-Meier estimates for time to first event, Nelson-Aalen estimates for cumulative number of events over time and regression analysis for estimates of total volume of ascites. Results: Both time to first paracentesis (relative risks 0.60-0.79) and recurrent event methods (relative risks 0.63-0.90) demonstrated treatment effects on risk of paracentesis. Estimations of the total cumulative volume of ascites (ratios of slopes 0.66-0.97) also demonstrated treatment differences. Number of paracenteses proved less sensitive when there was an imbalance in the mean volume of ascites removed at each procedure.

Conclusion: There are good alternatives to assessing time to first recurrence of ascites in studies of chronic ascites treatment. Sensitivity was greater with analyses of all paracenteses, not only first event, and estimations of the cumulative volume of ascites may avoid bias from paracentesis of different volumes and may also be appropriate to studies in populations undergoing less frequent paracentesis.

\section{PP23-40}

Splenic Artery Embolization (SAE) and Selective Partial Splenic Embolization (SPSE) to Treat Complications of Portal Hypertension. A Preliminary Study

P. Quaretti ${ }^{1}$, T. Cascella ${ }^{1}$, N. Cionfoli ${ }^{1}$, M. Torresi ${ }^{1}$, G. Poggi ${ }^{2}$, G. Ghittoni ${ }^{3}$ ${ }^{1}$ Interventional Radiology, Fondazione IRCCS Policlinico San Matteo; ${ }^{2}$ Oncology II, Fondazione IRCCS Salvatore Maugeri; ${ }^{3}$ Interventional Ultrasound, Fondazione IRCCS Policlinico San Matteo, Pavia, Italy

Background and aims: To evaluate the safety and efficacy of SAE and SPSE as new materials and devices allow either to decrease the portal pressure than to induce a predictable amount of spleen infarction.

Patients and methods: Twenty patients (m/f 13/7; age 38-81 years) were treated with SAE by coils or plugs in splenic artery and with SPSE by ultraselective glue injection. Bleeding varices or ascites were treated by SAE and SPSE if associated with thrombocytopenia (T) $(<30.000 / \mathrm{ml})$. SAE alone was performed in absence of T. Standalone SPSE was applied for isolated T. The resolution of haemorrage or ascites or a threefold increase of platelet count were considered as clinical success. Necrotic spleen volume was evaluated by computed tomography (NSVCT).

Results: Twenty-four procedures were performed (SAE with SPSE in 9/20 patients). Thirteen had cirrhosis and liver cancer; four had portal thrombosis not-cirrhosis related. One had mesenteric aneurysms (Kadir syndrome) and hypersplenism. SAE alone was performed in five patients with liver cancer. Only one stand-alone SPSE was attempted for $\mathrm{T}$ in myelofibrosis related splenomegaly associated to splenic vein thrombosis. Technical success was $100 \%$ (24/24), while clinical success in 19/20 as platelets count does not improved significantly after SPSE alone (1/1). NSVCT was always $>50 \%$ after SPSE (14/14). All SPSE-patients experienced a mild postembolization syndrome $(100 \%)$. Transitory severe ascites and hydrothorax occurred after SPSE alone (1/1). No complications were detected after SAE alone (8/8).

Conclusions: SAE and SPSE could be feasible and effective therapeutic options in the management of complicated severe portal hypertension and thrombocytopenia. Further studies are warranted.

\section{PP23-41}

Procalcitonin Documents a Proinflammatory State in Cirrhotics with Ascites Not Present in Nonascitic Cirrhotics

D.H. van Thiel ${ }^{1}$, C. Moore ${ }^{1}$, N.I. Nedelcu ${ }^{2}$, M. George ${ }^{1}$, A. Bashar ${ }^{3}$, A. Zacharian ${ }^{3}$, S. Dhillon ${ }^{1}$, G. Ramadori ${ }^{4}$, J. Fareed ${ }^{5}$

${ }^{1}$ Medicine/Hepatology/Liver Transplant, Rush University Med

Center, Chicago, IL, USA; ${ }^{2}$ Medicine/Infection Diseases/ 
Epidemiology, Victor Babes infection Clinic, Bucharest, Romania;

${ }^{3}$ Hepatology/GI, Cook County Hospital, Chicago, IL, USA;

${ }^{4}$ Hepatology/Transplant/GI, August Georg University, Gottingen,

Germany; ${ }^{5}$ Pharmacology/Pathology, Loyola University Medical

Center, Maywood, IL, USA

Background: Cytokines, interleukins and growth factors modulate liver disease progression. Procalcitonin (PCT) a prohormone released by cells during an inflammatory increases logarithmically as the inflammation process progresses from a local condition to organ failure. Aims:

1) To assess the level of PCT in the serum, plasma and ascetic fluid of cirrhotics with and without ascites.

2) Compare the levels of cytokines and growth factors in the serum, plasma and ascetic fluid of cirrhotics.

Methods: 41 cirrhotics with massive ascites and 47 cirrhotics without ascites were studied. A complete medical evaluation MELD, Child Pugh Scores were calculated. PCT was assayed utilizing the BioMerieux assay. Cytokines and growth factors were assayed utilizing biochips from Randox. Control values were defined by results obtained using normal plasma.

Results: The 47 nonascitic subjects consisted of 23 with HCV, 13 with alcohol and 11 with NASH. The 41 ascitic subjects consisted of 21 with alcoholic cirrhosis, 8 with extrahepatic malignancy, 4 with cardiac cirrhosis and 8 with $\mathrm{HCV}$, three of whom had hepatoma.The PCT levels in the noncirrhotics was $0.10+0.01$, in cirrhotics with ascites, it was $1.38+0.04$ and in normal pooled serum it was $<0.10$. The levels of IL-4, IL-6, IL-8, IL-10, IL-1b, VEGF, TNF- $\alpha$ and MCP1 were all significantly greater in ascites fluid than plasma and serum. EGF levels were greater in plasma/serum than in ascitic fluid. No difference between plasma/serum and ascitic fluid for levels of IL-2, IL-1a and IFN- $\gamma$.

\section{Conclusions:}

1) Ascites is associated with a proinflammatory state manifested by an increased PCT level.

2) Levels of IL-4, IL-6, IL-8, IL-10, IL-1b, VEGF, TNF- $\alpha$, and MCP-1, are greater in ascetic fluid than plasma/serum in cirrhotics with ascites.

3) EGF levels are greater in plasma/serum than in ascitic fluid suggesting a primary extraperitonial or nonhepatic origin.

PP23-42

Survival of Alcoholic Cirrhosis and Cryptogenic Cirrhosis where Liver Transplantation Is Not an Option

S.M. Senanayake ${ }^{1}$, A. Kasturiratne ${ }^{2}$, J.P. de Alwis ${ }^{3}$, S.D. Nandasiri ${ }^{3}$, S.P. Hewawasam ${ }^{1}$, R.S. Kumarasena ${ }^{1}$, A.P. de Silva ${ }^{3}$, A.S. Dassanayake ${ }^{4}$, H.J. de Silva ${ }^{3}$

${ }^{1}$ University Medical Unit, Colombo North Teaching Hospital;

${ }^{2}$ Department of Public Health; ${ }^{3}$ Department of Medicine;

${ }^{4}$ Department of Pharmacology, Faculty of Medicine University of

Kalaniya, Ragama, Sri Lanka

Background/aims: The main causes of cirrhosis in Sri Lanka are alcohol induced or cryptogenic. This study compared the survival of alcoholic cirrhosis and cryptogenic cirrhosis in a setting where liver transplantation is not an option.

Methods: Patients with alcoholic cirrhosis and cryptogenic cirrhosis whose clinical and follow-up details were available were identified from our cirrhosis database, maintained since 1995. For those who had not attended out-patient clinics within 4 weeks, the patient or families were contacted to obtain survival status or details of death. The cause of death was ascertained from death certificates and patient records. Survival was compared using Kaplan-Meier curves. Patients were not considered for liver transplantation abroad, mainly due to financial constraints.
Results: Details of 424 patients (alcoholic cirrhosis 224, cryptogenic cirrhosis 200) were available for analysis $(80.15 \%$ (424/529) of all registered patients from 1995). There were 64 deaths (58 liver related) in the alcoholic cirrhosis group and 64 deaths (60 liver related) in the cryptogenic cirrhosis group. Kaplan-Meier survival curves showed no significant difference in survival between the two groups (logrank test statistic 0.807) [Figure]. Multivariate analysis using the Cox proportional hazards model showed that the only predictor of survival was Child's grade $(p<0.001)$. $71 \%$ of cryptogenic cirrhotics had components of the metabolic syndrome, suggesting a possible diagnosis of non-alcoholic steatohepatitis related cirrhosis.

Conclusion: In this cohort, the outcome of alcoholic cirrhosis seems to be similar to cryptogenic cirrhosis (many of whom were possible NASH related), and deaths in both groups were predominantly liver related.

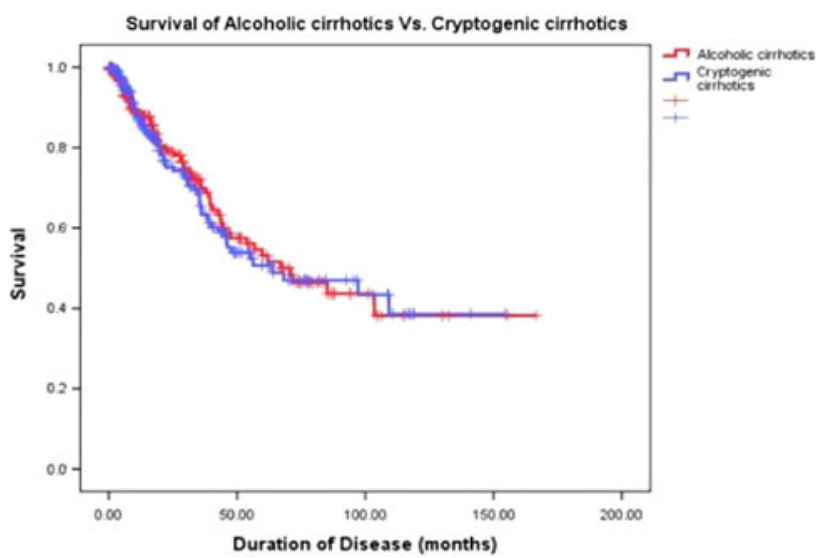

Survival-Alcoholic versus Cryptogenic cirrhosis

\section{PP23-43}

Hepatic Vein Transit Time (HVTT) in Assessing Portal Hypertension and Response to Treatment with Beta-Blockers. Preliminary Results L. Siciliani, G. Vitale, S. Racco, G. Marrone, M. Pompili, G.L. Rapaccini Catholic University of Rome, Rome, Italy

Background: It has been demonstrated that alterations in Doppler hepatic vein $(\mathrm{HV})$ waveform are associated with cirrhosis and portal hypertension. Hepatic vein flow becomes flat when cirrhosis worsens. We prospectively evaluated the correlation between the extent of Doppler transfiguration in cirrhotics expressed by an hepatic vein waveform index (HVWI) and the time employed by an ultrasound contrast agent of II generation to cross the liver, before and after acute oral administration of propranolol.

Methods: We enrolled 37 participants: 33 cirrhotics and 4 healthy controls; a subgroup of 15 patients received acute oral administration of $40 \mathrm{mg}$ propranol. Doppler shifts signals were obtained from right hepatic vein. HVWI was calculated by the maximum velocity minimum velocity / maximum velocity. We recorded out a clip from $20 \mathrm{~s}$ before to $2 \mathrm{~min}$ after a peripheral intravenous bolus injection of $2.4 \mathrm{ml}$ of SonoVue ${ }^{\circledR}$. The time employed by contrast agent to cross liver from hepatic artery and portal vein to hepatic vein was defined as HA-HVTT and PV-HVTT.

Results: Cirrhotics with low HVWI showed an earlier transit times; participants with higher HVWI had longer transit times. We found a significant difference of transit times between participants with HVWI $>1$ and patients with HVWI $<1$ ( $p<0.01$ for PV-HVTT and HA-HVTT). 
HVWI increased by $42.60 \%$ after propranolol administration with significant difference $(p<0.05)$. HV-HATT becomes significantly $(p<0.01)$ longer after propranolol (mean : $6.55 \mathrm{~s}$; mean after propranolol $9.74 \mathrm{~s}$ ).

HV-PVTT becomes longer after propranolol (mean: $1.79 \mathrm{~s}$; mean after propranolol: $3.29 \mathrm{~s}$ ) without a significant difference.

Conclusions: Our data suggest that a haemodynamic effect of intrahepatic shunts rather than a fixed structural abnormality is the pathogenic mechanism responsible for the transfiguration of hepatic vein waveform in cirrhosis. Transit times could be useful tool in the evaluation of response to treatment with propranolol.

PP23-44

Is Acoustic Radiation Force Impulse Elastography (ARFI) a Good Method for the Evaluation of Ascites Syndrome?

S. Bota, I. Sporea, R. Sirli, A. Popescu, M. Danila, M. Sendroiu Gastroenterology and Hepatology, University of Medicine and Pharmacy, Timisoara, Romania

Introduction: ARFI elastography is a new method used for the assessment of liver fibrosis, offered by Siemens and integrated into an ACUSON S2000 ultrasound system.

Aim: To assess the feasibility of ARFI elastography in patients with ascites and its predictive value for the cirrhotic our non-cirrhotic etiology of the ascites.

Methods: Our study included 153 patients with ascites, mean age $58.8 \pm 3.1$ years, 92 men and 61 women. $115(75.2 \%)$ patients had ascites in the context of cirrhosis (diagnosed by clinical, ultrasound, endoscopic and/or laparoscopic criteria). 29 (18.9\%) of patients had noncirrhotic ascites (20 laparoscopic demonstrated peritoneal carcinomatosis, 7 acute pancreatitis with ascites which was later resolved and one case each of lymphatic ascites and ascites in the context of a liver abscess). In nine (5.9\%) cases we could not establish the etiology of ascites. We performed ten ARFI measurements and a median value was calculated, expressed in meters/second $(\mathrm{m} / \mathrm{s})$.

Results: We could not obtain ARFI measurements in five patients (3.2\%). The mean liver stiffness measurements by means of ARFI were statistically significant higher in patients with cirrhotic ascites, than in those with non-cirrhotic ascites: $3.04 \pm 0.70$ versus $1.45 \pm 0.59 \mathrm{~m} / \mathrm{s}(p<0.001-E S)$. For a cut-off value of $1.8 \mathrm{~m} / \mathrm{s}$ for predicting cirrhosis (and ascites in the context of cirrhosis), ARFI had $98.1 \% \mathrm{Se}, 86.2 \% \mathrm{Sp}, 96.4 \% \mathrm{PPV}, 92.5 \% \mathrm{NPV}$ and $95.6 \%$ accuracy for predicting cirrhotic ascites.

Conclusion: ARFI elastography is feasible in $96.8 \%$ of cases with ascites and has a very good accuracy $(95.6 \%)$ for predicting the cirrhotic or non-cirrhotic etiology of ascites.

\section{PP23-45}

The Utility of Transient Elastography in Predicting Complications of Chronic Liver Disease

T. Zakareya, N. Allam, W. Abdel-Razek, A. Abogabal, E. Rewisha, I. Waked Hepatology, National Liver Institute, Menoufiya University, Shebeen Al-koam, Egypt

Background: Transient elastography (TE) is a non-invasive and reproducible method for assessment of liver fibrosis by measuring liver stiffness (LSM). Several studies in chronic liver disease have determined good accuracy of TE in predicting significant fibrosis and an optimal accuracy in predicting cirrhosis, and showed that liver stiffness varies widely after histological diagnosis of cirrhosis $(15-75 \mathrm{kPa})$.

The aim of the present study was to evaluate the utility of TE in predicting the development of complications of chronic liver disease. Methods: 75 Patients ( $75 \%$ males, mean age 44.3 years) presenting with one or more of the complications of liver cirrhosis had LSM assessed by Fibroscan. The correlation between LSM and each complication was studied. For each complication, a cut-off value of LSM was determined at which negative predictive value was $100 \%$ to include all cases with the complication.

Results: The underlying etiology of liver cirrhosis was HCV in 94.7\%. $66.7 \%$ of patients were Child A; $16 \%$ were Child B and $17.3 \%$ were Child C. There was a significant correlation between LSM and all complications of cirrhosis. Cut off values of 15.05, 17.75, 18.3, 39.9 , and $32.35 \mathrm{kPa}$ predicted the presence of esophageal varices, large varices, variceal bleeding, Child-Pugh class $\mathrm{C}$ and hepatocellular carcinoma (HCC) respectively with $100 \%$ NPV and sensitivity. Conclusion: Transient elastography appears a promising non-invasive tool for predicting the occurrence of complications of cirrhosis. LSM beyond $15 \mathrm{kPa}$ indicates the risk of developing varices and above 32 for $\mathrm{HCC}$ and patients with measurements above these values should be screened and closely monitored. Prospective longitudinal studies are needed to validate these results.

\section{PP23-46}

Capsule Endoscopy in Diagnose of Alteration of Vessel Chanel and Gastrointestinal Bleeding in Patients with Portal Hypertension with Cirhosis

D. Mareeva, I. Kovyazina, P. Shcherbakov, L. Lazebnik Central Scientific Research Institute of Gastroenterology, Moscow, Russia

Introduction: Portal hypertension is characterized by wide development of collateral blood circulations. Usually it occurs in area of cardioesafageal transition, hemorrhoidal veins in rectum, vascular texture of anterior wall of belly, however it is frequent together that takes part in small bowel veins. Diagnostics of intestines vascular channel condition and intestinal bleedings was limited of standard endoscope long or was made according to indirect laboratory indicators.

\section{Aim:}

1. To estimate capsule endoscopy possibilities for estimation of condition of a vascular channel of a small intestine at patients with portal hypertension.

2. To do a comparative estimation of changes of small intestine mucous.

Materials and methods: We investigated 17 patients at the age from 19 to 68 years ( 12 men and 5 women) with portal hypertension and varicose expanded veins of esophagus and colon various degree. For all patients after preparation by Flit-fosfosoda or PEG was given video capsule with help of two capsules: 1 . Given Image SB2 ${ }^{\circledR}$. 2. MiroCam ${ }^{\circledR}$. After carrying out CE was made a comparative analysis researched from standard endoscopy.

Results: It is possible to confirm that at all of patients with portal hypertension have changes of a vascular channel in a kind of varicose expansions was defined in 15 arterivenous malformation in 6 angyodisplasia in 5, Delafua defects in 3, bleedings in 2 patients. CE is an indicative and authentic method of diagnostics of changes of a vascular channel at patients with a portal hypertension.

\section{PP23-47}

Portal Hypertension in Patients with Liver Cirrhosis in Small Bowel

D. Mareeva, I. Kovyazina, P. Shcherbakov, L. Lazebnik Central Scientific Research Institute of Gastroenterology, Moscow, Russia

Liver cirrhosis is one of the most serious diseases in gastroenterology. This problem is going up because of several different reasons: (1) increase of quantity of people getting much alcoholic drinks, (2) spreading of viral hepatitis. And of course one of the most serious complications of liver cirrhosis is portal hypertension. It is well known that portal hypertension characterized by wide development of collateral blood circulations. 
Aim:

1. To evaluate development of portal hypertension in patients with liver cirrhosis in small bowel.

2. To evaluate the possibility of bleeding in such patients.

3. To do a correlation between class of liver cirrhosis and expression of portal hypertension in veins of small bowel.

Materials and methods: We investigated 17 patients at the age from 19 to 68 years (12 men and 5 women) with portal hypertension and varicose expanded veins of esophagus and colon various degree. For all patients after preparation by Flit-fosfosoda or PEG was given video capsule with help of two capsules: 1.Given Image SB2 ${ }^{\circledR}$. 2. MiroCam ${ }^{\circledR}$. After carrying out CE was made a comparative analysis researched from standard endoscopy and class of liver cirrhosis.

Results: It is possible to confirm that at all of patients with portal hypertension have changes of a vascular channel in a kind of varicose expansions was defined in 15 arteriovenous malformation in 6 angyodisplasia in 5, Delafua defects in 3, bleedings in 2 patients.

Conclusion: It is possible to say that all of the patients with liver cirrhosis and portal hypertension have different alterations in small bowel. These changes doesn't correlate with class of liver cirrhosis. $\mathrm{CE}$ is an indicative and authentic method of diagnostics of changes of a vascular channel at patients with a portal hypertension and doesn't influence quality of live of patients.

\section{PP23-48}

Hassab's Operation in Patient with Hepatocellular Carcinoma

T. Shiraki, J. Kita, Y. Iso, M. Kato, M. Shimoda, T. Sawada, K. Kubota

The Second Surgery, Dokkyo Medical University, Tochigi-Ken, Japan

Backgrounds: Hassab's operation was introduced clinically in early 1970's by Hassab MAF,to treat esophageal varices and hypersplenism secondary to portal hypertension. Hassab's operation consists of splenectomy and devascularization of upper part of stomach and lower part of esophagus.It is not rare to have patients having esophageal varices and hypersplenism as well as hepatocellular carcinoma (HCC). One interesting question in these patients is the timing of Hassab's operation. In this study, we retrospectively reviewed the clinical data and analyzed outcome of patients with HCC, who underwent hepatectomy and Hassab's operation simultaneously, and who underwent Hassab's operation followed by hepatectomy.

Methods: Between July 2000 and April 2010,15 patients with HCC underwent Hassab's operation and hepatectomy at our department. There were 13 males and 2 females with a mean age of 62-year-old. There were one HBV-positive, five HCV-positive, seven HBV- and $\mathrm{HCV}$ - positive, and two HBV- and HCV-negative patients. Patients were divided into two groups, Group A $(n=10)$ : patients who underwent hepatectomy and Hassab's operation simultaneously, Group B $(n=5)$ : patients who underwent Hassab's operation followed by hepatectomy. Our indication for simultaneous operation is the patients with HCC having preoperative platelet count more than $5.0 \times 10,000 / \mathrm{mm}$. Preoperative,operative and postoperative data were compared between the two groups.

Results: Mean preoperative platelet counts of Group A and B were $5.0 \times 10,000 / \mathrm{mm}$ and $23.6 \times 10,000 / \mathrm{mm}$, respectively $(p<0.01)$. There were no significant differences between the two groups,in preoperative total AST,ALT,bilirubin, and albumin. Mean blood loss were 1,204 and $912 \mathrm{ml}(p=0.26)$. No post-operative complication, such as bleeding from surgical sites and rupture of esophageal varices, were observed in the two groups. In Group B, median interval between Hassab's operation and hepatectomy was 5 weeks.

Conclusion: Simultaneous operation of hepatectomy and Hassab's operation can be performed safely, as long as the indication is observed and operation is performed with meticulous techniques.
PP23-49

Short Term Nasogastric Feeding for Hospitalised Asian Patients with Liver Cirrhosis

M.-L.S. Tai ${ }^{1}$, H. Razlan ${ }^{2}$, K.-L. Goh $^{1}$, S.H.M. Taib ${ }^{3}$, A.T.M. Huzaini ${ }^{3}$, S. Rampal ${ }^{4}$, S. Mahadeva ${ }^{1}$

${ }^{1}$ Medicine, University of Malaya; ${ }^{2}$ Medicine, Hospital University Kebangsaan Malaysia; ${ }^{3}$ Dietetics; ${ }^{4}$ Social and Preventive Medicine, University of Malaya, Kuala Lumpur, Malaysia

Background: Malnutrition in patients with advanced cirrhosis is common. The benefit of short-term naso-gastric (NG) feeding over oral supplementation remains uncertain.

Methods: A prospective, dual-centre, randomized trial of NG versus oral feeding was conducted in Asian patients with decompensated liver cirrhosis. Nutritional intervention was conducted for 2 weeks with a follow up observation at 6 weeks. The primary outcome related to changes in nutritional parameters whilst alteration in Child-Pugh (CP) score was a secondary end-point.

Results: A total of 52 patients (mean age $58.9 \pm 12.2$ years, $38.5 \%$ females, main aetiology: alcohol $36.5 \%$ and viral hepatitis $44.2 \%$ ) were recruited between August 2006 and May 2010. 28 cases were randomized to NG feeding and 24 to oral feeding. No significant differences were noted between the two groups at baseline in terms of $\mathrm{CP}$ score, anthropometry and Global Subjective Assessment. At 2 weeks, there was a non-significant improvement in anthropometry (TST mean difference $0.013 \pm 1.184$, MAMC mean difference $0.3513 \pm 1.81997$ ) and biochemistry (serum transferrin mean difference $0.12 \pm 1.20$ ) in the NG group compared to the oral group. Improvement in $\mathrm{CP}$ scores at 2 weeks were not significantly different between both groups (NG mean difference $-0.68 \pm 1.14 \mathrm{vs}$. oral mean difference $-0.43 \pm 1.29, p=\mathrm{NS}) .12 / 28(42.9 \%)$ patients had poor tolerance (VAS score $<50$ ) to NG tube placement. Nine patients (four NG, five oral) suffered mortality prior to the 6 week follow up. No significant differences in both nutritional and clinical parameters were observed between both groups in the remaining patients at 6 weeks.

Conclusion: Short-term NG tube feeding is poorly tolerated and confers little benefit over oral feeding in Asian patients with advanced cirrhosis.

\section{PP23-50}

Contrast Research of Nucleoside Analogs on Treatment for Cirrhosis due to Chronic Hepatitis B

D.-W. Zeng, J. Dong, W.-J. Xu, Y.-Y. Zhu, J.-J. Jiang

Center of Liver Disease, The First Affiliated Hospital of Fujian Medical University, Fuzhou, China

Objective: The aim of the research was to evaluate the therapy efficacy and safety of adefovir dipivoxil (ADV), lamivudine (LAM) and entecavir (ETV) on the treatment of decompensated cirrhosis due to chronic hepatitis B.

Methods: Patients with cirrhosis of hepatitis B virus infection were divided into three groups. Group A were given ADV $10 \mathrm{mg} / \mathrm{d}$, group B LAM $100 \mathrm{mg} / \mathrm{d}$, and group C were ETV $0.5 \mathrm{mg} / \mathrm{d}$. Child-Pugh scores and HBV DNA were analyzed, retrospectively.

Result: After the treatment of 12 weeks, 48-96 weeks, the negative rate of HBV DNA in group A was $34.3,57.7,71.5 \%$, respectively, in the group B was $47.8,50,60 \%$, respectively; at weeks $12-48$, the negative rate of HBV DNA in group C was $54.5,76 \%$, respectively. The ChildPugh scores of group A was $8.40 \pm 0.43$ at the pretreatment, $6.86 \pm 0.38,5.66 \pm 0.33,5.26 \pm 0.65$ at weeks $12,48,96$, respectively; That of group B was $8.65 \pm 0.35,6.47 \pm 0.38,5.91 \pm 0.37$, $5.59 \pm 0.43$ respectively; That of group $C$ was $8.25 \pm 0.41$, $6.47 \pm 0.47,5.62 \pm 0.41$ at the pretreatment and at weeks 12,48 , respectively. During follow-up did not find the virus mutation. Found no obvious side effects.

Conclusion: ADV, LAM and ETV can inhibit HBV replication in cirrhosis patients due to hepatitis $\mathrm{B}$, impove the liver function and 
decrease in Child-Pugh score. Its long-term efficacy and early virologic response in patients with a positive correlation exists, the virological response sooner, the more stable long-term effects.

\section{PP23-51}

Long-term Result of Endoscopic Histoacryl ${ }^{\circledR}$ ( $N$-butyl-2-cyanoacrylate) Injection for Treatment of Gastric Varices: Focus on Primary Prophylaxis J.Y. Jang, S.W. Jeong, E.J. Kang, S.W. Hong, J.Y. Cho, S.H. Lee, S.G. Kim, Y.S. Kim, Y.K. Cheon, Y.D. Cho, H.S. Kim, B.S. Kim Institute for Digestive Research, Department of Internal Medicine, Soon Chun Hyang University Hospital, Seoul, Republic of Korea

Background and aim: Endoscopic obliteration with Histoacryl ${ }^{\circledR}$ ( $N$-butyl-2-cyanoacrylate) is the most commonly used treatment for gastric variceal bleeding. However data regarding the long-term efficacy and safety of this procedure are lacking. Thus we evaluated the long-term efficacy and safety of endoscopic obliteration with Histoacry $l^{\circledR}$ for treatment of gastric variceal bleeding. Especially we focused on the Histoacry ${ }^{\circledR}$ injection therapy for gastric varices which carry a high risk of bleeding.

Methods: A total of 127 patients with gastric varices received Histoacryl $^{\circledR}$ injections endoscopically. Of these, 27 received such injections as a prophylactic procedure.

Results: The rate of initial hemostasis was $98.4 \%$ and recurrent bleeding within 1 year occurred in $18.1 \%$ of patients. Successful hemostasis during episodes of rebleeding was achieved in $73.9 \%$ of cases. Median survival was 50 months (95\% CI 30.5-69.5). Major complications occurred in four patients $(3.1 \%)$. The rebleeding rate in patients with hepatocellular carcinoma (HCC) was higher than in those with other conditions. None of the 27 subjects who were treated prophylactically experienced treatment-related complications. Cumulative survival rates of the 127 patients at 6 months, 1, 3, and 5 years were $92.1,84.2,64.2$, and $45.3 \%$, respectively. The 6-month cumulative survival rate of the 27 patients treated prophylactically was $75 \%$.

Conclusion: Histoacryl ${ }^{\circledR}$ injection therapy is an effective treatment for gastric varices and has a high rate of successful hemostasis and low incidence of complications. Furthermore, Histoacryl ${ }^{\circledR}$ injection therapy is an effective prophylactic treatment of gastric varices which carry high risk of bleeding.

\section{PP23-52}

Estimating GFR with Creatinine and Cystatin C-based Equations in Cirrhotic Patients

C. Luangchosiri ${ }^{1}$, A. Sobhonslidsuk ${ }^{1}$, P. Klyprayong ${ }^{1}$, P. Meemaew ${ }^{2}$,

A. Sangcakul ${ }^{3}$, A. Chittamma ${ }^{2}$, C. Kuhiran ${ }^{1}$, S. Vanavanan ${ }^{2}$, C. Kitiyakara ${ }^{1}$

${ }^{1}$ Medicine; ${ }^{2}$ Pathology; ${ }^{3}$ Research Center, Ramathibodi Hospital,

Mahidol University, Bangkok, Thailand

Background: In patients without liver disease, glomerular filtration rate (GFR) can be estimated by equations based on serum creatinine $(\mathrm{Cr})$ such as MDRD or the Cockcroft-Gault $(\mathrm{C}-\mathrm{G})$ equations. Serum $\mathrm{Cr}$ is an inaccurate indicator and has many limitations in decompensate cirrhotic patients who generally have low muscle mass and depleted $\mathrm{Cr}$ production. Moreover, serum $\mathrm{Cr}$ measurement is interfered by high level of plasma bilirubin. Cystatin C (Cys), a peptide which has been used as an alternative marker of GFR measurement, may be more sensitive in patients with cirrhosis.

Objective: To compare the accuracy of estimated GFR (eGFR) equations based on serum $\mathrm{Cr}$ and Cys with measured GFR (mGFR) in cirrhotic patients.

Methods: This study was performed in 30 cirrhotic patients who had no history of recent acute kidney injury. $5 \mathrm{ml}$ of iohexol (Omnipaque 300) was injected to each study patient. Plasma iohexol concentration was performed by HPLC technique at 10-time points between 5-300 min after injection. mGFR was calculated from iohexol dose divided by area under the concentration-time curve using two- compartment model method. Baseline $\mathrm{Cr}$ and Cys were transformed into different eGFR equations, and were compared with mGFR.

Results (The final results will be presented in Feb 2011): Until now, we have obtained the results of six cirrhotic patients (five males and one female aged between 46 and 65 years). Mean single plasma $\mathrm{Cr}$ was $0.7 \mathrm{mg} / \mathrm{dl}(+0.24)$. $\mathrm{mGFR}$ was $115.14+15.58 \mathrm{ml} / \mathrm{min} / 1.73$ $\mathrm{m}^{2}$. MDRD (132.0 $\left.\overline{2} \pm 41.39\right)$ tended to overestimate mGFR. C-G $(111.85+35.48)$ and $\mathrm{Cys}(119.34+19.43)$ provided the closest estimate to mGFR.

Conclusions: In patients with cirrhosis, MDRD equation tends to overestimate mGFR. C-G and Cys equations may be more accurate to estimate mGFR. More studies with greater number of patients are necessary to confirm if Cys equations are superior to Cr-based equations.

\section{PP23-53}

Clinical Features and 1-year Mortality of Cirrhotic Patients with and without Portal Vein Thrombosis

K. Liaw, J. Chang

Gastroenterology and Hepatology, Singapore General Hospital, Singapore, Singapore

Background: Portal vein thrombosis (PVT) is an important complication of liver cirrhosis that is associated with poor outcome.

Aim: To investigate the clinical features and outcome of cirrhotic patients with and without PVT.

Methods: Retrospective cohort study of 292 patients with liver cirrhosis seen in our department from September 2006 to January 2009. Clinical, radiological and endoscopic data were retrieved from computerized hospital records. Clinical features and outcome at 1 year were compared between those with and without PVT.

Results: Eighteen patients $(6.2 \%)$ were identified to have PVT. Demographic data and Child class were similar in both groups. Hepatitis B was less common in those with PVT versus those without (38.9 vs. $59.1 \%, p=0.014$ ). Alphafetoprotein levels were significantly higher $(2,808 \pm 11,861$ vs. $64 \pm 495, p<0.001)$ and platelet counts significantly lower in the PVT group $(115 \pm 61$ vs. $154 \pm 78$, $p=0.038$ ). Hemoglobin levels were significantly lower and urea levels significantly higher in the PVT group, reflecting the higher frequency of variceal bleeding. On radiology, patients with PVT had significantly higher frequency of splenomegaly and varices. Gastroscopy was performed more frequently in PVT patients (95 vs. 63\%, $p=0.006$ ). Esophageal and gastric varices were present in 100 and $35 \%$ of patients with PVT, compared to 46 and $11 \%$ in those without ( $p<0.001$ and 0.011 , respectively). Large varices were more common in the PVT group (93.8 vs. $49.2 \%, p<0.05$ ). Outcome at 1 year was poorer amongst those with PVT, with a higher rate of decompensation ( 83.3 vs. $20.8 \%, p<0.001)$, variceal bleeding (33.3 vs. $6.9 \%, p<0.001)$, ascites (61.1 vs. $11.3 \%, p<0.001)$, encephalopathy (33.3 vs. $9.0 \%, p=0.002)$ and spontaneous bacterial peritonitis (22.2 vs. $1.5 \%, p<0.001$ ). Cirrhotics with PVT had a higher 1-year mortality compared to those without (27.8 vs. $9.5 \%, p=0.015)$. Spontaneous resolution of thrombosis was seen in five (27.8\%) with median time to resolution of 11.5 months (range 2-24).

Conclusion: The development of portal vein thrombosis in cirrhotics is a poor prognostic factor and is associated with higher rate of variceal bleeding and a significantly higher 1 -year mortality rate.

\section{PP23-54}

Screening of Minimal Hepatic Encephalopathy in Asymptomatic, Apparently Healthy, Ambulant, Non-shunted, Egyptian Drivers with Liver Cirrhosis

Y.Z. Gad

Internal Medicine, Mansoura University, Mansoura, Egypt

Background/aim: Minimal hepatic encephalopathy (MHE) represents a portion of the spectrum of hepatic encephalopathy (HE). MHE 
can have a far-reaching impact on quality of life, ability to function in daily life and progression to overt HE. This study was designed to screen for MHE in Mansoura drivers with liver cirrhosis.

Patients and methods: 174 consecutive drivers, with viral markers positive serology attending the hepatology outpatient clinic of Mansoura Specialized Medical Hospital for assessment prior to deciding Interferon therapy, were screened for MHE. Questionnaires, and standard psychometric tests and well informed consent were performed at the same setting. The diagnosis of MHE was made when one or both SDT and NCT appeared abnormal.

After overnight fasting, venous blood was taken for haematologic tests and routine liver function tests by conventional methods. Arterial ammonia was measured also.

Results: No significant differences are present apart from a significantly elevated arterial ammonia level $(p<0.001)$ and a bad selfreported driving history $(p<0.05)$ in MHE +ve group when compared with MHE-ve group. Results exhibit a significant statistical differences $(p<0.05)$ when comparing MHE+ve group with the other groups (control and MHE-ve ones). Multivariate logistic relationship revealed that advanced Child-Pugh grade $(p<0.001)$, HBVrelated etiology $(p<0.001)$ and smoking are significant risk factors for MHE. MHE is significantly common among Child's C $(p<0.05)$ when compared with the other Child-Pugh grades. Results of the psychometric tests correlated significantly with stage of fibrosis and advanced stage of fibrosis is associated with a significantly worse psychometric test performance $(p<0.001)$.

Conclusions: Our data revealed that a high prevalence of MHE (47\%) among Egyptian drivers with liver cirrhosis. The driving history and regular psychometric testing for evaluating those at risk, at least at the outpatient setting, are advised.

\section{PP23-55}

Study of Serum Procalcitonine in Patients with Liver Cirrhosis and its Prognostic Significance

N.A. Dubale ${ }^{1}$, P.N. Rao ${ }^{2}$, R. Gupta ${ }^{2}$, G. Dipika ${ }^{3}$, P. Rao ${ }^{4}$, D. Nageshwar Reddy ${ }^{1}$

${ }^{1}$ Medical Gastroenterology; ${ }^{2}$ Hepatology; ${ }^{3}$ Biochemistry;

${ }^{4}$ Microbiology, Asian Institute of Gastroenterology, Hyderabad, India

Background and aims: Serum procalcitonine, produced in the liver is an accepted biomarker for bacterial sepsis in noncirrhotic population. This study was aimed to evaluate its diagnostic and prognostic value as a predictor of 30-day mortality in cirrhotic patients with sepsis.

Methods: Consecutive patients admitted to intensive care unit were evaluated prospectively and followed up till 30 days. Those already receiving antibiotics were excluded and divided into three groups, group A (45) patients with cirrhosis and no sepsis, group B (45) patients with cirrhosis and sepsis and group C (52) patients with sepsis without cirrhosis.

Serum procalcitonine levels were estimated by semiquantitative method on admission and after $48 \mathrm{~h}$. All with sepsis received antibiotics. Child's, MELD, APACHE II and SOFA were calculated. Logistic regression analysis was used for predicting outcomes.

Results: Serum procalcitonine was found to be elevated significantly above the cut off $(0.5 \mathrm{ng} / \mathrm{ml})$ in group B than in group A both on admission $(p<0.0001)$ and after $48 \mathrm{~h}(p<0.0001)$.It did not differ significantly between groups B and C. Higher values were observed in group $\mathrm{B}$ and $\mathrm{C}$ than in $\mathrm{A}$. Mortality at 30 days was found to be more in group B than in A (33 vs. $15 \% ; p=0.04)$ and C (33 vs. $28 \%$; $p=0.633$ ). In group B, SOFA score, initial and values after $48 \mathrm{~h}$ of procalcitonine, while in group A, only SOFA score were found to be predictors of mortality. In group C, SOFA, initial and procalcitonine values after $48 \mathrm{~h}$, were found to be predictors of 30-day mortality.

Conclusion: Serum procalcitonine is a marker for sepsis in patients of liver cirrhosis. Initial and persistently elevated levels after $48 \mathrm{~h}$ can redict short term mortality.
PP23-56

Precipitating Factors of Hepatic Encephalopathy: an Experience at Mansoura Specialized Medical Hospital, Egypt

Y.Z. Gad

Internal Medicine, Mansoura University, Mansoura, Egypt

Background/aim: Hepatic encephalopathy (HE) is a frequent complication of liver cirrhosis and its development heralds a poor prognosis. This work was designed to study the precipitating factors of HE in patients with liver cirrhosis at Mansoura Specialized Medical Hospital, Egypt.

Patients and methods: A total of 237 consecutive chronic liver disease patients, presented with signs and symptoms of hepatic encephalopathy, were admitted in the hepatology unit of Mansoura Specialized Medical Hospital . The precipitating factors of hepatic encephalopathy were identified with the aid of clinical examination and appropriate investigations. Grades of HE and Child-Pugh scores were determined.

Results: The precipitating factors of HE in our studied cases were upper GI bleeding in 87 patients (36.7\%), advanced HCC in 43 patients $(18.14 \%)$, infections in 37 patients $(15.61 \%)$, excess protein intake in 27 patients $(11.39 \%)$, electrolyte imbalance in 7 patients $(2.95 \%)$, and altered bowel habits in 33 patients (13.92\%). Males were commonly affected by HCC $(p<0.05)$ and upper GI bleeding more than female patients $(p<0.05)$. Male patients were commonly presented with HE (63.29\%) and this gender difference reached a statistical significance among those presented with upper GI bleeding $(p<0.05)$ and HCC $(p<0.05)$. Upper GI bleeding, Advanced HCC and infections were the leading causes of death with a higher prevalence in grade IV comatose $(p<0.05)$, Child C $(p<0.05)$, male patients $(p<0.05)$.

Conclusions: Our data revealed that upper GI bleeding, advanced $\mathrm{HCC}$ and infections are the main precipitating factors of HE as well as the major leading causes of death in patients with liver cirrhosis. Priority should be given to control these factors. Implementation of an effective screening program for early HCC detection all over the country should be on the top of the official health concerns.

\section{PP23-57}

Can Cyanoacrylate Cause Early Rebleeding in Patients with Gastric Varices? Tertiary Care Experience

S. Karim ${ }^{1}$, A. Faryal ${ }^{2}$, A. Memon ${ }^{3}$, S. Zaigham ${ }^{3}$, R. Mirza ${ }^{3}$, W. Jafri ${ }^{1}$

${ }^{1}$ Gastroenterology, Department of Medicine, Aga Khan University Hospital; ${ }^{2}$ Medicine, Jinnah Medical College Hospital; ${ }^{3}$ Medicine, Aga Khan University Hospital, Karachi, Pakistan

Background: Gastro-esophageal variceal hemorrhages are the complication of cirrhosis induced portal hypertension and raised hepatic venous pressure gradient. Cyanoacrylate and TIPS are considered as treatment of choice. Controversies are there about early rebleeding post cyanoacrylate injection into gastric varices. Our aim was to find the association of cyanoacrylate with early rebleeding.

Methods: Retrospective analysis, from 2005 to 2009 , those found to have gastric varices in esophagogastroduodenoscopy (EGD) at gastroenterology department of Aga Khan University hospital,were included. Early rebleeding was assessed and defined as upper gastrointestinal bleeding, melena or drop of hemoglobin within 5 days $(120 \mathrm{~h})$ of EGD, done at admission. They were grouped on the basis of cyanoacrylate whether they received or not.

Results: Two hundred twelve patient's data were reviewed. Mean age was $48.11 \pm 13.54$ years with male predominance $(62.26 \%)$. One fifty six $(73.6 \%)$ patients presented with upper gastrointestinal bleeding \pm melena. Gastroesophageal varices I (GOVI) were most common $67.9 \%$ (144) and $30.1 \%$ (52) of them were with recent sign of bleeding. One ninety four $(91.5 \%)$ patients had esophageal varices, $58.5 \%$ of them were large and $37.7 \%$ (80) had sign of recent bleeding. Mean Child's and MELD score were $8.7 \pm 1.94$ and $11.71 \pm 5.99$, 
respectively. Out of 212, seventy two patients (34\%) were injected with cyanoacrylate into gastric varices. (Mean cyanoacrylate and lipoidal were $0.48 \pm 0.76$ and $0.61 \pm 1.25 \mathrm{cc}$, respectively), while other $140(66 \%)$ gastric varices were not injected (i.e. no need). Rebleeding was observed in 16 subjects within $120 \mathrm{~h}$. Out of sixteen, $12 / 72(16.67 \%)$ were received cyanoacrylate and 4/140 (2.85\%) were those did not received (OR 6.8, 95\%CI 2.11-21.95). Male gender, presence of HCC, UGIB at admission, Child's $\mathrm{C}$ and visible clot on gastric varices were found to be associated predictor for early rebleeding.

Conclusions: In presence of above mentioned predictor, cyanoacrylate injection can be associated with early rebleeding.

\section{PP23-58}

Comparison of 2 Days and 5 Days Terlipressin with Endoscopic Band Ligation for Esophageal Variceal Bleed in Patients of Cirrhosis

N.S. Choudhary, Y.K. Chawla, A. Duseja, R.K. Dhiman

Hepatology, PGIMER, Chandigarh, India

Background: Combined endoscopic and pharmacological (Terlipressin) therapy is preferred therapy for esophageal variceal bleed. Cost of Terlipressin for 5 days is significant limiting factor.

Methods: Twenty-eight patients of cirrhosis with esophageal variceal bleed randomized to either 2 days (group A) or 5 days (group B) Terlipressin treatment (1 mg 6 hourly) with endoscopic variceal banding (first day and then 3 weekly as required). Exclusion criteria were grade 3 or 4 encephalopathy, gastric variceal bleed or hepatocellular carcinoma. Primary outcome was absence of early rebleed (first 5 days) and secondary outcome was absence of bleed at 6 weeks.

Results: Study comprised of 12 males and 2 females in each group. Mean age (SD) was 41.6 (11.9) and 46.8 (11.03) for group A and B, respectively. Etiology of cirrhosis was alcohol in ten and nine patients of group A and B. CTP score was not significantly different between groups, MELD was significantly higher in group B. None of the patients has early rebleed, rebleed after 5 days occurred in four patients in group B (one died) and two patients of group A ( $p=n s$ ); two patients of group B and one patient of group A were died due to late rebleed $(p=\mathrm{ns})$.

Conclusion: Combined with endoscopic variceal ligation, 2 days Terlipressin therapy is as effective as 5 days therapy.

\section{PP23-59}

Can Acoustic Radiation Force Impulse Elastography be a Predictor of Liver Cirrhosis Complications?

R. Sirli, I. Sporea, S. Bota, O. Ciof, A. Popescu, M. Danila

Gastroenterology, University of Medicine and Pharmacy Timisoara, Timisoara, Romania

The aim of this study was to find out if ARFI liver stiffness measurements can predict the presence of complications in liver cirrhosis (LC) [portal hypertension, hepatocellular carcinoma (HCC) and decompensation].

Materials and methods: 299 cases with proven LC (clinically, by ultrasound, endoscopy, morphopathology or laparoscopy) were evaluated by means of ARFI (114 women, 185 men; mean age $59.8 \pm 5.6$ years). Ultrasound examination and ARFI (Siemens Acuson S2000 ${ }^{\mathrm{TM}}$ ultrasound system: median value of ten measurements, results in $\mathrm{m} / \mathrm{s}$ ) were performed in all patients in the same session. Recent gastroscopies (6 months old) were available in 263 subjects. In HCC patients, ARFI measurements were made outside the tumor.

Results: Valid ARFI measurements were obtained in 290/299 patients (97\%).

There were no statistically significant differences between the mean values of ARFI measurements in patients with grade 2-3 esophageal varices (EV) (122 subjects) versus no or small EV (133 subjects):
$2.98 \pm 0.71$ versus $2.78 \pm 0.71 \mathrm{~m} / \mathrm{s}(p=0.27)$; in patients with a history of variceal bleeding (43 subjects) versus no bleeding (79 subjects): $2.83 \pm 0.74$ versus $2.92 \pm 0.7 \mathrm{~m} / \mathrm{s}(p=0.50)$; in patients with HCC (39 subjects) versus no HCC (208 subjects): $2.71 \pm 0.69$ versus $2.85 \pm 0.72 \mathrm{~m} / \mathrm{s}(p=0.13)$.

In patients without ascites (154 subjects), the mean value of ARFI measurements was statistically significant lower than in those with ascites (118 subjects): $2.68 \pm 0.69$ versus $3 \pm 0.71 \mathrm{~m} / \mathrm{s} \quad(p=$ $0.0007)$. For a cut-off value of $3.13 \mathrm{~m} / \mathrm{s}$, ARFI had $46 \% \mathrm{Se}, 72 \% \mathrm{Sp}$, $56 \% \mathrm{PPV}$ and $63 \% \mathrm{NPV}$ for predicting ascites (AUROC-0.61, $p=0.005$ ).

Conclusion: LS measurement by means of ARFI cannot predict the presence and severity of portal hypertension and $\mathrm{HCC}$, but it can be an indicator of imminent ascites.

\section{PP23-60}

Hyaluronic Acid and Other Non Endoscopic Parameters in Detection and Grading of Oesophageal Varices in Liver Cirrhosis

W.M. Elzefzafy ${ }^{1}$, R.S. Shahin ${ }^{2}$, S.M. Mahmoud ${ }^{3}$, S.A.-M. Abd-Alla ${ }^{4}$, H.M. Moustafa ${ }^{5}$

${ }^{1}$ Gastroenterology and Hepatology; ${ }^{2}$ Clinical Pathology, Al-Azhar University for Girls; ${ }^{3}$ Tropical Medicine; ${ }^{4}$ Clinical Pathology, ElSahel Teaching Hospita, Cairo; ${ }^{5}$ Gastroenterology and Hepatology, Al-Azhar University, Assiut, Egypt

Objectives: Two-third of all cirrhotic patients develop oesophageal varices during their life time. In this study we investigate the association of hyaluronic acid (HA) and other biochemical, ultrasonographic parameters as non invasive predictors for the presence and grading of oesophageal varices in patients with liver cirrhosis aiming to reduce medical, social and economic cost.

Methods: We measured serum hyaluronic acid levels in 80 patients with cirrhosis, (60 patients with variable grades of oesophageal varices, 20 patients without oesophageal varices served as a control group). We also determined other biochemical, ultrasound indicators of oesophageal varices.

Results: We found highly significant increase in hyaluronic acid levels in varices groups when compared to control group and when the varices groups were compared to each others. Also we found that HA sensitivity was $100 \%$, specificity $70 \%$, positive predictive value (PPV) $90.9 \%$ and negative predictive value (NPV) $100 \%$. A highly significant increase in the levels of total bilirubin were detected in patients with varices compared to control while, prothrombin concentration and platelets count were highly significantly decreased and serum albumin were highly significantly decreased in patients with large varices when compared to control group also there were highly significant increase in spleen diameter in patients with varices as compared to control group. A significantly higher portal vein diameter (PVD) and portal vein flow (PVF) and a significantly lower mean portal vein velocity (PVV) were recorded in patients with large oesophageal varices only. There was highly significant increase in Child's-Pugh score inpatients with varices in comparison to the control group.

Conclusion: Serum hyaluronic acid, platelets count, spleen diameter, PVD, PVV and PVF, serum bilirubin, serum albumin and Child'sPugh score can be used as simple, commonly available, non invasive and sensitive parameters for prediction of oesophageal varices.

\section{PP23-61}

Hepatic Pathologies and ACE Inhibitors

O. Mikheyeva, L. Lazebnik, I. Komissarenko, V. Drozdov Central Research Institute of Gastroenterology, Moscow, Russia

Enalapril and Lisinopril in hypertensive patients with hepatic pathologies with the goal of the treatment correction. As many as 120 hypertensive subjects with steatosis (Group 1), hepatic cirrhosis (HC - Group 2) and without any hepatic pathologies (Group 3) - 40 
patients per group, respectively - were enrolled in the study. Each group was subdivided into two subgroups (Enalapril or Lisinopril $10 \mathrm{mg}$ ). To assess the efficacy of the hypotensive drugs, daily monitoring of arterial pressure monitoring (DMAP) was conducted. The time index (TI) was assessed as the efficacy index. The resulting pharmacokinetic parameters point at the general trend towards decreasing the liver metabolic activity at steatosis and HC. The maximum concentration of the active Enalapril metabolite (Enalaprilate) decreased depending on the degree of hepatic functional state disorders. Thus, while the mean maximum Enalaprilate concentration $\left(\mathrm{C}_{\max }\right)$ made up $58.1 \pm 1.41 \mathrm{ng} / \mathrm{ml}$ in the patients from the control group, insignificant decrease in the Enalaprilate blood level $(54.7 \pm 4.19 \mathrm{ng} / \mathrm{ml})$ was revealed in hypertensive patients with hepatic steatosis; statistically significant decrease down to $38.8 \pm 9.3 \mathrm{ng} / \mathrm{ml}(p<0.001)$ was detected in patients with hypertension and HC. At that the time to maximum Enalaprilate concentration increased from $3.25 \pm 0.29 \mathrm{~h}$ in patients from Group 3 to $4.17 \pm 0.26 \mathrm{~h}$ in hypertensive patients with hepatic steatosis $(p<0.001)$ and $5.5 \pm 0.38 \mathrm{~h}(p<0.001)$ in people suffering from hypertension and HC. Thus, water soluble Lisinopril being a pharmacologically active form has the advantage related to its administration to patients with impaired hepatic function as compared to other ACE inhibitors. Against the background of Lisinopril $10 \mathrm{mg}$ therapy, the reduction in such average daily indices as arterial pressure and arterial pressure TI was observed in all of the three groups. A good antihypertensive effect was obtained in 55\% of $\mathrm{HC}$ patients with Lisinopril treatment.

\section{PP23-62}

Renal Hemodynamics Evaluated by Duplex Doppler Ultrasonography in Cirrhotic Patients before and after Sclerotherapy or Banding Ligation H.M. Moustafa ${ }^{1}$, H.A.-K. Younis ${ }^{2}$, M.A.-A. Nafady ${ }^{3}$, K.E. Abdel-Azeem ${ }^{1}$ ${ }^{1}$ Gastroenterology and Hepatology; ${ }^{2}$ General Medicine; ${ }^{3}$ Radiology, Al Azhar University, Assiut, Egypt

Background and Aim of Work : Cirrhosis of the liver is associated with significant morbidity and mortality especially if complicated by acute renal failure. The aim of the work is non-invasive evaluation of the renal hemodynamics by colour duplex Doppler ultrasonography in patients with liver cirrhosis (without primary renal diseases) and studying the effect of injection scleortherapy or banding ligation on the hemodynamics.

Subjects and methods: The study involved 50 cirrhotic patients, and 10 healthy persons as a control group, selected from the General Medicine Department, Al-Azhar Assiut University Hospital over a period of 1 year. History and clinical examination, routine investigations, liver functions and renal functions tests, urine $\mathrm{Na}$ fraction to exclude primary renal diseases, abdominal sonography, upper endoscopy, injection sclerotherapy or banding for varices and duplex Doppler ultrasonography on main renal arteries on both sides to detect pulsatility and resisitive indices were performed.

Results: The pulsatility and resisitive indices were significantily higher in cirrhotic patients compared to control group ( $p$ value $=$ 0.001). The renal hemodynamics were higher in Child -class $C$ than $B$ and in Child- class B than A ( $p$ value $=0.0001)$. The renal hemodynamics were high in patients with renal impairment,so the pulsatility and resistive indices were high in patients with elevated serum creatinine, blood urea and decreased creatinine clearance and vice virsa. ( $p$ value $=0.001,0.0001,0.002$ respectively). Renal hemodynamics were increased as plasma renin increased ( $p$ value $=0.0001)$. Pulsatility and resistitive indices were done before and one week after banding ligation or sclerotherapy and showed no significant difference.

Conclusion: The renal hemodynamics (the pulsatility and resistive indices) were significantly higher in cirrhotic patients than control group and were directly correlated with the severity of liver cirrhosis. No significant differences were detected in the pulsatility and resistive indices before and one week after banding ligation or sclerotherapy.

\section{PP23-63}

Therapeutic Interventions for the Secondary Prophylaxis of Esophageal Variceal Bleeding: A Meta-Analysis

K.M. Nomorosa, R. Romero, E. Comeily-Birjandi, M. Uy, F. Zaño,

P.M.J. Torres

Section of Gastroenterology and Digestive Endoscopy, Manila

Doctors Hospital, Manila, Philippines

Background: Recurrent esophageal variceal bleeding (EVB) increases mortality. Several methods are available for its prevention. Objective: To compare pharmacotherapy (PT), band ligation (BL), and PT + BL for secondary prevention of EVB.

Methodology: We searched PubMed, OVID, and the Cochrane Library for randomized controlled trials of PT, BL and PT $+\mathrm{BL}$ for cirrhotics. We also reviewed the reference lists of the retrieved studies. We did meta-analyses using Random Effects Model for the primary outcomes of rebleeding, mortality and complications.

Results: 12 RCTs were included, 7 PT versus BL, 3 PT + BL versus BL, 2 PT + BL versus PT. All trials used nonselective beta blockers \pm nitrates as PT. Among trials comparing PT and BL, mortality was significantly reduced [RR $1.29,(1.06,1.58)]$ with PT. There was no significant difference in rebleeding [RR $0.88,95 \% \mathrm{CI}(0.64,1.19)]$ and complications [RR 0.97, 95\%CI $(0.75,1.24)]$. Among trials comparing PT $+\mathrm{BL}$ versus $\mathrm{BL}$, rebleeding was significantly reduced with PT + BL [RR $0.54,95 \%$ CI $(0.36,0.81)]$, but there was only a trend toward less mortality [RR $0.59,95 \% \mathrm{CI}(0.33,1.04)]$. There was also an insignificant increase in complications with this intervention [RR 3.80, 95\%CI $(0.49,29.65)]$. Among trials comparing PT + BL versus $\mathrm{PT}$, there was only a trend toward less rebleeding with $\mathrm{PT}+\mathrm{BL}$ [RR $0.7695 \% \mathrm{CI}(0.56,1.04)]$. There was no significant difference in mortality [RR $1.1395 \% \mathrm{CI}(0.72,1.77)]$ and complications [RR $1.2695 \% \mathrm{CI}(0.55,2.86)]$.

Conclusions: This analysis showed that PT is better than BL in terms of mortality, and PT $+\mathrm{BL}$ is better than BL in terms of rebleeding, but PT + BL is only insignificantly better than PT. We suggest a large trial comparing $\mathrm{PT}$ versus $\mathrm{PT}+\mathrm{BL}$.

\section{PP23-64}

Meta Analysis: Propranolol versus Rubber Band Ligation in the Primary Prophylaxis of Esophageal Variceal Bleeding

R. Romero, K.M. Nomorosa, E. Comeily-Birjandi, P.M.J. Torres, M. Uy, F. Zano

Section of Gastroenterology and Digestive Endoscopy, Manila Doctors Hospital, Manila, Philippines

Background: Variceal bleeding remains to be a major complication of portal hypertension resulting from liver cirrhosis. Propranolol (PPL) and rubber band ligation of esophageal varices (RBL) have been used for primary prophylaxis of esophageal variceal bleeding (EVB). Studies on the use of these therapeutic options have shown conflicting results as to their effectiveness.

Objective: To compare propranolol (PPL) and rubber band ligation (RBL) in the primary prophylaxis of EVB.

Methods: We searched Pubmed and the Cochrane Library for randomized controlled trials comparing PPL with RBL in the primary prophylaxis of EVB among cirrhotics. We did meta-analyses using Random Effects Model for the primary outcome of bleeding and secondary outcomes of bleeding-related mortality and overall mortality.

Results: Ten studies (771 subjects) were included for the primary outcome of bleeding. Bleeding was significantly reduced with RBL compared with PPL [RR 1.51; 95\%CI $(1.06,2.15)]$. For the secondary outcomes of bleeding-related mortality and overall mortality, eight 
(710 subjects) and nine (741 subjects) studies were included, respectively. There was no significant difference in bleeding-related mortality [RR $1.40 ; 95 \%$ CI $(0.77,2.52)]$ and overall mortality [RR $0.88 ; 95 \%$ CI $(0.71,1.10)]$ between PPL and RBL. There was only a trend favoring the use of RBL in reducing bleeding-related mortality and PPL in reducing overall mortality.

Conclusions: RBL is more effective than PPL in reducing primary bleeding from esophageal varices among cirrhotics. There was no significant difference between PPL and RBL in reducing bleedingrelated mortality and overall mortality. More trials comparing PPL with RBL with these outcomes are needed.

\section{PP23-65}

Effect of Malnutrition on Morbidity and Survival in Patients with Liver Cirrhosis. Cuban Experience

M.I. Castellanos

Hepatology, Instituto de Gastroenterología, Havana, Cuba

Background/aims: Liver cirrhosis (LC) is the tenth cause of death in Cuba. The effect of malnutrition on morbidity and survival of patients with LC were investigated in this study.

Methods: 355 cirrhotic patients were included in a prospective study and were followed clinically for 2 year or until death. Child-Pugh score was used to assess the severity of cirrhosis. Malnourished were defined when midarm circumference and/or triceps skinfold thickness and/or subscapular skinfold thickness were below the 10th percentile of an age- and sex- matched population. Subjective global assessment (SGA) was used to recorded clinical and nutritional aspects. During follow up 29 patients died. Estimated survival rate was $90.3 \%$ at 1 year and $83.1 \%$ at 2 years. Multivariate analysis according to Cox's model assessed the predictive power of nutritional parameters on morbidity and mortality.

Results: Malnutrition was observed in $56.3 \%$.The highest risk was observed in male [OR: 1,9 (IC: $95 \% 1.2-2.9$ )], older than 60 years [OR: 1.9 (IC: 95\% 1.2-3.0)] and advanced Child-Pugh [Child B, OR: 3.7 (IC: 95\% 2.2-6.2), Child C, OR:17.4 (IC: 95\% 6.0-50.1)]. The ethiology of LC was independent of nutritional status. Ascitis, hepatic encephalopathy and spontaneous bacterial peritonitis were more frequent among undernourished. Subjective nutritional elements which better predicted these events were the loss of weight in the last 6 months, dysphagia, vomits and diminished functional capacity $(p<0.01)$. Anthropometrics parameters and SGA results were analyzed as possible predictors of survival. SGA in it's C stage (severely undernourished) $(P<0.05)$ was the stronger independent predictor $(P<0.05)$ as well as Child $\mathrm{B}$ and $\mathrm{C}$, hepatocellular carcinoma, hepatic encephalopathy and gastrointestinal bleeding $(P<0.001)$.

Conclusions: The progression of the illness took into account the commitment of the nutritional state and made a negative influence on survival. The SGA was the best nutritional indicator of survival in LC.

\section{PP23-66}

The Link between Immunological Dysfunction and Ethiology, Nutritional State and Severity of Liver Cirrhosis

M.I. Castellanos ${ }^{1}$, O. Seijas ${ }^{2}$, M. Ronquillo ${ }^{3}$

${ }^{1}$ Instituto de Gastroenterología; ${ }^{2}$ Gastroenterology; ${ }^{3}$ Immunology, Instituto de Gastroenterología, Havana, Cuba

Background/aims:Patients with liver cirrhosis (LC) are predisposed to complications due to their immunologic deficiencies. The relationship between immunological dysfunction and ethiology, nutritional state and severity of the cirrhosis were investigated in this study.

Methods: A prospective study was carried out in 76 cirrhotic of different causes, It were a $50.3 \pm 8.8$ year-old average, divided according to Child-Pugh classification in A: $52(68.4 \%)$; B: 17 (22.4\%); and C: 7 (9,2\%). Parameters of humoral immunity
(Components 3 and 4 of seric complement and immunoglobulins A, $\mathrm{M}, \mathrm{G}$ and $\mathrm{E}$ ) and cellular immunity (total leukocytes and lymphocytes in peripheral blood and intradermic tests of delayed hypersensitivity) as well as nutrititional parameters (anthropometric measures) were evaluated. Non parametric test were used to analyse the difference between means of groups.

Results: The results showed more immunological dysfunction in cellular than in humoral responses. Intradermic tests of delayed hypersensitivity did show greater frequency of anergy for candidin and respiratory bacteria's antigens. According to the etiology, only $\mathrm{C} 4$ showed statistical difference and it was lower in viral and alcoholic group. The severity of illness was the main factor who reveal differences. IgA was higher in Child $\mathrm{C}$ whereas $\mathrm{C} 3, \mathrm{C} 4$ and total leukocytes and lymphocytes in peripheral blood were lower with the progression of illness. Anergy was more evident for toxoid and intestinal bacteria's antigens in Child Pugh B and C. Depending on nutritional state there were no differences in immunological responses. Only C4 levels, were diminished in malnourished.

Conclusions: The variation in immunological responses in $\mathrm{LC}$ depends on severity of illness more than ethiology and nutritional state.

\section{PP23-67}

Prediction of esophageal varices in patients with HBV-related cirrhosis using artificial neural network

W.-D. Hong, Q.-H. Zhu, Q.-K. Huang

Department of Gastroenterology and Hepatology, The First Affiliated Hospital of Wenzhou Medical College, Wenzhou, China

Background: Prediction of esophageal varices in cirrhotic patients by noninvasive variables was still unsatisfactory. Artificial neural network has not been used in prediction of varices.

Objective: The aim of this study was to evaluate the accuracy of an ANN in predicting varices in patients with HBV-related cirrhosis.

Methods: An ANN was constructed with data from 197 patients with HBV-related cirrhosis. The candidates for input nodes of the ANN were assessed by univariate analysis and sensitivity analysis. Fivefold cross validation was performed to avoid overfitting. The performance of ANN was compared with that of other single parameters by using receiver operator characteristic (ROC) curve analysis.

Results: Prevalence of esophageal varices was $73.1 \%$. Fourteen variables were reduced by univariate analysis and sensitivity analysis, and an ANN was developed with three variables (platelet count, spleen width and portal vein diameter). Based on ROC analysis, the area under the receiver operator characteristic curve (AUC) of ANN model $(0.92 \pm 0.02)$ was statistically significantly higher than that of platelet count $(0.77 \pm 0.04, P<0.001)$, spleen width $(0.79 \pm 0.04$, $P<0.001)$ or portal vein diameter $(0.80 \pm 0.03, P<0.001)$. With a cutoff value of 0.2 , the ANN model has $96.5 \%$ sensitivity, $60.4 \%$ specificity, $86.9 \%$ positive predictive value, $86.5 \%$ negative predictive value and $86.8 \%$ diagnostic accuracy for the prediction of varices.

Conclusions: An artificial neural network may be useful for predicting presence of esophageal varices in patients with HBV-related cirrhosis.

\section{PP23-68}

The Influence of Helicobacter pylori Infection and Portal Hypertensive Gastropathy on Active Peptic Ulcer Disease in Patients with Liver Cirrhosis

R. Amaraa, B. Gegeebadrakh, U. Purevsuren, T. Jargalsaikhan

Department of Internal Medicine I, General Hospital II, Ulaanbaatar, Mongolia

Background/aims: Peptic ulcer disease is one of the most frequently observed complications in patients with liver cirrhosis. The aim of our study was to investigate the role of Helicobacter pylori infection and 
portal hypertension gastropathy in the prevalence of active peptic ulcer among patients with liver cirrhosis.

Methods: 399 patients with liver cirrhosis, 363 patients with nonulcer dyspepsia, 359 patients with peptic ulcer disease undergoing upper endoscopy were enrolled. H.pylori infection was determined by rapid urease test and/or histology.

Results: The prevalence of H.pylori infection in patients with cirrhosis, patients with non-ulcer dyspepsia, and peptic ulcer disease were $43.1,85.4$, and $89.9 \%$, respectively. The prevalence of H.pylori infection in patients with HBV, HCV, HBV- and HCV-related, alcohol-related liver cirrhosis was 50, 37.5, 42.8, 20.0\%, respectively. The prevalence of H.pylori infection in patients with Child-Pugh class A, B and C liver cirrhosis was 57.8, 49.6, 20\%, respectively. The prevalence of peptic ulcer disease in patients with cirrhosis was $9 \%$ (29 gastric ulcer, 7 gastric and duodenal ulcer). The prevalence of peptic ulcer disease in cirrhotic patients according to Child-Pugh class $\mathrm{A}, \mathrm{B}$ and $\mathrm{C}$ was $4.9,10.4,12.1 \%$, respectively. Peptic ulcer in patients with Child-Pugh class B, C liver cirrhosis accompanied with severe portal hypertensive gastropathy such as erosive or hemorrhagic gastritis.

Conclusions: The prevalence of H.pylori infection in patients with cirrhosis was lower than in non-ulcer dyspepsia $(p<0.001)$ or peptic ulcer disease patients $(p<0.001)$. In patients with virusrelated liver cirrhosis the prevalence of H.pylori infection was higher than in alcohol-related cirrhotic patients $(p<0.001)$. A negative correlation was noted between the prevalence of $H$. pylori infection and the severity of liver cirrhosis $(r=-0.75, p<0.01)$ The prevalence of peptic ulcer tends to be higher in patients with severe liver cirrhosis and associated with severe portal hypertensive gastropathy.

\section{PP23-69}

Prediction of Esophageal Variceal Bleeding in B-viral Liver Cirrhosis Using P2/MS Noninvasive Index Based on Complete Blood Counts

B.K. Kim, S.H. Ahn, K.H. Han, J.Y. Park, J.K. Kim, Y.H. Paik, K.S. Lee, C.Y. Chon, D.Y. Kim

Internal Medicine, Yonsei University College of Medicine, Seoul, Republic of Korea

Objectives: Periodic endoscopic screening for esophageal varices (EVs) and prophylactic treatment for high-risk EVs (HEVs; medium/ large EVs and small EVs with red sign or decompensated cirrhosis) are recommended for all cirrhotic patients. We assessed cumulative risks of esophageal variceal bleeding (EVB) using one of the simple, noninvasive tests based on complete blood counts, called P2/MS, (platelet count $)^{2} /($ monocyte fraction $[\%] \times$ segmented neutrophil fraction [\%]).

Methods: We prospectively enrolled 475 consecutive B-viral cirrhosis patients from 2008 to 2009, none of whom experienced EVB. All underwent laboratory workups, endoscopy, and ultrasonography. Those with HEVs took non-selective $\beta$-blockers as prophylaxis for EVB after diagnosis, if not contraindicated. The major endpoint was the first occurrence of EVB, which was examined using KaplanMeier and Cox regression methods.

Results: Among the whole population, $97.0 \%$ negative and $92.2 \%$ positive predictive value were provided at P2/MS cutoffs 25 and 11 for prediction of HEV. Among patients with HEV $(n=131), 25$ experienced their first EVBs during follow-up (median, 28.4 months). To differentiate EVB risk, we divided them into two subgroups according to P2/MS value (Subgroup 1: $\geq 9$ and Subgroup 2: $<9$ ). Risk increased significantly in association with lower P2/MS value between two subgroups $(p=0.029)$. From multivariate analysis, P2/MS $(p=0.040)$ remained a significant predictor, alongside variceal sizes $(p=0.015)$ and Child-Pugh classifications $(p=0.001)$. Subgroup 1 had a similarly low EVB risk, compared with patients with low-risk EVs $(p=0.164)$.
Conclusions: $\mathrm{P} 2 / \mathrm{MS}$ is a reliable predictor for EVB risk among patients with HEVs. According to risk stratification, different prophylactic treatments should be considered for subgroups with P2/MS $<9$.

\section{PP23-70}

Correlation of Platelet Count with Grading of Esophageal Varices in Cirrhotic Patients

N. Aqodad, H. Ighoudane, M. Elyousfi, I. Mellouki, M. Elabkari, D. Benajeh, A. Ibrahimi

Gastroenterology, Hospital University Hassan II, Fez, Morocco

The screening of esophageal varices (EV) by esophagogastroduodenoscopy (EGD) is recommended in all patients with cirrhosis. However, EGD remains an invasive and expensive tool; so many studies have focused on studying non-invasive tests to predict the presence of EV. The aim of this work is to study the correlation of platelet count with grading of esophageal varices in cirrhotic patients. Materials and methods: In this retrospective study (January 2001 and January 2010) we included all patients with cirrhosis and we excluded all portal hypertension due to portal vein thrombosis or Budd Chiari syndrome. The Chi square test was used for comparison of two variables and the ROC curve was used to determine a threshold of platelets predictive of the presence of EV.

Results: The total number of patients was 797 . Their average age was $52.5 \pm 16.9$ years, with a sex ratio of 0.9. The viral $\mathrm{C}$ cirrhosis was the most common etiology (28\%). Varices were present in $88 \%$ of patients and absent in $12 \%$ of patients. In the first group, the average platelet count was $118,230.54 \pm 74,624.15$ versus $153,852.7 \pm$ $125,713.3$ in the second group $(p<0.000)$. At the patients with EV grade I, II and III the average of platelets count was, respectively, $134,653.5 \pm 94,460.3 ; \quad 119,003.29 \pm 77642.9$ and $109,188.16 \pm$ $54884.9(p<0.009)$. A platelets count cutoff less than 106,000 was predictive of presence of varices with a sensitivity of $53 \%$ [95\% CI (50-57\%)], and specificity of $60 \%$ [95\% CI (50-69\%)].

Conclusion: The presence and grading of EV is statistically correlated with the degree of thrombocytopenia. A platelets count cutoff less than 106,000 is predictive of the presence of $\mathrm{EV}$ with a sensitivity of $53 \%$ and specificity of $60 \%$.

\section{PP23-71}

A Case of Acute Budd-chiari Syndrome with Hepatic Hydatid Cyst and Protein C Deficiency

H.S. Das ${ }^{1}$, M. Mohapatra ${ }^{2}$

${ }^{1}$ Gastroenterology, S.C.B.Medical College, Cuttack; ${ }^{2}$ Radiology, Kalinga Hospital, Bhubaneswar, India

Case report: Thirty-three-year-old lady presented with pain abdomen and abdominal distension of 3 weeks duration. There was no past history of hematemesis, melena, or jaundice. Ascitic fluid examination was exudative in nature. Ultrasound of abdomen was suggestive of mild hepatosplenomegaly with a well defined multicystic heterogenous lesion of $80 \mathrm{~mm}$ in right lobe of liver. Multiple small cystic areas of varying shapes and sizes were seen within the cyst. Patient had moderate amount of ascites. Endoscopy was suggestive of Grade one oesophageal varices. USG Doppler suggested patent IVC, compressed by enlarged caudate lobe and occlusion of all hepatic veins along with hydatid cyst of liver. Patient was negative for all viral marker and serology for hydatid (anti echinococcus IgG) was positive. Patient was investigated for procoagulant state and found to be protein $\mathrm{C}$ deficient. Thus a diagnosis of Budd-chiari syndrome with hepatic hydatid cyst in a case of protein $\mathrm{C}$ deficiency was made. Patient was put on anticoagulation therapy with a planning for radiological treatment.

Summary: Though Budd-chiari syndrome has been described in cases of hydatid cyst, we found the case with underlying Protein $\mathrm{C}$ deficient which is responsible for hepatic venous thrombosis. 


\section{PP23-72}

Profile of Acute and Chronic Budd-Chiari Syndrome in Coastal Orissa, a 6-Year Prospective Study

H.S. Das ${ }^{1}$, M. Mohapatra ${ }^{2}$, C.R. Panda ${ }^{1}$, P.K. Thatoi ${ }^{3}$, S.P. Singh ${ }^{1}$,

B.K. Mishra ${ }^{4}$

${ }^{\text {I}}$ Gastroenterology, S.C.B. Medical College, Cuttack, Orissa, Cuttack; ${ }^{2}$ Radiology, Kalinga Hospital, Bhubaneswar; ${ }^{3}$ Medicine, S.C.B.

Medical College, Cuttack, Orissa; ${ }^{4}$ Gastroenterology, S.C.B. Medical College, Cuttack, India

Background: Budd-chiari syndrome (BCS) though rare is an important cause of chronic liver disease. There are very limited data in the field from this region.

Objective: To study prospectively consecutive patients of diagnosed BCS over a period of 6 years with special reference to the nature, treatment outcome and course of disease.

Materials and methods: Patients with high suspicion of BCS underwent USG Doppler, IVC gram and hepatic venogram to diagnose BCS.

Observation: Sixty-eight cases of BCS were diagnosed in last 6 years with $\mathrm{M}: \mathrm{F}$ ratio of 29:39 (age range of 3-78 years), median age of 32 years . Ninety percent patients were of low socioeconomic status. Out of sixty-eight 40 had acute BCS . Symptoms ranged from 7 days to 20 years. Predisposing factors were pregnancy in five, postpartum in eight, hydatid cyst in two, HCC in four, renal carcinoma in two, pancreatic carcinoma in one case. Site of obstruction was IVC in 11, HV in 34 and IVC + HV in 23. Nature of obstruction was membrane, thrombosis and membrane with thrombosis in 8,36 and 22 , respectively. Three patients only had undergone workup for procoagulant state due to financial constraint. Two had protein c and one protein $\mathrm{S}$ deficiency. Treatment offered were radiolological in 14, only anticoagulation in 22, surgery in one. Three patients had complete recanalization of HV following anticoagulant therapy. Eight cases of acute BCS expired with extensive thrombosis during hospital stay. Six patients with chronic BCS expired during follow up.Eleven lost to follow up.

Summary: Budd-chiari syndrome in our region is mainly due to thrombotic lesion with acute presentation and HV is the commonest site of obstruction. At least anticoagulant therapy should be started in patients those who cannot afford other modality of treatment due to financial constraint

\section{PP23-73}

Epidemiological and Etiological Characteristics of Portal Hypertension in Fes- Morocco?

N. Aqodad ${ }^{1}$, B. Benyachou ${ }^{1}$, M. Elyousfi ${ }^{1}$, I. Mellouki ${ }^{1}$, D. Benajeh ${ }^{1}$,

M. Elabkari ${ }^{1}$, A. Ibrahimi ${ }^{1}$, A. Najdi ${ }^{2}$, C. Nejjari ${ }^{2}$

${ }^{1}$ Gastroenterology; ${ }^{2}$ Epidemiology, Hospital University Hassan II,

Fez, Morocco

Portal hypertension $(\mathrm{PH})$ is a common and severe disease of multiple etiologies, the most important is cirrhosis. Our aim is to report our results on the epidemiological, clinical, biological, radiological and etiological of $\mathrm{PH}$.

Materiel and methods: We retrospectively analyzed data from all patients with HP between 2001 and 2010 in gastroenterology center of Fez were included. We studied the epidemiological, clinical, biological, radiological and etiological data of patients. The analysis was done using the Epi Info.

Results: During this period we collected 1,107 cases of PH. Their average age was 52 (12-96 years old), with a slight female predominance $(52.2 \%)$. Bleeding from ruptured varices was the most common symptom $(40 \%)$. Ascites, splenomegaly and jaundice were found respectively in 55,35 , and $22 \%$ of cases. Biologically, the average hemoglobin was $9 \mathrm{~g} / \mathrm{dl}$, platelets at 106,000 , the prothrombin activity to $65 \%$, total bilirubin at $29 \mathrm{mg} / \mathrm{l}$ and albumin at $30 \mathrm{~g} / \mathrm{l}$. The ultrasound showed splenomegaly in $76 \%$ of cases, ascites in $71 \%$ of cases, and a dysmorphic liver in $52 \%$ of cases. The oesophageal varices were present in $80 \%$ of the cases: $49 \%$ were stage II and $33 \%$ were stage III. At diagnosis $20 \%$ of patients were Child A, 56 were Child B and $24 \%$ were child C. Cirrhosis was post-viral C in $41 \%$, post viral $\mathrm{B}$ in $18.5 \%$. Budd-chiari syndrome represented $2.3 \%$ of etiologies and portal thrombosis $9 \%$ of cases.

Conclusion: Portal hypertension is diagnosed in relatively young subjects, without sex difference. Hemorrhagic decompensation $(40 \%)$ and ascites $(55 \%)$ are the most common of presentation. Viral cirrhosis are the principal cause. The most patients $(80 \%)$ have a score of Child $>7$ at the time of diagnosis.

\section{PP23-74}

Risk Factors for Rebleeding and Mortality in Variceal Hemorrhage I. Ratiu, A. Goldis, D. Lazar, L. Olaru, D. Koukoulas, I. Sporea Emergency Clinical County Hospital Timisoara, Timisoara, Romania

Introduction: Upper digestive bleeding remains one of the problems commonly encountered in medical practice and, especially in patients with liver cirrhosis it has a major influence in morbidity and mortality. The key point in approaching these patients is to determine patients with major risk for bleeding.

Aims and methods: This study proposes to evaluate predictive factors for early mortality and rebleeding in patients with variceal hemorrhage. We evaluated a group of 447 patients with liver cirrhosis 352 males $(54.4 \%)$ and 204 females (45.5\%), with a mean age of $54.56 \pm 26.16$ years, admitted to hospital for an episode of variceal bleeding during 1st January 2004-31 December 2008. Dividing the patients into two categories as follows:

1. Patients without rebleeding $(N=289)$ versus patients who rebleed $(N=158)$

2. Survivors $(N=370)$ versus deceased $(N=77)$, we tried to identify possible risk factors for prediction of bleeding episode, rebleeding and early mortality by analyzing the following parameters: the degree of hepatic insufficiency (Child-Pugh classification and MELD score, albumin, cholinesterase, bilirubin), the severity of bleeding (anemia, hemorrhagic shock,), endoscopic parameters (variceal grade, active bleeding at endoscopy), coagulation disorders (platelets count and INR), etiology of cirrhosis and the decompensation of the underlying disease.

\section{Results:}

Rebleeding

Parameter P Value

age $0.01293 * \mathrm{~S}$

bilirubin $0.031679 * \mathrm{~S}$

MELD score $0.000897 * * *$ ES

ascites $0.000294 * * * \mathrm{ES}$

Mortality

Parameter $P$ Value

Male sex $0.038004 * \mathrm{~S}$

albumin $0.000139 * * *$ ES

rebleeding $0.005095 * *$ VS

MELD score $<0.0001 * * *$ ES

encephalopathy $<0.0001 * * *$ ES

ascites $0.028065 * \mathrm{~S}$

anemia $0.002801 * *$ VS

Conclusion: The factors which have the most powerful influence over mortality are: MELD score, albumin, encephalopathy, rebleeding and severe anemia and the main predictors of rebleeding are: MELD score and presence of large ascites. 
PP23-75

Predictors of Early Re-bleeding and Mortality after Acute Variceal Hemorrhage in Patients with Cirrhosis

N. Aqodad ${ }^{1}$, K. Saada ${ }^{1}$, I. Mellouki ${ }^{1}$, M. Elyousfi ${ }^{1}$, D. Benajeh ${ }^{1}$, M. Elabkari ${ }^{1}$, A. Ibrahimi ${ }^{1}$, K. Bendahou ${ }^{2}$, C. Nejjari ${ }^{2}$

${ }^{I}$ Gastroenterology; ${ }^{2}$ Epidemiology, Hospital University Hassan II, $\mathrm{Fez}$, Morocco

Background/aims: Hemorrhage from esophageal varices is one of the most feared complications of portal hypertension and is associated with significant mortality in patients with cirrhosis. This study was conducted to investigate the independent indicators of early rebleeding and mortality in cirrhotic patients after acute variceal hemorrhage.

Patients and Methods : We conducted a retrospective analysis about 215 cases collected between January, 2001 and July, 2010. All patients with cirrhosis and acute variceal hemorrhage were been included. univariate analysis was performed to find an association between rebleeding, mortality, and clinical, biological, and socio demographic characteristics of our patients. The data analysis has been performed with the software Epi-Info.

Results: The average age of our patients was 51 years. The sex ratio was one. Thirty percent of patients had viral cirrhosis, and $45 \%$ were Child-Pugh class B. The majority of patients $(79 \%)$ had only esophageal varices, and 3\% had only gastric varices. Fifty $(23 \%)$ patients had rebleeding. Univariate analysis showed that rebleeding was significantly associated with the presence of a lowered rate of prothrombin $(p=0.05)$, however, the other factors: ascites $(p=0.1)$, hemoglobin $(p=0.2)$, bilirubine $(p=0.41)$, Child-Pugh class $(p=0.2)$, and active bleeding at initial endoscopy $(p=0.3)$ were not associated with rebleeding. Twenty-five patients died during the first 10 days $(11.5 \%)$, of which $52 \%$ had rebleeding $(p=0.01)$. Univariate analysis showed that early mortality was significantly associated with the advanced age of patients $(p=0.018)$, low serum prothombin $(p=0.022)$, a low serum sodium $(p=0.03)$, a low platelet count $(p=0.05)$, and elevated transaminases $(p=0.02)$.

Conclusion: The low level of prothrombin was the only predictor of rebleeding. However, survival in patients with cirrhosis after acute variceal hemorrhage was influenced by advanced age, low serum prothrombin, serum sodium, the platet count, and elevated transaminases.

\section{PP23-76}

Spontaneous Bacterial Peritonitis (SBP): Prevalence and Risk Factors in Patients with Liver Cirrhosis in Upper Egypt

Y.M. Fouad ${ }^{1}$, E. Al Sharkawy ${ }^{2}$, H.A. Mokarab ${ }^{1}$, T. Hassanain ${ }^{1}$, H. Ibraheem ${ }^{1}$, A. El Sayed ${ }^{1}$, R. Yehia ${ }^{1}$

${ }^{I}$ Tropical Medicine; ${ }^{2}$ Clinical Pathology, Minia University, Minia, Egypt

Aim: To study the prevalence and risk factors of SBP in patients with liver cirrhosis and ascites at hospital admission in minya, upper Egypt.

Methods: Five hundred consecutive patients with liver cirrhosis and ascites were included. All patients subjected to full clinical history and examination and abdominal ultrasonography. Laboratory investigations included total serum proteins, serum albumin, transaminases, bilirubin, prothrombin time and concentration, creatinine, complete blood count. Diagnostic paracentesis done for all patients for type and count of cells, protein, albumin, glucose and culture.

Results: 120 patients $(24 \%)$ of 500 included patients had SBP. The classic type (culture positive neutrocytic bacterascites) was detected in 20 patients $(4 \%)$ and was gram negative strains in 15 and gram positive in 5 patients. Monomicrobial non-neutocytic bacterascites detected in 20 patients $(4 \%)$ and was gram positive strains in five cases and gram negative in 15 patients. Culture negative neutrocytic ascites in 80 patients (16\%). Recent history of hematemesis, tense ascites, leucocytosis, high serum bilirubin level, and ascitic fluid total proteins less than $1 \mathrm{~g}$ were significantly more frequent in patients with SBP.

Conclusion: SBP is a frequent complication of liver cirrhosis in our locality. Recent history of hematemesis, tense ascites, leucocytosis, high serum bilirubin level, and ascitic fluid total proteins less than $1 \mathrm{~g}$ put the patient of liver cirrhosis at a risk of SBP.

\section{PP23-77}

Oxidative Stress in the Portal Hypertensive Patients with Chronic Hepatitis B Virus Infection

J. Zhang ${ }^{1,2}$, X.-P. Fan ${ }^{1,3}$, L.-Y. Han $^{1,3}$, C.-X. Juan ${ }^{1,3}$, K. Wang ${ }^{1,3}$

${ }^{I}$ Department of Hepatology, Qilu Hospital of Shandong University; ${ }^{2}$ Hepatology Institute, Shandong University; ${ }^{3}$ Hepatology Institute of Shandong University, Jinan, China

Background and aims: Whether oxidative and antioxidative stress indicators can be the markers for liver fibrosis with portal hypertension remains obscure. The aim of the study was to investigate the oxidative stress profiles in portal hypertensive patients with hepatitis $B$ virus infection.

Methods: Forty-five portal hypertensive patients with hepatitis B virus infection were classified to three groups according to ChildPugh Grading. Oxidative stress indicators such as malondialdehyde (MDA) and antioxidative indicators vitamin C, Vitamin E, hydroxyl radical inhibitory ability were detected in the serum samples. Alanine aminotransferase and Hepatitis virus DNA were also determined.

Results: The levels of MDA were significantly increased in portal hypertensive patients compared with healthy controls $(P<0.05)$. And the levels in Child $\mathrm{C}$ groups was significantly higher than that in Child A and B classes $(P<0.05)$. The levels of Vitamin $\mathrm{C}$ and $\mathrm{E}$ in the patients with Child-Pugh B class were significantly higher than that with Child $\mathrm{A}$ and $\mathrm{C}$ classes and were both positively correlated with ALT $(r=0.63, r=0.72, P<0.05)$, negatively correlated with serum HBV DNA level $(r=-0.70, r=-0.66, P<0.05)$. Meanwhile, in patients with portal hypertension, Alanine aminotransferase levels were markedly increased compared with healthy controls $(P<0.05)$. The levels were higher In Child B than that in Child A and C. The HBV DNA value in Child B was the lowest in the portal hypertensive patients $(P<0.05)$. Significantly differences of hydroxyl radical inhibitory ability were not detected in the three classes. Conclusions: Oxidative stress plays an important role in the development of portal hypertensive cirrhosis with hepatitis B virus infection.

\section{PP23-78}

Propofol Sedation for Esophagogastroduodenoscopy in Cirrhotics S. Singh ${ }^{1}$, P. Sharma ${ }^{2}$, C.K. Pandey ${ }^{1}$, S.K. Sarin ${ }^{2}$

${ }^{I}$ Department of Anaesthesia and Critical Care, Institute of Liver and Biliary Sciences; ${ }^{2}$ Department of Hepatology, Institute of Liver and Biliary Science, New Delhi, India

Background: Cirrhotics are at increased risk of development of complications related to sedation.

Objectives: The primary aim was successful completion of esophagogastroduodenoscopy (EGD) and secondary aim was evaluation of complications related to propofol sedation in cirrhotics.

Methods: 108 cirrhotic patients aged 18-65 years, underwent EGD under propofol sedation. After a pre-anaesthetic check up, all patients underwent critical flicker frequency ( $\mathrm{CFF}$ ) test and a combination of psychometry tests [number connection test-A and B (NCT-A, B); digit symbol test (DST), line tracing test (LTT) and serial dotting test (SDT)]. Post Anaesthesia Discharge Score (PADS) assessed every $5 \mathrm{~min}$ and CFF done at $30 \mathrm{~min}$, and repeated every $30 \mathrm{~min}$ for $2 \mathrm{~h}$. Psychometry repeated at $2 \mathrm{~h}$. 25 non-cirrhotic patients served as control. 
Results: Of 108 patients[(age $40.7 \pm 11.8$ years, M:F 90:18), BMI (median $21.2 \mathrm{~kg} / \mathrm{m}^{2}$, range 16.4-34.7)], Child's status (A:B:C: 16:43:49), ASA (class $2, n=30$, class3, $n=78$ ) EGD was successfully completed in all. Ten patients in cirrhotic group (9\%) and 1 $(2.5 \%)$ in control group $(p>0.05)$ showed transient desaturation managed by increasing oxygen flow and chin lift maneuver. Hypotension seen in $18(17 \%)$ patients in cirrhotic group and in $2(8 \%)$ in control group $(p>0.05)$. PADS achieved at $15 \mathrm{~min}$ in all patients. Baseline CFF was $39.6 \pm 3.2 \mathrm{~Hz}$. Significant deterioration from baseline was seen in CFF at $30 \mathrm{~min}(38.3 \pm 3.5 \mathrm{~Hz}, p=0.001)$ and at $1 \mathrm{~h}(38.7 \pm 3.4 \mathrm{~Hz}, p=0.001)$ but not thereafter. No deterioration was observed on psychometry [NCT-A (65.2 \pm 44.4 vs. $62.4 \pm 43.6 \mathrm{~s}, p=0.01)$, NCT-B $(110.4 \pm 34.7$ vs. $109.6 \pm 44.6 \mathrm{~s}$, $p=0.45)$, DST $(26.2 \pm 10.0$ vs. $26.7 \pm 9.9 \mathrm{~s}, p=0.25)$, LTT $(116.6 \pm 55.2$ vs. $115.4 \pm 51.3 \mathrm{~s}, p=0.47)$, SDT $(88.6 \pm 47.5$ vs. $84.3 \pm 44.1 \mathrm{~s}, p=0.02)$ ]. Controls showed no deterioration in psychometry and CFF at $2 \mathrm{~h}$. No patients developed overt HE at $2 \mathrm{~h}$. Conclusions: Propofol sedation for EGD is safe in cirrhotics and does not precipitate hepatic encephalopathy.

\section{PP23-79}

Hepatic Osteodystrophy in Patients with Alcohol and Virus Related Cirrhosis of Liver

M. Tomar ${ }^{1}$, N. Choudhary ${ }^{1}$, Y.K. Chawla ${ }^{1}$, S. Bhadada ${ }^{2}$, N. Khandelwal ${ }^{3}$, R.K. Dhiman ${ }^{1}$, A. Duseja ${ }^{1}$, A. Bhansali ${ }^{2}$

${ }^{1}$ Hepatology; ${ }^{2}$ Endocrinology; ${ }^{3}$ Radiodiagnosis, PGIMER, Chandigarh, India

Background: Patients of cirrhosis are more prone to develop metabolic bone disease. Scanty data is available in the literature on osteodystrophy from India in patients with non cholestatic liver diseases.

Methods: Diagnosed patients of cirrhosis were prospectively evaluated for bone mineral density (BMD) as measured by Dual Energy X-ray absorptiometry at femoral neck, lumbar spine and left forearm. Correlation of BMD was studied with age, sex, etiology of cirrhosis, Child's class, serum bilirubin, alkaline phosphatase, albumin, calcium, phosphate, $25(\mathrm{OH}) \mathrm{D}$ and parathyroid hormone.

Results: Study group comprised of 115 cirrhotic patients; (107 M and $8 \mathrm{~F})$. Etiology was alcohol in $67(58.2 \%)$ and viral in $48(41.7 \%)$; Hepatitis B in 29 (25.2\%) and Hepatitis C in 19 (16.5\%); mean age was 49 (5.5) years. Prevalence of osteodystrophy was significantly higher in males as compared to females; 97.1 and $75 \%$, respectively $(p=0.038)$. Both alcoholic and viral groups had similar baseline characteristics except albumin levels. Child's class was B in 72 and C in 43 patients. Osteodystrophy was present in $97 \%$ of alcoholic cirrhosis and $93.7 \%$ of viral cirrhosis patients $(p>0.05)$. Low BMD was present at; femoral neck in $80.8 \%$ lumbar spine in $77.3 \%$, and forearm in $59.9 \%$ of patients. Correlation was significant only for PTH (negative correlation).

Conclusion: Osteodystrophy is common in alcoholic and viral cirrhosis patients.

\section{PP23-80}

Determination of Leukocyte Esterase Activity with Urine Strip in Spontaneous Bacterial Peritonitis Diagnosis, Peritoneal Carcinomatosis and Peritoneal Tuberculosis

Y. Colak ${ }^{1}$, B. Pinarbasi ${ }^{2}$, Y. Cakaloglu ${ }^{3}$, G. Adali ${ }^{1}$, F. Akyuz ${ }^{2}$, K. Demir ${ }^{2}$, S. Ozdil ${ }^{2}$, F. Besisik ${ }^{2}$, S. Kaymakoglu ${ }^{2}$, G. Boztas ${ }^{2}$, Z. Mungan ${ }^{2}$, A. Okten ${ }^{2}$ ${ }^{1}$ S.B. Istanbul Goztepe Education and Research Hospital, Department of Gastroenterology; ${ }^{2}$ Gastroenterology, Istanbul University, Istanbul Faculty of Medicine; ${ }^{3}$ Gastroenterology, Memorial Hospital, Istanbul, Turkey

Background: Spontaneous bacterial peritonitis (SBP) is a severe complication of cirrhosis with ascites and is associated with an immediate and high risk of death. A quick and reliable method to detect SBP is required. In our study, the diagnostic value of US for SBP has been investigated. The evaluation of US in patients with peritoneal carcinomatosis (PC) and peritoneal tuberculosis (PT) was also studied.

Methods: 301 AF samples in total were taken from 246 patients with portal hypertension $(\mathrm{PH}), \mathrm{PC}$ and $\mathrm{PT}$ induced ascites; in the AF samples, the PMN count was determined with automatic blood count, and leukocyte esterase activity (LEA) was also evaluated using US (Multistix ${ }^{\circledR} 10$ SG Bayer Diagnostics). Those who had a LEA value of $0-1(+)$ were considered as insignificant (LEA-negative), and 2-3(+) as SBP-compatible (LEA-positive). The correlation between the LEA and PMN counts was assessed methodologically.

Results: There were 190 patients in the $\mathrm{PH}$ group, paracentesis was performed on 56 of these patients and $246 \mathrm{AF}$ samples were evaluated in total. 47 and $8 \mathrm{AF}$ samples were examined respectively in the $\mathrm{PC}$ and PT groups. The analysis results of a total of $246 \mathrm{AF}$ samples taken from 190 patients in the PH group with US, were as follows: sensitivity of US in SIA diagnosis: $97 \%$, specificity: $99 \%(p<0.0001)$, positive predictive value: $98 \%$, negative predictive value: $97 \%$, accuracy: $98 \%$, kappa: 0.95 . In the PC group of 47 patients in total, out of 28 patients with AF PMN count $>250 / \mathrm{mm}^{3} 4$ had LEA-positive $(14.3 \%)$ and 24 had LEA negative $(85.7 \%)$, and out of 19 patients with AF PMN count $<250 / \mathrm{mm}^{3}$, all were found LEA negative. The PT group included 8 patients 6 of whom had PMN count $>250 / \mathrm{mm}^{3}$ and all whom were found LEA-negative.

Conclusions: LEA detection via Multistix ${ }^{\circledR}$ may be used as an alternative diagnosis method in SBP diagnosis in patients with $\mathrm{PH}$ ascites.

\section{PP23-81}

Colorimetric Grade as Fast Screening Test for SBP at Outpatient Setting R.P. Costa ${ }^{1}$, S. Carvalho ${ }^{1}$, S. Tavares ${ }^{1}$, E. Pinelo ${ }^{1}$, J. Presa ${ }^{2}$

${ }^{I}$ Internal Medicine; ${ }^{2}$ Hepatology, CHTMAD - Vila Real, Vila Real, Portugal

Aim: To evaluate the efficacy urinary reagent strips in diagnosis of spontaneous bacterial peritonitis (SBP) in cirrhotic outpatients who underwent paracentesis.

Methods: 407 ascitic fluid samples were collected from 112 outpatients with cirrhosis and ascites who underwent paracentesis between $01 / 01 / 2007$ and 31/06/2008. Fluid was manually analyzed by cytology for polymorphonuclear (PMN) cell count and with urinary reagent strips (Multistix 10SG). The diagnostic cut off level used for PMN cell count was $250 \mathrm{cell} / \mathrm{mm}^{2}$. The results of urinary reagent strip were graded for color change from 0 to $3+$. The sensitivity, specificity, positive and negative predictive values and accuracy of the three different colorimetric cut off scales $(1+, 2+, 3+)$ were calculated and compared.

Results: Mean age $60 \pm 12$ years; $68.8 \%$ male, $31.3 \%$ female; ChildPugh A $0.9 \%$, B $33.9 \%$, and C $65.2 \%$. Of the 407 paracentesis performed, SBP was diagnosed in $9.6 \%$. The sensitivity, specificity, positive and negative predictive values and accuracy of $1+$ were 84.62 , $98.10,82.50,98.37$, and $96.80 \%$, respectively, of $2+$ were 74.36 , $99.73,96.70,97.35$, and $97.30 \%$, respectively and of $3+$ were 25.6 , $100,100,92.70$, and $92.87 \%$, respectively.

Conclusion: The use of a urine reagent strip is highly specific and has a high negative predictive value, even in the lower cut off scales, therefore should be used as a routine screening test in all outpatients with ascitis and cirrhosis in order to exclude SBP.

\section{PP23-82}

Correlation of Platelet Count/Spleen Diameter Ratio with Presence of Esophageal Varices in Patients with Liver Cirrhosis

N. Aqodad, B. Benyachou, M. El Yousfi, I. Mellouki, D. Benajah, M. El Abkari, A. Ibrahimi

Gastroenterology, Hospital University Hassan II, Fez, Morocco 
Esophageal varices (EVs) are a serious consequence of portal hypertension in patients with liver diseases. Several studies have evaluated possible noninvasive markers of EVs to reduce the number of unnecessary endoscopies in patients with cirrhosis but without varices. Our aim is to identify clinical, biochemical, and ultrasonographic parameters which might non-invasively predict the presence of $\mathrm{OV}$ in patients with liver cirrhosis.

Materials and methods: We retrospectively analyzed data from all cirrhotic patients, collected between January, 2001 and May, 2010. We evaluated epidemiological data, clinical, laboratory and endoscopic finding. Platelet count/spleen diameter ratio was calculated for all patients. Analysis of the results has been performed with the software Epi-Info.

Results: We collected 311 patients underwent a complete biochemical workup, upper digestive endoscopy, and ultrasonographic measurement of spleen bipolar. The average age of our patients was 50.8 (15-96 years old) with a slight female predominance in $52 \%$ of cases. Major causes of cirrhosis were hepatitis C (35.1\%), hepatitis B (19.3\%), and three patients had primary biliar cirrhosis (1\%). $15 \%$ of patients had esophageal varices grade I, 50\% had grade II, and 35\% had grade III. In a univariate analysis we found that total bilirubin (26.6 mg/l; $p=0.04)$, prothrombin activity $(65 \% ; p=0.03)$, platelet count $\left(106,000 \mathrm{n} / \mathrm{mm}^{3} ; p=0.02\right)$ were significantly associated with the presence of EVs, however the platelet count/spleen diameter ratio ( $p=0.09$ ) was not associated to the presence of EVs.

Conclusion: The lower prothrombin activity, higher serum total bilirubin and lower platelet count were significantly associated with the presence of EVs. However the platelet count/spleen diameter ratio was not associated to the presence of EVs.

\section{PP23-83}

Predictors Factors of Bleeding Complication after Endoscopic Band Ligation (EBL) in Cirrhotic Patients

N. Aqodad, B. Benyachou, I. Mellouki, M. El Yousfi, D. Benajah, M. El Abkari, A. Ibrahimi

Gastroenterology, Hospital University Hassan II, Fez, Morocco

After EBL, patients are at risk of post interventional bleeding. This represents a rare and severe complication. The aim of our study is to determinate predictors factors of bleeding associated with this complication after EBL.

Materials and methods: We retrospectively analyzed data from all cirrhotic patients who underwent EBL, collected between January, 2001 and May, 2010. We evaluated epidemiological data, clinical, laboratory and endoscopic findings. Analysis of the results has been performed with the software Epi-Info.

Results: We collected 1,107 patients with cirrhotic portal hypertension with 365 who underwent EBL. Thirty-five of patients had presented bleeding complications after EBL, which was confirmed in all cases by endoscopy in the presence of bleeding in relation to one or more sites of ulceration esophageal after EBL. The average age of our patients was 51 (15-80 years old), with an equal number of women than men. Cirrhosis was post-viral $\mathrm{C}$ in $35.2 \%$ of cases, post viral B in $18.1 \%$ of cases, with a score of Child Pugh class A: $28.1 \%$, Class B: $57.9 \%$, Class C: $14 \%$. The EV were present in $100 \%$ with stage III were $52.3 \%$ and $44.5 \%$ were stage II. The bleeding complications after EBL occurred in 57.2\% after the first EBL, within 6.5 days (1-16 days). This complication was significantly associated with a lower prothrombin activity $<60 \%(p=0.02)$. However, other parameters namely ascites, history of gastrointestinal bleeding, leukocytes, Child score, and history of transfusion were not associated with this complication. Twelve patients $(34.2 \%)$ died by this complication.

Conclusion: Bleeding complications after EBL in patients with cirrhosis occurred in $10 \%$ of cases. Lower prothrombin activity is the only predictor factor of this complication.
PP23-84

Community-acquired versus Nosocomial Spontaneous Bacterial Peritonitis in Patients with Liver Cirrhosis

Y.E. Chon ${ }^{1}$, S.U. Kim ${ }^{1}$, C.K. Lee ${ }^{2}$, J.Y. Park ${ }^{1}$, D.Y. Kim ${ }^{1}$, K.-H. Han ${ }^{1}$, C.Y. Chon ${ }^{1}$, S. Kim ${ }^{1}$, K.S. Jung ${ }^{1}$, S.H. Ahn ${ }^{1}$

${ }^{I}$ Department of Internal Medicine, Yonsei University College of Medicine; ${ }^{2}$ Department of Internal Medicine, National Health

Insurance Corporation Ilsan Hospital, Seoul, Republic of Korea

Background and aim: Spontaneous bacterial peritonitis (SBP) is a common complication in patients with end-stage liver disease, but reports comparing community-acquired SBP (CA-SBP) with nosocomial SBP (N-SBP) are rare. The present study compared the clinical characteristics, microbiological characteristics, and treatment outcomes of patients with CA-SBP and N-SBP.

Methods: Records for 248 patients (173 men, 75 women) with cirrhosis who experienced SBP were retrospectively reviewed.

Results: The study population included $202(81.5 \%)$ patients with CA-SBP and 46 (18.5\%) patients with N-SBP (mean age, 56.1 years). Patients with CA-SBP or N-SBP showed no significant differences in baseline or microbiological characteristics, except for a high frequency of previous SBP history in the N-SBP population $(P=0.020)$. During hospitalization, antibiotic switching and in-hospital mortality were significantly higher for patients with N-SBP than those with CASBP $(35.6 \%$ vs. $8.9 \% ; P=0.001$; hazard ratio (HR), $4.943 ; 95 \%$ confidence interval (CI), 2.227-10.975 and $30.4 \%$ vs. $12.9 \%$, $P=0.028 ; \mathrm{HR}, 2.412 ; 95 \% \mathrm{CI}, 1.238-2.841)$. There were 202 $(81.5 \%)$ deaths during the follow-up period, with longer overall survival time in patients with CA-SBP than those with N-SBP (7.9 vs. 3.9 months; $P=0.041)$. However, time to recurrence was not significantly different between the two groups (4.7 vs. 3.6 months; $P=0.910)$.

Conclusions: N-SBP was significantly associated with increased antibiotic switching, higher in-hospital mortality and shorter overall survival than CA-SBP. Third-generation cephalosporin (cefotaxime) may be inappropriate as first-line empirical antibiotics for patients with N-SBP.

\section{PP23-85}

Influenza Vaccination in Patients with Cirrhosis and Inactive Carriers of Hepatitis B

B. Sayad ${ }^{1}$, S.M. Alavian ${ }^{2}$, F. Najafi ${ }^{3}$, M.H. Ari Tabarestani ${ }^{1}$, A. Janbakhsh ${ }^{1}$, F.A. Mansouri ${ }^{1}$, M. Afsharian ${ }^{1}$, S. Vaziri ${ }^{1}$, M. Shirvani ${ }^{1}$

${ }^{1}$ Kermanshah Liver Diseases and Hepatitis Research Center,

Kermanshah University of Medical Sciences, Kermanshah;

${ }^{2}$ Baqiyatallah Research Center for Gastroenterology and Liver

Disease, Baqiyatallah University of Medical Sciences, Tehran;

${ }^{3}$ Kermanshah Health Resarch Center, Kermanshah University of

Medical Sciences, Kermanshah, Iran

Background and aims: Influenza can cause morbidity and mortality that are greatly enhanced in patients with underlying chronic diseases such as cirrhotic patients. This study was performed to assess the immunogenicity of influenza vaccination in patients with cirrhosis and inactive carriers of hepatitis B.

Material and methods: In this clinical trial 93 enrolled persons divided in three groups: (1) Cirrhotic $(N=28)$ (2) Inactive carriers of hepatitis B $(N=31)$, (3) Healthy individuals $(N=34)$ and were immunized with Influenza vaccine (Influvac ${ }^{(B)}$ ). Serum samples were taken before and 4 weeks after vaccination and the humoral immunogenicity was assessed by the hemagglutination inhibition (HI) test. Descriptive data were compared by the Chi square test and the paired $t$ test.

Results: Four weeks after vaccination, seroconversion rates ranged between 71.4 and $100 \%$ in Group 1, 70.6 and $94.1 \%$ in Group 2, and 58.1 and $80.7 \%$ in Group 3. A significant $(P<0.05)$ difference was noted in the rates of seroconversion and GMTs against influenza B 
vaccine component between the three groups. No significant differences were seen in the rates of seroconversion and GMTs against influenza A (H1N1) and influenza A (H3N2) vaccine components among the three groups $(P>0.05)$. No significant $(P>0.05)$ differences in the rates of seroprotection were observed within the three groups. Antibody GMTs against all three strains of influenza vaccine were significantly higher $(P<0.05)$ than those measured at baseline in all three groups.

Conclusions: Influenza vaccination is effective in cirrhotic patients and inactive carriers of Hepatitis B as well as healthy individuals and we considered vaccination in these groups for reduced the morbidity and mortality of influenza.

\section{PP23-86}

Study of the Impact on Cellular Immune Function and Antiviral Effect with Zhenqifuzheng Capsule Combined Adefovir in Treating CHB H. Zhong

Wuxi Hospital for Infectious Diseases, Wuxi, China

Objective: Evaluation the efficacy of joint adefovir with Zhenqifuzhen Capsule and adefovir alone for the treatment of c CHB patients in cellular immune function and inhibit viral replication.

Methods: Seventy patients of CHB on the basis of treating liver were divided into two groups randomly. The therapeutic group (35) were treated with Adefovir orally $10 \mathrm{mg}$ once daily combined with Zhenqifuzhenjiaonang orally $1.4 \mathrm{~g}$ tid, while the control group (35) were treated with Adefovir orally $10 \mathrm{mg}$ once daily only. The course of treatment is 6 months.

Results: Immune function of peripheral blood lymphocytes: treatment 24 weeks, The $\mathrm{CD} 4^{+}, \mathrm{CD}^{+} / \mathrm{CD}^{+}, \mathrm{NK}$ values of the treatment group is higher than the control group, and the difference was significant $(P<0.05)$. HBV DNA load Answer: 12 weeks, the treatment group than the control group, the difference was not statistically significant $(P>0.05)$; but treatment of 24 weeks, the treatment group compared with the control group, the difference was significant $(P<0.05)$. E antigen seroconvertion rate Answer: At 24 weeks, the treatment group higher than the control group $(P<0.05)$.

Conclusion: Zhenqifuzhenjiaonang combined with adefovir for $\mathrm{CHB}$ is superior to adefovir alone in improving cellular immune function,reducing of the load of HBVDNA and enhancing $\mathrm{E}$ antigen seroconvertion rate.

\section{PP23-87}

Different Modalities in the Management of Junctional Varices

Y. Elsherif, N. Omar, A. Abu Gabal, H. Taha

National Liver Institute, Menofia University, Shebin Elkom, Egypt

Background: Junctional varices extend from the oesophagus to the stomach at the gastroesophageal junction. They are classified into GOV1, extending to the lesser curvature; and GOV2, extending to the greater curvature of the stomach. They account the majority of gastric varices. There are several endoscopic treatment options for junctional varices, however, there is no consensus for optimum endoscopic treatment. The aim of this study is to assess the efficacy of different endoscopic modalities in management of bleeding junctional varices. Patients and methods: Seventy-five patients with first bleeding from junctional varices were randomly classified into three endoscopic treatment groups (25 patients/group): band ligation (BL), ethanolamine oleate injection (EO) and $N$-Butyl cyanoacrylate injection (CyA). All patients had subsequent follow up for 6 months to assess rebleeding (bleeding within $48 \mathrm{~h}$ after initial control), recurrent bleeding (bleeding within 6 months after initial control) and death rates. Endoscopic failure cases underwent TIPPS and/or surgery and were excluded from the study.

Results: The groups were matched for age, sex, Child-Pugh score and grade of varices. Initial control of bleeding was significantly higher in the CyA group compared to the EO group $(23 / 25$ vs. $16 / 25, p<0.05)$.
CyA was significantly superior to both BL and EO groups with respect to variceal eradication (19/24 vs. $11 / 18$ and $6 / 13$ consequently, $p<0.01)$ and the number number of required endoscopy sessions, (means: $1.6 \pm 0.8,3.9 \pm 1.3$ and $4.2 \pm 1.9$ consequently, $p<0.01)$. There was no significant difference between the CyA and $\mathrm{BL}$ groups or between the BL and EO groups as regards initial control of bleeding. There was no significant difference between the three groups in term of rebleeding, recurrent bleeding or overall death rates. Conclusion: $N$-Butyl cyanoacrylate is an effective modality in the management of junctional varices. Band ligation may be used as an alternative.

\section{PP23-88}

Gastric Fundal Variceal Bleeding after Band Ligation Treated with Endoscopic Cyanoacrylate Injection Followed by Balloon-occluded Retrograde Transvenous Obliteration

K. Kanazawa ${ }^{1}$, H. Imazu ${ }^{1}$, H. Kakutani ${ }^{1}$, K. Matsunaga ${ }^{1}$, K. Ikeda ${ }^{1}$, K. Goda ${ }^{1}$, T. Miyazaki ${ }^{1}$, H. Tajiri ${ }^{2}$

${ }^{I}$ Department of Endoscopy; ${ }^{2}$ Division of Gastroenterology and Hepatology, Departement of Internal Medicine, The Jikei University School of Medicine, Tokyo, Japan

Introduction: Bleeding gastric fundal varices (FV) with gastro-renal shunt is difficult to control endoscopically, because of abundant blood flow. Cyanoacrylate (CA) injection therapy has been reported as firstline treatment for bleeding FV, although relatively high re-bleeding rate has been shown. Endoscopic variceal ligation (EVL) has been widely accepted as treatment of bleeding esophageal varices. However, its use for bleeding FV might have potential risk of bleeding from post-EVL ulcer. We report a case with FV bleeding due to ulcer caused with EVL successfully treated with CA injection followed by balloon-occluded retrograde transvenous obliteration (B-RTO).

Case report: 64-year-old man with HCV-related liver cirrhosis who underwent successful EVL therapy for FV bleeding was referred to our hospital. EGD showed post-EVL ulcer on a marked enlarged nodular FV, and $\mathrm{CT}$ revealed large FV with gastro-renal shunt. Therefore, B-RTO was scheduled as secondary prophylaxis. However, 2 days after hospitalization, he had a massive hematemesis, and emergent EGD showed spurting bleeding from post-EVL ulcer on FV. A total of $2.8 \mathrm{ml}$ of CA with lipiodol was endoscopically injected into $\mathrm{FV}$, and hemostasis was successfully achieved. Then, 1 week after CA injection therapy, B-RTO was performed with ethanolamine oleate for remaining FV without any complication. CT and EGD 1 month after B-RTO showed FV, feeder vein and gastro-renal shunt completely disappeared.

Conclusion: Although EVL for bleeding FV is useful for temporally hemostasis, post-EVL ulcer on FV can cause re-bleeding from FV. Therefore, CA injection therapy should be applied for bleeding FV as first-line treatment. Additional B-RTO is useful for complete obliteration of $\mathrm{FV}$ as well as feeding vein under temporally control of bleeding with CA injection.

\section{PP23-89}

Administration of Oral Lansoprazole Can Increase Healing of Post-banding Esophageal Ulcers after Endoscopic Variceal Ligation P. Pisespongsa ${ }^{1}$, P. Kulseth ${ }^{2}$, S. Thongsawat ${ }^{1}$, A. Leerapun ${ }^{1}$, T. Chitapanarux ${ }^{1}$, O.-A. Praisontarangkul ${ }^{1}$

${ }^{1}$ Chiang Mai University, Chiang Mai; ${ }^{2}$ Chiangrai Prachanukroh Hospital, Chiangrai, Thailand

Backgrounds/aims: Endoscopic variceal ligation (EVL) has been standard treatment for esophageal varices (EV). However, some patients have complications from post-banding esophageal ulcers such as chest pain, dysphagia and more seriously, bleeding ulcer. Since gastric acid may interfere ulcer healing, this study aim to investigate the effects of oral lansoprazole on post-banding ulcer healing. 
Methods: After the first variceal band ligation, patients were randomly assigned to receive $15 \mathrm{mg}$ lansoprazole twice a day or placebo for 10 days. Upper endoscopy was performed at 10 days after first EVL to assess size and number of residual banding ulcers.

Results: Sixteen patients were included, seven in lansoprazole group and nine in placebo group. In lansoprazole group, there were 31 postbanding ulcers: 11 were partially healed and 2 were completely healed. The mean ulcer sizes in lansoprazole group was $126.1+86.7 \mathrm{~mm}^{2}$. In control group, there were 39 ulcers: 5 were partially healed and none was completely healed. The mean ulcer sizes in control group was $124.2+77 \mathrm{~mm}^{2}$. The number of healing and healed ulcers were higher in lansoprazole group $(p=0.012)$; but there was no difference in mean ulcers sizes in both groups $(p=0.923)$.

Conclusion: Oral lansoprazole can increase the healing rate of postbanding ulcers but cannot decrease the mean ulcer sizes. Further large randomized control trial is need to confirm the clinical significance of this finding.

\section{PP23-90}

Salvage TIPS with Polytetrafluoroethylene-covered Stents in Patients with Cirrhosis and Refractory Variceal Bleeding

M. Rudler, P. Cluzel, J. Massard, P. Lebray, T. Poynard, D. Thabut Pitié-Salpêtrière Hospital Assistance Publique-Hôpitaux de Paris, Paris, France

Background: Salvage Transjugular Intrahepatic Portosystemic Shunt (TIPS) is efficient in variceal bleeding refractory to standard treatment. No data is available concerning feasibility, efficacy and complications with new polytetrafluoroethylene-covered stents.

Aims: To describe efficacy and tolerance of TIPS with covered stents in a population of non-selected patients with cirrhosis and refractory variceal bleeding.

Methods: We report our retrospective monocentric experience of salvage TIPS in refractory variceal bleeding in 41 non-selected consecutive patients (sex male $=78 \%$, mean age $=53.3$ years, shock at presentation $=58 \%$, aetiology: alcohol $n=27$, virus $n=7$, mixte $n=5$, other $n=2$ ) hospitalized in Intensive Care Unit between November 2002 and December 2009. Child score was A/B/C in 5/12/ 24 cases, and mean MELD score of patients was $19.0 \pm 8.8$. There was an active bleeding at initial endoscopy in $80 \%$ of cases. Bleeding was controlled in $76 \%$ of cases. Five patients presented early rebleeding. Six week-survival was $74 \%$ in whom TIPS was efficient versus $10 \%$ in the absence of control of bleeding $(p<0.0001)$. Twenty-six patients presented complications after TIPS: 1 hepatic hematoma, 23 episodes of hepatic encephalopathy, 1 early TIPS thrombosis, and 3 episodes of heart failure. In univariate analysis, factors associated with 6 weeks-mortality were MELD score, Child score, Prothrombin time, INR, creatinin, albumin, shock at presentation, primary TIPS failure, complication after TIPS, and infection. In multivariate analysis, the only factor was MELD score $(p=0.04)$. Eight patients had a cirrhosis with a Child score $>13 ; 6$ week-survival was 12 versus $69.7 \%$ in patients with Child score $\leq 13(p=0.003)$. Conclusion: Salvage TIPS was efficient in $76 \%$ of cases in our population of patients hospitalized for variceal bleeding refractory to standard treatment. Six-week survival was only $12 \%$ in patients with a Child score $>13$. An early TIPS placement could be proposed in these patients.

\section{PP23-91}

Soft Tissue Infections in Cirrhotics: A Prospective Study from a Tertiary Care Institute in Northern India

O. Goyal ${ }^{1}$, P. Goyal ${ }^{1}$, V. Midha ${ }^{2}$, N. Sood ${ }^{3}$, A. Sood

${ }^{1}$ Gastroenterology; ${ }^{2}$ Medicine, DMC and Hospital, India;

${ }^{3}$ Pathology, DMC and Hospital, Ludhiana, India

Aims: Bacterial infections are an important cause of morbidity and mortality in cirrhotics. Data on soft tissue infections (STI) in cirrhotics is scarce. This study was undertaken to evaluate the clinical features and outcome of cirrhotic with STIs.

Methods: Cirrhotics with STI admitted from September 2007 to August 2010 were studied. Blood and local site cultures were sent wherever possible. Exclusion criteria were- trauma or insect bite; or infections relating to diabetes, $\mathrm{CCF}, \mathrm{CRF}$, ischemia, deep vein thrombosis, malignancy, chemotherapy or radiotherapy.

Results: A total of $19.43 \%(271 / 1,395)$ cirrhotics presented with bacterial infections [SBP-45\%, STI-31.4\% $(n=85)$, UTI-24.5\%, bacteremia- $12.9 \%$, RTI- $8.9 \%$ and meningitis-0.74\%]. Patients with STI had mean age $49.8 \pm 10.2$ years (male:female $82: 3$ ). STI was one of the presenting complaints in $52(61.2 \%)$ patients, the sole presenting complaint in $16(18.8 \%)$, while it was diagnosed on routine examination in $17(20 \%)$ patients. The mean duration of presence of STI was $15.3 \pm 20.5$ days. Other presenting complaints were- fever $(n=31)$, abdominal distension $(n=33)$, gastrointestinal bleed $(n=18)$, jaundice $(n=21)$, altered sensorium $(n=14)$ and oliguria $(n=7)$. Sixty patients were known cirrhotics; alcohol and HCV were most common aetiologies. Most common sites of STI were- lower limbs $[n=74 ; 87 \%$, (unilateral in 65$)]$, abdominal wall $(n=5$; $5.9 \%)$, upper $\operatorname{limb}(n=2 ; 2.4 \%)$, face $(n=2 ; 2.4 \%)$ and gluteal region $(n=2 ; 2.4 \%)$. Types of STI were- cellulitis $(n=52 ; 61.2 \%)$, hemorrhagic bullae $(n=12 ; 14.1 \%)$, pustules $(n=9 ; 10.6 \%)$, ulcers ( $n=8 ; 9.4 \%)$, discharging sinuses $(n=2 ; 2.4 \%)$ and necrotising fascitis $(n=2 ; 2.4 \%)$. Cultures from the local site were positive in $15 / 28(53.6 \%)$ patients \{ gram negative bacilli in 13/15; gram positive cocci in 2/15). Most patients were managed conservatively. Six patients underwent fasciotomy/debridement. Mortality rate was $23.5 \%$ (20/85). Factors predicting mortality were- leucocytosis, renal failure and haemorrhagic bullae.

Conclusions: STIs are common in cirrhotics. STIs mostly involve lower limbs and are predominantly caused by gram negative bacilli. Leucocytosis, renal failure and haemorrhagic bullae predict poor outcome.

\section{PP23-92}

Peptic Ulcer Bleeding Is Mostly Idiopathic in Patients with Cirrhosis M. Rudler ${ }^{1}$, J. Massard ${ }^{1}$, L. Deforges ${ }^{2}$, T. Poynard ${ }^{1}$, D. Thabut ${ }^{1}$ ${ }^{1}$ Pitié-Salpêtrière Hospital Assistance Publique-Hôpitaux de Paris; ${ }^{2}$ Henri Mondor Hospital APHP, Paris, France

Background: Peptic ulcer bleeding (PUB) is the most important cause of upper gastrointestinal bleeding. PUB occurs more often is patients with cirrhosis. Physiopathology of PUB in cirrhosis has been weakly described.

Aims: To compare characteristics of PUB in cirrhotic and non cirrhotic patients.

Methods: All patients hospitalized in the Intensive Care Unit of Hepato-Gastroenterology of the Pitié-Salpêtrière Hospital (Paris, France) for PUB between September 2007 and July 2010 were treated with high dose proton pump inhibitors (PPIs), started before endoscopy, and a double endoscopic treatment. Helicobacter Pylori (HP) serology was assessed and systematic eradication of HP was performed.

Results: 188 patients were analyzed (sex male $=72.9 \%$, mean age $=60.6 \pm 15.9$ years, cirrhosis $=10.2 \%$ ). PUB were classified Forrest I/II/III in 33/61/24 patients. $45.8 \%$ of patients had been treated with non-steroidal anti-inflammatory (NSAIs) and $52.5 \%$ were positive for HP. Twenty-nine percent of patients had «idiopathic PUB», that is neither related to NSAIs nor to HP. We compared patients with cirrhosis $(n=16)$ and patients without cirrhosis $(n=102)$ : there was no difference in terms of shock at presentation, ulcer's size, sex, Forrest classification, localization. Patients with cirrhosis were significantly younger $(54.9 \pm 7.4$ vs. $61.4 \pm 16.7$ years, $p=0.01)$. Proportion of patients who had been treated with NSAIs was weaker $(12.5$ vs. $51 \% p=0.004)$, and HP positive serology rate was lower 
(18.8 vs. $57.8 \%, p<0.003)$ in patients with cirrhosis. Proportion of patients with idiopathic PUB was higher (71.2 vs. $18.8 \%, p<10-4)$. Fourteen patients had endoscopic stigmata of portal hypertension. Prognosis was not different in cirrhotic patients.

Conclusion: PUB is more frequently idiopathic and occurs in patients of younger age. These preliminary results suggest that the physiopathology of ulcers in the setting of cirrhosis has to be reassessed.

\section{PP23-93}

Assessment on the Risk of Bleeding in Patients with Esophageal Varices by Color Doppler Sonography

H. Xu, T. Tang, Y. Bai, Y. Zhu

Jilin University Affiliated No.1 Hospital, Changchun, China

Background/aim: Screening for esophageal varices with endoscopy is recommended in patients with cirrhosis. Non-invasive alternative measurements would be valuable. To evaluate the diagnosis performance of color Doppler sonography for prediction of esophageal varices.

Methods: 68 liver cirrhosis accompanied esophageal varices were divided into two groups according to the history of with or without hemorrage, 30 healthy people were as control. The portal hemodynamical parameter were detected by Doppler ultrasonography, including the internal diameter,blood flow velocity and blood flow direction of left gastric vein (LGV), splenic vein (SV) and portal vein (PV).

Results:

1) As compared to the normal controls, the diameter (D) of PV, SV and LGV was much wider; the blood flow velocity (V) was much lower, and the blood flow volume $(\mathrm{Q})$ was increased .The value of $\mathrm{Q}_{\mathrm{LGV}} / \mathrm{Q}_{\mathrm{PV}}$ was higher the controls.

2) The $\mathrm{D}_{\mathrm{PV}}, \mathrm{D}_{\mathrm{SV}}$ and $\mathrm{D}_{\mathrm{LGV}}$ were significantly wider $(p<0.01)$; The $\mathrm{Vpv}$ and Vsv were significantly slower $(p<0.01)$; $\mathrm{V}_{\mathrm{LGV}}$ were significantly higher $(p<0.05)$; the Qpv were not changed in all $\operatorname{groups}(p>0.05)$.

3) Flow direction of LGV was hepatopetal in all healthy controls, whereas it was hepatofugal in $83 \%$ of the patients with hemorrhage group; Flow direction of LGV was hepatopetal in $65 \%$ of the patients with without hemorrhage group.

Conclusion: Examination of PV, SV, LGV and hemodynamics in patients with cirrhosis can be used to evaluate the risk of varices.

\section{PP23-94}

Assessment of 80 Cases of Gastro-esophageal Variceal Bleeding with Advanced Hepatocellular Carcinoma

T. Kawai, S. Sato, S. Obi, T. Sato, M. Kanda

Liver Unit, Kyoundo Hospital, Tokyo, Japan

Background/aims: Endoscopic variceal ligation (EVL) have proven to be comparable in the prevention and acute bleeding of variceal rebleeding. However, Patients with advanced hepatocellular carcinoma (HCC) have limited survival. The treatment of bleeding from esophageal varices in such patients is controversial in the point of no evidence of the beneficial of survival. This study evaluated the efficacy of EVL in controlling acute bleeding from esophageal varices and the benefit of eradicate varices in patients with advanced HCC. Methodology: Between 2007 and 2009, eighty of 910 patients (8.7\%) treated for bleeding esophageal varices had cirrhosis and irresectable HCC. There were 65 men and 15 women; median age, 67 years (range: $30-79$ ). 45 patients were Child's-Pugh grade B and 30 grade C; 23 patients were stage III and 52 stage IV. We evaluated control rate of rupture of varices, re-rupture rate,

Results: Endoscopic variceal ligation (EVL) for rupture of esophageal varices was carried out in all 80 patients of liver cirrhosis with HCC. All patients were success for control of rupture of varices by
EVL. 24 out of 80 patients (30\%) re-ruptured within 30 days after EVL. Median survival time (MST) without re-rupture was 250 days after EVL. MST in all 80 patients was 67 days after EVL.

Conclusions: EVL controlled acute variceal bleeding in most patients with advanced HCC. We suggest that emergency EVL for rupture of esophageal varices should be performed in patients with relatively good general conditions, irrespective of the stage of HCC.

\section{PP23-95}

The Effects of Vasopressin Receptor Antagonists on Serum Sodium Concentration of Patients with Liver Cirrhosis: A Meta-analysis

A.J.F. Alejandre, I.H. Cua

Institute of Digestive and Liver Diseases, St. Luke's Medical Center, Quezon City, Philippines

Serum sodium concentration has been known to be of value in determining the prognosis of patients with cirrhosis. The latter stages of cirrhosis corresponds to progressive decrease in serum sodium concentration resulting in hypervolemic hyponatremia. The aim of this study is to determine the efficacy of selective vasopressin receptor antagonists in increasing the serum sodium concentration among hyponatremic patients with liver cirrhosis. The safety and tolerability of VRAs were also assessed.

Methodology: Literature search was done by use of MEDLINE, EMBASE, Cochrane and bibliographies of retrieved articles from 2002 up to the present. Randomized controlled trials involving hyponatremic patients with liver cirrhosis that were given VRAs and that measured the mean change serum concentration from baseline to endof-study period were included in this meta-analysis. Random effects model for combining quantitative data was used.

Results: Two studies with a total sample size of 143 patients were included. 107 patients received selective VRAs and 36 patients received placebo. The two VRAs (Satavaptan and VPA-985) that were used in the studies, produced significant increases in the mean serum concentration of sodium in patients with liver cirrhosis, as compared to placebo, when comparing baseline to end-of-study values with a $95 \%$ CI $1.32(0.81,1.82)$. These increases were doserelated. Thirst was more common in patients given the vasopressin receptor antagonists than placebo. The test for heterogeneity was also significant $(p<0.00001)$ among the included studies.

Conclusion: The vasopressin receptor antagonists, satavaptan and VPA-985, significantly increased the serum sodium concentration in patients with cirrhosis and hyponatremia.

\section{PP23-96}

Aetiology of Upper GI Bleeds in South India

K. Devadas, S. Devi

Medical Gastroenterology, Medical College, Kottayam, India

Introduction: Literature on aetiology of Upper Gastrointestinal (UGI) bleeds in the adult population in India is scarce. It may be at variance to that reported in the Western population.

Aims: To look at the aetiology and age related difference, of Upper GI bleeds in our population.

Materials and methods: The endoscopy records of the Department of Gastroenterology at a tertiary care centre, in South India over the past five years (2005 July-2010 June) were retrospectively perused to look for the cause of bleed in those patients who presented with hematemesis and/or malena. The causes of UGI bleed overall and in the age groups below 36 years, 36-65 years and above 65 years were analysed. Statistical analysis was done using the Chi square method when different causes were grouped and Odds ratio was used when same cause was analysed.

Results: Total of 2,524 bleeds with male to female ratio of 3.42:1. $65.4 \%$ of patients were 36-65 years of age. Portal Hypertensive (PHT) bleeds [Oesophageal and Gastric varix and Portal Hypertensive Gastropathy(PHG)] was the most common cause (39.82\%) followed 
by ulcer bleeds $(15.97 \%)$. Ulcer bleeds were more common than PHT in patients above 65 years $(P<0.0001)$ in both males $(P=0.00012)$ and females $(P=0.0067) .40 .43 \%$ of all ulcer bleeds occurred in this age group. There were significantly more DU bleeds than GU bleeds across all age groups. 9.5\% of endoscopies were normal with females significantly more likely to have a normal endoscopy (OR 3.75, CI 2.85-4.94, $P<0.0001)$. $11.93 \%$ had Mallory Weiss tear, $10.18 \%$ erosive gastropathy and $3.5 \%$ esophagitis.

Conclusions: Bleeds due to Portal Hypertension are more common in our population compared to ulcer bleeds. However ulcer bleeds are more common in the elderly. Upto $10 \%$ of endoscopies following an UGI bleed may be normal, especially in females.

\section{PP23-97}

Delta Meld Score in Predicting 3 Months Mortality among Egyptian Liver Cirrhosis Patients

H. Badran ${ }^{1}$, E. Rewisha ${ }^{1}$, N. Allam ${ }^{1}$, M. Hagar ${ }^{2}$

${ }^{1}$ National Liver Institute Menofeya University; ${ }^{2}$ Shebin Elkom

Teaching Hospital, Shebin Elkom, Egypt

Introduction/objectives: Studies proposed that delta MELD (might have additional prognostic value over MELD). There has been no report concerning the predictive DMELD scoring system in predicting the outcome in Egyptian cirrhotic patients.

Aims and methods: We aimed to assess $\triangle$ MELD in these patients and evaluating the factors associated with its increase. In addition, its predictive power was compared with that of both initial MELD and initial MELD Na. 200 cirrhotic patients were recruited at the inpatient department of the Liver Institute. Patients with concomitant malignancy,advanced organic renal, respiratory or cardiac diseases were excluded. At the initial visit, MELD score was calculated.Patients were prospectively followed up for 3 months with determination of MELD score monthly. Occurrence of gastrointestinal bleeding, spontaneous bacterial peritonitis (SBP), hepatic encephalopathy were reported.

Results: 55 patients $(27.5 \%)$ died during follow up; 10 in the first month, 18 in the second, 27 in the third. The mean initial MELD score was $23.12 \pm 7.61$ There was a statistically significant association between DMELD/month and initialMELD score. $(=0.49$; $P=<0.01)$. Taking 3-months mortality as the endpoint, At cut off level of 15 , AUC for the initial MELD score was 0.98 . it was $100 \%$ and, specificity $22 \%$ Regarding initial MELD sodium, AUC was 0.96 and at a cut off of 29 ,sensitivity was $96.4 \%$, specificity $72.4 \%$. AUC for mean DMELD/month was 0.96 . At a cutoff point of 3, sensitivity was $88.9 \%$ and specificity $85.5 \%$. In the subgroup with hepatic encephalopathy, sensitivity was highest at cut off point of 2. Multivariate analysis revealed that hepatic encephalopathy, SBP, initial MELD score $>15$ were independent predictors for elevated mean DMELD/month.

Conclusion: Initial and DMELD are significant predictive factors for 3-months mortality MELD $>15$ is the best predictor of short term survival . In hepatic encephalopathy, determining DMELD of more than 2 is of great predictive value of short term mortality.among liver cirrhosis patients MELD sodium.

\section{PP23-98}

Acute on Liver Failure in Hasan Sadikin General Hospital; During Periode 2008-2009

A. Djumhana, N. Agustanti, W. Yudi, S.A. Abdurachman Internal Medicine, Hasan Sadikin General Hospital, Bandung, Indonesia

Introduction: An Insult on patients who previously were in stable Chronic Liver Disease (CLD) may lead to Acute on Chronic Liver Disease (AoCLF) in many cases. .We studied short-term outcome of the patients with AoCLF during hospitalization in Dr. Hasan Sadikin
Hospital which has no liver support device facility nor referral system for liver transplantation.

Material and method: A retrospective study was conducted to 33 patients with AoCLF during period January 2008 to December 2009. The diagnosis of AoCLF based on criteria of APASL consensus 2008. Result: One hundred fifty six CLD patients with acute insult admitted to our Hospital during period January 2008 until December 2009 . AoCLF were diagnosed in 33 pattiens. Among of them: 23 were HBsAg positives, 6 anti $\mathrm{HCV}$ positives, $3 \mathrm{HBsAg}$ positives and anti $\mathrm{HCV}$ positives, and 1 due to alcohol . The precipitating factors were bleeding (16), infection (9), Herbal medicine (6) and HBV flare/ reactivation (2) . Nine of 33 AoCLF patients were died. In nonsurvivor mean INR was $2.44 \pm 0.69$, mean bilirubin was $15.38 \pm 10.71 \mathrm{mg} \%$, mean creatinin was $1.81 \pm 0.27 \mathrm{mg} \%$, and mean MELD score was $31.00 \pm 4.58$. The causes of death: bleeding in four patients, sepsis two patients, encephalopathy two patients and HRS 1 patient . In survivor group, mean INR was $2.12 \pm 0.78$, mean bilirubin was $8.74 \pm 5.96 \mathrm{mg} \%$, mean creatinin was $1.50 \pm$ $0.41 \mathrm{mg} \%$, and mean MELD score was $25.58 \pm 5.43$. Statistical analysis showed that the hospital mortality was associated to MELD score $(p=0.013)$ and serum creatinin $(p=0.044)$.

Conclusion: In this study,we conclude that hospital mortality in our hospital, is high. The mortality was associated to MELD score and serum creatinin.

\section{Poster Presentation 24: Portal Hypertension and Cirrhosis: Pathophysiology}

\section{PP24-01}

A PH-sensitive Gel Added Improves the Stability of Microemulsion for Orientating Removal of Colonic Ammonia in Gastrointestinal Tract

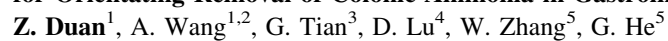

${ }^{1}$ Gastroenterology, The First Affiliated Hospital of Dalian Medical University, Dalian; ${ }^{2}$ Branch Hospital of Tangshan Gongren Hospital, Tangshan; ${ }^{3}$ College of Pharmacy, Dalian Medical University; ${ }^{4}$ Gastroenterology, the First Affiliated Hospital of Dalian Medical University; ${ }^{5}$ Membrane Science and Technology Center,Dalian University of Technology, Dalian, China

Background: We specially prepared an oral W/O type microemulsion for removal of ammonia in colon (ME-RA), but its effect was affected by digestion of gastrointestinal tract. In this paper, we aimed to prove the stability improved by adding gel to prepare microemulsion-based gels for removal of ammonia (MBGs-RA).

Methods: MBGs-RA was prepared based on ME-RA by adding sodium alginate. MBGs-RA and ME-RA were made to pass through simulative gastrointestinal process respectively, then colonic ammonia, appearances and $\mathrm{pH}$ values of gastrointestinal fluid were detected. 18 healthy rats were divided into three groups randomly. Three-way pipes were placed at gastroduodenal junction in Group I, and at terminal ileum in Group II. After intragastric administration with MERA or MBGs-RA, the stomach content in Group I, the effluent from terminal ileum in Group II and the drainage from anus in Group III were collected. PH values of gastrointestinal juice were measured by using $\mathrm{pH}$ test paper and those of colon were detected by using universal indicator.

Results: MBGs-RA had a better clearance rate for artificial colonic ammonia than ME-RA $(P<0.05)$. The lowing of $\mathrm{pH}$ in artificial small intestinal fluid because of ME-RA was recovered when MBGs-RA substituting it. In simulative gastrointestinal process MBGs-RA could keep more stable and release emulsion (ME-RA) in colon fluid. It showed the similar results in step tests of gastrointestinal tract on normal rats. MBGs-RA did not reduce $\mathrm{pH}$ of small intestine like ME-RA. 
Conclusions: MBGs-RA had a better stability in gastrointestinal tract which made it possible to achieve orientating removal of colonic ammonia.

PP24-02

Portal Bacteraemia in Shistosomal Hepatic Fibrosis

A.A.E.M. Soliman ${ }^{1}$, S.A. Zaki ${ }^{2}$, R. Zaher ${ }^{1}$, A.E.M. Sadek ${ }^{3}$, A. Ismail ${ }^{3}$

${ }^{1}$ Hepatology, Faculty of Medicine; ${ }^{2}$ Microbiology, Faculty of

Medicine; ${ }^{3}$ Surgery, Alexandria University, Alexandria, Egypt

Hepatic Shistosomal fibrosis is a continuous porcess. Bacteria may reach the liver via the portal venous blood from the intestinal tract and abnormalities in the liver circulation could ablish the filter effect of the liver.

Aim: To determine wheather bacteria are present or not,to know its species and evaluat its role in the pathogenesis of Schistosomal liver fibrosis.

Subjects and methods:

A 1-25 patients with Shistosomal hepatic fibrosis were submitted to clinical examination the concent was written by each patient before spleenectomy.

2. Portal pressure was measured

3. Excisional liver biopsy was taken for histopathology and culture.

4. $15 \mathrm{ml}$ of portal blood was aspirated by veinpuncture from the portal vein and $15 \mathrm{ml}$ venous blood was simultaneously taken from the anticubital vein

5 . Each $5 \mathrm{ml}$ of the portal and venous blood were added to $10 \mathrm{ml}$ of $0.05 \%$ sulphonated broth.

6 . The blood broth tubes were incubated aerobicaly, anaerobically and in $5 \% \mathrm{CO} 2$.

B. Portal and systemic blood samples were taken from six non-bilharzial patients as control group during peptic ulcer operations.

Results:

1. The systemic blood of all patients and the liver biopsy cultures proved to be sterile.

2. Three portal blood samples were excluded due to contamination.

3. $22.7 \%$ of portal blood samples from Shistosomal patients were positive for bacteria (Staphyloccus aureus from three patients, Escherichia coli from one patient and Bacteroides fragilis from one patient.

4. Samples of portal and systemic blood from controls were sterile. Conclucion:

1. Portal bacteraemia in Shistosomal hepatic fibrosis explain the occurrence of parenchymal involvement in such patients by the effect of the viable bacteria or its toxin on the hepatocytes.

2. Induction of an immune mechanism maintain an immune inflamatory reaction in the liver sharing in the pathogenesis of cirrhosis. 3. Patients showing unexpected deterioration in liver functions or decompensation of hepatic cirrhosis must do blood cultures and receive antibiotics.

\section{PP24-03}

Study of Some Biochemical Indices of Malignancy in Ascitic Fluid

H.A. Abd Elmoety, M. Abaza, S. El Hefni, A. Zaki

Chemical Pathology, Medical Research Institute, Alexandria

University, Alexandria, Egypt

Portal hypertension and hypoalbuminaemia leads to decrease in blood volume which stimulates renin-angiotensin system leading to hyperaldostronism and salt and water retention which triggers ascites formation .Often caused by chronic liver disease and malignancy. Cytological examination of ascitic fluid in malignancy revealed high incidence of false negative results.
Aim: Estimation of Cholesterol in the ascitic fluid to differentiate between malignant and non-malignant ascites.

Subjects: 50 patients with ascites divided into: A The non-malignant 25 patients were diagnosed as Schistosomal hepatic fibrosis, posthepatic cirrhosis, tuberclous peritonitis and nephrotic syndrome. B Malignant group: 25 patients diagnosed as hepatocellular carcinoma, liver metastasis, gall bladder cancer, anaplastic carcinoma from unknown primary, cancer colon and pancreatic carcinoma.

Methods: Full clinical examination-Laboratory investigations: Complete urine and stool examination. ascitic fluid included; Test for malignant cells and Protein, Fibrinogen, glucose, Chloride, Cholesterol, Carcinoembryonic Antigen, Bilirubin, Lactic Dehydrogenase, Alkaline Phosphatase, Gamma-Glytamyl Transpeptidase and aminotransferses. Results:

1. Ascitic fluid protein,cholesterol and Alkaline Phosphatase in the malignant group were significantly increased than the non-malignant group.

2. The ascitic fluid/serum ratios for protein and cholesterol significantly higher in malignant group than the non-malignant.

3. The diagnostic performances of ascitic fluid and ascitic fluid/serum ratio for protein and cholesterol including sensitivity, specificity, predictive value of positive and negative results and the efficiency for ascitic fluid/serum ratio for protein and cholesterol was calculated.

4. For ascitic fluid protein, it was 54.2, 96, 92.9, 68.6 and $75.5 \%$, respectively. For ascitic fluid protein ratio, it was 9.1,100, 100, 53.5 and $55.6 \%$.

5. For ascitic fluid cholesterol all the diagnostic characteristics were $100 \%$. For ascitic fluid/serum cholesterol ratio was 82.6, 100, 100, 88.5 and $93.3 \%$.

Conclusion: Ascitic fluid cholesterol level exceeding (100 mg/dl) or ascitic fluid/serum ratio above 0.7 are highly suggestive of malignancy even in the absence of positive cytological findings. Thus Ascitic fluid cholesterol level and ascitic fluid/serum cholesterol ratio should be added to monitor malignancy.

\section{PP24-04}

Relationship of Serum Resistin and Adiponectin to Insulin Resistance in Cirrhotic Patients

H.K. El-Shereef ${ }^{1}$, G.M.K. Galal ${ }^{2}$, H.G. Abdel-Azeem ${ }^{3}$

${ }^{1}$ Internal Medicine, Assiut Faculty of Medicine, Assiut University, Assiut; ${ }^{2}$ Tropical Medicine and Gastroenterology, Sohag Faculty of Medicine, Sohag University, Sohag; ${ }^{3}$ Clinical Pathology, Assiut Faculty of Medicine, Assiut University, Assiut, Egypt

Background and aim: Although hyperinsulinemia and insulin resistance are commonly found in patients with liver cirrhosis, their exact mechanisms are not fully understood. Among the candidate mediators, resistin and adiponectin have been incriminated in experimental animal models. Their exact role in cirrhotic patients remains a subject of considerable debate. Therefore, we studied serum levels of resistin and adiponectin in cirrhotic patients and their relationship to insulin resistance and pro-inflammatory cytokines.

Patients and methods: Thirty-eight non-diabetics with post-hepatitic liver cirrhosis (6 Child A, 14 Child B and 18 Child C) were included in the study as well as age, sex and BMI matched fifteen healthy controls. Fasting serum samples were collected from all participants for estimation of glucose, insulin, C-peptide, TNF- $\alpha$, IL-6, resistin and adiponectin. HOMA-IR was calculated for all of them.

Results: Cirrhotic patients showed significantly higher serum levels of insulin, resistin, adiponectin, IL- 6 and TNF- $\alpha$ than the control group. Resistin had significant positive correlation with IL-6 and TNF- $\alpha$, C-peptide, HOMA-IR and prothrombin time. Also, resistin correlated negatively with serum albumin and prothrombin concentration. Adiponectin significantly increased with the progression of 
Child grade. It significantly correlated with TNF- $\alpha$ but not with BMI, insulin level or HOMA-IR.

Conclusion: Resistin may contribute to insulin resistance in patients with liver cirrhosis. Adiponectin is increased dependent on the Child grade of the disease suggesting that it might be an indicator of severity of chronic liver disease reflecting one of the anti-inflammatory mechanisms in this disorder.

\section{PP24-05}

Infringements of the Immune Status and Hormonal Disbalance at a Hepar Cirrhosis

I. Nosova

Gastroenterology, Medical Clinical Research Centre 'Health Clinic', Vinnytsya, Ukraine

Background: Liver is the main link of metabolic processes. The important universal component of adaptation are endocrine reactions. Features of hormonal disorders are often used for research of pathogenesis, diagnostics and treatments of chronic liver diseases.

Methods: 70 patients with a cirrhosis (A and B class on Child-Pugh) have been divided into two groups: 1st with an alcoholic cirrhosis ( 38 foreheads), 2nd with a cirrhosis of a virus etiology. The control group was made by 20 foreheads which did not suffer liver diseases.

Results: Average values of indicators of the hormonal status of patients with a cirrhosis changed depending on activity of pathological process in a liver, etiological factor and were characterised by the following: content T3, testosteron authentically decreased, and estradiol and a parity estradioltestosteron raised in both groups, increase of a caption of antibodies to thyreoglobulin was observed. Patients with a virus hepatitis had a correlation communication between indicators of immunity and hormones of whey of blood: indicator T3 directly depended from T-lymphocytes $(r=+0,47)$, V- lymphocytes $(r=+0,46)$, T4from T-supressors $(r=+0,49), \mathrm{IgG}$ $(r=+0.49)$, and back depends from T-helpers $(r=-0.47)$, cortizol from $\mathrm{V}$ - lymphocytes $(r=-0.52)$, testosteron from T- lymphocytes $(\mathrm{r}=+0.46), \operatorname{Ig}$ And $(r=+0.51), \operatorname{IgM}(r=+0.46), \operatorname{IgG}(r=+0.41)$, estradiol from $\operatorname{IgA}(r=+0.42)$. At a virus cirrhosis strong positive correlation communication is established between insulin and T- lymphocytes, cortizol and $\operatorname{IgG}(r=+0.59)$.

Conclusions: The carried out researches allows to reveal features of a hormone profile changes of patients with a cirrhosis and interrelation of immune and hormonal factors that is the precondition for correction of hormonal shifts for the purpose of efficiency increase medical measures.

\section{PP24-06}

GRP78 Is Involved in Pulmonary Microvascular Remodeling in Development of HPS Induced by Multiple Pathogenic Factors

Z. Hui-Ying ${ }^{1}$, Z. Zhong-Fu ${ }^{2}$, J. Jing-Quan ${ }^{3}$, J. Jian-Tao ${ }^{3}$, Z. Li-Li ${ }^{3}$,

T. Xiao-Xia ${ }^{3}$, L. Min-Li ${ }^{4}$, G. Jian-Hong ${ }^{5}$, H. De-Wu ${ }^{5}$, J. Cheng ${ }^{6}$

${ }^{1}$ Pathophysiology, Changzhi Medical College, Chang Zhi City;

${ }^{2}$ Institute of Hepatology; ${ }^{3}$ Pathophysiology, Changzhi Medical College, Changzhi; ${ }^{4}$ Neurology, First Hospital of Shanxi Medical University; ${ }^{5}$ Institute of Hepatology, Shanxi Medical University, Taiyuan, China; ${ }^{6}$ Department of Medicine, University of Southern California, Los Angeles, CA, USA

Background: Hepatopulmonary syndrome (HPS) is characterized by a defect in arterial oxygenation induced by pulmonary microvascular dilatation and/or remodeling in the setting of liver disease. But its pathogenesis of remodeling remains elusive. We have demonstrated that intestinal endotoxemia (IETM) plays a central role in the development of HPS in the cirrhotic rat model induced by multiple pathogenic factors. Since endotoxin and hypoxia have been known to be stressors of endoplasmic reticulum (ER) stress, and GRP78 (78 kDa glucose regulated protein, a hallmark of ER stress) was found to be a leading pathogenesis of angiogenesis in many diseases, we speculate that GRP78 may function in vascular remodeling in the development of HPS.

Design: We developed a hepatic cirrhosis model induced by multiple pathogenic factors in rats. We measured levels of endotoxin, TNF $\alpha$, homocysteine in blood plasma with respective protocol, and expressions of mRNA and protein in GRP78 and protein of CD31 (indicative of microvessel density) in pulmonary architecture with RT-PCR or immunohistochemistry at 4,6 , and 8 weeks in the development of HPS.

Results: The endotoxin, $\mathrm{TNF} \alpha$, homocysteine in blood plasma and expressions of GRP78 and CD31 were significantly higher at every time point than respective normal control with development and progression of cirrhosis.. There were a close correlation between proteins of GRP78 $(0.08 \pm 0.01,0.13 \pm 0.02,0.22 \pm 0.02)$ and $\mathrm{CD} 31(0.048 \pm 0.013,0.065 \pm 0.015,0.090 \pm 0.017)$. And proteins of GRP78 and CD31 were positively related to increased endotoxin, $\mathrm{TNF} \alpha$, homocysteine in plasma.

Conclusion: These results suggest that GRP78 is markedly involved in pulmonary microvascular remodeling of HPS. It make them possible that a new theory for its pathogenesis wouldl be put forward and a new therapeutic strategy would also be developed for HPS.

\section{PP24-07}

Evidence for Early Astrocyte Activation, Cellular Stress and Compensatory Microglial Related TGF- $\alpha$ Responses in Bile-duct Ligated Rats G. Wright ${ }^{1}$, T. Newman ${ }^{2}$, Y. Sharifi ${ }^{1}$, N.A. Davis ${ }^{1}$, H. Perry ${ }^{2}$, R. Jalan ${ }^{1}$ ${ }^{1}$ UCL Hepatology, Royal Free Hospital, Upper Third UCL Medical School, London; ${ }^{2}$ School of Biological Sciences, Southampton University, Southampton, UK

Inflammation and ammonia are important mediators in the pathogenesis of hepatic encephalopathy (HE) with astrocytes thought to have a central role. Recently Microglia have been implicated in the brain effects of acute liver failure. This longitudinal study aimed to characterise early brain responses in bile-duct ligated (BDL) rats in the 4-weeks following ligation.

Methods: Twenty-four male Sprague-Dawley rats were studied after sham-operation or BDL and sacrificed at either 1-day or, 1-, 2- or 4weeks post-surgery ( $n=4$ /group). Consciousness, brain water, arterial ammonia, plasma biochemistry and proinflammatory (IL-6, TNF$\alpha$ and $\gamma$-IFN) and antiinflammatory (IL-4 and IL-10) cytokines, were analysed. Immunohistochemical markers of activated microglia (ED1, OX6 and Iba-1), astrocytes (GFAP), inflammatory responses (IL-1 $\beta$ and iNOS), cellular stress (HSP-25) and the antiinflammatory and alternative microglial activation marker TGF- $\beta$ (using RT-PCR), were also analysed.

Results: Compared to Shams, arterial and brain ammonia $(p<$ 0.001 ,respectively), bilirubin (from day-1), and TNF-a and IL-6 were significantly increased by 4 -weeks BDL $(p<0.001)$, which induced mild brain oedema $(p=0.07)$. There was no classical microglial activation or cellular infiltration (negative OX6 and perivascular ED1 staining), with observable resting microglia (Iba-1 staining) in all groups. iNOS and IL- $1 \beta$ protein expression increased. GFAP staining indicated significant astrocyte activation, primarily in the corpus callosum in all BDL groups. Hsp 25 peaked at 2-weeks post-BDL especially in the corpus callosum similar to activated astrocytes. TGF- $\beta$ was significantly upregulated by threefold in all BDL groups.

Conclusion: Regional astrocyte activation and cellular stress are early features of BDL and associated with increased brain proinflammatory cytokine and iNOS expression. This proinflammatory response is not from classical microglia activation, but consequent upon hyperammonemia, astrocyte activation and associated cell swelling. However, altered TGF- $\beta$ may reflect compensatory antiinflammatory microglial responses designed to limit the effect of astrocyte activation; with interventions targeting its brain expression potential novel therapies for HE. 


\section{PP24-08}

Prevalence of Hepatopulmonary Syndrome in Cirrhotic Patients without Intrinsic Cardiopulmonary Disease

W. Chaiphornphathana

Department of Internal Medicine, Bhumibol Adulyadej Hospital, Bangkok, Thailand

Background: HPS is defined as the triad of liver disease, hypoxemia or increased alveolar-arterial oxygen gradient, and intrapulmonary vasodilatation. The presence of HPS independently worsens prognosis of patients with cirrhosis.

Objective: To determine the prevalence of HPS in cirrhotic patients that has still not been reported in Thailand.

Patients and methods: One hundred and fifty cirrhotic patients (103 males, 47 females) were enrolled in this study. These patients underwent history taking, physical examination, laboratory data, pulse oximetry (upright position) chest radiograph, imaging study, arterial blood gas analysis, pulmonary function test, and transthoracic contrast echocardrogram. Diagnosis of HPS will be fulfilled and established with:

1) The presence of chronic liver disease.

2) Hypoxemia $\mathrm{PaO}(70 \mathrm{mmHg}$ or $270 \mathrm{mmHg}$ or an upper limitwidened age-corrected alveolar-arterialPaOgradient greater than normal.

3) Intrapulmonary vascular dilatation, detected by transthoracic contrastechocardiography, and

4) Absence of intrinsic cardiopulmonary disease.

Results: Total numbers of the 150 consecutive cirrhotic patients were enrolled. Only three cases were compatible with HPS. These three cases were distributed in all Child-Pugh classification. There was no significant difference in history, physical examination, laboratory tests to determine the predictor of HPS presentation, difference in history, physical examination, laboratory tests to determine the predictor of HPS presentation.

Conclusion: In our study, prevalence of HPS in cirrhotic patients without intrinsic cardiopulmonary disease is 2 percent.

\section{PP24-09}

Protective Effect of Caffeic Acid Phenethyl Ester in Cirrhotic Rats

M. Dianat, S. Saadat Fard, M. Badavi, A. Ahangarpour

Physiology Research Center, Department of Physiology, Faculty of Medicine, Ahvaz Jundishapur University of Medical Sciences, Ahvaz, Iran

Introduction: Patients with cirrhosis show an attenuated systolic and diastolic function, as well as, cardiac electrical abnormalities such as QT prolongation. Caffeic acid phenethyl ester (CAPE) is an active component of honeybee propolis extract. The aim was to investigate the effect of CAPE on systolic blood pressure (SBP) and QT interval in cirrhotic rats.

Material and methods: Sprague rats divided in three groups (sham, cirrhotic, cirrhotic treated with CAPE). Biliary cirrhosis in animals induced by chronic (5-weeks) bile duct-ligation (BDL). CAPE administrated $(1 \mu \mathrm{g} / \mathrm{kg} /$ day, ip) for 5 weeks. In all groups before BDL and 5 weeks after surgery, animals anesthetized and lead II electrocardiogram recorded for calculating QT interval. QT was corrected for RR interval using the Bazzet's formula. SBP measured by tail cuff in all groups.

Results: Results showed that not significant differences in SBP and QT interval between groups before intervention. After induced of BDL, QT interval in the cirrhotic group showed significantly increased compared to sham group $(p<0.001)$. QT interval was reduced in cirrhotic group treated with CAPE compared to cirrhotic group $(p<0.05)$.

Conclusion: Significant decrease in QT interval in cirrhotic group treated by CAPE suggests the protective effect of CAPE in cirrhotic patients. Since the hemodynamic disorders in cirrhotic patients are the result of increased free radicals, therefore the antioxidant effect of CAPE is probably mechanism of decreased QT interval.
PP24-10

Collagen in Space of Disse Is an Independent Predictor of the Presence of High Portal Pressure

A. Rastogi ${ }^{1}$, A. Kumar ${ }^{2}$, C. Bihari ${ }^{1}$, S.K. Sarin ${ }^{2}$

${ }^{1}$ Pathology; ${ }^{2}$ Hepatology, Institute of Liver and Biliary Sciences,

Delhi, India

Background: Cirrhotic liver biopsies show great variability in the degree and extent of histological features, few indicating more severe disease. Cirrhosis related architectural distortion causes increased intrahepatic resistance that in turn leads to portal hypertension, best assessed by hepatic venous pressure gradient (HVPG) which reflects the interaction between hepatic vascular resistance and blood flow.

Aim: To correlate various histological parameters with portal pressure as measured by HVPG.

Methods: Consecutive Forty patients with biopsy-proven cirrhosis and hepatic venous pressure gradient measurements within 1 month of biopsy were studied. Histological parameters analysed and scored semiquantitatively were: nodule size, loss of portal tracts and central veins, portal inflammation, periportal inflammation, bile duct proliferation, lobular inflammation, ballooning, fatty change, cholestasis, septal thickness and collagen IV immunostaining in space of Disse.

Results: Forty patients (mean age $45 \pm 11$ years, $70 \%$ males) were included in the study. Their mean HVPG was $16 \pm 5 \mathrm{~mm} \mathrm{Hg}$. There was significant correlation between HVPG and nodule size, septal thickness, and Disse space collagen $(p<0.05)$. When these variables were entered into multivariate analysis by binary logistic regression, to predict high HVPG ( $\geq 16 \mathrm{~mm} \mathrm{Hg}$ ), only Disse space collagen (odds ratio: 0.18 ; $95 \%$ confidence interval: $0.05-0.60, p=0.005$ ) was found to independently predict high HVPG.

Conclusion: Collagen in Space of Disse is independent predictor of the presence of high portal pressure and can be used to predict high HVPG.

\section{PP24-11}

Upper Gastrointestinal Bleeding Was Associated with Higher CRP Level in Liver Cirrhosis

K. Mariadi, D.N. Wibawa, N. Purwadi, G.A. Suryadarma

Internal Medicine, Udayana University Faculty of Medicine,

Denpasar, Indonesia

Background: Bacterial infections are frequently documented in cirrhotic patients presenting with upper gastrointestinal bleeding. Bacterial infection is independently associated with failure to control bleeding in cirrhotic patients with gastrointestinal bleeding. Bacterial infection may trigger the production of endotoxin, which in turn stimulates production proinflamatory cytokin (IL-6), IL-6 stimulates hepatocytes to produce CRP as a marker of inflammatory activity, and in the next step stimulates endothelin and expression of inducible nitric oxide synthase. Subsequently, endothelin may stimulate stellate cells to contract and results in elevation of variceal pressure. Production of nitric oxide reduces platelet aggregation and primary hemostasis. As a result, the chain reaction induces variceal bleeding. This study aim to know the association between CRP and upper gastrointestinal bleeding in liver cirrhosis patients.

Methods: We performed a cross-sectional analytic study in liver cirrhosis patients, in sanglah general hospital Bali. We used a student $t$ test to know the mean different of CRP level between patient with or without upper gastrointestinal bleeding.

Result: We collected 55 liver cirrhosis patients. Forty one (74.5\%) out of this was male and the rest was female. With range of age between 30 and 78 years old. Base on Child-tourcotte-pugh classification $56.4 \%$ with class C, only $5.5 \%$ with class A and the rest was class B. We found $12.7 \%$ and $65.4 \%$ out of patients with hepatic encephalopathy and ascites, consecutively. Thirty (54.5\%) out of 55 patients with acute bleeding. We found significant higher mean level of CRP in liver cirrhosis patient with upper gastrointestinal bleeding compared with those without upper gastrointestinal bleeding ( $25.2 \mathrm{mg} / \mathrm{L}$ vs. $6.5 \mathrm{mg} / \mathrm{L} ; p<0.01)$. 
Conclusions: Higher CRP level was associated with upper gastrointestinal bleeding.

\section{Poster Presentation 25: Autoimmune Hepatitis And Cholestatic Liver Disease}

\section{PP25-01}

Mycophenolate Mofetil (MMF) in Patients with Autoimmune Hepatitis (AIH) Intolerant to Azathioprine

D. Jothimani ${ }^{1}$, K. Sleigh ${ }^{2}$, M.E. Cramp ${ }^{1}$, J.D. Mitchell ${ }^{1}$, T.J. Cross ${ }^{1}$

${ }^{1}$ Southwest Liver Unit; ${ }^{2}$ Department of Histopathology, Derriford

Hospital, Plymouth, UK

Background: The majority of patients with $\mathrm{AIH}$ respond to the standard treatment combination of prednisolone and azathioprine. Twenty percent of patients are non-responders due to refractory disease, non-compliance or azathioprine intolerance. Several case series supports the use of MMF in AIH.

Aim: To evaluate the efficacy and tolerability of MMF in the management of AIH.

Methods: A retrospective case note review from January 2000 to May 2010 in patients diagnosed with AIH. Patients on MMF were identified and evaluated. Treatment response to MMF was defined as a biochemical remission in 4 weeks of treatment commencement and treatment failure as either a non-response to the standard therapy or relapse while on standard therapy.

Results: 117 patients with AIH were identified and $20(17 \%)$ received MMF. The median age was 56 (18-79 years) with male:female, 1:7. Three patients had 'overlap syndrome' and six had cirrhosis. All patients were commenced on prednisolone $30 \mathrm{mg}(7.5-40 \mathrm{mg})$ for induction and azathioprine within 3 months for remission maintenance. Azathioprine was discontinued due to intolerance in 18 patients within 4 months (0-24 months). Two patients were nonresponders to azathioprine. All these patients were commenced on MMF $500 \mathrm{mg}-1 \mathrm{~g}$ twice daily in addition to maintenance prednisolone. At a median follow-up of 47 months (5-83 months), MMF was well tolerated and 14/19 patients (1 lost follow-up) remained in remission including five patients with cirrhosis. Median aspartate aminotransferase levels pre-MMF and at the time of their recent clinic visit was 62 and $26 \mathrm{iu} / \mathrm{L}$, and immunoglobulin $\mathrm{G}$ was 17.0 and $12.4 \mathrm{~g} / \mathrm{L}$, respectively. MMF intolerance was seen in three patients (rash, hair loss) and poor response in two patients.

Conclusion: Our case series supports the use of MMF as a second line agent in $\mathrm{AIH}$ patients intolerant to Azathioprine, including those with cirrhosis.

\section{PP25-02}

Budesonide in the Management of Autoimmune Hepatitis (AIH) - A Short Term Analysis

D. Jothimani ${ }^{1}$, T.J. Cross ${ }^{1}$, M.E. Cramp ${ }^{1}$, K. Sleigh ${ }^{2}$, J.D. Mitchell ${ }^{1}$

${ }^{1}$ Southwest Liver Unit; ${ }^{2}$ Department of Histopathology, Derriford

Hospital, Plymouth, UK

Background: AIH is a chronic necroinflammatory condition of the liver. Corticosteroids (prednisolone) and azathioprine are the main stay of treatment. Budesonide is a synthetic corticosteroid with high first pass metabolism and causes fewer side effects. A few case series and a recent phase $2 \mathrm{~b}$ study supports budesonide for AIH patients.

Aim: To evaluate the efficacy of budesonide in the treatment of AIH. Methods: A retrospective case note review carried out from January 2009 to March 2010 in AIH patients (immune profile and liver biopsy). Patients on budesonide were identified and evaluated. Treatment response to budesonide was defined as improvements in biochemical parameters within 4 weeks of treatment commencement.
Results: We identified 13 patients with $\mathrm{AIH}$ on budesonide, with a median age 49 (16-79 years) and male:female, 3:10. Overlap syndrome in three, post liver transplantation recurrent $\mathrm{AIH}$ in one, and cirrhosis in one patient, were noted. All patients initially received prednisolone $30 \mathrm{mg}$ once daily for induction (except post transplant) and azathioprine within 3 months for remission. Prednisolone was substituted with budesonide (6 mg daily) for any of the following indications: frequent high dose prednisolone for $\mathrm{AIH}$ relapse, prednisolone/azathioprine intolerance or ongoing disease activity. At a median follow up of 6 months (1-14 months) budesonide was well tolerated and 11/13 (84.6\%) patients remain on budesonide, including three patients in combination with mycophenolate and one with tacrolimus. Budesonide was stopped in two patients due to inadequate treatment response. In responders, the median aspartate aminotransferase levels pre-budesonide and at 6 months of treatment was 40 and $35 \mathrm{iu} / \mathrm{L}$, and Immunglobulin $\mathrm{G}$ was 13 and $14.3 \mathrm{~g} / \mathrm{L}$, respectively. Conclusion: This short term analysis shows that budesonide is an effective alternative to prednisolone in patients with AIH. However, long-term data is required to confirm its efficacy and tolerability.

\section{PP25-03}

Clinical Features of Elderly Chinese Patients with Type 1Autoimmune Hepatitis

Y. Zhang ${ }^{1}$, F.-K. Zhang ${ }^{2}$, Y. Zhang ${ }^{1}$

${ }^{1}$ First Department of Liver Disease, Dalian Sixth People Hospital, Dalian; ${ }^{2}$ Liver Research Center, Beijing Friendship Hospital, Beijing, China

Aim: To study the clinical features in elderly Chinese patients with Type 1 autoimmune hepatitis.

Methods: The clinical features of 36 patients diagnosed at age $>60$ years were compared with those of 39 patients aged $<50$ years.

Results: The M:F ratio was 1:35 ( $>60$ years) versus 3:36 ( $<50$ years). The patients $>60$ years had a higher frequency of cirrhosis at presentation than the patients $<50$ years (50.0 vs. $15.4 \%, P=0.001$ ). The patients $>60$ years also had a significantly increased incidence of ascites at presentation ( 44.4 vs. $10.3 \%, P=0.001)$ and lower serum albumin levels $(P=0$.037). There were significant differences between older and younger patients with respect to the frequencies or titers of ANA ( 46.7 vs. $83.3 \%, P=0.011$ ). HLA DR3 positivity occurred more frequently in the patients $<50$ years than in those $>60$ years $(59.0$ vs. $19.4 \%, P<0.001)$, whereas HLA DR4 occurred more often in the patients $>60$ years $(63.9$ vs. $25.6 \%, P=0.001)$ Treatment failure occurred more frequently in the patients $<50$ years (28.2 vs. $9.1 \%, P=0.041$ ). However, there were no significant difference between the groups with respect to mode of onset, other clinical signs at presentation biochemical parameters and SMA positivity.

Conclusion: Elderly patients have a greater frequency of cirrhosis at presentation, ANA, HLA DR4 positivity than patients $<50$ years, and they have a lower occurrence of treatment failure Conventional corticosteroid regimens may be an effective management for older patients with AIH.

\section{PP25-04}

Autoimmune Hepatitis: A Tertiary Referral Centre Experience in Malaysia

K.H. Lam, S. Palaniappan

Medicine, Hospital Raja Permaisuri Bainun, Ipoh, Malaysia

Introduction: Autoimmune Hepatitis (AIH) is a chronic disease with hepatocellular inflammation and tends to progress to cirrhosis. Data from Asian population have indicated that AIH is relatively uncommon. In addition to that, there is paucity of data available in this region.

Objective: This study aims to analyse the profiles of AIH patients in a state hospital in Malaysia. 
Methods: Data regarding clinical manifestation, autoantibody, histology and response to therapy were collected and analysed retrospectively from clinic notes of $13 \mathrm{AIH}$ patients.

Results: The median age of diagnosis of AIH was 51 years old (range 25-73) with a male to female ratio of 1:12. Prevalence was highest among the Malays $(53.8 \%)$, followed by Chinese $(38.5 \%)$ and Indians (7.7\%). 35.8\% had other autoimmune diseases. Common presentations were cholestatic hepatitis $(92.3 \%)$, followed by non specific symptoms $(69.2 \%)$ i.e. fatigue and lethargy. Physical examination revealed that many patients had jaundice $(92.3 \%)$, followed by stigmata of chronic liver disease $(23.1 \%)$, hepatomegaly $(15.4 \%)$, splenomegaly (7.7\%), pruritic marks (7.7\%) and bruising (7.7\%). Anti Smooth Muscle Antibody was detected in $53.8 \%$ of patients, followed by Anti Nuclear Antibody in $30.8 \%$. $15.4 \%$ of patients were seronegative. Liver biopsy was performed in $46.2 \%$ of patients. All biopsied patients showed interphase hepatitis and lymphoplasmacytic infiltration. All the patients had negative viral markers. Response to treatment was complete in $77 \%$ while $7.7 \%$ had partial response. Steroid sparing agents were used in $77 \%$ of the patients, in which Azathioprine was the commonest used $(80 \%)$ followed by 6 Mercaptopurine $(10 \%)$ and Methotrexate $(10 \%)$

Conclusion: $\mathrm{AIH}$ affects a wide range of age groups, almost always in the female population. It is more common among the Malay race and is associated with other autoimmune diseases. Most patients presented with cholestatic hepatitis and had a complete response to treatment. This data is comparable to other literature.

\section{PP25-05}

Clinical, Anatomic Aspects, and Therapeutic Management of Auto Immune Chronic Hepatitis: Retrospective Study of 76 Cases

S. Berkane ${ }^{1}$, Y. Chikhi ${ }^{2}$, S. Cheraitia ${ }^{2}$, S. Chabi ${ }^{2}$, S. Ait Younes ${ }^{3}$, Z.C. Amir ${ }^{3}$, F. Asselah ${ }^{3}$, N. Ali Arous ${ }^{2}$, R. Ould Gougam ${ }^{2}$, H. Asselah ${ }^{2}$

${ }^{1}$ Bologhine Hospital, Algiers University; ${ }^{2}$ Hepatogastroenterology,

Bologhine Hospital, Algiers University; ${ }^{3}$ Pathological Anatomy,

Mustapha Hospital, Algiers University, Algiers, Algeria

Objectives: To analyse clinical characteristics, therapeutics and evolutive of auto-immune hepatitis.

Method: Retrospective study of 76 patients with auto-immune hepatitis, included from January 1995 to December 2009. The diagnosis was established according to the revised international grades in 1999 after exclusion of the other etiology of the hepatic disease (viral, drug use, genetics).

Results: 76 patients studied, medium age of 40 years [18-77], sexratio $0.35(20 \mathrm{H} / 56 \mathrm{~F})$. The main diagnosis circumstance was: jaundice $(n=31)$, decompensated cirrhosis $(n=21)$ with ascitis in 14 cases, bleeding oesophagel varices in five cases, hepatosplenomegaly $(n=11)$, biological abnormality $(n=13)$; raised ALAT and cholestasis eight cases, pruritus two cases, asthenia three cases. The main extra hepatic manifestations associated were: diabetes $(n=6)$, celiac disease $(n=3)$, dysthyroïdy $(n=4)$, LED $(n=2)$, Gougerot Sjögren syndrome $(n=3)$, Sharp syndrom $(n=1)$, Raynaud $(n=2)$, idiopathic thrombopenic purpura $(n=1)$, Lichen planus $(n=1)$. Immunologicaly, wether il is about type 1 autoimmune (AML and / or $\mathrm{AN}+)$ in 55 cases $(72.3 \%)$, type 2 in one case, seronegative in ten cases, anti-mitochandrial antibodies associated in the frame of overlap syndrome in 13 cases. Histogical proof is obtained in 56 cases with advanced fibrosis F3-F4 in 38 cases. The patients received bitherapy with corticosteroid + Imurel, the overlap syndrom received association ursolvan-corticosteroid-Imurel. After medium follow-up of 21 months, ten patients were dead, mainly from cirrhosis complications.

Conclusion: Autoimmune hepatitis is relatively frequent, it come in the second position after viral infection. The diagnosis remains late, at cirrhosis stage, raising the interest to make early diagnosis more responsive to well done immunosuppressed treatment.
PP25-06

HLA- DRB1 as a Risk Factor in Children with Autoimmune Hepatitis and its Relation to Hepatitis A Infection

A. Elfaramawy ${ }^{1}$, R. Elhossiny ${ }^{1}$, A. Abbas ${ }^{2}, \mathrm{H}$. Abdel Aziz ${ }^{1}$

${ }^{1}$ Pediatrics; ${ }^{2}$ Clinical Pathology, Ain Shams University, Cairo, Egypt

Background: The human leukocyte antigens (HLAs) are proteins found in the membranes of nearly all nucleated cells. People with certain HLA antigens are more likely to develop certain autoimmune diseases.

Aim of the work: Was to determine the frequency of HLA -DRB1 in children with autoimmune hepatitis (AIH) as a risk factor for occurrence, its relation to preceding hepatitis A infection and treatment outcome.

Subjects and methods: 25 children with autoimmune hepatitis were subjected to HLA-DRB 1 typing performed by sequence specific oligonucleotide probe technique and compared to HLA-DRB1found in 548 normal populations.

Results: The most frequent alleles found in our children with AIH were HLA-DRB1*13 (36\%), HLA-DRB1*04 (18\%) and HLADRB $1 * 03(14 \%)$. HLA-DRB $1 * 13$ was significantly more frequent in the studied cases when compared to the control group. In type I AIH patients HLA-DRB $1 * 13$ was the most frequent allele $(32.4 \%)$, followed by HLA-DRB $1 * 04$ in $(20.6 \%)$ and HLA-DRB $1 * 03$ in $(14.7 \%)$, While in type II, the most frequent alleles were HLA-DRB $1 * 13$ in $(40 \%)$, HLA-DRB $1 * 07(20 \%)$ and HLA-DRB $1 * 15$ in $(20 \%)$. HLA-DRB $1 * 12$ was significantly more frequent in AIH patients with positive Hepatitis $\mathrm{A}(\mathrm{HAV}) \mathrm{IgM}$ than in patients with negative hepatitis A IgM. No statistically significant difference between partial responders and complete responders to treatment as regards HLA-DRB1 subtypes.

Conclusion: It is concluded from the previous study that HLADRB $1 * 13$ is susceptibility allele for the occurrence of autoimmune hepatitis in our population. HLA-DRB $1 * 07$ and HLA-DRB $1 * 15$ are susceptibility alleles for occurrence of autoimmune hepatitis type 2 . HLA-DRB $1 * 12$ could be a risk factor for AIH in patients triggered by Hepatitis A.

\section{PP25-07}

Screening for Liver Disease in Parents of Children with Autoimmune Hepatitis

M. Woźniak ${ }^{1}$, M. Woynarowski ${ }^{1}$, B. Cukrowska ${ }^{2}$, H. Gregorek ${ }^{3}$ ${ }^{1}$ Gastroetnerology Hepatology and Immunology; ${ }^{2}$ Department of Pathology; ${ }^{3}$ Department of Immunology, Children's Memorial Health Institute, Warsaw, Poland

Background: It is known that different autoimmune diseases may coexist in patient or in patient's family. Autoimmune hepatits (AIH) develops as occult disease and the clinical manifestation occurs when liver is already damaged.

Aim: The aim of our study was to detect AIH risk factors in parents of children affected with AIH.

Material and methods: Liver function tests (bilirubin, ALT, AST, GGTP, AP, INR), and autoimmune disease features (gammaglobulins, IgG, ANA, SMA, LKM) were tested in 126 parents (mothers-68, fathers-58) from 69 families with AIH children. The AIH risk score based on the presence of increased ALT, increased gammaglobulins or $\mathrm{IgG}$, presence of autoantibodies and family history of autoimmune disease was calculated.

Results: ALT was increased in $14(11.1 \%)$ of parents (mothers-2, fathers-12, $p<0.01)$. IgG was increased in $8(6.3 \%)$ of parents (mothers-6, fathers-2), and gammaglobulins in $14(11 \%)$ of parents (mothers-10, fathers-4). $53(42.1 \%)$ of patients were positive for ANA (mothers-28, fathers-25). SMA was present in 71 (56.5\%) of parents (mothers-41, fathers-30) and LKM was present in one mother and one father. EmA antibodies were present in two parents (one mother and one father). Thyroiditis was present in 17 families, diabetes mellitus 
in 12 and rheumatoid arthritis in 8. Vitiligo, ulcerative colitis, celiac disease and autoimmune anemia were present in a few cases. None of the AIH risk factors was noted in $22(17.5 \%)$ parents, one risk factor was present in $77(61.1 \%)$ two in $23(18.1 \%)$ and three in $4(3.2 \%)$ parents. No differences in risk factors distribution was observed between mothers and fathers.

Conclusion: Presence of autoantibodies is the most common risk factor for AIH in parents of children affected with $\mathrm{AIH}$, however majority of parents do not present more than one risk factor for liver disease development.

\section{PP25-08}

The Frequency of Osteoporosis and Osteopenia in Patients With Japanese Female Primary Biliary Cirrhosis

K. Takaguchi ${ }^{1}$, S. Takashima ${ }^{2}$, N. Baba ${ }^{1}$, T. Seno ${ }^{1}$, T. Nagano ${ }^{1}$, K. Kawai ${ }^{2}$, K. Miyatake ${ }^{3}$, S. Fujioka ${ }^{4}$, R. Okamoto ${ }^{5}$, Y. Iwasaki ${ }^{6}$, H. Kobashi ${ }^{6}$, K. Yamamoto ${ }^{6}$

${ }^{1}$ Department of Hepatology; ${ }^{2}$ Department of Gastroenterology, Kagawa Prefecutual Central Hospital, Takamatsu; ${ }^{3}$ Department of Gastroenterology, Mitoyo General Hospital, Kanonji; ${ }^{4}$ Department of Gastroenterology, Okayama Saiseikai General Hospital, Okayama; ${ }^{5}$ Department of Gastroenterology, Hiroshima City Hospital, Hiroshima; ${ }^{6}$ Department of Gastroenterology and Hepatology, Okayama University Graduate School of Medicine, Dentistry, and Pharmaceutical Sciences, Okayama, Japan

Background: Several reports have suggested patients with primary biliary cirrhosis (PBC) have increased risk risk of osteoporosis or osteopenia. We examined prevalences of osteoporosis and osteopenia among Japanese women with PBC and factors for low bone mineral density (BMD).

Materials and methods: The study was a cross-sectional, prospective, multicenter, clinical study in Okayama University Hospital and their affiliated hospital, including Kagawa Prefectural Central Hospital. In outpatients with PBC, BMD of lumber spine was measured by dual energy X-ray absorption (DXA). The diagnostic criteria for primary osteoporosis by the Japanese Society for Bone and Mineral Research were used to define osteoporosis or osteopenia. The following measurements were obtained: age, menopausal status, BMD, duration of $\mathrm{PBC}$, stage of $\mathrm{PBC}$, accompanying symptom of $\mathrm{PBC}$, medications for PBC and osteoporosis (including UDCA, bezafibrate, vitamin $\mathrm{D}$, or bisphosphonates). We examined an association between BMD and these symptomatic factors.

Results: The prevalence of osteoporosis in outpatients with PBC was $23.8 \%(15 / 63)$, the rate of osteopenia was $20.6 \%(12 / 63)$. There was no significant difference in the rate of osteoporosis and osteopenia between outpatients with $\mathrm{PBC}$ and control group without PBC. Patients with low BMD (patients diagnosed osteoporosis or osteopenia) had older age, shorter height, and lower weight than those of the historical control. The multiple regression analysis on these parameters suggested that low BMD was associated with age and bezafibrate use.

Conclusions: The prevalence of osteoporosis and osteopenia in patients with PBC was slightly higher than control group, but there was no significant difference. Low BMD in patients with PBC may associate with not only age but also bezafibrate use.

\section{PP25-09}

Inteferon/ribavirine Suppression of Autoimmune Hepatitis Misdiagnosed as Hepatitis $\mathbf{C}$

V. Csibova ${ }^{1}$, M.R. Pijak ${ }^{2}$

${ }^{1}$ University Hospital, ${ }^{2}$ Slovak Medical University, Bratislava, Slovak Republic

Background: Latent autoimmune hepatitis $(\mathrm{AIH})$ triggered during interferon (IFN) treatment in patients with chronic hepatitis $\mathrm{C}$ (CHC) is well known. Here, we report for the first time improvement of $\mathrm{AIH}$ using IFN and ribavirin (RBV) in a patient initially incorrectly diagnosed as having $\mathrm{CHC}$.

Case report: 32 years old female was referred because of fluctuating elevations of aminotransferases (AT) (4-19-fold normal) known last 6 months. She had positive HCV-RNA by RT-PCR an an untypable genotype. Liver biopsy revealed chronic active hepatitis grade 2, stage 2. She also had Hashimoto's thyroiditis with high titres of antithyroid antibodies. After 12 weeks of treatment with Intron A $3 \mathrm{MU}$ TIW and RBV $1000 \mathrm{mg} / \mathrm{d}$, HCV RNA was negative and AT fluctuated between normal and twofold normal. One week after completing 48 weeks of treatment, AT showed 11-fold increase, but $\mathrm{HCV}$ remained negative. Indirect immunofluorescence became positive for ASMA (3+) and ANA (4+). A repeated liver biopsy showed no improvement. A therapeutic trial with RBV was started and within 11 weeks of this treatment her symptoms markedly improved and AT decreased to twofold. Unfortunately, cessation of RBV resulted in immediate reversion of symptoms and elevation of AT to its previous state. After restarting RBV therapy no response was observed within 2 weeks and the treatment was stopped. She was then put on prednisone $30 \mathrm{mg} / \mathrm{d}$ and azathioprine $50 \mathrm{mg} / \mathrm{d}$. Within 6 weeks her symptoms resolved and AT and IgG became completely normal. The patient remains in remission with prednisone $5 \mathrm{mg}$ and azathioprine $50 \mathrm{mg} / \mathrm{d}$. A re-evaluation of the primary diagnosis of hepatitis $\mathrm{C}$ was warranted because of unusually high AT activity and repeatedly negative anti-HCV using a third generation assay.

Conclusions: This case may provide support for the hypothesis that RBV may dampen autoimmune reaction induced by IFN alpha and potentiate its anti-inflammatory effects.

\section{PP25-10}

The Haplotype of Killer Cell Immunoglobulin-like Receptors Does Not Determine the Course of Autoimmune Hepatitis in Children M. Woynarowski ${ }^{1}$, M. Woźniak ${ }^{1}$, B. Piątosa ${ }^{1}$, H. Gregorek ${ }^{2}$

${ }^{1}$ Department of Gastroenterology Hepatology and Immunology;

${ }^{2}$ Department of Immunology, Children's Memorial Health Institute, Warsaw, Poland

Introduction: Killer cells immunoglobulin-like receptors (KIR) may play a role in the immunological response in many autoimmune diseases. The role of KIR in autoimmune hepatitis has not yet been studied.

Aim: The aim of the study was to assess the KIR haplotype in children with AIH and to compare the course of AIH in subjects with different KIR haplotypes.

Methods: Blood samples taken from 41 children (M-9, F-32) with AIH diagnosed at the age 10.2 \pm 3.7 years were analyzed by a PCRbased sequence-specific priming method for $16 \mathrm{KIR}$ genes. Three KIR haplotypes were defined: $\mathrm{AA}, \mathrm{AB}$ and $\mathrm{BB}$. Case records of all patients were reviewed and the data about the liver function tests and immunological parameters at disease presentation, at 2 years of treatment and at the last patient's visits were recorded and compared among children with different KIR haplotypes

Results: 37 patients had AIH type I and 4 had AIH type II. Haplotype AA was found in $16(39 \%), \mathrm{AB}$ in $13(31,7 \%)$ and $\mathrm{BB}$ in $12(29,3 \%)$ of children. At the disease presentation ALT activity was $717 \pm 765 \mathrm{U} / \mathrm{l}$, IgG $2,587 \pm 1,470 \mathrm{mg} / \mathrm{dl}$. Liver biopsy showed marked inflammation and fiborsis (mean grading $2.7 \pm 0.8$, staging $2.4 \pm 1.0$ ). After 2 years of prednisone and azathioprine treatment mean ALAT decreased to $101 \pm 148 \mathrm{U} / \mathrm{l}$ and $\mathrm{IgG}$ to $1,455 \pm 733 \mathrm{mg} / \mathrm{dl}$. Liver biopsy done after 2 years of therapy showed grading $1.5 \pm 1.0$ and staging $2.0 \pm 1.2$. The final evaluation was done at the age of $15.2 \pm 3$ years. Mean ALT at this stage of the study was $33 \pm 34 \mathrm{U} / 1$ and mean IgG was $1,458 \pm 46 \mathrm{mg} / \mathrm{dl}$. No differences in liver function tests, IgG or gammaglobulins among children with KIR haplotype AA, $\mathrm{AB}$ or $\mathrm{BB}$ were observed at any stage of the study. 
Conclusion: Our results do not confirm that KIR haplotype may alter the course of AIH in children.

\section{PP25-11}

Coexistence of Autoimmune Hepatitis and Rheumatoid Diseases in Children

M. Woźniak ${ }^{1}$, I. Szczygielska ${ }^{2}$, M. Woynarowski ${ }^{1}$

${ }^{I}$ Department of Gastroenterology Hepatology and Immunology,

Children's Memorial Health Institutel; ${ }^{2}$ Department of Pediatric Rheumatology, Rheumatology Institute, Warsaw, Poland

Introduction: Autoimmune hepatitis (AIH) and rheumatoid diseases (RD) can coexist in the same patient.

Aim: The aim of the study was to identify children with AIH and RD and to describe the relationship of both diseases.

Methods: We retrospectively reviewed 224 records of patients with $\mathrm{AIH}(\hat{\jmath}-63 \stackrel{+}{-}-161$, mean age at AIH presentation 11.5 years) treated from 1989 to 2009 and selected patients with signs or symptoms of RD. Results: 15 children with AIH presented signs and symptoms suggesting RD (fever, bone pain and joints edema), however RD was finally diagnosed in six children (Mixed Connective Tissue Disease (MCTS) was diagnosed in one boy and Juvenile Idiopathic Arthritis (JIA) was present in one boy and four girls). The diagnosis of RD was confirmed by morning stiffness (5), typical joints appearance on ultrasound examination (6), presence of HLAB27 (6), aCCP (5) and Anty-RNP (1). In four children RD was diagnosed before AIH presentation. In these four patients RD started at the age of 10.5 years. AIH occurred within 7 months from RD and presented as jaundice, hepatomegaly and ALT flare. In two patients with AIH diagnosed at the age of 11.5 years the liver disease had been present for 3.5 years before RD presentation. All children were treated with prednisone and azathioprine. Two children did not respond to prednisone and were switched to cyclosporine. Two children were treated with hydrochlorochine and four with metotrexat. The remission of both diseases was achieved in one child only. Four patients continue therapy. Liver transplantation was performed in one child with subsequent remission of RD.

Conclusions: The collaboration of hepatologist and rheumatologist revealed several cases of simultaneous AIH and RD. Patients with liver disease with extrahepatic manifestations should be referred to rheumatology department for checking for RD.

\section{PP25-12}

Diagnostic Value and Utility of the Simplified International Autoimmune Hepatitis Group (IAIHG) Criteria for Autoimmune Hepatitis in Korea Y.N. Lee ${ }^{1}$, Y.S. Kim ${ }^{1}$, S.G. Kim ${ }^{1}$, H.G. Yoon ${ }^{1}$, S.W. Jeong ${ }^{1}$, S.H. Lee ${ }^{1}$, H.S. Kim ${ }^{1}$, H.K. Kim ${ }^{2}$

${ }^{1}$ Internal Medicine; ${ }^{2}$ Pathology, Soon Chun Hyang University School of Medicine, Bucheon Si, Republic of Korea

Background and aim: Diagnostic criteria for autoimmune hepatitis (AIH) have been created and revised by the International Autoimmune Hepatitis Group (IAIHG) in 1999. A simplified scoring criteria has been proposed recently that is based on four clinical components. The aim of this study is to assess diagnostic value and usefulness of the simplified scoring criteria in Korean.

Methods: We applied the simplified scoring criteria to 22 AIH patients diagnosed according to the original revised scoring criteria proposed in 1999. Furthermore, in order to compare predictabilities between these two diagnostic criteria, we included 84 patients with other liver disease [toxic hepatitis $(n=50)$, nonalcoholic fatty liver disease $(n=18)$, primary biliary cirrhosis (PBC) $(n=11)$, PBC/AIH overlap syndrome $(n=5)]$ than AIH.

Results: 20 (90.9\%) patients with AIH and $5(100 \%)$ patients with $\mathrm{PBC} / \mathrm{AIH}$ overlap syndrome were diagnosed with $\mathrm{AIH}$ according to the simplified scoring criteria. Four $(36.4 \%)$ patients with PBC were false positive AIH according to the simplified scoring criteria. These patients diagnosed according to the simplified scoring criteria showed higher frequency of antinuclear antibodies and/or smooth muscle antibodies of 1:80 or greater $(p=0.491)$ and higher frequency of serum levels of immunoglobulin $\mathrm{G}$ of upper normal limit or greater than non-diagnostic patients with $\operatorname{PBC}(p=0.006)$. The sensitivity and specificity of simplified scoring criteria for the diagnosis of $\mathrm{AIH}$ were 92 and $95 \%$.

Conclusions: The simplified scoring criteria is a reliable diagnostic criteria in Korean and simple tool for excluding AIH. But, this new criteria may have some limitations for the diagnosis of patients with atypical features, especially patients with lower autoantibodies and immunoglobulin G.

\section{PP25-13}

Paris Criteria is Not Appropriate for Diagnosis of Asian Primary Biliary Cirrhosis- autoimmune Hepatitis Overlap Syndrome

J.M. Byun ${ }^{1}$, S.G. Kim ${ }^{1}$, Y.S. Kim ${ }^{1}$, H.K. Kim ${ }^{2}$, S.W. Jung ${ }^{1}$, J.Y. Jang ${ }^{1}$, S.H. Lee ${ }^{1}$, J.H. Moon ${ }^{1}$, H.S. Kim ${ }^{1}$, M.S. Lee ${ }^{1}$, B.S. Kim ${ }^{1}$

${ }^{1}$ Internal Medicine; ${ }^{2}$ Pathology, Soon Chun Hyang University School of Medicine, Bucheon Si, Republic of Korea

Background: The most commonly utilized criteria for the diagnosis of primary biliary cirrhosis-autoimmune hepatitis overlap syndrome (PBC-AIH OS) is Paris criteria proposed by Chazouilleres. In this study we aimed to evaluate the efficacy of Paris criteria and characteristics in the diagnosis of Korean PBC-AIH OS.

Method: From January 1994 to December 2009 at Soon Chun Hyang University hospital, we retrospectively analyzed 36 patients suspected with PBC-AIH OS. The patients suspected with OS were included on the basis of either histological assessment (bile duct damage and hepatic lesions in the same biosy specimen) or serological and biochemical data(presence of both AMA and other autoantibodies; presence of both cholestatic enzyme and aminotransferase elevation). Results: Mean age was $48.9 \pm 9.3$ years and 33 patients $(91.7 \%)$ were female. Positivity for AMA and ANA was observed in each $29(80.5 \%)$ and $32(88.9 \%)$. But only $1(3.7 \%)$ of 28 patients was positive for anti-SMA and $1(11.1 \%)$ of nine patients was positive for anti-LKM Ab. Double positivity for AMA and other autoantibodies was present in 25 (69.4\%) of 36 patients. Laboratory findings were AST $115.1 \pm 94.5 \mathrm{IU} / \mathrm{L}, \quad$ ALT $407.3 \pm 90.7 \mathrm{IU} / \mathrm{L}, \quad$ ALP $407.3 \pm 274.1 \mathrm{IU} / \mathrm{L}, \gamma-\mathrm{GTP} 480.1 \pm 405.9 \mathrm{IU} / \mathrm{L}$, and total bilirubin $2.2 \pm 3.3 \mathrm{mg} / \mathrm{dL}$. IgG and $\mathrm{IgM}$ elevations were seen in each 10 $(34.5 \%)$ and $26(89.7 \%)$ of 29 patients. In comparison of Paris criteria, proportions of patients with ALP $>2$ ULN, $\gamma$-GTP $>5$ ULN were $21(58.3 \%), 22(61.1 \%)$ among all patients, and those of patients with ALT $>5$ ULN, IgG $>2$ ULN were 5 (13.9\%), 1(2.8\%). Only $6(16.7 \%)$ of 36 patients were exactly satisfied with Paris criteria.

Conclusions: In our study, most of patients suspected with PBC-AHI OS were not satisfied with Paris criteria, because of very low percentage of positivity for SMA and insufficient IgG, ALT elevations. On the other hands, percentages of positivity for ANA and IgM elevations were very high. Further studies will be required to set more appropriate criteria for the diagnosis of PBC-AIH OS for Asian.

\section{PP25-14}

A Retrospective Study of Autoimmune Hepatitis with Sjogren's Syndrome Y. Zhang, Y. Liu

Gastroenterology Department, Peking University People's Hospital, Beijing, China

Objective: The purpose of this study was to describe the clinical features of autoimmune hepatitis (AIH) patients with Sjogren's syndrome(SS).

Methods and materials: We retrospectively reviewed the clinical findings in 38 patients who were diagnosed as autoimmune hepatitis in Peking University People's Hospital between 2002 and 2009. 
Results: There were 34 females and 4 males with the mean age of $54.8 \pm 12.6$ years. Among the 25 AIH patients accompanied with other autoimmune diseases, Sjogren's syndrome was found in 15 patients. We devided the 38 patients into two groups, one was AIH patients with SS, and another without SS. The mean age of AIH with SS was $54.80 \pm 12.161$, while that of AIH without SS was $54.79 \pm 13.062$ showing no significance. Clinical manifestations of AIH such as fatigue, nausea, icterus and bloating showed no difference in two groups, as well as 5 cirrhosis cases $(33.3 \%), 2$ varices $(13.33 \%)$ in $\mathrm{AIH}$ with SS and 12 cirrhosis, 9 varices $(39.13 \%)$ in another group. There was no significant difference between the two groups in the level of ALT, AST, ALB, ALP, GGT, TBIL, DBIL, cholesterol, $\gamma$-globulin, IgG, IgM, IgA. The positive rate of antinuclear antibody (ANA), antismooth muscle antibody (SMA) in AIH with SS group was 93.33 and $26.67 \%$, while in the group without SS was 86.96 and $13.04 \%$, in which no difference was found. The positive rate of SSA and (or) SSB in two groups were significant different $\left(\chi^{2}=5.294, P=0.021\right)$. Interestingly, celiac lymphadenectasis was found by CT scan in $5 / 15$ cases AIH with SS, while $1 / 23$ case in AIH without SS $\left(\chi^{2}=3.764, P=0.052\right)$.

Conclusion: Autoimmune hepatitis is often accompanied with other autoimmune diseases, especially with Sjogren's disease. There was no significant difference in clinical features between AIH patients with or without SS.

\section{PP25-15}

Significant Role of Intrahepatic Activated NKT Cells to Induce Autoreactive T Cells in the Liver in Experimental AIH Model Mouse

M. Nakano, C. Saeki, T. Oikawa, H. Takahashi, S. Homma, M. Zeniya Jikei University School of Medicine, Tokyo, Japan

Background/aim: The role of NKT cells in the pathogenesis of autoimmune hepatitis (AIH) remains unknown. We evaluated the significance of NKT cell in AIH by using mouse AIH model which we previously established.

Methods: AIH model was made by subcutaneous vaccination of fusion cell (FC) of dendritic cells and well differentiated hepatoma cells (Hepa1-6) followed by intraperitoneal injection of IL-12. The number of intrahepatic and splenic NKT cells were measured by FACS. Surface expression of CD69, CXCR6 and production of IFN- $\gamma$ of NKT cells were analyzed by FACS and intracellular staining. Intrahepatic CXCL16 mRNA expression was analyzed by qRT-PCR. All analyses were performed on day 0, day 21 (inflammation phase) and day 23 (improved phase). To evaluate the role of activated NKT cell in the pathogenesis of this model, $\alpha$ GalCer $(0.5 \mu \mathrm{g}$ /mouse) was injected intraperitoneally to FC pretreated and untreated mouse. Serum level of ALT and CD62L expression on $\mathrm{CD}^{+} \mathrm{T}$ cells were assessed $48 \mathrm{~h}$ after $\alpha$-GalCer administration.

Results: Intrahepatic NKT cells significantly increased on day 21 compared with day 0 and day 23. On the contrary, splenic NKT cells decreased on day 21. Expression of CD69, CXCR6 on intrahepatic NKT cells, intrahepatic CXCL16 mRNA expression and the number of IFN- $\gamma$ producing intrahepatic NKT cells were up-regulated on day 21. $\alpha$-GalCer administration induced remarkable increase of serum ALT and abundant $\mathrm{CD}_{2} \mathrm{~L}^{-} \mathrm{CD} 8^{+}$effector $\mathrm{T}$ cells infiltration in FC pre-treated but not in FC untreated mouse.

Conclusion: Intrahepatic activated NKT cells which secrete IFN- $\gamma$ may participate in the liver damage in $\mathrm{AIH}$. In addition, activated NKT cells may recruit $\mathrm{CD}_{2} \mathrm{~L}^{-} \mathrm{CD}^{+}$effector $\mathrm{T}$ cell into the liver. These results indicated that activated NKT has a significant role in the pathogenesis of this AIH model.
PP25-16

Clinical Features in Primary Biliary Cirrhosis in Sapporo, Japan H. Shida, J.-H. Kang, T. Kin, T. Matsui, K. Tsuji, T. Fukuda, Y. Kodama, Y. Sakurai, H. Maguchi

Center for Gastroenterology, Teine KeijInkai Hospital, Sapporo, Japan

Background and aims: Primary biliary cirrhosis (PBC) is recognized as chronic liver disease characteristic of progressive cholestasis based on genetic background, however, in Asian countries, their clinical impact has remained obscure. The aim of this study was to clarify the clinical features of PBC in Sapporo, Japan.

Methods: Authors reviewed the medical records and retrospectively analyzed clinical feature in the patients with PBC diagnosed from 1997 to September, 2010.

Result: A total of 114 patients (104 females, median age 59 years) were diagnosed as PBC in the last 14 years. At presentation, 44 patients $(38.6 \%)$ were asymptomatic, $53(46.5 \%)$ showed only pruritis and remaining $17(14.9 \%)$ had other symptom but pruritis. Among 103 patients undergone liver biopsy, 53 were diagnosed in Scheuer I or II, 21 in III or IV, respectively. Anti mitochondrial antibody was positive in 97 patients by immunofluorescece or enzyme linked immunoassay for M2. Ursodeoxycholic acid (UDCA) was administered in 105 patients and bezafibrate was also used with UDCA in 19. During the followed-up periods (74 months in median), 5 patients (4.4\%) developed liver failure, 7 (5.9\%) had hepatocellular carcinoma (HCC) and $15(13.2 \%)$ showed gastro-esophageal varices. All patients complicated with HCC had advanced liver cirrhosis with decreased platelet counts $(89000 /$ micrlL). Liver transplantation was undergone in three patients who developed liver failure. Out of eight patients died during follow-up periods, six had liver-related cause; two hepatic failure, two HCC, two variceal rupture.

Conclusions: Almost patients with PBC are asymptomatic or manifest mild symptom at presentation, and show good prognosis during 6 years observation. Nine of 114 patients $(7.9 \%)$ showed fatal disease progression. Main cause of death is hepatic failure.

\section{PP25-17}

How Do We Assess Evolution of Primary Biliary Cirrhosis under UDCA Treatment

I. Copaci, L. Micu, L. Iliescu, M. Voiculescu

Center of Internal Medicine, Fundeni Clinical Institute, Bucharest, Romania

Introduction: Assessment of the efficacy of ursodeoxycholic acid (UDCA) in the treatment of primary biliary cirrhosis (PBC) is mostly based on hepatic biochemical tests. The aim of our study was to identify a combination of hepatic biochemical tests measured under UDCA, allowing for a better discrimination between patients regarding survival.

Patients and methods: The inclusion criteria were: histologically proven $\mathrm{PBC}$, treatment with UDCA $13-15 \mathrm{mg} / \mathrm{kg} / \mathrm{day}$ and availability of the main hepatic biochemical tests before and after 1 year of treatment. The major evaluation criterion was survival in the absence of liver transplantation (LT). The predictive value of a combination of three hepatic biochemical tests measured after 1 year of UDCA therapy.

Results: Of the 78 patients screened, 62 patients respected the inclusion criteria (mean age 53 years, females $92 \%$, cirrhosis $27 \%$ ). Mean survival was $6.1 \pm 4.3$ years (1.2-21.5 years). 12 deaths ( 9 due to $\mathrm{PBC}$ ) and $5 \mathrm{LT}$ were observed during survival. Transplant-free survival at 10 years was $75 \%$ (CI 95\%: 67-81\%). After one year of therapy, $\mathrm{AP}<3 \mathrm{xN}$, AST $<2 \mathrm{xN}$ and serum bilirubin $<1.5 \mathrm{mg} / \mathrm{dL}$ discriminated patients with risk of death or transplant. When these 
parameters were respected ( $62 \%$ of cases), transplant-free survival at 10 years was $90 \%$ (CI 95\%: 81-95\%) compared to 51\% (CI 95\%: $38-64 \%)$ in their absence $(p<0.0001)$. Independent factors associated with risk of death or liver transplant were: histological stage $\geq 3$ on initial biopsy (RR: 1.5), bilirubin before therapy $>1.5 \mathrm{mg} / \mathrm{dL}$ (RR: $1.7)$, presence of piecemeal necrosis of moderate/severe intensity (RR: 1.9) and absence of biochemical response after 1 year (RR: 2.3). Conclusions: In patients with PBC treated with UDCA, combined measurement of alkaline phosphatase, AST and bilirubin after 1 year of treatment is a simple evaluation of long-term prognosis. The histological stage and grade of piecemeal necrosis remain independent prognostic factors.

\section{PP25-18}

Subcellular Peculiarities of Cholestasis-induced Hepatocyte Damage in Pediatric Patients and Estimation of their Relevance

V. Groma ${ }^{1}$, V. Ozolins ${ }^{2}$, S. Skuja ${ }^{1}$, L. Smane ${ }^{1}$, A. Leidere-Reine ${ }^{3}$

${ }^{1}$ Laboratory of Electron Microscopy; ${ }^{2}$ Children's University

Hospital; ${ }^{3}$ Riga Stradins University, Riga, Latvia

Background/aim: The liver of pediatric patients is relatively sensitive to injury, and its response is more limited. Liver biopsy still is assumed to appear the single most useful test to determine the cause of cholestasis. The canalicular membrane of the cholestatic hepatocyte reveals significant changes in its organization initially appearing as a loss of membrane asymmetry. Relevance of cytoskeleton changes in estimation of cholestatic liver disease is demonstrated in small cohorts of adult patients. The cellular and molecular peculiarities of the cholestatic hepatocyte damage are not entirely clear in pediatric patients. Here we assess cholestasis-induced ultrastructural changes of hepatocytes demonstrated in pediatric liver biopsy material.

Methods: Biopsy liver specimens were examined by light and transmission electron microscopy. Routine laboratory studies included detection of serum bilirubin, bile salt concentration, cholesterol, lipoprotein, alkaline phosphatase, gamma-glutamyl transferase levels. Results: The hepatocytes displayed notable changes of the internal organization. The cell shape and the polar fashion of arrangement were changed. The canalicular surface revealed a prominent smoothening of the external contour and disappearance of the microvilli. Bleb formation was registered as well. Intercellular junctions remained intact in a majority of cases. The canalicular space was irregularly dilated. The cortical, actin microfilaments containing, layer reflecting the involvement of peripheral membrane proteins showed a prominent thickening. The presence of cytoskeleton changes was graded and estimated using semiquantitative criteria applied separately for three zones within the hepatocyte - pericanalicular, perinuclear, and perivascular.

Conclusions: Cell surface and polarity changes as well as rearrangement of the hepatocyte cytoskeleton are indicative of cholestatic damage, in general, and in pediatric patients, in particular. Cytoskeleton changes may be reversible and correlate with ability of the hepatocyte create and maintain protein synthesis on polyribosomes, whereas, sustained function of the rough endoplasmic reticulum contributes to biological membrane molecules manufacturing capacity.

\section{PP25-19}

fgl2 Gene Expression Contributes to Liver Injury in Human and Experimental Autoimmune Hepatitis

H. Yu, G. Ai, F. Xiao, W. Yan, X. Wang, X. Luo, J. Huang, Q. Ning

Tongji Hospital, Huazhong Science and Technology University, Wuhan, China

Objective: To investigate the role of fgl 2 expression in the development of liver injury in human and experimental autoimmune hepatitis $(\mathrm{EAH})$.
Method: A gene expression microarray between peripheral mononuclear cells (PBMCs) in patients with autoimmune hepatitis (AIH) and healthy control were conducted to screen the differentially expressed genes. The fgl 2 expression were confirmed by Real-time PCR and immnunohistochemistry. Subsequently an EAH model was established and the expression of $\mathrm{mfg} 12$ gene in liver was examined. An fgl2 antisense plasmid was constructed and the effect of this plasmid specifically targeting on fgl2 gene was evaluated. Furthermore, the sfgl2 expression in total population of $\mathrm{T}$ cells and CD4+CD25+ Tregs was also examined and the effect of expression of fgl2 molecule on CD8+ T cells proliferation and cytokine production was observed.

Result: Compared with healthy controls, fgl2 gene was overexpressed in patients with $\mathrm{AIH}$ as displayed in microarray. Hfgl 2 overexpression was further evidenced to be in liver tissue and PBMCs in patients with AIH. An mfgl2 protein expression was found to be localized in nonparenchymal cells of liver tissues in EAH. An mfgl2 antisense plasmid significantly reduced hepatic mfgl2 expression, improved liver function, and markedly ameliorated hepatocyte necrosis compared with animals treated with sense plasmid. This upregulated fgl2 protein was identified to be sfgl 2 by flow cytometer analysis of spleen $\mathrm{T}$ cells and measurement of the supernantants from culture $\mathrm{T}$ lymphocytes. Furthermore, upregulated sfgl2 expression was found in CD4+CD25+ Tregs and its expression strongly promoted the proliferation and enhanced cytokine production of CD8+ T cells.

Conclusion: This current study demonstrates that upregulated sfgl2 expression in liver plays an important role in the pathogenesis of AIH and this effect was due to its high expression in CD4+CD25+ Tregs which functioned as an effective molecule, promoting the $\mathrm{CD} 8+\mathrm{T}$ cells proliferation and enhancing its cytokine production.

\section{PP25-20}

Primary Biliary Cirrhosis in FEZ-Morocco: How Special?

N. Aqodad, W. Zerhoune, I. Mellouki, M. El Yousfi, D.A. Benajah,

M. El Abkari, S.A. Ibrahimi

Gastroenterology, Hospital University Hassan II, Fez, Morocco

Primary biliary cirrhosis (PBC) is a chronic cholestatic disease characterized by inflammation and destruction of interlobular bile ducts. The aim is to study the epidemiology, clinicopathologic, histologic and progressive $\mathrm{PBC}$.

Materials: Retrospective descriptive study, which included all cases of PBC collected on a 5-year period between 2005 and 2010. The diagnosis of PBC was asked about the presence of a syndrome of cholestatic clinical, laboratory, immunoassay with the presence of Anti-mitochondrial antibody (AMA) and/or the presence of histological lesions suggestive in liver biopsy.

Results: Of a total of 1,107 patients with cirrhosis, 18 cases of PBC were diagnosed or $1.6 \%$ of chronic liver disease. The average patient age was 50 years, a female predominance was noted $83 \%$. Pruritus was the mode of presentation most common among the majority of patients $78 \%$, associated with jaundice in 55\%. PBC was diagnosed with cirrhosis and decompensated hemorrhagic ascites in four cases $(22 \%)$ and incidentally discovered during a review of cholestasis in two cases. Biological cholestasis found in all patients, associated with moderate cytolysis and hypercholesterolemia in $55 \%$, the AMA was positive in all patients, the dosage of other antismooth muscle antibody, anti-nuclear, anti LKM1 were negative. PBC was associated with hypertension in two cases, hypothyroidism in two cases, sarcoidosis multi visceral in one case, myeloproliferative disorder (jack 2 mutation positive, myelofibrosis in the BOM) in one case. Liver biopsy realised in 12 cases (67\%), 75\% were in stage 3 Ludwig, debutante form was found in $25 \%$. All patients received ursodeoxycholic acid at doses of $13-15 \mathrm{mg} / \mathrm{g} / \mathrm{d}$ with a clinical and biological improvement, with an average decline of 12 months. 
Conclusion: PBC is a rare, affecting mainly middle-aged woman. Pruritus is the most mode of revelation. Evolution is slow, $72 \%$ of our patients diagnosed at an advanced stage. Treatment and prognosis improved with ursodeoxycholic acid.

\section{PP25-21}

Characterization of Autoimmune Hepatitis and Chronic Hepatitis C on Clinical, Laboratory and Therapeutic Bases

S. Agha ${ }^{1}$, W. El-Emshaty ${ }^{1}$, D. Raafat ${ }^{1}$, M. El-Bendary ${ }^{2}$, M. Abd El-Maksoud ${ }^{2}$, H. Elalfy ${ }^{2}$

${ }^{1}$ Clinical Pathology; ${ }^{2}$ Tropical Medicine, Mansoura University, Mansoura, Egypt

Background: Difficulty in differentiating autoimmune hepatitis (AIH) from chronic hepatitis $\mathrm{C}$ (CHC) with non organ specific autoantibodies (NOSA) which consequently determine the treatment selection triggered us to conduct this study.

Aim of the study: To characterize the clinical, biochemical and serological variables as well as response to combined interferon / ribavirin (IFN/ribavirin) or steroid therapy that could help in differentiating autoimmune and $\mathrm{HCV}$ related liver diseases, with evaluation of the IAHG diagnostic criteria of AIH.

Subject and methods: Fifty-three chronic hepatitis patients not attributed to hepatitis B infection, drugs, alcohol or metabolic liver diseases were subjected to thorough clinical examination, liver function tests, NOSA, anti HCV antibodies and HCV RNA detection with calculation of International Autoimmune Hepatitis Group (IAHG) scoring.

Results: The obtained variables classified them into, 12 patients with $\mathrm{AIH}$ and $39 \mathrm{CHC}$, while 2 patients not fulfill criteria for either AIH or $\mathrm{CHC}$ and were considered of AIH outlier syndrome. False anti HCV positivity was recorded in $33.3 \%$ of $\mathrm{AIH}$ while $56.4 \%$ of $\mathrm{CHC}$ patients had NOSA. The scoring system of IAHG gave sensitivity of $85.7 \%$ and specificity of $100 \%$. Anti $\mathrm{HCV}$ antibodies titers were significantly higher in $\mathrm{CHC}$ patients than in $\mathrm{AIH}$ patients while NOSA titers were significantly higher in $\mathrm{AIH}$ patients than in $\mathrm{CHC}$ patients. There was no significant difference between auto-antibodies negative and positive $\mathrm{CHC}$ patients in response to combined antiviral therapy. Moreover anti HCV positivity in $\mathrm{AIH}$ patients had no impact on response to steroid therapy.

Conclusion: Scoring system is helpful in determining AIH particularly in cases with overlap features, also the titers of anti HCV, and NOSA are more reliable than qualitative results in differentiating $\mathrm{AIH}$ from $\mathrm{CHC}$.

\section{PP25-22}

Safety and Efficacy of Second Line Therapies for Autoimmune Hepatitis L.Y. Lim ${ }^{1}$, K. Kowdley ${ }^{2}$

${ }^{1}$ Gastroenterology, Indiana University, Indianapolis, IN; ${ }^{2}$ Virginia

Mason Medical Center, Seattle, WA, USA

Introduction: Minority of patients with Autoimmune Hepatitis (AIH) do not respond to standard therapy due to inability to taper steroids or inability to tolerate steroids or azathioprine/6-MP.Such patients are often treated with second line agents such as mycophenolate mofetil (MMF), cyclosporine (CSA) or tacrolimus (FK506).

Objective: Goals of this study were to examine the efficacy and safety of second-line agents(MM, CSA, FK506) in refractory AIH.

Methods: Consecutive retrospective review of patients with autoimmune hepatitis treated at Virginia Mason Medical Center Hepatology clinic between 1999 and 2010. All patients meet Hennes criteria score $\geq 6$.

Results: Total of 68 patients were included. 47 were in the control group (patients who receive corticosteroids + azathioprine/6-MP). 21 patients in the study group who received second line agents: $\mathrm{MMF} \pm \mathrm{FK506}$. There are no significant differences in their clinical characteristics between the two. 90\% of patients have Type I Autoimmune Hepatitis. Mean time to followup is $25 \pm 7$ months in the study group and $59 \pm 7.8$ months in the control group. There was a $76 \%$ corticosteroids dosage reduction in the study group. Only 2 (9\%) AIH patients in the study group did not respond to second line therapy. Three predictors found to be associated with non-response to second line agents (cirrhosis, age $>30$ and younger age at onset of disease). We found no clinically significant change in serum BUN, creatinine, WBC or PLT count throughout duration of therapy in the study group.

Conclusions: Second line agents (FK506 and MMF) are safe alternative long term therapies for autoimmune hepatitis. $90 \%$ of patients with refractory AIH were able to achieve biochemical remission on second line agents. Limitations of this study include the retrospective review and small sample size. We also relied on biochemical response rather than histological response.

\section{Poster Presentation 26: Case Report/Case Series}

\section{PP26-01}

Hepatocellular Carcinoma Associated with Paraneoplastic Erythema Nodosum and Polyarthritis

S. Glinkov ${ }^{1}$, I. Krasnaliev ${ }^{2}$, V. Glinkova ${ }^{3}$

${ }^{1}$ Department of Surgery, St. Anna Hospital, Medical University;

${ }^{2}$ Department of Pathology; ${ }^{3}$ Department of Hepatogastroenterology, Medical University, Varna, Bulgaria

Introduction: Hepatocellular carcinoma (HCC) may manifest various paraneoplastic syndromes. Such syndromes have been reported to be present in 10-20\% of the patients with HCC. Sometimes combination of paraneoplastic syndromes in one HCC patient has been observed. We report a rare case of HCC associated with erythema nodosum and polyarthritis.

Methods: Case report

Results: A 23-year-old woman was hospitalized for a liver tumour detected at abdominal ultrasonography. She had a 2-year history of erythema nodosum and polyarthritis, which poorly responded to corticosteroids and NSAIDs and were still present at admission. Fine needle aspiration biopsy revealed moderately differentiated HCC. No other reasons for erythema nodosum were revealed. Atypical resection of liver segments III, IV, V and part of segment II was performed and the diagnosis of HCC was confirmed by histology. Erythema nodosum and the polyarthritis disappeared completely 10 days after surgery without any treatment. Eight years after the operation the patient is free of any symptoms.

Conclusion: The clinical course of the disease strongly suggests that erythema nodosum and polyarthritis in this patient appeared as paraneoplastic syndromes of HCC. The case reminds the necessity of taking into account $\mathrm{HCC}$ as a possible cause for inexplicable and poorly controlled erythema nodosum and polyarthritis.

\section{PP26-02}

Cerebral Lipiodol Embolism Following Transcatheter Arterial Chemoembolization for Hepatocellular Carcinoma

L. Wu, Y.-F. Yang

The First Department of Interventional Radiology, Eastern Hepatobiliary Surgery Hospital, Second Military Medical University,

Shanghai, China

Cerebral lipiodol embolism (CLE) is an extremely rare complication of transcatheter arterial chemoembolization for hepatocellular carcinoma (HCC). The authors present a case of CLE that occurred after the second hepatic arterial chemoembolization for HCC, and attempt to introduce several plausible mechanisms of CLE, after reporting the clinical and radiological findings and reviewing the medical literature. 
published on World J Gastroenterol 2010 January 21; 16(3): 398-402 doi:10.3748/wjg.v16.i3.398

\section{PP26-03}

Analysis of High-flow Hepatic Hemangioma Using Parametric Imaging Method with Sonazoid-enhanced Ultrasonography

N. Wakui ${ }^{1}$, R. Takayama ${ }^{2}$, Y. Sumino ${ }^{1}$, N. Kamiyama ${ }^{3}$

${ }^{I}$ Toho University Omori Medical Center; ${ }^{2}$ Division of

Gastroenterology and Hepatology, Toho University Omori Medical

Center, Tokyo; ${ }^{3}$ Toshiba Medical Systems Corporation, Otawara,

Japan

Case: Abdominal ultrasonography detected a hypoechogenic nodule of $\varphi 32 \mathrm{~mm}$ at $\mathrm{S} 5$ in the liver of a 62-year-old man, who was then hospitalized in our hospital. During the early vascular phase, Levovist-enhanced ultrasonography showed perfusion from the region surrounding the tumor straight to the central part of the tumor. Images taken in the parenchymal phase were weaker than the images of the surrounding liver. Abdominal plain CT depicted the lesion as a low-density area (LDA). The blood velocity was too quick for Levovist-enhanced ultrasonography to visualize the detailed hemodynamics. Subsequently, abdominal MRI was performed and the subject was diagnosed with hepatic hemangioma. In March 2008, image-analyzing software developed by Kamiyama and his colleagues at Toshiba Medical Systems was used to obtain images of the tumor to follow up the clinical course. These images were analyzed using a parametric imaging method based on Sonazoid-enhanced ultrasonography. The analyzing software enabled color differentiation of perfused liver sections following the time course, which helped us to roughly judge the starting point of sectional perfusion and the orders to be perfused.

Conclusion: The software for analyzing hepatic tumors permits highly detailed visual identification of perfusion kinetics, which may be useful for characterizing liver tumors.

\section{PP26-04}

Does Sorafenib Really Improves Survival in Metastatic Hepatocellular Carcinoma?

F.G. Nery $^{1}$, L. Graça ${ }^{2}$, M. Ribeiro ${ }^{3}$, H. Guimarães ${ }^{3}$, H.P. Miranda ${ }^{1}$

${ }^{1}$ Internal Medicine Dep; ${ }^{2}$ Surgery Dep; ${ }^{3}$ Radiology, Centro

Hospitalar do Porto - Hospital Sto António, Porto, Portugal

Background and aims: Sorafenib is a multikinase inhibitor with many properties, approved for the treatment of advanced hepatocellular carcinoma (HCC) since 2007. There are case reports that show benefit also in metastatic HCC.

Methods: A case report is presented, with a retrospective review of data.

Results: A women, with a chronic hepatitis B diagnosed in 1997, presented in 2001, when she was 45 years-old, with a hepatic tumor on the right lobe. The liver tests were ASAT $=155$ UI, ALA$\mathrm{T}=192 \mathrm{UI}, \mathrm{ALP}=130 \mathrm{UI}, \mathrm{GGT}=170 \mathrm{UI}$. AFP was $60,7 \mathrm{ng} / \mathrm{mL}$. Serum HB surface antigen and anti-HBe antibody were positive with a serum HBV DNA undetected. Diagnosis of HCC was done based on different imaging techniques, and confirmed after a right hepatectomy. The histology showed a moderately differentiated HCC and a chronic hepatitis with mild fibrosis of the remnant liver. AFP returned to normal levels $(3.4 \mathrm{ng} / \mathrm{mL})$. In 2004 a pulmonary solitary metastatic disease was found, and the pathology of the lesion, after metastectomy demonstrated a HCC metastasis. During surveillance, the AFP levels were normal, till January $2007(13.5 \mathrm{ng} / \mathrm{mL})$. A rising AFP contrasted with the absence of demonstration of any tumor in repeatedly imaging techniques. In December 2008 the AFP peaked at $365.4 \mathrm{ng} / \mathrm{mL}$. At that time, a dynamic computed tomography identified an inferior mediastinal mass of $3.5 \times 3.3 \mathrm{~cm}$, with a vena cava thrombus associated. Sorafenib (400 mg twice a day) was started in February 2009, resulting in a decline of AFP values, a reduction of more than $50 \%$ of the metastatic mass (15 mm wide in March 2010) and vena cava thrombus size. The patient is asymptomatic, reporting only hair loss during the treatment.

Conclusions: The protracted time course of the HCC in this patient was very uncommon. Sorafenib seems a very exciting therapy for advanced HCC, but may also have very important benefits in particular patients with metastatic HCC.

\section{PP26-05}

Investigation of Heavy Metal Load in Liver Disease Patients at Coal Fired Industrial Area in Central India

B. Gurugubelli

Pt Ravishankar Shukla University, Raipur, India

The components and quantities of heavy metals load has been reported to be the pollution indicator of large urban areas. The multiplicity and complexity of sources of heavy metals in urban regions (e.g. industrial complexes composed of a variety of industrial processes, automobiles, construction activities etc.) has put forward the need of investigation of these sources indicating their contribution to specific anthropogenic health. The study presented here is focused on investigation of heavy metal load in liver disease patients at coal fired industrial area of central India. Six sampling sites have been identified on the basis of land use for development plan of anthropogenic activities. A random sampling plan has been conducted at various hospitals in the study area, 220 samples has been collected form various sites and perform chemical analysis for Arsenic and Lead. The patients from Coal-fired industrial area have shown highest amount of heavy metals and in savior condition in compared to other area patients.

\section{PP26-06}

A Rare Consequence of Acute Hepatitis A Virus Infection - Case Report D. Jothimani ${ }^{1}$, M.E. Cramp ${ }^{1}$, J.D. Mitchell ${ }^{1}$, K. Sleigh ${ }^{2}$, T.J. Cross ${ }^{1}$ ${ }^{1}$ Southwest Liver Unit; ${ }^{2}$ Department of Histopathology, Derriford Hospital, Plymouth, UK

Introduction: Hepatitis A virus (HAV) infection is a common cause of acute viral hepatitis. Resolution of acute infection leads to a complete recovery with no long-term liver damage in the majority of patients. We report a case of immune mediated hepatitis following acute HAV infection.

Case description: A 21-year-old Indian student presented with 10 days history of fever, jaundice and right upper quadrant pain following a trip to India. Blood tests showed aspartate aminotransferase (AST) $93 \mathrm{iu} / \mathrm{L}$ (10-37), alkaline phosphatase (ALP) $139 \mathrm{iu} / \mathrm{L}$ (40-120), gamma glutamyl transferase (GGT) 34 iu/L (10-60), bilirubin $82 \mu \mathrm{mol} / \mathrm{L}(3-20)$, albumin $40 \mathrm{~g} / \mathrm{L}(35-50)$ and prothrombin time (PT) $15.9 \mathrm{~s}(11-15)$. An ultrasound abdomen was normal. His liver screen revealed acute hepatitis A infection (IgG and IgM antibodies positive). His autoimmune serology was unremarkable. He was discharged home. Two weeks later he was re-admitted with worsening jaundice, pruritus and fatigue. There were no features of acute liver failure. Blood tests revealed AST 1,343 iu/L, ALP $118 \mathrm{iu} / \mathrm{L}$, GGT $26 \mathrm{iu} / \mathrm{L}$, bilirubin $563 \mu \mathrm{mol} / \mathrm{L}$, albumin $40 \mathrm{~g} / \mathrm{L}$ and PT $17 \mathrm{~s}$. Antinuclear (ANA), smooth muscle (SMA) and mitochondrial (AMA) antibodies were negative. IgG 27.1 (6-16 g/L), IgM 2.84 (0.5-1.9 g/L) and $\operatorname{IgA} 2.32(0.8-2.8 \mathrm{~g} / \mathrm{L})$. Hepatitis A IgG and IgM remained positive. A transjugular liver biopsy showed portal tract inflammation by plasma cells, eosinophils and neutrophils, interface hepatitis, focal apoptotic hepatocytes but no fibrosis. Based on these features the patient was commenced on prednisolone $40 \mathrm{mg}$ once a day and tapered over months. He achieved good clinical and biochemical remission. His prednisolone was tapered gradually and currently on $2.5 \mathrm{mg}$ daily. This case highlights that autoimmune hepatitis can be triggered by $\mathrm{HAV}$ infection and patients with this infection must be followed up in the clinic after resolution of their acute illness. 


\section{PP26-07}

Newly Defined Clinical Features and Treatment Experience of Seventh Day Syndrome Following Living Donor Liver Transplantation

Z. Zhang

Sichuan University, Chengdu, China

Liver transplantation has become an established treatment option for end-stage liver diseases. Post liver transplantation, acute liver failure resulting from acute rejection, infection and Seventh Day Syndrome (7DS), can be life-threatening. The mortality rate from 7DS has historically been rated the highest. Since 2005, eight cases of 7DS were identified in our center. Previously reported features for 7DS include a rapid deterioration of liver function followed by renal failure and sudden peak of liver enzyme levels around day 7. In addition, the following attributes were observed in our patients:

1) All recipients were male while donor tissue included both genders;

2) Most patients showed increased levels of irritability;

3) Color Doppler showed a reduction of blood flow or bidirectional blood flow in the portal vein;

4) Coagulative necrosis with disruption of lobular architecture was observed in all examined patients;

5) After onset, steroids pulse with or without OKT3 therapy showed minimal effect;

6) An abrupt increase of liver enzymes was observed 1-2 days after intravenous (i.v.) methylprednisolone was switched to oral immunosuppressants were administered;

7) Extension of i.v. methylprednisolone treatment delayed occurrence from 8 to 11 days post-operative.

Previous studies suggest that 7DS is not related to acute rejection. In contrast, our study supports the hypothesis that 7DS might be associated with an undefined immune response. Our experience extending i.v methylprednisolone treatment up to 14 days posttransplantation provides a possible therapeutic prospect for 7DS, intended to delay occurrence and reduce mortality.

\section{PP26-08}

Successful Treatment of HBV HDV Coinfection Related Cirhhosis by Contemporary Antiviral Treatment with Peginterferon-alpha 2a and Entecavir: Case Report

A. Izzi $^{1}$, B. Fimiani ${ }^{2}$

${ }^{1}$ Deparment of Emergency Infectious Diseases, 'D. Cotugno' Hospital, Naples; ${ }^{2}$ Internal Medicine Unit, Umberto $1^{\circ}$ Hospital, Nocera Inferiore, Italy

Background: HBV and HDV coinfection is often a progressive serious and life-threatening condition leading rapidly towards an endstage liver disease with hepatic decompensation and onset of HCC. Case description: In June 2008, a caucasian patient, male 66 years old, with a long lasting active liver disease and previous hepatobiopsy showing in 2004 a moderate $\mathrm{CAH}$, went at our observation: patient was HBsAg positive, Anti-HBs negative, Anti-HBe positive, AntiHDV IgG positive, Anti-HDV IgM positive, Anti-HCV negative, anti-HIV negative, HBV-DNA positive $134 \mathrm{IU} / \mathrm{ml}$ in RT-PCR, HDVRNA positive by qualitative PCR assay. Serum ALT were six times the ULN values at baseline. An abdominal ultrasound check revealed an important coarse nodular pattern of the liver, showing therefore a liver cirrhosis, without decompensation. In July 2008 patient started a combination treatment of PEGIFN $\alpha-2 \mathrm{a}$ and Entecavir (ETV) at the dosage of $180 \mu \mathrm{g}$ subcutaneously once a week and $0.5 \mathrm{mg}$ qd given orally, respectively. Altough PEGIFN $\alpha-2 \mathrm{a}$ has by itself an antiviral activity against both HBV and HDV, ETV treatment was contemporary started in the aim to obtain, in synergistic effect with PEGIFN action, a prompt, deep and persistent suppression of both HBV replication and HBsAg production, being the HBV surface antigen essential to allow the HDV replication and it's survival too.

Results: Since the first month of treatment ALT levels felt within the normal values, HBV-DNA and HDV-RNA were undetectable by RT-PCR $(<12 \mathrm{IU} / \mathrm{ml})$ and qualitative PCR, respectively. Anti-HDV IgM dropped below the cut-off of positivity. Such treatment was effective throughout all the duration of therapy with persistent suppression for 18 months of both viral replications, and then was discontinued. Actually, after 8 months from the treatment withdrawal, neither a biochemical or virological relapse were observed. Treatment was safe and well tolerated by the patient without significant side effects.

\section{PP26-09}

HBV Suppression with Adefovir/Entecavir Association for HBEAG Positive Reactivation after Lamivudine Induced YMDD Mutation in HBEAG Negative Chronic Hepatitis B

A. Izzi $^{1}$, V. Iovinella ${ }^{2}$, B. Fimiani ${ }^{3}$

${ }^{I}$ Department of Emergency Infectious Diseases, 'D. Cotugno'

Hospital; ${ }^{2}$ Internal Medicine Unit, 'San Paolo' Hospital, Naples;

${ }^{3}$ Internal Medicine Unit, 'Umberto $1^{\circ}$ ' Hospital, Nocera Inferiore, Italy

Background: HBV reactivation in long-term lamivudine (LAM) treated patients with $\mathrm{CAH}$ is a potential life-threatening situation that can lead to fatal liver failure.

Case description: In 2002, a 46-year-old patient, male, caucasian, went at our observation for a chronic HBV infection with a 1 year elevation of serum ALT.

Features: HBsAg positive, Anti-HBe positive, Anti-HDV $\mathrm{IgG} / \mathrm{IgM}$ negative, Anti-HCV negative, anti-HIV negative, HBV-DNA $>200,000$ copies $/ \mathrm{ml}$, genotype D of HBV. A liver biopsy showed a moderate $\mathrm{CAH}$ with a global Ishak score 12 . An IFN- $\alpha 2$ a based treatment (10 MU TIW) was started. After 6 months of treatment patient resulted non responder. On January 2003 a LAM treatment (100 mg qd orally) was started. Patient showed a persistent normalization of serum ALT and undetectable HBV-DNA till October 2004, when a virological and biochemical breakthrough was observed. A direct sequencing assay (Trugene Bayer) showed YMDD M204V mutation. Patient was switched to Adefovir (ADV) $10 \mathrm{mg} /$ day orally, with a new prompt response that lasted till October 2006, when a new breakthrough due to $\mathrm{N} 236 \mathrm{~T}$ mutation was found with no evidence of the previous M204V mutation. LAM re-treatment resulted in viral rebound with serum HBV-DNA $>4 \times 10^{6} \mathrm{IU} / \mathrm{ml}$, serum ALT arose tenfold ULN values, HBeAg positivity, anti-HBc IgM positivity, as seroreversion of viral population towards wild type due to M204I mutation. ADV was firstly added on to LAM, and LAM was then replaced by Entecavir (ETV, $1 \mathrm{mg} /$ day orally).

Results: A prompt normalization of ALT was obtained, while serum HBV became undetectable within few months. Treatment mantains it's efficacy over time due to the contemporary use of drugs with a different spectrum of resistance, particularly ETV with it's potent and rapid antiviral activity and high genetic barrier too. Interestingly, patient continue to be $\mathrm{HBeAg}$ positive despite full suppression of HBV replication

\section{PP26-10}

Simultaneous Development of Hepatocellular Carcinoma and Non-Hodgkin Lymphoma in a Patient with Chronic Hepatitis C

I. Subortseva ${ }^{1,2}$, S. Lepkov $^{3}$, I. Poddybnaya ${ }^{2}$, G. Storogakov ${ }^{2}$, A. Kovrigina ${ }^{1}$, O. Kolomiycev ${ }^{1}$, S. Kosura ${ }^{3}$, O. Malihova ${ }^{1}$

${ }^{I}$ Blokhin Cancer Research Centre; ${ }^{2}$ Russian Academy for Postgraduate Medical Education Minisry of Health of Russia, Clinical Oncology Chair; ${ }^{3}$ The Moscow Medical University of N.N. Pirogov, Moscow, Russia 
Backgrounds: Pathogenetic link between of a virus hepatitis $\mathrm{C}$ and a primary cancer of a liver is known for a long time. According to classification WHO DLBCL is a virus hepatitis C-associated tumour. Methods: We report a clinical case of simultaneous development of hepatocellular carcinoma and non-Hodgkin lymphoma in a patient with chronic hepatitis $\mathrm{C}$ and a cirrhosis.

Results: A 67-year-old man in 2005 was admitted to cancer research center with formation in the field of the left shoulder. Within 15 years of the patient the suffers from a virus hepatitis $C$, genotype $1 \mathrm{~b}$. According to ultrasonography the cirrhosis was diagnosed. Treatment has been appointed: Peg - intron and Rebetol within 6 months. A computerized tomography showed a plural centres of defeat from 3 to $10 \mathrm{sm}$ in diameter, located in both shares of a liver. Level $\alpha$-FP in blood was 44,000 IU. It was diagnosed histopathologically as DLBCL by fine niddle biopsy of tumoral formation in the field of the left shoulder. The diagnosis of a hepatocellular carcinoma was established by fine needle biopsy of liver tumors. A virus of a hepatitis $\mathrm{C}$ proteins were expressed on cells of both tumors. The patient was treated with cyclophosphamide, adriamycin, vincristin and prednisolone in combination with rituximab. Treatment was with antiviral agents. It was efficiency as PR of DLBCL. There was stabilization of liver tumors. But toxicity (IV grade), acute hepatonephritic insufficiency were reason of patient's death.

Conclusions: Screening of the persons infected HCV is necessary for revealing of early stages of tumours, timely appointment for therapy. The rarity of the disorders does not allow to speak about optimal terapeutical strategy in case of simultaneous two virus-associated tumours. However, the expediency of the combined treatmentis is obvious.

\section{PP26-11}

A Case of Vanishing Bile Duct Syndrome after Administration of Amoxycillin/Clavulanic Acid

J. Silasuwan ${ }^{1}$, A. Sobhonslidsuk ${ }^{1}$, P. Sornmayura ${ }^{2}$, N. Wisedopas ${ }^{3}$

${ }^{1}$ Medicine; ${ }^{2}$ Pathology, Ramathibodi Hospital, Mahidol University;

${ }^{3}$ Pathology, Chulalongkorn University, Bangkok, Thailand

A 47-year-old female with 4-month old pregnancy presented in Dec 2003 with a history of skin rash, jaundice, and pruritus which occurred one day after receiving intravenous amoxicillin/clavulanic acid. A diagnosis of drug-induced liver injury was made. Symptomatic treatment was given. However, her jaundice progressed with increased pruritis. She denied significant medical illness, alcohol or herbal use. Blood tests revealed aspartate aminotransferase (AST) $94 \mathrm{U} / \mathrm{L}$, alanine aminotransferase (ALT) $114 \mathrm{U} / \mathrm{L}$, alkaline phosphatase (ALP) $406 \mathrm{U} / \mathrm{L}$, gamma-glutamyl transpeptidase (GGT) $456 \mathrm{U} / \mathrm{L}$, total bilirubin (TB) $11.1 \mathrm{U} / \mathrm{L}$ and direct bilirubin (DB) $9.5 \mathrm{U} / \mathrm{L}$. Anti-mitochondrial, anti-nuclear and anti-smooth muscle antibodies were negative. Because of prolonged jaundice in the second trimester, a liver biopsy was done and it showed scattered fatty changes, marked swelling and dispersed hepatic necrosis and cholestasis with a few inflammatory cells in portal tracts. Ursodeoxycholic acid $750 \mathrm{mg}$ per day was given uninterruptedly but cholestasis persisted until two years later with AST 74 U/L, ALT 113 U/L, ALP $347 \mathrm{U} / \mathrm{L}$, GGT 1,389 U/L, TB 2.1 U/L and DB 2.0 U/L repeated liver biopsy showed cholestasis, bile duct proliferation and elongation, and portal fibrosis. Ursodeoxycholic acid $750 \mathrm{mg}$ was continued with a short course of prednisolone $15 \mathrm{mg} /$ day. Five years later, blood tests showed mild abnormality with ALP 202 U/L, GGT 272 U/L, TB $0.5 \mathrm{U} / \mathrm{L}, \mathrm{DB} 0.3 \mathrm{U} / \mathrm{L}$. The third liver biopsy performed in June 2010 showed focal loss of bile ducts and presence of focal bridging fibrosis without cholestasis, consistent with vanishing bile duct syndrome.
Discussion: Amoxicillin/clavulanic acid is an uncommon cause of drug-induced liver injury. The majority of case reports exhibited a predominantly cholestatic pattern that often occurred during the few weeks following drug withdrawal, and the outcome was usually benign. Herein, we report a rare case of vanishing bile duct syndrome after administration of amoxycillin/clavulanic acid.

\section{PP26-12}

Benign Hepatic Diseases Mimicking Malignant Tumors on Imaging D. Choi

Department of Radiology, Samsung Medical Center, Sungkyunkwan University School of Medicine, Seoul, Republic of Korea

Hepatocellular carcinoma (HCC) has various histological types and enhancement patterns, but the early enhancement with washout is the most common imaging feature of multiphasic CT scan. However if the HCC may present unusual imaging features, it may lead to the delayed diagnosis and treatment. Whereas if any other benign hepatic lesions show similar enhancement pattern with $\mathrm{HCC}$, we tend to diagnose them as HCC first. Our center is in an endemic area for Hepatitis B which is strong etiological factor for HCC, so we occasionally misdiagnose benign liver lesions as HCCs. Here are several cases which were misinterpreted as malignant tumors in the radiologic reports and were resected. This exhibition aims to show the imaging features of the resected cases of benign hepatic diseases that mimic malignant tumors, and to learn the important imaging findings that can be overlooked or misinterpreted. During a recent 10 -year period, 141 patients underwent hepatectomy for benign liver lesions in our institution. 54 benign liver lesions were misinterpreted as malignant tumors in the radiologic reports such as HCC or cholangiocarcinoma. Two most common benign tumors were hepatocelluar adenomas $(n=13)$ and cavernous hemangiomas $(n=10)$. Focal nodular hyperplasia $(n=7)$, angiomyolipoma $(n=6)$, abscess $(n=6)$, biliary cystadenoma $(n=4)$, dysplastic nodule $(n=2)$, simple cyst $(n=2)$, infarcted nodule $(n=1)$, inflammatory pseudotumor $(n=1)$, sclerosing hemangioma $(n=1)$, mature teratoma $(n=1)$ were followed.

\section{PP26-13}

Drug Induced Hepatic Injury Associated with Sitagliptin - Ultrastructural Findings

M. Toyoda ${ }^{1}$, H. Yokomori ${ }^{1}$, F. Kaneko ${ }^{1}$, Y. Shimizu ${ }^{1}$, H. Yoshida ${ }^{1}$, H. Takeuchi ${ }^{1}$, K. Tahara ${ }^{1}$, T. Motooir ${ }^{2}$, M. Ohbu ${ }^{3}$, M. Oda ${ }^{4}$, H. Toshifumi $^{5}$ ${ }^{1}$ Internal Medicine; ${ }^{2}$ Pathology, Kitasato University, Kitasato Institute Medical Center Hospital, Saitama; ${ }^{3}$ Pathology, School of Allied Health Sciences, Kitasato University, Kanagawa; ${ }^{4}$ Organized Center of Clinical Medicine, International University of Health and Welfare; ${ }^{5}$ Internal Medicine, School of Medicine, Keio University, Tokyo, Japan

In daily clinical practice, drug induced liver injury can always be a cause of liver injury in patients taking medications. We reported a rarely drug induced liver injury due to Sitagliptin. A 58-year-old man with type II diabetes mellitus for 10 years presented with a clinical picture of progressive jaundice 3 days before admission. A thorough and detailed history revealed that the patient had started on a new medication, sitagliptin, 1 month earlier for the treatment of diabetes mellitus. Laboratory investigations revealed severe liver dysfunction(total/direct bilirubin was $11.8 / 9.4 \mathrm{mg} / \mathrm{dl}$, Serum aspartate aminotransferase (AST) was 1,318 IU/L, alanine aminotransferase (ALT) was 1,653 IU/L, alkaline phosphatase (ALP) was $814 \mathrm{IU} / \mathrm{L}$, and $\gamma$-GTP was $369 \mathrm{IU} / \mathrm{L})$. Blood urea nitrogen was $8.3 \mathrm{mg} / \mathrm{dl}$, creatinin was $0.7 \mathrm{mg} / \mathrm{dl}$, BS246 (70-109 mg/dl), HbA1c11.3 
(4.3-5.8\%). Viral hepatitis serology was performed and was negative for hepatitis A virus (HAV), hepatitis B virus (HBV), but positive hepatitis $\mathrm{C}$ virus (HCV). HCV Taq man-PCR: $7.6(<1.2 \mathrm{log} \mathrm{IU} / \mathrm{ml})$. Ultrasonography revealed no extrahepatic biliary duct dilatation or stones in the gall bladder. Abdominal computed tomography was excluded pancreatic or hepatic focal lesions. Drug-induced lymphocyte stimulation (DLST) test was negative. Liver biopsy revealed edematous and pigmentation of hepatocytes around central vein. Acute hepatocellular injury can result in necrosis affecting single (spotty necrosis) and confluent necrosis around zone 3 or confluent necrosis in portal tracts and piecemeal necrosis and confluent droplet in portal tract. Evidence of hepatitis $\mathrm{C}$ infection was seen in the form of portal inflammation and periportal piece meal necrosis. We investigated the hepatocyte pigmentation by electron microscopy. Electron micrographs showed a pseudo-bile ductule, dilated bile canaliculi with loss of microvilli, and huge diverticula. He improved liver function upon discontinuation of Sitagliptin. Drugs are an important, often unrecognized, cause of acute liver injury. Among the rare causes responsible for this complication is Sitagliptin.

PP26-14

The First Case of Living Donor Liver Transplantation for Acute Liver Failure in Thailand

P. Intaraprasong ${ }^{1}$, A. Sobhonslidsuk ${ }^{1}$, S. Treepongkaruna ${ }^{2}$, S. Teeraratkul ${ }^{3}$, S. Leela-Udomlipi ${ }^{3}$, A. Vijitpavan ${ }^{4}$, A. Sarovath ${ }^{4}$, S. Sriphojanart ${ }^{3}$

${ }^{1}$ Division of Gastroenterology and Hepatology; ${ }^{2}$ Pediatrics;

${ }^{3}$ Surgery; ${ }^{4}$ Anesthesiology, Mahidol University, Bangkok, Thailand

Introduction: Living donor liver transplantation (LDLT) was begun in Thailand in 2001. All previous LDLTs were performed for chronic liver disease, which have surplus time for donor evaluation. Here, we present the first case of living donor liver transplantation for acute liver failure in a pediatric patient that had never been performed in Thailand.

Case description: A 26-year-old female potential donor from Bangkok was referred to our hospital for emergency LDLT evaluation for her daughter. Her daughter was 11 months old who suffered from acute liver failure. The cause of her liver failure was unknown. The blood group of the donor and recipient were B positive. The result of donor blood tests and imaging study were within the normal range. Her BMI was $20.28 \mathrm{~kg} / \mathrm{m}^{2}$. The donor and recipient were urgently evaluated by pediatric gastroenterologist and surgeon, transplant hepatologist and surgeon, psychiatrist, radiologist, anesthesiologist and transplant co-ordinator nurse. The process was completed within $48 \mathrm{~h}$. No contraindication was found. The donor underwent left lateral hepatectomy to donate left lobe liver graft to her daughter. She was discharged from the hospital 4 days later. Both donor and recipient completed 5 months follow up without any complication.

Conclusion: LDLT for acute liver failure is feasible in Thailand. The process should be done based on balanced a decision-making process and needs to be carried out by a well organized transplantation team in order to shorten the duration of evaluation for patients to undergoing LDLT.

\section{PP26-15}

Acute Intrahepatic Cholestasis Accompanied with Chlamydophila Pneumoniae Infection

M. Toyoda ${ }^{1}$, H. Yokomori ${ }^{1}$, F. Kaneko ${ }^{1}$, Y. Shimizu ${ }^{1}$, H. Yoshida ${ }^{1}$, H. Takeuchi ${ }^{1}$, K. Tahara ${ }^{1}$, T. Motoori ${ }^{2}$, M. Ohbu ${ }^{3}$, M. Oda ${ }^{4}$, T. Hibi $^{5}$ ${ }^{1}$ Internal Medicine; ${ }^{2}$ Pathology, Kitasato University, Kitasato Institute Medical Center Hospital, Saitama; ${ }^{3}$ Pathology, School of Allied Health Sciences, Kitasato University, Kanagawa; ${ }^{4}$ Organized Center of Clinical Medicine, International University of Health and Welfare; ${ }^{5}$ Internal Medicine, School of Medicine, Keio University, Tokyo, Japan
Chlamydophila(c) pneumoniae pneumoniae is a common cause of respiratory infections in humans, although asymptomatic infection or unrecognized, mildly symptomatic illnesses are the most common results of infection. We report a case of $C$. pneumoniae infection presenting with fever and rapidly intrahepatic cholestasis in a patient. A 63-year-old man had a week-long history of intermittent high fever and rapidly progressive jaundice with atypical erythema. Blood tests revealed a white cell count of $10.50 \times 10^{3}$ cells $/ \mathrm{mm}^{3}$ (Neu 87.0\%, Lym 3.5\%, Mono 9.0\%, and Eo 0.5\%), CRP; $14.11 \mathrm{mg} / \mathrm{dl}$, total/direct bilirubin was $4.9 / 2.7 \mathrm{mg} / \mathrm{dl}$, Serum aspartate aminotransferase(AST) was $44 \mathrm{IU} / \mathrm{L}$, alanine aminotransferase (ALT) was $44 \mathrm{IU} / \mathrm{L}$, alkaline phosphatase (ALP) was $896 \mathrm{IU} / \mathrm{L}$, and $\gamma$-GTP was $520 \mathrm{IU} / \mathrm{L}$. The results of all serological tests were negative; the $\operatorname{IgM}, \operatorname{IgG}$, and $\operatorname{IgA}$ titers for $C$. pneumoniae had increased, which is indicative of a $C$. pneumoniae infection. A computed tomography (CT) scan revealed segmental consolidation with partially intermingled areas of consolidation. Minocycline was administered, the patient's fever and liver dysfunction improved. The patient's fever and liver dysfunction improved upon administration of minocycline. Light microscopic findings showed the presence of enlarged liver cells with clear cytoplasm, a few mitotic figures, multinucleated cells, and bile cholestasis. The electron microscopic appearance of liver biopsy showed that bile canaliculi exhibited intrahepatic forms of cholestasis. From results of light and electron microscopy, we inferred atypical intrahepatic cholestasis, probably resulting from $C$. pneumoniae infection.

\section{PP26-16}

Add on Peg-interferon Alpha and Ribavirin for 24 Weeks during Continuous Lamivudine Treatment in Choronic Hepatitis B and C Co-infection

S.H. Kim, S.Y. Lee, Y.J. Kim, E.S. Lee, B.S. Lee, H.Y. Lee Internal Medicine, Chungnam National University, Medical School, Daejeon, Republic of Korea

Introduction: $\mathrm{Co}-$ infrections of chronic hepatitis $\mathrm{B}$ and $\mathrm{C}$ are rare. We knew the fact that one type of chronic viral hepatits is more predominant than the other type in patient with co-infection of chronic hepatitis $\mathrm{B}$ and $\mathrm{C}$. We usually need to treat only one predominant type of chronic viral hepatitis instead of both chronic viral hepatitis simultaneoulsy. We teated a rare case by add on peginterferon alpha and ribavirin for 24 weeks during continous Lamivudine treatment in a patients with chronic hepatitis $\mathrm{B}$ and $\mathrm{C}$ coinfection.

Patient and methods: A male patient with 39 years had co-infection with chronic hepatitis $\mathrm{B}$ and $\mathrm{C}$. $\mathrm{HBe} \mathrm{Ag}$ was negative. AntiHCV and HCV-RT-PCR were positive and HBV DNA level was $42,500 \mathrm{IU} / \mathrm{ml}$. Also he had thrombocytopenia, esophageal varix without bleeding histroy and cirrhotic liver in abodminal CT finding. At first, we treated him for HBV by Lamivudine without adding any treatment for HCV. After 3 months, transaminase level decreased but was not noraml level despite achieving undetectable HBV DNA level. After 12 month, transaminase level increased 5 times more than normal level. HCV-RNA was 393,000 IU/ml and genotype of HCV was 2a, but HBV DNA was still undetectable. We treated simultaneously him for HCV by combinant peginterferon alpha and ribavrin for 24 weeks during treating him for HBV by Lamivudine. He had SVR for HCV and also undetectable HBV DNA and his transaminase level was normal. But, he has taken Lamivudine continuously, and the outcomes of treatment about HBV and HCV have been maintained until now (for 12 months).

Conclusions: We need to treat the both chronic hepatitis $\mathrm{B}$ and $\mathrm{C}$ simultaneously in some patient with co-infection of chronic hepatitis 
B and C. And also we can successfully treat the chronic hepatitis B and $\mathrm{C}$ co-infection at the same time.

\section{PP26-17}

Hepatic Adenomas: A Single Tertiary Center Approach to Management C. Bunchorntavakul ${ }^{1}$, R. Bahirwani ${ }^{2}$, D. Drazek ${ }^{1}$, K. Olthoff ${ }^{1}$, A. Shaked ${ }^{1}$, R.K. Reddy ${ }^{1}$

${ }^{I}$ Department of Surgery; ${ }^{2}$ Department of Medicine, University of Pennsylvania, Philadelphia, PA, USA

Background: Hepatic adenoma is a benign liver tumor. Because of the risk of bleeding and malignant transformation, therapeutic intervention may need to be considered. However, the best therapeutic strategy remains controversial.

Methods: Records of 60 consecutive patients (between 2005 and 2010) with a diagnosis of HA from a single tertiary center were reviewed and analyzed.

Results: 58/60 patients were women, median age was 36 years, $75 \%$ had history of contraceptive use and $55 \%$ were obese (BMI $\geq$ $\left.30 \mathrm{~kg} / \mathrm{m}^{2}\right)$. Majority $(63 \%)$ were asymptomatic. Single $(28 \%)$ and multiple adenomas $(72 \%)$ were encountered; the size ranged from 1 to $19.7 \mathrm{~cm}$; median follow-up was 2.6 years. 34 patients(Group A) underwent therapeutic intervention (size $2.4-19.7 \mathrm{~cm}$, mean $7.5 \mathrm{~cm}) ; 17$ had surgical resection, 9 underwent transarterial embolization (TAE), and 8 had both interventions sequentially. In 26 patients (Group B; size of lesions 1-5.1 cm, mean $3.0 \mathrm{~cm}$; Group A vs. Group B comparison of size $p<0.01$ ) who had not intervention, $80 \%$ had stability or regression of lesions while $20 \%$ showed enlargement. A significant predictor of progression was obesity $(p=0.05)$. Seven patients who presented with bleeding were successfully managed by either urgent TAE/resection $(N=5)$, or initially conservative with subsequent resection $(N=2)$. There was no bleeding, malignant transformation, and death during our follow-up.

Conclusions: Majority of the patients had obesity (55\%) and had used contraceptive steroids $(75 \%)$. A significant predictor of progression was obesity. Therapeutic intervention was often pursued in those with larger lesions, and those who presented with bleeding. TAE is an option for high risk surgical candidates or multiple adenomas, and also can be an adjuvant intervention to surgery.

\section{PP26-18}

Primary Entecavir Resistance Induced by Lamivudine and Adefovir Sequential Therapy in 3 Chinese Chronic Hepatitis B Patients

S. Yang, X. Wang, W. Ju, Q. Wang, H. Xing, J. Cheng

Beijing Ditan Hospital, Capital Medical University, Beijing, China

Objectives: To investigate the multiple drug resistance (MDR) profiles in patients who took sequential lamivudine therapy after adefovir treatment failure.

Methods: 210 patients' clinical data and serum samples who took sequential lamivudine therapy as rescue therapy after adefovir treatment failure were collected from five hospitals in China during December 2007 to December 2009. PCR product population sequencing was used for screening MDR. PCR product cloning sequencing was used for further resistance profiles analyzing.

Results: Three MDR patients were identified with population sequencing. All three patients are genotype $\mathrm{C}$ patients. Population sequencing showed rtM204V + rtL180M + rtA181V mutation in all three patients. And 64 clones were get from three patients. Cloning sequencing results were showed in Chart1. All clones carried mutations resistant to lamivudine and adefovir. rtM204V + rtL180M + rtA181V is the major mutation type in three MDR patients $(67.2 \%)$.
Besides, all three patients carried clones with entecavir resistance mutation (rtM250V/H, rtS202I, rtI169V), though in minority (23.4\%).

Table 1 Cloning sequencing of 3 MDR chronic hepat

\begin{tabular}{lll}
\hline $\begin{array}{l}\text { Treatment } \\
\text { History }\end{array}$ & Mutation Mode & $\begin{array}{l}\text { Number } \\
\text { of } \\
\text { Clones }\end{array}$ \\
\hline $\begin{array}{cl}\text { ADV(36 m) } \rightarrow \\
\text { LAM(12 m) }\end{array}$ & M204V + A181V + L180M & 19 \\
& M204I + A181T & 2 \\
& M204V + A181T & 1 \\
M204V + A181V + L180M + M250V & 11 \\
LAM(15 m) & M204V + A181V + L180M & 8 \\
& M204V + A181V + L180M + S202I & 1 \\
& M204V + A181V & 1 \\
ADV(12 m $\rightarrow \rightarrow$ & M204V + A181V + L180M & 1 \\
LAM $(12 ~ m)$ & M204V + A181V + L180M + I169V & 1 \\
& M204V + A181V + L180M + M250V & 1 \\
& M204V + A181V + L180M + M250H & 1 \\
& M204V + A181I & 1 \\
\hline
\end{tabular}

Conclusions: Single lamivudine as rescue therapy for adefovir treatment failure may cause MDR. rtM204V + rtL180M + rtA181V is the major form of mutation in these patients. Entecavir resistance mutation maybe induced with sequential therapy.

\section{PP26-19}

Acute Obstructive Jaundice by Necrotic Tumor Fragment after Transarterial Chemoembolization

J. Suh, K. Kim, J. Park

Internal Medicine, Dongguk University Gyeongju Hospital, Gyeongju, Republic of Korea

Introduction: Transcatheter arterial chemoembolization (TACE) is a widely accepted treatment option for hepatocellular carcinoma (HCC). But, several complications after TACE, such as pulmonary embolism, abscess formation, tumor lysis syndrome, biloma and postembolization syndrome have been reported. Herein, we report a case of a acute obstructive jaundice by necrotic migrating tumor fragment after TACE and successfully treated with endoscopic removal.

Case: A 48-year-old male was admitted due to fever and jaundice for 3 days. He had a chronic hepatitis B related hepatocellular carcinoma. Prior admission 6 days ago, He was treated by TACE for HCC treatment. Physical examination revealed icteric sclera. Liver funtion test showed AST $82 \mathrm{IU} / \mathrm{L}$, ALT $103 \mathrm{IU} / \mathrm{L}$, total bilirubin $9.0 \mathrm{mg} / \mathrm{dL}$. Ultrasonography revealed gall bladder (GB) stone with GB wall thickening and left intrahepatic duct (IHD) dilatation. Abdominal CT showed $18 \mathrm{~mm}$ densely lipidol uptaked HCC and multiple tiny lipiodol uptaked HCCs in S4 with parenchymal high density change due to parenchymal lipiodol uptake and distal common bile duct (CBD) stone. ERCP showed several filling defect on CBD and left IHD dilatation. Some gelantinous material were extracted from CBD by snare. Biopsy finding for extracted material revealed necroinflammatory exudate and thick collagenous tissue lined by degenerated 
cells of uncertain nature. Clinical and laboratory findings improved after ERCP procedure.

\section{PP26-20}

A Case of Gallbladder Stone Fortunately Removed by ERCP

J.I. Suh, K. Kim, J.B. Park

Internal Medicine, Dongguk University Gyeongju Hospital, Gyeongju, Republic of Korea

Introduction: For treatment of gallbladder (GB) stone, there are several treatment modalities such as laparoscopic cholecystectomy, open cholecystectomy, and medical treatment. But, endoscopically retrograde gallbladder stone removal is nearly impossible because of difficult anatomical approach. We report a case of successfully treated by retrograde endoscopic removal for GB stone.

Case: A 70-year-old male was admitted due to right upper quadrant pain, fever for 1 day duration. Prior admission 1 year ago, $\mathrm{He}$ already treated with endoscopically retrograde common bile duct (CBD) stone removal. At admission, he appeared acute ill-looking and body temperature was $38^{\circ} \mathrm{C}$. Laboratory findings were as follows: white blood cell count, $8,370 / \mathrm{mm} 3$, total bilirubin, $1.76 \mathrm{mg} /$ $\mathrm{dL}$; gamma-glutamyl transferase, $174 \mathrm{IU} / \mathrm{L}$; alkaline phosphatase, $94 \mathrm{IU} / \mathrm{L} ;$ AST/ALT, 1,128/512 IU/L; and amylase/lipase, 51/131 IU/ L. Ultrasonography showed a stone shadowing in GB with wall thickening. Computed tomography showed high-density material in a dependent position of the GB and CBD. The patient was diagnosed with $\mathrm{GB}$ and $\mathrm{CBD}$ stone. An endoscopic retrograde cholangiography (ERCP) revealed movable filling defect at the distal CBD and GB. First of all, CBD stone was removed by stone basket. and then, basket easily could be introduced into GB and successfully removed stone. After then, clinical finding of the patient was improved dramatically.

\section{PP26-21}

Hemochromatosis Due to Excessive Parenteral Iron Loading J.H. Kim

Internal Medicine, Yeoeuido St. Mary's Hospital Catholic Univ. Medical College, Seoul, Republic of Korea

Hemochromatosis is an inherited or acquired disease of iron metabolism in which an inappropriate increase in iron absorption leads to deposition of excessive amounts of iron in parenchymal cells with eventual tissue damage and impaired organ function. But it is very rarely caused by excessive parenteral iron loading. We report a case a 44-year-old female patient who experienced hemochromatosis after infusion of parenteral iron to control iron deficiency anemia. She was admitted to the emergency room for right abdominal discomfort. On physical examination, five fingers breadth hepatomegaly and hyperpigmented skin were observed. On laboratory findings, the hemoglobin was $10.0 \mathrm{~g} / \mathrm{dL}$, the hematocrit was $31.2 \%$, the MCV was $75 \mathrm{fL}$, the $\mathrm{MCH}$ was $24 \mathrm{pg}$, the platelet count was $28,000 / \mathrm{mm}^{3}$, the iron was $187 \mu \mathrm{g} / \mathrm{dL}$, and the ferritin was $7,680 \mu \mathrm{g} / \mathrm{dL}$. She got total gastrectomy 10 years ago due to advanced gastric cancer, and got 5,400 mg parenteral iron complex (Venoferrum ${ }^{\circledR}$ ) weekly due to iron deficiency anemia since 5 years ago. An abdominal CT scan showed hepatosplenomagly with diffuse high density at pre-enhance phage. A liver biopsy revealed chronic hepatitis with cirrhosis and marked hemosiderin (Figure 1). Deferosirox (Exjade ${ }^{\circledR}$ ) was administrated immediately. In 10 days, she complained of dyspnea, and chest radiograph revealed both pleural effusions with cardiomegaly. Electrocardiograph showed atrial fibrillation, and echocardiograph showed severe left ventricular systolic dysfunction with dilated end systolic dimension. Her vital sign and general condition deteriorated gradually even with intensive care. She died of heart failure with ventricular tachycardia due to systemic hemochromatosis.

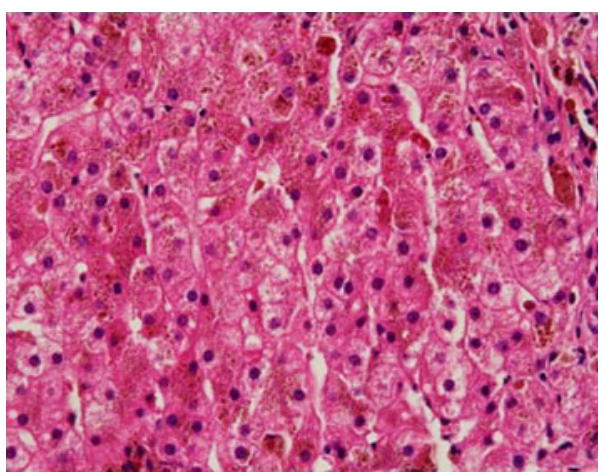

Fig. 1

\section{PP26-22}

Preoperative Predictive Factors of Intrabiliary Rupture of Hepatic Hydatid Cyst

R. Bedoui, H. Maghrebi, W. Dougaz, C. Dziri

Department B of General Surgery, Charles Nicolle Hospital, Tunis, Tunisia

Introduction: Hydatid cysts are common in Tunisia and liver is the most commonly infested organ. Intrabiliary rupture of a hepatic hydatid cyst is a common complication and early diagnosis and treatment are mandatory.

Aim: To identify preoperative predictive factors associated with asymptomatic intrabiliary rupture of a liver hydatid cyst.

Patients and methods: The medical records of 56 patients who underwent surgery for hepatic hydatidosis between January 1, 2008, and December 31, 2009, at the Department B of General Surgery of Charles Nicolle Hospital, Tunis, Tunisia, were reviewed retrospectively. Patients with cyst-biliary communication were divided into two groups according to an occult perforation or a frank intrabiliary rupture. Preoperative demographic, clinical, biological and radiological characteristics were recorded in all patients. All the collected data were analyzed using the SPSS (v.11.5) statistical program (SPSS, Chicago, IL, USA). A $p$ value less than 0.05 was considered significant. Receiver operating characteristic (ROC) curve were used to evaluate optimal cut-off values.

Results: A cysto-biliary communication was detected in $20(36 \%)$ patients. It was an occult communication in four cases (20\%) and a frank intrabiliary rupture in 16 cases $(80 \%)$. Univariate analysis identified preoperative predictive factors associated with the presence of a frank intrabiliary rupture: jaundice, and size of the cyst. Those of occult intrabiliary rupture were ultrasonography Gharbi type II or III cyst, recurrent cyst, and size of the cyst. As concern the size of cyst, ROC curve was used to determine the most appropriate cut-off point which was $6.5 \mathrm{~cm}$ with a sensitivity of $83 \%$, a specificity of $60 \%$, and a negative predictive value of $92 \%$.

Conclusion: Cysto-biliary communication should be suspected preoperatively and searched carefully intraoperatively in the presence of preoperative predictive factors identified by this study especially size of hydatid cyst greater than $6.5 \mathrm{~cm}$.

\section{PP26-23}

Resection of Hepatocellular Carcinoma Recurring in the Diaphragm after Right Hepatic Lobectomy

J. Ueda, H. Yoshida, N. Taniai, M. Yoshioka, S. Mineta, Y. Kawano,

E. Uchida

Surgery, Nippon Medical School, Tokyo, Japan

We describe a 35-year-old man with hepatocellular carcinoma (HCC) that recurred in the diaphragm after right hepatic lobectomy. The 
patient had undergone right hepatic lobectomy for $\mathrm{HCC}$ with chronic hepatitis B virus infection 1 year previously. On pathological examination, the tumor measured $15 \times 14.4 \times 11 \mathrm{~cm}$ and was moderately well differentiated. The surgical margins were negative. After 1 year, computed tomography of the abdomen revealed a mass extending from the right side of the diaphragm to the retroperitoneal space. The mass was enhanced in the early phase and washed out in the late phase. Extrahepatic recurrence of HCC in the diaphragm was diagnosed. We performed tumor resection with partial resection of the right side of the diaphragm and wedge resection of the right lower lobe of the lung. The diaphragm was reconstructed with a sheet of artificial pericardium. The histopathological diagnosis was recurrence of HCC in the diaphragm with invasion of the right lung. The postoperative course was uneventful, and the patient was discharged on postoperative day 8 . He underwent chemotherapy with cisplatin and 5-fluorouracil. After 9 months, the patient died of unresectable recurrence of HCC in the brain. No recurrence was detected in the right subphrenic area.

\section{PP26-24}

A Case of Spontaneous Rupture of a Simple Hepatic Cyst

M. Shibata, N. Taniai, J. Ueda, H. Yoshida, S. Mineta, M. Yoshioka, Y.

Kawano, E. Uchida

Surgery, Nippon Medical School, Tokyo, Japan

We describe the spontaneous rupture of a simple hepatic cyst associated with a benign course. A 64-year-old woman was admitted for right upper quadrant pain. The patient denied having a history of abdominal trauma. Abdominal computed tomography (CT) showed a huge solitary hepatic cyst (diameter, $10 \mathrm{~cm}$ ) in the right lobe. Part of the cyst surface was irregular, and the internal echo was heterogeneous. Fluid retention was detected under the capsule of the liver. Spontaneous rupture of a non-parasitic hepatic cyst was suspected. She was admitted to our hospital for further evaluation and treatment. Examination of the abdomen revealed right upper quadrant pain, but no signs or symptoms of peritonitis. One week after admission, CT revealed that the volume of fluid retained under the liver capsule had decreased. Percutaneous puncture was performed with the use of a needle and an 8-French pigtail catheter under ultrasonographic guidance. Serous, brown fluid was aspirated. After percutaneous aspiration, the patient's symptoms resolved. Minocycline hydrochloride was given daily by intravenous injection for 7 days. The catheter was then removed. There has been no evidence of recurrence after 1 year.

\section{PP26-25}

Successful Treatment of Complications after ABO Incompatible Liver Transplantation

M. Zhang, W. Zhang, M. Zhang, L. Zhou, S. Zheng

Division of Hepatobiliary and Pancreatic Surgery, Department of

Surgery, Key Laboratory of Combined Multi-Organ Transplantation,

Ministry of Public Health, First Affiliated Hospital, Zhejiang

University School of Medicine, Hangzhou, China

Introduction: In principle, $\mathrm{ABO}$ blood group matching is required for allogeneic liver transplantation. This patient was in the urgent need of a transplant and $\mathrm{ABO}$ incompatible liver transplantation was necessary. Currently, combined rutuximab pretreatment with splenoectomy, plasmapheresis, and immunoglobulin infusion is the protocol for humoral rejection prevention.

Case report: The case we present here is a late stage HCC patient, who had multiple lesions in the liver (Fig.1) with elevated serum $\alpha$ fetoprotein level (119.5 ng/l), and histology finding showed moderately-low differentiated HCC. He had bile duct infection and stenosis, also acute rejection happened (Fig. 2) after receiving ABO incompatible liver transplantation despite following the above protocol strictly. Repeated plasmapheresis and immunoglobulin infusion after transplantation significantly decreased the alanine aminotransferase (ALT), aspartate transaminase (AST), and total bilirubin (TB) level, (Fig. 3) which proved to be the effective therapy and successfully salvaged the patient eventually.

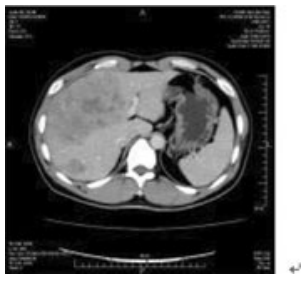

Fig. 1 CT scan showed multiple lesion in the liver pre-operation
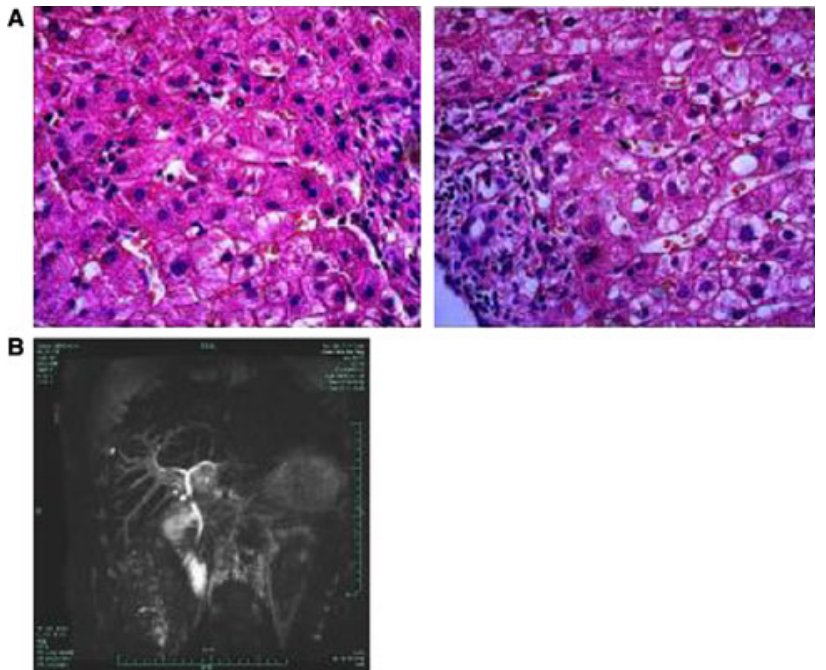

Fig. 2 (a) Histology image showed acute rejection in the liver after transplantation. (b) MR CP showed bile duct stenosis after liver transplantation 

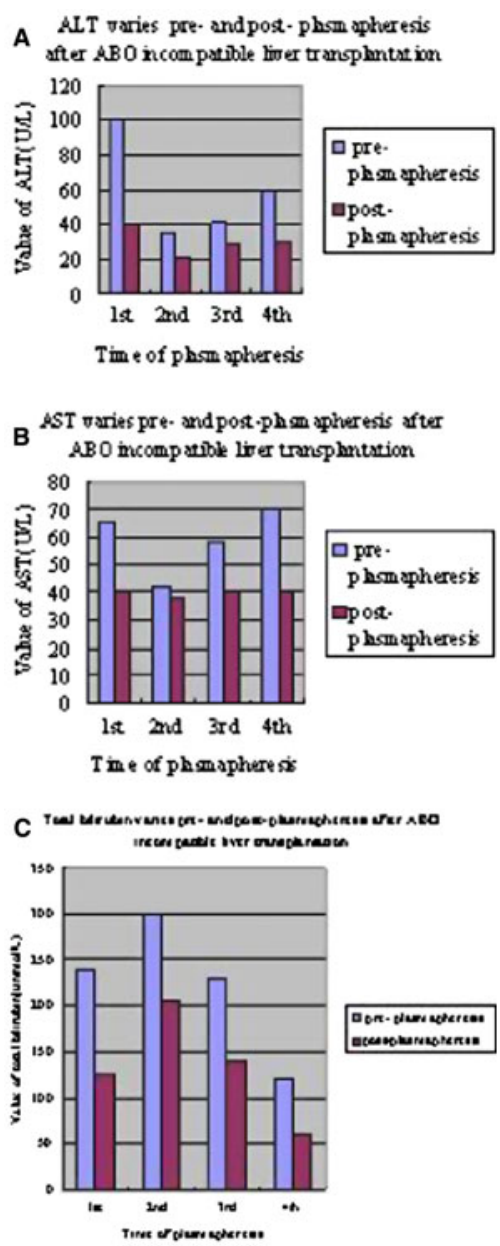

Fig. 3 ALT(a), AST(b), TB(C) varies pre- and post- plasmapheresis after $\mathrm{ABO}$ incompatible liver trtansplantation

Conclusion: This case encourages us to adopt plasmapheresis and immunoglobulin infusion as rescue therapy in the serious complications after $\mathrm{ABO}$ incompatible liver transplantation.

\section{PP26-26}

Case Report of Isolated Cryptococcal Meningitis in a Sri Lankan Nonalcoholic Cirrhosis

R.L. Satarasinghe, A.C.R. Navaratne, A.T. Jayalal, A.N.R. Fernandopulle Sri Jayewardenepura General Hospital, Colombo, Sri Lanka

Introduction: Cryptococcal infections are said to be very rare infections of cirrhotics affecting either peritoneum or brain. Cryptcoccal meningitis is not reported in non-alcoholic cirrhotics from the Indian subcontinent to the best of our knowledge.

Case description: A 53-year-old Sri Lankan male who was diagnosed having nonalcoholic cirrhosis, diabetes mellitus, hypothyroidism, asymptomatic cholelithiasis and a strong history of familial cirrhosis presented to the principal author's unit at Sri Jayewardenepura General Hospital, Kotte, Sri Lanka on 11th August 2010 with one week history of headache, vomiting and fever. His level of consciousness gradually deteriorated from GCS level 15/15 to 5/15. Examination revealed stigmata of chronic liver disease, mild neck stiffness, bilateral equal pupillary reaction and oedema of right optic disc with hemorrhages. Rest of the examination was unremarkable. The investigations revealed, persistently elevated ESR $(>100 \mathrm{~mm} / 1 \mathrm{st} \mathrm{h}), \mathrm{C}-\mathrm{RP}=48$ $\mathrm{mg} / \mathrm{dl}$, a neutrophil leucocytosis of $88 \%, \mathrm{WBC}=19,000 / \mathrm{mm}^{3}$, a reactive blood picture with evidence of chronic liver disease. liver function test- SGOT $99 \mathrm{iu} / 1$, SGPT-526 iu/l, ALP-526 iu/l, total bilirubin $41 \mathrm{mg} / \mathrm{dl}$ (direct $2.6 \mathrm{mg} / \mathrm{dl}$ ). serum creatinine- $165 \mathrm{mmol} / \mathrm{L}$, blood urea- $74 \mathrm{mg} / \mathrm{dl}$.normal electrolytes, negative urine and blood cultures. Cerebro spinal fluid examination -cloudy appearance, positive Pandy's test, RBC- $10 / \mathrm{mm}^{3}$, WBC- $145 / \mathrm{mm}^{3}$, neutrophils $60 \%$, lymphocytes $40 \%$, glucose- $48 \mathrm{mg} / \mathrm{dl}$, protein- $70 \mathrm{mg} / \mathrm{dl}$, TB-PCR negative, culture-Cryptococcal organism was isolated with positive Cryptococcal antigen titre of $1 / 64$, negative HIV, hepatitis and autoimmune screenings, normal TSH, normal serum iron studies, CT brain-left lacunae infarction. EEG-consistent with encephalopathy or focal encephalitis. USS -liver parenchymal disease, 2D Echocardiogram-mild to moderate LVH.The final diagnosis of cryptococcal meningitis, on a background of nonalcoholic cirrhosis was made. The patient died in spite of appropriate therapy.

Conclusion: Primary cryptococcal meningitis should be considered in nonalcoholic cirrhosis as well in the differential diagnosis of refractory hepatic encephalopathy like syndromes. This seems to be the first reported case in the Indian subcontinent.

\section{PP26-27}

\section{A Rare Type of Budd-Chiari Syndrome}

C. Lin, W. Cheng-Lin, Y. Zhi-Dong, G. Xue-Jun, Z. Hai-Yan, R. Xiao-Xuan Perking University Shenzhen Hospital, Shenzhen, China

We report a rare case of Budd-Chiari syndrome. A 66-year-old female was admitted to the hospital for intestinal obstruction. The patient has no portal vein hypertension, hepatomegaly, lower extremity venous stasis or other clinical symptoms and signs. The size of left lobe of liver was mildly increased on plain CT scan. Enhanced CT scan showed delay enhancement of the lateral segment of left lobe, anterior segment of right lobe and the dilatation of left portal vein. On CT Angiography, there was a narrow of the inferior vena cava, about $0.6 \mathrm{~cm}$ in length. The openings of middle and right hepatic vein were upstream the narrow, but the opening left hepatic vein was downstream the narrow of the inferior vena cava. There was no abnormal collateral circulation around the hepatic hilar region and in the abdominal cavity. According to clinical and CT appearance of this case, the different types of Budd-Chiari syndrome, this case belong to a rare type of Budd-Chiari syndrome - narrow without obstruction.

\section{PP26-28}

Maging Diagnosis of Hepatic Ectopic Pregnancy: A Report of One Case C. Lin, W. Cheng-Lin, Y. Zhi-Dong, Z. Hai-Yan, R. Xiao-Xuan Perking University Shenzhen Hospital, Shenzhen, China

This article is about a case of hepatic ectopic pregnancy. A patient suffered from acute abdomen with 14-day vaginal bleeding. Serum, HCG $8,988 \mathrm{~min} / \mathrm{ml}$ revealed a bit of pelvic effusion. CT plain scan displayed a polygonal, moderate density shadow of left liver lobe. Enhanced CT had no sign of intensification. MRI plain scan was undergone. On $\mathrm{T}_{1} \mathrm{WI}$, the lesion appeared to be low signal; on $\mathrm{T}_{2} \mathrm{WI}$, the lesion appeared to be high signal. With enhanced MRI, the lesion showed irregular mild plague-like intensification during the venous phase. It was excised by operation and chorionic tissue was seen under a microscope. The result of pathological diagnosis was hepatic ectopic pregnancy.

\section{PP26-29}

Radiofrequency Thermal Ablation of Liver Carcinoma. Prospective Study of 82 Lesions

E. Lermite ${ }^{1}$, J. Lebigot ${ }^{2}$, F. Oberti ${ }^{3}$, P. Pessaux ${ }^{4}$, C. Aubé $^{2}$, J.-P. Arnaud ${ }^{1}$

${ }^{1}$ Digestive Surgery; ${ }^{2}$ Radiology; ${ }^{3}$ Hepatology, CHU Angers, Angers; ${ }^{4}$ Digestive Surgery, CHU Hautepierre, Strasbourg, France

Aim: The aim of this prospective study was to evaluate the feasibility of radiofrequency ablation of hepatic tumors. We studied the rates of 
mortality, morbidity, and recurrence and recorded overall and disease-free survival.

Methods: All patients aged over 18 years with histologically proven malignant liver disease inaccessible to surgical treatment (one to four tumor localizations) were eligible for' inclusion in the study. ChildPugh score $>$ B9, platelets count $\leq 50.10^{3} / \mathrm{mm}^{3}$, prothrombin index $\leq 50 \%$ and pregnancy were exclusion factors.

Results: Between July 2001 and June 2004, 51 patients (13 women, 38 men, mean age $66 \pm 9$ years) were included and underwent 61 radiofrequency thermal ablation procedures for 82 hepatic lesions: hepatocarcinoma $(n=44)$, cholangiocarcinoma $(n=1)$, metastatic tumors $(n=37)$. Hospital morbidity and mortality were 11.5 and $2 \%$ respectively. Recurrence rate at 3 years was $20.6 \%$. In multivariate analysis only tumor size $>3 \mathrm{~cm}$ was correlated significantly with recurrence $(p=0.03)$. Actuarial 1-, 2- and 3-year survival rates in the hepatocarcinomas group $(n=31)$ were $84.5,57.7$ and $36.4 \%$ respectively. Actuarial 1-, 2-, 3-year survival rates in the metastasis from colorectal carcinoma group $(n=14)$ were 90,54 , and $54 \%$ respectively ( $p=0.72)$.

Conclusion: Radiofrequency thermal ablation is an effective treatment for hepatic tumors measuring less than $3 \mathrm{~cm}$. There is low risk of complications and open surgery can be associated.

\section{PP26-30}

Prognostic Factors of Survival in Repeat Liver Resection for Recurrent Colorectal Metastases

E. Lermite ${ }^{1}$, D. Brachet ${ }^{1}$, G. Lorimier ${ }^{2}$, S. Mucci ${ }^{1}$, A. Hamy ${ }^{1}$, A. Roch ${ }^{1}$, J.-P. Arnaud ${ }^{1}$

${ }^{1}$ Digestive Surgery, CHU Angers; ${ }^{2}$ Surgery, Centre Paul Papin, Angers, France

Aim: Liver metastases develop in 50\% of patients with colorectal carcinoma. Recurrent liver disease is usual. Repeat liver resection remains the only curative treatment. The aim of the study was to review our data on repeat hepatectomy and to analyse potential prognostic factors of survival.

Methods: Patients who underwent repeat liver resection for metastases of colorectal carcinoma between January 1992 and August 2007 were identified from a prospective database and their medical records were analyzed.

Results: Of 62 patients who underwent a second hepatectomy, 15 underwent a third hepatectomy, and two underwent a fourth hepatectomy. There was no perioperative mortality. Morbidity was less than 20 percent for the first and second hepatectomies. Overall 5-year survival rate after first hepatectomy was 40 percent. Univariate analysis identified three risk factors confirmed by log-rank test and multivariate Cox regression analysis: serum carcinoembryonic antigen concentrations $>5 \mathrm{ng} / \mathrm{ml}$ at first hepatectomy $(\mathrm{HR}=2.265$; $\mathrm{CI}=1.140-4.497 ; p=0.020)$, anatomic resection $(\mathrm{HR}=2.124$; $\mathrm{CI}=1.069-4.218 ; p=0.031)$, and tumors $\geq 3 \mathrm{~cm}$ at the second resection $(\mathrm{HR}=2.039 ; \mathrm{CI}=1.013-4.103 ; p=0.046)$.

Conclusion: Our study shows that repeat hepatectomy for liver metastases of colorectal carcinoma may be performed with low mortality and morbidity. Preoperative concentration of carcinoembryonic antigen at first hepatectomy, tumor size and type of anatomic resection are independent prognostic factors

\section{PP26-31}

Multiple Liver Abscesses Caused by Streptococcus constellatus

T. Shinha ${ }^{1}$, K. Perumareddi ${ }^{2}$, P. Nautiyal ${ }^{2}$

${ }^{1}$ Internal Medicine, ${ }^{2}$ Long Island College Hospital, New York, NY, USA

Introduction: Streptococcus milleri's is an important cause of pyogenic liver abscesses. Although there are many reports confirming liver abscesses secondary to $S$. milleri's species, only few cases have been reported indicating $S$. constellatus as the cause of such infections.
Case description: A 54-year-old African American woman with a history of asthma presented with 3 weeks history of left lower quadrant (LLQ) pain associated with subjective fever, nausea and vomiting. Her past medical history was unremarkable except for diverticulosis diagnosed in 2008 by routine colonoscopy. There was no history of illicit drug use or alcohol abuse. Vital signs were significant for a temperature of $101.4^{\circ} \mathrm{F}$. On physical examination, her heart sounds were regular and lungs were clear to auscultation. The abdomen was soft, but there was tenderness over LLQ without rebound tenderness or guarding. Labs revealed leukocytosis and elevated liver enzymes. Serologic investigation for hepatitis B, C, $\mathrm{HIV}$ and Entamoeba histolytica antigen were all negative. Abdominal CT revealed multiple hypodense lesions in the liver. The patient was started on intravenous antibiotics and underwent percutaneous drainage. Culture of the purulent and foul-smelling material yielded $S$. constellatus.

Discussion: S. anginosus, S. intermedius and S. constellatus form the $S$. milleri's group. These organisms colonize in the intestinal tract and are not considered to be pathogenic except when any invasive manipulation is performed. In our case, possible diverticulitis with subsequent intestinal mucosal damage acted as an entry for the dissemination of the organism. Interestingly, this group of microorganisms is characterized by the tendency to form abscesses in different organs. Also, S. milleri's abscess is clinically distinct from other forms of pyogenic liver abscesses due to its torpid nature and longer duration of symptoms. Therefore, it is important to recognize $S$. constellatus as a causative agent of liver abscesses.

\section{PP26-32}

A 8 Years Old Boy with Hepatocellular Carcinoma with Skin Metastasis C.R. Debnath

Hepatology, Mymensingh Medical College Hospital, Mymensingh, Bangladesh

Introduction: Hepatocellular carcinoma (HCC) in childhood is rare and accounts for less than $0.5 \%$ of all pediatric malignancies. It is the second most common malignant liver neoplasm after hepatoblastoma (HB) in children. Its relative frequency is 0.5 to 1.0 cases per million children. It is more common in older children and teenagers than in infants. The most common clinical sign is hepatomegaly. Sometimes HCC is associated with abdominal pain. HCC often develops in the presence of underlying liver disease, such as in cirrhosis (esp HBV and $\mathrm{HCV}$ related), and inborn metabolic errors. Compared with HB, the prognosis in $\mathrm{HCC}$ is poor.

Hepatitis B viral infection is the most common cause in endemic areas. Distal metastasis occurs in $30 \%$ cases and it indicate bad prognosis.

Case description: A 8-year-old boy with HBV related hepatocellular carcinoma with skin metastasis is being presented. The boy presented with yellow colouration of urine and sclera for one month, abdominal pain for 15 days. On clinical examination patient was icteric and on abdominal examination there was tender hepatomegaly. Ultrasonography of upper abdomen shows SOL in liver. FNAC from liver and skin shows well differentiated hepatocellular carcinoma. Chemotherapy was given and the patient expired after 1 month

\section{PP26-33}

A Report of Two Thai Patients with Ferroportin Disease

S. Chirnaksorn ${ }^{1}$, P. Sornmayura ${ }^{2}$, T. Kitiyakara ${ }^{1}$, T. Sura ${ }^{1}$

${ }^{1}$ Medicine; ${ }^{2}$ Pathology, Ramathibodi Hospital, Mahidol University, Bangkok, Thailand

Case report: Ferroportin disease or type IV hereditary hemochromatosis is an autosomal-dominant, non-HFE-related, clinically and genetically heterogeneous iron overload syndrome. We report two cases of ferroportin disease in Thailand. The first case is a 74-year-old male who presented with chronic hepatitis for one year. On 
examination the patient had skin hyper pigmentation and complicated diabetes mellitus. Investigation revealed hyperferritinemia of $5,436 \mathrm{ng} / \mathrm{ml}$ with $108 \%$ transferrin iron saturation. The second case is 54-year-old male with a similar presentation of chronic hepatitis for a decade without definite diagnosis despite investigations. Hyperferritinemia of $4,272 \mathrm{ng} / \mathrm{ml}$ with $32 \%$ transferrin iron saturation was discovered in this patient. Iron stain yields deposition of iron granules in the liver parenchyma. Both patients had no history of acquired iron overload or any hemoglobinopathy. A heterozygous mutation of TGC $>$ TAC (Cys326Tyr) in exon 7 of SLC40Al (Ferroportin) gene was found without any other mutations of hemochromatosis genes in both patients. Initial treatment with phlebotomy followed by oral iron chelator, normalized liver function tests and improved the level of serum ferritin in both patients.

Conclusion: Ferroportin disease may be more prevalent than HFErelated hereditary hemochromatosis in Thailand. We report two cases, one with non-selective ferroportin disease and another with selective or classic ferroportin disease.

\section{PP26-34}

Prophylaxis of Hepatitis B Virus in HBsAg Negative Recipient from Donor with High HBV DNA after Allogenic Stem Cell Transplantation

S.B. Cho, H.K. Jeong, Y.J. Kim, W.S. Lee, Y.E. Joo, S.K. Choi

Internal Medicine, Chonnam National University Medical School, Gwang Ju, Republic of Korea

Bone marrow transplantation (BMT) from HBsAg positive donor is occasionally unavoidable. BMT with $\mathrm{HBsAg}$-positive hematopoietic stem cells can lead to de novo hepatitis B virus (HBV) infection and severe hepatitis if no effective prophylactic measures are taken prior to and after transplantation. However, prophylactic methods are little information about the use of hepatitis B immunoglobulin (HBIg) and antiviral drug in recipients from HBsAg positive donor of BMT. We report a case of successful HBV prevention who received IV HBIg and lamivudine (LAM) in recipient from donor with high HBV DNA titer in BMT for myelodysplastic syndrome (MDS). A 60-year-old man with MDS and negative of all HBV serologic marker was planned to receive allogenic peripheral blood stem cell transplantation (PBSCT) from his sole HLA-identical silbing, his sister, who had been a known chronic hepatitis B. she was normal level of aminotransferase, anti-HBe positive and had HBV DNA titer of 13,000 IU/ L. LAM was started both donor and recipient before 3 weeks of BMT. LAM prophylaxis was continued to 6 months after BMT. Hepatitis B vaccine was given to him as regular schedule $(0,1,6$ month). From the day before to 8th day after BMT, HBIg 1,000 IU was injected intramuscularly daily. The anti- HBs titer of patient was maintained to 500-600 IU/mL during HBIg treatment. He remains in well clinical condition at 1 year after BMT and his blood test showed that he was HBsAg negative, antiHBs positive (at $85 \mathrm{IU} / \mathrm{L}$ ) and undectable HBV DNA.

\section{PP26-35}

Clinical Diagnosis of 5 Patients with Hepatic Amyloid Degeneration

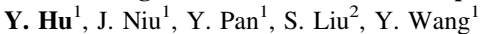

${ }^{1}$ The First Hospital of Jilin Universityp; ${ }^{2}$ The Second Hospital of Jilin University, Changchun, China

Backgrounds and aims: Hepatic amyloidosis is a rare group of metabolic disorders which often lead to the misdiagnois for the complex and unspecific clinical manifestation. We aim to analyze the clinical features of patients diagnosed as hepatic amyloid degeneration to improve the current understanding of the disease and decrease misdiagnosis.

Methods: A total of five patients were diagnosed with hepatic amyloid degeneration based on the pathology results gained from the aspiration samples of liver or kidney and specific dyeing .From the five patients, two were female and three were male for age 43-56.
Liver function tests, blood fat, routine urine tests, abdominal ultrasound and CT or MRI of the patients were performed.

Results: All patients had hepatomegaly, significant elevation of AKP (183-729 $\mu$ ) and r-GT $(216-572 \mu)$, hypoproteinemia and hyperlipidemia,while the serum levels of ALT and AST are often normal or slightly elevated. Four patients had serious hypoalbuminemia and ascites respectively. Three patients suffered from weight loss. Hepatic ultrasound and CT showed an enlarged liver and with low density, and the hepatic echo was fine and closely similar with the features of fatty liver. Liver blood vessel of three patients was mildly thin in CT image. Kidney ultrasound showed enhanced echo, with unclear stucture and reduced blood flow. Pathologic results of liver or kidney aspirate showed sedimentation of pink amyloid matters. Congo red dye test was positive and pathological consequence was hepatic and renal amyloidosis.

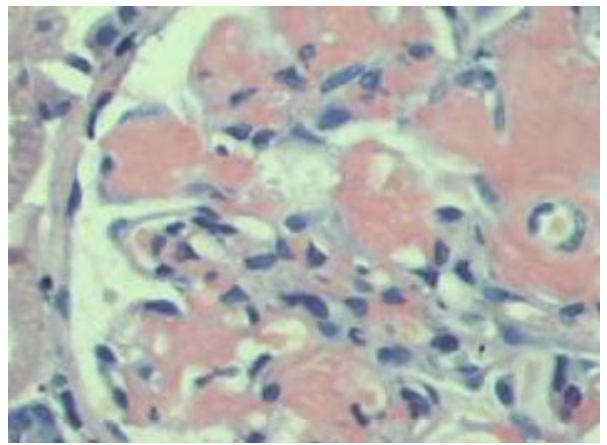

Conclusion: Liver or kidney aspirate examination and specific dyeing (Congo red dye) is necessary in patients suspected with hepatic amyloid degeneration.

\section{PP26-36}

Chronic Hepatitis B Virus Infection in an Immune-competent Patient Previously Vaccinated Sucessfully against Hepatitis B Virus

J.A. O'Halloran ${ }^{1}$, C.F. De Gascun², M.J. Carr², R. Howard ${ }^{1}$, L. Dunford ${ }^{2}$, W.W. Hall ${ }^{2}$, J.S. Lambert ${ }^{1,3}$

${ }^{I}$ Department of Infectious Diseases, Mater Misericordiae University Hospital; ${ }^{2}$ National Virus Reference Laboratory; ${ }^{3}$ School of Medicine and Medical Science, University College Dublin, Dublin, Ireland

Introduction: Immune-competent patients with documented antiHBs titres $>10 \mathrm{mI} \mathrm{U} / \mathrm{mL}$ on completion of a course of hepatitis $B$ vaccination are considered to be protected against natural infection with hepatitis B virus (HBV). We report a case of chronic HBV infection in a patient 2 years post documentation of protective antiHBs levels.

Case description: A 38-year-old man who has sex with men (MSM) attended the sexually transmitted infections clinic in December 2009. The patient had negative HBV serology in April 2006 and a course of recombinant hepatitis $B$ vaccine was then completed $(0,1$ and 6 months) using Engerix- ${ }^{\circledR}$. Anti-HBs titres were not preformed one month post course completion. Seven months later his anti-HBs titres were rechecked and found to be $<10 \mathrm{mI} \mathrm{U} / \mathrm{mL}$. A booster dose was administered and titres measured 2 months after this were $13 \mathrm{mI} \mathrm{U} / \mathrm{mL}$ (Abbott Architect). A further booster dose was administered and antiHBs titres in November 2007 were $161 \mathrm{mI} \mathrm{U} / \mathrm{mL}$ and $62 \mathrm{mI} \mathrm{U} / \mathrm{mL}$ on the Biomerieux VIDAS and Abbott Architect anti-HBs assays respectively.

On return in Dec 2009, the patient complained of non-specific symptoms including fatigue and myalgia. HBV serology was preformed and revealed positive HBsAg, anti-Hep B core IgM, anti-Hep $\mathrm{B}$ core (total) and $\mathrm{HBeAg}$ consistent with acute $\mathrm{HBV}$ infection, confirmed on repeat sample. HBV DNA $>70,000,000 \mathrm{IU} / \mathrm{mL}$ (Roche 
AmpliPrep) was detected. Phylogenetic analysis of the entire 3,215bp genome determined the virus was $>99 \%$ (3,211/3,215-bp) similar to a HBV subgenotype F1 detected in Buenos Aires, Argentina.The patient had visited Argentina on two occasions and reported having unprotected sexual intercourse with multiple partners. Repeat serology in August 2010 demonstrated progression to chronic HBV infection. Analysis of the $S$ gene did not reveal the presence of any previously described vaccine escape mutations. This report describes a case of chronic HBV infection in the setting of vaccine failure.

\section{PP26-37}

Congenital Hepatic Fibrosis: Is the Early Decades of Life Associated with Pure Liver Disease?

S. Karim ${ }^{1}$, A. Faryal ${ }^{2}$, A. Fahim ${ }^{3}$, W. Jafri ${ }^{1}$

${ }^{1}$ Gastroenterology, Depart of Medicine, Aga Khan University

Hospital; ${ }^{2}$ Medicine, Jinnah Medical College Hospital; ${ }^{3}$ Medicine, Aga Khan University Hospital, Karachi, Pakistan

Introduction: Congenital hepatic fibrosis (CHF) is an autosomal recessive inherited malformation defined pathologically by a variable degree of periportal fibrosis and irregularly shaped proliferating bile ducts. Clinically it is characterized by hepatic fibrosis, portal hypertension, and renal cystic disease. CHF is known to occur in association with a range of both inherited and non-inherited disorders, with multi-organ involvement, as a result of ductal plate malformation.

Case description: We report here the clinicopathological features of three cases 15 -year-old boy and 16 and 18 year old two girls with all having liver biopsies revealing enlarged portal tract, distorted bile duct with fibrosis(Fig: Biopsy attached). Clinical presentation of a boy and a girl was non-specific intermittent abdominal pain, while abdominal distention and jaundice in second girl. 15-year-old boy and 16-year-old girl had grade 1 varices with three columns and radiology showing mild hepato-splenomegaly, splenic varices and biliary prominence (Fig: CT scan attached) i.e. with compensated cirrhosis child's '(MELD score 17 and 10). Eighteen old female had large varices, radiology showed shrunken and nodular liver, splenomegally and ascites (Fig: CT scan attached) i.e. with decompensated cirrhosis child's C (MELD score 25). Literature showed congenital hepatic fibrosis is commonly associated with multi-organ involvement especially in third to fifth decades. But this case series showed a rather pure picture of congenital hepatic fibrosis which is very rare and is known to be first reported cases in Pakistan of this age group. Are we genetically different or is it the age associated with pure liver disease. It needs to be answered.

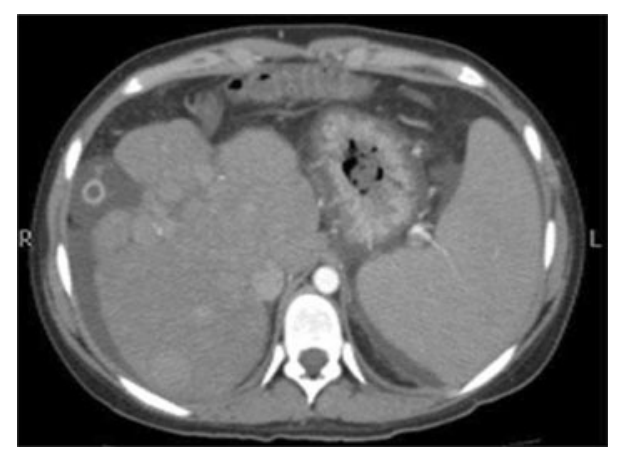

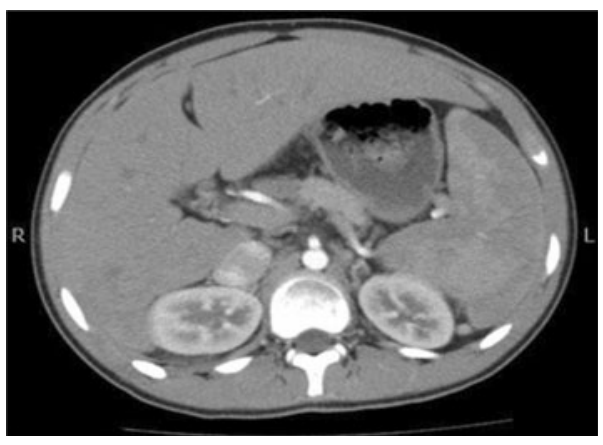

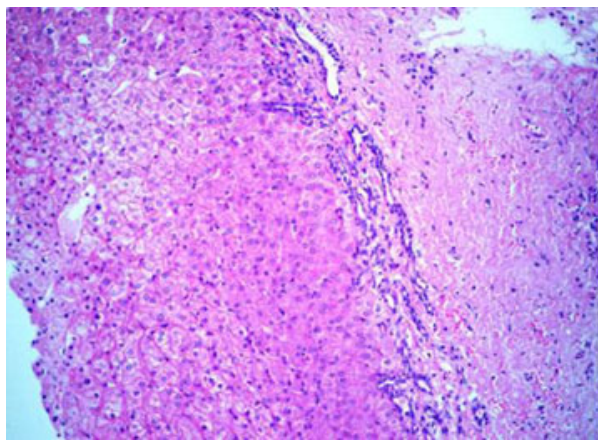

PP26-38

Extrahepatic Portal Venous Obstruction due to a Giant Hepatic Hemangioma Associated with Kasabach-Merritt Syndrome

H. Koh, H. Yoshida, N. Taniai, S. Mineta, M. Yoshioka, Y. Kawano, J. Ueda Surgery, Nippon Medical School, Tokyo, Japan

We describe a patient with extrahepatic portal venous obstruction due to a giant hepatic hemangioma associated with Kasabach-Merritt syndrome. A 67-year-old woman presented with upper abdominal distension and appetite loss. The medical history was not relevant to the current disorder. Initial laboratory tests revealed the following: serum platelet count, $9.9 \times 10^{4} / \mu \mathrm{L}$; serum fibrinogen degradation products, $12 \mu \mathrm{g} / \mathrm{mL}$; prothrombin time, 1.26 ; and serum fibrinogen, $111 \mathrm{mg} / \mathrm{dL}$. Computed tomography demonstrated homogenous lowdensity areas, $15 \mathrm{~cm}$ in diameter, in the left lobe of the liver. Common hepatic arteriography revealed a hypervascular tumor with pooling of contrast medium in the delayed phase. The portal venous phase of supra-mesenteric arteriography revealed obstruction and cavernous transformation of the portal vein. We diagnosed extrahepatic portal venous obstruction due to a giant hepatic hemangioma associated with Kasabach-Merritt syndrome. Laparotomy was performed, and the liver was found to be severely enlarged. After mobilization of the left lobe, a left hepatectomy was performed with intermittent clamping. After resection, Doppler ultrasonography revealed recovery of the portal venous flow. The cavernous transformation shrank. Pathologic examination of the surgical specimen confirmed the presence of a giant benign hepatic cavernous hemangioma. The patient was discharged 16 days after operation. Laboratory data and complications improved after 2 months. 


\section{PP26-39}

Hemobilia and Hemocholecyst Following Radiofrequency Ablation Therapy for Hepatocellular Carcinoma

S.Y. Park, Y.O. Kweon, W.Y. Tak, K.Y. Shin, H.G. Park, D.W. Lee, H.S. Lee, J. Heo

Gastroenterology and Hepatology, Kyungpook National University

Hospital, Daegu, Republic of Korea

Introduction: Radiofrequency ablation (RFA) is an simple, effective, and safe treatment for hepatocellular carcinoma (HCC). However RFA may cause early and late complications from 8 to $9.5 \%$. As we experienced hemobilia accompanied with hemocholecyst after RFA for treatment of $\mathrm{HCC}$, we report clinical course and management of the present case.

Case report: A 69-year-old man visited hospital due to right upper abdominal pain 2 weeks after RFA for $14 \mathrm{~mm}$ sized HCC in segment 8. Ultrasound guided RFA was performed under local anesthesia using cool-tip electrode in overlapping ablation method. Patient had Murphy's sign with fever and laboratory finding showed increased serum ALT (242 IU/L from $24 \mathrm{IU} / \mathrm{L})$, bilirubin $(3.6 \mathrm{mg} / \mathrm{dL}$ from $0.4 \mathrm{mg} / \mathrm{dL})$, and decreased hemoglobin level $(12.1 \mathrm{~g} / \mathrm{dL}$ from $15.1 \mathrm{~g} /$ $\mathrm{dL}$ ). Abdomenl computed tomogram (CT) showed new onset gallbladder filled with high density material. Abdominal ultrasonography reveals heterogenous echogenic mass in the gall bladder. Endoscopic retrograde cholangio-pancreatography (ERCP) showed an irregular shaped filling defect in gallbladder. The patient was diagnosed as hemocholecyst associated with hemobilia. Abdominal pain gradually decreased with antibiotic therapy after endoscopic sphincterectomy to decompress the bile duct. The follow up CT scan 3 days after ERCP showed a decreased mass-like lesion within gallbladder. Due to persistent pain, laparoscopic cholecystectomy was performed. There were blood clots in gallbladder wall. The pathologic examination showed hemorrhage and diffuse infiltration of inflammatory cells in GB wall. After cholecystectomy, patient's symptom and laboratory finding improved. The patient was discharged 5 days after cholecystectomy. In conclusion, although hemobilia with hemocholecyst is a very rare complication of RFA, we should suspect possibility of this complication. Early recognition and management is crucial not to miss the chance for the opportune treatment. Prompt surgical management should be considered when medical therapy fails to improve clinical course.

\section{PP26-40}

Ruptured Liver Tumors - Outcomes of a Multidisciplinary Approach

V. Velayutham, J. Sathyanesan, S. Ramaswamy, J. Damodaran, S. Eswaran, R. Palaniappan, S. Rajagopal

Dept of Surgical Gastroenterology and Liver Transplantation, Government Stanley Medical College Hospital, Chennai, India

Background: Spontaneous rupture of hepatic tumors (which includes both benign and malignant liver tumors) is a life-threatening presentation, with an incidence of 3-14\%, which carries significant morbidly and mortality. The aim of this study was to report a single center's experience of patients with ruptured liver tumors during a 10year period.

Materials and methods: A retrospective review was performed of all patients who presented with ruptured HCC between 1999 and 2008. Data on clinical features, treatment strategies, and survival outcomes were collected.

Results: A cohort of 24 patients (18 male and 6 female) was identified. 21 patients had malignant tumors ( $20 \mathrm{HCC}, 1$ angiosarcoma), 3 patients had benign lesions ( 2 adenomas and 1 Focal nodular hyperplasia). Initial bleeding control was attempted by transarterial embolization (TAE) in $8(33 \%)$ and it was successful in 7 patients. surgery was the primary modality of management in 16 patients. The procedures performed were Rt hepatectomy in three, Lt hepatectomy in three patients, Lt lateral segmentectomy in two cases, non anatomical resection in two patients and segmentectomy in one. Hepatic artery ligation was done in two and perihepatic packing in two patients. Morbidity observed were bile leak in two, intra abdominal sepsis in two,Pulmonary complications in two and posthepatectomy liver failure in one. Mean hospital stay was 14 days (10-28 days.), 8/ $24(33.3 \%)$ died. The causes of death were Coagulopathy in four patients, sepsis in three and post hepatectomy liver failure in one. The follow up ranged from 6 months to 10 years.

Conclusion: Ruptured liver lesions is a life threatening event and requires a multidisciplinary approach. Primary hemostasis, followed by emergency or staged hepatic resection, is the treatment of choice. Patients who had no underlying liver disease had better prognosis than those who had cirrhosis.

\section{PP26-41}

A Chronic Hepatitis B Patient with 3 Primary Malignancies: Hepatocellular Carcinoma, Non Hodgkin Lymphoma and Breast Cancer S. Kiziltas ${ }^{1}$, L. Doganay ${ }^{1}$, Y. Colak ${ }^{1}$, M. Gulluoglu ${ }^{2}$, M.B. Bilgiç ${ }^{2}$, O. Ozturk ${ }^{1}$, I. Tuncer ${ }^{1}$

${ }^{1}$ Goztepe Teaching and Research Hospital; ${ }^{2}$ Istanbul University School of Medicine, Istanbul, Turkey

Introduction: The association between hepatocellular carcinoma (HCC) and hepatitis B is well known. Non Hodgkin Lymphoma (NHL) is more frequent in patients infected with HBV. Here, we present a patient with HCC who developed NHL and breast carcinoma later.

Case: Fifty-three years old female was referred to our clinic with the diagnosis of $\mathrm{HBeAg}$ negative hepatitis B in 2004. She was put on lamivudine. Follow up sonography in the first year revealed a mass with $6 \mathrm{~cm}$ diameter in the right posterior segment of the liver. AFP was $9 \mathrm{ng} / \mathrm{ml}$. The blood chemistry tests were in the limits. Dynamic MRI revealed arterial hypervascularity of the mass and delayed venous wash out of the contrast. The needle biopsy was concordant with $\mathrm{HCC}$ and right posterior sectionectomy was performed. Surgical margins were free of the tumor and pathology also revealed advanced fibrosis. Before surgery, virologic breakthrough and lamivudine resistance were noted. Adefovir was added on. Her follow up was insignificant till postoperative third year when thoraco-abdominal CT scan revealed a mass of $2 \mathrm{~cm}$ diameter in right breast. During the work up for surgery, a right submandibular mass with $2 \mathrm{~cm}$ diameter detected. The surgical excision revealed follicular lymphoma of the submandibular gland. The needle biopsy from the breast revealed adenocarcinoma and mastectomy was performed. Finally, she has Stage Ia NHL and Stage IIa breast cancer four years after HCC diagnosis. She is on adjuvant chemo-radiotherapy at present and antiviral treatment is switched to tenofovir plus lamivudine because of detected adefovir resistance.

Conclusion: Chronic hepatitis B patients should undergo HCC surveillance. Regarding the higher incidence of NHL, any suspicious lymph node should prompt a detailed investigation. During the follow up of a patient with operated HCC, the possibility of de novo malignancies in other potential sites, like breast in our patient, should not be underestimated. 


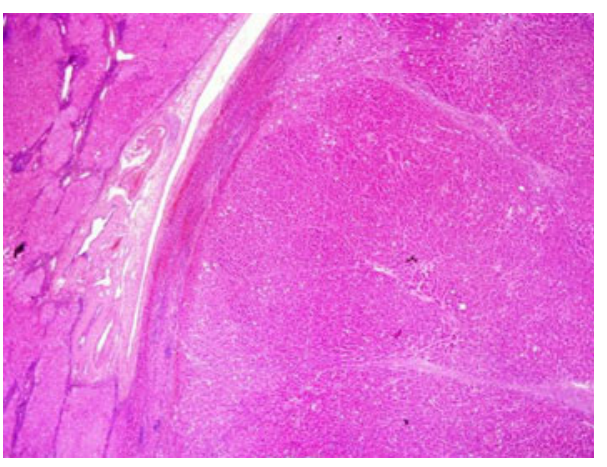

Fig. 1

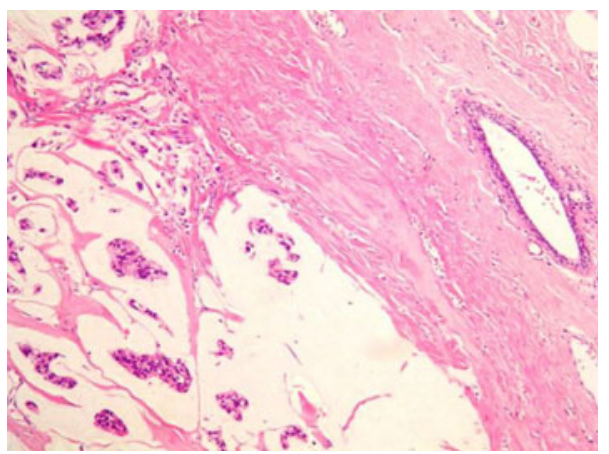

Fig. 2

PP26-42

Molecular Target Therapy after Palliative Resection Was Effected for Huge HCC with Multiple Lung Metastasis: A Case Report H. Kijima

Second Department of Surgery, Dokkyo University School of Medicine, Tochigi, Japan

Sorafenib (Soraf) is worldwide using molecular targeted drug for unresected HCC. Here, we reported that use of Soraf after palliative resection of the huge $\mathrm{HCC}$ was very effective for multiple lung metastases.

Case: The patient is 66-year-old female. The chief complaint was the right shoulder pain. CT and US revealed a huge tumor, $10 \mathrm{~cm}$ in diameter, located in the segment 4 and 8 with invasion to the diaphragm and also she had multiple metastatic lesions in the bilateral lungs. We diagnosed the patient as having huge $\mathrm{HCC}$ with multiple lung metastases.

Operation: In order to control her shoulder pain, we performed resection of ventral part of segment 8 and cranial part of segment 4 with partial resection of the diaphragm. Post operative course was uneventful. On the 17th PO, we started Soraf of $800 \mathrm{mg} /$ day. fiftyfour days after treatment, CT revealed that numbers of lung metastases were extremely decreasing before treatment(rt; $180 \rightarrow 64$, 1 t; $148 \rightarrow 65)$.

Conclusion: Soraf was effective for controlling the lung metastases after palliative resection of huge HCC.

PP26-43

A Case of Autoimmune Cholangitis with Sjogren's Syndrome

Y.N. Kim, H.C. Lee, J.D. Kim, B.H. Cha, K.H. Song, J.R. Lee, W.J. Jeon, S.J. Lee

Cheju Halla General Hospital, Jeju, Republic of Korea

Autoimmune cholangitis (AIC) is characterized by chronic progressive cholestasis, high titer antinuclear antibody (ANA), but negative
Antinuclear antibody (ANA). It has clinical courses and histologic findings similar to primary biliary cirrhosis (PBC), but differs from the disease by showing high titer ANA positivity instead of AMA. Histopathologic features of AIC is nonsupprative cholangitis in bile duct with lymphoplasmacytic infiltration. Sjogren's syndrome is systemic chronic inflammatory disorder characterized by lymphocytic infiltrates in exocrine organs. AIC and Sjogren's syndrome share similar histopatholoigic findings and ANA postivity, these two diseases may have similar pathogenesis. We report a case of Autoimmune cholangitis associated with Sjogren's syndrome in 48year-old woman who visited Halla hospital. She was successfully treated with ursodeoxycholic acid and steroid.

\section{PP26-45}

A Case of Adenoendocrine Cell Carcinoma of the Gallbladder

K. Suzumura, T. Okada, Y. Iimuro, Y. Asano, Y. Yoshida, N. Kuroda,

T. Hirano, K. Oh, S. Saito, N. Uyama, M. Satake, Y. Kondo, N. Nakai, Y. Uda, J. Fujimoto

Hyogo College of Medicine, Hyogo, Japan

A 75-year-old man was seen at another hospital because of right upper abdominal pain and fever. The patient was admitted to our hospital with a diagnosis of cholecystitis and cholangitis. Abdominal ultrasonography showed a thick wall of the gallbladder with irregular elevated lesion. Computed tomography showed stones in the common bile duct. PTCD was performed, and laparoscopic cholecystectomy and choledochotomy were performed after resolution of the inflammation. There was a tumor in the fundus of the gallbladder. The pathological diagnosis was adenoendocrine cell carcinoma. The diagnosis of gallbladder cancer was made and the S4a,5 bisegmentectomy of the liver with regional lymph node resection (D2) was performed for the purpose of curative operation. The patient had multiple metastasis to the liver and lymph node 22 months after the operation and died 24 months after the operation. Since adenoendocrine cell carcinoma of the gallbladder is rare, we report on this case with some bibliographical comments.

\section{PP26-46}

No-limits Tace in Intrahepatic Cholangiocarcinoma Conditioning Jaundice without Biliary Tree Dilatation. A Case Report

T. Cascella, N. Cionfoli, M. Torresi, P. Quaretti

Interventional Radiology, Fondazione IRCCS Policlinico San Matteo, Pavia, Italy

Cholangiocarcinoma is the second most common primary liver malignancy. The only curative therapy for cholangiocarcinoma is surgical resection; however, only approximately $30 \%$ of patients with cholangiocarcinoma are suitable for surgical resection because of the advanced nature of this disease at the time of diagnosis. We present a case of a 70-year-old woman with jaundice, referred to our department by the surgeon. MRI scans showed a huge enhanced lesion at right lobe with the involvement of IV segment and V-VIII biliary and portal branches, suggestive for intrahepatic cholangiocarcinoma (after confermed with biopsy). She was cirrhotic and her's bilirubin levels was about $9 \mathrm{mg} / \mathrm{dl}$, but there wasn't dilatation of biliary tree. She didn't present other comorbidities. Within 6 weeks we performed two subsequent session of precision DEB-TACE with two vial DCBE$\mathrm{ADS}^{\mathrm{TM}} 100-300$ micron loaded with $100 \mathrm{mg}$ epirubicin the first time and one vial DCBEADS ${ }^{\text {TM }} 100-300$ micron loaded with $50 \mathrm{mg}$ epirubicin the second time respectively, each followed by injection of $25 \%$ Glubran in the interested segmental arterial branches, because a hemobilious fistula was suspected. During treatments we saw regression of jaundice until normal level of bilirubin and CT scan at follow up showed complete necrosis of the lesion, with a thin ring enhancement. The surgeon, in front of these findings, decided to perform an extended right hepatectomy, but she died one month later for biliary complications. 
Conclusions: DEB TACE is safe and effective even in cholangiocarcinoma, especially when a clear contrast enhancement is present on imaging. If bilirubin levels are increased, consider the possibility of a connection between biliary tree and the blood stream.

\section{PP26-47}

Phenotypes and Genotypes of Alagille Syndrome in Thai Children: Report of 4 Cases

C. Lertudomphonwanit ${ }^{1}$, S. Treepongkaruna ${ }^{1}$, D. Charoenpipop ${ }^{2}$,

C. Tocharoenthanaphol ${ }^{3}$, D. Wattanasirichaigoon ${ }^{4}$

${ }^{1}$ Division of Gastroenterology, Department of Pediatrics; ${ }^{2}$ Research

Center, Faculty of Medicine, Ramathibodi Hospital, Mahidol

University; ${ }^{3}$ Chulabhorn Research Institute; ${ }^{4}$ Division of Medical

Genetics, Department of Pediatrics, Faculty of Medicine,

Ramathibodi Hospital, Mahidol University, Bangkok, Thailand

Introduction: Alagille syndrome (AGS) is a highly variable, multisystem, autosomal dominant disorder that mainly affects liver, heart, eyes, face and skeletal system. Mutations in JAG1 and NOTCH2 gene have been identified as an etiology of this disorder. Herein we describe phenotypes and genotypes of AGS in Thai children.

Case report: Four patients, two males and two females, presented with cholestatic jaundice during infancy. Age of diagnosis ranged from 6 months to 2.8 years. Liver biopsies showed intrahepatic bile duct paucity in 3 out of 4 patients. Other associated findings identified in these patients included peripheral pulmonary stenosis (2/4), butterfly vertebrae (4/4), characteristic facie of frontal bossing, deep-set eyes and pointed chin (3/4) and posterior embryotoxon (3/4). All patients had elevated triglyceride and cholesterol levels with xanthomas. Fluorescence in situ hybridization (FISH) of 20p12 was performed as initial genetic screening, followed by genomic DNA and mRNA analysis of the JAGI gene if the FISH study yielded negative result. One patient was found to have microdeletion detected by FISH analysis. Two patients were heterozygote for novel point mutations of JAG1 gene which were IVS4-1G $>$ A and c. $247 \mathrm{C}>\mathrm{T}$ (p.Q83X). The last patient's result is pending. Two patients died at age of 3.5 and 4.8 years due to sepsis and post liver transplant hepatic vein obstruction complicated by sepsis, respectively. One patient remains well following liver transplantation at 3.8 years of age, while the other is stable without liver transplantation.

\section{PP26-48}

Intrahepatic Sarcomatoid Cholangiocarcinoma

H.J. Jang ${ }^{1}$, J.H. Ahn ${ }^{2}$, J.H. Kwak ${ }^{1}$, G.J. Cheon ${ }^{3}$

${ }^{1}$ Surgery; ${ }^{2}$ Radiology; ${ }^{3}$ Medicine, Gangneung Asan Hospital,

University of Ulsan, Gangneung, Republic of Korea

Sarcomatoid features are occasionally seen in various types of epithelial tumors, and it is sometimes difficult to differentiate between sarcomatoid carcinoma and true sarcoma. Most sarcomatoid carcinomas in the liver are thought to be sarcomatoid hepatocellular carcinomas. Recently, there have been reports demonstrating sarcomatous changes in cholangiocarcinomas. This type of tumor is defined as "sarcomatous intrahepatic cholangiocarcinoma" in the WHO classification of tumors. We report herein a patient with intrahepatic sarcomatous cholangiocarcinoma who underwent a hepatic resection. The main part of the tumor consisted of pleomorphic spindle and giant cells. A 63-year-old male patient presented with a liver mass. Computed tomography revealed a well-demarcated, low-attenuated mass in the left lobe of the liver. On laparotomy, 3 and 4 segmentectomies were performed. The tumor was $3 \times 4 \mathrm{~cm}$ in size. Microscopically, the tumor cells were contained in the sarcomatous component, and adenocarcinoma component on histological mapping.
Immunohistochemical staining for cytokeratin was positive in the sarcomatous components. The diagnosis confirmed a sarcomatoid cholangiocarcinoma. 4 months after surgery, the patient died of pneumonia. To the best of our knowledge, only 17 cases of intrahepatic sarcomatoid cholangiocarcinoma have been reported in the English-language literature. It has been reported that the prognosis for intrahepatic sarcomatoid cholangiocarcinoma is worse than that for intrahepatic cholangiocarcinoma. We report herein a patient with intrahepatic sarcomatoid cholangiocarcinoma who underwent a hepatic resection. The main part of the tumor consisted of pleomorphic spindle and giant cells.

\section{PP26-49}

Surgical Resection of Bilateral Adrenal Metastasis from Hepatocellular Carcinoma

H.J. Jang ${ }^{1}$, J.H. Ahn ${ }^{2}$, J.H. Kwak ${ }^{1}$, G.J. Cheon ${ }^{3}$

${ }^{1}$ Surgery; ${ }^{2}$ Radiology; ${ }^{3}$ Medicine; Gangneung Asan Hospital, University of Ulsan, Gangneung, Republic of Korea

Hepatocellular carcinoma (HCC) frequently metastasizes to the adrenal glands. The surgical strategy for HCC associated with bilateral adrenal gland metastasis is complicated because of the possibility of adrenal insufficiency. We report a patient with HCC who had bilateral adrenal gland metastasis that was treated successfully. A 71year-old man with $\mathrm{HCC}(8 \mathrm{~cm}$ in diameter) in the right lobe of the liver was admitted to our hospital. Right hemihepatectomy was performed. One year after surgery, the patient with bilateral adrenal gland metastasis (right, $5 \mathrm{~cm}$ in diameter; left, $4 \mathrm{~cm}$ in diameter) was admitted to our hospital. Bilateral adrenalectomy was performed simultaneously. Hydrocortisone supplement was given, and the postoperative course was uneventful. No recurrence was observed during the 6-month follow-up period. According to our experience, we suggest that surgery should be considered as one of the treatment strategies for patients with HCC and bilateral adrenal metastasis, to provide safer surgery and longer survival

\section{PP26-50}

Hepatic Recurrence of Intrahepatic Cholangiocarcinoma Eight Years after Initial Hepatectomy

S. Yamashita, S.-I. Ariizumi, M. Yamamoto, Y. Kotera

Surgery, Gastroenterology, Tokyo Women's Medical University,

Tokyo, Japan

We present a case of hepatic recurrence of intrahepatic cholangiocarcinoma in a 76-year-old man. He had a history of distal gastrectomy in 1998. In 2002, a hypervascular tumor, measuring $2 \mathrm{~cm}$ in diameter, was found in the left lobe of the liver on computed tomography (CT). The tumor marker CEA was slightly increased. He was given a diagnosis of hepatocellular carcinoma (HCC), and left hepatectomy was performed. The resected specimen showed a massforming type tumor $25 \mathrm{~mm}$ in diameter. The pathological findings showed moderately papillary differentiated adenocarcinoma. After surgery, immunotherapy with dendritic cell vaccine was performed for 8 years. In 2010, a new liver tumor $2 \mathrm{~cm}$ in diameter was found in segment 5. CT revealed a hypervascular tumor in the arterial phase. The enhanced CT imaging patterns resembled those of the first tumor. The tumor marker CEA was within normal limits. He was given a diagnosis of intrahepatic recurrence of intrahepatic cholangiocarcinoma. Resection of segment 7 was performed. The resected specimen showed a mass forming type tumor, $25 \mathrm{~mm}$ in diameter. The pathological findings showed well differentiated papillary adenocarcinoma. This is a rare case of intrahepatic recurrence of intrahepatic cholangiocarcinoma 8 years after the initial hepatectomy. 


\section{PP26-51}

Caroli Disease and Hereditary Pancreatitis: A Case Report

D. Murphy, R. Leen, M. Iqbal, J. Hegarty

Department of Hepatology, ST Vincent's University Hospital, Dublin, Ireland

Introduction: Caroli's disease is a rare hereditary condition characterised by segmental saccular intrahepatic duct dilatation. Hereditary (Familial) Pancreatitis is a rare autosomal dominant disorder characterised by chronically recurring bouts of Pancreatitis often beginning in early childhood. We are reporting a case of young girl with two rare disorders.

Case description: A 16-year-old girl presented to hospital with a 2-week history of central abdominal pain and jaundice. She was a known case of hereditary chronic pancreatitis which was diagnosed 8 years prior and she is a confirmed carrier of the R122H mutation. On this occasion her abdominal pain was accompanied with jaundice, which had never happened previously. She described having dark urine, pale stools and a mild itch. Physical examination revealed $1 \mathrm{~cm}$ palpable hepatomegaly and a mildly tender epigastrium. Initial biochemistry showed an elevated bilirubin in a obstructive pattern with good synthetic function. (ALB $29 \mathrm{~g} / \mathrm{L}$, Bili $134 \mu \mathrm{mol} / \mathrm{l}$, ALP $618 \mathrm{IU} / \mathrm{L}$, GGT $588 \mathrm{IU} / \mathrm{L}$, ALT $212 \mathrm{IU} / \mathrm{L}$, INR 1.23). Her MRCP revealed gross diffuse saccular dilatation of the intrahepatic bile ducts marked centrally and in the left lobe of the liver. There was no evidence of extra hepatic bile duct obstruction. These findings were diagnostic of Caroli disease. She was treated with ursodeoxycholic acid and analgesia and her pain and jaundice improved over the course of her inpatient stay. On discharge her liver function tests had improved. (ALB 30, Bili 71, ALP 574,GGT 101, ALT 146). She was reviewed 2 months after discharge and she had no further episodes of jaundice.

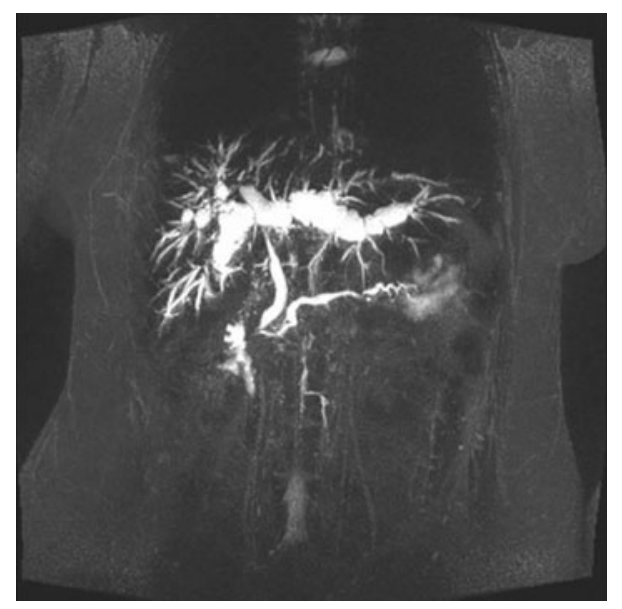

MRCP

\section{PP26-52}

Eosinophilic Liver Abscess Presenting as Acute Abdomen

H.G. Park, D.W. Lee, H.S. Lee, S.Y. Park, W.Y. Tak, Y.O. Kweon Kyungpook National University Hospital, Daegu, Republic of Korea

We report a case of a patient with eosinophilic liver abscess who presented as acute abdomen due to hemoperitoneum and subcapsular hematoma of the liver. A 67-year-old man with an unremarkable medical history was admitted with a complaint of sudden onset of right upper quadrant abdominal pain and mild abdominal distension. Computed tomography (CT) of the abdomen showed about $3 \mathrm{~cm}$ sized ill defined low density lesion in the right lobe of liver with subcapsular liver hematoma and ascites. Laboratory findings included leukocytosis and eosinophilia. Abdominal paracentesis revealed hemoperitoneum and liver biopsy of irregular low-attenuated lesion proved it to be eosinophilic abscess. He was treated with supportive care alone. Follow up CT scan after 2 months demonstrated that previously identified hepatic lesions were marked improved.

\section{PP26-53}

Primary Malignant Melanoma of the Liver: The Description of Treatment of a Case Report

A. Gettueva, S.V. Lepkov, I.N. Subortceva, A.M. Kovrigina, G.I. Storogakov, T.T. Kondratieva, I.S. Stilidi, D.V. Podlujniy, E.S. Chuchev, Y.I. Patutko, S.D. Kosura

The Moscow Medical University of N.N. Pirogov, Moscow, Russia

Primary isolated melanoma of liver is an exceedingly rare disease. Only 13 cases have been reported in the literature. We describe a case of isolated malignant melanoma a liver at the Russian patient of 32 years. Abdominal computer tomography revealed a $20 \times 17 \mathrm{~cm}$ tumor into the right hepatic lobe. Histopathological examination of biopsy revealed malignant melanoma. Immunostaining researches on tumour cells have been revealed positive markers: HMB-45, S-100 a protein, melanoma-pan and vimentin. The diagnosis was confirmed by electronic microscopy. Full inspection of a skin, brain, lung gastrointenstinal has not revealed the primary locus of a lesion. The patient had a chronic hepatitis $\mathrm{C}$ with a high level RNA - $5.7 \times 10^{7}$ and high level of transaminaz ALT- 570 IU/l, AST - 450 IU/l, GTP-850 IU/l. CEA, CA-19-9, AFP were normal level. The first was antiviral treatment-alpha interferon on $6 \mathrm{ME}$ daily + ribaverin $1,000 \mathrm{mg}$. After a month of therapy level of ferments has decreased to 80 and 65. Level RNA has decreased to $2.3 \times 10^{4}$. The size tumour was without change. To the patient three times has been executed chemioembolisetion. The size of a tumour has decreased to $5 \times 6 \mathrm{~cm}$. Complete methabolic response by FDG-PET was achived. Patient was treated systemic chemotherapy DAC (dacarbasin, cisplatin, tamoxifen) and antiviral therapy. Remission was conserved 14 months. After there has come progressing of the basic disease with a diffusive lesion of all liver and there has come death of the patient. Rarity desease described by us cannot allow to speak about optimal therapy malignant melanoma of liver. All cases described earlier at the first stage of treatment used a surgical method of treatment but all described patients has not live more then 8 months after operation. Probably chemioembolisetion, immunotherapy and chemotherapy will allow to receive more good results.

\section{PP26-54}

Sorafenib for Hepatocellular Carcinoma - Does it Lead to a Long Term Survival Benefit?

S. Khanna ${ }^{1}$, N. Saraf ${ }^{2}$, A. Gupta ${ }^{3}$, R. Puri ${ }^{2}$, M. $\operatorname{Kumar}^{4}$, R. Sud $^{2}$

${ }^{1}$ Gastroenterology and Hepatology, Dr BL Kapur Memorial Hospital;

${ }^{2}$ Gastroenterology and Hepatology, Medanta, The Medicity;

${ }^{3}$ Interventional Radiology; ${ }^{4}$ Gastroenterology and Hepatology, Sir Ganga Ram Hospital, New Delhi, India

Introduction: Sorfenib currently is recommended for advanced Hepatocellular Carcinoma (HCC) but without any significant long term survival benefit. We present a case who is currently on sofafenib for $1 \frac{1}{2}$ years. 
Case description: A 54 years male presented with complaints of pain right hypochondrium for past $1 \frac{1}{2}$ months associated with decreased appetite and weight loss. He had received blood transfusion 12 years back following an orthopaedic surgery. Examination revealed a nodular hepatomegaly of $17 \mathrm{~cm}$ but no jaundice, splenomegaly or ascites. He was HBsAg and HBeAg positive with HBV DNA load of 3,500,000 IU/ml and alphafetoprotein levels of $1,100 \mathrm{ng} / \mathrm{ml}$. Aminotransferases were mildly elevated. Ultrasonography and triple phase CT scan of the liver revealed a $10 \times 7 \mathrm{~cm}$ solitary mass lesion in segments 5,6 and 8 of the liver consistent with HCC, a normal portal venous system and a metastatic nodule in the left lung. The patient was a CHILD-A cirrhotic with an excellent performance status. He underwent transarterial chemoembolisation (TACE) along with oral entacavir and sorafenib therapy. Repeat TACE was done $1 \frac{1}{2}$ months later due to partial radiological response to the first procedure and sorafenib was continued. Endoscopy was done 1 month later for dysphagia and revealed an extrinsic compression in the lower esophagus. PET CT scan showed presence of FDP avid paraesophageal nodes. Endoultrasound guided FNA from these nodes revealed metastatic HCC. Patient was subjected to 3rd TACE four months later for recurrence of primary tumor, the patient still having a CHILD-A status and no increase of extrahepatic disease. The patient is currently doing well and is on sorafenib for the past $1 \frac{1}{2}$ years. Our case highlights the survival benefit of sorafenib even in the presence of disseminated HCC, provided the patient has a good performance status and the primary is adequately dealt with as the secondaries respond well to sorafenib.

\section{PP26-55}

Recovery of Fulminant Hepatic Failure after Gastric Bypass Surgery H.E. Elsiesy, A. Alshammari

Medicine, King Fahad Specialist Hospital-Dammam, Dammam, Saudi Arabia

Introduction: Fulminant hepatic failure (FHF) is a rare complication after gastric bypass surgery. The few cases reported either died or required liver transplantation. We report a 23 years old morbidly obese Saudi female (BMI 54) had gastric bypass surgery in April 2008 at a community hospital in an underserved area. The postoperative course was complicated by anastomotic leak and sepsis, requiring re-operation and antibiotics. Two month later, she developed jaundice (bilirubin $261.5 \mathrm{mmol} / \mathrm{L}$ and high INR of 1.6). She was receiving vitamin supplements but no other medication. Viral seology for hepatitis A, B, C, autoimmune hepatitis, Wilson and A1AT were all negative, here BMI dropped to 41, Abdominal sonogram and MRCP showed marked hepatomegally with no evidence of biliary dilation. A month later she was admitted to ICU at our facility with FHF, she received antibiotics for sepsis and high calorie enteral feeds. Liver biopsy showed diffuse severe liver fatty change (Fig. 1) The patient recovered after 6 weeks, but had repeated admission to the hospital for sepsis or ascites management, Bilirubin peaked to 993 but normalized completely after 8 month, the main therapy was high caloric diet to reduce rapid weight loss. Repeat imaging a year later showed marked decrease in liver size and repeat liver biopsy showed improvement of the previous fatty liver changes (Fig 2). Her current bilirubin is $8.9 \mathrm{mmol} / \mathrm{L}$, BMI is 28 . To our knowledge, this is the first case reported in the literature of FHF after gastric bypass recovering without liver transplant.

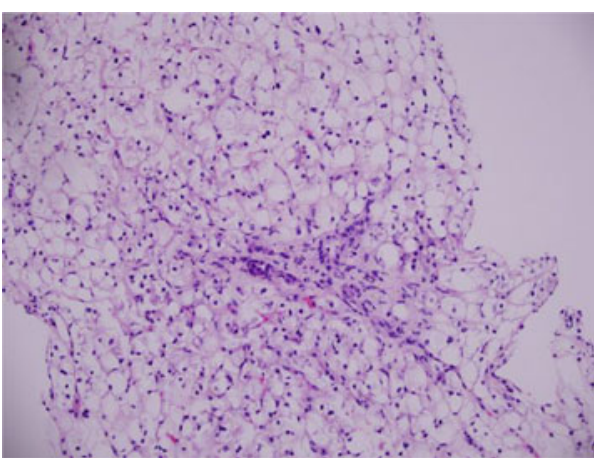

Fig. 1

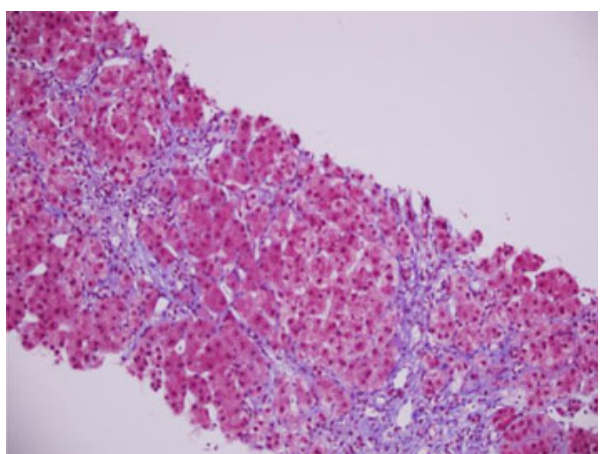

Fig. 2

\section{PP26-56}

A Case of Mirizzi Syndrome

L. Alrubaiy ${ }^{1}$, J. Sutton ${ }^{2}$

${ }^{1}$ Swansea University, Swansea; ${ }^{2}$ Gastroenterology, Ysbyty Gwynedd, Bangor, UK

Mirizzi's syndrome is a rare complication of prolonged cholelithiasis with presence of large, impacted gallstone into the Hartman's pouch, causing chronic extrinsic compression of common bile duct (CBD). We report here a 65 -year-old patient referred by her General Practitioner with the complaint of right upper quadrant pain which she has been experiencing for 3 months. She said that she had been feeling nauseous but without vomiting. She also had itching all over her body which automatically ceased. Her past medical history was not significant apart from total hip replacement. On examination, patient was jaundices with no signs of chronic liver disease. Abdomen was soft but tender in the right upper quadrant. No organomegally was present. Routine blood tests showed a white cell count of 6.7, Hb $12.4 \mathrm{~g} / \mathrm{l}$, PT 15, Bilirubin 61, Alkaline phosphatase 2618, ALT 250, AST 204. She had an ultrasound abdomen which showed a contracted calculus gall bladder and dilated intra-hepatic biliary tree. Common bile duct (CBD) measured $10 \mathrm{~mm}$ and appeared to contain an $8 \mathrm{~mm}$ calculus in the distal portion. Endoscopic retrograde cholangio-pancreatography (ERCP) showed a smooth narrowing of the mid CBD caused by what appeared to be a calcified mass. A stent was 
placed in the CBD to relieve obstruction. She had a CT scan which showed a large laminated stone in the gall bladder which compressing the CBD which is consistant with the diagnosis of Mirizzi Syndrome. The patient was referred to the surgeons to have a cholycystectomy. On a follow up visit 5 months later her liver function returned to normal.

Lessons:

1. Mirizzi's syndrome refers to an extrinsic compression of the CBD by an impacted gall stone in the Gall bladder.

2. Stenting of the $\mathrm{CBD}$ will relieve the obstructive jaundiced symptoms but the definitive treatment is cholycystectomy.

\section{PP26-57}

Sclerosing Cholangitis after Transcatheter Arterial Chemoembolization K. Qu, C. Liu, Q.F. Wu

The First Affiliated Hospital of Medical College, Xi'an Jiaotong University, Xi'an, China

Transarterial chemoembolization (TACE) for hepatocellular carcinoma is widely used but secondary sclerosing cholangitis as a serious complaction is rare reported. A 23-year-old male who was diagnosed with hepatocellular carcinoma accepted TACE for four times in 2 years. About one month after the last TACE, he developed fever and jaundice. Both imagings and biopsy supported the diagnosis of serious sclerosing cholangitis. Injury of the blood supply of bile duct caused by chemotherapeutic and embolization agents might be the cause. The patient received endoscopic naso-biliary drainage plus oral ursodeoxycholic acid and is in a relatively stable condition in 6 months fellow-up.

\section{PP26-58}

Hepatitis in Pregnancy with Mycoplasma

G. Cerasari ${ }^{1}$, A. Rossi ${ }^{2}$, R. Chiodi ${ }^{3}$, A. Cerasari ${ }^{4}$, S. Cerasari ${ }^{4}$, C. Donadio ${ }^{5}$, G. Puglisi ${ }^{2}$

${ }^{1}$ Liver Unit; ${ }^{2}$ Pneumology Unit; ${ }^{3}$ Pediatric Unit, S.Camillo Forlanini Hospital Rome; ${ }^{4}$ Sapienza University of Rome;

${ }^{5}$ Gynecology, S.Camillo - Forlanini Hospital Rome, Rome, Italy

Introduction: Mycoplasma is actually recognized as a real bacterium. Only one, $M$. pneumoniae has an important role in human disease, particularly in respiratory disease. In exceptional cases is held responsible for non respiratory infections such as hepatitis, pericarditis and others.

Case report: A woman of 35 years to 34th week of gestation comes to our observation in June 2010. Onset of fever and dry cough for about 1 week. She was treated with Amoxicillin antibiotic for few days. She was underwent to a chest X-ray that shows "thickening of the right ilar lung. Thickening in the medial segment of the middle. It was established a treatment with ceftriaxone and ursodessossicolic acid and performed sampling for hepatitis viruses, respiratory viruses, mycoplasma and chlamydia. The values of transaminases at admission were: GOT:113; GPT:156 U/L (reference values 5-40 U/L). After a few days, with the persistence of the fever it was carried a new haematochemical control that shows further increase in transaminases: GOT:183; GPT:308. Subsequently we received the answers of the serological tests that show negativity for respiratory viruses and hepatitis viruses, but positive IgG and IgM to Mycoplasma : $\operatorname{IgG}=1: 64, \operatorname{IgM}=1: 12$ (meth. IFA) We start treatment with macrolides. We are witnessing to a rapid disappearance of cough and fever and reduction of transaminases, that after 10 days since the last inspection is GOT:67; GTP:118 U/L. Chest X-ray control after 13 days shows: "regression of the inflammation".
Conclusions: In pregnancy, the increase in transaminases poses serious problems of differential diagnosis. We must therefore always suspect the involvement of this pathogen in the course of diseases that affecting the respiratory system and to a chance, not always rare, of complications in other organs, particularly the liver.

\section{PP26-59}

Hepatitis C-associated Hepatocellular Carcinoma in an African Woman with Non-cirrhotic Liver

L. Nosotti, A. Morrone, T. D'Arca

National Institute for Health, Migration and Poverty, Roma, Italy

Background: Hepatocellular carcinoma (HCC), on the rise in many countries, is of multifactorial etiology: cirrhosis from any cause is one of the strongest known risk factors for HCC. However, HCC may develop in non-cirrhotic livers: in particular, HBV can be directly oncogenic, and liver cirrhosis in HBV-infected patients is not a prerequisite for hepatocarcinogenesis. In contrast, the general belief is that presence of cirrhosis determines the increased risk for $\mathrm{HCC}$ in individuals with chronic HCV infection. Very little data exist on HCC arising in HCV infected, but noncirrhotic, livers.

Methods: A 55-year-old ethiopian women was treated for chronic hepatitis $\mathrm{C}$ in 2007 by a 48 -week peginterferon-alpha-2a and ribavirin course, and she achieved sustained virological response. A follow up ultrasound in September 2009 showed a $5 \mathrm{~cm}$ nodule in the right liver lobe. The patient had no relevant history of alcohol use, cigarette smoking and iron overload, and she was negative for serological evidence of HBV co-infection.

Results: CT scan confirmed the lesion, and biopsy established the pathological diagnosis of HCC in a non-cirrhotic liver (stage 1 fibrosis by Ishak score). Tumor transarterial chemoembolization followed by portal embolization of the right liver lobe were performed to induce left lobe hypertrophy with the aim to allow for the successive surgical resection of the tumor. Intrahepatic HBV-DNA was negative. After 45 days of interval, a right hepatectomy was performed without any complications.

Conclusions: This case shows that HCC can develop in livers with chronic hepatitis $\mathrm{C}$ infection without cirrhosis and without the evidence of other risk factors (HBV infection, alcohol intake, smoking habits, iron overload). Our findings highlight a previously underrecognized risk for $\mathrm{HCC}$ in non cirrhotic $\mathrm{HCV}$-infected individuals.

\section{References:}

1. Trevisani F, Frigerio M, Santi V, Grignaschi A and Bernardi M. Hepatocellular carcinoma in non-cirrhotic liver: a reappraisal. Dig Liv Dis 2010 May Vol. 42 (5): 341-347.

\section{PP26-60}

A Successfully Resected Case of Hepatic Epithelioid Hemangioendothelioma with Lung Metastases

M. Inagaki, N. Tokunaga, Y. Kimura, M. Nishie, R. Hamano, H. Miyaso, Y. Tsunemitsu, S. Otsuka, K. Iwakawa, H. Iwagaki

Department of Surgery, National Hospital Organization Fukuyama Medical Center, Fukuyama, Japan

Introduction: Epithelioid hemangioendothelioma (EHE) is a rare neoplasm of vascular origin that occurs in the liver. We experienced a successfully resected case of hepatic EHE with lung metastases.

Case report: A 38-year-old man was found to have multiple small lung nodules and those nodules enlarged in 2 years. Abdominal CT scan was performed and liver tumor was pointed out. He was 
admitted to our hospital. Abdominal CT and MRI scan revealed a $4 \mathrm{~cm}$ tumor in the S5 plus S8 segments of the liver with a hypovascular staining. Angiography showed pale nodule stained with hypervascularity at the margin. Although a fine-needle biopsy of the liver was performed, it was impossible to make a definite diagnosis. Video-assisted thoracoscopic surgery was performed to enable an excisional biopsy and histological examination revealed cytoplasmic vacuoles representing vascular lumina, some of which contained erythrocytes and diagnosed as EHE. Those suggested a liver tumor was a primary EHE and small nodules of lung were metastases. We performed a right lobectomy of the liver and histological examination showed same findings as lung nodules and tumor cells invaded into surrounding hepatic veins. Immunohistochemical studies revealed that the tumor cells were positive for factor VIII-related antigen and CD34. Although no increase in the size of lung metastases had been observed, new two lesions in the remnant liver were found 5 years after the operation. Percutaneous radiofrequency ablation was performed and recurrent tumors were treated.

Conclusions: EHE is a very rare clinical entity. We herein presented a successfully treated case of hepatic EHE with multiple lung metastases. Although multiple lung metastases exist, tumor growth of EHE is very slow and metastases and recurrences have been controlled to date. Surgical treatment offers good long-term results in patients suffering from EHE when tumor is presented with monolobular intrahepatic disease.

\section{PP26-61}

Management of Intractable Hepatic Hydrothorax with Pleuro-venous Shunt: A Case Report

Y. Kimura, M. Inagaki, N. Tokunaga, M. Nishie, R. Hamano, H. Miyaso, Y. Tsunemitsu, S. Otsuka, K. Iwakawa, H. Iwagaki

Department of Surgery, National Hospital Organization Fukuyama Medical Center, Fukuyama, Japan

Introduction: Hepatic hydrothorax is a rare, but can be observed as a complication of portal hypertension in less than $10 \%$ of the patients with ascites secondary to advanced liver cirrhosis. Intractable hepatic ascites and hydrothorax deteriorates the quality of life (QOL) in patients with liver cirrhosis. We experienced a case of intractable hepatic hydrothorax successfully treated by pleurovenous shunt.

Case report: A 67-year-old woman with type C liver cirrhosis was admitted to our hospital due to hepatic ascites and pleural effusion. Ascites was controlled by diuretics, but pleural effusion was intractable. Chest-X-ray films showed a massive pleural effusion in right hemithorax. The patient suffered from dyspnea in spite of repetitive thoracenteses and traditional medical treatment. A pleuro-venous shunt was inserted into the right-sided pleural cavity to drain effusion into the subclavian vein. Initially, pleural effusion was unchangeable, but subsequently decreased by increasing pumping times and mandatory thoracocentesis. Pleural effusion resolved and symptom improved. Chest-X-ray films showed a full expansion of right lung and few pleural effusion. She could stay at home and there were no shunt troubles. Pleuro-venous shunt remained patent without significant pleural effusion until her death due to liver failure 5 years after the operation.

Conclusion: We experienced a successfully managed case of intractable hepatic hydrothorax with pleuro-venous shunt. Pleurovenous shunting offers an alternative procedure with minimal invasiveness for high-risk patients with liver cirrhosis and may provide satisfactory palliation and improve the QOL for a patient with massive pleural effusion which failed to respond to medical therapy.
PP26-62

Sjogren's Syndrome Secondary to Anti Viral Treatment for Chronic Hepatitis C

N. Aqodad, M. Lahbabi, R. Zamharir, M. Elyousfi, I. Mellouki, D. Benajah, M. Elabkari, A. Ibrahimi

Gastroenterology Unit, Hospital University Hassan II, Fez, Morocco

Introduction and aim of study: The Sjogren's syndrome (SMS) is a chronic autoimmune disease responsible for a sicca syndrome. Interferon potentially provider of autoimmunity may induce sicca syndrome in patients with hepatitis C. We describe a case of Sjogren's syndrome secondary to pegylated interferon for hepatitis $\mathrm{C}$ virus.

Observation: A patient of 51 years, followed in our department for chronic hepatitis C virus (HCV): cytolytic (GPT two times the upper limit of normal, GOT: four times the upper limit of normal), replicative (high viral load at 2,740,104 U/ml $(6.44 \mathrm{log})$ with genotype $1 \mathrm{~b}$. An abdominal ultrasound was normal. A liver biopsy objectified a chronic viral hepatitis c METAVIR score A1F4 without detectable signs of autoimmunity. The decision was to treat the patient with interferon + ribavirin. At the 16th week of treatment she presented mouth eye and nasal mucosa dryness with arthralgia. The velocity sedimentation was high: 59/100 mm. Immunological analysis showed an GSS showing antinuclear antibodies (ANA) positive (1/320) with the presence of anti-DNA, anti-SSA $(>240 \mathrm{IU} / \mathrm{ml})$ and anti-SSB $(>47 \mathrm{UI} / \mathrm{ml})$. Salivary gland biopsy showed chronic sialadenitis Chisholm grade 4. The HCV viral load at this time was negative, which could exclude the accountability of HCV. The diagnosis of Sjogren's syndrome secondary to interferon was retained. The patient has received symptomatic treatment and anti-inflammatory drugs with good clinical improvement. We had maintained the same therapeutic doses that resulted in the complete negativity of his viral load at W24. Conclusion: Our observation suggests that the IFN alpha-inducing autoimmunity may be responsible for induction of sicca syndrome in patients with hepatitis $\mathrm{C}$ treated. The symptoms of this syndrome may be regressive.

\section{PP26-63}

A Case of Liver Damage Following Chemotherapy for Hepatic Colorectal Metastases

J. Takasaki, S. Ariizumi, Y. Kotera, S. Katagiri, Y. Takahashi, T. Kato,

K. Kitagawa, K. Hayashi, M. Yamamoto

Gastroenterology, Tokyo Women's Medical University, Tokyo, Japan

70-year-old man with sigmoid colon cancer with multiple liver synchronous metastases underwent sigmoid colon resection. He received postoperative chemotherapy with s-1, oxaliplatin (sox: s-1 $100 \mathrm{mg}$ / day (day1-14), L-OHP $100 \mathrm{mg}$ (day1, 14) 4 week/1 course) for hepatic colorectal metastases. Before chemotherapy, nine colorectal liver metastases about $10 \mathrm{~mm}$ in diameter were seen in the right lobe on computed tomography(CT). After three cycles of chemotherapy, CT showed only a tiny unclear space occupying lesion. However, about a year later, nine metastatic liver tumors were noted again on $\mathrm{CT}$, and right hepatectomy was performed. The resected specimen showed hard consistency similar to that seen with early cirrhosis. Pathologic review of the liver specimens revealed fine hepatic cord around central veins, sinusoidal dilatation, hepatic tissue atrophy and collagenization of the perisinusoidal space.

\section{PP26-64}

Hepatocellular Carcinoma Down-staged by Combination TACE + RFA Followed by Hepatic Resection

I. Mohamed Rose ${ }^{1}$, R. Zakaria ${ }^{2}$, N.Y. Yaacob ${ }^{2}$, Z. Mohamed ${ }^{2}$, B. Jan Jing ${ }^{3}$ ${ }^{1}$ Pathology; ${ }^{2}$ Radiology; ${ }^{3}$ Surgery, Universiti Kebangsaan Malaysia (UKM), Kuala Lumpur, Malaysia 
Introduction: Hepatocellular carcinoma (HCC) is still a major complication of chronic HBV infection that presents a problem in management. Conventional trans-arterial chemo-embolisation (TACE) has been the mainstay for treating intermediate stage inoperable tumours. Recently, drug eluting beads using doxorubicin (DEB TACE) has been added that slowly release the drug into the blood while avoiding the systemic effects of chemotherapy.

Case report: A 61-year-old Chinese male with chronic HBV infection for 20 years and Child-Pugh A was diagnosed with HCC in segment V on CT scan in September 2009. The 1st TACE was done in February 2010 and post-TACE CT showed residual enhancing tumour. His $\alpha$-fetoprotein level then was $>35,000 \mathrm{ng} / \mathrm{ml}$. A hepatectomy planned in April was abandoned as intra-operatively a large HCC was found involving the anterior section and segments IVA and IVB. An open radio frequency ablation (RFA) was performed instead. A 2nd TACE was done in May using doxorubicin $50 \mathrm{mg} / 25 \mathrm{ml}$ with lipiodol to segment V lesions. Repeat CT showed reduction in tumour size from previous $13.2 \times 9.7 \times 8.4 \mathrm{~cm}$ to current $5.1 \times 8.9 \times$ $10.6 \mathrm{~cm}$ size. A 3rd DEB TACE was done in July using $75 \mathrm{mg}$ doxorubicin and 100-300 $\mu \mathrm{m}$ size DC beads. Subsequently, a central hepatectomy done in August showed an extensively necrotic tumour at segments V and VIII extending to IVB. Histopathological examination confirmed a $\mathrm{HCC}$ with almost $80 \%$ necrosis, surrounding fibrosis and variable ischaemic changes in adjacent cirrhotic nodules. The beads appeared as rounded basophilic materials within blood vessels in surrounding liver.

Conclusion: We present a case of a large hepatocellular carcinoma managed by a combination of TACE and RFA that down-sized the tumour sufficiently for a hepatectomy to be performed. Patient's followup so far has been uneventful without any significant liver complications.

\section{PP26-65}

Unusual Complications of Expanded Polytetrafluoroethylene Patch Graft in the Venoplasty of Middle Hepatic Veins in Living Donor Liver Transplantation

J.-I. Choi ${ }^{1}$, D.G. Kim ${ }^{2}$, J.Y. Byun ${ }^{1}$, M.J. Kim ${ }^{3}$, S.H. Kim ${ }^{4}$, H.B. Kim ${ }^{5}$, J.K. $\mathrm{Han}^{6}$, Y.H. So ${ }^{7}$, K.S. $\mathrm{Suh}^{8}$

${ }^{1}$ Radiology; ${ }^{2}$ Surgery, Seoul Saint Mary's Hospital, The Catholic University of Korea, Seoul; ${ }^{3}$ Radiology; ${ }^{4}$ Center for Liver Cancer; ${ }^{5}$ Radiology, Center for Liver Cancer, Research Insitute and Hospital, National Cancer Center, Goyang; ${ }^{6}$ Radiology, Seoul National University, College of Medicine; ${ }^{7}$ Radiology, Seoul Metropolitan Government/Seoul National University Boramae Medical Center; ${ }^{8}$ Surgery, Seoul National University, College of Medicine, Seoul, Republic of Korea

Introduction: Congestion in the anterior section in a right hemiliver without a middle hepatic vein could cause outflow obstruction and might lead to graft dysfunction in living donor liver trasplatation. Various methods of venoplasty were applied to solve this problem including introduction of draining middle hepatic vein branches with augologous grafts. An expanded polytetrafluoroethylene (ePTFE) patch graft may be used for anterior section drainage in cases of unavailability of autologous vessels and applying ePTFE patch graft also can reduce operation time because preparation of autologous veins is time-consuming. To our knowledge, no complication by applying ePTFE patch grafts to transplanted liver was reported. However, we experienced four cases of the complications after applying ePTFE.

Case report: Four cases were from three institutes. In two cases, ePTFE patch graft was partially detached from the surface of transplanted liver and made erosion of wall of gastric antrum. Thickening, erosion and air bubbles in gastric wall were noted on CT scans. On follow-up CT, grafts eventually perforated gastric lumen and in one case, graft was totally detatched from liver and migrated to small bowel loop. In another case, ePTFE patch graft perforated duodenal bulb. In these three cases, no clinical symptom related to the perforation was observed except the dilatation of intrahepatic bile ducts by other cause in two patients. In another case, hyperbilirubinemia was noted and percutaneous transhepatic biliary drainage (PTBD) tube was inserted. On cholangiogram, the filling defect was found in anterior branch of intrahepatic duct and thought to be a stone. Stone removal was performed through PTBD tract with stone basket and the fragmented segment of ePTFE patch graft was found. In conclusion, ePTFE patch graft in living donor liver transplantation can cause erosion and perforation of adjacent bowels or bile ducts, though clinical symptom is not common.

\section{PP26-66}

Malignant Hepatic Epithelioid Hemangioendothelioma with Abdominal Pain due to Rapid Progression

J. Harada ${ }^{1}$, N. Taniai ${ }^{1}$, J. Ueda ${ }^{1}$, H. Yoshida ${ }^{1}$, S. Mineta ${ }^{1}$, M. Yoshioka ${ }^{1}$, Y. Kawano ${ }^{1}$, M. Mizuse $^{2}$, E. Uchida ${ }^{1}$

${ }^{1}$ Surgery; ${ }^{2}$ Nippon Medical School, Tokyo, Japan

Epithelioid hemangioendothelioma (EHE) is an uncommon neoplasm of the vascular endothelium. It is an uncommon tumor of vascular origin that may develop in the soft tissue, lung, bone, brain, and small intestine. Rarely it occurs in the liver. We report a patient underwent hepatectomy for malignant hepatic epithelioid hemangioendothelioma with rapid progression. An 83-year-old man was admitted to our hospital because of acute sever epigastralgia. She had been detected hepatic tumor $3 \mathrm{~cm}$ in diameter in left lobe before seven months. Abdominal computed tomography $(\mathrm{CT})$ and enhanced ultrasonography and magnetic resonance image (MRI) revealed hypervascular tumor in $9 \mathrm{~cm}$ in diameter in left lobe of the liver and tumor stain remained at late phase. Abdominal angiography revealed cotton wool appearance in this tumor. We diagnosed this tumor as cavernous hemangioma, but not ruled out of malignant potential. We underwent left hepatic lobectomy because she complicated abdominal pain, and this tumor was not ruled malignancy. Pathological finding was that a lot of necrosis was spread in the tumor, and showed slightly pleomorphic neoplastic cells with rounded and spindle nuclei and scant cytoplasm occasionally arranged in vascular channel. Neoplastic cells were obviously positive for factor VIII-related antigen and CD31, CD34. And Mib-1 index was high. This tumor was diagnosed as malignant hepatic epithelioid hemangioendothelioma. He had no eventful postsurgical course and discharged on postoperative day 11. After 3 months, multiple metastasic tumors appeared in both hepatic lobes. We performed transcatheter arterial chemoembolization. We should suspect malignant hepatic epithelioid Hemangioendothelioma when the hepatic tumor looked like cavernous hemanigoma progresses rapidly.

\section{PP26-67}

Effective Trastuzumab Treatment and Chemotherapy with S-1 for Hepatic Metastasis of Gastric Cancer

S. Yamashita ${ }^{1}$, S.-I. Ariizumi ${ }^{1}$, Y. Kotera ${ }^{1}$, Y. Takahashi ${ }^{1}$, T. Katou ${ }^{1}$, J. Takasaki ${ }^{1}$, N. Ooshima ${ }^{1}$, S. Katagiri ${ }^{1}$, K. Shimizu ${ }^{2}$, M. Yamamoto ${ }^{1}$ ${ }^{I}$ Department of Surgery, Institution of Gastroenterology, Tokyo Women's Medical University; ${ }^{2}$ Shin- Itabashi Clinic, Tokyo, Japan

Trastuzumab treatment for hepatic metastasis of gastric cancer was performed in a 56-year-old man. He underwent distal gastrectomy for gastric cancer in 2005. In 2006, a liver metastasis, $2 \mathrm{~cm}$ in diameter, was detected in segment 4 on computed tomography (CT). He underwent chemotherapy with various anticancer drugs. However, the tumor size increased to $5 \mathrm{~cm}$ in diameter and another recurrent tumor was detected in the liver. In 2009, he underwent central bisegmentectomy and immunotherapy with dendritic cell vaccine after surgery. In 2010, multiple metastatic tumors were detected in the remnant liver. He underwent chemotherapy with TS-1 and Trastuzumab, since the cancer cells were positive for immunohistochemical staining of human epidermal growth factor receptor 2. After 8 months, no changes in tumor size or number were seen on CT, and the serum level of CEA was decreased. Trastuzumab treatment was effective for hepatic metastasis of gastric cancer. 


\section{PP26-68}

Findings of ${ }^{11} \mathrm{C}$-acetate and ${ }^{18} \mathrm{~F}$-FDG PET/CT for a Case of Hepatic Inflammatory Pseudotumor

C.-L. Ho, S. Chen, T. Cheng, Y.L. Leung, K.K. Wong

Hong Kong Sanatorium and Hospital, Hong Kong, Hong Kong SAR

Inflammatory pseudotumor (IPT) of the liver is a benign pathology of unknown cause, consisting of fibrous stroma and chronic inflammatory infiltrates without anaplasia. It is difficult to differentiate hepatic IPT from malignant tumors by radiological imaging because findings are usually variable and non-specific. We performed PET/CT on a case of hepatic mass presented with indeterminate findings on contrast CT.

Case description: A 40-year-old male patient had fever and chills, leucocytosis, CRP of $155 \mathrm{mg} / \mathrm{L}$, and mildly deranged liver enzymes. Abdominal CT showed a 5-cm hypervascular lesion in segment IV. ${ }^{11} \mathrm{C}$ acetate and ${ }^{18} \mathrm{~F}$-FDG PET/CT showed a hypermetabolic mass in the same location, more avid for ${ }^{18} \mathrm{~F}$-FDG than ${ }^{11} \mathrm{C}$-acetate (lesion-to-liver ratio: 6.9 and 2.7, respectively). The degree of increased ${ }^{18} \mathrm{~F}-\mathrm{FDG}$ uptake was thought to be too intense for hepatocellular carcinoma or common hepatic secondaries (lesion-to-liver ratio usually $<4$ ), but could be found in pure cholangioadenocarcinoma or sarcoma. However the latter two types of malignant tumors were shown to be negative for ${ }^{11} \mathrm{C}$-acetate from our past experience. Therefore, our impression favored benign pathology such as an organized abscess or other inflammatory/ infectious causes. Patient was conservatively treated by antibiotics for 1 month before ultrasound-guided core biopsy subsequently confirmed IPT. Follow-up PET/CT after 2 months of antibiotics treatment suggested dramatic improvement by demonstrating decreased lesion size and intensity with both tracers (Figure). This example, though just a single case, suggests that the degree and pattern of dual-tracer $\left({ }^{11} \mathrm{C}\right.$-acetate and ${ }^{18} \mathrm{~F}$-FDG) uptake might have potential in differentiating IPT from malignant liver masses and accessing treatment effect.

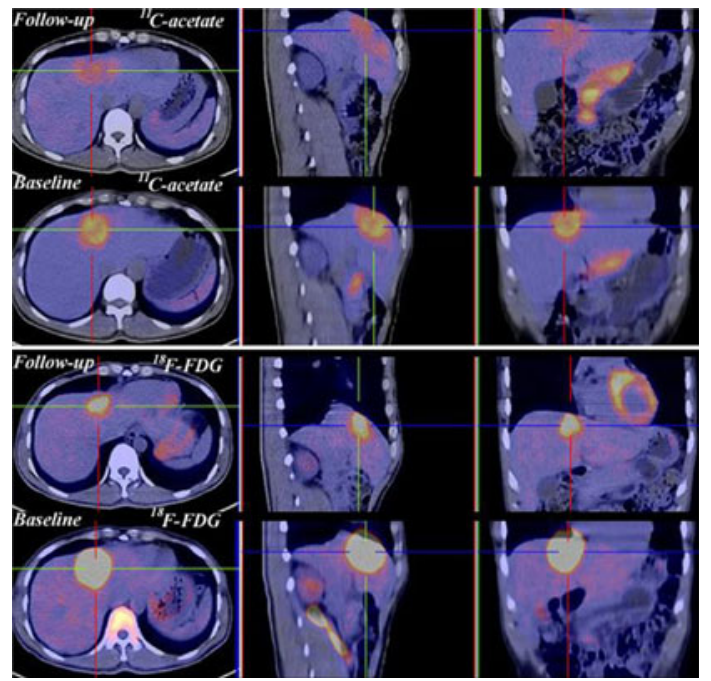

IPT

\section{PP26-69}

A Rare Etiology of Portal Hypertension in an Adult: Infantile Hemangioendothelioma

S. Kiziltas ${ }^{1}$, Y. Colak ${ }^{1}$, M. Gulluoglu ${ }^{2}$, O. Ozturk ${ }^{1}$, E. Yorulmaz ${ }^{1}$, G. Adali ${ }^{1}$, L. Doganay ${ }^{1}$, C. Ulasoglu ${ }^{1}$, F. Yilmaz Enc ${ }^{1}$, G. Tasan ${ }^{1}$, I. Tuncer ${ }^{1}$

${ }^{I}$ S.B. Istanbul Goztepe Education and Research Hospital, Department of Gastroenterology; ${ }^{2}$ Pathology, Istanbul University, Istanbul Faculty of Medicine, Istanbul, Turkey

Infantile hemangioendothelioma is the most frequent primary mesenchymal tumor of liver in the first year of life. It frequently presents as abdominal mass and hepatomegaly and it rarely seen in the adulthood. Although it is benign, it complicated by congestive heart failure secondary to hepatic arteriovenous shunts, disseminated intravasculary coagulation and subsequently hemorrhagic diathesis, hemoperitoneum secondary to rupture, portal hypertension and rarely malign transformation (angiosarcoma). Here we report a case which a 43-year-old man was diagnosed with infantile hemangioendothelioma while investigated for etiology of portal hypertension.

Case description: A 43-year-old man presented with progressively increasing malaise and abdominal swelling for 6 months. He was referred to gastroenterology when hepatosplenomegaly and ascites detected on physical examination. On admission the laboratory findings were as follows: hemoglobin $9.9(13.5-17.5) \mathrm{g} / \mathrm{dl}$, leukocytes 3,900/ $\mathrm{mm}^{3}\left(4,000-10,000 / \mathrm{mm}^{3}\right)$, platelet $38,000 / \mathrm{mm}^{3}\left(150,000-40,000 / \mathrm{mm}^{3}\right)$, alkaline phosphatase $799(30-136) \mathrm{IU} / \mathrm{L}$, total bilirubin $5.3(0-1.5) \mathrm{mg} /$ $\mathrm{dl}$, direct bilirubin $3.7(0-8) \mathrm{mg} / \mathrm{dl}$, albumin $3.8(3.5-5.5) \mathrm{g} / \mathrm{dl}$, globulin $2.7(2.3-3.5) \mathrm{g} / \mathrm{dl}$, prothrombin time long $>1 \mathrm{~s}$. Viral and autoimmune hepatitis markers, ceruloplasmin, urine copper, ferritin and other routine biochemical investigations were normal. An abdominal CT revealed lesions compatible with multiple metastases in liver, splenomegaly and ascites. Endoscopic investigations were normal except grade 2 esophageal varices. Malign FDG activity was not detected in PET/CT scan. Laparoscopic examination showed numerous purplish, small speckles over the liver surface. A liver biopsy revealed vascular channels in the liver parenchyma with different sizes. Immunohistochemical staining revealed that the endothelial cells lining these vascular channels were CD34 (+) (Fig. 1). Based on these findings, the case was diagnosed as portal hypertension secondary to infantile hemangioendothelioma. The liver transplantation was offered to patient but he did not accept. The patient died due to variceal hemorrhage 2 years after the diagnosis.

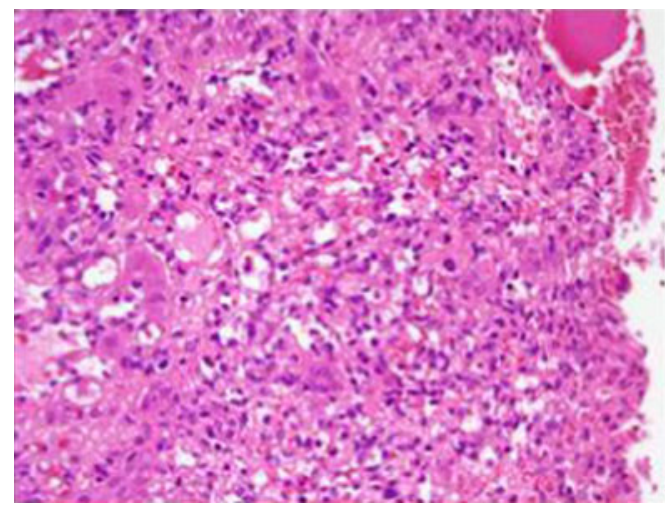

Fig. 1 Areas composed of small vascular channels lined by endothelial cells (H\&E)

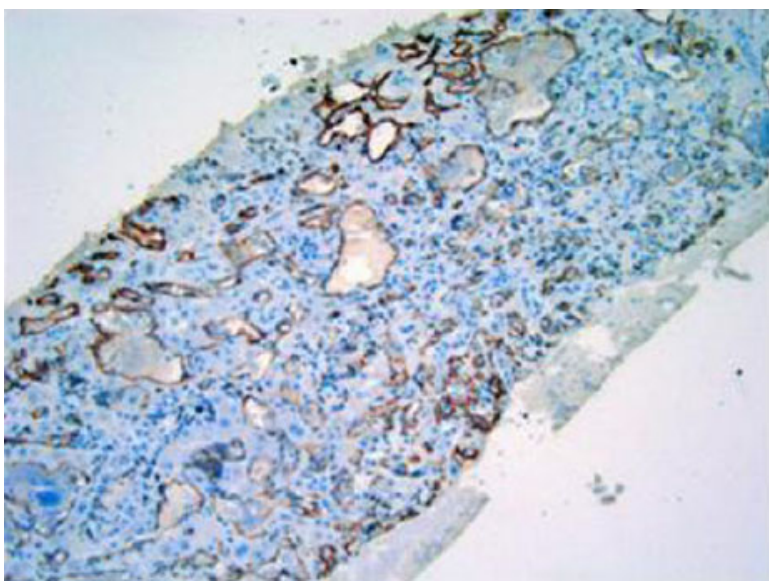

Fig. 2 The endothelial cells lining the vascular channels were CD34 positive immunohistologically (CD34, counterstain Mayer hematoxylin) 


\section{PP26-70}

Successful Management of a Symptomatic Splenic Artery Aneurysm with Transcatheter Embolization

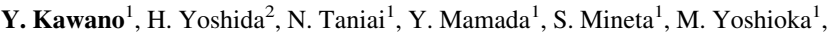
A. Hirakata ${ }^{2}$, J. Ueda ${ }^{1}$, K. Hara ${ }^{1}$, E. Uchida ${ }^{1}$

${ }^{1}$ Department of Surgery, Nippon Medical School; ${ }^{2}$ Department of Surgery, Nippon Medical School, Tama Nagayama Hospital, Tokyo, Japan

We describe a symptomatic splenic artery aneurysm (SAA) with occasional left-sided abdominal pain which was successfully treated with transcatheter embolization. A 65-year-old man was referred to a nearby clinic because of left shoulder and abdominal pain developing the day after blunt trauma to the shoulder and abdomen. Radiography revealed no fracture, and the patient went home. He stopped working for 7 months. Left-sided abdominal pain then developed several times after strenuous physical labor, and the patient was referred to a nearby hospital. The patient had a history of asthma and untreated hypertension; the use of iodinated contrast material was therefore avoided. Unenhanced computed tomography (CT) and magnetic resonance imaging (MRI) of the abdomen revealed a hematoma in the splenic hilum. The patient was referred to Nippon Medical School Hospital. His neurologic status was stable. Unenhanced CT of the abdomen at a nearby hospital revealed a low-density area in the splenic hilum. Fat-suppressed, T1-weighted images showed a hyperintense lesion adjacent to the splenic hilum. Fat-suppressed, T2weighted images showed a dark hemosiderin rim, a bright ring, and an intermediate central core, which indicated a recurrent chronic hematoma in the abdomen. Dynamic contrast-enhanced MRI revealed a small hyperenhanced lesion adjacent to the hematoma, which appeared to be an SAA. After an injection of corticosteroids, selective splenic arteriography was performed. The splenic artery was catheterized via a right femoral approach. Arteriography showed an SAA, $1 \mathrm{~cm}$ in diameter with extravasation of contrast material. The neck of the SAA was catheterized with a microcatheter, and coils were placed successfully into the SAA to prevent recurrent bleeding. Postembolization angiography showed total occlusion of the SAA. The patient was discharged 7 days after embolization and has since resumed normal activities, with no residual symptoms.

\section{PP26-71}

Infected Hepatic Cyst Treated with Percutaneous Transhepatic Drainage Y. Kawano ${ }^{1}$, H. Yoshida ${ }^{2}$, N. Taniai ${ }^{1}$, Y. Mamada ${ }^{1}$, S. Mineta ${ }^{1}$, M. Yoshioka ${ }^{1}$, A. Hirakata ${ }^{2}$, J. Ueda $^{1}$, E. Uchida ${ }^{1}$

${ }^{1}$ Department of Surgery, Nippon Medical School; ${ }^{2}$ Department of Surgery, Nippon Medical School Tama Nagayama Hospital, Tokyo, Japan

Simple hepatic cysts are common benign lesions that are usually asymptomatic and require no treatment. However, complications can occur. This report describes a patient with an infected hepatic cyst treated with percutaneous transhepatic drainage. A 64-year-old woman presented at a nearby hospital because of acute right-upper-quadrant pain, mild leftlower-quadrant pain, diarrhea, and fever. She was admitted and received intravenous antibiotics for 1 week, but symptoms persisted. She was, therefore, referred to our hospital. On admission, ultrasonography demonstrated multiple hepatic cysts. One 13-cm lesion was hypoechoic, unlike the other simple cysts, which were anechoic. Computed tomography showed that the density of the hypoechoic cyst was slightly higher than that of the other cysts. The wall of the cyst was thickened and showed contrast enhancement. On initial laboratory tests the serum C-reactive protein concentration was $18.49 \mathrm{mg} / \mathrm{dL}$, and the white-cell count was $13,300 / \mu \mathrm{L}$. An infected hepatic cyst was suspected, and percutaneous transhepatic drainage of the cyst was performed. A catheter was inserted into the cyst, and dark red fluid was obtained. The right-upper-quadrant pain gradually resolved after drainage. An infected hepatic cyst was diagnosed, and system antibiotics were administered. However, the mild left-lowerquadrant pain persisted. No pathogens were isolated from the drainage fluid. Minocycline hydrochloride (200 mg) was injected, and the catheter was clamped for $30 \mathrm{~min}$, once daily for 3 days. The serum C-reactive protein concentration was $1.78 \mathrm{mg} / \mathrm{dL}$, and the white-cell count was $5,700 /$ $\mu \mathrm{L}$. The left-lower-quadrant pain resolved, and colonoscopic examination revealed multiple diverticula of the sigmoid colon. Infection has not recurred, and the hepatic cyst has not become larger.

\section{PP26-72}

Invasive Aspergillus and Mucor Infections in Organ Transplant Patiens: Report of Two Cases and Review of the Literature

L. Lei, C. Liu, S. Huang, X.F. Zhang, X.G. Zhang, B. Wang, X.M. Liu, L. Yu, Y. Lv

Hepatobiliary Surgery, First Affiliated Hospital of Medical College of Xi'an Jiaotong University, Xi'an, China

Introduction: Invasive fungal infections(INF) are a recognized cause of death in patients who have undergone organ transplantation (OT). The incidence of combined with deep Aspergillus and Mucor infections after OT is rare compared with common fungal infection (Candida species).

Cases description: Herein we present two special cases combined with Aspergillus and Mucor infections after OT in our center. Among them, liver and kidney transplantation was done in one case, the other undergone orthotopic liver transplantation (OLT). In the first patient, the hepatic and renal arteries suddenly ruptured caused by Aspergillus and Mucor infectsions on the 8th day post op.

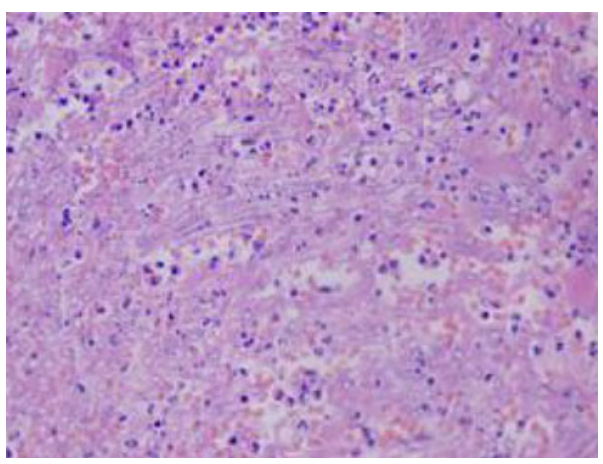

Fig. 1

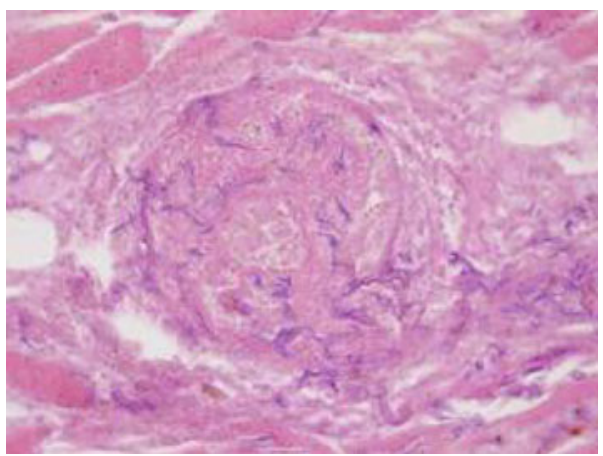

Fig. 2

Oweing to excessive bleeding in a short time,the patient died 4 days after the second operation due to multiple organ failure. In the other recipient, operative incision developed Mucor infection which spread rapidly from the muscle layer to the skin surface and consecutive sputum culture results indicated that lower respiratory tract Aspergillus infection. The patient eventually died of systemic severe infection and respiratory failure. In summation, INF is usually occult in onset in OT recipients, and the prognosis is poor. As the possible cause for INF in OT patients,potential deep Aspergillus or Mucor 
infections ought to be considered. Meanwhile,selective prophylaxis and individualized combination anti-fungal treatment might be useful to improve patients's prognosis.

\section{PP26-73}

Hepatocellular Carcinoma Presenting with Multiple Bone and Soft Tissue Metastases and Atypical Cytomorphology-A Rare Case Report A. Rastogi ${ }^{1}$, C. Bihari ${ }^{1}$, D. Jain ${ }^{2}$, S.K. Sarin ${ }^{3}$

${ }^{1}$ Pathology; ${ }^{2}$ Radiology; ${ }^{3}$ Hepatology, Institute of Liver \& Biliary Sciences, Delhi, India

Hepatocellular carcinoma (HCC) presenting with bone and soft tissue metastasis is rare and concomitant presence of atypical cytological features such as multinucleated osteoclast-like giant cells and intracytoplasmic hyaline bodies (IHBs) has never been reported in literature. We hereby report a patient of clinically and radiologically unsuspected hepatocellular carcinoma (HCC) presenting with bone metastases accompanied by soft tissue formation and displaying uncommon cytomorphological features such as abundant multinucleated osteoclast-like giant cells and very prominent IHBs. A 65-year-old male patient of HCC who presented with bone and soft tissue metastases and was clinically and radiologically suspected to have a soft tissue sarcoma. The patient presented with severe cervical pain with palpable masses in right scapular, nape of neck and occiput area of scalp. Radiologically, these were large, bulky soft tissue masses expansile, destructive and lytic in nature. Cytomorphologic studies revealed HCC with uncommon features of multinucleated osteoclast-like giant cell and very prominent intracytoplasmic Hyaline Bodies (IHBs). Cytology, immunohistochemistry on cell block preparation, rising serum $\alpha$ fetoprotein (AFP) levels $(1,121.93-5,000 \mathrm{ng} / \mathrm{ml})$ and PIVKA II levels confirmed the diagnosis. The patient has been on follow up on sorafinib for 2 months and is doing well. This case emphasizes the need for systematic approach in cases of HCC with atypical clinical presentation and unusual cytomorphology.

\section{PP26-74}

Adenoendocrine Carcinoma of Gallbladder in a Patient with Primary Sclerosing Cholangitis and Ulcerative Colitis

A. Rastogi ${ }^{1}$, V. Bhatia ${ }^{2}$, C. Bihari ${ }^{1}$, S. Singh ${ }^{3}$, S.K. Sarin ${ }^{2}$

${ }^{1}$ Pathology; ${ }^{2}$ Hepatology; ${ }^{3}$ Hepatobilary and Pancreatic Surgery, Institute of Liver and Biliary Sciences, Delhi, India

Adenoendocrine carcinoma of gall bladder is a rare histological type. Carcinomas of the gallbladder have been described in PSC but are less common than bile duct carcinomas. Adenoendocrine carcinoma of the gallbladder associated with Primary sclerosing Cholangitis (PSC) and Ulcerative Colitis (UC) has not been reported so far. We report a 48-year-old female, case of UC (Pancolitis) 20 years, PSC with chronic liver disease and portal hypertension 7 years, diagnosed to have a mass lesion in Gall bladder fossa for 4 months. CECT abdomen showed uniform mural enhancement of biliary ductal system, heterogenous ill-defined soft tissue enhancement along the gall bladder fossa and features of secondary biliary cirrhosis. Endoscopic ultrasound guided FNA showed features of neuroendocrine carcinoma with Xanthgranulomatous inflammation. PET CT showed FDG avid heterogeneously enhancing mass in the gall bladder fossa with contiguous infiltration of adjacent segment IVB and V of liver with an absence of FDG avid regional lymphadenopathy or distant metastatic disease. Extended cholecystectomy and segmentectomy performed and histopathologic examination showed adenocarcinoma (Pan- Cytokeratin positive), neuroendocrine carcinoma (Chromogranin A and Synaptophysin positive) and foci of xanthogranulomatous cholecystitis. Patient has been on follow-up for 4 months and is doing well.
PP26-75

Spontaneous Necrosis of Hepatocellular Carcinoma with Focal Peritumoral Vasculitis, a Case Report

T. Sricharunrat ${ }^{1}$, S. Termmathurapoj ${ }^{2}$, C. Laohapand ${ }^{3}$, T. Tanwandee ${ }^{4}$

${ }^{1}$ Pathology, Chulabhorn Hospital; ${ }^{2}$ Pathology, Phramongkutklao College of Medicine; ${ }^{3}$ Chulabhorn Hospital; ${ }^{4}$ Division of

Gastroenterology, Department of Medicine, Siriraj Hospital, Mahidol University, Bangkok, Thailand

Introduction: Hepatocellular carcinoma (HCC) with spontaneous necrosis is a rare phenomenon with uncertain mechanism. Previously, proposed mechanisms of regression include tumor ischemia by rapid growth of tumor or cirrhosis, immunological mechanism, alcohol abstinence, radiation and post intervention and unexplained mechanism. We present a case of HCC patient with histological feature of extensive necrosis of tumor cell who has spontaneously regressed without specific treatment for $\mathrm{HCC}$ and without known metastatic site. Case description: A 61-year-old woman with chronic viral hepatitis $\mathrm{B}$, incidentally found $1.8 \mathrm{~cm}$ liver mass with cystic degeneration at segment V by CT scan with cirrhosis. She also had high alpha-fetoprotein level of $327.2 \mathrm{ng} / \mathrm{ml}$. She received no prior treatment except lamivudine for chronic viral hepatitis B infection. She had no known metastatic site. She received wedge resection of segment $\mathrm{V}$ of liver. The gross specimen showed a $1.8 \mathrm{~cm}$, well encapsulated lesion with near total necrosis and presence of liver with cirrhosis. The microscopic findings are a nodule with central area of necrosis $(75 \%)$ and surrounding several nests of viable hepatocellular carcinoma $(25 \%)$. The viable tumor cell has lymphocyte (predominant CD8+ T cell) and plasma cells infiltrate between the tumor cell and granuloma surrounding the tumor nest. There is an arteriole near tumor capsule, showing fibrinoid necrosis in tunica intima with surrounding granuloma and lymphocyte (CD8+ T cell) in the outer layer. This case we proposed of immunological mechanism especially by cell mediated immune response in the underlying mechanism of our findings.

\section{PP26-76}

Liver Damage after Breast Plastic Surgery-Clinical Case Report

I. Tolmane ${ }^{1,2}$, I. Strumfa ${ }^{3}$, J. Keiss ${ }^{1}$, B. Rozentale ${ }^{4,5}$, R. Simanis ${ }^{5}$

${ }^{1}$ Hepatology, SA Latvian Infectology Center; ${ }^{2}$ Doctoral Studies;

${ }^{3}$ Pathology Department, Riga Stradins University; ${ }^{4}$ SA Latvian Infectology Center; ${ }^{5}$ Infectology and Dermatology Department, Riga Stradins University, Riga, Latvia

Introduction: Since silicone implants were introduced in the early 1960s, those have been widely used for cosmetic and reconstructive breast surgery. Although a recent review has shown no relationship between the silicone breast implant and systemic complications, leakage of the silicone into the tissues and migration to the regional lymph nodes remains a clinical problem. This was the first case in our practice when possibly breast implant material was found in the liver tissue.

Case description: 38 -year-old women presents with discomfort in the upper right quadrant and epigastric region. Complaints last for approximately 5 years, periodically. The patient reports no itching, bleeding, jaundice, nausea or other dyspeptic complaint. Laboratory tests within normal ranges. 16 years ago patient had plastic operation for breast enlargement. The mammary prosthesis consisted of polyurethane cover and filled with synthetic thermostable rubber of low molecular structure. There were episodes of allergic reactions with face edema, Quincke's edema and lymphadenopathy starting about 9 years ago. Lymph node biopsy: infiltration of macrophages and atrophy of lymph node. Breast implants were removed 8 years ago. After that there was an episode of hepatosplenomegaly and enlarged lymph nodes 5 years ago.

Ultrasound examination: Right lobe of liver slightly enlarged with multiple hypoechogenic focuses $3-5 \mathrm{~mm}$ diameter.

Liver biopsy: Accumulation of unknown material (fat, air, lypophile radiopaque) in the portal tracts without significant damage of liver 
parenchyma. Spear-shaped holesterol or fatty acid crystals are not found, there is no damage of hepatocytes or Kupffer cells. There are no data of inflammatory or neoplastic process.

Conclusion: It is possible that material of breast implant has been spread into organism tissues including lymph nodes and liver.

\section{Poster Presentation 27: Cholangioca and Other Liver Neoplasm}

\section{PP27-01}

Comparison of Serum Tumor Markers for Intrahepatic Cholangiocarcinoma and Hepatocellular Carcinoma

L.-Y. Tao $^{1}$, X.-D. $\mathrm{He}^{2}$

${ }^{1}$ China Rehabilitation Research Center; ${ }^{2}$ Peiking Union Medical College Hospital, Beijing, China

Aims: Serum tumor markers such as alpha-fetoprotein (AFP), carcinoembryonic antigen (CEA), carbohydrate antigen 19-9 (CA19-9), CA242, and CA50 were analyzed to evaluate their diagnostic values in single and combined tests for distinguishing intrahepatic cholangiocarcinoma (ICC) form hepatocellular carcinoma (HCC).

Methods: Preoperative serum levels of AFP, CEA, CA19-9, CA242, and CA50 were measured in $45 \mathrm{ICC}$ and $76 \mathrm{HCC}$ patients.

Results: The serum levels and the positive rate of AFP, CA19-9, and CA242 were significantly different between the ICC patients and HCC patients. Although AFP (-) was the most sensitive assay for distinguishing ICC from HCC $(91.1 \%)$, its specificity was significantly lower than that of CA242 (+) and CA19-9 (+). The combination of AFP (-) and CA242 (+) afforded a high specificity of $94.3 \%$ and showed highest accuracy $(78.5 \%)$. Evaluation of patients without liver cirrhosis also showed similar results.

Conclusion: The diagnostic value of CA242 (+) is better than that of CA19-9 (+) and AFP (-) in distinguishing ICC from HCC. Combined detection of AFP (-) and CA242 (+) can improve the specificity and accuracy of diagnosis of ICC.

\section{PP27-02}

Hepatic Hemangiomas: Possible Association with Female Sex Hormones V. Glinkova ${ }^{1}$, O. Shevah ${ }^{2}$, M. Boaz ${ }^{3}$, H. Shirin ${ }^{4}$

${ }^{1}$ Department of Hepatogastroenterology, Medical University, Varna, Bulgaria; ${ }^{2}$ Department of Gastroenterology, The Edith Wolfson

Medical Center, Sackler Faculty of Medicine, Tel - Aviv University;

${ }^{3}$ Epidemiology Unit, The Edith Wolfson Medical Center, Sackler

Faculty of Medicine, Tel - Aviv University, Israel., Tel Aviv, Israel;

${ }^{4}$ Department of Gastroenterology, The Edith Wolfson Medical Center, Sackler Faculty of Medicine, Tel - Aviv University, Israel., Tel Aviv, Bulgaria

Background: Hemangiomas are the most common benign tumors of the liver. Although a relationship between estrogens and liver tumors has been reported, the association of hepatic hemangiomas with female sex hormones is not entirely clear.

Aims: To prospectively evaluate the natural history of liver hemangiomas and the impact of female sex hormones.

Methods: We studied 94 women with 181 hemangiomas that were diagnosed by ultrasound with a follow-up period of 1-17 years $(7.3 \pm 5.5)$. All patients underwent imaging follow-up every three months in the first year and after that yearly. Location, number, size, and ultrasonographic pattern of the lesions were evaluated. Patients were evaluated by questionnaire for the age of the first period and menopause, number of pregnancies and deliveries and hormonal therapy. We also compared the change in number and size of hemangiomas in patients who received or did not receive exogenous hormonal treatment.

Results: Age at first period was inversely associated with the size of hemangiomas $(r=0.181, p=0.015)$ while age at menopause was positively correlated to number of hemangiomas detected at first ultrasound $(r=0.542, p<0.0001)$. During follow-up no change in the ultrasonographic pattern or number of the hemangiomas was observed. An increase in the size of the lesions was demonstrated in 13/94 (13.8\%) patients. Three variables, ultrasonographic pattern, number of hemangiomas and hormonal therapy, predicted whether or not a given hemangioma will increase in size. Hypoechoic pattern increased the risk of progression, while a hyperechoic pattern decreases that risk $(p=0.03)$, hemangioma number was inversely associated with the likelihood of progression $(p=0.017)$ and finally hormonal therapy increased the risk of hemangioma enlargement $(p=0.05)$.

Conclusions: Hepatic hemangiomas seem to be influenced by both endogenous and exogenous female sex hormones. Consequently we suggest that liver ultrasound follow-up should be performed routinely in women with hepatic hemangiomas receiving hormonal therapy.

\section{PP27-03}

Segment 3 Biliary Bypass for Carcinoma Gallbladder with Obstructive Jaundice

\section{Pandey}

Department of Surgical Oncology, Institute of Medical Sciences, Banaras Hindu University, Varanasi, India

Background: Obstructive jaundice in gallbladder cancer is usually a manifestation of advanced disease. Palliative options for jaundice include endoscopic stenting, transhepatic biliary stenting, or surgical biliary bypass. As the site of biliary block in gallbladder cancer is near the hilum, endoscopic stenting is generally not feasible.

Method: This is an analysis of a prospective database of patients who underwent segment 3 cholangiojejunostomy for palliation of jaundice due to advanced gallbladder cancer. The patients who underwent segment 3 bypass had significant pruritus, a failed attempt at endoscopic biliary stenting, and were highly motivated for this palliative surgery. The surgical procedure involved Roux-en- $Y$ anastomosis of jejunum with the segment 3 duct.

Result: Between January 2008 and March 2010, twenty five (25) patients with advanced gallbladder cancer with obstructive jaundice underwent palliative segment 3 bypass. Six of these patients also had associated gastric outlet obstruction for which they underwent gastrojejunostomy as well. Serum bilirubin levels ranged between 9 and $42 \mathrm{mg} / \mathrm{dl}$ (median $26 \mathrm{mg} / \mathrm{dl}$ ). There were two postoperative deaths: one because of biliary leak and subsequent sepsis, and the other due to acute myocardial infarction on second postoperative day. Additionally, three patients had postoperative complications in form of bleeding (1) and wound infection (2). The median hospital stay was 7 days (range 5-13 days). All the patients were relieved of pruritus. The reduction of serum bilirubin was complete in ten patients who subsequently completed six cycles of palliative chemotherapy. All surviving patients had a subjective feeling of a better quality of life after the surgery.

Conclusion: In carefully selected patients, segment 3 bypass is a useful palliative procedure for gallbladder cancer with obstructive jaundice who has failed endoscopic stenting. This is an important alternative in regions with high incidence of gallbladder cancer where the expertise in interventional radiology is limited.

\section{PP27-04}

Accuracy of Bile Duct Brushing and Forceps Biopsy in the Evaluation of Biliary Strictures

E.G. Santi, A. Ong-Go, S. Wong, A. Ismael

Internal Medicine - Gastroenterology, University of Santo Tomas Hospital, Manila, Philippines

Background: Confirming malignancy in biliary strictures during endoscopic retrograde cholangiopancreatography (ERCP) can be challenging. Brush cytology and forceps biopsy are two diagnostic methods that can be employed during ERCP. 
Objective: To compare the sensitivity and specificity of brush cytology and forceps biopsy among those suspected to have malignant biliary strictures.

Methods: Consecutive patients with suspected malignant biliary strictures who underwent ERCP from March 2008 to July 2009 at the University of Santo Tomas Hospital were included in the study. Brushing and biopsy were performed immediately after dilation using a $10 \mathrm{Fr}$ biliary balloon dilator. Gold standard for diagnosis was established by surgery, biopsy or follow-up.

Results: Among 48 patients with suspected malignant strictures, brush cytology and forceps biopsy were performed in 20 . The procedures were not done in 46 patients for various reasons: ampullary carcinoma $(60.9 \%)$, duodenal mass which precluded visualization of the ampulla (36.9\%) and CBD stone $(2.2 \%)$. The sensitivity, negative predictive value and accuracy of brush cytology and forceps biopsy are as follows: 53 versus 26 $(p=0.21), 41$ versus $31(p=0.09)$ and 65 versus $45,(p=0.114)$. Specificity and positive predictive values of both test is $100 \%$.

Conclusions: There is a trend of endoscopic bile duct brushing being more sensitive and accurate than forceps biopsy. It is a useful technique in the assessment of patients with suspected malignant bile duct strictures, and has high diagnostic yield which may contribute to clinical decisions.

\section{PP27-05}

Predictors and Outcome of Early Recurrence after Resection of Hepatic Metastases of Colorectal Cancer

M. Kaibori, K. Matsui, M. Ishizaki, S. Iwamoto, A.-H. Kwon

Surgery, Kansai Medical University, Hirakata, Japan

Background: This study investigated risk factors for early recurrence and examined the outcome after recurrence in patients undergoing R0 resection for liver metastases of colorectal cancer.

Methods: A total of 119 patients were divided into two groups, i.e., 54 patients who had recurrence within 2 years of surgery (early recurrence group) and 65 patients who remained disease-free for at least 2 years (2-year recurrence-free group).

Results: Only $4 / 65$ patients $(6 \%)$ from the 2-year recurrence-free group died during the initial 5-year period after surgery, while 29/54 patients $(54 \%)$ died in the early recurrence group. Multivariate analysis showed that postoperative morbidity was an independent predictor of the early recurrence of liver metastases of colorectal cancer.

Conclusions: Early recurrence is the leading cause of death within 5 years after R0 resection of liver metastases of colorectal cancer. Postoperative morbidity increases the risk of early recurrence in these patients. Therefore, if perioperative morbidity can be decreased, this will not only reduce medical costs but may also improve the outcome of $\mathrm{R} 0$ resection.

\section{PP27-06}

Timing of Resection for Synchronous Liver Metastases from Colorectal Cancer

M. Kaibori, M. Ishizaki, K. Matsui, S. Iwamoto, A.-H. Kwon

Surgery, Kansai Medical University, Hirakata, Japan

Background: This study aimed to compare the surgical outcome and long-term survival between simultaneous and delayed resection of liver metastases from colorectal cancer (LM), and to identify the factors influencing hepatic disease-free survival in patients with synchronous LM. Methods: Seventy-four patients with LM were divided into two groups, i.e., 32 patients who underwent hepatectomy at the time of colorectal surgery (simultaneous group) and 42 patients who underwent delayed liver resection (delayed group).

Results: The hepatic disease-free survival rates of patients from the delayed group with either $\geq 3$ or $<3$ liver metastases were significantly better than that of the simultaneous group. Mutivariate analysis showed that simultaneous resection was one of three independent prognostic indicators with an influence on hepatic disease-free survival. In 13 of the $42(31 \%)$ patients from the delayed group, new metastatic lesions were found in the same and/or different segments after re-evaluation during the interval between operations. These patients had a higher incidence of poorly differentiated or mucinous adenocarcinoma, shorter interval between procedures, and larger tumors than patients without tumor progression.

Conclusions: Tumor progression could be recognized and occult metastases were detected during the interval between operations. Delayed resection of synchronous LM may be useful to reduce the risk of rapid recurrence in the remnant liver. Patients with poorly differentiated or mucinous adenocarcinoma and those with larger tumors who undergo delayed liver resection should receive neoadjuvant chemotherapy during the interval between operations.

\section{PP27-07}

Combination of $\mathrm{Y}$ and Zilver Stents for Bilateral Stenting in Patients with Hilar Cholangiocarcinoma

D.H. Kang ${ }^{1}$, C.W. Choi ${ }^{2}$, H.W. Kim² ${ }^{2}$ S.B. Park ${ }^{2}$, M.H. Kim² ${ }^{2}$, C.W. Chung ${ }^{2}$, Y.I. Jeong ${ }^{2}$

${ }^{I}$ Gastroenterology; ${ }^{2}$ Pusan National University Hospital, Yangsan Hospital, Yangsan, Republic of Korea

Introduction: Although endoscopic bilateral metal stenting in a "stent in stent" fashion using a Y stent is currently used to treat patients with unresectable hilar cholangiocarcinoma, this method has limited application in cases of tight strictures, and inserting stents into the $\mathrm{Y}$ stent side is problematic in cases of tumor recurrence.

Objective: To evaluate the clinical efficacy of Y configured bilateral metal stenting using a $\mathrm{Y}$ and Zilver stent combination.

Design: Prospective, uncontrolled, single-center.

Setting: Tertiary referral university hospital.

Patients and methods: Seven patients with unresectable Bismuth type III or IV hilar cholangiocarcinoma.For endoscopic bilateral metal stenting, we first inserted a Y stent with central wide open mesh. An open cell type Zilver stent, which is preloaded in a slim delivery system (7 Fr), was placed into the contralateral hepatic duct through the central portion of the Y stent.

Results: Both technical and functional success was achieved in all seven patients (100\%). The early complication rate was $0 \%$, and late complications due to tumor recurrence occurred in 2 out of $7(28.6 \%)$ patients. These patients were managed by placement of additional stents or percutaneous transhepatic biliary drainage.

Conclusions: We suggest that a combination technique using $\mathrm{Y}$ and Zilver stents improves bilateral stenting for patients with advanced hilar cholangiocarcinoma and facilitates stent reinsertion in cases of tumor recurrence.

\section{PP27-08}

A Granulin-like Growth Factor Secreted by the Carcinogenic Liver Fluke, Opisthorchis viverrini, Promotes Proliferation of Host Bile Duct Cells M. Smout

James Cook Univeristy, Cairns, QLD, Australia

Background and aims: The human liver fluke, Opisthorchis viverrin, infects millions of people throughout South-East Asia and is a major cause of cholangiocarcinoma (bile duct cancer). The mechanisms by which the parasite causes cancer are multi-factorial, but one process is the secretion of mitogenic parasite proteins into the bile ducts, driving cell hyperproliferation and creating a tumorigenic environment.

Results: Using proteomics, we identified a homologue of human granulin, a growth factor involved in cell proliferation and would healing, in the secretions of the parasite. Opisthorchis granulin, termed $O v$-GRN-1, was expressed in most parasite tissues, particularly the gut and tegument. Furthermore, $O v$-GRN-1 was detected in situ on the surface of biliary epithelial cells of hamsters experimentally infected with Opisthorchis. Recombinant $O v$-GRN-1 stimulated proliferation of human cholangiocarcinoma and non-cancerous bile 
duct cell lines at nanomolar concentrations and antibodies against $O v$ GRN-1 inhibited the ability of Opisthorchis secretions to induce proliferation of fibroblasts and a human cholangiocarcinoma cell line in vitro, indicating that $O v-\mathrm{GRN}-1$ is the major growth factor in Opisthorchis secretions.

Conclusions: This is the first report of a secreted growth factor from a parasitic worm that induces proliferation of host cells, and supports a role for this fluke protein in establishment of a tumorigenic environment that may ultimately manifest as cholangiocarcinoma.

\section{PP27-09}

Outcome of Hepatectomy of Liver Metastasis from Colorectal Cancer before Bevacizumab and Cetuximab

N. Oba, J. Izeki, M. Takagi, K. Ohata, M. Watanabe, S. Sato, K. Nakagami, M. Tsuneizumi, K. Oshima, T. Watanabe, M. Sakata, Y. Hanaoka, K. Date,

T. Sato, H. Kosaka

Department of Surgery, Shizuoka General Hospital, Shizuoka, Japan

Background: The prognostic factors of hepatectomy for liver metastasis from colorectal carcinoma (LM) remain unconfirmed. We reviewed our series retrospectively.

Patients and methods: From 1997 through 2005, 79 patients with LM underwent hepatectomy. All had no local recurrence and unresectable other distant metastasis and follow-up for more than 5 years. For synchronous patients, except some advanced cases, the primary lesions were resected first, then liver metastases 1-3month later.

Table 1 Patient and tumor characteristics $(n=79)$

\begin{tabular}{ll}
\hline Age (year) & $63.0(33-87)$ \\
\hline Gender (M:F) & $54: 25$ \\
Number of lesions & $2.9(1-25)$ \\
Hepatic lesion & \\
Maximum diameter (cm) & $4.1(0.9-19)$ \\
Intrahepatic distribution & \\
$\quad$ Unilateral:bilateral & $64: 15$ \\
Primary lesion & \\
$\quad \begin{array}{l}\text { Tumor location (colon: rectum) } \\
\text { Temporal relationship }\end{array}$ & $48: 31$ \\
Synchronous:metachronous & \\
Lymph node metastasis & $35: 44$ \\
Yes:no & \\
\hline
\end{tabular}

Table 2 Univariate and multivariate analysis of prognostic factors

\begin{tabular}{|c|c|c|c|c|}
\hline \multirow[t]{2}{*}{$* p<0.05$} & \multicolumn{2}{|c|}{ Univariate } & \multicolumn{2}{|c|}{ Multivariate } \\
\hline & $\begin{array}{l}\text { Relative } \\
\text { risk }\end{array}$ & $P$ value & $\begin{array}{l}\text { Hazard } \\
\text { ratio }\end{array}$ & $P$ value \\
\hline \multicolumn{5}{|l|}{ Hepatic lesion } \\
\hline $\begin{array}{l}\text { Temporal relationship } \\
\text { metachoronous: synchornous }\end{array}$ & 1.372 & 0.173 & 1.117 & 0.651 \\
\hline $\begin{array}{l}\text { Number of lesion } \\
\text { solitary:multiple }\end{array}$ & 1.616 & $0.033 *$ & 0.953 & 0.975 \\
\hline $\begin{array}{l}\text { Maximum diameter } \\
\quad<5 \mathrm{~cm}:>5 \mathrm{~cm}\end{array}$ & 1.675 & $0.041^{*}$ & 1.158 & 0.747 \\
\hline $\begin{array}{l}\text { Intrahepatic distribution } \\
\text { unilateral:bilateral }\end{array}$ & 1.561 & 0.074 & 2.322 & 0.087 \\
\hline $\begin{array}{l}\text { Primary lesion Site } \\
\text { (colon:rectum) }\end{array}$ & 1.183 & 0.559 & 0.922 & 0.830 \\
\hline $\begin{array}{l}\text { Lymph node metastasis } \\
\text { yes:no }\end{array}$ & 7.708 & $<0.001^{*}$ & 28.505 & $<0.001 *$ \\
\hline
\end{tabular}

Results: The postoperative survival time after hepatectomy was 58.0 (1-156) month. Survival rates of 3, 5 and 10 years by Kaplan-Meier method were $62.7,44.4$ and $34.8 \%$ and disease free survival rates $40.8,34.7$ and $34.7 \%$. Number of lesion, tumor size and lymph node metastasis of the primary lesion (LNM) were significant prognostic factors in univariate analysis, but only LNM was significant in multivariate analysis. In 5-year survivors $(n=35)$, the recurrent patients had LMN more frequently than ones without it $(p<0.05)$. Some recurrent cases with chemotherapy survived long time.

Conclusion: Our result of hepatic resection for LM is valid and not inferior to other's reports. LNM is an important factor so chemotherapy by Bevacizumab or Cetuximab for the cases with LNM and recurrent cases may improve the operative result.

PP27-10

Hepatic Resection for Cholangioca

M.A. Wahab

Gastroentrology Center, Mansoura - Egypt, Mansoura, Egypt

Background and aim: Hilar cholangiocarcinoma still a challenging problem for surgeons and resectional surgery is the treatment of choice for long-term survival. In this study we tried to evaluate different prognostic factors after resection.

Methodology: From January 1995 to Augest 2010, 264 patients with hilar cholangiocarcinoma admitted to Gastroenterology Surgical Center. Mansoura University Egypt. Of these patients 240 underwent potentially curative resection the remaining 24 patients underwent non-surgical treatment because of advanced disease, advanced cirrhosis and poor general condition. Of the 24patients, (48\%) underwent localized hepatic resection and $(52 \%)$ patients underwent major hepatic resection. Various prognostic factors for survival were evaluated by univariate and multivariate analysis.

Results: Hospital mortality occurred in (11\%) patients. The most common post-operative complications were: bile leak, liver cell failure and wound infection 23.2, 17.8 and $9.5 \%$ respectively. The survival rates at 1, 2, 3, 4, 5 years were $79,32.6,18.5,137$ and $13 \%$ respectively. The result of univariate analysis revealed that radicality of resection, lymph nodes status, tumor differentiation, modified Bismuth staging, underlying liver pathology, HCV viral infection, blood transfusion, preoperative serum bilirubin $<10 \mathrm{mg}$ and $\mathrm{CA}^{19-9}$ are dependent prognostic factors. By multivariate Cox analysis radicality of resection, lymph nodes status, serum bilirubin below $10 \mathrm{mg} /$ $\mathrm{dL}$ level of $\mathrm{CA}^{19-9}$ and hepatitis viral infection were independent predictor factors.

Conclusion: From this study we found that aggressive surgical procedure to obtain curative resection with preoperative serum bilirubin below $10 \mathrm{mg}$ and $\mathrm{HCV}$ infective negative especially in non cirrhotic liver may bring a better prognosis in hilar cholangiocarcinoma.

\section{PP27-11}

ABCC3 as a Novel Prognostic Marker in Cholangiocarcinoma

U. Srimunta ${ }^{1,2}$ K. Sawanyawisuth ${ }^{1,2}$, C. Wongkham ${ }^{1,2}$, S. Wongkham ${ }^{1,2}$, K. Vaeteewoottacharn ${ }^{1,2}$

${ }^{1}$ Department of Biochemistry; ${ }^{2}$ Liver Fluke and Cholangiocarcinoma Research Center, Faculty of Medicine, Khon Kaen University, Khon Kaen, Thailand

Background/aim: The incidence of cholangiocarcinoma (CCA) in Northeastern Thailand is the highest in the world. Resistance to several chemotherapeutic drugs is a major obstacle for CCA treatment. High expression of drug efflux pumps especially ATPbinding cassette $(\mathrm{ABC})$ transporter has been demonstrated to play a role in multidrug resistant phenotype of cancers. In this study, we aimed to determine the expressions of $\mathrm{ABC}$ transporter genes in CCA tissues. 
Methods: RNA from 28 hepatectomized tissues were isolated and converted to cDNA by standard methods. cDNA was used as template for SYBR Green-based quantitative real-time PCR (qRT-PCR) amplification of $\mathrm{ABCC} 1, \mathrm{ABCC} 2, \mathrm{ABCC} 3$ and $\mathrm{ABCG} 2$. Gene expression levels were determined and categorized into low and high expressions by dichotomizing at median $\Delta \mathrm{C}_{\mathrm{t}}$ value. Clinical data of the patients was analyzed by multivariate analysis using Cox proportional hazard regression model. Patient survival was calculated from the time of surgical resection to death and the survival curves were constructed according to Kaplan-Meier, with a Log-Rank test. Results: From 28 CCA samples, $60.7 \%$ were men and $39.3 \%$ were women with an average age of 55 years. The median survival time of patients was 320 days. $46.4 \%$ were histologically classified as papillary type. Among four genes tested, only expression of ABCC3 was significantly associated with survival time $(p<0.05)$ in which high expression associated with long survival.

Conclusion: Our data indicated the relationship between ABCC3 expression and survival times of patients. This finding is the first to suggest role of $\mathrm{ABCC} 3$ as a prognostic marker in CCA. Functional study of $\mathrm{ABCC} 3$ in vitro is being investigated.

Acknowledgment: This work was supported by The Office of the Higher Education Commission and National Research University Program, Khon Kaen University, Thailand. US received a scholarship from the Liver Fluke and Cholangiocarcinoma Research center, Faculty of Medicine, Khon Kaen University, Thailand.

\section{PP27-12}

In vivo Liver and Lung Metastasis Cholangiocarcinoma Model K. Vaeteewoottacharn ${ }^{1,2}$, C. Subimerb ${ }^{1,2}$, S. Wongkham ${ }^{1,2}$, S. Okada ${ }^{3}$ ${ }^{I}$ Department of Biochemistry; ${ }^{2}$ Liver Fluke and Cholangiocarcinoma Research Center, Faculty of Medicine, Khon Kaen University, Khon Kaen, Thailand; ${ }^{3}$ Division III, Center for AIDS Research, Kumamoto University, Kumamoto, Japan

Background/aim: Cholangiocarcinoma (CCA) or bile duct cancer is the most common cancer in Northeastern Thailand. The incidence of CCA in this area is the highest in the world. Slow growing, high metastasis and resistance to multiple chemotherapeutic drugs are typical characteristics of CCA. To date, there is no standard regimen for CCA treatment. Development and screening of novel drugs are urgently needed. Therefore, in this study, we aimed to develop an animal model for CCA treatment response assessment in vivo.

Methods: Red fluorescent protein, mCherry, stably expressed KKUM213 CCA cell line (mCherry-KKU-M213) was previously developed in our lab. Fluorescent expressed cells were injected intrasplenically into NOD/SCID Jak deficient (NOJ) mice. Mice weights were monitored twice a week and mice were scarified at 3 week post-injection. In vivo fluorescent images of whole body and internal organs were captured using Meastro ${ }^{\mathrm{TM}}$ in vivo imaging system. Organs were weighted and were analyzed histologically for mCherry expression using standard protocol.

Results: In our model, body weights of mCherry-KKU-M213 injected mice were comparable to control group. Jaundice was detected in one mCherry-KKU-M213 injected mouse. Spleens, livers and lungs of mCherry-KKU-M213 injected group were significantly larger than those of control. In vivo fluorescent signal and immunohistochemistry staining using mCherry antibody showed multiple nodules in livers and lungs consistent liver and lung metastasis, respectively.

Conclusion: we have developed mCherry-KKU-M213 intrasplenic injected NOJ mice which liver and lung metastatic tumors were detected grossly and histologically. This might be a useful in vivo CCA model for metastatic study and for verification of anti-cancer/ anti-metastatic treatment outcome.

Acknowledgment: This work was supported by the Khon Kaen Research Fund, Thailand (grant no. 980-1-1055402) and KV was supported by the Matsumae International Foundation, Japan.
PP27-13

Clinical Presentation and Management of Patients with Cholangiocarcinoma

C.R.A. Lesmana ${ }^{1,2}$, L.A. Lesmana ${ }^{1,2}$, L.S. Pakasi ${ }^{2}$, B. Angga ${ }^{2}$

${ }^{1}$ Internal Medicine, Division of Hepatology, University of Indonesia;

${ }^{2}$ Internal Medicine, Digestive Disease Centre, Medistra Hospital, Jakarta, Indonesia

Background: Patients with cholangiocarcinoma (CCA) commonly remained undiagnosed until advanced stage. Currently, there is no established therapeutic option for unresectable cholangiocarcinoma.

Objective: To evaluate clinical characteristics and management of patients with CCA.

Method: We reviewed medical record data of patients with primary CCA between 2008 and 2010 in Medistra Hospital, Jakarta. Site of obstruction, blood chemistry, and tumor marker data were presented descriptively. Patients' management and survival were analyzed using Kaplan Meier's curve and Cox regression analyses.

Results: There were 23 cases of CCA, 14 (60.9\%) of them are women. Patients' median age was 64 years. Fifteen $(65.2 \%)$ patients showed hilar obstruction (Klatskin tumor), others were middle onethird in six patients, distal and proximal CBD each in one patient. All patients came with obstructive jaundice; mean bilirubin level was $18.2+8.89 \mathrm{mg} / \mathrm{dL}$. The mean CA19-9 tumor marker was 5,276.9 U/ $\mathrm{mL}$. Biliary drainage using stent was applied to $19(82.6 \%)$ of patients, $9(39.1 \%)$ of them had bilateral drainage. Mean patients' survival was $10 \pm 8.7$ months. There was no significant difference of survival between patients underwent nasobiliary tubing vs stenting (hazard ratio $[\mathrm{HR}]=1.074 \quad(95 \%$ confidence interval $[C I]=0.569-2.025$. Survival was also not differed between patients underwent unilateral vs bilateral stenting $\quad(\mathrm{HR}=2.012$; 95\% CI $=0.584-6.932$ ).

Conclusion: Most patients with CCA come with advanced stage with survival less than 1 year. Bilateral drainage has no added survival advantage over unilateral drainage.

\section{PP27-14}

Two Cases of Atypical Hepatic Hemangioma: Management Indication by ${ }^{11} \mathrm{C}$-acetate PET/CT

C.-L. Ho, S. Chen, Y.L. Leung, K.C. Cheng, K.K. Wong Hong Kong Sanatorium and Hospital, Hong Kong, China

Hepatic hemangiomas are venous malformations, often asymptomatic and managed conservatively. We reported two unusual cases of asymptomatic hepatic hemangiomas (negative HBV, normal alphafetoprotein), initially suspected as hepatocellular carcinoma (HCC) on contrast CT, presented for dual-tracer $\left({ }^{11} \mathrm{C}\right.$-acetate and $\left.{ }^{18} \mathrm{~F}-\mathrm{FDG}\right)$ PET/CT. Case description: 1. A 60-year-old man had a large lowattenuation mass in the left lobe of liver which was hypometabolic for ${ }^{11} \mathrm{C}$-acetate and isometabolic for ${ }^{18} \mathrm{~F}$-FDG (I). This dual-tracer pattern was not suggestive of HCC or other pathologies such as cholangiocarcinoma, liver metastases, FNH, angiomyolipoma or adenoma. The diagnosis of atypical hemangioma was suggested. Wedge biopsy confirmed the diagnosis and conservative management was followed. 2. A 41-year-old man had an intensely ${ }^{11} \mathrm{C}$-acetate-avid lesion (lesionto-liver ratio 6.4:1) in the same location as contrast CT with no abnormal ${ }^{18} \mathrm{~F}$-FDG activity (II). This intense degree of ${ }^{11} \mathrm{C}$-acetate uptake was previously found in angiomyolipoma, which was significantly higher than well-differentiated HCC or FNH. In view of its high metabolic rate that suggested a potential risk of bleeding, surgical removal was advised. Resection pathology was consistent with hemangioma of arteriovenous type (arteriovenous malformations) with areas of previous and impending hemorrhage. Microscopy showed marked proliferation of blood vessels of various types with capillary anastomoses in the fibrous stroma. Resection was considered a right decision for this patient. ${ }^{11} \mathrm{C}$-acetate PET/CT appears to be helpful in differentiating the arteriovenous type of hemangioma from 
the pure venous type, thus suggesting the correct way of management by the metabolic character of ${ }^{11} \mathrm{C}$-acetate in these seemingly malignant pathologies seen on CT.
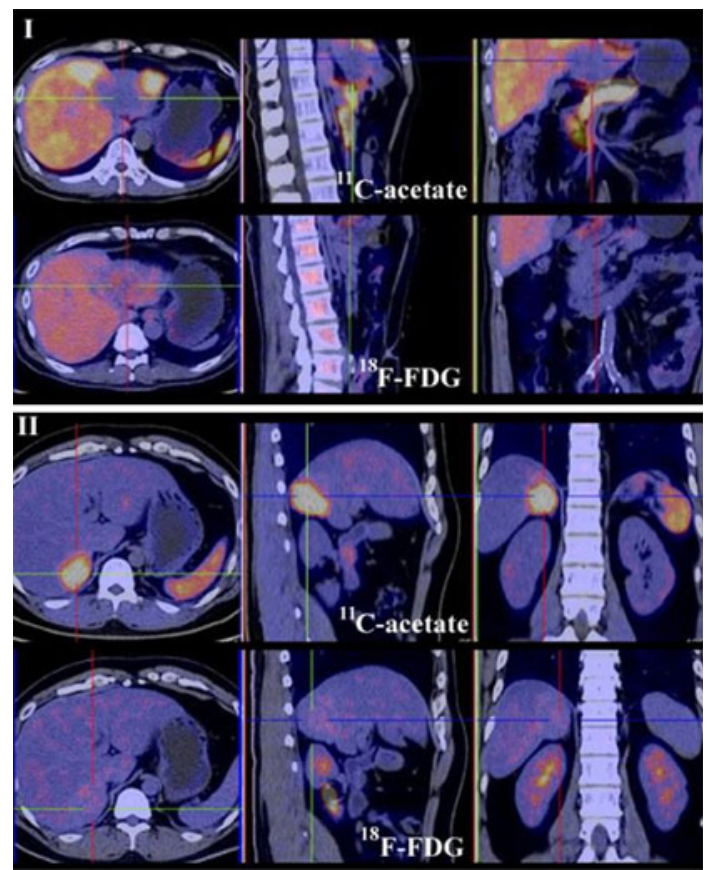

Hemangioma

\section{PP27-15}

Primary Extranodal Hepatic Diffuse Large B cell Lymphoma (DLBCL): Study of Eight Cases

I. Subortseva ${ }^{1}$, A.M. Kovrigina ${ }^{2}$, S.V. Lepkov ${ }^{3}$, G.S. Tymyan ${ }^{1}$,

G.I. Storogakov ${ }^{3}$, T.T. Kondratieva ${ }^{2}$

${ }^{1}$ Clinical Oncology, Russian Academy for Postgraduate Medical Education Minisry of Health of Russia; ${ }^{2}$ Blokhin Cancer Research Centre, ${ }^{3}$ The Moscow Medical University of N.N. Pirogov, Moscow, Russia

Primary hepatic lymphoma represents less than $1 \%$ of all extranodal lymphomas and there is no consensus on the best approach for management. We report eight cases of primary hepatic lymphoma diagnosed in Blokhin canser research center in the last 5 years. There were seven $(87.5 \%)$ men and one $(12.5 \%)$ woman, median age 47 years. The diagnosis DLBCL was made from biopsy specimens in all cases. Three patients had bones involvement, one patient was diagnosed from bone marrow biopsies, two patients had hilar lymph node involvement, and in two cases diagnosis was made incidentally from surgically removed spesiments-the patient was operated with hepatocellular carcinoma and other one was operated with intestinal impassability. All patients had non specific complains. Abdominal ultrasound combined with CT demonstrates a great variety of appearances. In four cases of primary lymphoma, single well-defined lesion was seen. Lymphoma occurred as multiple lesion in three cases. It appeared as a diffusely infiltrating lesion in only one case. There was no peripheral lymphadenopathy. Therapy depend of age, general condition, complications. In two cases the consensus was to treat with R-CODOX-M/IVAC. Radical resection with subsequent chemotherapy R-CHOP has been executed in one case. This patients are alive in complete remission after 3 months. One patient had systemic disease and intestinal impassability. He was undergo palliative operation, than chemotherapy CHOP. He died of acute hepato - nephritic insufficiency. Four patients were treated CHOP. With median of 38 months of follow-up, four patients are alive.

Conclusion: Primary hepatic lymphoma is rare entity with nonspecific clinical features that must be considered in the differential diagnosis of space-occupying lesions in the liver. Although no one imaging finding appear to be diagnostic of hepatic lymphoma. The rarity of hepar lymphoma does not allow to speak about presence of optimal therapeutical strategy.

\section{PP27-16}

Percutaneous Ultrasound-Guided Thermal Ablation of Intrahepatic Cholangiocarcinoma

H.-X. Xu

Sun Yat-Sen University, Guangzhou, China

Objective: To evaluate the value of percutaneous ultrasound (US) guided thermal ablation for intrahepatic cholangiocarcinoma (ICC). Methods: 17 patients with 24 histologically proven nodules of intrahepatic cholangiocarcinoma underwent US-guided thermal ablation with curative intention. The local treatment response, complication and survival were analyzed.

Results: 15 (88.2\%) patients had complete ablation, while 2 (11.8\%) patients with large tumors (6.4 and $6.9 \mathrm{~cm}$ in diameter) had residual tumor, so the complete ablation rate of all nodules was $91.7 \%(22 / 24)$. The mortality associated with thermal ablation was 0 , and $1(5.9 \%)$ patient had portal vein thrombosis after ablation, which resolved spontaneously. The follow-up period ranged from 1.1 to 80.4 months (mean $19.5 \pm 25.4$ months; median 8.8 months). 11 (64.8\%) patients were followed up for less than 1 year; $3(17.6 \%)$ for $1-5$ years; and 3 $(17.6 \%)$ for more than 5 years. $13(76.5 \%)$ patients developed recurrence in follow-up. The disease-free survival ranged from 0.5 to 80.4 months (mean $15.3 \pm 26.4$ months; median 4.0 months). 12 (70.6\%) died during the observation period. The cause of death included tumor progression $(10 / 12,83.3 \%)$, liver failure $(1 / 12,8.3 \%)$ and others $(1 / 12,8.3 \%)$.

Conclusion: Percutaneous US-guided thermal ablation therapy by means of MWA or RFA is an safe and effective therapeutic technique for intrahepatic cholangiocarcinoma.

\section{PP27-17}

Clinicopathologic Characteristics of Intrahepatic Cholangiocarcinoma Patients with Hepatitis B Virus Infection

L.-Y. Tao ${ }^{1}$, X.-D. $\mathrm{He}^{2}$

${ }^{1}$ China Rehabilitation Research Center; ${ }^{2}$ Peiking Union Medical College Hospital, Beijing, China

Background:To identify the role of HBV in the pathogenesis and prognosis of intrahepatic cholangiocarcinoma (ICC) by a retrospective study.

Methods: Ninety-seven ICC patients (59 males and 38 females) were divided into three groups:

Group I: hepatitis B virus surface antigen ( $\mathrm{HBsAg}$ )-positive and Hepatitis B core antibody (anti-HBc)-positive $(n=26)$;

Group II: HBsAg -negative and anti-HBc-positive $(n=50)$;

Group III: HBsAg -negative and anti-HBc-negative $(n=21)$.

The clinicopathologic features were analyzed and compared.

Results: Compared with Group III, the mean age $(P=0.018)$ and the positive rate of CA19-9 $(>37 \mathrm{U} / \mathrm{ml})(P=0.000)$ of Group Iwere significantly lower, while the positive rate of AFP $(>25 \mathrm{ng} / \mathrm{ml})$ $(P=0.012)$, the mean PT value $(P=0.030)$, hepatic cirrhosis $(P=0.001)$, the account of poor differentiation $(P=0.028)$ and weight loss $(P=0.041)$ were significantly higher. In addition, ICC patients in Group Itend to develop right liver tumor, and Group III tend to left liver tumor $(P=0.000)$. Furthermore, patients of Group Ihad better outcome compared to Group II and Group III $(P=0.010)$. Conclusion: ICC patients infected by HBV tend to develop tumor in the right liver tumor, at earlier age, be AFP positive, be CA19-9 
negative, get hepatic cirrhosis, have high PT mean value, be poor differentation, develop right liver ICC, get weightless and worsen outcome.

\section{PP27-18}

Discovery of a New Carbohydrate Marker for Diagnosis and Prognosis of Cholangiocarcinoma

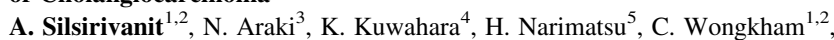
C. Pairojkul ${ }^{2,6}$, N. Sakaguchi ${ }^{4}$, S. Wongkham ${ }^{1,2}$

${ }^{1}$ Department of Biochemistry; ${ }^{2}$ Liver Fluke and Cholangiocarcinoma Research Center, Faculty of Medicine, Khon Kaen University, Khon Kaen, Thailand; ${ }^{3}$ Department of Tumor Genetics and Biology;

${ }^{4}$ Department of Immunology, Gradate School of Medical Sciences,

Kumamoto University, Kumamoto; ${ }^{5}$ Research Center of Medical Glycosciences, National Institute of Advanced Industrial Science and Technology, Ibaraki, Japan; ${ }^{6}$ Department of Pathology, Faculty of Medicine, Khon Kaen University, Khon Kaen, Thailand

Background: The current serum markers for cholangiocarcinoma (CCA) have low sensitivity and specificity to CCA. Our study was aimed to use monoclonal antibody (mAb) approach to detect a new CCA-associated marker with a better diagnostic parameters.

Methods: S121 mAb was obtained by using crude CCA tissueextracts as an immunogen. The $\mathrm{mAb}$ was used to detect $\mathrm{S} 121$-antigen in tissues and sera from CCA patients by immunohistiochemistry and lectin-captured ELISA, respectively. Protein digestion by protease and sugar oxidation by periodate were used to determine the biochemical component of the S121-mAb epitope. The specific glycan moiety for S121-mAb was identified by glyco-conjugate microarray and the carrier protein in CCA patient serum was identified by LC/ MS/MS.

Results: S121-mAb reacted strongly with neoplastic bile duct epithelium but not normal bile ducts and hepatocyte in CCA tissues. The S121-antigen was detected significantly higher in sera from CCA patients than those from the control groups, with $87 \%$ sensitivity and $89 \%$ specificity. High level of serum S121-antigen was related to poor survival of CCA patients and was significantly decreased after tumor removal. The S121-mAb recognized the carbohydrate moiety modified on serum MUC5AC mucin. However, S121-mAb did not react with any of 98 known carbohydrate antigens in the glyco-conjugate microarray, suggesting a new CCA associated marker of S121 antigen.

Conclusions: We have established S121-mAb which recognized a new carbohydrate marker related to CCA. The marker is useful for both diagnosis and prognostic prediction of CCA.

Acknowledgment: This works were co-supported by the Office of the Higher Education Commission, National Research Council of Thailand, and National Research University Program of Khon Kaen University (SHeP-GMS), and Kumamoto University.

\section{PP27-19}

Two Cases of G-CSF-producing Hepatobiliary Malignant Tumor with Diffused FDG Accumulation in Bone Marrow on PET-CT

T. Okamoto, Y. Iimuro, K. Suzumura, Y. Yoshida, Y. Asano, S. Saito, T. Okada, N. Kuroda, T. Hirano, J. Fujimoto

Surgery, Hyogo College of Medicine, Hyogo, Japan

We experienced two rare cases of granulocyte-colony-stimulating factor (G-CSF) producing hepatobiliary malignant tumor with characteristic views of 18F-fluorodeoxyglucose positron emission tomography (FDG-PET).

Case1: A 78-year-old man was admitted to our hospital with complaints of continuous high fever $\left(38-40^{\circ} \mathrm{C}\right)$ and appetite loss. He presented with marked leukocytosis $(30,000 / \mu 1)$ and high concentrations of serum C-reactive protein (CRP; $20-25 \mathrm{mg} / \mathrm{dl}$ ) and G-CSF. Abdominal computed tomography (CT) revealed a gall bladder tumor invading into the liver. PET/CT showed diffused FDG accumulation into the bone marrows, in addition to the tumor. Gall bladder carcinoma was confirmed by histological examination of the needle biopsy. Central bisectionectomy of the liver and lymphadenectomy were performed. After the surgery, WBC, CRP, and G-CSF levels were normalized and the performance status improved from 2 to 0 . $\mathrm{He}$ is healthy with no signs of recurrence 4 months after the surgery.

Case 2: A 61-year-old woman was admitted with complaints of the sharp pain in the epigastrium and left hypochondrium. She also presented with marked leukocytosis $(25,000 / \mu 1)$ and high concentrations of serum CRP (7-8 mg/dl) and G-CSF. Abdominal CT revealed an appearance of multiple intrahepatic cholangiocarcinoma (ICC) in the left lobe. Similar to Case1, FDG-PET showed diffused FDG accumulation into the bone marrows as well as in the tumors. Left hepatic trisectionectomy, lymphadenectomy, and hepaticojejunostomy were performed. The leukocytosis was improved transiently just after the operation, however 1 month later, it again became prominent accompanied by tumor recurrence in the remnant liver. She died of cancer metastases to multiple organs 3 months after the operation.

Discussion: The G-CSF-producing malignant tumors generally possess very poor prognosis. With early diagnosis employing FDG-PET, curative treatment could be performed in some of the patients. Thus it seems important to recognize the characteristic appearance of FDGPET in G-CSF producing tumors.

\section{PP27-20}

Microarray Analysis of 9 Intrahepatic Cholangiocarcinoma Cases H. Chen ${ }^{1}$, R. Hua ${ }^{2}$, F. Wang ${ }^{1}$

${ }^{I}$ Infectious Disease; ${ }^{2}$ Hepatopathy, First Hospital, Jilin University, Changchun, China

Background: Intrahepatic cholangiocarcinoma(CC) is an aggressive malignancy with poor prognosis. The causes of this disease lethality are not only its rapid growth but also its tendency to invade adjacent organs and metastasize. Genomic changes linked with CC progression remains unclarified. In this study whole genomic DNA microarray (Nimblegen 385k) were performed on nine intrahepatic CC cases to reveal Chromosome abnormalities that may responsible for $\mathrm{CC}$ development, progression or outcome.

Methods: A total of 15 intrahepatic CC tumor FFPE samples were collected from the First Hospital of Jilin University, Changchun, P.R. China. This study was approved by Ethnic Committee of the Hospital. Genomic DNA was extracted from FFPE tissues according to the optimized protocol which Roch recommended. To quality control the DNA samples,we use NanoDrop Spectrophotometer and run 1\% agarose gel. Whole genomic DNA microarray (Nimblegen 385k) were performed on nine good quality DNA samples. Then we draw CNC DNA MAP according to array images.

Results: The CNC DNA MAP shows gain of 8q24.3 (6/9), 11p15.3 (3/9), 16p13.3 (3/9), 19p13.3 (3/9), 20q13.3 (4/9), 22q13.3 (3/9) and loss of $18 \mathrm{q} 12.2(3 / 9)$.

Conclusion: Imbalance, either gain and loss are not the same as those have been reported before. Further tests are needed to confirm if these DNA bands harbor some genes that are related to intrahepatic CC.

PP27-21

Extracellular Cyclophilin A, via Interacting with its Membrane Receptor CD147, Enhances Cell Proliferation in Cholangiocarcinoma

S. Obchoei ${ }^{1,2}$, C. Chen ${ }^{2}$, Q. Yao ${ }^{2}$, C. Wongkham ${ }^{1}$, K. Sawanyawisuth ${ }^{1}$, S. Wongkham ${ }^{1}$

${ }^{I}$ Department of Biochemistry, The Liver Fluke and Cholangiocarcinoma Research Center, Faculty of Medicine, Khon Kaen Universiy, Khon Kaen, Thailand; ${ }^{2}$ Molecular Surgeon Research Center, Michael E. DeBakey Department of Surgery, Baylor College of Medicine, Houston, TX, USA

Background: We have previously shown that cyclophilin A (CypA) and its membrane receptor CD147, were over-expressed in 
cholangiocarcinoma (CCA) patients' tissues. Suppression of CypA expression significantly reduces cancer cell proliferation in vitro and tumor growth in nude mice, suggesting the possible roles of these molecules in carcinogenesis of CCA. In this study, we further investigated the effects and potential mechanisms of extracellular CypA and CD147 on cell proliferation of CCA cells.

Methods: The conditioned media (CM) were collected from 6 distinct CCA cell lines, CypA knockdown, and scramble control cells. Concentrated CM were subjected to western blot analysis for CypA detection. KKU100 and/or M139 cells were treated with CM or purified human recombinant CypA (hrCypA) for $48 \mathrm{~h}$. The cell proliferation assay was then performed using sulforhodamine B (SRB) staining.

Results: CypA was significantly detected in CM from CCA cell lines in correlation with intracellular CypA expression. CM from CypA knockdown cells significantly decreases cell proliferation as compared to the control CM $(p<0.05)$. Administration of purified hrCypA to CCA KKU100 and M139 cell cultures significantly increased the cell proliferation in dose dependent fashion $(p<0.05)$. This mitogenic effect of hrCypA was abolished when cells were pretreated with monoclonal antibody against CD147 but not with the IgG isotype control antibody.

Conclusions: In conclusions, we demonstrate that CypA enhances CCA cell proliferation. One of the possible mechanisms is that CypA is expressed and secreted by CCA cells and acts as autocrine/paracrine at least via interacting with its receptor CD147 on the cell membrane. Further characterization of CypA-CD147 interactions and the downstream signaling may eventually aid the development of targeted therapy for CCA.

Acknowledgment: Supported by the RGJ PhD Program, TRF (PHD/ 0210/2548) to SO and SW, grant of Faculty of Medicine, KKU, and Micheal E. DeBakey Department of Surgery at the Baylor

\section{PP27-22}

Cyclin D1, p16 and pRB Expression in Carcinoma of the Gallbladder V. Srivastava ${ }^{1}$, B. Kumar ${ }^{2}$, M. Shukla ${ }^{3}$, M. Kumar ${ }^{3}$, M. Pandey ${ }^{2}$

${ }^{I}$ Surgical Oncology, Institute of Medical Sciences, Banaras Hindu University; ${ }^{2}$ Surgical Oncology, Institute of Medical Sciences;

${ }^{3}$ Pathology, Institute of Medical Sciences, Banaras Hindu University, Varanasi, India

Background: In cell-cycle progression there are restrictions points called the cell-cycle check points that tightly regulate and finely orchestrate sequence of a normal cell cycle. Alteration in the expression of these proteins may play a critical role in tumorigenesis. Our study focused on the expression of cyclin D1, p16 and $\mathrm{pRB}$ in carcinoma of gallbladder to evaluate their relationship with pathogenesis, development and stage of the gallbladder carcinoma.

Methods: Between November 2007 and July 2009, 25 patients had gallbladder carcinoma (group I), 25 with cholelithiasis (group II) and 10 normal subjects were recruited. Median age was $47.40 \pm 8.119$ years. All the patients with cancer were staged using AJCC 2002 TNM classification, Majority of the patients $(n=17$, $68 \%$ ) were in stage II, while there were $16 \%$ patients in stage III $(n=4), 12 \%$ in stage IV $(n=03)$. Following immunohistochemical staining, quantitative immunohistochemistry (IHC) was performed. The data analyzed using percentages, categorical data was analyzed using Chi square test, and correlation was carried out by spearman correlation.

Result: All the three cases of distant metastasis (stage IV) expressed Cyclin D1 and it significantly correlated with metastatic disease ( $p$ value $=0.012)$. A positive correlation between $p 16$ expression and nodal involvement was observed in carcinoma group ( $p$ value $=$ $0.035)$. The difference in expression in any group was however not significant for Retinoblastoma protein $(p$ value $=0.327$ )
Conclusion: Cyclin D1 and p16 expression may indicate invasive and advance carcinoma of the gallbladder hence can be used as a biomarker in gallbladder malignancy and in prognosticating the patients.

\section{Poster Presentation 28: Cholelithiasis and Biliary Tract Disease}

\section{PP28-01}

Investigation on Mice of Sharp Toxicity of Methyl-tret-Buthyl Ether R.G. Saifutdinov, E.V. Trifonova

Chair of Tharapy, Kazan Medical Academy for Postdegree

Education, Kazan, Russia

Introduction: Methyl-tret-butyl ether (MTBE) is used at contact chemical dissolution (CCD) of cholesterol gallstones since 1981 (Jarrett L.N., et al., 1981; Leuschner U., et al., 1981). However no abroad, no in Russia on MTBE the sanction as on a medical product is not received.

Aim: To determine parameters of sharp toxicity MTBE at white mice. Materials and methods: MTBE entered per se to white mice intrastomach (Litchfield-Wilcocson). Calculations of average lethal dozes carried out (V.B.Prozorovski et al., 1978). Batching carried out on weight of substance, taking into account its density of $0,74 \mathrm{~g} / \mathrm{sm}^{2}$, and expressed in $\mathrm{mg} / \mathrm{kg}$ of weight of a body of animals. Research carried out together with «Institute of toxicology (Saint Petersburg, Russia). Results: The period of supervision was 14 days. Lethal, time of destruction, semiology of a poisoning carried out before introduction, for 7 and 14 days of supervision, opening and macroscopical research of all perishing and survived animals - at the end of research. The death of animals at intrastomach introduction of fatal dozes MTBE (more than $5 \mathrm{~g} / \mathrm{kg}$ ) was observed within 1.5-5 h from the moment of introduction at the phenomena of slackness, devocalization and a paralysis. Semiology was of the deep narcotic intoxication. Opening of the died animals has revealed: attributes venous full bloods of the internal bodies, subpleural haemorrhages, fine haemorrhages in an oblong brain and the big hemispheres of a bark. At the animals receiving less $5 \mathrm{~g} / \mathrm{kg}$, in the first day block, slackness, drowsiness, decrease of consumption of a forage and water were marked. In other days they did not differ from those at animals of control group.

Conclusion: Sharp intrastomach introduction MTBE does not cause macroscopical changes internal and glandulars bodies, a brain, and also mucous of a stomach and an intestines.

\section{PP28-02}

Surgical Indications for Gallbladder Polyp Are Need to Change? J.H. Shin ${ }^{1}$, Y.K. Cho ${ }^{2}$

${ }^{1}$ Surgery; ${ }^{2}$ Gastroenterology, Sungkyunkwan University, Seoul, Republic of Korea

Using the clinical and radiological data to differentiate true versus cholesterol Gall bladder (GB) polyps, can be useful to decide the treatment of the patient. 187 patients underwent cholecystectomy for GB polyps, divided into two groups, cholesterol polyps (146) and non-cholesterol polyps (41) based on the pathological findings. Gender, age, BMI, laboratory findings, size, number of polyps, present of stone, maximum diameter measured by preoperative radiological findings of the USG, CT and pathological diameter were comparative analysis. All the data were retrospectively analyzed, and the statistical analyses were performed with the Chi-square test and Mann-Whitney $U$ test. As a result patients who were diagnosed as cholesterol polyps had younger age and higher BMI than the other group. It was notable that $28.6 \%$ of the cholesterol polyps were not found in the preoperative CT, which were found on the USG, but the percentage of the undetectable rate was significantly lower $(8 \%)$ on 
the non-cholesterol polyp group. There is a discrepancy of maximum diameters between the two radiological methods in both groups but the discrepancy between the maximum diameters of the USG and CT was larger in the cholesterol polyp group than the non-cholesterol polyp group, which is statistically significant. Though a laparoscopic cholecystectomy can be considered as a less complicated operation, the necessity of the operation for $10 \mathrm{~mm}$ polyps should be reviewed once again. The clinical signs which can be helpful to diagnose whether it is a cholesterol polyp or not, before the operation, includes younger patients who have high BMI, polyps which are detectable on the USG but not on the CT, and the maximum diameters of the polyp between the USG and CT has a larger difference. If the polyp is considered as a cholesterol polyp the patient may have a chance to delay the operation and follow up.

\section{PP28-03}

Endoscopic Sphincterotomy Plus Endoprostheses in the Treatment of Large or Multiple Common Bile Duct Stones

W.-D. Hong, Q.-H. Zhu, Q.-K. Huang

Department of Gastroenterology and Hepatology, The First Affiliated Hospital of Wenzhou Medical College, Wenzhou, China

Background: Little information is available on the outcomes of endoscopic sphincterotomy plus biliary stents placement without stones extraction as primary therapy at the initial ERCP in the treatment of large or multiple common bile duct stones.

Aim: The aim of this study was to study the effect of biliary stents and sphincterotomy as primary therapy for patients with choledocholithiasis.

Methods: Patients with large ( $\geq 20 \mathrm{~mm}$ ) or multiple ( $\geq 3$ ) CBD stones were retrospectively studied. The patients underwent endoscopic sphincterotomy and placement of plastic stents in the bile duct without stone extraction at the initial ERCP. Three or more months later, a second ERCP was performed and stone removal was attempted. Differences in stone size and the largest CBD diameter before and after stenting were compared. Stone clearance, complications were also evaluated.

Results: 35 patients were enrolled. After a median 123 days of biliary plastic stents placement. The mean stone size decreased from 15 to $9.1 \mathrm{~mm}(P<0.01)$. The mean CBD diameter also decreased from 14.5 to $10.6 \mathrm{~mm}(P<0.01)$ The total stone clearance at second ERCP was $94.3 \%$, only $2.9 \%$ of which needed mechanical lithotripsy. Complications: pancreatitis in $1(2.9 \%)$ at initial ERCP, cholangitis in $1(2.9 \%)$ after 84 days of placement of stent. No complications were recorded at second ERCP.

Conclusions: Biliary plastic stents plus endoscopic sphincterotomy without stones extraction as primary therapy at the initial ERCP is a safe and effective method in the management of large or multiple CBD stone.

\section{PP28-04}

Evaluation of the Aetiological Spectrum of Obstructive Jaundice K. Siddique ${ }^{1,2}$, S. Mirza ${ }^{3}$, Q. $\mathrm{Ali}^{3}$, P.A. Zafar ${ }^{2}$

${ }^{1}$ Upper GI Surgery, East Kent Hospitals NHS Trust, Canterbury, UK; ${ }^{2}$ Holy Family Hospital, Punjab University; ${ }^{3}$ General Surgery, Holy Family Hospital, Rawalpindi, Pakistan

Objective: Evaluation of aetiological spectrum of obstructive jaundice.

Hypothesis: Malignant obstructive jaundice is more common than benign.

Study design: A prospective, descriptive study was done.

Place and duration of study: The study was conduted at Surgical Units-II Holy Family Hospital, Rawalpindi, Pakistan (May 2007 till April 2008).

Patients and methods: Sixty patients, who presented at the outpatient department were included in the study. Data including patients demographics, presentating complaints, examination findings, biochemical tests and other investigations like abdominal ultrasound scan, ERCP, CT-Scan, and MRCP and histopathology were collected. The analysis was performed using SPSS V.14.0.

Results: Out of the toatal 60 patients; $40(66.66 \%)$ were male and the rest were female [ $20(33.33 \%)$ ]. The mean age was 49.50 years. Malignant obstructive jaundice was seen in $34(56.66 \%)$ patients while $26(43.33 \%)$ had benign etiology. Amongst the commonest symptom; clay coloured stools $(75 \%)$ was more frequent in patients with malignant disease whereas abdominal pain $(51.66 \%)$ was most common in benign conditions. Commonest malignancy was Carcinoma $(\mathrm{Ca})$ of the head of pancreas 18/60 (30\%) followed by Ca gall bladder $8 / 60$ (13.33\%), cholangiocarcinoma $7 / 60$ (11.66\%), and periampullary carcinoma $1 / 60(1.66 \%)$. Choledocholithiasis $21 / 60$ (35\%) was the commonest benign cause followed by stricture of common bile duct $3 / 60(5 \%)$ and acute pancreatitis $2 / 60$ (3.33\%).

Conclusion: Malignant obstructive jaundice is more common than benign casuesproving our hypothesis. $\mathrm{Ca}$ head of pancreas is the commonest malignancy while Choledocholithiasis is the commonest benign cause. USG, ERCP and CT-Scan are important diagnostic modalities for evaluation of patient with obstructive jaundice with ERCP having the additional advantage of being therapeutic as well. Take home message: Obstructive jaundice should always be investigated and treated aggressively due to a high risk of malignancy. ERCP is the invastigation of choice and should be done as soon as possible.

\section{PP28-05}

Cholangiocyte Response after Injury Mediates the Temporal Dependence of the Experimental Model of Biliary Atresia

S.K. Mohanty ${ }^{1}$, M. Jafri ${ }^{1}$, B. Donnelly ${ }^{1}$, A. Bondoc ${ }^{1}$, S.R. Allen ${ }^{1}$, M. Mcneal ${ }^{2}$, R. Ward ${ }^{2}$, D. Witte ${ }^{3}$, G.M. Tiao ${ }^{1}$

${ }^{1}$ Department of Pediatric and Thoracic Surgery; ${ }^{2}$ Division of Infectious Diseases, ${ }^{3}$ Department of Pathology, Cincinnati Childrens Hospital Medical Center, Cincinnati, OH, USA

Background: Biliary atresia (BA) is the most common cause of pediatric end stage liver disease characterized by bile duct injury and obstruction of unknown etiology. The disease is usually seen in infants within 1-3 month of life and not in older children or adults. A murine model of BA has been established in which infection of newborn mice with rhesus rotavirus (RRV) leads to an obstructive cholangiopathy that mirrors the human disease. The purpose of the present study was to characterize the temporal relationship between viral infection and the induction of this model.

Methods: BALB/c mice were infected with RRV on day of life (DOL) $0,3,5$, and 7. Groups were characterized as early infection (infection before DOL 3) or late infection (infection after DOL 5). Subsets of early and late infection mice were sacrificed, and their extrahepatic biliary trees were harvested and assayed for viral antigen, replication competent virus, cytokine expression, histological evaluations, or immunohistochemical staining. Murine cholangiocytes were isolated ex vivo, cultured, infected with RRV, and the yield of replication competent virus was determined after one viral life cycle. Results: Early RRV infection caused symptoms in 95\% of pups and resulted in a mortality rate of $80 \%$. In contrast, late infection caused symptoms in only $50 \%$ of mice, and $100 \%$ of pups survived. These clinical findings correlated with histological analysis of extrahepatic biliary trees from the corresponding cohorts. Yield of replication competent RRV was higher in early cholangiocytes as was production of pro-inflammatory cytokines.

Conclusion: The degree of injury sustained by relatively immature cholangiocytes due to more robust RRV replication and pro-inflammatory cytokine production correlated with more severe clinical manifestations of cholangiopathy, including mortality. These findings establish a temporal dependence of RRV infection in murine BA and 
begin to define the cholangiocyte's physiologic role in this relationship.

\section{PP28-06}

Could Somatostatin Prevent Post-ERCP Pancreatitis or Hyperamylasemia? A Randomized, Double Blind, Controlled Preliminary Clinical Trial Z. Wang, W. Li

Department of Gastroenterology and Hepatology, Chinese PLA General Hospital, Beijing, China

Aims: To study the effect of somatostatin (SS) on preventing postERCP pancreatitis (PEP) and hyperamylasemia.

Methods: Patients who were indicated for ERCP were included in the study from December 2008 to May 2010. They were randomly divided into three groups: preoperative Group $(n=34)$-before ERCP (1-3 h), SS was administrated by continuously intravenous infusion $(0.5 \mathrm{mg} / \mathrm{h})$ for $24 \mathrm{~h}$; Postoperative Group $(n=49)$-after ERCP $1 \mathrm{~h}$, SS were administrated by continuously intravenous infusion $(0.5 \mathrm{mg} / \mathrm{h})$ for $24 \mathrm{~h}$; Placebo $\operatorname{Group}(n=42)$-saline were continuously intravenous infused until PEP was established. Once PEP occurred, SS was administrated by continuously intravenous infusion $(0.5 \mathrm{mg} / \mathrm{h})$. Anti-acid and anti-inflammatory drugs were avoided in all three groups. Patients' symptoms and signs were recorded and serum amylase and lipase levels were measured before ERCP and at time point 6,24 and $48 \mathrm{~h}$ after ERCP respectively.

Results: 125 patients were involved in the study. There were no significant difference between the three groups in items of age, gender, past medical history and ERCP procedures $(p>0.05)$. The overall incidence of PEP was $11.2 \%(14 / 125)$ and the individual incidence was comparable among the three groups, i.e. Preoperative Group $14.7 \%$ (5/34), Postoperative Group 8.2\% (4/49) and Placebo Group $11.9 \%(5 / 42)(p=0.6391)$. However, there is a significant difference in the incidence of hyperamylasemia that was defined as elevated amylase level without abdominal pain among the three groups, $23.5 \%$ (8/34) in Preoperative Group, 18.4\% (9/49) in Postoperative Group and 45.2\% (19/42) in Placebo Group ( $p=0.0136)$. Prophylactic SS used after ERCP procedures was significantly more effective to decrease the hyperamylasemia than placebo (18.4 vs. $45.2 \%, p<0.05)$, but there was no statistically significant difference of the incidence of PEP between the Preoperative Group and the Placebo Group (23.5 vs. $45.2 \%, p>0.05$ ).

Conclusion: SS did not seem to be effective in reducing PEP, however, it was useful in preventing hyperamylasemia in patients undergoing ERCP.

\section{PP28-07}

Effects of Relief of Obstructice Jaundice by Different Biliary Drainage on Expression of Bile Acid Receptor-TGR5 by Rat Kupfer Cells Z. Wang, W. Li

Department of Gastroenterology and Hepatology, Chinese PLA General Hospital, Beijing, China

Background: Our precious study demonstrated that internal biliary drainage (ID) could reverse the endotoxemia, releasing of cytokines and the expression of iNOSmRNA and CD14mRNA by Kupffer cells $(\mathrm{KCs})$ in rats with Obstructive jaundice (OJ), but external biliary drainage (ED) could not; furthermore, the hydrophilic bile acid salt could prevent $\mathrm{KCs}$ from the affection of endotoxin and cytokines. We presume that bile acid (BA) as a signaling molecule could play a protective role in improving $\mathrm{KCs}$ ' immune function by activating $\mathrm{BA}$ receptor-TGR5.

Aims: To detect TGR5 and TGR5 mRNA by rat KCs and then to explain the mechanism whether ID is superior to ED in relief of OJ. Methods: Male adult SD rats were randomly assigned to four groups: OJ $(n=17)$; SH $(n=20)$; ID $(n=14)$ and $\mathrm{ED}(n=15)$. KCs were isolated by in situ hepatic perfusion and digestion with collagen IV, density gradient centrifuged by Percoll reagent and purified by cell culture attachment. The TGR5 expression in liver tissue was determined by IHC and the expression of TGR5 mRNA by the KCs was detected by RTQ-PCR.

Results: The expression of TGR5 by KCs was significantly stronger in OJ group (OJ vs. SH $P<0.01$ ). The expression of TGR5 was significantly downregulated by ID (ID vs. OJ, $P<0.01$ ) (ID vs. SH $P>0.05$ ), but it could not be inhibited by ED (ED vs. OJ, $P>0.05$ ). The expression of TGR 5 mRNA by KCs was significantly stronger in $\mathrm{OJ}$ (OJ vs. SH $P<0.01$ ). Compared between ID and SH group, there was not statistically significant $(P>0.05)$. It was significantly stronger in ED group compared with that in SH group $(P<0.01)$, and there was still statistically significant between ED group and OJ group $(P<0.01)$.

Conclusions: The mechanism of ID is superior to ED in relief of OJ seems to be based on the regulation process of TGR5 genes.

\section{PP28-08}

An Improved Rendezvous Technique for Difficult Cannulation of the Bile Duct: A Report of 43 Cases

Z. Wang, W. Li

Department of Gastroenterology and Hepatology, Chinese PLA General Hospital, Beijing, China

Aims: To explore the effect of the improved rendezvous technique of PTBD and ERCP procedures to treat refractory obstructive jaundice caused by difficult cannulation of the bile duct.

Methods: Between 2003 and 2010, the clinical data of patients who underwent attempted endoscopic biliary therapy using the improved rendezvous technique were analyzed. These patients had failed endoscopy-alone procedures and had contraindications to surgery. The first procedure was percutaneous transhepatic access to the biliary tree guided under color Doppler ultrasound. Subsequent attempts were to introduce a guidewire or a cannula through the cannula or the guidewire accordingly into the duodenum. A duodenoscope is then positioned in the duodenum immediately or 2-3 days later after the percutaneous procedures. Lastly the wire or the cannula from the percutaneous transhepatic access guided the endoscopic catheter or wire to access the common bile duct accordingly, no need to grasp and extract the guide wire perorally.

Results: A total of 43 patients ( 29 male, 14 female, mean age $62.67 \pm 13.23$ years) were involved. The mean causes included: bile duct carcinoma $41.8 \%$ (18/43), carcinoma ofhead of pancreas $27.9 \%$ (12/43), duodenal papilla carcinoma $14 \%$ (6/43), primary liver cancer invading portal $9.3 \%$ (4/43), sclerosing cholangitis $7 \%$ (3/43). The PTBD approach to the biliary tree was successful in all cases. Subsequent attempts via common bile duct and papilla access to the duodenum were successful in all but three cases (93.0\%, 40/43). 23 cases succeeded in one time, and 17 cases in another time. The serum total bilirubin and direct bilirubin decreased 41.89 and $39.06 \%$ in 4 days after operation. Procedure-related morbidity and mortality were 7.7 and $0 \%$ respectively.

Conclusions: The improved rendezvous technique combined PTBD and ERCP approach is feasible, safe and effective in patients with difficult access to the bile duct. Procedure-related morbidity and mortality is low along with the improved rendezvous technique.

\section{PP28-09}

The Role of ERCP in the Era of Laparoscopic Cholecystectomy for the Evaluation of Choledocholithiasis in Sickle Cell Anemia

H. Issa ${ }^{1}$, A. Al-Salem ${ }^{2}$

${ }^{I}$ Internal Medicine, King Fahad Specialist Hospital-Dammam;

${ }^{2}$ Maternity and Children Hospital-Dammam, Dammam, Saudi Arabia

Aim: To evaluate the role of ERCP for choledocholithiasis in patients with sickle cell anemia (SCA) in the era of laparoscopic cholecystectomy (LC). 
Methods: 224 patients with SCA underwent ERCP as part of their evaluation for cholestatic jaundice (CJ). There were 144 males and 80 females. Their mean age was 22.4 years (5-70 years). The indications for ERCP were: CJ only in 97, CJ and dilated bile ducts on ultrasound in 103 and CJ and bile duct stones (BDS) on ultrasound in 42.

Results: In total, BDS were found in $88(39.3 \%)$ patients and in 16 , there was evidence of recent stone passage. Fifteen were post-LC patients. These had endoscopic sphincterotomy and stone extraction. The remaining 73 had endoscopic sphincterotomy (ES) and stone extraction followed by LC without an IC.

Conclusions: In patients with SCA and cholelithiasis, ERCP is valuable whether pre or post-operatively and in none there was a need to do IC. Sequential ES and stone extraction followed by LC is beneficial in these patients. ES may also prove to be useful in these patients as it may prevent the future development of biliary sludge and bile duct stones.

\section{PP28-10}

Endoscopic Palliative Treatment of Bile Duct Obstruction in Ampullary Cancer

S.B. Park, D.H. Kang, J.H. Kang, H.W. Kim, C.W. Choi

Pusan National University Hospital, Yangsan Hospital, Yangsan, Republic of Korea

Introduction: Ampullary cancer is a relatively uncommon tumor, and causes biliary obstruction. Endoscopic biliary drainage has been considred as a palliative treatment of choice in patients with inoperable ampullary cancer. However, there is no data which stent is superior in patients with inoperable ampullary cancer.

Objective: To compare the efficacy of metallic and plastic stents for biliary obstruction in patient with ampullary cancer.

Patients and methods: This study included all patients with ampullary cancer diagnosed by radiologic findings (CT and MRCP) and endoscopic finding with biopsy between January 2005 and January 2010. Among 45 patients, 22 patients underwent palliative drainage for ampullary cancer. Uncovered SEMSs were placed in 10 patients and double pigtail plastic stents were placed in 12 patients, respectively.

Results: Clinical success was achieved in all patients (100\%) in both groups. The median period of stent patency was 131.5 days in metallic stent group, and 126.5 days in plastic stent group $(p=0.665)$. Stent occlusion occurred $9(90 \%)$ and $8(67 \%)$ patients in metallic and plastic stent groups, respectively $(p=0.218)$. Stent migration occurred 1 $(10 \%)$ and $3(25 \%)$ patients in metallic and plastic stent groups, respectively $(p=0.368)$. Reintervention rate was 100 and $92 \%$ in metallic and plastic stent groups, respectively $(p=0.545)$.

Conclusion: Endoscopic biliary drainage using metallic or plastic stent is effective in patients with obstructive jaundice by ampullary cancer. Although metallic and plastic stent showed similar clinical effect, it seems to be reasonable to choose plastic stent as the first option in patients with ampullary cancer considering cost-effectiveness.

\section{PP28-11}

Endoscopic Bilateral Stenting and Endoscopic Revision in Patients with Hilar Cholangiocarcinoma

C.W. Choi, D.H. Kang, M.H. Kim, C.W. Chung, Y.I. Jeong

Pusan National University Hospital, Yangsan Hospital, Yangsan, Republic of Korea

Introduction: Endoscopic biliary drainage is one option of palliative treatments in patients with unresectable hilar cancer. Endoscopic bilateral drainage is very difficult and technically challenging.

Aims and methods: The aim of this study was to evaluate the clinical efficacy of bilateral metal stenting with the use of more slimmer (7 Fr) and open-cell design stent. This study was prospective, uncontrolled, single center study and tertiary referral university hospital.Thirty four patients with unresectable hilar cholangiocarcinoma (Bismuth type II to IV). The endoscopic bilateral metal stenting in "stent in stent" method was performed. At first, Y stent with central wide open mesh was inserted and then, Zilver stent, which is preloaded in a slimmer ( $7 \mathrm{Fr}$ ) delivery system than those (7.5-8.5 Fr) of other conventional stents, was placed into the contralateral hepatic duct through the central portion of $\mathrm{Y}$ stent. Zilver stent is open-cell design stent and it can be dilated easily. Thus, revision with bilateral plastic stents was tried in cases of stent obstruction.

Results: Technical success was achieved in 29 (85.3\%) patients. Functional success was noted in 29 (100.0\%) patients among 29 patients. Early complications such as pancreatitis and cholecystitis occurred in $3(10.3 \%)$ patients. Late complications occurred in 11 (37.9\%) patients. Cholecystitis, which occurred in two patients, was managed by percutaneous transhepatic gallbladder drainage. Stent obstruction by tumor ingrowth or overgrowth occurred in 9 out of 29 $(31.0 \%)$ patients. These patients were managed by placement of bilateral plastic stents (4/9), percutaneous transhepatic biliary drainage (4/9), and combined method (1/9). Of the five patients in whom endoscopic revision was attempted, four $(80 \%)$ patients were managed endoscopically by bilateral plastic stents.

Conclusions: A slimmer ( $7 \mathrm{Fr}$ ) and open-cell design stent is effective in endoscopic bilateral stenting for advanced hilar cholangiocarcinoma and endoscopic revision in case of tumor recurrence.

\section{PP28-12}

The Clinical Epidemiological Study of the Willingness to Pay Associated with Screening for Gallstone Disease

C.-T. Hsu, J.-H. Liu

Cheng-Hsin General Hospital, Taipei, Taiwan R.O.C.

Purpose: A cross-sectional study was conducted to assess the willingness-to-pay values (WTP) associated with varying degrees of gallstone disease based on the OPD patients at Cheng-Hsin General Hospital, Taipei, Taiwan.

Methods: The WTP values were measured by using discrete-choice method. A total of 120 OPD patients (30 subjects with no gallstone disease, 30 subjects with single stone, 30 subjects with multiple stones, and 30 subjects with cholecystectomy) evaluated WTP values from in January 1, 2007 to December 31, 2007. The diagnosis of gallstone disease was performed by a panel of specialists using ultrasound sonography.

Results: Seventy-six out of 120 subjects (63.3\%) answered that they would be willing to pay of the ultrasound sonography screening for reducing cholecystectomy. The overall mean WTP value was NTD $359.4 \pm 227.6$. Age was borderline significant related to WTP values. The subjects with severe stage of gallstone disease have higher WTP values for screening than those subjects with mild stage.

Conclusions: In conclusion, our results found that different degrees of gallstone disease were the independent factors to affect the WTP values of screening for gallstone disease.

\section{PP28-13}

Cholecystitis and Cholelithiasis in Patients on Total Parenteral Nutrition: Systematic Evaluation of 518 Adult Patients with Qualitative Meta-analysis

N.S. Mann

Gastroenterology-Hepatology, University of California,Davis School of Medicine,Sacramento, FOLSOM, CA, USA

Purpose: Many adult patients on total parenteral nutrition (TPN) develop cholelithiasis,cholecystitis, and biliary sludge. To evaluate this problem we reviwed the literature and performed qualitative meta-analysis (QMA) on 518 adult patients on TPN.

Methods: Pubmed search using terms TPN,cholelithiasis, cholecystis, biliary sludge without time or language barrier was performed. Additional papers manually added from reference lists of key aricles. To facilitate QMA,summary sheets of all papares were created. QMA was performed using known methods of Qualitative Research e.g. 
Diagramming, Theme Repitition Without Serious Contradiction, Theme Saturation and Investigator Refelexivity (Eval Rev 1985;9:627-643,the Lancet 2001;358:483-488). Quantitative data can be used to perform QMA.

Results: The search produced 518 adult patients on TPN for systematic evaluation.Information about gender available in $276 / 518$ (53.2\%).There were 177/276 (64.1\%) men and 99/276 (35.9\%) women. Information about age available in 434/518 (83.7\%). Mean age was 39.5 years (range 16-84). Information about duration of TPN available in $339 / 518(65.4 \%)$ cases; Mean duration of TPN 406.3 days(range $7-2,100$ ). About $25 \%$ develop symptomatic cholelithiasis,more develop biliary sludge.of this subset $16 \%$ developed acalculous cholecystitis(ACC). G.B.stasis is implicated in formation of GB stones. G.B ischemia may be responsible for ACC.Anticholinergic drugs and narcotics may be controbutory factors. US of abdomen and cholescintigraphy are complementary diagnostic modalities. Long acting Cholecystokinin infusion or infusion of ceruletid may prevent it. Early Cholecystectomy is definitive treatment. ACC patients can be managed by cholecystotomy.

Conclusion: About 25\% adults on TPN develop symptomatic cholelithiasis; of these $16 \%$ have ACC. Early cholecystectomy is recommended; ACC can be managed by cholecystotomy. The criteria for ACC are GB wall thicness of $3.5 \mathrm{~mm}$ and presence of pericholecystic fluid. In ACC if absence of gall-stones is confirmed, interval cholecystectomy may not be needed.

\section{PP28-14}

Endoscopic Treatment of Bile Leak Following Cholecystectomy

L. Kozumplík ${ }^{1}$, J. Ochmann ${ }^{2}$, J. Maršová ${ }^{1}$, V. Krass ${ }^{1}$

${ }^{1}$ Surgical Department; ${ }^{2}$ Endoscopy, Traumatological Hospital, Brno, Czech Republic

Aim: Bile leak is among the common complication of open and laparoscopic cholecystectomy. The aim of our retrospective study was to evaluate the efficacy of endoscopic approach in treatment of bile leakage following cholecystectomy.

Methods: We identified 12 patients with bile leak in standart postoperative drain of hepatic "bed" after laparoscopic (9 patients) and open (3 patients) cholecystectomy from January 2008 to September 2010. The presence of bile leak was confirmed by ERC (endoscopic retrograde cholangiography) with following therapeutic endoscopic procedures - sfincterotomy, extraction of remaining stone and biliary stenting (size $10 \mathrm{~F}$ ). The abdominal drainage was removed after bile leak stopped and stent was removed after interval 6-8 weeks.

Results: 12 patients ( 9 women, 3 men) underwent ERC for bile leak of abdominal drain after cholecystectomy within 5-16 days after surgery. The cholangiography identified the leak of cyst duct in nine cases, of duct of Luschka, of right hepatic duct and common bile duct in one case. All patients underwent sfincterotomy following by stenting ( $10 \mathrm{~F}$ stent). In three cases the remaining stone was extracted. The abdominal drain was removed within 3-12 days after stenting. There were no complications related to ERC, the stents were removed after 6-8 weeks endoscopically without problems.

Conclusions: ERC is safe and effective method for detection of bile leak. The most common bile leak after cholecystectomy is in cystic duct and endoscopic therapy-sfincterotomy with stenting is most effective in the management of bile leak after cholecystectomy.

\section{PP28-15}

PTC-CT in the Diagnosis of Biliary Obstruction

W. Cheng-Lin ${ }^{1}$, W. Yi-Xiang ${ }^{2}$, C. Lin ${ }^{1}$, S. Qiao ${ }^{1}$, X. Xian-Jun ${ }^{1}$, Y. Zhi-Dong ${ }^{1}$ ${ }^{1}$ Peking University Shenzhen Hospital, Shenzhen; ${ }^{2}$ Department of Organ Imaging, Chinese University of Hong Kong, Hong Kong, China

Objective: To explore a new method to show Biliary system to improve the diagnosis of Biliary Obstruction.
Materials and method: Seven cases of Biliary Obstruction took PTCCT examination in our hospital, five males and two females, aged between 24-74 years old, 47.7 years old on average, five of which were Hilar cholangiocarcinoma, one of which was Sclerosing cholangitis, one of which was inferior segment of common bile duct adenoma cancerization. First,all subjects were introduced PTC tube to percutaneous transhepatic cholangiography,injected contrast material (1.5-2\% iodine solution $30 \mathrm{ml}$ ), took CT scans after biliary tract filling; Then mainlined $300 \mathrm{mgI} / \mathrm{ml}$ iohexol contrast medium, at the speed of $3-5 \mathrm{ml} / \mathrm{s}$ and $1.5 \mathrm{ml} / \mathrm{kg}$, and scan during arterial phase, venous phase, delayed phase seperately; And finally upload the original data to Vitrea2 workstation for MPI,MIP and VR post-processing

Result: The procedure was successful in seven cases, six cases clearly showed the position of Biliary obstruction. Through CT scan, seven cases all show biliary dilatation ,but not the position of Lesion and Obstruction; During arterial phase, three cases show the slight enhancement of the lesion; During venous phase, seven cases have enhancement of the Lesion in different state, showing features like tram-track sign, eccentricity, irregularity stenosis. Bile ducts parietes thickening, intracavitary deletion. The MPI, MIP, VR reconstructions shows the Lesion in a much better way than Constant enhancement CT, MIP images can display the lesions most clearly, VR images can show the position of Lesion but failed to show what the Lesion in details for patients who suffer from severe biliary obstruction, PTCD treatment can be progressed with PTC-CT at the same time.

Conclusion: PTC-CT technology which has not been reported yet,is simple, practical, easy to promote, and is one of the advanced method to diagnose biliary obstruction.

\section{PP28-16}

Autopsy Features of Pancreaticobiliary Ductal Junction of Chinese People in MRCP

W. Cheng-Lin, C. Lin, Z. Hai-Yan, R. Xiao-Xuan

Perking University Shenzhen Hospital, Shenzhen, China

Objective: To study the autopsy features of pancreaticobiliary ductal junction of Chinese people,by statistically analyze a group of MRCP imaging sample in ShenZhen City which is made up of over $10,000,000$ domestic immigrants.

Method: Retrospective analyze 250 patients (140 males and 110 females) who received MRCP examines in our hospital. Autopsy of PDJ is divided into type of normal and abnormal. Abnormal type is divided into type of opening separately into the bulb and type of a common channel, including B-P type and P-B type. Imagines were determined by experienced doctors, and the converged angle between the bile duct and the pancreatic duct,the length of the co-duct and the height of the choledochal opening were also measured.

Results: There are 116 cases of nomal type and 134 cases of abnomal type-31 malfusion of pancreatobiliary ducts, 85 common channel, 54 B-P type, 31 P-B type. 85 cases of common channel have a length of $0.3-1.6 \mathrm{~cm}$ (average of $0.76 \mathrm{~cm}$ ). The convergered angle of common channel type is $5-135^{\circ}\left(45^{\circ}\right.$ on average). 66 are less than $45^{\circ}$. The height of the choledochal opening measuerd $0.8-4.2 \mathrm{~cm}$. Nine of 250 are pancreaticobiliary anomaly, two are right accessory hepatic duct, one is left accessory hepatic duct, four are common hepatic duct and left branch of hepatic proper artery, five are accessory pancreatic duct. Conclusion: According to the autopsy definition of normal PDJ, type of normal PDJ takes $46.4 \%$.The abnormal type are in a high frequency $(53.6 \%)$, however type of opening separately in to the bulb seldom has pancreatic secretion reflux, therefore it can be count as type of normal.The result of this group data indicates that among the type of a common channel, $77.6 \%$ of cases has a less than $45^{\circ}$ converged angle between the bile duct and the pancreatic duct, $50 \%$ of the cystic duct opening within a distance $2.6 \mathrm{~cm}$ from the bifurcation of the left and right hepatic ducts.This autopsy feature might be related to the occurrence of biliary or pancreatic diseases. 


\section{PP28-17}

Endoscopic Large Balloon Dilation for Revion of Old Sphincterotomy in Recurrent CBD Stones

S.H. Dong, C.H. Cho, Y. Hwangbo, J. Shim

Kyung Hee University College of Medicine, Seoul, Republic of Korea

Introduction: The rate of recurrent common bile duct (CBD) stones after CBD stone removal with endoscopic sphincterotomy (EST) is $5.8-24 \%$. The main cause of stone recurrence is post-EST ampullary stenosis. Endoscopic papillary large balloon dilation (EPLBD) could be used for the removal of recurrent CBD stones. We investigated the role of EPLBD in lowering the recurrence rate in patients with CBD stones.

Methods: Between 2001 and 2009, 577 patients with CBD stones underwent EST for bile duct stones at Kyung Hee Medical center. CBD stones recurred at least once in 56 patients. For the purpose of removing the recurrent $\mathrm{CBD}$ stone in 56 patients, 34 patients were treated with EPLBD and 22 patients were treated with EST. We compared the recurrence rates of CBD stones between the EPLBD group and the EST group.

Results: Among 56 patients with a first recurrence of CBD stones, 11 patients $(19.6 \%)$ had a second recurrence. The median duration of recurrence was $24.7 \pm 12.0$ months. The recurrence rate of CBD stones was $8.8 \%$ ( 3 of 34 ) in the EPLBD group and $36.3 \%$ ( 8 of 22 ) in the EST group. These rates were significantly different in multivariate analysis (logistic regression analysis; $p$-value $=0.019$ ). There were no significant differences between two groups with regard to other risk factors such as CBD diameter, diverticulum, or cholecystectomy history. In the EPLBD group, there was no significant complication such as massive bleeding or post ERCP pancreatitis. During the follow-up period, the cumulative rate for second recurrent CBD stones was also lower in the EPLBD treatment group compared to that in the EST group (Kaplan-Meier's method; $p$-value $=0.028$ ).

Conclusions: The EPLBD lowered the second recurrence rate of CBD stones in patients who were previously treated for CBD stones with EST. Therefore, EPLBD is an effective and safe treatment for recurrent CBD stones after EST.

\section{PP28-18}

Peribiliary Fibrotic Markers in Experimental Opisthorchiasis and Cholangiocarcinogenesis: A Promising Target for Predictive Risk of Human Cholangiocarcinoma

S. Prakobwong ${ }^{1,2}$, L. Charoensuk ${ }^{1,2}$, P. Yongvanit ${ }^{2,3}$, C. Pairojkul ${ }^{2,4}$ Y. Hiraku ${ }^{5}$, P. Sithithaworn ${ }^{1,2}$, P. Pinlaor ${ }^{2,6}$, S. Pinlaor ${ }^{1,2}$

${ }^{1}$ Department of Parasitology, Faculty of Medicine, Khon Kaen University; ${ }^{2}$ Liver Fluke and Cholangiocarcinoma Research Center, Faculty of Medicine, Khon Kaen University; ${ }^{3}$ Department of Biochemistry; ${ }^{4}$ Department of Pathology, Faculty of Medicine, Khon Kaen University, Khon Kaen, Thailand; ${ }^{5}$ Department of

Environmental and Molecular Medicine, Mie University Graduate

School of Medicine, Mie, Japan; ${ }^{6}$ Centre for Research and

Development in Medical Diagnostic Laboratory, Faculty of

Associated Medical Sciences, Khon Kaen University, Khon Kaen,

Thailand

Background/aim: Persistence of peribiliary fibrosis caused by chronic Opisthorchis viverrini infection is a risk factor of cholangiocarcinoma (CCA), which is a health problem in Northeast, Thailand. We aim to clarify the molecular mechanism of $O$. viverrin-induced peribiliary fibrosis involved cholangiocarcinogenesis in animal and applied to evaluate the fibrotic markers to predict the carcinogenic risk of opisthorchiasis-associated CCA in patients.

Methods: We determined the time profile of fibrosis with O. viverrini infection, either or $\mathrm{N}$-nitrosodimethylamine-treated hamster induced cholangiocarcinogenesis. The molecules linked between inflammation, fibrogenesis and carcinogenesis such as MMPs, TIMPs, collagens, hydroxyproline levels, and MMP-9 mediated Rac1- dependent DNA lesion formations were investigated. The plasma candidate fibrotic markers levels were determined in human opisthorchiasis, benign biliary disease (BBD), CCA, other tumor types, and healthy individuals (HA).

Results: The peribiliary fibrosis increased with time-dependently in $O$. viverrini-infected hamsters, and this correlated to experimental cholangiocarcinogenesis. The fibrotic markers including collagen I, MMP-7 hydroxyproline, MMP-9, and TIMP-2 were positively correlated with the degree of hepatobiliary disease and staging of CCA. MMP-9 expression was correlated with fibrosis level, iNOS and Rac1 expressions that potentiate DNA damage and CCA development. Plasma level of collagen I, MMP-7 and hydroxyproline were significantly increased in the order of CCA $>$ opisthorchiasis $>$ HA. Among these, only collagen I level was statistically different between CCA and BBD. ROC curve analysis revealed that collagen I, MMP-7, and hydroxyproline levels were significantly distinguished between opisthorchiasis and BBD. Interestingly, odds ratio analysis showed these fibrotic markers in opisthorchiasis were useful to predict the risk for BBD, and only collagen I level in BBD was a significant risk factor for CCA.

Conclusion: The peribiliary fibrosis was significantly increased with time-dependently in opisthorchiasis associated CCA. Collagen I, MMP-7, and hydroxyproline levels are the novel markers of opisthorchorsis, and collagen I is a potential marker to predict the hepatobiliary disease-associated CCA.

\section{PP28-19}

Repeated Rendezvous Treatment of PTBD and ERCP in Patients with Recurrent Obstructive Jaundice

Y. Liu, Y. Yang

Gastroenterology and Hepatology, Military General Hospital

of China, Beijing, China

Background and aims: Repeated applications of rendezvous technique combining percutaneous transhepatic biliary drainage (PTBD) with endoscopic retrograde cholangiopancreatography (ERCP) (PE) in patients with recurrent obstructive jaundice have not been reported. We aimed to evaluate treatment effects of this technique in patients who previously received the same rendezvous treatment.

Patients and methods: Repeated PE rendezvous procedure was performed in 27 patients who received the same procedure previously and had recurrent obstructive jaundice. Twenty-two patients were treated by second-time rendezvous procedure and five patients by third-time. The clinical characteristics and therapeutic effects were retrospectively analyzed.

Results: By means of repeated rendezvous technique, Twenty-six patients gained access to the bile duct and were successfully implanted new stents, only one failed with stent implantation. Total serum bilirubin level decreased within one week from $221.89 \pm 64.70 \mu \mathrm{mol} / \mathrm{L}$ to $156.0 \pm 32.2 \mu \mathrm{mol} / \mathrm{L}$ in patients with second-time treatment and from $297.07 \pm 109.12 \mu \mathrm{mol} / \mathrm{L}$ to $238.77 \pm 36.81 \mu \mathrm{mol} / \mathrm{L}$ in patients with third-time treatment. There was no severe complications observed that are associated with repeated PE procedure.

Conclusion: Rendezvous procedure of PTBD and ERCP could be used repeatedly and effectively for patients who present recurrent obstructive jaundice after ERCP failure.

\section{PP28-20}

Serum Adiponectin, Retinol Binding Protein-4, Hyaluronic Acid, and Transient Elastography $\left(\right.$ FibroScan $\left.{ }^{\circledR}\right)$ as Fibrosis Markers for Predicting Severity in Biliary Atresia

S. Honsawek ${ }^{1,2}$, M. Chayanupatkul ${ }^{2,3}$, V. Chongsrisawat ${ }^{2,4}$, P. Vejchapipat ${ }^{5}$,

L. Vimolket ${ }^{2}$, Y. Poovorawan ${ }^{2,4}$

${ }^{1}$ Biochemistry; ${ }^{2}$ Center of Excellence in Clinical Virology;

${ }^{3}$ Physiology; ${ }^{4}$ Pediatrics; ${ }^{5}$ Surgery, Faculty of Medicine,

Chulalongkorn University, Bangkok, Thailand 
Background: Biliary atresia (BA) is a neonatal liver disorder characterized by chronic inflammatory obliteration of extrahepatic bile ducts. Adiponectin and retinol binding protein-4 (RBP-4) are known to be adipokines that play essential roles in chronic liver diseases. The purpose of this study was to investigate relationship of serum adiponectin, RBP-4, hyaluronic acid (HA), and liver stiffness in BA patients.

Methods: Forty-eight BA patients post Kasai operation and 24 healthy controls were enrolled. BA patients were classified into two groups according to their serum total bilirubin levels (TB $<2$, no jaundice vs. TB $\geq 2 \mathrm{mg} / \mathrm{dl}$, jaundice). Adiponectin, RBP-4, and HA levels were determined by enzyme-linked immunosorbent assay. Liver stiffness scores were measured by transient elastography (FibroScan ${ }^{\circledR}$ ).

Results: BA patients had significantly higher adiponectin and HA levels, but lower RBP-4 levels than controls. Serum adiponectin and HA levels were significantly elevated in BA patients with jaundice compared with those without jaundice. In contrast, serum RBP-4 levels were significantly decreased in BA patients with jaundice compared with those without jaundice (controls, BA, non-jaundice, jaundice; adiponectin $11.1 \pm 1.0, \quad 15.8 \pm 1.2, \quad 12.7 \pm 1.3$, $20.9 \pm 1.9 \mu \mathrm{g} / \mathrm{ml}, \quad$ HA $25.0 \pm 1.5, \quad 53.3 \pm 8.4, \quad 29.0 \pm 3.1$, $93.6 \pm 18.4 \mathrm{ng} / \mathrm{ml}, \quad$ RBP-4 $18.7 \pm 1.0, \quad 14.9 \pm 1.0, \quad 18.2 \pm 1.2$, $9.5 \pm 0.9 \mathrm{ng} / \mathrm{ml}$, liver stiffness $5.0 \pm 0.5,29.5 \pm 3.3,17.1 \pm 2.7$, $50.1 \pm 4.4 \mathrm{kPa}, P<0.001$, respectively). BA patients with jaundice had significantly higher serum bilirubin, aminotransferases, alkaline phosphatase, aspartate aminotransferase to platelets ratio index, and liver stiffness value compared to those without jaundice. Area under receiver operating characteristic (ROC) curve of adiponectin, RBP-4, and HA for liver fibrosis were $0.721,0.793$, and 0.795 , respectively. Further analysis showed that adiponectin and HA significantly correlated with liver stiffness of BA in contrast with RBP-4 inversely correlated $(P<0.001)$

Conclusion: Adiponectin, RBP-4, HA, and liver stiffness might be useful as biomarkers reflecting the severity of liver dysfunction and could be predictive of prognosis with respect to the progression of postoperative BA.

\section{PP28-21}

Effects of Early Enteral Nutrition on Acute Inflammatory Response Syndrome after the Operations for Obstructive Jaundice

Q. Chen

Surgery, Binzhou Medical College, Binzhou, China

Objectives: To observe the effects of early enteral nutrition (EN) on system inflammatory response syndrome(SIRS) after the operations for obstructive jaundice (OJ).

Methods: According to random table, 60 cases of postoperative patients with obstructive jauncdice were divided into three groups accepting early enteral nutrition, parenteral nutrition or general IV infusion respectively. CRP, IL- 6 and TNF- $\alpha$ were measured on the day before operation and on the postoperative day 1,3, 5, 7 and 9 . At the same time, the clinical conditions of patients with SIRS, MODS and postoperative complications were observed and analyzed.

Results: Patients in all groups had SIRS after operations. The levels of CRP, IL- 6 and TNF- $\alpha$ on the day 3, 5, 7 and 9 after operations were lower in the EN group than those in the PN group and contral group. The duration of SIRS in the EN group was significantly shorter than that in the PN group and contral group. The morbidity of MODS and postoperative complications was lower significantly in the EN group than that in the PN group and contral group.

Conclusion: Early EN after operations on the patients with OJ can positively modulate SIRS and decrease the morbidity of MODS and postoperative complications.
PP28-22

The Role of Meteorological Factors in the Incidence of Cholecystectomy: A Population Study in Taiwan

C.-M. Liu ${ }^{1}$, N. Huang ${ }^{2}$, C.-T. Hsu ${ }^{3}$, Y.-J. Chou ${ }^{4}$

${ }^{1}$ General Surgery, Cheng Hsin General Hospital; ${ }^{2}$ Institute of

Hospital and Health Care Administration, National Yang-Ming

University; ${ }^{3}$ Division of Gastroenterology, Cheng Hsin General

Hospital; ${ }^{4}$ Institute of Public Health, National Yang-Ming University, Taipei, Taiwan R.O.C.

Introduction: We systematically investigated the relationship between meteorological factors and cholecystectomy incidence using data from cholecystectomy cases and meteorological factors from 1996 to 2008. We determined the statistical significance of the time series data using cross-correlation coefficients and autoregressive integrated moving average (ARIMA) model. Sunshine hours and winter affected cholecystectomy incidence more significantly than temperature and atmospheric pressure.

Method: We performed a retrospective longitudinal study of 194,518 patients who underwent cholecystectomies (ICD Operation Code 51.2) from 1996 to 2008; their data was collected using Taiwan National Health Insurance Research Database. We included patients who were aged $\leq 20$ or $\geq 80$ years, and $45.69 \%$ of patients were men. Meteorological data (ambient temperature, relative humidity, atmospheric pressure, and monthly hours of sunshine) from 1996 to 2008 was obtained from the Central Weather Bureau of Taiwan. The mean number of cholecystectomies performed in each season was calculated for different genders and age groups.

Results: The cross-correlation meteorological coefficients showed that temperature, atmospheric pressure, and sunshine hours contributed significantly to the incidence of cholecystectomy. In patients aged $\geq 80$ years, relative humidity was the only factor significantly associated with seasonal cholecystectomy rates, which was in stark contrast to the significant factors found in other age or gender categories. Adjustment by ARIMA regression model revealed that only sunshine hours consistently showed a significant association with the incidence of cholecystectomy for both genders and all age groups.

\section{Conclusions:}

1. Women and patients above 65 years of age had higher cholecystectomy incidences.

2. Sunshine hours was identified as the most significant factor influencing cholecystectomy incidence by using the ARIMA regression model.

3. Winter was identified to be mainly associated with cholecystectomy incidence by using ARIMA model to eliminate multiple variables within meteorological factors.

4. Summer or autumn may be the key seasons for exacerbation of gallstone disease.

\section{PP28-23}

The Effect of Obstructive Jaundice on the Tight Junction Protein ZO-1 and the Permeability of the Intestinal Mucosa in Rats

Q. Chen, P. Wu, X. Zhang, F. Zhang

Binzhou Medical College, Binzhou, China

Objectives: To study the effect of obstructive jaundice on the tight junctionprotein ZO-1 and the permeability of the intestinal mucosa in rats.

Methods: 30 male Wistar ratswere assigned randomly to three groups: group control, sham and OJ. In group OJ, thecommon bile duct was ligated to establish the animal model. 7 days later, the level of portal vein blood D-lactate was detected and immunohistochemistry was used to examine the distribution and expression of tight junction proteins (ZO-1). 
Results: The level of portal vein blood D-lacate was significantly higher than group control and sham (all $P<0.05$ ), (Table 1) and ZO1 staining appeared discontinuous and vague with rough edges, strong positive express ratio decreased significantly.

Conclusions: The distribution of ZO-1 confused and the expression reduced, the intestinal mucosal permeability increased, injured the intestinal mucosal barrier under the pathophysiological condition of OJ.

Level of portal vein blood D-lacate in the obstruc

\begin{tabular}{lll}
\hline Group & $n$ & D-lacate \\
\hline Control & 10 & $2.96 \pm 0.19$ \\
Sham & 10 & $3.03 \pm 0.22$ \\
OJ & 10 & $4.53 \pm 0.71^{*}$ \\
\hline
\end{tabular}

\section{PP28-24}

Expression of Farnesoid X Receptor, Angiogenin 1 and Carbonic Anhydrase 12 mRNA in Ileum Mucosa after Relief from Obstructive Jaundice L. Wu, W. Li

Department of Gastroenterology and Hepatology, Chinese PLA General Hospital, Beijing, China

Background and aims: Our previous study demonstrated that internal biliary drainage (ID) could inhibit bacterial overgrowth, translocation and mucosal injury in ileum of rats with obstructive jaundice (OJ), but external drainage (ED) could not. The aim of the present study is to clarify the mechanism that ID is superior to $\mathrm{ED}$ in reversing the intestinal dysfunction in rats with OJ by investigating the expression of farnesoid $\mathrm{X}$ receptor (FXR), angiogenin 1 (ANG1) and carbonic anhydrase 12 (CAR12) mRNA in the ileum mucosa.

Methods: Sixty male adult Sprague-Dawley rats were randomly assigned to four groups: OJ $(n=15), \mathrm{ID}(n=15), \mathrm{ED}(n=15)$ and sham operation $(\mathrm{SH})(n=15)$. The ileal mucosa was scraped and stored in liquid nitrogen. The expression of FXR, ANG1 and CAR12 mRNA in the ileal mucosa were detected by reverse transcription polymerase chain reaction (RT-PCR).

Results: The expression of FXR, ANG1 and CAR12 mRNA in the ileal mucosa were decreased in OJ group $(0.231 \pm 0.039$, $0.190 \pm 0.064,0.236 \pm 0.054)$ and ED group $(0.266 \pm 0.058$, $0.205 \pm 0.057,0.257 \pm 0.050)$ compared with that in SH group $(0.528 \pm 0.090,0.385 \pm 0.072,0.490 \pm 0.074)(P<0.01)$ and ID group $(0.485 \pm 0.094,0.365 \pm 0.068,0.470 \pm 0.066)(P<0.01)$ (Figs. 1, 2, 3).
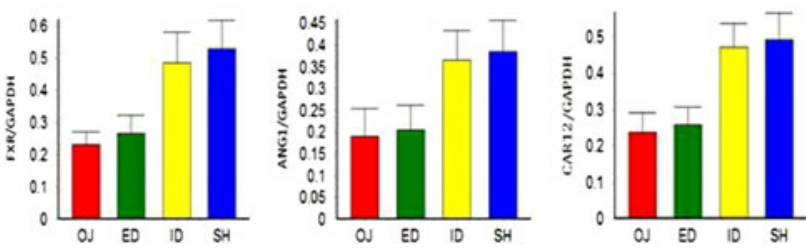

Figs. 1-3 Expression of FXR, ANG1 and CAR12 mRNA

Conclusion: The expression of FXR, ANG1 and CAR12 mRNA in ileal mucosa were decreased in OJ rats. ID could reverse the changes, but ED could not. The mechanism that internal biliary drainage is superior to external drainage in relief of obstructive jaundice bases on the regulation of FXR, ANG1 and CAR12 genes.
PP28-25

Internal Biliary Drainage Is Superior to External Drainage in Reversing Intestinal Mucosal Dysfunction in Rats with Obstructive Jaundice

L. Wu, W. Li, Z. Wang, Z. Yuan

Department of Gastroenterology and Hepatology, General Hospital of the Chinese People's Liberation Army, Beijing, China

Background and aims:Our previous studies demonstrated that internal biliary drainage (ID) is superior to external biliary drainage (ED) in terms of reversing Kupffer cell dysfunction in rats with obstructive jaundice (OJ). We assumed that the mechanism were related to intestinal dysfunction. So the present study was to investigate the mechanism by measurement of intestinal mucosal morphology, occludin, farnesoid X receptor (FXR) and bacterial translocation rate(BTR)with OJ and its relief by ID and ED.

Methods: Fifty-nine male Sprague Dawley rats were randomly assigned to four groups: $\mathrm{OJ}(n=14), \mathrm{ID}(n=16), \mathrm{ED}(n=15)$ and sham operation( $\mathrm{SH}, n=14)$. Blood from portal vein and inferior vena cava were collected for bacterial culture. Level of serum total bile acid (TBA)and white blood cell count (WBC) were tested. The terminal ileum was harvested for study of routen histology. Occludin and FXR were determined by immunohistochemical method.

Results:After bile duct ligation, WBC and TBA were significantly increased $\left(11.4 \pm 2.7 \times 10^{9} / \mathrm{L}\right.$ and $118.9 \pm 39.0 \mu \mathrm{mol} / \mathrm{L}$ respectively) compared with that in $\mathrm{SH}$ rats $\left(5.5 \pm 1.0 \times 10^{9} / \mathrm{L}\right.$ and $38.0 \pm 15.0 \mu \mathrm{mol} / \mathrm{L}$ respectively, both $P \leq 0.05$ ). The ileum villous became the lowest but thickest. The mucosal lesion grade according to Chiu was high. The expression of FXR was increased while occludin decreased. The BTR was $57.1 \%$, but increased to $80 \%$ in ED and $62.5 \%$ in ID. After ED,WBC reduced lightly while TBA greatly. The ileum villous became higher and thinner, and the mucosal lesion grade was lower than OJ. The expression of FXR was the weakest but the occludin not changed(ED vs. OJ, $P \geq 0.05$ ). After ID, not only WBC decreased,but also TBA, ileum structure, mucosal lesion grade, expression of FXR and occludin were almost same as SH $(P \geq 0.05)$. The bacterial species and BTRs were same in the portal vein and inferior vena cava in each group.

Conclusions: Compared with ED, ID can recover the enterohepatic circulation, increase expression of FXR, repair structure of intestinal and decrease infection more better.

\section{PP28-26}

The Effects of HMG-CoA Reductase Inhibitors (Statins) on Expression of COX-2 in Gallbladder Epithelial Cells (GBEC)

M.H. Choi, S.H. Park, D.H. Koh, H.J. Jang, S.H. Kae, J. Lee Internal Medicine, Hallym University College of Medicine, Seoul, Republic of Korea

Background: The preservation of gallbladder function could contribute to prevention of gallstone formation. Cyclooxygenase-2, a protein acts as an enzyme and specifically catalyzes the production of prostaglandins for promoting inflammation. Statins lower cholesterol by inhibiting the enzyme HMG-CoA reductase, improve endothelial function and modulate inflammatory responses. However, It has been not studied that the effect of statin on the expression of COX-2 in cultured GBEC yet.

Aims: This study was performed to demonstatrate effect of statin on the expression of COX-2 and production of prostagladin in GBEC, and to suggest pathway of statin to prevent inflammation in GBEC. Methods: In GBECs were cultured on dishes and MTT assays were performed to decide appropriate concentration of reagents to be used. The protein expression of COX-2 was measured by western blotting following treatment of various concentrations of LPS or statins. The mRNA expression of COX-2 was estimated by RT-PCR and production of PGE2 was measured by ELISA test following treatment of various concentrations of LPS or statins. The pathway of statin were studied by using Mevalonate, FPP, GGPP. The protein expression of 
ERK $1 / 2$ or p38 was measured by western blotting following treatment of various concentrations of LPS or statins.

Results: In cultured GBECs, Statins and LPS (1) activates expression of COX-2, (2) increase COX-2 mRNA, (3) increases production of PGE2. (4) The activation of expression of COX-2 by statin is not associated with blocking of cholesterol synthesis. (5) The pathway of expression of COX-2 by statin is associated with ERK1/2 and p38 MAPK pathway.

Conclusion: In cultured GBECs, statin increases the expression of COX-2, activates the expression of COX-2 mRNA. It is not associated with blocking of cholesterol synthesis and is associated with ERK1/2 and p38 MAPK pathway. It can be suggested that statin contributes th the prevention of gallstone formation.

\section{PP28-27}

Role of Wnt/ $\beta$-catenin Signaling Deregulation in Development of Gall Gladder Diseases and Carcinoma in Northeast India

R.K. Deblakshmi ${ }^{1}$, S. Bose ${ }^{1}$, M. Bose ${ }^{1}$, B. Baruah ${ }^{1}$, M. Deka ${ }^{1}$, D. Tiwari ${ }^{1}$, A. Saikia ${ }^{2}$, D. Thangjam ${ }^{3}$, B. Thangjam ${ }^{3}$

${ }^{1}$ Department of Biotechnology, Gauhati University; ${ }^{2}$ Central

Hospital, N.F. Railway, Maligaon, Guwahati, Assam, Guwahati;

${ }^{3}$ Babina Diagnostic Centre, Imphal, Imphal, India

Background: Gallbladder carcinoma (CaGB), a common malignancy in India, with case numbers increasing alarmingly in North eastern India, tends to be an aggressive tumor with poor prognosis. Majority of the CaGB cases have either Cholelithiasis or Cholecystitis background. The underlying molecular mechanism of development of CaGB is unknown.

Aim: To study deregulation of $\mathrm{Wnt} / \beta$-catenin in the progression of gall bladder diseases to carcinoma.

Method: Surgically ressected tissues of clinically and histopathologically proven cases of Gallbladder disease patients [Cholelithiasis $(n=15)$, Cholecystitis $(n=10)$ and $\mathrm{CaGB}(n=10)$ along with controls $(n=5)]$ were collected from Central Railway Hospital, Guwahati, and BDC,Imphal; since these places have higher incidences of gallbladder diseases Mutation analysis of $\beta$-catenin exon3 region was done by PCR-RFLP/sequencing analysis. mRNA expression analysis of $\beta$-catenin was studied by semiquantitative analysis followed by densitometry analysis, using $\beta$-actin as internal control. $\beta$-catenin protein expression was checked by immunohistochemistry on $5 \mu \mathrm{m}$ paraffin embedded sections.

Results: Amongst the enrolled patients, $\beta$-catenin mutation was only found in only one cholelithiasis case. The difference in the distribution of mutation was non-significant when compared between cholelithiasis and cholecystitis $(p=\mathrm{NS})$ or between cholelithiasis and $\operatorname{GBC}(p=\mathrm{NS})$ cases. But importantly, $\beta$-catenin mRNA expression was upregulated chronologically in the gallbladder diseases (control $<$ Cholelithiasis $<$ Cholecystitis $<\mathrm{CaGB}$ ). $\beta$-catenin protein expression was noticeably higher in $\mathrm{CaGB}$ cases compared to controls. Distinct positive $\beta$-catenin staining was localized more in cell membrane and cytoplasm in CaGB compared to faint membranous expression in controls.

Conclusion: Aberration in $\mathrm{Wnt} / \beta$-catenin pathway irrespective of the $\beta$-catenin mutation status appears to be a crucial step in the progression of gallbladder diseases to cancer.

\section{PP28-28}

Essential Phospholipids in Complex Therapy of Hepatic Cellular Impairment in Patients with Cholelithiasis Developing Biliary Obstruction

L. Palgova ${ }^{1}$, N. Kozybayev ${ }^{2}$, O. Reiman. ${ }^{3}$, I. Menshikova ${ }^{3}$, L.K. Palgova, N.K. Kozybayev, O.V. Reiman. MC

${ }^{I}$ Private Medical Centre 'EVELINA Med', Private Kazakh-Russian Medical Institute; ${ }^{2}$ DPA Central Clinical Hospital; ${ }^{3}$ Private Medical Centre 'EVELINA Med', Almaty, Kazakhstan
Background: Prolonged obstructive jaundice is accompanied by the development of hepatic impairment syndrome aggravating the decompressive surgery prognosis.

Aims: Study of the efficacy and safety of essential phospholipids in complex preoperative preparation and postoperative period of the patients with cholelithiasis and biliary obstruction syndrome.

Results and conclusion: 22 patients were enrolled in the study. Inclusion criteria: abdominal pain syndrome caused by biliary obstruction, verified cholelithiasis, age 16-75. Exclusion criteria: biliary obstruction caused by cancer, acute viral hepatitis, hepatic cirrhosis of B and C class as per Child-Pugh. Clinical characteristics of patients: Average age - 51.2, number of men - 7, women - 15 . Background pathology: type 2 diabetes mellitus -5 , arterial hypertension - 4, psoriasis -2, bronchial asthma - 2, sclerocystic ovarian disease -1, uterine fibromatosis -2, HCV-associated B-cell lymphoma - 1. Biliary obstruction was resolved by endoscopic papillosphincterotomy - 7, laparoscopic cholecystectomy - 9, laparotomic cholecystectomy -7, endoscopic papillosphincterectomy was ineffective in four cases, so laparotomic cholecystectomy (3) and classical cholecystectomy (1) were performed. All the patients in preoperative, early postoperative periods were administered intravenous infusions of Essentiale forte $10 \mathrm{mg} /$ day, in recovery period - Essentiale $\mathrm{N} 2$ capsules t.i.d. during 2 months. Cytolytic syndrome regression was observed on the 14th day of therapy up to 1.5 norms as well as normalization in transaminases activity, GGTP, bilirubin, albuminafter two months of therapy in patients without background pathology, in patients with type 2 diabetes mellitus analogous parameters exceeded the norm 2.3 times on the 14th day of therapy, 1.5 timesafter 2 months, treatment with EPL was prolonged up to 6 months with the achievement of biochemical response. Adverse side effects were not registered. EPL show a good clinical effect and high safety profile in treatment of biliary obstruction syndrome.

\section{PP28-29}

Comparison of Internal Biliary Drainage and External Biliary Drainage in Patients of Bile Duct Carcinoma

L. Wu, W. Li

Department of Gastroenterology \& Hepatology, General Hospital of the Chinese People's Liberation Army, Beijing, China

Background and Aims: Our previous studies demonstrated that internal biliary drainage (ID) is superior to external biliary drainage (ED) in terms of reversing Kupffer cell dysfunction and intestinal dysfunction in rats with obstructive jaundice (OJ). We assumed that the condition may same with clinical patients. So the present study was to investigate the patients conditions after ID and ED.

Methods: Forty patiens of bile duct carcinoma of advanced stage were randomly assigned to two groups. One was treated by endoscopic retrograde cholangiopancreatography (ID group) and the other was treated by percutaneous transhepatic biliary drainage guided by ultrasound (ED group). Serum alanine transarninase (ALT), aspartate aminotransferase (AST), gamma-glutamyl transpeptidase, alkaline phosphatase, total bilirubin, total bile acid and white cell count were detected before the operation day, the first day and the eighth day after operation respectively. The rate of complications, the time of hospitalization and cost were also compared.

Results: Serum indexes of both groups were fell down after operations, and there were no differences between them $(P<0.05)$. These decrease range of indexes of 1 week after operations were not more than one day after operations. However, two patient ALT and AST were increased after ED. One patient tube was blocked 21 days later and replaced another tuble. Though the ID therapeutic charge was higher than ED, the time of hospitalization were lower than ED $(P<0.05)$. 
Conclusions: Both groups serum indexes decreased sharply in the first day while torpidly later. There were no difference in the restore of the hepatic function and alleviate inflammatory reaction. But ID can improve the nutritional condition of the patients suffering from obstructive jaundice more effectively and more quickly, low down the occurrence of complication the time of hospitalization.

PP28-30

Placement of a Percutaneous Transhepatic Biliary Stent Using BLAKE Drain $^{\mathrm{TM}}$ for the Treatment of a Stenotic Hepaticojejunostomy

Y. Kawano ${ }^{1}$, H. Yoshida ${ }^{2}$, N. Taniai ${ }^{1}$, Y. Mamada ${ }^{1}$, S. Mineta ${ }^{1}$, M. Yoshioka ${ }^{1}$, A. Hirakata ${ }^{2}$, J. Ueda ${ }^{1}$, E. Uchida ${ }^{1}$

${ }^{1}$ Department of Surgery, Nippon Medical School; ${ }^{2}$ Department of Surgery, Nippon Medical School Tama Nagayama Hospital, Tokyo, Japan

Background: This report describes a method for percutaneous transhepatic biliary stenting with a BLAKE Silicon Drain and discusses the usefulness of placement of the drain connected to a J-VAC Suction Reservoir for the treatment of a stenotic hepaticojejunostomy. Methods: Percutaneous transhepatic biliary drainage was performed under ultrasonographic guidance in a patient with a stenotic hepaticojejunostomy after hepatectomy for hepatic hilum malignancy. The technique used was as follows. After dilatation of the drainage root, an 11-Fr tube (11Fr PTCS tube, Sumitomo Bakelite, Tokyo, Japan) with several side holes is passed through the stenosis of the hepaticojejunostomy. A 10-Fr BLAKE Silicon Drain is flexible, precluding one-step insertion. One week after insertion of the 11-Fr tube, a 0.035 -inch guidewire is inserted into the tube. After removal of the 11-Fr tube, the guidewire is put into the channel of a 10-Fr BLAKE Silicon Drain. The drain is inserted into the jejunal limb through the intrahepatic bile duct and is connected to a J-VAC Suction Reservoir. Low-pressure continued suction is applied. Patients can be discharged after insertion of the 10-Fr BLAKE Silicon Drain connected to the J-VAC Suction Reservoir.

Conclusions: Placement of a percutaneous transhepatic biliary stent using a 10-Fr BLAKE Silicon Drain connected to a J-VAC Suction Reservoir is useful for the treatment of a stenotic hepaticojejunostomy.

\section{PP28-31}

The Association between Gallstones and Chronic Hepatitis C Virus Infection: Ultrasonographic Survey in a Hepatitis C and B Hyperendemic Township

C.-Y. Dai ${ }^{1,2,3}$, M.-L. Yeh ${ }^{1}$, C.-I. Huang ${ }^{1}$, C.-Y. Lin ${ }^{2}$, C.-F. Huang ${ }^{2}$,

M.-Y. Hsieh ${ }^{1,4}$, J.-F. Huang ${ }^{1,3}$, M.-H. Hsieh ${ }^{2,5}$, M.-L. Yu ${ }^{1,3,4}$, J.-S. Chen ${ }^{6}$, W.-L. Chuang ${ }^{1,3}$

${ }^{1}$ Department of Internal Medicine; ${ }^{2}$ Department of Occupational and Environmental Medicine, Kaohsiung Medical University Hospital;

${ }^{3}$ Faculty of Internal Medicine, College of Medicine, Kaohsiung Medical University; ${ }^{4}$ Department of Internal Medicine, Kaohsiung Municipal Ta-Tung Hospital; ${ }^{5}$ Department of Preventive Medicine; ${ }^{6}$ Department of Surgery, Kaohsiung Medical University Hospital, Kaohsiung, Taiwan ROC

Background and aims: Gall bladder stone (GBS) disease has been reported associated with liver diseases. This community-based study aimed to investigate the prevalence of the GBS and the associated factors in a hepatitis B virus (HBV)/hepatitis $\mathrm{C}$ virus $(\mathrm{HCV})$ endemic township based on a screening projects in southern Taiwan.

Patients and methods: We have conducted a study in an HBV/HCV hyperendemic community located in southern Taiwan. A total of 1,701 residents ( 689 males, mean age of $51.2 \pm 16.0$ years) participated our screening project. They were all checked serum biochemistry including serum aspartate transaminase (AST), alanine aminotransferase (ALT), viral markers including hepatitis B surface antigen (HBsAg), antibody to $\mathrm{HCV}$ (anti-HCV) and examined by hepatobiliary ultrasonography (US).

Results: Of 1,701 residents, $243(14.3 \%)$ and 475 (27.9\%) residents were positive for HBsAg and anti-HCV, respectively. The US findings showed the prevalence of the GBS, GB polyp (GBP), and fatty liver (FL) were 6.8, 5.9 and $55.6 \%$, respectively. In univariate analyses, subjects with GBS have significantly higher proportions of male gender, age higher than 50 years and positive anti-HCV $(p=0.001$, $p<0.001, p=0.001$, respectively), and higher mean age and ALT level ( $p=<0.001$ and $p=0.048)$ than those without GBS. In multivariate analyses, male gender, positive anti-HCV and higher age ( $>50$ year) are factors associated with GBS (Odd ratio/ 95\% confidence interval/ p: 1.972/1.341-2.898/ 0.001, 1.539/1.023-2.317/0.039 and 2.360/1.499-3.714/<0.001, respectively).

Conclusions: The prevalence of the GBS, in the community-based study, was $6.8 \%$ in this HCV/HBV hyperendemic township. The GBS was associated with chronic hepatitis $\mathrm{C}$ but not $\mathrm{B}$ virus infection.

Poster Presentation 29: Hepatocellular Carcinoma: AntiNeoplastic Agent

\section{PP29-01}

Assessment of Hepatoprotective and Anticancer Properties of Aqueous Olive Leaf Extract in Chemically Induced Hepatocellular Preneoplasia in Rats

N.M. Abdel-Hamid ${ }^{1}$, M.A. Moselhy ${ }^{2}$, A.I. Abdel-Baky ${ }^{3}$, M.A. Fawzy ${ }^{3}$

${ }^{1}$ Biochemistry, Pharmacy; ${ }^{2}$ Pharmacology, Pharmacy;

${ }^{3}$ Biochemistry, Minia University, Minia, Egypt

Hepatocellular carcinoma (HCC) accounts for $80-90 \%$ of primary liver cancer and it is a major health problem worldwide, it is the fifth most common cancer in the world. In the present study we aimed to examine if aqueous olive leaf extract $(500 \mathrm{mg} / \mathrm{kg})$ has a role as a herbal medicine in modulating the metabolic disorders occurring in liver premalignancy induced by TCA $(500 \mathrm{mg} / \mathrm{kg})$. Eight groups of rats were used in this study as results showed that treatment with TCA induced a significant increase in ALT, AST and ALP activities, also in plasma total bilirubin, triglycerides, serum total glycosaminoglycans, alpha-fetoprotein, and acetyl CoA synthase (ACAS) activity. On the other hand, it induced a significant reduction in serum lipoprotein lipase activity (LPL). The histopathological results showed that damaged neoplastic hepatic tissue by TCA was treated and reversed towards normal cellular architecture by treatment with AOLE and 5FU. Treatment with AOLE especially in combination with 5-FU produced a pronounced improvements in most of the studied parameters up to or towards the normal range. It can be concluded that aqueous olive leaf extract has hepatoprotective and anticancer properties especially in combination with 5 -FU.

\section{PP29-02}

Synthesis of $\mathrm{N}, \mathrm{N}, \mathrm{N}$-trimethyl Chitosan and Encapsulation of Anti-HSPG for the Development of Antibody-based Drug Delivery System for Hepatocellular Carcinoma

P. Vongchan

Medical Technology, Associated Medical Science, Chiang Mai University, Muang, Thailand

Background/aims: 1E4-1C2 MAb was raised against HSPG isolated from human liver. It could react with and inhibited proliferation of HepG2 cells in vitro as well as reduce tumor size and weight in athymic mice implanted with HepG2 cells. The objective of this study 
was to synthesize $N, N, N$-trimethyl chitosan (TMChC) and develop nanocomplex for antibody-based drug delivery system.

Methods: Methyl groups were introduced into $\mathrm{NH}_{2}$ groups of chitosan to obtain TMChC. Nanocomplex of TMChC and 1E4-1C2 $\mathrm{MAb}$ was formed. Product obtained was studied for its toxicity to normal peripheral blood cells and macrophage by cultivation of those cells in presence or absence of various final concentrations of compounds. Cytotoxicity was then analyzed by MTT assay after 3 days of cultivation. Uptake of FITC-free 1E4-1C2 MAb or FITC-nanocomplex of HepG2 cells were investigated by analysis of total and intracellular fluorescent intensity detected before and after $\mathrm{CoCl}_{2}$ treatment.

Results: TMChC:MAb of 5:0.3 was the best ratio with resulting in optimal size $(64-65 \mathrm{~nm})$ and membrane zeta potential (9-11). Study for target cell specificity demonstrated that free antibody reacted and entered cells rapidly with peak at 3-h incubation while nanocomplex could much slower enter cell membrane. Intracellular level, however, was observed in the longer time. Nanocomplex could enter cell membrane mainly via facilitation and less effective via antigenantibody reaction. Moreover, it could inhibit HepG2 proliferation in vitro. Both nanocomplex and TMChC had no effect on normal peripheral blood cells.

Conclusion: Nanocomplex obtained could be attracted through facilitation by macrophage but with no side effect since $1 \mathrm{E} 4-1 \mathrm{C} 2$ was specific and inhibited proliferation of only target HepG2 cells. Nanocomplex crossed membrane slowly but stay longer intracellular when compared to free antibody. In vivo assay should be further on studied to investigate its efficiency in antibody-based drug delivery system.

Acknowledgment: This work was supported by NRCT (2008-2009).

\section{PP29-03}

Selenium Approach for Hepatocellular Carcinoma in Experimental Animals

N. Alwahaibi ${ }^{1}$, S.B. Budin ${ }^{2}$, J. Mohamed ${ }^{2}$, A. Alhamadani ${ }^{3}$

${ }^{1}$ Pathology, Sultan Qaboos University, Muscat, Oman; ${ }^{2}$ Biomedical Sciences, Universiti Kebangsaan Malaysia (UKM), Kuala Lumpur, Malaysia; ${ }^{3}$ Pathology, Sultan Qaboos University Hospital, Muscat, Oman

Background/aims: Hepatocellular carcinoma is common in Asia and it is increasing in the developed countries. Various studies on selenium as an anti-cancer agent have conflicting results and the molecular mechanism of selenium is unknown. The aim of this study was to investigate the overall effects of selenium on chemically induced hepatocarcinogenesis in rats.

Methods: Forty four male Sprague- Dawley rats (120-190 g) were obtained from the Laboratory Animal Resource Unit, Faculty of Medicine, Universiti Kebangsaan Malaysia (UKM), Malaysia, and divided randomly into six groups: negative control, positive control [Diethyl nitrosamine (DEN) + 2-Acetylaminofluorene (2-AAF)], preventive group (selenium at a dose of $4 \mathrm{mg} / \mathrm{L}$ for 4 weeks, then DEN + 2-AAF), preventive control (respective control for preventive group), therapeutic group (selenium after 4 weeks of DEN injection) and therapeutic control (respective control for therapeutic group). Histological, immunohistochemical, biochemical and molecular investigations were carried out.

Results: The numbers and sizes of hepatic foci were reduced in the preventive and therapeutic groups. Microscopic analysis of these two groups showed that the majority of nodules were hyper-plastic with preserved liver architecture whereas the positive control was full of neo-plastic nodules with a completely disrupted liver architecture. The expression of NF- $\kappa \mathrm{B}$ in the preventive group and its respected control was significantly lower when compared with both the negative and positive controls.
Conclusions: Preventive and therapeutic selenium groups were able to reduce, but not inhibit, the extent of liver cancer on chemically induced hepatocarcinogenesis in male rats. Selenium chemoprevention could be through the inhibition of NF- $\kappa \mathrm{B}$ pathway in the preventive group. However, selenium chemoprevention in the therapeutic group could be through another mechanism.

\section{PP29-04}

Efficacy of Transcatheter Arterial Chemo-embolization with a Finepowder Formulation of Cisplatin for Hepatocellular Carcinoma

Y. Tamura, K. Kasai, K. Sawara, Y. Kasai, A. Usio, Y. Miyamoto, J. Oikawa, K. Oikawa, H. Kuroda, Y. Takikawa, K. Suzuki

Hepato-Gastroenterology, Iwate Medical University, Morioka, Japan

Purpose: In this study, we evaluated the efficacy of transcatheter arterial chemoembolization (TACE) using a suspension of a finepowder formulation of cisplatin (DDPH) in lipiodol (LPD) in the treatment of hepatocellular carcinoma (HCC).

Methods: The study population was comprised of 164 patients who were treated by TACE alone. Of these patients, 76 had undergone TACE using a suspension of DDPH in LPD [DDPH group], and the remaining 88 had undergone TACE with an emulsion of doxorubicin (ADM) with LPD [ADM group]. We compared the DDPH group with $\mathrm{ADM}$ group in terms of the response rate (RR), progression free survival (PFS) and overall survival (OS).

Results: The objective response rate in the DDPH group was significantly higher than that in the ADM group (54.0 vs. $23.9 \%$, $P<0.0001)$. The PFS rate in the DDPH group was also significantly higher than that in the ADM group $(P<0.0001)$. Moreover, the OS in the DDPH group was significantly higher than that in the ADM group $(P=0.0463)$. Although the incidence rate of nausea or vomiting in the DDPH group was higher than that in the ADM group, the ADM group showed a higher incidence rate of the adverse events of the hepatic artery occlusion and leucopenia. No other serious complications were observed in either group.

Conclusion: We conclude that TACE using a suspension of DDPH in LPD could be a useful treatment for HCC.

\section{PP29-05}

Impact of Antiviral Therapy on the Survival Outcome of Patients after Major Hepatectomy for Hepatitis B-related Hepatocellular Carcinoma

A.C.Y. Chan, K. Chok, W.K. Yuen, R. Poon, C.M. Lo, S.T. Fan Department of Surgery, Queen Mary Hospital, The University of Hong Kong, Hong Kong, Hong Kong SAR

Background: Although the therapeutic benefit of anti-viral treatment in the management of chronic hepatitis B infection (HBV) is wellestablished, it remains uncertain if commencement of anti-viral therapy after hepatectomy in anti-viral naïve patients with hepatocellular carcinoma (HCC) would improve their survival outcome.

Methods: From September 2003 to December 2007, 42 patients (i.e. treatment group) were given Lamivudine $(n=39)$ or Enteca$\operatorname{vir}(n=4)$ after hepatectomy for HBV-related HCC. All these patients were anti-viral naïve before hepatectomy. Their preoperative data, tumor characteristics and survival outcome were compared with 94 patients without any antiviral treatment after hepatectomy (i.e. control group).

Results: The median age for treatment and control group were 57 and 55 years old respectively $(P=0.77)$. Patient demographics, preoperative liver function, tumor characteristics, liver function at the time of tumor recurrence were comparable between the two groups. Both disease-free and overall survival rates were significantly improved in the treatment group. The 1-, 3-, and 5-year overall survival rate in the treatment group was $88.1,79.1$, and $71.2 \%$ respectively, and in the 
control group was $76.5,47.5$, and $43.5 \%$ respectively $(P=0.005)$. The 1-, 3- and 5-year disease-free survival rate in the treatment group was $66.5,51.4$, and $51.4 \%$ respectively, and in the control group was 48.9, 33.8 , and $33.8 \%$ respectively $(P=0.05)$. Subgroup analysis stratified against tumour stage and portal vein status showed that post-hepatectomy antiviral treatment confers a significant survival benefit in AJCC stage I/II tumours or HCCs without portal vein invasion.

Conclusion: Anti-viral naïve HBV carriers could still benefit from the therapeutic effect of anti-viral treatment after curative treatment for hepatocellular carcinoma.

\section{PP29-06}

Targeting Prominin-1 on Hepatocellular Carcinoma Exhibits Tumor Cytotoxicity

H.Y. Chen, P. Zhu, Y.W. Qian, W. Sun, J. Fu, D.P. Hu, H.Y. Wang, Z. Li International Cooperation Laboratory on Signal Transduction, Eastern Hepatobiliary Surgery Hospital,The Second Military Medical University, Shanghai, China

Background: Cancer stem cells are believed to be important in tumor recurrence and chemoresistance that are key mortal causes of human cancer. Prominin-1(CD133) is identified to be expressed on cell surface of cancer stem or stem/progenitor cells and represents a potential therapeutic marker for targeting tumor. Applied prominin-1 antibody (CD133Ab) or drug-conjugated prominin-1 antibody has previously demonstrated potential cytotoxic effect on melanoma or hepatocellular and gastric cancer cells, respectively.

Aim: In this study we investigated therapeutic effect of CD133Ab on hepatocellular carcinoma cells (HCC) and the possible molecular mechanism of CD133 in cytotoxicity.

Methods: Several HCC lines (LM3, SMMC7721, Huh-7) and normal liver cells (LO2) were observed after treatment with different doses of CD133Ab. Cell morphology, proliferation, apoptosis, CD133 protein and mRNA expression, and tumor xenograft model were detected.

Results: CD133Ab added to medium retarded proliferation of LM3 and Huh-7 but not SMMC 7721 and LO2 cells because the latter had no or very low percentage of prominin-1 positive cells. Compared with control IgG1 treated cells, the antibody treatment apparently caused cell shrinking and reduced $30-60 \%$ of proliferation in LM3 and Huh-7 cells by cytotoxicity CCK-8 assay. Although CD133Ab did not dramatically affect CD133 protein and mRNA levels within $48 \mathrm{~h}$ treatment, antibody treated LM3 cells significantly delayed tumor growing in nude mice, suggesting CD133-linked internal signaling might be critical in cell growth. We also found apoptotic small bodies were increased in CD133 expressing cells after CD133Ab-treated cells. Conclusion: CD133-linked signaling may play an important role in tumor growth. Applied CD133Ab specific for the prominin-1 positive liver cancer cells could be considered as a direct targeting approach in treatment of HCC.

\section{PP29-07}

Sorafenib in the Treatment of Advanced Hepatocellular Carcinoma: A Meta-analysis of Randomized Trials

R.U.C. Cinco, J.G. Ignacio, F. Domingo

Department of Medicine, Section of Gastroenterology, Veterans Memorial Medical Center, Quezon City, Philippines

Background: There are only limited numbers of randomized controlled trials (RCTs) published to assess the therapeutic advantage of palliative treatments in cases of advanced hepatocellular carcinoma. Until recently, no standard systemic therapy that prolongs survival has been identified.

Objectives: This review aims to assess efficacy and safety of Sorafenib in advanced hepatocellular carcinoma.
Methods: Fully published RCTs on Sorafenib use in advanced cases of HCC in four electronic databases (MEDLINE on Pubmed, Cochrane Library, Ovid and HERDIN) were systematically reviewed and meta-analyzed. The primary end points were overall survival and time to symptomatic progression (TTSP). The secondary endpoints were tumor progression according to Response Evaluation Criteria in Solid Tumors (RECIST) and common drug-related adverse events of any grade. Revman V. 5 was used in the analysis.

Results: Two out of nine eligible fully published RCTs on Sorafenib use in advanced HCC were selected with a total of 828 patients studied. Overall mortality was significantly reduced with an RR of 0.81 (CI 0.73-2.091). Likewise, overall survival time showed statistically significant differences with $p$ value of $<0.001$ with overall survival time of 2.43 months (CI 1.54-3.33). However, no significant difference was noted in TTSP $(p=0.24)$. No complete response was seen on both studies with marginally significant $(p=0.07)$ result on partial response. Most common drug-related adverse events were hand and foot skin reaction, diarrhea and fatigue, all with significant results compared to placebo $(p<0.05)$.

Conclusion: Sorafenib showed improvement in overall mortality and also in extending survival time. However, drug related adverse events are common and disabling. Furthermore, cost of treatment is very expensive and both studies included patients only with good liver reserves (Child-Pugh A) thus, outcome is expected to be better.

\section{PP29-08}

Saffron Protects against Chemically-induced Liver Cancer in Rats: Molecular Mechnism

A. Amin ${ }^{1}$, D. Mahmoud-Ghoneim ${ }^{2}$, P. Corr ${ }^{3}$, A. Hamza ${ }^{1}$, S. Daoud ${ }^{4}$

${ }^{1}$ Biology; ${ }^{2}$ Physics; ${ }^{3}$ Radiology, UAE University; ${ }^{4}$ Histopathology,

Twaam Hospital, Al-Ain, United Arab Emirates

Saffron is a natural product that is a promising chemo-preventive agent against different cancer. The aim of the present work was to investigate the chemo-protective effect of saffron ethanol extract on chemically-induced liver cancer in rats. Hepatocarcinogenesis was induced by a single intraperitoneal injection of diethyl nitrosamine (DEN) (200 mg/kg body weight) and 2 weeks later, the carcinogenic effect was promoted by 2-acetylaminofluorene (2-AAF) (0.05\%). 42 Wister rats were divided into seven groups: negative control, positive control (DEN+2-AAF), saffron-alone group (saffron for 22 weeks, no $\mathrm{DEN}$ or 2-AAF) and three preventive groups (where saffron was administered at 300,150 and $75 \mathrm{mg} / \mathrm{kg}$ for 2 weeks before and 20 weeks after inducing cancer). The incidence and multiplicity of liver tumors as well as the expression of cancer marker, glutathione S-transferase placental form, were significantly reduced in preventive groups. This hepatopreventive effect was confirmed by the decreasing in cancer marker, $\alpha$ feto protein in plasma. Immunohistochemical analyses showed a decrease in cell proliferation marker (Ki67) and an increase of TUNEL-and caspase 3-positive apoptotic cells along with strong expressions of p53 in liver of preventive groups. These protective effect found to be mediated by anti-inflammatory and antioxidant effects. The anti-inflammatory activity was confirmed by saffron-based inhibition of inducible nitric oxide synthase (iNOS) and cyclooxygenase-2 (COX-2) protein expression. The effect of saffron on the expression of nuclear factor nuclear factor-Kappa $\mathrm{B}$ (NF-kappaB) was also assessed. The expression of NF-kappaB in the preventive groups was significantly low compared to positive control. The in vitro antioxidant capacity of saffron (FRAP Assay, DPPH Scavenging activity and $\mathrm{ABST}^{+}$Assay) was significantly high in ethanol-based extract. Finally, this study shows that NF- $\kappa$ B transcription factor seems to regulate the saffron chemo-protective effects. 


\section{PP29-09}

Effect of Sorafenib and Intermittent Hepatic Arterial Infusion Chemotherapy Using Cisplatin for Advanced Hepatocellular Carcinoma with Portal Vein Tumor Thrombus

M. Ishizaki, M. Kaibori, K. Matsui, A.-H. Kwon

Surgery, Kansai Medical University, Hirakata, Japan

Introduction: We evaluate the effect of sorafenib and intermittent hepatic arterial infusion chemotherapy (HAIC) using cisplatin for unresectable advanced hepatocellular carcinoma (HCC).

Methods: Five patients ( 4 male/ 1 female, median age: 73.0 years) with unresectable advanced HCC included portal vein tumor thrombus were treated with soraenib (400 mg/body, day 1-28) and cisplatin $\left(20 \mathrm{mg} / \mathrm{m}^{2}\right.$ HAIC, day $\left.1,8,15\right)$. We assess efficacy and safety after one course of this therapy.

Results: Grade 3 adverse events occurred in two patients (one serum bilirubin and aspartate aminotransferase (AST) elevation, one serum bilirubin elevation and hepatic encephalopathy). Grade2 adverse events occurred in four patients (one hypertention and nertropenia, one hypertention and thrombocytopenia, one hypertention and serum amylase elevation, and one abdominal pain). Grade 3 adverse events were improved by suspending this therapy, and completion of this therapy was achieved in all patients. After one course of this therapy, the clinical response were rated as one partial response (PR), two stable disease (SD), and one progressive disease (PD).

Conclusion: Sorafenib and intermittent HAIC using Cisplatin for unresectable advanced $\mathrm{HCC}$ was expected to be safe and effective treatment.

\section{PP29-10}

Influence of Arsenious Acid Chemotherapy on Tumor Recurrence in Liver Transplant Recipients with Primary Hepatocellular Carcinoma beyond Milan Criteria

L. Wu, X. He, Z. Guo, D. Wang, Q. Tai, X. Zhu, W. Ju

Organ Transplantation, The First Affiliated Hospital of Sun Yat-sen University, Guangzhou, China

Introduction/objective: About $40 \%$ of liver transplantation (LT) recipients are patients with primary hepatocellular carcimoma (HCC) in China. LT is the most effective therapy for the treatment of HCC theoretically, but high tumor reoccurrence rate after transplantation has a negative influence on the prognosis of these patients significantly. Intravenous arsenious acid chemotherapy is thought to be effective in patients with middle and end stage HCC to some extent, but there is no report of the usage of arsenious acid chemotherapy after LT. In this study, we aimed to explore the role of arsenious acid on tumor recurrence and patients survival in LT patients with HCC extending Milan criteria.

Methods: Twenty- three patients with HCC extending Milan criterion received intravenous arsenious acid chemotherapy after orthotopic liver transplantation (OLT), while the other 16 patients did not received chemotherapy. The difference of patients' survival and tumor recurrence were compared.

Results: All patients were routinely followed up for 3-32 months. Thirty recipients were found with tumor recurrence, 16 in chemotherapy group and 14 in non-chemotherapy group. Tumor recrrenced in lung, liver graft and bones in most cases. The total recurrence rate was similar in these two groups, but chemotherapy can delay recurrence after transplantation $(14.5 \pm 7.5 \mathrm{~m} \quad$ vs. $10.2 \pm 4.6 \mathrm{~m}$, $p=0.026$ ). 6-month, 1-year survival rate were similar (91.3 vs. $93.7 \%$ and 87.0 vs. $87.5 \%$, respectively), while 2-year survival in chemotherapy group was higher (73.9 vs. $50.0 \%, p=0.037)$; 6 -mon tumor-free survival rates in the two groups were similar ( 87.0 vs. $81.2 \%), 1$ - and 2-year tumor-free in chemotherapy group were sig- nificantly higher than those in non-chemotherapy group (82.6 vs. $50.0 \%, p=0.030$ and 65.2 vs. $43.7 \%, p=0.023$ ).

Conclusions: Intravenous arsenious acid chemotherapy can delay tumor recurrence and prolong survival in liver transplant patients with HCC extending Milan criteria.

\section{PP29-11}

Therapeutic RNA Interference of Malignant Melanoma by Small Interfering RNA Targeting Heparanase

\section{S. Zhou}

The 2nd Affiliated Hospital of Bengbu Medical College, Bengbu City, China

Heparanase is the predominant enzyme responsible for the degradation of heparan sulfate, the principal polysaccharide component of the basement membrane and extracellular matrix. Expression of the heparanase gene is associated with the invasive, angiogenic, and metastatic potential of diverse malignant tumors. We used RNA interference method to evaluate the role of heparanase in malignancy and to explore the therapeutic potential of its specific targeting. We designed short interfering RNAs (siRNA) duplexes corresponding to the mouse heparanase mRNAs. The mouse melanoma (B16-F10) tumor cell line was transfected with anti-heparanase siRNA. Semiquantitative reverse transcription-polymerase chain reaction (RT-PCR) was used to confirm the efficient silencing of heparanase gene expression. Cells transfected with the anti-heparanase siRNA were tested for invasiveness in vitro and experimental hepatic metastasis in vivo. The cell proliferation and melanogenesis of B16F10 cells were also evaluated. Compared with cells transfected with control siRNAs, cells transfected with the anti-heparanase siRNA had profoundly reduced invasion in vitro. In vivo, metastatic liver tumors produced by cells transfected with the anti-heparanase siRNA were less than that produced by cells transfected with the control siRNAs. While the cell proliferation ability and melanogenesis characters remain stable after the anti-heparanase siRNA transfection. Downregulation of the heparanase expression by anti-heparanase siRNAmediated gene-silencing is associated with the low invasiveness and metastasis of malignant cells suggests that heparanase is important in cancer progression. Heparanase gene silencing has potential use as a target for anticancer drug development. Gene silencing targeting heparanase might be a potential anticancer strategy.

\section{PP29-12}

Can Vitamin K2 Be Applied to Prevent the Recurrence of Hepatocellular Carcinoma Solely?

W. Meng ${ }^{1}$, Y. $\mathbf{L i}^{2}$, X. Li ${ }^{1}$, W. Zhou ${ }^{1}$, X. Zhu ${ }^{1}$, W. He ${ }^{1}$, Y. Liu $^{3}$

${ }^{I}$ The Second General Surgery Department, The First Hospital of Lanzhou University, Key Laboratory of Digestive System Tumors;

${ }^{2}$ General Surgery Department, The Second Hospital of Lanzhou University, Key Laboratory of Digestive System Tumors; ${ }^{3}$ EvidenceBased Medicine Center of Lanzhou University, LanZhou, China

Background/aims: Vitamin K2 used to be studied as a chemoprevention drug for hepatocellular carcinoma. Several studies have suggested that it can prevent the recurrence after curative treatment of HCC. We did a meta-analysis using available evidence from randomized controlled trials (RCTs) and systematically reviewed the efficacy of vitamin K2.

Methods: The Cochrane Library, PUBMED, EMBASE, EBM Review databases, Science Citation Index Expanded, and several Chinese databases up to October 2010 were searched. RCTs that compared the vitamin $\mathrm{K} 2$ with placebo for patients after curative treatment of hepatocellular carcinoma were analysed.

Result: Four RCTs comprising a total of 209 participants were included in the final meta-analysis. Overall, there were significant 
differences in both recurrence and survival during follow-up observation between the two groups. Significant differences have shown in all recurrence: 12 months (RR $0.42 ; 95 \%$ CI $0.25-0.71 ; P=0.0010$ ); 24 months (RR 0.59; 95\% CI 0.44-0.79; $P=0.0004$ ); and 36 months (RR 0.58; 95\% CI 0.46-0.72; $P<0.00001$ ). With the respect of the survival, unfortunately, significant difference was only shown in 24 months (RR 1.14; 95\% CI 1.04-1.24; $P=0.004$ ). There were no statistically significant differences in 12 months (RR, 1.05; 95\% CI, $0.99-1.11 ; P=0.10)$ and in 36 months (RR, $1.10 ; 95 \% \mathrm{CI}$, $0.96-1.27 ; P=0.16)$.

Conclusion: Administration of vitamin $\mathrm{k} 2$ either reduces recurrence in 12, 24 and 36 months, or enhances survival in 24 months compared with the placebo after curative treatment of hepatocellular carcinoma.

\section{PP29-13}

Clinical Experience of Sorafenib Treatment for Advanced Hepatocellular Carcinoma

H.Y. Woo, J. Heo, K.T. Yoon, M. Cho, J.H. Cheong, B.E. Lee, D.Y. Ryu, D.U. Kim, G.H. Kim, D.H. Kang, G.A. Song

Department of Internal Medicine, Pusan National University School of Medicine, Busan, Republic of Korea

Background/aims: Sorafenib has been shown to improve survival of patients with advanced hepatocellular carcinoma (HCC). However, its efficacy and tolerance in real clinical field has not been evaluated so far. Here, we investigated the efficacy as well as the safety and tolerance of sorafenib in advanced HCC within a single liver cancer centre.

Methods: Between March 2008 and September 2009, we prospectively registered the patients with advanced HCC who were administered sorafenib and evaluated tumor response (modified RECIST), time to progression (TTP) and tolerability.

Results: A total of 48 patients was treated with sorafenib. Mean age was $53.68 \pm 8.58$ years, $77.1 \%$ males, $83.3 \%$ HBV positive. $77.1 \%$ Child-Pugh A, BCLC stage C in $81.2 \%$. Sorafenib was the first therapy in $19(39.6 \%)$ and the second line therapy in $29(60.4 \%)$. Eighteen $(37.5 \%)$ had concomitant treatments (transarterial chemoembolization in 10, radiation treatment in 8 . After treatment, 3 $(6.25 \%)$ showed partial response and $20(41.6 \%)$ was stable response. However, 14 of 23 patients showing disease control showed progressive disease after mean $4.76 \pm 3.23$ days. Overall progression rate was $47.9 \%$ and median TTP was 5.1 months (95\% CI, 2.8-7.3 months). After progression, eight continued sorafenib, five stopped all treatment for HCC and ten changed the treatment. During the treatment, a total 66 cases of adverse events was reported in 37 (77\%) and the most common adverse event was hand-foot skin reaction $(16.5 \%)$. Eight patients $(16 \%)$ experienced dose modification and $5(10 \%)$ stopped the treatment due to adverse events. Median survival was 8.8 months (95\% CI, 2.8-14.7 months) and baseline predictor associated with prolonged survival were tumor response with sorafenib treatment $(P<0.001)$.

Conclusions: In real clinic field, sorafenib might benefit some HCC patients but showed modest efficacy in advanced HCC patients. Hence, more powerful and safer treatment options for advanced HCC need to be established.

\section{PP29-15}

Matrine-induced Autophagy Inhibits Apoptosis in Human Hepatoma Cells S.B. Xie, C.H. Zhao, K.S. Yao

China-Japan Friendship Hospital, Ministry of Health, Beijing, China

Aims: Matrine, one of the main components extracted from Sophora flavescens, has a wide range of pharmacological effects including anti-tumor activities on a number of cancer cell lines. However, the mechanism of matrine anti-tumor's effect has been remained elusive. In this study, we investigate the mechanism of matrine in the treatment of human hepatoma cells, and new target for the development of therapeutic strategies for hepatocellular carcinoma.

Methods: In HepG2 and SMMC-7721 cell lines, induction of apoptosis by matrine was initially measured by annexin-V-FITC/ PI analysis. We then confirmed activation of autophagy in the two cell lines after matrine treatment through MDC staining, electron microscopy and using LC3, a specific marker for detecting autophagy. Furthermore, we explored the function of the autophagic activity using 3-methyladenine (3MA) cotreatment to inhibit autophagy in HepG2 cells. Finally, we investigated the role of mammalian target of rapamycin (mTOR) in autophagic signaling of matrine processing in HepG2 cells.

Results: We indicated that autophagy was exclusively stimulated by matrine treatment at low dose, induction of apoptosis and autophagy concurrently at moderate dose, a remarked increase of apoptosis at high dose. Moreover, inhibition of autophagy using 3MA triggered induction of apoptosis by matrine at low and moderate dose in HepG2 cells. Finally, phosphorylation of ribosomal S6 protein kinase (S6K1, also known as p70S6K) at Thr389, has been widely described to reflect mTOR activity, was detected in HepG2 cells after exposure to matrine with different dose.

Conclusion: Matrine-induced autophagy inhibits apoptosis in human hepatoma cells, and promotes cell adaptation and survival. We also demonstrate that the autophagic activity is underlying an mTORindependent manner in HepG2 cells.

Acknowledgements: This study was supported by National Natural Science Foundation of China (Grant number: 30772859).

\section{PP29-16}

The Effect and Mechanism of siRNA MDM2 Inhibit HepG2 Cell Proliferation

Y. Zhao ${ }^{1}$, Y. Li ${ }^{2}$, Z. Yang ${ }^{3}$, D. Zhang ${ }^{4}$

${ }^{1}$ Department of Gastroenterology; ${ }^{2}$ Department of Surgery, The Fourth Hospital of Jilin University, Changchun City; ${ }^{3}$ Department of Surgery, China-Japan Union Hospital, Jilin University, Chanchun City; ${ }^{4}$ China-Japan Union Hospital, Jilin University, Changchun, China

Background: The mouse double minute gene 2 (MDM2) represents a pathway that the ubiquitin ligase involved in the degradation of the oncosuppressor $\mathrm{p} 53$. Overexpression of MDM2 is associated with the progression of hepatocellular carcinoma.Our aim to observe the effect of siRNA on MDM2 expression to hepatocellular carcinoma cell hepG2: the influence of cell proliferation and gene espression in hepG2 and investigate the mechanism.

Methods: MDM2-siRNAs against the MDM2 gene were designed and transfected into hepG2 cells respectively, meanwile,transfecting scramble plasmid as negative control siRNA. Using RT-PCR and western blot measured the gene and protein expression of MDM2,P53,Bcl2 and P21.Cell proliferation was measured by MTT assay,cell apoptosis and cell cycle were analysed by flow cytometer.

Results: RT-PCR and western blot showed that MDM2,Bcl2 mRNA and protein expression were down-regulated but P53, P21 mRNA and protein expression were up-regulated after transfecting with two siRNA-MDM2 respectively. Compareing with control group, MDM2, $\mathrm{Bcl} 2$ gene expression down-regrulated $64.7,41.1 \%$ and protein downregrulated 59.2, 36.8\%. P53, P21 gene expression up-regrulated 49.1, $42.8 \%$ and protein up-regrulated $46.9 .7,38.2 \%$. After transfecting with siRNA MDM2,MTT showed the proliferation of HepG2 cell was inhibited and the inhibitory rate got higher with time. Meanwhile, FCW suggested si RNA MDM2 can induce cell apoptosis and cell 
cycle change:the proportion of $S$ decreased but $\mathrm{G}_{0} / \mathrm{G}_{1}$ increased.However, negative control siRNA group did not have above-mentioned effect.

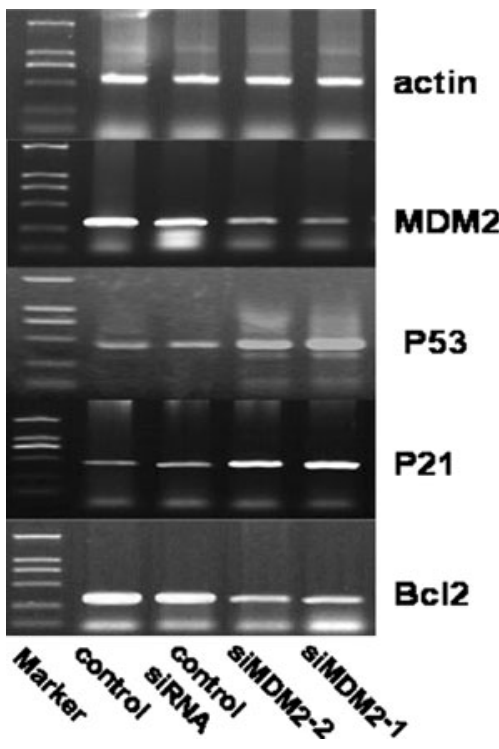

The gene expression change of MDM2, P53, P21 and Bcl

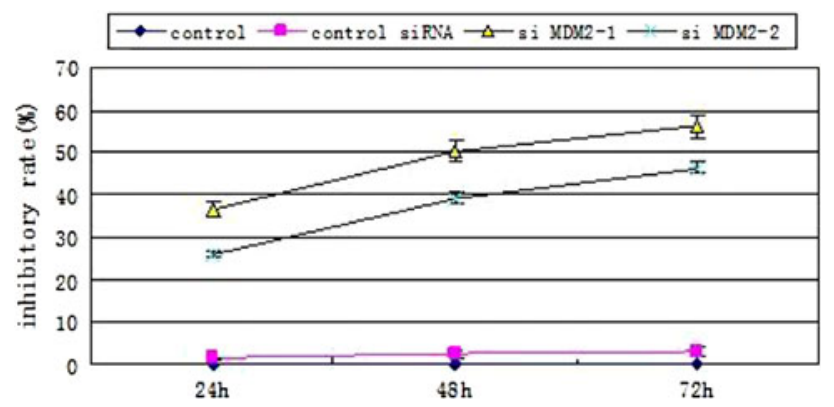

The interhibiory rate of hepG2 after transfection

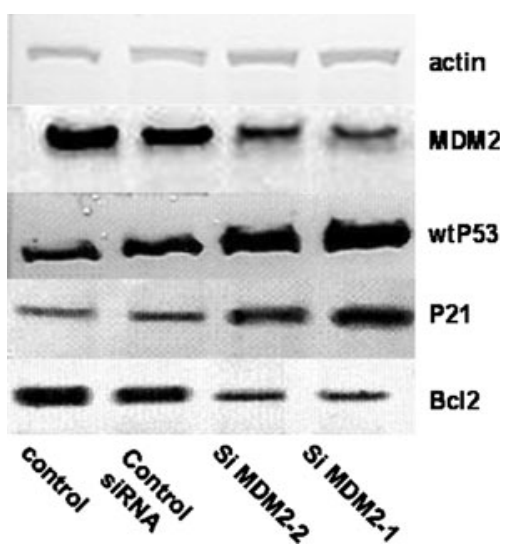

The Protein expression change of MDM2, P53, P21 and
Conclusions: siRNA against MDM2 could inhibit cell proliferation and induce cell apoptosis in hepG2 cell line effectively, which may be associated with the downregulation of MDM2 expression,thereby influence downstream gene expression:upregulating P53,P21 downregulating $\mathrm{Bcl} 2$ expression.

\section{PP29-17}

Lovastatin May Enhance Cytostatic Effects of Sorafenib in Hepatic Carcinoma. Primary Results of a Pilot Study

E.F. Georgescu ${ }^{1,2}$, F. Badulescu ${ }^{3}$, D. Dumitrescu ${ }^{4}$, L. Streba ${ }^{1,2}$,

R. Teodorescu ${ }^{1}$, M. Tataru Abagiu ${ }^{1}$

${ }^{1}$ Internal Medicine/Gastroenterology, Filantropia University

Hospital; ${ }^{2}$ Internal Medicine, University of Medicine and Pharmacy

Craiova, Craiova; ${ }^{3}$ Oncology, University of Medicine and Pharmacy Craiova, craiova; ${ }^{4}$ Radioimagistics, University of Medicine and Pharmacy Craiova, Craiova, Romania

Reports suggest that lovastatin and sorafenib have synergistic cytostatic effects against tumor cell lines in vitro. Our aim was to evaluate if this observation has relevance in 72 patients $(52 \mathrm{M} / 20 \mathrm{~F})$ with advanced primary inoperable hepatic carcinoma having no metastases, according to the NCCN guidelines.

Patients and methods: Mean age at inclusion was $62.19 \pm 1.08$. Child-Pugh A cirrhosis preexisted in 52 patients $(72.2 \%)$. The patients were randomly assigned either to group $\mathrm{S}$ receiving $400 \mathrm{mg}$ sorafenib (33 patients, $10 \mathrm{~F} / 23 \mathrm{M}$ ) or to group $\mathrm{S} / \mathrm{L}$ (39 patients, $10 \mathrm{~F} /$ $29 \mathrm{M}$ ) receiving $400 \mathrm{mg}$ sorafenib and $40 \mathrm{mg}$ lovastatin daily. Tumor response was evaluated every 8 weeks by triphasic helical computed tomography according to the RECIST algorithm revision 1.1 and quantified as complete response (CR); partial response (PR), progressive disease (PD) and stable disease (SD). The rate of alphafetoprotein increase per month $\delta(\mathrm{AFP})$ was also considered, as well as the time to disease progression (TDP) and the median survival (MS).

Results: Mean AFP value at inclusion was $923.93 \mathrm{ng} / \mathrm{ml}$ in group $\mathrm{S}$ and 980.76 in group $\mathrm{S} / \mathrm{L}$ with no difference between groups $(p=0.68)$. The $\delta(\mathrm{AFP})$ was significantly higher in group $\mathrm{S}$ $(184.79 \pm 30.77$ vs. $114.45 \pm 9.44 \mathrm{ng} / \mathrm{ml}, \quad p=0.02)$. CT scans observed significantly higher $\left(p=0.04, \mathrm{Chi}^{2}=4.119\right) \mathrm{PD}$ rate in group S (18/33 patients. i.e. $54.54 \%$ vs. $11 / 39$ patients. i.e. $28.20 \%)$, while PR rate was significantly higher $\left(p=0.05, \mathrm{Chi}^{2}=3.74\right)$ in group S/L (15/39 patients. i.e. $38.46 \%$ vs. $10 / 33$ pts. i.e. $15.15 \%)$. TDP was also higher in group S/L (6.33 \pm 0.32 vs. $5.42 \pm 0.29$ mo., $p=0.04)$. No difference was noticed regarding SD rates and MS, although this was slightly higher in group $\mathrm{S} / \mathrm{L}(12.15 \pm 0.76$ vs. $10.85 \pm 0.82 \mathrm{mo}$ ).

Conclusion: Lovastatin seems to increase the response to sorafenib in advanced hepatocellular carcinoma in terms of slowing the disease progression, perhaps by an impairment of the protein prenylation. However, the median survival is not significantly prolonged.

\section{PP29-18}

A Case of Multiple Hepatocellular Carcinoma Successfully Treated by Transcatherter Arterial Infusion Chemotherapy Using Cisplatin (IA-call ${ }^{\circledR}$ )

S. Ando, K. Hamaoka, K. Yoshino, Y. Mizuno, K. Sugawara, Y. Imai, S. Nagoshi, S. Mochida

Gastroenterology and Hepatology, Saitama Medical University, Saitama, Japan

We report on a case of hepatocellular carcinoma (HCC) with tumor thrombus successfully treated by transcatheter arterial infusion chemotherapy (TAI) followed by transcatheter arterial chemoembolization (TACE) using cisplatin. The patients was a 71- 
years-old male with a chronic hepatitis $\mathrm{C}$ showing multiple HCC in the liver complicating tumor thrombus extending to the main trunk of the portal vein. We treated the patients with TAI using cisplatin. CT examination done one month following TAI revealed that the sizes of tumors were markedly reduced with disappearance of tumor thrombus in the portal vein. Then, we performed TACE with cisplatin again, and complete remission of HCC was obtained in the patients. Although a single recurrent nodule of HCC was found on MRI imaging 27 months later, the nodule was successfully treated by?radiofrequency ablation therapy. Then later, serum AFP levels were maintained within the normal range, and no recurrence of HCC was found on CT and/or MRI imaging for 50 months. The factor affecting the therapeutic efficacy of TAI and TACE using cisplatin should be further investigated in a large scale of patients.

\section{PP29-19}

Sorafenib for Advanced Hepatocellular Carcinoma-Japanese Experience S. Obi, S. Sato, T. Sato, T. Kawai, M. Kanda Liver Unit, Kyoundo Hospital, Tokyo, Japan

Objectives: Hepatocellular carcinoma (HCC) is the common cancer in Japan. Sorafenib is considered the standard of care for patients with advanced HCC. Sorafenib was available in Japan since May 2009. We evaluated Sorafenib in Japanese.

Methods: We conducted a retrospective analysis of our center's experience. Patients with inoperable advanced HCC and Child-Pugh A received continuous oral sorafenib $400 \mathrm{mg}$ bid in 4 week cycles. Tumor response was assessed every two cycles.

Results: Forty-four patients (median age $=67$ years) who were treated at our center with sorafenib were included in this study. Forty-three percent had exposure to prior chemo therapy. On the basis of independent assessment, two patients achieved a partial response, 18 patients had stable disease for at least 12 weeks. Investigator assessed median time to progression (TTP) was 3.4 months, and median overall survival was 6.8 months. Grade 5 drug-related toxicities were in three patients. Grade 3/4 drug-related toxicities included hand-foot skin reaction (18\%), hypertension $(18 \%)$ and eruption $(7 \%)$.

Conclusion: Sorafenib has interesting activity. Adverse events were more severe than SHARP trial in Japanese patients.

\section{Poster Presentation 30: Hepatocellular Carcinoma: Basic Carcinogenesis}

\section{PP30-01}

$\alpha$-fetoprotein Is a Transcriptional Co-repressor of RAR- $\beta$ in Regulating the Expression of CXCR4 in Hepatoma Cells

M. $\mathrm{Li}^{1}, \mathrm{M} . \mathrm{Zhu}^{1}$, X. Xie ${ }^{2}, \mathrm{~S} . \mathrm{Fu}^{3}$, S. Zhou ${ }^{1}, \mathrm{G} . \mathrm{Li}^{4}$

${ }^{1}$ Key Laboratory of Molecular Biology; ${ }^{2}$ Department of Pathophysiology; ${ }^{3}$ Department of Physiology, Hainan Medical College, Haikou; ${ }^{4}$ Department of Biochemistry and Molecular Biology, Peking University Health Science Center, Beijing, China

Background: $\alpha$-fetoprotein(AFP) was a cellular factor for promoting the migration and resisting the apoptosis induced by all trans retinoic acid(ATRA) of hepatoma cells. The present study explored influence of AFP on apoptosis and expression of CXC chemokine receptor 4 (CXCR4) in hepatoma cells.

Methods: Human hepatoma cells line, Bel 7402 and HepG2 (AFPproducing), or HLE (non-AFP-producing) were selected for performing this investigation; Confocal laser microscopy was used to observed the co-location of AFP with RAR- $\beta$, and the expression of CXCR4 in membrane of the cancer cells; siRNA was applied to knockdown the expression of AFP; pcDNA3.1 was used to constructe AFP expressed vector (named pcDNA3.1-afp); Interaction of AFP with RAR- $\beta$ was analyzed by co-immunoprecipitation (Co-IP); RAR$\beta$ regulated CXCR4 gene expressed was detected by Chromatin immunoprecipitation (ChIP)-PCR.

Results: Observed indicated that co-location of AFP with RAR- $\beta$ in cytochylema; Co-IP revealed that AFP has a property to interact with RAR- $\beta$ in Bel 7402 or in HepG2 cells, and the same effect also generated after HLE cells were transfected with pcDNA3.1-afp for $36 \mathrm{~h}$; Blockdown AFP expressed by siRNA in Bel 7402 or in HepG2 cells led to expression of CXCR4 was repressed, but the expression of CXCR4 was enhanced while HLE cells was transfected with pcDNA3.1-afp, and co-treatment with Ly294002, the expression of CXCR4 was partial inhibited. Analyzed by ChIP-PCR indicated that AFP was able to impeded RAR- $\beta$ binding to the promoter of CXCR4, treated with ATRA $(160 \mu \mathrm{mol} / \mathrm{L})$ for $12 \mathrm{~h}$, the coupling of RAR- $\beta$ with CXCR4 promoter was increased, and western blotting analyzed showed that the expression of AFP was suppressed.

Conclusions: This was first evidences to show that AFP up-regulated the expression of CXCR4 through blocking the transcriptional activity of RAR- $\beta$; AFP has a property to stimulate the expression of CXCR4 was the crucial event that promote the metastasis of hepatoma cells.

\section{PP30-02}

Roles of TM4SF5 in Hepatocellular Carcinogenesis

J.W. Lee

Department of Pharmacy, College of Pharmacy, Seoul National University, Seoul, Republic of Korea

Transmembrane 4 L6 family member 5 (TM4SF5) homologues to a tumor-associated antigen L6 is highly expressed in liver tumor tissues, compared to normal counterparts. We also found that expression of TM4SF5 in hepatocytes causes cytosolic p27 $7^{\text {Kip1 }}$ stabilization, FAK activation, and RhoA inactivation, aberrant actin reorganization, epithelial-mesenchymal transition, which in turn correlates with loss of contact inhibition and enhanced migration and invasion. TM4SF5 overexpression in Korean HCC patients correlated with other molecular events for EMT and angiogenesis, indicating diverse roles of TM4SF5 in abnormal cell proliferation, EMT, migration, and invasion. We also screened and identified a small synthetic compound that antagonize the TM4SF5-mediated effects but did not affect TM4SF5-negative cells, presumably through an influence in the extracellular loop 2 of TM4SF5 critical for protein-protein interactions. Therefore, targeting TM4SF5 in hepatocytes can be a promising approach to deal with liver cancer and our compound appears to be promising for the purpose [This work was supported by a grant (2010-00150029) from NRF, Korea to JW Lee].

\section{PP30-03}

Study of Some Associated Factors with Hepatocellular Carcinoma in Egypt

A.A.E.m. Soliman ${ }^{1}$, N. Mashaal ${ }^{1}$, R. Mashali ${ }^{2}$

${ }^{1}$ Hepatology, Faculty of Medicine, Alexandria University;

${ }^{2}$ Dairy Science, Faculty of Agriculture Alexandria University, Alexandria, Egypt 
Background: With more concern to liver disease and with the provided new technology in diagnostic procedures, Egyptian physicians are facing increasing number of patients with hepatic tumors. Food mycotoxins, malnutrition, viral hepatitis, and various parasitic infestations are frequently found to co-exist with hepatocellular carcinoma and there is evidence that these factors interact in the genesis of liver cancer.

Objectives: Find the association between dietary Aflatoxins (AFs), hepatitis B virus infection (HBV), Schistosomal hepatic fibrosis (SHF), and hepatocellular carcinoma (HCC) in Egypt.

Methods: Thirty six patients with HCC, together with ten HBV seropositive cirrhotic patients, ten patients with SHF, and 20 age and sex matched healthy controls. Liver function tests, serum alpha fetoprotein,abdominal ultrasonography and liver biopsy was performed to patients. Qualitative and quantitative detection of AFs in the sera and in the liver tissue was carried out using thin layer chromatography (TLC) and TLC scanner. An in vitro study of aflatoxin B1 (AFB1) metabolism by normal human liver tissue versus human liver tissue with SHF.

Results: Ten out of $36(27.77 \%)$ patients with HCC had AFs in their sera and/or liver biopsy. The concentration of different types of AFs in the sera ranged between 5.66 and $41.88 \mathrm{ng} / \mathrm{ml}$, while the range was between 34.0 and $71.75 \mathrm{ng} / \mathrm{g}$ in liver tissue. Hepatitis B Virus was positive in 22 patients, and SHF was present in 25 patients. The in vitro metabolism of AFB1 revealed that normal human liver produced five derivatives composing $20.71 \%$ from the original dose of AFB 1 , while human liver with SHF produced seven derivatives composing $41.38 \%$ from the original dose of AFB1.

Conclusion: Hepatocellular carcinoma significantly associated with HBV infection. However, the presence of SHF together with AFs Contamination of foods should be considered as etiologic and promoting factors for liver cancer in Egypt.

\section{PP30-04}

Cannabinoid-2 Receptor Agonist Confers Doxorubicin Resistance in Hepatocellular Carcinoma Cells by Inducing p42/44 MAPK Activation and NF $\kappa$ B-dependent IL-8 Production

E.S. Jang, J.-H. Lee, J.-H. Yoon, M.-S. Kwak, E.J. Cho, S.J. Yu, Y.J. Kim, C.Y. Kim, H.-S. Lee

Department of Internal Medicine and Liver Research Institute, Seoul National University College of Medicine, Seoul, Republic of Korea

Background/aims: The production of endogenous cannabinoids (CB) increases in hepatocellular carcinoma (HCC) patients with/without cirrhosis as compared to normal liver. However, the function of $\mathrm{CB}$ in HCC cells remains unclear. In this study, we aimed to evaluate the role of $\mathrm{CB}-2$, those receptors predominantly exist on hepatocyte or blood cell but not on central nerve system, in chemo-resistance of HCC cells.

Methods: Human HCC cell line Huh-7 was used in this study. Cell growth was measured by an MTS assay. Apoptotic and kinase signaling were evaluated by immunoblot analysis. Nuclear factor $\kappa \mathrm{B}$ $(\mathrm{NF}-\kappa \mathrm{B})$ activation was assessed by immunofluorescent staining and ELISA. IL-8 expression was evaluated by real-time quantitative PCR. Results: JWH-133, a selective CB-2 agonist, significantly attenuated doxorubicin-induced HCC cell growth suppression in a dose-dependent manner. JWH-133 treatment induced activation of survival signaling including p42/44 MAPK and NF- $\kappa \mathrm{B}$ in HCC cells, and consequently attenuated doxorubicin-induced activation of caspase 7 and 9. The inhibition of CB-2 receptors (by AM-630, a selective CB-2 antagonist), phoryphorylated p42/44 (by U0126) or activated NF- $\kappa \mathrm{B}$ (by IKK2 inhibitor) reversed JWH-133-induced resistance to doxorubicin cytotoxicity. JWH- 133 treatment also induced transcription of IL-8, a major cytokine involved in angiogenesis, tumorigenicity, metastasis and chemo-resistance of tumors, and this induction was dependent on NF- $\kappa$ B activation by JWH-133.
Conclusions: This study demonstrates that CB-2 agonist may confer resistance to doxorubicin cytotoxicity in $\mathrm{HCC}$ cells by up-regulating p42/44 MAPK and NF- $\kappa$ B. In addition, CB-2 agonist can increase IL-8 production in $\mathrm{HCC}$ cells, thereby participating in multistep hepatocarcinogenic process. Thus, the inhibition of $\mathrm{CB}-2$ receptors may therapeutically be useful for interrupting $\mathrm{HCC}$ progression as well as overcoming its chemo-resistance.

\section{PP30-05}

Expression and Effects of Tansforming Growth Factor- $\beta 1$ on Human Primary Hepatocellular Carcinoma

J. Zhu, R. Gao, R. Zhang, F. He, X. Zhang, J. Niu

Dept of Hepatology, First Hospital, Jilin University, Changchun,

China

Objective: To determine the expression characteristics of TGF- $\beta 1$ in human hepatocellular carcinoma (HCC) in histology and to elucidate the roles of TGF- $\beta 1$ in the growth and metastasis of HCC in vitro.

Methods: Liver samples from 36 patients (who underwent hepatic resection for the first HCC between 2006 and 2009) and 6 normal individuals were examined for TGF- $\beta 1$ mRNA by in situ hybridization. Computer imaging analysis was performed to measure IOD of TGF- $\beta 1$ mRNA-positive cells in carcinoma foci and surrounding stroma respectively. TGF- $\beta 1$ was assessed for its stimulation of HepG2 cell migration, invasion by using commercial kits.

Results: In situ hybridization analysis showed that a significance increase of TGF- $\beta 1$ mRNA was mainly detected in connective tissue and vesculatures arounding carcinoma foci. Incubation of HepG2 cells with recombinant TGF- $\beta 1$ at concentration of $20 \mathrm{ng} / \mathrm{ml}$ can induced a significant migratory and invasion effect $(p<0.01)$, however, TGF- $\beta 1$-induced cell invasion was shown in a CTGF-dependent manner because it was fully abrogated in the presence of neutrolizing CTGF antibody.

Conclusion: Recombinant TGF- $\beta 1$ can induce hepatoma cell migration and invasiong, taken together with its over-expression in surrounding stroma of carcinoma foci, suggesting that TGF- $\beta 1$ may play an important role in the development of HCC.

\section{PP30-06}

Transcriptional Coactivator AIB1 Promotes Human Hepatocellular Carcinoma Progression by Enhancing Cell Proliferation and Invasiveness Q. Chen ${ }^{1,2}, \mathrm{Y} . \mathrm{Xu}^{1}, \mathrm{~W} \cdot \mathrm{Li}^{2}, \mathrm{X} . \mathrm{Su}^{1,2}, \mathrm{~T} . \mathrm{Chen}^{1}$, Y. Zhao ${ }^{1}, \mathrm{C} \cdot \mathrm{Yu}^{1}$

${ }^{I}$ Key Laboratory of Ministry of Education for Cell Biology and Tumor Cell Engineering, School of Life Sciences, Xiamen University;

${ }^{2}$ The First Affiliated Hospital of Xiamen University, Xiamen, China

Background/aims: Amplified in breast cancer 1 (AIB1) is a transcriptional coactivator for nuclear receptors and other transcription factors. AIB1 has been identified to play an important role in malignancy of several cancers such as breast and prostate cancers; however, its involvement in the progression of hepatocellular carcinoma (HCC) remains unclear. The aim of this study is to investigate the expression and possible role of AIB1 in HCC.

Methods: Expression of AIB1 protein was examined by Western blot analysis and immunohistochemical analysis in 22 HCC specimens. Functional studies on AIB1 knockdown HCC cells were performed to reveal the role of AIB1 in HCC progression.

Results: AIB1 protein was significantly increased in 15 of $22 \mathrm{HCC}$ specimens (68\%). Down-regulation of AIB1 reduced cell proliferation rate, cell migratory/invasive ability, colony formation ability, and tumorigenic potential in nude mice. Marked increase of cell cycle inhibitor p21 Cip1/Waf1 expression and dramatic decrease of the expression of phosphorylated Akt, proliferating cell nuclear antigen (PCNA), and matrix metallopeptidase MMP-9 in AIB1 knockdown cells contributed to these phenotypic changes. Consistent with these results, clinical AIB1-positive HCC exhibited higher levels of PCNA protein expression compared with AIB1-negative HCC. Further 
statistical calculation revealed a positive and tight correlation between the levels of AIB1 protein and PCNA protein in HCC, indicating that AIB1 contributes significantly to HCC cell proliferation.

Conclusion: Our results demonstrate that AIB1 promotes human HCC progression by enhancing cell proliferation and AIB1 can be a potential therapeutic target for HCC treatment.

\section{PP30-07}

Implication of NM23-H1 Metastasis Suppressor in Maintenance of Adherens Junctions and Limitation of the Invasive Potential in Human Cancer Cells

M. Boissan ${ }^{1}$, O. De Wever ${ }^{2}$, D. Wendum ${ }^{3}$, P. Chavrier ${ }^{4}$, M.-L. Lacombe ${ }^{5}$, C. Gespach ${ }^{6}$

${ }^{1}$ INSERM U938, Paris 6 University, Paris, France; ${ }^{2}$ Laboratory of Experimental Cancerelogy, Ghent University Hospital, Ghent, Belgium; ${ }^{3}$ INSERM U938, Paris 6 University, Service d'Anatomie Pathologique; ${ }^{4}$ Dynamique de la Membrane et du Cytosquelette, Institut Curie, CNRS, UMR144, Paris; ${ }^{5}$ INSERM U938, Paris 6 University, Paris University; ${ }^{6}$ Molecular and Clinical Oncology, Inserm and Paris 6 University, Paris, France

Loss of NM23-H1 expression correlates with the degree of metastasis and with unfavorable clinical prognosis in several types of human carcinoma. However, the mechanistic basis for the metastasis suppressor function of $N M 23-H 1$ is obscure. We silenced NM23-H1 expression in human hepatoma and colon carcinoma cells and methodologically investigated effects on cell-cell adhesion, migration, invasion and signaling linked to cancer progression. NM23-H1 silencing disrupted cell-cell adhesion mediated by E-cadherin resulting in beta-catenin nuclear translocation and TCF/ LEF-1 transactivation. Further, NM23-H1 silencing promoted cellular scattering, motility, and extracellular matrix invasion by promoting invadopodia formation and upregulating several matrix metalloproteases including MT1-MMP. In contrast, silencing the related $N M 23-H 2$ gene was ineffective at promoting invasion. NM23-H1 silencing activated pro-invasive signaling pathways involving Rac1, MAP kinases, PI3K/Akt, and Src kinase. Conversely, $N M 23-H 1$ was dispensable for cancer cell proliferation in vitro and liver regeneration in NM23-M1 null mice, instead inducing cellular resistance to chemotherapeutic drugs in vitro. Analysis of NM23-H1 expression in clinical specimens revealed high expression in premalignant lesions (liver cirrhosis, colon adenoma) and the central body of primary liver or colon tumors, but downregulation at the invasive front of tumors. Our findings reveal that NM23-H1 is critical for control of cell-cell adhesion and cell migration at early stages of the invasive program in epithelial cancers, orchestrating a barrier against conversion of in situ carcinoma into invasive malignancy.

\section{PP30-08}

Pitavastatin Suppresses Diethylnitrosamine-induced Liver Preneoplastic Lesions in Male C57BL/KsJ- $d b / d b$ Obese Mice

Y. Yasuda ${ }^{1}$, M. Shimizu ${ }^{1}$, H. Sakai ${ }^{1}$, M. Kubota ${ }^{1}$, Y. Osawa ${ }^{1}$, T. Tanaka ${ }^{2}$, H. Moriwaki ${ }^{1}$

${ }^{1}$ Department of Gastroenterology, Gifu University Graduate School of Medicine, Gifu; ${ }^{2}$ Department of Oncologic Pathology, Kanazawa Medical University, Ishikawa, Japan

Introduction: Obesity and related metabolic abnormalities, including a lipid accumulation and a state of inflammation in the liver, play a role in liver carcinogenesis. Imbalance of adipocytokine, such as decreasing serum adiponectin levels, is also involved in obesityrelated liver tumorigenesis.

Aim: In the present study, we examined the effects of pitavastatin, which is used for the treatment of hyperlipidemia, on the development of diethylnitrosamine (DEN)-induced liver preneoplastic lesions in $\mathrm{C} 57 \mathrm{BL} / \mathrm{KsJ}-\mathrm{db} / \mathrm{d} b(\mathrm{~d} b / \mathrm{d} b)$ obese mice.
Methods: Male $d b / d b$ mice were given tap water containing $40 \mathrm{ppm}$ DEN for 2 weeks and then they were subsequently fed a diet containing $1 \mathrm{ppm}$ or $10 \mathrm{ppm}$ pitavastatin for 14 weeks.

Results: At sacrifice, feeding with either dose of pitavastatin significantly inhibited the development of hepatic premalignant lesions, foci of cellular alteration, in comparison to the control pitavastatinuntreated group. Pitavastatin improved liver steatosis and activated the AMPK protein in the liver. It also decreased the levels of free fatty acid, total cholesterol, and aminotransferases, while increasing the levels of adiponectin in the serum. The serum levels of tumor necrosis factor (TNF)-a and the expression of TNF- $a$ and interleukin-6 mRNAs in the livers were decreased by pitavastatin treatment, suggesting attenuation of the chronic inflammation induced by excess adipose tissues.

Conclusion: These findings suggest that pitavastatin is effective in inhibiting obesity-related liver tumorigenesis and, therefore, may be useful in the chemoprevention of liver cancer in obese individuals.

\section{PP30-09}

Polymorphisms of Interferon Gamma Gene and Risk Ofhepatocellular Carcinoma in Korean Patients with Chronic Hepatitis B Viral Infection H.P. Shin, J.I. Lee, J.W. Jeon, J.J. Park, J.M. Cha, K.R. Joo

Internal Medicine, Kyung Hee University School of Medicine, Seoul, Republic of Korea

Increasing evidence supports the contribution of the pro-/antiinflammatory cytokine balance and genetic factors to hepatocellular carcinoma (HCC). Here, we investigated whether genetic IFNG polymorphisms were associated with $\mathrm{HCC}$ in Korean patients with chronic hepatitis B (CHB). We genotyped a single nucleotide polymorphism (SNP, rs2430561, +874A/T) and a microsatellite $\left(\mathrm{rs} 3138557,(\mathrm{CA})_{\mathrm{n}}\right.$ repeat), located in the first intron of the IFNG gene, by direct sequencing and the genescan method. A populationbased case-control study of HCC was conducted and included 180 patients with $\mathrm{CHB}$ and HCC, and $181 \mathrm{CHB}$ patients without $\mathrm{HCC}$ in a Korean population. Genotype and allele distributions of the IFNG SNP were associated with HCC. The frequencies of the AA genotype and the A allele were significantly increased in HCC subjects $(P<0.05)$. Combined analysis using the genotype of rs 2430561 and the number of microsatellites revealed that the frequencies of AT- $\mathrm{CA}_{12}$ and TT-CA 12 increased significantly in $\mathrm{HCC}$ subjects $(P<0.0001)$. Our results suggest that the IFNG gene may be a susceptibility gene and a risk factor for HCC in the Korean population.

\section{PP30-10}

HLA-G 14-bp Insertion/Deletion Polymorphism Is Not Associated with Hepatocellualr Carcinoma in Korean Patients with Chronic Hepatitis B Virus Infection

H.P. Shin, J.I. Lee, J.W. Jeon, J.J. Park, J.M. Cha, K.R. Joo

Internal Medicine, Kyung Hee University School of Medicine, Seoul, Republic of Korea

The aim of present study was to determine whether major histocompatibility complex, class I, G $(H L A-G)$ gene was associated with hepatocellualr carcinoma (HCC). Abnormal HLA-G expression shows in various diseases such as renal cell carcinoma, asthma, and classical Hodgkin's lymphoma. To investigate the possible association with susceptibility to HCC, 181 hepatitis patients and $180 \mathrm{HCC}$ patients were enrolled in this study. $H L A-G$ 14-bp insertion/deletion polymorphism is located in $3^{\prime}$ untranslated region of $H L A-G$ gene. $H L A-G$ 14-bp insertion/deletion polymorphism was analyzed using polymerase chain reaction (PCR). For analysis of genetic data, SNPStats, HapAnalyzer, Helixtree, SNPAnalyzer, and SPSS 18.0 were used. Logistic regression models were performed for odds ratio, $95 \%$ confidence interval, and $P$ value. Allele and genotype frequencies of $H L A$ $G$ 14-bp insertion/deletion polymorphism in the hepatitis group were 
similar pattern, compared to those in the HCC group. Furthermore, no differences were observed between liver cirrhosis and non liver cirrhosis. These results suggest that $H L A-G$ 14-bp insertion/deletion polymorphism may not be associated with susceptibility to HCC and development of liver cirrhosis in Korean population.

\section{PP30-11}

High Expression of MicroRNA-345 Predicts a Low Risk of Tumor Recurrence Following Curative Resection of Hepatocellular Carcinoma G.E. Chung ${ }^{1}$, J.-H. Yoon ${ }^{2}$, J.-H. Lee ${ }^{2}$, S.J. Yu ${ }^{2}$, Y.J. Kim ${ }^{2}$, H.-S. Lee ${ }^{2}$ ${ }^{1}$ Department of Internal Medicine and Healthcare Research Institute, Seoul National University Hospital Healthcare System Gangnam Center; ${ }^{2}$ Department of Internal Medicine and Liver Research Institute, Seoul National University College of Medicine, Seoul, Republic of Korea

Background and aims: MicroRNAs (miRNAs) has recently been implicated in carcinogenesis and tumor progression. Hepatocellular carcinoma (HCC) is well known for frequent relapses following curative resection. In this study, we attempted to identify the miRNAs associated with HCC recurrence and their biological functions.

Methods: We evaluated miRNA expression profiles in 25 pairs of $\mathrm{HCC}$ and adjacent non-tumor liver tissues from HCC patients using miRNA microarray and defined a target gene. To evaluate the function of miRNAs, expressions of which significantly differ between tumor and not-tumor tissues, we performed in vitro study using three immortalized human HCC cell lines (Huh-BAT, SNU475 and SNU761). MiRNA expressions were up- and down-regulated by its mimics and inhibitors, respectively. Cellular proliferation was measured by MTS assay.

Results: Out of 449 miRNAs assayed, we identified 21 miRNAs differentially expressed in HCC tissues by the false discovery rate procedure. Among them, high expression of miR-345 was significantly associated with longer disease-free survival $(P=0.01)$. In vitro study showed that inhibition of miR-345 in cultured HCC cells significantly increased $\mathrm{HCC}$ cell proliferation, while enhancement of miR-345 expression significantly decreased HCC proliferation (both $P<0.05$ ). In addition, enforced miR-345 expression caused multidrug resistance-associated protein 1 down regulation, whereas antimiR 345 induced its up-regulation.

Conclusions: These results indicate that miR-345 expression in HCC tissues may predict a low risk of $\mathrm{HCC}$ recurrence. In addition, the modulation of miR-345 expression may be useful as an anti-proliferation strategy for HCC treatment.

\section{PP30-12}

No Associations of Polymorphisms in ADPRT with HBV Clearance and HCC Occurrence in a Korean Population

J.S. Lee ${ }^{1}$, J.-H. Kim ${ }^{1}$, B.-L. Park ${ }^{2}$, H.S. Cheong ${ }^{2}$, J.Y.-H. Kim ${ }^{1}$, T.J. Park ${ }^{1}$, C.F. Pasaje ${ }^{1}$, J.S. Bae ${ }^{1}$, H.-S. Lee ${ }^{3}$, Y.J. Kim ${ }^{3}$, H.D. Shin ${ }^{1}$

${ }^{1}$ Sogang University; ${ }^{2}$ SNP Genetics, Inc; ${ }^{3}$ Department of Internal Medicine and Liver Research Institute, Seoul National University College of Medicine, Seoul, Republic of Korea

Background: The human adenosine diphosphate ribosyl transferase (ADPRT) gene might significantly affect cancer by encoding poly [ADP-ribose] polymerase 1 enzyme (PARP-1) and promoting an important role in cellular responses to DNA-damage, genomic stabilization and regulation of tumor suppressor genes.

Aim: We explored whether polymorphisms of ADPRT affect clearance of Hepatitis B virus (HBV) infection or risk of HCC occurrence in Korean HBV cohort.

Methods: Genotyping was performed in a total of 1,066 subjects composed of 434 spontaneously recovered (SR) subjects as normal controls and 632 chronic carriers (CC) of HBV who were further classified into 325 patients with liver cirrhosis (LC)/chronic hepatitis (CH) and 307 patients with hepatocellular carcinoma (HCC).
Results: Logistic analyses of six common single nucleotide polymorphisms (SNPs) and its haplotypes revealed that none of the polymorphisms were significantly associated with clearance of HBV infection and HCC occurrence, except for a nominal evidence of association between haplotype $2(h t 2)$ with $\mathrm{HBV}$ clearance $(P$ value $=0.05)$. In the analysis of age of HCC occurrence which is an important factor in disease progression to $\mathrm{HCC}$, results from Cox proportional hazards showed that none of the variants were significantly associated with onset age of HCC occurrence, although a nominal signal in $h t 4\left(P=0.03\right.$, but $\left.P^{\text {corr }}>0.05\right)$ was initially detected.

Conclusions: Although $A D P R T$ is an important gene for cellular responses and tumor regulations, our study provides evidence that $A D P R T$ variations do not affect $\mathrm{HBV}$ clearance and $\mathrm{HCC}$ occurrence.

\section{PP30-13}

TGFBR3 Polymorphisms and its Haplotypes Associated with Chronic Hepatitis B Virus Infection and Age of Hepatocellular Carcinoma Occurrence

J.-H. Kim ${ }^{1}$, B.-L. Park ${ }^{2}$, H.S. Cheong ${ }^{2}$, C.F.A. Pasaje ${ }^{1}$, J.S. Bae ${ }^{1}$, H.-S. Lee ${ }^{3}$, H.D. Shin ${ }^{1}$, Y.J. Kim ${ }^{3}$

${ }^{1}$ Sogang University; ${ }^{2}$ SNP Genetics, Inc; ${ }^{3}$ Department of Internal Medicine and Liver Research Institute, Seoul National University College of Medicine, Seoul, Republic of Korea

Objective: Hepatocellular carcinoma (HCC) is one of most common cancer and is mainly caused by viral infections including hepatitis B virus (HBV). Recently, the decreased expression level of the transforming growth factor, beta receptor III (TGFBR3) gene has been implicated in HCC and other human cancers. This study investigated whether TGFBR3 polymorphisms might be associated with $\mathrm{HBV}$ clearance and HCC occurrence.

Methods: This study identified 27 single nucleotide polymorphisms (SNPs) in the exon, promoter, and exon-intron boundary regions of TGFBR 3 by resequencing in 24 individuals. Then, nine SNPs in the promoter and exons of the gene were genotyped from 1,065 hepatitis patients composed of 637 chronic carriers (CC) and 428 spontaneously recovered (SR) subjects.

Results: Two SNPs, rs1805113 (Phe676Phe) in exon 13 and $r s 1805117$ in 3'UTR $(P=0.009$ and $P=0.008$, respectively) were significantly associated with HBV clearance. In addition, Cox relative hazards analyses revealed that haplotype $B L 2 \_h t 2$ showed a significant association with the age of HCC occurrence among chronic HBV patients (Relative Hazard $=1.38 ; P=0.007$ ).

Conclusion: Our findings suggest that TGFBR3 polymorphisms and its haplotypes might be associated with HBV clearance and age of HCC occurrence.

\section{PP30-14}

CCR1, Syndecan-1 and -4 Are Involved in RANTES/CCL5-mediated Migration or Invasion of Human Hepatoma Cells

F. Charni, N. Charnaux, A. Sutton

Université Paris 13, Bobigny, France

Background and aims: In addition of their physiologic effects in inflammation and angiogenesis, chemokines such as the CC-chemokine: regulated on activation, normal T-cell expressed, and secreted (RANTES)/CCL5 are involved in cancer pathology. We recently demonstrated that the human Huh7 hepatoma cells express RANTES/ CCL5 G protein-coupled receptor CCR1 but not CCR3 nor CCR5. RANTES/CCL5 binding to these cells depends on CCR1 and glycosaminoglycans (GAGs). Moreover, RANTES/CCL5 strongly stimulates the migration and the invasion of Huh7 cells at a $3 \mathrm{nmol} / \mathrm{L}$ physiological concentration. The aim of this study is to elucidate some of the molecular mechanisms including the involvement of signalling pathways or the role of two membrane proteoglycans belonging to the syndecans (SDC) family (SDC-1 and SDC-4), in RANTES/CCL5induced migration and invasion of human hepatoma cells. 
Methods: Semi-quantitative RT-PCR and flow cytometry experiments were performed to analyse the expression of CCR1, SDC-1 and SDC-4 by Huh7 hepatoma cells. We studied Huh7 migration or invasion by using Bio-coat cell migration chambers. A spreading experiment was performed after cell incubation with anti-CCR1, antiSDC-1, or anti-SDC-4 antibodies or specific RNA interference.

Results: RANTES/CCL5-induced migration, invasion and spreading of Huh7 cells are both strongly inhibited by anti-CCR1, anti-SDC-1 and anti-SDC-4 antibodies. Reducing CCR-1, SDC-1 and SDC-4 expression by RNA interference also decreased RANTES/CCL5mediated migration, invasion and spreading of Huh7 cells. Therefore, CCR1 and the two syndecans involved in these main events of HCC progression. RANTES/CCL5 also induces the phosphorylation of focal adhesion kinase, JNK/SAPK and MAPK. These transduction pathways, PKC and Pi3K are involved in RANTES/CCL5-mediated migration, invasion and spreading as treatments of the cells with specifics inhibitors strongly reduced these effects.

Conclusions: Our data indicate that the RANTES/CCL5 may play an important role in the pathogenesis of human hepatocellular carcinoma (HCC). Targeting the RANTES-glycosaminoglycan interaction could be a new therapeutic approach for HCC.

\section{PP30-15}

Association of TNF Alpha Promoter Polymorphism with an Increased Risk of Hepatocellular Carcinoma in North Indian Population

M. Asim, A. Malik, I. Ahmad, N.A. Khan, P. Kar

Medicine, Maulana Azad Medical College, University of Delhi, New Delhi, India

Background/aims: Tumor necrosis factor-a (TNF-a) is a proinflammatory cytokine that may act as an endogenous tumor promoter. A genetic polymorphism of TNF-a gene at position_308 promoter region is involved in the regulation of expression level and has been found to be associated with susceptibility tovarious types of cancer. The present study was undertaken to investigate the association between polymorphisms of tumor necrosis factor (TNF)- $\alpha$ and the risk for Hepatocellular carcinoma.

Methods: One hundred eight pairs of age and gender-matched patients with $\mathrm{HCC}$ and unrelated healthy controls were genotyped for TNF308 and TNF238 alleles with polymerase chain reaction and direct sequencing.

Results: The frequency of TNF308 genotype in cases was higher compared to controls [odds ratio $(\mathrm{OR})=4.37$ ]. whereas, TNFA -238 carrier A (GA/AA) genotype distribution revealed statistically nonsignificant trend from HCC cases with respect to controls (OR = 1.79). Multivariate analysis indicated that TNF308 allele (OR =3.23), hepatitis B surface antigen $(\mathrm{OR}=17.17)$ and antibodies to hepatitis $\mathrm{C}$ virus $(\mathrm{OR}=45.52)$ were independent risk factors for HCC. Surrogate markers for significant fibrosis implied that cases with the TNF308 allele have more advanced liver fibrosis. Moreover, multivariate analysis indicated that cirrhosis with Child-Pugh grade $\mathrm{C}$, low serum albumin, and low platelet count were independent risk factors for carrying the TNF308 allele.

Conclusion: TNF308 allele carriage and chronic hepatitis B virus/ hepatitis $\mathrm{C}$ virus infection are independent risk factors for HCC. Carriage of the TNF308 allele correlates with disease severity and hepatic fibrosis, which may contribute to a higher risk for HCC.

\section{PP30-16}

Gene Mutation and Altered Expression of NBS1 in HBV Associated Hepatocellular Carcinoma

Y. Wang ${ }^{1}$, Y.P. Zhao ${ }^{1}$, J.X. Zhang ${ }^{2}$, J. Long ${ }^{3}$, H. Li ${ }^{1}$, C.Z. Lu' ${ }^{2}$, H. You ${ }^{1}$, J.D. Jia ${ }^{1}$, J. Huang ${ }^{1}$

${ }^{1}$ Liver Research Center, Beijing Friendship Hospital,Capital Medical University, Beijing, China; ${ }^{2}$ Department of Hepatology, Tianjin Infectious Disease Specialty Hospital, Tianjin, China; ${ }^{3}$ Minimally
Invasive Hepatobiliary Cancer Center, Beijing You-An Hospital, Capital Medical University, Beijing, China

Introduction: As a key DNA repair associated gene for DNA double strand breakage (DSB), NBS1 plays an important role in the development of cancer. DSB is common event in HBV associated hepatocellular carcinoma (HCC) and it was shown recently in animal trials nbs1 heterozygous mice exhibited a high incidence of HCC, and there may be functional interactions between NBS1 and TP53. The objective of current study is to assess whether NBS1 play a role in the pathogenesis of HBV associated HCC.

Methods: With 43 cases of cirrhosis as control, 40 cases of $\mathrm{HBV}$ associated HCC were screened for mutations in NBS1 gene by single-stranded conformational polymorphism analysis followed by direct sequencing. Consequence of identified mutations was analyzed by detection of phosphorylated NBS1 level and binding state of MRE11-RAD50-NBS1 complex. NBS1 expression was analyzed in above samples, including 23 pairs of HCC and corresponding adjacent tissue, by RT-PCR, Real-time PCR, Western-blots and immunohistochemistry analysis. Genetic alteration in TP53 pathway including TP53 mutation, MDM2 amplification, p14ARF homozygous deletion and promoter methylation were carried out in the cases of HCC.

Results: Seven of 40(18\%) HCC carried a total of 13 NBS1 mutations in which 8 were missense mutation and 5 were intronic splicing mutations, whereas only 1 synonymous mutation was identified in 43 cases of cirrhosis (2\%). All HCC with NBS1 mutation contained at least one genetic alteration in the TP53 pathway. Of 7 HCC with TP53 mutation, 5(71\%) contained NBS1 mutations, and there was a significant association between TP53 mutations and NBS1 mutation $(\mathrm{P}<0.01)$. Increased NBS1 expression was shown in $80 \%$ advanced HCC compared to adjacent tissues. Initial result shows impaired function of NBS1 in the cases with NBS1 mutation.

Conclusion: Alteration of $N B S 1$ gene maybe involved in the development of HBV associated HCC, and is associated with inactivation of TP53 function.

\section{PP30-17}

Maintenance of Growth Inhibiton of Hepatoma Cells by Survivin-targeted ShRNA Cloning Vector-mediated Transfection

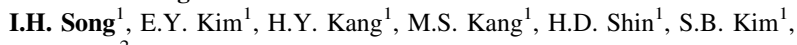
S.H. Yang ${ }^{2}$

${ }^{1}$ Division of Hepatology, Department of Internal Medicine, Dankook University College of Medicine, Dankook University Hospital Institute of Medical Science, Cheonan, Republic of Korea;

${ }^{2}$ Department of Internal Medicine, Seoul Veterans Hospital, Seoul, Republic of Korea

Background/aims: Induction of RNA interference, a phenomenon of post-transcriptional gene silencing, is currently used as an effective and powerful tool for functional analysis of its target gene. Survivin, a member of inhibitor of apoptosis protein, is considered to be a representative target for gene therapy for hepatocellular carcinoma. We investigated the impact of shRNA-induced survivin-knockout on growth regulation of hepatoma cells by survivin-targeted shRNA cloning vector-mediated transfection.

Methods: After the cloning of survivin exon 1-targeted shRNA to pENTR/U6 vector and transformation of cloning vector to E. coli, kanamycin-selected colonies were picked up for the plasmid DNA sequencing and the transfection to Hep3B hepatoma cells. After transfection of shRNA cloning vector into Hep3B cells, the regulation of survivin expression was analyzed using RT-PCR and Western blotting, and growth pattern of hepatoma cells was assessed by MTT cell viability assay and flow cytometry.

Results: Transfection efficiency at $24-72 \mathrm{~h}$ after treatment of cloning vector with survivin-targeted shRNA into cells was $50-70 \%$. The microscopic finding of hepatoma cells treated with 
survivin-targeted shRNA cloning vector revealed an inhibitory growth pattern on the time-dependent manner up to $72 \mathrm{~h}$ after transfection to hepatoma cells, but the growth pattern of hepatoma cells was different according to the status of serum deprivation. Also, survivin-targeted shRNA cloning vector induced a downregulation of survivin transcript and protein up to $72 \mathrm{~h}$ after transfection. The growth inhibition of hepatoma cells accompanied with cell cycle redistribution to increased fraction of sub-G1 phase, indicating the growth inhibition by apoptosis. Cell viability did not increase after transfection of shRNA cloning vector into hepatoma cells up to $72 \mathrm{~h}$.

Conclusions: Survivin-targeted shRNA cloning vector induced a growth inhibition of hepatoma cells with a stable down-regulation of survivin, although it did not reveal a complete knockout of survivin expression.

\section{PP30-18}

Expression of FGFs and FGFRs in Human Hepatocellular Carcinoma B.I. Yeh ${ }^{1}$, J. Namkung ${ }^{1}$, J. Kim ${ }^{2}$

${ }^{1}$ Biochemistry; ${ }^{2}$ Internal Medicine, Yonsei University, Wonju College of Medicine, Wonju, Republic of Korea

FGFs and FGFRs play important roles in angiogenesis, wound repair, and development. It is known that FGF signaling in cancers promote cell cycle progression and inhibit apoptosis. This study aimed to establish the first quantitative profiles of expression targeting all known FGFs and FGFRs in hepatocellular carcinomas and hepatoblastomas compared with normal liver. We used 6 hepatocellulcar carcinoma cell lines (SNU-398, SNU-449, SNU-739, SNU761, SNU-886, and Hep3B) and two hepatoblastoma cell lines (HepG2 and PLC/PRF/5). Normal hepatocyte cDNA was also used as a control. To compare the mRNA level real-time RT-PCR was performed. GAPDH levels were checked as controls and we compared delta Ct values of FGFs and FGFRs. It is reported there are 22 kinds of FGFs and 8 FGFRs so far. Among them, FGF-16 and 22 were not expressed in all cell lines. FGF-3, 4, 5, 8, and 20 were not expressed in normal hepatocyte, but in hepatoma cells and FGF-1, 9, $11,12,13,18,19$, and FGFR-4 and 5 were increased in hepatoma cells compared with those in normal cells. FGF-6, 7, 21, and 23 were increased in normal hepatocyte, and/or not expressed in hepatoma cells. In conclusion, we prepared the expression profiles of FGFs and FGFRs in hepatocellular carcinoma cells for the first time and we're going to study to develop new therapeutic target with these profiles.

\section{PP30-19}

Possible Role of P 21 RAS and IGF 2 in the Pathogenesis of Hepatocellular Carcinoma Occurring on Top of HCV

N.M. Baddour ${ }^{1}$, A. Zeid ${ }^{2}$, E. Farrag ${ }^{3}$, Y. Taher ${ }^{2}$

${ }^{1}$ Pathology; ${ }^{2}$ Hepatology, Internal Medicine, Alexandria Faculty of Medicine; ${ }^{3}$ Pathology, Institute of Research, Alexandria, Egypt

Introduction: $\mathrm{HCC}$ is one of the most common cancers worldwide representing app. Four percent of all malignancies. Hepatitis $\mathrm{C}$ is a major risk factor and a major health problem in Egypt having the highest seroprevalence in the world affecting app. 10-20\% of the population.

Objectives: The present work focused on studying the role of $\mathrm{p} 21$ RAS and IGF II in the pathogenesis of HCV related HCC. 15 cases of HCV hepatitis, 13 cases of HCV related cirrhosis and 15 cases of $\mathrm{HCC}$ on top of chronic HCV hepatitis were stained with monoclonal antibodies.

Results: P 21 Ras expression increased gradually from chronic HCV to a peak in the cirrhosis group and then its expression returned to baseline HCV levels. While IGF II expression increased gradually from $\mathrm{HCV}$ cases to a peak in cirrhosis and remained highly expressed in the HCC cases as well.
A significant positive correlation was observed between p21 RAS expression and the stage in both cirrhosis $(r=0.99, p=0.0001)$ and HCC cases $(r=0.99, p=0.0001)$. A significant positive correlation was observed between IGF II expression and each of grade $(r=0.409, p=0.01)$ and stage $(r=0.404, p=0.022)$ in the cirrhosis group.

Conclusions: Thus it may be deduced that $\mathrm{p} 21$ RAS has an important role in the initiation of HCC, however, it has no role in its progression. On the other hand, IGF II may have an important role in both the initiation and progression of $\mathrm{HCC}$ occurring on top of $\mathrm{HCV}$ hepatitis. Also, the significant positive correlations observed between each of p21 Ras and IGF II with the stage of the included cases, incriminates fibrosis in the pathogenesis of HCC. Increasing grade also plays a role as evidenced by the positive correlation between $\mathrm{p} 21$ RAS expression and grade of the studied cases.

\section{PP30-20}

Reduced Expression of ArgBP2, a Candidate Tumor Suppressor Gene at the Hot Allelic Deletion Region 4q35.1, in Human Hepatocellular Carcinoma

L. Zhou, W. Zhou, C. Xing, H. Xie, X. Yu, K. Chen, L. Pan, L. Wu, S. Zheng Key Lab of Combined Multi-organ Transplantation, Ministry of Public Health, Department of Hepatobiliary and Pancreatic Surgery, First Affiliated Hospital, School of Medicine, Zhejiang University, Hangzhou, China

Background and aims: Tumor suppressor genes (TSGs), which could be inactivated by loss of heterozygosity ( $\mathrm{LOH})$, are involved in hepatocarcinogenesis. In this study, we aim to assess deletion and expression pattern of $\mathrm{ArgBP} 2$ gene at the specific region chromosome 4q35.1 in hepatocellular carcinoma (HCC).

Methods: Twenty-one microsatellite markers across chromosome 4q21.2-q35.2 were selected for LOH analysis in 29 HCCs. mRNA expression of $\operatorname{ArgBP} 2$ gene was investigated in $\mathrm{HCC}$ cell lines. We further validated the deletion and the expression of ArgBP2 in 42 HCCs and studied the clinicopathological correlation with the expression of this gene.

Results: The frequency of $\mathrm{LOH}$ at each microsatellite locus was $46.1 \%$ in HCCs. Among these loci, D4S2920 had the highest LOH frequency (11/19 HCCs, 57.9\%) and could represent the preferential deletion region $4 \mathrm{q} 35.1$. The gene $\mathrm{ArgBP} 2$ was mapped at this region and its LOH frequency was $45.2 \%$ (14/31 HCCs). The mRNA expression of ArgBP2 was reduced in HCC cell lines, and was significantly lower in tumor tissues than in corresponding non-tumor tissues $(P=0.0106)$ in HCCs. Consistently, the expression of $\mathrm{Ar}$ gBP2 in HCC tissues was decreased or lost at protein level. Moreover, we found that the mRNA expression of $\mathrm{ArgBP} 2$ was down-regulated more frequently in tumors with size no more than $5 \mathrm{~cm}(P=0.0201)$. Conclusions: Chromosome 4q21.2-q35.2 is the common deletion region and some putative important candidate TSGs likely harbor at this region. $\operatorname{ArgBP} 2$ at the chromosome $4 \mathrm{q} 35.1$ may be a novel candidate TSG involved in HCC.Acknowledgements: This study is supported by National S\&T Major Project (No. 2008ZX10002-026) and National Key Technology R\&D Program (No. 2008BAI60B03). We thanks for Ahmed Taki Eldin's valuable advice.

\section{PP30-21}

TGF $\beta 2$ Effects on Tumorigenic and Metastatic Potential of HCC Cells N. Donner ${ }^{1}$, M. Makarova ${ }^{1}$, A. Makarova ${ }^{1,2}$, O. Morozova $^{3}$, N. Lazarevich ${ }^{1}$ ${ }^{I}$ Immunochemistry, Russian Cancer Research Center, Institute of Carcinogenesis; ${ }^{2}$ Moscow State University, ${ }^{3}$ Russian Cancer Research Center, Institute of Carcinogenesis, Moscow, Russia

Background and aims: Transforming Growth Factor (TGF) $\beta$ plays crucial role in hepatocellular carcinoma (HCC) tumorigenesis. Increased levels of TGF $\beta 1$ were detected in serum and urine of patients with advanced stages of HCC. While the role of TGF $\beta 1$ in 
hepatocarcinogenesis is under extensive investigation, the impact of other isoforms in this process is underestimated. We had investigated the role of TGF $\beta 2$ in HCC progression on the experimental model of mouse highly invasive fast-growing $\mathrm{HCC}$ (fgHCC) overexpressing TGF $\beta 2$.

Methods: To inhibit production of TGF $\beta 2$ in $\mathrm{H} 33$ cell culture obtained from fgHCC we used shRNA technique. The amount of TGF $\beta 2$ was determined by ELISA. The repression of $\mathrm{N}$ - and E-cadherin was achieved by siRNA transient transfection and confirmed by RT-PCR and Western blotting. The effects of TGF $\beta 2$ and $\mathrm{N}$-cadherin inactivation in $\mathrm{H} 33$ cells in vitro were studied by RT-PCR, proliferation and cell motility tests. To assess effect of TGF $\beta 2$ inactivation on metastatic and tumorogenic potential $\mathrm{H} 33$ cells in vivo we injected H33-shTGF $\beta 2$ cells in syngenic recipient mice.

Results: 3.5 -fold decrease of the amount of TGF $\beta 2$ in H33cells led to growth retardation and decrease of cell motility in vitro. After subcutaneous injection into mice H33-shTGF $\beta 2$ cells showed delay in tumor formation and decrease of metastatic potential. TGF $\beta 2$ repression also resulted in $\mathrm{N}$ - and E-cadherin upregulation and formation of $\mathrm{N}$ - and E-cadherin adherence junctions in H33-shTGF $\beta 2$ culture. siRNA-mediated $\mathrm{N}$-cadherin knockdown in these cells resulted in reversion of cell motility.

Conclusion: TGF $\beta 2$-induced activation of TGF $\beta$ signaling in $\mathrm{HCC}$ cells can contribute to tumor progression increasing tumorigenic and metastatic potential of tumor cells. It can be explained by involvement of TGF $\beta$ signaling in regulation of cell motility, at list partly through regulation of cadherin genes expression.

Acknowledgment: The work was supported by RFBR grants 10-0401504 and 09-04-13901-ofi-c.

\section{PP30-22}

Chronic High-fat Diet Postpones Diethylnitrosamine-induced Liver Tumorigenesis in Rats

X.Y. Duan, J.G. Fan, Q. Pan

Xinhua Hospital,Shanghai Jiao-tong University School of Medicine, Shanghai, China

It is well known that high-fat diet (HFD) feeding can make rats and mice develop obesity related fatty liver and accelerates chemically induced tumorigenesis. In this study, we investigated the effects of chronic HFD on diethylnitrosamine (DEN)-induced liver tumorigenesis in rats. We hypothesized that a HFD would promote chemically induced hepatocarcinogenesis in this model. Sixty-seven male SD rats were divided into 4 groups: control diet (CD) group $(n=10)$, HFD group $(n=10), \mathrm{CD}+\mathrm{DEN}$ group $(n=23)$ and HFD+DEN group $(n=24)$, which were given a standard chow diet or HFD with or without DEN $(0.2 \%, 10 \mathrm{mg} / \mathrm{kg}, 5$ times/week, ig) treatment. After 10 and 12 weeks, 7 rats respectively from $\mathrm{CD}+\mathrm{DEN}$ and HFD+DEN group were killed. The rest underwent sacrifice after 14 weeks. The body weight was recorded every week. Liver histology and liver function markers were investigated. All rats, but except for $\mathrm{CD}+\mathrm{DEN}$, gained weight at the end of experiment. And the rats treated with DEN showed lower weight than CD rats. Serum albumin levels decrease more significant in CD+DEN group than that in HFD+DEN group. After 10 and 12 weeks, 3 and 5 out of 7 rats in $\mathrm{CD}+\mathrm{DEN}$ group developed hepatocellular carcinoma (HCC), whereas none or only 2 of 7 showed HCC in HFD+DEN group. Conclusively, the incidence of HCC in CD+DEN group was higher than that in HFD+DEN group $(P<0.05)$. All rats suffering from DEN treatment were confirmed to have HCC with frank cirrhosis after 14 weeks. These results suggest that the detrimental effects of malnutrition induced low body weight, comparatively speaking, are more important for HCC development than high-fat diet per se. Similarly, correct problems related to malnutrition may have important implications for prevention and treatment of HCC in humans.
PP30-23

Actopaxin: A Novel Regulator of Cell Migration and Invasion in Human Hepatocellular Carcinoma

L. $\mathbf{N g}^{1}$, B.C.Y. Wong ${ }^{1,2}$, R. Pang ${ }^{1,2}$

${ }^{1}$ Department of Medicine; ${ }^{2}$ Centre for Cancer Research, The

University of Hong Kong, Hong Kong, Hong Kong S.A.R.

Background: The high mortality rate of hepatocellular carcinoma (HCC) is partly due to development of metastasis, which involves cell spreading, lamellipodia formation and cell migration. Formation of focal adhesions is a key regulator of cell motility and cell invasion. Actopaxin, a focal adhesion and cytoskeleton-associated protein, is required for such processes. The aim of this study was to examine the role of Actopaxin in HCC cell migration, invasion and development of metastasis.

Methods: The expression of Actopaxin in primary and metastatic liver cancer cell-lines was examined by western blot and RT-PCR. The functional and molecular effects of Actopaxin expression were studied by enforced expression or down-regulation in HCC cell-lines. The clinicopathological significance of Actopaxin expression in tumorous tissues of 119 HCC patients was also investigated.

Results: High protein level of Actopaxin was found in the metastatic HCC cell-lines compared with other non-metastatic HCC cell-lines. Expression of a shorter form of Actopaxin was also detected in some of these cell-lines. Comparing to Actopaxin (LF-Actopaxin), the unreported short form (SF-Actopaxin) lacks a fragment in the $\mathrm{C}$-terminal and hence results in an incomplete second $\mathrm{CH}$ domain which consists binding sites for its downstream activation targets. Expression of LF-Actopaxin but not SF-Actopaxin in HCC cell-lines was positively correlated with their migration and invasion potential in vitro, accompanied by altered expressions of several focal adhesion proteins as well as EMT proteins. Study in clinical samples showed that LF-Actopaxin expression was positively correlated with tumor size while SF-Actopaxin expression was lower in HCC tumor compared with corresponding nontumorous liver and lower in $\mathrm{HCC}$ with metastasis compared with HCC without metastasis.

Conclusion: This study demonstrated for the first time the promigratory effects of Actopaxin in human $\mathrm{HCC}$, and the existence of SF-Actopaxin which displays different expression pattern as well as functional and molecular effects.

\section{PP30-24}

ASS Is Down-regulated by Promoter CpG Methylation in Hepatocellular Carcinoma

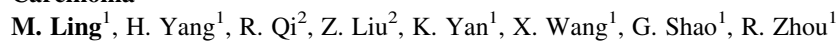
${ }^{1}$ Peking University; ${ }^{2}$ The 302 Hospital of People's Liberation Army, Beijing, China

Purpose: Argininosuccinate synthetase (ASS) is a key enzyme in the synthesis of arginine from citrulline, and its activity and expression levels in most cancer tissues are significantly decreased in comparison with the corresponding noncancerous tissues. However, its precise mechanisms remain unclear. So, this study was aimed to investigate the reason for the loss of ASS activity in hepatocellular carcinoma (HCC).

Methods: Western blot assay was used to evaluate the expression of ASS in HCC tissues and paired noncancerous liver samples from 72 patients. The correlation of ASS expression with selected clinicopathologic parameters was assessed with the chi-squared test. Promoter methylation analysis was performed to test the $\mathrm{CpG}$ methylation status of ASS promoter.

Results: ASS expression was down-regulated greatly in 38 of $65(58.5 \%)$ hepatocellular carcinoma tissues and the expression was significantly associated with TNM stage and portal vein invasion $(P<0.05)$. Further nested methylation-specific PCR (MSP) showed that ASS was methylated in 52 of $72(72.2 \%) \mathrm{HCC}$ and this may account for the low ASS activity in HCCs. 
Conclusion: ASS is down-regulated by promoter $\mathrm{CpG}$ methylation in hepatocellular carcinoma and it may be helpful for the therapy of HCC patients.

\section{PP30-25}

The Decreased Expression of CD27 on B Lymphocytes in Patients with Primary Hepatocellular Carcinoma

X.-D. Fang ${ }^{1}$, X.-D. Wang ${ }^{1}$, P.-W. Zhao ${ }^{2}$

${ }^{I}$ The 2nd Hospital, Jilin University; ${ }^{2}$ The First Hospital, Jilin

University, Changchun, China

Objective: The diversity of B cell response to HBV can lead to different outcome,whose mechanism is poorly understood. We hypothesized that the abnormity of memory(CD27+) B lymphocyte in CHB infection. Our aims to study the phenotype of peripheral B cells and B cell-related cytokine (IL-2,4,6,10) in CHB patients.

Methods: B cell phenotyping was performed by FACS in $104 \mathrm{CHB}$ patients (30 hepatitis, 46 cirrhosis, and 28 were primary hepatocellular carcinoma) and 30 healthy controls. Serum IL-2,4,6,10 were measured by CBA.

Results: The ratio of memory (CD27+) B cells to naïve (CD27-) B cells was lower in chronic HBV infected patients due to a decrease in the number of $\mathrm{CD} 27+\mathrm{B}$ cells and relative increase in the number of CD27- B cells ( $p=0.049,0.014,0.025$, respectively). A significant inverse correlation was obtained between CD27-B cells and HBV DNA levels $(R=-0.258, p=0.035)$. The level of IL-10 in CHB patients was increased $(p=0.048)$. IL-10 and B cells did not show statistically significant difference between the chronic HBV patients $(p=0.34)$. There was no correlation between hepatic inflammation, HBeAg status and the percentage of B lymphocyte subsets, the level of IL-10. IL-2,4,6 level did not show difference between the patients and $\operatorname{controls}(p=0.89,0.093,0.12$, respectively).

Conclusions: $\mathrm{CD} 27+\mathrm{B}$ cells are depleted and naïve (CD27-)/ memory $(\mathrm{CD} 27+) \mathrm{B}$ ratio are imbalance in peripheral blood of HBVinfected subjects, which predicts a humoral immune defect. The loss of memory B cells may contribute to unresponsiveness or low responsiveness to HBV and apoptosis induced by IL-10.

\section{PP30-26}

Role of Genetic Alterations in CYP2E1 and BER Pathway Genes in HBV Related Liver Diseases and Carcinoma in North India

S. Bose ${ }^{1,2}$, S. Beweja ${ }^{1}$, P. Sakhuja ${ }^{3}$, S.K. Sarin ${ }^{1,4}$

${ }^{1}$ Gastroenterology, G.B.Pant Hospital, New Delhi; ${ }^{2}$ Biotechnology, Gauhati University, Guwahati; ${ }^{3}$ Pathology, G B Pant Hospital;

${ }^{4}$ Institute for Liver and Biliary Sciences, New Delhi, India

Both viral and host genetic factors plays a critical role in determining the Hepatitis B related liver disease severity. Base excision repair pathway is a primary mechanism for treating DNA abducts because of assaults by different external agents like viruses, etc. A population based case-control study was designed to explore the genetic risk factors for HBV related liver disease susceptibility in Northern India. Methods: A total of 424 subjects comprising $\{210$ controls, 50 acute HBV (AVH), 84 chronic HBV (CHBV), $25 \mathrm{HBV}$ related cirrhosis and $55 \mathrm{HBV}$ related hepatocellular carcinoma (HCC) subjects $\}$ were included in the study from Department of Gastroenterology, GB Pant hospital, New Delhi. PCR-RFLP was used for the genotyping of Cyp2E1*5B, hOGG1-codon326 and XRCC1codon399. Statistical analysis was performed using SPSSv13 software.

Results: Compared to controls, Cyp2E1rsaI variant c2 genotype increased the risk of HBV related liver disease severity by 2.68 folds, highest for HCC cases (3.981 folds, $p=0.106$ ); and was associated with higher HAI $(p<0.001)$ in CHBV patients. Cyp2E1 and hOGG1 variants were independently associated with significantly higher fibrosis-score in CHBV group. Analysis of gene-gene interaction studies showed, (a) significant increased risk of HCC, cirrhosis and CHBV in Cyp2E1variants + XRCC1variants combination ( $\mathrm{p}<0.001)$; (b) hOGG1 variants $+X R C C 1$-variants was associated with significantly higher risk in HCC and cirrhosis cases but with lower risk in AVH $(p=0.004)$; (c) mutually independent heterozygous hOGG1 and XRCC1 combination resulted in decreased risk of HBV related liver disease; (d) wild-type hOGG1 and XRCCl combination was associated with significant higher risk of AVH $(p=0.010)$ but a significant lower risk of CHBV $(p=0.032)$ and $\mathrm{HCC}(p=0.006)$. The gene-gene interactions were also associated with significant increase in HAI and fibrosis score in CHBV patients.

Conclusion: Cyp2E1, hOGG1 and XRCCl genotypes significantly alter the risk of $\mathrm{HBV}$ related liver disease susceptibility and severity independently or through gene-gene interaction.

\section{PP30-27}

The Expression of Foxp3 in Hepatocellular Carcinoma Can Directly Participate in Tumor Cells Migration and Invasion Via Up-regulating MMP1

Y.H. Chen, H.H. Zhang, X.W. Xie, R. Fei, Y.X. Wang, L. Wei, H.S. Chen Peking University People's Hospital, Peking University Hepatology Institute, Beijing, China

Background/Aims: The forkhead transcription factor Foxp3 is a key molecular mediating regulatory $\mathrm{T}$ cells immune suppression. Recently we found that Foxp3 was also expressed in hepatocellular carcinoma cell lines, and HCC cell lines with high metastasis potential could express higher level of Foxp3 mRNA and protein than those with low metastasis potential. Our objective is to find out whether Foxp3 expression in hepatoma cell lines is directly involved in tumor metastasis and invasion or not and how.

Methods: Two hepatoma cell lines HepG2 and MHCC-97H with low/high metastasis potential were used in this study. HepG2, MHCC-97H, and HepG2 that over-expressed Foxp3 were transfected with Foxp3 full-length cDNA plasmid, Foxp3 shRNA plasmid, and matrix metalloproteinase-1 (Mmp-1) shRNA plasmid. Then, Foxp3 and Mmp-1 were detected in mRNA and protein levels. And Wound healing assay and Transwell invasion assay were used to assess the ability of tumor cells migration and invasion.

Result: Up-regulation of Foxp3 in HepG2 cells could increase the expression of $\mathrm{Mmp}-1$, and improved the tumor cells migration and invasion. Simultaneously, down-regulation of Foxp3 in MHCC-97H cells decreased the expression of Mmp-1, and reduced the tumor cells migration and invasion at the same time. Moreover, down-regulation of Mmp-1 in HepG2 which overexpressing Foxp3 and subsequently Mmp-1 expression increased could obviously decrease the expression of $\mathrm{Mmp}-1$ and tumor cells migration and invasion.

Conclusion: These results suggested that Foxp3 expression in hepatocellular carcinoma cell lines could directly involved the tumor cells migration and invasion through regulating the Mmp-1 expression.

Acknowledgment: Supported by the National Natural Science Foundation of China(No. 30772499, 81001072), National Key SciTech Special Project of China (no. 2008ZX10002-019), National 863 plans projects2007AA021103, Program for New Century Excellent Talents (985-2-099-113).

\section{PP30-28}

Expression of the Pin1 in the Hepatitis B, Cirrhosis and Hepatocelluar Carcinoma Tissues by Using Tissue Microarray

X. Xu ${ }^{1}$, D. Zhang ${ }^{1}, \mathrm{H} . \mathrm{Hu}^{1}$, Y. liao ${ }^{2}$

${ }^{I}$ Department of Infectious Disease, The Second Affiliated Hospital of Chongqing Medical University, Chongqing, China; ${ }^{2}$ Department of Experimental Therapeutics, The University of Texas M.D. Anderson Cancer Center, Houston, TX, USA

Aims: By using tissue microarray technique to detect the expressions of peptidyl-proplyl isomerase 1(pin1) in human hepatitis B, cirrhosis, hepatocelluar carcinoma(HCC) and normal liver tissue, discussing its 
role in different stage of oncogenesis of HCC and its relationship with clinicopathological parameter.

Methods: Immunohistochemistry was used to detect the expressions of pin 1 in 10 cases of hepatitis B, 40 cases of cirrhosis, 19 cases of HCC and 34 cases of normal liver tissue on tissue microarray.

Results: The positive expression rate and intensity of pin1 was stronger in the HCC than that in the hepatitis B, cirrhosis and normal liver tissue $(p<0.001)$, but there was no significant difference in the latter three tissuses $(p>0.001)$. The expression of pin1 was not correlated with clinicopathological parameter $(p>0.05)$.

Conclusion: pin1 plays a pivotal role in different stages of HCC.

\begin{tabular}{lrrrrll}
\hline & $N$ & - & + & ++ & +++ & Positive rate $(\%)$ \\
\hline Normal liver & 34 & 15 & 19 & 0 & 0 & 55.9 \\
Hepatitis B & 10 & 2 & 7 & 1 & 0 & 80 \\
Cirrhosis & 40 & 7 & 31 & 2 & 0 & 82.9 \\
HCC & 19 & 0 & 1 & 13 & 6 & $100^{*}$ \\
\hline
\end{tabular}

$* p<0.001$ comparing with normal tissue

Expression of pin1 in the four liver tissues

\section{PP30-29}

Development of a Liver Cancer Model via Hydrodynamic Transfection

W.S. Ro ${ }^{1}$, H.-L. Ju' ${ }^{2}$ J.Y. Park ${ }^{2}$, D.Y. Kim², S.H. Ahn' ${ }^{2}$, K.-H. Han ${ }^{2}$

${ }^{1}$ Liver Cirrhosis Clinical Research Center; ${ }^{2}$ Internal Medicine, Yonsei University School of Medicine, Seoul, Republic of Korea

Background/aims: Liver cancer is a major health concern worldwide. Nevertheless, its pathogenesis and underlying mechanisms are incompletely understood. To study liver cancer, various mouse models have been developed, among which transgenic mouse models are favored precisely mimicking human cancers. However, development of transgenic mouse models by the traditional method requires considerable time and resources. In this study, a transgenic liver cancer mouse model has been developed using hydrodynamic transfection. This strategy

(1) uses the Sleeping Beauty transposon system,

(2) reduces cost and time significantly, and

(3) can detect tumors noninvasively in vivo.

Methods: A transposon vector was constructed that encodes oncogenes and the firefly luciferase as a reporter. Its expression level was confirmed by Western blotting and a luciferase assay. The tumorigenic potential of the oncogenes was evaluated by a soft agar colony formation assay and a serum-independent growth test. To induce liver cancer, $50 \mu \mathrm{g}$ of plasmids encoding the Sleeping Beauty transposase and transposon vectors were diluted in $2.5 \mathrm{ml}$ of $0.9 \%$ Ringer's solution and then injected into the lateral tail veins of 6- to 8-weekold ICR and C57BL/6 mice. Tumors were observed via bioluminescence imaging.

Results: Liver cancer was induced by the stable expression of the oncogenes from the transposable vector. The tumor growth was observed via repeated bioluminescence imaging of mice over time. Whereas control mice that stably expressed only luciferase showed no significant changes in the luciferase signal intensities over time, the mice expressing oncogenes in the liver exhibited continuous tumor growth over months, based on the luciferase signal intensities and histological analyses.
Conclusion: We have established a transgenic mouse model for liver cancer using a transposable vector encoding oncogenes and luciferase. This model could be an efficient alternative to the traditional transgenic mouse model for liver cancer with significantly reduced time and cost.

\section{PP30-30}

Hepatitis C Virus and Alcohol: Same Mitotic Targets but Different Signaling Pathways

A. Alisi ${ }^{1}$, M. Ghidinelli ${ }^{2}$, A. Zerbini ${ }^{3}$, G. Missale ${ }^{3}$, C. Balsano ${ }^{2}$

${ }^{1}$ Units of Metabolic and Autoimmune Liver Diseases, Bambino Gesù Children's Hospital, Rome; ${ }^{2}$ Fondazione Andrea Cesalpino, Rome;

${ }^{3}$ Laboratory of Viral Immunopathology, Azienda Ospedaliero-

Universitaria di Parma, Parma, Italy

Background and aims: Chromosomal aberrations frequently present in hepatitis $\mathrm{C}$ virus (HCV)- and alcohol-related hepatocellular carcinomas (HCCs). The mechanisms by which chromosomal aberrations occur during hepatocarcinogenesis are still unknown. However, it is considered the results of some mitotic molecules deregulation, including the altered expression of cyclin B1 and Aurora kinase A, and phosphorylation of gamma-tubulin. Our study aims at investigating changes in expression and of the above refereed proteins and related intracellular pathways in in vitro and in vivo models of both HCV- and alcohol- dependent HCCs.

Methods: In this study, molecular defects and mechanisms involved in deregulation of mitotic machinery were studied in human hepatoma cells expressing HCV proteins and treated or not with ethanol; and, in liver tissues from control subjects $(n=10)$ and patients with HCV$(n=10)$ or alcohol-related $(n=10)$ HCCs.

Results: Expression of cyclin B1, Aurora kinase A and tyrosine-phosphorylated gamma-tubulin were analyzed in models reproducing $\mathrm{HCV}$ infection and ethanol treatment in HCC cells. Interestingly, HCV and alcohol increased the expression of cyclin B, Aurora kinase A and tyrosine-phosphorylated gammatubulin also in tissues from patients with $\mathrm{HCV}$ - or alcohol-related HCCs. In vitro models suggest that HCV requires the expression of PKR (RNA-activated protein kinase) as well as JNK (c-Jun N-terminal kinase) and p38MAPK (p38 mitogen-activated protein kinase) activities; while, ethanol bypasses all these pathways.

Conclusions: Our results support the idea that HCV and alcohol may promote oncogenesis acting through the same mitotic proteins, but via different signaling pathways; providing a good starting point to develop innovative combined therapeutic strategies.

\section{PP30-31}

Absence of Hotspot Mutations in Exons 9 and 20 of the PIK3CA Gene in Hepatocellular Carcinoma in Chinese Population

X. $\mathbf{L i}^{1}$, W. He ${ }^{1}$, Q. Zhang ${ }^{1}$, J. Yan ${ }^{1}$, T. Liu ${ }^{2}$, Y. $\mathrm{Li}^{2}$

${ }^{I}$ The First Hospital of Lanzhou University; ${ }^{2}$ Second Hospital of Lanzhou University, Lanzhou, China

Objective: Phosphatidylinositol-3 kinases (PI3K) as heterodimeric lipid kinases could regulate many cellular processes. Many studies have reported high frequencies of hotspot mutations in the PIK3CA gene, which encodes p110 catalytic subunit, in several human solid tumors, including hepatocellular carcinoma (HCC). The aim of this study was to determine the frequency of hotspot mutations in exons 9 and 20 of the PIK3CA gene in HCC in the Chinese population.

Methods: We examined the PIK3CA gene mutation in $72 \mathrm{HCC}$ specimens using PCR amplification followed by direct sequencing of DNA samples.

Results: No hotspot mutations were detected in any of the samples. Conclusions: These findings indicate that PI3K mutations seem not to contribute to HCC and other mechanisms of PIK3CA activation could be involved in the pathogenesis of the disease. 


\section{PP30-32}

Genes Associated with Recurrence of Hepatocellular Carcinoma

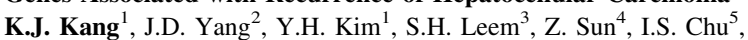
L. Roberts ${ }^{2}$

${ }^{1}$ Surgery, Keimyung University Dong-San Hospital, Daegu, Republic of Korea; ${ }^{2}$ Gastroenterology, College of Medicine Mayo Clinic, Rochester, MN, USA; ${ }^{3}$ Department of Biosciences, Dong-A University, Busan, Republic of Korea; ${ }^{4}$ Division of Biomedical Statistics and Informaticsc, College of Medicine, Mayo Clinic, Rochester, MN, USA, ${ }^{5}$ Korea Research Institute of Bioscience \& Biotechnology, Daejeon, Republic of Korea

Background and aims: The goal of this study is to identify genes whose methylation status and mRNA expression are associated with recurrence-free survival after surgical resection for hepatocellular carcinoma (HCC).

Methods: Sixty-two surgically resected HCCs were examined by both whole genome DNA methylation and transcriptome analysis to identify genes whose methylation status and mRNA expression are associated with recurrence-free survival after surgical resection for HCC. Genome wide gene expression, and methylation profiling was performed by Illumina chip. The Cox-Proportional hazards model was used to select genes whose methylation status or gene expression was significantly associated with recurrence-free survival. An independent validation of the association of gene expression with recurrence-free survival was performed using a separate cohort of 66 patients.

Results: Recurrence developed in $60 \%$ of patients with a median follow up of 20.2 months. In the training set, fifty-nine genes were associated with recurrence by both methylation and gene expression. Both increased $\mathrm{CpG}$ island methylation and decreased mRNA expression were associated with earlier recurrence for 12 genes (Group A), whereas decreased $\mathrm{CpG}$ island methylation and increased mRNA expression were associated with earlier recurrence for 25 genes (Group B). CFH, myosin VIIA and MYRIP in group A and PSRC1, MRE11A, and myosin IE (MYO1E) in group B were associated with recurrence by gene expression in the validation data set.

Conclusion: Our study suggests potential novel oncogenes and tumor suppressor genes implicated in hepatocarcinogenesis that may be regulated by promoter methylation, and may affect prognosis after resection for HCC. Several of the identified genes have been reported as tumor suppressor genes or oncogenes regulated by methylation in other cancers but are novel in HCC. The regulation of individual genes by promoter methylation and their prognostic role in HCC will need to be further validated using functional studies.

\section{PP30-33}

Recurrent SAV1 Mutations in Hepatocellular Carcinoma

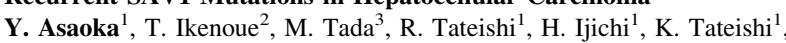
F. Kanai ${ }^{3}$, S. Shiina ${ }^{1}$, H. Yoshida ${ }^{1}$, M. Omata ${ }^{4}$, K. Koike ${ }^{1}$

${ }^{1}$ Gastroenterology, Graduate School of Medicine, University of

Tokyo; ${ }^{2}$ Division of Clinical Genome Research, Institute of Medical Science, University of Tokyo, Tokyo; ${ }^{3}$ Medicine and Clinical Oncology, Graduate School of Medicine, Chiba University, Chiba, ${ }^{4}$ Yamanashi Prefectural Hospital Organization, Kofu, Japan

Introduction: The Hippo pathway plays a key role in organ size control by regulating cell proliferation and apoptosis in Drosophila. Several recent studies using mouse model have revealed that liverspecific abrogation of this pathway leads to hepatomegaly and hepatocarcinogenesis by activating YAP oncogene. Components of this pahway were reported to be mutated in several cancers, but specifc gene alteration has not yet been detected in hepatocellular carcinoma (HCC).

Aim: The aim of this study is to discover the specific component altered in HCC using genome-wide analysis.
Material and methods: Genomic DNA from 13 HCC cell lines were applied to high-density single-nucleotide polymorphism genotyping microarrays and the data were analyzed by CNAG. Genetic alterations of the components in Hippo pathway were investigated. Mutational analysis of the altered gene was performed using genomic DNA from 36 specimens obtained by surgical resection in addition to 13 cell lines. Reporter assay representing YAP activity was performed to investigate the effect of the alteration.

Results: Six of 13 cell lines have loss of heterozygosity (LOH) of SAV1 located in Chr.14q. This gene was homozygously deleted in one cell line. Mutational analysis of this gene revealed that additional one cell line harbored nonsense mutation and two missense mutations were detected in clinical samples. Reporter assay showed that the mutation of SAV1 reduced the inhibitory activity against YAP.

Conclusion: SAV1 mutations were discovered in HCC, and these muations abrogate Hippo pathway activity and will lead to hepatocarcinogenesis.

\section{PP30-34}

The Anticancer Effect of Huaier Is Associated with the Down-regulation of P53

X.Y. Wei, Q. Wei, K. Wang, X. Xu, H.Y. Xie, L. Zhou, S.S. Zheng Division of Hepatobiliary and Pancreatic Surgery, Department of Surgery, the First Affiliated Hospital, Zhejiang University School of Medicine, Hangzhou, China

Bakeground/objective: Hepatocellular carcinoma (HCC) is the leading fetal disease in China. In recent years, the Traditional Chinese Medicine named Huaier is used in treatment of HCC. This study was designed to determine the mechanism of Huaier's anticancer effect on the experimental HCC.

Methods: $N$-nitrosodiethylamine (DEN) was used to make the rat HCC model. Seventy five rats were divided into five groups. Rats in group $\mathrm{A}, \mathrm{B}, \mathrm{C}$ and $\mathrm{D}$ were administrated with DEN. Rat in group $\mathrm{E}$ were administered natural saline instead of DEN as control. Group B and $\mathrm{D}$ were also given Huaier $(2 \mathrm{~mL} /$ rat once, original liquid diluted for 1:1 with ddH2O). Rats in Group A, B and E were sacrificed after 15 weeks for pathological observation and the other groups were followed up for survival investigation.

Results: The incidence of HCC in group B is more lower than group A $(54.5$ vs. $100 \%, P<0.05)$. There were significant differences of serum AFP concentration between group A and group E, group B and group $\mathrm{E}(39.96 \pm 3.47$ vs. $19.00 \pm 1.74 \mathrm{ng} / \mathrm{ml}, 27.98 \pm 2.52 \mathrm{ng} / \mathrm{ml}$ vs. $19.00 \pm 1.74 \mathrm{ng} / \mathrm{ml} . P<0.05$ for both). There were significant differences of serum ALT concentration between group A and group E $(221.20 \pm 14.20 \mathrm{U} / \mathrm{L}$ vs. $65.67 \pm 5.24 \mathrm{U} / \mathrm{L}, P<0.01)$, group $\mathrm{B}$ and group A $(155.67 \pm 12.14 \mathrm{U} / \mathrm{L}$ vs. $221.20 \pm 14.20 \mathrm{U} / \mathrm{L}, P<0.05)$. The mRNA level of p53 was increased $98.9 \%$ in group A compared to group $\mathrm{E}(P<0.01)$. In group $\mathrm{B}$, liver P53 mRNA was decreased $23.2 \%$ compared with group A $(P<0.05)$. There was no difference of 1-month survival rate between group $\mathrm{D}$ and $\mathrm{E}$.

Conclusion: Application of Huaier showed a significant effect on supressing HCC and it also had protective effect on chemical liver injury. The anticancer effect of Huaier is associated with the downregulation of $\mathrm{P} 53$.

\section{PP30-35}

HCV Infection Promotes Cells Invasiveness through Focal Adhesion Molecules

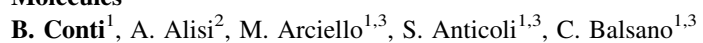

${ }^{1}$ Andrea Cesalpino Foundation; ${ }^{2}$ Units of Metabolic and Autoimmune Liver Diseases, Bambino Gesù Children's, Rome; ${ }^{3}$ Department of Internal Medicine, University of L'Aquila, L'Aquila, Italy

Background and aims: Hepatocellular carcinoma (HCC) is a major cause of death in many countries. Hepatitis $\mathrm{C}$ virus $(\mathrm{HCV})$ is the main causative viral agent of cirrhosis and HCC development. There are 
several clinical and basic reports about intra-hepatic spread of transformed cells during HCC course; however the HCV-related mechanisms, responsible for deregulation of cell adhesion and motility, implicated in HCC invasiveness, are not fully understood. We aim to elucidate the molecular mechanisms characterizing the development of intra-hepatic metastases in HCV-dependent HCC.

Methods: In order to investigate the role of $\mathrm{HCV}$ in regulating the invasiveness of HCC cell lines, we used an in vitro infection model (HuH7 5.1-JFH1). We performed a migration assay, western blotting, immunoprecipitation (IP) assays and immunofluorescence (IF) investigations by confocal microscopy, silencing gene expression with specific siRNA molecule for Focal Adhesion Kinase (FAK).

Results: HuH7 5.1-JFH1 infected cells showed increased cell invasiveness correlated with an enhanced expression levels of focal adhesion molecules (FAK, paxillin and beta1-integrin), which resulted re-distributed in cytosol. Interestingly, FAK siRNA targeting was only partially able to revert the $\mathrm{HCV}$ effects, thus suggesting the involvement of other molecular signaling pathways. Our preliminary results obtained using Wortmannin, a PI3K inhibitor, suggested a critical role for this kinase in HCV-related modulation of focal adhesion molecules.

Conclusions: Our findings demonstrate the role of $\mathrm{HCV}$, in promoting cell invasiveness through the deregulation of focal adhesion molecules. Furthermore our work suggests that alternative treatments, like drugs able to target intracellular pathways such as PI3K/AKT, could represent a new therapeutic strategy to counteract tumor progression.

\section{PP30-36}

Pattern of Chromosomal Aberrations in Hepatomas (Hepatocellular Carcinoma) Identified in Peripheral Blood Lymphocytes

S. Mohana Devi ${ }^{1}$, V. Balachandar ${ }^{1}$, K. Sasikala ${ }^{2}$, M. Arun ${ }^{2}$,

B. Balamuralikrishnan ${ }^{2}$, S. Mustaq Ahamad ${ }^{2}$, S.N. Dharwadkar ${ }^{3}$,

Human Genetics Laboratory

${ }^{1}$ Zoology (Human Genetics); ${ }^{2}$ Zoology, Bharathiar University;

${ }^{3}$ KLE Medical University, Coimbatore, India

Hepatoma or Hepatocellular Carcinoma (HCC) is the fourth leading cause of cancer deaths in the world, with a high incidence in developing country. It was estimated that 14,120 new patients will develop HCC in India. The aim of the present study was to identify the Chromosomal aberrations in hepatoma patients to assess whether peripheral blood had non-random cytogenetic aberrations as observed in tumor samples. The study was conducted on the peripheral blood of 93 hepatoma patients (aged 30-85 years male) undergoing hepatic resection of liver tumour with curative intent. All the patients and controls were analyzed for chromosomal alterations using conventional G-banging. We sought to identify those changes that may be associated with development and progression of HCC. In the present investigation, HCC patients had significantly increased aberrant metaphases compared to controls. Hepatoma samples revealed frequent aberrations in chromosomes 1, 8, 17, 13, 16 and 20. Our finding of a high incidence of 1q gain and frequent deletion in the short arm of chromosome 8 strongly suggested this aberration was associated with the development of this disease. Chromatid breaks were seen on chromosomes 1, 2, and 4 while chromatid gaps were on chromosomes 1,2 and 3. The identified altered chromosomal regions may harbour tumour suppressor genes or Oncogenes that are involved in the multistep process of carcinogenesis or disease pathology. Comprehensive elucidation of the specific genes and molecular pathways involved in progression from pre neoplastic lesion to frank neoplastic in the protracted process of hepatocarcinogenesis will facilitate development of new strategies for prevention and therapy. Identification of molecular pathways that drive the proliferation of neoplastic hepatocytes may enable development of drugs that can specifically target and kill those cells.
PP30-37

Alterations of Nuclear Receptors and Related Genes Expression by HBV in Human Hepatoma Cell Lines

S. Wu, T. Kanda, F. Imazeki, S. Nakamoto, A. Tawada, M. Arai, K. Fujiwara, O. Yokosuka

Medicine and Clinical Oncology, Chiba University, Graduate School of Medicine, Chiba, Japan

Background and aims: Nuclear receptors (NRs) are ligand-activated transcription factors that regulate gene expression. Recent studies implicated that NRs are involved in hepatocarcinogenesis. We assumed that HBV alters NRs and coregulators expression, and we compared the expression of 84 related genes between in HepG2 and HepG2.2.15 cells using real-time RT-PCR.

Methods: Gene expression profiling for $84 \mathrm{NRs}$ and related genes was performed by specific primers using real-time RT-PCR with $\mathrm{SyBr}$ green. Gene expression was normalized to beta-actin to determine the fold change in gene expression between HpeG2.2.15 and HepG2 by 2ddCT methods. Genes were annotated by Entrez Gene (NCBI, USA). Results: Fifty-two genes and five genes, respectively, were significantly up-regulated and down-regulated in HepG2.2.15, HepG2 with 1.3-fold HBV (serotype adw) genome, compared with HepG2 cells. Seven genes of $10(70 \%)$ in androgen receptors and related molecules were significantly up-regulated in HepG2.2.15, and among them NR1I3, MED16, MED4 were more than 3-fold changes. Six of 9 $(66.6 \%)$ and twelve of $19(63 \%)$, respectively, were significantly upregulated, in retinoic acid receptors and in thyroid hormone receptors and related genes in HepG2.2.15. Among transcription factors, 75\% of ligand-dependent NR transcription coactivators and $66.6 \%$ of positive regulators of transcription were up-regulated. In chromatine modification molecules, up-regulations of histone deacetylase mRNA were remarkable.

Conclusions: We found up-regulations of several NRs and related gene expression in the presence of HBV. These results suggest that HBV affects NRs and their related signal transduction and NRs play important roles in HBV-related hepatocarcinogenesis.

\section{Poster Presentation 31: Hepatocellular Carcinoma: Diagnosis and Biomarker}

\section{PP31-01}

YKL-40 Expression in Human Hepatocellular Carcinoma: A Potential Biomarker?

J. Chen

The Second Xiangya Hospital, Changsha, China

Background: YKL-40 is a new biomarker with a diagnostic value in many different cancers.

Objective: To examine the expression of YKL-40 in the serum and liver tissues of hepatocellular carcinoma (HCC) patients and in HCC cell lines, in comparison with that in non-HCC liver disease patients and non-tumor hepatic cell lines.

Methods: Immunohistochemical staining was used to detect YKL-40 protein expression in liver biopsy specimens from $8 \mathrm{HCC}$ patients. ELISA was used to detect the serum YKL-40 level in $30 \mathrm{HCC}$ patients, 30 inactive HBsAg carrier (IHC) patients with normal liver functions, and 30 liver cirrhosis patients. Real-time PCR was employed to examine YKL-40 mRNA expression in three HCC cell lines and two non-tumor hepatic cell lines.

Results: Immunohistochemcal staining of liver biopsy specimens from HCC patients showed that YKL-40 protein expression in tumor tissue was higher than that in adjacent normal tissues. ELISA revealed that the YKL-40 serum level in the HCC group was significantly higher than that in the IHC group $(P<0.01)$, but without significant 
difference from that in the cirrhosis group $(P>0.05)$. Real-time PCR showed that YKL-40 mRNA levels in HCC cell lines were significantly higher than those in non-tumor hepatic cells $(P<0.01)$.

Conclusion: YKL-40 is highly expressed in HCC at the molecular, cellular and tissue levels. However, it may not serve as a serum biomarker for HCC because detection of the serum YKL-40 level can not distinguish HCC from cirrhosis.

\section{PP31-02}

Glypican-3 Amino Terminal Marker for Early Detection of HCC H.A. Abd El Moety ${ }^{1}$, A.A. Abd El Moety ${ }^{2}$, Y. Rostom ${ }^{3}$, M. El Sawy ${ }^{4}$ ${ }^{1}$ Chemical Pathology, Medical Research Institue, Alexandria University; ${ }^{2}$ Hepatology, Faculty of Medicine, Alexandria University; ${ }^{3}$ Radiology, Faculty of Medicine, Alexandria University; ${ }^{4}$ Clinical Pathology, Faculty of Medicine, Alexandria University, Alexandria, Egypt

Introduction: $\mathrm{HCC}$ is the sixth common cancer. Global increase of hepatitis B and C infection, the incidence of $\mathrm{HCC}$ has been steadily increasing. Egypt seroprevalence of HCV in Nile delta was $20-35 \%$. AFP had limited sensitivity $60 \%$ and specificity $90 \%$ for small HCC. GPC-3 oncofetal protein over expressed in HCC.

Aim: Evaluating the validity of Glypican-3 as an early detector of $\mathrm{HCC}$

Material: 10 healthy controls and $40 \mathrm{HCV}$ positive patients: 10 patients with chronic hepatitis $\mathrm{C}$ virus infection.10 patients with compensated cirrhosis (child-Pugh class A and B). 10 patients with decompensated cirrhosis [child-Pugh class C]. 10 patients with HCC. Methods: Liver functions: ALT, AST, Bilirubin(T), Albumin, $\gamma$ GT.Tumor markers: AFP and GPC-3.Viral markers: HCV antibodies, $\mathrm{HBs} \mathrm{Ag}$ and $\mathrm{HBc} \mathrm{Ab}$.

Results: The median value of GPC- 3 in HCC, DC, CC was significantly higher than chronic hepatitis and control groups. No significant correlation found between AFP and GPC-3. AUROC of AFP was 0.85 and AUROC of GPC-3 was 0.84. The diagnostic Sensitivity of AFP (20 ng/ml) was $70 \%$ with PPV 53.8\%. The specificity was $85 \%$ with NPV $91.9 \%$. While the diagnostic Sensitivity of GPC-3 $(2 \mathrm{ng} / \mathrm{ml})$ was $100 \%$ with PPV $27 \%$. The specificity was $42.2 \%$ with NPV $100 \%$. Combined serial approach of AFP and GPC-3 improved the specificity to $87.5 \%$.

Conclusion: GPC-3 although it is a serological test for early detection of HCC, it showed limited specificity, where It is detected in different stages of chronic liver disease, as it is an oncofetal protein produced by regenerating liver cells. The diagnostic signature approach for simultaneous determination of AFP and GPC-3 may improve the prediction accuracy of $\mathrm{HCC}$ patients in those showing seronegativity to AFP.

\section{PP31-03}

Study of the Association of Hepatitis B Surface Antigen and HLA with Hepatocellular Carcinoma

S.A. Zaki ${ }^{1}$, A.A.E.M. Soliman ${ }^{2}$, M.F. Mohasseb ${ }^{3}$

${ }^{1}$ Microbiology, Faculty of Medicine; ${ }^{2}$ Hepatology, Faculty of

Medicine; ${ }^{3}$ Microbiology, Alexandria University, Alexandria, Egypt

Introduction: Primary liver cancer accounts for only $1-2 \%$ of malignant tumors found at autopsy in western countries. However, in some parts of Africa and Asia it may account for up to $20-30 \%$ of all types of malignancy. The high frequency of $\mathrm{HBsAg}$ positivity in individuals with Hepatocellular Carcinoma (HCC) strongly suggests that chronic HBV infection may contribute in some way towards the development of $1^{\text {ry }}$ liver cell carcinoma. Although HCC is probably caused by one or more environmental carcinogens, a hereditary predisposition to the tumor has not been excluded.

Aim: Determine the incidence of HBsAg carriers patients suffering from primary $\mathrm{HCC}$ and detect the association between primary HCC and HLA.
Subjects and methods: The material consisted of twenty patients diagnosed as HCC based on Ultrasonographic examination and by Histopathology, laboratory investigations; liver biopsy, HBsAg, Anti$\mathrm{HBcAg}(\mathrm{IgM})$ and HLA typing using lymphocyte microcytotoxicity technique.

Results: The 20 studied cases were negative for $\mathrm{HBcAb}$ IgM and 10 patients were positive for HBsAg. HLA typing revealed significant rise of HLA-A9, HLA-B5 among HCC patients. HLA-A9 was significantly increased in HBsAg positive and negative cases. HLA-B5 was significantly increased in HBsAg positive cases only while HLAB8 was significantly increased in HBsAg negative cases only.

In cases with mixed hepatic cirrhosis and Schistosomal hepatic fibrosis which were negative for $\mathrm{HBsAg}$ showed significant increase in HLA-A9.

Conclusion:

1. Significant association of $\mathrm{HBsAg}$ positivity in patients with $\mathrm{HCC}$. 2. Significant association of HLA-A9 and HLA-B5 with HCC

3. HLA-A9 in patients mixed cirrhosis and Schistosomal hepatic fibrosis may be predisposing to HCC.

\section{PP31-04}

Detection of Single Tumor Maker for Early Diagnosis of Liver Tumors (HCC)

F.U.H. Subhani

N/A, Lahore, Pakistan

Background: Primary liver cancer, almost entirely Hepatocellular Carcinoma (HCC) constitutes approximately $5.4 \%$ of cancers and in some populations is the most common cancer. The largest numbers of cases are found in Asia (76\% of all HCC), followed by Africa. An early diagnosis at stage $\mathrm{Ia}$ or $\mathrm{Ib}$ have very good prognosis in the treatment regimes. Different tumor markers are in clinical practice or clinical trials for the early detection of liver tumors.

Objectives: To trace out the common sequence of amino acid among different liver tumor markers.

Methods: It is a review in which we will discuss the structural common fragment of current tumor markers of liver carcinoma (HCC) to attain a common amino acid sequence as a single tumor marker for HCC diagnosis at stage Ia and Ib.

Results: Different liver tumor maker are studied for their structural fragment which can be concluded as single common sequence in multiple tumor markers to achieve a single marker which have more specificity and sensitivity. The tumor makers Integrins, $\beta$-glucuronidase, capecitabine, CA19-9, DU-PAN 2, SPAN 1 and $\alpha$-feto protein are compared for their structural sequences. These all tumor markers showed some structural common fragments but a specific common serological fragment is seems to be more realistic in the structural resemblance of antibody.

Conclusion: Although different liver tumor markers have some common molecular sequences but these sequences need further verification for the hypothesis of common fragment detection on serological test. But an antibody titer can be more appropriate as common tumor marker through the common fragments of above mentioned liver tumor markers.

\section{PP31-05}

Prognostically Significant Phenotypic Clusters Amongst Unresectable HCC Patients

B.I. Carr ${ }^{1}$, P. Pancoska ${ }^{2}$, R.A. Branch ${ }^{2}$

${ }^{1}$ Medical Oncology, Thomas Jefferson University, Philadelphia;

${ }^{2}$ Center for Clinical Pharmacology, University of Pittsburgh, Pittsburgh, PA, USA

Aims: To identify prognostically significant HCC phenotypic clusters.

Methods: Using graph theory to clinically identify discrete HCC patient prognostic subsets, taking into account stochastic variation, 
ambiguity and coherence, to identify biologically interpretable coherent structures in 790 patients with unresectable HCC who were followed from diagnosis to death, we ordered them according to survival and then examined data trends.

Results: We found that tumor size adversely influenced survival in older patients ( $>65$ years) but not younger ones ( $>45$ years). In younger patients, there was a subset with multicentric tumors and a high probability of hepatitis $\mathrm{B}$ and/or $\mathrm{C}$ that survived longer than similarly aged patients with large tumors. There was no separation of tumor characteristics from tests of impaired liver function in their effects on survival, implying interaction of the processes of tumor growth and liver dysfunction, consistent with the idea of a 'field' defect in the livers of patients bearing HCCs. Focussing on patients with bilirubin $<1.5 \mathrm{mg} / \mathrm{dL}$ and AFP $<200 \mathrm{ng} / \mathrm{ml}$, due to their huge prognostic impact when elevated, we found two broad categories of patients, one with typical serum GGTP levels of $<900 \mathrm{U} / 100 \mathrm{ml}$ and the other with GGTP levels above this. One patient cohort with GGTP 0-900 U/100 ml, normal bilirubin and AFP $<50 \mathrm{ng} / \mathrm{ml}$ had survival upto 1,250 days and comprised mainly females. Another cohort with high GTTP levels had mainly males and survival of 400-550 days. Ordering patients by survival, we found a linear curve for GGTP, with longest survivors having lowest GGTP levels and vice versa. The low AFP patients with GGTP 0-500 U/100 ml provided a linear correlation between GGTP levels and tumor mass (max size $\times$ number).

Conclusions: Clinical analysis provides evidence for an HCC liver 'field defect' and GGTP may be a useful early tumor marker.

\section{PP31-06}

Serum Levels of Soluble $\beta$-Satenin, SICAM-1E-Cadherin, MMP-2,VEGF and Osteopontin as Predictors of Hepatocellular Carcinoma in Egyptian Patients with HCV Genotype-4

A.-R.N. Zekri

Molecular Virology and Immunology, Cancer Biology, National Cancer Institute, Cairo University, Cairo, Egypt

Background: The rising incidence of hepatocellular carcinoma (HCC), which parallels the increase of HCV prevalence, has sparked a renewed interest in HCC serum markers, especially at early stages of chronic hepatitis $(\mathrm{CH})$.

Methods: We aimed study the serum levels of soluble epithelial cadherin ( $\mathrm{s}-\mathrm{E}$ cadherin), intercellular adhesion molecule (sICAM1), matrix metalloproteinase 2 (total MMP-2), vascular endothelial growth factor (VEGF), osteopontin (OPN), and $\beta$-catenin by quantitative ELISA assay in $32 \mathrm{HCV}$ - associated HCC, 28 chronic hepatitis $(\mathrm{CH})$ patients, 11 asymptomatic $\mathrm{HCV}$ carriers (ASC) and 20 subjects negative for $\mathrm{HCV}$. All cases and controls were negative for HBV.

Results: The median serum concentration of $\beta$-catenin was $1,379.5$ $\mathrm{pg} / \mathrm{ml}$ in $\mathrm{HCC}, 737 \mathrm{pg} / \mathrm{ml}$ in $\mathrm{CH}, 112.9 \mathrm{pg} / \mathrm{ml}$ in ASC and $30.8 \mathrm{pg} / \mathrm{ml}$ in healthy controls $(P<0.001)$. $M M P-2$ was significantly higher in $\mathrm{CH}(1261.2 \mathrm{ng} / \mathrm{ml})$ than in $\mathrm{HCC}(865.2 \mathrm{ng} / \mathrm{ml})(P<0.001)$. The median concentration of SICAM in $\mathrm{HCC}$ and $\mathrm{CH}$ were (73.9 and $63.1 \mathrm{ng} / \mathrm{ml}, P>0.05$ ), those of E-cadherin and $O P N$ were 14.47 and $36.41 \mathrm{ng} / \mathrm{ml}$, respectively in $\mathrm{CH}$ and 13.69 and $26.3 \mathrm{ng} / \mathrm{ml}$, respectively, in HCC $(P>0.05)$. VEGF did not differ significantly between studied groups. Exclusion of HCC in $\mathrm{CH}$ was predicted with $92.6 \%$ specificity and $96.9 \%$ sensitivity at $\geq 997.3 \mathrm{pg} / \mathrm{ml} \beta$-catenin serum level.

Conclusions: Serum $\beta$-catenin level of $\geq 997.3 \mathrm{pg} / \mathrm{ml}$ could be used for early detection of $\mathrm{HCC}$ in $\mathrm{HCV}$-associated $\mathrm{CH}$ patients with high sensitivity $(96.9 \%)$ and specificity $(92.6 \%)$. Larger studies are still required to confirm the clinical significance of serum $\beta$-catenin in HCC patients.
PP31-07

Clinical and Prognostic Significance of Pain at Presentation of HCC B.I. Carr $^{1}$, L. Pujol ${ }^{2}$

${ }^{1}$ Medical Oncology; ${ }^{2}$ Psychology, Pain Division, Thomas Jefferson University, Philadelphia, PA, USA

Aims: Abdominal pain at HCC presentation is a poorly-studied predictor of survival. We examined our large database of unresectable, biopsy-proven HCC patients for clinical correlates and significance for survival of pain at presentation.

Methods: We examined a database of 3,417 patients seen over 20 years. All were followed from diagnosis till death. Statistical analyses were performed using SPSS v.16, with ANOVA for differences between Pain and No Pain patients, and for short-and long-term survival groups. Kaplan-Meier analysis was used for overall survival. Results: $22 \%$ of patients had self-reported abdominal Pain at presentation and $78 \%$ had No Pain. Pain patients had shorter survival, larger tumor size, and higher GGTP, ALKP and AFP than those who with No Pain. Bilirubin and ALB were not different between groups. Max tumor size was larger for Pain versus No Pain patients 7.9 versus $5.0 \mathrm{~cm}, p<0.05$, as were AFP levels, $55 \mathrm{~K}$ versus $33 \mathrm{~K}, p<05$. Survival was significantly decreased in the Pain versus No Pain group, 325 versus 498 days median, $p<0.05$. Patients with Pain had shorter survival $\left(\chi^{2}=10.6,1, p<0.001\right)$ than with No Pain by Kaplan-Meier survival analysis. The plot showed an inflection point at around 1,000 days survival, suggesting the presence of two biological groups of patients, based upon shorter or longer survival. Patients with survival $>1,000$ days compared to shorter survival had less pain (38 vs. $58 \%$ ), smaller tumors (6 vs. $8 \mathrm{~cm}$ median diameter) and much lower AFP levels ( 9 vs. $46 \mathrm{~K}$ in the Pain group for long vs. short survivors, and 484 vs. $20 \mathrm{~K}$ for the No Pain group, for long vs. short survivors). Conclusions: Pain at presentation in unresectable HCC negatively impacts survival, with patients having smaller tumors and lower AFP levels. Very long survival is found even in Pain patients with low AFP.

\section{PP31-08}

Immunohistochemical Differentiation on Hepatocellular Tumors and Intrahepatic Cholangiocarcinoma

W.-M. Cong, H. Dong, X.-Y. Lu, Z.-H. Xian

Department of Pathology, Eastern Hepatobiliary Surgery Hospital, Shanghai, China

Background/aims: It is always difficult for pathologists to distinguish benign helpatocellular nodules from hepatocellular carcinoma (HCC), as well as to distinguish HCC from intrahepatic cholangiocarcinoma (ICC). The present study aims to improve the diagnostic accuracy of hepatocellular tumors among focal nodular hyperplasia (FNH), hepatocellular adenoma (HCA) and well-differentiated HCC (w-HCC) through the determination of the difference in microvessel density and distribution by using immunocytochemical staining of $\mathrm{CD} 34$, and to evaluate the significance of a panel of immunohistochemical markers for diagnosing and distinguishing between HCC and ICC.

Methods: Based on the comparative study of general clinicopathological features of 30 patients in each group of FNH, HCA, w-HCC and ICC, focus was placed on the observation of unique difference in microvessel density and distribution illustrated by immunostaining of CD34 among above three hepatocellular tumors, and on the expression difference between $\mathrm{w}-\mathrm{HCC}$ and ICC in the 10 antibodies including Hep Par 1,polyclonalCEA, Glypican-3, CD10,CD105, MRP-3,COX-2,MUC-1,AQP-1 and CK19. The positive rates among these markers were statistically compared.

Results: In the classical microvessel distribution models, FNH showed a focal-type distribution around both sides of the fibrous scar; 
HCA showed a patch-type distribution with a mixture between microvessel area and no vascular area; and w-HCC showed a diffusetype distribution throughout the whole tumor tissue, revealing a significant difference in each of the 3 entities. HCC was most sensitivity for Par 1, polyclonal CEA, Glypican-3 and CD34, whereas ICC was most sensitivity for CK19 and MUC-1.

Conclusions: CD34 could be used as a valuable assist diagnostic marker for differentiation among hepatocellular tumors by evaluation their characterized microvessel density and distribution. Hep Par 1 and CD34 can be used as the first line antibodies for HCC, and MUC1 and CK19 are the first line antibodies for the diagnosis of ICC.

\section{PP31-09}

Serum Level of Hepatocyte Growth Factor in Patients with Hepatocellular Carcinoma

L.A. Eissa $^{1}$, L. Farok ${ }^{2}$, A.A. El-Gayar ${ }^{3}$

${ }^{1}$ Biochemistry, Faculty of Pharmacy; ${ }^{2}$ Biochemistry, Mansoura, Egypt; ${ }^{3}$ Biochemistry, Faculty of Pharmacy, Mansoura University, Mansoura, Egypt

Hepatocellular carcinoma (HCC) is a common hepatic malignancy that carries a high mortality and dismal prognosis. Hepatocyte growth factor (HGF) is considered the most potent mitogen for hepatocytes. Vascular endothelial growth factor (VEGF) is a key regulator of angiogenesis. Previous reports indicated that HCC was associated with increased levels of HGF and VEGF.

Aim of the work: This study investigated the role of these growth factors as tumor markers for HCC besides their prognostic role. Thus, serum levels of AFP, VEGF and HGF were measured in $42 \mathrm{HCC}$ patients, 30 cirrhotic patients in addition to 15 healthy subjects were taken as a control group.

Results: The mean serum levels of HGF and VEGF were elevated significantly in both $\mathrm{HCC}$ and cirrhotic groups as compared to control group but, there was no significant difference between these two groups. Both growth factors had no relation with age, tumor size, number of lesions, AFP level, ascites, smoking and multiplicity of lesions. HGF had a significant correlation with total bilirubin concentration and Child Pugh classification in HCC and cirrhotic patients together. HCC patients with serum HGF level below the median exhibited significantly better survival than those with serum level above the median.

Conclusion: Serum level of HGF reflects the degree of hepatic dysfunction. Measuring serum VEGF and HGF levels is not helpful in screening of HCC and not useful as tumor markers. Serum HGF level could be a prognostic indicator, where serum HGF level above the median is suggestive of a grave prognosis, indicating that HGF may play an important role in disease progression.

\section{PP31-10}

Down-Regulation of iASPP in Human Hepatocellular Carcinoma Cells Inhibits Cell Proliferation

B. Lin, D. Xie, Y. Yan, J. Xie, Z. Gao

The liver diseases hospital of SUN Yat-sen University, Guangzhou, China

Background/aims: Oncoprotein inhibitory member of the ASPP family (iASPP) is a key inhibitor of tumor suppressor p53 and is found to be upregulated in patients with acute leukemia and breast carcinomas. The research aims is to investigate the effect of the iASPP RNAi on the proliferation of Hepatocellular carcinoma cells. Methods: Sequences of iASPP shRNA were inserted into the pGCLGFP lentivirus RNAi expression system; vectors were transfected together into 293T cells with pHelper1.0 and the lentiviral helper plasmid pHelper2.0 to generate the respective lentiviruses. Viral stocks were made and these were used to infect HepG2 cells and Hep3B cells. Results: The expressions of iASPP mRNA and protein in cells transfected with iASPP siRNA were significantly lower than that in parental cells or cells with nonsense vector. To investigate whether reduction of iASPP had effect on cell growth and proliferation, the MTT assay was performed. A striking variation in the growth pattern was observed among transfectants from the second day to the fifth day in both HepG2 and Hep3B cells. The iASPP siRNA transfected cells showed lower growth rate compared with the controls. And in Brdu assay, both HepG2 and Hep3B cells showed lower proliferation activation after being treated with iASPP siRNA compared to blank control or nonsense shRNA for $72 \mathrm{~h}$. Comparing with the controls, both the HepG2 and Hep3B cells transfected with iASPP siRNA formed significantly less colonies.

Conclusions: Down-regulation of iASPP could be a target for Hepatocellular carcinoma therapy.
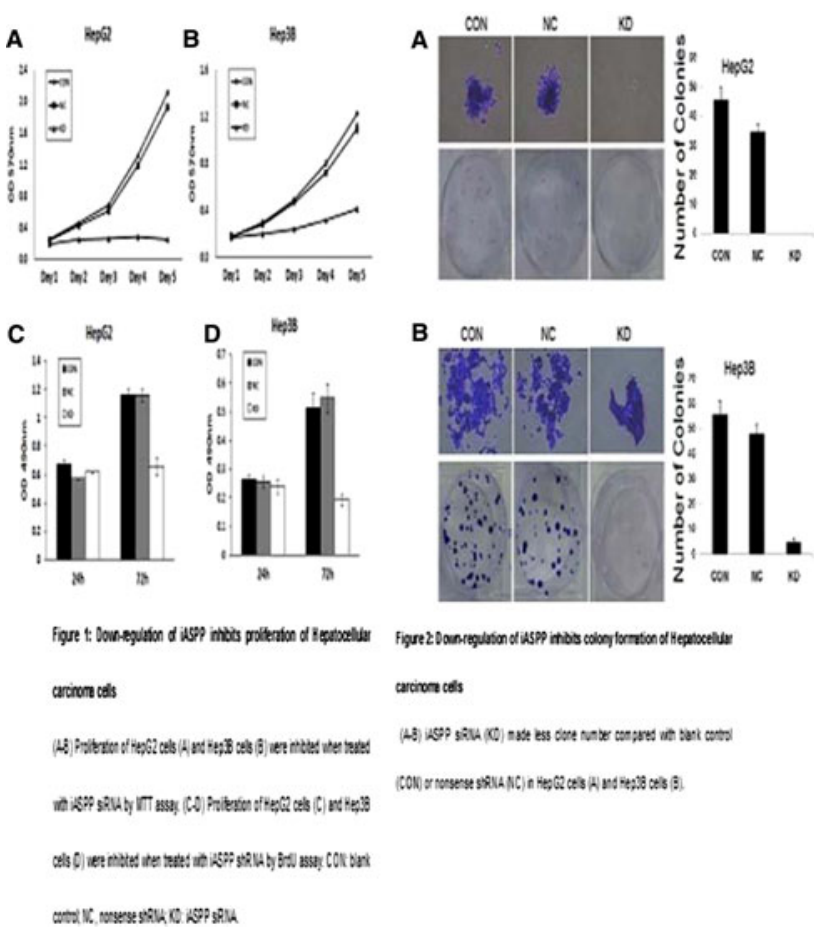

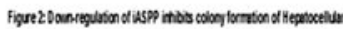

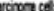

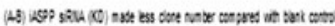

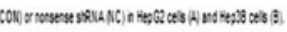

$$
\text { (1) }
$$

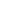

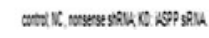

\section{PP31-11}

Evaluation of Nitric Oxide as a Novel Diagnostic Marker for Hepatocellular Carcinoma

H.A. Abd El Moety ${ }^{1}$, A.A. Abd El Moety ${ }^{2}$

${ }^{1}$ Chemical Pathology, Medical Research Institue, Alexandria

University; ${ }^{2}$ Hepatology, Faculty of Medicine, Alexandria University, Alexandria, Egypt

Background: Liver cancer is the sixth most common cancer worldwide. HCC is the most common primary tumour of the liver. The National Comprehensive Cancer Network ( $\mathrm{NCCN}$ ) clinical practice guidelines for treatment of hepatobiliary cancers propose surveillance for the early detection of HCC by liver ultrasonography every 3-6 months and evaluation of AFP. AFP $>200 \mathrm{ng} / \mathrm{ml}$ is considered diagnostic for HCC, although fewer than half of patients of HCC may generate levels that are high, so that the specificity of AFP is close to $100 \%$ but the sensitivity is $45 \%$. Nitrite/nitrate is a stable end product of nitric oxide increase in patients with HCC.

Aim: Evaluation of nitric oxide as a novel diagnostic marker for hepatocellular carcinoma.

Material and methods: 80 pateints and 15 normal individuals enrolled in the study:

Group-1: 15 normal individuals.

Group-2: 30 pateints with chronic liver disease without HCC. 
Group-3: 50 pateints with HCC. History taking,clinical examination, (detection of liver masses, ascites, spleen size, grade of encepathalopathy), Child-pugh scoring. Laboratory investigation: (AlT, AST, Bilirubin, Albumin, Prothrombin, GGT, platelet count, AFP, Nitric oxide, HBs-Ag, HCV-Ab). Abdominal ultrasonography and spiral CT

Results: The median level of nitric oxide was significantly higher in Group(3) $(170 \mu \mathrm{mol} / \mathrm{l})$ than in Group(2) $(56 \mu \mathrm{mol} / \mathrm{l})$ than in Group(1) $(22 \mu \mathrm{mol} / \mathrm{l})$, with a sensitivity of $(68 \%)$ and specificity of $(90 \%)$ at a cutoff level of $110 \mu \mathrm{mol} / \mathrm{l}$ and area under the curve of (0.810). While AFP; at a cutoff level of $200 \mathrm{ng} / \mathrm{ml}$ had a Sensitivity of $(52 \%)$, specificity of $(100 \%)$ and area under the curve $(0.855)$. Indeed Nitric oxide was high in $42 \%$ of AFP-negative HCC patients. Conclusion: Nitric oxide is a novel diagnostic marker for hepatocellular carcinoma, the simultaneous determination of serum Nitric oxide and AFP gave significant improvement in detection of HCC patients compared to that of AFP alone.

\section{PP31-13}

Study on the Correlation Between the Imaging Signs and Serological Index of 50 Cases HBV Related HCC

\section{Q. Zeng}

Department of Infectious Diseases, Affiliated Hospital of Yan'an University, Yan'an, China

Objective: To investigate the correlation between the imaging signs and serological index of HBV related HCC.

Methods: To collect 50 cases $\mathrm{HBV}$ related $\mathrm{HCC}$, to detect the serological index, such as the quantity of HBV M, HBV DNA, AFP, HA, ALT and so on; to do the imaging tests, such as B ultrosound, CT and MRI; Then do some statistical analysis about the correlation between the serological index and imaging signs of HBV related HCC.

Results: $\mathrm{HBsAg}, \mathrm{HBeAb}, \mathrm{HBcAb}$ positive mode is the most common mode in HBV related HCC, this mode always with HBVDNA replication $\left(\chi^{2}=38.093, P=0.000\right)$, statistical analysis showed that $\mathrm{HBe} \mathrm{Ab}$ has positive correlation with HBV DNA $(r=0.374$, $P=0.018)$; statistical analysis showed that AFP has positive correlation with the size of tumor $(\mathrm{r}=0.516, \mathrm{P}=0.000)$, and so was $\mathrm{HA}(r=0.328, P=0.002)$.

Conclusions: The tumor were more likely occurred in the mode of $\mathrm{HBsAg}, \mathrm{HBeAb}, \mathrm{HBcAb}$ positive $\mathrm{HBV}$ related HCC cases which always with HBV DNA replication, so we should be careful about this mode of HBV infection in the surveillance for HCC.

\section{PP31-14}

Aberrant Methylation of SPARC in Human Hepatocellular Carcinoma and its Clinical Implication

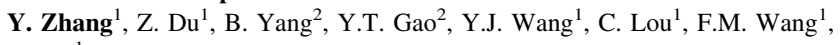
T. Bai ${ }^{1}$

${ }^{1}$ Department of Hepatobiliary Surgery, Third Central Hospital of

Tianjin; ${ }^{2}$ Key Laboratory of Artificial Cell of Tianjin, Tianjin, China

Background and aim: Secreted protein acidic and rich in cysteine $(S P A R C)$ gene is a putative tumor suppressor gene and frequently epigenetically silenced through aberrant methylation in cancers. $S P A R C$ is currently reported as a promising epigenetic marker in many cancers, while it has not been evaluated in hepatocellular carcinoma (HCC). This study was aimed to investigate the methylation status of SPARC and its clinical implication in HCC.

Methods: The methylation status of $S P A R C$ was analyzed in one HCC cell line (SMMC-7721) and 60 pairs of HCC and corresponding nontumorous tissues by methylation-specific PCR and bisulfite sequencing. The expression of SPARC was examined by reverse transcription PCR and immunohistochemistry. The correlations were analyzed between methylation status and gene expression, clinicopathological parameters, as well as prognosis after surgery.
Results: Methylation frequency of SPARC in HCC was significantly higher than that in corresponding nontumorous tissues (45/60 vs. 7/ 60, $P<0.001)$, and correlated with pathological classification $(P=0.019)$. The downregulation of SPARC mRNA expression in HCC was correlated with SPARC methylation $(P=0.040)$. In SMMC-7721 cell line, the loss of SPARC expression was correlated with aberrant methylation and could be reactivated by treatment with the demethylating agent 5-aza-2'-deoxycytidine. The patients with methylated SPARC had a poorer overall survival than those without methylated SPARC (28.0 vs. 41.0 month, $P=0.043$ ).

Conclusion: Aberrant methylation is an important mechanism for SPARC inactivation in HCC and SPARC methylation may be a promising biomarker for prognosis of HCC.

\section{PP31-15}

A Study on Examining the Role of PIVKA-II Indiagnosis of HCC in HBV Infected vietnamese: the first report from Vietnam

T.X. Bui

Department of Gastroenterology, Bach Mai Hospital, Hanoi, Vietnam

Background/aims: $\mathrm{HCC}$ is a common cancer lesions with a high rate of mortality. Alpha-fetoprotein (AFP) is the only marker for diagnosis of HCC in Vietnam and studies on examining the role of other biomarkers such as PIVKA-II (protein induced by vitamin $\mathrm{K}$ absence) are very limited. The aim of the study is to assess the role of PIVKAII in diagnosis of HCC in HBV infected Vietnamese.

Methods: A total of $104 \mathrm{HBV}$ infected patients were categorized into asymptomatic carriers (ASC), chronic hepatitis $(\mathrm{CH})$, liver cirrhosis (LC) and HCC. AFP level was examined by ELISA method and PIVKA-II level was confirmed by PIVKA-II Eitest. HBV-DNA level and HBV genotype was analyzed by real-time PCR and PCR-RFLP, respectively. The diagnosis of HCC was determined by histo-pathologic analysis.

Results: Comparison to AFP, PIVKA-II had a higher prevalence of sensitivity but lower prevalence of specificity in all levels analysis. The combination of these two markers showed a higher prevalence of specificity and a better accurate diagnosis. The level of both AFP and PIVKA-II were progressively elevated from ASC to $\mathrm{CH}, \mathrm{LC}$ and having the highest concentration in HCC group, $p<0.05$. The data of univariate analysis reported that age ( $\geq 50$ years), HBV genotype C, AFP $(\geq 20 \mathrm{ng} / \mathrm{mL})$ and PIVKA-II $(\geq 40 \mathrm{mAU} / \mathrm{mL})$ were the factors for prognosis of LC and HCC. However, in multivariate analysis only age ( $\geq 50$ years), HBV genotype $\mathrm{C}$ and PIVKA-II ( $\geq 40 \mathrm{mAU} / \mathrm{mL}$ ) were the independent factors for prognosis of LC and HCC.

Conclusions: PIVKA-II is the valuable cancer bio-marker and it could be complementary AFP in the detection of HCC.

\section{PP31-16}

Recent Trends in Hepatocellular Carcinoma (HCC) Practice in Taiwan: A Report from the Global HCC BRIDGE Study

P. Chen ${ }^{1}$, J. Liang ${ }^{1}$, W.S. Harmsen ${ }^{2}$, F.T. Enders ${ }^{2}$, L. Orsini ${ }^{3}$

${ }^{1}$ National Taiwan University and Hospital, Taipei, Taiwan ROC;

${ }^{2}$ Mayo Clinic, Rochester, MN, USA; ${ }^{3}$ Bristol-Myers Squibb, Wallingford, CT, USA

Background/aims: The global incidence of HCC has been annually increasing. More than two-thirds of global HCC cases occur in the Asia-Pacific region including Taiwan. The global BRIDGE study is a prospective, large sample-size and multinational cohort study of newly diagnosed HCC patients designed to assess trends in treatment and clinical outcomes. We analyzed treatment patterns for HCC patients treated at a single Taiwanese referral center (the National Taiwan University Hospital) participating in the global HCC BRIDGE study.

Methods: Patients with HCC diagnosed from January 2008 to December 2009 according to EASL or AASLD guidelines were 
identified from medical records. Assessments included patient characteristics, disease etiology, tumor stage, and treatment. Patient survival was updated as of September 15, 2010.

Results: A total of 768 patients ( $72 \%$ male) were analyzed. Mean age was 61 years. HBV (62\%) was the most common etiology, followed by $\mathrm{HCV}(32 \%)$, idiopathic disease $(11 \%)$, alcoholic liver disease (4\%), and nonalcoholic steatohepatitis (4\%). Estimated 1- and 2-year survival was $88 \%$ and $83 \%$, respectively. 400 patients $(53 \%)$ were diagnosed during HCC surveillance. Based on available data, $55 \%$ of patients were within Milan criteria, 92\% had Child-Turcotte-Pugh (CTP) disease stage A, and 75\% had Barcelona Liver Clinic (BCLC) stage 0 or 1 HCC. Most initial treatment approaches were locoregional (95\%)-surgical resection (50\%), local ablation (14\%), transarterial chemoembolization $(31 \%)$-followed by systemic therapy with sorafenib (1\%). Overall, 473 (69\%) patients received potentially curative treatment; this proportion was significantly higher in patients with $\mathrm{HCC}$ detected during surveillance $(78 \% ; p \leq 0.001)$. Conclusions: At this single Taiwanese referral center, hepatitis virus infection was the predominant HCC etiology (94\%). Based on Milan, CTP, and BCLC staging, HCC was diagnosed mostly at early stages. Comparison of these results with those of other sites can provide important information about global diversity in patient management and outcomes.

\section{PP31-17}

CpG Island Methylation of Tumor Suppressor Genes and Oncogenes in Viral-related Hepatocellular Carcinoma in India

M. Kiran ${ }^{1}$, Y.K. Chawla ${ }^{2}$, J. Kaur ${ }^{1}$, Hepatitis Patients, HCC Patients

${ }^{1}$ Biochemistry, Postgraduate Institute of Medical Education and

Research; ${ }^{2}$ Hepatology, Postgraduate Institute of Medical Education \& Research, Chandigarh, India

Background: Hepatocellular carcinoma (HCC) is the fifth most common cancer, and hepatitis B virus and hepatitis $\mathrm{C}$ virus infections are major risk factors. DNA methylation alterations have been linked to various carcinomas in different populations. Aberrant $\mathrm{CpG}$ island methylation of genes has been recognized in HCC, information is limited for hepatitis viruses related hepatocarcinogenesis. HCC risk has not previously been studied with gene-specific DNA methylation in India.

Aim: To study the role of promoter region methylation of tumor suppressor and oncogenes genes in pathogenesis of viral related HCC in India.

Methods: Promoter region methylation of a panel of six tumor suppressor genes (CDKN2A, CDKN2B, CDH1, GSTP1, SOCS1, and APC) and three oncogenes (MYC, HRAS, and KRAS) was determined by methylation-specific PCR among 23 HCC samples and 20 control hepatitis samples.

Results: CDKN2B methylation frequency in HCC was double that for hepatitis, and methylation allele density of APC, GSTP1, and CDKN2B increased 2.2-, 2.3-, and 7.6-fold, respectively. Epigenetic silencing of tumor suppressor genes starts during viral infection and progresses toward HCC with the chronicity of the disease. Findings of altered methylation status support involvement of these tumor suppressor genes in HCC. MYC showed decreased methylation in HCC, relative to hepatitis.

Conclusions: These observations on DNA methylation suggest the involvement of CDKN2B, SOCS1, CDH1, GSTP1, and MYC in pathogenesis of HCC in India and implicate altered DNA methylation in the molecular pathogenesis.

\section{PP31-18}

Expression and Clinical Significance of Interleukin-33 in Serum and Carcinoma Tissues of Patients with Hepatocellular Carcinoma

P. Zhang, X.-K. Liu, P.-W. Zhao, Y.-F. Jiang

The First Hospital, Jilin University, Changchun, China
Aim: To determine the expression of intercellular IL-33 and its clinical significance in hepatocellular carcinoma (HCC).

Methods: Immunohistochemical staining and image analysis were performed to detect the expression of IL-33 in tissues of 30 hepatocellular carcinoma patients, 30 liver tumor patients and 6 patients with non-neoplastic liver. Expression of serum IL-33 in $30 \mathrm{HCC}$ and 6 cases of non-neoplastic liver patients were detected by ELISA. In addition, the results of studding by IL-33 in HCC patients were analyzed with HCC histological grade, depth of invasion, and the relationship between lymph node metastasis.

Results: IL-33 was localized in cell cytoplasm and weakly positivity was also seen in mesenchyma. The expression of IL-33 among HCC, liver tumor and normal liver groups were 80,35 and $13 \%$, respectively. Tissue IL-33 was higher in HCC patients than liver tumor and non-neoplastic liver groups $(P<0.05)$, and was also higher in HCC with metastasis than those without metastasis $(P<0.05)$. The IL-33 positivity rate was significantly correlated with the histological grade, however, no significant correlation was observed in liver tumor and non-neoplastic liver groups $(P>0.05)$. The expression of IL-33 in infiltrated hepatocellular carcinoma was much higher than the noninfiltrated HCC $(P<0.05)$. Serum IL-33 was highly expressed in HCC patients than hepatic cirrhosis or non-neoplastic control groups $(P<0.05)$. The serum IL-33 expression was also higher in patients with metastasis than those without metastasis $(P<0.05)$

Conclusion: There is an enhanced expression of tissue and serum IL-33 in hepatocellular carcinoma. IL-33 may be used as a marker in the development and metastasis of hepatocellular carcinoma.

\section{PP31-19}

Screening and Clinical Evaluation of Auto-Antibody Based Biomarkers for Early Diagnosis of Hepatocellular Carcinoma

Y.P. Zhao ${ }^{1}$, Y. Wang ${ }^{1}$, H. Yan ${ }^{2}$, J. Long ${ }^{3}$, J.X. Zhang ${ }^{4}$, X.J. Ou ${ }^{1}$, H. You ${ }^{1}$, S.H. Chen ${ }^{2}$, C.Z. $\mathrm{Lu}^{4}$, J.D. Jia ${ }^{1}$, S.Q. Wang ${ }^{2}$, J. Huang ${ }^{1}$

${ }^{1}$ Liver Research Center, Beijing Friendship Hospital,Capital Medical University; ${ }^{2}$ Department of Biotechnology, Beijing Institute of Radiation Medicine; ${ }^{3}$ Minimally Invasive Hepatobiliary Cancer Center, Beijing You-An Hospital, Capital Medical University, Beijing; ${ }^{4}$ Department of Hepatology, Tianjin Infectious Disease Specialty Hospital, Tianjin, China

Objective: Recent study shows auto-antibodies are potential serum biomarkers for cancer at early stage. In the present study, the screening and clinical evaluation of novel auto-antibody based biomarkers for early diagnosis of hepatocellular carcinoma (HCC) was carried out.

Method: Total proteins extracted from HCC cells were separated by two-dimensional gel electrophoresis and subsequently incubated with serum from HCC patients and normal controls. Antigenic proteins (auto-antigen) were selected that react to auto-antibody in HCC but not normal control. Auto-antibodies with early diagnostic value were screened through detection of clinical samples including $20 \mathrm{HCC}$ of TNMI. To compare the sensitivity and specificity of screened autoantibodies based biomarkers with current used antigen based biomarkers for HCC, protein microarray which can be used for simultaneous detection of auto-antibodies and antigens was prepared with the screened auto-antigens and monoclonal antibodies to AFP, AFP-L3 and DCP. By detection with the prepared microarray, clinical evaluation was carried out with serum from 129 HCC of TNMI including 50 cases with AFP negative, 168 cases of cirrhosis or hepatitis with AFP positive or negative, and 90 cases of other cancers. Result: 13 HCC-associated auto-antibodies based biomarkers were identified with 6 showing diagnostic value for early HCC. The sensitivity and specificity for detection of HCC of TNMI ranged from 50 to $85 \%$ and 69.8 to $82.6 \%$. In particular, 8 of $20 \mathrm{HCC}$ of TNMI with AFP negative had positive result with any of the six auto-antibodies. The initial result of clinical evaluation with the microarray containing 
the above six auto-antigens and the three monoclonal antibodies showed sensitivity ranged from 50 to $80 \%$ of auto-antibodies, higher than AFP, AFP-L3 and DCP(15-50\%)in detection of HCC of TNMI. Further analysis of the clinical evaluation is ongoing.

Conclusion: The screened auto-antibody based biomarkers may have great potential value on early diagnosis of HCC.

\section{PP31-20}

Treatment Patterns for Hepatocellular Carcinoma (HCC) in China: Findings from the Global HCC BRIDGE Study

B. Cui ${ }^{1}$, M. Chen ${ }^{1}$, W.S. Harmsen ${ }^{2}$, F.T. Enders ${ }^{2}$, L. Orsini ${ }^{3}$

${ }^{1}$ Cancer Center, Sun Yat-Sen University, Guangzhou, China; ${ }^{2}$ Mayo Clinic, Rochester, MN, USA; ${ }^{3}$ Bristol-Myers Squibb, Wallingford, CT, USA

Background/aims: $\mathrm{HCC}$ is the third leading cause of cancer-related death worldwide. Approximately 55\% of global HCC cases occur in China. The HCC BRIDGE study, a large, multinational cohort study of newly diagnosed HCC patients, was designed to help develop an understanding of HCC treatment and associated clinical outcomes across the Asia-Pacific, European and North American regions. As part of the global HCC BRIDGE study, we assessed HCC treatment patterns at a single Chinese referral center, the Sun Yat-Sen University Cancer Center.

Methods: Medical records were used to identify patients with HCC diagnosed from January 2008 to December 2009 according to EASL or AASLD guidelines. We evaluated patient characteristics, disease risk factors, tumor stage at time of diagnosis, and initial treatment. Survival was updated as of September 15, 2010.

Results: A total of 772 patients were analyzed. Mean age was 50 years and $92 \%$ were male. The most common HCC etiologies were hepatitis B virus (78\%), other (26\%), iodiopathic disease (17\%), hepatitis $\mathrm{C}$ virus (2\%), and nonalcoholic fatty liver disease (1\%). Nine patients $(1 \%)$ were diagnosed during HCC surveillance. Four percent $(n=22 / 552)$ of patients were within Milan criteria and $95 \%$ ( $n=703 / 744)$ were Child-Turcotte-Pugh (CTP) stage A. The most common initial treatment was transarterial chemoembolization $(60 \%)$, followed by surgical resection (25\%), and local ablation (14\%). 237 (40\%) patients received potentially curative treatment. Estimated 1and 2-year survival was 63 and $51 \%$, respectively. Outcomes by viral etiology were also investigated.

Conclusions: At this single Chinese referral center, $\mathrm{HCC}$ was diagnosed mostly at advanced stages as indicated by Milan Criteria, although most patients had early stage liver disease. Hepatitis B viral infection was the predominant HCC etiology $(78 \%)$. These results provide an insight into modern treatment trends in China and further contribute to a more comprehensive overview of global HCC treatment patterns and outcomes.

\section{PP31-21}

Identification of Two Portal Vein Tumor Thrombosis Related Proteins in Hepatocellular Carcinoma: PDI A6 and Apolipoprotein A-I

X.Y. Wei, X. Xu, Q. Ling, J. Cheng, H.Y. Xie, S.S. Zheng

Division of Hepatobiliary and Pancreatic Surgery, Department of Surgery, the First Affiliated Hospital, Zhejiang University School of Medicine, Hangzhou, China

Background: Portal vein tumor thrombosis (PVTT) is one of the key risk factors influencing post-operative recurrence-free survival in patients with hepatocellular carcinoma (HCC). However, no predictive or prognostic biological indicator for PVTT has been revealed. In this study, we aim to find molecular biomarkers related to PVTT using proteomics analysis.

Methods: HCC tissues with PVTT and without PVTT were compared using the method of two-dimensional electrophoresis. Differentially expressed protein spots were identified with MALDI-TOF MS. Another verification study enrolling additional 40 HCC tissues (PVTT group, $n=20$; non-PVTT group, $n=20$ ) were conducted using realtime fluorescence quantitative PCR and western blot to verify quantitative expression differences of possible target protein.

Results: A total of 39 unique proteins ( $>$ two-fold change, $P<0.01$ ) were identified. HCC tissues with PVTT showed 10 up-regulated proteins including protein disulfide-isomerase A6 (PDI A6) and chloride intracellular channel protein 1(CLIC1), and tissues without PVTT showed 29 up-regulated proteins including human seleniumbinding protein 1 (HSP56) and apolipoprotein A-I (ApoA-I). In the verification study, PDI A6 protein expression was significantly up-regulated in PVTT group $(P<0.01)$, while ApoA-I protein expression was significantly down-regulated $(P<0.05)$.

Conclusion: Up-regulation of PDI A6 and down-regulation of ApoAI are closely related to PVTT in HCC.

\section{PP31-22}

Cell Death-Associated Biomarkers May Not Be Useful to Discriminate Between Displasia and Hepatocellular Carcinoma: A Pilot Study Y. Yilmaz ${ }^{1}$, E. Ulukaya ${ }^{2}$, D. Nart ${ }^{3}$, Y. Funda ${ }^{3}$, Z. Karasu ${ }^{3}$, S. Ulukaya ${ }^{3}$ ${ }^{1}$ Gastroenterology, Marmara University School of Medicine, Istanbul; ${ }^{2}$ Uludag University, School of Medicine; Bursa, ${ }^{3}$ Ege University School of Medicine, Izmir, Turkey

Hepatocellular carcinoma (HCC) is one of the most common diseases worldwide, with extremely poor prognosis because there is no reliable biomarker(s) to screen and diagnose it early. Alpha-fetoprotein (AFP) is the only available biomarker for HCC diagnosis. However, to discriminate displasia from HCC is difficult. Furthermore, there is currently no biomarker for this aim. Therefore, we investigated the possible role of cell death-associated novel biomarkers (M30 antigen and M65) to discriminate displasia from HCC. 15 HCC, 13 displasia patients and 40 healthy controls were enrolled the study. The histologic material contained primary $\mathrm{HCC}$ and surrounding liver tissue from 28 patients who underwent hepatectomy at the time of liver transplantation. M30 antigen levels were significantly different between displasia patients (median $228 \mathrm{U} / \mathrm{L}$, range 32.1-838.98 U/L) and controls (median 77,725 U/L, range 53.38-129.86 U/L) $(p=0.03)$. However, there was no significant difference in between HCC patients (median 69.14 U/L, range 11.6-538.92 U/L) and healthy controls. Importantly, the difference was not statistically significant between HCC patients and displasia $(p>0.05)$. The median M65 was 630,29 U/L (290.2-1020.5) in HCC patients, while it was $723.6 \mathrm{U} / \mathrm{L}(291.25-1582.5)$ in displasia patients with no statistically difference between the groups $(p>0.05)$. In addition, we analysed the correlations between these two biomarkers and the classical tumor markers (AFP, CA-125, CEA, CA-19.9). There was no statistically significant correlations although there was a moderate correlation $(r=0.495)$ between AFP and M30 antigen in HCC patients. In conclusion, because neither M30 antigen nor M65 are significantly changed between HCC and displasia in liver, the cell death-related parameters may not be useful to discriminate these two neoplastic processes of liver.

\section{PP31-23}

New Lesions Detected within Resecting Specimen by Intraoperative Ultrasound during Hepatic Resection for Hepatocellular Carcinoma: IOUS Findings and Pathologic Correlation

J.H. Ahn ${ }^{1}$, H.H. Jang ${ }^{2}$, J.H. Kwak ${ }^{2}$, N.K. Choi ${ }^{2}$, G.J. Cheon ${ }^{3}$

${ }^{1}$ Radiology; ${ }^{2}$ Surgery; ${ }^{3}$ Medicine, Gangneung Asan Hospital,

University of Ulsan, Gangneung, Republic of Korea

Purpose: Our purpose is to demonstrate IOUS findings of new lesions within resecting specimen detected by IOUS during hepatectomy for $\mathrm{HCC}$ and correlation with pathologic findings.

Materials and methods: From 2007 to 2010, 68 patients underwent hepatectomy and IOUS for HCC in our hospital. We reviewed IOUS findings of resecting part of liver and pathologic findings from 2009, 
and analyzed prospectively IOUS findings of resecting part of liver and pathologic findings. Of these 68 patients, 48 nodules in 22 patients were included. IOUS findings of 48 nodules were analyzed into three groups: type I, low echoic and very low echoic; type II, hyperechoic; type III, mosaic pattern (grade I: less than 30\% internal heterogenity, grade II: more than $30 \%$ ). Pathologic correlation was performed by US-guided localization for nodules in resected specimen.

Results: Number of nodules for each type was type I(low echoic:4 and very low echoic: 6), type II (hyperechoic: 20), and type III (mosaic grade I:11 and mosaic grade II:7). 48 nodules ranges from 5 to $12 \mathrm{~mm}$. Six very low echoic nodules were diagnosed five HCCs and one dysplastic nodule. Eight low echic nodules were diagnosed regenerative nodule. Twenty hyperechoic nodules were diagnosed three HCCs, one dysplastic nodule, and 16 regenerarive nodules. Eleven Mosaic grade I nodules were diagnosed six HCCs, two dysplastic nodules, and three regenerative nodules. Three mosaic grade II nodules were diagnosed all HCC.

Conclusion: IOUS during hepatectomy in patient of HCC should be done thoroughly not to overlook new nodules before deciding the resecting part. Although most of the new nodules are too small and lack of typical US findings, very low echoic nodules and mosaic nodules have a relative good chance of being HCC. We hope to become more familiar to IOUS findings of LC-related nodules.

\section{PP31-24}

Intraoperative Ultrasound(IOUS) Findings of Hepatocellular Carcinoma (HCC) and correlation with CT, MR, and pathology

J.H. Ahn ${ }^{1}$, H.J. Jang ${ }^{2}$, J.H. Kwak ${ }^{2}$, N.K. Choi ${ }^{2}$, G.J. Cheon ${ }^{3}$

${ }^{1}$ Radiology; ${ }^{2}$ Surgery; ${ }^{3}$ Medicine, Gangneung Asan Hospital,

University of Ulsan, Gangneung, Republic of Korea

Purpose: Our purpose is intended to demonstrate IOUS findings of HCC and correlation with CT and MR, and pathology.

Materials and methods: From january 2007 to april 2010, 68 patients underwent hepatic resection and IOUS for HCC in out hospital. IOUS was performed by using 5.0 and $7.5 \mathrm{MHz}$ after mobilization of liver. 98 HCCs were diagnosed in pathology. Of these 98 HCCs, we analysed interesting IOUS findings of HCC: 1, nodule-within-nodule appearance and Mosaic pattern; 2, diffuse HCC with portal vein tumor thrombus; 3 , infiltrative $\mathrm{HCC}$ with portal vein tumor thrombus; $4, \mathrm{HCC}$ with bile duct invasion; 5, HCC with satellite nodules.

Results: Interesting IOUS findings of HCC were nodule-withinnodule (6 lesions), Mosaic pattern (42 lesions), diffuse HCC with portal vein tumor thrombus ( 2 cases), infiltrative HCC with portal vein tumor thrmobus (4cases), and HCC with satellite nodules (9cases).

Conclusion: If we become more familiar to IOUS findings of interesting cases of HCC and correlation with preoperative CT, MR, and pathology, we could be helpful to understand sonographic findings of $\mathrm{HCC}$ and to perform transabdominal ultrasonography of HCC screening.

\section{PP31-25}

The Clinical Usefulness of Gadoxetic acid (Gd-EOB-DTPA, Primovist ${ }^{\circledR}$ )Enhanced MRI for Diagnosis of Hepatocellular Carcinoma

J.M. Byun ${ }^{1}$, Y.S. Kim ${ }^{1}$, S.G. Kim ${ }^{1}$, S.J. Park ${ }^{1}$, H.K. Lee ${ }^{2}$, S.W. Jung ${ }^{1}$, J.Y. Jang ${ }^{1}$, S.H. Lee ${ }^{1}$, J.H. Moon ${ }^{1}$, H.S. Kim ${ }^{1}$, M.S. Lee ${ }^{1}$, B.S. Kim ${ }^{1}$ ${ }^{1}$ Department of Hepatology and Gastroenterology; ${ }^{2}$ Department of Radiology, Soon Chun Hyang University Bucheon Hospital, Bucheon Si, Republic of Korea

Objective: The aim of this study was to evaluate the efficacy of Gadoxetic acid (Gd-EOB-DTPA, Primovist ${ }^{\circledR}$ )-enhanced magnetic resonance imaging (MRI) in the diagnosis of focal liver lesions through comparing with triple-phase multi-detector row computed tomography (MDCT).

Method: From January 2009 to March 2010 at Soon Chun Hyang University Bucheon hospital, total 31 patients suspected with hepatocellular carcinoma (HCC) underwent Gadoxetic acid-enhanced MRI and MDCT within a mean interval of 9 days (range $1-45$ days). Two experienced radiologists independently interpreted the MRI and MDCT images in random order, separately, and without patient identifiers.

Results: There was no diagnostic difference between the two methods in 22 patients $(70.9 \%)$. But in 6 patients $(19.4 \%), 8$ HCC nodules that couldn't be verified on MDCT were newly detected on MRI. Among these 6 patients, two should be changed treatment methods from liver lobectomy or transplantation to non-surgical treatment because of another HCC nodule. The most of newly detected HCC nodules on MRI were smaller than $20 \mathrm{~mm}(3-15 \mathrm{~mm})$. However, 2 (6.4\%) of total 31 patients suspected with HCC on MDCT were finally diagnosed as dysplastic nodules on MRI. Another 1 patients (3.2\%) suspected with multiple dysplastic nodules or cysts on MDCT, was finally diagnosed with multiple HCCs on MRI.

Conclusions: Gadoxetic acid-enhanced MRI was superior in the detection of small HCC ( $<$ or $=20 \mathrm{~mm}$ in diameter) and differentiation of dysplastic nodule compared with triple-phase MDCT. Gadoxetic acid-enhanced MRI could be an appropriate additional imaging modality for patients suspected with HCCs on triple-phase MDCT.

\section{PP31-26}

Overexpression of Human Carcinoma-Associated Antigen in Intrahepatic Cholangiocarcinoma

D. Zhou, H. Wang, H. Zhou, H. Hu

Eastern Hepatobiliary Surgery Hospital, Shanghai, China

Objective: To investigate the expression status of human carcinoma antigen (HCA) in human cholangiocellular carcinomas, and to explore the relationship between HCA and clinical features.

Methods: ICC tissues from 60 ICC patients and normal liver tissues from 20 hemangioma patients were assayed for the expression of HCA by immunohistochemistry and western-blot with anti-HCA IgG monoclonal antibody BCE075 and BDD048. Areas, each from different cases, of low-differntiated $(n=20)$, moderately-differentiated $(n=30)$, high-differentiated tumors $(n=10)$. Results were recorded as positive $(\geq 5 \%$ of cells staining and staining intensity $2+$ or $3+$ ) or negative $(<5 \%$ of cells staining and staining intensity $<2+)$ and analyzed using the $\chi^{2}$ test.

Result: These two antibodies showed similar staining pattens. The positive immnunostaining of BCE075 mainly localized in luminal cell cytoplasm and secretions. Their staining was positive in $15 \%$ of lowdifferntiated ICC, $72 \%$ of moderately differentiated, $100 \%$ of high differentiated tumors, but it is not detected in adjacent normal tissue. The difference in HCA expression among these tissues was statistically significant. Also we found expression of HCA is closely associated with differentiation degree of ICC and the shape tumor cell arranged. There was a correlation between expression of HCA and serum CA19-9.

Conclusion: It suggests that HCA potential markers for the diagnosis of Cholangiocellular carcinomas.

\section{PP31-27}

Identification and Verification of Human Carcinoma-Associated Antigen (HCA) in Hepatocellular Carcinoma (HCC)

D. Zhou, H. Zhou, H. Wang, H. Hu

Eastern Hepatobiliary Surgery Hospital, Shanghai, China

Background: Human carcinoma-associated antigen (HCA) is a cell surface mucin-like molecule observed primarily in cancers of 
epithelial origin. It is amplified in sera and cancer tissues of patients with various carcinomas detected by $\operatorname{IgM}$ antibody against HCA. The objective of this study is to produce new monoclonal IgG antibodies against HCA suitable for diagnostic use in order to determine if HCA is overexpressed in HCC and to identify HCCassociated HCA.

Methods: Three groups of HCA glycoprotein compounds originating from prostatic carcinoma cell line PC3, as well as prostatic carcinoma tissue and lung cancer tissue by HAE3 affinity chromatography, were immunized into BALB/c mice for the production of IgG antibodies. Western blot and immunohistochemistry analyses were conducted on normal livers (25 cases) and HCC tissues (32 cases), utilizing these antibodies against HCA. The antibody-recognized proteins were identified by mass spectrometry following immunoprecipitation.

Results: Sixty-two hybridoma cell strains secreting murine mAbs against human HCA by hybridoma technique were generated. The proteins recognized by BCE075 mAbs obviously increased in liver cancer, the positive immunostaining localized in cholangiole and interlobular bile duct. BCD021 mAbs increased in liver cancer, the positive immunostaining localized in liver cell. BCD021 can also recognize hepG2 cell and other liver cells, but not L-02 cells. As a result, CLIMP63 recognized by both monoclonal antibody BCD021 and BCE075 was elevated in cancer tissue.

Conclusions: We believe these well-characterized antibodies will be useful in finding cancer biomarkers, and more proteins specific to cancer will be found in the near future.

\section{PP31-28}

Ascitic Cholesterol Levels as a Marker of Malignant Transformation in Alcoholic Cirrhosis

R.P. Ponnusamy, R. Kannan

Gastroenterology, Govt. Theni Medical College, Theni, India

Introduction: Alcoholic liver disease is associated with dyslipidemia, hepatic steatosis, and acceleration of fibrosis, enhancing the development of cirrhosis and hepatocellular carcinoma.

Aims and methods: To study the usefulness of ascitic cholesterol levels in the diagnosis of malignant transformation in alcoholic cirrhosis. A prospective cohort study wherein alcoholic cirrhotics with hepatic decompensation (CTP-B and C) were enrolled. Undiagnosed hepatic SOL, known HCC, alcoholic cirrhotics without ascites (CTPA), other causes like viral, NAFLD, metabolic causes and cryptogenic were excluded. Serum AFP, ultrasound abdomen, ascitic cholesterol and cytology were done at baseline and repeated 6monthly. Follow-up was until the development of HCC or death. Contrast CT scan of the abdomen was done at baseline and at the event of suspicion of malignancy.

Results: Among the 68 patients included, 12(18.8\%) developed malignancy over a mean follow-up of 23 months. Malignant transformation was seen at a rate of 3.7/year after a mean duration of 5.6 years of cirrhosis. These 12 patients were deemed as cases and the remaining as controls. Baseline mean ascitic cholesterol level was $46.5 \mathrm{mg} / \mathrm{dL}$ among the controls and was $44.7 \mathrm{mg} / \mathrm{dL}$ among the cases, against a lab cut-off of $70 \mathrm{mg} / \mathrm{dL}$. The levels at 6,12, 18, 24, 32 and 36 months were 50.6 and $46.0 \mathrm{mg} / \mathrm{dL}, 48.9$ and $45.7 \mathrm{mg} / \mathrm{dL}, 52.3$ and $50 \mathrm{mg} / \mathrm{dL}, 51.6$ and $66.9 \mathrm{mg} / \mathrm{dL}, 51.2$ and $89.2 \mathrm{mg} / \mathrm{dL}$ and 53.5 and $98 \mathrm{mg} / \mathrm{dL}$, respectively, among controls and cases. Serial estimations revealed a significantly increasing trend among cases $(P<0.0001)$. Overall mean value of ascitic cholesterol among cases was $86.2 \mathrm{mg} / \mathrm{dL}$. These levels correlated with the occurrence of malignancies by other criteriae in $10(83.33 \%)$ cases.
Conclusion: Incidence of malignancy in alcoholic cirrhosis was $20 \%$ after a mean duration of 7.25 years of alcohol consumption. Ascitic cholesterol levels correlated well with the onset of malignant transformation, and at a screening level above $86.2 \mathrm{mg} / \mathrm{dL}$ malignant transformation should be suspected.

\section{PP31-29}

Alterations in Lipoprotein Patterns and Lipid Peroxidation in Egyptian Patients with Hepatocellular Carcinoma: Correlation with Child-pugh and MELD Score

N.H. Mousa ${ }^{1}$, T.A. Besheer ${ }^{1}$, Y.Z. Gad $^{2}$, E.A. Abd Elaal ${ }^{3}$

${ }^{1}$ Tropical Medicine; ${ }^{2}$ Internal Medicine; , ${ }^{3}$ Clinical Pathology, Mansoura University, Mansoura, Egypt

Background/aim: Hepatocellular carcinoma (HCC) constitutes the fifth most frequent cancer worldwide. Liver is one of the most important organs in energy metabolism and plays a critical role in both the production and catabolism of lipids, lipoproteins and apolipoproteins. This study was aimed to analyze the plasma lipid profile as well as C-reactive protein (CRP) and Malondialdehyde (MDA) among Egyptian patients with chronic HCV infection suffering from HCC and its correlation with Child-pugh and MELD score.

Methods: This study was carried out on 91 patients (52males, 39 females) with HCC and 90 cirrhotic controls. The patients were classified according to Child-pugh classification into Child A $(n=26), \mathrm{B}(n=31)$ and $\mathrm{C}(n=34)$, according to MELD score into 32 patients $\leq 9$ and $59>9$ and according to size of HCC into 33 patients $\leq 5 \mathrm{~cm}$ and 58 patients $>5 \mathrm{~cm}$. Plasma lipid profiles, MDA and CRP were determined by conventional methods.

Results: Plasma levels of Cholesterol, triglycerides (TG), low-density lipoproteins (LDL), high-density lipoproteins (HDL) were significantly decreased in patients with HCC than control $(p=0.005,0.005$, 0.008 and 0.009), respectively, however CRP and MDA are significantly increased compared to control $(p=0.006)$. With progression from Child A to Child $\mathrm{C}$ there were significant decrease in lipids profiles and significant increase in CRP and MDA. In MELD score $\leq 9$ lipids profiles were significant increases when compared with MELD score $>9$. Whereas in CRP and MDA showed significant decreases in MELD score $\leq 9$ than MELD score $>9(p=0.045$ and $0.001)$. Lipids profiles were significant increase in $\mathrm{HCC} \leq 5 \mathrm{~cm}$ than $\mathrm{HCC}>5 \mathrm{~cm}$; however, CRP and MDA showed significant decreases in $\mathrm{HCC} \leq 5 \mathrm{~cm}$ than $\mathrm{HCC}>5 \mathrm{~cm}$.

Conclusion: There were significant alteration in the plasma lipid profiles, MDA and CRP parameters in patients with HCC and could be used as prognostic value.

\section{PP31-30}

The Role of mRNA GP-73 in Diagnosis of Hepatocellular Carcinoma in Patients Infected with HBV

S.H. Le, H.T.T. Tran

Military Central Hospital, Hanoi, Vietnam

Golgi protein 73 (GP-73) is one of biomarkers that has been extensive studied, however, the result is still controversy. Using Real Time RTPCR we evaluated the expression of GP-73 gene in 190 hepatitis B virus (HBV) infected patients including 90 hepatocellular carcinoma (HCC), 50 chronic hepatitis B and 50 asymptomatic chronic HBV carriers and 90 healthy control. GP-73 gene was higher expressed in HBV infected patients compared to healthy control $(P<0.0001)$. GP73 gene was highest expressed in HCC group, following by chronic B hepatitis, asymptomatic chronic HBV carriers. It showed that GP-73 gene expression is a potential biomarker for diagnosis of HCC with sensitivity is $82.1 \%$ and specificity is $90.9 \%$. 
PP31-31

Significance of pro2000 Epxression, a Novel Proliferation-associated Protein in Hepatocellular Carcinoma

L. Guo ${ }^{1}, \mathrm{H} . \mathrm{Gao}^{1}, \mathrm{H} . \mathrm{Chen}^{2}$, J. Huang ${ }^{3}$, J. Yang ${ }^{3}$

${ }^{1}$ Pathology, Zhujiang Hosptial/ Nanfang Medical University,

Guangzhou, China; ${ }^{2}$ University of California, Davis Cancer Center, Sacromento, CA, USA; ${ }^{3}$ Zhujiang Hosptial/ Nanfang Medical

University, Guangzhou, China

Background: pro2000 may be an important candidate gene which located within a region of chromosome $8 \mathrm{q}$ in HCC. However, its significance is still unknown. In the present study, we examined the significancer of pro2000 protein and its relationships to ki-67, cyclin D1, p53 and p21 WAF1/Cip1 in HCCs.

Methods: Seventy-seven of human HCC along with corresponding non-tumor tissues were examined immunohistohemically to assess pro2000 expression and its relationships to clinicopathologic features, ki-67, cyclin D1, p53 and p21 WAF1/Cip1

Results: pro2000 expression was present in 65 of $107(60.75 \%)$ HCC specimens in which 17 of $37(45.95 \%)$ in well differentiated tumors and 48 of $70(68.57 \%)$ in poorly differentiated tumors respectively, while $11(10.28 \%)$ in adjacent non-tumor tissues with scattered positive cells. pro2000 expression was associated with clinicopathological features such as histological differentiation, tumor size and metastasis, but not with age, cirrhosis, HBV infection and serum fetoprotein (AFP) level. There was a closely relationship between pro2000 and ki-67 and cyclin D1 in HCC. pro2000 immunopositivity was independent of p53 and p $21^{\text {WAF1i }}$ Cip1

Conclusion: We conclude that increased expression of pro2000 is associated with adverse outcome in patients with $\mathrm{HCC}$ and is a predictor of poor prognosis for HCC. pro2000 may be involved in the development of HCC and might promote cell proliferation through a

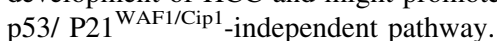

Acknowledgment: This work was supported by the National Natural Science Foundation of China (81071987).

\section{PP31-32}

Correlation of Alpha Fetoprotein Level with the Tumor Size in Hepatocellular Carcinoma

A. Abbasi ${ }^{1}$, N. Butt ${ }^{1,2}$, A.-R. Bhutto ${ }^{1}$, S.M. Munir ${ }^{1}$

${ }^{1}$ Hepatology Section, Medical Unit III, Jinnah Postgraduate Medical Centre; ${ }^{2}$ Gastroenterology, Aga Khan University Hospital, Karachi, Pakistan

Introduction: Patients with chronic liver (CLD) disease are at increased risk for hepatocellular carcinoma. Although serum Alpha fetoprotein (AFP) is often used to detect hepatocellular carcinoma (HCC) in CLD patients, its utility is unclear.

Objective: To find out the correlation of serum AFP with the tumor size in hepatocellular carcinoma.

Design: Cross sectional

Patients and methods: This study was conducted in Hepatology section of Medical Unit-III, Jinnah Post Graduate Medical Centre Karachi, Pakistan from January 2009 to December 2009. Patients enrolled in study were known cases of CLD complicated with the development of HCC.On the basis of AFP level the patients were divided into three groups; Group I with normal AFP $(<20 \mathrm{IU} / \mathrm{ml})$, Group II with moderately elevated AFP (20-399 IU/ml), Group III with markedly elevated AFP ( $>400 \mathrm{IU} / \mathrm{ml})$ and also on the basis of size of tumor patients were divided in three groups; group A with size of $\leq 3 \mathrm{~cm}$, group B $3-5 \mathrm{~cm}$ and group C $>5 \mathrm{~cm}$.

Results: A total of 62 patients were reviewed. On the basis of AFP level $13(21 \%)$, patients were in group I, $18(29 \%)$ in group II and $31(50 \%)$ in group III. While on the basis of tumor size the $15(24.2 \%)$ patients were in group A, 16(25.8\%) were in group B and $31(50 \%)$ were in group C. Twenty (32.25\%) of patients had AFP level of
$>400 \mathrm{IU} / \mathrm{l}$ as well as size of tumor $>5 \mathrm{~cm}$, while only $2(3.22 \%)$ patients had normal AFP and tumor size of $\leq 3 \mathrm{~cm}$.

Conclusion: Our study suggests that HCC patients with high AFP level had larger tumor size. But nonetheless we could not establish a correlation between increase AFP level and tumor size as one group of patients have smaller tumor size but markedly elevated AFP levels.

\section{PP31-33}

The Prognostic Roles of Preoperative AFP and PIVKA-II in Patients with Hepatocellular Carcinoma Undergoing Hepatectomy (Curative Resection)

M.H. Lee ${ }^{1}$, G.H. Choi ${ }^{2}$, J.S. Choi ${ }^{2}$, S.H. Ahn ${ }^{1}$, C.Y. Chon ${ }^{1}$, K.-H. Han ${ }^{1}$, J.Y. Park ${ }^{1}$

${ }^{1}$ Internal Medicine; ${ }^{2}$ Department of Surgery, Division of Gastroenterology and Hepatology Yonsei University College of Medicine, Seoul, Republic of Korea

Background: The clinical role of $\alpha$-fetoprotein (AFP) and protein induced by vitamin $\mathrm{K}$ absence or antagonist-II (PIVKA-II) as predictors of treatment outcomes in patients with hepatocellular carcinoma (HCC) undergoing hepatectomy are unclear. This study investigated whether preoperative AFP and PIVKA-II can predict treatment outcomes.

Methods: We evaluated 348 patients with HCC who underwent hepatectomy between January 1998 and December 2007. The patients received dynamic computed tomography or magnetic resonance imaging every 3 months for at least the first 5 years after hepatectomy. We divided the 348 patients into three groups according to the preoperative cutoff values of AFP $(100 \mathrm{ng} / \mathrm{mL})$ and PIVKA-II (100 $\mathrm{mAU} / \mathrm{mL}$ ): both values low (Group I), one value high (Group II), and both values high (Group III).

Results: The median follow-up period was 49.7 months (range 3.2-105.1). Ninety six patients (27.8\%) died, and 192 patients $(55.7 \%)$ had recurrence during follow-up. Group I, II, and III were comprised of 126, 150, and 72 patients, respectively. The median AFP and PIVKA-II levels were $36.0 \mathrm{ng} / \mathrm{mL}(0.6-99,600)$ and 64.0 $\mathrm{mAU} / \mathrm{mL}(0.5-2,000)$ at baseline. Univariate and subsequent multivariate analyses identified differences observed in overall survival (OS) among the groups (median OS; Group I 66.7, Group II 62.6, Group III 50.9 months, HR 1.627, $P=0.025$ ).

Conclusion: Group I had the highest OS in our study; Group II had a OS between I and III; and Group III the lowest OS. Hence preoperative AFP and PIVKA-II measurement could be useful for predicting OS in HCC patients undergoing hepatectomy.

\section{PP31-34}

A Non-smooth Tumor Margin in the Hepatobiliary Phase of Gd-EOBDTPA- MRI Predicts Microscopic Portal Invasion in Patients with Hepatocellular Carcinoma

S. Ariizumi, K. Kitagawa, Y. Kotera, Y. Takahashi, S. Katagiri, M. Yamamoto Department of Surgery, Institute of Gastroenterology, Tokyo Women's Medical University, Tokyo, Japan

Objective: The value of the hepatobiliary phase of gadoxetic acid disodium (Gd-EOB-DTPA)-enhanced magnetic resonance imaging (MRI) in patients with hepatocellular carcinoma (HCC) has not been evaluated in detail.

Methods: Between 2008 and 2009, 61 patients with HCC within the Milan criteria underwent Gd-EOB-DTPA-enhanced MRI and hepatectomy. The tumor margin was determined preoperatively based on hepatobiliary phase images. Microscopic portal vein invasion (MPVI) and intrahepatic metastasis (IM) of HCC were evaluated in 24 patients with non-smooth margins at the periphery of the tumor and 37 patients with smooth margins.

Results: The number of patients with MPVI and IM of HCC was significantly higher among those with non-smooth margins (42 and 38\%, respectively) than among those with smooth margins (3\%; $p=0.0002$ 
and $5 \% ; p=0.0042$, respectively). A non-smooth margin was identified as a significant predictor of MPVI (odds ratio 18.814, $p=0.024$ ) and IM (odds ratio 6.498, $p=0.036$ ) of HCC on multivariate analysis. Conclusions: A non-smooth tumor margin in the hepatobiliary phase of Gd-EOB-DTPA-enhanced MRI is useful to predict MPVI and IM of HCC.

\section{PP31-35}

Early On-treatment Predictions of Clinical Outcomes Using AFP and PIVKA-II Response in Patients with Advanced Hepatocellular Carcinoma H.J. Ryu ${ }^{1,2}$, M.H. Lee ${ }^{1,2}$, S.U. Kim ${ }^{1,2}$, D.Y. Kim ${ }^{1,2,3}$, S.H. Ahn ${ }^{1,2,3,4}$, E.H

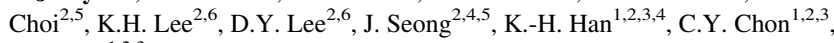
J.Y. Park ${ }^{1,2,3}$

${ }^{1}$ Department of Internal Medicine, Yonsei University College of Medicine; ${ }^{2}$ Liver Cirrhosis Clinical Research Center; ${ }^{3}$ Institute of Gastroenterology, Yonsei University College of Medicine; ${ }^{4}$ Brain Korea 21 Project for Medical Science; ${ }^{5}$ Department of Radiation Oncology, Yonsei University College of Medicine; ${ }^{6}$ Department of Radiology, Yonsei University College of Medicine, Seoul, Republic of Korea

Background and aims: The clinical role of alpha-fetoprotein (AFP) and prothrombin induced by vitamin $\mathrm{K}$ absence or antagonist-II (PIVKA-II) as a predictor of treatment outcome in patients with advanced hepatocellular carcinoma (HCC) receiving hepatic artery infusional chemotherapy (HAIC) or concurrent chemoradiation therapy (CCRT) has been poorly defined. This study investigated whether early on-treatment response using AFP and PIVKA-II is a good indicator for prognosis in patients with advanced HCC.

Methods: Between January 2003 and December 2007, we enrolled 127 treatment-naïve patients who received HAIC $(n=60)$ or CCRT $(n=67)$ as an initial treatment modality. AFP and PIVKA-II response was defined as a reduction of each level more than $20 \%$ from baseline.

Results: AFP responders showed a significantly better OS than nonresponders in patients with HAIC (17.3 vs. 6.4 months, $P<0.001)$ and with CCRT (17.6 vs. 8.7 months, $P=0.014)$. PIVKA-II responders also showed significantly better PFS than non-responders in the CCRT group. Multivariate Cox regression hazard model analyses showed that AFP response independently predicted OS in both groups $(P=0.009$ in HAIC and $P=0.008$ in CCRT) whereas PIVKA-II only predicted PFS in patients with CCRT $(P=0.015)$.

Conclusion: Early on-treatment response using AFP can predict OS in treatment-naïve patients with advanced HCC receiving HAIC and CCRT as an initial treatment modality. Furthermore, early on-treatment response using PIVKA-II is useful for predicting PFS in patients with CCRT.

\section{PP31-36}

Survivin mRNA Expression in High-grade Hepatocellular Carcinoma E. El-Ahwany ${ }^{1}$, S. Zada ${ }^{2}$, M. Fawzy ${ }^{2}$, M. Moussa ${ }^{3}$, M. Youssef ${ }^{4}$

${ }^{1}$ Immunology Department, Theodor Bilharz Research Institute, Giza;

${ }^{2}$ Biology Department, The American University in Cairo, New Cairo;

${ }^{3}$ Pathology Department; ${ }^{4}$ Gastroenterology and Hepatology

Department, Theodor Bilharz, Research Institute, Giza, Egypt

Background/aim: Hepatocellular carcinoma (HCC) is a liver cancer that has several tumor markers. The most common causes of $\mathrm{HCC}$ are hepatitis $\mathrm{C}$ virus (HCV), hepatitis B virus (HBV), and liver cirrhosis. These affect the balance between cellular proliferation and apoptosis (programmed cell death). When there is a low rate of apoptosis, cancer cells have a higher rate of surviving. However, in HCC, even the increased rates of apoptosis do not counterbalance the high proliferation rates of the cancer cells. Survivin is a tumor marker, an inhibitorof-apoptosis protein (IAP), unique in that it cannot be detected in noncancerous adult tissue, but its mRNA and protein are explicitly expressed in tumors. Survivin not only inhibits apoptosis, but also stimulates cell proliferation, highly disrupting the homeostasis between these two processes. It allows for the release of $\mathrm{p} 21$ from $\mathrm{CdK} 4$, which causes an arrest at the $\mathrm{G}_{1}$ phase of the cell cycle, preventing apoptosis of tumor cells. Because it facilitates cancer cell growth, it is expressed in hepatocellular carcinoma (HCC). The objective of this observational study was to determine if the levels of survivin protein could be used to diagnose the progression of hepatocellular carcinoma.

Method: Messenger RNA was extracted from 120 blood samples of three groups of patients (40 cases in each group): low-grade HCC, high-grade $\mathrm{HCC}$, and healthy patients serving as a control group. Survivin mRNA expression was determined by gel electrophoresis after using reverse transcription polymerase chain reaction (RT-PCR). The results showed that in patients with low-grade HCC, survivin mRNA was not expressed, whereas patients with high-grade HCC presented varying degrees of survivin mRNA expression.

Conclusions: Therefore, a correlation between survivin mRNA expression and the malignancy of HCC was observed. This suggests that the levels of survivin may serve as a prognostic bioindicator for the extent of liver cancer malignancy.

\section{PP31-37}

Vascular Endothelial Growth Factor A and its Isoforms Expression in Hepatocellular Carcinoma

B. Sharma ${ }^{1}$, R. Srinivasan ${ }^{2}$, Y.K. Chawla ${ }^{1}$, A. Chakraborti ${ }^{3}$, A. Duseja ${ }^{1}$, N. Kalra ${ }^{4}$, R.K. Dhiman ${ }^{1}$

${ }^{1}$ Hepatology; ${ }^{2}$ Cytopathology; ${ }^{3}$ Eperimental Medicine and Biotechnology; ${ }^{4}$ Radiodiagnosis, PGIMER, Chandigarh, India

Patients and methods: Expression of VEGF and presence of its different isoforms namely VEGF165, 121, 189 and 206 were analyzed by quantitative real time-polymerase chain reaction(qRT-PCR) and reverse transcriptase - polymerase chain reaction(RT-PCR) in 67 $\mathrm{HCC}$ patients $(23 \mathrm{HBV}, 22 \mathrm{HCV}, 22 \mathrm{NBNC})$ with mean age $59.09 \pm 11.13$ years $(60 \mathrm{M}: 7 \mathrm{~F}), 9$ cirrhosis patients $(2 \mathrm{HBV}, 2 \mathrm{HCV}$, 5ALD) with mean age $48.33 \pm 11.31$ years $(8 \mathrm{M}: 1 \mathrm{~F})$ and 14 chronic hepatitis patients $(7 \mathrm{HBV}, 7 \mathrm{HCV})$ with mean age $48.33 \pm 7.17$ years (10 M: $4 \mathrm{~F}$ ), respectively. HCC was diagnosed on the basis of EASL (European Association for the study of the liver) criteria.

Results: Expression of VEGF was found to be significantly higher in patients with HCC compared with cirrhosis and chronic hepatitis.VEGF isoforms were found in all three groups of chronic liver disease patients but not associated with severity of liver disease. The difference in the expression of VEGF in cirrhosis and chronic hepatitis were not significant $(p=0.243)$. A positive correlation was seen between the tumor size and VEGF expression $(\rho=0.347, p=0.003)$. Conclusion: High expression of VEGF is found in HCC patients. However the patterns of VEGF isoforms were similar in all three groups of chronic liver diseases. Further studies with a larger number of patients need to be done.

\section{PP31-38}

Chromosomal segments copy number alterations of the hepatocellular carcinoma patients of different levels of differentiation

H. Chen ${ }^{1}$, F. Wang ${ }^{1}$, M. Jin ${ }^{2}$

${ }^{I}$ Infectious Disease; ${ }^{2}$ Pathology, First Hospital, Jilin University, Changchun, China

Background: Hepatocellular carcinoma (HCC) is one of the most common malignant tumors. Multiple factors are relevant to hepatocarcinogenesis. Identification of gene(s) responsible for HCC is challenge and little is known about exact mechanism of HCC at molecular level. In this study whole genomic DNA microarray (Nimblegen 385k) were performed on 9 well differentiated HCC cases and 8 poorly differentiated HCC cases to reveal Chromosome abnormalities that may responsible for HCC differentiation.

Methods: Good quality DNA of 9 well differentiated HCC cases and 8 poorly differentiated HCC cases were collected from the First Hospital 
of Jilin University, Changchun, P.R. China. This study was approved by Ethnic Committee of the Hospital. All the cases were diagnosed by experienced pathology doctor. To quality control the DNA samples,we use NanoDrop Spectrophotometer and run 1\% agarose gel. Whole genomic. DNA microarray (Nimblegen 385k) were performed on these DNA samples. Then we draw CNC DNA MAP of well differentiated HCC and poorly differentiated HCC according to array images.

Results: Low differentiated patients have more gain and loss in chromosome 2, but high differentiated patients have no significant gain and loss. 50\% low differentiated patients have large region loss in chromosome $14 q$ and $38 \%$ patients have gain in chromosome 20 . In addition, low differentiated patients have loss in chromosome 11q and gain in chromosome 12 and 16.

Conclusion: Some chromosomal segments such as chr2, 14q and chr20 may harbor some genes that are related to HCC differentiation. Further tests about genetic expression are needed.

\section{PP31-39}

Serum Hyaluronic Acid and Serum Sialic Acid for Diagnosis of Hepatocellular Carcinoma in Patients with Chronic Liver Disease

T. Khuhaprema ${ }^{1}$, O.-A. Praisontarangkul ${ }^{2}$, P. Pisespongsa ${ }^{2}, \mathrm{~W}$.

Sumetchotimaytha ${ }^{1}$, P. Kongtawelert ${ }^{3}$, S. Ong-chai ${ }^{3}$, A. Leerapun ${ }^{2}$,

S. Thongsawat ${ }^{2}$, K. Grudpan ${ }^{4}$

${ }^{1}$ National Cancer Institute of Thailand, Bangkok; ${ }^{2}$ Internal Medicine;

${ }^{3}$ Biochemistry; ${ }^{4}$ Chemistry, Chiang Mai University, Chiang Mai,

Thailand

Backgrounds/aims: Although alpha-fetoprotein (AFP) is widely used as a biomarker of hepatocellular carcinoma (HCC), there is still a need for additional biomarkers to aid in diagnosis and surveillance of HCC. Hyaluronic acid (HA) has association with cirrhosis and sialic acid (SA) was associated with various cancers so this study aim was to evaluate the diagnostic value of HA and SA for diagnosis of HCC. Methods: The serum of HCC patients and chronic hepatitis patients without HCC (control) in Chiang Mai University hospital were tested for HA and SA level and were compared.

Results: There were 208 HCC patients and 181 controls. The mean serum HA in HCC patients $(773.82 \mathrm{ng} / \mathrm{ml})$ was significantly higher than in control group $(136.84 \mathrm{ng} / \mathrm{ml})(p<0.001)$ whereas mean serum SA in HCC patients $(37.76 \mathrm{nmol} / \mathrm{ml})$ was significantly lower than in control group $(773.82 \mathrm{ng} / \mathrm{ml})(\mathrm{p}<0.001)$. The serum HA cut-off level at $150 \mathrm{ng} / \mathrm{ml}$ for diagnosis of HCC has sensitivity and specificity at 78.4 and $74.6 \%$, consequently.

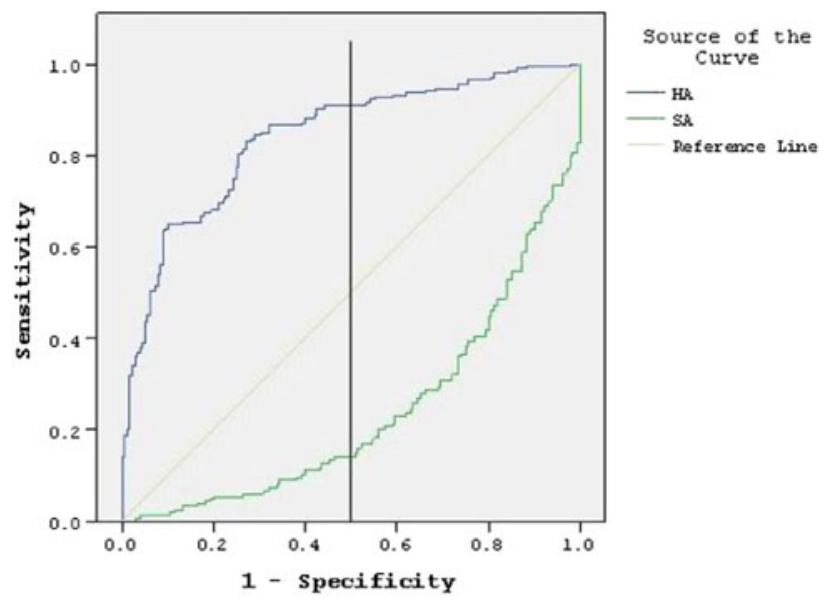

[The ROC of serum HA and SA for diagnosis of HCC]

Conclusion: Serum HA has diagnostic value in diagnosis of HCC while serum SA has negative correlation. The further studies are need to validate the clinical significant of these two markers.
PP31-40

Clinical Significance of Alpha-fetoprotein as a Tumor Marker in Hepatitis B-Related Hepatocellular Carcinoma (HCC)

K. Sasikala, V. Balachandar, S. Mohana Devi

Zoology, Bharathiar University, Coimbatore, India

Hepatocellular carcinoma (HCC) is one of the most prevalent human cancers, ranking the fifth most common worldwide The prevalence of hepatitis B virus (HBV) is reportedly the main cause of HCC in India, where hepatitis $\mathrm{C}$ virus (HCV)-associated $\mathrm{HCC}$ is believed to be relatively less prevalent. We verified the usefulness of alpha-fetoprotein (AFP) as a tumor marker and analyzed the influence of viral etiology on AFP levels in HCC. Of a total of 250 cases with liver disease, who were investigated for the presence of AFP (153 HCC cases, 32 cirrhosis cases, and 65 chronic liver disease (CLD) cases). In addition, equal number of serum samples from healthy patients were included as controls. AFP was quantitatively determined using a commercial ELISA. In HBV and HCV co-infected HCC cases, the AFP positivity was $80.5 \%$. In HBV alone associated $\mathrm{HCC}$, the positivity was $60.7 \%$, and $52.5 \%$ of AFB1 positive HCC cases showed AFP. Based on the data analyzed and investigations performed, we postulate that serum AFP levels in HCC patients are influenced by HBV pattern and could be used in HCC diagnosis. We conclude that the overall positivity pattern of AFP in HCC does indicate that higher levels of AFP are observed with hepatitis virus positivity, especially with HBV. Further studies are required to correlate the serum levels of AFP with the size, number, and degree of differentiation of HCC nodules.

\section{PP31-41}

MAGE-1 and MAGE-3 mRNA Expressions as Molecular Biomarkers in Patients with Hepatitis C Virus-related Hepatocellular Carcinoma H.A. El Aggan ${ }^{1}$, S. Mahmoud ${ }^{2}$, W. El Delgawy ${ }^{2}$, N. El Deeb ${ }^{3}$, R. Shams El Din ${ }^{2}$

${ }^{1}$ Department of Medicine (Hepatobiliary Unit); ${ }^{2}$ Department of Medical Biochemistry; ${ }^{3}$ Department of Pathology, Faculty of Medicine, Alexandria University, Alexandria, Egypt

Background/aim: The melanoma antigen (MAGE) family members are tumor-specific antigens exclusively expressed in neoplastic cells. Therefore, the present work was designed to study the expression of MAGE-1 and MAGE-3 mRNAs in the peripheral blood and cancerous tissues of patients with hepatitis $\mathrm{C}$ virus (HCV)-related hepatocellular carcinoma (HCC).

Methods: Thirty patients with HCV-related cirrhosis (15 patients with $\mathrm{HCC}$ and 15 patients without HCC) and 15 healthy subjects were enrolled in the present study. Expression of MAGE-1 and MAGE-3 mRNAs in peripheral blood samples, HCC specimens and surrounding non-neoplastic liver tissues, were studied by a reversetranscription polymerase chain reaction (RT-PCR) with the specific primers after RNA extraction. The sensitivity and specificity of MAGE-1 and MAGE-3 mRNAs as markers for the diagnosis of HCC have been assessed.

Results: In HCC patients, the positive rate of MAGE-1 and MAGE-3 mRNA expression was 53.3 and $33.3 \%$ in peripheral blood samples respectively, whilst the positive rate was 53.3 and $40.0 \%$ in HCC tissue samples, respectively. By contrast, MAGE-1 and MAGE-3 mRNA were not detected in the adjacent non-neoplastic liver tissues or in the peripheral blood samples of cirrhotic patients without HCC and healthy subjects. No relationship was found between MAGE-1 and MAGE-3 mRNA expression and age, gender, Child-Pugh score, tumor size, clinical stage and histopathological grade $(P>0.05)$. The sensitivity and specificity of MAGE-1 mRNA as a marker for the diagnosis of HCC was 53.3 and $100 \%$, respectively while MAGE-3 mRNA has a sensitivity of $40 \%$ and a specificity of $100 \%$.

Conclusions: MAGE-1 and MAGE-3 mRNA are highly expressed in HCV-related HCCs and may play a role in hepatocarcinogenesis. 
These tumor-specific antigens can be used as molecular markers for early diagnosis of $\mathrm{HCC}$ and detection of disseminated tumor cells and may act as a potential target for immunotherapy in HCC patients.

\section{PP31-42}

Claudin Expression Profile Differentiates Colorectal Liver Metastasis from Hepatocellular Carcinoma

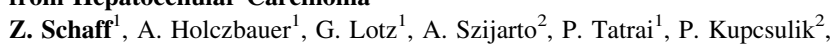
I. Kovalszky ${ }^{3}$, A. Kiss ${ }^{1}$

${ }^{1}$ 2nd Department of Pathology; ${ }^{2} 1$ st Department of Surgery; ${ }^{3} 1$ st

Department of Pathology, Semmelweis University Budapest,

Budapest, Hungary

Background: Claudins (CLDN) (1-24) have been recently identified as integral proteins of tight junction strands and are widely implicated in carcinogenesis. They have been reported to be differentially expressed not only in normal tissues but also in malignancies deriving therefrom. CLDN-4 expression separates cholangiocellular carcinoma from hepatocellular carcinoma (HCC). Claudin-3 and 4 can function as the receptor of Clostridium perfringens enterotoxin. Further, benign and malignant tumors of several organs can be differentiated based on the CLDN expression profile.

Aims: The objective of the current study was to characterize mRNA and protein expression of claudins in HCC and in liver metastasis of colorectal carcinoma (MET) as well as in their corresponding nontumorous liver samples and also in normal livers.

Methods: Expression of claudin-1, -2, -3, -4-, and -7 was measured by real-time RT-PCR, immunohistochemistry (IHC), and Western blotting in 6 normal livers and in thirty MET as well as in thirty HCCs with their surrounding non-tumorous tissues. Result of IHC was evaluated by morphometrical analysis. Beta-actin was used as reference gene for relative quantification of mRNA expression.

Results: Claudin-1, $-3,-4,-7$ protein expression was found elevated in MET with high significancy (0.0001) in comparions to HCC. Similarly, all investigated claudins were significantly higher expressed in MET compared to both normal and surrounding non-tumorous livers. mRNA expression analysis found similar changes in CLDN expression as IHC. Western blot analysis confirmed IHC results.

Conclusion: Claudin expression profile might help to differenciate HCC from colorectal liver metastasis. Biological role and significance of upregulated claudin expression in the progression of colorectal liver metastasis should be elucidated. The high claudin expression in MET might enable target therapy for these tumors based not only on Clostridium perfringens enterotoxin but also on antibody treatment.

\section{Poster Presentation 32: Hepatocellular Carcinoma: Epidemiology}

\section{PP32-01}

Glycated Haemoglobin A1c and Antidiabetic Strategies as Risk Factors for Hepatocellular Carcinoma

V. Donadon, M. Balbi

Internal Medicine, Pordenone Hospital, Pordenone, Italy

Background/aims: Previous studies have reported the association between type 2 diabetes mellitus (DM2) and hepatocellular carcinoma (HCC), but it is unclear how diabetes mellitus and its control influence hepatocarcinogenesis. Therefore, we evaluate the relationship between glycemic control (assessed by glycated hemoglobin HbAlc values), antidiabetic therapies and the risk of hepatocellular carcinoma.
Methods: We recruited 465 HCC patients, 618 cases with liver cirrhosis (LC) and 490 controls. Among subjects with DM2, the associations between the antidiabetic strategies and $\mathrm{HbA} 1 \mathrm{c}$ level with HCC were determined through 2 series of multivariate logistic regression models using cirrhotic patients and controls as comparison groups.

Results: DM2 prevalence was $31.2 \%$ in patients with HCC, $23.2 \%$ in cirrhotic patients and $12.6 \%$ in controls $(P<0.0001)$. In $86 \%$ of study subjects, DM2 had been diagnosed for more than 1 year before the HCC diagnosis. HCC patients with DM2 had a 1.5-2.5-fold increased risk of liver cancer. The HbA1c mean levels were significantly higher in DM2 patients with HCC than in cirrhotic and control DM2 patients. Antidiabetic treatment with metformin was more common among cirrhotic and control DM2 subjects than among cases with HCC. In both series of multivariate analyses, treatment with metformin significantly reduced the risk of HCC by more than $80 \%$ compared with sulphonylureas and insulin therapy. No significant differences were seen between sulphonylureas and insulin treatment. Elevated $\mathrm{HbAlc}$ levels were positively related to the risk for $\mathrm{HCC}$ in diabetic patients, with a $26-50 \%$ increase in risk for each $1 \%$ increase in $\mathrm{HbAlc}$ values.

Conclusions: In patients with preexisting DM2 the risk of HCC is positively associated with poor chronic glycemic control evaluated by A1c levels and significantly decreased by metformin therapy.

PP32-02

Clinical Profile of Hepatocellular Carcinoma at Veterans Memorial Medical Center

R.U.C. Cinco, J.G. Ignacio

Department of Medicine, Section of Gastroenterology, Veterans Memorial Medical Center, Quezon City, Philippines

Background: Philippines ranks fourth among Southeastern Asian countries with high incidence of hepatocellular carcinoma (HCC). There is a paucity of published literature on profile of HCC in the Philippines and we rely mostly on international data.

Objectives: To determine the clinical profile and prognosis of HCC among patients at Veterans Memorial Medical Center.

Methods: A prospective, analytical study of 33 patients seen at VMMC from January 2008 to Nov 2009 was done. Cases were included if they met the diagnostic criteria of European Association for the Study of Liver for HCC. Clinical profile of patients, risk factors, degree of liver function impairment, biochemical, radiologic and endoscopic features, extrahepatic metastases, treatment given, date and cause of death were recorded and analyzed.

Results: The prevalence of HCC was $38.4 \%$. Mean age of onset was 63.4 with preponderance of male at $87.9 \%$. Cirrhosis was present at $84.7 \%$ and the primary risk factor for HCC was HBV (39.4\%).Tumor size of $\geq 4 \mathrm{~cm}$ was $93.9 \%$ and $32 \%$ of them had relatively low AFP level. Fourteen had extrahepatic metastases on diagnosis with pulmonary $(30.3 \%)$ as the most common site. Overall mortality was $36.4 \%$ with liver failure $(58.3 \%)$ as the leading cause of death. Out of $51.5 \%$ patients who received treatment only $23.5 \%$ died. There were no significant differences in mortality between patients with HCC with and without treatment $(p$ value $=0.1198)$ however, there's a highly significant difference $(p$ value $=0.01004)$ in the length of survival with 27 months for those with treatment and 8.6 months for those without treatment.

Conclusion: Poor prognosis is attributed to the compounding problems of HCC, cirrhosis and viral hepatitis. Since cirrhosis is present in the vast majority of these patients, treatment choices are limited but beneficial in prolonging survival. 
PP32-03

The Features of Non-B Non-C Hepatocellular Carcinoma: Ten Years Experience of Single Medical Center

M. Kondo, T. Ohki, K. Saito, S. Ihara, K. Takagi, M. Kakiuchi, T. Nakatsuka, M. Seki, N. Toda, K. Tagawa

Gastroenterology, Mitsui Memorial Hospital, Tokyo, Japan

Backgrounds: Recently, the number of non-B non-C hepatocellular carcinoma ( $\mathrm{nBnC} \mathrm{HCC}$ ) patients has been increasing in proportion to various metabolic diseases. We investigated the clinical features of $\mathrm{nBnC} \mathrm{HCC}$.

Methods: A total of $256 \mathrm{HCC}$ patients were curatively treated with radiofrequency ablation (RFA) from February 2000 to January 2009. They were divided into two groups: those who were positive for hepatitis B surface antigen or positive for hepatitis C virus antibody (virus HCC group: $n=211$ ), and those negative for both of them (nBnC HCC group: $n=45$ ). We compared patient backgrounds, liver function-related ones, and tumor factors between the two groups. We also assessed the prognostic values of survival rates and risk factors of recurrence using multivariate analysis.

Results: The proportion of male patients, obese patients, comorbidity with diabetes, and heavy alcohol consumption were significantly higher in $\mathrm{nBnC}$ HCC group. Serum albumin level and platelet counts were significantly higher in $\mathrm{nBnC} \mathrm{HCC}$ group and aspartate aminotransferase level was significantly lower in $\mathrm{nBnC}$ HCC group. There were no significant differences in age, Child-Pugh score, and tumor related factors. The cumulative recurrence rates significantly differed between the two groups $(P=0.0017) ; 11.9,32.4$, and $58.0 \%$ at 1,2 , and 3 years, respectively, in $\mathrm{nBnC} \mathrm{HCC}$ group, and 29.2, 56.1, and $73.7 \%$ in virus HCC group. On the other hand, there were no significant differences in overall survival rate $(P=0.39) ; 91.0,83.3$, and $61.8 \%$ at 1,3 , and 5 years, respectively, in $\mathrm{nBnC}$ HCC group, and 94.5, 74.5, and $55.3 \%$, in virus HCC group.

Conclusions: Non-B non-C HCC group had higher rate of comorbidity with metabolic factors compared to virus HCC group. The recurrence rate after RFA was significantly lower in the $\mathrm{nBnC}$ HCC group. However, there were no significant differences in overall survival rate between the two groups.

\section{PP32-04}

Clinical Profile and Etiology of Hepatocellular Carcinoma in a Tertiary Level Hospital in Bangladesh

M.H.-O.- Rashid ${ }^{1}$, A. $\mathrm{Alim}^{2}$, K. Rahman ${ }^{3}$, M.M.H. Chowdhary ${ }^{3}$,

M.A. Haque ${ }^{3}$, S. Ekram ${ }^{3}$

${ }^{1}$ Hepatology Department; ${ }^{2}$ Gastroenterology Department; ${ }^{3}$ Medicine Department, Rajshahi Medical College, Rajshahi, Bangladesh

Background/aim: Clinical and etiological profile among HCC patients in Bangladesh are limited. Our aim to study clinical profile and to treat etiology and enhance the conciousness of peoples about HCC.

Method: It was a prospective study in Inpatient department of Rajshahi Medical College Hospital from July 2007 to July 2009. Patients of child's A/B cirrhosis were included. Total number of patients were 65 . Most of the patients had abdominal pain, weight loss, anorexia and fever.

Result: Among 65 patients male 55(84.6\%), female 10(15.4\%) Mean age 55 years \pm 15 . Of these HBV alone was 38 . HCV only 9, Alcohol 02, combined etiology (HBV + HCV) 04, unknown 12. AFP level was normal $(<20 \mathrm{ng} / \mathrm{ml})$ in $05(7.7 \%), 20-400 \mathrm{ng} / \mathrm{ml}$ in 25 (38.4\%) and $>400 \mathrm{ng} / \mathrm{ml}$ in $35(53.8 \%)$. Mean tumour size was $7.5 \mathrm{~cm}$, portal vein thrombosis was seen in $40 \%$, Okuda stage 1 in $28 \%$, II in $62 \%$, III in $10 \%$. Patients of HCC who received therapy had survival benefit.

Conclusion: HBV infection is the predominant cause of HCC. AFP has good sensitivity in diagnosis Most patients present late. Treated patients have survival benefit.
PP32-05

Characteristics of HCV Related Hepatocellular Carcinoma in India: A Case Control Study

M.P. Sarma, M. Asim, S. Medhi, P. Kar

Medicine, Maulana Azad Medical College,University of Delhi,

New Delhi, India

Background and aim: $\mathrm{HCV}$ is the major risk factors for Hepatocellular Carcinoma (HCC) in India after HBV. The study was designed to investigate the clinical and molecular profile of $\mathrm{HCV}$ related HCC cases in Indian patients.

Method: In a retrospective study, $68 \mathrm{HCV}$ related HCC (GP I), 55 $\mathrm{HCV}$ related chronic hepatitis (GP II) and $68 \mathrm{HCV}$ related cirrhosis (GP III) cases were included. GST gene polymorphism was analyzed in all the cases.

Results: The sex ratios were 5.18:1, 1.39:1 and 0.83:1 with mean age of $50.57 \pm 12.47,39.41 \pm 13.34$, and $46.08 \pm 15.06$, respectively, in 3 groups. Genotype 3 was the most common followed by 1 and 4 . Older age (OR $=2.66$; GP II] and $[\mathrm{OR}=2.04$; GP III $)$ patients, Poor socioeconomic status $(\mathrm{OR}=2.00$; GP II) and $(\mathrm{OR}=1.81$; GP III) HCV genotype $4(\mathrm{OR}=2.73$; GP II: OR $=3.55$; GP III $)$, high HCV viral load $(\mathrm{OR}=1.0 ; \mathrm{GP}$ II $)$, smoking $(\mathrm{OR}=4.89$; GPII $)$ and $(\mathrm{OR}=9.50$; GPIII $)$, alcohol intake $(p=0.0001)$ and GSTT1 null genotype $(\mathrm{OR}=2.3$; GP II $)$ and $(\mathrm{OR}=4.40$; GP III $)$ were significantly associated with HCC. $34(50.0 \%)$ cases had tumor in right lobe while in $28(41.17 \%)$ cases it was bilobar. In $38(55.88 \%)$ cases the size of the tumor was $\geq 5 \mathrm{~cm}$ while in $26(38.23 \%)$ cases the size was between 2 and $5 \mathrm{~cm}$. Okuda stage I was seen in $30(49.18 \%)$ and stage II in $21(34.43 \%)$ cases.

Conclusion: Older age, poor standard of living, High HCV viral load, Genotype 4, smoking and null genotypes of GST are risk factors for development of HCC.

\section{PP32-06}

Decreased Hepatocellular Carcinoma Risk in Chinese Cirrhotic Patients with HBV Infection and Diabetes Mellitus

C. Gao, L. Fang, H.-C. Zhao, J.-T. Li, S.-K. Yao

Department of Gastroenterology, China-Japan Friendship Hospital, Ministry of Health, Beijing, China

Objectives: Chronic hepatitis B and cirrhosis are the two major risk factors for hepatocellular carcinoma (HCC). Diabetes mellitus (DM) was recently identified as a new risk factor. We hypothesized that the risk of HCC would be increased among patients with both DM and hepatitis B virus (HBV)-related cirrhosis. Our study was designed to determine the role of DM in the progression of cirrhosis to HCC among Chinese HBV infection patients.

Methods: A total of 1028 patients, treated at the China-Japan Friendship Hospital, Ministry of Health, Beijing, China, and with a hospital discharge diagnosis of HCC and/or cirrhosis, were included. The demographic, metabolic, virological, biochemical, radiological and pathological features was analyzed and the multiple factor unconditional logistic regression was used to study the association of HCC with any factor, especially DM.

Results: One hundred and fifteen patients were diagnosed with diabetes, including 39 in the HCC group and 76 in the cirrhosis group $(39 / 224=17.4 \%$ vs. $76 / 248=30.6 \%, P=0.001)$. Whether the HCC patients $(n=224)$ were compared with the cirrhotic patients $(n=248)$, or the HCC patients with clinical cirrhosis $(n=122)$ were compared with the simple cirrhotic patients $(n=248)$, or the HCC patients with decompensated cirrhosis $(n=67)$ were compared with the simple decompensated cirrhotic patients $(n=147)$, DM was independently associated with the presence of $\mathrm{HCC}$ [odds ratio (OR) $0.307,95 \%$ confidence interval $(\mathrm{CI}) 0.159-0.592, P<0.001$; OR $0.376,95 \%$ CI $0.175-0.807, P=0.012$; OR $0.192,95 \%$ CI 0.054-0.679, $P=0.010$ ]. Moreover, after the 50 patients with hepatogenous diabetes were excluded, DM was continued to be 
shown as an independent factor (OR 0.298 , 95\% CI $0.132-0.675$, $P=0.004$; OR 0.236 , 95\% CI $0.080-0.694, P=0.009$; OR 0.157 , $95 \%$ CI $0.026-0.956, P=0.045)$.

Conclusions: DM was inversely associated with the presence of HCC in Chinese cirrhotic patients with HBV infection, especially in those with decompensated cirrhosis.

\section{PP32-07}

Reduced Expression of ASS Is Closely Related to Clinicopathological Features and Post-resectional Survival of Hepatocellular Carcinoma

H. Yang, M. Lin, F. Xiong, Y. Yang, G. Shao

Peking University, Beijing, China

Purpose: Argininosuccinate synthetase (ASS) has previously been proven to be reductively expressed in hepatocellular carcinoma (HCC) and various types of HCC cell lines. Moreover, arginine, the product of ASS, has been used as a target in HCC by recombinant human arginase (rhArg) or Arginine deiminase (ADI) which is now in phase II clinical trial stage. This study aimed to present the levels of ASS expression in HCCs and its correlation with clinicopathological features and prognosis of HCC patients.

Methods: Immunohistochemistry assay was used to determine the expression of ASS in normal and HCC tissues from 71 patients. The correlations of ASS expression with clinicopathological parameters, including gender, age, background liver, viral status, tumor size, portal vein invasion, histopathological differentiation, serum AFP level, TNM staging and tumor recurrence of HCC were assessed by Chi-squared test. Patient survival and their differences were determined by KaplanMeier method and log-rank test. Cox regression (Proportional hazard model) was adopted for multivariate analysis of prognostic factors.

Results: ASS immunoreactivity was positive or high in normal liver tissues, but low in HCC tissues $(50 / 71,70.4 \%)$. The reduced expression of ASS was significantly associated with gender, background liver, histopathological differentiation, tumor recurrence, TNM staging and portal vein invasion $(P<0.05)$, but not with age, viral status, tumor size and serum AFP level. Patients with low ASS expression had significantly poorer overall survival (OS) and diseasefree survival (DFS) (both $P<0.001$ ). On multivariate analysis, ASS expression was found to be an independent prognostic factor for OS and DFS ( $P=0.004$ and $P=0.015$, respectively).

Conclusions: ASS might be a novel maker of prognosis of patient with HCC.

\section{PP32-08}

Etiology of HBsAg- and Anti-HCV-negative Hepatocellular Carcinoma in Korea: Prior HBV Infection or Metabolic Syndrome?

E.J. Cho, E.S. Jang, M.-S. Kwak, S.J. Yu, J.-H. Lee, Y.J. Kim, J.-H. Yoon, H.-S. Lee

Seoul National University Hospital, Seoul, Republic of Korea

Background: Chronic HBV, HCV infections and alcohol are wellknown risk factors for hepatocellular carcinoma(HCC). However, $5-30 \%$ of HCC cases do not have these risk factors. Recently, metabolic syndrome is emphasized as an important risk factor in non-B non-C HCC(NBNC-HCC). This study was carried out to assess the relative etiologic role of prior $\mathrm{HBV}$ infection and metabolic syndrome in the development of NBNC-HCC in Korea, one of HBV endemic areas.

Methods: A retrospective, hospital-based analysis was done in 6,058 HCC patients diagnosed from January 2000 to May 2010. The clinical characteristics of patients and potential risk factors for HCC were assessed. Prior HBV infection was defined as the presence of isolated anti-HBc IgG and metabolic syndrome was defined according to the NCEP ATP III.

Results: The major leading cause of $\mathrm{HCC}$ was chronic $\mathrm{HBV}$ infection(72\%), followed by HCV infection(12.1\%) and cryptogenic $(10.6 \%)$. The proportion of NBNC-HCC increased from $9.1 \%$ in 2000 to $12.3 \%$ in 2010 but the difference was not significant. The proportion of HBsAg-positive HCC patients decreased with age while that of isolated anti-HBc-positive HCC patients increased with age, which suggests that isolated anti-HBc-positivity is a result of $\mathrm{HBsAg}$ clearance in hepatitis B infection. In 389 NBNC-HCC patients, 338 patients had serologic evidence of past $\mathrm{HBV}$ infection, and 51 patients did not. Of these, 25 patients had metabolic syndrome, and 26 patients had no apparent risk factors. The mean age distribution of HCC varies significantly according to the cause(HBsAg-positive HCC:53.8 years,NBNC-HCC:61.5 years,HCV-HCC:65.9 years, $P<0.05)$. The same trend was also showed in NBNC-HCC group, but was not statistically significant (prior HBV infection: 60.5 years,metabolic syndrome: 63.5 years).

Conclusions: Prior HBV infection is more important than metabolic syndrome in the development of NBNC-HCC in Korea, a high HBV endemic area. Periodic medical surveillances for patients with prior HBV infection could be considered for diagnosis and curative therapy of HCC.

\section{PP32-09}

Epidemiological Study of Risk Factor of Hepatocellular Carcinoma (HCC) from the Hepatitis B Virus (HBV)-related Cirrhotic Patients

E.-Y. Cho, H.-C. Kim

Wongkwang University, Iksan, Republic of Korea

Background/Aim: It is well-known evidences that liver cirrhosis (LC) is one of the end results of HBV infection and closely related to HCC development. However, its prevalence from the HBV-related liver cirrhosis is variable.

Subjects/Methods: We selected HBsAg-positive 408 LC patients (Pts) those were visited to our hospital from January 2004 to December 2006. We evaluated from them of their laboratory examinations every 3-4 months and radiological examinations 6-9 months interval. However we lossed of 59 Pts. So we analysed from 349 LC Pts at september 2010.

Results: 1 . The mean age was differed accoring to gender. $(p<0.001$ male; $50.43 \pm 9.98$ vs female;54.34 \pm 10.34 ) And 17 cirrhotic patients were expired due to non-hepatic disease.

2. 54 patients were developed HCC. The prevalence of HCC development were not differed according to the severity of cirrhotic grade of Child classification ( $p=0.26$ Child A; 38/228, B; 10/57, C; 6/58). 3. However, the mortality of LC Pts were differed according to Child classification during their examination period $(p<0.001$, Child A; $4.82 \%$ B; 33.34\% C; $81.04 \%$ ). Among in Child A group, 7 among 190 Pts were expired due to hepatic failure (HF) without HCC, but 4 among 38 Pts were died hepatic failure with HCC. There were different results accoring to different group.(Child B; 16/48 Pts in HF without HCC vers 3/9 Pts in HF with HCC, Child C; 45/52 Pts in HF without $\mathrm{HCC}$ vers $3 / 6$ Pts in $\mathrm{HF}$ with $\mathrm{HCC}$ )

4. During the follow-up period, HCC were more significantly developed in the patient of Child A those had active hepatic injury (1.2X UNL either of AST or ALT) $(p<0.005)$.

5. Age and gender were also the risk factors of hepatocarcinogenesis in HBV-related LC $(p<0.05)$

Conclusion: These results suggested that the severity of liver cirrhosis and active hepatic injury are important in the devolopment of $\mathrm{HCC}$ in HBV-related LC.

\section{PP32-10}

Epidemiology of Hepatocellular Carcinoma Based on the Family History of Primary Liver Cancer in Korea

C.-H. Park ${ }^{1}$, H.Y. Kim ${ }^{1}$, M.J. Song ${ }^{1}$, S.-H. Jeong ${ }^{2,3}$, H.-W. Yim ${ }^{2,3}$, N.I. Han ${ }^{1}$, S.W. Choi ${ }^{1}$, Y.S. Lee ${ }^{1}$, C.D. Lee ${ }^{1}$, S.H. Bae ${ }^{1}$, J.Y. Choi ${ }^{1}$, S.K. Yoon ${ }^{1}$ ${ }^{1}$ Division of Hepatology, Dept. of Internal Medicine, The Catholic Univ. of Korea; ${ }^{2}$ Department of Preventive Medicine, The Catholic University of Korea, ${ }^{3}$ Clinical Research Coordinating Center of Catholic Medical Center, Seoul, Republic of Korea 
Background and aims: This study was to evaluate the relations between positive family history of primary liver cancer and hepatocellular carcinoma(HCC) development in Korean HCC patients.

Method: 2,242 patients diagnosed of HCC at Seoul St. Mary's Hospital between January 1990 and July 2008, and whose family history of primary liver cancer was clearly described in medical records were studied retrospectively. The positive family history was defined as having primary liver cancer in their family members.

Results: Positive family history was observed in $159(7.1 \%)$ patients with male to female ratio of 3.3:1. Their median age at diagnosis of HCC was 52.0(range 29.0-79.0), and major causes of HCC were hepatitis B virus(HBV) in 129(81.1\%), hepatitis C virus(HCV) in $16(10.1 \%)$ and alcohol in $2(1.3 \%)$ patients. Those who did not have positive family history were $2083(92.9 \%$ ) with male to female ratio of 3.7:1, and their median age at diagnosis was 57.0 (range 18.0-89.0). The major causes of HCC in these patients were HBV in $1554(74.6 \%), \mathrm{HCV}$ in $280(13.4 \%)$ and alcohol in $68(3.3 \%)$ patients. The median age at diagnosis in patients with positive family history was younger than in those without family history by five years with statistical significance $(p<0.0001)$. Among those with HBV infection, the positive family history was observed in $24(18.5 \%)$ patients at ages under forty-five, and $106(81.5 \%)$ at 45 years or more. Patients who do not have HBV infec$\operatorname{tion}(n=529)$ were sub-analyzed by family history, and their median ages at diagnosis were 57 versus 61 in those having positive family history and those who do not, respectively, but without statistical significance.

Conclusion: The positive family history of primary liver cancer seems to be associated with earlier development of HCC in Korean population. More intensive surveillance maybe recommended to those with positive family history of $\mathrm{HCC}$ for earlier diagnosis and proper management especially when associated with $\mathrm{HBV}$ infection.

PP32-11

Underlying Hepatic Diseases of HCC in Okinawa, Southern Part of Japan, Are Different from these in Mainland

H. Sunagawa, N. Oshiro

Surgery, Nakagami Hospital, Okinawa, Japan

Background: Hepatitis $\mathrm{C}$ virus (HCV)-related $\mathrm{HCC}$ represents $75 \%$ of all HCC in Japan. Negative virus markers for both hepatitis B and $\mathrm{C}$ (non-BC) are recognized from 17 to $28 \%$. The purpose of this study is to determine the distribution of underlying diseases of HCC in Okinawa, southern part of Japan.

Methods: A total of 114 HCC patients diagnosed from January 2000 to September 2010 were enrolled. The distribution of underlying hepatic disease were examined.

Results: HCV related HCC represented $33.3 \%$ of all HCC in our hospital. Hepatitis B virus (HBV) related HCC represented $14.9 \%$. Non-B and $\mathrm{C}$ represents $59 \%$. $49.1 \%$ of non-BC patients were habitual alchol drinkers, $8.4 \%$ had nonalcoholic fatty liver disease (NAFLD), and $42.3 \%$ had no apparent etiology. The age of the nonBC patients was higher (69.8) than that of HBV patients (65.5) but was identical to that of the HCV patients. The clinical stages (defined based on the criteria of the Japanese Association of Hepatocellular Carcinoma) were no difference among the groups (B, C, non-BC).

Conclusion: In Okinawa, non-BC patients account for 59\%. The distribution of underlying diseases of HCC patients in Okinawa, is different from that in Mainland of Japan.
PP32-12

Type 2 Diabetes and Hepatocellular Carcinoma: A Case-control Study in Patients with Chronic Hepatitis B

Q. Li $^{1}$, W.-W. Li $^{2}$, X. Yang ${ }^{3}$, W.-B. Fan ${ }^{4}$, J.-H. Yu ${ }^{1}$, S.-S. Xie ${ }^{1}$, L. Liu ${ }^{1}$, S.-J. Chen ${ }^{1}$

${ }^{1}$ Division of Liver Disease, Jinan Infectious Disease Hospital, Shandong University, Jinan, China; ${ }^{2}$ Unit of Disease Control Genome Medicine, Institute of Medical Science, University of Tokyo, Tokyo, Japan; ${ }^{3}$ Unit of Hyperbaric Medicine, Shandong Province Hospital, Jinan; ${ }^{4}$ Unit of Infectious Disease, Dezhou People's Hospital, Dezhou, China

Type 2 diabetes has been suggested as an independent risk factor for the development of hepatocellular carcinoma (HCC). However, the role of type 2 diabetes on the development of (HCC) in the presence of chronic hepatitis B (CHB) has not been well documented. We conducted a prospective case-control study, which included 1,410 eligible hospitalized patients with hepatitis B virus (HBV)-related HCC and 2,810 hospitalized controls with $\mathrm{CHB}$, to investigate the association between type 2 diabetes and HCC risk in men and women, in patients with and without cirrhosis in the presence of $\mathrm{CHB}$. We used multivariate logistic regression models to control for the confounding effects of established HCC risk factors. Type 2 diabetes and alcohol consumption were associated with a significant high risk of $\mathrm{HCC}$ in female patients; after adjusting for age, family history of $\mathrm{HCC}$, cirrhosis status, and $\mathrm{HBeAg}$ status, the odds ratio(OR) were 2.0 [95\% confidence interval (CI) 1.1-3.9) and 7.0 (95\% CI, 1.7-29.5), respectively. Restricted analyses among female patients without cirrhosis, indicated a strong association between type 2 diabetes, alcohol consumption and HCC risk, with an adjusted OR 6.5 (95\% CI, 2.2-19.2) and 54.3(6.7-443.4), respectively. In conclusion, type 2 diabetes and alcohol consumption are independently associated with the increased HCC risk in women in the presence of CHB. Gender differences in HCC risk and known risk factors are substantial and warrant further study.

\section{PP32-13}

Chronic Viral Hepatitis-Related Hepatocellular Carcinoma: Prevalence in Municipality of Petrópolis, RJ, Brazil

K.M. do $\mathbf{O}^{1}$, L.H. Spielmann ${ }^{2}$, F.R. d'Albuquerque e Castro ${ }^{3}$

${ }^{1}$ Hepatologia, Fundação Oswaldo Cruz, Petrópolis; ${ }^{2}$ Radiology, Hospital Alcides Carneiro; ${ }^{3}$ Medicina, Faculdade de Medicina de Petrópolis, Rio de Janeiro, Brazil

Hepatocellular Carcinoma (HCC) is the most frequently occuring cancer world-wide and is responsible for approximately one million deaths each year. It is frequently found in patients with chronic liver disease resulting from infection with hepatitis $\mathrm{C}$ virus, and as many 170 million or more people may infected with this virus. Epidemiological studies have identified the risk of HCC in patients infected with the Hepatitis $\mathrm{C}$ virus and the routes of transmission of the disease. The World Health Organization has estimated that approximately 1-2\% of patients with acute hepatitis $\mathrm{C}$ viral infection will progress to HCC.

Objectives: The purpose of this review is to determine which risk factors interact with hepatitis $\mathrm{C}$ infection among persons who develop HCC.

Methods: To determine trends in $\mathrm{HCC}$ incidence in Petrópolis, RJ, we analyzed data for the period 2003-2009. A total of 340 patients, 2 $\mathrm{AgHBs}$ positive and 18 anti-HCV positive consisting of 11 males and 9 females. The presence of anti-HCV antibodies and AgHBs was tested using kit AXSYM. 
Conclusion: HCV plays a significant role in the etiology of Hepatocellular Carcinoma in Petrópolis. Therefore, patients with chronic hepatitis $\mathrm{C}$ should be screened for $\mathrm{HCC}$ on regular basis.

\section{PP32-14}

Clinico-etiological Spectrum and Outcome of Hepatocellular Carcinoma in Northern India: A Prospective Analysis of 128 Cases

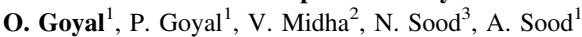

${ }^{I}$ Gastroenterology, ${ }^{2}$ Medicine, ${ }^{3}$ Pathology, DMC and Hospital, Ludhiana, India

Aim: Hepatocellular carcinoma (HCC) has variable presentation and etiological factors in different regions of world. Though $\mathrm{HCC}$ is a major health problem in India, studies on the profile and outcome of HCC in India are limited. Hence, this prospective study was undertaken.

Methods: Patients with HCC presenting between September 2007 and August 2010 were enrolled. Diagnosis of HCC was based on the BCLC criteria using radiology (ultrasonography, triphasic CT or MRI), serum AFP and/or histology. HBsAg, HBV DNA, total Ab-HBc, anti HCV and HCV RNA were done for viral etiology. Work up for Wilsons, NAFLD and autoimmune liver disease was done where indicated.

Results: HCC was diagnosed in 128 patients (mean age $=$ $59 \pm 9.7$ years; male:female $=4.8: 1$ ). $99.2 \%$ patients had underlying cirrhosis; $57.8 \%$ were known cirrhotics and $41.4 \%$ were diagnosed as cirrhotics for the first time. Decompensation of cirrhosis was the most common presentation. Ascites was present in $78.1 \%$, icterus in $55.5 \%$, and hepatomegaly in $50.8 \%$. Aetiological factors were (1) HCV, 52.3\% (HCV alone, 21.9\%; alcohol and HCV, 16.4\%; HCV and HBV, 8.6\%); (2) alcohol 43.8\% (alcohol alone, 18.8\%); (3) HBV, $28.1 \%$ (HBV alone, 10.9\%); (4) cryptogenic/NASH in $14.1 \%$ patients. Mean CTP score was $11.3 \pm 3.2$. Serum AFP was normal $(<20 \mathrm{IU} / \mathrm{ml})$ in $19.5 \%$ and diagnostic $(>400 \mathrm{IU} / \mathrm{ml})$ in $57.8 \%$. HCC was multi-centric in $57 \%$; and with portal vein thrombosis in $34.4 \%$ patients. The mean size of SOL was $5.3 \pm 2.9 \mathrm{~cm}$. Median survival without treatment was 2 months.

Conclusions: In northern India, almost all patients of HCC have underlying cirrhosis. $\mathrm{HCV}$ is the major etiological factor in contrast to HBV in other parts of India. Significant number of cirrhotics $(41 \%)$ present for the first time with HCC. AFP is diagnostic in only about half of the cases. Most patients present at advanced stage with large or multi-centric tumours.

Poster Presentation 33: Hepatocellular Carcinoma: LocoRegional Treatment

\section{PP33-01}

Tumor Size Is an Independent Predictor of Complete Ablation after Radiofrequency Ablation of Malignant Liver Tumors E.G. Santi ${ }^{1}$, S. Wong ${ }^{1,2}$

${ }^{1}$ Internal Medicine-Gastroenterology, University of Santo Tomas Hospital; ${ }^{2}$ Internal Medicine-Gastroenterology, Chinese General Hospital, Manila, Philippines

Background: Radiofrequency ablation (RFA) is a widely used technique for primary and metastatic liver cancers. However, the complete ablation rate (CA) is not $100 \%$ and is affected by multiple factors. We aimed to determine independent predictors of $\mathrm{CA}$ after RFA.

Methods: RFA was performed in 55 consecutive patients with hepatocellular carcinoma $(n=36)$ and liver metastasis $(n=19)$ from
March 2007 to November 2009. Complete ablation was defined as the absence of contrast enhancement (enhancing tumors) or hypodensity that encompasses the original tumor location (non-enhancing tumors) on CT-Scan performed 1 month post-RFA. Primary effectiveness pertains to the ability to completely ablate a tumor after $\geq 1$ session. Tumors that were within $5 \mathrm{~mm}$ of a large vessel or vital structure were considered high-risk (HR).

Results: Sixty-nine (69) RFA sessions were performed for 148 tumors with a mean size of $2.9 \pm 2 \mathrm{~cm}$. CA after 1 session $(<3 \mathrm{~cm}=93.2 \% ; 3.1-6 \mathrm{~cm}=74.1 \% ;>6 \mathrm{~cm}=27.3 \%)$ and primary effectiveness rates $(<3 \mathrm{~cm}=97.7 \% ; 3.1-6 \mathrm{~cm}=92.3 \%$; $>6 \mathrm{~cm}=45.5 \%)$ were higher for smaller tumors $(p<0.05)$. Highrisk tumors had lower $\mathrm{CA}$ after 1 session (HR $=78.6$ vs. non$\mathrm{HR}=92.9 \% ; \quad p=0.043)$ and primary effectiveness rates $(\mathrm{HR}=87.8 \%$ vs. non-HR $=100 \% ; \quad p=0.030)$. Other predictive factors for $\mathrm{CA}$ and primary effectiveness on univariate analysis include concomitant ethanol injection, RFA time, and number of overlaps and breaks during RFA. However, the only independent predictor of both CA after 1 session [OR $(95 \% \mathrm{CI})=<3 \mathrm{~cm}=37$ (7.6-166.7); 3.1-6 $\mathrm{cm}=7.6(1.6-37)]$ and primary effectiveness $[\mathrm{OR}(95 \% \mathrm{CI})=<3 \mathrm{~cm}=50(8-333.3) ; 3.1-6 \mathrm{~cm}=14.5(2.2-90.9)]$ on multivariate analysis was tumor size $(p<0.05)$.

Conclusion: RFA results in satisfactory local tumor control of malignant liver tumors. Complete tumor ablation is substantial in lesions that do not exceed $6 \mathrm{~cm}$.

\section{PP33-02}

Younger Hepatocellular Carcinoma Patients Have Better Prognosis after Percutaneous Radiofrequency Ablation Therapy

C.-W. Su ${ }^{1}$, Y.-Y. Chiou ${ }^{2}$, H.-H. Hung ${ }^{1}$, J.-C. Wu ${ }^{3}$, T.-I. Huo ${ }^{1}$, Y.-H. Huang ${ }^{1}$, H.-C. Lin $^{1}$, S.-D. Lee ${ }^{1}$

${ }^{I}$ Division of Gastroenterology, Department of Medicine; ${ }^{2}$ Department of Radiology, Taipei Veterans General Hospital; ${ }^{3}$ Institute of Clinical Medicine, National Yang-Ming University, Taipei, Taiwan, ROC.

Background and aims: The impact of age on the clinico-pathologic manifestations and outcomes of hepatocellular carcinoma (HCC) remains controversial.

Methods: Two hundred and fifty-eight HCC patients who underwent percutaneous radiofrequency ablation (RFA) were enrolled. Patients aged $\leq 65$ years $(n=100)$ were defined as the younger group and those aged $>65$ years $(n=158)$ were the elderly group. Their clinico-pathologic features and prognosis were compared.

Results: Younger patients had a higher male-to-female ratio, higher prevalence of hepatitis B virus, and smaller tumor size than older patents. After median follow-up of $28.5 \pm 18.7$ months, 45 patients died and the remaining 213 remained alive on the last visit. The cumulative 5-year survival rates were 81.3 and $65.4 \%$ in younger and older HCC patients, respectively. Multivariate analysis disclosed that age $>65$ years $(p=0.032)$, serum albumin level $\leq 3.7 \mathrm{~g} / \mathrm{dL}$ $(p<0.001)$, prothrombin time international normalized ratio $>1.1$ $(p=0.003)$, alfa-fetoprotein (AFP) $>20 \mathrm{ng} / \mathrm{ml}(p=0.030)$, creatinine $>1.5 \mathrm{mg} / \mathrm{dL}(p=0.002)$, and no antiviral therapy after RFA $(p=0.003)$ were independent risk factors associated with poor overall survival. Besides, there were 163 patients with tumor recurrence after RFA. Multivariate analysis showed that age $>65$ years $(p=0.032)$, platelet count $\leq 10^{5} / \mathrm{mm}^{3}(p=0.023)$ AFP $>20 \mathrm{ng} / \mathrm{ml}(p=0.034)$, multi-nodularity $(p=0.013)$, and tumor size $>2 \mathrm{~cm}(p<0.001)$ were the independent risk factors predicting recurrence. For 92 patients in the Barcelona-Clinic Liver Cancer stage 0, younger HCC patients had higher overall survival rate than the older patients $(p=0.027)$.

Conclusions: Younger HCC patients have better overall survival and lower recurrence rate after RFA compared to older patients. 
PP33-03

High Intensity Focused Ultrasound Therapy: A New Treatment on the Horizon for Hepatocellular Carcinoma

A. Chan, S.T. Fan, T.T. Cheung, R. Poon, C.M. Lo

Department of Surgery, Queen Mary Hospital, The University

of Hong Kong, Hong Kong, Hong Kong S.A.R.

Background: High intensity focused ultrasound (HIFU) therapy has emerged to be a new and innovative loco-regional treatment for hepatocellular carcinoma (HCC) in recent years. It is an extracorporeal conformal therapy. By targeting a high intensity ultrasound beam at a focal point within the tumor, hyperthermia is induced leading to coagulation necrosis of the tumor cells. We herein report our experience in its clinical application for treatment of recurrent HCC.

Methods: From October 2006 to October 2009, 27 patients received HIFU therapy for recurrent HCC in our center. Their postoperative and survival outcomes were compared with 76 patients who received radiofrequency ablation (RFA) within the same period. $P$ value $<0.05$ was considered to be significant.

Results: The median age of patients receiving HIFU and RFA was 63 and 62 years old respectively. There was no difference in the frequency of co-morbid illness and hepatitis B positivity between the two groups. The median tumor size in HIFU and RFA group was 1.8 and $1.7 \mathrm{~cm}$ respectively $(P=0.28)$. Operative morbidity rate in the HIFU and RFA group was 7.4 and $22.4 \%$ respectively $(P=0.06)$. Skin burn and liver abscess were the two complications associated with HIFU therapy. There was no hospital mortality in the HIFU group while two deaths were observed in the RFA group. The 1-, 2- and 3-year tumor-free survival rates for HIFU group were 33.3, 22.2 and $0 \%$, respectively, and for RFA group were $26.9,0,0 \%$, respectively $(P=0.22)$. The 1 -, 2 - and 3-year overall survival rates for HIFU group were 100, 100 and $85.7 \%$, respectively, and for RFA group were $89.1,71.9$ and $62.7 \%$, respectively $(P=0.19)$.

Conclusion: Our preliminary experience in utilizing HIFU therapy for treatment of recurrent HCC is promising. Further studies are needed to define its role as a primary treatment of HCC.

\section{PP33-04}

Continuing Control for Recurrent Tumors Provide Survival Benefits in Early Stage Hepatocellular Carcinoma Patients Treated by Radiofrequency Ablation

T.-M. Chen ${ }^{1,2,3}$, C.-C. Lin $^{1}$

${ }^{1}$ Institute of Medicine, Chung Shan Medical University; ${ }^{2}$ Department of Hepato-Gastroenterology, Tungs' Taichung Metro Harbor

Hospital, Taichung; ${ }^{3}$ Department of Internal Medicine, School of

Medicine, Taipei Medical University, Taipei, Taiwan ROC

Background: Radiofrequency ablation (RFA) is an effective local therapy for patients with hepatocellular carcinoma (HCC). However, new recurrences frequently occur in the remnant liver.

Aims: We evaluated the efficacy of RFA with/without combined transcatheter arterial chemoembolization (TACE) as first-line therapies for patients with early HCC. We also appraised the benefits of continuing tumor control by iterative therapies when subsequent HCC recurrences occurred.

Methods: A total of 135 patients with early HCC underwent index therapies, and received iterative treatments for subsequent 126 new recurrences. All patients had a follow-up period of more than 8 months. The primary endpoint of this study was overall survival (OS), and its independent prognosticator was determined using Cox proportional hazards regression analysis.

Results: After a mean follow-up of $26.2( \pm 17.0)$ months, 35 $(25.9 \%)$ patients died. The cumulative OS at 1,3 and 5 years were $88.7,69.0$, and $59.7 \%$, respectively. Combined TACE did not provide survival benefit compared to RFA alone $(P=0.190)$. With multivariate analysis, Child-Pugh class B [hazard ratio (HR), 3.39; 95\% confidence interval $(\mathrm{CI}), 1.09-10.60, P=0.036)$, maximum tumor size more than $2.5 \mathrm{~cm}$ (HR, 4.57; 95\% CI, 1.87-11.19; $P=0.001)$ and having any treatment failure (HR, 2.94; 95\% CI, 1.39-6.23; $P=0.005$ ) were found as independent unfavorable predictors of OS. Patients who experienced any recurrence but finally remained tumor-free after successful treatment had similar survival outcome compared to those without any recurrence (1 year, 97.6 vs. $90.5 \%$; 3 years, 80.9 vs. $81.3 \%$; 5 years, 80.9 vs. $81.3 \%$; $P=0.380$ ), but had better survival outcome than those with treatment failure (1 year, 97.6 vs. $81.3 \%$; 3 years, 80.9 vs. $51.8 \%$; 5 years, 80.9 vs. $25.8 \% ; P=0.001)$.

Conclusions: Continuing tumor control by iterative therapies for subsequent recurrence provided survival benefit in these patients with early stage HCC.

\section{PP33-05}

Radiofrequency Ablation in Malignant Liver Masses: Marginal Recurrence Patterns and Prognostic Factors for Distant Recurrence

J.K. Kim ${ }^{1}$, K.M. Lee ${ }^{2}$

${ }^{1}$ Radiology; ${ }^{2}$ Gastroenterology, Ajou University School of Medicine, Suwonsi, Republic of Korea

Introduction: Radiofrequency ablation (RFA) is an effective technique for the local destruction of tumors. RFA is an increasingly accepted treatment for controlling both hepatocellular carcinomas (HCCs) and hepatic metastases especially from colorectal cancers

Objective: This study analyzed the therapeutic results of RFA and the factors that predict recurrence after RFA in patients with hepatic metastases due to a variety of primary cancers.

Material and methods: 33 patients who had no extrahepatic metastases underwent curative RFA. All patients (23 men and 10 women; mean age: 57 years, range 34-77 years) had a minimum of 6 months follow-up.

Results: Local tumor recurrence was seen at the ablation margin in $13(25 \%)$ of 52 lesions. There was no significant differences in sex, age, tumor location, the presence of contiguous blood vessels, time interval, site or differentiation of the primary cancer, or previous treatment. Distant recurrence occurred in $18(54.5 \%)$ of 33 patients. The 6-, 12-, and 24-month cumulative distant metastasis-free intervals were $75.3,53.3$, and $30.5 \%$, respectively. Patients with primary colorectal cancer had a significantly longer distant recurrence-free interval compared to patients with non-colorectal cancers.

Conclusions: Percutaneous RFA is an effective alternative modality for the treatment of hepatic metastases from various primary cancers, and has a relatively low recurrence rate. The presence of an ablative margin of less than $5 \mathrm{~mm}$ was a significant prognostic factor for marginal tumor recurrence. In addition, distant metastasis (a new hepatic lesion or extrahepatic metastasis) was significantly influenced by the site of the primary cancer.

\section{PP33-06}

Ultraselective Transcatheter Infusion of Epirubicin in Water-in-Oil-in Water Emulsion for Hepatocellular Carcinoma

I. Ikushima ${ }^{1}$, S. Higashi ${ }^{2}$, A. Ishii ${ }^{3}$, K. Seguchi ${ }^{2}$, Y. Iryo ${ }^{1}$, Y. Ota $^{2}$, M. Shima ${ }^{2}$, K. Kobayashi ${ }^{4}$

${ }^{1}$ Radiology, Miyakonojo Medical Association Hospital; ${ }^{2}$ Surgery;

${ }^{3}$ Radiology; ${ }^{4}$ Internal Medicine, Medical City East Hospital, Miyakonojo, Japan

Background: We have previously reported the clinical efficacy of water-in-oil-in-water $(\mathrm{W} / \mathrm{O} / \mathrm{W})$ emulsion containing anticancer agent, especially by non-selective transcatheter arterial infusion (TAI) therapy. W/O/W emulsion limits the damage of normal hepatic parenchyma, because of minimal embolic effect on the peripheral artery and slow release of anticancer agent. The purpose of this study is to evaluate the safety and effectiveness of ultraselective TAI of W/ $\mathrm{O} / \mathrm{W}$ emulsion for hepatocellular carcinoma. 
Methods: Eleven patients with HCC were performed ultraselective TAI therapy of W/O/W at our institute. The response was assessed using the Response Evaluation Criteria in Solid Tumors [complete response (CR), partial response (PR), stable disease (SD), and progressive disease (PD)]. Survival time was defined as the time from the date of first TAI to the date of death or last follow-up and the survival curve was estimated using the Kaplan-Meier survival method.

Results: All 11 patients exhibited a CR. The 12 and 36-month cumulative survival rates for 11 patients were 90 and $60 \%$, respectively.

Conclusion: Ultraselective TAI therapy using W/O/W for the patients with HCC can obtain good local response.

\section{PP33-07}

Comparing Treatment Outcomes between TACE with Adjuvant PEI and TACE alone in Patients with Intermediate-to Advanced-stage Hepatocellular Carcinoma

A. Sirinawasatien

Rajavithi Hospital, Bangkok, Thailand

Objective: To compare rates of survival of patients with intermediate to advanced stage of hepatocellular carcinoma who received treatment with TACE combined with PEI therapy (TACE-PEI), or TACEalone.

Methods: Retrospective analysis was performed among 215 patients with hepatocellular carcinoma who were not eligible for curative treatment (single HCC larger than $5 \mathrm{~cm}$ or more than three nodules, each nodule larger than $3 \mathrm{~cm}$ ) treated at Songklanagarind Hospital between 1 January 2004 and 30 June 2009.

Intervention: Patients received treatment with combined TACE-PEI $(n=117)$ or TACE-alone $(n=98)$.

Main outcome measures: The primary outcome was survival. The secondary outcome was parameters predicting survival.

Results: Of these 215 patients, 170 were male and 45 were female. The mean age was 58.7 years. There were $84 \%$ with evidence of cirrhosis and 70, 25 and 5\% were in Child's class A, B and C, respectively. HBsAg was positive in $60 \%$. Regarding CLIP score for tumor staging, $11,30,24,19,12$ and $4 \%$ had score $0,1,2,3,4$, 5-6, respectively. Overall median survival times were 18.2 months in the TACE-PEI group, and 6.6 months in the TACE-alone group. Patients who were treated with TACE-PEI combination therapy had significantly better overall survival than those who were treated with TACE-alone $(P=0.02)$. The survival benefit of combination therapy over TACE monotherapy was seen only in patients with CLIP score less than 3 . Multivariate analysis revealed that only treatment modality and CLIP score were independent factors determining survival.

Conclusion: TACE-PEI combination therapy was superior to TACE monotherapy in improving survival for patients with intermediate stage of hepatocellular carcinoma.

\section{PP33-08}

Doubling Time of AFP-L3 is Predictive for the Short Term Recurrence of Hepatocellular Carcinoma in TACE Treated Patients

H. Mochizuki ${ }^{1}$, S. Hirose ${ }^{1}$, F. Iwamoto ${ }^{1}$, Y. Tsukui ${ }^{1}$, T. Yoshida ${ }^{1}$, K. Hosoda ${ }^{1}$, Y. Suzuki ${ }^{1}$, K. Hosoda ${ }^{1}$, Y. Kojima ${ }^{1}$, Y. Hirose ${ }^{1}$, M. Omata ${ }^{1,2}$

${ }^{1}$ Gastroenterology, Yama-Nashi Central Hospital, Yamanashi;

${ }^{2}$ Gastroenterology, University of Tokyo, Tokyo, Japan

Background and aims: Following transarterial chemoembolization (TACE) of hepatocellular carcinoma (HCC), making it important to detect the recurrence by expensive enchanced CT and MRI. Thus, simpler and less expensive measures are needed. We assessed the treatment effect by calculating doubling and halfning time of tumor markers after TACE. Halfning time (HT) was calculated as $\mathrm{HT}=\mathrm{d} \times \log 0.5 / \log (\mathrm{b} / \mathrm{a})$ [HT: halfning time (days), d: interval (days), a: pre-value, b; post-value] Doubling time as $\mathrm{DT}=\mathrm{d} \times \log 2 / \log (\mathrm{b} / \mathrm{a})$
[DT: doubling time (days), d: interval (days), a: pre-value, b; postvalue]

Methods: This prospective study included 18 patients who have raised values in either or both of two tumor markers. The two markers are AFP ( $\alpha$-fetoprotein) and AFP-L3 (lens culinaris aggulutininreactive fraction of $\alpha$-fetoprotein). Post-TACE doubling time was calculated a monthly interval as each others. Follow up image study after TACE was performed by dynamic CT or EOB-MRI for 2 or 3 month intervals for 1 year.

Results: In 1 year period, tumor recurrence was observed in 10 cases by CT/MRI, whereas 8 were non-recurrent. In the recurrent 10 cases, doubling time calculation by AFP-L3 levels suggested continuous growth, evidenced by doubling time over 22 days or over 170 halfning time in 5 cases. None showed negative turn of AFP-L3 at any time in any of the recurrent 10 cases.In the 8 non-recurrent cases, 3 showed negative turn of AFP-L3 right after treatment. In the remaining 2 cases, halfning time showed less than 100 days after TACE. In contrast to AFP-L3, classical AFP doubling and halfning time calculation could not discriminate the recurrent and non-recurrent cases.

Conclusions: Post-TACE tumor marker doubling/halfning time, especially not that of classical AFP measurement but that of AFP-L3 after TACE closely related to the short term recurrence of HCC.

\section{PP33-09}

Doubling and Halfning Time of Serum Tumor Markers in Hepatocellular Carcinoma

H. Mochizuki ${ }^{1}$, S. Hirose ${ }^{1}$, F. Iwamoto ${ }^{1}$, Y. Tsukui ${ }^{1}$, T. Yoshida ${ }^{1}$, K. Hosoda ${ }^{1}$, Y. Suzuki ${ }^{1}$, K. Hosoda ${ }^{1}$, Y. Kojima ${ }^{1}$, Y. Hirose ${ }^{1}$, M. Omata ${ }^{1,2}$

${ }^{I}$ Gastroenterology, Yama-Nashi Central Hospital, Yamanashi;

${ }^{2}$ Gastroenterology, University of Tokyo, Tokyo, Japan

Background and aims: There have been studies on doubling time of tumor growth by Ultrasound and Computed Tomography. However, these methods are either expensive or time/cost consuming, Here, we report a simple method to measure Doubling (growth) and Halfning (retard) time of HCC.

Methods: This prospective study included 19 patients who have serial determinations of two tumor markers; AFP ( $\alpha$-fetoprotein) and AFPL3(lens culinaris aggulutinin-reactive fraction of $\alpha$-fetoprotein. Doubling and halfning time was calculated based on the tumor marker before and after the treatment of $\mathrm{HCC}$ as, Doubling Time [DT $=\mathrm{d} \times \log 2 / \log (\mathrm{b} / \mathrm{a}) \quad$ DT: doubling time (days), d: interval (days), a: pre-value, b; post-value] and Halfning Time $[\mathrm{HT}=\mathrm{d} \times \log 0.5 / \log (\mathrm{b} / \mathrm{a}) \mathrm{HT}$ : halfning time (days), $\mathrm{d}$ : interval (days), a: pre-value, b; post-value].

Results: Doubling time before HCC treatment, e.g., untreated HCC growth, was 121 days in average. The fastest growing tumor showed one months (29 days) of doubling time, whereas the slowest was over 2 years (709 days). After treatment, the therapeutice effect was expressed from 5 to 76 days as Halfning time, suggesting the treatment efficacy could be quantitatively measured.

Conclusions: These doubling and halfning time may have wide range of clinical applications including the understanding of natural growth rate and its prognosis to the effects by various treatments.

PP33-10

Radiofrequency Thermal Ablation of Large HCC during Stop Flow (RFTA- SF)

F. Garbagnati ${ }^{1}$, G. Di Tolla ${ }^{2}$, P. Quaretti ${ }^{3}$, G. Ghittoni ${ }^{4}$, S. Rossi ${ }^{4}$

${ }^{1}$ Intralesional Treatment Unit - Radiology; ${ }^{2}$ Radiology, National

Cancer Institute Milano, Milano; ${ }^{3}$ Radiology; ${ }^{4}$ Medicine, Policlinico San Matteo Pavia Foundation, Pavia, Italy

We describe a simple Radiofrequency technique to destroy in one step,in one day hospitalization and with local anestesia very large HCC. The reduction or complete suspension of the arterial 
vascularization during RF procedure, permits to have bigger lesions areas reducing the heating dissipation,increasing the heating diffusion inside the tumoral capsule and reducing tissutal impedance. In the treatment of Hcc, the occlusion of only hepatic artery is indicated because the arterial tumoral irroration is prevalent and with only one insertion of the RF needle electrode it is possible to obtain very big lesion volumes. The RFTA during arterial stop flow conditions is useful in the treatment of $\mathrm{Hcc}$ with a diameter bigger than $4 \mathrm{~cm}$. After diagnostic angiographic study, to evaluate the vascularization of the tumor,the patency of portal vein and the anatomy of hepatic artery, before positioning theRF needle electrode into the tumoral area, gelfoam in injected into the hepatic artery to obtain arterial flow reduction into the tumor. The RF probe is inserted with localanestesia,percutaneously into the tumor. The combination of both procedures RFTA and arterial stop flow, can obtain thermal lesions with a diameter up to $10 \mathrm{~cm}$ in only one session, also utilizing very thin 19 G Radiofrequency electrodes

Conclusions: At CT evaluation after 24 hours RFTA -SF $100 \%$ of the tumors treated have a necrotic volume with the same shape of the tumoral areas. CT repeated after 3-6-12 months after the procedure can better evaluate the possible residual tumor. Percutaneous RFTA associated with arterial stop flow embolization (RFTA-SF) is very effective tecnique to destroy in one session very large HCC

\section{PP33-11}

Reperfusion Ultrasound Imaging Using Sonovue: Novel Tool in Assessing Response to Locoregional Treatments for Hepatocellular Carcinoma L. Siciliani, A.M. De Gaetano, L. Riccardi, M. Garcovich, M. Pompili, G.L. Rapaccini

Catholic University of Rome, Rome, Italy

Backgrounds and aims: Contrast Enhanced Ultrasound (CEUS) has been recognized as useful tool in assessing residual/recurrent HCC after locoregional treatments. The most important finding that indicates complete ablation is the disappearance of any previously visualized intralesional enhancement.A novel ultrasound technique called Defect Reperfusion Ultrasound Imaging, which uses Sonazoid, has been previously described (Kudo et al.). Our aim was to prospectively evaluate the response after locoregional treatments using for the first time SonoVue and the Reperfusion Imaging.

Material and methods: We enrolled three participants who received diagnosis of typical HCC by imaging, according to AASLD guidelines and submitted to locoregional treatments. All the patients were submitted to reperfusion techniques: a bolus of $2.4 \mathrm{~mL}$ of SonoVue (Milan, Italy) was injected intraveounosly. The probe was subsequently applied to the area of necrosis inducted by treatment and during the late phase (after $3 \mathrm{~min}$, in the postinjection time) SonoVue $(2.4 \mathrm{~mL})$ was reinjected. The presence of arterial blood flow entering the area of necrosis was determined and defined as residual of disease; volume of residual was calculated. The day after, all patients underwent MRI, as control of the procedure.

Results: In two patients $\mathrm{HCC}$ was monofocal and in one bifocal. A total of two nodules were treated by percutaneous ethanol injection (PEI); one patient was treated with transcutaneous arterial chemioembolization (TACE). After treatments all images were difficulty depicted by B-Mode ultrasound, because nodules appeared iso-echoic to parenchyma.None of the nodules showed viable tissue after the first bolus. Reinjection detected residual enhanced area with a respective volume of $0.70 \mathrm{cc}, 0.08 \mathrm{cc}, 1.08 \mathrm{cc}$.MRI confirmed local residuals disease with an overlap of data for dimensions and localization.

Conclusions: Reperfusion Imaging with SonoVue could rappresent a reliable method in assessing response to treatment for HCC when BMode ultrasound is limited in the evaluation of lesions and minimal residual. Further comparative studies are needed.
PP33-12

Percutaneous Radiofrequency Thermal Ablation of Large Unresectable Hepatocellular Carcinoma after Occlusion of Tumor Blood Supply: Preliminary Study

G. Ghittoni ${ }^{1}$, P. Quaretti ${ }^{2}$, F. Torello Viera ${ }^{1}$, F. Garbagnati ${ }^{3}$, V. Ravetta ${ }^{1}$, T. Cascella ${ }^{2}$, L. Rosa ${ }^{1}$, S. Macchi ${ }^{1}$, L. Veronese ${ }^{1}$, S. Rossi ${ }^{1}$

${ }^{I}$ Medicina IV, Interventional Ultrasound; ${ }^{2}$ Radiology, IRCCS

Policlinico San Matteo, Pavia; ${ }^{3}$ Intralesional Treatment

Unit-Radiology, National Cancer Institute Milano, Milan, Italy

Background and aims: To evaluate the effectiveness of percutaneous radio-frequency (RF) thermal ablation after occlusion of tumor arterial supply in the treatment of large, unresectable hepatocellular carcinoma (HCC).

Materials and methods: One hundred twenty-two conscious cirrhotic patients with single HCC $3.5-10.4 \mathrm{~cm}$ in diameter (mean $5.9 \mathrm{~cm}$ ) underwent RF ablation after angiographically-assisted occlusion of the tumor arterial blood supply by balloon catheter $(n=40)$ or gelfoam embolization $(n=82)$. Ablation procedures were performed with commercial radiofrequency systems and expandable-tip 19-gauge needle electrodes. Total RF procedure time ranged 24-64 min (mean $35 \mathrm{~min}$ ). Findings at spiral CT were used to establish the outcome of therapy.

Results: Spiral CT performed after the first RF procedure showed a non-enhancing area reproducing HCC nodule shape, suggesting complete tumor necrosis, in 108 of 122 patients (88.9\%). In the remaining 14 patients, apparently complete necrosis was achieved after a second RF procedure. No major complications occurred. After $\mathrm{RF}$ treatment patients were followed up for 3-23 months (mean 12.1 months). Local recurrence was demonstrated in 24 (19.6\%) of 122 treated HCC nodules, whereas the remaining 98 (80.4\%) showed progressive reduction in size without recurrence.

Conclusions: RF thermal ablation after occlusion of tumor arterial supply results in a large volume of necrosis selectively involving HCC tissue and sparing surrounding non-tumoral tissue. This technique could be useful for single-session ablation of large unresectable HCC in cirrhosis.

\section{PP33-13}

Safety and Tolerance of High Intensive Focused Ultrasound Ablaion for Hepatocellular Carcinoma in $\mathbf{1 0 0}$ Consecutive Patients

T.T. Cheung ${ }^{1}$, R.T.P. Poon ${ }^{2}$, C.M. Lo $^{2}$, S.T. Fan ${ }^{2}$

${ }^{1}$ Surgery, ${ }^{2}$ The University of Hong Kong, Hong Kong, Hong Kong S.A.R.

High intensive focused ultrasound ablation (HIFU) is a novo noninvasive ablation methods for hepatocellular carcinoma (HCC). Emerging evidence has shown that it is effective in the treatment of HCC particularly in patient with poor liver function. Unlike partial hepatectomy or RFA for HCC on patients with cirrhosis, there are no data on the safety limit of HIFU. However, information is vital for the selection of appropriate patients for the procedure. In this study, we analyzed the results of HIFU on HCC patients and determined the lower limit of liver function and other patient's factors with which HCC patients can tolerate HIFU.

Method: Preoperative variables of consecutive 100 patients who underwent HIFU for HCC were analyzed to identify the risk factors in HIFU intolerance in terms of morbidity associated with stress-induced complications.

Results: 15 patients were in Child Pugh grade B liver cirrhosis and 1 patient was in Pugh Child C liver cirrhosis. 15 patients presented with ascites before treatment. Thirteen (13\%) patients developed intolerance to HIFU. Postoperative morbidity was mainly due to skin and subcutaneous injury $(n=9)$, five patients developed first degree skin burn, one patient developed second degree skin burn and three patients developed third degree skin burn. Two patients developed pneumothorax, one patient developed hyperbilirubinemia and six 
patients developed other complications. Univariated analysis only showed that age $(p=0.022)$ is the single independent factor that predict the development of intolerance.

Conclusions: HIFU is generally well tolerated inpatients with cirrhosis. This is a safe method for patients even when TACE is considered risky as a treatment modality. This ablative treatment modality can be used in selected patients with Pugh Child C liver cirrhosis.

\section{PP33-14}

Early Complications Following TACE for HCC Performed with Nonresorbables Microspheres

J. Palma, F. Pilleul

Imagerie Digestive, Hôpital Edouard Herriot, Lyon, France

Purpose: To evaluate complications after transcatheter arterial chemoembolization (TACE) of hepatocellular carcinoma (HCC) performed with nonresorbables microspheres.

Materials and method: We retrospectively reviewed 50 TACE performed in 37 patients between January 2003 and February 2007. Same procedure was performed and consisted in injection of anticancer drugs mixed with lipiodol into the hepatic artery followed by administration of embolizing agents (embospheres; $300-1,200 \mu \mathrm{m})$. Complications occurred within thirty days following procedures were listed and compared to which previously reported.

Results: Serious complications requiring extra-days of hospitalization or specific treatment were identified in 17 procedures. We listed three $(6 \%)$ major acute liver failure, identified in patients with a prior poor liver function and/or after proximal non selective TACE. Others expected complications observed were: two (4\%) severe post-embolization syndrome, five (10\%) ischemic cholecystitis, two (4\%) splenic infarcts. These incidences of complication were equivalent to these previously reported with classic embolic agents. No complication such as biliary necrosis or bilioma, gastro-intestinal bleeding, pulmonary embolism or death within 30 days, were observed.

Conclusion: Use of embospheres was not associated with an increased risk of complication during TACE compared to other embolizing agents. Strict selection of candidate for TACE and selective or superselective embolization are necessary to avoid important postprocedural complications.

\section{PP33-15}

Excellent Outcomes with Angiographic Subsegmentectomy in the Treatment of Typical Hepatocellular Carcinoma

S. Iwamoto ${ }^{1}$, T. Yamaguchi ${ }^{1}$, O. Hongo ${ }^{1}$, H. Iwamoto ${ }^{2}$

${ }^{1}$ Iwamoto Hospital, Kitakyushu; ${ }^{2}$ Kurume University, Kurume, Japan

Background: We have successfully adopted an interesting and effective treatment method for hepatocellular carcinoma(HCC) referred to as angiographic sub-segmentectomy (AS). The peripheral feeding artery and portal vein were embolized simultaneously. Therefore, almost all of the HCC and peripheral liver parenchyma developed complete necrosis anatomically.

Objectives: In the methods.

Methods: To determine the effectiveness of this method, we have retrospectively studied the local recurrence rates of 49 solitary HCCs and the long-term survival rates of 120 patients with HCC between 2000 and 2008.

Results: Results showed that in 31 small, solitary HCCs $(<2.0 \mathrm{~cm})$, the local recurrence rate was only $9.6 \%$ and in 10 slightly larger HCCs $(<3.0 \mathrm{~cm})$ the local recurrence rate was only $10 \%$. The 5-, 8-, and 10 -year survival rates in stage 1 (stage $1 \&$ Child A) were $74.27 \%$ (77.65\%), 53.05\% (51.76\%), and 53.0\% (51.76\%), respectively, and the 5-, 8-, and 10-year survival rates in stage 2 (stage $2 \&$ Child A) were $66.21 \%(71.41 \%), 39.9 \%(39.60 \%)$, and $29.92 \%(25.0 \%)$, respectively. There were no severe complications.
Conclusions: AS should be further investigated as a potential firstline therapy for the treatment in stages 1 and $2 \mathrm{HCC}$.

I want to see the short video.

If you saw this video. you can understand the blood systems of HCC. Now, we use this treatment method to treat advanced HCC and metastatic live cancers and have the greater success.

References: Excellent outcomes with angiographic subsegmentectomy in the treatment of typical hepatocellular carcinoma: a retrospective study of local recurrence and long-term survival rates in 120 patients with hepatocellular carcinoma.

\section{PP33-16}

Advanced Hepatocellular Carcinoma: Treatment with Transcatheter Arterial Chemo Embolization

M.H. Othman ${ }^{1}$, M.A.-A. Nafady ${ }^{2}$, M.A. El -Sharkawy ${ }^{1}$, H. El Amin ${ }^{3}$, H.M. Moustafa ${ }^{4}$

${ }^{1}$ Radiology, Assiut University; ${ }^{2}$ Radiology, Al-Azhar University;

${ }^{3}$ General Medicine, Assiut University; ${ }^{4}$ Gastroenterology and

Hepatology, Al-Azhar University, Assiut, Egypt

Purpose: To evaluate the efficacy and safety of transarterial chemoemboization (TACE) in the treatment of hepatocellular carcinoma (HCC).

Materials and methods: This study included 60 patients with HCC. All patients were followed-up for one month, six months and one year after TACE treatment. The tumor response was evaluated by triphasic $\mathrm{CT}$ examination and tumor markers (AFP). By triphasic CT criteria, the response to TQCE therapy, was by disappearance of enhancement within the neoplastic tissue and lipidol trapment within the necrotic tissue and when this same picture was confirmed by scans performed at successive follow-up.

Results: This study was done in the Radiology Department of Assiut University Hospitals in the period between July 2008 and March 2010. This study included 60 patients with hepatocellular carcinoma which proved by high level of AFP and early arterial enhancement in triphasic CT. Totally, 74 sessions of TACE were performed to 60 patients. Single session was sufficient for treatment 46 patients, and two sessions were performed to treat the other 14 patients because in the early follow-up after 1 month dynamic CT revealed residual tumor tissue (residual enhancement with minimal response), so a second session was performed for completion of the treatment . Only 40 of 60 patients came for the second follow-up after 6 months with no tumor recurrence(no residual enhancement). Tumor size was reduced in 50 patients in first follow-up. Lipidol trapment was good in 42 patients and poor in 18 patients initially following the first session. Serum AFP values were elevated in all patients before TACE, (mean value was $1,150 \mathrm{mng} / \mathrm{ml}$ ). It was significantly decreased in all patients in follow up after ablation (mean value was $57 \mathrm{ng} / \mathrm{ml}$.)

Conclusion: TACE is a safe and effective tool for local control of disease in hepatocellular carcinoma (HCC).

\section{PP33-17}

Radiofrequency Ablation of Hepatocellular Carcinoma M.H. Othman ${ }^{1}$, M.A.-A. Nafady ${ }^{2}$, M.A. El -Sharkawy ${ }^{1}$, H.M. Moustafa ${ }^{2}$ ${ }^{1}$ Radiology, Assiut University, ${ }^{2}$ Gastroenterology and Hepatology, Al-Azhar University, Assiut, Egypt

Purpose: To evaluate the efficacy and safety of radiofrequency ablation (RFA) in the treatment of hepatocellular carcinoma (HCC). Materials and methods: This study included 30 patients with hepatocellular carcinoma (HCC). All patients were followed-up for one month, six months and one year after RFA treatment. The tumour response was evaluated by triphasic CT examination and tumor markers (AFP). By triphasic CT criteria, the response to RFA therapy, was considered complete when CT scans showed total disappearance 
of enhancement within the neoplastic tissue and when this same picture was confirmed by scans performed at successive follow-up.

Results: This study was done in the Radiology Department of Upper Egypt Cancer Institute in the period between July 2008 and October 2009. This study included 30 patients with age range from 30 to 70 years They included 22 men $(73.3 \%)$ and 8 women $(26.7 \%)$ At the time of reference, epigastric pain was the complaint of 20 patients $(66.7 \%)$, abdominal distension of 6 patients $(20 \%), 4$ patients were discovered during follow up of liver cirrhosis, palpable mass was associated sign in 6 patients $(20 \%)$ and jaundice in 6 patients $(20 \%)$. All these patients had liver cirrhosis. At the time of RFA treatment, 20 patients $(66.7 \%)$ were within Child-Pugh class A, and 10 patients (33.3\%) were within Child-Pugh class B. Totally, 34 radiofrequency ablation sessions were performed to treat 30 patients. Single session was sufficient for ablation in 26 patients, and two sessions were performed to treat two lesions in one patient. Three patients needed another session for complete treatment of the lesion.

Conclusion: RFA of HCC is a safe and effective tool for local control of disease in hepatocellular carcinoma (HCC).

\section{PP33-18}

Usefulness of 3D Imaging of US, CT and MRI for the Planning and Monitoring of Hepatocellular Carcinoma Treatment Using FUS

H. Fukuda ${ }^{1}$, K. Numata ${ }^{1}$, A. Nozaki ${ }^{1}$, M. Kondou ${ }^{1}$, M. Morimoto ${ }^{1}$, K. Tanaka ${ }^{1}$, S. Maeda ${ }^{2}$, M. Ohto ${ }^{3}$, H. Zhu ${ }^{4}$, Z.-B. Wang ${ }^{5}$

${ }^{1}$ Gastroenterological Center, Yokohama City University Medical Center; ${ }^{2}$ Gastroenterology, Yokohama City University, Yokohama; ${ }^{3}$ Sanmu Medical Center, Sanmu, Japan; ${ }^{4}$ Clinical Center of Tumor Therapy of 2nd Affiliated Hospital; ${ }^{5}$ Institute of Ultrasonic Engineering in Medicine, Chongqing, China

Background: B-mode conventional sonography is used to monitor the HCC during focused ultrasound surgery (FUS), but tumors are often obscured on conventional monitor sonography.

Purpose: We evaluated the usefulness of FUS assisted by threedimensional (3D) imaging of ultrasound, computed tomography (CT) and magnetic resonance imaging (MRI) for the treatment of hepatocellular carcinoma (HCC).

Methods: FUS ablation was performed in 12 patients with small HCC ( $\leq 3$ lesions, $\leq 3 \mathrm{~cm}$ in diameter). The FUS system (Chongqing Haifu Tech) was used under ultrasound guidance. ZioM900 was used for multiplanar reconstruction images.

Results: In 5 of the 12 patients, multi-reflections were responsible for the poor visualization. In 2 cases, the tumor was poorly visualized because of a rib shadow. In one case, the margin of the tumor was too unclear to be detected using ultrasonography. The 3D US images obtained as part of the 3D imaging had a high resolution and were useful for examining the area of HCC invasion and for determining the extent of the ablation area. The CT and MRI images, which are not influenced by bone shadows or multi-reflections, were useful for detecting the tumors and for visualizing the presence of the intestines in the sonication zone. All cases were successfully treated under the guidance of 3D imaging.

Conclusion: 3D imaging of US, CT and MRI are useful for FUS treatment for HCC, compensating for the occasionally poor visualization provided by US monitor.

\section{PP33-20}

Iodized Lenoleic Acid and its Fluorodeoxyuridine Ester Inhibite Both in vitro and vivo Growth of Hepatocellular Cells

Z. Dai

Gastroenterology, Fudan University, Shanghai, China

Objective: Based on successfully synthesized iodized lenoleic acid (ILA) and its covalently combined 5-fluoro-deoxyuridine ester (IFU), we evaluated their inhibitive efficacies on hepatocellular carcinoma (HCC) in both vitro and vivo.

Methods: Both new compounds of ILA and IFU were aided by DMSO $(1 \mathrm{mg} / 0.1 \mathrm{ml})$ to become soluable. When SMMC-7721 and QGY-7703 cells were treated with $0-200 \mu \mathrm{M}$ ILA and IFU for $48 \mathrm{~h}$, compared with 5-fluorouracil (5-FU),their survival rates of HCC cell line were determined by MTT assay, respectively, then values of IC50 of ILA,IFU and 5-FU were all calculated.After subcutaneous inoculation of $\mathrm{H} 22$ cells in mice for 1 week, the 24 cases of Kunmin mice models implanted with $\mathrm{H} 22$ evenly grown tumor masses were selected to randomised into ILA,IFU,DMSO, and control groups with 6 cases of mice in each.The palpable masses were injected with $0.1 \mathrm{ml}$ of DMSO solution of ILA $(5 \mathrm{mg})$, IFU $(2.67 \mathrm{mg})$, the solutions with and without DMSO, respectively. After 2 weeks of interference, the mice and the exploited tumor masses were weighed.

Results: Exposure of both SMMC-7721 and QGY-7703 cells to DMSO solution of ILA for 48 hours results in the significant decrease in the survival of the cells in dose-dependent manner with IC50 of about 134.38 and $109.55 \mu \mathrm{M}$ respectively,those of IFU about 17.55 and $44.78 \mu \mathrm{M}$, as compared 5-FU about 7.38 and $98.06 \mu \mathrm{M}$. The palpabled tumors were given above different intratumor injection alone according to different groups respectively. After 3 weeks, ultimately the mean weights of tumor masses in the ILA, IFU,the DMSO and the control group in mice were $2.77 \pm 0.40, \quad 2.89 \pm 0.80, \quad 3.77 \pm 0.77$, and $3.78 \pm 0.92$ grams, respectively. There is remarked statistically differences between ILA to control group $(p<0.05)$. Compared with control group, the ratio of tumor growth inhibition were 26.74 and $23.61 \%$, respectively, in ILA and IFU group.

Conclusions: Our study demonstrated that ILA could inhibite growth of HCC.

\section{PP33-21}

Preliminary Anti-tumor Effects of Dinitrofluorobenzene (DNFB) on Hepatocellular Carcinoma(HCC) in Both vitro and vivo

\section{Z. Dai}

Gastroentology, Fudan University, Shanghai, China

Objective: 2,4-Dinitro-fluorobenzen(DNFB) is one of the most renowned small molecular hapten, which could induce great immunue reaction of T cell mediated delayed hypersensitivity (TDH) in skin or bowel of animal model. This study investigated preliminary effects of DNFB against hepatocellular carcinoma (HCC) in the cellular and vivo models.

Methods: Growth-inhibition effects of DNFB in vitro were evaluated in SMMC5521 by MTT assay. Intra-tumor injection in $\mathrm{H} 22$ tumor implanted mice models were used to analysed the effects on HCC by tumor weights with PBS solution of DNFB $(1 \mathrm{mg} / 0.1 \mathrm{ml}) 2$ times a week for 2 weeks(DNFB group), compared to the same dose of both PBS solution without DNFB (control group) and the ethanol without water (ethanol group), 6 mice in each group.

Results: Exposure of SMMC-7721 cells to PBS solution of DNFB for $48 \mathrm{~h}$ results in the significant decrease in the survival of the cells in dose-dependent manner with IC50 of about $32.22 \mu \mathrm{M}$, IC90 of about $49.53 \mu \mathrm{M}$, while to the compared 5-fluorouracil,whose IC50 is about $3.62 \mu \mathrm{M}$, but whose IC90 is more than $200 \mu \mathrm{M}$. After percutaneous inoculation of $\mathrm{H} 22$ cells and interference of intra-tumor injection with above 3 different solutions in mice model for 3 weeks, ultimately the tumor weights in the DNFB group, the ethanol group and the control group were $0.97 \pm 0.53,1.55 \pm 0.76$ and $3.79 \pm 0.75 \mathrm{~g}$, respectively. There is remarked statistically differences between any group of the DNFB or ethanol group to the control group $(p<0.01)$. The ratio of tumor growth inhibition were 74.43 and 59.20, respectively, in the DNFB group and the ethanol group. 
Conclusions: Our study demonstrated that DNFB could inhibite growth of $\mathrm{HCC}$ in both vitro and vivo.

\section{PP33-22}

Should Locoregional Treatments Be Provided before Liver Transplantation in Hepatocelluar Carcinoma Patients within Milan Criteria?

J.M. Kim, C.H.D. Kwon, J.-W. Joh, S. Song, M. Shin, S.J. Kim, S.-K. Lee Department of Surgery, Samsung Medical Center, Sungkyunkwan University School of Medicine, Seoul, Republic of Korea

Background: Many patients are diagnosed hepatocellular carcinoma (HCC) within Milan criteria, but these are preferentially treated by locoregional treatments instead of liver transplantation. Many studies did not consider the interval from diagnosis to transplantation that patients received locoregional treatments before transplantation, and we considered this interval as a covariate factor. We identified the effect of locoregional treatments in liver transplant recipients within Milan criteria at the diagnosis of $\mathrm{HCC}$ and investigated the risk factors of HCC recurrence and mortality.

Methods: We reviewed the medical records of patients with hepatocellular carcinoma within Milan criteria at diagnosis. We classified the patients by three groups; Group 1 was defined as primary liver transplantation without locoregional treatments; Group 2, patients within Milan criteria at liver transplantation who received locoregional treatments; Group 3, patients beyond Milan criteria at liver transplantation who received locoregional treatments. We analyzed the effects of variables by time-dependent Cox-regression analysis. Results: The microvascular invasion in group 3 was higher than group 1 and $2(P=0.010)$. The HCC disease-survival rate in group 3 was lower than group 1 and $2(P=0.017)$. The risk factors of patient survival was HCC recurrence (HR, 9.474; 95\% CI 3.797-23.640; $P<0.001)$ by multivariate analysis. MELD (HR, 0.839; $95 \% \mathrm{CI}$, $0.743-0.947 ; P=0.005$ ) and microvascular invasion (HR, 15.950; 95\% CI, 4.504-56.486; $P<0.001)$ are the significant risk factors of $\mathrm{HCC}$ recurrence in multivariate analysis.

Conclusion: We identified that the incidence of HCC recurrence was higher in progressed HCC patients despite locoregional treatments. Patients, who were diagnosed within Milan HCC received locoregional treatments, wasted their time and costs before transplantation. If patients had suitable living donor, patients within Milan criteria should receive liver transplantation as soon as possible.

\section{PP33-23}

The Related Study on PEI Affecting HCC Patients' Cellular Immunity Functions

G. Wang

Hepatic Cirrhosis Diseases, Dalian Sixth People Hospital, Dalian, China

Objective: To investigate the percutaneous ethanol injections(PEI) affecting hepatocellular carcinoma (HCC)patients' cell immunity functions.

Methods: 24 cases of hepatocellular carcinoma patients from our hospital were performed with PEI,and it was observed that the patients'peripheral blood CD4 CD8 T-lymphocyte changing before and after PEI respectively.

Results: CD4, CD4/CD8 of 4 weeks, 8 weeks after the patients' PEI were obviously more than controls and before therapy, and had significant differences $(P<0.05$, there were not the complications in the operations.

Conclusions: PEI can improve HCC patients' cellular immunity functions, and is one of safe and efficient measures in treating HCC, but the further study will be explored to its long term therapical effects.
PP33-24

Complications after Radiofrequency Ablation for Hepatocellular Carcinoma: An Analysis of 1843 Ablations in a Single-center Experience for 10 Years

S.Y. Park ${ }^{1}$, W.Y. Tak ${ }^{1}$, Y.O. Kweon ${ }^{1}$, M.K. Jung ${ }^{1}$, S.W. Jeon ${ }^{1}$, C.M. Cho ${ }^{1}$, S.K. Kim ${ }^{1}$, K.C. Kim ${ }^{2}$, H.G. Park ${ }^{1}$, D.W. Lee ${ }^{1}$, H.S. Lee ${ }^{1}$

${ }^{1}$ Gastroenterology and Hepatology; ${ }^{2}$ Interventional Radiology,

Kyungpook National University Hospital, Daegu, Republic of Korea

Background and aims: As minimally invasive technique, Radiofrequency ablation (RFA) is regarded as a safe treatment modality for hepatocellular carcinoma with favorable clinical results comparable to those of surgical treatment. However, the risk profile of RFA has not been well described due to its low incidence of adverse events.

Methods: We analyzed RFA therapies for hepatocellular carcionma performed in Kyungpook National University Hospital from January 2000 to July 2010.

Results: A total number of 1211 patients, 845 men (70.5\%) and 366 women $(30.0 \%)$, with median age of 68 years (range $27-88$ years) underwent 1843 cases of RFA. The incidence rate of mortality was $0.3 \%$ ( 3 cases of hepatic failure, 2 case of sepsis, and one case of renal failure). Overall incidence of complications was $6.8 \%$ (125 cases). Major complications (36 cases, 2.0\%) included liver abscess (15, $0.8 \%)$, intraperitoneal bleeding $(8,0.4 \%)$, liver failure $(5,0.3 \%)$, variceal bleeding $(3,0.2 \%)$, hemothorax $(2,0.1 \%)$, cholecystitis $(2$, $0.1 \%$ ), and bowel perforation $(1,0.1 \%)$. Among minor complications $(89,4.8 \%)$, post-RFA syndrome including pain and fever was most common $(75,4.1 \%)$. Other minor complications were pleural effusion $(7,0.4 \%)$, skin wound infection $(4,0.2 \%)$ and skin burn $(3,0.2 \%)$. Occurrence of major complications were significantly increases with tumor size $(\mathrm{OR}=1.5$, CI 1.131-8.423, $p=0.02)$, and Child classification $(\mathrm{OR}=1.3$, CI 1.031-6.486, $p=0.03)$. Thrombocytopenia $(<100,000 / \mu \mathrm{l})$ and tumor location were significantly related to postRFA bleeding episode and post-RFA syndrome respectively. $(p=0.03$, and 0.02).

Conclusion: RFA is a safe and effective local treatment for hepatocellular carcinoma. Careful selection of patient with appropriate RFA planning can decrease procedure related.

\section{PP33-25}

Significantly Improved Prognosis of Hepatitis B-related Hepatocellular Carcinoma

R. Tateishi ${ }^{1}$, T. Goto ${ }^{1}$, S. Shiina ${ }^{1}$, K. Uchino ${ }^{1}$, T. Arano ${ }^{1}$, K. Enooku ${ }^{1}$, E. Goto ${ }^{1}$, R. Masuzaki ${ }^{1}$, H. Nakagawa ${ }^{1}$, Y. Kondo ${ }^{1}$, H. Fujie ${ }^{1}$, M. Omata ${ }^{2}$, H. Yoshida ${ }^{1}$, K. Koike ${ }^{1}$

${ }^{1}$ Department of Gastroenterology, The University of Tokyo, Tokyo; ${ }^{2}$ Yamanashi Prefectural Hospital Organization, Yamanashi, Japan

Background and aims: Nucleos(t)ide analogs significantly improved the prognosis of hepatitis B-related cirrhosis. The aim of this study was to investigate whether hepatitis B-related hepatocellular carcinoma (B-HCC) was also improved.

Methods: We enrolled 142 B-HCC patients who underwent percutaneous ablation between 1990 and 2007. The patients were divided into 3 groups according to year of treatment: 1990-1995 (cohort 1, $n=21$ ), 1996-2001 (cohort 2, $n=24$ ), 2002-2007 (cohort 3, $n=97)$. We enrolled 1,086 hepatitis C-related HCC (C-HCC) patients underwent percutaneous ablation in the same period as a control. The use of interferon and nucleos(t)ide analogs was investigated. The improvement of prognosis across the ages was evaluated using Kaplan-Meier estimation and multivariate Cox proportional hazard regression in each viral group.

Results: The proportion of Child A patients increased in C-HCC (49, 63 and $70 \%, p<0.001)$ whereas did not change in B-HCC $(71,70$ and 
$79 \%)$. Two (10\%), $9(38 \%)$ and $62(63 \%)$ of B-HCC patients received nucleos(t)ide analogs during follow-up period in cohort 1, 2 and 3, respectively. Thirty-four (17\%), $9(4.1 \%)$ and $52(7.1 \%)$ of C-HCC patients received interferon therapies in cohort 1,2 and 3 , respectively. Among them 18, 3 and 20 patients achieved sustained virological response. Five-year survival rate was dramatically improved in B-HCC (47.6, 54.2 and $71.3 \%$ for cohort 1,2 and 3, respectively; $p=0.002$ ) whereas the improvement in survival rate was rather moderate in CHCC $(51.2,53.1$, and $60.0 \%, p=0.01)$. The prognosis of B-HCC adjusted by age, Child-Pugh score, and size and number of nodules was significantly improved [hazard ratio (HR), 0.374 for cohort 3 vs. 1; 95\% CI, $0.195-0.715 ; p=0.003]$ across the ages whereas the prognosis of C-HCC did not change (HR, 0.897; 95\% CI, 0.725-1.11).

Conclusions: The prognosis of hepatitis B-related HCC treated by percutaneous ablation was dramatically improved probably due to nucleos(t)ide analogs.

\section{PP33-26}

Usefulness of Artificial Ascites in Protecting Abdominal Wall from Burn during the Procedure of Percutaneous Radiofrequency Ablation

J. Imamura, K. Kimura, S. Saeki, S. Hayashi

Division of Hepatology, Department of Internal Medicine, Tokyo

Metropolitan Komagome Hospital, Tokyo, Japan

Background: Percutaneous radiofrequency ablation (RFA) for subsurface hepatic tumors is sometimes difficult because tumors are adjacent to abdominal organs or abdominal wall. Artificial ascites is reported to be useful in protecting abdominal organs from ablation. We assessed the effectiveness of artificial ascites in treating hepatic tumors adjacent to abdominal wall.

Patients and methods: A total of 166 patients with 240 nodules were treated by RFA in our institute from April 2009 to October 2010. Some of those with subsurface nodules were treated using artificial ascites because they were assumed to be at high risk for complications. Five-percent glucose solution was infused into the abdominal cavity using a 14 gauge needle and then ultrasound-guided RFA was performed. Prior to ablation, we confirmed that abdominal wall is located out of the range of ablation.

Results: Artificial ascites was used in 51 of 166 (31\%) patients. The procedure was successfully completed in 50 of $51(98 \%)$ patients. In one patient $(2 \%)$, glucose solution pooled under peritoneum. 39 of $166(23 \%)$ patients had subsurface nodules adjacent to abdominal wall. 23 of $39(59 \%)$ patients were treated using artificial ascites, and one of them (2.6\%) was diagnosed as peritoneal burn because low intensity area appeared on CT scan at the thin area of peritoneum after RFA. In 16 of $39(41 \%)$ patients, no artificial ascites was used, and two of them (13\%) were diagnosed as peritoneal burn. Complete ablation was achieved in 39 of 39 $(100 \%)$ patients. No adverse events were observed related to artificial ascites technique.

Conclusion: Artificial ascites is safe and effective method in treating hepatic tumors adjacent to abdominal wall as well as those adjacent to abdominal organs in RFA.

\section{PP33-27}

Thrombocytopenia before Radiofrequency Ablation (RFA) Is a Risk Factor for Aggravation of Liver Function, Not for Bleeding after RFA Therapy

D.W. Lee, Y.O. Kweon, W.Y. Tak, S.Y. Park, S.K. Kim, S.W. Jeon, M.K. Jung, H.S. Lee, H.K. Park

Gastroenterology and Hepatology, Kyung Pook National University Hospital, Daegu, Republic of Korea

Background and aims: To evaluate changes in liver function parameters and risk factors 12 months after percutaneous radiofrequency ablation (RFA) therapy in patients with hepatocellular carcinoma (HCC).
Methods: Subjects in this retrospective study comprised 55 patients with HCC underwent RFA therapy and showed no recurrence of HCC 12 months after RFA. Serial changes in serum total bilirubin, albumin, prothrombin time and Child-Pugh score were evaluated before and after RFA. Aggravation of liver function after RFA therapy was defined when Child-Pugh score was increased by 2 or more at 12 months after RFA therapy. In addition, logistic regression analysis was used to clarify risk factors for aggravation of liver function after RFA therapy.

Results: Serum albumin level showed a significant decrease from before $(3.52 \pm 0.67 \mathrm{~g} / \mathrm{dL})$ to 12 months after RFA therapy $(3.25 \pm 0.72 \mathrm{~g} / \mathrm{dL} ; P<0.05)$. Child-Pugh score was significantly increased from before $(6.24 \pm 1.47)$ to 12 months after RFA therapy (7.24 $\pm 2.07 ; P<0.05)$. Based on stepwise multivariate analysis, preRFA thrombocytopenia $\left(<75,000 / \mathrm{mm}^{3}\right)$ was selected as a significant risk factor for long term aggravation of liver function after RFA and there were no patients with bleeding evidence as a complication of RFA. Conclusion: Liver function parameters, particularly serum albumin level, gradually decreased in HCC patients over the course of 1 year after RFA therapy. A pre-RFA thrombocytopenia represents a major risk factor for the aggravation of liver function, not for bleeding after RFA therapy.

\section{PP33-28}

Percutaneous Radiofrequency Ablation for Unresectable Metastatic Liver Tumor Compared with Treated HCC

S. Sato, S. Obi, T. Sato, T. Kawai, M. Kanda

Liver Unit, Kyoundo Hospital, Tokyo, Japan

Purpose: To evaluate the therapeutic efficacy and safety of percutaneous radiofrequency ablation (RFA) for unresectable Metastatic liver tumor (Meta) in the liver compared with HCC treated RFA.

Materials and methods: Between 2004 and 2009, 220 patients with 428 liver neoplasms underwent percutaneous RFA with cool-tip electrode under ultrasonographic guidance. Of these, 60 patients with 207 lesions were diagnosed as metastatic liver tumor, 160 patients with 221 lesions were hepatocellural carcinoma (HCC). The patients with metastatic liver tumor had a history of resection for primary cancer. The mean diameter of tumors was $2.1 \mathrm{~cm}$ (range $0.8-4.0 \mathrm{~cm}$ ). Overall survival rates and local recurrence rate and distant recurrence rate were calculated.

Results: All the lesions (428 tumors) have shown as complete ablation by enhanced CT. Local recurrence rate after 2 years was $13 \%$ in the Meta-treated RFA group, $11 \%$ in the HCC-treated RFA group $(p=0.23)$. Distant recurrence rate was significantly superior in the Meta-treated RFA group than HCC-treated RFA group (1 and 3 years; $49,66 \% / 15,41 \%)$. Meta-treated RFA group was significantly inferior than HCC-treated RFA group in overall survival rates (1 and 3 years; $73,20 \% / 87,64 \%)$. No deaths or complications requiring further treatment occurred as a result of RFA.

Conclusion: Percutaneous RFA is an effective and safe method for treating Metastatic liver tumors, with a good overall patient survival rate and low local recurrence rate compared with HCC treated RFA.

\section{PP33-29}

Risk Factors for Recurrence after Radiofrequency Ablation of Hepatocellular Carcinoma

A.A.F.A.H. Zytoon, A.M. El-Wakil

Radiology, Faculty of Medicine-Menoufiya University, Shebin ElKoom, Egypt

Purpose: To evaluate risk factors for intrahepatic recurrence after RFA for HCC.

Patients and methods: We studied 40 patients with $48 \mathrm{HCCs}(\leq 3.5 \mathrm{~cm})$ who were treated with percutaneous RFA. The mean follow-up period was $24.1 \pm 15.7$ months. We evaluated the cumulative disease-free survival of overall intrahepatic recurrence, local tumor progression (LTP) and intrahepatic distant recurrence (IDR). Thirty host, tumoral 
and therapeutic risk factors were reviewed for significant tie-in correlation with recurrence; Age, gender, whether RFA was the initial treatment for HCC or not, severity of liver disease, cause of liver cirrhosis, contact of tumor to major hepatic vessels and liver capsule, degree of approximation of tumor to the liver hilum of, ablation time, degree of benign periablational enhancement, sufficient safety margin, tumor multinodularity, tumor histological differentiation, tumor segmental location, maximum tumor diameter, degree of tumor preablational enhancement at arterial phase CT, MRI or CT-angiography, laboratory markers pre- and post ablation (AFP, PIVKA II, TP, AST, ALT, ALP, TB).

Results: The incidence of overall recurrence, LTP and IDR was 65, 23 and $52.5 \%$, respectively. The cumulative disease-free survival rates were $54.6,74.8$ and $78.3 \%$ at 1 year, $27.3,71.9$ and $46.3 \%$ at 2 years, 20, 71.9 and $29.4 \%$ at 3 years, respectively. The significant risk factors for LTP were: tumor size $\geq 2.3 \mathrm{~cm}$, insufficient safety margin, multinodular tumor, tumors located at segments 8 and 5, and patient's age $>65$ years.

Conclusion: Our results would have clinical implication for advance warning and appropriate management of patients scheduled for RFA. Patients at risk of LTP should be closely monitored in the first year. Furthmore, regular long term surveillance is essential for early detection and eradication of IDR.

\section{Poster Presentation 34: Hepatocellular Carcinoma: Others}

\section{PP34-01}

Limitations of Antineoplastic Therapy for Hepato Cellular Carcinoma in a Low Resource Developing Country like India

\section{Thiruvenkatachari}

Medical Oncology, N.P Cancer Institute, Rajkot, India

Background: Advances in diagnostic imaging, surgical techniques, locoregional interventional therapies and transplantation have improved results of HCC dramatically in developed countries. However the lack of such resources are seen in a majority of Indian population. Not only that they present at advanced stages, even targeted therapies like sorafenib are possible in only a few of them.

Methods: The medical records of 40 patients of hepato cellular carcinoma (registered in our hospital between Jan 2007 and June 2009) were reviewed. Retrospective analysis of 40 patients (25 male, 15 female, mean age: 55 years) was done. Chemotherapy and survival patterns were evaluated. Overall survival was calculated from date of diagnosis till death.

Results: 25 patients tested positive for the hepatitis virus (10: both HBSAg and HCV positive, 15: positive for HBSAg alone). 10 patients had alcoholic liver disease. Therapy given was surgical resection: 3 , locoregional therapies: none, supportive care: 6 and chemotherapy: 31 patients. The most common chemotherapeutic agents used were single agent doxorubicin: 8, doxorubicin combinations: 9, 5FU: 5, capecitabine: 3, gemcitabine based combinations: 3 and Sorafenib: 3 . Patients with early disease and who underwent surgical resection had survival duration beyond 12 months. Maximum survival among sorafenib treated patients was 9 months and $<6$ months for other agents. Conclusions: The emphasis should be on preventive strategies like vaccination and early detection. Chemotherapeutic agents apart from sorafenib do not seem to have much impact in this disease. Efforts must be made to provide targeted chemotherapeutic agents at a low cost to this population.
PP34-02

Clinical Features of over 5 Year Survival Patients after Radiofrequency Ablation as an Initial Treatment for Early-stage Hepatocellular Carcinoma

T. Ohki, K. Saito, S. Ihara, M. Kondo, K. Takagi, M. Kakiuchi, T. Nakatsuka, M. Seki, N. Toda, K. Tagawa

Gastroenterology, Mitsui Memorial Hospital, Tokyo, Japan

Background: We elucidated the clinical features of over 5-year survival HCC patients after curative treatment with radiofrequency ablation (RFA).

Methods: Between January 2001 and February 2005, 126 HCC patients were curatively treated with RFA. The last follow up date was February 2010. These patients were divided into over 5 year survival group $(n=61)$ and the controls $(n=65)$. Their clinical data, recurrence rate, and time to first recurrence were compared. We also assessed the prognostic values of survival using multivariate analysis.

Results: Serum albumin level, platelet counts, and prothrombin activity time were significantly higher in over 5 year survival group. There were no significant differences about tumor sizes and numbers between the two groups. However, AFP and AFP-L3 level were significantly higher in the controls. During the follow up period ( $4.7 \pm 2.1$ years), 50 patients $(82.0 \%)$ of over 5-year survival group and 51 patients $(78.5 \%)$ of controls recurred HCC. There was significant difference about time to first recurrence between the two groups (over 5-year survival group: 3.2 years vs. controls: 1.6 years, $P<0.001$ ). The average frequency of RFA during the follow up period was 2.3 times in over 5-year survival group and 1.8 times in the controls $(P=0.035)$. The average frequency of TACE during the follow up period was 3.5 times in over 5 year survival group and 2.1 times in the controls $(P=0.045)$. Adjusting for significant prognostic factors in univariate analysis, multivariate analysis only indicated platelet counts (HR 0.85 , per $1.0 \times 10^{4} / \mu \mathrm{L}, P=0.008$ ) as an independent prognostic factor of long-term survival.

Conclusions: In early-stage HCC patients, only platelet counts is an independent prognostic factor of over 5-year survival. Even if recurrence occurred, repeat treatment with RFA or TACE might affect long-term survival.

\section{PP34-03}

Early Treatment of Preneoplastic Hepatitis B Virus-associated Hepatocellular Carcinoma with Compound Phyllanthus Urinaria L (PPUL)

G. Tong ${ }^{1}$, X. Zhang ${ }^{1}$, L. Tong ${ }^{2}$, D. Zhou ${ }^{1}$, J. He ${ }^{1}$, C. Xiao ${ }^{1}$, X. Liu ${ }^{1}$, Y. Zheng ${ }^{1}$, H. Tang ${ }^{1}$, S. Chen ${ }^{1}$

${ }^{1}$ Liver Disease, Guang Zhou TCM University Affiliated Shenzhen TCM Hospital, Shenzhen, China; ${ }^{2}$ North West University, Chicago, IL, USA

Background/aim: Dysplastic nodules and atypical adenomatous hyperplasia with cirrhosis are believed to be the precancerous HCC. In this study, we provide a simple diagnostic method, a treatment regime with a novel PPUL, and new efficacy assessment markers for preneoplastic HCC.

Methods: In the transfected HBx cell line, up-regulated genes URG4, URG7, URG11, URG12 and URG19, and a down-regulated gene DRG2 were identified. Sera of CBH patients were tested for the presence of these six proteins by ELISA. 102 cirrhosis patients who had regenerative or dysplastic nodules and whose sera were tested positive for at least one of these six proteins were randomly divided into treatment group (TG, $n=52$ ) and control group (CG, $n=50$ ). PPUL treatment was lasted for three years in the TG.

Results: Among the 102 patients, two in TG and nine in CG developed HCC, and significant difference between the two groups 
$(P=0.0212)$ was observed. Compared with the CG, the number of antigens that were tested positive in the patients in the TG was significantly lower after 24 months of drug treatment $(P<0.001)$. The positive rates for anti-URG11 and anti-URG19 were both over $60 \%$ in the two groups, and were higher than the positive rates of other antibodies tested. After drug treatment, the positive rates for these two antibodies were both decreased $(P=0.0417)$ in the TG, but were increased in the CG $(P=0.0436)$. Follow-up studies on the 11 patients who developed HCC were performed, during which URG11 and URG19 were always tested positive, while DRG2 was negative. Conclusions: URG11, URG19 and DRG2 could be used as the markers in the prediction of the therapeutic efficacy of PPUL in treating preneoplastic HCC. PPUL may be able to prevent or delay the development of HBV-associated cirrhosis to HCC.

\section{PP34-04}

Perioperative Rehabilitation with Diet and Exercise in Cirrhotic Patients with Hepatocellular Carcinoma Undergoing Hepatectomy

M. Kaibori, M. Ishizaki, K. Matsui, A.-H. Kwon

Surgery, Kansai Medical University, Hirakata, Japan

Objective: There are no details on the effect of exercise therapy in the management of hepatectomy. We examined the clinical efficacy of rehabilitation with combined diet and exercise in cirrhotic patients with HCC undergoing hepatectomy.

Materials and methods: Exercise $(n=32)$ or control $(n=34)$ group received exercise combined with diet or only diet, respectively. Before exercise therapy, the patients underwent cardiopulmonary exercise test (CPX). Exercise was started 1 month preoperatively, resumed from 1 week postoperatively and continued for 6 months (post 6-months) at an intensity based on the anaerobic threshold (AT) of each patient.

Results: Postoperative hospital stay in exercise group had significantly shorter than control. The entire body and fat mass by dual energy X-ray absorptiometry in exercise group had significantly decreased (POM6). In exercise group, fasting serum insulin showed a significant decrease (POM6) and there was also a significant difference in the HOMA score as index of insulin resistance. The BTR in exercise group also had significantly high. CPX showed that the maximal oxygen uptake at AT level was higher in exercise group (POM6). Although disease-free survival rate was not significantly between two groups, the exercise group was better than control.

Conclusion: When rehabilitation included exercise in HCC patients with hepatic impairment, there was 1) postoperative improvement of insulin resistance associated with hepatic damage; 2) maintenance of physical strength after hepatectomy and early recovery of daily activities; but 3) not enough a preventive effect on skeletal muscle atrophy.

\section{PP34-05}

Current Developments in Surgicopathological Classification of Primary Hepatic Space-occupying Lesions

W.-M. Cong, H. Dong, L. Tan, X.-Y. Lu

Department of Pathology, Eastern Hepatobiliary Surgery Hospital, Shanghai, China

Introduction: Liver neoplasm is one of the most common tumors worldwide, especially in China and other developing countries. Along with the rapid development of hepatic surgery, the operation numbers and histological types of primary hepatic space-occupying lesions (PHSOLs) have increased dramatically, which lead to many changes in the surgicopathological spectrum of PHSOLs, and constitute a theoretical basis for modern hepatic surgery and hepatic oncological pathology.
Background/aims: To the best of our knowledge, there is no such report thoroughly assessing the broad spectrum of hepatobiliary tumors in the English literature. The overall surgicopathological type features of PHSOLs are not full proposed. In the present study, we try to collect the type spectrum of PHSOLs as comprehensive as possible.

Methods: A systematic literature research was performed with regards to our experience at Eastern Hepatobiliary Surgery Hospital (EHBH), a total of 31901 patients have been diagnosed and operated for PHSOLs between 1982 and 2009.

Results: Of the 31901 PHSOLs in EHBH, the histological types could be classified into 3 categories, including tumor-like PHSOLs $(n=1,370,4.3 \%)$, benign PHSOLs $(n=3,847,12.1 \%)$ and malignant PHSOLs $(n=26,684,83.6 \%)$, and 6 subtypes derived from hepatocytes; bile ducts; vascular, lymphoid and haemopoietic tissues; muscular, fibrous and adipose tissues; neurogenic and neuroendocrine tissues; and miscellaneous tissues. Combined with review of literatures, we summarized more than 100 types of PHSOLs in the current classification.

Conclusions: We describe a surgicopathological classification of 3 categories and 6 subtypes containing more than 100 entities for PHSOLs. To our knowledge, this is the most complete pathological classification for PHSOLs in the current pathological classification systems of hepatic tumors. We think that the present classification may provide useful information as current reference for clinicians and pathologists to make correct diagnosis and differentiated diagnosis among varied PHSOLs.

\section{PP34-06}

Antioxidant Associated Chemoprevention by Garlic Oil in Hepatocarcinoma Induced by $\mathrm{N}$-nitrosodiethylamine in Rats

C. Zhang, T. Zeng, X. Zhao, K. Xie

Institute of Toxicology, Shandong University, Jinan, China

Aims: To investigate the protective effects of garlic oil (GO) on hepatocarcinoma induced by N-nitrosodiethylamine (NDEA) and related mechanisms.

Methods: Wistar male rats were pretreated with GO $(20$ or $40 \mathrm{mg} / \mathrm{kg})$ for 1 week, and then were given GO and NDEA (10 mg/kg, i.g., 5 time/week) for 20 weeks. The serum biochemical indices, histopathological changes and DNA oxidative damage were examined to evaluate the protective effects. Lipid peroxidation (LPO), antioxidant defense system were measured to investigate potential mechanisms. Results: NDEA administration induced a significant elevation in serum alanine aminotransferase (ALT), aspartate aminotransferase (AST), alkaline phosphatase (ALP) and gamma-glutamyl transpeptidase (GGT) activities. However, GO treatment restored or reduced these serum biochemical indices. Histopathological observations of liver were paralleled well with these indicators. Furthermore, NDEA increased the content of 8-hydroxy-2'- deoxyguanosine (8-OHdG) by $141 \%(P<0.01)$, which was counteracted by GO. GO significantly inhibited the NDEA-induced increase of MDA levels. Compared with those in NDEA group, the levels of GSH and the activities of superoxide dismutase (SOD), catalase (CAT), glutathione reductase (GR), glutathione Peroxidase (GPx) and Glutathione-S- transferase (GST) were enhanced by $143,29,47,122,27,75 \%(P<0.05$ or $P<0.01)$, respectively, in NDEA+GO $(20 \mathrm{mg} / \mathrm{kg} . \mathrm{bw})$ group and $636,24,98,187,34,94 \%(P<0.01)$ in NDEA+GO $(40 \mathrm{mg} / \mathrm{kg}$ bw $)$ group, respectively.

Conclusions: GO effectively attenuated hepatocarcinogenesis induced by NDEA in rats, which was partially due to the increase of the hepatic GSH level and the elevation of the antioxidant enzymes activities. 


\section{PP34-07}

Assessment of Liver Function by Methacetin C13 Breath Test Pre-and Post Liver Resection in HCC Patients in Mongolia

L. Nemekhbaatar ${ }^{1,2}$, S. Batmunkh ${ }^{1}$, A. Jazag ${ }^{1}$, M. Urtnasan ${ }^{2}$, C. Jigjidsuren ${ }^{3}$, T. Tseveg ${ }^{2}$

${ }^{I}$ Mongolian Association for the Study of Liver Diseases (MASLD), laanbaatar; ${ }^{2}$ Health Sciences University of Mongolia; ${ }^{3}$ National

Cancer Center of Mongolia, Ulaanbaatar, Mongolia

Aim: Our aim was to assess the feasibility of Methacetin breath tests in decreasing mortality during or after liver resection, predict complications and liver failure after the resection.

Method: Breath test was performed on the day before operation, post operation days 1 and 10. Patients with projected resection of more than $20 \%$ of the liver were included in the study. Additional parameters like liver enzymes, Child-Pugh score, platelets, albumin, CT volumetry were assessed. Total of 42 patients were included in the study, out of which 5 were healthy controls. 23 patients canceled operation because of insufficient liver function. 14 patients participated until the last measurement of day 10 at NCC.

Result: 5 healthy controls had the mean DOB peak value of 29.056 , CUM30 of 91.56 and liver function 114. All patients were Child-Pugh $\mathrm{A}$, and mean albumin was $3.8 \mathrm{~g} / \mathrm{dl}$ and platelets were $229 \times 10^{9} / \mathrm{L}$, ALT 179IU/L and AST 162 IU/L, respectively. In terms of predicting complications we observed DOB peak has the most meaningful results with 5 out of 6 patients with post operative complications had a value of $<20$, while 6 out of 7 patients without complications had values $>20$.

Conclusion: The closest parameter to the amount of liver resected was the highest concentration of methacetin DOB peak, whereas CUM 30 and liver function did not match the amount as that of DOB peak. We suggest the cut off value of DOB peak at 20. This method is non-invasive, non-toxic, direct method to assess liver function with high predictive and dynamic range in regard to residual liver function. The sensitivity of the methacetin test to predict post-operative complications were $83 \%$, and the specificity stands at $85 \%$ with PPV (Positive Predictive Value) $=83 \%$, and NPV (Negative Predictive Value $)=85 \%$, respectively. The most common post-operative complications we encountered were ascitis and wound infections.

\section{PP34-08}

Hepatitis B Infection History is a Risk Factor of Hepatocellular Carcinoma in the Patients with Negative HBsAg

T.-E. Wang, J.-H. Lai, S.-C. Lin, H.-Y. Wang, C.-H. Chu, S.-C. Shih Mackay Memorial Hospital, Taipei, Taiwan R.O.C.

Introduction/objectives: Hepatocellular carcinoma (HCC) is usually associated with chronic hepatitis B and C. But few HCC patients had negative finding of hepatitis $\mathrm{B}$ surface antigens and hepatitis $\mathrm{C}$ antibodies (NBNC-HCC). The clinical manifestation and other viral markers are rarely discussed.

Aims and methods: To clarify the etiologies, clinical manifestations, and prognosis of NBNC-HCC at a medical center, we reviewed the patients with HCC (ICD-9 code:1550) from Jan 2008 to Jan 2010. The patients who had positive HBsAg and anti-HCV were excluded. The diagnosis of HCC was basing on image finding plus serum AFP or pathology. The etiology, cancer stage, other serum virus marker, treatment and prognosis were analyzed.

Results: 54 patients were enrolled, 41 male and 13 female, with an average age of $68.9 \pm 12.5$ year old. 20 patients had been checked serum anti-HBc or Anti-HBs, and 10 patients were positive finding, meaning HBV exposure history. Seven patients were alcoholic liver disease and the remaining patients were unknown etiology. The most co-morbid diseases were hypertension and Diabetes Mellitus. 34 patients had liver cirrhosis. 17 patients had AFP level more than 200ng/mL. The most common Okuda stage was 2 (21/54) and CLIP score was $1(14 / 54)$. Portal vein involvement is noted in 18 patients and distal metastasis in 6 patients. 22 patient received transcatheter artery embolization, 8 patients received surgical resection, and 12 patients received palliative care. Other local treatments and target therapy were used in some patients.

Conclusion: Almost half of these patients had positive finding of hepatitis B infection history, if patient had been checked serum anti$\mathrm{HBs}$ or anti-HBc. The result may indicate that HBV exposure might still play an important unexpected factor for HCC. Anti-HBc and antiHBs should be regularly checked for hepatitis B surface antigens and anti-HCV negative patients with HCC.

\section{PP34-09}

Aqueous Extract of Aloe Barbadensis Mill. Protects against Acetaminophen and Galactosamine Induced Hepatotoxicity

B.K. Chandan ${ }^{1}$, A.K. Saxena ${ }^{2}$, S. Shukla ${ }^{3}$, N. Sharma ${ }^{2}$, K. Singh ${ }^{2}$, D.K. Gupta ${ }^{4}$

${ }^{I}$ Pharmacology, Indian Institute of Integrative Medicine, India;

${ }^{2}$ Department of Pharmacology, Indian Institute of Integrative Medicine, Jammu Tawi; ${ }^{3}$ School of Studies in Zoology, Jiwaji University, Gwalior; ${ }^{4}$ Department of Chemistry, Indian Institute of Integrative Medicine, Jатти Tawi, India

Background/aims of study: The use of Aloe barbadensis Mill. (Family- Liliaceae) in variety of diseases including liver disorders is well documented in traditional Indian system of medicine in India. In continuation of our previous significant findings indicating hepatoprotective potential of aqueous extract of aerial parts of aloe barbadensis against carbon tetrachloride induced hepatotoxicity, the aim of the present study was to validate its hepatoprotective efficacy against acetaminophen and galactosamine induced hepatotoxicity.

Materials and methods: Aqueous extracts (AB-4) of Aloe barbadensis was evaluated for hepatoprotective efficacy against acetaminophen (APAP) and D-galactosamine (GalN) induced hepatotoxicity in mice and rats respectively. Prophylactic and curative treatments with $\mathrm{AB}-4$ against APAP and curative treatment against GalN induced hepatotoxicity were used to determine the prophylactic and therapeutic efficacy of AB-4. Alanine aminotransferase (ALT), aspartate aminotransferase (AST), lactate dehydrogenase (LDH), alkaline phosphatase (ALP), triglycerides (TG), protein \& bilirubin in serum; lipid peroxidation (LP), glutathione (GSH), glucose-6-phosphatase (G-6-P), triglycerides (TG) \& protein in whole liver homogenate and amidopyrine $\mathrm{N}$-demethylase (N-de) \& aniline hydroxylase $(\mathrm{AH})$ in microsomes were estimated for the evaluation of hepatoprotective activity.

Results: AB-4 exhibited significant hepatoprotective activity against APAP and GalN induced hepatotoxicity as evident by restoration of ALT, AST, LDH, ALP, bilirubin, TG and protein. Hepatoprotective potential was further confirmed by recoupment in the levels of LP, GSH, G-6-P and microsomal AH \& N-de towards near normal. Conclusion: Present study exhibited significant efficacy of the aqueous extract of Aloe barbadensis against APAP and GalN induced hepatotoxicity. It appears that AB-4 is orally absorbed, well tolerated and safe at therapeutic doses, thus may be of significant therapeutic value in the management of hepatic diseases. 
PP34-10

Complications of Cryoablation: 320 Cases of Liver Cancer

K.-C. Xu, L.-Z. Niu, J. Wang, L.-H. He, L. Zhou

GIHB Affiliated Fuda Hospital Oncology, Chinese Academy of

Sciences, Guangzhou, China

Objective: To summarize the complications that occurred due to cryoablation for advanced liver cancer.

Methods: 320 liver cancer patients of 231 male cases and 89 female cases, aged 22-79 years, with advanced liver cancer, were analyzed. Freezing probes of $1.7 \mathrm{~mm}$ in diameter were guided by CT and (or) B ultrasound. 2-4 freezing probes were selected according to tumor size, shape, location, etc. Cryoabation cycle was set as $15 \mathrm{~min}$ freezing phase of $-140 \pm 10{ }^{\circ} \mathrm{C}$ with argon and 5 min thawing phase of $15 \pm 5^{\circ} \mathrm{C}$ with helium. Second cyoablation circle was applied conditionally when the extension of ice balls wasn't wide enough to cover the tumor. Cardiovascular system was closely observed, and all appropriate measures were taken if complications arose.

Results: The total mortality was $1.5 \%$ with 5 deaths. Mild complications such as chills, fever, liver pain and so on, were good controlled by symptomatic treatment. Elevated serum enzymes automatically fell back to normal level in around 2-3 weeks. 32 cases of thrombocytopenia was adjusted well by drugs, in which 5 severe cases were recovered from platelet transfusion. Major complications was $11.9 \%$, with 15 cases represented Pleural effusion who improved after paracentesis, 14 cases occured abdominal hemorrhage, in which two cases were controlled by surgical hemostasis and three were dead due to liver rupture. Drainage was supplied and showed effect in a patient presented bile leakage, and anti-infection and drainage treatment helped 1 patient went through hepatopostema. Besides, 2 patients showed acute renal failure after cryosurgery, in who hemodialysis had turned out well effect.

Conclusions: Cryoablation for liver cancer is a minimally invasive, safe and effective therapy, but attention should be paid to the possibly complications during surgery.

\section{PP34-11}

Transarterial Chemoembolization as a Curative Therapy for Patients with Barcelona Clinic Liver Cancer Stage 0 and Stage A Hepatocellular Carcinoma

K.T. Suk ${ }^{1}$, D.J. Kim ${ }^{1}$, H.C. Kim ${ }^{2}$, H.S. Kim ${ }^{1}$, S.H. Jun ${ }^{1}$, C.W. Kim ${ }^{1}$

${ }^{1}$ Gastroenterology Internal Medicine; ${ }^{2}$ Radiology, Chuncheon Sacred

Hospital Hallym University College of Medicine, Chuncheon,

Republic of Korea

Patients with BCLC stage 0 and stage A hepatocellular carcinoma (HCC) are candidates for curative therapies such as resection, transplantation, or ablation. Despite the lack of rigorous documentation, guidelines recommend not using TACE as a first-line therapy for those patients. We evaluated the efficacy of TACE as a first line therapy for patients with BCLC stage 0 and stage A HCC. Between April 2002 and April 2010, 131 patients with BCLC stage $0(n=40)$ and stage A HCC $(n=91)$ who underwent TACE were retrospectively enrolled. All of those patients were unable or refused to do resection, transplantation, and ablation therapy. Patient characteristics, scheduled pre-post CT or MRI findings, TACE findings, and 1, 5, 8-year survivals were assessed. The mean size of HCC was
$2.4 \pm 1.1 \mathrm{~cm}$ and the mean number of TACE was $1.1 \pm 0.5$. The mean overall survival time and 1, 5, 8-year survival rates were $78.3 \pm 6.6$ months, 94, 59, and 42\%, respectively. In the Cox regression analysis, Child-Pugh score $>5(p<0.01$, OR 10.02), presence of arterio-venous shunt $(p=0.01$, OR 9.69), amount of used lipiodol $>7 \mathrm{cc}(p<0.01$, OR 10.49), and gelfoam use $(p<0.01$, OR 11.71) during TACE were risk factors of death. The mean survival times of patients without risk factor, with 1 risk factor, and with more than 2 risk factors were $92.6 \pm 3.3$ months, $68.4 \pm 9.5$ months, and $46.8 \pm 12.8$ months, respectively. 1, 5, 8-year survival rates according to the number of risk factors were $96 \%, 96 \%$, and $96 \%$ (without risk factor), $98 \%, 51 \%$, and $25 \%$ (1 risk factor), $85 \%, 44 \%$, and $0 \%$ (more than 2 risk factors), respectively $(p<0.01)$.

Conclusions: In patients with low Child-Pugh score (5) and specific TACE findings (no visible arterio-venous shunt, amount of lipiodol $\leq 7 \mathrm{cc}$, without using gelfoam), TACE might be a curative therapy for patients with BCLC stage 0 and stage A HCC.

\section{PP34-12}

Added Value of Liver Gd-EOB-DTPA-enhanced MRI in Detection of Small $(<\mathbf{2} \mathbf{~ c m}) \mathbf{H C C}$ in Cirrhosis

V. Lucidi, M. Renzulli, B. Corcioni, R. Golfieri

Radiology Unit, Department of Digestive Diseases and Internal Medicine, Sant' Orsola-Malpighi Hospital, University of Bologna, Bologna, Italy

Background/aims: The aim of this single center prospective study is to demonstrate the added value of the hepatobiliary (HB) phase of Gd-EOB-DTPA-enhanced MRI in identifying small $\mathrm{HCC}(<2 \mathrm{~cm})$ undetected at Dynamic phase by itself due to their atypical pattern, according to the AASLD criteria.

Methods: 127 MRI of cirrhotic patients (pts), performed between May 2008 and November 2009, were prospectively evaluated: 215 nodules were detected, 149 of which $(69 \%)$ demonstrate typical HCC pattern in 81 pts $(64 \%), 4$ (2\%) with "mosaic" HCC pattern in 4 pts (3\%) and 62 nodules (29\%) with atypical pattern for HCC in 42 pts $(36 \%)$. The gold standard was the histological assessment in all atypical nodules.

Results: Atypical nodules at Dynamic MRI (absence of arterial hypervascularity or portal-venous washout) were found in $29 \%$ (36\% of pts) and they represented High-grade dysplastic Nodules (DN)/early HCC $(n=20)$, low-grade DN $(n=21)$, regenerative nodules $(n=17)$ and nodular regenerative hyperplasia $(n=4)$. The HB phase identified 19/20 High-grade DN/hypointense small HCC (mean diameter $16 \mathrm{~mm}$, range $11-19 \mathrm{~mm}$ ) in 10 pts unidentified by dynamic MRI. The sensitivity of Dynamic MRI for High grade DN/early HCC was $88 \%(149 / 169)$ and that of combined Dynamic and HB phases was $99 \%$ (168/169), with an added value of $11 \%$.

Conclusions: Gd-EOB-DTPA-enhanced MRI of the cirrhotic liver is $11 \%$ more sensitive in the detection of High grade DN/early HCC (99\%) as compared to dynamic MRI alone (88\%). The high incidence of atypical nodules in our series (36\% of pts), suggest the extensive employment of Gd-EOB-DTPA-enhanced MRI in the follow-up of small nodules detected at screening ultrasound. In conclusion, a high prevalence $(36 \%)$ of atypical pattern among $\mathrm{HCC}<2 \mathrm{~cm}$, and the superior sensitivity of Gd-EOB-DTPA-MRI than Dynamic-MRI in HGDN/early HCC detection of these nodules was demonstrated. 


\section{PP34-13}

Our Experience with Follow-up of Hepatocellular Carcinoma after Chemoembolisation using Contrast-enhanced Ultrasonography (CEUS) Compared with Computer Tomography (CT)

F. Charvat, H. Parobkova

Department of Radiology, Central Military Hospital, Prague, Czech Republic

Aims: A transarterial chemoembolisation (TACE) is often used for treatment of unresecable hepatocellular carcinoma. Our aims are to evaluate the contribution of CEUS for follow-up treatments effect and to compare it with the CT imaging.

Methods: A group of nine patiens with solitary, unresecable hepatocellular carcinoma underwent TACE with the aim of tumor necrosis during 16 months long period. A necrotic part of tumor evaluated as the lack of contrast enhancement at CEUS and CT images performed one month after chemoembolisation was considered as success criteria of used treatment.

Results: The control imaging embolised tumors of four patients depicted complete lack of contrast enhancement. The same results were recorded in second control imaging 3 months ago. In other five cases was described the incomplete necrosis with residual enhancing tumor parts. These patients underwent second TACE and imaging follow-up after 1 month. In one case was performed for very small tumor necrosis the third TACE, two patiens had the complete tumor necrosis. Last patient with incomplete necrosis underwent control imaging after five months. The result showed the complete lack of enhancement.

Conclusion: In the evaluation of tumor necrosis after TACE seems to be CEUS a fully comparable imaging method to the CT. Its major advantages are in possibility to image the vascularisation of lesions in real time and the lack of radiation exposure.

\section{PP34-14}

Spontaneous Regression of HCC: A Case Report

M. Kohla ${ }^{1}$, I. Waked ${ }^{1}$, M. El-Warraky ${ }^{2}$, A. Ibrahim ${ }^{1}$, R. Gish ${ }^{3}$

${ }^{1}$ Hepatology; ${ }^{2}$ Radiology, Nationa Liver Institute, Shebeen El-Kom, Egypt; ${ }^{3}$ Hepatology, UCSD, San Diego, CA, USA

Introduction: Spontaneous regression of $\mathrm{HCC}$ is extremely rare. This event could be explained by strong and persistent activation of the immune system directed against the neoplastic cells or sudden change in vascular inflow resulting in autoinfarction.

Case description: We report herein a case of 51-year old man presented in March 2010 with HCV-induced Child A compensated cirrhosis. Ultrasound revealed an ill-defined right lobe lesion approximately $7 \times 8 \mathrm{~cm}$ in diameter, involving segments VII and VIII with portal vein thrombosis, AFP was $19,309 \mathrm{ng} / \mathrm{ml}$. Triphasic CT (Fig. 1) revealed a large invasive right lobe mass in segment IV,V,VI,VIII with an arterial blush and rapid washout pattern diagnostic for $\mathrm{HCC}$, and right portal vein thrombosis. The patient did not receive any specific treatment. Triphasic CT in June (Fig. 2), demonstrated a marked decrease in tumor size $(4 \times 3.8 \mathrm{~cm})$. The thrombosed right portal vein showed cavernous transformation. AFP was $1,923 \mathrm{ng} / \mathrm{ml}$. In conclusion, we report a rare spontaneous event in a patient with HCC, that resulted in a marked decrease in the tumor size and neovascularization. This rare event, which can change patients prognosis substantially, needs to be recognized by clinicians who monitor HCC patients both on and off treatment as well as in clinical trials.

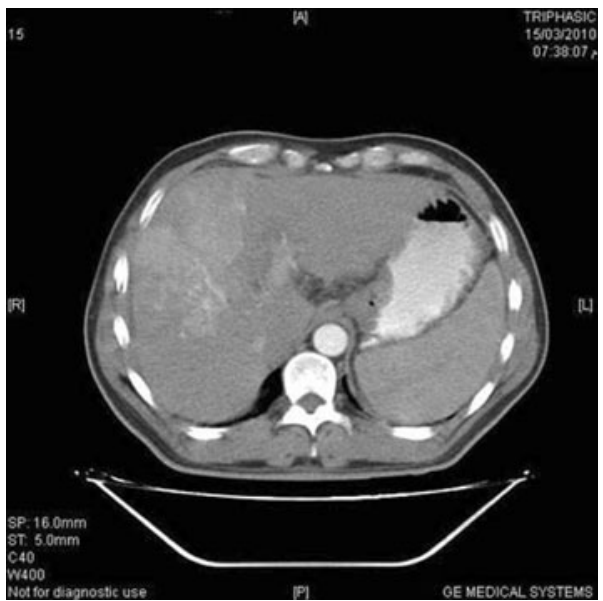

Fig. 1

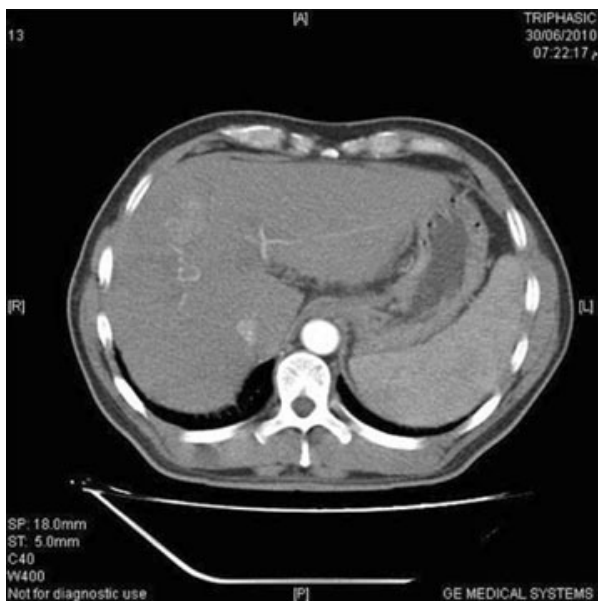

Fig. 2

\section{PP34-15}

Metastasis versus Focal Eosinophilic Infiltration in the Liver: Comparison of MRI Findings

K.M. Lee, J.K. Kim

Ajou University School of Medicine, Suwonsi, Republic of Korea

Aims: To compare the MRI findings of focal hepatic eosinophilic infiltration and hepatic metastasis.

Methods: Contrast enhanced MR images of 40 lesions in 15 patients with focal hepatic eosinophilic infiltration and 34 lesions in 17 patients with liver metastasis were reviewed retrospectively. The size, number and location of focal lesions were analyzed. The lesion-toliver contrast difference of each lesion was recorded using region of interest (ROI) curve on T1 - and T2-weighted images. Enhancement pattern was classified into homogenous, rim-enhancement and heterogeneous enhancement. All the images were reviewed by two gastrointestinal radiologists in consensus. 
Results: Focal eosinophilic infiltration and hepatic metastasis showed predominantly low signal intensity on T1WI and high signal intensity on T2WI. However, focal eosinophilic infiltration manifested as iso signal intensity, more frequently than hepatic metastasis on T1WI (42.5 vs. 0\%). Metastasis showed more frequently high signal intensity on portal (94.1 vs. $57.5 \%$ ) and delayed phase (94.1 vs. $45.0 \%$ ) comparing with focal eosinophilic infiltration. The lesion-toliver contrast was greater in metastasis on both images(19 \pm 17 vs. $8 \pm 6$ on T1WI and $21 \pm 19$ vs. $13 \pm 10$ on T2WI). Rim enhancement pattern is more frequently seen in metastasis on all phases. Seventeen (43\%) focal eosinophilic infiltrations showed rim enhancement on arterial or portal venous phase and homogenous enhancement on delayed phase.

Conclusion: MRI findings to favor eosinophilic infiltration include subcapsular location, lesser lesion-to-liver contrast on T1 and T2 weighted image and early rim enhancement with layered homogenous enhancement.

\section{PP34-16}

Identification of Helicobacter Hepaticus Infection in Various Strains Mice from China

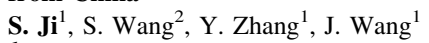

${ }^{1}$ China-Japan Union Hospital, Jilin University; ${ }^{2} J i$ Lin University, Changchun, China

Objective: To identity the prevalence of Helicobacter hepaticus $(H$. hepaticus) infection in various strains mice from commercial and academic institutions in different regions of China.

Methods: We tested the prevalence of serum anti-H.hepaticus-IgG and feces H.hepaticus antigen in 114 various strains mice from different regions of China. The feces received from all mice were srceened for Helicobacter genus-specific 16SrRNA and H.hepaticus species-specific 16SrRNA by PCR and were cultured and identitied for Helicobacter species. With above methods, we could analyze the H.hepaticus infection.

Results: 25 (21.9\%) mice were infected with Helicobacter species in 114 mice and $44.0 \%(11 / 25)$ were H.hepaticus infection, and SCID mice and $\mathrm{C} 3 \mathrm{H}$ mice were the susceptible strains. At the same time, the other positive PCR amplicons of Helicobacter genus-specific 16SrRNA with $H$. hepaticus species-specific 16SrRNA negative were sequenced and it was found that other Helicobacter species could infect the laboratory mice.

Conclusions: In China, Helicobacter species and H.hepaticus could infect various strains mice, and H.hepaticus was the main strain infected mice, but also there might be exist other Helicobacter species infected mice.

\section{PP34-17}

Evaluation of Predictive Value of MELD, Child-trcotte-Pugh, Okuda, CLIP and TNM Staging for Prediction of Survival in Hepatocellular Carcinoma

P. Pruksakorn, A. Leerapun, S. Thongsawat, T. Chitapanarux, P. Pisespongsa, O. Praisontarangkul

Internal Medicine, Chiang Mai University, Chiangmai, Thailand

Background: Presentation of HCC is very heterogeneous and different in prognosis. The number systems have been proposed to predict the prognosis for hepatocellular carcinoma (HCC), no consensus as to which staging system is best. The aim of this study was to evaluate the predictor of survival among HCC patients.

Method: A retrospective analysis of the 220 patients with HCC diagnoses from 2000 to 2006 was preformed. Predictors of survival were identified using Kaplan-Meir method and Cox model for comparing those of clinical staging system including; MELD, CTP score, Okuda, CLIP and TNM staging.
Result: Overall median survival was 5 months and survival rate at 1 year was only $33.4 \%$. The majority of patients had Okuda stage II, CLIP stage III-V and TNM stage III. Patients with MELD score $\leq 10$ had significantly better survival compare with those who had MELD score $>10(P=0.004)$. The MELD score, Okuda staging system, presence of AFP $>400 \mathrm{ng} / \mathrm{ml}$ and ALT $>40 \mathrm{U} / \mathrm{L}$ are independent predictors of survival in HCC patients. $(P<0.05)$

Conclusion: The system that utilize the liver function factors along with the tumor factor should do the best in predicting outcome of HCC, whether the survival or the response to any modalities of treatment, systemic or loco-regional.

\section{PP34-18}

Correlation of Quality of Life of Hepatocellular Carcinoma Patients with Severity of Underlying Chronic Liver Disease-A Prospective Study L.V. $\mathrm{Li}^{1}$, W. Yeo ${ }^{2}$, S.L. Chan ${ }^{2}$, J. Koh ${ }^{2}$, E.P. Hui ${ }^{1}$, F.K. $\mathrm{Mo}^{2}$

${ }^{1}$ Prince of Wales Hospital; ${ }^{2}$ Chinese University of Hong Kong, Hong Kong, Hong Kong S.A.R.

Background: Patients with advanced hepatocellular carcinoma (HCC) have been reported to have poor prognosis. For the majority, only palliative treatment could be offered. One of the main objectives in palliative therapy is to maintain or improve patients' quality of life (QOL), and hence QOL assessment is an important integral in the care of these patients. Apart from a general QOL assessment tool for cancer patients irrespective of their primary malignancies, a more disease-specific QOL assessment tool may increase the sensitivity of individual disease assessment. In HCC, the EORTC QLQHCC18 questionnaire is an HCC-specific tool which may have a better reflection of QOL in HCC patients with chronic liver disease. The objectives of this prospective study are to assess correlation between QOL factors using QLQ-HCC18 and the severity of cirrhosis.

Methods: Two hundred patients with newly diagnosed HCC were prospectively recruited. Patients' baseline QOL assessment, clinical factors, staging and treatment were collected prior to any interventional therapy. The correlations between QOL factors and stage of $\mathrm{HCC}$ and severity of cirrhosis were analysed by Wilcoxon rank-sum test.

Results: Median overall survival of the 200 patients was 8.1 months (95\% CI 6.2-9.6 months). Six out of 8 domains in EORTC QLQHCC18 were significantly correlated with Child's classification $(p<0.04)$; these included fatigue, body image, jaundice, nutrition, pain and abdominal swelling.

Conclusions: This study was the first to report the QOL data of untreated HCC patients using EORTC QLQ-HCC18 module. There were significant correlations between worse QOL scores in QLQHCC18 and advanced cirrhosis (Child-Pugh's classification). Worse QOL scores correlate significantly with advanced cirrhosis.

\section{PP34-19}

The Decreased Expression of TLR9 on NK Lymphocytes in Patients with Primary Hepatocellular Carcinoma

W. Li, Q. Jin, Y. Gao, M. Wang, J. Niu

The First Hospital of Jilin University, Changchun, China

Objective: The mechanism of NK cell dysfunction in primary hepatocellular carcinoma(HCC) is poorly understood.We hypothesized that there was the abnormity of TLR9 ${ }^{+} \mathrm{NK}$ lymphocyte in HCC.Our aims to study the phenotype of peripheral B cells and NK cell-related cytokine IFN-gamma in HCC patients.

Methods: NK cell phenotyping (CD3-CD56+TLR9+)and intracellular IFN-gamma were performed by FACS in $35 \mathrm{HCC}$ patients $(15$ $\mathrm{HBV}$ infection, $10 \mathrm{HCV}$ infection, and 10 alcohol-associated liver disease) and 30 healthy controls. Clinical parameters including serum 
ALT,AST,viral loads,AFP as well as blood routine examinations were evaluated.

Results: TLR9 ${ }^{+} \mathrm{NK}$ lymphocytes of HCC patients was much less than that of healthy controls $(\mathrm{p}=0.035)$.A inverse correlation was obtained between TLR $9^{+} \mathrm{NK}$ lymphocyte cells and ALT levels $(R=-0.536$, $p=0.033)$. The level of intracellular IFN-gamma in HCC patients was decreased dramatically $(p=0.048)$. There was no correlation between the percentage of TLR9 ${ }^{+} \mathrm{NK}$ lymphocytes and the level of IFN-gamma. The TLR9 ${ }^{+} \mathrm{NK}$ lymphocytes and IFN-gamma level showed no difference between the HCC patients of different causes (Table 1).

Table 1 TLR9+NK lymphocytes percentage and intrace

\begin{tabular}{|c|c|c|c|}
\hline Indicators & $\mathrm{HCC}$ & Healthy control & $P$ \\
\hline $\begin{array}{l}\text { TLR9+NK } \\
\text { lymphocytes (\%) }\end{array}$ & $32.64(18.03-49.71)$ & $40.1(20.63-72.6)$ & 00 \\
\hline IFN-gamma (\%) & $20.23(10.1-37.5)$ & $30.3(19.91-45.64)$ & 0.0 \\
\hline
\end{tabular}

Data are expressed as median(range).

Conclusions: TLR9 ${ }^{+} \mathrm{NK}$ lymphocytes are depleted in peripheral blood of HCC subjects, which predicts a innate immune defect. The loss of TLR9 ${ }^{+} \mathrm{NK}$ lymphocytes may contribute to $\mathrm{HCC}$ instead of virus or alcohol,and has no relation with the cytokine secretion.

\section{PP34-20}

Tissue Expression of Squamous Cellular Carcinoma Antigen (SCCA) and Ki67 in Hepatocellular Carcinoma - Correlation with Prognosis Z. Ben Ari ${ }^{1,2}$, A. Tovar ${ }^{3}$, M. Halpern ${ }^{4}$, I. Levy ${ }^{5}$, E. Shabtai ${ }^{6}$, H. SchmilovitzWeiss $^{2,7}$

${ }^{1}$ Liver Institute, Rabin Medical Center, Beilinson Hospital, Petah

Tiqwa; ${ }^{2}$ Sackler Faculty of Medicine, Tel Aviv University, Tel Aviv; ${ }^{3}$ Histopathology, Rabin Medical Center, Beilinson Hospital;

${ }^{4}$ Histopathology, Rabin Medical Center, Hasharon Hospital, Petah Tiqwa; ${ }^{5}$ Liver Unit, Hadassah Medical Center, The Hebrew University School of Medicine, Jerusalem; ${ }^{6}$ Statistical Service, Tel Aviv Sourasky Medical Center, Tel Aviv; ${ }^{7}$ Gastroenterology Unit, Rabin Medical Center, Hasharon Hospital, Petah Tiqwa, Israel

Dysregulation of the balance between proliferation and cell death represents a pro-tumorigenic principle in human hepatocarcinogenesis. Squamous cellular carcinoma antigen (SCCA), has been reported to be over-expressed in HCC tissue from patients with HCC. Ki-67 (a marker of cell proliferation) is associated with higher mitotic activity in HCC. The aim of this study was to assess hepatic SCCA and Ki67 immunohistochemistry in a large series of HCCs and correlate findings with prognosis. Sixty one HCC patients were included in the study. Serum $\alpha$-fetoprotein (AFP) level was determined and liver specimens for SCCA and Ki67 immunohistochemistry were obtained in all patients either by ultrasound-guided for diagnostic purposes $(n=17)$ or surgically in patients who underwent hepatic resection $(n=27)$ or liver transplantation $(n=17)$. Hepatic immunohistochemistry staining for AFP and the TUNEL assay (for the detection of apoptosis) were performed as well. Hepatic SCCA immunostaining was detected in $83.6 \%$ of HCCs specimens. Mean hepatic ki67 score was significantly higher in negative versus positive hepatic SCCA immunostaining $(p=0.017)$. A statistical significant correlation was found between negative SCCA staining and mortality $(p=0.026)$. Positive hepatic SCCA staining was associated with well and moderate differentiated tumors $(p=0.022)$. Using multiple logistic regression analysis, hepatic Ki67 immunostaining and TUNEL assay were significant independent predictors of negative hepatic SCCA immunostaining. The $\mathrm{C}$ parameter (area under the ROC curve) was 0.87. Kaplan Meier survival analysis revealed a significant difference between the two patient groups (positive and negative hepatic SCCA immunostaining) in survival time $(p=0.0106)$. Cox proportional hazard regression analysis demonstrated that hepatic ki67 immunostaining, liver transplantation and hepatic resection as treatment modalities were independently associated with mortality. In conclusion, SCCA is overexpressed in HCC and could represent a useful marker for the detection of HCC. Its status is associated with cell proliferation, apoptosis and survival. Hepatic ki67 immunostaining can predict survival.

\section{PP34-21}

Rapid Measurement of Indocyanine Green Clearance by Pulse Spectrophotometry-A Validation Study in 70 Patients with Child-pugh A Liver Function

T.T. Cheung, S.C. Chan, R.T.P. Poon, C.M. Lo, S.T. Fan Surgery, the University of Hong Kong, Hong Kong, Hong Kong S.A.R.

Background: Inadequate liver functional reserve is the greatest barrier for major hepatectomy in patients with hepatocellular carcinoma and liver cirrhosis. The development of pulse spectrophotometry provides a faster alternative for ICG measurement but its accuracy in for preoperative assessment is not well documented as most of the previous studies consisted of a heterogenous group of patients in the critically ill patients. This study compared the result of non-invasive liver function monitoring using the LiMON (Pulsion Medical System, Munich, Germany) and traditional spectrophotometry by serial blood sampling in patient with Pugh Child A score before major hepatic resection.

Study design: From September 2008 to Jan 2009, 70 patients with Pugh Child A score were enrolled for investigations. Their ICG clearance at $15 \mathrm{~min}$ were measured by traditional spectrophotometry with serial blood sampling and the LiMON. The results were compared.

Results: The median ICG retention at 15 min by spectrophotometry was $14.748 \%$. The median ICGR15 measured by LiMON was 10.38 . The mean difference between the LiMON and spectrophotometry was -4.3606 . There was a strong correlation between the 2 measurement methods (correlation coefficient $0.844,95 \% \mathrm{CI}=0.762,0.899$ ). The ICGR 15 measured by LiMON, the ICG15 measured by traditional method can be calculated using the following formula. ICG15= $1.16 *$ ICGR $15+2.73$

Conclusions: The LiMON is a portable unit which provides a fast and repeatable method to evaluate the ICG clearance at 15 min for patient with Pugh Child A Score. Adjustment of result is necessary in order to achieve a comparable result obtained by spectrophotometry.

\section{PP34-22}

The Natural History and Growth of Hepatocellular Carcinoma in the Elderly

S. Uegaki, M. Sasaki, A. Akahide, K. Nakajima, M. Tobari, Y. Ushio, K. Kino Department of Gastroenterology, Tokyo Metropolitan Geriatric Hospital, Tokyo, Japan

Background and aims: As patients with chronic liver disease are rapidly getting older in Japan, it is not uncommon that hepatocellular carcinoma (HCC) are found in the elderly. However the natural history of HCC in the elderly has not been well clarified. In the current study we examined natural history of HCC in the elderly who were found to develop HCC and to be followed without any treatment, due to various clinical reasons.

Patients and methods: Fifty-five cases with HCC, clinically diagnosed and confirmed by autopsy, were noted in our hospital from 
1995 to 2009. Among them 9 HCC cases were followed up from diagnosis without any therapeutic procedures for HCC due to other concomitant diseases or denial for treatment, and clinical characteristics of these 9 cases were closely examined in the current study.

Results: Male/female were $6 / 3$, and the mean age at the diagnosis of $\mathrm{HCC}$ was $72 \pm 11$. The etiology for HCC included $2 \mathrm{HBV}, 4$ $\mathrm{HCV}, 2$ alcoholics and 1 undetermined. The observation period from diagnosis until autopsy was 37.2 months [8-166]. Staging of HCC (I/II/III/IV) at diagnosis and at autopsy was $1 / 0 / 2 / 6$ and $0 / 0 / 2 /$ 7. The diameter of the maximal tumor at diagnosis and at autopsy was $5.9 \mathrm{~cm} \mathrm{[1-15]} \mathrm{and} 10.3 \mathrm{~cm} \mathrm{[5-20],} \mathrm{respectively,} \mathrm{indicating}$ remarkable growth of the tumors. The average growing rate was $2.9 \mathrm{~cm}$ per a year, and the maximum was $6 \mathrm{~cm}$ per a year in a female patient. The cause for mortality was hepatic failure in 5 patients, rupture of $\mathrm{HCC}$ in 2, rupture of varices in 1 and GI bleeding in 1 .

Conclusion: In our experiences, HCC patients in the elderly could survive for 3 years in average without any treatment, even diagnosed at stage IV. The growth of the tumor was remarkable, $3 \mathrm{~cm}$ per a year, and was very rapid in some cases.

\section{PP34-23}

Population-level Prevention of Hepatitis B-related HCC in Australia-The Primary Care Perspective

M. Robotin ${ }^{1,2}$, Y. Patton ${ }^{3}$, V. Putha ${ }^{4}$, J. George ${ }^{1,5}$

${ }^{1}$ School of Medicine, University of Sydney; ${ }^{2}$ Research and Scientific Development, Cancer Council NSW; ${ }^{3}$ Biostatistics, Macquarie University; ${ }^{4}$ Community Medicine, University of NSW; ${ }^{5}$ Storr Liver Unit, Millenium Institute, Sydney, NSW, Australia

Background: Approximately $70 \%$ of hepatitis-B related hepatocellular cancers (HCC) cases in Australia are diagnosed in people born in hepatitis B-endemic infection countries. Informed by economic modeling suggesting that antiviral therapy is more cost-effective than routine hepatitis care, we developed a pilot community-based program aiming to optimize the detection and management of chronic hepatitis B $(\mathrm{CHB})$ in at-risk populations in Sydney, Australia.

Methods: Free hepatitis B (HBV) educational seminars were offered to all general practitioners (GPs) in the program area. Participants completed a baseline knowledge survey on CHB and HCC, using a validated, anonymous questionnaire. Overall knowledge scores were compared, using the 2-sample t- test. Subsequently, we conducted semi-structured interviews with seminar participants, seeking their views on determinants of effective GP-based programs and used the responses to inform program evaluation.

Results: 69 GPs attended $\geq 1$ educational seminars; questionnaire response rates exceeded $95 \%$. Most $(92 \%)$ participants self-identified as Asian-Australians, worked full time (67\%), had graduated $\geq 20$ years previously $(68 \%)$ and cared for few patients with $\mathrm{CHB}$ $(57 \%)$. Mean knowledge score (64\%) did not vary by career stage, work pattern or number of CHB patients cared for. Most GPs (90\%) knew that $\mathrm{CHB}$ prevalence is higher in Chinese migrants; fewer $(42 \%)$ knew the test used to screen for CHB. 61 participants (response rate $88 \%$ ) were subsequently interviewed about program recruitment. Expected clinical benefit (83\%) and GP interest in research $(40 \%)$ were deemed important facilitators; patient disinterest (51\%), time constraints (39\%) and complex paperwork (33\%) were key disincentives. Additional suggestions included web-based enrolment (83\%), provision of administrative support (43\%) and offering financial incentives (23\%).

Conclusion: The HBV knowledge survey suggested additional GP support and CHB information are critical to improve CHB screening and management in primary care. GPs feedback led to program redesign, closing the loop from research to program implementation.
PP34-24

Symptom Experience of Patients with Advanced Hepatocellular Carcinoma (HCC) in Hong Kong

C. Chan ${ }^{1}$, J.Y.C. Tong ${ }^{2}$, S.Y. Chair ${ }^{1}$, K.C. Choi ${ }^{1}$, W.Y. ${ }^{1}{ }^{1}$, J.W.H. Sit ${ }^{1}$, W.K.W. So ${ }^{1}$

${ }^{1}$ The Chinese University of Hong Kong; ${ }^{2}$ Prince of Wales Hospital, Hong Kong, Hong Kong S.A.R.

Background and aim: Hepatocellular Carcinoma (HCC) is one of the major causes of cancer deaths in Hong Kong. Palliative treatment modalities are commonly used in about $80 \%$ of HCC patients. Although these palliative treatments have increased patient's survival rate, they damage normal liver function during the treatment. This study aimed to assess the symptoms and health problems among advanced HCC patients and identify factors (demographics, treatments, clinical data, and laboratory results) affecting their symptom experience.

Methods: A cross-sectional quantitative design was adopted to examine descriptive, comparison and association data related to symptoms and health problems. Data were collected from 85 HCC patients in a public funded hospital using The European Organization for Research and Treatment of Cancer (EORTC) HCC18 Chinese Version. It consisted of 18 questions assessing fatigue, body image, jaundice, nutrition, pain, fever, sexual life and abdominal swelling.

Results: Patients experienced greatest distress in sexual life (mean $54.9 / 100$ ), followed by fatigue (mean $44.8 / 100$ ). When comparing the four treatment groups of patients: Transarterial Chemoembolization (TACE), Percutaneous Ethanol Injection (PEI), Radiofrequency Ablation (RFA), and Supportive Care, patients in Supportive Care Group had significant poorer symptoms/problems in all items $(p<0.005$, Kruskal Wallis test). Patients below age 60 experienced more sexual problems $(p<0.001)$ but less fever $(p<0.05)$. Elevation of Alpha Feto Protein (AFP) and bilirubin, and lower albumin level were factors associated with poorer symptoms/problems $(p<0.05$, Spearman's rank order correlations).

Conclusion: Group differences (e.g. treatment, age, liver function) found in this study can be used by health care professionals to identify at risk groups and stratify the patients for unique plan of care. Interventions focusing on promoting liver functions and sexual health are suggested.

\section{PP34-25}

GIDEON (Global Investigation of Therapeutic Decisions in HCC and of its Treatment with Sorafenib) Interim Results: Child-pugh Status Subgroup Analysis

S.-L. Ye ${ }^{1}$, J. Marrero ${ }^{2}$, R. Lencioni ${ }^{3}$, A. Venook ${ }^{4}$, K. Nakajima ${ }^{5}$, M. Kudo ${ }^{6}$ ${ }^{1}$ Liver Cancer Institute, Zhongshan Hospital, Fudan University, Shanghai, China; ${ }^{2}$ Multidisciplinary Liver Tumor Clinic, University of Michigan, Ann Arbor, MI, USA; ${ }^{3}$ Division of Diagnostic Imaging and Intervention, Department of Liver Transplantation, Hepatology and Infectious Diseases, Pisa University School of Medicine, Pisa, Italy; ${ }^{4}$ University of California, San Francisco, CA, USA; ${ }^{5}$ Global Medical Affairs, Bayer HealthCare Pharmaceuticals, Montville, NJ, USA; ${ }^{6}$ Department of Gastroenterology and Hepatology, Kinki University School of Medicine, Osaka, Japan

Purpose: GIDEON is an ongoing, global, prospective, non-interventional study of patients with unresectable HCC (uHCC). The aim of GIDEON is to evaluate safety/efficacy of sorafenib in real-life clinical settings and evaluate patient subgroups where data are currently limited.

Materials and methods: From Jan 2009 to Oct 2010, >2,300 patients were enrolled of the planned 3000 patients; the first pre-planned interim analysis was triggered at $\geq 4$ months follow-up in approximately the initial 500 treated patients. Demographic data and medical/ 
disease history are recorded at study entry; adverse events are collected during the study. A pre-specified subanalysis by Child-Pugh status was performed.

Results: Demographics, disease characteristics and safety by ChildPugh status are presented (Tables). Of the 479 patients valid for safety, 28\%, had Child-Pugh B status at the start of sorafenib therapy. In these early data, the incidences of overall and drug-related adverse events were similar between Child-Pugh A and B subgroups, while Child-Pugh B patients had a higher incidence of serious adverse events.

Conclusions: GIDEON is providing valuable information about patients with uHCC and varying liver dysfunction. The sorafenib adverse event profile in patients with moderate liver dysfunction is as expected.

\section{Table 1}

\begin{tabular}{llll}
\hline $\begin{array}{l}\text { Demographic } \\
\text { and disease } \\
\text { characteristics }\end{array}$ & $\begin{array}{l}\text { Child-Pugh A } \\
(58 \%) \mathrm{a}\end{array}$ & $\begin{array}{l}\text { Child-Pugh B } \\
(28 \%) \mathrm{a}\end{array}$ & $\begin{array}{l}\text { Total, } \\
n=479 \mathrm{~b}\end{array}$ \\
\hline $\begin{array}{l}\text { Age, years mean } \\
(\text { range })\end{array}$ & $62(18-88)$ & $61(23-84)$ & $61(18-89)$ \\
$\begin{array}{c}\text { ECOG PS 0-1, } \\
n(\%)\end{array}$ & $236(85)$ & $102(76)$ & $380(79)$ \\
$\begin{array}{c}\text { BCLC Staging } \\
\text { B/C }, n(\%)\end{array}$ & $60 / 151(22 / 54)$ & $25 / 71(19 / 53)$ & $92 / 253(19 / 53)$ \\
\hline
\end{tabular}

Table 2

\begin{tabular}{llllll}
\hline $\begin{array}{l}\text { Adverse Events, } \\
n(\%)^{\mathrm{c}}\end{array}$ & $\begin{array}{l}\text { Child-Pugh A } \\
\begin{array}{l}n=278 \\
(58 \%)^{\mathrm{a}}\end{array}\end{array}$ & $\begin{array}{l}\text { Child-Pugh B } \\
n=134 \\
(28 \%)^{\mathrm{a}}\end{array}$ & $\begin{array}{l}\text { Total, } \\
n=479^{\mathrm{b}}\end{array}$ \\
\hline $\begin{array}{l}\text { All grades: } \\
\text { overall/drug } \\
\text { related }\end{array}$ & $193(69)$ & $122(91)$ & $84(63)$ & $415(87)$ & $319(67)$ \\
$\begin{array}{l}\text { Grade 3/4: } \\
\text { overall/drug } \\
\text { related }\end{array}$ & $65(23)$ & $52(39)$ & $31(23)$ & $161(34)$ & $109(23)$ \\
$\begin{array}{l}\text { Serious, all grades : } \\
\text { overall/drug } \\
\text { related }\end{array}$ & $28(10)$ & $80(60)$ & $22(16)$ & $201(42)$ & $51(11)$ \\
$\begin{array}{l}\text { Sorafenib discontinued } \\
\text { due to adverse } \\
\text { events, } n \text { (\%) }\end{array}$ & $53(40)$ & & $133(28)$ & \\
$\begin{array}{l}\text { Deaths during sorafenib } \\
\text { and within 30 days of } \\
\text { last dose, } n \text { (\%) }\end{array}$ & $50(37)$ & & $114(24)$ & \\
\hline
\end{tabular}

$B C L C$ Barcelona clinic liver cancer

${ }^{\text {a }}$ Percentage of total patients

${ }^{\mathrm{b}}$ Child-Pugh C pts $(n=11.2 \%)$ and pts with missing or not evaluable Child-Pugh status $(\mathrm{n}=56,12 \%)$ not included

${ }^{c}$ Treatment emergent adverse events of all grades using NCI CTCAE, v3.0 ECOG PS, Eastern Cooperative Oncology Group performance status
PP34-26

Overexpression of Tyro3 and Implications on HCC Progression

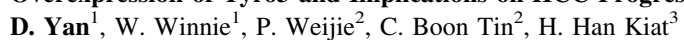

${ }^{1}$ Department of Pharmacy, National University of Singapore; ${ }^{2}$ Singapore OncoGenome Laboratory, Institute of Medical Biology, $A * S T A R ;{ }^{3}$ Department of Pharmacy, National University of Singpore, Singapore, Singapore

Background/aims: Hepatocellular carcinoma (HCC) is the sixth most common malignancy and the third leading cause of cancer deaths worldwide. The recent success of sorafenib implicated that progression of HCC may involve multiple kinases. In the search for novel multi-kinase inhibitors, our group found a very potent compound which acts on a relatively uncharacterized receptor tyrosine kinase, Tyro3. To explore the role of tyrosine kinase Tyro3 in HCC, we examined the expression of Tyro3 in HCC tumors and correlated it with clinical outcomes.

Methods: cDNAs derived from HCC patients tumor-adjacent normal tissue paired samples were quantified for Tyro3 expression by quantitative PCR. Expression profile was correlated with clinical data. Subsequent Tyro3 silencing was performed on HCC cell lines to better understand the mechanistic effect of Tyro3 on HCC progression.

Results: $44.64 \%$ of patients' tumor tissues shown overexpression of Tyro3, with more than two-fold increase compared with adjacent normal tissues. This overexpression demonstrated significant correlation with tumor size, HBV infection, AFP, AST and ALT level. Notably, patients with HBV infection have significantly higher expression of Tyro3, with more than two-fold increase compared with patients without HBV infection. Subsequent in vitro investigation revealed that Tyro3 silencing suppressed hallmarks of cancer such as a decreased cell proliferation[p1].

Conclusion: Our finding of elevated Tyro3 expression in HCC tissues implicates Tyro3 may play role in HCC progression, especially for HCC caused by HBV infection. The decrease in proliferation with Tyro3-silencing, qualifies it as a potential target for further characterization.

\section{PP34-27}

Effects of Nanosecond Pulsed Electric Field (nsPEF) on Human Liver Cancer Cells in vitro

D. Xiao, J. Cheng, L. Tang

Key Laboratory of Biorheological Science and Technology, Ministry of Education, Chongqing University, Chongqing, China

Pulsed electric fields play an important role in cancer therapy due to their special effects on cancer cells. In this study, a nanosecond electric pulsed field with high strength of $8 \mathrm{KV} / \mathrm{cm}$, durations of $450 \mathrm{~ns}$ and frequency of $1 \mathrm{HZ}$ was applied to adherent liver cancer cell line HepG2 and the liver cell line L02. Cell viability, cell apoptosis, mitochondria membrane potential $\left(\boldsymbol{\Delta} \boldsymbol{\Psi}_{\boldsymbol{m}}\right)$, and electroporation were detected using Trypan Blue exclusion, flow cytometry with AnnexinV-FITC and Propidium iodide (PI), spectrophotofluorimetry with mitochondrial membrane potential assay kit (JC-1), and fluorescence imager with Propidium iodide (PI), respectively. Results showed that electric pusles decreased cells viability significantly in both cells lines (67.3\% for HepG2 and $78.9 \%$ for L02) compared to control cells (96.2\% for HepG2 and $95.6 \%$ for L02). Results of cell apoptosis showed that electric pulses also induced cell apoptosis in both cell lines (16.94\% early apoptic cells for HepG2 and $8.92 \%$ for L02). These results suggests that electric pulses can kill cancer cells and 
HepG2 cells have more sensibility to electric field than L02 cells. To better understand the mechanism of the apoptosis induced by electric pulsed fields, changes of $\boldsymbol{\Delta} \boldsymbol{\Psi} \boldsymbol{m}$ were investigated. From our results, an obvious time dependent decrease in $\Delta \boldsymbol{\Psi} \boldsymbol{m}$ in response to nsPEFs was observed. In electroporation experiment, PI uptake in HepG2 cells emerged at $7 \mathrm{~min}$ after exposure to nsPEF and lasted for at least 4 hours, indicating the formation of electroporation on cytolemma. In conclusion, electric pulses with high strength and nanosecond durations can kill cancer cells companied with a series intracellular changes, providing a strong evidence for the application of pulsed electric fields on cancer treatment.

\section{PP34-28}

New-drug-Development for Hepatobiliary-diseases in Developing Nations: NGO's Role in Improving Access to Hepatitis Treatment \& Vaccines P. Sankpal

Community Medicine, Health Alert Organisation of India [NGO], Dhule, India

Issues: Treatment agents/drugs for hepatocellular-carcinoma out of reach of $>92 \%$ populations of Asia. Price reduction, appropriate drugsupply-chain, monitoring of chemotherapeutic-agents necessary. we need to address access to affordable treatment. Resource-poor-nations have no platform for raising these issues of hepatitis-sufferers.

Description: In-developing-countries unaffordable-chemo-cost leads to poor therapeutic-compliance. Since 2005 schemes offer discounted Anti-hepatitis-drugs, diagnostics/technical assistance scrutinized. common methodology to facilitate development of sound/sustainable Low-cost vaccine supply chain needed

Results: In last 4 years, 66 patients diagnosed for hepatobiliary-carcinoma in rural/tribal India. 14 NGOs, 28 governments, 24 privateentities. Major Lacunae is absence of co-ordination between specialized centers and primary-healthcare-workers. Drug distribution/ cost/nurses-training are neglected issues [Our NGO-OperationalPerforma handouts available to 21st APASL-Congress-Participants] Lessons learned: must identify/design newer-treatments available to masses. Specific-NGO-FORUM needed to implement/expand costcutting-measures. We Plan to expand our-NGO-Chemo-advocacyprogram to larger-population.

Recommendations: New-drug-development for hepatobiliary-carcinoma in infantile stage \& expensive in developing-nations. Vaccines hope for future. But Promoting dialogue between public-hospitals/ NGO/Pharmaceuticals must improve access to chemo-therapy. NGO's/Funding-agencies representative at APASL2011-conference need to address-this-issue. We NGO-representatives from developingnations need exposure to research treatments used by APASL seniors/ experts. Do we all failed in addressing socio-economic issues of cancer-sufferers? We need to address these socio-economic issues of affected population in resource-poor-nations.

\section{PP34-29}

The Related Study on Hepatocellular Carcinoma and Cellular Immunity Function

G. Wang

Hepatic Cirrhosis Diseases, Dalian Sixth People Hospital, Dalian, China

Objective: To investigate the clinical significance of hepatocellular carcinoma (HCC) patients' cellular immunity functions.

Methods: There were 54 cases of HCC patients from our hospital and 24 cases of sound blood donors' peripheral blood $\mathrm{CD}^{+}, \mathrm{CD}^{+}$ T-lymphocyte with APAAP, liver function, and AFP, respectively. Results: $\mathrm{CD}^{+}$and $\mathrm{CD}^{+} / \mathrm{CD}^{+}$of $\mathrm{HCC}$ patients were obviously more than control group, $P<0.05$; and $\mathrm{CD}^{+}$lower than control group, $P<0.05$. There were 18 cases of portal cancer embolus in 54 cases of HCC patients, T-lymphocytes subset significantly lower than 36 cases of non-portal cancer embolus, $P<0.05$; befor therapy, and hadn't statistically significant differences between liver cirrhosis and non- liver cirrhosis, several AFP groups.

Conclusions: HCC patients' cellular immunity functions is worse than that of sound blood donors, and it has correlation with invasive activity of tumor cell.

\section{PP34-30}

Changes of Serum HBV DNA Titer and Hepatitis Flare-up after Everolimus, a mTOR Inhibitor, in HBsAg-seropositive Advanced Hepatocellular Carcinoma Patients

C.-Y. Dai ${ }^{1,2,3}$, H.-S. Shiah ${ }^{4,5}$, J.-Y. Chang ${ }^{4}$, W.-L. Chuang ${ }^{1,3}$, L.-T. Chen ${ }^{1,3,4}$ ${ }^{I}$ Department of Internal Medicine; ${ }^{2}$ Department of Occupational Medicine, Kaohsiung Medical University Hospital, Kaohsiung

Medical University; ${ }^{3}$ Faculty of Medicine, College of Medicine, Kaohsiung Medical University, Kaohsiungl; ${ }^{4}$ National Institute of Cancer Research, National Health Research Institutes; ${ }^{5}$ Department of Internal Medicine, National Cheng Kung University Hospital, Tainan, Taiwan, $R O C$

Background: The phosphatidylinositol 3-kinase (PI3K)-protein kinase B (Akt)-mammalian target-of-rapamycin (mTOR) signaling pathway is frequently activated in hepatocellular carcinoma (HCC). A correlation exists between activation of the PI3K-Akt pathway and inhibition of hepatitis B virus (HBV) replication during hepatocarcinogenesis. We investigated the effect of everolimus on $\mathrm{HBV}$ replication in patients with advanced HCC.

Materials and methods: Serum HBV DNA level was quantified using RT-PCR assay $\left(\right.$ COBAS $^{\circledR}$ TaqMan $^{\circledR}$ ) in serial serum samples from 22 HBsAg-seropositive HCC patients receiving everolimus in a Phase I study. The median baseline ALT level was 40 (range 13-148) IU/L. Except for one patient with entecavir, none had concurrent anti-HBV therapy.

Results: Baseline HBV DNA levels was undetectable, $<10^{4}, 10^{4}-<$ $10^{6},>10^{6} \mathrm{IU} / \mathrm{mL}$ in $9,7,1$, and 5 patients, respectively. During everolimus treatment, the serum HBV DNA levels fluctuated within one $\log$ in $9(40.9 \%)$ and increased more than one $\log$ in $13(59.1 \%)$ patients. Of the latte group, HBV DNA level increased by $10^{1}-10^{2}$, $10^{2}-10^{4}$, and $>10^{4}$ folds in 8,2 , and 3 patients, respectively, and ALT level increased by 101-200 (including the one with concurrent entecavir) and $>200 \mathrm{IU} / \mathrm{L}$ in 3 and 4 patients $(53.8 \%, 7 / 13)$, respectively. Two of former three patients with increase of HBV DNA $>4 \log$ and fluctuated ALT (absolute increase value $<200$ IU/L) did not receive any anti-HBV therapy; while the latter 4 patients, everolimus was withheld and lamivudine $100 \mathrm{mg} /$ day was initiated upon absolute increase of ALT $>200$ (median serum ALT level: 470, ranged 255-656) IU/L, which resulted in a return of ALT and HBV DNA levels to baseline within $4-8$ weeks.

Conclusions: Everolimus therapy was associated with increased HBV replication in $59.1 \%$ HBsAg-seropositive HCC patients, which resulted in overt hepatitis flare-up in $53.8 \%$ patients. Prophylactic anti-HBV therapy has to be considered before starting everolimus in $\mathrm{HBsAg}$-seropositive patients.

\section{PP34-31}

Predictive Factors of Developing Hepatocellular Carcinoma in Treated HCV-carriers

Y. Cherradi, A. Rajaa, B. Hanae, M. Benazzouz, A. Essaid Department of Hepatogastroenterology-Médecine “C”, Ibn Sina Hospital, Rabat, Morocco

Background and purpose: Hepatitis "C" infection (HCV) is a real public health problem. In Morocco, it's the first major cause for hepatocellular carcinoma (HCC). Antiviral treatment limits fibrosis progression and reduces risk of developing $\mathrm{HCC}$ but few cases of 
HCC in HCV-treated patients were reported. The aim of this study is to define this population's features and to identify predictive factors of developing HCC.

Patients and methods: It's a retrospective and analytic study. It concerns all HCV-carriers who developed HCC after antiviral treatment. We compare HCV-treated patients with no developed HCC to HCC population using khi-2 and Ficher Exact analysis.

Results: $369 \mathrm{HCV}$-treated patients were considered from January 2002 to April 2010. $20 \mathrm{HCC}$ were reported with 12 female (60\%) and 8 male (40\%), ranging in age between 40 and 72 years; the mean age is 61 years. The mean time of HCC occurrence is $5 \pm 2$ years. $53 \%$ of patients were genotye $1,47 \%$ were genotype 2 and $94.6 \%$ had severe fibrosis at the beginning of treatment. SVR was achieved in $32 \%$ of patients. The risk of HCC was not significant according to gender and genotypes (respectively $p=0.63$ and $p=0.87$ ). Advanced age and severe fibrosis were significant risk factors (respectively $p=0.003$ and $p=0.0001$ ). The comparison of non-SVR patients to sustained virological responders shows significant results: $\mathrm{HCC}$ was reported in $2.6 \%$ of SVR versus $12.5 \%$ of non responders $(p=0004)$ which demonstrate that SVR reduces considerably the risk of developing HCC but screening is indicated even after SVR.

Conclusion: In our series, $5 \%$ of previously treated patients developed an HCC. Advanced age and severe fibrosis $(F>2)$ at $\mathrm{HCV}$ diagnosis are predictive factors of HCC occurrence. SVR reduces considerably the risk of developing HCC but screening is indicated even after SVR.

\section{PP34-32}

Efficacy of Prophylactic Antiviral before Transcatheter Arterial Chemoembolization for HBV DNA Negative Hepatitis B Virus-related Hepatocellular Carcinoma

J. Zhou ${ }^{1}$, H. Xiang ${ }^{2}$

${ }^{1}$ Department of Artificial Cell Key Laboratory, Third Central Hospital of Tianjin Medical University, Tianjiin; ${ }^{2}$ Third Central Hospital of Tianjin Medical University, Tianjin, China

Aims: To explore whether prophylactic antiviral is beneficial in patients before transcatheter arterial chemoembolization(TACE) for HBV DNA negative hepatitis B virus -related hepatocellular carcinoma(HCC).

Method: Sixty consecutive patients with HBV-related HCC who received TACE were retrospectively enrolled in this study. 30 patients received antiviral drugs before TACE, others did not. All patients were HBV DNA negative. Compare the HBV reactivation, the HBV DNA level, how long the HBV reactivation after TACE, the incidence of hepatitis, alanine aminotransferase (ALT) and the cumulative survival rate between these two groups.

Results: After TACE, 5 (16.7\%) of 30 patients in the prophylactic antiviral group and $12(40 \%)$ of 30 in the control group developed HBV reactivation $(P=0.045)$; $4(13.3 \%)$ patients developed hepatitis due to HBV reactivation in the prophylactic antiviral group, while no patient developed hepatitis due to HBV reactivation in the control group $(P=0.121)$. In addition, the number of overall hepatitis in the prophylactic antiviral group is $7(23.3 \%)$ patients, the control group is $14(46.7 \%)$ $(P=0.058)$. The HBV DNA level of the reactivation patients in the prophylactic antiviral group is lower than control group $(P=0.073)$, reactivation time after TACE is longer than the control group $(P=0.461)$. The ALT value in the prophylactic antiviral group is significantly lower than that in the control group $(P=0.006)$. The cumulative survival rate of patients in the prophylactic antiviral group is significantly higher than those patients in the control group, $(P=0.025)$. Conclusion: It is suggested that antiviral therapy is beneficial for patients before TACE for HBV DNA negative hepatitis B virusrelated $\mathrm{HCC}$ because it contributes to reducing the reactivation of hepatitis B virus, decreasing the incidence and the severity level of hepatitis and prolonging survival.
PP34-33

Risk of Hepatocellular Carcinoma Occurrence in Sustained Virological Responders

Y. Cherradi ${ }^{1}$, R. Afifi ${ }^{1}$, H.O. El Malki ${ }^{2}$, A. El Akkaoui ${ }^{1}$, H. Benbrahim ${ }^{1}$, M. Benazzouz $^{1}$, A. Essaid ${ }^{1}$

${ }^{1}$ Department of Hepatogastroenterology -Médecine" C", Ibn Sina Hospital, ${ }^{2}$ Laboratory of Clinical Research, Mohammed V University, Rabat, Morocco

Introduction: Sustained virological response (SVR) is the major endpoint of antiviral treatment in hepatitis " $C$ " population. It limits fibrosis progression and reduces significantly risk of developing hepatocellular carcinoma (HCC). In our series, HCC was reported in $12.5 \%$ of non-SVR patients vs. $2.6 \%$ of sustained virological responders $(p=0.004)$.The aim of this study is to determinate risk factors of developing HCC after SVR.

Patients and methods: It's a monocentric study. We included consecutives sustained virological responders with at least 03 years follow-up. We considered age of HCV diagnosis, gender, baseline viral loads (BVL), genotype, necro-inflammatory activity and fibrosis degrees at the beginning of antiviral treatment. We compare sustained virological responders with no developed HCC to HCC population using Pearson chi-square test.

Results: On $369 \mathrm{HCV}$-carriers treated in our department since 2002-2010, SVR was achieved in $70 \%$. on the 20 patients who developed HCC, 06 were previously sustained virological responders. They were 3 male and 3 female, ranging in age between 51 and 70 years. Mean age is 60 years. 5 patients were HCV-2 and one patient was HCV-1. All patients were in severe fibrosis (F3-F4). Mean time of follow up was 6 years [3-9]. The risk of HCC occurrence in sustained virological responders was not significant according to gender, genotypes and initial high viral loads (respectively $p=1, p=0.4$ and $p=0.57)$. High necro-inflammatory activity didn't influence hepatocarcinogenesis. Severe fibrosis was the only significant risk factor $(p=0.01)$.

Conclusion: SVR reduces considerably HCC occurrence. Screening is indicated even after SVR; patients with severe fibrosis (F3-F4) must be considered carefully.

\section{PP34-34}

Meta-analysis of 111 Patients with Nonalcoholic Steatohepatitis-associated Hepatocellular Carcinoma

X.Y. Duan, J.G. Fan

Xinhua Hospital, Shanghai Jiao-tong University School of Medicine, Shanghai, China

Objective: To summarize the clinical features of patients with nonalcoholic steatohepatitis (NASH)-associated hepatacellular carcinoma (HCC).

Methods: Systematic analysis of clinical data of 111 patients with NASH-associated HCC from 22 studies published between 1990 and 2010 by searching Pubmed, Medline, OVID databases.

Results: 69 patients were male (62\%). Mean age at onset of abnormal liver function test and diagnosis of NASH and HCC was respectively 60, 63.9 and 66.7 years. Most patients were obese (79\%) and diabetic (78\%), $33 \%$ of patients had dyslipidemia and $52 \%$ had hypertension. $98.6 \%(71 / 72)$ of cases complicated with at least one metabolic disease. $71 \%$ of patients had a single nodule, the tumors ranged from 1.0 to $13 \mathrm{~cm}$ in diameter (mean $3.3 \mathrm{~cm}$ ) and often showed well to moderately-differentiated HCC with steatosis and Mallory bodies. Most nodules located in the right lobe of liver. 33\% of cases showed non-cirrhosis in non-cancerous areas. Most patients had no abnormal clinical manifestations but found tumor by routine health examination. $66 \%$ of cases exhibited elevation of AFP and/or PIVKAII. 51\% (38/74) of cases underwent hepatectomy, 25\% (19/74) received liver transplantation. During the mean follow-up of 24 months, $21 \%$ of patients died (11/51), 15\% recurred. 
Conclusions: NASH-associated HCC was common in male. These patients were usually accompanied by metabolic diseases. Although most patients had good tumor differentiation and high radical resection rate, they might not find tumor until late stage, which caused relatively poor prognosis because of indolent progressive course of NASH. Regular screening for HCC is necessary for NASH patients, especially the elderly men with multiple metabolic disorders.

\section{PP34-35}

To Reveal the Genetic Commonness of Hepatocellular Carcinoma Cases Using Pooled DNA

H. Chen ${ }^{1}$, M. Jin ${ }^{2}$, F. Wang ${ }^{1}$

${ }^{1}$ Infectious Disease; ${ }^{2}$ Pathology, First Hospital, Jilin University, Changchun, China

Background: Hepatocellular carcinoma (HCC) has the nature of the heterogeneous. It is necessary to establish robust methods capable of of quickly identifying chromosome regions associated to hepatocarcinogenesis and prognostic. In this study, we constructed DNA pool of 42 Chinese HCC patients and then applied microarray analysis to estimate possible common genetic variation of Chinese HCC cases by searching whole genome level and to reveal if the pooling-based genomewide analysis work well in tumor study.

Methods: DNA samples of $42 \mathrm{HCC}$ cases were obtained. The DNA pool was constructed by mixing equal amount of DNA from each sample, and then mixed well. Then do microarray analysis of the pooled DNA and the individual 42 DNA samples. We drew CNV DNA MAP of pooled DNA and CNV DNA MAP of all the 42cases together.

Result: From the CNV DNA MAP of pooled DNA we can find Loss of $4 \mathrm{q}, 8 \mathrm{p}, 13 \mathrm{q}, 16 \mathrm{q}, 17 \mathrm{p}, \mathrm{chr} 21$, gain of 1q, 5p, 6p, 7q, 8q, 17q 19q, 20q, all these imbalance show significant changes in the CNV DNA MAP of all the 42cases together. Compare the two maps, we can found that among all the HCC cases, if more than $24-26 \%$ cases have loss of the same area and more than $17 \%$ cases have gain of same area, the changes can be detect in the pooling DNA map.

Conclusion: Microarray analysis of HCC pooled DNA has the potential to provide reliability of detecting significant genomic aberrations.

\section{PP34-36}

Microarray Analysis of Hepatocellular Carcinoma Using FFPE Samples H. Chen ${ }^{1}$, F. Wang ${ }^{1}$, M. Jin ${ }^{2}$

${ }^{1}$ Infectious Disease; ${ }^{2}$ Pathology, First Hospital, Jilin University, Changchun, China

Background:Formalin-fixed, paraffin-embedded (FFPE) tissues represent an invaluable resource for cancer research. Array comparative genomic hybridization (aCGH) is a valuable tool for identifying DNA copy number changes in tumor genomes. Our study's aim was to reveal possible common chromosomal regions of Hepatocellular Carcinoma (HCC) DNA that may harbor genes responsible for HCC by searching whole genome level using oligoarray $\mathrm{CGH}$, and to reveal the efficiant of FFPE samples.

Methods: A total of $120 \mathrm{HCC}$ tumor FFPE samples were collected from the First Hospital of Jilin University, Changchun, P.R. China. This study was approved by Ethnic Committee of the Hospital. Genomic DNA was extracted from FFPE tissues according to the optimized protocol which Roch recommended. To quality control the DNA samples,we use NanoDrop Spectrophotometer and run 1\% agarose gel.Whole genomic DNA microarray (Nimblegen $385 \mathrm{k}$ ) were performed on good quality DNA samples.

Results: 72 Genomic DNA of good quality were obtained from 120 FFPE samples. 42 DNA samples were labeled successfully and get good array image. Imbalance, either loss or gain were observed in all 42 cases studied. Some DNA band may harbor genes that may responsible for HCC development, progression or outcome of disease.
Conclusion: FFPE samples provide a large source of potentially useful data for cancer research.DNA exrracted from FFPE samples is often degraded or crosslinks and therefore more challenging to use for aCGH. DNA of sufficient quality is of great importance for aCGH when using FFPE material.

\section{PP34-37}

A Gene-wide Investigation on Polymorphism in the XRCC1, p53 Gene and Hepatocellular Carcinoma (HCC) Patients Risks in South Indian Population

V. Balachandar ${ }^{1}$, S. Mohana Devi ${ }^{2}$, P. Velmurugan ${ }^{3}$, K. Sasikala ${ }^{2}$,

M. Ahamed ${ }^{2}$, B. Balamuralikrishnan ${ }^{2}$, A. Karthick Kumar², S. Suresh Kumar ${ }^{4}$, P. Manikantan ${ }^{4}$, S. Dharwadkar ${ }^{5}$, Human Genetics Laboratory

${ }^{1}$ Zoology (Human Genetics); ${ }^{2}$ Zoology; ${ }^{3}$ Environmental Science;

${ }^{4}$ Bharathiar University, Coimbatore; ${ }^{5}$ KLE Medical University,

Bangalore, India

Polymorphism in p53 and XRCC1 genes were associated with increased risk of early-onset HCC. We thus hypothesized that these polymorphism could be more likely in South Indian population. However the status of these mutations in South Indian HCC had not been studied. In the present study 93 HCC patients and 93 control subjects were analyzed for known mutations in the p53 and XRCC1 genes. In Arg194Trp polymorphism of p53 gene, we found 21 subjects $(13.21 \%$ ) with Arg399Trp (heterozygous variant) genotype and 4 subjects $(0.92 \%)$ with $399 \mathrm{Trp}$ (homozygous variant) in comparison to controls who exhibited 81 subjects $(82.95 \%)$ with wild type genotype. In Arg399Gln polymorphism of XRCC1 gene, 45 subjects (45.64\%) were found to have Arg399Gln (heterozygous variant) genotype and 12 subjects $(8.33 \%)$ were found to have 399Gln (homozygous variant) genotype in comparison to controls who exhibited $51(48.34 \%)$ individuals with heterozygous variant genotype and 10 individuals (15.12\%) with homozygous variant genotype. This polymorphic incidence revealed significant association with advanced stages of the tumor and also well differentiated tumor grade. Our study is the first report of p53 and XRCC polymorphisms from South Indian ethnicity, with relatively high incidence of mutations. The results suggest that these genes could play an important role in the tumorigenesis of HCC.

\section{Poster Presentation 35: Hepatocellular Carcinoma:} Radiotherapy

\section{PP35-01}

Hepatic Arterial Lodine-131-Labeled Metuximab Injection Combined with Chemoembolization for Unresectable Hepatocellular Carcinoma: Interim Safety and Survival Data from 110 Patients

W. Lu, Y.-F. Yang

The First Department of Interventional Radiology, Eastern HepatoBiliary Surgery Hospital, Second Military Medical University, Shanghai, China

Few options are available to treat patients with hepatocellular carcinoma (HCC). We tested whether the combination of ${ }^{131}$ I-metuximab with chemoembolization could improve outcomes in patients with intermediate HCC. Between April 2008 and April 2009, 110 patients with unresectable HCC were treated with 113 intra-arterial ${ }^{131} \mathrm{I}$-metuximab injections combined with chemoembolization (mean 1.03 per patient; median 1; range 1-2), followed by 264 sessions of transcatheter arterial chemoembolization (TACE) (mean, 2.4 per patient; median, 3 ; range, 1-6). The survival rates at 6,12 , and 18 months were $88.2,79.1$, and $57.4 \%$, respectively, by the Kaplan-Meier method. Of these patients, $12 \%$ exhibited grade $3 / 4$ bilirubin toxicity, $5 \%$ exhibited grade 3/4 WBC (white blood count) toxicity, and 7\% exhibited grade $3 / 4$ platelet toxicity. Response rates based on WHO 
and EASL criteria were 42.73 and $61.82 \%$, respectively. The combination of ${ }^{131}$ I-metuximab and chemoembolization appeared to extend survival in patients with unresectable HCC compared with historical controls, as well as being well-tolerated by Child-Pugh A and $\mathrm{B}$ patients. This combination may represent a promising treatment modality for patients with intermediate HCC.

\section{PP35-02}

Comparison of Efficacy and Safety of Short-course Carbon Ion Radiotherapy for Patients with Hepatocellular Carcinoma Depending on the Histological Differentiation

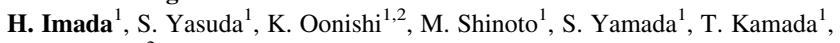
O. Yokosuka ${ }^{2}$

${ }^{1}$ Research Center Hospital for Charged Particle Therapy, National Institute of Radiological Sciences; ${ }^{2}$ Medicine and Clinical Oncology, Graduate School of Medicine, Chiba University, Chiba, Japan

Background/aims: To compare the efficacy and safety of the shortcourse carbon ion radiotherapy (C-ion RT) for hepatocellular carcinoma (HCC) in terms of tumor histological differentiation.

Methods: The study consisted of 63 patients undergoing C-ion RT of 52.8 GyE in 4 fractions between April 2000 and March 2003. Eligibility criteria were as follows: biopsy-proven HCC; recurrent or residual tumor after other ineffective treatments or no indication for any other treatment; no tumor thrombosis of main trunk of portal vein; no other viable lesion outside the planning target volume; no prior radiotherapy for target tumors; hepatic disorder of Child-Pugh classification A or B; ECOG performance status of $0-2$; no other active cancer; and digestive tract not in contact with clinical target volume. Maximum tumor diameter was $4.0 \mathrm{~cm}$ (range $1.2-12.0 \mathrm{~cm}$ ). Four were well differentiated tumor, 2 were well to moderately-differentiated, 51 were moderately differentiated, 4 were moderately to poorly-differentiated, and 2 were poorly differentiated. The total dose delivered was $52.8 \mathrm{GyE}$ in 4 fractions for 1 week. The dose per fraction was $13.2 \mathrm{GyE}$.

Results: The 5-year local control rates were $100.0 \%$ in the well differentiated group, $96.0 \%$ in the moderately differentiated group, $83.3 \%$ in the poorly differentiated group, respectively. The 5-year overall survival and cause-specific survival rates were 33.3 and $33.3 \%$ in the well differentiated group, 23.4 and $32.8 \%$ in the moderately differentiated group, and 25.0 and $25.0 \%$ in the poorly differentiated group, respectively. There were no significant differences ( $P=0.710, P=0.977$, respectively). Further, there was no significant differences in late liver toxicity, and no hepatic failure resulting from the therapy and no treatment-related death occurred.

Conclusions: Excellent local control was obtained independent of tumor histological differentiation. Short-course C-ion RT appears to be an effective and safe treatment modality for all histological type of HCC.

\section{PP35-03}

Outcomes after Short-course Carbon Ion Radiotherapy for Patients with Hepatocellular Carcinoma According to Tumor Size

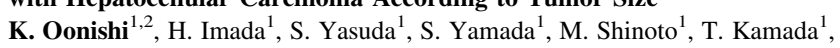
O. Yokosuka ${ }^{2}$

${ }^{I}$ National Institute of Radiological Sciences; ${ }^{2}$ Medicine and Clinical Oncology, Chiba University, Chiba, Japan

Purpose: Since 1995, carbon ion radiotherapy (C-ion RT) has been performed for treatment of HCC, and clinical trials were initiated at the National Institute of Radiological Science (NIRS).

Even though the early detection has progressed, HCC that exceeds $5 \mathrm{~cm}$ exists in no small way, too. This time,we compared the treatment results in terms of tumor size.

Materials and methods: The study consisted of 69 patients undergoing C-ion RT of 52.8 GyE in four fractions between April 2000 and March 2003. We divided into two groups (more than $5 \mathrm{~cm}$ or not) and compared local control, survival, and adverse events between the two groups.

Results: The enrolled patients consisted of 52 males and 17 females. Median age was 69 years (range 37-84). Median maximum tumor diameter was $40 \mathrm{~mm}, 5 \mathrm{~cm}$ or less was 53 examples, $5-10 \mathrm{~cm}$ was 14 examples, and $10-15 \mathrm{~cm}$ was 2 examples.

When the patients divided into two groups according to tumor $\operatorname{size}(\leq 5$ vs. $>5 \mathrm{~cm})$, there were no significant differences in late liver toxicity. No hepatic failure resulting from the therapy and no treatment-related death occurred. The 5-year local tumor control rates were $94 \%$ in two groups. The overall 5-year survival rate was $42 \%$ $(\leq 5 \mathrm{~cm})$ and $13 \%(>5 \mathrm{~cm})$. There were no significant differences between the two groups.

Conclusions: The 5-year local control rate was $94 \%$ in two groups $(<5 \mathrm{~cm}$ vs. $>5 \mathrm{~cm})$ and the effectiveness of the $\mathrm{C}$-ion RT as the local treatment was shown. Short-course C-ion RT appears to be an effective and safe therapeutic option for HCC regardless of tumor size.

\section{PP35-04}

Safety and Response of Hypofractionated Radiotherapy in Locally Advanced Hepatocellular Carcinoma

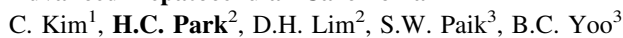

${ }^{1}$ Department of Radiation Oncology, Samsung Medical Center;

${ }^{2}$ Department of Radiation Oncology; ${ }^{3}$ Department of Medicine,

Samsung Medical Center, Sungkyunkwan University, Seoul, Republic of Korea

Purpose: To evaluate the response to hypofractionated radiotherapy (HFRT) for unresectable hepatocellular carcinoma (HCC) and safety of this treatment.

Methods and materials: Between January 2008 and December 2009, a total of 140 patients who were treated with HFRT for unresectable HCC at Samsung Medical Center, were evaluated. Radiotherapy (RT) was delivered with a curative (planned) or palliative intent combined with transhepatic arterial chemoembolization (TACE) and the other local modalities. RT dose was $30-50 \mathrm{~Gy}$ in 10 or 11 fractions. Tumor response was evaluated by RECIST amendments on serial CT or MRI scans. Treatment-related gastro-intestinal morbidity was evaluated by the Common Terminology Criteria for Adverse Events (CTCAE) v3.0 and hepatic toxicity was evaluated by the change of Child-Pugh score before and after RT.

Results: The median follow-up time was 17.8 months after RT. Objective tumor response (CR + PR) was achieved in 86 of 140 patients $(61.4 \%)$. The 1 -year overall survival rate was $89.4 \%$ and 1 year local control was $84.0 \%$. Median survival was not reached yet. Overall survival and local control were affected by RT dose, RT field, treatment aim, tumor response at post-RT 1 month, hepatic toxicity. Intra-hepatic distant metastasis and distant metastasis were most common failure patterns. 5 patients (3.6\%) developed grade 3 gastrointestinal toxicity (ulcer bleeding).

Conclusions: Hypofractionated radiotherapy was safe and feasible. The response rate was equivalent to that of conventional fractionated radiotherapy.

\section{PP35-05}

Accuracy and Efficacy of the Gated Liver MRI Fusion Planning for Radiation Therapy of the Patients with Hepatocellular Carcinoma J.I. Yu ${ }^{1}$, H.C. Park ${ }^{2}$, D.H. Lim², J.S. Kim ${ }^{1}$, Y. Han ${ }^{2}$

${ }^{1}$ Department of Radiation Oncology, Samsung Medical Center;

${ }^{2}$ Department of Radiation Oncology, Samsung Medical Center, Sungkyunkwan University, Seoul, Republic of Korea

Purpose: We designed this study to measure the discrepancies and efficacies of the fusion planning between gated liver MRI and gated planning CT for the radiation therapy (RT) in the patients with hepatocellular carcinoma (HCC). 
Methods and materials: From April to December, 2009, gated liver MRI and planning CT were obtained in the 53 inoperable HCC patients accrued to this study. At the same day, gated MRI and planning CT were conducted with the RPM signal and full inspiratory phase images were obtained. Image fusion between MRI and CT was performed by manually used the vertebral bodies. Internal target volume (ITV) was determined by all phase CT images and gated MRI images. Liver volumes and three specific anatomic landmarks were contoured at each modality according to full inspiratory phase images, and centers of the liver and landmarks were compared.

Results: Eleven patients (20.8\%) showed new tumor lesion compared with last evaluated imaging modality. In these patients, recurrent nodules were not detected by CT in seven patients, definitely. The average differences of the volume of the liver, centers of the liver and landmarks were $25.11 \mathrm{cc}( \pm 27.77$, range $0.69-113.17), 3.6 \mathrm{~mm}$ ( \pm 2.5 , range $0.1-11.1), 3.7 \mathrm{~mm}( \pm 2.4$, range $0.5-11.6), 3.7 \mathrm{~mm}$ ( \pm 2.5 , range $0.4-10.9)$ and $3.6 \mathrm{~mm}( \pm 2.5$, range $0.1-11.1)$. The differences of the centers of the liver and landmarks were statistically significant correlated with positive radiation response $(p<0.05)$.

Conclusion: Gated MRI fusion liver MRI could be provided more information to RT planning. The difference of the gated liver MRI and planning CT was the prognostic factor for the good response.

\section{PP35-06}

Combination Treatment with Transcatheter Arterial Chemoembolisation and Image-guided Radiotherapy for Cirrhotic Patients with Unresectable Hepatocellular Carcinoma

L. NG ${ }^{1}$, T.W. Li ${ }^{1}$, W.C. Lao ${ }^{1}$, Y.W. Luk ${ }^{1}$, C.M. Leung ${ }^{1}$, I.S. Leong ${ }^{1}$, W.T. $\mathrm{Ng}^{2}$, L.Y. Law ${ }^{2}$, K.H. Fung ${ }^{3}$, K.N. Kung ${ }^{1}$, W.H. Li ${ }^{1}$, L.F. Tai ${ }^{1}$, H. $\mathrm{Ng}^{1}$, T.L. Lee $^{1}$

${ }^{1}$ Medicine; ${ }^{2}$ Clinical Oncology; ${ }^{3}$ Radiology, Pamela Youde Nethersole Eastern Hospital, Hong Kong, Hong Kong S.A.R.

Background and aim: Transcatheter arterial chemoembolisation (TACE) used to be the treatment of choice for unresectable hepatocelluar carcinoma (HCC). Recently, there was increased evidence that image-guided radiotherapy (IGRT) showed survival benefits in selected patients. Our aim is to evaluate the treatment efficacy of combined treatment modalities of TACE and IGRT for unresectable HCC.

Method: Patients who had received both IGRT and TACE for unresectable HCC between May 2006 and October 2008 were retrospectively reviewed. All patients received at least one session of TACE, either before or after radiotherapy. IGRT was given in the form of three-dimensional conformal radiotherapy (CRT) at a dose of 4.5-5.5 Gy per fraction, 5 fractions per week for a total of 10 fractions. Tumour response (decrease in tumour size or reduction of AFP) and survival rates were evaluated in our patient cohort.

Result: A total of 14 patients were included. The mean age at treatment was 65.5 years (range 48-76). All patients had chronic viral hepatitis related liver cirrhosis (Child-Pugh A or B). All had unresectable tumour; four were classified as stage A, 10 as stage B according to the Barcelona Clinic Liver Cancer (BCLC) staging. Twelve patients received TACE before given radiotherapy, two received CRT before TACE. Nine patients continued to receive TACE for multifocal disease after CRT. A median of 4 sessions of TACE had been given (range 1-16). The mean tumour size was $3.5 \mathrm{~cm}$ (range $1-8.7 \mathrm{~cm}$ ). Tumour response rate to CRT was $85.7 \%$. Complete response was achieved by combination therapy in 3 patients $(21.4 \%)$ leading to termination of therapy. The median survival was 17 months (range 7-38 months). One-year survival rate was $83.3 \%$. Conclusion: IGRT is an alternative treatment for those who have failed TACE. Combination therapy of IGRT with TACE appears to be a promising treatment modality for selected patients with unresectable HCC.
PP35-07

Stereotactic Body Radiation Therapy for Primary Hepatocellular Carcinoma: The Clinical Outcomes

S.M. Yoon ${ }^{1}$, Y.-S. Lim ${ }^{2}$, J.H. Kim ${ }^{1}$, H.J. Won ${ }^{3}$, J.-H. Park ${ }^{1}$, J.H. Shim ${ }^{2}$, K.M. Kim ${ }^{2}$, H.C. Lee', Y.-H. Chung' ${ }^{2}$, Y.S. Lee', D.J. Suh ${ }^{2}$

${ }^{I}$ Radiation Oncology; ${ }^{2}$ Gastroenterology; ${ }^{3}$ Radiology, Asan Medical Center, University of Ulsan College of Medicine, Seoul, Republic of Korea

Background/aims: To evaluate the clinical results of stereotactic body radiation therapy (SBRT) for hepatocellular carcinoma (HCC). Methods: Between March 2007 and December 2009, 95 patients (105 lesions) with HCC were treated. Inclusion criteria were HCC not suitable for surgery or other local ablative therapy, tumor diameter $<5 \mathrm{~cm}$, adequate hepatic function, and no evidence of major vascular invasion. Four-dimensional computed tomography simulation was performed for the evaluation of respiratory movement. A dose of 10-20 Gy (median, $15 \mathrm{~Gy}$ ) per fraction was given over 3-4 consecutive days to a total dose of 30-60 Gy (median, 45 Gy). Local failure was defined as a recurrence in the treated lesion, and regional failure as a recurrence within the liver outside the treated lesion. Radiation-induced hepatic toxicity was graded according to the Common Terminology Criteria for Adverse Events version 3.0 or a decline in liver function using Child-Pugh score.

Results: The median follow-up was 22 months. The overall response rate was $69.5 \%$, with 32 complete response $(30.5 \%)$ and 41 partial response $(39.5 \%)$ at 3 months after the end of SBRT. Local failure, regional failure, and distant metastasis were observed in 8 (7.6\%), 50 (47.6\%), and $15(14.3 \%)$ patients, respectively. Local recurrence-free survival rates at 1 and 2 years were both $90 \%$, and progression-free survival rates at 1 and 2 years were 43 and $28 \%$, respectively. Overall survival rates at 1 and 2 years were 81 and $51 \%$, respectively. Grade $\geq 2$ hepatic toxicity was observed in $10(10.5 \%)$ and progression of Child-Pugh score by 2 or more was developed in 5 (5.3\%) patients. Conclusions: SBRT was feasible with acceptable toxicity and encouraging local control. The main patterns of failure after SBRT were not local, but regional failures. Further study of additional combined modalities is needed to investigate the improvement of treatment result.

Poster Presentation 36: Hepatocellular Carcinoma: Surgery

PP36-01

Salvage Liver Transplantation for Patients with Recurrent Hepatocellular Carcinoma after Curative Resection

L. Wu, X. He, Z. Guo, Q. Tai, W. Ju, D. Wang, X. Zhu

The First Affiliated Hospital of Sun Yat-sen University, Guangzhou, China

Introduction/objective: The incidence of hepatocellular carcinoma (HCC) is increasing in China mainland and accounting about $40 \%$ of orthotopic liver transplantation (OLT) candidates. However, under the pressure of donor organ shortage and the high medical cost, the application of OLT for early HCC is limited. Patients with early HCC might suffer from HCC recurrence or deterioration of liver function after primary liver resection. Our purpose is to summarize the experience with salvage liver transplantation for patients with recurrent HCC after primary liver resection.

Methods: From 2004 to 2008, 376 patients with HCC received liver transplantation in our single center. Among these patients, $36(9.6 \%)$ underwent salvage liver transplantation after primary liver curative resection due to intrahepatic recurrence. 29 were male and 7 were female, the mean age was 46 years old. 16 received right lobectomy, 10 received left lobectomy and the others received sectionectomy or 
segmentectomy. The mean interval between initial liver resection and salvage transplantation was $34.9 \pm 16.2$ months (1-63 months). As a control group for comparison, we used clinical data of the 291 patients who underwent primary OLT for HCC within Milan criteria. Results: Intraoperative bleeding volume, transfusion volume and operative time in the salvage group was significantly different $(p<0.05)$ from those in control group. But the post-operative complications, tumor recurrence rate, patient survival rate and tumor-free survival between these two groups had no difference $(p>0.05)$.

Conclusions: Comparing with primary OLT, although salvage liver transplantation would increase the operation difficulties; it still remains a good option for patients with $\mathrm{HCC}$ recurrence after curative resection.

\section{PP36-02}

Fibrolamellar Carcinoma of the Liver: A Clinical Review M. Soloviy

Lviv National University of Medicine, Lviv, Ukraine

Introduction: Fibrolamellar carcinoma (FLC) is a malignant hepatic tumor that differs from hepatocellular carcinoma (HCC) in some pathological and clinical features.Aim of the study was to investigate most commonly described pathological and clinical characteristics of the FLC of the liver.

Methods: Medline search for the words FLC was performed. 151 abstracts and 23 full papers were identified and studied for the period from 2000 to 2010.

Results: FLC is most commonly described as a rare disease of adolescents and young adults without gender predominance. Etiology is unknown and typical HCC risk factors such as viral hepatitis or cirrhosis are absent.Typically FLC patients present with nonspecific abdominal pain, discomfort, malaise and weight loss or even can be asymptomatic and have their tumors revealed incidentally. The tumors are usually large, heterogenous lesions with well defined margins and areas of hypervascularity on CT scan. A triad of histological features is commonly used for diagnosis - tumor cells with eosinophilic cytoplasm, lamellar fibrosis and large vesiculated nucleoli.Many studies report FLC to be less aggressive with better 5year survival reaching up to $76 \%$ in resected cases compared to conventional HCC. One of the major explanation for this can be the absence of cirrhosis in FLC patients. However, several other studies state FLC to be aggressive tumor with $20 \%$ to $45 \%$ 5-year survival in resected cases, decreasing to $15 \%$ in patients with metastatic disease. Conclusions: FLC differs from HCC in demographics, condition of the liver and prognosis. Although many studies emphasize the better survival in FLC compared to HCC, these results can be similar to that in HCC without cirrhosis, thus referring FLC to be though an aggressive neoplasms.

\section{PP36-03}

Evaluation of Metabolic Factors on the Prognosis of Patients Undergoing Resection of Hepatocellular Carcinoma

M. Kaibori, M. Ishizaki, K. Matsui, A.-H. Kwon

Surgery, Kansai Medical University, Hirakata, Japan

Objectives: The metabolic factors including obesity, diabetes, and hypertension have been implicated as risk factors of hepatocellular carcinoma (HCC) in patients with chronic hepatitis. The effects of metabolic factors was investigated on the prognosis of patients undergoing resection of HCC.

Methods: A total of $469 \mathrm{HCC}$ patients were classified into three groups; hepatitis B virus (HBV)-, hepatitis C virus (HCV)-, and non-HBV/HCV (NBC)-related HCC. Further the patients with HCVrelated HCC were sub-classified into three groups; the patients who did not have documented hypertension, hypertensive patients who received angiotensin II-blocking agents (ABA), and hypertensive patients who received without ABA.
Results: There were no significant difference of survival in the HBVHCC and NBC-HCC patients with or without obesity, diabetes, and hypertension. In the patients with HCV-related HCC, however, hypertensive patients were significantly worse on both disease-free and overall survivals than non-hypertensive patients. Among the HCV-HCC patients with chronic hepatitis, hypertensive patients with ABA had significantly better preoperative liver function, and hypertensive patients without ABA were significantly worse on both disease-free and overall survivals than those of hypertensive patients with $\mathrm{ABA}$ and non-hypertensive patients.

Conclusions: Results suggest that hypertension is a risk factor for a poor prognosis after resection of HCV-related HCC. Angiotensin II blockade may improve the prognosis of hypertensive patients with early hepatic fibrosis after resection in HCV-related HCC.

\section{PP36-04}

Postoperative Infectious and Noninfectious Complications after Hepatectomy for Hepatocellular Carcinoma

M. Kaibori, K. Matsui, M. Ishizaki, A.-H. Kwon

Surgery, Kansai Medical University, Hirakata, Japan

Background and aim: Relatively high morbidity rates are problematic after hepatic resection for hepatocellular carcinoma (HCC). This study investigated risk factors for morbidity after resection of $\mathrm{HCC}$ that were related to perioperative management and operative techniques.

Methods: Five hundred and thirty HCC patients who underwent hepatectomy between 1992 and 2008 were divided into three groups, i.e., 51 patients with infectious complications during their hospital stay (infectious group), 67 patients with noninfectious complications (Noninfectious group), and 412 patients without complications who were discharged within 21 days after hepatectomy (uncomplicated group).

Results: Noninfectious complications decreased significantly over time. Although infectious complications also decreased, the change was not significant. The overall survival rate of the groups with complications was significantly worse than that of the uncomplicated group $(p<0.0005)$. Univariate and multivariate analyses showed that an operating time $>300 \mathrm{~min}$ and bile leakage were independent risk factors for infectious complications, while a platelet count $\leq 13 \times 10^{4} / \mu$ l, cirrhosis, and operative blood loss $>1,000 \mathrm{ml}$ were risk factors for noninfectious complications.

Conclusions: To achieve zero morbidity, it is important to avoid bile leakage and minimize blood loss during resection of $\mathrm{HCC}$ in patients with cirrhosis.

\section{PP36-05}

Hepatic Resection for Hepatocellular Carcinoma in the Elderly

M. Kaibori, K. Matsui, M. Ishizaki, A.-H. Kwon

Surgery, Kansai Medical University, Hirakata, Japan

Background and objectives: Aging of the population has significantly increased the number of elderly patients undergoing surgery for hepatocellular carcinoma (HCC). We aimed to compare the results of hepatectomy for HCC in patients $\geq 70$ years old with those for younger patients.

Methods: Clinicopathological data and outcomes for 155 elderly patients and 333 younger patients with HCC who underwent hepatectomy between 1992 and 2007 were retrospectively compared.

Results: The elderly group had a higher incidence of associated diseases, but had better preoperative liver function. Although postoperative delirium was more common in the elderly group, there were no significant differences between the 2 groups with regard to operative morbidity, hospital death, disease-free survival, and overall survival. The overall recurrence rate was significantly higher in the elderly patients with alcohol abuse than in younger patients with alcohol abuse. Multivariate analysis revealed that 
preoperative alcohol abuse was a prognostic factor for elderly patients.

Conclusions: The short-term and long-term outcomes of surgery for HCC were similar in elderly and younger patients. Elderly patients with preoperative alcohol abuse should be followed closely, even after R0 surgery, because alcohol abuse is strongly correlated with postoperative recurrence and worse survival.

\section{PP36-06}

Predictors of Microvascular Invasion before Hepatectomy for Hepatocellular Carcinoma

M. Ishizaki, M. Kaibori, K. Matsui, A.-H. Kwon

Surgery, Kansai Medical University, Hirakata, Japan

Background and objectives: Microvascular invasion (MVI) is difficult to detect before resection of hepatocellular carcinoma (HCC). Methods: Clinicopathological and outcome data were retrospectively compared between 213 HCC patients with MVI and 221 patients without MVI who underwent hepatectomy.

Results: MVI risk was selected as an independent prognostic indicator for both disease-free and overall survival in our $434 \mathrm{HCC}$ patients. Univariate and multivariate analyses showed that an age under 65 years, a protein induced by vitamin $\mathrm{K}$ absence/antagonism II (PIVKA-II) level $\geq 200 \mathrm{mAU} / \mathrm{ml}$, a preoperative tumor size $\geq 5.0 \mathrm{~cm}$, and poorly differentiated carcinoma were independent predictors of MVI. When age, PIVKA-II level, and tumor size (data available before surgery) were scored as a combined index, the total score demonstrated a significant correlation with the extent of vascular invasion and with survival after hepatic resection.

Conclusions: An age under 65 years, increase of PIVKA-II, and larger tumor size were preoperative predictors of MVI in HCC patients undergoing potentially curative resection. Our combined score based on the age, serum PIVKA-II, and preoperative tumor size is a reliable predictor of MVI and survival in patients with HCC.

\section{PP36-07}

Defining an Optimal Surgical Strategy for Treatment of Recurrent Hepatocellular Carcinoma after Hepatectomy: Re-resection or Radiofrequency Ablation?

A. Chan, R. Poon, T.T. Cheung, C.M. Lo, S.T. Fan

Department of Surgery, Queen Mary Hospital, The University of

Hong Kong, Hong Kong, Hong Kong S.A.R.

Background: Tumor recurrence after resection of hepatocellular carcinoma $(\mathrm{HCC})$ is a common phenomenon with a 5 -year recurrence rate over $50 \%$. Aggressive salvage treatment policy in the form of reresection or radiofrequency ablation (RFA) improves the prognosis of patients with recurrent disease. However, the choice of treatment to achieve better survival benefit in the management of recurrent $\mathrm{HCC}$ is not yet clear.

Methods: From January 2001 to December 2008, 179 patients developed intrahepatic recurrences after hepatectomy for HCC in our center. 29 patients received re-resections and 45 patients received RFA as salvage treatment. Patient characteristics, clinicopathological data, and survival outcomes were reviewed, and compared between the two groups. $P$ value $<0.05$ was considered to be significant.

Results: Patients in the RFA group were older than those in the re-resection group (59 vs. 52-year-old). Nonetheless, the liver function status, staging of the initial tumor and time to develop first recurrence after hepatectomy for initial tumor (12.2 vs. 8.7 months, $p=0.84$ ) were comparable between the two groups. There was no difference in recurrent tumor size $(2.1$ vs. $2.1 \mathrm{~cm}, p=0.73)$ and tumor multi-focality. The time to develop further tumor recurrence after re-resection and RFA were 5.9 and 4.0 months $(p=0.30)$. The 1-, 3- and 5-year tumor-free survival rates after re-resection were $41.4,24.2$ and $24.2 \%$, respectively, and after RFA were $32.2,12.4$ and $9.3 \%$ respectively $(P=0.14)$. The $1-, 3-$, and 5 -year overall survival rates after re-resection were $89.7,56.5$ and $35.2 \%$, respectively, and after RFA were $83.7,43.1$ and $29.1 \%$, respectively $(P=0.48)$. The 1-, 3- and 5-year overall survival rates after hepatectomy for initial tumor in the re-resection group were $89.7,82.3$ and $55.7 \%$, and in the RFA group were $95.6,68.2$ and $44.5 \%$, respectively.

Conclusion: Both RFA and re-resection attain similar survival benefit in the management of recurrent HCC after hepatectomy.

\section{PP36-08}

Usefulness of Endoscopic Surgical Spacer (SECUREA ${ }^{\mathrm{TM}}$ ) for Laparoscopic Liver Resection

Y. Kawano, N. Taniai, Y. Nakamura, S. Matsumoto, S. Mineta, M. Yoshioka, J. Ueda, Y. Mamada, H. Yoshida, E. Uchida

Department of Surgery, Nippon Medical School, Tokyo, Japan

Introduction: Although laparoscopic approaches have become increasingly used in selected patients with liver resection, there're still some technical difficulties and / or irritating procedures; e.g. suction of blood and fluid and gently retraction of the liver. For reducing these difficulties, we've developed a new device, sponge spacer, for laparoscopic surgery in cooperation with Hogy Medical Co., Ltd. We demonstrate effectiveness of the device in laparoscopic liver resection.

Material: The composition of this sponge is polyurethane foam. The sponges have an oval-columnar shape with the 3 type of sizes, $65 \times 35 \times 20 \mathrm{~mm}, \quad 50 \times 30 \times 15 \mathrm{~mm}$ and $40 \times 25 \times 15 \mathrm{~mm}$, respectively, and have an attached radiopaque string which is easily held by laparoscopic forceps. The sponges were easily inserted into the peritoneal cavity via a $10-12 \mathrm{~mm}$ laparoscopic port.

Advantages: The sponge was considered having advantages during laparoscopic liver resection such as the capability of gentle retraction of any organs, without exchange another laparoscopic instrument as a retractor, the ability to absorb bodily fluid, irrigation saline, and the flow of blood and to take off the absorption fluid easily by suction to keep operative field dry and clear, the capability as a safety spacer between resecting tissue and preserving tissue and the convenience of attaching hemostatic agents different from surgical gauze.

Conclusion: This new device might have utilities in various scene and any kinds of operation of laparoscopic surgery, especially in liver resection.

\section{PP36-09}

The Association of Family History of HBV Infection with Resectability of Hepatocellular Carcinoma among Asian Patients

Z.H. Hu, Z.W. Li, W.L. Wang, L. Zhou, M. Zhang, Y. Shen, S.Y. Ye, S.S. Zheng

Hepatobiliary and Pancreatic Surgery Unit, The First Affiliated

Hospital, School of Medicine, Zhejiang University, Hangzhou, China

Aims: This study aims to address the relationship between a positive family history of liver cancer or hepatitis B virus (HBV) infection and the operative resectability of hepatocellular carcinoma.

Methods: Two hundred and sixty-two hepatocellular carcinoma patients, either pathologically confirmed or clinically and visually diagnosed, underwent operative or nonoperative therapies in the Hepatobiliary and Pancreatic Surgery Unit, the First Affiliated Hospital, School of Medicine, Zhejiang University and were investigated. One hundred and eighteen of the patients were clinically considered as resectable patients, that is the liver tumor could be radically resected and liver function successfully reserved. These patients underwent subsequent hepatic resection. The remaining 144 patients were clinically unresectable, and were used as the control group. All patient information, including a family history of malignant liver tumors and HBV infection, was collected from case files. This includes information regarding the number of relatives with liver cancer or carrying HBV, the type of liver malignant tumor, the extent 
of HBV infection and the details of the individual's relationship with the relative.

Results: A family history of HBV infection is significantly associated with the resectability of hepatocellular carcinoma $(\mathrm{AOR}=2.332$ [95\% CI, 1.051-5.177]), and with the same manner, there also has a significant association between the first degree relatives infected with $\mathrm{HBV}$ and the resectability of hepatocellular carcinoma $(\mathrm{AOR}=2.334$ [95\% CI, 1.050-5.190]), especially in siblings, the patient's sibling with a history of HBV infection being significantly related to the resectability of hepatocellular carcinoma $(\mathrm{AOR}=2.727[95 \% \mathrm{CI}$, 1.092-6.808]).

Conclusions: A family history of HBV infection is associated with the resectability of hepatocellular carcinoma, and in particular, if the patient's sibling has a history of HBV infection, it means that the patient has a less opportunity to receive curative liver resection. Further research exploring HBV and the environmental interactions associated with the unresectability of liver cancer is required.

\section{PP36-10}

Anatomical Variations of Hepatic Veins: A 3-dimensional Computerized Tomography Scan on 200 Subjects

F. Chi-Hua ${ }^{1}$, Y. Jin-Hua ${ }^{1}$, L. Wan Yee $^{2}$, L.C.H. Eric ${ }^{2}$, F. Ying-Fang ${ }^{1}$, Z. Shi-Zhen ${ }^{3}$, L. Ke-Xiao ${ }^{1}$, C. Zhi-Xiang ${ }^{1}$, S. Zhong-He ${ }^{1}$, B. Su-Su ${ }^{4}$ ${ }^{1}$ Department of Hepatobiliary Surgery, Zhujiang Hospital, The Southern Medical University, Guangzhou; ${ }^{2}$ Faculty of Medicine, The Chinese University of Hong Kong, Prince of Wales Hospital, Hong Kong; ${ }^{3}$ The Clinical Anatomy Institute, Southern Medical University; ${ }^{4}$ The Computer School, South China Normal University, Guangzhou, China

Background: The impact of hepatic venous anatomical variations on hepatic resection and transplantation is the least understood aspect of liver surgery.

Methods: A prospective Three-dimensional computerized tomography scan study was undertaken on 200 consecutive subjects with normal livers to determine the prevalence of surgically significant hepatic venous anatomical variations.

Results: The prevailing pattern of the 3 hepatic veins was a right hepatic vein (RHV), and a common trunk of the middle (MHV) and left hepatic veins $(\mathrm{LHV})(122 / 200=61 \%)$. The remaining patients had the right, middle, and left hepatic veins draining independently into the inferior vena cava (IVC). In 39\% of patients, the RHV was small and was compensated by a large right inferior hepatic vein (21\%), an accessory RHV (8.5\%) or a well-developed MHV $(6.5 \%)$. A segment 4 vein was seen in $51.5 \%$ of patients. This segment 4 vein joined the LHV (26\%), the MHV (17.5\%) or the IVC $(8 \%)$. An umbilical vein and a segment 4 vein were seen in $(3.5 \%)$ of patients. Both these 2 veins either joined the LHV (2\%) or the MHV (1.5\%). Conclusions: Knowing the variations of hepatic veins before surgery is useful in partial hepatectomy and in donor operations for living related liver transplantation.

\section{PP36-11}

Usefulness of Glove Method with Pringle Maneuver for Laparoscopic Liver Resection

Y. Kawano, N. Taniai, Y. Nakamura, S. Matsumoto, S. Mineta, M. Yoshioka, J. Ueda, Y. Mamada, H. Yoshida, E. Uchida

Department of Surgery, Nippon Medical School, Tokyo, Japan

Introduction: Although marked development of laparoscopic surgery for liver resection has been seen in recent years, it has been important to decrease the bleeding during the operation. We have conducted laparoscopic liver resection using Glove method with Pringle maneuver, and demonstrate effectiveness of the procedure in laparoscopic liver resection.

Methods: After the examination of abdominal cavity using the laparoscope, a small abdominal wall incision, $5 \mathrm{~cm}$ in diameter, is made which would be able to retrieve the resected liver at the adequate place. Applied Alexis ${ }^{\mathrm{TM}}$, which is a wound protector and retractor, is placed at the wound and covered with a sterile disposable glove, of which one finger part is cut and to which a trocar is attached. After encircle of hepatoduodenal ligament using Nelaton catheter, Kelly clamp is inserted via one finger part of the Glove and clamp the catheter as Pringle maneuver. After the procedure, liver resection was done mainly using Harmonic scalpel.

Results: We have done laparoscopic liver resection in 2 cases with the procedure (partial resection of the S2/3 for metastatic liver tumor and partial resection of the S4 for solitary HCC). Total blood loss of these operations were 120 and $100 \mathrm{ml}$ and operative durations were 330 and $272 \mathrm{~min}$. No post-operative complication were recognized in each case.

Conclusion: Laparoscopic liver resection using Glove method with Pringle maneuver could be done easily and effective to decrease blood loss during the operation. Furthermore, this procedure seems to make laparoscopic liver resection safer and easier because of ease conversion to open method and manipulation via the wound covered by Glove at need.

\section{PP36-12}

Surgical Outcomes and Prognostic Factors of Elder Patients 75 Years of Age or Older) with Hepatocellular Carcinoma Undergoing Hepatectomy

N. Taniain, H. Yoshida, Y. Kawano, J. Ueda, E. Uchida

Surgery, Nippon Medical School, Tokyo, Japan

Backgroud and aims: The present study aimed to evaluate the surgical outcome and prognostic factors of elderly patients with hepatocellular carcinoma (HCC) undergoing hepatectomy.

Methods: Overall survival rates and disease-free survival rates of HCC in 48 patients 70 years of age or older undergoing hepatectomy (old group) were clarified and compared with those of 312 patients younger than 70 years of age (young group) in this retrospective study. And the prognostic factors were evaluated by multivariate analysis using Cox's proportional hazards model.

Results: The overall 3- and 5- year survival rates of old group were 58.5 and $45.9 \%$ and those of young group were 63.0 and $46.8 \%$, respectively. And the disease-free 3- and 5- survival rates of old group were 38.5 and $32.1 \%$ and those of young group were 30.5 and $20.0 \%$, respectively. Statistically significant differences between two groups were not found in the overall survival rates and disease-free survival rates. Multivariate analysis revealed a statistically significant association between presence of venous invasion $(P=0.002)$ and TMN stage by the Liver Cancer Study Group of Japan $(P=0.015)$ and AFP level $(P=0.013)$ and blood loss $(P=0.001)$ and prognosis.

Conclusions: Selected elderly patients with HCC benefited from adequate and safety hepatectomy as much as young patients.

\section{PP36-13}

Preliminary Experience of Laparoscopic Hepatectomy for Hepatocellular Carcinoma

C.H.D. Kwon ${ }^{1}$, G.C. Lee ${ }^{1}$, J.W. Joh ${ }^{1}$, J.S. Heo ${ }^{1}$, J.M. Kim ${ }^{1}$, M.J. Shin ${ }^{1}$, S.H. Song ${ }^{1}$, M.S. Choi ${ }^{2}$

${ }^{1}$ Surgery, Samsung Medical Center, Sungkyunkwan University;

${ }^{2}$ Internal Medicine, Samsung Medical Center, Sungkyunkwan

University School of Medicine, Seoul, Republic of Korea

Purpose: Laparoscopic liver resection has gained much popularity in the recent years, but relatively few centers have performed hepatectomies in hepatocellular carcinoma (HCC) patients due to the technical difficulties faced with underlying liver cirrhosis. We herein present the early experience of laparoscopic liver resection in HCC performed in a single institution. 
Methods: From October 2003 until March 2009, 39 laparoscopic liver resections were performed on HCC patients among whom 26 had underlying liver cirrhosis.

Results: The location of the tumor was in the left lateral section in 15, segment 5 or 6 in 20 , segment 4 in 3 and caudate lobe in 1 . Resection involving less than monosegment was done in 26 and more than 2 segments in 13 . Tumor size ranged from $0.8 \mathrm{~cm}$ to $6.6 \mathrm{~cm}$ (median 2.35 ) and the resection margin from 0.1 to $6 \mathrm{~cm}$ (median $1.5 \mathrm{~cm}$ ). All patients were either stage I (29) or II (10). There was no difference between cirrhotic and non-cirrhotic patients in operation time (median $210 \mathrm{~min}$, range $60-637)$, change of hematocrit value $(4.8 \%$, $-1-19.6 \%$ ), hospital stay (8 days, 3-67 days). The median follow up duration was 15.1 months, and 2-year recurrence free survival rate was $48.3 \%$.

Conclusion: Laparoscopic liver resection in HCC with or without underlying cirrhosis seems to be feasible with minimal morbidity especially in well selected cases with early stage HCC.

\section{PP36-14}

Role of Portal Vein Embolisation in Hepatocellular Carcinoma Management and its Effect on Recurrence: An Intention-to-Treat Analysis R.C. Siriwardana, C.M. Lo, S.C. Chan, K.S. Chok, S. Tsang, T.T. Cheung, W. Sharr, A. Chan, S.T. Fan

Department of Heparobiliary and Transplant Surgery, University of Hong Kong, Hong Kong, China

Background: Portal vein embolisation (PVE) for hepatocellular carcinoma is complicated due to the presence of background liver disease, issue of progression of the primary tumor, and possible effect on micrometastasis on the contralateral side.

Patients and methods: Fifty-four patients with inadequate functional residual liver volume (FRLV) were offered PVE during a seven-year period. Clinicopathological features were compared between 34 (63\%) patients who had undergone curative resection and others. A control group was selected by matching each patient who underwent surgery after PVE (PVE group) with three controls having surgery without PVE. Cases were matched for vascular invasion, type of resection and year of surgery. Postoperative complication pattern of recurrence and survival were compared.

Results: PVE increased the FRLV in $66 \%$ of the cirrhotic patients. Combination of PVE and surgery was effective in $60 \%(n=32)$ of patients. Minor (control, 29\%; PVE, 24\%; $p=0.12$ ) and major (PVE, $18 \%$; control, $15 \% ; p=0.78)$ complications were similar between PVE and control groups. After a follow-up of 35 months (standard deviation 25 months), extrahepatic recurrence was detected in 10 (31\%) and $41(40 \%)$ cases in the PVE group and the control group, respectively $(p=0.25)$ and intrahepatic recurrence in $10(29 \%)$ and $47(46 \%)$ cases, respectively $(p=0.09)$. In the PVE group, $41 \%$ $(n=14)$ of the recurrences were detected before one year, compared to $42 \%(n=43)$ in the control group $(p=1)$. Disease-free survival at 1,3 and 5 years was 57,29 and $26 \%$, respectively, in the control group and 60, 42 and $42 \%$, respectively, in the PVE group (log-rank, $p=0.33$ ). On multivariate analysis, PVE was not an individual factor affecting survival $(p=0.82)$.

Conclusion: PVE increases resectability and gives patients with hepatocellular carcinoma comparable outcomes.

\section{PP36-15}

Reducing Hepatitis B Viral Load with Lamivudine and Foscarnet Sodium Injection in the Perioperative Period of Hepatectomy for Hepatocellular Carcinoma

Q. Ling, X. Xu, B. Zhou, H.Y. Xie, L. Zhou, S.S. Zheng

Division of Hepatobiliary and Pancreatic Surgery, Department of Surgery, the First Affiliated Hospital, Zhejiang University School of Medicine, Hangzhou, China
Background/aims: The hepatitis B viral load has been demonstrated as a predictor of hepatocellular carcinoma (HCC) recurrence after hepatectomy. Surgical resection may put patients at high risk of hepatitis B virus (HBV) reactivation. We aim to evaluate the efficacy of different antiviral therapies on the perioperative HBV control.

Methods: Forty two patients who were initially found high serum HBV DNA levels ( $>4 \log _{10}$ copies/ml) and never received antiviral therapy and at list for curative hepatectomy for HBV-related HCC were included in this study. According to perioperative antiviral protocol, they were divided into Group I ( $n=14$, lamivudine), Group II ( $n=16$, lamivudine and foscarnet sodium $)$ and Group III $(n=12$, lamivudine and Adefovir dipivoxil). The immune function, dynamic change of serum HBV DNA levels and the HBV reactivation rate were compared.

Results: Patients characteristics did not differ significantly among three groups. The serum IgG, IgM, IgA, C3 and C4 values showed a sharp decline immediately after hepatectomy $(P<0.001)$ and gradually increased during the next two weeks independent of operation types. After 8 days treatment, an obvious decrease of HBV DNA levels were found in Group I ( $4.2 \pm 0.4$ vs. $5.3 \pm 0.9 \log _{10}$ copies/ $\mathrm{ml}, P<0.05)$, Group II $\left(3.8 \pm 0.5\right.$ vs. $5.5 \pm 0.7 \log _{10}$ copies $/ \mathrm{ml}$, $P<0.05)$ and Group III $\left(4.1 \pm 0.7\right.$ vs. $5.6 \pm 1.1 \log _{10}$ copies $/ \mathrm{ml}$, $P<0.05)$. Group II showed the sharpest decline of HBV DNA level (vs. Group I, $P<0.05$ ). During the first post-operative week, the Group I showed higher HBV reactivation rate compared with Group II and Group III.

Conclusion: A surgical strike may result in a low immunity and reactivation of viral replication. Lamivudine combined with foscarnet sodium can't only promptly reduce viral load before operation but also effectively prevent HBV reactivation after hepatectomy.

\section{PP36-16}

Characteristics of Long Term Disease Free Survivors after Surgical Resection for Hepatocellular Carcinoma

I. Kirino, H. Ikeda, M. Okabe, A. Tsuruta, K. Sano, K. Kawamoto, Y. Morimoto, T.B. Park, Y. Yoshida, T. Ito, K. Ogasawara Department of Surgery, Kurashiki Central Hospital, Kurashiki, Japan

Aim: The aim of this study is to clarify the characteristics of longterm disease free survivors after surgical resection for hepatocellular carcinoma (HCC).

Patients and methods: Clinicopathologic features and perioperative results of 5-year disease free survivors are compared to those of patients who had developed recurrent disease within 5 years after surgery. Statistical analyses were performed by Chi-square test and logistic regression model.

Results: Between 1987 and 2006, 202 patients underwent hepatic resection for HCC in the Department of Surgery, Kurashiki Central Hospital. One hundred and seventy four patients were followed up for more than 5 years, over the time of developing recurrent disease, or to the death. Within median follow up period of 69.1 months, 133 patients had developed recurrent disease and 26 of them occurred over 5 years after surgery. Median time to recurrence was 23.3 months. Ninety eight patients survived for more than 5 years and 54 patients were free of disease at 5 years. TNM stage I,II $(P=0.038)$ and hepatitis $\mathrm{B}$ related liver disease $(P=0.002)$ were significant predictive factor for 5 year disease free survival by both univariate and multivariate analysis. Tumor size less than $5 \mathrm{~cm}$ $(P=0.028)$, encapsulated tumor without capsular infiltration $(P=0.04)$, and surgery without blood transfusion $(P=0.005)$ were associated with 5 year disease free survival by univariate analysis.

Conclusion: Clinicopathological features associated with long term disease free survival after resection for HCC are, hepatitis B related HCC with early TNM stage (I or II), favorable tumor characteristics, and surgery without blood transfusion. 


\section{PP36-17}

Heptocelluar Carcinoma with Portal Vein Thrombus-Surgical Techniques and Outcome

J. Kita

Department of Second Surgery, Dokyo Medical University, Tochigi, Japan

The patient was 59-year-old male with positive for HBV antigen. $\mathrm{He}$ was pointed-out to have a HCC in the posterior segment of the liver and the HCC grew rapidly. Preoperative evaluations revealed that HCC invaded the right branch of the portal vein and formed thrombus, which extended to left branch of the portal vein. ICG R15 was $13 \%$. He had a portal hypertension and severe esophageal varices with red color sign. CT showed the occlusion of right branch of the portal vein by tumor thrombus, and the left lobe of the liver showed compensatory enlargement. CT-volumetry demonstrated that the remnant liver was to be $836 \mathrm{~cm}^{3}$, which was $61 \%$ of the total liver volume. We decided to perform right hepatectomy and removal of portal vein thrombus. At the operation, the intraoperative ultrasonography demonstrated that tumor thrombus was located in right branch of the portal vein, extending to umbilical portion of the left portal vein through bifurcation. The thrombus did not extend distally to the portion of confluence of the splenic vein. The right and left portal veins were taped. Small orifice was placed in right portal vein and whole thrombus was simply removed from the orifice. Confirming that whole thrombus was removed by ultrasound, right lobectomy of the liver was performed. Postoperative clinical course was uneventful. Endoscopy performed on postoperative day 14 showed the improvement of the esophageal varices. Between April 2000 and August 2009, 315 hepatectomies for $\mathrm{HCC}$ were performed. Over all 5-year survival rate was $64.0 \%$. However, in 4 cases with portal thrombus, the prognosis was relatively poor with 1-year survival rate of $66.1 \%$ and 2-year survival rate of $0 \%$. After aggressive removal of portal thrombus by surgical procedure, intensive adjuvant therapies are needed to improve the outcome.

\section{PP36-18}

Clinical Outcome of Repeated Hepatectomy for Recurrent Hepatocellular Carcinoma

J. Kita

Department of Second Surgery, Dokyo Medical University, Tochigi, Japan

Purpose: We retrospectively reviewed our outcome for patients with recurrent $\mathrm{HCC}$ who had undergone hepatectomy.

Methods: Between April 2000 and May 2010, 370 patients with HCC underwent curative hepatectomy. Among these patients, 218 of 370 patients, who had recurrence of HCC, were included in the present study. Patients were divided into two groups, Group A $(n=76)$; patients who were treated with repeated hepatectomy, Group B ( $n=142)$; patients who were treated with other treatment modalities. Patients' age, ICG R15 (\%), operation time (min), intraoperative blood loss (IOB; ml),AFP doubling time (AFP-DT; day), tumor size $(\mathrm{TS} ; \mathrm{cm})$, survival rate $(\mathrm{SR} ; \%)$ and disease-free survival rate (DFS; $\%)$ were compared between the two groups. Data were expressed as Group A versus Group B.

Results: There were no significant differences in age (67 vs. 67; $P=0.33$ ), ICG R15 (15 vs. $16 ; P=0.96$ ). On the other hand, there were significant differences in operation time ( 270 vs. $330 ; P<0.05)$, IOB (416 vs. $600 ; P<0.01)$, TS (2.5 vs. $4.5 ; P<0.01)$ and AFP DT (23.1 vs. $12.9 ; P<0.03$ ). The 5 -year SR of Group A and B were 76.5 and $37.8 \%$, respectively $(P<0.01)$, and the 5 -year DFS were 2.6 and $1.4 \%$, respectively $(P<0.01)$. By multivariate analysis, Stage II $(P<0.03)$ and recurrence with less than 3 nodules $(P<0.05)$ were significant prognostic factors in Group A. On the other hand, in Group $\mathrm{B}$, recurrence only in one organ was a significant prognostic factor
$(P<0.01)$. In Group B, most frequent treatment was TACE $(73.2 \%)$. In Group B, there was no significant difference in overall survival rates between TACE-treated patients and the patients treated with other than TACE $(P=0.55)$.

Conclusion: Outcome of repeated hepatectomy for recurrent HCC is better than that of other treatment, as long as the indication of Makuuchi's criteria is observed.

\section{PP36-19}

Is Wider Surgical Margin Justified for Better Clinical Outcomes for Patients with Resectable Hepatocellular Carcinoma

K.-T. Lee, S.-N. Wang

Department of Surgery, Kaohsiung Medical University Hospital, Kaohsiung, Taiwan, ROC

Background and Aim: Surgical resection for hepatocellular carcinoma is regarded as a curable treatment, however, the postoperative recurrence still pose a challenge to surgeons. The effect of surgical margin on long-term outcome of is still controversial, although it has been considered as the treatment-related risk factor for recurrence. Precise assessment of the effects of surgical margin on clinical outcome is required to clarify the issue.

Methods: A retrospective study was conducted on 402 patients with microscopically complete resection of $\mathrm{HCC}$, they were divided into three groups with surgical margin negative by $1-5 \mathrm{~mm}$ (Group A, $n=156$ ); $6-10 \mathrm{~mm}$ (Group B, $n=109$ ); and wider than $10 \mathrm{~mm}$ (Group C, $n=142$ ).The groups were compared for clinicopathologic characteristics, perioperative features, postoperative recurrence and long term outcome.

Results: All patients were with a median follow-up period of 72.97 months. Recurrence rate were similar among these patients. There is no significant difference of the overall $1,3,5$ year actural survival rate for the groups on the long-rank test $(p=0.073)$. After controlling the independent risks for disease-free survial, there is also no significant difference of the 1,3,5 year disease-free survival for the groups $(p=0.354)$. The patients with wider surgical margin had worse perioperative outcomes, more patients in this group needed blood transfusion $(p<0.001)$ and more patients suffered from postoperative complications $(p=0.020)$. They also had higher in-hospital mortality rate than that of other groups (1.41 vs. $0.64 \%)$.

Conclusion: No superiority was seen in patients with wider surgical margin in either perioperative features or long term outcome $(0.001)$.

\section{PP36-20}

Preceded Laparoscopic Splenectomy and Hepatic Resection Could Make an Alternative Option for Surgical Treatment of HCC with Severe Hypersplenism

Y. Iimuro, T. Okada, T. Hirano, S. Saito, Y. Asano, N. Uyama, Y. Yoshida, K. Suzumura, Y. Kondo, J. Fujimoto

Surgery, Hyogo College of Medicine, Nishinomiya, Japan

Background: Indication of liver resection for hepatocellular carcinoma (HCC) is sometimes limited by impaired liver function. Meanwhile, we have experienced laparoscopic splenectomy in patients with hypersplenism due to HCV-related chronic hepatitis or cirrhosis for the purpose of IFN therapy, and have observed that the impaired liver function has been improved in many of the patients by splenectomy per se. Thus, we have tried preceded laparoscopic splenectomy in two HCC patients with Child B or C, and could successfully perform anatomical liver resection in these patients.

\section{Patients:}

Case1: 61 years old, male, S6 HCC (6 cm in diameter; HBV-positive) with severely impaired liver function (Child-Pugh score 11; Alb 2.7 $\mathrm{mg} / \mathrm{dl}$, T.Bil 1.1, PT $46 \%$, ICGR15 35\%, Plt $\left.35 \times 10^{3} / \mu \mathrm{L}\right)$. He had received twice hepatic resection for HCC in 1997 and 2003, and was introduced to our department. We first performed laparoscopic 
splenectomy in this patient. One month later, the liver function significantly improved (Child-Pugh score 7; Alb $3.1 \mathrm{mg} / \mathrm{dl}$, T.Bil $0.5 \mathrm{mg} / \mathrm{dl}$, PT $54 \%$, ICGR15 $21 \%$, Plt $299 \times 10^{3} / \mu \mathrm{L}$ ), and S6 subsegmentectomy could be successfully performed. He is now healthy and tumor-free for 5 months.

Case 2: 64 years old, male, S1\&S8 HCC (HCV-positive) with impaired liver function (Child-Pugh score 8; Alb $3.1 \mathrm{mg} / \mathrm{dl}$, T.Bil 1.4 $\mathrm{mg} / \mathrm{dl}$, PT $66 \%$, ICGR15 42\%, Plt $\left.31 \times 10^{3} / \mu \mathrm{L}\right)$. Laparoscopic splenectomy and partial resection of S1 were done, and TACE was selected for S8 HCC (1 cm diameter). However, 4 months after TACE local recurrence and expansion of S8 HCC was detected with improved liver function (Child-Pugh score 6; Alb $3.9 \mathrm{mg} / \mathrm{dl}$, T.Bil 0.7 $\mathrm{mg} / \mathrm{dl}$, PT $78 \%$, ICGR $1522 \%$, Plt $60 \times 10^{3} / \mu \mathrm{L}$ ). We could perform anterior segmentectomy in this patient and he successfully discharged, but recurrence with portal thrombus appeared $3 \mathrm{M}$ later.

Conclusion: Preceded laparoscopic splenectomy could make an alternative option for surgical treatment of HCC with impaired liver function.

\section{PP36-21}

Da Vinci Robot - Assisted Liver Resection

G.H. Choi ${ }^{1}$, M.K. Ju ${ }^{1}$, J.Y. Park ${ }^{2}$, J.S. Choi ${ }^{1}$, W.J. Lee

${ }^{I}$ Department of Surgery; ${ }^{2}$ Department of Internal Medicine,

Yonsei University Health System, Seoul, Republic of Korea

Background: Since the minimally invasive surgery has been developed in all surgical fields, laparoscopic liver resection has been more frequently performed by many surgeons. However, laparoscopy has the limitations such as 2-dimensional imaging and restricted instrument motion. The da Vinci Robotic system provides 3-dimensional images and EndoWrist with a 360-degree range of motion.

Methods: From November 2008 to July 2010, 19 patients underwent robot- assisted liver resection in Yonsei University Health System, Seoul, Korea. We analyzed perioperative and short-term outcomes after robot-assisted liver resection.

Results: The mean age was 49.5 years (range: $32-68$ ) and 10 $(52.6 \%)$ of 19 patients were male. There were 13 malignant tumors ( 8 hepatocellular carcinomas, 2 cholangiocellullar carcinomas and 3 liver metastases from GI tract) and 6 benign lesions (4 intrahepatic stones, 1 recurrent liver cyst and 1 schwannoma). Right hepatectomy was performed in 5 patients, left hepatectomy in 9 patients, left lateral sectionectomy in 3 patients and wedge resection in 2 patients. Two patients (1 right hepatectomy and 1 wedge resection) underwent robotic colon resection simultaneously. The average operating times of right and left hepatectomy were 739 min (range: 704-812) and $551 \mathrm{~min}$ (range: 460-763), respectively. The average estimated blood loss of right and left hepatectomy were $625 \mathrm{cc}$ (range: 100-1,500) and $370 \mathrm{cc}$ (range: 150-900), respectively. Four patients $(20.1 \%)$ received perioperative transfusion. There were 2 conversions to open surgery (1 right hepatectomy and 1 left hepatectomy). The morality rate was $0 \%$ and the overall rate of complications was $31.6 \%$. Only two patients with bile leakage underwent endoscopic and percutaneous drainage procedures. The remaining four complications were managed conservatively without further procedure. The median days of hospitalization were 9 days (range: 5-46).

Conclusions: From our preliminary experience, robot-assisted liver resection seems to be a feasible and safe procedure.

\section{PP36-22}

Safe Hepatic Resection for Hepatocellular Carcinoma in a 90-year-Old Woman with Liver Cirrhosis

N. Oshima, S.-I. Ariizumi, Y. Kodera, Y. Takahashi, S. Yamashita, T. Kato, J. Takasaki, S. Katagiri, M. Yamamoto

Institute of Gastroenterology/Department of Surgery/Tokyo Womens Medical University, Tokyo, Japan
Hepatic resection for hepatocellular carcinoma (HCC) has become a safe procedure with low operative mortality. However, hepatic resection is often avoided in elderly patients because of the stress involved. We present the case of a 90-year-old woman who underwent hepatic resection for HCC with liver cirrhosis. In 2010, liver tumors were detected at a local hospital during follow-up of chronic hepatitis $\mathrm{C}$ and hypertension. Computed tomography showed 2 HCCs, 3 and $1.8 \mathrm{~cm}$ in diameter, respectively, in the anterior section. The indocyanine green retention rate at 15 minutes was $28 \%$, and the Child-Pugh classification was class A. The tumor markers AFP and PIVKA II were elevated to $650 \mathrm{ng} / \mathrm{ml}$ and $17 \mathrm{mAU} / \mathrm{ml}$, respectively. Based on the diagnosis of HCC, we performed resection of segment 5. The postoperative course was uneventful, and she was discharged 18 days after hepatic resection. To the best of our knowledge, this is the third report of hepatic resection in a patient with HCC aged 90 or over in Japan.

\section{PP36-23}

Continuous Hepatic Arterial Infusion Chemotherapy Combined with Intraportal Infusion Chemotherapy in Patients with Hepatocellular Carcinoma Complicating Portal Vein Tumor Thrombosis

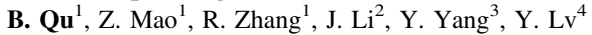

${ }^{I}$ Department of Oncological Surgery, Affiliated Hospital of Medical College of Chinese People's Armed Police Force, Tianjin;

${ }^{2}$ Department of Oncological Surgery, Shannxi Provincial People's Hospital; ${ }^{3}$ Department of General Surgery, The Second Affiliated Hospital of Medical College, Xi'an Jiaotong University; ${ }^{4}$ Department of Hepatobiliary Surgery, The First Affiliated Hospital of Medical College, Xi'an Jiaotong University, Xi'an, China

Background/aims: The prognosis of patients with hepatocellular carcinoma (HCC) complicating portal vein tumor thrombosis (PVTT) is extremely poor. The aim of this study was to evaluate the efficacy of postoperative continuous hepatic arterial infusion chemotherapy (CHAIC) combined with continuous intraportal infusion chemotherapy (CIPIC) for patients with HCC complicating PVTT.

Methods: 32 patients with gross PVTT in the portal trunk and/or the first-order branch underwent hepatic resection plus portal thrombectomy were divided into groups $\mathrm{A}(n=18)$ and $\mathrm{B}(n=14)$. Patients in group A received postoperative CHAIC combined with CIPIC via subcutaneously implanted injection port. The chemobiotherapy regimen consisted of 5-fluorouracil, doxorubicin, cisplatin, and leucovorin. Treatment was repeated every 4 weeks for six cycles. Patient outcomes of group A were compared with that in group B receiving CHAIC alone.

Results: The patients of group A and group B received an average of 4.8 and 5.2 cycles of chemotherapy respectively, starting from the 14th to the 24th day after surgery. For patients with low EdmondsonSteiner tumor grade (grade I and II), there was no significantly difference of survival rate and recurrence rate between the two groups. For patients with high Edmondson-Steiner grade (grade III and IV), 1-, 2-, and 3-year survival rates of group A (45.5, 27.3 and 9.1\%, respectively) were significantly higher than those of group B $(25,12.5$, and $0 \%$, respectively) $(p<0.05)$.

Conclusions: Preoperative CHAIC combined with CIPIC could benefit patients with high Edmondson-Steiner tumor grade HCC complicating PVTT more than using CHAIC alone.

\section{PP36-24}

Predictors of Recurrence after Hepatectomy for HCV Related Small Solitary Hepatocellular Carcinoma: A Retrospective Study

I. Kirino, H. Ikeda, M. Okabe, A. Tsuruta, K. Sano, K. Kawamoto, Y. Morimoto, T.B. Park, Y. Yoshida, T. Ito, K. Ogasawara Department of Surgery, Kurashiki Central Hospital, Kurashiki, Japan

Background and aim: Surgical resection for small $(<5 \mathrm{~cm})$ solitary hepatocellular carcinoma (HCC) is generally thought to be associated 
with a good prognosis. However, we experience substantial variability in postoperative survival even among those patients with early stage and preserved liver function. This study aims to investigate factors affecting disease recurrence after resection of $\mathrm{HCV}$ related small solitary HCC.

Patients and methods: Patients with clinically and pathologically confirmed HCV related solitary HCC less than $5 \mathrm{~cm}$ in size who underwent hepatic resection were evaluated using Kaplan-Meier curve and Cox proportional hazard model.

Results: From 1991 to 2006, 202 patients underwent hepatic resection for hepatocellular carcinoma $(\mathrm{HCC})$ in a single institution. Of these, 99 patients were with hepatitis $\mathrm{C}$ related, small $(<5 \mathrm{~cm})$ solitary HCC. Median age at surgery was 67 years and 82 patients $(82 \%)$ were male. Within median follow up of 60.0 months, 65 patients $(65 \%)$ developed recurrent disease and 62 $(95 \%)$ of them were intrahepatic. Corresponding disease free survival rates at 1, 3, 5 years were $88.9,59.4,47.5 \%$, respectively. Liver cirrhosis and capsular infiltration of the tumor were statistically significant risk factor for HCC recurrence on multivariate analysis. Capsular infiltration of the tumor was also statistically significant as the risk factor of early recurrence $(<2$ years after surgery) on univariate analysis. AFP, pathological grade, R1 resection, and satellite nodules were not statistically significant as the risk factors of recurrence.

Conclusions: In patients with $\mathrm{HCV}$ related relatively early stage $\mathrm{HCC}$, factors related to tumor characteristics and background liver cirrhosis were most strongly contributing to HCC recurrence. Although surgical curability is another factor that we usually consider, influence of $\mathrm{R} 1$ resection was not clear in this study.

\section{PP36-25}

Clinic Feature of Patients with Large Hepatocellular Carcinoma Larger than $10 \mathrm{~cm}$ in Diameter and Longterm Survival after Hepatic Resection A. Li, Z. Weiping, W. Mengchao

The Third Department of Hepatic Surgery, Eastern Hepatobiliary Surgery Hospital, Shanghai, China

Background: The role of hepatic resection for large hepatocellular carcinoma (HCC) larger than $10 \mathrm{~cm}$ remains unclear. Few data are available on longterm outcomes after this operation. We are investigating the safety and longterm survival in with large HCC.

Study design: Perioperative and long term outcomes of Between June 1996 and and july 1999, a total of 40 patients underwent resection for HCC larger than $10 \mathrm{~cm}$ at our group. Date were analyzed with regard to patient characteristics, intraoperative detail, pathologic findings, perioperative morbidity and mortality, and outcomes as determined by survival.

Results: 40 patients under went major hepatic resection. Macroscopic residual tumor, macroscopic venous invasion, and multiple tumors were identified as independent prognostic factors. The median survival of patients with curative resection of solitary HCC larger than $10 \mathrm{~cm}$ without macroscopic venous invasion was 64.0 months; that of patients with both macroscopic venous invasion and multiple tumors was only 15.5 months. 10-year survival rates was $20 \%$

Conclusions: Hepatic resection is a safe and effective treatment for $\mathrm{HCC}$ larger than $10 \mathrm{~cm}$ when liver function reserve is satisfactory and when curative resection can be expected. Patients with solitary HCC larger than $10 \mathrm{~cm}$ without macroscopic venous invasion can enjoy longterm survival after surgery. Multiple tumors were found after TACE. So we propose hepatic resection as a standard treatment for solitary HCC.
PP36-26

Laparoscopy-assisted Hepatectomy for Hepatocellular Carcinoma O. Itano ${ }^{1,2}$, M. Tanabe ${ }^{1}$, S. Kawachi ${ }^{1}$, M. Shinoda ${ }^{1}$, G. Oshima ${ }^{1}$, Y. Ono ${ }^{1}$, N. Tani ${ }^{1}$, R. Nishiyama ${ }^{1}$, H. Fujisaki ${ }^{1}$, K. Mihara ${ }^{1}$, T. Fujimura ${ }^{1}$, Y. Kadota ${ }^{1}$, K. Aiura ${ }^{1}$, M. Ueda ${ }^{1}$, S. Aikou ${ }^{2}$, Y. Kitagawa ${ }^{1}$

${ }^{1}$ Department of Surgery, Keio University, School of Medicine;

${ }^{2}$ Department of Surgery, Eiju General Hospital, Tokyo, Japan

Purpose: The purpose of this work was to evaluate the short term and mid term results of laparoscopy-assisted hepatectomy (LAH) for hepatocelluar carcinoma (HCC).

Methods: We retrospectively analyzed the clinical outcomes of 22 patients who had undergone LAH for HCC among 93 cases of laparoscopic hepatectomy from January 2006 to September 2010. The patients mean age was $67 \pm 9$ years. Tumor mean size was $2.8 \pm 1.0$ $\mathrm{cm}$ (range $1.2-5.5 \mathrm{~cm}$ ). A total of 10 patients were $\mathrm{HCV}$-positive and 5 were $\mathrm{HBV}$-positive. Others without viral infection consisted of 5 alcoholic hepatitis and 2 NASH. Child-pugh scores were Child-pugh $\mathrm{A}$ in 16 patients and $\mathrm{B}$ in 4 patients. The operation consisted of laparoscopic mobilization of the target liver lobe, followed by open liver resection through a $8-12 \mathrm{~cm}$ extraction site.

Results: There were 2 subsectionectomies, 7 anterior sectionectomies, 1 right hemihepatectomy and 12 partial hepatectomy. The mean operative time was $385 \pm 112 \mathrm{~min}$. The mean blood loss was $620 \pm 605 \mathrm{ml}$ and blood transfusion was required for two patients. There were no intraoperative complications. Postoperative complications developed in 4 cases $(18.2 \%)$, all of which were improved by conservative management. However, there was no postoperative mortality. Median postoperative hospital stay was 8.0 days. Recurrence was detected in 7 cases $(31.8 \%)$ after a mean period of 32.7 months from operation.

Conclusion: LAH for HCC is safe and feasible. More accumulation of date may be needed for evaluation of long-term outcome.

\section{Poster Presentation 37: Imaging Modalities}

PP37-01

Functional Computed Tomography with Perfusion Imaging in the Assessment of Tumor Vascularization in Cirrhotic Liver Disease

J. Huijie

Department of Radiology, Second Affiliated Hospital, Harbin Medical University, Haerbin, China

Introduction: Hepatocellular carcinoma (HCC) is a common malignant tumour in China, and early diagnosis is critical for patient outcome. In patients with liver cirrhosis, developing from benign regenerative nodules and dysplastic nodules to HCC lesions; differences in their respective blood supplies can assist in their detection and characterization. These haemodynamic and physiological properties can be measured serially using functional CT perfusion techniques Perfusion imaging of the liver has been invoked as a means of improving the sensitivity and specificity of diagnostic liver imaging.

Objectives: The aim of the present study was to evaluate the physiological characteristics of tumor angiogenesis in cirrhotic liver disease by functional CT perfusion.

Methods: Perfusion CT was performed in 30 volunteers without liver disease (control subjects) and 49 patients with liver cirrhosis, including 27 patients with HCC and 22 patients with only liver cirrhosis, proved by clinical and laboratory parameters. All patients underwent pre-contrast triple-phase CT and perfusion CT study using 
low-dose 64-slice VCT. A deconvolution mathematical model was applied to provide hepatic blood flow (HBF), hepatic blood volume (HBV), mean transit time (MTT), permeability of capillary vessel surface (PS), hepatic arterial index (HAI), hepatic artery perfusion (HAP) and hepatic portal perfusion (HPP) data.

Results: Tumor had significantly higher HAP, HAI $(P<0.05)$ and lower HPP $(P<0.05)$ than background liver parenchyma adjacent to $\mathrm{HCC}$ and control. The value of HBF in the tumor rim was significantly higher than control $(P<0.05)$. For cirrhotic liver parenchyma, except MTT and PS, such as HBF, HAP,HPP, HAI and HBV were significantly higher $(P<0.05)$ at cirrhotic liver parenchyma with HCC than control. Relatively satisfied image quality and perfusion parameters of liver had been acquired with low-dose scan.

Conclusions: PCT can be used to assessment of tumor vascularization in cirrhotic liver disease.

\section{PP37-02}

Intraoperative Indocyanine Green Fluorescent Imaging for Prevention of Bile Leakage after Hepatic Resection

M. Kaibori, M. Ishizaki, K. Matsui, A.-H. Kwon

Surgery, Kansai Medical University, Hirakata, Japan

Background: Bile leakage is one of most common complications, and is associated with an increase of sepsis and liver failure. There are no standard preventive methods against bile leakage after hepatic surgery. The aim of the present randomized clinical trial was to evaluate the application of indocyanine green (ICG) fluorescent cholangiography for preventing postoperative bile leakage.

Method: The subjects were 102 patients who underwent hepatic resection without biliary reconstruction. They were divided into two groups. The control group $(n=50)$ underwent a leak test with ICG dye alone, and the experiment group underwent a leak test with ICG dye, followed by ICG fluorescent cholangiography using the Photodynamic Eye (PDE group, $n=52$ ).

Results: Among 42 patients with fluorescence in the PDE group, 25 patients had insufficient closure of bile duct stumps on the cut surface of the liver, and these stumps were closed by suture or ligation. There were five cases of postoperative bile leakage in the control group versus no bile leakage in the PDE group (10 vs. 0\%, $p=0.019$ ).

Conclusion: ICG fluorescent cholangiography could detect insufficiently closed bile duct stumps that could not be identified by a standard bile leak test. ICG fluorescent cholangiography may have useful potential for prevention of bile leakage after hepatic resection.

\section{PP37-03}

Common Bile Duct Size in Patients with Cirrhosis

C. Wongjitrat ${ }^{1}$, A. Pattanapraphapan ${ }^{1}$, N. Wongjitrat ${ }^{2}$, W. Burivong ${ }^{3}$

${ }^{1}$ Internal Medicine; ${ }^{2}$ Pediatric; ${ }^{3}$ Radiology, Srinakharinwirot

University, Ongkarak, Thailand

Background: Cirrhosis is a common condition in general practice. The cirrhotic patients have increased risk for hepatocellular carcinoma (HCC) and require HCC surveillance by ultrasonography and alpha-fetoprotein (AFP) every 6 months. There is no data about common bile duct (CBD) size in cirrhotic patients compare with noncirrhosis.

Objective: The purpose of this study was to evaluate the common bile duct size in cirrhotic patients.

Methods: This was a pilot study on 100 patients age $>18$ years old including 50 cirrhosis and 50 non-cirrhosis. They had no evident of gallstone or pancreatic disease and no history of cholecystectomy or currently opioid use. Ultrasonography was performed after $12 \mathrm{~h}$ fasting to measure $\mathrm{CBD}$ diameter. Fasting blood samples were obtained and albumin, total bilirubin (TB), aspartate aminotransferase (AST), alanine aminotransferase (ALT) and alkaline phosphatase (ALP) were measured.
Results: There was no significant difference in mean age between cirrhotic and non-cirrhotic groups (56.4 vs. $55.6 \mathrm{yr} ; p=0.741)$. CBD size in cirrhotic group was significantly increased compared to noncirrhotic group (0.56 vs. $0.35 \mathrm{~cm} ; p<0.001)$. CBD dilatation $(\mathrm{CBD} \geq 0.8 \mathrm{~cm})$ was seen in 8 and $0 \%$ of cirrhotic and non-cirrhotic groups, respectively.

Conclusion: Cirrhosis is associated with CBD dilatation and should be considered in the differential diagnosis of asymptomatic CBD dilatation. Follow up by ultrasounography should be considered over further investigation (endoscopic retrograde cholangiopancreatography, magnetic resonance cholangiopancreatography) in cirrhotic patients without clinical or laboratory evidence suggesting biliary obstruction.

\section{PP37-04}

Can We Rely on Transient Elastography? The First 1000 Cases Performed by the New Zealand Liver Transplant Unit

A. Raj, E. Gane

New Zealand Liver Transplant Unit, Auckland, New Zealand

Aim: Transient elastography (FibroScan ${ }^{\mathrm{TM}}$ ) is an attractive noninvasive assessment of liver fibrosis, but appropriate use is important to obtain a clinically meaningful result. It is best validated for discriminating cirrhosis (F4) from $\leq \mathrm{F} 3$, and in patients with chronic viral hepatitis. Criteria for reliability of the result have recently been established. In this audit, we evaluate the reliability and validity of the first 1,000 FibroScans performed by the NZ Liver Transplant Unit according to these defined criteria.

Methods: Consecutive FibroScans (June 2009-August 2010) were retrospectively assessed for reliability, defined as

(i) at least 10 valid shots and

(ii) success rate $>60 \%$ and

(iii) interquartile range $(\mathrm{IQR}) /$ median $<30 \%)$.

Subgroup analyses were performed in patients with $\mathrm{HCV}$ (value of $>12.5 \mathrm{kPa}$ equivalent to cirrhosis), HBV and non-viral aetiology.

Results: 1000 FibroScan $^{\mathrm{TM}}$ procedures were performed, $50 \%$ for $\mathrm{HCV}, 36 \%$ for $\mathrm{HBV}, 7 \%$ for NAFLD, $2 \%$ ALD, $5 \%$ other. In $2.7 \%$ of cases, no result could be obtained. In the total population, $8.3 \%$ had $<10$ valid shots, $10.4 \%$ had $<60 \%$ success rate, and $18.2 \%$ had IQR $>30 \%$. Overall $74.4 \%$ met criteria for reliability. The reliability was similar amongst the 3 subgroups: HCV (76.7\%), HBV (72.5\%, $p$ value $=0.38$ vs. $\mathrm{HCV}$ ) and non-viral $71.9 \%$ ( $p$ value 0.19 vs. $\mathrm{HCV}$ ). Of the HCV group, $17.6 \%$ had cirrhosis.

Conclusion: Transient elastography is a useful test when referred appropriately. However, in $1 / 4$ of cases the result may be unreliable and in a small percentage, no result can be obtained.

\section{PP37-05}

Multicenter, Randomized Comparison Study to EVALUatE Outcomes and Resource Needs to Evaluate Metachronous Colorectal Liver Metastases - An Interim Analysis

M.-J. Kim ${ }^{1}$, C.J. Zech ${ }^{2}$, On Behalf of the VALUE Study Group

${ }^{1}$ Department of Radiology, Severance Hospital, Yonsei University, Seoul, Republic of Korea; ${ }^{2}$ Department of Clinical Radiology,

University of Munich, Munich, Germany

Purpose: To perform an economic evaluation of Gd-EOB-DTPAenhanced MRI (PV-MRI) compared to MRI with extracellular contrast-media (ECCM-MRI) and contrast-enhanced MDCT (CE-MDCT) as initial diagnostic modalities in the work-up of patients with colorectal liver metastases.

Methods and materials: 35 study centres prospectively recruited 296 patients from $10 / 2008$ to $07 / 2010$, which were referred for preoperative staging of the liver in case of suspected liver metastases. The initial imaging study was randomized. A consensus between the investigating surgeon and radiologist was reached 
whether further imaging was required to obtain a therapeutic decision. The primary efficacy variable was the proportion of patients for whom further imaging was required after initial imaging of the liver with PV-MRI as compared to ECCM- MRI and CE-MDCT. The results were compared using a one-sided approximate test for equality at a significance level of $2.5 \%$. The diagnostic confidence was rated by the investigator on a four-point scale.

Results: 98 patients received PV-MRI, 95 ECCM-MRI and 91 CE-MDCT. Further imaging was required in $0(0 \%), 15(15.8 \%)$ and 33 cases $(36.3 \%)$, respectively. These results are statistically significant. Diagnostic confidence was high/very high in 96 (98\%), 84 $(88.4 \%)$ and 51 cases $(67.1 \%)$, respectively.

Conclusion: The results show superiority of PV-MRI over CE-MDCT and ECCM-MRI with regard to the requirement for further imaging. With PV-MRI as the initial staging strategy, no further tests to assess operability were needed, which has implications for work-flow and costs. Further evaluation of the collected data with regard to diagnostic efficacy of each method compared to surgery and an exact cost calculation is planned.

\section{PP37-06}

Gd-EOB-DTPA-enhanced MRI of the Liver in Pre-capillary Pulmonary Hypertension: Incidence of Large Regenerative Nodules (LRN). Preliminary Data

M. Renzulli ${ }^{1}$, V. Lucidi ${ }^{1}$, E. Gotti ${ }^{2}$, C. Mosconi ${ }^{1}$, N. Galiè ${ }^{2}$, R. Golfieri ${ }^{1}$ ${ }^{1}$ Radiology Unit, Department of Digestive Diseases and Internal Medicine, Sant' Orsola-Malpighi Hospital, University of Bologna, Bologna, Italy; ${ }^{2}$ Cardiology Unit, Cardiovascular Department, Sant' Orsola-Malpighi Hospital, University of Bologna, Bologna, Italy

Background/aims: In this single centre prospective study, we evaluated the incidence of large regenerative nodules (LRN) in precapillary pulmonary hypertension (PI) with Gd-EOB-DTPA (Primovist $\left.^{\circledR}\right)$-enhanced MRI. The correlation with pre-capillary PI severity has been also assessed.

Methods: From September to December 2009, 24 patients (17 F, $70 \%$ ) aged between 24 and 86 years (mean 48 years) with different degrees of pre-capillary IP underwent Primovist ${ }^{\circledR}$-MRI: a blinded images evaluation was performed by two radiologists.

Results. A pattern of LRN was observed in 4 patients (16.7\%) (3 females, a mean age of $36 \mathrm{yrs}$ ). The total lesions number was 11 (range 1-5, mean 3 ) with average diameter of $8 \mathrm{~mm}$ (range 3-18 mm). The nodules appeared iso-hyperintense on T1-weighted images, isohypo-hyperintense on T2-weighted images, uniformly hypervascular in arterial phase without washout in portovenous phase and hyperintense (more rarely isointense) in hepatobiliary phase, reflecting hepatocellular composition and delayed clearance of bile from the nodules. The right atrial pressure of patients bearing LRN nodules tends to be higher than subjects without those characteristic lesions (13 vs. $10 \mathrm{mmHg}, p=0.19$ ). The interobserver diagnostic agreement was $100 \%$.

Conclusions: The incidence of LRN in patients suffering from precapillary PI (16.7\%) is higher than that reported in other diseases associated with LRN, with lower age onset (36 aa). MRI with hepatobiliary agent Gd-EOB-DTPA demonstrated high diagnostic accuracy in identification and characterization of LRN, with high degree of interobserver concordance. The trend towards higher right atrial pressure in patients with LRN suggests the involvement of an hemodynamic mechanism.In conclusion, the clinical relevanceapplication of our study is that pre-capillary PI has higher incidence of LRN than the other associated diseases and correlates with higher right atrial pressure and that Gd-EOB-DTPA enhanced-MRI is highly accurate in LRN characterization.
PP37-07

The Retrospective Study of the Discrepancy Cases between ICG Test and ${ }^{99 m}$ Tc-GSA Scintigraphy before Hepatectomy - Which Do You Trust Y. Ohkura, T. Hamada, K. Kumamoto, I. Ohsawa, M. Kishiwada, S. Mizuno, M. Usui, M. Tabata, S. Isaji, H. Sakurai

Department of Hepatobiliary Pancreatic and Transplant Surgery, Mie University Hospital, Tsu City, Japan

Background: The indocyanine green retention rate at $15 \mathrm{~min}$ (ICGR15) and technetium-99m galactosyl human serum albumin ( $\left.{ }^{99 \mathrm{~m}} \mathrm{Tc}-\mathrm{GSA}\right)$ scintigraphy have been widely used to assess preoperative evaluation of hepatic function, however the discrepancy between these tests are occasionally observed. The aim of this study is to evaluate the clinical reliability of the ICGR 15 and the hepatic uptake ration of ${ }^{99 \mathrm{~m}} \mathrm{Tc}-\mathrm{GSA}$ (LHL15).

Methods: The 158 patients who underwent hepatectomy at Mie University Hospital between 2006 and 2009 were enrolled in this study. We evaluated ICGR15, LHL15, hyaluronic acid(HA) and other liver function parameters before operation, pathological findings, operation method and postoperative course.

Results: A significant correlation was observed between ICGR15 and LHL15 (ICGR15 = 121.52-116.32 $\times$ LHL15, $r=-0.580$ ). However, there were no significant difference in any other liver function parameters. Both ICGR15 and LHL15 were significantly associated with fibrotic degree at pathological findings of liver. The discrepancy between ICGR 15 and LHL15 in twenty-three patients were observed, we divided three groups, patients in whom LHL15 was worse than ICGR15 (groupA, $n=9$ ), patients in whom LHL15 was better than ICGR15 (groupB, $n=13$ ), and patients in whom were not observed discrepancy (groupC, $n=136$ ). In groupA, the levels of serum HA tended to higher. The moderate and severe fibrotic degree in liver were pathologically proven in 8 cases $(89 \%)$ in groupA and in 7 cases $(54 \%)$ in groupB. In groupA, all pateints were perfomed underwent partial hepatectomy or segmentectomy, in groupB, four patients (31\%) were perfomed resection of two or more segments. However, there were no differences in postoperative complication.

Conclusions: Our data suggests that LHL15 is refrected more exactly pathological finding of background liver and pre-operative evaluation of hepatic function compared with ICGR15, and HA level is useful index with the discrepancy between ICGR15 and LHL15.

\section{PP37-08}

Liver Tumors-The Vascularisation Pattern Assessed by ContrastEnhanced Ultrasound

C. Cijevschi Prelipcean, I. Pintilei, O. Nedelciuc, D. Chirita, M. Dranga, C. Mihai

University of Medicine and Pharmacy 'Gr. T. Popa', Iasi, Romania

The development of contrast-enhanced ultrasound (CEUS) has considerably improved the possibilities of ultrasound in the assessment of liver tumors, based on tumor- specific vascularization pattern.

Aim: To investigate the ability of CEUS to differentiate between benign and malignant focal liver tumors in clinical practice.

Material and methods: In a prospective study between 1st September 2009 and 1st September 2010 we examined 120 patients with focal liver lesions diagnosed by conventional ultrasound. All patients were examined by CEUS with the contrast agent Sonovue, $5 \mathrm{~min}$, using low-MI ultrasound with contrast-specific software (Hitachi). The final diagnostic was made by computed tomography, magnetic resonance imaging and in some cases histological exam.

Results: There were 64 men and 56 women, aged between 27 and 79 years. We found 76 malignant lesions (38 hepatocarcinoma, 34 metastasis, 4 colangiocarcinoma) (group A) and 44 benign tumors (18 hemangiomas, 12 regenerative nodules, 6 focal steatosis, 4 focal 
nodular hyperplasia, 2 adenoma, 2 abcess)(group B). Hyperenhancement in the arterial phase with diffuse complete enhancement was found in 50 patients in group A and only in 4 patients in group B $(p<0.01)$. Hyperenhacement in the late phase was found in 2 patients group A and 16 patients group B. Hypoenhacement or no enhacement in the late phase was found in 72 patients group $\mathrm{A}$ and 12 patients group B $(p<0.01)$.

Conclusions: Hypoenhacement in the late phase is the best CEUS parameter to differentiate benign from malignant liver tumors.

\section{PP37-09}

Pain and Tolerance to Ultrasound-guided Percutaneous Liver Biopsy in Asian Patients with Diffuse Liver Disease

S. Mahadeva ${ }^{1}$, A.S. Mahfudz ${ }^{2}$, A. Vijayanathan ${ }^{2}$

${ }^{1}$ Medicine; ${ }^{2}$ Radiology, University of Malaya, Kuala Lumpur, Malaysia

Background: Percutaneous liver biopsy remains vital in the management of chronic liver disease but pain and tolerance among Asians is relatively unknown.

Methods: Consecutive adult patients undergoing ultrasound (US)guided percutaneous liver biopsy for diffuse liver disease had their worst pain sensation, post procedure, reported using a visual analogue scale (VAS) from 0 to 10 . Willingness to undergo a repeat biopsy was examined 2 weeks later.

Results: A total of 205 patients (median age 50 years; $43.9 \%$ female; ethnicity: Chinese $40 \%$, Malay $41.5 \%$ and Indian $18 \%$; disease: NAFLD $89 \%$, viral hepatitis $11 \%$ ) underwent USS guided liver biopsy from April 2009 to June 2010. No (i.e. 0\%) complications occurred in all patients. Post procedure VAS pain scores were categorized and reported as follows: none (score $0=39.9 \%$ ), mild (score $1-3=27.6 \%$ ), moderate (score $4-6=25.1 \%$ ) and severe (score $7-10=7.4 \%$ ). 55 $(27.1 \%)$ patients required analgesia (mostly Tramadol) for post-procedure pain, and this corresponded to the severity of pain scores $(73.3 \%$ severe, $45.1 \%$ moderate and $23.2 \%$ mild, $p<0.0001$ ). Independent risk factors for significant (i.e. moderate $\&$ severe) pain were explored and were identified as follows: age $<50$ years (OR 3.0, 95\% $\mathrm{CI}=1.4-6.3)$, female gender (OR 3.7, 95\% CI=1.8-7.5), Malay (OR $2.7,95 \% \mathrm{CI}=1.3-5.9)$ and Indian (OR 2.9, 95\% CI $=1.1-7.4)$ ethnicity. Education levels, number of needle passes, BMI and waist circumference were not found to be predictive of significant pain. $60.9 \%$ of patients with significant pain compared to $82.8 \%$ of patients with no/ mild pain were willing to undergo a repeat biopsy $(p=0.001)$ Conclusion: USS-guided liver biopsy is safe and well tolerated by the majority of Asian patients. Approximately one-third of patients experienced significant pain and this was more prominent in adults who were younger, of female gender and non-Chinese ethnicity.

\section{PP37-10}

Focal Liver Lesions Assessment by Acoustic Radiation Force Impulse Elastography (ARFI)

M. Sendroiu, S. Bota, I. Sporea, M. Danila, A. Popescu, R. Sirli UMFT V. Babes, Timisoara, Romania

Introduction: ARFI elastography is a new method used for the assessment liver fibrosis, thyroid gland nodules, breast nodules, liver and kidney tumors characterization. It is offered by Siemens and integrated into an ACUSON S2000 ultrasound system.

Aim: To assess the feasibility and the value of ARFI technique for the differentiation between focal liver lesions (FLL) and normal liver parenchyma and between malignant and benign FLL.

Methods: We evaluated 55 FLL: 38 hepatocellular carcinomas (HCCs), 9 liver metastases, 6 hemangiomas, 1 adenoma and 1 focal fatty lesion. To evaluate tissue stiffness (TS), we performed 10 ARFI measurements each in the tumor and in the surrounding liver parenchyma. Median values were calculated, expressed in meters/second $(\mathrm{m} / \mathrm{s})$.
Results: We could not obtain valid ARFI measurements in the tumor in 7 cases $(12.7 \%)$ and in the liver in 2 cases (3.6\%). The mean values of TS were similar in benign vs. malignant tumors: $1.76 \pm 0.76 \mathrm{~m} / \mathrm{s}$ vs. $2.43 \pm 1.01 \mathrm{~m} / \mathrm{s}(p=0.35)$, also in hemangiomas vs. surrounding liver $(1.60 \pm 0.64 \mathrm{~m} / \mathrm{s}$ vs. $1.62 \pm 0.64 \mathrm{~m} / \mathrm{s}, p=0.95)$. TS was statistically significant lower in HCCs than in the surrounding liver parenchyma $(2.31 \pm 0.99 \mathrm{~m} / \mathrm{s}$ vs. $2.74 \pm 0.67 \mathrm{~m} / \mathrm{s}, p=0.04)$, but statistically significant higher in metastases than in the liver parenchyma $(3.15 \pm 0.78 \mathrm{~m} / \mathrm{s}$ vs. $1.78 \pm 0.62 \mathrm{~m} / \mathrm{s}, p=0.007)$. TS was statistically significant higher in metastases than in hemangiomas $(3.15 \pm 0.78 \mathrm{~m} / \mathrm{s}$ vs. $1.60 \pm 0.64 \mathrm{~m} / \mathrm{s}, p=0.004)$, but similar in HCCs versus metastases $(2.31 \pm 0.99 \mathrm{~m} / \mathrm{s}$ vs. $3.15 \pm 0.78 \mathrm{~m} / \mathrm{s}$, $p=0.05)$ and HCCs versus hemangiomas $(2.31 \pm 0.99 \mathrm{~m} / \mathrm{s}$ vs. $1.60 \pm 0.64 \mathrm{~m} / \mathrm{s}, p=0.10)$.

Conclusion: Using ARFI we found statistically significant differences between the surrounding liver parenchyma and malignant FLL, but this method can't distinguish malignant from benign FLL.

\section{PP37-11}

Early Response Monitoring of Chemotherapy by Flyoxyglycose Positron Emission Tomography (FDG-PET) in Hepar Lymphoma

I. Subortseva ${ }^{1}$, A.M. Kovrigina ${ }^{2}$, S.V. Lepkov ${ }^{3}$, G.I. Storogakov ${ }^{3}$, T.T. Kondratieva ${ }^{2}$

${ }^{1}$ Clinical Oncology, Russian Academy for Postgraduate Medical Education Minisry of Health of Russia; ${ }^{2}$ Blokhin Cancer Research Centre; ${ }^{3}$ The Moscow Medical University of N.N. Pirogov, Moscow, Russia

Purpose: FDG-PET is more accurate than CT (computer tomography) alone in determining the extend of lymphoma. This study was performed to evaluate the impact of functional imaging of chemotherapy in patients with hepar lymphoma.

Patients and methods: Patients were eligible if they: had (morphological) cytological evidence of hepar lymphoma, had pre-treatment staging CT and FDG-PET, had no resent history of other malignances, had no prior treatment of lymphoma. Each patient underwent sequential CT and FDG-PET. For each patient individual treatment plan was made with CT and FDG-PET based. There were five patients with these criteria: 3 patients with primary extranodal lymphoma had hepar and bones involvement. One patient had hepar and lymph nodes involvement and one patient had hepar, pancreas, kidney, mediastinum involvement. All patients reserved systemic chemotherapy. After finish treatment tumor metabolic response was in 3 patients: 2 with primary extranodal and one with secondary hepar lymphoma. CT showed remainder nodes in hepar and bones. It has been regarded as CR (Complete Remission). These patients are alive in complete remission after 3 months. In two cases FDG-PET on completion of chemotherapy showed a partial metabolic response. Patient with residual tumor mass in left femur bone received involved field radiation therapy. And other patient with residual tumor mass in hepar was treated allogenic stem cell transplantation. Median follow up is one month.

Conclusion: FDG-PET has been shown to discriminate between "active" lymphoma and scar tissue following therapy leading to a redefinition of the response criteria, eliminating the category of " $\mathrm{CRu}$ ". It may be possible to individualise therapy on the basic of functional imaging after finish chemotherapy.

\section{PP37-12}

How Useful Is Contrast Enhanced Ultrasound (CEUS) for the Characterization of Focal Liver Lesions?

I. Sporea ${ }^{1}$, R. Badea ${ }^{2}$, A. Popescu ${ }^{1}$, A. Martie ${ }^{1}$, M. Socaciu ${ }^{2}$, R. Sirli ${ }^{1}$, M. Danila ${ }^{1}$, M. Sendroiu ${ }^{1}$, S. Bota $^{1}$

${ }^{1}$ Department of Gastroenterology and Hepatology, University of Medicine and Pharmacy Timisoara, Timisoara; ${ }^{2}$ IIIrd Medical Clinic, University of Medicine Iuliu Hatieganu Cluj Napoca, Cluj-Napoca, Romania 
Focal liver lesions (FLL) are quite frequently discovered in daily practice, at routine ultrasound.

The aim of our study is to present a bicentric experience (Department of Gastroenterology and Hepatology Timisoara and IIIrd Medical Clinic Cluj-Napoca) concerning the use of contrast enhanced ultrasound (CEUS) for the characterization of FLL and to find when we can avoid using other expensive imaging modalities as contrast enhanced CT or MRI.

Material and method: We performed a prospective, bicentric study, between September 2009-May 2010, and we evaluated 577 FLL (406 examinations in Timisoara Center and 171 examinations in ClujNapoca Center). A CEUS examination was considered conclusive if the FLL had a typical enhancement pattern after contrast injection according to the EFSUMB guidelines.

Results: From the 577 cases with FLL, 289 (50.1\%) were patients without known liver disease and 288 (49.9\%) with known chronic liver disease; in $473 / 577$ cases (82\%) CEUS was conclusive for the diagnosis. CEUS allowed the positive diagnosis of benign vs. malignant lesion in 517/577 (89.6\%) of all FLL, while in $60(10.4 \%)$ cases it was inconclusive and could not differentiate between benign or malignant lesions. The most frequent FLL in patients without chronic liver disease were: liver metastases-98 cases, hemangiomas-66 cases, focal nodular hyperplasia-21 cases, liver adenomas -8 cases, abscesses -7 cases, hydatid cysts-6 cases, biliary cysts- 12 cases, liver hematomas- 2 cases, etc

Conclusion: In our study, CEUS was conclusive in approx. $82 \%$ of the FLL and the benign or malignant character of a lesion were demonstrated in approximately $90 \%$ of cases. Thus, when faced to an uncharacteristic FLL on standard ultrasound examination, our local strategy is to perform CEUS as first-line investigation (thus avoiding other expensive examinations) and only in unclear cases to perform contrast enhanced CT or MRI.

\section{PP37-13}

Contrast Enhanced Ultrasonography (CEUS) Helps Characterization of Focal Liver Lesions in HIV Positive Patients

E. Dumitru ${ }^{1}$, I.M. Dumitru ${ }^{2}$, L. Alexandrescu' ${ }^{2}$, S. Rugina ${ }^{2}$

${ }^{1}$ Faculty of Medicine; ${ }^{2}$ Ovidius University, Constanta, Romania

Introduction: Patients infected with HIV frequently have abnormal liver biochemistry, leading to imagistic evaluation for hepatic lesions. The conventional sonography is frequently used to detect incidental focal liver lesions, but it has limited accuracy.

Aim: To determine the potential application of CEUS in the characterization of focal liver lesions encountered in clinical practice in HIV positive patients, and to evaluate whether CEUS could differentiate malignant focal liver lesions from benign lesions.

Method: All consecutive HIV positive patients with focal liver lesions detected by conventional ultrasonography during standard follow-up in a period of 15 months were included in the study. These patients were further referred for CEUS examination with SonoVue using low mechanical index. CEUS images were obtained and each lesion was characterized as malignant or benign and given specific lesion type diagnosis. The CEUS diagnosis was compared to that indicated by other imaging modalities, histopathology, and clinical follow up.

Results: Four hundred thirty-two HIV positive patients were studied, of witch $36(8.3 \%)$ patients were diagnosed with focal liver lesions based on conventional ultrasonography. CEUS correctly identified malignant liver lesions in 3 patients (malignant liver tumor-2 cases, focal liver infiltration due to lymphoma-1 case), with the final diagnosis confirmed by histopathology. 33 patients were correctly identified with benign liver lesions on CEUS imaging, with all these cases confirmed on clinical and radiological follow up (hemangioma -5 cases, focal nodular hyperplasia -1 case, focal steatosis -27 cases). In the detection of malignancy, the sensitivity and the specificity of CEUS was $100.0 \%$. In comparison with unenhanced US, CEUS markedly improves sensitivity and specificity for the characterization malignant/benign) of focal liver lesions.

Conclusions: In HIV positive patients, CEUS with SonoVue improves differentiation between malignant and benign focal liver lesions, and also provides improved lesion type diagnosis.

PP37-14

Lesion Volumetry: A New Tool for Evaluation and Follow-up of Liver Metastases

F. Vandenbroucke, B. Ilsen, J. de Mey

Radiology, UZ Brussel, Brussels, Belgium

Purpose: To evaluate the performance of a software solution for semi-automated volumetry and follow-up of liver metastases, and to investigate its impact on the RECIST.

Methods and materials: 70 liver metastases of colorectal carcinoma in 18 patients with a total of $54 \mathrm{CT}$-scans were included. With at least 2 follow-up exams per patient, 178 lesions were evaluated. For each lesion 2 readers (R1, R2) manually measured the longest axial diameter (LAD) and determined the volume after manual lesion delineation. In addition, LAD and volume were calculated by a semiautomatic software. Volumes obtained by both methods were compared for each lesion. RECIST was used to evaluate treatment response in each follow-up using the sum of the measured LADs as well as the sum of the automated calculated volumes.

Results: $90 \%$ of al the cases were classified as 'good' or 'average' of which $60 \%$ were 'good'. For lesions with a 'good' semi-automated segmentation, mean volume difference between the manual (average of volume measured by R1 and R2) and semi automated method was $28 \%(p<0.01)$ whereas between the 2 readers it was $16 \%(p<0.01)$. Response assessments done with volume in comparison to LAD lead to differences in response categories (progression, stability, regression) of $14.7,11.8$ and $8.8 \%$ in follow-up of patients; evaluated by $\mathrm{R} 1, \mathrm{R} 2$, and the software solution, respectively.

Conclusion: The software solution with automatic liver segmentation showed performances comparable to the manual delineation of lesions. In the near future treatment response could be assessed according to volume instead of LAD.

\section{PP37-15}

Immediate Low Dose Chest Computed Tomography after Trascatheter Arterial Chemoembolization. Benefits and Radiation Dose

J.-I. Choi ${ }^{1}$, H.B. Kim ${ }^{2}$, J.S. Lee ${ }^{3}$, M.J. Kim ${ }^{3}$, J.-W. Park ${ }^{4}$, H.-K. Ko ${ }^{3}$, S.B. $\mathrm{An}^{3}$ ${ }^{1}$ Radiology, Seoul Saint Mary's Hospital, The Catholic University of Korea, Seoul; ${ }^{2}$ Radiology, Center for Liver Cancer; ${ }^{3}$ Radiology;

${ }^{4}$ Center for Liver Cancer, Research Institute and Hospital, National Cancer Center, Goyang, Republic of Korea

Background/aims: As the effectiveness of combined therapies of hepatocellular carcinoma (HCC) by multiple treatment modalities such as radiofrequency ablation (RFA) and transcatheter arterial chemoembolization (TACE), immediate response evaluation after treatment gets more importance recently. We performed low dose chest CT (LDCT) including upper abdomen immediate after TACE for the evaluation of appropriateness of embolization on the basis of the idea that the contrast between lipiodol uptakes and liver parenchyma is so prominent that noisy LDCT images might be enough for the evaluation of extent of lipiodol uptake to HCC masses. The purpose of this study was to evaluate the benefits of LDCT immediate after TACE and compare radiation dose of LDCT to that of conventional chest CT.

Methods: From 2006, we performed LDCT immediate after TACE for the most of the patients who were treated by TACE. LDCT scans were done within $2 \mathrm{~h}$ of embolization. We retrospectively reviewed the benefits of LDCT immediate after TACE in terms of 
(1) Completeness of treatment,

(2) Presence of immediate complication of TACE,

(3) Detection of new pulmonary metastases.

We calculated mean dose-length product (DLP) of LDCT of 100 patients who performed LDCT recently and compare mean DLP to that of conventional chest CT of HCC patients for the evaluation of radiation exposure by LDCT

Results: We experienced cases that

(1) early detected incomplete embolization and immediate additional RFA or PEIT,

(2) complications including lipiodol uptakes to diaphragm, lung or gallbladder,

(3) effective embolization of metastatic lymphadenopathies.

Mean DLP of LDCT and conventional chest CT was 137.8 and $660.3 \mathrm{mGy} \mathrm{cm}$ and the difference was statistically significant.

Conclusion: LDCT after TACE has benefits in immediate decision marking for unsuccessful treatment after TACE and early detection of TACE related complications. Radiation dose of LDCT was very low and additional LDCT after TACE might be justified.

\section{PP37-16}

Magnetic Resonance Elastography of Liver as an Alternative to Percutaneous Liver Biopsy in Chronic Hepatitis B Patients-initial Clinical Experience

S.K. Venkatesh ${ }^{1}$, H.S. Alsaif ${ }^{1}$, A.M. $\mathrm{Oo}^{2}$, V. Lai ${ }^{2}$, S.G. Lim ${ }^{2}$

${ }^{I}$ Diagnostic Imaging; ${ }^{2}$ Gastroenterology and Hepatology, National University Health System, Singapore, Singapore

Purpose: To describe our initial experience with MR Elastography (MRE) for as an alternative to percutaneous liver biopsy for evaluation of liver fibrosis in chronic hepatitis B patients.

Methods: Fifty-two patients with chronic hepatitis B infection underwent MRE of the liver to assess for underlying liver fibrosis for further management of their disease. Liver MRE was performed in 36 patients (Group A) who had received no treatment before; in 12 patients who were on anti-viral treatment (AVT) (Group B) and in 3 patients (Group C) had completed or discontinued AVT. MRE was performed on a $1.5 \mathrm{~T}$ MRI scanner using a specialized sequence known as MRE sequence Four sections through the entire crosssection of the largest part of the liver were obtained and mean liver stiffness was estimated. Stiffness values more than $2.93 \mathrm{kPa}$ was reported as suggestive of fibrosis. Clinical follow up was performed to see if MRE was useful for further management of these patients.

Results: Liver stiffness values and hepatitis B viral load determined the management plan of the study group. In Group A, 17 patients with significant fibrosis $(>3.5 \mathrm{kPa})$ and/or elevated hepatitis B viral load $\left(>10^{5}\right.$ copies $\left./ \mathrm{ml}\right)$ were started on AVT. Six patients with mild stiffness and normal viral load, and 14 patients with normal stiffness did not receive any AVT. In Group B, 11 patients were continued on AVT as they had either high liver stiffness or high viral load. One patient was discontinued on AVT as liver stiffness and viral load was normal. In Group C, all patients showed normal liver stiffness and were followed up. The liver function tests did not influence clinical decision for antiviral treatment.

Conclusions: MRE of the liver is an useful alternative to liver biopsy in the management of chronic hepatitis B patients.

PP37-17

Comparison of MR Elastography and Fibro-C Index for Quantification of Liver Fibrosis in Chronic Hepatitis B Patients

S.K. Venkatesh ${ }^{1}$, D. Tai ${ }^{2}$, A. Wee ${ }^{3}$

${ }^{1}$ Diagnostic Imaging, National University Health System; ${ }^{2}$ Institute of Infocommunications and Research, ASTAR; ${ }^{3}$ Pathology, National

University Health System, Singapore, Singapore
Purpose: Comparison of MR Elastography (MRE) and Fibro-C index for quantification of liver fibrosis in chronic hepatitis B.

Methods: A retrospective comparison of quantification of liver fibrosis with MRE and Fibro-C index was performed in 30 patients with chronic hepatitis B. Stiffness of the liver was estimated by placing regions of interest on the stiffness maps MRE and a mean value in kilopascals $(\mathrm{kPa})$ obtained. The liver biopsy samples were processed with standard histology technique and an experienced hepatopathologist graded for fibrosis using METAVIR score. On the same biopsy sample, quantification of the amount of collagen with Fibro-C index was performed with Gaussian mixture model segmentation method and quantification index representing total aggregated collagen amount was obtained. The stiffness values, METAVIR scores and quantification index scores were correlated. Receiver operator curve (ROC) analysis was performed for accuracy of MRE and Fibro-C index for detection of fibrosis.

Results: Liver stiffness values with MRE and quantification by Fibro$\mathrm{C}$ index correlated well $\left(R^{2}=0.64, p<0.01\right)$. There was no significant difference between the accuracies of MRE and Fibro-C index for detection of fibrosis $(0.94$ vs. $0.90, P=0.5)$, significant fibrosis (0.95 vs. $0.90, P=0.41)$ and cirrhosis $(0.99$ vs. $0.94, P=0.2)$. However, MRE performed better than Fibro-C index in differentiating the different grades of fibrosis from normal liver.

Conclusion: MRE derived stiffness of liver correlates well with fibrous content of liver and differentiates fibrotic liver and normal liver, therefore MRE may be suitable alternative for non-invasive assessment of liver fibrosis burden in chronic hepatitis B patients.

\section{PP37-18}

Non-invasive Detection of Liver Fibrosis in Chronic Hepatitis B: Comparison of Magnetic Resonance Elastography with Serum Markers of Fibrosis

S.K. Venkatesh ${ }^{1}$, H.S. Alsaif ${ }^{1}$, L.L.S. Teo ${ }^{1}$, S.G. Lim ${ }^{2}$

${ }^{I}$ Diagnostic Imaging; ${ }^{2}$ Gastroenterology and Hepatology, National University Health System, Singapore, Singapore

Purpose: To compare magnetic resonance elastography (MRE) and serum markers of fibrosis for non-invasive detection of liver fibrosis in patients with chronic Hepatitis B (CHB)

Methods and materials: Fifty two patients with known chronic hepatitis B infection and who underwent liver biopsy or surgery were evaluated with MRE and routinely available serum markers of fibrosis: aspartate aminotransferase (AST)-to-platelet ratio index (APRI), AST/alanine aminotransferase (ALT) ratio, prothrombin time/international normalized ratio (INR). All patients underwent MRE on a standard 1.5T MRI scanner. MRE was performed prior to commencement of therapy or surgical resection using modified phasecontrast gradient-echo sequences, with TR/TE $=100 / 27 \mathrm{~ms}$, matrix $96 \times 256,4 \times 10 \mathrm{~mm}$ slices, interslice gap $5 \mathrm{~mm}$. Regions of interest (ROI) were drawn on the elastograms to exclude large vessels, liver edges and artifacts wherever possible. Mean stiffness values were expressed in kilopascals $(\mathrm{kPa})$. The histopathologic grading of liver fibrosis was performed by an experienced hepatopathologist. The histopathologic grading was used as gold standard. The stiffness values in $\mathrm{kPa}$ were compared with serum markers using histopathologic examination as the reference standard. Patients with normal liver tissue confirmed on tissue samples taken from resections performed for other causes were also included as normal controls. Receiver operating characteristic (ROC) analysis was performed for determining accuracy and compared. Accuracy for detection of liver fibrosis and clinically significant fibrosis ( $\Rightarrow$ METAVIR F2 stage).

Results: MRE was successful in all the 52 patients. ROC analysis showed that the area under curve (AUC) values for MRE, APRI, AAR and PI for detection of fibrosis was 0.975, 0.66, 0.46 and 0.70, respectively. Similarly the accuracy for detection of significant fibrosis was $0.975,0.75,0.45,0.70$, respectively. 
Conclusion: MRE is a better predictor of fibrosis and significant fibrosis in patients with chronic hepatitis B as compared to routine serum markers.

\section{PP37-19}

Magnetic Resonance Elastography of Liver-What, Why, How and When?

S. Venkatesh ${ }^{1}$, S.G. Lim $^{2}$

${ }^{I}$ Diagnostic Imaging; ${ }^{2}$ Gastroenterology and Hepatology, National University Health System, Singapore, Singapore

Introduction: Magnetic resonance elastography is a new, non-invasive technique for quantitative evaluation of stiffness of tissues in vivo. MRE provides quantitative parameters that can be useful for characterization of disease processes of liver. Chronic liver diseases irrespective of the cause finally leads to liver fibrosis and if progressive to cirrhosis. Fibrosis of the liver leads to increased stiffness of the liver which can be detected by the elastography.

Objective: To introduce the new technique to hepatologists and answer the following questions. What is this technique? Why do we need this technique? How is this technique performed? and when is this indicated?

Methods: The principles of the MRE will be described followed by the steps involved in performing this technique. The strengths and limitations of this technique will be described. Indications for this technique will be illustrated with suitable clinical examples. A comparison with Fibroscan will also be presented.

Conclusion: An overview of the new technique of MR Elastography of Liver is presented.

\section{PP37-20}

Focal Nodular Hyperplasia and Adenoma of the Liver: Contrast Enhanced Ultrasound Performance

P. Taimr ${ }^{1}$, R.J. de Knegt ${ }^{1}$, R. de Man ${ }^{1}$, R. Dwarkasing ${ }^{2}$, H.L.A. Janssen ${ }^{1}$ ${ }^{1}$ Hepatogastroenterology; ${ }^{2}$ Radiology, Erasmus Medical Centre, Rotterdam, The Netherlands

Aims: The purpose of this prospective single centre study was to assess the clinical value and impact of contrast-enhanced ultrasound (CEUS) imaging in the characterization of focal nodular hyperplasia $(\mathrm{FNH})$ and hepatocellular adenoma in a tertiary referral centre. FNH and adenoma imaging have different typical findings, but some atypical nodules may still be difficult to differentiate without biopsy. Methods: One-hundred-and-nine consecutive patients with FNH or adenoma were assessed with CEUS, using contrast agent Sonovue (Bracco) The reference imaging included MRI (82\%) and CT (18\%). The final diagnosis was based on consensus interpreting all examinations, including histology (18 pts, 17\%) and clinical follow-up. CEUS and MRI diagnosis was based on standard criteria (FNH: central scar, centrifugal arterial filling, adenoma: arterial filling, both lesions without wash-out during late phase).

Results: In these 109 patients the MRI and/or CT diagnosis was FNH in 51 pts, adenoma in 52 pts and not discernible (adenoma/FNH) in 6 patients. Average lesion diameter was $60.6(20-120) \mathrm{mm}$. CEUS resulted in concordance with MRI in 92 out of 109 patients (84\%) and falsely interpreted 17 patients (16\%). CEUS correctly diagnosed 14 of the 18 biopsy proven lesions and MRI/CT group 12 of 18 lesions. Results against final diagnosis were for CEUS 93 out of 109 correct (85\%) and for MRI 102 out of 109 (94\%). If we regard both methods as complementary, the diagnosis was correct at least by one imaging in $106 / 109$ patients $(97 \%)$.

Conclusion: CEUS has a comparable performance as MRI in the diagnosis of FNH and adenoma of the liver. Therefore, CEUS could be used as the first line imaging method. In patients, where CEUS findings are unclear, MRI is the method of choice. Only in patients with contradictory results from both imaging methods histological assessment is needed.
Poster Presentation 38: Liver Transplantation

\section{PP38-01}

Rapamycin Induces Heme Oxygenase-1 in Liver

A. Kist ${ }^{1}$, J. Wakkie ${ }^{1}$, A. Nikolic ${ }^{1}$, R. Versteeg ${ }^{1}$, J. Ten Berge $^{1}$, V. Nieuwenhuijs ${ }^{2}$, R.T. Padbury ${ }^{3}$, G. Barritt ${ }^{1}$

${ }^{I}$ Medical Biochemistry, Flinders University, Adelaide, SA, Australia;

${ }^{2}$ Surgery, University Medical Centre, Groningen, The Netherlands;

${ }^{3}$ The HPB \& Liver Transplant Unit, Flinders Medical Centre and School of Medicine, Flinders University, Adelaide, SA, Australia

Background/aims: Liver surgery is associated with ischemia and reperfusion (IR) injury resulting in loss of liver function. Reactive oxygen species (ROS) play a major role in hepatocyte damage. This can be reduced by induction of the anti-oxidant enzyme heme oxygenase-1 (HO-1). Rapamycin, an immunosuppressant, induces the synthesis of HO-1 in non-liver tissues. The aim was to test whether rapamycin will induce HO-1 in liver and protect against IR injury.

Methods: An established rat liver model of segmental IR in which the rate of bile flow is measured gravimetrically was employed. Hepatocytes were isolated by collagenase digestion. Expression of HO-1 was measured by quantitative PCR and western blot.

Results: Incubation of hepatocytes with rapamycin or the established inducer cobalt protoporphyrin (CoPP) increased expression of $\mathrm{HO}-1$ mRNA and protein. Maximal effects $(7.7 \pm 3$ cf $0.9 \pm 0.2$, mean \pm SEM, $n=4, p<0.01$, relative expression mRNA) were observed at $36 \mathrm{~h}$, and half-maximal at $10-100 \mathrm{ng} / \mathrm{ml}$ rapamycin. In rats, rapamycin or CoPP increased HO-1 expression in livers removed immediately after laparotomy, and in livers subject to sham surgery. Livers subject to a sham surgery alone exhibited higher expression of HO-1 mRNA than livers removed immediately. The effects of rapamycin or CoPP on HO-1 were associated with a 3-fold decrease in the rate of bile flow recovery immediately following IR.

Conclusions: Rapamycin induces the expression of HO-1 in the liver. The effects of rapamycin on bile flow require investigation to further evaluate the capacity of rapamycin pre-treatment to protect against IR injury.

\section{PP38-02}

Assessment of Prognostic Factors for Patients with Fulminant Hepatitis B Underwent Emergency Living Donor Liver Transplantation

L. Lu ${ }^{1}$, F. Zhang ${ }^{2}$, X. Wang ${ }^{1}$, X. Li ${ }^{1}$, L. Kong ${ }^{1}$, B. Sun ${ }^{1}, \mathrm{G} \mathrm{Li}^{1}$

${ }^{1}$ First Affiliated Hospital of Nanjing Medical University, ${ }^{2}$ Liver

Transplantation Center, First Affiliated Hospital of Nanjing Medical University, Nanjing, China

Background: The high mortality rate associated with acute fulminant hepatitis B combined with the limited availability of cadaveric organs requires consideration of alternatives to conventional cadaveric transplantation. Living donor transplantation holds promise in a variety of circumstances, including high-acuity situations. The impact of LDLT as a proactive approach on the outcome of patients with acute fulminant hepatitis B in the urgency condition was analyzed. Methods: From September 2002 to May 2008, emergency LDLT was performed to consecutive 20 patients with fulminant hepatitis B. Variables that may affect patient survival following LDLT were analyzed. The resulting patient survival model was examined and refined in emergency and selective operation for hepatic failure secondary of $\mathrm{HBV}$ in the model for end-stage liver disease (MELD) database. Kaplan-Meier methods, univariate comparisons, and multivariate Cox proportional hazard regression were employed for analysis.

Results: The main complications after transplantation included pulmonary infection in 2, acute renal failure in 2 and transplantationrelated encephalopathy in 1 . No primary liver function failure, vascular or biliary complications occurred. The 1-year survival rate was $75 \%$. There were no donor serious complications or death. 
Conclusion: Postransplant patient survival can be accurately predicted based on eight straight forward factors. The balanced application of a model for liver transplant survival estimate, in addition to disease severity, as estimated by the model for fulminant hepatitis B, would markedly improve survival outcomes and reassessment indications for emergency liver transplantation.

\section{PP38-03}

Diagnosis and Treatment of Acute Appendicitis Immediately after Orthotopic Liver Transplantation in Adult

L. Wu, X. He, Z. Guo, D. Wang, W. Ju, Q. Tai, X. Zhu

The First Affiliated Hospital of Sun Yat-sen University, Guangzhou, China

Objective: The incidence rate of acute appendicitis (AA) after orthotopic liver transplantation (OLT) was low, but it is difficult to be distinguished from other complications after transplantation. This study aimed to summarize the clinical characteristics, diagnosis and treatment of AA during the early post-operative stage after OLT.

Methods: The clinical data of 4 liver transplant recipients with AA immediately after transplantation and another 4 patients with misdiagnosed appendicitis, between January 2000 and December 2007, were studied retrospectively.

Results: The incidence rate of AA immediately after OLT was $0.49 \%$ (4/817) in our single center. The 4 patients complained right lower quadrant abdominal pain with fixed tenderness and rebound tenderness and fever on the 8 th, 13th, 11th and 9th day post-transplantation, respectively. The white blood cells counts were elevated in all patients. Appendectomy was performed on day $1(2 / 4)$ or day $2(2 / 4)$ of the onset. No appendicular perforation was found during the operation. The patients recovered well and now have been followed up for 84, 62, 40 and 29 months, respectively. The other 4 misdiagnosed patients were diagnosed with duodenal diverticulum perforation in 1 , jejunum perforation in 1 and bile leakage in 2 after exploratory laparotomy. 2 of the misdiagnosed patients died even after aggressive surgical intervention.

Conclusions: The incidence rate of AA immediately after OLT is low, which should be differentiated from other surgical complications, such as gastrointestinal perforation and bile leakage. Appendectomy are recommended in this group of patients as early as possible.

\section{PP38-04}

The Evaluation of Living Donor for Liver Transplantation-A Retrospective Study

A. Sobhonslidsuk ${ }^{1}$, P. Intaraprasong ${ }^{1}$, S. Leelaudomlipi ${ }^{2}$, S. Teeraratkul ${ }^{2}$, S. Treepongkaruna ${ }^{3}$, P. Pootracool ${ }^{2}$, S. Jongjirasiri ${ }^{4}$, S. Viengteerawat ${ }^{5}$, P. Sornmayura ${ }^{6}$, P. Junchotikul ${ }^{7}$, K.R. Reddy ${ }^{8}$, S. Sriphojanart ${ }^{2}$

${ }^{1}$ Medicine; ${ }^{2}$ Surgery; ${ }^{3}$ Pediartics; ${ }^{4}$ Radiology; ${ }^{5}$ Anaesthesiology;

${ }^{6}$ Pathology; ${ }^{7}$ Nursing, Ramathibodi Hospital, Mahidol University,

Bangkok, Thailand; ${ }^{8}$ Gastroenterology, University of Pennsylvania, Philadelphia, PA, USA

Background: Living donor liver transplantation (LDLT) has been performed in Thailand for nearly ten years. Pediatric LDLT using left lobe graft constitutes the majority of cases. The evaluation of living donors is to exclude donors who have high risks of morbidity and mortality, and prevent graft dysfunction in recipients.

Method: A retrospective review of potential donors referred to the Ramathibodi liver transplant program between 1 Mar 2001 and 30 Sep 2010 was performed. Our three-phase protocol for living donor evaluation was implemented in 2005. Fifty-three potential donors were evaluated according to this protocol. Seven donor records could not be found.

Result: Forty-six potential donors for forty-three pediatric recipients were referred for evaluation. Biliary atresia was the most common $(60.9 \%)$ indication for pediatric LDLT. Donor age was 30.8 years.
The dominant blood group was O (52.2\%). Medical diseases (viz. dyslipidemia, diabetes mellitus) were found in 11 (23.9\%) cases. Regular alcohol drinking was reported in $4(8.7 \%)$ cases. Body mass index (BMI) was $21.7 \mathrm{~kg} / \mathrm{m}^{2}$. Overweight, obesity and morbid obesity were found in $10(21.7 \%), 5(10.9 \%)$ and $3(6.5 \%)$ cases. Liver biopsy was done in $12(26.1 \%)$ cases, with high BMI being the most common indication of biopsy (83.3\%). Significant fatty liver changes (15 and $20 \%$ ) was seen in two cases; repeat biopsy after weight reduction, life-style changes and alcohol discontinuation revealed resolution of fatty change. LDLT was not pursued in $5(10.9 \%)$ cases due to financial constraint (2), positivity for HBsAg (1), anti-HBc IgG (1) and anti-HCV antibody (1). There were no serious complications after LDLT.

Conclusion: The significant number of living donors had medical diseases and high body weight that might contraindicate donation. Careful and comprehensive evaluation of donors helps in good donor and recipient outcomes. Protocol for living donor evaluation comprising laboratory, radiological and histological study is crucial.

\section{PP38-05}

Health-related Quality of Life of Filipino Pediatric Liver Transplant Recipients and Children with Chronic Liver Disease using PedsQL4.0 ${ }^{\mathrm{TM}}$ Tagalog Version

K.M. Calixto-Mercado ${ }^{1}$, G.V. Gregorio ${ }^{2}$

${ }^{1}$ Pediatrics, Makati Medical Center, Makati; ${ }^{2}$ Pediatrics, University of the Philippines College of Medicine-Philippine General Hospital, Manila, Philippines

Background: The pediatric quality of life (PedsQL4.0 ${ }^{\mathrm{TM}}$ ) scale has been shown to be reliable and valid in assessing health related quality of life (HRQOL). However, its Tagalog version has not been fieldtested. The HRQOL of Filipino patients pre- and post-liver transplant has never been reported.

Aims: To determine the reliability and validity of the PedsQL4.0 $0^{\mathrm{TM}}$ Tagalog version and to compare the HRQOL of Filipino LT recipients, children with chronic liver disease (CLD) and healthy controls. Methodology: This is a cross-sectional study that included Filipinos $2-18$ years who had undergone LT and those with CLD. PedsQL4.0 ${ }^{\mathrm{TM}}$ Tagalog version was administered as a proxy-report for caregivers and child-report for children $>5$ years.

Results: 237 PedsQL4.0 questionnaires were completed. Reliability was demonstrated for psychosocial (Cronbach $\alpha=0.86-0.88$ ), physical $(\alpha=0.86-0.88)$ and total $(\alpha=0.89-0.92)$ health summary scores. Construct validity showed a medium to large effect size (0.39-1.34) between patients and controls. No difference was noted on the total health summary scores and the individual domains between LT recipients and controls. The scores of patients with CLD were significantly lower compared to LT recipients and healthy subjects.

Conclusions: The PedsQL4.0 ${ }^{\mathrm{TM}}$ Tagalog version is a valid and reliable HRQOL tool. The HRQOL of LT recipients is similar to healthy children while CLD patients had poorer HRQOL.

\section{PP38-06}

Orthotopic Liver Transplantation for Hepatocellar Carcinoma Exceeding Hangzhou Criteria: Challenge and Experience

W. Zhang, M. Zhang, M. Zhang, L. Wu, L. Zhou, S. Zheng

Division of Hepatobiliary and Pancreatic Surgery, Department of

Surgery, Key Laboratory of Combined Multi-Organ Transplantation, Ministry of Public Health, First Affiliated Hospital, Zhejiang

University School of Medicine, Hangzhou, China

Introduction: Hepatocellar carcinoma (HCC) is the most common malignant hepatic tumor which can be cured by hepatic resection and liver transplantation. Orthotopic liver transplantation (OLT) is considered much preferable method since it may eradicate both the 
tumor and the primary hepatic disease such as cirrhosis. Shortages of donor and tumor recurrence make considerable limitations in choosing liver carcinoma recipients for this treatment. Milan or UCSF criteria are adopted by many transplantation centers, also Hangzhou criteria which expand the above standard is recently established.

Case report: We report a case of hepatocellar carcinoma (HCC) exceeding Hangzhou criteria which was treated with orthotopic liver transplantation 6 years ago. The patient, 59-year-old man, had multiple lesions in the liver, histology showed moderately-low differentiated HCC (Fig. 1), with elevated serum $\alpha$-fetoprotein level (10380.1 ng/l), but weekly expressed for MMP9, BCL-2, Cerb-B2, and P53 by immunohistochemical examination (Fig. 2), has no tumor recurrence can be found so far after transplantation. (Fig. 3)

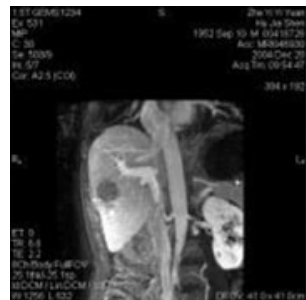

A

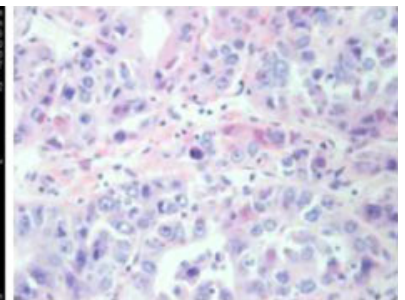

B (magnification, X400)
Fig. 1 a MRCP showed big occupation(size: $8.5 \mathrm{~cm} \times 8 \mathrm{~cm}$ ) in right liver before liver transplantation, cirrhosis also can be observed. b Giant and bizarre tumor cells by H\&E (hematoxylin-eosin) staining
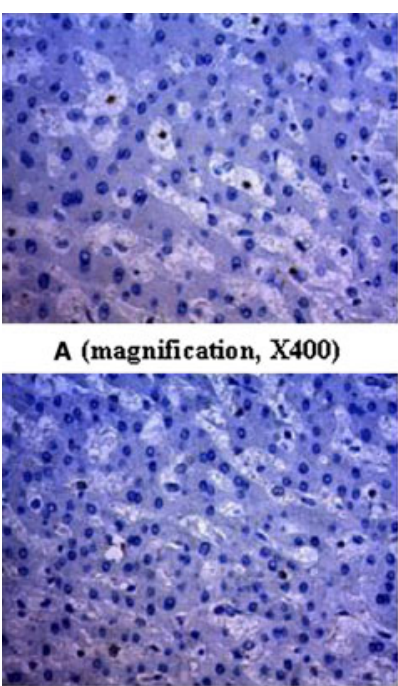

C (magnification, $\mathrm{X} 400$ )

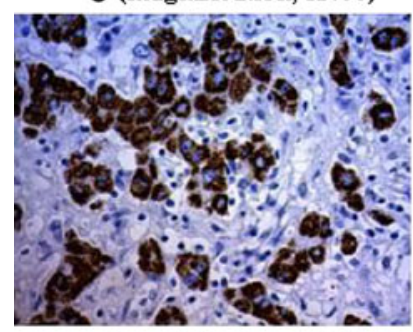

E (magnification, X200)
A (magnification, X400)

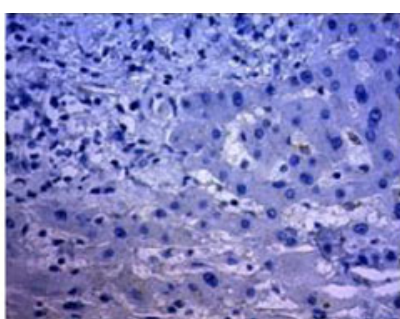

B (magnification, $\mathrm{X} 400$ )

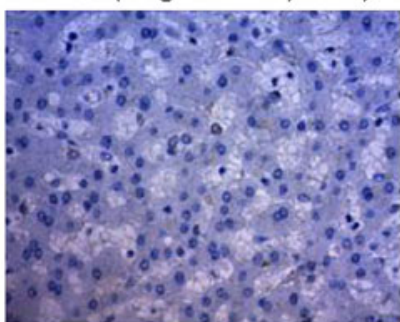

D (magnification, X400)
Fig. 2 Immunohistochemical assessment for a Bcl-2, b Cerb-B2, c MMP-9, d P53 and e Hepatocyte expression in liver carcinome tissue
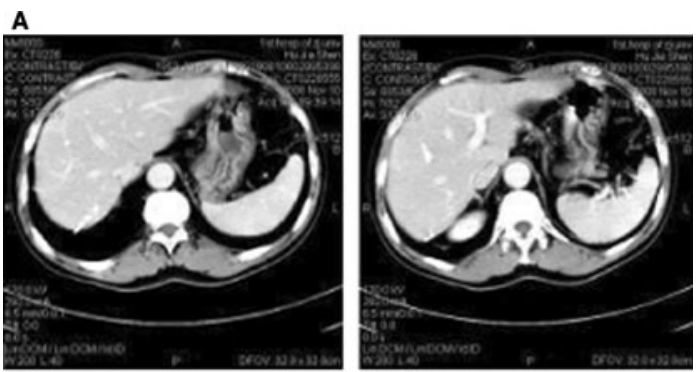

B
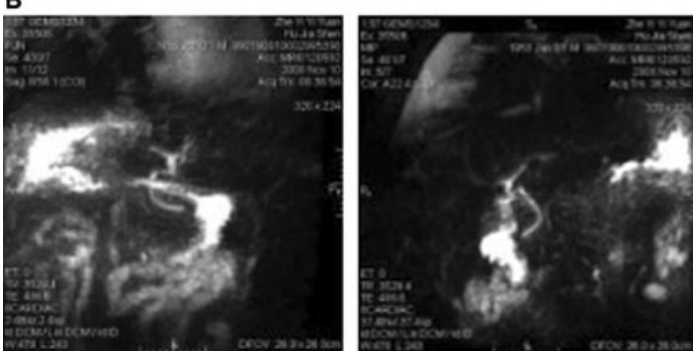

Fig. 3 a CT scan showed no tumor in the graft 6 years after liver transplantation. b MRCP scan showed good state of bile duct tree, slight stenosis in anatomy, no abnormal signal in the liver 6 after liver transplantation

Conclusion: This case suggests that late stage HCC also can be cured by OLT in spit of the high risk of tumor recurrence and the shortage of organ donation. Some tumor related biomarker expression of HCC may be helpful for judging the prognosis of liver transplantation.

\section{PP38-07}

Steroid-Resistant Acute Rejection after Cadaveric Liver Transplantation: Experience from One Single Center

L. Wu, X. He, D. Wang, W. Ju, Q. Tai, Z. Guo, X. Zhu

Organ Transplantation, The First Affiliated Hospital of Sun Yat-sen University, Guangzhou, China

Objective: Over $90 \%$ of acute rejection after liver transplantation can be reversed by steroids bolus treatment. Steroid-resistant acute rejection (SRAR) will lead to liver graft failure if can not be reversed timely, although its incidence is low, and retransplantation remains the only option to rescue the patient. We aimed to explore the outcome and treatment for recipients with SRAR after cadaveric orthotropic liver transplantation (OLT).

Methods: From Jan 2004 to Dec 2007, 596 adult patients received OLT in the Organ Transplantation Center, the First Affiliated Hospital of Sun Yat-Sen University. Among these patients, 96 recipients experienced 113 episodes of acute rejection (AR) after the operation, 11 recipients had no response to steroid bolus treatment, 10 were male and 1 was female. Six patients among them had hepatitis B related cirrhosis, 4 had primary hepatcellular carcinoma and 1 had acute liver failure. Immunosuppressive regimen in this group of patients included IL-2 induction in 1 case, bigeminy strategy: steroids + calcinerium inhibitors (CNIs) in 6 cases and trigeminy strategy: steroids + CNIs + mycophenolate mofetil (MMF) in 4 cases. The clinical data of this group of patients was analyzed retrospectively. Results: Incidence rate of AR after OLT in our single center was $16.1 \%$ (96/596), among them $9.7 \%$ (11/113) were steroid-resistant. SRAR occurred on 19 days (6-72 days) after the operation, 3 were controlled by OKT3 treatment, 4 were reversed by IL-2 receptor inhibitors combined MMF treatment. Four were not reversed and 2 received retransplantation. Mortality associated with SRAR was $36.4 \%$ (4/11, 1 died from acute liver failure, 1 from chronic liver 
failure, 1 from renal failure after retransplantation and 1 from pulmonary infection after OKT3 treatment).

Conclusion: SRAR is a severe complication with high mortality after liver transplantation, OKT3 and IL-2 receptor inhibitors can reverses such rejections to some extend.

\section{PP38-08}

Hepatic Artery Thrombosis after Orthotopic Liver Transplantation: A Review of the Same Institute in 5 Years

L. Wu, X. He, D. Wang, Q. Tai, Z. Guo, W. Ju, Y. Ma, X. Zhu, A. Hu Organ Transplantation, The First Affiliated Hospital of Sun Yat-sen University, Guangzhou, China

Objective: Hepatic artery thrombosis (HAT) remains an important risk factor for graft loss and (or) death of recipients after orthotopic liver transplantation (OLT). We aimed to summarize the experience of the management of patients with HAT after OLT in one single center.

Methods: A total of 726 adult patients who received liver transplantation at the Department of Organ Transplantation, the First Affiliated Hospital of Sun Yat-sen University from January 2004 to December 2009 were selected. Fourteen patients suffered from HAT after the operation, the clinical data of these patients were analyzed retrospectively.

Results: The incidence rate of HAT was $1.9 \%$ (14/726), mean time of the onset was 10 days (1-41 days) postoperatively. Six of them had acute deterioration of liver function, 4 had bile leakage, 1 had hepatic abscess and 3 had no symptoms. Three patients received urgent rearteriarization, two received intra-arterial thrombolysis, three received combined urgent rearteriarization and intra-arterial thrombolysis, and six patients received retransplantation. Mortality rate associated with HAT was $42.9 \%$ (6/14), 2 from biliary necrosis and secondary hepatic failure after urgent rearteriarization, 1 from recurrence of HAT and multiple organ failure after intra-arterial thrombolysis, 1 from renal failure and severe infection after combined urgent rearteriarization and intra-arterial thrombolysis, 2 from severe infection after retransplantation. The other patients recovered and were followed up 18-66 months. Their liver grafts were all functioning well with patent artery, 2 died from tumor recurrence at 18 and 29 month after transplantation.

Conclusion: HAT is a severe complication after liver transplantation, which leads to graft loss and recipients death. Rearteriarization as early as possible before irreversible biliary and liver parenchyma damage can avoid retransplantation.

\section{PP38-09}

Liver Transplantation for Metabolic Diseases: Single Center Experience N. Allam ${ }^{1,2}$, H. Khalaf ${ }^{2}$, Y. Medhat ${ }^{2}$, K. Alawi ${ }^{2}$, H. Bahili ${ }^{2}$, M. Al-Sofayan ${ }^{2}$, W. Alhamoudi ${ }^{2}$, F. Abaalkhail ${ }^{2}$, A. Abdo ${ }^{2}$, M. Al-Sebayel ${ }^{2}$

${ }^{1}$ Hepatology, National Liver Institute, Menoufiya University, Shebeen Al-Koam, Egypt; ${ }^{2}$ King Faisal Specialist Hospital, Riyadh, Saudi Arabia

Background and aims: Liver transplantation (LT) has become an accepted treatment for several metabolic liver diseases. The objective of this study was to analyse our experience in LT for metabolic diseases.

Methods: Since the start of liver transplant program in Saudi Arabia in 2001, a total of 15 patients with an underlying metabolic defect were transplanted in our center.

Results: The patients had a variety of underlying metabolic defects: Wilson's disease $(46 \%)$, primary hyperoxaluria with endstage renal disease $(33 \%)$ and glycogen storage disease $(20 \%)$. Female to male ratio was: 7/8. Eight patients were children (median age 14); seven were adults (median age 25). Fifty-three percent underwent living related donor liver transplantation one of whom had a combined simultaneous liver kidney transplant and another received a kidney transplant a few months later. Forty-seven percent underwent deceased donor transplant (DDLT), three of whom received a kidney transplant later. Average waiting time was 345 days in the case of DDLT. Three patients were considered highly urgent and were transplanted within a week of listing. Median blood transfusion was $3 \mathrm{U}$ (range 1-12) and the median hospital stay was 29 days. Postoperative complications were vascular in two patients (hepatic artery thrombosis and portal vein thrombosis); biliary in three LDLT patients (biliary stricture, bile leak and biliary obstruction requiring retransplantation). One patient (Wilson) suffered from exacerbation of neurologic symptoms immediately postoperative but stabilized on follow up. Perioperative mortality rate was $13 \%$ (due to vascular complications). Cumulative graft and patient survival rate at 5 years was $86.7 \%$.

Conclusion: Analysis of our series reveals only low rate of perioperative complications due to technical complications and generally excellent long term survival rates and quality of life. Overall results of the transplantation for metabolic diseases in our center are encouraging.

\section{PP38-10}

Mycophenolate Mofetil (MMF) Use in Liver Transplantation in New Zealand

J.C. Hsiang, R. Harry

NZ Liver Transplant Unit, Auckland, New Zealand

Aim: To audit the use of MMF in liver transplantation in New Zealand.

Methods: Patients treated with MMF between 2004 and February 2010 were identified retrospectively from Pharmac databases and divided into 2 groups; A: MMF as primary immunosuppression as part of a renal protection protocol with IL2 receptor antibody, steroids and delayed tacrolimus and $\mathrm{B}$ : those switched to MMF based second line therapy (B). Clinical data was retrieved from NZLTU database. Results: 61 patients were identified for study; (13 Group A, 48 group B) with most (79\%) started on MMF since 2008.

In group $\mathrm{A}$, the renal protection protocol was adhered to in each case in the first week but none received therapy to one year. During MMF therapy (93 days IQR 148), 2 patients (15.4\%) developed 4 episodes of acute rejection (ACR). None developed CMV. In group B, MMF was started 361 days (IQR 1617) after transplant. In 29 patients (60\%) this was for renal impairment. 8 of these $(28 \%)$ underwent renal US, $14(48 \%)$ MSU and $6(20 \%)$ formal creatinine clearance as per switching protocol. During MMF therapy (441 days IQR 105), 11 patients $(22.9 \%)$ had 21 episodes of ACR and 4 patients $(8 \%)$ developed CMV.

Conclusion: Use of MMF post liver transplant in New Zealand is increasing. ACR and CMV occur during its use. Further study is required to assess the clinical importance of these findings.

\section{PP38-12}

Development of a New Auxiliary Heterotopic Partial Liver Transplantation Technique Based on Liver Cirrhosis in Minipigs: Preliminary Report of 8 Transplants

J. Zhang, G. Yue, H. Zhong, X. Meng

Inner Mongolia Medical College, Huhhot, China

This study aims to develop a new auxiliary heterotopic partial liver transplantation (AHPLT) technique in minipigs on the basis of liver cirrhosis. According to previous study, 14 minipigs was induced to cirrhosis by intraperitoneal injection administration of carbon tetrachloride $\left(\mathrm{CCl}_{4}\right)$. All of the cirrhotic animals were used as recipients. The donor's liver was placed on the recipient's splenic bed, and the anastomosis was performed as below: end-to-end anastomosis between donor's portal vein and recipient's splenic vein, end-to-side anastomosis between donor's suprahepatic vena cava and recipient's suprahepatic vena cava, and end-to-end anastomosis between donor's 
hepatic artery and recipient's splenic artery. The donor's common bile duct was intubated and bile was collected with an extracorporeal bag. Throughout the transplantation process, vital signs, portal vein pressure (PVP), hepatic venous pressure (HVP) and portal vein pressure gradient (PVPG) were monitored. All of 8 minipigs developed liver cirrhosis were used to establish new AHPLT; 7 cases of which survived. After surgical intervention, the PVP and PVPG of the recipient were lower than before operation $(P<0.05)$, and the PVP and PVPG of the donor increased significantly compared with those of the normal ones $(P<0.05)$. This study successfully described a new operative technique for AHPLT based on liver cirrhosis.

\section{PP38-13}

Does the Recipient Age Affect the Correction of Hypophosphataemia and Hypomagnesaemia after Living-Donor Liver Transplantation? H. Elgendy ${ }^{1,2}$, W. El Moghazy ${ }^{3}$, K. Fukuda ${ }^{1}$, S. Uemoto ${ }^{3}$

${ }^{1}$ Anesthesia, Kyoto University, Kyoto, Japan; ${ }^{2}$ Anesthesia and Intensive Care, Assiut U Hospital, Assiut, Egypt; ${ }^{3}$ Hepato-PancreatoBiliary Surgery and Transplantation, Kyoto University, Kyoto, Japan

Background/aims: In living donor liver transplantation (LDLT), preoperative electrolyte derangements are not uncommon as a result of debilitating hepatic pathology. Several studies have shown electrolytes changes after LDLT, but long-term follow-up data have been lacking. To clarify the implications of hypophosphataemia and hypomagnesaemia on the recipient outcome at different ages.

Methods: We retrospectively reviewed pre and postoperative laboratory data. Of 1296 cases of LDLT, 867 were selected. Recipients with chronic end-stage liver disease were designated as children or adults.

Results: All patients had a significant decrease of both phosphate and magnesium during the early postoperative course. The maximal serum P decrease was observed on the 3rd and 5th PO days in children and adults, respectively. In children, serum $P$ level recovered to normal levels by the 3rd PO month, whereas in adults, the serum P level recovered by the 1st PO month and was maintained at its normal level until the 5th PO year. The incidence of hypophosphataemic cases was greater in the child subgroups than in adults; this difference was marked on the 5th PO day. We observed a reciprocal decrease of hypophosphataemic cases with increasing age of the children. Infants with pre-operative hypophosphataemia showed significant growth retardation. Prior to transplantation, hypomagnesaemia occurred more frequently in adults; whereas, it became higher in children in the 5th postoperative year. Recipients with hypomagnesaemia during the early postoperative period exhibited better patient survival compared with those with normomagnesaemia. Hypomagnesaemia group had higher tacrolimus trough level than that of normomagnesaemia, $p<0.05$. Hypophosphataemia had an impact only on infant survival, but had no effect on adult outcome.

Conclusions: We concluded that magnesium has an impact on patient survival and that phosphate contributes essentially to infant growth and survival.

\section{PP38-14}

What Increases the Risk of Infection in Children after Living-Donor Liver Transplantation?

H. Elgendy ${ }^{1}$, H. Nafady-Hego ${ }^{2}$, W. El Moghazy ${ }^{2}$, K. Fukuda ${ }^{1}$, S. Uemoto ${ }^{2}$

${ }^{1}$ Anesthesia; ${ }^{2}$ Hepatobiliary, Pancreas and Transplant Surgery, Kyoto University, Kyoto, Japan

Background/aims: Infection after pediatric living donor liver transplantation (LDLT) is a major cause of morbidity and mortality. Herein, we aimed to determine the incidence, timing, location and risk factors for infection.

Methods: We retrospectively investigated infection for 3 post-operative months in 345 consecutive pediatric patients (43.8\% males), who underwent primary LDLT in Kyoto university hospital.
Results: A total of 209 patients $(60.6 \%)$ developed at least one infection episode, with an infection rate of 1.6/patient. The predominant infection site was the surgical site (53\%). Most of the bacterial and fungal infections occurred within the first month, while viral infection occurred in the second month. Enterococcus species and Cytomegalovirus were the predominant bacterial and viral pathogens, respectively. All fungal isolates were Candida species. Prolonged preoperative hospital stay (PPOHS) $>7$ days was an independent predictor of infection $(p=0.004)$. PPOHS $(p=0.009)$, repeated surgery $(p=0.043)$ and bile leak $(p=0.026)$ were independent predictors of bacterial infection. Preoperative hyponatremia $(p=0.019)$, fulminant hepatic failure (FHF) $(p=0.019)$, prolonged abdominal drain insertion $(p=0.014)$, steroid pulse therapy $(p=0.012)$ and high trough levels of tacrolimus $(p=0.045)$ were independent predictors of viral infection. PPOHS $(p=0.049)$ and preoperative ascites $(p=0.002)$ independently predicted fungal infections.

Conclusions: Infection was responsible for $52.4 \%$ of the causes of deaths in our study. To avoid infectious complications after LDLT, broader spectrum antibiotics, early drain removal, correction of preoperative hyponatremia, limiting steroid pulse therapy and careful adjustment of the tacrolimus loading dose should be considered.

\section{PP38-15}

Liver Transplantation in Crigler-Najjar Type 1 Disease: A Successful Case Report of Reversable Neurological Symptoms

Z. Tu ${ }^{1,2}$, H. Lou ${ }^{3}$, J. Jiang ${ }^{1}$, S. Zheng ${ }^{1,2}$

${ }^{1}$ Division of Hepatobiliary and Pancreatic Surgery, Department of Surgery; ${ }^{2}$ Key Laboratory of Combined Multi-Organ Transplantation, Ministry of Public Health \& Key Laboratory of Organ

Transplantation of Zhejiang Province; ${ }^{3}$ Department of Radiology, First Affiliated Hospital, Zhejiang University, School of Medicine, Hangzhou, China

Crigler-Najjar syndrome type 1 (CNS 1 ) is a very rare disease characterized by severe indirect hyperbilirubinemia from birth with normal liver function. This disease is due to lack of activity of the hepatic enzyme uridine diphosphate-glucuronosyltransferase which is an autosomal recessive inherited disease. Patients with one normal allele have a normal bilirubin metabolism. The children suffering from this hereditary disease present with a high serum level of unconjugated bilirubin. In the early term of infant, phototherapy and phenobarbital seem effective to reduce the bilirubin level, but children are still at high risk of kernicterus and neurological sequels, which are considered as inreverseable even post liver transplantation (LTx), and in turn ultimately lead to severe disability or death. Currently, LTx is a lifesaving procedure for patients with chronic end-stage liver disease and acute liver failure when there are not any other available medical and surgical treatment options. LTx is still an attractive technique to treat liver-based inborn errors of metabolism. We admitted a 18-month-old patient diagnosed as CNS 1, both by clinical and genetic characteristics. The patient suffered from kernicterus before operation and then LTx was performed about 5 days later. Magnetic resonance imaging (MRI) showed positive alteration, accompanied with obvious improvement of growth evaluation, 3 months post LTx. Reversable neurological symptoms were detected, successfully.

\section{PP38-16}

The Effect of Reactive Oxygen Species on Hepatocyte Xanthine Oxidase Activity and Importance of Effective Scavenging

H. Kocic

Medical Faculty Nis, Nis, Serbia

Background/aim: The interruption of hepatic inflow represents a common procedure during trauma surgery, liver transplantation, and resectional surgery. The acute phase response is characterized by activation of the Kupffer cells, subsequent production and release of 
reactive oxygen species (ROS), leading to the oxidative shift. Reperfusion is associated with activation of xanthine oxidase, redox-sensitive transcription factors (NF- $\kappa \mathrm{B}$ ), the expression of proinflammatory cytokines (IL-12 and TNF- $\alpha$ ), activation of neutrophilattracting chemokines and endothelial cell adhesion molecules. Xanthine oxidase (XO) has a cental importance in the pathobiochemistry of liver injury, because of its notable property of generating ROS during I/R injury and the aim was to obtain evidence about protective effect of radical scavengers and chelators against the ROS generated during $\mathrm{XO}$ activation.

Methods: Primary cultures of rat hepatocytes, isolated by collagenase technique (1mg of RPMI 1640 medium) were exposed to reactive ROS conditions $(0.5 \mathrm{mM} \mathrm{FeCl}$ ) alone and simultaneously with a-lipoic acid $(2.5 \mathrm{mg} / \mathrm{mL})$, melatonin $(2 \mathrm{mM})$, desferoxamine $(10 \mathrm{mM} / \mathrm{mL})$, or their combination.

Results: During exposure of isolated ral liver cells to ROS, XO activity increased $\left(10.04 \pm 1.21 * * * \mathrm{U} / 10^{8}\right.$ cells vs. $7.35 \pm 1.32$ of control), resulting in increased lipid peroxidation (LP: $13.61 \pm$ $1.08 * * *$ nmolMDA $/ 10^{8}$ cells vs. $7.62 \pm 1.18$ of control). The protective effect against $\mathrm{XO}$-induced superoxide and $\mathrm{OH}$ production was documented for lipoic acid (XO: $8.04 \pm 1.40 * *$; LP: $9.05 \pm$ $1.47 * * *)$, melatonin (XO: $8.27 \pm 1.33^{*}$; LP: $\left.9.11 \pm 2.62 * * *\right)$ and deferoxamine (XO: $8.42 \pm 1.03^{* * *}$; LP: $9.35 \pm 0.88$ ). Given together they decrease more significantly than any of them alone (XO: $6.64 \pm 1.40^{* *}$; LP: $\left.7.65 \pm 1.47 * * *\right)$. Each given to intact hepatocytes decreased XO and LP (lipoic acid: XO: $6.62 \pm 1.43 * * * ;$ LP: $6.06 \pm 1.70 *$; melatonin: XO: $3.8 \pm 0.65^{* * *}$; LP: $6.51 \pm 2.25$; desferoxamine: XO:5.4 $\pm 0.83^{* * *}$; LP: $5.53 \pm 0.83^{* * *}$ ).

Conclusion: Combined "cocktail" of radical scavengers and chelators may be the optimal therapeutic strategy during different clinical conditions, such as trauma surgery, liver transplantation or treatment of inflammation, in order to reduce possible hepatic failure, prolong organ storage or improve graft function.

\section{PP38-17}

Effect of Donor Steatosis on Liver Biochemistry and Significance of Body Mass Index and Alanine Aminotransferase in Predicting Steatosis R.C. Siriwardana ${ }^{1}$, S.C. Chan ${ }^{1}$, C.M. Lo $^{1}$, K.S. Chok ${ }^{1}$, S.T. Fan ${ }^{2}$ ${ }^{1}$ Hepatobiliary and Transplant Surgery; ${ }^{2}$ Department of Surgery, University of Hong Kong, Hong Kong, China

Background: Hepatic steatosis in living-donor liver transplantation is a major concern on donor safety and graft function.

Methods: Data of 329 Asian donors who underwent right hepatectomy were analyzed. The donors were categorized as having no steatosis (G0, $n=178),<10 \%$ steatosis $(\mathrm{G} 1, n=128)$, and $>10 \%$ (G2, $n=19)$ steatosis. Pattern of change and factors affecting aspartate aminotransferase (AST), alanine aminotransferase (ALT), total bilirubin, and prothrombin time on days 1 to 7 and day 30 were assessed. Factors predicting steatosis were also assessed.

Results: A rise in ALT level was noted till day 3 in G1 and day 6 in G2 compared with G0 $(p<0.05)$. AST levels were different till day 7 in $\mathrm{G} 2$ but similar in G1 $(p<0.05)$. Bilirubin level was higher only in day 1 in $\mathrm{G} 2(p<0.05)$. On multivariate analysis, apart from fatty change, body mass index (BMI) and remnant left lobe volume showed a significant correlation with ALT levels $(p<0.05)$. By day 30, the median level of enzymes and number of patients having levels above the reference range were similar $(p>0.05)$. Receiver operating characteristic area under the curve (AOC) for BMI on predicting steatosis was $0.75(\mathrm{CI}=69-80)$. Seventy-five percent of patients in the fourth quartile of BMI $\left(23.5-29 \mathrm{~kg} / \mathrm{m}^{2}\right)$ had steatosis. Seventy percent of G2 were in this category. In patients with BMI over $23.5 \mathrm{~kg} / \mathrm{m}^{2}$, multivariate analysis showed a significant correlation between ALT and steatosis $(p=0.05, \mathrm{OR}=1.14, \mathrm{CI}=1-1.3)$. ALT level $>17.5 \mu / \mathrm{L}$ predicted steatosis with $66 \%$ sensitivity $(\mathrm{CI}=52-77)$ and $78 \%$ $(\mathrm{CI}=52-92)$ specificity $(\mathrm{AOC}-0.71, \mathrm{CI}=56-85)$.
Conclusions: Donors showed a short-lasting rise in ALT, AST and bilirubin level dependent on the degree of steatosis. For Asian donors, an ALT level above $17.5 \mu / \mathrm{L}$ and a BMI above $23.5 \mathrm{~kg} / \mathrm{m}^{2}$ are useful guides in the decision on liver biopsy.

\section{PP38-18}

The Efficacy of Anti Viral Therapy to HCV Recurrence after Orthotopic Liver Transplantation

H. Su, M. Zhang, Z. Liu

Liver Transplantation Center, 302 Hospital of PLA, Beijing, China

Objectives: To analysis the efficacy of anti viral therapy to HCV recurrence after orthotopic liver transplantation (OLT).

Patients and methods: 12 cases with HCV recurrence after liver transplantation were treated with interferon (IFN) plus ribavirin and low-accelerating dose regimen was used since 2005 in our hospital. HCVRNA loads, rapid viral respond (RVR), early viral respond (EVR), end of treatment viral respond (ETVR), sustained viral respond (SVR) and side effects of anti viral therapy were observed.

Results: The mean time from OLT to the start of treatment was 15 months (2-36 months) and 7 patients (58.3\%) completed standard anti viral treatment duration in 12 patients. RVR was achieved in 3 patients (25\%), EVR in 9 patients (75\%), ETVR in 8 patients $(58.3 \%)$ and SVR in 3 patients $(25 \%)$ among 12 patients. Among 3 patients with SVR, HCVRNA genotype 1 was isolated in 2 patients and genotype 2 in 1 patient, RVR was achieved in 2 patients and EVR in 3 patients. Anti viral therapy had to be stopped due to neutropenia in 1 patient, acute rejection in 2 patients, severe infection in 1 patient and allergy in 1 patient.

Conclusions: Anti viral therapy achieves lower SVR in patients with $\mathrm{HCV}$ recurrence after OLT. Side effects of anti viral therapy should be closely monitored. Use of colony stimulating factor is beneficial to the completion of anti viral therapy.

\section{PP38-19}

To Study the Patients with Liver Transplant Associated Hyperglycemia Z. Liu, H. Su, M. Zhang, Y. Gao, Y. Xiang, R. Tang, X. He Liver Transplantation Center, 302 Hospital of PLA, Beijing, China

Objectives: Retrospective study the characteristic of patients with orthotopic liver transplant associated hyperglycemia (TAH).

Methods: The data of all patients with normal fast glucose before liver transplantation (LT) in our hospital from 2003 to 2009 were collected. TAH was defined by American diabetes association criteria.

Results: Among 81 patients with normal fast glucose before LT, 24 patients' fast glucose was high than normal and 10 patients were diagnosed as diabetes mellitus 1 year after LT. Fast glucose is highest 1 week after LT and maintained 3 weeks after LT. Serum triglycerides (TG) and total cholesterol (TC) after LT were increased significantly than pre transplantation in patients with TAH $(1.27 \pm 0.68$ vs. $0.88 \pm 0.55 ; 3.88 \pm 1.07$ vs. $2.78 \pm 1.22)$. Body mass index (BMI) was associated with TAH.

Conclusion: The occurrence of TAH is high in liver transplantation patients which should be given high attention and proper intervention.

\section{PP38-20}

Liver Biopsy for Potential Liver Donors

N. Debzi ${ }^{1}$, N. Guessab ${ }^{1}$, H. Ait Kaci ${ }^{2}$, N. Afredj ${ }^{1}$, A. Nani ${ }^{1}$, S.A. Faraoun ${ }^{3}$, T. Benathmane ${ }^{4}$, S.E. Bendib ${ }^{3}$, O. Chafa ${ }^{5}$, N. Terki ${ }^{2}$, T. Boucekkine ${ }^{1}$ ${ }^{1}$ Hepatology Unit, Mustapha Hospital, Algiers University; ${ }^{2}$ Service d'Anatomie Pathologique; ${ }^{3}$ Service de Radiologie, Centre Pierre et Marie Curie; ${ }^{4}$ Service de Psychiatrie; ${ }^{5}$ Centre de Transfusion Sanguine, Mustapha Hospital, Algiers University, Algiers, Algeria

Introduction: The indication of liver biopsy (LB) in evaluation and selection of donors for living donor liver transplantation (LDLT) is 
still controversial. The main objective of LB should be to ensure the donor's safety.

Patients and methods: Our selection of donors and recipients did not differ from others teams. These donor's evaluation comprised 3 steps: clinical, non invasive and invasive morphology (LB), and multidisciplinary team evaluation.

LB was obtained in a blind percutaneous fashion by means of Menghini needle.

Results: From january 2007 to january 2010, 16 potentials donors underwent LB, they were 8 women and 8 men, mean age 21 years (19-58). They were all relatives, 1 st degree in $87.5 \%$ of cases $(n=14)$ and 2 nd degree in $12.5 \%(n=2)$. The preoperative imaging liver donor evaluation by abdominal doppler sonography, multidetector computed tomography scan and cholangiopancreatography MRI did not show any abnormalities. No major complications related to LB were observed. 4 (25\%) of 16 candidates who underwent LB had a positive finding at histological examination that induced their exclusion from donation, of whom 1 had steatohepatitis, 3 had chronic hepatitis.

Conclusion: These findings suggest that all potential candidates for LDLT should undergo screening liver biopsies. The precise significance of these changes remains to be determined, including which of these changes are contraindications to liver transplantation.

\section{PP38-21}

Influenza Vaccination in Liver Transplant Children-A Decreased Antibody Response

R. Shapiro ${ }^{1}$, Y. Avitzur ${ }^{2}$, E. Mor ${ }^{3}$, I. Hoyjak ${ }^{4}$, R. Shamir ${ }^{5}$, Y. Haimi-Cohen ${ }^{5}$, Z. Zakay-Rones ${ }^{6}$, D. Wolf ${ }^{7}$

${ }^{1}$ Gastroenterology, Nutrition and Liver Diseases, Schneider Children's Medical Center of Israel, Petah-Tikva, Israel; ${ }^{2}$ Hospital for Sick Children, Toronto, ON, Canada; ${ }^{3}$ Rabin Medical Center, Beilinson Hospital, Petah-Tikva, Israel; ${ }^{4}$ Referral Center for Pediatric Gastroenterology and Nutrition, Children's Hospital Zagreb, Zagreb, Croatia; ${ }^{5}$ Schneider Children's Hospital, and Sackler Faculty of Medicine, Tel-Aviv University, Petah-Tikva; ${ }^{6}$ Virology Department The Hebrew University, Hadassah School of Medicine; ${ }^{7}$ Clinical Virology Unit, The Hebrew University, Hadassah School of Medicine, Jerusalem, Israel

Background: Annual Influenza vaccination is recommended to all immunocompromized hosts including solid organ transplant recipients. There is little data published regarding response to immunizations in liver transplant children. Our hypothesis was that liver transplanted children on a Tacrolimus based immunosuppression have decreased antibody response and immunity post influenza vaccination.

Aim: To study prospectively the immune response to trivalent subvirion influenza vaccine in 2 different vaccination protocols conducted in liver transplant children.

Methods: Study subjects: pediatric liver transplant recipient were immunized with inactivated influenza vaccine during the influenza seasons of 2005 (one dose) and 2006 (two doses over an interval of 4-6 weeks).

Seroresponse: Fourfold increase in antibody titer.

Seroproetection: Antibody titer $>$ or $=1: 40$. The antibody titer was measured by haemaglutinin inhibition enzyme-linked immunosorbent spot assays for A/H1N1 (New Caledonia), A/H3N2 (New York), and $\mathrm{B}$ (Shanghai) or A/H1N1 (New Caledonia), A/H3N2 (Wisconsin), and B (Malaysia) in 2005 and 2006, respectively. Measurements were performed at baseline 4-6 weeks after each vaccination. All patients were treated in one center. The immunosuppression protocol included Tacrolimus alone or in combination with Cellcept, Prednison or sirolimus

Results: Eighteen patients were vaccinated in 2005. Seroresponse rates for $\mathrm{A} / \mathrm{H} 1 \mathrm{~N} 1, \mathrm{~A} / \mathrm{H} 3 \mathrm{~N} 2$ and Shanghai serotypes were 35.3, 23.5, and $41.2 \%$, respectively. In 2006, 30 patients were immunized with dose doses and seroresponse rates after one immunization dose for $\mathrm{A} / \mathrm{H} 1 \mathrm{~N} 1, \mathrm{~A} / \mathrm{H} 3 \mathrm{~N} 2$ and $\mathrm{B}$, were $43.3,70.0$, and 32.3\%, respectively. GMT (Geometric Mean Titer) increased significantly $(p<0.01)$ in all serotypes investigated. There was no significant increase in response rate or geometric mean titers after the second immunization dose. No cases of influenza or vaccine-related serious adverse events were documented in the study.

Conclusions: Immuncompromized liver transplant children have a diminished response rate to influenza vaccine. No statistical significant was found between a single and a repeated vaccination.

\section{PP38-22}

Changes of Platelet Count in Liver Transplant Recipients with History of Spelenectomy.Doc

T. Shen, L. Wu, M. Zhang, W. Wang, L. Zhou, S. Zheng Division of Hepatobiliary and Pancreatic Surgery, Department of Surgery, Key Laboratory of Combined Multi-Organ Transplantation, Ministry of Public Health, First Affiliated Hospital, Zhejiang University School of Medicine, Hangzhou, China

Aims: Herein we discuss the changes of platelet count in liver transplant recipient who had undergone splenectomy before transplantation.

Methods: Between June 2005 and September 2010, 26 patients received liver transplantation in our center, all of whom had history of splenectomy. The platelet count before transplantation, 1 day, 3 day, 1 week, 2 week, 1 month, 3 month and 6 month after transplantation were investigated.

Results: The time between splenectomy and liver transplantation was $6.3 \pm 3.4$ years. The platelet count pre-liver transplantation was $158 \pm 61 \times 10^{9} / \mathrm{L}\left(80-251 \times 10^{9} / \mathrm{L}\right)$, and platelet count of 1 day, 3 day, 1 week, 2 week, 1 month, 3 month and 6 month post-liver transplantation were $52 \pm 24$ (24-107), $53 \pm 29$ (20-142), $104 \pm 49$ (44-215), $267 \pm 176$ (55-316), $394 \pm 133$ (194-714), $263 \pm 96$ (89-399), $195 \pm 32(86-328) \times 10^{9} / \mathrm{L}$, respectively. Statistically significant changes of platelet count were seen between pre-transplantation and post-transplantation. The platelet count decreased rapidly in early stage after liver transplantation, but increased in 1 month rapidly. 1 month after transplantation, these patients' platelet count usually reached a highest level up to $714 \times 10^{9} / \mathrm{L}$. 6 months after transplantation their platelet count gradually dropped to normal. There were 20 patient received anti-platelet treatments, with aspirin or dextran. Thrombosis of hepatic artery and portal vein were observed in 1 and 2 patients respectively.

Conclusions: In liver transplant recipients who had history of splenectomy, platelet count will decrease in early stage after transplantation, but will increase to an abnormal high level 1 month after transplantation. Most of them need therapy of anti-platelet to prevent complication such as thrombosis of portal vein or hepatic artery.

\section{PP38-23}

Increased Expression of Peroxiredoxin 1 and a Novel Lipid Metabolising Enzyme in the Early Phase of Liver Ischemia Reperfusion Injury C.H. Wilson ${ }^{1}$, S. Zeile ${ }^{1}$, T. Chataway ${ }^{2}$, V.B. Nieuwenhuijs ${ }^{3}$, R.T.A. Padbury ${ }^{4}$, G. Barritt ${ }^{1}$

${ }^{1}$ Medical Biochemistry; ${ }^{2}$ Physiology, Flinders University, Adelaide, SA, Australia; ${ }^{3}$ Surgery, University Medical Centre, Groningen, The Netherlands; ${ }^{4}$ The HPB \& Liver Transplant Unit, Flinders Medical Centre and School of Medicine, Flinders University, Adelaide, SA, Australia

Background/aims: Warm ischemia reperfusion (IR) injury of the liver is associated with changes in the expression and/or modification of numerous proteins. However, only a few of these have been identified. The aim was to identify cytosolic proteins altered in the early phase of IR injury. 
Methods: An established rat model of segmental hepatic ischemia was employed. Liver cytosol was prepared by differential centrifugation, proteins minimally covalently labelled with CyDyes, and analysed by 2D-DIGE (differential in gel-electrophoresis). Proteins were identified by in-gel tryptic digestion, followed by LC-MS/MS. Results: In total, 1200 cytosolic proteins were detected. Comparison of ischemic liver with non-ischemic liver revealed 33 differentially expressed and/or covalently modified proteins (change greater than 1.5 times and $P \leq 0.05$ ). Sixteen of these abundant proteins were identified, including 12 enzymes involved in glucose and lipid metabolism, protein degradation, or peptide cleavage. One, isoamyl acetate hydrolysing enzyme (confirmed by western blot), has not previously been characterised in liver. The two proteins which showed the greatest change (3-fold increase, $P \leq 0.05$ ) were the reduced and oxidised forms of peroxiredoxin 1 (confirmed by western blot). A 1.6-fold increase $(P \leq 0.05)$ in expression of peroxiredoxin 1 mRNA was observed following IR.

Conclusions: Increased expression of peroxiredoxin 1 is a rapid response to IR and may represent a component of mechanisms induced to protect hepatocytes against IR damage.

\section{PP38-24}

De Novo Malignancies after Orthotopic Liver Transplantation (OLT) do Not Compromise Outcome

J. Spicak ${ }^{1}$, S. Frankova ${ }^{1}$, J. Sperl ${ }^{1}$, P. Trunecka ${ }^{1}$, M. Adamec ${ }^{2}$

${ }^{1}$ Hepatogastroenterology; ${ }^{2}$ Department of Transplant and Vascular Surgery, Institute for Clinical and Experimental Medicine, Prague, Czech Republic

Introduction: Solid organ transplant recipients are at a high risk to develop malignancies, representing generally a major cause of late death after transplantation. The aim of our study was to assess the impact of the malignancies on the outcome of our OLT program.

Patients and methods: A cohort of all consecutive 699 adult liver transplant recipients from cadaveric donors performed from 1994 to 2010 was reviewed retrospectively. Patients underwent a screening program consisting of yearly skin examination, chest X-ray, abdominal ultrasound, mammography, gynecology exam and colonoscopy tailored according to individual risks.

Results: Among the 699 patients after orthotopic liver transplantation (OLT) with a mean follow-up of 74 months (1-192 months), 67 patients developed de novo malignancy (overall incidence of 9.5\%). The mean time from OLT to tumor diagnosis was 55 months (1-149 months). Non-melanoma skin cancer appeared in 17 patients, posttransplant lymphoproliferative disorder was diagnosed in 14 patients, head and neck cancer in 10 patients, lung cancer in 7 patients, 6 women presented with cervical cancer and 4 with breast cancer. Thirty-four tumors were diagnosed during regular screening examinations. Twenty patients with de novo malignancy died, from whom only 12 patients $(1.7 \%$ of all recipient) due to malignant disease progression. Five patients died of head and neck cancer, 4 patients of lymphoma, 2 patients of lung cancer a 1 patient of pancreatic cancer. None of the patients who were diagnosed through a screening procedure died.

Conclusion: OLT recipients are at a considerable risk to develop de novo malignancies. They can be efficiently prevented by tailored surveillance programs for selected groups of patients. When applied, de novo malignancies do not influence the outcome of OLT.

\section{PP38-25}

Health Related Quality of Life in Egyptian Liver Transplant Recipients M. Abdel-Rahman ${ }^{1}$, A. Omar ${ }^{1}$, M. EL-Sahhar ${ }^{2}$, S. Awny ${ }^{2}$, N. Zayed ${ }^{1}$ ${ }^{I}$ Endemic Medicine and Hepatology Department, Cairo University; ${ }^{2}$ Hepatology and Gastroenterology Department, Police Authority Hospital, Agouza, Cairo, Egypt

Introduction/Aim With improvements in patient and graft survival after liver transplantation (LTx), recipient health related quality of
Life (HRQOL) has become an important focus of patient care and clinical outcomes research. The current study was designed to evaluate different aspects and changes of HRQOL using the liver disease quality of life 1.0 (LDQOL 1.0) Questionnaire among recipients following either cadaveric or living donor liver transplantation (LDLT) in comparison to a non-transplant population.

Methods: The LDQOL 1.0 questionnaire includes a 111-item containing the generic Short Form-36 Health Survey version-2.0 (SF36v2) supplemented by disease-targeted scales was applied to ambulatory patients, either in the transplant list with end stage liver disease (ESLD) $(n=50)$ or at least after 6 months; mean $5 \pm 4$ years in transplant recipients $(n=103)$ who had underwent either living donor or cadaveric LTx at 3 different hospitals in Egypt. Pre-transplant model for end-stage liver disease (MELD), Child-Pugh scores, co-morbidities in addition to the reported post operative complications were analyzed.

Results: Liver transplant recipients had significant higher HRQOL than patients with ESLD. In patients awaiting LTx, MELD scores $\geq 15$, Child-Pugh class $\mathrm{C}$, the presence of hepatocellular carcinoma (HCC) demonstrated worse HRQOL, using both the SF-36 and LDQOL questionnaires with a statistically significant difference with respect to HCC presence among transplant population. Cadaveric recipients with documented surgical complications had a statistically better HRQOL using LDQOL questionnaire, but not the generic SF36. LDLT recipients with evidence of infection episodes had lower HRQOL while those with rejection had better scores. Deterioration of HRQOL after recurrence of hepatitis $\mathrm{C}$ in both transplant population was evident as assessed by both components.

Conclusion: Generally HRQOL improves dramatically after LTx, compared to prior health status. Specific questionnaires; LDQOL; can provide more accurate information about liver symptoms and could demonstrate with better reliability, impairment before and after liver transplantation.

\section{PP38-26}

Endoscopic Management of Biliary Complications after Liver Transplantation

Y. Qin, Q. Li, F. Yang, S. Zheng

First Affiliated Hospital, College of Medicine, Zhejiang University, Hangzhou, China

Background: Although ERCP has become the preferred method of treatment for treating anastomotic strictures, bile leaks in patients after liver transplantation (OLT). A standard approach has not yet been established. We attempted to evaluate the efficacy of endoscopic management of those complications.

Materials and methods: This study retrospectively reviewed 57 patients, who received liver transplants and developed persistently abnormal liver function tests and presented biliary stricture or bile leakage suggested by MRCP, underwent ERCP from January 2003 to July 2009. If the stricture was confirmed, dilation was performed and a plastic stent was placed. If endoscopic therapy failed, percutaneous transhepatic drainage was performed to dilate the stricture or underwent surgery. If the leakage was confirmed, a plastic stent was placed. Clinical and biochemical evaluation was done every three months. Resolution of symptoms and normalization or $\geq 50 \%$ reduction of at least one liver function test were needed to consider the problem as clinically relevant. Patients were followed up for a median of 20 months.

Results: ERCP was performed on all 57 patients, and $51(89.5 \%)$ were successfully treated. 34 patients were confirmed the anastomotic biliary stricture and underwent endoscopic dilation and stenting. 7 patients were confirmed the bile leaks and underwent stenting. 4 of 6 patients in whom endoscopic therapy failed were treated by percutaneous transhepatic drainage. The other two patients underwent surgery. The data suggested the problem of the leakage to be 
clinically relevant in all patients, while the stricture always to be dilated repeatedly and prolonged stenting. Patients treated by percutaneous transhepatic drainage and dilation recovered smoothly. Procedure-related mortality rate was $0 \%$.

Conclusions: ERCP is a safe and preferred procedure in the treatment of biliary complications. In failed cases, percutaneous transhepatic drainage and dilation is an effective rescue therapy. But surgery is still necessary.

\section{PP38-27}

Survival Advantage of Primary Liver Transplantation for Resectable Hepatocellular Carcinoma with Vascular Invasion

S.C. Chan, C.M. Lo, K.S.H. Chok, W.W. Sharr, A.C.Y. Chan, S.T. Fan, Key State Laboratory for Liver Research

Surgery, University of Hong Kong, Hong Kong, Hong Kong S.A.R.

Background: Vascular invasion is a poor prognostic factor of surgical resection (LR) and liver transplantation (LT) for hepatocellular carcinoma (HCC). Microvascular invasion in the LR specimen is paradoxically considered an indication for LT in some centers.

Methods: From July 2000 to June 2009, adult patients underwent primary LT and LR for HCC were included. Those who received neoadjuvant systemic chemotherapy, targeted therapy, with positive resection margins, with direct or metastatic spread of HCC were also excluded.

Results: This study included 95 LT recipients and 620 LR patients. Among the LT recipients $81 \%$ were within up-to 7 criteria, $77 \%$ within UCSF criteria. Slightly over one quarter (26.3\%) had HCC with either macro- or micro-vascular invasion. About three-quarter (73.7\%) were of LDLT. 5-year overall survival was $82 \%$. When within up-to-7 criteria $(n=75)$, the 5 -year survival improved to $85.3 \%$. The presence or absence of either macro- or micro-vascular invasion was did not affect survival (5-year overall survival, 88.2 vs. $84.7 \%$, respectively). LT recipients with HCC with vascular invasion had survival comparable to LR patients with HCC without vascular invasion ( 88.2 vs. $78.9 \%, p=0.828)$ LR patients had 5-year survivals much better than those with vascular invasion who under LR $(47.3 \%)$. Thus, the significant survival advantage of LT over LR for treatment of HCC with vascular invasion. For the entire cohort of 95 LT recipients and $620 \mathrm{LR}$ patients, the overall 5-year survivals were compromised by: multiple tumors $(\mathrm{OR}=1.592, \mathrm{CI}=1.196-2.119$, $p=0.001)$, presence of vascular invasion $(\mathrm{OR}=2.332$, $\mathrm{CI}=1.782-3.052, \quad p<0.0001)$ and beyond up-to-7 criteria $(\mathrm{OR}=1.526, \mathrm{CI}=1.152-2.023, p=0.003)$. Liver transplantation was a favorable factor for survival $(\mathrm{OR}=0.410, \mathrm{CI}=0.246-0.684$, $p=0.001)$.

Conclusions: Primary LT for HCC within up-to-7 criteria irrespective of vascular invasion has 5-year survival over $80 \%$. Those with microvascular invasion has the survival advantage of twice as likely a cure.

\section{PP38-28}

End-stage Nonalcoholic Fatty Liver Disease: Evaluation of Pathomorphologic Features and Relationship to Cryptogenic Cirrhosis

N. Vasdev

Pathology, Medanta the Medicity, Gurgaon, India

Summary In a proportion of liver cirrhosis, the etiology continues to remain elusive. It is uncertain whether and to what extent cirrhosis evolving from nonalcoholic fatty liver disease contributes to this group of cryptogenic cirrhosis because the clinicopathologic features of nonalcoholic fatty liver disease cirrhosis are largely unknown. We explored these facets by examining the explant livers and clinical data in living donor liver transplant recipients. Among 163 adult liver transplant recipients with different types of chronic liver disease, 50 had a pre-liver transplant diagnosis of cryptogenic cirrhosis. A final categorization of the native liver disease was attempted in these cases on the basis of detail pathomorphological findings in adequately sampled explant liver correlated with careful review of preliver transplant clinical data. Of the 50 cryptogenic cirrhosis cases, $(65.0 \%)$ finally labeled as nonalcoholic fatty liver disease cirrhosis showed histologic features in several respects different from those reported for the early and established phases of nonalcoholic fatty liver disease. Steatosis was infrequent and focal or even absent, whereas variable grades of Mallory hyaline and inflammation were consistently present. Ductular proliferation and hydropic change of hepatocytes were also frequent. Only $50 \%$ of the cases had nonalcoholic fatty liver disease associated risk factors like diabetes and obesity. It was concluded that appreciation of quantitative and qualitative differences in hepatic morphology between the cirrhotic and the early/established stage of nonalcoholic fatty liver disease will help in making a correct diagnosis of nonalcoholic fatty liver disease cirrhosis in the proper clinical setting. When appropriate criteria are used, nonalcoholic fatty liver disease appears to account for close to two thirds of cases currently labeled as cryptogenic cirrhosis.

\section{PP38-29}

Extramedullar B Cell Development in the Spleens Induced by Anti-CD3\& Antibody: An Assessment of B Cell Homeostasis, Phenotypes and Alloantibodies

G. $\mathrm{Wu}^{1}, \mathrm{~T}$. Todo ${ }^{1}$, Y. $\mathrm{He}^{2}$, A. Klein ${ }^{1}$

${ }^{1}$ Comprehensive Transplant Center, Cedars-Sinai Medical Center, Los Angeles, CA, USA; ${ }^{2}$ Department of Digestive Diseases, First Affiliated Hospital of Zhongshan University, GuangZhou, China

Objective: Antithymocyte globulin (ATG) and anti-CD3 (OkT3) have been in use in induction therapy for steroid-free liver transplantation or hepatitis $\mathrm{C}$ positive liver transplant patients. It is well known that these anti-T cell antibodies can trigger cytokine release syndrome that may affect the homeostatic balance of immune cells. Methods: We have recently developed a mouse model of allogenic immunization to study the effect of anti-TCR/CD3 antibodies on B and $\mathrm{T}$ cell homeostasis.

Results: A surge in the blood sera of Th1 cytokines (IFN $\gamma$, IL-2, TNF) and B-cell growth factors (IL-6, IL-5) occurred at 6-24 h after anti-CD3 $\varepsilon$ treatment. Pre-mature B cells expressing a phenotype c$\mathrm{kit}^{-} \mathrm{CD} 19^{+} \mathrm{CD}^{ \pm} \mathrm{CD} 11 \mathrm{~b}^{-} \mathrm{CD} 40^{-} \mathrm{B}_{22} 0^{\mathrm{lo}} \mathrm{sIgM}^{+} \mathrm{sIgD}^{-}$proliferated in the spleens 14 days after anti-CD $3 \varepsilon$ induction. The splenic B220 $0^{\text {lo }}$ cells, in contrast to the $\mathrm{B} 220^{\mathrm{hi}}$ cells, were refractory to anti-CD20 depletion. Flow cytometric analysis revealed that the B220 ${ }^{\text {lo }}$ cells in the spleens were phenotypically similar to the B220 ${ }^{\mathrm{lo}} \operatorname{sIgM}^{+} \operatorname{sIgD}^{-} \mathrm{B}$ cells presented in the bone marrow of naïve mice. Despite the presence of the $\mathrm{B} 220^{\mathrm{lo}}$ cells in the spleens mice treated with combined anti-CD3\&/CD20 produced little alloantibody after primary immunization by skin allografts.

Conclusions: $\mathrm{T}$ cell targeting by antibody to TCR/CD3 $\varepsilon$ complex causes extramedullar expansion of developing B cells (pre-B and immature B) through a pathway possibly involving B cell growth/ differentiation cytokines released by the stimulated $\mathrm{T}$ cells. The findings provide a mechanism for B-cell proliferation and alloantibody production in the setting of anti-T cell antibody use in liver transplantation.

\section{PP38-30}

'Down-regulation' for Patients with High MELD Scores before Liver Transplantation? Pro

X. Xu, Q. Ling, Z.L. He, Q. Wei, L. Zhou, S.S. Zheng

Division of Hepatobiliary and Pancreatic Surgery, Department of Surgery, the First Affiliated Hospital, Zhejiang University School of Medicine, Hangzhou, China

Background/aims: High model for end-stage liver diseases (MELD) score before liver transplantation (LT) indicated poor prognosis. Artificial liver support system (ALSS) has been proved to be an 
efficient way to improve liver and kidney functions, and to salvage patients with end-stage severe hepatitis. It has been considered as a bridge to LT nowadays. We aim to evaluate the efficacy of ALSS on reducing MELD score and assess its impact on patient survival.

Methods: One hundred and eighteen patients with end-stage liver diseases and MELD score $>30$ were include in this prospective study. Of 118 liver transplant candidates, 36 gained appropriate donor grafts and received LT within five days after their admission (Group I) and other 82 received ALSS as salvaging treatment. Of the 82 patients, 43 were found down-regulated MELD score $(\leq 30)$ at the time of operation (Group II), while the other 39 underwent LT with MELD score $>30$ (Group III).

Results: Patients characteristics were comparable among the three groups. MELD scores at admission and at operation were $34.7 \pm 4.0$ and $36.7 \pm 3.6, \quad 34.0 \pm 3.5$ and $26.3 \pm 2.6, \quad 34.9 \pm 3.7$ and $36.2 \pm 3.9$ in Group I, II and III, respectively. The median interval time from ALSS to LT was 1 day and 3 day in Group II and III, respectively. The 6-month and 1-year survival rates in Group I, II and III were 75.0 and $66.7 \%, 88.4$ and $83.7 \%, 78.2$, and $71.5 \%$, respectively. Group II showed remarkably higher cumulative patient survival than Group II and III (both $P<0.05$ ).

Conclusion: Down-regulation of MELD score before LT for patients with especially high MELD score $(>30)$ was essential for the improvement of prognosis. Prompt ALSS treatment before LT could be beneficial.

\section{PP38-31}

Post-Liver-Transplantation Infection of 6 Years: Single Center Experience in China

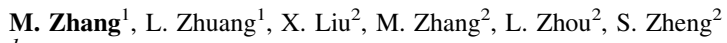

${ }^{I}$ Division of Hepatobiliary and Pancreatic Surgery, Department of

Surgery, Key Laboratory of Combined Multi-organ Transplantation,

Ministry of Public Health, First Affiliated Hospital, Zhejiang

University School of Medicine; ${ }^{2}$ Division of Hepatobiliary and

Pancreatic Surgery, Department of Surgery, Hangzhou, China

Background: Infection is one of the most common complications after liver transplantation, and is closely associated with recipient mortality rate, especially in early stage post-transplantation (0-1 month). In recent years, thanks to senior antibiotics, the infection rate in recipients was decreased significantly. However, a growing number of bacterial resistance and high-resistance bacteria turned out to be thorny problems.

Methods: We chose the recent 6 years liver transplantation recipients as research objects, and collected the body fluid culture data and image assessments from Jan. 2004 to Dec. 2009, 564 cases in all. All cases were analyzed to study the infections after liver transplantation. Results: Of all 564 recipients studied, 396 got at least one kind of bacterial infection, incidence $70.4 \%$, and 41 died from infectionassociated diseases, mortality rate $7.2 \%$. At 2004 , the incidence was $96.4 \%(106 / 110)$, and infection related mortality rate was $9.1 \%$ (10/110). While at 2009, incidence was $43.2 \%$ (41/95), and mortality rate was $6.3 \%(6 / 95)$. Of those 396 infected cases, 374 got more than one bacterial infection, while only 22 got single infection, the incidences were 94.4 versus $5.6 \%$. There were 1773 infections found in those 376 infected cases, including 264 fungal infections (14.9\%), $497 \mathrm{G}+$ bacterial (28\%) and $1012 \mathrm{G}-$ bacterial infections $(57.1 \%)$, 4.7 times per people in average. The most common bacteria were Stenotrophomonas maltophilia, Acinetobacter baumannii, Pseudomonas aeruginosa, Klebsiella pneumoniae, and Staphylococcus, and the majority of those were highly drug-resistant.The most common fungal infections were Candida and Aspergillus.

Conclusions: With more and more senior antibiotics adventured, the infection incidence after liver transplants decreased year by year, while on the other hand, infection related mortality showed no significant decreasing, result from antibiotic overuse caused high bacteria resistance. In fungal infections, comparing with the early years, Candida infection slightly decreased in recent 2 years, while Aspergillus infection increased significantly.

\section{PP38-32}

Inaccurate Preoperative Imaging Assessment on Biliary Anatomy Does Not Increase Biliary Complications after Living Donor Liver Transplantation

Q. Ling, X. Xu, H.W. Bao, K. Wang, H.Y. Xie, L. Zhou, S.S. Zheng Division of Hepatobiliary and Pancreatic Surgery, Department of Surgery, the First Affiliated Hospital, Zhejiang University School of Medicine, Hangzhou, China

Backgrounds/aims: Accurate assessment of graft bile duct is important to plan surgical procedure. We aim to estimate the efficacy of preoperative magnetic resonance cholangiopancreatography (MRCP) on depicting biliary anatomy, and assess the importance of preoperative biliary assessment on the prevention of biliary complications.

Methods: The data of 118 cases living donor liver transplantation (LDLT) were recorded. Information from preoperative MRCP was assessed using intraoperative cholangiography (IOC) as the gold standard. The possible risk factors of recipient biliary complications were analyzed.

Results: Of 118 donors, 84 had normal anatomy (type A) and 34 had anatomic variants (19 cases of type B, 9 cases of type C, 1 case of type E, 2 cases of type $\mathrm{F}$ and 3 cases of type I) confirmed by IOC. MRCP correctly predicted all 84 normal cases and 17 of 34 variant cases, and showed an accuracy of $85.6 \%$ (101/118). The incidence of biliary complications was comparable between cases with accurate and inaccurate information from MRCP, and between cases with normal and variant anatomy of bile duct. While cases with graft duct opening $\leq 5 \mathrm{~mm}$ showed a significant higher incidence of total biliary complications $(19.3$ vs. $6.6 \%, P=0.038)$ and bile duct stricture $(10.5$ vs. $1.6 \%, P=0.041)$ compared with cases with large duct opening $>5$ $\mathrm{mm}$.

Conclusion: MRCP could correctly predict normal but not variant biliary anatomy. Inaccurate information from MRCP not increases the rate of biliary complications, while small-sized graft duct may cause an increase in biliary complications particularly bile duct stricture after LDLT.

\section{PP38-33}

Steroid-free Immunosuppression in Liver Transplantation for Hepatocellular Carcinoma: Hangzhou Experience

Q. Wei, X. Xu, Q. Ling, L. Zhuang, L. Zhou, S.S. Zheng

Division of Hepatobiliary and Pancreatic Surgery, Department of Surgery, the First Affiliated Hospital, Zhejiang University School of Medicine, Hangzhou, China

Background/aims: Glucocorticoids are commonly used as the immunosuppressant after liver transplantation (LT), however, they are associated with considerable side effects like tumor recurrence, diabetes mellitus and hypertension. This retrospective study was designed to investigate the effects of immunosuppression protocol without glucocorticoid after LT for HCC.

Methods: Sixty-six patients undergoing LT for HCC between December 2007 and February 2010 were included. According to the different immunosuppression protocol, they were divided into steroid group (tacrolimus + mycophenolate mofetyl + steroid, $n=44$ ) and steroid-free group (tacrolimus + mycophenolate mofetyl + basiliximab, $n=22)$. Methylprednisolone (1 g, during operation) and basiliximab (during operation and 4 days post-LT) were given in steroid-free group. The study end points were the occurrences of acute rejection, HCC recurrence, HBV recurrence, infectious complications, metabolic complications, and patient death. 
Results: The patient characteristics were comparable between the two groups. The median follow-up time was 17.2 months. Compared with steroid group, steroid-free group showed significantly lower plasma cholesterol concentration at 4 -week $(2.94 \pm 0.21$ vs. $3.85 \pm 0.20$ $\mathrm{mmol} / \mathrm{L}, P<0.05)$, and lower overall lung infection rate $(9.1 \mathrm{vs}$. $31.8 \%, P<0.05)$. The incidences of acute rejection, new-onset diabetes mellitus, hypertension and HBV recurrence were not found significant difference between steroid group and steroid-free group. Further more, patient cumulative survival and tumor-free survival were comparable between the two groups.

Conclusion: Liver transplantation for HCC can be performed safely without using steroids in the postoperative course. Immunosuppression without steroids can reduce the plasma concentration of cholesterol after LT.

\section{PP38-34}

Prophylactic Treatment of Sorafenib Improved the Survival after Liver Transplantation for Hepatocellular Carcinoma

M.L. Zhang, L. Zhuang, M. Zhang, Z.H. Tu, L. Zhang, J.L. Fu, S.S. Zheng Division of Hepatobiliary and Pancreatic Surgery, Department of Surgery, the First Affiliated Hospital, Zhejiang University School of Medicine, Hangzhou, China

Background/aims: The approval of Sorafenib as the first effective drug for the treatment of HCC represents a milestone. The identification of Sorafenib (Sor) after LT for HCC remains poorly defined. In this study, we evaluated the Sor' security and outcome of patients in our center.

Methods: We analyzed the data of 14 patients who underwent LT for HCC (BCLC B/C) from 11/ 2007 to 10/2010. The patients were divided into prophylactic treatment group (PT group, 7 cases) and salvage treatment group (ST group, 7 cases), according to Sor received before or after tumor recurrent or metastasis.

Results: The characteristics of the patients showed no differences between PT and ST groups. All patients had not abnormal graft function related to Sorafenib. The adverse reaction of Sor were just I-II stage. For PT group, the mortality was $28.5 \%(2 / 7), 2$ cases had $\mathrm{SD}$, the mean disease-free survival (mDFS) after LT was 190 days (58-387). But in ST group, all patients died, mDFS was 146 days (34-397). After taking Sor, in PT group, the median overall survival (mOS) had not reach, the survival rate of 3, 6, 9 and over 12 months were $100 \%$ (7/7), $71.4 \%$ (5/7), $42.9 \%$ (3/7), $42.9 \%$ (3/7). The mOS of ST group was 128 days, the survival rate were $85.7 \%(6 / 7), 42.9 \%$ (3/7), 28.6\%(2/7), $14.3 \%$ (1/7), respectively.

Conclusion: Sor was effective after LT for HCC (BCLC B/C), and prophylactic treatment is better than salvage treatment.

\section{PP38-35}

Liver Stiffness Measurement in Post Liver Transplantation Children A. Wongteerasut ${ }^{1}$, V. Chongsrisawat ${ }^{1}$, S. Nivatvong ${ }^{2}$, B. Nonthasoot ${ }^{2}$, K. Setrkraising ${ }^{1}$, R. Lorchatchawankul ${ }^{1}$, P. Sintusek ${ }^{1}$, Y. Poovorawan ${ }^{1}$

${ }^{I}$ Department of Pediatrics; ${ }^{2}$ Department of Surgery, Chulalongkorn University, Bangkok, Thailand

Introduction: Children post liver transplantation may develop various complications including rejection, infection, biliary tract obstruction, and vascular thrombosis. These complications can lead to liver injury and fibrosis. Transient elastography (FibroScan) is a novel, non-invasive tool to assess liver fibrosis by measuring liver stiffness. The purpose of this study was to evaluate liver stiffness in post liver transplantation children.

Methods: Post liver transplantation patients in King Chulalongkorn Memorial Hospital and normal controls were enrolled to this study. Liver stiffness measurement (LSM) was performed by FibroScan ${ }^{\circledR}$. Data was expressed as mean $\pm \mathrm{SD}$.

Results: Twelve patients (age 12.0 \pm 5.11 years) and forty-seven normal controls (age $11.03 \pm 3.47$ years) were enrolled in this study.
LSM score of post transplantation patients was significantly higher than the controls $(11.49 \pm 7.44$ vs. $4.7 \pm 1.15 \mathrm{kPa}, p=0.01)$. There were 3 out of 6 patients with serum alanine transaminase (ALT) level $<40 \mathrm{U} / \mathrm{L}$ who had LSM score $>7 \mathrm{kPa}$ (mean $+2 \mathrm{SD}$ of normal value). The characteristics and post operative complications of 12 patients were shown in Table 1.

Table 1 Characteristics of twelve patients in the study

\begin{tabular}{|c|c|c|c|c|c|c|}
\hline No. & $\begin{array}{l}\text { Age } \\
\text { (year) }\end{array}$ & $\begin{array}{l}\text { Duration } \\
\text { aflcr } \\
\text { liver } \\
\text { transplan- } \\
\text { tation } \\
\text { (year) }\end{array}$ & $\begin{array}{l}\text { LSM } \\
\text { score }\end{array}$ & $\begin{array}{l}\text { Inter- } \\
\text { quartile } \\
\text { range } \\
(\mathrm{kPa})\end{array}$ & $\begin{array}{l}\text { Serum } \\
\text { ALT } \\
(\text { LVL) }\end{array}$ & $\begin{array}{l}\text { Post } \\
\text { operative } \\
\text { complication }\end{array}$ \\
\hline 1 & 14 & 1 & 21.8 & 4.6 & 137 & Chronic rejection \\
\hline 2 & 7 & 4 & 27 & 0.7 & 134 & $\begin{array}{l}\text { Chronic rejection, } \\
\text { bile duct stricture at } \\
\text { hepaticojejunostomy } \\
\text { anastomosis }\end{array}$ \\
\hline 3 & 16 & 10 & 7.8 & 1.5 & 45 & Chronic rejection \\
\hline 4 & 16 & 13 & 18.5 & 3.1 & 69 & Chronic rejection \\
\hline 5 & 2 & 1 & 4.6 & 1.4 & 91 & Bile duct stricture \\
\hline 6 & 13 & 12 & 16 & 3.7 & 51 & Bile duct stricture \\
\hline 7 & 7 & 6 & 8.9 & 1.1 & 35 & - \\
\hline 8 & 16 & 11 & 7.4 & 1.2 & 13 & $\begin{array}{l}\text { Renal insufficiency } \\
\text { (IgA nephropathy) }\end{array}$ \\
\hline 9 & 6 & 2 & 86 & 11 & 24 & - \\
\hline 10 & 15 & 12 & 5.9 & 0.1 & 10 & $\begin{array}{l}\text { HCV genotype } 3 \\
\text { infection }\end{array}$ \\
\hline 11 & 14 & 11 & 6.6 & 0.9 & 13 & - \\
\hline 12 & 18 & 16 & 4.8 & 0.8 & 16 & - \\
\hline
\end{tabular}

Conclusions: The results show that FibroScan may be more sensitive than liver biochemistry test to detect liver abnormality. FibroScan is a rapid and non-invasive technique to detect liver graft fibrosis after liver transplantation. It might be an alternative tool and thus avoiding protocol biopsies after pediatric liver transplantation.

\section{PP38-36}

Long-term Outcome and Causes of Late Death after Adult-to-Adult Liver Transplantation: University of Tokyo Experience

N. Yamashiki ${ }^{1,2}$, Y. Sugawara ${ }^{2,3}$, S. Tamura ${ }^{3}$, J. Kaneko ${ }^{3}$, T. Aoki ${ }^{3}$, K. Hasegawa ${ }^{3}$, Y. Beck ${ }^{3}$, K. Nojiri², K. Koike ${ }^{1}$, N. Kokudo ${ }^{2,3}$

${ }^{1}$ Department of Gastroenterology, University of Tokyo; ${ }^{2}$ Organ Transplantation Service, University of Tokyo Hospital; ${ }^{3}$ The Artificial Organ and Transplantation Division, Department of Surgery, University of Tokyo, Tokyo, Japan

Aims: To clarify the cases of late death after living donor liver transplantation (LDLT) in adult (age $\geq 18$ years) recipients.

Methods: Between 1996 and 2009, 365 primary adult-to-adult LDLT were performed at the University of Tokyo. The causes of early ( $<1$ year) and late $(1 \geq$ year) death were reviewed. The association between pretransplant characteristics and late death were analyzed.

Results: The median age of recipients was 51 years and $54 \%$ were male. The median follow up was $5.4(2.8-7.4)$ years. The cumulative survival rate was $91.4,83.7$, and $79.1 \%$ at 1,5 , and 10 years after LDLT. There were 33 early death and 29 late death; the causes of late death were infection $(n=6)$, de novo malignancy (8), graft failure (5), recurrence of HCC (4), cardiovascular (2) and others (4). None of the pre-transplant patient/ donor characteristics were associated with late death. Causes of graft failure were recurrent diseases in 3 (PSC 1, 
HCV 2), hepatic vein obstruction (1), and acute rejection in 1. De novo malignancy occurred in 8 ; lymphoma in 2 , acute myeloid leukemia in 1 , esophageal cancer in 1 , gastric cancer in 1 , colon cancer in 1 , Merkel tumor in 1 , and histiocytosis in 1 . Their median age at LDLT was 58 years, and background liver disease were fulminant hepatic failure (3), $\operatorname{PBC}(2), \operatorname{HCV}(1), \operatorname{HBV}(1)$, and $\operatorname{PSC}(1)$.

Conclusions: The long-term survival of LDLT recipients was excellent. Although the recurrent primary disease is the cause of graft-related death, the majority of non graft-related death were due to de novo malignancy, which is similar to reported long-term experience from countries performing deceased donor liver transplantation.

\section{PP38-37}

Effects of Smoking on Length of Hospital Stay in Patients Surviving an Orthotopic Liver Transplant

S. Riyaz, M. Aldersley, G. Robbins

Liver Unit, St James University Hospital, Leeds, UK

Background and aims: Smoking in orthotopic liver transplant (OLT) recipients has been associated with increased risk of vascular complications, cardiac events, graft failure and oesophageal cancer. The aim of this study was to determine the effect of smoking on length of hospital stay post OLT and also to audit support provided to these patients.

Methods: We invited 200 consecutive patients attending the adult post transplant clinic to complete an anonymous standardised questionnaire. Answers were analyzed using relevant statistical methods including chi-square, crosstabs and linear-by-linear association.

Results: 174 patients responded by answering at least 1 question (mean age 52.2 years). 80 patients had a smoking history (eversmoked) while 94 were non smokers (never-smoked). Among the smokers the median pack year history was 13 (range 0.1-56.25). Out of 56 patients with a smoking history, $46 \%(n=26)$ were offered advice and only $18 \%(n=10)$ were offered any support. In the year prior to the OLT when the patients were likely to have been under the care of the transplant centre, out of 11 patients $64 \%(n=7)$ had been offered advice and only $27 \%(n=3)$ had been offered support. Of the 26 patients smoking at the time of OLT, $58 \%(n=15)$ were offered advice and only $19 \%(n=5)$ had been offered support. There was no significant difference in length of hospital stay post OLT between patients who had never-smoked and ever-smoked $(p=0.780)$. In addition, smoking at the time of OLT had no significant impact on length of hospital stay $(p=0.922)$.

Conclusions: Smoking per se does not seem to increase hospital stay post OLT. Patients are not adequately counselled about smoking. Various support mechanisms, including better awareness among doctors and allied health care workers should be made available to tackle this problem.

\section{PP38-38}

Growth and Cognitive Function of Pediatric Living Donor Liver Transplantation Recipients

S. Treepongkaruna ${ }^{1}$, R. Charoenmaung ${ }^{1}$, J. Chuthapisith $^{1}$,

N. Ruengdarakanon ${ }^{1}$, S. Teeraratkul ${ }^{2}$, S. Leelaudomlipi ${ }^{2}$, S. Viengteeravat ${ }^{3}$, S. Sriphojanart ${ }^{2}$

${ }^{1}$ Pediatrics; ${ }^{2}$ Surgery; ${ }^{3}$ Anesthesiology, Ramathibodi Hospital, Mahidol University, Bangkok, Thailand

Background: Liver transplantation (LT) is the standard treatment for patients with end-stage liver diseases with the survival rate over $80 \%$ in most centers. Living donor liver transplantation (LDLT) has been accepted worldwide for an alternative for deceased donor liver transplantation.

Objective: To evaluate a long-term outcome of pediatric LDLT recipients at Ramathibodi Hospital in the aspects of growth and cognitive function.
Study design: Retrospective cohort study.

Patients and methods: All pediatric patients who underwent LDLT for more than 1 year at Ramathibodi Hospital from January 2001 to November 2009 were enrolled. Growth was assessed using standardization $\mathrm{Z}$ scores for height age (H/A). Cognitive function was evaluated in the patients over 2 years of age. Weschler Intelligence Scale for Children III (WISC III) and Standford-Binet Intelligence Scale IV (SBIV) were used for patients aged 6 years and below 6 years, respectively.

Results: Twenty-one patients were eligible for growth evaluation. Mean ZH/A improved from -2.29 at baseline (pre LT) to -2.07 , $-1.99,-1.52$ at $1,1.5$ and 2 years following LT, respectively. At 2 years following LT, normal growth, defined as ZH/A more than -2 , was achieved in 11 patients $(52.3 \%)$. Factors affecting growth delay included duration of prednisolone therapy, duration of re-admission and PELD score before LT. Cognitive functions of the patients $(n=18)$ were as follows: superior $(n=2)$, average $(n=4)$, low average $(n=6)$ and borderline $(n=6)$. There were no identified factors affecting cognitive function.

Conclusion: Pediatric LDLT recipients have a good long-term outcome especially for growth and fair cognitive function.

\section{PP38-39}

Anatomy of the Right Posterior Bile Duct of Donor Is Important in Bile Duct Complication in Living Donor Liver Transplantation

K.-W. Lee, Y.M. Jeon, K.-S. Suh, N.-J. Yi, G. Hong, K.U. Lee

Department of Surgery, Seoul National University, Seoul, Republic of Korea

Background and aims: Biliary complication is still the Achilles heel in liver transplantation (LT), especially in living donor LT. Among type I and II bile duct, the right posterior duct (RPD) goes left side of the right anterior duct in some cases. In these cases, the RPD can be exposed or close to dissection plane in right donor hepatectomy. The rate of biliary complication can increase in this condition because of potential bile duct injury. In this study, we evaluated the relationship between the anatomy of the RPD and the incidence of biliary complication in LDLT using right hemilver.

Methods: From Jan 2002 to Dec 2009, 167 LDLT in which MR cholangiogram of the donors were available were included. When the RPD goes in right side of the anterior duct, it was defined as Type A. If the RPD goes left side of the anterior duct and near the parenchymal dissection plane, it was defined as Type B. Among Type B, if the exposed length of the RPD to the parenchymal plane is short $(\leq 5 \mathrm{~mm})$, it was Type B-S. If the exposed length is long $(>5 \mathrm{~mm})$, it was defined as Type B-L. 56 patients were Type A and 111 patients were Type B. Type B-S was 54 and B-L was 57.

Results: The rate of biliary complication was higher in Type B (5.36\% in Type A and $29.73 \%$ in Type B, $p<0.01$ ). Among Type B, biliary complication occurred more in Type B-L (12.96\% in Type B$\mathrm{S}, 45.61 \%$ in Type B-L, $p<0.01)$.

Conclusion: The detail anatomy of the RPD was important risk factor for biliary complication in LDLT using right hemiliver from the donors with biliary Type I and II. Cautions are needed in this type to prevent damage or ischemic injury of the RPD.

\section{PP38-40}

Usefulness of Novel Criteria Based on AFP, PIVKA II, and 18FDG-PET in Prediction of Recurrence after Liver Transplantation for HCC K.-S. Suh, K.-W. Lee, G. Hong, N.-J. Yi, Y.M. Jeon, K.U. Lee Department of Surgery, Seoul National University, Seoul, Republic of Korea

Background and aims: Hepatocellular carcinoma (HCC) became an important indication for liver transplantation. However, Milan criteria is considered too restrictive, especially in living donor liver transplantation (LDLT). Recently, several biological markers were introduced to refine selection criteria. In this study, we evaluated the 
usefulness of three biologic markers (AFP, PIVKA II, and 18FDGPET) in prediction of recurrence after LT and suggested new selection criteria based on combination of three biologic markers.

Methods: Among 130 LDLT for HCC from Feb 1991 to Dec 2007, 50 patients who underwent all three test (AFP, PIVKA II, and 18FDGPET) before transplantation were included. 43 patients were male. 38 patients were within Milan and 12 were outside Milan. 10 patients showed PET positivity. 11 patients were AFT $>200 \mathrm{mg} / \mathrm{dl}$. 11 patients were PIVKAII $>100 \mathrm{mg} / \mathrm{dl}$. Patients were divided into 4 groups according to the number of risk factors (Group A; all negative, Group B; one positive, Group C; two positive, Group D; three positive).

Results: 2 year disease free survivals of each group were $96.2 \%$ (Group A, reference), 90\% (Group B, HR; 2.44, $p>0.01$ ), 33.3\% (Group C, HR; 32.86, $p<0.05$ ), 0\% (Group D, HR; 248.4, $p<0.05$ ) Conclusion: The novel criteria based on three biological markers (AFP, PIVKA II, and 18FDG-PET) excluding conventional markers (size and number of tumor) showed good differentiation of the risk of recurrence. This new criterion can be used to select good candidate for LT, especially in outside Milan.

\section{PP38-41}

Quality of Life of Pediatric Living Donor Liver Transplantation Recipients

R. Charoenmuang ${ }^{1}$, S. Treepongkaruna ${ }^{1}$, S. Teeraratkul ${ }^{2}$, S. Leelaudomlipi ${ }^{2}$, P. Junchotikul ${ }^{3}$, J.W. Varni ${ }^{4}$, S. Sriphojanart ${ }^{2}$

${ }^{1}$ Department of Pediatrics, ${ }^{2}$ Department of Surgery, ${ }^{3}$ Department of Nursing, Faculty of Medicine, Ramathibodi Hospital, Mahidol University, Bangkok, Thailand, ${ }^{4}$ Department of Pediatrics, College of Medicine, Texas A\&M University, College Station, TX, USA

Background: Liver transplantation (LT) is the standard treatment for patients with end-stage liver diseases with the survival rate over $80 \%$ in most centers. Living donor liver transplantation (LDLT) has been accepted worldwide for an alternative for deceased donor liver transplantation with the better outcome. Quality of life (QL) of children underwent LDLT in Thailand have not been studied.

Objective: To evaluate the quality of life (QL) of children underwent LDLT at Ramathibodi Hospital.

Study design: Retrospective cohort study.

Patients and methods: All pediatric patients aged over 2 years who underwent LDLT for more than 1 year at Ramathibodi Hospital from January 2001 to November 2009 were enrolled. QL was assessed by PedsQL 4.0 Generic Core Scale (Thai version) which composed of physical, emotional, social and school domains. The questionnaires were designed for parental report for patients aged 2-4 years and 5-12 years, and child self-report for patients aged 5-12 years. Healthy children, age and sex matching, who attended a Well-baby Clinic were served as controls.

Results: Total 21 children were eligible for study. Total scores, physical, social and school scores of the patients aged 2-4 years were lower than controls as well as patients aged 5-12 years (child report). Only physical scores of patients aged 5-12 years reported by parents were significantly lower than controls. Factors affecting low physical scores were age over 2 years at transplantation, re-admission duration and duration of prednisolone therapy $(P<0.05)$.

Conclusion: Pediatric LDLT recipients has a good long-term outcome, however, quality of life remains lower than healthy children.

\section{PP38-42}

Living Liver Donor Mortality and Morbidity Demand Genuine Transparency: The Situation in Egypt

H. Abdel-dayem ${ }^{1}$, N. Allam ${ }^{2}$, A. Helmy ${ }^{1}$

${ }^{1}$ Hepatobiliary Surgery; ${ }^{2}$ Hepatology, National Liver Institute,

Menoufiya University, Shebeen Al-koam, Egypt

Background: The reluctance to publish a complete account of any death or serious complication among living-liver donors, although understandable in a fraught medicolegal environment, is not good for patient care or liver transplantation. A donor death or serious complication will have a devastating effect not only on the families of the donor and recipient but also on the clinical staff involved. The impact may spread to other potential donors and recipients, and lead to negative publicity and potential economic damage to the transplant center.

Objective: To discuss the mortality and morbidity within living liver donors in Egypt and to try answering the question: what level of risk are the potential donors and the society willing to accept?

Methods: By personal communication and reviewing all published data and reports from centers performing LDLT in Egypt, morbidity and mortality within the donors were analyzed.

Results: From August 2002 to January 2010, the number of living donor liver transplants performed in Egypt topped out to more than 1,000 procedures in 11 centers. There were two reported donor mortality $(0.29 \%)$. The first was a 45 year-old male who donated a right lobe to his brother and died of sepsis from bile leak 1 month after donation. The second was a 22 year-old male who donated his right lobe to his father; he suffered from massive intraoperative bleeding from the stump of the portal vein and died of multisystem organ failure after 10 days. Donor morbidity included: portal vein thrombosis $(0.5 \%)$, biliary complications (2\%), bleeding (3\%), intraabdominal collections (6\%), pneumonia (4\%), pleural effusion (22\%), and depression (12\%).

Conclusion: Live liver donor morbidity and mortality remains underreported. LDLT programs are best served by collaborative effort between centers reporting accurate information characterized by genuine transparency.

\section{PP38-43}

Study on COX-2, TLR4 and HO-1 in Ischemia Reperfusion Injury of Whole or Partial Liver Transplantation in Rat

X.-L. Zhu ${ }^{1}$, Y.-M. Li ${ }^{2}$, X. Li ${ }^{3}$, W.-C. Zhou ${ }^{3}$

${ }^{1}$ General Surgery, The First Hospital of Lanzhou University;

${ }^{2}$ The Second Hospital of Lanzhou University; ${ }^{3}$ The First Hospital of Lanzhou University, Lanzhou, China

Aim: To investigate the expression and role of COX-2, TLR4, HO-1 in ischemia reperfusion injury of liver transplantation of rat.

Methods: Male Wistar rat were divided to 4 groups at random: normal group (O), liver transplantation group (LT), 50\% partial liver transplantation group (PLT) and NS398 administrated group (NS). Whole/partial liver transplantation models of rats were built up successfully, and experimental animals were sacrificed after grafts ischemia/reperfusion 3, 6, 12 and $24 \mathrm{~h}$. The expression of COX-2, TLR4, HO-1 mRNA and COX-2 protein were detected by RT-PCR and immunohistochemistry, respectively. And the level of TNF- $\alpha$ and IL-10 were detected by ELISA after operation.

Results: The amount of COX-2 mRNA in different time after reperfusion in $50 \%$ partial liver transplantation group (PLT) was all higher than whole liver transplantation group (LT). And expression of ALT/AST and histopathologic damage, level of harmful TNF- $\alpha$ and protective IL-10 all higher than LT group too. Whereas, even if the expression of COX-2 was inhibited by NS398 in intervention group (NS), there were still higher ALT/AST and the more severe histopathologic damage, and the higher level of TNF- $\alpha$ and IL-10 than LT $\operatorname{group}(P<0.05)$. The inhibition of NS398 to COX-2 had no influence with TLR4. TLR4 was sensitive to high endotoximia (LPS) in group PLT. HO-1 is a protective endogenous factor, which includes in mechanism of IRI in liver transplantation. And NS398 can decrease expression of HO-1.

Conclusion: There was a high level expression of COX-2 in partial liver transplantation group (PLT), but the inhibition to COX-2 also increased ischemia reperfusion injury after liver transplantation. So, it may be a protective role in liver transplantation to reduce IRI. TLR4 has no obviously correlation with COX-2, and HO-1 may be a product downstream of COX-2. 


\section{PP38-44}

Quality of Life in Liver Transplantation

S. Radfar, S.A. Tavallaii, S. Nouhi, G. Karami, A.-R. Adibzadeh, M. Radfar Baqiyatallah University of Medical Sciences-Research Center of Behavioral Sciences, Tehran, Iran

Quality of life (QOL) is a term used most frequently in the context of medicine and healthcare and refer to an individual's emotional, social and physical wellbeing, including their ability to function in the ordinary tasks of living. Understanding quality of life is recognized as increasingly important in healthcare, where the relationship between cost and value raises complex problems. Health related quality of life (HRQOL) concerns the patient's overall physical and psycho-social well-being. The properties of the HRQOL questionnaire make it appropriate for use in clinical trials designed to evaluate its responsiveness to treatment among patients with liver transplantation. HRQOL is easy-to-use instrument and self-administered questionnaires. This is a review literature study. The literature suggests that during the organ-waiting period patients experience significant difficulties, including high levels of emotional distress, and physical and functional disability. Liver transplantation is the treatment of choice for end-stage liver disease. Following transplantation, most patients appear to enjoy a dramatic improvement in quality of life. Most studies have shown that the overall quality of life of transplant recipients improves markedly after transplant in all areas. Post-transplant patients reported significantly higher levels of physical and psychological functioning compared with pre-transplant patients.

\section{PP38-45}

Occult Hepatitis B Virus Infection in Living Donors Egyptian Liver Transplant Candidates

Y.Z. Gad

Internal Medicine, Mansoura University, Mansoura, Egypt

Background/aim: The prevalence of occult HBV infection in healthy people, who may represent the potential liver donors, is still unknown in Egypt. The aim of this study was to investigate the prevalence of occult HBV infection among healthy subjects.

Subjects and methods: Serum samples for serological assays and liver tissue specimens were taken from 107 apparently healthy individuals, prepared as liver transplant donors at the Gastroenterology Surgical Centre during 2008-2009. HBsAg and anti-HBs and antiHbc were determined in the participants who had normal liver enzymes and histology and were negative for HBsAg and IgM anti$\mathrm{HBc}$. DNA was extracted from the frozen liver specimens of each case by standard procedure. The presence of occult HBV infection was considered if at least two of the three nested-PCR amplifications were HBV DNA-positive.

Results: HBV DNA sequences were detected in liver tissue specimens of 21/107 (19.62\%) (15 Anti-HBc-positive and 6 AntiHBcnegative) apparently healthy subjects. There were no age statistical difference $(31.3 \pm 10.4)$ versus $(30.1 \pm 9.7),(p<0.005)$ or sex statistical difference $(10 / 8)$ versus(47/42) $(p<0.005)$ when comparing the occult-positive cases and the occult-negative cases. Occult HBV status was strongly related to the anti-HBc antibody status; where HBV DNA was detected in 15 of the $21(71.43 \%)$ positive cases versus 6 of the $86(6.99 \%)$ HBV marker negative cases $(p<0.001)$

Conclusion: Occult HBV infection will remain as an overlooked source of HBV transmission without a highly sensitive PCR for HBV DNA testing in the context of orthotopic liver transplantation especially, in areas of high HBV endemicity.
PP38-46

In Vitro Expanded Adult Human Liver Progenitor Cells are Able to Regenerate the Liver

D.N. Khuu, O. Nyabi, M. Najimi, E. Sokal

Université Catholique de Louvain, Brussels, Belgium

Adult derived human liver progenitor cells (ADHLPCs) with selfrenewal capacity and pre-determined for in vitro hepatogenic differentiation are attractive candidates for liver cell based therapy. ADHLPCs were isolated from 3 different healthy cadaveric donor livers and intrasplenically transplanted into female SCID mice at six week-old to investigate their engraftment capacity and their hepatic regeneration and differentiation potency. ADHLPCs were able to migrate from the splenic site of injection towards the liver parenchyma and engrafted mostly in the peri portal vein areas. They maintained human albumin expression as well as human Alu sequences and acquired in less than one month hepatocyte-like morphology. Analysis of serial sections revealed that these cells lost the mesenchymal marker vimentin, characteristic of their initial mesenchymal phenotype from day seven and acquired ornithine transcarbamylase (OTC) enzyme, a mature hepatocyte maker one month post transplantation. Co-expression of human albumin and OTC observed after 1 and 2 months supports the in vivo hepatogenic differentiation of ADHLPCs. Human albumin positive cell clusters grew up in size and number between one and two months post transplantation. To confirm self-renewal potency of implanted human cells, we performed 5-bromo-2'-deoxyuridine (BrdU) uptake on one month transplanted SCID mice and demonstrated cell regeneration levels at $11.42 \pm 3.48$ to $18.18 \pm 1.35 \%$ after 14 and 28 days, respectively. In physiological condition, proliferation of implanted human cells was similar to mice hepatocytes. Following 20\% partial hepatectomy, implanted human cells participated to regeneration from day 3 and acquired rapidly hepatogenic differentiation as host hepatocytes from day 14. In vivo hepatogenic differentiation capacity and response to liver regenerative stimulus confirm that ADHLPCs can be considered for the development of clinical liver regenerative medicine in humans.

\section{PP38-47}

Diagnosis and Management of Paralysis of the Right Hemidiaphragm after Liver Transplantation

T. Chen, J.-P. Liu, Y.-L. Wan, H.-M. Lin, J. Wang

General Surgery, Second Hospital, Sun Yat-sen University, Guangzhou, China

Objective: To investigate the diagnosis and management of paralysis of the right hemidiaphragm after liver transplantation.

Material and methods: 60 cases adult patients who received liver transplantation from Feb 2001 through March 2007 in 2nd affiliated hospital, Sun Yat-sen University were retrospectively analyzed. 5 cases of them developed posttransplant paralysis of the right hemidiaphragm. 2 of whom received radiotherapy for hepatocellular carcinoma and the other 2 cases received repeated hepatectomy for hepatocellular carcinoma before transplant. 1 of the 5 cases suffered from severely HBV related liver failure.

Results: 101 episodes of postoperative respiratory complications in 40 cases were found which included pleural effusion, atelectasis, acute respiratory distress syndrome (ARDS), hepatopulmonary syndrome (HPS), pulmonary interstitial edema, pneumonia and tracheorrhagia. 5 cases of patients developed paralysis of the right hemidiaphragm. Ventilator supporting times were 14,16, 34, 45 and 60 days, respectively. 4 survived post transplant, well the other case with severely liver fail dead on 31 day postoperatively. Treatments for those patients were complicated and tremendous which resulted in big economy burden to the patients. 
Conclusions: The strictness of monitoring of the respiratory function and the accurate using of respirator for paralysis of the right hemidiaphragm post-liver-transplant is important. For patients with previous radiotherapy and/or repeated hepatectomy history, attention should be paid on difficulty of diaphragm isolation and postoperative dysfunction of diaphragm.

\section{PP38-49}

Hemodynamic Changes in the Liver and Kidney after Orthotopic Liver Transplantation Using Piggy-Back Technique

A. Gregusova ${ }^{1}$, S. Hrusovsky ${ }^{2}$, M. Zigrai ${ }^{2}$, F. Danninger ${ }^{3}$, P. Nemec ${ }^{4}$, J. Ondrasek ${ }^{4}$, E. Christova ${ }^{5}$, M. Pauliny ${ }^{5}$

${ }^{1}$ Internal Medicine I, University Hospital of Bratislava; ${ }^{2}$ Internal Medicine; ${ }^{3}$ Liver Transplant Centre, Slovak Medical University, Bratislava, Slovak Republic; ${ }^{4}$ Transplant Centre, Brno, Czech Republic; ${ }^{5}$ ICU, University Hospital of Bratislava, Bratislava, Slovak Republic

Background: In advanced stages of portal hypertension with ascites, the renal artery flow decreases in peripheral parts of the renal cortex. After liver transplant, important changes in blood flow occur. Acute kidney injury is a current condition.

Patients and methods: In Bratislava Liver Transplant Centre, we measured flow changes in liver and both kidneys in 20 consecutive patients (12 men and 8 women aged 18-60 years) during orthotopic liver transplantation (OLTx) using piggy-back technique, by duplex ultrasonography: max. portal vein flow, hepatic artery pulsatility index (PI) and resistive index (RI), and RI and PI in small renal arteries of the right and left kidney, before OLTx and daily in next seven days. Parameters were compared in patients with or without splenectomy, with or without MELD score higher than 15, with or without portal vein and/or hepatic artery desobstruction.

Results: Six patients underwent splenectomy, seven patients had MELD $>15$, eleven patients had hepatorenal syndrome. Three patients underwent reconstruction of hepatic artery, one desobstruction of hepatic artery, and three desobstruction of portal vein. In nine patients kidney flow improved $12 \mathrm{~h}$ after OLTx. In five patients from 6 who underwent splenectomy, the right kidney flow improved and in 4 of them the left kidney flow worsened. In four patients from 8 with MELD $>15$, the right kidney flow worsened within $12 \mathrm{~h}$ after OLTx and in six of them the left kidney flow improved. Increase of hepatic artery RI occurred in sixteen patients immediately after the OLTx, and fell back on fourth day. The portal vein flow increased in seventeen patients immediately after OLTx and remained high during the next 7 days.

Conclusion: Our results suggest that OLTx using piggy-back technique decreases temporarily the right kidney flow, and concomitant splenectomy decreases the left kidney flow, with compensatory increase of flow in right kidney.

\section{PP38-50}

Risk Factors Associated with Development of Biliary Complication after Liver Transplantation

G. Seven ${ }^{1}$, K. Çınar ${ }^{1}$, R. İdilman ${ }^{1}$, A. Tüzüner ${ }^{2}$, S. Hazinedaroğlu ${ }^{2}$, S. Karayalçın ${ }^{1}$, K. Bahar ${ }^{1}$, K. Karayalçın ${ }^{1}$, S. Ersöz ${ }^{2}$

${ }^{1}$ Gastroenterology; ${ }^{2}$ Hepatobiliary Surgery, Ankara University Medical Faculty, Ankara, Turkey

Introduction: Although a small number of studies suggest that the incidence of biliary complications is higher in living donor liver transplantation (LDLT) than deceased donor liver transplantation (DDLT), the data are contradictory. Aim of this study is to determine if LDLT is an independent risk factor for biliary complications.

Methods: 176 liver recipients (118 living liver donors, 57 deceased liver donors) who underwent surgery in Ankara University Faculty of Medicine Hepatobiliary Surgery and Transplantation Unit between years 1994 and 2010 were retrospectively examined. Patients under age 18 , dead in the first 30 days after surgery and patients without adequate follow up datas were excluded, 119 liver recipients were analyzed. Patients were followed up until August 1st, 2010. Reconstruction types were duct-to-duct choledocho-choledochostomy $(n=68)$ and Rouxen-Y choledochojejunostomy $(n=51)$ depending on the donor's native bile duct condition. With univariable analyze, seven variables related with biliary complications were analyzed. These variables were age, sex, donor type (living or deceased donors), biliary anastomosis type (duct-to-duct choledocho-choledochostomy or Roux-en-Y choledochojejunostomy), ABO incompatibility, Rhesus antigen status, primary disease (malign or benign, biliary or nonbiliary).

Results: 119 liver recipients (mean age was $43 \pm 12.7,72$ were male) were analyzed. Mean follow up after liver transplantation was 49 months (range 3-180). Living and deceased donor liver transplantation were performed in 71 and 48 patients; respectively. Incidence of biliary complication was $36.1 \%$ ( 29 male; mean age was $44.2 \pm 12.1)$. In univariable analyze, only D-D anastomosis type was an important risk factor for biliary complications (Odds ratio 3.346; 95\% CI, $1.409-7.946 ; p=0.005)$. 81.3\% (34/43) of biliary complications were treated successfully by endoscopic and percutan techniques.

Conclusion: No correlation was observed between LDLT and development of biliary complications. Duct-to-duct choledocho-choledochostomy was a risk factor for biliary complications either for LDLT and DDLT. Most of the complications were successfully treated by endoscopic and percutan techniques.

\section{PP38-51}

Incidence, Pattern of Infectious Problems Following Living Donor Liver Transplantation in Egypt

A.O. Abdelaziz ${ }^{1}$, M. Mostafa ${ }^{2}$, M. Nashaat ${ }^{2}$, A. Yosry ${ }^{3}$, W. Doss ${ }^{3}$, G. Esmat ${ }^{3}$, M. El-Serafy ${ }^{3}$, H. El-Gabaly ${ }^{4}$, A.A. El Kholy ${ }^{5,6}$

${ }^{I}$ Tropical Medicine Department, Cairo University and Dar Al Fouad Hospital, Cairo; ${ }^{2}$ Endemic and Infectious Medicine Department, Suez Canal University, Ismailia; ${ }^{3}$ Tropical Medicine Department, Cairo University, Cairo; ${ }^{4}$ Liver Transplantation Unit Dar Al Foud Hospital, Dar Al Fouad Hospital, th of October City; ${ }^{5}$ Clinical Pathology Department, Cairo University, Cairo; ${ }^{6}$ Microbiology and Infection Control Departments, Dar Al Fouad Hospital, 6th of October City, Egypt

Background: Living donor liver transplantation (LDLT) is a successful therapy for patients with end-stage liver disease. Infection is currently a life-threatening complication for these patients.

Aim of work: To determine the incidence of various infections in patients with LDLT during early (1st month) and intermediate (2nd to 6th month) postoperative periods and to investigate the risk factors associated with first episodes of bacterial, fungal and viral infections including cytomegalovirus (CMV) reactivation.

Methods: The study included 128 LDLTs performed in 128 recipients from October 2001 to January 2007; those patients were followed up for six months after liver transplantation operation. A descriptive analysis estimating the $95 \%$ confidence interval was performed with 100 variables stratified according to preoperative, operative and postoperative conditions. Cox regression analysis was used to identify the variables associated with infection.

Results: Among the total, 53.1 and $27.3 \%$ of patients developed infection during early and intermediate postoperative periods respectively. During early postoperative period; $40.6 \%$ of patients had bacterial infections (BI); 7\% had fungal infections (FI) and 5\% CMV. $\mathrm{HCV}$ infection recurrence was universal. During intermediate postoperative period; $18.7 \%$ of patients had BI, $4.7 \%$ FI and 3.9\% CMV. Though HCV infection recurrence was universal, yet recurrence of chronic hepatitis C occurred in $19.53 \%$. Multivariate analyses identified the following risk factors for infections: spontaneous bacterial 
peritonitis (SBP), plural effusion, MELD score, preoperative bilirubin level, long usage of indwelling devices (CV and urinary catheters), long ICU and hospital stay, operation time, reoperation, raised immunosuppressive level and frequent pulse steroids.

In conclusion: Infectious problems were frequently faced after LDLT operation.

\section{PP38-52}

Impact of Infectious Complications on Patient Survival Following Living Donor Liver Transplantation in Egypt: A 5 Years Follow-up A. Abdelaziz ${ }^{1}$, A. Yosry $^{1}$, G. Esmat ${ }^{2}$, M. El-Serafy ${ }^{2}$, H. El-Gabaly ${ }^{3}$, A. El Kholy ${ }^{4}$

${ }^{1}$ Tropical Medicine Department, , Cairo University, Egypt;

${ }^{2}$ Cairo University, Cairo; ${ }^{3}$ Dar Al Fouad Hospital, th of October City; ${ }^{4}$ Clinical Pathology and Infection Control, Cairo University and Dar Al Fouad Hospital, Cairo, Egypt

Background: Liver transplantation (LT) has been an accepted treatment for end-stage liver disease since the 1980s. Currently it is a highly successful treatment for this indication. Infection is currently a life-threatening complication for these patients.

Aim of work: To determine the impact of infectious problems on the survival of patients after living donor liver transplantation (LDLT).

Methods: The study includes 128 LDLTs performed from October 2001 to January 2007; those patients were followed up for six months after liver transplantation operation. A descriptive analysis estimating the $95 \%$ confidence interval was performed with 100 variables stratified according to preoperative, operative and postoperative conditions. Cox regression analysis was used to identify the variables associated with decreased survival. Kaplan Meier survival analysis was done.

Results: 53.1 and $27.3 \%$ of patients developed infection during early and intermediate postoperative periods respectively. Mean survival was decreased significantly in recipients who caught early infections (1063.09 days compared to 1534.83 for non-infected), intermediate infections (1046.39 vs. 1403.21 days for non-infected). Mortality was more frequent among recipients who had infections in the early $(P<0.001)$, intermediate $(P=0.01)$ periods postoperatively, or in both periods $(P=0.001)$. Mortality was more frequent in recipients infected with MSSA $(p=0.03)$, MRSA $(p=0.001)$, Acinetobacter $(p=0.02)$, Enterococci $(p=0.03)$, Fungi $(0.008)$ and CMV $(p=0.047)$ early period after transplant. Likewise during intermediate period mortality was more frequent in recipients infected with CMV $(p=0.047)$ and Klebsiella $(p=0.04)$.

In conclusion: Infectious problems remain a remarkable threat and have serious impact on patient survival after living donor liver transplantation.

\section{PP38-53}

Liver Transplant for Alcoholic Steatohepatitis (ASH)

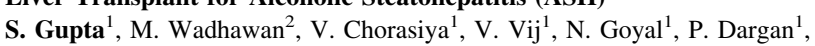
K. Vasudevan ${ }^{1}$

${ }^{1}$ Liver Transplant Surgery, Indraprastha Apollo Hospital, New Delhi;

${ }^{2}$ Gastroenterology, Hepatology \& Liver Transplantation, Indraprastha Apollo Hospital, Delhi, India

Background: Alcoholic hepatitis is considered a strict contraindication for liver transplant in the west for the fear of recidivism. A substantial subset of ASH patients remains non-responsive to medical therapy, on applying the Lille Model, this group reveals a 6-months mortality of up to $75 \%$. The 6 month rule is a very poor predictor of post transplant abstinence. There is a debate that 6 month rule should be relaxed for patients suffering from severe ASH. We report liver transplant for severe alcoholic hepatitis at our centre.
Methods: All patients of ASH were admitted for conservative treatment. Those having worsening bilirubin \& INR, hepatic encephalopathy or renal failure at 4 weeks after admission were given the option of liver transplant. All patients taken for transplant underwent pre-transplant intensive psychiatric evaluation and family counseling regarding need for abstinence.

Results: Fifteen patients of severe alcoholic hepatitis underwent LRLT at our centre between Sep 2006 to Aug 2010. Baseline characteristics-age $48 \pm 7.4 \mathrm{M}: \mathrm{F}$ 14:1, Childs' Score 13 (Range 10-14) $\mathrm{S}$ bil $25.6 \pm 12.6 \mathrm{~S}$ creatinine $1.2 \pm 1.1$ INR $2.2 \pm 0.4$ Meld score 28 (Range 22-44). HPE of explant liver showed evidence of alcoholic hepatitis in all, 11 patients had underlying cirrhosis, 2 had incomplete cirrhosis and 2 had massive hepatic necrosis. Two patients died in the peritransplant period (sepsis, hyponatremia-cpm). Four patients had early morbidity post transplant (SFSS in 1-underwent splenic artery ligation, delayed recovery in renal function-1, ACR in 1 \& prolonged ascitic drainage which settled over 4 weeks. Thirteen patients are alive at last FU (14.6 \pm 10.1 months). One out of these has had recidivism, all others are abstinent at last FU.

Conclusions: Liver Transplant for severe ASH has results comparable to transplants for ESLD. In view of high mortality in this subgroup, LT should be considered in the treatment algorithm.

\section{PP38-54}

Post-liver Transplantation(LT) Outcome in HIV/HCV Co-infected Patients. Comparison with Monoinfected Patients with HCV-cirrhosis V. Aguilera ${ }^{1,2}$, C. Vinaixa ${ }^{1}$, M. Blanes ${ }^{3}$, A. Rubín ${ }^{1,2}$, F. San Juan ${ }^{4}$, R. López-Andujar ${ }^{4}$, M. Berenguer ${ }^{1,2}$, M. Prieto ${ }^{1,2}$

${ }^{1}$ Hepatology Unit, Hospital Universitario La Fe;

${ }^{2}$ CIBEREHD, Instituto de Salud Carlos III; ${ }^{3}$ Infectious Diseases Unit; ${ }^{4}$ Liver Transplant and Surgery Unit, Hospital Universitario La Fe, Valencia, Spain

LT in HIV/HCV co-infected patients is still controversial.

Aim: To compare the post-LT outcome of HIV/HCV co-infected with HCV mono-infected controls.

Methods: Between 2004-2010, 23 HIV/HCV LT were performed; of these, 16 were positive HCV RNA at LT and were matched to $32 \mathrm{HCV}$ mono-infected controls with regards to LT date, age and sex. Data analyzed included: baseline features, hepatocellular carcinoma (HCC), MELD at transplantation, donor features, mean CD4, histological outcome, infections pre and post-LT, antiviral treatment and survival. Results: HIV/HCV group versus control group: 84 versus $81 \%$ male, median age: 46 versus 48 , donor age: 53 versus 50.5 , Meld: 19 versus 17 , HCC 19 versus $44 \%$, Genotype 1: 50 versus $75 \%(p=0.01)$. Opportunistic infections occurred in 4 HIV patients. Baseline immunosuppression: Tac-Prednisone or CsA-prednisone \pm mycophenolate mofetil (90\% controls and $81 \%$ HIV/HCV, pNS). Post-transplant infections: $44 \% \mathrm{HIV} / \mathrm{HCV}$ versus $25 \%$ in controls (pNS). Mean follow up (days): 1266 in HIV versus 1961 in controls $(p=0.121)$. GGT, FA and Bilirrubin at 6 month were higher in HIV/HCV vs controls $(p<0.05)$. A 1 year liver biopsy was performed 14 HIV patients and in 24 non-HIV. Fibrosis $>1$ at 1 year was $42 \%$ in HIV/HCV versus $55 \%$ in controls $(p=0.4)$. Antiviral therapy was started in $37 \%$ in HIV/HCV versus $65 \%$ of controls $(p=0.06)$. Patient survival at 1 and 3 years: 93 and $81 \%$ in controls versus 71 and $59 \%$ in HIV/HCV group $(p=0.121)$. Five $(31 \%)$ patients died in HIV/HCV group (3 related to HCV-recurrence and 2 rejection, because of low adherence) and $4(12.5 \%)$ in the control group (1 HCC recurrence, 1 haemorrhage, 2 sepsis).

Conclusions: Preliminary results in HIV/HCV LT co-infected patients are inferior to controls although better baseline features (less HCC and genotype1). Those results could be explained because a lower use of antiviral therapy in HIV group due to co-morbidities. Adherence to treatment is fundamental in HIV group. 


\section{PP38-55}

Cmv Infection Does Not Always Lead to Disease Cmv Infection Does Not Always Lead to Disease

K. Makki, M. Wadhawan, N. Goyal, V. Vij, V. Chorasiya, S. Gupta Center for Liver and Biliary Surgery, Apollo Hospital, New Delhi, India

Background: Most of the data available on CMV disease is based on western population. 18-29\% of all liver transplant recipients are at risk of developing CMV disease. To prevent CMV disease, prophylactic or preemptive strategies are used.

Aim: To study prevalence of CMV disease among patients with CMV infection in population from the Indian subcontinent.

Methodology: All recipients negative for CMV IgG pretransplant are given prophylaxis if donors are positive. Rest do not receive any prophylaxis but undergo CMV-PCR and PP65-antigen testing at two weeks post transplant and then only if symptomatic. Only symptomatic patients with increasing CMV DNA titres are treated with Intravenous (IV) Gancyclovir and Valgancyclovir (oral) for 3 months. Asymptomatic patients with stable/decreasing titres are observed. As all our recipients were CMV IgG positive, none received prophylaxis. Results: Of 319 consecutive liver transplant patients from Indian subcontinent, between September 2006 and June 2010, 277 (87\%) tested negative for CMV DNA at two weeks and none developed CMV disease on follow up. Forty two patients were positive for CMV DNA. Of these 31 patients had DNA $<500(10 \%)$. 29/31 remained asymptomatic, did not develop disease on follow up and did not receive treatment. 2/31 on follow up developed CMV disease and required treatment. Of 11 patients with CMV DNA $>500,4$ were asymptomatic, not treated and on follow up did not develop CMV disease. Seven developed symptoms $(5=$ bone marrow depression, $1=$ steroid bolus, $1=$ diarrhea) and required treatment. Of $9 / 42$ patients developed CMV disease requiring treatment (21\%)

Conclusion: Though CMV IgG positivity is almost universal in Indian subcontinent among recipients, disease incidence is low. CMV DNA positivity does not correlate with disease. Both preemptive and prophylactic strategies may not be required for patients from Indian subcontinent.

\section{PP38-56}

Indication of Orthotopic Liver Transplantation for Hepatocellular Carcinoma: A Retrospective Analysis with Hangzhou Criteria, Single Center Experience for 8 Years

L. Lei, C. Liu, R.T. Wang, S. Huang, X.G. Zhang, B. Wang, X.M. Liu,

L. Yu, Y. Lv

Hepatobiliary Surgery, First Affiliated Hospital, School of Medicine, $X i$ 'an Jiaotong University, Xi'an, China

Background and aims: Hangzhou criteria (HC) have been recently proposed to identify suitable candidates with hepatocellular carcinoma (HCC) for orthotopic liver transplantation (OLT) in China. The purpose of this study was to evaluate the role and significance of $\mathrm{HC}$ compared with other recognized criteria (Milan, University of California San Francisco).

Methods: A retrospective analysis was performed in a cohort of 54 patients with HCC among 184 consecutive OLTs at our institution from 2002 to 2009. Survival and tumor-free survival rates were calculated by the Kaplan-Meier method and log-rank test. The difference of tumor recurrence rates between the Milan criteria (MC) and the $\mathrm{HC}$ groups were examined by Chi-squared test.

Results: Among the 54 OLT patients with HCC, mostly in association with hepatitis B virus infection, at a mean follow-up of 33.2 months. 24 cases complied with the MC; 4 patients beyond the MC within the UCSF; 10 patients exceeded the MC and the UCSF but within the HC. Upon survival analysis, the 1-, 3-, and 5-years survival and disease-free survival of the HC group ( $n=34 ; 82,69,50 \%$ and $73,63,46 \%$, respectively) compared with the MC $(n=24 ; 83,73,68 \%$ and $75,70,70 \%$, respectively) and the UCSF ( $n=28 ; 82,74,59 \%$ and $75,67,62 \%$, respectively) groups were not statistically significant $(P=0.441$, $P=0.721$ and $P=0.332, P=0.597$, respectively). Tumor recurrence rates at 1-, 3- and 5-years between groups MC and $\mathrm{HC}$ did not show statistically significant difference $(P=0.869 . P=0.179$ and $P=0.118)$. It was found that more patients $(n=10)$, could underwent OLT without influence overall outcomes. However, the survival and tumor-free survival rates of group beyond the $\mathrm{HC}(n=10 ; 60,15,5 \%$ and $30,15 \%, 5 \%$, respectively) compared with the MC, UCSF and HC decreased significantly $(P<0.001)$.

Conclusions: The HC do not worsen the survival rates at 1-, 3-, and 5-years of follow-up and show a reliable and feasible candidates selection and prognostic criteria of OLT in HCC patients. Importantly, more HCC patients are given opportunities to receive OLT and achieved favorable long-term survival.

\section{PP38-57}

Cyclooxygenase-2 Regulate PGI2/IP and TxA2/TP in Ischemia-Reperfusion Injury after Liver Transplantation in Rat

H. Zhang, M.Y. Li

Lanzhou University, Lanzhou, China

Methods: 80 Sprague Dawley Rats were divided into three groups: control group(E1), orthotropic liver transplantation group(E2), NS398 treatment before orthotropic liver transplantation group(E3). To obtain samples on 3, 6, 12, $24 \mathrm{~h}$ after operation or Portal vein opened; Radioimmunity assay was used to detect 6-Keto-PGF-1a, TxB 2 (stable metabolites of the $\mathrm{TxA}_{2}$ and $\mathrm{PGI}_{2}$ ) in tissue and Plasma; The expressions of COX-2, IP and TP mRNA were detected by RT-PCR. Hematoxylin Eosin staining was used to classify the injury extent of liver.

Results: The expression of Cox-2 and the value of 6-Keto-PGF-1a, TxB2 in E2, E3 group were significantly higher than in the E1 group $(P<0.05)$, especially the $\mathrm{TxB}_{2}$, The expression of the value of 6-Keto-PGF-1a, TxB2 in the E3 were significantly lower than in the E2 group $(P<0.05)$, Hematoxylin Eosin staining examination showed the liver injury was significantly attenuated in E3 group compared with the E2 group; Expressions of IP mRNA and TP mRNA in E3 were significantly lower than that in E2 group at $6 \mathrm{~h}$ and $12 \mathrm{~h}$ after operation $(P<0.05)$, In E2 group liver injury was obvious by HE staining, and more severe than that in E3. Serum AST (each time) and ALT (3, 6 and 12 h) levels E2 group were significantly higher than that in E1 and E3 $(P<0.05)$.

Conclusion: NS398 has beneficial effects for IR injury in livertransplantation, or Protect the liver. The mechanism was Partly reduction of $\mathrm{TxB}_{2}$ and regulating balance of $\mathrm{TxA}_{2} / \mathrm{GI}_{2}$. The balance of IP/TP takes part in and plays an important role in the ischemia reperfusion injury of liver transplantation. Changing imbalance of IP/ TP may reduce liver transplantation ischemia reperfusion injury by inhibiting COX-2 expression.

\section{PP38-58}

Morphology of the Caudate Lobe's Biliary Ducts System. Study on Corrosion Casts

P. Matusz

Department of Anatomy, 'Victor Babes' University of Medicine and Pharmacy, Timisoara, Romania

The system of intraparenchymatous biliary ducts is associated to the elements of the afferent liver's pedicle (portal hepatic vein and hepatic artery proper). The study concerning the morphology of the intrahepatic biliary ducts system of the caudate liver was performed on a number of 150 hepatic corrosion casts. The hepatic corrosion casts were realized by injection of the hepatic vasculo-ductal elements with plastic, followed by the corrosion of the hepatic parenchyma. In the first step was analyzed the modality of constitution of the superior biliary confluence, and in the second step was revealed the biliary branching manner of the caudate lobe. There have been revealed three 
construction manners of the superior biliary confluence: the confluence between the right hepatic duct and the left hepatic duct $(85.33 \%)$, the confluence of the left hepatic duct with the anterior branch and the posterior branch $(6.00 \%)$ and the confluence of the left hepatic duct with the anterior branch $(8.67 \%)$; in this case the posterior branch drains in the left hepatic duct. On the 150 hepatic corrosion casts were revealed 307 biliary ducts of the caudate lobe (an average of 2.05 ducts of the caudate lobe/cast). The number of the biliary ducts of the caudate lobe ranged between 1 and 3, mostly often 4 ducts $(68.67 \%)$. The biliary ducts of the caudate lobe drained in : the right hepatic duct $(32.90 \%)$, the right left duct $(54.72 \%)$, the anterior branch $(0.98 \%)$, the posterior branch $(9.77 \%)$, the medial branch $(0.32 \%)$ and the lateral branch $(1.30 \%)$. The drainage of the posterior branch in the left hepatic duct enables the increase of the number of the caudate lobe's ducts; in these cases the left hepatic duct becomes the main hepatic duct of biliary drainage of the liver (Supported by CNMP 41092/2007).

\section{PP38-59}

Exercise Training to Increase Liver Volume in Donors with Smaller Liver Volume

V.K. Chorasiya, K. Makki, V. K R, P. Dargan, M. Wadhawan, N. Goyal, V. Vij, S. Gupta

Centre for Liver and Biliary Surgery, Indraprastha Apollo Hospital, New Delhi, India

Background: Optimal utilization of available donor pool in LRLT is of growing concern owing to limited donor availability. Large majority of donors are found unfit and are rejected because of smaller liver graft (providing insufficient GRWR) or insufficient remnant. This has direct implications on patient's treatment especially in country like India where liver donation is legally restricted to blood relatives. Physical training with muscle building has shown to increase liver volume according to the increased body requirement. This can be utilized to increase liver volume among donors with smaller liver volume.

Aim and objective: To study effect of exercise training on liver volume.

Methods: Two only available donors of patients with liver cirrhosis who had small liver volume were subjected to supervised exercise therapy including moderate weight training and diet for 6 weeks. Liver volumetry was performed before and after 6 weeks and result studied.

Result: After 6 weeks of exercise training both donors had increase in the total liver volume and LAI (Liver attenuation index). Total liver volume increase by $341 \mathrm{~g}(43.3 \%)$ and $393 \mathrm{~g}$ (35.6\%) respectively. LAI increased from 11 to 19 and 7 to 15 , respectively. Both donor subsequently underwent right lobe donor hepatectomy and provided sufficient GRWR for recipient and had adequate remnant. No complications were reported in either of the donor recipient combination. Conclusion: Supervised exercise training programme with weight training is a good technique to increase liver volume and help expand donor pool.

\section{PP38-61}

Triptolide Protects Mice from Hepatic Ischemia/Reperfusion Injury by Inhibition of IL-17 Production

F. Zhang ${ }^{1}$, X. Wang ${ }^{2}$, L. Lu ${ }^{2}$

${ }^{1}$ Liver Transplantation Center, Nanjing Medical University; ${ }^{2}$ Liver Transplantation Center, First Affiliated Hospital of Nanjing Medical University, Nanjing, China

Ischemia and reperfusion have been identified as a complex cascade of inflammatory mediators that are involved in the pathogenesis of hepatic injury. Triptolide (diterpenoid triepoxide), was extracted from a purified component of a traditional Chinese Medicine, Tripterygium wilfondii Hook F. Currently, triptolide has been shown to have antiinflammatory, immunosuppressive, and antineoplastic activity. Accumulated data have shown that $\mathrm{T}$ helper 17 cells (Th17) might contribute to the pathogenesis of liver diseases. Triptolide has been shown to reduce interleukin (IL)-17 expression in inflammatory bowel disease and arthritis. However, the role of triptolide in liver ischemia/reperfusion (I/R) and whether it can attenuate injury and the potential mechanism have not been investigated. Mice were treated with triptolide $(0.1 \mathrm{mg} / \mathrm{kg})$ for 1 week or IL-17 antibody $(50 \mu \mathrm{g} /$ mouse) 2 days before ischemic insult. Partial warm ischemia was produced in the hepatic lobes of C57BL/6 mice for 90 minutes, followed by various periods of reperfusion. We demonstrated that IL-17 was involved in the inflammatory response to hepatic I/R injury, and that triptolide inhibited IL-17 generation and suppressed neutrophil migration after liver I/R injury through downregulation of signal transducer and activator of transcription 3 (STAT3) transcription. Also, triptolide pretreatment protected the liver from warm I/R injury, at least in part, mediated by upregulation of Foxp3 expression. These results could pave the way for the use of triptolide as a novel agent to attenuate hepatic I/R injury.

\section{PP38-62}

Acute Cellular Rejection and Cytomegalovirus Infection in Pediatric Living Donor Liver Transplant Recipients

S. Treepongkaruna ${ }^{1}$, P. Sornmayura ${ }^{2}, S$. Teeraratkul ${ }^{3}$, R. Charoenmuang ${ }^{1}$, A. Sobhonslidsuk $^{4}$, P. Pootrakul ${ }^{3}$, S. Sriphojanart ${ }^{3}$

${ }^{1}$ Department of Pediatrics; ${ }^{2}$ Department of Pathology; ${ }^{3}$ Department of Surgery; ${ }^{4}$ Department of Medicine, Faculty of Medicine, Ramathibodi Hospital, Mahidol University, Bangkok, Thailand

Background: Liver transplantation (LT) is the standard treatment for patients with end-stage liver diseases. Acute cellular rejection (ACR) and cytomegalovirus (CMV) infection are common complications in pediatric LT recipients and they are related to immunosuppression therapy.

Objective: To evaluate the prevalence and outcome of ACR and CMV infections in pediatric living donor liver transplant (LDLT) recipients at Ramathibodi Hospital.

Study design: Retrospective study.

Patients and methods: All pediatric patients underwent LDLT at Ramathibodi Hospital were enrolled. ACR was diagnosed by characteristic histological examination. CMV infection was defined as peripheral blood CMV-DNA PCR over 600 copies/mL and/or symptomatic CMV disease, proven by histology of tissue biopsies. CMV prophylaxis was given in all high-risk patients (donor+/recipient-) and patients who were at risk for CMV infection. Preemptive therapy was given in all patients with CMV-DNA PCR over 600 copies/mL.

Results: From January 2001 to September 2010, 34 pediatric patients underwent LDLT. Eighteen ACR episodes occurred in 17 patients (50 $\%)$ at the duration of 30 days $(n=9),>30$ days -3 months $(n=4)$, $>3-12$ months $(n=2)$ and $>12-24$ months $(n=3)$ following LT. All except one ACR episodes were successfully treated with pulse methylprednisolone. Anti-thymocyte globulin was used in one ACR. None had graft-loss due to ACR and none developed chronic rejection. CMV infection developed in $13(38.2 \%)$ and only 2 had symptomatic CMV disease (hepatitis 1 , colitis 1). Five patients (14.7 \%) had both ACR and CMV infection. CMV diseases were successfully treated with ganciclovir and reducing immunosuppression. There was no mortality related to these complications.

Conclusion: Acute cellular rejection and CMV infection are common in pediatric LDLT recipients but can be successfully treated without graft loss and mortality. 


\section{PP38-63}

Chylous Ascites is Benign Post Liver Transplant

K. Makki ${ }^{1}$, V. Chorasiya ${ }^{2}$, N. Goyal ${ }^{2}$, V. Vij ${ }^{2}$, M. Wadhawan ${ }^{2}$, S. Gupta ${ }^{2}$

${ }^{I}$ Liver Transplant, Center for Liver and Biliary Surgery, Apollo

Hospital, Delhi; ${ }^{2}$ Center for Liver and Biliary Surgery, Apollo

Hospital, New Delhi, India

Background: Chylous ascites is defined as ascitic fluid with triglyceride level of $>200 \mathrm{mg} / \mathrm{dl}$ or as ratio of ascitic fluid triglyceride level to plasma triglyceride level of $>2$. After major abdominal surgery, $1.1 \%$ patients develop chylous ascites. Chylous ascites is seen in 1-3\% patients after orthotopic liver transplant and usually resolves spontaneously. Sporadic cases of chylous ascites have also been reported after living related liver transplant and one case has been reported in a living donor.

Method: In this study, we have included 319 consecutive cases of liver transplants at our centre from September 2006 to June 2010. Of these, 27 were pediatric cases, 292 were adults. We did 310 living related liver transplants and 9 cadaveric transplants. Sixty patients were females and 259 were males. All liver transplant recipients with chylous drains were evaluated for their drain triglyceride levels. Drains of patients with chylous ascites were not removed if output was chylous and more than $50 \mathrm{ml} / \mathrm{day}$. All patients with chylous drain output received a high protein, low fat, medium chain triglyceride based diet.

Results: Of 319 consecutive liver transplants done at our centre, eight recipients $(2.5 \%)$ developed chylous ascites. Of these patients, 2 had chylous ascites pre transplant also. Three other recipients had chyliform ascites. Of our 310 living donors, only one developed chylous ascites post donor hepatectomy. All patients recovered with conservative management and diet modification.

Conclusion: Chylous ascites in liver transplant recipients is not uncommon and benign. All of our patients recovered with dietary management with none requiring any intervention.

\section{PP38-64}

Living Donor Liver Transplantation for Adult Type-II Citrullinemia from Heterozygotic Donor: Report of Two Cases

N. Kimura ${ }^{1}$, M. Umehara ${ }^{2}$, K. Ishido ${ }^{1}$, D. Kudo ${ }^{1}$, N. Wajima ${ }^{1}$, Y. Toyoki ${ }^{1}$, S. Narumi $^{1}$, K. Hakamada ${ }^{1}$

${ }^{1}$ Gastroenterological Surgery; ${ }^{2}$ Pediatric Surgery, Hirosaki

University, Hirosaki, Japan

Introduction: Citrin deficiency caused by SLC25A13 gene mutations develops into adult-onset type II citrullinemia (CTLN2) presenting with hepatic encephalopathy. We herein report two cases of CTLN2 treated with living donor liver transplantation (LRLT) for repetitive encephalopathy.

Case 1: A 30-year-old Japanese man was diagnosed as CTLN2 from repetitive episodes of encephalopathy.

Case 2: A 40-year-old man was diagnosed as having CTLN2 because of abnormal behavior associated with hyperammonemia. The two patients were diagnosed as CTLN2 definitely by DNA analysis and received LRLT from their siblings in whom heterozygotic gene expression was confirmed. In the postoperative course, both patients recovered well without any food restriction.

Conclusion: Recent studies have suggested that excessive loading of carbohydrates is harmful in citrin-deficient individuals and intensive nutritional support may improve patient survival. However, liver transplantation is still a definite procedure to inverse metabolic abnormality without any food restriction and should be performed before irreversible encephalopathy occurs. Use of a graft from heterozygotic donor is permissible treatment for CTLN2.
PP38-65

Recurrent Hepatitis C Post OLT Requiring Retransplantation

M. Iqbal, J. Hegarty

Liver Unit, St. Vincent's University Hospital, Dublin, Ireland

Background: Recurrence of Hepatitis C post liver transplantation is universal although disease progression following recurrent infection is highly variable. Retransplantation for hepatitis C related graft failure usually needed in $10 \%$ of patients.

Aim: The aim of this study was to give an overview of the patients who had liver transplantation for hepatitis $\mathrm{C}$ related end stage liver disease and required retransplantation for recurrent hepatitis $\mathrm{C}$ and to examine their outcome.

Method: This is a retrospective review of all the patients who were transplanted for HCV related cirrhosis between January 1993 and October 2008 at St Vincent's University Hospital, Dublin.

Results: A total of 483 patients underwent 561 OLT at St Vincent's University Hospital between 1993 and 2008. Of these, 61 (12.6\%) patients had cirrhosis secondary to chronic hepatitis $\mathrm{C}$ infection and underwent $73(13 \%)$ transplants. There were 38 male (62\%) and 23 female $(38 \%)$ patients. The mean age was 49.8 years. Fifty patients $(82 \%)$ received a single graft. Eleven patients were retransplanted (10 patients underwent 1 retransplantation and 1 patient underwent retransplantation twice). The causes of allograft failure were recurrent hepatitis $\mathrm{C}$ in 5 patients, chronic rejection in 5 patients, hepatic artery thrombosis in 1 patient and pseudo aneurysm of hepatic artery in 1 patient. Retransplantation for HCV related graft failure was required in 5 patients $(8 \%)$.Two patients died; the cause of death was subarachnoid haemorrhage in one and sepsis in another. The time for retransplantation for $\mathrm{HCV}$ related graft failure varied from 1 to nine years; mean 4 years. Overall 1,2 and 5 year patient survival rate were 92,83 and $73 \%$, respectively.

Conclusion: Liver transplantation for hepatitis $\mathrm{C}$ related cirrhosis has been done in Ireland successfully. Retransplantation was required in small number of patients. Overall patient survival is comparable to the other centres.

\section{Poster Presentation 39: Miscellaneous}

\section{PP39-02}

Biochemical Alterations in Malignant Obstructive Jaundice: Effect of Pre-operative Drainage

M.A. Khalil ${ }^{1}$, N. Samy ${ }^{2}$, A. Yehia ${ }^{3}$, N. Abdelmaksoud ${ }^{1}$, H. Ragab $^{1}$

${ }^{I}$ Biochemistry Department, National Research Centre, Giza;

${ }^{2}$ Biochemistry Department, National Research Centre; ${ }^{3}$ Surgical Department, Faculty of Medicine, Banha University, Cairo, Egypt

The benefit of preoperative biliary drainage in jaundiced patients undergoing pancreaticoduodenectomy for a suspected cancer head of pancreas is still under debate. This study evaluated the postoperative outcomes of preoperative biliary drainage with delayed surgery and immediate surgery. This study conducted on 42 patients who had malignant obstructive jaundice, serum bilirubin level was $>8$ and $<20 \mathrm{mg} / \mathrm{dL}$, besides 25 healthy subjects. Patients were divided into group (I) was taken initially for preoperative biliary drainage and stent insertion for a period of 3 weeks followed by surgery and group (II) received immediate surgical decompression. We determined liver functions, immunoglobulins ( $\operatorname{IgA}, \operatorname{IgG}$, and $\operatorname{IgM}$ ) and cytokines (IL6, IL-8, and IL-10). Results, mean serum bilirubin in-group (I) was $16.3 \mathrm{mg} / \mathrm{dL}$ at admission and fell to $2.7 \mathrm{mg} / \mathrm{dL}$ by three week post drainage. While in-group (II), it was $15.9 \mathrm{mg} / \mathrm{dL}$ at admission and 
fell to nearly normal after 1 week post-operatively in both groups, ALP was significantly reduced after three weeks of drainage and one week post-operative in both groups. Liver enzymes were significantly high at admission and decreased after drainage or surgery. The morbidity and mortality were significantly reduced in-group (I) compared to group (II). The serum immunoglobulin $\operatorname{IgA}$ level was significantly increased at admission in both groups (I and II). Its level was significantly decreased in-group (I) three weeks after drainage, and one-week post-operative in both groups. At admission, the circulating IL- 8 concentrations were significantly high at admission in both groups (I and II) and reduced significantly after drainage in group (I), and one week post-operative in both groups (I and II). In conclusion, our study point at the clinical significance of the preoperative biliary drainage when major surgery is required in patients with malignant obstructive jaundice, since it improves the overall morbidity and mortality.

\section{PP39-03}

Disturbance of Cell Signal Pathways and Genes in Nrf2 Kockout Mice in Cholestasis

W. Xu, H. Wang

The Second Hospital of Shandong University, Jinan, China

Aim: To study nuclear factor-E2-related factor2 (Nrf2) in cholestasis in mice after common bile duct ligation (BDL). Methods Wild type (WT) and Nrf2 knockout (KO) mice suffered BDL for 4 weeks. Liver function, histology, MAPK signal and some mRNA were studied.

Results: After BDL, levels of serum total bilirubin (TBIL), alanine aminotransferase (ALT) increased significantly compared with Sham (no BDL) group, and the level of ALT was higher in KO mice, but TBIL is reverse. Fibronectine (FN) expressed stronger in $\mathrm{KO}$ mice than WT mice. Western blot showed phosphorylation of JNK, stat3, AKT, p38 after BDL, and stronger in WT mice, but phosphorylation of ERK is stronger in KO mice. RNAase protection assay (RPA) showed that the mRNA levels of some Nrf2 target genes were upregulated after BDL. Induction of NQO1, GST-Ya, GST-pa expression were attenuated in $\mathrm{KO}$ mice. Expression of TGF- $\beta 1$, TIMP-1, Collagen1 were upregulated after BDL, and greater in KO mice.

Conclusion: Nrf2 have some effect on MAPK signals, inflammation, fibrosis in liver injury during cholestasis, and partly via the upregulation of anti-oxidation gene for cell protection.

Acknowledgments: We grateful to Prof. Dr. Sabine Werner, Zurich, Switzerland for her suggestions. The mice and most of experiments of this study were done in her laboratory. And this study was supported by the China Scholarship Council.

\section{PP39-04}

Synthesis and Antihepatotoxic Activity of Thiazolidinone Derivatives of 1,4-Benzodioxane Ring System

H. Khalilullah ${ }^{1,2}$, B. Ahmed ${ }^{2}$

${ }^{1}$ Department of Pharmaceutical Chemistry, Alwar Pharmacy College, Alwar; ${ }^{2}$ Department of Pharmaceutical Chemistry, Jamia Hamdard, New Delhi, India

Liver is considered to be one of the most vital organs that functions as a centre of metabolism of nutrients such as carbohydrates, proteins and lipids and excretion of waste metabolites. Additionally, it is also involved in the metabolism and excretion of drugs and other xenobiotics from the body thereby providing protection against foreign substances by detoxifying and eliminating them. Herbal-based therapeutics for liver disorders has been in use in India for a long time. Traditional drugs used in the treatment of liver diseases are sometimes inadequate to cater the need of large population. In spite of tremendous strides in the modern medicine, there are not much drugs available for the treatment of liver disorders.
Silymarin isolated from seeds of Silybum marianum commonly known as 'milk thistle' has been found most potent antihepatotoxic agent against a variety of toxicants. The Silymarin has been found to be a mixture of three isomers of flavonolignan i.e. Silybin, Silychristin and Silydianin. The Silybin is the most potent component containing 1,4-dioxan ring system. Other isomer Silychristin and Silydianin do not possess 1,4-dioxan ring system and thus do not display significant antihepatotoxic activity. On the basis of the above hypothesis we taught that 1,4-dioxane ring system plays an important role in exhibiting antihepatotoxic activity, and thus we prepared some new hydrazone and thiazolidinone derivatives containing 1,4-dioxane ring system and evaluated them for antihepatotoxic activity against $\mathrm{CCl}_{4}{ }^{-}$induced hepatotoxicity in rats. Among them, some compounds have shown significant antihepatotoxic activity as comparable to standard drug Silymarin. Other compounds of the series showed moderate antihepatotoxic activity.

\section{PP39-05}

Use of Antioxidant Enzymes and Oxidative Stress as Potential Biomarkers for Pesticide Contamination

\section{F.M. El-Demerdash}

Department of Environmental Studies, University of Alexandria, Institute of Graduate Studies and Research, Alexandria, Egypt

Pesticides are being extensively used in agriculture and public health to control insects, weeds, animals and vectors of diseases. Pesticides may cause toxicity through several different mechanisms; direct damage to structure of cells, interference with biochemical processes necessary for normal cell function and biotransformation metabolites. Oxidative stress by increased production of reactive oxygen species has been implicated in the toxicity of many pesticides. Therefore, the aim of the present study was to investigate the effect of a broad spectrum insecticide Encore (Fenitrothion 25\%, Lambda cyhalothrin 2.5\% and Piperonyl butoxide 6\%) on antioxidant status and oxidative stress biomarkers in different rat organs. The insecticide was incubated at $37^{\circ} \mathrm{C}$ with different concentrations $(0,0.1,1,10,100$ and $1000 \mathrm{mM})$ for various times $(0,30,60,120,180$ and $240 \mathrm{~min})$. All tested insecticide concentrations significantly $(p<0.05)$ increased thiobarbituric acid reactive substances (TBARS), which might be associated to decreased levels of reduced glutathione (GSH) and superoxide dismutase (SOD), catalase (CAT), and glutathione $S$-transferase (GST) activities in rat brain, liver, kidney and testes and the response was concentration and time dependent. Results showed that the used insecticide had the propensity to cause significant oxidative damage in rat organs, which is associated with marked perturbations in antioxidant defense system and suggesting the use of these antioxidant enzymes as a potential biomarkers of toxicity associated with pesticides exposure.

\section{PP39-06}

Immunization against Viral Hepatitis B among Prisoners in Iran: A Comparison Between Accelerated vs. Classic Vaccination Protocols A.A. Zolghadr Asli ${ }^{1}$, M. Moghadami ${ }^{2}$, N. Zamiri ${ }^{2}$, S.T. Heidari ${ }^{2}$, H.R. Tolide-ei ${ }^{3}$, K.B. Lankarani ${ }^{2}$

${ }^{1}$ Islamic Azad University, Istahban Branch, Istahban; ${ }^{2}$ Health Policy Research Center; ${ }^{3}$ Gastroenterology Research Center, Shiraz. University of Medical Sciences, Shiraz, Iran

Background and aims: Prisoners and injecting drug users are at constant risk of hepatitis B (HBV) infection. Hence implementation of classic 6 months HBV vaccination regimen might not provide immunization rapidly enough and vaccination in shorter durations seem reasonable. In this clinical randomized trial we compared the efficacy of two rapid vaccination protocols and the classic schedule among prisoners in Iran regarding seroprotection rate and compliance. Methods: 304 prisoners were randomized into 3 vaccination groups; group A with accelerated HBV vaccination at $0,1,4$ and 8 weeks, group B with a 0,4 and 16 weeks schedule and group $\mathrm{C}$ with classic 0 , 
1 and 6 month regimen. Anti HBS antibody was assessed at baseline, 1, 2, 6 and 8 months after the first vaccine dose using immunoenzymatic assays. Anti HBS Ab titer of $10 \mathrm{IU} / \mathrm{L}$ or more was considered seroprotective. Anti HBC antibody and HBS Ag was measured at baseline and 8 months for evaluation of new HBV infection and failure of vaccination.

Results: Overall compliance was $100,97.6$ and $90.6 \%$ in groups A, B and $\mathrm{C}$, respectively. Seroprotection at one month was significantly higher in groups A $(28.7 \%)$ and B $(23.2 \%)$ with $P<0.001$. At the 8th month seroprotection rate was $80.9,76.3$ and $92.9 \%$ in groups $\mathrm{A}, \mathrm{B}$ and $\mathrm{C}$, respectively. $(P<0.002)$

Conclusion: Compared to classic HBV vaccination regimen, an accelerated $0,1,4$ and 8 weeks vaccination schedule more rapidly achieves early seroprotection, provides clinically sufficient seroprotection rate along with higher compliance in prisoners and can be suggested in situations that rapid immunization against HBV infection is warranted.

\section{PP39-07}

Proteomic Patterns in Serum and Urine of Patients Infected with Schistosoma mansoni: Basis for a Biomarker in Liver Disease

Y. Elsherif ${ }^{1}$, S. Hamilton ${ }^{1}$, M. Salama ${ }^{2}$, P. Langford ${ }^{1}$, S. Sharaf ${ }^{2}$, I. Waked ${ }^{2}$, M. Thursz ${ }^{1}$

${ }^{1}$ Imperial College, London, UK; ${ }^{2}$ National Liver Institute, Shebin

Elkom, Egypt

Introduction: Schistosomiasis is one of the most important parasitic liver diseases in tropical and subtropical areas, affecting over 200 million. Diagnosis of $S$. mansoni infection is carried out by the detection of eggs in faeces. This method has low sensitivity, especially in acute phase of the illness and low-intensity infection. Serological tests cannot differentiate between active and past infection. Rectal biopsy is the definitive gold standard for diagnosis. Non invasive diagnostic tests for active disease and monitoring response to treatment are required.

Aim: To identify proteomic based biomarkers in S. mansoni infection before and after therapeutic intervention using Surface Enhanced Laser Desorption/Ionization Time Of Fight-Mass Spectrometry (SELDI TOF-MS).

Methods: Serum were collected from 30 patients and urine from 14 patients both prior to and four weeks after treatment with praziquantel. All patients had a confirmed diagnosis of active infection on rectal biopsy. Serum and urine proteomic profiles were obtained by SELDI TOF-MS using cation capture (CM10) and immobilized metal affinity (IMAC30) ProteinChip ${ }^{\mathrm{TM}}$ arrays.

Result: All patients were recruited from Delta region of Egypt. In urine samples, nine protein peaks demonstrated significant differences between pre and post treatment samples: 46, 44, 34, 13.3, 10.8, 19.7, $15.9,18.1,4.7 \mathrm{KDa}(P<0.05)$. On ROC curve analysis, the protein at $4.7 \mathrm{kDa}$ gave a significant diagnostic signal (AUROC $=0.77)$ indicating that it has potential as biomarker for active infection. In serum samples, four peaks were significantly different between pre and post treatment groups $(p<0.01)$. However no serum peak demonstrated significance on ROC curve analysis.

Conclusions: These results suggest that urinary proteomic testing may provide a non-invasive diagnostic test for $S$. mansoni. Development of an ELISA based test will be needed to convert this into a practical diagnostic assay.

\section{PP39-08}

Adolescents' Vaccination for Viral Hepatitis B: An Updated Report of a National Program in Iran

S.M. Alavian ${ }^{1}$, N. Zamiri $^{2}$, M.M. Gooya ${ }^{3}$, A. Tehrani ${ }^{1}$, S.T. Heydari ${ }^{2}$, K.B. Lankarani ${ }^{2}$

${ }^{1}$ Baqiyatallah Research Center for Gastroenterology and Liver Disease, Tehran; ${ }^{2}$ Health Policy Research Center, Shiraz, University of Medical Sciences, Shiraz; ${ }^{3}$ Center for Disease Control, Ministry of Health and Medical Education, Tehran, Iran
Introduction: In Iran universal hepatitis B vaccination program for newborns has been taken into action since 1993 and since 2007 a nationwide HBV vaccination campaign is being implemented for 17 year-old adolescents. In this survey we aim to report the vaccination coverage rate of this campaign in 1990 to 1991-born adolescents in Iran (second and third stages of the campaign) which has been implemented in 2008 and 2009.

Methods: A passive approach toward mass HBV vaccination was chosen by project experts at National Committee for Hepatitis. Target population included 1,709,337 and 1,673,571 adolescents in 2008 and 2009. In each year 3 rounds of vaccination were executed throughout the country; from March 5th to March 18th, April 4th to April 19th and August 5th to September 21st. At the end of each round the information were collected by officials and referred to Ministry of Health for final analysis

Results: Overall national coverage rate was $74.9 \%$ for receiving one dose of vaccination and $62.76 \%$ for full dose vaccination in 2008 and $75.7 \%$ and $55.6 \%$ in 2009 respectively. Coverage rates in rural areas and in girls were significantly higher than urban areas and boys. $(P<$ $0.001)$. The lowest coverage rates were reported in Tehran and Qom provinces in each year.

Discussion: Overall the current passive (media education) approach of this campaign has led to acceptable coverage rates in Iran considering the large number of target population. However the gradual decline in coverage rates warrants constant performance monitoring and perhaps a new catch-up vaccination campaign to expand the coverage.

\section{PP39-09}

Bacteriological Studies of Pityriasis Alba

S.A. Zaki ${ }^{1}$, M. Elmansy ${ }^{2}$, M. Elshazly ${ }^{3}$, F. Elsehrawy ${ }^{3}$

${ }^{1}$ Microbiology, Faculty of Medicine; ${ }^{2}$ Dermatology Faculty of Medicine; ${ }^{3}$ Public Health, Alexandria University, Alexandria, Egypt

Introduction: Pityriasis alba is one of the most common skin diseases seen in Egypt and in several other countries mostly affecting children. Various accused etiologic factors. Climatic, environmental, hygienic, nutritional, bacterial, fungal and immunologic factors are blamed.

Aim: To determine the role of bacteria \& fungai in Pityriasis alba.

Subjects and methods: Fifty patients with clinically evident Pityriasis alba and control group of 15 normal individuals. 1-Skin sampling: An area of $1 \mathrm{~cm}^{2}$ over the lesion was marked by a sterile metal frame using a sterile scalpel, twenty five upward strokes were done using even pressure. A similar specimen was taken from the control area nearby the lesion and from controls. The scrapings were inoculated in a tube of $1 \mathrm{ml}$. nutrient broth with glass beads and specimens were cultured for bacteria and fungi and for Quantitative estimation. They were counted first as total bacterial counts $/ 1 \mathrm{~cm}^{2}$ of the skin and then differential counting of the various species of bacteria. 2-Urine and Stools analysis. Results:

1-The most frequently isolated organism from the lesions was Staphylococcus epidermides in $57.14 \%$.

2 - There was higher counts of bacteria in the patches of Pityriasis alba than in the uninvolved skin and skin of normal controls this increase is not statistically significant.

3-The incidence of Parasites among Pityriasis alba cases was $56.00 \%$, while it was $44.00 \%$ in the control group. Ascariasis was the most prevalent amounting to $46.00 \%$ in cases of Pityriasis alba, compared with $30.00 \%$ in normal controls.

4-All scraping did not give any fungal growth.

Conclusion: Bacteria may play a role in the etiopathogenesis of Pityriasis alba it can be assumed that the condition of the skin in Pityriasis alba lesion is more susceptible to bacterial over-growth, this increased susceptibility may be predisposed by non-specific factors such as parasitic infestation, atopy, ultraviolet rays or unknown factors. 


\section{PP39-10}

Histocompatibility Antigens in Relation to Hepatic Schistosomiasis S.A. Zaki ${ }^{1}$, A.A.E.M. Soliman ${ }^{2}$, R. Zaher ${ }^{3}$

${ }^{1}$ Microbiology, Faculty of Medicine; ${ }^{2}$ Hepatology, Faculty of Medicine; ${ }^{3}$ Hepatology, Faculty of Medicine, Alexandria University, Alexandria, Egypt

Hepatic Schistosomiasis is one of the most prevalent chronic liver disease in Egypt. Great variation in disease severity occurs among infested individuals dueto the wide range of intensity and duration of tissue egg deposition.

Aim: To determine the association between hepatic Schistosomiasis and antigens of the HLA system.

Materials: 52 hepatic Schistosomiasis divided into Group I mild, Group II moderate, Group III severe liver fibrosis. 10 patients active intestinal Schistosomiasis no hepatic involvement. 300 healthy controls.

Methods: Clinical examination, ultrasonography, liver function tests, Viral markers for HBV and HCV, HlA testing by two stages lymphocyte microcytotoxicity technique.

Results: No significant association between active intestinal Schisto and HLA antigens. Significant association between HLA-AW 19 $(\mathrm{X} 2=19.593$, corrected $P=>0.00115, \mathrm{RR}=4.31)$ and hepatic Schisto. (38.46\%) compared to controls (12.67\%). Similarly HLA-B5 significantly higher $(\mathrm{X} 2=31.219$, corrected $P=>0.00023$, RR$5.68)$ in patients $(48.08 \%)$ than in controls $(14 \%)$. In group I HLA-B5 significantly increased in patients $(60 \%)$ as compared to controls (14\%). In group II HLA-B5 significantly higher in patients $(45.46 \%)$ than controls (14\%) also HLA-AW19 significantly higher $(40.91 \%)$ in patients than controls (12.67\%). In group III HLA-AW19 significantly increased in patients $(46.67 \%)$ compared to controls. No significant association between HLA antigens and cases with HBV or $\mathrm{HCV}$ infection.

Conclusion: The significantly high association of HLA-AW19 and HLA-B5 in patients with hepatic Schistosomiasis as compared to normal controls together with the lack of any association with active intestinal Schisto. Antigens predispose to liver affection. Individuals possessing HLA-AW19 appear to be more prone to severe form of liver disease

\section{PP39-11}

The Value of Ascitic Adenosine Deaminase Activity and Interferon Gamma Level in Discriminating Tuberculous from Non-tuberculous Ascites

A.A.E.m. Soliman ${ }^{1}$, H. Elaggan ${ }^{1}$, A. Elhenawy ${ }^{2}$, S. Abo Deya ${ }^{1}$, S. Abo Deya ${ }^{1}$ ${ }^{1}$ Hepatology, Faculty of Medicine; ${ }^{2}$ Microbiology, Faculty of Medicine, Alexandria University, Alexandria, Egypt

Introduction: Tuberculosis is still an important cause of ascites particularly in developing countries. The early diagnosis of tuberculous ascites is difficult because of its insidious onset, moreover the bacteriologic culture requires weeks to provide diagnosis, so a simple, rapid and safe screening test with high sensitivity and specificity would be most desirable.

Aim: To assess the value of adenosine deaminase activity (ADA) and interferon gamma level (IFN-g) of the ascitic fluid in discriminating tuberculous from other causes of ascites.

Subjects and methods: Fifty patients with ascites. Diagnostic paracentesis was performed and ascitic fluid samples were examined for; 1-protein and glucose concentration. 2-Bacteriologic study: a Gram and Zeihl-Neelsen stained smears and culture on blood agar plates. Sterile samples were inoculated on Lowenstein Jensen medium slopes which were incubated at $37^{\circ}$ and examined daily fo the appearance for visible colonies, they were identified by growth on media containing $500 \mathrm{mg} / 1$ paravitrobenzoic acid, niacin production, rate of growth, pigment production and growth at $35^{\circ}$ and $37^{\circ}$ and growth on nutrient agar. 3-Adenosine Deaminase Activity. 4Interferon-Gamma Level.

Results: Of the fifty patients with ascites, tuberculosis was suspected clinically in $13(26 \%)$ patients. By bacteriologic culture, tuberculous ascites was proven in 17 (34\%) patients and 33 patients non- tuberculous. Nine $(52.9 \%)$ patients with tuberculous ascites had underlying SHF. Ascitic ADA activity was significantly higher in tuberculous than in other causes of ascites $(P<0.001)$ regardless of the presence of liver disease. A cut-off of $28 \mathrm{U} / \mathrm{L}$ reached sensitivity $94.4 \%$, specificity and PPV100\%, and NPV97.1\%. Ascitic IFN-g level was also significantly higher in tuberculous ascites than in other causes of ascites $(P<0.05)$. A cut-off $26 \mathrm{pg} / \mathrm{ml}$ reached a sensitivity of $81 \%$, specificity and PPV $100 \%$ and NPV $89.2 \%$. There was no correlation between ascitic ADA activity and IFN-g level in the tuberculous group $(r=0.329)$.

Conclusion: Tuberculous ascites should be considered as an important cause of ascites. Ascitic ADA was more sensitive than ascitic IFN-g in detecting tuberculous ascites.

\section{PP39-13}

CD25-A Good Marker for Cholangiole of Human Liver Cell

Z. Lu

Department of Epidemiology, Wuxi Infectious Disease Hospital, Wuxi, China

Objective: To search a better marker of cholangiole of human liver for observing the distribution and morphology of cholangiole.

Methods: We observe the expressions of CD25 on cholangiole of liver by immunohistochemistry, immunofluorescence and Immunomicroscope compared with CEA marker and with Tonsil tissue and CD4 CD8 as positive control. To confirm furtherly the specificity of the expressions of CD25 on cholangiole of human liver by blocking test.

Results: The cholangiole displays yellowish-brown strip funicular structure by immunohistochemistry and showed blue fluorescence by immunofluorescence staining with CD25 antibodies. This method is better than by CEA antibodies which is generally standard method at present. The blocking test confirmed furtherly the specificity of the expressions of CD25 on cholangiole of human liver. Enzyme labelling immunemicroscopy revealed obvious electron-dense deposition in cholangioles.

Conclusions: Cholangiole of human liver cell staining by CD25 marker displays more clear and specific than using CEA marker.

\section{PP39-14}

Proposed Recommendations to Optimize MARS Therapy in Acute and Chronic Liver Disease

G. Novelli ${ }^{1}$, V. Morabito ${ }^{1}$, S. Novelli ${ }^{2}$, F. Pugliese ${ }^{3}$, M. Rossi ${ }^{1}$, M.C. Annessini $^{4}$, P.B. Berloco ${ }^{1}$

${ }^{I}$ General Surgery and Organs Transplant, Sapienza University of Rome, Rome; ${ }^{2}$ Bioclinical Engineering, Sapienza University of Rome, Roma; ${ }^{3}$ Anaesthetic Sciences and Critical Care Medicine;

${ }^{4}$ Biochemical Engineering, Sapienza University of Rome, Rome, Italy

Molecular Adsorbent Recirculating System (MARS) first proposed by Stange et al. in the early 1990s, has been extensively investigated as to its clinical efficiency. In this paper, the consolidated approach of chemical engineers' to process design is applied to the analysis of the performance of a MARS treatment. From 1999 MARS has been used in our Department as bridging therapy either to optimize the clinical status for Liver Transplant (LT) or resolution without necessity to LT with excellent results. 2.707 treatments on 288 patients; 152 Acute on Chronic Liver Failure (AoCHF), 48 Fulminant Hepatitis (FH); 24 Delayed Function (DF); 18 Primary No Function (PNF); 26 acute liver failure after surgery and 20 Intractable Pruritus (IP). Sapienza University of Rome's mono-centre experience has obliged us to focus our attention on four different aspects in order to optimize this 
treatment and consequently improve clinical results: safety, coagulopathy, pump volume flow rate and albumin function. Currently the collaboration with engineers allows us to optimize detoxification in MARS treatment to improve the survival with a decries of management costs. Furthermore it can also provide us with predictive criteria. Ideally the application of mathematical model can indicate whether MARS should be carried out in acute liver disease. Predictive criteria should be individual and not subject to etiologies or single parameters but should take into account all these variables in one process which determines clinical entity. This one process is the application of mathematical model.

\section{PP39-15}

MARS and Polymyxin-B Based Hemoperfusion in AoCHF Patients with SIRS

G. Novelli ${ }^{1}$, V. Morabito ${ }^{1}$, S. Novelli ${ }^{2}$, F. Ruberto ${ }^{3}$, G. Ferretti ${ }^{4}$, M. Rossi ${ }^{1}$, P.B. Berloco ${ }^{1}$

${ }^{1}$ General Surgery and Organs Transplant, Sapienza University of

Rome, Rome; ${ }^{2}$ Bioclinical Engineering, Sapienza University of Rome, Roma; ${ }^{3}$ Anaesthetic Sciences and Critical Care Medicine; ${ }^{4}$ Infectious and Tropical Diseases, Sapienza University of Rome, Rome, Italy

Introduction: The aim of our study is the making of early diagnosis of endotoxin activity in patients with acute liver on chronic liver disease (AoCLF) by the test of Endotoxin Activity Assay (EAA) and treated with Polymyxin-B based hemoperfusion (PMX-DHP) and albumin dialysis Molecular Adsorbent Recirculating System (MARS).

Material and methods: Since February 2008, ten AoCLF patients with SIRS and EAA positive test $(>0.60)$ were included in the study. These patients showed similar Model End-Stage Liver Disease (MELD) score of 19-25. Five patients received treatment to remove endotoxins (PMX-DHP) and MARS treatment (Group A). While the other five patients received MARS treatment only (Group B).

Results: In Group A, two PMX-DHP treatments were performed on 4 patients (mean EAA $=0.66)$, three treatments for 1 patient $(\mathrm{EAA}=$ 0.82 ) and these 5 patients also underwent a mean of 3.5 MARS treatments. After PMX-DHP, the mean level of EAA was 0.31. MARS resulted in improved hemodynamic and liver parameters in association with reduced levels of pro-inflammatory cytokines. At 30 days from the observation, all five patients treated with MARS and PMX-DHP are alive. In Group B, a mean of 7.5 MARS treatments were performed. We observed an improvement in hemodynamic and liver functions with reduced levels of pro-inflammatory cytokines in 4 patients. One patient showed no improvement in clinical status with the development of sepsis and subsequent MOF after 24 days.

Conclusions: The possibility of an early diagnosis with EAA test in AoCLF patients with SIRS could prevent the progression of sepsis cascade. The use of PMX-DHP and MARS in these patients, could lead to resolution of clinical status in a short time with a particular improvement in hemodynamic parameters.

\section{PP39-16}

Psychiatric Profiles in Chronic Liver Disease Patients

O. Osman, W. Hassan, S. Kamel, M. Al-Attar, Y. El-Serogey, E. Hassan Asyut University, Asyut, Egypt

Background and aim: Health-related quality of life (HRQOL) has become an important outcome measure in patients with chronic liver disease (CLD). The aim of this study was to assess the health related quality of life (HRQOL) in subjects with chronic liver disease and to evaluate the common psychiatric disorders and their effects on HRQOL in these patients.

Patients and methods: Quality of life was prospectively assessed by administration of The SF-36 questionnaire to 300 patients with chronic liver disease (100 with chronic hepatitis and 200 with liver cirrhosis) and 200 control volunteers. HRQOL scores were compared among the different study groups. Their psychiatric disorders were investigated using Symptom Checklist-90 revised (SCL-90-R), Hamilton anxiety rating scale, and Hamilton Checklist of Symptoms of depressive illness and Modified form of social scale assessment.

Results: Compared with healthy controls, CLD patients had lower HRQOL on all scales of the SF-36 $(P<0.001)$. Increasing severity of liver cirrhosis (based on the Child-Pugh score) was associated with a decrease in scores of SF-36. Compared with the control group, CLD patients were more likely to have depression, anxiety and increased mean scores of some SCL-90 subscales (e.g. somatization, depression and hostility). Multiple regression analysis found that older age, severity of liver disease and psychiatric comorbidities as depression and anxiety were risk factors for lowering HRQOL scores of both the physical and mental component summary scales.

Conclusion: Patients with CLD (chronic viral hepatitis and cirrhosis) showed significant impairment of HRQOL, which was further affected by worsening disease severity. Comorbid psychiatric disorders were associated with both lower physical and mental components of HRQOL.

\section{PP39-17}

Protective Mechanisms of Anthocyanin-rich Purple Sweet Potato Extract against Tert-butyl Hydroperoxide-Induced Oxidative Damage Y.P. Hwang ${ }^{1}$, J.H. Choi ${ }^{1,2}$, G.Y. Song ${ }^{1}$, Y.C. Chung ${ }^{3}$, H.G. Jeong ${ }^{1}$ ${ }^{1}$ Pharmacy, Chungnam National University, Daejeon; ${ }^{2}$ Pharmacy, Chosun University, Gwangju; ${ }^{3}$ Food Science, Korea International University, Jinju, Republic of Korea

Background/aims: There was growing interest in the pharmacological potential of natural products such as anthocyanins. Recently, purple sweet potato color, which is a natural anthocyanin pigment derived from purple sweet potato storage roots, has received a great deal of attention because of its unique color, nutrition and healthpromoting benefits. Anthocyanins of purple sweet potato possess biological functions such as scavenging free radicals, hepatoprotective effects, anti-mutagenicity and anti-carcinogen activity. In the present work, we investigated the protective effects of an anthocyanin fraction (AF) obtained from purple-fleshed sweet potato against tertbutyl hydroperoxide ( $t$-BHP)-induced hepatotoxicity in HepG2 cells. Methods: Human HepG2 cells were incubated with AF and changes in expression of HO-1 and $\mathrm{Nrf} 2$ were determined on mRNA, protein and functional level. Transient transfection assays were performed to gene promoter activities, and immunoblot analysis to study its molecular mechanisms of action.

Results: Pretreatment of HepG2 cells with AF significantly reduced $t$ BHP-induced generation of ROS, caspase-3 activation, and subsequent cell death. Also, AF up-regulated heme oxygenase-1 (HO-1) expression, which conferred cytoprotection against oxidative in-jury induced by $t$-BHP. Moreover, AF in-duced nuclear translocation of the transcription factor NF-E2-related factor 2 (Nrf2), which is upstream of AF-induced HO-1 expression, and PI3K/Akt activation, a pathway that is involved in induced Nrf2 nuclear translocation, HO-1 expression and cytoprotection.

Conclusions: Taken together, these results suggest that the protective effects of AF against $t$-BHP-induced cytotoxicity may be due, at least in part, to its ability to scavenge ROS and to regulate the antioxidant enzyme HO-1 via the PI3K/Akt-Nrf2 signaling pathways.

\section{PP39-18}

Glycine Protection of Human Hepatocytes from Toxicity: Functional Models with Hepatocyte Cell Lines

C. Kantamool, A. Howard, B.H. Hirst

Institute for Cell and Molecular Biosciences/Newcastle University, Newcastle Upon Tyne, UK

Background and aims: Glycine has been reported to have hepatoprotective properties and reduce damage caused by a range of 
oxidative challenges. To determine if it acts directly on hepatocytes, we have studied the protective effect of glycine against tert-butyl hydroperoxide (tBOOH)-induced oxidative stress in the human hepatocyte-like cell lines HepG2 and $\mathrm{HuH} 7$ and examined the role of the specific glycine transporter GLYT1.

Methods: Cell viability and generation of reactive oxygen species (ROS) were evaluated by the lactate dehydrogenase (LDH) leakage and carboxy- $\mathrm{H}_{2}$ DCFDA assays, respectively. Intracellular glutathione (GSH) levels were quantified using the bioluminescent GSH-Glo ${ }^{\mathrm{TM}}$ glutathione assay (Promega).

Results: Pre-treatment with 1 or $5 \mathrm{mM}$ glycine prevented the increase of LDH leakage and ROS production and decrease of GSH concentration induced by $\mathrm{tBOOH}$. In contrast, glycine had no beneficial effect when given either concurrent with or after $\mathrm{tBOOH}$. The protective effects of glycine were dependent on GLYT1 activity, being inhibited by a selective GLYT1 inhibitor, ALX-5407.

Conclusions: These results suggest that the hepatoprotection by glycine in both cell lines results from a decrease in ROS production and maintenance of GSH. In addition, this study has demonstrated that glycine may protect liver through direct effects on hepatocytes as well as by modulation of macrophage activity. The data support previous studies in human intestinal cells indicating a role for GLYT1 in protection of cells exposed to oxidative stress.

\section{PP39-19}

The Benefit of Ultrasound-guided Liver Biopsy on Decision Making A. Sobhonslidsuk

Medicine, Ramathibodi Hospital, Mahidol University, Bangkok, Thailand

Background: Liver biopsy is the gold standard for assessment of liver pathology, with rare mortality rate of $0.01-0.1 \%$. The practice of ultrasound (US) guided liver biopsy is becoming more and more prevalent worldwide. The benefit of US-guided liver biopsy has never been proved. We aimed to assess if US could result in changing decision of liver biopsy.

Method: A prospective study of patients who underwent a liver biopsy was done between March 2008 and April 2010. The point of liver biopsy was assessed by percussion first, and then followed by using US to confirm or change the biopsy site. If the biopsy site identified from US was far from that indicated by percussion by over $1 \mathrm{~cm}$, "biopsy site changing" was defined. The reason of biopsy site changing was recorded.

Result: One hundred and ninety patients with 117 (61.6\%) male were enrolled. Mean age was 47.1 (10.5) years. Chronic viral hepatitis was the most common causes of liver diseases $(56.8 \%)$. The most common indication of liver biopsy was the assessment of disease severity $(69.5 \%)$. The intercostal spaces (ICS) that liver biopsy should be done indicated by percussion or US guided were at the 8th and 7th. Ultrasound changed the biopsy site in $37(19.5 \%)$ cases, leading to the cancellation of liver biopsy in one case because of ascites. The risk of organ or vascular puncture and small size of liver were the most common reasons for the biopsy site changing [19 (51.4\%) and $16(43.2 \%)]$. There was no mortality in this study except for hemothorax in one case. Conclusion: Using US in liver biopsy leads to changing of the biopsy site in about $20 \%$. Though there has not been good evidence to support US-guided liver biopsy, US can help find the proper site for liver biopsy.

\section{PP39-20}

Factors Responsible for Frank Intrabiliary Rupture of Hepatic Hydatid Cysts

A.H. Al Ani ${ }^{1}$, F.F. Al- Rubaye ${ }^{2}$

${ }^{1}$ General Surgery, Al Bashir Teaching Hospital, Amman;

${ }^{2}$ Department of Pathology, Al Zarqa Teaching Hospital, Al Zarqa, Jordan
Introduction: Frank intra hepatic biliary rupture is a complication that occurs in 3-17\% of patients with hepatic hydatid cyst with all it's drawbacks.

Aims: The aim of this study is to identify Factors Responsible For frank intrabiliary Rupture Of Hepatic Hydatid Cysts.

Methods: Total of 150 patients with hepatic hydatid cysts where studied retrospectively in the period between March 1998 to December 2004 in Gastroenterology \& Hepatology Teaching center in Baghdad. Patient and cyst characteristics were subjected to analysis to identify risk factors responsible for frank intra hepatic biliary rupture of hepatic hydatid cysts.

Results: Frank Intra hepatic biliary rupture of hepatic hydatid cysts has statistically significant association with multivesicular cysts and Non infected cysts. It has a non statistically significant association with younger age, female gender, shorter duration of symptoms, smaller cysts solitary, and right lobe cysts .

Conclusion: Patients with non infected, multivesicular hepatic hydatid cysts, are at increasing risk of frank intra hepatic biliary rupture, so as smaller, solitary, right lobe cysts and cysts in patients with prolonged history.

\section{PP39-21}

Experimental Research on Brain Death Donor Management with Prostaglandin $E_{1}$ to Improve the Graft Quality

L. Wu, X. He, D. Wang, Z. Guo, X. Zhu, Y. Ma, W. Ju, A. Hu, Q. Tai

Organ Transplantation, The First Affiliated Hospital of Sun Yat-sen University, Guangzhou, China

Objective: We establish an gradual onset BD rat model, to investigate the role of $\mathrm{PGE}_{1}$ on improving the quality of liver grafts from $\mathrm{BDD}$ and to explore the relative mechanism.

Methods: SD rats were inducted brain death by gradual on-set method. All rats were divided into 3 groups (20 in each group): BD group, $\mathrm{PGE}_{1}$ group and control group. 2 and $4 \mathrm{~h}$ after BD induction, 10 rats in each group were sacrificed to obtain the serum and liver specimen. Difference of liver function parameters, histological and subcellular structure change, serum hyaluronic acid (HA) and apoptosis index (AI) between the groups were compared.

Results: Two hours after $\mathrm{BD}$, liver enzymes in $\mathrm{BD}$ group and $\mathrm{PGE}_{1}$ group were higher than in control group, but no difference was found between these two groups. $4 \mathrm{~h}$ after BD, ALT in control group, BD group and $\mathrm{PGE}_{1}$ group were $48.2 \pm 12.6,210.6 \pm 42.8$ and $156.5 \pm 33.6 \mathrm{U} / \mathrm{L}, \quad$ AST were $116.5 \pm 18.2, \quad 310.6 \pm 60.8$ and $226.8 \pm 45.4 \mathrm{U} / \mathrm{L}$, respectively, liver enzymes in $\mathrm{PGE}_{1}$ group was significantly improved compared with BD group. After BD, electron microscope showed the microstructure of the hepatocytes and sinusoids endothelial cells were damaged, kupffer cells were activated, $\mathrm{PGE}_{1}$ can relieve the damage of liver cells and inhibit the activation of kupffer cells. $2 \mathrm{~h}$ after BD, AI in control group, BD group and $\mathrm{PGE}_{1}$ group were $1.2 \pm 0.4,8.3 \pm 3.7$ and $5.2 \pm 2.8 \%$, $4 \mathrm{~h}$ after brain death, AI in each group were $1.5 \pm 0.4,13.6 \pm 4.8$ and $9.5 \pm 3.6 \%$, respectively. $\mathrm{PGE}_{1}$ can relieve the apoptosis of liver cells caused by brain death significantly.

Conclusions: $\mathrm{PGE}_{1}$ management on the brain death donor can improve the microcirculation of liver, inhibit apoptosis of liver cells and activation of kupffer cells, then it can improve the quality of liver grafts from brain death donors.

\section{PP39-22}

Immunosuppressive Regimen after Simultaneous Pancreas and Kidney Transplantation

L. Wu, X. He, Q. Tai, X. Zhu, D. Wang, W. Ju, Y. Ma, Z. Guo, A. Hu Organ Transplantation, The First Affiliated Hospital of Sun Yat-sen University, Guangzhou, China

Introduction/objective: The incidence of rejection after pancreas transplantation, including simultaneous pancreas and kidney 
transplantation (SPK) is higher than that of other abdominal organ transplantation due to high immunogenicity of pancreas. Feasible immunosuppressive regimen is critical to the success of pancreas transplantation as well as SPK. Our center performed the first case of SPK in 2005, until now, 9 cases have been successfully performed. We retrospectively analyzed the clinical data of this group of patients to investigate a feasible immunosuppressive regimen after SPK.

Methods: From January 2005 to June 2009, 9 patients with diabetic nephropathy and end stage uremia received SPK. The pancreatic allograft exocrine secretion was drained into the proximal jejunum via a side-to-side duodenojujunostomy. Quadruple immunosuppressive regime including IL-2 receptor monoclonal antibody induction, tacrolimus, mycophenolate mofetil (MMF) and steroid were used, then converted to tacrolimus monotherapy. The clinical data of the 9 patients were analyzed retrospectively.

Results: SPK was successfully applied to all patients without serious surgical complications such as pancreatitis, graft dysfunction and pancreatic fistula. One patient died of cardiovascular accident in the early stage after SPK. The other 8 patients were followed up for $4-50$ months. Serum creatinine decreased to normal range within 1 week after the operation. The 8 patients achieved euglycemia with insulin independence about 10 days after the operation. Acute rejection of the renal graft occurred in 4 patients, 1 patient died due to cardiovascular accident and the other 3 recovered after ATG or steroids bolus treatment. No rejection was noted in pancreatic grafts.

Conclusions: SPK is an effective treatment for patients with DM related uremia. Quadruple immunosuppressive regime including IL-2 receptor monoclonal antibody induction is feasible after SPK, and such regimen can be safely converted to tacrolimus monotherapy.

PP39-23

Nutritional Parameters in Cirrhotic Patients due to Hepatitis B and C in a Referral Center of Tehran

M. Abbasinazari

Clinical Pharmacy, Shahid Beheshti University of Medical Sciences, Tehran, Iran

Objectives: Protein-calorie malnutrition (PCM) is a frequent finding in patients with chronic liver diseases. Investigations about nutritional status have been rarely performed in Iranian patients with liver cirrhosis. This study was done to evaluate nutritional status in cirrhotic patients due to hepatitis B and C in a GI and Liver diseases ward of Talighani hospital, a referral center in Tehran, Iran.

Methods: Nutritional status was evaluated in 50 hospitalized patients with cirrhosis (36 due to hepatitis B and 14 due to hepatitis C). Measured anthropometric parameters consist of Actual Body weight (ABW), height, Triceps Skinfold Thickness (TSF), Mid Arm Circumference (MAC), Mid Arm Muscle Circumference (MAMC), Ideal Body Weight (IBW) and Body Mass Index (BMI). Also serum albumin and prealbumin were measured as biochemical parameters of nutrition in patients.

Results: In $18 \%$ of patients ABW/IBW (Actual body weight/ Ideal Body Weight) ratio was less than $70 \%$ (malnourished). $8 \%$ of patients had BMI below $18.5 \mathrm{~kg} / \mathrm{m}^{2}$. Non-caloric stores, evaluated by TSF were extremely low in $18(36 \%)$ of the cases, while muscular stores evaluated by MAMC were depleted in all 50 patients. TSF values were correlated with BMI $(p=0.001)$ and IBW $(p=0.038)$. Also MAMC values were correlated with BMI $(p=0.001)$. Mean values of serum albumin and prealbumin concentration in plasma showed significant decrease compared with normal ranges. There was no difference between plasma concentration of these proteins among two groups (hepatitis B and C).

Conclusion: PCM is a common complication of evaluated cirrhotic patients. There is not any difference between hepatitis B and hepatitis $\mathrm{C}$ patients regarding malnutritional parameters. TSF and MAMC can use for prediction of nutritional status in cirrhotic patients.
PP39-24

Liver Injury in Experimental Sepsis: The Late Phase Effects of Hyperbaric and Normobaric Oxygen Therapies

M. Mas ${ }^{1}$, H. Erttürk ${ }^{2}$, I. Taşçı ${ }^{2}$, L. Yamanel ${ }^{2}$, A. Ozturk ${ }^{2}$, B. Uysal ${ }^{2}$, U. Kaldırım ${ }^{2}$, T. Topal ${ }^{2}$, N. Mas ${ }^{3,4}$

${ }^{1}$ Internal Medicine; ${ }^{2}$ GATA Medical University, Ankara; ${ }^{3}$ Anatomy; ${ }^{4}$ Afyonkocatepe University, Afyon, Turkey

Introduction: Liver is one of the most frequently affected site in sepsis. Hyperbaric oxygen therapy reverses many pathogenic events involved in the mechanism of sepsis. However, its use in an intensive care unit is currently not available. Some studies have reported normobaric oxygen treatment as an alternative to $\mathrm{HBO}$ with similar effects. In the present study, the effect of HBO versus NBO treatment on tissue oxidative stress parameters and histological injury was compared in a rat sepsis model.

Methods: Forty 40 male wistar rats were divided into four with 10 animals in each group; Group $1=$ sham, group $2=$ Sepsis plus cefepim (control), group $3=$ Sepsis plus cefepim plus HBO, group $4=$ Sepsis plus cefepim plus NBO. Sepsis was induced via i.p. viable Escherichia coli injection. All treatments were started $6 \mathrm{~h}$ after the induction of sepsis. Cefepim was administrated i.p. HBO was administered $90 \mathrm{~min}$ b.i.d at $2.4 \mathrm{~atm}$. NBO was administered $90 \mathrm{~min}$ b.i.d with $5 \mathrm{l} / \mathrm{min}$ oxygen at $1 \mathrm{~atm}$. All animals were killed by the 5 th day of treatment.

Results: Liver tissue malondialdehide and superoxide dismutase levels were lower in group $3(0.33 \pm 0.05 \mathrm{mmol} / \mathrm{g}, 47.88 \pm 9.34$ $\mathrm{U} / \mathrm{g})$ and group $4(0.39 \pm 0.09 \mathrm{mmol} / \mathrm{g}, \quad 70.48 \pm 11.48 \mathrm{U} / \mathrm{g})$ compared to group $2(0.43 \pm 0.11 \mathrm{mmol} / \mathrm{g} 61.39 \pm 14.88 \mathrm{U} / \mathrm{g})$. Glutathione peroxidase level was higher in all sepsis groups with no difference in between. Tissue myeloperoxidase (MPO) level was increased in all sepsis groups but it was relatively lower in HBO treated group $3(12.53 \pm 2.54 \mathrm{U} / \mathrm{L})$ compared to $\mathrm{NBO}$ treated group $4(14.65 \pm 2.42 \mathrm{U} / \mathrm{L})$. It was similar in groups 2 and 3 $(13.30 \pm 2.77 \mathrm{U} / \mathrm{L})$.

Conclusions: In rat sepsis, oxidative liver injury is reduced after HBO administration but not after NBO. Both options have no effect over antibiotherapy in terms of histological recovery. The role of bedside $\mathrm{NBO}$ as an alternative to HBO therapy needs more research.

\section{PP39-25}

Changes in Expression of Hepatic Drug Metabolizing Enzymes in Rat Inhaled with Nano Carbon Particles

H.C. Kwak ${ }^{1}$, C.S. Ryu ${ }^{1}$, M.Y. Lee ${ }^{2}$, B.H. Kim ${ }^{1}$, S.K. Kim ${ }^{1}$

${ }^{1}$ College of Pharmacy and RCTCP, Chungnam National University, Daejeon; ${ }^{2}$ College of Pharmacy, Chunnam National University, Gwangju, Republic of Korea

In the present study we determined whether expression of hepatic drug metabolizing enzymes was affected in rat inhaled with nano carbon particles at the concentrations of approximately $10^{4}, 10^{5}$ or $10^{6}$ particles $/ \mathrm{ml}$. Rats were treated with nano carbon particles by inhalation for four weeks and hepatic expression of cytochrome P450 (CYP)-dependent drug metabolizing enzymes including CYP1A1, CYP1A2, 1B1, 2B1, 2C12, 2E1, 3A1, 3A2, 4A1, cytochrome $b_{5}$ and NADPH-dependent CYP reductase was determined by immunoblot assay. Hepatic microsomal protein contents, total CYP contents and NADPH-dependent CYP reductase activity were also determined using spectrophotometric methods. There is no significant difference in hepatic expression of CYP-dependent drug metabolizing enzymes except for CYP1A1 of which expression was significantly increased in rats treated with nano carbon particles at the high concentration. Further study to elucidate mechanism(s) involved in induction of CYP1A1 observed in rats treated with nano carbon particles is being conducted in this laboratory. 


\section{PP39-26}

Expression of HO-1 in Liver Tissue of Rats with Carbon Dioxide Pneumoperitoneum

G.S. Xu ${ }^{1}$, X.L. $\mathrm{Wu}^{2}$, H.N. Liu ${ }^{3}$, X.K. Yang ${ }^{1}$, G.X. Bai ${ }^{1}$, Q. Cai ${ }^{1}$, X.Y. Yang ${ }^{1}$ ${ }^{1}$ Emergency Ward; ${ }^{2}$ Department of Digestion, Chengdu Military

General Hospital, Chengdu 610083, Sichuan Province, China,

Chengdu; ${ }^{3}$ Department of Anesthesia, PLA General Hospital, Beijing, 100001, Beijing, China

Aim: To observe the gene expression of heme oxygenase-1 (HO-1) in liver tissue of rats with carbon dioxide pneumoperitoneum and explore its potential clinical significance.

Methods: Thirty rats were randomly divided into three groups: sham-operated group (A, $n=10$ ), experimental group of sham operation with carbon dioxide pneumoperitoneum $(\mathrm{B}, n=10)$ and experimental group of haemin-induction before sham operation with carbon dioxide pneumoperitoneum $(\mathrm{C}, n=10)$. The expression of heme oxygenase-1(HO-1)mRNA was detected with in situ hybridization (ISH) and HO-1 protein in liver was assayed with Western Blot.

Results: In group B, ALT and AST were increased to $195.2 \pm 7.1$ and $186.9 \pm 6.2 \mathrm{U} / \mathrm{L}$, respectively. In group C, ALT and AST were increased to $69.6 \pm 7.5$ and $61.3 \pm 5.8 \mathrm{U} / \mathrm{L}$, respectively. The concentration of MDA in the homogenate of liver in group A was only $3.86 \pm 1.73 \mu \mathrm{mol} / \mathrm{L}$. In group B and C, it was increased to $8.25 \pm 2.16$ and $6.49 \pm 2.01 \mu \mathrm{mol} / \mathrm{L}$, respectively. In group $\mathrm{A}$, the GSH in liver homogenate was only $53.17 \pm 14.12 \mu \mathrm{mol} / \mathrm{L}$, it was decreased to $27.61 \pm 10.63 \mu \mathrm{mol} / \mathrm{L}$ in group $\mathrm{B}$ and $35.58 \pm 12.07 \mu \mathrm{mol} / \mathrm{L}$ in group $\mathrm{C}$, respectively. The expression of HO-1 mRNA in group A was very feeble, it was enhanced more significantly in group $\mathrm{C}$. The $\mathrm{OD}$ value of $\mathrm{HO}-1$ protein was $0.48 \pm 0.13$ in group B and $0.79 \pm 0.25$ in group C, respectively. Conclusion: Carbon dioxide pneumoperitoneum could induce mild liver injury in experimental rats. The expression of HO-1 in liver was enhanced after sham operation with carbon dioxide pneumoperitoneum. Haemin-stimulated expression of $\mathrm{HO}-1$ in liver tissue alleviated the liver injury, which suggested a self-protective mechanism in liver. Promoting the expression of HO-1 may be an effective way in protecting liver against the damage induced by anoxia.

\section{PP39-27}

Type III Giant Para-oesophageal Hernias: Our Technique of Gastropexy with Biologic Prosthesis (Permacol)

K. Siddique ${ }^{1,2}$, B. Aravind ${ }^{3}$, Esophageal Disease

${ }^{1}$ Upper GI Surgery, East Kent Hospitals NHS Trust, Ashford, ${ }^{2}$ Lower GI Surgery, East Kent Hospitals NHS Trust, Margate, ${ }^{3}$ Upper GI

Surgery, East Kent Hospitals NHS Trust, Canterbury, UK

Aim: Laparoscopic primary cruraplasty for giant para-oesophageal hernias are associated with high crural disruption, recurrent herniation and return of symptoms. Aim to review whether a novel technique of laparoscopic permacol placement and fixation to the crura with anterior fundoplication (not previously described) reduces recurrence and improves patient outcome.

Patient and methods: Study included patients referred to the upper gastrointestinal unit with symptomatic, endoscopic (oesophagogastroduodenoscopy) and radiologically (barium study and computerised tomography) confirmed type III giant para-oesophageal hernias between September 2007 and December 2009.

Patients had $5-10 \mathrm{~cm}$ hiatal defects with $>50 \%$ of stomach in chest. Following sac dissection, oesophageal mobilisation and anterior/ posterior hiatal repairs [6* (4-10) 0 ethibond stitches] without tension, hiatal defect $>2-3 \mathrm{~cm}$ was still evident in the right anterolateral aspect of the oesophagus. A $3-4 \mathrm{~cm}$ tennis-racket shaped transverse gap was created from the centre of the shorter border of a $7.5 \times 5 \mathrm{~cm}$ permacol. The handle-end of the gap in the permacol introduced from the right towards the left crus helped bridge the hiatal defect and simultaneously allowed the oesophagus to lie loose in the space created by the tennis-racket gap. Mesh was then fixed to the diaphragm followed by a $180^{\circ}$ anterior fundoplication (5 interrupted 0 ethibond stitches between the fundus and diaphragm/ right crus).

Results: Study included 8 patients with a F:M ratio of 7:1, age of $74^{*}$ (69-87) years and ASA 3*. Presentations included dysphagia (4), heartburn (3), regurgitation (3), vomiting (3), chest pain (3) and postprandial pain (2). Six and two patients underwent elective and emergency procedures respectively. Operative time was $210^{*}$ (150-240) min. Follow up was $10^{*}(1-18)$ months and all patients were asymptomatic with a good quality of life.

Conclusion: Our technique of laparoscopic fixation of type III giant para-oesophageal hernia with permacol is a challenging but unique procedure with a good outcome.

\section{PP39-28}

Can the White Cell Count Predict the Severity of Acute Appendicitis in Pediatric Population? Experience at a District General Hospital K. Siddique ${ }^{1}$, S. Mirza ${ }^{2}$, G. Harinath ${ }^{3}$

${ }^{I}$ Upper GI Surgery, East Kent Hospitals NHS Trust, Canterbury, UK; ${ }^{2}$ Holy Family Hospital, Punjab University, Rawalpindi, Pakistan; ${ }^{3}$ Lower GI Surgery, East Kent Hospitals NHS Trust, Ashford, UK

Objective: To determine the diagnostic predictability of white cell count for severity of acute appendicitis in children at a DGH.

Methods: A cross-sectional study was performed on all children who underwent appendicectomy from January 2007 to January 2009. Data regarding history, clinical examination and investigations were collected retrospectively. After computing descriptive statistics, multiple linear regression analysis was performed taking white cell count as the outcome variable and appendicitis at histopathology as the main exposure variable. Correlation coefficients were also computed.

Results: Out of a total of 204 patients, 112 (54.9\%) were female. Age was $12.8 *$ years, duration of symptoms was $2.641 *$ days, white cell count was $12.18^{*}\left(4-29 \times 10^{6} / \mathrm{L}\right)$. $82.3 \%$ of patients were operated within $24 \mathrm{~h}$ (0-4 days). Grossly inflamed appendix was seen in $82 \%$ including perforation (19\%) and localized abscess (27.2\%) Histopathology showed that $52.7 \%$ patients had suppurative, $15.4 \%$ gangrenous, $21.7 \%$ normal and $5.3 \%$ had lymphoid hyperplasia. Linear regression analyses revealed that suppurative and gangrenous appendicitis were strongly associated with high white cell count (eta: 0.9 and 0.88). Post-operative complications included ileus ( 8 patients), pelvic collection (4 patients) and abdominal pain (7 patients). 5.3\% patients were re-admitted. All managed conservatively.

Conclusion: Linear regression analysis showed that white cell count is a strong predictor of severity of acute appendicitis in children and should be used to support the clinical diagnosis of appendicitis. This study also shows that management of pediatric appendicitis at a DGH is safe with promising results.

*Mean.

\section{PP39-29}

Life-style Analysis of Amoebic Liver Abscess Patients in a Tertiary Level Hospital in Bangladesh

H.-O.- Rashid ${ }^{1}$, A. Alim ${ }^{2}$, K. Rahman ${ }^{3}$, M.R. Khan ${ }^{3}$, A.S. Alam ${ }^{3}$, S. Ekram ${ }^{3}$ ${ }^{1}$ Hepatogy Department; ${ }^{2}$ Gastroenterology Department; ${ }^{3}$ Medicine Department, Rajshahi Medical College, Rajshahi, Bangladesh

Background/aim: Amoebic liver abscess is the most common cause of amoebiasis out side the intestine. Our aim to study the risk factors of amoebic liver abscess and manage it accordingly.

Method: It was a prospective study in Inpatient department of Rajshahi Medical College Hospital from May 2007 to May 2009. 100 amoebic liver abscess patients diagnosed clinically and by 
USG, CT-scan, Liver aspirates (Macroscopy/ Microscopy/Culture/ E. histolytica antigen detection) were included. Most of the patients had fever, upper abdominal pain, anorexia, nausea and weight loss.

Result: Among 100 patients 80 (80\%) male, 20(20\%) female. Mean age $45 \pm 20$ years. Occupations of the patients were farmer, day labourer, teacher, house wife and others. Mostly affected. occupation is farmer and age group is $25-40$ years. Most of the patients took tube- well water and use sanitary latrines. Smoker 70 (70\%), non smoker 30 (30\%). Ethnic origin was Aborigine 38, Bengali 62. Literates were 45 and illiterates were 55. History of Alcohol (crude) intake was present in 85 patients both in regular and irregular basis. Non alcoholic were 15 patients only. Other addictions were present in 07 patients along with alcohol.

Conclusion: Alcohol (crude) is the predominant cause of amoebic liver abscess in Bangladesh especially in illiterates and lower socioeconomic group. We try to build up awareness against alcohol intake.

\section{PP39-30}

Peg-interferon Alfa-2a Induces Less IP10 Release from Human Lung Endothelial Cells Compared to Non-pegylated Interferon Alfa or Peginterferon Alfa-2b

T. Manigold ${ }^{1}$, R. Badiger ${ }^{2}$, F. Tatsch ${ }^{1}$, C. Atkin ${ }^{2}$, H. Gashaw ${ }^{2}$, H.J. Hinton ${ }^{1}$, T.T. Hansel ${ }^{3}$, T. Singer ${ }^{1}$, J.A. Mitchell ${ }^{2}$

${ }^{1}$ Roche, Basel, Switzerland; ${ }^{2}$ Cardiothoracic Pharmacology, National Heart and Lung Institute, Imperial College; ${ }^{3}$ Imperial Clinical Respiratory Research Unit, ICRRU, St. Mary's Hospital, Paddington, London, UK

Background: Interferon (IFN)-based therapies are the current standard of care for patients infected with hepatitis $\mathrm{C}$ virus. IFN $\alpha$ therapy is associated with rare, but potentially serious lung toxicity for which the underlying mechanism remains unknown. We studied effects of IFN $\alpha$ preparations on the release of chemokine IP10-a potentially important biomarker of toxicity-by human lung microvascular endothelial cells (HLMVEC) and human lung epithelial cells (A549).

Methods: HLMVEC or A549 cells were grown under standard conditions. Cells were stimulated with various concentrations of different IFNs in hydrocortisone-free medium and IP10 protein measured after $24 \mathrm{~h}$ by ELISA in conditioned medium. Pre-treatment of cells with $10 \mathrm{ng} / \mathrm{ml}$ tumour necrosis factor $\alpha(\mathrm{TNF} \alpha)$ was performed in a second series of experiments.

Results: When compared on an IFN mass/volume basis, IP10 release was lower with PegIFN $\alpha 2$ a than PegIFN $\alpha 2 b$ or non-pegylated IFN $\alpha$ preparations (Figure; $* * p<0.05$ ). Similar results were seen when IP10 release was measured from A549 or HLMVEC cells pre-treated with TNFa. Pegylation reduces in vitro biological activity due to steric hindrance, an effect related to size/shape of the PEG moiety. However, it is interesting that whilst plasma PEGIFN $\alpha 2 \mathrm{a}$ IFN levels are up to 10 -fold higher than for PEGIFN $\alpha 2 \mathrm{~b}$ IFN levels, our assay suggests a greater than 10-fold difference in the apparent potency of PEGIFN $\alpha 2 b$ in the induction of IP10 release from HLMVECs.

Conclusion: IFN $\alpha$ preparations activate human lung endothelial and epithelial cells to release IP10 which may explain some of the pulmonary side effects associated with IFN $\alpha$ therapies.

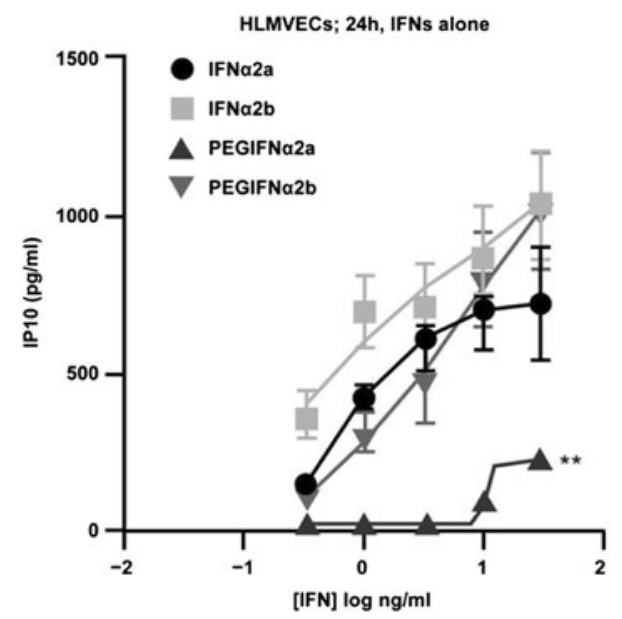

Acknowledgments: Funded by Roche, Basel, Switzerland

\section{PP39-31}

Assessment of Nutritional Status in Patients with Hepatitis C Virus Liver Disease

F. Ismail, R. Khan, A. Ahmad, H. Shah, S. Hamid, W. Jafri Aga Khan University Hospital, Karachi, Pakistan

Background: Hepatitis C virus liver disease spans a spectrum from chronic hepatitis $\mathrm{C}$, to compensated cirrhosis, and finally decompensated cirrhosis. While malnutrition associated with decompensated cirrhosis has been well documented in literature, there is little data regarding the nutritional status of patients with simple chronic hepatitis or the compensated cirrhotic, as compared to the normal population. This data is extremely relevant in the developing world, where lack of education and awareness, can lead to unnecessary dietary restrictions which initiate or worsen nutritional status.

Aims: To assess the nutritional status of patients with hepatitis $\mathrm{C}$ virus liver disease

Methods: Patients were enrolled from the out-patient hepatology clinics prospectively, and categorized into 4 distinct populations of 100 patients each: normal controls (NC), those with chronic hepatitis $\mathrm{C}$ infection (CHC), compensated cirrhotics (CC) and decompensated cirrhotics (DC). The subjective global assessment (SGA) tool was used to assess nutritional status, which combines multiple elements of nutritional assessment to classify malnutrition. Date analysis was performed using SPSS version 16.0.

Results: 400 patients were enrolled, divided amongst the 4 groups. Most of the patients in the NC group were class A (best nutritional status) according to the SGA, and there were none in class $\mathrm{C}$ (worst nutritional status). In contrast the majority (68\%) in the DC group were in the class $\mathrm{C}$, while only $4 \%$ were in the A category. Importantly, while $86 \%$ of patients in the CHC group had a class A SGA score, only $10 \%$ of the $\mathrm{CC}$ did, suggesting that malnutrition starts early in the disease process. The nutritional status showed a gradual transition from Class A to $\mathrm{C}$ through the 4 groups which was statistically significant $(p$ value $<0.001)$.

Conclusion: Malnutrition exists throughout the spectrum of $\mathrm{HCV}$ disease. It occurs early, and progresses relentlessly through the disease process. 
PP39-32

Ultrasonic Hepato-Biliary Involvement in Epidemic Dengue Affecting Adult Sri Lankans and its Clinico-pathological Significance

R.L. Satarasinghe ${ }^{1}$, A.T. Jayalal ${ }^{1}$, A.C.R. Navaratne ${ }^{1}$, C. Subasinghe ${ }^{1}$, P.A. Daluwatte ${ }^{1}$, S.K.Y.I. Kodikara ${ }^{2}$, S.H. Egodage ${ }^{2}$, P. Udayakumaran ${ }^{2}$, A.N. Umakanthan ${ }^{2}$, P.K. Arambepola ${ }^{2}$, D.J. Wickramarathne ${ }^{2}$

${ }^{1}$ Medicine; ${ }^{2}$ Radiology, Sri Jayewardenepura General Hospital, Colombo, Sri Lanka

Aims: To elucidate the ultrasonic hepato- biliary involvement in epidemic dengue affecting adult Sri Lankans and its clinico pathological significance.

Methods: Case notes of patients suffering from suspected dengue fever having a platelet count less than $100000 / \mathrm{mm}^{3}$, who were subsequently serologically confirmed, admitted to the principal author's unit at Sri Jayewardenepura General Hospital, Kotte, Sri Lanka, during the recent dengue epidemics from 01.06.2009 to 30.09.2010 were retrospectively analyzed to obtain the required data.

Results: There were 144 patients with an age range of 14-76 years. and a sex distribution of male:female $=106: 60.97 \%$ had elevated SGOT (range 16-1033 iu/l), and 94\% had elevated SGPT (range 8-1858 iu/l), respectively. No significant ultrasonic hepatomegaly was noted except change of hepatic echo pattern in spite of transaminitis. The relationship between transaminases values, abdominal pain and ultrasonic findings are depicted in the table below.

\begin{tabular}{lll}
\hline & AST $>40(p$ value $)$ & ALT $>40(p$ value $)$ \\
\hline Abdominal pain & 0.89 & 0.43 \\
Pericholicystic fluid & 0.25 & 0.46 \\
Gall bladder thickened & 0.01 & 0.14 \\
Ascitis & $\mathbf{0 . 0 5}$ & $\mathbf{0 . 0 3}$ \\
\hline
\end{tabular}

Conclusions: Prevalence of ultrasonic ascitis had clearly correlated with transaminitis. The gall bladder thickening and abdominal pain also have shown some relationship with elevated SGOT, which may serve as a predictor for organ involvement mentioned.

\section{PP39-33}

Imbalance Between Th17 and T Regulatory Cell in Patients with Chronic Hepatitis B

P. Hu, W. Gao, H.-D. Hu, H. Ren

Department of Infectious Diseases, Institute for Viral Hepatitis,

Key Laboratory of Molecular Biology for Infectious Diseases,

Ministry of Education, The Second Affiliated Hospital of Chongqing Medical University, Chongqing, China

Background and aims: Recent studies have demonstrated $\mathrm{T}$ regulatory cells (Treg) and Th17 cells play a critical role in inflammationassociated disease, Th17/Treg balance controls inflammation and may be important in the pathogenesis of autoimmunity. However, its role in chronic hepatitis B virus (HBV) infection remains unknown. The aim of this study was to investigate whether the Th17/Treg balance was broken in the patients with chronic hepatitis B.

Methods: A total of 60 patients with chronic hepatitis B were enrolled in this study, including 25 with chronic hepatitis B (CHB), 20 with HBV-associated acute-on-chronic liver failure (ACLF), and 15 healthy subjects as controls. We detected Th17/Treg functions on different levels including cell frequencies, related cytokine secretion. Peripheral Th17 and Treg cells were analyzed by flow cytometry. The serum concentrations of the cytokines were measured by ELISA.

Results: The frequency of peripheral Th17 cells increased with disease progression from CHB (mean, 3.11\%) to ACLF (mean, 20.55\%) patients versus healthy controls (mean, 2.21\%). Th17 related cytokines (IL-17, IL-6) was also increased in CHB and ACLF patients. In contrast, patients with ACLF obvious decrease in Treg number (mean, $6.85 \%$ ) as compared with patients with CHB (mean, $8.56 \%$ ), but higher than healthy controls (mean, $5.0 \%$ ).

Conclusions: Th17/Treg functional imbalance exists in patients with $\mathrm{CHB}$, and exhibit a potential to exacerbate liver damage during chronic HBV infection.

This study was supported by: The National Key Technologies Research and Development Program of China during the 11th Fiveyear Plan Period (2008ZX10002-006); Program for Changjiang Scholars and Innovative Research Team in University(IRT0872); The Nature Science Foundation of China, NSFC(30671869, 30771923, 30930082, 30972584, 30872250).

\section{PP39-34}

Hepato-renal Leptospirosis in Northern India- Clinical Profile and Outcome of 216 Cases

D.K. Chhina ${ }^{1}$, P. Goyal ${ }^{2}$, O. Goyal ${ }^{2}$, R.S. Chhina ${ }^{3}$, R.S. Chhina ${ }^{2}$

${ }^{1}$ Department of Microbiology, DMC and Hospital, Ludhiana;

${ }^{2}$ Department of Gastroenterology, DMC and Hospital, India;

${ }^{3}$ Community Medicine, DMC and Hospital, Ludhiana, India

Aim: Leptospirosis is an emerging zoonotic disease with world-wide distribution. Though well-reported from southern India, data regarding leptospirosis in northern India is scarce. This study was undertaken to analyse the clinical spectrum, prognostic indicators and outcome of hospitalised cases of leptospirosis.

Methods: Records of serologically confirmed cases of leptospirosis (positive IgM anti-leptospira antibody by ELISA), admitted from January to December 2008 were screened. 'Good outcome' was defined as recovery and discharge from hospital; and 'poor outcome' as non-survival or leaving against medical advice in sick condition. Results: Of the total 260 patients with leptospirosis, 44 had co-infections, and were excluded. Mean age was $46.1 \pm 13.9$ years (male:female $=4.2: 1)$. Maximum admissions $(31 \%)$ were from July to September. Common presenting symptoms were-fever (74.5\%), myalgias $(72.2 \%)$, jaundice $(71.7 \%)$, altered sensorium $(41.6 \%)$, abdominal distension (35.6\%), abdominal pain (28.2\%), oliguria $(27.7 \%)$, haemorrhagic manifestations $(22.2 \%)$ and breathlessness $(16.2 \%)$. Ascites $(48.1 \%)$, hepatomegaly $(46.3 \%)$, splenomegaly $(25 \%)$ and pleural effusion (15.8\%) were important physical signs. Eighty five percent patients had icteric leptospirosis and $15 \%$ had anicteric leptospirosis. Serum AST and ALT were deranged in $85 \%$ patients; while ALP was deranged in $72.2 \%$. Mean $( \pm \mathrm{SD})$ AST and ALT values were not significantly different $(230.7 \pm 728$ vs. $171.7 \pm 491 \mathrm{U} / \mathrm{L} ; p>0.05)$. Renal involvement was seen in $65.8 \%$ (121/184) patients of icteric leptospirosis and $50 \%(16 / 32)$ of anicteric cases $(p=0.456)$. Pulmonary manifestations were present in $36.1 \%$ patients. Ten percent patients needed haemodialysis and $7.4 \%$ needed artificial ventilation. Poor outcome was seen in $28.7 \%$ patients. Factors predicting poor outcome were presence of encephalopathy, renal failure and respiratory failure.

Conclusions: Hepato-renal leptospirosis is common in northern India. One-fourth patients may not present with fever. There is mild and equal rise in ALT and AST. Renal failure is equally common in icteric and non-icteric cases. Encephalopathy, renal failure and respiratory failure predict poor outcome.

\section{PP39-35}

Prevalence of Hepato Renal Syndrome in adult Sri Lankans Having Chronic Hepatic Dysfunction Admitted to a Tertiary Referral Center R.L. Satarasinghe, A.T. Jayalal, C. Subasinghe, A.C.R. Navaratne Sri Jayewardenepura General Hospital, Colombo, Sri Lanka

Aims: To elucidate prevalence of Hepato Renal Syndrome (HRS) and other related matters in a cohort of adult Sri Lankans having chronic liver cell disease admitted to a tertiary referral center seeking treatment. 
Methods: Case notes of 249 patients admitted to the principal author's unit at Sri Jayewardenepura General Hospital, Kotte, Sri Lanka, from 01.01.2007 to 30.09.2010 having chronic liver cell disease, were retrospectively analysed.

Results: The age range was $14-87$ years comprising 169 alcoholics and 80 non-alcoholics. The sex distribution in alcoholics; male:female 168:1, in nonalcoholics 42:38. The comparison of important laboratory parameters of alcoholics and nonalcoholics are shown below.

Conclusions: The prevalence of HRS is about $18 \%$ in alcoholics and $8 \%$ in nonalcoholics. The significant leucocytosis and high creatinine level in alcoholics could be due to increased prevalence of sepsis and muscle damage respectively contributing towards HRS.

\begin{tabular}{lcl}
\hline & Alcoholic group & Non-alcoholic group \\
\hline Age (years) & $56.6 \pm 12.2$ & $63.3 \pm 12.6$ \\
Urea $(\mathrm{mg} / \mathrm{dl})$ & $87.1 \pm 32.1$ & $86.8 \pm 46.1$ \\
Creatinine $(\mu \mathrm{mol} / \mathrm{L})$ & $173.0 \pm 101.3$ & $156.3 \pm 112.7, P=0.002$ \\
SGOT $(\mathrm{U} / \mathrm{L})$ & $89.8 \pm 66.6$ & $71.4 \pm 65.3$ \\
SGPT $(\mathrm{U} / \mathrm{L})$ & $50.5 \pm 36.5$ & $40.6 \pm 32.0$ \\
Albumin $(\mathrm{g} / \mathrm{dl})$ & $2.8 \pm 0.5$ & $2.6 \pm 0.6$ \\
WBC $\left(10^{9} / \mathrm{L}\right)$ & $8.5 \pm 4.9$ & $8.0 \pm 5.6, P=0.007$ \\
Platelets $(10 / \mathrm{L})^{9}$ & $111.4 \pm 8.2$ & $126.4 \pm 87.5$ \\
Haemoglobin $(\mathrm{g} / \mathrm{dl})$ & $9.4 \pm 2.1$ & $10.1 \pm 2.3$ \\
\hline
\end{tabular}

\section{PP39-36}

Prevalence of Hepatic Encephalopathy in Adult Sri Lankans Having Evidence of Chronic Hepatic Dysfunction Admitted to a Tertiary Care Center

R.L. Satarasinghe, A.C.R. Navaratne, C. Subasinghe, A.T. Jayalal Sri Jayewardenepura General Hospital, Colombo, Sri Lanka

Aims: To elucidate prevalence of Hepatic Encephalopathy and other related matters in a cohort of adult Sri Lankans having chronic liver cell disease admitted to a tertiary referral center seeking treatment.

Methods: Case notes of 332 patients, admitted to the principal author's unit at Sri Jayewardenepura General Hospital, Kotte, Sri Lanka, from 01.01.2007 to 30.09.2010 having chronic liver cell disease, were retrospectively analysed to identify those had Hepatic Encephalopathy.

\begin{tabular}{lll}
\hline & Alcoholic group (66) & Nonalcoholic group (32) \\
\hline Age (years) & $55.5 \pm 10.8$ & $65.1 \pm 10.4$ \\
BloodUrea $(\mathrm{mg} / \mathrm{dl})$ & $53.3 \pm 39.5$ & $49.6 \pm 38.1$ \\
Creatinine $(\mu \mathrm{mol} / \mathrm{L})$ & $123.2 \pm 61.2$ & $118.5 \pm 87.3, P=0.04$ \\
SGOT $(\mathrm{U} / \mathrm{L})$ & $90.9 \pm 61.8$ & $68.6 \pm 35.5$ \\
SGPT $(\mathrm{U} / \mathrm{L})$ & $58.4 \pm 65.2$ & $45.6 \pm 19.9$ \\
Albumin $(\mathrm{g} / \mathrm{dl})$ & $2.7 \pm 0.6$ & $3.0 \pm 0.8$ \\
Platelets $\left(10^{9} / \mathrm{L}\right)$ & $101.4 \pm 56.5$ & $98.0 \pm 40.1$ \\
Haemoglobin $(\mathrm{g} / \mathrm{dl})$ & $10.1 \pm 1.9$ & $10.8 \pm 2.4$ \\
Deaths & $15(23 \%)$ & $5(22 \%)$ \\
\hline
\end{tabular}

Results: The age range was $28-85$ years comprising 66 alcoholics and 32 non-alcoholics. Sex distribution; alcoholics; male:female = 65:1, nonalcoholics; 20:12. Hepatic Encephalopathy was seen in $19.8 \%$ of alcoholics and $9.6 \%$ of non-alcoholics. Evidence of gastrointestinal bleeding was noted in 30 alcoholics and 9 non-alcoholics.
Conclusions: The prevalence of Hepatic Encephalopathy was 19.8 and $9.6 \%$ in alcoholics and non-alcoholics, respectively. The aetiology had not affected death. Alcoholic myopathy may explain high creatinine in alcoholics.

\section{PP39-37}

Prevalence of Oesophageal Varices in Nonalcoholic Liver Disease in Adult Sri Lankans Seeking Treatment at a Tertiary Care Referral Center R.L. Satarasinghe, A.C.R. Navaratne, C. Subasinghe, A.T. Jayalal Sri Jayewardenepura General Hospital, Colombo, Sri Lanka

Aims: To assess the prevalence of oesophageal varices in nonalcoholic liver disease in a cohort of adult Sri Lankans seeking treatment at a tertiary care referral center

Methods: Case notes of patients who had undergone consented upper GI endoscopy for varices surveillance having chronic liver disease due to nonalcoholic aetiology from 01.01. 2007 to 30.09.2010, admitted to principal author's unit at Sri Jayewardenepura General Hospital, Sri Lanka, were retrospectively analysed.

Results: Male:female $=51: 31$.Age range $14-93$ years. 23,28 and $49 \%$ had no varices, early varices (grade 1-11) advanced varices (grade $111-1 \mathrm{v}$ ), respectively. $32 \%$ had clinical evidence of bleeding. $88.5 \%$ of bleeders had advanced varices while this was seen in $30.4 \%$ of nonbleeders .The comparison of important laboratory parameters between nonvariceal and variceal groups are depicted below.

\begin{tabular}{lll}
\hline & Alcoholic group (66) & Nonalcoholic group (32) \\
\hline Age (years) & $55.5 \pm 10.8$ & $65.1 \pm 10.4$ \\
BloodUrea $(\mathrm{mg} / \mathrm{dl})$ & $53.3 \pm 39.5$ & $49.6 \pm 38.1$ \\
Creatinine $(\mu \mathrm{mol} / \mathrm{L})$ & $123.2 \pm 61.2$ & $118.5 \pm 87.3, \quad P=0.04$ \\
SGOT $(\mathrm{U} / \mathrm{L})$ & $90.9 \pm 61.8$ & $68.6 \pm 35.5$ \\
SGPT $(\mathrm{U} / \mathrm{L})$ & $58.4 \pm 65.2$ & $45.6 \pm 19.9$ \\
Albumin $(\mathrm{g} / \mathrm{dl})$ & $2.7 \pm 0.6$ & $3.0 \pm 0.8$ \\
Platelets $\left(10^{9} / \mathrm{L}\right)$ & $101.4 \pm 56.5$ & $98.0 \pm 40.1$ \\
Haemoglobin $(\mathrm{g} / \mathrm{dl})$ & $10.1 \pm 1.9$ & $10.8 \pm 2.4$ \\
Deaths & $15(23 \%)$ & $5(22 \%)$ \\
\hline
\end{tabular}

Conclusions: Prevalence rate of oesophageal varices in a nonalcoholic liver disease is about 77 and 50\% needed intervention. No laboratory parameters could predict presence of varices.

\section{PP39-38}

Effects of IL-21 on Stimulating Production of HBeAb-Ig G

P. Hu, L.-Z. Liu, X. An, H.-D. Hu, D.-Z. Zhang, H. Ren Department of Infectious Diseases, Institute for Viral Hepatitis, Key Laboratory of Molecular Biology for Infectious Diseases, Ministry of Education, The Second Affiliated Hospital of Chongqing Medical University, Chongqing, China

Objective: To judge the effects of IL-21 on stimulating production of $\mathrm{HBe} A b-\mathrm{IgG}$ and proliferation of B cells in peripheral blood monouclear cells from patients infected with hepatitis B virus.

Materails and methods: Mononuclear cells were isolated from fresh peripheral blood of CHB patients by Ficoll-Hypaque density gradient centrifugation and were stained with CFSE, then cultured with the stimulus of IL-4, IL-10, and IL-21 for 1 week in vitro. Flow cytometry was used to detect cell proliferation of B cells was detected, and ELISA was used to detect the level of $\mathrm{HBeAb}$ in supernatant.

Results: Compared with controls, all groups of the stimulus could produce more antibody; in addition IL-21 show more power in promoting proliferation of $\mathrm{B}$ cells and production of HBeAb than IL-4 
and IL-10; No difference was observed in proliferation of B cells and production of $\mathrm{HBeAb}$ between IL-4 and IL-10.

Conclusion: IL-21could promote proliferation of $\mathrm{B}$ cells and production of $\mathrm{HBeAb}$ deeply, which may show some insight for inducing $\mathrm{HBeAb}$ seroconversion during the treatment for chronic hepatitis B. This study was supported by: the National Key Technologies Research and Development Program of China during the 11th Fiveyear Plan Period (2008ZX10002-006); Program for Changjiang Scholars and Innovative Research Team in University(IRT0872); The Nature Science Foundation of China, NSFC(30671869, 30771923, 30930082, 30972584, 30872250).

PP39-39

What Influences on Gastric Emptying Time in Patients with Liver Cirrhosis?

J.H. Kim, J.I. Kim

Internal Medicine, Yeoeuido St. Mary's Hospital Catholic University Medical College, Seoul, Republic of Korea

Background/aims: Although patients with cirrhosis often complain of gastrointestinal symptoms such as indigestion, little attention has been paid to the possible effects of portal hypertension on gut function. Frequently, endoscopic feature of patients with cirrhosis included congestive gastropathy. However, it has not been shown that whether gastric emptying time is delayed in cirrhotic patients with congestive gastropathy. The aim of this study was to evaluate the gastric emptying in cirrhotic patients with congestive gastropathy and to understand factors causing the delay of gastric emptying time in patients with cirrhosis. Figure 1

Methods: 30 cirrhotic patients with congestive gastropathy, 27 cirrhotic patients without congestive gastropathy, and 15 controls were participated in this study. All patients were subjected to evaluation of gastrointestinal symptom score, endoscopy, Child-Pugh score, and gastric emptying time with solid meal.

Results: The gastric emptying time was longer in cirrhotic patients with congestive gastropathy than in controls (105.6 \pm 9.58 vs. $72.4 \pm 6.02 \mathrm{~min}$ ), but there was no significant difference between cirrhotic patients without congestive gastropathy and controls (93.7 \pm 7.93 vs. $72.4 \pm 6.02 \mathrm{~min})$. Among factors influencing gastric emptying time in cirrhosis such as congestive gastropathy, ascites, gastric varix, AST, ALT, Child-Pugh score, prothrombin time, age, albumin level, esophageal varix, and bilirubin, congestive gastropathy was the only statistically significant factor $(p<0.05)$.

Conclusions: The gastric emptying time as delayed in cirrhotic patients who had congestive gastropathy. Thus, congestive gastropathy may contribute to the pathogenesis of delayed gastric emptying in patient with liver cirrhosis.

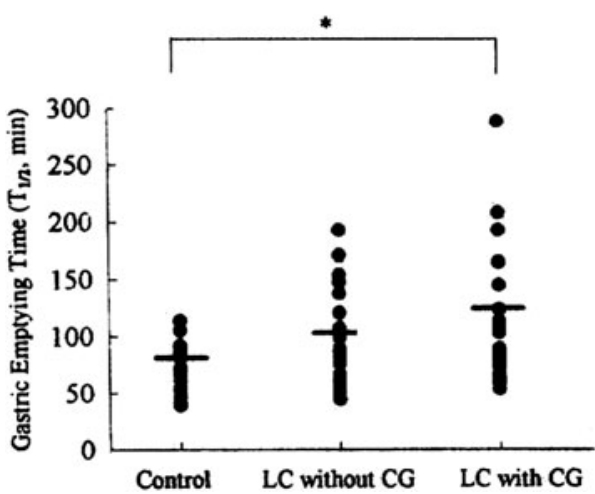

Fig. 1 Gastric emptying time in controls, cirrhosis without CG, and cirrhosis with CG. LC, liver cirrhosis; CG, congestive gastropathy; *, LC with CG versus control, $p=0.0312$
PP39-40

Aetiology and Other Major Associated Factors in Adult Sri Lankans Having Nonalcoholic Liver Disease

R.L. Satarasinghe, A.T. Jayalal, A.C.R. Navaratne

Sri Jayewardenepura General Hospital, Colombo, Sri Lanka

Aims: To elucidate aetiology and other major associated factors in adult Sri Lankans nonalcoholic liver disease

Methods: Case notes of patients having non alcoholic liver disease admitted to the principal authors unit at Sri Jayewardenepura general hospital, Kotte, Sri Lanka from 01.01.2007 to 30.09.2010 were retrospectively analyzed to obtained the required information.

Results: There were 82 patients in the study population with an age range of $14-93$ years. Mean age $61.1 \pm$ SD 13.3 years, sex distribution was male: female $=51: 31$. The accountable aetiological factors were as follows; Haemacromatosis $3.6 \%$ Wilson's disease, chronic active hepatitis $2.4 \%$, hepatitis B $2.4 \%$, secondary biliary cirrhosis $1.2 \%$, respectively. In $86.8 \%$ no aetiology was found. Diabetics was found in $66.7 \%$ whose random blood sugar values were more than $180 \mathrm{mg} / \mathrm{dl}$ ( $p=0.001$ in comparison to an alcoholic cohort). $24 \%$ had a history of usage of herbal medicine at some point. 35,30 and $25 \%$ have used native treatment for more than 6 months, $1-3$ and 3-6 months respectively. The components of herbs were largely unknown but comprised variety of plant products.

Conclusion: The aetiology of nonalcoholic liver disease in adult Sri Lankan remains largely unknown. The diabetes mellitus was a major association while usage of herbal medicine probably had a causal relationship. An in-depth study is needed to identify the responsible plant.

\section{PP39-41}

Pain Score within Twenty-four Hours Post-endoscopic Ultrasonography: A Comparison of Imaging Study with or without Fine Needle Aspiration Procedure

S. Amornyotin, S. Kongphlay

Faculty of Medicine Siriraj Hospital, Mahidol, Bangkok, Thailand

Aim: To compare the pain score within twenty-four hours postendoscopic ultrasonography (EUS) between the diagnostic with or without fine needle aspiration (FNA) procedure in adult patients.

Methods: We prospectively analyzed the patients who underwent EUS from January, 2009 to December, 2009. Pain score was compared by using visual analog scale (VAS, 0-100) and pain relief medications at 2, 6, 12,18, $24 \mathrm{~h}$ post-procedure.

Results: One hundred and twenty-four patients, 40 patients only with a diagnostic procedure (group D) and 84 patients with a diagnostic and FNA procedure (group F), were enrolled. All procedures were completed successfully. Sedative agents in both groups were propofol, midazolam and fentanyl and were comparable dose among the two groups. The mean procedural time in $\mathrm{D}$ and $\mathrm{F}$ was $39.8 \pm 19.1$ and $60.6 \pm 26.0 \mathrm{~min}$, respectively. Mean pain score at base line and at 2, 6, 12, 18 and $24 \mathrm{~h}$ post-EUS was not significantly different between the two groups. Additionally, total dose of pethidine used for pain control after ERCP in both groups was not significantly different. Conclusion: EUS-induced abdominal pain is mild and mainly occurs within $6 \mathrm{~h}$ after the procedure. Pain score within twenty-four hours post-EUS after the diagnostic with or without fine needle aspiration procedure is comparable.

\section{PP39-42}

Clinical Efficacy of Deep Sedation for Endoscopic Retrograde Cholangiopancreatography: A Comparison between Clinical Assessment and Narcotrend $^{\mathrm{TM}}$ Monitoring

S. Amornyotin, W. Chalayonnavin, S. Kongphlay

Faculty of Medicine Siriraj Hospital, Mahidol, Bangkok, Thailand

Background and aim: Moderate to deep sedation is generally used for endoscopic retrograde cholangiopancreatography (ERCP). The 
depth of sedation is usually judged by clinical assessment and EEGguided monitoring. The aim of this study was to compare the clinical efficacy of clinical assessment and Narcotrend ${ }^{\mathrm{TM}}$ monitoring during sedated ERCP.

Methods: 138 patients who underwent ERCP in a single year, were randomly assigned to either group $\mathrm{C}$ or N. Patients in group C (90) were sedated by using MOAA/S scale. Patients in group N (48) were sedated by using the Narcotrend ${ }^{\mathrm{TM}}$ system. The MOAA/S scale 1 or 2 and the Narcotrend stage D0-E0 (index 27-36 to 57-64) were maintained during procedure. The primary outcome variable of the study was the total dose of propofol used during the procedure. The secondary outcome variables were complications during and immediately after procedure, and recovery time.

Results: All endoscopies were completed successfully. Mean total dose of propofol in group $\mathrm{C}$ was significantly lower than in group N. However, the mean dose of propofol, expressed as dose $/ \mathrm{kg}$ or dose $/ \mathrm{kg} / \mathrm{h}$ in both groups, was not significantly different $(p=0.400,0.227)$. Recovery time, patient tolerance and satisfaction, and endoscopist satisfaction was comparable among the two groups. Sedation- related adverse events during and immediately after the procedure, such as hypotension, hypertension, tachycardia, bradycardia, transient hypoxia, or upper airway obstruction in group C $(62.2 \%)$, were significantly higher than in group $\mathrm{N}(37.5 \%)(p=0.006)$.

Conclusion: Clinical assessment and Narcotrend-guided sedation using propofol for deep sedation demonstrated comparable propofol dose and recovery time. Both monitorings were equally safe and effective. However, the Narcotrend-guided sedation showed lower hemodynamic changes and complications as compare to the clinical assessment-guided sedation.

\section{PP39-43}

Clinical Efficacy of Propofol-based Deep Sedation for ERCP and EUS Procedures in Geriatric Patients in a Developing Country

S. Amornyotin, U. Kachintorn, S. Kongphlay

Faculty of Medicine Siriraj Hospital, Mahidol, Bangkok, Thailand

Background and aim: Deep sedation in geriatric patients has been established as a safe and effective technique in Western countries. It is uncertain if the situation holds true among Asians. This study aimed to evaluate the outcome of propofol-based deep sedation (PBDS) for ERCP and EUS procedures in geriatric patients ( $\geq 65$ years old) and to compare the clinical efficacy of PBDS between the very old patients ( $>80$ years old) and those younger $(\leq 80$ years old) for these procedures.

Methods: We undertook a retrospective review of the sedation service records of patients who underwent GIE procedures. All procedures were performed by senior endoscopists and fellows in GI endoscopy. All sedations were administered by anesthetic personnel in the endoscopy room.

Results: Sedation was provided for 1,779 patients in 2,061 GIE procedures. Of these, 252 patients were in the younger than 65 years old group, 209 patients were in the age 65-85 years old group, and 30 patients were in 81 years of age and older group. Fentanyl, propofol and midazolam were the most common intravenous sedative drugs used in all three groups. Mean dose of propofol and midazolam in the very old patients was statistically significantly lower than the other young groups. The combination of propofol, midazolam and fentanyl as well as propofol and fentanyl were frequently been used in all patients. Mean sedation time was comparable between the three groups. Sedation related adverse events and procedure related complications were not statistically significantly different among the three groups. Most of them were hypotension (42.5\%).

Conclusion: In the setting of the developing country, PBDS for ERCP and EUS procedures in geriatric patients by trained anesthetic personnel with appropriate monitoring is relatively safe and effective.
All adverse events are usually transient and mild degree as well as easily treated, with no sequelae.

\section{PP39-44}

Parecoxib for Analgesia after Endoscopic Retrograde Cholangiopancreatography Procedure

S. Amornyotin, S. Kongphlay

Faculty of Medicine Siriraj Hospital, Mahidol, Bangkok, Thailand

Background and aim: Parecoxib is generally used for analgesia in post-procedural patients. The clinical efficacy of parecoxib used for endoscopic retrograde cholangio-pancreatography (ERCP) is controversy. The aim of this study was to determine and compare the clinical efficacy of parecoxib and placebo used for pre-medication in sedated ERCP patients.

Methods: 99 patients who underwent ERCP in a single year, were randomly assigned to $\mathrm{C}$ and $\mathrm{P}$ groups. Patients in group $\mathrm{C}$ (57) were received normal saline and patients in group $\mathrm{P}(42)$ were received 40 $\mathrm{mg}$ of parecoxib before the procedure. All patients were monitored for the depth of sedation by using the Narcotrend maintaining index 27-36 to 57-64 (Narcotrend stage D0-E0) during ERCP. The primary outcome variable of the study was the visual analog scale (VAS, $0-10)$ at 2, 12 and $24 \mathrm{~h}$ post-ERCP. The secondary outcome variables were the total number and dose of pethidine used post-procedure, patient and endoscopist satisfaction as well as complications during and immediately after procedure.

Results: All endoscopies were completely successful. The sedative agents used during the procedure, pain at $24 \mathrm{~h}$ post-ERCP, endoscopist satisfaction and complications in both groups were comparable. The total number of pethidine used post-ERCP in group $\mathrm{C}$ was significantly higher than in group P. Additionally, pain at 2 and $12 \mathrm{~h}$ post-ERCP in group $\mathrm{C}$ was significantly greater than in group $\mathrm{P}$. Patient satisfaction in group $\mathrm{P}$ was significantly higher than group $\mathrm{C}$. Conclusions: Parecoxib has a clinical efficacy for ERCP patients. The analgesic efficacy of parecoxib is clearly demonstrated during 12 $\mathrm{h}$ post-procedure. Additionally, the parecoxib has also higher patient satisfaction and seems to have fewer complications than the placebo.

\section{PP39-45}

Experience of Intravenous Sedation for Gastrointestinal Endoscopy in Extremely Elderly Patients in a Teaching Hospital in Thailand

S. Amornyotin ${ }^{1}$, W. Srikureja ${ }^{2}$, N. Pausawasdi ${ }^{1}$, U. Prakanrattana ${ }^{1}$,

U. Kachintorn ${ }^{1}$

${ }^{I}$ Faculty of Medicine Siriraj Hospital, Mahidol, Bangkok, Thailand;

${ }^{2}$ Loma Linda University Medical Center, Loma Linda, CA, USA

Background and aim: To evaluate the outcome of intravenous sedation (IVS) for gastrointestinal endoscopy (GIE) procedures in very old patients ( $\geq 86$ years old) and to compare the clinical efficacy of IVS between very elderly and those younger ( $<86$ years old).

Methods: We undertook a retrospective review of patients underwent GIE procedures by using IVS technique. The patients were divided into three groups: Group 1, age $<65$ years; Group 2, age $65-85$ years; and Group 3, age $\geq 86$ years. All procedures were performed by senior endoscopists and fellows in GI endoscopy. All sedations were administered by anesthetic personnel in the endoscopy room.

Results: All sedations were completed successfully. Fentanyl, midazolam and propofol were the most common sedatives used in all three groups. Most of them were used in combination. The combination of propofol, fentanyl and midazolam was commonly employed (32.3, 36.5 and $59.1 \%$ in Group 1, 2 and 3, respectively). Patients in Group 3 required lower mean doses of sedatives than Group 1 or 2 $(p<0.001)$. Mean sedation time in Group 3 was longer than Group 1 or $2(p=0.010)$. Complications occurred frequently and were medication or airway related. However, all complications were transient and easily treated, with no adverse sequelae. Adverse events in Group 
3 occurred more frequently when compared to Group 1 or $2(p<$ $0.001)$.

Conclusion: In the setting of the developing country, IVS for GIE procedure in very old patients is relatively safe and effective when carried out by trained anesthetic personnel with appropriate monitoring.

\section{PP39-46}

Deep Sedation for Therapeutic Upper Gastrointestinal Endoscopy in Pediatric Patients in Thailand

S. Amornyotin, P. Aanpreung

Faculty of Medicine Siriraj Hospital, Mahidol, Bangkok, Thailand

Background and aim: Sedation practice in pediatric patients undergoing TUGIE procedure is highly variable. A number of sedation protocols have been shown to be effective. The aim of this study is to evaluate the clinical efficacy of deep sedation for therapeutic upper gastrointestinal endoscopy (TUGIE) in pediatric patients in a teaching hospital in Thailand.

Methods: We undertook a retrospective review of the sedation service records of pediatric patients who underwent TUGIE. All sedation was administered by staff anesthesiologist or anesthetic personnel in the gastroenterology procedure room.

Results: Deep sedation was provided for 161 GIE procedures in 154 patients during the study period. Of these, 70 UGIE procedures in 65 patients ranged in age from 1 to 17 years and in weight from 9.5 to $55 \mathrm{~kg}$ were reviewed. Most common indications for the procedure were esophageal varices (88.6\%), esophageal stricture (10.0\%) and foreign body $(1.4 \%)$. The majority of therapeutic interventions were banding $(47.1 \%)$, sclerosing injection $(41.4 \%)$, and dilatation (5.7\%). Propofol, fentanyl and midazolam were frequently used. Mean dose of propofol was $3.3 \pm 1.7 \mathrm{mg} / \mathrm{kg}$, fentanyl was $0.001 \pm 0.000 \mathrm{mg} / \mathrm{kg}$, and the mean dose of midazolam was $0.05 \pm 0.02 \mathrm{mg} / \mathrm{kg}$. Most of them were used in combination. The combination of propofol, fentanyl and midazolam was commonly employed $(42.9 \%)$. The mean sedation time of all procedures was $25.1 \mathrm{~min}$. All complications were easily treated, with no adverse sequelae.

Conclusions: In the setting of the developing country, deep sedation for pediatric TUGIE by trained anesthetic personnel with appropriate monitoring was safe and effective. Serious adverse events were rare in our population.

\section{PP39-47}

Vaccination Practices against Hepatitis B Virus(HBV) and Immune Response Following HBV Vaccine among Health Care Workers M. Hoque ${ }^{1}$, M.A. Latif ${ }^{2}$

${ }^{1}$ Hepatology, Comilla Medical College; ${ }^{2}$ Medicine, Comolla Medical College, Comilla, Bangladesh

Introduction: The risk of acquiring HBV infection through exposure to blood and blood products is highest among the Health Care Workers(HCWs). Despite the potential risks, a proportion of HCWs never vaccinated. This study was carried out to investigate the HBV vaccination practices and immune response following HBV vaccine among HCWs.

Methods: This hospital based cross sectional study was carried out among HCWs(Doctors, Dentists, Nurse, Lab technicians, Cleaners) of Comilla Medical college Hospital, Bangladesh. Among 320 HCWs, 260 participate in this study. All were screened for HBsAg, Anti HCV and 123 vaccinated HCWs for Anti HBs antibody in their sera by ELISA.

Result: Among $260 \mathrm{HCWs}$, 4(1.53\%) were HBsAg positive. None were Anti $\mathrm{HCV}$ positive. $123 \mathrm{HCWs}(47.30 \%)$ were vaccinated against $\mathrm{HBV}$ and none were $\mathrm{HBsAg}$ positive in this group. Protective titre of Anti HBs antibody (>10 mIU/ml) was present in $114(92.68 \%)$ HCWs. Reasons for nonvaccination were negligence in $80(58.4 \%)$ cases. 45(32.9\%) HCWs were unaware of their need for HBV vaccination.

Conclusion: Despite the beneficial outcome of HBV vaccine, a major proportion of HCWs never get vaccinated and awareness practices are incomplete. Mandatory vaccination of HCWs against HBV should be implemented in every hospital to reduce HBV burden.

\section{PP39-48}

Hepatoprotective Role of Syzygium cumini Linn. Extract along with Vitamin $E$ and $C$ against Silica Induced Biochemical and Histopathological Alterations

S. Johri ${ }^{1}$, S. Shukla ${ }^{2}$, S.K. Nirala ${ }^{2}$, M. Bhadauria ${ }^{2}$

${ }^{I}$ Department of Biochemistry, Boston College for Professional

Studies; ${ }^{2}$ SOS in Zoology, Jiwaji University, Gwalior, India

Background and aim: Silica is one of the most documented workplace contaminants. Long-term occupational exposure to silica is associated with an increased risk for respiratory diseases such as silicosis, tuberculosis and chronic liver diseases. No antidote is available for silica induced hepatic abnormalities. Present study was aimed to protect silica induced hepatic damage by ethanolic extract of stem bark of Syzygium cumini with and without combination of vitamin $\mathrm{E}$ and vitamin $\mathrm{C}$.

Methods: Female albino rats were exposed to silica $\left(\mathrm{SiO}_{2} ; 40 \mathrm{mg} /\right.$ $\mathrm{kg}$; ip for 28 days) followed by treatment of Syzygium cumini (SC extract, $1 \mathrm{~g} / \mathrm{kg}$, po) with and without combination of vitamin $\mathrm{E}(50$ $\mathrm{mg} / \mathrm{kg}$, ip) and vitamin C (100 mg/kg, po) for five consecutive days. All the animals were euthanized after $24 \mathrm{~h}$ of last treatment and various biochemical and histopathological parameters were performed.

Results: Toxicological consequences were evident by enhanced level of serum AST, ALT, ALP, protein, cholesterol and blood sugar after silica administration. Tissue biochemistry revealed raise in acid phosphatase whereas fall in alkaline phosphatase, adenosine triphosphatase and total protein content in liver after silica toxicity. Significant raise in lipid peroxidation and decreased level of reduced glutathione after silica exposure showed oxidative stress consequences in liver. Histopathological observations of liver substantiated the biochemical findings after exposure to silica followed by different treatments.

Conclusion: Combination of vitamin $\mathrm{E}$ and $\mathrm{C}$ was found to be most effective in reversal of silica-induced oxidative stress, biochemical and histopathological alterations in liver.

\section{PP39-49}

Patient Perception and Knowledge of Diet in Liver Cirrhosis; Are They Different with the Level of Education

R. Khan, Hepatology and Nutrition

Medicine, Aga Khan University, Karachi, Pakistan

Background: Malnutrition is a common problem in cirrhosis of liver and adversely effects the survival in these patients. In this regard patient's knowledge and perception of diet are not well studied.

Aim: To determine the current patient's perceptions and knowledge regarding diet in cirrhosis and to find out its relationship with the level of education.

Methods: Consecutive adult patients with compensated cirrhosis presented to three different sites of two University hospitals were enrolled. After taking written consent, demographic data, level of education, type of food the patients were restricting and reason for avoiding these dietary components and source of dietary information was asked. Base line laboratory test done and nutritional status of the patient was assessed by using modified body mass index (BMI) normogram for Asians.

Results: Out of 90 cirrhotic patients enrolled forty five were in each educated and uneducated class. Fifty two patients (58\%) were male. Mean age of the patients was $49 \pm$ SD 11 years. Mean BMI was 
$21.4 \pm 3.51$. Overall $66(73 \%)$ of the patients were restricting fats, meat, fish and eggs in their diet $31(47 \%)$ of them were in educated and $35(53 \%)$ of uneducated group (CI, 0.24-1.62, $p, 0.34)$. Twenty two $(62.8 \%)$ patients in uneducated and $21(68 \%)$ in educated group were restricting diet on the advise of their doctors whereas $13(37 \%)$ in uneducated group and 11(32) in educated group believes these dietary components are harmful for the liver.

Conclusions: Both educated and uneducated classes of the society have improper knowledge and perception of diet in cirrhosis. Patients with cirrhosis who restrict the diet had relatively low BMI, Hemoglobin and albumin as compared to those who do not restrict. Main source of dietary information to cirrhotic patients are health care personals and there is need to educate both.

\section{PP39-50}

Clinical Profile of Patients with Acute-on-Chronic Liver Failure with Comparison of Severity Scores in Predicting Prognosis - A Prospective Study M. Rangan, V.A. Saraswat

Gastroenterology, Sanjay Gandhi Post Graduate Institute of Medical Sciences, Lucknow, India

Background: ACLF occurs when an acute insult in a patient with chronic liver disease results in liver failure within 4 weeks of onset. There are limited data on ACLF.

Patients and methods: All patients admitted with liver failure from January to November 2009 to this hospital were screened for ACLF. Inclusion criteria included an acute hepatic insult (serum bilirubin $\geq 5$ $\mathrm{mg} / \mathrm{dL}$ ), liver failure (INR $\geq 1.5$, ascites and/or hepatic encephalopathy; HE) and underlying chronic liver disease. Acute insult was categorized as non-hepatitic, hepatitic, both or unidentifiable. Severity of liver failure was assessed using Child-Turcotte Pugh (CTP), model for end-stage liver disease (MELD), Na-MELD and sequential organ failure assessment (SOFA) scores at the time of admission. All patients with ACLF were followed for 3 months. The predictive prognosis according to various scores was determined by the area under the receiver operating characteristic curve (AUROC).

Results: ACLF was diagnosed in 100 patients (mean age $45.6 \pm 13.4$ years). Underlying liver cirrhosis was due to alcohol (26), HBV (36), HCV (9), AIH (5), Budd-Chiari syndrome (2) and cryptogenic (22). The cause of acute insult was non-hepatitic (33), hepatitic (39), both hepatitic and non-hepatitic (16) and unidentifiable (12). Non-hepatitic causes included sepsis (30), UGI bleeding (13), HCC (3) and metabolic factors (3) while hepatitic causes included HEV (21), alcohol (6), drugs (6), HAV (2) and HBV (11). 93\% had Child's C liver function. Ascites alone was present in 29 patients, HE alone in 5 patients and both ascites and HE in 65 patients. Renal failure in 39, SBP in 26 and hypoxemia in 18 patients were other complications. Inhospital mortality was $41 \%$ including those discharged pre-terminally. Mean scores were CTP 13, MELD 27, Na-MELD 36, and SOFA 8. SOFA score had highest AUROC in predicting in-hospital as well as 3 month mortality.

Conclusions: ACLF is a common cause of liver failure with high inhospital mortality without liver transplantation. SOFA score is most useful in predicting outcome.

\section{PP39-51}

Association of Toll-like Receptor 3 with Disease Severity in Indian Patients with Chronic Hepatitis C Virus Infection

S. Medhi ${ }^{1,2}$, M. Deka ${ }^{2}$, P. Kar ${ }^{1}$

${ }^{I}$ Medicine, Maulana Azad Medical College, New Delhi;

${ }^{2}$ Biotechnology, Gauhati University, Guwahati, India

Objective: TLR-3 involved in innate immunity has been identified to respond to dsRNA, a molecular signature of viruses like hepatitis $\mathrm{C}$ virus $(\mathrm{HCV})$. The aim of the study was to assess genetic variations of promoter region and the reported five SNPs viz rs3775290, rs3775296, rs5743305, rs3775291 and rs5743316 of TLR3 gene and its expression associated with disease severity in patients with chronic $\mathrm{HCV}$ (CHC) infection.

Methods: Genetic polymorphism of TLR3 was investigated in 180 chronic HCV patients who were HCV-RNA positive and 180 controls by PCR-RFLP, TaqMan assay, SSCP and direct sequencing. Expression analysis of TLR3 was done by real time PCR and Immunohistochemistry.

Results: Two new variants $-288 \mathrm{G} / \mathrm{A}$ and $-705 \mathrm{~A} / \mathrm{G}$ were observed in TLR3 promoter region by direct sequencing. The results were significant in -705 allele $(P<0.05)$ and were associated with high risk of HCV infection OR 2.79(1.46-5.42). In silico sequence analysis showed the presence of Ectropic viral integration site 1 encoded factor, in which $\mathrm{G}$ at -705 results in the loss of this site. The $-7 \mathrm{C} / \mathrm{A}$ (rs3775296) polymorphism was not found in our cohort. No significant variation in genotype and allele frequency was observed in c.1377T (rs3775290), L412F (rs3775291), rs3775296 and T976A (rs5743305) SNPs. There were no significant correlation with respect to HCV genotype distribution, histological manifestation and TLR3 SNPs. Except in N284I (rs5743316) the results were significant $(p=0.01)$ but were not a risk factor for progression to cirrhosis OR $0.52(0.29-0.93)$. The expression of TLR3 is upregulated in chronic HCV patients $\left(\Delta \Delta \mathrm{C}_{\mathrm{T}}=2.33\right)$ compared to controls $\left(\Delta \Delta \mathrm{C}_{\mathrm{T}}=4.54\right)$. At rs5743316 the minor allele A expression $\left(\Delta \Delta \mathrm{C}_{\mathrm{T}}=2.54\right)$ is comparatively less from the major allele $\mathrm{T}\left(\Delta \Delta \mathrm{C}_{\mathrm{T}}=3.13\right)$.

Conclusion: TLR3 SNP of -705 and rs5743316 is associated with chronic HCV infection. rs5743316 was found to be associated with histological manifestation and mRNA expression was downregulated among the patients with minor allele A.

\section{PP39-52}

Fascioliasis Liver Abscess: An Unrecognized and Fatal but Curable Disease

N. Waisayanand ${ }^{1}$, A. Leerapun ${ }^{1}$, N. Morakot ${ }^{2}$, S. Thongsawat ${ }^{1}$, P. Pisespongsa ${ }^{1}$, T. Chitapanarux ${ }^{1}$, O. Praisontarangkul ${ }^{1}$

${ }^{1}$ Internal Medicine; ${ }^{2}$ Parasitology, Chiang Mai University, Chiangmai, Thailand

Objective: Fascioliasis is one of the unrecognized zoonosis. Humans are infected by taking aquatic vegetable and water contaminated with metacercariae. Patients mostly presented with eosinophilic liver abscess. We aim to review demography, clinical features, radiologic manifestations, treatments and their response in human fascioliasis liver abscess at our hospital.

Method: We retrospectively studied 29 patients with fascioliasis liver abscess. Some sera were sent for immunoblot analysis for a specific $27 \mathrm{kDa}$ band of Fasciola gigantica excretory-secretory (ES) antigens. Results: Women were predominating (79.3\%), mean age was 45.8 years. There was variety of occupation and residence. History of raw, uncooked vegetable ingestion was $55.2 \%$. Disease was mostly acquired in the rainy season. The three most common symptoms were abdominal distension (68.9\%), abdominal pain (65.5\%) especially of right upper quadrant and weight loss $(65.5 \%)$ and signs were abdominal tenderness (58.6\%), right upper quadrant predominantly, fever $(58.6 \%)$ and hepatomegaly $(51.7 \%)$. Hypereosinophilia, anemia and hyperglobulinemia were present in 89.7, 75.8, and $86.2 \%$, respectively. The common radiologic findings were conglomerated hypodense $(100 \%)$, rim enhancing lesions located commonly in subcapsular region, $92 \%$, with parasitic tracts found nearly half of cases. The immunoblot assay for specific $27 \mathrm{kDa}$ diagnostic band was positive in 18 of 20 patients. Fasciola eggs were detected in three patients and adult Fasciola gigantica in other three. Main treatment was triclabendazole with good response in all patients. Two patients received artesunate with poor response and showed adverse events, leukopenia. All 19 followed up patients had full resolution within 6 months. In subgroup of triclabendazole monotherapy, response rate at 3 months was $85.7 \%$. 
Conclusion: When encountering with eosinophilic liver abscess, physicians should remind themselves of this fatal disease and employ typical clinical clues and radiologic findings presented in our study. Triclabendazole provided satisfactory treatment result.

\section{PP39-53}

Impact of Different Sources of Chromium(III) Dietary Supplementation on Lipids Content in Muscles and Liver of Chicken Broilers

B. Debski, M. Gralak

Department Physiological Sciences, Warsaw University of Life Sciences, Warsaw, Poland

Background and aims: Proponents of chromium supplementation assume that chromium addition to the diet will enhance insulin function, improve glucose tolerance and lipid profile. Chromium salts and enriched yeast are used in several supplements, however results of many experiments not confirm biological efficiency of $\mathrm{Cr}$ in humans and animals. Experiments performed on poultry demonstrated rather consistent effects of $\mathrm{Cr}$ (III) on improvement of serum lipid profile and occasionally cholesterol concentration. The aim of experiment was to chack the influence of different $\mathrm{Cr}$ sources on hepatic lipids content. Table 1

Table 1

\begin{tabular}{lrllll}
\hline $\begin{array}{l}\text { Feed } \\
\text { supplement }\end{array}$ & \multicolumn{2}{l}{$\begin{array}{l}\text { Total Fat }(\mathbf{g} / \mathbf{1 0 0 g} \\
\text { wet tissue) }\end{array}$} & & \multicolumn{2}{l}{$\begin{array}{l}\text { Total Cholesterol } \\
(\mathbf{m g} / \mathbf{1 0 0 g} \text { wet tissue) }\end{array}$} \\
\cline { 2 - 3 } & \multicolumn{1}{l}{ Liver } & Muscle & & Liver & Muscle \\
\hline Control & $9.2 \pm 0.85$ & $2.60 \pm 0.15$ & & $286 \pm 45$ & $\mathbf{9 5} \pm 18$ \\
Cr chloride & $\mathbf{6 . 9 7} \pm 0.67$ & $\mathbf{1 . 7 1} \pm 0.17$ & & $\mathbf{2 6 4} \pm 51$ & $\mathbf{7 2} \pm 15$ \\
Cr picolinate & $\mathbf{7 . 3 2} \pm 0.92$ & $\mathbf{1 . 8 3} \pm 0.14$ & & $\mathbf{2 0 7} \pm 28$ & $\mathbf{4 6} \pm 12$ \\
Cr yeast & $\mathbf{1 0 . 1 3} \pm 1.21$ & $\mathbf{1 . 8 5} \pm 0.21$ & & $\mathbf{4 7 6} \pm 56$ & $\mathbf{4 3} \pm 13$ \\
\hline
\end{tabular}

Material and methods: We used $\mathrm{Cr}$ as chloride, picolinate and $\mathrm{Cr}$ yeast in dose $0.5 \mathrm{ppm}$ for enrichment of chicken broilers diet. 28 days old ISA broilers were given 28 days standard diet supplemented with mentioned above $\mathrm{Cr}$ sources.

Results: Obtained results confirm that $\mathrm{Cr}$ supplements regardless used source of $\mathrm{Cr}(\mathrm{III})$ are lowering total fat and cholesterol concentration in muscles (estimations performed in pectoral muscles). In the liver, $\mathrm{Cr}$ effect depends on used source of this microelement. $\mathrm{CrCl}_{3}$ and $\mathrm{Cr}$-picolinate supplementation are leading to decrease of hepatic fat content. Decrease of cholesterol concentrations in the liver was statistically significant only in case of Cr-picolinate. Use of Cr-yeast as nutritional supplement however, may lead to increase of fat and cholesterol concentration in the liver of broilers.

Conclusions:

1/ Chromium dietary supplementation ( $28 \mathrm{~d}, 0.5 \mathrm{ppm}$ of $\mathrm{Cr}$ ) caused decrease of total fat and cholesterol content in muscles of broilers.

2/ Effect of Cr supplementation on hepatic fat and cholesterol content depends on

Cr source.

\section{PP39-54}

Identification of H.pylori cagA, vacA and glmM Genes and Helicobacter Species 16SrRNA in Liver Samples of Patients with HCV

Y.G. Zhang, S.W. Ji, J.B. Wang

China-Japan Union Hospital of Jilin University, Changchun, China

Amis: To investigate the the role of H.pylori in the development of chronic hepatitis $\mathrm{C}(\mathrm{HCV})$.

Methods: Serum anti-H.pylori-IgG was tested by ELISA in 34 patients with HCV. H.pylori-related genes(cagA, vacA and glmM) were detected by PCR in liver samples of serum anti-H.pylori-IgG positive. Helicobacter genus-special 16SrRNA gene was detected by PCR in liver samples of serum anti-H.pylori-IgG negative. Then, the amplified products of helicobacter genus-special 16SrRNA gene positive were identified by sequencing. The liver samples of Helicobacter genus-special 16SrRNA gene or helicobacter genus-special 16SrRNA gene was positive were isolated and cultured of bacteria.

Results: Serum anti-H.pylori-IgG were positive in $67.6 \%(23 / 34)$ and the seroprevalence of H.pylori was higher in patients with cirrhosis $(10 / 14,71.4 \%)$ and hepatocellular carcinoma $(5 / 6,83.3 \%)$ than that in chronic hepatitis $(8 / 14,57.1 \%)(p<0.05)$. H.pylori associated genes were found in 7 of $23(30.4 \%)$ liver samples of $\mathrm{HCV}$ patients with serum anti-H.pylori-IgG positive. The positive rate of H.pylori-related genes in patients with hepatocellular carcinoma(4/5,80.0\%) was higher than that in patients with chronic hepatitis $(1 / 8,12.5 \%)$ and cirrhosis $(2 / 10,20.0 \%)(p<0.05)$, and the glmM gene was the main gene. Therefore, Helicobacter genusspecial 16SrRNA gene was found in one liver samples of serum anti-H.pylori-IgG negative. Then, the sample amplified products of helicobacter genus-special 16SrRNA gene positive were sequenced and were found that the sample was similar to H.hepaticus with $92.0 \%$.

Conclusion: H. pylori-related genes were existed in liver samples of $\mathrm{HCV}$ patients with H.pylori infection, and the positive rate of $\mathrm{H}$. pylori-related genes in patients with hepatocellular carcinoma was higher than that in patients with chronic hepatitis and cirrhosis. Other helicobacter 16SrRNA genes were also existed in liver samples of patients with HCV. H.pylori and other helicobacter infection might play an important synergic role with $\mathrm{HCV}$ in the development of $\mathrm{HCC}$.

\section{PP39-55}

Influence of Low-protein Diet, Training and Riboflavin Supplementation on Liver Mineral Concentration in Rats

M.A. Gralak ${ }^{1}$, B. Debski ${ }^{1}$, J. Bertrandt ${ }^{2}$, A. Klos ${ }^{2}$, A.B. Stryczek ${ }^{1}$, A. Piastowska-Ciesielska ${ }^{3}$, A. Sikorska ${ }^{1}$

${ }^{1}$ Department of Physiological Sciences, Faculty of Veterinary

Medicine, Warsaw University of Life Sciences; ${ }^{2}$ Military Institute of Hygiene and Epidemiology, Warszawa; ${ }^{3}$ Department of Comparative Endocrinology, Medical University of Lodz, Lodz, Poland

The objective was to study the effect of dietary protein and riboflavin (vitamin $\mathrm{B}_{2}$ ) content, and training on hepatic $\mathrm{Mg}, \mathrm{Fe}, \mathrm{Zn}, \mathrm{Mn}$, $\mathrm{Cu}$ and $\mathrm{Cr}$ concentration in rats. The experiment was performed on 41 growing male Wistar rats divided into six groups fed ad libitum for 90 days with semipurified diets. Two diets contained 14.7 MJ gross energy per $1 \mathrm{~kg}(350 \mathrm{kcal} / 100 \mathrm{~g})$ and $20 \%$ of energy originated from protein. Animals of another four groups were fed isocaloric diet $(14.7 \mathrm{MJ} / \mathrm{kg})$ but only $4.5 \%$ of the energy derived from protein. Two of four protein restricted diets were supplemented with riboflavin in amount of $75 \mathrm{mg} / \mathrm{kg}$ diet as fed. It was the 25 fold higher concentration than in groups without supplementation. Rats of three groups were trained for one hour daily: one fed diet with $20 \%$ protein content, second fed diet with $4.5 \%$ protein content without addition of riboflavin and the other supplemented with riboflavin. Generally low protein diet increased $(p \leq 0.05)$ liver $\mathrm{Cu}$ content from $4.15 \pm 0.22(n=15)$ to $4.42 \pm 0.17 \mathrm{mg} / \mathrm{kg}(n=14)$. In trained rats as compared to non-trained ones, higher $(p \leq 0.05)$ liver concentration of iron $(35.7 \pm 8.3 ; n=13$ v. $30.3 \pm 6.3 \mathrm{mg} /$ $\mathrm{kg} ; n=16)$ and lower $(p \leq 0.05)$ of chromium content $(4.00$ $\pm 0.29 ; n=13$ v. $4.27 \pm 0.35 \mathrm{mg} / \mathrm{kg} ; n=16)$ were observed. Liver $\mathrm{Zn}$ concentration was significantly lower in animals supplemented with riboflavin $(163 \pm 16 \mathrm{mg} / \mathrm{kg} ; n=12)$ than in rats fed diet with normal riboflavin content $(192 \pm 28 \mathrm{mg} / \mathrm{kg} ; n=14)$. 


\begin{tabular}{lcccccc}
\hline Group/Mineral & $\begin{array}{l}20 \% \mathrm{GE} \text { from } \\
\text { protein (control) } \\
(n=10)\end{array}$ & $\begin{array}{l}20 \% \mathrm{GE} \text { from } \\
\text { protein 1 hour } \\
\text { running }(n=6)\end{array}$ & $\begin{array}{l}4,5 \% \mathrm{GE} \text { from } \\
\text { protein }(n=6)\end{array}$ & $\begin{array}{l}4,5 \% \text { GE from protein } \\
1 \text { hour running }(n=8)\end{array}$ & $\begin{array}{l}4,5 \% \mathrm{GE} \text { from } \\
\text { protein }+ \text { vit.B2 } \\
(n=6)\end{array}$ & $\begin{array}{l}4,5 \% \mathrm{GE} \text { from } \\
\text { protein }+ \text { vit.B2 } \\
1 \text { hour running }(n=5)\end{array}$ \\
\hline $\mathrm{Mg}(\mathrm{g} / \mathrm{kg})$ & $2.93 \pm 0.15$ & $2.85 \pm 0.24$ & $3.01 \pm 0.39$ & $2.89 \pm 0.19$ & $3.01 \pm 0.20$ & $3.09 \pm 0.29$ \\
$\mathrm{Fe}(\mathrm{mg} / \mathrm{kg})$ & $30.5 \pm 6.4$ & $40.9 \pm 11.6$ & $30.8 \pm 3.6$ & $32.4 \pm 3.3$ & $30.3 \pm 3.3$ & $34.5 \pm 4.3$ \\
$\mathrm{Zn}(\mathrm{mg} / \mathrm{kg})$ & $182 \pm 9$ & $175 \pm 21$ & $187 \pm 26$ & $195 \pm 31$ & $166 \pm 14$ & $161 \pm 19$ \\
$\mathrm{Cu}(\mathrm{mg} / \mathrm{kg})$ & $4.20 \pm 0.16$ & $4.07 \pm 0.32$ & $4.49 \pm 0.22$ & $4.37 \pm 0.12$ & $4.43 \pm 0.23$ & $4.37 \pm 0.14$ \\
$\mathrm{Mn}(\mathrm{mg} / \mathrm{kg})$ & $0.365 \pm 0.090 \mathrm{ab}$ & $0.315 \pm 0.055 \mathrm{~b}$ & $0.325 \pm 0.055 \mathrm{~b}$ & $0.400 \pm 0.100 \mathrm{ab}$ & $0.480 \pm 0.080 \mathrm{a}$ & $0.345 \pm 0.120 \mathrm{ab}$ \\
$\mathrm{Cr}(\mathrm{mg} / \mathrm{kg})$ & $4.23 \pm 0.40$ & $3.83 \pm 0.34$ & $4.34 \pm 0.28$ & $4.11 \pm 0.21$ & $4.33 \pm 0.22$ & $4.34 \pm 0.29$ \\
\hline
\end{tabular}

[Liver mineral concentration in rats] means not sharing the same letter differ at $P \leq 0.05$ (Test F Ryan-Einot-Gabriel-Welsch)

\section{PP39-56}

Neuroendocrine Liver Metastases: Results of a Multidisciplinary Approach

V. Velayutham, J. Sathyanesan, S. Ramaswamy, J. Damodaran, S. Eswaran, R. Palaniappan, S. Rajagopal

Dept of Surgical Gastroenterology and Liver Transplantation,

Government Stanley Medical College Hospital, Chennai, India

Background: In patients with neuroendocrine liver metastases, liver surgery or radiofrequency ablation (RFA) may result in apparently total clearance of metastases. However, it is not clear whether such therapy will provide symptom reduction or increased survival. Our objective was to analyse the options in the management of neuroendocrine tumours with liver metastases and we also sought to determine the effect of surgical resection, ablation, embolization or combination of these modalities in patients with neuroendocrine metastases.

Materials and methods: Between January 2004 and May 2010, 42 Patients undergoing operations or interventions for neuroendocrine liver metastases from any primary were identified from a prospective liver database. Recorded data and medical record review were used to analyse the type of procedure, length of hospital stay, peri-operative morbidity, tumour recurrence, progression, and survival.

Results: The median age was 40.8 years (range 16-64 years). 42 patients included 28 carcinoids, 12 islet cell tumors, 2 nonfunctioning tumors from unknown origin. Thirty-six patients underwent surgical therapy, including anatomical liver resection $(n=22)$, ablation $(n=4)$, or combined resection and ablation $(n=10)$. Hepatic artery embolization was performed in 6 patients. No difference was noted in the percentage of liver involved with tumor between the 2 groups. An association of improved survival was noted in patients treated surgically, with a 3-year survival of $83 \%$ for patients treated by surgical resection alone or in combination, compared with $31 \%$ in patients treated with medical therapy or embolization.

Conclusion: Early and aggressive surgical management of hepatic metastases from neuroendocrine tumours is associated with significant long-term survival rates. Radiofrequency ablation either alone or in combination with surgery is a reasonable option if a lesion is unresectable.

\section{PP39-57}

Serum Trehalase is a New Interesting Marker for Cholestasis M. Yoshiba, K. Inoue

Gastroenterology, Showa University Fujigaoka Hospital, Yokohama, Japan

Introduction: Trehalase, a disaccharidase which specifically hydrolyzes one molecule of trehalose into two molecules of glucose, is localized on mammalian intestinal brush border membrane where it serves in the absorption of trehalose which is mainly contained in mushrooms. A person who lacks trehalase in intestine is known to show intolerance to mushrooms. Human serum trehalase activity was investigated by several authors, and was reported to be elevated in diabetes mellitus and liver cirrhosis. However, clinical implication of these phenomena was not explained. In the present study we have measured serum trehalase activity in various liver diseases.

Material and method: Subjects of the present study was 40 nonalcoholic healthy adults giving normal liver function tests including $\gamma$ GTP, eight patients with acute viral hepatitis, four with fulminant hepatitis, 32 with chronic hepatitis, 30 with non-alcoholic liver cirrhosis, 15 with cholestatic liver diseases (three with primary biliary cirrhosis, one with drug-induced intrahepatic cholestasis and 11 with obstructive jaundice) and 11 with alcoholic liver injury (eight with fatty liver and three with liver fibrosis).

Results: The activity was elevated in cholestasis and alcoholic liver diseases, while it was lowered in acute and fulminant hepatitis. In chronic hepatitis and liver cirrhosis the activity was not significantly elevated, although it was elevated above normal range about in $30 \%$ of patients with both diseases. Trehalase activity changed in a close correlation with that of $\gamma$-glutamyl transpeptidase in cholestasis and alcoholic liver diseases.

Conclusion: These observations suggest that the induction of enzyme activities in cholestasis and alcoholic liver diseases is common to several brush border membrane enzymes.

\section{PP39-58}

Proinflammatory Cytokines Play a Role in the Development of Cerebral Edema in Acute on Chronic Liver Failure

M. Rangan ${ }^{1}$, S.K. Yadav ${ }^{2}$, V.A. Saraswat ${ }^{1}$, R.K.S. Rathore ${ }^{3}$, M.A. Thomas ${ }^{4}$ K.N. Prasad ${ }^{5}$, C.M. Pandey ${ }^{6}$, R.K. Gupta ${ }^{2}$

${ }^{l}$ Gastroenterology, Sanjay Gandhi Post Graduate Institute of Medical Sciences; ${ }^{2}$ Radiology, Sanjay Gandhi Postgraduate Institute of Medical Sciences, Lucknow; ${ }^{3}$ Mathematics and Statistics, Indian Institute of Technology, Kanpur, India; ${ }^{4}$ Radiological Sciences, UCLA School of Medicine, Los Angeles, CA, USA; ${ }^{5}$ Microbiology; ${ }^{6}$ Biostatistics, Sanjay Gandhi Post Graduate Institute of Medical Sciences, Lucknow, India

Background: Acute-on-chronic liver failure (ACLF) is defined as appearance of hepatic encephalopathy (HE) and/or ascites within 4 weeks of onset of jaundice in patients with underlying chronic liver disease. While hyperammonemia is considered to play a major role in the pathogenesis of cerebral edema (CE) and HE, recent reports suggest that inflammation derived cytokines also contribute significantly. Data supporting this remain scanty. This study looked at the relationship between cytokines and glutamate/glutamine ratio (Glx) on ${ }^{1} \mathrm{H}$-MR spectroscopy $\left({ }^{1} \mathrm{H}-\mathrm{MRS}\right)$, a measure of cerebral ammonia elevation, and diffusion tensor imaging (DTI) derived metrics for CE, 
namely mean diffusivity (MD) and spherical anisotropy (CS), which are decreased and increased respectively in CE of ACLF patients. Methods: Seventeen patients with ACLF and 14 controls, matched for age and gender, were included in this study. Apart from clinical and biochemical evaluations, all patients had undergone MRI $\left({ }^{1} \mathrm{H}-\right.$ MRS and DTI). Serum pro-inflammatory cytokines (IL- 6 and TNF- $\alpha$ ), blood ammonia and Glx were measured in both groups and compared using independent t-test. Correlations between cytokines and MR derived metrics were assessed using Pearson's correlation coefficient. Results: Levels of cytokines, ammonia and brain Glx were significantly increased in ACLF patients as compared to controls $(p<0.001)$. Significant positive correlation was present between cytokines and Glx $[(r=0.667, p=0.003)$ for TNF- $\alpha$ and $(r=0.502, p=0.04)$ for IL-6] as well as with spectroscopy voxel (SV) derived CS $[(r=0.578, p=0.015)$ for TNF- $\alpha$ and $(r=0.681$, $p=0.003$ ) for IL-6], while a negative correlation was noted with SV derived MD [ $r=-0.506, p=0.038)$ for TNF- $\alpha$ and $(r=-0.619$, $p=0.008$ ) for IL-6]. These cytokines also had positive and negative correlations with CS and MD respectively, in various specific brain regions.

Conclusions: These results support a role for pro-inflammatory cytokines in the pathogenesis of CE in ACLF patients. DTI derived metrics and $1 \mathrm{H}-\mathrm{MRS}$ are very good non-invasive tools for understanding the pathogenesis of CE in ACLF.

\section{PP39-59}

Is Obesity a Risk Factor Associated with Amebic Liver Abscess? L.E. Moreno Luna ${ }^{1}$, M. Cortez-Navarrete ${ }^{2}$, L. Cernichiaro-Espinoza ${ }^{2}$, J.E. Segura-Ortega ${ }^{2}$

${ }^{1}$ Clinica Médica, Universidad de Guadalajara; ${ }^{2}$ Departamento de Gastroenterología, Hospital Civil de Guadalajara Fray Antonio Alcalde, Guadalajara, Mexico

Amebic liver abscess (ALA) is a severe form of invasive amebiasis caused by Entamoeba histolytica. It is more common in developing countries. Immunosuppresion is associated with ALA. It is known that malnourished patients have a higher risk of infections and potentiate the severity of the disease associated to immunosuppression. Obesity has not been previously associated as a risk factor for ALA. The aim of this study was to evaluate the nutritional status of patients with ALA.

Materials and methods: Patients gave informed consent. Patients with ALA diagnosis from February 2008 to August 2010 were included. Liver function tests, cytometry, hepatic ultrasound and percutaneous abscess drainage with its analysis were performed for the diagnosis. Patients received conventional treatment for ALA. Nutritional status was assessed using anthropometric, dietary and clinical evaluations.

Results: Forty-four patients were included $73 \%$ men, mean age 40 age (range 16-70), 79\% $(n=35)$ had all home services. The hygienic conditions were considered as good in $7 \%(n=3)$, regular in $66 \%$ $(n=27)$ and poor in $14 \%(n=6)$ of the patients. Sixty one percent $(n=27)$ use alcohol and 29\% $(n=13)$ use drugs. Patients' nutritional status was considered as normal in $25 \%$, malnourished in $40 \%$ and obese or overweight in 35\%. Mean BMI for normal nutritional status was 23.8 , for malnourished 18.5 and for obese 30.77 . Seventy five percent $(n=33)$ of all patients were considered as having malnutrition. Conclusions: In this group all patients had similar social, hygienic and addictive conditions. Malnourishment was the most common nutritional status but $35 \%$ of patients were obese. Immunosuppression is present in malnourished and obese patients. Obesity in this group of patients seems to be a predisposing factor for ALA. We are conducting another study to evaluate the relationship between ALA and fatty liver disease.
PP39-60

Association of Serum Gamma-glutamyl Transferase and Total Bilirubin Levels with Metabolic Syndrome in Korean Men and Women

S.V. Ahn ${ }^{1}$, K.H. Ha ${ }^{2}$, H.C. Kim ${ }^{1}$, M. Suh ${ }^{1}$, D.P. Choi ${ }^{1}$, I. Suh ${ }^{1}$

${ }^{I}$ Department of Preventive Medicine, Yonsei University College of Medicine; ${ }^{2}$ Department of Public Health, Graduate School of Yonsei University, Seoul, Republic of Korea

Objective: The purpose of this study was to assess the association of serum gamma-glutamyl transferase (GGT) and total bilirubin levels with metabolic syndrome.

Methods: The data used for this study was obtained from the Korean Genome and Epidemiology Study (KoGES)-Kangwha. In 2006 and 2007, We measured fasting serum GGT and total bilirubin levels and presence of metabolic syndrome for 975 men and 1670 women aged 40 years or older who lived in rural areas of Korea. Metabolic syndrome was defined by the modified criteria of National Cholesterol Education Program Adult Treatment Panel III (NCEP-ATPIII).

Results: According to the increasing number of metabolic abnormality, serum GGT concentration tended to increase (men, $p$ for trend $<0.001$; women, $p$ for trend $<0.001$ ), whereas serum total bilirubin concentration tended to decrease (men, $p$ for trend $=0.0353$; women, $p$ for trend $<0.001)$. After adjusting for age, smoking status, alcohol consumption, aspartate transaminase, alanine transaminase and total bilirubin, higher quartiles of serum GGT levels were associated with increased risk of metabolic syndrome in men and women; adjusted odds ratio (95\% CI) were 1.00 (reference), 1.09 (0.66-1.81), $2.21(1.33-3.67)$ and 2.28 (1.34-3.90) in men and 1.00 (reference), 1.65 (1.18-2.31), 2.36 (1.71-3.26) and $4.12(2.92-5.82)$ in women, respectively. However, higher quartiles of serum total bilirubin levels were associated with decreased risk of metabolic syndrome in women; adjusted odds ratio $(95 \% \mathrm{CI})$ were 1.00 (reference), 0.79(0.50-1.23), $1.00(0.64-1.56)$ and $0.78(0.50-1.23)$ in men and 1.00 (reference), 0.53 (0.39-0.71), 0.56 (0.41-0.77) and $0.55(0.41-0.75)$ in women, respectively.

Conclusions: Increased serum GGT and decreased serum total bilirubin levels were associated with metabolic syndrome in men and women. Hepatic enzymes could be used as clinical markers that identify metabolic abnormalities.

\section{PP39-61}

The Importance of Colonoscopic Screening in the Patients with "Cryptogenic" Pyogenic Liver Abscess - 7 Year Retrospective Analysis S.W. Jeong, J.Y. Jang, S.W. Hong, S.H. Lee, S.G. Kim, Y.K. Cheon, Y.S. Kim, Y.D. Cho, H.S. Kim, B.S. Kim Institute for Digestive Research, Department of Internal Medicine, Soonchunhyang University Hospital, Seoul, Republic of Korea

Background and aim: It has been believed that mucosal defects present on the colonic lesions or compromised mucosal barrier separating enteric bacteria from the circulation allows a route for bacteria invasion into the portal system with subsequent hematogenous spread to the liver. However, there is rare data about colonoscopic screening in patients with "cryptogenic" pyogenic liver abscess. We investigated retrospectively the relation of the colonic disease to the pyogenic liver abscess using colonscopic investigation.

Methods: A total of 230 patients who were diagnosed with pyogenic liver abscess between May 2003 and May 2010 were investigated retrospectively. Colonoscopic screening was performed at 3 to 4 weeks after diagnosis of pyogenic liver abscess. Colonoscopy evaluation was performed only to cryptogenic pyogenic liver abscess and not performed to patients who were untolerable to colonoscopy or refuse it.

Results: The causes of pyogenic liver abscess in 230 patients were pancreato-biliary disease $(n=135)$, cryptogenic origin including diabetes $\operatorname{mellitus}(n=79)$, and $\operatorname{others}(n=16$; metastatic liver 
cancer-7, appendicitis-5, HIV infection-1, post kidney transplantation -1 , prostate abscess-1, colon cancer with post operation-1). Thirty six patients of 79 patients with cryptogenic pyogenic liver abscess were performed with colonoscopic evaluation. In 36 colonoscopic screening, colon cancer was 5(13.9\%), lateral spreading tumor (LST) was $3(8.3 \%)$, colon polyp was $17(47.2 \%)$, diverticul was $2(5.6 \%)$. Mucosal defects and easy touch bleeding was noted in 4 patients with colon cancer, 3 patients with LST, and 2 patients with colonic adenoma.

Conclusion: Five patients (6.3\%) in 79 cryptogenic pyogenic liver abscess was colon cancer and 9 patients $(11.4 \%)$ exhibited mucosal defects in colonic lesions. Colonoscopic screening is essential for the detection of colonic origin in patients with cryptogenic pyogenic liver abscess.

\section{PP39-62}

Hepatobiliary Complications in Inflammatory Bowel Disease

N. Patel ${ }^{1}$, D. Amarapurkar ${ }^{2}$, M. Padaravat ${ }^{2}$, M. Choksi ${ }^{2}$, S. Joshi ${ }^{2}$, N. Bhatt ${ }^{2}$

${ }^{1}$ Jivandeep Hospital, Anand; ${ }^{2}$ Bombay Hospital and Medical

Research Centre, Mumbai, India

Introduction: Hepatobiliary manifestations are well known in inflammatory bowel disease (IBD). Exact prevalence of them in Indian patients is not well studied.

Methods: This prospective study was carried out on all the consecutive patients of IBD (ulcerative colitis[UC] and Crohn's disease[CD]) during study period from June 2005 to June 2010. All the patients were evaluated with clinical history- examination, complete blood count, liver biochemistry (including bilirubin, AST, ALT, GGTP, Alkaline phosphatase, proteins, LDH and prothrombin time) and ultrasonography to find out hepatobiliary abnormalities. In those with abnormal test results, further testing was carried out in form of HBsAg, AntiHCV, other viral markers, HOMA, lipids, ferritin, autoimmune hepatitis markers, protein electrophoresis, ceruloplasmin, CT scan, MRCP and liver biopsy as and when indicated.

Results: Of 644 UC patients (age $=34.1 \pm 14.2(6-83$ ) years; 402 males), 199 (30.9\%) patients had hepatobiliary abnormalities $n(\%)$ : elevated transaminases 76(11.8) [14 herbal drug-induced], fatty liver 35 (5.4), cryptogenic cirrhosis $3(0.46)$, autoimmune hepatitis 2 $(0.31)$, primary sclerosing cholangitis $4(0.62)$, hepatomegaly 17 (2.6), acute pancreatitis 3 (0.46) [2 azathioprine-induced], chronic pancreatitis $3(0.46)$, gall stone 10 (1.5), liver metastasis from unknown primary $2(0.31)$, hepatobiliary tuberculosis $1(0.15)$, hepatitis B 5 (0.77), hepatitis C $1(0.15)$, hepatitis E $1(0.15)$, liver hemangioma $2(0.31)$, simple liver cyst $2(0.31)$, gall bladder polyp 2 (0.31) and sepsis-related cholestasis 4 (0.62). Of $162 \mathrm{CD}$ patients $($ age $=40.6 \pm 11.2(6-82)$ years; 88 males $), 42(25.92 \%)$ patients had hepatobiliary abnormalities $n(\%)$ : elevated transaminases 6(3.7) [2 herbal drug-induced], fatty liver 14 (8.64) [3 methotraxate-induced], cryptogenic cirrhosis $2(1.23)$, autoimmune hepatitis 2 (1.23), hepatomegaly 11 (6.79), acute pancreatitis 1 (0.61) [azathioprine-induced], gall stone 10 (6.17) and liver abscess 1 (0.61).

Conclusions: More than one forth patients of IBD do have hepatobiliary abnormalities. Association with autoimmune liver disease needs special attention to prevent progressive liver damage. Presence of viral hepatitis is important to find out when immunosuppressant therapy is planned.

\section{PP39-63}

Hepatobiliary Complications in Celiac Disease

N. Patel ${ }^{1}$, D. Amarapurkar ${ }^{2}$, N. Bhatt ${ }^{2}$, M. Choksi ${ }^{2}$, S. Joshi ${ }^{3}$, M. Padaravat ${ }^{2}$

${ }^{1}$ Jivandeep Hospital, Anand; ${ }^{2}$ Bombay Hospital and Medical

Research Centre; ${ }^{3}$ Gastroenterology, Bombay Hospital and Medical

Research Centre, Mumbai, India
Introduction: Celiac disease (CD) is associated with nonspecific and autoimmune hepatobiliary manifestations. Exact prevalence is not well studied in Indian patients of CD.

Methods: This prospective study was carried out on all the consecutive patients of $C D$ during study period from June 2005 to June 2010. All the patients were evaluated with clinical history- examination, complete blood count, liver biochemistry (including bilirubin, AST, ALT, GGTP, Alkaline phosphatase, proteins, LDH and prothrombin time) and ultrasonography to find out hepatobiliary abnormalities. In those with abnormal test results, further testing was carried out in form of HBsAg, AntiHCV, other viral markers, HOMA, lipids, ferritin, autoimmune hepatitis markers, protein electrophoresis, ceruloplasmin, CT scan, MRCP and liver biopsy as and when indicated.

Results: Of $58 \mathrm{CD}$ patients (age $=32.5 \pm 16.2$ (4-68) years; 28 males), $23(39.6 \%)$ patients had hepatobiliary abnormalities $n(\%)$ : elevated transaminases 15 (25.8), fatty liver 6 (10.3), cryptogenic cirrhosis 1 (1.7), autoimmune hepatitis 1 (1.7), and gall stone 1 (1.7). Conclusions: More than one third patients of $\mathrm{CD}$ do have hepatobiliary abnormalities. Association with autoimmune liver disease needs special attention to prevent progressive liver damage.

\section{PP39-64}

Abnormal Liver Biochemistry in Vitamin B12 Deficiency

N. Patel ${ }^{1}$, D. Amarapurkar ${ }^{2}$

${ }^{1}$ Jivandeep Hospital, Anand; ${ }^{2}$ Gastroenterology, Bombay Hospital and Medical Research Centre, Mumbai, India

Introduction: Vitamin B12 deficiency (VB12D) is associated with hemolysis, which is evidenced on liver biochemistry by high indirect bilirubin, AST and LDH. Liver biochemistry in VB12D is not systematically studied.

Methods: This prospective study was planned on patients of VB12D during study period(June 2005-June 2010). VB12D was established on presence of megaloblastic anemia, high homocystiene and/or low vitamin B12 level. Cause of VB12D was established. All the patients were evaluated with clinical history- examination, complete blood count, liver biochemistry (including bilirubin, AST, ALT, GGTP, Alkaline phosphatase, proteins, LDH and prothrombin time) and ultrasonography. In those with abnormal test results, further testing was carried out in form of $\mathrm{HBsAg}$, AntiHCV, other viral markers, HOMA, lipids, ferritin, autoimmune hepatitis markers, protein electrophoresis, ceruloplasmin, CT scan, MRCP and liver biopsy as and when indicated to establish etiology of liver disease. Patients with gastrointestinal disease which can cause liver abnormalities or known co-existing liver disease were excluded. All the patients were treated with vitamin B12 supplementation. ALT-AST was obtained at 1 month of follow up.

Results: Of 1962 VB12D patients, 452 patients were excluded (36 hepatitis B, 4 hepatitis C, 122 non-alcoholic fatty liver disease, 20 cryptogenic cirrhosis, 2 autoimmune hepatitis, 110 alcoholic liverpancreatic disease, 10 pernicious anemia, 15 celiac disease, 37 Crohn's disease, 42 tuberculosis, 4 intestinal surgery, 50 HIV). Cause of VB12D was nutritional in $1,170(77.4 \%)$ and tropical sprue in 340 $(22.5 \%)$ patients. Of 1,510 patients, 380 (25.2\%) patients had abnormal liver biochemistry. ALT > AST was present in 342 (90\%), whereas AST > ALT in $38(10 \%)$ patients (of which 35 patients had evidence of ongoing hemolysis). Liver biopsy in 6 patients showed near-normal histology. In $362(95.2 \%)$ patients, ALT-AST normalized within first month of therapy.

Conclusions: Around one forth patients of VB12D do have abnormal liver biochemistry. ALT elevation in VB12D responds promptly to vitamin B12 supplementation. 


\section{PP39-65}

Adipocytokines and Steatosis in Chronic Hepatitis B and C

G.T. Kumar ${ }^{1}$, S.B. Rooge ${ }^{1}$, S.S. Hissar ${ }^{2}$, A. Rastogi ${ }^{3}$, A. Kumar ${ }^{2}$, R. Gondal ${ }^{1}$, S.K. Sarin ${ }^{2}$, P. Sakhuja ${ }^{1}$

${ }^{1}$ Pathology, G.B.Pant Hospital; ${ }^{2}$ Gastroenterology; ${ }^{3}$ Pathology,

ILBS, New Delhi, India

Introduction: Adipocytokines are adipose tissue-derived secretary proteins. Obesity and visceral fat accumulation impair adipocyte function and adipocytokine secretion. Apart from NASH, CHB and $\mathrm{CHC}$ are also frequently associated with hepatic steatosis which may be related to viral or host metabolic factors and may have altered adipocytokine levels influencing histologic activity and fibrosis progression.

Aim: We aimed to assess the prevalence of steatosis its relationship and correlation with biochemical, metabolic, and histologic parameters in patients with $\mathrm{CHB}, \mathrm{CHC}$ and NASH.

Patients and methods: Well characterized patients of CHB (70), CHC (46), NASH (7), and HC (13) were included in the study. ELISA for an Adiponectin $(n=116)$, Leptin $(n=136)$, Resistin $(n=104)$ and IL-6 $(n=101)$ was randomly performed in all the groups.

Results: As expected steatosis percent was found to be greatest in NASH followed by HCV, then HBV. Steatosis percent was significantly higher with raised BMI $\left(\geq 23 \mathrm{Kg} / \mathrm{m}^{2}\right)$ in CHB $(P=0.004)$ but not in CHC. The histologic activity and fibrosis scores were significantly higher in $\mathrm{CHC}$ than $\mathrm{CHB}$. This was consistent with higher steatosis in CHC as compared to HBV. In CHB and CHC the histologic activity scores and fibrosis stage were higher with higher steatosis. Patients with CHB showed significant correlation of serum Leptin levels with HBV DNA $(P=0.029)$ and Fibrosis $(P=0.005)$. Patients with $\mathrm{CHC}$ showed significant correlation of serum adiponectin levels with Triglyceride $(P=0.001)$, while serum IL-6 levels with Fibrosis $(P=0.045)$ and steatosis $(P=0.002)$ and serum resistin levels with cholesterol $(P=0.008)$ and steatosis $(P=0.044)$. $70 \%$ of patients with $\mathrm{CHC}$ had genotype 3 .

Conclusion: Steatosis may also be seen in patients with $\mathrm{CHB}$ and $\mathrm{CHC}$ and correlates with increased histologic activity and fibrosis. The altered adipocytokine levels influenced metabolic factors mostly fibrosis and steatosis in chronic viral hepatitis.

\section{PP39-66}

Cystatin C: A Predictor of Hepatorenal Syndrome in Patient with HCV Related Liver Cirrhosis

E. Shawky ${ }^{1}$, M. Sharawy ${ }^{1}$, L. Hamdy ${ }^{2}$, H. Hassan ${ }^{1}$, A. Ali ${ }^{1}$, Y. Fouad ${ }^{3}$

${ }^{1}$ Internal Medicine; ${ }^{2}$ Clinical Pathology; ${ }^{3}$ Tropical Medicine, Minia University, Minia, Egypt

Background: Recent studies suggest that serum cystatin C (CysC) is a more sensitive marker of renal functions serum creatinine.

Aim: Evaluation of the clinical significance of cystatin $\mathrm{C}$ in patients with liver cirrhosis, ascites and normal serum $\mathrm{Cr}$ level as a predictor of hepatorenal syndrome.

Methods: Eighty patients with cirrhotic ascites were enrolled in the study (53 men and 27 women; age, $59.5 \pm 7.5$ years). All patients were subjected to full clinical assessment and laboratory investigations focussing on renal functions and glomelular filtration rate and measurement of serum Cystatin level.

Results: All 80 patients were in Child-Pugh classes C. Serum $\mathrm{Cr}$ and CysC levels were $1.04 \pm 0.1$ and $1.8 \pm 0.8 \mathrm{mg} / \mathrm{L}$, respectively. The serum CysC level was weakly associated with the serum $\mathrm{Cr}$ level (Spearman's correlation coefficient, 0.22). HRS developed in 18 patients during the follow-up period $(6$ months $)$. Age $(p<0.001)$, Albumin $(p<0.001)$, Sodium $(p<0.005)$, Cystatin C $(p<0.001)$, and e-GFR $\mathrm{MDRD}_{\mathrm{M}}(p<0.007)$ were significant dependent predictive factor for the development of HRS. The CysC level was the most independent predictive factor for $\mathrm{HRS}(\mathrm{OR}, 2.1$; 95\% CI, 0.75-0.97; $P<0.002)$. Eighteen patients died during the follow-up period. Age $(p<0.001)$, INR $(p<0.001)$, e-GFR MDRD $(p<0.03)$, sodium $(p<0.01)$, meld score $(p<0.05)$, albumin $(p<0.001)$ and CysC $(p<0.001)$ levels were significant dependent factors for predicting mortality . Cys C (OR, 5.3; $P<0.006$ ) level and INR (OR, 1.01; $P<0.006)$ were the most independent factors for predicting mortality. Conclusion: Serum CysC level may be considered a predictor of hepatorenal syndrome and mortality in patients with cirrhotic ascites.

\section{PP39-67}

Hepatitis C Virus (Genotype 4)-Associated Mixed Cryoglobulinemia Vasculitis: Effects of Antiviral Treatment

H.R. El Khayat ${ }^{1}$, Y.M. Fouad ${ }^{2}$, E. Ahmed ${ }^{3}$, H. El Amin ${ }^{4}$, A. Rizk ${ }^{5}$, F. Esmael ${ }^{6}$ ${ }^{I}$ Gastroenterology, Theodor Bilharz Research Institute, Cairo;

${ }^{2}$ Tropical Medicine, Minia University, Minia; ${ }^{3}$ Gastroenterology, Alexandria University, Alexandria; ${ }^{4}$ Internal Medicine, Assiut Faculty of Medicine, Assiut University, Assuit; ${ }^{5}$ Rheumatology, Cairo University, Cairo; ${ }^{6}$ Rheumatology, Minia University, Minia, Egypt

Objective: Type II mixed cryoglobulinemia (MC) is a systemic vasculitis usually associated with hepatitis $\mathrm{C}$ virus (HCV), which may involve the skin, kidneys, and nervous system. The present trial was established to investigate the efficacy of therapy with Pegylated interferon alfa-2a (PEG-IFN alfa-2a) plus ribavirin in patients with $\mathrm{HCV}$-related $\mathrm{MC}$ vasculitis and evaluate the factors associated with clinical remission of MC.

Methods: Forty-six consecutive patients with $\mathrm{HCV}$ related MC received PEG-IFN alfa-2a (standard dose $180 \mathrm{mg} /$ week) subcutaneously plus oral ribavirin (800-1,200 mg/day) for 1 year. The response to treatment was analyzed by comparing clinical, immunologic, and virologic parameters at the initial evaluation with those observed during follow up. Logistic regression was used to assess the factors associated with clinical remission.

Result: Twenty two patients (48\%) had a sustained virologic response and were complete clinical responders. Serum cryoglobulin disappeared in 20 of 46 patients (43\%), and complement levels normalized in $70 \%$ of the patients. One patient had a partial virologic response with a complete clinical response. In another patient, a clinical relapse of MC vasculitis occurred in association with the reappearance of HCV RNA. Treatment was well tolerated, and no patient had side effects for which discontinuation of therapy was required.

Conclusion: Treatment with PEG-IFN alfa-2a plus ribavirin can achieve a complete clinical response in patients with HCV-related MC. vasculitis. Complete clinical response correlates with the eradication of HCV.

\section{PP39-68}

Role of MTHFR Gene Mutation and Altered Homocysteine Levels in Predisposition to Hepatobiliary Anomalies

B. Baruah ${ }^{1}$, D. Tiwari ${ }^{1}$, M. Ghaznavi ${ }^{1}$, M. Bose ${ }^{1}$, S. Bose ${ }^{1}$, A. Saikia ${ }^{2}$, R. Deblakshmi ${ }^{1}$, M. Sharma ${ }^{1}$, M. Deka ${ }^{1}$ ${ }^{1}$ Department of Biotechnology, Gauhati University; ${ }^{2}$ Department of Gastroeterology, Central Railway Hospital, Maligaon, Guwahati, India

Alterations in the genes involved in the folate pathway, may result in the predisposition to high disease risk of different etiologies.

Aim: Evaluate genetic alteration in MTHFR gene involved in folate metabolism and altered plasma homocysteine levels in predisposing patients to hepatobiliary anomalies.

Methods: Hepatobiliary cases $\{$ acute liver disease $(n=10)$, chronic liver disease $(n=10)$, cholelithiasis $(n=20)$, cholecystitis $(n=20)$, 
and CaGB $(n=10)\}$ admitted to Central Railway Hospital, Guwahati, and age and sex matched community controls $(n=30)$ were enrolled for the study. Genomic DNA was extracted from blood, and MTHFR677C-T genotyping was performed by PCR-RFLP method using HinfI digestion. Plasma homocysteine levels were determined by chemiluminescence's method.

Results: Prevalence of MTHFR677C-T mutant genotypes was higher in $\operatorname{CaGB}(n=9 / 10,90 \%)$ and chronic liver disease cases $(n=9 / 10$, $90 \%)$ compared to acute liver disease $(n=8 / 10,80 \%)$, cholelithia$\operatorname{sis}(n=7 / 20, \quad 35 \%), \quad$ cholecystitis $\quad(n=11 / 20, \quad 55 \%) \quad$ and $\operatorname{controls}(n=7 / 30,23.33 \%)$. Presence of mutant genotype non-significantly increased the risk of cholelithiasis and cholecystitis. Presence of mutant genotype increased CaGB risk in cholelithiasis patients significantly $\{\mathrm{OR}=13.0(1.319-128.106), p=0.033\}$. The risk of acute $\{\mathrm{OR}=12.0(0.773-186.362)\}$ and chronic liver disease $\{\mathrm{OR}=11.0(1.697-71.282)\}$ was higher in presence of mutant genotype compared to controls. Wilcoxon-Signed-Ranks test of correlation showed that homocysteine levels was significantly higher in the MTHFR mutated cases $(p=0.012)$. Elevated level of homocysteine was found for both MTHFR homozygous $(p=0.026)$ and heterozygous mutant $(p<0.001)$ cases; which correspond to the moderate risk group for homocysteine levels. Interestingly, the homocysteine levels were higher for wild type $\mathrm{CaGB}$ cases compared to wildtype of other groups.

Conclusion: MTHFR gene mutation and its influence on homocysteine levels predispose patients to hepatobiliary diseases and even play a role in disease progression and severity.

\section{PP39-69}

\section{Status of Viral Hepatitis in North India}

A. Singh, S. Singh, M.A. Ansari, P. Nag, M. Irshad

Laboratory Medicine, All India Institute of Medical Sciences,

New Delhi, India

Background/aim: Viral hepatitis is a major health problem and is an important cause of morbidity and mortality throughout the world. Present study is aimed to assess the etiological spectrum of viral hepatitis in different forms of liver diseases in north India.

Methods: A total number of 370 adult patients from various liver diseases were analyzed for different hepatitis viral marker including hepatitis virus A (IgM anti-HAV), hepatitis virus B (HBsAg and IgM anti-HBc), hepatitis virus $\mathrm{C}$ (IgG anti-HCV, HCV core-Ag and HCVRNA), hepatitis virus D (IgM anti-HDV) and hepatitis virus E (IgM anti-HEV) infections.

Results: The results of this study demonstrate Hepatitis A virus (HAV) infection to be rare $(8.1 \%)$ in acute viral hepatitis (AVH) cases. Hepatitis B virus (HBV) and hepatitis C virus (HCV) infections, as the predominant causes of acute liver diseases, were detected in 12.3 and $10.6 \%$ cases of AVH respectively. Both HBV and $\mathrm{HCV}$ were reported in chronic liver diseases in more than $36 \%$ cases. Similarly, Hepatitis E virus (HEV) infection is quite common in acute viral hepatitis patients (25.3\%). Hepatitis E virus (HEV) infection in fulminant hepatic failure patients was found in $44.4 \%$ cases. Hepatitis D virus (HDV) infection could not be detected in these patients.

Conclusion: The data from this study indicates that HAV infection to be rare in adult population. HBV and $\mathrm{HCV}$ are the major cause of acute and chronic liver diseases. HEV infection is an important cause of acute liver diseases. HDV infection is quite uncommon in India. Acknowledgement: We thank ICMR for the financial grant to conduct the study.
PP39-70

Anti-thyroid Auto-antibodies in Egyptian Patients with Chronic HCV Infection: The Relation to Human Leucocyte Antigens

Y.Z. Gad

Internal Medicine, Mansoura University, Mansoura, Egypt

Background/aim: The role of hepatitis $\mathrm{C}$ virus (HCV) have been demonstrated in many autoimmune diseases; including the autoimmune thyroid disease. The exact mechanism of development of a such extra-hepatic autoimmune phenomenon is still unknown.

This work was to study the prevalence of anti-thyroid auto-antibodies in patients with chronic $\mathrm{HCV}$ infection and their possible relation to the human leukocyte antigen (HLA) status.

Patients and methods: Sera from, 147consecutive patients (75 males and 72 females) with chronic HCV infection and prepared for INF therapy at the Mansoura University Hospitals during 2008-2009, were analyzed.

The detection of HCV RNA in sera was performed by the nested polymerase chain reaction (PCR) technique. Thyroid microsomal and thyroglobulin autoantibodies were determined by the hemagglutination tests. Ig-G type anti-GOR were measured using an ELISA assay. HLA$\mathrm{A},-\mathrm{B},-\mathrm{C}$, and -DR were determined using the standard complementdependent microdroplet lymphocyte cytotoxicity test. HLA allele frequencies of $126 \mathrm{HCV}$-infected individuals also served as controls.

Results: Anti-thyroid antibodies were detected in $21 \mathrm{HCV}$ RNA positive patients [group 1:(18) females, (3) males] and remaining 126 patients [group 2;(72) males and (54) females].

Comparing prevalences in the studied groups, a statistically significant difference was observed regarding anti-GOR antibodies in group $1(p<0.001)$, anti-thyroid auto-antibodies among the studied females $(p<0.001)$ and HLA-A2 antigen in group $1(<0.05)$.

In conclusion: Our data revealed that HLA-A2 may be regarded as an immunologic risk factor for the development of anti-thyroid autoantibodies in patients with chronic HCV infection and these autoantibodies should be evaluated prior to initiating INF- $\alpha$ therapy.

\section{PP39-71}

Spectrum of Liver Disease in Two Tertiary Care Centers of Western India N. Patel $^{1}$, D. Amarapurkar ${ }^{2}$, S. Joshi ${ }^{2}$, M. Padaravat ${ }^{2}$

${ }^{1}$ Jivandeep Hospital, Anand; ${ }^{2}$ Bombay Hospital and Medical Research Centre, Mumbai, India

Introduction: Epidemiological data on spectrum of liver disease is sparsely reported from India.

Methods: Retrospective analysis of case records (1995-2009) was carried out to find out etiological spectrum liver disease in two tertiary care centers of western India. Cases with inadequate information or diagnosis were excluded. In 2010, etiological spectrum was prospectively defined.

Results: A total of 11,604 patients (3,219 females, 2,150 acute disease, 688 pediatric age group $[<18$ years], age $=43.2 \pm 22.4$ years) were included in retrospective analysis. A total of 647 patients ( 175 females, 136 acute disease, 53 pediatric age group, age $=46.2 \pm 18.6$ years) were included in prospective analysis. Commonest etiologies of each category are tabulated in descending order. (Abbreviations: $\mathrm{HAV}=$ hepatitis $\mathrm{A}, \mathrm{HEV}=$ hepatitis $\mathrm{E}, \mathrm{HBV}=$ hepatitis $\mathrm{B}$, $\mathrm{HCV}=$ hepatitis $\mathrm{C}$, DILI $=$ drug induced liver injury, ALA = amebic liver abscess, $\mathrm{AH}=$ acute hepattis, NAFLD = non-alcoholic fatty liver disease, $\mathrm{ALD}=$ alcoholic liver disease, $\mathrm{AIH}=$ autoimmune hepatitis, $\mathrm{HCC}=$ hepatocellular carcinoma, $\mathrm{BCS}=$ Budd Chiari syndrome, EHPVO = extrahepatic portal vein obstruction) Table 1 
Table 1

\begin{tabular}{|c|c|c|c|}
\hline Category & Subcategory & $\begin{array}{l}\text { Retrospective } \\
\text { analysis }\end{array}$ & $\begin{array}{l}\text { Prospective } \\
\text { analysis }\end{array}$ \\
\hline $\begin{array}{c}\text { Acute liver } \\
\text { disease }\end{array}$ & Pediatric & $\begin{array}{l}\mathrm{HAV}, \mathrm{HEV}, \\
\text { hemolytic anemia }\end{array}$ & $\begin{array}{l}\text { HAV, undetermined } \\
\text { AH, HEV }\end{array}$ \\
\hline $\begin{array}{c}\text { Acute liver } \\
\text { disease }\end{array}$ & Adult & $\begin{array}{l}\mathrm{HEV}, \text { ALA, HAV, } \\
\text { malaria, DILI }\end{array}$ & $\begin{array}{l}\mathrm{HEV}, \mathrm{HBV}, \mathrm{HAV}, \\
\text { undetermined AH, } \\
\text { ALA, malaria }\end{array}$ \\
\hline $\begin{array}{c}\text { Acute liver } \\
\text { disease }\end{array}$ & Male & $\begin{array}{l}\text { HEV, ALA, } \\
\text { undetermined AH, } \\
\text { HAV, CBD calculi }\end{array}$ & $\begin{array}{l}\mathrm{HEV}, \mathrm{HBV}, \\
\text { undetermined } \mathrm{AH}\end{array}$ \\
\hline $\begin{array}{c}\text { Acute liver } \\
\text { disease }\end{array}$ & Female & $\begin{array}{l}\mathrm{HEV}, \mathrm{HAV}, \\
\quad \text { undetermined } \mathrm{AH}\end{array}$ & $\mathrm{HEV}, \mathrm{HBV}, \mathrm{HAV}$ \\
\hline $\begin{array}{l}\text { Chronic liver } \\
\text { disease }\end{array}$ & Pediatric & $\begin{array}{l}\text { WD, NAFLD, } \\
\text { EHPVO }\end{array}$ & HBV, AIH, BCS \\
\hline $\begin{array}{l}\text { Chronic liver } \\
\text { disease }\end{array}$ & Adult & $\begin{array}{r}\text { NAFLD, HBV, } \\
\text { ALD, HCV, } \\
\text { Cryptogenic }\end{array}$ & $\begin{array}{c}\text { ALD, NAFLD, HBV, } \\
\text { HCV, cryptogenic }\end{array}$ \\
\hline $\begin{array}{l}\text { Chronic liver } \\
\text { disease }\end{array}$ & Male & $\begin{array}{l}\text { HBV, ALD, } \\
\text { NAFLD, HCV, } \\
\text { cryptogenic }\end{array}$ & $\begin{array}{l}\text { ALD, HBV, } \\
\text { NAFLD, HCV }\end{array}$ \\
\hline $\begin{array}{l}\text { Chronic liver } \\
\text { disease }\end{array}$ & Female & $\begin{array}{l}\text { NAFLD, HBV, } \\
\text { HCV, cryptogenic, } \\
\text { AIH }\end{array}$ & $\begin{array}{c}\text { NAFLD, HCV, } \\
\text { HBV, AIH }\end{array}$ \\
\hline $\begin{array}{l}\text { Space occupying } \\
\text { lesion in } \\
\text { liver }\end{array}$ & - & $\begin{array}{l}\text { ALA, HCC, } \\
\text { hemangioma }\end{array}$ & $\begin{array}{l}\text { ALA, HCC, liver } \\
\text { metastasis }\end{array}$ \\
\hline
\end{tabular}

Conclusions: There is age-wise and gender-wise different spectrum of etiology in liver diseases. In hepatology practice, chronic liver diseases form major part, which lead to great economic burden. Strategies to prevent chronic liver disease should be developed.

\section{PP39-72}

Dynamics Analysis of an HBV Infection Model and Prediction of Longterm Therapy Results

Y. Zheng ${ }^{1}$, L. $\mathrm{Min}^{2}, \mathrm{Y} . \mathrm{Ye}^{3}$

${ }^{1}$ Information Engineering School; ${ }^{2}$ Mathematics and Physics School, University of Science and Technology Beijing; ${ }^{3}$ Traditional Chinese International Medicine Key Laboratory of China, Capital Medical University, Beijing, China

Background: In an international co-operation study on anti-HBV infection treatment reported by G. K. Lau et al., one group of chronic HBV patients were assigned to receive peginterferonalfa- $2 a$ once weekly plus $100 \mathrm{mg}$ of lamivudine once daily. The study was comprised of 48 weeks of treatment and 24 weeks of treatment-free follow-up. Based on an anti-virus infection model (AITMM) for patients with abnormal ALTs (see Y.Zheng, L.Min, X.Chen, Y.Ye, Hepatology International, 4(1), (2010), p. 52), We chose the above patients' mean HBV DNA data for simulating.

Methods: To describe the dynamics of anti-HBV infection treatment for above patients, a basic virus infection model with an additional immune variable (AITMM) is used. We show that if the basic infection reproductive number of the AITMM is $\mathrm{R} 0<1$, then the infection-free equilibrium of the AITMM is globally attractive.

Results: The number simulation (solid lines)of the treatment model in which the clinical data are marked by dots.

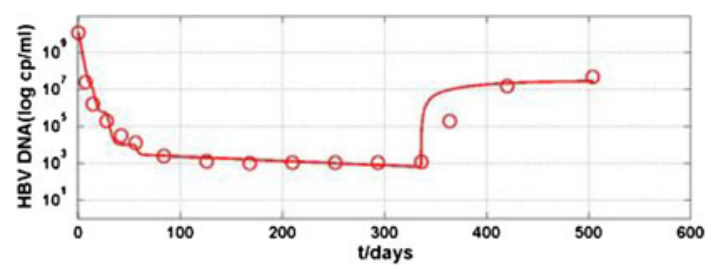

Observe that the dynamics can describe the rapid reduction and relapse of patients' mean HBV DNA levels.

Conclusions: This result implies that an infected individual with $\mathrm{R} 0<1$ will eventually recover even if infected with a large amount of virus. A clinical numerical simulation result shows that 11 years of treatment is required to clear the hepatitis B virus from all hepatocytes.

Acknowledgments: This work is jointly supported by the 11th 5Year Plan Key Research Project of China (No. 2008ZX10502-006) and the NNSF of China (No. 61074192).

\section{PP39-73}

Prevalence of Hepatitis B and C Co Infection among Ahwaz Blood Donors in $2006-2010$

S.M. Sajjadi ${ }^{1}$, M.A. Jalali $\mathrm{Far}^{2}$, G.A. Kaydani ${ }^{3}$, J. Torabi ${ }^{4}$

${ }^{I}$ Medical Laboratory Science, Paramedical School, Birjand University of Medical Sciences, Birjand; ${ }^{2}$ Medical Laboratory

Science Dep. Paramedical School, Ahwaz, Jondishapour University of Medical Sciences, Thalasemia and Hemoglubinopathy Center;

${ }^{3}$ Ahwaz, Jondishapour University of Medical Science; ${ }^{4}$ Iranian Blood Transfusion Organization Research Centre (IBTO), Ahwaz, Iran

Hepatitis B and C are the most viral infection that infected human in all worldwide. Globally, it's estimated that 1-2 million deaths per year as a consequence of the HBV infection and $20 \%$ of HCV patients progress to liver cirrhosis with an increased risk for the development of hepatocellular carcinoma. The HCV-HBV co infected patients are more likely to progress to liver cirrhosis.

In this cross sectional descriptive, we study the prevalence of HCV-HBV co infection among the 1,128 initially reactive for HBsAg and 1,236 samples of blood donors that admitted to Ahwaz blood transfusion service during March 2008-2010. The confirmatory tests were done for repeatedly reactive samples based on neutralization tests and RIBA, respectively. The data analyzed by using SPSS.

We found 625 and 270 blood donors confirmed for HBs-Ag and HCV-Ab, respectively. We found $0.45 \%$ (4/895) samples co infected with $\mathrm{HCV}$ and HBs-Ag.

According to our findings, because the success in selection low risk donors, the rate of confirmed samples among initially samples was less and the HBV-HCV co infected people are significantly less than other population and study. Counseling the infected donors to prevent the transmission that infections is recommended.

PP39-74

Safety of Anti Tumor Necrosis Factor- $\alpha$ Therapy in Psoriatic Patients with Hepatitis B Virus (HBV) Infection

L. Nosotti ${ }^{1}$, A. Morrone ${ }^{1}$, C. Bonifati ${ }^{2}$, E. Berardesca ${ }^{2}$

${ }^{1}$ National Institute for Health, Migration and Poverty; ${ }^{2}$ San Gallicano Institute, Roma, Italy

Background: The risk of reactivation of hepatitis B virus (HBV) replication in inactive hepatitis surface antigen (HBsAg) carriers undergoing therapy with anti-TNF- $\alpha$ drugs is well known. The 
majority of cases of $\mathrm{HBV}$ reactivation during anti-TNF- $\alpha$ therapy has been reported in HBsAg carriers treated for diseases such as: rheumatoid arthritis, ankylosing spondilitis or Crohn's disease. At the moment no data is available on the safety of TNF- $\alpha$ inhibitors in psoriatic subjects with occult HBV infection.

Methods: The present study reports our experience with psoriatic patients undergoing anti-TNF- $\alpha$ therapy found to be HBV-infected. Since October 2006 at our Department all consecutive patients (130 subjects) with psoriasis candidates to receive immunosuppressive therapy underwent an evaluation of HBV markers.

Results: We found 6 potential occult HBV carriers and 1 inactive HBV carrier. The patients received anti-TNF- $\alpha$ therapy with etanercept or adalimumab. To verify a possible HBV reactivation after starting the anti-TNF- $\alpha$ therapy, the 6 potential occult carriers were evaluated for HBsAg, HBV DNA, AST and ALT every 3 months. During the periods considered, none of the potential occult carriers showed the appearance of HBsAg or detectable amounts of HBV DNA in the serum. With regard to patient 7 (inactive carrier), we used a prophylaxis with lamivudine throughout the treatment period considered.

Conclusions: Our data indicate that for psoriatic patients who are also either occult or inactive carriers, it is safe to administer anti-TNF- $\alpha$ drugs.In particular, occult HBV carriers can simply be monitored with HBsAg determinations every 3 months during the treatment and continued with the same timing for the subsequent 6 months after stopping anti-TNF- $\alpha$ therapy. Concerning HBV inactive carriers, prophylaxis with lamivudine should be started 2 weeks before the anti-TNF- $\alpha$ therapy, continued during the course of treatment and extended for 6-12 months after therapy to prevent HBV flares due to immune reconstitution.

\section{PP39-75}

Clinical Features and Comprehensive Diagnosis of Autoimmune Pancreatitis

L. Wu, W. Li

Department of Gastroenterology \& Hepatology, General Hospital of the Chinese People's Liberation Army, Beijing, China

Background and Aims: The research on autoimmune pancreatitis (AIP) were mainly reported in European countries, United States and Japan. However, there was little case reported in China and had high misdiagnosis. It is of great importance to raise awareness of the characteristics of AIP and to avoid misdiagnosis and even mistreatment. This study is to search the key factor to avoid misdiagnosing AIP as pancreatic carcinoma.

Methods: Clinical, imaging,laboratory and pathological data about AIP were collected and analyzed from the General Hospital of the Chinese People's Liberation Army from 1995 to 2009.

Results: 33 patients (male 29, female 4) aged 35 to 76 years (mean $52.3 \pm 9.3$ years) were diagnosed as AIP. The misdiagnosis rate was as high as $95.7 \%(22 / 23)$ with a mis-laparotomy rate $91.3 \%(21 / 23)$ before 2005. After 2005, however, AIP became awareness and the misdiagnosis rate significantly decreased to $20.0 \%(2 / 10)(P=0.000)$ with a mis-laparotomy rate $0.0 \%(P=0.000)$. The major symptom is jaundice in 24/33 (72.7\%) and abdominal pain in 12/33 (36.4\%). A half of the patients were accompanied with other autoimmune disorders. Laboratory test showed elevated level of serum $\operatorname{IgG}$ in $9 / 11$ $(81.8 \%)$ and increased eosinophil count in 16/33 (48.5\%). CT scan showed a low-contrast margin characterized as a "banana peel-like" rim in 24/32 (75.0\%). Pathologic examination showed fibrosis and massive lymphocytes and plasma cells infiltration in pancreas,salivary glands, and hepatic. Prednisone was given to the patients and 12/12 $(100 \%)$ of them had very good response.

Conclusions: AIP seems to be a systemic autoimmune disease rather than an isolated disorder, markedly overlapping with other autoimmune diseases. Awareness-raising is the key to avoid misdiagnosing AIP as malignant diseases.

\section{PP39-76}

Protective Properties of Laggera alata Extract and its Principle Components against D-Galactosamine-induced Hepatocyte Damage

Y.-H. Wu ${ }^{1}$, B.-J. Hao ${ }^{1}$, E. Shen ${ }^{2}$, Q.-L. Meng ${ }^{1}$, M.-H. Hu ${ }^{3}$, Y. Zhao ${ }^{3}$

${ }^{I}$ Department of Pharmacy, College of Life Sciences, China Jiliang

University; ${ }^{2}$ Zhejiang Provincial People's Hospital; ${ }^{3}$ Zhejiang University, Hangzhou, China

Background/aims: Laggera alata, as a traditional chinese medicine, has been used for over three hundred years, especially for the treatment of some ailments associated with hepatitis. The aim of this work was to investigate hepatoprotective effect of $L$. alata and its substance basis responsible for the protective potential.

Methods: L. alata extract (LAE) was quantitatively analyzed and its principle components isochlorogenic acids were isolated and authenticated. Protective properties of LAE were studied using a dgalactosamine (d-GalN)-induced injury model in neonatal rat hepatocytes and a d-GalN-induced acute liver damage model in mice. Meanwhile, the effect of isochlorogenic acids (4,5-O-dicaffeoylquinic acid, 3,5- $O$-dicaffeoylquinic acid and 3,4- $O$-dicaffeoylquinic acid) derived from $\mathrm{LAE}$ on $\mathrm{d}-\mathrm{GalN}$-induced hepatocyte injury were also measured in vitro.

Results: LAE at concentrations of $10-100 \mu \mathrm{g} / \mathrm{ml}$ significantly reduced cellular leakage of aspartate aminotransferase (AST) and alanine aminotransferase (ALT) and improved cell viability. And isochlorogenic acids at concentrations of $1-100 \mu \mathrm{g} / \mathrm{ml}$ also improved remarkably viability of hepatocyte. The oral treatment of LAE at doses of 50, 100 and $200 \mathrm{mg} / \mathrm{kg}$ markedly reduced the serum AST and ALT activity of mice and resulted in significant recovery of hepatocytes in liver sections.

Conclusions: The study firstly verifies the potent hepatoprotective effect of $L$. alata extract containing isochlorogenic acids against dGalN-induced hepatocyte injury in vitro and in vivo. Isochlorogenic acids may be its substance basis responsible for the hepatoprotective potential. Hepatoprotective effect of LAE is probably associated with scavenging free radicals to ameliorate oxidate stess. These data provide a scientific explanation for the folkloric uses of $L$. alata in the treatment of some ailments associated with inflammation including hepatitis.

Acknowledgements: This work was supported by the National Natural Science Foundation of China (Grant No. 30701049), the Experimental Animal Science and Technology Project of Zhejiang Province (Grant No. 2008F800169) and the foundation from zhejiang university.

\section{PP39-77}

Development of Newer Hepatitis-vaccines and Ethical Issues: NGO Efforts to Protect Human Rights of Hepatitis Patients

P. Sankpal

Community Medicine, Health Alert Organsiation of India [NGO], Dhule, India

Issue/argument: Asia has rising cases of hepatitisB/C. Alcohol/ Food-habits cause high prevalence in rural/tribal areas. Lack of monitoring/follow-up complicates management. vaccines emerge as hope. Clinical-trials of vaccines debated-issue. Design of hepatitisvaccine-trials in developing-countries complex ethical-issue. We focus on controversies identified in international/regional/local CME/ pharma programs as vulnerability of volunteers to exploitation by foreign/local research-groups/funding-agencies. Critical task is protect interests of vaccine-subjects in face of substantial-risks. Determine if hepatitis-Vaccine-volunteers will have access to treatment during trial. Access to Vaccine-trial-outcome. Interaction with 
seniors at 21st APASL-Congress will give voice to such burningissues.

Methodology: Researchers/pharmaceuticals/Govt-policy planners need to develop forum to solve these problems. NGO's can play pivotal role. Obligation on part of researchers to create mechanisms to offset anticipated risks of participation in controversial, Risky vaccine-development.

Conclusion: Counselling/right to withdraw from trial be made basic guideline. Apart from monetary aspects unsuspected adverse reactions/deaths be properly evaluated/monitored. Researchers need to evolve policy-guidelines to overcome barriers as variation in interpretation of essential ethical ideas, legal-system-differences, educational/economic-status. Need to develop common consensus between research-community/pharma sector to reduce suffering of hepatitis-affected patients community.

Recommendations: Researchers/NGOs should come together at 21st APASL-congress platform to form workgroup to settle these issues. We shall raise our this burning issue \& present HepatitisPrevention-Advocacy plan of our NGO graphically to APASL2011participants.

\section{PP39-78}

Improving QOL Hepatitis Patients in Resource Poor Developing Nations: Supportive Care Efforts by an Non-govt-Organisation [NGO] in Rural/ Tribal India

P. Sankpal

HIV Field Projects, Health Alert Organsiation of India [NGO], Dhule, India

Issues: Fatigue/sexual-dysfunction/depression commonly seen in Hepatitis-sufferers. Most patients of hepatitis treated symptomatically. Active treatment very expensive. Palliative-care inaccessible in rural/tribal areas. Hence our NGO-nurses took initiatives to improve QOL with alternative-medicines since October 2005.

Objective: Around 112 patients die each-year from Hepatitis. Statistically over $90 \%$ express sexual-dysfunction, $68 \%$ experience fatigue/weakness; $70 \%$ suffer social neglect/humiliation; $54 \%$ sleeplessness, nausea/vomiting; 64\% depression. Our NGO-nurses followed-up Hepatitis-sufferers unable to afford Rx or who needed of palliative care.

Methods: 81 patients [58 hepatitis-B and 23 hepatitis-C] included. After 14 weeks-Rx-with alternative-medicines. Counseling/palliative support with nutrition, we noted QOL improved to statistically. Assessment performed on weekly-basis. Traditional-faith-healers involved for giving traditional Indian-medicinal-plant extracts [noncommercial].

Results: 53\% patients expressed that alternative-medicines-Rx most important factor to cope with hepatitis. higher scores of QOL (ANOVA $p<0.001$ ) correlated with alternative-medicines-Rx. Our NGO-initiative suggests that over $70 \%$ patients will need well trained specialist for home-based-care unit.

Conclusions: Life-span/QOL of Hepatitis-sufferers depends on appropriate-palliative-care. NGO-personals should be trained in $\mathrm{Pal}-$ liative-care-services. Our data is being used for palliative care advocacy. Field of Spiritual/psycho-social/community support is fertile ground for further investigations. Such use of complimentary Indian medicinal plant extracts needs further evaluation in a large group in multicentre trial.

\section{PP39-79}

Disease Spectrum of Infectious Patients in Rui Jin Hospital in Shanghai during the Last Six Years

H.Q. Wu, H. Wang, Q. Xie, G.D. Zhao, H.J. Zhou, H.L. Gui, J.D. Xie Department of Infectious Diseases, Rui Jin Hospital, Shanghai Jiao Tong University School of Medicine, Shanghai, China
Background and aims: In recent years, the disease spectrum of infectious diseases has changed a lot in shanghai. The purpose of this study was to investigate the changes in the numbers of cases and the disease spectrum of the infectious patients in Rui jin hospital during the last 6 years.

Methods: The data of hospitalized patients in the department of infectious disease from January 2005 to September 2010 were arranged and analyzed.

Results: The sum of the infectious patients was 12,961 , which was based mainly on category B infectious disease with a ratio of $84.50 \%$. The category A infectious disease was rare, which occupied $0.09 \%$ and there were only 11 cases of influenza A virus subtype H1N1 in 2010 . The category $C$ infectious disease occupied $1.30 \%$, of which the overall trend of the proportion was declining. The proportion of the hospitalized patients of hepatitis, central nervous system infection, measles, intestinal infection and parasites since 2005 ranked into the top five in the list of all infectious diseases. Viral hepatitis B was in the majority which occupied $48.89 \%$ among all types of viral hepatitis, followed by viral hepatitis $\mathrm{C}$ with a ratio of $40.33 \%$. The number of cases of viral hepatitis $\mathrm{C}$ significantly increased in the last six years, rising from No.4 to No.1 in the annual ranking. Viral hepatitis B gave priority to chronic type which occupied $65.32 \%$.

Conclusions: In Shanghai, influenza A virus subtype H1N1 had become an epidemic over the past year and the infectious diseases gave priority to category B infectious disease, among which the viral hepatitis ranked number one, especially chronic hepatitis $B$. The number of cases of viral hepatitis $\mathrm{C}$ significantly increased while the others were under control.

\section{PP39-80}

Effect of Ramadan Fasting on Muslim Patients with Chronic Liver Diseases

M.H. Elnadry, I.A. Nigm, I.M. Abdel Aziz, A.M. Elshafee, S.S. Elazhary, M.A. Abdel Hafeez, S.M. Mohii, D.M. Elteeby

Tropical Medicine, Al Azhar University, Cairo, Egypt

Background: Ramadan is the ninth month of the Islamic calendar; it is the Islamic month of obligatory fasting, during this month Muslims around the world fast from dawn until sunset

Aim of study: To evaluate the efficacy of fasting Ramadan on the clinical, laboratory and ultrasonographic parameters of patients with chronic liver disease

Methods: The studied patients were selected from the departments of Tropical medicine and outpatient clinics of Al-Azhar University hospitals-Cairo Egypt from the period of 26th of July till 30th of September 2010. Patients submitted to complete clinical, laboratory, ultrsonographic and endoscopic evaluation pre, during, and post Ramadan. Patients were divided into two groups fasting group and non fasting group and each group is subdivided into two subgroup Chronic hepatitis group and Cirrhotic group

Results: A total of 202 patients were included in the study, fasting group involved $103(51 \%)$ and non fasting group 99 (49\%) patients, both group were matched,non fasting group showed significantly good adherence to therapy $(43.4 \%)$ compared to $(27.2 \%)$ in fasting group $(p=0.16)$.

Dyspeptic symptoms was significantly higher in fasting $(53.4 \%)$ compared to $(38.4 \%)$ in non fasting ( $p=0.032)$.

G.I. bleeding during Ramadan was significantly higher in fasting group $(17.5 \%)$ compared to $(14.1 \%)$,in non fasting, bleeding due to o.v $(1 \%)$ in fasting group compared to $(9.1 \%)$ in non fasting group ( $p=0.004)$

Chronic hepatitis fasting group showed non significant changes pre, during and post Ramadan regarding liver function. Fasting cirrhotic group patients Child class $\mathrm{C}$ was increased to (13\%) during and $(32.6 \%)$ after Ramadan compared to $(0 \%)$ before Ramadan $(p=0.001)$ 
Conclusion: Ramadan fasting is prohibited for cirrhotic patients as it enhance the complication of liver cirrhosis. Patients with chronic hepatitis must be thoroughly investigated prior to Ramadan fasting.

\section{PP39-81}

Serum Level of Interleukin-1 $\beta$ (IL-1 $\beta$ ) Is Correlated with the Severity of Chronic Liver Diseases

Y.M. Fouad ${ }^{1}$, M. Makhlouf ${ }^{1}$, T. Sleem ${ }^{2}$, H. Mokarab ${ }^{1}$, T. Hassanain ${ }^{1}$, A. El Sayed ${ }^{1}$, R. Yehia ${ }^{3}$

${ }^{I}$ Tropical Medicine, Minia University; ${ }^{2}$ Biochemistry, Assiut University; ${ }^{3}$ Minia University, Minia, Egypt

IL-1 $\beta$ is a proinflammatory cytokine, synthesized by many hepatic cells, such as Kupffer cells, hepatocytes and stellate cells. It may contribute to occurrence and aggravation of the liver diseases.

Aim: Estimation of the serum level of interleukin 1 beta (IL-1 $\beta$ ) in patients with chronic liver diseases and correlating its level with the severity of liver diseases.

Patients and methods: Seventy patients with chronic liver diseases were included in this study. They were classified into 3 groups: group 1 (30 patients with chronic hepatitis), group 2 (20 patients with liver cirrhosis) and group 3 (20 patients with hepatocellular carcinoma). Ten healthy individuals served as controls. All the patients and controls were subjected to full history and clinical examination, abdominal ultrasonography and collection of blood samples for routine investigations and serological assay for the IL-1 $\beta$. Liver biopsy and histopathological assessment were done for 31 patients.

Results: The mean serum level of IL $1 \beta$ was significantly higher in the patients with chronic liver diseases than the controls $(p=0.001)$. It was significantly higher in males than in females $(p=0.04)$ and there was a significant positive correlation between the mean serum levels of IL- $1 \beta$ and the age of the patients $(p=0.01)$. The highest mean serum level was in group 3 (HCC) followed by group 2 (liver cirrhosis) and then group 3 (chronic hepatitis) $(P=0.0001,0.0001$, 0.001 , respectively). The mean serum level of IL1 $\beta$. was significantly higher in severe than mild hepatitis $(p=0.01)$ and was significantly higher in Child's class C than B ( $p$ value $=0.01$ and 0.01 , respectively).

Conclusion: Serum level of interleukin- $\beta$ is elevated in patients with chronic liver diseases than healthy controls and its level is correlated with the severity of liver diseases.

\section{PP39-82}

Single Nucleotide Polymorphisms (SNPs) of $I L-28 B$ Gene in Thai Populations

S. Payungporn ${ }^{1}$, Y. Tanaka ${ }^{2}$, P. Tangkijvanich ${ }^{1}$, S. Akkarathamrongsin ${ }^{3}$, S. Kani ${ }^{2}$, W. Sopipong ${ }^{1}$, P. Nilyanimit ${ }^{1}$, P. Komolmit ${ }^{4}$, Y. Poovorawan ${ }^{3}$ ${ }^{I}$ Department of Biochemistry, Faculty of Medicine, Chulalongkorn University, Bangkok, Thailand; ${ }^{2}$ Virology and Liver Unit, Nagoya City University Graduate School of Medical Sciences, Nagoya, Japan; ${ }^{3}$ Center of Excellence in Clinical Virology; ${ }^{4}$ Department of Medicine, Faculty of Medicine, Chulalongkorn University, Bangkok, Thailand

Background/aims: Pegylated interferon-a (PEG-IFN) plus ribavirin (RBV) are the recommended treatment for patients with chronic hepatitis C. Recent study revealed that treatment responses against HCV infection by PEG-IFN and RBV are significantly associated with the single nucleotide polymorphisms (SNPs) of interleukin-28B $(I L-28 B)$ gene. Until now, there is no information about the SNPs within this gene in Thai population. Thus, this study focused on the SNP patterns of the $I L-28 B$ gene in Thai populations.

Methods: DNA sample was extracted from PBMCs or sera of Thai patients with chronic hepatitis $(N=156)$. Informed consent was obtained from each patient who participated in the study. The study protocol conforms to the relevant ethical guidelines as reflected in a priori approval by the ethics committees of all the participating universities and hospitals. Then SNPs within $I L-28 B$ gene were detected by TaqMan real-time PCR (rs8099917 and rs12979860) and Invader Plus assay (rs11881222 and rs8103142). The data were analyzed by allelic discrimination (AD) software on the ABI$7900 \mathrm{HT}$

Results: The SNPs of $I L-28 B$ gene in Thai population were shown in the table below.

\begin{tabular}{lcccc}
\hline SNP & rs8099917 & rs12979860 & rs11881222 & rs8103142 \\
\hline Major allele & T 134/156 & C $133 / 156$ & A $133 / 156$ & T 133/156 \\
& $(85.90 \%)$ & $(85.26 \%)$ & $(85.26 \%)$ & $(85.26 \%)$ \\
Heterozygous & T/G 20/156 & C/T 19/156 & A/G 19/156 & T/C 19/156 \\
& $(12.82 \%)$ & $(12.18 \%)$ & $(12.18 \%)$ & $(12.18 \%)$ \\
Minor allele & G 2/156 & T 4/156 & G 4/156 & C 4/156 \\
& $(1.28 \%)$ & $(2.56 \%)$ & $(2.56 \%)$ & $(2.56 \%)$ \\
\hline
\end{tabular}

[The SNPs of IL-28B gene in Thai population]

Conclusions: The data obtained from this study provide the prevalence of 4 SNPs within the $I L-28 B$ gene in Thai population which might be useful for further application in terms of chronic hepatitis $\mathrm{C}$ treatment in Thailand.

Acknowledgement: We would like to thank the Hitachi Scholarship Foundation and Faculty of Medicine, Chulalongkorn University for funding support.

\section{PP39-83}

Rectal Snip versus Microscopic Stool Examination for the Diagnosis of Schistosma Mansoni

Y. Elsherif, A. Higazy, M. Salama, S. Sharaf, I. Waked

National Liver Institute, Menofia University, Shebin Elkom, Egypt

Background: Schistosoma mansoni (S. mansoni) has been one of the major causes of liver diseases. It is estimated that portal hypertension due to $S$ Mansoni produces 130,000 fatalities each year. Diagnosis is pivotal to all aspects of schistosomiasis. In spite of new knowledge in the area of $S$ mansoni, there remains the unsolved controversy associated with the basic diagnosis of this important disease. This study was designed to assess the performance of parasitological microscopy that is widely used for the diagnosis of active $S$. mansoni infection. Methods: 651 (55 children and 596 adults) individuals in the National Liver Institute of Egypt, coming from three governorates mainly endemic for $S$ mansoni in Lower Egypt, were examined for $S$ mansoni ova by microscopic stool examination (MSE) alone ( $n$ : 166; 111 adults and 55 children), rectal snip (RS) alone ( $n: 32$ adults) or both MSE and RS ( $n$ : 453 adults).

Results: Although all patients came from same background, infection rates substantially changed according to method of diagnosis. It was significantly lower in MSE alone group $(9 \% ; 15 / 166)$ compared to RS alone group $(40.6 \% ; 13 / 32)$. When both diagnostic methods were used, only $23 / 453(5 \%)$ patients were positive by stool examination. By contrast, 169/453 (37.3\%) (148 snip only positive +21 stool and snip positive) patients were positive by rectal snip in the same group. MSE failed to diagnose $86.5 \%$ (148/171) of positive cases. It is also noted that adding MSE to RS did not increase the prevalence than RS alone, 37.3 vs. $40.6 \%$. Using the summation of both MSE and RS tests as gold standard, sensitivity of MSE was significantly lower than RS, 13.5 vs. $98.8 \%$.

Conclusion: Our data suggests that true prevalence in endemic communities may be considerably underestimated. The use of simple microscopic stool examination for monitoring infection is ineffective because of low sensitivity. 


\section{PP39-84}

Five-year Cumulative Risk of Severe ALT Elevation in HIV-HBV Co-infected Adults on Antiretroviral Treatment (HAART) in Thailand

G. Jourdain $^{1,2,3}$, N. Ngo-Giang-Huong ${ }^{1,2,3}$, W. Khamduang ${ }^{1,3,4}$, S. Prommas ${ }^{5}$, N. Winiyakul ${ }^{6}$, P. Pathipavanich ${ }^{7}$, P. Chirawatthanaphan ${ }^{8}$, P. Maharom ${ }^{9}$, S. Thongpaen ${ }^{10}$, M. Lallemant ${ }^{1,2,3}$, Program for HIV Prevention and Treatment Cohort, Thailand (NCT00433030)

${ }^{1}$ IRD UMI 174, Institut de Recherche pour le Développement (IRD), Chiang Mai, Thailand; ${ }^{2}$ Immunology and Infectious Diseases, Harvard School of Public Health, Boston, MA, USA; ${ }^{3}$ Faculty of Associated Medical Sciences, Chiang Mai university, Chiang Mai, Thailand; ${ }^{4}$ INSERM U966, Université François-Rabelais, Tours, France; ${ }^{5}$ Bhumibol Adulyadej Hospital, Bangkok; ${ }^{6}$ Regional Health Promotion Centre 6, Khon Kaen; ${ }^{7}$ Lampang Provincial Hospital, Lampang; ${ }^{8}$ Phaholpolphayuhasena Hospital, Kanjanaburi; ${ }^{9}$ Somdej Prapinklao Hospital, Sattaheep; ${ }^{10}$ Mahasarakam Hospital, Mahasarakam, Thailand

Introduction: Some antiretroviral drugs are associated with a risk of hepatotoxicity, which may increase in HBV-HIV co-infected patients. Methods: Between February 2000 and August 2005, 915 HIVinfected antiretroviral naïve adults initiated an NNRTI or Proteaseinhibitor based regimen in 46 sites of the multicenter PHPT cohort (NCT00433030). The risk of severe elevation of Alanine-AminoTransferase (sev-ALT) ( $>5$ times upper limit of normal values, ACTG Grade III) after initiation of HAART was estimated using Kaplan Meier (KM) curves, log rank and Cox models. Intent-to-treat analysis.

Results: $20 \%$ were male, $8.2 \% \mathrm{HBsAg}+$ At treatment initiation, median age was 30.7 years [IQR: 27.0-35.7], BMI 20.3 [18.4-22.7] $\mathrm{kg} / \mathrm{m}^{2}$, CD4 count 104 cells/mm3 [34-194], HIV RNA load 4.84 $\log _{10}$ copies/mL [4.26-5.24], ALT $30 \mathrm{IU}$ [20-38], hemoglobin $11.3 \mathrm{~g} /$ dL [10.2-12.2], 62\% were at CDC clinical stage A, 13\% B, and 25\% $\mathrm{C}$, with no difference according to HBsAg status. First line combinations contained nevirapine in 59\% in $\mathrm{HBsAg}+$ versus $75 \%$ in HBsAg-; efavirenz $21 \%$ versus $11 \%$; protease inhibitors in $20 \%$ versus $14 \%$; lamivudine $79 \%$ versus $98 \%$; didanosine $21 \%$ versus $3 \%$; zidovudine $41 \%$ versus $29 \%$; stavudine $59 \%$ versus $71 \%$, respectively. Nevirapine was discontinued within 3 months of initiation in $23 \%$ of $\mathrm{HBsAg}+$ versus $13 \%$ of $\mathrm{HBsAg}-(P=0.07)$.

At 5 years, the KM risk of sev-ALT was 7.8\% [95\% CI 6.1-10\%]. In the univariate analysis, risk factors for increased risk were: HBsAg+ (HR 4.8, $P<0.0001$ ), baseline ALT above median (HR 2.7, $P=0.003$ ), first line use of didanosine instead of lamivudine (HR $3.5, P=0.002$ ), and male sex (HR 2.1, $P=0.008$ ). Upon multivariate analysis, ALT above median $(P=0.01)$, use of didanosine instead of lamivudine $(P=0.04$ in HBsAg-, $P<0.001$ in HBsAg+) and HBsAg+ $(P=0.04)$ remained independently associated with an increased risk of sev-ALT.

Conclusion: This supports avoiding didanosine and including antiHBV drugs in the treatment of HBV-HIV co-infected patients.

\section{PP39-85}

Intensive Nutritional Intervention Improves Hypoproteinemia in Decompensated Liver Cirrhosis

P. Srivastava, G. Choudhuri, P. Singh, D. Singh

Gastroenterology, Sanjay Gandhi Post Graduate Institute of Medical Sciences, Lucknow, India

Background and aim: Hypoalbuminemia is frequent and often severe in patients with Liver Cirrhosis (LC). We evaluated whether intensive nutritional intervention could improve albumin synthesis and blood levels in patients with advanced LC.

Method: 495 patients (male 381, mean age $45 \pm 10.9$, Child A: 142, Child B: 133, Child C: 109) of LC recruited for the study were stratified according to Child-Pugh class and randomized to receive one of 3 nutritional inputs: (a) usual nutritional counselling with the help of a leaflet,

(b) intensive nutritional counselling by a trained nutritionist and dietary intervention with homemade feeds

(c) Intensive nutritional counseling as above in with additional supplementation with protein formula feeds to account for atleast $25 \%$ of total daily calories.

Prealbumin and albumin levels were measured at baseline and at 3 months. Paired ' $t$ ' test was used for analysis.

Result: Type "c" diet showed maximum increase, over baseline, in prealbumin (Child A: 10\%, Child B: 17\%, Child C: 16.6\%) and albumin levels (A: 3\%, B: 7\%, C: 4\%). The corresponding values for "b" diet for pre-albumin (A: 9.5\%, B: 13\%, C: $8 \%$ ) and albumin (A: $0 \%$, B: $3.2 \%$, C: $0 \%$ ) were less. The "a" diet fared worst with minimal increase in prealbumin (A: 5\%,B: $4 \%, \mathrm{C}: 3.4 \%$ ) and albumin (A: 0\%,B: 3.3\%,C: 0\%) levels.

Conclusion: Our study showed that intensive nutritional intervention by a trained nutritionist and feeding of high protiein diet supplemented with formula-feeds produced the maximum increase in serum levels of pre-albumin and albumin. The benefit was most evident in patients with decompensated (Child B \&C) liver Cirrhosis.

\section{PP39-86}

Frequency and Pattern of Impairment in Health-related Quality of Life (HRQL) in Liver Cirrhosis

P. Srivastava, G. Choudhuri, P. Singh, D. Singh

Gastroenterology, Sanjay Gandhi Post Graduate Institute of Medical Sciences, Lucknow, India

Background and aims: Liver Cirrhosis (LC) is a chronic progressive disease that affects health related quality of life (HRQL). We studied the frequency and pattern of HRQL impairment in different grades of LC.

Method: We evaluated the clinical, demographic and laboratory data of 495 patients of Liver Cirrhosis (male 381, mean age $45 \pm 10.9$, Child A: 142, Child B: 133, Child C: 109) and measured their HRQL by Short Form 36 and Nottingham health Profile questionnaires. Apart from noting the number who reported poor HRQL in each Child-Pugh class, we analysed the $8 \mathrm{HRQL}$ parameters that were most frquently affected in them.

Result: Poor HRQL was noted in 18\% (26/142) of patients with Child A, and increased progressively to 50\% (66/133) in Child B and $87 \%$ (95/109) in Child C patients. The most common parameters to be impaired were emotional well-being and social functioning Child A patients, whereas physical functioning and general health were the ones commonly impaired in Child B. Poor HRQL in Child C patients showed impairment of several parameters such as low energ, fatigue, pain, role limitation due to emotional problem and physical health. Pain was specifically observed in Child C.

Conclusion: HRQL is significantly affected in LC, the frquency increasing with the stage of disease. $18 \%$ of Child A cirrhotics have poor HRQL, largely due to emotional and social consequences. Loss of energy and fatigue were additional factors in Child B, whereas pain, physical weakness nd role limitation were the main factors in patients with Child C.

\section{PP39-87}

Neonatal Jaundice, G6PD Deficiency and Hemolysis M. Rezaie, S.H. Nabavi Zadeh, M. Zoladl, F. Hashemi Paediatrics, Yasouj University of Medical Science, Yasouj, Iran

Introduction: G6PD deficiency lead to anemia due to hemolysis related to drug and fovea and also is one of the causes of severe and prolonged neonatal jaundice. Studies show that neonatal hyperbilirubinemia in G6PD deficiency is due to lever immaturity and also hemolysis. In this study we want to see the role of hemolysis in jaundice of newborns with G6PD deficiency. 
Material and methods: We studied 234 newborns with jaundice admitted in Imam Sajjad hospital of Yasouj in 2010 for signs of hemolysis, which from, 107 G6PD deficient neonates and 127 neonates with normal level of G6PD . Datas (age, days of admission, level of hemoglobin, bilirubin, reticulocyte, urinalysis and comb's test) were collected in patient's forms. Then all of them analyzed with SPSS.

Results: In both groups there was no meaningful difference in age of admission, level of hemoglobin, reticulocyte and urinalysis $(p>0.05)$, but there was a significant difference in level of bilirubin (13-23.2 in G6PD deficient neonates and 11.2-21.6 in neonates with normal G6PD) . Despite of non-significant difference in hemoglobinuria, relative risk for hemoglobinuria in G6PD deficient neonates was 1.24 .

Conclusion: This study shows that hemolysis has an ignorable role as a cause of severe jaundice in G6PD deficient neonates. We suggest more studies to determine role of liver immaturity and other causes of jaundice in neonates with G6PD deficiency.

\section{PP39-88}

Effect of Byesukar on Lipid Profile and Hepatic Enzymes of Glucose Metabolism in Diabetic Rats

\section{Guruvayoorappan}

Biotechnology, Karunya University, Coimbatore, India

Many anti-diabetic herbal preparations have been recommended in alternative systems of medicine for the treatment of diabetes. No systematic study has been done on the anti-diabetic efficacy of Byesukar, a polyherbal formulation to treat diabetes. The antidiabetic efficacy of byesukar ethanol extract was evaluated in an animal model of diabetes induced by alloxan. Male Wistar rats were divided in to four groups. Group 1 was normal control group; group 2 and 3 received alloxan. After inducing experimental diabetes group 2 served as diabetic control; group 3 received byesukar (500 $\mathrm{mg} / \mathrm{kg}$ body weight) orally for 30 consecutive days. Group 4 were normal rats which received byesukar extract alone. The effect of byesukar on glucose level in diabetic rats was studied and the level of glucose metabolizing enzymes (Hexokinase, glucose-6phosphatase and fructose 1, 6-bisphosphatase) in the liver and kidney were estimated. The effect of byesukar on the serum and tissue lipid profile (Cholesterol, triglycerides, phospholipids and free fatty acids) were also estimated in diabetic rats. Our results indicate that treatment with byesukar resulted in significant reduction of blood glucose, tissue glucose-6-phosphatase and fructose 1, 6-bisphosphatase activity. The decreased tissue hexokinase activity in diabetes state was found to be significantly increased by byesukar treatment. Also the byesukar treated diabetic rats showed a significant decrease in the tissue lipid profile compared to the diabetic rats. In conclusion the decreased blood glucose accompanied with decreased lipid profile and changes in the activities of the glucose metabolizing enzymes shows the antidiabetic effect of byesukar. 


\section{Authors Index}

Aamir, U.B. PP12-35, PP12-45

Aanpreung, P. PP39-46

Abaalkhail, F. PP38-09

Abas, A. PP16-35

Abaza, M. PP24-03

Abbas, A. PP25-06

Abbas, Z. PP08-17, PP09-25, PP12-29

Abbasi, A. PP13-41, PP13-52, PP31-32

Abbasi, P. PP01-40, PP09-23, PP09-24

Abbasinazari, M. PP39-23

Abbott, W.G.H. PP06-60

Abd, El, Baky, M. PP23-03

Abd, El, Hamied, M.I. PP11-03

Abd, El, Moety, H.A. PP07-01, PP11-01, PP12-02, PP31-02, PP31-11

Abd, El, Moety, H.A. PP07-01, PP11-01,

PP11-02, PP12-02, PP23-03, PP24-03, PP31-02, PP31-11

Abd, Elaal, E.A. PP31-29

Abd, El-Maksoud, M. PP25-21

Abd, Elmoety, A.A. PP08-03, PP13-07

Abd, Elmoety, H.A. PP24-03

Abd-Alla, S.A.-M. PP23-60

Abdel, Aziz, H. PP25-06

Abdel, Aziz, I.M. PP39-80

Abdel, Fattah, M. PP13-23

Abdel, Hafeez, M.A. PP39-80

Abdelaal, S.M. PP14-03

Abdelal, E.A. PP14-15

Abdel-Azeem, H.G. PP24-04

Abdel-Azeem, K.E. PP23-62

Abdelaziz, A. PP38-52

Abdel-Baky, A.I. PP29-01

Abdel-dayem, H. PP38-42

Abdel-Hafeez, H.A. PP16-17

Abdel-Hamid, N.M. PP29-01

Abdel-Hammed, M. PP12-06

Abdelmaksoud, N. PP39-02

Abdel-Moneim, A. PP05-143

Abdel-Naby, B. PP16-52

Abdel-Rahman, M. PP38-25

Abdel-Razek, W. PP23-45

Abdo, A. PP38-09

Abdou, L. PP22-08

Abdulkhakov, S. PP10-23, PP10-24

Abdurachman, S.A. PP23-98

Abdurahman, S.A. PP02-27

Abdurakhmanov, D. PP13-50

Abdusalamov, S.N. PP01-43

Abe, K. PP13-22, PP13-78

Abedi, F. PP14-13

Abergel, A. PP13-47

Abo, Deya, S. PP39-11

Abogabal, A. PP23-45

Abou, Khatwa, M. PP04-03, PP06-03

Abramishvili, N. PP10-25

Abu, Gabal, A. PP23-87

Abutidze, A. PP13-53

Acar, A. PP02-22, PP04-33, PP05-20, PP06-26

Acharya, S.K. PP05-15, PP05-18,

PP06-10, PP16-37, PP23-07

Adali, G. PP23-80, PP26-69

Adamcova-Selcanova, S. PP04-42

Adamec, M. PP38-24
Adar, P. PP05-133

Adda, N. PS02-03

Adeeb, U. PP05-83, PP05-84, PP13-10,

PP13-37, PP23-31

Adibi, P. PP12-11, PP12-12, PP12-13

Adibzadeh, A.-R. PP38-44

Adorini, L. PP07-22

Adwan, Z. PP10-01

Aejaz, Habeeb, M. PP22-09

Afdhal, N.H. PP13-89

Afifi, R. PP04-47, PP06-34, PP34-33

Afify, S. FC05-05

Afredj, N. PP38-20

Afsharian, M. PP23-85

Agarwal, A. PP13-63

Agarwal, K. PP13-40

Agarwal, A.K. PP23-29

Aggarwal, R. C18-03

Agha, S. PP25-21

Aghayeva, G.S. PP13-54

Aguilar, J.C. PP05-127

Aguilera, V. PP38-54

Agustanti, N. 71, PP02-27, PP23-98

Ahamed, M. PP34-37

Ahangarpour, A. PP24-09

Ahlén, G. PP05-64, PP13-114

Ahmad, A. PP39-31

Ahmad, I. PP30-15

Ahmad, N. PP05-54, PP05-124, PP13-49

Ahmad, U.S. PP09-32

Ahmed, B. PP39-04

Ahmed, E. PP39-67

Ahmed, M. PP13-68, PP14-09, PP16-24

Ahn, J.H. PP26-48, PP26-49, PP31-23, PP31-24

Ahn, S.H. PP03-26, PP13-18, PP16-46,

PP16-47, PP16-50, PP16-58, PP19-04,

PP20-12, PP23-69, PP23-84, PP30-29,

PP31-33, PP31-35

Ahn, S.V. PP39-60

Ahn, Y.-J. PP05-154

Ai, G. 173, PP06-50, PP25-19

Ai, Z.-L. PP20-11

Aiad, H. PP21-07

Aikou, S. PP36-26

Ain, N.U. PP12-35

Aït, Kaci, H. PP38-20

Ait, Younes, S. PP04-06, PP25-05

Aiura, K. PP36-26

Akahide, A. PP34-22

Akaike, J. PP03-08

Akbar, F. PP01-06, PP05-98

Akbar, I. PP13-70

Akbar, S.M.F. PP05-127

Akbari, A. PP12-04

Akcali, K.C. PP21-03

Akhan, S. PP14-24

Akın, M.S. PP05-109, PP05-110,

PP05-111, PP13-62

Akita, T. PP04-08

Akkarathamrongsin, S. PP12-41,

PP12-42, PP39-82

Akkiz, H. PP07-31

Akl, M. PP14-27

Aksoy, K. PP07-31

Aksoy, N. PP05-17,PP05-144

Akyol, G. PP16-05
Akyuz, F. PP09-29, PP23-80

Akyuz, U. PP09-29

Al, Akwaa, A.M. PP08-50

Al, Ani, A.H. PP39-20

Al-, Rubaye, F.F. PP39-20

Al, Sharkawy, E. PP23-76

Alahdab, Y.O. PP08-21

Alam, K. PP05-54, PP05-154, PP13-49

Alam, A.S. PP39-29

Alam, M.S. PP05-13, PP05-54,

PP05-124, PP13-49

Alaoui, R. PP02-17

Al-Attar, M. PP39-16

Alavian, S. PP13-20, PP14-13

Alavian, S.M. FC05-04, PP04-14, PP04-15,

PP04-43, PP06-24, PP09-27, PP11-04,

PP11-12, PP12-26, PP13-101, PP17-14,

PP23-85, PP39-08

Alavian, S.-M. PP13-09

Alawi, K. PP38-09

Alberti, A. C01-03, FC05-02, PP03-10

Alboraie, M. PP09-02

Albrecht, J. PS02-02, PP13-75, PP13-90

Al-Bulushi, I.S. PP13-69

Aldersley, M. PP38-37

Alejandre, A.J.F. PP23-95

Alexandrescu, L. PP10-17, PP10-18, PP37-13

Al-Falahy, Y. PP03-40

Al-Freah, M. PP13-40

Alhamadani, A. PP29-03

Alhamoudi, W. PP38-09

Al-Harthy, S.A. PP13-69

Ali, A. PP39-66

Ali, Arous, N. PP04-06, PP25-05

Ali, Q. PP28-04

Ali, S. PP01-40, PP09-24

Aliev, B. PP06-11

Alim, M.A. PP08-39, PP32-04, PP39-29

Alinejadi, M. PP04-35, PP12-39

Alishah, H. FC06-05, PP23-23

Alisi, A. PP30-30, PP30-35

Al-Khalidy, N. PP03-40

Allam, N. PP05-143, PP16-52, PP23-45,

PP23-97, PP38-09, PP38-42

Allameh, A. PP01-25, PP10-20, PP22-07

Allen, S. PP28-05

Allweiss, L. PP09-06

Al-Mahtab, M. PP05-77, PP05-127

Al-Naagi, M. PP13-111

AL-Ofairy, B. PP03-40

Alpini, G. PP22-13

Alric, L. FC05-01

Alrubaiy, L. PP26-56

Alsaif, H. PP37-16, PP37-18

Al-Salem, A. PP28-09

Al-Sebayel, M. PP38-09

Alshammari, A. PP26-55

Alshinnawy, H. PP13-04

Al-Sofayan, M. PP38-09

Altuntas, Aydin, O. PP04-21, PP06-05

Alves, T. PP12-01

Alwahaibi, N. PP29-03

Alwakeel, H. PP13-04

Amaraa, R. PP23-68

Amarapurkar, A.D. PP23-26 
Amarapurkar, D. AA-03, PP01-23, PP04-31, PP08-41, PP39-62, PP39-63, PP39-64, PP39-71

Amarapurkar, D.N. PP02-24

Ambreen, S. PP13-68, PP16-24

Amer, A. PP09-03

Amien, A. PP11-10

Amin, A. PP29-08

Amin, N. PP16-17

Amir, Z.C. PP04-06, PP25-05

Amornyotin, S. PP39-41, PP39-42,

PP39-43, PP39-44, PP39-45, PP39-46

An, S.B. PP37-15

An, P. PP05-121

An, X. PP39-38

Anand, S. PP14-05

Anderson, F. PP13-64, PP13-75

Anderson, J. PS01-02, PP05-91, PP05-99

Ando, S. PP23-32, PP29-18

Andrade, D.L. PP12-01, PP12-28

Andreone, P. PS02-04, FC05-03

Andriulli, A. PP14-08

Angga, B. PP01-20, PP27-13

Anisimova, S. PP02-15

Anjum, S. PP12-46, PP13-12

Annessini, M.C. PP39-14

Ansari, K. PP01-40, PP09-23, PP09-24

Ansari, M.A. PP09-36, PP12-33,

PP14-18, PP39-69

Antaki, N. C22-02

Anticoli, S. PP30-35

Antonas, K. PP07-20

Anwar, G. PP17-13

Ao, F. PP13-42

Aoki, T. FC02-04, C21-02, PP38-36

Aoki, Y. PP13-122, PP13-123

Aqodad, N. PP04-36, PP12-36, PP12-43,

PP13-25, PP13-108, PP13-109, PP16-41, PP23-70, PP23-73, PP23-75, PP23-82,

PP23-83, PP25-20, PP26-62

Arafa, M. PP14-22

Arai, M. PP01-05, PP03-39, PP04-46,

PP05-80, PP12-40, PP13-27, PP13-36, PP30-37

Arakawa, T. PP03-08

Araki, N. PP27-18

Arambepola, P.K. PP39-32

Arano, T. PP33-25

Arasli, M. PP05-58

Aravind, B. PP39-27

Arciello, M. PP08-52, PP30-35

Ardelean, A. PP08-05, PP10-04

Ardelean, M. PP13-107

Arellano-Olivera, I. PP16-51

Arguillas, M.O. C08-03

Ari, A. PP05-78, PP05-79

Ari, Tabarestani, M.H. PP23-85

Arican, K. PP02-22

Arican, N. PP05-17, PP05-144

Arif, M. PP13-68

Ariizumi, S. PP26-63, PP31-34

Ariizumi, S.-I. PP26-50, PP26-67, PP36-22

Armendariz, Borunda, J. PP15-48

Armendariz-Borunda, J. PP15-45, PP20-07

Armendáriz-Borunda, J. PP15-49, PP16-51

Arnaud, J.-P. PP26-29, PP26-30
Arora, S.K. PP02-06

Arshiy, E.I. PP16-09

Artacho, A. PP06-08

Arthur, Lee, Kiat, S. PP13-29

Arun, M. PP30-36

Asano, Y. PP26-45, PP27-19, PP36-20

Asaoka, Y. PP30-33

Asatryan, T. PP13-08, PP13-18

Aselah, T. PP03-14, PP03-17

Asim, M. PP06-29, PP16-19, PP30-15, PP32-05

Aslan, T. PP06-25

Asoyan, A. PP13-08, PP13-118

Asselah, F. PP04-06, PP25-05

Asselah, H. PP04-06, PP25-05

Asselah, T. PS02-01, PP06-43, PP13-57,

PP13-120, PP14-16

Assem, M. PP13-23

Assy, N. PP08-06

Asuvapongpatana, S. PP15-17

Ataei, B. PP09-15, PP12-11, PP12-12,

PP12-13, PP12-16, PP12-19, PP13-71, PP14-13

Ataie, M. PP09-21, PP12-13, PP12-16,

PP12-19, PP13-71

Atak, A. PP16-05

Atef, S. PP16-42

Atkin, C. PP39-30

Atta, R. PP11-07, PP11-14

Attar, B.M. PP10-19

Attasaranya, S. PP05-94

Aubé, C. PP26-29

Aung, M.O. PP04-50, PP05-40, PP05-66,

PP05-72, PP05-114, PP13-29

Avahingsanon, A. PP15-33

Avci, I. PP05-78, PP05-79, PP13-35

Avc1, I.Y. PP13-105

Avila, C. PP04-44

Avitzur, Y. PP38-21

Awad, M.M. PP16-16

Awny, S. PP38-25

Ayaşlığlu, E. PP06-52

Aydemir, S. PP05-58

Aydinli, M. PP05-103

Ayres, A. PP05-113, PP05-159

Azam, I. PP23-12

Azam, M.G. PP05-54, PP05-124,

PP08-20, PP13-49

Azar-Keyvan, A. PP11-04

Azemoto, R. PP13-36

Azizee, R. PP12-44

Azuma, T. PP05-145

Baba, N. PP25-08

Babak, A. PP09-15, PP09-21, PP12-11, PP12-12, PP12-13, PP12-16, PP12-19, PP13-71

Babany, G. FC05-01

Babic, I. PP05-70

Bachani, S. PP01-16

Baci, A. PP12-22

Bacon, B. PS02-02, PP13-90

Badavi, M. PP24-09

Badawy, A.A. PP11-07

Baddour, N.M. PP22-08, PP30-19

Badea, R. PP37-12

Bader, H. PP11-10

Badiger, R. PP39-30
Badran, H. PP23-97

Badridze, N. PP06-15, PP14-17

Badulescu, F. PP29-17

Badur, S. PP09-29

Bae, H. PP05-99, PP05-101

Bae, J.S. PP30-12, PP30-13

Bae, S.H. PP05-51, PP22-12, PP32-10

Bagir, G.S. PP17-03

Baha, W. PP04-04

Bahar, K. PP38-50

Bahili, H. PP38-09

Bahirwani, R. PP26-17

Bahrami, H. PP11-05

Bai, G.X. PP39-26

Bai, T. PP31-14

Bai, X. PP05-152

Bai, Y. PP23-93

Baik, S.K. FC06-02, C15-04, PP23-38

Bain, V. FC05-03

Bains, H.S. PP09-37

Baioumy, S. PP11-01, PP23-06

Bajraktarevic, A. PP17-02

Bakulin, I. PP13-48, PP13-50

Balachandar, V. PP30-36, PP31-40, PP34-37

Balamuralikrishnan, B. PP30-36, PP34-37

Balamurugan, K. PP02-01

Balbi, M. PP32-01

Ballot, E. PS04-02

Balsano, C. PP08-52, PP30-30, PP30-35

Baltadzhiev, I.G. PP12-24

Banez, V. PP23-11

Bang, B.W. PP07-03

Bani, A. PP13-111

Bao, H.W. PP38-32

Bao, J. PS03-04, PP19-14, PP22-01, PP22-02

Bao, S. PP06-02

Bao, W. PP05-119

Baran, B. PP09-29

Barbano, R. PP14-08

Barnard, R. PP13-75

Barone, M. PP16-27

Barreyro, F. PP16-01

Barritt, G. PP07-34, PP38-01, PP38-23

Baruah, B. FC04-05, PP28-27,

PP39-68

Basaranoglu, M. PP15-06

Bashar, A. PP23-41

Bashir, H. PP12-35, PP12-45

Bashir, Q. PP12-35, PP12-45

Basu, K. PP15-29, PP15-30, PP19-20

Basu, P.P. PP13-79, PP13-83, PP13-87, PP15-32

Batmunkh, S. PP34-07

Bayatian, A. PP13-60

Baykam, N. PP06-09

Bayramov, N.Y. PP08-38

Bazinet, M. PP05-77

Beck, Y. FC02-04, C21-02, PP38-36

Bedossa, P. PS02-01, PP14-16

Bedoui, R. PP26-22

Beebe, S. PP13-104

Beeching, N.J. PP04-25

Behnava, B. FC05-04, PP11-12,

PP12-26, PP13-09, PP13-20, PP13-101, PP17-14

Beilfuss, A. PP07-31 
Beinhardt, S. PP12-34, PP13-61, PP13-84, PP13-88

Beleoken, E. PS04-02

Belinda, M. PP05-66, PP05-72, PP05-114

Ben, Ari, Z. PP34-20

Ben, Mustapha, N. PP04-13

Benajah, D. PP04-36, PP12-36, PP12-43, PP13-108, PP13-109, PP16-41, PP23-82, PP23-83, РP26-62

Benajah, D.A. PP13-25, PP25-30

Benajeh, D. PP23-70, PP23-73, PP23-75

Benathmane, T. PP38-20

Benayed, R. PP13-57

Benazzouz, M. PP04-47, PP06-34,

PP13-16, PP34-31, PP34-33

Benbrahim, H. PP13-16, PP34-33

Bendahou, K. PP23-75

Bendib, S.E. 38-20

Bengtsson, L. PSO2-03

Benjelloun, S. PP04-47, PP06-34

Bennani, A. PP04-04

Bensalem, A. PP04-06

Benyachou, B. PP23-73, PP23-82, PP23-83

Berardesca, E. PP39-74

Berenguer, M. PP38-54

Berg, T. PS01-03

Berger, A. PP12-27

Berkane, S. PP04-06, PP25-05

Berkowitz, D. PP23-02

Berloco, P. PP39-14, PP39-15

Bernard, P.-H. PP16-13

Bernstein, B. PP13-95

Bertasso, A. PP13-45

Bertoletti, A. C02-02, PP09-41

Bertrandt, J. PP39-55

Besheer, T.A. PP06-45, PP14-03,

PP16-11, PP31-29

Beşirbellioğlu, A.B. PP13-35, PP13-105

Besisik, F. PP09-29, PP23-80

Bessone, F. FC01-01

Bestari, B. PP02-27

Beuers, U. C10-03

Beumont, M. PS02-04

Beweja, S. PP30-26

Bhadada, S. PP23-79

Bhadauria, M. PP01-14, PP07-19,

PP15-26, PP39-48

Bhansali, A. PP23-79

Bhardwaj, A. PP01-14

Bharti, P. PP13-55

Bhat, D. PP09-37

Bhatia, V. PP16-36, PP26-74

Bhatt, N.R. PP02-24, PP23-26, PP39-62, PP39-63

Bhatt, S.P. PP08-27

Bhatti, T. PP09-24

Bhuiyan, T. PP08-20

Bhutto, A.-R. PP31-32, PP13-41, PP13-52

Bialkowska, J. PP13-104

Bian, L. PP05-121

Bièche, I. PS02-01, PP14-16

Bifano, M. PP13-76

Bihari, C. PP24-10, PP26-73, PP26-74

Bilgiç, M.B. PP26-41

Binesh, N. PP10-12

Bisheya, A. PP13-111
Bishnu, D. PP02-10, PP02-11

Biswas, A. PP02-10, PP02-11, PP15-29, PP15-30, PP19-02

Bitetto, D. PP05-42

Blanes, M. PP38-54

Block, T.M. PP14-23

Bo, H. PP13-73

Boaz, M. PP27-02

Bocobo, J. PP08-59, PP08-60

Bogomolov, P.O. PP03-38

Boissan, M. PP30-07

Bojovic, K. PP13-94

Bokharaei, Salim, F. PP06-24

Bolondi, L. PS03-01, PP19-07

Bomfim, E. PP12-01, PP12-28

Bondarenko, L. PP02-15

Bondoc, A. PP28-05

Bonifati, C. PP39-74

Bonino, F. C14-03, PP03-24, PP06-56

Boon, Tin, C. PP34-26

Boonsirichan, R. PP04-49

Boparai, N. PS02-02, PP13-90

Bornscheuer, T. PP09-06

Borovik, V.V. PP05-95

Borroto-Esoda, K. PS01-01, PS01-03, PP05-91, PP05-125

Bose, M. FC04-05, PP28-27, PP39-68

Bose, P.D. PP09-40

Bose, S. FC04-05, PP28-27, PP30-26, PP39-68

Bota, S. PP16-30, PP16-31, PP23-44, PP23-59, PP37-10, PP37-12

Boubaker, J. PP04-13

Boucekkine, T. PP38-20

Bouhris, J.-H. PS04-02

Bourliere, M. PP13-46

Bouvier-Alias, M. FC05-01

Bowden, S. PP03-24, PP05-87

Bowen, D. PP21-04

Boyer, N. PP03-14, PP06-43

Boyer, T.D. C04-03, SA01-01

Bozaci, M. PP09-29

Bozic, M. PP13-94

Boztas, G. PP09-29, PP23-80

Brachet, D. PP26-30

Brahim, I. PP06-34

Branca, N. PP16-15

Branch, R.A. PP31-05

Braß, A. PP13-114

Brass, C. PS02-02, PP13-75, PP13-90

Brenndörfer, E.D. PP13-114

Brescia, L. PP16-27

Bronowicki, J.-P. FC05-01, PP13-90

Brown, C.M. PP06-57

Brown, Jr, R.S. PP13-104

Brown, R.J. PP13-79, PP13-83, PP13-87, PP15-32

Bruix, J. C17-04

Brunetti, V. PP14-08

Brunetto, M. C07-02, PP03-24

Brunetto, M.R. PP06-56

Bruno, S. PP13-90

$\mathrm{Bu}$, H. PS03-04, PP19-14, PP21-10,

PP21-11, PP22-01, PP22-02

$\mathrm{Bu}$, Q. PP09-13, PP09-31

Buachidze, T. PP01-09, PP17-09
Budihusodo, U. PP03-11, PP06-28

Budin, S.B. PP29-03

Bugdaci, M.S. PP06-25, PP06-26

Bugdayci, G. PP03-29

Bui, T.X. PP31-15

Bujanover, Y. PP23-02

Bunchorntavakul, C. PP04-49, PP26-17

Buranabanjasatean, S. PP06-41

Buranawanitchakorn, Y. PP06-41

Burganova, G. PP10-23, PP10-24

Burivong, W. PP37-03

Burnet, M. PP07-23

Burroughs, A.K. FC06-01

Burroughs, M. PS02-02

Burwaiss, A. PP13-111

Buti, M. PP05-91, PP06-08, PP13-59

Butt, A.S. PP01-44, PP13-34

Butt, N. PP13-41, PP13-52, PP31-32

Butt, T. PP12-18

Butt, Z. PP13-70

Büyükberber, M. PP06-09

Byun, H.J. PP08-15

Byun, J.M. PP25-13, PP31-25

Byun, J.Y. PP26-65

Byun, K.S. PP09-34

Bzowej, N.H. PS02-03

Cagatay, A. PP06-25, PP06-26

Cai, Q. PP39-26

Cakaloglu, Y. PP23-80

Calabro, S. PP15-41, PP15-42

Calixto-Mercado, K. PP38-05

Cammà, C. PP08-52

Campanale, F. PP05-88

Canale, C. FC03-04

Canataroglu, A. PP17-03

Canbay, A. PP07-31

Cao, C.-Y. PP08-28, PP08-33, PP08-34

Cao, H. PP05-49, PP05-46, PP22-04, PP22-05

Cao, W. PP04-41

Cao, Y. PP16-02

Cao, Y.-X. PP05-121

Cardoso, A.C. PP03-14

Carey, I. PP13-40

Carr, B. PP31-05, PP31-07

Carr, M. PP26-36

Carvalho, S. PP23-81

Carvalho-Filho, R. PP06-43

Cascella, T. PP23-40, PP26-46, PP33-12

Castellaneta, S. PP16-27

Castellanos, M. PP23-65, PP23-66

Castellon, S. PP10-12

Castelnau, C. PP06-43

Castro, J. PP07-34

Causevic, A. PP17-02

Cavallo, L. PP16-27

Cavallone, D. PP06-56

Çavdar, G. PP05-133, PP06-52, PP13-119

Cavuslu, S. PP05-20

Cecinati, V. PP16-27

Ceranic, D. PP02-05

Cerasari, A. PP26-58

Cerasari, G. PP26-58

Cerasari, S. PP26-58

Cernat, R.C. PP10-11

Cernichiaro-Espinoza, L. PP39-59

Cervantes, J. PP08-60 
Ceylan, B. PP06-25, PP06-26

Cha, B.H. PP26-43

Cha, J.M. PP30-09, PP30-10

Chabi, S. PP25-05

Chadha, D.S. PP08-27

Chae, H.B. PP05-30

Chafa, O. PP38-20

Chaiear, K. PP12-42

Chainuvati, S. C16-03, PP13-11

Chainuwatti, S. PP03-31, PP03-32, PP05-48

Chaiphornphathana, W. PP24-08

Chair, S.Y. PP34-24

Chakraborti, A. PP31-37

Chakraborty, B.C. PP15-29, PP15-30

Chakravarthi, S. PP02-16

Chakravarty, R. PP06-22

Chalasani, N. FC04-04

Chalayonnavin, W. PP39-42

Chalermwat, C. PP18-01

Chamroonkul, N. PP05-94, PP13-15

Chan, A. PP33-03, PP36-07, PP36-14

Chan, C. PP34-24

Chan, C.W. PP08-26

Chan, F. C16-01

Chan, H. L.Y. PS01-04, C07-01, PP04-05, PP05-119

Chan, H.L. FC01-03, PP05-08

Chan, H.L.-Y. FC01-01, PP05-19,

PP05-102, PP08-19, PP16-07

Chan, H.-Y. PP05-102

Chan, K.H. PP08-26

Chan, R. PP04-50

Chan, S. PS01-03, PP05-99, PP05-101

Chan, S.C. PP34-21, PP36-14, PP38-17, PP38-27

Chandan, B. PP34-09

Chandrasekaran, E. PP23-29

Chang, J. PP16-28

Chang, J.-Y. PP34-30

Chang, K.-C. PP05-151, PP16-53

Chang, K.-K. PP13-98

Chang, M.-H. C19-02, PP04-44, PP08-14

Chang, M.-L. PP08-42

Chang, T.-T. PP05-65, PP05-129, PP05-141

Chang, Y. PP09-13

Chao, K. PP04-16, PP04-17

Chao, Y.C. PP05-139

Chao, Y.-C. PP05-129

Chao, Y.-J. PP17-05, PP17-17

Chaojin, T. PP23-22

Chappell, B. PS01-01

Charaf-Eddine, M. FC05-01

Charatcharoenwitthaya, P. C10-04,

PP03-31, PP03-32, PP05-48, PP13-11

Charnaux, N. PP30-14

Charni, F. PP30-14

Charoenmaung, R. PP38-38

Charoenmuang, R. PP38-41, PP38-62

Charoenpipop, D. PP26-47

Charoensuk, L. PP28-18

Charvat, F. PP34-13

Chataway, T. PP38-23

Chattopadhyay, T.K. PP23-07

Chaturvedi, N.K. PP23-29

Chaudhary, G.M.-U.-d. PP12-46, PP13-12

Chaudhri, E. PP13-75
Chaunchaiyakul, S. PP15-07

Chavrier, P. PP30-07

Chawla, Y.K. C11-03, PP10-13, PP14-05, PP23-58, PP23-79, PP31-17, PP31-37

Chayanupatkul, M. PP28-20

Che, H.-H. PP08-11

Che, X.-H. PP15-43, PP19-06

Che, Y.H. PP05-05

Chebli, A. PP05-04, PP08-01

Cheinquer, H. FC01-01, PP13-50,

PP13-104

Chen, A. PP05-136, PP13-114

Chen, C. PP05-05, PP05-22, PP05-27,

PP05-150, PP18-01, PP27-21

Chen, C.-H. PP05-140

Chen, C.-J. PP04-28

Chen, C.-L. LT2-02, PP04-07

Chen, D. PP06-49, PP15-46

Chen, D.S. S01-01, C20-04

Chen, D.-S. PP04-07, PP06-27, PP13-93

Chen, E.-Q. PP16-14

Chen, F.-L. PP17-05, PP17-17

Chen, G. PP05-45, PP23-20

Chen, G.-F. PP16-54

Chen, H. PP06-14, PP15-20, PP15-25, PP27-20, PP31-31, PP31-38, PP34-35, PP34-36

Chen, H.-S. PP22-15

Chen, H.Y. PP05-05, PP05-27, PP29-06

Chen, J.J. PP05-36, PP05-68, PP05-69,

PP06-20, PP16-43, PP16-55

Chen, J.-D. PP04-28

Chen, J.-H. PP13-93

Chen, J.-L. PP14-11

Chen, J.-S. PP28-31

Chen, J.-Y. PP17-05, PP17-17

Chen, K. PP30-20

Chen, K.-C. PP17-05, PP17-17

Chen, L. FC02-01, PP01-33, PP03-18, PP05-05, PP05-27, PP06-18, PP06-62

Chen, L.-B. PP05-49

Chen, L.-M. PP05-163

Chen, L.-T. PP34-30

Chen, L.Y. PP01-36

Chen, L.-Y. PP01-38, PP16-14

Chen, M. PP04-16, PP04-17, PP05-21,

PP13-114, PP31-20

Chen, M.Q. PP06-01, PP05-09

Chen, P. PP31-16

Chen, P.J. S01-01, S03-01

Chen, P.-J. PP04-07, PP06-27, PP13-93

Chen, Q. PP28-21, PP28-23, PP30-06

Chen, R. PP05-76

Chen, S. PP05-122, PP15-24, PP26-68, PP27-14, PP34-03

Chen, S.H. PP31-19

Chen, S.-J. PP32-12

Chen, T. PP05-45, PP05-47, PP05-53, PP05-107, PP06-50, PP20-08, PP20-09, PP30-06, PP38-47

Chen, T.-M. PP33-04

Chen, W. FC01-05, PP05-60, PP05-76, PP05-153

Chen, X. FC03-04, PP04-24, PP05-61, PP05-76, PP06-63, PP08-04, PP12-03, PP12-31, PP39-74

Chen, X.-B. PP16-14
Chen, Y. PP05-22, PP05-107, PP05-117,

PP13-26, PP16-07, PP20-01

Chen, Y.-C. PP05-129, PP13-104

Chen, Y.H. PP30-27

Chen, Y.J. PP03-16

Chen, Y.-P. PP04-11

Chen, Y.-W. C03-02, PP07-35

Cheng, A.-L. C06-03

Cheng, D.Y. PP02-25, PP19-09

Cheng, H.-R. PP06-27

Cheng, J. PP05-44, PP05-134, PP12-20,

PP14-10, PP14-11, PP14-12, PP15-08,

PP15-10, PP24-06, PP26-18, PP31-21, PP34-27

Cheng, K.C. PP27-14

Cheng, M. PP05-152

Cheng, T. PP26-68

Cheng-Lin, W. PP26-26, PP26-28, PP28-15, PP28-16

Cheon, G.J. FC06-02, PP23-38, PP26-48, PP26-49, PP31-23, PP31-24

Cheon, Y.K. PP23-51, PP39-61

Cheong, H.S. PP30-12, PP30-13

Cheong, J. PP16-23

Cheong, J.H. PP29-13

Cheong, J.Y. FC06-04, PP05-120,

PP16-49, PP23-35

Cheraitia, S. PP04-06, PP25-05

Cherkaoui, A. PP02-17

Cherkashova, E.A. PP08-13

Chernyvscay, Z.T. PP01-43

Cherradi, Y. PP04-47, PP06-34,

PP13-16, PP34-31, PP34-33

Cherubini, B. PP06-56

Chetkina, T. PP17-15, PP17-16

Cheung, S.P. PP08-26

Cheung, T.T. PP33-03, PP33-13,

PP34-21, PP36-07, PP36-14

Chevaliez, S. FC05-01, PP05-135

Chhina, D.K. PP39-34

Chhina, R.S. PP10-09, PP39-34

Chiang, B. PP05-99

Chiba, T. PP01-05, PP03-39, PP04-46

Chibout, S.-D. PP06-38

Chichkina, M. PP10-21

Chicykina, M. PP10-22

Chieco, P. PS03-01, PP19-07

Chi-Hua, F. PP36-10

Chikayama, T. PP23-32

Chikhi, Y. PP04-06, PP25-05

Chim, A.M. PP05-08

Chim, A.M.-L. PP05-102

Chin, D. PP13-57

Chiodi, R. PP26-58

Chiou, Y.-Y. PP33-02

Chirawatthanaphan, P. PP39-84

Chirita, D. PP37-08

Chirnaksorn, S. PP26-33

Chitapanarux, T. PP23-89, PP34-17, PP39-52

Chittamma, A. PP23-52

Chiu, C.-T. PP08-42

Chiu, W.-H. PP04-14

Chkhartishvili, N. PP13-53

Cho, C.H. PP28-17

Cho, C.M. FC04-01, PP04-29, PP15-37, PP33-24 
Cho, D.H. PP02-08, PP13-100

Cho, E.J. PP04-18, PP07-15, PP15-03,

PP16-18, PP16-20, PP30-04, PP32-08

Cho, E.Y. PP09-33

Cho, E.-Y. PP32-09

Cho, H.C. PP03-21, PP03-23, PP05-14, PP05-160

Cho, J.Y. PP23-51

Cho, M. PP05-139, PP12-25, PP16-23,

PP16-49, PP29-13

Cho, S.B. PP05-123, PP13-72, PP26-34

Cho, S.-H. PP08-15

Cho, S.W. FC06-04, PP05-120, PP16-23,

PP16-49, PP23-35

Cho, W.K. PP08-24

Cho, Y.D. PP23-51, PP39-61

Cho, Y.K. PP05-55, PP09-33, PP23-05, PP28-02

Choe, W.H. PP09-34, PP23-28

Choi, B.I. S05-01

Choi, B.J. PP13-102

Choi, B.K. PP23-37

Choi, C.S. PP09-33

Choi, C.W. PP27-07, PP28-10, PP28-11

Choi, D. PP26-12

Choi, D.J. PP05-57

Choi, D.P. PP39-60

Choi, E.H. PP16-58, PP31-35

Choi, G.H. PP16-47, PP31-33, PP36-21

Choi, H.-K. PP07-18

Choi, J.H. PP07-02, PP07-03, PP07-04,

PP15-04, PP39-17

Choi, J.-I. PP26-65, PP37-15

Choi, J.S. PP13-102, PP16-47, PP31-33, PP36-21

Choi, J.W. PP05-115, PP13-67

Choi, J.Y. PP05-51, PP22-12, PP32-10

Choi, K.C. PP34-24

Choi, K.-Y. PP19-10

Choi, M.H. PP28-26

Choi, M.K. PP01-22, PP15-23

Choi, M.S. PP03-21, PP03-23, PP05-15,

PP05-160, PP06-13, PP09-17, PP36-13

Choi, N.K. PP31-23, PP31-24

Choi, S.B. PP13-102

Choi, S.H. PP19-04, PP20-12

Choi, S.K. PP05-123, PP13-72, PP26-34

Choi, S.W. PP32-10

Choi, S.Y. PP04-29

Choi, W. PP05-139

Choi, Y.M. PP08-26

Chok, K. PP29-05

Choksi, M.J. PP02-24, PP23-26,

PP39-62, PP39-63

Chon, C.Y. PP03-26, PP13-18, PP16-46,

PP16-47, PP16-50, PP16-58, PP23-69,

PP23-84, PP31-33, PP31-35

Chon, Y.E. PP23-84

Chong, D. PP22-11

Chong, L.-W. PP07-24

Chong, Y.-T. PP03-01, PP04-02, PP22-14

Chongsrisawat, V. PP08-31, PP23-10,

PP28-20, PP38-35

Chonprasertsuk, S. PP04-49

Choo, S. PP12-25

Chook, J.B. PPO6-39
Chooklin, S. PP16-21, PP23-24

Chorasiya, V. PP38-53, PP38-55, PP38-59, PP38-59

Chotiyaputta, W. PP13-11

Chou, Y.-J. PP28-22

Choudhary, N.S. PP23-58, PP23-79

Choudhuri, G. PP05-73, PP05-74, PP05-75, PP13-30, PP13-31, PP13-33, PP13-106, PP39-85, PP39-86

Chow, W.H. PP08-26

Chowdhary, M.M.H. PP32-04, PP08-39

Chowdhury, A. PPO2-10, PP02-11, PP15-29, PP15-30, PP19-02

Chowdhury, M.A.J. PP08-39

Chowdhury, S. FC07-01, PP15-53

Christova, E. PP38-49

Chu, C.-H. PP34-08

Chu, I.S. PP30-32

Chu, S.H.-T. PP05-102

Chu, T. PP13-57

Chu, Y.C. PPO5-104

Chuang, P.-H. PP05-106

Chuang, W. FC01-03

Chuang, W.L. PP12-25

Chuang, W.-L. FC05-03

Chuang, W.-L. PP28-31

Chuang, W.-L. PP34-30

Chuchev, E.S. PP26-53

Chung, C.W. PP27-07, PP28-11

Chung, G.E. PP16-18, PP30-11,

Chung, K.W. PPO5-51, PP05-67

Chung, W.J. PP13-28

Chung, Y.C. PP07-03, PP07-04,

PP15-04, PP39-17

Chung, Y.-H. PP35-07

Chupin, I.D. PP07-09, PP08-12

Chutaputti, A. AA-05

Chuthapisith, J. PP38-38

Ciaccioli, G. PPO7-22

Ciccorossi, P. PP06-56

Cichoż-Lach, H. PP10-02

Cijevschi, Prelipcean, C. PP16-32, PP37-08

Çınar, K. PP38-50

Cinco, R.U.C. PP29-07, PP32-02

Ciof, O. PP23-59

Cionfoli, N. PP23-40, PP26-46

Clark, P. PP12-25

Clayton, F. FC03-04

Cloherty, G. PP03-06

Clouston, A. PP15-21, 21-04

Cluzeaud, F. PP06-43

Cluzel, P. PP23-90

Cobb, B. PP06-46

Coco, B. PP06-56

Cohen, D. PP05-65, PP05-146, PP05-152, PP05-155, PP13-95

Colak, Y. PP23-80, PP26-41, PP26-69

Coldea, L. PP11-11

Cole, L. PP06-02

Colombatto, P. PP06-56

Colombo, M. C17-03, PP09-07

Comeily-Birjandi, E. PP23-63, PP23-64

Cong, M. FC03-05, PP06-14, PP15-36,

PP15-40, PP22-06

Cong, W.-M. PP31-08, PP34-05

Cong, X. PP03-22, PP05-148, PP22-15
Connell, E. PP13-47

Connor, S. PP13-103

Conti, B. PP08-52, PP30-35

Cooksley, W.G.E. PP13-47

Coombs, D. PS01-02, PP05-99, PP05-101

Coombs, D.H. PP05-91

Cooney, E. PP13-104

Copaci, I. PP10-14, PP25-17

Corcioni, B. FC04-02, PP34-12

Cordoba, S. PP15-53

Coric, M. PP13-24

Cornianu, M. PP16-39

Corr, P. PP29-08

Cortez-Navarrete, M. PP39-59

Coskun, O. PP13-35

Coşkun, Ö. PP13-105

Costa, R. PP23-81

Costes, B. FC05-01

Cotoraci, C. PP10-04

Cotrim, H. PP08-51

Cottone, M. PP13-13, PP14-21

Couzigou, P. FC05-01

Cowen, U. PP06-46

Cramp, M.E. PP25-01, PP25-02, PP26-06

Crane, M. PP15-33

Crawford, D. C21-04, PP23-19

Craxì, A. PP08-52

Cross, T.J. PP25-01, PP25-02, PP26-06

Csibova, V. PP04-42, PP05-11, PP05-100, PP25-09

Cua, I.H. PP08-59, PP08-60, PP23-95

Cui, B. PP31-20

Cui, Q. PP12-03

Cui, Y. FC01-03, PP23-25

Cukrowska, B. PP25-07

Cun, Y.L. PP05-05

Curescu, M. PP13-46

Curtis, M. PP05-125

Cvetkovic, T. PP01-08

Daddiego, G. PP09-08

D'Addiego, G. PP05-88

Dai, C.-Y. PP28-31, PP34-30

Dai, L. PP16-07

Dai, W. PP05-59

Dai, Z. PP33-20, PP33-21

d'Albuquerque, e, Castro, F.R. PP17-19,

PP32-13

Daluwatte, P.A. PP39-32

Damodaran, J. PP26-40, PP39-56, FC02-03

Dan, I. PP16-30

Dan, Y.Y. PP02-07, PP02-21, PP05-40, PP05-66, PP05-72, PP05-114, PP05-158, PP22-16

D'Andrea, M. PP12-38

Dandri, M. PP09-06

Dang, S. PP04-44

Danila, M. PP16-30, PP16-31, PP16-39,

PP23-44, PP23-59, PP37-10, PP37-12

Danninger, F. PP38-49

Daoud, S. PP29-08

D'Arca, T. PP04-38, PP26-59

Dargan, P. FC08-01, PP38-53, PP38-59

D'Armiento, J. PP15-38

Daruich, J. FC01-01

Darwish, M. PP09-03

Das, A. PP14-05 
Das, B.C. PP09-40, PP13-55

Das, H.S. PP05-63, PP23-71, PP23-72

Dasgupta, J. PP08-27

Dassanayake, A.S. PP23-42

Date, K. PP27-09

Datta, I.K. PP08-20

Datta, S. PP06-22

Datz, C. PP13-61

Davasaz, Tabrizi, A. PP02-29

Davis, M. PP13-75, PP24-07

Davis, N.A. PP24-07

Davison, S. PP13-103

D'Avolio, A. PP05-42

de, Alwis, J.P. PP23-42

de, Franco, F. PP07-22

De, Gaetano, A.M. PP33-11

De, Gascun, C.F. PP26-36

de, Knegt, R. PP37-20

De, la, Cruz, N. PP07-05

de, Ledinghen, V. PS04-04, PP16-15, PP16-13

De, Lusong, M.A. PP08-02, PP23-11

de, Man, R. PP37-20

de, Man, R.A. PP05-91

de, Mey, J. PP37-14

de, Meyer, S. PS02-04

De, Meyer, S. FC05-02

De, Muynck, S. PS02-01, PP14-16

de, Silva, A.P. PP23-42

de, Silva, H.J. PP23-42

De, Smedt, G. PP13-59

De, U. PP07-07

De, Wever, O. PP30-07

De-Almeida, A.J. PP14-25

Deblakshmi, R. FC04-05, PP39-68

Deblakshmi, R.K. PP28-27

Debnath, C.R. PP04-30, PP26-32

Debnath, M.R. PP04-30

Debski, B. PP39-53, PP39-55

Debzi, N. PP38-20

Dede, S. PP05-40, PP05-66, PP05-72,

PP05-114

Deforges, L. PP23-92

Deka, M. FC04-05, PP28-27, PP39-51, PP39-68

Deka, P. PP09-11

Dekonenko, E. PP09-39

Dela, Cruz, E. PP08-02

Dela, Cruz, E.A. PP23-11

Deleanu, A. PP16-30

Delgado, Rizo, V. PP23-16

Delikanli, B. PP05-58

Dembinski, S. PP16-27

Demes, M. PP01-26

Demir, K. PP09-29, PP23-80

Deng, B.-C. PP01-35

Deng, G. FC01-05, PP20-09

Derbala, M. PP12-18

Derdabi, O. PP04-47, PP06-34

Dersi, N. PP04-04

Deshpande, M. PP13-63

Deuschle, U. PP07-23

Devadas, K. PP23-96

Devi, S. PP23-96

Devrajani, B. PP16-10

De-Wu, H. PP24-06

Dhali, G. PP02-10, PP02-11, PP15-30
Dhana, N. PP05-150

Dharwadkar, S. PP34-37

Dharwadkar, S.N. PP30-36

Dhillon, S. PP10-19, PP23-41

Dhiman, R. PP23-58, PP23-79, PP31-37

Dhooria, G. PP09-37

Di, Bisceglie, A. PS02-03

Di, Bonaventura, M. PP12-10

Di, Perri, G. PP05-42

Di, Tolla, G. PP33-10

Diago, M. PS02-04, PP13-47, PP13-56

Dianat, M. PP24-09

Diao, N. PP03-37

Dias, K. PP10-10

Diculescu, M. PP16-12

Dieterich, D. PP13-47

Diktas, H. PP02-22, PP04-33

Dimitroulopoulos, D. PP13-06

Ding, H. PP04-12, PP05-61, PP16-44

Ding, K. PP12-03

Ding, L. PP05-53

Ding, M. PP20-01

Ding, X.-M. PP13-117

Dipika, G. PP23-55

Dizaji, R. PP02-26

Djogic, V. PP05-70

Djumhana, A. PP02-27, PP23-98

do, Ó, K.M. PP32-13

Dobbins, T. PP13-103

Dofiles, M. PP08-59

Dogan, Ü.B. PP13-62, PP17-03

Dogan, U.B. PP09-14

Doganay, L. PP26-41, PP26-69

Dökmeci, A. PP13-19

Dolatkhah, R. PP12-08, PP12-09

Dolmazashvili, E. PP13-53

Domingo, E. PP14-13

Domingo, F. PP29-07

Donadio, C. PP26-58

Donadio, L. PP07-22

Donadon, V. PP32-01

Dong, H. PP31-08, PP34-05

Dong, J. PP03-34, PP05-60, PP05-137, PP07-28, PP23-50

Dong, M. PP05-44, PP06-38

Dong, S.H. PP28-17

Dong, Y. FC01-01, PP05-50, PP05-117

Donnelly, B. PP28-05

Donner, N. PP30-21

Dore, G.J. PP13-56, PP13-59, PP15-33

Dorobat, C. PP05-03

Dosoki, T.M. PP16-16

Doss, W. FC05-05, PP38-51

Dou, X. PP05-155

Dougaz, W. PP26-22

Doustar, Y. PP02-29, PP17-04

Doutreloigne, J. PP03-10

Dovlo, A. PP13-69

Doyuk, Kartal, E. PP05-131

Dranga, M. PP16-32, PP37-08

Drapkina, O.M. PP07-11, PP07-12,

PP07-13, PP07-14, PP08-10

Drazek, D. PP26-17

Drozdov, V. PP23-61

$\mathrm{Du}, \mathrm{S}$. PP03-22

Du, X. PP03-37
Du, Z. PP31-14

Duan, J. PP04-41

Duan, X.Y. PP07-26, PP30-22, PP34-34

Duan, Y. PP02-13

Duan, Z. PP05-152, PP16-08, PP20-01,

PP24-01

Duan, Z.-J. PP16-03

Dubale, N.A. PP23-55

Duces, A. PP14-16

Ducès, A. PS02-01

Duclos-Vallée, J.-C. PS04-02

Dufour, J.-F. AE-04

Duh, P.-D. PP19-08

Dumas, E.O. PP13-95

Dumitrescu, D. PP29-17

Dumitru, E. PP10-11, PP10-17, PP10-18, PP16-22, PP37-13

Dumitru, I.M. PP10-11, PP10-17, PP10-18, PP16-22, PP37-13

Dunford, L. PP26-36

Dunn, D. FC03-04

Dunne, A.M. PS02-03

Dupas, E. PS02-01

Durgapal, H. FC03-02, PP06-10, PP09-09, PP09-18

Duseja, A. PP23-58, PP23-79, PP31-37

Dusheiko, G.M. PS02-03, PP13-46

Dutta, A.K. PP10-13

Duttagupta, S. PP05-18, PP16-37

Duyun, C. PP05-116

Dwarkasing, R. PP37-20

Dweik, N.A. PP12-18

Dziri, C. PP26-22

Edano, J. PP08-60

Edwards, R. PP05-159

Egesel, T. PP17-03

Egodage, S.H. PP39-32

Ehsan, N. PP21-07

Eissa, L.A. PP31-09

Ekram, S. PP17-07, PP32-04, PP39-29

El, Abkari, M. PP13-25, PP23-82, PP23-83, PP25-20

El, Aggan, H.A. PP31-41

El, Akkaoui, A. PP34-33

El, Amin, H. PP33-16, PP39-67

El, Azab, G. PP13-23

El, Deeb, N. PP31-41

El, Delgawy, W. PP31-41

El, Feydi, Essaid, A. PP04-47

El, -Gandour, M. PP07-01

El, Ghamry, F. PP09-02

El, Ghazzawy, I. PP23-06

El, Hefni, S. PP24-03

El, Karaksy, H. PP17-12

El, Khayat, H.R. PP39-67

El, Kholy, A.A. PP38-52, PP38-51

El, Malki, A. PP04-04

El, Malki, H.O. PP13-16, PP34-33

El, Moghazy, W. PP38-13, PP38-14

el, Rafie, M.S. PP11-03

El, Raziky, M.E.S. PP08-53

El, Sawy, M. PP31-02

El, Sayed, A. PP23-76, PP39-81

El, -Sharkawy, M.A. PP33-16, PP33-17

El, Sheikh, R. PP08-03

El, Younis, C.M. PP14-14 
El, Yousfi, M. PP13-25, PP23-82, PP23-83, PP25-20

Elabkari, M. PP12-36, PP12-43, PP13-108, PP13-109, PP16-41, PP23-70, PP23-73, PP23-75, PP26-62

Elaggan, H. PP39-11

El-Ahwany, E. PP11-14, PP31-36 Elalfy, H. PP14-22, PP21-09, PP25-21 El-Attar, M.M. PP07-16, PP11-06, PP12-06 Elazhary, S.S. PP39-80

El-Baz, M.A.H. PP16-17

Elbendary, M.M. PP06-45, PP14-03, PP16-11, PP21-09

El-Bendary, M. PP25-21

El-Deek, B.S. PP14-22

El-Demerdash, F.M. PP39-05

Elefsiniotis, I. PP13-06

El-Emshaty, W. PP25-21

Elesily, N. PP11-07

Eletreby, S. PP14-22

Elewa, A. PP09-38

Eley, T. PP13-76

El-Fakkarany, E. PP13-05

Elfaramawy, A. PP25-06

El-Gabaly, H. PP38-51, PP38-52

El-Gayar, A.A. PP31-09

El-Gendy, A.A. PP14-03

Elgendy, H. PP38-13, PP38-14

Elgendy, M. PP23-03

Elhadad, B. PP13-02

Elhassi, A.F. PP13-02

Elhenawy, A. PP39-11

El-Hennawy, A. PP17-12, PP17-13

Elhossiny, R. PP25-06

El-Karaksy, H. PP17-13

El-Kiki, H. PP17-12

El-Koofy, N. PP17-12, PP17-13

El-Lakkany, N. PP08-46

Elmansy, M. PP39-09

El-Mlegy, N.I. PP07-16

El-Mougy, F. PP17-13

Elnadry, M.H. PP39-80

El-Raziky, M. PP17-12, PP17-13

EL-Sahhar, M. PP38-25

El-Sayed, R. PP17-12

Elsehrawy, F. PP39-09

El-Serafy, M. PP38-51, PP38-52

El-Serogey, Y. PP39-16

Elshafee, A.M. PP39-80

Elshamy, M. PP08-46

Elshazly, M. PP39-09

El-Sherbeeny, W. PP06-45

El-Shereef, H.K. PP24-04

Elsherif, Y. PP23-87, PP39-07, PP39-83

Elsiesy, H.E. PP26-55

Elteeby, D.M. PP39-80

El-Wakil, A.M. PP33-29

El-Warraky, M. PP34-14

Elyamany, A. PP13-17

Elyousfi, M. PP04-36, PP12-36, PP12-43,

PP13-108, PP13-109, PP16-41, PP23-70, PP23-73, PP23-75, PP26-62, PP04-36

Elzefzafy, W.M. PP23-60

Emerson, S. FC03-03

Enders, F. PP31-16, PP31-20

Endo, R. PP13-22, PP13-39
Enooku, K. PP33-25

Eo, S.J. PP09-17

Er, Y. PP04-41

Erben, N. PP05-131

Erdem, L. PP06-25, PP06-26

Eren, F. PP08-21

Ergen, N. PP08-25

Eric, L. PP36-10

Eric, W. PP13-29

Ersoz, F. PP05-78, PP05-79, PP13-35

Ersöz, F. PP13-105

Ersoz, G. PP12-30

Ersöz, S. PP38-50

Erttürk, H. PP39-24

Esen, S. PP06-09

Eshraghi, H. PP12-44

Esmael, F. PP39-67

Esmat, G. FC05-05, PP38-51, PP38-52

Essaid, A. 253, PP13-16, PP34-31, PP34-33

Essaid, El, Feydi, A. PP04-47, PP06-34

Essioux, L. PP13-57

Esteban, R. PS02-02, PP06-08

Estrabaud, E. PS02-01, PP06-43, PP14-16

Eswaran, S. FC02-03, PP26-40, PP39-56

Eun, C.S. PP16-33

Eun, J.R. PP23-36

Evans, K.A. FC07-01, PP15-53

Everson, G. PP13-64, PP13-76, PP13-89

Eyigun, C.P. PP05-78, PP05-79, PP13-35

Eyigün, C.P. PP13-105

Ezzikouri, S. PP06-34

Fabris, C. PP05-42

Fadaei, Nobari, R. PP12-11

Fadaly, G. PP23-03

Fafutis-Morris, M. PP23-16

Fagan, E. PP05-99, PP05-101

Faheem, M. PP14-01

Fahim, A. PP26-37

Fallon, M.B. C11-02, SA03-01

Fan, J. PP07-36

Fan, J.G. PP07-26, PP30-22, PP34-34

Fan, J.-G. C03-02, PP07-35

Fan, K. PP04-26, PP05-46, PP05-147

Fan, S.T. PP29-05, PP33-03, PP33-13, PP34-21, PP36-07, PP36-14, PP38-17, PP38-27

Fan, T. PP08-19

Fan, W.-B. PP32-12

Fan, X. PP01-33

Fan, X.-P. PP01-31, PP01-38, PP23-77

Fan, Y.C. PP01-28

Fan, Y.-C. PP01-31, PP01-38, PP04-11

Fang, J.L. PP14-06

Fang, L. PP17-10, PP32-06

Fang, X.-D. PP30-25

FanXiao, T. PP05-90

Farag, R.E.-S. PP14-22

Farahani, M. PP12-22

Farahat, N. PP13-07

Faraoun, S.A. PP38-20

Fareed, J. PP10-19, PP23-41

Farid, S. PP23-06

Farkasova, A. PP01-26

Farley, J.D. PP12-37, PP13-14

Farok, L. PP31-09

Farrag, E. PP30-19
Farrell, G. AS-03

Faryal, A. PP23-57, PP26-37

Fassio, E. FC01-01

Fathi, M. PP13-111

Fawzy, M. PP29-01, PP31-36

Fazio, V. PP05-88, PP09-08, PP13-44, PP14-08

Fede, G. FC06-01

Fedorov, I. PP09-39, PP10-22

Fedosina, E. PP23-01, PP23-34

Fei, R. PP15-27, PP22-15, PP30-27

Fekih, M. PP04-13

Feng, B. PP14-06

Feng, J.-Y. PP04-22, PP04-23, PP12-21

Feng, L. PP05-116

Feng, X. PP05-05

Feng, X.-S. PP13-117

Ferderigou, A. PP13-06

Ferenci, P. PP12-34, PP13-46, PP13-59, PP13-61

Fernandas, M. PP05-40, PP05-66, PP05-72, PP05-114

Fernandez-Fernandez, PP06-08, PP13-84, PP13-84

Fernandopulle, A. PP01-10, PP26-26

Ferretti, G. PP39-15

Feutren, G. C05-03

Filali, A. PP04-13

Fimiani, B. PP05-10, PP26-08

Firpo, M. FC03-04

Firuzmandi, M. PP11-04

Flamm, S. PP13-89

Fletcher, L. PP23-19

Flisiak, R. FC05-03, PP12-15

Florentino, A. PP08-51

Florentino, G. PP08-52

Flores-Contreras, L. PP16-51

Floresvillar-Mosqueda, J. PP15-45

Focaccia, R. PS02-04

Focsa, M. PP16-30

Foglieni, B. PP09-07

Fontana, D. PP13-64

Fornari, F. PS03-01

Forroza, R. PP08-02, PP23-11

Forughifar, M. PP09-15

Foster, G. FC05-03

Foster, G.R. PS02-04

Fouad, Y. PP08-14

Foucher, J. PP16-13, PP16-15

Foulstin, S. PP13-79, PP15-32

Foustin, S. PP13-83, PP13-87

Francavilla, R. PP16-27

Francis, H. PP22-13

Francois, P. PP06-38

Franklin, M. PP23-19

Frankova, S. PP38-24

Frederick, D. PS01-03

Freitas, L. PP15-02

Freitas, L.A. PP17-19

Frelin, L. PP05-64, PP05-186

Frentiu, A. PP08-05

Fried, M. PP13-57

Fried, M.W. PP13-57

Friedman, S. PP15-24

Fu, J.-L. PP37-02

Fu, S. PP38-34

Fu, X. PP19-36 
Fujie, H. PP35-25

Fujimoto, J. PP26-45, PP27-79, PP36-20

Fujimoto, M. PP04-08

Fujimura, T. PP36-26

Fujioka, S. PP25-08

Fujisaki, H. PP36-26

Fukuda, H. PP38-13

Fukuda, K. PP38-14

Fukuda, T. PP01-05

Fukuda, Y. PP25-16

Fukushima, K. PP14-20

Funda, Y. PP31-22

Fung, J. PP06-37

Fung, K.H. PP35-06

Fung, S. PP05-157

Gabr, M. PP13-111

Gadd, V. PP16-28

Gadelha, C. PP08-51

Galal, G. PP16-17

Galati, J. PP13-75

Galiè, N. PP13-78

Gall, M. PP19-10

Galstyan, T. PP113-118

Gan, J.-H. PP06-62

Gan, W.-Q. PP06-47

Gane, E. C04-02, PS01-02

Gane, E.J. PS04-05

Gang, L. PP05-108

Gangwar, D. PP12-27

Gani, R. PP03-11

Gao, C. PP03-12

Gao, F. PP12-31

Gao, H. PP13-27

Gao, L. PP13-01

Gao, R. PP16-04

Gao, S. PP13-02

Gao, W. PP33-150

Gao, X. PP05-148

Gao, Y. PP012-13, PP04-32

Gao, Y.-Q. PP05-39

Gao, Z.L. PP03-01, PP13-03, PP22-14

Garbagnati, F. PP03-10, PP33-12

Garcia-Bañuelos, J.J. PP15-45

Garcia-Tsao, G. AS-08

Garcovich, M. FC06-01, PP33-11

Gardiner, D.F. PP13-76

Garg, H.K. PS01-05, PP16-34, PP16-36

Gashaw, H. PP39-30

Gatserelia, L. PP06-15, PP13-53, PP14-17

Gaudy-Graffin, C. PP06-41

Gaultier, I.A. PP13-95

Gaur, K.J.B.S. PP08-30

Gayatri, P. PP04-10, PP04-39

Gazizov, I. PP10-23, PP10-24

Ge, F.S. PP05-92, PP06-12

Ge, J. PP01-38

Gegeebadrakh, B. PP23-68

Gegeshidze, T. PP01-09, PP17-09

Gehring, A.J. PP.09-41

Geng, J. PP09-13, PP09-20, PP09-31

George, J. C03-01, PS01-02, PP15-38, PP15-39, PP34-23

George, M. PP10-19, PP23-41

George, S. PS02-03, PP13-89

Georgescu, E.F. PP29-17

Gera, A. PP13-40
Gerada, E. PP23-04

Gerada, J. PP23-04

Gerasimova, O. PP05-95

Gerhardt, D. PP16-38

Gerken, G. PP07-31

Germer, S. PP13-57

Gershwin, M.E. PS04-01

Gerzova, L. PP23-21

Gespach, C. PP30-07

Gettueva, A. PP26-53

Gettueva, A.A. PP01-43, PP13-19

Ghaderi, M. PP22-07

Ghaffar, A. PP05-138

Ghalib, R. PP13-64, PP13-76

Ghamari, S. PP14-13

Ghandforoush-Sattari, M. PP12-08, PP12-09

Ghane, M. PP12-22

Ghanem, A. PP23-06

Ghatak, S.L. PP02-10, PP02-11,

PP15-29, PP15-30, PP19-02

Ghazinyan, H.L. PP13-08, PP13-118

Ghaznavi, M. PP39-68

Gheorghe, C. PP16-12

Gheorghe, L. PP16-12

Gherhardt, D. PP16-39

Ghidinelli, M. PP30-30

Ghittoni, G. PP23-40, PP33-10, PP33-12

Gholam, Abas, K. PP04-35, PP12-39

Ghosh, S. PP19-02

Ghufran, A. PP23-23

Giannuoli, G. PP13-13

Gill, M.L. PP05-83, PP05-84, PP13-10,

PP13-37, PP23-31

Gill, S. PP01-40, PP09-23, PP09-24

Gines, P. PP23-15, PP23-39

Ginhoux, F. PP09-41

Giovannini, C. PS03-01, PP19-07

Gish, R. PP05-141, PP34-14

Glaser, S. PP22-13

Glass, A. PP05-113

Glinkov, S. PP26-01

Glinkova, V. PP26-01, PP27-02

Goda, K. PP23-88

Goda, M. PP09-02

Godec, M. PP02-05

Goh, K.L. EC-02

Goh, K.-L. PP23-49

Gokak, V.P. EC-01

Gokturk, K. PP06-25, PP06-26

Goldis, A. PP23-74

Golfieri, R. FC04-02, PP34-12, PP37-06

Golovanova, E. PP10-16

Golovchenko, O. PP13-82

Gondal, M. FC08-02

Gondal, R. PP39-65

Gong, G. PP03-18, PP04-44, PP19-09

Gong, G.-M. PP11-13

Gong, X. PP04-16, PP04-17

Gonzalez-Cuevas, J. PP15-45

Goodman, Z.D. PP05-65

Goodwin, D. PP05-101

Gooya, M.M. PP39-08

Gopal, M. PS03-05

Gopalakrishnan, D. PP06-33

Gopez-Cervantes, J. PP08-59

Gorbunova, N.V. PP01-43
Gordillo-Bastidas, D. PP15-49

Gordon, S. PS02-02, PP13-75

Gorenek, L. PP02-22, PP04-33, PP05-20, PP06-25

Gorrell, M.D. FC07-01, PP15-53, PP19-10

Goto, E. PP33-25

Goto, N. PP05-80

Goto, T. PS02-05, PP33-25

Gotti, E. PP37-06

Goudeau, A. PP06-41

Goyal, N. FC08-01, PP38-53, PP38-55, PP38-59, PP38-63

Goyal, O. PP10-09, PP23-91, PP32-14, PP39-34

Goyal, P. PP23-91, PP32-14, PP39-34

Goyal, R. PP05-15, PP05-18, PP16-37

Gözaydın, A. PP04-20, PP13-119

Graça, L. PP26-04

Gracía-Trevijano, E.R. PP21-06

Graf, T.N. PP14-04

Graham, C.S. FC05-02

Grahovac, B. PP14-26

Gralak, M.A. PP39-53, PP39-55

Gramantieri, L. PS03-01, PP19-07

Gramatyuk, S. PP13-110

Granov, D.A. PP05-95

Grare, M. PP05-135

Grasela, D. PP13-76

Gray, T. PP13-64

Grazioli, L. FC04-02

Gregorek, H. PP25-07, PP25-10

Gregorio, G.V. PP38-05

Gregusova, A. PP01-26, PP38-49

Grenet, O. PP06-38

Grgic, D. PP05-70

Griffiths, W.J. PP23-04

Groma, V. PP25-18

Grudpan, K. PP31-39

Gruengreiff, K. PP23-13

Gschwantler, M. PP12-34, PP13-61

Gu, L.-L. PP11-13

Guan, R. AA-06

Gudsoorkar, V.S. PP23-26

Guessab, N. PP38-20

Guettier, C. PS04-02

Gui, H. PP06-02, PP06-36

Gui, H.L. PP39-79

Guimarães, H. PP26-04

Gul, H.C. PP05-78, PP05-79, PP13-35

Gül, H.C. PP13-105

Gulluoglu, M. PP26-41, PP26-69

Gumei, M. PP22-08

Gumerova, A. PP10-23, PP10-24

Gumma, P.K. PP13-55

Gumurdulu, Y. PP09-14

Gümürdülü, Y. PP05-10, PP05-11

Gümüşay, Ö. PP16-05

Gunal, E. PP05-78, PP05-79, PP13-35

Günal, E. PP13-105

Gunardi, H. PP04-10, PP04-39

Gunduz, A. PP06-26

Guner, R. PP06-09

Guo, F. PP14-06, PP14-07

Guo, H. PP05-130

Guo, H.-H. PP13-80

Guo, J. PP14-11, PP14-12, PP15-24, PP16-02, PP16-43, PP16-55 
Guo, J.-C. PP05-24

Guo, J.-T. PP14-23

Guo, L. PP31-31

Guo, S. PP19-05

Guo, T. PP13-76

Guo, W.W. PP05-45, PP05-47, PP05-53, PP05-92, PP06-12

Guo, X. PP04-44, PP05-61, PP05-128

Guo, Z. PP01-33, PP29-10, PP36-01,

PP38-03, PP38-07, PP38-08, PP39-21, PP39-22

Gupta, A. PP26-54

Gupta, D.K. PP10-09, PP34-09

Gupta, E. PP06-23

Gupta, P. PP09-18

Gupta, R.K. PP10-12, PP23-55, PP39-58

Gupta, S. C11-03, FC08-01, PP13-65,

PP13-66, PP38-53, PP38-55, PP38-59,

PP38-63, PP39-58

Gurel, S. PP05-91

Gursel, I. PP21-03

Gurugubelli, B. PP26-05

Guruvayoorappan, C. PP39-88

Gusejnzade, M. PP10-16

Gustiananda, M. PP06-30

Guvener, Demirag, N. PP08-25

Gwak, G.-Y. PP03-21, PP03-23,

PP05-14, PP05-160, PP06-13, PP09-17

Ha, K.H. PP39-60

Habbua, P.V. PP02-12

Habeeb, M.A. PP22-10

Hacharoen, P. PP12-41, PP12-42

Hadziyannis, S.J. PP13-56

Hagar, M. PP23-97

Haghdoost, A.A. PP04-14, PP04-15

Hahn, T.H. FC06-04

Haimi-Cohen, Y. PP38-21

Hairulhisyam, M.N. PP21-05

Hai-Yan, Z. PP26-27, PP26-28, PP28-16

Hajarizadeh, B. PP04-14, PP04-15

Hajibeigi, B. PP11-04, PP11-12

Hakamada, K. FC08-04, PP21-12, PP38-64

Haleagrahara, N. PP02-16

Hall, W.W. PP26-36

Halota, W. PP12-15

Halpern, M. PP34-20

Halue, G. PP06-41

Hamada, T. PP37-07

Hamano, R. PP26-60, PP26-61

Hamaoka, K. PP23-32, PP29-18

Hambruch, E. PP07-23

Hamdy, L. PP39-66

Hamed, M.A. PP14-15

Hamid, S. FC06-05, PP01-16, PP01-44,

PP09-32, PP11-09, PP12-45, PP13-21,

PP13-34, PP23-23, PP39-31

Hamilton, M. PP01-26

Hamilton, S. PP39-07

Hammam, O.A. PP11-07

Hammond, R. PP03-24

Hamy, A. PP26-30

Hamza, A. PP29-08

Hamzah, H.A. PP12-05

Han, D.S. PP16-33

Han, E.H. PP07-03

Han, J. PP01-34, PP05-148
Han, J.H. PP05-30

Han, J.K. PP26-65

Han, J.Y. PP22-12

Han, K.H. FC01-03, FC06-04, ET2-03, PP05-12, PP05-139, PP12-25, PP23-69

Han, K.-H. PP03-26, PP05-65, PP13-18, PP16-46, PP16-47, PP16-50, PP16-58, PP19-04, PP20-12, PP23-84, PP30-29, PP31-33, PP31-35

Han, Kiat, H. PP34-26

Han, L. FC01-02, PP01-33

Han, L.-Y. PP23-77

Han, M. PP05-45, PP06-49, PP06-50, PP20-09

Han, N.I. PP32-10

Han, P. PP16-54

Han, S.-H. PP10-12

Han, T. PP01-42, PP06-64

Han, W.K. PP16-47

Han, Y. PP03-20, PP05-23, PP05-25, PP05-26, PP06-59, PP11-13, PP22-13, PP35-05

Han, Z. FC04-03

Hanae, B. PP34-31

Hanaoka, Y. PP27-09

Handa, T. PP23-32

Haniffa, M. PP09-41

Hanina, E. PP10-16

Hansel, T.T. PP39-30

Hao, B.-J. PP39-76

Hao, Y. PP05-16, PP05-93

Haque, M.A. PP17-07, PP32-04

Haque, M.Z. PP17-07

Hara, K. PP26-70

Harada, J. PP26-66

Harahap, A. PP06-30

Harahap, A.R. PP04-19, PP06-31

Harinath, G. PP39-28

Harmandar, F. PP05-58

Harmsen, W.S. PP31-16, PP31-20

Harrison, S.A. PP13-50, PP13-56

Harry, R. PP38-10

Hasan, H.E. PP11-06

Hasan, I. PP03-11, PP06-28, PP16-25

Hasegawa, H. PP05-52

Hasegawa, K. FC02-04, C21-02, PP38-36

Hashemi, F. PP39-87

Hashimoto, K. PP13-32

Hasmoni, M.H. PP12-05, PP16-35

Hassan, E. PP39-16

Hassan, H. PP39-66

Hassan, M. PP14-27

Hassan, M.S. PP08-46, PP09-38

Hassan, S.M. PP08-17

Hassan, W. PP39-16

Hassanain, T. PP23-76, PP39-81

Hassanein, T. PP13-45, PP13-46

Hassar, M. PP06-34

Hassouna, M. PP05-143

Hasuike, S. PP13-121

Hata, K. FC08-03

Hatipoglu, M. PP04-33

Hayashi, K. PP26-63

Hayashi, S. PP33-26

Hazam, R.K. PP09-11

Hazinedaroğlu, S. PP38-50

$\mathrm{He}$, C. PP01-18

He, F. PP05-90, PP16-06, PP30-05
He, G. PP24-01

He, H.W. PP15-09

He, J. PP34-03

He, J.S. PP03-16

He, L.-H. PP34-10

$\mathrm{He}, \mathrm{Q} . \mathrm{PP} 13-42$

He, W. PP03-03, PP29-12, PP30-31

He, W.H. PP15-08, PP15-10, PP15-11

He, X. PP29-10, PP36-01, PP38-03, PP38-07,

PP38-08, PP38-19, PP39-21, PP39-22

He, X.-D. PP04-01, PP27-01, PP27-17

He, Y. PP01-15, PP04-44, PP38-29

He, Z.L. PP38-30

Heathcote, E.J. PS01-01, PS01-02, PP05-91

Heathcote, J. PP05-99

Hegarty, J. PP26-51, PP38-65

Heidari, S.T. PP39-06

Hellstrom, U.B. PP05-41

Helmy, A. PP38-42

Heo, J. FC04-01, PP15-37, PP26-39, PP29-13

Heo, J.S. PP36-13

Hernández, Ortega, L.D. PP15-48

Herrera*, K. PP07-05

Hewawasam, S.P. PP23-42

Heydari, S.T. PP39-08

Hézode, C. FC05-01, PP05-135

Hibi, T. PP26-15

Hidirov, B. PP06-11

Hien, T.T. PP07-18

Higami, K. PP13-122, PP13-123

Higashi, S. PP33-06

Higazy, A. PP39-83

Hillas, E. FC03-04

Hillson, J. PP13-64

Hindes, R. PP13-76

Hinkin, C.H. PP10-12

Hinton, H.J. PP39-30

Hirahara, K. PP23-32

Hirakata, A. PP26-70, PP26-71, PP28-30

Hiraku, Y. PP28-18

Hirankarn, N. FC01-04

Hirano, T. PP26-45, PP27-19, PP36-20

Hirose, S. PP23-17, PP33-08, PP33-09

Hirose, Y. PP33-08, PP33-09

Hirsch, K.R. PS01-03, PP05-101

Hirst, B.H. PP39-18

Hissar, S.S. PP39-65

Hitesh, J. PP19-12, PP19-13

Hizel, K. PP06-09

Hlaing, N.K.T. PP14-02

Hliwa, W. PP02-17

Hmeda, N. PP13-111

Ho, C.-L. PP26-68, PP27-14

Ho, H.K. PP02-21

Ho, Y.-C. PP03-35

Hofer, H. PP12-34, PP13-61, PP13-84, PP13-88

Hohmann, M. PP13-50

Holczbauer, A. PP15-55, PP31-42

Holden, A. PP23-18

Hollinger, F.B. C18-01

Holmström, F. PP13-114

Holzmann, H. PP12-34

Homma, S. PP25-15

Homs, M. PP06-08

Hong, C. PP09-22

Hong, G. PP38-39, PP38-40 
Hong, S.T. PP06-04

Hong, S.M. PP05-115

Hong, S.P. PP05-120

Hong, S.-S. PP15-22

Hong, S.W. PP23-51, PP39-61

Hong, S.-W. PP15-22

Hong, W.-D. PP23-08, PP23-67, PP28-03

Hong, X. PP22-04

Hong-Li, S. PP22-11

Hongo, O. PP33-15

Honsawek, S. PP28-20

Hooshmand, B. PP11-04

Hopkins, M. PP04-25

Hoque, M. PP39-47

Hoque, N. PP08-20

Horasan, E.S. PP12-30

Horban, A. PS02-04, PP12-15, PP13-46

Horga, M.A. PP13-64

Horlenko, F.V. PP16-09

Horlenko, O.M. PP16-09

Hosoda, K. PP33-08, PP33-09

Hosseyn, Pour, Feizi, A. PP12-08, PP12-09

Hou, J. PP05-22, PP05-44, PP05-146,

PP05-152, PP05-155, PP06-60,

PP12-17, PP12-47, PP13-117, PP16-07

Hou, W. PP01-32

Houssein, M.Z. PP11-03

Howard, A. PP39-18

Howard, R. PP26-36

Hoyjak, I. PP38-21

Hrstic, I. PP05-70, PP13-24, PP14-26

Hrusovsky, S. PP01-26, PP38-49

Hsiang, J. PP23-18, PP38-10

Hsieh, M.-H. PP28-31

Hsieh, M.-Y. PP28-31

Hsu, C.-S. PP04-07

Hsu, C.-T. PP17-05, PP28-12, PP28-22

Hsu, C.-W. PP05-12

Hsu, H.-W. PP05-129

Hsu, S.-J. PP13-93

Hu, A. PP38-08, PP39-21, PP39-22

Hu, C.-T. PP05-96

Hu, D.P. PP29-06

Hu, G.J. PP05-92, PP06-12

Hu, H. PP30-28, PP31-26, PP31-27

Hu, H.-D. PP39-33, PP39-38

Hu, J. PP03-03

Hu, K.-Q. PP05-65

Hu, L.H. PP01-36

Hu, M.-H. PP39-76

Hu, P. PP39-33, PP39-38

$\mathrm{Hu}$, P.X. PP03-16

Hu, T.-H. PP05-140, PP05-151, PP16-53

Hu, X. PP06-60

$\mathrm{Hu}$, Y. PP26-35

$\mathrm{Hu}, \mathrm{Z}$. PS02-05, PP03-18

Hu, Z.H. PP36-09

Hua, R. PP27-20

Huang, A. PP05-163

Huang, C. PP20-04

Huang, C.-F. PP28-31

Huang, C.H. PP05-104

Huang, C.-I. PP28-31

Huang, C.-M. PP08-29, PP08-32

Huang, H. PP12-17, PP12-47, PP15-46
Huang, J. PP05-45, PP05-76, PP08-18,

PP25-19, PP30-16, PP31-19, PP31-31

Huang, J.-F. PP28-31

Huang, J.H. PP05-05, PP05-27

Huang, K.-W. PP20-06

Huang, M. PP13-63

Huang, M.L. PP05-05

Huang, N. PP28-22

Huang, Q. PP05-24

Huang, Q.-K. PP23-67, PP28-03

Huang, S. PP26-72, PP38-56

Huang, S.Y. PP06-59

Huang, X. PP06-60, PP18-02

Huang, Y. PP05-45, PP22-05

Huang, Y.-H. PP33-02

Huang, Y.-T. PP07-24

Huang, Z. PP05-23

Huda, A. PP10-12

Hughes, E. PP13-65, PP13-66

Hui, A.J. PP04-09

Hui, E.P. PP34-18

Huibin, G. PP22-11

Huijie, J. PP37-01

Hui-Ying, Z. PP24-06

Hujii, Y. PP23-32

Hujimori, K. PP23-32

Hung, C.-H. PP05-140, PP16-53

Hung, H.-H. PP33-02

Hunyady, B. PP16-29

Huo, T.-I. PP33-02

Huong, D.D.1. PP05-139

Hussain, T. FC06-05

Hutson, W. FC03-04

Huzaini, A.T.M. PP23-49

Hwang, J.S. PP13-28

Hwang, S. LT1-02

Hwang, S.G. PP05-139, PP16-23, PP16-49

Hwang, S.-G. PP05-119

Hwang, S.Y. PP16-20

Hwang, Y.P. PP07-02, PP07-03,

PP07-04, PP15-04, PP39-17

Hwangbo, Y. PP28-17

Hyun, C. PP05-101

Iannucci, G. PP08-52

Ibraheem, H. PP23-76

Ibraheim, Y. PP08-53

Ibrahim, A. PP34-14

Ibrahim, T. PP16-48

Ibrahimi, A. PP04-36, PP12-36, PP12-43, PP13-25, PP13-108, PP13-109, PP16-41, PP23-70, PP23-73, PP23-75, PP23-82,

PP23-83, PP26-62

Ibrahimi, S.A. PP25-20

İdilman, R. PP38-50

Ie, S.I. PP04-19, PP06-31

Ighoudane, H. PP23-70

Ignacio, J. PP29-07, PP32-02

Iguer, F. PP04-06

Ihara, S. PP32-03, PP34-02

Iida, T. FC08-03

Iimuro, Y. PP26-45, PP27-19, PP36-20

Ijaz, S. PP06-42

Ijichi, H. PP30-33

Ikeda, H. PP36-16, PP36-24

Ikeda, K. PP23-88

Ikeda, M. C21-02
Ikenoue, T. PP30-33

Ikushima, I. PP33-06

Ilcenko, L.Y. PP10-22

Ilchenko, L. PP09-39, PP10-21

Iliescu, L. PP25-17

Iloeje, U.H. PP04-28

Ilsen, B. PP37-14

Ilyankova, A. PP13-81

Ilyassova, B.S. PP08-36, PP15-44

Im, J.H. PP07-03

Imada, H. PP35-02, PP35-03

Imai, Y. PP23-32, PP29-18

Imamura, J. PP33-26

Imamura, M. PP13-122, PP13-123

Imanieh, M. PP12-04

Imazeki, F. PP01-05, PP03-39, PP04-46,

PP05-80, PP12-40, PP13-27, PP13-36, PP30-37

Imazu, H. PP23-88

Imran, A. PP12-46, PP13-12

Inagaki, M. PP26-60, PP26-61

İnan, D. PP06-09

Inao, M. PP23-32

Inci, A. PP06-25, PP06-26

Indrio, F. PP16-27

Inoue, J. PP14-20

Inoue, K. PP01-41, PP39-57

Inoue, $\mathrm{M}$. PP03-07

Inoue, Y. FC02-04, C21-02

Intaraprasong, P. PP26-14, PP38-04

Invernizzi, P. PS04-01

Iovinella, V. PP05-10, PP26-09

Ip, W.Y. PP34-24

Iqbal, M. PP26-51, PP38-65

Iqbal, R. PP23-12

Irhoudane, H. PP13-25

Irshad, M. PP09-36, PP12-33, PP14-18, PP39-69

Iryo, Y. PP33-06

Isaji, S. PP37-07

Isakov, V. PP08-40

Isakova, G.B. PP08-36

Ishido, K. FC08-04, PP38-64

Ishii, A. PP33-06

Ishii, K. PP09-10, PP09-19

Ishizaki, M. PP27-05, PP27-06, PP29-09,

PP34-04, PP36-03, PP36-04, PP36-05,

PP36-06, PP37-02

Ishizawa, T. FC02-04, C21-02

Iskandar, M.Y. PP04-10, PP04-39

Islam, M.A. PP17-07

Islam, M.B. PP05-13

Islam, Q.T. PP08-39

Ismaeili, S. PP22-07

Ismael, A. PP27-04

Ismail, A. PP24-02

Ismail, F. PP39-31

Ismail, U. PP16-24

Iso, Y. PP23-48

Issa, H. PP13-17, PP13-58, PP28-09

Itano, O. PP36-26

Ito, K. PP13-122, PP13-123

Ito, T. PP36-16, PP36-24

Itoi, T. EC-06

Itthikul, K. PP05-62

Ivanikov, I. PP13-81

Ivashkin, V. PP23-01, PP23-34 
Ivashkin, V.T. PP07-11, PP07-12,

PP07-13, PP07-14, PP08-10

Iwagaki, H. PP26-60, PP26-61

Iwakawa, K. PP26-60, PP26-61

Iwakiri, H. PP13-121

Iwamoto, F. PP33-08, PP33-09

Iwamoto, H. PP33-15

Iwamoto, S. PP27-05, PP27-06, PP33-15

Iwasaki, Y. PP25-08

Izatullayev, E.A. PP08-36, PP08-37

Izeki, J. PP27-09

Izumi, N. PP13-78

Izzi, A. PP05-10, PP26-08, PP26-09

Jaafar, K.A. PP16-35

Jaafar, M.H. PP16-35

Jabbari, H. PP13-60

Jacobson, I. PS02-03, PP13-59, PP13-64, PP13-75, PP13-90

Jafarzadeh, Y. PP02-23

Jafri, M. PP28-05

Jafri, W. FC06-05, PP01-44, PP09-32, PP13-34, PP23-12, P23-23, PP23-57, PP26-37, PP39-31

Jain, D. PP26-73

Jain, P. PP23-29

Jalali, Far, M.A. PP04-35, PP12-39, PP39-73

Jalan, R. PP24-07

Jamali-Zavarei, M. PP10-20

Jan, Jing, B. PP26-64

Janbakhsh, A. PP23-85

Janevski, M. PP07-20

Jang, B.K. PP13-28

Jang, E.S. PP04-18, PP07-15, PP15-03,

PP16-18, PP16-20, PP30-04, PP32-08

Jang, H.H. PP31-23

Jang, H.J. PP26-48, PP26-49, PP28-26, PP31-24

Jang, H.W. PP16-58

Jang, J.S. PP23-37

Jang, J.W. PP05-51, PP05-67

Jang, J.Y. PP02-04, PP23-51, PP25-13, PP31-25, PP39-61

Jang, M.K. PP09-16, PP09-28

Jang, Y.O. FC06-02, PP23-38

Janssen, H. C07-02

Janssen, H.L.A. C02-03, PP37-20

Jardí, R. PP06-08

Jardin-Watelet, B. PS02-01

Jargalsaikhan, T. PP23-68

Javadi, Goavgani, A. PP02-29

Jayalal, A.T. PP01-10, PP10-10, PP26-26,

PP39-32, PP39-35, PP39-36, PP39-37, PP39-40

Jazag, A. PP34-07

Jazayeri, S. PP14-13

Jeamsripong, W. PP04-49

Jee, S.R. PP13-102

Jeffers, L. PP13-104

Jensen, D. PP13-56, PP13-57

Jeon, B.-H. PP15-43, PP19-06

Jeon, B.J. PP15-28

Jeon, H.J. PP08-24

Jeon, H.K. FC06-02, PP23-38

Jeon, J.W. PP30-09, PP30-10

Jeon, S.W. FC04-01, PP04-29, PP15-37, PP33-24, PP33-27
Jeon, W.J. PP26-43

Jeon, Y.C. PP16-33

Jeon, Y.M. PP38-39, PP38-40

Jeong, C.U. PP02-08, PP13-100

Jeong, H.G. PP07-02, PP07-03, PP07-04, PP15-04, PP39-17

Jeong, H.K. PP05-123, PP13-72, PP26-34

Jeong, J.Y. PP16-33

Jeong, S. PP07-30

Jeong, S.H. PP09-34, PP13-67

Jeong, S.-H. PP09-04, PP32-10

Jeong, S.W. PP23-51, PP25-12, PP39-61

Jeong, Y.I. PP27-07, PP28-11

Jevtovic, T. PP01-08

Ji, D. PP16-54

Ji, F. PP05-116

Ji, G. PP07-17, PP07-29, PP10-15

Ji, S. PP05-130, PP34-16

Ji, S.W. PP39-54

Ji, S.-W. PP13-80

Jia, J. FC01-03, FC03-05, PP01-07, PP05-12, PP05-22, PP05-152, PP06-14, PP15-20, PP15-25, PP15-36, PP15-40

Jia, J.D. PP30-16, PP31-19

Jian, L. PP08-23

Jiang, C.Z. PP05-92

Jiang, D. PP14-07

Jiang, J. PP12-23, PP38-15

Jiang, J.-J. PP03-09, PP03-34, PP05-60, PP05-137, PP07-28, PP23-50

Jiang, L. PP06-44, PP08-04, PP22-04

Jiang, M.D. PP15-13

Jiang, N.T. PP05-92, PP06-12

Jiang, S. PP19-05

Jiang, T. PP07-22, PP12-21, PP12-23

Jiang, W.-Y. PP15-43, PP19-06

Jiang, $X$. PP06-60

Jiang, Y. PP03-18, PP06-18, PP08-47, PP21-08

Jiang, Y.-F. PP04-22, PP04-23, PP05-34, PP12-21, PP19-09, PP31-18

Jiang, Y.-M. PP05-24

Jian-Hong, G. PP24-06

Jian-Tao, J. PP24-06

Jiao, J. PP05-130, PP13-73, PP13-80

Jie, H. PP05-43

Jie, J. PP05-116

Jigjidsuren, C. PP34-07

Jin, H. PP03-30

Jin, M. PP31-38, PP34-35

Jin, Q. PP12-23, PP14-06, PP14-07, PP34-19

Jin, R. PP05-61, PP05-128

Jin, X. PP07-27

Jing, W. PP05-101

Jing, Z. PP22-11

Jing-Quan, J. PP24-06

Jin-Hua, Y. PP36-10

Jiraskova, A. FC07-03

Jo, S.H. PP22-12

Joh, J.W. PP36-13

Joh, J.-W. PP33-22

Joh, T. PP03-07, PP13-78

Johanet, C. PS04-02

John, A. FC07-02, PP12-18

Johnson, N. C03-01

Johri, S. PP39-48
Jonaidi, N. PP04-43

Jongboonyanuparp, T. PP13-15

Jongjirasiri, S. PP38-04

Jonsson, J. PP15-21

Joo, K.R. PP30-09, PP30-10

Joo, Y.E. PP05-123, PP26-34

Joshi, D. PP01-03, PP13-40

Joshi, S. PP08-41, PP39-62, PP39-63, PP39-71

Jothimani, D. PP25-01, PP25-02, PP26-06

Jourdain, G. PP06-41, PP39-84

Jovanovska, J. FC07-03

Joy, Hoskeri, H. PP10-06

Ju, H.-L. PP30-29

Ju, M.K. PP36-21

Ju, W. PP12-20, PP14-10, PP26-18, PP29-10, PP36-01, PP38-03, PP38-07, PP38-08, PP39-21, PP39-22

Juan, C.-X. PP23-77

Juanjuan, X. PP05-01

Judaki, M. PP14-13

Judkins, J. FC03-04

Judson, J.P. PP02-16

Jullian, N. PS02-01

Jun, D.W. PP08-24, PP16-33

Jun, S.H. PP23-30, PP34-11

Jun, S.W. FC06-04

Junchotikul, P. PP38-04, PP38-41

Jung, E.U. PP05-89, PP13-102

Jung, G.M. PP09-33

Jung, H.S. PP05-67

Jung, K.H. PP15-22

Jung, K.S. PP23-84

Jung, M. PP05-56, PP05-161

Jung, M.K. FC04-01, PP04-29, PP15-37,

PP33-24, PP33-27

Jung, S.W. PP02-04, PP25-13, PP31-25

Jung, Y.J. PP03-12

Jung, Y.K. PP05-57, PP16-57

Jurankova, J. PP23-21

Justa, S. PP14-05

Juszczyk, J. PP12-15

K, R, V. PP38-59

K.C., S. PP01-39

Kaabi, S.A. PP12-18

Kabir, M. PP08-20

Kachintorn, U. PP03-31, PP03-32, PP05-48, PP39-43, PP39-45

Kadota, Y. PP36-26

Kae, S.H. PP28-26

Kaganov, B. PP08-40

Kagawa, T. PP23-17

Kahraman, A. PP07-31

Kaibori, M. PP27-05, PP29-09, PP34-04,

PP36-03, PP36-04, PP36-05, PP36-06,

PP37-02

Kaibullayeva, D.A. PP08-36

Kaido, T. FC08-03

Kaita, K. PS01-02

Kakazu, E. PP14-20

Kakiuchi, M. PP32-03, PP34-02

Kakutani, H. PP23-88

Kaldırım, U. PP39-24

Kallel, L. PP04-13

Kalra, N. PP05-15, PP31-37

Kalyon, S. PP05-17, PP05-144

Kamada, T. PP35-02, PP35-03 
Kamae, I. PP12-10

Kamel, S. PP39-16

Kamezaki, H. PP05-80

Kamiyama, N. PP26-03

Kamiyama, T. PP01-24

Kanai, F. PP30-33

Kanazawa, K. PP23-88

Kanda, M. PP23-94, PP29-19, PP33-28

Kanda, T. PP01-05, PP03-39, PP04-46,

PP12-40, PP13-27, PP13-36, PP30-37

Kande, T. PP05-80

Kandemir, Ö. PP06-09

Kandil, H.M. PP13-04

Kandil, M.H. PP23-03, PP21-07

Kaneko, F. PP26-13, PP26-15

Kaneko, J. PP38-36

Kang, D.H. PP27-07, PP28-10, PP28-11, PP29-13

Kang, E.J. PP23-51

Kang, E.M. PP23-37

Kang, H.Y. PP08-58, PP30-17

Kang, J.H. PP28-10

Kang, J.-H. PP03-08, PP25-16

Kang, J.M. PP08-15

Kang, K.J. PP30-32

Kang, K.W. PP07-18

Kang, M.S. PP08-58, PP30-17

Kani, S. PP39-82

Kannan, R. PP31-28

Kansal, S.S. PP08-30

Kantamool, C. PP39-18

Kao, J.-H. AS-01, PP04-07, PP06-27, PP13-93

Kao, J.-T. PP05-106

Kapur, N. FC03-02

Kar, P. PP04-44, PP09-11, PP09-35,

PP13-55, PP30-15, PP32-05, PP39-51

Kara, B. PP05-109, PP05-110, PP05-111,

PP09-14, PP09-40, PP13-62

Karaagac, M. PP05-17, PP05-144

Karabay, M. PP03-13

Karabay, O. PP03-13

Karacaer, Z. PP05-20

Karahocagil, M. PP06-09

Karami, G. PP38-44

Karapetyan, G.R. PP21-01

Karasu, Z. PP31-22

Karayalçın, K. PP38-50

Karayalçın, S. PP38-50

Karchava, M. PP06-15, PP13-53, PP14-17

Karim, S. PP23-57, PP26-37

Karimi, Elizee, P. FC05-04, PP11-04,

PP11-12, PP13-101

Karimi, M. PP12-04

Karimzadeh, H. PP14-13

Karino, Y. PP03-08

Karki, A. PP15-01

Karoui, S. PP04-13

Karthick, Kumar, A. PP34-37

Karunanayake, S.A.A.P. PP04-25

Kasai, K. PP29-04

Kasai, Y. PP29-04

Kasapoglu, B. PP08-07, PP08-62, PP08-63

Kassaian, N. PP09-15, PP12-11,

PP12-12, PP12-13, PP12-19

Kasserra, C. PP13-65, PP13-66

Kasturiratne, A. PP23-42
Katagiri, S. PP26-63, PP26-67, PP31-34, PP36-22

Katayama, K. PP04-08

Kato, A. PP13-39

Kato, M. PP23-48

Kato, N. PS02-05

Kato, T. PP26-63, PP36-22

Katou, T. PP26-67

Kauffman, R.S. PS02-03, PP13-89

Kaur, G. FC07-04, FC07-05

Kaur, J. PP31-37

Kavalar, R. PP02-05

Kawachi, S. PP36-26

Kawai, K. PP25-08

Kawai, T. PP23-94, PP29-19, PP33-28

Kawamoto, K. PP36-16, PP36-24

Kawano, Y. PP26-23, PP26-24, PP26-38, PP26-66, PP26-70, PP26-71, PP28-30, PP36-08, PP36-11, PP36-12

Kaya, A. PP12-30

Kaya, R. PP05-111

Kaydani, G.A. PP39-73

Kaymakoglu, S. PP09-29, PP23-80

Kchomerici, S.G. PP08-13

$\mathrm{Ke}$, Q. PP21-11

Ke, W.-M. PP06-47

Keane, F.M. PP15-53

Kee, W.J. PP05-123

Keiss, J. PP17-18, PP26-36

Kemper, T. PP06-63

Kench, J. PP21-04

Keshvari, M. FC05-04, PP11-04,

PP11-12, PP13-20, PP13-101, PP17-14

Kevorkian, A. PP12-24

Kevorkian, G.A. PP21-01

Kew, S.T. PP08-16

Ke-Xiao, L. PP36-10

Keyter, M. PP06-33

Keyvani, H. PP06-24, PP09-27,

PP11-04, PP12-22

Khadka, S. PP01-39

Khalaf, H. PP38-09

Khalid, A.B. FC06-05, PP23-23

Khalil, M.A. PP39-02

Khalil, Z. PP12-35

Khalilullah, H. PP39-04

Khamduang, W. PP06-41, PP39-84

Khan, A.A. PP22-09, PP22-10

Khan, M. PP05-54, PP05-124, PP13-49

Khan, M.A. PP08-17

Khan, M.M.R. PP08-39

Khan, M.R. PP17-07, PP39-29

Khan, N.A. PP30-15

Khan, R. PP39-31, PP39-40

Khandelwal, N. PP23-79

Khanna, R. PP16-36

Khanna, S. PP26-54

Khawaja, A. PP09-32

Khawar, I. PP12-35, PP12-45

Khedive, A. PP14-13

Khow-Ean, U. PP05-94

Khuhaprema, T. PP31-39

Khunvirojpanich, M. PP15-16

Khuu, D.N. PP38-46

Ki, S.H. PP07-30

Kiassov, A. PP10-23
Kieffer, T.L. FC05-02

Kijima, H. PP26-42

Kikuchi, E. PP13-32

Kikuchi, K. PS04-01

Kim, B.H. PP05-89, PP07-06

Kim, B.I. PP05-55, PP23-05

Kim, B.K. PP23-69

Kim, B.S. PP02-04, PP23-51, PP25-13, PP31-25, PP39-61

Kim, C. PP35-04

Kim, C.H. PP07-06

Kim, C.W. FC06-04, PP23-30, PP34-11

Kim, C.Y. PP30-04

Kim, D. PP08-15

Kim, D.G. PP15-28, PP26-65

Kim, D.-G. PP15-04

Kim, D.J. FC06-04, PP09-28, PP16-23, PP16-49, PP23-30, PP34-11

Kim, D.K. PP05-57

Kim, D.U. PP29-13

Kim, D.Y. PP03-26, PP13-18, PP16-46, PP16-47, PP16-50, PP16-58, PP19-04, PP20-12, PP23-69, PP23-84, PP30-29, PP31-35

Kim, E.-C. PP16-20

Kim, E.S. PP13-113

Kim, E.Y. PP30-17

Kim, G.H. PP29-13

Kim, H.B. PP26-65, PP37-15

Kim, H.C. PP34-11, PP39-60

Kim, H.-C. PP32-09

Kim, H.G. PP34-11, PP39-60

Kim, H.I. PP15-28

Kim, H.J. PP23-05, PP05-55, PP08-58

Kim, H.K. PP07-03

Kim, H.S.FC06-04, PP02-04, PP03-26, PP07-07, PP09-16, PP09-28, PP09-34, PP13-72, PP23-30, PP23-51, PP25-12, PP25-13, PP31-25, PP34-11, PP34-61

Kim, H.W. PP27-07, PP28-10

Kim, H.Y. PP09-16, PP22-12, PP32-10

Kim, I.H. PP09-33, PP15-14, PP15-28

Kim, J. PP30-18

Kim, J.D. PP26-43

Kim, J.H. PP05-57, PP13-102, PP16-57, PP23-37, PP26-21, PP35-07, PP39-39

Kim, J.-H. PP30-12, PP30-13

Kim, J.-H. PP30-12, PP30-13

Kim, J.I. PP39-39

Kim, J.K. PP23-69, PP33-05, PP34-15

Kim, J.M. PP33-22, PP36-13

Kim, J.S. PP09-04, PP16-20, PP35-05

Kim, J.-W. PP09-04

Kim, J.Y. PP07-03, PP09-04

Kim, K. PP26-19, PP26-20

Kim, K.A. PP05-115, PP22-03

Kim, K.-A. PP05-156

Kim, K.-A. PP05-156

Kim, K.C. PP33-24

Kim, K.H. C13-04

Kim, K.M. PP09-17, PP3507

Kim, M. PP05-101

Kim, M.H. PP27-07, PP28-11

Kim, M.J. PP26-65, PP37-15

Kim, M.-J. PP09-05, PP37-05

Kim, M.-J. PP09-05, PP37-05 
Kim, M.N. PP13-18

Kim, M.Y. FC06-02, PP30-17

Kim, R. PP13-86

Kim, S. PP23-84

Kim, S.B. PP08-58, PP30-17

Kim, S.G. PP02-04, PP23-51, PP25-12,

PP25-13, PP31-25, PP39-61

Kim, S.H. PP05-123, PP13-113, PP15-28,

PP19-04, PP23-37, PP26-16, PP26-65

Kim, S.J. PP33-22

Kim, S.K. FC04-01, PP04-29, PP07-06,

PP15-37, PP33-24, PP33-27, PP39-25

Kim, S.-O. PP05-120

Kim, S.S. PP05-120, PP16-23, PP23-35

Kim, S.U. PP03-26, PP16-46, PP16-47,

PP16-50, PP16-58, PP23-84, PP31-35

Kim, S.W. PP15-28

Kim, T.G. PP16-49

Kim, T.H. PP07-07

Kim, T.-J. PP01-37

Kim, T.Y. PP08-24, PP16-33

Kim, U.-H. PP15-14

Kim, W. PP03-12, PP08-15

Kim, W.R. C20-02, S04-01

Kim, W.-S. PP19-10

Kim, Y.D. FC06-02, PP23-38

Kim, Y.H. PP23-69, PP30-32, PP23-51

Kim, Y.J. FC-06-02, PP13-28, PP13-72,

PP13-113, PP15-03, PP16-18, Pp16-20,

PP23-38, PP26-16, PP26-34, PP30-04,

PP30-11, PP30-13, PP32-08

Kim, Y.-J. PP22-03

Kim, Y.M. PP09-04

Kim, Y.N. PP26-43

Kim, Y.S. PP23-51, PP39-61, PP16-57, PP25-12, PP25-13, PP31-25, PP39-61

Kimura, K. PP33-26

Kimura, N. FC08-04, PP21-12, PP38-64

Kimura, O. PP14-20

Kimura, Y. PP26-60, PP26-61

Kin, T. PP03-08, PP26-16

Kino, K. PP34-22

Kiran, M. PP31-17

Kirbas, I. PP08-17

Kirino, I. PP36-16, PP36-24

Kishiwada, M. PP37-07

Kiss, A. PP15-55, PP31-42

Kist, A. PP38-01

Kita, J. PP23-48, PP36-17

Kita, K. PP13-36

Kitab, B. PP06-34

Kitagawa, K. PP26-63, PP31-34, PP36-18

Kitagawa, Y. PP36-26

Kitikomonkun, P. PP03-31

Kitiyakara, C. C11-04, PP23-52

Kitiyakara, T. C11-04, PP26-33

Kitrinos, K. PS01-01, PP05-125

Kittichaisri, K. PP05-150

Kiykım, A. PP12-30

Kiziltas, S. PP26-41, PP26-69

Kleber, K. PP13-89

Klein, A. PP38-29

Klos, A. PP39-55

Klyprayong, P. PP23-52

Knighton, S. PP13-40

Ko, H.-K. PP37-15
Ko, S.Y. PP23-28

Kobashi, H. PP25-08

Kobayashi, K. PP14-20, PP33-06

Kocak, F. PP06-25, PP06-26

Kocak, H. PP21-03

Kocic, G. PP01-08

Kocic, H. PP38-16

Kocic, R. PP01-08

Kodama, Y. PP25-16

Kodera, Y. PP36-22

Kodikara, S.K.Y.J PP39-32

Koga, H. PP13-38

Koh, D.H. PP28-26

Koh, H. PP26-38

Koh, J. PP34-18

Koh, K.C. PP03-21, PP03-23, PP05-14, PP05-160, PP06-13, PP09-17

Koh, Y.Y. PP13-112

Kohla, M. PP34-14

Kohli, K. K. PP10-13

Koike, K. PS02-05, PP30-33, PP33-25, PP38-36

Kojima, Y. PP33-08, PP33-09

Kojiro, M. OR-01

Köksal, I. PP06-09

Kokudo, N. FC02-04, C21-02, PP38-36

Kolaric, B. PP14-26

Kolesnikova, O. V. PP07-08, PP07-09,

PP07-10, PP08-12

Kolomiycev, O. PP26-10

Kolomiycev, O.A. PP13-91

Komada, N. PP13-121

Komissarenko, I. PP10-16, PP23-61

Komolmit, P. FC01-01, PP04-49, PP06-55, PP39-82

Kondo, M. PP32-03, PP34-02

Kondo, Y. PP14-20, PP26-45, PP33-25, PP36-20

Kondou, M. PP33-18

Kondratieva, T.T. PP13-91, PP26-53, PP27-15, PP37-11

Konduru, S. PP09-41

Kong, L. PP05-27, PP15-54, PP38-02

Kong, L.-B. PP08-11

Kongphlay, S. PP39-41, PP39-42,

PP39-43, PP39-44

Kongtawelert, P. PP31-39

Konovalova, S. PP19-03

Koo, R.M. PP08-16

Korenaga, T. PS04-03

Korkolis, D. PP13-06

Korkusuz, R. PP06-05

Korneeva, O.N. PP08-10

Korotkov, S.M. PP01-04

Koruk, I. PP05-103

Koruk, M. PP05-103

Kosaka, H. PP27-09

Köse, S. PP04-20, PP05-133, PP06-52

Kosinska, A. PP20-10

Kosura, S. PP26-10

Kosura, S.D. PP01-43, PP13-91, PP26-53

Kosyura, S. PP09-39, PP10-21, PP10-22

Kotera, Y. PP26-50, PP26-63, PP26-67, PP31-34

Koukoulas, D. PP23-74

Koutoubi, Z. PP05-04, PP08-01
Koutsounas, S. PP13-06

Kovalenko, V. PP02-15

Kovalszky, I. PP15-55, PP31-42

Kovrigina, A. PP26-10

Kovrigina, A.M. PP01-43, PP13-91,

PP26-53, PP27-15, PP37-11

Kovyazina, I. PP23-46, PP23-47

Kowatari, N. PS02-05

Kowdley, K. PP05-65, PP25-22

Kozumplík, L. PP28-14

Kozybayev, N. PP28-28

Kraev, S. PP05-56

Kramvis, A. PP06-33

Krasnaliev, I. PP26-01

Krass, V. PP28-14

Krastev, Z. PP05-91

Kraus, M.R. PP12-27

Kreil, A. PP12-34

Kreile, M. PP17-18

Kremoser, C. PP07-23

Kriener, S. PP12-27

Krishna, V. PP10-06

Krishnaswamy, N. PP13-79, PP13-83,

PP13-87, PP15-32

Krisnuhoni, E. PP06-28, PP08-22

Krumina, A. PP17-18

Kubota, K. PP23-48

Kubota, M. PP30-08

Kudo, D. FC08-04, PP38-64

Kudo, M. FC02-05, PP34-25

Kuhiran, C. PP23-52

Kukurtchyan, N.S. PP21-01

Kulhara, P. PP10-13

Kulkarni, V.H. PP02-12

Kulseth, P. PP23-89

Kumada, S. PS04-03

Kumagai, I. PP13-22

Kumamoto, K. PP37-07

Kumar, A. PS01-05, FC03-02, FC08-01,

C16-02, PP05-15, PP05-18, PP09-09,

PP09-35, PP09-40, PP16-37, PP24-10, PP39-65,

Kumar, B. PP27-22

Kumar, G.T. PP39-65

Kumar, M. PP16-34, PP16-36, PP26-54, PP27-22

Kumar, P. PP01-40

Kumar, R. PP16-36

Kumarasena, R.S. PP23-42

Kumarr, A. PP06-23

Kumbasar, H.K. PP06-25, PP06-26

Kumbasar Karaosmanoglu, H. PP04-21, PP06-05

Kung, K.N. PP35-06

Kunkongkapan, S. PP06-41

Kuo, J.-J. PP13-98

Kuo, Y.-H. PP04-32

Kupcsulik, P. PP15-55, PP31-42

Kurbanov, F. PP03-07, PP06-40

Kurbanova, M.G. PP08-36

Kuroda, H. PP16-56, PP29-04

Kuroda, N. PP26-45, PP27-19

Kurosaki, M. PP13-78

Kurt, R. PP08-21

Kurtagic, S. PP17-02

Kusakabe, A. PP03-07 
Kusumoto, K. PP13-121

Kutoglu, K. PP05-17, PP05-144

Kuwahara, K. PP27-18

Kuzmina, O.S. PP03-38

Kuzushita, N. PP05-52

Kwac, M.S. PP05-115

Kwak, H.C. PP39-25

Kwak, J.H. PP26-48, PP26-49, PP31-23, PP31-24

Kwak, M.S. PP15-03, PP16-18

Kwak, M.-S. PP04-18, PP07-15, PP16-20,

PP30-04, PP32-08

Kwan, W.K. PP08-26

Kweon, Y.O. FC04-01, PP04-29, PP05-139,

PP15-37, PP26-39, PP26-52, PP33-24,

PP33-27

Kwo, P. PP13-75

Kwon, A.-H. PP27-05, PP27-06, PP29-09,

PP34-04, PP36-03, PP36-04, PP36-05,

PP36-06, PP37-02

Kwon, C.H.D. PP33-22, PP36-13

Kwon, H.J. PP08-24

Kwon, J.H. PP05-51, PP05-67

Kwon, J.-H. PP16-20

Kwon, O.K. PP23-30

Kwon, O.S. PP05-57, PP09-34, PP16-57

Kwon, S.Y. PP09-34, PP23-28

Kypreos, D. PP13-06

Laalakusolvong, S. PP05-48

Labbadia, G. PP08-52

Lacatusu, C. PP16-32

Lace, B. PP17-18

Lacombe, M.-L. PP30-07

Lada, O. PS02-01, PP03-14, PP03-17,

PP06-43, PP14-16

Laferl, H. PP13-61

Laghari, S. PP01-40, PP09-23, PP09-24

Lahbabi, M. PP04-36, PP12-36, PP12-43,

PP13-25, PP13-108, PP13-109, PP16-41, PP26-62

Lai, C.-L. PP05-65, PP05-141, PP06-37

Lai, H.-C. PP05-106

Lai, J. PP06-47

Lai, J.-H. PP34-08

Lai, M.Y. PP05-139, PP13-47

Lai, Q. PP06-60

Lai, R.C. PP02-21

Lai, S.-W. PP05-104

Lai, V. PP05-158, PP37-16

Laique, S.N. PP01-16

Lal, N. PP01-40, PP09-23

Lallemant, M. PP06-41, PP39-84

Lalouel, J.-M. FC03-04

Lam, K. PP04-09

Lam, K.H. PP25-04

Lambert, J.S. PP26-36

Lambert, N. PS02-01

Lampe, E. PP14-25

Lampertico, P. C05-02

Lanerolle, R.D. PP04-25

Lang, Y.-X. PP13-80

Lang, Z.-W. PP05-134

Langford, P. PP39-07

Langley, P. PP12-10

Lankarani, K.B. FC05-04, PP11-12,

PP13-101, PP39-06, PP39-08
Lao, W.C. PP35-06

Laohapand, C. PP04-48, PP26-75

Laohaphand, C. PP04-45

Laouénan, C. PP13-120

Lapalus, M. PS02-01, PP03-14, PP03-17, PP13-120, PP14-16

Larsen, L.M. PP13-95

Lashin, S. PP13-77

Lata, J. PP23-21

Latif, M.A. PP39-47

Lau, G. K. C14-02, PP05-19

Laughlin, M. PP13-57

Laune, D. PS02-01

Lavrynenko, O. PP08-08

Law, L.Y. PP35-06

Lawitz, E. PS02-02, PS02-04, PP13-64, PP13-75, PP13-76

Lawrence, G. PP07-33

Lazaar, F. PP04-04

Lazar, D. PP23-74

Lazarevich, N. PP30-21

Lazebnik, L. PP10-16, PP23-46, PP23-47, PP23-61

le, Bail, B. PS04-04

Le, Bail, B. PP16-15

Le, Caer, J.-P. PS04-02

Lebigot, J. PP26-29

Lebray, P. PP23-90

Lecorps, G. PP23-39

Leduc, T.-S. PP05-101

Lee, A. PP15-41, PP15-42, PP21-04

Lee, B.E. PP29-13

Lee, B.S. PP13-113, PP26-16

Lee, C.D. PP32-10

Lee, C.H. PP09-34, PP23-28

Lee, C.K. PP05-115, PP23-84

Lee, C.M. PP05-139, PP12-25

Lee, C.-M. FC05-03, PP05-129, PP05-140

Lee, C.P. PP19-08

Lee, D. PP05-120

Lee, D.H. PP06-13, PP07-30

Lee, D.W. FC04-01, PP15-37, PP26-39, PP26-52, PP33-24, PP33-27

Lee, D.Y. PP31-35

Lee, E.S. PP13-113, PP26-16

Lee, G.C. PP36-13

Lee, G.H. PP04-50, PP05-40, PP05-66, PP05-72, PP05-114, PP05-158

Lee, G.-Y. PP15-22

Lee, H. PP15-22

Lee, H.C. PP23-28, PP23-36, PP26-43, PP35-07

Lee, H.J. PP23-36

Lee, H.K. PP31-25

Lee, H.S. FC04-01, PP13-102, PP15-37, PP23-35, PP26-39, PP26-52, PP33-24, PP33-27

Lee, H.-S. PP04-18, PP05-89, PP06-53, PP07-15, PP08-15, PP15-03, PP16-18, PP16-20, PP22-03, PP30-04, PP30-11, PP30-12, PP30-13, PP32-08

Lee, H.Y. PP13-113, PP26-16

Lee, H.-Y. PP03-12

Lee, J. PP28-26

Lee, J.D. PP23-28
Lee, J.H. PP03-21, PP03-23, PP03-26, PP05-14, PP05-160, PP06-13, PP09-04, PP09-16, PP09-17, PP16-50

Lee, J.-H. PP04-18, PP07-15, PP08-15, PP15-03, PP15-22, PP16-18, PP16-20, PP22-03, PP30-04, PP30-11, PP32-08

Lee, J.I. PP07-30, PP13-102, PP30-09, PP30-10

Lee, J.J. PP05-57, PP16-57

Lee, J.M. C17-02

Lee, J.R. PP26-43, PP06-46

Lee, J.S. PP05-115, PP05-156, PP30-12, PP37-15

Lee, J.W. PP09-34, PP13-67, PP30-02

Lee, J.-W. PP07-30

Lee, K. PP01-11, PP05-19, PP12-25

Lee, K.H. PP31-35

Lee, K.I. PP13-28

Lee, K.M. PP33-05, PP34-15, PP34-15

Lee, K.S. PP23-69

Lee, K.-T. PP36-19

Lee, K.U. PP38-39, PP38-40

Lee, K.-W. PP38-39, PP38-40

Lee, K.Y. PP15-04

Lee, M.H. PP31-33, PP31-35

Lee, M.S. PP09-28, PP13-67, PP20-12, PP23-30, PP25-13, PP31-25

Lee, M.Y. PP39-25

Lee, N.H. PP15-23

Lee, S.-D. PP33-02

Lee, S.H. PP02-04, PP13-102, PP15-43, PP19-06, PP23-51, PP25-12, PP25-13, PP31-25, PP39-61

Lee, S.J. PP05-57, PP26-43

Lee, S.-K. PP33-22

Lee, S.O. PP15-28

Lee, S.T. PP15-28

Lee, S.W. PP05-55

Lee, S.Y. PP13-113, PP26-16

Lee, T.L. PP35-06

Lee, W.G. PP04-05, FC06-03

Lee, W.J. PP36-21

Lee, W.S. PP26-34

Lee, Y.J. PP05-156, PP07-07, PP13-102, PP16-23, PP16-49

Lee, Y.-J. PP05-89

Lee, Y.M. PP05-40, PP05-66, PP05-72, PP05-114, PP05-158

Lee, Y.-M. PP01-37

Lee, Y.S. PP32-10, PP35-07

Leelakusolvong, S. PP03-31, PP03-32

Leelaudomlipi, S. PP38-04, PP38-38, PP38-41

Leela-Udomlipi, S. PP26-14

Leem, S.H. PP30-32

Leen, R. PP26-51

Leerapun, A. C03-03, PP23-89, PP31-39,

PP34-17, PP39-52

Lei, H. PP05-27

Lei, L. PP26-72, PP38-56

Lei, N. PP12-03

Leidere-Reine, A. PP25-18

Lekcharoen, K. PP02-20

Lencioni, R. FC02-05, PP34-25

Lengyel, G. PP13-46

Lenz, O. PP13-59

Leo, Hartono, J. PP05-158

Leong, I.S. PP35-06 
Leoni, V. PP08-52

Lepkov, S. PP01-43, PP13-91, PP26-10 Lepkov, S.V. PP26-53, PP27-15, PP37-11 Lermite, E. PP26-29, PP26-30

Leroy, V. FC05-01

Lertkupinit, C. PP05-62

Lertudomphonwanit, C. PP26-47

Lesmana, C. PP06-28

Lesmana, L. AA-04

Letinas, E. PP10-19

Leung, C.M. PP35-06

Leung, G.S.P. PP04-09

Leung, N. PP13-104, C19-04

Leung, Y.L. PP26-68, PP27-14

Levi, M. PP07-22

Levy, I. PP34-20

Levy, M.T. PP05-113, PP13-103

Lewandowski, P. PP07-20

Lewin, S. PP15-33

$\mathrm{Li}$, A. PP36-25

Li, B. PP02-13, PP15-09, PP15-10, PP15-11, PP16-54

Li, B.-S. PP05-163

Li, C. PP12-47

$\mathrm{Li}, \mathrm{C} . \mathrm{K}$. PP04-44

Li, F. PP01-27, PP01-33, PP04-11, PP15-15, PP16-54, PP21-08

Li, F.C. PP01-36

Li, F.-C. PP01-31, PP01-38

Li, G. PP30-01, PP38-02

Li, H. PP01-07, PP04-40, PP06-14,

PP14-10, PP15-20, PP15-25, PP30-16

Li, J. PP01-32, PP03-30, PP05-23, PP05-25, PP05-26, PP05-34, PP05-101, PP06-44, PP06-46, PP12-47, PP15-35, PP36-23 Li, J.G. PP03-01

Li, J.-G. PP04-02, PP05-85, PP05-86

Li, J.M. PP05-05, PP05-27

Li, J.-T. PP32-06

Li, L. PP05-118, PP06-38, PP09-20, PP15-46, PP22-01, PP22-04

Li, L.V. PP34-18

Li, M. PP30-01, PP05-39, 05-101, FC03-01

Li, N. PP06-01

Li, P. PP03-27

Li, Q. PP32-12, PP38-26, PP06-01, PS02-05

Li, Q.-H. PP15-15

Li, S.-L. PP16-40

Li, T. PP01-08, PP03-30, PP04-12, PP06-21, PP09-10

Li, T.W. PP35-06

Li, W. PS02-05, PP01-12, PP06-49, PP12-17, PP12-47, PP14-10, PP15-31, PP16-04, PP18-02, PP20-01, PP28-06, PP28-07, PP28-08, PP28-24, PP28-25, PP28-29, PP30-06, PP34-19, PP39-75 Li, W.H. PP35-06

Li, W.-W. PP32-12

Li, X. FC02-02, FC04-03, PP03-27, PP05-16, PP05-61, PP05-93, PP29-12, PP30-31, PP38-02, PP38-43

Li, X.-H. PP03-20, PP06-32, PP11-13

Li, Y. PP09-30, PP13-80, PP13-117,

PP15-31, PP16-04, PP29-12, PP29-16,
PP30-31, PP05-130, PP06-49, PP06-64,

PP06-60, FC05-03

Li, Y.-B. PP03-34

Li, Y.L. PP05-27

Li, Y.-M. PP38-43

Li, Y.-Y. PP08-28, PP08-29, PP08-32, PP08-33, PP08-34

Li, Z. PP29-06

Li, Z.W. PP36-09

Liang, C.-C. PP13-93

Liang, J. PP08-47, PP31-16

Liang, P. FC04-03

Liang, X. PP16-07

Liang, Z. PP04-24

Liangpunsakul, S. FC04-04

Liao, K.-F. PP05-104

Liao, X.-W. PP06-32, PP11-13

liao, Y. PP30-28

Liao, Y. PP13-05

Liaw, K. PP23-53

Liaw, Y.-F. FC01-03, KL04, PP04-28, PP13-104

Likitlersuang, S. PP03-32

Li-Li, Z. PP24-06 GRP78

Lim, D.H. PP35-04, PP35-05

Lim, H.J. PP07-07

Lim, J.H. PP02-04

Lim, L.Y. PP25-22

Lim, S.G. LT2-03, C14-04, C20-01, PP02-07, PP04-50, PP05-40, PP05-66, PP05-72, PP05-114, PP05-158, PP13-29,

PP22-16, PP37-16, PP37-18, PP37-19

Lim, S.K. PP02-21

Lim, T.H. PP02-18, PP02-19

Lim, Y.-S. PP35-07

Lin, A. PP13-47

Lin, B. PP06-07, PP22-05, PP31-10

Lin, B.-B. PP03-09

Lin, B.-L. PP05-02

Lin, C. PP26-27, PP26-28, PP28-15, PP28-16

Lin, C.-C. PP33-04

Lin, C.-L. PP13-93

Lin, C.S. PP03-01, PP13-03, PP22-14

Lin, C.-S. PP04-02, PP13-01

Lin, C.Y. FC01-02, PP05-104

Lin, C.-Y. PP28-31

Lin, F. PP05-116

Lin, G. PP02-09

Lin, H.-C. PP33-02

Lin, H.-M. PP38-47

Lin, M. PP32-07

Lin, M.-T. PP05-151

Lin, R.-C. PP13-98

Lin, S. PP01-12, PP01-15, PP02-21, PP05-60, PP05-71, PP05-107, PP16-26

Lin, S.-C. PP34-08

Lin, T.-H. PP08-14

Lin, X.-F. PP04-11

Lin, X.-Y. PP07-28

Lin, Y. PP11-13

Lin, Y.Y. PP05-104

Lindor, K. AS-06

Ling, M. PP30-24

Ling, Q. PP05-149, PP31-21, PP36-15,

PP38-30, PP38-32, PP38-33
Lis, E. PP10-02, PP10-03

Litalien, G. PP13-86

L'italien, G.J. PP12-10

Littlejohn, M. PP05-159

Liu, B. PP05-25, PP05-26

Liu, B.-M. PP04-12

Liu, C. FC04-03, PP16-14, PP26-57, PP26-72, PP38-56

Liu, C.-G. PP22-13

Liu, C.-H. PP04-07, PP13-93

Liu, C.J. S01-01

Liu, C.-J. ET1-02, PP04-07, PP06-27, PP13-93

Liu, C.-M. PP28-22

Liu, F. PP06-59, PP12-14, PP14-06, PP14-07,

PP15-27, PP23-25, PP22-15

Liu, G.G. PP12-10

Liu, H. PP01-02

Liu, H.N. PP39-26

Liu, J. PP05-85, PP05-86, PP12-31, PP13-01,

PP13-03, PP20-08, PP23-25

Liu, J.-H. PP08-14, PP28-12

Liu, J.P. PP17-08

Liu, J.-P. PP38-47

Liu, L. PP16-02, PP16-14, PP32-12

Liu, L.-Z. PP39-38

Liu, M. PP05-50, PP05-107

Liu, P. PP01-35, PP09-31

Liu, S. FC04-03, PP12-17, PP12-47, PP14-10, PP26-35

Liu, T. FC03-05, PP07-29, PP15-36, PP15-40, PP22-06, PP30-31

Liu, X. PP06-21, PP08-47, PP20-09, PP34-03, PP38-31

Liu, X.-E. PP04-12

Liu, X.-K. PP31-18

Liu, X.M. PP26-72, PP38-56

Liu, Y. FC02-02, PP01-42, PP02-14, PP05-16, PP05-23, PP05-26, PP05-93, PP10-08, PP15-39, PP25-14, PP28-19, PP29-12

Liu, Y.-B. PP21-02

Liu, Y.-R. PP03-34

Liu, Y.-X. PP15-35

Liu, Y.-Z. PP19-09

Liu, Z. PP02-28, PP30-24, PP38-18, PP38-19

Liu, Z.L. PP17-08

Llamoso, C. PP05-141, PP05-146,

PP05-152, PP05-155

Lo, C. LT1-04

Lo, C.M. KL02, PP29-05, PP33-03, PP33-13, PP34-21, PP36-07, PP36-14, PP38-17, PP38-27

Lo, L. FC07-01, PP15-53

Lo, S. EC-09

Locarnini, S. C05-01, C20-03, PP05-87, PP05-159

Lohse, A.W. PP09-06

Lok, A. PP13-76

Long, J. PP16-26, PP30-16, PP31-19

Long, Q. PP03-19

Long, S.-J. PP15-35

Lönnerdal, B. PP13-05

López-Andujar, R. PP38-54

Lopez-Talavera, J.C. PP13-64 
Lorchatchawankul, R. PP08-31, PP23-10, PP38-35

Lorimier, G. PP26-30

Lotz, G. PP15-55, PP31-42

Lou, C. PP31-14

Lou, H. PP38-15

Lou, L. PP05-99, PP05-101

Louisirirotchanakul, S. PP03-24

Lounes, F. PP04-06

Lovrantova, E. PP04-42

Lu, C.Z. PP30-16, PP31-19

Lu, D. PP24-01

Lu, F. PP04-24, PP12-31

Lu, F.-M. PP03-30

Lu, H. PP05-28, PP06-12

Lu, L. PP08-04, PP38-02, PP38-61

Lu, M. PP06-17, PP06-63, PP20-10

Lu, R. PP21-11

Lu, S. PP06-21

Lu, S.-N. PP04-28, PP04-32, PP05-140, PP16-53

Lu, W. PP35-01

Lu, X.-Y. PP31-08, PP34-05

Lu, Y. PP05-47

Lu, Z. PP39-13

Luangchosiri, C. PP23-52

Lu-Biao, C. PP05-108

Lucano-Landeros, S. PP16-51

Lucic, D. PP03-06

Lucidi, V. FC04-02, PP34-12, PP37-06

Luck, N.H. PP08-17

Luekamlung, N. PP06-41

Lufiandati, A. PP03-33

Luk, J.M. PP15-24

Luk, Y.W. PP35-06

Luketic, V. ET2-02

Luo, D. PS02-04, PP05-44

Luo, X. FC02-02, PP05-76, PP06-49, PP06-50, PP20-09, PP25-19

Luo, X.-R. PP05-21

Lütgheetmann, M. PP09-06

Lv, D. PP05-121

Lv, X. PP15-50, PP15-51, PP15-52

Lv, Y. PP26-72, PP36-23, PP38-56

Ma, H. PP03-22, PP14-06, PP15-36

$\mathrm{Ma}, \mathrm{K}$. PP05-45, PP16-44

$\mathrm{Ma}$, S. PP06-60

Ma, X. PP05-76, PP05-101

Ma, Y. PP38-08, PP39-21, PP39-22

Ma, Y.L. PP05-05, PP05-27

Ma, Z. PP06-17, PP06-63

Mabrouk, M. FC05-05

Mabuchi, A. PP21-05

Macchi, S. PP33-12

Macievich, M.V. PP03-38

Mackiewicz, V. PP06-43

Madjd, Z. PP06-24

Madonia, S. PP13-13, PP14-21

Maeda, S. PP33-18

Maekawa, Y. PP13-32

Maeno, H. PP13-43

Maertens, R. PP03-10

Maeshiro, T. PP06-40

Maevskaya, M. PP23-01, PP23-34

Maggio, A. PP13-13, PP14-21

Maghrebi, H. PP26-22
Maglajlija, S. PP17-02

Magour, G.H. PP11-02, PP23-03

Maguchi, H. PP03-08, PP25-16

Mahachai, V. C11-01, PP06-54,

PP06-55, PP12-25

Mahadeva, S. PP23-49, PP37-09

Mahadevan, K.M. PP02-12

Mahanta, M. PP05-18, PP16-37

Maharem, D.A. PP07-01, PP11-02

Maharom, P. PP39-84

Maher, D. PP21-07

Mahfudz, A.S. PP37-09

Mahinic, A. PP17-02

Mahmoud, S. PP31-41

Mahmoud-Ghoneim, D. PP29-08

Mahtab, M. PP05-98

Mai, L. PP13-01

Maieron, A. PP12-34, PP13-61

Majid, S. FC06-05

Majstorovic, S. PP17-02

Mak, B. PP04-50, PP05-158

Makarova, A. PP30-21

Makarova, M. PP30-21

Makhlouf, M. PP39-81

Makki, K. PP38-55, PP38-59, PP38-63

Malekzadeh, R. PP14-13

Maley, M. PP05-113, PP13-103

Malhotra, A.K. PP23-29

Malihova, O. PP26-10

Malik, A. PP06-29, PP16-19, PP30-15

Malik, I.A. PP01-17

Malik, I.R. PP05-136, PP05-138

Malik, N.A. PP05-138

Malishev, N. PP09-39

Malizia, G. PP13-13

Mallard, B. PP21-05

Mallat, A. FC05-01

Mallick, I. PP38-11

Mallikarjuna Rao, C. PP19-12, PP19-13

Malov, I.V. PP13-74

Mamada, Y. PP26-70, PP26-71, PP28-30, PP36-08, PP36-11

Mamoon, A.A. PP08-20

Mananwatte, S. PP04-25

Manatsathit, S. PP03-31, PP03-32, PP05-48

Manciuc, C. PP05-03

Mancke, L.V. PP09-06

Mangia, A. PP13-46

Manigold, T. PP39-30

Manikantan, P. PP34-37

Mann, N.S. PP28-13

Manns, M. PS01-03, PP13-90

Manns, M.P. C10-01, C18-04, PP05-161, PP19-01

Manoharan, S. PP05-113

Mansoir-Ghanai, F. PP14-13

Mansouri, F.A. PP23-85

Mao, D. PP13-26

Mao, Q. PP04-26, PP04-44, PP05-46, PP05-147

Mao, R. PP06-48

Mao, Y. PP05-105, PP05-112

Mao, Z. PP36-23

Marcellin, P. PS01-01, PS01-02, PS01-03, PS02-01, PS02-02, PS02-03, C05-03, PP03-14, PP03-17, PP05-91, PP05-99,
PP06-43, PP13-56, PP13-57, PP13-59,

PP13-120, PP14-16

Marcu, K.B. PP19-07

Mareeva, D. PP23-46, PP23-47

Marengo, A. PP05-42

Mari, H. PP11-14

Mariadi, K. PP12-48, PP24-11

Marinho, A. PP08-51

Marinozzi, F. PP15-12

Marlini, M. PP21-05

Marrero, J. FC02-05, PP34-25

Marrone, G.PP23-43

Marrone, R. PP04-38

Maršová, J. PP28-14

Martie, A. PP13-107, PP37-12

Martin, E. FC05-02

Martin, J.T. PP05-126

Martin, M. PP13-89

Martinot-Peignoux, M. PS02-01, PP03-14,

PP03-17, PP06-43, PP13-120, PP14-16

Martorell, C. PP13-45, PP13-76

Maruoka, D. PP12-40

Marwaha, N. PP10-13

Marzano, A. PP05-42

Mas, M. PP39-24

Mas, N. PP39-24

Masaki, N. PP13-122, PP13-123

Mashaal, N. PP30-03

Mashali, R. PP30-03

Mashall, N. PP08-03

Mashayekhi, S.O. PP02-03, PP12-08, PP12-09

Mason, W. PP20-10

Massard, J. PP23-90, PP23-92

Mastroleo, C. PP19-07

Masuzaki, R. PP33-25

Matar, K. PP12-18

Matarese, L.E. PP13-04

Mathur, R. PP15-26

Mathurin, P. FC05-01

Matsui, K. PP27-05, PP27-06, PP29-09,

PP34-04, PP36-03, PP36-04, PP36-05,

PP36-06, PP37-02

Matsui, T. PP03-8, PP25-16

Matsumoto, S. PP36-08, PP36-11

Matsunaga, K. PP23-88

Matsuo, H. PP13-32

Matsuo, J. PP04-08

Matsuura, K. PP03-15, PP13-78

Matthews, G. PP15-33

Matusz, P. PP38-58

Maung Maung, T. PP12-37, PP13-14

Maylin, S. PP13-120

Mayronne, S. PP12-37, PP13-14

Mazur, W. PP13-50

Mazzoccoli, G. PP14-08

Mazzulli, T. PP05-157

Mc Hutchison, J.G. PP12-25

McCall, J.L. PS04-05, FC06-03

McCaughan, G. PP21-04

McCaughan, G.W. LT1-01, C13-03, FC07-01, PP15-53, PP19-10

Mccone, J. PP13-90

McCone, J. PP13-75

McDonald, C. FC07-02

McEwan, P. PP13-86

Mchedlishvili, I. PP09-12 
McHutchison, J.G. PS02-03, FC05-02

McLennan, S. PP15-41, PP15-42

McLennan, S.V. FC07-01, PP15-53

Mcneal, M. PP28-05

McPhee, F. PP13-76

Medhat, Y. PP38-09

Medhi, S. PP09-11, PP32-05, PP39-51

Meemaew, P. PP23-52

Mehmood, N. PP05-83, PP05-84, PP23-31

Mehrnoosh, L. PP11-12

Melik-Andreasyan, G. PP13-08

Melino, M. PP15-21

Melkova, K.N. PP01-43

Mellouki, I. PP04-36, PP12-36, PP12-43, PP13-25, PP13-108, PP13-109, PP16-41, PP23-70, PP23-73, PP23-75, PP23-82, PP23-83, PP25-20, PP26-62

Memon, A. PP01-16, PP23-57

Memon, M.S. PP09-23, PP09-24

Memon, S. PP01-40, PP09-32, PP22-33

Mena-Enriquez, M.G. PP15-45, PP20-07

Menaka, N. PP10-07

Mendes, A. PP13-40

Mendez, P. PP13-75

Meng, F. PP22-13

Meng, F.-L. PP01-38

Meng, J. PP08-04

Meng, M. PP15-54

Meng, P.P. PP02-13

Meng, Q.H. PP01-28

Meng, Q.-H. PP01-32

Meng, Q.-L. PP39-76

Meng, W. PP29-12

Meng, X. PP38-12

Meng, Z. PP20-10

Mengchao, W. PP36-25

Meno, K. PS04-03

Menshikova, I. PP28-28

Merat, S. PP13-60

Merrouche, W. PS04-04, PP16-13, PP16-15

Mert, G. PP13-35, PP13-105

Merzlikina, N. PP10-22

Meshkati, M. PP12-11, PP12-13

Messinger, D. PP13-56

Mete, B. PP06-25, PP06-26

Metreveli, D. PP10-25

Meysami, P. PP09-27

Mi, L.-J. PP05-101

Micu, L. PP10-14, PP25-17

Midha, V. PP23-91, PP32-14

Miette, V. PS04-04

Migdalski, P. PP13-92

Mihai, B. PP16-32

Mihai, C. PP16-32, PP37-08

Mihara, K. PP36-26

Mikail, H. PP06-03

Mikami, S. PP13-36

Mikheyeva, O. PP23-61

Miki, A. PP05-145

Milich, D.R. PP13-114

Miller, M. PP05-125

Milosevic, I. PP13-94

Min, B.-H. PP06-13

Min, L. PP05-29, PP39-72

Mine, T. PP23-17
Mineta, S. PP26-23, PP26-24, PP26-38, PP26-66, PP26-70, PP26-71, PP28-30, PP36-08, PP36-11

Minguzzi, M. PS03-01, PP19-07

Minhas, Z. PP14-09

Minini, P. PP23-39

Min-Li, L. PP24-06

Minz, M. PP14-05

Minz, R.W. PP14-05

Miokovic, M. PP17-02

Miranda, H.P. PP26-04

Mirandola, S. PP03-10

Miri, S.M. FC05-04, PP11-04, PP11-12, PP13-101

Mirza, R. PP23-57

Mirza, S. PP11-09, PP13-21, PP28-04, PP39-28

Miseta, A. PP16-29

Mishiro, S. PP05-127

Mishra, B.K. PP23-72

Mishra, S.R. PP16-34

Misra, A. PP08-27

Missale, G. PP30-30

Mita, E. PP05-52

Mitchell, J.A. PP39-30

Mitchell, J.D. PP25-01, PP25-02, PP26-06

Mithani, H. PP09-25

Mittal, D.K. PP05-07

Miura, T. PP21-12

Miyakawa, Y. PP06-40

Miyamoto, Y. PP16-56, PP29-04

Miyasaka, A. PP13-22, PP13-39, PP16-56

Miyaso, H. PP26-60, PP26-61

Miyatake, K. PP25-08

Miyazaki, T. PP23-88

Mizokami, M. KL03-01, PP03-07, PP03-15, PP06-40, PP06-51, PP12-41, PP13-78,

PP13-122, PP13-123

Mizuno, S. PP37-07

Mizuno, Y. PP23-32, PP29-18

Mizuse, M. PP26-66

Mo, A. PP02-07

Mo, F.K. PP34-18

Mo, L.-R. PP13-98

Moawad, M.M. PP01-29

Mobasherizadeh, S. PP12-12

Mochida, S. PP23-32, PP29-18

Mochizuki, H. PP33-08, PP33-09

Moghadami, M. PP39-06

Mohajeri, D. PP020-02, PP02-23, PP02-26

Mohamadkhani, A. PP06-16

Mohamadpour, M. PP06-06

Mohamed, A.H. PP11-02

Mohamed, J. PP29-03

Mohamed, R. C08-02, PP06-39

Mohamed, Rose, I. PP26-64

Mohamed, S.Z. PP08-53

Mohamed, Z. PP26-64

Mohammad Alizadeh, A.H.M.A. PP06-24

Mohammed, G. PP03-40

Mohammed, H. PP04-04

Mohammed, M.A. PP12-06

Mohammed, A.A.-E. FC08-05

Mohana Devi, S. PP30-36, PP31-40, PP34-37

Mohannadi, M.A. PP12-18

Mohanty, S.K. PP28-05

Mohapatra, M. PP23-71, PP23-72
Mohar, B. PP14-26

Mohasseb, M.F. PP31-03

Mohhammadzadeh, M. PP12-16

Mohii, S.M. PP39-80

Mohsen, N. PP17-12

Mohsenifar, A. PP01-25, PP10-20

Mokarab, H.A. PP23-76, PP39-81

Molina, I. PS02-01

Moller, B. PS01-03

Monaharan, S. PP13-103

Monavari, S.H.R. PP09-27, PP06-24

Mondou, E. PS01-02, PP05-91, PP05-99

Montaser-Kouhsari, L. PP06-16

Montazeri, G. PP06-16, PP14-13

Moon, J.H. EC-07, PP25-13, PP31-25

Moon, S.H. PP08-58

Moore, C. PP23-41

Mor, E. PP38-21

Morabito, V. PP15-12, PP39-14, PP39-15

Morad, W.S. PP12-07

Morakot, N. PP39-52

Moreau, A. PP06-41

Moreno Luna, L.E. PP39-59

Moreno-Luna, L.E. PP23-16

Morgan, A. PP15-41, PP15-42

Mori, A. FC08-03

Moriconi, F. PP03-24

Morimoto, M. PP33-18

Morimoto, Y. PP36-18, PP36-24

Morishima, C. PP14-04

Moriwaki, H. PP17-06, PP30-08

Morovic, M. PP13-24

Morozov, S. PP08-40

Morozova, O. PP30-21

Morrone, A. PP04-38, PP12-38, PP26-59, PP39-74

Mos, L. PP10-04

Mos, L.M. PP08-05

Mosaad, Y.M. PP14-22

Mosconi, C. PP37-06

Moselhy, M.A. PP29-01

Moskal, O.M. PP16-09

Moslemi, E. PP03-04

Mostafa, G. PP13-49

Mostafa, M. PP38-51

Motooir, T. PP26-13

Motoori, T. PP26-15

Motoya, D. PP23-32

Mourad, Z. PP13-07

Mousa, N. PP08-46, PP09-38, PP14-15, PP31-29

Mousavi, G. PP02-29

Moussa, M. PP31-36

Moustafa, H.M. PP23-27, PP23-60,

PP23-62, PP33-16, PP33-17

Mozer-Lisewska, P. PP13-92

Mucci, S. PP26-30

Muhammad, N. PP03-36

Muharrmi, Z. PP13-69

Muir, A.J. PS02-03, PP13-64

Mukaide, M. PP13-122, PP13-123

Mukarjee, R.K. PP07-32

Mukhopadhyay, C.K. PP06-23

Muljono, D.H. PP04-10, PP04-19,

PP04-39, PP06-30, PP06-31 
Mumtaz, K. FC08-02, PP09-32,

PP13-34, PP23-23

Mungan, Z. PP09-29, PP23-80

Munir, S.M. PP13-41, PP13-52, PP31-32

Murad, A.F. PP23-27

Murata, K. PP13-122, PP13-123

Murmu, M. PP23-29

Muroyama, R. PS02-05

Murphy, D. PP26-51

Murphy, R. FC06-03

Murugan, K. PP02-01

Mustaq Ahamad, S. PP30-36

Muto, T. PP13-27

Muzzamil-Uddin, S. PP01-16

Myers, R.P. PP13-104

Myrick, F. PS01-01

Myung, S.J. PP22-03

Nabavi Zadeh, S.H. PP39-87

Nadir, S. PP02-17

Nadvi, N.A. PP15-53

Naeem, A. PP13-68, PP13-70, PP14-09, PP16-24

Nafady, M.A.-A. PP23-27, PP23-62,

PP33-16, PP33-17

Nafady-Hego, H. PP38-14

Nag, P. PP09-36, PP12-33, PP14-18, PP39-69

Nagaki, M. PP17-06

Nagano, T. PP25-08

Naganuma, H. PP06-51

Nagarajan, R. PP10-12

Nagashima, R. PS04-03

Nagata, K. PP13-121

Nagata, N. PP23-17

Nagaty, A. PP09-03

Nageshwar Reddy, D. PP23-55

Nagoshi, S. PP23-32, PP29-18

Najafi, F. PP23-85

Najdi, A. PP13-25, PP23-73

Najimi, M. PP38-46

Nakagami, K. PP27-09

Nakagawa, H. PP33-25

Nakai, M. FC08-04

Nakai, N. PP26-45

Nakajima, K. FC02-05, PP34-22, PP34-25

Nakamoto, S. PP05-80, PP13-27,

PP13-36, PP30-37

Nakamura, K. PP13-121

Nakamura, Y. PP36-08, PP36-11

Nakano, M. PP25-15

Nakashima, A. PP04-08

Nakata, R. PP13-115

Nakatani, T. PP13-32

Nakatsuka, T. PP32-03, PP34-02

Nakayama, N. PP23-32

Nakayoshi, T. PP06-40

Nakazawa, M. PP23-32

Nakazuru, S. PP05-52

Nalini, N. PP10-07

Nam, S.W. PP05-30

Namaei, M.H. PP06-06, PP12-44

Namasivayam, N. PS03-05

Namkung, J. PP30-18

Nanashima, N. PP21-12

Nandasiri, S.D. PP23-42

Nani, A. PP38-20

Naoumov, N.V. PP05-19, PP05-161
Naqvi, S.A.A. PP08-17, PP12-29

Narimatsu, H. PP27-18

Nart, D. PP31-22

Narumi, S. FC08-04, PP38-64

Naseem, S. PP05-83, PP05-84, PP13-37, PP23-31

Nashaat, M. PP38-51

Nasir, Z. PP01-16

Nasr, A.M. PP12-06

Nassiri Toosi, M. PP06-24, PP13-60

Nath, S. PP06-23

Nathaphan, S. PP02-20

Nautiyal, P. PP26-31

Navaratne, A.C.R. PP01-10, PP10-10, PP26-26, PP39-32, PP39-35, PP39-36, PP39-37, PP39-40

Nayman Alpat, S. PP05-131

Naz, F. PP01-40

$\mathrm{Naz}, \mathrm{N}$. PP01-17

Nazar, A.L.M. PP04-25

Nazari, M. PP12-08, PP12-09

Nazlican, O. PP04-21, PP06-05

Nedelciuc, O. PP37-08

Nedelcu, N.I. PP10-19, PP23-41

Negi, T.S. PP13-30, PP13-31, PP13-33, PP13-106

Negravi, S. PP04-35

Nejatizadeh, A. PP14-13

Nejjari, C. PP13-25, PP23-73, PP23-75

Nelson, D. PP13-46, PP13-89

Nemec, P. PP38-49

Nemekhbaatar, L. PP38-49

Nemtsova, V.D. PP07-08, PP07-09, PP07-10, PP08-12

Nersesov, A.V. PP08-36, PP08-37

Nery, F.G. PP26-04

Netinatsunton, N. PP13-15

Newman, T. PP24-07

Nezu, S. PS04-01

$\mathrm{Ng}, \mathrm{H}$. PP35-06

Ng, K.L. PP08-26

Ng, L. PP30-23

NG, L. PP35-06

Ng, W. PP05-113, PP13-103

Ng, W.T. PP35-06

Ngeow, Y.F. PP06-39

Ngo-Giang-Huong, N. PP06-41, PP39-84

Nguyen, R. PP13-103

Nguyen, T. PP05-141, PP12-37, PP13-14

Niazi, U. PP14-01

Nicolas, E. PS02-01

Nicolau, C. PP05-03

Nie, J.-J. PP03-30

Nie, Y.-Q. PP08-28, PP08-29, PP08-32, PP08-33, PP08-34

Nieuwenhuijs, V.B. PP38-01, PP38-23

Nigam, P. PP08-27

Nigm, I.M. PP39-80

Nikanfar, A. PP12-08, PP12-09

Nikolic, A. PP38-01

Nilbu-Nga, S. PP15-07, PP15-16

Nilova, T.V. PP08-13

Nilyanimit, P. PP39-82

Nimanong, S. PP03-31, PP03-32, PP05-48, PP13-11

Ninashvili, N. PP09-12
Ning, Q. FC02-02, PP05-44, PP05-45, PP05-47, PP05-53, PP06-49, PP06-50, PP16-44, PP20-09, PP25-19

Nirala, S.K. PP07-19, PP15-26, PP39-48

Nishida, N. PP13-78

Nishie, M. PP26-60, PP26-61

Nishiyama, R. PP36-26

Nitesh, K. PP19-12, PP19-12

Niu, J. PP05-22, PP05-117, PP05-122,

PP05-155, PP12-23, PP26-35, PP30-05, PP34-19

Niu, J.-Q. PP04-23, PP04-37, PP05-34, PP08-43, PP08-44, PP08-45, PP12-21

Niu, L.-Z. PP34-10

Nivatvong, S. PP38-35

Nojiri, K. PP38-36

Nojiri, S. PP03-07, PP03-15

Nokhodian, Z. PP12-11, PP12-12,

PP12-13, PP12-16, PP12-19

Nomorosa, K.M. PP23-63, PP23-64

Nonthasoot, B. PP38-35

Norouzi, M. PP14-13

Nosotti, L. PP04-38, PP12-38, PP26-59, PP39-74

Nosova, I. PP24-05

Nosseir, M.M.F. PP08-53

Nouhi, S. PP38-44

Novelli, G. PP15-12, PP39-14, PP39-15

Novelli, S. PP15-12, PP39-14, PP39-15

Novianti, T. PP03-33

Novruzov, A. PP08-38

Nozaki, A. PP33-18

Nseir, W. PP08-06

Nugmanova, M.N. PP08-37

Numata, K. PP33-18

Nunes, V.S. PP14-19, PP15-02

Nursanty, N.K.D. PP06-30

Nyabi, O. PP38-46

Oba, N. PP27-09

Obchoei, S. PP18-01, PP27-21

O'Beirne, J. FC06-01

Oberlies, N.H. PP14-04

Oberti, F. PP26-29

Obi, S. PP23-94, PP29-19, PP33-28

Oceguera-Contreras, E. PP15-49

Ocepek, A. PP02-05

Ochmann, J. PP28-14

Oda, M. PP26-13, PP26-15

Odintcova, A. PP10-23, PP10-24

Ogasawara, K. PP36-16, PP36-24

Ogura, Y. FC08-03

Ogutlu, A. PP03-13

Oh, B.S. PP05-51, PP05-67

Oh, J.E. PP02-08, PP13-100

Oh, J.M. PP07-06

Oh, K. PP26-45

Oh, S.J. PP07-06

O'Halloran, J.A. PP26-36

Ohata, K. PP27-09

Ohayon, O. PS03-03

Ohbu, M. PP26-13, PP26-15

Ohira, H. PS04-01

Ohki, T. PP32-03, PP34-02

Ohkura, Y. PP37-07

Ohmura, T. PP03-08

Ohsawa, I. PP37-07 
Ohtani, O. PP15-07

Ohto, M. PP33-18

Oikawa, J. PP29-04

Oikawa, K. PP16-56, PP29-04

Oikawa, T. PP13-39, PP25-15

Okabe, M. PP36-16, PP36-24

Okada, S. PP27-12

Okada, T. PP26-45, PP27-19, PP36-20

Okamoto, R. PP25-08

Okamoto, T. PP27-19

Okanoue, T. PS04-03

Oksuz, M. PP17-03

Okten, A. PP23-80

Ola, R.P. PP02-06

Olaru, L. PP23-74

Oliveira, A.C.P. PP12-28, PP14-19, PP15-02

Oliveri, F. PP06-56

Olivo, M. PP13-13

Olthoff, K. PP26-17

Omar, A. PP38-25

Omar, M. PP14-27

Omar, N. PP13-04, PP23-87

O'Mara, E. PP13-65, PP13-66

Omata, M. PS02-05, AE-01, PP13-115,

PP30-33, PP33-08, PP33-09, PP33-25

Oncul, O. PP02-22, PP04-33, PP05-20

Ondrasek, J. PP38-49

Ong, A. PS01-04, PP04-05

Ong, J. PP08-02

Ong-chai, S. PP31-39

Ong-Go, A. PP27-04

Onji, M. 05-127

Ono, Y. PP36-26

Ono-Nita, S. FC01-01

Oo, A.M. PP05-158, PP37-16

Oonishi, K. PP35-02, PP35-03

Ooshima, N. PP26-67

Oota, M. PP01-24

Oralndi, S. PP07-22

Orlando, E. FC04-02

Orlova, L.S. PP13-74

Örmeci, N. PP13-19

Orr, D. PP02-18, PP02-19

Ors, F. PP08-54

Orsini, L. PP31-16, PP31-20

Ortega, I. PP06-08

Osawa, Y. PP17-06, PP30-08

Oshakbayev, K. PP08-36, PP08-37

Oshima, G. PP36-26

Oshima, K. PP27-09

Oshima, N. PP36-22

Oshiro, N. PP32-11

Osman, O. PP39-16

Osman, W. PP05-143

Ostini, L. FC07-02

Ostojic, R. PP05-70, PP13-24, PP14-26

Ota, Y. PP33-06

Othman, M.H. PP23-27, PP33-16, PP33-17

Otsuka, S. PP26-60, PP26-61

Ou, W.N. PP02-13

Ou, X.J. PP31-19

Ouaz, A. PP04-13

Ould Gougam, R. PP25-05

Ou-Yang, L. PP20-04

Ovartlarnporn, B. PP05-94

Ow, M.M.G. PP04-27
Ozaras, R. PP06-25, PP06-26

Ozdil, S. PP09-29, PP23-80

Ozeki, I. PP03-08

Özenirler, S. PP16-05

Ozgunes, I. P05-131

Ozgunes, N. PP06-25, PP06-26

Ozkan, H. PP06-35, PP13-19

Özkan, S. PP16-05

Ozol, D. PP08-07

Ozolins, V. PP25-18

Ozturk, A. PP39-24

Öztürk, N.A. PP05-111

Ozturk, O. PP26-41, PP26-69

Pabich, E. PP03-06

Pacana, T. PP13-79, PP13-83, PP13-87, PP15-32

Padaravat, M. PP08-41, PP39-62, PP39-63, PP39-71

Padavarat, M. PP02-24

Padbury, R.T.A. PP38-01, PP38-23

Padidarcheep, W. PP15-07

Padilha, C. PP08-51

Paik, S.W. PP03-21, PP03-23, PP05-14,

PP05-160, PP06-13, PP09-17, PP35-04

Paik, Y.H. PP09-26, PP23-69

Pairojkul, C. PP27-18, PP28-18

Paizi, M. PS03-03

Pajno, C. PP04-38

Pajor, L. PP16-29

Pakasi, L.S PP01-20, PP03-11, PP08-22, PP27-13

Pal, S. PP23-07

Palaniappan, R. FC02-03, PP26-40, PP39-56

Palaniappan, S. PP25-04

Palgova, L. PP28-28

Palma, J. PP33-14

Pamornpiboon, P. PP13-11

Pan, C. PP01-15, PP05-65, PP05-99, PP05-101

Pan, L. PP30-20

Pan, L.-L. PP06-62

Pan, Q. PP07-26, PP07-36, PP30-22

Pan, X. PP05-148

Pan, X.-B. PP14-23

Pan, X.-M. PP13-117

Pan, Y. PP12-21, PP26-35

Pancoska, P. PP31-05

Panda, C.R. PP05-63, PP23-72

Panda, S.K. FC03-02, PP06-10, PP09-09, PP09-18

Pande, C. PP06-23

Pandey, C.K. PP23-78

Pandey, C.M. PP39-58

Pandey, D. PP27-03

Pandey, M. PP27-22

Pandeya, D. PP06-04

Pandhi, P. PP02-06

Pang, M. FC05-03

Pang, R. PP30-23

Pang, R.W. C06-01

Pang, X. FC01-02

Panjaworayan, N. PP06-57

Par, A. PP16-29

Par, G. PP16-29

Paradis, V. PS02-01

Paraná, R. PP12-01, PP12-28, PP14-19, PP15-02
Paraskevas, E. PP13-06

Parildar, H. PP08-25

Paripovic, E. PP12-37, PP13-14

Park, B.H. PP07-04

Park, B.-L. PP30-12

Park, C.H. PP13-72

Park, C.-H. PP22-12, PP32-10

Park, C.K. PP09-28, PP13-67, PP23-30

Park, C.W. PP13-28

Park, G.T. PP02-08, PP13-100

Park, H. PP03-26, PP16-47

Park, H.C. PP35-04, PP35-05

Park, H.G. FC04-01, PP15-37, PP26-39, PP26-52, PP33-24

Park, H.J. FC06-02, PP07-03, PP15-23, PP23-38

Park, H.K. PP33-27

Park, H.W. PP09-16

Park, J. PP26-19

Park, J.B. PP26-20

Park, J.-H. PP35-07

Park, J.J. PP30-09

Park, J.-W. PP37-15

Park, J.Y. PP03-26, PP13-18, PP16-46, PP16-47, PP16-50, PP16-58, PP19-04, PP20-12, PP23-69, PP23-84, PP30-29, PP31-33, PP31-35, PP36-21

Park, K.-H. PP15-14

Park, K.J. PP05-123

Park, M.S. FC06-04

Park, S.B. PP27-07, PP28-10

Park, S.H. FC06-04, PP09-28, PP09-34,

PP13-67, PP16-49, PP23-30, PP28-26

Park, S.J. PP05-89, PP13-102, PP13-112, PP31-25

Park, S.-M. PP05-30

Park, S.Y. PP15-37, PP23-38, PP26-39, PP26-52, PP33-24, PP33-27, FC04-01, FC06-02, PP04-29

Park, T.B. PP36-16, PP36-24

Park, T.J. PP30-12

Park, Y.M. PP13-67, PP13-112

Park, Y.N. PP16-46, PP16-58

Parkash, O. PP01-16, PP23-12

Parobkova, H. PP34-13

Parrella, P. PP14-08

Parveen, N. PP22-09

Parvez, M. FC03-03

Parwati, T. PP12-48

Pasaje, C. PP30-12

Paschwingel, P.A. PP08-51

Pasic, F. PP12-18

Pasquinelli, C. PP13-76

Passeri, D. PP07-22

Patanchon, M. PP16-13

Patcharatrakul, T. PP04-49

Patel, K. PP12-25

Patel, N. PP01-23, PP04-31, PP08-41, PP39-62, PP39-63, PP39-64, PP39-71

Pathipavanich, P. PP39-84

Patil, B.S. PP02-12

Patra, N. PP07-07

Patra, S. PP06-23

Patsavela, S. PP13-06

Pattanapraphapan, A. PP37-03

Patton, Y. PP34-23 
Patutko, Y.I. PP26-53

Pauliny, M. PP38-49

Pausawasdi, N. PP03-31, PP03-32, PP05-48

Pavan, G. PP05-113

Pavlova, T.V. PP03-38

Pavlovic, V. PP17-02

Pawlotsky, J. C09-04

Pawlotsky, J.-M. FC05-01, PP05-135

Pawlowska, M. PP12-15

Payungporn, S. PP06-51, PP39-82

Pazienza, V. PP14-08

Pécheur, E.-I. PP14-04

Pecoraro, L. PP04-38

Pedicone, L. PP13-75

Peeters, M. PP13-59

Pei, R. PP06-63

Pei, X. PP02-14

Peipei, Z. PP05-01

Peng, C.Y. PP12-25

Peng, C.-Y. PP05-106, PP13-104

Peng, J. PP16-07

Peng, L. PP05-02, PP05-85, PP05-86,

PP13-01, PP15-18, PP16-02, PP22-14

Peng, M.-M. PP04-11

Peng, Z. PP12-03

Peng, Z.-T. PP15-35

Pepanyan, A. PP13-116

Peprny, M. PP04-42

Peres-da-Silva, A. PP14-25

Perricone, G. PP13-13, PP14-21

Perry, H. PP24-07

Persohn, E. PP06-38

Persson, A. PP13-76

Perumareddi, K. PP26-31

Peschell, K. PS01-03, PP05-125

Pessaux, P. PP26-29

Pessoa, M. FC01-01, PP13-47

Petersen, J. PP09-06

Petta, S. PP08-52

Petti, M.C. PP12-38

Pevzner, A. PS03-03

Phisalprapa, P. PP03-24, PP05-48

Phua, K. 48

Pi, B. PP20-09

Pianko, S. FC05-03

Piastowska-Ciesielska, A. PP39-55

Piątosa, B. PP25-10

Piazza, A. PP14-21

Picchio, G. PS02-04, FC05-02

Pichoud, C. PP06-32

Piekuse, L. PP17-18

Pijak, M. PP04-42, PP05-11, PP05-100, PP25-09

Pilleul, F. PP33-14

Pinarbasi, B. PP09-29, PP23-80

Pinelo, E. PP23-81

Ping, Q. PP05-116

Pinlaor, P. PP28-18

Pinlaor, S. PP28-18

Pinney, J. PP26-44, PP38-60

Pintilei, I. PP37-08

Piratvisuth, T. KL06, FC01-01, FC01-03, FC05-03, PP04-49, PP05-62, PP05-94, PP13-15

Piro, S. FC06-01

Pirvulescu, I. PP16-12
Pisespongsa, P. PP23-89, PP31-39, PP34-17, PP39-52

Plank, L. PS04-05, FC06-03

Plano, S. PP13-13, PP14-21

Poddybnaya, I. PP26-10

Podlujniy, D.V. PP26-53

Podsadecki, T. PP13-95

Poggi, G. PP23-40

Pol, S. PS02-04, PP13-46

Polyak, S. PP14-04

Pompa, J. PP12-01, PP15-02

Pompili, M. PP23-43, PP33-11

Pongmayteegul, S. PP15-17

Pongprasobchai, S. EC-05, PP03-31, PP03-32, PP05-48

Ponnusamy, R.P. PP31-28

Poon, R. PP29-05, PP33-03, PP36-07

Poon, R.T. C21-03

Poon, R.T.P. PP33-13, PP34-21

Poon, W.H. PP04-09

Poonkhum, R. PP15-07

Poordad, F. PS02-02, PP13-89, PP13-90

Pootracool, P. PP38-04

Pootrakul, P. PP38-62

Poovorawan, K. PP06-55

Poovorawan, Y. C19-03, PP06-51, PP06-54, PP06-55, PP08-31, PP12-41, PP12-42,

PP23-10, PP28-20, PP38-35, PP39-82

Pop, C. PP10-04

Popescu, A. PP13-107, PP16-30,

PP16-31, PP16-38, PP16-39, PP23-44,

PP23-59, PP37-10, PP37-12

Popescu, A.M. PP13-85

Popovic, V. PP05-157

Popow-Kraupp, T. PP13-88

Pornvoranunt, A. PP08-09

Potapov, A. PP17-15

Pothiraju, D. PP19-01

Potter, A. PP15-41, PP15-42

Pound, D. PP13-75

Powell, E. C12-01, C12-02, C12-03, PP15-21

Powell, L. C08-01

Poynard, T. PP23-90, PP23-92

Prabowo, T. PP05-31

Prachayakul, V. PP03-31, PP03-32, PP05-48

Pradidarcheep, W. PP15-16, PP15-17

Praianantathavorn, K. PP12-42

Praianantathavorn, K.P. PP12-41

Praisontarangkul, O. PP34-17, PP39-52

Praisontarangkul, O.-A. PP23-89, PP31-39

Prakanrattana, U. PP39-45

Prakobwong, S. PP28-18

Prakoso, E. PP21-04

Pramoolsinsap, C. C18-02, PP12-25

Prasad, K.N. PP39-58

Prasad, R. FC07-04, FC07-05

Prati, D. PP09-07

Presa, J. PP23-81

Pribac, G. PP08-05, PP10-04

Pribramska, V. PP23-21

Prieto, M. PP38-54

Prisacaru, L.J. PP05-03

Privitera, G. FC06-01

Promlikitchai, P. PP05-62

Prommas, S. PP39-84

Prom-on, S. FC01-04
Promrat, K. AS-04

Pruksakorn, P. PP34-17

Pruzanski, M. PP07-22

Pugliese, F. PP39-14

Puglisi, G. PP26-58

Pujol, L. PP31-07

Pulkstenis, E. PP12-25

Punamiya, S. EC-03, EC-08

Puoti, M. PP13-46

Purcell, R.H. FC03-03

Purevsuren, U. PP23-68

Puri, R. PP26-54

Purrello, F. FC06-01

Purwadi, N. PP24-11

Putha, V. PP34-23

Putica, S. PP17-02

Qadri, I. PP04-34, PP14-01

Qazi, M.A. PP12-46, PP13-12

Qi, J. PP05-45, PP16-44

Qi, R. PP30-24

Qi, W. PP13-99

Qi, Y.F. PP05-34

Qi, Z.-X. PP01-38

Qian, F. PP10-08

Qian, Y.W. PP29-06

Qian, Z.P. PP06-01

Qiao, F. PP01-18

Qiao, S. PP28-15

Qi-Feng, X. PP05-108

Qi-Huan, X. PP05-108

Qin, B. PP06-63

Qin, H. PP14-06, PP14-07

Qin, J.P. PP15-13

Qin, Y. PP38-26

Qingshou, S. PP05-37

Qingxian, C. PP06-61

Qiu, J. PP01-19

Qiu, M. PP03-16

Qiu, N. PP06-21

Qiu, P. PP13-75

Qu, B. PP36-23

Qu, K. PP26-57

Quak, S.H. C19-01

Quan, Z.-W. PP21-02

Quaretti, P. PP23-40, PP26-46, PP33-10, PP33-12

Quer, J. PP06-08

Quin, J.W. PP13-103

Qureshi, J.A. PP05-136, PP05-138

Raafat, D. PP25-21

Rabuazzo, A.M. FC06-01

Racco, S. PP23-43

Radfar, M. PP38-44

Radfar, S. PP38-44

Radhika, G. PP07-32

Radic, D. PP05-70, PP13-24

Radicheva, M. PP17-01

Rafatpanah, F. PP14-13

Raffaele, L. PP09-07

Rafiei, B. PP02-23, PP02-26

Ragab, H. PP39-02

Raghavendran, H.B. PP15-23

Ragheb, M.M. PP16-16

Rahimi, H. PP09-21

Rahman, A. PP08-20

Rahman, K. PP32-04, PP39-29 
Rahman, M. PP05-136, PP05-138, PP08-20

Rahman, M.K. PP08-39

Rahman, S. PP01-06, PP05-98

Raj, A. PP37-04

Rajaa, A. PP13-16, PP34-31

Rajagopal, S. FC02-03, PP26-40, PP39-56

Rajamanthri, R.A.M. PP01-10

Rajasuriar, R. PP15-33

Rajendraprasad, A. PP22-09, PP22-10

Rajender Reddy, K. LT2-04

Ralston, R. PP13-75

Ramachandran, V. PP23-07, PP23-09

Ramadori, G. PP01-17, PP10-19, PP23-41

Ramaswamy, S. FC02-03, PP26-40, PP39-56

Ramos, E. PP13-64

Rampal, S. PP23-49

Ramzy, I.I. PP08-53

Rana, S.V. PP02-06

Rangan, M. PP39-50, PP39-58

Rao, H. PP11-08

Rao, H.Y. PP12-14, PP14-06, PP14-07, PP15-27, PP22-15

Rao, P. PP23-55

Rao, P.N. PP07-32, PP23-55

Raouf, A.A. FC08-05

Rapaccini, G.L. PP23-43, PP33-11

Raptopoulou-Gigi, M. PP13-104

Rashed, H.G. PP11-106

Rashed, M. PP17-12, PP23-06

Rashid, M.H.-O.- PP08-39, PP17-07, PP32-04, PP39-29

Rashidian, A. PP04-14, PP04-15

Rastgar, Jazii, F. PP06-16

Rastogi, A. PP24-10, PP26-73, PP26-74, PP39-65

Rastogi, M. FC08-01

Ratanachu-ek, T. EC-04

Ratanakorn, P. PP06-51

Rathore, R.K.S. PP39-58

Ratiu, I. PP16-38, PP23-74

Ratziu, V. C12-01, C12-02, C12-03, C15-01

Ravendhran, N. PP13-75

Ravetta, V. PP33-12

Raza, H. PP12-46, PP13-12

Raza, S. PP09-25

Razavi-Azarkhiavi, K. PP01-25, PP10-20

Razlan, H. PP23-49

Razon-Gonzalez, E.V.B. PP08-02, PP23-11

Rebbani, K. PP06-34

Reddy, D.N. EC-01, PP07-32

Reddy, K.R. ET1-01, PP13-50, PP13-56, PP38-04

Reddy, R. PP02-03, PP13-90

Reddy, R.K. PP26-17

Redwan, E.M. PP13-05

Reif, S. PP23-02

Reiman., O.V. PP28-28

Reindollar, R. PP13-76

Ren, G.Y. PP06-18

Ren, H. PP05-22, PP05-105, PP05-112,

PP05-117, PP05-119, PP05-146,

PP39-33, PP39-38

Renda, D. PP14-21

Renda, M.C. PP13-13

Renzulli, M. FC04-02, PP34-12, PP37-06

Restivo, G. PP14-21
Rew, J.S. PP05-123, PP13-72

Rewisha, E. PP13-04, PP23-45, PP23-97

Rezaei, Moghadam, A. PP02-23,

PP02-26, PP02-29

Rezaie, M. PP39-87

Ribeiro, M. PP26-04

Riccardi, L. PP33-11

Riesing, K. PP03-06

Rios, M. PP14-19

Ripault, M.P. PP03-14

Ripault, M.-P. PP06-43

Ripoli, M. PP14-08

Riyaz, S. PP38-37

Rizk, A. PP39-67

Rizvi, S.A.-U.-H PP08-17, PP12-29

Rizzetto, M. PP05-42, PP13-56

Rizzo, G. PP07-22

Ro, W.S. PP19-04, PP20-12, PP30-29

Robbins, G. PP38-37

Roberts, L. PP30-32

Roberts, S. PS02-04

Robotin, M. PP34-23

Roch, A. PP26-30

Roche, B. PS04-02

Rodriguez-Frias, F. PP06-08

Rodriguez-Torres, M. PP13-45, PP13-50, PP13-64

Roggendorf, M. PP06-17, PP20-10

Rohrwasser, A. FC03-04

Romeo, R. PP09-07

Romero, R. PP23-63, PP23-64

Roni, M. PP06-31

Ronquillo, M. PP23-66

Rooge, S.B. PP39-65

Rosa, I. FC05-01

Rosa, L. PP33-12

Rossaro, L. PP13-75

Rossi, A. PP26-58

Rossi, M. PP15-12, PP39-14, PP39-15

Rossi, S. PP33-101, PP33-12

Rostom, Y. PP31-02

Rouanet, S. FC05-01

Rousseau, F. PP05-101

Rozentale, B. PP26-76

Rtskhiladze, I. PP10-25

Ruan, Y. PP17-08

Ruberto, F. PP39-15

Rubín, A. PP38-54

Rubin, R. PP13-75

Rudler, M. PP23-90, PP23-92

Ruengdarakanon, N. PP38-38

Ruggeri, R.F. PP13-13, PP14-21

Rugina, C.N. PP10-11

Rugina, S. PP10-11, PP16-22, PP37-13

Ruiz, Corro, L.A. PP15-48

Rustgi, V. PP13-76

Rusyn, V.I. PP16-09

Rutter, K. PP12-34, PP13-64, PP13-84, PP13-88

Ruxrungtham, K. PP15-33

Ryan*, J. PP07-05

Rychkov, G.Y. PP07-34

Ryu, C.S. PP39-25

Ryu, D.Y. PP29-13

Ryu, H.J. PP31-35

Saada, K. PP23-75
Saadat, Fard, S. PP24-09

Saadat, J.A. PP12-35, PP12-45

Saadatjoo, S.A. PP06-06

Saba, I. PP01-40

Saber, M.A. PP14-27

Sadeghi, S. PP12-16

Sadeghpour, B. PP07-22

Sadek, A.E.M. PP24-02

Sadhewa, A. PP04-19

Sadik, M.A. PP07-32

Saeed, A. PP08-17, PP12-29, PP13-69

Saeedi, S.-Y. PP11-12, PP12-26

Saeki, C. PP25-15

Saeki, S. PP33-26

Safadi, R. PP05-119

Safi, S.W. PP04-34, PP12-35

Sagar, Gang, S. PP19-12, PP19-13

Sagayaraj, A. PP22-16

Sahni, P. PP23-07

Saidalieva, M. PP06-11

Saiedi, Hosseini, S.Y. PP04-43

Saiedi, S. PP13-20

Saifutdinov, R.G. PP28-01

Saikia, A. FC04-05, PP28-27, PP39-68

Sainokami, S. PP13-22

Saito, H. PP13-122

Saito, K. PP13-27, PP13-123, PP32-03, PP34-02

Saito, M. PP05-145

Saito, S. PP26-45, PP27-19, PP36-20

Sajjad, M. PP12-35

Sajjadi, S.M. PP39-73

Sakaguchi, N. PP27-18

Sakai, H. PP30-08

Sakakibara, Y. PP05-52

Sakamoto, M. C06-02

Sakata, M. PP27-09

Sakhuja, P. PP06-23, PP30-26, PP39-65

Saki, N. PP04-35, PP12-39

Sakota, B. PP13-121

Sakurai, H. PP37-07

Sakurai, Y. PP25-16

Salahuddin, M. PP05-138

Salam, G.D. PP09-35

Salama, H. PS03-02

Salama, M. PP39-07, PP39-83, FC08-05,

PP16-52

Salama, O.S. PP16-11

Salazar, Montes, A.M. PP15-48

Salazar-Montes, A. PP15-49

Saleh, S.M. FC08-05

Salih, Alj, H. PP06-34

Salim, S. PP16-25

Salimi, S. PP13-20

Sallberg, M. PP05-136, PP05-138

Sällberg, M. PP05-64, PP13-114

Salleh, R. PP12-05, PP16-35

Sallenave, B. PP02-01

Salman, T. FC08-05, PP16-52

Salvana, A.D. PP08-02, PP23-11

Samaka, R. PP21-07

Samir, W. PP11-10

Samsonova, N.G. PP08-13

Samuel, D. PSO4-02

Samy, N. PP39-02

San, Juan, F. PP38-54 
Sanborn, M. PP03-06

Sandoval-, Rodriguez, A. PP15-45

Sandoval-Rodriguez, A. PP16-51, PP20-07

Sandrin, L. PP04-04

Sanei, E. PP14-13

Sang, X. PP15-54

Sangcakul, A. PP23-52

Sa-nguanmoo, P. PP06-51, PP06-54

Sankoh, A.J. PP13-89

Sankpal, P. PP34-28, PP39-77, PP39-78

Sannuk, S. PP13-111

Sano, K. PP36-16, PP36-24

Sanpajit, T. PP04-49, PP05-62

Santi, E.G. PP27-04, PP33-01

Santiago-Tripon, E. PP23-11

Santillan-Montes, J. PP20-07

Santos, J.F.B. PP12-28

Santra, A. PP02-10, PP02-11, PP15-29,

PP15-30, PP19-02

Santra, S. PP02-10

Sanyal, A.J. AS-04, C13-04, ET2-01

Saraf, N. PP26-54

Saraswat, V.A. PP39-50, PP39-58

Saravanan, N. PP10-07

Sargsyan, V. PP13-116

Sargsyants, N. PP13-116

Sarin, S.K. PS01-05, AS07, C13-04,

FC05-03, C15-04, C16-04, PP05-142,

PP06-23, PP13-104, PP16-34, PP16-36,

PP23-78, PP24-10, PP26-73, PP26-74, PP30-26, PP39-65

Sarizade, G. PP14-13

Sarma, M.P. PP09-11, PP10-12, PP32-05

Sarovath, A. PP26-14

Sasaki, M. PP34-22

Sasikala, K. PP31-40, PP34-37, PP30-36

Sasikala, M. PP07-32

Sasso, M. PP04-04

Satake, M. PP26-45

Satarasinghe, R.L. PP01-10, PP10-10, PP26-26, PP39-32, PP39-35, PP39-36, PP39-37, PP39-40

Sathyanesan, J. FC02-03, PP26-40, PP39-56

Sato, S. PP23-94, PP27-09, PP29-19,

PP33-28

Sato, T. PP03-08, PP23-94, PP27-09, PP29-19, PP33-28

Sato, Y. PP13-32, PP13-122, PP13-123

Satpathy, G. PP06-10

Savina, M. PP19-03

Sawada, T. PP23-48

Sawada, Y. PP13-32

Sawanyawisuth, K. PP27-11, PP27-21

Sawara, K. PP13-39, PP16-56, PP29-04

Saxena, A.K. PP34-09

Sayad, B. PP23-85

Sayan, M. PP06-25, PP06-26, PP14-24

Sayyad, B. PP14-13

Schäfer, A. PP12-27

Schaff, Z. PP15-55, PP31-42

Schaper, M. PP06-08

Schawalder, J. PP01-01

Scherzer, T.M. PP12-34, PP13-61, PP13-84, PP13-88

Scheuerlen, M. PP12-27

Schiff, E. AS-02, PP13-75
Schinoni, M. PP12-01, PP12-28, PP14-19, PP15-02

Schlaak, J. PP06-17

Schmilovitz-Weiss, H. PP34-20

Schmitz, M. PP13-46

Schwartz, J. FC03-04

Scotto, G. PP05-88, PP09-08, PP13-44

Sebagh, M. PS04-02

Segarra, I. PP02-06

Seguchi, K. PP33-06

Segura-Ortega, J. PP23-16, PP39-59

Seijas, O. PP23-66

Seishima, M. PP17-06

Sekar, V. PP13-59

Seki, K. PP13-32

Seki, M. PP32-03, PP34-02

Seldin, M. PS04-01

Seliverstova, S. PP08-13

Semela, D. SA02

Semyatichko, E. PP07-08, PP07-10

Sen, S. PP23-04

Senanayake, S. PP23-42

Sendroiu, M. PP16-30, PP16-31, PP16-38, PP23-44, PP37-10

Sengupta, S. PP06-10

Seno, T. PP25-08

Senturk, O. PP14-24

Senyakovitch, V. PP17-15

Seo, E.H. PP16-49

Seo, J.H. PP03-26

Seo, J.W. PP23-37

Seo, Y. PP05-145

Seo, Y.S. PP09-34, PP16-23

Seol, S.Y. PP13-102

Seong, J. PP31-35

Sepand, M.-R. PP01-25

Serban, C. PP13-107

Serebour, E.F. P13-69

Serevina, D. PP06-28

Serghini, M. PP04-13

Serrão, R. PP13-45

Seth, D. PP16-37, PP05-15

Seto, W.-K. PP06-37

Setrkraising, K. PP08-31, PP23-10, PP38-35

Setthaudom, C. PP08-07

Seven, G. PP38-50

Seydel, G.S. PP07-31

Sha, S.A. PP09-01

Sha, W.H. PP08-35

Shaat, E. PP08-03

Shaban, A.I. PP13-111

Shaban, E. PP13-111

Shabtai, E. PP34-20

Shackel, N. PP21-04

Shah, H. PP39-31

Shah, N.J. PP13-79, PP13-83, PP13-87

Shah, S. FC05-03

Shah, S.R. PP12-25

Shah, V. PP13-76

Shahhosseiny, M.H. PP03-04

Shahi, D. PP02-26

Shahidullah, M. PP04-30

Shahin, R.S. PP23-60

Shaik, M. PP22-10

Shaked, A. T1-0, P13-01, PP26-17

Shaker, M. PP16-48
Shaker, O.G. PP08-53

Shakil, O. FC08-02

Shalimar, S. PP05-08

Shamir, R. PP38-21

Shams, El, Din, R. PP31-41

Shao, G. PP30-24, PP32-07

Shao, L. PP03-37

Shao, Q. PP16-54

Shao, Y.-L. PP06-62

Shapina, M. PP13-81

Shapiro, R. PP23-02, PP38-21

Sharabanov, A. PP13-48

Sharaf, S. PP39-07, PP39-83

Sharawy, M. PP39-66

Sharbeen, G. PP19-10

Sharif, H. PP13-68, PP16-24

Sharifi, Y. PP24-07

Sharma, A. PP14-05

Sharma, B. PP16-34, PP31-37

Sharma, D. PP01-39

Sharma, M. PP05-142, PP12-18, PP39-68

Sharma, N. FC07-05, PP34-09

Sharma, P. PP16-34, PP16-36, PP23-78

Sharma, R. PP23-07

Sharma, S. PP02-06

Sharma, V. PP05-142

Sharr, W. PP36-14, PP38-27

Sharvadze, L. PP06-15, PP13-53

Shastry, R. PP02-12, PP14-17

Shaw, T. PP05-87

Shawky, E. PP39-66

Shayakhmetova, G. PP02-15

Shcherbakov, P. PP23-46

She, L.H. PP13-03

She, L.-H. PP04-02

Shehata, M. PP12-06

Sheikh, N. PP01-17, PP07-25

Shen, C. PP03-25, PP08-11

Shen, E. PP39-76

Shen, L. PP63-37

Shen, T. PP12-31, PP38-22, PP06-19

Shen, Y. PP36-09

Shenoy, K.T. PP06-33

Shenoy, S. PP23-09

Sheriff, M.H.R. PP04-25

Sherman, D. PP13-76

Sherman, K. PP13-89, FC05-02

Sherman, M. IC17-01

Shevah, O. PP27-02

Sheykhi, D. PP12-08, PP12-09

Shi, F. PP05-162, PP05-164

Shi, G. PP05-119, PP05-82

Shi, G.-F. PP05-09, PP05-134, PP06-01

Shi, H. PP16-02

Shi, K.-Q. PP04-11

Shi, M. PP10-08

Shi, Q. PP13-26

Shi, X. PP12-23

Shi, X.-D. PP12-21

Shi, Y. PS03-04, PP19-14, PP21-10, PP22-01, PP22-02

Shiah, H.-S. PP34-30

Shibata, M. PP26-24

Shibuya, A. PP04-01

Shida, H. PP03-08

Shiffman, M. PP13-46, PP13-47, PP13-50 
Shih, H.-C. PP08-14

Shih, S.-C. PP34-08

Shiha, G. C22-01, PP11-10, PP16-48

Shiina, S. PP30-33, PP33-25

Shim, J. PP28-17

Shim, J.H. PP35-07

Shim, S.G. PP02-08, PP13-100

Shima, M. PP33-06

Shimamura, T. PP01-24

Shimizu, A. FC02-04

Shimizu, K. PP26-67

Shimizu, M. PP30-08

Shimizu, T. PP21-12

Shimizu, Y. PP26-13, PP26-15

Shimoda, K. PP13-121

Shimoda, M. PP23-48, PP36-26

Shimosegawa, T. PP14-20

Shin, C.S. PP08-15

Shin, H.D. PP08-58, PP30-12, PP30-13, PP30-17

Shin, H.P. PP30-09, PP30-10

Shin, H.S. PP16-58

Shin, J.E. PP08-58

Shin, J.H. PP28-02

Shin, J.W. PP01-22, PP15-23, PP26-39

Shin, K.Y. FC04-01, PP15-37

Shin, M. PP33-22

Shin, M.J. PP36-13

Shin, S.R. PP09-28, PP23-30

Shin, W.G. PP09-16

Shin, Y.W. PP07-30

Shinha, T. PP26-31

Shinkai, N. PP03-15

Shinoda, M. PP36-26

Shinoto, M. PP35-02, PP35-03

Shinozaki, M. PP05-80

Shiraishi, K. PP23-17

Shiraki, K. PP04-03

Shiraki, T. PP23-48

Shirasawa, H. PP13-27

Shirin, H. PP27-02

Shirvani, M. PP23-85

Shi-Zhen, Z. PP36-10

Shoaei, P. PP09-15, PP09-21, PP12-13,

PP12-16, PP13-71, PP12-19

Showpittapornchai, U. PP15-16, PP15-17

Shteyer, E. PP23-02

Shu, J. PP15-50, PP15-51, PP15-52

Shukla, M. PP27-22

Shukla, S. PP34-09, PP39-48

Shulman, N. PP13-57

Shum, W. PP12-37, PP13-14

Shunsuke, N. PP13-78

Siciliani, L. PP23-43, PP33-11

Sidarta, E. PP04-19, PP06-31

Siddique, K. PP28-04, PP39-27, PP39-28

Siddiqui, R. PP13-21

Sidhu, S.S. PP10-09

Sievert, W. PS01-02

Sigalas, A. FC06-01

Sikorska, A. PP39-55

Silasuwan, J. PP26-11

Silsirivanit, A. PP27-18

Silva, C.A.C. PP12-28, PP14-19

Silva, G.F. PP13-46, PP13-50

Silva, M. PP13-66
Simanis, R. PP26-76

Simon, K. PP12-15

Sinagra, E. PP13-13, PP14-21

Singer, T. PP39-30

Singh, A. PP09-36, PP12-33, PP14-18, PP39-69

Singh, B. PP12-32

Singh, D. PP39-85, PP39-86

Singh, G. PP12-32

Singh, K. PP02-06, PP05-73, PP05-74,

PP05-75, PP12-32, PP34-09

Singh, P. PP39-85, PP39-86

Singh, R. FC07-04, PP12-18

Singh, S. PP09-36, PP12-33, PP14-18,

PP23-78, PP26-74, PP39-69

Singhal, A. PP10-12

Singla, P. PP10-09

Sinha, S.K. PP02-06

Sintusek, P. PP23-10, PP38-35, PP08-31

Siqing, T. PP05-130

Siqing, T.-N.-L. PP13-80

Sirinawasatien, A. PP33-07

Sirirungsi, W. PP06-41

Sirivatanauksorn, Y. C21-01

Siriwardana, R.C. PP36-14, PP38-17

Sirli, R. PP16-30, PP16-31, PP16-38,

PP16-39, PP23-59, PP37-10, PP37-12

Sirmatel, F. PP03-29

Sirmatel, O. PP03-29

Sirmatelli, F. PP05-78, PP05-79

Sithithaworn, P. PP28-18

Sitthimongkhonkarn, P. PP05-150

Skacelova, H. PP23-21

Skinner, N. PP15-33

Skoien, R. PP15-21

Skok, P. PP02-05

Skrha, J. FC07-03

Skuja, S. PP25-18

Sleem, T. PP39-81

Sleigh, K. PP25-01, PP25-02, PP26-06

Slim, J. PP13-45

Słomka, M. PP10-02, PP10-03

Smane, L. PP25-18

Smout, M. PP27-08

Sniukiene, V. PP13-90

Snow-Lampart, A. PS01-01, PS01-03, PP05-101

So, T.M.-K. PP05-19

So, W.K.W. PP34-24

So, Y.H. PP26-65

Sobesky, R. PS04-02

Sobhonslidsuk, A. T2-01, PP08-09, PP16-45, PP23-52, PP26-11, PP26-14, PP38-04, PP38-62, PP39-19

Sobrevilla, Navarro, A. PP15-48

Socaciu, M. PP37-12

Söderholm, J. PP13-114

Sodsai, P. FC01-04

Soedarmono, Y.S.M PP04-19

Soeroto, A. Y. PP02-27

Sofia, S. PP08-52

Sohn, D.H. PP15-43, PP19-06

Sohn, J.H. PP08-24, PP16-33

Sokal, E. PP38-46

Sokolovic, D. PP01-08

Solà, R. PP13-45

Solangi, S. PP09-23, PP09-24
Soliman, A. PP04-03, PP11-03, PP12-02,

PP13-07, PP14-15, PP23-06, PP24-02,

PP30-03, PP31-03, PP39-10, PP39-11

Soloviy, M. PP36-02

Somaweera, G.N. PP08-16

Somi, M. PP14-13

Somia, A. PP12-48

Sommanustweechai, A. PP06-51

Son, C.G. PP01-22, PP15-23

Son, C.Y. PP16-47

Son, M.-K. PP15-22

Sondore, V. PP17-18

Song, C.-H. PP05-162

Song, G. PP05-53

Song, G.A. PP29-13

Song, G.Y. PP07-04, PP39-17

Song, H. PP07-29, PP10-15

Song, I.H. PP08-58, PP30-17

Song, J. PP03-27, PP05-45, PP16-44

Song, J.C. PP09-04

Song, K.H. PP26-43

Song, M.J. PP22-12, PP32-10

Song, S. FC07-01, PP09-10, PP15-53, PP33-22

Song, S.H. PP36-13

Song, W.Y. PP05-115

Song, Y.A. PP13-72

Song, Y.J. PP13-102

Sönmez, C. PP16-05

Sood, A. PP10-09, PP23-91, PP32-14

Sood, N. PP23-91, PP32-14

Sopipong, W. PP39-82

Sorensen, J. FC03-04

Sornmayura, P. PP08-09, PP16-45, PP26-11, PP26-33, PP38-04, PP38-62

Sottisuporn, J. PP13-15

Soulier, A. FC05-01

Souza, T.F.P. PP12-01, PP12-28, PP15-02

Soylu, A. PP05-110

Sozzi, T. PP05-87

Spadaro, L. FC06-01

Spellman, M. PP05-126

Sperl, J. PP38-24

Spicak, J. PP38-24

Spielmann, L.H. PP17-19, PP32-13

Spira, G. PS03-03

Sporea, I. PP16-30, PP16-31, PP16-38,

PP16-39, PP23-44, PP23-59, PP23-74,

PP37-10, PP37-12

Spreafico, M. PP09-07

Sprinzl, K. PP12-27

Sricharunrat, T. PP26-75

Srikureja, W. PP39-45

Srimunta, U. PP27-11

Srinivasan, R. PP31-37

Sripa, B. S02-01

Sripariwuth, E. PP04-49

Sriphojanart, S. PP26-14, PP38-04, PP38-38, PP38-41, PP38-62

Sriprayoon, T. PP04-45, PP04-48

Srivastava, P. PP05-73, PP05-74, PP05-75,

PP39-85, PP39-86

Srivastava, V. PP27-22

Stabile, A. PP06-56

Staettermayer, A. PP12-34, PP13-61, PP13-84, PP13-88

Stancic, S. PP13-45 
Standish, R. PP07-20

Stauber, R. PP13-61

Stefanescu, G. PP16-32

Steindl-Munda, P. PP12-34, PP13-84, PP13-88

Sterling, R.K. PP13-45

Stilidi, I.S. PP26-53

Stokes, A. PP22-13

Storogacov, G.I. PP01-43

Storogakov, G. PP26-10

Storogakov, G.I. PP13-91, PP26-53,

PP27-15, PP37-11

Storozakov, G.I. PP10-22

Storozhakov, G. PP09-39, PP10-21

Stosova, T. PP23-21

Strain, M. PP16-30

Strasser, M. PP12-34

Strassl, R. PP12-34

Streba, L. PP29-17

Strumfa, I. PP26-76

Stryczek, A.B. PP39-55

Su, C.-W. PP33-02

Su, H. PP38-18, PP38-19

Su, J. PP04-28, PP12-03

Su, L. PP15-39

Su, T.-H. PP13-93

Su, W.-P. PP05-106

Su, X. PP05-49, PP30-06

Suarez, E. PS01-03

Subasinghe, C. PP10-10, PP39-32,

PP39-35, PP39-36, PP39-37

Subhani, F.U.H. PP31-04

Subimerb, C. PP27-12

Subortceva, I.N. PP01-43, PP26-53

Subortseva, I. PP26-10, PP27-15, PP37-11

Subortseva, I.N. PP13-91

Subramanian, G.M. PP12-25

Suceveanu, A.I. PP10-17, PP10-18

Suceveanu, A.P. PP10-17, PP10-18

Suciu, S. PP16-12

Sud, R. PP26-54

Suddle, A. PP13-40

Suemura, S. PP05-52

Sugauchi, F. PP06-40, PP13-78

Sugawara, K. PP23-32, PP29-18

Sugawara, Y. FC02-04, C21-02, PP38-36

Sugimoto, K. PS04-03

Sugiyama, M. PP13-78, PP13-122, PP13-123

Suh, D.J. PP35-07

Suh, I. PP39-60

Suh, J. PP26-19

Suh, J.I. PP26-20

Suh, K.S. PP26-65

Suh, K.-S. PP38-39, 38-40

Suh, M. PP39-60

Sui, Y. PP01-18, PP09-13

Suk, K.T. FC06-04, PP09-28, PP23-30, PP34-11

Sukarom, I. PP13-11

Sukeepaisarnjaroen, W. FC01-01, PP04-49, PP05-62

Sulaiman, A. PP07-33

Sulaiman, A.S. PP03-11, PP06-08, PP16-25

Sulejmanpasic, G. PP17-02

Sulkowski, M.S. FC05-02, PP13-45,

PP13-89, PP13-90

Sullivan, J.C. FC05-02
Sumetchotimaytha, W. PP31-39

Sumino, Y. PP26-03

Sun, B. PP38-02

Sun, C.-A. PP04-28

Sun, D.G. PP14-06, PP14-07

Sun, H. PS03-04, PP19-14, PP21-10, PP22-01

Sun, J. PP06-60, PP12-23

Sun, K.-X. PP03-30, PP06-46

Sun, L.-J. PP01-21, PP13-96, PP13-97

Sun, M.-S. PP03-30

Sun, W. PP29-06

Sun, X. PP16-08

Sun, X.-H. FC03-01, PP05-39

Sun, Y. PP05-47, PP15-54, PP23-20

Sun, Z. PP30-32

Sunaga, M. PP13-36

Sunagawa, H. PP32-11

Sung, C.-M. PP08-42

Sung, J.J.-Y. PP08-19

Sung, J.Y. PP05-12

Sura, T. PP26-33

Sureau, C. PP06-41

Suresh Babu, P. PP10-06

Suresh Kumar, S. PP34-37

Suryadarma, G.A. PP24-11

Sussman, N. PP13-64

Su-Su, B. PP36-10

Sutedja, D. PP05-158

Sutton, A. PP30-14

Sutton, J. PP26-56

Suzuki, H. PS04-03

Suzuki, K. PP01-12, PP13-22, PP13-39,

PP16-56, PP29-04

Suzuki, T. PP01-24

Suzuki, Y. PP33-08, PP33-09

Suzumura, K. PP26-45, PP27-19, PP36-20

Svarovskaia, E. PP05-125

Svay, S. PP04-08

Swain, M.G. PP13-47

Sweefy, E.M. PP11-06

Syed, A.A. PP07-25

Sylvan, S.P.E. PP05-41

Szczygielska, I. PP25-11

Szijarto, A. PP15-55, PP31-42

Szypulska, R. PP06-42

Tabak, F. PP06-25, PP06-26

Tabata, M. PP37-07

Tabatabaee, H. PP12-04

Tabatabaei, S.V. FC05-04, PP13-09,

PP13-101, PP17-14

Tabernero, D. PP06-08

Tabuchi, A. PP04-08

Tada, M. PP30-33

Tae, J.W. PP02-04

Tag Eldin, L.A. PP16-16

Tagawa, K. PP32-03, PP34-02

Taha, A.A. PP13-04

Taha, H. PP23-87

Taha, M. PP05-04, PP08-01

Tahara, K. PP26-13, PP26-15

Taher, Y. PP22-08, PP30-19

Tahiliani, G.P. PP02-24

Tai, D. PP37-17

Tai, L.F. PP35-06

Tai, M.-L.S. PP23-49
Tai, Q. PP29-10, PP36-01, PP38-03,

PP38-07, PP38-08, PP39-21, PP39-22

Taib, S.H.M. PP23-49

Taie, D.M. PP21-07

Taimr, P. PP37-20

Tajiri, H. PP23-88

Tak, W.Y. FC04-01, PP04-29, PP15-37, PP26-39, PP26-52, PP33-24, PP33-27

Takagi, K. PP32-03, PP34-02

Takagi, M. PP27-09

Takaguchi, K. PP25-08

Takahashi, H. PP25-15

Takahashi, N. FC03-04

Takahashi, Y. PP26-63, PP26-67,

PP31-34, PP36-22

Takamatsu, S. PP13-38

Takasaki, J. PP26-63, PP26-67, PP36-22

Takashi, M. PP13-36

Takashima, S. PP25-08

Takayama, R. PP26-03

Takeda, N. PP09-10

Takemura, N. FC02-04

Takeuchi, H. PP26-13, PP26-15

Takikawa, H. PS04-01

Takikawa, Y. PP01-12, PP13-22,

PP13-39, PP16-56, PP29-04

Tamori, A. PP13-78

Tamura, S. PP38-36

Tamura, Y. PP29-04

Tan, C.Y. PP02-21

Tan, D. PP03-18, PP05-22, PP05-117,

PP05-146, PP16-28

Tan, J.R. PP02-16

Tan, L. PP34-05

Tan, N. PP04-44

Tan, T.C.H. PP23-19

Tan, T.C. PP06-39

Tan, Z. PP05-47

Tanabe, M. PP36-26

Tanaka, A. PS04-01

Tanaka, H. PP13-38

Tanaka, J. PP04-08

Tanaka, K. PP33-18

Tanaka, T. PP30-08

Tanaka, Y. C09-01, PP03-07, PP03-08, PP03-15, PP06-40, PP06-51, PP12-41, PP13-78, PP13-122, PP39-82

Tang, B.Z. PP05-05, PP05-27

Tang, C. PP13-79, PP13-83, PP13-87, PP15-32

Tang, H. PP05-65, PP05-155, PP16-14, PP21-02, PP34-03

Tang, H.H. PP03-16

Tang, K. PP01-07

Tang, L. PP34-27

Tang, R. PP38-19

Tang, S. PP13-104

Tang, T. PP09-30, PP23-93

Tang, W. PP13-42

Tang, X. PP05-152

Tang, Y.T. PP01-01, PP23-20

Tang, Z.-H. PP21-02

Tangkijvanich, P. FC01-04, C22-03, PP06-54, PP06-55, PP12-41, PP12-42, PP39-82

Tangkitvanich, P. PP15-33 
Tani, N. PP36-26

Taniai, N. PP26-23, PP26-24, PP26-38, PP26-66, PP26-70, PP26-71, PP28-30, PP36-08, PP36-11

Taniain, N. PP36-12

Taniguchi, H. PP13-115

Taniguchi, M. PP01-24

Tantry, B.V. PP23-09

Tanwandee, T. FC01-01, ET1-03, PP03-24, PP03-31, PP03-32, PP04-45, PP04-48, PP04-49, PP05-12, PP05-48, PP05-62, PP05-119, PP05-139, PP05-150, PP12-25, PP13-11, PP13-104, PP26-75

Tao, L.-Y. PP04-01, PP27-01, PP27-17

Tao, W. PP05-116

Tarar, S. PP09-23

Tarik, H. PP17-07

Tas, I.F. PP21-03

Tasan, G. PP26-69

Taşç1, I. PP39-24

Taşdoğan, B. PP05-111

Tasdogan, B.E. PP09-14

Tasyaran, M.A. PP06-09

Tataru, Abagiu, M. PP29-17

Tateishi, K. PP30-33

Tateishi, R. AE-03, PP30-33, PP33-25

Tatematsu, K. PP03-07, PP06-40, PP06-51

Tatrai, P. PP15-55, PP31-42

Tatsch, F. PP13-46, PP13-47, PP13-50,

PP13-56, PP13-57, PP39-30

Tavallai, J. PP04-43

Tavallaii, S.A. PP38-44

Tavares, S. PP23-81

Tavis, J.E. PP14-04

Tawada, A. PP01-05, PP03-39, PP04-46, PP13-27, PP13-36, PP30-37

Tawhid, Z. PP14-22

Taylor, P. PP23-19

Tedder, R. PP06-42

Teeraratkul, S. PP26-14, PP38-04, PP38-38, PP38-41, PP38-62

Tehrani, A. PP39-08

Teker, B. PP03-13

Ten, Berge, J. PP38-01

Teng, L. PP04-41

Teo, L.L.S. PP37-18

Teodorescu, R. PP29-17

Terki, N. PP38-20

Termmathurapoj, S. PP26-75

Terrault, N. C13-02

Tessier, E. PP14-04

Teuber, G. PP12-27

Thabut, D. PP23-90, PP23-92

Thakkinstian, A. PP16-45

Thames, A.D. PP10-12

Thangjam, B. PP28-27

Thangjam, D. PP28-27

Thapa, B.R. FC07-04, FC07-05

Tharwa, E. PP06-45

Thatoi, P.K. PP23-72

Theamboonlers, A. PP06-54, PP12-41, PP12-42

Thedja, M. PP04-19, PP06-30, PP06-31

Theise, N. C01-01, KL01

Thiruvenkatachari, M. PP34-01

Thitichettrakul, Y. PP16-45

Thomas, M.A. PP10-12, PP39-58
Thommes, J. PP13-57

Thongam, N. PP12-32

Thongpaen, S. PP39-84

Thongsawat, S. PP04-49, PP05-62,

PP05-119, PP12-25, PP23-89, PP31-39,

PP34-17, PP39-52

Thongtae, N. PP06-57

Thursz, M. PP39-07

Tian, G. PP24-01

Tian, P.-X. PP13-117

Tian, X. PP23-25

Tian, X.-H. PP13-117

Tian, Y.-H. PP06-46

Tiao, G.M. PP28-05

Tien, P. PP05-164

Tilak, K. PP07-04

Tine', F. PP13-13

Titova, M. PP10-23, PP10-24

Tiwari, D. PP28-27, PP39-68

Tobari, M. PP34-22

Tocharoenthanaphol, C. PP26-47

Toda, N. PP32-03, PP34-02

Todo, S. PP01-24

Todo, T. PP38-29

Togo, S. PP03-39, PP04-46

Tokcaer-Keskin, Z. PP21-03

Tokunaga, K. PP13-78

Tokunaga, N. PP26-60, PP26-61

Tolide-ei, H.R. PP39-06

Tolmane, I. PP26-76

Tomaka, F. FC05-02

Tomar, M. PP23-79

Tomar, V. PP16-36

Tomaselli, T. FC06-01

Tong, G. PP34-03

Tong, G.D. PP03-16

Tong, J.Y.C. PP03-03, PP34-24

Tong, L. PP05-61, PP34-03

Tong, M.J. PP05-65

Tong, X. PP13-75

Toniutto, P. PP05-42

Topal, T. PP39-24

Topaloğlu, S. PP04-20

Topilskaya, N. PP08-40

Torabi, J. PP39-73

Torbenson, M. PP12-25

Torello, Viera, F. PP33-12

Torres, L. PP21-06

Torres, P.M.J. PP23-63, PP23-64

Torresi, M. PP23-40, PP26-46

Toshifumi, H. PP26-13

Totolyan, G. PP09-39, PP10-22

Tovar, A. PP34-20

Toyama, T. PP05-52

Toyoda, J. PP03-08

Toyoda, M. PP26-13, PP26-15

Toyoki, Y. FC08-04, PP38-64

Toyota, H. PS04-03

Tragulpiankit, P. PP02-20, PP05-150

Tran, A. FC05-01

Tran, H.T.T. PP31-30

Tran, S.V. PP05-101

Treepongkaruna, S. PP04-44, PP26-14, PP26-47, PP38-04, PP38-38, PP38-41, PP38-62

Treitel, M. PP13-65, PP13-66
Trifonova, E.V. PP28-01

Trinh, H. PS01-02, PS01-03, PP05-99,

PP05-101

Tripon, E.S. PP08-02

Trunecka, P. PP38-24

Truong, A. PP12-37, PP13-14

Truong, T.B. PP05-97

Trylesinski, A. FC01-01, PP04-44,

PP05-19, PP05-56, PP05-119

Tryvitz, A. PS03-03

Tsai, M.-C. PP05-151

Tsai, N. PP05-65, PP13-104

Tsamakidis, K. PP13-06

Tsang, K.P.K. PP04-09

Tsang, S.W.-C. PP08-19, PP36-14

Tseng, K.-C. PP03-35

Tseng, P.-L. PP05-151

Tseng, T.-C. PP04-07, PP13-93

Tsertsvadze, T. PP06-15, PP13-53, PP14-17

Tseveg, T. PP34-07

Tsochatzis, E. FC06-01

Tsubouchi, H. PP13-78

Tsuchida, S. PP21-12

Tsuchimochi, M. PP13-121

Tsuchiya, T. EC-06

Tsugane, S. PP03-07

Tsuji, K. PP03-08, PP25-16

Tsukui, Y. PP33-08, PP33-09

Tsuneizumi, M. PP27-09

Tsunemitsu, Y. PP26-60, PP26-61

Tsuruta, A. PP36-16, PP36-24

Tsutsumi, M. PP15-38

Tu, C. PP15-24

Tu, Y. PP05-37, PP08-47

Tu, Z.H. PP38-15, PP38-34

Tudora, A. PP16-30

Tufail, B. PP09-23

Tumanova, E. PP17-15

Tumyan, G.S. PP13-91

Tuna, N. PP03-13

Tuncel, A.F. PP16-05

Tuncer, I. PP26-41, PP26-69

Tung, T.-H. PP08-14, PP17-05

Tuntipanichteerakul, S. PP04-49

Tupas-Edano, J. PP08-59

Turcanu, A. PP05-06

Turgunov, A. PP06-11

Turhan, N. PP15-06

Turhan, V. PP02-22, PP04-33, PP05-20

Türkay, C. PP08-07, PP08-62, PP08-63

Türken, M. PP04-20, PP06-52

Turyadi, T. PP04-19, PP04-39, PP06-31

Tüzüner, A. PP38-50

Twigg, S.M. FC07-01, PP15-53

Tyagi, P. PP23-29

Tymyan, G.S. PP27-15

Uccella, I. PP04-38

Uchida, E. PP26-23, PP26-24, PP26-66, PP26-70, PP26-71, PP28-30, PP36-08,

PP36-11, PP36-12

Uchida, K. PS04-03

Uchida, Y. PP23-32

Uchino, K. PP33-25

Uchiya, H. PP23-32

Uda, Y. PP26-45

Udayakumaran, P. PP39-32 
Uddin, A. PP04-44, PP05-54, PP05-56, PP05-117, PP05-161

Udompanthurak, S. PP03-31, PP03-32, PP05-48

Udupa, N. PP19-12, PP19-13

Ueda, J. PP26-23, PP26-24, PP26-38,

PP26-66, PР26-70, PP26-71, PP28-30, PP36-08, PP36-11, PP36-12

Ueda, M. PP36-26

Uegaki, S. PP34-22

Uemoto, S. FC08-03, PP38-13, PP38-14

Ueno, Y. PP14-20

Ulasoglu, C. PP26-69

Ulu, Y. PP04-20

Ulukaya, E. PP08-48, PP31-22

Ulukaya, S. PP31-22

Um, S. PP12-25

Um, S.H. PP16-23

Umakanthan, A.N. PP39-32

Umar, M. AA-02, PP11-09, PP13-21,

PP13-68, PP13-70, PP14-09, PP16-24, PP16-34

Umehara, M. PP38-64

Unal, A. PP08-25

Unal, H. PP08-25

Ural, O. PP06-09

Urtnasan, M. PP34-07

Ushio, Y. PP34-22

Usio, A. PP29-04

Usluer, G. PP05-131, PP06-09

Usmani, M.T. PP09-32

Ustundag, Y. PP05-58

Usui, M. PP37-07

Uy, D.C. PP08-60

Uy, M. PP23-63, PP23-64

Uyama, N. PP26-45, PP36-20

Uyanikoglu, A. PP09-29

Uysal, B. PP39-24

Vaeteewoottacharn, K. PP27-11, PP27-12

Vaidya, M. PP08-27

Vaillant, A. PP05-77

Vairappan, B. PS03-05

Vali, M. PP22-09

Valla, D. PS02-01, PP14-16

van, Baelen, B. FC05-02

Van, Heeswijk, R. PS02-04

van, Thiel, D.H. PP23-41

Van, Thiel, D.H. PP10-19

Vanavanan, S. PP23-52

Vandenbroucke, F. PP37-14

Vanithakumari, G. PP02-01

Varma, A. PP08-30

Varma, S.P.K. FC03-02

Varni, J.W. PP38-41

Vasanth, Raj, P. PP19-12, PP19-13

Vasdev, N. PP38-28

Vashakidze, E. PP01-09, PP17-09

Vasu, K. FC08-01

Vasudevan, K. PP38-53

Vata, A. PP05-03

Vaziri, S. PP23-85

Vejapipat, P. PP23-10

Vejchapipat, P. PP28-20

Velasquez, M.E. PP08-02, PP23-11

Velayutham, V. FC02-03, PP26-40, PP39-56

Velmurugan, P. PP34-37
Venkata, Rao, J. PP19-12, PP19-13

Venkatesh, S.K. C15-02, PP37-16,

PP37-17, PP37-18, PP37-19

Venook, A. FC02-05, PP34-25

Venter, J. PP22-13

Venugopal, S. PP08-16

Vergniol, J. PP16-13, PP16-15

Verma, A. PP05-73, PP05-74,

PP05-75, PP13-30, PP13-31, PP13-33,

PP13-106

Vernic, C. PP16-39

Veronese, L. PP33-12

Versteeg, R. PP38-01

Vidaud, M. PS02-01, PP06-43, PP14-16

Viengteeravat, S. PP38-38

Viengteerawat, S. PP38-04

Vierling, J. PS02-02, PP13-75

Vij, V. FC08-01, PP38-53, PP38-55, PP38-59, PP38-63

Vijayanathan, A. PP37-09

Vijitpavan, A. PP26-14

Vilstrup, H. PP23-15

Vimolket, L. PP28-20

Viña, J.R. PP21-06

Vinaixa, C. PP38-54

Vincent, L. PP05-40, PP05-66, PP05-72, PP05-114

Vinciguerra, M. PP14-08

Vincze, A. PP16-29

Visvanathan, K. C02-01, PP15-33

Vitale, G. PP23-43

Vítek, L. FC07-03

Vivante, A. PP23-02

Vogel, A. PP19-01

Voiculescu, M. PP10-14, PP13-85, PP25-17

Volynec, G. PP17-16

Volz, T. PP09-06

Vongchan, P. PP29-02

Vorobev, A.N. PP01-43

Voronina, A. PP02-15

Voronkova, N.V. PP03-38

Vucelic, B. PP05-70, PP13-24, PP14-26

Vujnovic, G. PP02-05

Wadhawan, M. FC08-01, PP38-53, PP38-55, PP38-59, PP38-63

Wagner, J.-S. PP12-10

Wagoner, J. PP14-04

Wahab, M.A PP27-10

Wahid, S. PP07-33

Wai, S.N. PP04-25

Waisayanand, N. PP39-52

Wajima, N. FC08-04, PP38-64

Waked, I. PP05-143, PP23-45, PP34-14, PP39-07, PP39-83

Wakita, T. PP09-10

Wakkie, J. PP38-01

Wakui, N. PP26-03

Wakui, Y. PP14-20

Wakuta, M. PP06-40

Walker, G. PP15-21

Wallace, D. FC07-02

Wan, M. PP05-22, PP05-117, PP05-119, PP05-155

Wan, Y.-L. PP38-47

Wan, Yee, L. PP36-10

Wang, A. PP24-01
Wang, B. PP01-31, PP01-36, PP05-149,

PP15-36, PP26-72, PP38-56

Wang, B.-S. PP19-08

Wang, C. PP01-18

Wang, C.-C. PP04-07

Wang, C.-H. PP13-98

Wang, D. FC02-01, PP12-23, PP29-10, PP36-01, PP38-03, PP38-07, PP38-08, PP39-21, PP39-22

Wang, F. PP27-20, PP31-38, PP34-35, PP34-36

Wang, F.M. PP31-14

Wang, F.S. C14-01

Wang, F.-S. PP05-38

Wang, F.-S. PP05-162

Wang, F.-S. PP05-163

Wang, F.-S. PP05-164

Wang, G. PP01-30, PP06-58, PP33-23, PP34-29

wang, H. PP03-03

Wang, H. PP04-40, PP06-02, PP06-36,

PP06-50, PP15-20, PP18-01, PP31-26,

PP31-27, PP39-03, PP39-79

Wang, H.Y. PP29-06

Wang, H.-Y. PP05-21

Wang, H.-Y. PP34-08

Wang, J. FC02-02, PP03-03, PP04-22, PP04-23, PP05-34, PP05-81, PP05-130, PP12-17,

PP12-47, PP13-73, PP13-99, PP14-06,

PP15-09, PP15-24, PP15-39, PP16-02,

PP17-10, PP20-02, PP34-10, PP34-16, PP38-47

Wang, J.B. PP39-54

Wang, J.-B. PP13-80

Wang, J.H. PP01-22, PP14-06, PP14-07,

PP15-23, PP15-27

Wang, J.-H. PP01-32

Wang, J.-H. PP05-140

Wang, J.-H. PP16-53

Wang, J.Y. PP01-27, PP02-09

Wang, J.-Y. PP15-15

Wang, K. PP01-28, PP01-31, PP01-33,

PP01-36, PP01-38, PP23-77, PP30-34,

PP38-32, PP01-28, PP01-31, PP01-33, PP01-36, PP01-38

Wang, K.Y. PP23-20

Wang, L. PP01-33, PP03-30, PP04-40, PP09-13, PP09-20, PP09-31, PP10-15

Wang, L.-C. PP16-14

Wang, L.-H. PP05-21

Wang, L.-Y. PP04-28

Wang, M. PP01-18, PP01-32, PP04-26, PP05-22, PP05-147, PP06-21, PP12-23, PP06-50, PP09-13, PP34-19, PP38-61

Wang, M.-L. PP12-21

Wang, N.-Y. PP03-02, PP03-05

Wang, P. PP15-35, PP15-36, PP15-40, PP22-06

Wang, Q. PP05-22, PP12-20, PP14-10,

PP23-25, PP26-18

Wang, Q.Q. PP05-05, PP05-27

Wang, R.T. PP38-56

Wang, S. PP05-101, PP21-08, PP34-16

Wang, S.-L. PP15-05

Wang, S.-N. PP36-19

Wang, S.Q. PP31-19

Wang, T.-E. PP34-08

Wang, W. PP01-35, PP03-25, PP05-107,

PP08-11, PP38-22

Wang, W.L. PP36-09

Wang, W.-Q. PP16-40 
Wang, X. FC02-02, PP01-32, PP05-46, PP05-47, PP06-49, PP06-50, PP09-13, PP05-53, PP09-20, PP09-31, PP25-19, PP17-10, PP12-20, PP30-34, PP30-24, PP26-18, PP38-02

Wang, X.-D. PP30-25

Wang, Y. FC04-03, PP02-14, PP02-28, PP04-22, PP04-23, PP04-26, PP05-22, PP05-44, PP05-46, PP05-117, PP05-146, PP05-147, PP20-08, PP20-09, PP26-35, PP30-16

Wang, Y.D. PP03-25

Wang, Y.-D. PP08-11

Wang, Y.-G. PP16-40

Wang, Z. PP02-28, PP15-13, PP15-20, PP28-06, PP28-07, PP28-08, PP28-25

Wang, Z.-B. PP33-18

Wang2, X.X. PP07-22

Wannason, K. PP15-17

Ward, R. PP28-05

Warda, O. PP08-46

Wardhan, H. PP23-29

Warner, F.J. PP15-41, PP15-42

Wat, C. FC01-03, PP05-12

Watanabe, K. PP23-32

Watanabe, M. PP27-09

Watanabe, T. PP27-09

Watson, H.R. PP23-15, PP23-39

Wattanasirichaigoon, D. PP26-47

Wattanasirichaigoon, S. PP15-16

Watters, D. FC07-02

Weaver, M. PP06-38

Wedemeyer, H. AE-01, PP03-24, PP05-141, PP05-161

Wee, A. PP37-17

Wei, C.-J. PP17-05, PP17-17

Wei, J. PP12-17

Wei, L. PP03-22, PP05-22, PP05-119, PP05-148, PP05-155, PP11-08, PP12-14, PP12-31, PP14-06, PP14-07, PP14-23, PP15-27, PP22-15, PP30-27

Wei, L.-J. FC05-02

Wei, L.R. PP02-13

Wei, Q. PP30-34, PP38-30, PP38-33

Wei, X.Y. PP30-34, PP31-21

Weijie, P. PP34-26

Weiping, Z. PP22-11, PP36-25

Weiss, B. PP23-02

Weiss, R. FC03-04

Weizman, Z. PP23-02

Weltman, M. PP13-56

Wen, V. PP15-41, PP15-42

Wendelburg, P. PP13-76

Wendum, D. PP30-07

Wenjin, D. PP08-49

Wheatley, A. PP21-05

Wibawa, D.N. PP12-48, PP24-11

Wickramarathne, D.J. PP39-32

Wilson, C.H. PP07-34, PP38-23

Wilson, N. PP10-12

Win, K.M. PP14-02

Wind-Rotolo, M. PP13-76

Winiyakul, N. PP39-84

Winnie, W. PP34-26

Winslow, Rasiah, M. PP13-29

Wisedopas, N. PP08-09, PP16-45, PP26-11
Witeerungrot, T. PP13-15

Witte, D. PP28-05

Wolf, A. PP06-38

Wolf, D. PP38-21

Won, H.J. PP35-07

Won, I.S. PP05-57, PP16-57

Won, Y.B. PP19-04

Wong, B.C.Y. PP30-23

Wong, C.W.K. PP08-26

Wong, D.K.-H. PP06-37

Wong, F. PP05-157, PP23-15, PP23-39

Wong, G.L. PP05-08

Wong, G.L.H. PS01-04, PP04-05

Wong, G.L.-H. PP05-102, PP08-19

Wong, K.K. PP26-68, PP27-14

Wong, S. PP27-04, PP33-01

Wong, V.W. PP05-08

Wong, V.W.S. PP01-04, PP04-05

Wong, V.W.-S. C01-02, PP08-19, PP05-102

Wong, W.K. PP08-26

Wongitrat, C. PP37-03

Wongjitrat, N. PP37-03

Wongkham, C. PP18-01, PP27-11, PP27-18, PP27-21

Wongkham, S. PP18-01, PP27-11, PP27-12, PP27-18, PP27-21

Wongteerasut, A. PP08-31, PP23-10, PP38-35

Woo, H.Y. PP29-13

Woynarowski, M. PP25-07, PP25-10, PP25-11

Woźniak, M. PP25-07, PP25-10, PP25-11

Wrba, F. PP13-84

Wright, C. PP13-89

Wright, G. PP24-07

Wu, C. PP05-16, PP05-93, PP06-17

Wu, D. PP05-101, PP06-50

Wu, G. PP20-02, PP38-29

Wu, H.C. PP05-122, PP06-20

Wu, H.-L. PP06-27

Wu, H.Q. PP39-79

Wu, J. FC01-02, PP16-44

Wu, J.-C. PP33-02

Wu, J.-H. PP05-21, PP06-62

Wu, L. PP26-02, PP28-24, PP28-25, PP28-29, PP29-10, PP30-20, PP36-01, PP38-03, PP38-06, PP38-07, PP38-08, PP38-22, PP39-21, PP39-22, PP39-75

Wu, M. PP05-146, PP05-152, PP05-155

$\mathrm{Wu}, \mathrm{N}$. PP14-06, PP22-15

$\mathrm{Wu}, \mathrm{P} . \mathrm{PP} 28-23$

Wu, Q. PP17-08, PP21-11

Wu, S. PP05-22, PP05-80, PP13-27, PP13-36, PP23-14, PP30-37

Wu, S.-F. PP03-35

Wu, S.-S. PP05-65

Wu, T. PP16-44

Wu, X. PP05-121

Wu, X.L. PP15-13, PP39-26

Wu, Y. PP12-17, PP12-47, PP20-03

Wu, Y.-H. PP39-76

Wu, Y.-S. PP17-05

Wu, Z. PP05-45, PP05-47, PP05-53, PP16-44, PP20-09

Wursthorn, K. PP05-161, PP03-24

Xia, J. PP05-16, PP05-93, PP21-10, PP22-01

Xia, P. PP19-10

Xian, Z.-H. PP31-08
Xiang, H. PP34-32

Xiang, H.-L. PP13-117

Xiang, J. PP05-24

Xiang, $\mathrm{X}$. PP06-02

Xiang, Y. PP38-19

Xian-Jun, X. PP28-15

Xiao, C. PP34-03

Xiao, D. PP34-27

Xiao, D.-D. PP04-11

Xiao, F. PP25-19

Xiao, H. PP05-81

Xiao, J. PP18-02

Xiao, X.-Q. PP19-09

Xiaohong, Z. PP09-22

Xiaojing, S. PP22-11

Xiao-Xia, T. PP24-06

Xiao-Xuan, R. PP26-27, PP26-28, PP28-16

Xiaozhen, X. PP05-116

Xibing, G. PP03-28

Xie, C. PP05-02, PP15-18

Xie, D. PP06-07, PP22-05, PP31-10, PP39-79

Xie, D.Y. PP22-14

Xie, D.-Y. PP05-02

Xie, F. PP05-23

Xie, H. PP30-20

Xie, H.Y. PP30-34, PP31-21, PP36-15, PP38-32

Xie, J. PP06-07, PP22-05, PP31-10

Xie, J.D. PP39-79

Xie, J.-Q. PP05-02

Xie, K. PP34-06

Xie, K.-Q. PP10-05

Xie, Q. PP05-12, PP05-22, PP05-44,

PP05-119, PP05-146, PP05-153, PP06-02,

PP06-36, PP12-31, PP19-05, PP39-79

Xie, S.B. PP22-14, PP29-15

Xie, S.-B. PP05-02

Xie, S.-S. PP32-12

Xie, W. 71, PP02-25, PP12-20

Xie, X. PP30-01

Xie, X.W. PP30-27

Xie, Y. PP12-20

Xiernayi, H. PP05-90

Xin, J. PP22-04

Xin, S. PP01-02, PP05-108

Xing, C. PP30-20

Xing, H. PP12-20, PP26-18

Xing, Y. PP05-50

Xing, Z. PP06-14, PP15-20, PP15-25

Xinopoulos, D. PP13-06

Xiong, F. PP32-07

Xirouchakis, E. FC06-01

Xiu, D. PP06-21

$\mathrm{Xu}, \mathrm{D}$. PP05-22, PP05-44, PP05-105, PP05-112

$\mathrm{Xu}$, D.-Z. PP05-117

Xu, G.G. PP06-18

Xu, G.S. PP15-13, PP39-26

Xu, H. PP15-13, PP23-14, PP23-20, PP23-93

$\mathrm{Xu}, \mathrm{H} . \mathrm{-W}$. PP16-53

$\mathrm{Xu}$, H.-X. PP27-16

$\mathrm{Xu}, \mathrm{J} . \mathrm{PP} 01-18$

Xu, K.-C. PP34-10

Xu, L. PP05-47, PP15-19

$\mathrm{Xu}, \mathrm{Q}$. PP05-23, PP05-25, PP05-26, PP12-31

Xu, Q.-H. PP05-49

Xu, W. PP15-46, PP39-03 
Xu, W.J. PP05-36, PP05-69

Xu, W.-J. PP23-50

Xu, X. PP05-35, PP05-149

Xu, X.-S. PP05-163, PP30-28, PP30-34,

PP31-21, PP36-15, PP38-30, PP38-32,

PP38-33

Xu, Y. PP05-130, PP13-80, PP20-10, PP30-06

$\mathrm{Xu}, \mathrm{Z}$. PP05-49, PP13-51

Xue, B.Y. FC01-05

Xue, W.-J. PP13-117

Xuebing, Y. PP05-01

Xue-Jun, G. PP26-27

Xun, Y.-H. PP05-24

Yaacob, N.Y. PP15-13

Yacoub, R. PP12-18

Yadav, S.K. PP39-58

Yagi, S. FC08-03

Yahyaoğlu, M. PP03-13

Yakut, M. PP06-35, PP13-19

Yalçın, K.S. PP08-62, PP08-63

Yamada, S. PP35-02, PP35-03

Yamada, T. PP21-12

Yamaguchi, T. PP33-15

Yamahata, N. PP13-27

Yamamoto, K. PP25-08

Yamamoto, M. PP26-50, PP26-63,

PP26-67, PP31-34, PP36-22

Yamana, D. PP21-12

Yamanel, L. PP39-24

Yamaoka, M. PP38-24

Yamashiki, N. PP38-36

Yamashita, K. PP01-24

Yamashita, S. PP26-50, PP26-67, PP36-22

Yamazhan, T. PP06-09

Yan, C.-H. PP04-12

Yan, D. PP34-26

Yan, H. PP13-117, PP31-19

Yan, J. PP30-31

Yan, K. PP30-24

Yan, L. PP03-30, PP04-12

Yan, M.X. PP06-19

Yan, M.-X. PP16-40

Yan, S.M. PP05-05, PP05-27

Yan, W. PP05-47, PP05-53, PP06-49,

PP06-50, PP25-19

Yan, X. PP05-16, PP05-93

Yan, Y. PP31-10

Yang, A. PP13-26, PP15-20, PP22-06

Yang, B. PP08-11, PP14-10, PP31-14

Yang, C.Q. PP07-21

Yang, C.-Q. PP15-34

Yang, D. FC02-02, PP05-45, PP20-10, PP34-26

Yang, F. PP16-26, PP38-26

Yang, H. PP30-24, PP32-07

Yang, H.-I. PP04-28

Yang, J. PP31-31

Yang, J.D. PP30-32

Yang, J.H. PP07-03

Yang, J.I. PP08-15

Yang, J.M. PP16-23, PP16-49, PP22-12

Yang, J.-X. PP04-72

Yang, K.-C. PP07-24

Yang, L. FC02-01, PP05-50, PP07-29

Yang, Q. PP09-10

Yang, R. PP03-22, PP11-08, PP21-11, PP22-01

Yang, S. PP12-20, PP26-18
Yang, S.C. PP16-47

Yang, S.H. PP23-37, PP30-17

Yang, S.-S. PP13-93

Yang, X. PP21-08, PP32-12

Yang, X.K. PP39-26

Yang, X.Y. PP39-26

Yang, Y. PP02-14, PP05-43,

PP06-04, PP06-63, PP28-19, PP32-07, PP36-23

Yang, Y.-F. PP26-02, PP35-01

Yang, Z. PP29-16

Yano, Y. PP05-145

Yao, A. PP05-99, PP05-101

Yao, J.G. PP01-13

Yao, L. PP13-27

Yao, Q. PP18-01, PP27-21

Yao, Q.-W. PP01-32

Yao, S.-K. PP20-11, PP32-06

Yao, T.-W. PP15-53, PP19-10

Yao, Y.T. PP08-35

Yap, S.F. PP06-39

Yaran, M. PP09-15, PP09-21, PP12-11, PP12-19

Yasuda, S. PP35-02, PP35-03

Yasuda, Y. PP17-06, PP30-08

Yasui, S. PP01-05

Yavaş, S. PP05-133

Ye, F. PP05-107, PP21-11

Ye, S.-L. FC02-05, PP34-25

Ye, S.Y. PP36-09

Ye, Y. PP05-29, PP39-72

Yee, M.M. PP05-66

Yeh, B.I. PP30-18

Yeh, C.-T. PP08-42

Yeh, M.-L. PP23-76, PP39-81

Yehia, A. PP09-29

Yehia, R. PP23-76, PP39-81

Yenen, S. PP09-29

Yenice, N. PP05-17, PP05-144

Yeo, W. PP34-18

Yeon, J.E. PP09-34

Yermekova, L.Y. PP15-44

Yesmembetov, K.I. PP15-44

Yeung, N.C. PP08-26

Yi, N.-J. PP38-39, PP38-40

Yilmaz, Enc, F. PP26-69

Y1lmaz, G. PP16-05

Yilmaz, T. PP10-23, PP10-24

Yilmaz, Y. PP08-21, PP08-48, PP31-22

Yim, H.J. PP13-67

Yim, H.-W. PP32-10

Yin, B. PP05-121

Yin, H.Z. PP03-25

Yin, L. PP05-43

Yin, Y. FC05-03

Yin, Y.P. PP01-36

Ying, Z. PP09-22

Ying-Fang, F. PP36-10

Yi-Xiang, W. PP28-15

Yokomori, H. PP26-13, PP26-15

Yokosuka, O. PP01-05, PP03-39,

PP04-46, PP05-80, PP12-40, PP13-27,

PP13-36, PP30-37, PP35-02, PP35-03

Yonal, O. PP08-21

Yoneyama, H. PP07-23

Yongvanit, P. PP28-18
Yoo, B.C. PP03-21, PP03-23, PP05-14, PP05-139, PP05-160, PP06-13, PP09-17, PP35-04

Yoo, G.J. PP02-08

Yoo, S.H. PP05-51, PP05-67

Yoo, S.-H. PP05-30

Yoon, H.G. PP25-12

Yoon, J.-H. PP04-18, PP05-89, PP05-156, PP07-15, PP08-15, PP15-03, PP16-18, PP16-20, PP22-03, PP30-04, PP30-11, PP32-08

Yoon, K.T. PP29-13

Yoon, S.K. FC02-05, PP05-51,

PP05-139, PP22-12, PP32-10

Yoon, S.-K. PP05-65

Yoon, S.M. PP35-07

Yorulmaz, E. PP26-69

Yoshiba, M. PP01-41, PP39-57

Yoshida, H. PS02-05, PP13-115,

PP26-13, PP26-15, PP26-23, PP26-24, PP26-38, РP26-66, РP26-70, РP26-71, PP28-30, PP30-33, PP33-25, PP36-08, PP36-11, PP36-12

Yoshida, T. PP33-08, PP33-09

Yoshida, Y. PP13-39, PP26-45, PP27-19, PP36-16, PP36-20, PP36-24

Yoshino, K. PP23-32, PP29-18

Yoshio, T. PP05-52

Yoshioka, M. PP26-23, PP26-24, PP26-38, PP26-66, PP26-70, PP26-71, PP28-30, PP36-08, PP36-11

Yoshizawa, A. FC08-03

Yosry, A. PP38-51, PP38-52

You, H. FC03-05, PP06-14, PP15-20, PP15-36, PP15-40, PP22-06, PP30-16, PP31-19

You, J. PP05-05, PP05-27

You, S. PP01-02

You, S.-L. PP04-28

Youn, S.J. PP05-30

Young, T.P. PP03-06

Younis, H.A.-K. PP23-62

Younossi, Z. PS01-02, PS02-04

Youssef, G. PP16-52

Youssef, M. PP31-36

Yu, C. PP30-06

Yu, D.M. FC07-01, PP06-59, PP15-53, PP19-10

Yu, D.-M. PP06-32

Yu, G.J. PP13-100

Yu, G.-R. PP15-14

Yu, H. PP25-19

Yu, H.-C. PP05-151

Yu, H.-W. PP01-32

Yu, J. PP06-21, PP09-30, PP13-26, PP15-46

Yu, J.-H. PP32-12

Yu, J.I. PP35-05

Yu, J.-W. PP01-21, PP13-96

Yu, L. PP26-72, PP38-56

Yu, L.-J. PP13-97

Yu, M.-L. PP28-31

Yu, P. PP12-23

Yu, R. PP12-03

Yu, S.J. PP04-18, PP06-53, PP07-15, PP15-03, PP16-20, PP30-04, PP30-11, PP32-08

Yu, S.-J. PP22-03 
Yu, X. PP30-20

Yu, Y. PP05-28

Yu, Z.-J. PP05-134

Yuan, Q.H. PP13-27

Yuan, Y. PP12-10, PP13-86

Yuan, Z. PP28-25

Yuan, Z.-H. PP03-20

Yudi, W. PP23-98

Yue, G. PP38-12

Yuen, J. PP06-37

Yuen, L. PP05-159

Yuen, M.F. PP05-139

Yuen, M.-F. C04-01, PP06-37

Yuen, W.K. PP29-05

Yuguchi, K. PP05-52

Yun, C.O. PP19-04

Yun, H. PP09-04

Yun, S.M. PP05-30

Yu-Sheng, J. PP05-108

Yu-Tian, C. PP05-108

Zacharian, A. PP23-41

Zacher, B. PP03-24

Zada, S. PP11-14, PP31-36

Zafar, M.N. PP08-17

Zafar, P.A. PP28-04

Zaghla, H.E. PP13-04

Zaghloul, M.H. PP06-45

Zaher, R. PP04-03, PP24-02, PP39-10

Zaidi, L. PP02-17

Zaidi, S. PP14-01

Zaigham, S. PP23-57

Zakareya, T. PP23-45

Zakaria, R. PP26-64

Zakay-Rones, Z. PP38-21

Zaki, A. PP24-03

Zaki, M. PP01-40, PP09-23, PP09-24

Zaki, S.A. PP04-03, PP06-03, PP09-03,

PP11-01, PP11-03, PP12-02, PP13-07, PP23-06, PP24-02, PP31-03, PP39-09, PP39-10

Zalata, K. PP16-48, PP21-09

Zamani, F. PP13-20

Zamharir, R. PP13-108, PP26-62

Zamiri, N. PP39-06, PP39-08

Zano, F. PP23-64

Zaño, F. PP23-63

Zarina, A. PP17-18

Zayed, N. FC05-05, PP38-25

Zech, C.J. PP37-05

Zeid, A. PP30-19

Zeidabadi Nejad, L. PP09-15, PP13-71

Zeile, S. PP38-23

Zekri, A.-R.N. PS03-02, PP31-06

Zeng, D.-W. PP23-50

Zeng, F. PP13-26

Zeng, M. PP05-105, PP05-112, PP05-155

Zeng, Q. FC02-02, PP31-13

Zeng, T. PP10-05, PP34-06

Zeng, W.Z. PP15-13

Zeng, Y. PP12-17, PP12-47

Zeng, Z. PP05-164

Zeniya, M. AS-05, PP25-15

Zerbini, A. PP30-30

Zerhoune, W. PP25-20

Zeuzem, S. PS02-02, PS02-03, PS02-04,

FC01-01, FC05-02, FC05-03, PP12-27,

PP13-47, PP13-59
Zeynalova, P.A. PP13-91

Zhamutashvili, M. PP13-53

Zhang, A.L. PP17-08

Zhang, B. PP13-63

Zhang, C. PP23-25, PP34-06

Zhang, C.-L. PP10-05

Zhang, D. PP03-02, PP05-35, PP29-16, PP30-28

Zhang, D.-Z. PP39-38

Zhang, E. PP06-17, PP20-10

Zhang, F. PP12-17, PP28-23, PP38-02, PP38-61

Zhang, F.-K. PP25-03

Zhang, F.-Y. PP01-32

Zhang, G. PP05-50, PP20-05

Zhang, G.-L. PP06-47, PP15-18

Zhang, H. PP04-44, PP05-34, PP05-61,

PP05-65, PP08-43, PP08-44, PP08-45,

PP08-47, PP20-01, PP38-57

Zhang, H.-F. PP05-134

Zhang, J. PP01-32, PP05-16, PP16-54,

PP23-14, PP23-77, PP38-12

Zhang, J.X. PP30-16, PP31-19

Zhang, J.-Y. PP05-162, PP05-163, PP05-164

Zhang, K.H. PP15-08, PP15-09,

PP15-10, PP15-11

Zhang, L. PP07-17, PP20-04, PP22-04, PP38-34

Zhang, M. PP26-25, PP36-09, PP38-06, PP38-18, PP38-19, PP38-22, PP38-31, PP38-34

Zhang, P. PP12-23, PP31-18

Zhang, Q. PP16-07, PP30-31

Zhang, R. PP16-06, PP20-09, PP30-05, PP36-23

Zhang, S. PP04-16, PP04-17, PP05-38, PP05-107, PP05-128, PP20-05

Zhang, S.C. PP02-09

Zhang, S.J. PP20-03

Zhang, S.-Q. PP05-02

Zhang, T. PP12-17

Zhang, W. PP03-37, PP12-14, PP12-31, PP22-15, PP24-01, PP26-25, PP38-06

Zhang, W.-P. PP21-02

Zhang, X. PP01-18, PP05-27, PP05-107, PP05-122, PP06-07, PP06-17, PP06-63, PP15-54, PP16-06, PP20-10, PP28-23, PP30-05, PP34-03

Zhang, X.F. PP26-72

Zhang, X.G. PP26-72, PP38-56

Zhang, X.-H. PP13-51

Zhang, X.X. PP06-59

Zhang, X.-X. PP03-20, PP06-32, PP11-13

Zhang, Y. PP05-50, PP05-128, PP05-130, PP12-03, PP14-14, PP25-03, PP25-14, PP31-14, PP34-16

Zhang, Y.F. PP03-01, PP05-05, PP22-14

Zhang, Y.-F. PP04-02, PP06-47

Zhang, Y.G. PP39-54

Zhang, Y.-G. PP13-80

Zhang, Y.S. PP05-05

Zhang, Z. PP05-38, PP05-162, PP05-163,

PP07-36, PP15-19, PP16-02, PP26-07

Zhang, Z.J. PP15-34

Zhanghong, H. PP04-37

Zhao, C.H. PP29-15

Zhao, C.Y. PP03-25

Zhao, C.-Y. PP04-12
Zhao, C.-Y. PP04-12, PP08-11

Zhao, G. PP05-61, PP06-36

Zhao, G.D. PP39-79

Zhao, G.-M. PP05-134

Zhao, H.-C. PP32-06

Zhao, J. PP19-11

Zhao, P. PP05-130, PP13-73, PP13-80

Zhao, P.-W. PP04-22, PP30-25, PP31-18

Zhao, W. PP03-02, PP05-146, PP06-62

Zhao, X. PP05-45, PP34-06

Zhao, X.-L. PP10-05

Zhao, Y. PP05-122, PP29-16, PP30-06, PP39-76

Zhao, Y.P. PP30-16, PP31-19

Zhao, Y.-Z. PP15-43, PP19-06

Zhao, Z. PP05-50, PP23-14

Zhao, Z.X. PP13-03, PP22-14

ZheBin, W. PP09-22

Zhen, Y.J. PP03-16

Zheng, J.M. PP05-09, PP06-01

Zheng, L. PP05-43

Zheng, M.-H. PP04-11

Zheng, P. PP07-17, PP07-29

Zheng, Q. PP05-137, PP21-08

Zheng, R. PP08-04

Zheng, S. PP05-107, PP26-25, PP30-20, PP38-06, PP38-15, PP38-22, PP38-26, PP38-31

Zheng, S.S. PP30-34, PP31-21, PP36-09, PP36-15, PP38-30, PP38-32, PP38-33, PP38-34

Zheng, Y. PP15-54, PP34-03, PP39-72

Zherebtsov, F.K. PP05-95

Zhi-Dong, Y. PP26-27, PP26-28, PP28-15

ZhiLiang, G. PP09-22

Zhi-Xiang, C. PP36-10

Zhong, B. PP04-16, PP04-17

Zhong, H. PP23-86, PP38-12

Zhong, W. PP01-15

Zhong-Fu, Z. PP24-06

Zhong-He, S. PP36-10

Zhongyang, S. PP22-11

Zhou, B. PP12-17, PP36-15

Zhou, C. PP05-130

Zhou, C.-Y. 275

Zhou, D. PP31-26, PP31-27, PP34-03

Zhou, D.F. PP03-25

Zhou, D.Q. PP03-16

Zhou, H. PP19-05, PP31-26, PP31-27

Zhou, H.J. PP39-79

Zhou, J. PP34-32

Zhou, J.Y. PP03-25

Zhou, L. PP05-149, PP26-25, PP30-20, PP30-34, PP34-10, PP36-09, PP36-15, PP38-06, PP38-22, PP38-30, PP38-31, PP38-32, PP38-33

Zhou, P. PS03-04, FC04-03

Zhou, Q. PP07-35

Zhou, R. PP30-24

Zhou, S. PP29-11, PP30-01

Zhou, T. PP20-10

Zhou, W. PP29-12, PP30-20

Zhou, W.-C. PP38-43

Zhou, X. PP03-27, PP19-05

Zhou, Y. PP01-35, PP05-81, PP12-17, PP12-47, PP20-09 
Zhou, Y.-J. PP08-28, PP08-29, PP08-32, PP08-33, PP08-34

Zhou, Y.-L. PP16-03

Zhu, C. PP16-04

Zhu, C.-W. PP05-21

Zhu, F.S. PP07-21

Zhu, G.-T. PP05-24

Zhu, H. PP15-50, PP15-51, PP15-52, PP33-18

Zhu, J. PP16-06, PP17-11, PP30-05

Zhu, K. PP13-76

Zhu, L. PP05-21, PP20-09

Zhu, M. PP30-01

Zhu, M.Q. PP06-01

Zhu, P. PP29-06

Zhu, Q. PP17-10

Zhu, Q.-H. PP23-08, PP23-67, PP28-03

Zhu, Q.M. PP05-92, PP06-12
Zhu, X. PP15-08, PP15-09, PP15-10, PP15-11, PP16-14, PP29-10, PP29-12, PP36-01, PP38-03, PP38-07, PP38-08, PP39-21, PP39-22

Zhu, X.-J. FC03-01, PP05-39

Zhu, X.-L. PP38-43

Zhu, Y. PP05-125, PP09-13, PP09-20, PP09-31, PP16-07, PP23-93

Zhu, Y.-L. PP06-62

Zhu, Y.-Y. PP03-09, PP05-137,

PP07-28, PP23-50

Zhuang, H. PP03-30, PP04-12, PP04-24, PP06-21, PP06-46, PP09-13, PP09-20, PP09-31, PP12-31, PP14-06

Zhuang, L. PP05-05, PP05-27, PP38-31, PP38-33, PP38-34

Zhuo, Y.H. PP05-68, PP16-43
Zhuravlyova, L. PP08-08

Ziaee, M. PP06-06, PP12-44

Ziai, M. PP14-13

Zigrai, M. PP01-26, PP38-49

Zinober, K. PP13-84

Zoladl, M. PP39-87

Zolghadr Asli, A.A. PP39-06

Zorila, C. PP08-05, PP10-04

Zou, M. Y. PP01-13

Zou, Y. PP06-50

Zou, Z.Q. PP01-28

Zou, Z.-S. PP05-163

Zoulim, F. FC05-02, PP06-32

Zuñiga, V. PP23-16

Zvenigorodskaja, L. A. PP08-13

Zvizdic, Z. PP17-02

Zytoon, A.A.F.A.H. PP33-29 